$\operatorname{Argonne} \underset{\bigotimes}{\bigotimes}$

\title{
Phase II Investigation at the Former CCC/USDA Grain Storage Facility in Savannah, Missouri
}

\author{
Environmental Science Division
}


About Argonne National Laboratory

Argonne is a U.S. Department of Energy laboratory managed by UChicago Argonne, LLC under contract DE-AC02-06CH11357. The Laboratory's main facility is outside Chicago, at 9700 South Cass Avenue, Argonne, Illinois 60439. For information about Argonne and its pioneering science and technology programs, see www.anl.gov.

\section{Availability of This Report}

This report is available, at no cost, at http://www.osti.gov/bridge. It is also available on paper to the U.S. Department of Energy and its contractors, for a processing fee, from:

U.S. Department of Energy

Office of Scientific and Technical Information

P.O. Box 62

Oak Ridge, TN 37831-0062

phone (865) 576-8401

fax (865) 576-5728

reports@adonis.osti.gov

\section{Disclaimer}

This report was prepared as an account of work sponsored by an agency of the United States Government. Reference herein to any specific commercial product, process, or service by trade name, trademark, manufacturer, or otherwise, does not necessarily constitute or imply its endorsement, recommendation, or favoring by the United States Government or any agency thereof. The views and opinions of document authors expressed herein do not necessarily state or reflect those of the United States Government or any agency thereof, Argonne National Laboratory, or UChicago Argonne, LLC. 


\section{Phase II Investigation at the Former CCC/USDA Grain Storage Facility in Savannah, Missouri}

by

Applied Geosciences and Environmental Management Section

Environmental Science Division, Argonne National Laboratory

May 2012

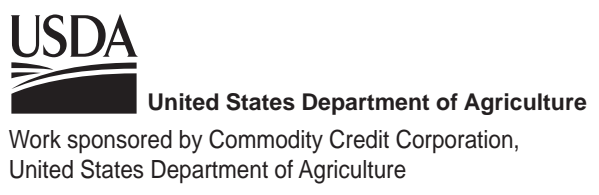




\section{Contents}

Notation.

viii

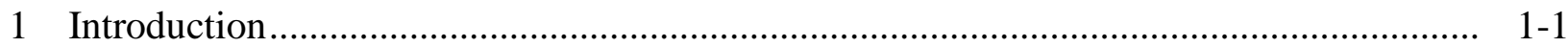

1.1 Overview of the Phase I Study at Savannah ...................................................... 1-2

1.2 Phase II Investigation Objectives..................................................................... 1-6

2 Investigative Methods .............................................................................................. 2-1

2.1 Overview of the Phase II Field Program..................................................................... 2-2

2.2 Methods to Investigate the Site-Specific Hydrogeology near the Former

CCC/USDA Facility

2.3 Methods to Investigate for Carbon Tetrachloride in the Vadose Zone and Deeper Soils beneath the Former CCC/USDA Facility and the MoDOT Property ................ 2-5

2.3.1 Phase I Findings for Soils …………………............................................ 2-5

2.3.2 Phase II Soil Boring Locations ................................................................... 2-6

2.3.3 Handling and Analysis of Soil Samples........................................................ 2-6

2.4 Methods to Investigate the Distribution of Carbon Tetrachloride in

Groundwater near the Former CCC/USDA Facility................................................. 2- 2-7

2.4.1 Sampling at Phase II Investigation Locations in Late June 2010 .................. 2-8

2.4.2 Sampling of Phase II Monitoring Wells in Early July 2010 ......................... 2-8

2.4.3 Sampling in Late August 2010, after Seven Weeks of Groundwater Accumulation...................................................................................... 2- 2-9

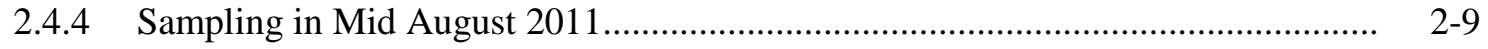

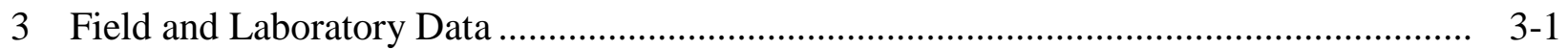

3.1 Analytical Data for Soil Samples.............................................................................. 3-1

3.1.1 Carbon Tetrachloride Concentrations above the Quantitation Limit............. 3-1

3.1.2 Carbon Tetrachloride Concentrations above the Default Target Level .......... 3-2

3.1.3 Carbon Tetrachloride Concentrations at Phase II Boring SB44 and Phase I Boring SB42 ....................................................................... 3-2

3.1.4 Vertical Profile of Carbon Tetrachloride Concentrations at Boring SB46 .... 3-3

3.1.5 Trace Detections of Chloroform ................................................................ 3-3

3.2 Lithologic and Physical Property Data for Soils....................................................... 3-4

3.3 Installation of Permanent Monitoring Wells............................................................. 3-4

3.4 Analytical Data for Groundwater Samples ......................................................... 3-5

3.4.1 Results for Shallow Monitoring Wells SB24 and SB43S-SB49S ................. 3-6

3.4.2 Results for the Morgan and MoDOT Private Wells ...................................... 3-7

3.4.3 Results for Deep Monitoring Wells SB43D-SB49D and SB38 …................ 3-8

3.5 Groundwater Level Data............................................................................... 3-9 


\section{Contents (Cont.)}

3.6 Coordinates Survey Data .............................................................................. 3-10

3.7 Abandonment of the MoDOT Private Well ............................................................... 3-10

3.8 Results of Quality Control Activities..................................................................... 3-11

3.9 Waste Characterization, Handling, and Disposal ..................................................... 3-13

4 Interpretation of Results.......................................................................................

4.1 Site Geology and Hydrostratigraphy .................................................................. 4-1

4.1.1 Stratigraphy near the Former CCC/USDA Facility ....................................... 4-1

4.1.2 Porosities and Permeabilities .................................................................... 4-3

4.1.3 Hydrostratigraphy near the Former CCC/USDA Facility …......................... 4-4

4.2 Distribution of Carbon Tetrachloride in Soils at the Former CCC/USDA Facility and the MoDOT Property ……………............................................................. 4-10

4.3 Distribution of Carbon Tetrachloride in Groundwater ................................................ 4-12

4.3.1 Carbon Tetrachloride in the Shallow Monitoring Interval ............................ 4-13

4.3.2 Carbon Tetrachloride in the Deep Monitoring Interval .................................. 4-15

4.3.3 Chloroform Distribution and Possible Degradation of Carbon

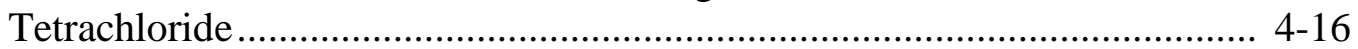

4.4 Preliminary Consideration of Human Health and Environmental Risks Associated with the Carbon Tetrachloride Contamination........................................ 4-17

4.4.1 Contamination in Soils..................................................................... 4-18

4.4.2 Contamination in Groundwater................................................................ 4-18

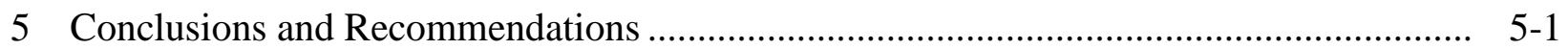

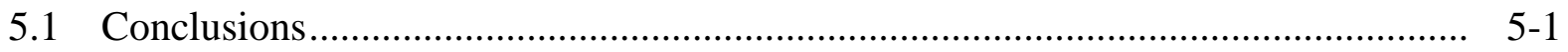

5.2 Recommendations.................................................................................... $5-5$

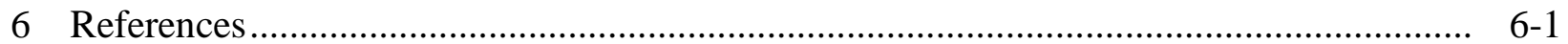

Appendix A: Sequence of Sampling Events in Phase II (2010-2011).................................... A-1

Appendix B: Soil Sampling Data.............................................................................. B-1

Appendix C: Lithologic Logs ……………………................................................ C-1

Appendix D: Well Certification and Registration Forms ………........................................... D-1

Appendix E: Groundwater Sampling Data ……………..................................................... E-1 


\section{Contents (Cont.)}

Appendix F: Coordinates Survey Data

F-1

Supplement 1: Automatically Recorded Groundwater Level Data and Rainfall Data on $\mathrm{CD}$

Supplement 2: Quality Control for Sample Collection, Handling, and Analysis on CD

Supplement 3: Verification Laboratory Data on CD

Supplement 4: Waste Characterization Data. on $\mathrm{CD}$

\section{Figures}

1.1 Location of Savannah, Missouri

1.2 Phase I investigation locations inside the boundaries of the former CCC/USDA grain storage facility

1.3 Vertical distribution of carbon tetrachloride in soil cores collected during Phase I at locations SB24, SB29, SB38, and SB42

1.4 Phase I investigation locations outside the former CCC/USDA grain storage facility, with carbon tetrachloride concentrations in water samples collected in October 2007-March 2008

1.5 Water level contours constructed with data measured manually on February 6, 2009

2.1 Locations of Phase II investigative borings

2.2 Monitoring wells and private wells sampled and/or equipped with groundwater level recorders in the Phase II studies....

3.1 Vertical distribution of the carbon tetrachloride concentrations identified in soils in borings SB43-SB46

3.2 Areal distribution of the maximum carbon tetrachloride concentrations identified in soils in Phase I and Phase II work .... 


\section{Figures (Cont.)}

3.3 Lateral distribution of carbon tetrachloride in groundwater, as determined in the June-July 2010 sampling of shallow monitoring points SB24 and SB43-SB49, plus the Morgan and MoDOT private wells.

3.4 Lateral distribution of carbon tetrachloride in groundwater, as determined in the August 2010 sampling of shallow monitoring points SB24 and SB43-SB49

3.5 Lateral distribution of carbon tetrachloride in groundwater, as determined in the August 2011 sampling of shallow monitoring points SB24 and SB43-SB49

3.6 Grout being pumped into the MoDOT private well, located beneath the floor of the MoDOT vehicle maintenance building, during well abandonment on June 27, 2010

3.7 The grouting operation at the MoDOT well on June 27, 2010.

4.1 Locations of hydrogeologic cross sections A-A' and B-B', with the distribution of current surface features in the vicinity of the former CCC/USDA facility

4.2 Locations of hydrogeologic cross sections A-A' and B-B', with the surface topography in the vicinity of the former CCC/USDA facility.

4.3 West-to-east hydrogeologic cross section A-A', showing the distribution of groundwater levels identified in shallow and deep monitoring wells.

4.4 North-to-south hydrogeologic cross section B-B', showing the distribution of groundwater levels identified in shallow and deep monitoring wells

4.5 Hydrographs for wells in the private well monitoring network, January to December 2009

4.6 Hydrographs for wells in the private well monitoring network, January to December 2010

4.7 Hydrographs for wells in the private well monitoring network, January to December 2011 


\section{Figures (Cont.)}

4.8 Water level contours constructed with data measured automatically in selected private wells on August 10, 2011

4.9 Hydrographs for shallow monitoring wells SB24 and SB43S-SB49S, July 2010 to December 2011

4.10 The temporary stick-up surface housings installed at wells SB46S and SB46D from April to September, 2011

4.11 Water level contours constructed with data measured automatically in shallow monitoring wells SB24 and SB43S-SB49S on August 10, 2011

4.12 Water level contours constructed with data measured automatically in selected private wells and shallow monitoring wells SB24 and SB43S-SB49S on August 10, 2011

4.13 Hydrographs for deep monitoring wells SB38 and SB43D-SB49D, July 2010 to December 2011

4.14 Water level contours constructed with data measured automatically in deep monitoring wells SB43D-SB49D on August 10, 2011.

4.15 West-to-east hydrogeologic cross section A-A' showing the distribution of maximum carbon tetrachloride concentrations identified in soils and groundwater ... 4-35

4.16 North-to-south hydrogeologic cross section B-B' showing the distribution of maximum carbon tetrachloride concentrations identified in soils and groundwater

4.17 Water level contours for the private wells and shallow monitoring wells at 8:00 a.m. on August 10, 2011, constructed with data obtained from the automatic recorders, and maximum carbon tetrachloride concentrations identified in groundwater during the Phase II sampling events.

4.18 Water level contours for the private wells and shallow monitoring wells at 8:00 a.m. on August 10, 2011, constructed with data obtained from the automatic recorders, with locations of the maximum concentrations of carbon tetrachloride identified in groundwater and locations of private domestic wells in the vicinity of the former CCC/USDA facility 


\section{Tables}

3.1 Summary of purge-and-trap analysis results for carbon tetrachloride in soil samples collected in Phase II

3.2 Construction data for monitoring wells

3.3 Chronological compilation of analytical results from the AGEM Laboratory for carbon tetrachloride and chloroform in groundwater samples collected in 2010-2011

3.4 Location-depth compilation of analytical results from the AGEM Laboratory for carbon tetrachloride and chloroform in groundwater samples collected in 2010-2011

3.5 Construction data for selected private wells

3.6 Hand-measured groundwater levels in June 2010 to December 2011.

A.1 Sequence of sampling activities during the Phase II investigation

B.1 Results from the AGEM Laboratory for analyses of carbon tetrachloride and chloroform in soil samples

B.2 Results of grain size analyses for soil samples

B.3 Results of physical properties analysis for soil samples.

E.1 Results from the AGEM Laboratory for water samples collected during the Phase II investigation.

E.2 Field measurements made during collection of groundwater samples in 2007-2011

F.1 Coordinates survey data for the Phase II investigation. 


\section{Notation}

\begin{tabular}{ll} 
AGEM & Applied Geosciences and Environmental Management \\
AMSL & above mean sea level \\
BGL & below ground level \\
${ }^{\circ}$ C & degree(s) Celsius \\
CCC & Commodity Credit Corporation \\
CD & compact disc \\
CLP & Contract Laboratory Program \\
cm & centimeter(s) \\
COC & chain of custody \\
CPT & cone penetrometer \\
d & day(s) \\
DF & dilution factor \\
DTL & default target level \\
DO & dissolved oxygen \\
EPA & U.S. Environmental Protection Agency \\
ft & foot (feet) \\
g & gram(s) \\
gal & gallon(s) \\
GC-MS & gas chromatograph-mass spectrometer \\
hr & hour(s) \\
I.D. & inner diameter \\
in. & inch(es) \\
$\mu \mathrm{g} / \mathrm{kg}$ & microgram(s) per kilogram \\
$\mu \mathrm{g} / \mathrm{L}$ & microgram(s) per liter \\
$\mu \mathrm{S} / \mathrm{cm}$ & microsiemen(s) per centimeter \\
MCL & maximum contaminant level \\
MDNR & Missouri Department of Natural Resources \\
mg/L & milligram(s) per liter \\
mi & mile(s) \\
min & minute(s) \\
mL & milliliter(s) \\
mm & millimiter(s) \\
MoDOT & Missouri Department of Transportation \\
MUE & Missouri University Extension \\
\hline
\end{tabular}


Savannah Phase II Report

Version 00, 05/21/12

NAD North American Datum

NAVD North American Vertical Datum

ng nanogram(s)

ORP oxidation-reduction potential

PID photoionization detector

ppb part(s) per billion

PVC polyvinyl chloride

QA quality assurance

QC quality control

RPD relative percent difference

SDG sample delivery group

USDA U.S. Department of Agriculture

VOC volatile organic compound 


\section{Phase II Investigation at the Former CCC/USDA Grain Storage Facility in Savannah, Missouri}

\section{Introduction}

From approximately 1949 until 1970, the Commodity Credit Corporation of the U.S. Department of Agriculture (CCC/USDA) operated a grain storage facility on federally owned property approximately $0.25 \mathrm{mi}$ northwest of Savannah, Missouri (Figure 1.1). During this time, commercial grain fumigants containing carbon tetrachloride were commonly used by the CCC/USDA and the private grain storage industry to preserve grain in their facilities. In November 1998, carbon tetrachloride was detected in a private well (Morgan) roughly $50 \mathrm{ft}$ south of the former CCC/USDA facility, as a result of statewide screening of private wells near former CCC/USDA facilities, conducted in Missouri by the U.S. Environmental Protection Agency (EPA 1999).

The 1998 and subsequent investigations by the EPA and the Missouri Department of Natural Resources (MDNR) confirmed the presence of carbon tetrachloride in the Morgan well, as well as in a second well on property currently owned by the Missouri Department of Transportation (MoDOT), directly east of the former CCC/USDA facility. The identified concentrations in these two wells were above the EPA maximum contaminant level (MCL) and the Missouri risk-based corrective action default target level (DTL) values of $5.0 \mu \mathrm{g} / \mathrm{L}$ for carbon tetrachloride in water used for domestic purposes (EPA 1999; MDNR 2000a,b, 2006).

Because the observed contamination in the Morgan and MoDOT wells might be linked to the past use of carbon tetrachloride-based fumigants at its former grain storage facility, the CCC/USDA is conducting an investigation to (1) characterize the source(s), extent, and factors controlling the subsurface distribution and movement of carbon tetrachloride and (2) evaluate the potential risks to human health, public welfare, and the environment posed by the contamination. This work is being performed in accord with an Intergovernmental Agreement established in 2007 between the Farm Service Agency of the USDA and the MDNR, to address carbon tetrachloride contamination potentially associated with a number of former CCC/USDA grain storage facilities in Missouri. The site characterization at Savannah is being conducted on behalf of the CCC/USDA by the Environmental Science Division of Argonne National Laboratory. 
The investigation is being conducted in phases, so that information obtained and interpretations developed during each incremental stage can be used most effectively to guide subsequent phases of the program.

\subsection{Overview of the Phase I Study at Savannah}

With the approval of the MDNR (2007), the Phase I investigation was conducted in October-November 2007 and January 2008. Automated measurement and recording of the groundwater levels in selected private wells and two monitoring points installed at the former CCC/USDA facility (SB24 and SB38) was also initiated at this time (Argonne 2007). The key findings of the Phase I studies (Argonne 2010a) are summarized as follows:

- Refusal depths encountered during vertical probing with the cone penetrometer (CPT) unit ranged from $91.8 \mathrm{ft}$ to $96.3 \mathrm{ft}$ below ground level (BGL), suggesting a relatively consistent bedrock surface elevation beneath the former CCC/USDA facility. The observed refusal depths were comparable to depths to bedrock reported previously for the Savannah area; however, actual contact with the bedrock surface could not be confirmed during Phase I.

- Two predominantly fine-grained lithologic units were identified at the former CCC/USDA facility. The uppermost interval consists of approximately 58-65 ft of generally dense, stiff to slightly softer, non-plastic and noncalcareous silty clay to clayey silt, with possible traces of very fine sand. The second, deeper unit consists of approximately 26-30 ft of generally firm and dense, uniformly light gray silty clay till with some fine to medium sand.

- Thin zones (up to approximately $1.1 \mathrm{ft}$ thick) of damp-to-dry, coarser-grained deposits were penetrated directly above the refusal depth at three locations at the former CCC/USDA facility (SB24, SB38, and SB42; Figure 1.2). No other distinctly coarse-grained intervals were identified, however, in the overlying silty clay and till units at the locations investigated during Phase I.

- The observed physical, textural, and water-producing characteristics of the silty clay to clayey silt and till units suggest that the ability of these deposits to 
transmit groundwater is limited. Occasional thin, discontinuous intervals of relatively softer and lighter-colored materials, observed most commonly at depths of 50-70 ft BGL, suggested the presence of preferred pathways for horizontal groundwater migration in these units. Locally enhanced porosity and/or permeability might occur in association with calcareous nodules and patches observed in the till, or with the sporadically observed coarser-grained deposits noted above, if these materials are locally saturated and the hydraulic communication among them is sufficient.

- No carbon tetrachloride contamination was identified at concentrations at or above either the DTL of $79.6 \mu \mathrm{g} / \mathrm{kg}$ or the quantitation limit of $10 \mu \mathrm{g} / \mathrm{kg}$ for analyses according to EPA Methods 5030B and 8260B (purge-and-trap sample preparation with analysis on a gas chromatograph-mass spectrometer [GC-MS] system), in surficial and shallow subsurface soils collected to depths of $12 \mathrm{ft} \mathrm{BGL}$ at 42 locations on the former CCC/USDA property (Figure 1.2).

- With the approval of the MDNR, deeper subsurface soils were sampled for analyses for volatile organic compounds (VOCs) at four locations (SB24, SB29, SB38, and SB42; Figure 1.2). Traces of carbon tetrachloride (below the purge-and-trap method quantitation limit of $10 \mu \mathrm{g} / \mathrm{kg}$ ) were detected at each location; however, quantifiable concentrations of carbon tetrachloride were identified only at SB38 and SB42. Carbon tetrachloride concentrations of $10-42 \mu \mathrm{g} / \mathrm{kg}$ were identified at SB38, at depths of 20-64 ft BGL. Concentrations of $15-171 \mu \mathrm{g} / \mathrm{kg}$ were identified at SB42, over the restricted depth interval at 44-56 ft BGL (Figure 1.3).

- Only the two highest carbon tetrachloride concentrations in soil samples identified during the Phase I investigation, at SB42 $(126 \mu \mathrm{g} / \mathrm{kg}$ and $171 \mu \mathrm{g} / \mathrm{kg}$, at $44-48 \mathrm{ft}$ BGL in the zone of saturated soils), were greater than the DTL for this contaminant $(79.6 \mu \mathrm{g} / \mathrm{kg})$ in vadose zone soils. No concentrations detected in the subsurface (vadose) zone as defined by the MDNR (2006) exceeded the DTL. (The MDNR defines the "subsurface" as the vadose zone from $3 \mathrm{ft}$ BGL to the water table [saturation].) For carbon tetrachloride in soils, the DTL reflects risk that might be associated with the indoor inhalation pathway. Because the former CCC/USDA property has been used only for 
non-residential purposes since the CCC/USDA grain storage operations were terminated (by 1974), the indoor inhalation pathway is incomplete at the former facility, and no receptors have historically been or presently are at risk of exposure by this route.

- Samples of groundwater recovered at locations SB24 and SB38 at the former CCC/USDA facility (Figure 1.2) contained carbon tetrachloride at concentrations of 3.8-6.4 $\mu \mathrm{g} / \mathrm{L}$. The identified concentrations were either slightly less or slightly greater than the MCL and the DTL values for carbon tetrachloride $(5.0 \mu \mathrm{g} / \mathrm{L})$ in groundwater used for domestic purposes.

- Samples of both surface water obtained by the city of Savannah from a municipal reservoir approximately $0.75 \mathrm{mi}$ southwest of the former CCC/USDA facility (Figure 1.4) and water from the Savannah public distribution system were confirmed to be free of carbon tetrachloride contamination.

- Sixteen private wells were identified within approximately $0.5 \mathrm{mi}$ of the former CCC/USDA facility (Figure 1.4). Three of these wells were confirmed to be damaged or otherwise inaccessible, and nine are not used for any purpose. Two wells (Morgan and Clizer "old") are currently used for lawn and garden watering, and two (Moore and Clizer "new") are used for domestic purposes. Access for sampling was denied for one well (Vanschoiack) identified as not in use.

- Except for the Moore residence, all other occupied residential and commercial properties within approximately $0.5 \mathrm{mi}$ of the former CCC/USDA facility have confirmed access to and obtain water for domestic purposes from a public water supply. (The Clizer "new" private well is used to supplement the water obtained from the public supply at this residence.)

- Only two of the private wells tested in Phase I contained carbon tetrachloride: the Morgan well $(13 \mu \mathrm{g} / \mathrm{L})$ and the MoDOT well $(150 \mu \mathrm{g} / \mathrm{L})$. The carbon tetrachloride concentrations at these locations had decreased since the wells were originally sampled by the MDNR in 1998 and 2000, respectively. The 
Moore well and the Clizer "new" well (used for domestic purposes) were confirmed to be free of carbon tetrachloride contamination.

- The carbon tetrachloride concentration identified in the Morgan well, to the south of the former CCC/USDA facility, was generally comparable to the levels detected in the groundwater at the former facility. The identified carbon tetrachloride concentration in the MoDOT well, to the east of the former CCC/USDA facility, was greater by approximately an order of magnitude than the concentrations at the former facility.

- Groundwater level monitoring conducted in private wells in the vicinity of the former CCC/USDA facility suggested a general pattern of semi-radial groundwater flow in the study area, from a relative high west-northwest of the former facility (Figure 1.5). The observed pattern qualitatively mirrored the dominant local topographic trends, supporting a preliminary interpretation of groundwater flow primarily toward the south or southeast beneath the former CCC/USDA facility. The data suggested only a weak component of groundwater flow from the former CCC/USDA facility directly east, in the direction of the contaminated MoDOT well.

- Monitoring well SB24 (on the former CCC/USDA property) penetrates the subsurface to a stratigraphic level (elevation above mean sea level [AMSL]) that is comparable to the levels reached in the nearby McPike, Morgan, and MoDOT wells (Figure 1.5). Despite differences in the construction of these wells, the extended monitoring data indicated that the groundwater elevations observed at SB24 were generally consistent with those in the McPike, Morgan, and MoDOT wells.

- Groundwater level relationships observed in deeper on-site monitoring point SB38 (Figure 1.2) further suggested that both vertical (downward) and horizontal hydraulic gradients affect the movement of groundwater in the study area. 


\subsection{Phase II Investigation Objectives}

On the basis of the above findings, and in consultation with the MDNR and CCC/USDA project managers (MDNR 2008, 2009), a second phase of investigation was implemented at the Savannah site in 2010-2011. The specific technical objectives developed for the Phase II program were as follows (Argonne 2010b):

- Investigate the more detailed characteristics of groundwater flow in the vicinity of the former CCC/USDA facility and the contaminated Morgan and MoDOT private wells.

- Obtain additional information on the vertical and lateral distribution and concentrations of carbon tetrachloride in groundwater in the vicinity of the former CCC/USDA facility and the contaminated Morgan and MoDOT private wells.

- Investigate further for possible evidence of carbon tetrachloride in the subsurface (vadose zone) and deeper soils beneath the former CCC/USDA facility, as well as in the vicinity of the contaminated MoDOT private well.

This report presents the technical findings of the Phase II studies. The Phase II investigation was undertaken in June-July 2010, in accord with the final site-specific Phase II Work Plan (Argonne 2010b; approved by the MDNR [2010]).

The Phase II work was implemented in accord with the standard operating procedures, quality assurance/quality control (QA/QC) measures, and general health and safety policies outlined in the Master Work Plan (Argonne 2002) for operations in Kansas, which has been reviewed by the MDNR and accepted for current use. The work was also fully consistent with applicable Missouri regulations. Consequently, the site-specific Work Plan (Argonne 2010b), the Master Work Plan (Argonne 2002), and germane Missouri regulations together form the complete documentation that guided the Phase II investigations at Savannah.

Section 2 of this report describes the investigative methods used in the Phase II studies, and Section 3 presents a summary of the resulting field and laboratory results. These data, in conjunction with information presented previously (Argonne 2007, 2010a,b), are interpreted and 
integrated in Section 4 to (1) update and refine the preliminary conceptual hydrogeologic model developed previously for this site and (2) further assess the contaminant levels and potential exposure pathways that might be of concern in the evaluation of risks to human health, public welfare, and the environment. The working conclusions developed from the combined Phase I and Phase II studies are presented in Section 5. 


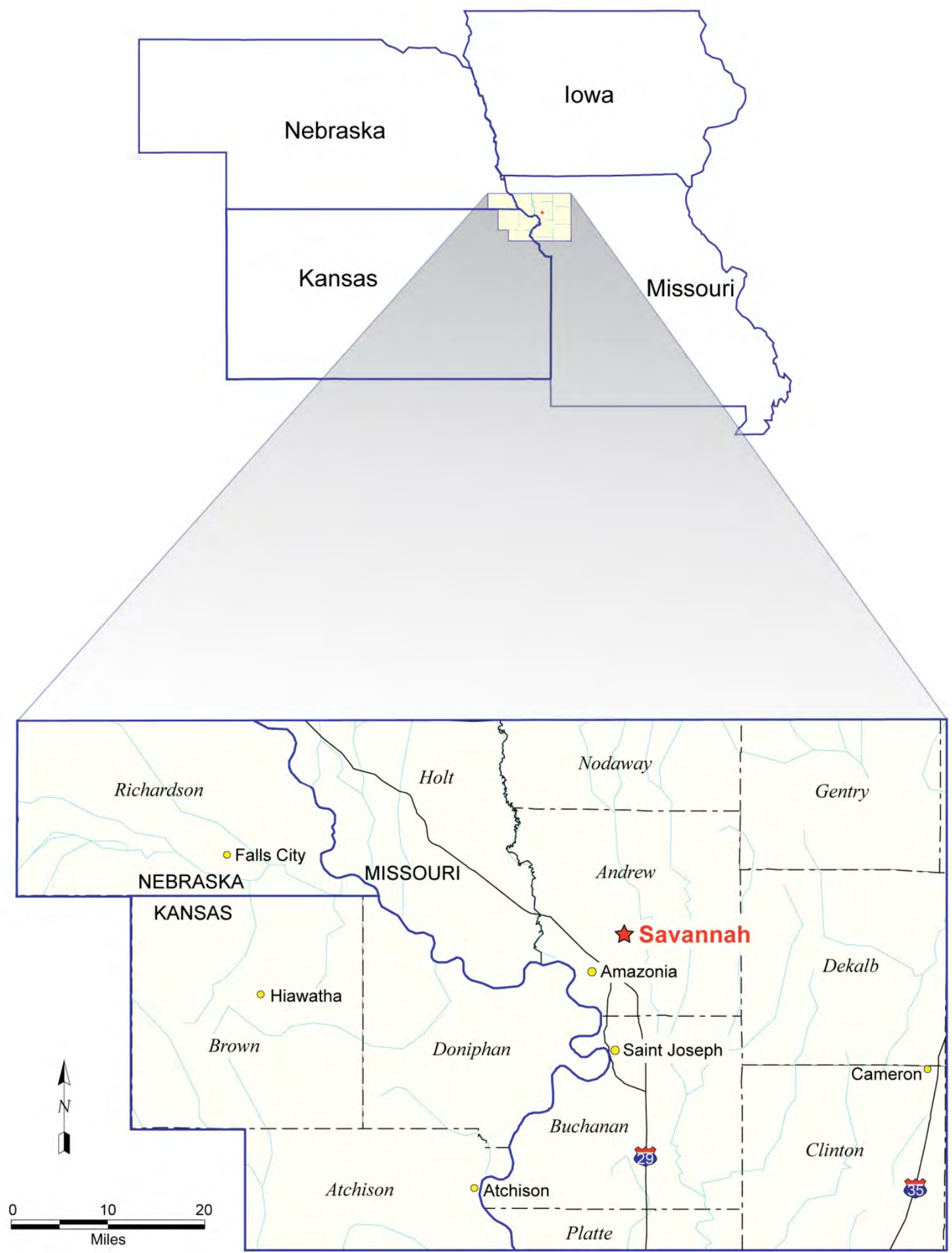

FIGURE 1.1 Location of Savannah, Missouri. 


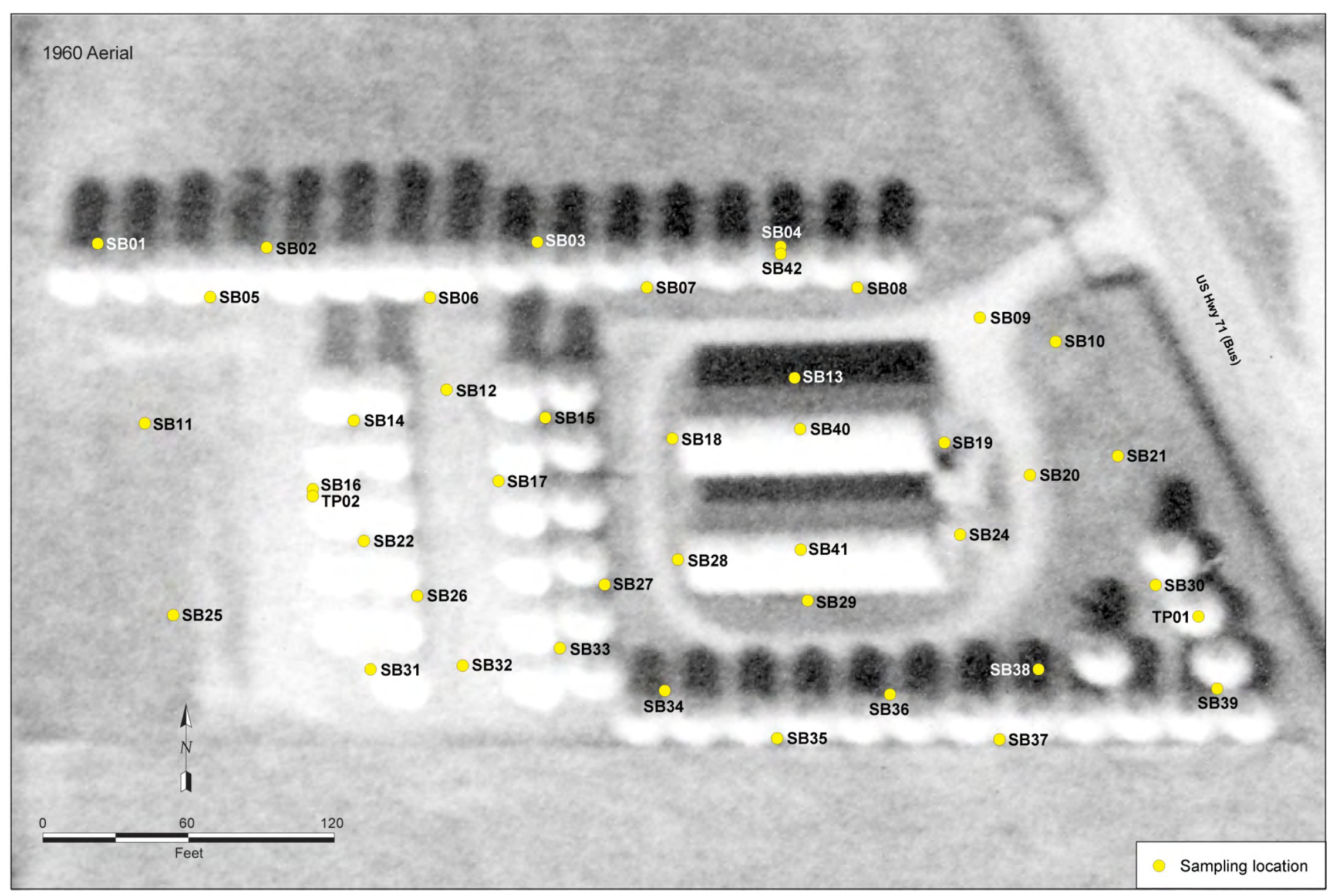

FIGURE 1.2 Phase I investigation locations inside the boundaries of the former CCC/USDA grain storage facility. Source of aerial photograph: USGS (1960). 


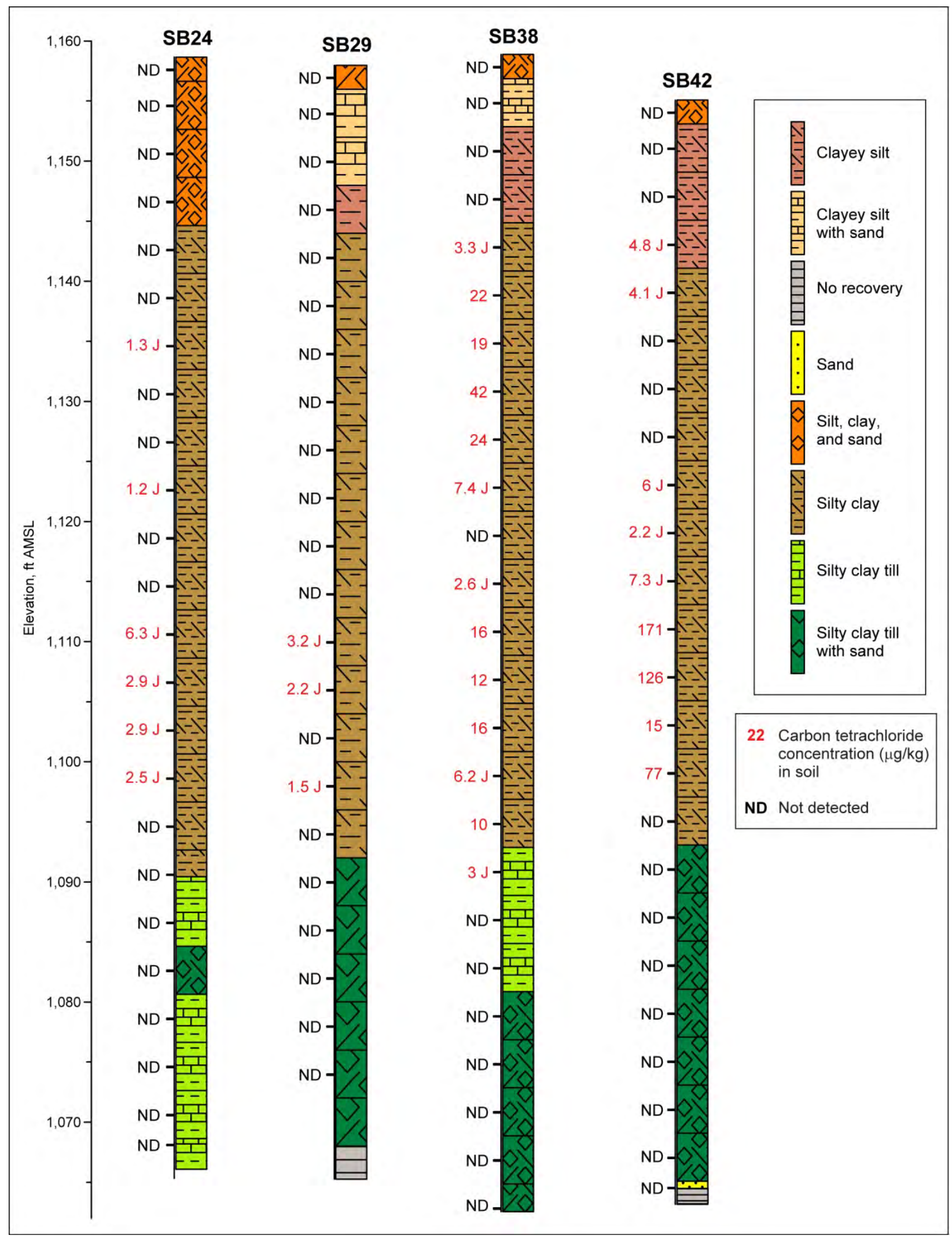

FIGURE 1.3 Vertical distribution of carbon tetrachloride in soil cores collected during Phase I at locations SB24, SB29, SB38, and SB42. 


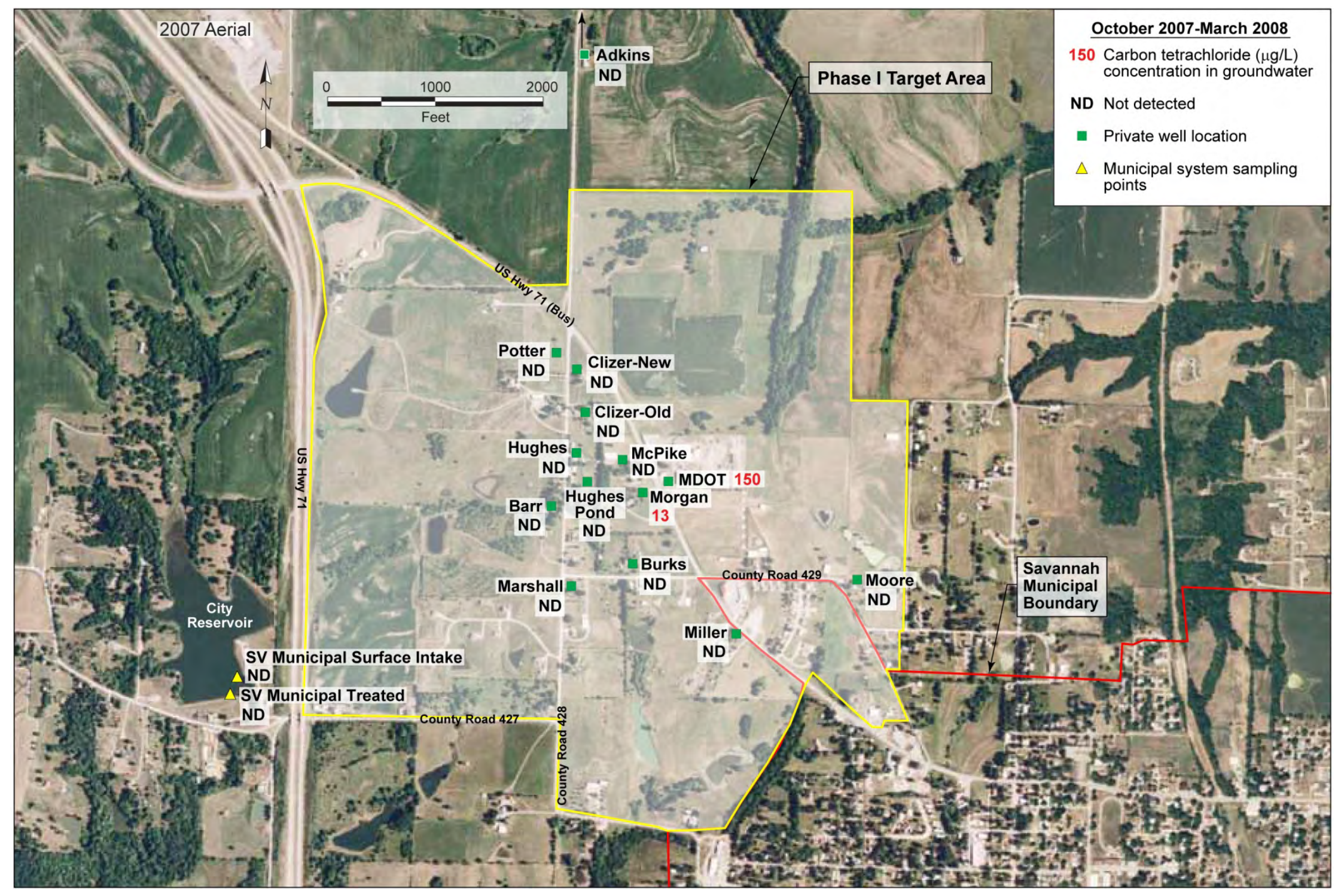

FIGURE 1.4 Phase I investigation locations (private wells and municipal water system sampling points) outside the former CCC/USDA grain storage facility, with carbon tetrachloride concentrations in water samples collected in October 2007-March 2008. Source of aerial photograph: NAIP (2007). 


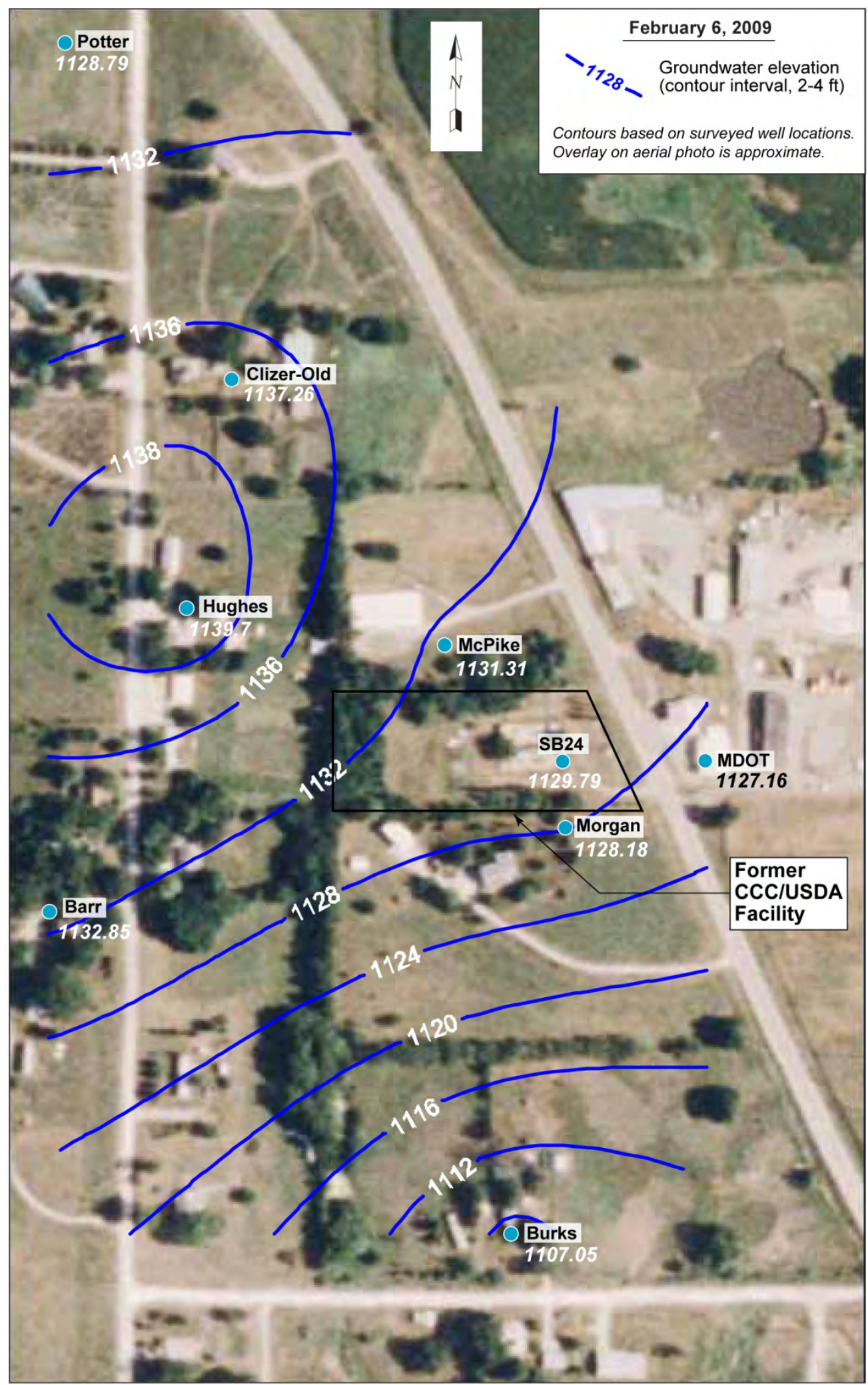

FIGURE 1.5 Water level contours constructed with data measured manually on February 6, 2009. The level for the Clizer "old" well is from continuously recorded data; the well was not accessible for manual measurement on this date. Source of aerial photograph: NAIP (2007). 


\section{Investigative Methods}

The Phase II studies at Savannah were performed by using an iterative process of data collection, evaluation, and interpretation during the field activities, to ensure acquisition of the information necessary to address the technical objectives outlined in Section 1. The results were reviewed as they were obtained and were discussed with the CCC/USDA and MDNR program managers to obtain their input and approval for decisions affecting the direction of the field efforts.

Throughout the Phase II activities, a comprehensive QA/QC program was employed to confirm the reliability of all information as it was accumulated. The detailed field and laboratory procedures followed are described in the Master Work Plan (Argonne 2002) and the site-specific Work Plan (Argonne 2010b).

The field activities during the Phase II investigation included the following:

- The collection of continuous soil cores from the surface to bedrock - through the use of conventional drilling — for lithologic characterization and VOCs analyses.

- Installation of permanent monitoring wells to facilitate groundwater sampling and the measurement of groundwater levels, both manually and through use of automatic water level recording devices.

- Collection of groundwater samples for VOCs analyses at monitoring wells and selected private wells in the vicinity of the former CCC/USDA facility.

- $\quad$ Plugging and abandonment of the private well at the MoDOT facility.

The locations investigated during the Phase II studies are shown in Figures 2.1 and 2.2. 


\subsection{Overview of the Phase II Field Program}

The Phase II studies at Savannah were implemented during one main field session and three additional, more limited mobilizations to the site. The field activities during these events were as follows:

- June 21-July 3, 2010 - Main Field Session

The main field session at Savannah began on June 21 and ended on July 3, 2010. The activities defined in the approved Phase II Work Plan (Argonne 2010b) were largely completed during this event. These activities included the following:

- Use of conventional (sonic) drilling techniques to recover continuous soil cores from the surface to the top of bedrock at seven locations selected in consultation with the CCC/USDA and MDNR program managers, to determine the lithologic and hydrogeologic properties of the sediments in the vicinity of the former CCC/USDA facility (SB43-SB49; Figure 2.1).

- Collection of soil samples for VOCs analyses at four boring locations (SB43-SB46; Figure 2.1), at 4-ft intervals from $4 \mathrm{ft}$ BGL to the top of bedrock.

- Installation of paired shallow and deep monitoring wells at each of the seven boring locations (SB43-SB49; Figure 2.2).

- Observation of slow rates of groundwater accumulation in the newly installed monitoring wells, as previously noted for the private and Phase I monitoring wells at this site (Argonne 2010a). Initial groundwater samples for VOCs analyses were therefore collected in conjunction with the development of the new wells, to ensure that sufficient volume for analysis could be recovered at each well location. 
- Collection of groundwater samples from the Morgan and MoDOT private wells and Phase I monitoring points SB24 and SB38 (Figure 2.2) for contemporary comparison with results for the Phase II monitoring wells.

- Plugging and abandonment of the (contaminated) private well at the MoDOT facility, directly east of the property formerly occupied by the CCC/USDA facility (Figure 2.2), at the request of the MDNR. Plugging and abandonment were in accord with MDNR regulations and were approved by the MoDOT facility management.

- July 7-11, 2010 - Minor Field Visit 1 (and Follow-up Data Retrieval)

On July 7 and July 11, 2010, automatic water level measuring and recording devices were installed in the 14 Phase II monitoring wells to supplement the 10 units deployed in Phase I (at SB24, SB38, and 8 private wells in the vicinity of the former CCC/USDA facility; Figure 2.2). Periodic brief followup visits to the site were subsequently made to measure groundwater levels manually and to retrieve data stored by the recording units.

- August 24-25, 2010 - Minor Field Visit 2

On August 24-25, 2010, when groundwater had accumulated in the Phase II monitoring wells for roughly seven weeks after installation and development, groundwater samples were collected for VOCs analyses from the shallow and deep monitoring points at locations SB43-SB49, as well as from Phase I monitoring points SB24 and SB38 (Figure 2.2). At each well, a grab sample was first obtained from the accumulated water in the casing, to ensure the recovery of sufficient water for analysis. At SB43S-SB49S and SB43DSB49D, samples were then collected by using the low-flow sampling methodology, with the approval of the MDNR program manager. At SB24 and SB38, insufficient recovery prevented further sampling. 
- August 10-11, 2011 - Minor Field Visit 3

Groundwater monitoring at Savannah demonstrated that by midsummer 2011, the hydraulic heads in all monitoring wells had reached apparently ambient static levels. On August 10-11, 2011, groundwater samples for VOCs analyses were therefore collected again from the shallow and deep monitoring wells at locations SB43-SB49, as well as from Phase I monitoring points SB24 and SB38 (Figure 2.2). An initial grab sample and a low-flow sample were obtained from each location.

A chronological summary of the sampling activities during the Phase II investigations is in Appendix A, Table A.1.

\subsection{Methods to Investigate the Site-Specific Hydrogeology near the Former CCC/USDA Facility}

To obtain detailed information on the lithologic and hydrogeologic characteristics of the unconsolidated deposits in the vicinity of the former CCC/USDA facility, continuous soil cores were collected from the ground surface to the top of bedrock at seven locations (SB43-SB49; Figure 2.1) selected with the approval of the CCC/USDA and MDNR program managers (MDNR 2010). The investigative borings were advanced, under the direction of Argonne personnel, by Boart-Longyear Drilling Company (Missouri Certified Driller's License Permit \#4484-M). A sonic drilling rig was used to obtain nominal 4-in.-diameter cores. All cores were described in accord with procedures in the Master Work Plan (Argonne 2002, Sections 4.3.1.3 and 6.4.1.5) and archived at the Argonne equipment storage facility in Lincoln, Nebraska. Selected core intervals from borings SB43, SB44, and SB45 that reflected the range of sediments penetrated at the site were also shipped to Alfred Benesch and Company, Lincoln, Nebraska, for laboratory analyses of soil physical parameters including particle size, moisture content, porosity, bulk density, total organic carbon, and vertical permeability (Argonne 2002, Sections 6.1.2 and 6.3.1).

At each of the seven boring locations, paired shallow and deep monitoring wells were installed to facilitate investigation of the occurrence and possible direction(s) of groundwater flow at the site. In keeping with the approved Work Plan (Argonne 2010b), the shallow wells were screened to intersect a consistent interval near the base of the previously identified silty- 
clay to clayey-silt unit, at an elevation of approximately 1,098-1,110 ft AMSL. To achieve this objective, the depths of the shallow wells were adjusted, as necessary, to accommodate variations in the topographic elevations of the individual boring locations. The associated deep wells were completed directly above bedrock, which occurred at 1,040-1,045 ft AMSL.

Groundwater levels were measured periodically by hand (in conjunction with the activities noted in Section 2.1) in all of the Phase I and Phase II monitoring wells, as well as in selected private wells near the former CCC/USDA facility. Groundwater levels were determined manually by using an electronic water level meter to measure the depth to water from a designated (surveyed) point at the top of each well casing. Self-contained, downhole pressuresensing and data-logging units (Instrumentation Northwest, Inc. Model PT2X ${ }^{\mathrm{TM}}$ sensor/loggers) were also installed in the 14 Phase II monitoring wells in July 2010. These units augment the network of 10 water level recorders established at the site during Phase I (in monitoring wells SB24 and SB38, and in the McPike, Potter, Morgan, MoDOT, Clizer "old," Hughes, Barr, and Burks private wells; Figure 2.2). With one exception, these recorders continue to gather data at Savannah; in June 2010, the recorder at the MoDOT well was removed and the well was plugged and abandoned at the request of the MDNR. The units in all wells are programmed to measure and record water levels once every $4 \mathrm{hr}$.

To assist in the evaluation of possible variations or trends in the groundwater levels at Savannah, daily rainfall data for the weather monitoring station in nearby St. Joseph, Missouri (approximately $7 \mathrm{mi}$ south of Savannah), were obtained from the Missouri Historical Agricultural Weather Database maintained by the Missouri University Extension (MUE) office. These data are available online (MUE 2011).

\subsection{Methods to Investigate for Carbon Tetrachloride in the Vadose Zone and Deeper Soils beneath the Former CCC/USDA Facility and the MoDOT Property}

\subsubsection{Phase I Findings for Soils}

No carbon tetrachloride contamination was identified in surficial or shallow subsurface soils (to a depth of $12 \mathrm{ft} \mathrm{BGL)} \mathrm{collected} \mathrm{at} 41$ locations across the former CCC/USDA facility in Phase I (Argonne 2010a), at concentrations at or above the DTL of $79.6 \mu \mathrm{g} / \mathrm{kg}$ or the quantitation limit of $10 \mu \mathrm{g} / \mathrm{kg}$ for analyses according to EPA Methods 5030B and 8260B (purge-and-trap 
sample preparation with analysis by GC-MS). Carbon tetrachloride concentrations greater than the DTL were identified in only 2 of the 79 deeper soil samples collected from $12 \mathrm{ft}$ BGL to the depth of CPT refusal at Phase I investigation locations SB24, SB29, SB38, and SB42 (Figures 1.2 and 1.3). Concentrations of $171 \mu \mathrm{g} / \mathrm{kg}$ and $126 \mu \mathrm{g} / \mathrm{kg}$ were detected at $44 \mathrm{ft}$ BGL and $48 \mathrm{ft}$ BGL, respectively, in the saturated zone at SB42.

\subsubsection{Phase II Soil Boring Locations}

To obtain additional information on the possible occurrence of a soil source of carbon tetrachloride contamination to the groundwater in the vicinity of the former CCC/USDA facility, soil samples were collected for VOCs analyses, at 4-ft intervals from $4 \mathrm{ft}$ BGL to the top of bedrock, at Phase II boring locations SB43-SB46 (Figure 2.1). Borings SB43 and SB44 were positioned along the northern margin of the property formerly occupied by the CCC/USDA grain storage facility, to potentially lie upgradient from the carbon tetrachloride contamination previously identified in groundwater to both the south (at the Morgan private well and Phase I monitoring points SB24 and SB38) and the east (at the MoDOT private well). Boring SB46 was located on the MoDOT property immediately to the west and potentially upgradient of the contaminated MoDOT well; SB45 was inferred to lie downgradient of the MoDOT well (Figures 1.4 and 2.1).

\subsubsection{Handling and Analysis of Soil Samples}

Soil samples for VOCs analyses were taken from the soil cores immediately, as they were recovered from the core barrels and split for examination. At the request of the MDNR, each core segment was quickly screened by using a calibrated, portable photoionization detector (PID) instrument (ppbRAE $2000^{\mathrm{TM}}$ ) as a possible aid in the prioritization of intervals to be sampled for analysis. The use of the PID proved ineffective for this purpose, although it continued to be used for air monitoring to ensure worker safety.

Each sample for VOCs analysis was obtained by quickly cutting an approximately 1.5-in.-long segment from the center portion of the (approximately 4-in.-diameter) core, centered at the chosen target depth. The core segments were immediately placed in laboratory-approved 125-mL glass sample jars, sealed, labeled, and preserved by placement in a cooler on dry ice. 
The samples were shipped, at the required holding temperature, by overnight service to the Applied Geosciences and Environmental Management (AGEM) Laboratory at Argonne for rapid-turnaround analysis (generally within $24 \mathrm{hr}$ for headspace analyses), so that interim results could be evaluated on-site as the Phase II field session progressed. All soil samples were analyzed by both a headspace method with a gas chromatograph and electron capture detector (modified EPA Method 5021), and by a purge-and-trap sample preparation method with analysis by GC-MS (EPA Methods 5030B and 8260B; Argonne 2002, Sections 6.1.1, 6.2, and 6.3.1).

\subsection{Methods to Investigate the Distribution of Carbon Tetrachloride in Groundwater near the Former CCC/USDA Facility}

The Phase I studies (Argonne 2010a) identified carbon tetrachloride contamination in groundwater at only four locations in the vicinity of the former CCC/USDA facility. Carbon tetrachloride concentrations of 3.8-6.4 $\mu \mathrm{g} / \mathrm{L}$ were detected at monitoring points SB24 and SB38 at the former CCC/USDA facility. Concentrations of $13 \mu \mathrm{g} / \mathrm{L}$ and $150 \mu \mathrm{g} / \mathrm{L}$ were identified in the Morgan and MoDOT wells, respectively, located to the south and east of the former facility (Figure 1.4). To obtain a more detailed picture of the potential areal extent and vertical distribution of carbon tetrachloride contamination near the former CCC/USDA facility, groundwater samples were collected for VOCs analyses from the Phase II monitoring wells.

A complete chronological listing of the groundwater sampling events in the 2010-2011 investigations and the conditions under which each sample was collected is in Appendix A, Table A.1.

The groundwater samples collected for VOCs analyses were preserved on ice at $4^{\circ} \mathrm{C}$ and shipped by an overnight service to the AGEM Laboratory for purge-and-trap sample preparation with analysis on a GC-MS system (EPA Methods 5030B and 8260B; Master Work Plan [Argonne 2002], Section 6.3.2.) To ensure reproducibility, aliquots from 29 locations (representing approximately 15\% of the total samples collected) were selected in the field for verification analyses by a second laboratory (TestAmerica, Inc., South Burlington, Vermont) with the EPA's Contract Laboratory Program (CLP) methods. An index of the EPA methods is online at http://www.epa.gov/epahome/index/. 


\subsubsection{Sampling at Phase II Investigation Locations in Late June 2010}

Groundwater samples were collected for VOCs analyses during the main field session (on June 23-28, 2010) from monitoring wells SB24 and SB38, as well as from the Morgan and MoDOT private wells, to provide contemporary results from these Phase I investigation locations for comparison with the initial Phase II well data (Figure 2.2). With the verbal approval of the MDNR program manager, a low-flow methodology was employed to sample each of these wells. In this approach, a minimal pumping rate is used to purge the well of a relatively small volume of water. The sample is collected after the satisfactory stabilization of several diagnostic groundwater parameters (temperature, conductivity, $\mathrm{pH}$, dissolved oxygen [DO] content, and oxidation-reduction potential [ORP]) that are monitored continuously in the purge water stream.

At the Morgan and MoDOT wells and at well SB38, low-flow samples were collected both near the bottom of the well and at the midpoint of the (initial) static water column. The objective was to identify possible stratification of the contaminant distributions in these wells, all of which are open to the surrounding formations over long ( $\geq 40-\mathrm{ft}$ ) vertical intervals. A single sample was collected near the bottom of monitoring point SB24, which is screened over a more conventional 10-ft interval. Finally, for comparison purposes, the Morgan and MoDOT wells were resampled after completion of the low-flow sampling, according to the conventional purging of approximately three casing volumes of groundwater from each well.

\subsubsection{Sampling of Phase II Monitoring Wells in Early July 2010}

As noted in Section 2.1, during the Phase I investigation an extended time period was required for the accumulation of sufficient groundwater in monitoring wells SB24 and SB38 to permit sampling for VOCs analyses (Argonne 2010a). Similarly slow groundwater accumulation rates were observed in the shallow Phase II monitoring wells. The result was that limited quantities of groundwater were present in a majority of these wells by the end of the main Phase II field session (in early July 2010).

To obtain an initial indication of the possible occurrence and concentrations of carbon tetrachloride, a grab sample of groundwater was collected from each of the shallow Phase II monitoring points (SB43S-SB49S; Figure 2.2) as these wells were being developed, on July 1-3, 2010. The samples were collected immediately before the wells went "dry," upon purging of approximately one casing volume from each well by using a Waterra inertial pump. 
At each deep monitoring point SB43D-SB49D (Figure 2.2), an initial groundwater sample for VOCs analysis was collected immediately after the well was developed by purging of three casing volumes with the Waterra pump.

\subsubsection{Sampling in Late August 2010, after Seven Weeks of Groundwater Accumulation}

On August 24-25, 2010, when groundwater had accumulated in the Phase II monitoring wells for roughly seven weeks after installation and development, groundwater samples were again collected for VOCs analyses from the Phase II shallow and deep monitoring points (SB43SB49), as well as from Phase I wells SB24 and SB38 (Figure 2.2). A grab sample was first obtained from each well without purging, to ensure sufficient water recovery for analysis. Samples were then collected at the SB43-SB49 shallow and deep wells, from near the base of each well, by using the low-flow sampling methodology (Section 2.4.1), with the approval of the MDNR program manager.

\subsubsection{Sampling in Mid August 2011}

Monitoring of groundwater levels demonstrated that the heads in all of the monitoring wells had recovered from the August 2010 sampling and had generally stabilized at apparently ambient levels by midsummer 2011. Phase II monitoring wells SB43-SB49 and Phase I wells SB24 and SB38 were therefore resampled on August 10-11, 2011. An initial grab sample and a sample collected with the low-flow methodology were obtained from each well. 


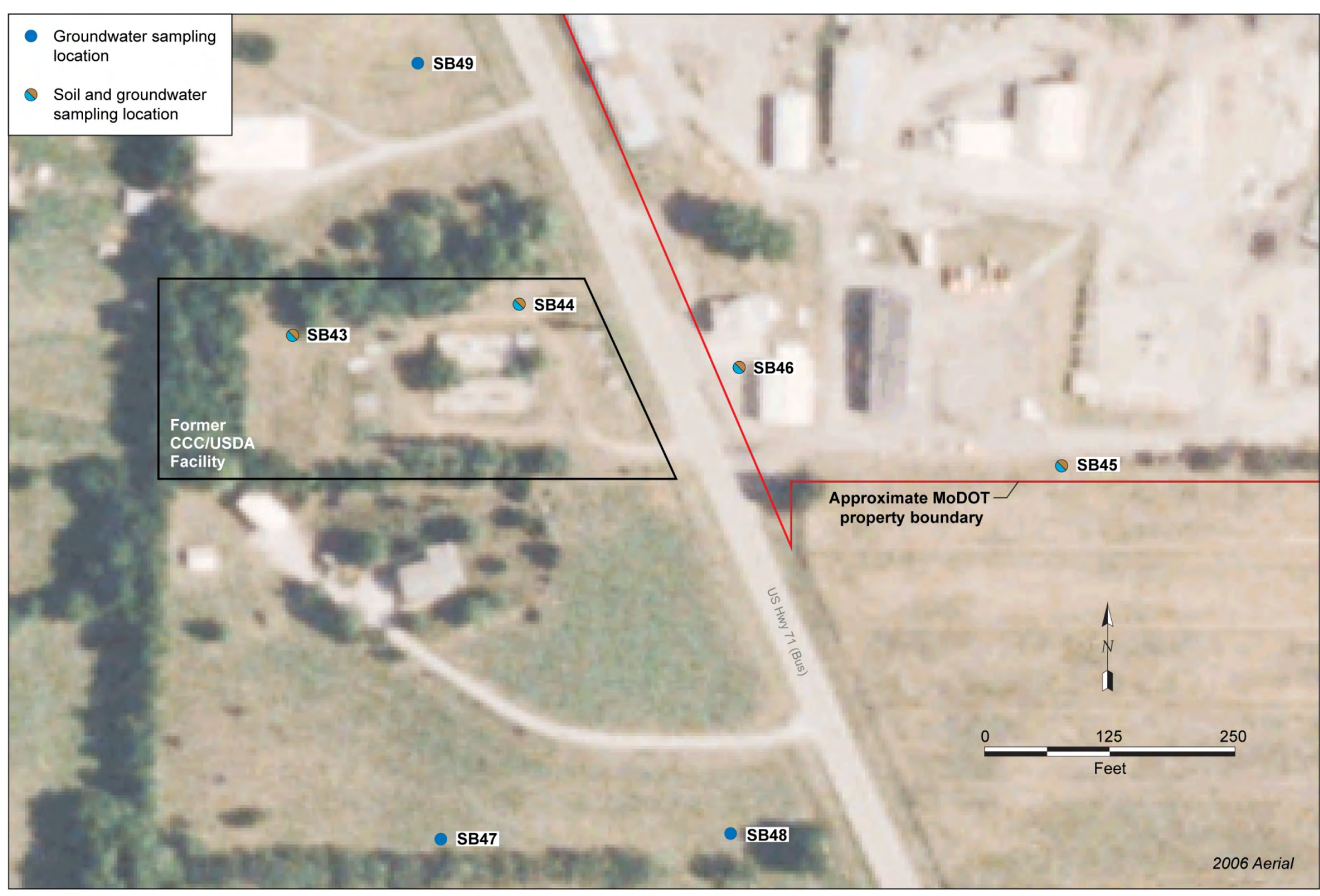




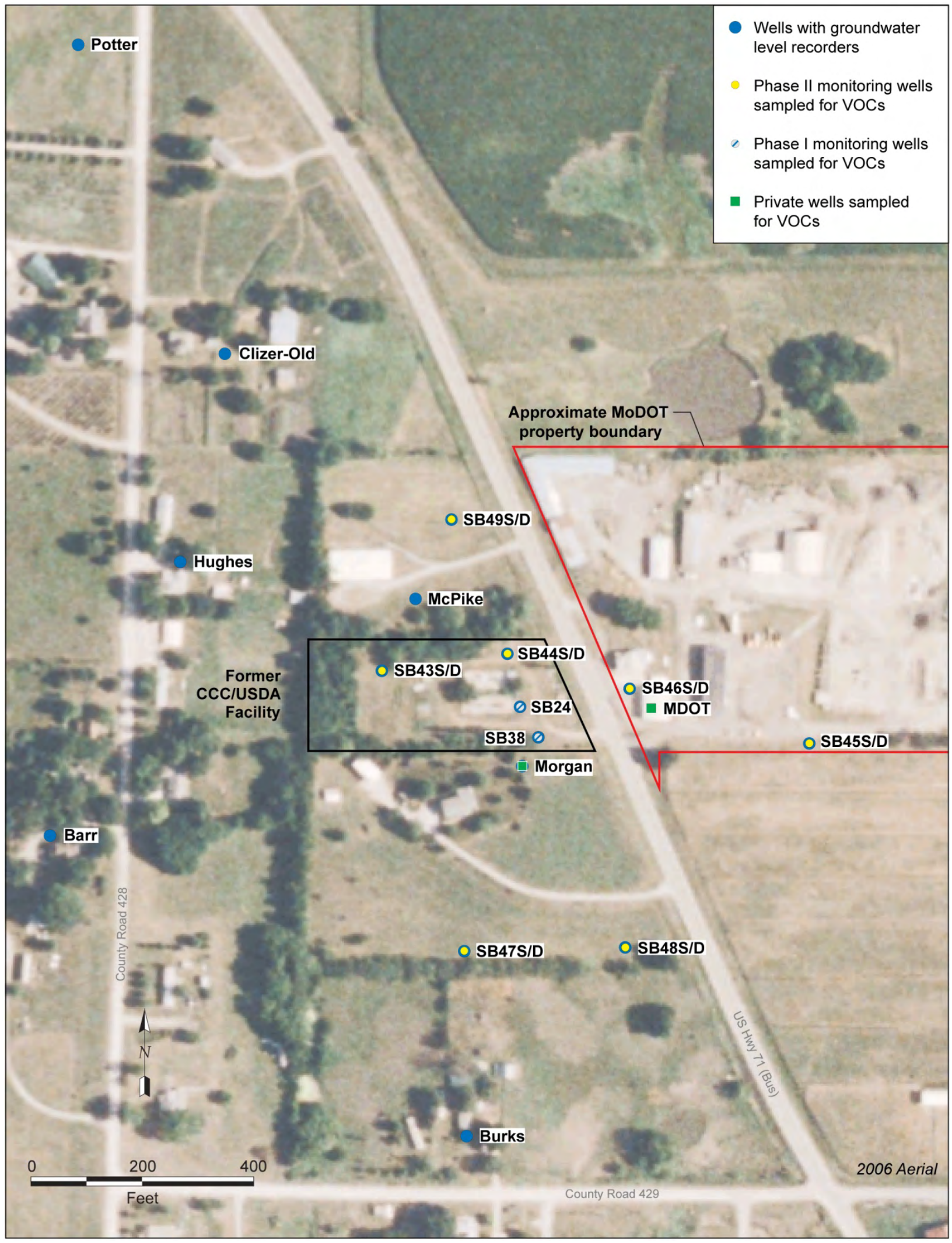

FIGURE 2.2 Monitoring wells and private wells sampled and/or equipped with groundwater level recorders in the Phase II studies. Source of aerial photograph: NAIP (2006). 


\section{Field and Laboratory Data}

This section presents the field and laboratory data generated during the Phase II investigation. A chronological summary of the sampling activities is in Appendix A, Table A.1. The methods and procedures employed in collecting these data are described in Section 2, in the Master Work Plan (Argonne 2002), and in the site-specific Work Plan (Argonne 2010b).

\subsection{Analytical Data for Soil Samples}

A total of 109 soil samples were collected for VOCs analyses during the Phase II field program, at four locations (SB43-SB46; Figure 2.1) selected on the basis of the Phase I investigation findings summarized in Section 1.1. All of the soil samples were analyzed by the purge-and-trap method described in Section 2.3.3. The samples were also analyzed by a screening headspace method to assist with decision making in the field; these results are not used, however, for purposes requiring quantitative data.

The purge-and-trap analytical data for carbon tetrachloride in the soil samples are summarized in Table 3.1. The complete analytical data are in Appendix B, Table B.1. Figure 3.1 illustrates the distribution of carbon tetrachloride with depth (purge-and-trap results) in each of the Phase II borings sampled for VOCs analyses (SB43-SB46). The highest concentrations of carbon tetrachloride identified in soils at the deep boring locations to date, in both Phase I and Phase II, are shown in Figure 3.2.

\subsubsection{Carbon Tetrachloride Concentrations above the Quantitation Limit}

Carbon tetrachloride concentrations at or above the quantitation limit of $10 \mu \mathrm{g} / \mathrm{kg}$ for the purge-and-trap method were identified during Phase II in 22 soil samples collected at three locations: SB44 on the former CCC/USDA property and SB45 and SB46 on the MoDOT property (Figures 3.1 and 3.2). Briefly, the results for carbon tetrachloride (Table 3.1) were as follows:

- At boring SB44, in the northeast corner of the former CCC/USDA facility, concentrations were $14-364 \mu \mathrm{g} / \mathrm{kg}$ at $40-60 \mathrm{ft}$ BGL. No carbon tetrachloride was detected at depths $>60 \mathrm{ft}$ BGL. 
- At boring SB45, at the southern edge of the MoDOT property, concentrations were $12 \mu \mathrm{g} / \mathrm{kg}$ at $12 \mathrm{ft}$ BGL and 25-63 $\mu \mathrm{g} / \mathrm{kg}$ at 25-44 ft BGL. No carbon tetrachloride was detected at depths $>44 \mathrm{ft} \mathrm{BGL}$.

- At SB46, near the western edge of the MoDOT property, concentrations were $12-82 \mu \mathrm{g} / \mathrm{kg}$ at 8-48 ft BGL. No carbon tetrachloride was detected at depths $>48 \mathrm{ft}$ BGL.

- At SB43, no carbon tetrachloride was detected (at an instrumental detection limit of $1.0 \mu \mathrm{g} / \mathrm{kg}$ for the purge-and-trap method).

The maximum depths of the contamination in soils identified in borings SB43-SB46 (44-60 ft BGL) correspond relatively closely to the stratigraphic interval targeted for groundwater sampling in the Phase II shallow monitoring wells (1,099-1,109 ft AMSL; see Sections 2.2 and 3.2).

\subsubsection{Carbon Tetrachloride Concentrations above the Default Target Level}

Carbon tetrachloride concentrations above the DTL for this contaminant in soils $(79.6 \mu \mathrm{g} / \mathrm{kg})$ were identified in seven soil samples collected at restricted depth intervals, as follows (Table 3.1): (1) in five samples from SB44 (106-364 $\mu \mathrm{g} / \mathrm{kg}$ at 44-56 ft BGL) and (2) in 2 samples from SB46 $(81-82 \mu \mathrm{g} / \mathrm{kg}$ at $40-44 \mathrm{ft}$ BGL). The maximum carbon tetrachloride concentrations identified to date in soils at Savannah occurred in boring SB44, at $44 \mathrm{ft}$ BGL (364 $\mu \mathrm{g} / \mathrm{kg})$ and $48 \mathrm{ft}$ BGL (212 $\mu \mathrm{g} / \mathrm{kg})$.

\subsubsection{Carbon Tetrachloride Concentrations at Phase II Boring SB44 and Phase I Boring SB42}

Boring location SB44 on the former CCC/USDA property was selected (at the request of the MDNR program manager) to lie in close proximity to Phase I deep boring location SB42 (Figure 3.2), which returned the highest identified carbon tetrachloride concentrations in soils during Phase I (Figure 1.3). At SB42, carbon tetrachloride levels of 15-171 $\mu \mathrm{g} / \mathrm{kg}$ were identified over the depth interval $44-56 \mathrm{ft}$ BGL, with the highest concentrations detected at $44 \mathrm{ft}$ BGL $(171 \mu \mathrm{g} / \mathrm{kg})$ and $48 \mathrm{ft}$ BGL $(126 \mu \mathrm{g} / \mathrm{kg})$. The vertical distribution of carbon tetrachloride 
identified in Phase II boring SB44 (Figure 3.1) therefore mirrors that observed at SB42; however, the range of concentrations detected at SB44 is consistently higher.

\subsubsection{Vertical Profile of Carbon Tetrachloride Concentrations at Boring SB46}

Except at SB46, no carbon tetrachloride was detected in Phase I or Phase II sampling (above the instrumental limit of $1.0 \mu \mathrm{g} / \mathrm{kg}$ for the purge-and-trap analysis method) at depths of $8 \mathrm{ft} \mathrm{BGL}$ or less. Furthermore, concentrations exceeding the quantitation limit of $10 \mu \mathrm{g} / \mathrm{kg}$ have been observed in only two of the soil samples collected at depths of $20 \mathrm{ft}$ BGL or less. In contrast, at SB46 a continuous distribution of carbon tetrachloride concentrations, ranging from trace levels to a maximum of $82 \mu \mathrm{g} / \mathrm{kg}$, was observed from $2 \mathrm{ft}$ to $48 \mathrm{ft}$ BGL (Table 3.1 and Figure 3.1).

The results of ongoing groundwater level monitoring (Section 4.1.3) indicate that the apparent upper extent of saturation in the soils in the vicinity of the former CCC/USDA facility and the present MoDOT facility (as represented by the groundwater levels at monitoring points SB24 and SB43S-SB46S) occurred at depths of about 18-22 ft BGL during the period of review. These observations therefore suggest that the concentrations of carbon tetrachloride detected in many of the deeper soil samples (greater than approximately $20 \mathrm{ft}$ BGL) reflect contamination that is (1) adsorbed to the solid soil particles, (2) dissolved in the pore water contained in the soils, or (3) in a combination of these two physical states. The shallower (less than approximately $20 \mathrm{ft}$ BGL) carbon tetrachloride present at SB46 might, however, reflect residual contamination of the subsurface (vadose) zone soils at this location.

\subsubsection{Trace Detections of Chloroform}

No chloroform was detected in any of the soil samples from borings SB43-SB46, at concentrations greater than the DTL $(76.6 \mu \mathrm{g} / \mathrm{kg})$ for this contaminant or the quantitation limit of $10 \mu \mathrm{g} / \mathrm{kg}$ for the purge-and-trap analysis method. Trace levels of chloroform ( $<10 \mu \mathrm{g} / \mathrm{kg})$ were detected in a total of 15 samples (excluding quality control samples), however, recovered at various depths in these borings (Appendix B, Table B.1). 


\subsection{Lithologic and Physical Property Data for Soils}

Lithologic descriptions for the continuous soil cores recovered at borings SB43-SB49 (Figure 2.1) are in Appendix C.

The results of grain size and physical property analyses for selected core intervals from borings SB43, SB44, and SB45 are in Appendix B, Tables B.2 and B.3. The core intervals submitted for laboratory analyses were chosen to reflect the predominant sediment types penetrated, as well as several minor, thin, more coarsely grained horizons (see Section 4.1.1) identified by visual inspection. Bulk dry density, moisture content, specific gravity, porosity, total organic carbon content, and sediment particle size distribution determinations were completed by the physical testing laboratory (Alfred Benesch and Company, Lincoln, Nebraska) for most of the samples submitted. Vertical permeability determinations were obtained only for three samples from boring SB44, because of difficulties reported by the laboratory in the preparation of suitable sediment plugs for analysis.

\subsection{Installation of Permanent Monitoring Wells}

As outlined in Section 2.2, paired shallow (S) and deep (D) permanent monitoring wells were installed at each of the seven Phase II soil boring locations (Figures 2.1 and 2.2). All wells were constructed with nominal 2-in.-diameter, Schedule 40 (for shallow wells) or Schedule 80 (for deep wells) polyvinyl chloride (PVC) casing and 0.010-in.-slot PVC screens, in accord with MDNR requirements. Primary and secondary sand packs were placed around each screened interval, and the remaining annulus was grouted by using a tremie pipe. All wells were completed at the surface with flush-mounted well housings, at the request of the individual property owners. The construction details for the wells are in Table 3.2; copies of the MDNR certification forms for the wells are in Appendix D.

In keeping with the approved Work Plan (Argonne 2010b), shallow wells SB43S-SB49S were installed to facilitate groundwater sampling and the measurement of hydraulic heads at a consistent elevation in the previously identified silty-clay to clayey-silt unit. On the basis of the groundwater level responses and identified carbon tetrachloride contamination in Phase I monitoring point SB24 and the Morgan and MoDOT private wells (Argonne 2010a), SB43SSB49S were screened and sand packed (within $\pm 1 \mathrm{ft}$ ) over the 10 -ft target interval at 1,099-1,109 ft BGL. 
Deep monitoring points SB43D-SB49D were completed within the silty clay till and/or weathered bedrock materials identified directly above the more competent bedrock at each boring location (Section 4.1.1). Well SB43D was completed with a 10-ft screen and 14-ft sand pack, and well SB44D was constructed with a 10 -ft screen and 20-ft sand pack, with the verbal approval of the MDNR program manager. Deep wells SB45D-SB49D were all constructed with $20-\mathrm{ft}$ screens and 20-ft (or greater) sand pack intervals, at the request of the MDNR, in an attempt to enhance the potential for recovery of groundwater from these wells (Table 3.2).

\subsection{Analytical Data for Groundwater Samples}

During the Phase II investigation, 86 groundwater samples were collected for VOCs analyses. The sampling was conducted, to the extent possible (as discussed further below), in accordance with the procedures outlined in Section 2.4, in the site-specific Work Plan (Argonne 2010b), and in the Master Work Plan (Argonne 2002).

The specific conditions under which each groundwater sample was collected are in Appendix A, Table A.1. The complete analytical results for the VOCs in water samples are in Appendix E, Table E.1. Results for carbon tetrachloride and chloroform are summarized in Table 3.3 (compiled chronologically) and Table 3.4 (compiled by location and depth). The groundwater parameter measurements obtained for these samples in the field are in Appendix E, Table E.2.

As noted in Section 2.4, slow groundwater accumulation rates observed in the Phase II shallow monitoring wells resulted in the accumulation of only limited quantities of groundwater in most of these wells by the end of the main Phase II field session (in early July 2010). To obtain an indication of the possible contaminant levels at each location, grab samples of groundwater were collected in conjunction with the development of monitoring points SB43SSB49S and SB43D-SB49D (Figure 2.2) on July 1-3, 2010.

Groundwater samples were also collected from Phase I monitoring wells SB24 and SB38 and from the Morgan and MoDOT private wells during the main field session (on June 23-28, 2010; Figure 2.2). With the verbal approval of the MDNR program manager, a low-flow methodology was employed to sample each of these wells. Samples were collected from the bottoms of the Morgan, MoDOT, and SB38 wells and at the midpoint of each (initial) static 
water column. A single sample was collected near the bottom of monitoring point SB24. Following completion of the low-flow sampling, the Morgan and MoDOT wells were resampled after purging of approximately three well casing volumes of groundwater from each well (Section 2.4).

In August 2010, roughly seven weeks after installation and development, the SB43-SB49 shallow and deep monitoring points, SB24, and SB38 (Figure 2.2) were resampled for VOCs analyses. A grab sample was first obtained from each well without purging. Samples were then collected in the SB43-SB49 shallow and deep wells (from near the base of each well) by using the low-flow sampling methodology. The complete suite of wells was again sampled in August 2011, by collecting an initial grab sample and a low-flow sample at each well, following apparent stabilization of the groundwater levels in all wells during the intervening period (August 2010 to August 2011; see Section 2.4).

\subsubsection{Results for Shallow Monitoring Wells SB24 and SB43S-SB49S}

The results of VOCs analyses for the groundwater samples from the shallow monitoring wells (SB24 and SB43S-SB49S, completed in the depth interval at 1,098 ft-1,110 ft AMSL) are summarized in Tables 3.3 and 3.4 and illustrated in Figures 3.3-3.5.

Carbon tetrachloride was identified (at concentrations above the analytical method detection limit of $0.1 \mu \mathrm{g} / \mathrm{L}$ ) in 24 of the 39 samples collected from the shallow monitoring interval, at concentrations ranging from $<1 \mu \mathrm{g} / \mathrm{L}$ to $273 \mu \mathrm{g} / \mathrm{L}$. For locations with detectable levels of carbon tetrachloride, the concentrations for samples collected with the low-flow methodology were typically comparable to or greater than the values for corresponding grab samples collected in August 2010 and August 2011 without purging (Figures 3.4 and 3.5). The low-flow and grab sample results generally demonstrated similar concentration trends between the two sampling events, however, at each location.

Carbon tetrachloride concentrations above the DTL $(5.0 \mu \mathrm{g} / \mathrm{L})$ for this contaminant in groundwater were identified in 19 samples from the shallow monitoring interval, at wells SB24 and SB43S-SB47S (Table 3.4 and Figures 3.3-3.5). Levels above the DTL were consistently observed at wells SB24, SB44S, SB45S, and SB46S. However, carbon tetrachloride was detected at SB43S only in the grab sample collected in August 2010, and at SB47S only in the 
initial grab sample recovered in July 2010. No carbon tetrachloride was detected in any other groundwater samples from SB43S or SB47S. No carbon tetrachloride was detected at SB49S, and only trace levels $(<3 \mu \mathrm{g} / \mathrm{L}$ ) were identified at SB48S (Figures 3.3-3.5).

The maximum carbon tetrachloride concentrations identified in the shallow monitoring interval occurred at wells SB44S (at the northeast corner of the former CCC/USDA property) and SB45S (at the southern edge of the MoDOT property). The levels ranged from $59 \mu \mathrm{g} / \mathrm{L}$ to $273 \mu \mathrm{g} / \mathrm{L}$ at SB44S and from $5.7 \mu \mathrm{g} / \mathrm{L}$ to $86 \mu \mathrm{g} / \mathrm{L}$ at SB45S. The carbon tetrachloride concentrations detected at SB44S increased from August 2010 to August 2011 (Table 3.4), while those at SB45S decreased slightly during this period. Well SB46S is located roughly midway between SB44S and SB45S, near the western margin of the MoDOT property. Carbon tetrachloride concentrations of 1.4-17 $\mu \mathrm{g} / \mathrm{L}$ were identified at SB46S (Figures 3.3-3.5 and Table 3.4). The concentrations at this well showed little change during the review period.

Chloroform was detected in 33 of the 39 groundwater samples collected from the shallow monitoring interval (wells SB24 and SB43S-SB49S), at concentrations ranging from $1.4 \mu \mathrm{g} / \mathrm{L}$ to $27 \mu \mathrm{g} / \mathrm{L}$ (Tables 3.3 and 3.4). No chloroform was detected at a concentration above the DTL ( $80 \mu \mathrm{g} / \mathrm{L}$ ) for this contaminant in groundwater. Although the absolute levels of chloroform identified are generally low, the chloroform concentrations are significant relative to the associated levels of carbon tetrachloride observed at wells SB24 (47-133\%, calculated as chloroform concentration in $\mu \mathrm{g} / \mathrm{L}$ divided by carbon tetrachloride concentration in $\mu \mathrm{g} / \mathrm{L}$ ), SB44S (10-44\%), SB45S (10-133\%), and SB46S (12-47\%). Chloroform was also detected in the absence of carbon tetrachloride at wells SB43S, SB47S, and (in samples from the June-July 2010 and August 2010 sampling events) SB49S.

\subsubsection{Results for the Morgan and MoDOT Private Wells}

Construction information obtained in Phase I indicates that the private wells in the investigation area might intercept groundwater from the full depth interval penetrated by each boring (Argonne 2010a). The Morgan and MoDOT private wells are, however, completed to depths representing elevations of approximately 1,097 ft AMSL and 1,108 ft AMSL, respectively; Table 3.5. These elevations are comparable to those of the shallow monitoring wells (Table 3.2). The analytical results for groundwater samples obtained from the Morgan and MoDOT wells in the June-July 2010 sampling event are therefore included, for comparison, in 
Figure 3.3. The data for these wells (Tables 3.3 and 3.4) showed little variation with sampling methodology.

The carbon tetrachloride concentration identified in the Morgan private well in June-July 2010 (13 $\mu \mathrm{g} / \mathrm{L})$ was identical to the level determined for this well during the Phase I (2007) investigation (Argonne 2010a). Carbon tetrachloride concentrations of 179-210 $\mu \mathrm{g} / \mathrm{L}$ detected in the MoDOT well during the Phase II work are greater than the concentration of $150 \mu \mathrm{g} / \mathrm{L}$ measured during Phase I (Argonne 2010a), but the Phase II values remain lower than the original concentration of $321 \mu \mathrm{g} / \mathrm{L}$ reported for this well in 2000 (MDNR 2000b).

Low concentrations of chloroform $(3.4-6.8 \mu \mathrm{g} / \mathrm{L})$ were detected at both the Morgan and MoDOT wells in the Phase II investigation. These concentrations yield chloroform-to-carbon tetrachloride ratios that are low (3\%) at the MoDOT well but are significant (47-133\%) at the Morgan well.

\subsubsection{Results for Deep Monitoring Wells SB43D-SB49D and SB38}

\subsubsection{Monitoring Wells SB43D-SB49D}

With one exception, no carbon tetrachloride was identified (at the analytical detection limit of $0.1 \mu \mathrm{g} / \mathrm{L}$ ) in the 35 samples of groundwater recovered from deep monitoring wells SB43D-SB49D. A trace of carbon tetrachloride (estimated at $0.3 \mu \mathrm{g} / \mathrm{L}$; below the method quantitation limit of $1.0 \mu \mathrm{g} / \mathrm{L}$ ) was detected in the initial grab sample collected from well SB44D in July 2010; this value was not corroborated, however, by any subsequent analyses.

In contrast to the results for carbon tetrachloride, trace to low concentrations of chloroform were detected in the groundwater samples from deep monitoring wells SB43D, SB44D, and SB46D-SB49D. The highest chloroform concentrations were identified at wells SB44D (2.4-4.1 $\mu \mathrm{g} / \mathrm{L})$ and SB49D (3.0-3.4 $\mu \mathrm{g} / \mathrm{L})$ during the July 2010 and August 2010 sampling events; however, the levels detected at all locations have decreased with time. With one exception (the grab sample obtained at wells SB44D; Tables 3.3 and 3.4), no chloroform was detected in the deep monitoring wells during the August 2011 sampling event. 


\subsubsection{Monitoring Well SB38D}

Monitoring point SB38 was installed on the former CCC/USDA property during Phase I (Argonne 2010a), with a 40-ft screened interval intended to mimic the construction approach employed in many local private wells. Well SB38 intersects the depth interval (at elevations of approximately 1,098-1,110 ft AMSL) represented by the shallow monitoring wells (Section 3.4.1) and penetrates an additional $30 \mathrm{ft}$ into the silt-clay and till units identified at this location. The well does not, however, intersect the interval immediately overlying bedrock that is screened in the Phase II deep monitoring wells (SB43D-SB49D; Table 3.2). The construction of SB38 is therefore unique.

Carbon tetrachloride concentrations of $1.9-20 \mu \mathrm{g} / \mathrm{L}$ were identified in the groundwater samples from well SB38, with the higher values (11-20 $\mu \mathrm{g} / \mathrm{L})$ detected in August 2010 and August 2011 (Tables 3.3 and 3.4). These results are higher than the carbon tetrachloride concentration identified at this location in Phase I (4.9 $\mu \mathrm{g} / \mathrm{L}$ in March 2008; Argonne 2010a). Low levels of chloroform also consistently detected at SB38 (2.1-3.2 $\mu \mathrm{g} / \mathrm{L})$ showed little distinct change during the Phase II sampling events. The data therefore indicate that the chloroform-tocarbon tetrachloride ratios at this location decreased, from approximately $86-100 \%$ in June-July 2010 to 12-22\% in August 2010 and August 2011.

\subsection{Groundwater Level Data}

To obtain information on the occurrence and possible direction(s) of groundwater flow in the vicinity of the former CCC/USDA facility, static water levels were periodically measured manually in the eight selected private wells (Morgan, McPike, MoDOT, Clizer "old," Potter, Barr, Hughes, Burks), eight shallow monitoring wells (SB24, SB43S-SB49S), and eight deep monitoring wells (SB38, SB43D-SB49D) illustrated in Figure 2.2. The specific dates on which groundwater levels were measured at each monitoring point and the results of the manual measurements are in Table 3.6.

In conjunction with the Phase I investigations, automated measurement of groundwater levels was initiated in 2007-2008 in monitoring wells SB24 and SB38 and in the Morgan, McPike, MoDOT, Clizer “old,” Potter, Barr, Hughes, and Burks private wells (Argonne 2010a). Except at the MoDOT well, the automated monitoring at these locations continued during the 
Phase II studies. The MoDOT well was abandoned as part of the Phase II field program, as discussed in Section 3.7.

The groundwater level records obtained from the data loggers through early May 2009 were presented and discussed previously (Argonne 2010a,b). The complete water level records for these wells for the period May 2009 through December 2011 are on the compact disc (CD) inside the back cover of this report, in Supplement 1, Table S1.1.

To augment these observations that began during Phase I, water level sensing and data logging units were installed in shallow monitoring wells SB43S-SB49S and deep monitoring wells SB43D-SB49D in July 2010. The complete water level records for the Phase II shallow and deep monitoring wells, respectively, are in Supplement 1, Tables S1.2 and S1.3. The patterns of water level variations, the apparent hydraulic gradients, and groundwater flow directions developed from the data logger records and manual measurements are discussed in Section 4.1.3.

Information on precipitation in the study area during the groundwater monitoring period was acquired to assist in evaluation of the observed groundwater level trends. Rainfall data for the nearest available recording station (Saint Joseph, located approximately $7 \mathrm{mi}$ from Savannah) were obtained from the Missouri Historical Agricultural Weather Database (MUE 2011). The precipitation data are on CD, in Supplement 1, Table S1.4.

\subsection{Coordinates Survey Data}

Phase II monitoring wells SB43S-SB49S and SB43D-SB49D were surveyed by SchwabEaton, P.A., Manhattan, Kansas, to provide horizontal and vertical control for the interpretation and correlation of stratigraphic and groundwater level data. The coordinates for the surveyed Phase II locations are in Appendix F, Table F.1.

\subsection{Abandonment of the MoDOT Private Well}

In discussions among the CCC/USDA and MDNR program managers and Argonne staff prior to implementation of the approved Phase II Work Plan (Argonne 2010b), the MDNR program manager expressed concerns regarding the observed physical condition of the MoDOT private well. Previous sampling (MDNR 2000; Argonne 2010a) demonstrated that the highest 
concentrations of carbon tetrachloride contamination in groundwater identified at Savannah occurred in this well $(150-321 \mu \mathrm{g} / \mathrm{L})$. The well was not in use by the MoDOT staff as a water source at the time of the field activities reported here.

The MoDOT well was a large-diameter (30-in.), concrete-cased well located in an unsealed, cement-lined vault below the floor of the MoDOT vehicle maintenance building, near the western edge of the MoDOT property (Figure 2.2). Access to the well vault was via a manhole in the building floor that had been capped by the MoDOT with a perforated cover. The vehicle maintenance building is well ventilated and is not permanently occupied; however, on the basis of the well's condition, the MDNR suggested that it might serve as an unacceptable source of carbon tetrachloride vapor intrusion to the building air.

With the approval of the CCC/USDA and MDNR program managers and representatives of the MoDOT, the MoDOT well was plugged and abandoned on June 27, 2010, after the well had been sampled for VOCs analyses as described in Sections 2.4 and 3.4.2. The uppermost segment of the concrete casing projecting above the well vault floor was removed, and the full length of the well was sealed flush with the vault floor, with cement grout tremied into the casing through use of the high-volume grout pump on the sonic drilling unit (Figures 3.6 and Figure 3.7). The MDNR Abandonment Registration Record for the MoDOT well is in Appendix D.

\subsection{Results of Quality Control Activities}

The QA/QC procedures followed for sample collection, handling, and analysis are described in detail in the Master Work Plan (Argonne 2002). A detailed report addressing QA/QC activities related to sample collection, handling, and analysis is on CD, in Supplement 2.

The results of the QA/QC activities are summarized as follows:

- Sample integrity was maintained successfully throughout the collection, shipping, and analysis activities by documenting samples as they were collected and using custody seals and chain-of-custody records. 
- All samples were received with custody seals intact and at the appropriate preservation conditions. Samples were analyzed within the required holding times.

- Carbon tetrachloride and chloroform, primary contaminants of concern in the investigation, were not detected in laboratory method blanks.

- Quality control samples (field blanks, equipment rinsates, and trip blanks) were collected to monitor sample-handling activities. Carbon tetrachloride was not detected. Chloroform was detected at low concentrations in 2 of the 4 field blanks and 2 of the 20 trip blanks. The chloroform concentrations were consistent with chlorination of the public water supply water used during the investigation, and cross-contamination is not indicated.

- Soil and groundwater samples were analyzed for carbon tetrachloride and chloroform at the AGEM Laboratory by using the purge-and-trap method (on a GC-MS system) for a quantitative determination of contaminant distribution. Dual analyses of samples indicated consistency in the sampling and analytical methodologies. Dual analyses were accomplished through either analysis of replicate samples submitted to the laboratory or duplicate analysis of samples selected by the laboratory. Consistency in both the sampling and analytical methodologies is indicated by average relative percent difference values of $7 \%$ for carbon tetrachloride and 6\% for chloroform for the dual analyses with the contaminants present above the method quantitation limit.

- The analyses of soil and groundwater samples by the AGEM Laboratory were verified by a second laboratory (TestAmerica). Agreement in the results from the two laboratories was good over the range of contaminant concentrations detected. The concentrations detected in soil and groundwater in analysis at the AGEM Laboratory are supported by the verification analysis results from TestAmerica. The verification data from TestAmerica are in Supplement 3 (on CD). 


\subsection{Waste Characterization, Handling, and Disposal}

Purge water and equipment decontamination water generated as potentially contaminated investigation-derived waste was containerized on-site. The accumulated waste water was sampled on October 31, 2011 (along with wastewaters from several CCC/USDA sites in Kansas), and analyzed by Pace Analytical Services, Inc., Lenexa, Kansas, on November 4, 2011. Methods used were EPA Method 5030/8260 for VOCs, EPA Method 504.1 for ethylene dibromide, and EPA Method 353.2 for nitrate/nitrite nitrogen. Carbon tetrachloride and chloroform were detected at $6.9 \mu \mathrm{g} / \mathrm{L}$ and $3.3 \mu \mathrm{g} / \mathrm{L}$, respectively. Ethylene dibromide was not detected. Nitrate/nitrite nitrogen was present at $0.41 \mathrm{mg} / \mathrm{L}$. The laboratory results are in Supplement 4 (on CD). The water was delivered on December 19, 2011 (together with purge water from several CCC/USDA investigation sites in Kansas), for disposal at the Sabetha, Kansas, publicly owned wastewater treatment plant. 
TABLE 3.1 Summary of purge-and-trap analysis results for carbon tetrachloride in soil samples collected in Phase II. $a, b$

\begin{tabular}{|c|c|c|c|c|}
\hline Location & $\begin{array}{l}\text { Sample } \\
\text { Date }\end{array}$ & $\begin{array}{c}\text { Depth } \\
\text { (ft BGL) }\end{array}$ & $\begin{array}{c}\text { Number } \\
\text { of Samples }\end{array}$ & $\begin{array}{c}\text { Carbon } \\
\text { Tetrachloride } \\
(\mu \mathrm{g} / \mathrm{kg})\end{array}$ \\
\hline SB43 & $6 / 21 / 10$ & $4-112$ & 28 & $N^{c}$ \\
\hline \multirow[t]{12}{*}{ SB44 } & $6 / 23 / 10$ & $4-24$ & 6 & ND \\
\hline & $6 / 23 / 10$ & 28 & 1 & $2.3 \mathrm{~J}^{\mathrm{d}}$ \\
\hline & $6 / 23 / 10$ & 32 & 1 & $4.8 \mathrm{~J}$ \\
\hline & $6 / 23 / 10$ & 36 & 1 & $4.7 \mathrm{~J}$ \\
\hline & $6 / 23 / 10$ & 40 & 1 & 14 \\
\hline & $6 / 23 / 10$ & 44 & 1 & 364 \\
\hline & $6 / 23 / 10$ & 45 & 1 & 135 \\
\hline & $6 / 23 / 10$ & 48 & 1 & 212 \\
\hline & $6 / 23 / 10$ & 52 & 1 & 139 \\
\hline & $6 / 23 / 10$ & 56 & 1 & 106 \\
\hline & $6 / 23 / 10$ & 60 & 1 & 18 \\
\hline & $6 / 23 / 10$ & 64-105 & 14 & ND \\
\hline \multirow[t]{12}{*}{ SB45 } & $6 / 24 / 10$ & $2.5-8$ & 2 & ND \\
\hline & $6 / 24 / 10$ & 12 & 1 & 12 \\
\hline & $6 / 24 / 10$ & 16 & 1 & ND \\
\hline & $6 / 24 / 10$ & 20 & 1 & $8.6 \mathrm{~J}$ \\
\hline & $6 / 24 / 10$ & 22.4 & 1 & $7.7 \mathrm{~J}$ \\
\hline & $6 / 24 / 10$ & 25 & 1 & 32 \\
\hline & $6 / 24 / 10$ & 28 & 1 & 28 \\
\hline & $6 / 24 / 10$ & 32 & 1 & 38 \\
\hline & $6 / 24 / 10$ & 36 & 1 & 41 \\
\hline & $6 / 24 / 10$ & 40 & 1 & 25 \\
\hline & $6 / 24 / 10$ & 44 & 1 & 63 \\
\hline & $6 / 24 / 10$ & $48-100$ & 14 & ND \\
\hline \multirow[t]{11}{*}{ SB46 } & $6 / 25 / 10$ & 2 & 1 & $2.8 \mathrm{~J}$ \\
\hline & $6 / 25 / 10$ & 8 & 1 & 22 \\
\hline & $6 / 25 / 10$ & 12 & 1 & 16 \\
\hline & $6 / 26 / 10$ & 16 & 1 & 31 \\
\hline & $6 / 26 / 10$ & 20 & 1 & 45 \\
\hline & $6 / 25 / 10$ & 28 & 1 & 12 \\
\hline & $6 / 25 / 10$ & 36 & 1 & $8.7 \mathrm{~J}$ \\
\hline & $6 / 25 / 10$ & 40 & 1 & 81 \\
\hline & $6 / 25 / 10$ & 44 & 1 & 82 \\
\hline & $6 / 25 / 10$ & 48 & 1 & 30 \\
\hline & $6 / 25 / 10$ & $52-108$ & 15 & ND \\
\hline
\end{tabular}

a Complete results are in Appendix B, Table B.1.

b Gray highlights mark concentrations above the DTL of $79.6 \mu \mathrm{g} / \mathrm{kg}$ for carbon tetrachloride in soil.

c ND, not detected at an instrument detection limit of $1 \mu \mathrm{g} / \mathrm{kg}$.

d Qualifier J indicates an estimated concentration below the method quantitation limit of $10 \mu \mathrm{g} / \mathrm{kg}$. 
TABLE 3.2 Construction data for monitoring wells.

\begin{tabular}{|c|c|c|c|c|}
\hline \multirow[b]{2}{*}{ Well } & \multicolumn{2}{|c|}{ Screened Interval } & \multicolumn{2}{|c|}{ Primary Sand Pack Interval } \\
\hline & Depth (ft BGL) ${ }^{a}$ & $\begin{array}{l}\text { Elevation } \\
\text { (ft AMSL) }^{\mathrm{b}}\end{array}$ & Depth (ft BGL) & Elevation (ft AMSL) \\
\hline SB43S & $45-55$ & 1099.81-1109.81 & $45-55$ & 1099.81-1109.81 \\
\hline SB43D & $95-105$ & 1049.99-1059.99 & $95-109$ & 1045.99-1059.99 \\
\hline SB44S & $46.5-56.5$ & 1099.10-1109.10 & $46.5-56.5$ & 1099.10-1109.10 \\
\hline SB44D & $92-102$ & $1053.77-1063.77$ & $92-112$ & 1043.77-1063.77 \\
\hline SB45S & $37-47$ & 1098.03-1108.03 & $37-47$ & 1098.03-1108.03 \\
\hline SB45D & $79.5-99.5$ & 1045.84-1065.84 & 79.5-101 & 1044.34-1065.84 \\
\hline SB46S & $42-52$ & 1099.96-1109.96 & $42-52$ & 1099.96-1109.96 \\
\hline SB46D & $90-110$ & 1041.89-1061.89 & $90-111$ & 1040.89-1061.89 \\
\hline SB47S & $25-35$ & 1099.68-1109.68 & $25-35$ & 1099.68-1109.68 \\
\hline SB47D & $77-97$ & 1038.18-1058.18 & $77-103$ & 1032.18-1058.18 \\
\hline SB48S & $25-35$ & 1099.88-1109.88 & $25-35$ & 1099.88-1109.88 \\
\hline SB48D & $77-97$ & 1037.94-1057.94 & $77-101$ & 1033.94-1057.94 \\
\hline SB49S & $29-39$ & 1098.72-1108.72 & $29-39$ & 1098.72-1108.72 \\
\hline SB49D & $77-97$ & $1040.92-1060.92$ & $77-99$ & 1038.92-1060.92 \\
\hline $\mathrm{SB} 24^{\mathrm{C}}$ & $50-60$ & 1098.65-1108.65 & $50-60$ & $1098.65-1108.65$ \\
\hline SB38 ${ }^{\mathrm{C}}$ & $50-90$ & $1068.87-1108.87$ & $50-90$ & 1068.87-1108.87 \\
\hline
\end{tabular}

a BGL, below ground level.

b AMSL, above mean sea level.

c Data for Phase I monitoring wells SB24 and SB38 are included for comparison. 
TABLE 3.3 Chronological compilation of analytical results from the AGEM Laboratory for carbon tetrachloride and chloroform in groundwater samples collected in 2010-2011.

\begin{tabular}{|c|c|c|c|c|c|c|}
\hline \multirow[b]{2}{*}{ Location } & \multirow[b]{2}{*}{ Sample } & \multirow[b]{2}{*}{$\begin{array}{l}\text { Sample } \\
\text { Date }\end{array}$} & \multirow[b]{2}{*}{$\begin{array}{l}\text { Depth } \\
\text { (ft BGL) }\end{array}$} & \multirow[b]{2}{*}{ Type $^{a}$} & \multicolumn{2}{|c|}{ Concentration $(\mu \mathrm{g} / \mathrm{L})$} \\
\hline & & & & & $\begin{array}{c}\text { Carbon } \\
\text { Tetrachloride }\end{array}$ & Chloroform \\
\hline \multicolumn{7}{|l|}{ June-July 2010} \\
\hline MoDOT & SVMODOTLFM-W-25776 & $6 / 25 / 10$ & 44 & LFM & 210 & 6.8 \\
\hline MoDOT & SVMODOTLFB-W-25775 & $6 / 25 / 10$ & 44 & LFB & 179 & 6.2 \\
\hline MoDOT & SVMODOT3X-W-25778 & $6 / 27 / 10$ & 44 & $3 \mathrm{VP}$ & 196 & 6.8 \\
\hline Morgan & SVMORGANLEM-W-25771 & $6 / 23 / 10$ & 60.58 & LFM & 13 & 5.0 \\
\hline Morgan & SVMORGANLFB-W-25770 & $6 / 23 / 10$ & 60.58 & LFB & 13 & 5.0 \\
\hline Morgan & SVMORGAN3X-W-25779 & $6 / 28 / 10$ & 60.58 & $3 \mathrm{VP}$ & 13 & 3.4 \\
\hline SB24 & SVSB24LFB-W-25774 & $6 / 25 / 10$ & $50-60$ & LFB & 4.5 & 6.0 \\
\hline SB38 & SVSB38LFM-W-25773 & $6 / 24 / 10$ & $50-90$ & LFM & 3.7 & 3.2 \\
\hline SB38 & SVSB38LFB-W-25772 & $6 / 23 / 10$ & $50-90$ & LFB & 1.9 & 2.1 \\
\hline SB43 & SVSB43-W-25666 & $6 / 21 / 10$ & $95-100$ & GNP & $N D^{b}$ & ND \\
\hline SB43S & SVSB43S-W-25812 & $7 / 2 / 10$ & $45-55$ & DEV & ND & 27 \\
\hline SB43D & SVSB43D-W-25813 & $7 / 3 / 10$ & 95-105 & DEV & ND & 2.3 \\
\hline SB44S & SVSB44S-W-25811 & $7 / 2 / 10$ & $46.5-56.5$ & DEV & 59 & 26 \\
\hline SB44D & SVSB44D-W-25810 & $7 / 2 / 10$ & $92-102$ & $3 \mathrm{VP}$ & $0.3 \mathrm{Jc}^{\mathrm{c}}$ & 4.1 \\
\hline SB45S & SVSB45S-W-25802 & $7 / 1 / 10$ & $37-47$ & DEV & 5.7 & 7.6 \\
\hline SB45D & SVSB45D-W-25803 & $7 / 1 / 10$ & $79.5-99.5$ & $3 \mathrm{VP}$ & ND & ND \\
\hline SB46S & SVSB46S-W-25801 & $7 / 1 / 10$ & $42-52$ & DEV & 14 & 6.6 \\
\hline SB46D & SVSB46D-W-25800 & $7 / 1 / 10$ & $90-110$ & $3 \mathrm{VP}$ & ND & 1.4 \\
\hline SB47S & SVSB47S-W-25809 & $7 / 2 / 10$ & $25-35$ & DEV & 6.8 & 6.1 \\
\hline SB47D & SVSB47D-W-25808 & $7 / 2 / 10$ & $77-97$ & 3VP & ND & 1.0 \\
\hline SB48S & SVSB48S-W-25807 & $7 / 2 / 10$ & $25-35$ & DEV & ND & 16 \\
\hline SB48D & SVSB48D-W-25806 & $7 / 2 / 10$ & $77-97$ & $3 \mathrm{VP}$ & ND & $0.5 \mathrm{~J}$ \\
\hline SB49S & SVSB49S-W-25805 & $7 / 2 / 10$ & $29-39$ & DEV & ND & 18 \\
\hline SB49D & SVSB49D-W-25804 & $7 / 1 / 10$ & $77-97$ & $3 \mathrm{VP}$ & ND & 1.4 \\
\hline \multicolumn{7}{|l|}{ August 2010} \\
\hline SB24 & SVSB24-W-25854 & $8 / 25 / 10$ & $50-60$ & GNP & 8.3 & 6.4 \\
\hline SB38 & SVSB38-W-25855 & $8 / 25 / 10$ & $50-60$ & GNP & 12 & 2.3 \\
\hline SB43S & SVSB43S-W-25815 & $8 / 24 / 10$ & $45-55$ & GNP & 12 & ND \\
\hline SB43S & SVSB43S-W-25830 & $8 / 24 / 10$ & $45-55$ & LFB & ND & 9.6 \\
\hline SB43D & SVSB43D-W-25816 & $8 / 24 / 10$ & $95-105$ & GNP & ND & $0.6 \mathrm{~J}$ \\
\hline SB43D & SVSB43D-W-25831 & $8 / 24 / 10$ & 95-105 & LFB & ND & 1.3 \\
\hline
\end{tabular}


TABLE 3.3 (Cont.)

\begin{tabular}{|c|c|c|c|c|c|c|}
\hline \multirow[b]{2}{*}{ Location } & \multirow[b]{2}{*}{ Sample } & \multirow[b]{2}{*}{$\begin{array}{l}\text { Sample } \\
\text { Date }\end{array}$} & \multirow[b]{2}{*}{$\begin{array}{c}\text { Depth } \\
\text { (ft BGL) }\end{array}$} & \multirow[b]{2}{*}{ Type $^{a}$} & \multicolumn{2}{|c|}{ Concentration ( $\mu \mathrm{g} / \mathrm{L})$} \\
\hline & & & & & $\begin{array}{c}\text { Carbon } \\
\text { Tetrachloride }\end{array}$ & Chloroform \\
\hline \multicolumn{7}{|c|}{ August 2010 (cont.) } \\
\hline SB44S & SVSB44S-W-25817 & $8 / 24 / 10$ & $46.5-56.5$ & GNP & 60 & 8.3 \\
\hline SB44S & SVSB44S-W-25832 & $8 / 24 / 10$ & $46.5-56.5$ & LFB & 84 & 10 \\
\hline SB44D & SVSB44D-W-25818 & $8 / 24 / 10$ & $92-102$ & GNP & ND & 3.4 \\
\hline SB44D & SVSB44D-W-25833 & $8 / 24 / 10$ & $92-102$ & LFB & ND & 2.4 \\
\hline SB45S & SVSB45S-W-25819 & $8 / 24 / 10$ & $37-47$ & GNP & 51 & 6.9 \\
\hline SB45S & SVSB45S-W-25834 & $8 / 25 / 10$ & $37-47$ & LFB & 86 & 8.3 \\
\hline SB45D & SVSB45D-W-25820 & $8 / 24 / 10$ & $79.5-99.5$ & GNP & ND & ND \\
\hline SB45D & SVSB45D-W-25835 & $8 / 24 / 10$ & $79.5-99.5$ & LFB & ND & ND \\
\hline SB46S & SVSB46S-W-25821 & $8 / 24 / 10$ & $42-52$ & GNP & 1.4 & ND \\
\hline SB46S & SVSB46S-W-25836 & $8 / 25 / 10$ & $42-52$ & LFB & 15 & 1.8 \\
\hline SB46D & SVSB46D-W-25822 & $8 / 24 / 10$ & $90-110$ & GNP & ND & 1.0 \\
\hline SB46D & SVSB46D-W-25837 & $8 / 24 / 10$ & $90-110$ & LFB & ND & ND \\
\hline SB47S & SVSB47S-W-25823 & $8 / 24 / 10$ & $25-35$ & GNP & ND & 5.0 \\
\hline SB47S & SVSB47S-W-25838 & $8 / 25 / 10$ & $25-35$ & LFB & ND & 5.6 \\
\hline SB47D & SVSB47D-W-25824 & $8 / 24 / 10$ & $77-97$ & GNP & ND & $0.2 \mathrm{~J}$ \\
\hline SB47D & SVSB47D-W-25839 & $8 / 24 / 10$ & $77-97$ & LFB & ND & ND \\
\hline SB48S & SVSB48S-W-25825 & $8 / 24 / 10$ & $25-35$ & GNP & 2.2 & 1.7 \\
\hline SB48S & SVSB48S-W-25840 & $8 / 25 / 10$ & $25-35$ & LFB & 2.9 & 2.4 \\
\hline SB48D & SVSB48D-W-25826 & $8 / 24 / 10$ & $77-97$ & GNP & ND & ND \\
\hline SB48D & SVSB48D-W-25841 & $8 / 24 / 10$ & $77-97$ & LFB & ND & ND \\
\hline SB49S & SVSB49S-W-25827 & $8 / 24 / 10$ & 29-39 & GNP & ND & 3.1 \\
\hline SB49S & SVSB49S-W-25842 & $8 / 25 / 10$ & $29-39$ & LFB & ND & 4.2 \\
\hline SB49D & SVSB49D-W-25828 & $8 / 24 / 10$ & $77-97$ & GNP & ND & 3.4 \\
\hline SB49D & SVSB49D-W-25843 & $8 / 24 / 10$ & $77-97$ & LFB & ND & 3.0 \\
\hline \multicolumn{7}{|l|}{ August 2011} \\
\hline SB24 & SVSB24GRAB-W-25856 & $8 / 10 / 11$ & $50-60$ & GNP & 8.2 & 4.3 \\
\hline SB24 & SVSB24-W-25872 & $8 / 11 / 11$ & $50-60$ & LFB & 10 & 4.7 \\
\hline SB38 & SVSB38GRAB-W-25857 & $8 / 10 / 11$ & $50-90$ & GNP & 20 & 2.3 \\
\hline SB38 & SVSB38-W-25873 & $8 / 11 / 11$ & $50-90$ & LFB & 11 & 2.4 \\
\hline SB43S & SVSB43SGRAB-W-25858 & $8 / 10 / 11$ & $45-55$ & GNP & ND & 3.2 \\
\hline SB43S & SVSB43S-W-25874 & $8 / 10 / 11$ & $45-55$ & LFB & ND & 3.2 \\
\hline SB43D & SVSB43DGRAB-W-25859 & $8 / 10 / 11$ & $95-105$ & GNP & ND & ND \\
\hline SB43D & SVSB43D-W-25875 & $8 / 10 / 11$ & $95-105$ & LFB & ND & ND \\
\hline SB44S & SVSB44SGRAB-W-25860 & $8 / 10 / 11$ & $46.5-56.5$ & GNP & 158 & 26 \\
\hline SB44S & SVSB44S-W-25876 & $8 / 11 / 11$ & $46.5-56.5$ & LFB & 273 & 28 \\
\hline SB44D & SVSB44DGRAB-W-25861 & $8 / 10 / 11$ & $92-102$ & GNP & ND & 2.1 \\
\hline SB44D & SVSB44D-W-25877 & $8 / 11 / 11$ & $92-102$ & LFB & ND & ND \\
\hline
\end{tabular}


TABLE 3.3 (Cont.)

\begin{tabular}{|c|c|c|c|c|c|c|}
\hline \multirow[b]{2}{*}{ Location } & \multirow[b]{2}{*}{ Sample } & \multirow[b]{2}{*}{$\begin{array}{l}\text { Sample } \\
\text { Date }\end{array}$} & \multirow[b]{2}{*}{$\begin{array}{l}\text { Depth } \\
\text { (ft BGL) }\end{array}$} & \multirow[b]{2}{*}{ Type $^{\mathrm{a}}$} & \multicolumn{2}{|c|}{ Concentration $(\mu \mathrm{g} / \mathrm{L})$} \\
\hline & & & & & $\begin{array}{c}\text { Carbon } \\
\text { Tetrachloride }\end{array}$ & Chloroform \\
\hline \multicolumn{7}{|c|}{ August 2011 (cont.) } \\
\hline SB45S & SVSB45SGRAB-W-25862 & 8/10/11 & $37-47$ & GNP & 40 & 11 \\
\hline SB45S & SVSB45S-W-25878 & $8 / 11 / 11$ & $37-47$ & LFB & 53 & 12 \\
\hline SB45D & SVSB45DGRAB-W-25863 & 8/10/11 & $79.5-99.5$ & GNP & ND & ND \\
\hline SB45D & SVSB45D-W-25879 & 8/11/11 & $79.5-99.5$ & LFB & ND & ND \\
\hline SB46S & SVSB46SGRAB-W-25864 & $8 / 10 / 11$ & $42-52$ & GNP & 12 & 5.9 \\
\hline SB46S & SVSB46S-W-25880 & 8/11/11 & $42-52$ & LFB & 17 & 7.8 \\
\hline SB46D & SVSB46DGRAB-W-25865 & 8/10/11 & $90-110$ & GNP & ND & ND \\
\hline SB46D & SVSB46D-W-25881 & $8 / 11 / 11$ & $90-110$ & LFB & ND & ND \\
\hline SB47S & SVSB47SGRAB-W-25866 & 8/10/11 & $25-35$ & GNP & ND & 1.4 \\
\hline SB47S & SVSB47S-W-25882 & $8 / 10 / 11$ & $25-35$ & LFB & ND & 1.4 \\
\hline SB47D & SVSB47DGRAB-W-25867 & 8/10/11 & $77-97$ & GNP & ND & ND \\
\hline SB47D & SVSB47D-W-25883 & $8 / 10 / 11$ & $77-97$ & LFB & ND & ND \\
\hline SB48S & SVSB48SGRAB-W-25868 & 8/10/11 & $25-35$ & GNP & ND & ND \\
\hline SB48S & SVSB48S-W-25884 & $8 / 11 / 11$ & $25-35$ & LFB & $0.7 \mathrm{~J}$ & ND \\
\hline SB48D & SVSB48DGRAB-W-25869 & 8/10/11 & $77-97$ & GNP & ND & ND \\
\hline SB48D & SVSB48D-W-25885 & $8 / 10 / 11$ & $77-97$ & LFB & ND & ND \\
\hline SB49S & SVSB49SGRAB-W-25870 & 8/10/11 & $29-39$ & GNP & ND & ND \\
\hline SB49S & SVSB49S-W-25886 & 8/10/11 & 29-39 & LFB & ND & ND \\
\hline SB49D & SVSB49DGRAB-W-25871 & 8/10/11 & $77-97$ & GNP & ND & 1.3 \\
\hline SB49D & SVSB49D-W-25887 & 8/10/11 & $77-97$ & LFB & ND & ND \\
\hline
\end{tabular}

a GNP - Grab sample collected without purging.

LFM - Low-flow sample collected from the approximate midpoint of the initial water column.

LFB - Low-flow sample collected from the base of the water column.

3VP - Sample collected after conventional purging of three well casing volumes.

DEV - Sample collected during initial well development, after purging of one or two casing volumes.

b ND, not detected at an instrument detection limit of $0.1 \mu \mathrm{g} / \mathrm{L}$.

c Qualifier $\mathrm{J}$ indicates an estimated concentration below the purge-and-trap method quantitation limit of $1.0 \mu \mathrm{g} / \mathrm{L}$. 
TABLE 3.4 Location-depth compilation of analytical results from the AGEM Laboratory for carbon tetrachloride and chloroform in groundwater samples collected in 2010-2011.

\begin{tabular}{|c|c|c|c|c|c|c|}
\hline \multirow[b]{2}{*}{ Location } & \multirow[b]{2}{*}{ Sample } & \multirow[b]{2}{*}{$\begin{array}{l}\text { Sample } \\
\text { Date }\end{array}$} & \multirow[b]{2}{*}{$\begin{array}{l}\text { Depth } \\
\text { (ft BGL) }\end{array}$} & \multirow[b]{2}{*}{ Type $^{a}$} & \multicolumn{2}{|c|}{ Concentration $(\mu \mathrm{g} / \mathrm{L})$} \\
\hline & & & & & $\begin{array}{c}\text { Carbon } \\
\text { Tetrachloride }\end{array}$ & Chloroform \\
\hline MoDOT & SVMODOTLFM-W-25776 & $6 / 25 / 10$ & 44 & LFM & 210 & 6.8 \\
\hline MoDOT & SVMODOTLFB-W-25775 & $6 / 25 / 10$ & 44 & LFB & 179 & 6.2 \\
\hline MoDOT & SVMODOT3X-W-25778 & $6 / 27 / 10$ & 44 & $3 V P$ & 196 & 6.8 \\
\hline Morgan & SVMORGANLEM-W-25771 & $6 / 23 / 10$ & 60.58 & LFM & 13 & 5.0 \\
\hline Morgan & SVMORGANLFB-W-25770 & $6 / 23 / 10$ & 60.58 & LFB & 13 & 5.0 \\
\hline Morgan & SVMORGAN3X-W-25779 & $6 / 28 / 10$ & 60.58 & $3 V P$ & 13 & 3.4 \\
\hline SB24 & SVSB24LFB-W-25774 & $6 / 25 / 10$ & $50-60$ & LFB & 4.5 & 6.0 \\
\hline SB24 & SVSB24-W-25854 & $8 / 25 / 10$ & $50-60$ & GNP & 8.3 & 6.4 \\
\hline SB24 & SVSB24GRAB-W-25856 & $8 / 10 / 11$ & $50-60$ & GNP & 8.2 & 4.3 \\
\hline SB24 & SVSB24-W-25872 & $8 / 11 / 11$ & $50-60$ & LFB & 10 & 4.7 \\
\hline SB38 & SVSB38LFM-W-25773 & $6 / 24 / 10$ & $50-90$ & LFM & 3.7 & 3.2 \\
\hline SB38 & SVSB38LFB-W-25772 & $6 / 23 / 10$ & $50-90$ & LFB & 1.9 & 2.1 \\
\hline SB38 & SVSB38-W-25855 & $8 / 25 / 10$ & $50-60$ & GNP & 12 & 2.3 \\
\hline SB38 & SVSB38GRAB-W-25857 & $8 / 10 / 11$ & $50-90$ & GNP & 20 & 2.3 \\
\hline SB38 & SVSB38-W-25873 & $8 / 11 / 11$ & $50-90$ & LFB & 11 & 2.4 \\
\hline SB43 & SVSB43-W-25666 & $6 / 21 / 10$ & $95-100$ & GNP & $N D^{b}$ & ND \\
\hline SB43S & SVSB43S-W-25812 & $7 / 2 / 10$ & $45-55$ & GNP & ND & 27 \\
\hline SB43S & SVSB43S-W-25815 & $8 / 24 / 10$ & $45-55$ & GNP & 12 & ND \\
\hline SB43S & SVSB43S-W-25830 & $8 / 24 / 10$ & $45-55$ & LFB & ND & 9.6 \\
\hline SB43S & SVSB43SGRAB-W-25858 & $8 / 10 / 11$ & $45-55$ & GNP & ND & 3.2 \\
\hline SB43S & SVSB43S-W-25874 & $8 / 10 / 11$ & $45-55$ & LFB & ND & 3.2 \\
\hline SB43D & SVSB43D-W-25813 & $7 / 3 / 10$ & $95-105$ & GNP & ND & 2.3 \\
\hline SB43D & SVSB43D-W-25816 & $8 / 24 / 10$ & $95-105$ & GNP & ND & $0.6 \mathrm{Jc}^{\mathrm{c}}$ \\
\hline SB43D & SVSB43D-W-25831 & $8 / 24 / 10$ & $95-105$ & LFB & ND & 1.3 \\
\hline SB43D & SVSB43DGRAB-W-25859 & $8 / 10 / 11$ & $95-105$ & GNP & ND & ND \\
\hline SB43D & SVSB43D-W-25875 & $8 / 10 / 11$ & $95-105$ & LFB & ND & ND \\
\hline SB44S & SVSB44S-W-25811 & $7 / 2 / 10$ & $46.5-56.5$ & GNP & 59 & 26 \\
\hline SB44S & SVSB44S-W-25817 & $8 / 24 / 10$ & $46.5-56.5$ & GNP & 60 & 8.3 \\
\hline SB44S & SVSB44S-W-25832 & $8 / 24 / 10$ & $46.5-56.5$ & LFB & 84 & 10 \\
\hline SB44S & SVSB44SGRAB-W-25860 & $8 / 10 / 11$ & $46.5-56.5$ & GNP & 158 & 26 \\
\hline SB44S & SVSB44S-W-25876 & $8 / 11 / 11$ & $46.5-56.5$ & LFB & 273 & 28 \\
\hline SB44D & SVSB44D-W-25810 & $7 / 2 / 10$ & $92-102$ & GNP & $0.3 \mathrm{~J}$ & 4.1 \\
\hline SB44D & SVSB44D-W-25818 & $8 / 24 / 10$ & $92-102$ & GNP & ND & 3.4 \\
\hline SB44D & SVSB44D-W-25833 & $8 / 24 / 10$ & $92-102$ & LFB & ND & 2.4 \\
\hline SB44D & SVSB44DGRAB-W-25861 & 8/10/11 & $92-102$ & GNP & ND & 2.1 \\
\hline SB44D & SVSB44D-W-25877 & $8 / 11 / 11$ & $92-102$ & LFB & ND & ND \\
\hline SB45S & SVSB45S-W-25802 & $7 / 1 / 10$ & $37-47$ & GNP & 5.7 & 7.6 \\
\hline SB45S & SVSB45S-W-25819 & $8 / 24 / 10$ & $37-47$ & GNP & 51 & 6.9 \\
\hline SB45S & SVSB45S-W-25834 & $8 / 25 / 10$ & $37-47$ & LFB & 86 & 8.3 \\
\hline SB45S & SVSB45SGRAB-W-25862 & $8 / 10 / 11$ & $37-47$ & GNP & 40 & 11 \\
\hline SB45S & SVSB45S-W-25878 & $8 / 11 / 11$ & $37-47$ & LFB & 53 & 12 \\
\hline
\end{tabular}


TABLE 3.4 (Cont.)

\begin{tabular}{|c|c|c|c|c|c|c|}
\hline \multirow[b]{2}{*}{ Location } & \multirow[b]{2}{*}{ Sample } & \multirow[b]{2}{*}{$\begin{array}{c}\text { Sample } \\
\text { Date }\end{array}$} & \multirow[b]{2}{*}{$\begin{array}{c}\text { Depth } \\
\text { (ft BGL) }\end{array}$} & \multirow[b]{2}{*}{ Type $^{a}$} & \multicolumn{2}{|c|}{ Concentration $(\mu \mathrm{g} / \mathrm{L})$} \\
\hline & & & & & $\begin{array}{c}\text { Carbon } \\
\text { Tetrachloride }\end{array}$ & Chloroform \\
\hline SB45D & SVSB45D-W-25803 & $7 / 1 / 10$ & $79.5-99.5$ & GNP & ND & ND \\
\hline SB45D & SVSB45D-W-25820 & $8 / 24 / 10$ & $79.5-99.5$ & GNP & ND & ND \\
\hline SB45D & SVSB45D-W-25835 & $8 / 24 / 10$ & $79.5-99.5$ & LFB & ND & ND \\
\hline SB45D & SVSB45DGRAB-W-25863 & $8 / 10 / 11$ & $79.5-99.5$ & GNP & ND & ND \\
\hline SB45D & SVSB45D-W-25879 & $8 / 11 / 11$ & $79.5-99.5$ & LFB & ND & ND \\
\hline SB46S & SVSB46S-W-25801 & $7 / 1 / 10$ & $42-52$ & GNP & 14 & 6.6 \\
\hline SB46S & SVSB46S-W-25821 & $8 / 24 / 10$ & $42-52$ & GNP & 1.4 & ND \\
\hline SB46S & SVSB46S-W-25836 & $8 / 25 / 10$ & $42-52$ & LFB & 15 & 1.8 \\
\hline SB46S & SVSB46SGRAB-W-25864 & $8 / 10 / 11$ & $42-52$ & GNP & 12 & 5.9 \\
\hline SB46S & SVSB46S-W-25880 & $8 / 11 / 11$ & $42-52$ & LFB & 17 & 7.8 \\
\hline SB46D & SVSB46D-W-25800 & $7 / 1 / 10$ & $90-110$ & GNP & ND & 1.4 \\
\hline SB46D & SVSB46D-W-25822 & $8 / 24 / 10$ & $90-110$ & GNP & ND & 1.0 \\
\hline SB46D & SVSB46D-W-25837 & $8 / 24 / 10$ & $90-110$ & LFB & ND & ND \\
\hline SB46D & SVSB46DGRAB-W-25865 & $8 / 10 / 11$ & $90-110$ & GNP & ND & ND \\
\hline SB46D & SVSB46D-W-25881 & $8 / 11 / 11$ & $90-110$ & LFB & ND & ND \\
\hline SB47S & SVSB47S-W-25809 & $7 / 2 / 10$ & $25-35$ & GNP & 6.8 & 6.1 \\
\hline SB47S & SVSB47S-W-25823 & $8 / 24 / 10$ & $25-35$ & GNP & ND & 5.0 \\
\hline SB47S & SVSB47S-W-25838 & $8 / 25 / 10$ & $25-35$ & LFB & ND & 5.6 \\
\hline SB47S & SVSB47SGRAB-W-25866 & $8 / 10 / 11$ & $25-35$ & GNP & ND & 1.4 \\
\hline SB47S & SVSB47S-W-25882 & $8 / 10 / 11$ & $25-35$ & LFB & ND & 1.4 \\
\hline SB47D & SVSB47D-W-25808 & $7 / 2 / 10$ & $77-97$ & GNP & ND & 1.0 \\
\hline SB47D & SVSB47D-W-25824 & $8 / 24 / 10$ & $77-97$ & GNP & ND & $0.2 \mathrm{~J}$ \\
\hline SB47D & SVSB47D-W-25839 & $8 / 24 / 10$ & $77-97$ & LFB & ND & ND \\
\hline SB47D & SVSB47DGRAB-W-25867 & $8 / 10 / 11$ & $77-97$ & GNP & ND & ND \\
\hline SB47D & SVSB47D-W-25883 & $8 / 10 / 11$ & $77-97$ & LFB & ND & ND \\
\hline SB48S & SVSB48S-W-25807 & $7 / 2 / 10$ & $25-35$ & GNP & ND & 16 \\
\hline SB48S & SVSB48S-W-25825 & $8 / 24 / 10$ & $25-35$ & GNP & 2.2 & 1.7 \\
\hline SB48S & SVSB48S-W-25840 & $8 / 25 / 10$ & $25-35$ & LFB & 2.9 & 2.4 \\
\hline SB48S & SVSB48SGRAB-W-25868 & $8 / 10 / 11$ & $25-35$ & GNP & ND & ND \\
\hline SB48S & SVSB48S-W-25884 & $8 / 11 / 11$ & $25-35$ & LFB & $0.7 \mathrm{~J}$ & ND \\
\hline SB48D & SVSB48D-W-25806 & $7 / 2 / 10$ & $77-97$ & GNP & ND & $0.5 \mathrm{~J}$ \\
\hline SB48D & SVSB48D-W-25826 & $8 / 24 / 10$ & $77-97$ & GNP & ND & ND \\
\hline SB48D & SVSB48D-W-25841 & $8 / 24 / 10$ & $77-97$ & LFB & ND & ND \\
\hline SB48D & SVSB48DGRAB-W-25869 & 8/10/11 & $77-97$ & GNP & ND & ND \\
\hline SB48D & SVSB48D-W-25885 & $8 / 10 / 11$ & $77-97$ & LFB & ND & ND \\
\hline SB49S & SVSB49S-W-25805 & $7 / 2 / 10$ & 29-39 & GNP & ND & 18 \\
\hline SB49S & SVSB49S-W-25827 & $8 / 24 / 10$ & $29-39$ & GNP & ND & 3.1 \\
\hline SB49S & SVSB49S-W-25842 & $8 / 25 / 10$ & $29-39$ & LFB & ND & 4.2 \\
\hline SB49S & SVSB49SGRAB-W-25870 & $8 / 10 / 11$ & $29-39$ & GNP & ND & ND \\
\hline SB49S & SVSB49S-W-25886 & $8 / 10 / 11$ & 29-39 & LFB & ND & ND \\
\hline
\end{tabular}


TABLE 3.4 (Cont.)

\begin{tabular}{|c|c|c|c|c|c|c|}
\hline \multirow[b]{2}{*}{ Location } & \multirow[b]{2}{*}{ Sample } & \multirow[b]{2}{*}{$\begin{array}{l}\text { Sample } \\
\text { Date }\end{array}$} & \multirow[b]{2}{*}{$\begin{array}{c}\text { Depth } \\
\text { (ft BGL) }\end{array}$} & \multirow[b]{2}{*}{ Type $^{a}$} & \multicolumn{2}{|c|}{ Concentration $(\mu \mathrm{g} / \mathrm{L})$} \\
\hline & & & & & $\begin{array}{c}\text { Carbon } \\
\text { Tetrachloride }\end{array}$ & Chloroform \\
\hline SB49D & SVSB49D-W-25804 & $7 / 1 / 10$ & $77-97$ & GNP & ND & 1.4 \\
\hline SB49D & SVSB49D-W-25828 & $8 / 24 / 10$ & $77-97$ & GNP & ND & 3.4 \\
\hline SB49D & SVSB49D-W-25843 & $8 / 24 / 10$ & $77-97$ & LFB & ND & 3.0 \\
\hline SB49D & SVSB49DGRAB-W-25871 & $8 / 10 / 11$ & $77-97$ & GNP & ND & 1.3 \\
\hline SB49D & SVSB49D-W-25887 & $8 / 10 / 11$ & $77-97$ & LFB & ND & ND \\
\hline
\end{tabular}

a GNP - Grab sample collected without purging.

LFM - Low-flow sample collected from the approximate midpoint of the initial water column

LFB - Low-flow sample collected from the base of the water column.

3VP - Sample collected after purging of three well casing volumes.

b ND, not detected at an instrument detection limit of $0.1 \mu \mathrm{g} / \mathrm{L}$.

c Qualifier J indicates an estimated concentration below the purge-and-trap method quantitation limit of $1.0 \mu \mathrm{g} / \mathrm{L}$. 
TABLE 3.5 Construction data for selected private wells.

\begin{tabular}{|c|c|c|c|c|}
\hline Location & $\begin{array}{c}\text { Casing } \\
\text { Diameter }^{\mathrm{a}} \text { (in.) }\end{array}$ & $\begin{array}{c}\text { Ground Surface } \\
\text { Elevation }^{\mathrm{b}} \text { (ft AMSL) }\end{array}$ & $\begin{array}{c}\text { Well } \\
\operatorname{Depth}^{\mathrm{a}}(\mathrm{ft} \text { BGL) }\end{array}$ & $\begin{array}{c}\text { Bottom of Well } \\
\left.\text { Elevation }^{\mathrm{b}} \text { ( } \mathrm{ft} \mathrm{AMSL}\right)\end{array}$ \\
\hline Barr & 30 & 1153.05 & 34.5 & 1118.6 \\
\hline Burks & 18 & 1126.11 & 37.8 & 1088.3 \\
\hline Clizer "old" & 18 & 1153.76 & 35.7 & 1118.1 \\
\hline Hughes & 48 & 1149.65 & 56.4 & 1093.3 \\
\hline McPike & 18 & 1148.76 & 48.5 & 1100.3 \\
\hline MoDOT & 30 & 1151.94 & 44.0 & 1107.9 \\
\hline Morgan & $36 / 25^{c}$ & 1158.04 & 60.6 & 1097.4 \\
\hline Potter & 30 & 1134.80 & 51.3 & 1083.5 \\
\hline
\end{tabular}

a Measurements made by Argonne staff during the Phase I field program.

b Phase I surveyed elevations.

c Shallow and deep casing sizes were provided by the well owner; shallow casing size (36 in.) was confirmed in the field. 
TABLE 3.6 Hand-measured groundwater levels in June 2010 to December 2011.

Recorded Water Level (ft below reference point) on Indicated Date

\begin{tabular}{|c|c|c|c|c|c|c|c|c|c|c|c|c|c|c|c|}
\hline \multirow[b]{2}{*}{ Location } & \multirow[b]{2}{*}{$\begin{array}{l}\text { Reference } \\
\text { Elevation } \\
\text { (tt AMSL) }\end{array}$} & \multicolumn{14}{|c|}{ Recorded Water Level (ft below reference point) on Indicated Date } \\
\hline & & $\begin{array}{c}\text { June } 23-25^{a} \\
2010\end{array}$ & $\begin{array}{c}\text { June } 28 \\
2010\end{array}$ & $\begin{array}{l}\text { July 1-3 } \\
2010\end{array}$ & $\begin{array}{l}\text { July } 7 \\
2010\end{array}$ & $\begin{array}{l}\text { July } 11 \\
2010\end{array}$ & $\begin{array}{c}\text { August } 20 \\
2010\end{array}$ & $\begin{array}{c}\text { August } 24-25^{a} \\
2010\end{array}$ & $\begin{array}{c}\text { October } 29 \\
2010\end{array}$ & $\begin{array}{c}\text { November } 16 \\
2010\end{array}$ & $\begin{array}{l}\text { April } 1 \\
2011\end{array}$ & $\begin{array}{l}\text { June } 23 \\
2011\end{array}$ & $\begin{array}{c}\text { August 10-11 a } \\
2011\end{array}$ & $\begin{array}{c}\text { September } 23 \\
2011\end{array}$ & $\begin{array}{l}\text { December } 2 \\
2011\end{array}$ \\
\hline \multicolumn{16}{|l|}{ Private wells } \\
\hline McPike & 1148.76 & $-^{\mathrm{b}}$ & 7.61 & - & - & - & - & - & - & 13.95 & 12.91 & 15.62 & - & 14.96 & 17.31 \\
\hline Potter & 1134.80 & - & 4.38 & - & - & - & - & - & - & 5.98 & 5.78 & 6.53 & - & 6.40 & 6.82 \\
\hline Hughes & 1149.65 & - & 6.20 & _ & _ & - & - & - & - & 9.70 & 8.57 & 10.15 & - & 1036 & 1079 \\
\hline Barr & 1153.05 & - & 13.96 & - & - & - & - & - & - & 18.68 & 17.82 & 18.66 & - & 19.66 & 20.61 \\
\hline Burks & 1126.11 & - & 6.92 & - & - & - & - & - & - & 12.05 & 11.08 & 12.09 & - & 15.44 & 21.78 \\
\hline MoDOT & 1150.29 & 12.58 & & - & - & - & - & - & - & & & & - & & \\
\hline Clizer "old" & 1149.44 & & 5.84 & - & - & - & - & - & - & $N A^{c}$ & NA & 12.98 & - & & NA \\
\hline Morgan & 1159.27 & 14.49 & 22.84 & - & - & - & - & - & - & 27.50 & 26.83 & 29.46 & - & 28.28 & 30.58 \\
\hline \multicolumn{16}{|c|}{ Monitoring wells } \\
\hline SB24 & 1158.06 & 19.71 & 45.81 & - & - & - & 23.21 & 26.45 & 30.63 & - & 25.34 & 26.16 & & 32.19 & 28.66 \\
\hline SB38 & 1158.40 & 35.73 & 62.48 & - & - & - & 37.38 & 40.50 & 44.09 & - & 37.26 & 37.79 & & 55.18 & 41.28 \\
\hline SB43S & 1154.44 & - & - & 11.40 & 50.29 & - & 40.98 & 40.70 & 31.93 & - & 23.48 & 21.96 & 24.30 & 31.93 & 27.71 \\
\hline SB43D & 1154.48 & - & - & 55.60 & $61.54^{d}$ & - & 56.46 & 57.43 & 58.04 & - & 57.95 & 58.53 & 60.85 & 58.88 & 60.13 \\
\hline SB44S & 1155.12 & - & - & 13.26 & Dry & - & 17.01 & 18.28 & 21.13 & - & 20.61 & 23.28 & 24.10 & & 24.81 \\
\hline SB44D & 1155.37 & - & - & 58.25 & 58.07 & - & 59.14 & 59.72 & 60.50 & - & 60.71 & 60.94 & 61.70 & 61.23 & 62.30 \\
\hline $\begin{array}{l}\text { SB45S } \\
\text { SRA5D }\end{array}$ & 1144.66 & - & - & 11.10 & - & 40.30 & 23.88 & 23.92 & $\begin{array}{l}19.05 \\
-177\end{array}$ & - & 18.45 & 18.66 & 20.60 & 21.88 & 19.76 \\
\hline $\begin{array}{l}\text { SB45D } \\
\text { SB46S }\end{array}$ & $\begin{array}{l}1144.98 \\
115159\end{array}$ & & - & $\begin{array}{l}67.45 \\
40.81\end{array}$ & $\overline{-}$ & 49.32 & $\begin{array}{l}50.45 \\
1208\end{array}$ & $\begin{array}{l}50.91 \\
1117\end{array}$ & $\begin{array}{l}51.75 \\
2.8\end{array}$ & - & 51.89 & $\begin{array}{l}52.19 \\
2039\end{array}$ & $\begin{array}{r}52.60 \\
21.72\end{array}$ & $\begin{array}{l}52.44 \\
22.27\end{array}$ & 53.47 \\
\hline $\begin{array}{l}\text { SB46S } \\
\text { SB46D }\end{array}$ & $\begin{array}{l}1151.59 \\
1151.50\end{array}$ & $\begin{array}{lll}- & & \end{array}$ & $\begin{array}{lll}- & & \end{array}$ & $\begin{array}{l}40.81 \\
54.72\end{array}$ & $\begin{array}{l}\text { Dry } \\
5577\end{array}$ & - & $\begin{array}{l}12.08 \\
56.86\end{array}$ & $\begin{array}{l}11.17 \\
57.72\end{array}$ & $\begin{array}{r}2.48 \\
58.17\end{array}$ & - & $\begin{array}{l}11.65 \\
58.24\end{array}$ & $\begin{array}{l}20.39 \\
5862\end{array}$ & $\begin{array}{l}21.72 \\
5868\end{array}$ & $\begin{array}{l}22.27 \\
5887\end{array}$ & $22.833^{\mathrm{d}}$ \\
\hline $\begin{array}{l}\text { SB47S } \\
\text { SB4S }\end{array}$ & $\begin{array}{l}1151.50 \\
1134.27\end{array}$ & - & $\begin{array}{ll}- \\
-\end{array}$ & $\begin{array}{r}54.12 \\
4.70\end{array}$ & 55.11 & $\overline{31.28}$ & $\begin{array}{l}25.11 \\
27.11\end{array}$ & $\begin{array}{l}51.12 \\
27.33\end{array}$ & $\begin{array}{l}58.17 \\
26.72\end{array}$ & $\begin{array}{ll}- \\
-\end{array}$ & $\begin{array}{l}58.24 \\
15.94\end{array}$ & $\begin{array}{l}58.62 \\
13.77\end{array}$ & $\begin{array}{l}58.68 \\
15.51\end{array}$ & $\begin{array}{l}28.81 \\
26.16\end{array}$ & $\begin{array}{l}5.83 \\
2214\end{array}$ \\
\hline SB47D & 1134.79 & - & - & 36.17 & - & 36.41 & 37.51 & 39.85 & 39.14 & - & 38.97 & 39.66 & 40.53 & 40.06 & $\begin{array}{l}22.1 .24 \\
41.24\end{array}$ \\
\hline SB48S & 1134.45 & - & - & 9.16 & - & Dry & 20.70 & 20.63 & 15.89 & - & 9.76 & 9.82 & 12.13 & 22.45 & 15.74 \\
\hline SB48D & 1134.48 & - & - & 36.90 & - & 36.26 & 37.37 & 38.25 & 38.90 & - & 38.88 & 39.43 & 39.51 & 39.77 & 40.93 \\
\hline SB49S & 1137.16 & - & - & 10.43 & - & 30.71 & 14.31 & 14.50 & 8.57 & - & 6.39 & 6.52 & 4.59 & 12.25 & 8.53 \\
\hline SB49D & 1137.36 & - & - & 40.00 & - & 40.20 & 41.34 & 42.31 & 42.68 & - & 42.82 & 43.12 & 45.20 & 43.38 & 44.46 \\
\hline
\end{tabular}

a Levels measured in conjunction with groundwater sampling event.

b No measurement.

c NA, not accessible; well winterized by owner.

d Water level changing during measurement period. 


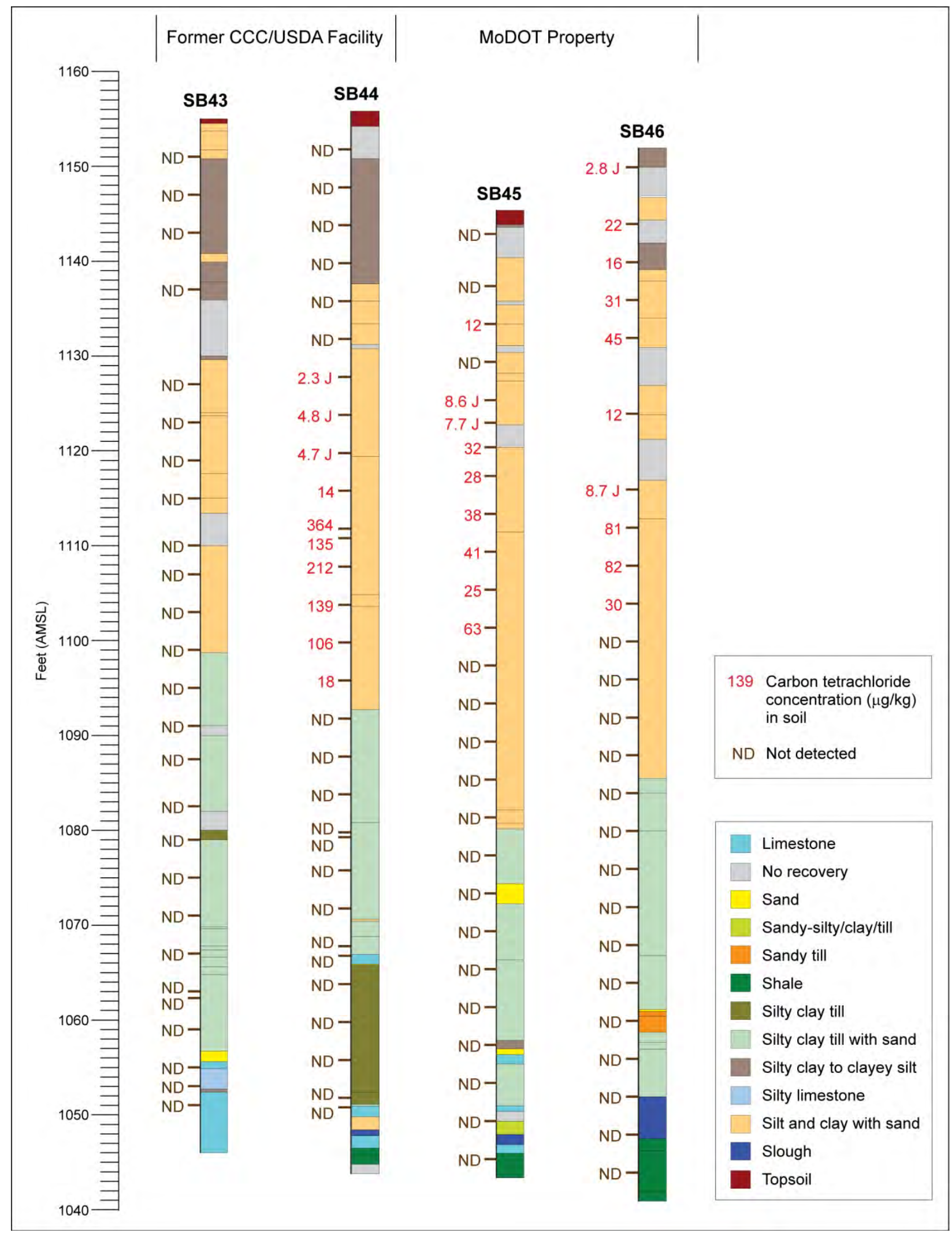

FIGURE 3.1 Vertical distribution of the carbon tetrachloride concentrations identified in soils in borings SB43-SB46. 


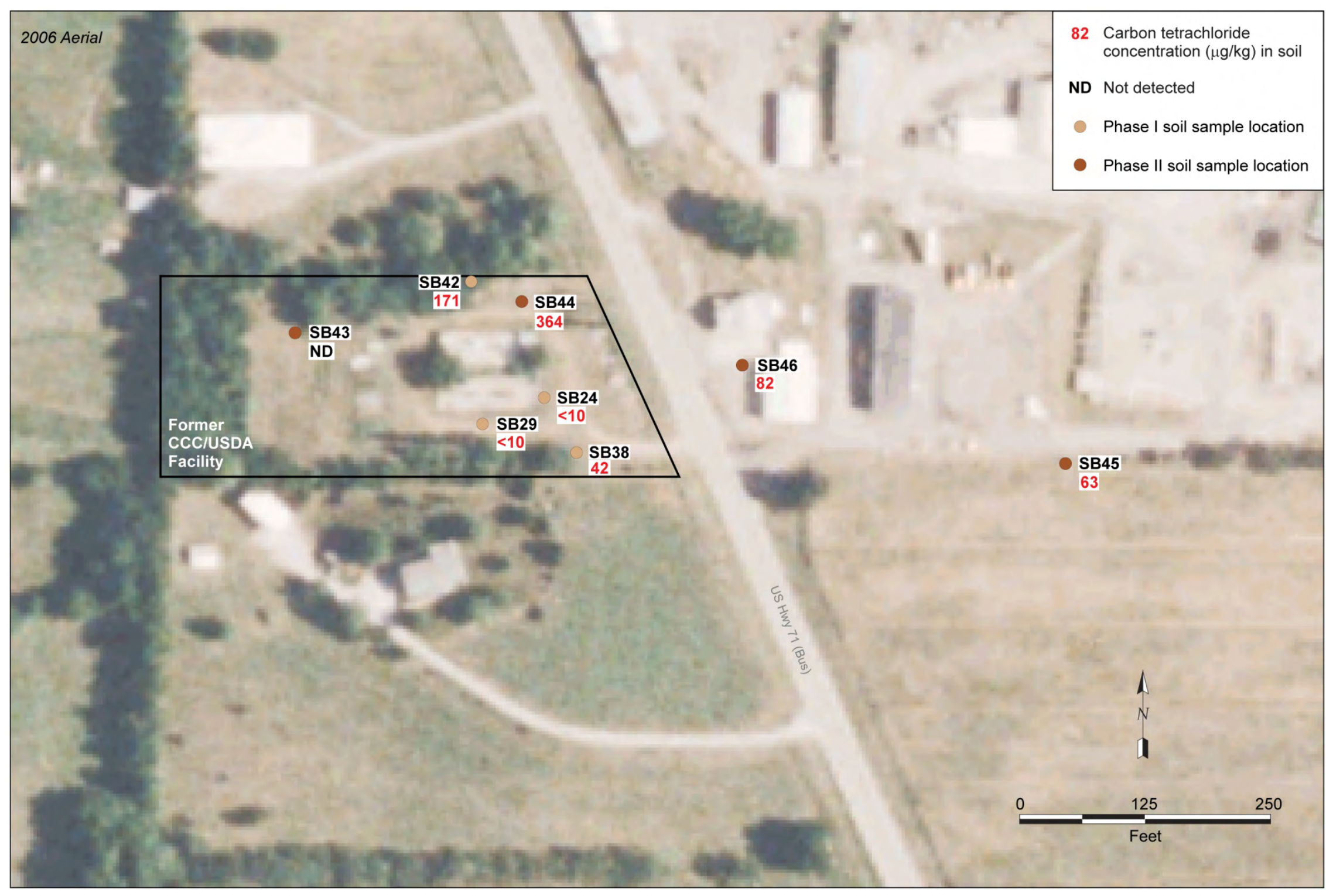

FIGURE 3.2 Areal distribution of the maximum carbon tetrachloride concentrations identified in soils in Phase I and Phase II work. Source of aerial photograph: NAIP (2006). 


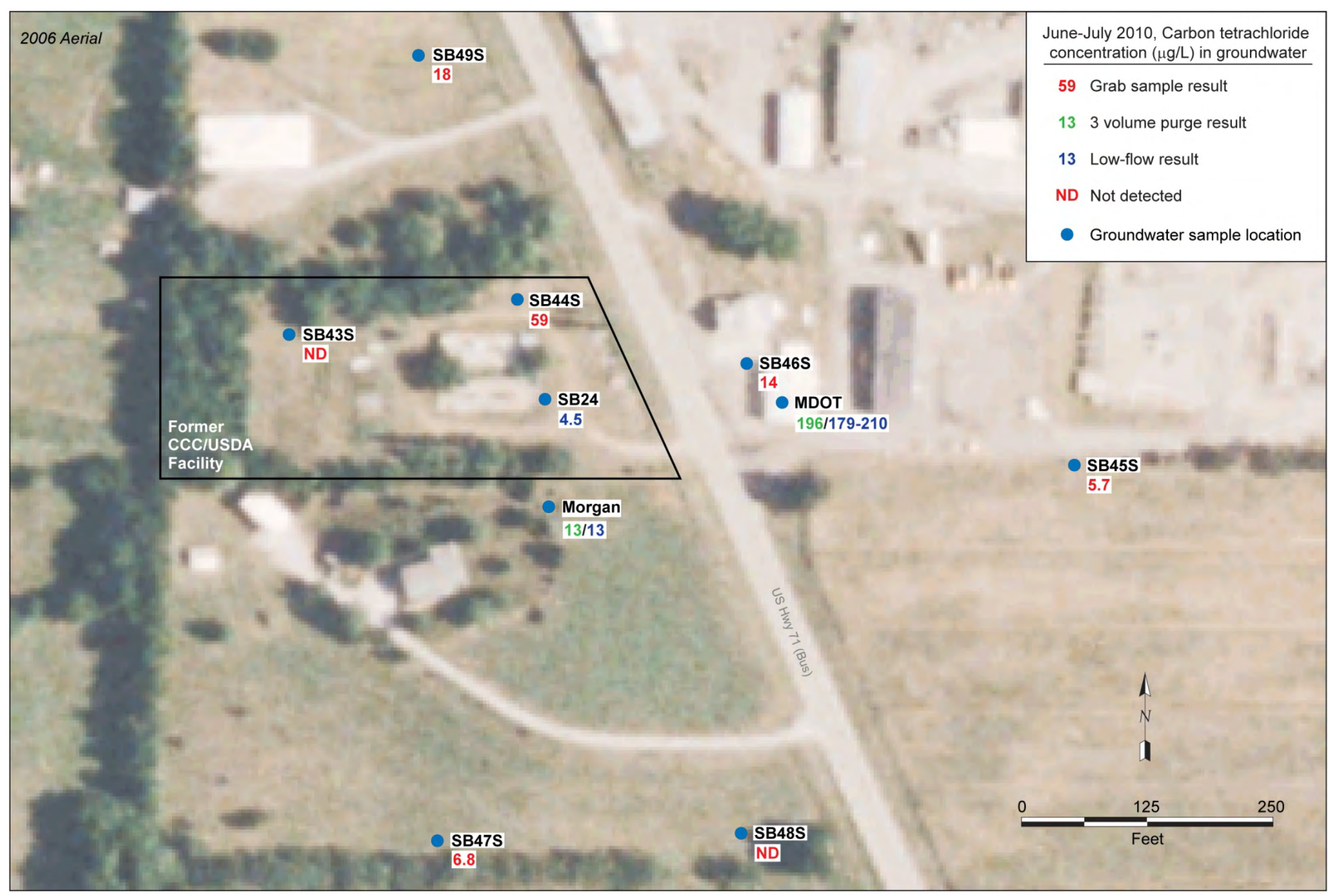

FIGURE 3.3 Lateral distribution of carbon tetrachloride in groundwater, as determined in the June-July 2010 sampling of shallow monitoring points SB24 and SB43-SB49, plus the Morgan and MoDOT private wells. Source of aerial photograph: NAIP (2006). 


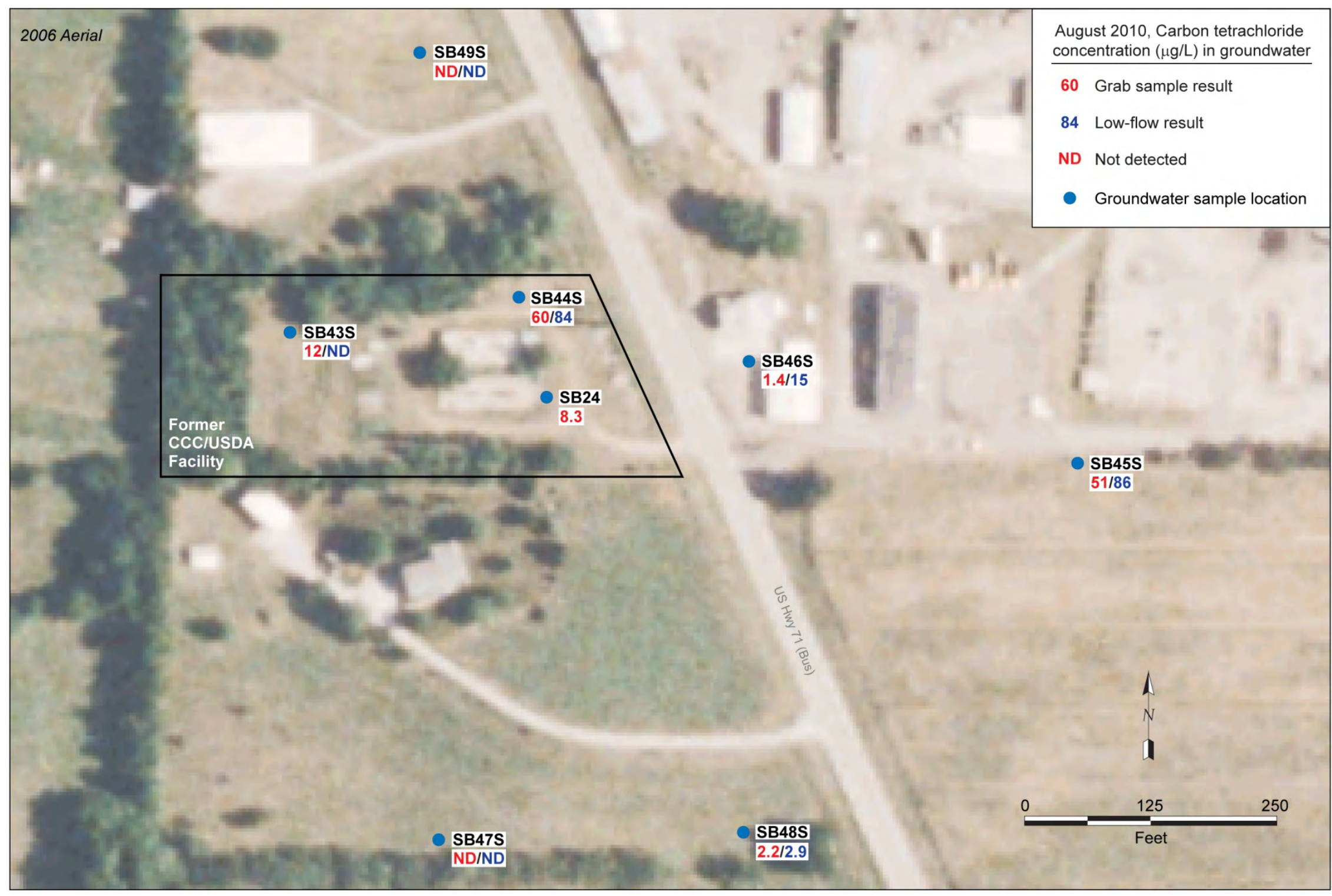

FIGURE 3.4 Lateral distribution of carbon tetrachloride in groundwater, as determined in the August 2010 sampling of shallow monitoring points SB24 and SB43-SB49. Source of aerial photograph: NAIP (2006). 


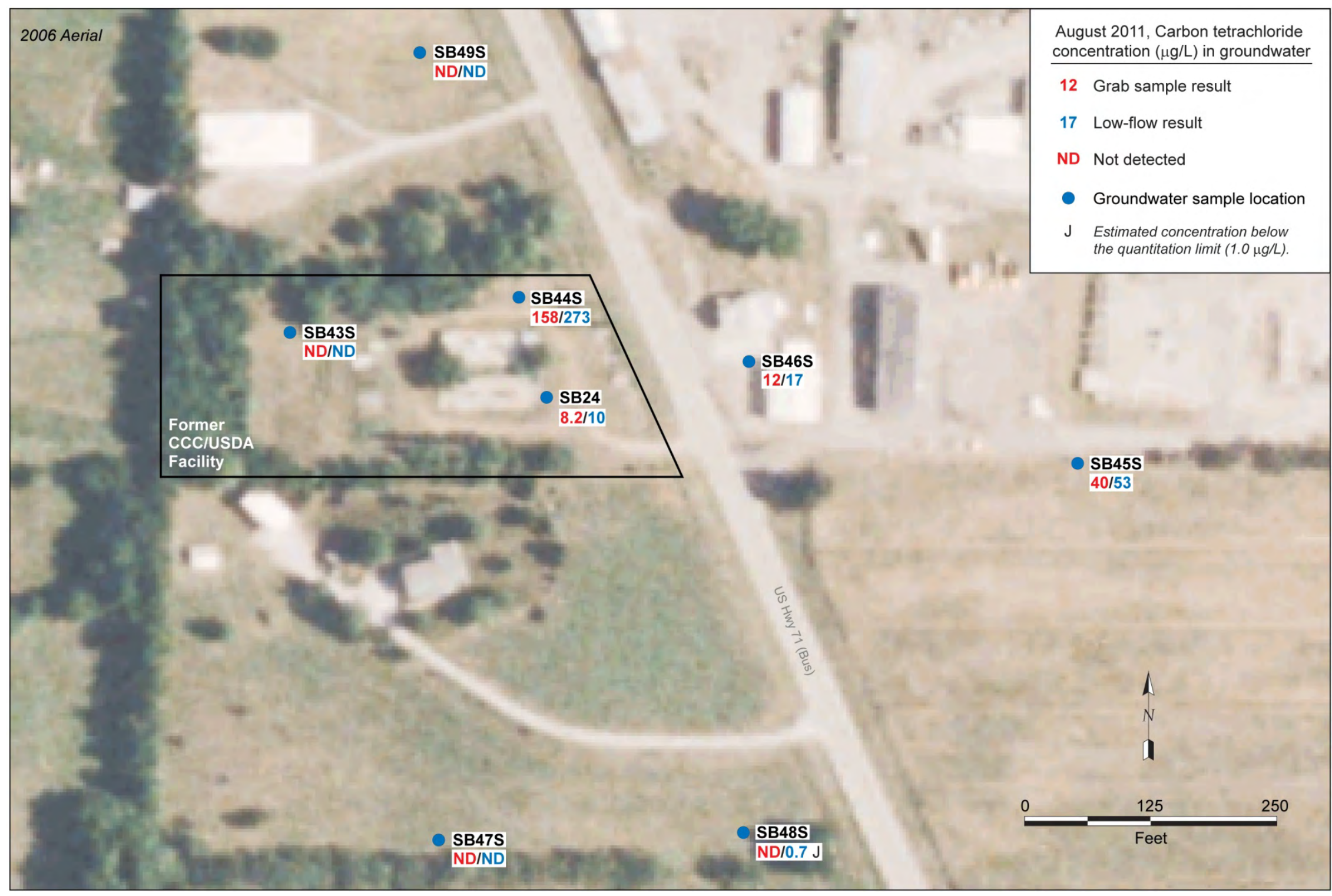

FIGURE 3.5 Lateral distribution of carbon tetrachloride in groundwater, as determined in the August 2011 sampling of shallow monitoring points SB24 and SB43-SB49. Source of aerial photograph: NAIP (2006). 


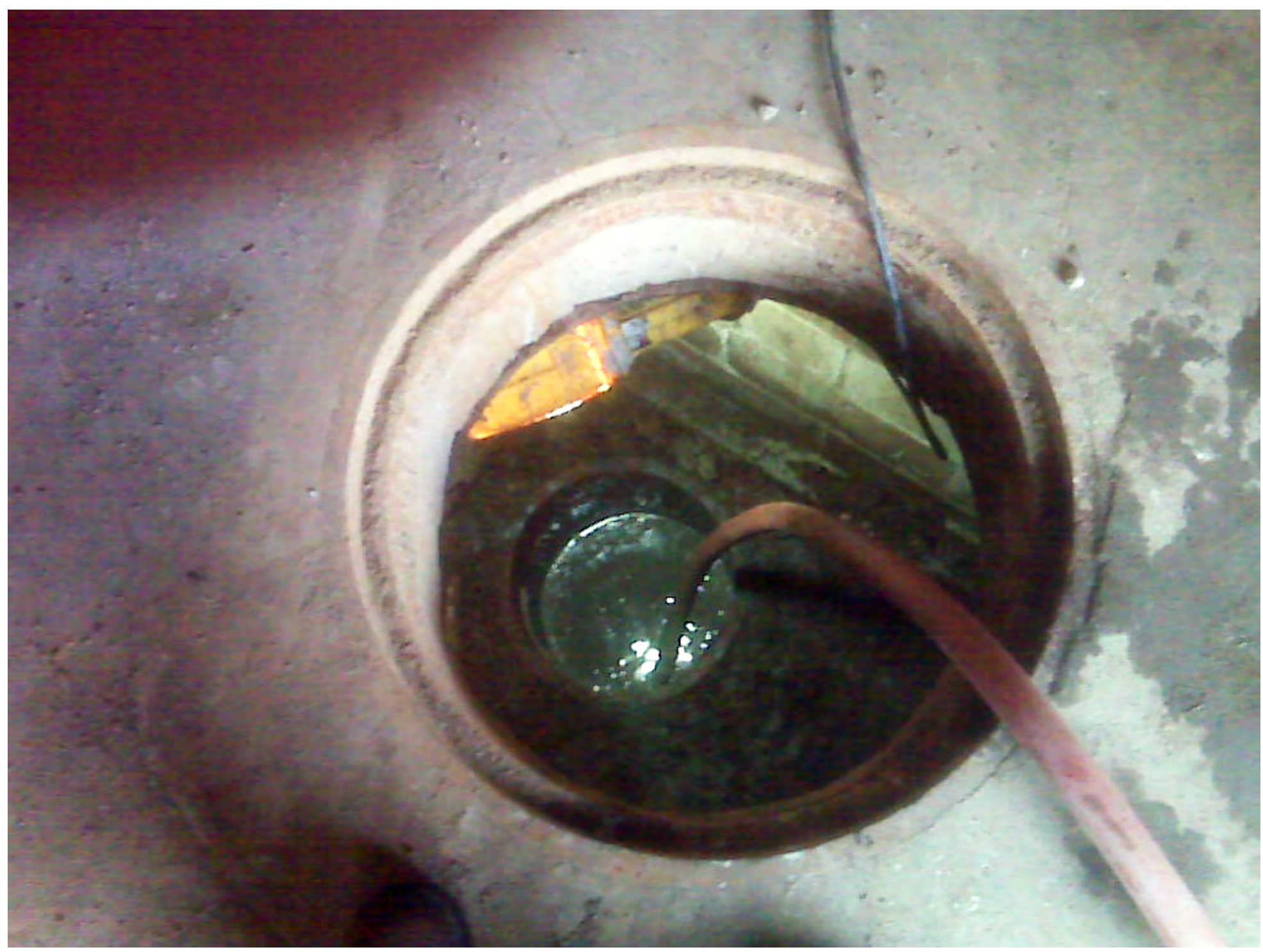

FIGURE 3.6 Grout being pumped into the MoDOT private well, located beneath the floor of the MoDOT vehicle maintenance building, during well abandonment on June 27,2010 . The uppermost segment of concrete well casing had been removed from the well. 


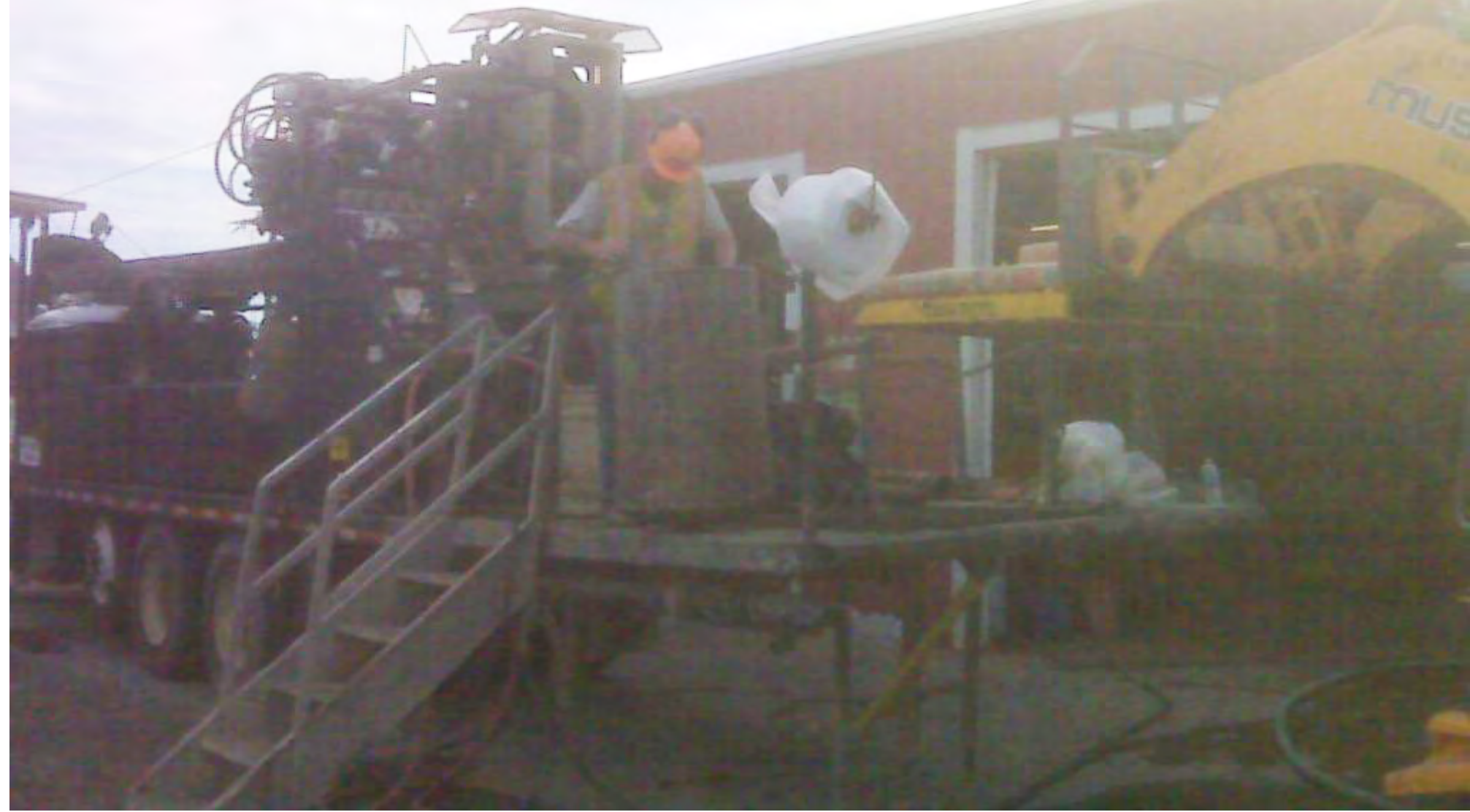

FIGURE 3.7 The grouting operation at the MoDOT well on June 27, 2010. 


\section{Interpretation of Results}

In this section, the investigation results summarized in Section 3 are reviewed in the context of the primary Phase II technical objectives identified in Section 1.2. The results are then used in the consideration of potential risks to human health, public welfare, and the environment associated with the contaminant concentrations and distribution identified in the soils and groundwater at Savannah.

The MDNR has established DTL values for chemicals of concern, against which the results of site characterization studies in Missouri are to be compared, as outlined in MDNR (2006) guidance. The DTL values for carbon tetrachloride in groundwater and in soils of all types are $5.0 \mu \mathrm{g} / \mathrm{L}$ and $79.6 \mu \mathrm{g} / \mathrm{kg}$, respectively. The DTL values for chloroform in groundwater and in soils of all types are $80 \mu \mathrm{g} / \mathrm{L}$ and $78.8 \mu \mathrm{g} / \mathrm{kg}$, respectively.

\subsection{Site Geology and Hydrostratigraphy}

\subsubsection{Stratigraphy near the Former CCC/USDA Facility}

Direct-push techniques were used in Phase I to acquire information on the unconsolidated soil sequence underlying the former CCC/USDA grain storage facility, to the refusal depth of the CPT probing equipment. In Phase I, CPT borings were advanced at six locations, and continuous soil cores were recovered with depth at four locations (SB24, SB29, SB38, and SB42; Figures 1.2 and 1.3). The observed refusal depths appeared comparable to depths to bedrock reported previously for the Savannah area; however, actual contact with the bedrock surface could not be confirmed during Phase I.

On the basis of these Phase I data, two predominant lithologic units were identified beneath an approximately 2-ft-thick layer of modern soil. The uppermost interval (Unit 1) consisted of approximately 58-65 ft of generally dense, stiff to slightly softer, non-plastic and non-calcareous silty clay to clayey silt, with traces of very fine sand. The second, deeper unit (Unit 2) consisted of approximately 26-30 ft of generally firm and dense, locally calcareous, uniformly light gray silty clay till with some fine to medium sand and sporadic coarse inclusions. 
In keeping with the approved site-specific Work Plan (Argonne 2010b), conventional (sonic) drilling techniques were employed in Phase II to expand the hydrogeologic study laterally to the north, south, and east of the former CCC/USDA facility (in the inferred directions of groundwater flow) and also vertically to confirm the lithologic sequence to bedrock. Information on the site-specific stratigraphy near the former CCC/USDA facility was obtained from continuous cores collected at investigation locations SB43-SB49 (Figure 2.1). Lithologic descriptions for these borings are in Appendix $\mathrm{C}$, and data regarding the physical properties of selected cored intervals are in Appendix B, Tables B.2 and B.3.

Two hydrostratigraphic cross sections were developed from the Phase II boring results, together with information from the Phase I studies, at the locations shown in Figures 4.1 and 4.2. The cross sections are in Figures 4.3 and 4.4.

The results of the present study confirm that the two stratigraphic units previously identified beneath the former CCC/USDA facility (Units 1 and 2 in Figures 4.3 and 4.4) are laterally continuous across the expanded investigation area. Both the upper silt-clay unit (Unit 1) and the underlying calcareous till (Unit 2) are composed primarily of clay (29-70\%) and silt (24-69\%), with trace to minor amounts of fine to medium sand (1-6\%). Unit 1 is generally yellowish brown to olive brown with variably abundant rusty orange to black patchy and streaky staining in its upper reaches, transitioning to a more uniform grayish brown with occasional staining becoming fainter with depth. The underlying till (Unit 2) is grayish brown to light gray, but it is generally less stained than Unit 1 and is distinguished primarily by the presence of variably sparse to abundant, small to large (approximately $1 \mathrm{~mm}$ to $2-3 \mathrm{~cm}$ or more), white calcareous nodules and patches, along with occasional non-calcareous pebbles to approximately $4 \mathrm{~cm}$ in diameter.

Figure 4.3 indicates that the thicknesses of both Unit 1 and Unit 2 are relatively consistent along the line of cross section A-A' (approximately $56-72 \mathrm{ft}$ and $30-44 \mathrm{ft}$, respectively), which roughly follows the axis of the northwest-southeast trending topographic high that underlies the former CCC/USDA and MoDOT facilities (Figure 4.2). Figure 4.4 demonstrates that the thickness of Unit 1 decreases to the north and south of the former CCC/USDA facility (to a minimum of approximately $37 \mathrm{ft}$ at boring SB48), as a result of local downcutting along the flanks of this topographic high. 
As noted above, refusal of the direct-push probes employed in Phase I was encountered at relatively consistent depths (approximately 92-96 ft BGL or 1,060-1,065 ft AMSL) beneath the former CCC/USDA property (Argonne 2010a). At Phase I borings SB24, SB38, and SB42 (Figure 1.3), thin deposits (0.5-1.1 ft) of coarser-grained material, ranging from clayey sand to coarse sand or gravel, were contacted at refusal. Thin deposits of medium-coarse sand, clayey to cemented sand, or sand and limestone fragments (up to approximately $2 \mathrm{ft}$ in thickness) were also noted at similar depths in several of the Phase II borings (SB44, SB46, SB49), suggesting that these deposits might represent a locally continuous interval. Figures 4.3 and 4.4 illustrate, however, that these sediments occur within the till unit (Unit 2) and not at the bedrock interface. The results obtained at borings SB43, SB45, and SB48 further demonstrate that the apparent areal extent of the thin deposits at this stratigraphic level is limited.

Competent bedrock - consisting of mildly weathered, dense, gray to greenish gray shale (Unit 4 in Figures 4.3 and 4.4) — was penetrated in six of the Phase II investigative borings, at depths ranging from approximately $95 \mathrm{ft}$ BGL at SB48 to $110 \mathrm{ft}$ BGL at SB46. At SB43, drilling was terminated at a total depth of $109 \mathrm{ft}$ BGL because of excessive "chatter" of the drilling string experienced during the attempt to penetrate several feet of hard, dry limestones and other calcareous materials at this location (see below). Figures 4.3 and 4.4 indicate that the identified depths to the shale define a relatively flat bedrock surface beneath the study area, at an elevation of approximately 1,040-1,045 ft AMSL.

The shale bedrock (Unit 4) is directly overlain by a heterogeneous interval of dense, hard, platy to shattered, pale yellow limestones, sandy limestones, sandy dolomites, highly weathered shale, silt, and clay interpreted as a weathered "rubble" zone (Unit 3) developed below the till unit (Unit 2). The apparent thickness of the Unit 3 rubble zone varies, from approximately $4 \mathrm{ft}$ (at SB44) to $24 \mathrm{ft}$ (at SB48).

\subsubsection{Porosities and Permeabilities}

Porosities determined from cores representing Unit 1 varied from 31\% to 40\%; values for the till (Unit 2) were similar but slightly greater, ranging from $34 \%$ to $46 \%$. These ranges include results for samples collected in both Phase I (Argonne 2010a) and Phase II. Analyses of samples from several of the thin, sandier horizons in the till (Unit 2) indicated greater content of coarsergrained material but somewhat lower porosity values of 24-31\% (Appendix B, Tables B.2 and B.3). 
Vertical permeability determinations were obtained for only 3 samples (of 13 total) submitted for this evaluation in Phase II, because of difficulties reported by the testing laboratory (Alfred Benesch and Company, Lincoln, Nebraska) in the preparation of suitable sediment plugs for analysis. These limited results, for samples collected from boring SB44 on the former CCC/USDA property (Appendix B, Table B.3), suggest that the vertical hydraulic conductivity values for both Unit $1\left(3.4 \times 10^{-4}\right.$ to $\left.4.0 \times 10^{-4} \mathrm{ft} / \mathrm{d}\right)$ and a sandier horizon in the till (Unit 2) $\left(7.3 \times 10^{-4} \mathrm{ft} / \mathrm{d}\right)$ are quite low.

\subsubsection{Hydrostratigraphy near the Former CCC/USDA Facility}

\subsubsection{Groundwater Level Monitoring in Phase I}

Groundwater level monitoring was initiated in Phase I in a network of selected private wells in the vicinity of the former CCC/USDA facility (McPike, Potter, Morgan, MoDOT, Clizer “old,” Hughes, Barr, and Burks private wells), as well as in monitoring wells SB24 and SB38 on the former CCC/USDA property. The data suggested a general pattern of semi-radial groundwater flow in the study area, from a relative high west-northwest of the former CCC/USDA facility (Figure 1.5; Argonne 2010a,b). The observed pattern qualitatively mirrored the dominant local topographic trends, suggesting groundwater flow primarily toward the south or southeast beneath the former facility (Argonne 2010a,b). Data for several wells in the immediate vicinity (McPike, Morgan, MoDOT, and SB24; Figure 2.2) suggested that the onset of groundwater saturation in the soils beneath the former facility occurred at approximately 18-27 ft BGL at the time of the Phase I field studies (Argonne 2010a).

Monitoring of the private wells and monitoring wells SB24 and SB38 continued in Phase II. However, the construction of the private wells, which permits groundwater entry over the full depth intervals penetrated, might mask influences of lateral and vertical hydraulic gradients on the movement of groundwater in the local hydrostratigraphic sequence. 


\subsubsection{Installation of Shallow and Deep Monitoring Wells at Locations SB43-SB49 in Phase II}

To obtain a more detailed understanding of the three-dimensional pattern(s) of groundwater flow, paired shallow and deep monitoring wells were installed at each of the seven Phase II boring locations (SB43-SB49; Figure 2.2). The shallow wells were screened to intersect a consistent interval near the base of the previously identified silty clay to clayey silt (Unit 1), at an elevation of approximately 1,098-1,110 ft AMSL. This interval was targeted, on the basis of preliminary data obtained in Phase I, for the following reasons:

- Occasional thin, slightly softer intervals observed in Unit 1 beneath the former CCC/USDA facility, most commonly at depths of 50-70 ft BGL, suggested the possibility of preferred horizontal migration pathways at this level.

- A small show of free water at this level during the coring of location SB24 was the only occurrence of free water during the Phase I studies (Argonne 2010a).

- This interval corresponds roughly to the maximum depths penetrated by the private wells in the immediate vicinity of the former CCC/USDA facility.

The deep monitoring wells at SB43-SB49 were completed in the basal till (Unit 2) and weathered rubble zone (Unit 3) directly above bedrock. The construction details for the Phase II monitoring wells are in Table 3.2.

\subsubsection{Groundwater Level Monitoring in the Private Well Network in Phase II}

The results of manual measurements of groundwater levels at all monitoring points during Phase II are in Table 3.6. The complete data recovered from the automatic measurement and data logging units installed in the private wells and the shallow and deep monitoring wells are in Supplement 1, Tables S.1-S.3 (on CD).

Figures 4.5-4.7, respectively, present hydrographs for 2009, 2010, and 2011 generated from the water level data recorded automatically in the private wells. Daily precipitation data obtained from the weather station in nearby St. Joseph, Missouri, approximately $7 \mathrm{mi}$ south of 
Savannah (Supplement 1, Table S.4), are shown in conjunction with the water level traces. Figures 4.5-4.7 demonstrate that transient groundwater level fluctuations of up to approximately $8 \mathrm{ft}$ have occurred in many of the private wells during the period of record, in apparent response to both individual precipitation events and the longer-term seasonal influence of wetter periods in late spring to early fall and drier periods in late fall to early spring.

The construction of the private wells (Section 4.1.3.1), coupled with the generally finegrained nature of the surrounding silt-clay and till units, makes these wells susceptible to the interception and temporary storage of surface or shallow subsurface runoff, in addition to groundwater received via slow infiltration from the deeper saturated sediments. These factors might contribute to the rapid and substantial water level increases observed in the wells in response to individual rainfall events, as well as the subsequent slower decline in levels following such events. Figures 4.5-4.7 illustrate, however, little to no net change in the observed groundwater levels in the private wells during the period of record.

Figure 4.8 shows the estimated configuration of the potentiometric surface near the former CCC/USDA facility, constructed from water level measurements obtained from the automatic recorders on August 10, 2011. (See further discussion in Section 4.1.3.4.) The pattern of groundwater level contours depicted in Figure 4.8 is generally similar to those previously documented for the private wells (for example, Figure 1.5; Argonne 2010a,b); however, a more distinct southward direction of groundwater flow beneath the former CCC/USDA facility is suggested by the current data. This observation is, in part, an artifact of the loss of the MoDOT well (abandoned at the MDNR's request in June 2010) as a control point for mapping of the private well water levels.

\subsubsection{Groundwater Level Monitoring in the Shallow Monitoring Wells in Phase II}

As noted in Section 2.2 and Section 4.1.3.2, shallow wells SB43S-SB49S and Phase I monitoring point SB24 were screened at a consistent elevation near the base of the previously identified silty clay to clayey silt (Unit 1). The wells were thus installed to permit the investigation of hydraulic head relationships on the basis of water level measurements obtained along an approximate horizontal "plane” in Unit 1. Hydrographs generated from the automatic water level monitoring data for the shallow wells are in Figure 4.9. 
The groundwater trace in Figure 4.9 for monitoring well SB46S, near the western edge of the MoDOT property (Figure 2.2) is incomplete, and it displays anomalously high and erratic apparent water levels shortly after installation of the automatic recording unit. Because of logistic limitations imposed by surface features at the MoDOT site, the SB46 well pair was installed near the northwest edge of a concrete apron in front (west) of the MoDOT vehicle maintenance building in which the MoDOT well was formerly located. Flush-mounted well completions were used at the request of the property owner. At this location, the SB46 well housings are periodically subject to heavy surface runoff in response to rainfall events, which can cause transient flooding of the well housings and result in erroneous data from the recording units. To prevent damage to the SB46 recorders, the instruments were removed from the shallow and deep wells in late October 2010. With the permission of the MoDOT, the recorders were reinstalled in April 2011, by constructing a temporary, aboveground ("stick-up”) housing and extended well casing at each well (Figure 4.10). At the request of the MoDOT, the temporary stick-ups and recorders were again removed in September 2011, to prevent possible interference with winter snow plowing activities at the vehicle maintenance facility.

The water level traces for most of the shallow monitoring points indicate transient drawdowns in the well casings in response to the 2010 and 2011 groundwater sampling events, despite use of the low-flow sampling technique to minimize this effect (Section 2.4). Figure 4.9 illustrates that the durations of the recovery trends in conventional (2-in.-diameter) wells SB43SSB49S following these events are similar to or longer than those observed in the 1-in.-diameter well SB24, suggesting that the fine-grained nature of Unit 1 (not the boring size) represents the primary factor controlling the slow accumulation of groundwater in these wells. The apparent recovery periods ranged from roughly 3 months at SB24 to 10 months at SB47S. Because of the slow rates of groundwater accumulation in these wells, the trends depicted in Figure 4.9 suggest that relatively stable, ambient groundwater levels at the shallow monitoring points were not achieved until summer 2010, prior to the August 2010 sampling event. Except for SB44S, the traces for the shallow monitoring wells show little sensitivity to short-term or seasonal rainfall events.

In light of these observations, groundwater level data automatically recorded on August 10, 2011, immediately prior to the 2011 sampling event, were selected for illustration of the potentiometric surface configuration for the shallow monitoring horizon. The resulting groundwater level interpretation is in Figure 4.11. The results confirm that the former CCC/USDA facility overlies a local divide in the groundwater flow pattern. Relatively little 
hydraulic gradient is apparent directly beneath the former facility. Figure 4.11 shows that semiradial groundwater flow is expected to the north, east, and south from this area, with the hydraulic gradient increasing most rapidly to the south-southwest.

\subsubsection{Evaluation of Groundwater Flow in the Silt-Clay Unit}

Figures 4.7 and 4.9 demonstrate that the stabilized groundwater levels documented in the shallow monitoring wells at the former CCC/USDA facility (SB24, SB43, SB44) and the adjacent private wells (McPike, Morgan) are very consistent with each other, despite the recognized differences in the construction of these wells. The McPike and Morgan wells permit infiltration of groundwater over the entire saturated length of each boring, while monitoring wells SB24, SB43, and SB44 are each constructed with a discrete, 10-ft screened interval. All of these wells, however, penetrate the upper portion of Unit 1 to similar elevations (1,098-1,110 ft AMSL). Together, these observations suggest that the impacts of vertical hydraulic gradients are minor in the upper portion of Unit 1, above the elevation range indicated.

On the basis of this hypothesis, the groundwater level data obtained for the private wells and shallow monitoring wells were combined to generate an estimate of the potential groundwater flow patterns in the upper portion of the Unit 1 across the study area. The interpreted potentiometric surface, shown in Figure 4.12, closely mimics the local topographic trends inferred previously (Argonne 2010a,b). Figure 4.12 indicates that groundwater beneath most of the former CCC/USDA facility is expected to flow primarily to the south-southwest. Flow to the southeast, east, or northeast is possible, however, from the eastern portion of the former facility, which lies along the approximate crest of the groundwater (and topographic) divide that extends beneath the CCC/USDA and MoDOT facilities.

\subsubsection{Groundwater Level Monitoring and Evaluation of Groundwater Flow in the Deep Monitoring Interval in Phase II}

Deep monitoring wells SB43D-SB49D were completed at the base of the till unit (Unit 2), in the heterogeneous, weathered rubble zone (Unit 3) that overlies shale bedrock. Hydrographs generated from the groundwater level data automatically recorded in these wells, plus Phase I deep monitoring point SB38 (which has a unique pattern), are in Figure 4.13. 
The groundwater levels in wells SB43D-SB49D (but not in well SB38) showed relatively little net change during the period of record. Figure 4.13 indicates that subtle fluctuations in the groundwater levels in each of these wells appeared in response to seasonal periods of rainfall. Most notably, however, the traces for these wells show no discernible drawdown response to the groundwater sampling events in 2010 and 2011, in comparison to the associated shallow monitoring points (Figure 4.9). This observation suggests that the deep rubble zone (Unit 3) has a greater capacity to transmit groundwater to wells than the shallower Unit 1. No private wells in the vicinity of the former CCC/USDA facility are known to penetrate the rubble zone (Unit 3) (Argonne 2010a,b).

Figure 4.14 shows the estimated configuration of the potentiometric surface for the deep monitoring interval, constructed from water level measurements obtained from the automatic recorders on August 10, 2011. The interpreted groundwater level contours do not show the clear relationship to the local topographic trends noted for the shallow monitoring points and private wells in Unit 1 (Section 4.1.3.5). The existing data suggest that groundwater flow in the deep monitoring interval (base of Unit 2 and Unit 3) is predominantly to the east in the vicinity of the former CCC/USDA and MoDOT facilities, under hydraulic gradients that are low in comparison to those observed across most of the study area in Unit 1. The lower observed hydraulic gradients are empirically consistent with the inferred possibility, noted in Section 4.1.3.6, of somewhat higher hydraulic conductivity in the basal rubble zone (Unit 3).

The groundwater elevations observed in the deep monitoring interval were approximately 25-40 ft below the levels in the associated shallow monitoring points. These results indicate that significant vertical hydraulic gradients exist between the upper portion of Unit 1 and the rubble zone (Unit 3) at the base of the unconsolidated sequence. The low vertical hydraulic conductivities noted in Section 4.1.2 suggest, however, that the rates of vertical groundwater movement in response to these hydraulic driving forces may be limited.

As noted in Section 3.4.3.2, monitoring point SB38 is unique in that it is constructed with a 40 -ft screen (at $50-90 \mathrm{ft}$ BGL) that spans the 10-ft interval represented by the shallow monitoring wells, as well as $30 \mathrm{ft}$ of the lower portion of Unit 1 and the upper portion of Unit 2. Well SB38 does not intersect the interval screened in deep monitoring wells SB43D-SB49D (Table 3.2 and Figure 4.4). The groundwater level trace for monitoring point SB38 is included in Figure 4.13 with those for deep monitoring wells SB43D-SB49D; however, the distinct character of the SB38 hydrograph is evident. Well SB38 shows drawdown responses similar to those 
observed in the shallow monitoring wells (Figure 4.9) in association with the 2010 and 2011 groundwater sampling events, suggesting a low capacity to transmit groundwater to wells for the deeper silty clay to clayey silt of Unit 1 and the till of Unit 2. The stabilized groundwater levels observed at SB38 are, however, generally 10-15 ft below those in the nearby shallow monitoring wells and approximately $25 \mathrm{ft}$ higher than those in the corresponding deep monitoring points, confirming the interpretation that vertical hydraulic gradients exist in the deeper portion of Unit 1 and the underlying till (Unit 2).

\subsection{Distribution of Carbon Tetrachloride in Soils at the Former CCC/USDA Facility and the MoDOT Property}

In Phase I, carbon tetrachloride concentrations greater than the DTL were identified in only 2 of 79 soil samples collected in vertical profiles at 4-ft intervals to the depth of CPT refusal at 4 locations (SB24, SB29, SB38, and SB42; Figures 1.2 and 1.3). Concentrations of $171 \mu \mathrm{g} / \mathrm{kg}$ and $126 \mu \mathrm{g} / \mathrm{kg}$ were detected at $44 \mathrm{ft}$ BGL and $48 \mathrm{ft}$ BGL, respectively, in the saturated zone at SB42.

In Phase II, soil samples were collected for VOCs analyses at 4-ft intervals, from $4 \mathrm{ft}$ BGL to the top of bedrock, at boring locations SB43-SB46 (Figure 2.1). Borings SB43 and SB44 are located near the northern edge of the former CCC/USDA facility, upgradient from the carbon tetrachloride contamination previously identified in groundwater to the south (at the Morgan private well and monitoring points SB24 and SB38) and to the east (at the MoDOT private well). Boring SB46 is located on the MoDOT property immediately upgradient of the contaminated MoDOT well; SB45 lies downgradient of the MoDOT well (Figures 2.2, 4.12, and 4.14). The analytical results for VOCs in soil samples are summarized in Table 3.1, and the complete analytical data are in Appendix B, Table B.1.

The vertical distribution of carbon tetrachloride identified in soils from borings SB43SB46 is displayed in Figures 4.15 and 4.16, in conjunction with the results of the soil analyses for Phase I borings SB24, SB38, and SB42. No quantifiable concentrations of carbon tetrachloride were detected in Phase I boring SB29 (Figure 1.3), which does not appear in Figure 4.15 or 4.16 .

The results discussed in Section 4.1.3 demonstrate that transient fluctuations in groundwater levels have been observed in the private wells around the former CCC/USDA 
facility in response to rainfall events; however, the apparent distribution of saturated conditions in the unconsolidated sediments has changed little during the investigations. The data presently available therefore suggest that the groundwater levels depicted for the shallow monitoring wells in Figures 4.3 and 4.4 might reasonably approximate the upper extent of saturated conditions during this period. These data suggest depths to saturation of approximately 18-22 ft BGL across the former CCC/USDA facility, as well as at locations SB45 and SB46 on the adjacent MoDOT property.

On the basis of this interpretation, no carbon tetrachloride has been detected in the subsurface (vadose zone) soils at Savannah as defined by the MDNR (2006), at concentrations greater than the DTL $(79.6 \mu \mathrm{g} / \mathrm{kg})$ for this contaminant. Further, with only two exceptions, no carbon tetrachloride has been detected in the subsurface soils at concentrations above the quantitation limit of $10 \mu \mathrm{g} / \mathrm{kg}$ for the purge-and-trap analysis method. At SB45 (on the MoDOT property), carbon tetrachloride was detected at $12 \mu \mathrm{g} / \mathrm{kg}$ in a soil sample collected at $12 \mathrm{ft}$ BGL; however, no contamination was detected (at a method detection limit of $1.0 \mu \mathrm{g} / \mathrm{kg}$ ) in the soils immediately above and below this interval. At SB46 (also on the MoDOT property), carbon tetrachloride concentrations of $16-45 \mu \mathrm{g} / \mathrm{kg}$ were identified in the vadose zone soils at $8-20 \mathrm{ft}$ BGL, and a trace concentration of carbon tetrachloride (estimated at $2.8 \mu \mathrm{g} / \mathrm{kg}$ ) was detected in a soil sample from $2 \mathrm{ft} \mathrm{BGL}$ at this location (Figure 4.15).

In the saturated zone, the presence of carbon tetrachloride in the soils might reflect contamination that is (1) adsorbed to the solid soil particles, (2) dissolved in the pore water contained in the soils, or (3) in a combination of these two physical states. Carbon tetrachloride was identified at concentrations above the DTL value in the deeper soils at two of the Phase II boring locations: SB44 and SB46 (Figure 4.15).

At SB44, carbon tetrachloride concentrations of $106-364 \mu \mathrm{g} / \mathrm{kg}$ were detected over the depth interval at 44-56 ft BGL. The occurrence of carbon tetrachloride in SB44 corroborates the elevated levels of this contaminant $(77-171 \mu \mathrm{g} / \mathrm{kg}$ ) previously identified in nearby Phase I boring SB42 at these depths (Figure 4.15), thus confirming the presence of a localized carbon tetrachloride "hot spot" in the deeper, saturated soils at the northeast corner of the former CCC/USDA facility (Figure 3.2). Figure 4.15 illustrates, however, that no quantifiable concentrations of carbon tetrachloride were identified in the soils above or below this interval at boring SB42 or SB44. 
At SB46, carbon tetrachloride was identified at concentrations of $81-82 \mu \mathrm{g} / \mathrm{kg}$ in the two soil samples collected at 40-44 ft BGL (Figure 4.15). No contamination was detected in the deeper soils (> $48 \mathrm{ft}$ BGL) in this boring; however, the SB46 location is unique in that it is the only boring in which a continuous distribution of carbon tetrachloride was documented from the ground surface to the depth of maximum contamination, suggesting that this location might represent a former area of carbon tetrachloride entry into the vadose zone (and deeper) soils.

Figures 4.15 and 4.16 show that no carbon tetrachloride was detected in soils at any location, at depths corresponding to elevations of less than approximately 1,095 ft AMSL, in the deeper portion of Unit 1 at SB45 and SB46 or in the underlying till (Unit 2) and rubble zone (Unit 3). These observations are qualitatively consistent with the groundwater level relationships discussed in Section 4.1.3, which document hydraulic head differences between the upper portion of Unit 1 and the basal rubble zone (Unit 3). Together with the fine-grained character of the intervening deposits, these data support a hypothesis that the lower portion of Unit 1 and the underlying till (Unit 2) restrict vertical contaminant migration from the shallower sediments into the deeper portions of the unconsolidated sequence.

\subsection{Distribution of Carbon Tetrachloride in Groundwater}

Carbon tetrachloride contamination was identified in groundwater at only four locations during Phase I (Argonne 2010a). Concentrations of 3.8-6.4 $\mu \mathrm{g} / \mathrm{L}$ were detected at monitoring points SB24 and SB38 at the former CCC/USDA facility. Concentrations of $13 \mu \mathrm{g} / \mathrm{L}$ and $150 \mu \mathrm{g} / \mathrm{L}$ were identified in the Morgan and MoDOT wells, respectively, south and east of the former facility (Figure 1.4). To obtain a more detailed picture of the distribution of carbon tetrachloride in groundwater near the former CCC/USDA facility, 86 groundwater samples were collected for VOCs analyses during the Phase II investigation.

As outlined in Sections 2.4 and 3.4, slow groundwater accumulation rates, particularly in the private wells and shallow monitoring wells, complicated the sampling of these wells for VOCs analysis. To ensure that sufficient groundwater could be recovered for analysis, samples were generally obtained during the three groundwater sampling events in June-July 2010, August 2010, and August 2011 by bailing from each monitoring well, without purging, prior to subsequent sampling with the low-flow technique. The discussions in Section 4.1.3 indicate that sampling under apparently equilibrium groundwater level conditions was possible only during the August 2011 sampling event, because of the prolonged time frame required for stabilization 
of the levels in many wells. The specific conditions under which each groundwater sample was collected during the Phase II program are in Appendix A, Table A.1.

The results of the Phase II groundwater analyses for carbon tetrachloride and chloroform are summarized in Tables 3.3 and 3.4 and are discussed in detail in Section 3.4. Although variability is noted in the results obtained at several locations over time, or in possible association with the sampling methods employed in any one event, the results presented in Section 3.4 demonstrate that the patterns of carbon tetrachloride distribution identified across the study area have been consistent among the sampling events. Consequently, as a conservative measure, the maximum carbon tetrachloride concentrations detected at the groundwater sampling points are used as the basis for the evaluation here. The maximum values are illustrated for the groundwater sampling points included in Figures 4.15 and 4.16, as well as for the shallow monitoring and private wells in Figure 4.17. As discussed below, no quantifiable concentrations of carbon tetrachloride were detected in the deep monitoring wells.

\subsubsection{Carbon Tetrachloride in the Shallow Monitoring Interval}

Figures 4.15 and 4.16 demonstrate that at each of the boring locations sampled for soil analyses (except for SB38), the highest concentrations of carbon tetrachloride identified in soils correlated closely with the presence of the contaminant in groundwater samples from the shallow monitoring wells. The completion depths of the shallow monitoring wells were selected primarily because of the inferred possibility of slightly greater water-producing capacity from the silty clay to clayey silt (Unit 1) at approximately 50-70 ft BGL in the vicinity of the former CCC/USDA facility, although no distinct variations in the lithologic characteristics of the sediments in this interval were readily identified (Argonne 2010a). The results of the Phase II study support this hypothesis, suggesting that horizontal contaminant migration might also occur preferentially in Unit 1, in a zone at elevations of approximately 1,098-1,110 ft AMSL.

Figure 4.17 confirms that the carbon tetrachloride contamination hot spot identified in soil at 44-56 ft BGL in Unit 1 at borings SB42 and SB44, in the northeast corner of the former CCC/USDA facility (Section 4.2), is also reflected in groundwater in shallow monitoring well SB44S. A maximum carbon tetrachloride concentration of $273 \mu \mathrm{g} / \mathrm{L}$, representing the highest concentration detected in groundwater (by Argonne) to date, was identified at SB44S. As noted in Section 3.4.1, this concentration was identified in the August 2011 sampling event; however, 
elevated carbon tetrachloride concentrations were also detected at SB44S (59-84 $\mu \mathrm{g} / \mathrm{L})$ during the June-July 2010 and August 2010 sampling events.

Figure 4.17 illustrates rapid decreases in carbon tetrachloride concentrations in groundwater in Unit 1 with distance away from the SB44S hot spot in the apparent directions of groundwater flow and potential contaminant transport, with low maximum concentrations at nearby shallow monitoring well SB24 $(10 \mu \mathrm{g} / \mathrm{L})$ and the Morgan private well $(13 \mu \mathrm{g} / \mathrm{L})$ to the south, as well as at SB46S $(17 \mu \mathrm{g} / \mathrm{L})$ to the east. A similar low maximum concentration $(20 \mu \mathrm{g} / \mathrm{L})$ was detected at monitoring point SB38, which intersects the portion of Unit 1 penetrated in the adjacent shallow monitoring wells. (Although the screened interval in SB38 extends deeper into the underlying till of Unit 2, the results of the soil analyses discussed in Section 4.2 and illustrated in Figure 4.16 indicate that no carbon tetrachloride was detected in this deeper portion of the unconsolidated sequence.) Trace concentrations of carbon tetrachloride (estimated at $2.9 \mu \mathrm{g} / \mathrm{L}$ or less) at shallow monitoring well SB48S suggest that low-level contamination in Unit 1 extends beneath the Morgan property to the south-southeast of the SB44S hot spot, in qualitative agreement with the expected patterns of groundwater flow in this direction.

With only two possible exceptions, no carbon tetrachloride was identified in the shallow monitoring or private wells to the north, west, or southwest of the contamination at SB44S (Figure 4.17). At SB43S, carbon tetrachloride was reported at a concentration of $12 \mu \mathrm{g} / \mathrm{L}$ in a single grab sample, collected without purging, during the August 2010 sampling event. This apparent detection was not substantiated by the corresponding low-flow sample collected during this event or by any samples collected during the June-July 2010 and August 2011 sampling events. Similarly, a carbon tetrachloride concentration of $6.8 \mu \mathrm{g} / \mathrm{L}$ was reported for the initial (June-July 2010) grab sample obtained from shallow monitoring well SB47S. This value was not corroborated by any of the subsequent sampling results at this location. The significance of these two apparent detections of carbon tetrachloride is therefore uncertain and subject to further evaluation.

In light of these relationships, the relatively high and persistent concentrations of carbon tetrachloride identified at the MoDOT well and at shallow monitoring point SB45S to the eastsoutheast of the MoDOT well appear potentially anomalous. Concentrations of $179-210 \mu \mathrm{g} / \mathrm{L}$ were detected in the groundwater samples collected from the MoDOT well during the Phase II study, prior to the abandonment of this well (at the request of the MDNR) in June 2010. These 
values are comparable to the concentrations identified in Phase I (150 $\mu \mathrm{g} / \mathrm{L}$; Argonne 2010a) and in earlier sampling by the MDNR (321 $\mathrm{g} / \mathrm{L}$; MDNR 2000b). Concentrations of 40-86 $\mu \mathrm{g} / \mathrm{L}$ were identified at SB45S during the sampling in both August 2010 and August 2011 (Table 3.4).

Figure 4.17 indicates that both the MoDOT and SB45S wells lie downgradient from the carbon tetrachloride hot spot identified in soils and groundwater at SB42/SB44 in the northeast corner of the former CCC/USDA property (Figure 4.15); a possible association with this contaminant source area cannot therefore be discounted. The consistently lower carbon tetrachloride concentrations identified at intervening well SB46S (lower by approximately an order of magnitude than those at SB44S and the MoDOT well) do not, however, support the presence of a continuous trend of high concentrations in groundwater in Unit 1, extending eastward from the SB44S area to the MoDOT well location.

The specific origin of the increased carbon tetrachloride concentrations noted downgradient of SB46S at the MoDOT and SB45S wells and the potential areal limits of this contamination beyond the SB45S location cannot be determined from the presently available data. The identification of residual carbon tetrachloride contamination in the vadose zone soils at SB46S (discussed in Sections 3.1 and 4.2), coupled with the proximity of this boring to the MoDOT well location, might, however, suggest the present or former existence of an additional source of carbon tetrachloride contamination to the groundwater in Unit 1 in the vicinity of these borings.

\subsubsection{Carbon Tetrachloride in the Deep Monitoring Interval}

The results of the soil analyses for VOCs outlined in Section 4.2 support the hypothesis that no vertical migration of carbon tetrachloride contamination into the till (Unit 2) or basal rubble zone (Unit 3) has occurred. With only one possible exception, the results of the analyses of groundwater samples from deep monitoring wells SB43D-SB49D are consistent with this interpretation.

Tables 3.3 and 3.4 indicate that no carbon tetrachloride was detected (at the method detection limit of $0.1 \mu \mathrm{g} / \mathrm{L}$ ) in the groundwater samples recovered from wells SB43D and SB45D-SB49D during the 2010 and 2011 sampling events. At well SB44D, a trace of carbon tetrachloride $(<1 \mu \mathrm{g} / \mathrm{L}$ ) was reported in the initial grab sample recovered in July 2010; however, 
no carbon tetrachloride was detected in any of the samples collected from this well during the subsequent 2010 and 2011 sampling events.

\subsubsection{Chloroform Distribution and Possible Degradation of Carbon Tetrachloride}

Chloroform represents the initial degradation product of the inorganic or biologically mediated reductive dechlorination of carbon tetrachloride under anaerobic reducing conditions. Subsequent degradation products generated via this pathway may include methylene chloride, chloromethane, and methane. The presence of relatively elevated concentrations of chloroform (or further degradation products) in association with carbon tetrachloride might therefore indicate naturally occurring degradation of carbon tetrachloride in soils or groundwater.

The complete analytical data for the soil samples analyzed for VOCs in Phase II are in Appendix B, Table B.1. No concentrations of chloroform were detected above the analytical method quantitation limit of $10 \mu \mathrm{g} / \mathrm{kg}$ for this contaminant in the vadose zone or saturated soils collected at borings SB43-SB49; however, trace levels of chloroform were detected sporadically in association with the carbon tetrachloride contamination identified at borings SB44-SB46, as well as in the absence of carbon tetrachloride in boring SB43. The very low levels of chloroform observed in these samples provide no evidence of carbon tetrachloride degradation in the soils at this site.

In contrast, chloroform was detected in a majority of the groundwater samples collected from the shallow monitoring wells. The absolute concentrations of chloroform present were generally low, and no chloroform was detected at concentrations above the DTL (80 $\mu \mathrm{g} / \mathrm{L})$ for this contaminant in groundwater. No evidence of the more advanced degradation products associated with reductive dechlorination of carbon tetrachloride was detected in any of the groundwater samples. Significant levels of chloroform relative to carbon tetrachloride were, however, identified at several of the shallow monitoring wells and at the Morgan private well (Tables 3.3 and 3.4). Ratios of chloroform to carbon tetrachloride $\geq 10 \%$ (calculated as chloroform concentration in $\mu \mathrm{g} / \mathrm{L}$ divided by carbon tetrachloride concentration in $\mu \mathrm{g} / \mathrm{L}$ ) were derived at the following locations:

- $\quad$ SB24 (47-133\%) 
- $\operatorname{SB} 44 \mathrm{~S}(10-44 \%)$

- $\quad$ SB45S (10-133\%)

- $\quad$ SB46S (12-47\%)

- Morgan well (47-133\%)

These results suggest that natural degradation processes might have affected the carbon tetrachloride concentrations in groundwater in Unit 1. As indicated, however, the ratios of chloroform to carbon tetrachloride for the groundwater samples obtained to date at each of these locations vary significantly.

The identification of low DO values (generally less than $2 \mathrm{mg} / \mathrm{L}$ ) and low ORP values (near zero or ideally negative) in groundwater can together provide preliminary evidence for the occurrence of the oxygen-depleted, chemically reducing conditions that are conducive to the possible reductive dechlorination of carbon tetrachloride (EPA 1998a). The results of DO and ORP measurements for the groundwater samples collected in 2010-2011 (Appendix E, Table E.2) do not support the general presence of oxygen-depleted, reducing conditions in Unit 1, although the DO and ORP measurements for several individual groundwater samples satisfy the criteria outlined above. Together, the VOCs, DO, and ORP data generated in the 2010-2011 sampling events suggest limited evidence for natural degradation of the carbon tetrachloride in groundwater in Unit 1.

\subsection{Preliminary Consideration of Human Health and Environmental Risks Associated with the Carbon Tetrachloride Contamination}

As discussed in the preceding sections, carbon tetrachloride was identified in soils and groundwater at Savannah, at concentrations that exceed the respective DTL values for this contaminant $(79.6 \mu \mathrm{g} / \mathrm{kg}$ and $5.0 \mu \mathrm{g} / \mathrm{L})$. This section presents a preliminary discussion of potential health and environmental risks associated with the contamination. 


\subsubsection{Contamination in Soils}

The analytical results indicate no concentrations above the DTL for carbon tetrachloride in soils in the subsurface (vadose zone) soils as defined by the MDNR (2006). Carbon tetrachloride concentrations above the DTL value were identified at two locations, over restricted depth intervals in the saturated zone, as follows:

- Carbon tetrachloride concentrations of $106-364 \mu \mathrm{g} / \mathrm{kg}$ at depths of $44-56 \mathrm{ft}$ BGL at borings SB42 and SB44, at the northeast corner of the former CCC/USDA facility.

- Carbon tetrachloride concentrations of $81-82 \mu \mathrm{g} / \mathrm{kg}$ at depths of $40-44 \mathrm{ft}$ BGL at boring SB46, at the southeast edge of the MoDOT property.

The DTL for soils reflects the maximum risk-based concentration in unsaturated soils of any lithologic type that is considered acceptable for unrestricted use of a property affected by a contaminant of concern. For carbon tetrachloride, the DTL reflects risk associated with the indoor inhalation pathway. Because the former CCC/USDA facility and the present MoDOT facility are used only for non-residential purposes, and no habitable buildings are present on either property within $100 \mathrm{ft}$ of the identified contamination in soils, the indoor inhalation pathway is incomplete. Thus, no present or reasonably anticipated future receptors are at risk of exposure by this route. The elevated concentrations of carbon tetrachloride in the soils at these locations might, however, continue to contribute this contaminant to the nearby groundwater.

\subsubsection{Contamination in Groundwater}

The DTL for carbon tetrachloride in groundwater $(5.0 \mu \mathrm{g} / \mathrm{L})$ reflects risk associated with potential exposure via the use of water for domestic purposes, including consumption.

Carbon tetrachloride at concentrations exceeding the DTL for this contaminant was confirmed by the groundwater sampling conducted in Phase II, at seven locations on and near the former CCC/USDA facility, at the following maximum concentrations:

- $\quad \operatorname{SB} 44 S(273 \mu \mathrm{g} / \mathrm{L})$ 
- MoDOT well $(210 \mu \mathrm{g} / \mathrm{L})$

- $\quad \mathrm{SB} 45 \mathrm{~S}(86 \mu \mathrm{g} / \mathrm{L})$

- $\operatorname{SB38}(20 \mu \mathrm{g} / \mathrm{L})$

- $\quad \operatorname{SB} 46 \mathrm{~S}(17 \mu \mathrm{g} / \mathrm{L})$

- Morgan well (13 $\mu \mathrm{g} / \mathrm{L})$

- $\mathrm{SB} 24(10 \mu \mathrm{g} / \mathrm{L})$

To the south of the former CCC/USDA facility, the downgradient extent of the carbon tetrachloride contamination in groundwater (at concentrations above the DTL value) appears to be defined by the non-detectable to trace concentrations identified at shallow monitoring wells SB47S and SB48S, along the southern boundary of the Morgan private property. The potential downgradient extent of the contamination to the east and southeast, however, cannot be constrained by the presently available data (Figure 4.17).

At the request of the MDNR, the MoDOT private well was plugged and abandoned (in accord with applicable MDNR requirements) on June 27, 2010, in conjunction with the main Phase II field session (see Section 3.7). The contaminated Morgan well is used solely for lawn and garden purposes.

A survey in conjunction with the Phase I studies identified only two private wells within approximately $0.5 \mathrm{mi}$ of the former CCC/USDA facility that are used for domestic purposes. These wells are located at the Clizer "new" and Moore residences (Figure 4.18). Both the Clizer "new" and Moore wells were sampled during the Phase I field program and were found to be free of carbon tetrachloride. These results indicate no current risks to these wells associated with the groundwater contamination identified at the former CCC/USDA and MoDOT facilities (Argonne 2010a).

The Clizer "new" well is located to the northwest and effectively upgradient of the former CCC/USDA and MoDOT facilities, and therefore it is not at future risk from the identified groundwater contamination. The Moore well is located approximately $0.4 \mathrm{mi}$ southeast 
of the former CCC/USDA facility, in the apparent direction of groundwater flow from the site. Figure 4.18 illustrates, however, that the Moore well lies on the eastern flank of a second, northsouth trending topographic divide to the southeast of the former CCC/USDA and MoDOT facilities. The presence of this feature supports the interpretation that the Moore well does not, therefore, lie along the probable migration pathway of the presently identified groundwater contamination.

On the basis of these observations, the potential future human health risks associated with possible domestic use of the contaminated groundwater identified at the former CCC/USDA and MoDOT facilities are believed to be minimal.

Figure 4.17 shows that three buildings on the MoDOT property overlie or are within $100 \mathrm{ft}$ of the elevated concentration of carbon tetrachloride $(210 \mu \mathrm{g} / \mathrm{L})$ identified in the Unit 1 groundwater at and near the former MoDOT well. One of these buildings is used solely for equipment storage; the other two are used, during normal working hours, for the parking and maintenance of vehicles. Ventilation is normally provided via multiple garage doors when the buildings are in use.

Figure 4.17 also indicates that the Morgan residence might lie within $100 \mathrm{ft}$ of the relatively low levels of carbon tetrachloride (10-20 $\mu \mathrm{g} / \mathrm{L})$ detected in the Unit 1 groundwater south of the former CCC/USDA facility.

The proximity of the identified structures to the footprint of the contamination in groundwater suggests that vapor intrusion to indoor air might represent a potential exposure pathway at these locations. As of this date, however, the MDNR has not established target contaminant levels for the evaluation of potential indoor air contamination by carbon tetrachloride. 


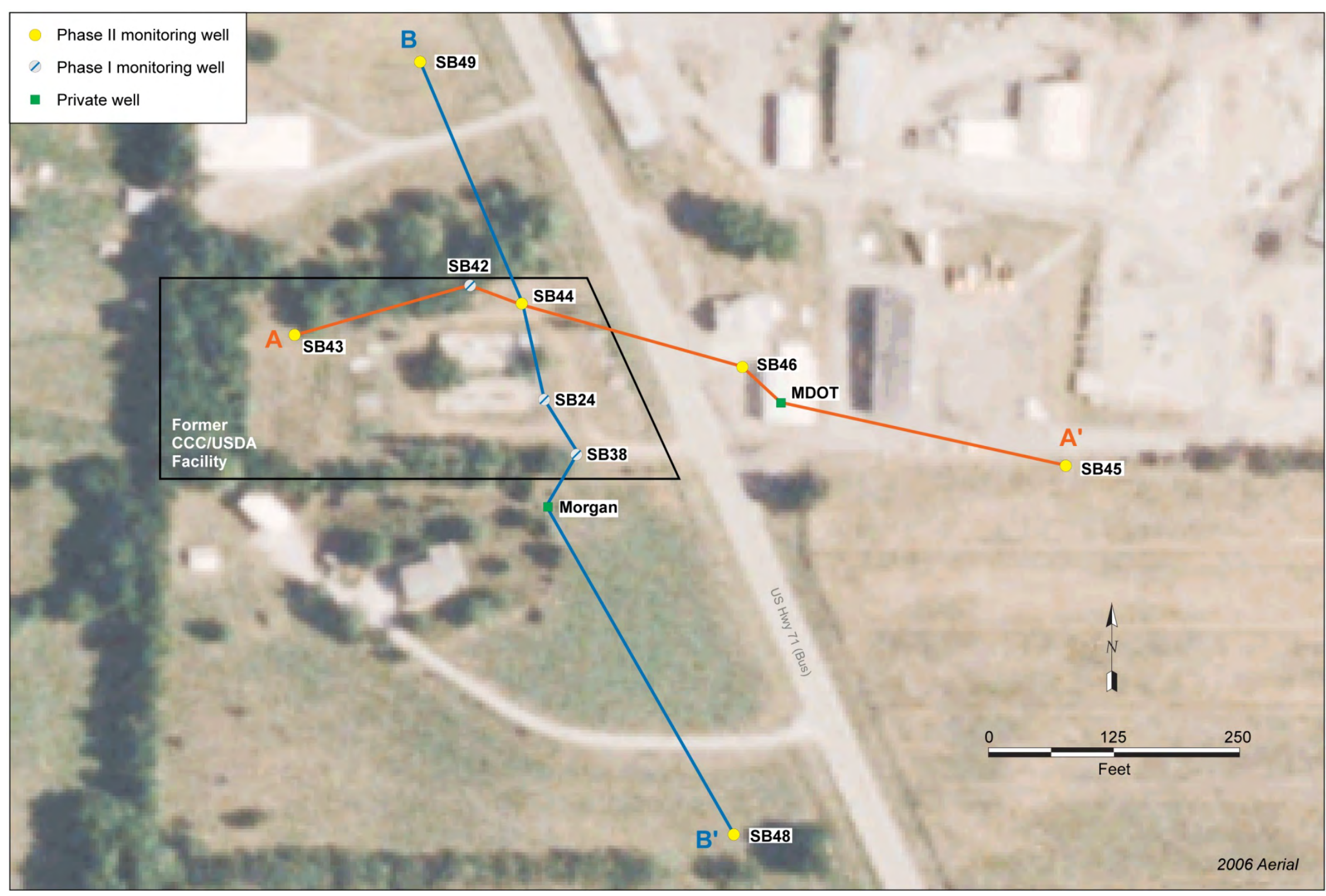

FIGURE 4.1 Locations of hydrogeologic cross sections $A-A^{\prime}$ and $B-B^{\prime}$, with the distribution of current surface features in the vicinity of the former CCC/USDA facility. Source of aerial photograph: NAIP (2006). 


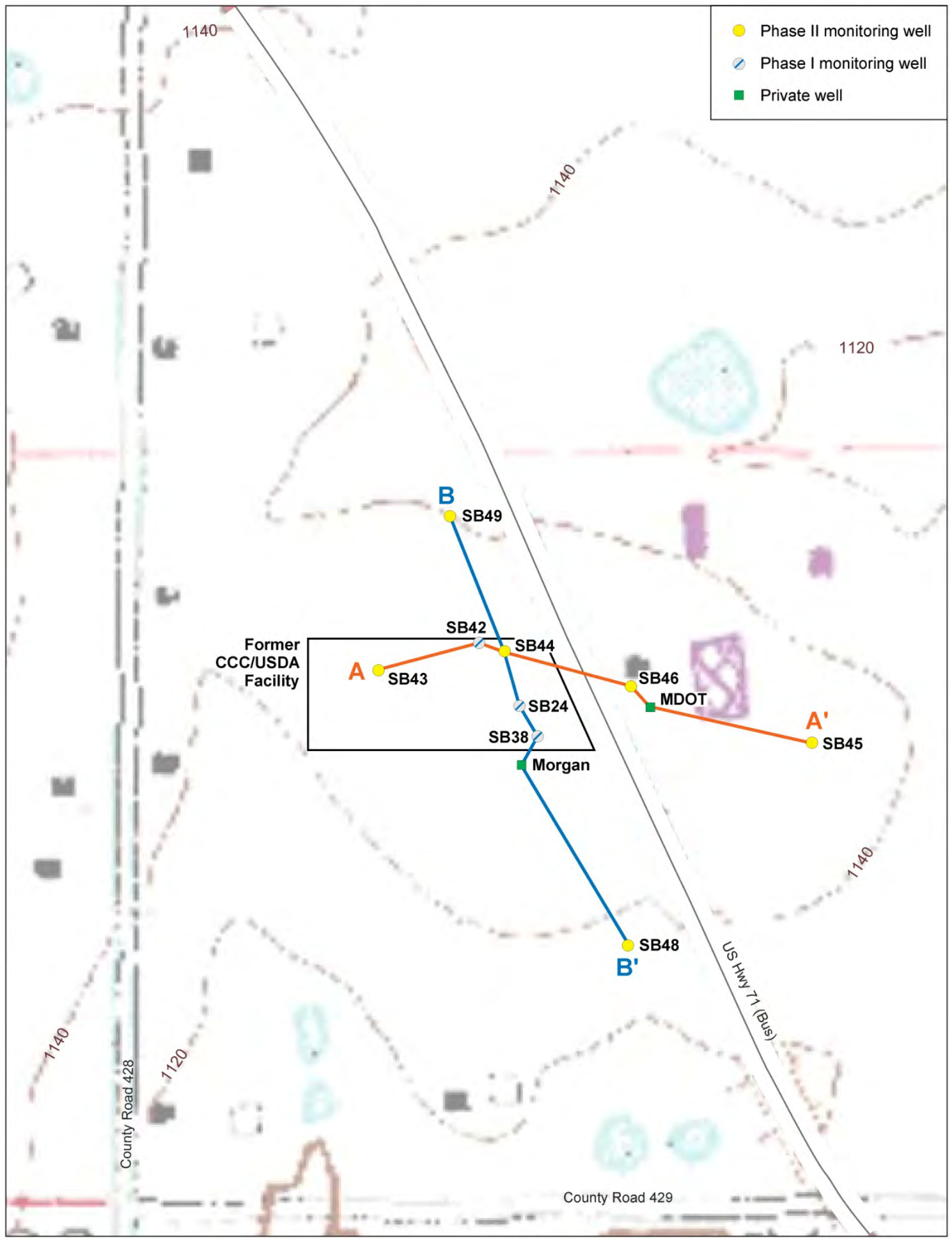

FIGURE 4.2 Locations of hydrogeologic cross sections A-A' and B-B', with the surface topography in the vicinity of the former CCC/USDA facility. Source of topographic map: USGS (1996). 


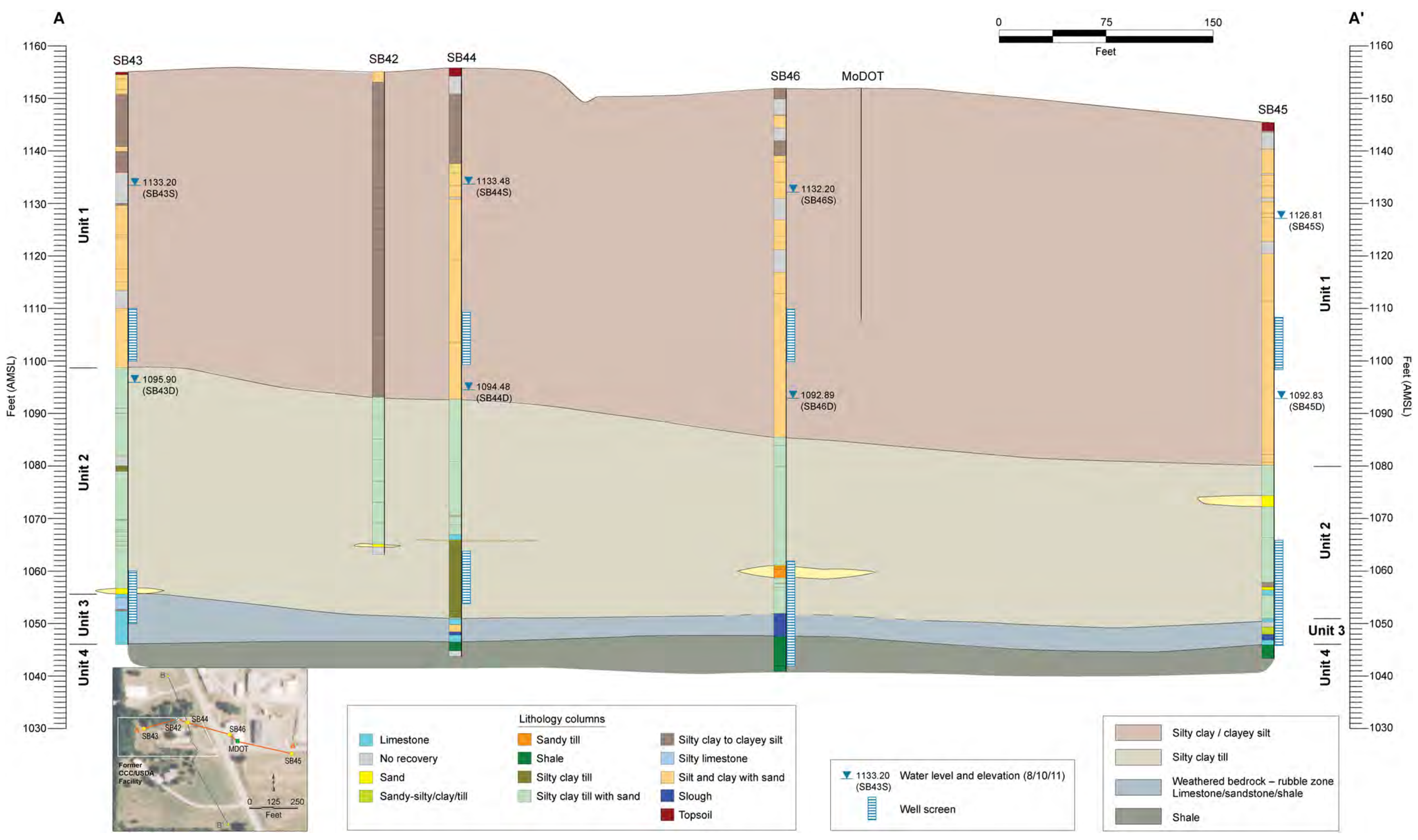

FIGURE 4.3 West-to-east hydrogeologic cross section A-A' (vertically exaggerated), showing the distribution of groundwater levels identified in shallow and deep monitoring wells. 


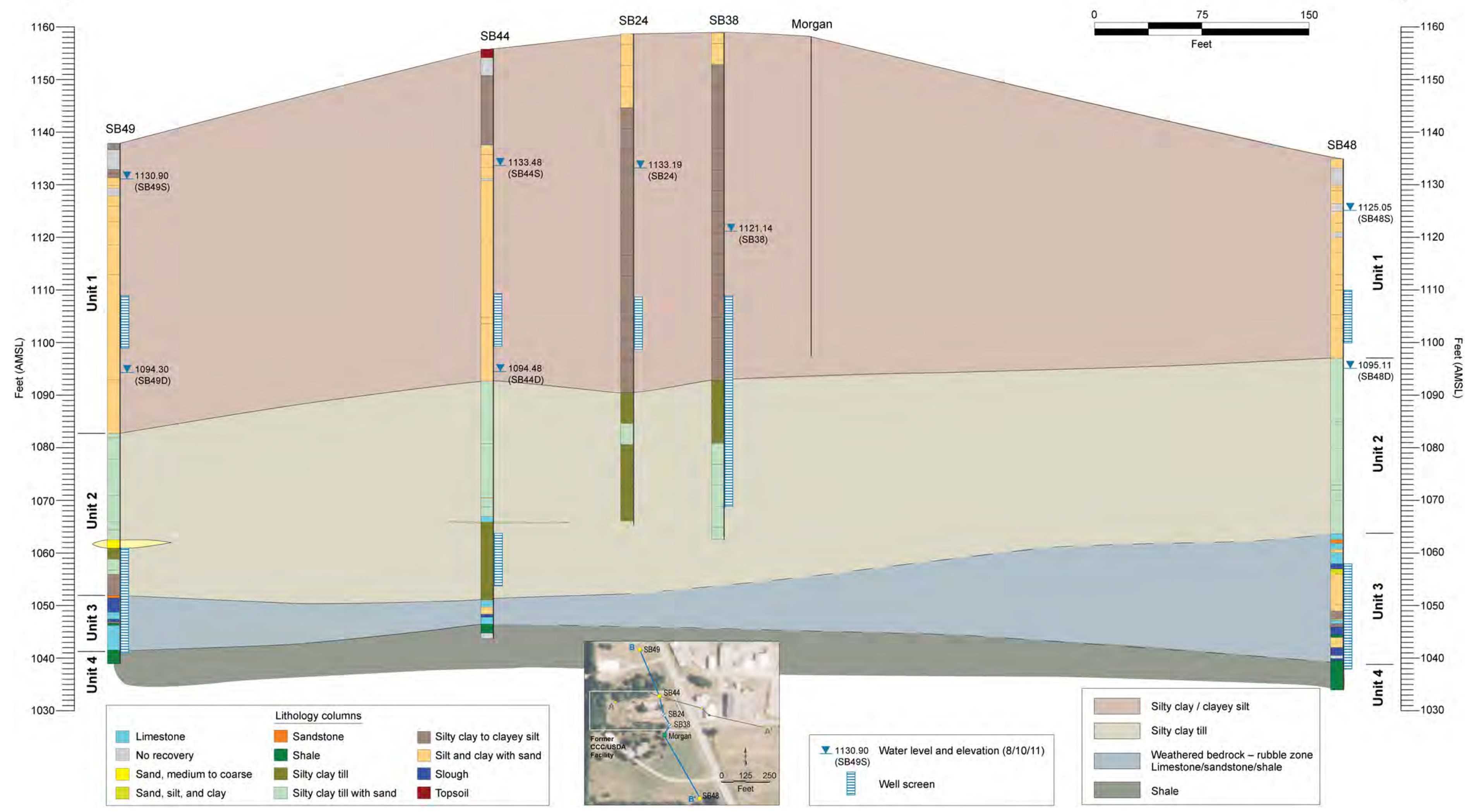

FIGURE 4.4 North-to-south hydrogeologic cross section B-B' (vertically exaggerated), showing the distribution of groundwater levels identified in shallow and deep monitoring wells. 
Savannah Private Well Water Levels - 2009

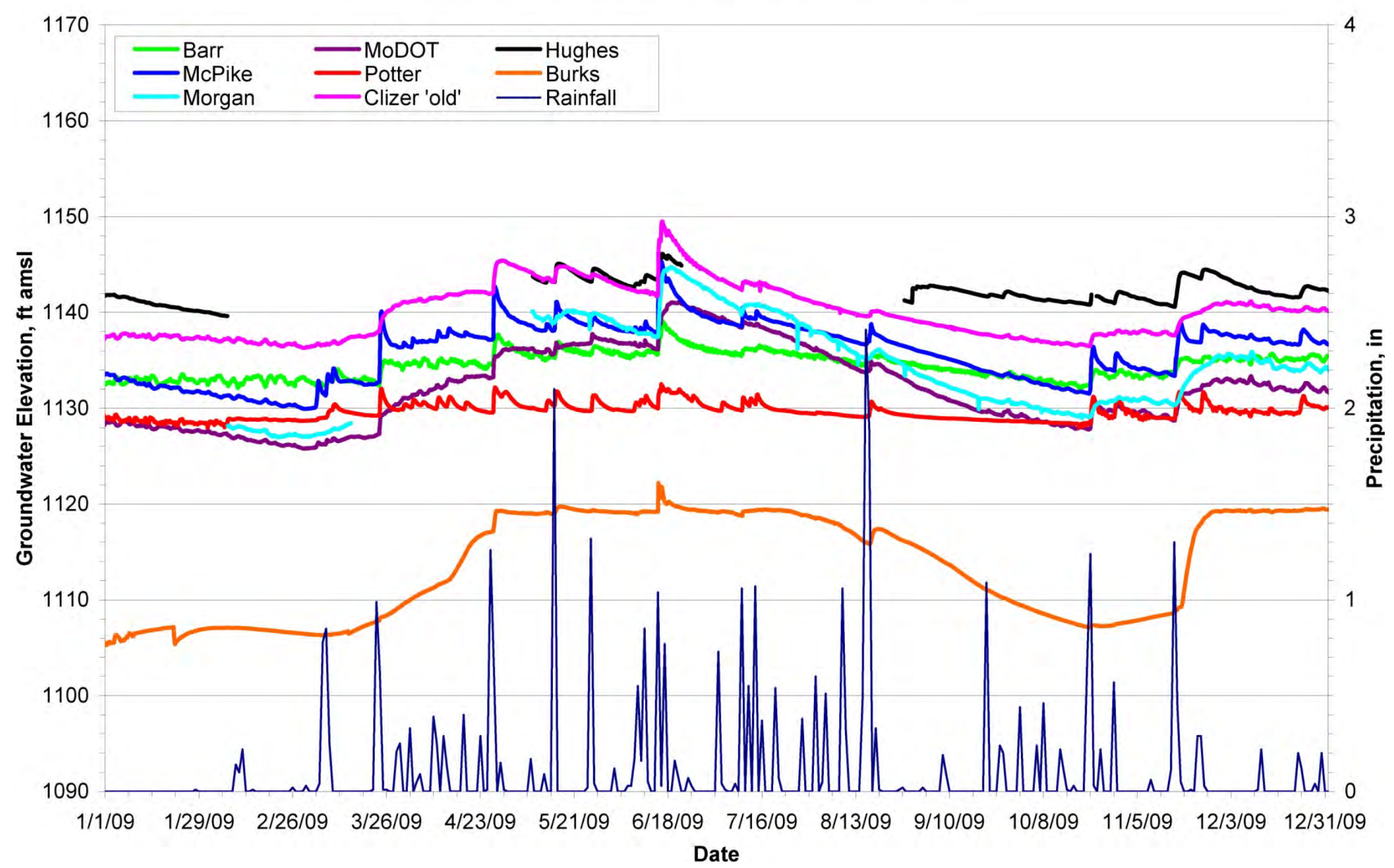

FIGURE 4.5 Hydrographs for wells in the private well monitoring network, January to December 2009. 
Savannah Private Well Water Levels - 2010

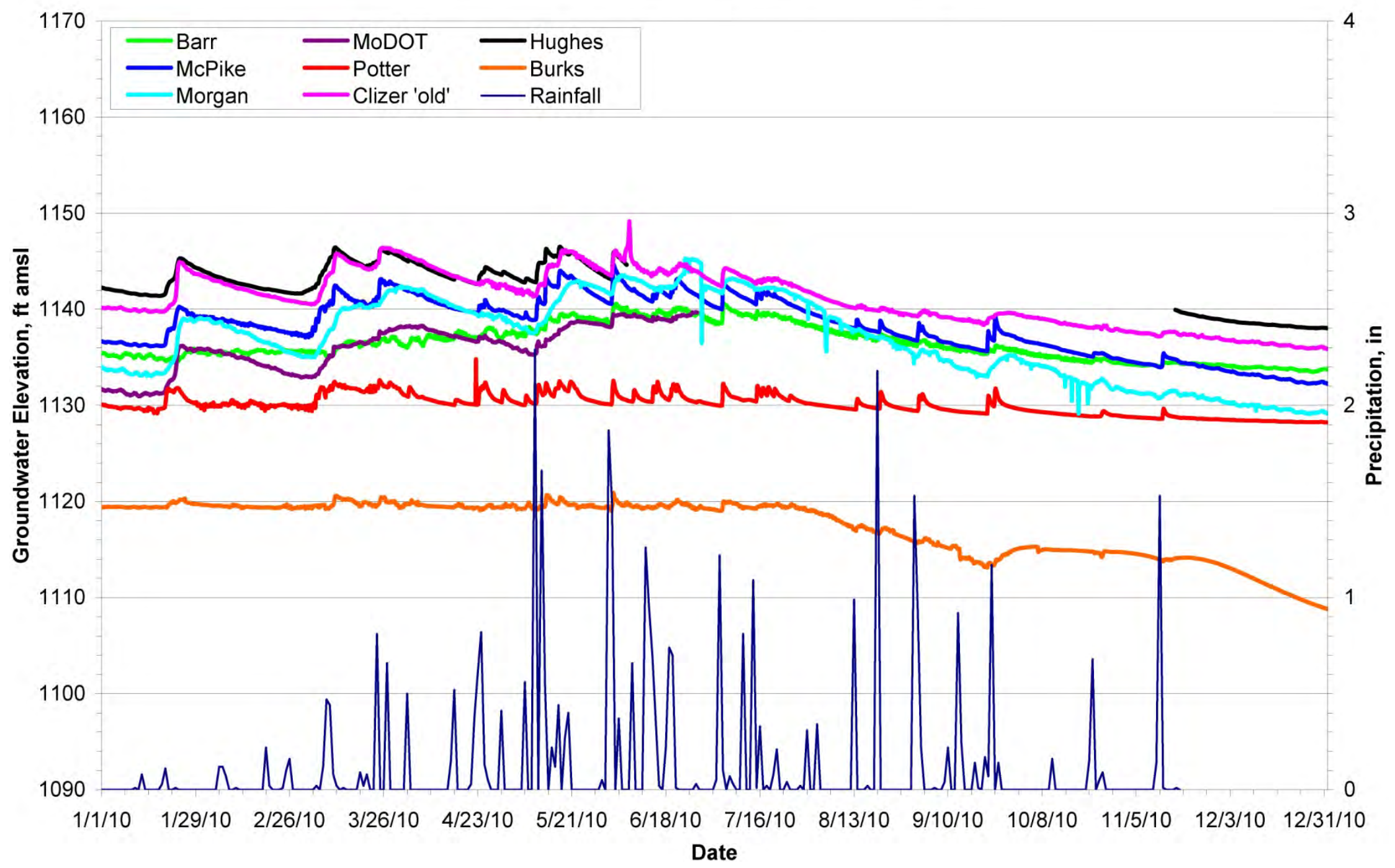

FIGURE 4.6 Hydrographs for wells in the private well monitoring network, January to December 2010. 
Savannah Private Well Water Levels - 2011

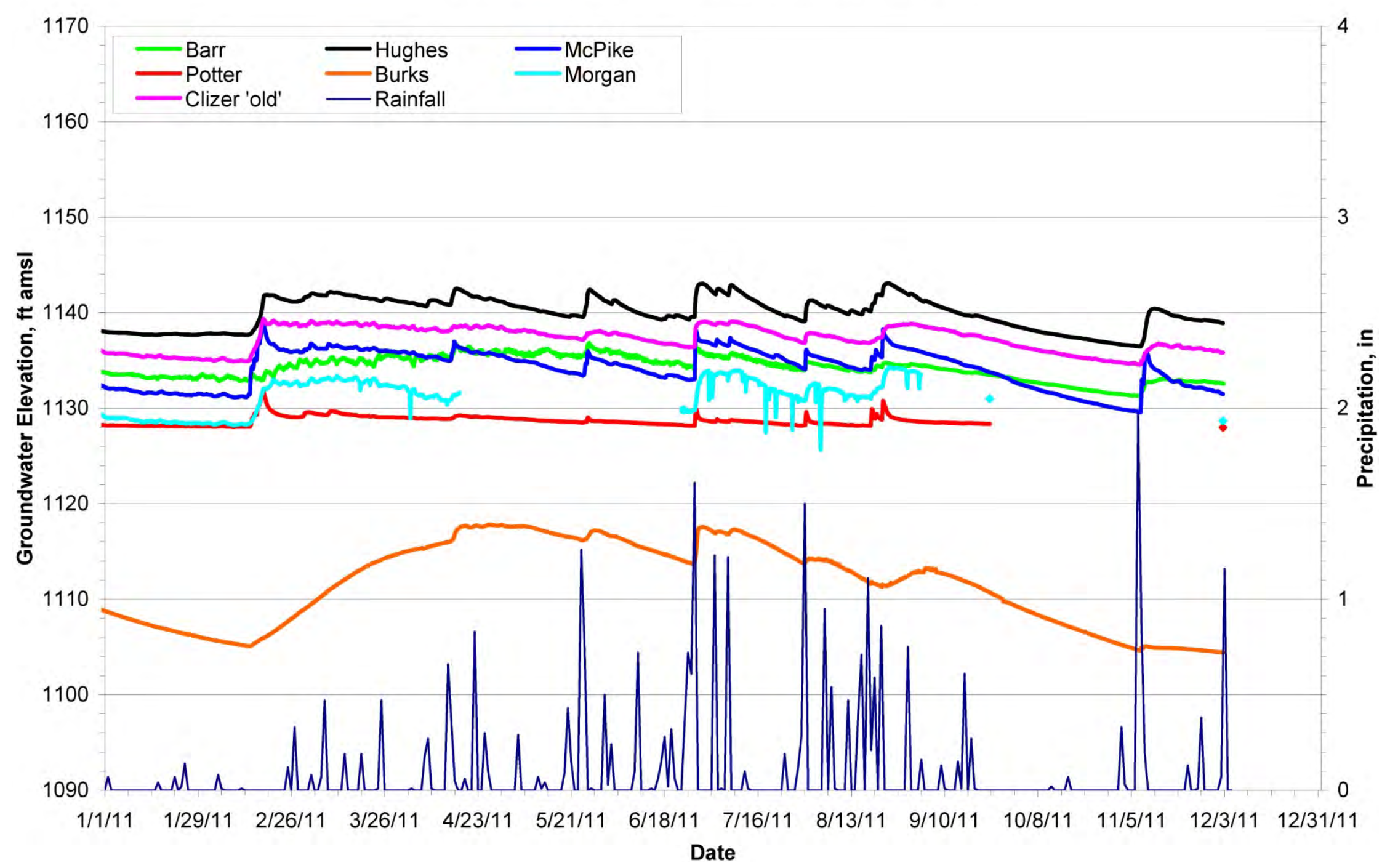

FIGURE 4.7 Hydrographs for wells in the private well monitoring network, January to December 2011. 


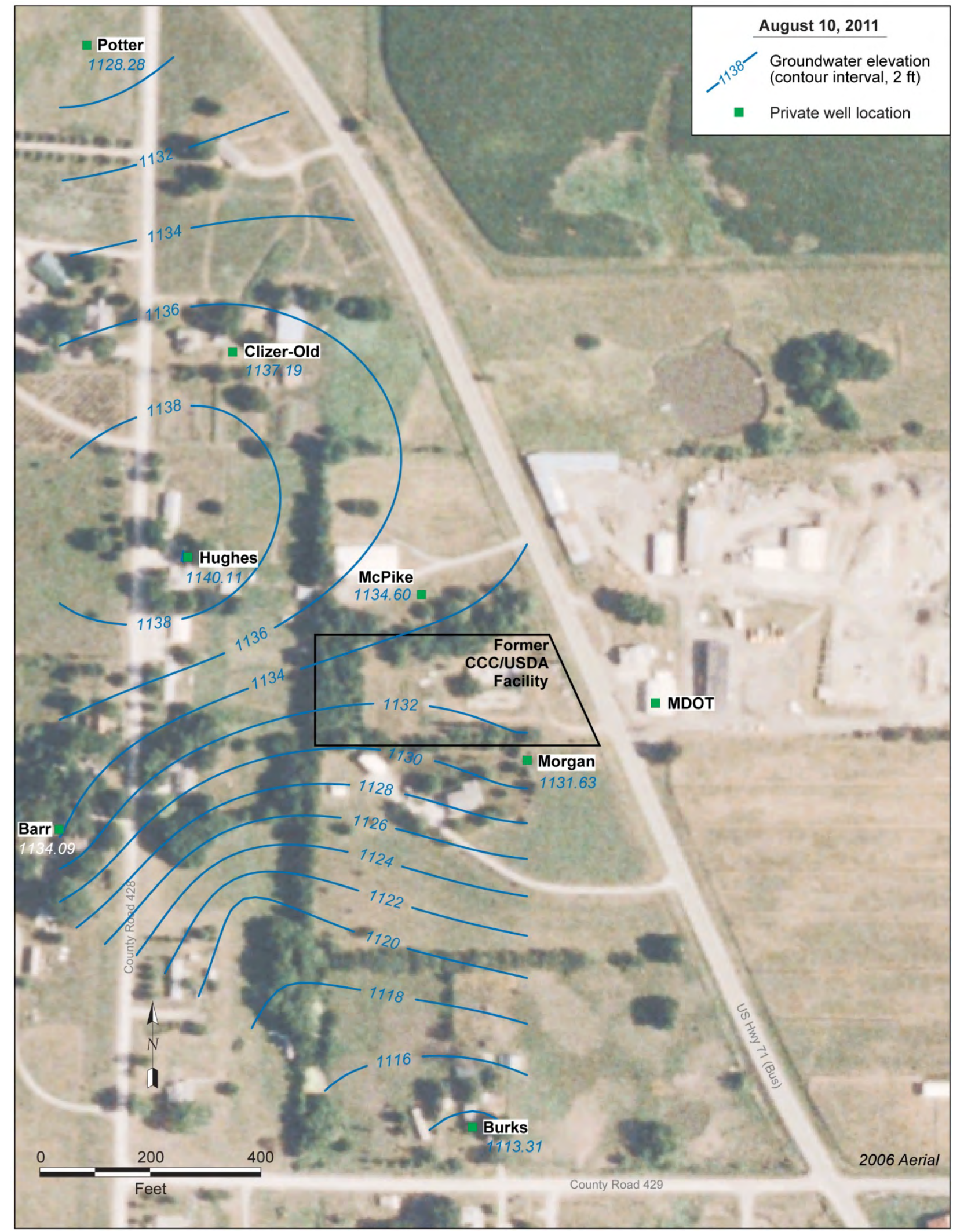

FIGURE 4.8 Water level contours constructed with data measured automatically in selected private wells on August 10, 2011. Source of aerial photograph: NAIP (2006). 
Savannah Shallow Monitoring Wells

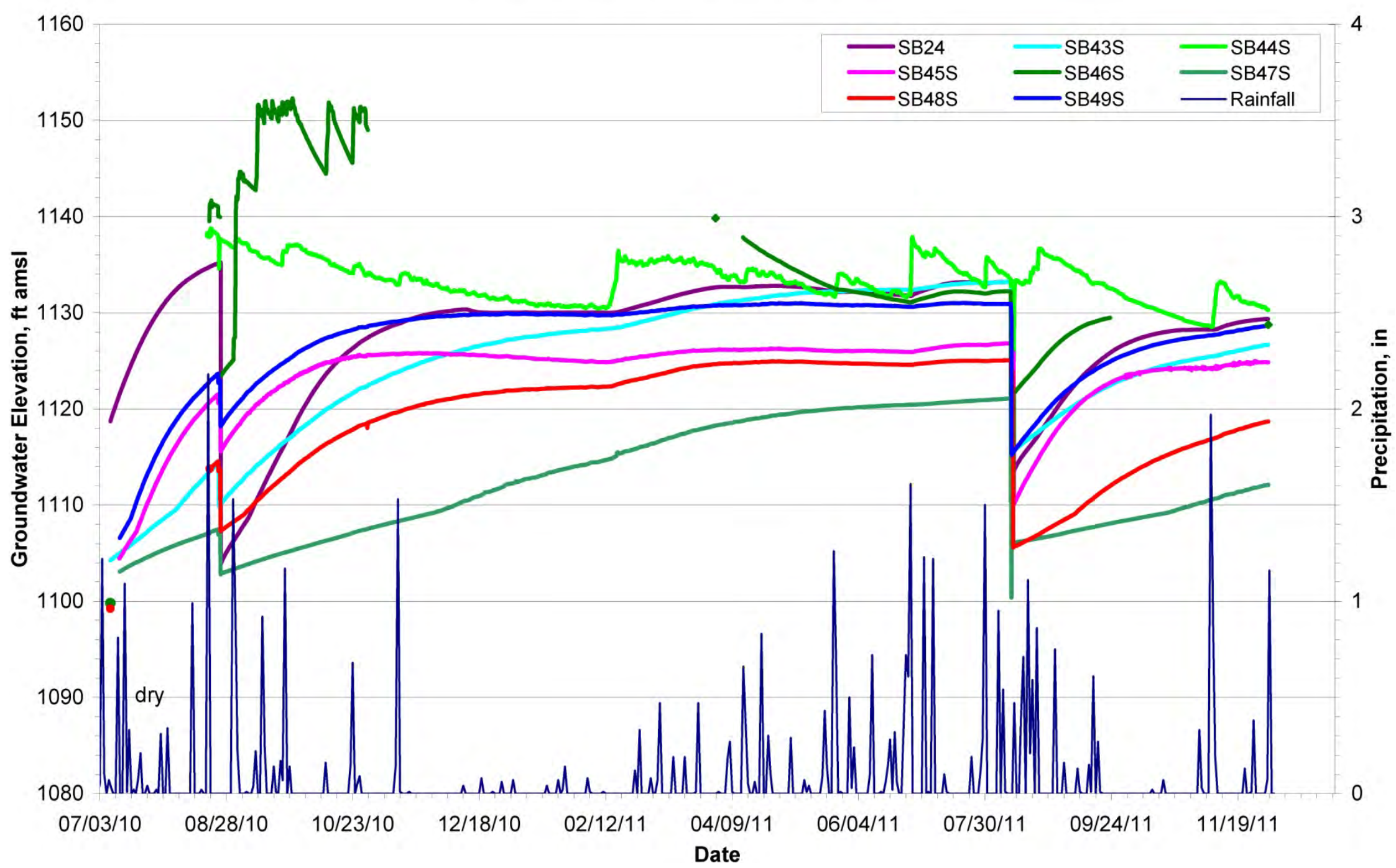

FIGURE 4.9 Hydrographs for shallow monitoring wells SB24 and SB43S-SB49S, July 2010 to December 2011. 


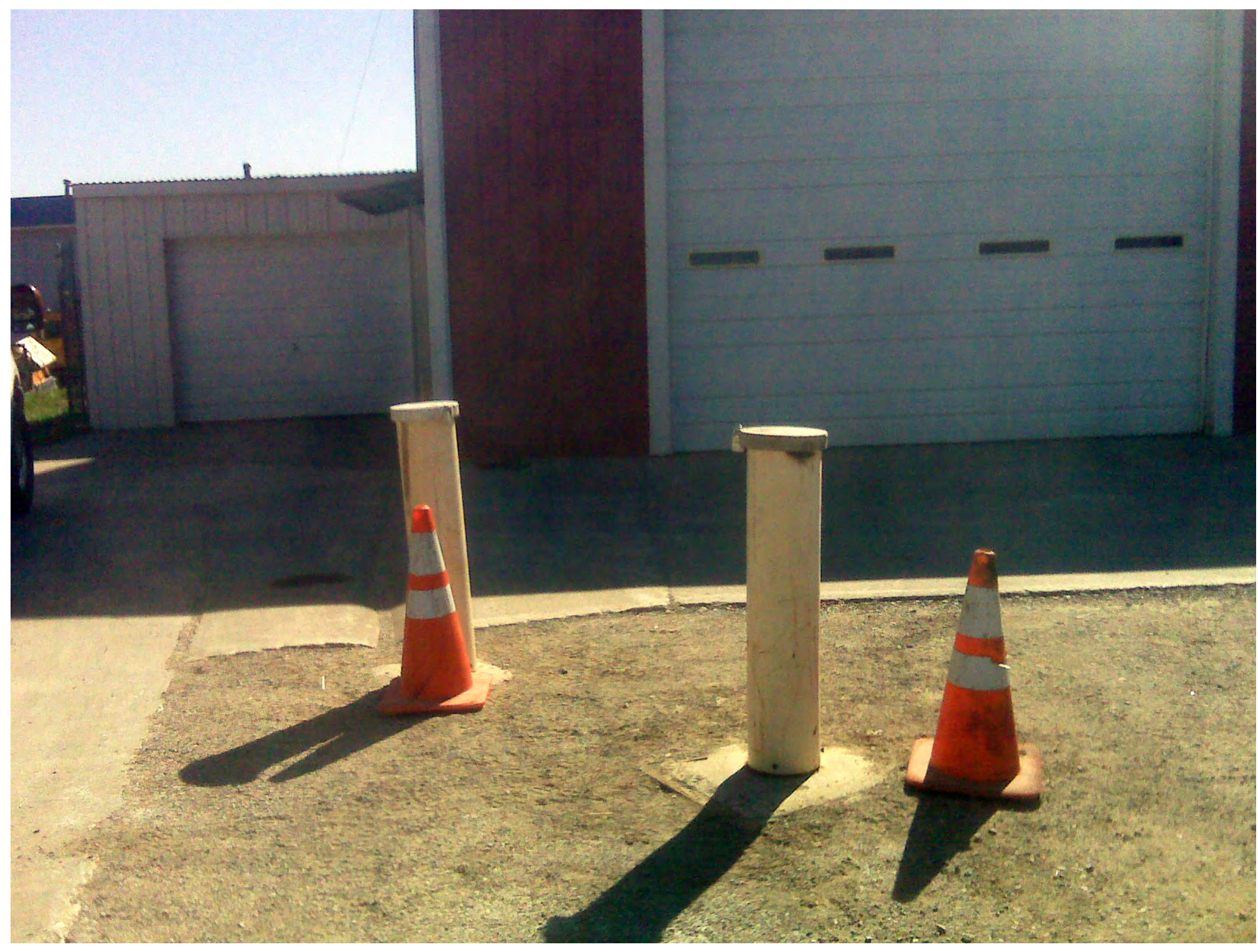

FIGURE 4.10 The temporary stick-up surface housings installed at wells SB46S and SB46D from April to September, 2011. 


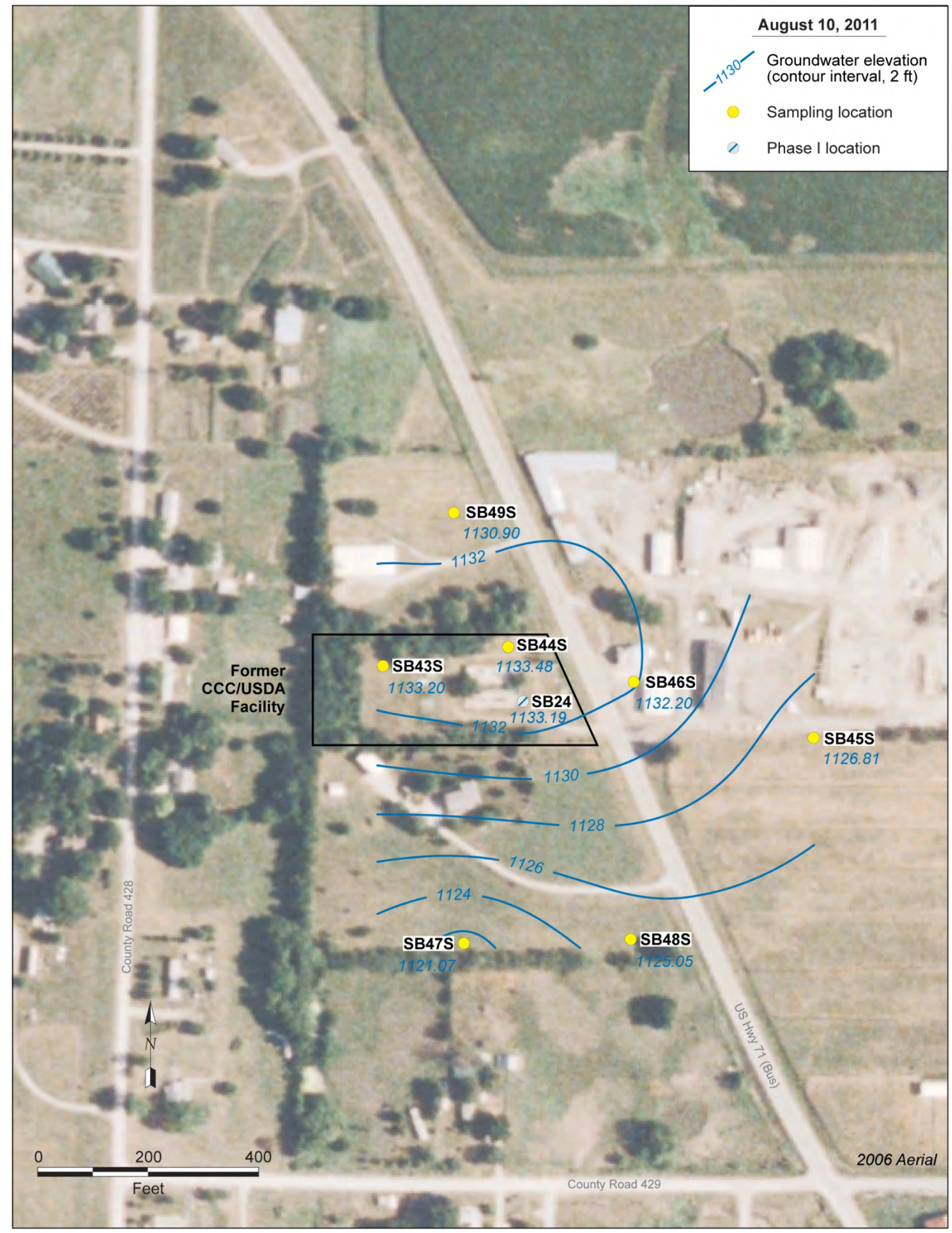

FIGURE 4.11 Water level contours constructed with data measured automatically in shallow monitoring wells SB24 and SB43S-SB49S on August 10, 2011. Source of aerial photograph: NAIP (2006). 


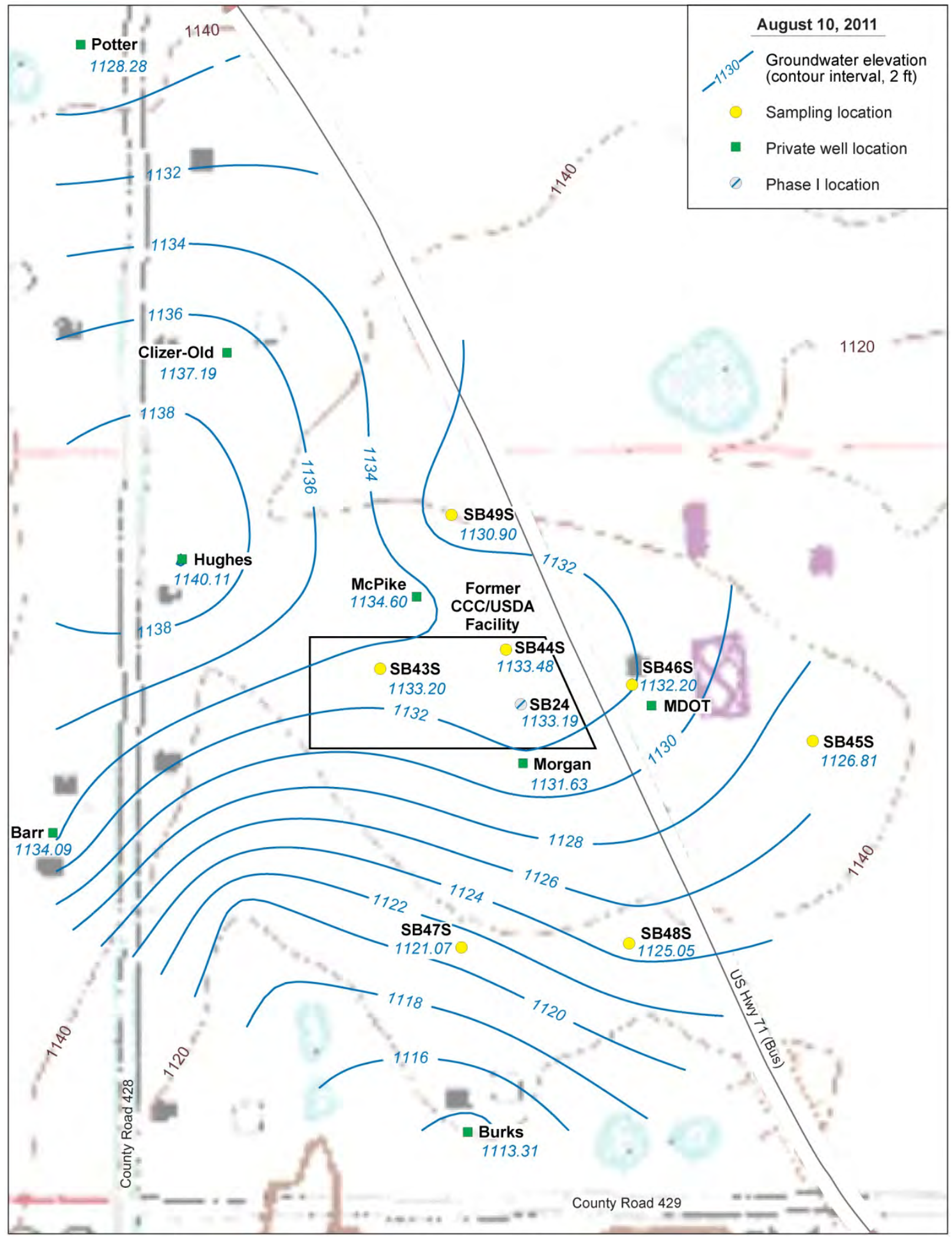

FIGURE 4.12 Water level contours constructed with data measured automatically in selected private wells and shallow monitoring wells SB24 and SB43S-SB49S on August 10, 2011. Source of topographic map: USGS (1996). 
Savannah Deep Monitoring Wells

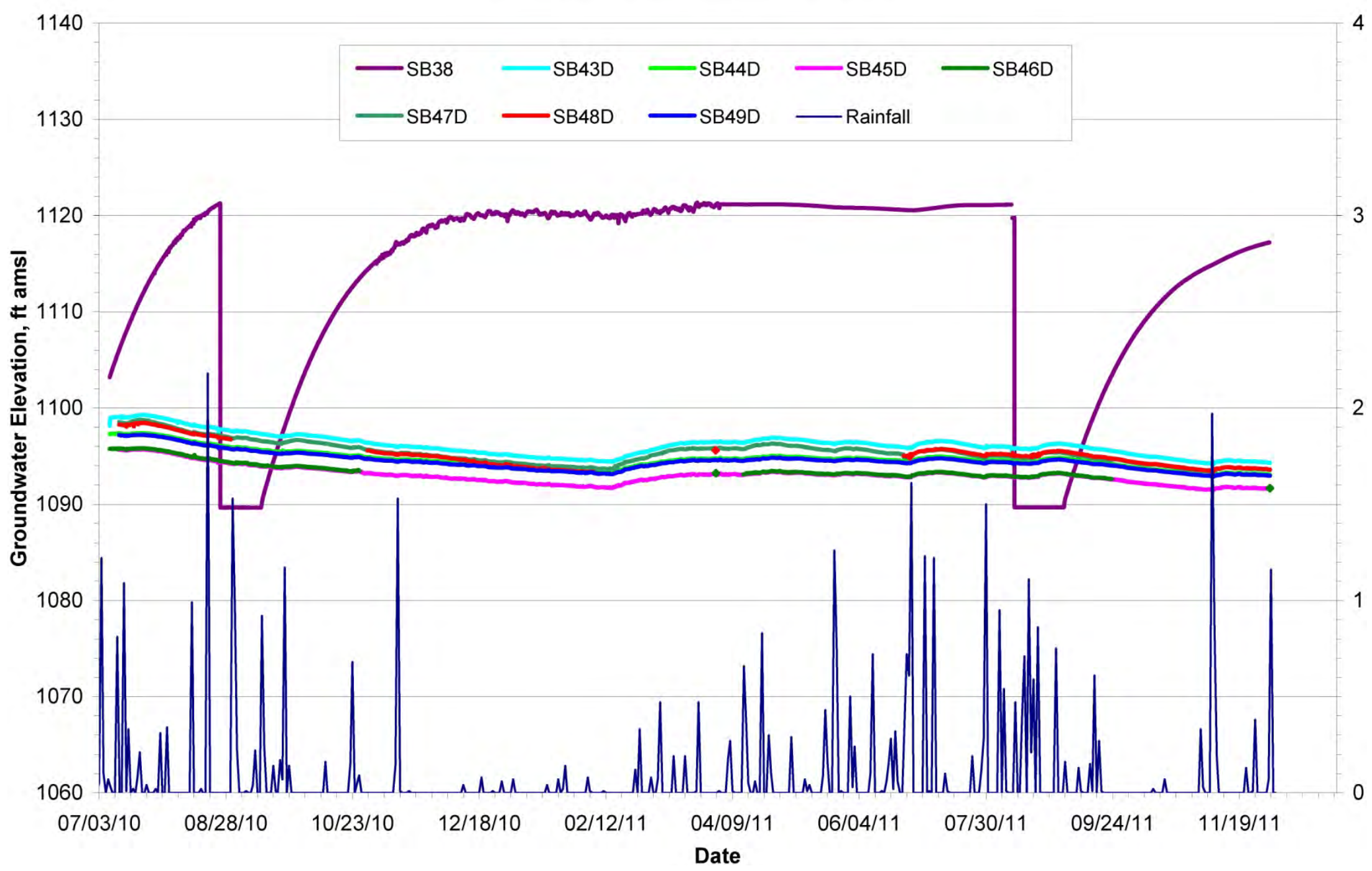

FIGURE 4.13 Hydrographs for deep monitoring wells SB38 and SB43D-SB49D, July 2010 to December 2011. 


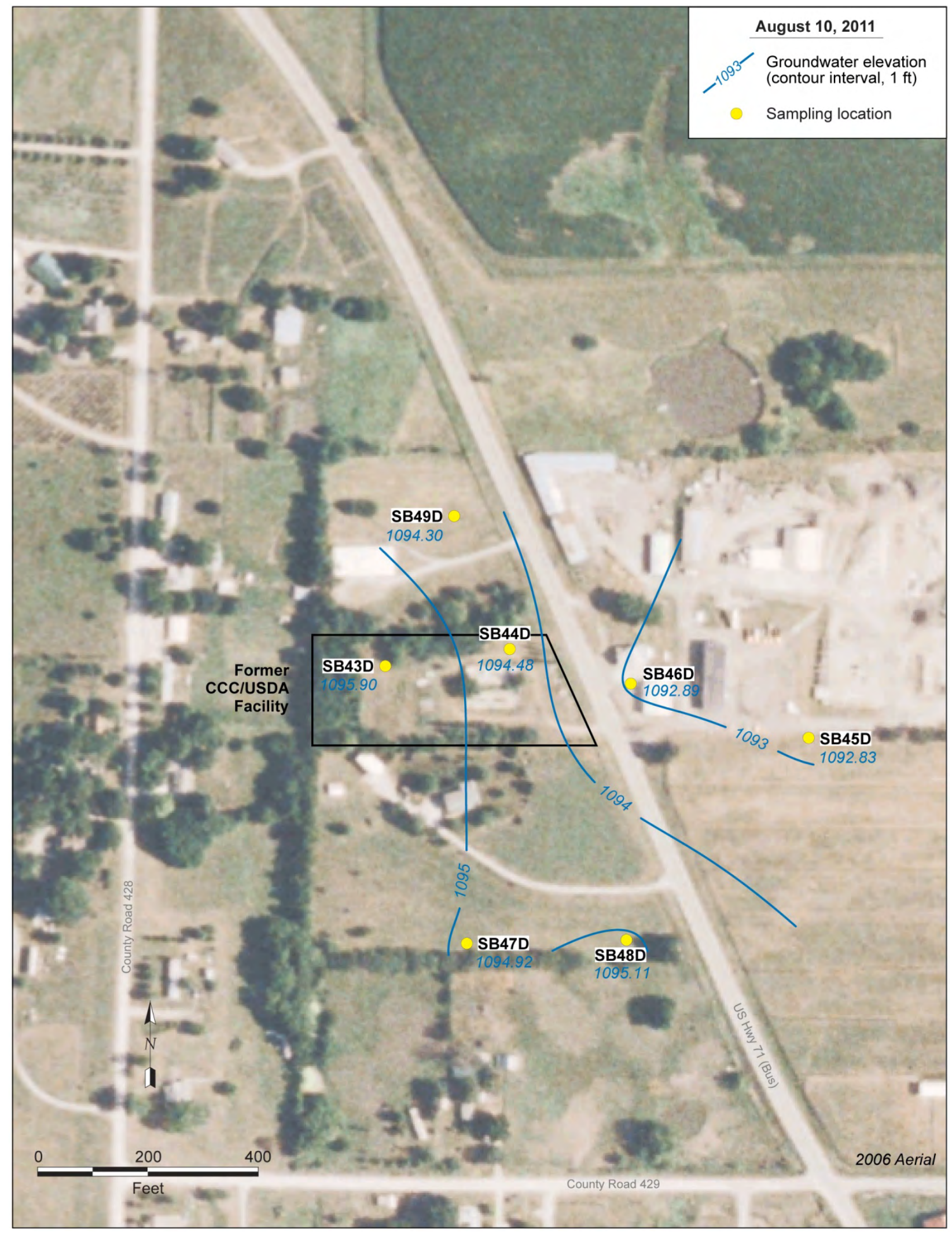

FIGURE 4.14 Water level contours constructed with data measured automatically in deep monitoring wells SB43D-SB49D on August 10, 2011. Source of aerial photograph: NAIP (2006). 
A

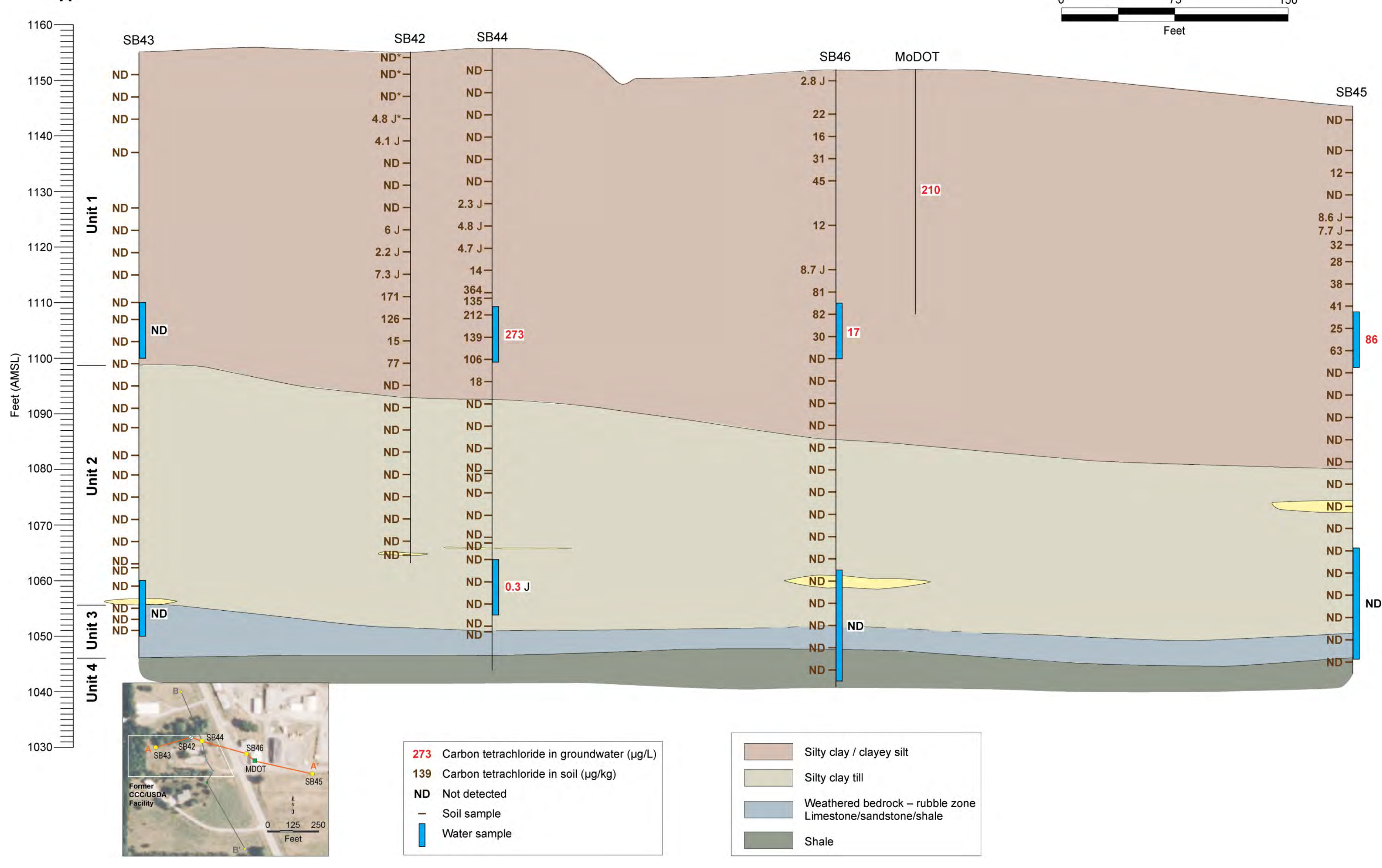


B

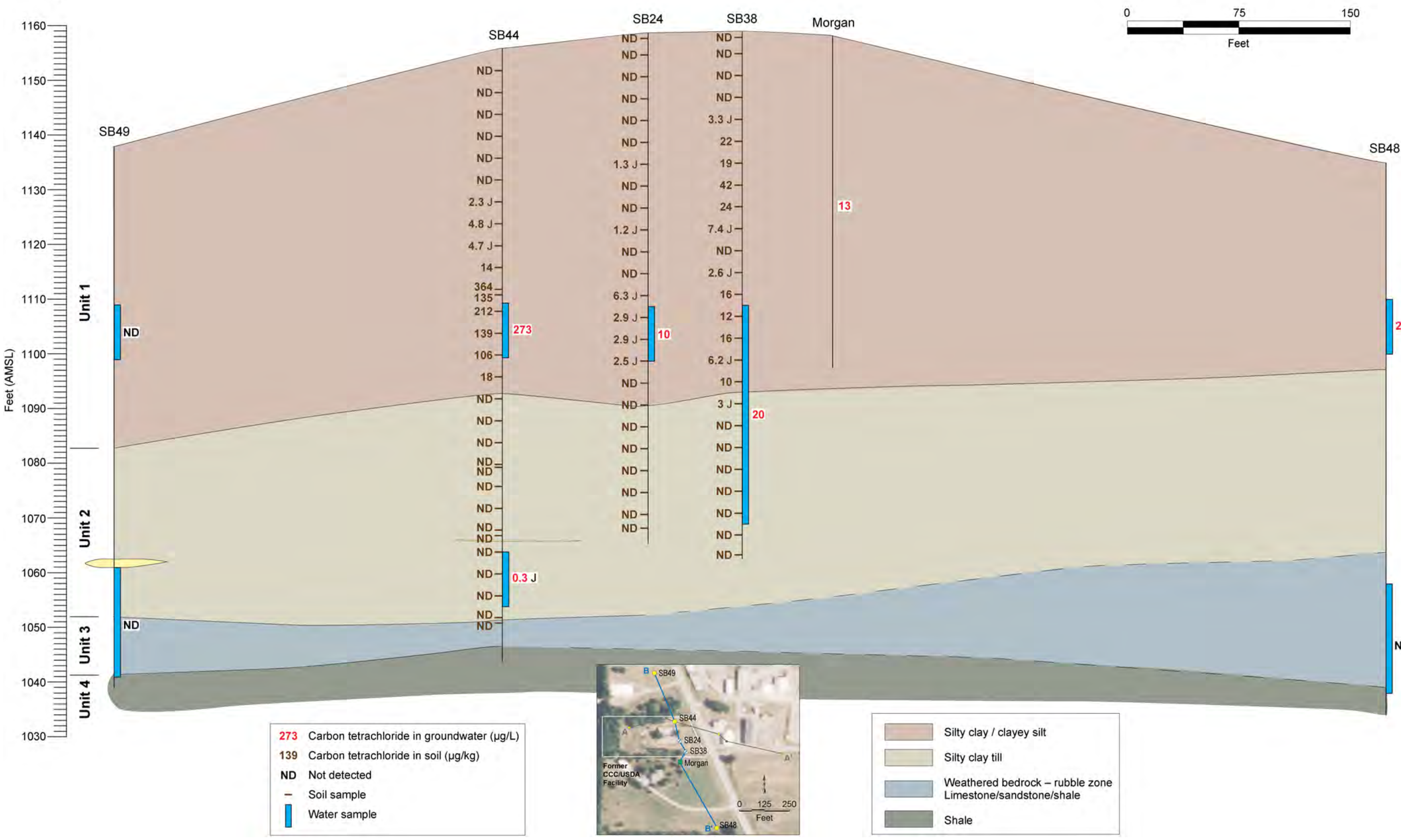

B'

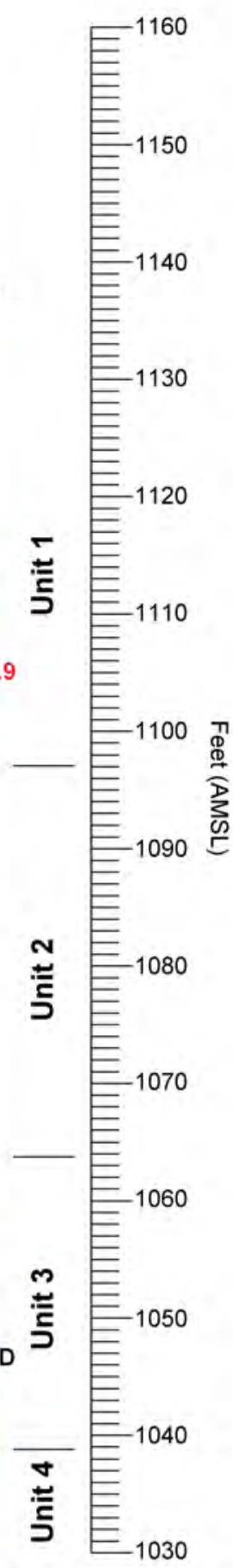

FIGURE 4.16 North-to-south hydrogeologic cross section B-B' (vertically exaggerated), showing the distribution of maximum carbon tetrachloride concentrations identified in soils and groundwater. 


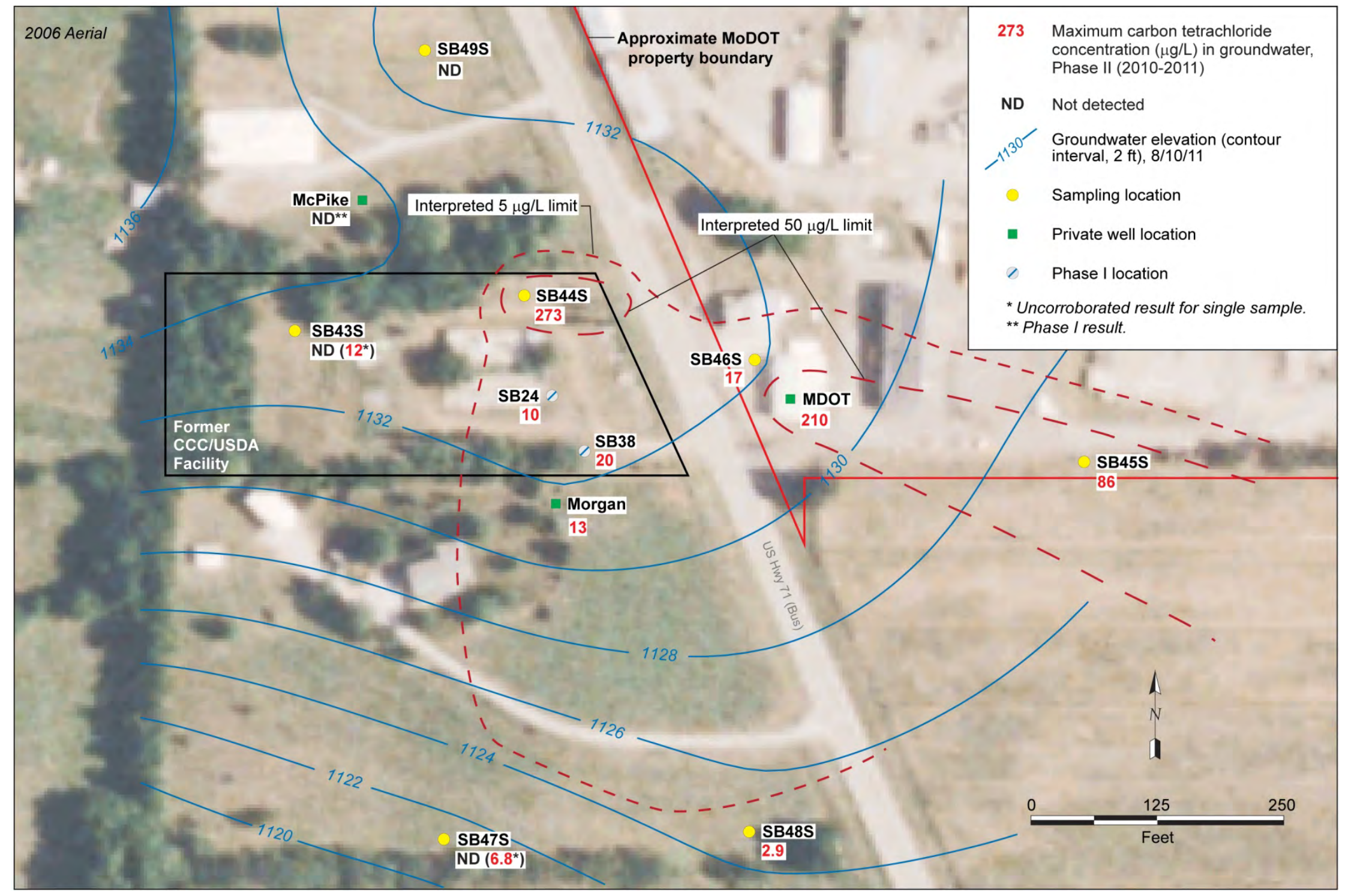

FIGURE 4.17 Water level contours for the private wells and shallow monitoring wells at 8:00 a.m. on August 10, 2011, constructed with data obtained from the automatic recorders, and maximum carbon tetrachloride concentrations identified in groundwater during the Phase II (20102011) sampling events. Source of aerial photograph: NAIP (2006). 


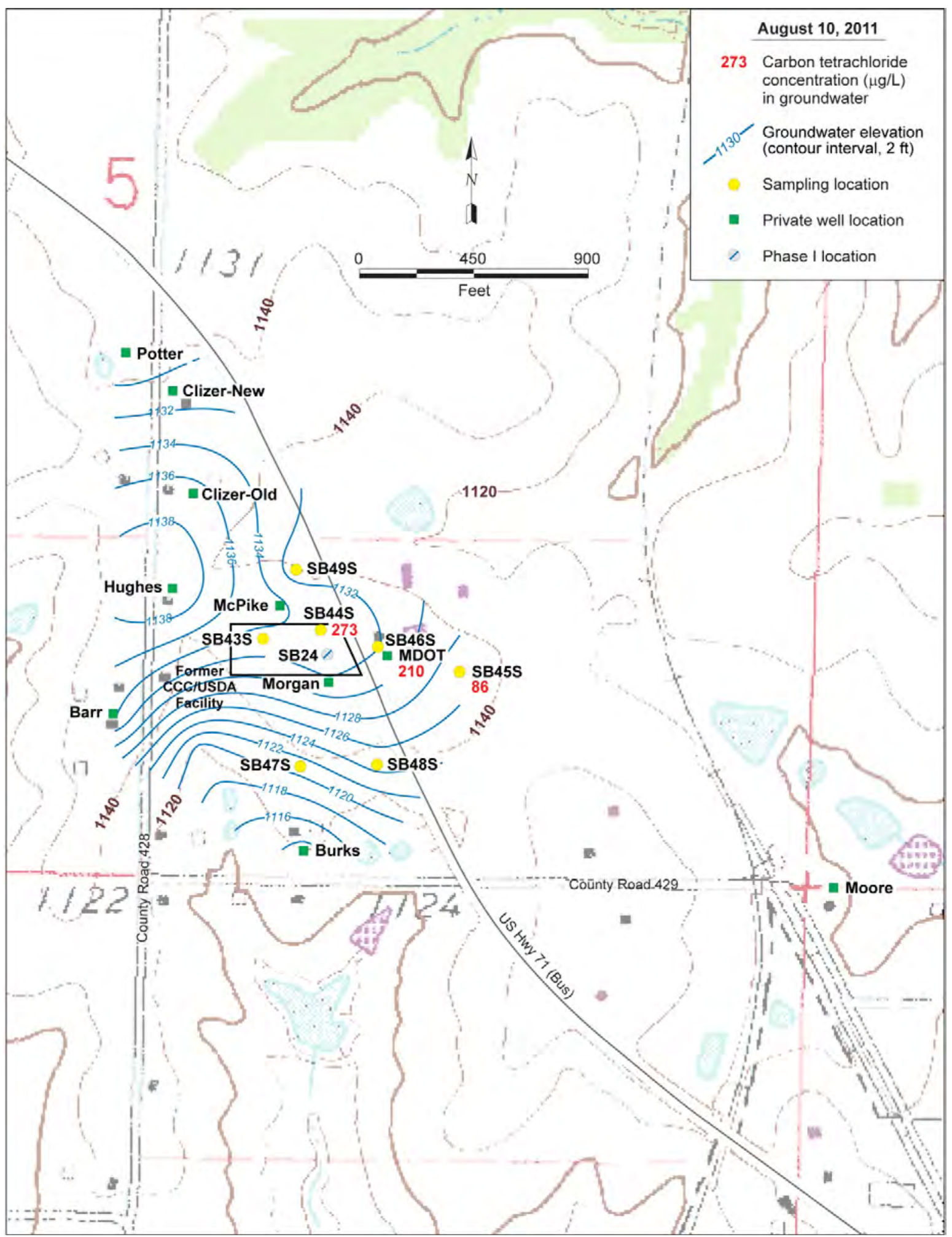

FIGURE 4.18 Water level contours for the private wells and shallow monitoring wells at 8:00 a.m. on August 10, 2011, constructed with data obtained from the automatic recorders, with locations of the maximum concentrations of carbon tetrachloride identified in groundwater and locations of private domestic wells in the vicinity of the former CCC/USDA facility. Source of topographic map: USGS (1996). 


\section{Conclusions and Recommendations}

\subsection{Conclusions}

The site-specific information obtained in the Phase II investigation at the former CCC/USDA grain storage facility at Savannah provides a basis for refinement of the preliminary conceptual model of the hydrogeologic setting and distribution of carbon tetrachloride contamination developed in Phase I. The principal features of the revised conceptual model are summarized as follows:

- The unconsolidated sequence above the shale bedrock in the study area is composed of three primary stratigraphic units. The upper two of these unconsolidated intervals are dominated by fine-grained and poorly sorted deposits, representing an upper unit of silty clay to clayey silt (37-72 ft thick; Unit 1) overlying a unit of calcareous glacial till (30-44 ft thick; Unit 2). Below the till, a weathered, heterogeneous rubble zone (4-24 ft thick) composed of platy to shattered limestones, sandy limestones, sandy dolomites, soft shale, silt, and clay (Unit 3) was identified overlying more competent shale bedrock (Unit 4).

- No areally extensive, coarse-grained intervals were identified in Unit 1 or Unit 2 above the basal rubble zone of Unit 3. One or more thin, discontinuous sandy horizons were observed in the till (Unit 2).

- The fine-grained character of Units 1 and 2, coupled with observed slow rates of groundwater accumulation in monitoring and private wells completed in these intervals, suggests that that the ability of these units to transmit groundwater is limited.

- The results of the Phase II study suggest that horizontal groundwater flow (and contaminant migration) occur preferentially in Unit 1 in a zone at elevations of approximately 1,098-1,110 ft AMSL. This zone approximately corresponds to the completion depths of many private wells in the vicinity of the former CCC/USDA facility. No distinctive variations in the lithologic 
characteristics of the sediments in this interval were readily identified, however.

- Groundwater level responses observed in the deep monitoring wells suggest that the hydraulic conductivity of the basal rubble zone (Unit 3) is greater than that of Units 1 and 2; however, no known private wells in the vicinity of the former CCC/USDA facility penetrate the Unit 3 rubble.

- Extended automated groundwater monitoring indicated relatively little net change in the distribution of saturated conditions in the unconsolidated sediments throughout the investigation, with depths to saturation of approximately 18-22 ft beneath the former CCC/USDA facility and the adjacent MoDOT property.

- Lateral groundwater flow patterns in the upper portion of Unit 1 closely mirror the local ground surface topography. The former CCC/USDA facility lies near the crest of a northwest-southeast trending topographic and groundwater divide that extends beneath the former CCC/USDA facility and the adjacent MoDOT property. Groundwater flow in this interval is predominantly to the south-southwest beneath much of the former CCC/USDA facility; flow to the southeast, east, or northeast is also possible from the eastern portion of the former facility.

- Groundwater flow in the rubble zone (Unit 3) is predominantly to the east in the vicinity of the former CCC/USA facility.

- Groundwater level relationships among the shallow and deep monitoring wells confirm the presence of vertical hydraulic gradients between the upper portion of the silty clay to clayey silt (Unit 1) and the basal rubble zone (Unit 3). Limited vertical hydraulic conductivity data suggest, however, that the potential rates of vertical groundwater flow in response to these driving forces are limited.

- No carbon tetrachloride has been detected in the subsurface (vadose zone) soils as defined by the MDNR (2006), at concentrations greater than the DTL 
(79.6 $\mu \mathrm{g} / \mathrm{kg}$ ) for this contaminant - or, with only two exceptions, at levels above the quantitation limit of $10 \mu \mathrm{g} / \mathrm{kg}$ for the purge-and-trap analysis method. The MDNR defines the "subsurface" as the vadose zone from $3 \mathrm{ft}$ BGL to the water table (saturation).

- In the saturated zone, the presence of carbon tetrachloride in the soils might reflect contamination that is (1) adsorbed to the solid soil particles, (2) dissolved in the pore water contained in the soils, or (3) in a combination of these two physical states. Carbon tetrachloride was identified at concentrations above the DTL value in the deeper soils at two of the Phase II boring locations, SB44 and SB46, and at Phase I location SB42.

- At SB44, carbon tetrachloride concentrations of $106-364 \mu \mathrm{g} / \mathrm{kg}$ in soil were detected at 44-56 ft BGL, confirming the elevated levels of this contaminant (77-171 $\mu \mathrm{g} / \mathrm{kg}$ ) previously identified at these depths in adjacent boring SB42. No quantifiable concentrations of carbon tetrachloride were identified in the soils above or below this depth interval at SB42 or SB44.

- At SB46, carbon tetrachloride was identified in soil at concentrations of $81-82 \mu \mathrm{g} / \mathrm{kg}$ at $40-44 \mathrm{ft}$ BGL. No contamination was detected in the deeper soils (> $48 \mathrm{ft}$ BGL) at this location; however, a continuous distribution of carbon tetrachloride was documented from the ground surface to the depth of maximum contamination at SB46, suggesting possible evidence of carbon tetrachloride entry into the vadose zone (and deeper) soils in this vicinity.

- No carbon tetrachloride was detected in soils at any location in the deeper portion of Unit 1 or the underlying till (Unit 2) and rubble zone (Unit 3), suggesting that these intervals might impede contaminant migration from the shallower sediments into the deeper portions of the unconsolidated sequence. In keeping with this interpretation, no carbon tetrachloride was detected in groundwater in the deep monitoring wells.

- The carbon tetrachloride contamination hot spot identified in the Unit 1 soils at borings SB42 and SB44 (at 44-56 ft BGL) is reflected in the groundwater at 
shallow monitoring well SB44S, where a maximum carbon tetrachloride concentration of $273 \mu \mathrm{g} / \mathrm{L}$ was identified.

- The maximum carbon tetrachloride concentrations identified at monitoring wells SB24 $(10 \mu \mathrm{g} / \mathrm{L})$, SB38 $(20 \mu \mathrm{g} / \mathrm{L})$, and SB46S $(17 \mu \mathrm{g} / \mathrm{L})$, as well as at the Morgan private well $(13 \mu \mathrm{g} / \mathrm{L})$, suggest that the levels of this contaminant in groundwater in Unit 1 decrease rapidly away from the SB42/SB44 hot spot, in the identified directions of groundwater flow.

- Relatively high maximum concentrations of carbon tetrachloride in groundwater were identified in Unit 1 at the MoDOT well $(210 \mu \mathrm{g} / \mathrm{L})$ and shallow monitoring well SB45S $(86 \mu \mathrm{g} / \mathrm{L})$, to the south-southeast and downgradient from the former CCC/USDA facility. An association of this contamination with the hot spot identified at borings SB42/SB44 cannot be ruled out; however, the identification of residual carbon tetrachloride contamination in the vadose zone soils at SB46S, coupled with the proximity of this boring to the MoDOT well, suggests the possibility of an additional source of carbon tetrachloride contamination to the groundwater in the vicinity of the MoDOT and SB46S wells.

- Data for VOCs, DO, and ORP generated in the 2010-2011 sampling events provide limited evidence for natural degradation of carbon tetrachloride in groundwater in Unit 1.

- A preliminary evaluation of the carbon tetrachloride concentrations and distribution at Savannah suggests that the contamination identified in soils poses no current risks and only minimal potential future risks to human health. The identified contamination in groundwater also poses no current risks and minimal potential future risks to human health via exposure pathways associated with the possible domestic use of groundwater. The present data suggest, however, that the two vehicle maintenance buildings on the MoDOT property and the Morgan residence might be susceptible to vapor intrusion to indoor air from the contaminated groundwater. As of this date, however, the MDNR has not established target contaminant levels for the evaluation of potential indoor air contamination by carbon tetrachloride. 


\subsection{Recommendations}

The results of the Phase I and Phase II studies at Savannah demonstrate that carbon tetrachloride is present in soils and groundwater at concentrations that exceed the respective DTL values for these media. The data further suggest, however, that the identified contamination poses little risk to human health under present or reasonably anticipated future conditions of land use and groundwater use for potentially domestic purposes.

On the basis of these observations, periodic monitoring of the contaminant distribution in groundwater is recommended, at locations and with a frequency to be established in consultation with the MDNR and CCC/USDA program managers.

Investigation of the potential vapor intrusion pathway, particularly at the Morgan private residence, may also be warranted as the MDNR establishes criteria for the quantitative evaluation of this contaminant in indoor air. 


\section{References}

Argonne, 2002, Final Master Work Plan: Environmental Investigations at Former CCC/USDA Facilities in Kansas, 2002 Revision, ANL/ER/TR-02/004, prepared for the Commodity Credit Corporation, U.S. Department of Agriculture, Washington, D.C., by Argonne National Laboratory, Argonne, Illinois, December.

Argonne, 2007, Final Work Plan: Phase I Investigation of Potential Contamination at the Former CCC/USDA Grain Storage Facility in Savannah, Missouri, ANL/EVS/AGEM/TR-0709, prepared for the Commodity Credit Corporation, U.S. Department of Agriculture, Washington, D.C., by Argonne National Laboratory, Argonne, Illinois, September.

Argonne, 2010a, Final Report: Phase I Investigation at the Former CCC/USDA Grain Storage Facility in Savannah, Missouri, ANL/EVS/AGEM/TR-08-12, prepared for the Commodity Credit Corporation, U.S. Department of Agriculture, Washington, D.C., by Argonne National Laboratory, Argonne, Illinois, June.

Argonne, 2010b, Final Work Plan: Phase II Investigation of Potential Contamination at the Former CCC/USDA Grain Storage Facility in Savannah, Missouri, ANL/EVS/AGEM/TR-0907, prepared for the Commodity Credit Corporation, U.S. Department of Agriculture, Washington, D.C., by Argonne National Laboratory, Argonne, Illinois, June.

EPA, 1994, USEPA Contract Laboratory Program National Functional Guidelines for Organic Data Review, EPA 540/R-94/012, U.S. Environmental Protection Agency, Office of Emergency and Remedial Response, Washington, D.C., February.

EPA, 1995, Method 524.2: Measurement of Purgeable Organic Compounds in Water by Capillary Column Gas Chromatography/Mass Spectrometry, Revision 4.1, edited by J.W. Munch, National Exposure Research Laboratory, Office of Research and Development, U.S. Environmental Protection Agency, Cincinnati, Ohio.

EPA, 1998a, Technical Protocol for Evaluating Natural Attenuation of Chlorinated Solvents in Groundwater, EPA/600/R-98/128, U.S. Environmental Protection Agency, Office of Research and Development, Washington, D.C. 
EPA, 1998b, Test Methods for Evaluating Solid Waste: Physical/Chemical Methods, EPA SW846, 3rd edition, Draft Update IVA, U.S. Environmental Protection Agency, January (available from National Technical Information Service, 5285 Port Royal Road, Springfield, Virginia, 22161).

EPA, 1999, private well sampling data pages for Savannah, Missouri, U.S. Environmental Protection Agency, Washington, D.C.

MDNR, 2000a, Preliminary Assessment Report, Savannah Former CCC/USDA Grain Bins Andrew County, Missouri MOSFN0703496, Hazardous Waste Program, Division of Environmental Quality, Missouri Department of Natural Resources, Jefferson City, Missouri, March 31.

MDNR, 2000b, Site Inspection Report, Savannah Former CCC/USDA Grain Bins Andrew County, Missouri MOSFN0703496, Hazardous Waste Program, Division of Environmental Quality, Missouri Department of Natural Resources, Jefferson City, Missouri, September 28.

MDNR, 2006, Missouri Risk-Based Corrective Action (MRBCA): Departmental Technical Guidance, Division of Environmental Quality, Missouri Department of Natural Resources, Jefferson City, Missouri, final draft with updates. (http://www.dnr.mo.gov/env/hwp/mrbca/ mrbca.htm).

MDNR, 2007, letter from S. Muenks (Hazardous Waste Program, Missouri Department of Natural Resources, Jefferson City, Missouri) to S. Gilmore (Commodity Credit Corporation, U.S. Department of Agriculture, Washington, D.C.), indicating MDNR approval of the Phase I Work Plan for the Savannah investigation, September 26.

MDNR, 2008, letter from S. Muenks (Hazardous Waste Program, Missouri Department of Natural Resources, Jefferson City, Missouri) to S. Gilmore (Commodity Credit Corporation, U.S. Department of Agriculture, Washington, D.C.), regarding the draft report for the Savannah Phase I investigation, October 1.

MDNR, 2009, letter from S. Muenks (Hazardous Waste Program, Missouri Department of Natural Resources, Jefferson City, Missouri) to S. Gilmore (Commodity Credit Corporation, 
U.S. Department of Agriculture, Washington, D.C.), regarding CCC/USDA responses to MDNR comments on the draft report for the Savannah Phase I investigation, May 6.

MDNR, 2010, letter from S. Muenks (Hazardous Waste Program, Missouri Department of Natural Resources, Jefferson City, Missouri) to S. Gilmore (Commodity Credit Corporation, U.S. Department of Agriculture, Washington, D.C.), indicating MDNR approval of the Phase II Work Plan for the Savannah investigation, June 16.

MUE, 2011, archived daily rainfall data for the St. Joseph, Missouri, weather station, Missouri Historical Agricultural Weather Database, Missouri University Extension (http://agebb.missouri.edu/weather/history/index.asp).

NAIP, 2006, Compressed County Mosaic, Andrew County, Missouri, naip_11_2n_s_mo003_2006_1, National Agriculture Imagery Program, Aerial Photography Field Office, Farm Service Agency, U.S. Department of Agriculture, Salt Lake City, Utah, August 16.

NAIP, 2007, Compressed County Mosaic, Andrew County, Missouri, ortho_11_1n_s_mo003_2007_1, National Agriculture Imagery Program, Aerial Photography Field Office, Farm Service Agency, U.S. Department of Agriculture, Salt Lake City, Utah, August 21.

USDA, 1960, aerial photograph CCC-YAA-1960C, U.S. Department of Agriculture, Washington, D.C., November 17.

USGS, 1996, Savannah, digital raster graphic of 7.5-minute topographic map dated 1978, U.S. Geological Survey, Reston, Virginia, processed December 19. 


\section{Appendix A:}

Sequence of Sampling Events in Phase II (2010-2011) 
TABLE A.1 Sequence of sampling activities during the Phase II investigation.

\begin{tabular}{|c|c|c|c|c|c|c|c|c|c|}
\hline $\begin{array}{l}\text { Sample } \\
\text { Date }\end{array}$ & $\begin{array}{c}\text { Sample } \\
\text { Time }\end{array}$ & Sample & $\begin{array}{l}\text { Sample } \\
\text { Medium }\end{array}$ & $\begin{array}{c}\text { Sample } \\
\text { Type }\end{array}$ & $\begin{array}{l}\text { Sample } \\
\text { Location }\end{array}$ & $\begin{array}{c}\text { Depth } \\
\text { (ft BGL) }\end{array}$ & $\begin{array}{l}\text { Chain of } \\
\text { Custody }\end{array}$ & $\begin{array}{l}\text { Shipping } \\
\text { Date }\end{array}$ & Sample Description \\
\hline $6 / 21 / 10$ & $12: 11$ & SVSB43-S-25640 & Soil & СРТ & SB43 & 4 & 2873 & $6 / 21 / 10$ & \\
\hline $6 / 21 / 10$ & $12: 17$ & SVSB43-S-25641 & Soil & СРТ & SB43 & 12 & 2873 & $6 / 21 / 10$ & \\
\hline $6 / 21 / 10$ & $12: 23$ & SVSB43-S-25642 & Soil & СРТ & SB43 & 8 & 2873 & $6 / 21 / 10$ & \\
\hline $6 / 21 / 10$ & $12: 30$ & SVSB43-S-25643 & Soil & $\mathrm{CPT}$ & SB43 & 18 & 2873 & $6 / 21 / 10$ & \\
\hline $6 / 21 / 10$ & $12: 40$ & SVSB43-S-NO REC 20 & Soil & СРТ & SB43 & 20 & - & - & No recovery. \\
\hline $6 / 21 / 10$ & $12: 45$ & SVSB43-S-NO REC 24 & Soil & СРТ & SB43 & 24 & - & - & No recovery. \\
\hline $6 / 21 / 10$ & $13: 00$ & SVSB43-S-25644 & Soil & СРT & SB43 & 28 & 2873 & $6 / 21 / 10$ & \\
\hline $6 / 21 / 10$ & $13: 02$ & SVSB43-S-25645 & Soil & CPT & SB43 & 32 & 2873 & $6 / 21 / 10$ & \\
\hline $6 / 21 / 10$ & $13: 28$ & SVSB43-S-25646 & Soil & СРТ & SB43 & 36 & 2873 & $6 / 21 / 10$ & \\
\hline $6 / 21 / 10$ & $13: 32$ & SVSB43-S-25647 & Soil & $\mathrm{CPT}$ & SB43 & 40 & 2873 & $6 / 21 / 10$ & \\
\hline $6 / 21 / 10$ & $13: 40$ & SVSB43-S-NO REC 44 & Soil & СРТ & SB43 & 44 & - & - & No recovery. \\
\hline $6 / 21 / 10$ & $14: 15$ & SVSB43-S-25649 & Soil & СРТ & SB43 & 45 & 2873 & $6 / 21 / 10$ & \\
\hline $6 / 21 / 10$ & $14: 18$ & SVSB43-S-25648 & Soil & $\mathrm{CPT}$ & SB43 & 48 & 2873 & $6 / 21 / 10$ & \\
\hline $6 / 21 / 10$ & $14: 18$ & SVSB43-S-25650 & Soil & CPT & SB43 & 48 & 2873 & $6 / 21 / 10$ & Replicate of sample SVSB43-S-25648. \\
\hline $6 / 21 / 10$ & $14: 27$ & SVSB43-S-25651 & Soil & СРТ & SB43 & 52 & 2873 & $6 / 21 / 10$ & \\
\hline $6 / 21 / 10$ & $15: 00$ & SVSB43-S-25652 & Soil & CPT & SB43 & 56 & 2873 & $6 / 21 / 10$ & \\
\hline $6 / 21 / 10$ & $15: 03$ & SVSB43-S-25653 & Soil & $\mathrm{CPT}$ & SB43 & 60 & 2873 & $6 / 21 / 10$ & \\
\hline $6 / 21 / 10$ & $15: 08$ & SVSB43-S-25654 & Soil & СРT & SB43 & 64 & 2874 & $6 / 21 / 10$ & \\
\hline $6 / 21 / 10$ & $15: 32$ & SVSB43-S-25655 & Soil & СРT & SB43 & 70 & 2874 & $6 / 21 / 10$ & \\
\hline $6 / 21 / 10$ & $15: 37$ & SVSB43-S-25656 & Soil & CPT & SB43 & 65 & 2874 & $6 / 21 / 10$ & \\
\hline $6 / 21 / 10$ & $16: 09$ & SVSB43-S-25657 & Soil & СРТ & SB43 & 76 & 2874 & $6 / 21 / 10$ & \\
\hline $6 / 21 / 10$ & $16: 10$ & SVSB43-S-25658 & Soil & СРТ & SB43 & 80 & 2874 & $6 / 21 / 10$ & \\
\hline $6 / 21 / 10$ & $16: 10$ & SVQCTB-S-25671 ${ }^{\mathrm{a}}$ & Soil & TB & $\mathrm{QC}$ & - & 2874 & $6 / 21 / 10$ & $\begin{array}{l}\text { Trip blank sent to the AGEM } \\
\text { Laboratory for organic analysis with } \\
\text { soil samples listed on chain-of- } \\
\text { custody forms (COCs) } 2873 \text { and } \\
2874 \text {. }\end{array}$ \\
\hline $6 / 21 / 10$ & $16: 18$ & SVSB43-S-25659 & Soil & СРТ & SB43 & 84 & 2874 & $6 / 21 / 10$ & \\
\hline $6 / 21 / 10$ & $16: 43$ & SVSB43-S-25660 & Soil & СРT & SB43 & 88 & 2874 & $6 / 21 / 10$ & \\
\hline $6 / 21 / 10$ & $16: 52$ & SVSB43-S-25661 & Soil & СРТ & SB43 & 92 & 2874 & $6 / 21 / 10$ & \\
\hline $6 / 21 / 10$ & $16: 55$ & SVSB43-S-25662 & Soil & СРT & SB43 & 92.7 & 2874 & $6 / 21 / 10$ & \\
\hline $6 / 21 / 10$ & $17: 11$ & SVSB43-S-25663 & Soil & CPT & SB43 & 96 & 2874 & $6 / 21 / 10$ & \\
\hline $6 / 21 / 10$ & $17: 15$ & SVSB43-S-25664 & Soil & СРТ & SB43 & 100 & 2874 & $6 / 21 / 10$ & "100 A" in log. \\
\hline $6 / 21 / 10$ & $17: 22$ & SVSB43-S-25665 & Soil & СРТ & SB43 & 100 & 2874 & $6 / 21 / 10$ & "100 B" in log. \\
\hline $6 / 21 / 10$ & $17: 35$ & SVTRUCK-W-25667a & Water & FB & QC & - & 2875 & $6 / 21 / 10$ & Blank of water in field support truck. \\
\hline $6 / 21 / 10$ & $17: 45$ & SVTAP1-W-25668 & Water & $\mathrm{FB}$ & $\mathrm{QC}$ & - & 2875 & $6 / 21 / 10$ & $\begin{array}{l}\text { Blank of water from city tap for field } \\
\text { support truck. }\end{array}$ \\
\hline
\end{tabular}


TABLE A.1 (Cont.)

\begin{tabular}{|c|c|c|c|c|c|c|c|c|c|}
\hline $\begin{array}{l}\text { Sample } \\
\text { Date }\end{array}$ & $\begin{array}{l}\text { Sample } \\
\text { Time }\end{array}$ & Sample & $\begin{array}{l}\text { Sample } \\
\text { Medium }\end{array}$ & $\begin{array}{l}\text { Sample } \\
\text { Type }\end{array}$ & $\begin{array}{l}\text { Sample } \\
\text { Location }\end{array}$ & $\begin{array}{l}\text { Depth } \\
\text { (ft BGL) }\end{array}$ & $\begin{array}{l}\text { Chain of } \\
\text { Custody }\end{array}$ & $\begin{array}{l}\text { Shipping } \\
\text { Date }\end{array}$ & Sample Description \\
\hline $6 / 21 / 10$ & $17: 55$ & SVSB43-W-25669a & Water & TB & $\mathrm{QC}$ & - & 2875 & $6 / 21 / 10$ & $\begin{array}{l}\text { Trip blank sent to the AGEM } \\
\text { Laboratory for organic analysis with } \\
\text { water samples listed on COC } 2875 \text {. }\end{array}$ \\
\hline $6 / 21 / 10$ & $18: 00$ & SVSB43-S-25670 & Soil & СРТ & SB43 & 102 & 2874 & $6 / 21 / 10$ & \\
\hline $6 / 21 / 10$ & $18: 32$ & SVSB43-W-25666 & Water & СРT & SB43 & $95-100$ & 2875 & $6 / 21 / 10$ & $\begin{array}{l}\text { Collected from open borehole via bailer } \\
\text { during recovery of core. }\end{array}$ \\
\hline $6 / 22 / 10$ & $7: 57$ & SVSB43-S-25672 & Soil & СРТ & SB43 & 104 & 2876 & $6 / 22 / 10$ & Silt. \\
\hline $6 / 22 / 10$ & $7: 58$ & SVSB43-S-25673 & Soil & СРT & SB43 & 104 & 2876 & $6 / 22 / 10$ & Lime. \\
\hline $6 / 22 / 10$ & $17: 09$ & SVSB43-S-25675a & Soil & TB & $\mathrm{QC}$ & - & 2876 & $6 / 22 / 10$ & $\begin{array}{l}\text { Trip blank sent to the AGEM } \\
\text { Laboratory for organic analysis with } \\
\text { soil samples listed on COC } 2876 \text {. }\end{array}$ \\
\hline $6 / 23 / 10$ & $7: 28$ & SVSB44-S-25676 & Soil & СРТ & SB44 & 4 & 2878 & $6 / 23 / 10$ & \\
\hline $6 / 23 / 10$ & $7: 32$ & SVSB44-S-25677 & Soil & СРТ & SB44 & 8 & 2878 & $6 / 23 / 10$ & \\
\hline $6 / 23 / 10$ & $7: 38$ & SVSB44-S-25678 & Soil & СРТ & SB44 & 12 & 2878 & $6 / 23 / 10$ & \\
\hline $6 / 23 / 10$ & $7: 48$ & SVSB44-S-25679 & Soil & СРТ & SB44 & 16 & 2878 & $6 / 23 / 10$ & \\
\hline $6 / 23 / 10$ & $7: 51$ & SVSB44-S-25680 & Soil & СРТ & SB44 & 20 & 2878 & $6 / 23 / 10$ & \\
\hline $6 / 23 / 10$ & $7: 55$ & SVSB44-S-25681 & Soil & СРТ & SB44 & 24 & 2878 & $6 / 23 / 10$ & \\
\hline $6 / 23 / 10$ & $8: 00$ & SVSB44-S-25682 & Soil & СРТ & SB44 & 28 & 2878 & $6 / 23 / 10$ & \\
\hline $6 / 23 / 10$ & $8: 05$ & SVSB44-S-25686 & Soil & СРT & SB44 & 44 & 2881 & $6 / 25 / 10$ & \\
\hline $6 / 23 / 10$ & $8: 06$ & SVSB44-S-25683 & Soil & СРT & SB44 & 32 & 2878 & $6 / 23 / 10$ & \\
\hline $6 / 23 / 10$ & $8: 20$ & SVSB44-S-25684 & Soil & СРТ & SB44 & 36 & 2878 & $6 / 23 / 10$ & \\
\hline $6 / 23 / 10$ & $8: 23$ & SVSB44-S-25685 & Soil & СРT & SB44 & 40 & 2878 & $6 / 23 / 10$ & \\
\hline $6 / 23 / 10$ & $8: 36$ & SVSB44-S-25696 & Soil & CPT & SB44 & 45 & 2877 & $6 / 23 / 10$ & $\begin{array}{l}\text { Collected at top of } 45-\mathrm{ft} \text { core because } \\
\text { of poor recovery at } 35-45 \mathrm{ft} \text {. } \\
\text { Subsequently returned to depth to } \\
\text { collect sample at } 44 \mathrm{ft} \text {. }\end{array}$ \\
\hline $6 / 23 / 10$ & $8: 47$ & SVSB44-S-25687 & Soil & СРТ & SB44 & 48 & 2878 & $6 / 23 / 10$ & \\
\hline $6 / 23 / 10$ & $8: 53$ & SVSB44-S-25688 & Soil & СРТ & SB44 & 52 & 2878 & $6 / 23 / 10$ & \\
\hline $6 / 23 / 10$ & 9:03 & SVSB44-S-25689 & Soil & СРТ & SB44 & 56 & 2878 & $6 / 23 / 10$ & \\
\hline $6 / 23 / 10$ & $9: 17$ & SVSB44-S-25690 & Soil & СРТ & SB44 & 60 & 2877 & $6 / 23 / 10$ & \\
\hline $6 / 23 / 10$ & $9: 18$ & SVSB44-S-25691 & Soil & СРТ & SB44 & 64 & 2877 & $6 / 23 / 10$ & \\
\hline $6 / 23 / 10$ & $9: 24$ & SVSB44-S-25692 & Soil & СРT & SB44 & 68 & 2877 & $6 / 23 / 10$ & \\
\hline $6 / 23 / 10$ & $9: 35$ & SVSB44-S-25693 & Soil & СРТ & SB44 & 72 & 2877 & $6 / 23 / 10$ & \\
\hline $6 / 23 / 10$ & $9: 51$ & SVSB44-S-25694 & Soil & СРТ & SB44 & 76 & 2877 & $6 / 23 / 10$ & \\
\hline $6 / 23 / 10$ & $9: 56$ & SVSB44-S-25704 & Soil & СРT & SB44 & 76.5 & 2879 & $6 / 23 / 10$ & \\
\hline $6 / 23 / 10$ & $10: 07$ & SVSB44-S-25695 & Soil & СРT & SB44 & 80 & 2877 & $6 / 23 / 10$ & \\
\hline $6 / 23 / 10$ & $10: 07$ & SVSB44-S-25705a & Soil & СРT & SB44 & 80 & 2879 & $6 / 23 / 10$ & Replicate of SVSB44-S-25695. \\
\hline $6 / 23 / 10$ & $10: 08$ & SVSB44-S-25697 & Soil & СРT & SB44 & 84 & 2877 & $6 / 23 / 10$ & \\
\hline
\end{tabular}


TABLE A.1 (Cont.)

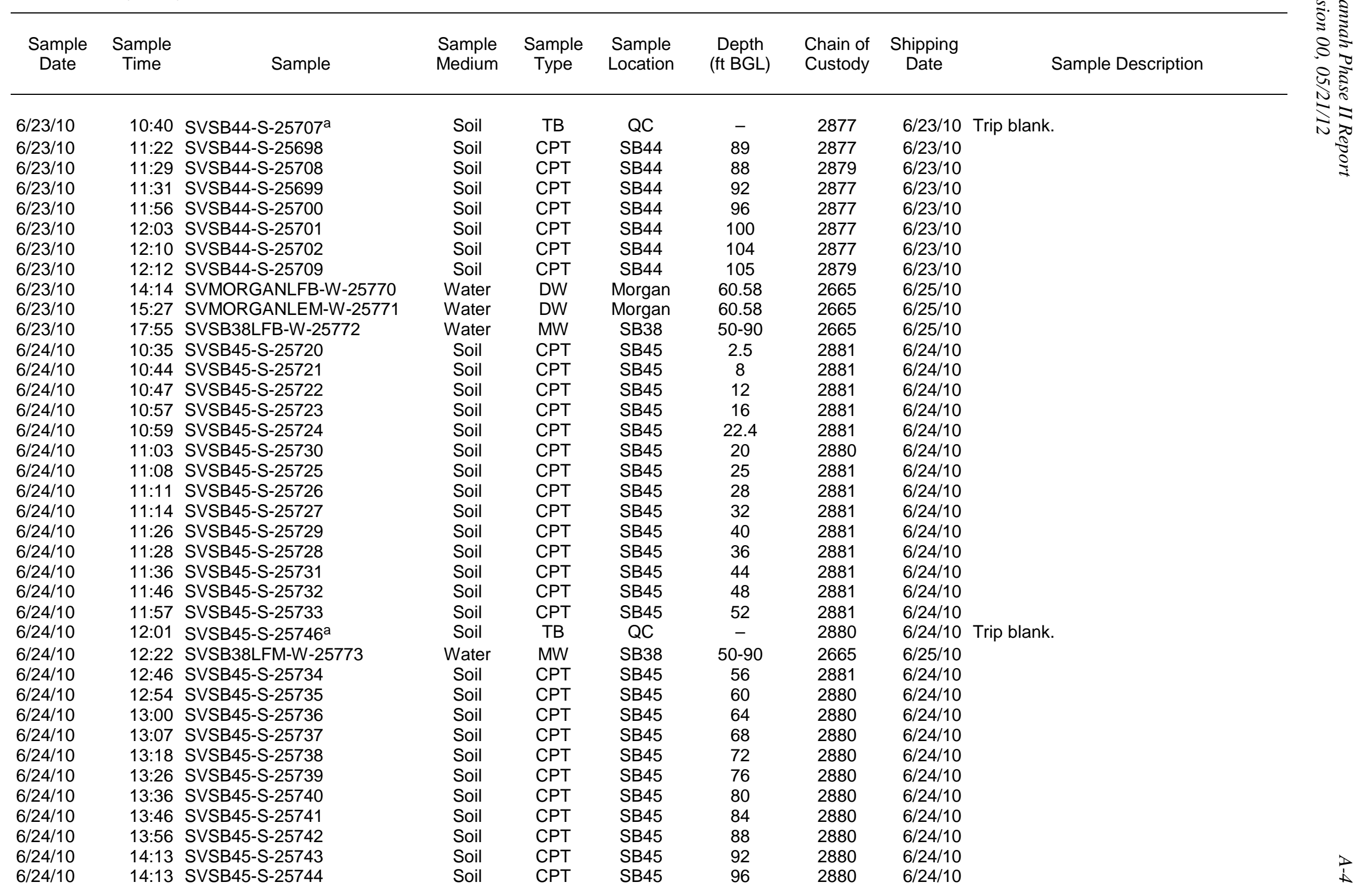


TABLE A.1 (Cont.)

\begin{tabular}{|c|c|c|c|c|c|c|c|c|c|}
\hline $\begin{array}{l}\text { Sample } \\
\text { Date }\end{array}$ & $\begin{array}{l}\text { Sample } \\
\text { Time }\end{array}$ & Sample & $\begin{array}{l}\text { Sample } \\
\text { Medium }\end{array}$ & $\begin{array}{c}\text { Sample } \\
\text { Type }\end{array}$ & $\begin{array}{l}\text { Sample } \\
\text { Location }\end{array}$ & $\begin{array}{c}\text { Depth } \\
\text { (ft BGL) }\end{array}$ & $\begin{array}{l}\text { Chain of } \\
\text { Custody }\end{array}$ & $\begin{array}{l}\text { Shipping } \\
\text { Date }\end{array}$ & Sample Description \\
\hline $6 / 24 / 10$ & $15: 00$ & SVSB45-S-25745 & Soil & CPT & SB45 & 100 & 2880 & $6 / 24 / 10$ & \\
\hline $6 / 24 / 10$ & $15: 00$ & SVSB45-S-25747a & Soil & CPT & SB45 & 100 & 2880 & $6 / 24 / 10$ & Replicate of sample SVSB45-S-25745. \\
\hline $6 / 25 / 10$ & $10: 03$ & SVSB46-S-25710 & Soil & СРТ & SB46 & 2 & 2883 & $6 / 25 / 10$ & \\
\hline $6 / 25 / 10$ & 10:07 & SVSB46-S-25711 & Soil & CPT & SB46 & 8 & 2883 & $6 / 25 / 10$ & \\
\hline $6 / 25 / 10$ & $10: 11$ & SVSB46-S-25712 & Soil & СРT & SB46 & 12 & 2883 & $6 / 25 / 10$ & \\
\hline $6 / 25 / 10$ & $10: 38$ & SVSB46-S-25716 & Soil & CPT & SB46 & 28 & 2883 & $6 / 25 / 10$ & \\
\hline $6 / 25 / 10$ & $10: 38$ & SVSB46-S-25717 & Soil & СРТ & SB46 & 32 & 2883 & $6 / 25 / 10$ & \\
\hline $6 / 25 / 10$ & $10: 54$ & SVSB46-S-25718 & Soil & СРT & SB46 & 36 & 2883 & $6 / 25 / 10$ & \\
\hline $6 / 25 / 10$ & $10: 54$ & SVSB46-S-25766 ${ }^{a}$ & Soil & CPT & SB46 & 36 & 2882 & $6 / 25 / 10$ & Replicate of sample SVSB46-S-25718. \\
\hline $6 / 25 / 10$ & $10: 58$ & SVSB46-S-25719 & Soil & СРТ & SB46 & 40 & 2883 & $6 / 25 / 10$ & \\
\hline $6 / 25 / 10$ & $11: 02$ & SVSB46-S-25750 & Soil & СРТ & SB46 & 44 & 2883 & $6 / 25 / 10$ & \\
\hline $6 / 25 / 10$ & $12: 02$ & SVSB46-S-25751 & Soil & СРТ & SB46 & 48 & 2883 & $6 / 25 / 10$ & \\
\hline $6 / 25 / 10$ & $12: 12$ & SVSB46-S-25752 & Soil & СРТ & SB46 & 52 & 2883 & $6 / 25 / 10$ & \\
\hline $6 / 25 / 10$ & $12: 18$ & SVSB46-S-25753 & Soil & СРT & SB46 & 56 & 2883 & $6 / 25 / 10$ & \\
\hline $6 / 25 / 10$ & $12: 23$ & SVSB46-S-25754 & Soil & СРТ & SB46 & 60 & 2883 & $6 / 25 / 10$ & \\
\hline $6 / 25 / 10$ & $12: 28$ & SVSB46-S-25755 & Soil & СРТ & SB46 & 64 & 2883 & $6 / 25 / 10$ & \\
\hline $6 / 25 / 10$ & $12: 34$ & SVSB46-S-25756 & Soil & СРT & SB46 & 68 & 2883 & $6 / 25 / 10$ & \\
\hline $6 / 25 / 10$ & $12: 39$ & SVSB46-S-25757 & Soil & СРТ & SB46 & 72 & 2883 & $6 / 25 / 10$ & \\
\hline $6 / 25 / 10$ & $13: 33$ & SVSB46-S-25758 & Soil & СРТ & SB46 & 76 & 2882 & $6 / 25 / 10$ & \\
\hline $6 / 25 / 10$ & $13: 39$ & SVSB46-S-25759 & Soil & СРТ & SB46 & 80 & 2882 & $6 / 25 / 10$ & \\
\hline $6 / 25 / 10$ & $14: 00$ & SVSB46-S-25765 & Soil & TB & $\mathrm{QC}$ & - & 2882 & $6 / 25 / 10$ & \\
\hline $6 / 25 / 10$ & $14: 33$ & SVSB46-S-25760 & Soil & СРТ & SB46 & 84 & 2882 & $6 / 25 / 10$ & \\
\hline $6 / 25 / 10$ & $14: 48$ & SVSB46-S-25761 & Soil & СРT & SB46 & 88 & 2882 & $6 / 25 / 10$ & \\
\hline $6 / 25 / 10$ & $14: 54$ & SVSB24LFB-W-25774 & Water & MW & SB24 & $50-60$ & 2665 & $6 / 25 / 10$ & \\
\hline $6 / 25 / 10$ & $14: 56$ & SVSB46-S-25762 & Soil & СРТ & SB46 & 92 & 2882 & $6 / 25 / 10$ & \\
\hline $6 / 25 / 10$ & $15: 39$ & SVSB46-S-25764 & Soil & СРТ & SB46 & 96 & 2882 & $6 / 25 / 10$ & \\
\hline $6 / 25 / 10$ & $15: 40$ & SVSB46-S-25763 & Soil & СРТ & SB46 & 100 & 2882 & $6 / 25 / 10$ & \\
\hline $6 / 25 / 10$ & $15: 49$ & SVSB46-S-25767 & Soil & СРT & SB46 & 104 & 2882 & $6 / 25 / 10$ & \\
\hline $6 / 25 / 10$ & $16: 14$ & SVSB46-S-25768 & Soil & СРТ & SB46 & 108 & 2882 & $6 / 25 / 10$ & \\
\hline $6 / 25 / 10$ & $16: 26$ & SVMODOTLFB-W-25775 & Water & DW & MoDOT & 44 & 2665 & $6 / 25 / 10$ & \\
\hline $6 / 25 / 10$ & $17: 58$ & SVMODOTLFM-W-25776 & Water & DW & MoDOT & 44 & 2665 & $6 / 25 / 10$ & \\
\hline $6 / 25 / 10$ & $18: 30$ & SVQCTB-W-25777a & Water & TB & QC & - & 2665 & $6 / 25 / 10$ & $\begin{array}{l}\text { Trip blank sent to the AGEM } \\
\text { Laboratory for organic analysis with } \\
\text { water samples listed on COC } 2665 \text {. }\end{array}$ \\
\hline $6 / 26 / 10$ & & SVSB46-S-25713 & Soil & CPT & SB46 & 16 & 2670 & $6 / 28 / 10$ & $\begin{array}{l}\text { Collected on June } 24 \text { because of poor } \\
\text { recovery in prior sampling. }\end{array}$ \\
\hline $6 / 26 / 10$ & & SVSB46-S-25714 & Soil & CPT & SB46 & 20 & 2670 & $6 / 28 / 10$ & $\begin{array}{l}\text { Collected on June } 24 \text { because of poor } \\
\text { recovery in prior sampling. }\end{array}$ \\
\hline
\end{tabular}


TABLE A.1 (Cont.)

\begin{tabular}{|c|c|c|c|c|c|c|c|c|c|}
\hline $\begin{array}{l}\text { Sample } \\
\text { Date }\end{array}$ & $\begin{array}{l}\text { Sample } \\
\text { Time }\end{array}$ & Sample & $\begin{array}{l}\text { Sample } \\
\text { Medium }\end{array}$ & $\begin{array}{c}\text { Sample } \\
\text { Type }\end{array}$ & $\begin{array}{l}\text { Sample } \\
\text { Location }\end{array}$ & $\begin{array}{l}\text { Depth } \\
\text { (ft BGL) }\end{array}$ & $\begin{array}{l}\text { Chain of } \\
\text { Custody }\end{array}$ & $\begin{array}{l}\text { Shipping } \\
\text { Date }\end{array}$ & Sample Description \\
\hline $6 / 26 / 10$ & & SVQCTB-S-25715a & Soil & TB & QC & - & 2670 & $6 / 28 / 10$ & $\begin{array}{l}\text { Trip blank sent to the AGEM } \\
\text { Laboratory for organic analysis with } \\
\text { soil samples listed on COC } 2670 \text {. }\end{array}$ \\
\hline $6 / 27 / 10$ & $16: 44$ & SVMODOT3X-W-25778 & Water & DW & MoDOT & 44 & 2663 & $6 / 28 / 10$ & \\
\hline $6 / 28 / 10$ & $14: 56$ & SVMORGAN3X-W-25779 & Water & DW & Morgan & 60.58 & 2663 & $6 / 28 / 10$ & \\
\hline $6 / 28 / 10$ & 19:14 & SVQCTB-W-25780a & Water & TB & QC & - & 2663 & $6 / 28 / 10$ & $\begin{array}{l}\text { Trip blank sent to the AGEM } \\
\text { Laboratory for organic analysis with } \\
\text { water samples listed on COC } 2663 \text {. }\end{array}$ \\
\hline $7 / 1 / 10$ & $12: 30$ & SVSB46D-W-25800 & Water & MW & SB46D & $90-110$ & 2865 & $7 / 6 / 10$ & $\begin{array}{l}\text { In front of MoDOT shop building. Depth } \\
\text { to water }=547.72 \mathrm{ft} \mathrm{BGL} \text {. Depth of } \\
\text { well = } 109.75 \mathrm{ft} \text {. Collected by using } \\
\text { Waterra pump after purging of } \\
55.14 \text { gal during development. }\end{array}$ \\
\hline $7 / 1 / 10$ & $14: 00$ & SVSB46S-W-25801 & Water & MW & SB46S & $42-52$ & 2865 & $7 / 6 / 10$ & $\begin{array}{l}\text { Depth to water }=40.81 \mathrm{ft} \text { BGL. Depth of } \\
\text { well }=51.5 \mathrm{ft} \text {. Sample collected by } \\
\text { using Waterra pump after purging of } \\
2.5 \mathrm{gal} \text { during development, when } \\
\text { the well went dry. }\end{array}$ \\
\hline $7 / 1 / 10$ & $15: 00$ & SVSB45S-W-25802 & Water & MW & SB45S & $37-47$ & 2865 & $7 / 6 / 10$ & $\begin{array}{l}\text { Depth to water = } 11.10 \mathrm{ft} \mathrm{BGL} \text {. Depth of } \\
\text { well }=47.235 \mathrm{ft} \text {. Sample collected by } \\
\text { using Waterra pump after purging of } \\
6-7 \text { gal during development, when } \\
\text { the well went dry. }\end{array}$ \\
\hline $7 / 1 / 10$ & $16: 54$ & SVSB45D-W-25803 & Water & MW & SB45D & $79.5-99.5$ & 2865 & $7 / 6 / 10$ & $\begin{array}{l}\text { Behind the MoDOT building, south of } \\
\text { fuel tanks. Depth to water }=67.45 \mathrm{ft} \\
\text { BGL. Depth of well }=99.05 \mathrm{ft} \text {. } \\
\text { Sample collected by using Waterra } \\
\text { pump after purging of } 32 \text { gal during } \\
\text { development. }\end{array}$ \\
\hline $7 / 1 / 10$ & 19:00 & SVSB49D-W-25804 & Water & MW & SB49D & $77-97$ & 2865 & $7 / 6 / 10$ & $\begin{array}{l}\text { Depth to water }=40.0 \mathrm{ft} \text { BGL. Depth of } \\
\text { well }=96.30 \mathrm{ft} \text {. Sample collected by } \\
\text { using Waterra pump after purging of } \\
57 \mathrm{gal} \text { during development. }\end{array}$ \\
\hline
\end{tabular}

14.56 SVMORGAN3X-W-25779

0.58

$6 / 28 / 10$

Laboratory for organic analysis with . o water $=547.72 \mathrm{ft} \mathrm{BGL}$. Depth of well $=109.75 \mathrm{ft}$. Collected by using Waterra pump after purging of pth to water $=40.81 \mathrm{ft} \mathrm{BGL}$. Depth of = $51.5 \mathrm{ft}$. Sample collected by the well went dry. 6-7 gal during development, when well went dry.

el tanks. Depth to water $=67.45 \mathrm{ft}$ Depth of well $=99.05 \mathrm{ft}$ pump after purging of $32 \mathrm{gal}$ during 57 gal during development. 
TABLE A.1 (Cont.)

\begin{tabular}{|c|c|c|c|c|c|c|c|c|c|}
\hline $\begin{array}{c}\text { Sample } \\
\text { Date }\end{array}$ & $\begin{array}{c}\text { Sample } \\
\text { Time }\end{array}$ & Sample & $\begin{array}{l}\text { Sample } \\
\text { Medium }\end{array}$ & $\begin{array}{c}\text { Sample } \\
\text { Type }\end{array}$ & $\begin{array}{l}\text { Sample } \\
\text { Location }\end{array}$ & $\begin{array}{c}\text { Depth } \\
\text { (ft BGL) }\end{array}$ & $\begin{array}{l}\text { Chain of } \\
\text { Custody }\end{array}$ & $\begin{array}{l}\text { Shipping } \\
\text { Date }\end{array}$ & Sample Description \\
\hline $7 / 2 / 10$ & $8: 40$ & SVSB49S-W-25805 & Water & MW & SB49S & $29-39$ & 2865 & $7 / 6 / 10$ & $\begin{array}{l}\text { McPike ground, east of storage units. } \\
\text { Depth to water }=10.43 \mathrm{ft} \text { BGL. } \\
\text { Depth of well = } 38.22 \mathrm{ft} \text {. Sample } \\
\text { collected by using Waterra pump } \\
\text { after purging of } 4.5 \text { gal during } \\
\text { development. Well went dry at } \\
6.5 \text { gal. }\end{array}$ \\
\hline $7 / 2 / 10$ & $12: 00$ & SVSB48D-W-25806 & Water & MW & SB48D & $77-97$ & 2865 & $7 / 6 / 10$ & $\begin{array}{l}\text { Southwest corner of pasture field. } \\
\text { Depth to water }=36.90 \mathrm{ft} \text { BGL. } \\
\text { Depth of well }=97.55 \mathrm{ft} \text {. Sample } \\
\text { collected by using Waterra pump } \\
\text { after purging of } 61 \text { gal during } \\
\text { development. }\end{array}$ \\
\hline $7 / 2 / 10$ & $13: 10$ & SVSB48S-W-25807 & Water & MW & SB48S & $25-35$ & 2865 & $7 / 6 / 10$ & $\begin{array}{l}\text { Southwest corner of pasture field. } \\
\text { Depth to water = 9.16 ft BGL. Depth } \\
\text { of well = } 34.64 \mathrm{ft} \text {. Sample collected } \\
\text { by using Waterra pump after } \\
\text { purging of } 4.5 \text { gal during } \\
\text { development. Well went dry at } 5 \text { gal. }\end{array}$ \\
\hline $7 / 2 / 10$ & $15: 05$ & SVSB47D-W-25808 & Water & MW & SB47D & $77-97$ & 2865 & $7 / 6 / 10$ & $\begin{array}{l}\text { Southeast corner of pasture field. } \\
\text { Depth to water }=36.17 \mathrm{ft} \mathrm{BGL} \text {. } \\
\text { Depth of well }=96.70 \mathrm{ft} \text {. Sample } \\
\text { collected by using Waterra pump } \\
\text { after purging of } 61 \text { gal during } \\
\text { development. }\end{array}$ \\
\hline $7 / 2 / 10$ & $15: 35$ & SVSB47S-W-25809 & Water & MW & SB47S & $25-35$ & 2865 & $7 / 6 / 10$ & $\begin{array}{l}\text { Southeast corner of pasture field. } \\
\text { Depth to water = } 4.7 \mathrm{ft} \text { BGL. Depth } \\
\text { of well = } 35 \mathrm{ft} \text {. Sample collected by } \\
\text { using Waterra pump after purging of } \\
5.5 \text { gal during development when } \\
\text { well went dry. }\end{array}$ \\
\hline $7 / 2 / 10$ & $17: 15$ & SVSB44D-W-25810 & Water & MW & SB44D & $92-102$ & 2865 & $7 / 6 / 10$ & $\begin{array}{l}\text { Northeast corner of school property. } \\
\text { Depth to water }=58.25 \mathrm{ft} \text { BGL. } \\
\text { Depth of well = } 101.78 \mathrm{ft} \text {. Sample } \\
\text { collected by using Waterra pump } \\
\text { after purging of } 44 \text { gal during } \\
\text { development. }\end{array}$ \\
\hline
\end{tabular}


TABLE A.1 (Cont.)

\begin{tabular}{|c|c|c|c|c|c|c|c|c|c|}
\hline $\begin{array}{l}\text { Sample } \\
\text { Date }\end{array}$ & $\begin{array}{l}\text { Sample } \\
\text { Time }\end{array}$ & Sample & $\begin{array}{l}\text { Sample } \\
\text { Medium }\end{array}$ & $\begin{array}{c}\text { Sample } \\
\text { Type }\end{array}$ & $\begin{array}{l}\text { Sample } \\
\text { Location }\end{array}$ & $\begin{array}{l}\text { Depth } \\
\text { (ft BGL) }\end{array}$ & $\begin{array}{l}\text { Chain of } \\
\text { Custody }\end{array}$ & $\begin{array}{l}\text { Shipping } \\
\text { Date }\end{array}$ & Sample Description \\
\hline $7 / 2 / 10$ & $17: 45$ & SVSB44S-W-25811 & Water & MW & SB44S & $46.5-56.5$ & 2865 & $7 / 6 / 10$ & $\begin{array}{l}\text { Northeast corner of school property. } \\
\text { Depth to water }=13.26 \mathrm{ft} \mathrm{BGL} \text {. } \\
\text { Depth of well = } 55.31 \mathrm{ft} \text {. Sample } \\
\text { collected by using Waterra pump } \\
\text { after purging of } 7 \text { gal during } \\
\text { development. Well went dry at } 9 \text { gal. }\end{array}$ \\
\hline $7 / 2 / 10$ & 18:26 & SVSB43S-W-25812 & Water & MW & SB43S & $45-55$ & 2865 & $7 / 6 / 10$ & $\begin{array}{l}\text { Northwest corner of school property. } \\
\text { Depth to water }=11.40 \mathrm{ft} \mathrm{BGL} \text {. } \\
\text { Depth of well }=54.80 \mathrm{ft} . \text { Sample } \\
\text { collected by using Waterra pump } \\
\text { after purging of } 7.5 \text { gal during } \\
\text { development when well went dry. }\end{array}$ \\
\hline $7 / 3 / 10$ & 9:00 & SVSB43D-W-25813 & Water & MW & SB43D & 95-105 & 2865 & $7 / 6 / 10$ & $\begin{array}{l}\text { Northwest corner of school property. } \\
\text { Depth to water = } 55.60 \mathrm{ft} \text { BGL. } \\
\text { Depth of well = } 104.30 \mathrm{ft} \text {. Sample } \\
\text { collected by using Waterra pump } \\
\text { after purging of } 8.5 \text { gal during } \\
\text { development. Well went dry at } \\
20 \text { gal. }\end{array}$ \\
\hline $7 / 6 / 10$ & $12: 00$ & SVQCTB-W-25814 ${ }^{a}$ & Water & TB & QC & - & 2865 & $7 / 6 / 10$ & $\begin{array}{l}\text { Trip blank sent to the AGEM } \\
\text { Laboratory for organic analysis with } \\
\text { water samples listed on COC } 2865 \text {. }\end{array}$ \\
\hline $8 / 24 / 10$ & $8: 30$ & SVSB44D-W-25818 & Water & MW & SB44D & $92-102$ & 2678 & $8 / 24 / 10$ & Grab sample from unpurged well. \\
\hline $8 / 24 / 10$ & $8: 43$ & SVSB44S-W-25817 & Water & MW & SB44S & $46.5-56.5$ & 2678 & 8/24/10 & Grab sample from unpurged well. \\
\hline $8 / 24 / 10$ & $8: 57$ & SVSB43D-W-25816 & Water & MW & SB43D & $95-105$ & 2678 & $8 / 24 / 10$ & Grab sample from unpurged well. \\
\hline 8/24/10 & 9:08 & SVSB43S-W-25815 & Water & MW & SB43S & $45-55$ & 2678 & $8 / 24 / 10$ & Grab sample from unpurged well. \\
\hline 8/24/10 & $9: 23$ & SVSB49D-W-25828 & Water & MW & SB49D & $77-97$ & 2678 & $8 / 24 / 10$ & Grab sample from unpurged well. \\
\hline $8 / 24 / 10$ & 9:30 & SVSB49S-W-25827 & Water & MW & SB49S & $29-39$ & 2678 & $8 / 24 / 10$ & Grab sample from unpurged well. \\
\hline 8/24/10 & $9: 43$ & SVSB45D-W-25820 & Water & MW & SB45D & $79.5-99.5$ & 2678 & 8/24/10 & Grab sample from unpurged well. \\
\hline 8/24/10 & 9:51 & SVSB45S-W-25819 & Water & MW & SB45S & $37-47$ & 2678 & $8 / 24 / 10$ & Grab sample from unpurged well. \\
\hline 8/24/10 & 10:07 & SVSB46D-W-25822 & Water & MW & SB46D & $90-110$ & 2678 & $8 / 24 / 10$ & Grab sample from unpurged well. \\
\hline $8 / 24 / 10$ & 10:14 & SVSB46S-W-25821 & Water & MW & SB46S & $42-52$ & 2678 & $8 / 24 / 10$ & Grab sample from unpurged well. \\
\hline $8 / 24 / 10$ & $10: 36$ & SVSB48D-W-25826 & Water & MW & SB48D & $77-97$ & 2678 & $8 / 24 / 10$ & Grab sample from unpurged well. \\
\hline $8 / 24 / 10$ & $10: 43$ & SVSB48S-W-25825 & Water & MW & SB48S & $25-35$ & 2678 & $8 / 24 / 10$ & Grab sample from unpurged well. \\
\hline $8 / 24 / 10$ & $10: 53$ & SVSB47D-W-25824 & Water & MW & SB47D & $77-97$ & 2678 & $8 / 24 / 10$ & Grab sample from unpurged well. \\
\hline $8 / 24 / 10$ & $11: 02$ & SVSB47S-W-25823 & Water & MW & SB47S & $25-35$ & 2678 & $8 / 24 / 10$ & Grab sample from unpurged well. \\
\hline $8 / 24 / 10$ & $11: 42$ & SVSB45D-W-25835 & Water & MW & SB45D & $79.5-99.5$ & 2739 & $8 / 24 / 10$ & \\
\hline $8 / 24 / 10$ & $11: 42$ & SVSB49DDUP-W-25844 & Water & MW & SB49D & $77-97$ & 2739 & 8/24/10 & \\
\hline
\end{tabular}
collected by using Waterra pump after purging of $7 \mathrm{gal}$ during rthwest corner of school property. collected by using Waterra pump after purging of $7.5 \mathrm{gal}$ during . Depth to water $=55.60 \mathrm{ft} \mathrm{BGL}$. Depth of well $=104.30 \mathrm{ft}$. Sample colect by using Waterra pump ter purging of $8.5 \mathrm{gal}$ during 20 gal.

Laboratory for organic analysis with 8/24/10 Grab sample from unpurged well. $8 / 24 / 10$ Grab sample from unpurged well. 8/24/10 Grab sample from unpurged well. Grab sample from unpurged we Grab sample from unpurged well. 8/24/10 Grab sample from unpurged well. 8/24/10 Grab sample from unpurged well. Grab sample from unpurged well. $8 / 24 / 10$ Grab sample from unpurged well. 
TABLE A.1 (Cont.)

\begin{tabular}{|c|c|c|c|c|c|c|c|c|c|}
\hline $\begin{array}{l}\text { Sample } \\
\text { Date }\end{array}$ & $\begin{array}{l}\text { Sample } \\
\text { Time }\end{array}$ & Sample & $\begin{array}{l}\text { Sample } \\
\text { Medium }\end{array}$ & $\begin{array}{c}\text { Sample } \\
\text { Type }\end{array}$ & $\begin{array}{l}\text { Sample } \\
\text { Location }\end{array}$ & $\begin{array}{l}\text { Depth } \\
\text { (ft BGL) }\end{array}$ & $\begin{array}{l}\text { Chain of } \\
\text { Custody }\end{array}$ & $\begin{array}{l}\text { Shipping } \\
\text { Date }\end{array}$ & Sample Description \\
\hline $8 / 24 / 10$ & 13:08 & SVSB46D-W-25837 & Water & MW & SB46D & $90-110$ & 2739 & $8 / 24 / 10$ & \\
\hline 8/24/10 & 13:14 & SVSB44D-W-25833 & Water & MW & SB44D & $92-102$ & 2739 & 8/24/10 & \\
\hline 8/24/10 & 13:26 & SVQCIR-W-25847 & Water & $\mathrm{RI}$ & QC & - & 2739 & 8/24/10 & \\
\hline 8/24/10 & $15: 00$ & SVSB49D-W-25843 & Water & MW & SB49D & $77-97$ & 2739 & 8/24/10 & \\
\hline $8 / 24 / 10$ & $15: 28$ & SVSB48D-W-25841 & Water & MW & SB48D & $77-97$ & 2739 & $8 / 24 / 10$ & \\
\hline $8 / 24 / 10$ & $16: 18$ & SVSB43D-W-25831 & Water & MW & SB43D & $95-105$ & 2739 & $8 / 24 / 10$ & \\
\hline 8/24/10 & $16: 34$ & SVQCIR-W-25848 & Water & $\mathrm{RI}$ & $\mathrm{QC}$ & - & 2739 & 8/24/10 & \\
\hline $8 / 24 / 10$ & $17: 24$ & SVSB44S-W-25832 & Water & MW & SB44S & $46.5-56.5$ & 2739 & $8 / 24 / 10$ & \\
\hline $8 / 24 / 10$ & $18: 30$ & SVQCTB-W-25829a & Water & TB & QC & - & 2678 & $8 / 24 / 10$ & $\begin{array}{l}\text { Trip blank sent to the AGEM } \\
\text { Laboratory for organic analysis with } \\
\text { water samples listed on COC } 2678 \text {. }\end{array}$ \\
\hline $8 / 24 / 10$ & 18:30 & SVSB43S-W-25830 & Water & MW & SB43S & $45-55$ & 2739 & 8/24/10 & \\
\hline $8 / 24 / 10$ & $18: 42$ & SVSB47D-W-25839 & Water & MW & SB47D & $77-97$ & 2739 & $8 / 24 / 10$ & \\
\hline $8 / 24 / 10$ & 19:00 & SVQCTB-W-25851 ${ }^{\mathrm{a}}$ & Water & TB & QC & - & 2739 & $8 / 24 / 10$ & $\begin{array}{l}\text { Trip blank sent to the AGEM } \\
\text { Laboratory for organic analysis with } \\
\text { water samples listed on COC } 2739 \text {. }\end{array}$ \\
\hline $8 / 25 / 10$ & $9: 48$ & SVSB45S-W-25834 & Water & MW & SB45S & $37-47$ & 2740 & $8 / 25 / 10$ & \\
\hline $8 / 25 / 10$ & $9: 56$ & SVQCIR-W-25849 & Water & $\mathrm{RI}$ & $\mathrm{QC}$ & - & 2740 & $8 / 25 / 10$ & \\
\hline $8 / 25 / 10$ & $10: 10$ & SVSB47S-W-25838 & Water & MW & SB47S & $25-35$ & 2740 & $8 / 25 / 10$ & \\
\hline $8 / 25 / 10$ & $10: 33$ & SVQCIR-W-25850 & Water & RI & $\mathrm{QC}$ & - & 2740 & $8 / 25 / 10$ & \\
\hline $8 / 25 / 10$ & $10: 40$ & SVSB49S-W-25842 & Water & MW & SB49S & 29-39 & 2740 & $8 / 25 / 10$ & \\
\hline $8 / 25 / 10$ & $11: 13$ & SVSB48S-W-25840 & Water & MW & SB48S & $25-35$ & 2740 & $8 / 25 / 10$ & \\
\hline $8 / 25 / 10$ & $11: 13$ & SVSB48SDUP-W-25845 & Water & MW & SB48S & $25-35$ & 2740 & $8 / 25 / 10$ & \\
\hline $8 / 25 / 10$ & $12: 22$ & SVSB46S-W-25836 & Water & MW & SB46S & $42-52$ & 2740 & $8 / 25 / 10$ & \\
\hline $8 / 25 / 10$ & $12: 41$ & SVSB24-W-25854 & Water & MW & SB24 & $50-60$ & 2740 & $8 / 25 / 10$ & \\
\hline $8 / 25 / 10$ & $13: 58$ & SVSB38-W-25855 & Water & MW & SB38 & $50-90$ & 2740 & $8 / 25 / 10$ & \\
\hline $8 / 25 / 10$ & $15: 30$ & SVQCTB-W-25852 ${ }^{a}$ & Water & TB & QC & - & 2740 & $8 / 25 / 10$ & $\begin{array}{l}\text { Trip blank sent to the AGEM } \\
\text { Laboratory for organic analysis with } \\
\text { water samples listed on COC } 2740 .\end{array}$ \\
\hline $8 / 25 / 10$ & $15: 46$ & SVDIH2O-W-25846a & Water & FB & QC & - & 2740 & $8 / 25 / 10$ & \\
\hline $8 / 25 / 10$ & $15: 50$ & SVQCTB-W-25853a & Water & TB & QC & - & & & \\
\hline $8 / 10 / 11$ & $11: 12$ & SVSB44DGRAB-W-25861 & Water & MW & SB44D & 92-102 & 2988 & $8 / 10 / 11$ & $\begin{array}{l}\text { Grab sample collected without purging. } \\
\text { Bailer lowered to bottom of well and } \\
\text { withdrawn. }\end{array}$ \\
\hline $8 / 10 / 11$ & $11: 16$ & SVSB44SGRAB-W-25860 & Water & MW & SB44S & $46.5-56.5$ & 2988 & $8 / 10 / 11$ & $\begin{array}{l}\text { Grab sample collected without purging. } \\
\text { Bailer lowered to bottom of well and } \\
\text { withdrawn. }\end{array}$ \\
\hline
\end{tabular}


TABLE A.1 (Cont.)

\begin{tabular}{|c|c|c|c|c|c|c|c|c|c|}
\hline $\begin{array}{l}\text { Sample } \\
\text { Date }\end{array}$ & $\begin{array}{l}\text { Sample } \\
\text { Time }\end{array}$ & Sample & $\begin{array}{l}\text { Sample } \\
\text { Medium }\end{array}$ & $\begin{array}{l}\text { Sample } \\
\text { Type }\end{array}$ & $\begin{array}{l}\text { Sample } \\
\text { Location }\end{array}$ & $\begin{array}{l}\text { Depth } \\
\text { (ft BGL) }\end{array}$ & $\begin{array}{l}\text { Chain of } \\
\text { Custody }\end{array}$ & $\begin{array}{l}\text { Shipping } \\
\text { Date }\end{array}$ & Sample Description \\
\hline 8/10/11 & $11: 28$ & SVSB43DGRAB-W-25859 & Water & MW & SB43D & 95-105 & 2988 & $8 / 10 / 11$ & $\begin{array}{l}\text { Grab sample collected without purging. } \\
\text { Bailer lowered to bottom of well and } \\
\text { withdrawn. }\end{array}$ \\
\hline $8 / 10 / 11$ & $11: 30$ & SVSB43SGRAB-W-25858 & Water & MW & SB43S & $45-55$ & 2988 & $8 / 10 / 11$ & $\begin{array}{l}\text { Grab sample collected without purging. } \\
\text { Bailer lowered to bottom of well and } \\
\text { withdrawn. }\end{array}$ \\
\hline 8/10/11 & $11: 54$ & SVSB46SGRAB-W-25864 & Water & MW & SB46S & $42-52$ & 2988 & $8 / 10 / 11$ & $\begin{array}{l}\text { Grab sample collected without purging. } \\
\text { Bailer lowered to bottom of well and } \\
\text { withdrawn. }\end{array}$ \\
\hline $8 / 10 / 11$ & $12: 04$ & SVSB46DGRAB-W-25865 & Water & MW & SB46D & $90-110$ & 2988 & 8/10/11 & $\begin{array}{l}\text { Grab sample collected without purging. } \\
\text { Bailer lowered to bottom of well and } \\
\text { withdrawn. }\end{array}$ \\
\hline $8 / 10 / 11$ & $12: 12$ & SVSB45DGRAB-W-25863 & Water & MW & SB45D & $79.5-99.5$ & 2988 & 8/10/11 & $\begin{array}{l}\text { Grab sample collected without purging. } \\
\text { Bailer lowered to bottom of well and } \\
\text { withdrawn. }\end{array}$ \\
\hline $8 / 10 / 11$ & $12: 16$ & SVSB45SGRAB-W-25862 & Water & MW & SB45S & $37-47$ & 2988 & $8 / 10 / 11$ & $\begin{array}{l}\text { Grab sample collected without purging. } \\
\text { Bailer lowered to bottom of well and } \\
\text { withdrawn. }\end{array}$ \\
\hline $8 / 10 / 11$ & $12: 20$ & SVSB49SGRAB-W-25870 & Water & MW & SB49S & $29-39$ & 2989 & $8 / 10 / 11$ & $\begin{array}{l}\text { Grab sample collected without purging. } \\
\text { Bailer lowered to bottom of well and } \\
\text { withdrawn. }\end{array}$ \\
\hline $8 / 10 / 11$ & $12: 25$ & SVSB49DGRAB-W-25871 & Water & MW & SB49D & $77-97$ & 2989 & 8/10/11 & $\begin{array}{l}\text { Grab sample collected without purging. } \\
\text { Bailer lowered to bottom of well and } \\
\text { withdrawn. }\end{array}$ \\
\hline $8 / 10 / 11$ & $12: 30$ & SVSB24GRAB-W-25856 & Water & MW & SB24 & $50-60$ & 2988 & $8 / 10 / 11$ & $\begin{array}{l}\text { Grab sample collected without purging. } \\
\text { Bailer lowered to bottom of well and } \\
\text { withdrawn. }\end{array}$ \\
\hline $8 / 10 / 11$ & $12: 38$ & SVSB38GRAB-W-25857 & Water & MW & SB38 & $50-90$ & 2988 & $8 / 10 / 11$ & $\begin{array}{l}\text { Grab sample collected without purging. } \\
\text { Bailer lowered to bottom of well and } \\
\text { withdrawn. }\end{array}$ \\
\hline $8 / 10 / 11$ & $12: 40$ & SVQCIR-W-25891 ${ }^{a}$ & Water & RI & QC & - & 2989 & $8 / 10 / 11$ & $\begin{array}{l}\text { Rinsate of decontaminated sampling } \\
\text { bailer after collection of sample } \\
\text { SVSB38GRAB-W-25857. }\end{array}$ \\
\hline $8 / 10 / 11$ & $14: 43$ & SVSB48SGRAB-W-25868 & Water & MW & SB48S & $25-35$ & 2988 & $8 / 10 / 11$ & $\begin{array}{l}\text { Grab sample collected without purging. } \\
\text { Bailer lowered to bottom of well and } \\
\text { withdrawn. }\end{array}$ \\
\hline
\end{tabular}
withdrawn. 
TABLE A.1 (Cont.)

\begin{tabular}{|c|c|c|c|c|c|c|c|c|c|}
\hline $\begin{array}{l}\text { Sample } \\
\text { Date }\end{array}$ & $\begin{array}{l}\text { Sample } \\
\text { Time }\end{array}$ & Sample & $\begin{array}{l}\text { Sample } \\
\text { Medium }\end{array}$ & $\begin{array}{l}\text { Sample } \\
\text { Type }\end{array}$ & $\begin{array}{l}\text { Sample } \\
\text { Location }\end{array}$ & $\begin{array}{l}\text { Depth } \\
\text { (ft BGL) }\end{array}$ & $\begin{array}{l}\text { Chain of } \\
\text { Custody }\end{array}$ & $\begin{array}{l}\text { Shipping } \\
\text { Date }\end{array}$ & Sample Description \\
\hline 8/10/11 & $14: 49$ & SVSB48DGRAB-W-25869 & Water & MW & SB48D & $77-97$ & 2988 & $8 / 10 / 11$ & $\begin{array}{l}\text { Grab sample collected without purging. } \\
\text { Bailer lowered to bottom of well and } \\
\text { withdrawn. }\end{array}$ \\
\hline $8 / 10 / 11$ & $15: 04$ & SVSB47DGRAB-W-25867 & Water & MW & SB47D & $77-97$ & 2988 & $8 / 10 / 11$ & $\begin{array}{l}\text { Grab sample collected without purging. } \\
\text { Bailer lowered to bottom of well and } \\
\text { withdrawn. }\end{array}$ \\
\hline $8 / 10 / 11$ & $15: 09$ & SVSB47SGRAB-W-25866 & Water & MW & SB47S & $25-35$ & 2988 & $8 / 10 / 11$ & $\begin{array}{l}\text { Grab sample collected without purging. } \\
\text { Bailer lowered to bottom of well and } \\
\text { withdrawn. }\end{array}$ \\
\hline $8 / 10 / 11$ & $15: 25$ & SVQCTB-W-25895a & Water & TB & QC & - & 2988 & $8 / 10 / 11$ & $\begin{array}{l}\text { Trip blank sent to the AGEM } \\
\text { Laboratory for organic analysis with } \\
\text { water samples listed on COC } 2988 .\end{array}$ \\
\hline $8 / 10 / 11$ & $16: 08$ & SVSB43S-W-25874 & Water & MW & SB43S & $45-55$ & 2989 & $8 / 10 / 11$ & $\begin{array}{l}\text { Depth to water }=24.30 \mathrm{ft} \text {. Depth of } 2 \text {-in. } \\
\text { well }=55.03 \mathrm{ft} \text {. Sample collected by } \\
\text { using low-flow bladder pump after } \\
\text { purging of } 11 \mathrm{~L} \text {. Pump intake } \\
\text { positioned at } 53 \mathrm{ft} \text {. }\end{array}$ \\
\hline $8 / 10 / 11$ & $16: 59$ & SVSB47S-W-25882 & Water & MW & SB47S & $25-35$ & 2989 & $8 / 10 / 11$ & $\begin{array}{l}\text { Depth to water }=15.51 \mathrm{ft} \text {. Depth of 2-in. } \\
\text { well = } 34.9 \mathrm{ft} \text {. Sample collected by } \\
\text { using low-flow bladder pump after } \\
\text { purging of } 12.75 \mathrm{~L} \text {. Pump intake } \\
\text { positioned at } 33 \mathrm{ft} \text {. }\end{array}$ \\
\hline $8 / 10 / 11$ & $17: 14$ & SVSB43D-W-25875 & Water & MW & SB43D & $95-105$ & 2989 & $8 / 10 / 11$ & $\begin{array}{l}\text { Depth to water }=60.85 \mathrm{ft} \text {. Depth of } 2 \text {-in. } \\
\text { well = } 104.5 \mathrm{ft} \text {. Sample collected by } \\
\text { using low-flow bladder pump after } \\
\text { purging of } 7 \mathrm{~L} \text {. Pump intake } \\
\text { positioned at } 103 \mathrm{ft} \text {. }\end{array}$ \\
\hline $8 / 10 / 11$ & $17: 15$ & SVSB43DDUP-W-25888a & Water & MW & SB43D & $95-105$ & 2989 & $8 / 10 / 11$ & $\begin{array}{l}\text { Replicate of sample SVSB43D-W- } \\
25875 .\end{array}$ \\
\hline $8 / 10 / 11$ & $18: 14$ & SVSB47D-W-25883 & Water & MW & SB47D & $77-97$ & 2989 & $8 / 10 / 11$ & $\begin{array}{l}\text { Depth to water }=40.53 \mathrm{ft} \text {. Depth of } 2 \text {-in. } \\
\text { well }=97.65 \mathrm{ft} \text {. Sample collected by } \\
\text { using low-flow bladder pump after } \\
\text { purging of } 9 \mathrm{~L} \text {. Pump intake } \\
\text { positioned at } 95 \mathrm{ft} \text {. }\end{array}$ \\
\hline
\end{tabular}


TABLE A.1 (Cont.)

\begin{tabular}{|c|c|c|c|c|c|c|c|c|c|}
\hline $\begin{array}{l}\text { Sample } \\
\text { Date }\end{array}$ & $\begin{array}{l}\text { Sample } \\
\text { Time }\end{array}$ & Sample & $\begin{array}{l}\text { Sample } \\
\text { Medium }\end{array}$ & $\begin{array}{c}\text { Sample } \\
\text { Type }\end{array}$ & $\begin{array}{l}\text { Sample } \\
\text { Location }\end{array}$ & $\begin{array}{l}\text { Depth } \\
\text { (ft BGL) }\end{array}$ & $\begin{array}{l}\text { Chain of } \\
\text { Custody }\end{array}$ & $\begin{array}{l}\text { Shipping } \\
\text { Date }\end{array}$ & Sample Description \\
\hline $8 / 10 / 11$ & $18: 48$ & SVSB49D-W-25887 & Water & MW & SB49D & $77-97$ & 2989 & 8/10/11 & $\begin{array}{l}\text { Depth to water }=45.20 \mathrm{ft} \text {. Depth of 2-in. } \\
\text { well }=96.5 \mathrm{ft} \text {. Sample collected by } \\
\text { using low-flow bladder pump after } \\
\text { purging of } 8 \mathrm{~L} \text {. Pump intake } \\
\text { positioned at } 95 \mathrm{ft} \text {. }\end{array}$ \\
\hline $8 / 10 / 11$ & 19:00 & SVQCTB-W-25896a & Water & TB & QC & - & 2989 & 8/10/11 & $\begin{array}{l}\text { Trip blank with water samples to AGEM } \\
\text { Lab for VOC analysis listed on COC } \\
2989 \text {. }\end{array}$ \\
\hline $8 / 10 / 11$ & 19:36 & SVSB48D-W-25885 & Water & MW & SB48D & $77-97$ & 2989 & $8 / 10 / 11$ & $\begin{array}{l}\text { Depth to water }=39.51 \mathrm{ft} \text {. Depth of 2-in. } \\
\text { well }=97.65 \mathrm{ft} \text {. Sample collected by } \\
\text { using low-flow bladder pump after } \\
\text { purging of } 5 \mathrm{~L} \text {. Pump intake } \\
\text { positioned at } 95 \mathrm{ft} \text {. }\end{array}$ \\
\hline $8 / 10 / 11$ & $19: 38$ & SVSB49S-W-25886 & Water & MW & SB49S & $29-39$ & 2989 & 8/10/11 & $\begin{array}{l}\text { Depth to water }=9.59 \mathrm{ft} \text {. Depth of } 2 \text {-in. } \\
\text { well }=38.43 \mathrm{ft} \text {. Sample collected by } \\
\text { using low-flow bladder pump after } \\
\text { purging of } 9 \mathrm{~L} \text {. Pump intake } \\
\text { positioned at } 37 \mathrm{ft} \text {. }\end{array}$ \\
\hline $8 / 10 / 11$ & $19: 50$ & SVQCIR-W-25892 ${ }^{\mathrm{a}}$ & Water & $\mathrm{RI}$ & QC & - & 2989 & 8/10/11 & $\begin{array}{l}\text { Rinsate of decontaminated sampling } \\
\text { line after collection of sample } \\
\text { SVSB49S-W-25886. }\end{array}$ \\
\hline $8 / 11 / 11$ & $12: 10$ & SVSB44D-W-25877 & Water & MW & SB44D & $92-102$ & 2990 & $8 / 11 / 11$ & $\begin{array}{l}\text { Depth to water }=61.70 \mathrm{ft} \text {. Depth of 2-in. } \\
\text { well }=102.01 \mathrm{ft} \text {. Sample collected } \\
\text { by using low-flow bladder pump } \\
\text { after purging of } 9 \mathrm{~L} \text {. Pump intake } \\
\text { positioned at } 100 \mathrm{ft} .\end{array}$ \\
\hline $8 / 11 / 11$ & $12: 23$ & SVSB48S-W-25884 & Water & MW & SB48S & $25-35$ & 2990 & $8 / 11 / 11$ & $\begin{array}{l}\text { Depth to water }=12.13 \mathrm{ft} \text {. Depth of } 2 \text {-in. } \\
\text { well }=35.22 \mathrm{ft} \text {. Sample collected by } \\
\text { using low-flow bladder pump after } \\
\text { purging of } 14.50 \mathrm{~L} \text {. Pump intake } \\
\text { positioned at } 33 \mathrm{ft} \text {. }\end{array}$ \\
\hline $8 / 11 / 11$ & $13: 30$ & SVSB44S-W-25876 & Water & MW & SB44S & $46.5-56.5$ & 2990 & $8 / 11 / 11$ & $\begin{array}{l}\text { Depth to water }=24.10 \mathrm{ft} \text {. Depth of } 2 \text {-in. } \\
\text { well }=55.55 \mathrm{ft} \text {. Sample collected by } \\
\text { using low-flow bladder pump after } \\
\text { purging of } 14 \mathrm{~L} \text {. Pump intake } \\
\text { positioned at } 54.5 \mathrm{ft} \text {. }\end{array}$ \\
\hline
\end{tabular}

using low-flow bladder pump after

well $=97.65 \mathrm{ft}$. Sample collected by purging of $9 \mathrm{~L}$. Pump intake

line after collection of sample

. by using low-flow bladder pump after purging of $9 \mathrm{~L}$. Pump intake adder pump after purging of $14.50 \mathrm{~L}$. Pump intake positioned at $33 \mathrm{ft}$. purging of $14 \mathrm{~L}$. Pump intake 
TABLE A.1 (Cont.)

\begin{tabular}{|c|c|c|c|c|c|c|c|c|c|}
\hline $\begin{array}{l}\text { Sample } \\
\text { Date }\end{array}$ & $\begin{array}{l}\text { Sample } \\
\text { Time }\end{array}$ & Sample & $\begin{array}{l}\text { Sample } \\
\text { Medium }\end{array}$ & $\begin{array}{l}\text { Sample } \\
\text { Type }\end{array}$ & $\begin{array}{l}\text { Sample } \\
\text { Location }\end{array}$ & $\begin{array}{c}\text { Depth } \\
\text { (ft BGL) }\end{array}$ & $\begin{array}{l}\text { Chain of } \\
\text { Custody }\end{array}$ & $\begin{array}{l}\text { Shipping } \\
\text { Date }\end{array}$ & Sample Description \\
\hline $8 / 11 / 11$ & $13: 42$ & SVQCIR-W-25894a & Water & RI & QC & - & 2990 & $8 / 11 / 11$ & $\begin{array}{l}\text { Rinsate of decontaminated sampling } \\
\text { line after collection of sample } \\
\text { SVSB44S-W-25876. }\end{array}$ \\
\hline $8 / 11 / 11$ & $14: 02$ & SVSB46D-W-25881 & Water & MW & SB46D & $90-110$ & 2990 & $8 / 11 / 11$ & $\begin{array}{l}\text { Depth to water }=58.68 \mathrm{ft} \text {. Depth of 2-in. } \\
\text { well }=108.65 \mathrm{ft} \text {. Sample collected } \\
\text { by using low-flow bladder pump } \\
\text { after purging of } 10 \mathrm{~L} \text {. Pump intake } \\
\text { positioned at } 108 \mathrm{ft} \text {. }\end{array}$ \\
\hline $8 / 11 / 11$ & $14: 03$ & SVSB46DDUP-W-25889a & Water & MW & SB46D & $90-110$ & 2990 & $8 / 11 / 11$ & $\begin{array}{l}\text { Replicate of sample SVSB46D-W- } \\
25881 \text {. }\end{array}$ \\
\hline $8 / 11 / 11$ & $15: 23$ & SVQCIR-W-25893a & Water & RI & QC & - & 2990 & $8 / 11 / 11$ & $\begin{array}{l}\text { Rinsate of decontaminated sampling } \\
\text { line after collection of sample } \\
\text { SVSB46D-W-25881 and replicate } \\
\text { SVSB46DDUP-W-25889. }\end{array}$ \\
\hline $8 / 11 / 11$ & $15: 44$ & SVSB24-W-25872 & Water & MW & SB24 & $50-60$ & 2990 & $8 / 11 / 11$ & $\begin{array}{l}\text { Started to purge three well volumes } \\
\text { with } 3 / 8 \text {-in. Waterra tubing and } \\
\text { valve. Well went dry at first volume, } \\
\text { so the tubing was removed, and a } \\
\text { sample of the water in the tubing } \\
\text { was collected. }\end{array}$ \\
\hline $8 / 11 / 11$ & $16: 13$ & SVSB46S-W-25880 & Water & MW & SB46S & $42-52$ & 2990 & 8/11/11 & $\begin{array}{l}\text { Depth to water }=21.72 \mathrm{ft} \text {. Depth of } 2 \text {-in. } \\
\text { well }=51.8 \mathrm{ft} \text {. Sample collected by } \\
\text { using low-flow bladder pump after } \\
\text { purging of } 6 \mathrm{~L} \text {. Pump intake } \\
\text { positioned at } 50 \mathrm{ft} \text {. }\end{array}$ \\
\hline $8 / 11 / 11$ & $16: 26$ & SVSB38-W-25873 & Water & MW & SB38 & $50-90$ & 2990 & $8 / 11 / 11$ & $\begin{array}{l}\text { Started to purge three well volumes } \\
\text { with } 3 / 8 \text {-in. Waterra tubing and } \\
\text { valve. Well went dry at first volume, } \\
\text { so the tubing was removed, and a } \\
\text { sample of the water in the tubing } \\
\text { was collected. }\end{array}$ \\
\hline $8 / 11 / 11$ & $17: 52$ & SVSB45S-W-25878 & Water & MW & SB45S & $37-47$ & 2990 & 8/11/11 & $\begin{array}{l}\text { Depth to water }=20.60 \mathrm{ft} \text {. Depth of } 2 \text {-in. } \\
\text { well }=47.46 \mathrm{ft} \text {. Sample collected by } \\
\text { using low-flow bladder pump after } \\
\text { purging of } 10.5 \mathrm{~L} \text {. Pump intake } \\
\text { positioned at } 45 \mathrm{ft} \text {. }\end{array}$ \\
\hline
\end{tabular}
well $=108.65 \mathrm{ft}$. Sample collected by using low-flow bladder pump positioned at $108 \mathrm{ft}$ 25881. SVSB46D-W-25881 and replicate valve. Well went dry at first volume so the tubing was removed, and a sample of the water in the tubing .

well $=51.8 \mathrm{ft}$. Sample collected by using low-flow bladder pump after purging of $6 \mathrm{~L}$. Pump intake valve. Well went dry at first volume, tubing was removed, and a was collected positioned at $45 \mathrm{ft}$. 
TABLE A.1 (Cont.)

\begin{tabular}{|c|c|c|c|c|c|c|c|c|c|}
\hline $\begin{array}{c}\text { Sample } \\
\text { Date }\end{array}$ & $\begin{array}{l}\text { Sample } \\
\text { Time }\end{array}$ & Sample & $\begin{array}{l}\text { Sample } \\
\text { Medium }\end{array}$ & $\begin{array}{c}\text { Sample } \\
\text { Type }\end{array}$ & $\begin{array}{l}\text { Sample } \\
\text { Location }\end{array}$ & $\begin{array}{c}\text { Depth } \\
\text { (ft BGL) }\end{array}$ & $\begin{array}{l}\text { Chain of } \\
\text { Custody }\end{array}$ & $\begin{array}{l}\text { Shipping } \\
\text { Date }\end{array}$ & Sample Description \\
\hline $8 / 11 / 11$ & $18: 30$ & SVQCTB-W-25897ª & Water & TB & QC & - & 2990 & $8 / 11 / 11$ & $\begin{array}{l}\text { Trip blank sent to the AGEM } \\
\text { Laboratory for organic analysis with } \\
\text { water samples listed on COC } 2990 .\end{array}$ \\
\hline $8 / 11 / 11$ & 19:09 & SVSB45D-W-25879 & Water & MW & SB45D & $79.5-99.5$ & 2990 & 8/11/11 & $\begin{array}{l}\text { Depth to water }=52.60 \mathrm{ft} \text {. Depth of } 2 \text {-in. } \\
\text { well }=98.95 \mathrm{ft} \text {. Sample collected by } \\
\text { using low-flow bladder pump after } \\
\text { purging of } 6 \mathrm{~L} \text {. Pump intake } \\
\text { positioned at } 97.5 \mathrm{ft} \text {. }\end{array}$ \\
\hline $8 / 11 / 11$ & $19: 24$ & SVDIH2O-W-25890 & Water & FB & QC & - & 2990 & $8 / 11 / 11$ & $\begin{array}{l}\text { Blank of water used for equipment } \\
\text { decontamination. Distilled water } \\
\text { from Wal-Mart. }\end{array}$ \\
\hline
\end{tabular}

a Quality control sample. 


\section{Appendix B:}

\section{Soil Sampling Data}


TABLE B.1 Results from the AGEM Laboratory for analyses of carbon tetrachloride and chloroform in soil samples.

\begin{tabular}{|c|c|c|c|c|c|c|c|}
\hline \multirow[b]{3}{*}{ Location } & \multirow[b]{3}{*}{ Sample } & \multirow[b]{3}{*}{$\begin{array}{l}\text { Sample } \\
\text { Date }\end{array}$} & \multirow[b]{3}{*}{$\begin{array}{c}\text { Depth } \\
\text { (ft BGL) }\end{array}$} & \multicolumn{4}{|c|}{ Concentration $(\mu \mathrm{g} / \mathrm{kg})$} \\
\hline & & & & \multicolumn{2}{|c|}{ Headspace Analysis $^{\mathrm{a}}$} & \multicolumn{2}{|c|}{ Purge-and-Trap Analysis ${ }^{\mathrm{b}}$} \\
\hline & & & & $\begin{array}{l}\text { Carbon } \\
\text { Tetrachloride }\end{array}$ & Chloroform & $\begin{array}{c}\text { Carbon } \\
\text { Tetrachloride }\end{array}$ & Chloroform \\
\hline SB43 & SVSB43-S-25640 & $6 / 21 / 10$ & 4 & $N D^{c}$ & ND & ND & ND \\
\hline SB43 & SVSB43-S-25642 & $6 / 21 / 10$ & 8 & ND & ND & ND & ND \\
\hline SB43 & SVSB43-S-25641 & $6 / 21 / 10$ & 12 & ND & ND & ND & ND \\
\hline SB43 & SVSB43-S-25643 & $6 / 21 / 10$ & 18 & ND & ND & ND & ND \\
\hline SB43 & SVSB43-S-25644 & $6 / 21 / 10$ & 28 & ND & ND & ND & ND \\
\hline SB43 & SVSB43-S-25645 & $6 / 21 / 10$ & 32 & ND & ND & ND & ND \\
\hline SB43 & SVSB43-S-25646 & $6 / 21 / 10$ & 36 & ND & ND & ND & ND \\
\hline SB43 & SVSB43-S-25647 & $6 / 21 / 10$ & 40 & ND & ND & ND & $2.8 \mathrm{~J}^{\mathrm{d}}$ \\
\hline SB43 & SVSB43-S-25649 & $6 / 21 / 10$ & 45 & ND & ND & ND & ND \\
\hline SB43 & SVSB43-S-25648 & $6 / 21 / 10$ & 48 & ND & ND & ND & ND \\
\hline SB43 & SVSB43-S-25648DUPe & $6 / 21 / 10$ & 48 & $N A^{f}$ & NA & ND & ND \\
\hline SB43 & SVSB43-S-25650 & $6 / 21 / 10$ & 48 & ND & ND & ND & ND \\
\hline SB43 & SVSB43-S-25651 & $6 / 21 / 10$ & 52 & ND & ND & ND & $2.2 \mathrm{~J}$ \\
\hline SB43 & SVSB43-S-25652 & $6 / 21 / 10$ & 56 & ND & ND & ND & ND \\
\hline SB43 & SVSB43-S-25653 & $6 / 21 / 10$ & 60 & ND & ND & ND & ND \\
\hline SB43 & SVSB43-S-25654 & $6 / 21 / 10$ & 64 & ND & ND & ND & ND \\
\hline SB43 & SVSB43-S-25656 & $6 / 21 / 10$ & 65 & ND & ND & ND & ND \\
\hline SB43 & SVSB43-S-25655 & $6 / 21 / 10$ & 70 & ND & ND & ND & ND \\
\hline SB43 & SVSB43-S-25657 & $6 / 21 / 10$ & 76 & ND & ND & ND & $2.5 \mathrm{~J}$ \\
\hline SB43 & SVSB43-S-25658 & $6 / 21 / 10$ & 80 & ND & ND & ND & ND \\
\hline SB43 & SVSB43-S-25658DUPe & $6 / 21 / 10$ & 80 & NA & NA & ND & ND \\
\hline SB43 & SVSB43-S-25659 & $6 / 21 / 10$ & 84 & ND & ND & ND & ND \\
\hline SB43 & SVSB43-S-25660 & $6 / 21 / 10$ & 88 & ND & ND & ND & ND \\
\hline SB43 & SVSB43-S-25661 & $6 / 21 / 10$ & 92 & ND & ND & ND & ND \\
\hline SB43 & SVSB43-S-25662 & $6 / 21 / 10$ & 92.7 & ND & ND & ND & ND \\
\hline SB43 & SVSB43-S-25663 & $6 / 21 / 10$ & 96 & ND & ND & ND & ND \\
\hline SB43 & SVSB43-S-256649 & $6 / 21 / 10$ & 100 & ND & ND & ND & ND \\
\hline SB43 & SVSB43-S-25665 h & $6 / 21 / 10$ & 100 & ND & ND & ND & ND \\
\hline SB43 & SVSB43-S-25670 & $6 / 21 / 10$ & 102 & ND & ND & ND & ND \\
\hline SB43 & SVSB43-S-25672g & $6 / 22 / 10$ & 104 & ND & ND & ND & ND \\
\hline SB43 & SVSB43-S-25673 h & $6 / 22 / 10$ & 104 & ND & ND & ND & ND \\
\hline SB44 & SVSB44-S-25676 & $6 / 23 / 10$ & 4 & 0.1 & ND & ND & ND \\
\hline SB44 & SVSB44-S-25677 & $6 / 23 / 10$ & 8 & ND & ND & ND & ND \\
\hline SB44 & SVSB44-S-25678 & $6 / 23 / 10$ & 12 & ND & ND & ND & ND \\
\hline SB44 & SVSB44-S-25679 & $6 / 23 / 10$ & 16 & ND & ND & ND & ND \\
\hline SB44 & SVSB44-S-25680 & $6 / 23 / 10$ & 20 & 0.3 & ND & ND & ND \\
\hline SB44 & SVSB44-S-25681 & $6 / 23 / 10$ & 24 & 0.2 & ND & ND & ND \\
\hline SB44 & SVSB44-S-25682 & $6 / 23 / 10$ & 28 & 1.0 & ND & $2.3 \mathrm{~J}$ & $1.8 \mathrm{~J}$ \\
\hline SB44 & SVSB44-S-25683 & $6 / 23 / 10$ & 32 & 0.4 & ND & $4.8 \mathrm{~J}$ & ND \\
\hline SB44 & SVSB44-S-25684 & $6 / 23 / 10$ & 36 & 3.6 & ND & $4.7 \mathrm{~J}$ & ND \\
\hline SB44 & SVSB44-S-25685 & $6 / 23 / 10$ & 40 & 0.5 & ND & 14 & ND \\
\hline SB44 & SVSB44-S-25686 & $6 / 23 / 10$ & 44 & 263.4 & ND & 364 & $2.0 \mathrm{~J}$ \\
\hline SB44 & SVSB44-S-25696 & $6 / 23 / 10$ & 45 & 3.6 & ND & 135 & $5.2 \mathrm{~J}$ \\
\hline SB44 & SVSB44-S-25687 & $6 / 23 / 10$ & 48 & 60.6 & ND & 212 & $3.2 \mathrm{~J}$ \\
\hline SB44 & SVSB44-S-25688 & $6 / 23 / 10$ & 52 & 77.0 & ND & 139 & ND \\
\hline SB44 & SVSB44-S-25688DUPe & $6 / 23 / 10$ & 52 & NA & NA & 151 & ND \\
\hline SB44 & SVSB44-S-25689 & $6 / 23 / 10$ & 56 & 56.1 & ND & 106 & ND \\
\hline
\end{tabular}




\begin{tabular}{|c|c|c|c|c|c|c|c|}
\hline \multirow[b]{3}{*}{ Location } & \multirow[b]{3}{*}{ Sample } & \multirow[b]{3}{*}{$\begin{array}{l}\text { Sample } \\
\text { Date }\end{array}$} & \multirow[b]{3}{*}{$\begin{array}{c}\text { Depth } \\
\text { (ft BGL) }\end{array}$} & \multicolumn{4}{|c|}{ Concentration $(\mu \mathrm{g} / \mathrm{kg})$} \\
\hline & & & & \multicolumn{2}{|c|}{ Headspace Analysis $^{a}$} & \multicolumn{2}{|c|}{ Purge-and-Trap Analysis ${ }^{\mathfrak{b}}$} \\
\hline & & & & $\begin{array}{c}\text { Carbon } \\
\text { Tetrachloride }\end{array}$ & Chloroform & $\begin{array}{c}\text { Carbon } \\
\text { Tetrachloride }\end{array}$ & Chloroform \\
\hline SB44 & SVSB44-S-25690 & $6 / 23 / 10$ & 60 & 5.6 & ND & 18 & ND \\
\hline SB44 & SVSB44-S-25691 & $6 / 23 / 10$ & 64 & 0.4 & ND & ND & ND \\
\hline SB44 & SVSB44-S-25692 & $6 / 23 / 10$ & 68 & ND & ND & ND & $2.8 \mathrm{~J}$ \\
\hline SB44 & SVSB44-S-25693 & $6 / 23 / 10$ & 72 & ND & ND & ND & ND \\
\hline SB44 & SVSB44-S-25693DUPe & $6 / 23 / 10$ & 72 & NA & NA & ND & ND \\
\hline SB44 & SVSB44-S-25694 & $6 / 23 / 10$ & 76 & ND & ND & ND & ND \\
\hline SB44 & SVSB44-S-25704 & $6 / 23 / 10$ & 76.5 & ND & ND & ND & ND \\
\hline SB44 & SVSB44-S-25695 & $6 / 23 / 10$ & 80 & ND & ND & ND & ND \\
\hline SB44 & SVSB44-S-25705f & $6 / 23 / 10$ & 80 & ND & ND & ND & ND \\
\hline SB44 & SVSB44-S-25697 & $6 / 23 / 10$ & 84 & ND & ND & ND & ND \\
\hline SB44 & SVSB44-S-25708 & $6 / 23 / 10$ & 88 & ND & ND & ND & ND \\
\hline SB44 & SVSB44-S-25698 & $6 / 23 / 10$ & 89 & ND & ND & ND & ND \\
\hline SB44 & SVSB44-S-25699 & $6 / 23 / 10$ & 92 & ND & ND & ND & ND \\
\hline SB44 & SVSB44-S-25700 & $6 / 23 / 10$ & 96 & ND & ND & ND & ND \\
\hline SB44 & SVSB44-S-25701 & $6 / 23 / 10$ & 100 & ND & ND & ND & ND \\
\hline SB44 & SVSB44-S-25702 & $6 / 23 / 10$ & 104 & 0.3 & ND & ND & ND \\
\hline SB44 & SVSB44-S-25709 & $6 / 23 / 10$ & 105 & 0.1 & ND & ND & ND \\
\hline SB45 & SVSB45-S-25720 & $6 / 24 / 10$ & 2.5 & ND & ND & ND & ND \\
\hline SB45 & SVSB45-S-25721 & $6 / 24 / 10$ & 8 & 0.1 & ND & ND & ND \\
\hline SB45 & SVSB45-S-25722 & $6 / 24 / 10$ & 12 & ND & ND & 12 & ND \\
\hline SB45 & SVSB45-S-25723 & $6 / 24 / 10$ & 16 & 0.2 & ND & ND & ND \\
\hline SB45 & SVSB45-S-25730 & $6 / 24 / 10$ & 20 & 3.6 & ND & $8.6 \mathrm{~J}$ & ND \\
\hline SB45 & SVSB45-S-25724 & $6 / 24 / 10$ & 22.4 & 4.0 & ND & $7.7 \mathrm{~J}$ & ND \\
\hline SB45 & SVSB45-S-25725 & $6 / 24 / 10$ & 25 & 29.9 & ND & 32 & ND \\
\hline SB45 & SVSB45-S-25726 & $6 / 24 / 10$ & 28 & 15.5 & ND & 28 & ND \\
\hline SB45 & SVSB45-S-25727 & $6 / 24 / 10$ & 32 & 27.4 & ND & 38 & ND \\
\hline SB45 & SVSB45-S-25728 & $6 / 24 / 10$ & 36 & 15.4 & ND & 41 & $1.6 \mathrm{~J}$ \\
\hline SB45 & SVSB45-S-25729 & $6 / 24 / 10$ & 40 & 12.6 & ND & 25 & ND \\
\hline SB45 & SVSB45-S-25731 & $6 / 24 / 10$ & 44 & 39.9 & ND & 63 & $1.7 \mathrm{~J}$ \\
\hline SB45 & SVSB45-S-25731DUPe & $6 / 24 / 10$ & 44 & NA & NA & 65 & $1.9 \mathrm{~J}$ \\
\hline SB45 & SVSB45-S-25732 & $6 / 24 / 10$ & 48 & 1.4 & ND & ND & ND \\
\hline SB45 & SVSB45-S-25733 & $6 / 24 / 10$ & 52 & ND & ND & ND & ND \\
\hline SB45 & SVSB45-S-25734 & $6 / 24 / 10$ & 56 & 0.1 & ND & ND & ND \\
\hline SB45 & SVSB45-S-25735 & $6 / 24 / 10$ & 60 & 0.3 & ND & ND & ND \\
\hline SB45 & SVSB45-S-25736 & $6 / 24 / 10$ & 64 & 0.1 & ND & ND & ND \\
\hline SB45 & SVSB45-S-25737 & $6 / 24 / 10$ & 68 & ND & ND & ND & ND \\
\hline SB45 & SVSB45-S-25738 & $6 / 24 / 10$ & 72 & 0.3 & ND & ND & ND \\
\hline SB45 & SVSB45-S-25739 & $6 / 24 / 10$ & 76 & 0.1 & ND & ND & ND \\
\hline SB45 & SVSB45-S-25740 & $6 / 24 / 10$ & 80 & ND & ND & ND & ND \\
\hline SB45 & SVSB45-S-25741 & $6 / 24 / 10$ & 84 & ND & ND & ND & ND \\
\hline SB45 & SVSB45-S-25742 & $6 / 24 / 10$ & 88 & ND & ND & ND & ND \\
\hline SB45 & SVSB45-S-25743 & $6 / 24 / 10$ & 92 & 0.2 & ND & ND & ND \\
\hline SB45 & SVSB45-S-25744 & $6 / 24 / 10$ & 96 & ND & ND & ND & ND \\
\hline SB45 & SVSB45-S-25745 & $6 / 24 / 10$ & 100 & ND & ND & ND & ND \\
\hline SB45 & SVSB45-S-25747e & $6 / 24 / 10$ & 100 & ND & ND & ND & ND \\
\hline SB46 & SVSB46-S-25710 & $6 / 25 / 10$ & 2 & 3.2 & ND & $2.8 \mathrm{~J}$ & ND \\
\hline SB46 & SVSB46-S-25711 & $6 / 25 / 10$ & 8 & 9.2 & ND & 22 & $4.0 \mathrm{~J}$ \\
\hline SB46 & SVSB46-S-25712 & $6 / 25 / 10$ & 12 & 13.6 & ND & 16 & $2.1 \mathrm{~J}$ \\
\hline SB46 & SVSB46-S-25713 & $6 / 26 / 10$ & 16 & 41.2 & ND & 31 & $2.9 \mathrm{~J}$ \\
\hline
\end{tabular}




\begin{tabular}{|c|c|c|c|c|c|c|c|}
\hline \multirow[b]{3}{*}{ Location } & \multirow[b]{3}{*}{ Sample } & \multirow[b]{3}{*}{$\begin{array}{l}\text { Sample } \\
\text { Date }\end{array}$} & \multirow[b]{3}{*}{$\begin{array}{c}\text { Depth } \\
\text { (ft BGL) }\end{array}$} & \multicolumn{4}{|c|}{ Concentration $(\mu \mathrm{g} / \mathrm{kg})$} \\
\hline & & & & \multicolumn{2}{|c|}{ Headspace Analysis $^{a}$} & \multicolumn{2}{|c|}{ Purge-and-Trap Analysis ${ }^{\mathrm{b}}$} \\
\hline & & & & $\begin{array}{c}\text { Carbon } \\
\text { Tetrachloride }\end{array}$ & Chloroform & $\begin{array}{c}\text { Carbon } \\
\text { Tetrachloride }\end{array}$ & Chloroform \\
\hline SB46 & SVSB46-S-25714 & $6 / 26 / 10$ & 20 & 61.4 & ND & 45 & $3.1 \mathrm{~J}$ \\
\hline SB46 & SVSB46-S-25716 & $6 / 25 / 10$ & 28 & 2.2 & ND & 12 & $2.9 \mathrm{~J}$ \\
\hline SB46 & SVSB46-S-25718 & $6 / 25 / 10$ & 36 & 4.0 & ND & $8.7 \mathrm{~J}$ & ND \\
\hline SB46 & SVSB46-S-25766e & $6 / 25 / 10$ & 36 & 6.0 & ND & $7.2 \mathrm{~J}$ & ND \\
\hline SB46 & SVSB46-S-25719 & $6 / 25 / 10$ & 40 & 30.5 & ND & 81 & ND \\
\hline SB46 & SVSB46-S-25750 & $6 / 25 / 10$ & 44 & 34.1 & ND & 82 & ND \\
\hline SB46 & SVSB46-S-25751 & $6 / 25 / 10$ & 48 & 16.5 & ND & 30 & ND \\
\hline SB46 & SVSB46-S-25752 & $6 / 25 / 10$ & 52 & 0.9 & ND & ND & ND \\
\hline SB46 & SVSB46-S-25753 & $6 / 25 / 10$ & 56 & 0.2 & ND & ND & ND \\
\hline SB46 & SVSB46-S-25754 & $6 / 25 / 10$ & 60 & 0.2 & ND & ND & ND \\
\hline SB46 & SVSB46-S-25755 & $6 / 25 / 10$ & 64 & ND & ND & ND & ND \\
\hline SB46 & SVSB46-S-25756 & $6 / 25 / 10$ & 68 & ND & ND & ND & ND \\
\hline SB46 & SVSB46-S-25757 & $6 / 25 / 10$ & 72 & ND & ND & ND & ND \\
\hline SB46 & SVSB46-S-25758 & $6 / 25 / 10$ & 76 & ND & ND & ND & ND \\
\hline SB46 & SVSB46-S-25759 & $6 / 25 / 10$ & 80 & ND & ND & ND & ND \\
\hline SB46 & SVSB46-S-25760 & $6 / 25 / 10$ & 84 & 0.2 & ND & ND & ND \\
\hline SB46 & SVSB46-S-25761 & $6 / 25 / 10$ & 88 & ND & ND & ND & ND \\
\hline SB46 & SVSB46-S-25761DUPe & $6 / 25 / 10$ & 88 & NA & NA & ND & ND \\
\hline SB46 & SVSB46-S-25762 & $6 / 25 / 10$ & 92 & ND & ND & ND & ND \\
\hline SB46 & SVSB46-S-25764 & $6 / 25 / 10$ & 96 & 0.1 & ND & ND & ND \\
\hline SB46 & SVSB46-S-25763 & $6 / 25 / 10$ & 100 & ND & ND & ND & ND \\
\hline SB46 & SVSB46-S-25767 & $6 / 25 / 10$ & 104 & 0.2 & ND & ND & ND \\
\hline SB46 & SVSB46-S-25768 & $6 / 25 / 10$ & 108 & ND & ND & ND & ND \\
\hline $\mathrm{QC}$ & SVSB43-S-25675 & $6 / 22 / 10$ & - & ND & ND & ND & ND \\
\hline QC & SVSB46-S-25765 & $6 / 25 / 10$ & - & ND & ND & ND & ND \\
\hline QC & SVQCTB-S-25715 & $6 / 26 / 10$ & - & 0.2 & ND & ND & ND \\
\hline QC & SVSB45-S-25746 & $6 / 24 / 10$ & - & 0.3 & ND & ND & ND \\
\hline QC & SVSB44-S-25707 & $6 / 23 / 10$ & - & ND & ND & ND & ND \\
\hline $\mathrm{QC}$ & SVSB45-S-25746DUPe & $6 / 24 / 10$ & - & NA & NA & ND & ND \\
\hline QC & SVQCTB-S-25671 & $6 / 21 / 10$ & - & ND & ND & ND & ND \\
\hline
\end{tabular}

a Analyzed by monified EPA Method 5021 (headspace analysis by gas chromatography with electron capture detection).

b Analyzed by modified EPA Methods 5030B and 8260B (purge-and-trap sample preparation with analysis by gas chromatography-mass spectrometry).

c ND, not detected at the instrument detection limit of $0.1 \mu \mathrm{g} / \mathrm{kg}$ for carbon tetrachloride or $0.75 \mu \mathrm{g} / \mathrm{kg}$ for chloroform for headspace analysis or $1 \mu \mathrm{g} / \mathrm{kg}$ for purge-and-trap analysis.

d Qualifier J indicates an estimated concentration below the purge-and-trap method quantitation limit of $10 \mu \mathrm{g} / \mathrm{kg}$.

e Quality control sample (laboratory duplicate or field replicate).

f NA, not analyzed by the headspace method.

$\mathrm{g}$ Subsample of more silty material at the indicated depth.

h Subsample of more calcareous material at the indicated depth. 
TABLE B.2 Results of grain size analyses for soil samples. ${ }^{\text {a }}$

Percent Passing through Sieve of Indicated Size

Composition (\%)

\begin{tabular}{|c|c|c|c|c|c|c|c|c|c|c|c|c|c|c|c|c|c|c|c|c|c|c|c|}
\hline \multirow[b]{2}{*}{ Location } & \multirow[b]{2}{*}{ Sample } & \multirow{2}{*}{$\begin{array}{l}\text { Depth Interval } \\
\text { (ft BGL) }\end{array}$} & \multicolumn{17}{|c|}{ Percent Passing through Sieve of Indicated Size } & \multicolumn{4}{|c|}{ Composition (\%) } \\
\hline & & & $1.5 \mathrm{in.}$ & 1 in. & $3 / 4 \mathrm{in.}$ & $1 / 2 \mathrm{in}$. & $3 / 8 \mathrm{in.}$ & $\# 4$ & $\# 10$ & $\# 18$ & $\# 35$ & \#40 & $\# 60$ & $\# 100$ & $\# 120$ & $\# 140$ & \#200 & $\# 230$ & \#270 & Gravel & Sand & Silt & Clay \\
\hline \multirow[t]{4}{*}{ SB43 } & s & & & & & & & 0 & & & 99.9 & 9 & & 6 & & & 1.1 & & & 0.0 & 9 & 51.2 & 6.9 \\
\hline & & & & & & & & & & & & & & & & & & & & & & & \\
\hline & & & & & & & & & & & & & & & & & & & & & & & \\
\hline & & & & 100 & 100 & 100 & 00 & 100 & 100 & 5 & 99.4 & & 99 . & 0.1 & & & & & & & & J.J & 52.8 \\
\hline \multirow[t]{6}{*}{ SB44 } & s & $33.0-33$ & & 100.0 & 1 & 100.0 & & 100 & 100 & & 5 & 99.4 & 99.3 & 1 & & 99.0 & 4 & & & 0.0 & 2.6 & 68.9 & .5 \\
\hline & & & & & & & & & & & & & & & & & & & & & & & \\
\hline & & & & 10 & & & & & & & & & & & & & & & & & 1 & & \\
\hline & & & & & & & & & & & & & & & & & & & & & & & \\
\hline & & & & & & & & & & & & & & & & & & & & & & & \\
\hline & s) & $101.0-1$ & 100.0 & 100.0 & 100.0 & 100.0 & 100.0 & 98.9 & 97.3 & 95.4 & 92.2 & 91.2 & 80. & 10.1 & 11 & 10.5 & 71.9 & 10.0 & 00.0 & 1.1 & 27.0 & 37.7 & 4.2 \\
\hline \multirow[t]{2}{*}{ SB45 } & VSB45- & 41.0- & 100.0 & 100.0 & 100.0 & 100.0 & 100 & 100.0 & 100.0 & 99.9 & 99.7 & & 0 & .1 & 99.0 & 8 & 9 & 95.6 & & 0.0 & 2.5 & 51.1 & \\
\hline & $\begin{array}{l}\text { SVSB44-S } \\
\text { SVSB45-S }\end{array}$ & 101.7 & 100.0 & $\begin{array}{l}100.0 \\
100.0\end{array}$ & $\begin{array}{l}100.0 \\
100.0\end{array}$ & $\begin{array}{l}100.0 \\
100.0\end{array}$ & 100.0 & $\begin{array}{r}99.8 \\
100.0\end{array}$ & $\begin{array}{r}97.8 \\
100.0\end{array}$ & $\begin{array}{r}93.6 \\
100.0\end{array}$ & $\begin{array}{l}90.4 \\
99.9\end{array}$ & $\begin{array}{l}89.8 \\
99.9\end{array}$ & $\begin{array}{l}86.9 \\
99.9\end{array}$ & $\begin{array}{l}74.3 \\
99.8\end{array}$ & 99.8 & 99.8 & 99.7 & 99.6 & 99.5 & 0.0 & 0.3 & 369 & $\begin{array}{l}18.0 \\
62.8\end{array}$ \\
\hline
\end{tabular}

Analyses were by Alfred Benesch and Company, Lincoln, Nebraska, with ASTM D442-90, "Method for Particle Size Analysis of Soils," and ASTM D854, "Standard Test Method for Specific Gravity of Soil Solids by Water Pycnometer."

TABLE B.3 Results of physical properties analyses for soil samples. ${ }^{\text {a }}$

\begin{tabular}{|c|c|c|c|c|c|c|c|c|}
\hline Location & Sample & $\begin{array}{l}\text { Depth Interval } \\
\text { (ft BGL) }\end{array}$ & $\begin{array}{l}\text { Bulk Dry } \\
\text { Density } \\
\left(\mathrm{lb} / \mathrm{ft}^{3}\right)\end{array}$ & $\begin{array}{l}\text { Moisture } \\
\text { Content } \\
(\%)\end{array}$ & $\begin{array}{l}\text { Specific } \\
\text { Gravity }\end{array}$ & $\begin{array}{l}\text { Total } \\
\text { Organic } \\
\text { Carbon } \\
(\%)\end{array}$ & $\begin{array}{l}\text { Porosity } \\
\text { (\%) }\end{array}$ & $\begin{array}{l}\text { Hydraulic } \\
\text { Conductivity } \\
(\mathrm{cm} / \mathrm{s})\end{array}$ \\
\hline SB43 & $\begin{array}{l}\text { SVSB43-S-28983 } \\
\text { SVSB43-S-28984 } \\
\text { SVSB43-S-28986 } \\
\text { SVSB43-S-28985 }\end{array}$ & $\begin{array}{c}52.5-53.0 \\
62.5-63.0 \\
98.3-98.6 \\
102.5-102.8\end{array}$ & $\begin{array}{l}106.61 \\
103.04 \\
117.39 \\
118.71\end{array}$ & $\begin{array}{l}20.23 \\
23.46 \\
12.12 \\
14.28\end{array}$ & $\begin{array}{l}2.54 \\
2.49 \\
2.68 \\
2.68\end{array}$ & $\begin{array}{l}0.76 \\
1.04 \\
- \\
-\end{array}$ & $\begin{array}{l}36.7 \\
33.6 \\
29.8 \\
28.9\end{array}$ & $\begin{array}{l}- \\
- \\
- \\
-\end{array}$ \\
\hline SB44 & $\begin{array}{l}\text { SVSB44-S-28987 } \\
\text { SVSB44-S-28988 } \\
\text { SVSB44-S-28989 } \\
\text { SVSB44-S-29217 } \\
\text { SVSB44-S-28990 } \\
\text { SVSB44-S-28991 }\end{array}$ & $\begin{array}{r}33.0-33.5 \\
48.5-49.0 \\
54.5-55.0 \\
87.0-87.5 \\
93.2-93.5 \\
101.0-101.4\end{array}$ & $\begin{array}{r}98.56 \\
106.58 \\
107.13 \\
126.60 \\
114.03\end{array}$ & $\begin{array}{r}11.30 \\
16.52 \\
20.59 \\
8.59 \\
14.70 \\
12.90\end{array}$ & $\begin{array}{l}2.62 \\
2.58 \\
2.57 \\
2.68 \\
2.66 \\
2.65\end{array}$ & $\begin{array}{l}0.41 \\
0.64 \\
0.07 \\
0.41 \\
- \\
-\end{array}$ & $\begin{array}{l}39.7 \\
32.7 \\
33.1 \\
24.2 \\
31.2\end{array}$ & $\begin{array}{c}1.2 \times 10^{-7} \\
1.41 \times 10^{-7} \\
- \\
- \\
2.56 \times 10^{-7} \\
-\end{array}$ \\
\hline SB45 & $\begin{array}{l}\text { SVSB45-S-28992 } \\
\text { SVSB45-S-28993 } \\
\text { SVSB45-S-28994 }\end{array}$ & $\begin{array}{c}41.0-41.5 \\
88.5-89.0 \\
101.7-102.0\end{array}$ & $\begin{array}{l}108.82 \\
124.59 \\
120.11\end{array}$ & $\begin{array}{l}20.20 \\
12.45 \\
13.11\end{array}$ & $\begin{array}{l}2.52 \\
2.69 \\
2.63\end{array}$ & $\begin{array}{l}0.76 \\
0.29 \\
-\end{array}$ & $\begin{array}{l}30.9 \\
25.8 \\
26.8\end{array}$ & $\begin{array}{l}- \\
- \\
-\end{array}$ \\
\hline
\end{tabular}

a Bulk density and porosity were determined by the "Volumetric and Displacement Method" of the Corps of

Engineers, EM1110-2-1906, Appendix II Unit Weights, Void Ratio, and Porosity, by Alfred Benesch and Lampany, Lincoln, Nebraska. Tola 


\section{Appendix C:}

\section{Lithologic Logs}


Argonne National Laboratory Project: Savannah, MO Phase II

Geologist: Bob Sedivy

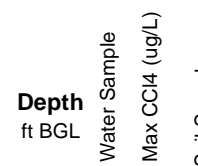

产
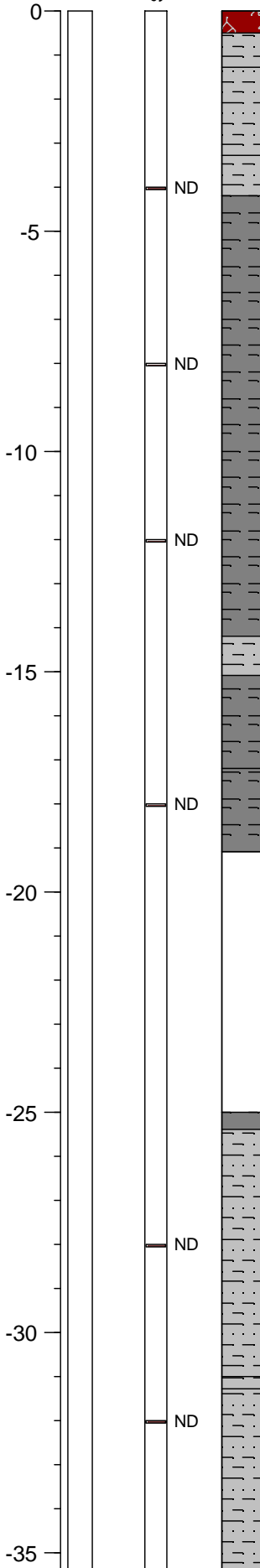
olive brown $2.5 \mathrm{Y}$. $2.5 Y$.

NO RECOVERY
Well ID: SB43

Log Date: 6/22/2010
Elevation: $1154.985 \mathrm{ft}$

Depth: $109 \mathrm{ft} \mathrm{BGL}$

TOPSOIL: With roots, very dark brown, 10YR.

SILTY CLAY WITH SAND: Fine sand, dry-damp, with faint orange (iron) and black (manganese?) streaks, dark yellowish brown 10YR.

SILTY CLAY WITH SAND: Fine sand, dry-damp, stiff to friable, occasional corroded calcareous grains/nodules, occasional coarse sand grains, very dark brown to black 10YR.

SILTY CLAY WITH SAND: Fine-medium sand, dry-damp, non-calcareous, occasional black streaks and corroded blebs, faint orange staining, brown 10YR.

SILTY CLAY: Uniform texture, damp, firm to soft-plastic, occasional patchy orange staining, light

SILTY CLAY WITH SAND: Fine sand, dry-friable to damp, streaky to mottled orange staining, dark yellowish brown 10YR.

SILTY CLAY: With trace fine sand, uniform texture, damp, firm to soft-plastic, light olive brown

SILTY CLAY: Damp, firm-stiff, dense, with fine sand 17.2'-18.2', patchy to streaky orange staining, occasional corroded black grains and streaks, yellowish brown 10YR.

SILTY CLAY: Damp, firm-stiff, dense, with patchy dark brown to black staining, brown 10YR

SILTY CLAY WITH SAND: Fine sand, damp, firm-stiff, dense, with light streaky orange to patchy black staining, brown to dark yellowish brown 10YR.

SILTY CLAY WITH SAND: As above, but with very dark brown to black (10YR) grains and patches, dark brown 2.5Y.

SILTY CLAY WITH SAND: Fine sand, damp, dense, firm-stiff to 35.3', slightly softer, more plastic 35.3'-37.4', occasional streaky to patchy orange staining and black grains, patches, and streaks, brown 10YR to dark grayish brown $2.5 \mathrm{Y}$. 


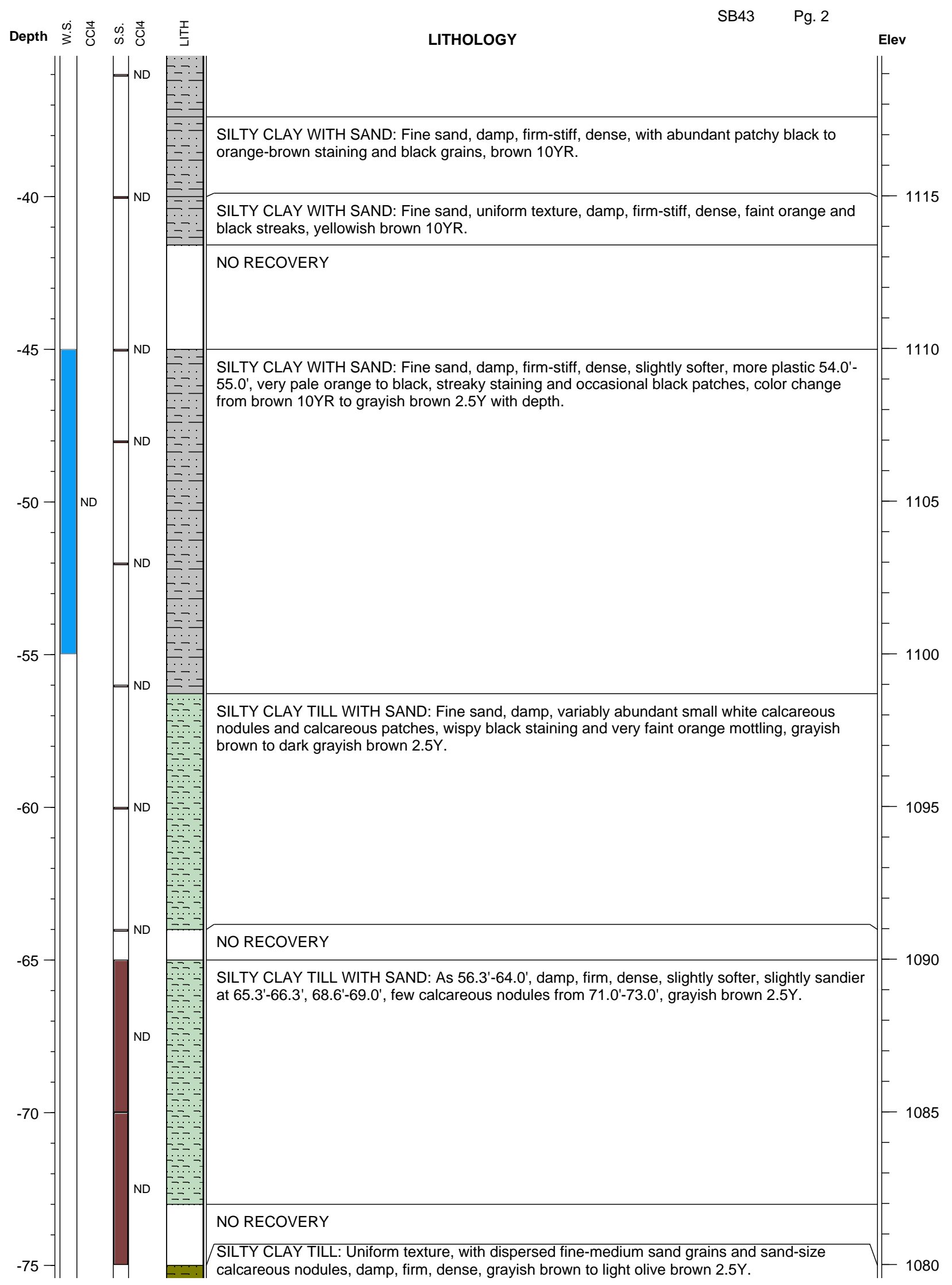




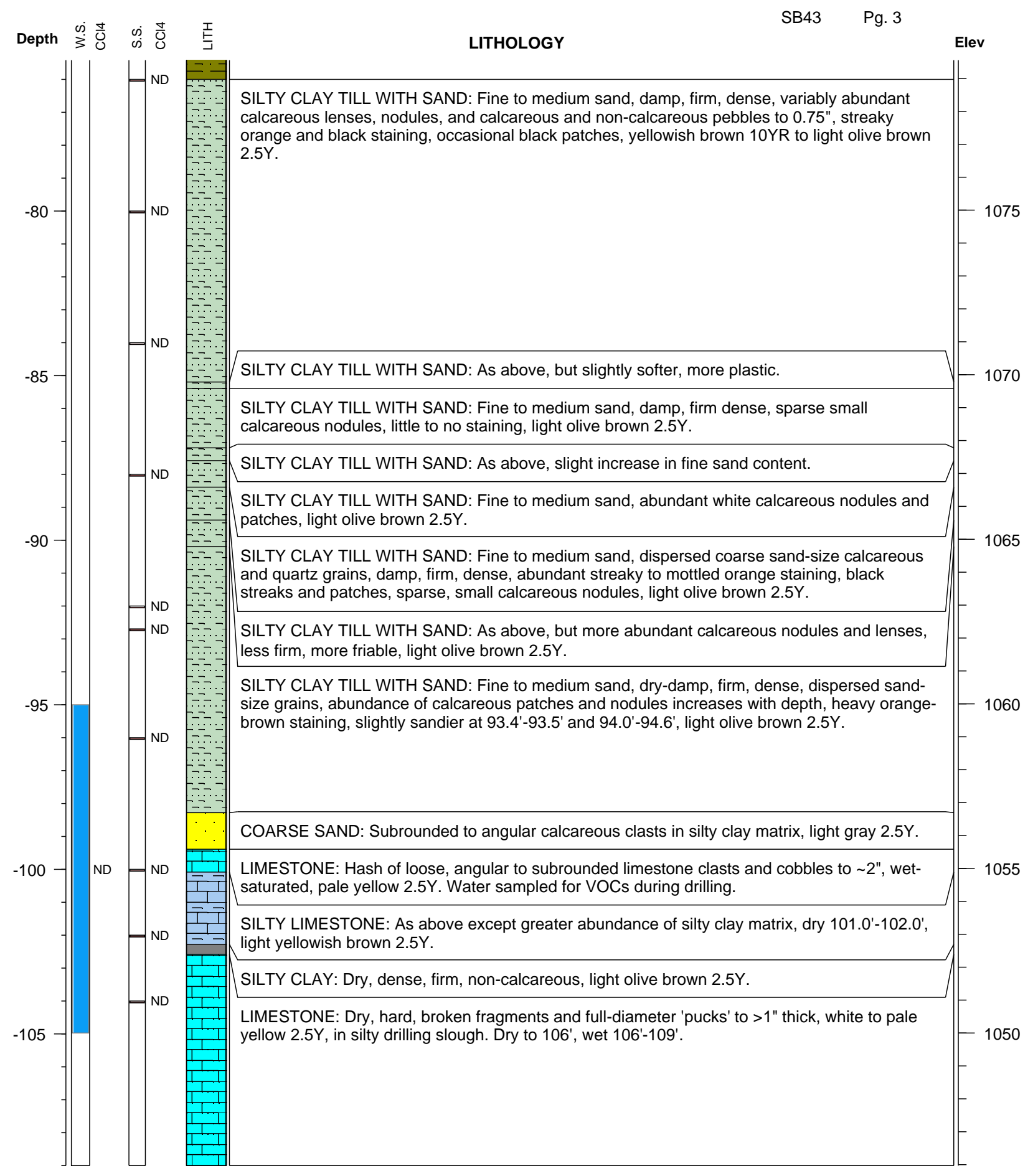




\section{Argonne National Laboratory} Project: Savannah, MO Phase II

Geologist: Bob Sedivy

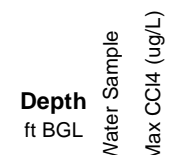

$\mathrm{ftBGL}$

$07 \square$

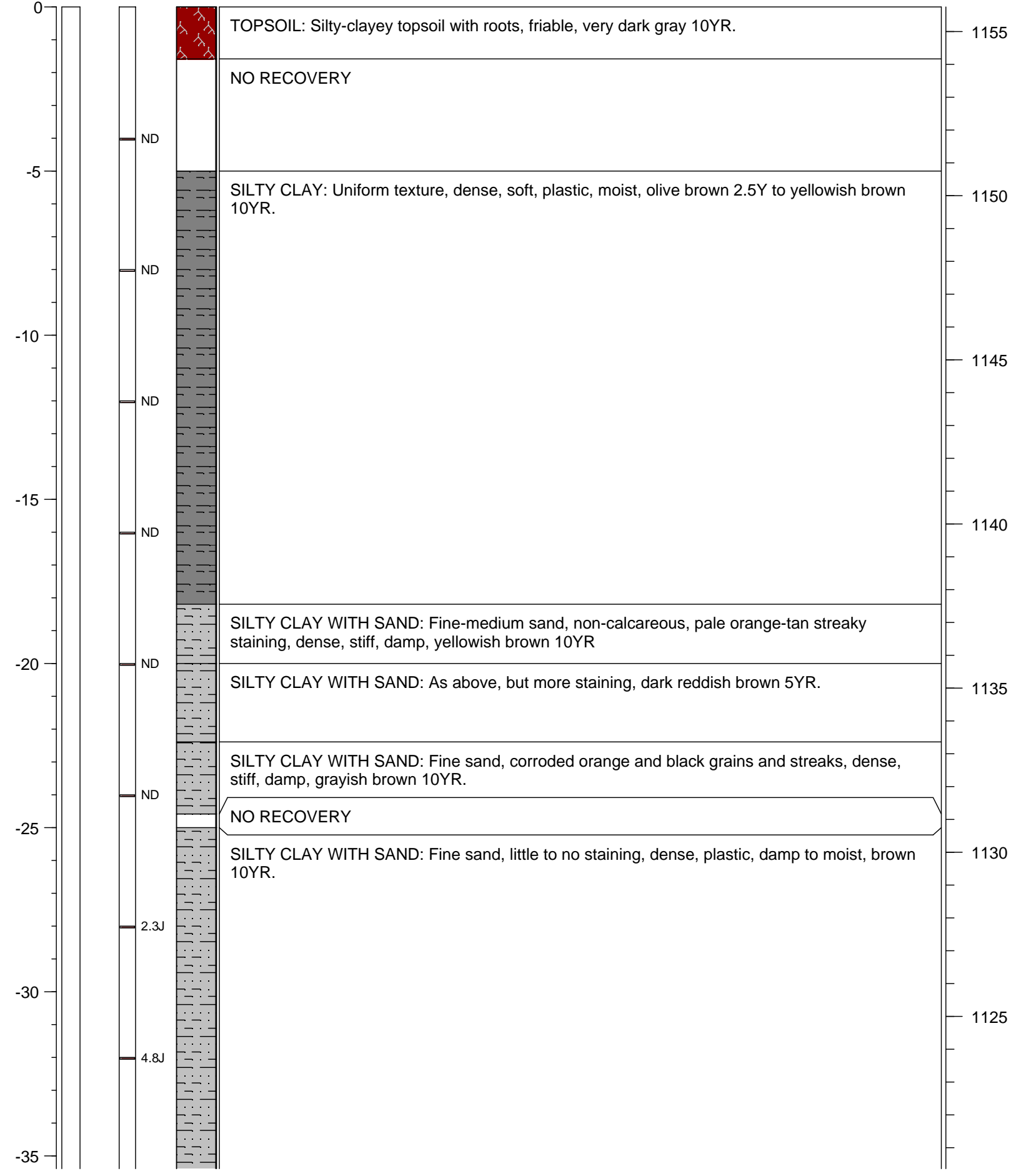

Well ID: SB44

Elevation: $1155.769 \mathrm{ft}$

Depth: $112 \mathrm{ft} \mathrm{BGL}$

Log Date: 6/23/2010

LITHOLOGY DESCRIPTION
Elev $\mathrm{ft}$ AMSL 


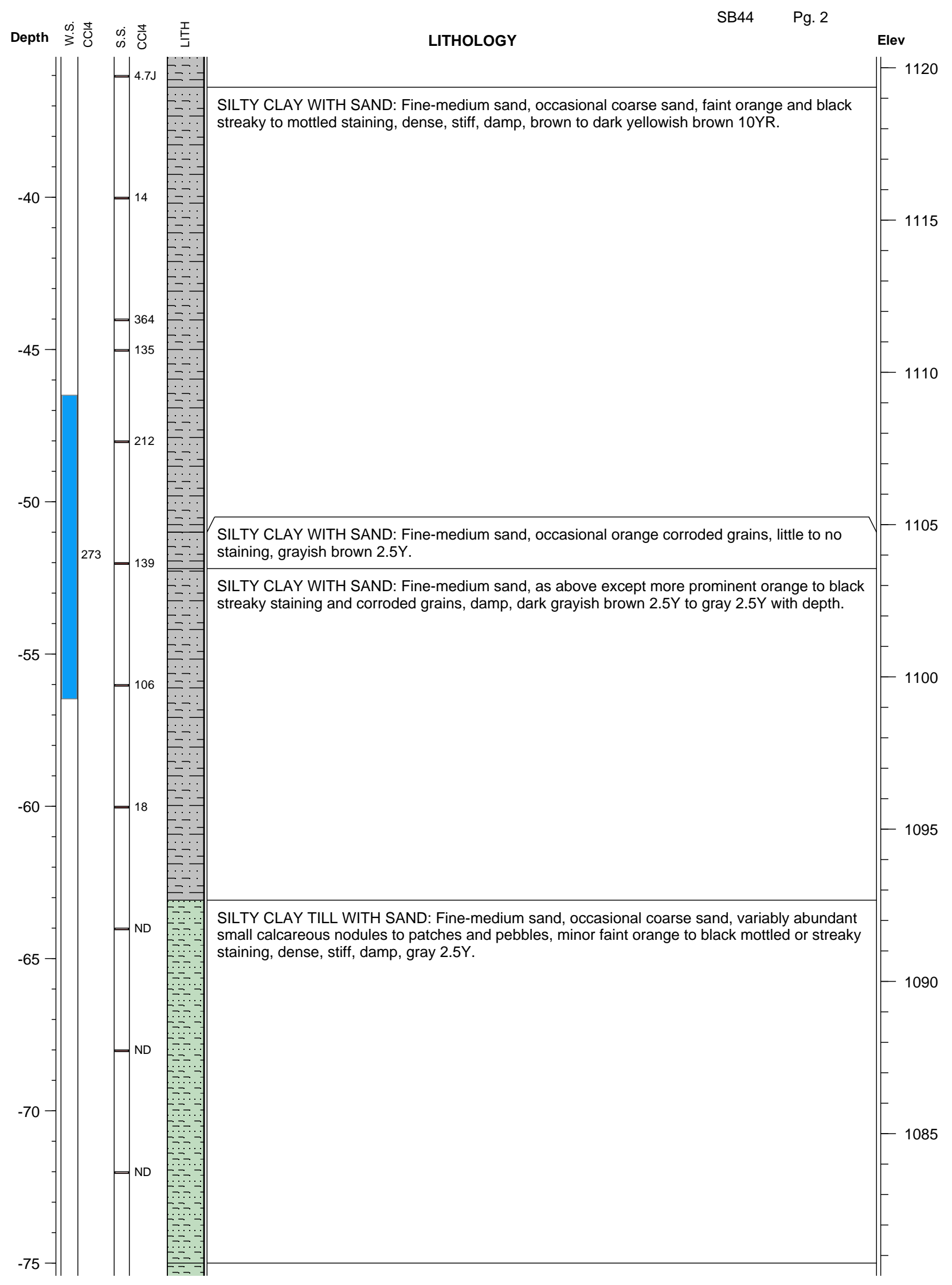




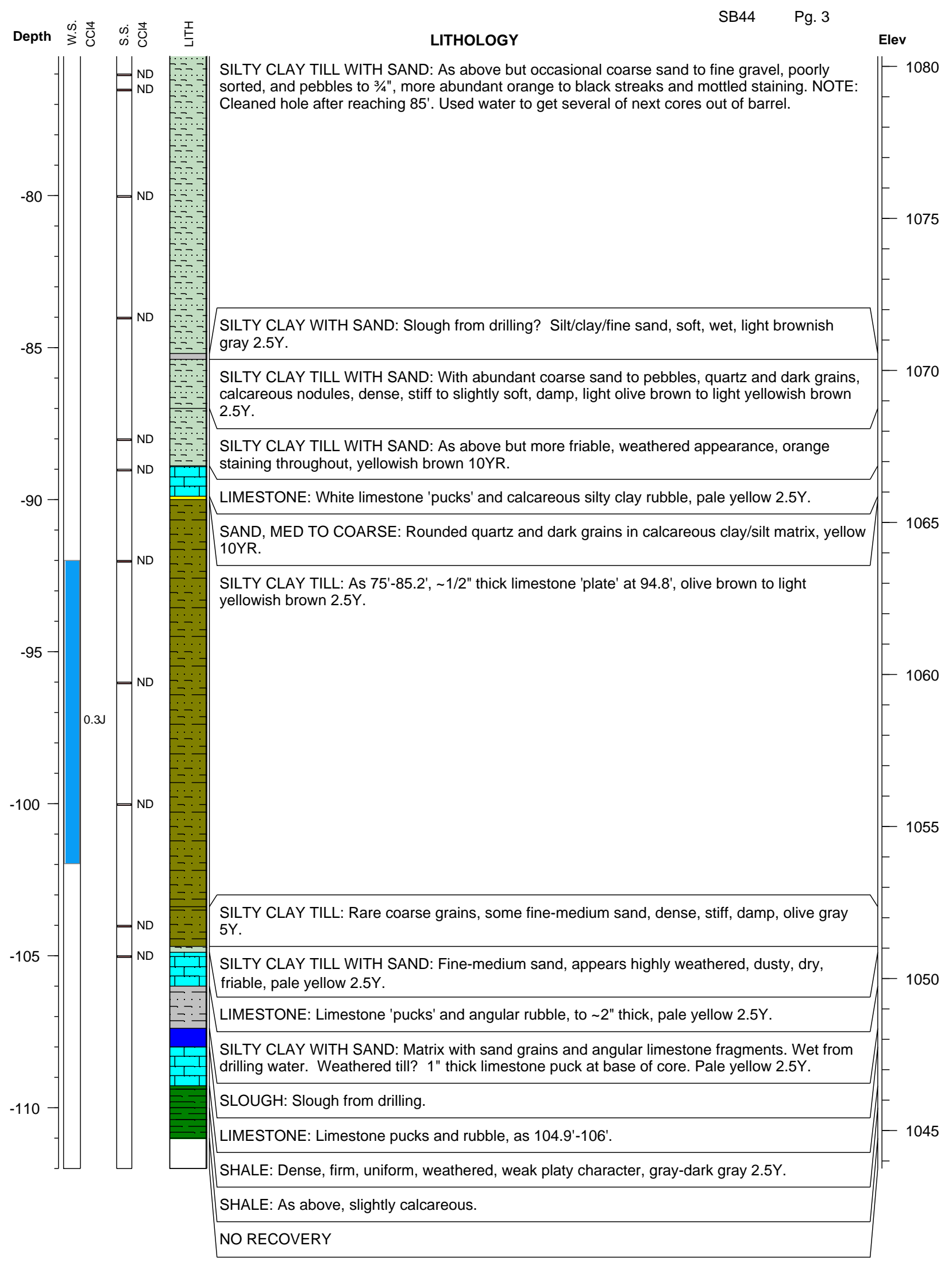




\section{Argonne National Laboratory} Project: Savannah, MO Phase II Geologist: Bob Sedivy

Elevation: $1145.341 \mathrm{ft}$

Depth: $102 \mathrm{ft}$ BGL
Well ID: SB45

Log Date: 6/24/2010

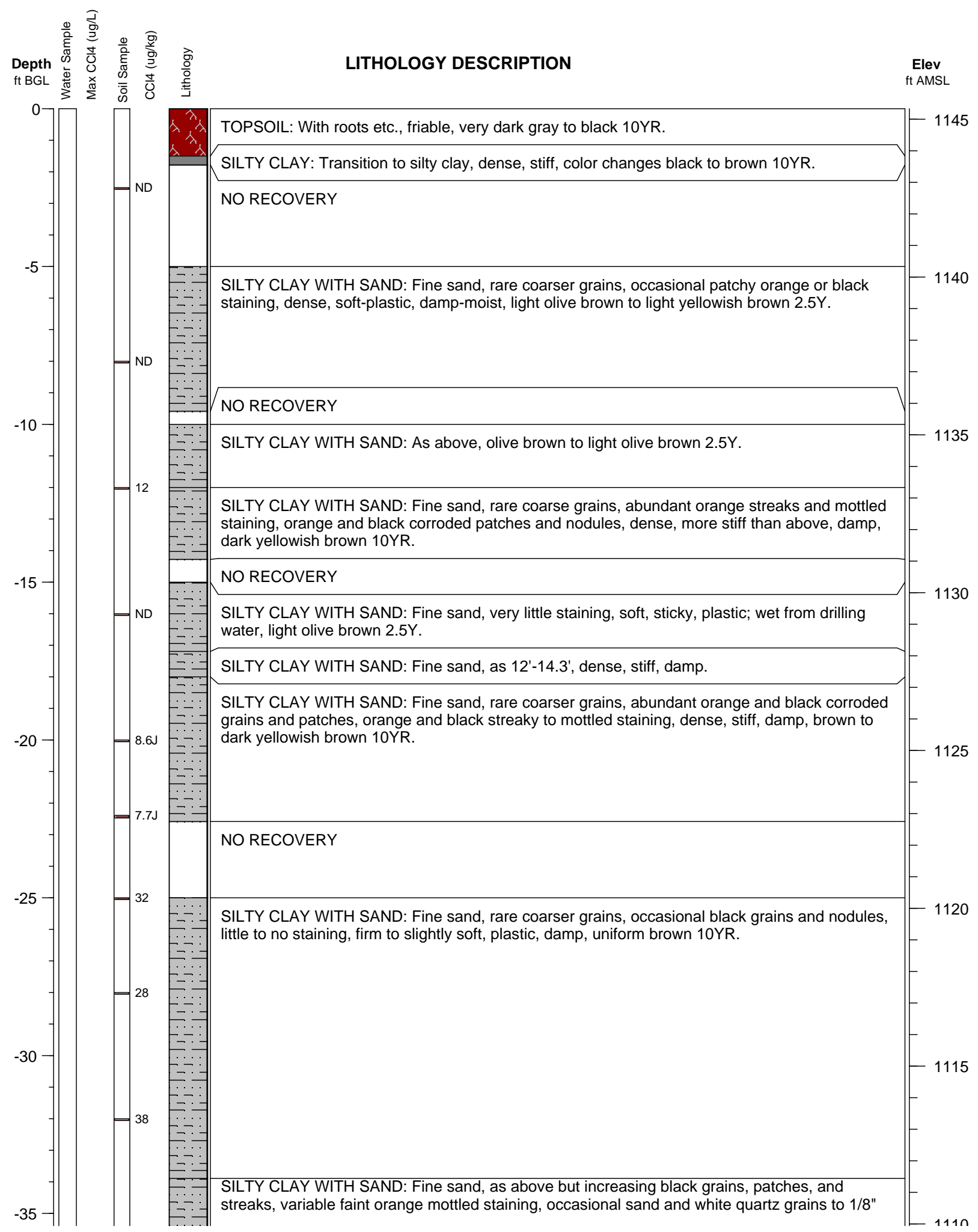




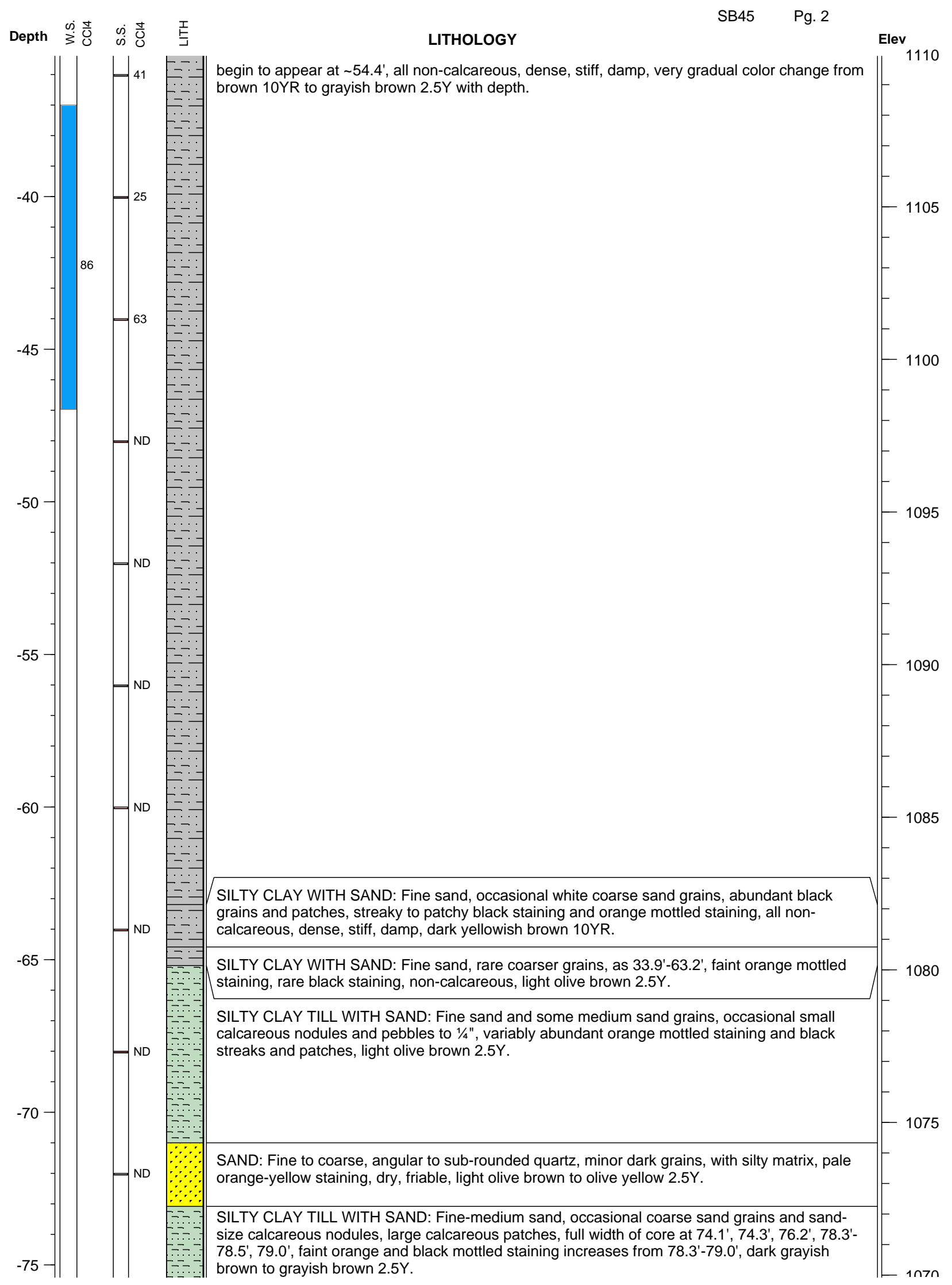




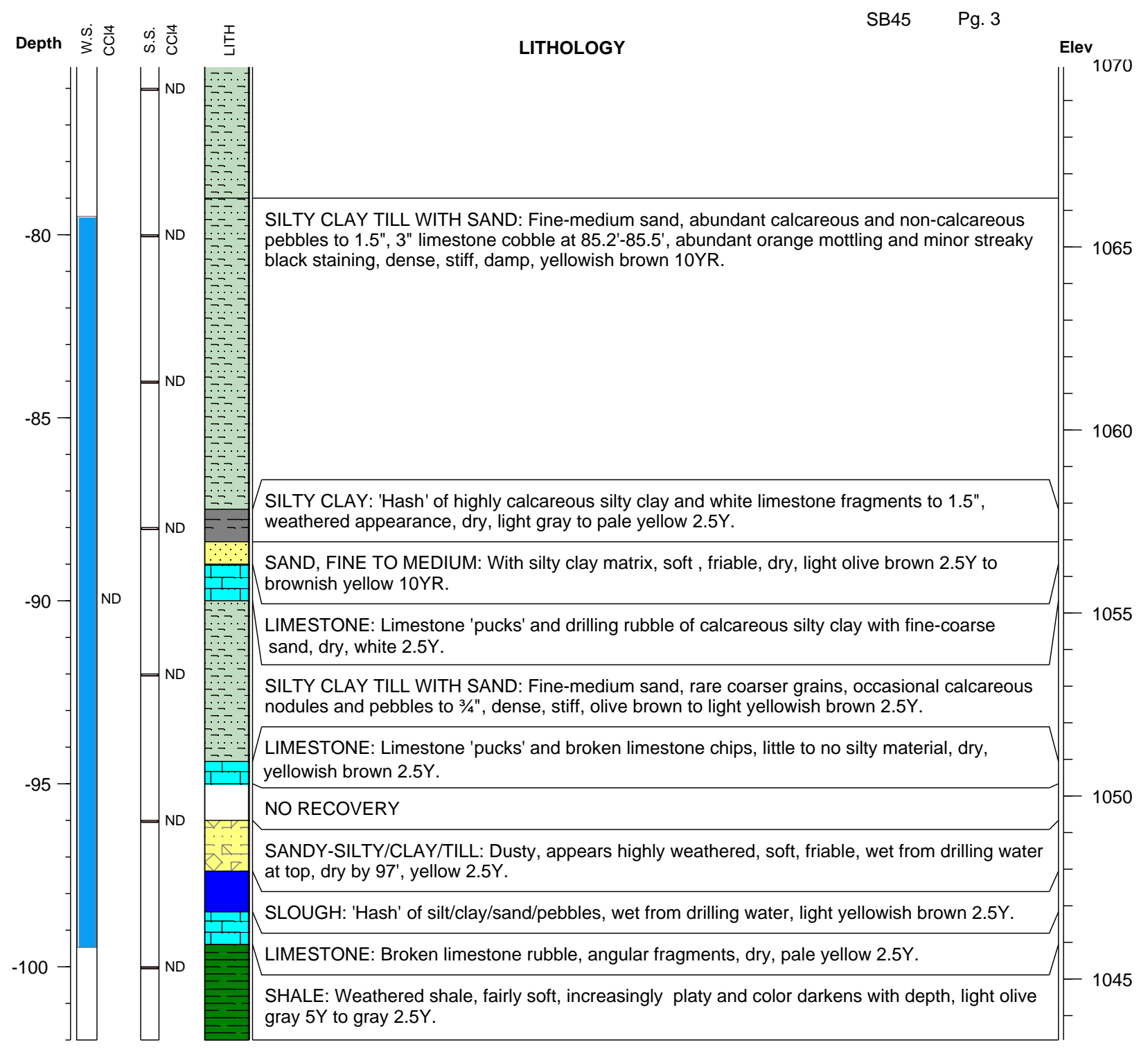




\section{Argonne National Laboratory} Project: Savannah, MO Phase II Geologist: Bob Sedivy

Elevation: $1151.891 \mathrm{ft}$

Depth: $111 \mathrm{ft} \mathrm{BGL}$
Well ID: SB46

Log Date: 6/25/2010

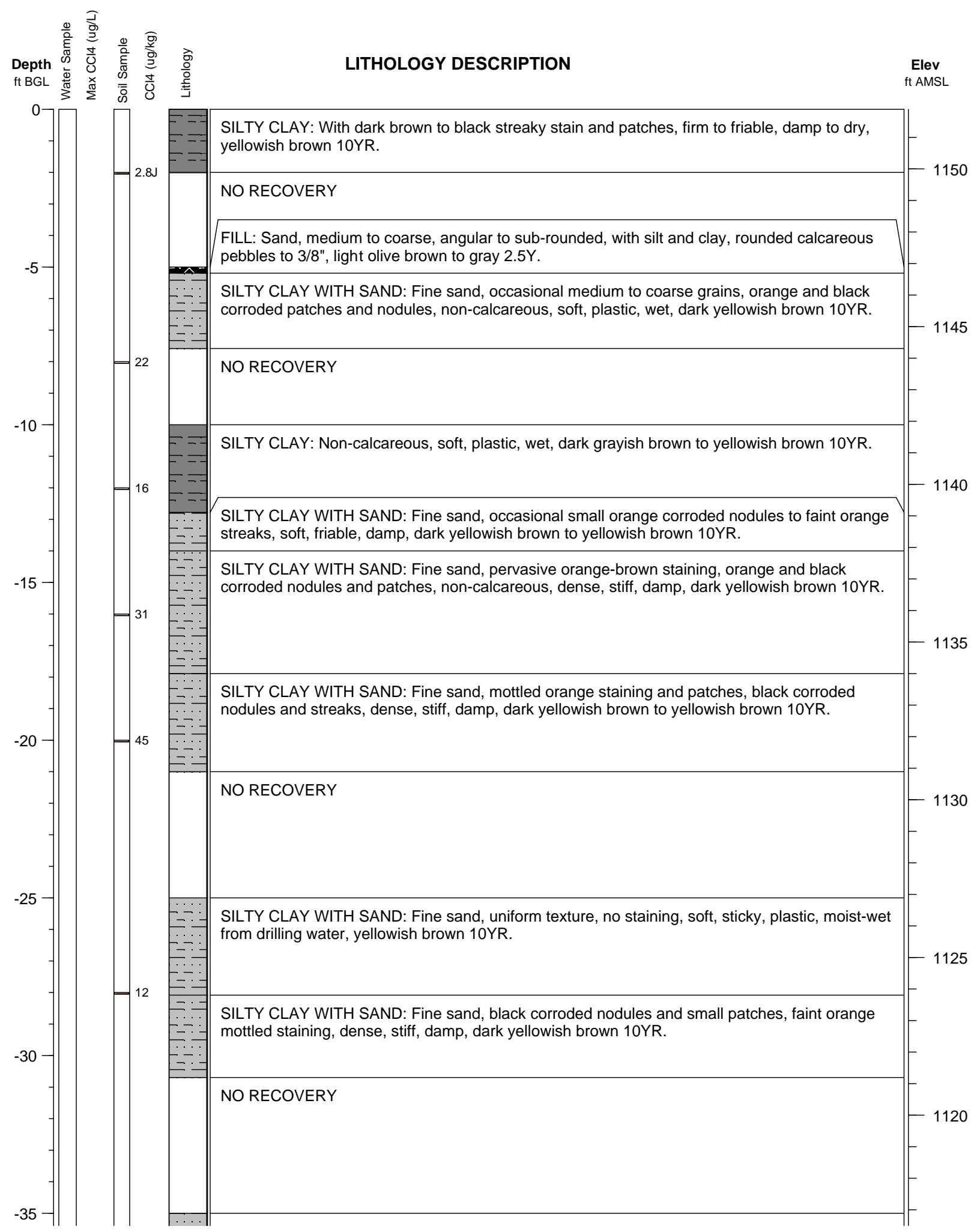




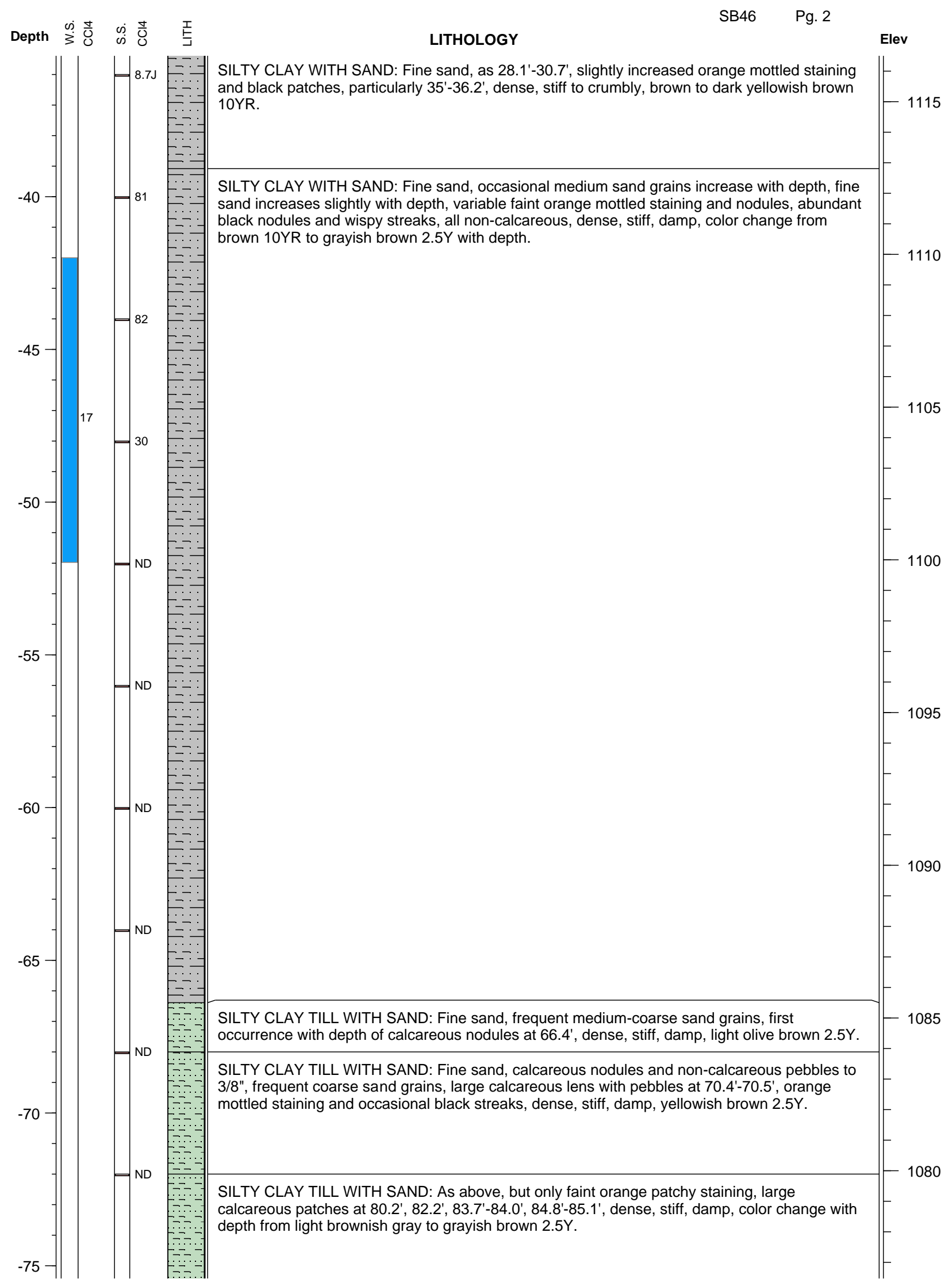




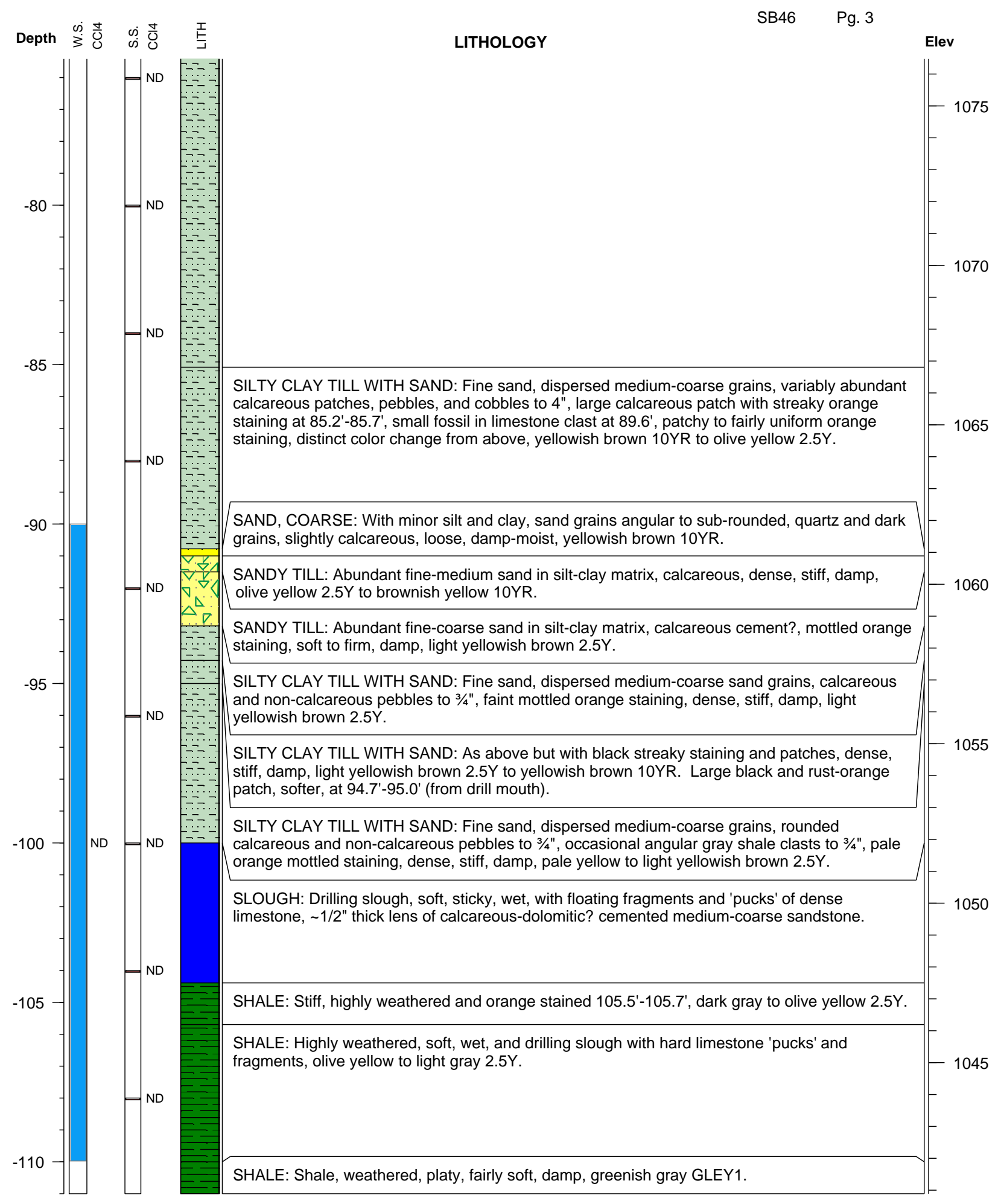




\section{Argonne National Laboratory} Project: Savannah, MO Phase II

Geologist: Bob Sedivy

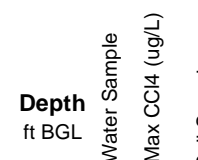

ft BGL

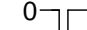

0
-5

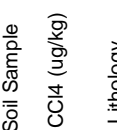

Well ID: SB47

Elevation: $1135.178 \mathrm{ft}$

Depth: $103 \mathrm{ft} \mathrm{BGL}$

Log Date: 6/26/2010

LITHOLOGY DESCRIPTION

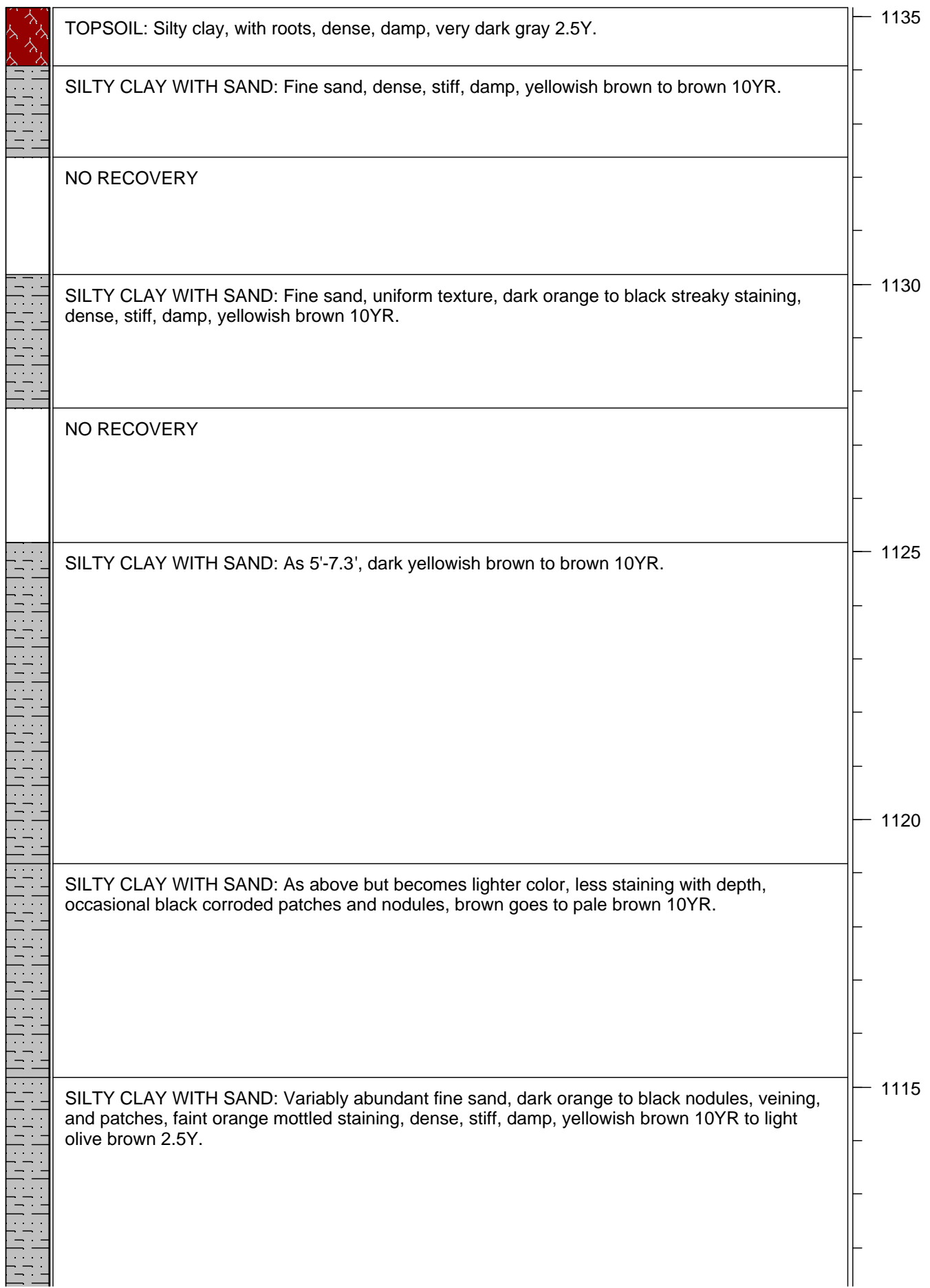

Elev ft AMSL 


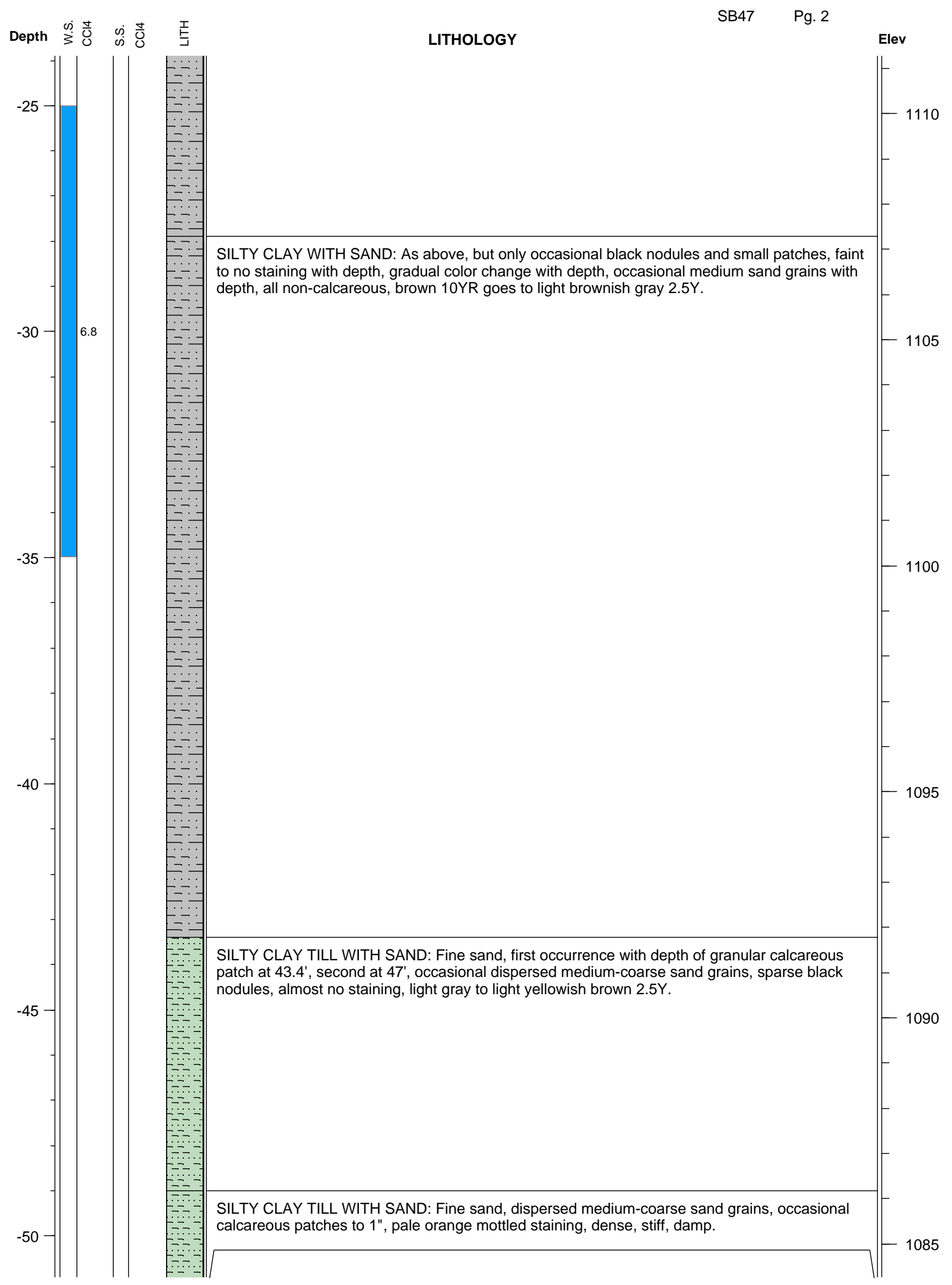




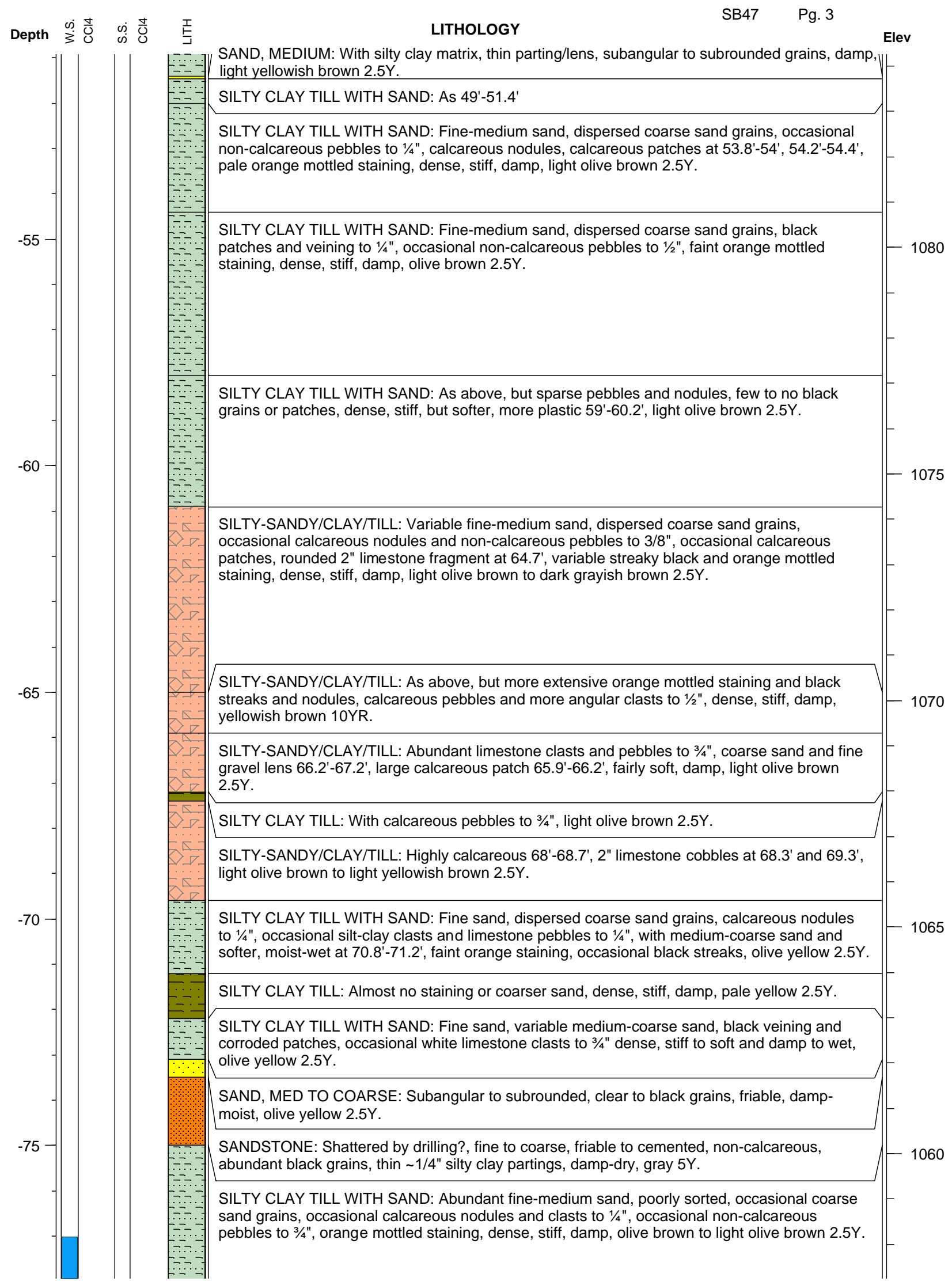




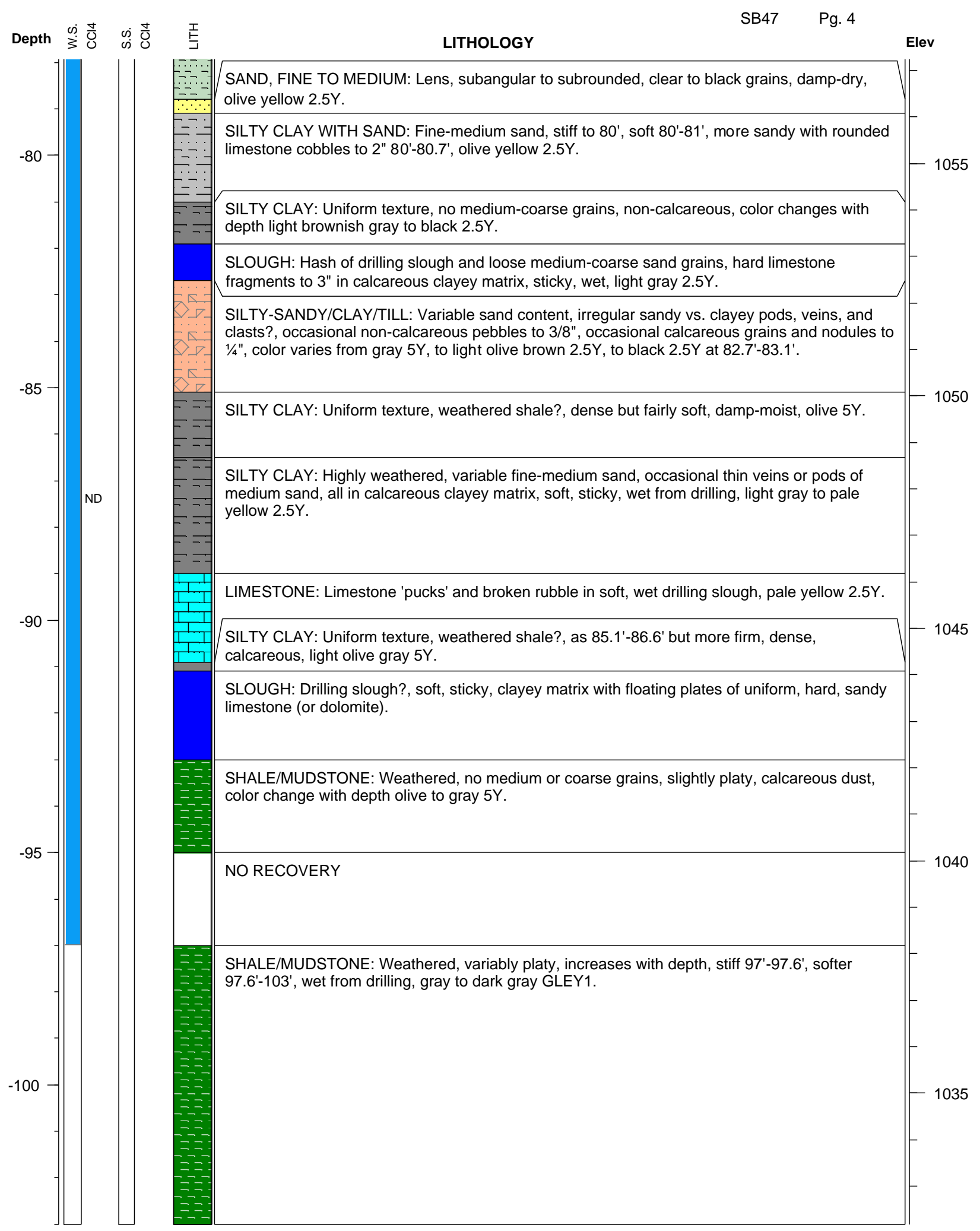




\section{Argonne National Laboratory} Project: Savannah, MO Phase II Geologist: Bob Sedivy

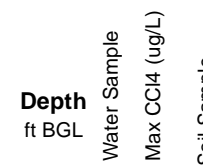

-
Elevation: $1134.938 \mathrm{ft}$

Depth: $101 \mathrm{ft} \mathrm{BGL}$
Well ID: SB48

Log Date: 6/27/2010

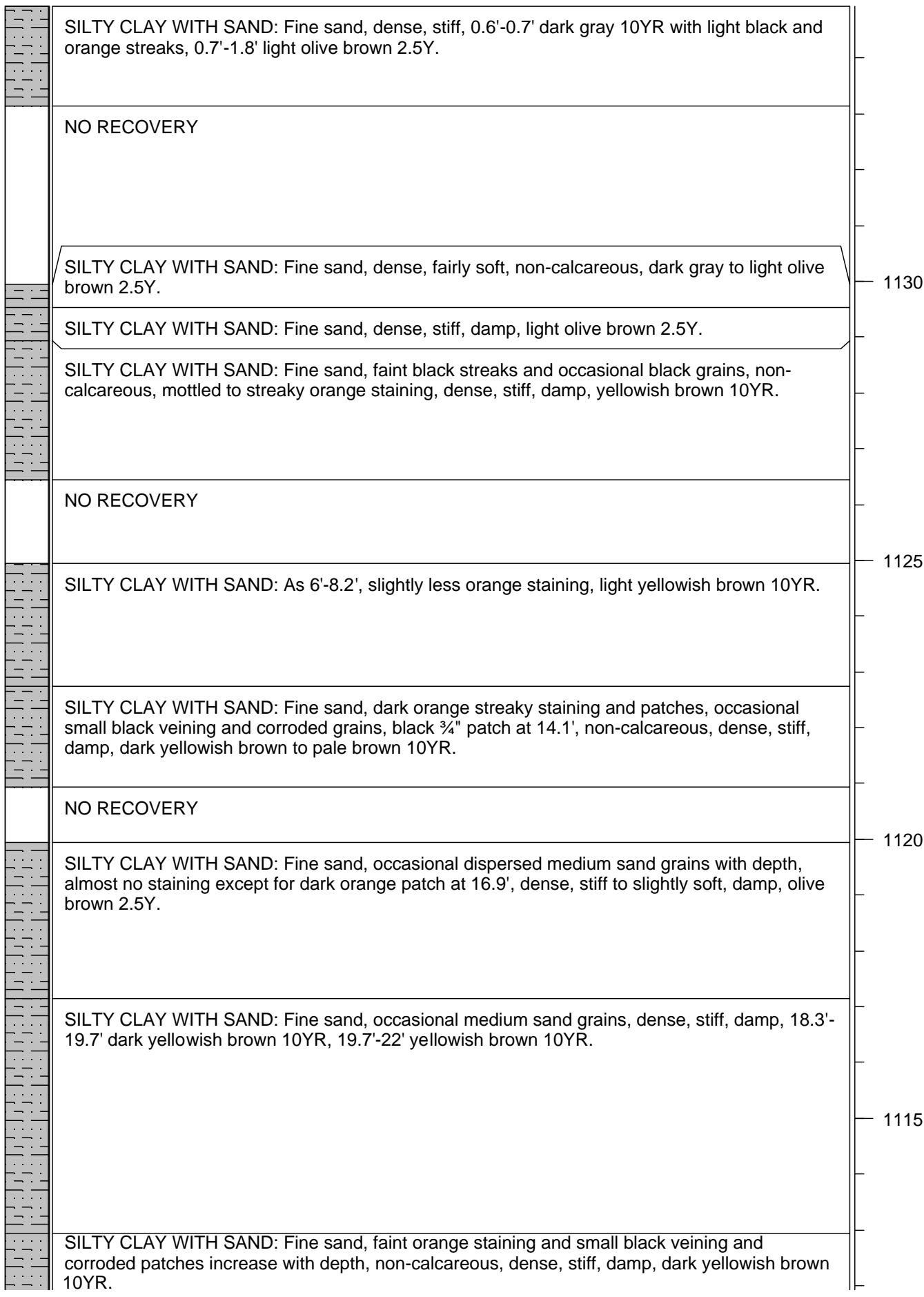




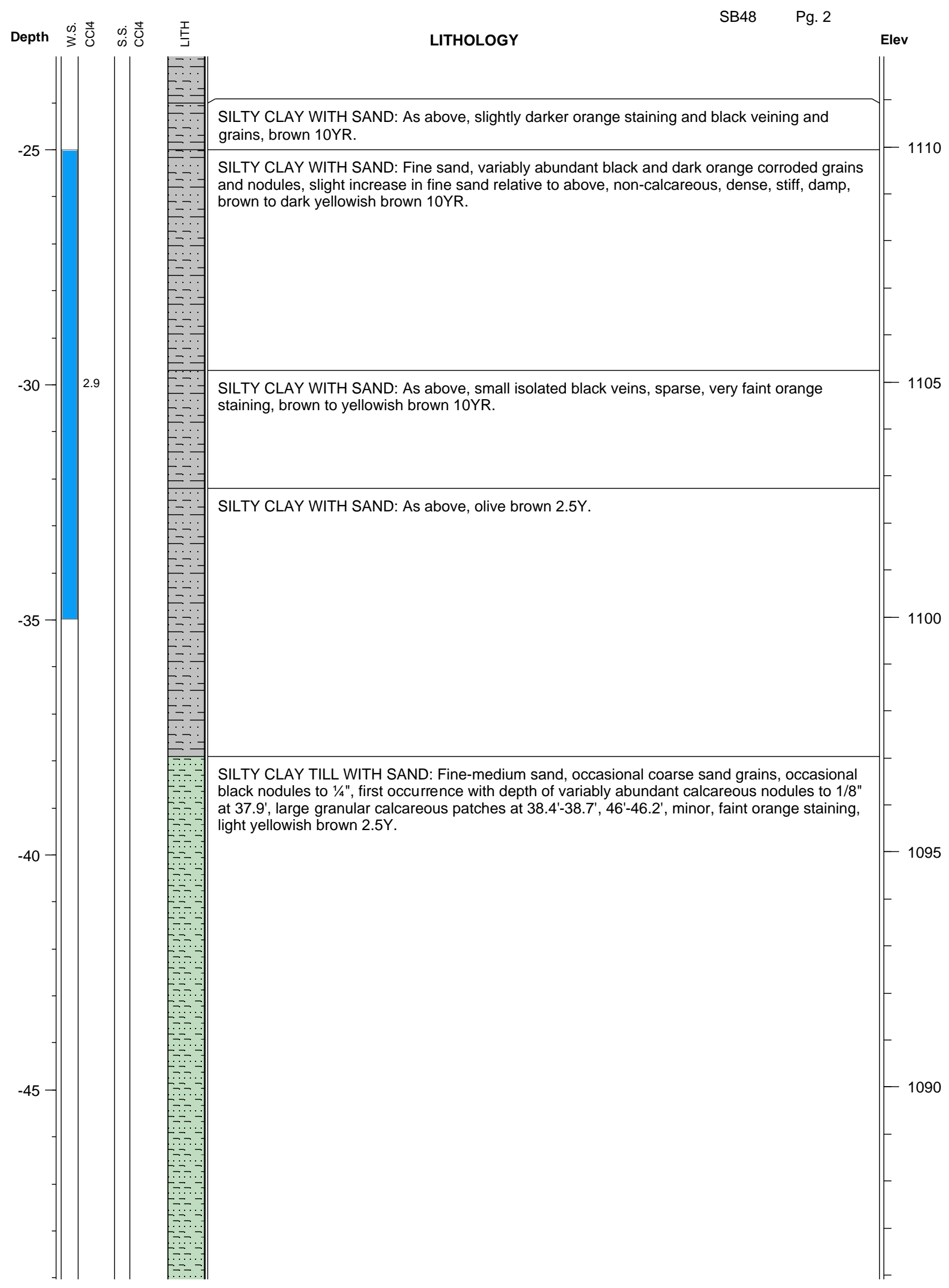




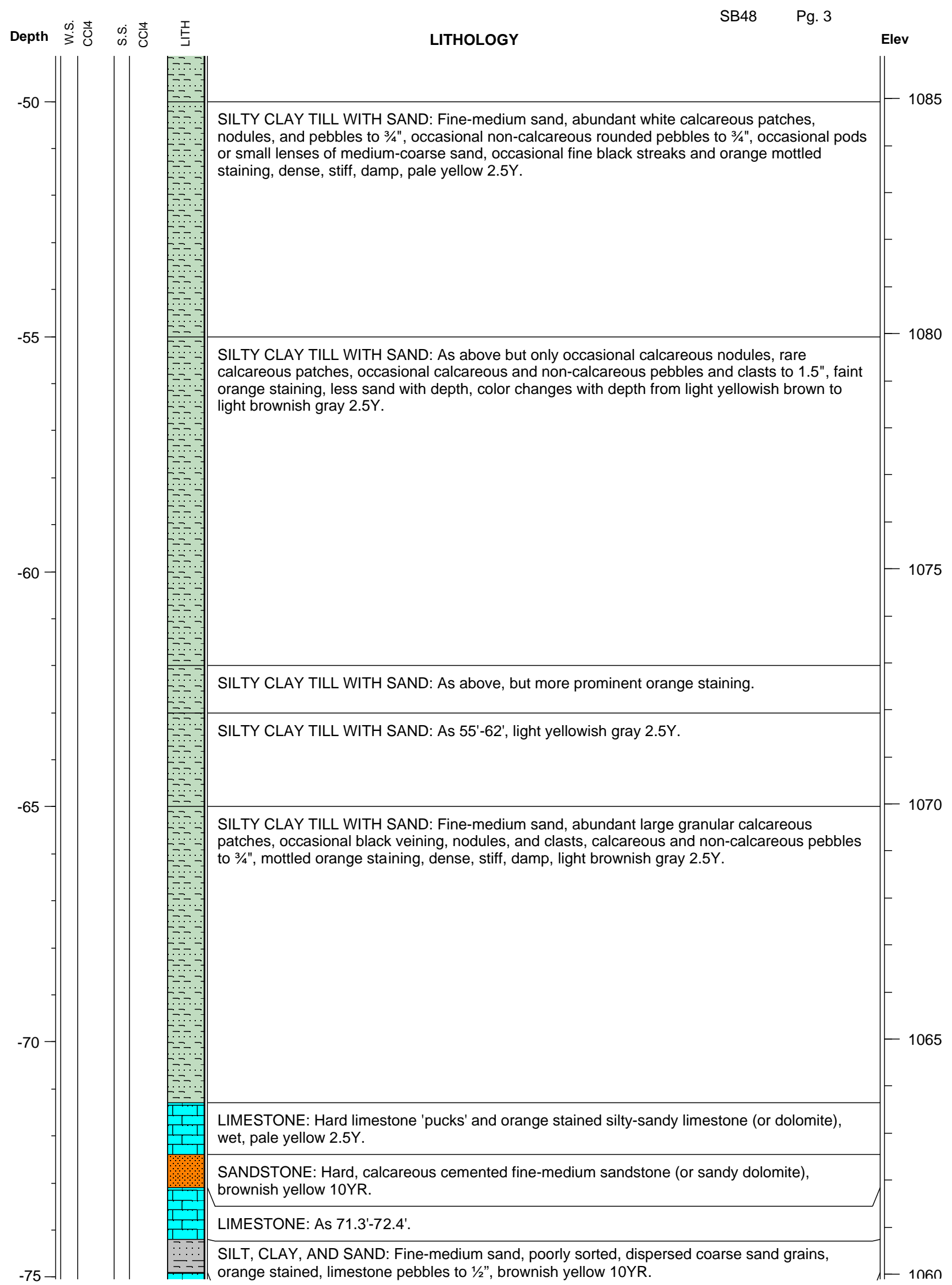




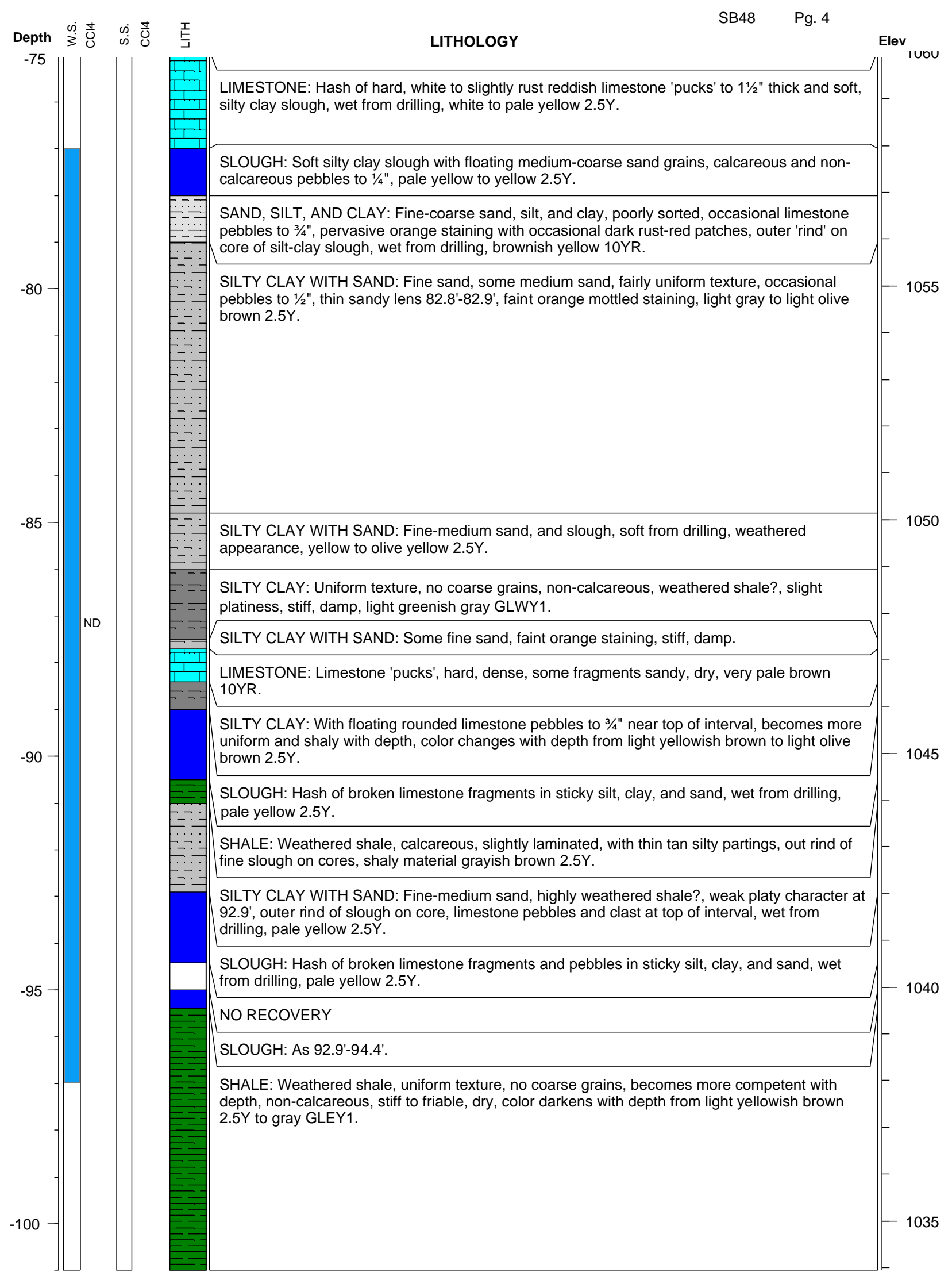




\section{Argonne National Laboratory} Project: Savannah, MO Phase II Geologist: Bob Sedivy

Elevation: $1137.923 \mathrm{ft}$

Depth: $99 \mathrm{ft}$ BGL
Well ID: SB49

Log Date: 6/28/2010

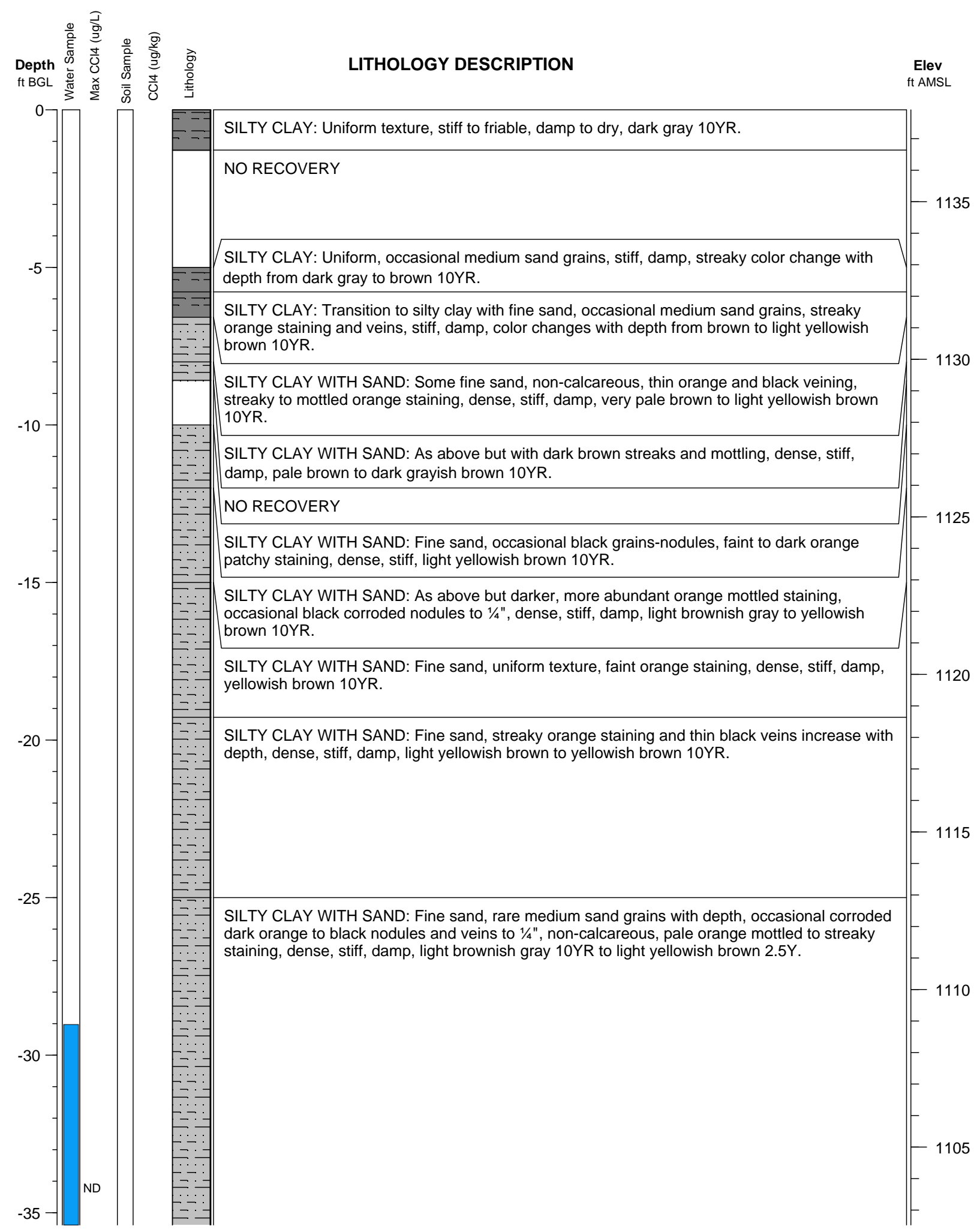




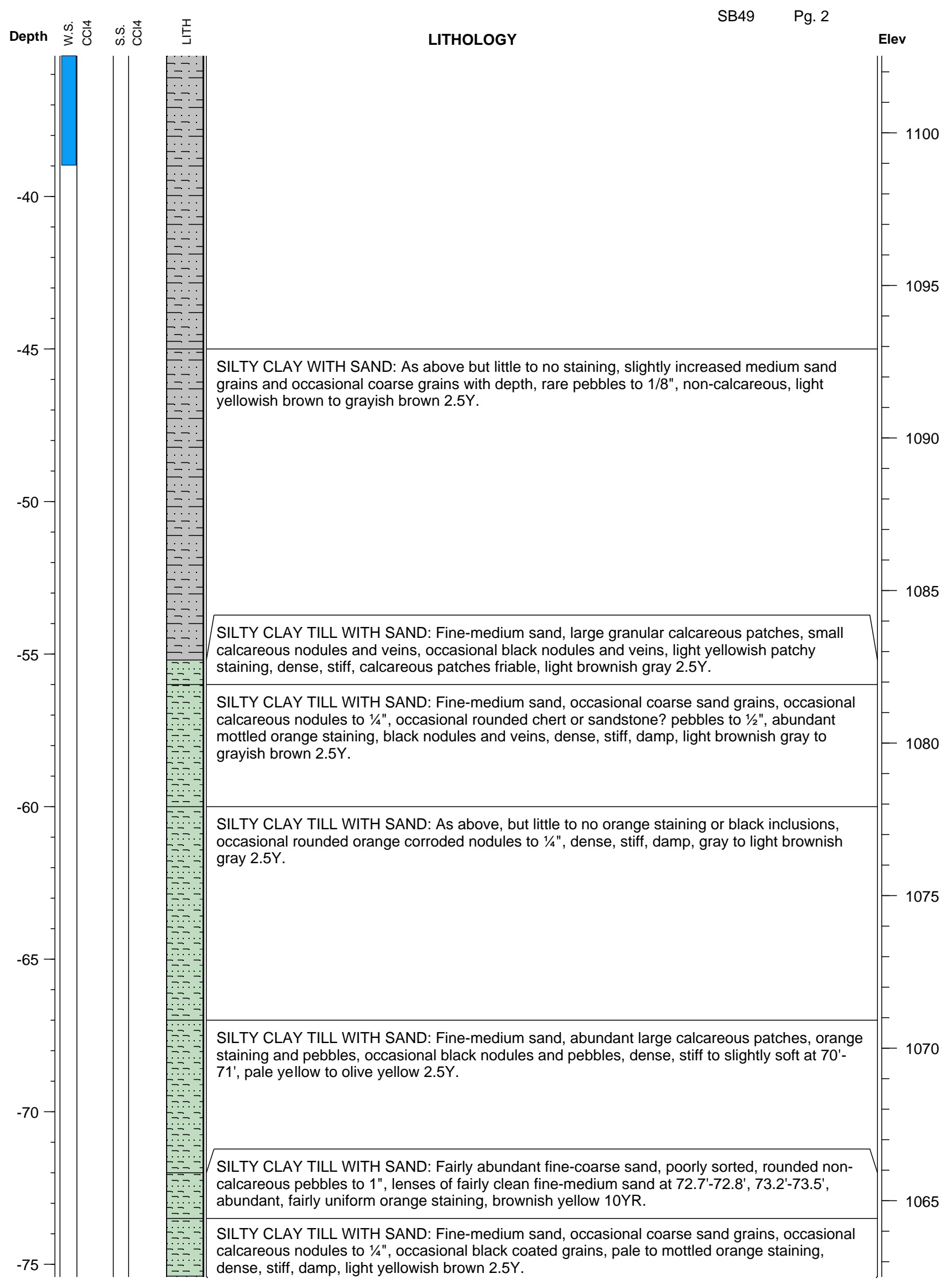




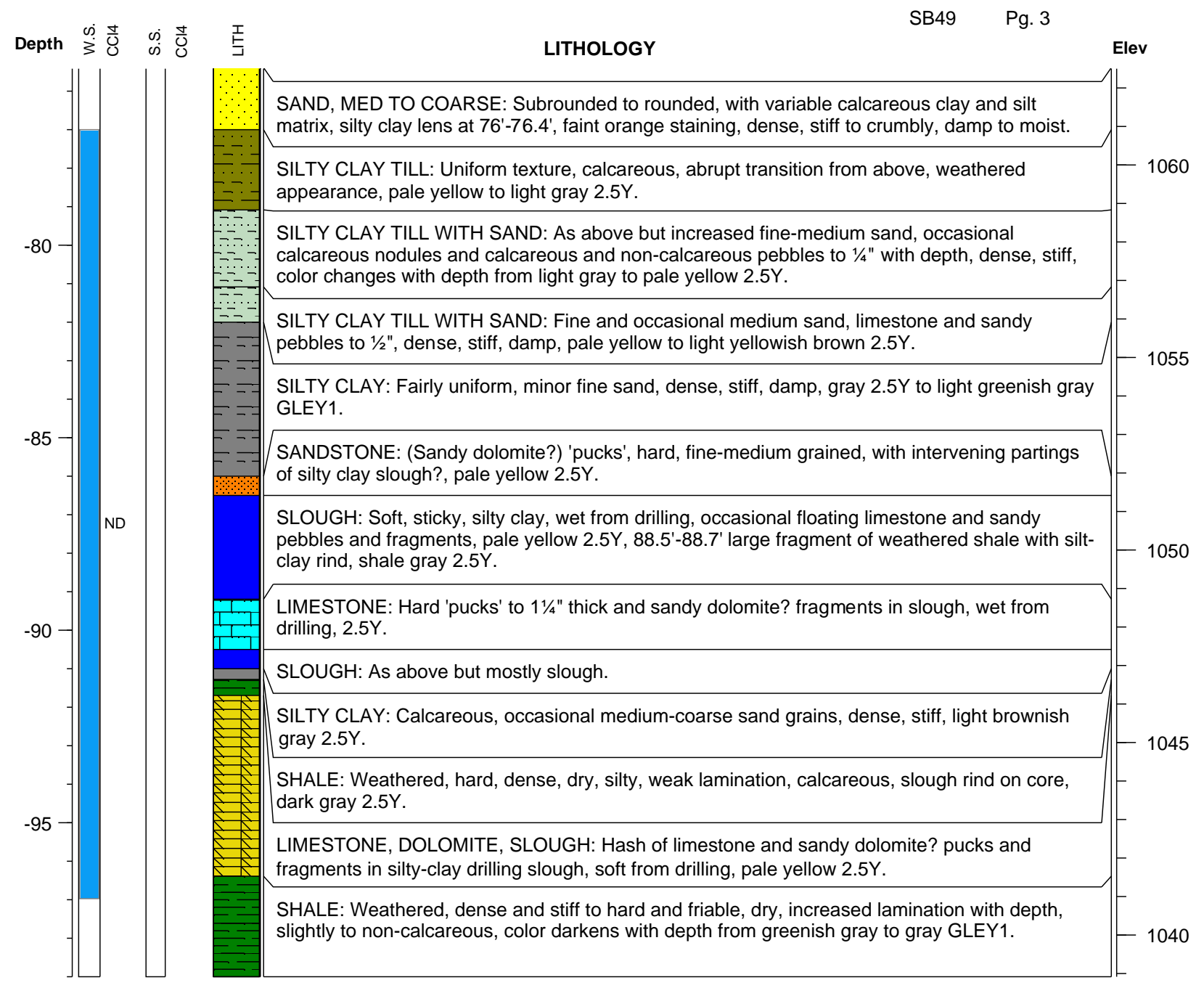




\section{Appendix D:}

\section{Well Certification and Registration Forms}




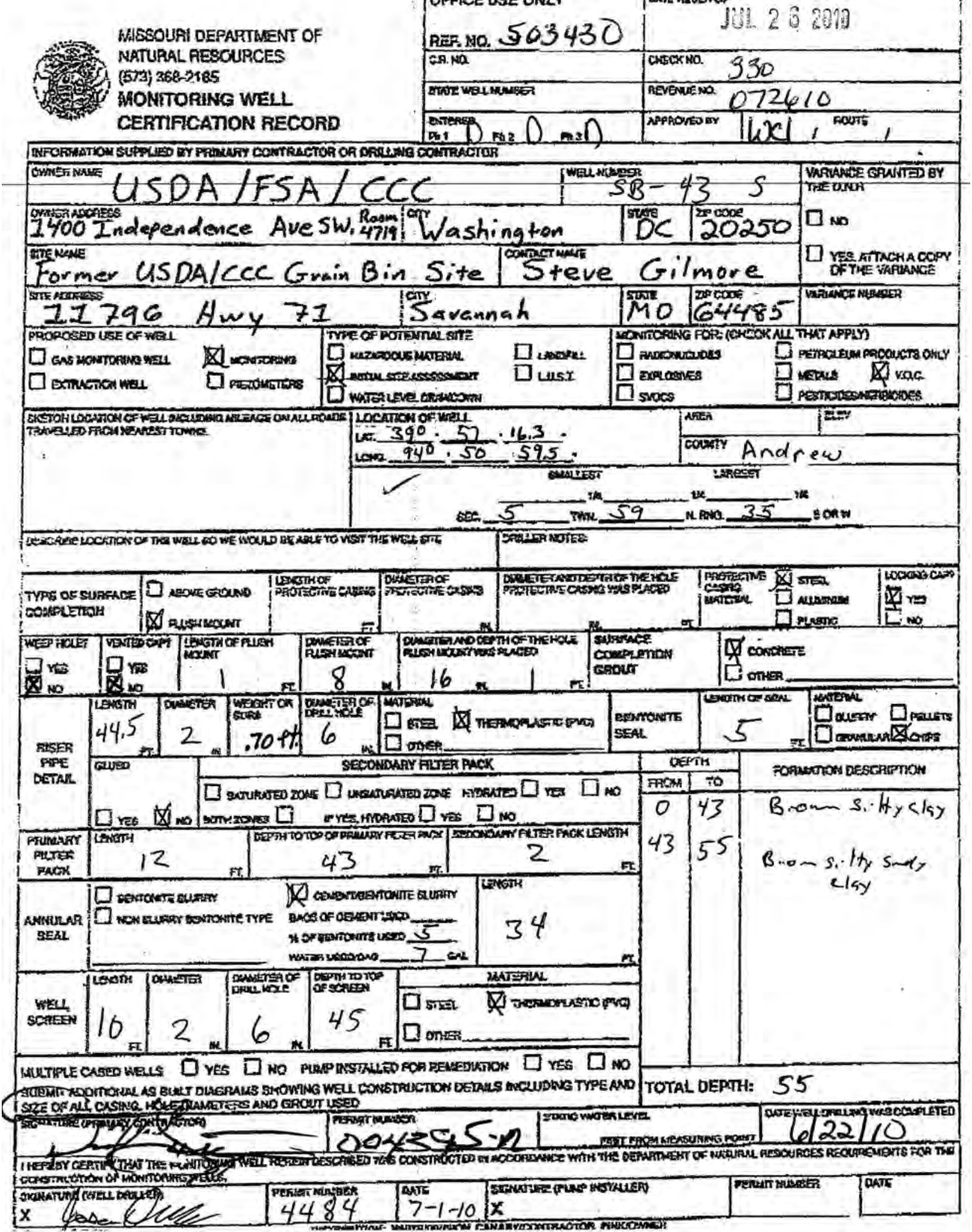




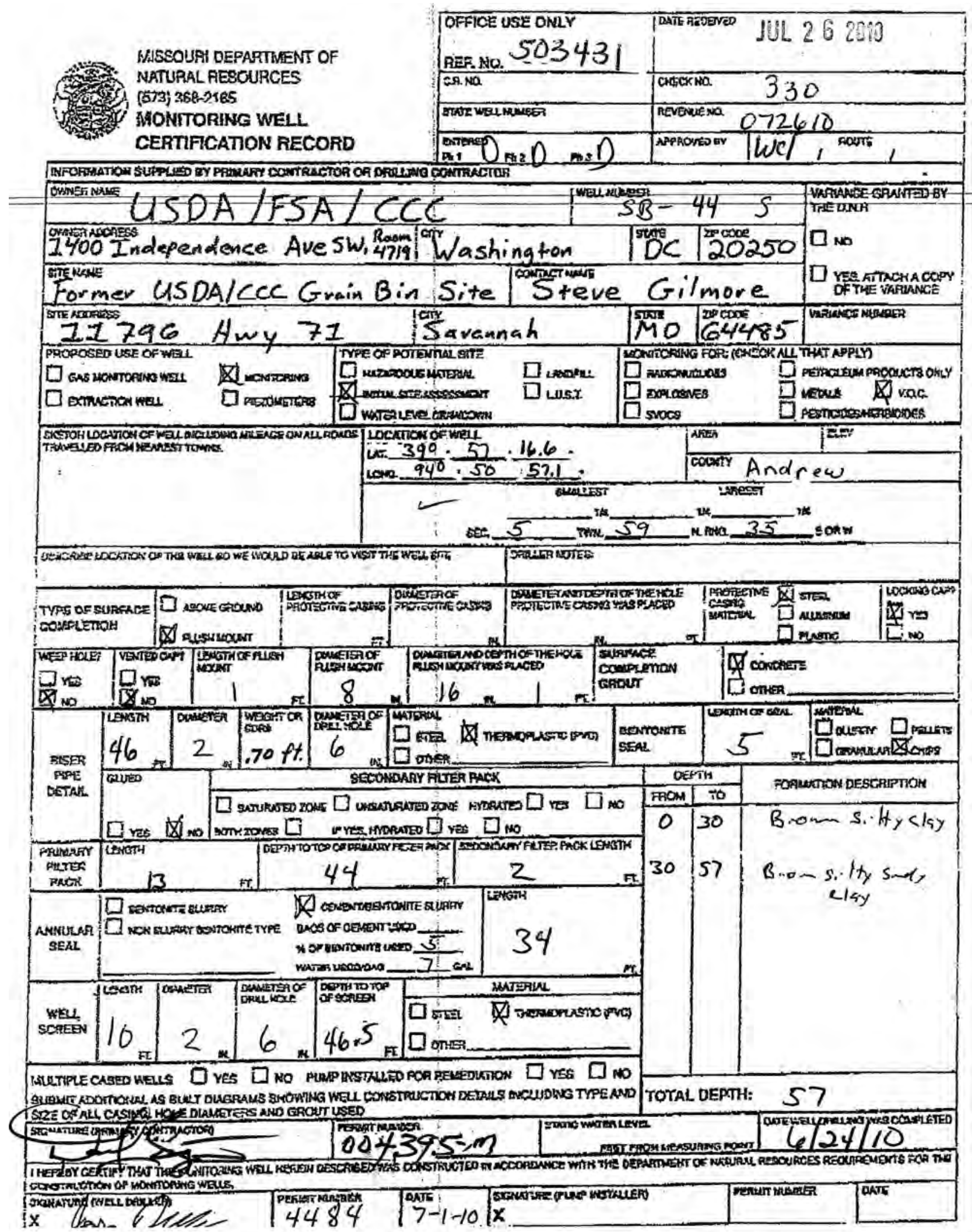




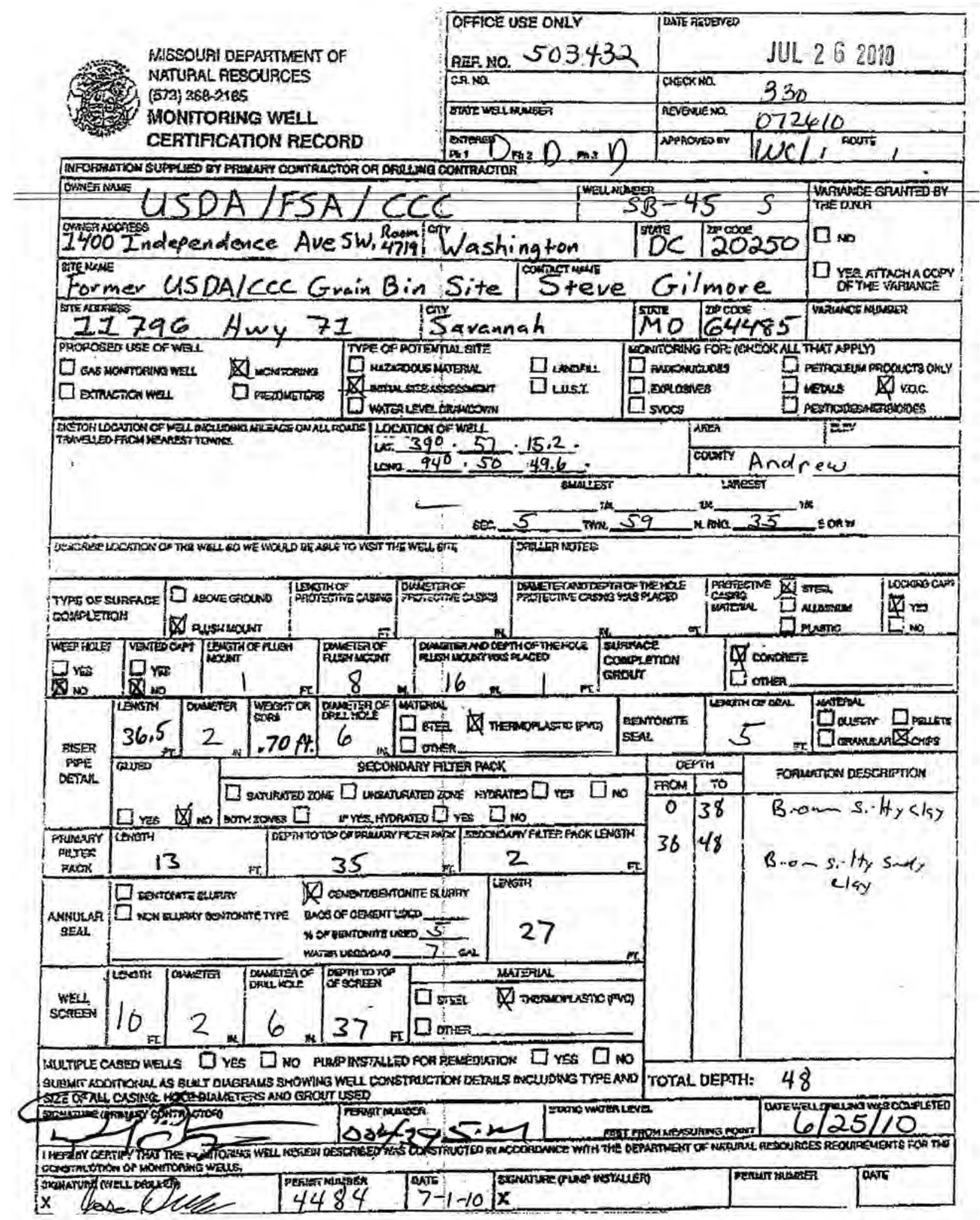




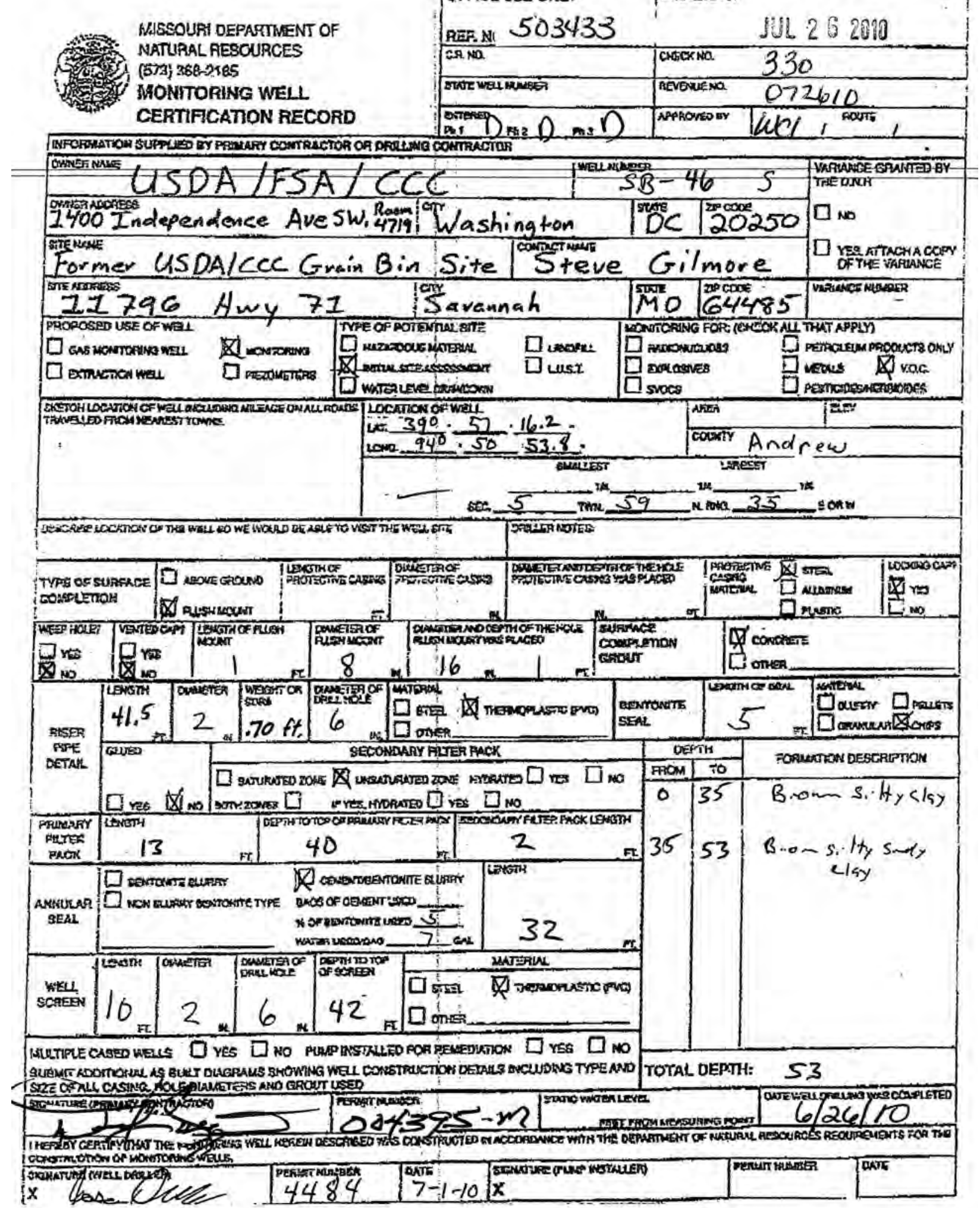




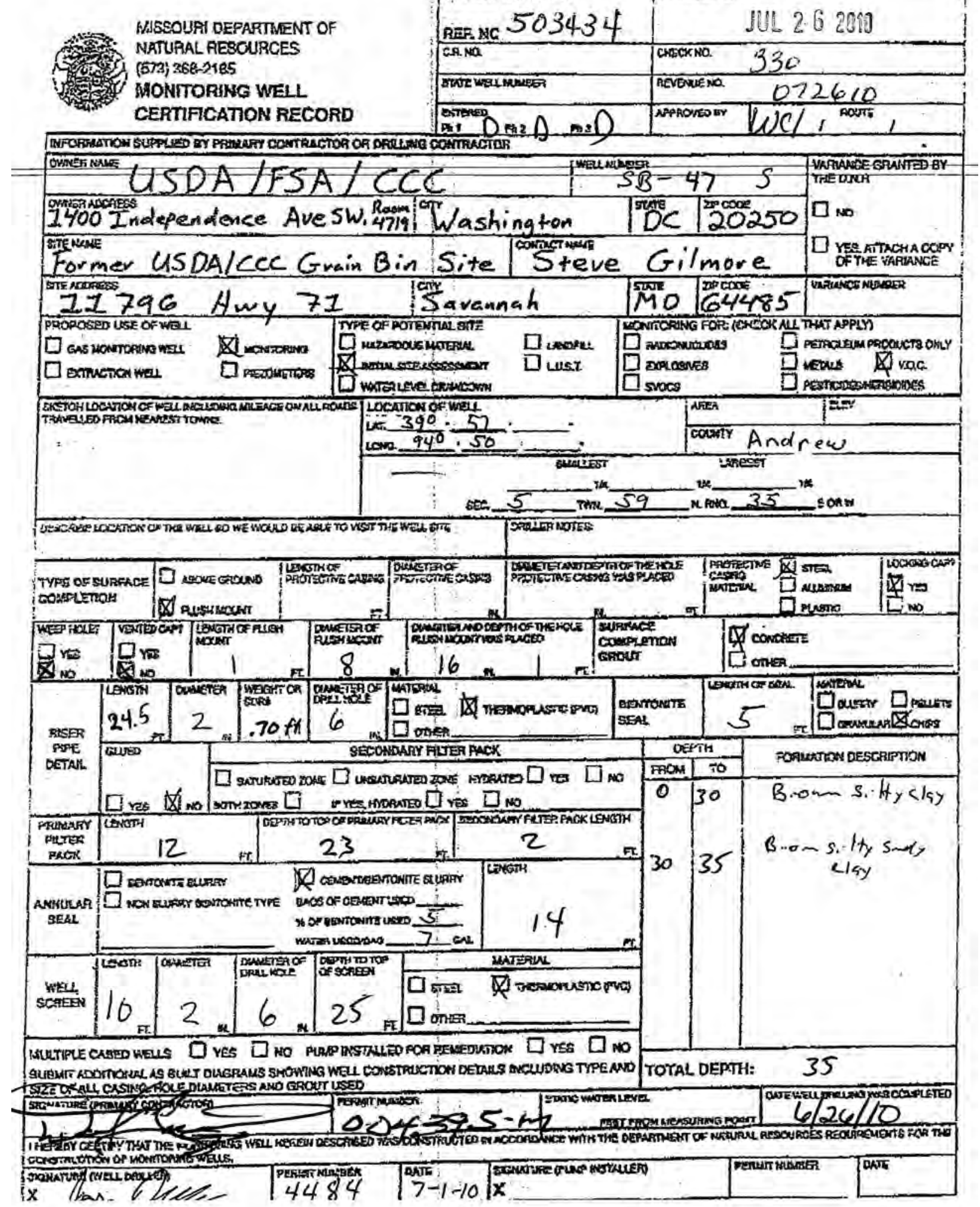




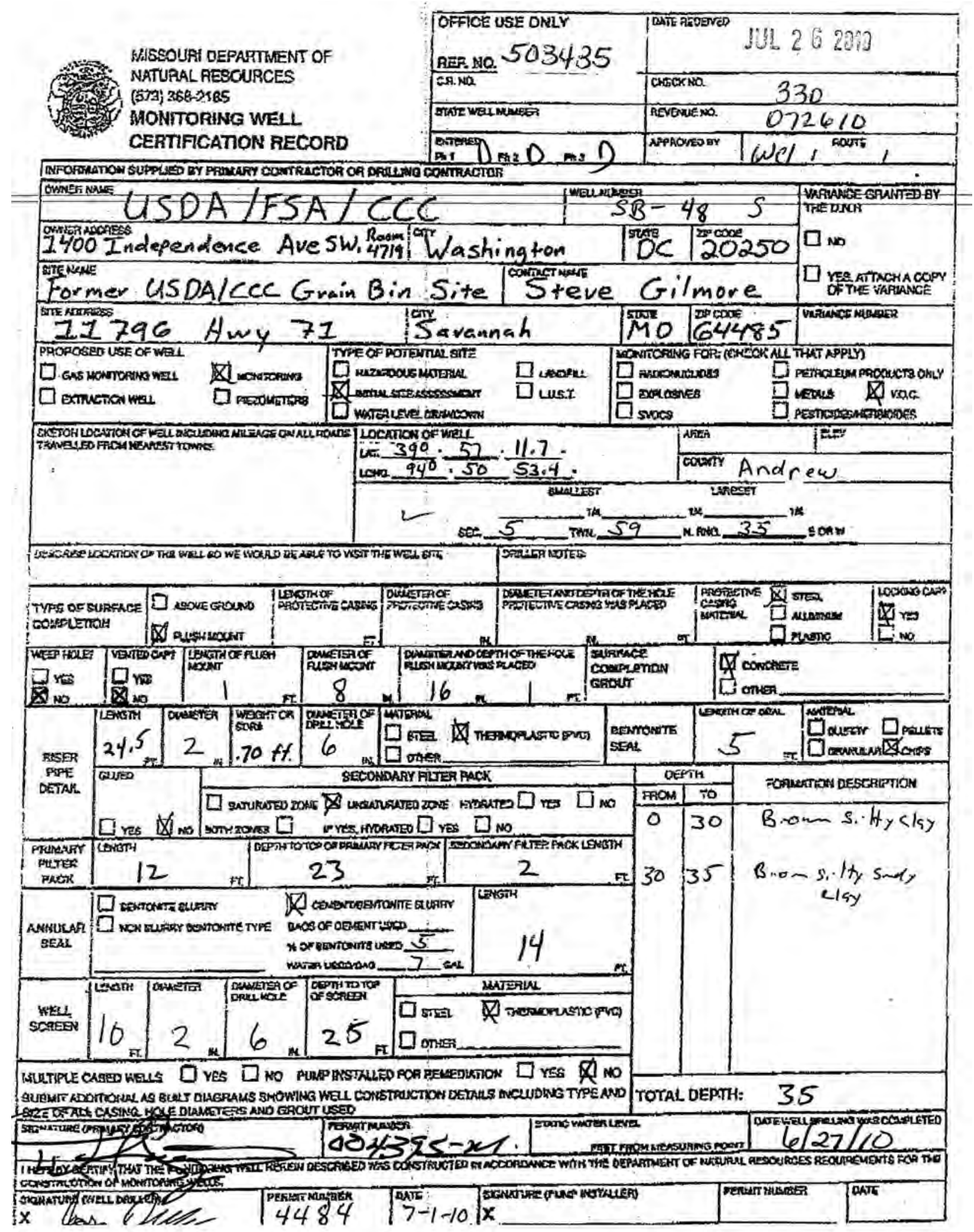




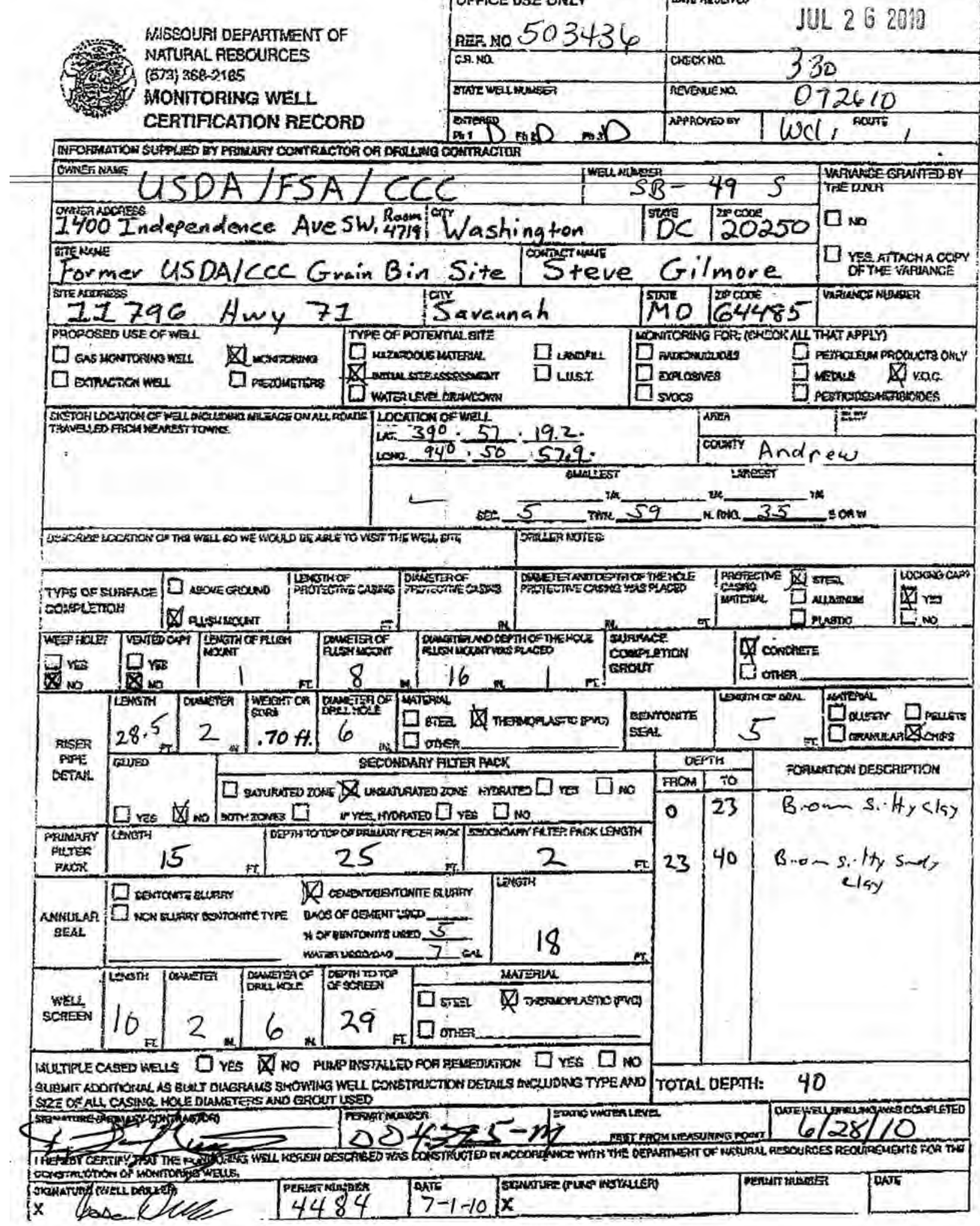




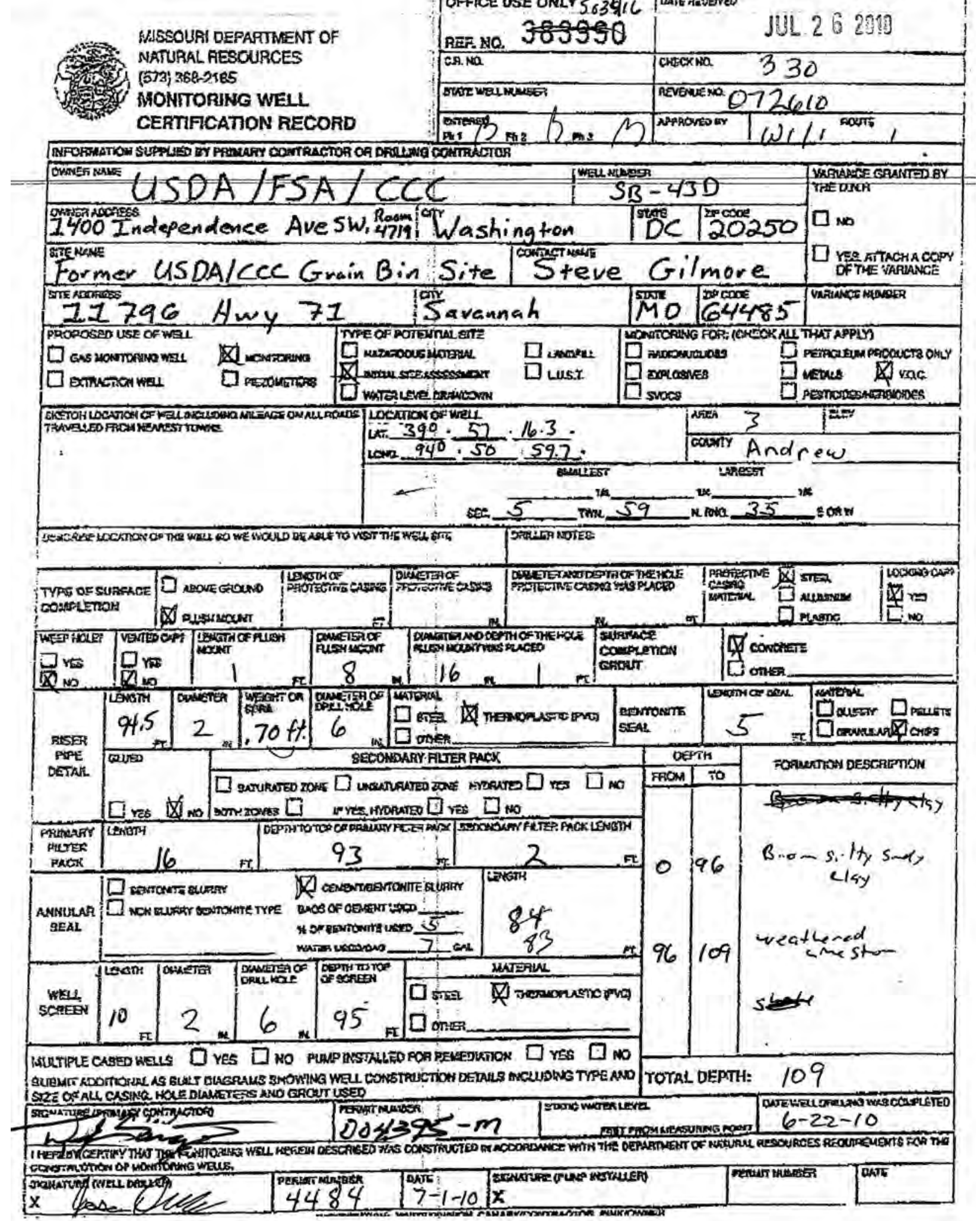




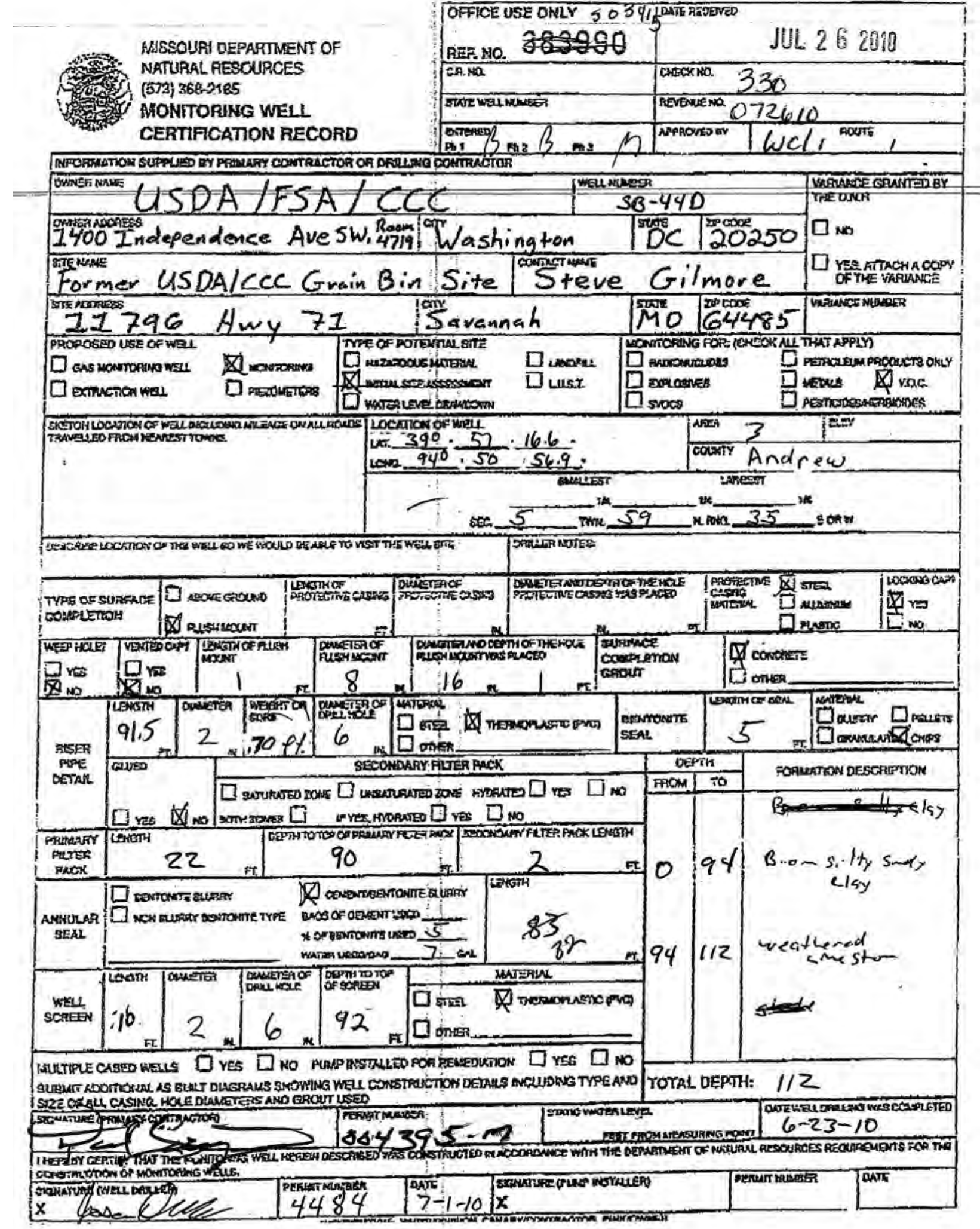




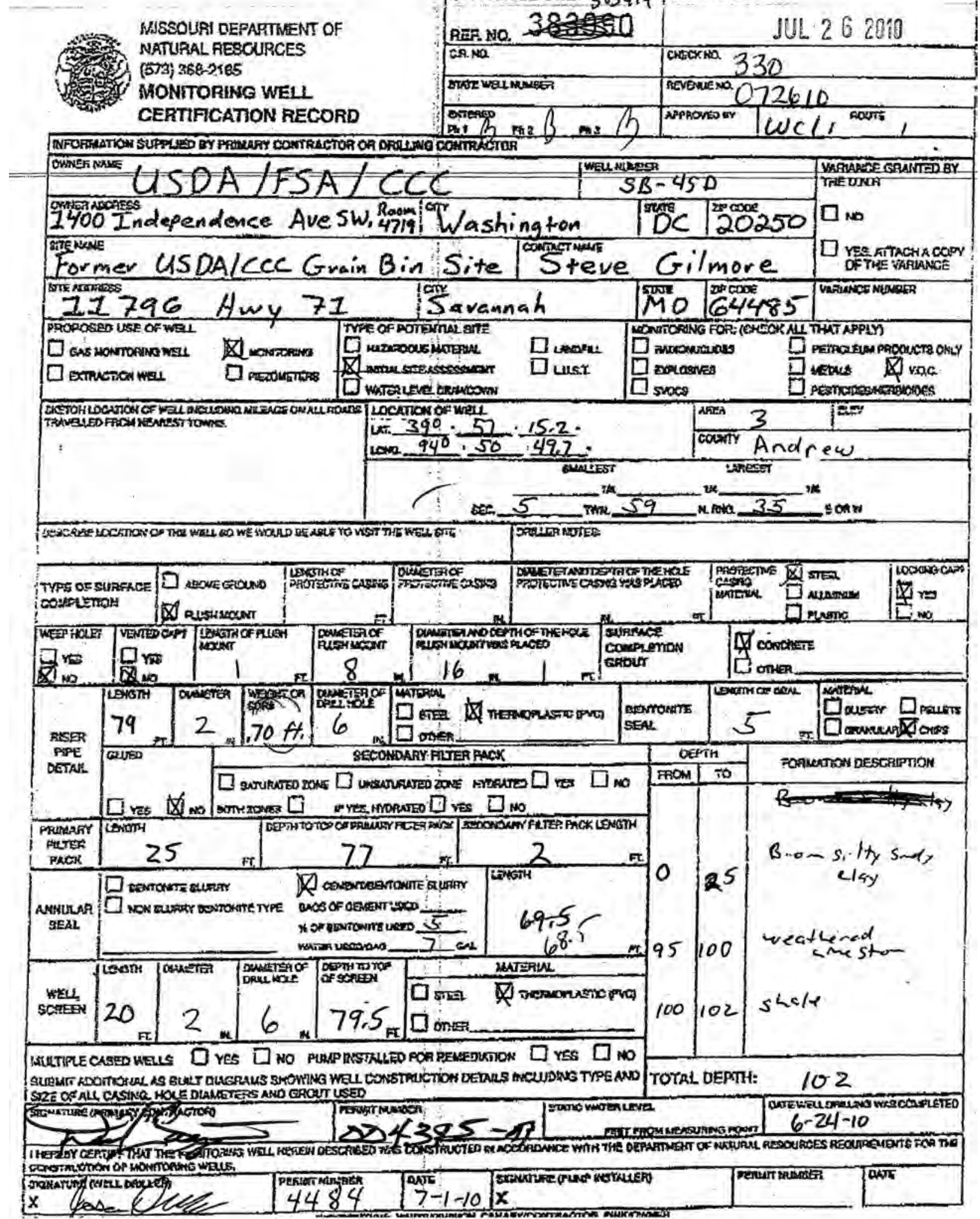




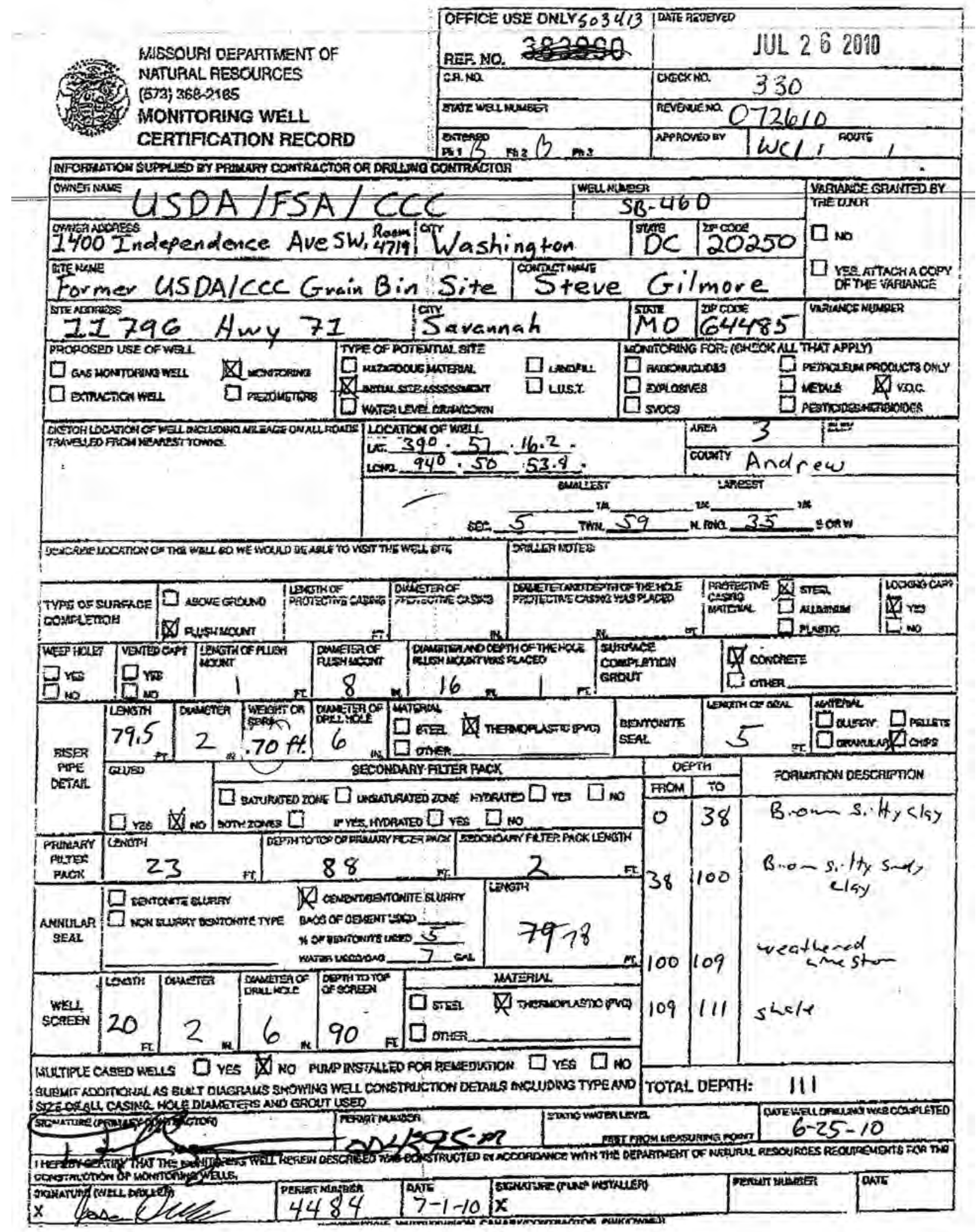




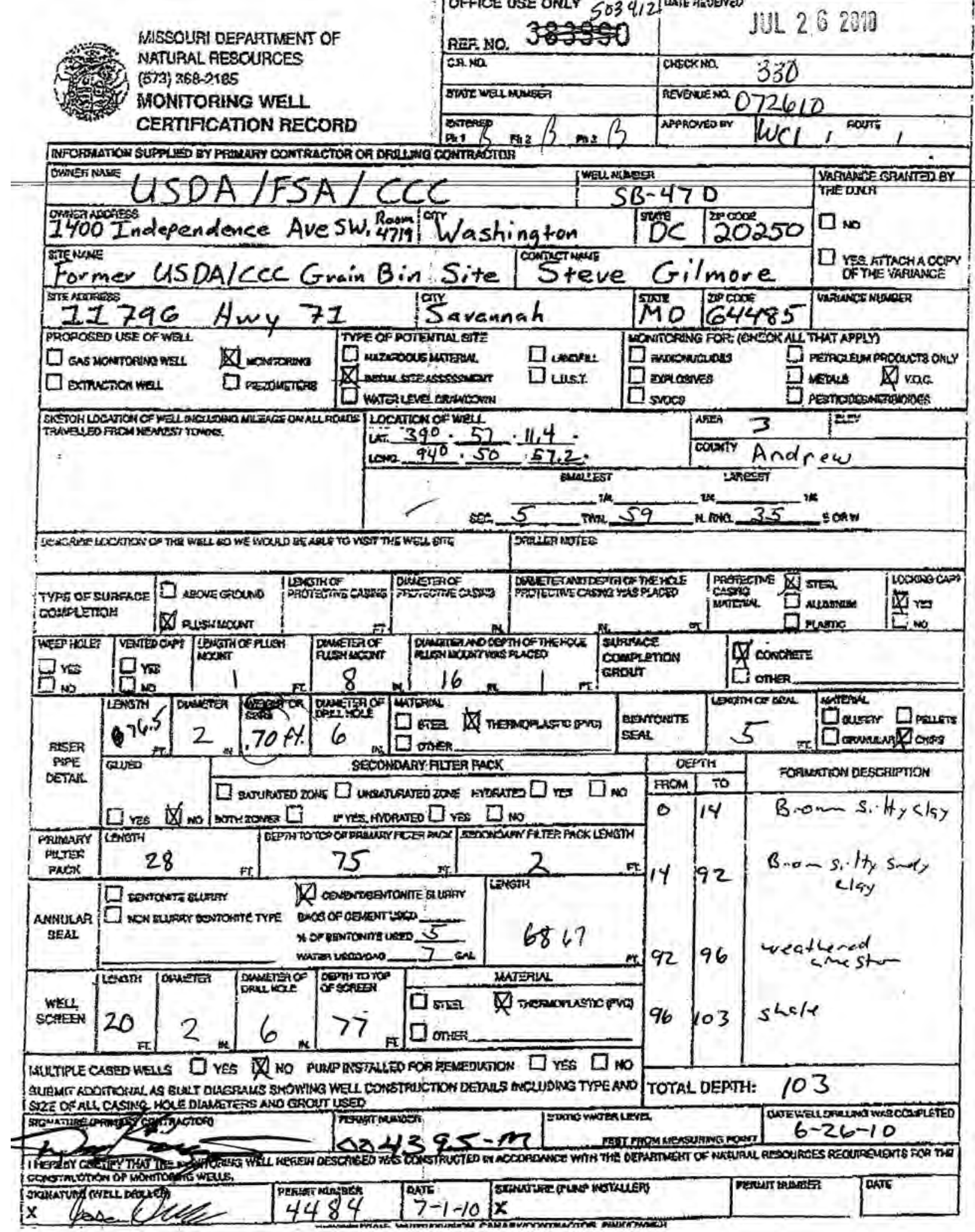


MASSOUAI DEFARTMENT OF NATURAL FESCURCES

MONITORING WELL CERTIFCATION RECORD (5)/3) $368-2165$

\begin{tabular}{|c|c|}
\hline $\begin{array}{l}\text { OFFICE USE ONLY SO34 } \\
\text { REF NO } 383900\end{array}$ & 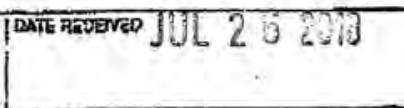 \\
\hline cos.sa & ChECKNA 330 \\
\hline 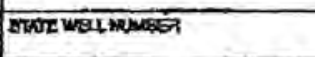 & neveruena 072610 \\
\hline 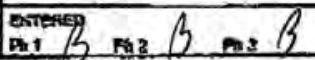 & 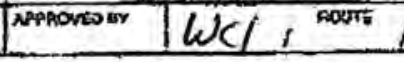 \\
\hline
\end{tabular}

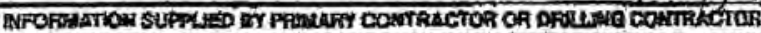

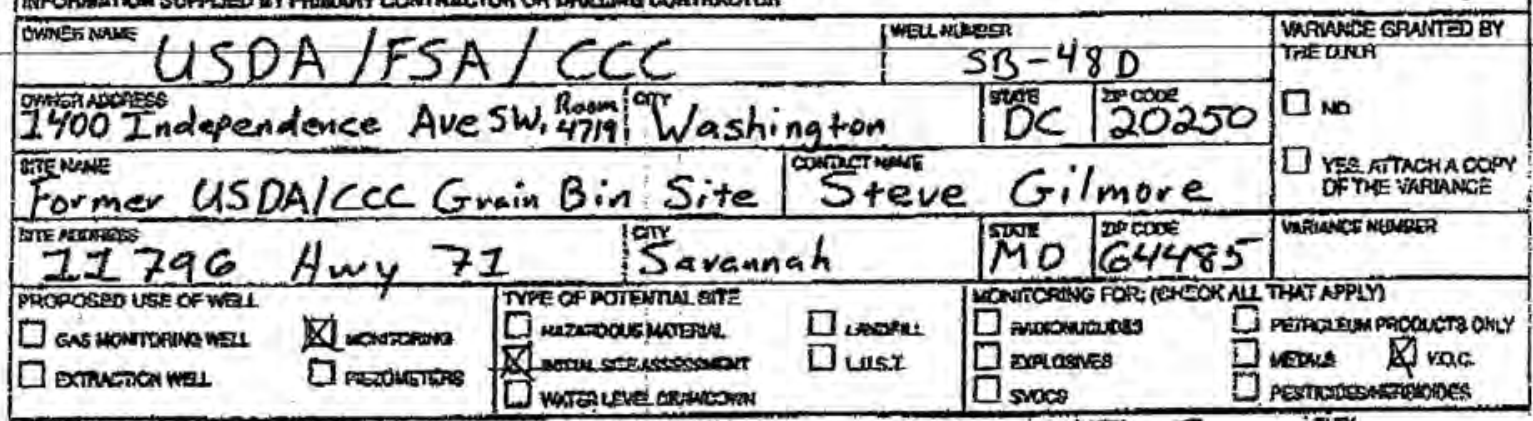

\begin{tabular}{|c|c|c|}
\hline \multirow{2}{*}{ 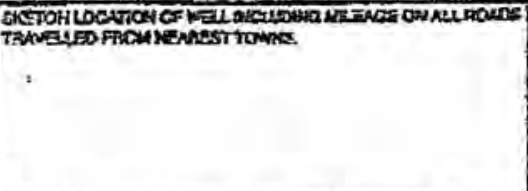 } & \multirow{2}{*}{ Lece $39^{\circ} \cdot \frac{57}{54^{\circ}} \cdot \frac{11.7}{53.5}$} & 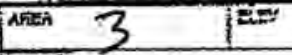 \\
\hline & & cownty Andrew \\
\hline
\end{tabular}

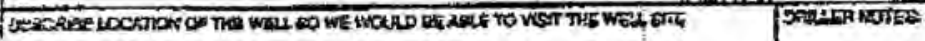

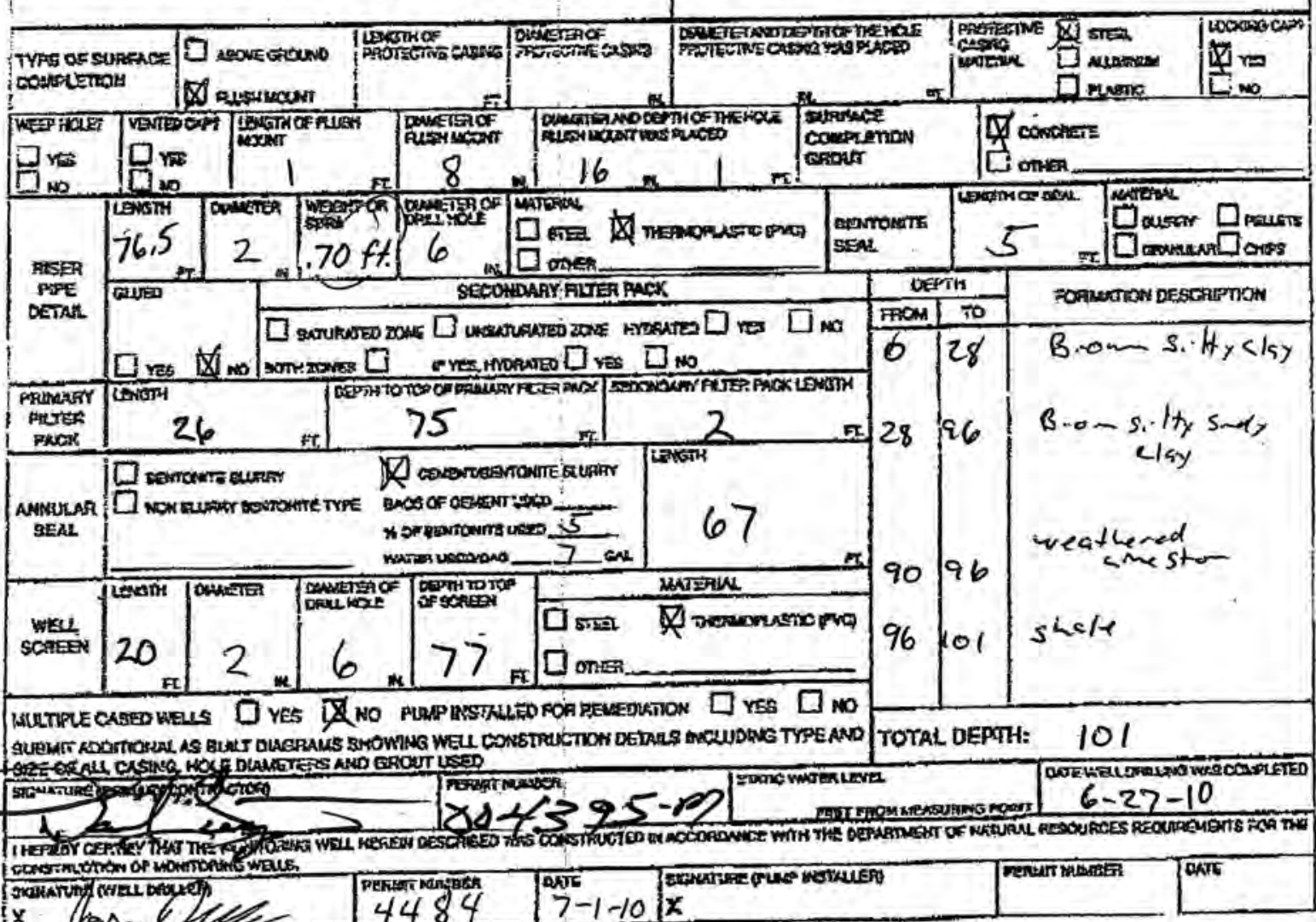


MABSOUAI DEPARTMENT OF NRTURAL RESCURCES

(53/2) 358-3105

MONITORING WEHL CERTIICATION RECORD

\begin{tabular}{|c|c|}
\hline OFFICE USE ONLYY & 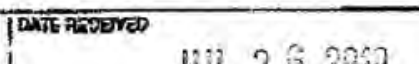 \\
\hline Aan No 383990 & 2010 \\
\hline CAN2 & Checkna \\
\hline 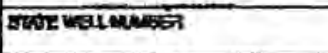 & nevoutena 072610 \\
\hline $\begin{array}{l}\text { Exteri } \\
\sin _{1} / 2\end{array}$ & 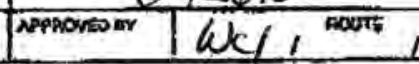 \\
\hline
\end{tabular}

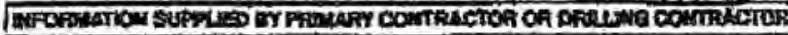

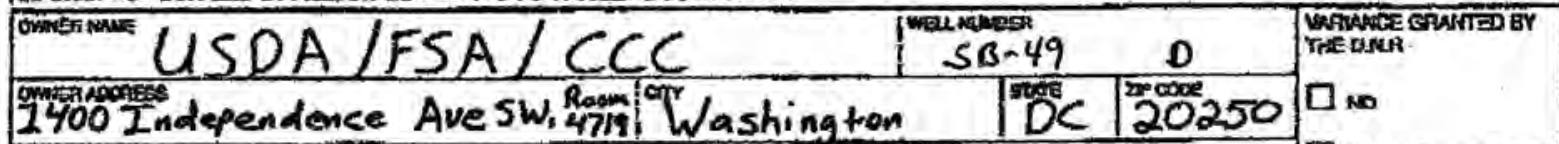

stevene Former USDA/CCC Grain Bin Site Steve Gilmore DFT:

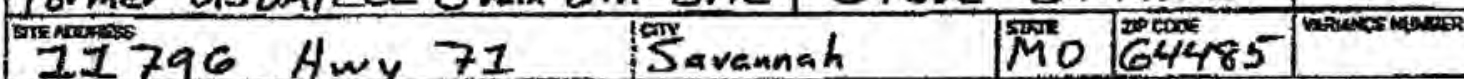

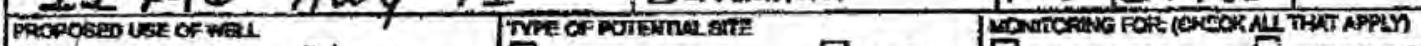

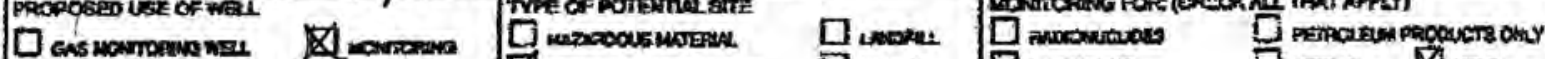

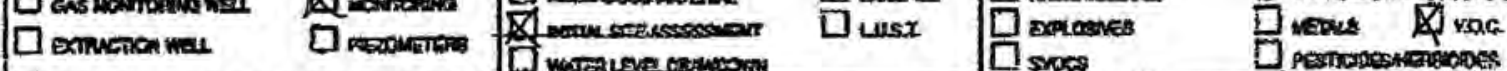

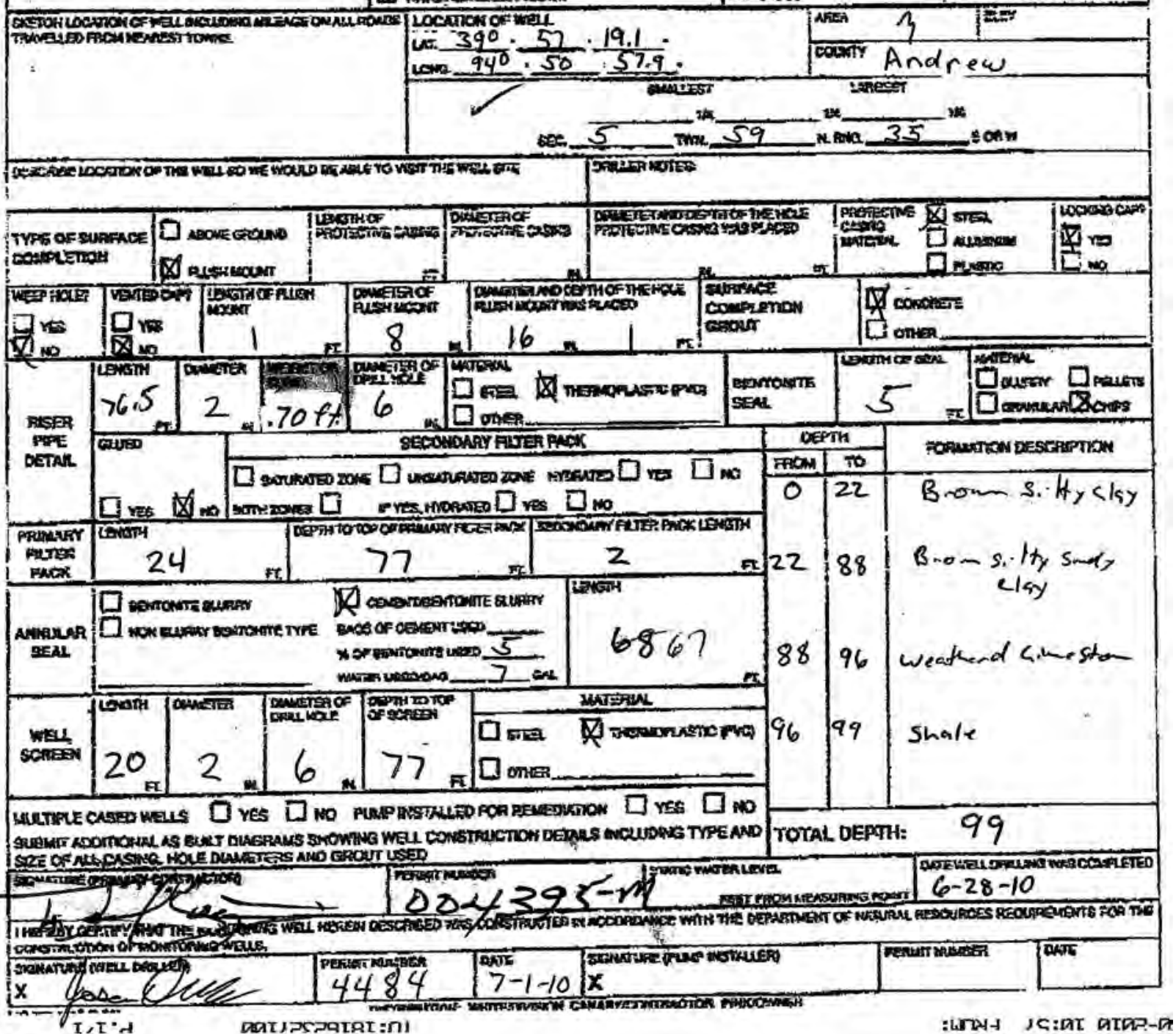




\section{\begin{tabular}{|l|l|}
\hline$Q$ & $\equiv$ \\
\hline A & \\
\hline
\end{tabular} \\ MISSOURI DEPARTMENT OF NATURAL RESOURCES GEOLOGICAL SURVEY PROGRAM WELL PLUGGING REGISTRATION RECORD}

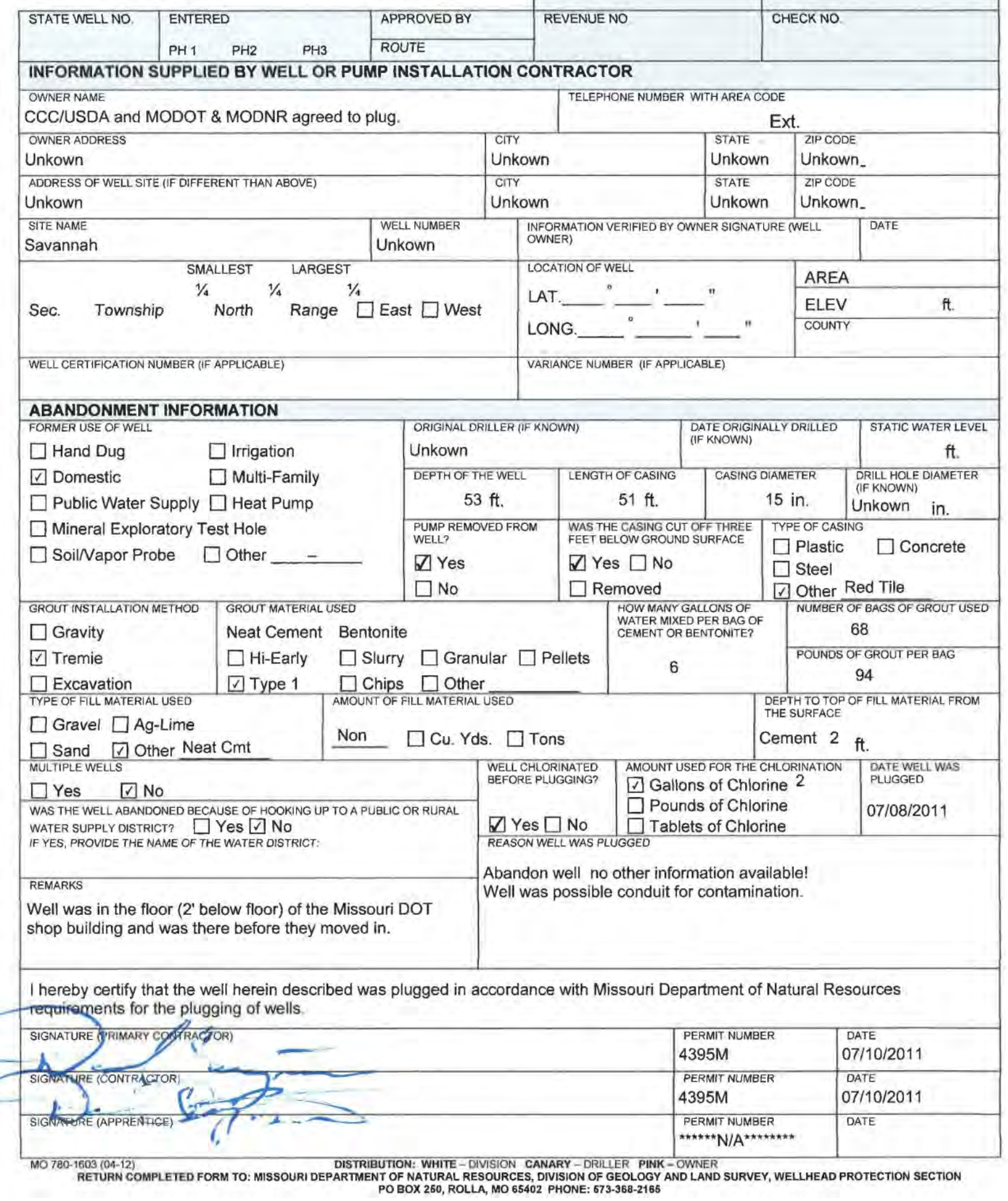




\section{Appendix E:}

\section{Groundwater Sampling Data}


Version 00, 05/21/12

TABLE E.1 Results from the AGEM Laboratory for water samples collected during the Phase II investigation.

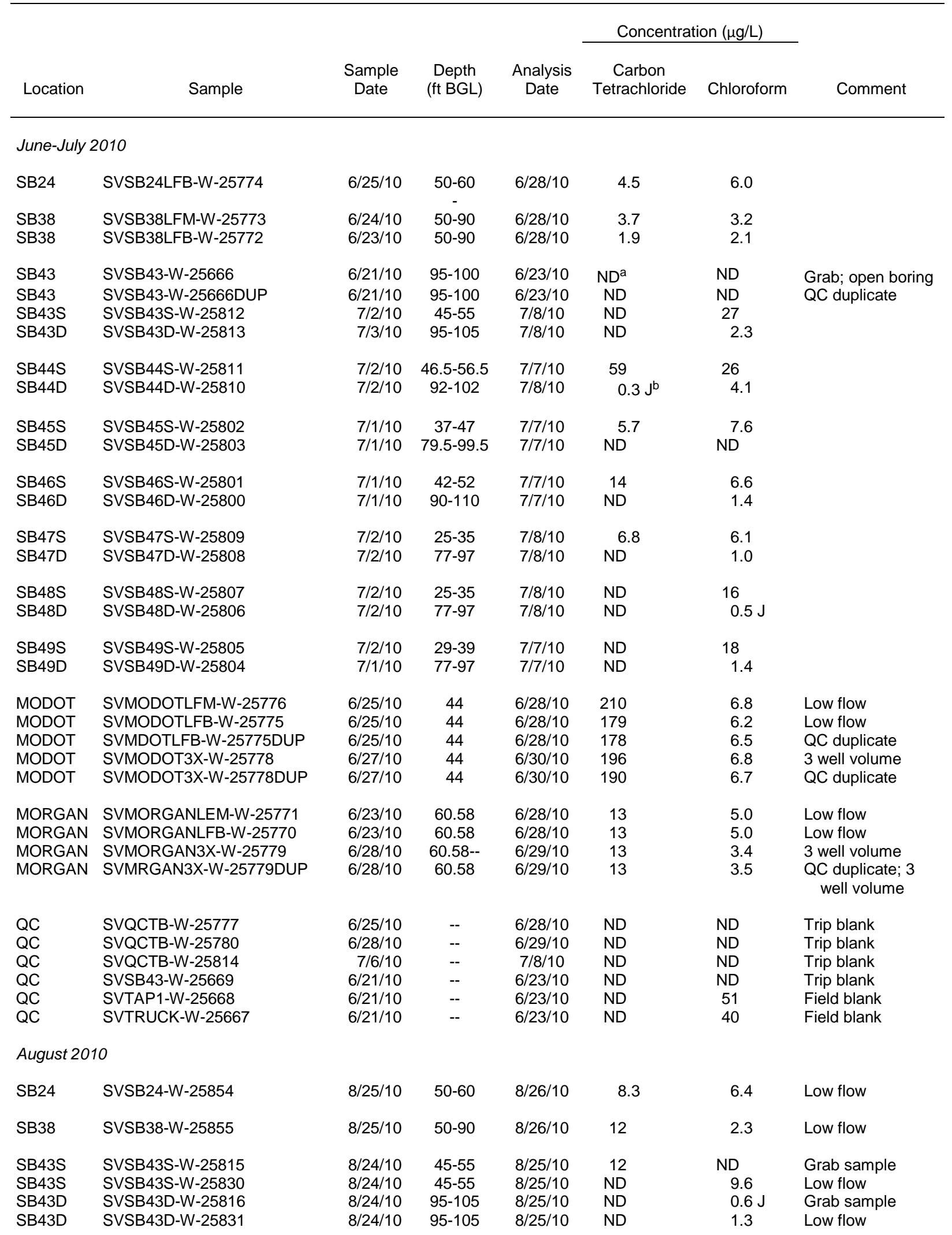


TABLE E.1 (Cont.)

\begin{tabular}{|c|c|c|c|c|c|c|c|}
\hline \multirow[b]{2}{*}{ Location } & \multirow[b]{2}{*}{ Sample } & \multirow[b]{2}{*}{$\begin{array}{l}\text { Sample } \\
\text { Date }\end{array}$} & \multirow[b]{2}{*}{$\begin{array}{l}\text { Depth } \\
\text { (ft BGL) }\end{array}$} & \multirow[b]{2}{*}{$\begin{array}{l}\text { Analysis } \\
\text { Date }\end{array}$} & \multicolumn{2}{|c|}{ Concentration $(\mu \mathrm{g} / \mathrm{L})$} & \multirow[b]{2}{*}{ Comment } \\
\hline & & & & & $\begin{array}{c}\text { Carbon } \\
\text { Tetrachloride }\end{array}$ & Chloroform & \\
\hline \multicolumn{8}{|c|}{ August 2010 (cont.) } \\
\hline SB44S & SVSB44S-W-25817 & $8 / 24 / 10$ & $46.5-56.5$ & $8 / 25 / 10$ & 60 & 8.3 & Grab sample \\
\hline SB44S & SVSB44S-W-25832 & $8 / 24 / 10$ & $46.5-56.5$ & $8 / 26 / 10$ & 84 & 10 & Low flow \\
\hline SB44S & SVSB44S-W-25832DUP & $8 / 24 / 10$ & $46.5-56.5$ & $8 / 26 / 10$ & 83 & 10 & $\begin{array}{l}\text { QC duplicate; low } \\
\text { flow }\end{array}$ \\
\hline SB44D & SVSB44D-W-25818 & 8/24/10 & $92-102$ & $8 / 25 / 10$ & ND & 3.4 & Grab sample \\
\hline SB44D & SVSB44D-W-25833 & $8 / 24 / 10$ & $92-102$ & $8 / 25 / 10$ & ND & 2.4 & Low flow \\
\hline SB45S & SVSB45S-W-25819 & $8 / 24 / 10$ & $37-47$ & $8 / 25 / 10$ & 51 & 6.9 & Grab sample \\
\hline SB45S & SVSB45S-W-25834 & $8 / 25 / 10$ & $37-47$ & $8 / 26 / 10$ & 86 & 8.3 & Low flow \\
\hline SB45D & SVSB45D-W-25820 & $8 / 24 / 10$ & $79.5-99.5$ & $8 / 25 / 10$ & ND & ND & Grab sample \\
\hline SB45D & SVSB45D-W-25835 & $8 / 24 / 10$ & $79.5-99.5$ & $8 / 25 / 10$ & ND & ND & Low flow \\
\hline SB46S & SVSB46S-W-25821 & 8/24/10 & $42-52$ & $8 / 25 / 10$ & 1.4 & ND & Grab sample \\
\hline SB46S & SVSB46S-W-25836 & $8 / 25 / 10$ & $42-52$ & $8 / 26 / 10$ & 15 & 1.8 & Low flow \\
\hline SB46D & SVSB46D-W-25822 & $8 / 24 / 10$ & $90-110$ & $8 / 25 / 10$ & ND & 1.0 & Grab sample \\
\hline SB46D & SVSB46D-W-25837 & $8 / 24 / 10$ & $90-110$ & $8 / 25 / 10$ & ND & ND & Low flow \\
\hline SB47S & SVSB47S-W-25823 & $8 / 24 / 10$ & $25-35$ & $8 / 25 / 10$ & ND & 5.0 & Grab sample \\
\hline SB47S & SVSB47S-W-25838 & $8 / 25 / 10$ & $25-35$ & $8 / 26 / 10$ & ND & 5.6 & Low flow \\
\hline SB47D & SVSB47D-W-25824 & 8/24/10 & $77-97$ & $8 / 25 / 10$ & ND & $0.2 \mathrm{~J}$ & Grab sample \\
\hline SB47D & SVSB47D-W-25839 & $8 / 24 / 10$ & $77-97$ & $8 / 25 / 10$ & ND & ND & Low flow \\
\hline SB47D & SVSB47D-W-25839DUP & $8 / 24 / 10$ & $77-97$ & $8 / 25 / 10$ & ND & ND & $\begin{array}{l}\text { QC duplicate; low } \\
\text { flow }\end{array}$ \\
\hline SB48S & SVSB48S-W-25825 & $8 / 24 / 10$ & $25-35$ & $8 / 26 / 10$ & 2.2 & 1.7 & Grab sample \\
\hline SB48S & SVSB48S-W-25840 & $8 / 25 / 10$ & $25-35$ & $8 / 26 / 10$ & 2.9 & 2.4 & Low flow \\
\hline SB48S & SVSB48S-W-25840DUP & $8 / 25 / 10$ & $25-35$ & $8 / 26 / 10$ & 2.5 & 2.0 & $\begin{array}{l}\text { QC duplicate; low } \\
\text { flow }\end{array}$ \\
\hline SB48S & SVSB48SDUP-W-25845 & 8/25/10 & $25-35$ & 8/26/10 & 3.0 & 2.5 & $\begin{array}{l}\text { QC replicate; low } \\
\text { flow }\end{array}$ \\
\hline SB48D & SVSB48D-W-25826 & $8 / 24 / 10$ & $77-97$ & $8 / 26 / 10$ & ND & ND & Grab sample \\
\hline SB48D & SVSB48D-W-25841 & $8 / 24 / 10$ & $77-97$ & $8 / 25 / 10$ & ND & ND & Low flow \\
\hline SB49S & SVSB49S-W-25827 & $8 / 24 / 10$ & 29-39 & $8 / 26 / 10$ & ND & 3.1 & Grab sample \\
\hline SB49S & SVSB49S-W-25842 & $8 / 25 / 10$ & $29-39$ & $8 / 26 / 10$ & ND & 4.2 & Low flow \\
\hline SB49D & SVSB49D-W-25828 & $8 / 24 / 10$ & $77-97$ & $8 / 26 / 10$ & ND & 3.4 & Grab sample \\
\hline SB49D & SVSB49D-W-25843 & $8 / 24 / 10$ & $77-97$ & $8 / 26 / 10$ & ND & 3.0 & Low flow \\
\hline SB49D & SVSB49DDUP-W-25844 & $8 / 24 / 10$ & $77-97$ & $8 / 25 / 10$ & ND & ND & $\begin{array}{l}\text { QC replicate; low } \\
\text { flow }\end{array}$ \\
\hline QC & SVDIH2O-W-25846 & $8 / 25 / 10$ & -- & $8 / 26 / 10$ & ND & ND & Field blank \\
\hline $\mathrm{QC}$ & SVQCIR-W-25847 & $8 / 24 / 10$ & -- & $8 / 25 / 10$ & ND & ND & Rinsate \\
\hline QC & SVQCIR-W-25848 & $8 / 24 / 10$ & -- & $8 / 26 / 10$ & ND & ND & Rinsate \\
\hline $\mathrm{QC}$ & SVQCIR-W-25849 & $8 / 25 / 10$ & -- & $8 / 26 / 10$ & ND & ND & Rinsate \\
\hline QC & SVQCIR-W-25850 & $8 / 25 / 10$ & -- & $8 / 26 / 10$ & ND & ND & Rinsate \\
\hline $\mathrm{QC}$ & SVQCTB-W-25829 & $8 / 24 / 10$ & -- & $8 / 25 / 10$ & ND & ND & Trip blank \\
\hline $\mathrm{QC}$ & SVQCTB-W-25852 & $8 / 25 / 10$ & -- & $8 / 26 / 10$ & ND & ND & Trip blank \\
\hline \multicolumn{8}{|c|}{ August 2011} \\
\hline SB24 & SVSB24GRAB-W-25856 & 8/10/11 & $50-60$ & 8/11/11 & 8.2 & 4.3 & Grab sample \\
\hline SB24 & SVSB24-W-25872 & 8/11/11 & $50-60$ & 8/12/11 & 10 & 4.7 & Low flow \\
\hline SB38 & SVSB38GRAB-W-25857 & 8/10/11 & $50-90$ & 8/11/11 & 20 & 2.3 & Grab sample \\
\hline SB38 & SVSB38-W-25873 & $8 / 11 / 11$ & $50-90$ & $8 / 12 / 11$ & 11 & 2.4 & Low flow \\
\hline
\end{tabular}


TABLE E.1 (Cont.)

\begin{tabular}{|c|c|c|c|c|c|c|c|}
\hline \multirow[b]{2}{*}{ Location } & \multirow[b]{2}{*}{ Sample } & \multirow[b]{2}{*}{$\begin{array}{l}\text { Sample } \\
\text { Date }\end{array}$} & \multirow[b]{2}{*}{$\begin{array}{l}\text { Depth } \\
\text { (ft BGL) }\end{array}$} & \multirow[b]{2}{*}{$\begin{array}{l}\text { Analysis } \\
\text { Date }\end{array}$} & \multicolumn{2}{|c|}{ Concentration $(\mu \mathrm{g} / \mathrm{L})$} & \multirow[b]{2}{*}{ Comment } \\
\hline & & & & & $\begin{array}{c}\text { Carbon } \\
\text { Tetrachloride }\end{array}$ & Chloroform & \\
\hline \multicolumn{8}{|c|}{ August 2011 (cont.) } \\
\hline SB43S & SVSB43SGRAB-W-25858 & 8/10/11 & $45-55$ & 8/11/11 & ND & 3.2 & Grab sample \\
\hline SB43S & SVSB43S-W-25874 & $8 / 10 / 11$ & $45-55$ & $8 / 11 / 11$ & ND & 3.2 & Low flow \\
\hline SB43D & SVSB43DGRAB-W-25859 & $8 / 10 / 11$ & $95-105$ & $8 / 11 / 11$ & ND & ND & Grab sample \\
\hline SB43D & SVSB43D-W-25875 & $8 / 10 / 11$ & $95-105$ & $8 / 11 / 11$ & ND & ND & Low flow \\
\hline SB43D & SVSB43DDUP-W-25888 & 8/10/11 & $95-105$ & $8 / 12 / 11$ & ND & ND & $\begin{array}{l}\text { QC replicate; low } \\
\text { flow }\end{array}$ \\
\hline SB44S & SVSB44SGRAB-W-25860 & 8/10/11 & $46.5-56.5$ & $8 / 15 / 11$ & 158 & 26 & Grab sample \\
\hline SB44S & SVSB44SGRAB-W-25860DUP & $8 / 10 / 11$ & $46.5-56.5$ & $8 / 15 / 11$ & 141 & 24 & QC duplicate; grab \\
\hline SB44S & SVSB44S-W-25876 & 8/11/11 & $46.5-56.5$ & $8 / 15 / 11$ & 273 & 28 & Low flow \\
\hline SB44D & SVSB44DGRAB-W-25861 & $8 / 10 / 11$ & $92-102$ & $8 / 11 / 11$ & ND & 2.1 & Grab sample \\
\hline SB44D & SVSB44D-W-25877 & 8/11/11 & $92-102$ & $8 / 12 / 11$ & ND & ND & Low flow \\
\hline SB45S & SVSB45SGRAB-W-25862 & 8/10/11 & $37-47$ & 8/11/11 & 40 & 11 & Grab sample \\
\hline SB45S & SVSB45S-W-25878 & 8/11/11 & $37-47$ & $8 / 12 / 11$ & 53 & 12 & Low flow \\
\hline SB45D & SVSB45DGRAB-W-25863 & $8 / 10 / 11$ & $79.5-99.5$ & $8 / 11 / 11$ & ND & ND & Grab sample \\
\hline SB45D & SVSB45D-W-25879 & 8/11/11 & $79.5-99.5$ & $8 / 15 / 11$ & ND & ND & Low flow \\
\hline SB46S & SVSB46SGRAB-W-25864 & 8/10/11 & $42-52$ & 8/11/11 & 12 & 5.9 & Grab sample \\
\hline SB46S & SVSB46S-W-25880 & 8/11/11 & $42-52$ & 8/15/11 & 17 & 7.8 & Low flow \\
\hline SB46D & SVSB46DGRAB-W-25865 & $8 / 10 / 11$ & $90-110$ & $8 / 11 / 11$ & ND & ND & Grab sample \\
\hline SB46D & SVSB46D-W-25881 & $8 / 11 / 11$ & $90-110$ & $8 / 15 / 11$ & ND & ND & Low flow \\
\hline SB46D & SVSB46DDUP-W-25889 & $8 / 11 / 11$ & $90-110$ & $8 / 15 / 11$ & ND & ND & $\begin{array}{l}\text { QC replicate; low } \\
\text { flow }\end{array}$ \\
\hline SB47S & SVSB47SGRAB-W-25866 & 8/10/11 & $25-35$ & 8/11/11 & ND & 1.4 & Grab sample \\
\hline SB47S & SVSB47S-W-25882 & 8/10/11 & $25-35$ & $8 / 11 / 11$ & ND & 1.4 & Low flow \\
\hline SB47D & SVSB47DGRAB-W-25867 & $8 / 10 / 11$ & $77-97$ & $8 / 11 / 11$ & ND & ND & Grab sample \\
\hline SB47D & SVSB47D-W-25883 & $8 / 10 / 11$ & $77-97$ & $8 / 12 / 11$ & ND & ND & Low flow \\
\hline SB48S & SVSB48SGRAB-W-25868 & 8/10/11 & $25-35$ & 8/11/11 & ND & ND & Grab sample \\
\hline SB48S & SVSB48SGRAB-W-25868DUP & 8/10/11 & $25-35$ & $8 / 11 / 11$ & ND & ND & QC duplicate; grab \\
\hline SB48S & SVSB48S-W-25884 & $8 / 11 / 11$ & $25-35$ & $8 / 15 / 11$ & $0.7 \mathrm{~J}$ & ND & Low flow \\
\hline SB48D & SVSB48DGRAB-W-25869 & 8/10/11 & $77-97$ & 8/11/11 & ND & ND & Grab sample \\
\hline SB48D & SVSB48D-W-25885 & 8/10/11 & $77-97$ & $8 / 12 / 11$ & ND & ND & Low flow \\
\hline SB49S & SVSB49SGRAB-W-25870 & 8/10/11 & $29-39$ & 8/11/11 & ND & ND & Grab sample \\
\hline SB49S & SVSB49S-W-25886 & $8 / 10 / 11$ & $29-39$ & $8 / 12 / 11$ & ND & ND & Low flow \\
\hline SB49D & SVSB49DGRAB-W-25871 & 8/10/11 & $77-97$ & 8/11/11 & ND & 1.3 & Grab sample \\
\hline SB49D & SVSB49DGRAB-W-25871DUP & 8/10/11 & $77-97$ & 8/11/11 & ND & 1.3 & QC duplicate; grab \\
\hline SB49D & SVSB49D-W-25887 & 8/10/11 & $77-97$ & $8 / 12 / 11$ & ND & ND & Low flow \\
\hline QC & SVQCIR-W-25891 & 8/10/11 & -- & 8/12/11 & ND & ND & Rinsate \\
\hline $\mathrm{QC}$ & SVQCIR-W-25892 & $8 / 10 / 11$ & -- & $8 / 12 / 11$ & ND & ND & Rinsate \\
\hline QC & SVQCIR-W-25893 & $8 / 11 / 11$ & -- & 8/15/11 & ND & ND & Rinsate \\
\hline QC & SVQCIR-W-25894 & 8/11/11 & -- & 8/15/11 & ND & ND & Rinsate \\
\hline QC & SVQCCTB-W-25895 & 8/10/11 & -- & 8/11/11 & ND & ND & Trip blank \\
\hline $\mathrm{QC}$ & SVQCTB-W-25896 & $8 / 10 / 11$ & -- & $8 / 12 / 11$ & ND & ND & Trip blank \\
\hline QC & SVQCTB-W-25897 & 8/11/11 & -- & 8/15/11 & ND & ND & Trip blank \\
\hline
\end{tabular}

a ND, not detected at an instrument detection limit of $0.1 \mu \mathrm{g} / \mathrm{L}$.

b J, estimated concetration below the purge-and-trap method quantitation limit of $1.0 \mu \mathrm{g} / \mathrm{L}$. 
TABLE E.2 Field measurements made during collection of groundwater samples in 2007-2011.

\begin{tabular}{|c|c|c|c|c|c|c|c|c|}
\hline Location & Sample & $\begin{array}{l}\text { Depth } \\
\text { (ft BGL) }\end{array}$ & $\begin{array}{l}\text { Sample } \\
\text { Date }\end{array}$ & $\begin{array}{c}\text { Temperature } \\
\left({ }^{\circ} \mathrm{C}\right)\end{array}$ & $\mathrm{pH}$ & $\begin{array}{c}\text { Conductivity } \\
(\mu \mathrm{S} / \mathrm{cm})\end{array}$ & $\begin{array}{c}\text { Dissolved } \\
\text { Oxygen } \\
\text { (mg/L) }\end{array}$ & $\begin{array}{c}\text { Oxidation- } \\
\text { Reduction } \\
\text { Potential } \\
\quad(\mathrm{mV})\end{array}$ \\
\hline SB24 & SVSB24-W-25854 & $50-60$ & $8 / 25 / 10$ & 26.0 & 6.73 & 387 & 4.47 & 57 \\
\hline SB38 & SVSB38-W-25855 & $50-90$ & $8 / 25 / 10$ & 23.7 & 6.96 & 346 & 5.35 & 1 \\
\hline SB43S & SVSB43S-W-25830 & $45-55$ & 8/24/10 & 16.1 & 6.79 & 417 & 0.21 & 8 \\
\hline SB43S & SVSB43S-W-25874 & $45-55$ & 8/10/11 & 15.3 & 6.61 & 319 & 2.24 & 55 \\
\hline SB43D & SVSB43D-W-25831 & $95-105$ & $8 / 24 / 10$ & 17.3 & 7.16 & 460 & 0.21 & -19 \\
\hline SB43D & SVSB43D-W-25875 & $95-105$ & 8/10/11 & 16.4 & 7.33 & 474 & 1.23 & 98 \\
\hline SB44S & SVSB44S-W-25832 & $46.5-56.5$ & $8 / 24 / 10$ & 16.3 & 7.12 & 302 & 0.20 & -12 \\
\hline SB44S & SVSB44S-W-25876 & $46.5-56.5$ & 8/11/11 & 15.1 & 6.76 & 226 & 2.69 & 64 \\
\hline SB44D & SVSB44D-W-25833 & $92-102$ & $8 / 24 / 10$ & 17.1 & 8.09 & 448 & 0.25 & -73 \\
\hline SB44D & SVSB44D-W-25877 & $92-102$ & $8 / 11 / 11$ & 15.7 & 7.30 & 450 & 3.03 & 55 \\
\hline SB45S & SVSB45S-W-25834 & $37-47$ & $8 / 25 / 10$ & 16.2 & 6.59 & 573 & 0.18 & 132 \\
\hline SB45S & SVSB45S-W-25878 & $37-47$ & 8/11/11 & 16.4 & 6.28 & 488 & 0.19 & 75 \\
\hline SB45D & SVSB45D-W-25835 & $79.5-99.5$ & $8 / 24 / 10$ & 16.9 & 7.52 & 371 & 0.39 & 90 \\
\hline SB45D & SVSB45D-W-25879 & $79.5-99.5$ & 8/11/11 & 16.2 & 7.65 & 389 & 2.17 & 249 \\
\hline SB46S & SVSB46S-W-25836 & $42-52$ & $8 / 25 / 10$ & 18.1 & 10.83 & 659 & 0.14 & -96 \\
\hline SB46S & SVSB46S-W-25880 & $42-52$ & 8/11/11 & 14.5 & 7.10 & 536 & 0.23 & 7 \\
\hline SB46D & SVSB46D-W-25837 & $90-110$ & $8 / 24 / 10$ & 18.0 & 6.80 & 455 & 3.60 & 27 \\
\hline SB46D & SVSB46D-W-25881 & $90-110$ & $8 / 11 / 11$ & 15.8 & 7.55 & 426 & 2.58 & 244 \\
\hline SB47S & SVSB47S-W-25838 & $25-35$ & $8 / 25 / 10$ & 14.7 & 6.42 & 234 & 4.97 & 51 \\
\hline SB47S & SVSB47S-W-25882 & $25-35$ & 8/10/11 & 13.0 & 6.59 & 173 & 2.60 & 145 \\
\hline SB47D & SVSB47D-W-25839 & $77-97$ & $8 / 24 / 10$ & 16.1 & 7.39 & 330 & 3.35 & 20 \\
\hline SB47D & SVSB47D-W-25883 & $77-97$ & $8 / 10 / 11$ & 13.7 & 7.89 & 320 & 18.42 & 307 \\
\hline SB48S & SVSB48S-W-25840 & $25-35$ & $8 / 25 / 10$ & 16.4 & 7.03 & 313 & 0.75 & 4 \\
\hline SB48S & SVSB48S-W-25884 & $25-35$ & 8/11/11 & 13.2 & 7.10 & 246 & 3.44 & 348 \\
\hline SB48D & SVSB48D-W-25841 & $77-97$ & $8 / 24 / 10$ & 18.9 & 7.10 & 370 & 4.50 & 31 \\
\hline SB48D & SVSB48D-W-25885 & $77-97$ & 8/10/11 & 14.2 & 7.92 & 362 & 3.81 & 339 \\
\hline SB49S & SVSB49S-W-25842 & $29-39$ & $8 / 25 / 10$ & 15.7 & 6.70 & 260 & 0.17 & 71 \\
\hline SB49S & SVSB49S-W-25886 & $29-39$ & 8/10/11 & 13.8 & 6.65 & 214 & 0.07 & -99 \\
\hline SB49D & SVSB49D-W-25843 & $77-97$ & $8 / 24 / 10$ & 17.1 & 7.73 & 474 & 0.17 & -145 \\
\hline SB49D & SVSB49D-W-25887 & $77-97$ & 8/10/11 & 15.0 & 7.27 & 490 & 2.37 & -111 \\
\hline
\end{tabular}




\section{Appendix F:}

\section{Coordinates Survey Data}


TABLE F.1 Coordinates survey data for the Phase II investigation.

\begin{tabular}{lccccc}
\hline & \multicolumn{2}{c}{ Horizontal Location $^{\mathrm{a}}(\mathrm{ft})$} & & \multicolumn{2}{c}{ Elevation $^{\mathrm{b}}$ (ft AMSL) } \\
\cline { 2 - 3 } \cline { 5 - 6 } Location & \multirow{2}{*}{ Easting } & Northing & & Ground & Top of Casing \\
& & & & & \\
\hline & & & & & \\
SB43S & 2690648.349 & 1379530.688 & & 1154.81 & 1154.44 \\
SB43D & 2690654.601 & 1379531.113 & & 1154.99 & 1154.48 \\
SB44S & 2690874.218 & 1379569.071 & & 1155.60 & 1155.12 \\
SB44D & 2690879.838 & 1379565.950 & & 1155.77 & 1155.37 \\
SB45S & 2691429.570 & 1379414.170 & & 1145.03 & 1144.66 \\
SB45D & 2691422.615 & 1379414.031 & & 1145.34 & 1144.98 \\
SB46S & 2691103.195 & 1379509.842 & & 1151.96 & 1151.59 \\
SB46D & 2691099.418 & 1379506.118 & & 1151.89 & 1151.50 \\
SB47S & 2690804.074 & 1379031.118 & & 1134.68 & 1134.27 \\
SB47D & 2690810.492 & 1379031.290 & & 1135.18 & 1134.79 \\
SB48S & 2691105.526 & 1379043.791 & & 1134.88 & 1134.45 \\
SB48D & 2691099.264 & 1379042.117 & & 1134.94 & 1134.48 \\
SB49S & 2690772.369 & 1379810.132 & & 1137.72 & 1137.16 \\
SB49D & 2690774.298 & 1379804.770 & & 1137.92 & 1137.36 \\
& & & & & \\
\hline
\end{tabular}

a Coordinates are in the Missouri State Plane, East Zone, North American Datum (NAD) 83.

b Vertical datum is North American Vertical Datum (NAVD) 88. 
Supplement 1:

Automatically Recorded Groundwater Level Data and Rainfall Data 
TABLE S1.1 Automatically recorded groundwater level data for selected private wells and monitoring wells SB24 and SB38, May 2009 to December 2011.

\begin{tabular}{|c|c|c|c|c|c|c|c|c|c|c|}
\hline \multirow[b]{2}{*}{ Date and Time } & \multicolumn{8}{|c|}{ Water Level (ft below reference point) } & \multirow[b]{2}{*}{ SB24 } & \multirow[b]{2}{*}{ SB38 } \\
\hline & McPike & Potter & Hughes & Barr & Burks & MoDOT & Clizer "Old" & Morgan & & \\
\hline 5/1/09 0:00 & 8.804 & 3.189 & $N A^{a}$ & 16.355 & 6.954 & 14.162 & 4.226 & NA & 23.610 & 36.878 \\
\hline 5/1/09 4:00 & 8.898 & 3.248 & & 16.278 & 6.966 & 14.192 & 4.275 & & 23.575 & 36.862 \\
\hline 5/1/09 8:00 & 8.999 & 3.315 & & 16.185 & 6.980 & 14.223 & 4.329 & & 23.538 & 36.845 \\
\hline 5/1/09 12:00 & 9.075 & 3.388 & & 16.194 & 6.987 & 14.219 & 4.362 & & 23.506 & 36.831 \\
\hline 5/1/09 16:00 & 9.117 & 3.477 & & 16.369 & 6.983 & 14.179 & 4.372 & & 23.468 & 36.812 \\
\hline 5/1/09 20:00 & 9.145 & 3.587 & & 16.469 & 6.971 & 14.139 & 4.381 & & 23.433 & 36.796 \\
\hline 5/2/09 0:00 & 9.200 & 3.697 & & 16.425 & 6.978 & 14.152 & 4.419 & & 23.399 & 36.782 \\
\hline 5/2/09 4:00 & 9.230 & 3.776 & & 16.448 & 6.980 & 14.121 & 4.428 & & 23.366 & 36.763 \\
\hline 5/2/09 8:00 & 9.256 & 3.816 & & 16.500 & 6.985 & 14.107 & 4.449 & & 23.331 & 36.746 \\
\hline 5/2/09 12:00 & 9.289 & 3.842 & & 16.572 & 6.997 & 14.087 & 4.461 & & 23.296 & 36.732 \\
\hline 5/2/09 16:00 & 9.287 & 3.896 & & 16.837 & 6.980 & 14.027 & 4.459 & & 23.262 & 36.713 \\
\hline 5/2/09 20:00 & 9.299 & 3.971 & & 16.917 & 6.980 & 13.997 & 4.473 & & 23.226 & 36.697 \\
\hline 5/3/09 0:00 & 9.353 & 4.055 & & 16.842 & 6.999 & 14.033 & 4.517 & & 23.191 & 36.680 \\
\hline 5/3/09 4:00 & 9.395 & 4.125 & & 16.819 & 7.009 & 14.045 & 4.550 & & 23.159 & 36.664 \\
\hline 5/3/09 8:00 & 9.450 & 4.181 & & 16.781 & 7.025 & 14.071 & 4.592 & & 23.126 & 36.647 \\
\hline 5/3/09 12:00 & 9.490 & 4.221 & & 16.882 & 7.028 & 14.065 & 4.611 & & 23.094 & 36.631 \\
\hline 5/3/09 16:00 & 9.518 & 4.270 & & 17.066 & 7.032 & 14.039 & 4.621 & & 23.059 & 36.614 \\
\hline 5/3/09 20:00 & 9.549 & 4.322 & & 17.115 & 7.032 & 14.039 & 4.651 & & 23.027 & 36.598 \\
\hline 5/4/09 0:00 & 9.603 & 4.373 & & 17.007 & 7.049 & 14.075 & 4.698 & & 22.994 & 36.581 \\
\hline 5/4/09 4:00 & 9.648 & 4.418 & & 16.979 & 7.049 & 14.094 & 4.733 & & 22.964 & 36.565 \\
\hline 5/4/09 8:00 & 9.699 & 4.457 & & 16.912 & 7.063 & 14.130 & 4.773 & & 22.931 & 36.548 \\
\hline 5/4/09 12:00 & 9.728 & 4.488 & & 16.970 & 7.058 & 14.116 & 4.790 & & 22.899 & 36.532 \\
\hline $5 / 4 / 09$ 16:00 & 9.728 & 4.513 & & 17.152 & 7.046 & 14.066 & 4.780 & & 22.869 & 36.515 \\
\hline $5 / 4 / 0920: 00$ & 9.747 & 4.544 & & 17.196 & 7.054 & 14.069 & 4.809 & & 22.839 & 36.499 \\
\hline 5/5/09 0:00 & 9.777 & 4.572 & & 17.131 & 7.061 & 14.089 & 4.839 & & 22.808 & 36.482 \\
\hline 5/5/09 4:00 & 9.796 & 4.593 & & 17.138 & 7.056 & 14.079 & 4.855 & & 22.775 & 36.468 \\
\hline 5/5/09 8:00 & 9.824 & 4.614 & & 17.091 & 7.065 & 14.108 & 4.891 & & 22.748 & 36.452 \\
\hline 5/5/09 12:00 & 9.836 & 4.626 & & 17.224 & 7.056 & 14.078 & 4.895 & & 22.715 & 36.435 \\
\hline 5/5/09 16:00 & 9.827 & 4.640 & & 17.408 & 7.046 & 14.040 & 4.893 & & 22.685 & 36.419 \\
\hline 5/5/09 20:00 & 9.834 & 4.656 & & 17.434 & 7.049 & 14.036 & 4.910 & & 22.655 & 36.402 \\
\hline 5/6/09 0:00 & 9.867 & 4.677 & & 17.362 & 7.068 & 14.062 & 4.942 & & 22.625 & 36.386 \\
\hline 5/6/09 4:00 & 9.865 & 4.687 & & 17.397 & 7.063 & 14.041 & 4.949 & & 22.597 & 36.372 \\
\hline 5/6/09 8:00 & 9.895 & 4.701 & & 17.329 & 7.079 & 14.077 & 4.985 & & 22.567 & 36.353 \\
\hline 5/6/09 12:00 & 9.916 & 4.708 & & 17.415 & 7.077 & 14.099 & 5.018 & & 22.537 & 36.339 \\
\hline 5/6/09 16:00 & 9.928 & 4.712 & & 17.494 & 7.079 & 14.075 & 5.018 & & 22.509 & 36.322 \\
\hline 5/6/09 20:00 & 9.961 & 4.724 & & 17.536 & 7.075 & 14.104 & 5.058 & & 22.479 & 36.306 \\
\hline 5/7/09 0:00 & 10.011 & 4.740 & & 17.441 & 7.091 & 14.142 & 5.100 & & 22.453 & 36.292 \\
\hline 5/7/09 4:00 & 10.041 & 4.747 & & 17.390 & 7.094 & 14.158 & 5.126 & & 22.425 & 36.273 \\
\hline 5/7/09 8:00 & 10.077 & 4.759 & & 17.341 & 7.098 & 14.190 & 5.166 & & 22.399 & 36.259 \\
\hline $5 / 7 / 09$ 12:00 & 10.077 & 4.761 & & 17.506 & 7.087 & 14.171 & 5.168 & & 22.370 & 36.245 \\
\hline 5/7/09 16:00 & 10.067 & 4.771 & & 17.739 & 7.077 & 14.121 & 5.161 & & 22.342 & 36.228 \\
\hline 5/7/09 20:00 & 10.070 & 4.789 & & 17.774 & 7.065 & 14.066 & 5.187 & & 22.316 & 36.212 \\
\hline 5/8/09 0:00 & 10.124 & 4.803 & & 17.620 & 7.091 & 14.087 & 5.238 & & 22.291 & 36.197 \\
\hline 5/8/09 4:00 & 10.140 & 4.804 & & 17.599 & 7.087 & 14.059 & 5.255 & & 22.263 & 36.181 \\
\hline 5/8/09 8:00 & 10.150 & 4.797 & & 17.592 & 7.075 & 14.038 & 5.276 & & 22.237 & 36.164 \\
\hline 5/8/09 12:00 & 10.173 & 4.773 & & 17.576 & 7.087 & 14.058 & 5.309 & 19.166 & 22.210 & 36.122 \\
\hline 5/8/09 16:00 & 10.183 & 4.736 & 5.882 & 17.769 & 7.082 & 14.038 & 5.318 & 19.157 & 22.183 & 36.106 \\
\hline $5 / 8 / 0920: 00$ & 10.268 & 4.748 & 5.923 & 17.625 & 7.112 & 14.152 & 5.410 & 19.256 & 22.157 & 36.091 \\
\hline 5/9/09 0:00 & 10.348 & 4.778 & 5.974 & 17.403 & 7.145 & 14.217 & 5.471 & 19.394 & 22.135 & 36.075 \\
\hline 5/9/09 4:00 & 10.404 & 4.804 & 6.011 & 17.233 & 7.145 & 14.273 & 5.520 & 19.484 & 22.109 & 36.061 \\
\hline 5/9/09 8:00 & 10.463 & 4.829 & 6.053 & 17.131 & 7.155 & 14.351 & 5.584 & 19.575 & 22.089 & 36.047 \\
\hline 5/9/09 12:00 & 10.515 & 4.857 & 6.093 & 17.191 & 7.160 & 14.394 & 5.631 & 19.669 & 22.066 & 36.030 \\
\hline 5/9/09 16:00 & 10.527 & 4.883 & 6.126 & 17.427 & 7.141 & 14.373 & 5.638 & 19.726 & 22.045 & 36.016 \\
\hline 5/9/09 20:00 & 10.541 & 4.907 & 6.154 & 17.443 & 7.124 & 14.378 & 5.671 & 19.702 & 22.023 & 36.004 \\
\hline 5/10/09 0:00 & 10.565 & 4.928 & 6.187 & 17.371 & 7.129 & 14.400 & 5.701 & 19.764 & 22.003 & 35.990 \\
\hline 5/10/09 4:00 & 10.560 & 4.939 & 6.212 & 17.387 & 7.115 & 14.376 & 5.704 & 19.825 & 21.979 & 35.974 \\
\hline 5/10/09 8:00 & 10.593 & 4.953 & 6.241 & 17.387 & 7.120 & 14.433 & 5.758 & 19.818 & 21.961 & 35.962 \\
\hline 5/10/09 12:00 & 10.607 & 4.960 & 6.271 & 17.487 & 7.120 & 14.432 & 5.776 & 19.858 & 21.939 & 35.948 \\
\hline $5 / 10 / 09$ 16:00 & 10.605 & 4.970 & 6.295 & 17.625 & 7.108 & 14.417 & 5.790 & 19.848 & 21.918 & 35.934 \\
\hline $5 / 10 / 09$ 20:00 & 10.619 & 4.979 & 6.320 & 17.653 & 7.105 & 14.441 & 5.819 & 19.881 & 21.898 & 35.919 \\
\hline 5/11/09 0:00 & 10.640 & 4.988 & 6.348 & 17.599 & 7.108 & 14.466 & 5.849 & 19.909 & 21.879 & 35.908 \\
\hline
\end{tabular}


Water Level (ft below reference point)

\begin{tabular}{|c|c|c|c|c|c|c|c|c|c|c|}
\hline Date and Time & McPike & Potter & Hughes & Barr & Burks & MoDOT & Clizer "Old" & Morgan & SB24 & SB38 \\
\hline 5/11/09 4:00 & 10.652 & 4.993 & 6.374 & 17.599 & 7.096 & 14.482 & 5.875 & 19.940 & 21.859 & 35.894 \\
\hline 5/11/09 8:00 & 10.661 & 4.996 & 6.397 & 17.639 & 7.089 & 14.497 & 5.899 & 19.989 & 21.841 & 35.879 \\
\hline 5/11/09 12:00 & 10.668 & 5.000 & 6.425 & 17.774 & 7.089 & 14.490 & 5.910 & 19.992 & 21.820 & 35.868 \\
\hline 5/11/09 16:00 & 10.647 & 5.005 & 6.444 & 17.965 & 7.072 & 14.445 & 6.117 & 20.135 & 21.802 & 35.853 \\
\hline 5/11/09 20:00 & 10.633 & 5.014 & 6.465 & 17.946 & 7.065 & 14.427 & 5.924 & 19.942 & 21.782 & 35.842 \\
\hline 5/12/09 0:00 & 10.647 & 5.026 & 6.486 & 17.844 & 7.068 & 14.453 & 5.950 & 19.975 & 21.763 & 35.828 \\
\hline 5/12/09 4:00 & 10.633 & 5.028 & 6.505 & 17.844 & 7.061 & 14.434 & 5.948 & 19.961 & 21.746 & 35.816 \\
\hline 5/12/09 8:00 & 10.633 & 5.031 & 6.521 & 17.846 & 7.063 & 14.444 & 5.964 & 19.959 & 21.724 & 35.804 \\
\hline 5/12/09 12:00 & 10.598 & 5.024 & 6.533 & 18.042 & 7.051 & 14.376 & 5.967 & 19.914 & 21.707 & 35.790 \\
\hline 5/12/09 16:00 & 10.407 & 4.897 & 6.528 & 17.956 & 6.950 & 14.237 & 5.866 & 19.832 & 21.675 & 35.773 \\
\hline $5 / 12 / 0920: 00$ & 9.957 & 4.320 & 6.344 & 17.918 & 6.973 & 14.117 & 5.833 & 19.771 & 21.666 & 35.759 \\
\hline 5/13/09 0:00 & 9.997 & 4.104 & 6.252 & 17.842 & 6.990 & 14.130 & 5.823 & 19.745 & 21.674 & 35.747 \\
\hline 5/13/09 4:00 & 10.034 & 4.013 & 6.205 & 17.858 & 6.987 & 14.122 & 5.814 & 19.684 & 21.745 & 35.736 \\
\hline 5/13/09 8:00 & 10.108 & 3.992 & 6.191 & 17.739 & 7.016 & 14.179 & 5.849 & 19.709 & 21.649 & 35.722 \\
\hline 5/13/09 12:00 & 10.148 & 3.997 & 6.189 & 17.807 & 7.018 & 14.192 & 5.861 & 19.756 & 21.734 & 35.710 \\
\hline 5/13/09 16:00 & 10.221 & 4.041 & 6.201 & 17.751 & 7.044 & 14.265 & 5.922 & 19.822 & 21.686 & 35.698 \\
\hline $5 / 13 / 0920: 00$ & 10.334 & 4.121 & 6.234 & 17.569 & 7.079 & 14.400 & 6.021 & 19.853 & 21.531 & 35.684 \\
\hline 5/14/09 0:00 & 10.473 & 4.212 & 6.283 & 17.317 & 7.131 & 14.563 & 6.124 & 20.064 & 21.232 & 35.672 \\
\hline 5/14/09 4:00 & 10.548 & 4.289 & 6.325 & 17.287 & 7.134 & 14.610 & 6.164 & 20.248 & 21.476 & 35.660 \\
\hline 5/14/09 8:00 & 10.628 & 4.364 & 6.367 & 17.245 & 7.145 & 14.696 & 6.227 & 20.307 & 21.336 & 35.646 \\
\hline 5/14/09 12:00 & 10.675 & 4.429 & 6.405 & 17.436 & 7.138 & 14.710 & 6.249 & 20.420 & 21.468 & 35.634 \\
\hline 5/14/09 16:00 & 10.666 & 4.476 & 6.428 & 17.732 & 7.096 & 14.642 & 6.225 & 20.382 & 21.651 & 35.623 \\
\hline 5/14/09 20:00 & 10.647 & 4.518 & 6.442 & 17.772 & 7.065 & 14.588 & 6.216 & 20.328 & 21.638 & 35.609 \\
\hline 5/15/09 0:00 & 10.654 & 4.561 & 6.461 & 17.723 & 7.061 & 14.586 & 6.227 & 20.365 & 21.513 & 35.599 \\
\hline 5/15/09 4:00 & 10.515 & 4.462 & 6.465 & 17.800 & 7.032 & 14.475 & 5.967 & 20.281 & 21.524 & 35.585 \\
\hline 5/15/09 8:00 & 9.433 & 3.887 & 5.911 & 17.501 & 6.884 & 13.992 & 5.727 & 20.191 & 21.495 & 35.568 \\
\hline 5/15/09 12:00 & 8.931 & 3.503 & 5.552 & 17.350 & 6.877 & 13.936 & 5.471 & 20.083 & 21.478 & 35.559 \\
\hline 5/15/09 16:00 & 7.640 & 3.117 & 4.942 & 17.054 & 6.723 & 13.433 & 5.006 & 19.841 & 21.482 & 35.545 \\
\hline $5 / 15 / 0920: 00$ & 7.663 & 3.035 & 4.691 & 16.772 & 6.579 & 13.613 & 4.830 & 19.702 & 21.370 & 35.531 \\
\hline 5/16/09 0:00 & 7.875 & 3.063 & 4.595 & 16.532 & 6.497 & 13.725 & 4.738 & 19.662 & 21.218 & 35.512 \\
\hline $5 / 16 / 09$ 4:00 & 8.101 & 3.148 & 4.560 & 16.271 & 6.452 & 13.861 & 4.719 & 19.702 & 20.996 & 35.503 \\
\hline 5/16/09 8:00 & 8.292 & 3.258 & 4.555 & 16.129 & 6.431 & 13.947 & 4.712 & 19.738 & 20.917 & 35.493 \\
\hline 5/16/09 12:00 & 8.429 & 3.367 & 4.563 & 16.208 & 6.415 & 13.973 & 4.696 & 19.782 & 21.013 & 35.479 \\
\hline 5/16/09 16:00 & 8.493 & 3.496 & 4.570 & 16.451 & 6.379 & 13.919 & 4.647 & 19.684 & 21.202 & 35.465 \\
\hline 5/16/09 20:00 & 8.552 & 3.648 & 4.579 & 16.465 & 6.355 & 13.893 & 4.632 & 19.604 & 21.155 & 35.453 \\
\hline 5/17/09 0:00 & 8.637 & 3.791 & 4.602 & 16.390 & 6.370 & 13.915 & 4.644 & 19.618 & 21.006 & 35.441 \\
\hline 5/17/09 4:00 & 8.693 & 3.896 & 4.626 & 16.402 & 6.377 & 13.889 & 4.635 & 19.545 & 20.981 & 35.429 \\
\hline 5/17/09 8:00 & 8.752 & 3.969 & 4.649 & 16.423 & 6.391 & 13.890 & 4.644 & 19.538 & 20.995 & 35.415 \\
\hline 5/17/09 12:00 & 8.785 & 4.015 & 4.675 & 16.590 & 6.400 & 13.841 & 4.632 & 19.479 & 21.170 & 35.401 \\
\hline 5/17/09 16:00 & 8.799 & 4.072 & 4.698 & 16.812 & 6.393 & 13.775 & 4.616 & 19.383 & 21.276 & 35.389 \\
\hline 5/17/09 20:00 & 8.839 & 4.149 & 4.724 & 16.763 & 6.398 & 13.761 & 4.632 & 19.310 & 21.187 & 35.375 \\
\hline 5/18/09 0:00 & 8.884 & 4.221 & 4.757 & 16.684 & 6.421 & 13.750 & 4.642 & 19.291 & 21.069 & 35.364 \\
\hline 5/18/09 4:00 & 8.912 & 4.280 & 4.782 & 16.686 & 6.429 & 13.730 & 4.649 & 19.246 & 21.018 & 35.349 \\
\hline 5/18/09 8:00 & 8.957 & 4.334 & 4.815 & 16.679 & 6.445 & 13.733 & 4.668 & 19.263 & 20.974 & 35.340 \\
\hline 5/18/09 12:00 & 8.976 & 4.378 & 4.851 & 16.835 & 6.452 & 13.680 & 4.661 & 19.211 & 21.115 & 35.326 \\
\hline 5/18/09 16:00 & 8.988 & 4.427 & 4.881 & 17.019 & 6.457 & 13.639 & 4.663 & 19.105 & 21.244 & 35.312 \\
\hline 5/18/09 20:00 & 9.035 & 4.483 & 4.916 & 16.900 & 6.474 & 13.645 & 4.698 & 19.086 & 21.114 & 35.300 \\
\hline 5/19/09 0:00 & 9.089 & 4.530 & 4.961 & 16.791 & 6.502 & 13.660 & 4.731 & 19.133 & 20.944 & 35.286 \\
\hline 5/19/09 4:00 & 9.132 & 4.568 & 4.996 & 16.751 & 6.516 & 13.664 & 4.755 & 19.117 & 20.869 & 35.276 \\
\hline 5/19/09 8:00 & 9.198 & 4.600 & 5.045 & 16.702 & 6.544 & 13.695 & 4.799 & 19.154 & 20.790 & 35.265 \\
\hline 5/19/09 12:00 & 9.233 & 4.629 & 5.087 & 16.889 & 6.556 & 13.668 & 4.816 & 19.152 & 20.927 & 35.250 \\
\hline 5/19/09 16:00 & 9.254 & 4.659 & 5.129 & 17.080 & 6.566 & 13.632 & 4.834 & 19.112 & 21.061 & 35.239 \\
\hline 5/19/09 20:00 & 9.287 & 4.692 & 5.174 & 16.993 & 6.575 & 13.611 & 4.858 & 19.086 & 20.965 & 35.227 \\
\hline 5/20/09 0:00 & 9.337 & 4.724 & 5.218 & 16.870 & 6.598 & 13.635 & 4.895 & 19.107 & 20.778 & 35.213 \\
\hline $5 / 20 / 094: 00$ & 9.365 & 4.746 & 5.256 & 16.847 & 6.606 & 13.623 & 4.912 & 19.110 & 20.713 & 35.203 \\
\hline $5 / 20 / 098: 00$ & 9.395 & 4.764 & 5.291 & 16.847 & 6.610 & 13.619 & 4.931 & 19.115 & 20.700 & 35.189 \\
\hline 5/20/09 12:00 & 9.412 & 4.781 & 5.328 & 17.042 & 6.620 & 13.584 & 4.947 & 19.230 & 20.845 & 35.177 \\
\hline 5/20/09 16:00 & 9.417 & 4.799 & 5.366 & 17.203 & 6.622 & 13.544 & 4.966 & 19.265 & 20.971 & 35.163 \\
\hline 5/20/09 20:00 & 9.445 & 4.825 & 5.408 & 17.084 & 6.638 & 13.530 & 4.992 & 19.065 & 20.871 & 35.154 \\
\hline 5/21/09 0:00 & 9.492 & 4.853 & 5.450 & 16.949 & 6.660 & 13.571 & 5.034 & 19.098 & 20.674 & 35.140 \\
\hline 5/21/09 4:00 & 9.539 & 4.877 & 5.492 & 16.889 & 6.676 & 13.592 & 5.069 & 19.105 & 20.572 & 35.130 \\
\hline 5/21/09 8:00 & 9.589 & 4.893 & 5.537 & 16.854 & 6.690 & 13.621 & 5.109 & 19.157 & 20.515 & 35.119 \\
\hline 5/21/09 12:00 & 9.636 & 4.907 & 5.581 & 16.986 & 6.712 & 13.626 & 5.147 & 19.220 & 20.608 & 35.104 \\
\hline 5/21/09 16:00 & 9.660 & 4.923 & 5.628 & 17.171 & 6.716 & 13.610 & 5.180 & 19.213 & 20.738 & 35.093 \\
\hline
\end{tabular}


Water Level (ft below reference point)

\begin{tabular}{|c|c|c|c|c|c|c|c|c|c|c|}
\hline Date and Time & McPike & Potter & Hughes & Barr & Burks & MoDOT & Clizer "Old" & Morgan & SB24 & SB38 \\
\hline 5/21/09 20:00 & 9.697 & 4.942 & 5.672 & 17.094 & 6.731 & 13.603 & 5.217 & 19.213 & 20.658 & 35.081 \\
\hline 5/22/09 0:00 & 9.737 & 4.958 & 5.714 & 17.012 & 6.742 & 13.618 & 5.250 & 19.270 & 20.514 & 35.069 \\
\hline $5 / 22 / 094: 00$ & 9.763 & 4.968 & 5.752 & 16.993 & 6.745 & 13.625 & 5.271 & 19.263 & 20.456 & 35.057 \\
\hline 5/22/09 8:00 & 9.799 & 4.977 & 5.787 & 16.972 & 6.759 & 13.644 & 5.304 & 19.286 & 20.426 & 35.048 \\
\hline $5 / 22 / 09$ 12:00 & 9.813 & 4.984 & 5.822 & 17.175 & 6.763 & 13.621 & 5.325 & 19.321 & 20.577 & 35.036 \\
\hline 5/22/09 16:00 & 9.815 & 4.991 & 5.857 & 17.336 & 6.749 & 13.581 & 5.347 & 19.270 & 20.704 & 35.024 \\
\hline 5/22/09 20:00 & 9.834 & 5.008 & 5.897 & 17.280 & 6.764 & 13.567 & 5.379 & 19.258 & 20.639 & 35.013 \\
\hline 5/23/09 0:00 & 9.865 & 5.022 & 5.927 & 17.177 & 6.771 & 13.587 & 5.410 & 19.281 & 20.494 & 35.003 \\
\hline 5/23/09 4:00 & 9.884 & 5.026 & 5.960 & 17.161 & 6.768 & 13.582 & 5.426 & 19.350 & 20.440 & 34.991 \\
\hline 5/23/09 8:00 & 9.912 & 5.036 & 5.995 & 17.136 & 6.780 & 13.604 & 5.457 & 19.331 & 20.394 & 34.980 \\
\hline $5 / 23 / 09$ 12:00 & 9.933 & 5.040 & 6.028 & 17.289 & 6.787 & 13.590 & 5.488 & 19.345 & 20.510 & 34.970 \\
\hline 5/23/09 16:00 & 9.931 & 5.047 & 6.063 & 17.471 & 6.799 & 13.538 & 5.513 & 19.312 & 20.644 & 34.958 \\
\hline 5/23/09 20:00 & 9.959 & 5.062 & 6.098 & 17.285 & 6.806 & 13.562 & 5.560 & 19.321 & 20.497 & 34.942 \\
\hline 5/24/09 0:00 & 9.990 & 5.071 & 6.131 & 17.184 & 6.822 & 13.570 & 5.584 & 19.357 & 20.348 & 34.935 \\
\hline $5 / 24 / 094: 00$ & 10.006 & 5.069 & 6.157 & 17.189 & 6.820 & 13.569 & 5.598 & 19.376 & 20.315 & 34.923 \\
\hline 5/24/09 8:00 & 10.037 & 5.068 & 6.187 & 17.168 & 6.832 & 13.594 & 5.626 & 19.441 & 20.272 & 34.916 \\
\hline $5 / 24 / 0912: 00$ & 10.049 & 5.066 & 6.213 & 17.350 & 6.839 & 13.580 & 5.650 & 19.423 & 20.347 & 34.904 \\
\hline $5 / 24 / 09$ 16:00 & 10.049 & 5.066 & 6.243 & 17.452 & 6.851 & 13.535 & 5.673 & 19.420 & 20.331 & 34.892 \\
\hline $5 / 24 / 09$ 20:00 & 10.058 & 5.073 & 6.274 & 17.385 & 6.860 & 13.533 & 5.699 & 19.451 & 20.318 & 34.881 \\
\hline $5 / 25 / 090: 00$ & 10.079 & 5.080 & 6.304 & 17.299 & 6.872 & 13.548 & 5.722 & 19.472 & 20.302 & 34.871 \\
\hline $5 / 25 / 094: 00$ & 10.082 & 5.078 & 6.332 & 17.296 & 6.865 & 13.524 & 5.725 & 19.470 & 20.289 & 34.859 \\
\hline $5 / 25 / 098: 00$ & 10.096 & 5.078 & 6.349 & 17.287 & 6.865 & 13.541 & 5.751 & 19.470 & 20.276 & 34.852 \\
\hline $5 / 25 / 0912: 00$ & 10.100 & 5.078 & 6.372 & 17.341 & 6.874 & 13.523 & 5.758 & 19.493 & 20.262 & 34.841 \\
\hline $5 / 25 / 09$ 16:00 & 10.082 & 5.076 & 6.391 & 17.515 & 6.863 & 13.466 & 5.762 & 21.019 & 20.249 & 34.829 \\
\hline 5/25/09 20:00 & 10.091 & 5.080 & 6.412 & 17.401 & 6.867 & 13.481 & 5.791 & 19.912 & 20.232 & 34.819 \\
\hline 5/26/09 0:00 & 10.103 & 5.087 & 6.435 & 17.369 & 6.870 & 13.479 & 5.800 & 19.648 & 20.219 & 34.810 \\
\hline $5 / 26 / 094: 00$ & 10.077 & 5.026 & 6.421 & 17.376 & 6.823 & 13.219 & 5.727 & 19.573 & 20.206 & 34.796 \\
\hline 5/26/09 8:00 & 9.568 & 4.224 & 5.911 & 17.133 & 6.726 & 12.538 & 5.464 & 19.575 & 20.192 & 34.784 \\
\hline $5 / 26 / 0912: 00$ & 8.854 & 3.627 & 5.321 & 16.886 & 6.735 & 12.539 & 5.452 & 19.439 & 20.177 & 34.772 \\
\hline $5 / 26 / 09$ 16:00 & 8.936 & 3.480 & 5.148 & 16.786 & 6.745 & 12.680 & 5.455 & 19.373 & 20.161 & 34.763 \\
\hline $5 / 26 / 0920: 00$ & 9.101 & 3.503 & 5.104 & 16.684 & 6.768 & 12.782 & 5.471 & 19.368 & 20.145 & 34.751 \\
\hline $5 / 27 / 090: 00$ & 9.228 & 3.464 & 5.076 & 16.628 & 6.780 & 12.792 & 5.483 & 19.336 & 20.129 & 34.739 \\
\hline $5 / 27 / 094: 00$ & 9.323 & 3.431 & 5.071 & 16.574 & 6.790 & 12.830 & 5.488 & 19.343 & 20.114 & 34.725 \\
\hline 5/27/09 8:00 & 9.415 & 3.466 & 5.085 & 16.541 & 6.801 & 12.887 & 5.518 & 19.378 & 20.097 & 34.718 \\
\hline $5 / 27 / 09$ 12:00 & 9.483 & 3.534 & 5.106 & 16.597 & 6.806 & 12.902 & 5.530 & 19.373 & 20.082 & 34.706 \\
\hline $5 / 27 / 09$ 16:00 & 9.532 & 3.627 & 5.125 & 16.665 & 6.806 & 12.915 & 5.546 & 19.392 & 20.068 & 34.697 \\
\hline 5/27/09 20:00 & 9.584 & 3.728 & 5.148 & 16.665 & 6.811 & 12.944 & 5.574 & 19.394 & 20.053 & 34.685 \\
\hline 5/28/09 0:00 & 9.636 & 3.824 & 5.179 & 16.672 & 6.823 & 12.969 & 5.598 & 19.425 & 20.040 & 34.676 \\
\hline 5/28/09 4:00 & 9.686 & 3.908 & 5.209 & 16.681 & 6.830 & 12.992 & 5.624 & 19.455 & 20.023 & 34.664 \\
\hline 5/28/09 8:00 & 9.740 & 3.985 & 5.244 & 16.677 & 6.841 & 13.037 & 5.661 & 19.498 & 20.010 & 34.655 \\
\hline $5 / 28 / 09$ 12:00 & 9.789 & 4.063 & 5.281 & 16.732 & 6.851 & 13.061 & 5.692 & 19.545 & 19.997 & 34.645 \\
\hline $5 / 28 / 09$ 16:00 & 9.811 & 4.128 & 5.322 & 16.854 & 6.851 & 13.045 & 5.706 & 19.554 & 19.983 & 34.636 \\
\hline $5 / 28 / 0920: 00$ & 9.844 & 4.201 & 5.364 & 16.863 & 6.863 & 13.064 & 5.746 & 19.566 & 19.970 & 34.624 \\
\hline $5 / 29 / 090: 00$ & 9.891 & 4.266 & 5.404 & 16.795 & 6.867 & 13.088 & 5.776 & 19.611 & 19.958 & 34.614 \\
\hline 5/29/09 4:00 & 9.917 & 4.315 & 5.439 & 16.798 & 6.860 & 13.100 & 5.795 & 19.655 & 19.945 & 34.603 \\
\hline 5/29/09 8:00 & 9.957 & 4.364 & 5.474 & 16.798 & 6.865 & 13.136 & 5.830 & 19.674 & 19.932 & 34.593 \\
\hline 5/29/09 12:00 & 9.987 & 4.411 & 5.516 & 16.956 & 6.877 & 13.146 & 5.863 & 19.724 & 19.920 & 34.584 \\
\hline $5 / 29 / 09$ 16:00 & 10.006 & 4.460 & 5.563 & 17.147 & 6.877 & 13.131 & 5.901 & 19.752 & 19.909 & 34.574 \\
\hline 5/29/09 20:00 & 10.027 & 4.507 & 5.607 & 17.075 & 6.882 & 13.120 & 5.924 & 19.735 & 19.899 & 34.567 \\
\hline 5/30/09 0:00 & 10.042 & 4.545 & 5.638 & 16.986 & 6.865 & 13.118 & 5.929 & 19.815 & 19.887 & 34.558 \\
\hline 5/30/09 4:00 & 10.034 & 4.570 & 5.663 & 17.003 & 6.844 & 13.097 & 5.917 & 19.742 & 19.872 & 34.546 \\
\hline 5/30/09 8:00 & 10.032 & 4.594 & 5.687 & 17.049 & 6.837 & 13.090 & 5.892 & 19.726 & 19.863 & 34.537 \\
\hline 5/30/09 12:00 & 10.079 & 4.631 & 5.729 & 17.136 & 6.867 & 13.157 & 6.033 & 19.775 & 19.852 & 34.527 \\
\hline 5/30/09 16:00 & 10.115 & 4.673 & 5.780 & 17.273 & 6.893 & 13.166 & 6.072 & 20.041 & 19.841 & 34.520 \\
\hline 5/30/09 20:00 & 10.157 & 4.715 & 5.836 & 17.119 & 6.917 & 13.188 & 6.117 & 19.886 & 19.831 & 34.508 \\
\hline 5/31/09 0:00 & 10.207 & 4.753 & 5.883 & 16.965 & 6.931 & 13.231 & 6.155 & 20.081 & 19.821 & 34.501 \\
\hline 5/31/09 4:00 & 10.237 & 4.781 & 5.921 & 16.945 & 6.929 & 13.254 & 6.176 & 20.029 & 19.812 & 34.494 \\
\hline 5/31/09 8:00 & 10.259 & 4.804 & 5.954 & 16.970 & 6.919 & 13.262 & 6.195 & 20.046 & 19.803 & 34.483 \\
\hline 5/31/09 12:00 & 10.261 & 4.825 & 5.993 & 17.196 & 6.917 & 13.232 & 6.204 & 20.050 & 19.792 & 34.475 \\
\hline 5/31/09 16:00 & 10.239 & 4.844 & 6.021 & 17.380 & 6.912 & 13.170 & 6.211 & 19.999 & 19.781 & 34.466 \\
\hline 5/31/09 20:00 & 10.228 & 4.865 & 6.054 & 17.376 & 6.924 & 13.143 & 6.223 & 20.001 & 19.772 & 34.457 \\
\hline 6/1/09 0:00 & 10.249 & 4.889 & 6.085 & 17.271 & 6.917 & 13.179 & 6.251 & 19.952 & 19.761 & 34.450 \\
\hline 6/1/09 4:00 & 10.289 & 4.912 & 6.117 & 17.177 & 6.924 & 13.252 & 6.296 & 20.008 & 19.754 & 34.438 \\
\hline 6/1/09 8:00 & 10.329 & 4.933 & 6.159 & 17.140 & 6.938 & 13.280 & 6.324 & 20.196 & 19.746 & 34.433 \\
\hline
\end{tabular}


Water Level (ft below reference point)

\begin{tabular}{|c|c|c|c|c|c|c|c|c|c|c|}
\hline Date and Time & McPike & Potter & Hughes & Barr & Burks & MoDOT & Clizer "Old" & Morgan & SB24 & SB38 \\
\hline 6/1/09 12:00 & 10.353 & 4.949 & 6.192 & 17.292 & 6.945 & 13.298 & 6.361 & 20.142 & 19.738 & 34.424 \\
\hline 6/1/09 16:00 & 10.346 & 4.961 & 6.237 & 17.429 & 6.959 & 13.222 & 6.368 & 20.154 & 19.728 & 34.412 \\
\hline 6/1/09 20:00 & 10.407 & 4.977 & 6.255 & 17.112 & 6.950 & 13.324 & 6.448 & 20.208 & 19.722 & 34.402 \\
\hline 6/2/09 0:00 & 10.454 & 4.940 & 6.248 & 17.066 & 6.983 & 13.369 & 6.486 & 20.314 & 19.716 & 34.398 \\
\hline $6 / 2 / 094: 00$ & 10.454 & 4.919 & 6.281 & 17.131 & 6.966 & 13.341 & 6.481 & 20.363 & 19.707 & 34.388 \\
\hline $6 / 2 / 098: 00$ & 10.478 & 4.914 & 6.305 & 17.108 & 6.964 & 13.358 & 6.507 & 20.389 & 19.701 & 34.379 \\
\hline $6 / 2 / 09$ 12:00 & 10.501 & 4.912 & 6.330 & 17.164 & 6.962 & 13.394 & 6.531 & 20.450 & 19.694 & 34.372 \\
\hline 6/2/09 16:00 & 10.520 & 4.914 & 6.351 & 17.217 & 6.962 & 13.408 & 6.556 & 20.511 & 19.688 & 34.365 \\
\hline 6/2/09 20:00 & 10.544 & 4.921 & 6.379 & 17.157 & 6.966 & 13.437 & 6.585 & 20.539 & 19.682 & 34.355 \\
\hline 6/3/09 0:00 & 10.563 & 4.924 & 6.408 & 17.180 & 6.959 & 13.451 & 6.603 & 20.575 & 19.675 & 34.348 \\
\hline 6/3/09 4:00 & 10.579 & 4.931 & 6.436 & 17.173 & 6.957 & 13.468 & 6.620 & 20.648 & 19.671 & 34.341 \\
\hline 6/3/09 8:00 & 10.605 & 4.938 & 6.464 & 17.203 & 6.957 & 13.513 & 6.657 & 20.659 & 19.665 & 34.334 \\
\hline 6/3/09 12:00 & 10.617 & 4.945 & 6.492 & 17.355 & 6.964 & 13.499 & 6.674 & 20.716 & 19.660 & 34.327 \\
\hline 6/3/09 16:00 & 10.612 & 4.954 & 6.522 & 17.536 & 6.969 & 13.473 & 6.690 & 20.697 & 19.654 & 34.322 \\
\hline 6/3/09 20:00 & 10.619 & 4.968 & 6.551 & 17.462 & 6.974 & 13.474 & 6.718 & 20.699 & 19.650 & 34.313 \\
\hline 6/4/09 0:00 & 10.647 & 4.982 & 6.581 & 17.364 & 6.978 & 13.512 & 6.751 & 20.768 & 19.643 & 34.306 \\
\hline 6/4/09 4:00 & 10.650 & 4.989 & 6.600 & 17.378 & 6.962 & 13.500 & 6.756 & 20.758 & 19.640 & 34.299 \\
\hline $6 / 4 / 09$ 8:00 & 10.659 & 4.996 & 6.621 & 17.387 & 6.955 & 13.515 & 6.772 & 20.784 & 19.635 & 34.294 \\
\hline 6/4/09 12:00 & 10.673 & 5.006 & 6.649 & 17.462 & 6.969 & 13.535 & 6.801 & 20.902 & 19.631 & 34.287 \\
\hline 6/4/09 16:00 & 10.669 & 5.015 & 6.679 & 17.657 & 6.976 & 13.502 & 6.812 & 20.824 & 19.625 & 34.280 \\
\hline 6/4/09 20:00 & 10.666 & 5.027 & 6.705 & 17.606 & 6.983 & 13.482 & 6.831 & 20.805 & 19.621 & 34.273 \\
\hline 6/5/09 0:00 & 10.683 & 5.038 & 6.731 & 17.501 & 6.983 & 13.513 & 6.855 & 20.819 & 19.617 & 34.268 \\
\hline 6/5/09 4:00 & 10.688 & 5.043 & 6.750 & 17.497 & 6.959 & 13.520 & 6.862 & 20.831 & 19.613 & 34.261 \\
\hline 6/5/09 8:00 & 10.704 & 5.052 & 6.768 & 17.469 & 6.957 & 13.552 & 6.885 & 20.892 & 19.608 & 34.254 \\
\hline 6/5/09 12:00 & 10.711 & 5.062 & 6.792 & 17.611 & 6.974 & 13.555 & 6.906 & 20.906 & 19.605 & 34.249 \\
\hline 6/5/09 16:00 & 10.709 & 5.071 & 6.820 & 17.788 & 6.992 & 13.532 & 6.925 & 21.144 & 19.600 & 34.242 \\
\hline 6/5/09 20:00 & 10.709 & 5.083 & 6.843 & 17.704 & 7.000 & 13.516 & 6.946 & 20.918 & 19.596 & 34.238 \\
\hline 6/6/09 0:00 & 10.728 & 5.095 & 6.871 & 17.597 & 7.007 & 13.552 & 6.972 & 20.925 & 19.593 & 34.231 \\
\hline 6/6/09 4:00 & 10.706 & 5.095 & 6.883 & 17.620 & 6.988 & 13.496 & 6.942 & 20.913 & 19.588 & 34.226 \\
\hline 6/6/09 8:00 & 10.711 & 5.095 & 6.895 & 17.574 & 6.978 & 13.502 & 6.958 & 20.984 & 19.584 & 34.219 \\
\hline 6/6/09 12:00 & 10.690 & 5.090 & 6.892 & 17.697 & 6.974 & 13.474 & 6.953 & 20.892 & 19.581 & 34.214 \\
\hline 6/6/09 16:00 & 10.685 & 5.095 & 6.909 & 17.841 & 7.009 & 13.476 & 6.989 & 20.852 & 19.576 & 34.207 \\
\hline 6/6/09 20:00 & 10.709 & 5.102 & 6.937 & 17.692 & 7.054 & 13.508 & 7.033 & 20.873 & 19.570 & 34.202 \\
\hline 6/7/09 0:00 & 10.744 & 5.113 & 6.963 & 17.576 & 7.058 & 13.586 & 7.085 & 20.927 & 19.568 & 34.195 \\
\hline 6/7/09 4:00 & 10.761 & 5.118 & 6.974 & 17.464 & 7.063 & 13.582 & 7.094 & 20.993 & 19.564 & 34.188 \\
\hline $6 / 7 / 09$ 8:00 & 10.779 & 5.111 & 6.970 & 17.417 & 7.063 & 13.555 & 7.094 & 21.057 & 19.563 & 34.181 \\
\hline $6 / 7 / 09$ 12:00 & 10.789 & 5.088 & 6.953 & 17.511 & 7.061 & 13.593 & 7.113 & 21.137 & 19.560 & 34.179 \\
\hline 6/7/09 16:00 & 10.794 & 5.078 & 6.963 & 17.613 & 7.075 & 13.600 & 7.141 & 21.078 & 19.556 & 34.172 \\
\hline 6/7/09 20:00 & 10.787 & 5.046 & 6.965 & 17.571 & 7.075 & 13.587 & 7.144 & 21.139 & 19.555 & 34.165 \\
\hline 6/8/09 0:00 & 10.784 & 4.917 & 6.937 & 17.485 & 7.077 & 13.583 & 7.148 & 21.116 & 19.550 & 34.160 \\
\hline 6/8/09 4:00 & 10.308 & 4.543 & 6.813 & 17.406 & 7.040 & 13.449 & 7.106 & 21.151 & 19.549 & 34.153 \\
\hline 6/8/09 8:00 & 10.315 & 4.257 & 6.691 & 17.229 & 7.047 & 13.615 & 7.179 & 21.221 & 19.548 & 34.148 \\
\hline 6/8/09 12:00 & 10.457 & 4.185 & 6.651 & 17.182 & 7.044 & 13.705 & 7.221 & 21.311 & 19.543 & 34.141 \\
\hline 6/8/09 16:00 & 10.553 & 4.210 & 6.644 & 17.336 & 7.188 & 13.736 & 7.240 & 21.370 & 19.544 & 34.136 \\
\hline 6/8/09 20:00 & 10.629 & 4.278 & 6.654 & 17.308 & 7.101 & 13.773 & 7.273 & 21.407 & 19.539 & 34.134 \\
\hline 6/9/09 0:00 & 10.690 & 4.351 & 6.672 & 17.278 & 7.061 & 13.815 & 7.299 & 21.454 & 19.540 & 34.127 \\
\hline 6/9/09 4:00 & 10.728 & 4.414 & 6.689 & 17.315 & 7.030 & 13.818 & 7.301 & 21.489 & 19.539 & 34.125 \\
\hline 6/9/09 8:00 & 10.761 & 4.475 & 6.705 & 17.345 & 7.011 & 13.850 & 7.318 & 21.515 & 19.537 & 34.117 \\
\hline 6/9/09 12:00 & 10.780 & 4.524 & 6.717 & 17.382 & 6.993 & 13.822 & 7.315 & 21.536 & 19.538 & 34.110 \\
\hline 6/9/09 16:00 & 10.452 & 4.241 & 6.574 & 17.487 & 6.934 & 13.553 & 7.231 & 21.464 & 19.536 & 34.110 \\
\hline 6/9/09 20:00 & 10.355 & 3.991 & 6.455 & 17.483 & 6.917 & 13.602 & 7.221 & 21.414 & 19.534 & 34.101 \\
\hline 6/10/09 0:00 & 10.433 & 3.927 & 6.410 & 17.403 & 6.919 & 13.666 & 7.238 & 21.435 & 19.530 & 34.096 \\
\hline 6/10/09 4:00 & 10.487 & 3.932 & 6.403 & 17.431 & 6.901 & 13.683 & 7.238 & 21.419 & 19.528 & 34.092 \\
\hline 6/10/09 8:00 & 10.553 & 3.979 & 6.408 & 17.389 & 6.908 & 13.745 & 7.268 & 21.447 & 19.529 & 34.089 \\
\hline 6/10/09 12:00 & 10.589 & 4.037 & 6.420 & 17.473 & 6.908 & 13.738 & 7.275 & 21.454 & 19.528 & 34.082 \\
\hline 6/10/09 16:00 & 10.619 & 4.112 & 6.438 & 17.515 & 6.917 & 13.765 & 7.299 & 21.449 & 19.525 & 34.080 \\
\hline 6/10/09 20:00 & 10.631 & 4.180 & 6.455 & 17.529 & 6.912 & 13.745 & 7.296 & 21.435 & 19.524 & 34.073 \\
\hline 6/11/09 0:00 & 10.664 & 4.248 & 6.473 & 17.473 & 6.919 & 13.776 & 7.320 & 21.480 & 19.523 & 34.066 \\
\hline $6 / 11 / 094: 00$ & 9.962 & 3.846 & 6.071 & 17.348 & 6.853 & 13.207 & 7.162 & 21.438 & 19.520 & 34.059 \\
\hline 6/11/09 8:00 & 9.712 & 3.523 & 5.835 & 17.156 & 6.863 & 13.348 & 7.186 & 21.431 & 19.519 & 34.056 \\
\hline 6/11/09 12:00 & 9.886 & 3.499 & 5.762 & 17.122 & 6.877 & 13.497 & 7.310 & 21.504 & 19.517 & 34.051 \\
\hline 6/11/09 16:00 & 10.042 & 3.605 & 5.755 & 17.143 & 6.896 & 13.558 & 7.348 & 21.440 & 19.515 & 34.047 \\
\hline 6/11/09 20:00 & 10.157 & 3.747 & 5.774 & 17.115 & 6.908 & 13.605 & 7.263 & 21.468 & 19.514 & 34.042 \\
\hline 6/12/09 0:00 & 10.271 & 3.876 & 5.802 & 16.998 & 6.910 & 13.683 & 7.224 & 21.499 & 19.512 & 34.037 \\
\hline
\end{tabular}


Water Level (ft below reference point)

\begin{tabular}{|c|c|c|c|c|c|c|c|c|c|c|}
\hline Date and Time & McPike & Potter & Hughes & Barr & Burks & MoDOT & Clizer "Old" & Morgan & SB24 & SB38 \\
\hline 6/12/09 4:00 & 10.339 & 3.979 & 5.818 & 17.000 & 6.896 & 13.704 & 7.254 & 21.492 & 19.510 & 34.033 \\
\hline 6/12/09 8:00 & 10.393 & 4.072 & 5.840 & 17.017 & 6.884 & 13.736 & 7.303 & 21.496 & 19.509 & 34.028 \\
\hline 6/12/09 12:00 & 10.426 & 4.159 & 5.860 & 17.145 & 6.882 & 13.739 & 7.470 & 21.518 & 19.506 & 34.023 \\
\hline 6/12/09 16:00 & 10.457 & 4.243 & 5.891 & 17.217 & 6.901 & 13.732 & 7.512 & 21.464 & 19.503 & 34.018 \\
\hline 6/12/09 20:00 & 10.492 & 4.320 & 5.923 & 17.196 & 6.910 & 13.759 & 7.435 & 21.461 & 19.504 & 34.016 \\
\hline 6/13/09 0:00 & 10.551 & 4.393 & 5.959 & 17.096 & 6.922 & 13.837 & 7.383 & 21.541 & 19.499 & 34.011 \\
\hline 6/13/09 4:00 & 10.596 & 4.449 & 5.992 & 17.075 & 6.919 & 13.880 & 7.386 & 21.555 & 19.502 & 34.007 \\
\hline 6/13/09 8:00 & 10.662 & 4.505 & 6.029 & 16.982 & 6.929 & 13.987 & 7.336 & 21.661 & 19.500 & 34.004 \\
\hline 6/13/09 12:00 & 10.711 & 4.554 & 6.066 & 17.045 & 6.931 & 14.037 & 7.442 & 21.734 & 19.501 & 34.000 \\
\hline 6/13/09 16:00 & 10.733 & 4.592 & 6.097 & 17.159 & 6.927 & 14.029 & 7.524 & 21.760 & 19.502 & 33.995 \\
\hline 6/13/09 20:00 & 10.742 & 4.627 & 6.120 & 17.238 & 6.917 & 14.013 & 7.484 & 21.748 & 19.500 & 33.993 \\
\hline 6/14/09 0:00 & 10.773 & 4.660 & 6.151 & 17.180 & 6.917 & 14.058 & 7.454 & 21.779 & 19.501 & 33.988 \\
\hline 6/14/09 4:00 & 10.777 & 4.683 & 6.169 & 17.219 & 6.898 & 14.047 & 7.496 & 21.781 & 19.502 & 33.986 \\
\hline 6/14/09 8:00 & 10.817 & 4.713 & 6.200 & 17.168 & 6.910 & 14.121 & 7.520 & 21.837 & 19.502 & 33.981 \\
\hline 6/14/09 12:00 & 10.824 & 4.735 & 6.219 & 17.285 & 6.889 & 14.128 & 7.707 & 21.833 & 19.500 & 33.978 \\
\hline 6/14/09 16:00 & 10.836 & 4.760 & 6.249 & 17.462 & 6.898 & 14.130 & 7.769 & 21.847 & 19.503 & 33.974 \\
\hline $6 / 14 / 0920: 00$ & 10.846 & 4.781 & 6.282 & 17.427 & 6.901 & 14.123 & 7.670 & 21.823 & 19.501 & 33.969 \\
\hline 6/15/09 0:00 & 10.857 & 4.805 & 6.305 & 17.382 & 6.896 & 14.135 & 7.630 & 21.840 & 19.505 & 33.967 \\
\hline 6/15/09 4:00 & 4.307 & 3.537 & 5.423 & 16.695 & 3.910 & 12.325 & 3.181 & 21.447 & 19.505 & 33.953 \\
\hline 6/15/09 8:00 & 4.159 & 3.060 & 4.744 & 16.080 & 4.351 & 11.655 & 1.833 & 19.491 & 19.485 & 33.945 \\
\hline 6/15/09 12:00 & 4.437 & 2.887 & 4.417 & 15.717 & 4.835 & 11.544 & 1.783 & 18.357 & 19.455 & 33.941 \\
\hline 6/15/09 16:00 & 4.762 & 2.954 & 4.295 & 15.640 & 5.191 & 11.458 & 1.922 & 17.725 & 19.422 & 33.936 \\
\hline 6/15/09 20:00 & 5.069 & 3.053 & 4.265 & 15.549 & 5.464 & 11.376 & 1.976 & 17.304 & 19.393 & 33.929 \\
\hline 6/16/09 0:00 & 3.530 & 2.274 & 3.834 & 15.043 & 5.035 & 10.749 & 0.475 & 16.871 & 19.366 & 33.915 \\
\hline 6/16/09 4:00 & 3.499 & 2.391 & 3.553 & 14.470 & 4.297 & 10.543 & -0.035 & 15.978 & 19.326 & 33.901 \\
\hline 6/16/09 8:00 & 3.793 & 2.510 & 3.537 & 14.184 & 4.736 & 10.467 & -0.047 & 15.458 & 19.282 & 33.889 \\
\hline 6/16/09 12:00 & 4.090 & 2.618 & 3.600 & 14.020 & 5.141 & 10.402 & 0.181 & 15.204 & 19.240 & 33.882 \\
\hline 6/16/09 16:00 & 4.376 & 2.737 & 3.679 & 14.163 & 5.438 & 10.306 & 0.430 & 15.042 & 19.199 & 33.868 \\
\hline 6/16/09 20:00 & 4.661 & 2.854 & 3.773 & 14.200 & 5.672 & 10.238 & 0.470 & 14.906 & 19.158 & 33.858 \\
\hline 6/17/09 0:00 & 4.911 & 2.924 & 3.853 & 14.232 & 5.828 & 10.187 & 0.590 & 14.880 & 19.115 & 33.844 \\
\hline $6 / 17 / 09$ 4:00 & 5.123 & 2.957 & 3.911 & 14.384 & 5.920 & 10.103 & 0.778 & 14.891 & 19.074 & 33.830 \\
\hline 6/17/09 8:00 & 5.337 & 2.985 & 3.965 & 14.491 & 6.000 & 10.051 & 1.003 & 14.819 & 19.038 & 33.818 \\
\hline 6/17/09 12:00 & 5.549 & 3.041 & 4.028 & 14.712 & 6.075 & 9.995 & 1.384 & 14.840 & 18.998 & 33.804 \\
\hline 6/17/09 16:00 & 5.330 & 3.008 & 4.038 & 14.843 & 6.085 & 9.753 & 1.017 & 14.903 & 18.961 & 33.783 \\
\hline 6/17/09 20:00 & 5.194 & 2.809 & 3.729 & 14.694 & 6.042 & 9.572 & 0.902 & 14.713 & 18.924 & 33.771 \\
\hline 6/18/09 0:00 & 5.191 & 2.800 & 3.708 & 14.731 & 5.988 & 9.490 & 0.959 & 14.724 & 18.885 & 33.757 \\
\hline 6/18/09 4:00 & 5.264 & 2.833 & 3.724 & 14.799 & 5.903 & 9.474 & 0.893 & 14.652 & 18.846 & 33.741 \\
\hline 6/18/09 8:00 & 5.377 & 2.875 & 3.775 & 14.813 & 5.877 & 9.449 & 1.032 & 14.640 & 18.808 & 33.726 \\
\hline 6/18/09 12:00 & 5.521 & 2.952 & 3.841 & 14.976 & 5.915 & 9.395 & 1.273 & 14.612 & 18.770 & 33.710 \\
\hline 6/18/09 16:00 & 5.684 & 3.067 & 3.921 & 15.157 & 5.990 & 9.332 & 1.438 & 14.569 & 18.735 & 33.696 \\
\hline 6/18/09 20:00 & 5.828 & 3.158 & 3.995 & 15.139 & 6.056 & 9.288 & 1.464 & 14.673 & 18.696 & 33.679 \\
\hline 6/19/09 0:00 & 5.964 & 3.193 & 4.063 & 15.078 & 6.099 & 9.286 & 1.473 & 14.645 & 18.664 & 33.665 \\
\hline 6/19/09 4:00 & 6.061 & 3.186 & 4.105 & 15.148 & 6.113 & 9.247 & 1.534 & 14.560 & 18.626 & 33.651 \\
\hline 6/19/09 8:00 & 6.188 & 3.191 & 4.159 & 15.134 & 6.160 & 9.268 & 1.621 & 14.605 & 18.592 & 33.635 \\
\hline 6/19/09 12:00 & 6.285 & 3.191 & 4.206 & 15.325 & 6.181 & 9.226 & 1.861 & 14.621 & 18.558 & 33.620 \\
\hline 6/19/09 16:00 & 6.405 & 3.203 & 4.265 & 15.339 & 6.233 & 9.237 & 1.910 & 14.630 & 18.525 & 33.604 \\
\hline 6/19/09 20:00 & 6.539 & 3.214 & 4.328 & 15.362 & 6.280 & 9.270 & 1.903 & 14.701 & 18.493 & 33.590 \\
\hline 6/20/09 0:00 & 6.645 & 3.207 & 4.379 & 15.355 & 6.302 & 9.282 & 1.917 & 14.757 & 18.464 & 33.576 \\
\hline 6/20/09 4:00 & 6.740 & 3.186 & 4.419 & 15.409 & 6.313 & 9.295 & 1.959 & 14.793 & 18.431 & 33.562 \\
\hline 6/20/09 8:00 & 6.834 & 3.170 & 4.461 & 15.439 & 6.332 & 9.322 & 2.098 & 14.847 & 18.401 & 33.545 \\
\hline 6/20/09 12:00 & 6.924 & 3.170 & 4.506 & 15.609 & 6.358 & 9.320 & 2.335 & 14.882 & 18.374 & 33.531 \\
\hline 6/20/09 16:00 & 7.006 & 3.184 & 4.562 & 15.679 & 6.391 & 9.307 & 2.375 & 15.054 & 18.344 & 33.514 \\
\hline 6/20/09 20:00 & 7.060 & 3.135 & 4.585 & 15.780 & 6.382 & 9.272 & 2.413 & 14.920 & 18.314 & 33.498 \\
\hline 6/21/09 0:00 & 7.133 & 3.102 & 4.613 & 15.789 & 6.391 & 9.296 & 2.441 & 14.955 & 18.286 & 33.484 \\
\hline $6 / 21 / 09$ 4:00 & 7.176 & 2.980 & 4.609 & 15.826 & 6.384 & 9.202 & 2.502 & 14.962 & 18.261 & 33.470 \\
\hline 6/21/09 8:00 & 7.228 & 2.828 & 4.550 & 15.831 & 6.398 & 9.222 & 2.594 & 14.985 & 18.234 & 33.458 \\
\hline 6/21/09 12:00 & 7.296 & 2.863 & 4.569 & 15.947 & 6.424 & 9.250 & 2.833 & 15.037 & 18.206 & 33.441 \\
\hline 6/21/09 16:00 & 7.372 & 2.983 & 4.630 & 16.045 & 6.467 & 9.270 & 2.953 & 15.049 & 18.179 & 33.425 \\
\hline 6/21/09 20:00 & 7.445 & 3.090 & 4.686 & 16.006 & 6.500 & 9.282 & 2.918 & 15.124 & 18.154 & 33.411 \\
\hline 6/22/09 0:00 & 7.525 & 3.151 & 4.733 & 15.938 & 6.502 & 9.344 & 2.880 & 15.131 & 18.128 & 33.397 \\
\hline $6 / 22 / 094: 00$ & 7.596 & 3.172 & NA & 15.924 & 6.505 & 9.385 & 2.913 & 15.197 & 18.103 & 33.383 \\
\hline 6/22/09 8:00 & 7.664 & 3.186 & & 15.929 & 6.507 & 9.433 & 3.007 & 15.298 & 18.080 & 33.368 \\
\hline 6/22/09 12:00 & 7.730 & 3.221 & & 16.064 & 6.531 & 9.452 & 3.289 & 15.289 & 18.055 & 33.354 \\
\hline $6 / 22 / 09$ 16:00 & 7.798 & 3.303 & & 16.236 & 6.566 & 9.472 & 3.423 & 15.326 & 18.033 & 33.340 \\
\hline
\end{tabular}


Water Level (ft below reference point)

\begin{tabular}{|c|c|c|c|c|c|c|c|c|c|c|}
\hline Date and Time & McPike & Potter & Hughes & Barr & Burks & MoDOT & Clizer "Old" & Morgan & SB24 & SB38 \\
\hline 6/22/09 20:00 & 7.876 & 3.390 & & 16.159 & 6.606 & 9.491 & 3.350 & 15.416 & 18.009 & 33.326 \\
\hline 6/23/09 0:00 & 7.944 & 3.444 & & 16.078 & 6.606 & 9.541 & 3.327 & 15.435 & 17.984 & 33.312 \\
\hline 6/23/09 4:00 & 7.999 & 3.462 & & 16.073 & 6.594 & 9.575 & 3.336 & 15.475 & 17.962 & 33.300 \\
\hline 6/23/09 8:00 & 8.062 & 3.458 & & 16.068 & 6.596 & 9.625 & 3.430 & 15.529 & 17.941 & 33.286 \\
\hline $6 / 23 / 09$ 12:00 & 8.126 & 3.469 & & 16.215 & 6.622 & 9.661 & 3.684 & 15.571 & 17.918 & 33.272 \\
\hline 6/23/09 16:00 & 8.178 & 3.539 & & 16.416 & 6.665 & 9.657 & 3.841 & 15.592 & 17.897 & 33.258 \\
\hline 6/23/09 20:00 & 8.263 & 3.659 & & 16.280 & 6.707 & 9.721 & 3.686 & 15.637 & 17.877 & 33.246 \\
\hline 6/24/09 0:00 & 8.331 & 3.750 & & 16.082 & 6.710 & 9.762 & 3.658 & 15.721 & 17.857 & 33.232 \\
\hline 6/24/09 4:00 & 8.352 & 3.795 & & 16.169 & 6.667 & 9.751 & 3.724 & 15.731 & 17.836 & 33.220 \\
\hline 6/24/09 8:00 & 8.406 & 3.827 & & 16.166 & 6.667 & 9.815 & 3.818 & 15.780 & 17.816 & 33.208 \\
\hline 6/24/09 12:00 & 8.453 & 3.853 & & 16.385 & 6.681 & 9.839 & 4.071 & 15.827 & 17.800 & 33.194 \\
\hline 6/24/09 16:00 & 8.484 & 3.909 & & 16.544 & 6.712 & 9.815 & 4.099 & 15.839 & 17.779 & 33.182 \\
\hline 6/24/09 20:00 & 8.517 & 3.977 & & 16.411 & 6.693 & 9.834 & 3.958 & 15.863 & 17.762 & 33.168 \\
\hline 6/25/09 0:00 & 8.543 & 4.010 & & 16.369 & 6.686 & 9.853 & 3.984 & 15.900 & 17.741 & 33.156 \\
\hline 6/25/09 4:00 & 8.578 & 4.024 & & 16.395 & 6.674 & 9.887 & 4.024 & 15.935 & 17.725 & 33.145 \\
\hline 6/25/09 8:00 & 8.378 & 3.743 & & 16.334 & 6.644 & 9.774 & 4.116 & 16.020 & 17.707 & 33.130 \\
\hline 6/25/09 12:00 & 8.390 & 3.490 & & 16.499 & 6.658 & 9.800 & 4.308 & 15.994 & 17.689 & 33.116 \\
\hline 6/25/09 16:00 & 8.484 & 3.502 & & 16.644 & 6.707 & 9.821 & 4.424 & 16.008 & 17.671 & 33.107 \\
\hline 6/25/09 20:00 & 8.552 & 3.584 & & 16.609 & 6.743 & 9.823 & 4.344 & 16.027 & 17.651 & 33.093 \\
\hline 6/26/09 0:00 & 8.614 & 3.654 & & 16.506 & 6.738 & 9.869 & 4.283 & 16.069 & 17.634 & 33.081 \\
\hline $6 / 26 / 094: 00$ & 8.654 & 3.708 & & 16.483 & 6.722 & 9.903 & 4.290 & 16.102 & 17.619 & 33.067 \\
\hline 6/26/09 8:00 & 8.701 & 3.750 & & 16.457 & 6.717 & 9.945 & 4.360 & 16.149 & 17.601 & 33.055 \\
\hline 6/26/09 12:00 & 8.746 & 3.795 & & 16.600 & 6.738 & 9.973 & 4.579 & 16.189 & 17.582 & 33.043 \\
\hline 6/26/09 16:00 & 8.774 & 3.874 & & 16.746 & 6.773 & 9.968 & 4.663 & 16.281 & 17.567 & 33.032 \\
\hline 6/26/09 20:00 & 8.805 & 3.970 & & 16.656 & 6.804 & 9.968 & 4.607 & 16.227 & 17.550 & 33.020 \\
\hline 6/27/09 0:00 & 8.826 & 4.064 & & 16.602 & 6.781 & 9.986 & 4.593 & 16.251 & 17.535 & 33.006 \\
\hline 6/27/09 4:00 & 8.842 & 4.138 & & 16.593 & 6.762 & 9.998 & 4.609 & 16.281 & 17.520 & 32.996 \\
\hline $6 / 27 / 09$ 8:00 & 8.873 & 4.197 & & 16.597 & 6.755 & 10.039 & 4.680 & 16.319 & 17.503 & 32.984 \\
\hline 6/27/09 12:00 & 8.906 & 4.241 & & 16.732 & 6.773 & 10.064 & 4.889 & 16.354 & 17.490 & 32.973 \\
\hline 6/27/09 16:00 & 8.972 & 4.307 & & 16.758 & 6.832 & 10.120 & 4.729 & 16.406 & 17.475 & 32.961 \\
\hline 6/27/09 20:00 & 9.024 & 4.368 & & 16.600 & 6.832 & 10.151 & 4.762 & 16.460 & 17.458 & 32.951 \\
\hline $6 / 28 / 09$ 0:00 & 9.078 & 4.424 & & 16.513 & 6.828 & 10.221 & 4.804 & 16.535 & 17.445 & 32.942 \\
\hline $6 / 28 / 094: 00$ & 9.113 & 4.471 & & 16.551 & 6.809 & 10.259 & 4.837 & 16.575 & 17.431 & 32.928 \\
\hline 6/28/09 8:00 & 9.151 & 4.508 & & 16.534 & 6.802 & 10.292 & 4.875 & 16.629 & 17.418 & 32.918 \\
\hline 6/28/09 12:00 & 9.168 & 4.539 & & 16.756 & 6.804 & 10.291 & 4.917 & 16.761 & 17.405 & 32.907 \\
\hline 6/28/09 16:00 & 9.168 & 4.564 & & 16.970 & 6.830 & 10.266 & 4.964 & 16.653 & 17.391 & 32.897 \\
\hline 6/28/09 20:00 & 9.163 & 4.590 & & 16.923 & 6.847 & 10.235 & 4.992 & 16.683 & 17.378 & 32.885 \\
\hline 6/29/09 0:00 & 9.175 & 4.614 & & 16.823 & 6.832 & 10.258 & 5.011 & 16.667 & 17.365 & 32.876 \\
\hline 6/29/09 4:00 & 9.172 & 4.635 & & 16.805 & 6.809 & 10.272 & 5.023 & 16.697 & 17.353 & 32.864 \\
\hline 6/29/09 8:00 & 9.201 & 4.658 & & 16.774 & 6.814 & 10.313 & 5.060 & 16.817 & 17.342 & 32.855 \\
\hline 6/29/09 12:00 & 9.220 & 4.679 & & 16.968 & 6.835 & 10.323 & 5.114 & 16.768 & 17.329 & 32.843 \\
\hline 6/29/09 16:00 & 9.243 & 4.705 & & 17.115 & 6.877 & 10.340 & 5.187 & 16.803 & 17.315 & 32.831 \\
\hline 6/29/09 20:00 & 9.264 & 4.728 & & 17.003 & 6.906 & 10.338 & 5.234 & 16.808 & 17.304 & 32.822 \\
\hline 6/30/09 0:00 & 9.288 & 4.752 & & 16.896 & 6.887 & 10.375 & 5.262 & 16.855 & 17.293 & 32.812 \\
\hline 6/30/09 4:00 & 9.302 & 4.770 & & 16.865 & 6.868 & 10.389 & 5.276 & 16.923 & 17.282 & 32.803 \\
\hline 6/30/09 8:00 & 9.330 & 4.789 & & 16.821 & 6.861 & 10.432 & 5.314 & 16.951 & 17.271 & 32.791 \\
\hline 6/30/09 12:00 & 9.366 & 4.808 & & 16.968 & 6.877 & 10.469 & 5.363 & 17.001 & 17.262 & 32.784 \\
\hline 6/30/09 16:00 & 9.387 & 4.826 & & 17.133 & 6.908 & 10.478 & 5.417 & 17.022 & 17.251 & 32.772 \\
\hline 6/30/09 20:00 & 9.415 & 4.850 & & 16.968 & 6.936 & 10.497 & 5.467 & 17.055 & 17.243 & 32.765 \\
\hline 7/1/09 0:00 & 9.444 & 4.871 & & 16.886 & 6.913 & 10.536 & 5.497 & 17.146 & 17.231 & 32.756 \\
\hline 7/1/09 4:00 & 9.462 & 4.887 & & 16.868 & 6.887 & 10.560 & 5.518 & 17.137 & 17.223 & 32.742 \\
\hline 7/1/09 8:00 & 9.498 & 4.906 & & 16.842 & 6.884 & 10.615 & 5.553 & 17.212 & 17.213 & 32.737 \\
\hline 7/1/09 12:00 & 9.524 & 4.922 & & 17.042 & 6.891 & 10.637 & 5.591 & 17.323 & 17.205 & 32.728 \\
\hline 7/1/09 16:00 & 9.540 & 4.936 & & 17.210 & 6.922 & 10.642 & 5.636 & 17.273 & 17.197 & 32.718 \\
\hline 7/1/09 20:00 & 9.571 & 4.955 & & 17.112 & 6.955 & 10.669 & 5.687 & 17.320 & 17.187 & 32.706 \\
\hline 7/2/09 0:00 & 9.604 & 4.971 & & 17.007 & 6.943 & 10.715 & 5.723 & 17.436 & 17.179 & 32.699 \\
\hline $7 / 2 / 094: 00$ & 9.625 & 4.983 & & 16.991 & 6.920 & 10.736 & 5.744 & 17.419 & 17.173 & 32.688 \\
\hline 7/2/09 8:00 & 9.670 & 4.997 & & 16.951 & 6.920 & 10.803 & 5.793 & 17.513 & 17.163 & 32.681 \\
\hline $7 / 2 / 09$ 12:00 & 9.696 & 5.009 & & 17.098 & 6.922 & 10.825 & 5.835 & 17.544 & 17.157 & 32.673 \\
\hline 7/2/09 16:00 & 9.717 & 5.021 & & 17.243 & 6.972 & 10.837 & 5.894 & 17.690 & 17.151 & 32.664 \\
\hline $7 / 2 / 09$ 20:00 & 9.736 & 5.032 & & 17.212 & 6.997 & 10.847 & 5.943 & 17.666 & 17.144 & 32.657 \\
\hline 7/3/09 0:00 & 9.767 & 5.046 & & 17.119 & 6.990 & 10.893 & 5.976 & 17.666 & 17.138 & 32.650 \\
\hline 7/3/09 4:00 & 9.778 & 5.053 & & 17.094 & 6.967 & 10.914 & 5.995 & 17.734 & 17.131 & 32.641 \\
\hline 7/3/09 8:00 & 9.807 & 5.060 & & 17.096 & 6.957 & 10.955 & 6.026 & 17.781 & 17.125 & 32.631 \\
\hline
\end{tabular}


Water Level (ft below reference point)

\begin{tabular}{|c|c|c|c|c|c|c|c|c|c|c|}
\hline Date and Time & McPike & Potter & Hughes & Barr & Burks & MoDOT & Clizer "Old" & Morgan & SB24 & SB38 \\
\hline 7/3/09 12:00 & 9.800 & 5.063 & & 17.135 & 6.943 & 10.935 & 6.021 & 17.817 & 17.119 & 32.624 \\
\hline $7 / 3 / 09$ 16:00 & 9.778 & 5.056 & & 17.217 & 6.901 & 10.883 & 6.026 & 17.805 & 17.112 & 32.615 \\
\hline 7/3/09 20:00 & 9.750 & 5.049 & & 17.264 & 6.889 & 10.888 & 6.014 & 17.800 & 17.108 & 32.608 \\
\hline 7/4/09 0:00 & 9.731 & 4.927 & & 17.250 & 6.847 & 10.793 & 5.983 & 17.814 & 17.102 & 32.598 \\
\hline 7/4/09 4:00 & 9.679 & 4.389 & & 17.294 & 6.849 & 10.779 & 5.967 & 17.784 & 17.095 & 32.593 \\
\hline 7/4/09 8:00 & 9.672 & 4.225 & & 17.238 & 6.863 & 10.841 & 5.997 & 17.812 & 17.089 & 32.584 \\
\hline $7 / 4 / 09$ 12:00 & 9.670 & 4.199 & & 17.271 & 6.861 & 10.873 & 6.033 & 17.840 & 17.085 & 32.575 \\
\hline 7/4/09 16:00 & 9.686 & 4.228 & & 17.257 & 6.872 & 10.918 & 6.077 & 17.873 & 17.079 & 32.567 \\
\hline 7/4/09 20:00 & 9.717 & 4.274 & & 17.166 & 6.889 & 10.965 & 6.115 & 17.936 & 17.075 & 32.560 \\
\hline 7/5/09 0:00 & 9.764 & 4.330 & & 17.105 & 6.901 & 11.037 & 6.164 & 18.016 & 17.070 & 32.551 \\
\hline 7/5/09 4:00 & 9.783 & 4.382 & & 17.117 & 6.896 & 11.056 & 6.183 & 18.063 & 17.066 & 32.544 \\
\hline 7/5/09 8:00 & 9.814 & 4.433 & & 17.105 & 6.898 & 11.116 & 6.221 & 18.122 & 17.056 & 32.537 \\
\hline 7/5/09 12:00 & 9.837 & 4.480 & & 17.212 & 6.913 & 11.147 & 6.261 & 18.183 & 17.051 & 32.530 \\
\hline $7 / 5 / 09$ 16:00 & 9.842 & 4.520 & & 17.359 & 6.929 & 11.155 & 6.300 & 18.176 & 17.047 & 32.523 \\
\hline 7/5/09 20:00 & 9.847 & 4.555 & & 17.341 & 6.946 & 11.162 & 6.329 & 18.193 & 17.043 & 32.516 \\
\hline 7/6/09 0:00 & 9.868 & 4.590 & & 17.259 & 6.943 & 11.213 & 6.362 & 18.292 & 17.039 & 32.509 \\
\hline 7/6/09 4:00 & 9.877 & 4.621 & & 17.240 & 6.920 & 11.235 & 6.376 & 18.287 & 17.035 & 32.502 \\
\hline 7/6/09 8:00 & 9.894 & 4.651 & & 17.217 & 6.913 & 11.273 & 6.401 & 18.346 & 17.033 & 32.497 \\
\hline 7/6/09 12:00 & 9.906 & 4.679 & & 17.357 & 6.929 & 11.300 & 6.439 & 18.350 & 17.032 & 32.490 \\
\hline 7/6/09 16:00 & 9.906 & 4.705 & & 17.478 & 6.967 & 11.298 & 6.472 & 18.397 & 17.028 & 32.483 \\
\hline 7/6/09 20:00 & 9.913 & 4.726 & & 17.436 & 7.005 & 11.310 & 6.505 & 18.348 & 17.026 & 32.476 \\
\hline 7/7/09 0:00 & 9.934 & 4.752 & & 17.329 & 7.014 & 11.363 & 6.538 & 18.423 & 17.022 & 32.471 \\
\hline 7/7/09 4:00 & 9.939 & 4.770 & & 17.301 & 7.002 & 11.377 & 6.545 & 18.447 & 17.023 & 32.466 \\
\hline 7/7/09 8:00 & 9.948 & 4.791 & & 17.285 & 6.997 & 11.418 & 6.568 & 18.477 & 17.018 & 32.459 \\
\hline 7/7/09 12:00 & 9.957 & 4.813 & & 17.436 & 7.019 & 11.440 & 6.601 & 18.508 & 17.019 & 32.454 \\
\hline $7 / 7 / 09$ 16:00 & 9.972 & 4.831 & & 17.536 & 7.073 & 11.455 & 6.653 & 18.529 & 17.015 & 32.447 \\
\hline 7/7/09 20:00 & 9.990 & 4.855 & & 17.441 & 7.127 & 11.477 & 6.695 & 18.545 & 17.013 & 32.440 \\
\hline 7/8/09 0:00 & 10.014 & 4.876 & & 17.361 & 7.144 & 11.534 & 6.730 & 18.593 & 17.014 & 32.436 \\
\hline 7/8/09 4:00 & 10.054 & 4.897 & & 17.180 & 7.174 & 11.575 & 6.751 & 18.703 & 17.012 & 32.431 \\
\hline 7/8/09 8:00 & 10.045 & 4.911 & & 17.264 & 7.151 & 11.559 & 6.754 & 18.712 & 17.010 & 32.424 \\
\hline 7/8/09 12:00 & 10.056 & 4.922 & & 17.296 & 7.155 & 11.604 & 6.782 & 18.757 & 17.011 & 32.419 \\
\hline 7/8/09 16:00 & 10.066 & 4.932 & & 17.490 & 7.177 & 11.628 & 6.808 & 18.759 & 17.009 & 32.412 \\
\hline 7/8/09 20:00 & 10.089 & 4.946 & & 17.459 & 7.226 & 11.665 & 6.857 & 18.802 & 17.010 & 32.407 \\
\hline 7/9/09 0:00 & 10.113 & 4.960 & & 17.385 & 7.240 & 11.705 & 6.885 & 18.884 & 17.008 & 32.403 \\
\hline 7/9/09 4:00 & 10.132 & 4.969 & & 17.352 & 7.236 & 11.753 & 6.914 & 18.905 & 17.009 & 32.396 \\
\hline 7/9/09 8:00 & 10.155 & 4.986 & & 17.343 & 7.241 & 11.815 & 6.949 & 18.976 & 17.012 & 32.393 \\
\hline 7/9/09 12:00 & 10.170 & 4.993 & & 17.499 & 7.257 & 11.832 & 6.972 & 19.011 & 17.012 & 32.388 \\
\hline $7 / 9 / 09$ 16:00 & 10.193 & 5.007 & & 17.571 & 7.316 & 11.840 & 7.017 & 19.084 & 17.013 & 32.384 \\
\hline 7/9/09 20:00 & 10.198 & 5.016 & & 17.569 & 7.349 & 11.850 & 7.050 & 19.510 & 17.014 & 32.379 \\
\hline 7/10/09 0:00 & 10.226 & 5.030 & & 17.478 & 7.363 & 11.876 & 7.080 & 19.279 & 17.014 & 32.374 \\
\hline 7/10/09 4:00 & 9.734 & 4.721 & & 17.427 & 7.335 & 11.731 & 6.709 & 19.310 & 17.017 & 32.363 \\
\hline 7/10/09 8:00 & 8.251 & 3.769 & & 16.998 & 6.986 & 11.269 & 6.315 & 19.004 & 17.015 & 32.360 \\
\hline 7/10/09 12:00 & 8.524 & 3.664 & & 17.003 & 6.891 & 11.284 & 6.366 & 18.818 & 17.004 & 32.355 \\
\hline 7/10/09 16:00 & 8.748 & 3.769 & & 16.986 & 6.880 & 11.296 & 6.322 & 18.743 & 16.996 & 32.348 \\
\hline 7/10/09 20:00 & 8.902 & 3.898 & & 16.919 & 6.887 & 11.295 & 6.242 & 18.578 & 16.987 & 32.344 \\
\hline 7/11/09 0:00 & 9.026 & 4.017 & & 16.821 & 6.870 & 11.340 & 6.174 & 18.543 & 16.978 & 32.339 \\
\hline 7/11/09 4:00 & 9.109 & 4.120 & & 16.807 & 6.851 & 11.369 & 6.181 & 18.513 & 16.972 & 32.332 \\
\hline 7/11/09 8:00 & 9.187 & 4.209 & & 16.791 & 6.844 & 11.412 & 6.207 & 18.564 & 16.965 & 32.327 \\
\hline 7/11/09 12:00 & 9.253 & 4.289 & & 16.814 & 6.842 & 11.448 & 6.261 & 18.489 & 16.961 & 32.323 \\
\hline 7/11/09 16:00 & 9.307 & 4.356 & & 16.877 & 6.844 & 11.484 & 6.347 & 18.494 & 16.955 & 32.318 \\
\hline 7/11/09 20:00 & 9.340 & 4.420 & & 16.902 & 6.868 & 11.485 & 6.300 & 18.513 & 16.948 & 32.313 \\
\hline 7/12/09 0:00 & 9.378 & 4.473 & & 16.854 & 6.856 & 11.521 & 6.284 & 18.475 & 16.945 & 32.308 \\
\hline 7/12/09 4:00 & 9.390 & 4.515 & & 16.895 & 6.830 & 11.528 & 6.324 & 18.484 & 16.940 & 32.301 \\
\hline 7/12/09 8:00 & 9.404 & 4.551 & & 16.914 & 6.823 & 11.555 & 6.324 & 18.470 & 16.936 & 32.297 \\
\hline 7/12/09 12:00 & 9.248 & 3.987 & & 16.872 & 6.788 & 11.438 & 6.387 & 18.428 & 16.930 & 32.292 \\
\hline 7/12/09 16:00 & 9.274 & 3.765 & & 16.956 & 6.804 & 11.476 & 6.496 & 18.447 & 16.928 & 32.282 \\
\hline 7/12/09 20:00 & 9.302 & 3.788 & & 16.956 & 6.816 & 11.500 & 6.453 & 18.426 & 16.922 & 32.278 \\
\hline 7/13/09 0:00 & 9.352 & 3.877 & & 16.854 & 6.816 & 11.560 & 6.397 & 18.456 & 16.918 & 32.273 \\
\hline 7/13/09 4:00 & 9.368 & 3.961 & & 16.851 & 6.797 & 11.579 & 6.411 & 18.461 & 16.914 & 32.268 \\
\hline 7/13/09 8:00 & 9.382 & 4.048 & & 16.884 & 6.788 & 11.599 & 6.439 & 18.468 & 16.910 & 32.261 \\
\hline 7/13/09 12:00 & 9.408 & 4.129 & & 16.905 & 6.790 & 11.632 & 6.514 & 18.489 & 16.905 & 32.257 \\
\hline 7/13/09 16:00 & 9.413 & 4.200 & & 16.989 & 6.788 & 11.637 & 6.611 & 18.487 & 16.901 & 32.252 \\
\hline 7/13/09 20:00 & 9.420 & 4.265 & & 17.019 & 6.804 & 11.646 & 6.571 & 18.475 & 16.898 & 32.247 \\
\hline 7/14/09 0:00 & 9.427 & 4.324 & & 16.993 & 6.795 & 11.655 & 6.585 & 18.491 & 16.895 & 32.242 \\
\hline
\end{tabular}

TABLE S1.1 Page 7 of 89 
Water Level (ft below reference point)

\begin{tabular}{|c|c|c|c|c|c|c|c|c|c|c|}
\hline Date and Time & McPike & Potter & Hughes & Barr & Burks & MoDOT & Clizer "Old" & Morgan & SB24 & SB38 \\
\hline 7/14/09 4:00 & 9.423 & 4.373 & & 16.998 & 6.783 & 11.656 & 6.620 & 18.574 & 16.894 & 32.238 \\
\hline 7/14/09 8:00 & 8.741 & 3.921 & & 16.835 & 6.734 & 11.480 & 6.449 & 18.482 & 16.888 & 32.231 \\
\hline $7 / 14 / 09$ 12:00 & 8.612 & 3.421 & & 16.772 & 6.719 & 11.476 & 6.456 & 18.470 & 16.883 & 32.224 \\
\hline 7/14/09 16:00 & 8.697 & 3.365 & & 16.823 & 6.705 & 11.495 & 6.503 & 18.381 & 16.880 & 32.219 \\
\hline 7/14/09 20:00 & 8.803 & 3.472 & & 16.777 & 6.717 & 11.536 & 6.441 & 18.428 & 16.875 & 32.214 \\
\hline 7/15/09 0:00 & 8.888 & 3.601 & & 16.681 & 6.712 & 11.579 & 6.380 & 18.400 & 16.871 & 32.207 \\
\hline 7/15/09 4:00 & 8.975 & 3.727 & & 16.586 & 6.708 & 11.652 & 6.310 & 18.444 & 16.868 & 32.205 \\
\hline 7/15/09 8:00 & 9.071 & 3.854 & & 16.490 & 6.717 & 11.736 & 6.270 & 18.567 & 16.863 & 32.198 \\
\hline 7/15/09 12:00 & 9.144 & 3.961 & & 16.534 & 6.724 & 11.781 & 7.170 & 18.583 & 16.862 & 32.193 \\
\hline 7/15/09 16:00 & 9.192 & 4.062 & & 16.704 & 6.741 & 11.798 & 6.498 & 18.602 & 16.858 & 32.188 \\
\hline 7/15/09 20:00 & 9.232 & 4.158 & & 16.679 & 6.752 & 11.809 & 6.416 & 18.635 & 16.853 & 32.184 \\
\hline 7/16/09 0:00 & 9.260 & 4.237 & & 16.630 & 6.724 & 11.828 & 6.364 & 18.625 & 16.852 & 32.179 \\
\hline 7/16/09 4:00 & 9.267 & 4.298 & & 16.644 & 6.696 & 11.821 & 6.416 & 18.621 & 16.850 & 32.176 \\
\hline 7/16/09 8:00 & 9.286 & 4.354 & & 16.655 & 6.684 & 11.862 & 6.444 & 18.614 & 16.846 & 32.169 \\
\hline 7/16/09 12:00 & 9.328 & 4.408 & & 16.723 & 6.691 & 11.903 & 6.467 & 18.654 & 16.847 & 32.165 \\
\hline 7/16/09 16:00 & 9.307 & 4.443 & & 16.856 & 6.672 & 11.877 & 6.587 & 18.604 & 16.844 & 32.160 \\
\hline 7/16/09 20:00 & 9.350 & 4.490 & & 16.767 & 6.703 & 11.923 & 6.465 & 18.628 & 16.841 & 32.158 \\
\hline 7/17/09 0:00 & 9.399 & 4.534 & & 16.669 & 6.708 & 11.971 & 6.394 & 18.696 & 16.842 & 32.153 \\
\hline 7/17/09 4:00 & 9.418 & 4.569 & & 16.665 & 6.689 & 11.985 & 6.437 & 18.738 & 16.837 & 32.141 \\
\hline 7/17/09 8:00 & 9.449 & 4.602 & & 16.667 & 6.682 & 12.034 & 6.491 & 18.736 & 16.838 & 32.143 \\
\hline 7/17/09 12:00 & 9.482 & 4.635 & & 16.816 & 6.698 & 12.062 & 6.683 & 18.781 & 16.837 & 32.139 \\
\hline 7/17/09 16:00 & 9.493 & 4.663 & & 16.998 & 6.719 & 12.055 & 6.754 & 18.825 & 16.834 & 32.136 \\
\hline 7/17/09 20:00 & 9.517 & 4.693 & & 16.902 & 6.745 & 12.083 & 6.655 & 18.769 & 16.835 & 32.132 \\
\hline 7/18/09 0:00 & 9.543 & 4.726 & & 16.833 & 6.736 & 12.114 & 6.660 & 18.823 & 16.835 & 32.127 \\
\hline 7/18/09 4:00 & 9.564 & 4.752 & & 16.812 & 6.719 & 12.147 & 6.829 & 18.879 & 16.836 & 32.120 \\
\hline 7/18/09 8:00 & 9.590 & 4.775 & & 16.805 & 6.715 & 12.184 & 6.742 & 18.877 & 16.835 & 32.120 \\
\hline 7/18/09 12:00 & 9.616 & 4.796 & & 16.965 & 6.719 & 12.200 & 6.759 & 18.931 & 16.835 & 32.118 \\
\hline 7/18/09 16:00 & 9.628 & 4.817 & & 17.114 & 6.741 & 12.208 & 6.787 & 18.919 & 16.836 & 32.113 \\
\hline 7/18/09 20:00 & 9.644 & 4.838 & & 17.038 & 6.767 & 12.226 & 6.817 & 18.926 & 16.837 & 32.111 \\
\hline 7/19/09 0:00 & 9.668 & 4.862 & & 16.972 & 6.757 & 12.259 & 6.838 & 18.962 & 16.837 & 32.106 \\
\hline 7/19/09 4:00 & 9.680 & 4.876 & & 16.968 & 6.741 & 12.276 & 6.848 & 18.978 & 16.838 & 32.103 \\
\hline 7/19/09 8:00 & 9.703 & 4.897 & & 16.944 & 6.738 & 12.322 & 6.871 & 19.016 & 16.841 & 32.099 \\
\hline 7/19/09 12:00 & 9.715 & 4.909 & & 17.098 & 6.743 & 12.322 & 6.885 & 19.035 & 16.841 & 32.096 \\
\hline 7/19/09 16:00 & 9.712 & 4.920 & & 17.243 & 6.762 & 12.314 & 6.907 & 19.013 & 16.842 & 32.094 \\
\hline 7/19/09 20:00 & 9.715 & 4.934 & & 17.194 & 6.778 & 12.309 & 6.928 & 19.002 & 16.842 & 32.089 \\
\hline 7/20/09 0:00 & 9.734 & 4.951 & & 17.094 & 6.769 & 12.352 & 6.951 & 19.056 & 16.846 & 32.087 \\
\hline 7/20/09 4:00 & 9.724 & 4.958 & & 17.112 & 6.743 & 12.346 & 6.942 & 19.006 & 16.847 & 32.082 \\
\hline $7 / 20 / 09$ 8:00 & 9.736 & 4.969 & & 17.091 & 6.741 & 12.391 & 6.963 & 19.037 & 16.847 & 32.082 \\
\hline 7/20/09 12:00 & 9.736 & 4.976 & & 17.180 & 6.736 & 12.382 & 6.965 & 19.086 & 16.850 & 32.080 \\
\hline 7/20/09 16:00 & 9.753 & 4.988 & & 17.203 & 6.767 & 12.417 & 7.001 & 19.051 & 16.853 & 32.075 \\
\hline 7/20/09 20:00 & 9.750 & 4.995 & & 17.184 & 6.757 & 12.396 & 6.998 & 19.070 & 16.853 & 32.070 \\
\hline 7/21/09 0:00 & 9.769 & 5.007 & & 17.107 & 6.745 & 12.396 & 7.024 & 19.138 & 16.857 & 32.068 \\
\hline 7/21/09 4:00 & 9.767 & 5.009 & & 17.107 & 6.736 & 12.376 & 7.008 & 19.107 & 16.857 & 32.061 \\
\hline 7/21/09 8:00 & 9.781 & 5.012 & & 17.073 & 6.738 & 12.438 & 7.029 & 19.159 & 16.860 & 32.059 \\
\hline 7/21/09 12:00 & 9.800 & 5.012 & & 17.073 & 6.741 & 12.480 & 7.045 & 19.190 & 16.861 & 32.059 \\
\hline 7/21/09 16:00 & 9.821 & 5.009 & & 17.112 & 6.752 & 12.515 & 7.069 & 19.220 & 16.863 & 32.056 \\
\hline 7/21/09 20:00 & 9.840 & 5.012 & & 17.103 & 6.776 & 12.536 & 7.092 & 19.270 & 16.867 & 32.052 \\
\hline 7/22/09 0:00 & 9.863 & 5.014 & & 17.063 & 6.774 & 12.579 & 7.120 & 19.284 & 16.868 & 32.052 \\
\hline $7 / 22 / 094: 00$ & 9.875 & 5.014 & & 17.047 & 6.767 & 12.601 & 7.132 & 19.324 & 16.872 & 32.047 \\
\hline $7 / 22 / 09$ 8:00 & 9.903 & 5.019 & & 17.026 & 6.771 & 12.661 & 7.165 & 19.392 & 16.876 & 32.047 \\
\hline $7 / 22 / 09$ 12:00 & 9.930 & 5.026 & & 17.145 & 6.785 & 12.699 & 7.198 & 19.420 & 16.878 & 32.042 \\
\hline $7 / 22 / 09$ 16:00 & 9.943 & 5.033 & & 17.319 & 6.818 & 12.698 & 7.228 & 19.455 & 16.882 & 32.040 \\
\hline 7/22/09 20:00 & 9.948 & 5.035 & & 17.273 & 6.830 & 12.691 & 7.250 & 19.441 & 16.885 & 32.037 \\
\hline 7/23/09 0:00 & 9.965 & 5.044 & & 17.198 & 6.811 & 12.731 & 7.273 & 19.486 & 16.887 & 32.035 \\
\hline 7/23/09 4:00 & 9.974 & 5.047 & & 17.152 & 6.795 & 12.754 & 7.285 & 19.484 & 16.891 & 32.033 \\
\hline 7/23/09 8:00 & 9.988 & 5.054 & & 17.147 & 6.790 & 12.796 & 7.304 & 19.519 & 16.896 & 32.028 \\
\hline 7/23/09 12:00 & 10.012 & 5.061 & & 17.266 & 6.807 & 12.827 & 7.334 & 19.578 & 16.899 & 32.028 \\
\hline 7/23/09 16:00 & 10.031 & 5.070 & & 17.406 & 6.844 & 12.843 & 7.367 & 19.575 & 16.904 & 32.026 \\
\hline 7/23/09 20:00 & 10.045 & 5.080 & & 17.333 & 6.870 & 12.843 & 7.395 & 19.829 & 16.908 & 32.026 \\
\hline 7/24/09 0:00 & 10.061 & 5.087 & & 17.259 & 6.851 & 12.874 & 7.416 & 19.698 & 16.912 & 32.023 \\
\hline 7/24/09 4:00 & 10.059 & 5.091 & & 17.261 & 6.828 & 12.875 & 7.412 & 19.669 & 16.916 & 32.021 \\
\hline $7 / 24 / 09$ 8:00 & 10.057 & 5.096 & & 17.275 & 6.811 & 12.894 & 7.416 & 19.660 & 16.920 & 32.019 \\
\hline 7/24/09 12:00 & 10.062 & 5.103 & & 17.420 & 6.830 & 12.904 & 7.433 & 19.658 & 16.926 & 32.019 \\
\hline 7/24/09 16:00 & 10.076 & 5.112 & & 17.525 & 6.884 & 12.929 & 7.470 & 19.658 & 16.929 & 32.016 \\
\hline
\end{tabular}

TABLE S1.1 Page 8 of 89 
Water Level (ft below reference point)

\begin{tabular}{|c|c|c|c|c|c|c|c|c|c|c|}
\hline Date and Time & McPike & Potter & Hughes & Barr & Burks & MoDOT & Clizer "Old" & Morgan & SB24 & SB38 \\
\hline 7/24/09 20:00 & 10.087 & 5.122 & & 17.431 & 6.920 & 12.929 & 7.499 & 19.660 & 16.932 & 32.014 \\
\hline 7/25/09 0:00 & 10.118 & 5.136 & & 17.303 & 6.917 & 13.003 & 7.538 & 19.709 & 16.935 & 32.014 \\
\hline $7 / 25 / 094: 00$ & 10.175 & 5.152 & & 17.180 & 6.922 & 13.103 & 7.595 & 19.804 & 16.941 & 32.012 \\
\hline 7/25/09 8:00 & 10.205 & 5.164 & & 17.161 & 6.920 & 13.129 & 7.614 & 19.895 & 16.945 & 32.012 \\
\hline $7 / 25 / 09$ 12:00 & 10.240 & 5.175 & & 17.287 & 6.941 & 13.179 & 7.733 & 19.956 & 16.951 & 32.009 \\
\hline 7/25/09 16:00 & 10.267 & 5.187 & & 17.441 & 6.986 & 13.192 & 7.703 & 19.999 & 16.954 & 32.007 \\
\hline 7/25/09 20:00 & 10.288 & 5.197 & & 17.373 & 7.026 & 13.204 & 7.729 & 20.025 & 16.945 & 32.009 \\
\hline 7/26/09 0:00 & 10.316 & 5.208 & & 17.275 & 7.033 & 13.264 & 7.759 & 20.079 & 16.948 & 32.009 \\
\hline $7 / 26 / 094: 00$ & 10.337 & 5.218 & & 17.261 & 7.035 & 13.298 & 7.773 & 20.133 & 16.953 & 32.007 \\
\hline 7/26/09 8:00 & 10.354 & 5.222 & & 17.271 & 7.031 & 13.336 & 7.790 & 20.175 & 16.959 & 32.007 \\
\hline 7/26/09 12:00 & 10.373 & 5.232 & & 17.431 & 7.057 & 13.364 & 7.816 & 20.222 & 16.965 & 32.005 \\
\hline 7/26/09 16:00 & 10.384 & 5.239 & & 17.597 & 7.109 & 13.373 & 7.841 & 23.063 & 16.972 & 32.004 \\
\hline 7/26/09 20:00 & 10.399 & 5.248 & & 17.525 & 7.160 & 13.385 & 7.870 & 20.770 & 16.975 & 32.005 \\
\hline 7/27/09 0:00 & 10.422 & 5.260 & & 17.424 & 7.172 & 13.437 & 7.900 & 20.582 & 16.982 & 32.005 \\
\hline 7/27/09 4:00 & 10.420 & 5.262 & & 17.448 & 7.158 & 13.438 & 7.896 & 20.443 & 16.990 & 32.005 \\
\hline 7/27/09 8:00 & 10.439 & 5.269 & & 17.403 & 7.165 & 13.495 & 7.921 & 20.445 & 16.998 & 32.005 \\
\hline $7 / 27 / 09$ 12:00 & 10.448 & 5.274 & & 17.559 & 7.184 & 13.510 & 7.935 & 20.450 & 17.003 & 32.004 \\
\hline $7 / 27 / 09$ 16:00 & 10.448 & 5.278 & & 17.746 & 7.234 & 13.502 & 7.954 & 20.429 & 17.011 & 32.005 \\
\hline 7/27/09 20:00 & 10.462 & 5.285 & & 17.655 & 7.269 & 13.525 & 7.989 & 20.420 & 17.019 & 32.004 \\
\hline 7/28/09 0:00 & 10.479 & 5.292 & & 17.545 & 7.274 & 13.566 & 8.008 & 20.457 & 17.026 & 32.005 \\
\hline 7/28/09 4:00 & 10.481 & 5.297 & & 17.541 & 7.262 & 13.579 & 8.011 & 20.476 & 17.034 & 32.005 \\
\hline 7/28/09 8:00 & 10.493 & 5.302 & & 17.529 & 7.252 & 13.624 & 8.027 & 20.500 & 17.042 & 32.007 \\
\hline $7 / 28 / 09$ 12:00 & 10.509 & 5.309 & & 17.543 & 7.257 & 13.676 & 8.051 & 20.528 & 17.049 & 32.007 \\
\hline 7/28/09 16:00 & 10.502 & 5.309 & & 17.508 & 7.245 & 13.581 & 8.036 & 20.565 & 17.059 & 32.007 \\
\hline 7/28/09 20:00 & 10.507 & 5.309 & & 17.506 & 7.236 & 13.607 & 8.048 & 20.591 & 17.066 & 32.005 \\
\hline 7/29/09 0:00 & 10.531 & 5.311 & & 17.413 & 7.257 & 13.680 & 8.074 & 20.721 & 17.074 & 32.007 \\
\hline 7/29/09 4:00 & 10.538 & 5.306 & & 17.436 & 7.259 & 13.703 & 8.076 & 20.666 & 17.085 & 32.007 \\
\hline $7 / 29 / 09$ 8:00 & 10.556 & 5.309 & & 17.417 & 7.259 & 13.767 & 8.107 & 20.709 & 17.089 & 32.009 \\
\hline $7 / 29 / 09$ 12:00 & 10.571 & 5.311 & & 17.550 & 7.285 & 13.788 & 8.121 & 20.800 & 17.098 & 32.009 \\
\hline $7 / 29 / 09$ 16:00 & 10.575 & 5.311 & & 17.711 & 7.328 & 13.785 & 8.145 & 20.749 & 17.108 & 32.009 \\
\hline 7/29/09 20:00 & 10.587 & 5.316 & & 17.643 & 7.354 & 13.816 & 8.173 & 20.763 & 17.115 & 32.009 \\
\hline 7/30/09 0:00 & 10.601 & 5.318 & & 17.576 & 7.368 & 13.842 & 8.184 & 20.845 & 17.125 & 32.009 \\
\hline 7/30/09 4:00 & 10.611 & 5.321 & & 17.564 & 7.358 & 13.883 & 8.203 & 20.819 & 17.132 & 32.012 \\
\hline 7/30/09 8:00 & 10.637 & 5.328 & & 17.485 & 7.370 & 13.945 & 8.217 & 20.883 & 17.143 & 32.012 \\
\hline 7/30/09 12:00 & 10.665 & 5.335 & & 17.548 & 7.387 & 14.004 & 8.260 & 20.970 & 17.153 & 32.014 \\
\hline 7/30/09 16:00 & 10.679 & 5.339 & & 17.730 & 7.427 & 14.024 & 8.283 & 21.654 & 17.162 & 32.014 \\
\hline 7/30/09 20:00 & 10.700 & 5.346 & & 17.639 & 7.476 & 14.053 & 8.318 & 21.435 & 17.171 & 32.014 \\
\hline 7/31/09 0:00 & 10.731 & 5.356 & & 17.550 & 7.495 & 14.122 & 8.356 & 21.261 & 17.181 & 32.019 \\
\hline 7/31/09 4:00 & 10.745 & 5.360 & & 17.552 & 7.495 & 14.142 & 8.365 & 21.290 & 17.193 & 32.019 \\
\hline $7 / 31 / 09$ 8:00 & 10.766 & 5.365 & & 17.536 & 7.498 & 14.206 & 8.389 & 21.245 & 17.203 & 32.019 \\
\hline 7/31/09 12:00 & 10.780 & 5.370 & & 17.671 & 7.519 & 14.221 & 8.403 & 21.306 & 17.212 & 32.023 \\
\hline 7/31/09 16:00 & 10.783 & 5.372 & & 17.883 & 7.564 & 14.210 & 8.417 & 21.278 & 17.223 & 32.021 \\
\hline 7/31/09 20:00 & 10.783 & 5.374 & & 17.827 & 7.606 & 14.187 & 8.429 & 21.254 & 17.233 & 32.023 \\
\hline 8/1/09 0:00 & 10.797 & 5.381 & & 17.744 & 7.625 & 14.233 & 8.455 & 21.268 & 17.243 & 32.026 \\
\hline 8/1/09 4:00 & 10.764 & 5.358 & & 17.625 & 7.587 & 13.983 & 8.403 & 21.299 & 17.253 & 32.021 \\
\hline 8/1/09 8:00 & 10.738 & 5.283 & & 17.508 & 7.545 & 14.070 & 8.417 & 21.297 & 17.263 & 32.021 \\
\hline 8/1/09 12:00 & 10.740 & 5.250 & & 17.520 & 7.526 & 14.150 & 8.436 & 21.313 & 17.273 & 32.026 \\
\hline 8/1/09 16:00 & 10.752 & 5.246 & & 17.629 & 7.557 & 14.183 & 8.464 & 21.325 & 17.281 & 32.026 \\
\hline 8/1/09 20:00 & 10.773 & 5.250 & & 17.545 & 7.592 & 14.207 & 8.490 & 21.334 & 17.291 & 32.028 \\
\hline 8/2/09 0:00 & 10.802 & 5.260 & & 17.487 & 7.601 & 14.267 & 8.518 & 21.377 & 17.300 & 32.028 \\
\hline 8/2/09 4:00 & 10.809 & 5.265 & & 17.494 & 7.583 & 14.279 & 8.520 & 21.393 & 17.311 & 32.035 \\
\hline 8/2/09 8:00 & 10.816 & 5.272 & & 17.501 & 7.561 & 14.308 & 8.525 & 21.407 & 17.320 & 32.035 \\
\hline 8/2/09 12:00 & 10.816 & 5.276 & & 17.653 & 7.564 & 14.309 & 8.527 & 21.410 & 17.331 & 32.037 \\
\hline 8/2/09 16:00 & 10.806 & 5.281 & & 17.853 & 7.606 & 14.295 & 8.532 & 21.367 & 17.341 & 32.040 \\
\hline 8/2/09 20:00 & 10.823 & 5.295 & & 17.744 & 7.653 & 14.317 & 8.565 & 21.362 & 17.350 & 32.042 \\
\hline 8/3/09 0:00 & 10.835 & 5.307 & & 17.648 & 7.672 & 14.346 & 8.579 & 21.393 & 17.361 & 32.042 \\
\hline 8/3/09 4:00 & 10.835 & 5.311 & & 17.678 & 7.668 & 14.354 & 8.579 & 21.384 & 17.369 & 32.047 \\
\hline 8/3/09 8:00 & 10.858 & 5.323 & & 17.620 & 7.686 & 14.425 & 8.612 & 21.431 & 17.381 & 32.047 \\
\hline 8/3/09 12:00 & 10.875 & 5.335 & & 17.771 & 7.705 & 14.459 & 8.628 & 21.461 & 17.391 & 32.049 \\
\hline 8/3/09 16:00 & 10.886 & 5.346 & & 17.916 & 7.755 & 14.476 & 8.654 & 21.478 & 17.400 & 32.052 \\
\hline 8/3/09 20:00 & 10.910 & 5.360 & & 17.795 & 7.814 & 14.511 & 8.692 & 21.504 & 17.411 & 32.054 \\
\hline 8/4/09 0:00 & 10.936 & 5.374 & & 17.711 & 7.835 & 14.569 & 8.722 & 21.562 & 17.421 & 32.056 \\
\hline 8/4/09 4:00 & 10.938 & 5.372 & & 17.641 & 7.795 & 14.565 & 8.758 & 21.647 & 17.433 & 32.056 \\
\hline 8/4/09 8:00 & 10.967 & 5.356 & & 17.538 & 7.833 & 14.579 & 8.802 & 21.696 & 17.443 & 32.054 \\
\hline
\end{tabular}


Water Level (ft below reference point)

\begin{tabular}{|c|c|c|c|c|c|c|c|c|c|c|}
\hline Date and Time & McPike & Potter & Hughes & Barr & Burks & MoDOT & Clizer "Old" & Morgan & SB24 & SB38 \\
\hline 8/4/09 12:00 & 10.990 & 5.330 & & 17.573 & 7.828 & 14.665 & 8.828 & 21.743 & 17.456 & 32.061 \\
\hline 8/4/09 16:00 & 11.014 & 5.325 & & 17.723 & 7.870 & 14.708 & 8.868 & 21.762 & 17.465 & 32.061 \\
\hline 8/4/09 20:00 & 11.035 & 5.330 & & 17.702 & 7.913 & 14.727 & 8.812 & 21.816 & 17.478 & 32.063 \\
\hline 8/5/09 0:00 & 11.068 & 5.342 & & 17.606 & 7.929 & 14.802 & 8.805 & 21.910 & 17.491 & 32.068 \\
\hline 8/5/09 4:00 & 11.087 & 5.351 & & 17.590 & 7.932 & 14.840 & 8.795 & 21.934 & 17.500 & 32.068 \\
\hline 8/5/09 8:00 & 11.125 & 5.360 & & 17.538 & 7.944 & 14.930 & 8.838 & 22.035 & 17.513 & 32.073 \\
\hline 8/5/09 12:00 & 11.136 & 5.379 & & 17.678 & 7.953 & 14.968 & & 22.082 & 17.597 & 32.312 \\
\hline 8/5/09 16:00 & 11.141 & 5.384 & & 17.865 & 7.951 & 14.958 & 8.873 & 22.080 & 17.608 & 32.312 \\
\hline 8/5/09 20:00 & 11.155 & 5.391 & & 17.830 & 7.986 & 14.965 & 8.901 & 22.136 & 17.618 & 32.314 \\
\hline 8/6/09 0:00 & 11.184 & 5.400 & & 17.737 & 8.007 & 15.021 & 8.934 & 22.148 & 17.632 & 32.317 \\
\hline 8/6/09 4:00 & 11.191 & 5.405 & & 17.751 & 8.012 & 15.043 & 8.932 & 22.193 & 17.641 & 32.319 \\
\hline 8/6/09 8:00 & 11.205 & 5.412 & & 17.751 & 8.010 & 15.075 & 8.951 & 22.258 & 17.655 & 32.322 \\
\hline 8/6/09 12:00 & 11.219 & 5.414 & & 17.867 & 8.024 & 15.106 & 8.965 & 22.249 & 17.665 & 32.326 \\
\hline 8/6/09 16:00 & 11.202 & 5.414 & & 18.095 & 8.151 & 15.059 & 8.943 & 22.230 & 17.679 & 32.326 \\
\hline 8/6/09 20:00 & 11.214 & 5.421 & & 18.023 & 8.135 & 15.059 & 8.969 & 22.235 & 17.688 & 32.329 \\
\hline 8/7/09 0:00 & 11.228 & 5.426 & & 17.970 & 8.135 & 15.080 & 8.988 & 22.223 & 17.702 & 32.331 \\
\hline 8/7/09 4:00 & 11.217 & 5.426 & & 17.988 & 8.130 & 15.073 & 8.969 & 22.223 & 17.712 & 32.336 \\
\hline 8/7/09 8:00 & 11.214 & 5.428 & & 18.002 & 8.120 & 15.085 & 8.974 & 22.237 & 17.726 & 32.338 \\
\hline 8/7/09 12:00 & 11.226 & 5.435 & & 18.051 & 8.130 & 15.122 & 8.993 & 22.230 & 17.735 & 32.340 \\
\hline 8/7/09 16:00 & 11.231 & 5.442 & & 18.226 & 8.168 & 15.115 & 9.002 & 22.228 & 17.749 & 32.343 \\
\hline 8/7/09 20:00 & 11.245 & 5.452 & & 18.121 & 8.219 & 15.118 & 9.023 & 23.173 & 17.758 & 32.347 \\
\hline 8/8/09 0:00 & 11.268 & 5.463 & & 18.014 & 8.255 & 15.174 & 9.052 & 22.468 & 17.770 & 32.350 \\
\hline 8/8/09 4:00 & 11.285 & 5.468 & & 17.972 & 8.283 & 15.209 & 9.056 & 22.381 & 17.780 & 32.352 \\
\hline 8/8/09 8:00 & 11.304 & 5.480 & & 17.939 & 8.307 & 15.261 & 9.479 & 22.409 & 17.794 & 32.357 \\
\hline 8/8/09 12:00 & 11.318 & 5.487 & & 18.058 & 8.328 & 15.279 & 9.138 & 22.439 & 17.808 & 32.359 \\
\hline 8/8/09 16:00 & 11.332 & 5.494 & & 18.216 & 8.385 & 15.286 & 9.138 & 22.486 & 17.819 & 32.362 \\
\hline 8/8/09 20:00 & 11.365 & 5.510 & & 18.060 & 8.460 & 15.329 & 9.181 & 22.465 & 17.831 & 32.366 \\
\hline 8/9/09 0:00 & 11.410 & 5.522 & & 17.946 & 8.512 & 15.406 & 9.221 & 22.557 & 17.845 & 32.369 \\
\hline 8/9/09 4:00 & 11.443 & 5.531 & & 17.897 & 8.545 & 15.469 & 9.249 & 22.670 & 17.857 & 32.373 \\
\hline 8/9/09 8:00 & 11.466 & 5.538 & & 17.904 & 8.568 & 15.521 & 9.268 & 22.715 & 17.871 & 32.376 \\
\hline 8/9/09 12:00 & 11.495 & 5.548 & & 18.042 & 8.604 & 15.572 & 9.284 & 22.776 & 17.883 & 32.380 \\
\hline 8/9/09 16:00 & 11.495 & 5.550 & & 18.247 & 8.649 & 15.549 & 9.279 & 22.773 & 17.894 & 32.383 \\
\hline 8/9/09 20:00 & 11.537 & 5.564 & & 17.921 & 8.712 & 15.636 & 9.331 & 22.832 & 17.909 & 32.383 \\
\hline 8/10/09 0:00 & 11.558 & 5.569 & & 17.923 & 8.734 & 15.596 & 9.345 & 22.886 & 17.923 & 32.385 \\
\hline 8/10/09 4:00 & 11.573 & 5.571 & & 17.942 & 8.762 & 15.626 & 9.350 & 22.940 & 17.937 & 32.390 \\
\hline 8/10/09 8:00 & 11.594 & 5.576 & & 17.956 & 8.781 & 15.683 & 9.366 & 22.994 & 17.951 & 32.395 \\
\hline 8/10/09 12:00 & 11.620 & 5.583 & & 18.023 & 8.800 & 15.737 & 9.383 & 23.055 & 17.965 & 32.399 \\
\hline 8/10/09 16:00 & 11.617 & 5.585 & & 18.170 & 8.828 & 15.704 & 9.376 & 23.055 & 17.979 & 32.404 \\
\hline 8/10/09 20:00 & 11.629 & 5.590 & & 18.168 & 8.866 & 15.739 & 9.387 & 23.126 & 17.995 & 32.406 \\
\hline $8 / 11 / 090: 00$ & 11.660 & 5.597 & & 18.086 & 8.894 & 15.816 & 9.420 & 23.140 & 18.009 & 32.413 \\
\hline 8/11/09 4:00 & 11.674 & 5.599 & & 18.086 & 8.915 & 15.847 & 9.430 & 23.197 & 18.023 & 32.416 \\
\hline 8/11/09 8:00 & 11.702 & 5.604 & & 18.058 & 8.936 & 15.915 & 9.453 & 23.295 & 18.038 & 32.420 \\
\hline 8/11/09 12:00 & 11.723 & 5.608 & & 18.165 & 8.965 & 15.962 & 9.470 & 23.305 & 18.054 & 32.425 \\
\hline 8/11/09 16:00 & 11.735 & 5.613 & & 18.359 & 9.009 & 15.966 & 9.477 & 23.331 & 18.070 & 32.430 \\
\hline 8/11/09 20:00 & 11.747 & 5.620 & & 18.314 & 9.066 & 15.955 & 9.491 & 23.361 & 18.084 & 32.435 \\
\hline 8/12/09 0:00 & 11.771 & 5.625 & & 18.205 & 9.113 & 16.009 & 9.517 & 23.371 & 18.101 & 32.439 \\
\hline 8/12/09 4:00 & 11.782 & 5.625 & & 18.212 & 9.146 & 16.027 & 9.526 & 23.406 & 18.115 & 32.444 \\
\hline 8/12/09 8:00 & 11.801 & 5.627 & & 18.179 & 9.175 & 16.074 & 9.540 & 23.474 & 18.129 & 32.449 \\
\hline 8/12/09 12:00 & 11.815 & 5.632 & & 18.305 & 9.205 & 16.112 & 9.552 & 23.488 & 18.145 & 32.453 \\
\hline 8/12/09 16:00 & 11.822 & 5.639 & & 18.487 & 9.252 & 16.105 & 9.552 & 23.514 & 18.162 & 32.458 \\
\hline 8/12/09 20:00 & 11.834 & 5.643 & & 18.405 & 9.309 & 16.091 & 9.566 & 23.476 & 18.176 & 32.463 \\
\hline 8/13/09 0:00 & 11.855 & 5.648 & & 18.300 & 9.361 & 16.130 & 9.587 & 23.516 & 18.190 & 32.468 \\
\hline 8/13/09 4:00 & 11.858 & 5.646 & & 18.296 & 9.403 & 16.142 & 9.578 & 23.559 & 18.206 & 32.475 \\
\hline 8/13/09 8:00 & 11.884 & 5.651 & & 18.221 & 9.441 & 16.213 & 9.752 & 23.681 & 18.223 & 32.479 \\
\hline 8/13/09 12:00 & 11.910 & 5.660 & & 18.356 & 9.479 & 16.259 & 9.683 & 23.660 & 18.237 & 32.484 \\
\hline 8/13/09 16:00 & 11.924 & 5.669 & & 18.515 & 9.531 & 16.273 & 9.676 & 24.680 & 18.253 & 32.489 \\
\hline 8/13/09 20:00 & 11.943 & 5.676 & & 18.435 & 9.594 & 16.266 & 9.691 & 24.518 & 18.270 & 32.494 \\
\hline 8/14/09 0:00 & 11.966 & 5.683 & & 18.345 & 9.649 & 16.318 & 9.712 & 23.951 & 18.286 & 32.501 \\
\hline 8/14/09 4:00 & 11.983 & 5.686 & & 18.331 & 9.696 & 16.356 & 9.723 & 23.867 & 18.303 & 32.505 \\
\hline 8/14/09 8:00 & 12.002 & 5.686 & & 18.317 & 9.733 & 16.402 & 9.733 & 23.881 & 18.319 & 32.512 \\
\hline 8/14/09 12:00 & 12.020 & 5.695 & & 18.424 & 9.776 & 16.445 & 9.745 & 23.921 & 18.335 & 32.517 \\
\hline 8/14/09 16:00 & 12.030 & 5.702 & & 18.582 & 9.825 & 16.440 & 9.756 & 23.961 & 18.352 & 32.522 \\
\hline 8/14/09 20:00 & 12.039 & 5.707 & & 18.533 & 9.880 & 16.421 & 9.763 & 23.888 & 18.368 & 32.529 \\
\hline $8 / 15 / 09$ 0:00 & 12.053 & 5.711 & & 18.442 & 9.927 & 16.449 & 9.777 & 23.916 & 18.382 & 32.536 \\
\hline
\end{tabular}

TABLE S1.1 Page 10 of 89 
Water Level (ft below reference point)

\begin{tabular}{|c|c|c|c|c|c|c|c|c|c|c|}
\hline Date and Time & McPike & Potter & Hughes & Barr & Burks & MoDOT & Clizer "Old" & Morgan & SB24 & SB38 \\
\hline 8/15/09 4:00 & 12.060 & 5.711 & & 18.421 & 9.969 & 16.473 & 9.784 & 23.968 & 18.399 & 32.541 \\
\hline 8/15/09 8:00 & 12.070 & 5.714 & & 18.405 & 10.009 & 16.503 & 9.789 & 23.963 & 18.415 & 32.548 \\
\hline 8/15/09 12:00 & 12.077 & 5.716 & & 18.540 & 10.047 & 16.513 & 9.792 & 23.977 & 18.432 & 32.552 \\
\hline 8/15/09 16:00 & 12.089 & 5.726 & & 18.592 & 10.092 & 16.524 & 9.803 & 24.125 & 18.448 & 32.557 \\
\hline 8/15/09 20:00 & 12.108 & 5.735 & & 18.531 & 10.141 & 16.550 & 9.824 & 24.219 & 18.464 & 32.564 \\
\hline 8/16/09 0:00 & 12.089 & 5.730 & & 18.414 & 10.099 & 16.470 & 9.827 & 24.132 & 18.483 & 32.569 \\
\hline 8/16/09 4:00 & 12.039 & 5.714 & & 18.424 & 10.139 & 16.449 & 9.817 & 24.048 & 18.497 & 32.574 \\
\hline 8/16/09 8:00 & 12.011 & 5.707 & & 18.261 & 10.130 & 16.409 & 9.815 & 24.081 & 18.514 & 32.574 \\
\hline 8/16/09 12:00 & 11.664 & 5.616 & & 18.195 & 10.134 & 16.238 & 9.801 & 24.088 & 18.528 & 32.583 \\
\hline 8/16/09 16:00 & 11.669 & 5.578 & & 18.263 & 10.184 & 16.292 & 9.794 & 24.076 & 18.544 & 32.588 \\
\hline 8/16/09 20:00 & 11.723 & 5.566 & & 18.205 & 10.231 & 16.356 & 9.801 & 24.029 & 18.558 & 32.595 \\
\hline 8/17/09 0:00 & 11.773 & 5.562 & & 18.161 & 10.276 & 16.405 & 9.808 & 24.055 & 18.575 & 32.600 \\
\hline 8/17/09 4:00 & 11.035 & 5.047 & & 18.044 & 10.207 & 16.152 & 9.770 & 24.052 & 18.589 & 32.602 \\
\hline 8/17/09 8:00 & 11.052 & 4.909 & & 17.969 & 10.238 & 16.236 & 9.749 & 24.003 & 18.603 & 32.609 \\
\hline 8/17/09 12:00 & 10.057 & 4.341 & & 17.732 & 10.106 & 15.469 & 9.442 & 23.958 & 18.617 & 32.609 \\
\hline $8 / 17 / 09$ 16:00 & 9.965 & 4.102 & & 17.608 & 9.896 & 15.427 & 9.345 & 23.777 & 18.624 & 32.616 \\
\hline 8/17/09 20:00 & 10.243 & 4.132 & & 17.529 & 9.592 & 15.528 & 9.317 & 23.613 & 18.633 & 32.621 \\
\hline 8/18/09 0:00 & 10.488 & 4.233 & & 17.385 & 9.333 & 15.636 & 9.329 & 23.552 & 18.640 & 32.628 \\
\hline 8/18/09 4:00 & 10.653 & 4.336 & & 17.373 & 9.125 & 15.685 & 9.319 & 23.521 & 18.650 & 32.632 \\
\hline 8/18/09 8:00 & 10.776 & 4.432 & & 17.375 & 8.960 & 15.715 & 9.315 & 23.538 & 18.659 & 32.635 \\
\hline 8/18/09 12:00 & 10.884 & 4.525 & & 17.431 & 8.866 & 15.760 & 9.324 & 23.446 & 18.671 & 32.642 \\
\hline 8/18/09 16:00 & 10.946 & 4.607 & & 17.613 & 8.816 & 15.730 & 9.301 & 23.401 & 18.680 & 32.647 \\
\hline 8/18/09 20:00 & 11.005 & 4.687 & & 17.611 & 8.795 & 15.704 & 9.310 & 23.364 & 18.687 & 32.651 \\
\hline 8/19/09 0:00 & 11.057 & 4.745 & & 17.571 & 8.781 & 15.727 & 9.317 & 23.314 & 18.699 & 32.654 \\
\hline 8/19/09 4:00 & 11.080 & 4.787 & & 17.622 & 8.750 & 15.685 & 9.291 & 23.255 & 18.706 & 32.658 \\
\hline 8/19/09 8:00 & 11.089 & 4.823 & & 17.664 & 8.719 & 15.659 & 9.265 & 23.211 & 18.715 & 32.665 \\
\hline 8/19/09 12:00 & 11.118 & 4.860 & & 17.667 & 8.698 & 15.650 & 9.272 & 23.239 & 18.725 & 32.668 \\
\hline 8/19/09 16:00 & 11.151 & 4.909 & & 17.760 & 8.715 & 15.685 & 9.289 & 23.150 & 18.732 & 32.672 \\
\hline 8/19/09 20:00 & 11.179 & 4.951 & & 17.695 & 8.731 & 15.680 & 9.305 & 23.145 & 18.741 & 32.677 \\
\hline 8/20/09 0:00 & 11.207 & 4.937 & & 17.550 & 8.731 & 15.708 & 9.324 & 23.211 & 18.751 & 32.677 \\
\hline 8/20/09 4:00 & 11.238 & 4.855 & & 17.483 & 8.724 & 15.699 & 9.338 & 23.180 & 18.760 & 32.684 \\
\hline 8/20/09 8:00 & 11.273 & 4.820 & & 17.438 & 8.717 & 15.767 & 9.359 & 23.241 & 18.769 & 32.689 \\
\hline $8 / 20 / 09$ 12:00 & 11.309 & 4.825 & & 17.508 & 8.722 & 15.828 & 9.383 & 23.312 & 18.779 & 32.694 \\
\hline 8/20/09 16:00 & 11.342 & 4.860 & & 17.604 & 8.750 & 15.859 & 9.399 & 23.316 & 18.788 & 32.696 \\
\hline 8/20/09 20:00 & 11.382 & 4.902 & & 17.576 & 8.781 & 15.898 & 9.437 & 23.361 & 18.800 & 32.701 \\
\hline 8/21/09 0:00 & 11.429 & 4.937 & & 17.520 & 8.814 & 15.978 & 9.472 & 23.432 & 18.809 & 32.708 \\
\hline 8/21/09 4:00 & 11.455 & 4.965 & & 17.564 & 8.826 & 15.994 & 9.484 & 23.465 & 18.819 & 32.710 \\
\hline 8/21/09 8:00 & 11.493 & 4.996 & & 17.536 & 8.842 & 16.053 & 9.510 & 23.514 & 18.830 & 32.713 \\
\hline 8/21/09 12:00 & 11.535 & 5.026 & & 17.608 & 8.863 & 16.135 & 9.540 & 23.592 & 18.842 & 32.720 \\
\hline $8 / 21 / 09$ 16:00 & 11.570 & 5.059 & & 17.771 & 8.896 & 16.166 & 9.561 & 23.627 & 18.854 & 32.722 \\
\hline $8 / 21 / 09$ 20:00 & 11.608 & 5.092 & & 17.711 & 8.939 & 16.210 & 9.594 & 23.690 & 18.865 & 32.727 \\
\hline 8/22/09 0:00 & 11.650 & 5.120 & & 17.664 & 8.976 & 16.278 & 9.629 & 23.824 & 18.875 & 32.734 \\
\hline 8/22/09 4:00 & 11.684 & 5.141 & & 17.688 & 9.005 & 16.330 & 9.651 & 23.796 & 18.889 & 32.736 \\
\hline 8/22/09 8:00 & 11.714 & 5.159 & & 17.690 & 9.031 & 16.381 & 9.669 & 23.864 & 18.898 & 32.743 \\
\hline $8 / 22 / 09$ 12:00 & 11.745 & 5.180 & & 17.816 & 9.054 & 16.431 & 9.686 & 23.940 & 18.908 & 32.746 \\
\hline 8/22/09 16:00 & 11.752 & 5.199 & & 18.030 & 9.087 & 16.414 & 9.674 & 23.904 & 18.919 & 32.748 \\
\hline 8/22/09 20:00 & 11.764 & 5.218 & & 18.016 & 9.130 & 16.405 & 9.686 & 23.904 & 18.931 & 32.753 \\
\hline 8/23/09 0:00 & 11.790 & 5.234 & & 17.921 & 9.167 & 16.452 & 9.712 & 23.958 & 18.943 & 32.762 \\
\hline 8/23/09 4:00 & 11.808 & 5.248 & & 17.916 & 9.200 & 16.485 & 9.726 & 23.977 & 18.955 & 32.762 \\
\hline 8/23/09 8:00 & 11.832 & 5.262 & & 17.911 & 9.227 & 16.527 & 9.740 & 24.031 & 18.969 & 32.769 \\
\hline 8/23/09 12:00 & 11.851 & 5.274 & & 18.021 & 9.252 & 16.550 & 9.749 & 24.095 & 18.980 & 32.776 \\
\hline $8 / 23 / 09$ 16:00 & 11.860 & 5.290 & & 18.200 & 9.285 & 16.550 & 9.754 & 24.041 & 18.992 & 32.779 \\
\hline 8/23/09 20:00 & 11.874 & 5.307 & & 18.119 & 9.325 & 16.545 & 9.766 & 24.052 & 19.004 & 32.783 \\
\hline 8/24/09 0:00 & 11.900 & 5.323 & & 18.014 & 9.366 & 16.595 & 9.789 & 24.081 & 19.018 & 32.790 \\
\hline 8/24/09 4:00 & 11.922 & 5.335 & & 18.004 & 9.396 & 16.632 & 9.803 & 24.123 & 19.030 & 32.793 \\
\hline 8/24/09 8:00 & 11.943 & 5.344 & & 17.993 & 9.425 & 16.672 & 9.817 & 24.179 & 19.041 & 32.802 \\
\hline $8 / 24 / 09$ 12:00 & 11.957 & 5.354 & & 18.126 & 9.450 & 16.700 & 9.824 & 24.252 & 19.058 & 32.807 \\
\hline 8/24/09 16:00 & 11.969 & 5.365 & & 18.293 & 9.484 & 16.696 & 9.831 & 24.184 & 19.069 & 32.812 \\
\hline 8/24/09 20:00 & 11.990 & 5.382 & & 18.205 & 9.524 & 16.707 & 9.848 & 24.205 & 19.081 & 32.816 \\
\hline 8/25/09 0:00 & 12.013 & 5.396 & & 18.119 & 9.564 & 16.749 & 9.869 & 24.269 & 19.095 & 32.823 \\
\hline 8/25/09 4:00 & 12.028 & 5.403 & & 18.119 & 9.597 & 16.775 & 9.881 & 24.269 & 19.109 & 32.826 \\
\hline 8/25/09 8:00 & 12.051 & 5.412 & & 18.098 & 9.625 & 16.827 & 9.895 & 24.321 & 19.123 & 32.835 \\
\hline 8/25/09 12:00 & 12.072 & 5.424 & & 18.202 & 9.653 & 16.860 & 9.907 & 24.393 & 19.135 & 32.840 \\
\hline $8 / 25 / 09$ 16:00 & 12.089 & 5.433 & & 18.289 & 9.686 & 16.876 & 9.916 & 24.386 & 19.149 & 32.844 \\
\hline
\end{tabular}


Water Level (ft below reference point)

\begin{tabular}{|c|c|c|c|c|c|c|c|c|c|c|}
\hline Date and Time & McPike & Potter & Hughes & Barr & Burks & MoDOT & Clizer "Old" & Morgan & SB24 & SB38 \\
\hline 8/25/09 20:00 & 12.110 & 5.447 & & 18.249 & 9.724 & 16.899 & 9.932 & 24.410 & 19.163 & 32.849 \\
\hline 8/26/09 0:00 & 12.136 & 5.457 & & 18.182 & 9.757 & 16.963 & 9.956 & 24.466 & 19.177 & 32.856 \\
\hline 8/26/09 4:00 & 12.150 & 5.459 & & 18.205 & 9.786 & 16.975 & 9.965 & 24.495 & 19.191 & 32.861 \\
\hline 8/26/09 8:00 & 12.178 & 5.468 & & 18.177 & 9.809 & 17.026 & 9.984 & 24.537 & 19.206 & 32.868 \\
\hline 8/26/09 12:00 & 12.183 & 5.471 & & 18.191 & 9.833 & 17.059 & 9.986 & 24.579 & 19.220 & 32.875 \\
\hline 8/26/09 16:00 & 12.202 & 5.478 & & 18.265 & 9.856 & 17.080 & 9.994 & 24.605 & 19.234 & 32.882 \\
\hline 8/26/09 20:00 & 12.211 & 5.485 & & 18.298 & 9.884 & 17.087 & 10.001 & 24.621 & 19.248 & 32.887 \\
\hline 8/27/09 0:00 & 12.228 & 5.489 & & 18.270 & 9.908 & 17.122 & 10.012 & 24.652 & 19.264 & 32.894 \\
\hline 8/27/09 4:00 & 12.235 & 5.492 & & 18.279 & 9.927 & 17.139 & 10.017 & 24.676 & 19.278 & 32.899 \\
\hline 8/27/09 8:00 & 12.252 & 5.496 & & 18.254 & 9.946 & 17.181 & 10.024 & 24.708 & 19.295 & 32.903 \\
\hline 8/27/09 12:00 & 12.277 & 5.506 & 8.391 & 18.226 & 9.965 & 17.256 & 10.048 & 24.777 & 19.309 & 32.913 \\
\hline 8/27/09 16:00 & 12.296 & 5.515 & 8.407 & 18.272 & 9.986 & 17.275 & 10.059 & 25.894 & 19.323 & 32.918 \\
\hline 8/27/09 20:00 & 12.313 & 5.520 & 8.424 & 18.265 & 10.010 & 17.307 & 10.071 & 24.979 & 19.339 & 32.925 \\
\hline 8/28/09 0:00 & 12.332 & 5.527 & 8.442 & 18.261 & 10.033 & 17.347 & 10.083 & 24.953 & 19.356 & 32.929 \\
\hline 8/28/09 4:00 & 12.339 & 5.529 & 8.457 & 18.296 & 10.052 & 17.357 & 10.085 & 24.991 & 19.372 & 32.936 \\
\hline 8/28/09 8:00 & 12.360 & 5.536 & 8.473 & 18.284 & 10.069 & 17.408 & 10.099 & 24.984 & 19.386 & 32.943 \\
\hline 8/28/09 12:00 & 12.379 & 5.541 & 8.492 & 18.347 & 10.085 & 17.450 & 10.111 & 25.019 & 19.403 & 32.950 \\
\hline 8/28/09 16:00 & 12.391 & 5.548 & 8.511 & 18.463 & 10.109 & 17.462 & 10.116 & 25.028 & 19.419 & 32.958 \\
\hline 8/28/09 20:00 & 12.409 & 5.557 & 8.529 & 18.431 & 10.137 & 17.476 & 10.130 & 25.047 & 19.435 & 32.965 \\
\hline 8/29/09 0:00 & 12.431 & 5.562 & 8.550 & 18.345 & 10.163 & 17.530 & 10.149 & 25.153 & 19.452 & 32.972 \\
\hline 8/29/09 4:00 & 12.445 & 5.567 & 8.567 & 18.326 & 10.186 & 17.556 & 10.158 & 25.158 & 19.466 & 32.979 \\
\hline 8/29/09 8:00 & 12.471 & 5.576 & 8.583 & 18.291 & 10.208 & 17.615 & 10.179 & 25.240 & 19.485 & 32.983 \\
\hline 8/29/09 12:00 & 12.490 & 5.583 & 8.597 & 18.373 & 10.229 & 17.664 & 10.196 & 25.230 & 19.499 & 32.991 \\
\hline 8/29/09 16:00 & 12.504 & 5.592 & 8.611 & 18.517 & 10.255 & 17.671 & 10.200 & 25.249 & 19.517 & 32.998 \\
\hline 8/29/09 20:00 & 12.527 & 5.602 & 8.623 & 18.438 & 10.288 & 17.708 & 10.221 & 25.315 & 19.534 & 33.007 \\
\hline 8/30/09 0:00 & 12.560 & 5.611 & 7.137 & 18.319 & 10.318 & 17.781 & 10.254 & 25.355 & 19.550 & 33.007 \\
\hline 8/30/09 4:00 & 12.586 & 5.618 & 7.116 & 18.300 & 10.347 & 17.837 & 10.278 & 25.409 & 19.567 & 33.016 \\
\hline 8/30/09 8:00 & 12.615 & 5.627 & 7.137 & 18.293 & 10.373 & 17.905 & 10.304 & 25.477 & 19.585 & 33.026 \\
\hline 8/30/09 12:00 & 12.636 & 5.632 & 7.156 & 18.424 & 10.401 & 17.945 & 10.320 & 25.546 & 19.604 & 33.033 \\
\hline 8/30/09 16:00 & 12.650 & 5.639 & 7.177 & 18.538 & 10.429 & 17.945 & 10.322 & 25.597 & 19.621 & 33.040 \\
\hline 8/30/09 20:00 & 12.669 & 5.646 & 7.170 & 18.512 & 10.460 & 17.969 & 10.334 & 25.569 & 19.639 & 33.047 \\
\hline 8/31/09 0:00 & 12.695 & 5.651 & 7.189 & 18.466 & 10.491 & 18.027 & 10.358 & 25.633 & 19.658 & 33.054 \\
\hline 8/31/09 4:00 & 12.711 & 5.656 & 6.978 & 18.487 & 10.517 & 18.051 & 10.367 & 25.691 & 19.675 & 33.064 \\
\hline 8/31/09 8:00 & 12.732 & 5.660 & 6.940 & 18.475 & 10.543 & 18.102 & 10.386 & 25.708 & 19.693 & 33.068 \\
\hline 8/31/09 12:00 & 12.754 & 5.665 & 6.954 & 18.529 & 10.571 & 18.144 & 10.398 & 25.767 & 19.712 & 33.075 \\
\hline 8/31/09 16:00 & 12.761 & 5.670 & 6.966 & 18.666 & 10.597 & 18.116 & 10.386 & 25.783 & 19.731 & 33.082 \\
\hline 8/31/09 20:00 & 12.772 & 5.677 & 6.978 & 18.657 & 10.627 & 18.114 & 10.393 & 25.743 & 19.747 & 33.092 \\
\hline 9/1/09 0:00 & 12.794 & 5.684 & 6.992 & 18.592 & 10.660 & 18.173 & 10.414 & 25.797 & 19.768 & 33.097 \\
\hline 9/1/09 4:00 & 12.808 & 5.686 & 7.008 & 18.582 & 10.689 & 18.196 & 10.426 & 25.847 & 19.785 & 33.108 \\
\hline 9/1/09 8:00 & 12.829 & 5.691 & 7.020 & 18.545 & 10.719 & 18.241 & 10.442 & 25.868 & 19.806 & 33.115 \\
\hline 9/1/09 12:00 & 12.845 & 5.695 & 7.027 & 18.645 & 10.745 & 18.273 & 10.452 & 25.929 & 19.822 & 33.120 \\
\hline 9/1/09 16:00 & 12.850 & 5.700 & 7.004 & 18.808 & 10.776 & 18.241 & 10.440 & 25.882 & 19.841 & 33.127 \\
\hline 9/1/09 20:00 & 12.862 & 5.705 & 6.943 & 18.762 & 10.811 & 18.236 & 10.447 & 25.879 & 19.858 & 33.137 \\
\hline 9/2/09 0:00 & 12.878 & 5.709 & 6.943 & 18.694 & 10.847 & 18.280 & 10.466 & 25.929 & 19.879 & 33.144 \\
\hline 9/2/09 4:00 & 12.888 & 5.712 & 6.947 & 18.689 & 10.877 & 18.297 & 10.470 & 25.985 & 19.895 & 33.151 \\
\hline 9/2/09 8:00 & 12.904 & 5.716 & 6.954 & 18.673 & 10.906 & 18.320 & 10.480 & 25.971 & 19.914 & 33.158 \\
\hline $9 / 2 / 09$ 12:00 & 12.923 & 5.721 & 6.971 & 18.685 & 10.939 & 18.374 & 10.496 & 26.014 & 19.933 & 33.167 \\
\hline 9/2/09 16:00 & 12.923 & 5.719 & 6.971 & 18.883 & 10.967 & 18.325 & 10.477 & 25.985 & 19.944 & 33.174 \\
\hline 9/2/09 20:00 & 12.937 & 5.726 & 6.968 & 18.815 & 11.000 & 18.348 & 10.494 & 25.997 & 19.961 & 33.184 \\
\hline 9/3/09 0:00 & 12.956 & 5.730 & 6.978 & 18.729 & 11.035 & 18.400 & 10.517 & 26.037 & 19.977 & 33.191 \\
\hline 9/3/09 4:00 & 12.968 & 5.735 & 6.990 & 18.731 & 11.068 & 18.402 & 10.520 & 26.044 & 19.996 & 33.200 \\
\hline 9/3/09 8:00 & 12.982 & 5.737 & 6.999 & 18.717 & 11.099 & 18.433 & 10.531 & 26.070 & 20.015 & 33.207 \\
\hline 9/3/09 12:00 & 13.001 & 5.742 & 7.011 & 18.703 & 11.130 & 18.489 & 10.550 & 26.115 & 20.031 & 33.217 \\
\hline 9/3/09 16:00 & 13.006 & 5.744 & 6.947 & 18.813 & 11.160 & 18.468 & 10.538 & 26.105 & 20.050 & 33.224 \\
\hline 9/3/09 20:00 & 13.020 & 5.747 & 6.900 & 18.799 & 11.193 & 18.477 & 10.550 & 26.110 & 20.066 & 33.233 \\
\hline 9/4/09 0:00 & 13.039 & 5.751 & 6.870 & 18.741 & 11.226 & 18.529 & 10.571 & 26.152 & 20.087 & 33.240 \\
\hline 9/4/09 4:00 & 13.053 & 5.751 & 6.858 & 18.755 & 11.257 & 18.548 & 10.578 & 26.171 & 20.106 & 33.250 \\
\hline 9/4/09 8:00 & 13.069 & 5.756 & 6.849 & 18.724 & 11.288 & 18.592 & 10.597 & 26.206 & 20.123 & 33.259 \\
\hline $9 / 4 / 09$ 12:00 & 13.088 & 5.761 & 6.851 & 18.762 & 11.318 & 18.630 & 10.607 & 26.244 & 20.141 & 33.266 \\
\hline 9/4/09 16:00 & 13.102 & 5.765 & 6.861 & 18.841 & 11.351 & 18.649 & 10.618 & 26.260 & 20.160 & 33.273 \\
\hline 9/4/09 20:00 & 13.121 & 5.772 & 6.868 & 18.820 & 11.389 & 18.672 & 10.637 & 26.284 & 20.179 & 33.280 \\
\hline 9/5/09 0:00 & 13.145 & 5.777 & 6.875 & 18.745 & 11.422 & 18.731 & 10.665 & 26.336 & 20.198 & 33.290 \\
\hline 9/5/09 4:00 & 13.161 & 5.782 & 6.882 & 18.741 & 11.455 & 18.756 & 10.677 & 26.364 & 20.219 & 33.299 \\
\hline 9/5/09 8:00 & 13.183 & 5.787 & 6.884 & 18.720 & 11.486 & 18.806 & 10.696 & 26.439 & 20.235 & 33.306 \\
\hline
\end{tabular}

TABLE S1.1 Page 12 of 89 
Water Level (ft below reference point)

\begin{tabular}{|c|c|c|c|c|c|c|c|c|c|c|}
\hline Date and Time & McPike & Potter & Hughes & Barr & Burks & MoDOT & Clizer "Old" & Morgan & SB24 & SB38 \\
\hline 9/5/09 12:00 & 13.204 & 5.791 & 6.893 & 18.785 & 11.516 & 18.860 & 10.715 & 26.446 & 20.256 & 33.316 \\
\hline 9/5/09 16:00 & 13.218 & 5.796 & 6.905 & 18.929 & 11.557 & 18.860 & 10.712 & 26.463 & 20.275 & 33.325 \\
\hline 9/5/09 20:00 & 13.237 & 5.801 & 6.917 & 18.878 & 11.597 & 18.871 & 10.733 & 26.505 & 20.294 & 33.334 \\
\hline 9/6/09 0:00 & 13.261 & 5.805 & 6.931 & 18.792 & 11.634 & 18.925 & 10.759 & 26.517 & 20.315 & 33.344 \\
\hline 9/6/09 4:00 & 13.279 & 5.808 & 6.943 & 18.787 & 11.672 & 18.949 & 10.769 & 26.552 & 20.334 & 33.349 \\
\hline 9/6/09 8:00 & 13.298 & 5.812 & 6.954 & 18.759 & 11.705 & 18.993 & 10.783 & 26.608 & 20.352 & 33.360 \\
\hline 9/6/09 12:00 & 13.317 & 5.815 & 6.964 & 18.866 & 11.741 & 19.026 & 10.795 & 26.620 & 20.371 & 33.370 \\
\hline 9/6/09 16:00 & 13.329 & 5.819 & 6.978 & 19.044 & 11.778 & 19.003 & 10.778 & 26.615 & 20.390 & 33.377 \\
\hline 9/6/09 20:00 & 13.340 & 5.822 & 6.992 & 18.992 & 11.818 & 18.998 & 10.792 & 26.627 & 20.409 & 33.386 \\
\hline 9/7/09 0:00 & 13.362 & 5.826 & 7.006 & 18.885 & 11.856 & 19.052 & 10.820 & 26.651 & 20.430 & 33.396 \\
\hline 9/7/09 4:00 & 13.374 & 5.826 & 7.015 & 18.894 & 11.891 & 19.061 & 10.818 & 26.677 & 20.449 & 33.405 \\
\hline 9/7/09 8:00 & 13.388 & 5.829 & 7.027 & 18.885 & 11.924 & 19.082 & 10.823 & 26.721 & 20.467 & 33.415 \\
\hline 9/7/09 12:00 & 13.402 & 5.831 & 7.039 & 18.915 & 11.957 & 19.117 & 10.827 & 26.731 & 20.486 & 33.424 \\
\hline 9/7/09 16:00 & 13.411 & 5.831 & 7.037 & 19.069 & 11.993 & 19.099 & 10.816 & 26.717 & 20.505 & 33.431 \\
\hline 9/7/09 20:00 & 13.421 & 5.833 & 7.046 & 19.037 & 12.031 & 19.085 & 10.823 & 26.702 & 20.524 & 33.440 \\
\hline 9/8/09 0:00 & 13.437 & 5.836 & 7.058 & 18.957 & 12.071 & 19.125 & 10.846 & 26.773 & 20.545 & 33.450 \\
\hline 9/8/09 4:00 & 13.451 & 5.838 & 7.069 & 18.946 & 12.108 & 19.148 & 10.849 & 26.749 & 20.564 & 33.457 \\
\hline 9/8/09 8:00 & 13.468 & 5.843 & 7.081 & 18.920 & 12.144 & 19.185 & 10.867 & 26.782 & 20.582 & 33.466 \\
\hline 9/8/09 12:00 & 13.487 & 5.847 & 7.090 & 18.948 & 12.177 & 19.228 & 10.886 & 26.839 & 20.603 & 33.476 \\
\hline 9/8/09 16:00 & 13.499 & 5.847 & 7.100 & 19.006 & 12.203 & 19.228 & 10.879 & 26.813 & 20.620 & 33.485 \\
\hline 9/8/09 20:00 & 13.513 & 5.850 & 7.112 & 19.020 & 12.238 & 19.225 & 10.893 & 26.841 & 20.641 & 33.495 \\
\hline 9/9/09 0:00 & 13.536 & 5.857 & 7.126 & 18.918 & 12.276 & 19.307 & 10.938 & 26.900 & 20.660 & 33.504 \\
\hline 9/9/09 4:00 & 13.555 & 5.857 & 7.137 & 18.932 & 12.311 & 19.331 & 10.943 & 26.928 & 20.678 & 33.513 \\
\hline 9/9/09 8:00 & 13.574 & 5.861 & 7.149 & 18.911 & 12.344 & 19.378 & 10.961 & 26.963 & 20.697 & 33.521 \\
\hline 9/9/09 12:00 & 13.598 & 5.866 & 7.163 & 18.932 & 12.380 & 19.432 & 10.982 & 27.015 & 20.718 & 33.532 \\
\hline 9/9/09 16:00 & 13.609 & 5.859 & 7.156 & 18.983 & 12.375 & 19.366 & 11.032 & 27.022 & 20.737 & 33.539 \\
\hline 9/9/09 20:00 & 13.621 & 5.852 & 7.126 & 18.983 & 12.415 & 19.394 & 10.959 & 27.032 & 20.756 & 33.549 \\
\hline 9/10/09 0:00 & 13.640 & 5.854 & 7.116 & 18.911 & 12.450 & 19.462 & 10.943 & 27.088 & 20.775 & 33.556 \\
\hline 9/10/09 4:00 & 13.654 & 5.852 & 7.114 & 18.939 & 12.488 & 19.472 & 10.928 & 27.107 & 20.796 & 33.568 \\
\hline 9/10/09 8:00 & 13.675 & 5.854 & 7.119 & 18.913 & 12.526 & 19.514 & 10.964 & 27.145 & 20.814 & 33.575 \\
\hline 9/10/09 12:00 & 13.694 & 5.859 & 7.128 & 18.995 & 12.561 & 19.554 & 11.112 & 27.180 & 20.833 & 33.584 \\
\hline 9/10/09 16:00 & 13.706 & 5.861 & 7.140 & 19.174 & 12.604 & 19.537 & 11.135 & 27.175 & 20.852 & 33.594 \\
\hline 9/10/09 20:00 & 13.722 & 5.864 & 7.152 & 19.125 & 12.648 & 19.542 & 11.004 & 27.177 & 20.871 & 33.603 \\
\hline 9/11/09 0:00 & 13.746 & 5.869 & 7.166 & 19.034 & 12.691 & 19.589 & 10.999 & 27.222 & 20.890 & 33.612 \\
\hline 9/11/09 4:00 & 13.755 & 5.866 & 7.177 & 19.058 & 12.731 & 19.579 & 11.018 & 27.222 & 20.908 & 33.622 \\
\hline 9/11/09 8:00 & 13.772 & 5.868 & 7.187 & 19.032 & 12.769 & 19.612 & 11.034 & 27.243 & 20.929 & 33.634 \\
\hline 9/11/09 12:00 & 13.791 & 5.871 & 7.198 & 19.097 & 12.809 & 19.638 & 11.227 & 27.269 & 20.948 & 33.641 \\
\hline 9/11/09 16:00 & 13.800 & 5.871 & 7.208 & 19.251 & 12.849 & 19.615 & 11.222 & 27.253 & 20.967 & 33.650 \\
\hline 9/11/09 20:00 & 13.817 & 5.875 & 7.220 & 19.167 & 12.894 & 19.626 & 11.086 & 27.260 & 20.986 & 33.659 \\
\hline 9/12/09 0:00 & 13.838 & 5.878 & 7.234 & 19.088 & 12.939 & 19.668 & 11.130 & 27.297 & 21.004 & 33.671 \\
\hline 9/12/09 4:00 & 13.852 & 5.880 & 7.243 & 19.088 & 12.979 & 19.671 & 11.130 & 27.304 & 21.023 & 33.678 \\
\hline 9/12/09 8:00 & 13.869 & 5.880 & 7.255 & 19.074 & 13.019 & 19.697 & 11.140 & 27.328 & 21.042 & 33.688 \\
\hline 9/12/09 12:00 & 13.890 & 5.885 & 7.271 & 19.132 & 13.061 & 19.741 & 11.159 & 27.361 & 21.063 & 33.697 \\
\hline 9/12/09 16:00 & 13.904 & 5.887 & 7.285 & 19.253 & 13.104 & 19.734 & 11.159 & 27.358 & 21.082 & 33.707 \\
\hline 9/12/09 20:00 & 13.923 & 5.892 & 7.302 & 19.181 & 13.148 & 19.751 & 11.189 & 27.375 & 21.101 & 33.716 \\
\hline 9/13/09 0:00 & 13.949 & 5.897 & 7.318 & 19.085 & 13.191 & 19.809 & 11.217 & 27.422 & 21.119 & 33.728 \\
\hline 9/13/09 4:00 & 13.970 & 5.899 & 7.332 & 19.083 & 13.233 & 19.830 & 11.224 & 27.445 & 21.138 & 33.737 \\
\hline 9/13/09 8:00 & 13.991 & 5.901 & 7.346 & 19.078 & 13.273 & 19.861 & 11.243 & 27.471 & 21.159 & 33.747 \\
\hline 9/13/09 12:00 & 14.015 & 5.903 & 7.358 & 19.141 & 13.311 & 19.903 & 11.253 & 27.511 & 21.180 & 33.756 \\
\hline 9/13/09 16:00 & 14.027 & 5.901 & 7.374 & 19.304 & 13.356 & 19.870 & 11.236 & 27.490 & 21.199 & 33.765 \\
\hline 9/13/09 20:00 & 14.041 & 5.903 & 7.389 & 19.253 & 13.401 & 19.875 & 11.255 & 27.493 & 21.216 & 33.775 \\
\hline 9/14/09 0:00 & 14.062 & 5.906 & 7.405 & 19.188 & 13.443 & 19.910 & 11.274 & 27.521 & 21.237 & 33.784 \\
\hline 9/14/09 4:00 & 14.081 & 5.906 & 7.417 & 19.188 & 13.486 & 19.926 & 11.276 & 27.540 & 21.255 & 33.794 \\
\hline 9/14/09 8:00 & 14.100 & 5.908 & 7.431 & 19.151 & 13.526 & 19.962 & 11.297 & 27.565 & 21.274 & 33.806 \\
\hline 9/14/09 12:00 & 14.118 & 5.910 & 7.445 & 19.225 & 13.566 & 19.987 & 11.297 & 27.594 & 21.293 & 33.815 \\
\hline 9/14/09 16:00 & 14.133 & 5.913 & 7.457 & 19.325 & 13.606 & 19.973 & 11.297 & 27.582 & 21.314 & 33.824 \\
\hline 9/14/09 20:00 & 14.149 & 5.915 & 7.471 & 19.284 & 13.648 & 19.987 & 11.316 & 27.594 & 21.333 & 33.834 \\
\hline 9/15/09 0:00 & 14.168 & 5.918 & 7.485 & 19.228 & 13.688 & 20.020 & 11.333 & 27.622 & 21.352 & 33.843 \\
\hline 9/15/09 4:00 & 14.189 & 5.920 & 7.497 & 19.207 & 13.729 & 20.046 & 11.347 & 27.645 & 21.370 & 33.853 \\
\hline 9/15/09 8:00 & 14.210 & 5.925 & 7.511 & 19.195 & 13.769 & 20.081 & 11.363 & 27.676 & 21.391 & 33.864 \\
\hline 9/15/09 12:00 & 14.234 & 5.929 & 7.522 & 19.244 & 13.809 & 20.114 & 11.377 & 27.763 & 21.410 & 33.874 \\
\hline 9/15/09 16:00 & 14.255 & 5.934 & 7.536 & 19.323 & 13.849 & 20.116 & 11.384 & 27.728 & 21.429 & 33.883 \\
\hline 9/15/09 20:00 & 14.279 & 5.941 & 7.553 & 19.291 & 13.891 & 20.151 & 11.415 & 27.817 & 21.448 & 33.893 \\
\hline 9/16/09 0:00 & 14.307 & 5.946 & 7.567 & 19.232 & 13.934 & 20.194 & 11.438 & 27.770 & 21.466 & 33.902 \\
\hline
\end{tabular}


Water Level (ft below reference point)

\begin{tabular}{|c|c|c|c|c|c|c|c|c|c|c|}
\hline Date and Time & McPike & Potter & Hughes & Barr & Burks & MoDOT & Clizer "Old" & Morgan & SB24 & SB38 \\
\hline 9/16/09 4:00 & 14.333 & 5.948 & 7.581 & 19.214 & 13.974 & 20.226 & 11.452 & 27.815 & 21.488 & 33.914 \\
\hline 9/16/09 8:00 & 14.359 & 5.953 & 7.597 & 19.193 & 14.012 & 20.276 & 11.476 & 27.899 & 21.506 & 33.921 \\
\hline 9/16/09 12:00 & 14.387 & 5.957 & 7.609 & 19.288 & 14.052 & 20.306 & 11.485 & 27.871 & 21.527 & 33.933 \\
\hline 9/16/09 16:00 & 14.404 & 5.960 & 7.623 & 19.447 & 14.092 & 20.283 & 11.480 & 27.890 & 21.546 & 33.942 \\
\hline 9/16/09 20:00 & 14.430 & 5.967 & 7.640 & 19.365 & 14.136 & 20.318 & 11.513 & 27.906 & 21.565 & 33.952 \\
\hline 9/17/09 0:00 & 14.455 & 5.969 & 7.656 & 19.307 & 14.177 & 20.351 & 11.532 & 27.939 & 21.586 & 33.961 \\
\hline 9/17/09 4:00 & 14.481 & 5.971 & 7.673 & 19.298 & 14.214 & 20.377 & 11.544 & 27.965 & 21.605 & 33.975 \\
\hline 9/17/09 8:00 & 14.507 & 5.976 & 7.684 & 19.272 & 14.252 & 20.409 & 11.565 & 27.993 & 21.626 & 33.980 \\
\hline 9/17/09 12:00 & 14.533 & 5.978 & 7.698 & 19.365 & 14.292 & 20.440 & 11.565 & 28.024 & 21.645 & 33.992 \\
\hline 9/17/09 16:00 & 14.547 & 5.981 & 7.712 & 19.510 & 14.330 & 20.412 & 11.553 & 28.012 & 21.666 & 34.003 \\
\hline 9/17/09 20:00 & 14.566 & 5.983 & 7.727 & 19.456 & 14.372 & 20.426 & 11.577 & 28.019 & 21.685 & 34.013 \\
\hline 9/18/09 0:00 & 14.590 & 5.986 & 7.743 & 19.384 & 14.412 & 20.459 & 11.596 & 28.050 & 21.706 & 34.025 \\
\hline 9/18/09 4:00 & 14.611 & 5.988 & 7.755 & 19.377 & 14.450 & 20.480 & 11.607 & 28.071 & 21.724 & 34.034 \\
\hline 9/18/09 8:00 & 14.639 & 5.993 & 7.769 & 19.332 & 14.486 & 20.529 & 11.633 & 28.106 & 21.746 & 34.043 \\
\hline 9/18/09 12:00 & 14.665 & 5.997 & 7.783 & 19.405 & 14.523 & 20.562 & 11.647 & 28.424 & 21.767 & 34.053 \\
\hline 9/18/09 16:00 & 14.684 & 6.002 & 7.797 & 19.572 & 14.559 & 20.545 & 11.636 & 29.357 & 21.788 & 34.062 \\
\hline 9/18/09 20:00 & 14.705 & 6.007 & 7.811 & 19.505 & 14.599 & 20.566 & 11.664 & 28.370 & 21.807 & 34.074 \\
\hline 9/19/09 0:00 & 14.736 & 6.011 & 7.825 & 19.433 & 14.639 & 20.609 & 11.685 & 28.313 & 21.828 & 34.086 \\
\hline 9/19/09 4:00 & 14.757 & 6.011 & 7.839 & 19.435 & 14.677 & 20.627 & 11.692 & 28.280 & 21.846 & 34.093 \\
\hline 9/19/09 8:00 & 14.781 & 6.016 & 7.851 & 19.430 & 14.710 & 20.646 & 11.706 & 28.276 & 21.868 & 34.105 \\
\hline 9/19/09 12:00 & 14.804 & 6.018 & 7.865 & 19.521 & 14.745 & 20.672 & 11.711 & 28.287 & 21.889 & 34.114 \\
\hline 9/19/09 16:00 & 14.814 & 6.018 & 7.877 & 19.694 & 14.783 & 20.637 & 11.690 & 28.259 & 21.907 & 34.121 \\
\hline 9/19/09 20:00 & 14.826 & 6.018 & 7.891 & 19.645 & 14.820 & 20.627 & 11.704 & 28.243 & 21.926 & 34.133 \\
\hline 9/20/09 0:00 & 14.842 & 6.021 & 7.905 & 19.570 & 14.858 & 20.653 & 11.718 & 28.276 & 21.947 & 34.145 \\
\hline 9/20/09 4:00 & 14.854 & 6.018 & 7.917 & 19.598 & 14.896 & 20.644 & 11.704 & 28.245 & 21.966 & 34.157 \\
\hline 9/20/09 8:00 & 14.868 & 6.018 & 7.926 & 19.572 & 14.922 & 20.663 & 11.718 & 28.254 & 21.985 & 34.166 \\
\hline 9/20/09 12:00 & 14.877 & 6.023 & 7.935 & 19.668 & 14.952 & 20.660 & 11.711 & 28.276 & 22.006 & 34.175 \\
\hline 9/20/09 16:00 & 14.882 & 6.023 & 7.945 & 19.768 & 14.985 & 20.625 & 11.692 & 28.283 & 22.025 & 34.185 \\
\hline 9/20/09 20:00 & 14.889 & 6.025 & 7.959 & 19.689 & 15.018 & 20.625 & 11.711 & 28.224 & 22.043 & 34.197 \\
\hline 9/21/09 0:00 & 14.906 & 6.030 & 7.971 & 19.591 & 15.054 & 20.665 & 11.727 & 28.254 & 22.062 & 34.206 \\
\hline 9/21/09 4:00 & 14.915 & 6.028 & 7.980 & 19.612 & 15.087 & 20.653 & 11.718 & 28.278 & 22.081 & 34.215 \\
\hline 9/21/09 8:00 & 14.929 & 6.032 & 7.989 & 19.570 & 15.118 & 20.677 & 11.741 & 28.261 & 22.102 & 34.225 \\
\hline 9/21/09 12:00 & 14.934 & 6.032 & 7.987 & 19.502 & 15.096 & 20.567 & 11.734 & 28.290 & 22.121 & 34.234 \\
\hline 9/21/09 16:00 & 14.925 & 6.032 & 7.921 & 19.440 & 15.118 & 20.498 & 11.727 & 28.283 & 22.137 & 34.246 \\
\hline 9/21/09 20:00 & 14.936 & 6.042 & 7.860 & 19.274 & 15.151 & 20.566 & 11.762 & 28.337 & 22.158 & 34.255 \\
\hline 9/22/09 0:00 & 14.948 & 6.046 & 7.827 & 19.274 & 15.179 & 20.604 & 11.772 & 28.330 & 22.177 & 34.263 \\
\hline 9/22/09 4:00 & 14.962 & 6.049 & 7.809 & 19.274 & 15.210 & 20.637 & 11.779 & 28.355 & 22.196 & 34.274 \\
\hline 9/22/09 8:00 & 14.974 & 6.053 & 7.802 & 19.300 & 15.240 & 20.653 & 11.793 & 28.391 & 22.215 & 34.284 \\
\hline 9/22/09 12:00 & 14.998 & 6.058 & 7.802 & 19.370 & 15.271 & 20.695 & 11.812 & 28.388 & 22.233 & 34.293 \\
\hline 9/22/09 16:00 & 15.016 & 6.060 & 7.806 & 19.449 & 15.306 & 20.717 & 11.819 & 28.405 & 22.252 & 34.305 \\
\hline 9/22/09 20:00 & 15.035 & 6.067 & 7.811 & 19.416 & 15.339 & 20.745 & 11.845 & 28.428 & 22.271 & 34.312 \\
\hline 9/23/09 0:00 & 15.064 & 6.072 & 7.820 & 19.393 & 15.370 & 20.785 & 11.866 & 28.492 & 22.290 & 34.324 \\
\hline 9/23/09 4:00 & 15.087 & 6.074 & 7.832 & 19.428 & 15.401 & 20.806 & 11.877 & 28.461 & 22.308 & 34.331 \\
\hline 9/23/09 8:00 & 15.111 & 6.077 & 7.844 & 19.395 & 15.431 & 20.848 & 11.899 & 28.499 & 22.330 & 34.343 \\
\hline 9/23/09 12:00 & 15.141 & 6.081 & 7.858 & 19.456 & 15.462 & 20.890 & 11.917 & 28.560 & 22.348 & 34.354 \\
\hline 9/23/09 16:00 & 15.160 & 6.084 & 7.872 & 19.598 & 15.492 & 20.878 & 11.913 & 28.520 & 22.367 & 34.364 \\
\hline 9/23/09 20:00 & 15.179 & 6.088 & 7.886 & 19.582 & 15.528 & 20.899 & 11.932 & 28.541 & 22.386 & 34.371 \\
\hline 9/24/09 0:00 & 15.203 & 6.091 & 7.903 & 19.542 & 15.559 & 20.923 & 11.946 & 28.572 & 22.405 & 34.385 \\
\hline 9/24/09 4:00 & 15.224 & 6.088 & 7.917 & 19.554 & 15.592 & 20.932 & 11.946 & 28.609 & 22.423 & 34.390 \\
\hline 9/24/09 8:00 & 15.240 & 6.093 & 7.931 & 19.561 & 15.622 & 20.951 & 11.962 & 28.579 & 22.445 & 34.404 \\
\hline 9/24/09 12:00 & 15.264 & 6.095 & 7.947 & 19.617 & 15.651 & 20.977 & 11.964 & 28.607 & 22.463 & 34.416 \\
\hline 9/24/09 16:00 & 15.273 & 6.095 & 7.959 & 19.789 & 15.681 & 20.956 & 11.962 & 28.605 & 22.480 & 34.425 \\
\hline 9/24/09 20:00 & 15.292 & 6.100 & 7.975 & 19.691 & 15.714 & 20.977 & 11.983 & 28.661 & 22.501 & 34.434 \\
\hline 9/25/09 0:00 & 15.311 & 6.100 & 7.987 & 19.666 & 15.745 & 20.988 & 11.988 & 28.616 & 22.520 & 34.444 \\
\hline 9/25/09 4:00 & 15.325 & 6.098 & 8.001 & 19.682 & 15.773 & 20.986 & 11.986 & 28.626 & 22.538 & 34.453 \\
\hline 9/25/09 8:00 & 15.342 & 6.102 & 8.013 & 19.640 & 15.801 & 21.024 & 12.004 & 28.663 & 22.555 & 34.467 \\
\hline 9/25/09 12:00 & 15.363 & 6.102 & 8.025 & 19.701 & 15.832 & 21.040 & 12.011 & 28.720 & 22.576 & 34.477 \\
\hline 9/25/09 16:00 & 15.375 & 6.105 & 8.036 & 19.854 & 15.860 & 21.024 & 12.004 & 28.645 & 22.592 & 34.486 \\
\hline 9/25/09 20:00 & 15.389 & 6.109 & 8.050 & 19.791 & 15.893 & 21.031 & 12.030 & 28.661 & 22.611 & 34.496 \\
\hline 9/26/09 0:00 & 15.382 & 6.105 & 8.027 & 19.659 & 15.879 & 20.914 & 11.997 & 28.701 & 22.630 & 34.500 \\
\hline 9/26/09 4:00 & 15.342 & 6.098 & 7.898 & 19.579 & 15.886 & 20.724 & 11.953 & 28.631 & 22.649 & 34.512 \\
\hline 9/26/09 8:00 & 15.295 & 6.093 & 7.748 & 19.526 & 15.910 & 20.693 & 11.922 & 28.616 & 22.665 & 34.517 \\
\hline 9/26/09 12:00 & 15.257 & 6.095 & 7.637 & 19.507 & 15.936 & 20.691 & 11.903 & 28.562 & 22.684 & 34.531 \\
\hline 9/26/09 16:00 & 15.226 & 6.093 & 7.569 & 19.591 & 15.964 & 20.656 & 11.877 & 28.553 & 22.698 & 34.538 \\
\hline
\end{tabular}


Water Level (ft below reference point)

\begin{tabular}{|c|c|c|c|c|c|c|c|c|c|c|}
\hline Date and Time & McPike & Potter & Hughes & Barr & Burks & MoDOT & Clizer "Old" & Morgan & SB24 & SB38 \\
\hline 9/26/09 20:00 & 15.207 & 6.095 & 7.525 & 19.577 & 15.990 & 20.646 & 11.873 & 28.525 & 22.717 & 34.550 \\
\hline 9/27/09 0:00 & 15.191 & 6.093 & 7.499 & 19.579 & 16.018 & 20.625 & 11.854 & 28.497 & 22.733 & 34.557 \\
\hline 9/27/09 4:00 & 15.172 & 6.088 & 7.478 & 19.605 & 16.042 & 20.588 & 11.826 & 28.461 & 22.749 & 34.566 \\
\hline 9/27/09 8:00 & 15.148 & 6.081 & 7.464 & 19.624 & 16.068 & 20.543 & 11.800 & 28.414 & 22.766 & 34.576 \\
\hline 9/27/09 12:00 & 15.123 & 6.074 & 7.457 & 19.719 & 16.094 & 20.494 & 11.765 & 28.363 & 22.780 & 34.585 \\
\hline 9/27/09 16:00 & 15.123 & 6.086 & 7.461 & 19.752 & 16.120 & 20.534 & 11.809 & 28.384 & 22.796 & 34.595 \\
\hline 9/27/09 20:00 & 15.172 & 6.109 & 7.485 & 19.430 & 16.150 & 20.681 & 11.917 & 28.426 & 22.813 & 34.604 \\
\hline 9/28/09 0:00 & 15.241 & 6.128 & 7.515 & 19.253 & 16.181 & 20.815 & 12.000 & 28.527 & 22.829 & 34.618 \\
\hline 9/28/09 4:00 & 15.292 & 6.138 & 7.541 & 19.304 & 16.209 & 20.871 & 12.033 & 28.591 & 22.846 & 34.625 \\
\hline 9/28/09 8:00 & 15.335 & 6.149 & 7.565 & 19.300 & 16.235 & 20.930 & 12.063 & 28.671 & 22.864 & 34.635 \\
\hline 9/28/09 12:00 & 15.372 & 6.152 & 7.593 & 19.407 & 16.264 & 20.972 & 12.077 & 28.795 & 22.881 & 34.644 \\
\hline 9/28/09 16:00 & 15.396 & 6.154 & 7.616 & 19.619 & 16.290 & 20.979 & 12.077 & 28.765 & 22.900 & 34.654 \\
\hline 9/28/09 20:00 & 15.422 & 6.159 & 7.644 & 19.561 & 16.316 & 21.012 & 12.098 & 28.692 & 22.916 & 34.661 \\
\hline 9/29/09 0:00 & 15.450 & 6.163 & 7.673 & 19.493 & 16.341 & 21.066 & 12.126 & 28.736 & 22.932 & 34.670 \\
\hline 9/29/09 4:00 & 15.483 & 6.166 & 7.698 & 19.521 & 16.367 & 21.096 & 12.141 & 28.786 & 22.951 & 34.682 \\
\hline 9/29/09 8:00 & 15.512 & 6.170 & 7.722 & 19.514 & 16.391 & 21.141 & 12.164 & 28.781 & 22.968 & 34.694 \\
\hline 9/29/09 12:00 & 15.542 & 6.173 & 7.745 & 19.633 & 16.417 & 21.164 & 12.166 & 28.868 & 22.984 & 34.701 \\
\hline 9/29/09 16:00 & 15.549 & 6.168 & 7.764 & 19.873 & 16.443 & 21.127 & 12.138 & 28.788 & 23.003 & 34.710 \\
\hline 9/29/09 20:00 & 15.559 & 6.166 & 7.785 & 19.845 & 16.469 & 21.120 & 12.143 & 28.788 & 23.019 & 34.719 \\
\hline 9/30/09 0:00 & 15.575 & 6.166 & 7.806 & 19.791 & 16.495 & 21.139 & 12.155 & 28.828 & 23.038 & 34.729 \\
\hline 9/30/09 4:00 & 15.587 & 6.166 & 7.827 & 19.791 & 16.521 & 21.143 & 12.155 & 28.798 & 23.054 & 34.741 \\
\hline 9/30/09 8:00 & 15.603 & 6.166 & 7.844 & 19.777 & 16.544 & 21.157 & 12.159 & 28.809 & 23.071 & 34.750 \\
\hline 9/30/09 12:00 & 15.615 & 6.163 & 7.865 & 19.845 & 16.570 & 21.164 & 12.148 & 28.828 & 23.087 & 34.757 \\
\hline 9/30/09 16:00 & 15.604 & 6.154 & 7.881 & 20.017 & 16.594 & 21.092 & 12.098 & 28.802 & 23.104 & 34.769 \\
\hline 9/30/09 20:00 & 15.592 & 6.152 & 7.896 & 19.989 & 16.622 & 21.052 & 12.082 & 28.776 & 23.120 & 34.776 \\
\hline 10/1/09 0:00 & 15.589 & 6.152 & 7.912 & 19.966 & 16.646 & 21.047 & 12.077 & 28.746 & 23.136 & 34.788 \\
\hline 10/1/09 4:00 & 15.568 & 6.145 & 7.921 & 19.952 & 16.643 & 20.960 & 12.051 & 28.715 & 23.153 & 34.797 \\
\hline 10/1/09 8:00 & 15.542 & 6.140 & 7.905 & 19.929 & 16.662 & 20.918 & 12.028 & 28.694 & 23.169 & 34.807 \\
\hline 10/1/09 12:00 & 15.519 & 6.135 & 7.889 & 19.957 & 16.683 & 20.890 & 12.004 & 28.661 & 23.183 & 34.818 \\
\hline 10/1/09 16:00 & 15.526 & 6.147 & 7.886 & 19.780 & 16.707 & 20.967 & 12.051 & 28.689 & 23.202 & 34.825 \\
\hline 10/1/09 20:00 & 15.571 & 6.163 & 7.900 & 19.628 & 16.731 & 21.066 & 12.119 & 28.755 & 23.214 & 34.835 \\
\hline 10/2/09 0:00 & 15.604 & 6.168 & 7.914 & 19.661 & 16.754 & 21.082 & 12.126 & 28.781 & 23.230 & 34.844 \\
\hline 10/2/09 4:00 & 15.606 & 6.163 & 7.924 & 19.738 & 16.778 & 21.052 & 12.103 & 28.760 & 23.247 & 34.849 \\
\hline 10/2/09 8:00 & 15.606 & 6.163 & 7.931 & 19.770 & 16.799 & 21.050 & 12.101 & 28.743 & 23.261 & 34.861 \\
\hline 10/2/09 12:00 & 15.622 & 6.170 & 7.945 & 19.761 & 16.825 & 21.089 & 12.119 & 28.760 & 23.275 & 34.870 \\
\hline 10/2/09 16:00 & 15.639 & 6.175 & 7.964 & 19.777 & 16.851 & 21.115 & 12.143 & 28.776 & 23.287 & 34.880 \\
\hline 10/2/09 20:00 & 15.674 & 6.185 & 7.985 & 19.698 & 16.872 & 21.185 & 12.190 & 28.830 & 23.305 & 34.891 \\
\hline 10/3/09 0:00 & 15.707 & 6.189 & 8.004 & 19.703 & 16.896 & 21.230 & 12.209 & 28.868 & 23.319 & 34.901 \\
\hline 10/3/09 4:00 & 15.736 & 6.194 & 8.022 & 19.715 & 16.919 & 21.263 & 12.230 & 28.896 & 23.336 & 34.913 \\
\hline 10/3/09 8:00 & 15.766 & 6.198 & 8.041 & 19.722 & 16.943 & 21.307 & 12.256 & 28.929 & 23.347 & 34.917 \\
\hline 10/3/09 12:00 & 15.806 & 6.205 & 8.060 & 19.745 & 16.964 & 21.366 & 12.286 & 28.974 & 23.364 & 34.927 \\
\hline 10/3/09 16:00 & 15.839 & 6.210 & 8.079 & 19.803 & 16.988 & 21.399 & 12.298 & 29.019 & 23.378 & 34.936 \\
\hline 10/3/09 20:00 & 15.872 & 6.215 & 8.095 & 19.838 & 17.011 & 21.425 & 12.317 & 29.108 & 23.394 & 34.946 \\
\hline 10/4/09 0:00 & 15.910 & 6.222 & 8.116 & 19.794 & 17.035 & 21.483 & 12.350 & 29.068 & 23.411 & 34.957 \\
\hline $10 / 4 / 094: 00$ & 15.945 & 6.224 & 8.133 & 19.826 & 17.058 & 21.507 & 12.359 & 29.110 & 23.427 & 34.967 \\
\hline 10/4/09 8:00 & 15.976 & 6.227 & 8.151 & 19.836 & 17.082 & 21.535 & 12.376 & 29.164 & 23.439 & 34.972 \\
\hline 10/4/09 12:00 & 16.009 & 6.231 & 8.168 & 19.889 & 17.103 & 21.572 & 12.392 & 29.155 & 23.455 & 34.983 \\
\hline 10/4/09 16:00 & 16.021 & 6.229 & 8.182 & 20.080 & 17.127 & 21.542 & 12.371 & 29.150 & 23.472 & 34.993 \\
\hline 10/4/09 20:00 & 16.040 & 6.231 & 8.198 & 20.052 & 17.150 & 21.554 & 12.387 & 29.176 & 23.488 & 35.002 \\
\hline 10/5/09 0:00 & 16.065 & 6.234 & 8.217 & 19.996 & 17.176 & 21.589 & 12.404 & 29.240 & 23.505 & 35.012 \\
\hline 10/5/09 4:00 & 16.080 & 6.234 & 8.231 & 20.022 & 17.200 & 21.586 & 12.394 & 29.193 & 23.519 & 35.023 \\
\hline 10/5/09 8:00 & 16.091 & 6.233 & 8.243 & 20.020 & 17.219 & 21.591 & 12.399 & 29.207 & 23.535 & 35.028 \\
\hline 10/5/09 12:00 & 16.098 & 6.229 & 8.257 & 20.120 & 17.242 & 21.575 & 12.376 & 29.221 & 23.552 & 35.040 \\
\hline 10/5/09 16:00 & 16.082 & 6.220 & 8.266 & 20.299 & 17.264 & 21.500 & 12.324 & 29.221 & 23.566 & 35.049 \\
\hline 10/5/09 20:00 & 16.068 & 6.213 & 8.276 & 20.288 & 17.290 & 21.474 & 12.307 & 29.127 & 23.582 & 35.059 \\
\hline 10/6/09 0:00 & 16.061 & 6.210 & 8.287 & 20.271 & 17.308 & 21.455 & 12.293 & 29.143 & 23.598 & 35.068 \\
\hline $10 / 6 / 094: 00$ & 16.047 & 6.210 & 8.292 & 20.222 & 17.325 & 21.425 & 12.289 & 29.122 & 23.615 & 35.078 \\
\hline 10/6/09 8:00 & 16.049 & 6.217 & 8.285 & 20.097 & 17.344 & 21.469 & 12.324 & 29.143 & 23.629 & 35.082 \\
\hline 10/6/09 12:00 & 16.075 & 6.231 & 8.287 & 19.999 & 17.367 & 21.535 & 12.368 & 29.183 & 23.643 & 35.094 \\
\hline 10/6/09 16:00 & 16.106 & 6.241 & 8.292 & 20.024 & 17.389 & 21.584 & 12.399 & 29.216 & 23.662 & 35.103 \\
\hline 10/6/09 20:00 & 16.143 & 6.252 & 8.306 & 19.913 & 17.410 & 21.659 & 12.444 & 29.270 & 23.678 & 35.115 \\
\hline 10/7/09 0:00 & 16.188 & 6.259 & 8.320 & 19.917 & 17.433 & 21.713 & 12.469 & 29.322 & 23.697 & 35.125 \\
\hline 10/7/09 4:00 & 16.219 & 6.264 & 8.332 & 19.964 & 17.452 & 21.734 & 12.481 & 29.348 & 23.713 & 35.134 \\
\hline 10/7/09 8:00 & 16.247 & 6.266 & 8.344 & 19.980 & 17.471 & 21.762 & 12.498 & 29.374 & 23.730 & 35.143 \\
\hline
\end{tabular}


Water Level (ft below reference point)

\begin{tabular}{|c|c|c|c|c|c|c|c|c|c|c|}
\hline Date and Time & McPike & Potter & Hughes & Barr & Burks & MoDOT & Clizer "Old" & Morgan & SB24 & SB38 \\
\hline 10/7/09 12:00 & 16.268 & 6.266 & 8.356 & 20.071 & 17.492 & 21.762 & 12.488 & 29.691 & 23.744 & 35.153 \\
\hline 10/7/09 16:00 & 16.266 & 6.262 & 8.365 & 20.264 & 17.516 & 21.708 & 12.462 & 29.562 & 23.760 & 35.160 \\
\hline 10/7/09 20:00 & 16.273 & 6.264 & 8.374 & 20.255 & 17.539 & 21.711 & 12.465 & 29.395 & 23.774 & 35.169 \\
\hline 10/8/09 0:00 & 16.287 & 6.266 & 8.386 & 20.211 & 17.561 & 21.741 & 12.481 & 29.402 & 23.791 & 35.179 \\
\hline 10/8/09 4:00 & 16.301 & 6.264 & 8.395 & 20.227 & 17.582 & 21.746 & 12.476 & 29.430 & 23.810 & 35.188 \\
\hline 10/8/09 8:00 & 16.304 & 6.264 & 8.405 & 20.255 & 17.601 & 21.736 & 12.481 & 29.376 & 23.824 & 35.198 \\
\hline 10/8/09 12:00 & 16.306 & 6.266 & 8.410 & 20.225 & 17.610 & 21.694 & 12.488 & 29.392 & 23.840 & 35.207 \\
\hline 10/8/09 16:00 & 16.301 & 6.271 & 8.391 & 20.139 & 17.627 & 21.676 & 12.493 & 29.416 & 23.856 & 35.217 \\
\hline 10/8/09 20:00 & 16.289 & 6.273 & 8.363 & 20.111 & 17.643 & 21.666 & 12.495 & 29.458 & 23.871 & 35.224 \\
\hline 10/9/09 0:00 & 16.289 & 6.278 & 8.337 & 20.099 & 17.664 & 21.683 & 12.498 & 29.423 & 23.887 & 35.235 \\
\hline 10/9/09 4:00 & 16.294 & 6.280 & 8.320 & 20.083 & 17.683 & 21.701 & 12.512 & 29.439 & 23.903 & 35.242 \\
\hline 10/9/09 8:00 & 16.315 & 6.285 & 8.313 & 20.064 & 17.705 & 21.741 & 12.526 & 29.472 & 23.917 & 35.252 \\
\hline 10/9/09 12:00 & 16.339 & 6.290 & 8.313 & 20.080 & 17.726 & 21.774 & 12.542 & 29.576 & 23.931 & 35.261 \\
\hline 10/9/09 16:00 & 16.353 & 6.292 & 8.316 & 20.222 & 17.747 & 21.776 & 12.540 & 29.566 & 23.948 & 35.271 \\
\hline 10/9/09 20:00 & 16.379 & 6.299 & 8.323 & 20.162 & 17.768 & 21.821 & 12.570 & 29.522 & 23.962 & 35.282 \\
\hline 10/10/09 0:00 & 16.412 & 6.304 & 8.337 & 20.132 & 17.792 & 21.863 & 12.587 & 29.573 & 23.981 & 35.290 \\
\hline 10/10/09 4:00 & 16.443 & 6.308 & 8.346 & 20.148 & 17.811 & 21.887 & 12.610 & 29.569 & 23.995 & 35.299 \\
\hline 10/10/09 8:00 & 16.487 & 6.318 & 8.363 & 20.057 & 17.832 & 21.962 & 12.648 & 29.639 & 24.009 & 35.308 \\
\hline 10/10/09 12:00 & 16.532 & 6.327 & 8.377 & 20.087 & 17.853 & 22.013 & 12.674 & 29.726 & 24.028 & 35.318 \\
\hline 10/10/09 16:00 & 16.553 & 6.325 & 8.386 & 20.229 & 17.874 & 21.997 & 12.662 & 29.658 & 24.042 & 35.330 \\
\hline 10/10/09 20:00 & 16.570 & 6.325 & 8.398 & 20.290 & 17.896 & 21.997 & 12.669 & 29.672 & 24.058 & 35.339 \\
\hline 10/11/09 0:00 & 16.591 & 6.327 & 8.407 & 20.281 & 17.912 & 22.025 & 12.678 & 29.708 & 24.072 & 35.351 \\
\hline 10/11/09 4:00 & 16.610 & 6.327 & 8.417 & 20.327 & 17.936 & 22.027 & 12.678 & 29.755 & 24.089 & 35.351 \\
\hline 10/11/09 8:00 & 16.629 & 6.332 & 8.426 & 20.339 & 17.955 & 22.051 & 12.690 & 29.722 & 24.103 & 35.363 \\
\hline 10/11/09 12:00 & 16.645 & 6.330 & 8.435 & 20.390 & 17.973 & 22.041 & 12.676 & 29.790 & 24.117 & 35.374 \\
\hline 10/11/09 16:00 & 16.634 & 6.323 & 8.440 & 20.502 & 17.995 & 21.985 & 12.634 & 29.684 & 24.133 & 35.384 \\
\hline 10/11/09 20:00 & 16.629 & 6.320 & 8.445 & 20.507 & 18.013 & 21.973 & 12.627 & 29.679 & 24.147 & 35.393 \\
\hline 10/12/09 0:00 & 16.631 & 6.318 & 8.452 & 20.532 & 18.032 & 21.962 & 12.615 & 29.689 & 24.161 & 35.403 \\
\hline 10/12/09 4:00 & 16.624 & 6.313 & 8.456 & 20.558 & 18.051 & 21.940 & 12.596 & 29.710 & 24.178 & 35.410 \\
\hline 10/12/09 8:00 & 16.631 & 6.318 & 8.464 & 20.537 & 18.070 & 21.947 & 12.610 & 29.646 & 24.192 & 35.419 \\
\hline 10/12/09 12:00 & 16.648 & 6.323 & 8.473 & 20.509 & 18.089 & 21.983 & 12.629 & 29.675 & 24.206 & 35.431 \\
\hline 10/12/09 16:00 & 16.659 & 6.325 & 8.480 & 20.542 & 18.108 & 21.990 & 12.634 & 29.708 & 24.220 & 35.438 \\
\hline 10/12/09 20:00 & 16.692 & 6.337 & 8.494 & 20.406 & 18.127 & 22.067 & 12.686 & 29.736 & 24.236 & 35.447 \\
\hline 10/13/09 0:00 & 16.733 & 6.344 & 8.510 & 20.351 & 18.146 & 22.121 & 12.716 & 29.776 & 24.253 & 35.457 \\
\hline 10/13/09 4:00 & 16.766 & 6.348 & 8.522 & 20.369 & 18.167 & 22.144 & 12.728 & 29.799 & 24.269 & 35.469 \\
\hline 10/13/09 8:00 & 16.794 & 6.353 & 8.534 & 20.383 & 18.183 & 22.166 & 12.742 & 29.823 & 24.283 & 35.476 \\
\hline 10/13/09 12:00 & 16.810 & 6.355 & 8.546 & 20.453 & 18.204 & 22.163 & 12.730 & 29.830 & 24.300 & 35.487 \\
\hline 10/13/09 16:00 & 16.803 & 6.348 & 8.553 & 20.584 & 18.221 & 22.114 & 12.697 & 29.802 & 24.314 & 35.494 \\
\hline 10/13/09 20:00 & 16.803 & 6.348 & 8.560 & 20.619 & 18.238 & 22.100 & 12.695 & 29.811 & 24.330 & 35.502 \\
\hline 10/14/09 0:00 & 16.808 & 6.351 & 8.569 & 20.535 & 18.252 & 22.112 & 12.704 & 29.889 & 24.347 & 35.511 \\
\hline 10/14/09 4:00 & 16.791 & 6.348 & 8.571 & 20.570 & 18.268 & 22.065 & 12.690 & 29.790 & 24.361 & 35.518 \\
\hline 10/14/09 8:00 & 16.782 & 6.351 & 8.571 & 20.544 & 18.287 & 22.079 & 12.695 & 29.806 & 24.377 & 35.530 \\
\hline 10/14/09 12:00 & 16.775 & 6.346 & 8.571 & 20.598 & 18.304 & 22.055 & 12.674 & 29.839 & 24.391 & 35.539 \\
\hline 10/14/09 16:00 & 16.754 & 6.339 & 8.569 & 20.665 & 18.320 & 22.013 & 12.636 & 29.771 & 24.405 & 35.549 \\
\hline $10 / 14 / 0920: 00$ & 16.740 & 6.337 & 8.567 & 20.649 & 18.334 & 22.008 & 12.627 & 29.759 & 24.419 & 35.556 \\
\hline 10/15/09 0:00 & 16.716 & 6.337 & 8.553 & 20.600 & 18.346 & 21.952 & 12.624 & 29.745 & 24.433 & 35.565 \\
\hline 10/15/09 4:00 & 16.695 & 6.339 & 8.534 & 20.581 & 18.363 & 21.952 & 12.617 & 29.740 & 24.447 & 35.577 \\
\hline 10/15/09 8:00 & 16.692 & 6.346 & 8.522 & 20.507 & 18.379 & 21.985 & 12.639 & 29.745 & 24.462 & 35.582 \\
\hline 10/15/09 12:00 & 16.709 & 6.351 & 8.518 & 20.472 & 18.398 & 22.023 & 12.660 & 29.771 & 24.478 & 35.593 \\
\hline 10/15/09 16:00 & 16.723 & 6.355 & 8.513 & 20.474 & 18.414 & 22.037 & 12.671 & 29.778 & 24.492 & 35.600 \\
\hline 10/15/09 20:00 & 16.751 & 6.362 & 8.515 & 20.437 & 18.431 & 22.083 & 12.702 & 29.834 & 24.506 & 35.610 \\
\hline 10/16/09 0:00 & 16.782 & 6.367 & 8.520 & 20.418 & 18.450 & 22.121 & 12.725 & 29.834 & 24.523 & 35.622 \\
\hline 10/16/09 4:00 & 16.813 & 6.372 & 8.525 & 20.402 & 18.469 & 22.156 & 12.747 & 29.853 & 24.534 & 35.631 \\
\hline 10/16/09 8:00 & 16.846 & 6.379 & 8.534 & 20.386 & 18.485 & 22.205 & 12.775 & 29.886 & 24.551 & 35.641 \\
\hline 10/16/09 12:00 & 16.881 & 6.383 & 8.546 & 20.413 & 18.502 & 22.245 & 12.796 & 29.957 & 24.565 & 35.648 \\
\hline 10/16/09 16:00 & 16.897 & 6.386 & 8.555 & 20.504 & 18.518 & 22.241 & 12.789 & 29.907 & 24.579 & 35.657 \\
\hline $10 / 16 / 0920: 00$ & 16.921 & 6.390 & 8.564 & 20.483 & 18.537 & 22.273 & 12.810 & 29.933 & 24.595 & 35.664 \\
\hline 10/17/09 0:00 & 16.949 & 6.395 & 8.576 & 20.472 & 18.556 & 22.306 & 12.829 & 29.966 & 24.612 & 35.676 \\
\hline 10/17/09 4:00 & 16.978 & 6.400 & 8.588 & 20.455 & 18.572 & 22.346 & 12.852 & 30.011 & 24.626 & 35.683 \\
\hline 10/17/09 8:00 & 17.013 & 6.407 & 8.602 & 20.420 & 18.589 & 22.400 & 12.888 & 30.072 & 24.640 & 35.690 \\
\hline 10/17/09 12:00 & 17.048 & 6.411 & 8.616 & 20.467 & 18.608 & 22.428 & 12.895 & 30.058 & 24.656 & 35.702 \\
\hline 10/17/09 16:00 & 17.058 & 6.409 & 8.625 & 20.602 & 18.624 & 22.402 & 12.883 & 30.056 & 24.670 & 35.711 \\
\hline 10/17/09 20:00 & 17.065 & 6.407 & 8.632 & 20.639 & 18.643 & 22.391 & 12.876 & 30.058 & 24.684 & 35.718 \\
\hline 10/18/09 0:00 & 17.070 & 6.404 & 8.642 & 20.644 & 18.660 & 22.393 & 12.869 & 30.077 & 24.701 & 35.728 \\
\hline
\end{tabular}


Water Level (ft below reference point)

\begin{tabular}{|c|c|c|c|c|c|c|c|c|c|c|}
\hline Date and Time & McPike & Potter & Hughes & Barr & Burks & MoDOT & Clizer "Old" & Morgan & SB24 & SB38 \\
\hline 10/18/09 4:00 & 17.070 & 6.432 & 8.647 & 20.688 & 18.676 & 22.372 & 12.857 & 30.086 & 24.715 & 35.735 \\
\hline 10/18/09 8:00 & 17.070 & 6.463 & 8.651 & 20.716 & 18.693 & 22.358 & 12.845 & 30.126 & 24.731 & 35.744 \\
\hline 10/18/09 12:00 & 17.062 & 6.680 & 8.658 & 20.768 & 18.709 & 22.341 & 12.822 & 30.030 & 24.745 & 35.756 \\
\hline 10/18/09 16:00 & 17.037 & 6.772 & 8.658 & 20.942 & 18.723 & 22.276 & 12.770 & 30.001 & 24.759 & 35.763 \\
\hline 10/18/09 20:00 & 17.025 & 6.519 & 8.663 & 20.891 & 18.742 & 22.259 & 12.761 & 29.971 & 24.773 & 35.772 \\
\hline 10/19/09 0:00 & 17.020 & 6.491 & 8.670 & 20.845 & 18.756 & 22.252 & 12.751 & 29.966 & 24.788 & 35.782 \\
\hline 10/19/09 4:00 & 17.013 & 6.470 & 8.675 & 20.835 & 18.773 & 22.234 & 12.740 & 29.966 & 24.802 & 35.791 \\
\hline 10/19/09 8:00 & 17.020 & 6.439 & 8.684 & 20.770 & 18.789 & 22.257 & 12.756 & 29.952 & 24.816 & 35.801 \\
\hline 10/19/09 12:00 & 17.037 & 6.636 & 8.691 & 20.740 & 18.806 & 22.287 & 12.775 & 30.272 & 24.830 & 35.810 \\
\hline 10/19/09 16:00 & 17.041 & 6.732 & 8.701 & 20.847 & 18.822 & 22.271 & 12.765 & 30.086 & 24.844 & 35.817 \\
\hline 10/19/09 20:00 & 17.055 & 6.404 & 8.715 & 20.765 & 18.839 & 22.297 & 12.787 & 30.079 & 24.856 & 35.827 \\
\hline 10/20/09 0:00 & 17.079 & 6.353 & 8.726 & 20.693 & 18.855 & 22.334 & 12.812 & 30.023 & 24.872 & 35.834 \\
\hline 10/20/09 4:00 & 17.091 & 6.388 & 8.736 & 20.698 & 18.870 & 22.346 & 12.819 & 30.041 & 24.886 & 35.843 \\
\hline 10/20/09 8:00 & 17.103 & 6.365 & 8.745 & 20.679 & 18.886 & 22.360 & 12.831 & 30.074 & 24.900 & 35.853 \\
\hline 10/20/09 12:00 & 17.119 & 6.552 & 8.757 & 20.688 & 18.903 & 22.381 & 12.848 & 30.032 & 24.914 & 35.862 \\
\hline 10/20/09 16:00 & 17.131 & 6.524 & 8.769 & 20.726 & 18.917 & 22.386 & 12.852 & 30.041 & 24.928 & 35.869 \\
\hline 10/20/09 20:00 & 17.147 & 6.376 & 8.780 & 20.698 & 18.933 & 22.409 & 12.871 & 30.063 & 24.942 & 35.878 \\
\hline 10/21/09 0:00 & 17.176 & 6.316 & 8.794 & 20.614 & 18.947 & 22.463 & 12.904 & 30.105 & 24.959 & 35.888 \\
\hline 10/21/09 4:00 & 17.192 & 6.339 & 8.804 & 20.630 & 18.964 & 22.473 & 12.906 & 30.138 & 24.971 & 35.895 \\
\hline 10/21/09 8:00 & 17.202 & 6.353 & 8.816 & 20.644 & 18.978 & 22.482 & 12.916 & 30.119 & 24.987 & 35.904 \\
\hline 10/21/09 12:00 & 17.221 & 6.486 & 8.825 & 20.646 & 18.995 & 22.510 & 12.930 & 30.143 & 25.001 & 35.914 \\
\hline 10/21/09 16:00 & 17.230 & 6.458 & 8.834 & 20.709 & 19.011 & 22.498 & 12.925 & 30.204 & 25.015 & 35.921 \\
\hline 10/21/09 20:00 & 17.020 & 6.397 & 8.827 & 20.656 & 18.995 & 22.445 & 12.923 & 30.171 & 25.029 & 35.930 \\
\hline 10/22/09 0:00 & 16.700 & 6.418 & 8.771 & 20.625 & 18.983 & 22.327 & 12.881 & 30.159 & 25.043 & 35.937 \\
\hline 10/22/09 4:00 & 15.019 & 5.639 & 8.417 & 20.381 & 18.841 & 21.762 & 12.547 & 30.124 & 25.057 & 35.942 \\
\hline 10/22/09 8:00 & 13.558 & 4.584 & 7.790 & 20.031 & 18.794 & 21.082 & 12.340 & 29.921 & 25.069 & 35.947 \\
\hline 10/22/09 12:00 & 13.336 & 4.388 & & 19.889 & 18.803 & 20.939 & 12.258 & 29.773 & 25.076 & 35.954 \\
\hline 10/22/09 16:00 & 12.773 & 3.986 & & 19.663 & 18.740 & 20.473 & 12.000 & 29.611 & 25.083 & 35.961 \\
\hline 10/22/09 20:00 & 12.332 & 3.604 & & 19.365 & 18.738 & 20.175 & 11.868 & 29.458 & 25.090 & 35.963 \\
\hline 10/23/09 0:00 & 12.424 & 3.623 & & 19.253 & 18.747 & 20.077 & 11.793 & 29.327 & 25.095 & 35.968 \\
\hline 10/23/09 4:00 & 12.709 & 3.815 & & 19.204 & 18.759 & 20.041 & 11.741 & 29.237 & 25.099 & 35.975 \\
\hline 10/23/09 8:00 & 12.987 & 3.939 & & 19.116 & 18.770 & 20.055 & 11.732 & 29.054 & 25.102 & 35.977 \\
\hline $10 / 23 / 0912: 00$ & 13.211 & 4.103 & & 19.046 & 18.782 & 20.072 & 11.732 & 29.007 & 25.107 & 35.984 \\
\hline 10/23/09 16:00 & 13.381 & 4.271 & & 19.057 & 18.794 & 20.100 & 11.730 & 28.939 & 25.111 & 35.989 \\
\hline 10/23/09 20:00 & 13.532 & 4.341 & 7.940 & 19.053 & 18.801 & 20.156 & 11.744 & 28.896 & 25.116 & 36.001 \\
\hline 10/24/09 0:00 & 13.650 & 4.561 & 7.937 & 19.113 & 18.813 & 20.161 & 11.727 & 28.845 & 25.118 & 36.001 \\
\hline 10/24/09 4:00 & 13.749 & 4.678 & 7.947 & 19.158 & 18.818 & 20.168 & 11.720 & 28.795 & 25.123 & 36.013 \\
\hline 10/24/09 8:00 & 13.838 & 4.818 & 7.961 & 19.188 & 18.825 & 20.182 & 11.720 & 28.758 & 25.125 & 36.017 \\
\hline 10/24/09 12:00 & 13.918 & 5.134 & 7.987 & 19.237 & 18.829 & 20.208 & 11.715 & 28.725 & 25.130 & 36.022 \\
\hline 10/24/09 16:00 & 13.975 & 5.237 & 8.013 & 19.414 & 18.834 & 20.152 & 11.676 & 28.656 & 25.130 & 36.027 \\
\hline 10/24/09 20:00 & 14.036 & 5.162 & 8.048 & 19.407 & 18.837 & 20.168 & 11.683 & 28.619 & 25.135 & 36.032 \\
\hline 10/25/09 0:00 & 14.100 & 5.144 & 8.085 & 19.358 & 18.841 & 20.224 & 11.694 & 28.609 & 25.137 & 36.039 \\
\hline 10/25/09 4:00 & 14.161 & 5.167 & 8.123 & 19.351 & 18.841 & 20.266 & 11.706 & 28.602 & 25.139 & 36.043 \\
\hline 10/25/09 8:00 & 14.223 & 5.181 & 8.158 & 19.337 & 18.844 & 20.318 & 11.727 & 28.602 & 25.144 & 36.050 \\
\hline $10 / 25 / 0912: 00$ & 14.286 & 5.371 & 8.203 & 19.339 & 18.846 & 20.388 & 11.744 & 28.624 & 25.146 & 36.055 \\
\hline 10/25/09 16:00 & 14.338 & 5.396 & 8.240 & 19.423 & 18.846 & 20.407 & 11.748 & 28.609 & 25.151 & 36.060 \\
\hline 10/25/09 20:00 & 14.404 & 5.209 & 8.285 & 19.353 & 18.846 & 20.496 & 11.805 & 28.645 & 25.153 & 36.067 \\
\hline 10/26/09 0:00 & 14.475 & 5.197 & 8.334 & 19.297 & 18.846 & 20.590 & 11.833 & 28.699 & 25.158 & 36.069 \\
\hline 10/26/09 4:00 & 14.531 & 5.212 & 8.358 & 19.346 & 18.839 & 20.616 & 11.840 & 28.711 & 25.163 & 36.072 \\
\hline 10/26/09 8:00 & 14.583 & 5.188 & 8.374 & 19.363 & 18.837 & 20.649 & 11.866 & 28.729 & 25.168 & 36.074 \\
\hline 10/26/09 12:00 & 14.630 & 5.443 & 8.400 & 19.428 & 18.834 & 20.681 & 11.849 & 28.743 & 25.172 & 36.083 \\
\hline 10/26/09 16:00 & 14.654 & 5.574 & 8.416 & 19.619 & 18.832 & 20.632 & 11.809 & 28.706 & 25.175 & 36.088 \\
\hline 10/26/09 20:00 & 14.675 & 5.464 & 8.442 & 19.647 & 18.827 & 20.625 & 11.791 & 28.718 & 25.179 & 36.095 \\
\hline 10/27/09 0:00 & 14.689 & 5.483 & 8.458 & 19.696 & 18.825 & 20.599 & 11.762 & 28.689 & 25.184 & 36.097 \\
\hline 10/27/09 4:00 & 14.699 & 5.553 & 8.470 & 19.742 & 18.820 & 20.564 & 11.734 & 28.694 & 25.189 & 36.107 \\
\hline 10/27/09 8:00 & 14.701 & 5.626 & 8.480 & 19.780 & 18.818 & 20.531 & 11.706 & 28.605 & 25.191 & 36.109 \\
\hline $10 / 27 / 0912: 00$ & 14.699 & 5.845 & 8.491 & 19.833 & 18.813 & 20.506 & 11.668 & 28.567 & 25.193 & 36.114 \\
\hline $10 / 27 / 0916: 00$ & 14.680 & 5.841 & 8.496 & 19.940 & 18.808 & 20.426 & 11.621 & 28.499 & 25.196 & 36.119 \\
\hline 10/27/09 20:00 & 14.682 & 5.712 & 8.505 & 19.875 & 18.801 & 20.426 & 11.621 & 28.471 & 25.200 & 36.123 \\
\hline 10/28/09 0:00 & 14.703 & 5.679 & 8.522 & 19.787 & 18.797 & 20.470 & 11.636 & 28.482 & 25.203 & 36.126 \\
\hline 10/28/09 4:00 & 14.720 & 5.672 & 8.538 & 19.770 & 18.792 & 20.496 & 11.640 & 28.504 & 25.205 & 36.133 \\
\hline 10/28/09 8:00 & 14.743 & 5.623 & 8.555 & 19.719 & 18.785 & 20.536 & 11.659 & 28.565 & 25.207 & 36.138 \\
\hline 10/28/09 12:00 & 14.767 & 5.815 & 8.578 & 19.712 & 18.780 & 20.578 & 11.661 & 28.497 & 25.212 & 36.142 \\
\hline 10/28/09 16:00 & 14.779 & 5.792 & 8.597 & 19.810 & 18.775 & 20.545 & 11.652 & 28.473 & 25.214 & 36.145 \\
\hline
\end{tabular}


Water Level (ft below reference point)

\begin{tabular}{|c|c|c|c|c|c|c|c|c|c|c|}
\hline Date and Time & McPike & Potter & Hughes & Barr & Burks & MoDOT & Clizer "Old" & Morgan & SB24 & SB38 \\
\hline 10/28/09 20:00 & 14.809 & 5.668 & 8.627 & 19.712 & 18.768 & 20.618 & 11.680 & 28.515 & 25.219 & 36.149 \\
\hline 10/29/09 0:00 & 14.842 & 5.651 & 8.658 & 19.675 & 18.761 & 20.665 & 11.697 & 28.537 & 25.221 & 36.156 \\
\hline 10/29/09 4:00 & 14.854 & 5.703 & 8.674 & 19.766 & 18.756 & 20.627 & 11.659 & 28.522 & 25.226 & 36.161 \\
\hline 10/29/09 8:00 & 14.307 & 5.279 & 8.529 & 19.680 & 18.669 & 20.578 & 11.598 & 28.515 & 25.228 & 36.163 \\
\hline 10/29/09 12:00 & 13.379 & 4.521 & 8.257 & 19.565 & 18.646 & 20.424 & 11.549 & 28.457 & 25.231 & 36.168 \\
\hline 10/29/09 16:00 & 13.110 & 4.287 & 8.029 & 19.568 & 18.627 & 20.198 & 11.445 & 28.363 & 25.233 & 36.175 \\
\hline 10/29/09 20:00 & 12.999 & 4.117 & 7.832 & 19.491 & 18.613 & 20.067 & 11.382 & 28.283 & 25.236 & 36.182 \\
\hline 10/30/09 0:00 & 13.030 & 4.084 & 7.686 & 19.447 & 18.603 & 19.997 & 11.340 & 28.210 & 25.236 & 36.189 \\
\hline 10/30/09 4:00 & 13.129 & 3.951 & 7.588 & 19.325 & 18.594 & 20.001 & 11.333 & 28.184 & 25.238 & 36.189 \\
\hline 10/30/09 8:00 & 13.230 & 3.946 & 7.529 & 19.225 & 18.587 & 20.006 & 11.330 & 28.170 & 25.238 & 36.189 \\
\hline 10/30/09 12:00 & 13.322 & 4.054 & 7.501 & 19.167 & 18.577 & 20.027 & 11.333 & 28.189 & 25.240 & 36.194 \\
\hline 10/30/09 16:00 & 13.398 & 4.163 & 7.482 & 19.209 & 18.568 & 20.013 & 11.328 & 28.097 & 25.238 & 36.196 \\
\hline 10/30/09 20:00 & 13.497 & 4.145 & 7.489 & 19.085 & 18.561 & 20.116 & 11.389 & 28.144 & 25.240 & 36.201 \\
\hline 10/31/09 0:00 & 13.581 & 4.278 & 7.501 & 19.113 & 18.551 & 20.161 & 11.398 & 28.189 & 25.245 & 36.203 \\
\hline 10/31/09 4:00 & 13.654 & 4.329 & 7.513 & 19.113 & 18.542 & 20.198 & 11.427 & 28.238 & 25.245 & 36.203 \\
\hline 10/31/09 8:00 & 13.744 & 4.334 & 7.538 & 19.060 & 18.537 & 20.311 & 11.497 & 28.226 & 25.247 & 36.211 \\
\hline 10/31/09 12:00 & 13.829 & 4.608 & 7.574 & 19.085 & 18.528 & 20.393 & 11.523 & 28.285 & 25.250 & 36.215 \\
\hline 10/31/09 16:00 & 13.888 & 4.835 & 7.599 & 19.244 & 18.521 & 20.395 & 11.506 & 28.311 & 25.252 & 36.218 \\
\hline 10/31/09 20:00 & 13.940 & 4.741 & 7.623 & 19.274 & 18.509 & 20.414 & 11.513 & 28.301 & 25.254 & 36.222 \\
\hline 11/1/09 0:00 & 13.987 & 4.819 & 7.642 & 19.309 & 18.502 & 20.428 & 11.504 & 28.306 & 25.254 & 36.225 \\
\hline $11 / 1 / 094: 00$ & 14.022 & 4.910 & 7.656 & 19.370 & 18.492 & 20.416 & 11.481 & 28.285 & 25.259 & 36.227 \\
\hline 11/1/09 8:00 & 14.053 & 4.961 & 7.667 & 19.423 & 18.483 & 20.400 & 11.466 & 28.264 & 25.259 & 36.232 \\
\hline 11/1/09 12:00 & 14.079 & 5.249 & 7.679 & 19.458 & 18.473 & 20.402 & 11.450 & 28.252 & 25.261 & 36.236 \\
\hline 11/1/09 16:00 & 14.093 & 5.401 & 7.691 & 19.598 & 18.464 & 20.360 & 11.417 & 28.210 & 25.261 & 36.239 \\
\hline 11/1/09 20:00 & 14.119 & 5.104 & 7.712 & 19.547 & 18.455 & 20.386 & 11.441 & 28.287 & 25.264 & 36.244 \\
\hline 11/2/09 0:00 & 14.154 & 5.022 & 7.733 & 19.498 & 18.445 & 20.428 & 11.459 & 28.224 & 25.266 & 36.248 \\
\hline 11/2/09 4:00 & 14.197 & 4.980 & 7.759 & 19.419 & 18.436 & 20.492 & 11.497 & 28.266 & 25.268 & 36.251 \\
\hline 11/2/09 8:00 & 14.251 & 4.910 & 7.792 & 19.332 & 18.426 & 20.581 & 11.560 & 28.334 & 25.273 & 36.255 \\
\hline 11/2/09 12:00 & 14.312 & 5.076 & 7.827 & 19.304 & 18.417 & 20.672 & 11.598 & 28.421 & 25.275 & 36.258 \\
\hline 11/2/09 16:00 & 14.359 & 5.155 & 7.860 & 19.407 & 18.407 & 20.695 & 11.603 & 28.482 & 25.278 & 36.260 \\
\hline 11/2/09 20:00 & 14.402 & 5.088 & 7.890 & 19.398 & 18.398 & 20.735 & 11.614 & 28.435 & 25.282 & 36.267 \\
\hline 11/3/09 0:00 & 14.442 & 5.088 & 7.916 & 19.430 & 18.386 & 20.754 & 11.619 & 28.459 & 25.285 & 36.269 \\
\hline $11 / 3 / 094: 00$ & 14.472 & 5.081 & 7.940 & 19.481 & 18.377 & 20.756 & 11.605 & 28.473 & 25.289 & 36.269 \\
\hline 11/3/09 8:00 & 14.496 & 5.258 & 7.956 & 19.528 & 18.367 & 20.752 & 11.591 & 28.480 & 25.294 & 36.274 \\
\hline 11/3/09 12:00 & 14.510 & 5.513 & 7.970 & 19.624 & 18.355 & 20.728 & 11.556 & 28.497 & 25.297 & 36.281 \\
\hline 11/3/09 16:00 & 14.491 & 5.616 & 7.970 & 19.819 & 18.344 & 20.616 & 11.469 & 28.363 & 25.299 & 36.284 \\
\hline 11/3/09 20:00 & 14.484 & 5.534 & 7.975 & 19.817 & 18.334 & 20.583 & 11.455 & 28.325 & 25.301 & 36.286 \\
\hline 11/4/09 0:00 & 14.494 & 5.459 & 7.987 & 19.773 & 18.323 & 20.585 & 11.455 & 28.320 & 25.304 & 36.291 \\
\hline 11/4/09 4:00 & 14.505 & 5.406 & 7.998 & 19.721 & 18.313 & 20.597 & 11.462 & 28.325 & 25.306 & 36.293 \\
\hline 11/4/09 8:00 & 14.536 & 5.319 & 8.022 & 19.621 & 18.301 & 20.670 & 11.513 & 28.381 & 25.311 & 36.298 \\
\hline 11/4/09 12:00 & 14.576 & 5.462 & 8.055 & 19.556 & 18.290 & 20.752 & 11.556 & 28.407 & 25.313 & 36.302 \\
\hline 11/4/09 16:00 & 14.604 & 5.567 & 8.078 & 19.647 & 18.280 & 20.768 & 11.556 & 28.431 & 25.315 & 36.305 \\
\hline 11/4/09 20:00 & 14.635 & 5.347 & 8.102 & 19.617 & 18.268 & 20.799 & 11.570 & 28.457 & 25.320 & 36.312 \\
\hline 11/5/09 0:00 & 14.661 & 5.321 & 8.127 & 19.614 & 18.259 & 20.817 & 11.577 & 28.482 & 25.322 & 36.317 \\
\hline $11 / 5 / 094: 00$ & 14.682 & 5.371 & 8.146 & 19.631 & 18.247 & 20.827 & 11.570 & 28.511 & 25.327 & 36.319 \\
\hline 11/5/09 8:00 & 14.696 & 5.490 & 8.163 & 19.682 & 18.235 & 20.808 & 11.556 & 28.471 & 25.329 & 36.321 \\
\hline 11/5/09 12:00 & 14.701 & 5.743 & 8.177 & 19.740 & 18.223 & 20.792 & 11.518 & 28.461 & 25.334 & 36.326 \\
\hline 11/5/09 16:00 & 14.673 & 5.930 & 8.177 & 19.938 & 18.209 & 20.686 & 11.443 & 28.412 & 25.336 & 36.328 \\
\hline 11/5/09 20:00 & 14.654 & 5.707 & 8.177 & 19.913 & 18.200 & 20.649 & 11.419 & 28.355 & 25.339 & 36.331 \\
\hline 11/6/09 0:00 & 14.642 & 5.691 & 8.181 & 19.894 & 18.186 & 20.620 & 11.398 & 28.323 & 25.343 & 36.335 \\
\hline 11/6/09 4:00 & 14.628 & 5.721 & 8.186 & 19.899 & 18.174 & 20.588 & 11.368 & 28.285 & 25.343 & 36.340 \\
\hline 11/6/09 8:00 & 14.614 & 5.733 & 8.188 & 19.892 & 18.162 & 20.557 & 11.354 & 28.250 & 25.346 & 36.342 \\
\hline 11/6/09 12:00 & 14.602 & 5.969 & 8.198 & 19.894 & 18.150 & 20.541 & 11.328 & 28.224 & 25.346 & 36.345 \\
\hline 11/6/09 16:00 & 14.578 & 6.012 & 8.195 & 19.947 & 18.134 & 20.480 & 11.293 & 28.170 & 25.348 & 36.347 \\
\hline 11/6/09 20:00 & 14.571 & 5.745 & 8.200 & 19.829 & 18.122 & 20.484 & 11.304 & 28.153 & 25.350 & 36.352 \\
\hline 11/7/09 0:00 & 14.578 & 5.633 & 8.214 & 19.745 & 18.110 & 20.508 & 11.316 & 28.158 & 25.350 & 36.354 \\
\hline 11/7/09 4:00 & 14.593 & 5.572 & 8.228 & 19.668 & 18.096 & 20.541 & 11.335 & 28.167 & 25.353 & 36.357 \\
\hline 11/7/09 8:00 & 14.618 & 5.504 & 8.250 & 19.584 & 18.084 & 20.595 & 11.372 & 28.285 & 25.357 & 36.361 \\
\hline 11/7/09 12:00 & 14.651 & 5.757 & 8.278 & 19.526 & 18.073 & 20.667 & 11.401 & 28.236 & 25.357 & 36.364 \\
\hline 11/7/09 16:00 & 14.668 & 5.909 & 8.296 & 19.610 & 18.058 & 20.681 & 11.401 & 28.259 & 25.362 & 36.366 \\
\hline 11/7/09 20:00 & 14.694 & 5.544 & 8.318 & 19.535 & 18.044 & 20.735 & 11.438 & 28.280 & 25.365 & 36.371 \\
\hline 11/8/09 0:00 & 14.717 & 5.488 & 8.341 & 19.509 & 18.032 & 20.763 & 11.450 & 28.341 & 25.367 & 36.373 \\
\hline $11 / 8 / 094: 00$ & 14.734 & 5.511 & 8.362 & 19.500 & 18.018 & 20.782 & 11.457 & 28.311 & 25.369 & 36.378 \\
\hline 11/8/09 8:00 & 14.753 & 5.551 & 8.383 & 19.488 & 18.007 & 20.810 & 11.471 & 28.334 & 25.374 & 36.380 \\
\hline
\end{tabular}


Water Level (ft below reference point)

\begin{tabular}{|c|c|c|c|c|c|c|c|c|c|c|}
\hline Date and Time & McPike & Potter & Hughes & Barr & Burks & MoDOT & Clizer "Old" & Morgan & SB24 & SB38 \\
\hline 11/8/09 12:00 & 14.769 & 5.806 & 8.400 & 19.523 & 17.992 & 20.829 & 11.466 & 28.360 & 25.379 & 36.383 \\
\hline $11 / 8 / 0916: 00$ & 14.774 & 5.689 & 8.414 & 19.633 & 17.981 & 20.820 & 11.466 & 28.363 & 25.379 & 36.387 \\
\hline 11/8/09 20:00 & 14.802 & 5.703 & 8.437 & 19.509 & 17.964 & 20.892 & 11.520 & 28.440 & 25.383 & 36.392 \\
\hline 11/9/09 0:00 & 14.840 & 5.717 & 8.465 & 19.430 & 17.955 & 20.967 & 11.563 & 28.438 & 25.386 & 36.394 \\
\hline 11/9/09 4:00 & 14.882 & 5.728 & 8.494 & 19.398 & 17.940 & 21.031 & 11.598 & 28.492 & 25.390 & 36.397 \\
\hline 11/9/09 8:00 & 14.927 & 5.740 & 8.524 & 19.344 & 17.929 & 21.099 & 11.647 & 28.553 & 25.395 & 36.401 \\
\hline 11/9/09 12:00 & 14.967 & 5.750 & 8.552 & 19.395 & 17.919 & 21.141 & 11.657 & 28.602 & 25.400 & 36.406 \\
\hline 11/9/09 16:00 & 14.991 & 5.754 & 8.573 & 19.495 & 17.905 & 21.141 & 11.654 & 28.626 & 25.404 & 36.408 \\
\hline 11/9/09 20:00 & 15.019 & 5.761 & 8.595 & 19.468 & 17.891 & 21.185 & 11.678 & 28.694 & 25.407 & 36.413 \\
\hline 11/10/09 0:00 & 15.043 & 5.766 & 8.616 & 19.486 & 17.879 & 21.202 & 11.685 & 28.647 & 25.414 & 36.415 \\
\hline 11/10/09 4:00 & 15.062 & 5.768 & 8.632 & 19.502 & 17.870 & 21.216 & 11.690 & 28.668 & 25.418 & 36.420 \\
\hline 11/10/09 8:00 & 15.088 & 5.775 & 8.653 & 19.500 & 17.858 & 21.246 & 11.711 & 28.701 & 25.423 & 36.423 \\
\hline 11/10/09 12:00 & 15.116 & 5.782 & 8.674 & 19.500 & 17.844 & 21.289 & 11.730 & 28.741 & 25.428 & 36.427 \\
\hline 11/10/09 16:00 & 15.130 & 5.785 & 8.691 & 19.621 & 17.834 & 21.270 & 11.708 & 28.758 & 25.433 & 36.432 \\
\hline 11/10/09 20:00 & 15.142 & 5.789 & 8.707 & 19.600 & 17.825 & 21.286 & 11.725 & 28.802 & 25.440 & 36.437 \\
\hline 11/11/09 0:00 & 15.161 & 5.792 & 8.721 & 19.586 & 17.813 & 21.305 & 11.725 & 28.758 & 25.442 & 36.439 \\
\hline 11/11/09 4:00 & 15.170 & 5.796 & 8.735 & 19.596 & 17.801 & 21.305 & 11.720 & 28.786 & 25.447 & 36.441 \\
\hline 11/11/09 8:00 & 15.182 & 5.799 & 8.749 & 19.593 & 17.792 & 21.312 & 11.730 & 28.788 & 25.454 & 36.448 \\
\hline 11/11/09 12:00 & 15.191 & 5.799 & 8.764 & 19.642 & 17.780 & 21.312 & 11.708 & 28.790 & 25.461 & 36.451 \\
\hline 11/11/09 16:00 & 15.170 & 5.794 & 8.764 & 19.805 & 17.771 & 21.242 & 11.661 & 28.760 & 25.463 & 36.453 \\
\hline 11/11/09 20:00 & 15.158 & 5.796 & 8.768 & 19.782 & 17.759 & 21.223 & 11.647 & 28.767 & 25.468 & 36.458 \\
\hline 11/12/09 0:00 & 15.149 & 5.799 & 8.773 & 19.773 & 17.747 & 21.204 & 11.631 & 28.699 & 25.472 & 36.463 \\
\hline 11/12/09 4:00 & 15.135 & 5.796 & 8.778 & 19.768 & 17.735 & 21.183 & 11.607 & 28.682 & 25.475 & 36.467 \\
\hline 11/12/09 8:00 & 15.118 & 5.794 & 8.778 & 19.787 & 17.724 & 21.141 & 11.579 & 28.656 & 25.479 & 36.470 \\
\hline 11/12/09 12:00 & 15.092 & 5.792 & 8.778 & 19.826 & 17.712 & 21.108 & 11.542 & 28.642 & 25.484 & 36.474 \\
\hline 11/12/09 16:00 & 15.055 & 5.785 & 8.771 & 19.903 & 17.700 & 21.021 & 11.488 & 28.598 & 25.486 & 36.474 \\
\hline 11/12/09 20:00 & 15.031 & 5.787 & 8.768 & 19.829 & 17.688 & 21.014 & 11.490 & 28.600 & 25.491 & 36.479 \\
\hline 11/13/09 0:00 & 15.019 & 5.792 & 8.773 & 19.763 & 17.679 & 21.014 & 11.485 & 28.520 & 25.494 & 36.484 \\
\hline 11/13/09 4:00 & 15.003 & 5.792 & 8.773 & 19.761 & 17.665 & 20.982 & 11.459 & 28.506 & 25.496 & 36.484 \\
\hline 11/13/09 8:00 & 14.989 & 5.794 & 8.775 & 19.714 & 17.655 & 20.972 & 11.466 & 28.473 & 25.501 & 36.491 \\
\hline 11/13/09 12:00 & 14.982 & 5.801 & 8.785 & 19.668 & 17.641 & 20.991 & 11.452 & 28.473 & 25.503 & 36.493 \\
\hline 11/13/09 16:00 & 14.974 & 5.806 & 8.787 & 19.677 & 17.629 & 20.979 & 11.455 & 28.457 & 25.505 & 36.496 \\
\hline 11/13/09 20:00 & 14.979 & 5.815 & 8.796 & 19.593 & 17.617 & 21.012 & 11.476 & 28.480 & 25.508 & 36.500 \\
\hline 11/14/09 0:00 & 15.008 & 5.829 & 8.813 & 19.470 & 17.606 & 21.085 & 11.535 & 28.537 & 25.512 & 36.503 \\
\hline $11 / 14 / 094: 00$ & 15.048 & 5.841 & 8.832 & 19.379 & 17.594 & 21.153 & 11.582 & 28.616 & 25.515 & 36.507 \\
\hline 11/14/09 8:00 & 15.090 & 5.852 & 8.857 & 19.300 & 17.584 & 21.225 & 11.636 & 28.602 & 25.517 & 36.510 \\
\hline 11/14/09 12:00 & 15.135 & 5.864 & 8.886 & 19.255 & 17.573 & 21.298 & 11.671 & 28.682 & 25.522 & 36.514 \\
\hline 11/14/09 16:00 & 15.168 & 5.871 & 8.904 & 19.290 & 17.563 & 21.329 & 11.692 & 28.703 & 25.526 & 36.517 \\
\hline 11/14/09 20:00 & 15.198 & 5.878 & 8.928 & 19.262 & 17.551 & 21.380 & 11.725 & 28.741 & 25.533 & 36.521 \\
\hline 11/15/09 0:00 & 15.234 & 5.885 & 8.951 & 19.242 & 17.542 & 21.434 & 11.758 & 28.835 & 25.536 & 36.526 \\
\hline 11/15/09 4:00 & 15.262 & 5.890 & 8.972 & 19.276 & 17.533 & 21.453 & 11.772 & 28.802 & 25.543 & 36.529 \\
\hline 11/15/09 8:00 & 15.293 & 5.897 & 8.991 & 19.242 & 17.523 & 21.507 & 11.809 & 28.847 & 25.547 & 36.533 \\
\hline 11/15/09 12:00 & 15.321 & 5.897 & 9.010 & 19.262 & 17.514 & 21.532 & 11.812 & 28.892 & 25.550 & 36.538 \\
\hline 11/15/09 16:00 & 15.330 & 5.897 & 9.024 & 19.318 & 17.504 & 21.525 & 11.788 & 28.962 & 25.557 & 36.540 \\
\hline 11/15/09 20:00 & 15.352 & 5.902 & 9.038 & 19.316 & 17.492 & 21.558 & 11.833 & 28.899 & 25.562 & 36.545 \\
\hline 11/16/09 0:00 & 15.375 & 5.904 & 9.052 & 19.335 & 17.485 & 21.582 & 11.838 & 28.932 & 25.566 & 36.547 \\
\hline $11 / 16 / 09$ 4:00 & 15.380 & 5.904 & 9.050 & 19.367 & 17.452 & 21.558 & 11.819 & 28.941 & 25.571 & 36.547 \\
\hline 11/16/09 8:00 & 14.536 & 5.600 & 8.742 & 19.251 & 17.318 & 21.167 & 11.708 & 28.929 & 25.576 & 36.550 \\
\hline 11/16/09 12:00 & 13.563 & 4.830 & 8.334 & 19.055 & 17.273 & 20.972 & 11.591 & 28.896 & 25.580 & 36.547 \\
\hline 11/16/09 16:00 & 13.485 & 4.578 & 8.087 & 18.999 & 17.235 & 20.921 & 11.525 & 28.750 & 25.583 & 36.547 \\
\hline 11/16/09 20:00 & 12.891 & 4.245 & 7.618 & 18.817 & 17.146 & 20.517 & 11.380 & 28.708 & 25.585 & 36.552 \\
\hline 11/17/09 0:00 & 12.363 & 3.871 & 7.214 & 18.647 & 17.089 & 20.288 & 11.246 & 28.652 & 25.585 & 36.545 \\
\hline 11/17/09 4:00 & 11.635 & 3.525 & 6.700 & 18.533 & 17.004 & 19.819 & 10.973 & 28.560 & 25.585 & 36.547 \\
\hline 11/17/09 8:00 & 10.914 & 3.289 & 6.231 & 18.354 & 16.943 & 19.371 & 10.783 & 28.438 & 25.583 & 36.545 \\
\hline 11/17/09 12:00 & 10.449 & 3.169 & 5.975 & 18.188 & 16.910 & 19.122 & 10.656 & 28.323 & 25.583 & 36.547 \\
\hline 11/17/09 16:00 & 10.025 & 3.101 & 5.825 & 18.130 & 16.889 & 19.066 & 10.534 & 28.090 & 25.578 & 36.547 \\
\hline 11/17/09 20:00 & 9.768 & 3.097 & 5.675 & 17.995 & 16.870 & 19.000 & 10.430 & 27.937 & 25.573 & 36.547 \\
\hline 11/18/09 0:00 & 9.803 & 3.200 & 5.571 & 17.946 & 16.849 & 18.944 & 10.329 & 27.768 & 25.566 & 36.552 \\
\hline $11 / 18 / 094: 00$ & 10.008 & 3.350 & 5.527 & 17.899 & 16.799 & 18.909 & 10.245 & 27.634 & 25.559 & 36.554 \\
\hline 11/18/09 8:00 & 10.242 & 3.509 & 5.503 & 17.890 & 16.643 & 18.855 & 10.170 & 27.462 & 25.555 & 36.552 \\
\hline 11/18/09 12:00 & 10.466 & 3.703 & 5.498 & 17.867 & 16.372 & 18.827 & 10.111 & 27.321 & 25.547 & 36.557 \\
\hline 11/18/09 16:00 & 10.645 & 3.843 & 5.489 & 17.906 & 15.985 & 18.761 & 10.048 & 27.163 & 25.540 & 36.557 \\
\hline 11/18/09 20:00 & 10.822 & 3.925 & 5.484 & 17.860 & 15.556 & 18.747 & 10.003 & 27.126 & 25.533 & 36.557 \\
\hline 11/19/09 0:00 & 10.977 & 3.981 & 5.491 & 17.841 & 15.127 & 18.738 & 9.970 & 26.921 & 25.526 & 36.559 \\
\hline
\end{tabular}


Water Level (ft below reference point)

\begin{tabular}{|c|c|c|c|c|c|c|c|c|c|c|}
\hline Date and Time & McPike & Potter & Hughes & Barr & Burks & MoDOT & Clizer "Old" & Morgan & SB24 & SB38 \\
\hline 11/19/09 4:00 & 11.114 & 4.049 & 5.506 & 17.818 & 14.719 & 18.740 & 9.942 & 26.820 & 25.519 & 36.557 \\
\hline 11/19/09 8:00 & 11.232 & 4.119 & 5.522 & 17.813 & 14.309 & 18.735 & 9.921 & 26.724 & 25.510 & 36.557 \\
\hline 11/19/09 12:00 & 11.347 & 4.281 & 5.548 & 17.790 & 13.920 & 18.771 & 9.911 & 26.667 & 25.503 & 36.559 \\
\hline 11/19/09 16:00 & 11.416 & 4.369 & 5.562 & 17.874 & 13.531 & 18.728 & 9.883 & 26.587 & 25.496 & 36.557 \\
\hline 11/19/09 20:00 & 11.505 & 4.264 & 5.588 & 17.820 & 13.160 & 18.759 & 9.874 & 26.488 & 25.489 & 36.557 \\
\hline 11/20/09 0:00 & 11.581 & 4.309 & 5.613 & 17.797 & 12.809 & 18.778 & 9.867 & 26.427 & 25.482 & 36.559 \\
\hline 11/20/09 4:00 & 11.628 & 4.412 & 5.637 & 17.839 & 12.460 & 18.754 & 9.848 & 26.340 & 25.472 & 36.562 \\
\hline 11/20/09 8:00 & 11.675 & 4.449 & 5.656 & 17.862 & 12.134 & 18.740 & 9.829 & 26.260 & 25.465 & 36.557 \\
\hline 11/20/09 12:00 & 11.722 & 4.662 & 5.684 & 17.874 & 11.828 & 18.757 & 9.820 & 26.211 & 25.456 & 36.557 \\
\hline 11/20/09 16:00 & 11.729 & 4.802 & 5.698 & 18.021 & 11.519 & 18.693 & 9.782 & 26.100 & 25.449 & 36.554 \\
\hline 11/20/09 20:00 & 11.753 & 4.617 & 5.717 & 18.009 & 11.227 & 18.674 & 9.756 & 26.025 & 25.440 & 36.557 \\
\hline 11/21/09 0:00 & 11.764 & 4.653 & 5.733 & 18.039 & 10.944 & 18.639 & 9.726 & 25.938 & 25.430 & 36.554 \\
\hline 11/21/09 4:00 & 11.767 & 4.756 & 5.750 & 18.079 & 10.670 & 18.595 & 9.691 & 25.861 & 25.421 & 36.552 \\
\hline 11/21/09 8:00 & 11.783 & 4.784 & 5.768 & 18.084 & 10.401 & 18.576 & 9.669 & 25.814 & 25.411 & 36.550 \\
\hline 11/21/09 12:00 & 11.790 & 4.945 & 5.790 & 18.109 & 10.146 & 18.557 & 9.646 & 25.720 & 25.402 & 36.550 \\
\hline 11/21/09 16:00 & 11.767 & 5.053 & 5.799 & 18.249 & 9.887 & 18.466 & 9.594 & 25.628 & 25.393 & 36.547 \\
\hline 11/21/09 20:00 & 11.786 & 4.947 & 5.820 & 18.200 & 9.616 & 18.468 & 9.583 & 25.567 & 25.383 & 36.547 \\
\hline 11/22/09 0:00 & 11.800 & 4.938 & 5.844 & 18.188 & 9.389 & 18.459 & 9.568 & 25.524 & 25.372 & 36.545 \\
\hline 11/22/09 4:00 & 11.816 & 4.912 & 5.869 & 18.156 & 9.194 & 18.456 & 9.559 & 25.487 & 25.362 & 36.543 \\
\hline 11/22/09 8:00 & 11.830 & 4.910 & 5.893 & 18.149 & 9.024 & 18.445 & 9.550 & 25.447 & 25.350 & 36.543 \\
\hline 11/22/09 12:00 & 11.842 & 5.024 & 5.923 & 18.137 & 8.882 & 18.456 & 9.543 & 25.515 & 25.341 & 36.540 \\
\hline 11/22/09 16:00 & 11.830 & 5.069 & 5.942 & 18.221 & 8.762 & 18.398 & 9.510 & 25.362 & 25.329 & 36.538 \\
\hline $11 / 22 / 09$ 20:00 & 11.842 & 4.992 & 5.968 & 18.188 & 8.666 & 18.395 & 9.500 & 25.317 & 25.320 & 36.536 \\
\hline 11/23/09 0:00 & 11.849 & 4.982 & 5.989 & 18.177 & 8.581 & 18.381 & 9.489 & 25.285 & 25.308 & 36.536 \\
\hline 11/23/09 4:00 & 11.849 & 5.006 & 6.012 & 18.170 & 8.498 & 18.367 & 9.472 & 25.261 & 25.297 & 36.533 \\
\hline 11/23/09 8:00 & 11.863 & 4.992 & 6.038 & 18.144 & 8.413 & 18.367 & 9.472 & 25.252 & 25.287 & 36.526 \\
\hline 11/23/09 12:00 & 11.870 & 5.158 & 6.069 & 18.139 & 8.340 & 18.370 & 9.463 & 25.275 & 25.275 & 36.529 \\
\hline 11/23/09 16:00 & 11.859 & 5.212 & 6.085 & 18.235 & 8.262 & 18.325 & 9.435 & 25.155 & 25.266 & 36.526 \\
\hline 11/23/09 20:00 & 11.868 & 5.046 & 6.111 & 18.207 & 8.203 & 18.318 & 9.432 & 25.141 & 25.254 & 36.524 \\
\hline 11/24/09 0:00 & 11.861 & 5.104 & 6.132 & 18.230 & 8.151 & 18.290 & 9.409 & 25.120 & 25.245 & 36.521 \\
\hline 11/24/09 4:00 & 11.234 & 4.734 & 5.900 & 18.235 & 8.045 & 18.128 & 9.329 & 25.040 & 25.231 & 36.517 \\
\hline 11/24/09 8:00 & 10.558 & 3.988 & 5.534 & 18.144 & 7.923 & 17.955 & 9.240 & 24.958 & 25.217 & 36.512 \\
\hline 11/24/09 12:00 & 10.190 & 3.422 & 5.367 & 17.939 & 7.833 & 17.889 & 9.204 & 24.981 & 25.207 & 36.510 \\
\hline 11/24/09 16:00 & 10.016 & 3.132 & 5.257 & 17.827 & 7.753 & 17.854 & 9.169 & 24.864 & 25.196 & 36.505 \\
\hline $11 / 24 / 0920: 00$ & 10.074 & 3.118 & 5.203 & 17.734 & 7.682 & 17.891 & 9.153 & 24.871 & 25.182 & 36.503 \\
\hline 11/25/09 0:00 & 10.150 & 3.237 & 5.182 & 17.725 & 7.609 & 17.884 & 9.120 & 24.871 & 25.170 & 36.498 \\
\hline 11/25/09 4:00 & 10.225 & 3.361 & 5.172 & 17.720 & 7.548 & 17.868 & 9.089 & 24.824 & 25.156 & 36.493 \\
\hline 11/25/09 8:00 & 10.322 & 3.546 & 5.179 & 17.678 & 7.503 & 17.884 & 9.073 & 24.807 & 25.144 & 36.489 \\
\hline 11/25/09 12:00 & 10.400 & 3.764 & 5.182 & 17.708 & 7.451 & 17.868 & 9.038 & 24.779 & 25.130 & 36.484 \\
\hline 11/25/09 16:00 & 10.461 & 3.848 & 5.170 & 17.720 & 7.411 & 17.837 & 9.007 & 24.734 & 25.118 & 36.479 \\
\hline 11/25/09 20:00 & 10.527 & 3.897 & 5.179 & 17.713 & 7.385 & 17.830 & 8.986 & 24.706 & 25.104 & 36.474 \\
\hline 11/26/09 0:00 & 10.586 & 3.979 & 5.193 & 17.706 & 7.331 & 17.819 & 8.965 & 24.676 & 25.090 & 36.470 \\
\hline 11/26/09 4:00 & 10.640 & 4.044 & 5.219 & 17.694 & 7.281 & 17.821 & 8.948 & 24.657 & 25.078 & 36.470 \\
\hline 11/26/09 8:00 & 10.694 & 4.086 & 5.247 & 17.666 & 7.227 & 17.821 & 8.937 & 24.687 & 25.067 & 36.465 \\
\hline 11/26/09 12:00 & 10.746 & 4.267 & 5.280 & 17.645 & 7.182 & 17.837 & 8.925 & 24.621 & 25.053 & 36.463 \\
\hline 11/26/09 16:00 & 10.749 & 4.449 & 5.299 & 17.802 & 7.121 & 17.760 & 8.871 & 24.549 & 25.041 & 36.458 \\
\hline $11 / 26 / 0920: 00$ & 10.760 & 4.468 & 5.318 & 17.837 & 7.064 & 17.716 & 8.838 & 24.497 & 25.027 & 36.453 \\
\hline 11/27/09 0:00 & 10.756 & 4.540 & 5.336 & 17.878 & 7.012 & 17.664 & 8.791 & 24.452 & 25.015 & 36.451 \\
\hline 11/27/09 4:00 & 10.734 & 4.606 & 5.351 & 17.937 & 6.963 & 17.587 & 8.737 & 24.323 & 25.001 & 36.446 \\
\hline 11/27/09 8:00 & 10.723 & 4.662 & 5.365 & 17.981 & 6.946 & 17.526 & 8.697 & 24.255 & 24.987 & 36.444 \\
\hline 11/27/09 12:00 & 10.723 & 4.870 & 5.388 & 18.004 & 6.930 & 17.488 & 8.664 & 24.288 & 24.971 & 36.439 \\
\hline 11/27/09 16:00 & 10.697 & 5.064 & 5.397 & 18.142 & 6.894 & 17.406 & 8.610 & 24.299 & 24.954 & 36.434 \\
\hline 11/27/09 20:00 & 10.706 & 4.826 & 5.421 & 18.111 & 6.880 & 17.376 & 8.587 & 24.201 & 24.942 & 36.430 \\
\hline 11/28/09 0:00 & 10.716 & 4.756 & 5.447 & 18.072 & 6.869 & 17.350 & 8.568 & 24.027 & 24.926 & 36.427 \\
\hline 11/28/09 4:00 & 10.734 & 4.739 & 5.475 & 18.028 & 6.871 & 17.340 & 8.551 & 23.982 & 24.912 & 36.423 \\
\hline 11/28/09 8:00 & 10.756 & 4.734 & 5.505 & 17.995 & 6.864 & 17.331 & 8.547 & 23.918 & 24.898 & 36.415 \\
\hline 11/28/09 12:00 & 10.774 & 4.940 & 5.538 & 17.969 & 6.861 & 17.336 & 8.528 & 23.961 & 24.881 & 36.413 \\
\hline 11/28/09 16:00 & 10.765 & 5.090 & 5.559 & 18.090 & 6.836 & 17.265 & 8.493 & 23.970 & 24.867 & 36.408 \\
\hline $11 / 28 / 0920: 00$ & 10.796 & 4.795 & 5.592 & 18.004 & 6.836 & 17.289 & 8.502 & 23.878 & 24.853 & 36.404 \\
\hline $11 / 29 / 090: 00$ & 10.819 & 4.784 & 5.625 & 17.974 & 6.833 & 17.291 & 8.493 & 23.817 & 24.837 & 36.399 \\
\hline 11/29/09 4:00 & 10.857 & 4.734 & 5.663 & 17.883 & 6.847 & 17.322 & 8.511 & 23.782 & 24.823 & 36.392 \\
\hline 11/29/09 8:00 & 10.925 & 4.646 & 5.705 & 17.788 & 6.871 & 17.390 & 8.565 & 23.735 & 24.809 & 36.390 \\
\hline 11/29/09 12:00 & 10.987 & 4.699 & 5.759 & 17.685 & 6.894 & 17.483 & 8.598 & 23.787 & 24.795 & 36.385 \\
\hline 11/29/09 16:00 & 11.013 & 4.788 & 5.792 & 17.769 & 6.876 & 17.465 & 8.582 & 23.864 & 24.780 & 36.380 \\
\hline
\end{tabular}


Water Level (ft below reference point)

\begin{tabular}{|c|c|c|c|c|c|c|c|c|c|c|}
\hline Date and Time & McPike & Potter & Hughes & Barr & Burks & MoDOT & Clizer "Old" & Morgan & SB24 & SB38 \\
\hline 11/29/09 20:00 & 11.060 & 4.678 & 5.834 & 17.699 & 6.892 & 17.521 & 8.610 & 23.829 & 24.766 & 36.373 \\
\hline 11/30/09 0:00 & 11.093 & 4.660 & 5.874 & 17.685 & 6.899 & 17.544 & 8.612 & 23.855 & 24.750 & 36.368 \\
\hline 11/30/09 4:00 & 11.107 & 4.756 & 5.907 & 17.701 & 6.890 & 17.544 & 8.594 & 23.911 & 24.738 & 36.368 \\
\hline 11/30/09 8:00 & 11.114 & 4.858 & 5.937 & 17.741 & 6.869 & 17.530 & 8.584 & 23.970 & 24.724 & 36.364 \\
\hline 11/30/09 12:00 & 11.109 & 5.006 & 5.965 & 17.792 & 6.847 & 17.502 & 8.551 & 23.996 & 24.710 & 36.357 \\
\hline 11/30/09 16:00 & 11.076 & 5.163 & 5.982 & 17.965 & 6.814 & 17.420 & 8.497 & 24.041 & 24.698 & 36.352 \\
\hline 11/30/09 20:00 & 11.062 & 5.034 & 6.001 & 17.979 & 6.810 & 17.387 & 8.474 & 23.956 & 24.682 & 36.345 \\
\hline 12/1/09 0:00 & 11.048 & 5.022 & 6.022 & 17.983 & 6.795 & 17.347 & 8.453 & 23.890 & 24.668 & 36.342 \\
\hline 12/1/09 4:00 & 11.022 & 5.050 & 6.038 & 18.025 & 6.779 & 17.289 & 8.417 & 23.871 & 24.654 & 36.338 \\
\hline 12/1/09 8:00 & 11.003 & 5.050 & 6.055 & 18.049 & 6.765 & 17.235 & 8.396 & 23.853 & 24.640 & 36.333 \\
\hline 12/1/09 12:00 & 10.994 & 5.221 & 6.078 & 18.049 & 6.772 & 17.228 & 8.380 & 23.827 & 24.626 & 36.328 \\
\hline 12/1/09 16:00 & 10.972 & 5.319 & 6.092 & 18.144 & 6.760 & 17.169 & 8.352 & 23.803 & 24.612 & 36.321 \\
\hline 12/1/09 20:00 & 10.987 & 5.043 & 6.116 & 18.072 & 6.781 & 17.181 & 8.370 & 23.672 & 24.595 & 36.317 \\
\hline 12/2/09 0:00 & 11.022 & 4.947 & 6.148 & 17.979 & 6.805 & 17.223 & 8.401 & 23.667 & 24.581 & 36.312 \\
\hline 12/2/09 4:00 & 11.048 & 4.924 & 6.184 & 17.904 & 6.819 & 17.258 & 8.413 & 23.582 & 24.567 & 36.305 \\
\hline 12/2/09 8:00 & 11.071 & 4.891 & 6.212 & 17.864 & 6.814 & 17.275 & 8.429 & 23.582 & 24.553 & 36.300 \\
\hline 12/2/09 12:00 & 11.083 & 5.018 & 6.247 & 17.855 & 6.812 & 17.291 & 8.415 & 23.648 & 24.539 & 36.295 \\
\hline 12/2/09 16:00 & 11.081 & 5.013 & 6.266 & 17.923 & 6.793 & 17.254 & 8.413 & 23.693 & 24.525 & 36.288 \\
\hline 12/2/09 20:00 & 11.121 & 4.917 & 6.301 & 17.823 & 6.817 & 17.319 & 8.457 & 23.669 & 24.511 & 36.286 \\
\hline 12/3/09 0:00 & 11.156 & 4.875 & 6.339 & 17.753 & 6.831 & 17.376 & 8.490 & 23.655 & 24.497 & 36.279 \\
\hline 12/3/09 4:00 & 11.192 & 4.870 & 6.374 & 17.722 & 6.836 & 17.425 & 8.516 & 23.686 & 24.483 & 36.277 \\
\hline 12/3/09 8:00 & 11.225 & 4.830 & 6.411 & 17.697 & 6.843 & 17.462 & 8.539 & 23.716 & 24.471 & 36.272 \\
\hline 12/3/09 12:00 & 11.258 & 4.980 & 6.449 & 17.666 & 6.854 & 17.514 & 8.561 & 23.841 & 24.457 & 36.267 \\
\hline 12/3/09 16:00 & 11.274 & 5.053 & 6.479 & 17.713 & 6.838 & 17.519 & 8.563 & 23.928 & 24.445 & 36.260 \\
\hline 12/3/09 20:00 & 11.305 & 5.006 & 6.512 & 17.687 & 6.845 & 17.568 & 8.594 & 23.951 & 24.433 & 36.258 \\
\hline 12/4/09 0:00 & 11.321 & 5.025 & 6.545 & 17.706 & 6.840 & 17.582 & 8.596 & 23.925 & 24.419 & 36.251 \\
\hline 12/4/09 4:00 & 11.333 & 5.001 & 6.573 & 17.748 & 6.831 & 17.587 & 8.591 & 23.956 & 24.408 & 36.246 \\
\hline 12/4/09 8:00 & 11.331 & 5.083 & 6.599 & 17.788 & 6.814 & 17.572 & 8.582 & 23.977 & 24.394 & 36.241 \\
\hline 12/4/09 12:00 & 11.326 & 5.158 & 6.623 & 17.823 & 6.810 & 17.563 & 8.561 & 24.076 & 24.382 & 36.236 \\
\hline 12/4/09 16:00 & 11.291 & 5.188 & 6.634 & 17.946 & 6.781 & 17.481 & 8.518 & 24.081 & 24.365 & 36.232 \\
\hline 12/4/09 20:00 & 11.269 & 5.184 & 6.651 & 17.990 & 6.784 & 17.446 & 8.493 & 24.001 & 24.354 & 36.227 \\
\hline 12/5/09 0:00 & 11.253 & 5.202 & 6.667 & 18.011 & 6.784 & 17.415 & 8.478 & 23.968 & 24.340 & 36.222 \\
\hline 12/5/09 4:00 & 11.234 & 5.226 & 6.684 & 18.037 & 6.779 & 17.380 & 8.455 & 23.963 & 24.326 & 36.218 \\
\hline 12/5/09 8:00 & 11.218 & 5.193 & 6.695 & 18.056 & 6.774 & 17.340 & 8.450 & 23.867 & 24.314 & 36.213 \\
\hline $12 / 5 / 0912: 00$ & 11.218 & 5.317 & 6.719 & 18.014 & 6.788 & 17.357 & 8.450 & 23.890 & 24.300 & 36.208 \\
\hline 12/5/09 16:00 & 11.196 & 5.401 & 6.731 & 18.123 & 6.767 & 17.282 & 8.413 & 23.907 & 24.286 & 36.201 \\
\hline 12/5/09 20:00 & 11.222 & 5.221 & 6.752 & 18.049 & 6.805 & 17.329 & 8.457 & 23.810 & 24.274 & 36.196 \\
\hline 12/6/09 0:00 & 11.258 & 5.170 & 6.785 & 17.958 & 6.836 & 17.399 & 8.500 & 23.810 & 24.260 & 36.192 \\
\hline 12/6/09 4:00 & 11.286 & 5.153 & 6.813 & 17.920 & 6.845 & 17.432 & 8.528 & 23.883 & 24.248 & 36.187 \\
\hline 12/6/09 8:00 & 11.305 & 5.179 & 6.841 & 17.906 & 6.845 & 17.451 & 8.539 & 23.838 & 24.236 & 36.182 \\
\hline 12/6/09 12:00 & 11.326 & 5.216 & 6.869 & 17.860 & 6.854 & 17.502 & 8.549 & 23.883 & 24.225 & 36.178 \\
\hline 12/6/09 16:00 & 11.284 & 5.233 & 6.878 & 18.035 & 6.812 & 17.385 & 8.488 & 23.963 & 24.213 & 36.173 \\
\hline 12/6/09 20:00 & 11.293 & 5.165 & 6.900 & 17.965 & 6.821 & 17.413 & 8.514 & 23.871 & 24.199 & 36.166 \\
\hline 12/7/09 0:00 & 11.317 & 5.151 & 6.925 & 17.911 & 6.836 & 17.451 & 8.546 & 23.867 & 24.187 & 36.161 \\
\hline 12/7/09 4:00 & 11.373 & 5.046 & 6.958 & 17.799 & 6.861 & 17.551 & 8.610 & 23.878 & 24.175 & 36.156 \\
\hline 12/7/09 8:00 & 11.413 & 5.008 & 6.991 & 17.757 & 6.869 & 17.608 & 8.645 & 23.925 & 24.164 & 36.152 \\
\hline $12 / 7 / 0912: 00$ & 11.451 & 5.097 & 7.026 & 17.729 & 6.871 & 17.666 & 8.666 & 24.001 & 24.154 & 36.147 \\
\hline 12/7/09 16:00 & 11.456 & 5.191 & 7.052 & 17.792 & 6.850 & 17.666 & 8.662 & 24.102 & 24.145 & 36.142 \\
\hline 12/7/09 20:00 & 11.453 & 5.198 & 7.071 & 17.827 & 6.838 & 17.657 & 8.650 & 24.128 & 24.133 & 36.135 \\
\hline 12/8/09 0:00 & 11.432 & 5.237 & 7.087 & 17.888 & 6.817 & 17.615 & 8.619 & 24.147 & 24.124 & 36.133 \\
\hline 12/8/09 4:00 & 11.397 & 5.261 & 7.097 & 17.995 & 6.793 & 17.551 & 8.572 & 24.161 & 24.114 & 36.128 \\
\hline 12/8/09 8:00 & 11.326 & 5.350 & 7.092 & 18.156 & 6.767 & 17.415 & 8.483 & 24.147 & 24.100 & 36.123 \\
\hline 12/8/09 12:00 & 11.236 & 5.556 & 7.076 & 18.328 & 6.734 & 17.242 & 8.380 & 24.099 & 24.091 & 36.116 \\
\hline 12/8/09 16:00 & 11.152 & 5.630 & 7.052 & 18.447 & 6.713 & 17.080 & 8.307 & 23.733 & 24.077 & 36.112 \\
\hline 12/8/09 20:00 & 11.088 & 5.658 & 7.036 & 18.489 & 6.715 & 16.958 & 8.265 & 23.599 & 24.065 & 36.107 \\
\hline 12/9/09 0:00 & 11.064 & 5.502 & 7.031 & 18.414 & 6.727 & 16.932 & 8.279 & 23.502 & 24.051 & 36.100 \\
\hline 12/9/09 4:00 & 11.140 & 5.263 & 7.062 & 18.109 & 6.800 & 17.087 & 8.408 & 23.378 & 24.039 & 36.093 \\
\hline 12/9/09 8:00 & 11.236 & 5.113 & 7.109 & 17.855 & 6.866 & 17.277 & 8.518 & 23.413 & 24.030 & 36.090 \\
\hline $12 / 9 / 09$ 12:00 & 11.324 & 5.055 & 7.156 & 17.662 & 6.911 & 17.453 & 8.605 & 23.533 & 24.016 & 36.083 \\
\hline $12 / 9 / 0916: 00$ & 11.378 & 5.041 & 7.196 & 17.643 & 6.904 & 17.512 & 8.650 & 23.681 & 24.004 & 36.079 \\
\hline 12/9/09 20:00 & 11.432 & 4.938 & 7.233 & 17.585 & 6.911 & 17.601 & 8.697 & 23.773 & 23.995 & 36.074 \\
\hline 12/10/09 0:00 & 11.472 & 5.015 & 7.264 & 17.571 & 6.904 & 17.657 & 8.720 & 23.878 & 23.983 & 36.072 \\
\hline 12/10/09 4:00 & 11.493 & 5.111 & 7.292 & 17.608 & 6.885 & 17.673 & 8.737 & 23.982 & 23.974 & 36.065 \\
\hline 12/10/09 8:00 & 11.517 & 5.146 & 7.318 & 17.606 & 6.878 & 17.704 & 8.756 & 24.083 & 23.964 & 36.057 \\
\hline
\end{tabular}


Water Level (ft below reference point)

\begin{tabular}{|c|c|c|c|c|c|c|c|c|c|c|}
\hline Date and Time & McPike & Potter & Hughes & Barr & Burks & MoDOT & Clizer "Old" & Morgan & SB24 & SB38 \\
\hline $12 / 10 / 09$ 12:00 & 11.552 & 5.102 & 7.346 & 17.576 & 6.885 & 17.784 & 8.779 & 24.135 & 23.957 & 36.053 \\
\hline 12/10/09 16:00 & 11.557 & 5.165 & 7.367 & 17.655 & 6.859 & 17.760 & 8.777 & 24.224 & 23.948 & 36.048 \\
\hline 12/10/09 20:00 & 11.581 & 5.146 & 7.390 & 17.652 & 6.857 & 17.805 & 8.800 & 24.283 & 23.939 & 36.046 \\
\hline 12/11/09 0:00 & 11.599 & 5.163 & 7.414 & 17.652 & 6.854 & 17.835 & 8.814 & 24.288 & 23.931 & 36.041 \\
\hline 12/11/09 4:00 & 11.618 & 5.163 & 7.437 & 17.655 & 6.854 & 17.873 & 8.831 & 24.321 & 23.922 & 36.036 \\
\hline 12/11/09 8:00 & 11.637 & 5.160 & 7.461 & 17.680 & 6.852 & 17.891 & 8.845 & 24.391 & 23.913 & 36.032 \\
\hline 12/11/09 12:00 & 11.647 & 5.181 & 7.480 & 17.706 & 6.847 & 17.919 & 8.842 & 24.476 & 23.906 & 36.024 \\
\hline 12/11/09 16:00 & 11.621 & 5.223 & 7.489 & 17.834 & 6.824 & 17.854 & 8.810 & 24.544 & 23.899 & 36.022 \\
\hline 12/11/09 20:00 & 11.621 & 5.188 & 7.503 & 17.862 & 6.829 & 17.859 & 8.812 & 24.478 & 23.889 & 36.015 \\
\hline 12/12/09 0:00 & 11.614 & 5.230 & 7.517 & 17.906 & 6.814 & 17.840 & 8.803 & 24.469 & 23.882 & 36.015 \\
\hline 12/12/09 4:00 & 11.606 & 5.251 & 7.527 & 17.944 & 6.807 & 17.828 & 8.795 & 24.483 & 23.875 & 36.008 \\
\hline 12/12/09 8:00 & 11.588 & 5.265 & 7.534 & 18.009 & 6.793 & 17.788 & 8.770 & 24.476 & 23.866 & 36.003 \\
\hline $12 / 12 / 09$ 12:00 & 11.559 & 5.296 & 7.541 & 18.051 & 6.779 & 17.755 & 8.734 & 24.537 & 23.859 & 36.001 \\
\hline $12 / 12 / 09$ 16:00 & 11.517 & 5.329 & 7.534 & 18.163 & 6.755 & 17.659 & 8.692 & 24.417 & 23.849 & 35.996 \\
\hline 12/12/09 20:00 & 11.503 & 5.343 & 7.538 & 18.139 & 6.767 & 17.650 & 8.697 & 24.356 & 23.840 & 35.989 \\
\hline 12/13/09 0:00 & 11.500 & 5.319 & 7.543 & 18.109 & 6.774 & 17.645 & 8.706 & 24.297 & 23.831 & 35.987 \\
\hline 12/13/09 4:00 & 11.512 & 5.298 & 7.557 & 18.032 & 6.793 & 17.680 & 8.730 & 24.262 & 23.824 & 35.984 \\
\hline 12/13/09 8:00 & 11.552 & 5.237 & 7.571 & 17.944 & 6.817 & 17.739 & 8.784 & 24.236 & 23.814 & 35.977 \\
\hline 12/13/09 12:00 & 11.576 & 5.294 & 7.590 & 17.878 & 6.838 & 17.793 & 8.800 & 24.311 & 23.805 & 35.975 \\
\hline 12/13/09 16:00 & 11.552 & 5.333 & 7.595 & 17.995 & 6.812 & 17.727 & 8.763 & 24.393 & 23.798 & 35.970 \\
\hline $12 / 13 / 09$ 20:00 & 11.526 & 5.383 & 7.592 & 18.032 & 6.795 & 17.678 & 8.737 & 24.389 & 23.788 & 35.966 \\
\hline 12/14/09 0:00 & 11.498 & 5.415 & 7.588 & 18.077 & 6.786 & 17.626 & 8.704 & 24.332 & 23.781 & 35.961 \\
\hline 12/14/09 4:00 & 11.479 & 5.399 & 7.574 & 18.069 & 6.772 & 17.572 & 8.697 & 24.281 & 23.772 & 35.959 \\
\hline 12/14/09 8:00 & 11.548 & 5.102 & 7.569 & 17.818 & 6.821 & 17.687 & 8.817 & 24.144 & 23.767 & 35.954 \\
\hline 12/14/09 12:00 & 11.665 & 4.868 & 7.597 & 17.548 & 6.894 & 17.903 & 8.936 & 24.158 & 23.756 & 35.949 \\
\hline $12 / 14 / 09$ 16:00 & 11.736 & 4.833 & 7.625 & 17.466 & 6.913 & 18.004 & 8.998 & 24.353 & 23.751 & 35.944 \\
\hline 12/14/09 20:00 & 11.807 & 4.844 & 7.656 & 17.410 & 6.927 & 18.100 & 9.054 & 24.403 & 23.746 & 35.940 \\
\hline 12/15/09 0:00 & 11.861 & 4.863 & 7.684 & 17.378 & 6.935 & 18.184 & 9.096 & 24.525 & 23.739 & 35.940 \\
\hline 12/15/09 4:00 & 11.913 & 4.844 & 7.712 & 17.359 & 6.942 & 18.269 & 9.134 & 24.659 & 23.734 & 35.933 \\
\hline 12/15/09 8:00 & 11.948 & 4.903 & 7.738 & 17.396 & 6.925 & 18.309 & 9.160 & 24.796 & 23.730 & 35.933 \\
\hline 12/15/09 12:00 & 11.981 & 5.099 & 7.764 & 17.401 & 6.923 & 18.377 & 9.181 & 24.955 & 23.725 & 35.926 \\
\hline 12/15/09 16:00 & 11.981 & 5.179 & 7.782 & 17.543 & 6.890 & 18.349 & 9.160 & 25.106 & 23.720 & 35.923 \\
\hline 12/15/09 20:00 & 11.986 & 5.107 & 7.799 & 17.606 & 6.871 & 18.353 & 9.157 & 25.073 & 23.718 & 35.918 \\
\hline 12/16/09 0:00 & 11.981 & 5.160 & 7.811 & 17.666 & 6.857 & 18.344 & 9.145 & 25.106 & 23.713 & 35.918 \\
\hline $12 / 16 / 094: 00$ & 11.955 & 5.170 & 7.818 & 17.760 & 6.833 & 18.306 & 9.106 & 25.141 & 23.706 & 35.914 \\
\hline 12/16/09 8:00 & 11.932 & 5.198 & 7.822 & 17.860 & 6.812 & 18.260 & 9.077 & 25.153 & 23.704 & 35.911 \\
\hline 12/16/09 12:00 & 11.915 & 5.242 & 7.827 & 17.899 & 6.807 & 18.248 & 9.066 & 25.144 & 23.699 & 35.907 \\
\hline 12/16/09 16:00 & 11.882 & 5.315 & 7.829 & 18.039 & 6.784 & 18.180 & 9.028 & 25.176 & 23.695 & 35.904 \\
\hline 12/16/09 20:00 & 11.873 & 5.282 & 7.834 & 18.062 & 6.786 & 18.163 & 9.028 & 25.132 & 23.687 & 35.902 \\
\hline 12/17/09 0:00 & 11.856 & 5.317 & 7.837 & 18.097 & 6.784 & 18.140 & 9.009 & 25.080 & 23.683 & 35.897 \\
\hline 12/17/09 4:00 & 11.842 & 5.322 & 7.841 & 18.111 & 6.779 & 18.114 & 9.002 & 25.028 & 23.678 & 35.895 \\
\hline 12/17/09 8:00 & 11.830 & 5.326 & 7.844 & 18.125 & 6.774 & 18.088 & 8.997 & 24.993 & 23.673 & 35.893 \\
\hline 12/17/09 12:00 & 11.831 & 5.394 & 7.851 & 18.104 & 6.789 & 18.102 & 9.002 & 24.998 & 23.666 & 35.890 \\
\hline 12/17/09 16:00 & 11.814 & 5.446 & 7.851 & 18.181 & 6.779 & 18.060 & 8.988 & 25.005 & 23.659 & 35.885 \\
\hline 12/17/09 20:00 & 11.828 & 5.350 & 7.858 & 18.097 & 6.793 & 18.093 & 9.014 & 24.925 & 23.657 & 35.883 \\
\hline 12/18/09 0:00 & 11.833 & 5.310 & 7.860 & 18.065 & 6.798 & 18.098 & 9.019 & 24.892 & 23.650 & 35.881 \\
\hline $12 / 18 / 094: 00$ & 11.835 & 5.280 & 7.860 & 18.049 & 6.798 & 18.093 & 9.026 & 24.868 & 23.645 & 35.876 \\
\hline 12/18/09 8:00 & 11.852 & 5.265 & 7.867 & 18.000 & 6.810 & 18.121 & 9.049 & 24.857 & 23.641 & 35.871 \\
\hline 12/18/09 12:00 & 11.859 & 5.312 & 7.874 & 17.986 & 6.807 & 18.142 & 9.054 & 24.890 & 23.636 & 35.869 \\
\hline 12/18/09 16:00 & 11.854 & 5.329 & 7.879 & 18.032 & 6.796 & 18.114 & 9.054 & 24.927 & 23.631 & 35.867 \\
\hline 12/18/09 20:00 & 11.880 & 5.273 & 7.893 & 17.944 & 6.807 & 18.170 & 9.087 & 24.875 & 23.627 & 35.864 \\
\hline 12/19/09 0:00 & 11.908 & 5.247 & 7.907 & 17.888 & 6.814 & 18.215 & 9.120 & 24.859 & 23.622 & 35.862 \\
\hline 12/19/09 4:00 & 11.939 & 5.228 & 7.921 & 17.827 & 6.824 & 18.271 & 9.148 & 24.878 & 23.617 & 35.857 \\
\hline 12/19/09 8:00 & 11.972 & 5.205 & 7.940 & 17.785 & 6.831 & 18.325 & 9.183 & 24.932 & 23.612 & 35.853 \\
\hline 12/19/09 12:00 & 12.005 & 5.221 & 7.961 & 17.746 & 6.843 & 18.393 & 9.211 & 25.038 & 23.608 & 35.853 \\
\hline 12/19/09 16:00 & 12.012 & 5.231 & 7.975 & 17.799 & 6.824 & 18.384 & 9.211 & 25.057 & 23.605 & 35.850 \\
\hline 12/19/09 20:00 & 12.031 & 5.219 & 7.991 & 17.788 & 6.833 & 18.426 & 9.228 & 25.089 & 23.601 & 35.848 \\
\hline 12/20/09 0:00 & 12.031 & 5.249 & 8.001 & 17.844 & 6.822 & 18.417 & 9.216 & 25.153 & 23.598 & 35.845 \\
\hline 12/20/09 4:00 & 12.024 & 5.282 & 8.008 & 17.874 & 6.814 & 18.407 & 9.207 & 25.174 & 23.596 & 35.841 \\
\hline 12/20/09 8:00 & 12.007 & 5.317 & 8.015 & 17.941 & 6.805 & 18.372 & 9.183 & 25.231 & 23.591 & 35.838 \\
\hline $12 / 20 / 09$ 12:00 & 12.007 & 5.338 & 8.020 & 17.948 & 6.803 & 18.377 & 9.185 & 25.282 & 23.587 & 35.836 \\
\hline 12/20/09 16:00 & 12.017 & 5.331 & 8.027 & 17.993 & 6.805 & 18.379 & 9.202 & 25.223 & 23.584 & 35.836 \\
\hline $12 / 20 / 09$ 20:00 & 12.061 & 5.242 & 8.043 & 17.862 & 6.840 & 18.480 & 9.256 & 25.183 & 23.580 & 35.834 \\
\hline 12/21/09 0:00 & 12.087 & 5.258 & 8.055 & 17.846 & 6.843 & 18.517 & 9.277 & 25.221 & 23.577 & 35.829 \\
\hline
\end{tabular}


Water Level (ft below reference point)

\begin{tabular}{|c|c|c|c|c|c|c|c|c|c|c|}
\hline Date and Time & McPike & Potter & Hughes & Barr & Burks & MoDOT & Clizer "Old" & Morgan & SB24 & SB38 \\
\hline 12/21/09 4:00 & 12.102 & 5.270 & 8.067 & 17.844 & 6.840 & 18.541 & 9.286 & 25.254 & 23.575 & 35.827 \\
\hline 12/21/09 8:00 & 12.106 & 5.305 & 8.074 & 17.867 & 6.831 & 18.543 & 9.286 & 25.313 & 23.573 & 35.824 \\
\hline 12/21/09 12:00 & 12.106 & 5.376 & 8.083 & 17.895 & 6.824 & 18.550 & 9.279 & 25.343 & 23.570 & 35.824 \\
\hline 12/21/09 16:00 & 12.078 & 5.453 & 8.078 & 18.021 & 6.795 & 18.489 & 9.246 & 25.402 & 23.566 & 35.820 \\
\hline $12 / 21 / 0920: 00$ & 12.052 & 5.380 & 8.062 & 18.051 & 6.788 & 18.445 & 9.223 & 25.346 & 23.561 & 35.817 \\
\hline 12/22/09 0:00 & 12.047 & 5.350 & 8.050 & 18.028 & 6.798 & 18.449 & 9.228 & 25.308 & 23.561 & 35.817 \\
\hline 12/22/09 4:00 & 12.045 & 5.350 & 8.038 & 18.023 & 6.791 & 18.431 & 9.221 & 25.296 & 23.558 & 35.812 \\
\hline 12/22/09 8:00 & 12.057 & 5.289 & 8.036 & 17.969 & 6.803 & 18.442 & 9.242 & 25.259 & 23.556 & 35.810 \\
\hline $12 / 22 / 09$ 12:00 & 12.080 & 5.312 & 8.043 & 17.909 & 6.821 & 18.494 & 9.270 & 25.299 & 23.552 & 35.808 \\
\hline $12 / 22 / 09$ 16:00 & 12.090 & 5.291 & 8.045 & 17.953 & 6.814 & 18.487 & 9.279 & 25.327 & 23.549 & 35.808 \\
\hline $12 / 22 / 0920: 00$ & 12.116 & 5.228 & 8.050 & 17.892 & 6.824 & 18.527 & 9.310 & 25.301 & 23.547 & 35.805 \\
\hline 12/23/09 0:00 & 12.128 & 5.242 & 8.053 & 17.869 & 6.826 & 18.550 & 9.315 & 25.322 & 23.544 & 35.803 \\
\hline 12/23/09 4:00 & 12.113 & 5.275 & 8.050 & 17.916 & 6.810 & 18.520 & 9.298 & 25.355 & 23.540 & 35.798 \\
\hline 12/23/09 8:00 & 12.087 & 5.261 & 8.043 & 17.972 & 6.798 & 18.487 & 9.284 & 25.390 & 23.540 & 35.798 \\
\hline 12/23/09 12:00 & 11.946 & 5.076 & 7.996 & 17.946 & 6.800 & 18.456 & 9.272 & 25.360 & 23.535 & 35.796 \\
\hline $12 / 23 / 09$ 16:00 & 11.717 & 4.983 & 7.919 & 17.986 & 6.788 & 18.398 & 9.235 & 25.332 & 23.533 & 35.791 \\
\hline 12/23/09 20:00 & 11.095 & 4.494 & 7.747 & 17.955 & 6.779 & 18.316 & 9.214 & 25.289 & 23.530 & 35.789 \\
\hline 12/24/09 0:00 & 10.822 & 4.129 & 7.602 & 17.909 & 6.786 & 18.283 & 9.207 & 25.240 & 23.528 & 35.787 \\
\hline 12/24/09 4:00 & 10.848 & 3.953 & 7.510 & 17.858 & 6.788 & 18.250 & 9.192 & 25.219 & 23.526 & 35.784 \\
\hline 12/24/09 8:00 & 10.706 & 3.752 & 7.374 & 17.797 & 6.769 & 18.163 & 9.169 & 25.179 & 23.521 & 35.782 \\
\hline $12 / 24 / 09$ 12:00 & 10.544 & 3.525 & 7.217 & 17.764 & 6.753 & 18.093 & 9.122 & 25.134 & 23.516 & 35.777 \\
\hline 12/24/09 16:00 & 10.574 & 3.518 & 7.116 & 17.832 & 6.715 & 17.985 & 9.054 & 25.104 & 23.512 & 35.775 \\
\hline $12 / 24 / 0920: 00$ & 10.650 & 3.612 & 7.051 & 17.816 & 6.696 & 17.931 & 9.004 & 24.998 & 23.509 & 35.772 \\
\hline 12/25/09 0:00 & 10.685 & 3.801 & 6.994 & 17.979 & 6.645 & 17.753 & 8.922 & 24.946 & 23.505 & 35.770 \\
\hline 12/25/09 4:00 & 10.716 & 4.052 & 6.947 & 17.993 & 6.630 & 17.669 & 8.868 & 24.887 & 23.500 & 35.765 \\
\hline 12/25/09 8:00 & 10.791 & 4.112 & 6.924 & 17.932 & 6.630 & 17.652 & 8.880 & 24.713 & 23.495 & 35.761 \\
\hline 12/25/09 12:00 & 10.871 & 4.208 & 6.915 & 17.844 & 6.647 & 17.695 & 8.878 & 24.664 & 23.488 & 35.758 \\
\hline $12 / 25 / 09$ 16:00 & 10.933 & 4.285 & 6.905 & 17.844 & 6.637 & 17.671 & 8.875 & 24.643 & 23.481 & 35.756 \\
\hline 12/25/09 20:00 & 11.001 & 4.351 & 6.903 & 17.797 & 6.642 & 17.692 & 8.882 & 24.624 & 23.479 & 35.751 \\
\hline 12/26/09 0:00 & 11.072 & 4.377 & 6.908 & 17.736 & 6.649 & 17.725 & 8.906 & 24.626 & 23.472 & 35.747 \\
\hline 12/26/09 4:00 & 11.145 & 4.384 & 6.919 & 17.669 & 6.661 & 17.784 & 8.939 & 24.570 & 23.467 & 35.744 \\
\hline 12/26/09 8:00 & 11.237 & 4.409 & 6.938 & 17.583 & 6.682 & 17.875 & 8.993 & 24.558 & 23.462 & 35.739 \\
\hline 12/26/09 12:00 & 11.319 & 4.444 & 6.959 & 17.515 & 6.699 & 17.955 & 9.028 & 24.596 & 23.458 & 35.737 \\
\hline $12 / 26 / 09$ 16:00 & 11.406 & 4.451 & 6.983 & 17.471 & 6.708 & 18.041 & 9.077 & 24.631 & 23.453 & 35.735 \\
\hline $12 / 26 / 0920: 00$ & 11.479 & 4.482 & 7.006 & 17.440 & 6.713 & 18.109 & 9.101 & 24.704 & 23.451 & 35.730 \\
\hline 12/27/09 0:00 & 11.522 & 4.522 & 7.023 & 17.487 & 6.696 & 18.119 & 9.098 & 24.788 & 23.446 & 35.728 \\
\hline 12/27/09 4:00 & 11.552 & 4.550 & 7.032 & 17.520 & 6.678 & 18.121 & 9.087 & 24.831 & 23.444 & 35.728 \\
\hline 12/27/09 8:00 & 11.607 & 4.526 & 7.046 & 17.489 & 6.687 & 18.177 & 9.129 & 24.840 & 23.441 & 35.723 \\
\hline 12/27/09 12:00 & 11.684 & 4.496 & 7.077 & 17.399 & 6.715 & 18.299 & 9.183 & 24.861 & 23.437 & 35.721 \\
\hline $12 / 27 / 09$ 16:00 & 11.732 & 4.538 & 7.098 & 17.445 & 6.703 & 18.330 & 9.190 & 24.984 & 23.434 & 35.716 \\
\hline 12/27/09 20:00 & 11.767 & 4.559 & 7.114 & 17.459 & 6.699 & 18.358 & 9.202 & 25.005 & 23.434 & 35.716 \\
\hline 12/28/09 0:00 & 11.795 & 4.578 & 7.131 & 17.508 & 6.687 & 18.379 & 9.199 & 25.066 & 23.430 & 35.711 \\
\hline 12/28/09 4:00 & 11.816 & 4.587 & 7.142 & 17.562 & 6.680 & 18.386 & 9.195 & 25.120 & 23.427 & 35.711 \\
\hline 12/28/09 8:00 & 11.835 & 4.587 & 7.154 & 17.594 & 6.670 & 18.384 & 9.192 & 25.160 & 23.425 & 35.706 \\
\hline 12/28/09 12:00 & 11.880 & 4.596 & 7.170 & 17.538 & 6.689 & 18.459 & 9.228 & 25.160 & 23.425 & 35.706 \\
\hline $12 / 28 / 09$ 16:00 & 11.911 & 4.589 & 7.189 & 17.550 & 6.687 & 18.492 & 9.244 & 25.205 & 23.423 & 35.704 \\
\hline $12 / 28 / 0920: 00$ & 11.955 & 4.571 & 7.210 & 17.522 & 6.699 & 18.555 & 9.277 & 25.209 & 23.423 & 35.702 \\
\hline 12/29/09 0:00 & 11.984 & 4.596 & 7.229 & 17.545 & 6.692 & 18.583 & 9.279 & 25.289 & 23.420 & 35.702 \\
\hline 12/29/09 4:00 & 11.993 & 4.622 & 7.241 & 17.576 & 6.680 & 18.576 & 9.268 & 25.322 & 23.420 & 35.697 \\
\hline 12/29/09 8:00 & 11.996 & 4.639 & 7.250 & 17.639 & 6.663 & 18.560 & 9.249 & 25.397 & 23.418 & 35.695 \\
\hline 12/29/09 12:00 & 11.993 & 4.704 & 7.257 & 17.683 & 6.656 & 18.541 & 9.223 & 25.449 & 23.418 & 35.692 \\
\hline 12/29/09 16:00 & 11.948 & 4.739 & 7.253 & 17.825 & 6.628 & 18.454 & 9.160 & 25.534 & 23.416 & 35.690 \\
\hline 12/29/09 20:00 & 11.915 & 4.765 & 7.248 & 17.937 & 6.609 & 18.374 & 9.117 & 25.395 & 23.415 & 35.692 \\
\hline 12/30/09 0:00 & 11.892 & 4.795 & 7.241 & 17.986 & 6.604 & 18.328 & 9.087 & 25.346 & 23.413 & 35.688 \\
\hline 12/30/09 4:00 & 11.854 & 4.823 & 7.234 & 18.063 & 6.592 & 18.255 & 9.042 & 25.336 & 23.411 & 35.685 \\
\hline 12/30/09 8:00 & 11.823 & 4.854 & 7.224 & 18.128 & 6.581 & 18.189 & 9.009 & 25.273 & 23.406 & 35.685 \\
\hline 12/30/09 12:00 & 11.805 & 4.865 & 7.222 & 18.128 & 6.585 & 18.163 & 8.993 & 25.198 & 23.404 & 35.681 \\
\hline $12 / 30 / 09$ 16:00 & 11.781 & 4.889 & 7.215 & 18.163 & 6.578 & 18.102 & 8.974 & 25.146 & 23.401 & 35.681 \\
\hline $12 / 30 / 0920: 00$ & 11.790 & 4.858 & 7.220 & 18.091 & 6.595 & 18.121 & 9.002 & 25.045 & 23.397 & 35.676 \\
\hline 12/31/09 0:00 & 11.826 & 4.828 & 7.239 & 17.990 & 6.621 & 18.180 & 9.047 & 24.995 & 23.394 & 35.676 \\
\hline 12/31/09 4:00 & 11.882 & 4.751 & 7.260 & 17.834 & 6.652 & 18.278 & 9.108 & 24.946 & 23.392 & 35.673 \\
\hline 12/31/09 8:00 & 11.937 & 4.680 & 7.285 & 17.748 & 6.670 & 18.351 & 9.162 & 24.962 & 23.390 & 35.669 \\
\hline 12/31/09 12:00 & 12.005 & 4.695 & 7.318 & 17.587 & 6.708 & 18.499 & 9.228 & 25.021 & 23.390 & 35.669 \\
\hline 12/31/09 16:00 & 12.033 & 4.725 & 7.342 & 17.604 & 6.696 & 18.532 & 9.242 & 25.127 & 23.387 & 35.669 \\
\hline
\end{tabular}

TABLE S1.1 Page 23 of 89 
Water Level (ft below reference point)

\begin{tabular}{|c|c|c|c|c|c|c|c|c|c|c|}
\hline Date and Time & McPike & Potter & Hughes & Barr & Burks & MoDOT & Clizer "Old" & Morgan & SB24 & SB38 \\
\hline 12/31/09 20:00 & 12.064 & 4.702 & 7.363 & 17.594 & 6.696 & 18.571 & 9.263 & 25.179 & 23.387 & 35.666 \\
\hline 1/1/10 0:00 & 12.087 & 4.702 & 7.384 & 17.592 & 6.694 & 18.604 & 9.279 & 25.238 & 23.385 & 35.664 \\
\hline 1/1/10 4:00 & 12.102 & 4.730 & 7.400 & 17.606 & 6.687 & 18.623 & 9.282 & 25.287 & 23.385 & 35.664 \\
\hline 1/1/10 8:00 & 12.120 & 4.741 & 7.417 & 17.618 & 6.684 & 18.646 & 9.291 & 25.339 & 23.383 & 35.662 \\
\hline $1 / 1 / 1012: 00$ & 12.144 & 4.741 & 7.438 & 17.601 & 6.694 & 18.698 & 9.312 & 25.383 & 23.383 & 35.657 \\
\hline 1/1/10 16:00 & 12.151 & 4.779 & 7.452 & 17.662 & 6.682 & 18.698 & 9.308 & 25.466 & 23.383 & 35.657 \\
\hline $1 / 1 / 10$ 20:00 & 12.170 & 4.751 & 7.469 & 17.634 & 6.689 & 18.736 & 9.324 & 25.435 & 23.383 & 35.657 \\
\hline $1 / 2 / 100: 00$ & 12.191 & 4.739 & 7.487 & 17.627 & 6.694 & 18.768 & 9.338 & 25.501 & 23.383 & 35.655 \\
\hline 1/2/10 4:00 & 12.196 & 4.769 & 7.501 & 17.646 & 6.687 & 18.775 & 9.331 & 25.487 & 23.383 & 35.652 \\
\hline $1 / 2 / 108: 00$ & 12.191 & 4.800 & 7.511 & 17.699 & 6.675 & 18.759 & 9.319 & 25.543 & 23.383 & 35.652 \\
\hline $1 / 2 / 1012: 00$ & 12.191 & 4.839 & 7.520 & 17.739 & 6.668 & 18.752 & 9.310 & 25.593 & 23.383 & 35.652 \\
\hline 1/2/10 16:00 & 12.151 & 4.872 & 7.522 & 17.874 & 6.642 & 18.682 & 9.256 & 25.644 & 23.383 & 35.648 \\
\hline 1/2/10 20:00 & 12.146 & 4.868 & 7.527 & 17.893 & 6.644 & 18.665 & 9.256 & 25.630 & 23.383 & 35.648 \\
\hline 1/3/10 0:00 & 12.153 & 4.879 & 7.537 & 17.881 & 6.656 & 18.679 & 9.268 & 25.562 & 23.383 & 35.645 \\
\hline 1/3/10 4:00 & 12.149 & 4.910 & 7.546 & 17.888 & 6.656 & 18.679 & 9.258 & 25.548 & 23.380 & 35.648 \\
\hline 1/3/10 8:00 & 12.161 & 4.893 & 7.555 & 17.883 & 6.658 & 18.693 & 9.275 & 25.539 & 23.380 & 35.643 \\
\hline $1 / 3 / 1012: 00$ & 12.184 & 4.910 & 7.572 & 17.839 & 6.677 & 18.745 & 9.303 & 25.543 & 23.383 & 35.645 \\
\hline 1/3/10 16:00 & 12.186 & 4.935 & 7.583 & 17.886 & 6.666 & 18.738 & 9.298 & 25.574 & 23.380 & 35.643 \\
\hline $1 / 3 / 10$ 20:00 & 12.208 & 4.905 & 7.598 & 17.853 & 6.677 & 18.764 & 9.322 & 25.553 & 23.380 & 35.641 \\
\hline $1 / 4 / 100: 00$ & 12.224 & 4.905 & 7.612 & 17.827 & 6.687 & 18.794 & 9.338 & 25.567 & 23.380 & 35.638 \\
\hline 1/4/10 4:00 & 12.231 & 4.910 & 7.626 & 17.834 & 6.684 & 18.801 & 9.343 & 25.586 & 23.380 & 35.638 \\
\hline $1 / 4 / 108: 00$ & 12.241 & 4.891 & 7.640 & 17.823 & 6.687 & 18.813 & 9.350 & 25.597 & 23.380 & 35.638 \\
\hline $1 / 4 / 1012: 00$ & 12.250 & 4.938 & 7.654 & 17.795 & 6.691 & 18.846 & 9.359 & 25.628 & 23.383 & 35.638 \\
\hline 1/4/10 16:00 & 12.234 & 4.977 & 7.659 & 17.876 & 6.673 & 18.808 & 9.333 & 25.675 & 23.383 & 35.636 \\
\hline 1/4/10 20:00 & 12.234 & 4.968 & 7.668 & 17.893 & 6.670 & 18.799 & 9.329 & 25.658 & 23.380 & 35.636 \\
\hline 1/5/10 0:00 & 12.234 & 4.975 & 7.677 & 17.895 & 6.673 & 18.794 & 9.329 & 25.644 & 23.383 & 35.633 \\
\hline 1/5/10 4:00 & 12.236 & 4.975 & 7.684 & 17.893 & 6.673 & 18.794 & 9.333 & 25.628 & 23.383 & 35.633 \\
\hline 1/5/10 8:00 & 12.241 & 4.980 & 7.696 & 17.890 & 6.675 & 18.796 & 9.338 & 25.626 & 23.383 & 35.633 \\
\hline $1 / 5 / 1012: 00$ & 12.245 & 5.015 & 7.706 & 17.867 & 6.680 & 18.822 & 9.345 & 25.635 & 23.383 & 35.631 \\
\hline 1/5/10 16:00 & 12.222 & 5.050 & 7.710 & 17.949 & 6.661 & 18.780 & 9.315 & 25.670 & 23.383 & 35.629 \\
\hline $1 / 5 / 1020: 00$ & 12.215 & 5.043 & 7.715 & 17.979 & 6.658 & 18.752 & 9.300 & 25.666 & 23.383 & 35.626 \\
\hline $1 / 6 / 100: 00$ & 12.203 & 5.071 & 7.722 & 17.981 & 6.658 & 18.743 & 9.291 & 25.680 & 23.383 & 35.629 \\
\hline 1/6/10 4:00 & 12.186 & 5.085 & 7.727 & 18.018 & 6.656 & 18.717 & 9.275 & 25.600 & 23.383 & 35.626 \\
\hline $1 / 6 / 108: 00$ & 12.174 & 5.092 & 7.729 & 18.065 & 6.647 & 18.675 & 9.263 & 25.590 & 23.380 & 35.629 \\
\hline 1/6/10 12:00 & 12.163 & 5.122 & 7.734 & 18.044 & 6.654 & 18.682 & 9.253 & 25.550 & 23.380 & 35.624 \\
\hline $1 / 6 / 1016: 00$ & 12.141 & 5.167 & 7.734 & 18.112 & 6.642 & 18.635 & 9.232 & 25.626 & 23.378 & 35.624 \\
\hline 1/6/10 20:00 & 12.160 & 5.115 & 7.745 & 18.037 & 6.663 & 18.675 & 9.270 & 25.473 & 23.378 & 35.622 \\
\hline 1/7/10 0:00 & 12.189 & 5.069 & 7.757 & 17.939 & 6.687 & 18.740 & 9.307 & 25.482 & 23.373 & 35.619 \\
\hline 1/7/10 4:00 & 12.196 & 5.090 & 7.769 & 17.946 & 6.682 & 18.740 & 9.305 & 25.548 & 23.376 & 35.619 \\
\hline 1/7/10 8:00 & 12.210 & 5.066 & 7.776 & 17.923 & 6.682 & 18.743 & 9.324 & 25.470 & 23.376 & 35.617 \\
\hline $1 / 7 / 1012: 00$ & 12.259 & 5.038 & 7.797 & 17.767 & 6.717 & 18.857 & 9.380 & 25.466 & 23.376 & 35.617 \\
\hline 1/7/10 16:00 & 12.288 & 5.031 & 7.816 & 17.750 & 6.717 & 18.888 & 9.409 & 25.534 & 23.378 & 35.615 \\
\hline $1 / 7 / 1020: 00$ & 12.318 & 5.008 & 7.832 & 17.695 & 6.727 & 18.951 & 9.437 & 25.579 & 23.378 & 35.617 \\
\hline 1/8/10 0:00 & 12.349 & 4.996 & 7.856 & 17.634 & 6.738 & 19.014 & 9.467 & 25.630 & 23.378 & 35.615 \\
\hline 1/8/10 4:00 & 12.370 & 4.982 & 7.872 & 17.629 & 6.739 & 19.047 & 9.486 & 25.720 & 23.378 & 35.615 \\
\hline $1 / 8 / 108: 00$ & 12.387 & 4.975 & 7.886 & 17.639 & 6.732 & 19.075 & 9.500 & 25.800 & 23.380 & 35.615 \\
\hline 1/8/10 12:00 & 12.413 & 4.996 & 7.905 & 17.599 & 6.743 & 19.141 & 9.524 & 25.767 & 23.380 & 35.612 \\
\hline $1 / 8 / 1016: 00$ & 12.405 & 5.026 & 7.917 & 17.702 & 6.717 & 19.120 & 9.510 & 25.851 & 23.383 & 35.612 \\
\hline $1 / 8 / 1020: 00$ & 12.422 & 5.017 & 7.929 & 17.713 & 6.720 & 19.132 & 9.521 & 25.875 & 23.385 & 35.610 \\
\hline 1/9/10 0:00 & 12.434 & 5.024 & 7.943 & 17.727 & 6.722 & 19.148 & 9.531 & 25.941 & 23.385 & 35.612 \\
\hline 1/9/10 4:00 & 12.443 & 5.024 & 7.952 & 17.727 & 6.727 & 19.172 & 9.540 & 25.941 & 23.387 & 35.610 \\
\hline 1/9/10 8:00 & 12.455 & 5.019 & 7.964 & 17.748 & 6.724 & 19.179 & 9.547 & 25.978 & 23.390 & 35.612 \\
\hline 1/9/10 12:00 & 12.476 & 5.005 & 7.978 & 17.711 & 6.741 & 19.244 & 9.571 & 25.985 & 23.392 & 35.610 \\
\hline 1/9/10 16:00 & 12.474 & 5.045 & 7.987 & 17.785 & 6.722 & 19.223 & 9.559 & 26.030 & 23.394 & 35.608 \\
\hline $1 / 9 / 1020: 00$ & 12.479 & 5.026 & 7.997 & 17.813 & 6.717 & 19.211 & 9.561 & 26.051 & 23.397 & 35.608 \\
\hline $1 / 10 / 100: 00$ & 12.479 & 5.050 & 8.006 & 17.832 & 6.717 & 19.204 & 9.557 & 26.065 & 23.397 & 35.610 \\
\hline $1 / 10 / 104: 00$ & 12.467 & 5.087 & 8.013 & 17.867 & 6.710 & 19.179 & 9.540 & 26.068 & 23.399 & 35.608 \\
\hline $1 / 10 / 108: 00$ & 12.446 & 5.101 & 8.013 & 17.939 & 6.691 & 19.122 & 9.510 & 26.058 & 23.399 & 35.610 \\
\hline 1/10/10 12:00 & 12.424 & 5.134 & 8.013 & 17.974 & 6.684 & 19.092 & 9.486 & 26.049 & 23.401 & 35.608 \\
\hline 1/10/10 16:00 & 12.375 & 5.169 & 8.004 & 18.121 & 6.651 & 18.986 & 9.423 & 26.014 & 23.404 & 35.608 \\
\hline 1/10/10 20:00 & 12.372 & 5.160 & 8.006 & 18.088 & 6.672 & 19.000 & 9.432 & 25.934 & 23.404 & 35.605 \\
\hline 1/11/10 0:00 & 12.365 & 5.178 & 8.008 & 18.086 & 6.682 & 18.998 & 9.432 & 25.917 & 23.404 & 35.605 \\
\hline $1 / 11 / 104: 00$ & 12.370 & 5.176 & 8.018 & 18.065 & 6.689 & 19.007 & 9.444 & 25.882 & 23.406 & 35.605 \\
\hline $1 / 11 / 108: 00$ & 12.403 & 5.129 & 8.029 & 17.967 & 6.713 & 19.071 & 9.491 & 25.835 & 23.406 & 35.605 \\
\hline
\end{tabular}


Water Level (ft below reference point)

\begin{tabular}{|c|c|c|c|c|c|c|c|c|c|c|}
\hline Date and Time & McPike & Potter & Hughes & Barr & Burks & MoDOT & Clizer "Old" & Morgan & SB24 & SB38 \\
\hline 1/11/10 12:00 & 12.457 & 5.099 & 8.051 & 17.844 & 6.746 & 19.181 & 9.554 & 25.837 & 23.408 & 35.605 \\
\hline 1/11/10 16:00 & 12.483 & 5.110 & 8.067 & 17.839 & 6.743 & 19.216 & 9.578 & 25.889 & 23.411 & 35.603 \\
\hline 1/11/10 20:00 & 12.516 & 5.087 & 8.086 & 17.767 & 6.757 & 19.289 & 9.613 & 25.936 & 23.413 & 35.603 \\
\hline 1/12/10 0:00 & 12.542 & 5.068 & 8.102 & 17.751 & 6.757 & 19.326 & 9.639 & 26.004 & 23.415 & 35.605 \\
\hline $1 / 12 / 104: 00$ & 12.556 & 5.068 & 8.114 & 17.753 & 6.753 & 19.343 & 9.648 & 26.101 & 23.415 & 35.605 \\
\hline 1/12/10 8:00 & 12.561 & 5.073 & 8.126 & 17.778 & 6.741 & 19.338 & 9.646 & 26.077 & 23.418 & 35.603 \\
\hline $1 / 12 / 1012: 00$ & 12.556 & 5.120 & 8.133 & 17.804 & 6.734 & 19.340 & 9.636 & 26.143 & 23.420 & 35.605 \\
\hline $1 / 12 / 1016: 00$ & 12.530 & 5.169 & 8.133 & 17.923 & 6.705 & 19.279 & 9.601 & 26.159 & 23.423 & 35.603 \\
\hline 1/12/10 20:00 & 12.507 & 5.192 & 8.133 & 17.993 & 6.694 & 19.240 & 9.568 & 26.148 & 23.425 & 35.603 \\
\hline $1 / 13 / 100: 00$ & 12.485 & 5.204 & 8.130 & 18.046 & 6.684 & 19.190 & 9.540 & 26.138 & 23.425 & 35.600 \\
\hline $1 / 13 / 104: 00$ & 12.467 & 5.218 & 8.126 & 18.072 & 6.679 & 19.153 & 9.517 & 26.119 & 23.429 & 35.603 \\
\hline 1/13/10 8:00 & 12.450 & 5.241 & 8.126 & 18.102 & 6.679 & 19.115 & 9.500 & 26.058 & 23.429 & 35.603 \\
\hline 1/13/10 12:00 & 12.443 & 5.311 & 8.128 & 18.079 & 6.694 & 19.129 & 9.498 & 26.021 & 23.432 & 35.600 \\
\hline 1/13/10 16:00 & 12.408 & 5.407 & 8.121 & 18.156 & 6.663 & 19.036 & 9.465 & 26.011 & 23.429 & 35.600 \\
\hline 1/13/10 20:00 & 12.410 & 5.288 & 8.119 & 18.109 & 6.677 & 19.022 & 9.479 & 25.931 & 23.432 & 35.600 \\
\hline 1/14/10 0:00 & 12.417 & 5.286 & 8.121 & 18.067 & 6.691 & 19.010 & 9.488 & 25.924 & 23.429 & 35.600 \\
\hline $1 / 14 / 104: 00$ & 12.412 & 5.265 & 8.116 & 18.042 & 6.691 & 18.982 & 9.488 & 25.929 & 23.432 & 35.598 \\
\hline 1/14/10 8:00 & 12.431 & 5.209 & 8.119 & 17.984 & 6.710 & 19.003 & 9.514 & 25.821 & 23.432 & 35.600 \\
\hline 1/14/10 12:00 & 12.476 & 5.183 & 8.130 & 17.869 & 6.741 & 19.090 & 9.566 & 26.223 & 23.434 & 35.600 \\
\hline $1 / 14 / 1016: 00$ & 12.490 & 5.372 & 8.133 & 17.883 & 6.736 & 19.061 & 9.585 & 25.971 & 23.437 & 35.603 \\
\hline 1/14/10 20:00 & 12.518 & 5.082 & 8.173 & 17.813 & 6.741 & 19.068 & 9.622 & 26.002 & 23.434 & 35.600 \\
\hline $1 / 15 / 100: 00$ & 12.537 & 5.035 & 8.180 & 17.790 & 6.743 & 19.101 & 9.650 & 26.049 & 23.434 & 35.603 \\
\hline $1 / 15 / 104: 00$ & 12.558 & 5.028 & 8.187 & 17.769 & 6.746 & 19.132 & 9.672 & 26.096 & 23.437 & 35.598 \\
\hline 1/15/10 8:00 & 12.568 & 5.047 & 8.192 & 17.795 & 6.738 & 19.132 & 9.676 & 26.141 & 23.437 & 35.600 \\
\hline 1/15/10 12:00 & 12.594 & 5.134 & 8.208 & 17.718 & 6.762 & 19.216 & 9.709 & 26.176 & 23.439 & 35.600 \\
\hline 1/15/10 16:00 & 12.584 & 5.239 & 8.208 & 17.846 & 6.727 & 19.153 & 9.690 & 26.176 & 23.441 & 35.600 \\
\hline 1/15/10 20:00 & 12.584 & 5.173 & 8.215 & 17.865 & 6.720 & 19.148 & 9.683 & 26.206 & 23.444 & 35.598 \\
\hline 1/16/10 0:00 & 12.587 & 5.164 & 8.217 & 17.902 & 6.712 & 19.134 & 9.681 & 26.110 & 23.444 & 35.600 \\
\hline $1 / 16 / 104: 00$ & 12.584 & 5.155 & 8.222 & 17.923 & 6.708 & 19.122 & 9.672 & 26.093 & 23.446 & 35.598 \\
\hline 1/16/10 8:00 & 12.575 & 5.222 & 8.224 & 17.972 & 6.705 & 19.094 & 9.660 & 26.072 & 23.448 & 35.600 \\
\hline 1/16/10 12:00 & 12.580 & 5.421 & 8.231 & 17.956 & 6.722 & 19.132 & 9.662 & 26.093 & 23.451 & 35.598 \\
\hline $1 / 16 / 1016: 00$ & 12.551 & 5.601 & 8.229 & 18.112 & 6.691 & 19.047 & 9.627 & 26.035 & 23.451 & 35.598 \\
\hline 1/16/10 20:00 & 12.540 & 5.477 & 8.227 & 18.130 & 6.689 & 19.033 & 9.615 & 26.014 & 23.451 & 35.598 \\
\hline 1/17/10 0:00 & 12.523 & 5.487 & 8.224 & 18.200 & 6.679 & 18.984 & 9.594 & 25.992 & 23.453 & 35.598 \\
\hline 1/17/10 4:00 & 12.516 & 5.489 & 8.224 & 18.212 & 6.682 & 18.979 & 9.587 & 25.887 & 23.455 & 35.598 \\
\hline $1 / 17 / 108: 00$ & 12.507 & 5.426 & 8.222 & 18.237 & 6.684 & 18.958 & 9.580 & 25.854 & 23.455 & 35.596 \\
\hline 1/17/10 12:00 & 12.514 & 5.555 & 8.229 & 18.205 & 6.698 & 18.993 & 9.596 & 25.868 & 23.455 & 35.598 \\
\hline 1/17/10 16:00 & 12.509 & 5.620 & 8.231 & 18.263 & 6.691 & 18.970 & 9.592 & 25.849 & 23.458 & 35.596 \\
\hline 1/17/10 20:00 & 12.523 & 5.159 & 8.234 & 18.189 & 6.715 & 19.017 & 9.620 & 25.908 & 23.458 & 35.596 \\
\hline 1/18/10 0:00 & 12.544 & 5.164 & 8.241 & 18.137 & 6.727 & 19.052 & 9.650 & 25.936 & 23.458 & 35.596 \\
\hline $1 / 18 / 104: 00$ & 12.549 & 5.162 & 8.241 & 18.137 & 6.724 & 19.047 & 9.658 & 25.945 & 23.460 & 35.598 \\
\hline 1/18/10 8:00 & 12.558 & 5.157 & 8.241 & 18.137 & 6.720 & 19.050 & 9.667 & 25.952 & 23.460 & 35.596 \\
\hline $1 / 18 / 1012: 00$ & 12.573 & 5.155 & 8.246 & 18.102 & 6.731 & 19.075 & 9.679 & 25.978 & 23.462 & 35.596 \\
\hline $1 / 18 / 1016: 00$ & 12.558 & 5.138 & 8.241 & 18.193 & 6.710 & 19.031 & 9.667 & 25.948 & 23.465 & 35.598 \\
\hline 1/18/10 20:00 & 12.563 & 5.134 & 8.232 & 18.154 & 6.722 & 19.045 & 9.676 & 25.962 & 23.465 & 35.598 \\
\hline $1 / 19 / 10$ 0:00 & 12.570 & 5.126 & 8.210 & 18.130 & 6.726 & 19.050 & 9.686 & 25.978 & 23.465 & 35.596 \\
\hline 1/19/10 4:00 & 12.563 & 5.112 & 8.189 & 18.144 & 6.719 & 19.024 & 9.679 & 25.971 & 23.467 & 35.596 \\
\hline $1 / 19 / 108: 00$ & 12.556 & 5.096 & 8.168 & 18.170 & 6.710 & 18.991 & 9.672 & 25.948 & 23.469 & 35.593 \\
\hline 1/19/10 12:00 & 12.556 & 5.082 & 8.154 & 18.177 & 6.710 & 18.991 & 9.669 & 25.945 & 23.469 & 35.593 \\
\hline 1/19/10 16:00 & 12.528 & 5.061 & 8.135 & 18.284 & 6.689 & 18.914 & 9.636 & 25.887 & 23.469 & 35.593 \\
\hline 1/19/10 20:00 & 12.481 & 5.012 & 8.095 & 18.284 & 6.686 & 18.888 & 9.618 & 25.851 & 23.469 & 35.593 \\
\hline 1/20/10 0:00 & 12.391 & 4.743 & 8.011 & 18.335 & 6.672 & 18.806 & 9.585 & 25.800 & 23.472 & 35.596 \\
\hline 1/20/10 4:00 & 12.045 & 4.186 & 7.830 & 18.431 & 6.712 & 18.524 & 9.524 & 25.694 & 23.472 & 35.596 \\
\hline 1/20/10 8:00 & 11.684 & 3.657 & 7.619 & 18.429 & 6.771 & 18.360 & 9.467 & 25.607 & 23.472 & 35.596 \\
\hline $1 / 20 / 1012: 00$ & 11.418 & 3.348 & 7.424 & 18.359 & 6.738 & 18.283 & 9.430 & 25.560 & 23.469 & 35.593 \\
\hline $1 / 20 / 1016: 00$ & 11.128 & 3.157 & 7.243 & 18.345 & 6.684 & 18.138 & 9.357 & 25.484 & 23.469 & 35.596 \\
\hline 1/20/10 20:00 & 10.913 & 3.068 & 7.091 & 18.256 & 6.550 & 18.051 & 9.300 & 25.440 & 23.467 & 35.593 \\
\hline $1 / 21 / 100: 00$ & 10.824 & 3.065 & 6.966 & 18.277 & 6.451 & 17.898 & 9.206 & 25.334 & 23.467 & 35.591 \\
\hline 1/21/10 4:00 & 10.819 & 3.119 & 6.873 & 18.242 & 6.370 & 17.812 & 9.120 & 25.226 & 23.465 & 35.591 \\
\hline 1/21/10 8:00 & 10.866 & 3.203 & 6.805 & 18.203 & 6.300 & 17.751 & 9.068 & 25.151 & 23.462 & 35.589 \\
\hline $1 / 21 / 1012: 00$ & 10.925 & 3.301 & 6.758 & 18.168 & 6.252 & 17.720 & 9.016 & 25.026 & 23.460 & 35.586 \\
\hline 1/21/10 16:00 & 10.859 & 3.313 & 6.713 & 18.226 & 6.281 & 17.629 & 8.960 & 24.901 & 23.458 & 35.584 \\
\hline 1/21/10 20:00 & 10.734 & 3.241 & 6.678 & 18.098 & 6.182 & 17.669 & 8.953 & 24.875 & 23.453 & 35.584 \\
\hline $1 / 22 / 100: 00$ & 10.720 & 3.243 & 6.636 & 18.035 & 6.109 & 17.648 & 8.922 & 24.838 & 23.451 & 35.582 \\
\hline
\end{tabular}


Water Level (ft below reference point)

\begin{tabular}{|c|c|c|c|c|c|c|c|c|c|c|}
\hline Date and Time & McPike & Potter & Hughes & Barr & Burks & MoDOT & Clizer "Old" & Morgan & SB24 & SB38 \\
\hline $1 / 22 / 104: 00$ & 10.729 & 3.297 & 6.589 & 18.049 & 6.130 & 17.577 & 8.859 & 24.774 & 23.448 & 35.579 \\
\hline 1/22/10 8:00 & 10.743 & 3.376 & 6.542 & 18.065 & 6.083 & 17.505 & 8.812 & 24.716 & 23.446 & 35.577 \\
\hline $1 / 22 / 1012: 00$ & 10.765 & 3.451 & 6.499 & 18.051 & 6.083 & 17.474 & 8.755 & 24.542 & 23.441 & 35.577 \\
\hline $1 / 22 / 1016: 00$ & 10.583 & 3.374 & 6.443 & 18.224 & 6.240 & 17.303 & 8.650 & 24.365 & 23.437 & 35.575 \\
\hline 1/22/10 20:00 & 10.239 & 3.189 & 6.309 & 18.240 & 6.255 & 17.141 & 8.518 & 24.224 & 23.432 & 35.572 \\
\hline 1/23/10 0:00 & 9.860 & 3.091 & 6.100 & 18.256 & 6.266 & 16.834 & 8.276 & 24.067 & 23.425 & 35.570 \\
\hline $1 / 23 / 104: 00$ & 9.544 & 3.056 & 5.878 & 18.310 & 6.217 & 16.450 & 7.947 & 23.857 & 23.420 & 35.567 \\
\hline $1 / 23 / 108: 00$ & 9.261 & 3.013 & 5.582 & 18.394 & 6.186 & 15.952 & 7.379 & 23.533 & 23.408 & 35.567 \\
\hline 1/23/10 12:00 & 9.011 & 2.985 & 5.258 & 18.403 & 6.198 & 15.420 & 6.583 & 22.957 & 23.399 & 35.567 \\
\hline $1 / 23 / 1016: 00$ & 8.785 & 2.983 & 4.932 & 18.494 & 6.163 & 14.834 & 5.563 & 22.329 & 23.385 & 35.570 \\
\hline 1/23/10 20:00 & 8.650 & 3.013 & 4.645 & 18.408 & 6.092 & 14.428 & 4.887 & 21.783 & 23.371 & 35.565 \\
\hline 1/24/10 0:00 & 8.537 & 3.067 & 4.460 & 18.345 & 6.033 & 14.213 & 4.614 & 21.409 & 23.354 & 35.556 \\
\hline 1/24/10 4:00 & 8.476 & 3.142 & 4.368 & 18.282 & 5.988 & 14.124 & 4.520 & 21.137 & 23.338 & 35.551 \\
\hline 1/24/10 8:00 & 8.509 & 3.240 & 4.340 & 18.151 & 5.906 & 14.140 & 4.530 & 20.977 & 23.322 & 35.544 \\
\hline 1/24/10 12:00 & 8.570 & 3.336 & 4.347 & 18.049 & 5.894 & 14.166 & 4.551 & 20.902 & 23.303 & 35.542 \\
\hline 1/24/10 16:00 & 8.591 & 3.418 & 4.354 & 18.088 & 5.948 & 14.121 & 4.562 & 20.634 & 23.282 & 35.537 \\
\hline 1/24/10 20:00 & 8.629 & 3.502 & 4.378 & 18.039 & 5.934 & 14.126 & 4.600 & 20.525 & 23.263 & 35.530 \\
\hline 1/25/10 0:00 & 8.645 & 3.577 & 4.399 & 18.039 & 5.948 & 14.093 & 4.621 & 20.412 & 23.242 & 35.525 \\
\hline $1 / 25 / 104: 00$ & 8.667 & 3.652 & 4.425 & 18.023 & 5.965 & 14.079 & 4.656 & 20.321 & 23.223 & 35.518 \\
\hline $1 / 25 / 108: 00$ & 8.721 & 3.734 & 4.462 & 17.949 & 5.906 & 14.112 & 4.732 & 20.234 & 23.200 & 35.513 \\
\hline 1/25/10 12:00 & 8.787 & 3.818 & 4.512 & 17.816 & 5.854 & 14.192 & 4.818 & 20.262 & 23.181 & 35.506 \\
\hline $1 / 25 / 1016: 00$ & 8.791 & 3.888 & 4.552 & 17.755 & 5.882 & 14.220 & 4.887 & 20.264 & 23.160 & 35.497 \\
\hline $1 / 25 / 1020: 00$ & 8.799 & 3.953 & 4.601 & 17.657 & 5.821 & 14.285 & 4.966 & 20.321 & 23.136 & 35.492 \\
\hline 1/26/10 0:00 & 8.810 & 4.021 & 4.645 & 17.576 & 6.156 & 14.328 & 5.035 & 20.368 & 23.115 & 35.483 \\
\hline 1/26/10 4:00 & 8.855 & 4.089 & 4.688 & 17.513 & 6.179 & 14.363 & 5.105 & 20.314 & 23.097 & 35.476 \\
\hline 1/26/10 8:00 & 8.921 & 4.166 & 4.739 & 17.429 & 6.212 & 14.428 & 5.185 & 20.373 & 23.075 & 35.473 \\
\hline 1/26/10 12:00 & 9.084 & 4.238 & 4.791 & 17.322 & 6.248 & 14.504 & 5.255 & 20.443 & 23.057 & 35.461 \\
\hline 1/26/10 16:00 & 9.284 & 4.280 & 4.819 & 17.413 & 6.231 & 14.445 & 5.258 & 20.410 & 23.035 & 35.452 \\
\hline $1 / 26 / 1020: 00$ & 9.183 & 4.313 & 4.850 & 17.438 & 6.243 & 14.436 & 5.298 & 20.389 & 23.012 & 35.445 \\
\hline 1/27/10 0:00 & 9.173 & 4.336 & 4.883 & 17.427 & 6.254 & 14.436 & 5.328 & 20.387 & 22.993 & 35.436 \\
\hline $1 / 27 / 104: 00$ & 9.218 & 4.360 & 4.913 & 17.448 & 6.259 & 14.421 & 5.347 & 20.387 & 22.972 & 35.426 \\
\hline $1 / 27 / 108: 00$ & 9.256 & 4.383 & 4.939 & 17.483 & 6.262 & 14.386 & 5.366 & 20.269 & 22.951 & 35.419 \\
\hline 1/27/10 12:00 & 9.279 & 4.411 & 4.972 & 17.462 & 6.281 & 14.410 & 5.406 & 20.269 & 22.930 & 35.412 \\
\hline $1 / 27 / 1016: 00$ & 9.333 & 4.430 & 4.995 & 17.543 & 6.281 & 14.375 & 5.434 & 20.224 & 22.909 & 35.403 \\
\hline 1/27/10 20:00 & 9.157 & 4.456 & 5.040 & 17.420 & 6.320 & 14.468 & 5.528 & 20.300 & 22.888 & 35.393 \\
\hline $1 / 28 / 100: 00$ & 9.218 & 4.475 & 5.089 & 17.329 & 6.349 & 14.536 & 5.582 & 20.394 & 22.869 & 35.388 \\
\hline 1/28/10 4:00 & 9.234 & 4.493 & 5.138 & 17.261 & 6.363 & 14.579 & 5.643 & 20.455 & 22.848 & 35.370 \\
\hline 1/28/10 8:00 & 9.272 & 4.514 & 5.181 & 17.205 & 6.377 & 14.607 & 5.695 & 20.509 & 22.827 & 35.370 \\
\hline $1 / 28 / 1012: 00$ & 9.444 & 4.542 & 5.228 & 17.147 & 6.391 & 14.649 & 5.730 & 20.577 & 22.810 & 35.360 \\
\hline 1/28/10 16:00 & 9.534 & 4.552 & 5.246 & 17.292 & 6.356 & 14.541 & 5.704 & 20.525 & 22.789 & 35.351 \\
\hline $1 / 28 / 1020: 00$ & 9.515 & 4.563 & 5.277 & 17.273 & 6.377 & 14.560 & 5.737 & 20.443 & 22.770 & 35.344 \\
\hline 1/29/10 0:00 & 9.477 & 4.453 & 5.303 & 17.287 & 6.382 & 14.543 & 5.760 & 20.422 & 22.749 & 35.334 \\
\hline $1 / 29 / 104: 00$ & 9.491 & 4.453 & 5.326 & 17.329 & 6.379 & 14.508 & 5.763 & 20.389 & 22.731 & 35.325 \\
\hline $1 / 29 / 108: 00$ & 9.494 & 4.474 & 5.345 & 17.389 & 6.368 & 14.454 & 5.758 & 20.325 & 22.710 & 35.315 \\
\hline 1/29/10 12:00 & 9.593 & 4.757 & 5.371 & 17.378 & 6.394 & 14.482 & 5.770 & 20.351 & 22.691 & 35.311 \\
\hline $1 / 29 / 1016: 00$ & 9.595 & 4.844 & 5.380 & 17.548 & 6.358 & 14.370 & 5.756 & 20.238 & 22.670 & 35.301 \\
\hline 1/29/10 20:00 & 9.538 & 4.785 & 5.404 & 17.534 & 6.379 & 14.384 & 5.779 & 20.215 & 22.648 & 35.292 \\
\hline $1 / 30 / 100: 00$ & 9.513 & 4.692 & 5.430 & 17.541 & 6.391 & 14.384 & 5.796 & 20.206 & 22.630 & 35.285 \\
\hline 1/30/10 4:00 & 9.491 & 4.762 & 5.453 & 17.543 & 6.401 & 14.377 & 5.814 & 20.191 & 22.611 & 35.273 \\
\hline 1/30/10 8:00 & 9.487 & 4.757 & 5.481 & 17.539 & 6.410 & 14.386 & 5.850 & 20.189 & 22.590 & 35.268 \\
\hline 1/30/10 12:00 & 9.609 & 4.928 & 5.516 & 17.492 & 6.431 & 14.424 & 5.880 & 20.229 & 22.571 & 35.257 \\
\hline 1/30/10 16:00 & 9.706 & 5.122 & 5.538 & 17.576 & 6.419 & 14.382 & 5.878 & 20.196 & 22.550 & 35.247 \\
\hline 1/30/10 20:00 & 9.571 & 4.958 & 5.566 & 17.571 & 6.434 & 14.400 & 5.915 & 20.208 & 22.529 & 35.240 \\
\hline 1/31/10 0:00 & 9.524 & 4.888 & 5.596 & 17.560 & 6.445 & 14.426 & 5.948 & 20.231 & 22.510 & 35.228 \\
\hline $1 / 31 / 104: 00$ & 9.546 & 4.879 & 5.627 & 17.546 & 6.453 & 14.443 & 5.972 & 20.260 & 22.491 & 35.219 \\
\hline $1 / 31 / 108: 00$ & 9.493 & 4.746 & 5.657 & 17.525 & 6.464 & 14.461 & 6.012 & 20.283 & 22.470 & 35.209 \\
\hline 1/31/10 12:00 & 9.593 & 4.870 & 5.692 & 17.497 & 6.476 & 14.492 & 6.042 & 20.323 & 22.451 & 35.202 \\
\hline $1 / 31 / 1016: 00$ & 9.748 & 5.309 & 5.716 & 17.597 & 6.462 & 14.457 & 6.040 & 20.328 & 22.433 & 35.193 \\
\hline $1 / 31 / 1020: 00$ & 9.510 & 4.708 & 5.742 & 17.597 & 6.474 & 14.475 & 6.073 & 20.304 & 22.414 & 35.184 \\
\hline 2/1/10 0:00 & 9.508 & 4.657 & 5.765 & 17.602 & 6.478 & 14.473 & 6.080 & 20.311 & 22.395 & 35.174 \\
\hline 2/1/10 4:00 & 9.522 & 4.755 & 5.786 & 17.632 & 6.474 & 14.454 & 6.087 & 20.302 & 22.374 & 35.167 \\
\hline 2/1/10 8:00 & 9.576 & 4.830 & 5.805 & 17.688 & 6.467 & 14.421 & 6.089 & 20.278 & 22.358 & 35.158 \\
\hline 2/1/10 12:00 & 9.663 & 4.916 & 5.824 & 17.716 & 6.469 & 14.417 & 6.099 & 20.271 & 22.337 & 35.146 \\
\hline $2 / 1 / 10$ 16:00 & 9.668 & 4.944 & 5.838 & 17.795 & 6.460 & 14.370 & 6.096 & 20.250 & 22.318 & 35.139 \\
\hline
\end{tabular}

TABLE S1.1 Page 26 of 89 
Water Level (ft below reference point)

\begin{tabular}{|c|c|c|c|c|c|c|c|c|c|c|}
\hline Date and Time & McPike & Potter & Hughes & Barr & Burks & MoDOT & Clizer "Old" & Morgan & SB24 & SB38 \\
\hline 2/1/10 20:00 & 9.616 & 4.865 & 5.861 & 17.767 & 6.478 & 14.405 & 6.131 & 20.290 & 22.299 & 35.127 \\
\hline 2/2/10 0:00 & 9.614 & 4.841 & 5.887 & 17.741 & 6.493 & 14.438 & 6.160 & 20.262 & 22.280 & 35.122 \\
\hline $2 / 2 / 104: 00$ & 9.616 & 4.809 & 5.913 & 17.720 & 6.502 & 14.464 & 6.188 & 20.295 & 22.261 & 35.111 \\
\hline $2 / 2 / 108: 00$ & 9.541 & 4.678 & 5.944 & 17.657 & 6.516 & 14.511 & 6.244 & 20.351 & 22.243 & 35.099 \\
\hline 2/2/10 12:00 & 9.588 & 4.668 & 5.981 & 17.557 & 6.540 & 14.586 & 6.289 & 20.450 & 22.226 & 35.092 \\
\hline 2/2/10 16:00 & 9.677 & 4.888 & 6.007 & 17.599 & 6.528 & 14.572 & 6.308 & 20.474 & 22.208 & 35.082 \\
\hline 2/2/10 20:00 & 9.595 & 4.643 & 6.033 & 17.553 & 6.544 & 14.616 & 6.348 & 20.539 & 22.189 & 35.073 \\
\hline $2 / 3 / 100: 00$ & 9.574 & 4.586 & 6.066 & 17.499 & 6.556 & 14.658 & 6.380 & 20.610 & 22.172 & 35.066 \\
\hline $2 / 3 / 104: 00$ & 9.611 & 4.584 & 6.091 & 17.501 & 6.549 & 14.658 & 6.397 & 20.673 & 22.156 & 35.056 \\
\hline $2 / 3 / 108: 00$ & 9.602 & 4.488 & 6.120 & 17.462 & 6.559 & 14.689 & 6.439 & 20.626 & 22.140 & 35.047 \\
\hline 2/3/10 12:00 & 9.776 & 4.706 & 6.150 & 17.441 & 6.563 & 14.715 & 6.442 & 20.673 & 22.123 & 35.040 \\
\hline $2 / 3 / 1016: 00$ & 9.852 & 4.954 & 6.169 & 17.522 & 6.549 & 14.677 & 6.449 & 20.652 & 22.107 & 35.030 \\
\hline 2/3/10 20:00 & 9.772 & 4.825 & 6.190 & 17.536 & 6.556 & 14.696 & 6.477 & 20.695 & 22.093 & 35.023 \\
\hline 2/4/10 0:00 & 9.786 & 4.830 & 6.213 & 17.555 & 6.561 & 14.705 & 6.484 & 20.704 & 22.076 & 35.014 \\
\hline $2 / 4 / 104: 00$ & 9.772 & 4.809 & 6.235 & 17.553 & 6.566 & 14.717 & 6.510 & 20.709 & 22.057 & 35.005 \\
\hline 2/4/10 8:00 & 9.800 & 4.825 & 6.253 & 17.604 & 6.556 & 14.691 & 6.512 & 20.697 & 22.043 & 34.997 \\
\hline 2/4/10 12:00 & 9.897 & 4.900 & 6.272 & 17.625 & 6.561 & 14.705 & 6.512 & 20.704 & 22.027 & 34.988 \\
\hline 2/4/10 16:00 & 9.946 & 4.933 & 6.282 & 17.748 & 6.535 & 14.632 & 6.491 & 20.648 & 22.011 & 34.981 \\
\hline 2/4/10 20:00 & 9.842 & 4.830 & 6.293 & 17.776 & 6.537 & 14.632 & 6.510 & 20.619 & 21.994 & 34.972 \\
\hline $2 / 5 / 100: 00$ & 9.826 & 4.809 & 6.307 & 17.786 & 6.540 & 14.630 & 6.517 & 20.615 & 21.978 & 34.962 \\
\hline 2/5/10 4:00 & 9.833 & 4.848 & 6.321 & 17.818 & 6.535 & 14.614 & 6.514 & 20.591 & 21.961 & 34.953 \\
\hline 2/5/10 8:00 & 9.823 & 4.855 & 6.333 & 17.849 & 6.533 & 14.600 & 6.519 & 20.572 & 21.947 & 34.943 \\
\hline 2/5/10 12:00 & 9.835 & 4.909 & 6.352 & 17.830 & 6.542 & 14.616 & 6.540 & 20.584 & 21.931 & 34.936 \\
\hline $2 / 5 / 1016: 00$ & 9.823 & 4.853 & 6.366 & 17.844 & 6.542 & 14.621 & 6.564 & 20.589 & 21.914 & 34.929 \\
\hline 2/5/10 20:00 & 9.753 & 4.694 & 6.392 & 17.730 & 6.573 & 14.719 & 6.629 & 20.671 & 21.900 & 34.917 \\
\hline 2/6/10 0:00 & 9.739 & 4.626 & 6.422 & 17.634 & 6.596 & 14.806 & 6.686 & 20.786 & 21.884 & 34.906 \\
\hline 2/6/10 4:00 & 9.753 & 4.584 & 6.450 & 17.590 & 6.603 & 14.848 & 6.719 & 20.864 & 21.870 & 34.896 \\
\hline 2/6/10 8:00 & 9.769 & 4.563 & 6.481 & 17.529 & 6.613 & 14.897 & 6.770 & 20.934 & 21.856 & 34.887 \\
\hline 2/6/10 12:00 & 9.894 & 4.654 & 6.509 & 17.492 & 6.615 & 14.937 & 6.801 & 21.007 & 21.842 & 34.875 \\
\hline 2/6/10 16:00 & 9.948 & 4.734 & 6.533 & 17.534 & 6.603 & 14.933 & 6.808 & 21.029 & 21.830 & 34.868 \\
\hline 2/6/10 20:00 & 9.904 & 4.652 & 6.551 & 17.527 & 6.606 & 14.944 & 6.829 & 21.050 & 21.816 & 34.861 \\
\hline $2 / 7 / 100: 00$ & 9.925 & 4.617 & 6.570 & 17.525 & 6.608 & 14.954 & 6.843 & 21.073 & 21.802 & 34.851 \\
\hline 2/7/10 4:00 & 9.930 & 4.586 & 6.584 & 17.553 & 6.599 & 14.940 & 6.846 & 21.104 & 21.788 & 34.842 \\
\hline 2/7/10 8:00 & 9.934 & 4.640 & 6.598 & 17.564 & 6.599 & 14.937 & 6.857 & 21.050 & 21.776 & 34.835 \\
\hline 2/7/10 12:00 & 9.981 & 4.729 & 6.615 & 17.585 & 6.599 & 14.940 & 6.862 & 21.059 & 21.762 & 34.830 \\
\hline 2/7/10 16:00 & 10.064 & 4.825 & 6.624 & 17.674 & 6.648 & 14.883 & 6.846 & 21.017 & 21.750 & 34.823 \\
\hline 2/7/10 20:00 & 9.993 & 4.738 & 6.634 & 17.669 & 6.669 & 14.897 & 6.871 & 21.019 & 21.738 & 34.816 \\
\hline 2/8/10 0:00 & 10.000 & 4.729 & 6.648 & 17.667 & 6.693 & 14.909 & 6.881 & 21.050 & 21.724 & 34.809 \\
\hline 2/8/10 4:00 & 10.007 & 4.708 & 6.659 & 17.676 & 6.719 & 14.909 & 6.895 & 21.071 & 21.710 & 34.802 \\
\hline 2/8/10 8:00 & 10.010 & 4.692 & 6.673 & 17.653 & 6.719 & 14.935 & 6.921 & 21.137 & 21.699 & 34.795 \\
\hline 2/8/10 12:00 & 9.918 & 4.437 & 6.692 & 17.588 & 6.627 & 14.991 & 6.970 & 21.101 & 21.685 & 34.788 \\
\hline 2/8/10 16:00 & 9.901 & 4.308 & 6.718 & 17.529 & 6.537 & 15.038 & 6.998 & 21.174 & 21.673 & 34.778 \\
\hline 2/8/10 20:00 & 9.922 & 4.242 & 6.739 & 17.476 & 6.455 & 15.085 & 7.036 & 21.240 & 21.661 & 34.771 \\
\hline 2/9/10 0:00 & 9.988 & 4.242 & 6.758 & 17.476 & 6.551 & 15.090 & 7.045 & 21.283 & 21.652 & 34.764 \\
\hline 2/9/10 4:00 & 10.038 & 4.464 & 6.767 & 17.536 & 6.499 & 15.043 & 7.019 & 21.285 & 21.638 & 34.760 \\
\hline 2/9/10 8:00 & 10.007 & 4.317 & 6.781 & 17.501 & 6.448 & 15.055 & 7.057 & 21.306 & 21.626 & 34.750 \\
\hline $2 / 9 / 1012: 00$ & 10.083 & 4.598 & 6.803 & 17.448 & 6.478 & 15.104 & 7.090 & 21.275 & 21.614 & 34.741 \\
\hline $2 / 9 / 1016: 00$ & 10.087 & 4.663 & 6.821 & 17.418 & 6.577 & 15.139 & 7.125 & 21.344 & 21.602 & 34.734 \\
\hline $2 / 9 / 1020: 00$ & 10.064 & 4.581 & 6.845 & 17.352 & 6.525 & 15.214 & 7.172 & 21.414 & 21.598 & 34.729 \\
\hline 2/10/10 0:00 & 10.073 & 4.549 & 6.871 & 17.301 & 6.516 & 15.273 & 7.214 & 21.496 & 21.584 & 34.722 \\
\hline 2/10/10 4:00 & 10.130 & 4.525 & 6.894 & 17.280 & 6.502 & 15.303 & 7.235 & 21.565 & 21.572 & 34.715 \\
\hline 2/10/10 8:00 & 10.168 & 4.520 & 6.915 & 17.268 & 6.509 & 15.324 & 7.257 & 21.591 & 21.563 & 34.705 \\
\hline $2 / 10 / 1012: 00$ & 10.240 & 4.644 & 6.936 & 17.259 & 6.544 & 15.355 & 7.282 & 21.638 & 21.553 & 34.701 \\
\hline 2/10/10 16:00 & 10.255 & 4.930 & 6.950 & 17.324 & 6.613 & 15.329 & 7.257 & 21.635 & 21.544 & 34.694 \\
\hline 2/10/10 20:00 & 10.158 & 4.642 & 6.960 & 17.355 & 6.615 & 15.317 & 7.271 & 21.647 & 21.534 & 34.689 \\
\hline $2 / 11 / 100: 00$ & 10.165 & 4.694 & 6.974 & 17.378 & 6.575 & 15.315 & 7.271 & 21.619 & 21.525 & 34.682 \\
\hline 2/11/10 4:00 & 10.217 & 4.752 & 6.983 & 17.420 & 6.603 & 15.298 & 7.261 & 21.595 & 21.516 & 34.675 \\
\hline 2/11/10 8:00 & 10.226 & 4.757 & 6.993 & 17.441 & 6.634 & 15.294 & 7.268 & 21.581 & 21.506 & 34.670 \\
\hline $2 / 11 / 10$ 12:00 & 10.276 & 4.825 & 7.002 & 17.471 & 6.681 & 15.303 & 7.275 & 21.576 & 21.497 & 34.663 \\
\hline 2/11/10 16:00 & 10.401 & 5.037 & 7.007 & 17.581 & 6.825 & 15.254 & 7.259 & 21.529 & 21.487 & 34.656 \\
\hline 2/11/10 20:00 & 10.273 & 4.850 & 7.019 & 17.576 & 6.764 & 15.294 & 7.294 & 21.551 & 21.478 & 34.651 \\
\hline $2 / 12 / 100: 00$ & 10.233 & 4.778 & 7.033 & 17.576 & 6.747 & 15.312 & 7.308 & 21.581 & 21.469 & 34.644 \\
\hline 2/12/10 4:00 & 10.252 & 4.750 & 7.044 & 17.592 & 6.782 & 15.322 & 7.313 & 21.602 & 21.459 & 34.637 \\
\hline 2/12/10 8:00 & 10.227 & 4.743 & 7.056 & 17.583 & 6.761 & 15.343 & 7.343 & 21.638 & 21.450 & 34.630 \\
\hline
\end{tabular}


Water Level (ft below reference point)

\begin{tabular}{|c|c|c|c|c|c|c|c|c|c|c|}
\hline Date and Time & McPike & Potter & Hughes & Barr & Burks & MoDOT & Clizer "Old" & Morgan & SB24 & SB38 \\
\hline 2/12/10 12:00 & 10.318 & 4.864 & 7.075 & 17.534 & 6.671 & 15.404 & 7.376 & 21.729 & 21.441 & 34.625 \\
\hline $2 / 12 / 1016: 00$ & 10.446 & 5.049 & 7.087 & 17.657 & 6.657 & 15.387 & 7.376 & 21.659 & 21.431 & 34.621 \\
\hline 2/12/10 20:00 & 10.306 & 4.733 & 7.103 & 17.618 & 6.667 & 15.427 & 7.402 & 21.692 & 21.422 & 34.613 \\
\hline 2/13/10 0:00 & 10.297 & 4.698 & 7.115 & 17.606 & 6.672 & 15.446 & 7.414 & 21.722 & 21.415 & 34.606 \\
\hline 2/13/10 4:00 & 10.321 & 4.717 & 7.124 & 17.641 & 6.662 & 15.437 & 7.409 & 21.734 & 21.408 & 34.599 \\
\hline 2/13/10 8:00 & 10.288 & 4.635 & 7.134 & 17.646 & 6.657 & 15.439 & 7.426 & 21.739 & 21.398 & 34.595 \\
\hline 2/13/10 12:00 & 10.424 & 4.761 & 7.148 & 17.662 & 6.662 & 15.458 & 7.430 & 21.769 & 21.391 & 34.590 \\
\hline 2/13/10 16:00 & 10.479 & 4.853 & 7.152 & 17.765 & 6.648 & 15.423 & 7.421 & 21.755 & 21.382 & 34.583 \\
\hline 2/13/10 20:00 & 10.349 & 4.719 & 7.164 & 17.725 & 6.660 & 15.465 & 7.461 & 21.805 & 21.375 & 34.578 \\
\hline 2/14/10 0:00 & 10.311 & 4.649 & 7.178 & 17.683 & 6.674 & 15.509 & 7.489 & 21.793 & 21.366 & 34.571 \\
\hline 2/14/10 4:00 & 10.194 & 4.436 & 7.197 & 17.576 & 6.688 & 15.575 & 7.539 & 21.863 & 21.358 & 34.566 \\
\hline 2/14/10 8:00 & 10.222 & 4.303 & 7.218 & 17.494 & 6.702 & 15.634 & 7.571 & 21.943 & 21.351 & 34.562 \\
\hline 2/14/10 12:00 & 10.372 & 4.448 & 7.232 & 17.504 & 6.693 & 15.638 & 7.578 & 21.976 & 21.344 & 34.555 \\
\hline 2/14/10 16:00 & 10.356 & 4.480 & 7.244 & 17.520 & 6.686 & 15.641 & 7.590 & 21.993 & 21.337 & 34.550 \\
\hline 2/14/10 20:00 & 10.290 & 4.380 & 7.258 & 17.452 & 6.700 & 15.699 & 7.625 & 22.056 & 21.330 & 34.545 \\
\hline 2/15/10 0:00 & 10.380 & 4.373 & 7.274 & 17.450 & 6.697 & 15.706 & 7.621 & 22.094 & 21.323 & 34.540 \\
\hline 2/15/10 4:00 & 10.417 & 4.457 & 7.281 & 17.497 & 6.679 & 15.676 & 7.616 & 22.084 & 21.319 & 34.533 \\
\hline 2/15/10 8:00 & 10.408 & 4.429 & 7.293 & 17.462 & 6.690 & 15.720 & 7.656 & 22.129 & 21.312 & 34.526 \\
\hline 2/15/10 12:00 & 10.460 & 4.588 & 7.314 & 17.390 & 6.709 & 15.788 & 7.696 & 22.242 & 21.305 & 34.524 \\
\hline 2/15/10 16:00 & 10.462 & 4.626 & 7.328 & 17.401 & 6.704 & 15.812 & 7.710 & 22.188 & 21.298 & 34.519 \\
\hline 2/15/10 20:00 & 10.431 & 4.590 & 7.345 & 17.378 & 6.714 & 15.849 & 7.733 & 22.240 & 21.293 & 34.512 \\
\hline 2/16/10 0:00 & 10.455 & 4.541 & 7.359 & 17.369 & 6.712 & 15.866 & 7.752 & 22.268 & 21.286 & 34.507 \\
\hline 2/16/10 4:00 & 10.488 & 4.590 & 7.373 & 17.338 & 6.719 & 15.906 & 7.773 & 22.322 & 21.281 & 34.503 \\
\hline 2/16/10 8:00 & 10.516 & 4.609 & 7.392 & 17.296 & 6.726 & 15.952 & 7.806 & 22.385 & 21.276 & 34.500 \\
\hline 2/16/10 12:00 & 10.561 & 4.719 & 7.408 & 17.252 & 6.735 & 16.011 & 7.837 & 22.461 & 21.269 & 34.496 \\
\hline 2/16/10 16:00 & 10.603 & 4.789 & 7.420 & 17.322 & 6.719 & 15.981 & 7.832 & 22.484 & 21.265 & 34.491 \\
\hline 2/16/10 20:00 & 10.561 & 4.747 & 7.432 & 17.329 & 6.716 & 16.002 & 7.844 & 22.524 & 21.260 & 34.489 \\
\hline 2/17/10 0:00 & 10.537 & 4.637 & 7.443 & 17.322 & 6.721 & 16.023 & 7.858 & 22.472 & 21.255 & 34.482 \\
\hline $2 / 17 / 104: 00$ & 10.566 & 4.637 & 7.457 & 17.322 & 6.723 & 16.039 & 7.865 & 22.496 & 21.253 & 34.479 \\
\hline 2/17/10 8:00 & 10.561 & 4.567 & 7.472 & 17.301 & 6.726 & 16.060 & 7.886 & 22.515 & 21.246 & 34.472 \\
\hline 2/17/10 12:00 & 10.665 & 4.782 & 7.483 & 17.273 & 6.733 & 16.100 & 7.910 & 22.562 & 21.244 & 34.470 \\
\hline 2/17/10 16:00 & 10.691 & 5.086 & 7.493 & 17.322 & 6.721 & 16.095 & 7.905 & 22.571 & 21.241 & 34.465 \\
\hline 2/17/10 20:00 & 10.589 & 4.801 & 7.504 & 17.338 & 6.726 & 16.117 & 7.928 & 22.595 & 21.234 & 34.460 \\
\hline 2/18/10 0:00 & 10.587 & 4.674 & 7.516 & 17.329 & 6.730 & 16.142 & 7.940 & 22.630 & 21.232 & 34.458 \\
\hline 2/18/10 4:00 & 10.653 & 4.759 & 7.530 & 17.320 & 6.733 & 16.163 & 7.954 & 22.658 & 21.229 & 34.456 \\
\hline 2/18/10 8:00 & 10.651 & 4.756 & 7.542 & 17.306 & 6.737 & 16.196 & 7.975 & 22.698 & 21.227 & 34.449 \\
\hline 2/18/10 12:00 & 10.735 & 4.932 & 7.556 & 17.303 & 6.735 & 16.220 & 7.985 & 22.738 & 21.222 & 34.446 \\
\hline 2/18/10 16:00 & 10.823 & 5.358 & 7.561 & 17.471 & 6.714 & 16.175 & 7.950 & 22.726 & 21.218 & 34.442 \\
\hline 2/18/10 20:00 & 10.677 & 4.915 & 7.561 & 17.522 & 6.711 & 16.182 & 7.975 & 22.738 & 21.215 & 34.437 \\
\hline 2/19/10 0:00 & 10.655 & 4.869 & 7.556 & 17.459 & 6.730 & 16.231 & 8.001 & 22.705 & 21.213 & 34.432 \\
\hline 2/19/10 4:00 & 10.717 & 4.920 & 7.551 & 17.492 & 6.726 & 16.229 & 7.999 & 22.747 & 21.208 & 34.430 \\
\hline 2/19/10 8:00 & 10.717 & 4.880 & 7.549 & 17.520 & 6.721 & 16.220 & 8.001 & 22.733 & 21.206 & 34.425 \\
\hline 2/19/10 12:00 & 10.745 & 5.030 & 7.549 & 17.520 & 6.723 & 16.229 & 8.004 & 22.743 & 21.201 & 34.423 \\
\hline 2/19/10 16:00 & 10.780 & 5.096 & 7.549 & 17.592 & 6.707 & 16.187 & 7.989 & 22.707 & 21.199 & 34.420 \\
\hline 2/19/10 20:00 & 10.745 & 5.035 & 7.554 & 17.564 & 6.721 & 16.227 & 8.025 & 22.722 & 21.197 & 34.416 \\
\hline $2 / 20 / 100: 00$ & 10.735 & 4.955 & 7.568 & 17.525 & 6.735 & 16.269 & 8.053 & 22.773 & 21.192 & 34.411 \\
\hline 2/20/10 4:00 & 10.750 & 4.824 & 7.579 & 17.494 & 6.745 & 16.316 & 8.081 & 22.827 & 21.190 & 34.409 \\
\hline 2/20/10 8:00 & 10.728 & 4.752 & 7.596 & 17.436 & 6.756 & 16.379 & 8.123 & 22.891 & 21.185 & 34.404 \\
\hline 2/20/10 12:00 & 10.792 & 4.859 & 7.612 & 17.418 & 6.759 & 16.426 & 8.133 & 22.952 & 21.183 & 34.399 \\
\hline 2/20/10 16:00 & 10.816 & 4.878 & 7.622 & 17.464 & 6.740 & 16.405 & 8.130 & 22.952 & 21.180 & 34.394 \\
\hline 2/20/10 20:00 & 10.804 & 4.829 & 7.631 & 17.450 & 6.744 & 16.428 & 8.147 & 22.973 & 21.178 & 34.392 \\
\hline 2/21/10 0:00 & 10.839 & 4.873 & 7.643 & 17.464 & 6.744 & 16.435 & 8.152 & 22.987 & 21.176 & 34.390 \\
\hline 2/21/10 4:00 & 10.848 & 4.869 & 7.650 & 17.508 & 6.733 & 16.414 & 8.133 & 22.973 & 21.176 & 34.387 \\
\hline 2/21/10 8:00 & 10.841 & 4.812 & 7.655 & 17.548 & 6.773 & 16.403 & 8.140 & 22.957 & 21.173 & 34.385 \\
\hline 2/21/10 12:00 & 10.849 & 4.808 & 7.659 & 17.564 & 6.768 & 16.403 & 8.130 & 22.952 & 21.171 & 34.380 \\
\hline 2/21/10 16:00 & 10.855 & 4.976 & 7.662 & 17.627 & 6.817 & 16.372 & 8.123 & 22.933 & 21.169 & 34.376 \\
\hline 2/21/10 20:00 & 10.856 & 4.927 & 7.676 & 17.571 & 6.825 & 16.440 & 8.168 & 22.987 & 21.166 & 34.371 \\
\hline $2 / 22 / 100: 00$ & 10.867 & 4.878 & 7.687 & 17.539 & 6.794 & 16.482 & 8.199 & 23.084 & 21.164 & 34.369 \\
\hline $2 / 22 / 104: 00$ & 10.924 & 4.845 & 7.699 & 17.532 & 6.737 & 16.513 & 8.213 & 23.041 & 21.164 & 34.369 \\
\hline 2/22/10 8:00 & 10.929 & 4.805 & 7.711 & 17.529 & 6.740 & 16.532 & 8.236 & 23.072 & 21.161 & 34.364 \\
\hline 2/22/10 12:00 & 10.995 & 4.810 & 7.725 & 17.483 & 6.733 & 16.588 & 8.257 & 23.138 & 21.161 & 34.359 \\
\hline $2 / 22 / 1016: 00$ & 11.028 & 4.815 & 7.734 & 17.522 & 6.770 & 16.578 & 8.255 & 23.150 & 21.159 & 34.357 \\
\hline 2/22/10 20:00 & 11.014 & 4.768 & 7.744 & 17.506 & 6.780 & 16.614 & 8.283 & 23.185 & 21.159 & 34.354 \\
\hline 2/23/10 0:00 & 11.030 & 4.747 & 7.755 & 17.487 & 6.759 & 16.646 & 8.288 & 23.237 & 21.159 & 34.350 \\
\hline
\end{tabular}

TABLE S1.1 Page 28 of 89 
Water Level (ft below reference point)

\begin{tabular}{|c|c|c|c|c|c|c|c|c|c|c|}
\hline Date and Time & McPike & Potter & Hughes & Barr & Burks & MoDOT & Clizer "Old" & Morgan & SB24 & SB38 \\
\hline 2/23/10 4:00 & 11.018 & 4.756 & 7.763 & 17.501 & 6.702 & 16.656 & 8.297 & 23.262 & 21.159 & 34.350 \\
\hline 2/23/10 8:00 & 11.009 & 4.752 & 7.772 & 17.518 & 6.688 & 16.656 & 8.309 & 23.284 & 21.157 & 34.347 \\
\hline 2/23/10 12:00 & 11.042 & 4.723 & 7.784 & 17.446 & 6.678 & 16.733 & 8.354 & 23.371 & 21.157 & 34.343 \\
\hline 2/23/10 16:00 & 11.065 & 4.773 & 7.798 & 17.436 & 6.690 & 16.759 & 8.368 & 23.345 & 21.157 & 34.340 \\
\hline 2/23/10 20:00 & 11.049 & 4.712 & 7.809 & 17.394 & 6.683 & 16.818 & 8.401 & 23.408 & 21.157 & 34.336 \\
\hline 2/24/10 0:00 & 11.044 & 4.674 & 7.826 & 17.343 & 6.631 & 16.876 & 8.433 & 23.509 & 21.157 & 34.338 \\
\hline 2/24/10 4:00 & 11.079 & 4.681 & 7.840 & 17.334 & 6.560 & 16.897 & 8.438 & 23.547 & 21.159 & 34.333 \\
\hline 2/24/10 8:00 & 11.063 & 4.656 & 7.852 & 17.301 & 6.558 & 16.933 & 8.469 & 23.582 & 21.159 & 34.328 \\
\hline 2/24/10 12:00 & 11.131 & 4.695 & 7.866 & 17.257 & 6.556 & 16.991 & 8.487 & 23.643 & 21.161 & 34.328 \\
\hline 2/24/10 16:00 & 11.167 & 4.812 & 7.871 & 17.324 & 6.657 & 16.970 & 8.466 & 23.643 & 21.161 & 34.326 \\
\hline $2 / 24 / 1020: 00$ & 11.150 & 4.749 & 7.880 & 17.364 & 6.681 & 16.958 & 8.471 & 23.634 & 21.164 & 34.324 \\
\hline 2/25/10 0:00 & 11.155 & 4.740 & 7.889 & 17.341 & 6.643 & 16.996 & 8.490 & 23.674 & 21.164 & 34.321 \\
\hline 2/25/10 4:00 & 11.157 & 4.780 & 7.896 & 17.364 & 6.402 & 16.989 & 8.480 & 23.697 & 21.166 & 34.321 \\
\hline 2/25/10 8:00 & 11.152 & 4.773 & 7.903 & 17.352 & 6.530 & 17.008 & 8.502 & 23.737 & 21.166 & 34.319 \\
\hline 2/25/10 12:00 & 11.209 & 4.854 & 7.913 & 17.350 & 6.664 & 17.033 & 8.513 & 23.693 & 21.169 & 34.319 \\
\hline 2/25/10 16:00 & 11.244 & 4.964 & 7.917 & 17.411 & 6.780 & 17.022 & 8.499 & 23.681 & 21.171 & 34.317 \\
\hline 2/25/10 20:00 & 11.202 & 4.871 & 7.925 & 17.406 & 6.676 & 17.038 & 8.527 & 23.695 & 21.171 & 34.317 \\
\hline 2/26/10 0:00 & 11.204 & 4.817 & 7.936 & 17.387 & 6.617 & 17.078 & 8.546 & 23.737 & 21.173 & 34.314 \\
\hline 2/26/10 4:00 & 11.223 & 4.824 & 7.946 & 17.380 & 6.603 & 17.097 & 8.560 & 23.770 & 21.176 & 34.312 \\
\hline $2 / 26 / 108: 00$ & 11.235 & 4.819 & 7.955 & 17.383 & 6.634 & 17.111 & 8.570 & 23.801 & 21.178 & 34.310 \\
\hline 2/26/10 12:00 & 11.291 & 4.915 & 7.964 & 17.359 & 6.787 & 17.155 & 8.588 & 23.848 & 21.178 & 34.310 \\
\hline 2/26/10 16:00 & 11.412 & 5.135 & 7.969 & 17.441 & 6.884 & 17.097 & 8.563 & 23.855 & 21.180 & 34.307 \\
\hline $2 / 26 / 1020: 00$ & 11.294 & 4.868 & 7.971 & 17.460 & 6.846 & 17.047 & 8.570 & 23.824 & 21.183 & 34.307 \\
\hline 2/27/10 0:00 & 11.244 & 4.803 & 7.974 & 17.460 & 6.846 & 17.078 & 8.577 & 23.831 & 21.185 & 34.307 \\
\hline 2/27/10 4:00 & 11.233 & 4.840 & 7.976 & 17.469 & 6.822 & 17.092 & 8.586 & 23.831 & 21.187 & 34.305 \\
\hline 2/27/10 8:00 & 11.244 & 4.803 & 7.983 & 17.439 & 6.747 & 17.129 & 8.612 & 23.916 & 21.190 & 34.300 \\
\hline 2/27/10 12:00 & 11.327 & 4.883 & 7.993 & 17.390 & 6.777 & 17.188 & 8.631 & 23.904 & 21.192 & 34.303 \\
\hline 2/27/10 16:00 & 11.395 & 5.007 & 8.000 & 17.420 & 6.822 & 17.190 & 8.631 & 23.928 & 21.194 & 34.300 \\
\hline $2 / 27 / 1020: 00$ & 11.214 & 4.831 & 7.997 & 17.448 & 6.801 & 17.162 & 8.638 & 23.940 & 21.197 & 34.300 \\
\hline 2/28/10 0:00 & 11.188 & 4.815 & 7.993 & 17.439 & 6.761 & 17.172 & 8.645 & 23.965 & 21.199 & 34.300 \\
\hline $2 / 28 / 104: 00$ & 11.223 & 4.798 & 7.988 & 17.446 & 6.725 & 17.172 & 8.650 & 23.980 & 21.201 & 34.298 \\
\hline $2 / 28 / 108: 00$ & 11.228 & 4.723 & 7.988 & 17.401 & 6.676 & 17.212 & 8.675 & 24.024 & 21.204 & 34.295 \\
\hline 2/28/10 12:00 & 11.372 & 4.911 & 7.995 & 17.383 & 6.718 & 17.244 & 8.682 & 24.085 & 21.206 & 34.295 \\
\hline $2 / 28 / 1016: 00$ & 11.492 & 5.203 & 8.000 & 17.460 & 6.796 & 17.221 & 8.671 & 23.996 & 21.208 & 34.295 \\
\hline 2/28/10 20:00 & 11.350 & 4.976 & 7.997 & 17.450 & 6.777 & 17.233 & 8.697 & 24.015 & 21.213 & 34.293 \\
\hline $3 / 1 / 100: 00$ & 11.308 & 4.878 & 7.993 & 17.383 & 6.699 & 17.284 & 8.729 & 24.064 & 21.215 & 34.293 \\
\hline 3/1/10 4:00 & 11.346 & 4.871 & 7.988 & 17.369 & 6.666 & 17.303 & 8.744 & 24.102 & 21.218 & 34.293 \\
\hline 3/1/10 8:00 & 11.322 & 4.852 & 7.988 & 17.334 & 6.645 & 17.333 & 8.765 & 24.142 & 21.222 & 34.291 \\
\hline 3/1/10 12:00 & 11.435 & 4.997 & 7.995 & 17.292 & 6.666 & 17.390 & 8.793 & 24.224 & 21.225 & 34.291 \\
\hline 3/1/10 16:00 & 11.530 & 5.093 & 7.997 & 17.406 & 6.735 & 17.359 & 8.776 & 24.208 & 21.229 & 34.291 \\
\hline $3 / 1 / 1020: 00$ & 11.454 & 4.934 & 7.964 & 17.383 & 6.685 & 17.366 & 8.793 & 24.219 & 21.232 & 34.291 \\
\hline 3/2/10 0:00 & 11.428 & 4.892 & 7.915 & 17.380 & 6.671 & 17.350 & 8.795 & 24.219 & 21.234 & 34.291 \\
\hline $3 / 2 / 104: 00$ & 11.435 & 4.857 & 7.875 & 17.399 & 6.640 & 17.324 & 8.788 & 24.205 & 21.239 & 34.288 \\
\hline $3 / 2 / 108: 00$ & 11.426 & 4.847 & 7.847 & 17.411 & 6.593 & 17.303 & 8.795 & 24.187 & 21.241 & 34.288 \\
\hline 3/2/10 12:00 & 11.584 & 4.995 & 7.835 & 17.394 & 6.732 & 17.315 & 8.800 & 24.184 & 21.246 & 34.291 \\
\hline $3 / 2 / 10$ 16:00 & 11.685 & 5.280 & 7.819 & 17.525 & 6.864 & 17.268 & 8.776 & 24.142 & 21.246 & 34.291 \\
\hline 3/2/10 20:00 & 11.501 & 4.936 & 7.770 & 17.534 & 6.817 & 17.251 & 8.795 & 24.158 & 21.251 & 34.293 \\
\hline 3/3/10 0:00 & 11.421 & 4.859 & 7.725 & 17.497 & 6.742 & 17.247 & 8.807 & 24.187 & 21.251 & 34.291 \\
\hline 3/3/10 4:00 & 11.423 & 4.861 & 7.697 & 17.469 & 6.669 & 17.261 & 8.816 & 24.130 & 21.255 & 34.288 \\
\hline 3/3/10 8:00 & 11.407 & 4.824 & 7.685 & 17.446 & 6.591 & 17.275 & 8.842 & 24.137 & 21.258 & 34.284 \\
\hline 3/3/10 12:00 & 11.567 & 4.955 & 7.690 & 17.380 & 6.725 & 17.331 & 8.870 & 24.189 & 21.260 & 34.288 \\
\hline 3/3/10 16:00 & 11.654 & 5.315 & 7.669 & 17.485 & 6.815 & 17.312 & 8.861 & 24.196 & 21.265 & 34.288 \\
\hline 3/3/10 20:00 & 11.395 & 4.887 & 7.603 & 17.513 & 6.765 & 17.294 & 8.863 & 24.201 & 21.267 & 34.286 \\
\hline 3/4/10 0:00 & 11.301 & 4.779 & 7.565 & 17.453 & 6.666 & 17.305 & 8.875 & 24.217 & 21.269 & 34.286 \\
\hline 3/4/10 4:00 & 11.320 & 4.843 & 7.547 & 17.448 & 6.638 & 17.303 & 8.875 & 24.236 & 21.272 & 34.284 \\
\hline 3/4/10 8:00 & 11.324 & 4.782 & 7.547 & 17.411 & 6.586 & 17.324 & 8.903 & 24.212 & 21.276 & 34.288 \\
\hline 3/4/10 12:00 & 11.511 & 5.027 & 7.558 & 17.385 & 6.678 & 17.369 & 8.910 & 24.255 & 21.279 & 34.286 \\
\hline 3/4/10 16:00 & 11.419 & 5.376 & 7.521 & 17.560 & 6.829 & 17.305 & 8.870 & 24.219 & 21.281 & 34.286 \\
\hline $3 / 4 / 10$ 20:00 & 10.820 & 4.941 & 7.425 & 17.590 & 6.763 & 17.249 & 8.854 & 24.191 & 21.286 & 34.286 \\
\hline 3/5/10 0:00 & 10.615 & 4.683 & 7.382 & 17.499 & 6.631 & 17.268 & 8.873 & 24.203 & 21.288 & 34.284 \\
\hline 3/5/10 4:00 & 10.678 & 4.597 & 7.368 & 17.450 & 6.546 & 17.282 & 8.870 & 24.215 & 21.290 & 34.286 \\
\hline 3/5/10 8:00 & 10.761 & 4.527 & 7.368 & 17.425 & 6.603 & 17.289 & 8.884 & 24.222 & 21.293 & 34.284 \\
\hline 3/5/10 12:00 & 10.992 & 4.857 & 7.378 & 17.422 & 6.591 & 17.294 & 8.870 & 24.248 & 21.298 & 34.286 \\
\hline $3 / 5 / 10$ 16:00 & 10.810 & 5.055 & 7.321 & 17.560 & 6.567 & 17.207 & 8.802 & 24.088 & 21.300 & 34.284 \\
\hline
\end{tabular}

TABLE S1.1 Page 29 of 89 
Water Level (ft below reference point)

\begin{tabular}{|c|c|c|c|c|c|c|c|c|c|c|}
\hline Date and Time & McPike & Potter & Hughes & Barr & Burks & MoDOT & Clizer "Old" & Morgan & SB24 & SB38 \\
\hline 3/5/10 20:00 & 10.087 & 4.143 & 7.141 & 17.571 & 6.539 & 17.087 & 8.729 & 23.994 & 21.302 & 34.286 \\
\hline 3/6/10 0:00 & 9.731 & 3.722 & 7.044 & 17.478 & 6.527 & 17.071 & 8.689 & 23.930 & 21.305 & 34.286 \\
\hline 3/6/10 4:00 & 9.714 & 3.680 & 7.005 & 17.450 & 6.506 & 17.047 & 8.633 & 23.860 & 21.307 & 34.284 \\
\hline 3/6/10 8:00 & 9.856 & 3.790 & 6.986 & 17.450 & 6.492 & 17.022 & 8.593 & 23.773 & 21.309 & 34.284 \\
\hline 3/6/10 12:00 & 10.066 & 4.028 & 6.976 & 17.436 & 6.563 & 17.019 & 8.558 & 23.716 & 21.312 & 34.286 \\
\hline 3/6/10 16:00 & 9.792 & 4.042 & 6.829 & 17.548 & 6.728 & 16.890 & 8.445 & 23.554 & 21.314 & 34.284 \\
\hline 3/6/10 20:00 & 9.257 & 3.560 & 6.563 & 17.604 & 6.737 & 16.712 & 8.250 & 23.331 & 21.314 & 34.284 \\
\hline $3 / 7 / 100: 00$ & 8.890 & 3.280 & 6.338 & 17.574 & 6.699 & 16.600 & 8.029 & 23.194 & 21.312 & 34.284 \\
\hline 3/7/10 4:00 & 8.727 & 3.071 & 6.171 & 17.543 & 6.626 & 16.459 & 7.799 & 23.112 & 21.312 & 34.281 \\
\hline 3/7/10 8:00 & 8.585 & 2.901 & 6.101 & 17.464 & 6.494 & 16.426 & 7.651 & 23.063 & 21.309 & 34.279 \\
\hline $3 / 7 / 10$ 12:00 & 8.639 & 3.078 & 6.052 & 17.427 & 6.492 & 16.412 & 7.550 & 23.032 & 21.307 & 34.281 \\
\hline $3 / 7 / 10$ 16:00 & 8.644 & 3.118 & 5.930 & 17.483 & 6.539 & 16.335 & 7.416 & 22.976 & 21.305 & 34.279 \\
\hline 3/7/10 20:00 & 8.491 & 2.968 & 5.782 & 17.481 & 6.511 & 16.253 & 7.261 & 22.797 & 21.302 & 34.279 \\
\hline 3/8/10 0:00 & 8.451 & 2.926 & 5.693 & 17.474 & 6.463 & 16.189 & 7.125 & 22.717 & 21.300 & 34.279 \\
\hline 3/8/10 4:00 & 8.552 & 3.022 & 5.634 & 17.483 & 6.444 & 16.140 & 7.015 & 22.630 & 21.298 & 34.277 \\
\hline 3/8/10 8:00 & 8.696 & 3.167 & 5.594 & 17.497 & 6.445 & 16.105 & 6.951 & 22.550 & 21.293 & 34.277 \\
\hline $3 / 8 / 10$ 12:00 & 8.981 & 3.707 & 5.550 & 17.555 & 6.629 & 16.042 & 6.855 & 22.449 & 21.288 & 34.274 \\
\hline 3/8/10 16:00 & 8.809 & 3.986 & 5.362 & 17.823 & 6.885 & 15.816 & 6.401 & 22.270 & 21.283 & 34.272 \\
\hline 3/8/10 20:00 & 8.514 & 3.569 & 5.202 & 17.886 & 6.824 & 15.702 & 6.199 & 22.105 & 21.279 & 34.270 \\
\hline 3/9/10 0:00 & 8.380 & 3.361 & 5.106 & 17.877 & 6.772 & 15.655 & 6.117 & 22.018 & 21.272 & 34.267 \\
\hline 3/9/10 4:00 & 8.314 & 3.247 & 4.993 & 17.944 & 6.801 & 15.505 & 6.019 & 21.903 & 21.267 & 34.267 \\
\hline 3/9/10 8:00 & 8.255 & 3.200 & 4.890 & 17.993 & 6.834 & 15.441 & 5.967 & 21.805 & 21.258 & 34.265 \\
\hline $3 / 9 / 10$ 12:00 & 8.342 & 3.310 & 4.808 & 18.014 & 6.850 & 15.430 & 5.922 & 21.661 & 21.253 & 34.265 \\
\hline 3/9/10 16:00 & 8.387 & 3.300 & 4.705 & 18.114 & 6.878 & 15.350 & 5.847 & 21.553 & 21.244 & 34.267 \\
\hline 3/9/10 20:00 & 8.029 & 2.971 & 4.461 & 18.037 & 6.749 & 15.078 & 5.767 & 21.480 & 21.237 & 34.265 \\
\hline 3/10/10 0:00 & 7.920 & 2.945 & 4.320 & 18.005 & 6.747 & 15.073 & 5.699 & 21.426 & 21.227 & 34.263 \\
\hline 3/10/10 4:00 & 7.875 & 2.776 & 4.252 & 17.935 & 6.650 & 15.113 & 5.662 & 21.379 & 21.218 & 34.260 \\
\hline 3/10/10 8:00 & 7.906 & 2.781 & 4.212 & 17.830 & 6.565 & 15.162 & 5.645 & 21.384 & 21.208 & 34.255 \\
\hline $3 / 10 / 1012: 00$ & 8.090 & 2.982 & 4.195 & 17.781 & 6.551 & 15.198 & 5.615 & 21.398 & 21.199 & 34.255 \\
\hline 3/10/10 16:00 & 8.250 & 3.181 & 4.167 & 17.837 & 6.577 & 15.165 & 5.577 & 21.346 & 21.190 & 34.246 \\
\hline $3 / 10 / 10$ 20:00 & 8.222 & 3.001 & 4.153 & 17.853 & 6.567 & 15.146 & 5.549 & 21.306 & 21.183 & 34.244 \\
\hline 3/11/10 0:00 & 7.974 & 2.687 & 4.001 & 17.790 & 6.463 & 14.968 & 5.389 & 21.257 & 21.171 & 34.237 \\
\hline 3/11/10 4:00 & 6.730 & 2.456 & 3.419 & 17.718 & 6.367 & 14.208 & 4.938 & 21.073 & 21.161 & 34.232 \\
\hline 3/11/10 8:00 & 6.353 & 2.346 & 3.259 & 17.532 & 5.886 & 14.164 & 4.374 & 20.890 & 21.150 & 34.222 \\
\hline 3/11/10 12:00 & 6.284 & 2.385 & 3.215 & 17.350 & 5.600 & 14.215 & 3.961 & 20.760 & 21.136 & 34.218 \\
\hline $3 / 11 / 1016: 00$ & 6.296 & 2.512 & 3.222 & 17.275 & 5.529 & 14.234 & 3.780 & 20.591 & 21.122 & 34.213 \\
\hline 3/11/10 20:00 & 6.315 & 2.556 & 3.266 & 17.182 & 5.520 & 14.257 & 3.707 & 20.415 & 21.108 & 34.208 \\
\hline 3/12/10 0:00 & 6.381 & 2.603 & 3.320 & 17.126 & 5.534 & 14.248 & 3.669 & 20.161 & 21.093 & 34.204 \\
\hline 3/12/10 4:00 & 6.480 & 2.697 & 3.367 & 17.140 & 5.588 & 14.192 & 3.637 & 19.959 & 21.079 & 34.199 \\
\hline 3/12/10 8:00 & 6.522 & 2.699 & 3.421 & 17.098 & 5.638 & 14.178 & 3.658 & 19.787 & 21.065 & 34.192 \\
\hline $3 / 12 / 1012: 00$ & 6.630 & 2.783 & 3.480 & 17.040 & 5.701 & 14.192 & 3.705 & 19.688 & 21.049 & 34.187 \\
\hline 3/12/10 16:00 & 6.687 & 2.807 & 3.541 & 16.982 & 5.720 & 14.201 & 3.747 & 19.622 & 21.035 & 34.185 \\
\hline $3 / 12 / 1020: 00$ & 6.677 & 2.643 & 3.595 & 16.903 & 5.706 & 14.220 & 3.810 & 19.587 & 21.021 & 34.175 \\
\hline $3 / 13 / 100: 00$ & 6.727 & 2.603 & 3.646 & 16.842 & 5.697 & 14.215 & 3.839 & 19.521 & 21.004 & 34.171 \\
\hline 3/13/10 4:00 & 6.791 & 2.683 & 3.682 & 16.872 & 5.751 & 14.145 & 3.848 & 19.408 & 20.988 & 34.166 \\
\hline 3/13/10 8:00 & 6.826 & 2.683 & 3.717 & 16.849 & 5.763 & 14.119 & 3.878 & 19.324 & 20.974 & 34.159 \\
\hline 3/13/10 12:00 & 6.956 & 2.851 & 3.754 & 16.837 & 5.838 & 14.098 & 3.907 & 19.253 & 20.957 & 34.152 \\
\hline $3 / 13 / 1016: 00$ & 7.024 & 2.938 & 3.787 & 16.877 & 5.947 & 14.063 & 3.930 & 19.183 & 20.941 & 34.147 \\
\hline 3/13/10 20:00 & 6.984 & 2.771 & 3.832 & 16.814 & 5.897 & 14.082 & 3.991 & 19.164 & 20.922 & 34.140 \\
\hline 3/14/10 0:00 & 7.022 & 2.718 & 3.876 & 16.744 & 5.871 & 14.100 & 4.038 & 19.166 & 20.906 & 34.131 \\
\hline 3/14/10 4:00 & 7.059 & 2.694 & 3.916 & 16.716 & 5.878 & 14.084 & 4.081 & 19.145 & 20.892 & 34.121 \\
\hline 3/14/10 8:00 & 7.104 & 2.673 & 3.966 & 16.632 & 5.855 & 14.114 & 4.142 & 19.178 & 20.875 & 34.114 \\
\hline 3/14/10 12:00 & 7.234 & 2.828 & 4.015 & 16.577 & 5.874 & 14.140 & 4.193 & 19.232 & 20.859 & 34.107 \\
\hline 3/14/10 16:00 & 7.382 & 3.024 & 4.055 & 16.602 & 5.952 & 14.119 & 4.219 & 19.150 & 20.843 & 34.102 \\
\hline $3 / 14 / 1020: 00$ & 7.295 & 2.863 & 4.095 & 16.577 & 5.937 & 14.128 & 4.283 & 19.145 & 20.826 & 34.095 \\
\hline $3 / 15 / 100: 00$ & 7.264 & 2.893 & 4.146 & 16.467 & 5.843 & 14.168 & 4.339 & 19.197 & 20.812 & 34.091 \\
\hline 3/15/10 4:00 & 7.356 & 2.907 & 4.186 & 16.439 & 5.843 & 14.154 & 4.369 & 19.199 & 20.796 & 34.083 \\
\hline 3/15/10 8:00 & 7.385 & 2.919 & 4.231 & 16.383 & 5.819 & 14.164 & 4.423 & 19.232 & 20.779 & 34.076 \\
\hline 3/15/10 12:00 & 7.509 & 2.933 & 4.275 & 16.353 & 5.822 & 14.173 & 4.463 & 19.234 & 20.765 & 34.069 \\
\hline 3/15/10 16:00 & 7.693 & 2.949 & 4.308 & 16.402 & 5.932 & 14.135 & 4.475 & 19.209 & 20.749 & 34.062 \\
\hline 3/15/10 20:00 & 7.587 & 2.987 & 4.341 & 16.427 & 5.940 & 14.119 & 4.515 & 19.185 & 20.732 & 34.055 \\
\hline 3/16/10 0:00 & 7.597 & 3.019 & 4.383 & 16.369 & 5.916 & 14.131 & 4.555 & 19.199 & 20.716 & 34.048 \\
\hline 3/16/10 4:00 & 7.641 & 3.026 & 4.418 & 16.367 & 5.921 & 14.105 & 4.576 & 19.183 & 20.702 & 34.041 \\
\hline 3/16/10 8:00 & 7.644 & 3.022 & 4.454 & 16.351 & 5.895 & 14.086 & 4.607 & 19.162 & 20.685 & 34.036 \\
\hline
\end{tabular}


Water Level (ft below reference point)

\begin{tabular}{|c|c|c|c|c|c|c|c|c|c|c|}
\hline Date and Time & McPike & Potter & Hughes & Barr & Burks & MoDOT & Clizer "Old" & Morgan & SB24 & SB38 \\
\hline 3/16/10 12:00 & 7.724 & 3.019 & 4.489 & 16.348 & 5.916 & 14.070 & 4.630 & 19.147 & 20.671 & 34.027 \\
\hline 3/16/10 16:00 & 7.823 & 3.015 & 4.515 & 16.420 & 6.006 & 14.006 & 4.625 & 19.089 & 20.655 & 34.020 \\
\hline 3/16/10 20:00 & 7.795 & 3.029 & 4.541 & 16.444 & 6.048 & 13.976 & 4.651 & 19.044 & 20.641 & 34.013 \\
\hline 3/17/10 0:00 & 7.823 & 3.041 & 4.573 & 16.444 & 6.069 & 13.962 & 4.665 & 19.027 & 20.624 & 34.006 \\
\hline 3/17/10 4:00 & 7.854 & 3.036 & 4.602 & 16.481 & 6.136 & 13.924 & 4.677 & 18.990 & 20.608 & 33.999 \\
\hline 3/17/10 8:00 & 7.847 & 3.029 & 4.634 & 16.460 & 6.131 & 13.934 & 4.717 & 18.985 & 20.592 & 33.992 \\
\hline 3/17/10 12:00 & 7.955 & 3.031 & 4.674 & 16.460 & 6.178 & 13.931 & 4.743 & 18.992 & 20.575 & 33.985 \\
\hline $3 / 17 / 1016: 00$ & 8.129 & 3.045 & 4.702 & 16.567 & 6.294 & 13.892 & 4.745 & 18.962 & 20.561 & 33.977 \\
\hline $3 / 17 / 10$ 20:00 & 7.988 & 3.082 & 4.726 & 16.644 & 6.338 & 13.856 & 4.766 & 18.922 & 20.545 & 33.970 \\
\hline 3/18/10 0:00 & 7.903 & 3.115 & 4.756 & 16.625 & 6.319 & 13.847 & 4.781 & 18.919 & 20.528 & 33.961 \\
\hline 3/18/10 4:00 & 7.896 & 3.129 & 4.785 & 16.630 & 6.310 & 13.821 & 4.795 & 18.908 & 20.512 & 33.954 \\
\hline 3/18/10 8:00 & 7.910 & 3.141 & 4.813 & 16.621 & 6.279 & 13.809 & 4.818 & 18.908 & 20.495 & 33.949 \\
\hline $3 / 18 / 10$ 12:00 & 8.167 & 3.136 & 4.843 & 16.660 & 6.428 & 13.793 & 4.828 & 18.931 & 20.479 & 33.940 \\
\hline $3 / 18 / 10$ 16:00 & 8.325 & 3.155 & 4.857 & 16.907 & 6.600 & 13.711 & 4.802 & 18.778 & 20.463 & 33.933 \\
\hline $3 / 18 / 10$ 20:00 & 8.115 & 3.209 & 4.876 & 16.984 & 6.614 & 13.681 & 4.820 & 18.736 & 20.448 & 33.926 \\
\hline 3/19/10 0:00 & 7.986 & 3.260 & 4.904 & 16.954 & 6.595 & 13.685 & 4.851 & 18.743 & 20.430 & 33.916 \\
\hline 3/19/10 4:00 & 7.964 & 3.295 & 4.937 & 16.900 & 6.534 & 13.704 & 4.877 & 18.769 & 20.416 & 33.909 \\
\hline 3/19/10 8:00 & 7.971 & 3.317 & 4.970 & 16.882 & 6.515 & 13.704 & 4.914 & 18.788 & 20.397 & 33.902 \\
\hline 3/19/10 12:00 & 8.136 & 3.333 & 5.008 & 16.849 & 6.558 & 13.742 & 4.947 & 18.839 & 20.380 & 33.895 \\
\hline $3 / 19 / 1016: 00$ & 7.969 & 3.366 & 5.045 & 16.823 & 6.435 & 13.760 & 4.994 & 18.884 & 20.366 & 33.886 \\
\hline 3/19/10 20:00 & 7.915 & 3.394 & 5.087 & 16.686 & 6.256 & 13.807 & 5.051 & 18.971 & 20.350 & 33.876 \\
\hline 3/20/10 0:00 & 7.962 & 3.422 & 5.132 & 16.570 & 6.159 & 13.859 & 5.093 & 19.075 & 20.336 & 33.860 \\
\hline $3 / 20 / 104: 00$ & 8.068 & 3.424 & 5.167 & 16.544 & 6.232 & 13.854 & 5.109 & 19.020 & 20.319 & 33.850 \\
\hline 3/20/10 8:00 & 8.092 & 3.384 & 5.200 & 16.497 & 6.194 & 13.868 & 5.156 & 19.046 & 20.305 & 33.839 \\
\hline 3/20/10 12:00 & 8.177 & 3.333 & 5.223 & 16.448 & 6.173 & 13.892 & 5.185 & 19.096 & 20.291 & 33.829 \\
\hline $3 / 20 / 10$ 16:00 & 8.290 & 3.298 & 5.221 & 16.467 & 6.166 & 13.861 & 5.199 & 19.089 & 20.275 & 33.817 \\
\hline $3 / 20 / 10$ 20:00 & 8.299 & 3.216 & 5.153 & 16.458 & 6.216 & 13.849 & 5.222 & 19.096 & 20.261 & 33.806 \\
\hline 3/21/10 0:00 & 8.330 & 3.134 & 5.118 & 16.444 & 6.227 & 13.842 & 5.236 & 19.107 & 20.247 & 33.803 \\
\hline $3 / 21 / 104: 00$ & 8.330 & 3.089 & 5.108 & 16.500 & 6.256 & 13.781 & 5.239 & 19.077 & 20.230 & 33.801 \\
\hline 3/21/10 8:00 & 8.334 & 3.045 & 5.120 & 16.495 & 6.263 & 13.765 & 5.262 & 19.077 & 20.214 & 33.791 \\
\hline $3 / 21 / 10$ 12:00 & 8.436 & 3.253 & 5.144 & 16.481 & 6.333 & 13.763 & 5.276 & 19.098 & 20.200 & 33.784 \\
\hline $3 / 21 / 1016: 00$ & 8.514 & 3.410 & 5.139 & 16.577 & 6.435 & 13.674 & 5.246 & 19.086 & 20.183 & 33.777 \\
\hline $3 / 21 / 1020: 00$ & 8.353 & 2.937 & 5.054 & 16.581 & 6.440 & 13.552 & 5.246 & 18.995 & 20.169 & 33.770 \\
\hline 3/22/10 0:00 & 8.231 & 2.743 & 5.017 & 16.523 & 6.402 & 13.523 & 5.231 & 18.983 & 20.153 & 33.761 \\
\hline $3 / 22 / 104: 00$ & 8.167 & 2.755 & 5.008 & 16.516 & 6.388 & 13.505 & 5.220 & 18.959 & 20.137 & 33.754 \\
\hline $3 / 22 / 108: 00$ & 8.148 & 2.883 & 5.010 & 16.490 & 6.376 & 13.512 & 5.236 & 18.945 & 20.122 & 33.747 \\
\hline $3 / 22 / 1012: 00$ & 8.415 & 3.410 & 5.019 & 16.525 & 6.482 & 13.509 & 5.224 & 18.938 & 20.104 & 33.740 \\
\hline $3 / 22 / 10$ 16:00 & 8.466 & 3.412 & 4.925 & 16.754 & 6.605 & 13.406 & 5.138 & 18.865 & 20.085 & 33.730 \\
\hline $3 / 22 / 10$ 20:00 & 8.014 & 2.977 & 4.829 & 16.800 & 6.600 & 13.310 & 5.048 & 18.816 & 20.069 & 33.723 \\
\hline 3/23/10 0:00 & 7.783 & 2.757 & 4.808 & 16.737 & 6.517 & 13.336 & 5.025 & 18.821 & 20.054 & 33.714 \\
\hline $3 / 23 / 104: 00$ & 7.781 & 2.778 & 4.803 & 16.726 & 6.484 & 13.336 & 5.006 & 18.813 & 20.040 & 33.709 \\
\hline 3/23/10 8:00 & 7.783 & 2.808 & 4.808 & 16.695 & 6.430 & 13.362 & 5.022 & 18.825 & 20.024 & 33.700 \\
\hline $3 / 23 / 10$ 12:00 & 8.042 & 3.339 & 4.820 & 16.667 & 6.499 & 13.413 & 5.037 & 18.896 & 20.010 & 33.692 \\
\hline $3 / 23 / 1016: 00$ & 7.993 & 2.790 & 4.742 & 16.826 & 6.654 & 13.315 & 4.884 & 18.785 & 19.993 & 33.683 \\
\hline $3 / 23 / 1020: 00$ & 7.538 & 2.902 & 4.656 & 16.844 & 6.586 & 13.336 & 4.743 & 18.804 & 19.977 & 33.676 \\
\hline 3/24/10 0:00 & 7.460 & 2.991 & 4.658 & 16.756 & 6.510 & 13.392 & 4.708 & 18.842 & 19.963 & 33.669 \\
\hline $3 / 24 / 104: 00$ & 7.542 & 3.026 & 4.663 & 16.775 & 6.494 & 13.366 & 4.661 & 18.825 & 19.949 & 33.662 \\
\hline 3/24/10 8:00 & 7.592 & 2.996 & 4.686 & 16.709 & 6.414 & 13.404 & 4.651 & 18.839 & 19.935 & 33.652 \\
\hline $3 / 24 / 10$ 12:00 & 7.552 & 2.642 & 4.606 & 16.665 & 6.399 & 13.284 & 4.508 & 18.842 & 19.921 & 33.643 \\
\hline $3 / 24 / 10$ 16:00 & 6.680 & 2.224 & 4.038 & 16.609 & 6.333 & 12.794 & 4.076 & 18.759 & 19.904 & 33.636 \\
\hline $3 / 24 / 10$ 20:00 & 5.824 & 2.186 & 3.562 & 16.390 & 6.180 & 12.581 & 3.625 & 18.677 & 19.888 & 33.624 \\
\hline 3/25/10 0:00 & 5.645 & 2.256 & 3.416 & 16.264 & 5.973 & 12.654 & 3.343 & 18.595 & 19.872 & 33.615 \\
\hline $3 / 25 / 104: 00$ & 5.626 & 2.359 & 3.348 & 16.232 & 5.824 & 12.694 & 3.153 & 18.477 & 19.855 & 33.608 \\
\hline 3/25/10 8:00 & 5.593 & 2.457 & 3.348 & 16.157 & 5.668 & 12.731 & 3.075 & 18.369 & 19.836 & 33.601 \\
\hline $3 / 25 / 10$ 12:00 & 5.843 & 2.540 & 3.388 & 16.113 & 5.737 & 12.766 & 3.040 & 18.277 & 19.820 & 33.591 \\
\hline $3 / 25 / 1016: 00$ & 5.979 & 2.640 & 3.428 & 16.199 & 5.796 & 12.761 & 3.030 & 18.164 & 19.801 & 33.582 \\
\hline $3 / 25 / 1020: 00$ & 5.812 & 2.748 & 3.475 & 16.199 & 5.763 & 12.771 & 3.052 & 18.092 & 19.782 & 33.575 \\
\hline 3/26/10 0:00 & 5.786 & 2.813 & 3.529 & 16.104 & 5.708 & 12.780 & 3.073 & 18.070 & 19.764 & 33.565 \\
\hline $3 / 26 / 104: 00$ & 5.826 & 2.829 & 3.576 & 16.076 & 5.685 & 12.757 & 3.084 & 17.920 & 19.747 & 33.558 \\
\hline 3/26/10 8:00 & 5.911 & 2.825 & 3.618 & 16.048 & 5.673 & 12.743 & 3.101 & 17.842 & 19.728 & 33.549 \\
\hline $3 / 26 / 10$ 12:00 & 6.213 & 2.806 & 3.661 & 16.076 & 5.892 & 12.724 & 3.101 & 17.774 & 19.712 & 33.542 \\
\hline 3/26/10 16:00 & 6.010 & 2.829 & 3.670 & 16.348 & 6.128 & 12.614 & 3.052 & 17.640 & 19.693 & 33.530 \\
\hline $3 / 26 / 10$ 20:00 & 6.031 & 2.895 & 3.689 & 16.406 & 6.164 & 12.579 & 3.059 & 17.520 & 19.672 & 33.523 \\
\hline 3/27/10 0:00 & 6.073 & 2.930 & 3.726 & 16.346 & 6.116 & 12.586 & 3.087 & 17.490 & 19.653 & 33.513 \\
\hline
\end{tabular}


Water Level (ft below reference point)

\begin{tabular}{|c|c|c|c|c|c|c|c|c|c|c|}
\hline Date and Time & McPike & Potter & Hughes & Barr & Burks & MoDOT & Clizer "Old" & Morgan & SB24 & SB38 \\
\hline $3 / 27 / 104: 00$ & 6.097 & 2.928 & 3.764 & 16.344 & 6.138 & 12.562 & 3.082 & 17.428 & 19.635 & 33.504 \\
\hline 3/27/10 8:00 & 6.128 & 2.914 & 3.792 & 16.372 & 6.164 & 12.527 & 3.106 & 17.367 & 19.614 & 33.495 \\
\hline 3/27/10 12:00 & 6.144 & 2.818 & 3.815 & 16.402 & 6.171 & 12.490 & 3.073 & 17.311 & 19.595 & 33.488 \\
\hline 3/27/10 16:00 & 5.918 & 2.481 & 3.651 & 16.334 & 6.251 & 12.332 & 3.061 & 17.283 & 19.576 & 33.478 \\
\hline 3/27/10 20:00 & 5.854 & 2.411 & 3.616 & 16.213 & 6.213 & 12.368 & 3.113 & 17.276 & 19.557 & 33.466 \\
\hline $3 / 28 / 100: 00$ & 5.901 & 2.457 & 3.623 & 16.125 & 6.180 & 12.393 & 3.146 & 17.280 & 19.538 & 33.455 \\
\hline 3/28/10 4:00 & 5.961 & 2.518 & 3.644 & 16.064 & 6.128 & 12.400 & 3.174 & 17.273 & 19.517 & 33.447 \\
\hline 3/28/10 8:00 & 6.038 & 2.574 & 3.679 & 15.950 & 6.086 & 12.436 & 3.237 & 17.287 & 19.499 & 33.438 \\
\hline 3/28/10 12:00 & 6.092 & 2.619 & 3.726 & 15.901 & 6.095 & 12.459 & 3.275 & 17.306 & 19.480 & 33.429 \\
\hline 3/28/10 16:00 & 6.114 & 2.668 & 3.759 & 15.996 & 6.185 & 12.417 & 3.279 & 17.264 & 19.459 & 33.419 \\
\hline 3/28/10 20:00 & 6.147 & 2.771 & 3.792 & 16.036 & 6.173 & 12.410 & 3.312 & 17.247 & 19.440 & 33.410 \\
\hline 3/29/10 0:00 & 6.184 & 2.848 & 3.832 & 15.971 & 6.126 & 12.407 & 3.338 & 17.243 & 19.421 & 33.400 \\
\hline 3/29/10 4:00 & 6.208 & 2.867 & 3.862 & 15.947 & 6.114 & 12.384 & 3.355 & 17.215 & 19.402 & 33.391 \\
\hline 3/29/10 8:00 & 6.241 & 2.865 & 3.900 & 15.927 & 6.090 & 12.372 & 3.385 & 17.196 & 19.384 & 33.382 \\
\hline 3/29/10 12:00 & 6.257 & 2.846 & 3.937 & 15.971 & 6.213 & 12.349 & 3.383 & 17.478 & 19.363 & 33.372 \\
\hline 3/29/10 16:00 & 6.238 & 2.886 & 3.947 & 16.232 & 6.376 & 12.279 & 3.352 & 17.250 & 19.344 & 33.363 \\
\hline 3/29/10 20:00 & 6.253 & 2.974 & 3.966 & 16.341 & 6.458 & 12.241 & 3.362 & 17.095 & 19.323 & 33.353 \\
\hline 3/30/10 0:00 & 6.279 & 3.042 & 3.996 & 16.313 & 6.444 & 12.239 & 3.383 & 17.073 & 19.302 & 33.344 \\
\hline 3/30/10 4:00 & 6.290 & 3.042 & 4.027 & 16.341 & 6.473 & 12.203 & 3.387 & 17.041 & 19.283 & 33.332 \\
\hline 3/30/10 8:00 & 6.326 & 3.024 & 4.059 & 16.327 & 6.451 & 12.215 & 3.425 & 17.055 & 19.264 & 33.325 \\
\hline 3/30/10 12:00 & 6.335 & 3.024 & 4.095 & 16.423 & 6.602 & 12.180 & 3.418 & 16.996 & 19.243 & 33.313 \\
\hline 3/30/10 16:00 & 6.331 & 3.098 & 4.106 & 16.679 & 6.779 & 12.112 & 3.406 & 17.012 & 19.222 & 33.304 \\
\hline 3/30/10 20:00 & 6.364 & 3.213 & 4.132 & 16.749 & 6.746 & 12.100 & 3.437 & 16.930 & 19.198 & 33.294 \\
\hline 3/31/10 0:00 & 6.396 & 3.319 & 4.170 & 16.719 & 6.734 & 12.110 & 3.453 & 16.909 & 19.177 & 33.285 \\
\hline 3/31/10 4:00 & 6.406 & 3.396 & 4.196 & 16.789 & 6.763 & 12.058 & 3.460 & 16.871 & 19.156 & 33.273 \\
\hline 3/31/10 8:00 & 6.470 & 3.471 & 4.238 & 16.698 & 6.647 & 12.107 & 3.528 & 16.897 & 19.135 & 33.266 \\
\hline 3/31/10 12:00 & 6.533 & 3.527 & 4.289 & 16.660 & 6.680 & 12.154 & 3.582 & 17.071 & 19.116 & 33.254 \\
\hline 3/31/10 16:00 & 6.571 & 3.585 & 4.332 & 16.831 & 6.699 & 12.140 & 3.604 & 16.993 & 19.095 & 33.245 \\
\hline $3 / 31 / 1020: 00$ & 6.616 & 3.648 & 4.367 & 16.863 & 6.654 & 12.140 & 3.646 & 16.986 & 19.074 & 33.238 \\
\hline 4/1/10 0:00 & 6.658 & 3.698 & 4.411 & 16.812 & 6.397 & 12.150 & 3.679 & 17.012 & 19.053 & 33.226 \\
\hline 4/1/10 4:00 & 6.670 & 3.726 & 4.440 & 16.858 & 6.482 & 12.105 & 3.686 & 16.989 & 19.032 & 33.217 \\
\hline 4/1/10 8:00 & 6.703 & 3.737 & 4.472 & 16.835 & 6.484 & 12.107 & 3.721 & 16.993 & 19.018 & 33.207 \\
\hline 4/1/10 12:00 & 6.731 & 3.747 & 4.510 & 16.884 & 6.357 & 12.114 & 3.745 & 17.012 & 18.994 & 33.198 \\
\hline 4/1/10 16:00 & 6.736 & 3.768 & 4.536 & 17.045 & 6.350 & 12.075 & 3.742 & 16.998 & 18.978 & 33.188 \\
\hline 4/1/10 20:00 & 6.776 & 3.815 & 4.557 & 17.070 & 6.355 & 12.058 & 3.792 & 16.996 & 18.954 & 33.177 \\
\hline 4/2/10 0:00 & 6.802 & 3.850 & 4.594 & 16.996 & 6.374 & 12.068 & 3.815 & 17.043 & 18.936 & 33.170 \\
\hline 4/2/10 4:00 & 6.835 & 3.878 & 4.632 & 16.900 & 6.383 & 12.089 & 3.841 & 16.979 & 18.917 & 33.160 \\
\hline 4/2/10 8:00 & 6.859 & 3.901 & 4.663 & 16.907 & 6.381 & 12.065 & 3.869 & 16.998 & 18.901 & 33.151 \\
\hline 4/2/10 12:00 & 6.698 & 3.347 & 4.468 & 16.719 & 6.388 & 12.025 & 3.843 & 17.031 & 18.882 & 33.137 \\
\hline 4/2/10 16:00 & 6.602 & 2.940 & 4.388 & 16.639 & 6.409 & 12.000 & 3.860 & 17.043 & 18.863 & 33.127 \\
\hline $4 / 2 / 1020: 00$ & 6.658 & 2.909 & 4.397 & 16.532 & 6.352 & 12.042 & 3.907 & 17.066 & 18.844 & 33.118 \\
\hline 4/3/10 0:00 & 6.755 & 2.994 & 4.444 & 16.327 & 6.201 & 12.103 & 3.979 & 17.125 & 18.828 & 33.108 \\
\hline 4/3/10 4:00 & 6.823 & 3.092 & 4.498 & 16.185 & 6.081 & 12.119 & 4.012 & 17.163 & 18.809 & 33.099 \\
\hline 4/3/10 8:00 & 6.889 & 3.185 & 4.550 & 16.048 & 5.973 & 12.157 & 4.057 & 17.203 & 18.793 & 33.087 \\
\hline 4/3/10 12:00 & 6.936 & 3.263 & 4.597 & 16.017 & 6.109 & 12.164 & 4.076 & 17.229 & 18.776 & 33.080 \\
\hline 4/3/10 16:00 & 6.955 & 3.351 & 4.627 & 16.199 & 6.232 & 12.121 & 4.081 & 17.203 & 18.760 & 33.071 \\
\hline 4/3/10 20:00 & 6.988 & 3.454 & 4.655 & 16.208 & 6.256 & 12.121 & 4.106 & 17.198 & 18.741 & 33.061 \\
\hline 4/4/10 0:00 & 7.019 & 3.543 & 4.688 & 16.150 & 6.223 & 12.117 & 4.130 & 17.207 & 18.725 & 33.052 \\
\hline 4/4/10 4:00 & 7.028 & 3.611 & 4.712 & 16.162 & 6.244 & 12.089 & 4.137 & 17.189 & 18.708 & 33.045 \\
\hline 4/4/10 8:00 & 7.071 & 3.672 & 4.745 & 16.097 & 6.211 & 12.114 & 4.184 & 17.210 & 18.692 & 33.035 \\
\hline 4/4/10 12:00 & 7.094 & 3.716 & 4.782 & 16.118 & 6.286 & 12.121 & 4.193 & 17.229 & 18.675 & 33.023 \\
\hline 4/4/10 16:00 & 7.106 & 3.766 & 4.803 & 16.288 & 6.430 & 12.093 & 4.210 & 17.210 & 18.657 & 33.014 \\
\hline $4 / 4 / 1020: 00$ & 7.174 & 3.831 & 4.841 & 16.234 & 6.369 & 12.152 & 4.278 & 17.252 & 18.640 & 33.005 \\
\hline $4 / 5 / 100: 00$ & 7.243 & 3.890 & 4.895 & 16.038 & 6.201 & 12.225 & 4.337 & 17.346 & 18.624 & 32.998 \\
\hline 4/5/10 4:00 & 7.276 & 3.927 & 4.932 & 15.987 & 6.145 & 12.220 & 4.362 & 17.384 & 18.610 & 32.986 \\
\hline 4/5/10 8:00 & 7.288 & 3.948 & 4.961 & 15.982 & 6.499 & 12.201 & 4.379 & 17.410 & 18.593 & 32.979 \\
\hline 4/5/10 12:00 & 7.257 & 3.948 & 4.979 & 16.078 & 6.477 & 12.147 & 4.346 & 17.339 & 18.577 & 32.969 \\
\hline 4/5/10 16:00 & 7.222 & 3.941 & 4.984 & 16.299 & 6.447 & 12.068 & 4.318 & 17.254 & 18.563 & 32.960 \\
\hline 4/5/10 20:00 & 7.217 & 3.946 & 4.991 & 16.474 & 6.444 & 12.049 & 4.344 & 17.210 & 18.546 & 32.950 \\
\hline 4/6/10 0:00 & 7.248 & 3.951 & 5.019 & 16.455 & 6.468 & 12.089 & 4.369 & 17.252 & 18.532 & 32.941 \\
\hline 4/6/10 4:00 & 7.219 & 3.927 & 5.029 & 16.630 & 6.444 & 12.011 & 4.355 & 17.205 & 18.514 & 32.934 \\
\hline 4/6/10 8:00 & 7.236 & 3.904 & 5.047 & 16.651 & 6.456 & 12.025 & 4.386 & 17.226 & 18.499 & 32.922 \\
\hline 4/6/10 12:00 & 7.255 & 3.897 & 5.073 & 16.733 & 6.463 & 12.032 & 4.405 & 17.207 & 18.483 & 32.915 \\
\hline 4/6/10 16:00 & 7.269 & 3.927 & 5.090 & 16.858 & 6.470 & 12.023 & 4.424 & 17.205 & 18.467 & 32.906 \\
\hline
\end{tabular}

TABLE S1.1 Page 32 of 89 
Water Level (ft below reference point)

\begin{tabular}{|c|c|c|c|c|c|c|c|c|c|c|}
\hline Date and Time & McPike & Potter & Hughes & Barr & Burks & MoDOT & Clizer "Old" & Morgan & SB24 & SB38 \\
\hline $4 / 6 / 1020: 00$ & 7.340 & 3.983 & 5.127 & 16.672 & 6.506 & 12.100 & 4.489 & 17.273 & 18.448 & 32.896 \\
\hline 4/7/10 0:00 & 7.389 & 4.025 & 5.172 & 16.486 & 6.527 & 12.152 & 4.534 & 17.358 & 18.434 & 32.887 \\
\hline 4/7/10 4:00 & 7.422 & 4.053 & 5.205 & 16.402 & 6.529 & 12.168 & 4.562 & 17.391 & 18.420 & 32.880 \\
\hline 4/7/10 8:00 & 7.469 & 4.077 & 5.237 & 16.325 & 6.534 & 12.199 & 4.609 & 17.478 & 18.403 & 32.870 \\
\hline 4/7/10 12:00 & 7.533 & 4.098 & 5.282 & 16.185 & 6.560 & 12.279 & 4.670 & 17.499 & 18.392 & 32.861 \\
\hline 4/7/10 16:00 & 7.594 & 4.112 & 5.324 & 16.064 & 6.574 & 12.314 & 4.722 & 17.584 & 18.377 & 32.854 \\
\hline 4/7/10 20:00 & 7.651 & 4.129 & 5.366 & 15.929 & 6.586 & 12.370 & 4.771 & 17.650 & 18.363 & 32.844 \\
\hline 4/8/10 0:00 & 7.691 & 4.140 & 5.402 & 15.822 & 6.586 & 12.391 & 4.809 & 17.701 & 18.352 & 32.835 \\
\hline 4/8/10 4:00 & 7.712 & 4.150 & 5.432 & 15.766 & 6.581 & 12.391 & 4.828 & 17.722 & 18.338 & 32.830 \\
\hline 4/8/10 8:00 & 7.750 & 4.159 & 5.460 & 15.677 & 6.588 & 12.419 & 4.867 & 17.760 & 18.326 & 32.821 \\
\hline 4/8/10 12:00 & 7.776 & 4.171 & 5.493 & 15.670 & 6.588 & 12.445 & 4.889 & 17.807 & 18.312 & 32.811 \\
\hline 4/8/10 16:00 & 7.769 & 4.185 & 5.512 & 15.894 & 6.567 & 12.403 & 4.889 & 17.765 & 18.298 & 32.804 \\
\hline 4/8/10 20:00 & 7.790 & 4.210 & 5.531 & 15.931 & 6.574 & 12.410 & 4.914 & 17.777 & 18.286 & 32.795 \\
\hline 4/9/10 0:00 & 7.813 & 4.234 & 5.554 & 15.852 & 6.586 & 12.431 & 4.945 & 17.807 & 18.274 & 32.786 \\
\hline 4/9/10 4:00 & 7.832 & 4.248 & 5.580 & 15.805 & 6.588 & 12.443 & 4.971 & 17.835 & 18.263 & 32.781 \\
\hline 4/9/10 8:00 & 7.868 & 4.262 & 5.611 & 15.731 & 6.600 & 12.480 & 5.006 & 17.882 & 18.249 & 32.771 \\
\hline 4/9/10 12:00 & 7.886 & 4.274 & 5.639 & 15.766 & 6.600 & 12.497 & 5.011 & 17.915 & 18.237 & 32.764 \\
\hline 4/9/10 16:00 & 7.886 & 4.290 & 5.657 & 15.985 & 6.588 & 12.475 & 5.013 & 17.913 & 18.225 & 32.755 \\
\hline 4/9/10 20:00 & 7.908 & 4.316 & 5.676 & 16.038 & 6.593 & 12.478 & 5.044 & 17.948 & 18.213 & 32.748 \\
\hline 4/10/10 0:00 & 7.953 & 4.339 & 5.707 & 15.933 & 6.616 & 12.534 & 5.086 & 18.009 & 18.202 & 32.741 \\
\hline $4 / 10 / 104: 00$ & 7.978 & 4.353 & 5.735 & 15.882 & 6.619 & 12.553 & 5.114 & 18.056 & 18.192 & 32.734 \\
\hline 4/10/10 8:00 & 8.018 & 4.369 & 5.765 & 15.801 & 6.628 & 12.595 & 5.156 & 18.122 & 18.180 & 32.727 \\
\hline 4/10/10 12:00 & 8.030 & 4.381 & 5.794 & 15.857 & 6.626 & 12.604 & 5.173 & 18.068 & 18.171 & 32.717 \\
\hline $4 / 10 / 1016: 00$ & 8.033 & 4.398 & 5.810 & 16.108 & 6.609 & 12.583 & 5.185 & 18.085 & 18.157 & 32.710 \\
\hline 4/10/10 20:00 & 8.063 & 4.421 & 5.833 & 16.132 & 6.633 & 12.600 & 5.224 & 18.082 & 18.148 & 32.703 \\
\hline 4/11/10 0:00 & 8.106 & 4.442 & 5.864 & 16.010 & 6.638 & 12.663 & 5.272 & 18.146 & 18.138 & 32.696 \\
\hline 4/11/10 4:00 & 8.129 & 4.454 & 5.894 & 15.966 & 6.638 & 12.672 & 5.295 & 18.181 & 18.127 & 32.689 \\
\hline 4/11/10 8:00 & 8.174 & 4.466 & 5.923 & 15.852 & 6.654 & 12.724 & 5.347 & 18.240 & 18.117 & 32.682 \\
\hline 4/11/10 12:00 & 8.202 & 4.477 & 5.956 & 15.864 & 6.657 & 12.752 & 5.373 & 18.315 & 18.108 & 32.675 \\
\hline 4/11/10 16:00 & 8.202 & 4.484 & 5.974 & 16.120 & 6.638 & 12.724 & 5.375 & 18.291 & 18.101 & 32.668 \\
\hline 4/11/10 20:00 & 8.221 & 4.501 & 5.995 & 16.178 & 6.635 & 12.722 & 5.401 & 18.360 & 18.089 & 32.663 \\
\hline 4/12/10 0:00 & 8.240 & 4.512 & 6.019 & 16.108 & 6.647 & 12.747 & 5.429 & 18.336 & 18.080 & 32.656 \\
\hline $4 / 12 / 104: 00$ & 8.252 & 4.519 & 6.042 & 16.062 & 6.652 & 12.759 & 5.450 & 18.357 & 18.070 & 32.649 \\
\hline 4/12/10 8:00 & 8.268 & 4.522 & 6.066 & 16.003 & 6.654 & 12.780 & 5.476 & 18.374 & 18.063 & 32.642 \\
\hline $4 / 12 / 1012: 00$ & 8.278 & 4.526 & 6.089 & 16.059 & 6.657 & 12.794 & 5.490 & 18.465 & 18.051 & 32.635 \\
\hline $4 / 12 / 1016: 00$ & 8.276 & 4.538 & 6.103 & 16.285 & 6.642 & 12.771 & 5.481 & 18.414 & 18.044 & 32.630 \\
\hline 4/12/10 20:00 & 8.292 & 4.554 & 6.120 & 16.302 & 6.645 & 12.771 & 5.523 & 18.378 & 18.035 & 32.623 \\
\hline 4/13/10 0:00 & 8.313 & 4.569 & 6.143 & 16.222 & 6.661 & 12.804 & 5.556 & 18.414 & 18.026 & 32.616 \\
\hline $4 / 13 / 104: 00$ & 8.323 & 4.576 & 6.164 & 16.173 & 6.657 & 12.808 & 5.575 & 18.426 & 18.019 & 32.611 \\
\hline 4/13/10 8:00 & 8.363 & 4.583 & 6.192 & 16.048 & 6.678 & 12.867 & 5.622 & 18.484 & 18.009 & 32.604 \\
\hline 4/13/10 12:00 & 8.379 & 4.590 & 6.216 & 16.099 & 6.680 & 12.883 & 5.636 & 18.524 & 18.002 & 32.599 \\
\hline $4 / 13 / 1016: 00$ & 8.386 & 4.601 & 6.239 & 16.264 & 6.668 & 12.872 & 5.643 & 18.557 & 17.995 & 32.592 \\
\hline 4/13/10 20:00 & 8.398 & 4.615 & 6.256 & 16.309 & 6.666 & 12.862 & 5.666 & 18.555 & 17.986 & 32.588 \\
\hline 4/14/10 0:00 & 8.431 & 4.629 & 6.279 & 16.213 & 6.682 & 12.900 & 5.709 & 18.595 & 17.979 & 32.581 \\
\hline $4 / 14 / 104: 00$ & 8.471 & 4.646 & 6.310 & 16.076 & 6.701 & 12.961 & 5.751 & 18.684 & 17.972 & 32.574 \\
\hline 4/14/10 8:00 & 8.511 & 4.655 & 6.340 & 15.961 & 6.715 & 13.010 & 5.800 & 18.757 & 17.965 & 32.569 \\
\hline $4 / 14 / 1012: 00$ & 8.532 & 4.665 & 6.369 & 16.020 & 6.718 & 13.031 & 5.812 & 18.842 & 17.958 & 32.566 \\
\hline 4/14/10 16:00 & 8.535 & 4.674 & 6.390 & 16.222 & 6.699 & 13.008 & 5.819 & 18.759 & 17.953 & 32.557 \\
\hline 4/14/10 20:00 & 8.554 & 4.690 & 6.408 & 16.236 & 6.699 & 13.008 & 5.849 & 18.748 & 17.944 & 32.552 \\
\hline 4/15/10 0:00 & 8.580 & 4.704 & 6.434 & 16.122 & 6.718 & 13.052 & 5.880 & 18.795 & 17.941 & 32.548 \\
\hline 4/15/10 4:00 & 8.587 & 4.709 & 6.455 & 16.076 & 6.713 & 13.052 & 5.899 & 18.809 & 17.932 & 32.543 \\
\hline 4/15/10 8:00 & 8.622 & 4.716 & 6.481 & 15.940 & 6.727 & 13.106 & 5.941 & 18.861 & 17.927 & 32.536 \\
\hline 4/15/10 12:00 & 8.641 & 4.723 & 6.507 & 15.994 & 6.730 & 13.125 & 5.958 & 18.901 & 17.920 & 32.534 \\
\hline 4/15/10 16:00 & 8.638 & 4.730 & 6.526 & 16.204 & 6.711 & 13.094 & 5.948 & 18.882 & 17.918 & 32.526 \\
\hline 4/15/10 20:00 & 8.667 & 4.744 & 6.549 & 16.141 & 6.723 & 13.125 & 5.988 & 18.908 & 17.911 & 32.522 \\
\hline 4/16/10 0:00 & 8.697 & 4.751 & 6.549 & 15.968 & 6.699 & 13.162 & 6.004 & 18.976 & 17.906 & 32.510 \\
\hline $4 / 16 / 104: 00$ & 8.698 & 4.667 & 6.399 & 15.824 & 6.659 & 13.022 & 5.990 & 18.999 & 17.899 & 32.501 \\
\hline 4/16/10 8:00 & 8.714 & 4.405 & 6.265 & 15.605 & 6.543 & 13.050 & 6.023 & 19.051 & 17.894 & 32.489 \\
\hline $4 / 16 / 1012: 00$ & 8.724 & 4.258 & 6.230 & 15.526 & 6.548 & 13.099 & 6.040 & 19.091 & 17.890 & 32.493 \\
\hline 4/16/10 16:00 & 8.719 & 4.211 & 6.228 & 15.551 & 6.567 & 13.099 & 6.047 & 19.091 & 17.887 & 32.486 \\
\hline 4/16/10 20:00 & 8.724 & 4.220 & 6.239 & 15.537 & 6.546 & 13.099 & 6.063 & 19.089 & 17.880 & 32.484 \\
\hline 4/17/10 0:00 & 8.742 & 4.246 & 6.268 & 15.416 & 6.473 & 13.137 & 6.089 & 19.082 & 17.876 & 32.477 \\
\hline 4/17/10 4:00 & 8.749 & 4.272 & 6.291 & 15.363 & 6.432 & 13.148 & 6.108 & 19.093 & 17.871 & 32.477 \\
\hline 4/17/10 8:00 & 8.780 & 4.304 & 6.324 & 15.279 & 6.364 & 13.195 & 6.148 & 19.133 & 17.866 & 32.470 \\
\hline
\end{tabular}

TABLE S1.1 Page 33 of 89 


\begin{tabular}{|c|c|c|c|c|c|c|c|c|c|c|}
\hline \multirow[b]{2}{*}{ Date and Time } & \multicolumn{10}{|c|}{ Water Level (ft below reference point) } \\
\hline & McPike & Potter & Hughes & Barr & Burks & MoDOT & Clizer "Old" & Morgan & SB24 & SB38 \\
\hline 4/17/10 12:00 & 8.799 & 4.335 & 6.361 & 15.309 & 6.451 & 13.221 & 6.167 & 19.178 & 17.864 & 32.465 \\
\hline $4 / 17 / 1016: 00$ & 8.782 & 4.363 & 6.385 & 15.561 & 6.567 & 13.191 & 6.155 & 19.147 & 17.859 & 32.460 \\
\hline 4/17/10 20:00 & 8.787 & 4.398 & 6.411 & 15.605 & 6.579 & 13.181 & 6.181 & 19.138 & 17.854 & 32.456 \\
\hline 4/18/10 0:00 & 8.811 & 4.428 & 6.439 & 15.514 & 6.508 & 13.226 & 6.207 & 19.169 & 17.852 & 32.451 \\
\hline 4/18/10 4:00 & 8.825 & 4.452 & 6.465 & 15.477 & 6.482 & 13.242 & 6.230 & 19.190 & 17.847 & 32.451 \\
\hline 4/18/10 8:00 & 8.858 & 4.475 & 6.498 & 15.381 & 6.418 & 13.301 & 6.275 & 19.272 & 17.845 & 32.444 \\
\hline 4/18/10 12:00 & 8.874 & 4.494 & 6.526 & 15.456 & 6.553 & 13.320 & 6.291 & 19.307 & 17.840 & 32.439 \\
\hline 4/18/10 16:00 & 8.865 & 4.510 & 6.549 & 15.684 & 6.671 & 13.294 & 6.286 & 19.350 & 17.836 & 32.435 \\
\hline 4/18/10 20:00 & 8.879 & 4.531 & 6.570 & 15.698 & 6.694 & 13.303 & 6.317 & 19.333 & 17.833 & 32.432 \\
\hline 4/19/10 0:00 & 8.898 & 4.550 & 6.594 & 15.631 & 6.758 & 13.336 & 6.343 & 19.373 & 17.831 & 32.428 \\
\hline 4/19/10 4:00 & 8.910 & 4.562 & 6.617 & 15.600 & 6.758 & 13.355 & 6.364 & 19.413 & 17.826 & 32.423 \\
\hline 4/19/10 8:00 & 8.938 & 4.574 & 6.641 & 15.551 & 6.770 & 13.395 & 6.399 & 19.460 & 17.824 & 32.420 \\
\hline 4/19/10 12:00 & 8.947 & 4.585 & 6.667 & 15.633 & 6.770 & 13.413 & 6.406 & 19.498 & 17.822 & 32.416 \\
\hline 4/19/10 16:00 & 8.938 & 4.599 & 6.685 & 15.898 & 6.751 & 13.392 & 6.423 & 19.420 & 17.817 & 32.411 \\
\hline 4/19/10 20:00 & 8.950 & 4.616 & 6.704 & 15.950 & 6.746 & 13.388 & 6.449 & 19.467 & 17.815 & 32.409 \\
\hline 4/20/10 0:00 & 8.966 & 4.627 & 6.725 & 15.864 & 6.753 & 13.420 & 6.477 & 19.477 & 17.810 & 32.404 \\
\hline 4/20/10 4:00 & 8.969 & 4.634 & 6.746 & 15.833 & 6.753 & 13.434 & 6.493 & 19.491 & 17.808 & 32.399 \\
\hline 4/20/10 8:00 & 8.983 & 4.641 & 6.765 & 15.782 & 6.753 & 13.467 & 6.519 & 19.517 & 17.808 & 32.397 \\
\hline 4/20/10 12:00 & 8.999 & 4.648 & 6.786 & 15.861 & 6.770 & 13.479 & 6.533 & 19.547 & 17.805 & 32.395 \\
\hline 4/20/10 16:00 & 8.997 & 4.660 & 6.803 & 16.057 & 6.746 & 13.460 & 6.535 & 19.557 & 17.803 & 32.390 \\
\hline 4/20/10 20:00 & 9.009 & 4.674 & 6.821 & 16.059 & 6.749 & 13.474 & 6.561 & 19.507 & 17.801 & 32.387 \\
\hline $4 / 21 / 100: 00$ & 9.018 & 4.681 & 6.843 & 16.036 & 6.751 & 13.491 & 6.580 & 19.524 & 17.798 & 32.383 \\
\hline $4 / 21 / 104: 00$ & 9.028 & 4.683 & 6.859 & 16.008 & 6.756 & 13.514 & 6.601 & 19.545 & 17.796 & 32.380 \\
\hline 4/21/10 8:00 & 9.042 & 4.688 & 6.880 & 15.959 & 6.760 & 13.540 & 6.627 & 19.575 & 17.793 & 32.376 \\
\hline 4/21/10 12:00 & 9.058 & 4.691 & 6.899 & 15.926 & 6.737 & 13.570 & 6.648 & 19.618 & 17.791 & 32.376 \\
\hline $4 / 21 / 1016: 00$ & 9.054 & 4.688 & 6.911 & 16.059 & 6.857 & 13.559 & 6.653 & 19.615 & 17.791 & 32.371 \\
\hline 4/21/10 20:00 & 9.065 & 4.691 & 6.927 & 16.071 & 6.862 & 13.566 & 6.674 & 19.618 & 17.789 & 32.366 \\
\hline 4/22/10 0:00 & 9.087 & 4.693 & 6.946 & 15.992 & 6.789 & 13.603 & 6.702 & 19.660 & 17.786 & 32.364 \\
\hline 4/22/10 4:00 & 9.091 & 4.693 & 6.962 & 15.978 & 6.760 & 13.613 & 6.719 & 19.684 & 17.786 & 32.362 \\
\hline 4/22/10 8:00 & 9.108 & 4.693 & 6.979 & 15.929 & 6.763 & 13.652 & 6.747 & 19.716 & 17.786 & 32.357 \\
\hline 4/22/10 12:00 & 9.134 & & 6.997 & 15.922 & 6.768 & 13.683 & 6.775 & 19.766 & NA & 32.359 \\
\hline $4 / 22 / 10$ 16:00 & 9.117 & 4.736 & 6.993 & 15.989 & 6.742 & 13.615 & 6.768 & 19.792 & & 32.357 \\
\hline $4 / 22 / 1020: 00$ & 9.110 & 4.718 & 6.972 & 16.003 & 6.744 & 13.615 & 6.770 & 19.874 & & 32.354 \\
\hline 4/23/10 0:00 & 9.115 & 4.694 & 6.969 & 15.994 & 6.746 & 13.641 & 6.784 & 19.914 & & 32.350 \\
\hline 4/23/10 4:00 & 9.098 & 4.670 & 6.960 & 16.015 & 6.772 & 13.624 & 6.827 & 19.966 & & 32.347 \\
\hline 4/23/10 8:00 & 8.834 & 4.034 & 6.699 & 15.966 & 6.805 & 13.397 & 6.693 & 20.003 & & 32.345 \\
\hline 4/23/10 12:00 & 8.375 & 3.158 & 6.265 & 15.885 & 6.904 & 13.132 & 6.775 & 19.923 & & 32.340 \\
\hline $4 / 23 / 1016: 00$ & 8.429 & 3.007 & 6.120 & 15.922 & 7.027 & 13.200 & 6.885 & 19.879 & & 32.336 \\
\hline 4/23/10 20:00 & 8.530 & 3.103 & 6.066 & 15.940 & 7.001 & 13.230 & 6.740 & 19.846 & & 32.331 \\
\hline 4/24/10 0:00 & 8.625 & 3.221 & 6.028 & 15.817 & 6.921 & 13.312 & 6.686 & 19.749 & & 32.329 \\
\hline 4/24/10 4:00 & 8.306 & 2.884 & 5.859 & 15.735 & 6.801 & 13.233 & 6.531 & 19.665 & & 32.322 \\
\hline 4/24/10 8:00 & 8.342 & 2.795 & 5.805 & 15.654 & 6.801 & 13.263 & 6.672 & 19.641 & & 32.319 \\
\hline 4/24/10 12:00 & 8.436 & 2.884 & 5.803 & 15.679 & 6.944 & 13.287 & 6.766 & 19.855 & & 32.317 \\
\hline 4/24/10 16:00 & 8.509 & 3.023 & 5.812 & 15.719 & 6.925 & 13.291 & 6.665 & 19.940 & & 32.312 \\
\hline $4 / 24 / 1020: 00$ & 8.530 & 2.901 & 5.744 & 15.693 & 6.904 & 13.237 & 6.646 & 19.867 & & 32.307 \\
\hline 4/25/10 0:00 & 8.346 & 2.677 & 5.606 & 15.565 & 6.822 & 13.179 & 6.582 & 19.818 & & 32.300 \\
\hline 4/25/10 4:00 & 7.778 & 2.413 & 5.336 & 15.453 & 6.801 & 12.996 & 6.526 & 19.848 & & 32.296 \\
\hline 4/25/10 8:00 & 7.781 & 2.418 & 5.249 & 15.281 & 6.694 & 13.113 & 6.453 & 19.799 & & 32.289 \\
\hline 4/25/10 12:00 & 7.927 & 2.583 & 5.249 & 15.195 & 6.678 & 13.200 & 6.477 & 19.860 & & 32.291 \\
\hline $4 / 25 / 1016: 00$ & 8.073 & 2.783 & 5.275 & 15.153 & 6.657 & 13.259 & 6.460 & 19.905 & & 32.289 \\
\hline 4/25/10 20:00 & 8.207 & 2.972 & 5.312 & 15.088 & 6.600 & 13.322 & 6.439 & 19.756 & & 32.284 \\
\hline 4/26/10 0:00 & 8.325 & 3.141 & 5.357 & 15.020 & 6.529 & 13.385 & 6.430 & 19.726 & & 32.281 \\
\hline 4/26/10 4:00 & 8.412 & 3.280 & 5.397 & 15.013 & 6.508 & 13.409 & 6.465 & 19.735 & & 32.279 \\
\hline 4/26/10 8:00 & 8.500 & 3.405 & 5.437 & 14.967 & 6.466 & 13.472 & 6.474 & 19.749 & & 32.277 \\
\hline 4/26/10 12:00 & 8.561 & 3.513 & 5.477 & 14.971 & 6.468 & 13.502 & 6.589 & 19.836 & & 32.274 \\
\hline 4/26/10 16:00 & 8.594 & 3.615 & 5.507 & 15.076 & 6.569 & 13.488 & 6.660 & 20.041 & & 32.270 \\
\hline $4 / 26 / 1020: 00$ & 8.634 & 3.716 & 5.540 & 15.097 & 6.534 & 13.502 & 6.542 & 20.036 & & 32.270 \\
\hline 4/27/10 0:00 & 8.676 & 3.801 & 5.575 & 15.055 & 6.470 & 13.545 & 6.580 & 19.912 & & 32.263 \\
\hline 4/27/10 4:00 & 8.721 & 3.874 & 5.606 & 15.046 & 6.459 & 13.577 & 6.582 & 19.879 & & 32.260 \\
\hline $4 / 27 / 108: 00$ & 8.778 & 3.935 & 5.648 & 14.992 & 6.753 & 13.643 & 6.676 & 19.895 & & 32.258 \\
\hline 4/27/10 12:00 & 8.832 & 3.994 & 5.690 & 15.022 & 6.765 & 13.704 & 7.062 & 20.097 & & 32.256 \\
\hline 4/27/10 16:00 & 8.856 & 4.060 & 5.728 & 15.237 & 6.753 & 13.704 & 6.954 & 20.321 & & 32.253 \\
\hline 4/27/10 20:00 & 8.877 & 4.119 & 5.761 & 15.300 & 6.749 & 13.699 & 6.784 & 20.206 & & 32.251 \\
\hline 4/28/10 0:00 & 8.900 & 4.166 & 5.793 & 15.276 & 6.751 & 13.725 & 6.752 & 20.161 & & 32.248 \\
\hline
\end{tabular}

TABLE S1.1 Page 34 of 89 
Water Level (ft below reference point)

\begin{tabular}{|c|c|c|c|c|c|c|c|c|c|c|}
\hline Date and Time & McPike & Potter & Hughes & Barr & Burks & MoDOT & Clizer "Old" & Morgan & SB24 & SB38 \\
\hline 4/28/10 4:00 & 8.905 & 4.197 & 5.817 & 15.304 & 6.739 & 13.716 & 6.782 & 20.135 & & 32.248 \\
\hline 4/28/10 8:00 & 8.919 & 4.223 & 5.843 & 15.302 & 6.739 & 13.737 & 6.904 & 20.133 & & 32.246 \\
\hline 4/28/10 12:00 & 8.907 & 4.246 & 5.866 & 15.416 & 6.735 & 13.723 & 7.238 & 20.111 & & 32.241 \\
\hline 4/28/10 16:00 & 8.867 & 4.272 & 5.883 & 15.719 & 6.711 & 13.652 & 7.266 & 20.027 & & 32.241 \\
\hline $4 / 28 / 1020: 00$ & 8.856 & 4.305 & 5.899 & 15.808 & 6.706 & 13.624 & 7.073 & 20.050 & & 32.237 \\
\hline 4/29/10 0:00 & 8.842 & 4.326 & 5.915 & 15.836 & 6.709 & 13.615 & 7.078 & 19.970 & & 32.234 \\
\hline 4/29/10 4:00 & 8.809 & 4.340 & 5.927 & 15.933 & 6.699 & 13.568 & 7.151 & 19.909 & & 32.234 \\
\hline 4/29/10 8:00 & 8.792 & 4.352 & 5.937 & 16.001 & 6.699 & 13.556 & 7.254 & 19.827 & & 32.232 \\
\hline 4/29/10 12:00 & 8.820 & 4.376 & 5.965 & 16.101 & 6.716 & 13.608 & 6.801 & 19.893 & & 32.230 \\
\hline 4/29/10 16:00 & 8.849 & 4.411 & 5.993 & 16.274 & 6.725 & 13.636 & 6.822 & 19.914 & & 32.227 \\
\hline 4/29/10 20:00 & 8.863 & 4.446 & 6.026 & 16.311 & 6.732 & 13.638 & 6.836 & 19.930 & & 32.225 \\
\hline 4/30/10 0:00 & 8.867 & 4.468 & 6.049 & 16.299 & 6.735 & 13.652 & 6.848 & 19.935 & & 32.223 \\
\hline 4/30/10 4:00 & 8.903 & 4.491 & 6.070 & 16.208 & 6.794 & 13.727 & 6.907 & 20.001 & & 32.223 \\
\hline 4/30/10 8:00 & 8.830 & 3.897 & 5.899 & 15.938 & 6.610 & 13.735 & 6.893 & 20.156 & & 32.216 \\
\hline 4/30/10 12:00 & 8.818 & 3.421 & 5.803 & 15.843 & 6.635 & 13.690 & 6.947 & 20.180 & & 32.211 \\
\hline 4/30/10 16:00 & 8.809 & 3.205 & 5.744 & 15.845 & 6.709 & 13.697 & 6.965 & 20.384 & & 32.211 \\
\hline $4 / 30 / 1020: 00$ & 8.856 & 3.252 & 5.744 & 15.782 & 6.673 & 13.735 & 6.883 & 20.351 & & 32.208 \\
\hline 5/1/10 0:00 & 8.919 & 3.384 & 5.779 & 15.591 & 6.518 & 13.831 & 6.850 & 20.177 & & 32.208 \\
\hline 5/1/10 4:00 & 8.957 & 3.513 & 5.812 & 15.512 & 6.442 & 13.856 & 6.831 & 20.123 & & 32.206 \\
\hline 5/1/10 8:00 & 9.002 & 3.638 & 5.852 & 15.407 & 6.367 & 13.910 & 6.909 & 20.119 & & 32.204 \\
\hline $5 / 1 / 1012: 00$ & 9.040 & 3.754 & 5.894 & 15.398 & 6.456 & 13.957 & 7.156 & 20.405 & & 32.204 \\
\hline 5/1/10 16:00 & 9.028 & 3.850 & 5.927 & 15.628 & 6.614 & 13.901 & 7.245 & 20.650 & & 32.201 \\
\hline $5 / 1 / 1020: 00$ & 9.051 & 3.942 & 5.960 & 15.642 & 6.638 & 13.908 & 7.085 & 20.471 & & 32.199 \\
\hline 5/2/10 0:00 & 9.073 & 4.025 & 5.995 & 15.596 & 6.612 & 13.941 & 7.080 & 20.474 & & 32.197 \\
\hline 5/2/10 4:00 & 9.075 & 4.083 & 6.021 & 15.582 & 6.591 & 13.941 & 7.088 & 20.460 & & 32.194 \\
\hline $5 / 2 / 108: 00$ & 9.120 & 4.142 & 6.056 & 15.488 & 6.544 & 14.013 & 7.125 & 20.509 & & 32.197 \\
\hline $5 / 2 / 1012: 00$ & 9.143 & 4.185 & 6.089 & 15.495 & 6.600 & 14.042 & 7.299 & 20.551 & & 32.192 \\
\hline 5/2/10 16:00 & 9.165 & 4.227 & 6.120 & 15.617 & 6.666 & 14.058 & 7.351 & 20.563 & & 32.192 \\
\hline $5 / 2 / 1020: 00$ & 9.202 & 4.277 & 6.157 & 15.603 & 6.657 & 14.091 & 7.160 & 20.586 & & 32.190 \\
\hline 5/3/10 0:00 & 9.252 & 4.319 & 6.188 & 15.456 & 6.494 & 14.173 & 7.174 & 20.678 & & 32.187 \\
\hline 5/3/10 4:00 & 9.278 & 4.345 & 6.218 & 15.414 & 6.482 & 14.196 & 7.170 & 20.725 & & 32.187 \\
\hline 5/3/10 8:00 & 9.329 & 4.366 & 6.251 & 15.281 & 6.371 & 14.276 & 7.177 & 20.798 & & 32.187 \\
\hline $5 / 3 / 1012: 00$ & 9.374 & 4.392 & 6.291 & 15.281 & 6.442 & 14.342 & 7.459 & 20.880 & & 32.185 \\
\hline $5 / 3 / 10$ 16:00 & 9.379 & 4.418 & 6.324 & 15.519 & 6.544 & 14.323 & 7.499 & 21.033 & & 32.187 \\
\hline $5 / 3 / 1020: 00$ & 9.407 & 4.449 & 6.357 & 15.530 & 6.553 & 14.323 & 7.299 & 20.904 & & 32.185 \\
\hline 5/4/10 0:00 & 9.405 & 4.472 & 6.380 & 15.488 & 6.506 & 14.330 & 7.285 & 20.904 & & 32.185 \\
\hline 5/4/10 4:00 & 9.393 & 4.484 & 6.401 & 15.507 & 6.527 & 14.304 & 7.332 & 20.897 & & 32.185 \\
\hline 5/4/10 8:00 & 9.400 & 4.496 & 6.425 & 15.498 & 6.579 & 14.321 & 7.475 & 20.890 & & 32.185 \\
\hline $5 / 4 / 1012: 00$ & 9.398 & 4.510 & 6.446 & 15.640 & 6.756 & 14.316 & 7.790 & 20.876 & & 32.183 \\
\hline 5/4/10 16:00 & 9.384 & 4.531 & 6.465 & 15.915 & 6.909 & 14.278 & 7.792 & 20.822 & & 32.183 \\
\hline $5 / 4 / 1020: 00$ & 9.398 & 4.555 & 6.490 & 15.950 & 6.789 & 14.274 & 7.562 & 20.796 & & 32.183 \\
\hline 5/5/10 0:00 & 9.457 & 4.583 & 6.526 & 15.777 & 6.827 & 14.389 & 7.376 & 20.887 & & 32.183 \\
\hline 5/5/10 4:00 & 9.497 & 4.609 & 6.561 & 15.675 & 6.836 & 14.450 & 7.405 & 20.979 & & 32.180 \\
\hline 5/5/10 8:00 & 9.544 & 4.625 & 6.594 & 15.568 & 6.846 & 14.515 & 7.503 & 21.057 & & 32.183 \\
\hline $5 / 5 / 1012: 00$ & 9.584 & 4.646 & 6.629 & 15.591 & 6.855 & 14.572 & 7.773 & 21.134 & & 32.183 \\
\hline 5/5/10 16:00 & 9.601 & 4.670 & 6.659 & 15.798 & 6.855 & 14.576 & 7.764 & 21.163 & & 32.183 \\
\hline $5 / 5 / 1020: 00$ & 9.636 & 4.696 & 6.692 & 15.731 & 6.864 & 14.602 & 7.470 & 21.196 & & 32.183 \\
\hline 5/6/10 0:00 & 9.676 & 4.717 & 6.727 & 15.584 & 6.881 & 14.665 & 7.367 & 21.275 & & 32.185 \\
\hline 5/6/10 4:00 & 9.692 & 4.727 & 6.758 & 15.528 & 6.878 & 14.679 & 7.449 & 21.311 & & 32.183 \\
\hline 5/6/10 8:00 & 9.702 & 4.738 & 6.784 & 15.463 & 6.874 & 14.698 & 7.637 & 21.332 & & 32.185 \\
\hline 5/6/10 12:00 & 9.692 & 4.745 & 6.807 & 15.579 & 6.867 & 14.691 & 7.921 & 21.334 & & 32.185 \\
\hline $5 / 6 / 1016: 00$ & 9.655 & 4.752 & 6.819 & 15.836 & 6.834 & 14.602 & 7.635 & 21.245 & & 32.185 \\
\hline $5 / 6 / 1020: 00$ & 9.643 & 4.767 & 6.835 & 15.852 & 6.827 & 14.581 & 7.644 & 21.191 & & 32.187 \\
\hline 5/7/10 0:00 & 9.605 & 4.771 & 6.840 & 15.878 & 6.864 & 14.534 & 7.602 & 21.139 & & 32.185 \\
\hline $5 / 7 / 104: 00$ & 9.389 & 4.635 & 6.741 & 15.850 & 6.796 & 14.499 & 7.616 & 21.118 & & 32.180 \\
\hline 5/7/10 8:00 & 9.082 & 3.892 & 6.497 & 15.479 & 6.704 & 14.485 & 7.637 & 21.163 & & 32.175 \\
\hline 5/7/10 12:00 & 9.247 & 3.772 & 6.432 & 15.337 & 6.645 & 14.600 & 7.635 & 21.243 & & 32.183 \\
\hline 5/7/10 16:00 & 9.393 & 3.855 & 6.429 & 15.279 & 6.579 & 14.689 & 7.647 & 21.337 & & 32.183 \\
\hline $5 / 7 / 1020: 00$ & 9.509 & 3.984 & 6.450 & 15.144 & 6.459 & 14.759 & 7.473 & 21.428 & & 32.183 \\
\hline 5/8/10 0:00 & 9.613 & 4.109 & 6.486 & 14.999 & 6.305 & 14.848 & 7.456 & 21.534 & & 32.185 \\
\hline 5/8/10 4:00 & 9.674 & 4.203 & 6.523 & 14.962 & 6.227 & 14.879 & 7.501 & 21.593 & & 32.185 \\
\hline 5/8/10 8:00 & 9.740 & 4.281 & 6.558 & 14.880 & 6.341 & 14.944 & 7.576 & 21.666 & & 32.187 \\
\hline $5 / 8 / 10$ 12:00 & 9.794 & 4.350 & 6.596 & 14.957 & 6.444 & 14.989 & 7.858 & 21.750 & & 32.187 \\
\hline $5 / 8 / 1016: 00$ & 9.808 & 4.404 & 6.626 & 15.160 & 6.506 & 14.977 & 7.891 & 21.734 & & 32.190 \\
\hline
\end{tabular}

TABLE S1.1 Page 35 of 89 
Water Level (ft below reference point)

\begin{tabular}{|c|c|c|c|c|c|c|c|c|c|c|}
\hline \multirow[b]{2}{*}{ Date and Time } & \\
\hline & McPike & Potter & Hughes & Barr & Burks & MoDOT & Clizer "Old" & Morgan & SB24 & SB38 \\
\hline $5 / 8 / 1020: 00$ & 9.822 & 4.451 & 6.652 & 15.220 & 6.676 & 14.951 & 7.741 & 21.703 & & 32.192 \\
\hline 5/9/10 0:00 & 9.853 & 4.491 & 6.683 & 15.169 & 6.567 & 14.994 & 7.731 & 21.732 & & 32.192 \\
\hline 5/9/10 4:00 & 9.869 & 4.519 & 6.709 & 15.179 & 6.874 & 15.010 & 7.769 & 21.748 & & 32.194 \\
\hline 5/9/10 8:00 & 9.884 & 4.545 & 6.734 & 15.186 & 6.871 & 15.019 & 7.863 & 21.755 & & 32.194 \\
\hline 5/9/10 12:00 & 9.879 & 4.566 & 6.756 & 15.307 & 6.864 & 15.001 & 8.107 & 21.753 & & 32.194 \\
\hline 5/9/10 16:00 & 9.855 & 4.588 & 6.774 & 15.465 & 6.850 & 14.961 & 8.067 & 21.725 & & 32.197 \\
\hline 5/9/10 20:00 & 9.853 & 4.604 & 6.791 & 15.507 & 6.845 & 14.949 & 7.980 & 21.682 & & 32.199 \\
\hline 5/10/10 0:00 & 9.848 & 4.623 & 6.807 & 15.537 & 6.841 & 14.947 & 7.990 & 21.670 & & 32.199 \\
\hline 5/10/10 4:00 & 9.829 & 4.635 & 6.821 & 15.586 & 6.831 & 14.928 & 8.022 & 21.635 & & 32.201 \\
\hline 5/10/10 8:00 & 9.808 & 4.642 & 6.828 & 15.614 & 6.860 & 14.904 & 8.018 & 21.600 & & 32.201 \\
\hline $5 / 10 / 10$ 12:00 & 9.450 & 4.496 & 6.634 & 15.644 & 6.850 & 14.820 & 7.839 & 21.529 & & 32.201 \\
\hline 5/10/10 16:00 & 8.319 & 3.452 & 5.782 & 15.502 & 6.839 & 13.995 & 7.632 & 21.346 & & 32.204 \\
\hline 5/10/10 20:00 & 8.179 & 3.063 & 5.509 & 15.519 & 6.937 & 14.004 & 7.513 & 21.113 & & 32.206 \\
\hline $5 / 11 / 100: 00$ & 7.463 & 2.686 & 4.998 & 15.370 & 6.895 & 13.636 & 7.174 & 20.918 & & 32.208 \\
\hline $5 / 11 / 104: 00$ & 7.477 & 2.669 & 4.852 & 15.213 & 6.838 & 13.807 & 6.994 & 20.822 & & 32.208 \\
\hline 5/11/10 8:00 & 7.628 & 2.816 & 4.812 & 15.027 & 6.702 & 13.992 & 6.907 & 20.850 & & 32.208 \\
\hline $5 / 11 / 10$ 12:00 & 7.781 & 2.999 & 4.798 & 14.939 & 6.624 & 14.105 & 6.895 & 20.880 & & 32.206 \\
\hline $5 / 11 / 1016: 00$ & 7.889 & 3.190 & 4.793 & 15.001 & 6.636 & 14.124 & 6.935 & 20.873 & & 32.206 \\
\hline 5/11/10 20:00 & 7.991 & 3.372 & 4.800 & 15.032 & 6.586 & 14.140 & 6.792 & 20.838 & & 32.206 \\
\hline 5/12/10 0:00 & 8.076 & 3.520 & 4.815 & 14.999 & 6.522 & 14.168 & 6.792 & 20.833 & & 32.208 \\
\hline $5 / 12 / 104: 00$ & 8.116 & 3.631 & 4.817 & 15.069 & 6.513 & 14.128 & 6.801 & 20.756 & & 32.206 \\
\hline 5/12/10 8:00 & 8.172 & 3.727 & 4.826 & 15.097 & 6.506 & 14.114 & 6.759 & 20.695 & & 32.204 \\
\hline $5 / 12 / 1012: 00$ & 8.198 & 3.699 & 4.793 & 15.069 & 6.511 & 14.117 & 6.843 & 20.688 & & 32.204 \\
\hline $5 / 12 / 1016: 00$ & 8.194 & 3.574 & 4.775 & 15.097 & 6.584 & 14.065 & 6.932 & 20.603 & & 32.206 \\
\hline 5/12/10 20:00 & 8.227 & 3.544 & 4.782 & 15.183 & 6.685 & 14.070 & 6.944 & 20.568 & & 32.208 \\
\hline $5 / 13 / 100: 00$ & 8.066 & 3.541 & 4.697 & 15.176 & 6.626 & 14.103 & 6.761 & 20.568 & & 32.208 \\
\hline $5 / 13 / 104: 00$ & 6.176 & 2.775 & 3.688 & 14.827 & 6.405 & 13.739 & 6.120 & 20.539 & & 32.206 \\
\hline 5/13/10 8:00 & 5.382 & 2.464 & 3.334 & 14.382 & 5.853 & 13.420 & 5.742 & 20.300 & & 32.201 \\
\hline 5/13/10 12:00 & 5.464 & 2.537 & 3.378 & 14.216 & 5.442 & 13.474 & 5.542 & 20.187 & & 32.201 \\
\hline $5 / 13 / 1016: 00$ & 5.594 & 2.686 & 3.465 & 14.093 & 5.444 & 13.493 & 5.370 & 20.062 & & 32.201 \\
\hline 5/13/10 20:00 & 5.740 & 2.889 & 3.559 & 14.004 & 5.536 & 13.481 & 5.081 & 19.926 & & 32.199 \\
\hline 5/14/10 0:00 & 5.869 & 3.070 & 3.655 & 13.874 & 5.525 & 13.498 & 4.917 & 19.832 & & 32.197 \\
\hline 5/14/10 4:00 & 5.957 & 3.209 & 3.728 & 13.860 & 5.579 & 13.453 & 4.825 & 19.688 & & 32.197 \\
\hline 5/14/10 8:00 & 6.051 & 3.339 & 3.801 & 13.792 & 5.603 & 13.463 & 4.844 & 19.594 & & 32.194 \\
\hline $5 / 14 / 10$ 12:00 & 6.136 & 3.447 & 3.866 & 13.837 & 5.716 & 13.451 & 4.999 & 19.519 & & 32.192 \\
\hline 5/14/10 16:00 & 6.181 & 3.548 & 3.923 & 14.046 & 5.886 & 13.373 & 5.009 & 19.354 & & 32.192 \\
\hline 5/14/10 20:00 & 6.226 & 3.638 & 3.967 & 14.158 & 5.994 & 13.294 & 4.868 & 19.185 & & 32.190 \\
\hline 5/15/10 0:00 & 6.282 & 3.713 & 4.019 & 14.109 & 5.973 & 13.296 & 4.802 & 19.157 & & 32.190 \\
\hline $5 / 15 / 104: 00$ & 6.306 & 3.767 & 4.059 & 14.135 & 5.997 & 13.252 & 4.778 & 19.079 & & 32.187 \\
\hline 5/15/10 8:00 & 6.350 & 3.807 & 4.096 & 14.151 & 6.030 & 13.221 & 4.830 & 18.978 & & 32.185 \\
\hline 5/15/10 12:00 & 6.388 & 3.843 & 4.139 & 14.202 & 6.140 & 13.198 & 4.959 & 18.908 & & 32.183 \\
\hline 5/15/10 16:00 & 6.407 & 3.881 & 4.176 & 14.314 & 6.235 & 13.144 & 4.915 & 18.799 & & 32.180 \\
\hline 5/15/10 20:00 & 6.310 & 3.614 & 4.075 & 14.275 & 6.263 & 12.991 & 4.842 & 18.703 & & 32.175 \\
\hline 5/16/10 0:00 & 6.296 & 3.195 & 4.052 & 14.200 & 6.244 & 12.998 & 4.809 & 18.658 & & 32.171 \\
\hline 5/16/10 4:00 & 6.325 & 3.046 & 4.056 & 14.247 & 6.289 & 12.954 & 4.818 & 18.574 & & 32.166 \\
\hline 5/16/10 8:00 & 6.365 & 3.004 & 4.080 & 14.265 & 6.329 & 12.935 & 4.832 & 18.508 & & 32.164 \\
\hline $5 / 16 / 1012: 00$ & 6.405 & 3.016 & 4.113 & 14.275 & 6.357 & 12.928 & 4.877 & 18.508 & & 32.161 \\
\hline 5/16/10 16:00 & 6.426 & 3.004 & 4.139 & 14.328 & 6.400 & 12.897 & 4.900 & 18.442 & & 32.159 \\
\hline 5/16/10 20:00 & 6.459 & 3.027 & 4.169 & 14.344 & 6.395 & 12.883 & 4.842 & 18.386 & & 32.157 \\
\hline 5/17/10 0:00 & 6.280 & 3.042 & 4.113 & 14.293 & 6.315 & 12.879 & 4.628 & 18.364 & & 32.150 \\
\hline $5 / 17 / 104: 00$ & 5.342 & 2.448 & 3.341 & 13.920 & 6.209 & 12.482 & 4.374 & 18.275 & & 32.142 \\
\hline 5/17/10 8:00 & 4.887 & 2.295 & 3.153 & 13.687 & 5.931 & 12.419 & 4.175 & 18.195 & & 32.135 \\
\hline 5/17/10 12:00 & 4.757 & 2.356 & 3.132 & 13.576 & 5.657 & 12.461 & 4.045 & 18.125 & & 32.133 \\
\hline $5 / 17 / 1016: 00$ & 4.752 & 2.476 & 3.174 & 13.615 & 5.673 & 12.447 & 3.803 & 18.028 & & 32.131 \\
\hline 5/17/10 20:00 & 4.807 & 2.608 & 3.249 & 13.631 & 5.730 & 12.415 & 3.536 & 17.859 & & 32.126 \\
\hline 5/18/10 0:00 & 4.866 & 2.690 & 3.329 & 13.576 & 5.756 & 12.393 & 3.381 & 17.772 & & 32.121 \\
\hline 5/18/10 4:00 & 4.927 & 2.742 & 3.397 & 13.536 & 5.801 & 12.368 & 3.294 & 17.659 & & 32.117 \\
\hline 5/18/10 8:00 & 4.976 & 2.764 & 3.453 & 13.538 & 5.890 & 12.321 & 3.383 & 17.537 & & 32.112 \\
\hline $5 / 18 / 1012: 00$ & 5.047 & 2.799 & 3.517 & 13.583 & 5.982 & 12.311 & 3.609 & 17.454 & & 32.107 \\
\hline 5/18/10 16:00 & 5.104 & 2.893 & 3.568 & 13.818 & 6.103 & 12.239 & 3.576 & 17.450 & & 32.102 \\
\hline 5/18/10 20:00 & 5.170 & 3.037 & 3.625 & 13.839 & 6.171 & 12.199 & 3.371 & 17.257 & & 32.098 \\
\hline 5/19/10 0:00 & 5.226 & 3.141 & 3.681 & 13.757 & 6.176 & 12.185 & 3.301 & 17.151 & & 32.093 \\
\hline 5/19/10 4:00 & 5.276 & 3.185 & 3.728 & 13.718 & 6.169 & 12.150 & 3.275 & 17.059 & & 32.088 \\
\hline 5/19/10 8:00 & 5.323 & 3.197 & 3.777 & 13.694 & 6.214 & 12.119 & 3.385 & 16.982 & & 32.081 \\
\hline
\end{tabular}

TABLE S1.1 Page 36 of 89 


\begin{tabular}{|c|c|c|c|c|c|c|c|c|c|c|}
\hline \multirow[b]{2}{*}{ Date and Time } & \multicolumn{8}{|c|}{ Water Level (ft below reference point) } & \multirow[b]{2}{*}{ SB24 } & \multirow[b]{2}{*}{ SB38 } \\
\hline & McPike & Potter & Hughes & Barr & Burks & MoDOT & Clizer "Old" & Morgan & & \\
\hline $5 / 19 / 1012: 00$ & 5.379 & 3.204 & 3.826 & 13.718 & 6.336 & 12.096 & 3.486 & 16.944 & & 32.077 \\
\hline 5/19/10 16:00 & 5.415 & 3.249 & 3.873 & 13.797 & 6.416 & 12.032 & 3.465 & 16.836 & & 32.072 \\
\hline 5/19/10 20:00 & 5.464 & 3.320 & 3.916 & 13.799 & 6.445 & 12.007 & 3.446 & 16.765 & & 32.067 \\
\hline 5/20/10 0:00 & 5.502 & 3.334 & 3.951 & 13.755 & 6.383 & 11.976 & 3.432 & 16.744 & & 32.060 \\
\hline $5 / 20 / 104: 00$ & 5.500 & 2.987 & 3.937 & 13.764 & 6.456 & 11.885 & 3.475 & 16.667 & & 32.055 \\
\hline 5/20/10 8:00 & 5.382 & 2.662 & 3.880 & 13.680 & 6.449 & 11.781 & 3.414 & 16.615 & & 32.046 \\
\hline 5/20/10 12:00 & 5.294 & 2.410 & 3.770 & 13.590 & 6.461 & 11.723 & 3.439 & 16.582 & & 32.041 \\
\hline 5/20/10 16:00 & 5.252 & 2.323 & 3.730 & 13.576 & 6.482 & 11.676 & 3.439 & 16.533 & & 32.039 \\
\hline 5/20/10 20:00 & 5.269 & 2.328 & 3.704 & 13.550 & 6.487 & 11.662 & 3.411 & 16.493 & & 32.032 \\
\hline 5/21/10 0:00 & 5.339 & 2.405 & 3.709 & 13.499 & 6.456 & 11.676 & 3.402 & 16.481 & & 32.022 \\
\hline 5/21/10 4:00 & 5.405 & 2.495 & 3.728 & 13.466 & 6.433 & 11.676 & 3.404 & 16.467 & & 32.018 \\
\hline 5/21/10 8:00 & 5.481 & 2.573 & 3.765 & 13.415 & 6.390 & 11.695 & 3.418 & 16.472 & & 32.008 \\
\hline 5/21/10 12:00 & 5.544 & 2.641 & 3.808 & 13.417 & 6.390 & 11.704 & 3.524 & 16.479 & & 32.006 \\
\hline 5/21/10 16:00 & 5.596 & 2.752 & 3.852 & 13.524 & 6.428 & 11.685 & 3.644 & 16.455 & & 31.999 \\
\hline 5/21/10 20:00 & 5.643 & 2.886 & 3.899 & 13.606 & 6.475 & 11.650 & 3.569 & 16.422 & & 31.992 \\
\hline 5/22/10 0:00 & 5.681 & 2.992 & 3.941 & 13.594 & 6.452 & 11.641 & 3.536 & 16.413 & & 31.985 \\
\hline $5 / 22 / 104: 00$ & 5.712 & 3.058 & 3.977 & 13.606 & 6.447 & 11.617 & 3.573 & 16.394 & & 31.978 \\
\hline 5/22/10 8:00 & 5.738 & 3.089 & 4.012 & 13.615 & 6.478 & 11.606 & 3.745 & 16.382 & & 31.973 \\
\hline $5 / 22 / 10$ 12:00 & 5.768 & 3.117 & 4.045 & 13.762 & 6.643 & 11.566 & 4.015 & 16.363 & & 31.966 \\
\hline $5 / 22 / 10$ 16:00 & 5.813 & 3.230 & 4.087 & 14.025 & 6.820 & 11.526 & 4.069 & 16.312 & & 31.959 \\
\hline 5/22/10 20:00 & 5.874 & 3.388 & 4.141 & 14.063 & 6.829 & 11.519 & 3.905 & 16.330 & & 31.952 \\
\hline 5/23/10 0:00 & 5.929 & 3.517 & 4.197 & 13.979 & 6.733 & 11.547 & 3.862 & 16.349 & & 31.945 \\
\hline $5 / 23 / 104: 00$ & 5.966 & 3.609 & 4.237 & 13.979 & 6.730 & 11.533 & 3.862 & 16.337 & & 31.938 \\
\hline 5/23/10 8:00 & 6.032 & 3.687 & 4.289 & 13.906 & 6.659 & 11.563 & 3.895 & 16.375 & & 31.933 \\
\hline 5/23/10 12:00 & 6.101 & 3.751 & 4.345 & 13.857 & 6.619 & 11.601 & 4.043 & 16.415 & & 31.926 \\
\hline 5/23/10 16:00 & 6.162 & 3.817 & 4.401 & 13.958 & 6.666 & 11.592 & 4.015 & 16.961 & & 31.919 \\
\hline 5/23/10 20:00 & 6.214 & 3.876 & 4.451 & 13.976 & 6.657 & 11.585 & 3.994 & 16.507 & & 31.912 \\
\hline 5/24/10 0:00 & 6.266 & 3.918 & 4.498 & 13.920 & 6.605 & 11.603 & 3.977 & 16.493 & & 31.905 \\
\hline $5 / 24 / 104: 00$ & 6.317 & 3.944 & 4.545 & 13.876 & 6.556 & 11.615 & 3.984 & 16.502 & & 31.898 \\
\hline 5/24/10 8:00 & 6.367 & 3.972 & 4.587 & 13.848 & 6.532 & 11.622 & 4.111 & 16.519 & & 31.893 \\
\hline 5/24/10 12:00 & 6.421 & 4.003 & 4.638 & 13.897 & 6.570 & 11.643 & 4.332 & 16.561 & & 31.886 \\
\hline $5 / 24 / 1016: 00$ & 6.466 & 4.041 & 4.692 & 14.125 & 6.685 & 11.613 & 4.393 & 16.526 & & 31.879 \\
\hline 5/24/10 20:00 & 6.518 & 4.083 & 4.746 & 14.144 & 6.678 & 11.596 & 4.287 & 16.749 & & 31.874 \\
\hline 5/25/10 0:00 & 6.565 & 4.114 & 4.791 & 14.067 & 6.629 & 11.615 & 4.266 & 16.589 & & 31.867 \\
\hline 5/25/10 4:00 & 6.603 & 4.132 & 4.829 & 14.037 & 6.605 & 11.615 & 4.262 & 16.570 & & 31.860 \\
\hline 5/25/10 8:00 & 6.666 & 4.149 & 4.878 & 13.927 & 6.530 & 11.667 & 4.363 & 16.606 & & 31.855 \\
\hline 5/25/10 12:00 & 6.709 & 4.165 & 4.925 & 14.004 & 6.577 & 11.662 & 4.492 & 16.638 & & 31.848 \\
\hline 5/25/10 16:00 & 6.751 & 4.187 & 4.979 & 14.111 & 6.638 & 11.655 & 4.597 & 16.624 & & 31.841 \\
\hline 5/25/10 20:00 & 6.810 & 4.217 & 5.030 & 14.083 & 6.622 & 11.667 & 4.471 & 16.660 & & 31.834 \\
\hline 5/26/10 0:00 & 6.860 & 4.243 & 5.075 & 13.972 & 6.515 & 11.699 & 4.438 & 16.693 & & 31.829 \\
\hline 5/26/10 4:00 & 6.895 & 4.250 & 5.110 & 13.941 & 6.483 & 11.688 & 4.447 & 16.723 & & 31.822 \\
\hline 5/26/10 8:00 & 6.944 & 4.260 & 5.152 & 13.846 & 6.435 & 11.732 & 4.572 & 16.768 & & 31.815 \\
\hline 5/26/10 12:00 & 6.982 & 4.271 & 5.190 & 13.962 & 6.532 & 11.725 & 4.844 & 16.756 & & 31.810 \\
\hline $5 / 26 / 1016: 00$ & 7.017 & 4.290 & 5.239 & 14.149 & 6.633 & 11.709 & 4.804 & 16.751 & & 31.803 \\
\hline 5/26/10 20:00 & 7.050 & 4.311 & 5.277 & 14.132 & 6.622 & 11.704 & 4.727 & 16.761 & & 31.799 \\
\hline 5/27/10 0:00 & 7.098 & 4.328 & 5.319 & 14.023 & 6.551 & 11.751 & 4.684 & 16.822 & & 31.792 \\
\hline 5/27/10 4:00 & 7.133 & 4.337 & 5.352 & 13.986 & 6.523 & 11.751 & 4.687 & 16.827 & & 31.784 \\
\hline 5/27/10 8:00 & 7.175 & 4.342 & 5.389 & 13.906 & 6.476 & 11.781 & 4.823 & 16.867 & & 31.782 \\
\hline 5/27/10 12:00 & 7.213 & 4.356 & 5.432 & 13.990 & 6.546 & 11.793 & 5.140 & 16.890 & & 31.775 \\
\hline 5/27/10 16:00 & 7.241 & 4.380 & 5.476 & 14.275 & 6.683 & 11.765 & 5.107 & 16.876 & & 31.768 \\
\hline 5/27/10 20:00 & 7.279 & 4.410 & 5.519 & 14.296 & 6.709 & 11.749 & 4.938 & 16.878 & & 31.763 \\
\hline 5/28/10 0:00 & 7.324 & 4.432 & 5.558 & 14.170 & 6.709 & 11.786 & 4.868 & 16.918 & & 31.756 \\
\hline 5/28/10 4:00 & 7.350 & 4.439 & 5.591 & 14.107 & 6.700 & 11.791 & 4.882 & 16.963 & & 31.749 \\
\hline 5/28/10 8:00 & 7.380 & 4.443 & 5.624 & 14.039 & 6.702 & 11.810 & 5.037 & 16.984 & & 31.744 \\
\hline 5/28/10 12:00 & 7.421 & 4.455 & 5.664 & 14.156 & 6.716 & 11.824 & 5.321 & 17.008 & & 31.740 \\
\hline 5/28/10 16:00 & 7.444 & 4.476 & 5.706 & 14.435 & 6.733 & 11.796 & 5.070 & 16.994 & & 31.733 \\
\hline $5 / 28 / 1020: 00$ & 7.477 & 4.502 & 5.746 & 14.433 & 6.740 & 11.781 & 5.107 & 16.998 & & 31.728 \\
\hline 5/29/10 0:00 & 7.517 & 4.519 & 5.784 & 14.293 & 6.735 & 11.814 & 5.140 & 17.038 & & 31.723 \\
\hline $5 / 29 / 104: 00$ & 7.543 & 4.526 & 5.812 & 14.226 & 6.723 & 11.821 & 5.159 & 17.057 & & 31.716 \\
\hline 5/29/10 8:00 & 7.574 & 4.526 & 5.842 & 14.149 & 6.728 & 11.849 & 5.192 & 17.090 & & 31.711 \\
\hline $5 / 29 / 1012: 00$ & 7.607 & 4.535 & 5.880 & 14.272 & 6.747 & 11.854 & 5.208 & 17.130 & & 31.707 \\
\hline 5/29/10 16:00 & 7.640 & 4.561 & 5.925 & 14.501 & 6.768 & 11.847 & 5.298 & 17.149 & & 31.700 \\
\hline 5/29/10 20:00 & 7.678 & 4.587 & 5.969 & 14.456 & 6.780 & 11.842 & 5.323 & 17.168 & & 31.695 \\
\hline 5/30/10 0:00 & 7.708 & 4.599 & 6.002 & 14.375 & 6.761 & 11.856 & 5.359 & 17.200 & & 31.690 \\
\hline
\end{tabular}

TABLE S1.1 Page 37 of 89 


\begin{tabular}{|c|c|c|c|c|c|c|c|c|c|c|}
\hline \multirow[b]{2}{*}{ Date and Time } & \multicolumn{8}{|c|}{ Water Level (ft below reference point) } & \multirow[b]{2}{*}{ SB24 } & \multirow[b]{2}{*}{ SB38 } \\
\hline & McPike & Potter & Hughes & Barr & Burks & MoDOT & Clizer "Old" & Morgan & & \\
\hline $5 / 30 / 104: 00$ & 7.737 & 4.601 & 6.030 & 14.300 & 6.756 & 11.880 & 5.377 & 17.238 & & 31.686 \\
\hline $5 / 30 / 108: 00$ & 7.786 & 4.601 & 6.061 & 14.179 & 6.766 & 11.932 & 5.431 & 17.229 & & 31.678 \\
\hline $5 / 30 / 1012: 00$ & 7.821 & 4.615 & 6.103 & 14.275 & 6.787 & 11.948 & 5.469 & 17.273 & & 31.674 \\
\hline 5/30/10 16:00 & 7.852 & 4.637 & 6.150 & 14.477 & 6.813 & 11.939 & 5.495 & 17.280 & & 31.669 \\
\hline 5/30/10 20:00 & 7.894 & 4.651 & 6.187 & 14.312 & 6.822 & 11.955 & 5.549 & 17.316 & & 31.660 \\
\hline 5/31/10 0:00 & 7.949 & 4.658 & 6.218 & 14.170 & 6.742 & 12.000 & 5.591 & 17.382 & & 31.650 \\
\hline $5 / 31 / 104: 00$ & 7.979 & 4.655 & 6.244 & 14.095 & 6.674 & 12.030 & 5.615 & 17.424 & & 31.648 \\
\hline 5/31/10 8:00 & 8.019 & 4.648 & 6.272 & 14.032 & 6.596 & 12.068 & 5.655 & 17.473 & & 31.645 \\
\hline $5 / 31 / 1012: 00$ & 8.045 & 4.648 & 6.300 & 14.170 & 6.659 & 12.082 & 5.699 & 17.506 & & 31.643 \\
\hline 5/31/10 16:00 & 8.066 & 4.658 & 6.335 & 14.428 & 6.747 & 12.082 & 5.746 & 17.513 & & 31.636 \\
\hline 5/31/10 20:00 & 8.102 & 4.674 & 6.375 & 14.433 & 6.782 & 12.079 & 5.786 & 17.527 & & 31.631 \\
\hline 6/1/10 0:00 & 8.137 & 4.679 & 6.406 & 14.333 & 6.714 & 12.112 & 5.824 & 17.603 & & 31.627 \\
\hline 6/1/10 4:00 & 8.149 & 4.674 & 6.429 & 14.335 & 6.700 & 12.114 & 5.831 & 17.612 & & 31.624 \\
\hline 6/1/10 8:00 & 8.175 & 4.667 & 6.453 & 14.309 & 6.683 & 12.135 & 5.864 & 17.643 & & 31.617 \\
\hline $6 / 1 / 1012: 00$ & 8.184 & 4.667 & 6.481 & 14.475 & 6.796 & 12.135 & 5.887 & 17.737 & & 31.612 \\
\hline 6/1/10 16:00 & 8.184 & 4.681 & 6.516 & 14.703 & 6.966 & 12.098 & 5.911 & 17.631 & & 31.610 \\
\hline 6/1/10 20:00 & 8.189 & 4.695 & 6.542 & 14.764 & 7.070 & 12.056 & 5.939 & 17.664 & & 31.605 \\
\hline 6/2/10 0:00 & 7.402 & 4.354 & 6.169 & 14.198 & 6.683 & 11.875 & 5.727 & 17.675 & & 31.591 \\
\hline 6/2/10 4:00 & 6.270 & 3.003 & 5.049 & 13.587 & 6.461 & 11.275 & 5.053 & 17.541 & & 31.580 \\
\hline 6/2/10 8:00 & 4.078 & 2.228 & 3.808 & 12.814 & 5.185 & 10.787 & 3.914 & 17.078 & & 31.568 \\
\hline $6 / 2 / 1012: 00$ & 4.114 & 2.337 & 3.685 & 12.592 & 5.341 & 10.900 & 3.623 & 16.796 & & 31.565 \\
\hline 6/2/10 16:00 & 4.274 & 2.641 & 3.721 & 12.620 & 5.631 & 10.914 & 3.531 & 16.617 & & 31.561 \\
\hline $6 / 2 / 1020: 00$ & 4.449 & 2.942 & 3.793 & 12.588 & 5.827 & 10.912 & 3.374 & 16.417 & & 31.556 \\
\hline 6/3/10 0:00 & 4.606 & 3.166 & 3.864 & 12.506 & 5.886 & 10.937 & 3.317 & 16.319 & & 31.549 \\
\hline 6/3/10 4:00 & 4.727 & 3.333 & 3.916 & 12.504 & 5.921 & 10.928 & 3.310 & 16.215 & & 31.544 \\
\hline 6/3/10 8:00 & 4.845 & 3.468 & 3.969 & 12.492 & 5.964 & 10.933 & 3.463 & 16.145 & & 31.537 \\
\hline 6/3/10 12:00 & 4.962 & 3.574 & 4.023 & 12.630 & 6.063 & 10.923 & 3.750 & 16.072 & & 31.530 \\
\hline 6/3/10 16:00 & 5.085 & 3.689 & 4.089 & 12.881 & 6.195 & 10.891 & 3.813 & 15.992 & & 31.523 \\
\hline $6 / 3 / 1020: 00$ & 5.189 & 3.786 & 4.150 & 12.905 & 6.280 & 10.844 & 3.728 & 16.241 & & 31.518 \\
\hline 6/4/10 0:00 & 5.278 & 3.859 & 4.207 & 12.828 & 6.268 & 10.858 & 3.731 & 15.921 & & 31.511 \\
\hline 6/4/10 4:00 & 5.342 & 3.904 & 4.249 & 12.790 & 6.277 & 10.853 & 3.780 & 15.862 & & 31.504 \\
\hline 6/4/10 8:00 & 5.417 & 3.932 & 4.289 & 12.800 & 6.315 & 10.839 & 3.890 & 15.926 & & 31.497 \\
\hline $6 / 4 / 10$ 12:00 & 5.500 & 3.967 & 4.345 & 12.951 & 6.384 & 10.830 & 4.151 & 15.830 & & 31.492 \\
\hline 6/4/10 16:00 & 5.599 & 4.017 & 4.425 & 13.147 & 6.473 & 10.808 & 4.243 & 15.754 & & 31.485 \\
\hline $6 / 4 / 10$ 20:00 & 5.691 & 4.069 & 4.502 & 13.158 & 6.546 & 10.792 & 4.116 & 15.726 & & 31.478 \\
\hline 6/5/10 0:00 & 5.743 & 4.106 & 4.561 & 13.040 & 6.483 & 10.813 & 4.118 & 15.818 & & 31.471 \\
\hline 6/5/10 4:00 & 5.816 & 4.123 & 4.603 & 13.026 & 6.499 & 10.808 & 4.074 & 15.785 & & 31.464 \\
\hline 6/5/10 8:00 & 5.893 & 4.139 & 4.660 & 12.916 & 6.445 & 10.862 & 4.088 & 15.818 & & 31.459 \\
\hline 6/5/10 12:00 & 5.938 & 4.149 & 4.702 & 13.012 & 6.487 & 10.830 & 4.313 & 15.804 & & 31.452 \\
\hline 6/5/10 16:00 & 6.002 & 4.163 & 4.751 & 13.121 & 6.551 & 10.806 & 4.410 & 15.761 & & 31.443 \\
\hline $6 / 5 / 1020: 00$ & 6.098 & 4.189 & 4.824 & 13.131 & 6.556 & 10.839 & 3.771 & 15.736 & & 31.438 \\
\hline 6/6/10 0:00 & 6.200 & 4.226 & 4.904 & 12.914 & 6.376 & 10.944 & 3.493 & 15.851 & & 31.433 \\
\hline 6/6/10 4:00 & 6.273 & 4.243 & 4.958 & 12.870 & 6.282 & 10.975 & 3.233 & 15.879 & & 31.424 \\
\hline 6/6/10 8:00 & 6.344 & 4.259 & 5.016 & 12.835 & 6.204 & 11.029 & 3.134 & 15.945 & & 31.419 \\
\hline 6/6/10 12:00 & 6.405 & 4.274 & NA & 13.035 & 6.289 & 11.031 & 2.984 & 15.947 & & 31.412 \\
\hline 6/6/10 16:00 & 6.464 & 4.306 & & 13.310 & 6.393 & 11.022 & 2.805 & 15.942 & & 31.405 \\
\hline 6/6/10 20:00 & 6.530 & 4.342 & & 13.326 & 6.468 & 10.998 & 2.476 & 15.935 & & 31.398 \\
\hline 6/7/10 0:00 & 6.589 & 4.365 & & 13.254 & 6.419 & 11.027 & 1.387 & 16.015 & & 31.393 \\
\hline 6/7/10 4:00 & 6.622 & 4.368 & & 13.282 & 6.431 & 11.008 & 0.290 & 15.999 & & 31.386 \\
\hline 6/7/10 8:00 & 6.666 & 4.365 & & 13.245 & 6.409 & 11.019 & 1.946 & 16.013 & & 31.379 \\
\hline 6/7/10 12:00 & 6.702 & 4.375 & & 13.294 & 6.457 & 11.045 & 3.599 & 16.093 & & 31.372 \\
\hline 6/7/10 16:00 & 6.742 & 4.384 & & 13.426 & 6.575 & 11.010 & 4.050 & 16.065 & & 31.368 \\
\hline 6/7/10 20:00 & 6.792 & 4.401 & & 13.464 & 6.636 & 11.001 & 4.196 & 16.147 & & 31.360 \\
\hline 6/8/10 0:00 & 6.822 & 4.415 & & 13.417 & 6.605 & 11.015 & 4.325 & 16.086 & & 31.353 \\
\hline 6/8/10 4:00 & 6.831 & 4.405 & & 13.464 & 6.648 & 10.982 & 4.501 & 16.058 & & 31.349 \\
\hline 6/8/10 8:00 & 6.681 & 4.141 & & 13.282 & 6.641 & 10.940 & 4.523 & 16.074 & & 31.342 \\
\hline 6/8/10 12:00 & 6.636 & 3.291 & & 13.214 & 6.700 & 10.792 & 4.678 & 16.039 & & 31.339 \\
\hline 6/8/10 16:00 & 6.719 & 3.199 & & 13.219 & 6.688 & 10.862 & 4.758 & 16.079 & & 31.327 \\
\hline 6/8/10 20:00 & 6.799 & 3.300 & & 13.235 & 6.688 & 10.876 & 4.685 & 16.086 & & 31.323 \\
\hline 6/9/10 0:00 & 6.869 & 3.427 & & 13.189 & 6.641 & 10.935 & 4.664 & 16.128 & & 31.316 \\
\hline 6/9/10 4:00 & 6.930 & 3.543 & & 13.151 & 6.563 & 10.973 & 4.647 & 16.161 & & 31.311 \\
\hline 6/9/10 8:00 & 6.990 & 3.644 & & 13.163 & 6.499 & 11.029 & 4.732 & 16.208 & & 31.304 \\
\hline 6/9/10 12:00 & 7.037 & 3.741 & & 13.338 & 6.537 & 11.064 & 4.997 & 16.241 & & 31.297 \\
\hline 6/9/10 16:00 & 7.079 & 3.845 & & 13.583 & 6.645 & 11.045 & 5.119 & 16.295 & & 31.290 \\
\hline
\end{tabular}

TABLE S1.1 Page 38 of 89 
Water Level (ft below reference point)

\begin{tabular}{|c|c|c|c|c|c|c|c|c|c|c|}
\hline Date and Time & McPike & Potter & Hughes & Barr & Burks & MoDOT & Clizer "Old" & Morgan & SB24 & SB38 \\
\hline 6/9/10 20:00 & 7.129 & 3.941 & & 13.517 & 6.678 & 11.048 & 5.011 & 16.269 & & 31.285 \\
\hline 6/10/10 0:00 & 7.169 & 4.005 & & 13.473 & 6.636 & 11.073 & 4.992 & 16.293 & & 31.278 \\
\hline 6/10/10 4:00 & 7.185 & 4.045 & & 13.517 & 6.653 & 11.052 & 5.072 & 16.290 & & 31.273 \\
\hline 6/10/10 8:00 & 7.216 & 4.075 & & 13.531 & 6.657 & 11.069 & 5.185 & 16.312 & & 31.266 \\
\hline $6 / 10 / 1012: 00$ & 7.239 & 4.106 & & 13.659 & 6.792 & 11.052 & 5.293 & 16.305 & & 31.259 \\
\hline 6/10/10 16:00 & 7.279 & 4.151 & & 13.701 & 6.818 & 11.078 & 5.396 & 16.333 & & 31.254 \\
\hline 6/10/10 20:00 & 7.312 & 4.193 & & 13.687 & 6.865 & 11.066 & 5.366 & 16.340 & & 31.247 \\
\hline 6/11/10 0:00 & 7.336 & 4.224 & & 13.629 & 6.843 & 11.099 & 5.371 & 16.370 & & 31.243 \\
\hline 6/11/10 4:00 & 7.369 & 4.238 & & 13.643 & 6.846 & 11.094 & 5.359 & 16.460 & & 31.238 \\
\hline 6/11/10 8:00 & 7.433 & 4.257 & & 13.562 & 6.759 & 11.184 & 5.326 & 16.514 & & 31.233 \\
\hline 6/11/10 12:00 & 7.459 & 4.276 & & 13.683 & 6.789 & 11.188 & 5.563 & 16.530 & & 31.226 \\
\hline 6/11/10 16:00 & 7.508 & 4.304 & & 13.757 & 6.846 & 11.214 & 5.563 & 16.547 & & 31.219 \\
\hline 6/11/10 20:00 & 7.562 & 4.337 & & 13.685 & 6.811 & 11.254 & 5.498 & 16.589 & & 31.214 \\
\hline 6/12/10 0:00 & 7.609 & 4.360 & & 13.662 & 6.759 & 11.298 & 5.493 & 16.636 & & 31.210 \\
\hline $6 / 12 / 104: 00$ & 7.640 & 4.368 & & 13.694 & 6.740 & 11.315 & 5.502 & 16.664 & & 31.205 \\
\hline $6 / 12 / 108: 00$ & 7.687 & 4.375 & & 13.666 & 6.671 & 11.362 & 5.472 & 16.711 & & 31.198 \\
\hline $6 / 12 / 1012: 00$ & 7.704 & 4.370 & & 13.750 & 6.667 & 11.352 & 5.528 & 16.730 & & 31.193 \\
\hline 6/12/10 16:00 & 7.760 & 4.382 & & 13.785 & 6.636 & 11.425 & 5.664 & 16.787 & & 31.188 \\
\hline 6/12/10 20:00 & 7.796 & 4.401 & & 13.811 & 6.650 & 11.441 & 5.629 & 16.820 & & 31.181 \\
\hline 6/13/10 0:00 & 7.829 & 4.410 & & 13.809 & 6.622 & 11.470 & 5.655 & 16.881 & & 31.177 \\
\hline $6 / 13 / 104: 00$ & 7.836 & 4.403 & & 13.888 & 6.671 & 11.446 & 5.709 & 16.867 & & 31.172 \\
\hline 6/13/10 8:00 & 7.871 & 4.401 & & 13.871 & 6.733 & 11.470 & 5.735 & 16.925 & & 31.167 \\
\hline 6/13/10 12:00 & 7.904 & 4.401 & & 13.906 & 6.735 & 11.498 & 5.852 & 16.961 & & 31.167 \\
\hline 6/13/10 16:00 & 7.914 & 4.405 & & 14.021 & 6.813 & 11.486 & 5.977 & 16.958 & & 31.158 \\
\hline 6/13/10 20:00 & 7.949 & 4.422 & & 14.016 & 6.851 & 11.507 & 5.913 & 16.986 & & 31.156 \\
\hline 6/14/10 0:00 & 7.954 & 4.426 & & 14.000 & 6.841 & 11.517 & 5.923 & 17.008 & & 31.148 \\
\hline 6/14/10 4:00 & 7.256 & 3.397 & & 13.676 & 6.686 & 11.085 & 5.641 & 16.994 & & 31.137 \\
\hline $6 / 14 / 108: 00$ & 7.254 & 2.810 & & 13.503 & 6.676 & 11.177 & 5.646 & 16.989 & & 31.127 \\
\hline $6 / 14 / 10$ 12:00 & 7.362 & 2.819 & & 13.520 & 6.714 & 11.254 & 5.709 & 17.010 & & 31.127 \\
\hline 6/14/10 16:00 & 7.459 & 2.987 & & 13.552 & 6.737 & 11.289 & 5.770 & 17.295 & & 31.123 \\
\hline $6 / 14 / 1020: 00$ & 7.536 & 3.168 & & 13.590 & 6.766 & 11.303 & 5.740 & 17.083 & & 31.118 \\
\hline 6/15/10 0:00 & 7.617 & 3.324 & & 13.557 & 6.723 & 11.352 & 5.704 & 17.099 & & 31.113 \\
\hline 6/15/10 4:00 & 7.591 & 3.449 & & 13.589 & 6.714 & 11.374 & 5.697 & 17.106 & & 31.108 \\
\hline 6/15/10 8:00 & 7.152 & 2.852 & & 13.436 & 6.657 & 11.298 & 5.521 & 17.128 & & 31.104 \\
\hline 6/15/10 12:00 & 6.563 & 2.346 & & 13.205 & 6.495 & 11.116 & 5.390 & 17.106 & & 31.097 \\
\hline 6/15/10 16:00 & 6.570 & 2.464 & & 13.207 & 6.502 & 11.165 & 5.413 & 17.273 & & 31.090 \\
\hline 6/15/10 20:00 & 6.631 & 2.732 & & 13.184 & 6.528 & 11.181 & 5.291 & 17.217 & & 31.085 \\
\hline $6 / 16 / 100: 00$ & 6.697 & 2.968 & & 13.100 & 6.447 & 11.240 & 5.187 & 17.012 & & 31.080 \\
\hline 6/16/10 4:00 & 6.735 & 3.145 & & 13.128 & 6.391 & 11.266 & 5.159 & 16.916 & & 31.075 \\
\hline 6/16/10 8:00 & 6.780 & 3.293 & & 13.149 & 6.344 & 11.301 & 5.232 & 16.904 & & 31.073 \\
\hline 6/16/10 12:00 & 6.829 & 3.434 & & 13.308 & 6.391 & 11.331 & 5.404 & 17.429 & & 31.066 \\
\hline 6/16/10 16:00 & 6.879 & 3.588 & & 13.426 & 6.473 & 11.310 & 5.399 & 17.106 & & 31.061 \\
\hline 6/16/10 20:00 & 6.909 & 3.694 & & 13.412 & 6.478 & 11.289 & 5.359 & 17.045 & & 31.057 \\
\hline $6 / 17 / 100: 00$ & 6.954 & 3.769 & & 13.352 & 6.450 & 11.329 & 5.303 & 17.019 & & 31.052 \\
\hline $6 / 17 / 104: 00$ & 6.966 & 3.816 & & 13.401 & 6.476 & 11.310 & 5.350 & 16.979 & & 31.047 \\
\hline 6/17/10 8:00 & 6.999 & 3.854 & & 13.401 & 6.495 & 11.329 & 5.432 & 16.961 & & 31.042 \\
\hline 6/17/10 12:00 & 7.039 & 3.898 & & 13.520 & 6.551 & 11.338 & 5.636 & 17.005 & & 31.040 \\
\hline 6/17/10 16:00 & 7.086 & 3.965 & & 13.678 & 6.638 & 11.329 & 5.695 & 17.012 & & 31.035 \\
\hline 6/17/10 20:00 & 7.143 & 4.038 & & 13.566 & 6.686 & 11.338 & 5.601 & 16.935 & & 31.031 \\
\hline 6/18/10 0:00 & 7.206 & 4.089 & & 13.438 & 6.610 & 11.399 & 5.514 & 16.951 & & 31.026 \\
\hline 6/18/10 4:00 & 7.237 & 4.120 & & 13.475 & 6.605 & 11.406 & 5.530 & 17.024 & & 31.021 \\
\hline 6/18/10 8:00 & 7.263 & 4.132 & & 13.513 & 6.620 & 11.406 & 5.610 & 16.986 & & 31.019 \\
\hline 6/18/10 12:00 & 7.312 & 4.162 & & 13.622 & 6.669 & 11.432 & 5.815 & 16.949 & & 31.014 \\
\hline 6/18/10 16:00 & 7.350 & 4.207 & & 13.776 & 6.796 & 11.413 & 5.857 & 16.916 & & 31.009 \\
\hline 6/18/10 20:00 & 7.400 & 4.254 & & 13.666 & 6.825 & 11.423 & 5.787 & 16.909 & & 31.005 \\
\hline 6/19/10 0:00 & 7.421 & 4.226 & & 13.464 & 6.686 & 11.460 & 5.667 & 16.874 & & 31.000 \\
\hline 6/19/10 4:00 & 7.459 & 3.920 & & 13.401 & 6.605 & 11.495 & 5.629 & 17.062 & & 30.998 \\
\hline 6/19/10 8:00 & 7.515 & 3.835 & & 13.422 & 6.551 & 11.545 & 5.704 & 17.083 & & 30.995 \\
\hline 6/19/10 12:00 & 7.166 & 3.849 & & 13.550 & 6.572 & 11.559 & 5.634 & 17.095 & & 30.984 \\
\hline 6/19/10 16:00 & 6.789 & 3.088 & & 13.347 & 6.459 & 11.352 & 5.636 & 16.963 & & 30.984 \\
\hline 6/19/10 20:00 & 6.914 & 3.013 & & 13.340 & 6.499 & 11.385 & 5.542 & 16.930 & & 30.979 \\
\hline 6/20/10 0:00 & 6.994 & 3.119 & & 13.207 & 6.372 & 11.458 & 5.366 & 16.947 & & 30.972 \\
\hline $6 / 20 / 104: 00$ & 6.497 & 2.635 & & 13.170 & 6.377 & 11.148 & 5.404 & 16.947 & & 30.967 \\
\hline $6 / 20 / 108: 00$ & 6.547 & 2.586 & & 12.986 & 6.238 & 11.298 & 5.249 & 16.925 & & 30.965 \\
\hline
\end{tabular}

TABLE S1.1 Page 39 of 89 
Water Level (ft below reference point)

\begin{tabular}{|c|c|c|c|c|c|c|c|c|c|c|}
\hline \multirow[b]{2}{*}{ Date and Time } & \\
\hline & McPike & Potter & Hughes & Barr & Burks & MoDOT & Clizer "Old" & Morgan & SB24 & SB38 \\
\hline 6/20/10 12:00 & 6.539 & 2.713 & & 13.142 & 6.256 & 11.275 & 5.380 & 16.852 & & 30.958 \\
\hline $6 / 20 / 1016: 00$ & 6.566 & 2.925 & & 13.256 & 6.259 & 11.256 & 5.455 & 16.765 & & 30.953 \\
\hline 6/20/10 20:00 & 6.589 & 3.137 & & 13.294 & 6.268 & 11.228 & 5.429 & 16.695 & & 30.951 \\
\hline 6/21/10 0:00 & 6.254 & 3.305 & & 13.135 & 6.193 & 11.308 & 5.258 & 16.690 & & 30.944 \\
\hline 6/21/10 4:00 & 5.696 & 2.697 & & 12.993 & 6.054 & 11.017 & 5.124 & 16.516 & & 30.936 \\
\hline 6/21/10 8:00 & 5.632 & 2.654 & & 12.849 & 5.955 & 11.062 & 4.997 & 16.453 & & 30.927 \\
\hline 6/21/10 12:00 & 5.583 & 2.727 & & 12.888 & 5.919 & 11.003 & 4.986 & 16.387 & & 30.925 \\
\hline 6/21/10 16:00 & 5.644 & 2.857 & & 13.051 & 6.028 & 10.989 & 4.990 & 16.222 & & 30.920 \\
\hline 6/21/10 20:00 & 5.722 & 3.071 & & 12.970 & 6.105 & 10.980 & 4.842 & 16.145 & & 30.915 \\
\hline 6/22/10 0:00 & 5.788 & 3.229 & & 12.893 & 6.110 & 10.989 & 4.713 & 16.126 & & 30.908 \\
\hline $6 / 22 / 104: 00$ & 5.832 & 3.347 & & 12.909 & 6.148 & 10.961 & 4.671 & 15.989 & & 30.906 \\
\hline 6/22/10 8:00 & 5.894 & 3.444 & & 12.865 & 6.155 & 10.982 & 4.673 & 15.893 & & 30.901 \\
\hline $6 / 22 / 10$ 12:00 & 5.960 & 3.545 & & 12.981 & 6.228 & 10.963 & 4.838 & 15.867 & & 30.896 \\
\hline $6 / 22 / 1016: 00$ & 6.035 & 3.656 & & 13.147 & 6.346 & 10.926 & 4.903 & 15.761 & & 30.892 \\
\hline 6/22/10 20:00 & 6.113 & 3.759 & & 13.093 & 6.405 & 10.883 & 4.821 & 15.684 & & 30.887 \\
\hline 6/23/10 0:00 & 6.165 & 3.830 & & 12.995 & 6.372 & 10.895 & 4.791 & 15.653 & & 30.882 \\
\hline $6 / 23 / 104: 00$ & 6.207 & 3.872 & & 13.002 & 6.396 & 10.865 & 4.781 & 15.611 & & 30.878 \\
\hline 6/23/10 8:00 & 6.271 & 3.913 & & 12.956 & 6.360 & 10.893 & 4.788 & 15.625 & & 30.873 \\
\hline 6/23/10 12:00 & 6.325 & 3.953 & & 13.107 & 6.426 & 10.909 & 4.964 & 15.573 & & 30.866 \\
\hline 6/23/10 16:00 & 6.415 & 4.011 & & 13.184 & 6.466 & 10.928 & 4.960 & 14.031 & & 68.736 \\
\hline 6/23/10 20:00 & 6.504 & 4.073 & & 13.040 & 6.436 & 10.935 & 4.805 & 13.998 & & 53.434 \\
\hline 6/24/10 0:00 & 6.573 & 4.125 & & 12.979 & 6.320 & 10.973 & 4.760 & 14.099 & & 53.232 \\
\hline 6/24/10 4:00 & 6.613 & 4.153 & & 13.033 & 6.282 & 10.966 & 4.774 & 14.064 & & 53.072 \\
\hline 6/24/10 8:00 & 6.660 & 4.176 & & 13.056 & 6.263 & 10.977 & 4.870 & 14.061 & & 52.928 \\
\hline 6/24/10 12:00 & 6.705 & 4.195 & & 13.252 & 6.337 & 10.968 & 5.141 & 14.172 & & 67.113 \\
\hline $6 / 24 / 1016: 00$ & 6.752 & 4.228 & & 13.443 & 6.473 & 10.937 & 5.211 & 14.064 & & 66.670 \\
\hline 6/24/10 20:00 & 6.794 & 4.264 & & 13.412 & 6.563 & 10.898 & 5.126 & 15.362 & & 66.411 \\
\hline 6/25/10 0:00 & 6.837 & 4.287 & & 13.315 & 6.509 & 10.909 & 5.075 & 14.160 & & 66.185 \\
\hline 6/25/10 4:00 & 6.863 & 4.294 & & 13.326 & 6.511 & 10.893 & 5.084 & 14.132 & & 65.973 \\
\hline 6/25/10 8:00 & 6.896 & 4.301 & & 13.315 & 6.499 & 10.905 & 5.159 & 14.106 & & 65.770 \\
\hline 6/25/10 12:00 & 6.933 & 4.313 & & 13.473 & 6.598 & 10.778 & 5.385 & 14.083 & & 65.573 \\
\hline 6/25/10 16:00 & 6.969 & 4.344 & & 13.652 & 6.752 & & 5.512 & 14.052 & & 65.379 \\
\hline 6/25/10 20:00 & 7.004 & 4.377 & & 13.589 & 6.834 & 10.609 & 5.286 & 14.106 & & 65.193 \\
\hline 6/26/10 0:00 & 7.049 & 4.396 & & 13.473 & 6.787 & 10.623 & 5.307 & 14.059 & & 65.007 \\
\hline 6/26/10 4:00 & 7.079 & 4.405 & & 13.436 & 6.757 & 10.630 & 5.324 & 14.134 & & 64.826 \\
\hline 6/26/10 8:00 & 7.122 & 4.410 & & 13.387 & 6.695 & 10.665 & 5.357 & 14.148 & & 64.652 \\
\hline 6/26/10 12:00 & 7.148 & 4.421 & & 13.550 & 6.766 & 10.642 & 5.385 & 14.137 & & 64.480 \\
\hline $6 / 26 / 10$ 16:00 & 7.181 & 4.440 & & 13.727 & 6.889 & 10.619 & 5.432 & 14.111 & & 64.308 \\
\hline 6/26/10 20:00 & 7.214 & 4.469 & & 13.664 & 6.969 & 10.588 & 5.467 & 14.181 & & 64.136 \\
\hline 6/27/10 0:00 & 7.256 & 4.485 & & 13.557 & 6.898 & 10.623 & 5.469 & 14.170 & & 63.968 \\
\hline $6 / 27 / 104: 00$ & 7.270 & 4.487 & & 13.594 & 6.943 & 10.586 & 5.465 & 14.137 & & 63.804 \\
\hline 6/27/10 8:00 & 7.287 & 4.462 & & 13.403 & 6.834 & 10.616 & 5.479 & 14.181 & & 63.639 \\
\hline 6/27/10 12:00 & 7.339 & 4.393 & & 13.436 & 6.811 & $A B^{b}$ & 5.538 & 14.221 & & 63.476 \\
\hline 6/27/10 16:00 & 7.383 & 4.372 & & 13.471 & 6.808 & & 5.580 & 14.329 & & 63.318 \\
\hline 6/27/10 20:00 & 7.438 & 4.372 & & 13.473 & 6.792 & & 5.631 & 14.325 & & 63.158 \\
\hline $6 / 28 / 100: 00$ & 7.489 & 4.381 & & 13.422 & 6.691 & & 5.667 & 14.376 & & 63.003 \\
\hline 6/28/10 4:00 & 7.520 & 4.381 & & 13.475 & 6.627 & & 5.688 & 14.423 & & 62.845 \\
\hline 6/28/10 8:00 & 7.570 & 4.388 & & 13.471 & 6.563 & & 5.730 & 14.475 & & 62.689 \\
\hline 6/28/10 12:00 & 7.605 & 4.398 & & 13.678 & 6.634 & & 5.784 & 14.506 & & 62.527 \\
\hline $6 / 28 / 1016: 00$ & 7.659 & 4.436 & & 13.839 & 6.695 & & 5.862 & 22.792 & 45.871 & 62.353 \\
\hline 6/28/10 20:00 & 7.711 & 4.464 & & 13.799 & 6.721 & & 5.918 & 17.787 & 45.746 & 62.214 \\
\hline 6/29/10 0:00 & 7.768 & 4.485 & & 13.694 & 6.823 & & 5.958 & 17.133 & 45.627 & 62.075 \\
\hline 6/29/10 4:00 & 7.813 & 4.492 & & 13.673 & 6.813 & & 5.984 & 16.922 & 45.507 & 61.929 \\
\hline 6/29/10 8:00 & 7.855 & 4.504 & & 13.699 & 6.816 & & 5.996 & 16.849 & 45.387 & 61.780 \\
\hline 6/29/10 12:00 & 7.897 & 4.513 & & 13.997 & 6.830 & & 6.057 & 16.825 & 45.268 & 61.636 \\
\hline 6/29/10 16:00 & 7.937 & 4.539 & & 14.130 & 6.853 & & 6.122 & 16.872 & 45.146 & 61.490 \\
\hline 6/29/10 20:00 & 7.982 & 4.563 & & 14.004 & 6.874 & & 6.174 & 17.263 & 45.024 & 61.344 \\
\hline 6/30/10 0:00 & 8.022 & 4.577 & & 13.913 & 6.853 & & 6.200 & 16.910 & 44.904 & 61.201 \\
\hline 6/30/10 4:00 & 8.055 & 4.584 & & 13.911 & 6.841 & & 6.223 & 16.938 & 44.782 & 61.057 \\
\hline 6/30/10 8:00 & 8.100 & 4.593 & & 13.885 & 6.849 & & 6.263 & 16.936 & 44.665 & 60.913 \\
\hline 6/30/10 12:00 & 8.135 & 4.600 & & 14.092 & 6.863 & & 6.284 & 16.941 & 44.541 & 60.772 \\
\hline 6/30/10 16:00 & 8.156 & 4.622 & & 14.283 & 6.886 & & 6.327 & 16.922 & 44.421 & 60.631 \\
\hline $6 / 30 / 10$ 20:00 & 8.194 & 4.640 & & 14.160 & 6.910 & & 6.369 & 16.969 & 44.299 & 60.489 \\
\hline 7/1/10 0:00 & 8.242 & 4.657 & & 14.050 & 6.900 & & 6.404 & 16.997 & 44.180 & 60.348 \\
\hline
\end{tabular}

TABLE S1.1 Page 40 of 89 
Water Level (ft below reference point)

\begin{tabular}{|c|c|c|c|c|c|c|c|c|c|c|}
\hline Date and Time & McPike & Potter & Hughes & Barr & Burks & MoDOT & Clizer "Old" & Morgan & SB24 & SB38 \\
\hline 7/1/10 4:00 & 8.270 & 4.664 & & 14.055 & 6.882 & & 6.423 & 17.021 & 44.060 & 60.209 \\
\hline 7/1/10 8:00 & 8.305 & 4.669 & & 14.060 & 6.879 & & 6.451 & 17.124 & 43.938 & 60.072 \\
\hline $7 / 1 / 10$ 12:00 & 8.340 & 4.678 & & 14.260 & 6.893 & & 6.496 & 17.115 & 43.816 & 59.933 \\
\hline 7/1/10 16:00 & 8.364 & 4.695 & & 14.430 & 6.926 & & 6.541 & 17.110 & 43.696 & 59.797 \\
\hline 7/1/10 20:00 & 8.397 & 4.714 & & 14.300 & 6.955 & & 6.585 & 17.122 & 43.577 & 59.660 \\
\hline 7/2/10 0:00 & 8.428 & 4.728 & & 14.220 & 6.950 & & 6.611 & 17.157 & 43.457 & 59.526 \\
\hline 7/2/10 4:00 & 8.446 & 4.730 & & 14.220 & 6.929 & & 6.620 & 17.183 & 43.340 & 59.389 \\
\hline 7/2/10 8:00 & 8.468 & 4.730 & & 14.241 & 6.912 & & 6.644 & 17.199 & 43.220 & 59.255 \\
\hline $7 / 2 / 10$ 12:00 & 8.484 & 4.739 & & 14.432 & 6.926 & & 6.681 & 17.268 & 43.101 & 59.121 \\
\hline 7/2/10 16:00 & 8.506 & 4.756 & & 14.593 & 6.978 & & 6.726 & 17.237 & 42.981 & 58.989 \\
\hline $7 / 2 / 10$ 20:00 & 8.527 & 4.772 & & 14.481 & 7.025 & & 6.764 & 17.235 & 42.864 & 58.852 \\
\hline 7/3/10 0:00 & 8.562 & 4.787 & & 14.358 & 7.032 & & 6.794 & 17.277 & 42.747 & 58.720 \\
\hline 7/3/10 4:00 & 8.567 & 4.787 & & 14.379 & 7.014 & & 6.794 & 17.369 & 42.629 & 58.586 \\
\hline $7 / 3 / 108: 00$ & 8.593 & 4.787 & & 14.370 & 7.004 & & 6.825 & 17.350 & 42.512 & 58.456 \\
\hline $7 / 3 / 10$ 12:00 & 8.612 & 4.796 & & 14.519 & 7.028 & & 6.865 & 17.373 & 42.397 & 58.325 \\
\hline 7/3/10 16:00 & 8.635 & 4.810 & & 14.577 & 7.065 & & 6.907 & 17.387 & 42.280 & 58.195 \\
\hline $7 / 3 / 1020: 00$ & 8.661 & 4.822 & & 14.481 & 7.077 & & 6.935 & 17.413 & 42.163 & 58.065 \\
\hline $7 / 4 / 100: 00$ & 8.682 & 4.827 & & 14.435 & 7.075 & & 6.959 & 17.449 & 42.045 & 57.936 \\
\hline 7/4/10 4:00 & 8.701 & 4.827 & & 14.446 & 7.058 & & 6.975 & 17.533 & 41.930 & 57.806 \\
\hline 7/4/10 8:00 & 8.715 & 4.827 & & 14.456 & 7.058 & & 6.992 & 17.568 & 41.815 & 57.677 \\
\hline $7 / 4 / 10$ 12:00 & 8.734 & 4.824 & & 14.481 & 7.058 & & 7.020 & 17.580 & 41.700 & 57.549 \\
\hline 7/4/10 16:00 & 8.755 & 4.824 & & 14.488 & 7.068 & & 7.046 & 17.611 & 41.586 & 57.422 \\
\hline $7 / 4 / 10$ 20:00 & 8.119 & 4.280 & & 14.013 & 6.905 & & 6.484 & 17.604 & 41.473 & 57.293 \\
\hline 7/5/10 0:00 & 5.889 & 2.579 & & 12.776 & 6.283 & & 5.732 & 17.249 & 41.353 & 57.166 \\
\hline 7/5/10 4:00 & 5.967 & 2.562 & & 12.527 & 6.120 & & 5.437 & 16.934 & 41.222 & 57.038 \\
\hline $7 / 5 / 108: 00$ & 6.085 & 2.774 & & 12.485 & 6.113 & & 5.277 & 16.743 & 41.091 & 56.914 \\
\hline $7 / 5 / 10$ 12:00 & 6.165 & 2.880 & & 12.522 & 6.179 & & 5.197 & 16.588 & 40.962 & 56.786 \\
\hline 7/5/10 16:00 & 6.217 & 2.944 & & 12.596 & 6.235 & & 5.152 & 16.456 & 40.830 & 56.662 \\
\hline $7 / 5 / 1020: 00$ & 6.295 & 3.048 & & 12.631 & 6.266 & & 5.162 & 16.358 & 40.701 & 56.537 \\
\hline 7/6/10 0:00 & 6.365 & 3.170 & & 12.627 & 6.214 & & 5.171 & 16.303 & 40.575 & 56.412 \\
\hline 7/6/10 4:00 & 6.417 & 3.274 & & 12.685 & 6.188 & & 5.180 & 16.313 & 40.446 & 56.289 \\
\hline 7/6/10 8:00 & 6.474 & 3.373 & & 12.736 & 6.150 & & 5.202 & 16.228 & 40.319 & 56.165 \\
\hline $7 / 6 / 10$ 12:00 & 6.533 & 3.474 & & 12.841 & 6.141 & & 5.244 & 16.195 & 40.192 & 56.044 \\
\hline 7/6/10 16:00 & 6.589 & 3.575 & & 12.944 & 6.174 & & 5.279 & 16.158 & 40.066 & 55.922 \\
\hline 7/6/10 20:00 & 6.644 & 3.667 & & 12.993 & 6.212 & & 5.310 & 16.125 & 39.941 & 55.802 \\
\hline 7/7/10 0:00 & 6.688 & 3.738 & & 12.972 & 6.143 & & 5.331 & 16.134 & 39.817 & 55.679 \\
\hline 7/7/10 4:00 & 6.721 & 3.787 & & 13.023 & 6.139 & & 5.338 & 16.108 & 39.693 & 55.559 \\
\hline 7/7/10 8:00 & 6.757 & 3.820 & & 13.058 & 6.155 & & 5.359 & 16.094 & 39.571 & 55.437 \\
\hline $7 / 7 / 10$ 12:00 & 6.801 & 3.856 & & 13.109 & 6.188 & & 5.392 & 16.092 & 39.451 & 55.319 \\
\hline 7/7/10 16:00 & 6.827 & 3.889 & & 13.226 & 6.290 & & 5.413 & 16.066 & 39.329 & 55.199 \\
\hline 7/7/10 20:00 & 6.872 & 3.915 & & 13.209 & 6.320 & & 5.451 & 16.118 & 39.210 & 55.079 \\
\hline 7/8/10 0:00 & 6.912 & 3.938 & & 13.181 & 6.290 & & 5.476 & 16.118 & 39.090 & 54.961 \\
\hline 7/8/10 4:00 & 6.933 & 3.943 & & 13.219 & 6.308 & & 5.486 & 16.104 & 38.970 & 54.841 \\
\hline 7/8/10 8:00 & 6.971 & 3.945 & & 13.191 & 6.275 & & 5.516 & 16.115 & 38.853 & 54.723 \\
\hline 7/8/10 12:00 & 7.011 & 3.952 & & 13.242 & 6.285 & & 5.554 & 16.139 & 38.736 & 54.605 \\
\hline 7/8/10 16:00 & 7.044 & 3.969 & & 13.337 & 6.363 & & 5.589 & 16.191 & 38.616 & 54.487 \\
\hline $7 / 8 / 1020: 00$ & 7.087 & 3.995 & & 13.342 & 6.384 & & 5.624 & 16.172 & 38.499 & 54.370 \\
\hline 7/9/10 0:00 & 7.127 & 4.011 & & 13.319 & 6.365 & & 5.650 & 16.186 & 38.382 & 54.254 \\
\hline 7/9/10 4:00 & 7.150 & 4.021 & & 13.333 & 6.351 & & 5.667 & 16.209 & 38.267 & 54.141 \\
\hline 7/9/10 8:00 & 7.186 & 4.028 & & 13.323 & 6.351 & & 5.697 & 16.231 & 38.152 & 54.023 \\
\hline 7/9/10 12:00 & 7.230 & 4.049 & & 13.459 & 6.412 & & 5.751 & 16.346 & 38.037 & 53.910 \\
\hline 7/9/10 16:00 & 7.273 & 4.087 & & 13.615 & 6.530 & & 5.805 & 16.308 & 37.922 & 53.797 \\
\hline $7 / 9 / 1020: 00$ & 7.318 & 4.122 & & 13.563 & 6.613 & & 5.852 & 16.280 & 37.807 & 53.684 \\
\hline 7/10/10 0:00 & 7.353 & 4.146 & & 13.489 & 6.568 & & 5.873 & 16.294 & 37.695 & 53.573 \\
\hline 7/10/10 4:00 & 7.379 & 4.157 & & 13.475 & 6.563 & & 5.883 & 16.303 & 37.582 & 53.458 \\
\hline 7/10/10 8:00 & 7.410 & 4.167 & & 13.470 & 6.558 & & 5.906 & 16.325 & 37.469 & 53.347 \\
\hline $7 / 10 / 10$ 12:00 & 7.443 & 4.181 & & 13.615 & 6.639 & & 5.951 & 16.381 & 37.359 & 53.237 \\
\hline 7/10/10 16:00 & 7.476 & 4.214 & & 13.743 & 6.747 & & 6.003 & 16.367 & 37.249 & 53.126 \\
\hline 7/10/10 20:00 & 7.506 & 4.244 & & 13.687 & 6.839 & & 6.036 & 16.353 & 37.136 & 53.015 \\
\hline 7/11/10 0:00 & 7.537 & 4.263 & & 13.596 & 6.801 & & 6.050 & 16.393 & 37.026 & 52.907 \\
\hline $7 / 11 / 104: 00$ & 7.549 & 4.266 & & 13.610 & 6.813 & & 6.040 & 16.383 & 36.916 & 52.798 \\
\hline 7/11/10 8:00 & 7.563 & 4.266 & & 13.591 & 6.672 & & 6.045 & 16.466 & 36.806 & 52.692 \\
\hline $7 / 11 / 10$ 12:00 & 7.584 & 4.268 & & 13.580 & 6.681 & & 6.059 & 16.442 & 36.698 & 52.582 \\
\hline 7/11/10 16:00 & 7.603 & 4.259 & & 13.629 & 6.705 & & 6.082 & 16.602 & 36.590 & 52.476 \\
\hline
\end{tabular}

TABLE S1.1 Page 41 of 89 
Water Level (ft below reference point)

\begin{tabular}{|c|c|c|c|c|c|c|c|c|c|c|}
\hline \multirow[b]{2}{*}{ Date and Time } & \\
\hline & McPike & Potter & Hughes & Barr & Burks & MoDOT & Clizer "Old" & Morgan & SB24 & SB38 \\
\hline 7/11/10 20:00 & 7.624 & 4.249 & & 13.591 & 6.709 & & 6.101 & 16.496 & 36.482 & 52.365 \\
\hline $7 / 12 / 100: 00$ & 7.674 & 4.244 & & 13.468 & 6.728 & & 6.144 & 16.534 & 36.374 & 52.259 \\
\hline $7 / 12 / 104: 00$ & 7.690 & 4.228 & & 13.517 & 6.712 & & 6.153 & 16.534 & 36.269 & 52.158 \\
\hline $7 / 12 / 108: 00$ & 7.718 & 4.216 & & 13.514 & 6.724 & & 6.181 & 16.567 & 36.163 & 52.049 \\
\hline $7 / 12 / 1012: 00$ & 7.763 & 4.223 & & 13.559 & 6.745 & & 6.235 & 16.675 & 36.057 & 51.943 \\
\hline $7 / 12 / 1016: 00$ & 7.775 & 4.223 & & 13.671 & 6.740 & & 6.252 & 16.785 & 35.952 & 51.842 \\
\hline $7 / 12 / 10$ 20:00 & 7.806 & 4.228 & & 13.647 & 6.757 & & 6.289 & 16.769 & 35.846 & 51.736 \\
\hline $7 / 13 / 10$ 0:00 & 7.841 & 4.233 & & 13.615 & 6.757 & & 6.317 & 16.750 & 35.743 & 51.632 \\
\hline 7/13/10 4:00 & 7.867 & 4.233 & & 13.633 & 6.752 & & 6.331 & 16.757 & 35.640 & 51.533 \\
\hline 7/13/10 8:00 & 7.907 & 4.235 & & 13.610 & 6.764 & & 6.367 & 16.793 & 35.539 & 51.432 \\
\hline $7 / 13 / 10$ 12:00 & 7.935 & 4.244 & & 13.754 & 6.769 & & 6.402 & 16.891 & 35.438 & 51.331 \\
\hline $7 / 13 / 1016: 00$ & 7.978 & 4.273 & & 13.887 & 6.804 & & 6.468 & 16.877 & 35.337 & 51.232 \\
\hline $7 / 13 / 1020: 00$ & 8.018 & 4.301 & & 13.843 & 6.830 & & 6.517 & 16.898 & 35.237 & 51.133 \\
\hline $7 / 14 / 100: 00$ & 8.062 & 4.322 & & 13.759 & 6.820 & & 6.559 & 16.943 & 35.136 & 51.034 \\
\hline $7 / 14 / 104: 00$ & 8.095 & 4.327 & & 13.764 & 6.809 & & 6.578 & 16.981 & 35.040 & 50.935 \\
\hline $7 / 14 / 10$ 8:00 & 8.133 & 4.336 & & 13.782 & 6.808 & & 6.613 & 17.025 & 34.941 & 50.836 \\
\hline $7 / 14 / 1012: 00$ & 8.171 & 4.348 & & 13.911 & 6.823 & & 6.656 & 17.258 & 34.843 & 50.737 \\
\hline $7 / 14 / 1016: 00$ & 8.206 & 4.372 & & 14.057 & 6.856 & & 6.721 & 17.122 & 34.744 & 50.638 \\
\hline $7 / 14 / 10$ 20:00 & 8.244 & 4.402 & & 13.969 & 6.879 & & 6.775 & 17.115 & 34.650 & 50.544 \\
\hline $7 / 15 / 100: 00$ & 7.417 & 4.127 & & 13.496 & 6.792 & & 6.745 & 17.213 & 34.554 & 50.443 \\
\hline $7 / 15 / 104: 00$ & 6.903 & 2.831 & & 13.274 & 6.705 & & 6.524 & 17.188 & 34.458 & 50.349 \\
\hline 7/15/10 8:00 & 7.051 & 2.765 & & 13.295 & 6.648 & & 6.451 & 17.133 & 34.357 & 50.250 \\
\hline $7 / 15 / 10$ 12:00 & 7.207 & 2.925 & & 13.426 & 6.615 & & 6.562 & 17.115 & 34.261 & 50.155 \\
\hline $7 / 15 / 1016: 00$ & 7.346 & 3.163 & & 13.622 & 6.629 & & 6.576 & 17.199 & 34.162 & 50.059 \\
\hline $7 / 15 / 1020: 00$ & 7.445 & 3.384 & & 13.622 & 6.658 & & 6.463 & 17.183 & 34.064 & 49.965 \\
\hline $7 / 16 / 100: 00$ & 7.527 & 3.545 & & 13.570 & 6.568 & & 6.397 & 17.148 & 33.970 & 49.870 \\
\hline $7 / 16 / 104: 00$ & 7.579 & 3.660 & & 13.608 & 6.537 & & 6.418 & 17.136 & 33.874 & 49.776 \\
\hline $7 / 16 / 108: 00$ & 7.624 & 3.752 & & 13.647 & 6.530 & & 6.503 & 17.138 & 33.780 & 49.684 \\
\hline $7 / 16 / 10$ 12:00 & 7.674 & 3.841 & & 13.778 & 6.611 & & 6.688 & 17.185 & 33.686 & 49.590 \\
\hline $7 / 16 / 10$ 16:00 & 6.783 & 3.516 & & 13.643 & 6.608 & & 6.569 & 17.150 & 33.595 & 49.496 \\
\hline $7 / 16 / 10$ 20:00 & 6.837 & 2.977 & & 13.458 & 6.596 & & 6.390 & 17.126 & 33.499 & 49.404 \\
\hline $7 / 17 / 100: 00$ & 6.940 & 2.995 & & 13.391 & 6.497 & & 6.287 & 17.089 & 33.402 & 49.310 \\
\hline 7/17/10 4:00 & 7.007 & 3.102 & & 13.396 & 6.415 & & 6.254 & 17.068 & 33.309 & 49.220 \\
\hline 7/17/10 8:00 & 7.049 & 3.217 & & 13.445 & 6.382 & & 6.292 & 17.042 & 33.215 & 49.124 \\
\hline $7 / 17 / 1012: 00$ & 7.108 & 3.351 & & 13.573 & 6.396 & & 6.487 & 17.042 & 33.123 & 49.032 \\
\hline $7 / 17 / 1016: 00$ & 7.169 & 3.512 & & 13.731 & 6.516 & & 6.498 & 17.046 & 33.030 & 48.940 \\
\hline $7 / 17 / 1020: 00$ & 7.221 & 3.643 & & 13.691 & 6.594 & & 6.418 & 17.009 & 32.938 & 48.846 \\
\hline 7/18/10 0:00 & 7.271 & 3.742 & & 13.633 & 6.556 & & 6.357 & 17.007 & 32.847 & 48.754 \\
\hline $7 / 18 / 104: 00$ & 7.021 & 3.771 & & 13.503 & 6.464 & & 6.317 & 17.014 & 32.758 & 48.664 \\
\hline $7 / 18 / 10$ 8:00 & 6.945 & 3.120 & & 13.384 & 6.462 & & 6.209 & 17.089 & 32.668 & 48.573 \\
\hline $7 / 18 / 10$ 12:00 & 7.021 & 2.904 & & 13.426 & 6.469 & & 6.270 & 17.044 & 32.577 & 48.481 \\
\hline $7 / 18 / 1016: 00$ & 7.096 & 2.913 & & 13.533 & 6.495 & & 6.364 & 17.018 & 32.488 & 48.391 \\
\hline $7 / 18 / 1020: 00$ & 7.174 & 3.054 & & 13.591 & 6.552 & & 6.285 & 16.999 & 32.399 & 48.302 \\
\hline $7 / 19 / 10$ 0:00 & 7.228 & 3.200 & & 13.559 & 6.493 & & 6.235 & 17.091 & 32.312 & 48.212 \\
\hline 7/19/10 4:00 & 7.238 & 3.314 & & 13.629 & 6.495 & & 6.275 & 17.037 & 32.225 & 48.123 \\
\hline 7/19/10 8:00 & 7.266 & 3.415 & & 13.626 & 6.500 & & 6.275 & 17.025 & 32.138 & 48.035 \\
\hline $7 / 19 / 10$ 12:00 & 7.306 & 3.521 & & 13.673 & 6.481 & & 6.355 & 17.021 & 32.052 & 47.946 \\
\hline $7 / 19 / 1016: 00$ & 7.330 & 3.620 & & 13.768 & 6.549 & & 6.508 & 17.004 & 31.965 & 47.861 \\
\hline $7 / 19 / 1020: 00$ & 7.370 & 3.719 & & 13.768 & 6.622 & & 6.432 & 17.011 & 31.880 & 47.772 \\
\hline $7 / 20 / 100: 00$ & 7.412 & 3.794 & & 13.698 & 6.596 & & 6.371 & 17.021 & 31.796 & 47.687 \\
\hline $7 / 20 / 104: 00$ & 7.450 & 3.851 & & 13.664 & 6.568 & & 6.226 & 17.042 & 31.711 & 47.600 \\
\hline $7 / 20 / 10$ 8:00 & 7.153 & 3.292 & & 13.486 & 6.478 & & 6.207 & 17.070 & 31.627 & 47.513 \\
\hline $7 / 20 / 1012: 00$ & 7.261 & 3.076 & & 13.517 & 6.457 & & 6.374 & 17.112 & 31.545 & 47.428 \\
\hline $7 / 20 / 1016: 00$ & 7.344 & 3.139 & & 13.645 & 6.488 & & 6.367 & 17.305 & 31.463 & 47.345 \\
\hline $7 / 20 / 1020: 00$ & 7.429 & 3.257 & & 13.608 & 6.457 & & 6.240 & 17.150 & 31.378 & 47.258 \\
\hline $7 / 21 / 10$ 0:00 & 7.473 & 3.372 & & 13.617 & 6.401 & & 6.228 & 17.148 & 31.296 & 47.176 \\
\hline $7 / 21 / 104: 00$ & 7.511 & 3.474 & & 13.645 & 6.349 & & 6.221 & 17.155 & 31.217 & 47.093 \\
\hline 7/21/10 8:00 & 7.546 & 3.561 & & 13.638 & 6.302 & & 6.230 & 17.204 & 31.137 & 47.011 \\
\hline $7 / 21 / 1012: 00$ & 7.584 & 3.639 & & 13.719 & 6.323 & & 6.343 & 17.225 & 31.055 & 46.928 \\
\hline $7 / 21 / 1016: 00$ & 7.596 & 3.707 & & 13.862 & 6.415 & & 6.428 & 17.209 & 30.977 & 46.846 \\
\hline $7 / 21 / 10$ 20:00 & 7.615 & 3.761 & & 13.871 & 6.516 & & 6.425 & 17.188 & 30.898 & 46.766 \\
\hline $7 / 22 / 100: 00$ & 7.631 & 3.808 & & 13.857 & 6.514 & & 6.449 & 17.199 & 30.818 & 46.683 \\
\hline $7 / 22 / 104: 00$ & 7.638 & 3.839 & & 13.873 & 6.549 & & 6.461 & 17.185 & 30.740 & 46.603 \\
\hline $7 / 22 / 108: 00$ & 7.667 & 3.872 & & 13.824 & 6.526 & & 6.491 & 17.204 & 30.663 & 46.523 \\
\hline
\end{tabular}

TABLE S1.1 Page 42 of 89 
Water Level (ft below reference point)

\begin{tabular}{|c|c|c|c|c|c|c|c|c|c|c|}
\hline \multirow[b]{2}{*}{ Date and Time } & \\
\hline & McPike & Potter & Hughes & Barr & Burks & MoDOT & Clizer "Old" & Morgan & SB24 & SB38 \\
\hline $7 / 22 / 1012: 00$ & 7.695 & 3.910 & & 13.955 & 6.603 & & 6.691 & 17.204 & 30.583 & 46.443 \\
\hline $7 / 22 / 1016: 00$ & 7.730 & 3.961 & & 14.062 & 6.698 & & 6.731 & 17.202 & 30.508 & 46.365 \\
\hline $7 / 22 / 1020: 00$ & 7.775 & 4.013 & & 13.971 & 6.733 & & 6.646 & 17.199 & 30.431 & 46.285 \\
\hline $7 / 23 / 100: 00$ & 7.815 & 4.060 & & 13.876 & 6.691 & & 6.597 & 17.230 & 30.356 & 46.205 \\
\hline $7 / 23 / 104: 00$ & 7.846 & 4.086 & & 13.878 & 6.686 & & 6.578 & 17.303 & 30.281 & 46.130 \\
\hline $7 / 23 / 108: 00$ & 7.893 & 4.117 & & 13.838 & 6.634 & & 6.613 & 17.322 & 30.208 & 46.050 \\
\hline $7 / 23 / 10$ 12:00 & 7.935 & 4.145 & & 13.957 & 6.672 & & 6.595 & 17.333 & 30.133 & 45.974 \\
\hline $7 / 23 / 1016: 00$ & 7.982 & 4.183 & & 14.109 & 6.757 & & 6.644 & 17.336 & 30.058 & 45.897 \\
\hline $7 / 23 / 10$ 20:00 & 8.027 & 4.221 & & 14.046 & 6.799 & & 6.689 & 17.343 & 29.985 & 45.821 \\
\hline 7/24/10 0:00 & 8.077 & 4.251 & & 13.969 & 6.745 & & 6.719 & 17.444 & 29.913 & 45.746 \\
\hline $7 / 24 / 104: 00$ & 8.096 & 4.270 & & 14.001 & 6.738 & & 6.712 & 17.434 & 29.840 & 45.668 \\
\hline $7 / 24 / 108: 00$ & 8.140 & 4.289 & & 13.985 & 6.698 & & 6.745 & 17.458 & 29.769 & 45.595 \\
\hline $7 / 24 / 1012: 00$ & 8.190 & 4.310 & & 14.015 & 6.677 & & 6.782 & 17.493 & 29.699 & 45.520 \\
\hline $7 / 24 / 10$ 16:00 & 8.223 & 4.331 & & 13.924 & 6.655 & & 6.867 & 17.552 & 29.629 & 45.442 \\
\hline $7 / 24 / 1020: 00$ & 8.152 & 4.011 & & 13.938 & 6.662 & & 6.801 & 17.599 & 29.556 & 45.369 \\
\hline $7 / 25 / 100: 00$ & 8.239 & 3.829 & & 13.873 & 6.523 & & 6.736 & 17.653 & 29.488 & 45.296 \\
\hline $7 / 25 / 104: 00$ & 8.251 & 3.785 & & 13.990 & 6.495 & & 6.733 & 17.644 & 29.418 & 45.223 \\
\hline $7 / 25 / 108: 00$ & 8.291 & 3.804 & & 13.980 & 6.405 & & 6.761 & 17.667 & 29.350 & 45.150 \\
\hline $7 / 25 / 10$ 12:00 & 8.329 & 3.858 & & 14.092 & 6.396 & & 6.933 & 17.712 & 29.284 & 45.079 \\
\hline $7 / 25 / 1016: 00$ & 8.355 & 3.924 & & 14.260 & 6.471 & & 7.027 & 17.738 & 29.214 & 45.006 \\
\hline 7/25/10 20:00 & 8.383 & 3.985 & & 14.246 & 6.540 & & 6.947 & 17.712 & 29.148 & 44.936 \\
\hline 7/26/10 0:00 & 8.416 & 4.037 & & 14.171 & 6.504 & & 6.975 & 17.766 & 29.080 & 44.865 \\
\hline $7 / 26 / 104: 00$ & 8.426 & 4.079 & & 14.213 & 6.523 & & 6.968 & 17.766 & 29.014 & 44.792 \\
\hline $7 / 26 / 10$ 8:00 & 8.454 & 4.115 & & 14.197 & 6.528 & & 6.992 & 17.792 & 28.949 & 44.724 \\
\hline $7 / 26 / 1012: 00$ & 8.470 & 4.150 & & 14.316 & 6.606 & & 7.008 & 18.187 & 28.883 & 44.653 \\
\hline $7 / 26 / 10$ 16:00 & 8.485 & 4.183 & & 14.477 & 6.743 & & 7.031 & 17.865 & 28.817 & 44.582 \\
\hline $7 / 26 / 1020: 00$ & 8.515 & 4.218 & & 14.404 & 6.818 & & 7.071 & 17.848 & 28.752 & 44.514 \\
\hline $7 / 27 / 100: 00$ & 8.551 & 4.251 & & 14.288 & 6.740 & & 7.095 & 17.886 & 28.688 & 44.446 \\
\hline $7 / 27 / 104: 00$ & 8.567 & 4.275 & & 14.281 & 6.728 & & 7.100 & 17.898 & 28.625 & 44.375 \\
\hline $7 / 27 / 10$ 8:00 & 8.586 & 4.291 & & 14.281 & 6.714 & & 7.116 & 17.926 & 28.562 & 44.333 \\
\hline $7 / 27 / 10$ 12:00 & 8.609 & 4.313 & & 14.404 & 6.776 & & 7.147 & 17.949 & 28.498 & 44.401 \\
\hline $7 / 27 / 10$ 16:00 & 8.638 & 4.343 & & 14.540 & 6.856 & & 7.191 & 17.949 & 28.435 & 44.172 \\
\hline $7 / 27 / 10$ 20:00 & 8.666 & 4.367 & & 14.481 & 6.901 & & 7.226 & 18.011 & 28.374 & 44.104 \\
\hline 7/28/10 0:00 & 8.708 & 4.393 & & 14.353 & 6.853 & & 7.262 & 18.039 & 28.313 & 44.040 \\
\hline $7 / 28 / 104: 00$ & 8.735 & 4.407 & & 14.339 & 6.823 & & 7.276 & 18.055 & 28.252 & 43.974 \\
\hline $7 / 28 / 10$ 8:00 & 8.770 & 4.426 & & 14.316 & 6.761 & & 7.299 & 18.100 & 28.191 & 43.909 \\
\hline $7 / 28 / 1012: 00$ & 8.801 & 4.442 & & 14.470 & 6.806 & & 7.335 & 18.178 & 28.130 & 43.843 \\
\hline $7 / 28 / 1016: 00$ & 8.836 & 4.463 & & 14.623 & 6.889 & & 7.379 & 18.253 & 28.071 & 43.779 \\
\hline $7 / 28 / 1020: 00$ & 8.871 & 4.482 & & 14.533 & 6.919 & & 7.421 & 18.239 & 28.013 & 43.713 \\
\hline $7 / 29 / 100: 00$ & 8.918 & 4.506 & & 14.442 & 6.858 & & 7.459 & 18.276 & 27.954 & 43.652 \\
\hline $7 / 29 / 104: 00$ & 8.944 & 4.518 & & 14.430 & 6.802 & & 7.471 & 18.316 & 27.896 & 43.586 \\
\hline $7 / 29 / 10$ 8:00 & 8.968 & 4.527 & & 14.444 & 6.771 & & 7.494 & 18.344 & 27.839 & 43.522 \\
\hline $7 / 29 / 10$ 12:00 & 8.989 & 4.539 & & 14.602 & 6.851 & & 7.513 & 18.375 & 27.781 & 43.461 \\
\hline $7 / 29 / 10$ 16:00 & 9.003 & 4.558 & & 14.775 & 6.979 & & 7.536 & 18.370 & 27.722 & 43.397 \\
\hline $7 / 29 / 10$ 20:00 & 9.015 & 4.572 & & 14.740 & 7.070 & & 7.565 & 18.377 & 27.668 & 43.336 \\
\hline 7/30/10 0:00 & 9.034 & 4.586 & & 14.665 & 7.054 & & 7.581 & 18.389 & 27.612 & 43.275 \\
\hline $7 / 30 / 104: 00$ & 9.034 & 4.593 & & 14.686 & 7.061 & & 7.576 & 18.439 & 27.555 & 43.214 \\
\hline $7 / 30 / 108: 00$ & 9.039 & 4.595 & & 14.696 & 7.063 & & 7.581 & 20.266 & 27.499 & 43.150 \\
\hline $7 / 30 / 1012: 00$ & 9.053 & 4.600 & & 14.775 & 7.101 & & 7.607 & 18.843 & 27.443 & 43.089 \\
\hline $7 / 30 / 10$ 16:00 & 9.046 & 4.607 & & 14.752 & 7.099 & & 7.600 & 18.631 & 27.389 & 43.028 \\
\hline $7 / 30 / 1020: 00$ & 9.069 & 4.614 & & 14.747 & 7.144 & & 7.630 & 18.551 & 27.333 & 42.966 \\
\hline 7/31/10 0:00 & 9.100 & 4.624 & & 14.696 & 7.087 & & 7.659 & 18.561 & 27.281 & 42.907 \\
\hline $7 / 31 / 104: 00$ & 9.119 & 4.628 & & 14.675 & 7.040 & & 7.675 & 18.582 & 27.227 & 42.846 \\
\hline 7/31/10 8:00 & 9.152 & 4.635 & & 14.637 & 6.964 & & 7.706 & 18.634 & 27.173 & 42.790 \\
\hline $7 / 31 / 1012: 00$ & 9.182 & 4.647 & & 14.733 & 6.962 & & 7.741 & 18.702 & 27.122 & 42.728 \\
\hline $7 / 31 / 1016: 00$ & 9.211 & 4.659 & & 14.861 & 7.033 & & 7.778 & 18.725 & 27.068 & 42.672 \\
\hline $7 / 31 / 10$ 20:00 & 9.237 & 4.671 & & 14.826 & 7.122 & & 7.816 & 18.732 & 27.016 & 42.613 \\
\hline 8/1/10 0:00 & 9.270 & 4.685 & & 14.724 & 7.082 & & 7.849 & 18.791 & 26.967 & 42.556 \\
\hline 8/1/10 4:00 & 9.286 & 4.690 & & 14.733 & 7.075 & & 7.863 & 18.862 & 26.915 & 42.488 \\
\hline 8/1/10 8:00 & 9.314 & 4.697 & & 14.726 & 7.047 & & 7.889 & 18.892 & 26.866 & 42.410 \\
\hline 8/1/10 12:00 & 9.333 & 4.704 & & 14.870 & 7.108 & & 7.910 & 18.909 & 26.814 & 42.486 \\
\hline $8 / 1 / 1016: 00$ & 9.352 & 4.718 & & 15.010 & 7.205 & & 7.945 & 18.909 & 26.765 & 42.490 \\
\hline 8/1/10 20:00 & 9.371 & 4.730 & & 14.938 & 7.280 & & 7.976 & 18.975 & 26.716 & 42.304 \\
\hline $8 / 2 / 100: 00$ & 9.397 & 4.739 & & 14.863 & 7.252 & & 7.997 & 19.193 & 26.667 & 42.161 \\
\hline
\end{tabular}

TABLE S1.1 Page 43 of 89 
Water Level (ft below reference point)

\begin{tabular}{|c|c|c|c|c|c|c|c|c|c|c|}
\hline \multirow[b]{2}{*}{ Date and Time } & \\
\hline & McPike & Potter & Hughes & Barr & Burks & MoDOT & Clizer "Old" & Morgan & SB24 & SB38 \\
\hline 8/2/10 4:00 & 9.404 & 4.744 & & 14.877 & 7.250 & & 8.004 & 19.052 & 26.617 & 42.137 \\
\hline $8 / 2 / 108: 00$ & 9.413 & 4.748 & & 14.861 & 7.280 & & 8.006 & 19.090 & 26.570 & 42.033 \\
\hline $8 / 2 / 1012: 00$ & 9.432 & 4.748 & & 14.943 & 7.290 & & 8.030 & 19.088 & 26.521 & 42.137 \\
\hline $8 / 2 / 1016: 00$ & 9.446 & 4.751 & & 15.120 & 7.372 & & 8.053 & 19.073 & 26.474 & 42.189 \\
\hline $8 / 2 / 1020: 00$ & 9.475 & 4.762 & & 15.045 & 7.424 & & 8.095 & 19.088 & 26.430 & 42.001 \\
\hline 8/3/10 0:00 & 9.501 & 4.770 & & 14.966 & 7.389 & & 8.124 & 19.184 & 26.380 & 41.864 \\
\hline 8/3/10 4:00 & 9.524 & 4.774 & & 14.936 & 7.360 & & 8.147 & 19.186 & 26.336 & 41.760 \\
\hline 8/3/10 8:00 & 9.559 & 4.784 & & 14.908 & 7.325 & & 8.175 & 19.238 & 26.291 & 41.697 \\
\hline 8/3/10 12:00 & 9.590 & 4.793 & & 15.038 & 7.356 & & 8.208 & 19.285 & 26.244 & 41.777 \\
\hline 8/3/10 16:00 & 9.616 & 4.807 & & 15.215 & 7.457 & & 8.241 & 19.360 & 26.200 & 41.678 \\
\hline 8/3/10 20:00 & 9.635 & 4.814 & & 15.141 & 7.537 & & 8.274 & 19.346 & 26.155 & 41.628 \\
\hline $8 / 4 / 100: 00$ & 9.651 & 4.824 & & 15.061 & 7.523 & & 8.286 & 19.525 & 26.111 & 41.574 \\
\hline $8 / 4 / 104: 00$ & 9.682 & 4.828 & & 14.994 & 7.495 & & 8.323 & 19.457 & 26.068 & 41.525 \\
\hline 8/4/10 8:00 & 9.701 & 4.833 & & 15.008 & 7.469 & & 8.323 & 20.762 & 26.026 & 41.513 \\
\hline $8 / 4 / 1012: 00$ & 9.736 & 4.840 & & 15.071 & 7.469 & & 8.366 & 21.735 & 25.984 & 41.452 \\
\hline $8 / 4 / 1016: 00$ & 9.755 & 4.850 & & 15.106 & 7.486 & & 8.377 & 23.193 & 25.939 & 41.405 \\
\hline $8 / 4 / 10$ 20:00 & 9.772 & 4.857 & & 15.164 & 7.551 & & 8.399 & 23.659 & 25.900 & 41.313 \\
\hline 8/5/10 0:00 & 9.795 & 4.866 & & 15.094 & 7.514 & & 8.424 & 20.947 & 25.857 & 41.183 \\
\hline 8/5/10 4:00 & 9.802 & 4.871 & & 15.103 & 7.502 & & 8.434 & 20.362 & 25.817 & 41.136 \\
\hline 8/5/10 8:00 & 9.831 & 4.878 & & 15.080 & 7.460 & & 8.460 & 20.244 & 25.778 & 41.075 \\
\hline 8/5/10 12:00 & 9.859 & 4.885 & & 15.206 & 7.488 & & 8.481 & 20.216 & 25.740 & 41.162 \\
\hline 8/5/10 16:00 & 9.868 & 4.892 & & 15.432 & 7.625 & & 8.502 & 20.094 & 25.700 & 41.216 \\
\hline 8/5/10 20:00 & 9.889 & 4.902 & & 15.334 & 7.712 & & 8.532 & 20.033 & 25.660 & 41.039 \\
\hline $8 / 6 / 100: 00$ & 9.920 & 4.911 & & 15.199 & 7.653 & & 8.563 & 20.056 & 25.620 & 40.879 \\
\hline 8/6/10 4:00 & 9.934 & 4.920 & & 15.178 & 7.634 & & 8.572 & 20.101 & 25.583 & 40.844 \\
\hline 8/6/10 8:00 & 9.960 & 4.927 & & 15.143 & 7.587 & & 8.596 & 20.110 & 25.545 & 40.802 \\
\hline $8 / 6 / 10$ 12:00 & 9.984 & 4.934 & & 15.294 & 7.648 & & 8.615 & 20.136 & 25.508 & 40.898 \\
\hline 8/6/10 16:00 & 9.996 & 4.942 & & 15.465 & 7.776 & & 8.631 & 20.181 & 25.470 & 40.941 \\
\hline 8/6/10 20:00 & 10.010 & 4.949 & & 15.413 & 7.865 & & 8.652 & 20.129 & 25.433 & 40.802 \\
\hline 8/7/10 0:00 & 10.033 & 4.956 & & 15.308 & 7.837 & & 8.678 & 20.148 & 25.398 & 40.646 \\
\hline 8/7/10 4:00 & 10.036 & 4.960 & & 15.327 & 7.839 & & 8.673 & 20.160 & 25.362 & 40.634 \\
\hline 8/7/10 8:00 & 10.050 & 4.965 & & 15.313 & 7.816 & & 8.687 & 20.176 & 25.325 & 40.615 \\
\hline 8/7/10 12:00 & 10.066 & 4.972 & & 15.437 & 7.886 & & 8.699 & 20.219 & 25.290 & 40.693 \\
\hline 8/7/10 16:00 & 10.076 & 4.984 & & 15.600 & 7.995 & & 8.718 & 20.200 & 25.252 & 40.726 \\
\hline 8/7/10 20:00 & 10.090 & 4.991 & & 15.518 & 8.078 & & 8.744 & 20.261 & 25.219 & 40.585 \\
\hline 8/8/10 0:00 & 10.116 & 4.998 & & 15.397 & 8.045 & & 8.770 & 20.393 & 25.182 & 40.420 \\
\hline 8/8/10 4:00 & 10.128 & 5.005 & & 15.395 & 8.045 & & 8.774 & 20.322 & 25.149 & 40.387 \\
\hline 8/8/10 8:00 & 10.142 & 5.008 & & 15.385 & 8.040 & & 8.786 & 20.327 & 25.114 & 40.359 \\
\hline $8 / 8 / 10$ 12:00 & 10.161 & 5.015 & & 15.462 & 8.054 & & 8.800 & 20.357 & 25.079 & 40.394 \\
\hline $8 / 8 / 1016: 00$ & 10.179 & 5.024 & & 15.674 & 8.104 & & 8.821 & 20.414 & 25.046 & 40.448 \\
\hline 8/8/10 20:00 & 10.201 & 5.031 & & 15.576 & 8.169 & & 8.857 & 20.400 & 25.013 & 40.295 \\
\hline 8/9/10 0:00 & 10.231 & 5.043 & & 15.472 & 8.203 & & 8.887 & 21.199 & 24.980 & 40.151 \\
\hline 8/9/10 4:00 & 10.257 & 5.045 & & 15.423 & 8.217 & & 8.906 & 20.748 & 24.947 & 40.060 \\
\hline 8/9/10 8:00 & 10.297 & 5.055 & & 15.353 & 8.233 & & 8.936 & 21.390 & 24.915 & 39.956 \\
\hline $8 / 9 / 10$ 12:00 & 10.316 & 5.059 & & 15.504 & 8.257 & & 8.939 & 21.096 & 24.884 & 40.071 \\
\hline 8/9/10 16:00 & 10.335 & 5.069 & & 15.695 & 8.306 & & 8.965 & 20.903 & 24.851 & 40.010 \\
\hline $8 / 9 / 1020: 00$ & 10.359 & 5.081 & & 15.607 & 8.384 & & 8.998 & 20.835 & 24.823 & 39.972 \\
\hline 8/10/10 0:00 & 10.396 & 5.092 & & 15.485 & 8.424 & & 9.030 & 20.915 & 24.790 & 39.930 \\
\hline 8/10/10 4:00 & 10.415 & 5.095 & & 15.471 & 8.436 & & 9.040 & 20.938 & 24.760 & 39.890 \\
\hline 8/10/10 8:00 & 10.436 & 5.099 & & 15.478 & 8.443 & & 9.049 & 20.945 & 24.732 & 39.852 \\
\hline $8 / 10 / 1012: 00$ & 10.460 & 5.107 & & 15.632 & 8.460 & & 9.063 & 20.973 & 24.703 & 39.812 \\
\hline $8 / 10 / 10$ 16:00 & 10.472 & 5.116 & & 15.837 & 8.502 & & 9.082 & 21.027 & 24.673 & 39.772 \\
\hline $8 / 10 / 1020: 00$ & 10.495 & 5.125 & & 15.709 & 8.568 & & 9.110 & 20.997 & 24.645 & 39.732 \\
\hline 8/11/10 0:00 & 10.526 & 5.135 & & 15.525 & 8.648 & & 9.129 & 21.053 & 24.617 & 39.694 \\
\hline $8 / 11 / 104: 00$ & 10.540 & 5.137 & & 15.504 & 8.596 & & 9.141 & 21.070 & 24.588 & 39.657 \\
\hline 8/11/10 8:00 & 10.564 & 5.144 & & 15.518 & 8.540 & & 9.153 & 21.171 & 24.563 & 39.619 \\
\hline 8/11/10 12:00 & 10.582 & 5.149 & & 15.676 & 8.573 & & 9.162 & 21.185 & 24.535 & 39.579 \\
\hline 8/11/10 16:00 & 10.594 & 5.154 & & 15.900 & 8.660 & & 9.176 & 21.180 & 24.506 & 39.544 \\
\hline $8 / 11 / 1020: 00$ & 10.616 & 5.163 & & 15.795 & 8.714 & & 9.202 & 21.263 & 24.478 & 39.506 \\
\hline 8/12/10 0:00 & 10.639 & 5.170 & & 15.683 & 8.693 & & 9.221 & 21.272 & 24.452 & 39.468 \\
\hline 8/12/10 4:00 & 10.651 & 5.172 & & 15.672 & 8.693 & & 9.221 & 21.284 & 24.427 & 39.431 \\
\hline 8/12/10 8:00 & 10.670 & 5.175 & & 15.670 & 8.677 & & 9.232 & 21.312 & 24.401 & 39.412 \\
\hline $8 / 12 / 1012: 00$ & 10.684 & 5.182 & & 15.844 & 8.754 & & 9.239 & 21.686 & 24.375 & 39.513 \\
\hline $8 / 12 / 1016: 00$ & 10.691 & 5.187 & & 16.045 & 8.901 & & 9.249 & 21.437 & 24.349 & 39.558 \\
\hline
\end{tabular}

TABLE S1.1 Page 44 of 89 
Water Level (ft below reference point)

\begin{tabular}{|c|c|c|c|c|c|c|c|c|c|c|}
\hline \multirow[b]{2}{*}{ Date and Time } & \\
\hline & McPike & Potter & Hughes & Barr & Burks & MoDOT & Clizer "Old" & Morgan & SB24 & SB38 \\
\hline 8/12/10 20:00 & 10.708 & 5.196 & & 15.912 & 9.026 & & 9.272 & 21.368 & 24.323 & 39.263 \\
\hline $8 / 13 / 100: 00$ & 10.731 & 5.205 & & 15.800 & 9.021 & & 9.291 & 21.448 & 24.300 & 39.179 \\
\hline $8 / 13 / 104: 00$ & 10.745 & 5.210 & & 15.777 & 9.061 & & 9.293 & 21.434 & 24.274 & 39.216 \\
\hline 8/13/10 8:00 & 10.759 & 5.215 & & 15.772 & 9.063 & & 9.301 & 21.446 & 24.251 & 39.176 \\
\hline $8 / 13 / 1012: 00$ & 10.776 & 5.222 & & 15.879 & 9.106 & & 9.319 & 21.542 & 24.225 & 39.230 \\
\hline $8 / 13 / 1016: 00$ & 10.788 & 5.227 & & 16.031 & 9.186 & & 9.326 & 21.526 & 24.202 & 39.261 \\
\hline 8/13/10 20:00 & 9.864 & 4.491 & & 15.430 & 8.870 & & 9.232 & 21.488 & 24.178 & 39.068 \\
\hline $8 / 14 / 100: 00$ & 9.828 & 4.119 & & 15.306 & 8.818 & & 9.138 & 21.418 & 24.152 & 38.785 \\
\hline 8/14/10 4:00 & 9.993 & 4.169 & & 15.360 & 8.780 & & 9.080 & 21.390 & 24.124 & 38.785 \\
\hline 8/14/10 8:00 & 10.123 & 4.272 & & 15.397 & 8.686 & & 9.092 & 21.354 & 24.098 & 38.811 \\
\hline 8/14/10 12:00 & 10.227 & 4.371 & & 15.523 & 8.681 & & 9.202 & 21.340 & 24.073 & 38.891 \\
\hline $8 / 14 / 10$ 16:00 & 10.314 & 4.463 & & 15.702 & 8.707 & & 9.207 & 21.347 & 24.044 & 38.891 \\
\hline $8 / 14 / 1020: 00$ & 10.392 & 4.539 & & 15.642 & 8.733 & & 9.089 & 21.444 & 24.019 & 38.747 \\
\hline $8 / 15 / 10$ 0:00 & 10.467 & 4.600 & & 15.551 & 8.698 & & 9.059 & 21.460 & 23.993 & 38.634 \\
\hline $8 / 15 / 104: 00$ & 10.535 & 4.647 & & 15.492 & 8.599 & & 8.998 & 21.514 & 23.969 & 38.580 \\
\hline 8/15/10 8:00 & 10.590 & 4.687 & & 15.513 & 8.563 & & 9.052 & 21.559 & 23.946 & 38.578 \\
\hline $8 / 15 / 1012: 00$ & 10.637 & 4.722 & & 15.716 & 8.601 & & 9.279 & 21.669 & 23.922 & 38.691 \\
\hline $8 / 15 / 1016: 00$ & 10.670 & 4.755 & & 15.833 & 8.691 & & 9.237 & 21.639 & 23.899 & 38.670 \\
\hline 8/15/10 20:00 & 10.696 & 4.781 & & 15.837 & 8.790 & & 9.190 & 21.634 & 23.875 & 38.594 \\
\hline 8/16/10 0:00 & 10.736 & 4.809 & & 15.751 & 8.757 & & 9.153 & 21.674 & 23.854 & 38.498 \\
\hline 8/16/10 4:00 & 10.764 & 4.826 & & 15.751 & 8.752 & & 9.155 & 21.752 & 23.831 & 38.498 \\
\hline 8/16/10 8:00 & 10.792 & 4.845 & & 15.774 & 8.750 & & 9.221 & 21.770 & 23.810 & 38.519 \\
\hline 8/16/10 12:00 & 10.814 & 4.861 & & 15.916 & 8.804 & & 9.432 & 21.792 & 23.786 & 38.618 \\
\hline $8 / 16 / 1016: 00$ & 10.823 & 4.880 & & 16.129 & 8.922 & & 9.460 & 21.829 & 23.763 & 38.651 \\
\hline $8 / 16 / 1020: 00$ & 10.830 & 4.894 & & 16.040 & 9.019 & & 9.385 & 22.316 & 23.742 & 38.568 \\
\hline $8 / 17 / 100: 00$ & 10.858 & 4.911 & & 15.907 & 8.976 & & 9.425 & 21.919 & 23.721 & 38.422 \\
\hline $8 / 17 / 104: 00$ & 10.873 & 4.925 & & 15.919 & 8.997 & & 9.423 & 21.944 & 23.702 & 38.432 \\
\hline $8 / 17 / 108: 00$ & 10.887 & 4.937 & & 15.907 & 8.990 & & 9.427 & 21.904 & 23.678 & 38.413 \\
\hline $8 / 17 / 1012: 00$ & 10.908 & 4.948 & & 15.944 & 8.986 & & 9.442 & 22.006 & 23.660 & 38.401 \\
\hline $8 / 17 / 1016: 00$ & 10.922 & 4.960 & & 16.007 & 9.047 & & 9.446 & 21.952 & 23.639 & 38.385 \\
\hline $8 / 17 / 10$ 20:00 & 10.929 & 4.972 & & 16.017 & 9.075 & & 9.449 & 22.029 & 23.620 & 38.352 \\
\hline $8 / 18 / 100: 00$ & 10.953 & 4.984 & & 15.963 & 9.033 & & 9.470 & 22.027 & 23.599 & 38.272 \\
\hline 8/18/10 4:00 & 10.962 & 4.996 & & 15.996 & 9.037 & & 9.465 & 22.034 & 23.580 & 38.293 \\
\hline 8/18/10 8:00 & 10.974 & 5.003 & & 16.000 & 9.033 & & 9.472 & 22.050 & 23.559 & 38.281 \\
\hline 8/18/10 12:00 & 10.986 & 5.012 & & 16.108 & 9.085 & & 9.477 & 22.133 & 23.538 & 38.309 \\
\hline $8 / 18 / 1016: 00$ & 10.988 & 5.022 & & 16.289 & 9.177 & & 9.474 & 22.090 & 23.519 & 38.352 \\
\hline $8 / 18 / 1020: 00$ & 11.000 & 5.036 & & 16.210 & 9.252 & & 9.486 & 22.081 & 23.500 & 38.250 \\
\hline 8/19/10 0:00 & 11.021 & 5.047 & & 16.082 & 9.221 & & 9.510 & 22.123 & 23.482 & 38.128 \\
\hline $8 / 19 / 104: 00$ & 11.028 & 5.055 & & 16.082 & 9.240 & & 9.507 & 22.142 & 23.463 & 38.126 \\
\hline 8/19/10 8:00 & 11.040 & 5.059 & & 16.056 & 9.228 & & 9.512 & 22.198 & 23.446 & 38.123 \\
\hline $8 / 19 / 1012: 00$ & 11.056 & 5.073 & & 16.173 & 9.285 & & 9.524 & 22.210 & 23.430 & 38.170 \\
\hline $8 / 19 / 10$ 16:00 & 11.063 & 5.085 & & 16.343 & 9.391 & & 9.528 & 22.191 & 23.411 & 38.194 \\
\hline $8 / 19 / 10$ 20:00 & 11.075 & 5.095 & & 16.245 & 9.394 & & 9.543 & 22.180 & 23.395 & 38.109 \\
\hline $8 / 20 / 100: 00$ & 11.096 & 5.106 & & 16.126 & 9.431 & & 9.564 & 22.267 & 23.376 & 38.001 \\
\hline 8/20/10 4:00 & 11.106 & 5.113 & & 16.124 & 9.455 & & 9.566 & 22.250 & 23.360 & 37.977 \\
\hline 8/20/10 8:00 & 11.122 & 5.121 & & 16.110 & 9.474 & & 9.575 & 22.267 & 23.343 & 37.949 \\
\hline $8 / 20 / 10$ 12:00 & 11.139 & 5.130 & & 16.177 & 9.490 & & 9.587 & 22.292 & 23.317 & 37.852 \\
\hline $8 / 20 / 1016: 00$ & 10.920 & 5.113 & & 15.996 & 9.405 & & 9.564 & 22.339 & 23.299 & 37.824 \\
\hline $8 / 20 / 1020: 00$ & 9.937 & 3.813 & & 15.826 & 9.349 & & 9.385 & 22.213 & 23.277 & 37.796 \\
\hline $8 / 21 / 100: 00$ & 9.965 & 3.398 & & 15.723 & 9.247 & & 9.326 & 22.158 & 23.256 & 37.768 \\
\hline 8/21/10 4:00 & 10.156 & 3.488 & & 15.688 & 9.129 & & 9.277 & 22.102 & 23.235 & 37.739 \\
\hline 8/21/10 8:00 & 10.316 & 3.667 & & 15.670 & 9.009 & & 9.265 & 22.107 & 23.214 & 37.716 \\
\hline $8 / 21 / 1012: 00$ & 10.451 & 3.853 & & 15.721 & 8.915 & & 9.373 & 22.158 & 23.195 & 37.687 \\
\hline $8 / 21 / 1016: 00$ & 10.550 & 4.027 & & 15.916 & 8.924 & & 9.404 & 22.173 & 23.177 & 37.662 \\
\hline $8 / 21 / 1020: 00$ & 10.630 & 4.176 & & 15.902 & 8.943 & & 9.315 & 22.187 & 23.158 & 37.636 \\
\hline 8/22/10 0:00 & 10.710 & 4.293 & & 15.819 & 8.886 & & 9.308 & 22.231 & 23.139 & 37.610 \\
\hline 8/22/10 4:00 & 10.764 & 4.378 & & 15.842 & 8.886 & & 9.322 & 22.304 & 23.123 & 37.584 \\
\hline 8/22/10 8:00 & 10.821 & 4.451 & & 15.819 & 8.825 & & 9.359 & 22.328 & 23.104 & 37.560 \\
\hline $8 / 22 / 1012: 00$ & 10.870 & 4.517 & & 15.951 & 8.842 & & 9.491 & 22.361 & 23.090 & 37.537 \\
\hline $8 / 22 / 1016: 00$ & 10.901 & 4.569 & & 16.147 & 8.943 & & 9.526 & 22.344 & 23.071 & 37.511 \\
\hline $8 / 22 / 10$ 20:00 & 10.929 & 4.616 & & 16.091 & 9.021 & & 9.453 & 22.394 & 23.055 & 37.485 \\
\hline 8/23/10 0:00 & 10.962 & 4.659 & & 16.014 & 9.021 & & 9.446 & 22.387 & 23.041 & 37.459 \\
\hline 8/23/10 4:00 & 10.988 & 4.689 & & 15.996 & 9.021 & & 9.453 & 22.389 & 23.024 & 37.435 \\
\hline 8/23/10 8:00 & 11.016 & 4.720 & & 15.982 & 8.990 & & 9.493 & 22.408 & 23.008 & 37.414 \\
\hline
\end{tabular}

TABLE S1.1 Page 45 of 89 
Water Level (ft below reference point)

\begin{tabular}{|c|c|c|c|c|c|c|c|c|c|c|}
\hline Date and Time & McPike & Potter & Hughes & Barr & Burks & MoDOT & Clizer "Old" & Morgan & SB24 & SB38 \\
\hline 8/23/10 12:00 & 11.040 & 4.743 & & 16.140 & 9.063 & & 9.681 & 22.492 & 22.994 & 37.388 \\
\hline 8/23/10 16:00 & 11.056 & 4.769 & & 16.306 & 9.162 & & 9.644 & 22.452 & 22.980 & 37.367 \\
\hline 8/23/10 20:00 & 11.080 & 4.795 & & 16.196 & 9.224 & & 9.568 & 22.706 & 22.963 & 37.341 \\
\hline 8/24/10 0:00 & 11.111 & 4.819 & & 16.073 & 9.205 & & 9.540 & 22.535 & 22.949 & 37.318 \\
\hline $8 / 24 / 104: 00$ & 11.148 & 4.840 & & 15.986 & 9.139 & & 9.484 & 22.617 & 22.935 & 37.296 \\
\hline 8/24/10 8:00 & 11.191 & 4.864 & & 15.986 & 9.094 & & 9.493 & 22.673 & 22.921 & 37.273 \\
\hline 8/24/10 12:00 & 11.233 & 4.885 & & 16.031 & 9.063 & & 9.674 & 22.732 & 22.907 & 37.249 \\
\hline 8/24/10 16:00 & 11.257 & 4.904 & & 16.233 & 9.136 & & 9.679 & 22.822 & 22.893 & 37.228 \\
\hline 8/24/10 20:00 & 11.290 & 4.920 & & 16.166 & 9.165 & & 9.695 & 23.198 & 22.879 & 37.205 \\
\hline 8/25/10 0:00 & 11.323 & 4.934 & & 16.077 & 9.467 & & 9.714 & 22.991 & 22.865 & 37.183 \\
\hline 8/25/10 4:00 & 11.346 & 4.951 & & 16.094 & 9.488 & & 9.726 & 22.949 & 22.851 & 37.160 \\
\hline 8/25/10 8:00 & 11.377 & 4.963 & & 16.059 & 9.500 & & 9.742 & 22.970 & 22.839 & 37.141 \\
\hline $8 / 25 / 1012: 00$ & 11.403 & 4.979 & & 16.268 & 9.514 & & 9.754 & 23.010 & 22.827 & 37.117 \\
\hline $8 / 25 / 1016: 00$ & 11.415 & 4.993 & & 16.494 & 9.533 & & 9.754 & 23.040 & 54.131 & 68.757 \\
\hline 8/25/10 20:00 & 11.431 & 5.010 & & 16.392 & 9.568 & & 9.763 & 23.416 & 54.000 & 68.750 \\
\hline 8/26/10 0:00 & 11.455 & 5.022 & & 16.268 & 9.596 & & 9.782 & 23.141 & 53.920 & 68.748 \\
\hline 8/26/10 4:00 & 11.471 & 5.033 & & 16.243 & 9.618 & & 9.792 & 23.118 & 53.836 & 68.750 \\
\hline 8/26/10 8:00 & 11.495 & 5.045 & & 16.229 & 9.632 & & 9.803 & 23.144 & 53.751 & 68.748 \\
\hline 8/26/10 12:00 & 11.516 & 5.059 & & 16.375 & 9.644 & & 9.975 & 23.221 & 53.669 & 68.745 \\
\hline 8/26/10 16:00 & 11.521 & 5.071 & & 16.581 & 9.662 & & 9.963 & 23.174 & 53.587 & 68.745 \\
\hline 8/26/10 20:00 & 11.530 & 5.080 & & 16.485 & 9.693 & & 9.897 & 23.400 & 53.507 & 68.745 \\
\hline 8/27/10 0:00 & 11.547 & 5.097 & & 16.373 & 9.719 & & 9.850 & 23.280 & 53.427 & 68.746 \\
\hline 8/27/10 4:00 & 11.558 & 5.104 & & 16.366 & 9.738 & & 9.838 & 23.240 & 53.348 & 68.743 \\
\hline $8 / 27 / 108: 00$ & 11.577 & 5.116 & & 16.331 & 9.750 & & 9.864 & 23.264 & 53.270 & 68.745 \\
\hline $8 / 27 / 1012: 00$ & 11.591 & 5.125 & & 16.459 & 9.761 & & 10.015 & 23.287 & 53.198 & 68.746 \\
\hline 8/27/10 16:00 & 11.596 & 5.137 & & 16.669 & 9.780 & & 10.059 & 23.266 & 53.125 & 68.746 \\
\hline 8/27/10 20:00 & 11.608 & 5.149 & & 16.534 & 9.816 & & 9.932 & 23.252 & 53.057 & 68.746 \\
\hline 8/28/10 0:00 & 11.631 & 5.161 & & 16.399 & 9.846 & & 9.869 & 23.292 & 52.991 & 68.743 \\
\hline 8/28/10 4:00 & 11.643 & 5.170 & & 16.396 & 9.870 & & 9.876 & 23.369 & 52.923 & 68.746 \\
\hline 8/28/10 8:00 & 11.660 & 5.179 & & 16.378 & 9.886 & & 9.897 & 23.376 & 52.860 & 68.745 \\
\hline 8/28/10 12:00 & 11.676 & 5.191 & & 16.527 & 9.901 & & 9.909 & 23.398 & 52.797 & 68.743 \\
\hline 8/28/10 16:00 & 11.683 & 5.201 & & 16.725 & 9.924 & & 9.914 & 23.393 & 52.733 & 68.743 \\
\hline 8/28/10 20:00 & 11.702 & 5.212 & & 16.592 & 9.957 & & 9.928 & 23.384 & 52.665 & 68.743 \\
\hline 8/29/10 0:00 & 11.728 & 5.226 & & 16.441 & 9.995 & & 9.951 & 23.438 & 52.600 & 68.745 \\
\hline 8/29/10 4:00 & 11.747 & 5.236 & & 16.436 & 10.023 & & 9.965 & 23.513 & 52.532 & 68.745 \\
\hline 8/29/10 8:00 & 11.773 & 5.248 & & 16.427 & 10.044 & & 9.984 & 23.553 & 52.466 & 68.743 \\
\hline $8 / 29 / 1012: 00$ & 11.792 & 5.260 & & 16.583 & 10.063 & & 9.996 & 23.593 & 52.403 & 68.743 \\
\hline 8/29/10 16:00 & 11.806 & 5.269 & & 16.732 & 10.087 & & 10.005 & 23.673 & 52.337 & 68.743 \\
\hline 8/29/10 20:00 & 11.830 & 5.281 & & 16.632 & 10.122 & & 10.022 & 23.656 & 52.276 & 68.743 \\
\hline 8/30/10 0:00 & 11.858 & 5.292 & & 16.506 & 10.158 & & 10.050 & 23.715 & 52.213 & 68.743 \\
\hline 8/30/10 4:00 & 11.884 & 5.302 & & 16.485 & 10.188 & & 10.069 & 23.760 & 52.154 & 68.746 \\
\hline 8/30/10 8:00 & 11.910 & 5.311 & & 16.466 & 10.212 & & 10.090 & 23.851 & 52.095 & 68.743 \\
\hline 8/30/10 12:00 & 11.924 & 5.323 & & 16.646 & 10.231 & & 10.097 & 23.985 & 52.034 & 68.743 \\
\hline 8/30/10 16:00 & 11.931 & 5.332 & & 16.818 & 10.254 & & 10.087 & 23.884 & 51.976 & 68.743 \\
\hline 8/30/10 20:00 & 11.940 & 5.340 & & 16.741 & 10.283 & & 10.083 & 24.905 & 51.915 & 68.743 \\
\hline 8/31/10 0:00 & 11.957 & 5.349 & & 16.648 & 10.313 & & 10.092 & 24.167 & 51.858 & 68.745 \\
\hline 8/31/10 4:00 & 11.969 & 5.356 & & 16.634 & 10.339 & & 10.099 & 24.112 & 51.800 & 68.743 \\
\hline 8/31/10 8:00 & 11.983 & 5.363 & & 16.608 & 10.363 & & 10.106 & 24.068 & 51.739 & 68.743 \\
\hline $8 / 31 / 1012: 00$ & 11.995 & 5.370 & & 16.741 & 10.382 & & 10.113 & 24.268 & 51.678 & 68.743 \\
\hline 8/31/10 16:00 & 12.004 & 5.377 & & 16.902 & 10.405 & & 10.118 & 24.178 & 51.617 & 68.743 \\
\hline 8/31/10 20:00 & 12.023 & 5.384 & & 16.797 & 10.436 & & 10.134 & 24.065 & 51.556 & 68.746 \\
\hline 9/1/10 0:00 & 11.523 & 5.368 & & 16.620 & 10.313 & & 10.165 & 24.159 & 51.497 & 68.743 \\
\hline 9/1/10 4:00 & 10.802 & 5.099 & & 16.452 & 10.299 & & 10.071 & 24.087 & 51.439 & 68.746 \\
\hline 9/1/10 8:00 & 10.194 & 4.006 & & 16.382 & 10.243 & & 9.979 & 23.938 & 51.378 & 68.745 \\
\hline 9/1/10 12:00 & 10.465 & 3.782 & & 16.352 & 10.238 & & 9.895 & 23.826 & 51.319 & 68.743 \\
\hline 9/1/10 16:00 & 10.663 & 3.872 & & 16.520 & 10.356 & & 9.961 & 23.675 & 51.256 & 68.743 \\
\hline 9/1/10 20:00 & 10.826 & 4.032 & & 16.448 & 10.339 & & 9.820 & 23.583 & 51.195 & 68.743 \\
\hline 9/2/10 0:00 & 10.955 & 4.192 & & 16.389 & 10.318 & & 9.801 & 23.605 & 51.136 & 68.743 \\
\hline 9/2/10 4:00 & 10.670 & 4.157 & & 16.378 & 10.283 & & 9.740 & 23.553 & 51.075 & 68.743 \\
\hline 9/2/10 8:00 & 10.522 & 3.631 & & 16.350 & 10.283 & & 9.754 & 23.471 & 51.014 & 68.743 \\
\hline $9 / 2 / 1012: 00$ & 10.708 & 3.643 & & 16.368 & 10.276 & & 9.730 & 23.468 & 50.953 & 68.743 \\
\hline 9/2/10 16:00 & 10.873 & 3.789 & & 16.287 & 10.160 & & 9.601 & 23.546 & 50.892 & 68.746 \\
\hline 9/2/10 20:00 & 10.998 & 3.945 & & 16.287 & 10.111 & & 9.580 & 23.560 & 50.831 & 68.743 \\
\hline 9/3/10 0:00 & 11.111 & 4.088 & & 16.257 & 9.988 & & 9.535 & 23.619 & 50.770 & 68.746 \\
\hline
\end{tabular}

TABLE S1.1 Page 46 of 89 
Water Level (ft below reference point)

\begin{tabular}{|c|c|c|c|c|c|c|c|c|c|c|}
\hline Date and Time & McPike & Potter & Hughes & Barr & Burks & MoDOT & Clizer "Old" & Morgan & SB24 & SB38 \\
\hline 9/3/10 4:00 & 11.196 & 4.208 & & 16.285 & 9.943 & & 9.543 & 23.729 & 50.707 & 68.745 \\
\hline 9/3/10 8:00 & 11.264 & 4.307 & & 16.296 & 9.910 & & 9.601 & 23.722 & 50.641 & 68.746 \\
\hline 9/3/10 12:00 & 11.321 & 4.397 & & 16.471 & 9.962 & & 9.752 & 23.727 & 50.578 & 68.746 \\
\hline 9/3/10 16:00 & 11.365 & 4.477 & & 16.650 & 10.040 & & 9.789 & 23.706 & 50.512 & 68.746 \\
\hline $9 / 3 / 1020: 00$ & 11.405 & 4.550 & & 16.604 & 10.096 & & 9.702 & 23.771 & 50.451 & 68.743 \\
\hline 9/4/10 0:00 & 11.453 & 4.611 & & 16.532 & 10.068 & & 9.683 & 23.762 & 50.390 & 68.743 \\
\hline 9/4/10 4:00 & 11.493 & 4.659 & & 16.529 & 10.066 & & 9.705 & 23.769 & 50.329 & 68.746 \\
\hline 9/4/10 8:00 & 11.526 & 4.699 & & 16.548 & 10.049 & & 9.775 & 23.783 & 50.271 & 68.745 \\
\hline 9/4/10 12:00 & 11.554 & 4.736 & & 16.685 & 10.113 & & 9.989 & 24.037 & 50.214 & 68.746 \\
\hline 9/4/10 16:00 & 11.549 & 4.765 & & 16.900 & 10.269 & & 10.050 & 23.835 & 50.156 & 68.746 \\
\hline 9/4/10 20:00 & 11.559 & 4.793 & & 16.813 & 10.351 & & 9.935 & 23.736 & 50.097 & 68.743 \\
\hline 9/5/10 0:00 & 11.577 & 4.821 & & 16.725 & 10.332 & & 9.914 & 23.729 & 50.038 & 68.746 \\
\hline 9/5/10 4:00 & 11.585 & 4.845 & & 16.737 & 10.363 & & 9.881 & 23.713 & 49.980 & 68.746 \\
\hline 9/5/10 8:00 & 11.594 & 4.864 & & 16.737 & 10.368 & & 9.876 & 23.701 & 49.919 & 68.746 \\
\hline 9/5/10 12:00 & 11.603 & 4.885 & & 16.832 & 10.450 & & 9.876 & 23.689 & 49.855 & 68.746 \\
\hline $9 / 5 / 1016: 00$ & 11.601 & 4.899 & & 16.928 & 10.603 & & 9.862 & 23.689 & 49.797 & 68.743 \\
\hline 9/5/10 20:00 & 11.618 & 4.920 & & 16.820 & 10.648 & & 9.869 & 23.635 & 49.736 & 68.746 \\
\hline 9/6/10 0:00 & 11.653 & 4.946 & & 16.734 & 10.599 & & 9.900 & 23.732 & 49.680 & 68.746 \\
\hline 9/6/10 4:00 & 11.669 & 4.960 & & 16.767 & 10.641 & & 9.911 & 23.670 & 49.621 & 68.746 \\
\hline 9/6/10 8:00 & 11.686 & 4.979 & & 16.762 & 10.637 & & 9.916 & 23.682 & 49.562 & 68.745 \\
\hline 9/6/10 12:00 & 11.712 & 4.995 & & 16.874 & 10.677 & & 9.932 & 23.774 & 49.504 & 68.746 \\
\hline 9/6/10 16:00 & 11.745 & 5.012 & & 16.888 & 10.712 & & 9.961 & 23.757 & 49.443 & 68.746 \\
\hline 9/6/10 20:00 & 11.830 & 5.045 & & 16.578 & 10.500 & & 10.033 & 23.903 & 49.377 & 68.743 \\
\hline 9/7/10 0:00 & 11.905 & 5.071 & & 16.643 & 10.328 & & 10.109 & 24.150 & 49.304 & 68.746 \\
\hline 9/7/10 4:00 & 11.950 & 5.087 & & 16.669 & 10.620 & & 10.146 & 24.204 & 49.222 & 68.746 \\
\hline 9/7/10 8:00 & 11.995 & 5.104 & & 16.650 & 10.639 & & 10.179 & 24.272 & 49.142 & 68.743 \\
\hline 9/7/10 12:00 & 12.035 & 5.120 & & 16.790 & 10.658 & & 10.207 & 24.540 & 49.060 & 68.745 \\
\hline $9 / 7 / 1016: 00$ & 12.056 & 5.135 & & 17.000 & 10.677 & & 10.214 & 24.446 & 48.969 & 68.746 \\
\hline 9/7/10 20:00 & 12.082 & 5.144 & & 16.930 & 10.703 & & 10.228 & 24.439 & 48.882 & 68.746 \\
\hline 9/8/10 0:00 & 12.103 & 5.160 & & 16.860 & 10.726 & & 10.243 & 24.477 & 48.795 & 68.748 \\
\hline 9/8/10 4:00 & 12.120 & 5.168 & & 16.860 & 10.747 & & 10.250 & 24.493 & 48.711 & 68.748 \\
\hline 9/8/10 8:00 & 12.141 & 5.177 & & 16.837 & 10.766 & & 10.259 & 24.573 & 48.626 & 68.745 \\
\hline $9 / 8 / 1012: 00$ & 12.157 & 5.189 & & 16.986 & 10.783 & & 10.261 & 24.583 & 48.537 & 68.746 \\
\hline 9/8/10 16:00 & 12.157 & 5.198 & & 17.142 & 10.797 & & 10.245 & 24.587 & 48.446 & 68.746 \\
\hline 9/8/10 20:00 & 12.164 & 5.210 & & 17.093 & 10.823 & & 10.243 & 24.705 & 48.359 & 68.746 \\
\hline 9/9/10 0:00 & 12.181 & 5.222 & & 17.016 & 10.844 & & 10.254 & 24.604 & 48.275 & 68.746 \\
\hline 9/9/10 4:00 & 12.181 & 5.231 & & 17.058 & 10.865 & & 10.247 & 24.571 & 48.183 & 68.746 \\
\hline 9/9/10 8:00 & 12.188 & 5.236 & & 17.053 & 10.882 & & 10.245 & 24.616 & 48.096 & 68.746 \\
\hline 9/9/10 12:00 & 12.195 & 5.248 & & 17.137 & 10.896 & & 10.243 & 24.597 & 48.007 & 68.746 \\
\hline 9/9/10 16:00 & 12.193 & 5.252 & & 17.200 & 10.910 & & 10.231 & 24.552 & 47.920 & 68.745 \\
\hline 9/9/10 20:00 & 12.202 & 5.264 & & 17.114 & 10.929 & & 10.243 & 24.585 & 47.836 & 68.746 \\
\hline 9/10/10 0:00 & 12.219 & 5.276 & & 17.060 & 10.948 & & 10.259 & 24.594 & 47.752 & 68.746 \\
\hline 9/10/10 4:00 & 12.226 & 5.283 & & 17.063 & 10.964 & & 10.266 & 24.620 & 47.667 & 68.743 \\
\hline 9/10/10 8:00 & 12.235 & 5.288 & & 17.065 & 10.976 & & 10.268 & 24.623 & 47.585 & 68.743 \\
\hline 9/10/10 12:00 & 12.242 & 5.299 & & 17.109 & 10.988 & & 10.273 & 24.674 & 47.501 & 68.746 \\
\hline 9/10/10 16:00 & 12.240 & 5.304 & & 17.186 & 11.047 & & 10.264 & 24.644 & 47.412 & 68.748 \\
\hline 9/10/10 20:00 & 12.256 & 5.311 & & 17.149 & 11.021 & & 10.278 & 24.663 & 47.329 & 68.746 \\
\hline 9/11/10 0:00 & 12.287 & 5.325 & & 17.014 & 10.927 & & 10.318 & 24.820 & 47.252 & 68.745 \\
\hline 9/11/10 4:00 & 12.320 & 5.342 & & 16.949 & 10.823 & & 10.355 & 24.884 & 47.179 & 68.746 \\
\hline 9/11/10 8:00 & 12.351 & 5.354 & & 16.923 & 10.745 & & 10.386 & 24.947 & 47.100 & 68.745 \\
\hline 9/11/10 12:00 & 12.379 & 5.361 & & 17.051 & 10.721 & & 10.412 & 25.048 & 47.022 & 68.745 \\
\hline 9/11/10 16:00 & 12.395 & 5.370 & & 17.247 & 10.783 & & 10.416 & 25.067 & 46.938 & 68.745 \\
\hline 9/11/10 20:00 & 12.419 & 5.380 & & 17.161 & 10.785 & & 10.437 & 25.107 & 46.856 & 68.746 \\
\hline 9/12/10 0:00 & 12.442 & 5.389 & & 17.074 & 10.754 & & 10.456 & 25.164 & 46.776 & 68.746 \\
\hline 9/12/10 4:00 & 12.461 & 5.396 & & 17.042 & 10.757 & & 10.468 & 25.201 & 46.699 & 68.748 \\
\hline 9/12/10 8:00 & 12.483 & 5.403 & & 17.018 & 10.731 & & 10.484 & 25.251 & 46.619 & 68.748 \\
\hline $9 / 12 / 1012: 00$ & 12.501 & 5.408 & & 17.189 & 10.820 & & 10.496 & 25.293 & 46.539 & 68.746 \\
\hline 9/12/10 16:00 & 12.508 & 5.417 & & 17.366 & 10.957 & & 10.484 & 25.283 & 46.452 & 68.741 \\
\hline 9/12/10 20:00 & 12.523 & 5.427 & & 17.284 & 11.030 & & 10.492 & 25.283 & 46.373 & 68.463 \\
\hline 9/13/10 0:00 & 12.539 & 5.436 & & 17.168 & 11.002 & & 10.513 & 25.328 & 46.293 & 68.185 \\
\hline 9/13/10 4:00 & 12.556 & 5.438 & & 17.149 & 11.023 & & 10.522 & 25.340 & 46.211 & 67.912 \\
\hline 9/13/10 8:00 & 12.574 & 5.446 & & 17.151 & 11.011 & & 10.543 & 25.472 & 46.133 & 67.759 \\
\hline 9/13/10 12:00 & 12.589 & 5.448 & & 17.319 & 11.752 & & 10.541 & 25.450 & 46.054 & 67.613 \\
\hline 9/13/10 16:00 & 12.593 & 5.453 & & 17.468 & 12.153 & & 10.515 & 25.389 & 45.969 & 67.471 \\
\hline
\end{tabular}

TABLE S1.1 Page 47 of 89 
Water Level (ft below reference point)

\begin{tabular}{|c|c|c|c|c|c|c|c|c|c|c|}
\hline Date and Time & McPike & Potter & Hughes & Barr & Burks & MoDOT & Clizer "Old" & Morgan & SB24 & SB38 \\
\hline 9/13/10 20:00 & 12.605 & 5.457 & & 17.354 & 12.151 & & 10.567 & 25.417 & 45.889 & 67.327 \\
\hline 9/14/10 0:00 & 12.615 & 5.464 & & 17.168 & 12.061 & & 10.520 & 25.450 & 45.814 & 67.184 \\
\hline 9/14/10 4:00 & 12.605 & 5.464 & & 17.200 & 12.037 & & 10.588 & 25.460 & 45.735 & 67.047 \\
\hline 9/14/10 8:00 & 12.619 & 5.471 & & 17.116 & 11.969 & & 10.513 & 25.443 & 45.657 & 66.908 \\
\hline $9 / 14 / 1012: 00$ & 12.629 & 5.479 & & 17.168 & 11.952 & & 10.602 & 25.474 & 45.580 & 66.769 \\
\hline 9/14/10 16:00 & 12.626 & 5.481 & & 17.338 & 12.018 & & 10.614 & 25.502 & 45.498 & 66.630 \\
\hline 9/14/10 20:00 & 12.636 & 5.490 & & 17.258 & 12.014 & & 10.510 & 25.462 & 45.416 & 66.494 \\
\hline 9/15/10 0:00 & 12.648 & 5.497 & & 17.186 & 11.964 & & 10.508 & 25.483 & 45.338 & 66.355 \\
\hline 9/15/10 4:00 & 12.650 & 5.500 & & 17.210 & 11.995 & & 10.529 & 25.486 & 45.256 & 66.218 \\
\hline 9/15/10 8:00 & 12.655 & 5.502 & & 17.233 & 11.995 & & 10.595 & 25.488 & 45.176 & 66.084 \\
\hline 9/15/10 12:00 & 12.652 & 5.495 & & 17.405 & 12.085 & & 10.585 & 25.427 & 45.087 & 65.950 \\
\hline 9/15/10 16:00 & 12.657 & 5.500 & & 17.335 & 12.054 & & 10.632 & 25.441 & 45.012 & 65.813 \\
\hline 9/15/10 20:00 & 12.666 & 5.504 & & 17.331 & 12.077 & & 10.614 & 25.439 & 44.930 & 65.678 \\
\hline 9/16/10 0:00 & 12.683 & 5.511 & & 17.244 & 12.007 & & 10.571 & 25.483 & 44.855 & 65.547 \\
\hline 9/16/10 4:00 & 12.699 & 5.516 & & 17.168 & 11.962 & & 10.494 & 25.530 & 44.780 & 65.415 \\
\hline 9/16/10 8:00 & 12.723 & 5.521 & & 17.170 & 11.898 & & 10.461 & 25.594 & 44.707 & 65.278 \\
\hline $9 / 16 / 10$ 12:00 & 12.749 & 5.528 & & 17.179 & 11.990 & & 10.513 & 25.678 & 44.635 & 65.146 \\
\hline 9/16/10 16:00 & 12.763 & 5.533 & & 17.324 & 12.134 & & 10.649 & 25.707 & 44.557 & 65.014 \\
\hline 9/16/10 20:00 & 12.772 & 5.535 & & 17.382 & 12.169 & & 10.618 & 25.688 & 44.475 & 64.882 \\
\hline 9/17/10 0:00 & 12.784 & 5.540 & & 17.328 & 12.139 & & 10.583 & 25.704 & 44.398 & 64.751 \\
\hline 9/17/10 4:00 & 12.796 & 5.542 & & 17.335 & 12.129 & & 10.611 & 25.723 & 44.320 & 64.618 \\
\hline 9/17/10 8:00 & 12.812 & 5.547 & & 17.319 & 12.297 & & 10.616 & 25.801 & 44.243 & 64.489 \\
\hline 9/17/10 12:00 & 12.824 & 5.547 & & 17.403 & 12.351 & & 10.745 & 25.805 & 44.168 & 64.360 \\
\hline 9/17/10 16:00 & 12.829 & 5.552 & & 17.550 & 12.547 & & 10.783 & 25.768 & 44.086 & 64.230 \\
\hline 9/17/10 20:00 & 12.841 & 5.554 & & 17.470 & 12.554 & & 10.703 & 25.768 & 44.011 & 64.100 \\
\hline 9/18/10 0:00 & 12.860 & 5.561 & & 17.370 & 12.490 & & 10.694 & 25.899 & 43.936 & 63.973 \\
\hline 9/18/10 4:00 & 12.871 & 5.563 & & 17.363 & 12.483 & & 10.703 & 25.881 & 43.858 & 63.846 \\
\hline 9/18/10 8:00 & 12.893 & 5.570 & & 17.352 & 12.417 & & 10.679 & 25.916 & 43.783 & 63.721 \\
\hline $9 / 18 / 1012: 00$ & 12.914 & 5.575 & & 17.424 & 12.408 & & 10.813 & 26.309 & 43.708 & 63.594 \\
\hline 9/18/10 16:00 & 12.926 & 5.580 & & 17.515 & 12.450 & & 10.773 & 26.139 & 43.633 & 63.467 \\
\hline 9/18/10 20:00 & 12.942 & 5.585 & & 17.438 & 12.431 & & 10.691 & 26.090 & 43.556 & 63.342 \\
\hline 9/19/10 0:00 & 12.956 & 5.589 & & 17.401 & 12.401 & & 10.722 & 26.076 & 43.483 & 63.217 \\
\hline 9/19/10 4:00 & 12.966 & 5.592 & & 17.424 & 12.443 & & 10.672 & 26.055 & 43.406 & 63.092 \\
\hline 9/19/10 8:00 & 12.982 & 5.596 & & 17.419 & 12.438 & & 10.722 & 26.163 & 43.331 & 62.970 \\
\hline 9/19/10 12:00 & 12.999 & 5.603 & & 17.484 & 12.459 & & 10.834 & 26.153 & 43.255 & 62.847 \\
\hline 9/19/10 16:00 & 13.006 & 5.601 & & 17.669 & 12.582 & & 10.914 & 26.142 & 43.176 & 62.725 \\
\hline 9/19/10 20:00 & 13.018 & 5.608 & & 17.606 & 12.613 & & 10.783 & 26.121 & 43.101 & 62.600 \\
\hline 9/20/10 0:00 & 13.030 & 5.610 & & 17.519 & 12.537 & & 10.804 & 26.137 & 43.028 & 62.480 \\
\hline 9/20/10 4:00 & 13.034 & 5.613 & & 17.573 & 12.636 & & 10.860 & 26.191 & 42.953 & 62.360 \\
\hline 9/20/10 8:00 & 13.046 & 5.617 & & 17.559 & 12.646 & & 10.905 & 26.163 & 42.880 & 62.237 \\
\hline $9 / 20 / 1012: 00$ & 13.058 & 5.622 & & 17.682 & 12.724 & & 11.060 & 26.158 & 42.805 & 62.117 \\
\hline 9/20/10 16:00 & 13.058 & 5.620 & & 17.699 & 12.872 & & 11.046 & 26.160 & 42.728 & 61.997 \\
\hline 9/20/10 20:00 & 13.065 & 5.622 & & 17.636 & 12.929 & & 10.964 & 26.111 & 42.650 & 61.877 \\
\hline 9/21/10 0:00 & 13.079 & 5.629 & & 17.545 & 12.891 & & 10.919 & 26.128 & 42.580 & 61.759 \\
\hline 9/21/10 4:00 & 13.091 & 5.634 & & 17.524 & 12.872 & & 10.844 & 26.146 & 42.507 & 61.641 \\
\hline 9/21/10 8:00 & 13.105 & 5.639 & & 17.522 & 12.867 & & 10.860 & 26.233 & 42.437 & 61.523 \\
\hline 9/21/10 12:00 & 13.119 & 5.641 & & 17.669 & 12.926 & & 10.860 & 26.198 & 42.364 & 61.408 \\
\hline 9/21/10 16:00 & 13.129 & 5.648 & & 17.669 & 12.967 & & 10.870 & 26.273 & 42.292 & 61.288 \\
\hline 9/21/10 20:00 & 11.703 & 4.727 & & 17.354 & 12.636 & & 10.550 & 26.231 & 42.221 & 61.165 \\
\hline 9/22/10 0:00 & 11.024 & 3.794 & & 17.223 & 12.575 & & 10.452 & 25.975 & 42.148 & 61.048 \\
\hline 9/22/10 4:00 & 11.269 & 3.853 & & 17.182 & 12.514 & & 10.393 & 25.775 & 42.071 & 60.930 \\
\hline 9/22/10 8:00 & 11.483 & 4.055 & & 17.161 & 12.523 & & 10.358 & 25.730 & 41.994 & 60.817 \\
\hline $9 / 22 / 1012: 00$ & 11.625 & 4.255 & & 17.212 & 12.507 & & 10.334 & 25.610 & 41.919 & 60.701 \\
\hline 9/22/10 16:00 & 11.714 & 4.425 & & 17.403 & 12.610 & & 10.292 & 25.450 & 41.837 & 60.586 \\
\hline 9/22/10 20:00 & 11.790 & 4.557 & & 17.382 & 12.667 & & 10.264 & 25.413 & 41.759 & 60.470 \\
\hline 9/23/10 0:00 & 11.863 & 4.665 & & 17.317 & 12.641 & & 10.261 & 25.349 & 41.684 & 60.355 \\
\hline 9/23/10 4:00 & 11.914 & 4.746 & & 17.317 & 12.665 & & 10.254 & 25.286 & 41.607 & 60.242 \\
\hline 9/23/10 8:00 & 11.957 & 4.809 & & 17.324 & 12.665 & & 10.250 & 25.241 & 41.532 & 60.127 \\
\hline 9/23/10 12:00 & 11.990 & 4.861 & & 17.405 & 12.717 & & 10.243 & 25.265 & 41.452 & 60.013 \\
\hline 9/23/10 16:00 & 12.011 & 4.899 & & 17.470 & 12.764 & & 10.224 & 25.161 & 41.374 & 59.900 \\
\hline 9/23/10 20:00 & 11.038 & 4.486 & & 17.240 & 12.667 & & 10.146 & 25.126 & 41.299 & 59.783 \\
\hline 9/24/10 0:00 & 9.433 & 3.334 & & 16.928 & 12.551 & & 10.036 & 25.053 & 41.229 & 59.662 \\
\hline 9/24/10 4:00 & 9.499 & 3.047 & & 16.860 & 12.360 & & 9.987 & 24.957 & 41.154 & 59.554 \\
\hline $9 / 24 / 108: 00$ & 9.855 & 3.207 & & 16.795 & 12.176 & & 9.965 & 24.942 & 41.084 & 59.443 \\
\hline
\end{tabular}

TABLE S1.1 Page 48 of 89 
Water Level (ft below reference point)

\begin{tabular}{|c|c|c|c|c|c|c|c|c|c|c|}
\hline Date and Time & McPike & Potter & Hughes & Barr & Burks & MoDOT & Clizer "Old" & Morgan & SB24 & SB38 \\
\hline 9/24/10 12:00 & 10.156 & 3.438 & & 16.837 & 12.153 & & 9.949 & 24.867 & 41.006 & 59.335 \\
\hline 9/24/10 16:00 & 10.373 & 3.662 & & 17.014 & 12.217 & & 9.921 & 24.761 & 40.929 & 59.220 \\
\hline 9/24/10 20:00 & 10.552 & 3.867 & & 16.958 & 12.214 & & 9.902 & 24.674 & 40.849 & 59.109 \\
\hline 9/25/10 0:00 & 10.703 & 4.025 & & 16.902 & 12.146 & & 9.895 & 24.684 & 40.774 & 58.998 \\
\hline $9 / 25 / 104: 00$ & 10.809 & 4.149 & & 16.944 & 12.158 & & 9.883 & 24.578 & 40.692 & 58.888 \\
\hline 9/25/10 8:00 & 10.882 & 4.251 & & 16.997 & 12.136 & & 9.867 & 24.517 & 40.615 & 58.779 \\
\hline 9/25/10 12:00 & 10.951 & 4.336 & & 17.023 & 12.101 & & 9.857 & 24.444 & 40.535 & 58.668 \\
\hline 9/25/10 16:00 & 11.026 & 4.409 & & 16.988 & 11.995 & & 9.855 & 24.463 & 40.460 & 58.558 \\
\hline 9/25/10 20:00 & 11.087 & 4.475 & & 16.981 & 11.969 & & 9.855 & 24.406 & 40.380 & 58.445 \\
\hline 9/26/10 0:00 & 11.149 & 4.536 & & 16.932 & 11.827 & & 9.867 & 24.392 & 40.303 & 58.334 \\
\hline 9/26/10 4:00 & 11.193 & 4.583 & & 16.977 & 11.794 & & 9.867 & 24.366 & 40.223 & 58.233 \\
\hline 9/26/10 8:00 & 11.243 & 4.630 & & 16.958 & 11.679 & & 9.876 & 24.418 & 40.148 & 58.124 \\
\hline 9/26/10 12:00 & 11.281 & 4.665 & & 17.063 & 11.665 & & 9.876 & 24.376 & 40.068 & 58.016 \\
\hline 9/26/10 16:00 & 11.290 & 4.696 & & 17.221 & 11.726 & & 9.855 & 24.293 & 39.986 & 57.908 \\
\hline 9/26/10 20:00 & 11.314 & 4.727 & & 17.161 & 11.707 & & 9.846 & 24.242 & 39.909 & 57.799 \\
\hline 9/27/10 0:00 & 11.335 & 4.760 & & 17.140 & 11.665 & & 9.841 & 24.279 & 39.829 & 57.696 \\
\hline $9 / 27 / 104: 00$ & 11.349 & 4.781 & & 17.128 & 11.622 & & 9.834 & 24.204 & 39.749 & 57.587 \\
\hline 9/27/10 8:00 & 11.368 & 4.804 & & 17.114 & 11.547 & & 9.831 & 24.181 & 39.672 & 57.484 \\
\hline 9/27/10 12:00 & 11.380 & 4.826 & & 17.172 & 11.521 & & 9.824 & 24.538 & 39.597 & 57.378 \\
\hline 9/27/10 16:00 & 11.373 & 4.845 & & 17.310 & 11.599 & & 9.801 & 24.218 & 39.515 & 57.272 \\
\hline 9/27/10 20:00 & 11.375 & 4.863 & & 17.272 & 11.592 & & 9.785 & 24.072 & 39.435 & 57.168 \\
\hline 9/28/10 0:00 & 11.375 & 4.878 & & 17.237 & 11.542 & & 9.773 & 24.091 & 39.357 & 57.064 \\
\hline 9/28/10 4:00 & 11.396 & 4.896 & & 17.186 & 11.478 & & 9.780 & 24.030 & 39.280 & 56.961 \\
\hline 9/28/10 8:00 & 11.429 & 4.915 & & 17.119 & 11.358 & & 9.799 & 24.037 & 39.210 & 56.855 \\
\hline 9/28/10 12:00 & 11.460 & 4.936 & & 17.179 & 11.318 & & 9.815 & 24.061 & 39.135 & 56.751 \\
\hline 9/28/10 16:00 & 11.469 & 4.953 & & 17.359 & 11.365 & & 9.810 & 24.096 & 39.055 & 56.650 \\
\hline 9/28/10 20:00 & 11.490 & 4.972 & & 17.277 & 11.320 & & 9.815 & 24.047 & 38.982 & 56.548 \\
\hline 9/29/10 0:00 & 11.512 & 4.986 & & 17.212 & 11.252 & & 9.827 & 24.047 & 38.909 & 56.447 \\
\hline $9 / 29 / 104: 00$ & 11.523 & 4.998 & & 17.212 & 11.224 & & 9.831 & 24.068 & 38.832 & 56.344 \\
\hline 9/29/10 8:00 & 11.542 & 5.012 & & 17.177 & 11.191 & & 9.843 & 24.075 & 38.762 & 56.242 \\
\hline 9/29/10 12:00 & 11.556 & 5.026 & & 17.254 & 11.165 & & 9.841 & 24.087 & 38.684 & 56.143 \\
\hline 9/29/10 16:00 & 11.561 & 5.035 & & 17.384 & 11.139 & & 9.827 & 24.054 & 38.609 & 56.042 \\
\hline 9/29/10 20:00 & 11.589 & 5.052 & & 17.298 & 11.118 & & 9.843 & 24.072 & 38.537 & 55.943 \\
\hline 9/30/10 0:00 & 11.644 & 5.075 & & 17.165 & 11.099 & & 9.883 & 24.164 & 38.471 & 55.844 \\
\hline 9/30/10 4:00 & 11.688 & 5.092 & & 17.214 & 11.082 & & 9.918 & 24.242 & 38.403 & 55.745 \\
\hline 9/30/10 8:00 & 11.731 & 5.104 & & 17.198 & 11.063 & & 9.951 & 24.317 & 38.335 & 55.646 \\
\hline 9/30/10 12:00 & 11.769 & 5.118 & & 17.263 & 11.044 & & 9.979 & 24.383 & 38.267 & 55.547 \\
\hline 9/30/10 16:00 & 11.785 & 5.130 & & 17.480 & 11.028 & & 9.984 & 24.385 & 38.194 & 55.451 \\
\hline 9/30/10 20:00 & 11.811 & 5.144 & & 17.405 & 11.016 & & 9.998 & 24.406 & 38.124 & 55.354 \\
\hline $10 / 1 / 100: 00$ & 11.837 & 5.158 & & 17.328 & 11.004 & & 10.019 & 24.451 & 38.058 & 55.255 \\
\hline $10 / 1 / 104: 00$ & 11.858 & 5.167 & & 17.328 & 10.993 & & 10.031 & 24.482 & 37.990 & 55.161 \\
\hline 10/1/10 8:00 & 11.879 & 5.174 & & 17.321 & 10.981 & & 10.045 & 24.512 & 37.922 & 55.064 \\
\hline 10/1/10 12:00 & 11.893 & 5.182 & & 17.394 & 10.964 & & 10.052 & 24.536 & 37.852 & 54.968 \\
\hline $10 / 1 / 1016: 00$ & 11.884 & 5.191 & & 17.603 & 10.955 & & 10.038 & 24.491 & 37.781 & 54.871 \\
\hline $10 / 1 / 10$ 20:00 & 11.893 & 5.198 & & 17.543 & 10.948 & & 10.034 & 24.832 & 37.713 & 54.777 \\
\hline 10/2/10 0:00 & 11.901 & 5.203 & & 17.473 & 10.941 & & 10.036 & 24.547 & 37.645 & 54.681 \\
\hline $10 / 2 / 104: 00$ & 11.936 & 5.217 & & 17.375 & 10.934 & & 10.066 & 24.557 & 37.582 & 54.591 \\
\hline 10/2/10 8:00 & 11.988 & 5.233 & & 17.347 & 10.927 & & 10.116 & 24.724 & 37.521 & 54.494 \\
\hline 10/2/10 12:00 & 12.025 & 5.252 & & 17.396 & 10.920 & & 10.156 & 24.759 & 37.458 & 54.402 \\
\hline 10/2/10 16:00 & 12.042 & 5.262 & & 17.585 & 10.912 & & 10.163 & 24.761 & 37.390 & 54.308 \\
\hline $10 / 2 / 1020: 00$ & 12.061 & 5.273 & & 17.517 & 10.908 & & 10.177 & 24.790 & 37.329 & 54.219 \\
\hline 10/3/10 0:00 & 12.084 & 5.283 & & 17.470 & 10.903 & & 10.193 & 24.816 & 37.265 & 54.127 \\
\hline $10 / 3 / 104: 00$ & 12.103 & 5.292 & & 17.463 & 10.894 & & 10.207 & 24.834 & 37.202 & 54.037 \\
\hline 10/3/10 8:00 & 12.127 & 5.302 & & 17.445 & 10.886 & & 10.226 & 24.870 & 37.141 & 53.943 \\
\hline 10/3/10 12:00 & 12.143 & 5.311 & & 17.531 & 10.877 & & 10.240 & 24.938 & 37.057 & 53.851 \\
\hline $10 / 3 / 1016: 00$ & 12.134 & 5.316 & & 17.731 & 10.870 & & 10.219 & 24.893 & 36.986 & 53.762 \\
\hline $10 / 3 / 10$ 20:00 & 12.139 & 5.323 & & 17.678 & 10.863 & & 10.212 & 24.872 & 36.921 & 53.670 \\
\hline 10/4/10 0:00 & 12.153 & 5.330 & & 17.627 & 10.858 & & 10.224 & 24.895 & 36.857 & 53.585 \\
\hline 10/4/10 4:00 & 12.165 & 5.337 & & 17.627 & 10.853 & & 10.236 & 24.912 & 36.794 & 53.496 \\
\hline 10/4/10 8:00 & 12.181 & 5.344 & & 17.608 & 10.849 & & 10.252 & 24.940 & 36.731 & 53.406 \\
\hline $10 / 4 / 1012: 00$ & 12.195 & 5.356 & & 17.641 & 10.844 & & 10.264 & 24.971 & 36.670 & 53.319 \\
\hline 10/4/10 16:00 & 12.186 & 5.358 & & 17.825 & 10.839 & & 10.245 & 25.462 & 36.604 & 53.229 \\
\hline 10/4/10 20:00 & 12.195 & 5.368 & & 17.759 & 10.837 & & 10.245 & 25.055 & 36.545 & 53.142 \\
\hline $10 / 5 / 100: 00$ & 12.212 & 5.377 & & 17.682 & 10.837 & & 10.261 & 25.004 & 36.484 & 53.055 \\
\hline
\end{tabular}

TABLE S1.1 Page 49 of 89 
Water Level (ft below reference point)

\begin{tabular}{|c|c|c|c|c|c|c|c|c|c|c|}
\hline Date and Time & McPike & Potter & Hughes & Barr & Burks & MoDOT & Clizer "Old" & Morgan & SB24 & SB38 \\
\hline 10/5/10 4:00 & 12.226 & 5.384 & & 17.669 & 10.835 & & 10.275 & 24.992 & 36.426 & 52.973 \\
\hline 10/5/10 8:00 & 12.242 & 5.391 & & 17.643 & 10.832 & & 10.294 & 25.006 & 36.365 & 52.883 \\
\hline 10/5/10 12:00 & 12.259 & 5.401 & & 17.682 & 10.830 & & 10.311 & 25.107 & 36.306 & 52.801 \\
\hline 10/5/10 16:00 & 12.256 & 5.405 & & 17.897 & 10.830 & & 10.297 & 25.037 & 36.243 & 52.714 \\
\hline $10 / 5 / 1020: 00$ & 12.268 & 5.410 & & 17.825 & 10.830 & & 10.301 & 25.008 & 36.184 & 52.631 \\
\hline 10/6/10 0:00 & 12.280 & 5.419 & & 17.766 & 10.832 & & 10.315 & 25.018 & 36.125 & 52.544 \\
\hline 10/6/10 4:00 & 12.282 & 5.424 & & 17.755 & 10.835 & & 10.313 & 25.041 & 36.067 & 52.459 \\
\hline 10/6/10 8:00 & 12.292 & 5.429 & & 17.738 & 10.832 & & 10.320 & 25.044 & 36.008 & 52.377 \\
\hline 10/6/10 12:00 & 12.294 & 5.434 & & 17.825 & 10.830 & & 10.318 & 25.039 & 35.950 & 52.292 \\
\hline 10/6/10 16:00 & 12.289 & 5.436 & & 17.913 & 10.835 & & 10.304 & 24.992 & 35.889 & 52.210 \\
\hline 10/6/10 20:00 & 12.306 & 5.443 & & 17.839 & 11.358 & & 10.315 & 24.992 & 35.832 & 52.127 \\
\hline 10/7/10 0:00 & 12.327 & 5.450 & & 17.752 & 11.299 & & 10.344 & 25.034 & 35.778 & 52.047 \\
\hline 10/7/10 4:00 & 12.346 & 5.457 & & 17.699 & 11.259 & & 10.367 & 25.079 & 35.722 & 51.962 \\
\hline 10/7/10 8:00 & 12.374 & 5.467 & & 17.687 & 11.226 & & 10.398 & 25.128 & 35.666 & 51.884 \\
\hline 10/7/10 12:00 & 12.395 & 5.476 & & 17.764 & 11.198 & & 10.419 & 25.171 & 35.612 & 51.800 \\
\hline 10/7/10 16:00 & 12.398 & 5.481 & & 17.953 & 11.177 & & 10.405 & 25.211 & 35.553 & 51.720 \\
\hline $10 / 7 / 1020: 00$ & 12.407 & 5.488 & & 17.890 & 11.158 & & 10.405 & 25.168 & 35.499 & 51.637 \\
\hline 10/8/10 0:00 & 12.419 & 5.492 & & 17.850 & 11.146 & & 10.416 & 25.161 & 35.443 & 51.562 \\
\hline 10/8/10 4:00 & 12.421 & 5.497 & & 17.867 & 11.132 & & 10.416 & 25.147 & 35.389 & 51.482 \\
\hline 10/8/10 8:00 & 12.433 & 5.499 & & 17.848 & 11.120 & & 10.426 & 25.154 & 35.333 & 51.402 \\
\hline 10/8/10 12:00 & 12.443 & 5.502 & & 17.904 & 11.108 & & 10.435 & 25.222 & 35.279 & 51.321 \\
\hline 10/8/10 16:00 & 12.445 & 5.509 & & 18.034 & 11.101 & & 10.423 & 25.166 & 35.223 & 51.244 \\
\hline 10/8/10 20:00 & 12.457 & 5.514 & & 17.950 & 11.101 & & 10.435 & 25.156 & 35.171 & 51.168 \\
\hline 10/9/10 0:00 & 12.476 & 5.523 & & 17.867 & 11.101 & & 10.459 & 25.187 & 35.119 & 51.088 \\
\hline 10/9/10 4:00 & 12.490 & 5.528 & & 17.836 & 11.101 & & 10.475 & 25.281 & 35.065 & 51.013 \\
\hline 10/9/10 8:00 & 12.504 & 5.532 & & 17.848 & 11.099 & & 10.489 & 25.269 & 35.011 & 50.935 \\
\hline 10/9/10 12:00 & 12.518 & 5.540 & & 17.895 & 11.099 & & 10.501 & 25.276 & 34.962 & 50.860 \\
\hline $10 / 9 / 1016: 00$ & 12.527 & 5.544 & & 18.086 & 11.099 & & 10.501 & 25.255 & 34.908 & 50.784 \\
\hline $10 / 9 / 1020: 00$ & 12.544 & 5.554 & & 17.962 & 11.103 & & 10.515 & 25.265 & 34.857 & 50.709 \\
\hline 10/10/10 0:00 & 12.563 & 5.563 & & 17.895 & 11.111 & & 10.539 & 25.359 & 34.805 & 50.634 \\
\hline 10/10/10 4:00 & 12.572 & 5.565 & & 17.883 & 11.113 & & 10.543 & 25.345 & 34.753 & 50.558 \\
\hline 10/10/10 8:00 & 12.591 & 5.573 & & 17.883 & 11.115 & & 10.567 & 25.363 & 34.707 & 50.481 \\
\hline 10/10/10 12:00 & 12.608 & 5.577 & & 17.953 & 11.120 & & 10.581 & 25.387 & 34.655 & 50.410 \\
\hline 10/10/10 16:00 & 12.615 & 5.582 & & 18.076 & 11.125 & & 10.574 & 25.429 & 34.603 & 50.334 \\
\hline 10/10/10 20:00 & 12.629 & 5.589 & & 18.013 & 11.130 & & 10.593 & 25.415 & 34.552 & 50.261 \\
\hline 10/11/10 0:00 & 12.645 & 5.596 & & 17.936 & 11.137 & & 10.611 & 25.443 & 34.505 & 50.188 \\
\hline 10/11/10 4:00 & 12.659 & 5.601 & & 17.950 & 11.141 & & 10.621 & 25.453 & 34.456 & 50.118 \\
\hline 10/11/10 8:00 & 12.671 & 5.606 & & 17.946 & 11.144 & & 10.637 & 25.549 & 34.406 & 50.042 \\
\hline 10/11/10 12:00 & 12.690 & 5.613 & & 17.953 & 11.146 & & 10.656 & 25.554 & 34.359 & 49.974 \\
\hline $10 / 11 / 1016: 00$ & 12.693 & 5.615 & & 18.044 & 11.146 & & 10.644 & 25.528 & 34.310 & 49.899 \\
\hline 10/11/10 20:00 & 12.709 & 5.620 & & 17.999 & 11.148 & & 10.670 & 25.549 & 34.263 & 49.828 \\
\hline 10/12/10 0:00 & 12.723 & 5.624 & & 17.964 & 11.141 & & 10.689 & 25.676 & 34.216 & 49.760 \\
\hline 10/12/10 4:00 & 12.740 & 5.631 & & 17.969 & 11.141 & & 10.708 & 25.660 & 34.169 & 49.687 \\
\hline $10 / 12 / 108: 00$ & 12.756 & 5.639 & & 17.950 & 11.137 & & 10.738 & 25.695 & 34.125 & 49.611 \\
\hline $10 / 12 / 1012: 00$ & 12.782 & 5.648 & & 17.983 & 11.139 & & 10.771 & 25.763 & 34.080 & 49.545 \\
\hline 10/12/10 16:00 & 12.801 & 5.653 & & 18.072 & 11.144 & & 10.780 & 25.791 & 34.036 & 49.475 \\
\hline 10/12/10 20:00 & 12.827 & 5.662 & & 17.997 & 11.146 & & 10.816 & 25.890 & 33.991 & 49.406 \\
\hline 10/13/10 0:00 & 12.858 & 5.669 & & 17.950 & 11.153 & & 10.865 & 25.961 & 33.951 & 49.338 \\
\hline 10/13/10 4:00 & 12.886 & 5.681 & & 17.969 & 11.158 & & 10.886 & 26.012 & 33.909 & 49.267 \\
\hline 10/13/10 8:00 & 12.914 & 5.686 & & 18.016 & 11.163 & & 10.921 & 26.076 & 33.865 & 49.204 \\
\hline 10/13/10 12:00 & 12.943 & 5.695 & & 18.067 & 11.165 & & 10.933 & 26.179 & 33.822 & 49.136 \\
\hline 10/13/10 16:00 & 12.954 & 5.700 & & 18.279 & 11.167 & & 10.907 & 26.149 & 33.775 & 49.065 \\
\hline $10 / 13 / 1020: 00$ & 12.973 & 5.707 & & 18.190 & 11.170 & & 10.928 & 26.153 & 33.736 & 48.999 \\
\hline 10/14/10 0:00 & 12.992 & 5.709 & & 18.123 & 11.172 & & 10.938 & 26.182 & 33.693 & 48.933 \\
\hline 10/14/10 4:00 & 13.006 & 5.714 & & 18.172 & 11.172 & & 10.926 & 26.247 & 33.649 & 48.862 \\
\hline $10 / 14 / 108: 00$ & 13.020 & 5.719 & & 18.158 & 11.172 & & 10.926 & 26.219 & 33.604 & 48.792 \\
\hline $10 / 14 / 1012: 00$ & 13.022 & 5.721 & & 18.244 & 11.170 & & 10.889 & 26.193 & 33.557 & 48.730 \\
\hline 10/14/10 16:00 & 13.011 & 5.714 & & 18.316 & 11.165 & & 10.837 & 26.102 & 33.508 & 48.662 \\
\hline 10/14/10 20:00 & 13.025 & 5.723 & & 18.211 & 11.165 & & 10.863 & 27.320 & 33.468 & 48.596 \\
\hline 10/15/10 0:00 & 13.044 & 5.728 & & 18.093 & 11.165 & & 10.912 & 26.459 & 33.431 & 48.532 \\
\hline 10/15/10 4:00 & 13.063 & 5.735 & & 18.081 & 11.170 & & 10.945 & 26.342 & 33.388 & 48.464 \\
\hline 10/15/10 8:00 & 13.086 & 5.745 & & 18.074 & 11.174 & & 10.980 & 26.342 & 33.349 & 48.401 \\
\hline 10/15/10 12:00 & 13.107 & 5.749 & & 18.137 & 11.177 & & 10.990 & 26.417 & 33.309 & 48.337 \\
\hline $10 / 15 / 1016: 00$ & 13.117 & 5.754 & & 18.344 & 11.177 & & 10.961 & 26.377 & 33.266 & 48.273 \\
\hline
\end{tabular}

TABLE S1.1 Page 50 of 89 
Water Level (ft below reference point)

\begin{tabular}{|c|c|c|c|c|c|c|c|c|c|c|}
\hline Date and Time & McPike & Potter & Hughes & Barr & Burks & MoDOT & Clizer "Old" & Morgan & SB24 & SB38 \\
\hline 10/15/10 20:00 & 13.126 & 5.756 & & 18.272 & 11.179 & & 10.971 & 26.342 & 33.227 & 48.210 \\
\hline 10/16/10 0:00 & 13.140 & 5.761 & & 18.228 & 11.181 & & 10.980 & 26.356 & 33.187 & 48.144 \\
\hline 10/16/10 4:00 & 13.150 & 5.763 & & 18.242 & 11.184 & & 10.978 & 26.351 & 33.144 & 48.080 \\
\hline 10/16/10 8:00 & 13.159 & 5.768 & & 18.246 & 11.184 & & 10.985 & 26.356 & 33.105 & 48.017 \\
\hline 10/16/10 12:00 & 13.171 & 5.773 & & 18.260 & 11.186 & & 11.001 & 26.398 & 33.065 & 47.958 \\
\hline 10/16/10 16:00 & 13.183 & 5.778 & & 18.400 & 11.189 & & 11.013 & 26.386 & 33.027 & 47.896 \\
\hline 10/16/10 20:00 & 13.204 & 5.787 & & 18.253 & 11.193 & & 11.048 & 28.808 & 32.992 & 47.833 \\
\hline 10/17/10 0:00 & 13.223 & 5.794 & & 18.162 & 11.198 & & 11.076 & 26.951 & 32.955 & 47.769 \\
\hline 10/17/10 4:00 & 13.242 & 5.801 & & 18.148 & 11.203 & & 11.093 & 26.732 & 32.919 & 47.713 \\
\hline 10/17/10 8:00 & 13.261 & 5.808 & & 18.172 & 11.207 & & 11.107 & 26.680 & 32.882 & 47.652 \\
\hline 10/17/10 12:00 & 13.272 & 5.811 & & 18.253 & 11.210 & & 11.095 & 26.704 & 32.844 & 47.593 \\
\hline 10/17/10 16:00 & 13.275 & 5.813 & & 18.435 & 11.207 & & 11.051 & 26.612 & 32.804 & 47.531 \\
\hline 10/17/10 20:00 & 13.282 & 5.815 & & 18.379 & 11.210 & & 11.058 & 26.577 & 32.767 & 47.472 \\
\hline 10/18/10 0:00 & 13.294 & 5.820 & & 18.326 & 11.212 & & 11.072 & 26.654 & 32.732 & 47.414 \\
\hline 10/18/10 4:00 & 13.303 & 5.822 & & 18.300 & 11.214 & & 11.086 & 26.619 & 32.694 & 47.359 \\
\hline 10/18/10 8:00 & 13.317 & 5.829 & & 18.235 & 11.217 & & 11.121 & 26.633 & 32.657 & 47.298 \\
\hline 10/18/10 12:00 & 13.334 & 5.836 & & 18.277 & 11.222 & & 11.140 & 26.668 & 32.621 & 47.242 \\
\hline 10/18/10 16:00 & 13.346 & 5.839 & & 18.393 & 11.238 & & 11.135 & 26.701 & 32.586 & 47.183 \\
\hline 10/18/10 20:00 & 13.364 & 5.848 & & 18.312 & 11.238 & & 11.168 & 30.118 & 32.553 & 47.124 \\
\hline 10/19/10 0:00 & 13.381 & 5.855 & & 18.263 & 11.240 & & 11.177 & 27.534 & 32.521 & 47.067 \\
\hline 10/19/10 4:00 & 13.395 & 5.855 & & 18.277 & 11.243 & & 11.185 & 27.179 & 32.485 & 47.013 \\
\hline 10/19/10 8:00 & 13.412 & 5.860 & & 18.279 & 11.245 & & 11.203 & 27.047 & 32.450 & 46.959 \\
\hline 10/19/10 12:00 & 13.426 & 5.865 & & 18.309 & 11.247 & & 11.206 & 27.014 & 32.415 & 46.902 \\
\hline 10/19/10 16:00 & 13.433 & 5.867 & & 18.496 & 11.247 & & 11.170 & 26.948 & 32.378 & 46.846 \\
\hline 10/19/10 20:00 & 13.440 & 5.869 & & 18.456 & 11.250 & & 11.187 & 26.901 & 32.345 & 46.789 \\
\hline 10/20/10 0:00 & 13.452 & 5.872 & & 18.398 & 11.252 & & 11.194 & 26.920 & 32.312 & 46.738 \\
\hline 10/20/10 4:00 & 13.463 & 5.874 & & 18.393 & 11.257 & & 11.206 & 26.918 & 32.279 & 46.681 \\
\hline 10/20/10 8:00 & 13.475 & 5.879 & & 18.363 & 11.259 & & 11.229 & 26.925 & 32.249 & 46.627 \\
\hline 10/20/10 12:00 & 13.492 & 5.884 & & 18.370 & 11.264 & & 11.243 & 26.960 & 32.213 & 46.570 \\
\hline 10/20/10 16:00 & 13.501 & 5.888 & & 18.558 & 11.269 & & 11.227 & 26.943 & 32.181 & 46.518 \\
\hline 10/20/10 20:00 & 13.513 & 5.895 & & 18.468 & 11.273 & & 11.253 & 26.939 & 32.150 & 46.464 \\
\hline 10/21/10 0:00 & 13.529 & 5.900 & & 18.388 & 11.280 & & 11.281 & 27.045 & 32.117 & 46.410 \\
\hline $10 / 21 / 104: 00$ & 13.551 & 5.905 & & 18.323 & 11.285 & & 11.304 & 27.054 & 32.087 & 46.358 \\
\hline 10/21/10 8:00 & 13.569 & 5.914 & & 18.321 & 11.295 & & 11.335 & 27.087 & 32.059 & 46.306 \\
\hline 10/21/10 12:00 & 13.591 & 5.921 & & 18.367 & 11.299 & & 11.342 & 27.110 & 32.030 & 46.255 \\
\hline 10/21/10 16:00 & 13.600 & 5.921 & & 18.554 & 11.304 & & 11.323 & 29.062 & 31.998 & 46.200 \\
\hline 10/21/10 20:00 & 13.614 & 5.924 & & 18.493 & 11.309 & & 11.340 & 27.625 & 31.967 & 46.149 \\
\hline 10/22/10 0:00 & 13.626 & 5.928 & & 18.465 & 11.316 & & 11.340 & 27.324 & 31.939 & 46.099 \\
\hline $10 / 22 / 104: 00$ & 13.638 & 5.933 & & 18.479 & 11.321 & & 11.349 & 27.235 & 31.904 & 46.045 \\
\hline 10/22/10 8:00 & 13.650 & 5.935 & & 18.472 & 11.325 & & 11.354 & 27.202 & 31.876 & 45.993 \\
\hline 10/22/10 12:00 & 13.661 & 5.940 & & 18.540 & 11.330 & & 11.356 & 27.240 & 31.847 & 45.946 \\
\hline 10/22/10 16:00 & 13.671 & 5.947 & & 18.626 & 11.335 & & 11.344 & 27.553 & 31.817 & 45.897 \\
\hline 10/22/10 20:00 & 13.680 & 5.947 & & 18.570 & 11.339 & & 11.363 & 27.266 & 31.787 & 45.845 \\
\hline 10/23/10 0:00 & 13.687 & 5.952 & & 18.540 & 11.368 & & 11.354 & 27.219 & 31.756 & 45.798 \\
\hline $10 / 23 / 104: 00$ & 13.685 & 5.950 & & 18.482 & 11.365 & & 11.344 & 27.176 & 31.728 & 45.746 \\
\hline 10/23/10 8:00 & 13.461 & 5.938 & & 18.335 & 11.342 & & 11.290 & 27.153 & 31.697 & 45.696 \\
\hline 10/23/10 12:00 & 13.348 & 5.933 & & 18.335 & 11.415 & & 11.368 & 27.049 & 31.667 & 45.647 \\
\hline 10/23/10 16:00 & 13.317 & 5.928 & & 18.344 & 11.502 & & 11.340 & 27.009 & 31.634 & 45.597 \\
\hline 10/23/10 20:00 & 13.315 & 5.933 & & 18.239 & 11.462 & & 11.253 & 26.934 & 31.606 & 45.548 \\
\hline 10/24/10 0:00 & 13.322 & 5.935 & & 18.188 & 11.415 & & 11.217 & 26.901 & 31.578 & 45.501 \\
\hline 10/24/10 4:00 & 13.324 & 5.935 & & 18.223 & 11.446 & & 11.236 & 26.845 & 31.543 & 45.451 \\
\hline 10/24/10 8:00 & 13.329 & 5.933 & & 18.232 & 11.448 & & 11.231 & 26.861 & 31.512 & 45.404 \\
\hline 10/24/10 12:00 & 13.341 & 5.940 & & 18.228 & 11.436 & & 11.271 & 26.826 & 31.482 & 45.357 \\
\hline 10/24/10 16:00 & 13.348 & 5.940 & & 18.286 & 11.476 & & 11.286 & 26.786 & 31.451 & 45.310 \\
\hline 10/24/10 20:00 & 13.360 & 5.942 & & 18.260 & 11.446 & & 11.243 & 26.802 & 31.421 & 45.261 \\
\hline 10/25/10 0:00 & 13.369 & 5.942 & & 18.284 & 11.457 & & 11.267 & 26.781 & 31.390 & 45.216 \\
\hline 10/25/10 4:00 & 13.367 & 5.935 & & 18.332 & 11.505 & & 11.302 & 26.737 & 31.357 & 45.169 \\
\hline 10/25/10 8:00 & 13.362 & 5.924 & & 18.381 & 11.573 & & 11.330 & 26.680 & 31.324 & 45.124 \\
\hline 10/25/10 12:00 & 13.350 & 5.895 & & 18.421 & 11.663 & & 11.476 & 26.633 & 31.289 & 45.075 \\
\hline 10/25/10 16:00 & 13.327 & 5.803 & & 18.230 & 11.875 & & 11.499 & 26.574 & 31.254 & 45.030 \\
\hline $10 / 25 / 1020: 00$ & 13.320 & 5.627 & & 18.221 & 11.877 & & 11.459 & 26.490 & 31.224 & 44.983 \\
\hline 10/26/10 0:00 & 13.331 & 5.459 & & 18.216 & 11.750 & & 11.283 & 26.560 & 31.195 & 44.936 \\
\hline $10 / 26 / 104: 00$ & 13.353 & 5.403 & & 18.195 & 11.545 & & 11.187 & 26.588 & 31.167 & 44.893 \\
\hline 10/26/10 8:00 & 13.374 & 5.398 & & 18.190 & 11.396 & & 11.119 & 26.643 & 31.139 & 44.846 \\
\hline
\end{tabular}

TABLE S1.1 Page 51 of 89 
Water Level (ft below reference point)

\begin{tabular}{|c|c|c|c|c|c|c|c|c|c|c|}
\hline Date and Time & McPike & Potter & Hughes & Barr & Burks & MoDOT & Clizer "Old" & Morgan & SB24 & SB38 \\
\hline 10/26/10 12:00 & 13.404 & 5.417 & & 18.200 & 11.280 & & 11.206 & 26.720 & 31.111 & 44.801 \\
\hline 10/26/10 16:00 & 13.430 & 5.457 & & 18.214 & 11.283 & & 11.220 & 26.755 & 31.085 & 44.757 \\
\hline 10/26/10 20:00 & 13.461 & 5.504 & & 18.232 & 11.292 & & 11.149 & 26.802 & 31.062 & 44.714 \\
\hline 10/27/10 0:00 & 13.494 & 5.547 & & 18.251 & 11.297 & & 11.138 & 26.922 & 31.036 & 44.669 \\
\hline $10 / 27 / 104: 00$ & 13.522 & 5.582 & & 18.272 & 11.304 & & 11.142 & 26.939 & 31.008 & 44.622 \\
\hline 10/27/10 8:00 & 13.553 & 5.615 & & 18.295 & 11.309 & & 11.387 & 26.986 & 30.982 & 44.580 \\
\hline 10/27/10 12:00 & 13.591 & 5.646 & & 18.319 & 11.316 & & 11.419 & 27.110 & 30.961 & 44.535 \\
\hline 10/27/10 16:00 & 13.619 & 5.674 & & 18.339 & 11.321 & & 11.455 & 27.150 & 30.933 & 44.490 \\
\hline 10/27/10 20:00 & 13.666 & 5.707 & & 18.367 & 11.328 & & 11.530 & 27.249 & 30.912 & 44.448 \\
\hline 10/28/10 0:00 & 13.704 & 5.733 & & 18.405 & 11.337 & & 11.567 & 27.341 & 30.893 & 44.406 \\
\hline 10/28/10 4:00 & 13.737 & 5.752 & & 18.437 & 11.342 & & 11.589 & 27.414 & 30.867 & 44.363 \\
\hline 10/28/10 8:00 & 13.767 & 5.773 & & 18.472 & 11.347 & & 11.626 & 27.515 & 30.844 & 44.325 \\
\hline 10/28/10 12:00 & 13.798 & 5.794 & & 18.507 & 11.354 & & 11.647 & 27.562 & 30.823 & 44.278 \\
\hline 10/28/10 16:00 & 13.819 & 5.806 & & 18.535 & 11.358 & & 11.643 & 27.550 & 30.801 & 44.236 \\
\hline 10/28/10 20:00 & 13.838 & 5.815 & & 18.558 & 11.358 & & 11.647 & 27.581 & 30.776 & 44.194 \\
\hline 10/29/10 0:00 & 13.855 & 5.825 & & 18.579 & 11.361 & & 11.645 & 27.590 & 30.752 & 44.154 \\
\hline $10 / 29 / 104: 00$ & 13.869 & 5.829 & & 18.598 & 11.361 & & 11.633 & 27.609 & 30.729 & 44.109 \\
\hline 10/29/10 8:00 & 13.878 & 5.836 & & 18.612 & 11.361 & & 11.614 & 27.574 & 30.703 & 44.069 \\
\hline 10/29/10 12:00 & 13.883 & 5.839 & & 18.626 & 11.358 & & 11.577 & 27.553 & 30.677 & 44.029 \\
\hline $10 / 29 / 1016: 00$ & 13.869 & 5.829 & & 18.628 & 11.354 & & 11.511 & 27.449 & 30.649 & 44.015 \\
\hline 10/29/10 20:00 & 13.864 & 5.834 & & 18.626 & 11.351 & & 11.490 & 27.400 & 30.623 & 43.972 \\
\hline 10/30/10 0:00 & 13.864 & 5.836 & & 18.626 & 11.347 & & 11.476 & 27.376 & 30.600 & 43.930 \\
\hline 10/30/10 4:00 & 13.857 & 5.839 & & 18.621 & 11.344 & & 11.450 & 27.327 & 30.572 & 43.890 \\
\hline 10/30/10 8:00 & 13.864 & 5.848 & & 18.617 & 11.344 & & 11.476 & 27.324 & 30.550 & 43.852 \\
\hline 10/30/10 12:00 & 13.876 & 5.853 & & 18.614 & 11.347 & & 11.485 & 27.418 & 30.527 & 43.810 \\
\hline 10/30/10 16:00 & 13.885 & 5.860 & & 18.614 & 11.351 & & 11.495 & 27.397 & 30.504 & 43.770 \\
\hline 10/30/10 20:00 & 13.899 & 5.872 & & 18.617 & 11.356 & & 11.535 & 27.409 & 30.482 & 43.732 \\
\hline $10 / 31 / 100: 00$ & 13.916 & 5.883 & & 18.624 & 11.361 & & 11.577 & 27.447 & 30.461 & 43.692 \\
\hline $10 / 31 / 104: 00$ & 13.932 & 5.893 & & 18.631 & 11.368 & & 11.596 & 27.480 & 30.438 & 43.654 \\
\hline 10/31/10 8:00 & 13.949 & 5.902 & & 18.638 & 11.372 & & 11.631 & 27.581 & 30.417 & 43.619 \\
\hline 10/31/10 12:00 & 13.965 & 5.909 & & 18.649 & 11.380 & & 11.643 & 27.604 & 30.396 & 43.579 \\
\hline 10/31/10 16:00 & 13.977 & 5.917 & & 18.654 & 11.384 & & 11.633 & 27.553 & 30.375 & 43.539 \\
\hline 10/31/10 20:00 & 13.994 & 5.926 & & 18.663 & 11.389 & & 11.666 & 27.595 & 30.353 & 43.501 \\
\hline 11/1/10 0:00 & 14.013 & 5.935 & & 18.675 & 11.396 & & 11.680 & 27.640 & 30.335 & 43.466 \\
\hline 11/1/10 4:00 & 14.029 & 5.940 & & 18.684 & 11.403 & & 11.690 & 27.640 & 30.314 & 43.426 \\
\hline 11/1/10 8:00 & 14.048 & 5.949 & & 18.696 & 11.410 & & 11.718 & 27.729 & 30.295 & 43.390 \\
\hline 11/1/10 12:00 & 14.067 & 5.957 & & 18.710 & 11.417 & & 11.732 & 27.748 & 30.274 & 43.355 \\
\hline $11 / 1 / 1016: 00$ & 14.081 & 5.961 & & 18.719 & 11.422 & & 11.732 & 27.736 & 30.250 & 43.317 \\
\hline $11 / 1 / 10$ 20:00 & 14.097 & 5.971 & & 18.731 & 11.429 & & 11.758 & 27.759 & 30.234 & 43.280 \\
\hline $11 / 2 / 100: 00$ & 14.119 & 5.980 & & 18.745 & 11.438 & & 11.772 & 27.858 & 30.213 & 43.244 \\
\hline $11 / 2 / 104: 00$ & 14.133 & 5.983 & & 18.757 & 11.443 & & 11.772 & 27.811 & 30.194 & 43.209 \\
\hline 11/2/10 8:00 & 14.149 & 5.985 & & 18.766 & 11.450 & & 11.786 & 27.884 & 30.173 & 43.171 \\
\hline $11 / 2 / 1012: 00$ & 14.163 & 5.989 & & 18.778 & 11.457 & & 11.786 & 27.875 & 30.154 & 43.320 \\
\hline $11 / 2 / 1016: 00$ & 14.168 & 5.987 & & 18.782 & 11.462 & & 11.751 & 27.828 & 30.133 & 43.395 \\
\hline $11 / 2 / 1020: 00$ & 14.175 & 5.992 & & 18.787 & 11.467 & & 11.760 & 27.802 & 30.112 & 43.167 \\
\hline 11/3/10 0:00 & 14.182 & 5.994 & & 18.789 & 11.472 & & 11.755 & 27.816 & 30.093 & 43.084 \\
\hline 11/3/10 4:00 & 14.187 & 5.994 & & 18.791 & 11.474 & & 11.746 & 27.788 & 30.072 & 43.086 \\
\hline 11/3/10 8:00 & 14.194 & 5.997 & & 18.791 & 11.481 & & 11.753 & 27.774 & 30.053 & 42.992 \\
\hline 11/3/10 12:00 & 14.203 & 6.001 & & 18.796 & 11.488 & & 11.779 & 27.863 & 30.032 & 43.105 \\
\hline 11/3/10 16:00 & 14.211 & 6.001 & & 18.798 & 11.493 & & 11.772 & 27.821 & 30.013 & 43.141 \\
\hline 11/3/10 20:00 & 14.220 & 6.004 & & 18.796 & 11.502 & & 11.786 & 27.828 & 29.995 & 42.943 \\
\hline 11/4/10 0:00 & 14.234 & 6.008 & & 18.801 & 11.512 & & 11.805 & 27.870 & 29.978 & 42.818 \\
\hline $11 / 4 / 104: 00$ & 14.246 & 6.013 & & 18.805 & 11.519 & & 11.816 & 27.872 & 29.957 & 42.797 \\
\hline 11/4/10 8:00 & 14.258 & 6.020 & & 18.808 & 11.526 & & 11.823 & 27.868 & 29.941 & 42.740 \\
\hline 11/4/10 12:00 & 14.269 & 6.022 & & 18.812 & 11.535 & & 11.821 & 27.875 & 29.922 & 42.874 \\
\hline $11 / 4 / 1016: 00$ & 14.274 & 6.022 & & 18.815 & 11.540 & & 11.812 & 27.853 & 29.898 & 42.891 \\
\hline $11 / 4 / 10$ 20:00 & 14.286 & 6.030 & & 18.817 & 11.549 & & 11.840 & 27.877 & 29.884 & 42.667 \\
\hline 11/5/10 0:00 & 14.300 & 6.034 & & 18.819 & 11.559 & & 11.856 & 27.955 & 29.868 & 42.556 \\
\hline 11/5/10 4:00 & 14.312 & 6.039 & & 18.826 & 11.571 & & 11.868 & 27.952 & 29.849 & 42.472 \\
\hline 11/5/10 8:00 & 14.328 & 6.044 & & 18.831 & 11.580 & & 11.892 & 27.969 & 29.835 & 42.545 \\
\hline 11/5/10 12:00 & 14.345 & 6.048 & & 18.840 & 11.592 & & 11.903 & 27.990 & 29.816 & 42.724 \\
\hline 11/5/10 16:00 & 14.352 & 6.051 & & 18.845 & 11.599 & & 11.889 & 28.023 & 29.800 & 42.783 \\
\hline 11/5/10 20:00 & 14.361 & 6.053 & & 18.847 & 11.608 & & 11.901 & 28.004 & 29.781 & 42.587 \\
\hline $11 / 6 / 100: 00$ & 14.373 & 6.058 & & 18.852 & 11.618 & & 11.922 & 28.025 & 29.765 & 42.476 \\
\hline
\end{tabular}

TABLE S1.1 Page 52 of 89 
Water Level (ft below reference point)

\begin{tabular}{|c|c|c|c|c|c|c|c|c|c|c|}
\hline Date and Time & McPike & Potter & Hughes & Barr & Burks & MoDOT & Clizer "Old" & Morgan & SB24 & SB38 \\
\hline 11/6/10 4:00 & 14.385 & 6.060 & & 18.859 & 11.630 & & 11.927 & 28.030 & 29.748 & 42.457 \\
\hline 11/6/10 8:00 & 14.399 & 6.065 & & 18.836 & 11.639 & & 11.950 & 28.042 & 29.732 & 42.354 \\
\hline 11/6/10 12:00 & 14.413 & 6.067 & & 18.843 & 11.651 & & 11.955 & 28.112 & 29.716 & 42.514 \\
\hline 11/6/10 16:00 & 14.418 & 6.067 & & 18.917 & 11.660 & & 11.929 & 28.049 & 29.697 & 42.596 \\
\hline $11 / 6 / 1020: 00$ & 14.427 & 6.074 & & 18.885 & 11.670 & & 11.939 & 28.049 & 29.683 & 42.443 \\
\hline 11/7/10 0:00 & 14.434 & 6.077 & & 18.868 & 11.681 & & 11.948 & 28.058 & 29.664 & 42.368 \\
\hline $11 / 7 / 104: 00$ & 14.442 & 6.077 & & 18.889 & 11.693 & & 11.950 & 28.053 & 29.648 & 42.323 \\
\hline 11/7/10 8:00 & 14.449 & 6.081 & & 18.889 & 11.705 & & 11.960 & 28.072 & 29.633 & 42.260 \\
\hline 11/7/10 12:00 & 14.458 & 6.086 & & 18.901 & 11.717 & & 11.960 & 28.077 & 29.617 & 42.377 \\
\hline 11/7/10 16:00 & 14.456 & 6.086 & & 18.880 & 11.729 & & 11.924 & 28.032 & 29.596 & 42.490 \\
\hline 11/7/10 20:00 & 14.458 & 6.086 & & 18.875 & 11.738 & & 11.927 & 28.011 & 29.582 & 42.354 \\
\hline 11/8/10 0:00 & 14.463 & 6.091 & & 18.871 & 11.750 & & 11.927 & 28.018 & 29.563 & 42.307 \\
\hline 11/8/10 4:00 & 14.458 & 6.093 & & 18.866 & 11.764 & & 11.913 & 28.016 & 29.544 & 42.300 \\
\hline 11/8/10 8:00 & 14.463 & 6.100 & & 18.859 & 11.776 & & 11.920 & 28.030 & 29.530 & 42.236 \\
\hline 11/8/10 12:00 & 14.468 & 6.103 & & 18.854 & 11.790 & & 11.934 & 28.016 & 29.514 & 42.309 \\
\hline 11/8/10 16:00 & 14.470 & 6.107 & & 18.852 & 11.802 & & 11.927 & 27.999 & 29.497 & 42.359 \\
\hline $11 / 8 / 1020: 00$ & 14.475 & 6.112 & & 18.847 & 11.818 & & 11.948 & 28.006 & 29.483 & 42.180 \\
\hline 11/9/10 0:00 & 14.482 & 6.114 & & 18.843 & 11.835 & & 11.969 & 28.011 & 29.467 & 42.088 \\
\hline 11/9/10 4:00 & 14.491 & 6.117 & & 18.843 & 11.849 & & 11.974 & 28.016 & 29.453 & 42.043 \\
\hline 11/9/10 8:00 & 14.498 & 6.119 & & 18.843 & 11.865 & & 11.988 & 28.079 & 29.436 & 41.968 \\
\hline 11/9/10 12:00 & 14.508 & 6.124 & & 18.843 & 11.882 & & 11.993 & 28.075 & 29.420 & 42.083 \\
\hline 11/9/10 16:00 & 14.512 & 6.124 & & 18.838 & 11.896 & & 11.988 & 28.044 & 29.406 & 42.118 \\
\hline 11/9/10 20:00 & 14.522 & 6.126 & & 18.838 & 11.915 & & 12.009 & 28.058 & 29.390 & 41.977 \\
\hline 11/10/10 0:00 & 14.536 & 6.129 & & 18.843 & 11.934 & & 12.035 & 28.159 & 29.375 & 41.862 \\
\hline 11/10/10 4:00 & 14.548 & 6.136 & & 18.847 & 11.950 & & 12.044 & 28.147 & 29.364 & 41.803 \\
\hline 11/10/10 8:00 & 14.560 & 6.138 & & 18.850 & 11.967 & & 12.056 & 28.145 & 29.350 & 41.744 \\
\hline 11/10/10 12:00 & 14.574 & 6.143 & & 18.854 & 11.986 & & 12.077 & 28.157 & 29.336 & 41.753 \\
\hline $11 / 10 / 1016: 00$ & 14.588 & 6.147 & & 18.861 & 12.004 & & 12.105 & 28.272 & 29.324 & 41.683 \\
\hline $11 / 10 / 1020: 00$ & 14.616 & 6.157 & & 18.875 & 12.023 & & 12.152 & 28.319 & 29.314 & 41.449 \\
\hline 11/11/10 0:00 & 14.647 & 6.164 & & 18.889 & 12.042 & & 12.190 & 28.361 & 29.305 & 41.291 \\
\hline $11 / 11 / 104: 00$ & 14.675 & 6.171 & & 18.908 & 12.064 & & 12.218 & 28.408 & 29.293 & 41.221 \\
\hline 11/11/10 8:00 & 14.703 & 6.178 & & 18.927 & 12.082 & & 12.251 & 28.455 & 29.282 & 41.127 \\
\hline 11/11/10 12:00 & 14.732 & 6.183 & & 18.948 & 12.106 & & 12.265 & 28.547 & 29.272 & 41.369 \\
\hline 11/11/10 16:00 & 14.748 & 6.185 & & 18.964 & 12.123 & & 12.263 & 28.512 & 29.261 & 41.430 \\
\hline 11/11/10 20:00 & 14.762 & 6.187 & & 18.978 & 12.141 & & 12.270 & 28.507 & 29.249 & 41.386 \\
\hline 11/12/10 0:00 & 14.772 & 6.190 & & 18.985 & 12.151 & & 12.251 & 28.488 & 29.235 & 41.348 \\
\hline $11 / 12 / 104: 00$ & 14.774 & 6.190 & & 18.992 & 12.165 & & 12.235 & 28.439 & 29.221 & 41.397 \\
\hline $11 / 12 / 108: 00$ & 14.779 & 6.197 & & 18.992 & 12.196 & & 12.237 & 28.491 & 29.211 & 41.376 \\
\hline 11/12/10 12:00 & 14.764 & 6.195 & & 18.929 & 12.200 & & 12.195 & 28.455 & 29.197 & 41.310 \\
\hline $11 / 12 / 1016: 00$ & 14.727 & 6.195 & & 18.908 & 12.337 & & 12.145 & 28.383 & 29.181 & 41.296 \\
\hline $11 / 12 / 1020: 00$ & 14.604 & 6.176 & & 18.836 & 12.269 & & 12.033 & 28.326 & 29.169 & 41.397 \\
\hline 11/13/10 0:00 & 13.475 & 5.337 & & 18.677 & 12.342 & & 11.903 & 28.263 & 29.153 & 41.388 \\
\hline 11/13/10 4:00 & 13.327 & 5.146 & & 18.605 & 12.257 & & 11.868 & 28.157 & 29.136 & 41.393 \\
\hline $11 / 13 / 108: 00$ & 13.416 & 5.226 & & 18.552 & 12.182 & & 11.859 & 28.110 & 29.124 & 41.301 \\
\hline $11 / 13 / 1012: 00$ & 13.508 & 5.353 & & 18.537 & 12.141 & & 11.854 & 28.072 & 29.110 & 41.282 \\
\hline 11/13/10 16:00 & 13.584 & 5.473 & & 18.605 & 12.186 & & 11.840 & 28.086 & 29.096 & 41.277 \\
\hline 11/13/10 20:00 & 13.647 & 5.579 & & 18.586 & 12.148 & & 11.842 & 28.035 & 29.080 & 41.190 \\
\hline 11/14/10 0:00 & 13.695 & 5.662 & & 18.593 & 12.132 & & 11.835 & 28.011 & 29.066 & 41.209 \\
\hline 11/14/10 4:00 & 13.730 & 5.726 & & 18.612 & 12.125 & & 11.821 & 27.959 & 29.049 & 41.209 \\
\hline $11 / 14 / 108: 00$ & 13.763 & 5.773 & & 18.621 & 12.115 & & 11.823 & 27.926 & 29.033 & 41.155 \\
\hline 11/14/10 12:00 & 13.786 & 5.813 & & 18.635 & 12.123 & & 11.805 & 27.901 & 29.019 & 41.270 \\
\hline 11/14/10 16:00 & 13.798 & 5.839 & & 18.626 & 12.236 & & 11.779 & 27.839 & 29.000 & 41.320 \\
\hline $11 / 14 / 1020: 00$ & 13.817 & 5.865 & & 18.610 & 12.207 & & 11.777 & 27.809 & 28.986 & 41.188 \\
\hline 11/15/10 0:00 & 13.831 & 5.883 & & 18.607 & 12.177 & & 11.772 & 27.783 & 28.970 & 41.134 \\
\hline 11/15/10 4:00 & 13.841 & 5.902 & & 18.612 & 12.207 & & 11.751 & 27.802 & 28.942 & 41.145 \\
\hline $11 / 15 / 108: 00$ & 13.852 & 5.916 & & 18.591 & 12.195 & & 11.755 & 27.755 & 28.925 & 41.075 \\
\hline $11 / 15 / 1012: 00$ & 13.871 & 5.928 & & 18.563 & 12.137 & & 11.772 & 27.759 & 28.909 & 41.103 \\
\hline 11/15/10 16:00 & 13.885 & 5.942 & & 18.610 & 12.198 & & 11.769 & 27.736 & 28.892 & 41.110 \\
\hline 11/15/10 20:00 & 13.904 & 5.952 & & 18.545 & 12.122 & & 11.791 & 27.825 & 28.878 & 40.959 \\
\hline 11/16/10 0:00 & 13.923 & 5.961 & & 18.556 & 12.096 & & 11.791 & 27.790 & 28.859 & 40.924 \\
\hline 11/16/10 4:00 & 13.935 & 5.968 & & 18.575 & 12.071 & & 11.781 & 27.757 & 28.843 & 40.898 \\
\hline 11/16/10 8:00 & 13.947 & 5.975 & & 18.551 & 12.052 & & 11.786 & 27.738 & 28.829 & 40.870 \\
\hline 11/16/10 12:00 & 13.956 & 5.982 & & 18.582 & 12.066 & & 11.779 & 27.818 & 28.815 & 40.952 \\
\hline $11 / 16 / 1016: 00$ & 13.963 & 5.999 & 9.697 & 18.556 & 12.010 & & 11.774 & 27.745 & 28.798 & 40.969 \\
\hline
\end{tabular}

TABLE S1.1 Page 53 of 89 
Water Level (ft below reference point)

\begin{tabular}{|c|c|c|c|c|c|c|c|c|c|c|}
\hline \\
\hline Date and Time & McPike & Potter & Hughes & Barr & Burks & MoDOT & Clizer "Old" & Morgan & SB24 & SB38 \\
\hline 11/16/10 20:00 & 13.980 & 6.006 & 9.711 & 18.554 & 12.005 & & 11.807 & 27.755 & 28.784 & 40.792 \\
\hline 11/17/10 0:00 & 14.008 & 6.018 & 9.735 & 18.556 & 12.003 & & 11.854 & 27.802 & 28.773 & 40.726 \\
\hline 11/17/10 4:00 & 14.039 & 6.032 & 9.761 & 18.565 & 11.998 & & 11.901 & 27.865 & 28.761 & 40.623 \\
\hline $11 / 17 / 108: 00$ & 14.079 & 6.041 & 9.789 & 18.577 & 11.999 & & 11.962 & 27.940 & 28.752 & 40.580 \\
\hline 11/17/10 12:00 & 14.128 & 6.058 & 9.820 & 18.596 & 11.994 & & 12.016 & 28.086 & 28.740 & 40.509 \\
\hline 11/17/10 16:00 & 14.161 & 6.065 & 9.846 & 18.612 & 11.991 & & 12.028 & 28.077 & 28.728 & 40.566 \\
\hline 11/17/10 20:00 & 14.199 & 6.074 & 9.872 & 18.631 & 11.989 & & 12.068 & 28.131 & 28.716 & 40.448 \\
\hline 11/18/10 0:00 & 14.237 & 6.084 & 9.898 & 18.652 & 11.987 & & 12.096 & 28.176 & 28.707 & 40.481 \\
\hline 11/18/10 4:00 & 14.270 & 6.086 & 9.922 & 18.673 & 11.984 & & 12.108 & 28.263 & 28.695 & 40.460 \\
\hline 11/18/10 8:00 & 14.296 & 6.093 & 9.945 & 18.689 & 11.982 & & 12.122 & 28.241 & 28.681 & 40.420 \\
\hline 11/18/10 12:00 & 14.322 & 6.096 & 9.967 & 18.705 & 11.975 & & 12.122 & 28.251 & 28.669 & 40.488 \\
\hline 11/18/10 16:00 & 14.333 & 6.093 & 9.978 & 18.717 & 11.970 & & 12.084 & 28.194 & 28.653 & 40.547 \\
\hline 11/18/10 20:00 & 14.336 & 6.091 & 9.990 & 18.722 & 11.963 & & 12.051 & 28.159 & 28.641 & 40.533 \\
\hline 11/19/10 0:00 & 14.336 & 6.088 & 9.997 & 18.722 & 11.958 & & 12.030 & 28.150 & 28.627 & 40.514 \\
\hline 11/19/10 4:00 & 14.326 & 6.084 & 10.004 & 18.717 & 11.951 & & 11.993 & 28.126 & 28.613 & 40.573 \\
\hline $11 / 19 / 108: 00$ & 14.319 & 6.079 & 10.014 & 18.708 & 11.949 & & 11.979 & 28.053 & 28.597 & 40.538 \\
\hline 11/19/10 12:00 & 14.333 & 6.086 & 10.028 & 18.705 & 11.947 & & 12.004 & 28.065 & 28.587 & 40.528 \\
\hline 11/19/10 16:00 & 14.340 & 6.091 & 10.042 & 18.701 & 11.947 & & 12.009 & 28.049 & 28.576 & 40.514 \\
\hline 11/19/10 20:00 & 14.357 & 6.100 & 10.057 & 18.701 & 11.949 & & 12.049 & 28.169 & 28.566 & 40.359 \\
\hline 11/20/10 0:00 & 14.380 & 6.107 & 10.073 & 18.708 & 11.951 & & 12.084 & 28.176 & 28.555 & 40.260 \\
\hline 11/20/10 4:00 & 14.399 & 6.112 & 10.092 & 18.710 & 11.954 & & 12.094 & 28.171 & 28.543 & 40.234 \\
\hline 11/20/10 8:00 & 14.413 & 6.117 & 10.106 & 18.715 & 11.956 & & 12.108 & 28.171 & 28.533 & 40.189 \\
\hline 11/20/10 12:00 & 14.425 & 6.121 & 10.120 & 18.717 & 11.956 & & 12.089 & 28.164 & 28.519 & 40.290 \\
\hline 11/20/10 16:00 & 14.423 & 6.117 & 10.125 & 18.712 & 11.956 & & 12.058 & 28.166 & 28.508 & 40.378 \\
\hline 11/20/10 20:00 & 14.423 & 6.119 & 10.137 & 18.708 & 11.958 & & 12.054 & 28.122 & 28.496 & 40.354 \\
\hline 11/21/10 0:00 & 14.428 & 6.117 & 10.146 & 18.708 & 11.961 & & 12.054 & 28.114 & 28.484 & 40.345 \\
\hline 11/21/10 4:00 & 14.428 & 6.119 & 10.153 & 18.745 & 11.965 & & 12.035 & 28.093 & 28.470 & 40.385 \\
\hline $11 / 21 / 108: 00$ & 14.423 & 6.112 & 10.161 & 18.726 & 11.968 & & 12.028 & 28.065 & 28.458 & 40.385 \\
\hline 11/21/10 12:00 & 14.432 & 6.119 & 10.172 & 18.694 & 11.975 & & 12.051 & 28.086 & 28.449 & 40.349 \\
\hline 11/21/10 16:00 & 14.442 & 6.124 & 10.184 & 18.696 & 11.980 & & 12.070 & 28.096 & 28.437 & 40.279 \\
\hline 11/21/10 20:00 & 14.458 & 6.136 & 10.201 & 18.649 & 11.989 & & 12.105 & 28.138 & 28.428 & 40.158 \\
\hline 11/22/10 0:00 & 14.472 & 6.136 & 10.210 & 18.680 & 11.996 & & 12.110 & 28.159 & 28.419 & 40.163 \\
\hline 11/22/10 4:00 & 14.475 & 6.138 & 10.220 & 18.717 & 12.001 & & 12.082 & 28.131 & 28.407 & 40.217 \\
\hline 11/22/10 8:00 & 14.470 & 6.133 & 10.227 & 18.666 & 12.010 & & 12.070 & 28.098 & 28.393 & 40.231 \\
\hline 11/22/10 12:00 & 14.489 & 6.145 & 10.241 & 18.666 & 12.020 & & 12.141 & 28.171 & 28.386 & 40.052 \\
\hline $11 / 22 / 1016: 00$ & 14.515 & 6.152 & 10.258 & 18.670 & 12.029 & & 12.169 & 28.225 & 28.379 & 39.989 \\
\hline $11 / 22 / 1020: 00$ & 14.545 & 6.166 & 10.276 & 18.680 & 12.036 & & 12.223 & 28.286 & 28.372 & 39.845 \\
\hline 11/23/10 0:00 & 14.581 & 6.176 & 10.300 & 18.694 & 12.048 & & 12.267 & 28.352 & 28.365 & 39.767 \\
\hline 11/23/10 4:00 & 14.616 & 6.183 & 10.319 & 18.710 & 12.057 & & 12.289 & 28.460 & 28.355 & 39.753 \\
\hline $11 / 23 / 108: 00$ & 14.651 & 6.192 & 10.340 & 18.729 & 12.069 & & 12.324 & 28.486 & 28.348 & 39.742 \\
\hline $11 / 23 / 1012: 00$ & 14.687 & 6.199 & 10.359 & 18.747 & 12.081 & & 12.340 & 28.519 & 28.336 & 39.786 \\
\hline $11 / 23 / 1016: 00$ & 14.703 & 6.197 & 10.371 & 18.759 & 12.086 & & 12.312 & 28.495 & 28.325 & 39.866 \\
\hline $11 / 23 / 1020: 00$ & 14.710 & 6.197 & 10.381 & 18.766 & 12.095 & & 12.293 & 28.486 & 28.313 & 39.845 \\
\hline 11/24/10 0:00 & 14.708 & 6.192 & 10.388 & 18.770 & 12.100 & & 12.263 & 28.437 & 28.304 & 39.890 \\
\hline $11 / 24 / 104: 00$ & 14.687 & 6.185 & 10.390 & 18.763 & 12.102 & & 12.213 & 28.364 & 28.290 & 39.989 \\
\hline 11/24/10 8:00 & 14.663 & 6.178 & 10.388 & 18.752 & 12.112 & & 12.171 & 28.293 & 28.278 & 40.055 \\
\hline 11/24/10 12:00 & 14.642 & 6.166 & 10.388 & 18.740 & 12.116 & & 12.136 & 28.303 & 28.266 & 40.135 \\
\hline 11/24/10 16:00 & 14.621 & 6.162 & 10.385 & 18.724 & 12.126 & & 12.112 & 28.225 & 28.254 & 40.154 \\
\hline $11 / 24 / 1020: 00$ & 14.630 & 6.176 & 10.397 & 18.715 & 12.138 & & 12.185 & 28.258 & 28.250 & 39.897 \\
\hline 11/25/10 0:00 & 14.677 & 6.192 & 10.416 & 18.717 & 12.154 & & 12.256 & 28.345 & 28.243 & 39.713 \\
\hline $11 / 25 / 104: 00$ & 14.717 & 6.204 & 10.435 & 18.726 & 12.171 & & 12.319 & 28.495 & 28.238 & 39.534 \\
\hline 11/25/10 8:00 & 14.746 & 6.211 & 10.451 & 18.738 & 12.187 & & 12.343 & 28.502 & 28.231 & 39.504 \\
\hline $11 / 25 / 1012: 00$ & 14.769 & 6.216 & 10.466 & 18.747 & 12.201 & & 12.354 & 28.517 & 28.224 & 39.522 \\
\hline 11/25/10 16:00 & 14.779 & 6.218 & 10.480 & 18.756 & 12.215 & & 12.359 & 28.507 & 28.214 & 39.551 \\
\hline $11 / 25 / 1020: 00$ & 14.795 & 6.220 & 10.494 & 18.766 & 12.232 & & 12.375 & 28.538 & 28.210 & 39.473 \\
\hline $11 / 26 / 100: 00$ & 14.814 & 6.227 & 10.508 & 18.775 & 12.246 & & 12.390 & 28.606 & 28.200 & 39.424 \\
\hline 11/26/10 4:00 & 14.828 & 6.227 & 10.520 & 18.782 & 12.260 & & 12.392 & 28.599 & 28.196 & 39.426 \\
\hline 11/26/10 8:00 & 14.833 & 6.227 & 10.530 & 18.787 & 12.274 & & 12.392 & 28.597 & 28.186 & 39.442 \\
\hline 11/26/10 12:00 & 14.845 & 6.232 & 10.541 & 18.796 & 12.289 & & 12.397 & 28.597 & 28.179 & 39.487 \\
\hline 11/26/10 16:00 & 14.849 & 6.230 & 10.548 & 18.798 & 12.305 & & 12.394 & 28.568 & 28.172 & 39.522 \\
\hline 11/26/10 20:00 & 14.861 & 6.235 & 10.560 & 18.803 & 12.319 & & 12.413 & 28.672 & 28.163 & 39.440 \\
\hline 11/27/10 0:00 & 14.875 & 6.237 & 10.572 & 18.810 & 12.336 & & 12.432 & 28.667 & 28.158 & 39.360 \\
\hline $11 / 27 / 104: 00$ & 14.892 & 6.239 & 10.584 & 18.819 & 12.350 & & 12.441 & 28.653 & 28.151 & 39.325 \\
\hline $11 / 27 / 108: 00$ & 14.906 & 6.239 & 10.596 & 18.829 & 12.366 & & 12.453 & 28.667 & 28.144 & 39.318 \\
\hline
\end{tabular}

TABLE S1.1 Page 54 of 89 
Water Level (ft below reference point)

\begin{tabular}{|c|c|c|c|c|c|c|c|c|c|c|}
\hline \\
\hline Date and Time & McPike & Potter & Hughes & Barr & Burks & MoDOT & Clizer "Old" & Morgan & SB24 & SB38 \\
\hline 11/27/10 12:00 & 14.923 & 6.242 & 10.610 & 18.838 & 12.381 & & 12.460 & 28.733 & 28.139 & 39.367 \\
\hline 11/27/10 16:00 & 14.927 & 6.237 & 10.617 & 18.840 & 12.397 & & 12.439 & 28.681 & 28.130 & 39.466 \\
\hline 11/27/10 20:00 & 14.930 & 6.239 & 10.622 & 18.838 & 12.414 & & 12.434 & 28.653 & 28.123 & 39.445 \\
\hline $11 / 28 / 100: 00$ & 14.932 & 6.239 & 10.629 & 18.866 & 12.428 & & 12.432 & 28.636 & 28.116 & 39.442 \\
\hline 11/28/10 4:00 & 14.930 & 6.239 & 10.634 & 18.885 & 12.444 & & 12.418 & 28.681 & 28.109 & 39.454 \\
\hline 11/28/10 8:00 & 14.932 & 6.239 & 10.638 & 18.882 & 12.461 & & 12.413 & 28.639 & 28.100 & 39.464 \\
\hline 11/28/10 12:00 & 14.927 & 6.234 & 10.641 & 18.880 & 12.477 & & 12.392 & 28.604 & 28.093 & 39.560 \\
\hline 11/28/10 16:00 & 14.906 & 6.227 & 10.638 & 18.824 & 12.494 & & 12.350 & 28.547 & 28.083 & 39.697 \\
\hline 11/28/10 20:00 & 14.894 & 6.230 & 10.641 & 18.812 & 12.510 & & 12.347 & 28.587 & 28.076 & 39.638 \\
\hline 11/29/10 0:00 & 14.904 & 6.232 & 10.648 & 18.805 & 12.529 & & 12.371 & 28.592 & 28.069 & 39.577 \\
\hline 11/29/10 4:00 & 14.908 & 6.239 & 10.653 & 18.798 & 12.546 & & 12.366 & 28.559 & 28.060 & 39.586 \\
\hline 11/29/10 8:00 & 14.908 & 6.237 & 10.655 & 18.789 & 12.564 & & 12.364 & 28.545 & 28.053 & 39.593 \\
\hline 11/29/10 12:00 & 14.908 & 6.239 & 10.660 & 18.784 & 12.583 & & 12.364 & 28.542 & 28.046 & 39.607 \\
\hline 11/29/10 16:00 & 14.904 & 6.242 & 10.664 & 18.775 & 12.605 & & 12.378 & 28.528 & 28.036 & 39.527 \\
\hline 11/29/10 20:00 & 14.941 & 6.251 & 10.679 & 18.777 & 12.626 & & 12.467 & 28.709 & 28.036 & 39.268 \\
\hline $11 / 30 / 100: 00$ & 14.970 & 6.260 & 10.695 & 18.784 & 12.654 & & 12.493 & 28.735 & 28.032 & 39.167 \\
\hline 11/30/10 4:00 & 14.993 & 6.268 & 10.707 & 18.794 & 12.675 & & 12.514 & 28.754 & 28.029 & 39.115 \\
\hline 11/30/10 8:00 & 15.014 & 6.272 & 10.721 & 18.808 & 12.699 & & 12.530 & 28.768 & 28.020 & 39.049 \\
\hline 11/30/10 12:00 & 15.038 & 6.277 & 10.735 & 18.819 & 12.720 & & 12.556 & 28.815 & 28.020 & 39.023 \\
\hline 11/30/10 16:00 & 15.059 & 6.279 & 10.747 & 18.836 & 12.741 & & 12.573 & 28.888 & 28.008 & 39.002 \\
\hline 11/30/10 20:00 & 15.080 & 6.286 & 10.759 & 18.847 & 12.765 & & 12.585 & 28.888 & 28.003 & 38.992 \\
\hline 12/1/10 0:00 & 15.097 & 6.289 & 10.771 & 18.861 & 12.786 & & 12.594 & 28.888 & 27.994 & 38.974 \\
\hline 12/1/10 4:00 & 15.113 & 6.291 & 10.783 & 18.873 & 12.807 & & 12.606 & 28.897 & 27.992 & 38.950 \\
\hline 12/1/10 8:00 & 15.132 & 6.293 & 10.794 & 18.887 & 12.831 & & 12.622 & 28.984 & 27.985 & 38.901 \\
\hline $12 / 1 / 1012: 00$ & 15.151 & 6.296 & 10.806 & 18.899 & 12.852 & & 12.636 & 28.980 & 27.982 & 38.978 \\
\hline 12/1/10 16:00 & 15.163 & 6.296 & 10.813 & 18.910 & 12.873 & & 12.629 & 28.959 & 27.968 & 39.021 \\
\hline 12/1/10 20:00 & 15.172 & 6.300 & 10.820 & 18.920 & 12.895 & & 12.636 & 28.947 & 27.966 & 38.964 \\
\hline $12 / 2 / 100: 00$ & 15.182 & 6.300 & 10.828 & 18.927 & 12.918 & & 12.639 & 28.947 & 27.959 & 38.976 \\
\hline $12 / 2 / 104: 00$ & 15.187 & 6.300 & 10.835 & 18.936 & 12.937 & & 12.634 & 28.992 & 27.954 & 38.988 \\
\hline 12/2/10 8:00 & 15.194 & 6.300 & 10.842 & 18.938 & 12.961 & & 12.639 & 28.963 & 27.949 & 38.962 \\
\hline $12 / 2 / 1012: 00$ & 15.205 & 6.303 & 10.851 & 18.945 & 12.984 & & 12.650 & 28.975 & 27.945 & 38.969 \\
\hline $12 / 2 / 1016: 00$ & 15.210 & 6.305 & 10.858 & 18.966 & 13.005 & & 12.650 & 28.966 & 27.940 & 38.992 \\
\hline 12/2/10 20:00 & 15.227 & 6.312 & 10.868 & 18.894 & 13.029 & & 12.676 & 29.001 & 27.938 & 38.889 \\
\hline 12/3/10 0:00 & 15.245 & 6.315 & 10.877 & 18.906 & 13.053 & & 12.688 & 29.093 & 27.935 & 38.851 \\
\hline 12/3/10 4:00 & 15.257 & 6.315 & 10.884 & 18.922 & 13.076 & & 12.685 & 29.069 & 27.928 & 38.856 \\
\hline 12/3/10 8:00 & 15.262 & 6.312 & 10.889 & 18.973 & 13.100 & & 12.681 & 29.027 & 27.924 & 38.882 \\
\hline $12 / 3 / 1012: 00$ & 15.264 & 6.310 & 10.894 & 19.034 & 13.123 & & 12.657 & 29.062 & 27.919 & 38.985 \\
\hline $12 / 3 / 1016: 00$ & 15.243 & 6.305 & 10.889 & 19.041 & 13.147 & & 12.606 & 28.984 & 27.910 & 39.143 \\
\hline 12/3/10 20:00 & 15.227 & 6.303 & 10.891 & 19.001 & 13.168 & & 12.606 & 28.933 & 27.907 & 39.087 \\
\hline $12 / 4 / 100: 00$ & 15.234 & 6.305 & 10.896 & 18.985 & 13.192 & & 12.620 & 28.926 & 27.903 & 39.044 \\
\hline $12 / 4 / 104: 00$ & 15.260 & 6.315 & 10.906 & 18.915 & 13.218 & & 12.674 & 28.966 & 27.900 & 38.875 \\
\hline 12/4/10 8:00 & 15.286 & 6.322 & 10.913 & 18.973 & 13.246 & & 12.723 & 29.081 & 27.900 & 38.740 \\
\hline $12 / 4 / 1012: 00$ & 15.326 & 6.334 & 10.934 & 18.989 & 13.272 & & 12.761 & 29.154 & 27.903 & 38.649 \\
\hline $12 / 4 / 1016: 00$ & 15.354 & 6.336 & 10.946 & 19.006 & 13.300 & & 12.777 & 29.166 & 27.898 & 38.656 \\
\hline 12/4/10 20:00 & 15.382 & 6.343 & 10.960 & 19.022 & 13.324 & & 12.801 & 29.203 & 27.898 & 38.587 \\
\hline 12/5/10 0:00 & 15.408 & 6.348 & 10.972 & 19.038 & 13.350 & & 12.815 & 29.231 & 27.893 & 38.547 \\
\hline 12/5/10 4:00 & 15.432 & 6.352 & 10.981 & 19.055 & 13.373 & & 12.822 & 29.250 & 27.891 & 38.554 \\
\hline 12/5/10 8:00 & 15.450 & 6.352 & 10.993 & 19.050 & 13.399 & & 12.838 & 29.267 & 27.888 & 38.524 \\
\hline $12 / 5 / 1012: 00$ & 15.472 & 6.357 & 11.003 & 19.064 & 13.425 & & 12.848 & 29.293 & 27.877 & 38.543 \\
\hline 12/5/10 16:00 & 15.479 & 6.357 & 11.010 & 19.141 & 13.449 & & 12.836 & 29.274 & 27.853 & 38.653 \\
\hline $12 / 5 / 1020: 00$ & 15.486 & 6.355 & 11.017 & 19.099 & 13.475 & & 12.840 & 29.278 & 27.846 & 38.644 \\
\hline 12/6/10 0:00 & 15.495 & 6.359 & 11.021 & 19.090 & 13.498 & & 12.845 & 29.285 & 27.842 & 38.637 \\
\hline 12/6/10 4:00 & 15.500 & 6.357 & 11.029 & 19.094 & 13.522 & & 12.838 & 29.330 & 27.837 & 38.660 \\
\hline 12/6/10 8:00 & 15.500 & 6.359 & 11.033 & 19.115 & 13.548 & & 12.833 & 29.295 & 27.832 & 38.674 \\
\hline $12 / 6 / 1012: 00$ & 15.502 & 6.357 & 11.038 & 19.146 & 13.571 & & 12.826 & 29.269 & 27.830 & 38.731 \\
\hline $12 / 6 / 1016: 00$ & 15.491 & 6.355 & 11.038 & 19.185 & 13.595 & & 12.794 & 29.222 & 27.818 & 38.839 \\
\hline 12/6/10 20:00 & 15.479 & 6.352 & 11.038 & 19.176 & 13.621 & & 12.777 & 29.189 & 27.802 & 38.832 \\
\hline 12/7/10 0:00 & 15.474 & 6.355 & 11.043 & 19.153 & 13.645 & & 12.786 & 29.241 & 27.797 & 38.795 \\
\hline 12/7/10 4:00 & 15.479 & 6.357 & 11.045 & 19.125 & 13.671 & & 12.789 & 29.220 & 27.792 & 38.785 \\
\hline 12/7/10 8:00 & 15.488 & 6.362 & 11.050 & 19.097 & 13.699 & & 12.810 & 29.213 & 27.790 & 38.729 \\
\hline 12/7/10 12:00 & 15.507 & 6.366 & 11.057 & 19.087 & 13.725 & & 12.836 & 29.234 & 27.788 & 38.700 \\
\hline 12/7/10 16:00 & 15.512 & 6.364 & 11.062 & 19.167 & 13.751 & & 12.826 & 29.220 & 27.785 & 38.736 \\
\hline 12/7/10 20:00 & 15.521 & 6.366 & 11.066 & 19.111 & 13.779 & & 12.840 & 29.290 & 27.778 & 38.674 \\
\hline $12 / 8 / 100: 00$ & 15.533 & 6.371 & 11.074 & 19.101 & 13.805 & & 12.848 & 29.281 & 27.778 & 38.651 \\
\hline
\end{tabular}

TABLE S1.1 Page 55 of 89 
Water Level (ft below reference point)

\begin{tabular}{|c|c|c|c|c|c|c|c|c|c|c|}
\hline Date and Time & McPike & Potter & Hughes & Barr & Burks & MoDOT & Clizer "Old" & Morgan & SB24 & SB38 \\
\hline 12/8/10 4:00 & 15.543 & 6.373 & 11.078 & 19.120 & 13.831 & & 12.845 & 29.274 & 27.774 & 38.665 \\
\hline 12/8/10 8:00 & 15.549 & 6.371 & 11.083 & 19.125 & 13.859 & & 12.848 & 29.264 & 27.771 & 38.719 \\
\hline 12/8/10 12:00 & 15.559 & 6.373 & 11.090 & 19.134 & 13.885 & & 12.850 & 29.342 & 27.769 & 38.672 \\
\hline 12/8/10 16:00 & 15.559 & 6.373 & 11.090 & 19.127 & 13.911 & & 12.831 & 29.288 & 27.762 & 38.750 \\
\hline 12/8/10 20:00 & 15.561 & 6.373 & 11.095 & 19.125 & 13.939 & & 12.831 & 29.285 & 27.759 & 38.752 \\
\hline 12/9/10 0:00 & 15.559 & 6.373 & 11.095 & 19.120 & 13.965 & & 12.822 & 29.269 & 27.755 & 38.769 \\
\hline 12/9/10 4:00 & 15.552 & 6.373 & 11.097 & 19.113 & 13.991 & & 12.801 & 29.248 & 27.750 & 38.830 \\
\hline 12/9/10 8:00 & 15.533 & 6.366 & 11.095 & 19.101 & 14.017 & & 12.770 & 29.213 & 27.745 & 38.905 \\
\hline 12/9/10 12:00 & 15.514 & 6.362 & 11.092 & 19.087 & 14.043 & & 12.751 & 29.187 & 27.741 & 38.964 \\
\hline 12/9/10 16:00 & 15.502 & 6.364 & 11.092 & 19.076 & 14.071 & & 12.751 & 29.170 & 27.738 & 38.950 \\
\hline 12/9/10 20:00 & 15.519 & 6.371 & 11.097 & 19.069 & 14.097 & & 12.791 & 29.201 & 27.738 & 38.811 \\
\hline 12/10/10 0:00 & 15.547 & 6.378 & 11.104 & 19.066 & 14.126 & & 12.824 & 29.236 & 27.736 & 38.722 \\
\hline 12/10/10 4:00 & 15.564 & 6.383 & 11.111 & 19.064 & 14.154 & & 12.843 & 29.257 & 27.734 & 38.698 \\
\hline 12/10/10 8:00 & 15.575 & 6.385 & 11.116 & 19.064 & 14.180 & & 12.855 & 29.269 & 27.731 & 38.670 \\
\hline 12/10/10 12:00 & 15.585 & 6.388 & 11.121 & 19.066 & 14.208 & & 12.857 & 29.285 & 27.729 & 38.703 \\
\hline 12/10/10 16:00 & 15.578 & 6.385 & 11.121 & 19.062 & 14.234 & & 12.826 & 29.815 & 27.724 & 38.821 \\
\hline $12 / 10 / 1020: 00$ & 15.573 & 6.385 & 11.121 & 19.055 & 14.262 & & 12.824 & 29.314 & 27.722 & 38.837 \\
\hline 12/11/10 0:00 & 15.564 & 6.383 & 11.123 & 19.045 & 14.286 & & 12.810 & 29.241 & 27.717 & 38.865 \\
\hline 12/11/10 4:00 & 15.557 & 6.381 & 11.123 & 19.038 & 14.317 & & 12.798 & 29.274 & 27.715 & 38.910 \\
\hline 12/11/10 8:00 & 15.547 & 6.381 & 11.126 & 19.027 & 14.343 & & 12.812 & 29.234 & 27.710 & 38.853 \\
\hline $12 / 11 / 1012: 00$ & 15.578 & 6.390 & 11.130 & 19.029 & 14.369 & & 12.859 & 29.264 & 27.710 & 38.684 \\
\hline $12 / 11 / 1016: 00$ & 15.594 & 6.395 & 11.137 & 19.027 & 14.399 & & 12.873 & 29.276 & 27.710 & 38.620 \\
\hline $12 / 11 / 1020: 00$ & 15.609 & 6.397 & 11.147 & 19.029 & 14.428 & & 12.883 & 29.290 & 27.708 & 38.573 \\
\hline 12/12/10 0:00 & 15.632 & 6.399 & 11.154 & 19.034 & 14.456 & & 12.911 & 29.372 & 27.710 & 38.470 \\
\hline 12/12/10 4:00 & 15.663 & 6.411 & 11.159 & 18.999 & 14.484 & & 12.949 & 29.434 & 27.713 & 38.347 \\
\hline 12/12/10 8:00 & 15.701 & 6.421 & 11.175 & 19.059 & 14.512 & & 12.981 & 29.459 & 27.710 & 38.281 \\
\hline $12 / 12 / 1012: 00$ & 15.741 & 6.425 & 11.189 & 19.076 & 14.543 & & 13.010 & 29.488 & 27.713 & 38.253 \\
\hline 12/12/10 16:00 & 15.767 & 6.430 & 11.196 & 19.097 & 14.569 & & 13.024 & 29.497 & 27.717 & 38.232 \\
\hline $12 / 12 / 1020: 00$ & 15.792 & 6.435 & 11.206 & 19.113 & 14.595 & & 13.038 & 29.528 & 27.729 & 38.217 \\
\hline 12/13/10 0:00 & 15.809 & 6.437 & 11.218 & 19.129 & 14.623 & & 13.042 & 29.535 & 27.738 & 38.250 \\
\hline 12/13/10 4:00 & 15.818 & 6.437 & 11.222 & 19.141 & 14.652 & & 13.042 & 29.525 & 27.752 & 38.288 \\
\hline 12/13/10 8:00 & 15.825 & 6.435 & 11.230 & 19.153 & 14.680 & & 13.047 & 29.554 & 27.767 & 38.300 \\
\hline $12 / 13 / 1012: 00$ & 15.837 & 6.437 & 11.237 & 19.164 & 14.703 & & 13.054 & 29.568 & 27.783 & 38.307 \\
\hline $12 / 13 / 1016: 00$ & 15.840 & 6.435 & 11.239 & 19.171 & 14.729 & & 13.040 & 29.549 & 27.795 & 38.364 \\
\hline $12 / 13 / 1020: 00$ & 15.840 & 6.435 & 11.244 & 19.178 & 14.758 & & 13.047 & 29.544 & 27.811 & 38.371 \\
\hline 12/14/10 0:00 & 15.847 & 6.435 & 11.248 & 19.183 & 14.781 & & 13.054 & 29.551 & 27.825 & 38.366 \\
\hline 12/14/10 4:00 & 15.846 & 6.435 & 11.251 & 19.185 & 14.810 & & 13.038 & 29.544 & 27.844 & 38.434 \\
\hline 12/14/10 8:00 & 15.840 & 6.430 & 11.251 & 19.187 & 14.835 & & 13.031 & 29.572 & 27.858 & 38.486 \\
\hline $12 / 14 / 1012: 00$ & 15.837 & 6.430 & 11.256 & 19.187 & 14.864 & & 13.035 & 29.556 & 27.874 & 38.491 \\
\hline 12/14/10 16:00 & 15.818 & 6.421 & 11.253 & 19.183 & 14.887 & & 12.996 & 29.495 & 27.872 & 38.611 \\
\hline $12 / 14 / 1020: 00$ & 15.807 & 6.421 & 11.251 & 19.176 & 14.916 & & 12.991 & 29.469 & 27.879 & 38.592 \\
\hline 12/15/10 0:00 & 15.804 & 6.423 & 11.253 & 19.169 & 14.942 & & 12.991 & 29.450 & 27.888 & 38.599 \\
\hline 12/15/10 4:00 & 15.795 & 6.421 & 11.251 & 19.160 & 14.968 & & 12.979 & 29.429 & 27.900 & 38.634 \\
\hline 12/15/10 8:00 & 15.783 & 6.421 & 11.251 & 19.153 & 14.994 & & 12.967 & 29.462 & 27.910 & 38.672 \\
\hline $12 / 15 / 1012: 00$ & 15.774 & 6.416 & 11.248 & 19.143 & 15.017 & & 12.953 & 29.424 & 27.919 & 38.738 \\
\hline $12 / 15 / 1016: 00$ & 15.759 & 6.414 & 11.248 & 19.129 & 15.041 & & 12.949 & 29.389 & 27.926 & 38.736 \\
\hline $12 / 15 / 1020: 00$ & 15.771 & 6.421 & 11.253 & 19.122 & 15.064 & & 12.977 & 29.401 & 27.935 & 38.639 \\
\hline 12/16/10 0:00 & 15.788 & 6.428 & 11.258 & 19.118 & 15.090 & & 13.003 & 29.415 & 27.945 & 38.583 \\
\hline 12/16/10 4:00 & 15.807 & 6.430 & 11.263 & 19.113 & 15.116 & & 13.026 & 29.481 & 27.952 & 38.531 \\
\hline 12/16/10 8:00 & 15.830 & 6.437 & 11.272 & 19.113 & 15.144 & & 13.059 & 29.490 & 27.961 & 38.429 \\
\hline $12 / 16 / 1012: 00$ & 15.861 & 6.442 & 11.279 & 19.120 & 15.170 & & 13.087 & 29.528 & 27.971 & 38.352 \\
\hline 12/16/10 16:00 & 15.884 & 6.447 & 11.289 & 19.129 & 15.196 & & 13.101 & 29.549 & 27.980 & 38.335 \\
\hline $12 / 16 / 1020: 00$ & 15.910 & 6.451 & 11.298 & 19.141 & 15.225 & & 13.125 & 29.582 & 27.982 & 38.265 \\
\hline 12/17/10 0:00 & 15.936 & 6.458 & 11.308 & 19.150 & 15.251 & & 13.141 & 29.669 & 27.987 & 38.229 \\
\hline 12/17/10 4:00 & 15.960 & 6.463 & 11.317 & 19.162 & 15.279 & & 13.158 & 29.681 & 27.994 & 38.199 \\
\hline 12/17/10 8:00 & 15.983 & 6.465 & 11.326 & 19.174 & 15.305 & & 13.174 & 29.676 & 28.001 & 38.166 \\
\hline $12 / 17 / 1012: 00$ & 16.009 & 6.472 & 11.336 & 19.190 & 15.331 & & 13.190 & 29.704 & 28.008 & 38.156 \\
\hline $12 / 17 / 1016: 00$ & 16.021 & 6.472 & 11.341 & 19.201 & 15.359 & & 13.188 & 29.699 & 28.010 & 38.210 \\
\hline $12 / 17 / 1020: 00$ & 16.030 & 6.475 & 11.348 & 19.211 & 15.383 & & 13.193 & 29.706 & 28.015 & 38.222 \\
\hline 12/18/10 0:00 & 16.042 & 6.477 & 11.355 & 19.220 & 15.409 & & 13.195 & 29.791 & 28.020 & 38.229 \\
\hline 12/18/10 4:00 & 16.045 & 6.475 & 11.360 & 19.232 & 15.434 & & 13.190 & 29.742 & 28.022 & 38.269 \\
\hline 12/18/10 8:00 & 16.047 & 6.475 & 11.362 & 19.243 & 15.460 & & 13.190 & 29.713 & 28.024 & 38.326 \\
\hline $12 / 18 / 1012: 00$ & 16.052 & 6.475 & 11.367 & 19.253 & 15.486 & & 13.188 & 29.706 & 28.027 & 38.347 \\
\hline 12/18/10 16:00 & 16.038 & 6.472 & 11.367 & 19.236 & 15.510 & & 13.165 & 29.669 & 28.029 & 38.378 \\
\hline
\end{tabular}

TABLE S1.1 Page 56 of 89 
Water Level (ft below reference point)

\begin{tabular}{|c|c|c|c|c|c|c|c|c|c|c|}
\hline Date and Time & McPike & Potter & Hughes & Barr & Burks & MoDOT & Clizer "Old" & Morgan & SB24 & SB38 \\
\hline $12 / 18 / 1020: 00$ & 16.035 & 6.472 & 11.369 & 19.236 & 15.536 & & 13.172 & 29.662 & 28.032 & 38.366 \\
\hline 12/19/10 0:00 & 16.038 & 6.475 & 11.371 & 19.236 & 15.559 & & 13.179 & 29.690 & 28.032 & 38.366 \\
\hline 12/19/10 4:00 & 16.042 & 6.475 & 11.376 & 19.236 & 15.583 & & 13.183 & 29.685 & 28.032 & 38.382 \\
\hline 12/19/10 8:00 & 16.045 & 6.477 & 11.378 & 19.236 & 15.607 & & 13.186 & 29.685 & 28.034 & 38.366 \\
\hline 12/19/10 12:00 & 16.052 & 6.479 & 11.381 & 19.253 & 15.633 & & 13.188 & 29.699 & 28.032 & 38.354 \\
\hline $12 / 19 / 1016: 00$ & 16.042 & 6.475 & 11.383 & 19.290 & 15.654 & & 13.174 & 29.676 & 28.032 & 38.375 \\
\hline 12/19/10 20:00 & 16.040 & 6.477 & 11.383 & 19.278 & 15.675 & & 13.181 & 29.683 & 28.032 & 38.448 \\
\hline $12 / 20 / 100: 00$ & 16.042 & 6.479 & 11.385 & 19.278 & 15.701 & & 13.188 & 29.678 & 28.034 & 38.422 \\
\hline $12 / 20 / 104: 00$ & 16.042 & 6.479 & 11.385 & 19.288 & 15.729 & & 13.176 & 29.666 & 28.034 & 38.467 \\
\hline $12 / 20 / 108: 00$ & 16.030 & 6.477 & 11.383 & 19.278 & 15.751 & & 13.162 & 29.671 & 28.032 & 38.510 \\
\hline $12 / 20 / 1012: 00$ & 16.021 & 6.475 & 11.381 & 19.213 & 15.776 & & 13.153 & 29.655 & 28.032 & 38.559 \\
\hline $12 / 20 / 1016: 00$ & 16.007 & 6.470 & 11.381 & 19.204 & 15.800 & & 13.144 & 29.631 & 28.031 & 38.580 \\
\hline 12/20/10 20:00 & 16.021 & 6.477 & 11.383 & 19.197 & 15.824 & & 13.183 & 29.659 & 28.034 & 38.458 \\
\hline $12 / 21 / 100: 00$ & 16.049 & 6.482 & 11.393 & 19.197 & 15.850 & & 13.209 & 29.718 & 28.036 & 38.385 \\
\hline 12/21/10 4:00 & 16.071 & 6.487 & 11.397 & 19.199 & 15.875 & & 13.230 & 29.735 & 28.039 & 38.298 \\
\hline $12 / 21 / 108: 00$ & 16.096 & 6.496 & 11.407 & 19.206 & 15.901 & & 13.256 & 29.756 & 28.041 & 38.201 \\
\hline $12 / 21 / 1012: 00$ & 16.134 & 6.501 & 11.416 & 19.218 & 15.927 & & 13.284 & 29.793 & 28.046 & 38.144 \\
\hline $12 / 21 / 1016: 00$ & 16.158 & 6.503 & 11.426 & 19.227 & 15.951 & & 13.301 & 29.800 & 28.046 & 38.130 \\
\hline $12 / 21 / 10$ 20:00 & 16.191 & 6.508 & 11.435 & 19.243 & 15.975 & & 13.329 & 29.895 & 28.050 & 38.029 \\
\hline $12 / 22 / 100: 00$ & 16.226 & 6.512 & 11.447 & 19.260 & 16.003 & & 13.353 & 29.902 & 28.053 & 37.968 \\
\hline $12 / 22 / 104: 00$ & 16.259 & 6.517 & 11.459 & 19.276 & 16.029 & & 13.371 & 29.918 & 28.055 & 37.935 \\
\hline $12 / 22 / 108: 00$ & 16.287 & 6.522 & 11.468 & 19.295 & 16.052 & & 13.392 & 29.963 & 28.057 & 37.940 \\
\hline $12 / 22 / 1012: 00$ & 16.320 & 6.527 & 11.480 & 19.316 & 16.078 & & 13.409 & 29.984 & 28.060 & 37.921 \\
\hline $12 / 22 / 1016: 00$ & 16.335 & 6.527 & 11.487 & 19.334 & 16.102 & & 13.411 & 29.984 & 28.062 & 37.944 \\
\hline $12 / 22 / 1020: 00$ & 16.351 & 6.529 & 11.494 & 19.353 & 16.128 & & 13.421 & 29.998 & 28.062 & 37.940 \\
\hline 12/23/10 0:00 & 16.365 & 6.531 & 11.501 & 19.367 & 16.152 & & 13.425 & 30.007 & 28.064 & 37.980 \\
\hline $12 / 23 / 104: 00$ & 16.372 & 6.529 & 11.506 & 19.381 & 16.177 & & 13.428 & 30.031 & 28.062 & 38.008 \\
\hline $12 / 23 / 108: 00$ & 16.372 & 6.529 & 11.508 & 19.390 & 16.201 & & 13.416 & 30.010 & 28.064 & 38.064 \\
\hline $12 / 23 / 1012: 00$ & 16.370 & 6.527 & 11.513 & 19.413 & 16.222 & & 13.409 & 30.003 & 28.062 & 38.123 \\
\hline $12 / 23 / 1016: 00$ & 16.349 & 6.522 & 11.511 & 19.469 & 16.246 & & 13.376 & 29.982 & 28.062 & 38.241 \\
\hline $12 / 23 / 10$ 20:00 & 16.332 & 6.522 & 11.513 & 19.451 & 16.269 & & 13.371 & 29.946 & 28.060 & 38.250 \\
\hline $12 / 24 / 100: 00$ & 16.325 & 6.520 & 11.513 & 19.458 & 16.293 & & 13.374 & 29.934 & 28.062 & 38.253 \\
\hline $12 / 24 / 104: 00$ & 16.325 & 6.522 & 11.515 & 19.416 & 16.316 & & 13.381 & 29.927 & 28.062 & 38.241 \\
\hline $12 / 24 / 108: 00$ & 16.323 & 6.522 & 11.518 & 19.441 & 16.340 & & 13.381 & 29.982 & 28.060 & 38.255 \\
\hline $12 / 24 / 1012: 00$ & 16.323 & 6.522 & 11.520 & 19.437 & 16.361 & & 13.383 & 29.939 & 28.060 & 38.253 \\
\hline $12 / 24 / 1016: 00$ & 16.323 & 6.524 & 11.523 & 19.439 & 16.383 & & 13.385 & 29.972 & 28.057 & 38.239 \\
\hline $12 / 24 / 10$ 20:00 & 16.342 & 6.526 & 11.527 & 19.379 & 16.406 & & 13.409 & 29.970 & 28.060 & 38.222 \\
\hline 12/25/10 0:00 & 16.365 & 6.531 & 11.534 & 19.353 & 16.427 & & 13.432 & 29.989 & 28.062 & 38.196 \\
\hline $12 / 25 / 104: 00$ & 16.391 & 6.538 & 11.541 & 19.414 & 16.451 & & 13.451 & 30.014 & 28.064 & 38.055 \\
\hline $12 / 25 / 108: 00$ & 16.415 & 6.541 & 11.546 & 19.420 & 16.474 & & 13.465 & 30.104 & 28.064 & 38.017 \\
\hline $12 / 25 / 1012: 00$ & 16.441 & 6.545 & 11.556 & 19.432 & 16.496 & & 13.479 & 30.087 & 28.067 & 37.998 \\
\hline $12 / 25 / 1016: 00$ & 16.448 & 6.543 & 11.560 & 19.434 & 16.519 & & 13.477 & 30.064 & 28.064 & 38.031 \\
\hline $12 / 25 / 10$ 20:00 & 16.462 & 6.545 & 11.567 & 19.397 & 16.543 & & 13.489 & 30.069 & 28.067 & 38.034 \\
\hline $12 / 26 / 100: 00$ & 16.474 & 6.545 & 11.570 & 19.411 & 16.564 & & 13.489 & 30.069 & 28.067 & 38.041 \\
\hline $12 / 26 / 104: 00$ & 16.478 & 6.545 & 11.575 & 19.414 & 16.588 & & 13.489 & 30.071 & 28.067 & 38.067 \\
\hline 12/26/10 8:00 & 16.483 & 6.548 & 11.579 & 19.411 & 16.609 & & 13.493 & 30.123 & 28.067 & 38.069 \\
\hline $12 / 26 / 1012: 00$ & 16.492 & 6.550 & 11.586 & 19.446 & 16.632 & & 13.498 & 30.167 & 28.069 & 38.076 \\
\hline $12 / 26 / 1016: 00$ & 16.485 & 6.548 & 11.586 & 19.523 & 16.654 & & 13.482 & 30.106 & 28.067 & 38.159 \\
\hline 12/26/10 20:00 & 16.474 & 6.545 & 11.586 & 19.518 & 16.677 & & 13.477 & 30.076 & 28.067 & 38.185 \\
\hline $12 / 27 / 100: 00$ & 16.462 & 6.543 & 11.589 & 19.525 & 16.701 & & 13.475 & 30.085 & 28.064 & 38.194 \\
\hline $12 / 27 / 104: 00$ & 16.462 & 6.545 & 11.591 & 19.518 & 16.722 & & 13.475 & 30.076 & 28.064 & 38.208 \\
\hline $12 / 27 / 108: 00$ & 16.459 & 6.545 & 11.593 & 19.544 & 16.746 & & 13.472 & 30.069 & 28.064 & 38.234 \\
\hline $12 / 27 / 1012: 00$ & 16.462 & 6.545 & 11.596 & 19.544 & 16.767 & & 13.477 & 30.071 & 28.064 & 38.241 \\
\hline $12 / 27 / 1016: 00$ & 16.441 & 6.538 & 11.591 & 19.574 & 16.788 & & 13.437 & 30.036 & 28.062 & 38.359 \\
\hline $12 / 27 / 1020: 00$ & 16.429 & 6.536 & 11.591 & 19.532 & 16.809 & & 13.451 & 30.033 & 28.062 & 38.300 \\
\hline $12 / 28 / 100: 00$ & 16.426 & 6.541 & 11.596 & 19.539 & 16.831 & & 13.456 & 30.038 & 28.060 & 38.305 \\
\hline $12 / 28 / 104: 00$ & 16.431 & 6.543 & 11.596 & 19.537 & 16.852 & & 13.465 & 30.043 & 28.060 & 38.286 \\
\hline 12/28/10 8:00 & 16.436 & 6.545 & 11.598 & 19.521 & 16.873 & & 13.479 & 30.050 & 28.062 & 38.283 \\
\hline $12 / 28 / 1012: 00$ & 16.450 & 6.548 & 11.603 & 19.509 & 16.897 & & 13.486 & 30.066 & 28.062 & 38.276 \\
\hline $12 / 28 / 1016: 00$ & 16.443 & 6.543 & 11.603 & 19.495 & 16.915 & & 13.463 & 30.045 & 28.060 & 38.335 \\
\hline $12 / 28 / 10$ 20:00 & 16.438 & 6.543 & 11.600 & 19.483 & 16.937 & & 13.463 & 30.038 & 28.057 & 38.345 \\
\hline $12 / 29 / 100: 00$ & 16.429 & 6.541 & 11.600 & 19.486 & 16.958 & & 13.446 & 30.026 & 28.055 & 38.401 \\
\hline $12 / 29 / 104: 00$ & 16.415 & 6.536 & 11.596 & 19.474 & 16.979 & & 13.428 & 30.007 & 28.055 & 38.453 \\
\hline $12 / 29 / 108: 00$ & 16.396 & 6.531 & 11.591 & 19.404 & 16.998 & & 13.411 & 29.989 & 28.053 & 38.481 \\
\hline
\end{tabular}

TABLE S1.1 Page 57 of 89 
Water Level (ft below reference point)

\begin{tabular}{|c|c|c|c|c|c|c|c|c|c|c|}
\hline Date and Time & McPike & Potter & Hughes & Barr & Burks & MoDOT & Clizer "Old" & Morgan & SB24 & SB38 \\
\hline $12 / 29 / 1012: 00$ & 16.382 & 6.529 & 11.589 & 19.390 & 17.019 & & 13.390 & 29.972 & 28.050 & 38.406 \\
\hline $12 / 29 / 1016: 00$ & 16.349 & 6.522 & 11.579 & 19.372 & 17.038 & & 13.348 & 29.932 & 28.046 & 38.323 \\
\hline $12 / 29 / 1020: 00$ & 16.325 & 6.520 & 11.572 & 19.351 & 17.057 & & 13.341 & 29.911 & 28.043 & 38.295 \\
\hline 12/30/10 0:00 & 16.313 & 6.522 & 11.570 & 19.334 & 17.078 & & 13.341 & 29.899 & 28.041 & 38.253 \\
\hline 12/30/10 4:00 & 16.297 & 6.519 & 11.565 & 19.316 & 17.100 & & 13.329 & 29.890 & 28.039 & 38.269 \\
\hline 12/30/10 8:00 & 16.283 & 6.519 & 11.563 & 19.297 & 17.118 & & 13.331 & 29.871 & 28.039 & 38.316 \\
\hline $12 / 30 / 1012: 00$ & 16.287 & 6.524 & 11.567 & 19.283 & 17.135 & & 13.345 & 29.871 & 28.039 & 38.985 \\
\hline $12 / 30 / 1016: 00$ & 16.292 & 6.527 & 11.558 & 19.267 & 17.161 & & 13.362 & 29.857 & 28.039 & 38.884 \\
\hline $12 / 30 / 1020: 00$ & 16.337 & 6.541 & 11.570 & 19.264 & 17.180 & & 13.444 & 29.949 & 28.041 & 38.557 \\
\hline 12/31/10 0:00 & 16.393 & 6.555 & 11.582 & 19.269 & 17.198 & & 13.493 & 30.003 & 28.043 & 38.404 \\
\hline $12 / 31 / 104: 00$ & 16.426 & 6.557 & 11.589 & 19.274 & 17.220 & & 13.491 & 30.019 & 28.043 & 38.420 \\
\hline $12 / 31 / 108: 00$ & 16.426 & 6.552 & 11.589 & 19.274 & 17.241 & & 13.479 & 29.998 & 28.039 & 38.368 \\
\hline $12 / 31 / 1012: 00$ & 16.431 & 6.557 & 11.593 & 19.274 & 17.260 & & 13.496 & 30.012 & 28.039 & 38.342 \\
\hline $12 / 31 / 1016: 00$ & 16.462 & 6.562 & 11.600 & 19.278 & 17.281 & & 13.538 & 30.078 & 28.043 & 38.248 \\
\hline $12 / 31 / 1020: 00$ & 16.502 & 6.574 & 11.612 & 19.290 & 17.300 & & 13.576 & 30.120 & 28.046 & 38.081 \\
\hline 1/1/11 0:00 & 16.537 & 6.576 & 11.622 & 19.306 & 17.323 & & 13.592 & 30.167 & 28.048 & 38.071 \\
\hline 1/1/11 4:00 & 16.563 & 6.578 & 11.631 & 19.320 & 17.342 & & 13.599 & 30.170 & 28.048 & 38.062 \\
\hline 1/1/11 8:00 & 16.582 & 6.583 & 11.643 & 19.334 & 17.364 & & 13.623 & 30.188 & 28.050 & 38.003 \\
\hline $1 / 1 / 11$ 12:00 & 16.617 & 6.588 & 11.653 & 19.353 & 17.387 & & 13.656 & 30.233 & 28.053 & 37.921 \\
\hline $1 / 1 / 11$ 16:00 & 16.641 & 6.590 & 11.662 & 19.374 & 17.404 & & 13.667 & 30.247 & 28.053 & 37.888 \\
\hline $1 / 1 / 1120: 00$ & 16.669 & 6.593 & 11.671 & 19.344 & 17.427 & & 13.691 & 30.275 & 28.055 & 37.843 \\
\hline 1/2/11 0:00 & 16.690 & 6.595 & 11.679 & 19.365 & 17.448 & & 13.698 & 30.294 & 28.055 & 37.869 \\
\hline 1/2/11 4:00 & 16.702 & 6.595 & 11.686 & 19.379 & 17.467 & & 13.693 & 30.386 & 28.057 & 37.916 \\
\hline $1 / 2 / 118: 00$ & 16.705 & 6.595 & 11.690 & 19.397 & 17.489 & & 13.693 & 30.346 & 28.057 & 37.940 \\
\hline $1 / 2 / 1112: 00$ & 16.712 & 6.590 & 11.697 & 19.439 & 17.512 & & 13.681 & 30.322 & 28.060 & 38.046 \\
\hline $1 / 2 / 11$ 16:00 & 16.695 & 6.583 & 11.695 & 19.516 & 17.526 & & 13.660 & 30.266 & 28.055 & 38.128 \\
\hline $1 / 2 / 1120: 00$ & 16.688 & 6.588 & 11.697 & 19.502 & 17.545 & & 13.674 & 30.261 & 28.055 & 38.109 \\
\hline 1/3/11 0:00 & 16.693 & 6.588 & 11.700 & 19.476 & 17.566 & & 13.688 & 30.266 & 28.055 & 38.095 \\
\hline $1 / 3 / 114: 00$ & 16.695 & 6.590 & 11.702 & 19.486 & 17.583 & & 13.686 & 30.261 & 28.053 & 38.154 \\
\hline 1/3/11 8:00 & 16.693 & 6.588 & 11.705 & 19.509 & 17.602 & & 13.679 & 30.315 & 28.053 & 38.192 \\
\hline $1 / 3 / 11$ 12:00 & 16.698 & 6.583 & 11.709 & 19.500 & 17.623 & & 13.677 & 30.278 & 28.055 & 38.206 \\
\hline $1 / 3 / 11$ 16:00 & 16.686 & 6.581 & 11.707 & 19.546 & 17.644 & & 13.665 & 30.247 & 28.053 & 38.217 \\
\hline $1 / 3 / 1120: 00$ & 16.698 & 6.588 & 11.712 & 19.455 & 17.663 & & 13.698 & 30.266 & 28.046 & 38.109 \\
\hline 1/4/11 0:00 & 16.719 & 6.592 & 11.719 & 19.458 & 17.682 & & 13.719 & 30.341 & 28.046 & 38.048 \\
\hline $1 / 4 / 114: 00$ & 16.740 & 6.597 & 11.721 & 19.516 & 17.703 & & 13.728 & 30.337 & 28.046 & 38.093 \\
\hline 1/4/11 8:00 & 16.754 & 6.600 & 11.726 & 19.523 & 17.724 & & 13.735 & 30.334 & 28.046 & 38.062 \\
\hline $1 / 4 / 1112: 00$ & 16.766 & 6.600 & 11.733 & 19.532 & 17.743 & & 13.735 & 30.337 & 28.048 & 38.093 \\
\hline $1 / 4 / 11$ 16:00 & 16.761 & 6.592 & 11.733 & 19.537 & 17.762 & & 13.705 & 30.393 & 28.046 & 38.170 \\
\hline 1/4/11 20:00 & 16.745 & 6.590 & 11.733 & 19.539 & 17.781 & & 13.702 & 30.334 & 28.046 & 38.177 \\
\hline 1/5/11 0:00 & 16.740 & 6.595 & 11.735 & 19.539 & 17.800 & & 13.707 & 30.308 & 28.046 & 38.180 \\
\hline 1/5/11 4:00 & 16.733 & 6.595 & 11.735 & 19.537 & 17.821 & & 13.695 & 30.287 & 28.043 & 38.276 \\
\hline 1/5/11 8:00 & 16.721 & 6.593 & 11.733 & 19.535 & 17.840 & & 13.684 & 30.268 & 28.043 & 38.307 \\
\hline $1 / 5 / 11$ 12:00 & 16.721 & 6.592 & 11.735 & 19.530 & 17.859 & & 13.686 & 30.292 & 28.041 & 38.293 \\
\hline $1 / 5 / 11$ 16:00 & 16.719 & 6.592 & 11.738 & 19.525 & 17.878 & & 13.691 & 30.278 & 28.041 & 38.269 \\
\hline $1 / 5 / 1120: 00$ & 16.728 & 6.600 & 11.742 & 19.525 & 17.897 & & 13.717 & 30.294 & 28.041 & 38.194 \\
\hline 1/6/11 0:00 & 16.742 & 6.602 & 11.745 & 19.528 & 17.915 & & 13.731 & 30.313 & 28.039 & 38.168 \\
\hline 1/6/11 4:00 & 16.754 & 6.604 & 11.747 & 19.528 & 17.934 & & 13.728 & 30.318 & 28.041 & 38.187 \\
\hline 1/6/11 8:00 & 16.754 & 6.604 & 11.747 & 19.528 & 17.953 & & 13.719 & 30.337 & 28.039 & 38.243 \\
\hline $1 / 6 / 1112: 00$ & 16.759 & 6.604 & 11.752 & 19.525 & 17.972 & & 13.717 & 30.318 & 28.039 & 38.269 \\
\hline 1/6/11 16:00 & 16.754 & 6.602 & 11.752 & 19.523 & 17.988 & & 13.712 & 30.294 & 28.036 & 38.321 \\
\hline 1/6/11 20:00 & 16.749 & 6.602 & 11.754 & 19.518 & 18.007 & & 13.705 & 30.283 & 28.036 & 38.328 \\
\hline 1/7/11 0:00 & 16.735 & 6.597 & 11.749 & 19.511 & 18.024 & & 13.688 & 30.257 & 28.032 & 38.394 \\
\hline 1/7/11 4:00 & 16.719 & 6.595 & 11.747 & 19.502 & 18.043 & & 13.672 & 30.299 & 28.029 & 38.437 \\
\hline 1/7/11 8:00 & 16.723 & 6.597 & 11.749 & 19.495 & 18.059 & & 13.700 & 30.292 & 28.027 & 38.340 \\
\hline $1 / 7 / 11$ 12:00 & 16.745 & 6.607 & 11.757 & 19.493 & 18.083 & & 13.728 & 30.292 & 28.029 & 38.276 \\
\hline $1 / 7 / 11$ 16:00 & 16.768 & 6.609 & 11.757 & 19.493 & 18.097 & & 13.749 & 30.325 & 28.029 & 38.210 \\
\hline $1 / 7 / 1120: 00$ & 16.787 & 6.614 & 11.764 & 19.500 & 18.116 & & 13.761 & 30.339 & 28.029 & 38.173 \\
\hline 1/8/11 0:00 & 16.811 & 6.621 & 11.771 & 19.504 & 18.135 & & 13.782 & 30.358 & 28.032 & 38.097 \\
\hline 1/8/11 4:00 & 16.830 & 6.623 & 11.778 & 19.511 & 18.154 & & 13.794 & 30.377 & 28.032 & 38.123 \\
\hline 1/8/11 8:00 & 16.851 & 6.628 & 11.785 & 19.472 & 18.170 & & 13.818 & 30.398 & 28.034 & 38.031 \\
\hline $1 / 8 / 1112: 00$ & 16.886 & 6.628 & 11.794 & 19.479 & 18.189 & & 13.846 & 30.428 & 28.036 & 37.968 \\
\hline $1 / 8 / 1116: 00$ & 16.905 & 6.628 & 11.799 & 19.539 & 18.205 & & 13.841 & 30.494 & 28.036 & 37.980 \\
\hline 1/8/11 20:00 & 16.917 & 6.633 & 11.806 & 19.483 & 18.222 & & 13.858 & 30.478 & 28.039 & 37.958 \\
\hline 1/9/11 0:00 & 16.931 & 6.635 & 11.813 & 19.458 & 18.243 & & 13.869 & 30.475 & 28.039 & 37.963 \\
\hline
\end{tabular}

TABLE S1.1 Page 58 of 89 
Water Level (ft below reference point)

\begin{tabular}{|c|c|c|c|c|c|c|c|c|c|c|}
\hline Date and Time & McPike & Potter & Hughes & Barr & Burks & MoDOT & Clizer "Old" & Morgan & SB24 & SB38 \\
\hline 1/9/11 4:00 & 16.945 & 6.637 & 11.818 & 19.442 & 18.260 & & 13.872 & 30.480 & 28.041 & 37.987 \\
\hline 1/9/11 8:00 & 16.959 & 6.640 & 11.823 & 19.439 & 18.279 & & 13.883 & 30.492 & 28.041 & 37.982 \\
\hline 1/9/11 12:00 & 16.978 & 6.642 & 11.830 & 19.602 & 18.297 & & 13.895 & 30.518 & 28.043 & 37.954 \\
\hline 1/9/11 16:00 & 16.978 & 6.637 & 11.835 & 19.635 & 18.316 & & 13.886 & 30.494 & 28.041 & 38.020 \\
\hline 1/9/11 20:00 & 16.985 & 6.640 & 11.839 & 19.598 & 18.333 & & 13.890 & 30.501 & 28.043 & 38.036 \\
\hline 1/10/11 0:00 & 16.985 & 6.640 & 11.844 & 19.598 & 18.349 & & 13.897 & 30.579 & 28.043 & 38.034 \\
\hline 1/10/11 4:00 & 17.002 & 6.642 & 11.849 & 19.549 & 18.368 & & 13.909 & 30.562 & 28.043 & 38.010 \\
\hline 1/10/11 8:00 & 17.009 & 6.640 & 11.854 & 19.586 & 18.385 & & 13.902 & 30.565 & 28.043 & 38.048 \\
\hline 1/10/11 12:00 & 17.014 & 6.642 & 11.858 & 19.584 & 18.404 & & 13.909 & 30.536 & 28.046 & 38.031 \\
\hline 1/10/11 16:00 & 17.006 & 6.637 & 11.861 & 19.642 & 18.420 & & 13.904 & 30.551 & 28.046 & 38.062 \\
\hline 1/10/11 20:00 & 17.018 & 6.642 & 11.865 & 19.602 & 18.437 & & 13.914 & 30.548 & 28.046 & 38.050 \\
\hline 1/11/11 0:00 & 17.027 & 6.642 & 11.870 & 19.572 & 18.453 & & 13.926 & 30.546 & 28.046 & 38.027 \\
\hline $1 / 11 / 114: 00$ & 17.042 & 6.644 & 11.875 & 19.567 & 18.470 & & 13.940 & 30.553 & 28.048 & 37.991 \\
\hline $1 / 11 / 118: 00$ & 17.056 & 6.647 & 11.880 & 19.581 & 18.486 & & 13.956 & 30.628 & 28.050 & 37.958 \\
\hline 1/11/11 12:00 & 17.077 & 6.654 & 11.887 & 19.642 & 18.503 & & 13.977 & 30.628 & 28.053 & 37.907 \\
\hline 1/11/11 16:00 & 17.103 & 6.656 & 11.896 & 19.661 & 18.521 & & 13.996 & 30.642 & 28.053 & 37.871 \\
\hline $1 / 11 / 1120: 00$ & 17.126 & 6.659 & 11.901 & 19.623 & 18.538 & & 14.013 & 30.645 & 28.055 & 37.850 \\
\hline $1 / 12 / 110: 00$ & 17.143 & 6.659 & 11.908 & 19.707 & 18.557 & & 14.022 & 30.647 & 28.057 & 37.845 \\
\hline 1/12/11 4:00 & 17.164 & 6.663 & 11.915 & 19.716 & 18.573 & & 14.043 & 30.743 & 28.060 & 37.805 \\
\hline $1 / 12 / 118: 00$ & 17.185 & 6.666 & 11.924 & 19.716 & 18.592 & & 14.052 & 30.718 & 28.062 & 37.798 \\
\hline 1/12/11 12:00 & 17.204 & 6.668 & 11.932 & 19.730 & 18.609 & & 14.062 & 30.720 & 28.062 & 37.798 \\
\hline 1/12/11 16:00 & 17.212 & 6.666 & 11.934 & 19.765 & 18.625 & & 14.055 & 30.696 & 28.064 & 37.867 \\
\hline 1/12/11 20:00 & 17.219 & 6.668 & 11.939 & 19.775 & 18.644 & & 14.052 & 30.699 & 28.064 & 37.909 \\
\hline 1/13/11 0:00 & 17.209 & 6.666 & 11.941 & 19.805 & 18.661 & & 14.038 & 30.758 & 28.064 & 37.991 \\
\hline $1 / 13 / 114: 00$ & 17.197 & 6.663 & 11.941 & 19.847 & 18.677 & & 14.027 & 30.706 & 28.064 & 38.036 \\
\hline $1 / 13 / 118: 00$ & 17.181 & 6.656 & 11.941 & 19.873 & 18.694 & & 14.001 & 30.668 & 28.064 & 38.128 \\
\hline 1/13/11 12:00 & 17.164 & 6.654 & 11.939 & 19.887 & 18.710 & & 13.975 & 30.642 & 28.062 & 38.232 \\
\hline 1/13/11 16:00 & 17.129 & 6.642 & 11.932 & 19.898 & 18.727 & & 13.937 & 30.666 & 28.060 & 38.312 \\
\hline $1 / 13 / 1120: 00$ & 17.115 & 6.642 & 11.932 & 19.912 & 18.743 & & 13.937 & 30.623 & 28.060 & 38.307 \\
\hline 1/14/11 0:00 & 17.103 & 6.645 & 11.932 & 19.917 & 18.762 & & 13.942 & 30.600 & 28.060 & 38.281 \\
\hline 1/14/11 4:00 & 17.098 & 6.649 & 11.932 & 19.908 & 18.779 & & 13.951 & 30.600 & 28.062 & 38.272 \\
\hline 1/14/11 8:00 & 17.098 & 6.649 & 11.934 & 19.896 & 18.793 & & 13.966 & 30.600 & 28.062 & 38.236 \\
\hline 1/14/11 12:00 & 17.117 & 6.654 & 11.939 & 19.861 & 18.812 & & 13.987 & 30.612 & 28.062 & 38.201 \\
\hline 1/14/11 16:00 & 17.115 & 6.652 & 11.939 & 19.901 & 18.826 & & 13.970 & 30.680 & 28.062 & 38.279 \\
\hline $1 / 14 / 1120: 00$ & 17.112 & 6.652 & 11.939 & 19.880 & 18.842 & & 13.975 & 30.595 & 28.060 & 38.276 \\
\hline 1/15/11 0:00 & 17.113 & 6.652 & 11.941 & 19.884 & 18.859 & & 13.975 & 30.588 & 28.062 & 38.288 \\
\hline $1 / 15 / 114: 00$ & 17.112 & 6.654 & 11.943 & 19.840 & 18.878 & & 13.994 & 30.654 & 28.060 & 38.246 \\
\hline $1 / 15 / 118: 00$ & 17.129 & 6.661 & 11.948 & 19.796 & 18.892 & & 14.017 & 30.647 & 28.060 & 38.187 \\
\hline 1/15/11 12:00 & 17.155 & 6.666 & 11.953 & 19.777 & 18.911 & & 14.038 & 30.659 & 28.062 & 38.135 \\
\hline 1/15/11 16:00 & 17.167 & 6.664 & 11.955 & 19.819 & 18.925 & & 14.038 & 30.649 & 28.060 & 38.159 \\
\hline $1 / 15 / 1120: 00$ & 17.188 & 6.668 & 11.960 & 19.772 & 18.941 & & 14.052 & 30.734 & 28.060 & 38.123 \\
\hline 1/16/11 0:00 & 17.200 & 6.671 & 11.965 & 19.786 & 18.958 & & 14.057 & 30.708 & 28.060 & 38.130 \\
\hline 1/16/11 4:00 & 17.207 & 6.673 & 11.969 & 19.779 & 18.974 & & 14.062 & 30.696 & 28.060 & 38.135 \\
\hline 1/16/11 8:00 & 17.207 & 6.671 & 11.972 & 19.826 & 18.988 & & 14.050 & 30.701 & 28.060 & 38.201 \\
\hline 1/16/11 12:00 & 17.209 & 6.673 & 11.974 & 19.831 & 19.007 & & 14.050 & 30.769 & 28.060 & 38.213 \\
\hline 1/16/11 16:00 & 17.190 & 6.661 & 11.972 & 19.980 & 19.024 & & 14.010 & 30.675 & 28.057 & 38.338 \\
\hline $1 / 16 / 1120: 00$ & 17.176 & 6.659 & 11.965 & 19.982 & 19.038 & & 13.996 & 30.652 & 28.057 & 38.385 \\
\hline 1/17/11 0:00 & 17.153 & 6.654 & 11.962 & 19.989 & 19.055 & & 13.973 & 30.628 & 28.055 & 38.467 \\
\hline $1 / 17 / 114: 00$ & 17.122 & 6.647 & 11.955 & 19.793 & 19.071 & & 13.937 & 30.598 & 28.053 & 38.578 \\
\hline $1 / 17 / 118: 00$ & 17.091 & 6.638 & 11.948 & 19.777 & 19.085 & & 13.902 & 30.560 & 28.053 & 38.682 \\
\hline 1/17/11 12:00 & 17.070 & 6.640 & 11.941 & 19.758 & 19.099 & & 13.895 & 30.546 & 28.050 & 38.660 \\
\hline 1/17/11 16:00 & 17.042 & 6.633 & 11.931 & 19.724 & 19.090 & & 13.893 & 30.525 & 28.050 & 38.653 \\
\hline $1 / 17 / 1120: 00$ & 17.004 & 6.638 & 11.922 & 19.707 & 19.102 & & 13.926 & 30.525 & 28.048 & 38.585 \\
\hline $1 / 18 / 110: 00$ & 16.981 & 6.643 & 11.913 & 19.703 & 19.116 & & 13.977 & 30.551 & 28.048 & 38.519 \\
\hline 1/18/11 4:00 & 16.999 & 6.636 & 11.903 & 19.703 & 19.130 & & 14.024 & 30.584 & 28.048 & 38.472 \\
\hline 1/18/11 8:00 & 17.049 & 6.633 & 11.896 & 19.707 & 19.144 & & 14.074 & 30.685 & 28.048 & 38.394 \\
\hline 1/18/11 12:00 & 17.101 & 6.638 & 11.891 & 19.719 & 19.161 & & 14.092 & 30.692 & 28.048 & 38.177 \\
\hline 1/18/11 16:00 & 17.122 & 6.640 & 11.887 & 19.728 & 19.172 & & 14.095 & 30.678 & 28.048 & 38.067 \\
\hline 1/18/11 20:00 & 17.150 & 6.654 & 11.882 & 19.738 & 19.189 & & 14.114 & 30.715 & 28.048 & 37.980 \\
\hline 1/19/11 0:00 & 17.169 & 6.659 & 11.882 & 19.747 & 19.203 & & 14.121 & 30.718 & 28.048 & 37.973 \\
\hline 1/19/11 4:00 & 17.181 & 6.666 & 11.879 & 19.754 & 19.220 & & 14.130 & 30.722 & 28.048 & 38.017 \\
\hline $1 / 19 / 118: 00$ & 17.193 & 6.671 & 11.877 & 19.763 & 19.234 & & 14.135 & 30.732 & 28.048 & 38.053 \\
\hline 1/19/11 12:00 & 17.209 & 6.673 & 11.877 & 19.772 & 19.250 & & 14.144 & 30.805 & 28.048 & 38.013 \\
\hline 1/19/11 16:00 & 17.207 & 6.666 & 11.875 & 19.782 & 19.264 & & 14.123 & 30.750 & 28.048 & 38.137 \\
\hline
\end{tabular}

TABLE S1.1 Page 59 of 89 
Water Level (ft below reference point)

\begin{tabular}{|c|c|c|c|c|c|c|c|c|c|c|}
\hline Date and Time & McPike & Potter & Hughes & Barr & Burks & MoDOT & Clizer "Old" & Morgan & SB24 & SB38 \\
\hline 1/19/11 20:00 & 17.216 & 6.673 & 11.875 & 19.789 & 19.278 & & 14.149 & 30.772 & 28.048 & 38.064 \\
\hline 1/20/11 0:00 & 17.235 & 6.676 & 11.877 & 19.800 & 19.295 & & 14.160 & 30.772 & 28.048 & 38.043 \\
\hline 1/20/11 4:00 & 17.249 & 6.676 & 11.879 & 19.810 & 19.309 & & 14.168 & 30.779 & 28.048 & 38.029 \\
\hline 1/20/11 8:00 & 17.261 & 6.680 & 11.879 & 19.821 & 19.323 & & 14.177 & 30.783 & 28.050 & 38.003 \\
\hline 1/20/11 12:00 & 17.273 & 6.678 & 11.879 & 19.814 & 19.340 & & 14.175 & 30.788 & 28.050 & 38.031 \\
\hline 1/20/11 16:00 & 17.263 & 6.676 & 11.879 & 19.880 & 19.354 & & 14.165 & 30.760 & 28.048 & 38.111 \\
\hline 1/20/11 20:00 & 17.263 & 6.680 & 11.879 & 19.884 & 19.368 & & 14.163 & 30.842 & 28.048 & 38.142 \\
\hline $1 / 21 / 110: 00$ & 17.259 & 6.678 & 11.877 & 19.894 & 19.382 & & 14.163 & 30.826 & 28.048 & 38.170 \\
\hline $1 / 21 / 114: 00$ & 17.244 & 6.676 & 11.875 & 19.894 & 19.399 & & 14.144 & 30.809 & 28.048 & 38.241 \\
\hline 1/21/11 8:00 & 17.233 & 6.671 & 11.872 & 19.908 & 19.413 & & 14.130 & 30.760 & 28.046 & 38.298 \\
\hline $1 / 21 / 11$ 12:00 & 17.219 & 6.669 & 11.868 & 19.947 & 19.427 & & 14.118 & 30.753 & 28.046 & 38.342 \\
\hline $1 / 21 / 11$ 16:00 & 17.200 & 6.664 & 11.865 & 19.889 & 19.444 & & 14.104 & 30.708 & 28.046 & 38.401 \\
\hline 1/21/11 20:00 & 17.197 & 6.669 & 11.865 & 19.859 & 19.458 & & 14.123 & 30.708 & 28.043 & 38.345 \\
\hline 1/22/11 0:00 & 17.202 & 6.666 & 11.863 & 19.826 & 19.472 & & 14.125 & 30.753 & 28.046 & 38.364 \\
\hline $1 / 22 / 114: 00$ & 17.202 & 6.669 & 11.863 & 19.852 & 19.486 & & 14.125 & 30.762 & 28.043 & 38.371 \\
\hline 1/22/11 8:00 & 17.200 & 6.671 & 11.863 & 19.854 & 19.503 & & 14.135 & 30.727 & 28.043 & 38.361 \\
\hline 1/22/11 12:00 & 17.204 & 6.676 & 11.865 & 19.835 & 19.517 & & 14.144 & 30.720 & 28.043 & 38.328 \\
\hline 1/22/11 16:00 & 17.204 & 6.671 & 11.865 & 19.840 & 19.531 & & 14.137 & 30.699 & 28.043 & 38.371 \\
\hline 1/22/11 20:00 & 17.219 & 6.678 & 11.865 & 19.798 & 19.547 & & 14.163 & 30.727 & 28.041 & 38.295 \\
\hline $1 / 23 / 110: 00$ & 17.240 & 6.680 & 11.872 & 19.763 & 19.559 & & 14.182 & 30.788 & 28.043 & 38.232 \\
\hline $1 / 23 / 114: 00$ & 17.266 & 6.680 & 11.879 & 19.763 & 19.573 & & 14.207 & 30.797 & 28.046 & 38.147 \\
\hline 1/23/11 8:00 & 17.299 & 6.687 & 11.889 & 19.768 & 19.587 & & 14.231 & 30.812 & 28.046 & 38.088 \\
\hline $1 / 23 / 11$ 12:00 & 17.322 & 6.692 & 11.896 & 19.782 & 19.599 & & 14.233 & 30.819 & 28.046 & 38.095 \\
\hline $1 / 23 / 11$ 16:00 & 17.322 & 6.688 & 11.896 & 19.866 & 19.613 & & 14.217 & 30.826 & 28.046 & 38.187 \\
\hline 1/23/11 20:00 & 17.322 & 6.687 & 11.901 & 19.884 & 19.627 & & 14.214 & 30.819 & 28.046 & 38.225 \\
\hline 1/24/11 0:00 & 17.320 & 6.688 & 11.903 & 19.891 & 19.642 & & 14.212 & 30.814 & 28.046 & 38.250 \\
\hline $1 / 24 / 114: 00$ & 17.310 & 6.685 & 11.903 & 19.891 & 19.658 & & 14.205 & 30.797 & 28.043 & 38.302 \\
\hline 1/24/11 8:00 & 17.313 & 6.688 & 11.908 & 19.861 & 19.672 & & 14.219 & 30.805 & 28.043 & 38.269 \\
\hline 1/24/11 12:00 & 17.325 & 6.690 & 11.913 & 19.817 & 19.684 & & 14.236 & 30.826 & 28.046 & 38.220 \\
\hline 1/24/11 16:00 & 17.334 & 6.690 & 11.917 & 19.817 & 19.698 & & 14.245 & 30.835 & 28.043 & 38.206 \\
\hline 1/24/11 20:00 & 17.353 & 6.692 & 11.924 & 19.761 & 19.708 & & 14.264 & 30.856 & 28.046 & 38.152 \\
\hline $1 / 25 / 110: 00$ & 17.369 & 6.697 & 11.929 & 19.759 & 19.722 & & 14.271 & 30.866 & 28.046 & 38.152 \\
\hline $1 / 25 / 114: 00$ & 17.381 & 6.697 & 11.936 & 19.747 & 19.734 & & 14.278 & 30.875 & 28.043 & 38.142 \\
\hline 1/25/11 8:00 & 17.388 & 6.699 & 11.939 & 19.789 & 19.750 & & 14.273 & 30.875 & 28.043 & 38.185 \\
\hline $1 / 25 / 1112: 00$ & 17.391 & 6.702 & 11.943 & 19.793 & 19.762 & & 14.273 & 30.880 & 28.043 & 38.196 \\
\hline 1/25/11 16:00 & 17.376 & 6.695 & 11.943 & 19.905 & 19.767 & & 14.245 & 30.852 & 28.043 & 38.302 \\
\hline $1 / 25 / 1120: 00$ & 17.358 & 6.692 & 11.943 & 19.926 & 19.778 & & 14.247 & 30.840 & 28.043 & 38.312 \\
\hline 1/26/11 0:00 & 17.351 & 6.692 & 11.943 & 19.926 & 19.790 & & 14.250 & 30.840 & 28.041 & 38.309 \\
\hline $1 / 26 / 114: 00$ & 17.351 & 6.695 & 11.943 & 19.910 & 19.804 & & 14.257 & 30.840 & 28.043 & 38.300 \\
\hline 1/26/11 8:00 & 17.360 & 6.699 & 11.946 & 19.859 & 19.818 & & 14.280 & 30.856 & 28.041 & 38.234 \\
\hline 1/26/11 12:00 & 17.384 & 6.702 & 11.953 & 19.756 & 19.833 & & 14.304 & 30.889 & 28.043 & 38.154 \\
\hline 1/26/11 16:00 & 17.400 & 6.704 & 11.953 & 19.803 & 19.847 & & 14.299 & 30.894 & 28.043 & 38.192 \\
\hline 1/26/11 20:00 & 17.407 & 6.706 & 11.960 & 19.796 & 19.859 & & 14.301 & 30.899 & 28.043 & 38.203 \\
\hline 1/27/11 0:00 & 17.407 & 6.702 & 11.960 & 19.863 & 19.875 & & 14.287 & 30.887 & 28.043 & 38.272 \\
\hline $1 / 27 / 114: 00$ & 17.388 & 6.697 & 11.957 & 19.957 & 19.889 & & 14.259 & 30.863 & 28.041 & 38.378 \\
\hline 1/27/11 8:00 & 17.362 & 6.692 & 11.953 & 20.043 & 19.901 & & 14.231 & 30.830 & 28.041 & 38.462 \\
\hline $1 / 27 / 1112: 00$ & 17.348 & 6.692 & 11.953 & 20.024 & 19.915 & & 14.238 & 30.826 & 28.041 & 38.434 \\
\hline $1 / 27 / 11$ 16:00 & 17.341 & 6.690 & 11.950 & 20.024 & 19.925 & & 14.247 & 30.826 & 28.041 & 38.422 \\
\hline 1/27/11 20:00 & 17.336 & 6.695 & 11.953 & 19.954 & 19.939 & & 14.273 & 30.842 & 28.041 & 38.364 \\
\hline 1/28/11 0:00 & 17.334 & 6.697 & 11.955 & 19.905 & 19.951 & & 14.285 & 30.852 & 28.041 & 38.338 \\
\hline $1 / 28 / 114: 00$ & 17.332 & 6.700 & 11.955 & 19.901 & 19.965 & & 14.285 & 30.856 & 28.041 & 38.352 \\
\hline 1/28/11 8:00 & 17.329 & 6.702 & 11.955 & 19.903 & 19.979 & & 14.290 & 30.856 & 28.041 & 38.356 \\
\hline 1/28/11 12:00 & 17.325 & 6.700 & 11.955 & 19.929 & 19.993 & & 14.276 & 30.852 & 28.041 & 38.406 \\
\hline 1/28/11 16:00 & 17.299 & 6.693 & 11.950 & 20.029 & 20.007 & & 14.269 & 30.830 & 28.041 & 38.429 \\
\hline 1/28/11 20:00 & 17.263 & 6.698 & 11.948 & 19.924 & 20.019 & & 14.285 & 30.828 & 28.039 & 38.411 \\
\hline 1/29/11 0:00 & 17.240 & 6.698 & 11.943 & 19.807 & 20.033 & & 14.301 & 30.835 & 28.041 & 38.389 \\
\hline $1 / 29 / 114: 00$ & 17.235 & 6.702 & 11.936 & 19.728 & 20.047 & & 14.311 & 30.842 & 28.041 & 38.371 \\
\hline 1/29/11 8:00 & 17.237 & 6.705 & 11.927 & 19.696 & 20.059 & & 14.308 & 30.845 & 28.041 & 38.291 \\
\hline $1 / 29 / 1112: 00$ & 17.247 & 6.705 & 11.920 & 19.684 & 20.073 & & 14.318 & 30.852 & 28.041 & 38.241 \\
\hline 1/29/11 16:00 & 17.247 & 6.705 & 11.910 & 19.721 & 20.087 & & 14.313 & 30.849 & 28.041 & 38.253 \\
\hline 1/29/11 20:00 & 17.249 & 6.707 & 11.901 & 19.633 & 20.099 & & 14.339 & 30.856 & 28.043 & 38.250 \\
\hline $1 / 30 / 110: 00$ & 17.266 & 6.709 & 11.894 & 19.539 & 20.113 & & 14.353 & 30.877 & 28.043 & 38.234 \\
\hline $1 / 30 / 114: 00$ & 17.287 & 6.714 & 11.886 & 19.521 & 20.125 & & 14.367 & 30.894 & 28.043 & 38.076 \\
\hline 1/30/11 8:00 & 17.308 & 6.716 & 11.879 & 19.479 & 20.142 & & 14.386 & 30.908 & 28.046 & 38.038 \\
\hline
\end{tabular}

TABLE S1.1 Page 60 of 89 
Water Level (ft below reference point)

\begin{tabular}{|c|c|c|c|c|c|c|c|c|c|c|}
\hline Date and Time & McPike & Potter & Hughes & Barr & Burks & MoDOT & Clizer "Old" & Morgan & SB24 & SB38 \\
\hline 1/30/11 12:00 & 17.327 & 6.719 & 11.875 & 19.502 & 20.153 & & 14.384 & 30.920 & 28.046 & 38.043 \\
\hline 1/30/11 16:00 & 17.327 & 6.714 & 11.868 & 19.609 & 20.168 & & 14.360 & 30.894 & 28.046 & 38.149 \\
\hline 1/30/11 20:00 & 17.325 & 6.712 & 11.863 & 19.623 & 20.182 & & 14.365 & 30.887 & 28.046 & 38.156 \\
\hline 1/31/11 0:00 & 17.327 & 6.714 & 11.856 & 19.649 & 20.194 & & 14.355 & 30.882 & 28.046 & 38.187 \\
\hline 1/31/11 4:00 & 17.320 & 6.709 & 11.849 & 19.691 & 20.208 & & 14.341 & 30.870 & 28.046 & 38.246 \\
\hline 1/31/11 8:00 & 17.306 & 6.705 & 11.839 & 19.793 & 20.219 & & 14.313 & 30.847 & 28.043 & 38.323 \\
\hline 1/31/11 12:00 & 17.303 & 6.705 & 11.834 & 19.789 & 20.231 & & 14.318 & 30.845 & 28.046 & 38.314 \\
\hline $1 / 31 / 11$ 16:00 & 17.294 & 6.702 & 11.827 & 19.849 & 20.243 & & 14.304 & 30.826 & 28.043 & 38.356 \\
\hline 1/31/11 20:00 & 17.303 & 6.709 & 11.825 & 19.772 & 20.257 & & 14.327 & 30.837 & 28.046 & 38.291 \\
\hline 2/1/11 0:00 & 17.327 & 6.712 & 11.823 & 19.682 & 20.269 & & 14.360 & 30.861 & 28.046 & 38.199 \\
\hline $2 / 1 / 114: 00$ & 17.343 & 6.714 & 11.820 & 19.679 & 20.281 & & 14.360 & 30.866 & 28.046 & 38.203 \\
\hline 2/1/11 8:00 & 17.353 & 6.714 & 11.820 & 19.689 & 20.295 & & 14.339 & 30.863 & 28.046 & 38.265 \\
\hline 2/1/11 12:00 & 17.351 & 6.714 & 11.818 & 19.752 & 20.304 & & 14.323 & 30.849 & 28.043 & 38.309 \\
\hline 2/1/11 16:00 & 17.341 & 6.707 & 11.815 & 19.798 & 20.316 & & 14.311 & 30.830 & 28.046 & 38.302 \\
\hline 2/1/11 20:00 & 17.346 & 6.707 & 11.811 & 19.768 & 20.328 & & 14.325 & 30.837 & 28.046 & 38.279 \\
\hline 2/2/11 0:00 & 17.372 & 6.712 & 11.816 & 19.651 & 20.337 & & 14.374 & 30.875 & 28.048 & 38.159 \\
\hline 2/2/11 4:00 & 17.407 & 6.719 & 11.820 & 19.633 & 20.349 & & 14.395 & 30.908 & 28.048 & 38.093 \\
\hline 2/2/11 8:00 & 17.440 & 6.721 & 11.827 & 19.630 & 20.361 & & 14.421 & 30.939 & 28.048 & 38.022 \\
\hline 2/2/11 12:00 & 17.471 & 6.728 & 11.837 & 19.670 & 20.373 & & 14.447 & 30.960 & 28.050 & 37.947 \\
\hline $2 / 2 / 11$ 16:00 & 17.499 & 6.731 & 11.844 & 19.698 & 20.382 & & 14.454 & 30.976 & 28.053 & 37.963 \\
\hline 2/2/11 20:00 & 17.516 & 6.733 & 11.853 & 19.796 & 20.396 & & 14.468 & 30.988 & 28.053 & 37.963 \\
\hline 2/3/11 0:00 & 17.527 & 6.733 & 11.858 & 19.826 & 20.406 & & 14.459 & 30.993 & 28.053 & 38.055 \\
\hline $2 / 3 / 114: 00$ & 17.523 & 6.733 & 11.860 & 19.873 & 20.418 & & 14.433 & 30.979 & 28.053 & 38.166 \\
\hline $2 / 3 / 118: 00$ & 17.516 & 6.728 & 11.858 & 19.908 & 20.427 & & 14.428 & 30.967 & 28.053 & 38.199 \\
\hline 2/3/11 12:00 & 17.501 & 6.726 & 11.860 & 19.926 & 20.439 & & 14.405 & 30.960 & 28.053 & 38.262 \\
\hline 2/3/11 16:00 & 17.483 & 6.719 & 11.858 & 20.045 & 20.451 & & 14.370 & 30.927 & 28.053 & 38.373 \\
\hline 2/3/11 20:00 & 17.466 & 6.714 & 11.856 & 20.066 & 20.462 & & 14.362 & 30.915 & 28.053 & 38.387 \\
\hline 2/4/11 0:00 & 17.450 & 6.714 & 11.853 & 20.078 & 20.472 & & 14.360 & 30.906 & 28.050 & 38.411 \\
\hline $2 / 4 / 114: 00$ & 17.428 & 6.712 & 11.849 & 20.180 & 20.484 & & 14.320 & 30.882 & 28.050 & 38.524 \\
\hline 2/4/11 8:00 & 17.414 & 6.712 & 11.846 & 20.171 & 20.493 & & 14.320 & 30.870 & 28.050 & 38.500 \\
\hline $2 / 4 / 11$ 12:00 & 17.398 & 6.710 & 11.846 & 20.190 & 20.505 & & 14.297 & 30.859 & 28.050 & 38.554 \\
\hline $2 / 4 / 11$ 16:00 & 17.374 & 6.698 & 11.837 & 20.099 & 20.517 & & 14.250 & 30.823 & 28.048 & 38.679 \\
\hline 2/4/11 20:00 & 17.351 & 6.693 & 11.832 & 20.080 & 20.524 & & 14.238 & 30.802 & 28.048 & 38.693 \\
\hline 2/5/11 0:00 & 17.322 & 6.695 & 11.825 & 20.017 & 20.536 & & 14.222 & 30.783 & 28.048 & 38.724 \\
\hline 2/5/11 4:00 & 17.296 & 6.691 & 11.818 & 20.026 & 20.545 & & 14.193 & 30.758 & 28.046 & 38.806 \\
\hline $2 / 5 / 118: 00$ & 17.273 & 6.688 & 11.813 & 20.006 & 20.554 & & 14.182 & 30.739 & 28.046 & 38.813 \\
\hline 2/5/11 12:00 & 17.266 & 6.688 & 11.811 & 19.973 & 20.564 & & 14.186 & 30.729 & 28.043 & 38.769 \\
\hline 2/5/11 16:00 & 17.259 & 6.691 & 11.808 & 19.985 & 20.573 & & 14.191 & 30.722 & 28.046 & 38.733 \\
\hline 2/5/11 20:00 & 17.266 & 6.695 & 11.808 & 19.917 & 20.583 & & 14.217 & 30.734 & 28.043 & 38.616 \\
\hline 2/6/11 0:00 & 17.277 & 6.700 & 11.811 & 19.917 & 20.592 & & 14.238 & 30.741 & 28.046 & 38.564 \\
\hline $2 / 6 / 114: 00$ & 17.289 & 6.705 & 11.815 & 19.877 & 20.602 & & 14.264 & 30.755 & 28.046 & 38.521 \\
\hline 2/6/11 8:00 & 17.310 & 6.717 & 11.823 & 19.787 & 20.611 & & 14.306 & 30.779 & 28.046 & 38.408 \\
\hline $2 / 6 / 11$ 12:00 & 17.351 & 6.724 & 11.832 & 19.698 & 20.623 & & 14.360 & 30.819 & 28.048 & 38.295 \\
\hline $2 / 6 / 11$ 16:00 & 17.381 & 6.728 & 11.839 & 19.696 & 20.630 & & 14.386 & 30.842 & 28.048 & 38.272 \\
\hline 2/6/11 20:00 & 17.419 & 6.733 & 11.849 & 19.647 & 20.639 & & 14.426 & 30.875 & 28.048 & 38.196 \\
\hline 2/7/11 0:00 & 17.454 & 6.735 & 11.858 & 19.710 & 20.646 & & 14.445 & 30.903 & 28.050 & 38.175 \\
\hline 2/7/11 4:00 & 17.478 & 6.738 & 11.865 & 19.717 & 20.658 & & 14.445 & 30.915 & 28.050 & 38.210 \\
\hline $2 / 7 / 118: 00$ & 17.490 & 6.738 & 11.870 & 19.712 & 20.668 & & 14.456 & 30.922 & 28.050 & 38.203 \\
\hline 2/7/11 12:00 & 17.506 & 6.740 & 11.875 & 19.693 & 20.679 & & 14.456 & 30.936 & 28.050 & 38.217 \\
\hline 2/7/11 16:00 & 17.508 & 6.738 & 11.877 & 19.894 & 20.689 & & 14.445 & 30.927 & 28.050 & 38.295 \\
\hline 2/7/11 20:00 & 17.518 & 6.738 & 11.882 & 19.828 & 20.698 & & 14.466 & 30.946 & 28.050 & 38.255 \\
\hline 2/8/11 0:00 & 17.539 & 6.743 & 11.889 & 19.761 & 20.710 & & 14.492 & 30.969 & 28.053 & 38.177 \\
\hline 2/8/11 4:00 & 17.560 & 6.745 & 11.896 & 19.719 & 20.719 & & 14.515 & 30.993 & 28.053 & 38.166 \\
\hline 2/8/11 8:00 & 17.589 & 6.752 & 11.905 & 19.861 & 20.731 & & 14.541 & 31.019 & 28.055 & 38.079 \\
\hline $2 / 8 / 11$ 12:00 & 17.610 & 6.754 & 11.917 & 19.826 & 20.741 & & 14.546 & 31.040 & 28.055 & 38.079 \\
\hline $2 / 8 / 11$ 16:00 & 17.615 & 6.747 & 11.919 & 19.924 & 20.750 & & 14.513 & 31.019 & 28.055 & 38.215 \\
\hline 2/8/11 20:00 & 17.617 & 6.750 & 11.924 & 19.873 & 20.762 & & 14.532 & 31.026 & 28.055 & 38.194 \\
\hline 2/9/11 0:00 & 17.624 & 6.750 & 11.929 & 19.859 & 20.774 & & 14.532 & 31.040 & 28.055 & 38.210 \\
\hline 2/9/11 4:00 & 17.617 & 6.743 & 11.931 & 19.961 & 20.783 & & 14.501 & 31.019 & 28.055 & 38.314 \\
\hline 2/9/11 8:00 & 17.607 & 6.740 & 11.934 & 19.966 & 20.790 & & 14.503 & 31.007 & 28.057 & 38.328 \\
\hline $2 / 9 / 11$ 12:00 & 17.607 & 6.743 & 11.938 & 19.957 & 20.804 & & 14.506 & 31.007 & 28.057 & 38.345 \\
\hline 2/9/11 16:00 & 17.603 & 6.736 & 11.938 & 20.006 & 20.811 & & 14.487 & 30.997 & 28.055 & 38.406 \\
\hline 2/9/11 20:00 & 17.598 & 6.738 & 11.941 & 20.015 & 20.821 & & 14.492 & 30.995 & 28.057 & 38.420 \\
\hline 2/10/11 0:00 & 17.586 & 6.738 & 11.941 & 20.038 & 20.833 & & 14.487 & 30.993 & 28.057 & 38.439 \\
\hline
\end{tabular}

TABLE S1.1 Page 61 of 89 
Water Level (ft below reference point)

\begin{tabular}{|c|c|c|c|c|c|c|c|c|c|c|}
\hline Date and Time & McPike & Potter & Hughes & Barr & Burks & MoDOT & Clizer "Old" & Morgan & SB24 & SB38 \\
\hline 2/10/11 4:00 & 17.574 & 6.736 & 11.941 & 20.089 & 20.842 & & 14.463 & 30.976 & 28.057 & 38.519 \\
\hline 2/10/11 8:00 & 17.551 & 6.731 & 11.938 & 20.166 & 20.852 & & 14.433 & 30.950 & 28.057 & 38.592 \\
\hline 2/10/11 12:00 & 17.532 & 6.729 & 11.936 & 20.138 & 20.861 & & 14.438 & 30.941 & 28.055 & 38.566 \\
\hline 2/10/11 16:00 & 17.525 & 6.724 & 11.936 & 20.183 & 20.870 & & 14.417 & 30.924 & 28.057 & 38.630 \\
\hline 2/10/11 20:00 & 17.525 & 6.724 & 11.938 & 20.143 & 20.880 & & 14.433 & 30.927 & 28.055 & 38.590 \\
\hline 2/11/11 0:00 & 17.525 & 6.726 & 11.938 & 20.150 & 20.892 & & 14.433 & 30.927 & 28.055 & 38.592 \\
\hline 2/11/11 4:00 & 17.518 & 6.724 & 11.941 & 20.157 & 20.901 & & 14.435 & 30.922 & 28.055 & 38.604 \\
\hline 2/11/11 8:00 & 17.513 & 6.729 & 11.943 & 20.122 & 20.910 & & 14.452 & 30.924 & 28.057 & 38.576 \\
\hline 2/11/11 12:00 & 17.520 & 6.736 & 11.946 & 20.087 & 20.922 & & 14.463 & 30.932 & 28.057 & 38.540 \\
\hline 2/11/11 16:00 & 17.532 & 6.733 & 11.950 & 20.101 & 20.932 & & 14.473 & 30.932 & 28.057 & 38.531 \\
\hline 2/11/11 20:00 & 17.551 & 6.736 & 11.955 & 20.017 & 20.939 & & 14.503 & 30.960 & 28.057 & 38.432 \\
\hline $2 / 12 / 110: 00$ & 17.577 & 6.743 & 11.962 & 19.957 & 20.951 & & 14.525 & 30.988 & 28.060 & 38.375 \\
\hline 2/12/11 4:00 & 17.596 & 6.745 & 11.967 & 19.954 & 20.960 & & 14.529 & 31.002 & 28.060 & 38.385 \\
\hline 2/12/11 8:00 & 17.603 & 6.745 & 11.972 & 19.978 & 20.969 & & 14.520 & 31.004 & 28.060 & 38.439 \\
\hline 2/12/11 12:00 & 17.600 & 6.743 & 11.974 & 20.017 & 20.979 & & 14.508 & 30.997 & 28.060 & 38.488 \\
\hline 2/12/11 16:00 & 17.579 & 6.736 & 11.972 & 20.159 & 20.988 & & 14.478 & 30.969 & 28.060 & 38.568 \\
\hline 2/12/11 20:00 & 17.504 & 6.738 & 11.969 & 20.113 & 21.000 & & 14.492 & 30.964 & 28.060 & 38.503 \\
\hline 2/13/11 0:00 & 17.419 & 6.738 & 11.964 & 20.115 & 21.010 & & 14.485 & 30.948 & 28.060 & 38.526 \\
\hline 2/13/11 4:00 & 17.360 & 6.733 & 11.948 & 20.166 & 21.019 & & 14.454 & 30.920 & 28.060 & 38.583 \\
\hline 2/13/11 8:00 & 17.320 & 6.731 & 11.931 & 20.218 & 21.028 & & 14.438 & 30.889 & 28.060 & 38.663 \\
\hline $2 / 13 / 11$ 12:00 & 17.299 & 6.729 & 11.915 & 20.234 & 21.033 & & 14.421 & 30.868 & 28.057 & 38.689 \\
\hline 2/13/11 16:00 & 16.983 & 6.719 & 11.891 & 20.336 & 21.033 & & 14.341 & 30.821 & 28.057 & 38.693 \\
\hline 2/13/11 20:00 & 15.255 & 6.630 & 11.841 & 20.052 & 21.002 & & 14.001 & 30.736 & 28.057 & 38.660 \\
\hline $2 / 14 / 110: 00$ & 14.508 & 6.173 & 11.766 & 19.763 & 20.962 & & 13.846 & 30.673 & 28.060 & 38.606 \\
\hline 2/14/11 4:00 & 14.395 & 5.845 & 11.685 & 19.663 & 20.918 & & 13.724 & 30.583 & 28.060 & 38.571 \\
\hline 2/14/11 8:00 & 14.489 & 5.756 & 11.617 & 19.575 & 20.875 & & 13.653 & 30.489 & 28.060 & 38.444 \\
\hline 2/14/11 12:00 & 14.659 & 5.803 & 11.560 & 19.502 & 20.833 & & 13.606 & 30.417 & 28.060 & 38.342 \\
\hline 2/14/11 16:00 & 14.442 & 5.852 & 11.498 & 19.616 & 20.793 & & 13.423 & 30.318 & 28.060 & 38.307 \\
\hline 2/14/11 20:00 & 13.832 & 5.539 & 11.404 & 19.682 & 20.752 & & 13.287 & 30.191 & 28.057 & 38.364 \\
\hline 2/15/11 0:00 & 13.594 & 5.322 & 11.305 & 19.712 & 20.712 & & 13.174 & 30.059 & 28.057 & 38.404 \\
\hline 2/15/11 4:00 & 13.568 & 5.294 & 11.217 & 19.805 & 20.675 & & 13.059 & 29.918 & 28.055 & 38.413 \\
\hline 2/15/11 8:00 & 13.645 & 5.369 & 11.139 & 19.856 & 20.635 & & 12.981 & 29.779 & 28.050 & 38.425 \\
\hline $2 / 15 / 11$ 12:00 & 13.768 & 5.485 & 11.073 & 19.894 & 20.602 & & 12.918 & 29.662 & 28.048 & 38.328 \\
\hline 2/15/11 16:00 & 13.304 & 5.501 & 10.973 & 19.994 & 20.564 & & 12.798 & 29.514 & 28.048 & 38.641 \\
\hline 2/15/11 20:00 & 12.460 & 4.995 & 10.803 & 19.978 & 20.526 & & 12.598 & 29.290 & 28.043 & 38.592 \\
\hline 2/16/11 0:00 & 12.036 & 4.632 & 10.647 & 19.898 & 20.493 & & 12.427 & 29.166 & 28.041 & 38.531 \\
\hline $2 / 16 / 114: 00$ & 11.894 & 4.528 & 10.524 & 19.896 & 20.460 & & 12.270 & 29.041 & 28.036 & 38.526 \\
\hline 2/16/11 8:00 & 11.889 & 4.460 & 10.401 & 19.887 & 20.427 & & 12.124 & 28.919 & 28.034 & 38.559 \\
\hline 2/16/11 12:00 & 11.998 & 4.443 & 10.297 & 19.889 & 20.392 & & 12.006 & 28.815 & 28.029 & 38.397 \\
\hline 2/16/11 16:00 & 11.623 & 4.269 & 10.117 & 20.054 & 20.359 & & 11.767 & 28.561 & 28.024 & 38.338 \\
\hline 2/16/11 20:00 & 11.145 & 3.932 & 9.838 & 20.085 & 20.323 & & 11.386 & 28.237 & 28.017 & 38.392 \\
\hline 2/17/11 0:00 & 10.848 & 3.680 & 9.569 & 20.057 & 20.290 & & 11.053 & 28.070 & 28.010 & 38.378 \\
\hline 2/17/11 4:00 & 10.536 & 3.369 & 9.237 & 20.110 & 20.257 & & 10.621 & 27.851 & 28.001 & 38.361 \\
\hline 2/17/11 8:00 & 10.324 & 3.105 & 8.902 & 20.094 & 20.224 & & 10.374 & 27.609 & 27.996 & 38.359 \\
\hline 2/17/11 12:00 & 10.192 & 2.978 & 8.523 & 20.071 & 20.191 & & 10.198 & 27.419 & 27.987 & 39.204 \\
\hline 2/17/11 16:00 & 10.070 & 3.002 & 8.123 & 20.071 & 20.161 & & 10.080 & 27.242 & 27.980 & 39.073 \\
\hline $2 / 17 / 1120: 00$ & 10.133 & 3.237 & 7.896 & 19.796 & 20.130 & & 10.162 & 27.207 & 27.973 & 38.736 \\
\hline 2/18/11 0:00 & 10.409 & 3.515 & 7.814 & 19.558 & 20.099 & & 10.306 & 27.223 & 27.964 & 38.521 \\
\hline $2 / 18 / 114: 00$ & 10.694 & 3.774 & 7.788 & 19.383 & 20.066 & & 10.419 & 27.237 & 27.954 & 38.380 \\
\hline $2 / 18 / 11$ 8:00 & 10.970 & 4.001 & 7.792 & 19.241 & 20.038 & & 10.512 & 27.245 & 27.947 & 38.260 \\
\hline 2/18/11 12:00 & 11.217 & 4.201 & 7.806 & 19.155 & 20.005 & & 10.585 & 27.263 & 27.938 & 38.239 \\
\hline 2/18/11 16:00 & 11.382 & 4.359 & 7.787 & 19.290 & 19.972 & & 10.588 & 27.212 & 27.928 & 38.356 \\
\hline 2/18/11 20:00 & 11.521 & 4.495 & 7.776 & 19.314 & 19.937 & & 10.592 & 27.153 & 27.917 & 38.345 \\
\hline 2/19/11 0:00 & 11.653 & 4.616 & 7.785 & 19.274 & 19.901 & & 10.599 & 27.125 & 27.907 & 38.333 \\
\hline 2/19/11 4:00 & 11.764 & 4.719 & 7.802 & 19.255 & 19.863 & & 10.604 & 27.089 & 27.898 & 38.359 \\
\hline 2/19/11 8:00 & 11.863 & 4.806 & 7.823 & 19.265 & 19.830 & & 10.597 & 27.038 & 27.888 & 38.359 \\
\hline 2/19/11 12:00 & 11.948 & 4.887 & 7.842 & 19.267 & 19.790 & & 10.592 & 27.005 & 27.877 & 38.394 \\
\hline 2/19/11 16:00 & 11.988 & 4.948 & 7.828 & 19.409 & 19.753 & & 10.543 & 26.911 & 27.865 & 38.528 \\
\hline 2/19/11 20:00 & 12.026 & 5.004 & 7.814 & 19.444 & 19.712 & & 10.512 & 26.831 & 27.856 & 38.568 \\
\hline 2/20/11 0:00 & 12.049 & 5.047 & 7.816 & 19.493 & 19.675 & & 10.473 & 26.741 & 27.844 & 38.627 \\
\hline 2/20/11 4:00 & 12.047 & 5.087 & 7.816 & 19.570 & 19.635 & & 10.414 & 26.633 & 27.832 & 38.759 \\
\hline $2 / 20 / 11$ 8:00 & 12.033 & 5.117 & 7.814 & 19.677 & 19.592 & & 10.357 & 26.490 & 27.820 & 38.823 \\
\hline 2/20/11 12:00 & 12.023 & 5.150 & 7.814 & 19.759 & 19.557 & & 10.308 & 26.374 & 27.806 & 38.903 \\
\hline 2/20/11 16:00 & 12.011 & 5.179 & 7.816 & 19.889 & 19.517 & & 10.266 & 26.262 & 27.790 & 38.981 \\
\hline
\end{tabular}

TABLE S1.1 Page 62 of 89 
Water Level (ft below reference point)

\begin{tabular}{|c|c|c|c|c|c|c|c|c|c|c|}
\hline Date and Time & McPike & Potter & Hughes & Barr & Burks & MoDOT & Clizer "Old" & Morgan & SB24 & SB38 \\
\hline 2/20/11 20:00 & 12.097 & 5.226 & 7.844 & 19.600 & 19.474 & & 10.320 & 26.306 & 27.783 & 38.717 \\
\hline 2/21/11 0:00 & 12.181 & 5.273 & 7.880 & 19.355 & 19.437 & & 10.390 & 26.391 & 27.774 & 38.550 \\
\hline 2/21/11 4:00 & 12.235 & 5.311 & 7.913 & 19.251 & 19.396 & & 10.421 & 26.426 & 27.762 & 38.519 \\
\hline 2/21/11 8:00 & 12.294 & 5.341 & 7.944 & 19.111 & 19.356 & & 10.458 & 26.459 & 27.752 & 38.401 \\
\hline 2/21/11 12:00 & 12.356 & 5.377 & 7.979 & 18.985 & 19.316 & & 10.505 & 26.523 & 27.741 & 38.354 \\
\hline 2/21/11 16:00 & 12.398 & 5.405 & 8.007 & 18.987 & 19.276 & & 10.524 & 26.546 & 27.731 & 38.354 \\
\hline 2/21/11 20:00 & 12.455 & 5.433 & 8.041 & 18.862 & 19.236 & & 10.569 & 26.600 & 27.720 & 38.236 \\
\hline $2 / 22 / 110: 00$ & 12.509 & 5.459 & 8.074 & 18.750 & 19.196 & & 10.609 & 26.668 & 27.710 & 38.170 \\
\hline 2/22/11 4:00 & 12.549 & 5.480 & 8.104 & 18.705 & 19.154 & & 10.628 & 26.704 & 27.698 & 38.147 \\
\hline 2/22/11 8:00 & 12.584 & 5.499 & 8.133 & 18.652 & 19.113 & & 10.649 & 26.739 & 27.689 & 38.107 \\
\hline $2 / 22 / 11$ 12:00 & 12.627 & 5.523 & 8.164 & 18.591 & 19.071 & & 10.679 & 26.793 & 27.680 & 38.130 \\
\hline 2/22/11 16:00 & 12.629 & 5.530 & 8.180 & 18.768 & 19.026 & & 10.635 & 26.755 & 27.663 & 38.288 \\
\hline 2/22/11 20:00 & 12.629 & 5.537 & 8.192 & 18.827 & 18.981 & & 10.602 & 26.704 & 27.654 & 38.288 \\
\hline 2/23/11 0:00 & 12.638 & 5.551 & 8.206 & 18.799 & 18.939 & & 10.592 & 26.694 & 27.640 & 38.295 \\
\hline 2/23/11 4:00 & 12.631 & 5.558 & 8.216 & 18.848 & 18.894 & & 10.559 & 26.645 & 27.630 & 38.359 \\
\hline 2/23/11 8:00 & 12.615 & 5.563 & 8.223 & 18.906 & 18.849 & & 10.527 & 26.581 & 27.619 & 38.429 \\
\hline 2/23/11 12:00 & 12.610 & 5.570 & 8.230 & 18.936 & 18.805 & & 10.510 & 26.539 & 27.607 & 38.470 \\
\hline 2/23/11 16:00 & 12.603 & 5.577 & 8.235 & 19.039 & 18.764 & & 10.494 & 26.494 & 27.595 & 38.526 \\
\hline 2/23/11 20:00 & 12.643 & 5.598 & 8.253 & 18.915 & 18.717 & & 10.545 & 26.544 & 27.583 & 38.359 \\
\hline $2 / 24 / 110: 00$ & 12.686 & 5.617 & 8.277 & 18.768 & 18.672 & & 10.599 & 26.624 & 27.574 & 38.260 \\
\hline 2/24/11 4:00 & 12.709 & 5.633 & 8.296 & 18.717 & 18.630 & & 10.613 & 26.659 & 27.562 & 38.250 \\
\hline 2/24/11 8:00 & 12.719 & 5.643 & 8.313 & 18.726 & 18.585 & & 10.606 & 26.657 & 27.551 & 38.281 \\
\hline $2 / 24 / 11$ 12:00 & 12.735 & 5.652 & 8.329 & 18.671 & 18.543 & & 10.620 & 26.678 & 27.541 & 38.250 \\
\hline 2/24/11 16:00 & 12.697 & 5.645 & 8.331 & 18.815 & 18.498 & & 10.552 & 26.591 & 27.527 & 38.359 \\
\hline 2/24/11 20:00 & 12.719 & 5.657 & 8.346 & 18.710 & 18.456 & & 10.574 & 26.596 & 27.518 & 38.347 \\
\hline 2/25/11 0:00 & 12.761 & 5.669 & 8.367 & 18.615 & 18.411 & & 10.632 & 26.664 & 27.509 & 38.328 \\
\hline 2/25/11 4:00 & 12.803 & 5.688 & 8.393 & 18.493 & 18.368 & & 10.691 & 26.758 & 27.497 & 38.309 \\
\hline 2/25/11 8:00 & 12.839 & 5.706 & 8.419 & 18.414 & 18.326 & & 10.738 & 26.831 & 27.487 & 38.295 \\
\hline $2 / 25 / 11$ 12:00 & 12.872 & 5.718 & 8.443 & 18.386 & 18.283 & & 10.761 & 26.889 & 27.476 & 38.276 \\
\hline 2/25/11 16:00 & 12.874 & 5.721 & 8.457 & 18.463 & 18.239 & & 10.728 & 26.875 & 27.466 & 38.265 \\
\hline $2 / 25 / 11$ 20:00 & 12.877 & 5.721 & 8.469 & 18.507 & 18.196 & & 10.712 & 26.857 & 27.457 & 38.253 \\
\hline 2/26/11 0:00 & 12.881 & 5.725 & 8.478 & 18.538 & 18.156 & & 10.700 & 26.847 & 27.445 & 38.241 \\
\hline 2/26/11 4:00 & 12.865 & 5.723 & 8.483 & 18.603 & 18.111 & & 10.663 & 26.802 & 27.433 & 38.234 \\
\hline $2 / 26 / 118: 00$ & 12.844 & 5.721 & 8.485 & 18.678 & 18.069 & & 10.628 & 26.737 & 27.422 & 38.227 \\
\hline 2/26/11 12:00 & 12.832 & 5.723 & 8.490 & 18.703 & 18.026 & & 10.611 & 26.697 & 27.410 & 38.222 \\
\hline 2/26/11 16:00 & 12.806 & 5.721 & 8.488 & 18.792 & 17.984 & & 10.571 & 26.624 & 27.398 & 38.220 \\
\hline 2/26/11 20:00 & 12.803 & 5.723 & 8.492 & 18.759 & 17.941 & & 10.578 & 26.605 & 27.387 & 38.217 \\
\hline 2/27/11 0:00 & 12.803 & 5.732 & 8.497 & 18.752 & 17.899 & & 10.581 & 26.603 & 27.375 & 38.215 \\
\hline 2/27/11 4:00 & 12.794 & 5.735 & 8.497 & 18.787 & 17.859 & & 10.562 & 26.563 & 27.365 & 38.210 \\
\hline 2/27/11 8:00 & 12.782 & 5.735 & 8.499 & 18.794 & 17.816 & & 10.550 & 26.527 & 27.354 & 38.210 \\
\hline 2/27/11 12:00 & 12.784 & 5.742 & 8.504 & 18.726 & 17.772 & & 10.557 & 26.532 & 27.342 & 38.208 \\
\hline 2/27/11 16:00 & 12.756 & 5.740 & 8.502 & 18.843 & 17.708 & & 10.527 & 26.464 & 27.328 & 38.208 \\
\hline 2/27/11 20:00 & 12.775 & 5.747 & 8.495 & 18.694 & 17.637 & & 10.557 & 26.471 & 27.321 & 38.201 \\
\hline 2/28/11 0:00 & 12.775 & 5.751 & 8.447 & 18.484 & 17.590 & & 10.635 & 26.591 & 27.309 & 38.196 \\
\hline 2/28/11 4:00 & 12.803 & 5.742 & 8.421 & 18.284 & 17.555 & & 10.710 & 26.685 & 27.300 & 38.152 \\
\hline $2 / 28 / 118: 00$ & 12.857 & 5.744 & 8.431 & 18.037 & 17.517 & & 10.830 & 26.840 & 27.293 & 37.895 \\
\hline 2/28/11 12:00 & 12.895 & 5.737 & 8.445 & 17.951 & 17.482 & & 10.876 & 26.936 & 27.283 & 37.878 \\
\hline $2 / 28 / 11$ 16:00 & 12.912 & 5.726 & 8.445 & 18.027 & 17.437 & & 10.867 & 26.974 & 27.272 & 37.921 \\
\hline 2/28/11 20:00 & 12.912 & 5.711 & 8.417 & 18.032 & 17.397 & & 10.848 & 26.969 & 27.262 & 37.930 \\
\hline 3/1/11 0:00 & 12.907 & 5.697 & 8.376 & 18.030 & 17.359 & & 10.818 & 26.946 & 27.250 & 37.951 \\
\hline 3/1/11 4:00 & 12.876 & 5.678 & 8.334 & 18.139 & 17.317 & & 10.750 & 26.871 & 27.241 & 38.074 \\
\hline 3/1/11 8:00 & 12.872 & 5.660 & 8.308 & 18.139 & 17.279 & & 10.750 & 26.831 & 27.229 & 38.034 \\
\hline 3/1/11 12:00 & 12.879 & 5.652 & 8.294 & 18.153 & 17.239 & & 10.750 & 26.817 & 27.220 & 38.050 \\
\hline 3/1/11 16:00 & 12.846 & 5.631 & 8.237 & 18.267 & 17.199 & & 10.728 & 26.786 & 27.208 & 38.116 \\
\hline $3 / 1 / 1120: 00$ & 12.683 & 5.506 & 8.126 & 18.223 & 17.161 & & 10.710 & 26.784 & 27.199 & 37.996 \\
\hline 3/2/11 0:00 & 12.603 & 5.372 & 8.057 & 18.111 & 17.123 & & 10.726 & 26.800 & 27.189 & 37.911 \\
\hline 3/2/11 4:00 & 12.598 & 5.294 & 8.024 & 18.004 & 17.083 & & 10.764 & 26.833 & 27.180 & 37.826 \\
\hline 3/2/11 8:00 & 12.626 & 5.261 & 8.010 & 17.934 & 17.048 & & 10.790 & 26.852 & 27.171 & 37.789 \\
\hline 3/2/11 12:00 & 12.657 & 5.252 & 8.005 & 17.932 & 17.008 & & 10.783 & 26.852 & 27.161 & 37.841 \\
\hline $3 / 2 / 11$ 16:00 & 12.641 & 5.245 & 7.986 & 18.165 & 16.965 & & 10.710 & 26.758 & 27.150 & 38.015 \\
\hline $3 / 2 / 1120: 00$ & 12.551 & 5.240 & 7.955 & 18.258 & 16.920 & & 10.660 & 26.791 & 27.138 & 38.024 \\
\hline 3/3/11 0:00 & 12.502 & 5.233 & 7.925 & 18.281 & 16.878 & & 10.627 & 26.666 & 27.126 & 38.064 \\
\hline 3/3/11 4:00 & 12.476 & 5.228 & 7.899 & 18.365 & 16.838 & & 10.583 & 26.565 & 27.114 & 38.142 \\
\hline 3/3/11 8:00 & 12.478 & 5.233 & 7.884 & 18.377 & 16.795 & & 10.573 & 26.492 & 27.103 & 38.130 \\
\hline
\end{tabular}

TABLE S1.1 Page 63 of 89 
Water Level (ft below reference point)

\begin{tabular}{|c|c|c|c|c|c|c|c|c|c|c|}
\hline Date and Time & McPike & Potter & Hughes & Barr & Burks & MoDOT & Clizer "Old" & Morgan & SB24 & SB38 \\
\hline $3 / 3 / 11$ 12:00 & 12.469 & 5.238 & 7.868 & 18.445 & 16.753 & & 10.545 & 26.426 & 27.091 & 38.222 \\
\hline $3 / 3 / 11$ 16:00 & 12.266 & 5.240 & 7.780 & 18.612 & 16.708 & & 10.437 & 26.313 & 27.079 & 38.342 \\
\hline 3/3/11 20:00 & 11.995 & 5.247 & 7.698 & 18.631 & 16.666 & & 10.322 & 26.224 & 27.068 & 38.267 \\
\hline 3/4/11 0:00 & 11.976 & 5.259 & 7.655 & 18.570 & 16.621 & & 10.320 & 26.208 & 27.054 & 38.246 \\
\hline 3/4/11 4:00 & 12.004 & 5.266 & 7.629 & 18.535 & 16.574 & & 10.329 & 26.165 & 27.042 & 38.217 \\
\hline 3/4/11 8:00 & 12.080 & 5.283 & 7.624 & 18.419 & 16.531 & & 10.374 & 26.248 & 27.030 & 38.102 \\
\hline $3 / 4 / 11$ 12:00 & 12.150 & 5.301 & 7.634 & 18.356 & 16.486 & & 10.421 & 26.273 & 27.021 & 38.062 \\
\hline 3/4/11 16:00 & 12.186 & 5.313 & 7.641 & 18.312 & 16.442 & & 10.444 & 26.273 & 27.009 & 38.015 \\
\hline 3/4/11 20:00 & 12.237 & 5.337 & 7.655 & 18.205 & 16.397 & & 10.487 & 26.313 & 26.995 & 37.904 \\
\hline 3/5/11 0:00 & 12.303 & 5.356 & 7.676 & 18.076 & 16.354 & & 10.550 & 26.393 & 26.985 & 37.810 \\
\hline 3/5/11 4:00 & 12.360 & 5.374 & 7.698 & 18.004 & 16.312 & & 10.590 & 26.464 & 26.976 & 37.756 \\
\hline 3/5/11 8:00 & 12.409 & 5.391 & 7.719 & 17.960 & 16.265 & & 10.623 & 26.492 & 26.967 & 37.709 \\
\hline 3/5/11 12:00 & 12.452 & 5.407 & 7.740 & 17.920 & 16.222 & & 10.649 & 26.532 & 26.955 & 37.756 \\
\hline $3 / 5 / 11$ 16:00 & 12.464 & 5.412 & 7.752 & 18.088 & 16.177 & & 10.620 & 26.497 & 26.943 & 37.867 \\
\hline 3/5/11 20:00 & 12.485 & 5.422 & 7.764 & 18.114 & 16.130 & & 10.625 & 26.504 & 26.934 & 37.805 \\
\hline 3/6/11 0:00 & 12.511 & 5.431 & 7.780 & 18.051 & 16.083 & & 10.635 & 26.516 & 26.922 & 37.793 \\
\hline 3/6/11 4:00 & 12.513 & 5.438 & 7.787 & 18.107 & 16.036 & & 10.606 & 26.485 & 26.910 & 37.845 \\
\hline 3/6/11 8:00 & 12.520 & 5.443 & 7.797 & 18.125 & 15.991 & & 10.597 & 26.452 & 26.901 & 37.855 \\
\hline $3 / 6 / 11$ 12:00 & 12.527 & 5.452 & 7.809 & 18.151 & 15.946 & & 10.590 & 26.440 & 26.889 & 37.899 \\
\hline $3 / 6 / 11$ 16:00 & 12.499 & 5.452 & 7.804 & 18.337 & 15.899 & & 10.536 & 26.358 & 26.875 & 38.053 \\
\hline 3/6/11 20:00 & 12.487 & 5.457 & 7.806 & 18.365 & 15.852 & & 10.517 & 26.292 & 26.864 & 38.017 \\
\hline 3/7/11 0:00 & 12.496 & 5.469 & 7.813 & 18.312 & 15.807 & & 10.519 & 26.283 & 26.852 & 38.005 \\
\hline 3/7/11 4:00 & 12.489 & 5.471 & 7.816 & 18.340 & 15.762 & & 10.503 & 26.245 & 26.840 & 38.034 \\
\hline 3/7/11 8:00 & 12.499 & 5.481 & 7.825 & 18.314 & 15.718 & & 10.508 & 26.226 & 26.828 & 37.994 \\
\hline 3/7/11 12:00 & 12.513 & 5.490 & 7.839 & 18.305 & 15.675 & & 10.522 & 26.257 & 26.817 & 38.008 \\
\hline $3 / 7 / 11$ 16:00 & 12.501 & 5.497 & 7.842 & 18.389 & 15.630 & & 10.501 & 26.210 & 26.805 & 38.086 \\
\hline 3/7/11 20:00 & 12.508 & 5.504 & 7.844 & 18.342 & 15.583 & & 10.505 & 26.229 & 26.793 & 38.133 \\
\hline 3/8/11 0:00 & 12.520 & 5.513 & 7.839 & 18.267 & 15.538 & & 10.522 & 26.240 & 26.781 & 38.144 \\
\hline 3/8/11 4:00 & 12.518 & 5.518 & 7.837 & 18.265 & 15.498 & & 10.512 & 26.233 & 26.770 & 38.067 \\
\hline 3/8/11 8:00 & 12.515 & 5.523 & 7.835 & 18.272 & 15.453 & & 10.505 & 26.208 & 26.758 & 37.909 \\
\hline 3/8/11 12:00 & 12.515 & 5.525 & 7.837 & 18.274 & 15.409 & & 10.501 & 26.208 & 26.744 & 37.899 \\
\hline 3/8/11 16:00 & 12.463 & 5.518 & 7.794 & 18.323 & 15.340 & & 10.461 & 26.142 & 26.732 & 37.970 \\
\hline 3/8/11 20:00 & 12.329 & 5.506 & 7.709 & 18.226 & 15.277 & & 10.449 & 26.116 & 26.720 & 37.965 \\
\hline 3/9/11 0:00 & 12.145 & 5.459 & 7.619 & 18.177 & 15.227 & & 10.437 & 26.097 & 26.706 & 37.940 \\
\hline 3/9/11 4:00 & 12.051 & 5.367 & 7.553 & 18.137 & 15.185 & & 10.423 & 26.052 & 26.695 & 37.935 \\
\hline 3/9/11 8:00 & 12.039 & 5.271 & 7.513 & 18.035 & 15.142 & & 10.442 & 26.130 & 26.683 & 37.878 \\
\hline 3/9/11 12:00 & 12.077 & 5.191 & 7.492 & 17.927 & 15.100 & & 10.482 & 26.163 & 26.674 & 37.779 \\
\hline $3 / 9 / 11$ 16:00 & 12.110 & 5.129 & 7.482 & 17.895 & 15.064 & & 10.501 & 26.222 & 26.659 & 37.744 \\
\hline 3/9/11 20:00 & 12.162 & 5.101 & 7.487 & 17.799 & 15.029 & & 10.543 & 26.250 & 26.650 & 37.657 \\
\hline 3/10/11 0:00 & 12.209 & 5.092 & 7.496 & 17.736 & 14.991 & & 10.573 & 26.283 & 26.638 & 37.605 \\
\hline 3/10/11 4:00 & 12.242 & 5.089 & 7.508 & 17.732 & 14.949 & & 10.590 & 26.304 & 26.627 & 37.586 \\
\hline 3/10/11 8:00 & 12.277 & 5.101 & 7.525 & 17.690 & 14.909 & & 10.613 & 26.327 & 26.617 & 37.546 \\
\hline 3/10/11 12:00 & 12.303 & 5.120 & 7.539 & 17.704 & 14.866 & & 10.623 & 26.351 & 26.606 & 37.596 \\
\hline 3/10/11 16:00 & 12.289 & 5.127 & 7.541 & 17.904 & 14.826 & & 10.578 & 26.297 & 26.594 & 37.742 \\
\hline 3/10/11 20:00 & 12.277 & 5.139 & 7.541 & 17.993 & 14.784 & & 10.550 & 26.236 & 26.582 & 37.746 \\
\hline 3/11/11 0:00 & 12.265 & 5.148 & 7.541 & 18.007 & 14.741 & & 10.529 & 26.222 & 26.570 & 37.772 \\
\hline $3 / 11 / 114: 00$ & 12.247 & 5.158 & 7.541 & 18.060 & 14.699 & & 10.496 & 26.156 & 26.559 & 37.834 \\
\hline 3/11/11 8:00 & 12.216 & 5.165 & 7.532 & 18.137 & 14.659 & & 10.456 & 26.066 & 26.545 & 37.895 \\
\hline 3/11/11 12:00 & 12.185 & 5.174 & 7.525 & 18.235 & 14.621 & & 10.418 & 25.991 & 26.530 & 38.027 \\
\hline $3 / 11 / 11$ 16:00 & 12.150 & 5.184 & 7.515 & 18.459 & 14.579 & & 10.381 & 25.883 & 26.519 & 38.111 \\
\hline 3/11/11 20:00 & 12.190 & 5.212 & 7.527 & 18.291 & 14.541 & & 10.428 & 25.921 & 26.507 & 37.895 \\
\hline 3/12/11 0:00 & 12.237 & 5.243 & 7.546 & 18.135 & 14.503 & & 10.480 & 25.998 & 26.495 & 37.796 \\
\hline 3/12/11 4:00 & 12.254 & 5.261 & 7.560 & 18.090 & 14.465 & & 10.496 & 26.052 & 26.483 & 37.775 \\
\hline $3 / 12 / 118: 00$ & 12.280 & 5.280 & 7.579 & 18.021 & 14.428 & & 10.522 & 26.076 & 26.472 & 37.695 \\
\hline 3/12/11 12:00 & 12.315 & 5.301 & 7.600 & 17.937 & 14.392 & & 10.559 & 26.144 & 26.460 & 37.671 \\
\hline 3/12/11 16:00 & 12.327 & 5.318 & 7.615 & 18.030 & 14.352 & & 10.559 & 26.156 & 26.448 & 37.725 \\
\hline 3/12/11 20:00 & 12.348 & 5.334 & 7.634 & 18.000 & 14.317 & & 10.581 & 26.182 & 26.439 & 37.631 \\
\hline 3/13/11 0:00 & 12.383 & 5.356 & 7.655 & 17.890 & 14.277 & & 10.616 & 26.252 & 26.430 & 37.553 \\
\hline $3 / 13 / 114: 00$ & 12.400 & 5.367 & 7.671 & 17.857 & 14.241 & & 10.625 & 26.283 & 26.418 & 37.553 \\
\hline 3/13/11 8:00 & 12.419 & 5.379 & 7.690 & 17.829 & 14.204 & & 10.637 & 26.304 & 26.406 & 37.520 \\
\hline 3/13/11 12:00 & 12.449 & 5.393 & 7.712 & 17.776 & 14.166 & & 10.670 & 26.367 & 26.399 & 37.492 \\
\hline $3 / 13 / 11$ 16:00 & 12.449 & 5.400 & 7.723 & 17.876 & 14.126 & & 10.644 & 26.363 & 26.387 & 37.586 \\
\hline 3/13/11 20:00 & 12.463 & 5.408 & 7.738 & 17.864 & 14.088 & & 10.656 & 26.365 & 26.376 & 37.523 \\
\hline 3/14/11 0:00 & 12.482 & 5.422 & 7.754 & 17.827 & 14.050 & & 10.672 & 26.407 & 26.366 & 37.506 \\
\hline
\end{tabular}

TABLE S1.1 Page 64 of 89 
Water Level (ft below reference point)

\begin{tabular}{|c|c|c|c|c|c|c|c|c|c|c|}
\hline Date and Time & McPike & Potter & Hughes & Barr & Burks & MoDOT & Clizer "Old" & Morgan & SB24 & SB38 \\
\hline $3 / 14 / 114: 00$ & 12.475 & 5.429 & 7.764 & 17.878 & 14.013 & & 10.646 & 26.374 & 26.354 & 37.584 \\
\hline 3/14/11 8:00 & 12.492 & 5.436 & 7.778 & 17.857 & 13.973 & & 10.658 & 26.391 & 26.347 & 37.513 \\
\hline 3/14/11 12:00 & 12.487 & 5.445 & 7.787 & 17.885 & 13.932 & & 10.642 & 26.405 & 26.336 & 37.563 \\
\hline 3/14/11 16:00 & 12.459 & 5.445 & 7.787 & 18.065 & 13.892 & & 10.597 & 26.335 & 26.324 & 37.711 \\
\hline 3/14/11 20:00 & 12.447 & 5.450 & 7.790 & 18.100 & 13.852 & & 10.585 & 26.295 & 26.315 & 37.683 \\
\hline 3/15/11 0:00 & 12.456 & 5.457 & 7.799 & 18.030 & 13.815 & & 10.592 & 26.295 & 26.303 & 37.636 \\
\hline 3/15/11 4:00 & 12.444 & 5.462 & 7.804 & 18.039 & 13.774 & & 10.571 & 26.276 & 26.291 & 37.678 \\
\hline 3/15/11 8:00 & 12.445 & 5.471 & 7.811 & 18.023 & 13.737 & & 10.573 & 26.266 & 26.282 & 37.631 \\
\hline 3/15/11 12:00 & 12.452 & 5.478 & 7.823 & 18.025 & 13.701 & & 10.578 & 26.273 & 26.270 & 37.685 \\
\hline 3/15/11 16:00 & 12.428 & 5.481 & 7.823 & 18.216 & 13.661 & & 10.548 & 26.264 & 26.258 & 37.805 \\
\hline 3/15/11 20:00 & 12.421 & 5.485 & 7.828 & 18.228 & 13.626 & & 10.543 & 26.219 & 26.249 & 37.753 \\
\hline 3/16/11 0:00 & 12.442 & 5.495 & 7.842 & 18.118 & 13.588 & & 10.574 & 26.255 & 26.237 & 37.664 \\
\hline 3/16/11 4:00 & 12.447 & 5.502 & 7.851 & 18.097 & 13.553 & & 10.573 & 26.233 & 26.228 & 37.669 \\
\hline 3/16/11 8:00 & 12.449 & 5.506 & 7.861 & 18.086 & 13.517 & & 10.576 & 26.304 & 26.216 & 37.652 \\
\hline 3/16/11 12:00 & 12.440 & 5.511 & 7.870 & 18.137 & 13.477 & & 10.562 & 26.236 & 26.207 & 37.751 \\
\hline 3/16/11 16:00 & 12.407 & 5.514 & 7.865 & 18.372 & 13.442 & & 10.517 & 26.139 & 26.193 & 37.897 \\
\hline 3/16/11 20:00 & 12.383 & 5.516 & 7.863 & 18.414 & 13.407 & & 10.496 & 26.078 & 26.183 & 37.885 \\
\hline 3/17/11 0:00 & 12.374 & 5.518 & 7.868 & 18.400 & 13.369 & & 10.489 & 26.055 & 26.169 & 37.885 \\
\hline 3/17/11 4:00 & 12.353 & 5.518 & 7.865 & 18.459 & 13.333 & & 10.463 & 26.001 & 26.160 & 37.940 \\
\hline 3/17/11 8:00 & 12.343 & 5.521 & 7.870 & 18.477 & 13.298 & & 10.458 & 26.031 & 26.146 & 37.909 \\
\hline 3/17/11 12:00 & 12.360 & 5.525 & 7.884 & 18.433 & 13.267 & & 10.487 & 26.090 & 26.136 & 37.848 \\
\hline 3/17/11 16:00 & 12.386 & 5.542 & 7.899 & 18.412 & 13.234 & & 10.524 & 26.109 & 26.125 & 37.786 \\
\hline 3/17/11 20:00 & 12.447 & 5.561 & 7.927 & 18.230 & 13.204 & & 10.606 & 26.217 & 26.113 & 37.600 \\
\hline 3/18/11 0:00 & 12.499 & 5.580 & 7.955 & 18.053 & 13.173 & & 10.675 & 26.332 & 26.106 & 37.475 \\
\hline 3/18/11 4:00 & 12.534 & 5.587 & 7.979 & 17.941 & 13.140 & & 10.712 & 26.410 & 26.099 & 37.424 \\
\hline 3/18/11 8:00 & 12.569 & 5.594 & 8.007 & 17.867 & 13.107 & & 10.757 & 26.476 & 26.087 & 37.332 \\
\hline 3/18/11 12:00 & 12.607 & 5.605 & 8.036 & 17.806 & 13.076 & & 10.792 & 26.570 & 26.080 & 37.325 \\
\hline 3/18/11 16:00 & 12.614 & 5.610 & 8.050 & 17.960 & 13.041 & & 10.768 & 27.376 & 26.071 & 37.419 \\
\hline 3/18/11 20:00 & 12.628 & 5.613 & 8.069 & 17.962 & 13.008 & & 10.780 & 26.809 & 26.061 & 37.379 \\
\hline 3/19/11 0:00 & 12.647 & 5.619 & 8.088 & 17.864 & 12.977 & & 10.799 & 26.741 & 26.052 & 37.329 \\
\hline 3/19/11 4:00 & 12.657 & 5.620 & 8.104 & 17.848 & 12.942 & & 10.797 & 26.715 & 26.045 & 37.337 \\
\hline 3/19/11 8:00 & 12.661 & 5.622 & 8.116 & 17.867 & 12.907 & & 10.785 & 26.711 & 26.036 & 37.362 \\
\hline 3/19/11 12:00 & 12.647 & 5.617 & 8.126 & 17.939 & 12.871 & & 10.747 & 26.664 & 26.026 & 37.475 \\
\hline 3/19/11 16:00 & 12.616 & 5.622 & 8.126 & 18.027 & 12.836 & & 10.693 & 26.603 & 26.017 & 37.546 \\
\hline 3/19/11 20:00 & 12.598 & 5.610 & 8.128 & 18.123 & 12.800 & & 10.675 & 26.539 & 26.007 & 37.605 \\
\hline 3/20/11 0:00 & 12.579 & 5.610 & 8.126 & 18.114 & 12.765 & & 10.653 & 26.480 & 25.996 & 37.624 \\
\hline $3 / 20 / 114: 00$ & 12.560 & 5.610 & 8.128 & 18.174 & 12.732 & & 10.630 & 26.452 & 25.986 & 37.629 \\
\hline 3/20/11 8:00 & 12.539 & 5.608 & 8.126 & 18.235 & 12.697 & & 10.604 & 26.386 & 25.979 & 37.541 \\
\hline 3/20/11 12:00 & 12.522 & 5.613 & 8.123 & 18.316 & 12.664 & & 10.590 & 26.339 & 25.970 & 37.822 \\
\hline 3/20/11 16:00 & 12.506 & 5.612 & 8.123 & 18.475 & 12.633 & & 10.581 & 26.292 & 25.958 & 37.867 \\
\hline 3/20/11 20:00 & 12.522 & 5.624 & 8.135 & 18.398 & 12.605 & & 10.618 & 26.313 & 25.946 & 37.728 \\
\hline 3/21/11 0:00 & 12.567 & 5.638 & 8.154 & 18.214 & 12.574 & & 10.686 & 26.438 & 25.939 & 37.584 \\
\hline 3/21/11 4:00 & 12.591 & 5.648 & 8.171 & 18.153 & 12.548 & & 10.710 & 26.490 & 25.930 & 37.556 \\
\hline 3/21/11 8:00 & 12.602 & 5.650 & 8.182 & 18.107 & 12.520 & & 10.717 & 26.504 & 25.921 & 37.537 \\
\hline $3 / 21 / 11$ 12:00 & 12.600 & 5.655 & 8.194 & 18.149 & 12.492 & & 10.707 & 26.854 & 25.914 & 37.607 \\
\hline 3/21/11 16:00 & 12.569 & 5.650 & 8.192 & 18.379 & 12.461 & & 10.651 & 26.751 & 25.902 & 37.768 \\
\hline $3 / 21 / 1120: 00$ & 12.548 & 5.650 & 8.192 & 18.445 & 12.433 & & 10.632 & 26.464 & 25.893 & 37.765 \\
\hline 3/22/11 0:00 & 12.553 & 5.652 & 8.201 & 18.382 & 12.409 & & 10.646 & 26.429 & 25.883 & 37.716 \\
\hline $3 / 22 / 114: 00$ & 12.527 & 5.648 & 8.199 & 18.456 & 12.378 & & 10.606 & 26.358 & 25.874 & 37.803 \\
\hline 3/22/11 8:00 & 12.499 & 5.645 & 8.197 & 18.505 & 12.348 & & 10.574 & 26.271 & 25.862 & 37.855 \\
\hline 3/22/11 12:00 & 12.470 & 5.638 & 8.192 & 18.594 & 12.322 & & 10.543 & 26.205 & 25.850 & 37.956 \\
\hline 3/22/11 16:00 & 12.444 & 5.638 & 8.185 & 18.682 & 12.296 & & 10.517 & 26.203 & 25.841 & 38.015 \\
\hline 3/22/11 20:00 & 12.437 & 5.641 & 8.190 & 18.636 & 12.272 & & 10.520 & 26.142 & 25.832 & 37.918 \\
\hline 3/23/11 0:00 & 12.477 & 5.657 & 8.204 & 18.370 & 12.249 & & 10.585 & 26.224 & 25.822 & 37.742 \\
\hline 3/23/11 4:00 & 12.494 & 5.667 & 8.218 & 18.253 & 12.227 & & 10.616 & 26.283 & 25.813 & 37.669 \\
\hline 3/23/11 8:00 & 12.529 & 5.676 & 8.242 & 18.062 & 12.209 & & 10.675 & 26.327 & 25.801 & 37.506 \\
\hline $3 / 23 / 11$ 12:00 & 12.579 & 5.693 & 8.265 & 17.925 & 12.190 & & 10.745 & 26.443 & 25.796 & 37.431 \\
\hline 3/23/11 16:00 & 12.616 & 5.702 & 8.294 & 17.881 & 12.171 & & 10.806 & 26.537 & 25.789 & 37.355 \\
\hline $3 / 23 / 1120: 00$ & 12.673 & 5.718 & 8.322 & 17.706 & 12.152 & & 10.884 & 26.661 & 25.780 & 37.193 \\
\hline 3/24/11 0:00 & 12.718 & 5.728 & 8.348 & 17.582 & 12.131 & & 10.928 & 26.772 & 25.775 & 37.113 \\
\hline $3 / 24 / 114: 00$ & 12.748 & 5.737 & 8.376 & 17.527 & 12.112 & & 10.952 & 26.833 & 25.768 & 37.087 \\
\hline $3 / 24 / 11$ 8:00 & 12.781 & 5.742 & 8.402 & 17.471 & 12.091 & & 10.985 & 26.889 & 25.761 & 37.042 \\
\hline 3/24/11 12:00 & 12.805 & 5.747 & 8.424 & 17.494 & 12.069 & & 10.982 & 26.991 & 25.756 & 37.148 \\
\hline 3/24/11 16:00 & 12.786 & 5.740 & 8.431 & 17.774 & 12.043 & & 10.907 & 26.922 & 25.747 & 37.339 \\
\hline
\end{tabular}

TABLE S1.1 Page 65 of 89 


\begin{tabular}{|c|c|c|c|c|c|c|c|c|c|c|}
\hline \multirow[b]{2}{*}{ Date and Time } & \multicolumn{10}{|c|}{ Water Level (ft below reference point) } \\
\hline & McPike & Potter & Hughes & Barr & Burks & MoDOT & Clizer "Old" & Morgan & SB24 & SB38 \\
\hline $3 / 24 / 1120: 00$ & 12.751 & 5.730 & 8.431 & 17.944 & 12.018 & & 10.851 & 26.833 & 25.740 & 37.410 \\
\hline 3/25/11 0:00 & 12.767 & 5.730 & 8.440 & 17.834 & 11.994 & & 10.869 & 26.868 & 25.733 & 37.431 \\
\hline $3 / 25 / 114: 00$ & 12.756 & 5.728 & 8.440 & 17.834 & 11.954 & & 10.832 & 26.915 & 25.724 & 37.483 \\
\hline 3/25/11 8:00 & 12.753 & 5.730 & 8.405 & 17.755 & 11.921 & & 10.867 & 26.868 & 25.719 & 37.381 \\
\hline 3/25/11 12:00 & 12.767 & 5.737 & 8.365 & 17.690 & 11.867 & & 10.874 & 26.854 & 25.709 & 37.259 \\
\hline 3/25/11 16:00 & 12.753 & 5.742 & 8.312 & 17.699 & 11.841 & & 10.860 & 26.828 & 25.702 & 37.296 \\
\hline $3 / 25 / 1120: 00$ & 12.751 & 5.742 & 8.256 & 17.638 & 11.822 & & 10.870 & 26.892 & 25.693 & 37.259 \\
\hline 3/26/11 0:00 & 12.756 & 5.742 & 8.218 & 17.573 & 11.801 & & 10.867 & 26.871 & 25.686 & 37.245 \\
\hline $3 / 26 / 114: 00$ & 12.732 & 5.737 & 8.187 & 17.608 & 11.782 & & 10.820 & 26.793 & 25.677 & 37.311 \\
\hline 3/26/11 8:00 & 12.723 & 5.730 & 8.171 & 17.596 & 11.763 & & 10.811 & 26.770 & 25.670 & 37.296 \\
\hline $3 / 26 / 11$ 12:00 & 12.734 & 5.733 & 8.163 & 17.531 & 11.744 & & 10.837 & 26.774 & 25.663 & 37.254 \\
\hline $3 / 26 / 11$ 16:00 & 12.744 & 5.733 & 8.163 & 17.492 & 11.725 & & 10.844 & 26.795 & 25.656 & 37.231 \\
\hline $3 / 26 / 1120: 00$ & 12.760 & 5.733 & 8.173 & 17.450 & 11.709 & & 10.872 & 26.814 & 25.646 & 37.183 \\
\hline 3/27/11 0:00 & 12.784 & 5.737 & 8.187 & 17.403 & 11.690 & & 10.905 & 26.929 & 25.639 & 37.143 \\
\hline $3 / 27 / 114: 00$ & 12.789 & 5.735 & 8.197 & 17.424 & 11.671 & & 10.888 & 26.918 & 25.632 & 37.176 \\
\hline 3/27/11 8:00 & 12.805 & 5.737 & 8.213 & 17.361 & 11.654 & & 10.935 & 26.969 & 25.625 & 37.103 \\
\hline $3 / 27 / 11$ 12:00 & 12.817 & 5.740 & 8.232 & 17.391 & 11.635 & & 10.931 & 26.934 & 25.618 & 37.165 \\
\hline $3 / 27 / 11$ 16:00 & 12.798 & 5.740 & 8.239 & 17.576 & 11.614 & & 10.886 & 26.941 & 25.609 & 37.294 \\
\hline 3/27/11 20:00 & 12.800 & 5.740 & 8.251 & 17.555 & 11.600 & & 10.905 & 26.913 & 25.599 & 37.247 \\
\hline 3/28/11 0:00 & 12.819 & 5.744 & 8.270 & 17.485 & 11.581 & & 10.931 & 26.929 & 25.595 & 37.198 \\
\hline $3 / 28 / 114: 00$ & 12.829 & 5.742 & 8.286 & 17.473 & 11.562 & & 10.940 & 26.944 & 25.588 & 37.174 \\
\hline 3/28/11 8:00 & 12.840 & 5.747 & 8.305 & 17.433 & 11.544 & & 10.961 & 26.953 & 25.578 & 37.139 \\
\hline $3 / 28 / 11$ 12:00 & 12.850 & 5.749 & 8.322 & 17.480 & 11.525 & & 10.954 & 26.974 & 25.571 & 37.207 \\
\hline $3 / 28 / 11$ 16:00 & 12.833 & 5.747 & 8.331 & 17.627 & 11.506 & & 10.916 & 27.007 & 25.564 & 37.308 \\
\hline 3/28/11 20:00 & 12.831 & 5.749 & 8.343 & 17.620 & 11.487 & & 10.931 & 27.007 & 25.557 & 37.259 \\
\hline 3/29/11 0:00 & 12.852 & 5.756 & 8.360 & 17.520 & 11.468 & & 10.963 & 27.012 & 25.552 & 37.212 \\
\hline 3/29/11 4:00 & 12.864 & 5.759 & 8.376 & 17.515 & 11.452 & & 10.980 & 27.028 & 25.545 & 37.186 \\
\hline 3/29/11 8:00 & 12.885 & 5.766 & 8.398 & 17.429 & 11.433 & & 11.020 & 27.078 & 25.541 & 37.117 \\
\hline $3 / 29 / 11$ 12:00 & 12.902 & 5.770 & 8.416 & 17.450 & 11.414 & & 11.022 & 27.132 & 25.534 & 37.148 \\
\hline $3 / 29 / 11$ 16:00 & 12.899 & 5.770 & 8.428 & 17.552 & 11.395 & & 10.994 & 27.108 & 25.527 & 37.219 \\
\hline 3/29/11 20:00 & 12.906 & 5.770 & 8.440 & 17.541 & 11.376 & & 11.010 & 27.108 & 25.520 & 37.188 \\
\hline 3/30/11 0:00 & 12.918 & 5.777 & 8.457 & 17.510 & 11.357 & & 11.025 & 27.136 & 25.515 & 37.174 \\
\hline 3/30/11 4:00 & 12.909 & 5.773 & 8.466 & 17.576 & 11.341 & & 10.987 & 27.103 & 25.508 & 37.231 \\
\hline 3/30/11 8:00 & 12.911 & 5.773 & 8.478 & 17.569 & 11.322 & & 11.006 & 27.150 & 25.503 & 37.205 \\
\hline 3/30/11 12:00 & 12.916 & 5.777 & 8.490 & 17.615 & 11.303 & & 10.992 & 27.158 & 25.496 & 37.271 \\
\hline 3/30/11 16:00 & 12.892 & 5.773 & 8.490 & 17.750 & 11.284 & & 10.945 & 27.080 & 25.489 & 37.377 \\
\hline $3 / 30 / 1120: 00$ & 12.873 & 5.770 & 8.492 & 17.799 & 11.263 & & 10.921 & 27.040 & 25.482 & 37.410 \\
\hline 3/31/11 0:00 & 12.866 & 5.770 & 8.499 & 17.778 & 11.246 & & 10.921 & 27.033 & 25.475 & 37.393 \\
\hline 3/31/11 4:00 & 12.854 & 5.766 & 8.502 & 17.799 & 11.228 & & 10.900 & 27.016 & 25.470 & 37.421 \\
\hline 3/31/11 8:00 & 12.843 & 5.766 & 8.506 & 17.825 & 11.211 & & 10.893 & 26.995 & 25.463 & 37.435 \\
\hline 3/31/11 12:00 & 12.836 & 5.770 & 8.511 & 17.878 & 11.194 & & 10.884 & 26.991 & 25.459 & 37.487 \\
\hline $3 / 31 / 11$ 16:00 & 12.826 & 5.770 & 8.514 & 17.934 & 11.176 & & 10.869 & 26.969 & 25.454 & 37.520 \\
\hline 3/31/11 20:00 & 12.826 & 5.770 & 8.521 & 17.906 & 11.161 & & 10.881 & 26.991 & 25.447 & 37.480 \\
\hline 4/1/11 0:00 & 12.836 & 5.777 & 8.528 & 17.839 & 11.143 & & 10.900 & 27.019 & 25.445 & 37.428 \\
\hline 4/1/11 4:00 & 12.843 & 5.780 & 8.537 & 17.829 & 11.128 & & 10.909 & 27.033 & 25.437 & 37.421 \\
\hline 4/1/11 8:00 & 12.871 & 5.787 & 8.554 & 17.725 & 11.114 & & 10.963 & 27.101 & 25.435 & 37.313 \\
\hline 4/1/11 12:00 & 12.899 & 5.792 & 8.573 & 17.725 & 11.102 & & 10.985 & 27.167 & 25.428 & 37.285 \\
\hline 4/1/11 16:00 & 12.913 & 5.794 & 8.582 & 17.857 & 11.088 & & 10.994 & 27.193 & 25.426 & 37.332 \\
\hline $4 / 1 / 1120: 00$ & 12.939 & 5.801 & 8.596 & 17.797 & 11.074 & & 11.034 & 27.331 & 25.421 & 37.256 \\
\hline 4/2/11 0:00 & 12.968 & 5.810 & 8.615 & 17.694 & 11.060 & & 11.072 & 27.397 & 25.419 & 37.179 \\
\hline 4/2/11 4:00 & 12.986 & 5.813 & 8.632 & 17.652 & 11.046 & & 11.076 & 27.468 & 25.414 & 37.167 \\
\hline 4/2/11 8:00 & 13.012 & 5.817 & 8.648 & 17.594 & 11.034 & & 11.109 & 27.506 & 25.412 & 37.117 \\
\hline $4 / 2 / 1112: 00$ & 13.031 & 5.820 & 8.665 & 17.629 & 11.020 & & 11.109 & 27.727 & 25.409 & 37.176 \\
\hline $4 / 2 / 11$ 16:00 & 13.015 & 5.818 & 8.665 & 17.918 & 11.003 & & 11.029 & 30.325 & 25.407 & 37.393 \\
\hline 4/2/11 20:00 & 12.998 & 5.810 & 8.665 & 18.041 & 10.987 & & 10.999 & 28.140 & 25.402 & 37.440 \\
\hline 4/3/11 0:00 & 12.986 & 5.808 & 8.665 & 18.086 & 10.973 & & 10.956 & 27.712 & 25.398 & 37.527 \\
\hline 4/3/11 4:00 & 12.953 & 5.794 & 8.655 & 18.221 & 10.951 & & 10.895 & 27.503 & 25.395 & 37.640 \\
\hline $4 / 3 / 118: 00$ & 12.925 & 5.787 & 8.644 & 18.351 & 10.933 & & 10.860 & 27.386 & 25.388 & 37.242 \\
\hline $4 / 3 / 11$ 12:00 & 12.911 & 5.787 & 8.641 & 18.447 & 10.919 & & 10.848 & 27.324 & 25.386 & 37.240 \\
\hline 4/3/11 16:00 & 12.892 & 5.787 & 8.637 & 18.650 & 10.904 & & 10.830 & 27.244 & 25.379 & 37.238 \\
\hline $4 / 3 / 1120: 00$ & 12.925 & 5.806 & 8.653 & 18.428 & 10.895 & & 10.947 & 27.292 & 25.376 & 37.238 \\
\hline 4/4/11 0:00 & 12.996 & 5.824 & 8.681 & 18.016 & 10.888 & & 11.050 & 27.470 & 25.379 & 37.233 \\
\hline 4/4/11 4:00 & 13.050 & 5.841 & 8.705 & 17.839 & 10.881 & & 11.114 & 27.578 & 25.379 & 37.235 \\
\hline $4 / 4 / 118: 00$ & 13.099 & 5.850 & 8.736 & 17.669 & 10.876 & & 11.177 & 27.679 & 25.377 & 37.235 \\
\hline
\end{tabular}

TABLE S1.1 Page 66 of 89 


\begin{tabular}{|c|c|c|c|c|c|c|c|c|c|c|}
\hline \multirow[b]{2}{*}{ Date and Time } & \multicolumn{8}{|c|}{ Water Level (ft below reference point) } & \multirow[b]{2}{*}{ SB24 } & \multirow[b]{2}{*}{ SB38 } \\
\hline & McPike & Potter & Hughes & Barr & Burks & MoDOT & Clizer "Old" & Morgan & & \\
\hline 4/4/11 12:00 & 13.142 & 5.860 & 8.762 & 17.603 & 10.869 & & 11.220 & 27.764 & 25.379 & 37.233 \\
\hline 4/4/11 16:00 & 13.173 & 5.862 & 8.783 & 17.680 & 10.859 & & 11.224 & 27.802 & 25.376 & 37.231 \\
\hline 4/4/11 20:00 & 13.199 & 5.869 & 8.800 & 17.662 & 10.848 & & 11.243 & 27.842 & 25.377 & 37.231 \\
\hline 4/5/11 0:00 & 13.229 & 5.872 & 8.819 & 17.582 & 10.841 & & 11.264 & 27.910 & 25.376 & 37.233 \\
\hline 4/5/11 4:00 & 13.246 & 5.872 & 8.833 & 17.589 & 10.829 & & 11.248 & 27.941 & 25.377 & 37.231 \\
\hline 4/5/11 8:00 & 13.255 & 5.865 & 8.840 & 17.622 & 10.817 & & 11.220 & 27.922 & 25.374 & 37.231 \\
\hline 4/5/11 12:00 & 13.255 & 5.862 & 8.849 & 17.736 & 10.805 & & 11.184 & 27.969 & 25.377 & 37.228 \\
\hline 4/5/11 16:00 & 13.241 & 5.860 & 8.847 & 18.016 & 10.786 & & 11.140 & 27.905 & 25.372 & 37.228 \\
\hline 4/5/11 20:00 & 13.236 & 5.857 & 8.849 & 18.079 & 10.775 & & 11.126 & 27.856 & 25.372 & 37.228 \\
\hline 4/6/11 0:00 & 13.236 & 5.857 & 8.852 & 18.072 & 10.758 & & 11.109 & 27.839 & 25.372 & 37.226 \\
\hline 4/6/11 4:00 & 13.239 & 5.855 & 8.852 & 18.079 & 10.749 & & 11.104 & 27.830 & 25.369 & 37.226 \\
\hline 4/6/11 8:00 & 13.262 & 5.862 & 8.868 & 17.958 & 10.739 & & 11.173 & 27.870 & 25.369 & 37.226 \\
\hline 4/6/11 12:00 & 13.295 & 5.872 & 8.887 & 17.909 & 10.838 & & 11.229 & 27.957 & 25.369 & 37.226 \\
\hline 4/6/11 16:00 & 13.328 & 5.883 & 8.904 & 18.000 & 10.815 & & 11.250 & 28.023 & 25.370 & 37.226 \\
\hline 4/6/11 20:00 & 13.359 & 5.895 & 8.923 & 17.948 & 10.796 & & 11.290 & 28.084 & 25.372 & 37.226 \\
\hline 4/7/11 0:00 & 13.392 & 5.900 & 8.942 & 17.843 & 10.782 & & 11.304 & 28.159 & 25.372 & 37.226 \\
\hline 4/7/11 4:00 & 13.418 & 5.905 & 8.956 & 17.769 & 10.768 & & 11.306 & 28.192 & 25.374 & 37.226 \\
\hline 4/7/11 8:00 & 13.437 & 5.905 & 8.972 & 17.743 & 10.751 & & 11.311 & 28.199 & 25.374 & 37.226 \\
\hline 4/7/11 12:00 & 13.453 & 5.905 & 8.980 & 17.739 & 10.735 & & 11.299 & 28.216 & 25.377 & 37.226 \\
\hline 4/7/11 16:00 & 13.429 & 5.890 & 8.920 & 17.827 & 10.652 & & 11.173 & 28.157 & 25.377 & 37.228 \\
\hline 4/7/11 20:00 & 13.411 & 5.886 & 8.781 & 17.683 & 10.635 & & 11.198 & 28.105 & 25.374 & 37.226 \\
\hline 4/8/11 0:00 & 13.404 & 5.883 & 8.677 & 17.676 & 10.624 & & 11.180 & 28.105 & 25.374 & 37.223 \\
\hline 4/8/11 4:00 & 13.387 & 5.883 & 8.563 & 17.634 & 10.595 & & 11.151 & 28.063 & 25.374 & 37.223 \\
\hline 4/8/11 8:00 & 13.390 & 5.886 & 8.476 & 17.503 & 10.588 & & 11.189 & 28.129 & 25.376 & 37.223 \\
\hline 4/8/11 12:00 & 13.397 & 5.890 & 8.419 & 17.454 & 10.579 & & 11.189 & 28.100 & 25.377 & 37.226 \\
\hline 4/8/11 16:00 & 13.389 & 5.888 & 8.384 & 17.559 & 10.565 & & 11.149 & 28.067 & 25.377 & 37.223 \\
\hline 4/8/11 20:00 & 13.378 & 5.883 & 8.355 & 17.631 & 10.553 & & 11.128 & 28.037 & 25.376 & 37.223 \\
\hline 4/9/11 0:00 & 13.378 & 5.883 & 8.343 & 17.578 & 10.539 & & 11.130 & 28.032 & 25.379 & 37.223 \\
\hline 4/9/11 4:00 & 13.375 & 5.879 & 8.334 & 17.601 & 10.522 & & 11.126 & 28.004 & 25.377 & 37.223 \\
\hline 4/9/11 8:00 & 13.382 & 5.879 & 8.339 & 17.550 & 10.508 & & 11.142 & 28.009 & 25.376 & 37.223 \\
\hline 4/9/11 12:00 & 13.382 & 5.879 & 8.341 & 17.659 & 10.492 & & 11.128 & 28.016 & 25.376 & 37.223 \\
\hline 4/9/11 16:00 & 13.361 & 5.872 & 8.336 & 17.939 & 10.470 & & 11.072 & 28.117 & 25.376 & 37.223 \\
\hline $4 / 9 / 1120: 00$ & 13.347 & 5.869 & 8.334 & 18.007 & 10.454 & & 11.064 & 27.943 & 25.372 & 37.223 \\
\hline 4/10/11 0:00 & 13.347 & 5.867 & 8.343 & 17.962 & 10.437 & & 11.083 & 27.901 & 25.370 & 37.223 \\
\hline 4/10/11 4:00 & 13.342 & 5.867 & 8.350 & 17.976 & 10.421 & & 11.062 & 27.905 & 25.369 & 37.223 \\
\hline 4/10/11 8:00 & 13.342 & 5.864 & 8.360 & 17.953 & 10.402 & & 11.069 & 27.891 & 25.369 & 37.226 \\
\hline 4/10/11 12:00 & 13.345 & 5.867 & 8.369 & 18.007 & 10.388 & & 11.083 & 27.872 & 25.369 & 37.223 \\
\hline 4/10/11 16:00 & 13.354 & 5.874 & 8.386 & 18.023 & 10.371 & & 11.109 & 27.879 & 25.370 & 37.223 \\
\hline 4/10/11 20:00 & 13.392 & 5.886 & 8.414 & 17.881 & 10.360 & & 11.194 & 27.952 & 25.370 & 37.226 \\
\hline 4/11/11 0:00 & 13.439 & 5.897 & 8.447 & 17.657 & 10.352 & & 11.262 & 28.044 & 25.369 & 37.226 \\
\hline 4/11/11 4:00 & 13.467 & 5.904 & 8.476 & 17.559 & 10.338 & & 11.271 & 28.072 & 25.372 & 37.226 \\
\hline 4/11/11 8:00 & 13.495 & 5.909 & 8.506 & 17.438 & 10.331 & & 11.311 & 28.185 & 25.372 & 37.226 \\
\hline $4 / 11 / 11$ 12:00 & 13.529 & 5.914 & 8.537 & 17.405 & 10.319 & & 11.337 & 28.251 & 25.374 & 37.228 \\
\hline $4 / 11 / 11$ 16:00 & 13.552 & 5.916 & 8.559 & 17.555 & 10.308 & & 11.344 & 28.279 & 25.374 & 37.228 \\
\hline $4 / 11 / 1120: 00$ & 13.578 & 5.921 & 8.585 & 17.541 & 10.296 & & 11.375 & 28.397 & 25.377 & 37.228 \\
\hline $4 / 12 / 110: 00$ & 13.609 & 5.926 & 8.613 & 17.401 & 10.289 & & 11.407 & 28.430 & 25.379 & 37.231 \\
\hline 4/12/11 4:00 & 13.635 & 5.926 & 8.639 & 17.340 & 10.277 & & 11.415 & 28.397 & 25.381 & 37.231 \\
\hline 4/12/11 8:00 & 13.661 & 5.928 & 8.665 & 17.266 & 10.265 & & 11.440 & 28.427 & 25.383 & 37.233 \\
\hline $4 / 12 / 11$ 12:00 & 13.684 & 5.930 & 8.686 & 17.345 & 10.253 & & 11.431 & 28.451 & 25.386 & 37.231 \\
\hline $4 / 12 / 11$ 16:00 & 13.689 & 5.928 & 8.698 & 17.624 & 10.239 & & 11.379 & 28.420 & 25.386 & 37.233 \\
\hline 4/12/11 20:00 & 13.696 & 5.928 & 8.708 & 17.678 & 10.225 & & 11.375 & 28.401 & 25.386 & 37.233 \\
\hline 4/13/11 0:00 & 13.705 & 5.928 & 8.724 & 17.608 & 10.213 & & 11.382 & 28.491 & 25.386 & 37.235 \\
\hline 4/13/11 4:00 & 13.717 & 5.923 & 8.738 & 17.580 & 10.199 & & 11.382 & 28.460 & 25.388 & 37.235 \\
\hline 4/13/11 8:00 & 13.731 & 5.926 & 8.755 & 17.517 & 10.187 & & 11.400 & 28.437 & 25.388 & 37.238 \\
\hline 4/13/11 12:00 & 13.743 & 5.928 & 8.769 & 17.580 & 10.173 & & 11.379 & 28.444 & 25.391 & 37.238 \\
\hline $4 / 13 / 11$ 16:00 & 13.743 & 5.928 & 8.779 & 17.829 & 10.159 & & 11.344 & 28.900 & 25.393 & 37.238 \\
\hline 4/13/11 20:00 & 13.752 & 5.933 & 8.790 & 17.818 & 10.147 & & 11.356 & 28.597 & 25.393 & 37.240 \\
\hline 4/14/11 0:00 & 13.764 & 5.937 & 8.802 & 17.720 & 10.135 & & 11.370 & 28.533 & 25.395 & 37.242 \\
\hline 4/14/11 4:00 & 13.778 & 5.937 & 8.816 & 17.657 & 10.124 & & 11.372 & 28.505 & 25.398 & 37.242 \\
\hline 4/14/11 8:00 & 13.781 & 5.937 & 8.812 & 17.617 & 10.102 & & 11.358 & 28.472 & 25.398 & 37.242 \\
\hline 4/14/11 12:00 & 13.778 & 5.930 & 8.805 & 17.692 & 10.091 & & 11.318 & 28.460 & 25.402 & 37.245 \\
\hline 4/14/11 16:00 & 13.748 & 5.926 & 8.788 & 17.927 & 10.072 & & 11.250 & 28.373 & 25.400 & 37.245 \\
\hline $4 / 14 / 1120: 00$ & 13.741 & 5.923 & 8.783 & 17.911 & 10.060 & & 11.260 & 28.336 & 25.402 & 37.245 \\
\hline 4/15/11 0:00 & 13.550 & 5.907 & 8.516 & 17.734 & 9.949 & & 11.107 & 28.333 & 25.402 & 37.245 \\
\hline
\end{tabular}

TABLE S1.1 Page 67 of 89 


\begin{tabular}{|c|c|c|c|c|c|c|c|c|c|c|}
\hline \multirow[b]{2}{*}{ Date and Time } & \multicolumn{10}{|c|}{ Water Level (ft below reference point) } \\
\hline & McPike & Potter & Hughes & Barr & Burks & MoDOT & Clizer "Old" & Morgan & SB24 & SB38 \\
\hline 4/15/11 4:00 & 12.982 & 5.838 & 8.213 & 17.573 & 9.937 & & 11.065 & 28.223 & 25.402 & 37.245 \\
\hline 4/15/11 8:00 & 12.876 & 5.817 & 8.010 & 17.466 & 9.902 & & 10.996 & 28.110 & 25.402 & 37.242 \\
\hline $4 / 15 / 1112: 00$ & 12.779 & 5.812 & 7.840 & 17.356 & 9.843 & & 10.949 & 28.009 & 25.400 & 37.242 \\
\hline $4 / 15 / 1116: 00$ & 12.378 & 5.789 & 7.620 & 17.193 & 9.711 & & 10.860 & 27.941 & 25.398 & 37.242 \\
\hline 4/15/11 20:00 & 11.810 & 5.704 & 7.402 & 16.904 & 9.520 & & 10.837 & 27.872 & 25.395 & 37.238 \\
\hline 4/16/11 0:00 & 11.841 & 5.652 & 7.272 & 16.706 & 9.322 & & 10.848 & 27.837 & 25.393 & 37.240 \\
\hline $4 / 16 / 114: 00$ & 11.987 & 5.631 & 7.189 & 16.541 & 9.140 & & 10.874 & 27.875 & 25.388 & 37.238 \\
\hline 4/16/11 8:00 & 12.095 & 5.617 & 7.139 & 16.366 & 9.017 & & 10.935 & 27.877 & 25.388 & 37.238 \\
\hline $4 / 16 / 11$ 12:00 & 12.190 & 5.605 & 7.121 & 16.320 & 8.925 & & 10.966 & 27.893 & 25.388 & 37.240 \\
\hline $4 / 16 / 11$ 16:00 & 12.242 & 5.586 & 7.111 & 16.529 & 8.840 & & 10.931 & 27.835 & 25.383 & 37.240 \\
\hline 4/16/11 20:00 & 12.284 & 5.574 & 7.116 & 16.641 & 8.784 & & 10.914 & 27.762 & 25.379 & 37.240 \\
\hline 4/17/11 0:00 & 12.352 & 5.572 & 7.142 & 16.581 & 8.746 & & 10.940 & 27.755 & 25.377 & 37.242 \\
\hline 4/17/11 4:00 & 12.390 & 5.565 & 7.168 & 16.618 & 8.706 & & 10.914 & 27.719 & 25.374 & 37.242 \\
\hline 4/17/11 8:00 & 12.414 & 5.560 & 7.194 & 16.667 & 8.666 & & 10.893 & 27.738 & 25.370 & 37.242 \\
\hline 4/17/11 12:00 & 12.423 & 5.556 & 7.218 & 16.804 & 8.621 & & 10.860 & 27.640 & 25.367 & 37.240 \\
\hline 4/17/11 16:00 & 12.409 & 5.551 & 7.232 & 17.002 & 8.567 & & 10.797 & NA & 25.362 & 37.240 \\
\hline 4/17/11 20:00 & 12.416 & 5.553 & 7.258 & 17.047 & 8.524 & & 10.794 & & 25.358 & 37.240 \\
\hline 4/18/11 0:00 & 12.435 & 5.558 & 7.289 & 17.002 & 8.498 & & 10.804 & & 25.355 & 37.240 \\
\hline 4/18/11 4:00 & 12.477 & 5.570 & 7.329 & 16.865 & 8.491 & & 10.844 & & 25.353 & 37.238 \\
\hline 4/18/11 8:00 & 12.519 & 5.582 & 7.376 & 16.788 & 8.494 & & 10.886 & & 25.351 & 37.240 \\
\hline $4 / 18 / 11$ 12:00 & 12.560 & 5.591 & 7.419 & 16.760 & 8.491 & & 10.895 & & 25.348 & 37.240 \\
\hline 4/18/11 16:00 & 12.564 & 5.591 & 7.449 & 16.963 & 8.470 & & 10.855 & & 25.344 & 37.240 \\
\hline 4/18/11 20:00 & 12.578 & 5.596 & 7.480 & 16.951 & 8.458 & & 10.851 & & 25.341 & 37.238 \\
\hline 4/19/11 0:00 & 12.590 & 5.603 & 7.511 & 16.942 & 8.444 & & 10.834 & & 25.339 & 37.238 \\
\hline 4/19/11 4:00 & 12.562 & 5.593 & 7.523 & 17.086 & 8.409 & & 10.754 & & 25.334 & 37.240 \\
\hline 4/19/11 8:00 & 12.569 & 5.596 & 7.542 & 16.960 & 8.402 & & 10.778 & & 25.334 & 37.238 \\
\hline 4/19/11 12:00 & 12.588 & 5.600 & 7.558 & 16.956 & 8.402 & & 10.787 & & 25.330 & 37.235 \\
\hline 4/19/11 16:00 & 12.618 & 5.610 & 7.589 & 16.904 & 8.437 & & 10.832 & & 25.325 & 37.235 \\
\hline 4/19/11 20:00 & 12.663 & 5.626 & 7.627 & 16.786 & 8.484 & & 10.898 & & 25.325 & 37.233 \\
\hline 4/20/11 0:00 & 12.722 & 5.643 & 7.672 & 16.646 & 8.529 & & 10.978 & & 25.323 & 37.233 \\
\hline 4/20/11 4:00 & 12.772 & 5.655 & 7.714 & 16.609 & 8.557 & & 11.015 & & 25.323 & 37.233 \\
\hline 4/20/11 8:00 & 12.817 & 5.664 & 7.757 & 16.585 & 8.581 & & 11.043 & & 25.320 & 37.238 \\
\hline $4 / 20 / 11$ 12:00 & 12.861 & 5.676 & 7.799 & 16.590 & 8.600 & & 11.079 & & 25.320 & 37.235 \\
\hline 4/20/11 16:00 & 12.887 & 5.680 & 7.830 & 16.774 & 8.600 & & 11.060 & & 25.318 & 37.235 \\
\hline $4 / 20 / 1120: 00$ & 12.909 & 5.683 & 7.861 & 16.870 & 8.595 & & 11.053 & & 25.315 & 37.235 \\
\hline 4/21/11 0:00 & 12.932 & 5.688 & 7.892 & 16.823 & 8.593 & & 11.072 & & 25.316 & 37.235 \\
\hline $4 / 21 / 114: 00$ & 12.953 & 5.692 & 7.920 & 16.844 & 8.586 & & 11.055 & & 25.315 & 37.235 \\
\hline 4/21/11 8:00 & 12.963 & 5.690 & 7.944 & 16.909 & 8.569 & & 11.034 & & 25.313 & 37.235 \\
\hline $4 / 21 / 11$ 12:00 & 12.953 & 5.685 & 7.955 & 17.054 & 8.536 & & 10.959 & & 25.311 & 37.235 \\
\hline 4/21/11 16:00 & 12.916 & 5.671 & 7.955 & 17.284 & 8.496 & & 10.888 & & 25.308 & 37.233 \\
\hline $4 / 21 / 1120: 00$ & 12.904 & 5.674 & 7.963 & 17.268 & 8.468 & & 10.877 & & 25.306 & 37.233 \\
\hline 4/22/11 0:00 & 12.894 & 5.673 & 7.972 & 17.284 & 8.444 & & 10.846 & & 25.306 & 37.233 \\
\hline 4/22/11 4:00 & 12.869 & 5.666 & 7.974 & 17.336 & 8.411 & & 10.799 & & 25.301 & 37.235 \\
\hline 4/22/11 8:00 & 12.847 & 5.662 & 7.970 & 17.340 & 8.385 & & 10.790 & & 25.299 & 37.235 \\
\hline $4 / 22 / 11$ 12:00 & 12.833 & 5.662 & 7.955 & 17.345 & 8.378 & & 10.771 & & 25.297 & 37.240 \\
\hline 4/22/11 16:00 & 12.836 & 5.671 & 7.958 & 17.310 & 8.390 & & 10.792 & & 25.294 & 37.240 \\
\hline $4 / 22 / 1120: 00$ & 12.842 & 5.676 & 7.965 & 17.315 & 8.399 & & 10.801 & & 25.292 & 37.235 \\
\hline 4/23/11 0:00 & 12.876 & 5.688 & 7.989 & 17.165 & 8.428 & & 10.851 & & 25.292 & 37.235 \\
\hline $4 / 23 / 114: 00$ & 12.892 & 5.695 & 8.008 & 17.133 & 8.442 & & 10.855 & & 25.292 & 37.235 \\
\hline 4/23/11 8:00 & 12.930 & 5.704 & 8.038 & 17.009 & 8.477 & & 10.938 & & 25.290 & 37.238 \\
\hline $4 / 23 / 11$ 12:00 & 12.970 & 5.716 & 8.069 & 16.965 & 8.508 & & 10.971 & & 25.290 & 37.235 \\
\hline 4/23/11 16:00 & 12.989 & 5.725 & 8.093 & 17.049 & 8.517 & & 10.968 & & 25.290 & 37.235 \\
\hline $4 / 23 / 1120: 00$ & 13.008 & 5.728 & 8.116 & 17.049 & 8.531 & & 10.987 & & 25.292 & 37.233 \\
\hline 4/24/11 0:00 & 13.031 & 5.732 & 8.142 & 16.986 & 8.541 & & 11.010 & & 25.292 & 37.233 \\
\hline 4/24/11 4:00 & 13.050 & 5.732 & 8.166 & 16.991 & 8.541 & & 11.010 & & 25.292 & 37.233 \\
\hline 4/24/11 8:00 & 13.059 & 5.735 & 8.185 & 17.009 & 8.536 & & 11.006 & & 25.290 & 37.235 \\
\hline $4 / 24 / 11$ 12:00 & 13.071 & 5.735 & 8.206 & 17.079 & 8.529 & & 10.996 & & 25.287 & 37.235 \\
\hline $4 / 24 / 11$ 16:00 & 13.064 & 5.737 & 8.216 & 17.310 & 8.505 & & 10.938 & & 25.287 & 37.235 \\
\hline $4 / 24 / 1120: 00$ & 13.057 & 5.735 & 8.223 & 17.366 & 8.484 & & 10.921 & & 25.285 & 37.235 \\
\hline 4/25/11 0:00 & 13.066 & 5.742 & 8.239 & 17.284 & 8.479 & & 10.935 & & 25.285 & 37.235 \\
\hline $4 / 25 / 114: 00$ & 13.059 & 5.737 & 8.249 & 17.322 & 8.456 & & 10.902 & & 25.280 & 37.233 \\
\hline 4/25/11 8:00 & 13.045 & 5.732 & 8.256 & 17.345 & 8.430 & & 10.886 & & 25.283 & 37.233 \\
\hline $4 / 25 / 11$ 12:00 & 13.031 & 5.730 & 8.251 & 17.387 & 8.392 & & 10.853 & & 25.280 & 37.238 \\
\hline $4 / 25 / 11$ 16:00 & 13.000 & 5.723 & 8.235 & 17.473 & 8.359 & & 10.799 & & 25.278 & 37.235 \\
\hline
\end{tabular}

TABLE S1.1 Page 68 of 89 


\begin{tabular}{|c|c|c|c|c|c|c|c|c|c|c|}
\hline \multirow[b]{2}{*}{ Date and Time } & \multicolumn{8}{|c|}{ Water Level (ft below reference point) } & \multirow[b]{2}{*}{ SB24 } & \multirow[b]{2}{*}{ SB38 } \\
\hline & McPike & Potter & Hughes & Barr & Burks & MoDOT & Clizer "Old" & Morgan & & \\
\hline $4 / 25 / 1120: 00$ & 12.975 & 5.718 & 8.223 & 17.503 & 8.338 & & 10.771 & & 25.276 & 37.235 \\
\hline 4/26/11 0:00 & 12.956 & 5.713 & 8.216 & 17.517 & 8.321 & & 10.754 & & 25.273 & 37.233 \\
\hline 4/26/11 4:00 & 12.937 & 5.713 & 8.180 & 17.487 & 8.298 & & 10.738 & & 25.271 & 37.233 \\
\hline $4 / 26 / 118: 00$ & 12.937 & 5.721 & 8.149 & 17.347 & 8.300 & & 10.766 & & 25.269 & 37.233 \\
\hline $4 / 26 / 1112: 00$ & 12.956 & 5.732 & 8.145 & 17.235 & 8.312 & & 10.808 & & 25.271 & 37.233 \\
\hline $4 / 26 / 1116: 00$ & 12.979 & 5.739 & 8.152 & 17.212 & 8.321 & & 10.837 & & 25.269 & 37.233 \\
\hline $4 / 26 / 1120: 00$ & 12.996 & 5.749 & 8.161 & 17.182 & 8.314 & & 10.830 & & 25.266 & 37.233 \\
\hline 4/27/11 0:00 & 13.010 & 5.754 & 8.178 & 17.112 & 8.326 & & 10.867 & & 25.266 & 37.233 \\
\hline $4 / 27 / 114: 00$ & 13.022 & 5.756 & 8.192 & 17.116 & 8.319 & & 10.853 & & 25.264 & 37.231 \\
\hline 4/27/11 8:00 & 13.038 & 5.758 & 8.211 & 17.051 & 8.328 & & 10.891 & & 25.264 & 37.231 \\
\hline $4 / 27 / 11$ 12:00 & 13.066 & 5.768 & 8.237 & 17.009 & 8.342 & & 10.926 & & 25.266 & 37.231 \\
\hline 4/27/11 16:00 & 13.081 & 5.770 & 8.256 & 17.065 & 8.345 & & 10.931 & & 25.264 & 37.228 \\
\hline 4/27/11 20:00 & 13.102 & 5.775 & 8.277 & 17.047 & 8.357 & & 10.963 & & 25.264 & 37.231 \\
\hline 4/28/11 0:00 & 13.130 & 5.784 & 8.306 & 16.944 & 8.380 & & 11.001 & & 25.266 & 37.231 \\
\hline 4/28/11 4:00 & 13.149 & 5.784 & 8.327 & 16.939 & 8.385 & & 11.001 & & 25.266 & 37.228 \\
\hline 4/28/11 8:00 & 13.170 & 5.791 & 8.353 & 16.881 & 8.401 & & 11.041 & & 25.266 & 37.231 \\
\hline 4/28/11 12:00 & 13.198 & 5.798 & 8.377 & 16.935 & 8.399 & & 11.057 & & 25.266 & 37.231 \\
\hline 4/28/11 16:00 & 13.213 & 5.803 & 8.395 & 17.082 & 8.394 & & 11.050 & & 25.269 & 37.231 \\
\hline $4 / 28 / 1120: 00$ & 13.231 & 5.808 & 8.417 & 17.086 & 8.399 & & 11.069 & & 25.266 & 37.231 \\
\hline 4/29/11 0:00 & 13.248 & 5.812 & 8.436 & 17.049 & 8.406 & & 11.079 & & 25.269 & 37.231 \\
\hline 4/29/11 4:00 & 13.260 & 5.808 & 8.452 & 17.047 & 8.404 & & 11.067 & & 25.269 & 37.231 \\
\hline 4/29/11 8:00 & 13.269 & 5.808 & 8.469 & 17.030 & 8.404 & & 11.067 & & 25.269 & 37.233 \\
\hline $4 / 29 / 11$ 12:00 & 13.267 & 5.805 & 8.476 & 17.196 & 8.380 & & 11.013 & & 25.269 & 37.231 \\
\hline 4/29/11 16:00 & 13.229 & 5.798 & 8.469 & 17.496 & 8.338 & & 10.917 & & 25.266 & 37.231 \\
\hline 4/29/11 20:00 & 13.220 & 5.801 & 8.476 & 17.487 & 8.326 & & 10.933 & & 25.264 & 37.231 \\
\hline 4/30/11 0:00 & 13.215 & 5.803 & 8.483 & 17.471 & 8.314 & & 10.912 & & 25.264 & 37.231 \\
\hline 4/30/11 4:00 & 13.210 & 5.805 & 8.490 & 17.452 & 8.309 & & 10.919 & & 25.264 & 37.233 \\
\hline 4/30/11 8:00 & 13.220 & 5.808 & 8.504 & 17.389 & 8.321 & & 10.963 & & 25.264 & 37.233 \\
\hline 4/30/11 12:00 & 13.257 & 5.817 & 8.528 & 17.310 & 8.347 & & 11.025 & & 25.266 & 37.233 \\
\hline 4/30/11 16:00 & 13.283 & 5.826 & 8.549 & 17.410 & 8.370 & & 11.046 & & 25.266 & 37.233 \\
\hline 4/30/11 20:00 & 13.309 & 5.841 & 8.573 & 17.315 & 8.396 & & 11.102 & & 25.269 & 37.233 \\
\hline 5/1/11 0:00 & 13.342 & 5.855 & 8.604 & 17.126 & 8.439 & & 11.170 & & 25.269 & 37.235 \\
\hline 5/1/11 4:00 & 13.378 & 5.862 & 8.630 & 17.005 & 8.467 & & 11.194 & & 25.273 & 37.238 \\
\hline 5/1/11 8:00 & 13.415 & 5.869 & 8.660 & 16.874 & 8.493 & & 11.257 & & 25.276 & 37.238 \\
\hline $5 / 1 / 1112: 00$ & 13.448 & 5.871 & 8.684 & 16.890 & 8.500 & & 11.267 & & 25.278 & 37.238 \\
\hline $5 / 1 / 11$ 16:00 & 13.470 & 5.876 & 8.703 & 17.051 & 8.495 & & 11.241 & & 25.280 & 37.238 \\
\hline $5 / 1 / 1120: 00$ & 13.484 & 5.878 & 8.719 & 17.126 & 8.491 & & 11.238 & & 25.283 & 37.238 \\
\hline 5/2/11 0:00 & 13.510 & 5.881 & 8.743 & 16.984 & 8.502 & & 11.290 & & 25.285 & 37.238 \\
\hline 5/2/11 4:00 & 13.531 & 5.881 & 8.762 & 16.986 & 8.502 & & 11.292 & & 25.287 & 37.242 \\
\hline 5/2/11 8:00 & 13.561 & 5.885 & 8.788 & 16.835 & 8.519 & & 11.358 & & 25.290 & 37.242 \\
\hline $5 / 2 / 1112: 00$ & 13.592 & 5.890 & 8.809 & 16.918 & 8.523 & & 11.361 & & 25.294 & 37.245 \\
\hline 5/2/11 16:00 & 13.609 & 5.897 & 8.826 & 17.156 & 8.516 & & 11.330 & & 25.297 & 37.245 \\
\hline 5/2/11 20:00 & 13.625 & 5.899 & 8.842 & 17.161 & 8.516 & & 11.342 & & 25.299 & 37.245 \\
\hline 5/3/11 0:00 & 13.644 & 5.899 & 8.859 & 17.061 & 8.521 & & 11.358 & & 25.301 & 37.247 \\
\hline 5/3/11 4:00 & 13.663 & 5.899 & 8.876 & 17.012 & 8.519 & & 11.368 & & 25.304 & 37.247 \\
\hline $5 / 3 / 118: 00$ & 13.682 & 5.902 & 8.894 & 16.949 & 8.524 & & 11.391 & & 25.309 & 37.249 \\
\hline $5 / 3 / 1112: 00$ & 13.703 & 5.907 & 8.911 & 17.030 & 8.519 & & 11.389 & & 25.311 & 37.252 \\
\hline $5 / 3 / 1116: 00$ & 13.712 & 5.911 & 8.920 & 17.259 & 8.507 & & 11.363 & & 25.316 & 37.252 \\
\hline $5 / 3 / 1120: 00$ & 13.722 & 5.916 & 8.932 & 17.268 & 8.505 & & 11.368 & & 25.315 & 37.252 \\
\hline 5/4/11 0:00 & 13.741 & 5.921 & 8.949 & 17.116 & 8.517 & & 11.400 & & 25.320 & 37.254 \\
\hline 5/4/11 4:00 & 13.755 & 5.921 & 8.963 & 17.086 & 8.514 & & 11.403 & & 25.323 & 37.256 \\
\hline 5/4/11 8:00 & 13.771 & 5.918 & 8.980 & 17.054 & 8.521 & & 11.417 & & 25.325 & 37.259 \\
\hline $5 / 4 / 1112: 00$ & 13.781 & 5.921 & 8.989 & 17.161 & 8.512 & & 11.379 & & 25.330 & 37.259 \\
\hline $5 / 4 / 11$ 16:00 & 13.771 & 5.923 & 8.991 & 17.422 & 8.491 & & 11.318 & & 25.330 & 37.261 \\
\hline 5/4/11 20:00 & 13.762 & 5.928 & 8.994 & 17.468 & 8.479 & & 11.297 & & 25.332 & 37.263 \\
\hline 5/5/11 0:00 & 13.776 & 5.932 & 9.006 & 17.356 & 8.481 & & 11.323 & & 25.334 & 37.263 \\
\hline 5/5/11 4:00 & 13.788 & 5.935 & 9.017 & 17.310 & 8.483 & & 11.335 & & 25.337 & 37.266 \\
\hline $5 / 5 / 118: 00$ & 13.800 & 5.935 & 9.029 & 17.242 & 8.486 & & 11.356 & & 25.341 & 37.266 \\
\hline $5 / 5 / 11$ 12:00 & 13.811 & 5.935 & 9.043 & 17.231 & 8.491 & & 11.356 & & 25.344 & 37.271 \\
\hline $5 / 5 / 1116: 00$ & 13.804 & 5.935 & 9.046 & 17.398 & 8.481 & & 11.314 & & 25.346 & 37.271 \\
\hline $5 / 5 / 1120: 00$ & 13.809 & 5.942 & 9.055 & 17.382 & 8.491 & & 11.325 & & 25.348 & 37.273 \\
\hline 5/6/11 0:00 & 13.818 & 5.942 & 9.067 & 17.254 & 8.495 & & 11.335 & & 25.351 & 37.273 \\
\hline 5/6/11 4:00 & 13.823 & 5.940 & 9.074 & 17.231 & 8.493 & & 11.321 & & 25.353 & 37.275 \\
\hline 5/6/11 8:00 & 13.821 & 5.937 & 9.081 & 17.207 & 8.493 & & 11.316 & & 25.355 & 37.275 \\
\hline
\end{tabular}

TABLE S1.1 Page 69 of 89 


\begin{tabular}{|c|c|c|c|c|c|c|c|c|c|c|}
\hline \multirow[b]{2}{*}{ Date and Time } & \multicolumn{8}{|c|}{ Water Level (ft below reference point) } & \multirow[b]{2}{*}{ SB24 } & \multirow[b]{2}{*}{ SB38 } \\
\hline & McPike & Potter & Hughes & Barr & Burks & MoDOT & Clizer "Old" & Morgan & & \\
\hline $5 / 6 / 1112: 00$ & 13.816 & 5.937 & 9.086 & 17.340 & 8.488 & & 11.269 & & 25.360 & 37.275 \\
\hline 5/6/11 16:00 & 13.795 & 5.940 & 9.084 & 17.601 & 8.474 & & 11.227 & & 25.355 & 37.278 \\
\hline 5/6/11 20:00 & 13.778 & 5.944 & 9.086 & 17.606 & 8.467 & & 11.215 & & 25.360 & 37.280 \\
\hline $5 / 7 / 110: 00$ & 13.785 & 5.954 & 9.096 & 17.459 & 8.472 & & 11.257 & & 25.360 & 37.280 \\
\hline 5/7/11 4:00 & 13.785 & 5.949 & 9.100 & 17.475 & 8.467 & & 11.234 & & 25.365 & 37.282 \\
\hline 5/7/11 8:00 & 13.802 & 5.956 & 9.114 & 17.333 & 8.481 & & 11.290 & & 25.365 & 37.285 \\
\hline $5 / 7 / 11$ 12:00 & 13.828 & 5.965 & 9.131 & 17.352 & 8.502 & & 11.323 & & 25.370 & 37.285 \\
\hline 5/7/11 16:00 & 13.840 & 5.977 & 9.145 & 17.538 & 8.512 & & 11.321 & & 25.370 & 37.287 \\
\hline 5/7/11 20:00 & 13.856 & 5.987 & 9.162 & 17.482 & 8.531 & & 11.358 & & 25.372 & 37.289 \\
\hline 5/8/11 0:00 & 13.877 & 5.994 & 9.181 & 17.326 & 8.549 & & 11.396 & & 25.377 & 37.289 \\
\hline 5/8/11 4:00 & 13.894 & 5.991 & 9.195 & 17.247 & 8.557 & & 11.398 & & 25.379 & 37.289 \\
\hline 5/8/11 8:00 & 13.910 & 5.994 & 9.209 & 17.170 & 8.566 & & 11.419 & & 25.384 & 37.294 \\
\hline $5 / 8 / 1112: 00$ & 13.920 & 5.998 & 9.216 & 17.333 & 8.568 & & 11.379 & & 25.386 & 37.294 \\
\hline 5/8/11 16:00 & 13.913 & 6.005 & 9.223 & 17.582 & 8.566 & & 11.344 & & 25.388 & 37.294 \\
\hline 5/8/11 20:00 & 13.913 & 6.010 & 9.230 & 17.557 & 8.573 & & 11.346 & & 25.391 & 37.299 \\
\hline 5/9/11 0:00 & 13.924 & 6.015 & 9.245 & 17.436 & 8.587 & & 11.372 & & 25.393 & 37.301 \\
\hline 5/9/11 4:00 & 13.929 & 6.012 & 9.252 & 17.396 & 8.592 & & 11.368 & & 25.395 & 37.301 \\
\hline 5/9/11 8:00 & 13.939 & 6.012 & 9.263 & 17.333 & 8.601 & & 11.379 & & 25.398 & 37.304 \\
\hline $5 / 9 / 11$ 12:00 & 13.941 & 6.015 & 9.273 & 17.452 & 8.606 & & 11.363 & & 25.402 & 37.304 \\
\hline 5/9/11 16:00 & 13.946 & 6.027 & 9.282 & 17.610 & 8.620 & & 11.363 & & 25.405 & 37.306 \\
\hline $5 / 9 / 1120: 00$ & 13.957 & 6.038 & 9.299 & 17.540 & 8.637 & & 11.389 & & 25.407 & 37.308 \\
\hline 5/10/11 0:00 & 13.979 & 6.048 & 9.320 & 17.363 & 8.658 & & 11.443 & & 25.409 & 37.311 \\
\hline 5/10/11 4:00 & 13.993 & 6.045 & 9.332 & 17.312 & 8.665 & & 11.438 & & 25.414 & 37.313 \\
\hline 5/10/11 8:00 & 14.012 & 6.045 & 9.346 & 17.226 & 8.684 & & 11.469 & & 25.416 & 37.315 \\
\hline 5/10/11 12:00 & 14.035 & 6.053 & 9.363 & 17.252 & 8.710 & & 11.497 & & 25.423 & 37.315 \\
\hline $5 / 10 / 1116: 00$ & 14.056 & 6.067 & 9.386 & 17.417 & 8.738 & & 11.506 & & 25.423 & 37.318 \\
\hline 5/10/11 20:00 & 14.085 & 6.076 & 9.410 & 17.314 & 8.774 & & 11.546 & & 25.428 & 37.320 \\
\hline 5/11/11 0:00 & 14.108 & 6.086 & 9.431 & 17.207 & 8.792 & & 11.567 & & 25.433 & 37.322 \\
\hline 5/11/11 4:00 & 14.139 & 6.088 & 9.453 & 17.100 & 8.809 & & 11.607 & & 25.437 & 37.325 \\
\hline 5/11/11 8:00 & 14.170 & 6.086 & 9.472 & 17.012 & 8.828 & & 11.633 & & 25.442 & 37.327 \\
\hline $5 / 11 / 11$ 12:00 & 14.195 & 6.090 & 9.491 & 17.112 & 8.849 & & 11.626 & & 25.447 & 37.327 \\
\hline 5/11/11 16:00 & 14.207 & 6.097 & 9.505 & 17.287 & 8.870 & & 11.603 & & 25.452 & 37.329 \\
\hline $5 / 11 / 1120: 00$ & 14.228 & 6.104 & 9.524 & 17.214 & 8.891 & & 11.626 & & 25.454 & 37.332 \\
\hline 5/12/11 0:00 & 14.245 & 6.102 & 9.509 & 17.042 & 8.887 & & 11.638 & & 25.461 & 37.332 \\
\hline $5 / 12 / 114: 00$ & 14.261 & 6.097 & 9.488 & 16.949 & 8.908 & & 11.654 & & 25.463 & 37.334 \\
\hline $5 / 12 / 118: 00$ & 14.283 & 6.097 & 9.488 & 16.865 & 8.929 & & 11.675 & & 25.470 & 37.337 \\
\hline $5 / 12 / 11$ 12:00 & 14.302 & 6.097 & 9.495 & 16.869 & 8.948 & & 11.680 & & 25.475 & 37.339 \\
\hline $5 / 12 / 11$ 16:00 & 14.313 & 6.102 & 9.500 & 17.063 & 8.967 & & 11.659 & & 25.480 & 37.341 \\
\hline 5/12/11 20:00 & 14.323 & 6.107 & 9.512 & 17.091 & 8.981 & & 11.647 & & 25.482 & 37.344 \\
\hline 5/13/11 0:00 & 14.332 & 6.104 & 9.524 & 17.044 & 8.995 & & 11.671 & & 25.489 & 37.346 \\
\hline $5 / 13 / 114: 00$ & 14.349 & 6.107 & 9.536 & 16.993 & 9.009 & & 11.685 & & 25.494 & 37.348 \\
\hline 5/13/11 8:00 & 14.365 & 6.107 & 9.552 & 16.930 & 9.028 & & 11.718 & & 25.496 & 37.351 \\
\hline $5 / 13 / 11$ 12:00 & 14.398 & 6.111 & 9.571 & 16.818 & 9.052 & & 11.767 & & 25.501 & 37.353 \\
\hline 5/13/11 16:00 & 14.426 & 6.114 & 9.590 & 16.851 & 9.068 & & 11.781 & & 25.508 & 37.355 \\
\hline $5 / 13 / 1120: 00$ & 14.452 & 6.116 & 9.604 & 16.832 & 9.087 & & 11.805 & & 25.515 & 37.358 \\
\hline 5/14/11 0:00 & 14.481 & 6.121 & 9.621 & 16.853 & 9.106 & & 11.816 & & 25.520 & 37.360 \\
\hline $5 / 14 / 114: 00$ & 14.500 & 6.121 & 9.632 & 16.897 & 9.118 & & 11.816 & & 25.524 & 37.362 \\
\hline 5/14/11 8:00 & 14.518 & 6.121 & 9.649 & 16.918 & 9.132 & & 11.830 & & 25.529 & 37.365 \\
\hline 5/14/11 12:00 & 14.540 & 6.123 & 9.661 & 16.995 & 9.146 & & 11.844 & & 25.534 & 37.369 \\
\hline 5/14/11 16:00 & 14.554 & 6.123 & 9.675 & 17.109 & 9.158 & & 11.830 & & 25.538 & 37.372 \\
\hline 5/14/11 20:00 & 14.568 & 6.128 & 9.687 & 17.144 & 9.170 & & 11.847 & & 25.543 & 37.374 \\
\hline 5/15/11 0:00 & 14.589 & 6.133 & 9.699 & 17.128 & 9.182 & & 11.861 & & 25.550 & 37.377 \\
\hline 5/15/11 4:00 & 14.601 & 6.130 & 9.711 & 17.198 & 9.189 & & 11.847 & & 25.555 & 37.379 \\
\hline 5/15/11 8:00 & 14.613 & 6.130 & 9.725 & 17.193 & 9.200 & & 11.863 & & 25.562 & 37.381 \\
\hline $5 / 15 / 11$ 12:00 & 14.634 & 6.135 & 9.734 & 17.210 & 9.215 & & 11.884 & & 25.564 & 37.384 \\
\hline $5 / 15 / 1116: 00$ & 14.653 & 6.137 & 9.748 & 17.256 & 9.226 & & 11.894 & & 25.569 & 37.388 \\
\hline 5/15/11 20:00 & 14.672 & 6.140 & 9.763 & 17.289 & 9.243 & & 11.908 & & 25.574 & 37.391 \\
\hline $5 / 16 / 110: 00$ & 14.695 & 6.147 & 9.777 & 17.242 & 9.259 & & 11.931 & & 25.578 & 37.393 \\
\hline $5 / 16 / 114: 00$ & 14.714 & 6.147 & 9.789 & 17.249 & 9.274 & & 11.936 & & 25.583 & 37.395 \\
\hline 5/16/11 8:00 & 14.738 & 6.151 & 9.805 & 17.207 & 9.290 & & 11.964 & & 25.588 & 37.398 \\
\hline $5 / 16 / 1112: 00$ & 14.761 & 6.154 & 9.819 & 17.333 & 9.309 & & 11.969 & & 25.590 & 37.400 \\
\hline 5/16/11 16:00 & 14.773 & 6.163 & 9.831 & 17.566 & 9.323 & & 11.945 & & 25.595 & 37.402 \\
\hline 5/16/11 20:00 & 14.780 & 6.168 & 9.843 & 17.545 & 9.335 & & 11.948 & & 25.599 & 37.402 \\
\hline 5/17/11 0:00 & 14.797 & 6.170 & 9.855 & 17.429 & 9.351 & & 11.967 & & 25.606 & 37.410 \\
\hline
\end{tabular}

TABLE S1.1 Page 70 of 89 


\begin{tabular}{|c|c|c|c|c|c|c|c|c|c|c|}
\hline \multirow[b]{2}{*}{ Date and Time } & \multicolumn{8}{|c|}{ Water Level (ft below reference point) } & \multirow[b]{2}{*}{ SB24 } & \multirow[b]{2}{*}{ SB38 } \\
\hline & McPike & Potter & Hughes & Barr & Burks & MoDOT & Clizer "Old" & Morgan & & \\
\hline $5 / 17 / 114: 00$ & 14.811 & 6.170 & 9.867 & 17.405 & 9.361 & & 11.971 & & 25.611 & 37.412 \\
\hline 5/17/11 8:00 & 14.825 & 6.168 & 9.878 & 17.373 & 9.373 & & 11.981 & & 25.616 & 37.414 \\
\hline 5/17/11 12:00 & 14.841 & 6.173 & 9.888 & 17.510 & 9.387 & & 11.974 & & 25.623 & 37.417 \\
\hline 5/17/11 16:00 & 14.839 & 6.180 & 9.895 & 17.734 & 9.399 & & 11.936 & & 25.628 & 37.419 \\
\hline 5/17/11 20:00 & 14.841 & 6.191 & 9.904 & 17.655 & 9.410 & & 11.941 & & 25.630 & 37.424 \\
\hline 5/18/11 0:00 & 14.853 & 6.189 & 9.914 & 17.517 & 9.425 & & 11.962 & & 25.637 & 37.424 \\
\hline 5/18/11 4:00 & 14.863 & 6.189 & 9.923 & 17.492 & 9.434 & & 11.957 & & 25.644 & 37.428 \\
\hline 5/18/11 8:00 & 14.877 & 6.184 & 9.938 & 17.431 & 9.446 & & 11.981 & & 25.649 & 37.433 \\
\hline 5/18/11 12:00 & 14.891 & 6.189 & 9.947 & 17.508 & 9.460 & & 11.981 & & 25.656 & 37.435 \\
\hline 5/18/11 16:00 & 14.900 & 6.198 & 9.957 & 17.566 & 9.474 & & 11.978 & & 25.660 & 37.438 \\
\hline 5/18/11 20:00 & 14.907 & 6.208 & 9.966 & 17.557 & 9.486 & & 11.990 & & 25.667 & 37.440 \\
\hline 5/19/11 0:00 & 14.929 & 6.210 & 9.980 & 17.466 & 9.502 & & 12.016 & & 25.672 & 37.445 \\
\hline 5/19/11 4:00 & 14.943 & 6.210 & 9.990 & 17.496 & 9.514 & & 12.014 & & 25.679 & 37.450 \\
\hline 5/19/11 8:00 & 14.959 & 6.210 & 10.004 & 17.450 & 9.526 & & 12.042 & & 25.684 & 37.452 \\
\hline 5/19/11 12:00 & 14.983 & 6.217 & 10.016 & 17.429 & 9.543 & & 12.058 & & 25.691 & 37.454 \\
\hline 5/19/11 16:00 & 15.002 & 6.220 & 10.027 & 17.492 & 9.557 & & 12.058 & & 25.698 & 37.459 \\
\hline 5/19/11 20:00 & 15.018 & 6.220 & 10.037 & 17.475 & 9.568 & & 12.068 & & 25.703 & 37.461 \\
\hline 5/20/11 0:00 & 15.037 & 6.222 & 10.046 & 17.454 & 9.580 & & 12.084 & & 25.710 & 37.464 \\
\hline 5/20/11 4:00 & 15.051 & 6.222 & 10.058 & 17.506 & 9.590 & & 12.077 & & 25.717 & 37.466 \\
\hline $5 / 20 / 118: 00$ & 15.065 & 6.222 & 10.068 & 17.485 & 9.599 & & 12.089 & & 25.721 & 37.471 \\
\hline 5/20/11 12:00 & 15.072 & 6.222 & 10.072 & 17.513 & 9.573 & & 12.086 & & 25.728 & 37.473 \\
\hline 5/20/11 16:00 & 15.068 & 6.217 & 10.011 & 17.540 & 9.580 & & 12.065 & & 25.735 & 37.478 \\
\hline 5/20/11 20:00 & 15.056 & 6.217 & 9.959 & 17.538 & 9.594 & & 12.046 & & 25.740 & 37.480 \\
\hline 5/21/11 0:00 & 15.058 & 6.215 & 9.933 & 17.485 & 9.604 & & 12.046 & & 25.747 & 37.483 \\
\hline 5/21/11 4:00 & 15.058 & 6.217 & 9.919 & 17.478 & 9.616 & & 12.049 & & 25.752 & 37.485 \\
\hline 5/21/11 8:00 & 15.065 & 6.220 & 9.912 & 17.422 & 9.627 & & 12.070 & & 25.756 & 37.485 \\
\hline 5/21/11 12:00 & 15.079 & 6.224 & 9.909 & 17.445 & 9.639 & & 12.077 & & 25.766 & 37.490 \\
\hline 5/21/11 16:00 & 15.077 & 6.229 & 9.909 & 17.634 & 9.656 & & 12.054 & & 25.768 & 37.494 \\
\hline 5/21/11 20:00 & 15.077 & 6.236 & 9.909 & 17.603 & 9.668 & & 12.051 & & 25.773 & 37.499 \\
\hline 5/22/11 0:00 & 15.079 & 6.236 & 9.914 & 17.534 & 9.684 & & 12.068 & & 25.780 & 37.499 \\
\hline $5 / 22 / 114: 00$ & 15.086 & 6.236 & 9.919 & 17.529 & 9.693 & & 12.065 & & 25.785 & 37.501 \\
\hline $5 / 22 / 118: 00$ & 15.091 & 6.238 & 9.926 & 17.547 & 9.703 & & 12.077 & & 25.789 & 37.506 \\
\hline $5 / 22 / 11$ 12:00 & 15.101 & 6.238 & 9.935 & 17.617 & 9.719 & & 12.086 & & 25.796 & 37.508 \\
\hline 5/22/11 16:00 & 15.108 & 6.253 & 9.947 & 17.755 & 9.741 & & 12.084 & & 25.799 & 37.511 \\
\hline $5 / 22 / 1120: 00$ & 15.122 & 6.262 & 9.961 & 17.683 & 9.762 & & 12.115 & & 25.806 & 37.516 \\
\hline 5/23/11 0:00 & 15.141 & 6.267 & 9.978 & 17.564 & 9.781 & & 12.145 & & 25.813 & 37.518 \\
\hline 5/23/11 4:00 & 15.167 & 6.269 & 9.994 & 17.471 & 9.800 & & 12.173 & & 25.817 & 37.520 \\
\hline 5/23/11 8:00 & 15.190 & 6.267 & 10.009 & 17.468 & 9.816 & & 12.190 & & 25.825 & 37.525 \\
\hline 5/23/11 12:00 & 15.216 & 6.274 & 10.027 & 17.524 & 9.840 & & 12.218 & & 25.829 & 37.527 \\
\hline 5/23/11 16:00 & 15.240 & 6.283 & 10.044 & 17.715 & 9.863 & & 12.220 & & 25.836 & 37.532 \\
\hline 5/23/11 20:00 & 15.256 & 6.293 & 10.058 & 17.694 & 9.887 & & 12.227 & & 25.843 & 37.534 \\
\hline 5/24/11 0:00 & 15.284 & 6.297 & 10.077 & 17.568 & 9.911 & & 12.260 & & 25.848 & 37.537 \\
\hline 5/24/11 4:00 & 15.308 & 6.293 & 10.091 & 17.531 & 9.927 & & 12.270 & & 25.855 & 37.541 \\
\hline 5/24/11 8:00 & 15.332 & 6.290 & 10.108 & 17.501 & 9.941 & & 12.286 & & 25.862 & 37.544 \\
\hline $5 / 24 / 1112: 00$ & 15.348 & 6.293 & 10.115 & 17.573 & 9.877 & & 12.274 & & 25.869 & 37.546 \\
\hline 5/24/11 16:00 & 15.313 & 6.283 & 10.020 & 17.526 & 9.887 & & 12.230 & & 25.874 & 37.549 \\
\hline 5/24/11 20:00 & 15.273 & 6.283 & 9.942 & 17.527 & 9.906 & & 12.211 & & 25.881 & 37.551 \\
\hline 5/25/11 0:00 & 14.999 & 6.260 & 9.692 & 17.391 & 9.823 & & 12.051 & & 25.888 & 37.551 \\
\hline 5/25/11 4:00 & 14.153 & 6.208 & 9.306 & 17.273 & 9.830 & & 11.950 & & 25.893 & 37.553 \\
\hline 5/25/11 8:00 & 14.061 & 6.208 & 9.060 & 17.170 & 9.835 & & 11.924 & & 25.893 & 37.553 \\
\hline $5 / 25 / 11$ 12:00 & 14.094 & 6.215 & 8.897 & 17.168 & 9.828 & & 11.894 & & 25.897 & 37.558 \\
\hline 5/25/11 16:00 & 14.078 & 6.224 & 8.658 & 17.070 & 9.715 & & 11.835 & & 25.900 & 37.553 \\
\hline 5/25/11 20:00 & 13.107 & 5.916 & 7.845 & 16.657 & 9.625 & & 11.572 & & 25.902 & 37.549 \\
\hline $5 / 26 / 110: 00$ & 12.788 & 5.770 & 7.509 & 16.359 & 9.576 & & 11.591 & & 25.904 & 37.541 \\
\hline 5/26/11 4:00 & 12.909 & 5.833 & 7.331 & 16.285 & 9.470 & & 11.581 & & 25.904 & 37.532 \\
\hline 5/26/11 8:00 & 13.059 & 5.920 & 7.253 & 16.215 & 9.352 & & 11.595 & & 25.904 & 37.551 \\
\hline $5 / 26 / 1112: 00$ & 13.191 & 5.993 & 7.241 & 16.315 & 9.250 & & 11.591 & & 25.907 & 37.556 \\
\hline 5/26/11 16:00 & 13.283 & 6.047 & 7.260 & 16.560 & 9.165 & & 11.556 & & 25.904 & 37.558 \\
\hline 5/26/11 20:00 & 13.349 & 6.085 & 7.296 & 16.595 & 9.097 & & 11.534 & & 25.904 & 37.560 \\
\hline 5/27/11 0:00 & 13.411 & 6.109 & 7.346 & 16.529 & 9.050 & & 11.546 & & 25.902 & 37.560 \\
\hline $5 / 27 / 114: 00$ & 13.455 & 6.120 & 7.393 & 16.576 & 9.005 & & 11.520 & & 25.902 & 37.563 \\
\hline 5/27/11 8:00 & 13.488 & 6.125 & 7.440 & 16.643 & 8.974 & & 11.506 & & 25.900 & 37.565 \\
\hline $5 / 27 / 11$ 12:00 & 13.517 & 6.130 & 7.487 & 16.702 & 8.948 & & 11.476 & & 25.897 & 37.565 \\
\hline 5/27/11 16:00 & 13.528 & 6.125 & 7.523 & 16.800 & 8.925 & & 11.445 & & 25.895 & 37.567 \\
\hline
\end{tabular}

TABLE S1.1 Page 71 of 89 


\begin{tabular}{|c|c|c|c|c|c|c|c|c|c|c|}
\hline \multirow[b]{2}{*}{ Date and Time } & \multicolumn{10}{|c|}{ Water Level (ft below reference point) } \\
\hline & McPike & Potter & Hughes & Barr & Burks & MoDOT & Clizer "Old" & Morgan & SB24 & SB38 \\
\hline $5 / 27 / 1120: 00$ & 13.545 & 6.125 & 7.570 & 16.818 & 8.918 & & 11.438 & & 25.895 & 37.567 \\
\hline 5/28/11 0:00 & 13.566 & 6.132 & 7.618 & 16.774 & 8.913 & & 11.443 & & 25.893 & 37.570 \\
\hline $5 / 28 / 114: 00$ & 13.583 & 6.125 & 7.662 & 16.790 & 8.908 & & 11.426 & & 25.890 & 37.570 \\
\hline 5/28/11 8:00 & 13.599 & 6.123 & 7.707 & 16.832 & 8.910 & & 11.433 & & 25.890 & 37.572 \\
\hline 5/28/11 12:00 & 13.616 & 6.125 & 7.752 & 16.842 & 8.913 & & 11.391 & & 25.888 & 37.572 \\
\hline 5/28/11 16:00 & 13.627 & 6.125 & 7.797 & 16.977 & 8.922 & & 11.400 & & 25.885 & 37.572 \\
\hline 5/28/11 20:00 & 13.642 & 6.132 & 7.845 & 16.956 & 8.934 & & 11.415 & & 25.885 & 37.574 \\
\hline 5/29/11 0:00 & 13.660 & 6.135 & 7.889 & 16.921 & 8.946 & & 11.415 & & 25.883 & 37.574 \\
\hline 5/29/11 4:00 & 13.660 & 6.128 & 7.920 & 17.005 & 8.944 & & 11.377 & & 25.881 & 37.574 \\
\hline $5 / 29 / 118: 00$ & 13.665 & 6.123 & 7.953 & 17.014 & 8.944 & & 11.377 & & 25.881 & 37.574 \\
\hline 5/29/11 12:00 & 13.675 & 6.125 & 7.989 & 17.058 & 8.953 & & 11.346 & & 25.878 & 37.574 \\
\hline 5/29/11 16:00 & 13.686 & 6.132 & 8.036 & 17.177 & 8.977 & & 11.354 & & 25.874 & 37.577 \\
\hline 5/29/11 20:00 & 13.703 & 6.139 & 8.088 & 17.156 & 9.012 & & 11.391 & & 25.874 & 37.577 \\
\hline 5/30/11 0:00 & 13.731 & 6.151 & 8.138 & 17.033 & 9.043 & & 11.436 & & 25.874 & 37.579 \\
\hline 5/30/11 4:00 & 13.752 & 6.153 & 8.178 & 17.037 & 9.064 & & 11.443 & & 25.874 & 37.579 \\
\hline 5/30/11 8:00 & 13.778 & 6.158 & 8.223 & 17.012 & 9.085 & & 11.469 & & 25.874 & 37.579 \\
\hline 5/30/11 12:00 & 13.800 & 6.161 & 8.268 & 17.075 & 9.113 & & 11.478 & & 25.869 & 37.579 \\
\hline 5/30/11 16:00 & 13.825 & 6.168 & 8.320 & 17.137 & 9.149 & & 11.497 & & 25.871 & 37.581 \\
\hline 5/30/11 20:00 & 13.851 & 6.175 & 8.365 & 17.128 & 9.182 & & 11.506 & & 25.871 & 37.581 \\
\hline 5/31/11 0:00 & 13.868 & 6.177 & 8.403 & 17.144 & 9.205 & & 11.506 & & 25.871 & 37.581 \\
\hline $5 / 31 / 114: 00$ & 13.903 & 6.186 & 8.445 & 16.907 & 9.222 & & 11.581 & & 25.874 & 37.581 \\
\hline 5/31/11 8:00 & 13.934 & 6.186 & 8.419 & 16.869 & 9.248 & & 11.605 & & 25.871 & 37.579 \\
\hline 5/31/11 12:00 & 13.981 & 6.194 & 8.436 & 16.802 & 9.288 & & 11.661 & & 25.874 & 37.581 \\
\hline $5 / 31 / 11$ 16:00 & 14.016 & 6.203 & 8.474 & 16.965 & 9.326 & & 11.668 & & 25.876 & 37.584 \\
\hline 5/31/11 20:00 & 14.049 & 6.212 & 8.514 & 16.949 & 9.363 & & 11.685 & & 25.878 & 37.581 \\
\hline 6/1/11 0:00 & 14.082 & 6.215 & 8.552 & 16.893 & 9.396 & & 11.708 & & 25.881 & 37.586 \\
\hline 6/1/11 4:00 & 14.106 & 6.210 & 8.582 & 16.930 & 9.418 & & 11.701 & & 25.881 & 37.586 \\
\hline 6/1/11 8:00 & 14.129 & 6.210 & 8.620 & 16.925 & 9.439 & & 11.720 & & 25.883 & 37.589 \\
\hline $6 / 1 / 11$ 12:00 & 14.153 & 6.212 & 8.656 & 17.095 & 9.465 & & 11.706 & & 25.883 & 37.589 \\
\hline $6 / 1 / 11$ 16:00 & 14.162 & 6.212 & 8.689 & 17.291 & 9.486 & & 11.673 & & 25.885 & 37.589 \\
\hline 6/1/11 20:00 & 14.153 & 6.208 & 8.705 & 17.354 & 9.495 & & 11.638 & & 25.883 & 37.591 \\
\hline 6/2/11 0:00 & 14.162 & 6.205 & 8.729 & 17.296 & 9.510 & & 11.640 & & 25.885 & 37.593 \\
\hline 6/2/11 4:00 & 14.165 & 6.201 & 8.729 & 17.205 & 9.502 & & 11.638 & & 25.888 & 37.591 \\
\hline 6/2/11 8:00 & 14.137 & 6.191 & 8.542 & 17.163 & 9.488 & & 11.586 & & 25.888 & 37.591 \\
\hline $6 / 2 / 11$ 12:00 & 14.111 & 6.191 & 8.398 & 17.133 & 9.495 & & 11.560 & & 25.888 & 37.593 \\
\hline 6/2/11 16:00 & 14.082 & 6.193 & 8.339 & 17.245 & 9.510 & & 11.523 & & 25.888 & 37.593 \\
\hline $6 / 2 / 1120: 00$ & 14.056 & 6.201 & 8.325 & 17.252 & 9.521 & & 11.502 & & 25.885 & 37.593 \\
\hline 6/3/11 0:00 & 14.042 & 6.198 & 8.329 & 17.191 & 9.529 & & 11.509 & & 25.885 & 37.596 \\
\hline 6/3/11 4:00 & 14.040 & 6.203 & 8.344 & 17.161 & 9.535 & & 11.506 & & 25.888 & 37.596 \\
\hline 6/3/11 8:00 & 14.042 & 6.205 & 8.370 & 17.123 & 9.543 & & 11.525 & & 25.885 & 37.596 \\
\hline $6 / 3 / 11$ 12:00 & 14.052 & 6.210 & 8.412 & 17.172 & 9.559 & & 11.539 & & 25.885 & 37.598 \\
\hline $6 / 3 / 11$ 16:00 & 14.066 & 6.219 & 8.462 & 17.284 & 9.585 & & 11.546 & & 25.885 & 37.598 \\
\hline 6/3/11 20:00 & 14.080 & 6.229 & 8.509 & 17.249 & 9.613 & & 11.579 & & 25.885 & 37.598 \\
\hline 6/4/11 0:00 & 14.106 & 6.236 & 8.556 & 17.151 & 9.637 & & 11.633 & & 25.885 & 37.598 \\
\hline 6/4/11 4:00 & 14.134 & 6.238 & 8.599 & 17.133 & 9.658 & & 11.657 & & 25.885 & 37.600 \\
\hline 6/4/11 8:00 & 14.165 & 6.241 & 8.644 & 17.079 & 9.679 & & 11.701 & & 25.885 & 37.600 \\
\hline $6 / 4 / 11$ 12:00 & 14.188 & 6.245 & 8.689 & 17.214 & 9.701 & & 11.706 & & 25.888 & 37.603 \\
\hline $6 / 4 / 11$ 16:00 & 14.205 & 6.250 & 8.736 & 17.303 & 9.731 & & 11.720 & & 25.888 & 37.603 \\
\hline 6/4/11 20:00 & 14.221 & 6.255 & 8.776 & 17.335 & 9.755 & & 11.729 & & 25.890 & 37.603 \\
\hline 6/5/11 0:00 & 14.240 & 6.257 & 8.812 & 17.284 & 9.778 & & 11.748 & & 25.888 & 37.605 \\
\hline 6/5/11 4:00 & 14.254 & 6.255 & 8.843 & 17.282 & 9.795 & & 11.751 & & 25.890 & 37.605 \\
\hline 6/5/11 8:00 & 14.269 & 6.252 & 8.873 & 17.263 & 9.809 & & 11.760 & & 25.893 & 37.605 \\
\hline $6 / 5 / 11$ 12:00 & 14.285 & 6.252 & 8.911 & 17.375 & 9.833 & & 11.765 & & 25.893 & 37.607 \\
\hline 6/5/11 16:00 & 14.297 & 6.262 & 8.951 & 17.601 & 9.861 & & 11.755 & & 25.893 & 37.607 \\
\hline 6/5/11 20:00 & 14.302 & 6.266 & 8.987 & 17.594 & 9.892 & & 11.760 & & 25.893 & 37.607 \\
\hline 6/6/11 0:00 & 14.311 & 6.266 & 9.013 & 17.508 & 9.913 & & 11.774 & & 25.895 & 37.610 \\
\hline 6/6/11 4:00 & 14.318 & 6.262 & 9.034 & 17.482 & 9.932 & & 11.765 & & 25.895 & 37.610 \\
\hline 6/6/11 8:00 & 14.330 & 6.262 & 9.060 & 17.417 & 9.948 & & 11.781 & & 25.897 & 37.612 \\
\hline 6/6/11 12:00 & 14.342 & 6.266 & 9.091 & 17.552 & 9.972 & & 11.786 & & 25.897 & 37.612 \\
\hline 6/6/11 16:00 & 14.351 & 6.278 & 9.126 & 17.743 & 10.005 & & 11.779 & & 25.897 & 37.614 \\
\hline $6 / 6 / 1120: 00$ & 14.360 & 6.288 & 9.160 & 17.692 & 10.038 & & 11.795 & & 25.897 & 37.614 \\
\hline 6/7/11 0:00 & 14.377 & 6.290 & 9.188 & 17.566 & 10.064 & & 11.828 & & 25.900 & 37.617 \\
\hline 6/7/11 4:00 & 14.391 & 6.288 & 9.212 & 17.515 & 10.087 & & 11.833 & & 25.902 & 37.617 \\
\hline 6/7/11 8:00 & 14.408 & 6.285 & 9.235 & 17.457 & 10.109 & & 11.849 & & 25.902 & 37.619 \\
\hline
\end{tabular}

TABLE S1.1 Page 72 of 89 


\begin{tabular}{|c|c|c|c|c|c|c|c|c|c|c|}
\hline \multirow[b]{2}{*}{ Date and Time } & \multicolumn{10}{|c|}{ Water Level (ft below reference point) } \\
\hline & McPike & Potter & Hughes & Barr & Burks & MoDOT & Clizer "Old" & Morgan & SB24 & SB38 \\
\hline 6/7/11 12:00 & 14.422 & 6.290 & 9.264 & 17.587 & 10.132 & & 11.844 & & 25.902 & 37.619 \\
\hline 6/7/11 16:00 & 14.431 & 6.304 & 9.297 & 17.783 & 10.165 & & 11.849 & & 25.902 & 37.622 \\
\hline 6/7/11 20:00 & 14.443 & 6.311 & 9.330 & 17.708 & 10.201 & & 11.877 & & 25.907 & 37.622 \\
\hline 6/8/11 0:00 & 14.462 & 6.316 & 9.356 & 17.596 & 10.231 & & 11.913 & & 25.909 & 37.622 \\
\hline 6/8/11 4:00 & 14.481 & 6.318 & 9.380 & 17.550 & 10.255 & & 11.929 & & 25.904 & 37.624 \\
\hline 6/8/11 8:00 & 14.504 & 6.318 & 9.401 & 17.506 & 10.279 & & 11.950 & & 25.909 & 37.626 \\
\hline $6 / 8 / 1112: 00$ & 14.528 & 6.325 & 9.432 & 17.592 & 10.307 & & 11.962 & & 25.911 & 37.626 \\
\hline 6/8/11 16:00 & 14.549 & 6.335 & 9.467 & 17.759 & 10.342 & & 11.978 & & 25.914 & 37.629 \\
\hline $6 / 8 / 1120: 00$ & 14.573 & 6.342 & 9.503 & 17.704 & 10.378 & & 12.007 & & 25.916 & 37.629 \\
\hline 6/9/11 0:00 & 14.603 & 6.344 & 9.529 & 17.592 & 10.408 & & 12.046 & & 25.918 & 37.631 \\
\hline 6/9/11 4:00 & 14.632 & 6.344 & 9.552 & 17.601 & 10.432 & & 12.056 & & 25.921 & 37.633 \\
\hline 6/9/11 8:00 & 14.662 & 6.344 & 9.578 & 17.480 & 10.453 & & 12.082 & & 25.923 & 37.633 \\
\hline 6/9/11 12:00 & 14.688 & 6.342 & 9.599 & 17.552 & 10.472 & & 12.079 & & 25.925 & 37.636 \\
\hline 6/9/11 16:00 & 14.681 & 6.344 & 9.621 & 17.820 & 10.495 & & 12.042 & & 25.928 & 37.636 \\
\hline 6/9/11 20:00 & 14.657 & 6.351 & 9.642 & 17.811 & 10.521 & & 12.054 & & 25.930 & 37.638 \\
\hline 6/10/11 0:00 & 14.648 & 6.351 & 9.661 & 17.764 & 10.542 & & 12.070 & & 25.932 & 37.640 \\
\hline $6 / 10 / 114: 00$ & 14.665 & 6.354 & 9.682 & 17.699 & 10.564 & & 12.105 & & 25.935 & 37.640 \\
\hline $6 / 10 / 118: 00$ & 14.700 & 6.354 & 9.711 & 17.606 & 10.583 & & 12.152 & & 25.937 & 37.643 \\
\hline 6/10/11 12:00 & 14.724 & 6.354 & 9.727 & 17.701 & 10.597 & & 12.147 & & 25.939 & 37.645 \\
\hline 6/10/11 16:00 & 14.747 & 6.354 & 9.746 & 17.785 & 10.613 & & 12.164 & & 25.944 & 37.645 \\
\hline 6/10/11 20:00 & 14.766 & 6.356 & 9.763 & 17.820 & 10.630 & & 12.173 & & 25.946 & 37.647 \\
\hline $6 / 11 / 110: 00$ & 14.792 & 6.363 & 9.782 & 17.792 & 10.646 & & 12.199 & & 25.949 & 37.650 \\
\hline $6 / 11 / 114: 00$ & 14.823 & 6.363 & 9.801 & 17.801 & 10.661 & & 12.220 & & 25.954 & 37.650 \\
\hline 6/11/11 8:00 & 14.853 & 6.365 & 9.822 & 17.783 & 10.677 & & 12.251 & & 25.956 & 37.652 \\
\hline $6 / 11 / 11$ 12:00 & 14.886 & 6.368 & 9.843 & 17.953 & 10.694 & & 12.256 & & 25.961 & 37.655 \\
\hline $6 / 11 / 11$ 16:00 & 14.910 & 6.384 & 9.864 & 18.125 & 10.717 & & 12.263 & & 25.965 & 37.657 \\
\hline $6 / 11 / 1120: 00$ & 14.926 & 6.391 & 9.886 & 18.055 & 10.736 & & 12.263 & & 25.968 & 37.657 \\
\hline 6/12/11 0:00 & 14.950 & 6.396 & 9.907 & 17.946 & 10.757 & & 12.293 & & 25.970 & 37.659 \\
\hline $6 / 12 / 114: 00$ & 14.971 & 6.391 & 9.923 & 17.927 & 10.774 & & 12.303 & & 25.975 & 37.662 \\
\hline $6 / 12 / 118: 00$ & 14.999 & 6.391 & 9.942 & 17.895 & 10.788 & & 12.345 & & 25.977 & 37.664 \\
\hline $6 / 12 / 11$ 12:00 & 15.025 & 6.389 & 9.957 & 17.997 & 10.802 & & 12.328 & & 25.982 & 37.666 \\
\hline $6 / 12 / 11$ 16:00 & 15.044 & 6.396 & 9.976 & 18.144 & 10.823 & & 12.340 & & 25.986 & 37.666 \\
\hline $6 / 12 / 1120: 00$ & 15.061 & 6.408 & 9.994 & 18.111 & 10.845 & & 12.352 & & 25.991 & 37.671 \\
\hline 6/13/11 0:00 & 15.086 & 6.415 & 10.011 & 18.058 & 10.863 & & 12.373 & & 25.993 & 37.671 \\
\hline $6 / 13 / 114: 00$ & 15.101 & 6.408 & 10.025 & 18.086 & 10.880 & & 12.373 & & 25.998 & 37.673 \\
\hline 6/13/11 8:00 & 15.117 & 6.405 & 10.039 & 18.118 & 10.894 & & 12.375 & & 26.000 & 37.673 \\
\hline $6 / 13 / 11$ 12:00 & 15.129 & 6.412 & 10.054 & 18.179 & 10.908 & & 12.375 & & 26.005 & 37.678 \\
\hline 6/13/11 16:00 & 15.141 & 6.419 & 10.068 & 18.235 & 10.929 & & 12.380 & & 26.010 & 37.678 \\
\hline $6 / 13 / 1120: 00$ & 15.155 & 6.427 & 10.082 & 18.158 & 10.946 & & 12.394 & & 26.014 & 37.680 \\
\hline 6/14/11 0:00 & 15.167 & 6.427 & 10.094 & 18.174 & 10.960 & & 12.397 & & 26.017 & 37.680 \\
\hline $6 / 14 / 114: 00$ & 15.176 & 6.422 & 10.106 & 18.186 & 10.974 & & 12.399 & & 26.021 & 37.685 \\
\hline $6 / 14 / 118: 00$ & 15.190 & 6.419 & 10.120 & 18.158 & 10.991 & & 12.413 & & 26.024 & 37.687 \\
\hline $6 / 14 / 11$ 12:00 & 15.200 & 6.427 & 10.129 & 18.333 & 11.005 & & 12.401 & & 26.029 & 37.687 \\
\hline 6/14/11 16:00 & 15.204 & 6.436 & 10.146 & 18.410 & 11.028 & & 12.404 & & 26.031 & 37.690 \\
\hline $6 / 14 / 1120: 00$ & 15.209 & 6.445 & 10.160 & 18.321 & 11.050 & & 12.420 & & 26.036 & 37.692 \\
\hline 6/15/11 0:00 & 15.233 & 6.445 & 10.181 & 18.142 & 11.071 & & 12.465 & & 26.040 & 37.692 \\
\hline $6 / 15 / 114: 00$ & 15.259 & 6.443 & 10.196 & 18.104 & 11.090 & & 12.486 & & 26.045 & 37.697 \\
\hline 6/15/11 8:00 & 15.289 & 6.443 & 10.217 & 18.060 & 11.111 & & 12.521 & & 26.050 & 37.699 \\
\hline $6 / 15 / 11$ 12:00 & 15.327 & 6.452 & 10.233 & 18.214 & 11.132 & & 12.544 & & 26.054 & 37.702 \\
\hline 6/15/11 16:00 & 15.353 & 6.467 & 10.255 & 18.351 & 11.161 & & 12.559 & & 26.059 & 37.702 \\
\hline $6 / 15 / 1120: 00$ & 15.377 & 6.481 & 10.276 & 18.281 & 11.189 & & 12.575 & & 26.064 & 37.704 \\
\hline 6/16/11 0:00 & 15.400 & 6.478 & 10.295 & 18.230 & 11.212 & & 12.594 & & 26.068 & 37.709 \\
\hline $6 / 16 / 114: 00$ & 15.421 & 6.469 & 10.311 & 18.244 & 11.234 & & 12.603 & & 26.073 & 37.711 \\
\hline $6 / 16 / 118: 00$ & 15.440 & 6.467 & 10.328 & 18.258 & 11.257 & & 12.622 & & 26.078 & 37.713 \\
\hline 6/16/11 12:00 & 15.464 & 6.467 & 10.345 & 18.214 & 11.276 & & 12.622 & & 26.085 & 37.716 \\
\hline 6/16/11 16:00 & 15.468 & 6.462 & 10.354 & 18.333 & 11.295 & & 12.627 & & 26.087 & 37.718 \\
\hline 6/16/11 20:00 & 15.478 & 6.469 & 10.366 & 18.398 & 11.316 & & 12.627 & & 26.094 & 37.720 \\
\hline $6 / 17 / 110: 00$ & 15.494 & 6.471 & 10.382 & 18.298 & 11.337 & & 12.662 & & 26.099 & 37.723 \\
\hline $6 / 17 / 114: 00$ & 15.497 & 6.469 & 10.371 & 18.347 & 11.319 & & 12.636 & & 26.101 & 37.725 \\
\hline 6/17/11 8:00 & 15.501 & 6.464 & 10.323 & 18.288 & 11.340 & & 12.646 & & 26.108 & 37.728 \\
\hline $6 / 17 / 1112: 00$ & 15.506 & 6.464 & 10.302 & 18.279 & 11.363 & & 12.667 & & 26.113 & 37.730 \\
\hline 6/17/11 16:00 & 15.520 & 6.478 & 10.304 & 18.419 & 11.392 & & 12.676 & & 26.118 & 37.732 \\
\hline 6/17/11 20:00 & 15.537 & 6.492 & 10.314 & 18.363 & 11.420 & & 12.700 & & 26.122 & 37.735 \\
\hline 6/18/11 0:00 & 15.558 & 6.495 & 10.326 & 18.281 & 11.446 & & 12.721 & & 26.127 & 37.737 \\
\hline
\end{tabular}

TABLE S1.1 Page 73 of 89 


\begin{tabular}{|c|c|c|c|c|c|c|c|c|c|c|}
\hline \multirow[b]{2}{*}{ Date and Time } & \multicolumn{10}{|c|}{ Water Level (ft below reference point) } \\
\hline & McPike & Potter & Hughes & Barr & Burks & MoDOT & Clizer "Old" & Morgan & SB24 & SB38 \\
\hline 6/18/11 4:00 & 15.572 & 6.488 & 10.335 & 18.344 & 11.467 & & 12.725 & & 26.132 & 37.739 \\
\hline $6 / 18 / 118: 00$ & 15.494 & 6.481 & 10.245 & 18.235 & 11.387 & & 12.685 & & 26.139 & 37.742 \\
\hline $6 / 18 / 1112: 00$ & 15.365 & 6.478 & 10.108 & 18.165 & 11.418 & & 12.702 & & 26.141 & 37.742 \\
\hline $6 / 18 / 11$ 16:00 & 15.320 & 6.488 & 10.039 & 18.277 & 11.448 & & 12.690 & & 26.146 & 37.744 \\
\hline 6/18/11 20:00 & 15.303 & 6.497 & 10.004 & 18.253 & 11.477 & & 12.690 & & 26.148 & 37.744 \\
\hline 6/19/11 0:00 & 15.296 & 6.492 & 9.985 & 18.232 & 11.500 & & 12.685 & & 26.153 & 37.746 \\
\hline $6 / 19 / 114: 00$ & 15.294 & 6.488 & 9.976 & 18.251 & 11.521 & & 12.667 & & 26.158 & 37.749 \\
\hline 6/19/11 8:00 & 15.294 & 6.483 & 9.973 & 18.207 & 11.540 & & 12.688 & & 26.160 & 37.753 \\
\hline 6/19/11 12:00 & 15.303 & 6.488 & 9.978 & 18.349 & 11.561 & & 12.681 & & 26.162 & 37.753 \\
\hline 6/19/11 16:00 & 15.299 & 6.497 & 9.990 & 18.496 & 11.585 & & 12.671 & & 26.165 & 37.756 \\
\hline 6/19/11 20:00 & 15.301 & 6.509 & 10.006 & 18.424 & 11.611 & & 12.678 & & 26.167 & 37.758 \\
\hline 6/20/11 0:00 & 15.301 & 6.509 & 10.016 & 18.407 & 11.630 & & 12.685 & & 26.169 & 37.761 \\
\hline 6/20/11 4:00 & 15.299 & 6.499 & 10.025 & 18.403 & 11.649 & & 12.657 & & 26.174 & 37.761 \\
\hline $6 / 20 / 118: 00$ & 15.313 & 6.499 & 10.042 & 18.284 & 11.668 & & 12.702 & & 26.179 & 37.763 \\
\hline 6/20/11 12:00 & 15.332 & 6.507 & 10.058 & 18.398 & 11.694 & & 12.707 & & 26.181 & 37.768 \\
\hline 6/20/11 16:00 & 15.348 & 6.516 & 10.082 & 18.463 & 11.719 & & 12.723 & & 26.183 & 37.770 \\
\hline 6/20/11 20:00 & 15.362 & 6.525 & 10.106 & 18.444 & 11.743 & & 12.737 & & 26.186 & 37.770 \\
\hline 6/21/11 0:00 & 15.377 & 6.530 & 10.075 & 18.223 & 11.731 & & 12.749 & & 26.190 & 37.768 \\
\hline $6 / 21 / 114: 00$ & 15.355 & 6.521 & 9.980 & 18.225 & 11.755 & & 12.718 & & 26.190 & 37.770 \\
\hline $6 / 21 / 118: 00$ & 15.339 & 6.511 & 9.928 & 18.163 & 11.776 & & 12.723 & & 26.193 & 37.775 \\
\hline $6 / 21 / 11$ 12:00 & 15.351 & 6.516 & 9.912 & 18.156 & 11.797 & & 12.749 & & 26.197 & 37.775 \\
\hline $6 / 21 / 11$ 16:00 & 15.362 & 6.528 & 9.912 & 18.242 & 11.826 & & 12.765 & & 26.202 & 37.782 \\
\hline $6 / 21 / 1120: 00$ & 15.384 & 6.535 & 9.919 & 18.146 & 11.849 & & 12.796 & & 26.207 & 37.779 \\
\hline 6/22/11 0:00 & 15.407 & 6.537 & 9.931 & 18.165 & 11.873 & & 12.817 & & 26.207 & 37.782 \\
\hline 6/22/11 4:00 & 15.431 & 6.535 & 9.947 & 18.216 & 11.892 & & 12.829 & & 26.212 & 37.784 \\
\hline $6 / 22 / 118: 00$ & 15.450 & 6.535 & 9.964 & 18.235 & 11.913 & & 12.848 & & 26.216 & 37.786 \\
\hline $6 / 22 / 11$ 12:00 & 15.473 & 6.542 & 9.985 & 18.274 & 11.937 & & 12.866 & & 26.216 & 37.789 \\
\hline $6 / 22 / 11$ 16:00 & 15.501 & 6.547 & 10.006 & 18.370 & 11.960 & & 12.878 & & 26.221 & 37.791 \\
\hline $6 / 22 / 1120: 00$ & 15.525 & 6.556 & 10.030 & 18.402 & 11.981 & & 12.897 & & 26.226 & 37.793 \\
\hline 6/23/11 0:00 & 15.551 & 6.558 & 10.054 & 18.384 & 12.003 & & 12.918 & & 26.230 & 37.793 \\
\hline $6 / 23 / 114: 00$ & 15.572 & 6.556 & 10.073 & 18.428 & 12.024 & & 12.927 & & 26.233 & 37.798 \\
\hline $6 / 23 / 118: 00$ & 15.598 & 6.554 & 10.096 & 18.424 & 12.043 & & 12.951 & & 26.237 & 37.801 \\
\hline $6 / 23 / 11$ 12:00 & 15.624 & 6.561 & 10.120 & 18.493 & 12.066 & & 12.967 & & 26.240 & 37.805 \\
\hline 6/23/11 16:00 & 15.645 & 6.573 & 10.148 & 18.631 & 12.090 & & 12.977 & 29.460 & 26.240 & 37.808 \\
\hline $6 / 23 / 1120: 00$ & 15.664 & 6.601 & 10.170 & 18.626 & 12.111 & & 12.981 & 29.553 & 26.242 & 37.810 \\
\hline 6/24/11 0:00 & 15.685 & 6.598 & 10.193 & 18.591 & 12.132 & & 13.000 & 29.558 & 26.244 & 37.810 \\
\hline $6 / 24 / 114: 00$ & 15.704 & 6.594 & 10.217 & 18.582 & 12.153 & & 13.012 & 29.551 & 26.247 & 37.815 \\
\hline $6 / 24 / 118: 00$ & 15.728 & 6.589 & 10.238 & 18.573 & 12.175 & & 13.031 & 29.563 & 26.251 & 37.817 \\
\hline $6 / 24 / 11$ 12:00 & 15.749 & 6.596 & 10.262 & 18.696 & 12.198 & & 13.024 & 29.572 & 26.256 & 37.819 \\
\hline 6/24/11 16:00 & 15.761 & 6.608 & 10.285 & 18.854 & 12.222 & & 13.010 & 29.619 & 26.258 & 37.822 \\
\hline $6 / 24 / 1120: 00$ & 15.772 & 6.617 & 10.309 & 18.801 & 12.248 & & 13.017 & 29.579 & 26.263 & 37.824 \\
\hline 6/25/11 0:00 & 15.787 & 6.617 & 10.333 & 18.719 & 12.271 & & 13.035 & 29.579 & 26.265 & 37.826 \\
\hline $6 / 25 / 114: 00$ & 15.801 & 6.613 & 10.352 & 18.740 & 12.293 & & 13.028 & 29.568 & 26.270 & 37.829 \\
\hline $6 / 25 / 118: 00$ & 15.820 & 6.617 & 10.371 & 18.587 & 12.274 & & 13.057 & 29.652 & 26.275 & 37.834 \\
\hline $6 / 25 / 11$ 12:00 & 15.794 & 6.603 & 10.274 & 18.659 & 12.278 & & 13.005 & 29.579 & 26.277 & 37.834 \\
\hline 6/25/11 16:00 & 15.770 & 6.613 & 10.179 & 18.701 & 12.304 & & 13.014 & 29.563 & 26.280 & 37.836 \\
\hline $6 / 25 / 1120: 00$ & 15.758 & 6.617 & 10.127 & 18.656 & 12.328 & & 13.024 & 29.619 & 26.287 & 37.836 \\
\hline 6/26/11 0:00 & 15.754 & 6.617 & 10.101 & 18.612 & 12.354 & & 13.033 & 29.579 & 26.289 & 37.841 \\
\hline 6/26/11 4:00 & 15.749 & 6.608 & 10.087 & 18.622 & 12.375 & & 13.038 & 29.549 & 26.294 & 37.841 \\
\hline $6 / 26 / 118: 00$ & 15.763 & 6.605 & 10.087 & 18.631 & 12.396 & & 13.047 & 29.549 & 26.296 & 37.843 \\
\hline 6/26/11 12:00 & 15.761 & 6.608 & 10.087 & 18.722 & 12.418 & & 13.028 & 29.572 & 26.301 & 37.848 \\
\hline 6/26/11 16:00 & 15.744 & 6.615 & 10.089 & 18.889 & 12.444 & & 13.003 & 29.509 & 26.303 & 37.848 \\
\hline 6/26/11 20:00 & 15.737 & 6.622 & 10.101 & 18.831 & 12.465 & & 13.014 & 29.518 & 26.305 & 37.850 \\
\hline $6 / 27 / 110: 00$ & 15.735 & 6.620 & 10.068 & 18.726 & 12.347 & & 12.977 & 29.509 & 26.305 & 37.855 \\
\hline $6 / 27 / 114: 00$ & 13.882 & 6.089 & 9.134 & 18.176 & 11.845 & & 11.960 & 29.424 & 26.312 & 37.848 \\
\hline $6 / 27 / 118: 00$ & 10.592 & 4.859 & 7.989 & 17.272 & 11.708 & & 11.025 & 28.987 & 26.303 & 37.850 \\
\hline $6 / 27 / 11$ 12:00 & 10.686 & 4.942 & 7.459 & 17.019 & 10.821 & & 10.851 & 28.394 & 26.280 & 37.850 \\
\hline $6 / 27 / 11$ 16:00 & 11.002 & 5.210 & 7.159 & 16.995 & 9.911 & & 10.710 & 27.851 & 26.256 & 37.850 \\
\hline 6/27/11 20:00 & 11.252 & 5.451 & 6.972 & 16.890 & 9.279 & & 10.637 & 27.421 & 26.233 & 37.848 \\
\hline 6/28/11 0:00 & 11.431 & 5.625 & 6.844 & 16.795 & 8.927 & & 10.592 & 27.099 & 26.212 & 37.845 \\
\hline $6 / 28 / 114: 00$ & 11.542 & 5.745 & 6.750 & 16.816 & 8.755 & & 10.541 & 26.816 & 26.190 & 37.843 \\
\hline 6/28/11 8:00 & 11.622 & 5.828 & 6.681 & 16.853 & 8.651 & & 10.513 & 26.586 & 26.172 & 37.843 \\
\hline $6 / 28 / 11$ 12:00 & 11.681 & 5.894 & 6.646 & 16.986 & 8.600 & & 10.494 & 26.567 & 26.153 & 37.841 \\
\hline 6/28/11 16:00 & 11.695 & 5.943 & 6.634 & 17.210 & 8.576 & & 10.454 & 26.276 & 26.134 & 37.838 \\
\hline
\end{tabular}

TABLE S1.1 Page 74 of 89 


\begin{tabular}{|c|c|c|c|c|c|c|c|c|c|c|}
\hline \multirow[b]{2}{*}{ Date and Time } & \multicolumn{8}{|c|}{ Water Level (ft below reference point) } & \multirow[b]{2}{*}{ SB24 } & \multirow[b]{2}{*}{ SB38 } \\
\hline & McPike & Potter & Hughes & Barr & Burks & MoDOT & Clizer "Old" & Morgan & & \\
\hline 6/28/11 20:00 & 11.709 & 5.981 & 6.622 & 17.175 & 8.574 & & 10.428 & 26.064 & 26.113 & 37.834 \\
\hline 6/29/11 0:00 & 11.735 & 6.007 & 6.613 & 17.107 & 8.571 & & 10.428 & 25.986 & 26.097 & 37.834 \\
\hline $6 / 29 / 114: 00$ & 11.744 & 6.019 & 6.601 & 17.133 & 8.564 & & 10.412 & 25.848 & 26.080 & 37.826 \\
\hline $6 / 29 / 118: 00$ & 11.761 & 6.030 & 6.598 & 17.147 & 8.559 & & 10.407 & 25.765 & 26.061 & 37.826 \\
\hline 6/29/11 12:00 & 11.773 & 6.040 & 6.617 & 17.291 & 8.567 & & 10.405 & 25.859 & 26.045 & 37.822 \\
\hline 6/29/11 16:00 & 11.770 & 6.056 & 6.648 & 17.431 & 8.581 & & 10.386 & 25.671 & 26.026 & 37.819 \\
\hline 6/29/11 20:00 & 11.768 & 6.068 & 6.676 & 17.398 & 8.602 & & 10.376 & 25.596 & 26.010 & 37.815 \\
\hline 6/30/11 0:00 & 11.782 & 6.078 & 6.698 & 17.335 & 8.607 & & 10.383 & 25.518 & 25.991 & 37.812 \\
\hline 6/30/11 4:00 & 11.792 & 6.078 & 6.719 & 17.312 & 8.609 & & 10.388 & 25.462 & 25.977 & 37.808 \\
\hline 6/30/11 8:00 & 11.810 & 6.082 & 6.747 & 17.289 & 8.618 & & 10.402 & 25.509 & 25.961 & 37.803 \\
\hline 6/30/11 12:00 & 11.820 & 6.087 & 6.799 & 17.429 & 8.637 & & 10.402 & 25.542 & 25.944 & 37.801 \\
\hline 6/30/11 16:00 & 11.827 & 6.101 & 6.868 & 17.573 & 8.675 & & 10.405 & 25.408 & 25.928 & 37.793 \\
\hline 6/30/11 20:00 & 11.832 & 6.115 & 6.932 & 17.494 & 8.717 & & 10.412 & 25.396 & 25.914 & 37.789 \\
\hline 7/1/11 0:00 & 11.858 & 6.127 & 6.977 & 17.389 & 8.746 & & 10.437 & 25.361 & 25.897 & 37.786 \\
\hline 7/1/11 4:00 & 11.876 & 6.132 & 7.015 & 17.359 & 8.765 & & 10.456 & 25.352 & 25.883 & 37.782 \\
\hline 7/1/11 8:00 & 11.905 & 6.134 & 7.062 & 17.324 & 8.781 & & 10.482 & 28.413 & 25.869 & 37.777 \\
\hline $7 / 1 / 11$ 12:00 & 11.926 & 6.144 & 7.131 & 17.473 & 8.805 & & 10.494 & 26.174 & 25.850 & 37.772 \\
\hline 7/1/11 16:00 & 11.949 & 6.158 & 7.211 & 17.606 & 8.857 & & 10.510 & 25.723 & 25.839 & 37.768 \\
\hline $7 / 1 / 1120: 00$ & 11.968 & 6.167 & 7.279 & 17.543 & 8.908 & & 10.527 & 25.596 & 25.825 & 37.765 \\
\hline 7/2/11 0:00 & 12.001 & 6.176 & 7.332 & 17.452 & 8.946 & & 10.564 & 25.631 & 25.810 & 37.761 \\
\hline 7/2/11 4:00 & 12.037 & 6.179 & 7.381 & 17.424 & 8.982 & & 10.597 & 25.631 & 25.799 & 37.756 \\
\hline 7/2/11 8:00 & 12.070 & 6.181 & 7.431 & 17.403 & 9.010 & & 10.628 & 28.126 & 25.785 & 37.751 \\
\hline $7 / 2 / 11$ 12:00 & 12.107 & 6.188 & 7.488 & 17.531 & 9.043 & & 10.654 & 26.266 & 25.773 & 37.746 \\
\hline $7 / 2 / 11$ 16:00 & 12.129 & 6.200 & 7.561 & 17.680 & 9.085 & & 10.661 & 25.942 & 25.761 & 37.742 \\
\hline $7 / 2 / 1120: 00$ & 12.140 & 6.210 & 7.622 & 17.652 & 9.135 & & 10.663 & 25.883 & 25.747 & 37.737 \\
\hline 7/3/11 0:00 & 12.152 & 6.210 & 7.663 & 17.599 & 9.168 & & 10.670 & 25.824 & 25.735 & 37.735 \\
\hline 7/3/11 4:00 & 12.159 & 6.205 & 7.693 & 17.582 & 9.184 & & 10.665 & 25.789 & 25.726 & 37.730 \\
\hline 7/3/11 8:00 & 12.188 & 6.205 & 7.734 & 17.510 & 9.203 & & 10.696 & 25.838 & 25.712 & 37.728 \\
\hline $7 / 3 / 11$ 12:00 & 11.867 & 6.122 & 7.511 & 17.540 & 9.060 & & 10.597 & 25.824 & 25.700 & 37.720 \\
\hline 7/3/11 16:00 & 11.584 & 5.964 & 7.268 & 17.575 & 9.062 & & 10.555 & 25.732 & 25.688 & 37.716 \\
\hline $7 / 3 / 1120: 00$ & 11.643 & 5.990 & 7.187 & 17.561 & 9.050 & & 10.524 & 25.629 & 25.674 & 37.709 \\
\hline 7/4/11 0:00 & 11.718 & 6.030 & 7.157 & 17.484 & 9.036 & & 10.517 & 25.605 & 25.660 & 37.704 \\
\hline $7 / 4 / 114: 00$ & 11.768 & 6.056 & 7.149 & 17.498 & 9.017 & & 10.503 & 25.587 & 25.649 & 37.702 \\
\hline 7/4/11 8:00 & 11.822 & 6.077 & 7.159 & 17.473 & 9.008 & & 10.515 & 25.561 & 25.637 & 37.695 \\
\hline $7 / 4 / 11$ 12:00 & 11.862 & 6.096 & 7.185 & 17.575 & 9.005 & & 10.494 & 25.544 & 25.623 & 37.690 \\
\hline $7 / 4 / 11$ 16:00 & 11.891 & 6.122 & 7.235 & 17.703 & 9.024 & & 10.489 & 25.563 & 25.609 & 37.685 \\
\hline $7 / 4 / 1120: 00$ & 11.916 & 6.141 & 7.282 & 17.645 & 9.048 & & 10.496 & 25.509 & 25.595 & 37.680 \\
\hline 7/5/11 0:00 & 11.957 & 6.150 & 7.320 & 17.559 & 9.064 & & 10.524 & 25.526 & 25.583 & 37.676 \\
\hline 7/5/11 4:00 & 11.987 & 6.155 & 7.355 & 17.564 & 9.076 & & 10.543 & 25.582 & 25.571 & 37.671 \\
\hline 7/5/11 8:00 & 12.013 & 6.153 & 7.384 & 17.580 & 9.081 & & 10.550 & 25.570 & 25.557 & 37.666 \\
\hline $7 / 5 / 11$ 12:00 & 12.041 & 6.160 & 7.429 & 17.696 & 9.095 & & 10.560 & 25.676 & 25.545 & 37.662 \\
\hline $7 / 5 / 11$ 16:00 & 12.058 & 6.174 & 7.488 & 17.834 & 9.123 & & 10.560 & 25.584 & 25.534 & 37.657 \\
\hline $7 / 5 / 1120: 00$ & 12.091 & 6.183 & 7.540 & 17.710 & 9.161 & & 10.588 & 25.584 & 25.522 & 37.652 \\
\hline 7/6/11 0:00 & 12.103 & 6.183 & 7.570 & 17.713 & 9.175 & & 10.588 & 25.645 & 25.510 & 37.647 \\
\hline 7/6/11 4:00 & 12.140 & 6.183 & 7.611 & 17.603 & 9.194 & & 10.625 & 25.657 & 25.499 & 37.643 \\
\hline 7/6/11 8:00 & 12.166 & 6.186 & 7.644 & 17.617 & 9.208 & & 10.642 & 25.683 & 25.487 & 37.638 \\
\hline $7 / 6 / 11$ 12:00 & 12.185 & 6.183 & 7.675 & 17.710 & 9.222 & & 10.644 & 25.751 & 25.477 & 37.636 \\
\hline $7 / 6 / 11$ 16:00 & 12.190 & 6.191 & 7.710 & 17.869 & 9.338 & & 10.625 & 25.716 & 25.466 & 37.629 \\
\hline $7 / 6 / 1120: 00$ & 12.199 & 6.193 & 7.753 & 17.853 & 9.316 & & 10.621 & 25.683 & 25.454 & 37.624 \\
\hline 7/7/11 0:00 & 12.218 & 6.195 & 7.788 & 17.780 & 9.319 & & 10.639 & 26.379 & 25.445 & 37.622 \\
\hline 7/7/11 4:00 & 12.211 & 6.186 & 7.809 & 17.836 & 9.314 & & 10.616 & 25.975 & 25.433 & 37.617 \\
\hline 7/7/11 8:00 & 12.185 & 6.186 & 7.606 & 17.690 & 9.229 & & 10.574 & 25.859 & 25.421 & 37.610 \\
\hline $7 / 7 / 11$ 12:00 & 11.723 & 6.153 & 7.142 & 17.522 & 9.158 & & 10.466 & 25.761 & 25.409 & 37.605 \\
\hline $7 / 7 / 11$ 16:00 & 11.398 & 6.059 & 6.863 & 17.457 & 9.090 & & 10.414 & 25.629 & 25.398 & 37.598 \\
\hline 7/7/11 20:00 & 11.476 & 6.035 & 6.769 & 17.380 & 9.015 & & 10.388 & 25.518 & 25.386 & 37.591 \\
\hline $7 / 8 / 110: 00$ & 11.577 & 6.035 & 6.745 & 17.293 & 8.949 & & 10.386 & 25.500 & 25.374 & 37.589 \\
\hline 7/8/11 4:00 & 11.652 & 6.040 & 6.755 & 17.258 & 8.895 & & 10.383 & 25.429 & 25.360 & 37.581 \\
\hline 7/8/11 8:00 & 11.714 & 6.047 & 6.781 & 17.247 & 8.850 & & 10.383 & 25.422 & 25.348 & 37.577 \\
\hline $7 / 8 / 11$ 12:00 & 11.766 & 6.056 & 6.828 & 17.347 & 8.826 & & 10.383 & 25.396 & 25.334 & 37.572 \\
\hline $7 / 8 / 11$ 16:00 & 11.796 & 6.073 & 6.894 & 17.491 & 8.826 & & 10.374 & 25.356 & 25.323 & 37.565 \\
\hline $7 / 8 / 1120: 00$ & 11.829 & 6.085 & 6.958 & 17.440 & 8.836 & & 10.376 & 25.319 & 25.309 & 37.563 \\
\hline 7/9/11 0:00 & 11.874 & 6.089 & 7.005 & 17.347 & 8.843 & & 10.405 & 25.394 & 25.294 & 37.556 \\
\hline 7/9/11 4:00 & 11.898 & 6.087 & 7.038 & 17.359 & 8.833 & & 10.407 & 25.335 & 25.283 & 37.551 \\
\hline 7/9/11 8:00 & 11.931 & 6.085 & 7.076 & 17.338 & 8.831 & & 10.426 & 25.333 & 25.271 & 37.546 \\
\hline
\end{tabular}

TABLE S1.1 Page 75 of 89 
Water Level (ft below reference point)

\begin{tabular}{|c|c|c|c|c|c|c|c|c|c|c|}
\hline Date and Time & McPike & Potter & Hughes & Barr & Burks & MoDOT & Clizer "Old" & Morgan & SB24 & SB38 \\
\hline 7/9/11 12:00 & 11.947 & 6.087 & 7.121 & 17.487 & 8.838 & & 10.419 & 25.394 & 25.257 & 37.541 \\
\hline 7/9/11 16:00 & 11.968 & 6.096 & 7.180 & 17.613 & 8.869 & & 10.419 & 25.326 & 25.245 & 37.537 \\
\hline 7/9/11 20:00 & 11.997 & 6.108 & 7.235 & 17.550 & 8.904 & & 10.435 & 25.335 & 25.233 & 37.532 \\
\hline 7/10/11 0:00 & 12.020 & 6.110 & 7.272 & 17.480 & 8.920 & & 10.452 & 25.335 & 25.222 & 37.527 \\
\hline $7 / 10 / 114: 00$ & 12.030 & 6.103 & 7.298 & 17.517 & 8.925 & & 10.444 & 25.330 & 25.210 & 37.520 \\
\hline 7/10/11 8:00 & 12.046 & 6.099 & 7.327 & 17.515 & 8.923 & & 10.452 & 25.328 & 25.198 & 37.518 \\
\hline 7/10/11 12:00 & 12.084 & 6.106 & 7.374 & 17.538 & 8.942 & & 10.480 & 25.377 & 25.187 & 37.511 \\
\hline $7 / 10 / 11$ 16:00 & 12.110 & 6.113 & 7.424 & 17.594 & 8.968 & & 10.494 & 25.391 & 25.177 & 37.506 \\
\hline 7/10/11 20:00 & 12.126 & 6.120 & 7.466 & 17.641 & 9.001 & & 10.499 & 25.455 & 25.165 & 37.501 \\
\hline 7/11/11 0:00 & 12.145 & 6.120 & 7.500 & 17.606 & 9.024 & & 10.510 & 25.427 & 25.154 & 37.497 \\
\hline $7 / 11 / 114: 00$ & 12.188 & 6.125 & 7.537 & 17.522 & 9.048 & & 10.555 & 25.464 & 25.144 & 37.492 \\
\hline $7 / 11 / 118: 00$ & 12.235 & 6.129 & 7.580 & 17.461 & 9.069 & & 10.595 & 26.433 & 25.135 & 37.487 \\
\hline 7/11/11 12:00 & 12.265 & 6.136 & 7.627 & 17.613 & 9.095 & & 10.609 & 26.327 & 25.126 & 37.480 \\
\hline 7/11/11 16:00 & 12.287 & 6.148 & 7.693 & 17.769 & 9.140 & & 10.618 & 25.965 & 25.116 & 37.475 \\
\hline 7/11/11 20:00 & 12.310 & 6.160 & 7.748 & 17.731 & 9.192 & & 10.635 & 25.829 & 25.107 & 37.471 \\
\hline 7/12/11 0:00 & 12.341 & 6.165 & 7.788 & 17.648 & 9.232 & & 10.668 & 25.798 & 25.100 & 37.466 \\
\hline $7 / 12 / 114: 00$ & 12.357 & 6.158 & 7.819 & 17.655 & 9.253 & & 10.675 & 25.873 & 25.090 & 37.464 \\
\hline $7 / 12 / 118: 00$ & 12.376 & 6.155 & 7.847 & 17.669 & 9.267 & & 10.682 & 27.538 & 25.083 & 37.459 \\
\hline $7 / 12 / 11$ 12:00 & 12.402 & 6.158 & 7.890 & 17.773 & 9.291 & & 10.698 & 26.689 & 25.074 & 37.454 \\
\hline $7 / 12 / 11$ 16:00 & 12.416 & 6.172 & 7.944 & 17.955 & 9.326 & & 10.698 & 26.170 & 25.067 & 37.450 \\
\hline $7 / 12 / 1120: 00$ & 12.440 & 6.181 & 7.991 & 17.787 & 9.369 & & 10.733 & 26.040 & 25.058 & 37.445 \\
\hline 7/13/11 0:00 & 12.482 & 6.186 & 8.029 & 17.683 & 9.399 & & 10.773 & 26.099 & 25.053 & 37.443 \\
\hline $7 / 13 / 114: 00$ & 12.496 & 6.179 & 8.058 & 17.722 & 9.416 & & 10.776 & 26.057 & 25.043 & 37.438 \\
\hline $7 / 13 / 11$ 8:00 & 12.525 & 6.179 & 8.088 & 17.717 & 9.435 & & 10.804 & 26.120 & 25.039 & 37.433 \\
\hline 7/13/11 12:00 & 12.543 & 6.176 & 8.112 & 17.822 & 9.451 & & 10.804 & 26.130 & 25.032 & 37.428 \\
\hline 7/13/11 16:00 & 12.551 & 6.183 & 8.145 & 17.978 & 9.472 & & 10.785 & 26.120 & 25.025 & 37.424 \\
\hline 7/13/11 20:00 & 12.562 & 6.193 & 8.183 & 17.946 & 9.501 & & 10.797 & 26.111 & 25.015 & 37.421 \\
\hline 7/14/11 0:00 & 12.584 & 6.195 & 8.214 & 17.876 & 9.527 & & 10.820 & 26.156 & 25.011 & 37.417 \\
\hline $7 / 14 / 114: 00$ & 12.595 & 6.193 & 8.235 & 17.906 & 9.541 & & 10.818 & 26.151 & 25.004 & 37.412 \\
\hline 7/14/11 8:00 & 12.612 & 6.188 & 8.259 & 17.913 & 9.550 & & 10.825 & 26.167 & 24.999 & 37.410 \\
\hline 7/14/11 12:00 & 12.626 & 6.188 & 8.280 & 17.983 & 9.562 & & 10.827 & 26.186 & 24.992 & 37.405 \\
\hline $7 / 14 / 11$ 16:00 & 12.633 & 6.200 & 8.313 & 18.139 & 9.585 & & 10.818 & 26.177 & 24.987 & 37.402 \\
\hline $7 / 14 / 11$ 20:00 & 12.643 & 6.209 & 8.349 & 18.093 & 9.614 & & 10.825 & 26.158 & 24.980 & 37.398 \\
\hline 7/15/11 0:00 & 12.668 & 6.216 & 8.382 & 17.974 & 9.640 & & 10.865 & 26.226 & 24.973 & 37.395 \\
\hline $7 / 15 / 114: 00$ & 12.687 & 6.214 & 8.405 & 17.967 & 9.659 & & 10.877 & 26.226 & 24.966 & 37.391 \\
\hline $7 / 15 / 118: 00$ & 12.711 & 6.212 & 8.434 & 17.950 & 9.673 & & 10.905 & 26.250 & 24.964 & 37.391 \\
\hline $7 / 15 / 11$ 12:00 & 12.732 & 6.219 & 8.469 & 18.081 & 9.692 & & 10.905 & 26.311 & 24.957 & 37.384 \\
\hline 7/15/11 16:00 & 12.749 & 6.235 & 8.516 & 18.223 & 9.725 & & 10.910 & 26.294 & 24.952 & 37.381 \\
\hline 7/15/11 20:00 & 12.765 & 6.247 & 8.557 & 18.139 & 9.760 & & 10.931 & 26.353 & 24.947 & 37.377 \\
\hline 7/16/11 0:00 & 12.801 & 6.254 & 8.592 & 17.997 & 9.795 & & 10.985 & 26.374 & 24.943 & 37.374 \\
\hline $7 / 16 / 114: 00$ & 12.826 & 6.249 & 8.621 & 17.988 & 9.819 & & 11.020 & 26.396 & 24.938 & 37.372 \\
\hline 7/16/11 8:00 & 12.855 & 6.252 & 8.649 & 17.978 & 9.840 & & 11.036 & 26.866 & 24.933 & 37.367 \\
\hline 7/16/11 12:00 & 12.881 & 6.254 & 8.684 & 18.086 & 9.861 & & 11.051 & 26.638 & 24.931 & 37.365 \\
\hline 7/16/11 16:00 & 12.907 & 6.271 & 8.734 & 18.193 & 9.897 & & 11.076 & 26.682 & 24.926 & 37.362 \\
\hline 7/16/11 20:00 & 12.932 & 6.285 & 8.779 & 18.151 & 9.935 & & 11.100 & 26.619 & 24.924 & 37.360 \\
\hline 7/17/11 0:00 & 12.963 & 6.289 & 8.812 & 18.074 & 9.968 & & 11.140 & 26.781 & 24.919 & 37.355 \\
\hline $7 / 17 / 114: 00$ & 12.989 & 6.287 & 8.838 & 18.083 & 9.989 & & 11.156 & 26.706 & 24.917 & 37.355 \\
\hline $7 / 17 / 118: 00$ & 13.020 & 6.285 & 8.869 & 18.051 & 10.012 & & 11.199 & 26.741 & 24.915 & 37.351 \\
\hline $7 / 17 / 11$ 12:00 & 13.055 & 6.294 & 8.909 & 18.174 & 10.038 & & 11.222 & 26.826 & 24.915 & 37.348 \\
\hline 7/17/11 16:00 & 13.081 & 6.311 & 8.959 & 18.337 & 10.074 & & 11.234 & 26.819 & 24.912 & 37.348 \\
\hline 7/17/11 20:00 & 13.102 & 6.322 & 8.999 & 18.281 & 10.114 & & 11.250 & 26.885 & 24.907 & 37.346 \\
\hline 7/18/11 0:00 & 13.128 & 6.320 & 9.025 & 18.223 & 10.147 & & 11.281 & 26.870 & 24.907 & 37.341 \\
\hline $7 / 18 / 114: 00$ & 13.149 & 6.313 & 9.044 & 18.204 & 10.168 & & 11.295 & 26.873 & 24.905 & 37.339 \\
\hline 7/18/11 8:00 & 13.175 & 6.306 & 9.068 & 18.167 & 10.189 & & 11.318 & 31.794 & 24.903 & 37.339 \\
\hline 7/18/11 12:00 & 13.199 & 6.315 & 9.101 & 18.316 & 10.215 & & 11.330 & 28.571 & 24.903 & 37.334 \\
\hline 7/18/11 16:00 & 13.218 & 6.332 & 9.138 & 18.498 & 10.246 & & 11.330 & 27.574 & 24.903 & 37.334 \\
\hline 7/18/11 20:00 & 13.234 & 6.348 & 9.172 & 18.423 & 10.284 & & 11.342 & 27.308 & 24.900 & 37.332 \\
\hline 7/19/11 0:00 & 13.258 & 6.344 & 9.193 & 18.337 & 10.314 & & 11.372 & 27.435 & 24.898 & 37.329 \\
\hline $7 / 19 / 114: 00$ & 13.274 & 6.332 & 9.209 & 18.321 & 10.336 & & 11.379 & 27.242 & 24.898 & 37.329 \\
\hline 7/19/11 8:00 & 13.295 & 6.325 & 9.228 & 18.286 & 10.354 & & 11.398 & 28.366 & 24.898 & 37.327 \\
\hline $7 / 19 / 11$ 12:00 & 13.317 & 6.334 & 9.259 & 18.428 & 10.378 & & 11.403 & 27.388 & 24.896 & 37.325 \\
\hline $7 / 19 / 11$ 16:00 & 13.333 & 6.358 & 9.297 & 18.587 & 10.416 & & 11.410 & 27.294 & 24.896 & 37.325 \\
\hline 7/19/11 20:00 & 13.350 & 6.370 & 9.328 & 18.514 & 10.453 & & 11.419 & 27.197 & 24.896 & 37.322 \\
\hline 7/20/11 0:00 & 13.364 & 6.362 & 9.344 & 18.444 & 10.484 & & 11.443 & 27.310 & 24.893 & 37.322 \\
\hline
\end{tabular}

TABLE S1.1 Page 76 of 89 


\begin{tabular}{|c|c|c|c|c|c|c|c|c|c|c|}
\hline \multirow[b]{2}{*}{ Date and Time } & \multicolumn{10}{|c|}{ Water Level (ft below reference point) } \\
\hline & McPike & Potter & Hughes & Barr & Burks & MoDOT & Clizer "Old" & Morgan & SB24 & SB38 \\
\hline $7 / 20 / 114: 00$ & 13.385 & 6.358 & 9.361 & 18.365 & 10.510 & & 11.466 & 27.308 & 24.893 & 37.320 \\
\hline $7 / 20 / 118: 00$ & 13.406 & 6.351 & 9.375 & 18.365 & 10.531 & & 11.476 & 27.282 & 24.896 & 37.320 \\
\hline $7 / 20 / 1112: 00$ & 13.427 & 6.360 & 9.406 & 18.482 & 10.559 & & 11.492 & 27.517 & 24.893 & 37.318 \\
\hline $7 / 20 / 1116: 00$ & 13.430 & 6.384 & 9.446 & 18.617 & 10.597 & & 11.509 & 27.277 & 24.893 & 37.318 \\
\hline 7/20/11 20:00 & 13.376 & 6.400 & 9.479 & 18.540 & 10.640 & & 11.537 & 27.280 & 24.893 & 37.313 \\
\hline 7/21/11 0:00 & 13.303 & 6.403 & 9.505 & 18.428 & 10.675 & & 11.579 & 27.261 & 24.893 & 37.315 \\
\hline $7 / 21 / 114: 00$ & 13.234 & 6.395 & 9.524 & 18.391 & 10.706 & & 11.598 & 27.334 & 24.891 & 37.313 \\
\hline $7 / 21 / 118: 00$ & 13.199 & 6.391 & 9.543 & 18.337 & 10.734 & & 11.635 & 30.202 & 24.893 & 37.311 \\
\hline $7 / 21 / 11$ 12:00 & 13.185 & 6.403 & 9.574 & 18.512 & 10.762 & & 11.650 & 29.335 & 24.893 & 37.308 \\
\hline $7 / 21 / 11$ 16:00 & 13.185 & 6.424 & 9.611 & 18.680 & 10.802 & & 11.657 & 27.966 & 24.893 & 37.308 \\
\hline $7 / 21 / 1120: 00$ & 13.197 & 6.440 & 9.645 & 18.603 & 10.842 & & 11.678 & 27.649 & 24.893 & 37.308 \\
\hline 7/22/11 0:00 & 13.222 & 6.438 & 9.666 & 18.500 & 10.878 & & 11.713 & 27.611 & 24.893 & 37.308 \\
\hline $7 / 22 / 114: 00$ & 13.258 & 6.438 & 9.687 & 18.423 & 10.909 & & 11.746 & 27.569 & 24.896 & 37.306 \\
\hline $7 / 22 / 118: 00$ & 13.293 & 6.428 & 9.706 & 18.472 & 10.937 & & 11.760 & 27.548 & 24.896 & 37.306 \\
\hline $7 / 22 / 11$ 12:00 & 13.328 & 6.438 & 9.737 & 18.584 & 10.970 & & 11.781 & 27.545 & 24.898 & 37.306 \\
\hline $7 / 22 / 11$ 16:00 & 13.364 & 6.459 & 9.775 & 18.761 & 11.008 & & 11.793 & 27.529 & 24.898 & 37.306 \\
\hline $7 / 22 / 1120: 00$ & 13.397 & 6.476 & 9.808 & 18.645 & 11.050 & & 11.826 & 27.515 & 24.898 & 37.304 \\
\hline 7/23/11 0:00 & 13.435 & 6.473 & 9.831 & 18.566 & 11.085 & & 11.861 & 27.559 & 24.900 & 37.304 \\
\hline $7 / 23 / 114: 00$ & 13.472 & 6.466 & 9.850 & 18.542 & 11.116 & & 11.882 & 27.569 & 24.903 & 37.304 \\
\hline $7 / 23 / 118: 00$ & 13.515 & 6.459 & 9.872 & 18.505 & 11.144 & & 11.915 & 27.738 & 24.905 & 37.304 \\
\hline $7 / 23 / 11$ 12:00 & 13.555 & 6.473 & 9.902 & 18.661 & 11.180 & & 11.936 & 27.729 & 24.905 & 37.306 \\
\hline $7 / 23 / 11$ 16:00 & 13.592 & 6.494 & 9.945 & 18.831 & 11.220 & & 11.957 & 27.696 & 24.905 & 37.304 \\
\hline $7 / 23 / 1120: 00$ & 13.630 & 6.511 & 9.983 & 18.733 & 11.267 & & 11.992 & 27.705 & 24.910 & 37.304 \\
\hline 7/24/11 0:00 & 13.673 & 6.520 & 10.009 & 18.631 & 11.305 & & 12.032 & 27.752 & 24.912 & 37.304 \\
\hline $7 / 24 / 114: 00$ & 13.713 & 6.508 & 10.030 & 18.589 & 11.335 & & 12.065 & 27.778 & 24.915 & 37.304 \\
\hline $7 / 24 / 118: 00$ & 13.743 & 6.499 & 10.006 & 18.561 & 11.307 & & 12.051 & 27.828 & 24.917 & 37.304 \\
\hline $7 / 24 / 11$ 12:00 & 13.781 & 6.490 & 9.947 & 18.605 & 11.347 & & 12.079 & 27.844 & 24.922 & 37.301 \\
\hline $7 / 24 / 11$ 16:00 & 13.807 & 6.504 & 9.945 & 18.787 & 11.387 & & 12.079 & 27.842 & 24.922 & 37.301 \\
\hline $7 / 24 / 1120: 00$ & 13.828 & 6.518 & 9.962 & 18.733 & 11.427 & & 12.096 & 27.820 & 24.924 & 37.301 \\
\hline $7 / 25 / 11$ 0:00 & 13.859 & 6.518 & 9.973 & 18.542 & 11.463 & & 12.096 & 27.891 & 24.931 & 37.304 \\
\hline $7 / 25 / 114: 00$ & 13.885 & 6.504 & 9.988 & 18.680 & 11.491 & & 12.117 & 27.853 & 24.931 & 37.304 \\
\hline $7 / 25 / 118: 00$ & 13.908 & 6.494 & 10.002 & 18.677 & 11.519 & & 12.138 & 27.867 & 24.933 & 37.301 \\
\hline $7 / 25 / 11$ 12:00 & 13.934 & 6.497 & 10.018 & 18.824 & 11.550 & & 12.143 & 27.957 & 24.936 & 37.301 \\
\hline $7 / 25 / 11$ 16:00 & 13.953 & 6.518 & 10.044 & 18.952 & 11.583 & & 12.131 & 27.882 & 24.936 & 37.304 \\
\hline $7 / 25 / 1120: 00$ & 13.967 & 6.530 & 10.068 & 18.880 & 11.619 & & 12.143 & 27.853 & 24.938 & 37.304 \\
\hline 7/26/11 0:00 & 13.991 & 6.525 & 10.087 & 18.787 & 11.649 & & 12.169 & 27.846 & 24.940 & 37.304 \\
\hline $7 / 26 / 114: 00$ & 14.010 & 6.511 & 10.101 & 18.801 & 11.675 & & 12.169 & 27.900 & 24.945 & 37.304 \\
\hline $7 / 26 / 118: 00$ & 14.029 & 6.499 & 10.118 & 18.794 & 11.701 & & 12.183 & 31.547 & 24.947 & 37.304 \\
\hline $7 / 26 / 11$ 12:00 & 14.052 & 6.508 & 10.139 & 18.922 & 11.732 & & 12.190 & 28.538 & 24.950 & 37.304 \\
\hline 7/26/11 16:00 & 14.071 & 6.534 & 10.170 & 19.027 & 11.767 & & 12.192 & 28.312 & 24.952 & 37.304 \\
\hline $7 / 26 / 1120: 00$ & 14.088 & 6.549 & 10.200 & 18.959 & 11.802 & & 12.204 & 28.056 & 24.954 & 37.306 \\
\hline 7/27/11 0:00 & 14.109 & 6.548 & 10.219 & 18.864 & 11.835 & & 12.232 & 28.025 & 24.957 & 37.306 \\
\hline $7 / 27 / 114: 00$ & 14.132 & 6.539 & 10.236 & 18.838 & 11.864 & & 12.244 & 28.009 & 24.959 & 37.306 \\
\hline 7/27/11 8:00 & 14.158 & 6.534 & 10.255 & 18.805 & 11.894 & & 12.267 & 28.018 & 24.961 & 37.306 \\
\hline $7 / 27 / 11$ 12:00 & 14.180 & 6.544 & 10.274 & 19.006 & 11.925 & & 12.258 & 27.990 & 24.966 & 37.308 \\
\hline $7 / 27 / 11$ 16:00 & 14.203 & 6.570 & 10.309 & 19.066 & 11.968 & & 12.284 & 27.992 & 24.966 & 37.308 \\
\hline $7 / 27 / 1120: 00$ & 14.234 & 6.591 & 10.345 & 18.950 & 12.010 & & 12.317 & 28.004 & 24.971 & 37.311 \\
\hline 7/28/11 0:00 & 14.269 & 6.600 & 10.368 & 18.836 & 12.050 & & 12.366 & 28.611 & 24.975 & 37.311 \\
\hline $7 / 28 / 114: 00$ & 14.307 & 6.598 & 10.392 & 18.955 & 12.085 & & 12.404 & 28.307 & 24.978 & 37.311 \\
\hline 7/28/11 8:00 & 14.349 & 6.593 & 10.413 & 18.971 & 12.121 & & 12.436 & 28.244 & 24.983 & 37.311 \\
\hline $7 / 28 / 1112: 00$ & 14.394 & 6.603 & 10.439 & 18.990 & 12.154 & & 12.467 & 28.312 & 24.990 & 37.313 \\
\hline $7 / 28 / 11$ 16:00 & 14.436 & 6.617 & 10.465 & 19.008 & 12.192 & & 12.490 & 28.288 & 24.992 & 37.313 \\
\hline $7 / 28 / 1120: 00$ & 14.472 & 6.626 & 10.491 & 19.027 & 12.225 & & 12.516 & 28.293 & 24.997 & 37.315 \\
\hline 7/29/11 0:00 & 14.516 & 6.622 & 10.513 & 19.043 & 12.260 & & 12.556 & 28.465 & 25.001 & 37.315 \\
\hline $7 / 29 / 114: 00$ & 14.552 & 6.607 & 10.527 & 19.050 & 12.276 & & 12.568 & 28.448 & 25.006 & 37.315 \\
\hline 7/29/11 8:00 & 14.585 & 6.600 & 10.536 & 19.048 & 12.305 & & 12.584 & 28.420 & 25.013 & 37.313 \\
\hline $7 / 29 / 11$ 12:00 & 14.615 & 6.591 & 10.546 & 19.057 & 12.331 & & 12.591 & 28.495 & 25.018 & 37.318 \\
\hline $7 / 29 / 11$ 16:00 & 14.639 & 6.586 & 10.555 & 19.071 & 12.364 & & 12.591 & 28.458 & 25.025 & 37.318 \\
\hline $7 / 29 / 1120: 00$ & 14.660 & 6.586 & 10.565 & 19.087 & 12.392 & & 12.599 & 28.441 & 25.029 & 37.320 \\
\hline 7/30/11 0:00 & 14.679 & 6.581 & 10.548 & 19.057 & 12.385 & & 12.599 & 28.484 & 25.034 & 37.315 \\
\hline $7 / 30 / 114: 00$ & 14.679 & 6.574 & 10.463 & 19.011 & 12.210 & & 12.420 & 28.451 & 25.039 & 37.308 \\
\hline 7/30/11 8:00 & 12.897 & 5.516 & 9.713 & 18.514 & 11.944 & & 11.901 & 28.359 & 25.043 & 37.304 \\
\hline $7 / 30 / 11$ 12:00 & 12.622 & 5.215 & 9.153 & 18.300 & 11.989 & & 11.812 & 27.980 & 25.036 & 37.308 \\
\hline $7 / 30 / 11$ 16:00 & 12.780 & 5.443 & 8.857 & 18.228 & 12.041 & & 11.704 & 27.621 & 25.029 & 37.308 \\
\hline
\end{tabular}

TABLE S1.1 Page 77 of 89 


\begin{tabular}{|c|c|c|c|c|c|c|c|c|c|c|}
\hline \multirow[b]{2}{*}{ Date and Time } & \multicolumn{8}{|c|}{ Water Level (ft below reference point) } & \multirow[b]{2}{*}{ SB24 } & \multirow[b]{2}{*}{ SB38 } \\
\hline & McPike & Potter & Hughes & Barr & Burks & MoDOT & Clizer "Old" & Morgan & & \\
\hline $7 / 30 / 1120: 00$ & 12.897 & 5.684 & 8.682 & 18.204 & 12.012 & & 11.645 & 27.421 & 25.022 & 37.308 \\
\hline 7/31/11 0:00 & 12.982 & 5.863 & 8.559 & 18.202 & 11.937 & & 11.624 & 27.247 & 25.013 & 37.311 \\
\hline 7/31/11 4:00 & 13.046 & 5.988 & 8.469 & 18.211 & 11.880 & & 11.600 & 27.176 & 25.006 & 37.308 \\
\hline 7/31/11 8:00 & 13.100 & 6.075 & 8.405 & 18.228 & 11.828 & & 11.600 & 27.061 & 24.999 & 37.308 \\
\hline $7 / 31 / 1112: 00$ & 13.140 & 6.138 & 8.368 & 18.246 & 11.868 & & 11.589 & 27.014 & 24.992 & 37.308 \\
\hline 7/31/11 16:00 & 13.168 & 6.193 & 8.360 & 18.265 & 11.899 & & 11.574 & 26.908 & 24.985 & 37.308 \\
\hline $7 / 31 / 1120: 00$ & 13.192 & 6.233 & 8.363 & 18.284 & 11.904 & & 11.577 & 26.873 & 24.980 & 37.308 \\
\hline 8/1/11 0:00 & 13.216 & 6.254 & 8.358 & 18.300 & 11.854 & & 11.596 & 26.833 & 24.973 & 37.306 \\
\hline 8/1/11 4:00 & 13.234 & 6.263 & 8.351 & 18.316 & 11.838 & & 11.593 & 26.800 & 24.966 & 37.304 \\
\hline 8/1/11 8:00 & 13.251 & 6.270 & 8.349 & 18.333 & 11.828 & & 11.591 & 26.840 & 24.961 & 37.304 \\
\hline $8 / 1 / 11$ 12:00 & 13.267 & 6.282 & 8.372 & 18.347 & 11.892 & & 11.586 & 26.772 & 24.954 & 37.301 \\
\hline 8/1/11 16:00 & 13.277 & 6.303 & 8.417 & 18.358 & 11.951 & & 11.577 & 26.692 & 24.947 & 37.299 \\
\hline 8/1/11 20:00 & 13.284 & 6.320 & 8.455 & 18.370 & 11.975 & & 11.588 & 26.682 & 24.940 & 37.299 \\
\hline 8/2/11 0:00 & 13.298 & 6.327 & 8.474 & 18.379 & 11.937 & & 11.612 & 26.652 & 24.936 & 37.299 \\
\hline 8/2/11 4:00 & 13.305 & 6.322 & 8.483 & 18.388 & 11.953 & & 11.603 & 26.659 & 24.931 & 37.296 \\
\hline 8/2/11 8:00 & 13.315 & 6.322 & 8.502 & 18.395 & 11.923 & & 11.612 & 28.615 & 24.924 & 37.294 \\
\hline $8 / 2 / 11$ 12:00 & 13.329 & 6.332 & 8.547 & 18.407 & 11.993 & & 11.612 & 26.986 & 24.919 & 37.294 \\
\hline 8/2/11 16:00 & 13.343 & 6.353 & 8.606 & 18.419 & 11.996 & & 11.635 & 26.798 & 24.915 & 37.292 \\
\hline 8/2/11 20:00 & 13.364 & 6.369 & 8.656 & 18.430 & 11.972 & & 11.680 & 26.751 & 24.910 & 37.292 \\
\hline 8/3/11 0:00 & 13.392 & 6.374 & 8.689 & 18.447 & 11.913 & & 11.720 & 26.779 & 24.905 & 37.289 \\
\hline 8/3/11 4:00 & 13.416 & 6.372 & 8.718 & 18.463 & 11.876 & & 11.746 & 26.774 & 24.903 & 37.289 \\
\hline 8/3/11 8:00 & 13.442 & 6.372 & 8.744 & 18.482 & 11.828 & & 11.769 & 26.835 & 24.898 & 37.287 \\
\hline $8 / 3 / 11$ 12:00 & 13.470 & 6.376 & 8.781 & 18.503 & 11.883 & & 11.786 & 28.145 & 24.896 & 37.285 \\
\hline 8/3/11 16:00 & 13.487 & 6.393 & 8.829 & 18.519 & 11.956 & & 11.774 & 32.476 & 24.891 & 37.285 \\
\hline 8/3/11 20:00 & 13.503 & 6.402 & 8.874 & 18.535 & 11.996 & & 11.783 & 33.638 & 24.889 & 37.280 \\
\hline 8/4/11 0:00 & 13.524 & 6.405 & 8.902 & 18.547 & 11.937 & & 11.816 & 29.462 & 24.886 & 37.280 \\
\hline 8/4/11 4:00 & 13.546 & 6.400 & 8.928 & 18.563 & 11.960 & & 11.828 & 28.039 & 24.886 & 37.280 \\
\hline 8/4/11 8:00 & 13.562 & 6.395 & 8.947 & 18.575 & 11.941 & & 11.837 & 27.651 & 24.886 & 37.278 \\
\hline $8 / 4 / 11$ 12:00 & 13.583 & 6.398 & 8.971 & 18.591 & 12.001 & & 11.840 & 27.435 & 24.884 & 37.278 \\
\hline 8/4/11 16:00 & 13.600 & 6.407 & 9.004 & 18.607 & 12.045 & & 11.845 & 27.376 & 24.882 & 37.278 \\
\hline 8/4/11 20:00 & 13.616 & 6.419 & 9.037 & 18.619 & 12.048 & & 11.861 & 27.273 & 24.882 & 37.278 \\
\hline 8/5/11 0:00 & 13.637 & 6.421 & 9.063 & 18.635 & 12.022 & & 11.887 & 27.428 & 24.879 & 37.278 \\
\hline 8/5/11 4:00 & 13.654 & 6.417 & 9.082 & 18.649 & 12.050 & & 11.882 & 27.315 & 24.879 & 37.275 \\
\hline 8/5/11 8:00 & 13.678 & 6.417 & 9.091 & 18.661 & 11.920 & & 11.906 & 27.277 & 24.877 & 37.278 \\
\hline $8 / 5 / 11$ 12:00 & 13.663 & 6.409 & 8.973 & 18.573 & 11.984 & & 11.870 & 27.254 & 24.875 & 37.275 \\
\hline 8/5/11 16:00 & 13.663 & 6.414 & 8.928 & 18.554 & 12.048 & & 11.854 & 27.303 & 24.875 & 37.273 \\
\hline 8/5/11 20:00 & 13.661 & 6.424 & 8.921 & 18.559 & 12.090 & & 11.837 & 27.211 & 24.875 & 37.273 \\
\hline 8/6/11 0:00 & 13.670 & 6.424 & 8.923 & 18.568 & 12.062 & & 11.854 & 27.228 & 24.872 & 37.271 \\
\hline 8/6/11 4:00 & 13.675 & 6.419 & 8.928 & 18.579 & 12.109 & & 11.835 & 27.164 & 24.870 & 37.271 \\
\hline 8/6/11 8:00 & 13.694 & 6.424 & 8.942 & 18.593 & 12.043 & & 11.880 & 27.233 & 24.870 & 37.273 \\
\hline 8/6/11 12:00 & 13.711 & 6.426 & 8.961 & 18.612 & 12.104 & & 11.870 & 27.200 & 24.868 & 37.271 \\
\hline 8/6/11 16:00 & 13.725 & 6.438 & 8.994 & 18.628 & 12.166 & & 11.870 & 27.218 & 24.868 & 37.268 \\
\hline 8/6/11 20:00 & 13.734 & 6.450 & 9.030 & 18.645 & 12.215 & & 11.880 & 27.235 & 24.865 & 37.268 \\
\hline 8/7/11 0:00 & 13.760 & 6.452 & 9.061 & 18.663 & 12.118 & & 11.931 & 27.251 & 24.865 & 37.268 \\
\hline 8/7/11 4:00 & 13.772 & 6.450 & 9.077 & 18.680 & 12.137 & & 11.924 & 27.230 & 24.863 & 37.268 \\
\hline 8/7/11 8:00 & 13.793 & 6.450 & 9.103 & 18.701 & 12.163 & & 11.946 & 27.249 & 24.865 & 37.268 \\
\hline 8/7/11 12:00 & 13.817 & 6.454 & 9.139 & 18.719 & 12.236 & & 11.964 & 27.275 & 24.863 & 37.268 \\
\hline 8/7/11 16:00 & 13.821 & 6.459 & 9.160 & 18.736 & 12.343 & & 11.936 & 27.256 & 24.863 & 37.266 \\
\hline 8/7/11 20:00 & 13.838 & 6.464 & 9.186 & 18.750 & 12.397 & & 11.948 & 27.287 & 24.863 & 37.266 \\
\hline 8/8/11 0:00 & 13.854 & 6.466 & 9.212 & 18.761 & 12.354 & & 11.983 & 27.254 & 24.863 & 37.266 \\
\hline 8/8/11 4:00 & 13.868 & 6.466 & 9.228 & 18.775 & 12.425 & & 11.981 & 27.345 & 24.863 & 37.268 \\
\hline 8/8/11 8:00 & 13.890 & 6.466 & 9.243 & 18.785 & 12.404 & & 12.002 & 27.320 & 24.863 & 37.263 \\
\hline 8/8/11 12:00 & 13.901 & 6.464 & 9.250 & 18.785 & 12.406 & & 12.011 & 27.291 & 24.861 & 37.266 \\
\hline 8/8/11 16:00 & 13.916 & 6.475 & 9.278 & 18.794 & 12.496 & & 12.009 & 27.362 & 24.861 & 37.263 \\
\hline 8/8/11 20:00 & 13.932 & 6.487 & 9.309 & 18.808 & 12.498 & & 12.028 & 27.313 & 24.861 & 37.263 \\
\hline 8/9/11 0:00 & 13.956 & 6.487 & 9.332 & 18.822 & 12.460 & & 12.065 & 27.371 & 24.861 & 37.266 \\
\hline 8/9/11 4:00 & 13.977 & 6.487 & 9.351 & 18.836 & 12.486 & & 12.079 & 27.437 & 24.863 & 37.263 \\
\hline 8/9/11 8:00 & 14.003 & 6.490 & 9.373 & 18.852 & 12.446 & & 12.105 & 27.437 & 24.863 & 37.263 \\
\hline 8/9/11 12:00 & 14.033 & 6.497 & 9.399 & 18.873 & 12.659 & & 12.124 & 27.548 & 24.865 & 37.263 \\
\hline 8/9/11 16:00 & 14.055 & 6.511 & 9.437 & 18.889 & 12.689 & & 12.131 & 27.484 & 24.868 & 37.263 \\
\hline 8/9/11 20:00 & 14.081 & 6.525 & 9.470 & 18.908 & 12.717 & & 12.157 & 27.644 & 24.865 & 37.261 \\
\hline 8/10/11 0:00 & 14.106 & 6.523 & 9.498 & 18.924 & 12.748 & & 12.192 & 27.597 & 24.868 & 37.263 \\
\hline 8/10/11 4:00 & 14.130 & 6.520 & 9.515 & 18.938 & 12.776 & & 12.204 & 27.569 & 24.868 & 37.261 \\
\hline 8/10/11 8:00 & 14.163 & 6.520 & 9.541 & 18.957 & 12.805 & & 12.244 & 27.644 & 24.870 & 37.263 \\
\hline
\end{tabular}

TABLE S1.1 Page 78 of 89 
Water Level (ft below reference point)

\begin{tabular}{|c|c|c|c|c|c|c|c|c|c|c|}
\hline Date and Time & McPike & Potter & Hughes & Barr & Burks & MoDOT & Clizer "Old" & Morgan & SB24 & SB38 \\
\hline 8/10/11 12:00 & 14.194 & 6.522 & 9.564 & 18.978 & 12.831 & & 12.256 & 27.764 & 24.872 & 68.788 \\
\hline 8/10/11 16:00 & 14.222 & 6.541 & 9.597 & 18.997 & 12.864 & & 12.265 & 27.705 & 27.717 & 38.658 \\
\hline 8/10/11 20:00 & 14.243 & 6.551 & 9.628 & 19.013 & 12.894 & & 12.289 & 27.722 & 27.701 & 38.646 \\
\hline 8/11/11 0:00 & 14.276 & 6.553 & 9.654 & 19.029 & 12.925 & & 12.326 & 28.479 & 27.689 & 38.634 \\
\hline $8 / 11 / 114: 00$ & 14.302 & 6.548 & 9.675 & 19.045 & 12.956 & & 12.343 & 28.018 & 27.677 & 38.625 \\
\hline 8/11/11 8:00 & 14.330 & 6.544 & 9.697 & 19.064 & 12.984 & & 12.366 & 27.971 & 27.666 & 38.618 \\
\hline $8 / 11 / 11$ 12:00 & 14.363 & 6.553 & 9.725 & 19.080 & 13.017 & & 12.385 & 27.997 & 27.654 & 38.611 \\
\hline $8 / 11 / 11$ 16:00 & 14.385 & 6.567 & 9.756 & 19.097 & 13.050 & & 12.375 & 28.239 & 44.567 & 68.717 \\
\hline 8/11/11 20:00 & 14.401 & 6.581 & 9.782 & 19.111 & 13.088 & & 12.385 & 27.964 & 44.463 & 68.738 \\
\hline 8/12/11 0:00 & 14.422 & 6.581 & 9.803 & 19.122 & 13.123 & & 12.413 & 27.940 & 44.374 & 68.736 \\
\hline 8/12/11 4:00 & 14.448 & 6.574 & 9.817 & 19.134 & 13.095 & & 12.446 & 27.952 & 44.292 & 68.738 \\
\hline 8/12/11 8:00 & 14.453 & 6.565 & 9.732 & 19.052 & 13.118 & & 12.411 & 27.917 & 44.210 & 68.738 \\
\hline 8/12/11 12:00 & 14.477 & 6.562 & 9.621 & 19.006 & 13.111 & & 12.411 & 27.961 & 44.133 & 68.738 \\
\hline 8/12/11 16:00 & 14.467 & 6.555 & 9.510 & 18.941 & 13.144 & & 12.390 & 27.889 & 44.058 & 68.736 \\
\hline 8/12/11 20:00 & 14.472 & 6.562 & 9.460 & 18.941 & 13.182 & & 12.387 & 27.872 & 43.985 & 68.736 \\
\hline 8/13/11 0:00 & 14.481 & 6.565 & 9.439 & 18.952 & 13.217 & & 12.406 & 27.882 & 43.912 & 68.738 \\
\hline 8/13/11 4:00 & 14.493 & 6.562 & 9.434 & 18.969 & 13.253 & & 12.415 & 27.903 & 43.844 & 68.736 \\
\hline 8/13/11 8:00 & 14.507 & 6.560 & 9.437 & 18.987 & 13.286 & & 12.429 & 27.900 & 43.774 & 68.736 \\
\hline $8 / 13 / 11$ 12:00 & 14.524 & 6.570 & 9.458 & 19.011 & 13.324 & & 12.448 & 27.919 & 43.706 & 68.736 \\
\hline 8/13/11 16:00 & 14.543 & 6.586 & 9.493 & 19.032 & 13.361 & & 12.460 & 27.922 & 43.640 & 68.736 \\
\hline $8 / 13 / 1120: 00$ & 14.557 & 6.598 & 9.526 & 19.055 & 13.399 & & 12.481 & 27.929 & 43.574 & 68.738 \\
\hline 8/14/11 0:00 & 14.578 & 6.598 & 9.557 & 19.078 & 13.437 & & 12.514 & 28.138 & 43.511 & 68.736 \\
\hline 8/14/11 4:00 & 14.599 & 6.593 & 9.581 & 19.099 & 13.472 & & 12.530 & 28.053 & 43.448 & 68.736 \\
\hline 8/14/11 8:00 & 14.620 & 6.588 & 9.607 & 19.122 & 13.512 & & 12.547 & 28.046 & 43.384 & 68.738 \\
\hline 8/14/11 12:00 & 14.642 & 6.593 & 9.635 & 19.146 & 13.550 & & 12.554 & 28.056 & 43.324 & 68.738 \\
\hline 8/14/11 16:00 & 14.653 & 6.610 & 9.671 & 19.164 & 13.590 & & 12.549 & 28.034 & 43.263 & 68.736 \\
\hline 8/14/11 20:00 & 14.661 & 6.617 & 9.704 & 19.181 & 13.630 & & 12.552 & 28.521 & 43.204 & 68.738 \\
\hline $8 / 15 / 110: 00$ & 14.672 & 6.612 & 9.730 & 19.195 & 13.668 & & 12.566 & 28.088 & 43.148 & 68.736 \\
\hline 8/15/11 4:00 & 14.684 & 6.605 & 9.753 & 19.209 & 13.710 & & 12.582 & 28.088 & 43.091 & 68.734 \\
\hline 8/15/11 8:00 & 14.701 & 6.600 & 9.772 & 19.220 & 13.746 & & 12.577 & 28.056 & 43.035 & 68.736 \\
\hline 8/15/11 12:00 & 14.712 & 6.595 & 9.787 & 19.232 & 13.779 & & 12.592 & 28.143 & 42.981 & 68.736 \\
\hline 8/15/11 16:00 & 14.719 & 6.588 & 9.782 & 19.209 & 13.814 & & 12.580 & 28.084 & 42.927 & 68.738 \\
\hline 8/15/11 20:00 & 14.724 & 6.591 & 9.791 & 19.209 & 13.852 & & 12.580 & 28.138 & 42.873 & 68.736 \\
\hline 8/16/11 0:00 & 14.736 & 6.588 & 9.803 & 19.218 & 13.892 & & 12.589 & 28.074 & 42.822 & 68.738 \\
\hline 8/16/11 4:00 & 14.741 & 6.588 & 9.815 & 19.227 & 13.930 & & 12.573 & 28.086 & 42.770 & 68.736 \\
\hline 8/16/11 8:00 & 14.741 & 6.586 & 9.787 & 19.209 & 13.920 & & 12.580 & 28.053 & 42.716 & 68.736 \\
\hline 8/16/11 12:00 & 14.712 & 6.577 & 9.550 & 19.111 & 13.949 & & 12.549 & 28.100 & 42.667 & 68.736 \\
\hline 8/16/11 16:00 & 14.663 & 6.581 & 9.403 & 19.078 & 13.989 & & 12.521 & 28.067 & 42.615 & 68.738 \\
\hline 8/16/11 20:00 & 14.635 & 6.588 & 9.332 & 19.083 & 14.026 & & 12.523 & 28.034 & 42.564 & 68.738 \\
\hline $8 / 17 / 110: 00$ & 14.630 & 6.593 & 9.299 & 19.099 & 14.066 & & 12.547 & 28.088 & 42.514 & 68.736 \\
\hline 8/17/11 4:00 & 14.637 & 6.591 & 9.288 & 19.118 & 14.104 & & 12.554 & 28.048 & 42.465 & 68.738 \\
\hline 8/17/11 8:00 & 14.651 & 6.593 & 9.290 & 19.139 & 14.142 & & 12.577 & 28.107 & 42.416 & 68.738 \\
\hline $8 / 17 / 11$ 12:00 & 14.675 & 6.598 & 9.302 & 19.164 & 14.182 & & 12.589 & 28.084 & 42.367 & 68.738 \\
\hline $8 / 17 / 11$ 16:00 & 14.689 & 6.612 & 9.330 & 19.188 & 14.222 & & 12.580 & 28.121 & 42.317 & 68.738 \\
\hline $8 / 17 / 1120: 00$ & 14.689 & 6.624 & 9.359 & 19.206 & 14.262 & & 12.575 & 28.048 & 42.266 & 68.736 \\
\hline 8/18/11 0:00 & 14.701 & 6.619 & 9.385 & 19.225 & 14.302 & & 12.601 & 28.058 & 42.216 & 68.738 \\
\hline 8/18/11 4:00 & 14.712 & 6.612 & 9.408 & 19.241 & 14.340 & & 12.599 & 28.046 & 42.170 & 68.736 \\
\hline 8/18/11 8:00 & 14.722 & 6.610 & 9.432 & 19.258 & 14.378 & & 12.615 & 28.074 & 42.120 & 68.738 \\
\hline 8/18/11 12:00 & 14.734 & 6.612 & 9.460 & 19.274 & 14.415 & & 12.603 & 28.048 & 42.071 & 68.738 \\
\hline 8/18/11 16:00 & 14.731 & 6.624 & 9.493 & 19.285 & 14.453 & & 12.573 & 28.030 & 42.022 & 68.738 \\
\hline 8/18/11 20:00 & 14.722 & 6.631 & 9.522 & 19.290 & 14.493 & & 12.575 & 28.279 & 41.970 & 68.738 \\
\hline 8/19/11 0:00 & 14.041 & 5.750 & 9.226 & 19.157 & 14.279 & & 12.507 & 28.048 & 41.928 & 68.738 \\
\hline 8/19/11 4:00 & 13.371 & 4.887 & 8.907 & 19.032 & 14.319 & & 12.460 & 27.837 & 41.874 & 68.738 \\
\hline 8/19/11 8:00 & 13.423 & 4.927 & 8.758 & 19.008 & 14.359 & & 12.455 & 27.806 & 41.822 & 68.738 \\
\hline 8/19/11 12:00 & 13.520 & 5.160 & 8.689 & 19.013 & 14.399 & & 12.436 & 27.724 & 41.773 & 68.738 \\
\hline $8 / 19 / 11$ 16:00 & 13.593 & 5.420 & 8.659 & 19.022 & 14.434 & & 12.397 & 27.689 & 41.719 & 68.738 \\
\hline $8 / 19 / 1120: 00$ & 13.649 & 5.648 & 8.649 & 19.034 & 14.474 & & 12.394 & 27.571 & 41.670 & 68.738 \\
\hline 8/20/11 0:00 & 13.701 & 5.816 & 8.630 & 19.045 & 14.500 & & 12.401 & 27.571 & 41.618 & 68.738 \\
\hline 8/20/11 4:00 & 13.357 & 5.891 & 8.301 & 18.927 & 14.458 & & 12.321 & 27.632 & 41.571 & 68.738 \\
\hline 8/20/11 8:00 & 12.650 & 5.552 & 7.980 & 18.703 & 14.489 & & 12.244 & 27.447 & 41.518 & 68.738 \\
\hline $8 / 20 / 11$ 12:00 & 12.714 & 5.417 & 7.831 & 18.617 & 14.522 & & 12.195 & 27.371 & 41.466 & 68.741 \\
\hline 8/20/11 16:00 & 12.839 & 5.441 & 7.762 & 18.593 & 14.550 & & 12.143 & 27.334 & 41.414 & 68.738 \\
\hline 8/20/11 20:00 & 12.947 & 5.528 & 7.741 & 18.593 & 14.578 & & 12.108 & 27.235 & 41.363 & 68.738 \\
\hline $8 / 21 / 110: 00$ & 13.046 & 5.622 & 7.734 & 18.603 & 14.606 & & 12.096 & 27.181 & 41.311 & 68.741 \\
\hline
\end{tabular}

TABLE S1.1 Page 79 of 89 
Water Level (ft below reference point)

\begin{tabular}{|c|c|c|c|c|c|c|c|c|c|c|}
\hline Date and Time & McPike & Potter & Hughes & Barr & Burks & MoDOT & Clizer "Old" & Morgan & SB24 & SB38 \\
\hline 8/21/11 4:00 & 13.121 & 5.700 & 7.729 & 18.617 & 14.635 & & 12.077 & 27.164 & 41.260 & 68.738 \\
\hline 8/21/11 8:00 & 13.190 & 5.766 & 7.731 & 18.635 & 14.661 & & 12.072 & 27.110 & 41.208 & 68.738 \\
\hline 8/21/11 12:00 & 13.251 & 5.830 & 7.750 & 18.661 & 14.684 & & 12.065 & 27.124 & 41.156 & 68.738 \\
\hline 8/21/11 16:00 & 13.294 & 5.893 & 7.783 & 18.682 & 14.710 & & 12.035 & 27.115 & 41.107 & 68.741 \\
\hline $8 / 21 / 1120: 00$ & 13.331 & 5.950 & 7.812 & 18.701 & 14.736 & & 12.030 & 27.030 & 41.056 & 68.738 \\
\hline 8/22/11 0:00 & 13.371 & 5.988 & 7.831 & 18.722 & 14.758 & & 12.037 & 27.080 & 41.006 & 68.738 \\
\hline $8 / 22 / 114: 00$ & 13.404 & 6.011 & 7.847 & 18.740 & 14.781 & & 12.032 & 27.033 & 40.955 & 68.738 \\
\hline 8/22/11 8:00 & 11.757 & 5.036 & 7.590 & 18.591 & 14.571 & & 11.809 & 27.042 & 40.905 & 68.741 \\
\hline $8 / 22 / 11$ 12:00 & 10.493 & 4.032 & 7.171 & 18.407 & 14.689 & & 11.584 & 26.734 & 40.851 & 68.741 \\
\hline 8/22/11 16:00 & 10.637 & 4.051 & 6.958 & 18.335 & 14.658 & & 11.422 & 26.377 & 40.788 & 68.741 \\
\hline $8 / 22 / 1120: 00$ & 10.873 & 4.270 & 6.828 & 18.307 & 14.663 & & 11.304 & 26.111 & 40.722 & 68.741 \\
\hline 8/23/11 0:00 & 11.066 & 4.498 & 6.733 & 18.300 & 14.668 & & 11.220 & 25.862 & 40.664 & 68.741 \\
\hline 8/23/11 4:00 & 11.193 & 4.692 & 6.660 & 18.302 & 14.682 & & 11.135 & 25.664 & 40.601 & 68.741 \\
\hline 8/23/11 8:00 & 11.288 & 4.856 & 6.599 & 18.309 & 14.706 & & 11.051 & 25.516 & 40.542 & 68.741 \\
\hline $8 / 23 / 11$ 12:00 & 11.373 & 5.012 & 6.575 & 18.316 & 14.706 & & 10.982 & 25.377 & 40.481 & 68.741 \\
\hline $8 / 23 / 11$ 16:00 & 11.443 & 5.158 & 6.577 & 18.326 & 14.701 & & 10.921 & 25.241 & 40.422 & 68.741 \\
\hline 8/23/11 20:00 & 11.530 & 5.290 & 6.582 & 18.335 & 14.642 & & 10.912 & 25.166 & 40.361 & 68.741 \\
\hline 8/24/11 0:00 & 11.594 & 5.375 & 6.570 & 18.344 & 14.680 & & 10.867 & 25.116 & 40.305 & 68.741 \\
\hline $8 / 24 / 114: 00$ & 11.641 & 5.436 & 6.561 & 18.353 & 14.670 & & 10.834 & 25.081 & 40.244 & 68.741 \\
\hline 8/24/11 8:00 & 11.705 & 5.490 & 6.563 & 18.358 & 14.588 & & 10.834 & 24.982 & 40.185 & 68.741 \\
\hline $8 / 24 / 11$ 12:00 & 11.778 & 5.545 & 6.592 & 18.370 & 14.557 & & 10.834 & 25.029 & 40.129 & 68.741 \\
\hline 8/24/11 16:00 & 11.851 & 5.613 & 6.646 & 18.384 & 14.489 & & 10.837 & 25.003 & 40.070 & 68.741 \\
\hline $8 / 24 / 1120: 00$ & 11.912 & 5.674 & 6.696 & 18.402 & 14.401 & & 10.844 & 25.034 & 40.012 & 68.741 \\
\hline $8 / 25 / 110: 00$ & 11.976 & 5.714 & 6.724 & 18.421 & 14.543 & & 10.863 & 25.041 & 39.953 & 68.738 \\
\hline 8/25/11 4:00 & 12.028 & 5.735 & 6.745 & 18.437 & 14.517 & & 10.858 & 25.083 & 39.895 & 68.741 \\
\hline 8/25/11 8:00 & 12.075 & 5.754 & 6.767 & 18.456 & 14.489 & & 10.860 & 25.142 & 39.841 & 68.741 \\
\hline $8 / 25 / 11$ 12:00 & 12.117 & 5.773 & 6.804 & 18.475 & 14.463 & & 10.846 & 25.128 & 39.784 & 68.741 \\
\hline 8/25/11 16:00 & 12.134 & 5.804 & 6.852 & 18.489 & 14.437 & & 10.804 & 25.116 & 39.726 & 68.741 \\
\hline $8 / 25 / 1120: 00$ & 12.150 & 5.830 & 6.889 & 18.500 & 14.409 & & 10.785 & 25.138 & 39.667 & 68.741 \\
\hline 8/26/11 0:00 & 12.172 & 5.849 & 6.916 & 18.510 & 14.378 & & 10.778 & 25.076 & 39.611 & 68.741 \\
\hline $8 / 26 / 114: 00$ & 12.195 & 5.855 & 6.937 & 18.517 & 14.345 & & 10.771 & 25.072 & 39.554 & 68.741 \\
\hline 8/26/11 8:00 & 12.223 & 5.867 & 6.958 & 18.526 & 14.312 & & 10.773 & 25.105 & 39.498 & 68.741 \\
\hline $8 / 26 / 11$ 12:00 & 12.238 & 5.879 & 6.996 & 18.586 & 14.279 & & 10.750 & 25.093 & 39.440 & 68.741 \\
\hline 8/26/11 16:00 & 12.250 & 5.900 & 7.046 & 18.593 & 14.248 & & 10.726 & 25.072 & 39.386 & 68.741 \\
\hline $8 / 26 / 1120: 00$ & 12.256 & 5.919 & 7.083 & 18.558 & 14.215 & & 10.715 & 25.055 & 39.327 & 68.741 \\
\hline 8/27/11 0:00 & 12.287 & 5.936 & 7.114 & 18.500 & 14.182 & & 10.729 & 25.062 & 39.271 & 68.743 \\
\hline $8 / 27 / 114: 00$ & 12.306 & 5.940 & 7.140 & 18.507 & 14.149 & & 10.724 & 25.114 & 39.214 & 68.741 \\
\hline 8/27/11 8:00 & 12.327 & 5.950 & 7.166 & 18.503 & 14.114 & & 10.724 & 25.116 & 39.160 & 68.741 \\
\hline $8 / 27 / 11$ 12:00 & 12.346 & 5.959 & 7.197 & 18.598 & 14.081 & & 10.715 & 25.156 & 39.104 & 68.741 \\
\hline 8/27/11 16:00 & 12.353 & 5.980 & 7.247 & 18.607 & 14.050 & & 10.689 & 25.112 & 39.048 & 68.741 \\
\hline 8/27/11 20:00 & 12.365 & 5.999 & 7.294 & 18.552 & 14.022 & & 10.679 & 25.109 & 38.992 & 68.741 \\
\hline 8/28/11 0:00 & 12.384 & 6.011 & 7.325 & 18.563 & 13.991 & & 10.689 & 25.161 & 38.938 & 68.741 \\
\hline 8/28/11 4:00 & 12.391 & 6.011 & 7.351 & 18.565 & 13.960 & & 10.675 & 25.123 & 38.884 & 68.743 \\
\hline 8/28/11 8:00 & 12.400 & 6.011 & 7.377 & 18.568 & 13.927 & & 10.668 & 25.149 & 38.825 & 68.741 \\
\hline $8 / 28 / 11$ 12:00 & 12.419 & 6.020 & 7.412 & 18.568 & 13.899 & & 10.665 & 25.215 & 38.771 & 68.741 \\
\hline 8/28/11 16:00 & 12.412 & 6.027 & 7.445 & 18.568 & 13.868 & & 10.632 & 25.145 & 38.715 & 68.743 \\
\hline $8 / 28 / 1120: 00$ & 12.417 & 6.037 & 7.481 & 18.565 & 13.843 & & 10.628 & 25.140 & 38.659 & 68.741 \\
\hline 8/29/11 0:00 & 12.454 & 6.046 & 7.519 & 18.566 & 13.814 & & 10.665 & 25.159 & 38.600 & 68.741 \\
\hline 8/29/11 4:00 & 12.476 & 6.054 & 7.549 & 18.570 & 13.786 & & 10.670 & 25.222 & 38.546 & 68.741 \\
\hline 8/29/11 8:00 & 12.497 & 6.056 & 7.580 & 18.572 & 13.760 & & 10.686 & 25.309 & 38.490 & 68.743 \\
\hline $8 / 29 / 11$ 12:00 & 12.523 & 6.065 & 7.623 & 18.579 & 13.734 & & 10.684 & 25.326 & 38.431 & 68.741 \\
\hline 8/29/11 16:00 & 12.525 & 6.079 & 7.670 & 18.584 & 13.710 & & 10.658 & 26.482 & 38.375 & 68.743 \\
\hline 8/29/11 20:00 & 12.535 & 6.096 & 7.710 & 18.584 & 13.689 & & 10.658 & 27.157 & 38.318 & 68.743 \\
\hline 8/30/11 0:00 & 12.551 & 6.103 & 7.741 & 18.586 & 13.666 & & 10.665 & 25.714 & 38.262 & 68.741 \\
\hline 8/30/11 4:00 & 12.554 & 6.101 & 7.765 & 18.589 & 13.644 & & 10.651 & 25.530 & 38.206 & 68.741 \\
\hline 8/30/11 8:00 & 12.563 & 6.101 & 7.786 & 18.586 & 13.588 & & 10.651 & 25.500 & 38.150 & 68.743 \\
\hline $8 / 30 / 11$ 12:00 & 12.549 & 6.098 & 7.687 & 18.491 & 13.543 & & 10.623 & 25.483 & 38.096 & 68.743 \\
\hline 8/30/11 16:00 & 12.542 & 6.098 & 7.649 & 18.447 & 13.541 & & 10.595 & 25.436 & 38.039 & 68.743 \\
\hline 8/30/11 20:00 & 12.549 & 6.110 & 7.653 & 18.435 & 13.470 & & 10.590 & 25.403 & 37.981 & 68.741 \\
\hline 8/31/11 0:00 & 12.570 & 6.115 & 7.672 & 18.435 & 13.385 & & 10.607 & 25.368 & 37.927 & 68.743 \\
\hline 8/31/11 4:00 & 12.577 & 6.115 & 7.687 & 18.440 & 13.354 & & 10.600 & 25.403 & 37.870 & 68.743 \\
\hline 8/31/11 8:00 & 12.591 & 6.115 & 7.710 & 18.444 & 13.307 & & 10.607 & 25.375 & 37.817 & 68.743 \\
\hline $8 / 31 / 11$ 12:00 & 12.605 & 6.119 & 7.748 & 18.451 & 13.331 & & 10.611 & 25.387 & 37.760 & 68.743 \\
\hline $8 / 31 / 11$ 16:00 & 12.617 & 6.133 & 7.800 & 18.461 & 13.335 & & 10.609 & 25.401 & 37.706 & 68.743 \\
\hline
\end{tabular}

TABLE S1.1 Page 80 of 89 


\begin{tabular}{|c|c|c|c|c|c|c|c|c|c|c|}
\hline \multirow[b]{2}{*}{ Date and Time } & \multicolumn{8}{|c|}{ Water Level (ft below reference point) } & \multirow[b]{2}{*}{ SB24 } & \multirow[b]{2}{*}{ SB38 } \\
\hline & McPike & Potter & Hughes & Barr & Burks & MoDOT & Clizer "Old" & Morgan & & \\
\hline 8/31/11 20:00 & 12.629 & 6.148 & 7.845 & 18.470 & 13.317 & & 10.616 & 25.387 & 37.650 & 68.743 \\
\hline 9/1/11 0:00 & 12.648 & 6.152 & 7.880 & 18.479 & 13.286 & & 10.637 & 25.448 & 37.596 & 68.743 \\
\hline 9/1/11 4:00 & 12.662 & 6.155 & 7.914 & 18.489 & 13.251 & & 10.651 & 25.417 & 37.542 & 68.741 \\
\hline 9/1/11 8:00 & 12.681 & 6.152 & 7.944 & 18.498 & 13.220 & & 10.665 & 25.469 & 37.488 & 68.743 \\
\hline 9/1/11 12:00 & 12.697 & 6.159 & 7.987 & 18.510 & 13.265 & & 10.670 & 25.469 & 37.432 & 68.743 \\
\hline 9/1/11 16:00 & 12.700 & 6.171 & 8.044 & 18.517 & 13.319 & & 10.656 & 25.474 & 37.378 & 68.743 \\
\hline 9/1/11 20:00 & 12.716 & 6.185 & 8.096 & 18.524 & 13.284 & & 10.677 & 25.483 & 37.324 & 68.741 \\
\hline 9/2/11 0:00 & 12.740 & 6.192 & 8.136 & 18.533 & 13.234 & & 10.717 & 25.525 & 37.272 & 68.743 \\
\hline 9/2/11 4:00 & 12.763 & 6.197 & 8.169 & 18.540 & 13.206 & & 10.736 & 25.544 & 37.221 & 68.743 \\
\hline 9/2/11 8:00 & 12.787 & 6.199 & 8.204 & 18.552 & 13.144 & & 10.769 & 27.174 & 37.169 & 68.743 \\
\hline 9/2/11 12:00 & 12.815 & 6.207 & 8.249 & 18.563 & 13.194 & & 10.785 & 26.040 & 37.115 & 68.665 \\
\hline 9/2/11 16:00 & 12.825 & 6.218 & 8.299 & 18.575 & 13.291 & & 10.776 & 25.801 & 37.064 & 68.404 \\
\hline 9/2/11 20:00 & 12.836 & 6.228 & 8.342 & 18.584 & 13.288 & & 10.783 & 25.753 & 37.010 & 68.152 \\
\hline 9/3/11 0:00 & 12.855 & 6.230 & 8.372 & 18.593 & 13.291 & & 10.816 & NA & 36.958 & 67.902 \\
\hline 9/3/11 4:00 & 12.869 & 6.228 & 8.401 & 18.600 & 13.288 & & 10.823 & & 36.907 & 67.759 \\
\hline 9/3/11 8:00 & 12.884 & 6.221 & 8.422 & 18.610 & 13.286 & & 10.827 & & 36.855 & 67.627 \\
\hline $9 / 3 / 11$ 12:00 & 12.905 & 6.225 & 8.457 & 18.619 & 13.288 & & 10.853 & & 36.803 & 67.497 \\
\hline 9/3/11 16:00 & 12.921 & 6.232 & 8.488 & 18.628 & 13.286 & & 10.856 & & 36.752 & 67.368 \\
\hline 9/3/11 20:00 & 12.940 & 6.237 & 8.491 & 18.624 & 13.142 & & 10.886 & & 36.702 & 67.231 \\
\hline 9/4/11 0:00 & 12.966 & 6.235 & 8.424 & 18.565 & 12.994 & & 10.921 & & 36.651 & 67.108 \\
\hline 9/4/11 4:00 & 12.985 & 6.237 & 8.403 & 18.556 & 12.920 & & 10.928 & & 36.602 & 66.981 \\
\hline 9/4/11 8:00 & 13.008 & 6.240 & 8.406 & 18.568 & 12.833 & & 10.959 & & 36.552 & 66.854 \\
\hline 9/4/11 12:00 & 13.030 & 6.244 & 8.424 & 18.586 & 12.906 & & 10.947 & & 36.501 & 66.724 \\
\hline 9/4/11 16:00 & 13.037 & 6.254 & 8.453 & 18.603 & 12.963 & & 10.928 & & 36.452 & 66.597 \\
\hline 9/4/11 20:00 & 13.051 & 6.265 & 8.486 & 18.621 & 12.939 & & 10.957 & & 36.400 & 66.472 \\
\hline 9/5/11 0:00 & 13.072 & 6.265 & 8.512 & 18.640 & 12.890 & & 10.980 & & 36.351 & 66.345 \\
\hline 9/5/11 4:00 & 13.086 & 6.263 & 8.533 & 18.656 & 12.895 & & 10.980 & & 36.301 & 66.223 \\
\hline 9/5/11 8:00 & 13.103 & 6.258 & 8.554 & 18.675 & 12.852 & & 10.999 & & 36.254 & 66.098 \\
\hline 9/5/11 12:00 & 13.122 & 6.263 & 8.578 & 18.691 & 12.951 & & 10.994 & & 36.205 & 65.973 \\
\hline 9/5/11 16:00 & 13.122 & 6.270 & 8.602 & 18.705 & 13.036 & & 10.959 & & 36.156 & 65.846 \\
\hline 9/5/11 20:00 & 13.129 & 6.277 & 8.630 & 18.719 & 13.045 & & 10.968 & & 36.107 & 65.726 \\
\hline 9/6/11 0:00 & 13.145 & 6.277 & 8.654 & 18.731 & 12.982 & & 11.001 & & 36.060 & 65.606 \\
\hline 9/6/11 4:00 & 13.164 & 6.275 & 8.680 & 18.745 & 12.930 & & 11.020 & & 36.011 & 65.478 \\
\hline 9/6/11 8:00 & 13.183 & 6.275 & 8.703 & 18.757 & 12.923 & & 11.044 & & 35.964 & 65.356 \\
\hline 9/6/11 12:00 & 13.202 & 6.277 & 8.730 & 18.773 & 13.012 & & 11.046 & & 35.917 & 65.233 \\
\hline 9/6/11 16:00 & 13.211 & 6.291 & 8.755 & 18.784 & 13.081 & & 11.027 & & 35.867 & 65.111 \\
\hline 9/6/11 20:00 & 13.223 & 6.298 & 8.784 & 18.794 & 13.053 & & 11.048 & & 35.821 & 64.991 \\
\hline 9/7/11 0:00 & 13.244 & 6.298 & 8.810 & 18.808 & 13.012 & & 11.081 & & 35.774 & 64.871 \\
\hline 9/7/11 4:00 & 13.263 & 6.294 & 8.831 & 18.819 & 12.979 & & 11.093 & & 35.729 & 64.753 \\
\hline 9/7/11 8:00 & 13.286 & 6.296 & 8.857 & 18.831 & 12.944 & & 11.126 & & 35.682 & 64.633 \\
\hline 9/7/11 12:00 & 13.310 & 6.301 & 8.881 & 18.847 & 13.291 & & 11.130 & & 35.635 & 64.510 \\
\hline 9/7/11 16:00 & 13.322 & 6.310 & 8.907 & 18.859 & 13.298 & & 11.114 & & 35.591 & 64.392 \\
\hline 9/7/11 20:00 & 13.336 & 6.308 & 8.933 & 18.871 & 13.307 & & 11.130 & & 35.546 & 64.272 \\
\hline 9/8/11 0:00 & 13.357 & 6.308 & 8.959 & 18.882 & 13.319 & & 11.161 & & 35.499 & 64.155 \\
\hline 9/8/11 4:00 & 13.374 & 6.303 & 8.975 & 18.894 & 13.328 & & 11.166 & & 35.457 & 64.039 \\
\hline 9/8/11 8:00 & 13.393 & 6.303 & 8.997 & 18.903 & 13.335 & & 11.184 & & 35.410 & 63.921 \\
\hline 9/8/11 12:00 & 13.412 & 6.306 & 9.018 & 18.917 & 13.345 & & 11.180 & & 35.366 & 63.804 \\
\hline 9/8/11 16:00 & 13.414 & 6.298 & 9.037 & 18.924 & 13.355 & & 11.133 & & 35.319 & 63.688 \\
\hline 9/8/11 20:00 & 13.419 & 6.294 & 9.058 & 18.927 & 13.369 & & 11.140 & & 35.274 & 63.573 \\
\hline 9/9/11 0:00 & 13.428 & 6.294 & 9.075 & 18.931 & 13.378 & & 11.149 & & 35.232 & 63.457 \\
\hline 9/9/11 4:00 & 13.435 & 6.291 & 9.089 & 18.931 & 13.390 & & 11.147 & & 35.185 & 63.342 \\
\hline 9/9/11 8:00 & 13.447 & 6.291 & 9.103 & 18.936 & 13.402 & & 11.159 & & 35.140 & 63.231 \\
\hline 9/9/11 12:00 & 13.459 & 6.291 & 9.120 & 18.938 & 13.416 & & 11.156 & & 35.098 & 63.116 \\
\hline 9/9/11 16:00 & 13.454 & 6.289 & 9.136 & 18.938 & 13.430 & & 11.121 & & 35.054 & 63.000 \\
\hline 9/9/11 20:00 & 13.466 & 6.289 & 9.158 & 18.938 & 13.446 & & 11.159 & & 35.011 & 62.887 \\
\hline 9/10/11 0:00 & 13.485 & 6.294 & 9.176 & 18.943 & 13.460 & & 11.194 & & 34.967 & 62.777 \\
\hline $9 / 10 / 114: 00$ & 13.499 & 6.298 & 9.193 & 18.945 & 13.475 & & 11.201 & & 34.925 & 62.664 \\
\hline 9/10/11 8:00 & 13.520 & 6.303 & 9.212 & 18.952 & 13.489 & & 11.234 & & 34.882 & 62.553 \\
\hline 9/10/11 12:00 & 13.546 & 6.306 & 9.236 & 18.959 & 13.503 & & 11.250 & & 34.840 & 62.440 \\
\hline 9/10/11 16:00 & 13.562 & 6.310 & 9.259 & 18.966 & 13.524 & & 11.243 & & 34.796 & 62.331 \\
\hline 9/10/11 20:00 & 13.579 & 6.315 & 9.283 & 18.973 & 13.541 & & 11.274 & & 34.756 & 62.221 \\
\hline 9/11/11 0:00 & 13.600 & 6.320 & 9.304 & 18.983 & 13.557 & & 11.300 & & 34.714 & 62.108 \\
\hline 9/11/11 4:00 & 13.621 & 6.317 & 9.321 & 18.992 & 13.576 & & 11.316 & & 34.674 & 61.999 \\
\hline 9/11/11 8:00 & 13.643 & 6.322 & 9.340 & 19.001 & 13.593 & & 11.344 & & 34.631 & 61.891 \\
\hline
\end{tabular}

TABLE S1.1 Page 81 of 89 


\begin{tabular}{|c|c|c|c|c|c|c|c|c|c|c|}
\hline \multirow[b]{2}{*}{ Date and Time } & \multicolumn{8}{|c|}{ Water Level (ft below reference point) } & \multirow[b]{2}{*}{ SB24 } & \multirow[b]{2}{*}{ SB38 } \\
\hline & McPike & Potter & Hughes & Barr & Burks & MoDOT & Clizer "Old" & Morgan & & \\
\hline 9/11/11 12:00 & 13.668 & 6.327 & 9.359 & 19.013 & 13.611 & & 11.356 & & 34.592 & 61.783 \\
\hline 9/11/11 16:00 & 13.685 & 6.329 & 9.382 & 19.022 & 13.628 & & 11.340 & & 34.552 & 61.672 \\
\hline 9/11/11 20:00 & 13.699 & 6.329 & 9.404 & 19.029 & 13.649 & & 11.363 & & 34.510 & 61.566 \\
\hline 9/12/11 0:00 & 13.718 & 6.329 & 9.425 & 19.038 & 13.670 & & 11.384 & & 34.470 & 61.460 \\
\hline 9/12/11 4:00 & 13.734 & 6.329 & 9.441 & 19.048 & 13.689 & & 11.387 & & 34.430 & 61.351 \\
\hline 9/12/11 8:00 & 13.751 & 6.327 & 9.458 & 19.055 & 13.706 & & 11.405 & & 34.392 & 61.245 \\
\hline 9/12/11 12:00 & 13.767 & 6.331 & 9.477 & 19.062 & 13.727 & & 11.387 & & 34.352 & 61.137 \\
\hline 9/12/11 16:00 & 13.770 & 6.324 & 9.498 & 19.066 & 13.748 & & 11.363 & & 34.310 & 61.031 \\
\hline 9/12/11 20:00 & 13.779 & 6.329 & 9.524 & 19.066 & 13.772 & & 11.387 & & 34.273 & 60.923 \\
\hline 9/13/11 0:00 & 13.796 & 6.331 & 9.543 & 19.071 & 13.795 & & 11.419 & & 34.233 & 60.817 \\
\hline 9/13/11 4:00 & 13.812 & 6.334 & 9.562 & 19.076 & 13.817 & & 11.448 & & 34.198 & 60.711 \\
\hline 9/13/11 8:00 & 13.841 & 6.338 & 9.583 & 19.085 & 13.840 & & 11.502 & & 34.160 & 60.609 \\
\hline 9/13/11 12:00 & 13.871 & 6.348 & 9.607 & 19.097 & 13.864 & & 11.530 & & 34.127 & 60.506 \\
\hline 9/13/11 16:00 & 13.892 & 6.353 & 9.633 & 19.106 & 13.887 & & 11.530 & & 34.090 & 60.402 \\
\hline 9/13/11 20:00 & 13.911 & 6.357 & 9.657 & 19.118 & 13.911 & & 11.556 & & 34.057 & 60.301 \\
\hline 9/14/11 0:00 & 13.932 & 6.357 & 9.678 & 19.132 & 13.935 & & 11.579 & & 34.022 & 60.200 \\
\hline 9/14/11 4:00 & 13.958 & 6.362 & 9.697 & 19.143 & 13.960 & & 11.610 & & 33.989 & 60.098 \\
\hline 9/14/11 8:00 & 13.982 & 6.364 & 9.716 & 19.155 & 13.979 & & 11.650 & & 33.951 & 59.997 \\
\hline 9/14/11 12:00 & 14.015 & 6.364 & 9.735 & 19.169 & 14.003 & & 11.680 & & 33.918 & 59.896 \\
\hline 9/14/11 16:00 & 14.043 & 6.374 & 9.751 & 19.183 & 14.027 & & 11.690 & & 33.883 & 59.797 \\
\hline 9/14/11 20:00 & 14.069 & 6.374 & 9.772 & 19.199 & 14.050 & & 11.722 & & 33.850 & 59.698 \\
\hline 9/15/11 0:00 & 14.107 & 6.381 & 9.796 & 19.218 & 14.076 & & 11.769 & & 33.820 & 59.601 \\
\hline 9/15/11 4:00 & 14.137 & 6.381 & 9.815 & 19.236 & 14.100 & & 11.784 & & 33.782 & 59.505 \\
\hline 9/15/11 8:00 & 14.168 & 6.381 & 9.834 & 19.255 & 14.123 & & 11.814 & & 33.747 & 59.406 \\
\hline 9/15/11 12:00 & 14.201 & 6.383 & 9.853 & 19.274 & 14.144 & & 11.826 & & 33.712 & 59.312 \\
\hline 9/15/11 16:00 & 14.220 & 6.381 & 9.869 & 19.290 & 14.170 & & 11.795 & & 33.675 & 59.215 \\
\hline 9/15/11 20:00 & 14.229 & 6.379 & 9.884 & 19.302 & 14.192 & & 11.784 & & 33.642 & 59.118 \\
\hline 9/16/11 0:00 & 14.246 & 6.376 & 9.898 & 19.311 & 14.215 & & 11.791 & & 33.609 & 59.022 \\
\hline 9/16/11 4:00 & 14.258 & 6.371 & 9.910 & 19.320 & 14.239 & & 11.793 & & 33.576 & 58.930 \\
\hline 9/16/11 8:00 & 14.274 & 6.369 & 9.924 & 19.327 & 14.253 & & 11.807 & & 33.543 & 58.831 \\
\hline 9/16/11 12:00 & 14.288 & 6.369 & 9.917 & 19.318 & 14.260 & & 11.800 & & 33.506 & 58.732 \\
\hline 9/16/11 16:00 & 14.295 & 6.364 & 9.914 & 19.302 & 14.281 & & 11.765 & & 33.471 & 58.635 \\
\hline 9/16/11 20:00 & 14.305 & 6.364 & 9.917 & 19.302 & 14.305 & & 11.762 & & 33.438 & 58.541 \\
\hline 9/17/11 0:00 & 14.317 & 6.364 & 9.924 & 19.309 & 14.328 & & 11.772 & & 33.402 & 58.445 \\
\hline 9/17/11 4:00 & 14.328 & 6.364 & 9.931 & 19.313 & 14.354 & & 11.760 & & 33.367 & 58.353 \\
\hline 9/17/11 8:00 & 14.340 & 6.364 & 9.938 & 19.320 & 14.378 & & 11.762 & & 33.334 & 58.259 \\
\hline 9/17/11 12:00 & 14.352 & 6.369 & 9.947 & 19.325 & 14.399 & & 11.767 & & 33.302 & 58.162 \\
\hline 9/17/11 16:00 & 14.359 & 6.364 & 9.955 & 19.327 & 14.425 & & 11.744 & & 33.266 & 58.068 \\
\hline 9/17/11 20:00 & 14.364 & 6.364 & 9.962 & 19.330 & 14.449 & & 11.737 & & 33.234 & 57.976 \\
\hline 9/18/11 0:00 & 14.373 & 6.364 & 9.971 & 19.332 & 14.475 & & 11.748 & & 33.201 & 57.882 \\
\hline 9/18/11 4:00 & 14.385 & 6.367 & 9.981 & 19.334 & 14.498 & & 11.751 & & 33.168 & 57.790 \\
\hline 9/18/11 8:00 & 14.394 & 6.369 & 9.990 & 19.337 & 14.522 & & 11.762 & & 33.137 & 57.698 \\
\hline 9/18/11 12:00 & 14.409 & 6.374 & 9.999 & 19.339 & 14.545 & & 11.774 & & 33.105 & 57.608 \\
\hline 9/18/11 16:00 & 14.420 & 6.374 & 10.004 & 19.341 & 14.571 & & 11.762 & & 33.074 & 57.514 \\
\hline 9/18/11 20:00 & 14.430 & 6.376 & 9.978 & 19.316 & 14.574 & & 11.784 & & 33.041 & 57.420 \\
\hline 9/19/11 0:00 & 14.446 & 6.381 & 9.943 & 19.283 & 14.597 & & 11.807 & & 33.011 & 57.328 \\
\hline 9/19/11 4:00 & 14.465 & 6.383 & 9.929 & 19.285 & 14.621 & & 11.816 & & 32.980 & 57.243 \\
\hline 9/19/11 8:00 & 14.484 & 6.388 & 9.926 & 19.290 & 14.647 & & 11.838 & & 32.950 & 57.151 \\
\hline 9/19/11 12:00 & 14.508 & 6.393 & 9.929 & 19.304 & 14.673 & & 11.842 & & 32.917 & 57.064 \\
\hline 9/19/11 16:00 & 14.517 & 6.395 & 9.938 & 19.316 & 14.703 & & 11.816 & & 32.887 & 56.975 \\
\hline 9/19/11 20:00 & 14.524 & 6.397 & 9.955 & 19.325 & 14.734 & & 11.826 & & 32.856 & 56.888 \\
\hline 9/20/11 0:00 & 14.536 & 6.395 & 9.966 & 19.334 & 14.765 & & 11.838 & & 32.826 & 56.798 \\
\hline 9/20/11 4:00 & 14.545 & 6.395 & 9.978 & 19.341 & 14.793 & & 11.845 & & 32.795 & 56.711 \\
\hline 9/20/11 8:00 & 14.557 & 6.397 & 9.990 & 19.351 & 14.819 & & 11.852 & & 32.765 & 56.626 \\
\hline 9/20/11 12:00 & 14.574 & 6.397 & 10.007 & 19.360 & 14.850 & & 11.866 & & 32.736 & 56.539 \\
\hline 9/20/11 16:00 & 14.583 & 6.402 & 10.026 & 19.367 & 14.880 & & 11.854 & & 32.704 & 56.452 \\
\hline $9 / 20 / 1120: 00$ & 14.599 & 6.404 & 10.047 & 19.374 & 14.911 & & 11.906 & & 32.675 & 56.367 \\
\hline 9/21/11 0:00 & 14.628 & 6.411 & 10.068 & 19.383 & 14.942 & & 11.950 & & 32.647 & 56.280 \\
\hline 9/21/11 4:00 & 14.661 & 6.414 & 10.085 & 19.397 & 14.972 & & 11.981 & & 32.619 & 56.197 \\
\hline 9/21/11 8:00 & 14.689 & 6.419 & 10.104 & 19.411 & 14.998 & & 12.004 & & 32.591 & 56.115 \\
\hline 9/21/11 12:00 & 14.720 & 6.418 & 10.125 & 19.425 & 15.024 & & 12.025 & & 32.560 & 56.030 \\
\hline 9/21/11 16:00 & 14.746 & 6.426 & 10.146 & 19.439 & 15.052 & & 12.030 & & 32.532 & 55.948 \\
\hline 9/21/11 20:00 & 14.769 & 6.430 & 10.170 & 19.456 & 15.083 & & 12.056 & & 32.502 & 55.861 \\
\hline 9/22/11 0:00 & 14.797 & 6.430 & 10.191 & 19.469 & 15.114 & & 12.087 & & 32.476 & 55.783 \\
\hline
\end{tabular}

TABLE S1.1 Page 82 of 89 


\begin{tabular}{|c|c|c|c|c|c|c|c|c|c|c|}
\hline \multirow[b]{2}{*}{ Date and Time } & \multicolumn{8}{|c|}{ Water Level (ft below reference point) } & \multirow[b]{2}{*}{ SB24 } & \multirow[b]{2}{*}{ SB38 } \\
\hline & McPike & Potter & Hughes & Barr & Burks & MoDOT & Clizer "Old" & Morgan & & \\
\hline $9 / 22 / 114: 00$ & 14.823 & 6.428 & 10.210 & 19.483 & 15.144 & & 12.094 & & 32.448 & 55.700 \\
\hline 9/22/11 8:00 & 14.852 & 6.430 & 10.229 & 19.497 & 15.175 & & 12.126 & & 32.422 & 55.616 \\
\hline 9/22/11 12:00 & 14.880 & 6.435 & 10.245 & 19.511 & 15.203 & & 12.134 & & 32.394 & 55.533 \\
\hline $9 / 22 / 1116: 00$ & 14.892 & 6.433 & 10.262 & 19.523 & 15.236 & & 12.105 & & 32.363 & 55.453 \\
\hline 9/22/11 20:00 & 14.903 & 6.435 & 10.279 & 19.532 & 15.267 & & 12.134 & & 32.335 & 55.373 \\
\hline 9/23/11 0:00 & 14.929 & 6.437 & 10.297 & 19.542 & 15.298 & & 12.159 & & 32.310 & 55.291 \\
\hline 9/23/11 4:00 & 14.951 & 6.435 & 10.314 & 19.551 & 15.328 & & 12.159 & & 32.284 & 55.208 \\
\hline 9/23/11 8:00 & 14.969 & 6.433 & 10.326 & 19.560 & 15.357 & & 12.180 & & 32.258 & 55.128 \\
\hline 9/23/11 12:00 & 14.993 & 6.437 & 10.345 & 19.572 & 15.387 & & 12.185 & & 32.227 & 55.076 \\
\hline 9/23/11 16:00 & 15.002 & NA & 10.354 & 19.579 & 15.415 & & 12.166 & & 32.188 & 54.991 \\
\hline 9/23/11 20:00 & 15.012 & & 10.371 & 19.586 & 15.446 & & 12.180 & & 32.157 & 54.909 \\
\hline 9/24/11 0:00 & 15.029 & & 10.387 & 19.591 & 15.477 & & 12.199 & & 32.131 & 54.827 \\
\hline 9/24/11 4:00 & 15.043 & & 10.402 & 19.595 & 15.505 & & 12.204 & & 32.105 & 54.751 \\
\hline 9/24/11 8:00 & 15.059 & & 10.416 & 19.600 & 15.536 & & 12.220 & & 32.077 & 54.671 \\
\hline 9/24/11 12:00 & 15.078 & & 10.430 & 19.609 & 15.564 & & 12.223 & & 32.054 & 54.593 \\
\hline 9/24/11 16:00 & 15.085 & & 10.444 & 19.612 & 15.595 & & 12.211 & & 32.026 & 54.513 \\
\hline 9/24/11 20:00 & 15.104 & & 10.463 & 19.616 & 15.623 & & 12.244 & & 32.002 & 54.436 \\
\hline 9/25/11 0:00 & 15.125 & & 10.480 & 19.623 & 15.654 & & 12.267 & & 31.979 & 54.363 \\
\hline 9/25/11 4:00 & 15.144 & & 10.494 & 19.628 & 15.682 & & 12.274 & & 31.953 & 54.282 \\
\hline 9/25/11 8:00 & 15.165 & & 10.508 & 19.635 & 15.710 & & 12.293 & & 31.930 & 54.207 \\
\hline 9/25/11 12:00 & 15.189 & & 10.520 & 19.644 & 15.739 & & 12.298 & & 31.906 & 54.132 \\
\hline 9/25/11 16:00 & 15.198 & & 10.534 & 19.647 & 15.769 & & 12.289 & & 31.880 & 54.054 \\
\hline 9/25/11 20:00 & 15.215 & & 10.551 & 19.651 & 15.798 & & 12.314 & & 31.857 & 53.981 \\
\hline 9/26/11 0:00 & 15.236 & & 10.565 & 19.656 & 15.826 & & 12.326 & & 31.833 & 53.906 \\
\hline $9 / 26 / 114: 00$ & 15.252 & & 10.579 & 19.663 & 15.854 & & 12.338 & & 31.810 & 53.833 \\
\hline 9/26/11 8:00 & 15.276 & & 10.593 & 19.668 & 15.882 & & 12.366 & & 31.787 & 53.757 \\
\hline 9/26/11 12:00 & 15.304 & & 10.607 & 19.679 & 15.911 & & 12.378 & & 31.763 & 53.684 \\
\hline 9/26/11 16:00 & 15.321 & & 10.621 & 19.684 & 15.934 & & 12.378 & & 31.742 & 53.609 \\
\hline 9/26/11 20:00 & 15.335 & & 10.638 & 19.689 & 15.965 & & 12.404 & & 31.721 & 53.536 \\
\hline 9/27/11 0:00 & 15.358 & & 10.652 & 19.695 & 15.996 & & 12.427 & & 31.697 & 53.463 \\
\hline 9/27/11 4:00 & 15.382 & & 10.666 & 19.702 & 16.022 & & 12.446 & & 31.676 & 53.392 \\
\hline 9/27/11 8:00 & 15.413 & & 10.683 & 19.712 & 16.050 & & 12.481 & & 31.653 & 53.321 \\
\hline $9 / 27 / 1112: 00$ & 15.448 & & 10.700 & 19.723 & 16.361 & & 12.502 & & 31.634 & 53.248 \\
\hline 9/27/11 16:00 & 15.474 & & 10.716 & 19.733 & 16.342 & & 12.500 & & 31.613 & 53.178 \\
\hline 9/27/11 20:00 & 15.493 & & 10.730 & 19.742 & 16.342 & & 12.519 & & 31.592 & 53.107 \\
\hline 9/28/11 0:00 & 15.519 & & 10.747 & 19.751 & 16.352 & & 12.535 & & 31.571 & 53.039 \\
\hline 9/28/11 4:00 & 15.538 & & 10.761 & 19.758 & 16.361 & & 12.547 & & 31.550 & 52.968 \\
\hline 9/28/11 8:00 & 15.564 & & 10.775 & 19.765 & 16.380 & & 12.566 & & 31.531 & 52.897 \\
\hline 9/28/11 12:00 & 15.585 & & 10.789 & 19.775 & 16.399 & & 12.573 & & 31.510 & 52.827 \\
\hline 9/28/11 16:00 & 15.601 & & 10.806 & 19.782 & 16.420 & & 12.573 & & 31.491 & 52.758 \\
\hline 9/28/11 20:00 & 15.620 & & 10.823 & 19.789 & 16.441 & & 12.594 & & 31.470 & 52.688 \\
\hline 9/29/11 0:00 & 15.646 & & 10.839 & 19.798 & 16.465 & & 12.622 & & 31.449 & 52.619 \\
\hline 9/29/11 4:00 & 15.670 & & 10.853 & 19.807 & 16.486 & & 12.636 & & 31.428 & 52.551 \\
\hline 9/29/11 8:00 & 15.700 & & 10.870 & 19.817 & 16.510 & & 12.676 & & 31.411 & 52.483 \\
\hline 9/29/11 12:00 & 15.747 & & 10.889 & 19.833 & 16.531 & & 12.716 & & 31.392 & 52.417 \\
\hline 9/29/11 16:00 & 15.785 & & 10.910 & 19.852 & 16.557 & & 12.737 & & 31.374 & 52.349 \\
\hline 9/29/11 20:00 & 15.821 & & 10.931 & 19.868 & 16.583 & & 12.768 & & 31.355 & 52.283 \\
\hline 9/30/11 0:00 & 15.860 & & 10.953 & 19.887 & 16.609 & & 12.805 & & 31.336 & 52.217 \\
\hline 9/30/11 4:00 & 15.900 & & 10.972 & 19.905 & 16.632 & & 12.831 & & 31.320 & 52.153 \\
\hline 9/30/11 8:00 & 15.936 & & 10.988 & 19.924 & 16.656 & & 12.857 & & 31.301 & 52.085 \\
\hline 9/30/11 12:00 & 15.969 & & 11.005 & 19.947 & 16.682 & & 12.869 & & 31.285 & 52.019 \\
\hline 9/30/11 16:00 & 15.988 & & 11.016 & 19.961 & 16.705 & & 12.852 & & 31.266 & 51.950 \\
\hline 9/30/11 20:00 & 15.997 & & 11.033 & 19.973 & 16.729 & & 12.864 & & 31.247 & 51.887 \\
\hline 10/1/11 0:00 & 16.019 & & 11.047 & 19.987 & 16.755 & & 12.888 & & 31.231 & 51.823 \\
\hline 10/1/11 4:00 & 16.047 & & 11.064 & 20.001 & 16.779 & & 12.909 & & 31.212 & 51.757 \\
\hline $10 / 1 / 118: 00$ & 16.077 & & 11.078 & 20.017 & 16.802 & & 12.937 & & 31.195 & 51.698 \\
\hline $10 / 1 / 11$ 12:00 & 16.110 & & 11.092 & 20.036 & 16.826 & & 12.956 & & 31.179 & 51.630 \\
\hline 10/1/11 16:00 & 16.129 & & 11.104 & 20.050 & 16.852 & & 12.944 & & 31.160 & 51.566 \\
\hline 10/1/11 20:00 & 16.141 & & 11.116 & 20.061 & 16.878 & & 12.951 & & 31.144 & 51.503 \\
\hline 10/2/11 0:00 & 16.160 & & 11.130 & 20.073 & 16.901 & & 12.967 & & 31.127 & 51.439 \\
\hline $10 / 2 / 114: 00$ & 16.181 & & 11.142 & 20.085 & 16.927 & & 12.979 & & 31.111 & 51.380 \\
\hline 10/2/11 8:00 & 16.205 & & 11.154 & 20.096 & 16.951 & & 12.998 & & 31.095 & 51.317 \\
\hline $10 / 2 / 1112: 00$ & 16.228 & & 11.168 & 20.110 & 16.974 & & 13.007 & & 31.078 & 51.256 \\
\hline 10/2/11 16:00 & 16.238 & & 11.180 & 20.120 & 16.998 & & 12.989 & & 31.059 & 51.192 \\
\hline
\end{tabular}

TABLE S1.1 Page 83 of 89 


\begin{tabular}{|c|c|c|c|c|c|c|c|c|c|c|}
\hline \multirow[b]{2}{*}{ Date and Time } & \multicolumn{8}{|c|}{ Water Level (ft below reference point) } & \multirow[b]{2}{*}{ SB24 } & \multirow[b]{2}{*}{ SB38 } \\
\hline & McPike & Potter & Hughes & Barr & Burks & MoDOT & Clizer "Old" & Morgan & & \\
\hline 10/2/11 20:00 & 16.247 & & 11.191 & 20.126 & 17.024 & & 13.003 & & 31.045 & 51.131 \\
\hline 10/3/11 0:00 & 16.263 & & 11.206 & 20.134 & 17.047 & & 13.019 & & 31.029 & 51.067 \\
\hline 10/3/11 4:00 & 16.285 & & 11.217 & 20.143 & 17.073 & & 13.033 & & 31.013 & 51.011 \\
\hline 10/3/11 8:00 & 16.306 & & 11.229 & 20.152 & 17.097 & & 13.047 & & 30.998 & 50.949 \\
\hline 10/3/11 12:00 & 16.330 & & 11.241 & 20.164 & 17.121 & & 13.061 & & 30.980 & 50.890 \\
\hline 10/3/11 16:00 & 16.339 & & 11.258 & 20.173 & 17.147 & & 13.050 & & 30.966 & 50.829 \\
\hline 10/3/11 20:00 & 16.353 & & 11.272 & 20.180 & 17.170 & & 13.068 & & 30.949 & 50.768 \\
\hline 10/4/11 0:00 & 16.372 & & 11.288 & 20.189 & 17.196 & & 13.085 & & 30.935 & 50.711 \\
\hline 10/4/11 4:00 & 16.393 & & 11.298 & 20.199 & 17.220 & & 13.099 & & 30.919 & 50.652 \\
\hline 10/4/11 8:00 & 16.414 & & 11.310 & 20.208 & 17.241 & & 13.118 & & 30.905 & 50.594 \\
\hline 10/4/11 12:00 & 16.436 & & 11.324 & 20.217 & 17.267 & & 13.122 & & 30.891 & 50.535 \\
\hline 10/4/11 16:00 & 16.445 & & 11.338 & 20.227 & 17.290 & & 13.115 & & 30.874 & 50.478 \\
\hline 10/4/11 20:00 & 16.457 & & 11.357 & 20.231 & 17.316 & & 13.129 & & 30.858 & 50.422 \\
\hline 10/5/11 0:00 & 16.478 & & 11.369 & 20.238 & 17.340 & & 13.148 & & 30.846 & 50.365 \\
\hline 10/5/11 4:00 & 16.497 & & 11.381 & 20.248 & 17.363 & & 13.162 & & 30.830 & 50.309 \\
\hline 10/5/11 8:00 & 16.521 & & 11.392 & 20.257 & 17.387 & & 13.181 & & 30.818 & 50.252 \\
\hline 10/5/11 12:00 & 16.539 & & 11.407 & 20.269 & 17.413 & & 13.186 & & 30.801 & 50.198 \\
\hline 10/5/11 16:00 & 16.546 & & 11.421 & 20.273 & 17.434 & & 13.176 & & 30.787 & 50.141 \\
\hline 10/5/11 20:00 & 16.554 & & 11.435 & 20.278 & 17.460 & & 13.191 & & 30.773 & 50.087 \\
\hline 10/6/11 0:00 & 16.572 & & 11.447 & 20.285 & 17.481 & & 13.209 & & 30.759 & 50.031 \\
\hline $10 / 6 / 114: 00$ & 16.591 & & 11.459 & 20.292 & 17.505 & & 13.219 & & 30.745 & 49.974 \\
\hline 10/6/11 8:00 & 16.610 & & 11.471 & 20.299 & 17.526 & & 13.233 & & 30.733 & 49.922 \\
\hline 10/6/11 12:00 & 16.629 & & 11.480 & 20.311 & 17.545 & & 13.240 & & 30.722 & 49.868 \\
\hline 10/6/11 16:00 & 16.634 & & 11.497 & 20.313 & 17.571 & & 13.233 & & 30.705 & 49.814 \\
\hline 10/6/11 20:00 & 16.643 & & 11.513 & 20.318 & 17.595 & & 13.245 & & 30.694 & 49.760 \\
\hline 10/7/11 0:00 & 16.662 & & 11.525 & 20.325 & 17.618 & & 13.263 & & 30.679 & 49.706 \\
\hline 10/7/11 4:00 & 16.683 & & 11.537 & 20.332 & 17.642 & & 13.282 & & 30.668 & 49.656 \\
\hline 10/7/11 8:00 & 16.707 & & 11.549 & 20.341 & 17.663 & & 13.306 & & 30.654 & 49.602 \\
\hline 10/7/11 12:00 & 16.733 & & 11.563 & 20.350 & 17.689 & & 13.320 & & 30.642 & 49.550 \\
\hline 10/7/11 16:00 & 16.749 & & 11.577 & 20.359 & 17.713 & & 13.310 & & 30.630 & 49.498 \\
\hline 10/7/11 20:00 & 16.766 & & 11.596 & 20.366 & 17.734 & & 13.329 & & 30.616 & 49.446 \\
\hline 10/8/11 0:00 & 16.789 & & 11.610 & 20.378 & 17.757 & & 13.353 & & 30.607 & 49.395 \\
\hline $10 / 8 / 114: 00$ & 16.813 & & 11.620 & 20.390 & 17.781 & & 13.369 & & 30.595 & 49.343 \\
\hline 10/8/11 8:00 & 16.836 & & 11.631 & 20.399 & 17.805 & & 13.388 & & 30.581 & 49.293 \\
\hline 10/8/11 12:00 & 16.865 & & 11.646 & 20.413 & 17.830 & & 13.407 & & 30.572 & 49.242 \\
\hline 10/8/11 16:00 & 16.876 & & 11.662 & 20.422 & 17.852 & & 13.402 & & 30.560 & 49.192 \\
\hline 10/8/11 20:00 & 16.895 & & 11.676 & 20.432 & 17.875 & & 13.423 & & 30.546 & 49.143 \\
\hline 10/9/11 0:00 & 16.921 & & 11.690 & 20.443 & 17.899 & & 13.447 & & 30.536 & 49.091 \\
\hline 10/9/11 4:00 & 16.945 & & 11.702 & 20.455 & 17.920 & & 13.458 & & 30.525 & 49.041 \\
\hline 10/9/11 8:00 & 16.971 & & 11.714 & 20.469 & 17.941 & & 13.479 & & 30.513 & 48.992 \\
\hline 10/9/11 12:00 & 16.990 & & 11.724 & 20.481 & 17.965 & & 13.482 & & 30.504 & 48.942 \\
\hline 10/9/11 16:00 & 16.994 & & 11.738 & 20.490 & 17.986 & & 13.468 & & 30.492 & 48.893 \\
\hline 10/9/11 20:00 & 17.006 & & 11.752 & 20.497 & 18.010 & & 13.487 & & 30.480 & 48.843 \\
\hline 10/10/11 0:00 & 17.025 & & 11.764 & 20.509 & 18.033 & & 13.501 & & 30.471 & 48.794 \\
\hline 10/10/11 4:00 & 17.039 & & 11.771 & 20.518 & 18.054 & & 13.505 & & 30.459 & 48.744 \\
\hline 10/10/11 8:00 & 17.053 & & 11.780 & 20.525 & 18.076 & & 13.517 & & 30.450 & 48.697 \\
\hline 10/10/11 12:00 & 17.070 & & 11.787 & 20.534 & 18.097 & & 13.526 & & 30.440 & 48.650 \\
\hline 10/10/11 16:00 & 17.079 & & 11.795 & 20.541 & 18.121 & & 13.517 & & 30.429 & 48.601 \\
\hline $10 / 10 / 1120: 00$ & 17.086 & & 11.806 & 20.548 & 18.142 & & 13.529 & & 30.417 & 48.554 \\
\hline 10/11/11 0:00 & 17.100 & & 11.811 & 20.553 & 18.161 & & 13.536 & & 30.407 & 48.507 \\
\hline 10/11/11 4:00 & 17.110 & & 11.818 & 20.560 & 18.182 & & 13.543 & & 30.398 & 48.457 \\
\hline 10/11/11 8:00 & 17.124 & & 11.821 & 20.567 & 18.203 & & 13.552 & & 30.389 & 48.412 \\
\hline 10/11/11 12:00 & 17.136 & & 11.825 & 20.574 & 18.227 & & 13.555 & & 30.377 & 48.365 \\
\hline 10/11/11 16:00 & 17.136 & & 11.832 & 20.576 & 18.248 & & 13.534 & & 30.368 & 48.318 \\
\hline 10/11/11 20:00 & 17.140 & & 11.842 & 20.576 & 18.269 & & 13.545 & & 30.358 & 48.273 \\
\hline 10/12/11 0:00 & 17.152 & & 11.849 & 20.581 & 18.290 & & 13.555 & & 30.346 & 48.226 \\
\hline $10 / 12 / 114: 00$ & 17.159 & & 11.856 & 20.583 & 18.312 & & 13.550 & & 30.337 & 48.182 \\
\hline 10/12/11 8:00 & 17.166 & & 11.861 & 20.583 & 18.333 & & 13.550 & & 30.328 & 48.134 \\
\hline $10 / 12 / 11$ 12:00 & 17.178 & & 11.868 & 20.585 & 18.354 & & 13.566 & & 30.318 & 48.090 \\
\hline 10/12/11 16:00 & 17.181 & & 11.877 & 20.585 & 18.375 & & 13.557 & & 30.309 & 48.047 \\
\hline $10 / 12 / 1120: 00$ & 17.195 & & 11.889 & 20.586 & 18.389 & & 13.590 & & 30.300 & 47.995 \\
\hline 10/13/11 0:00 & 17.216 & & 11.899 & 20.592 & 18.411 & & 13.611 & & 30.290 & 47.951 \\
\hline 10/13/11 4:00 & 17.235 & & 11.908 & 20.599 & 18.432 & & 13.625 & & 30.281 & 47.908 \\
\hline 10/13/11 8:00 & 17.254 & & 11.915 & 20.606 & 18.453 & & 13.646 & & 30.271 & 47.871 \\
\hline
\end{tabular}

TABLE S1.1 Page 84 of 89 


\begin{tabular}{|c|c|c|c|c|c|c|c|c|c|c|}
\hline \multirow[b]{2}{*}{ Date and Time } & \multicolumn{10}{|c|}{ Water Level (ft below reference point) } \\
\hline & McPike & Potter & Hughes & Barr & Burks & MoDOT & Clizer "Old" & Morgan & SB24 & SB38 \\
\hline 10/13/11 12:00 & 17.275 & & 11.927 & 20.616 & 18.474 & & 13.663 & & 30.264 & 47.821 \\
\hline 10/13/11 16:00 & 17.291 & & 11.939 & 20.623 & 18.496 & & 13.656 & & 30.255 & 47.776 \\
\hline 10/13/11 20:00 & 17.303 & & 11.953 & 20.630 & 18.517 & & 13.672 & & 30.246 & 47.734 \\
\hline 10/14/11 0:00 & 17.322 & & 11.965 & 20.639 & 18.538 & & 13.696 & & 30.239 & 47.694 \\
\hline 10/14/11 4:00 & 17.345 & & 11.974 & 20.651 & 18.561 & & 13.712 & & 30.229 & 47.647 \\
\hline 10/14/11 8:00 & 17.369 & & 11.984 & 20.662 & 18.583 & & 13.738 & & 30.222 & 47.602 \\
\hline 10/14/11 12:00 & 17.400 & & 11.996 & 20.676 & 18.604 & & 13.764 & & 30.215 & 47.564 \\
\hline 10/14/11 16:00 & 17.419 & & 12.010 & 20.690 & 18.625 & & 13.768 & & 30.208 & 47.517 \\
\hline 10/14/11 20:00 & 17.440 & & 12.024 & 20.702 & 18.646 & & 13.787 & & 30.199 & 47.477 \\
\hline 10/15/11 0:00 & 17.463 & & 12.036 & 20.716 & 18.670 & & 13.808 & & 30.192 & 47.435 \\
\hline 10/15/11 4:00 & 17.485 & & 12.048 & 20.732 & 18.689 & & 13.822 & & 30.185 & 47.395 \\
\hline 10/15/11 8:00 & 17.506 & & 12.055 & 20.746 & 18.712 & & 13.839 & & 30.175 & 47.355 \\
\hline 10/15/11 12:00 & 17.525 & & 12.064 & 20.763 & 18.731 & & 13.841 & & 30.168 & 47.308 \\
\hline 10/15/11 16:00 & 17.522 & & 12.074 & 20.772 & 18.753 & & 13.804 & & 30.161 & 47.263 \\
\hline 10/15/11 20:00 & 17.515 & & 12.085 & 20.774 & 18.774 & & 13.794 & & 30.149 & 47.223 \\
\hline 10/16/11 0:00 & 17.520 & & 12.093 & 20.779 & 18.795 & & 13.797 & & 30.142 & 47.183 \\
\hline 10/16/11 4:00 & 17.522 & & 12.100 & 20.781 & 18.816 & & 13.811 & & 30.133 & 47.143 \\
\hline 10/16/11 8:00 & 17.546 & & 12.109 & 20.786 & 18.840 & & 13.851 & & 30.126 & 47.105 \\
\hline 10/16/11 12:00 & 17.581 & & 12.121 & 20.800 & 18.861 & & 13.893 & & 30.121 & 47.063 \\
\hline 10/16/11 16:00 & 17.605 & & 12.135 & 20.814 & 18.882 & & 13.900 & & 30.114 & 47.025 \\
\hline 10/16/11 20:00 & 17.628 & & 12.149 & 20.825 & 18.903 & & 13.914 & & 30.107 & 46.985 \\
\hline 10/17/11 0:00 & 17.647 & & 12.159 & 20.837 & 18.925 & & 13.921 & & 30.100 & 46.945 \\
\hline 10/17/11 4:00 & 17.661 & & 12.166 & 20.849 & 18.948 & & 13.926 & & 30.093 & 46.910 \\
\hline 10/17/11 8:00 & 17.673 & & 12.173 & 20.858 & 18.967 & & 13.933 & & 30.086 & 46.867 \\
\hline 10/17/11 12:00 & 17.687 & & 12.180 & 20.870 & 18.988 & & 13.938 & & 30.079 & 46.832 \\
\hline 10/17/11 16:00 & 17.694 & & 12.190 & 20.877 & 19.010 & & 13.945 & & 30.074 & 46.794 \\
\hline 10/17/11 20:00 & 17.713 & & 12.199 & 20.888 & 19.028 & & 13.963 & & 30.067 & 46.756 \\
\hline 10/18/11 0:00 & 17.727 & & 12.206 & 20.898 & 19.050 & & 13.968 & & 30.060 & 46.719 \\
\hline 10/18/11 4:00 & 17.741 & & 12.211 & 20.905 & 19.071 & & 13.968 & & 30.053 & 46.681 \\
\hline 10/18/11 8:00 & 17.751 & & 12.218 & 20.914 & 19.092 & & 13.980 & & 30.049 & 46.643 \\
\hline 10/18/11 12:00 & 17.770 & & 12.225 & 20.926 & 19.111 & & 13.996 & & 30.042 & 46.603 \\
\hline 10/18/11 16:00 & 17.784 & & 12.234 & 20.935 & 19.135 & & 14.006 & & 30.035 & 46.568 \\
\hline 10/18/11 20:00 & 17.800 & & 12.244 & 20.947 & 19.156 & & 14.017 & & 30.027 & 46.533 \\
\hline 10/19/11 0:00 & 17.815 & & 12.253 & 20.956 & 19.172 & & 14.020 & & 30.023 & 46.495 \\
\hline 10/19/11 4:00 & 17.826 & & 12.261 & 20.965 & 19.196 & & 14.027 & & 30.016 & 46.457 \\
\hline 10/19/11 8:00 & 17.840 & & 12.265 & 20.975 & 19.217 & & 14.041 & & 30.011 & 46.410 \\
\hline $10 / 19 / 11$ 12:00 & 17.855 & & 12.270 & 20.986 & 19.236 & & 14.053 & & 30.002 & 46.375 \\
\hline 10/19/11 16:00 & 17.866 & & 12.277 & 20.993 & 19.257 & & 14.053 & & 29.997 & 46.337 \\
\hline 10/19/11 20:00 & 17.878 & & 12.287 & 21.005 & 19.278 & & 14.067 & & 29.992 & 46.299 \\
\hline 10/20/11 0:00 & 17.892 & & 12.291 & 21.012 & 19.297 & & 14.071 & & 29.985 & 46.269 \\
\hline 10/20/11 4:00 & 17.904 & & 12.298 & 21.021 & 19.321 & & 14.076 & & 29.981 & 46.231 \\
\hline 10/20/11 8:00 & 17.916 & & 12.303 & 21.028 & 19.340 & & 14.090 & & 29.974 & 46.198 \\
\hline 10/20/11 12:00 & 17.935 & & 12.308 & 21.040 & 19.361 & & 14.102 & & 29.966 & 46.158 \\
\hline 10/20/11 16:00 & 17.942 & & 12.315 & 21.044 & 19.382 & & 14.102 & & 29.959 & 46.123 \\
\hline 10/20/11 20:00 & 17.956 & & 12.322 & 21.054 & 19.403 & & 14.121 & & 29.955 & 46.087 \\
\hline 10/21/11 0:00 & 17.972 & & 12.331 & 21.063 & 19.422 & & 14.137 & & 29.950 & 46.052 \\
\hline 10/21/11 4:00 & 17.989 & & 12.339 & 21.075 & 19.444 & & 14.149 & & 29.945 & 46.021 \\
\hline 10/21/11 8:00 & 18.005 & & 12.346 & 21.084 & 19.465 & & 14.165 & & 29.941 & 45.986 \\
\hline $10 / 21 / 1112: 00$ & 18.022 & & 12.353 & 21.096 & 19.488 & & 14.165 & & 29.934 & 45.951 \\
\hline 10/21/11 16:00 & 18.027 & & 12.360 & 21.103 & 19.510 & & 14.156 & & 29.929 & 45.913 \\
\hline 10/21/11 20:00 & 18.038 & & 12.369 & 21.110 & 19.531 & & 14.168 & & 29.924 & 45.880 \\
\hline 10/22/11 0:00 & 18.050 & & 12.381 & 21.119 & 19.552 & & 14.177 & & 29.920 & 45.845 \\
\hline $10 / 22 / 114: 00$ & 18.062 & & 12.391 & 21.126 & 19.571 & & 14.184 & & 29.915 & 45.814 \\
\hline 10/22/11 8:00 & 18.079 & & 12.398 & 21.138 & 19.592 & & 14.203 & & 29.913 & 45.786 \\
\hline $10 / 22 / 11$ 12:00 & 18.095 & & 12.407 & 21.147 & 19.613 & & 14.210 & & 29.910 & 45.753 \\
\hline 10/22/11 16:00 & 18.104 & & 12.417 & 21.156 & 19.635 & & 14.205 & & 29.906 & 45.722 \\
\hline 10/22/11 20:00 & 18.116 & & 12.428 & 21.163 & 19.653 & & 14.208 & & 29.901 & 45.689 \\
\hline 10/23/11 0:00 & 18.126 & & 12.438 & 21.168 & 19.677 & & 14.210 & & 29.898 & 45.661 \\
\hline $10 / 23 / 114: 00$ & 18.137 & & 12.445 & 21.175 & 19.696 & & 14.210 & & 29.894 & 45.630 \\
\hline 10/23/11 8:00 & 18.147 & & 12.454 & 21.180 & 19.717 & & 14.219 & & 29.891 & 45.600 \\
\hline $10 / 23 / 1112: 00$ & 18.161 & & 12.464 & 21.187 & 19.738 & & 14.224 & & 29.887 & 45.569 \\
\hline 10/23/11 16:00 & 18.168 & & 12.473 & 21.191 & 19.760 & & 14.212 & & 29.884 & 45.539 \\
\hline $10 / 23 / 1120: 00$ & 18.178 & & 12.488 & 21.196 & 19.781 & & 14.229 & & 29.880 & 45.510 \\
\hline 10/24/11 0:00 & 18.199 & & 12.499 & 21.203 & 19.802 & & 14.264 & & 29.877 & 45.480 \\
\hline
\end{tabular}

TABLE S1.1 Page 85 of 89 


\begin{tabular}{|c|c|c|c|c|c|c|c|c|c|c|}
\hline \multirow[b]{2}{*}{ Date and Time } & \multicolumn{8}{|c|}{ Water Level (ft below reference point) } & \multirow[b]{2}{*}{ SB24 } & \multirow[b]{2}{*}{ SB38 } \\
\hline & McPike & Potter & Hughes & Barr & Burks & MoDOT & Clizer "Old" & Morgan & & \\
\hline 10/24/11 4:00 & 18.218 & & 12.509 & 21.215 & 19.821 & & 14.278 & & 29.875 & 45.449 \\
\hline 10/24/11 8:00 & 18.236 & & 12.518 & 21.224 & 19.842 & & 14.297 & & 29.873 & 45.423 \\
\hline 10/24/11 12:00 & 18.255 & & 12.530 & 21.238 & 19.863 & & 14.302 & & 29.870 & 45.390 \\
\hline 10/24/11 16:00 & 18.260 & & 12.540 & 21.243 & 19.885 & & 14.271 & & 29.866 & 45.362 \\
\hline 10/24/11 20:00 & 18.263 & & 12.551 & 21.247 & 19.906 & & 14.266 & & 29.866 & 45.331 \\
\hline 10/25/11 0:00 & 18.274 & & 12.563 & 21.250 & 19.925 & & 14.273 & & 29.863 & 45.301 \\
\hline 10/25/11 4:00 & 18.277 & & 12.570 & 21.254 & 19.943 & & 14.257 & & 29.859 & 45.272 \\
\hline 10/25/11 8:00 & 18.279 & & 12.582 & 21.254 & 19.967 & & 14.252 & & 29.859 & 45.244 \\
\hline 10/25/11 12:00 & 18.284 & & 12.592 & 21.252 & 19.986 & & 14.255 & & 29.856 & 45.216 \\
\hline 10/25/11 16:00 & 18.288 & & 12.601 & 21.252 & 20.005 & & 14.264 & & 29.856 & 45.190 \\
\hline 10/25/11 20:00 & 18.303 & & 12.615 & 21.257 & 20.026 & & 14.313 & & 29.854 & 45.164 \\
\hline 10/26/11 0:00 & 18.326 & & 12.627 & 21.266 & 20.045 & & 14.344 & & 29.856 & 45.140 \\
\hline 10/26/11 4:00 & 18.347 & & 12.639 & 21.277 & 20.064 & & 14.363 & & 29.856 & 45.115 \\
\hline 10/26/11 8:00 & 18.364 & & 12.648 & 21.289 & 20.085 & & 14.386 & & 29.856 & 45.086 \\
\hline 10/26/11 12:00 & 18.383 & & 12.655 & 21.301 & 20.106 & & 14.389 & & 29.856 & 45.063 \\
\hline 10/26/11 16:00 & 18.394 & & 12.665 & 21.312 & 20.125 & & 14.391 & & 29.856 & 45.037 \\
\hline 10/26/11 20:00 & 18.409 & & 12.674 & 21.322 & 20.146 & & 14.407 & & 29.854 & 45.013 \\
\hline 10/27/11 0:00 & 18.423 & & 12.684 & 21.333 & 20.168 & & 14.419 & & 29.856 & 44.987 \\
\hline 10/27/11 4:00 & 18.439 & & 12.691 & 21.345 & 20.189 & & 14.426 & & 29.854 & 44.961 \\
\hline 10/27/11 8:00 & 18.453 & & 12.698 & 21.354 & 20.208 & & 14.445 & & 29.854 & 44.938 \\
\hline 10/27/11 12:00 & 18.472 & & 12.705 & 21.368 & 20.229 & & 14.452 & & 29.854 & 44.910 \\
\hline 10/27/11 16:00 & 18.484 & & 12.712 & 21.380 & 20.250 & & 14.450 & & 29.852 & 44.884 \\
\hline 10/27/11 20:00 & 18.496 & & 12.722 & 21.389 & 20.269 & & 14.468 & & 29.852 & 44.860 \\
\hline 10/28/11 0:00 & 18.515 & & 12.731 & 21.401 & 20.288 & & 14.480 & & 29.849 & 44.832 \\
\hline 10/28/11 4:00 & 18.531 & & 12.741 & 21.413 & 20.309 & & 14.487 & & 29.849 & 44.811 \\
\hline 10/28/11 8:00 & 18.543 & & 12.748 & 21.422 & 20.328 & & 14.497 & & 29.849 & 44.780 \\
\hline 10/28/11 12:00 & 18.557 & & 12.752 & 21.434 & 20.349 & & 14.499 & & 29.847 & 44.752 \\
\hline 10/28/11 16:00 & 18.567 & & 12.762 & 21.443 & 20.368 & & 14.499 & & 29.845 & 44.724 \\
\hline 10/28/11 20:00 & 18.581 & & 12.769 & 21.450 & 20.389 & & 14.515 & & 29.842 & 44.700 \\
\hline 10/29/11 0:00 & 18.597 & & 12.778 & 21.459 & 20.410 & & 14.511 & & 29.840 & 44.676 \\
\hline 10/29/11 4:00 & 18.606 & & 12.786 & 21.466 & 20.429 & & 14.513 & & 29.840 & 44.655 \\
\hline 10/29/11 8:00 & 18.616 & & 12.793 & 21.473 & 20.451 & & 14.522 & & 29.837 & 44.622 \\
\hline $10 / 29 / 11$ 12:00 & 18.626 & & 12.800 & 21.480 & 20.472 & & 14.504 & & 29.837 & 44.596 \\
\hline 10/29/11 16:00 & 18.625 & & 12.804 & 21.480 & 20.491 & & 14.478 & & 29.833 & 44.570 \\
\hline 10/29/11 20:00 & 18.630 & & 12.812 & 21.480 & 20.509 & & 14.490 & & 29.830 & 44.547 \\
\hline 10/30/11 0:00 & 18.639 & & 12.821 & 21.483 & 20.531 & & 14.499 & & 29.833 & 44.523 \\
\hline 10/30/11 4:00 & 18.651 & & 12.830 & 21.483 & 20.552 & & 14.513 & & 29.830 & 44.500 \\
\hline 10/30/11 8:00 & 18.663 & & 12.840 & 21.487 & 20.571 & & 14.541 & & 29.830 & 44.476 \\
\hline 10/30/11 12:00 & 18.682 & & 12.849 & 21.492 & 20.592 & & 14.560 & & 29.828 & 44.455 \\
\hline 10/30/11 16:00 & 18.701 & & 12.856 & 21.499 & 20.611 & & 14.565 & & 29.828 & 44.434 \\
\hline 10/30/11 20:00 & 18.713 & & 12.868 & 21.510 & 20.632 & & 14.574 & & 29.828 & 44.410 \\
\hline 10/31/11 0:00 & 18.727 & & 12.878 & 21.517 & 20.651 & & 14.588 & & 29.828 & 44.387 \\
\hline 10/31/11 4:00 & 18.743 & & 12.887 & 21.527 & 20.672 & & 14.598 & & 29.828 & 44.368 \\
\hline 10/31/11 8:00 & 18.757 & & 12.897 & 21.536 & 20.691 & & 14.609 & & 29.830 & 44.344 \\
\hline $10 / 31 / 11$ 12:00 & 18.772 & & 12.904 & 21.543 & 20.710 & & 14.607 & & 29.826 & 44.318 \\
\hline 10/31/11 16:00 & 18.776 & & 12.911 & 21.550 & 20.731 & & 14.588 & & 29.823 & 44.295 \\
\hline 10/31/11 20:00 & 18.783 & & 12.920 & 21.552 & 20.750 & & 14.602 & & 29.823 & 44.274 \\
\hline 11/1/11 0:00 & 18.795 & & 12.930 & 21.557 & 20.769 & & 14.605 & & 29.821 & 44.250 \\
\hline $11 / 1 / 114: 00$ & 18.805 & & 12.939 & 21.562 & 20.788 & & 14.605 & & 29.821 & 44.229 \\
\hline 11/1/11 8:00 & 18.816 & & 12.949 & 21.564 & 20.804 & & 14.616 & & 29.821 & 44.205 \\
\hline $11 / 1 / 1112: 00$ & 18.826 & & 12.956 & 21.569 & 20.825 & & 14.616 & & 29.819 & 44.184 \\
\hline $11 / 1 / 11$ 16:00 & 18.833 & & 12.961 & 21.571 & 20.844 & & 14.612 & & 29.816 & 44.163 \\
\hline $11 / 1 / 1120: 00$ & 18.842 & & 12.972 & 21.576 & 20.863 & & 14.633 & & 29.819 & 44.142 \\
\hline $11 / 2 / 110: 00$ & 18.856 & & 12.982 & 21.580 & 20.880 & & 14.645 & & 29.819 & 44.123 \\
\hline 11/2/11 4:00 & 18.871 & & 12.991 & 21.585 & 20.901 & & 14.661 & & 29.819 & 44.102 \\
\hline $11 / 2 / 118: 00$ & 18.885 & & 13.001 & 21.592 & 20.922 & & 14.675 & & 29.821 & 44.080 \\
\hline $11 / 2 / 11$ 12:00 & 18.899 & & 13.010 & 21.601 & 20.939 & & 14.680 & & 29.821 & 44.059 \\
\hline $11 / 2 / 11$ 16:00 & 18.911 & & 13.015 & 21.611 & 20.948 & & 14.692 & & 29.821 & 44.040 \\
\hline $11 / 2 / 1120: 00$ & 18.918 & & 13.020 & 21.604 & 20.962 & & 14.701 & & 29.823 & 44.017 \\
\hline 11/3/11 0:00 & 18.925 & & 13.024 & 21.611 & 20.979 & & 14.703 & & 29.823 & 43.996 \\
\hline $11 / 3 / 114: 00$ & 18.927 & & 13.029 & 21.620 & 20.995 & & 14.717 & & 29.826 & 43.974 \\
\hline $11 / 3 / 118: 00$ & 18.937 & & 13.032 & 21.627 & 21.014 & & 14.739 & & 29.823 & 43.953 \\
\hline $11 / 3 / 11$ 12:00 & 18.953 & & 13.039 & 21.641 & 21.033 & & 14.746 & & 29.823 & 43.932 \\
\hline $11 / 3 / 1116: 00$ & 18.963 & & 13.041 & 21.653 & 21.052 & & 14.750 & & 29.823 & 43.909 \\
\hline
\end{tabular}

TABLE S1.1 Page 86 of 89 


\begin{tabular}{|c|c|c|c|c|c|c|c|c|c|c|}
\hline \multirow[b]{2}{*}{ Date and Time } & \multicolumn{8}{|c|}{ Water Level (ft below reference point) } & \multirow[b]{2}{*}{ SB24 } & \multirow[b]{2}{*}{ SB38 } \\
\hline & McPike & Potter & Hughes & Barr & Burks & MoDOT & Clizer "Old" & Morgan & & \\
\hline $11 / 3 / 1120: 00$ & 18.974 & & 13.048 & 21.662 & 21.071 & & 14.760 & & 29.823 & 43.892 \\
\hline 11/4/11 0:00 & 18.986 & & 13.055 & 21.674 & 21.090 & & 14.774 & & 29.826 & 43.868 \\
\hline 11/4/11 4:00 & 18.996 & & 13.060 & 21.685 & 21.106 & & 14.769 & & 29.823 & 43.852 \\
\hline 11/4/11 8:00 & 19.007 & & 13.067 & 21.694 & 21.125 & & 14.776 & & 29.826 & 43.828 \\
\hline $11 / 4 / 1112: 00$ & 19.017 & & 13.072 & 21.704 & 21.144 & & 14.774 & & 29.823 & 43.810 \\
\hline $11 / 4 / 11$ 16:00 & 19.017 & & 13.074 & 21.711 & 21.163 & & 14.743 & & 29.821 & 43.788 \\
\hline $11 / 4 / 1120: 00$ & 19.021 & & 13.079 & 21.713 & 21.182 & & 14.743 & & 29.821 & 43.767 \\
\hline 11/5/11 0:00 & 19.029 & & 13.084 & 21.715 & 21.200 & & 14.748 & & 29.821 & 43.748 \\
\hline 11/5/11 4:00 & 19.033 & & 13.091 & 21.720 & 21.217 & & 14.746 & & 29.821 & 43.727 \\
\hline 11/5/11 8:00 & 19.038 & & 13.093 & 21.720 & 21.236 & & 14.750 & & 29.819 & 43.708 \\
\hline 11/5/11 12:00 & 19.043 & & 13.095 & 21.722 & 21.252 & & 14.741 & & 29.819 & 43.689 \\
\hline 11/5/11 16:00 & 19.038 & & 13.098 & 21.722 & 21.271 & & 14.715 & & 29.819 & 43.671 \\
\hline 11/5/11 20:00 & 19.040 & & 13.102 & 21.715 & 21.290 & & 14.710 & & 29.816 & 43.652 \\
\hline 11/6/11 0:00 & 19.047 & & 13.107 & 21.713 & 21.307 & & 14.732 & & 29.816 & 43.633 \\
\hline 11/6/11 4:00 & 19.057 & & 13.112 & 21.711 & 21.323 & & 14.739 & & 29.819 & 43.614 \\
\hline 11/6/11 8:00 & 19.064 & & 13.119 & 21.713 & 21.342 & & 14.774 & & 29.819 & 43.598 \\
\hline $11 / 6 / 11$ 12:00 & 19.078 & & 13.126 & 21.720 & 21.361 & & 14.807 & & 29.821 & 43.581 \\
\hline 11/6/11 16:00 & 19.095 & & 13.131 & 21.727 & 21.380 & & 14.811 & & 29.821 & 43.562 \\
\hline 11/6/11 20:00 & 19.109 & & 13.138 & 21.736 & 21.396 & & 14.830 & & 29.823 & 43.548 \\
\hline 11/7/11 0:00 & 19.125 & & 13.145 & 21.746 & 21.415 & & 14.847 & & 29.826 & 43.529 \\
\hline $11 / 7 / 114: 00$ & 19.137 & & 13.152 & 21.757 & 21.434 & & 14.856 & & 29.823 & 43.510 \\
\hline 11/7/11 8:00 & 19.151 & & 13.159 & 21.769 & 21.450 & & 14.872 & & 29.826 & 43.494 \\
\hline 11/7/11 12:00 & 19.163 & & 13.166 & 21.778 & 21.469 & & 14.863 & & 29.828 & 43.475 \\
\hline 11/7/11 16:00 & 19.170 & & 13.171 & 21.785 & 21.486 & & 14.844 & & 29.828 & 43.459 \\
\hline 11/7/11 20:00 & 19.158 & & 13.164 & 21.757 & 21.396 & & 14.830 & & 29.828 & 43.440 \\
\hline 11/8/11 0:00 & 17.063 & & 13.079 & 21.385 & 21.189 & & 14.490 & & 29.826 & 43.412 \\
\hline 11/8/11 4:00 & 15.757 & & 12.923 & 21.208 & 21.203 & & 14.445 & & 29.823 & 43.393 \\
\hline 11/8/11 8:00 & 15.818 & & 12.774 & 21.163 & 21.219 & & 14.396 & & 29.819 & 43.376 \\
\hline 11/8/11 12:00 & 16.052 & & 12.651 & 21.138 & 21.233 & & 14.353 & & 29.814 & 43.357 \\
\hline 11/8/11 16:00 & 16.250 & & 12.530 & 21.089 & 21.203 & & 14.278 & & 29.809 & 43.341 \\
\hline 11/8/11 20:00 & 15.198 & & 12.329 & 20.909 & 21.099 & & 13.992 & & 29.805 & 43.317 \\
\hline 11/9/11 0:00 & 13.855 & & 11.918 & 20.627 & 21.038 & & 13.672 & & 29.798 & 43.294 \\
\hline $11 / 9 / 114: 00$ & 13.242 & & 11.535 & 20.439 & 21.040 & & 13.637 & & 29.791 & 43.268 \\
\hline 11/9/11 8:00 & 13.344 & & 11.180 & 20.322 & 21.040 & & 13.590 & & 29.779 & 43.240 \\
\hline $11 / 9 / 11$ 12:00 & 13.457 & & 10.832 & 20.292 & 21.024 & & 13.548 & & 29.769 & 43.216 \\
\hline 11/9/11 16:00 & 13.179 & & 10.475 & 20.271 & 21.033 & & 13.449 & & 29.758 & 43.202 \\
\hline 11/9/11 20:00 & 13.016 & & 10.170 & 20.276 & 21.047 & & 13.381 & & 29.746 & 43.185 \\
\hline 11/10/11 0:00 & 13.240 & & 9.936 & 20.287 & 21.061 & & 13.329 & & 29.732 & 43.167 \\
\hline 11/10/11 4:00 & 13.492 & & 9.754 & 20.299 & 21.075 & & 13.280 & & 29.720 & 43.148 \\
\hline 11/10/11 8:00 & 13.716 & & 9.617 & 20.308 & 21.092 & & 13.247 & & 29.706 & 43.124 \\
\hline 11/10/11 12:00 & 13.919 & & 9.517 & 20.318 & 21.106 & & 13.219 & & 29.692 & 43.103 \\
\hline 11/10/11 16:00 & 14.074 & & 9.439 & 20.322 & 21.116 & & 13.162 & & 29.673 & 43.082 \\
\hline 11/10/11 20:00 & 14.199 & & 9.378 & 20.320 & 21.127 & & 13.115 & & 29.659 & 43.061 \\
\hline 11/11/11 0:00 & 14.300 & & 9.333 & 20.315 & 21.137 & & 13.071 & & 29.643 & 43.042 \\
\hline $11 / 11 / 114: 00$ & 14.378 & & 9.290 & 20.306 & 21.146 & & 13.010 & & 29.626 & 43.025 \\
\hline $11 / 11 / 118: 00$ & 14.428 & & 9.255 & 20.287 & 21.153 & & 12.951 & & 29.612 & 43.002 \\
\hline $11 / 11 / 11$ 12:00 & 14.487 & & 9.238 & 20.266 & 21.163 & & 12.927 & & 29.598 & 42.985 \\
\hline $11 / 11 / 11$ 16:00 & 14.543 & & 9.231 & 20.250 & 21.170 & & 12.906 & & 29.584 & 42.964 \\
\hline $11 / 11 / 1120: 00$ & 14.602 & & 9.229 & 20.231 & 21.174 & & 12.890 & & 29.570 & 42.947 \\
\hline 11/12/11 0:00 & 14.661 & & 9.231 & 20.217 & 21.179 & & 12.880 & & 29.556 & 42.929 \\
\hline $11 / 12 / 114: 00$ & 14.706 & & 9.231 & 20.201 & 21.184 & & 12.850 & & 29.542 & 42.912 \\
\hline $11 / 12 / 118: 00$ & 14.739 & & 9.234 & 20.185 & 21.189 & & 12.822 & & 29.528 & 42.889 \\
\hline $11 / 12 / 11$ 12:00 & 14.765 & & 9.238 & 20.164 & 21.193 & & 12.794 & & 29.511 & 42.870 \\
\hline $11 / 12 / 1116: 00$ & 14.781 & & 9.241 & 20.143 & 21.196 & & 12.754 & & 29.497 & 42.851 \\
\hline $11 / 12 / 1120: 00$ & 14.800 & & 9.250 & 20.117 & 21.196 & & 12.737 & & 29.481 & 42.832 \\
\hline 11/13/11 0:00 & 14.831 & & 9.262 & 20.096 & 21.198 & & 12.726 & & 29.467 & 42.813 \\
\hline $11 / 13 / 114: 00$ & 14.833 & & 9.269 & 20.073 & 21.198 & & 12.674 & & 29.453 & 42.794 \\
\hline $11 / 13 / 118: 00$ & 14.866 & & 9.288 & 20.050 & 21.200 & & 12.711 & & 29.439 & 42.776 \\
\hline $11 / 13 / 11$ 12:00 & 14.923 & & 9.316 & 20.036 & 21.203 & & 12.742 & & 29.425 & 42.757 \\
\hline $11 / 13 / 11$ 16:00 & 14.979 & & 9.342 & 20.026 & 21.205 & & 12.758 & & 29.413 & 42.738 \\
\hline $11 / 13 / 1120: 00$ & 15.038 & & 9.371 & 20.019 & 21.205 & & 12.784 & & 29.399 & 42.719 \\
\hline $11 / 14 / 110: 00$ & 15.083 & & 9.394 & 20.015 & 21.205 & & 12.770 & & 29.387 & 42.698 \\
\hline $11 / 14 / 114: 00$ & 15.114 & & 9.413 & 20.008 & 21.208 & & 12.749 & & 29.375 & 42.684 \\
\hline $11 / 14 / 118: 00$ & 15.144 & & 9.437 & 20.001 & 21.205 & & 12.765 & & 29.361 & 42.662 \\
\hline
\end{tabular}

TABLE S1.1 Page 87 of 89 


\begin{tabular}{|c|c|c|c|c|c|c|c|c|c|c|}
\hline \multirow[b]{2}{*}{ Date and Time } & \multicolumn{10}{|c|}{ Water Level (ft below reference point) } \\
\hline & McPike & Potter & Hughes & Barr & Burks & MoDOT & Clizer "Old" & Morgan & SB24 & SB38 \\
\hline $11 / 14 / 1112: 00$ & 15.194 & & 9.468 & 19.998 & 21.210 & & 12.794 & & 29.350 & 42.646 \\
\hline $11 / 14 / 1116: 00$ & 15.239 & & 9.494 & 19.996 & 21.208 & & 12.796 & & 29.338 & 42.625 \\
\hline 11/14/11 20:00 & 15.286 & & 9.522 & 19.998 & 21.210 & & 12.817 & & 29.326 & 42.608 \\
\hline 11/15/11 0:00 & 15.328 & & 9.543 & 19.998 & 21.210 & & 12.808 & & 29.314 & 42.589 \\
\hline 11/15/11 4:00 & 15.352 & & 9.565 & 19.998 & 21.210 & & 12.791 & & 29.303 & 42.575 \\
\hline 11/15/11 8:00 & 15.380 & & 9.584 & 19.998 & 21.212 & & 12.798 & & 29.293 & 42.554 \\
\hline $11 / 15 / 1112: 00$ & 15.422 & & 9.612 & 20.001 & 21.212 & & 12.822 & & 29.282 & 42.538 \\
\hline 11/15/11 16:00 & 15.458 & & 9.633 & 20.001 & 21.212 & & 12.831 & & 29.272 & 42.516 \\
\hline 11/15/11 20:00 & 15.521 & & 9.671 & 20.010 & 21.215 & & 12.892 & & 29.263 & 42.500 \\
\hline 11/16/11 0:00 & 15.594 & & 9.707 & 20.024 & 21.215 & & 12.930 & & 29.256 & 42.479 \\
\hline 11/16/11 4:00 & 15.656 & & 9.737 & 20.040 & 21.215 & & 12.951 & & 29.244 & 42.460 \\
\hline 11/16/11 8:00 & 15.708 & & 9.768 & 20.057 & 21.219 & & 12.977 & & 29.235 & 42.448 \\
\hline $11 / 16 / 11$ 12:00 & 15.776 & & 9.801 & 20.075 & 21.219 & & 13.019 & & 29.228 & 42.429 \\
\hline $11 / 16 / 11$ 16:00 & 15.821 & & 9.827 & 20.094 & 21.222 & & 13.017 & & 29.218 & 42.410 \\
\hline 11/16/11 20:00 & 15.866 & & 9.856 & 20.110 & 21.224 & & 13.033 & & 29.209 & 42.394 \\
\hline 11/17/11 0:00 & 15.908 & & 9.882 & 20.126 & 21.226 & & 13.043 & & 29.202 & 42.380 \\
\hline $11 / 17 / 114: 00$ & 15.943 & & 9.905 & 20.143 & 21.229 & & 13.047 & & 29.195 & 42.361 \\
\hline 11/17/11 8:00 & 15.969 & & 9.924 & 20.159 & 21.229 & & 13.050 & & 29.185 & 42.337 \\
\hline $11 / 17 / 1112: 00$ & 15.993 & & 9.948 & 20.171 & 21.231 & & 13.036 & & 29.174 & 42.326 \\
\hline 11/17/11 16:00 & 15.986 & & 9.952 & 20.178 & 21.233 & & 12.989 & & 29.160 & 42.304 \\
\hline $11 / 17 / 1120: 00$ & 15.967 & & 9.962 & 20.180 & 21.236 & & 12.953 & & 29.150 & 42.290 \\
\hline 11/18/11 0:00 & 15.957 & & 9.969 & 20.178 & 21.236 & & 12.932 & & 29.143 & 42.269 \\
\hline $11 / 18 / 114: 00$ & 15.943 & & 9.976 & 20.173 & 21.241 & & 12.899 & & 29.132 & 42.253 \\
\hline 11/18/11 8:00 & 15.929 & & 9.981 & 20.166 & 21.245 & & 12.883 & & 29.122 & 42.234 \\
\hline $11 / 18 / 11$ 12:00 & 15.924 & & 9.993 & 20.159 & 21.248 & & 12.869 & & 29.113 & 42.222 \\
\hline $11 / 18 / 11$ 16:00 & 15.903 & & 9.993 & 20.145 & 21.248 & & 12.836 & & 29.103 & 42.205 \\
\hline $11 / 18 / 1120: 00$ & 15.906 & & 10.002 & 20.133 & 21.250 & & 12.843 & & 29.094 & 42.189 \\
\hline 11/19/11 0:00 & 15.924 & & 10.012 & 20.126 & 21.255 & & 12.852 & & 29.087 & 42.175 \\
\hline 11/19/11 4:00 & 15.924 & & 10.019 & 20.117 & 21.257 & & 12.829 & & 29.078 & 42.156 \\
\hline 11/19/11 8:00 & 15.915 & & 10.023 & 20.105 & 21.259 & & 12.815 & & 29.066 & 42.142 \\
\hline $11 / 19 / 11$ 12:00 & 15.915 & & 10.035 & 20.094 & 21.262 & & 12.815 & & 29.059 & 42.125 \\
\hline $11 / 19 / 11$ 16:00 & 15.950 & & 10.054 & 20.085 & 21.266 & & 12.892 & & 29.049 & 42.111 \\
\hline $11 / 19 / 1120: 00$ & 16.047 & & 10.087 & 20.094 & 21.269 & & 12.998 & & 29.047 & 42.097 \\
\hline 11/20/11 0:00 & 16.141 & & 10.118 & 20.112 & 21.274 & & 13.059 & & 29.040 & 42.078 \\
\hline $11 / 20 / 114: 00$ & 16.214 & & 10.149 & 20.131 & 21.278 & & 13.094 & & 29.035 & 42.066 \\
\hline 11/20/11 8:00 & 16.269 & & 10.175 & 20.150 & 21.283 & & 13.118 & & 29.028 & 42.055 \\
\hline $11 / 20 / 1112: 00$ & 16.316 & & 10.198 & 20.168 & 21.288 & & 13.129 & & 29.024 & 42.036 \\
\hline $11 / 20 / 11$ 16:00 & 16.337 & & 10.217 & 20.185 & 21.290 & & 13.118 & & 29.017 & 42.019 \\
\hline $11 / 20 / 1120: 00$ & 16.360 & & 10.234 & 20.196 & 21.295 & & 13.132 & & 29.010 & 42.008 \\
\hline 11/21/11 0:00 & 16.382 & & 10.250 & 20.208 & 21.299 & & 13.129 & & 29.005 & 41.991 \\
\hline 11/21/11 4:00 & 16.396 & & 10.265 & 20.217 & 21.304 & & 13.134 & & 28.998 & 41.977 \\
\hline $11 / 21 / 118: 00$ & 16.415 & & 10.279 & 20.227 & 21.309 & & 13.139 & & 28.991 & 41.963 \\
\hline $11 / 21 / 11$ 12:00 & 16.431 & & 10.295 & 20.238 & 21.316 & & 13.139 & & 28.986 & 41.951 \\
\hline $11 / 21 / 11$ 16:00 & 16.426 & & 10.303 & 20.241 & 21.318 & & 13.108 & & 28.979 & 41.935 \\
\hline $11 / 21 / 1120: 00$ & 16.424 & & 10.312 & 20.243 & 21.323 & & 13.097 & & 28.972 & 41.920 \\
\hline 11/22/11 0:00 & 16.438 & & 10.324 & 20.248 & 21.328 & & 13.115 & & 28.967 & 41.906 \\
\hline $11 / 22 / 114: 00$ & 16.436 & & 10.331 & 20.248 & 21.333 & & 13.094 & & 28.960 & 41.895 \\
\hline $11 / 22 / 118: 00$ & 16.426 & & 10.326 & 20.229 & 21.333 & & 13.090 & & 28.953 & 41.876 \\
\hline $11 / 22 / 1112: 00$ & 16.429 & & 10.319 & 20.203 & 21.335 & & 13.094 & & 28.949 & 41.864 \\
\hline $11 / 22 / 11$ 16:00 & 16.429 & & 10.317 & 20.199 & 21.340 & & 13.104 & & 28.942 & 41.850 \\
\hline $11 / 22 / 1120: 00$ & 16.455 & & 10.324 & 20.201 & 21.344 & & 13.139 & & 28.935 & 41.836 \\
\hline 11/23/11 0:00 & 16.495 & & 10.333 & 20.208 & 21.349 & & 13.169 & & 28.932 & 41.824 \\
\hline $11 / 23 / 114: 00$ & 16.521 & & 10.343 & 20.220 & 21.354 & & 13.176 & & 28.925 & 41.810 \\
\hline $11 / 23 / 118: 00$ & 16.547 & & 10.355 & 20.227 & 21.361 & & 13.191 & & 28.920 & 41.796 \\
\hline $11 / 23 / 11$ 12:00 & 16.573 & & 10.366 & 20.238 & 21.366 & & 13.202 & & 28.916 & 41.781 \\
\hline $11 / 23 / 11$ 16:00 & 16.577 & & 10.371 & 20.248 & 21.370 & & 13.179 & & 28.911 & 41.770 \\
\hline $11 / 23 / 1120: 00$ & 16.587 & & 10.381 & 20.255 & 21.377 & & 13.191 & & 28.906 & 41.756 \\
\hline $11 / 24 / 110: 00$ & 16.599 & & 10.390 & 20.259 & 21.382 & & 13.193 & & 28.899 & 41.746 \\
\hline $11 / 24 / 114: 00$ & 16.606 & & 10.397 & 20.264 & 21.389 & & 13.188 & & 28.895 & 41.730 \\
\hline $11 / 24 / 118: 00$ & 16.610 & & 10.407 & 20.269 & 21.394 & & 13.188 & & 28.890 & 41.718 \\
\hline $11 / 24 / 1112: 00$ & 16.620 & & 10.416 & 20.273 & 21.401 & & 13.181 & & 28.885 & 41.706 \\
\hline $11 / 24 / 11$ 16:00 & 16.603 & & 10.418 & 20.271 & 21.406 & & 13.153 & & 28.876 & 41.692 \\
\hline $11 / 24 / 1120: 00$ & 16.599 & & 10.426 & 20.271 & 21.410 & & 13.153 & & 28.871 & 41.680 \\
\hline $11 / 25 / 110: 00$ & 16.606 & & 10.430 & 20.269 & 21.417 & & 13.155 & & 28.864 & 41.666 \\
\hline
\end{tabular}

TABLE S1.1 Page 88 of 89 


\begin{tabular}{|c|c|c|c|c|c|c|c|c|c|c|}
\hline \multirow[b]{2}{*}{ Date and Time } & \multicolumn{8}{|c|}{ Water Level (ft below reference point) } & \multirow[b]{2}{*}{ SB24 } & \multirow[b]{2}{*}{ SB38 } \\
\hline & McPike & Potter & Hughes & Barr & Burks & MoDOT & Clizer "Old" & Morgan & & \\
\hline 11/25/11 4:00 & 16.601 & & 10.435 & 20.266 & 21.422 & & 13.137 & & 28.862 & 41.654 \\
\hline 11/25/11 8:00 & 16.596 & & 10.442 & 20.262 & 21.429 & & 13.139 & & 28.855 & 41.642 \\
\hline 11/25/11 12:00 & 16.603 & & 10.449 & 20.259 & 21.434 & & 13.141 & & 28.850 & 41.631 \\
\hline 11/25/11 16:00 & 16.589 & & 10.452 & 20.252 & 21.441 & & 13.111 & & 28.843 & 41.619 \\
\hline 11/25/11 20:00 & 16.587 & & 10.456 & 20.245 & 21.448 & & 13.120 & & 28.838 & 41.605 \\
\hline 11/26/11 0:00 & 16.601 & & 10.468 & 20.241 & 21.453 & & 13.139 & & 28.831 & 41.593 \\
\hline $11 / 26 / 114: 00$ & 16.608 & & 10.463 & 20.217 & 21.457 & & 13.144 & & 28.829 & 41.581 \\
\hline $11 / 26 / 118: 00$ & 16.615 & & 10.452 & 20.192 & 21.460 & & 13.186 & & 28.827 & 41.567 \\
\hline 11/26/11 12:00 & 16.641 & & 10.433 & 20.164 & 21.465 & & 13.231 & & 28.820 & 41.553 \\
\hline $11 / 26 / 1116: 00$ & 16.643 & & 10.411 & 20.164 & 21.467 & & 13.249 & & 28.817 & 41.541 \\
\hline $11 / 26 / 1120: 00$ & 16.674 & & 10.409 & 20.175 & 21.476 & & 13.289 & & 28.815 & 41.532 \\
\hline $11 / 27 / 110: 00$ & 16.702 & & 10.411 & 20.192 & 21.481 & & 13.303 & & 28.810 & 41.522 \\
\hline 11/27/11 4:00 & 16.724 & & 10.416 & 20.206 & 21.486 & & 13.310 & & 28.808 & 41.513 \\
\hline 11/27/11 8:00 & 16.747 & & 10.423 & 20.222 & 21.493 & & 13.327 & & 28.803 & 41.501 \\
\hline $11 / 27 / 1112: 00$ & 16.775 & & 10.430 & 20.236 & 21.498 & & 13.343 & & 28.801 & 41.487 \\
\hline $11 / 27 / 1116: 00$ & 16.787 & & 10.435 & 20.250 & 21.507 & & 13.334 & & 28.798 & 41.473 \\
\hline $11 / 27 / 1120: 00$ & 16.804 & & 10.442 & 20.266 & 21.512 & & 13.348 & & 28.791 & 41.466 \\
\hline 11/28/11 0:00 & 16.820 & & 10.447 & 20.276 & 21.519 & & 13.348 & & 28.789 & 41.456 \\
\hline $11 / 28 / 114: 00$ & 16.825 & & 10.456 & 20.287 & 21.524 & & 13.334 & & 28.784 & 41.445 \\
\hline $11 / 28 / 118: 00$ & 16.834 & & 10.463 & 20.297 & 21.531 & & 13.348 & & 28.780 & 41.435 \\
\hline $11 / 28 / 11$ 12:00 & 16.841 & & 10.468 & 20.303 & 21.538 & & 13.336 & & 28.775 & 41.421 \\
\hline $11 / 28 / 11$ 16:00 & 16.832 & & 10.470 & 20.306 & 21.542 & & 13.317 & & 28.768 & 41.409 \\
\hline $11 / 28 / 1120: 00$ & 16.839 & & 10.480 & 20.310 & 21.549 & & 13.332 & & 28.763 & 41.397 \\
\hline $11 / 29 / 110: 00$ & 16.851 & & 10.485 & 20.313 & 21.557 & & 13.346 & & 28.759 & 41.390 \\
\hline $11 / 29 / 114: 00$ & 16.877 & & 10.496 & 20.320 & 21.564 & & 13.374 & & 28.754 & 41.372 \\
\hline 11/29/11 8:00 & 16.912 & & 10.513 & 20.329 & 21.568 & & 13.407 & & 28.752 & 41.367 \\
\hline $11 / 29 / 1112: 00$ & 16.950 & & 10.530 & 20.343 & 21.575 & & 13.433 & & 28.749 & 41.355 \\
\hline $11 / 29 / 11$ 16:00 & 16.966 & & 10.539 & 20.352 & 21.582 & & 13.428 & & 28.747 & 41.343 \\
\hline $11 / 29 / 1120: 00$ & 16.983 & & 10.551 & 20.359 & 21.590 & & 13.440 & & 28.745 & 41.332 \\
\hline $11 / 30 / 110: 00$ & 16.999 & & 10.563 & 20.371 & 21.597 & & 13.442 & & 28.742 & 41.322 \\
\hline $11 / 30 / 114: 00$ & 17.004 & & 10.570 & 20.376 & 21.604 & & 13.432 & & 28.737 & 41.317 \\
\hline $11 / 30 / 118: 00$ & 17.002 & & 10.577 & 20.378 & 21.608 & & 13.425 & & 28.737 & 41.301 \\
\hline $11 / 30 / 11$ 12:00 & 17.004 & & 10.584 & 20.380 & 21.615 & & 13.416 & & 28.733 & 41.291 \\
\hline $11 / 30 / 11$ 16:00 & 16.985 & & 10.589 & 20.378 & 21.623 & & 13.383 & & 28.728 & 41.282 \\
\hline $11 / 30 / 1120: 00$ & 16.976 & & 10.593 & 20.376 & 21.632 & & 13.388 & & 28.723 & 41.273 \\
\hline $12 / 1 / 110: 00$ & 16.997 & & 10.603 & 20.373 & 21.634 & & 13.425 & & 28.721 & 41.261 \\
\hline $12 / 1 / 114: 00$ & 17.021 & & 10.615 & 20.378 & 21.641 & & 13.444 & & 28.719 & 41.254 \\
\hline $12 / 1 / 118: 00$ & 17.056 & & 10.631 & 20.383 & 21.649 & & 13.484 & & 28.719 & 41.242 \\
\hline $12 / 1 / 1112: 00$ & 17.096 & & 10.648 & 20.392 & 21.658 & & 13.522 & & 28.716 & 41.233 \\
\hline $12 / 1 / 1116: 00$ & 17.136 & & 10.667 & 20.401 & 21.663 & & 13.552 & & 28.714 & 41.223 \\
\hline $12 / 1 / 1120: 00$ & 17.186 & & 10.688 & 20.418 & 21.670 & & 13.599 & & 28.714 & 41.214 \\
\hline $12 / 2 / 110: 00$ & 17.228 & & 10.707 & 20.434 & 21.677 & & 13.627 & & 28.714 & 41.204 \\
\hline $12 / 2 / 114: 00$ & 17.256 & & 10.721 & 20.453 & 21.684 & & 13.627 & & 28.712 & 41.195 \\
\hline $12 / 2 / 118: 00$ & 17.268 & & 10.735 & 20.464 & 21.691 & & 13.625 & & 28.712 & 41.183 \\
\hline $12 / 2 / 1112: 00$ & 17.277 & & 10.747 & 20.476 & 21.696 & & 13.613 & & & \\
\hline
\end{tabular}

a NA, data from indicated date and time until next reported value are missing because of logger electrical malfunction.

b $A B$, MoDOT well was plugged and abandoned on June 27, 2010. 
TABLE S1.2 Automatically recorded groundwater level data for shallow monitoring wells SB43S-SB49S, July 2010 to December 2011.

\begin{tabular}{|c|c|c|c|c|c|c|c|}
\hline \multirow[b]{2}{*}{ Date and Time } & \multicolumn{7}{|c|}{ Water Level (ft below reference point) } \\
\hline & SB43S & SB44S & SB45S & SB46S & SB47S & SB48S & SB49S \\
\hline 7/7/10 16:00 & 50.178 & Dry $^{\mathrm{a}}$ & & Dry $^{a}$ & & Dry $^{\mathrm{a}}$ & \\
\hline $7 / 7 / 1020: 00$ & 50.150 & & & & & & \\
\hline $7 / 8 / 100: 00$ & 50.116 & & & & & & \\
\hline $7 / 8 / 104: 00$ & 50.082 & & & & & & \\
\hline 7/8/10 8:00 & 50.054 & & & & & & \\
\hline 7/8/10 12:00 & 50.029 & & & & & & \\
\hline 7/8/10 16:00 & 50.001 & & & & & & \\
\hline $7 / 8 / 1020: 00$ & 49.972 & & & & & & \\
\hline 7/9/10 0:00 & 49.944 & & & & & & \\
\hline 7/9/10 4:00 & 49.914 & & & & & & \\
\hline 7/9/10 8:00 & 49.889 & & & & & & \\
\hline $7 / 9 / 1012: 00$ & 49.859 & & & & & & \\
\hline 7/9/10 16:00 & 49.827 & & & & & & \\
\hline $7 / 9 / 1020: 00$ & 49.797 & & & & & & \\
\hline 7/10/10 0:00 & 49.768 & & & & & & \\
\hline 7/10/10 4:00 & 49.738 & & & & & & \\
\hline 7/10/10 8:00 & 49.706 & & & & & & \\
\hline $7 / 10 / 1012: 00$ & 49.676 & & & & & & \\
\hline $7 / 10 / 1016: 00$ & 49.646 & & & & & & \\
\hline $7 / 10 / 10$ 20:00 & 49.614 & & & & & & \\
\hline 7/11/10 0:00 & 49.584 & & & & & & \\
\hline 7/11/10 4:00 & 49.554 & & & & & & \\
\hline 7/11/10 8:00 & 49.522 & & & & & & \\
\hline $7 / 11 / 1012: 00$ & 49.495 & & & & & & \\
\hline 7/11/10 16:00 & 49.465 & & 40.202 & & & & \\
\hline $7 / 11 / 1020: 00$ & 49.435 & & 40.139 & & 31.185 & & 30.572 \\
\hline $7 / 12 / 100: 00$ & 49.408 & & 40.081 & & 31.164 & & 30.504 \\
\hline $7 / 12 / 104: 00$ & 49.378 & & 40.023 & & 31.141 & & 30.434 \\
\hline $7 / 12 / 108: 00$ & 49.351 & & 39.965 & & 31.117 & & 30.362 \\
\hline $7 / 12 / 10$ 12:00 & 49.323 & & 39.902 & & 31.094 & & 30.299 \\
\hline $7 / 12 / 1016: 00$ & 49.293 & & 39.832 & & 31.071 & & 30.224 \\
\hline $7 / 12 / 1020: 00$ & 49.263 & & 39.769 & & 31.050 & & 30.156 \\
\hline 7/13/10 0:00 & 49.236 & & 39.702 & & 31.024 & & 30.089 \\
\hline 7/13/10 4:00 & 49.208 & & 39.637 & & 30.996 & & 30.016 \\
\hline 7/13/10 8:00 & 49.181 & & 39.571 & & 30.975 & & 29.951 \\
\hline $7 / 13 / 10$ 12:00 & 49.153 & & 39.504 & & 30.949 & & 29.879 \\
\hline 7/13/10 16:00 & 49.124 & & 39.427 & & 30.930 & & 29.813 \\
\hline $7 / 13 / 1020: 00$ & 49.094 & & 39.339 & & 30.902 & & 29.743 \\
\hline $7 / 14 / 100: 00$ & 49.069 & & 39.274 & & 30.879 & & 29.678 \\
\hline 7/14/10 4:00 & 49.039 & & 39.211 & & 30.858 & & 29.606 \\
\hline 7/14/10 8:00 & 49.011 & & 39.148 & & 30.835 & & 29.536 \\
\hline $7 / 14 / 10$ 12:00 & 48.984 & & 39.085 & & 30.814 & & 29.463 \\
\hline $7 / 14 / 1016: 00$ & 48.954 & & 39.022 & & 30.790 & & 29.391 \\
\hline $7 / 14 / 1020: 00$ & 48.924 & & 38.960 & & 30.767 & & 29.321 \\
\hline 7/15/10 0:00 & 48.899 & & 38.906 & & 30.746 & & 29.260 \\
\hline 7/15/10 4:00 & 48.869 & & 38.846 & & 30.723 & & 29.185 \\
\hline $7 / 15 / 108: 00$ & 48.839 & & 38.785 & & 30.699 & & 29.118 \\
\hline $7 / 15 / 1012: 00$ & 48.812 & & 38.725 & & 30.678 & & 29.052 \\
\hline $7 / 15 / 1016: 00$ & 48.782 & & 38.659 & & 30.657 & & 28.982 \\
\hline $7 / 15 / 1020: 00$ & 48.750 & & 38.592 & & 30.634 & & 28.912 \\
\hline 7/16/10 0:00 & 48.723 & & 38.536 & & 30.613 & & 28.840 \\
\hline
\end{tabular}

TABLE S1.2 Page 1 of 58 


\begin{tabular}{|c|c|c|c|c|c|c|c|}
\hline \multirow[b]{2}{*}{ Date and Time } & \multicolumn{7}{|c|}{ Water Level (ft below reference point) } \\
\hline & SB43S & SB44S & SB45S & SB46S & SB47S & SB48S & SB49S \\
\hline $7 / 16 / 104: 00$ & 48.693 & & 38.478 & & 30.592 & & 28.777 \\
\hline 7/16/10 8:00 & 48.661 & & 38.417 & & 30.571 & & 28.707 \\
\hline 7/16/10 12:00 & 48.629 & & 38.359 & & 30.550 & & 28.637 \\
\hline 7/16/10 16:00 & 48.599 & & 38.296 & & 30.529 & & 28.567 \\
\hline $7 / 16 / 10$ 20:00 & 48.567 & & 38.241 & & 30.508 & & 28.485 \\
\hline 7/17/10 0:00 & 48.535 & & 38.180 & & 30.489 & & 28.371 \\
\hline 7/17/10 4:00 & 48.505 & & 38.124 & & 30.468 & & 28.254 \\
\hline 7/17/10 8:00 & 48.471 & & 38.066 & & 30.449 & & 28.130 \\
\hline $7 / 17 / 10$ 12:00 & 48.443 & & 38.010 & & 30.426 & & 28.016 \\
\hline 7/17/10 16:00 & 48.409 & & 37.954 & & 30.407 & & 27.890 \\
\hline $7 / 17 / 10$ 20:00 & 48.377 & & 37.894 & & 30.386 & & 27.766 \\
\hline 7/18/10 0:00 & 48.344 & & 37.838 & & 30.365 & & 27.654 \\
\hline $7 / 18 / 104: 00$ & 48.317 & & 37.782 & & 30.349 & & 27.542 \\
\hline 7/18/10 8:00 & 48.285 & & 37.722 & & 30.328 & & 27.437 \\
\hline 7/18/10 12:00 & 48.253 & & 37.664 & & 30.309 & & 27.323 \\
\hline $7 / 18 / 10$ 16:00 & 48.221 & & 37.605 & & 30.290 & & 27.213 \\
\hline 7/18/10 20:00 & 48.189 & & 37.543 & & 30.269 & & 27.096 \\
\hline 7/19/10 0:00 & 48.159 & & 37.484 & & 30.258 & & 26.986 \\
\hline 7/19/10 4:00 & 48.127 & & 37.422 & & 30.239 & & 26.870 \\
\hline 7/19/10 8:00 & 48.097 & & 37.335 & & 30.225 & & 26.755 \\
\hline $7 / 19 / 10$ 12:00 & 48.067 & & 37.231 & & 30.211 & & 26.650 \\
\hline 7/19/10 16:00 & 48.033 & & 37.114 & & 30.192 & & 26.534 \\
\hline $7 / 19 / 10$ 20:00 & 48.001 & & 37.003 & & 30.173 & & 26.417 \\
\hline 7/20/10 0:00 & 47.971 & & 36.900 & & 30.157 & & 26.312 \\
\hline $7 / 20 / 104: 00$ & 47.941 & & 36.800 & & 30.138 & & 26.214 \\
\hline 7/20/10 8:00 & 47.911 & & 36.705 & & 30.122 & & 26.116 \\
\hline 7/20/10 12:00 & 47.884 & & 36.621 & & 30.103 & & 26.029 \\
\hline $7 / 20 / 10$ 16:00 & 47.852 & & 36.512 & & 30.082 & & 25.922 \\
\hline $7 / 20 / 10$ 20:00 & 47.822 & & 36.414 & & 30.061 & & 25.824 \\
\hline 7/21/10 0:00 & 47.792 & & 36.316 & & 30.043 & & 25.726 \\
\hline $7 / 21 / 104: 00$ & 47.765 & & 36.219 & & 30.022 & & 25.626 \\
\hline 7/21/10 8:00 & 47.735 & & 36.126 & & 30.003 & & 25.534 \\
\hline $7 / 21 / 10$ 12:00 & 47.705 & & 36.030 & & 29.984 & & 25.434 \\
\hline $7 / 21 / 10$ 16:00 & 47.673 & & 35.916 & & 29.963 & & 25.324 \\
\hline $7 / 21 / 10$ 20:00 & 47.641 & & 35.802 & & 29.945 & & 25.215 \\
\hline 7/22/10 0:00 & 47.611 & & 35.700 & & 29.924 & & 25.117 \\
\hline $7 / 22 / 104: 00$ & 47.579 & & 35.588 & & 29.905 & & 25.005 \\
\hline 7/22/10 8:00 & 47.549 & & 35.493 & & 29.886 & & 24.911 \\
\hline $7 / 22 / 10$ 12:00 & 47.517 & & 35.383 & & 29.867 & & 24.804 \\
\hline $7 / 22 / 10$ 16:00 & 47.485 & & 35.274 & & 29.844 & & 24.699 \\
\hline $7 / 22 / 10$ 20:00 & 47.453 & & 35.172 & & 29.825 & & 24.598 \\
\hline 7/23/10 0:00 & 47.423 & & 35.078 & & 29.807 & & 24.503 \\
\hline $7 / 23 / 104: 00$ & 47.391 & & 34.978 & & 29.786 & & 24.409 \\
\hline 7/23/10 8:00 & 47.361 & & 34.892 & & 29.769 & & 24.328 \\
\hline $7 / 23 / 10$ 12:00 & 47.329 & & 34.797 & & 29.751 & & 24.237 \\
\hline 7/23/10 16:00 & 47.295 & & 34.697 & & 29.732 & & 24.138 \\
\hline $7 / 23 / 10$ 20:00 & 47.260 & & 34.597 & & 29.716 & & 24.043 \\
\hline 7/24/10 0:00 & 47.226 & & 34.508 & & 29.695 & & 23.957 \\
\hline $7 / 24 / 104: 00$ & 47.189 & & 34.406 & & 29.676 & & 23.861 \\
\hline 7/24/10 8:00 & 47.146 & & 34.322 & & 29.657 & & 23.777 \\
\hline $7 / 24 / 10$ 12:00 & 47.096 & & 34.236 & & 29.641 & & 23.697 \\
\hline $7 / 24 / 10$ 16:00 & 47.059 & & 34.159 & & 29.624 & & 23.620 \\
\hline $7 / 24 / 10$ 20:00 & 47.022 & & 34.059 & & 29.603 & & 23.525 \\
\hline 7/25/10 0:00 & 46.992 & & 33.992 & & 29.587 & & 23.462 \\
\hline
\end{tabular}

TABLE S1.2 Page 2 of 58 


\begin{tabular}{|c|c|c|c|c|c|c|c|}
\hline \multirow[b]{2}{*}{ Date and Time } & \multicolumn{7}{|c|}{ Water Level (ft below reference point) } \\
\hline & SB43S & SB44S & SB45S & SB46S & SB47S & SB48S & SB49S \\
\hline $7 / 25 / 104: 00$ & 46.958 & & 33.887 & & 29.568 & & 23.366 \\
\hline 7/25/10 8:00 & 46.928 & & 33.810 & & 29.552 & & 23.289 \\
\hline $7 / 25 / 10$ 12:00 & 46.898 & & 33.722 & & 29.533 & & 23.200 \\
\hline $7 / 25 / 10$ 16:00 & 46.866 & & 33.624 & & 29.515 & & 23.114 \\
\hline $7 / 25 / 1020: 00$ & 46.834 & & 33.527 & & 29.496 & & 23.018 \\
\hline 7/26/10 0:00 & 46.804 & & 33.445 & & 29.477 & & 22.941 \\
\hline $7 / 26 / 104: 00$ & 46.772 & & 33.345 & & 29.459 & & 22.845 \\
\hline 7/26/10 8:00 & 46.742 & & 33.261 & & 29.442 & & 22.766 \\
\hline $7 / 26 / 10$ 12:00 & 46.713 & & 33.171 & & 29.424 & & 22.680 \\
\hline $7 / 26 / 10$ 16:00 & 46.681 & & 33.068 & & 29.403 & & 22.584 \\
\hline $7 / 26 / 1020: 00$ & 46.646 & & 32.975 & & 29.384 & & 22.493 \\
\hline 7/27/10 0:00 & 46.619 & & 32.894 & & 29.367 & & 22.418 \\
\hline $7 / 27 / 104: 00$ & 46.587 & & 32.803 & & 29.349 & & 22.332 \\
\hline 7/27/10 8:00 & 46.557 & & 32.717 & & 29.328 & & 22.252 \\
\hline $7 / 27 / 10$ 12:00 & 46.525 & & 32.631 & & 29.314 & & 22.171 \\
\hline $7 / 27 / 10$ 16:00 & 46.493 & & 32.540 & & 29.295 & & 22.084 \\
\hline $7 / 27 / 10$ 20:00 & 46.461 & & 32.447 & & 29.276 & & 22.000 \\
\hline 7/28/10 0:00 & 46.435 & & 32.375 & & 29.260 & & 21.926 \\
\hline $7 / 28 / 104: 00$ & 46.403 & & 32.289 & & 29.241 & & 21.849 \\
\hline 7/28/10 8:00 & 46.374 & & 32.214 & & 29.225 & & 21.781 \\
\hline $7 / 28 / 10$ 12:00 & 46.346 & & 32.130 & & 29.206 & & 21.702 \\
\hline 7/28/10 16:00 & 46.316 & & 32.047 & & 29.190 & & 21.622 \\
\hline $7 / 28 / 10$ 20:00 & 46.286 & & 31.963 & & 29.174 & & 21.545 \\
\hline 7/29/10 0:00 & 46.259 & & 31.893 & & 29.160 & & 21.480 \\
\hline $7 / 29 / 104: 00$ & 46.229 & & 31.814 & & 29.141 & & 21.407 \\
\hline 7/29/10 8:00 & 46.202 & & 31.735 & & 29.124 & & 21.333 \\
\hline 7/29/10 12:00 & 46.174 & & 31.654 & & 29.106 & & 21.256 \\
\hline $7 / 29 / 10$ 16:00 & 46.142 & & 31.565 & & 29.092 & & 21.174 \\
\hline $7 / 29 / 10$ 20:00 & 46.110 & & 31.474 & & 29.073 & & 21.090 \\
\hline 7/30/10 0:00 & 46.082 & & 31.395 & & 29.054 & & 21.020 \\
\hline $7 / 30 / 104: 00$ & 46.053 & & 31.304 & & 29.036 & & 20.936 \\
\hline 7/30/10 8:00 & 46.021 & & 31.221 & & 29.024 & & 20.861 \\
\hline $7 / 30 / 10$ 12:00 & 45.991 & & 31.135 & & 29.003 & & 20.777 \\
\hline 7/30/10 16:00 & 45.961 & & 31.048 & & 28.984 & & 20.702 \\
\hline 7/30/10 20:00 & 45.931 & & 30.965 & & 28.970 & & 20.625 \\
\hline 7/31/10 0:00 & 45.901 & & 30.893 & & 28.954 & & 20.555 \\
\hline $7 / 31 / 104: 00$ & 45.876 & & 30.818 & & 28.938 & & 20.485 \\
\hline 7/31/10 8:00 & 45.849 & & 30.751 & & 28.921 & & 20.422 \\
\hline 7/31/10 12:00 & 45.819 & & 30.676 & & 28.907 & & 20.362 \\
\hline 7/31/10 16:00 & 45.791 & & 30.599 & & 28.893 & & 20.287 \\
\hline 7/31/10 20:00 & 45.759 & & 30.518 & & 28.874 & & 20.214 \\
\hline 8/1/10 0:00 & 45.734 & & 30.453 & & 28.858 & & 20.151 \\
\hline 8/1/10 4:00 & 45.704 & & 30.378 & & 28.842 & & 20.084 \\
\hline 8/1/10 8:00 & 45.677 & & 30.309 & & 28.825 & & 20.023 \\
\hline 8/1/10 12:00 & 45.647 & & 30.234 & & 28.811 & & 19.960 \\
\hline 8/1/10 16:00 & 45.617 & & 30.155 & & 28.795 & & 19.881 \\
\hline 8/1/10 20:00 & 45.587 & & 30.076 & & 28.779 & & 19.813 \\
\hline 8/2/10 0:00 & 45.560 & & 30.006 & & 28.762 & & 19.748 \\
\hline 8/2/10 4:00 & 45.530 & & 29.929 & & 28.746 & & 19.682 \\
\hline 8/2/10 8:00 & 45.500 & & 29.855 & & 28.730 & & 19.617 \\
\hline 8/2/10 12:00 & 45.473 & & 29.787 & & 28.716 & & 19.547 \\
\hline 8/2/10 16:00 & 45.443 & & 29.706 & & 28.697 & & 19.479 \\
\hline 8/2/10 20:00 & 45.416 & & 29.634 & & 28.683 & & 19.411 \\
\hline 8/3/10 0:00 & 45.388 & & 29.566 & & 28.667 & & 19.353 \\
\hline
\end{tabular}

TABLE S1.2 Page 3 of 58 


\begin{tabular}{|c|c|c|c|c|c|c|c|}
\hline \multirow[b]{2}{*}{ Date and Time } & \multicolumn{7}{|c|}{ Water Level (ft below reference point) } \\
\hline & SB43S & SB44S & SB45S & SB46S & SB47S & SB48S & SB49S \\
\hline 8/3/10 4:00 & 45.358 & & 29.492 & & 28.650 & & 19.285 \\
\hline 8/3/10 8:00 & 45.333 & & 29.431 & & 28.639 & & 19.229 \\
\hline 8/3/10 12:00 & 45.308 & & 29.369 & & 28.620 & & 19.171 \\
\hline 8/3/10 16:00 & 45.278 & & 29.294 & & 28.608 & & 19.103 \\
\hline 8/3/10 20:00 & 45.244 & & 29.222 & & 28.589 & & 19.040 \\
\hline 8/4/10 0:00 & 45.216 & & 29.155 & & 28.575 & & 18.984 \\
\hline 8/4/10 4:00 & 45.191 & & 29.089 & & 28.559 & & 18.917 \\
\hline 8/4/10 8:00 & 45.166 & & 29.027 & & 28.545 & & 18.865 \\
\hline $8 / 4 / 10$ 12:00 & 45.138 & & 28.966 & & 28.531 & & 18.809 \\
\hline 8/4/10 16:00 & 45.113 & & 28.899 & & 28.512 & & 18.751 \\
\hline 8/4/10 20:00 & 45.086 & & 28.829 & & 28.501 & & 18.688 \\
\hline 8/5/10 0:00 & 45.060 & & 28.768 & & 28.482 & & 18.632 \\
\hline 8/5/10 4:00 & 45.028 & & 28.701 & & 28.470 & & 18.574 \\
\hline 8/5/10 8:00 & 45.008 & & 28.645 & & 28.454 & & 18.520 \\
\hline 8/5/10 12:00 & 44.980 & & 28.584 & & 28.440 & & 18.471 \\
\hline 8/5/10 16:00 & 44.927 & & 28.510 & & 28.424 & & 18.401 \\
\hline 8/5/10 20:00 & 44.877 & & 28.440 & & 28.407 & & 18.340 \\
\hline 8/6/10 0:00 & 44.838 & & 28.384 & & 28.393 & & 18.289 \\
\hline 8/6/10 4:00 & 44.788 & & 28.319 & & 28.379 & & 18.230 \\
\hline 8/6/10 8:00 & 44.751 & & 28.263 & & 28.363 & & 18.184 \\
\hline 8/6/10 12:00 & 44.705 & & 28.203 & & 28.349 & & 18.135 \\
\hline 8/6/10 16:00 & 44.646 & & 28.131 & & 28.332 & & 18.065 \\
\hline 8/6/10 20:00 & 44.588 & & 28.063 & & 28.316 & & 18.004 \\
\hline 8/7/10 0:00 & 44.547 & & 28.005 & & 28.302 & & 17.950 \\
\hline 8/7/10 4:00 & 44.494 & & 27.940 & & 28.286 & & 17.894 \\
\hline 8/7/10 8:00 & 44.446 & & 27.879 & & 28.274 & & 17.843 \\
\hline 8/7/10 12:00 & 44.396 & & 27.814 & & 28.258 & & 17.782 \\
\hline 8/7/10 16:00 & 44.336 & & 27.747 & & 28.241 & & 17.724 \\
\hline 8/7/10 20:00 & 44.284 & & 27.679 & & 28.225 & & 17.665 \\
\hline 8/8/10 0:00 & 44.242 & & 27.626 & & 28.213 & & 17.616 \\
\hline 8/8/10 4:00 & 44.192 & & 27.561 & & 28.197 & & 17.565 \\
\hline 8/8/10 8:00 & 44.144 & & 27.500 & & 28.183 & & 17.511 \\
\hline 8/8/10 12:00 & 44.098 & & 27.442 & & 28.169 & & 17.458 \\
\hline 8/8/10 16:00 & 44.043 & & 27.379 & & 28.157 & & 17.404 \\
\hline 8/8/10 20:00 & 43.992 & & 27.319 & & 28.139 & & 17.348 \\
\hline 8/9/10 0:00 & 43.954 & & 27.265 & & 28.127 & & 17.306 \\
\hline 8/9/10 4:00 & 43.910 & & 27.212 & & 28.113 & & 17.257 \\
\hline 8/9/10 8:00 & 43.880 & & 27.165 & & 28.099 & & 17.217 \\
\hline 8/9/10 12:00 & 43.834 & & 27.109 & & 28.087 & & 17.166 \\
\hline 8/9/10 16:00 & 43.779 & & 27.046 & & 28.071 & & 17.112 \\
\hline 8/9/10 20:00 & 43.731 & & 26.991 & & 28.057 & & 17.063 \\
\hline 8/10/10 0:00 & 43.699 & & 26.946 & & 28.045 & & 17.019 \\
\hline 8/10/10 4:00 & 43.651 & & 26.888 & & 28.031 & & 16.972 \\
\hline 8/10/10 8:00 & 43.607 & & 26.837 & & 28.017 & & 16.928 \\
\hline 8/10/10 12:00 & 43.566 & & 26.784 & & 28.003 & & 16.881 \\
\hline 8/10/10 16:00 & 43.511 & & 26.723 & & 27.991 & & 16.825 \\
\hline 8/10/10 20:00 & 43.461 & & 26.665 & & 27.975 & & 16.776 \\
\hline 8/11/10 0:00 & 43.424 & & 26.618 & & 27.963 & & 16.736 \\
\hline 8/11/10 4:00 & 43.381 & & 26.565 & & 27.949 & & 16.687 \\
\hline 8/11/10 8:00 & 43.339 & & 26.516 & & 27.933 & & 16.648 \\
\hline 8/11/10 12:00 & 43.296 & & 26.465 & & 27.921 & & 16.601 \\
\hline 8/11/10 16:00 & 43.243 & & 26.407 & & 27.907 & & 16.550 \\
\hline 8/11/10 20:00 & 43.195 & & 26.351 & & 27.893 & & 16.501 \\
\hline 8/12/10 0:00 & 43.154 & & 26.304 & & 27.879 & & 16.456 \\
\hline
\end{tabular}

TABLE S1.2 Page 4 of 58 


\begin{tabular}{|c|c|c|c|c|c|c|c|}
\hline \multirow[b]{2}{*}{ Date and Time } & \multicolumn{7}{|c|}{ Water Level (ft below reference point) } \\
\hline & SB43S & SB44S & SB45S & SB46S & SB47S & SB48S & SB49S \\
\hline $8 / 12 / 104: 00$ & 43.108 & & 26.248 & & 27.863 & & 16.412 \\
\hline 8/12/10 8:00 & 43.064 & & 26.200 & & 27.851 & & 16.368 \\
\hline $8 / 12 / 1012: 00$ & 43.016 & & 26.146 & & 27.837 & & 16.321 \\
\hline $8 / 12 / 1016: 00$ & 42.959 & & 26.085 & & 27.823 & & 16.267 \\
\hline $8 / 12 / 1020: 00$ & 42.904 & & 26.030 & & 27.807 & & 16.218 \\
\hline 8/13/10 0:00 & 42.865 & & 25.981 & & 27.797 & & 16.174 \\
\hline $8 / 13 / 104: 00$ & 42.815 & & 25.927 & & 27.781 & & 16.127 \\
\hline 8/13/10 8:00 & 42.771 & & 25.878 & & 27.772 & & 16.085 \\
\hline $8 / 13 / 10$ 12:00 & 42.725 & & 25.827 & & 27.755 & & 16.041 \\
\hline 8/13/10 16:00 & 42.675 & & 25.774 & & 27.744 & & 15.994 \\
\hline $8 / 13 / 10$ 20:00 & 42.634 & & 25.727 & & 27.727 & & 15.950 \\
\hline 8/14/10 0:00 & 42.611 & & 25.692 & & 27.718 & & 15.922 \\
\hline 8/14/10 4:00 & 42.567 & & 25.646 & & 27.709 & & 15.882 \\
\hline 8/14/10 8:00 & 42.533 & & 25.602 & & 27.695 & & 15.845 \\
\hline 8/14/10 12:00 & 42.494 & & 25.557 & & 27.681 & & 15.805 \\
\hline $8 / 14 / 10$ 16:00 & 42.455 & & 25.513 & & 27.669 & & 15.763 \\
\hline 8/14/10 20:00 & 42.418 & & 25.469 & & 27.657 & & 15.726 \\
\hline 8/15/10 0:00 & 42.386 & & 25.429 & & 27.636 & & 15.691 \\
\hline 8/15/10 4:00 & 42.359 & & 25.392 & & 27.627 & & 15.660 \\
\hline 8/15/10 8:00 & 42.322 & & 25.350 & & 27.615 & & 15.623 \\
\hline 8/15/10 12:00 & 42.285 & & 25.306 & & 27.599 & & 15.583 \\
\hline 8/15/10 16:00 & 42.239 & & 25.259 & & 27.585 & & 15.539 \\
\hline 8/15/10 20:00 & 42.194 & & 25.211 & & 27.573 & & 15.494 \\
\hline 8/16/10 0:00 & 42.159 & & 25.171 & & 27.561 & & 15.459 \\
\hline $8 / 16 / 104: 00$ & 42.122 & & 25.127 & & 27.547 & & 15.424 \\
\hline 8/16/10 8:00 & 42.081 & & 25.085 & & 27.533 & & 15.382 \\
\hline 8/16/10 12:00 & 42.038 & & 25.036 & & 27.519 & & 15.338 \\
\hline 8/16/10 16:00 & 41.985 & & 24.985 & & 27.505 & & 15.294 \\
\hline 8/16/10 20:00 & 41.932 & & 24.931 & & 27.489 & & 15.242 \\
\hline 8/17/10 0:00 & 41.900 & & 24.897 & & 27.477 & & 15.210 \\
\hline $8 / 17 / 104: 00$ & 41.854 & & 24.848 & & 27.463 & & 15.168 \\
\hline 8/17/10 8:00 & 41.813 & & 24.806 & & 27.449 & & 15.126 \\
\hline 8/17/10 12:00 & 41.772 & & 24.764 & & 27.435 & & 15.086 \\
\hline 8/17/10 16:00 & 41.726 & & 24.715 & & 27.421 & & 15.046 \\
\hline 8/17/10 20:00 & 41.680 & & 24.668 & & 27.405 & & 15.002 \\
\hline 8/18/10 0:00 & 41.644 & & 24.629 & & 27.396 & & 14.967 \\
\hline 8/18/10 4:00 & 41.600 & & 24.585 & & 27.379 & & 14.918 \\
\hline 8/18/10 8:00 & 41.559 & & 24.541 & & 27.365 & & 14.885 \\
\hline 8/18/10 12:00 & 41.515 & & 24.499 & & 27.351 & & 14.846 \\
\hline 8/18/10 16:00 & 41.465 & & 24.447 & & 27.333 & & 14.801 \\
\hline 8/18/10 20:00 & 41.414 & & 24.401 & & 27.316 & & 14.755 \\
\hline 8/19/10 0:00 & 41.378 & & 24.361 & & 27.304 & & 14.717 \\
\hline 8/19/10 4:00 & 41.332 & & 24.315 & & 27.286 & & 14.680 \\
\hline 8/19/10 8:00 & 41.291 & & 24.273 & & 27.272 & & 14.640 \\
\hline 8/19/10 12:00 & 41.249 & & 24.233 & & 27.253 & & 14.605 \\
\hline 8/19/10 16:00 & 41.197 & & 24.182 & & 27.234 & & 14.554 \\
\hline 8/19/10 20:00 & 41.146 & & 24.138 & & 27.218 & & 14.512 \\
\hline 8/20/10 0:00 & 41.110 & & 24.098 & & 27.199 & & 14.477 \\
\hline $8 / 20 / 104: 00$ & 41.061 & & 24.054 & & 27.178 & & 14.442 \\
\hline 8/20/10 8:00 & 41.020 & & 24.015 & & 27.162 & & 14.400 \\
\hline 8/20/10 12:00 & 40.974 & 17.010 & 23.973 & 12.080 & 27.146 & & 14.285 \\
\hline 8/20/10 16:00 & 40.933 & 16.997 & 23.936 & 10.504 & 27.190 & 20.700 & 14.248 \\
\hline 8/20/10 20:00 & 40.885 & 16.699 & 23.889 & 10.250 & 27.092 & 20.645 & 14.204 \\
\hline 8/21/10 0:00 & 40.855 & 16.447 & 23.852 & 10.299 & 27.076 & 20.608 & 14.176 \\
\hline
\end{tabular}

TABLE S1.2 Page 5 of 58 


\begin{tabular}{|c|c|c|c|c|c|c|c|}
\hline \multirow[b]{2}{*}{ Date and Time } & \multicolumn{7}{|c|}{ Water Level (ft below reference point) } \\
\hline & SB43S & SB44S & SB45S & SB46S & SB47S & SB48S & SB49S \\
\hline $8 / 21 / 104: 00$ & 40.821 & 16.368 & 23.821 & 10.104 & 27.059 & 20.575 & 14.146 \\
\hline 8/21/10 8:00 & 40.791 & 16.380 & 23.789 & 9.925 & 27.043 & 20.545 & 14.117 \\
\hline $8 / 21 / 1012: 00$ & 40.766 & 16.438 & 23.761 & 10.232 & 27.031 & 20.512 & 14.089 \\
\hline $8 / 21 / 10$ 16:00 & 40.727 & 16.480 & 23.724 & 10.587 & 27.015 & 20.475 & 14.054 \\
\hline $8 / 21 / 1020: 00$ & 40.688 & 16.512 & 23.684 & 10.313 & 27.001 & 20.445 & 14.019 \\
\hline $8 / 22 / 100: 00$ & 40.663 & 16.573 & 23.656 & 10.336 & 26.987 & 20.410 & 13.989 \\
\hline $8 / 22 / 104: 00$ & 40.621 & 16.610 & 23.619 & 10.355 & 26.970 & 20.372 & 13.952 \\
\hline $8 / 22 / 108: 00$ & 40.592 & 16.661 & 23.586 & 10.290 & 26.963 & 20.342 & 13.926 \\
\hline $8 / 22 / 1012: 00$ & 40.555 & 16.720 & 23.556 & 10.343 & 26.947 & 20.305 & 13.900 \\
\hline $8 / 22 / 1016: 00$ & 40.509 & 16.743 & 23.510 & 10.360 & 26.933 & 20.265 & 13.851 \\
\hline $8 / 22 / 1020: 00$ & 40.466 & 16.757 & 23.468 & 10.376 & 26.919 & 20.225 & 13.812 \\
\hline 8/23/10 0:00 & 40.429 & 16.792 & 23.435 & 10.395 & 26.905 & 20.190 & 13.779 \\
\hline $8 / 23 / 104: 00$ & 40.385 & 16.827 & 23.398 & 10.413 & 26.891 & 20.153 & 13.742 \\
\hline 8/23/10 8:00 & 40.351 & 16.859 & 23.363 & 10.432 & 26.875 & 20.120 & 13.709 \\
\hline $8 / 23 / 10$ 12:00 & 40.310 & 16.899 & 23.326 & 10.448 & 26.861 & 20.081 & 13.671 \\
\hline 8/23/10 16:00 & 40.262 & 16.918 & 23.284 & 10.460 & 26.849 & 20.041 & 13.634 \\
\hline 8/23/10 20:00 & 40.218 & 16.936 & 23.244 & 10.478 & 26.833 & 20.006 & 13.594 \\
\hline 8/24/10 0:00 & 40.188 & 16.983 & 23.214 & 10.499 & 26.819 & 19.973 & 13.564 \\
\hline $8 / 24 / 104: 00$ & 40.158 & 17.046 & 23.186 & 10.522 & 26.809 & 19.947 & 13.539 \\
\hline 8/24/10 8:00 & 40.129 & 17.127 & 23.156 & 10.543 & 26.795 & 19.917 & 13.511 \\
\hline $8 / 24 / 1012: 00$ & 41.020 & 17.556 & 24.068 & 11.569 & 27.351 & 20.904 & 14.454 \\
\hline $8 / 24 / 10$ 16:00 & 40.977 & 17.339 & 24.022 & 11.578 & 27.335 & 20.860 & 14.412 \\
\hline $8 / 24 / 1020: 00$ & 44.533 & 20.518 & 23.984 & 11.594 & 27.318 & 20.827 & 14.379 \\
\hline 8/25/10 0:00 & 44.487 & 18.506 & 23.949 & 11.613 & 27.302 & 20.790 & 14.341 \\
\hline $8 / 25 / 104: 00$ & 44.439 & 17.770 & 23.908 & 11.627 & 27.286 & 20.755 & 14.306 \\
\hline 8/25/10 8:00 & 44.400 & 17.560 & 23.875 & 11.646 & 27.269 & 20.722 & 14.271 \\
\hline $8 / 25 / 10$ 12:00 & 44.357 & 17.535 & 29.082 & & 31.486 & 27.161 & 18.975 \\
\hline 8/25/10 16:00 & 44.297 & 17.519 & 29.006 & 28.134 & 31.402 & 27.121 & 18.903 \\
\hline $8 / 25 / 1020: 00$ & 44.244 & 17.523 & 28.938 & 28.061 & 31.374 & 27.091 & 18.842 \\
\hline 8/26/10 0:00 & 44.203 & 17.556 & 28.875 & 28.003 & 31.360 & 27.063 & 18.786 \\
\hline $8 / 26 / 104: 00$ & 44.155 & 17.586 & 28.810 & 27.947 & 31.346 & 27.033 & 18.732 \\
\hline 8/26/10 8:00 & 44.116 & 17.628 & 28.752 & 27.899 & 31.335 & 27.005 & 18.674 \\
\hline $8 / 26 / 10$ 12:00 & 44.070 & 17.665 & 28.684 & 27.847 & 31.320 & 26.977 & 18.618 \\
\hline 8/26/10 16:00 & 44.009 & 17.665 & 28.610 & 27.787 & 31.306 & 26.946 & 18.550 \\
\hline 8/26/10 20:00 & 43.951 & 17.658 & 28.543 & 27.731 & 31.290 & 26.916 & 18.487 \\
\hline 8/27/10 0:00 & 43.908 & 17.684 & 28.477 & 27.680 & 31.276 & 26.888 & 18.431 \\
\hline $8 / 27 / 104: 00$ & 43.860 & 17.707 & 28.417 & 27.629 & 31.267 & 26.858 & 18.370 \\
\hline 8/27/10 8:00 & 43.818 & 17.737 & 28.359 & 27.580 & 31.253 & 26.830 & 18.319 \\
\hline $8 / 27 / 10$ 12:00 & 43.772 & 17.768 & 28.296 & 27.531 & 31.239 & 26.799 & 18.261 \\
\hline $8 / 27 / 10$ 16:00 & 43.711 & 17.768 & 28.224 & 27.475 & 31.225 & 26.769 & 18.198 \\
\hline $8 / 27 / 1020: 00$ & 43.656 & 17.761 & 28.159 & 27.422 & 31.208 & 26.743 & 18.135 \\
\hline 8/28/10 0:00 & 43.614 & 17.796 & 28.105 & 27.375 & 31.197 & 26.713 & 18.086 \\
\hline $8 / 28 / 104: 00$ & 43.571 & 17.826 & 28.045 & 27.324 & 31.183 & 26.685 & 18.032 \\
\hline 8/28/10 8:00 & 43.530 & 17.854 & 27.986 & 27.278 & 31.169 & 26.659 & 17.978 \\
\hline $8 / 28 / 10$ 12:00 & 43.484 & 17.889 & 27.928 & 27.229 & 31.157 & 26.629 & 17.927 \\
\hline 8/28/10 16:00 & 43.426 & 17.891 & 27.861 & 27.173 & 31.141 & 26.598 & 17.869 \\
\hline $8 / 28 / 1020: 00$ & 43.376 & 17.900 & 27.798 & 27.127 & 31.127 & 26.573 & 17.813 \\
\hline 8/29/10 0:00 & 43.342 & 17.945 & 27.751 & 27.087 & 31.115 & 26.545 & 17.768 \\
\hline $8 / 29 / 104: 00$ & 43.298 & 17.982 & 27.693 & 27.038 & 31.101 & 26.521 & 17.717 \\
\hline 8/29/10 8:00 & 43.264 & 18.028 & 27.644 & 26.999 & 31.087 & 26.493 & 17.675 \\
\hline $8 / 29 / 1012: 00$ & 43.222 & 18.070 & 27.591 & 26.954 & 31.075 & 26.465 & 17.624 \\
\hline 8/29/10 16:00 & 43.167 & 18.085 & 27.526 & 26.908 & 31.059 & 26.437 & 17.565 \\
\hline 8/29/10 20:00 & 43.126 & 18.105 & 27.472 & 26.866 & 31.047 & 26.414 & 17.518 \\
\hline 8/30/10 0:00 & 43.096 & 18.161 & 27.426 & 26.824 & 31.033 & 26.386 & 17.476 \\
\hline
\end{tabular}

TABLE S1.2 Page 6 of 58 


\begin{tabular}{|c|c|c|c|c|c|c|c|}
\hline \multirow[b]{2}{*}{ Date and Time } & \multicolumn{7}{|c|}{ Water Level (ft below reference point) } \\
\hline & SB43S & SB44S & SB45S & SB46S & SB47S & SB48S & SB49S \\
\hline 8/30/10 4:00 & 43.057 & 18.208 & 27.375 & 26.787 & 31.021 & 26.360 & 17.432 \\
\hline 8/30/10 8:00 & 43.025 & 18.257 & 27.326 & 26.747 & 31.007 & 26.335 & 17.390 \\
\hline 8/30/10 12:00 & 42.982 & 18.294 & 27.272 & 26.703 & 30.993 & 26.304 & 17.339 \\
\hline 8/30/10 16:00 & 42.924 & 18.289 & 27.209 & 26.655 & 30.979 & 26.278 & 17.285 \\
\hline 8/30/10 20:00 & 42.874 & 18.282 & 27.149 & 26.608 & 30.965 & 26.251 & 17.229 \\
\hline 8/31/10 0:00 & 42.835 & 18.310 & 27.100 & 26.566 & 30.954 & 26.227 & 17.182 \\
\hline 8/31/10 4:00 & 42.794 & 18.336 & 27.046 & 26.527 & 30.940 & 26.199 & 17.136 \\
\hline 8/31/10 8:00 & 42.753 & 18.366 & 26.995 & 24.275 & 30.926 & 26.174 & 17.094 \\
\hline 8/31/10 12:00 & 42.709 & 18.392 & 26.942 & 24.317 & 30.912 & 26.145 & 17.047 \\
\hline 8/31/10 16:00 & 42.656 & 18.401 & 26.883 & 24.217 & 30.898 & 26.118 & 16.991 \\
\hline 8/31/10 20:00 & 42.613 & 18.399 & 26.832 & 24.187 & 30.884 & 26.094 & 16.947 \\
\hline 9/1/10 0:00 & 42.592 & 18.415 & 26.795 & 18.906 & 30.872 & 26.068 & 16.914 \\
\hline 9/1/10 4:00 & 42.553 & 18.194 & 26.751 & 13.908 & 30.860 & 26.040 & 16.872 \\
\hline 9/1/10 8:00 & 42.507 & 17.824 & 26.693 & 10.748 & 30.844 & 26.015 & 16.818 \\
\hline 9/1/10 12:00 & 42.475 & 17.630 & 26.649 & 9.490 & 30.830 & 25.989 & 16.776 \\
\hline 9/1/10 16:00 & 42.414 & 17.544 & 26.579 & 9.778 & 30.816 & 25.959 & 16.720 \\
\hline 9/1/10 20:00 & 42.372 & 17.518 & 26.532 & 9.881 & 30.804 & 25.933 & 16.673 \\
\hline 9/2/10 0:00 & 42.336 & 17.549 & 26.481 & 9.822 & 30.790 & 25.903 & 16.631 \\
\hline 9/2/10 4:00 & 42.299 & 17.560 & 26.425 & 8.927 & 30.776 & 25.877 & 16.578 \\
\hline 9/2/10 8:00 & 42.248 & 17.502 & 26.376 & 7.620 & 30.760 & 25.849 & 16.531 \\
\hline 9/2/10 12:00 & 42.212 & 17.504 & 26.325 & 7.681 & 30.750 & 25.826 & 16.491 \\
\hline 9/2/10 16:00 & 42.191 & 17.546 & 26.320 & 7.692 & 30.736 & 25.800 & 16.456 \\
\hline 9/2/10 20:00 & 42.152 & 17.595 & 26.248 & 7.099 & 30.725 & 25.772 & 16.419 \\
\hline 9/3/10 0:00 & 42.134 & 17.668 & 26.260 & 6.971 & 30.713 & 25.746 & 16.386 \\
\hline 9/3/10 4:00 & 42.097 & 17.721 & 26.195 & 6.943 & 30.697 & 25.718 & 16.347 \\
\hline 9/3/10 8:00 & 42.061 & 17.768 & 26.130 & 6.922 & 30.687 & 25.690 & 16.300 \\
\hline 9/3/10 12:00 & 42.022 & 17.803 & 26.041 & 7.015 & 30.673 & 25.662 & 16.260 \\
\hline 9/3/10 16:00 & 41.971 & 17.814 & 25.948 & 7.295 & 30.657 & 25.634 & 16.207 \\
\hline 9/3/10 20:00 & 41.932 & 17.824 & 25.902 & 7.381 & 30.643 & 25.604 & 16.162 \\
\hline 9/4/10 0:00 & 41.896 & 17.865 & 25.857 & 7.262 & 30.629 & 25.578 & 16.120 \\
\hline 9/4/10 4:00 & 41.857 & 17.898 & 25.811 & 7.206 & 30.617 & 25.550 & 16.078 \\
\hline 9/4/10 8:00 & 41.820 & 17.931 & 25.769 & 7.171 & 30.606 & 25.522 & 16.032 \\
\hline 9/4/10 12:00 & 41.774 & 17.961 & 25.720 & 7.353 & 30.589 & 25.492 & 15.985 \\
\hline 9/4/10 16:00 & 41.715 & 17.931 & 25.648 & 7.797 & 30.573 & 25.462 & 15.924 \\
\hline 9/4/10 20:00 & 41.659 & 17.898 & 25.604 & 7.939 & 30.561 & 25.436 & 15.870 \\
\hline 9/5/10 0:00 & 41.618 & 17.914 & 25.580 & 7.818 & 30.545 & 25.412 & 15.826 \\
\hline 9/5/10 4:00 & 41.570 & 17.921 & 25.511 & 7.799 & 30.528 & 25.363 & 15.777 \\
\hline 9/5/10 8:00 & 41.522 & 17.921 & 25.448 & 7.802 & 30.519 & 25.317 & 15.728 \\
\hline 9/5/10 12:00 & 41.476 & 17.938 & 25.411 & 7.974 & 30.505 & 25.258 & 15.681 \\
\hline 9/5/10 16:00 & 41.414 & 17.900 & 25.341 & 7.892 & 30.486 & 25.205 & 15.623 \\
\hline 9/5/10 20:00 & 41.369 & 17.900 & 25.322 & 7.927 & 30.472 & 25.160 & 15.574 \\
\hline 9/6/10 0:00 & 41.336 & 17.956 & 25.273 & 7.967 & 30.461 & 25.118 & 15.541 \\
\hline 9/6/10 4:00 & 41.286 & 17.982 & 25.213 & 7.999 & 30.447 & 25.065 & 15.485 \\
\hline 9/6/10 8:00 & 41.242 & 18.001 & 25.166 & 8.029 & 30.433 & 25.020 & 15.443 \\
\hline 9/6/10 12:00 & 41.203 & 18.033 & 25.097 & 8.067 & 30.419 & 24.976 & 15.399 \\
\hline 9/6/10 16:00 & 41.162 & 18.073 & 25.073 & 8.102 & 30.405 & 24.945 & 15.364 \\
\hline 9/6/10 20:00 & 41.164 & 18.208 & 25.134 & 8.155 & 30.395 & 24.936 & 15.345 \\
\hline 9/7/10 0:00 & 41.153 & 18.380 & 25.108 & 8.202 & 30.384 & 24.901 & 15.324 \\
\hline 9/7/10 4:00 & 41.123 & 18.457 & 25.076 & 8.239 & 30.370 & 24.866 & 15.289 \\
\hline 9/7/10 8:00 & 41.098 & 18.520 & 25.073 & 8.281 & 30.360 & 24.838 & 15.254 \\
\hline 9/7/10 12:00 & 41.068 & 18.594 & 25.008 & 8.320 & 30.349 & 24.794 & 15.222 \\
\hline 9/7/10 16:00 & 41.027 & 18.613 & 24.875 & 8.360 & 30.332 & 24.749 & 15.177 \\
\hline 9/7/10 20:00 & 40.988 & 18.629 & 24.831 & 8.397 & 30.321 & 24.710 & 15.140 \\
\hline 9/8/10 0:00 & 40.951 & 18.664 & 24.813 & 8.436 & 30.304 & 24.665 & 15.105 \\
\hline
\end{tabular}




\begin{tabular}{|c|c|c|c|c|c|c|c|}
\hline \multirow[b]{2}{*}{ Date and Time } & \multicolumn{7}{|c|}{ Water Level (ft below reference point) } \\
\hline & SB43S & SB44S & SB45S & SB46S & SB47S & SB48S & SB49S \\
\hline 9/8/10 4:00 & 40.912 & 18.683 & 24.782 & 8.471 & 30.293 & 24.626 & 15.068 \\
\hline 9/8/10 8:00 & 40.880 & 18.713 & 24.743 & 8.508 & 30.281 & 24.588 & 15.030 \\
\hline 9/8/10 12:00 & 40.839 & 18.746 & 24.708 & 8.543 & 30.267 & 24.539 & 14.990 \\
\hline 9/8/10 16:00 & 40.784 & 18.727 & 24.645 & 8.576 & 30.253 & 24.490 & 14.941 \\
\hline 9/8/10 20:00 & 40.738 & 18.713 & 24.610 & 8.611 & 30.236 & 24.446 & 14.897 \\
\hline 9/9/10 0:00 & 40.702 & 18.734 & 24.594 & 8.646 & 30.225 & 24.399 & 14.857 \\
\hline 9/9/10 4:00 & 40.656 & 18.737 & 24.580 & 8.681 & 30.211 & 24.355 & 14.815 \\
\hline 9/9/10 8:00 & 40.615 & 18.741 & 24.519 & 8.713 & 30.197 & 24.313 & 14.773 \\
\hline 9/9/10 12:00 & 40.573 & 18.755 & 24.408 & 8.746 & 30.185 & 24.261 & 14.734 \\
\hline 9/9/10 16:00 & 40.518 & 18.737 & 24.354 & 8.776 & 30.171 & 24.217 & 14.685 \\
\hline 9/9/10 20:00 & 40.479 & 18.743 & 24.326 & 8.811 & 30.157 & 24.178 & 14.650 \\
\hline 9/10/10 0:00 & 40.445 & 18.778 & 24.319 & 8.843 & 30.143 & 24.138 & 14.605 \\
\hline 9/10/10 4:00 & 40.404 & 18.802 & 24.264 & 8.455 & 30.131 & 24.091 & 14.570 \\
\hline 9/10/10 8:00 & 40.367 & 18.816 & 24.210 & 7.660 & 30.117 & 24.051 & 14.533 \\
\hline 9/10/10 12:00 & 40.326 & 18.830 & 24.170 & 7.634 & 30.103 & 24.007 & 14.496 \\
\hline 9/10/10 16:00 & 40.278 & 18.827 & 24.131 & 4.916 & 30.092 & 23.965 & 14.449 \\
\hline 9/10/10 20:00 & 40.243 & 18.853 & 24.119 & 2.723 & 30.078 & 23.937 & 14.419 \\
\hline 9/11/10 0:00 & 40.225 & 18.927 & 24.161 & 0.034 & 30.066 & 23.911 & 14.398 \\
\hline 9/11/10 4:00 & 40.207 & 19.023 & 24.129 & -0.035 & 30.054 & 23.879 & 14.374 \\
\hline 9/11/10 8:00 & 40.186 & 19.091 & 24.119 & 0.032 & 30.045 & 23.853 & 14.349 \\
\hline 9/11/10 12:00 & 40.158 & 19.160 & 24.059 & 0.118 & 30.033 & 23.816 & 14.314 \\
\hline 9/11/10 16:00 & 40.119 & 19.184 & 23.949 & 0.406 & 30.019 & 23.774 & 14.281 \\
\hline 9/11/10 20:00 & 40.085 & 19.209 & 23.917 & 0.527 & 30.007 & 23.741 & 14.246 \\
\hline 9/12/10 0:00 & 40.058 & 19.256 & 23.894 & 0.483 & 29.996 & 23.704 & 14.218 \\
\hline 9/12/10 4:00 & 40.023 & 19.284 & 23.863 & 0.476 & 29.984 & 23.669 & 14.183 \\
\hline 9/12/10 8:00 & 39.993 & 19.319 & 23.861 & 0.462 & 29.970 & 23.636 & 14.157 \\
\hline 9/12/10 12:00 & 39.957 & 19.358 & 23.812 & 0.620 & 29.958 & 23.594 & 14.120 \\
\hline 9/12/10 16:00 & 39.909 & 19.351 & 23.754 & 1.041 & 29.947 & 23.550 & 14.082 \\
\hline 9/12/10 20:00 & 39.870 & 19.347 & 23.749 & 1.209 & 29.933 & 23.515 & 14.043 \\
\hline 9/13/10 0:00 & 39.838 & 19.384 & 23.754 & 1.160 & 29.919 & 23.477 & 14.012 \\
\hline 9/13/10 4:00 & 39.803 & 19.407 & 23.721 & 1.164 & 29.909 & 23.440 & 13.977 \\
\hline 9/13/10 8:00 & 39.776 & 19.447 & 23.698 & 1.164 & 29.895 & 23.407 & 13.952 \\
\hline 9/13/10 12:00 & 39.739 & 19.477 & 23.600 & 1.378 & 29.881 & 23.365 & 13.919 \\
\hline 9/13/10 16:00 & 39.684 & 19.451 & 23.514 & 1.818 & 29.867 & 23.316 & 13.875 \\
\hline 9/13/10 20:00 & 39.650 & 19.428 & 23.545 & 1.888 & 29.856 & 23.293 & 13.837 \\
\hline 9/14/10 0:00 & 39.622 & 19.477 & 23.528 & -0.098 & 29.846 & 23.255 & 13.814 \\
\hline 9/14/10 4:00 & 39.583 & 19.482 & 23.489 & -0.375 & 29.832 & 23.213 & 13.779 \\
\hline 9/14/10 8:00 & 39.554 & 19.438 & 23.482 & -0.405 & 29.818 & 23.185 & 13.749 \\
\hline 9/14/10 12:00 & 39.526 & 19.451 & 23.454 & -0.191 & 29.809 & 23.146 & 13.725 \\
\hline 9/14/10 16:00 & 39.482 & 19.421 & 23.328 & 0.206 & 29.797 & 23.104 & 13.686 \\
\hline 9/14/10 20:00 & 39.446 & 19.400 & 23.305 & 0.395 & 29.783 & 23.071 & 13.655 \\
\hline 9/15/10 0:00 & 39.416 & 19.426 & 23.275 & 0.371 & 29.769 & 23.029 & 13.623 \\
\hline 9/15/10 4:00 & 39.377 & 19.414 & 23.272 & 0.420 & 29.758 & 22.994 & 13.588 \\
\hline 9/15/10 8:00 & 39.343 & 19.426 & 23.221 & 0.488 & 29.746 & 22.950 & 13.555 \\
\hline 9/15/10 12:00 & 39.285 & 19.379 & 23.198 & 0.697 & 29.727 & 22.910 & 13.508 \\
\hline 9/15/10 16:00 & 39.253 & 19.389 & 23.158 & 0.802 & 29.718 & 22.865 & 13.478 \\
\hline 9/15/10 20:00 & 39.219 & 19.391 & 23.116 & 0.927 & 29.706 & 22.833 & 13.448 \\
\hline 9/16/10 0:00 & 39.191 & 19.433 & 23.116 & 0.934 & 29.694 & 22.805 & 13.417 \\
\hline 9/16/10 4:00 & 39.164 & 19.489 & 23.107 & 0.934 & 29.683 & 22.777 & 13.394 \\
\hline 9/16/10 8:00 & 39.141 & 19.554 & 23.123 & 0.913 & 29.671 & 22.754 & 13.370 \\
\hline 9/16/10 12:00 & 39.123 & 19.631 & 23.109 & 0.937 & 29.662 & 22.719 & 13.357 \\
\hline 9/16/10 16:00 & 39.084 & 19.652 & 23.007 & 1.146 & 29.650 & 22.679 & 13.322 \\
\hline 9/16/10 20:00 & 39.045 & 19.649 & 22.942 & 1.334 & 29.638 & 22.646 & 13.284 \\
\hline 9/17/10 0:00 & 39.013 & 19.670 & 22.930 & 1.341 & 29.624 & 22.611 & 13.261 \\
\hline
\end{tabular}

TABLE S1.2 Page 8 of 58 


\begin{tabular}{|c|c|c|c|c|c|c|c|}
\hline \multirow[b]{2}{*}{ Date and Time } & \multicolumn{7}{|c|}{ Water Level (ft below reference point) } \\
\hline & SB43S & SB44S & SB45S & SB46S & SB47S & SB48S & SB49S \\
\hline 9/17/10 4:00 & 38.978 & 19.696 & 22.898 & 1.395 & 29.613 & 22.576 & 13.226 \\
\hline 9/17/10 8:00 & 38.948 & 19.715 & 22.884 & -0.422 & 29.601 & 22.543 & 13.200 \\
\hline 9/17/10 12:00 & 38.914 & 19.747 & 22.860 & -0.315 & 29.580 & 22.504 & 13.170 \\
\hline 9/17/10 16:00 & 38.868 & 19.731 & 22.830 & 0.065 & 29.566 & 22.464 & 13.132 \\
\hline 9/17/10 20:00 & 38.836 & 19.736 & 22.849 & 0.334 & 29.554 & 22.431 & 13.104 \\
\hline 9/18/10 0:00 & 38.809 & 19.782 & 22.826 & 0.337 & 29.545 & 22.401 & 13.079 \\
\hline 9/18/10 4:00 & 38.777 & 19.812 & 22.798 & 0.390 & 29.531 & 22.368 & 13.051 \\
\hline 9/18/10 8:00 & 38.751 & 19.864 & 22.779 & 0.411 & 29.522 & 22.343 & 13.032 \\
\hline 9/18/10 12:00 & 38.724 & 19.917 & 22.751 & 0.509 & 29.510 & 22.308 & 13.004 \\
\hline 9/18/10 16:00 & 38.689 & 19.936 & 22.695 & 0.739 & 29.498 & 22.275 & 12.976 \\
\hline 9/18/10 20:00 & 38.657 & 19.948 & 22.695 & 0.832 & 29.487 & 22.247 & 12.946 \\
\hline 9/19/10 0:00 & 38.628 & 19.985 & 22.672 & 0.837 & 29.475 & 22.207 & 12.925 \\
\hline 9/19/10 4:00 & 38.591 & 19.992 & 22.642 & 0.920 & 29.463 & 22.177 & 12.894 \\
\hline 9/19/10 8:00 & 38.563 & 20.024 & 22.619 & 0.955 & 29.454 & 22.151 & 12.869 \\
\hline 9/19/10 12:00 & 38.534 & 20.066 & 22.593 & 1.102 & 29.440 & 22.111 & 12.845 \\
\hline 9/19/10 16:00 & 38.492 & 20.055 & 22.521 & 1.430 & 29.426 & 22.074 & 12.808 \\
\hline 9/19/10 20:00 & 38.458 & 20.057 & 22.458 & 1.590 & 29.416 & 22.044 & 12.780 \\
\hline 9/20/10 0:00 & 38.426 & 20.087 & 22.425 & 1.585 & 29.402 & 22.006 & 12.754 \\
\hline 9/20/10 4:00 & 38.385 & 20.073 & 22.404 & 1.713 & 29.391 & 21.969 & 12.722 \\
\hline 9/20/10 8:00 & 38.353 & 20.083 & 22.390 & 0.704 & 29.379 & 21.936 & 12.696 \\
\hline 9/20/10 12:00 & 38.318 & 20.101 & 22.379 & 0.265 & 29.365 & 21.894 & 12.663 \\
\hline 9/20/10 16:00 & 38.266 & 20.073 & 22.302 & 0.674 & 29.353 & 21.857 & 12.626 \\
\hline 9/20/10 20:00 & 38.231 & 20.055 & 22.288 & 0.583 & 29.342 & 21.824 & 12.598 \\
\hline 9/21/10 0:00 & 38.201 & 20.085 & 22.290 & 0.588 & 29.330 & 21.794 & 12.570 \\
\hline 9/21/10 4:00 & 38.169 & 20.125 & 22.309 & 0.625 & 29.316 & 21.759 & 12.549 \\
\hline 9/21/10 8:00 & 38.139 & 20.143 & 22.286 & 0.809 & 29.307 & 21.731 & 12.519 \\
\hline 9/21/10 12:00 & 38.105 & 20.169 & 22.258 & 0.916 & 29.295 & 21.691 & 12.493 \\
\hline 9/21/10 16:00 & 38.071 & 20.171 & 22.218 & 1.023 & 29.283 & 21.663 & 12.465 \\
\hline 9/21/10 20:00 & 38.043 & 19.994 & 22.214 & -0.289 & 29.272 & 21.633 & 12.442 \\
\hline 9/22/10 0:00 & 38.020 & 19.109 & 22.193 & -0.138 & 29.262 & 21.609 & 12.420 \\
\hline 9/22/10 4:00 & 37.995 & 18.762 & 22.172 & -0.108 & 29.251 & 21.579 & 12.402 \\
\hline 9/22/10 8:00 & 37.972 & 18.713 & 22.165 & -0.228 & 29.241 & 21.549 & 12.378 \\
\hline 9/22/10 12:00 & 37.945 & 18.750 & 22.123 & -0.056 & 29.232 & 21.509 & 12.350 \\
\hline 9/22/10 16:00 & 37.901 & 18.741 & 22.000 & 0.253 & 29.216 & 21.467 & 12.318 \\
\hline 9/22/10 20:00 & 37.862 & 18.725 & 21.981 & 0.441 & 29.204 & 21.434 & 12.287 \\
\hline 9/23/10 0:00 & 37.832 & 18.760 & 21.962 & 0.460 & 29.194 & 21.399 & 12.264 \\
\hline 9/23/10 4:00 & 37.798 & 18.776 & 21.955 & 0.534 & 29.183 & 21.365 & 12.227 \\
\hline 9/23/10 8:00 & 37.766 & 18.795 & 21.930 & 0.623 & 29.171 & 21.327 & 12.206 \\
\hline 9/23/10 12:00 & 37.729 & 18.797 & 21.900 & 0.792 & 29.157 & 21.287 & 12.168 \\
\hline 9/23/10 16:00 & 37.683 & 18.776 & 21.860 & 0.995 & 29.148 & 21.250 & 12.133 \\
\hline 9/23/10 20:00 & 37.661 & 18.739 & 21.860 & -0.282 & 29.134 & 21.255 & 12.110 \\
\hline 9/24/10 0:00 & 37.644 & 18.436 & 21.920 & -0.342 & 29.127 & 21.243 & 12.091 \\
\hline 9/24/10 4:00 & 37.631 & 18.157 & 21.911 & -0.308 & 29.117 & 21.185 & 12.082 \\
\hline 9/24/10 8:00 & 37.617 & 18.103 & 21.888 & -0.361 & 29.108 & 21.154 & 12.066 \\
\hline 9/24/10 12:00 & 37.594 & 18.136 & 21.860 & -0.284 & 29.101 & 21.129 & 12.044 \\
\hline 9/24/10 16:00 & 37.560 & 18.126 & 21.767 & 0.025 & 29.087 & 21.091 & 12.009 \\
\hline 9/24/10 20:00 & 37.528 & 18.117 & 21.725 & 0.171 & 29.075 & 21.056 & 11.981 \\
\hline 9/25/10 0:00 & 37.500 & 18.140 & 21.734 & 0.167 & 29.066 & 21.021 & 11.951 \\
\hline 9/25/10 4:00 & 37.463 & 18.122 & 21.669 & 0.244 & 29.054 & 20.989 & 11.925 \\
\hline 9/25/10 8:00 & 37.427 & 18.122 & 21.665 & 0.348 & 29.043 & 20.949 & 11.890 \\
\hline 9/25/10 12:00 & 37.392 & 18.101 & 21.644 & 0.437 & 29.031 & 20.911 & 11.865 \\
\hline 9/25/10 16:00 & 37.363 & 18.105 & 21.611 & -0.354 & 29.019 & 20.879 & 11.834 \\
\hline 9/25/10 20:00 & 37.333 & 18.117 & 21.583 & -0.259 & 29.008 & 20.846 & 11.806 \\
\hline 9/26/10 0:00 & 37.308 & 18.143 & 21.585 & -0.215 & 28.998 & 20.814 & 11.781 \\
\hline
\end{tabular}

TABLE S1.2 Page 9 of 58 


\begin{tabular}{|c|c|c|c|c|c|c|c|}
\hline \multirow[b]{2}{*}{ Date and Time } & \multicolumn{7}{|c|}{ Water Level (ft below reference point) } \\
\hline & SB43S & SB44S & SB45S & SB46S & SB47S & SB48S & SB49S \\
\hline 9/26/10 4:00 & 37.276 & 18.157 & 21.530 & -0.563 & 28.989 & 20.783 & 11.753 \\
\hline 9/26/10 8:00 & 37.246 & 18.175 & 21.541 & -0.703 & 28.975 & 20.744 & 11.730 \\
\hline 9/26/10 12:00 & 37.216 & 18.185 & 21.485 & -0.526 & 28.966 & 20.706 & 11.699 \\
\hline 9/26/10 16:00 & 37.170 & 18.150 & 21.471 & -0.152 & 28.954 & 20.666 & 11.664 \\
\hline 9/26/10 20:00 & 37.133 & 18.126 & 21.441 & 0.106 & 28.942 & 20.631 & 11.634 \\
\hline 9/27/10 0:00 & 37.099 & 18.122 & 21.462 & 0.160 & 28.928 & 20.594 & 11.601 \\
\hline 9/27/10 4:00 & 37.062 & 18.112 & 21.383 & 0.232 & 28.916 & 20.559 & 11.573 \\
\hline 9/27/10 8:00 & 37.028 & 18.112 & 21.339 & 0.292 & 28.905 & 20.519 & 11.538 \\
\hline 9/27/10 12:00 & 36.991 & 18.115 & 21.327 & 0.437 & 28.893 & 20.480 & 11.510 \\
\hline 9/27/10 16:00 & 36.946 & 18.070 & 21.281 & 0.811 & 28.881 & 20.438 & 11.473 \\
\hline 9/27/10 20:00 & 36.904 & 18.040 & 21.262 & 1.006 & 28.867 & 20.398 & 11.442 \\
\hline 9/28/10 0:00 & 36.865 & 18.022 & 21.234 & 1.074 & 28.856 & 20.363 & 11.407 \\
\hline 9/28/10 4:00 & 36.833 & 18.031 & 21.225 & 1.095 & 28.844 & 20.333 & 11.379 \\
\hline 9/28/10 8:00 & 36.810 & 18.077 & 21.236 & 1.085 & 28.835 & 20.307 & 11.358 \\
\hline 9/28/10 12:00 & 36.781 & 18.122 & 21.201 & 1.211 & 28.825 & 20.267 & 11.335 \\
\hline 9/28/10 16:00 & 36.739 & 18.112 & 21.136 & 1.576 & 28.811 & 20.230 & 11.302 \\
\hline 9/28/10 20:00 & 36.705 & 18.115 & 21.141 & 1.692 & 28.800 & 20.199 & 11.279 \\
\hline 9/29/10 0:00 & 36.675 & 18.143 & 21.164 & 1.688 & 28.790 & 20.167 & 11.253 \\
\hline 9/29/10 4:00 & 36.638 & 18.157 & 21.120 & 1.723 & 28.776 & 20.132 & 11.223 \\
\hline 9/29/10 8:00 & 36.611 & 18.173 & 21.097 & 1.739 & 28.767 & 20.104 & 11.202 \\
\hline 9/29/10 12:00 & 36.579 & 18.196 & 21.027 & 1.906 & 28.755 & 20.062 & 11.176 \\
\hline 9/29/10 16:00 & 36.535 & 18.178 & 20.980 & 2.004 & 28.741 & 20.031 & 11.143 \\
\hline 9/29/10 20:00 & 36.508 & 18.199 & 20.994 & 2.057 & 28.732 & 20.008 & 11.129 \\
\hline 9/30/10 0:00 & 36.494 & 18.282 & 21.039 & 1.964 & 28.723 & 19.985 & 11.111 \\
\hline 9/30/10 4:00 & 36.471 & 18.359 & 21.025 & 2.139 & 28.716 & 19.961 & 11.092 \\
\hline 9/30/10 8:00 & 36.451 & 18.427 & 21.025 & 2.232 & 28.704 & 19.933 & 11.076 \\
\hline 9/30/10 12:00 & 36.428 & 18.485 & 20.997 & 2.323 & 28.695 & 19.903 & 11.057 \\
\hline 9/30/10 16:00 & 36.391 & 18.490 & 20.901 & 2.413 & 28.681 & 19.868 & 11.027 \\
\hline 9/30/10 20:00 & 36.361 & 18.504 & 20.880 & 2.502 & 28.669 & 19.840 & 11.008 \\
\hline 10/1/10 0:00 & 36.336 & 18.543 & 20.880 & 2.592 & 28.659 & 19.814 & 10.989 \\
\hline 10/1/10 4:00 & 36.304 & 18.571 & 20.880 & 2.678 & 28.648 & 19.784 & 10.966 \\
\hline 10/1/10 8:00 & 36.276 & 18.597 & 20.871 & 2.769 & 28.638 & 19.756 & 10.950 \\
\hline 10/1/10 12:00 & 36.247 & 18.620 & 20.811 & 2.853 & 28.627 & 19.719 & 10.922 \\
\hline 10/1/10 16:00 & 36.203 & 18.587 & 20.766 & 2.937 & 28.613 & 19.679 & 10.892 \\
\hline 10/1/10 20:00 & 36.166 & 18.569 & 20.762 & 3.020 & 28.603 & 19.649 & 10.864 \\
\hline 10/2/10 0:00 & 36.134 & 18.578 & 20.806 & 3.104 & 28.592 & 19.623 & 10.840 \\
\hline 10/2/10 4:00 & 36.114 & 18.629 & 20.797 & 3.197 & 28.578 & 19.606 & 10.831 \\
\hline 10/2/10 8:00 & 36.102 & 18.736 & 20.808 & 3.283 & 28.573 & 19.585 & 10.817 \\
\hline 10/2/10 12:00 & 36.082 & 18.813 & 20.801 & 3.371 & 28.564 & 19.555 & 10.807 \\
\hline 10/2/10 16:00 & 36.047 & 18.832 & 20.720 & 3.453 & 28.552 & 19.525 & 10.782 \\
\hline 10/2/10 20:00 & 36.020 & 18.841 & 20.704 & 3.539 & 28.540 & 19.499 & 10.761 \\
\hline 10/3/10 0:00 & 35.994 & 18.881 & 20.711 & 3.625 & 28.529 & 19.471 & 10.742 \\
\hline 10/3/10 4:00 & 35.967 & 18.909 & 20.692 & 3.706 & 28.517 & 19.445 & 10.721 \\
\hline 10/3/10 8:00 & 35.942 & 18.941 & 20.711 & 3.790 & 28.505 & 19.417 & 10.707 \\
\hline 10/3/10 12:00 & 35.912 & 18.978 & 20.669 & 3.871 & 28.496 & 19.385 & 10.688 \\
\hline 10/3/10 16:00 & 35.873 & 18.948 & 20.543 & 3.950 & 28.482 & 19.348 & 10.660 \\
\hline 10/3/10 20:00 & 35.836 & 18.923 & 20.550 & 4.030 & 28.468 & 19.320 & 10.635 \\
\hline 10/4/10 0:00 & 35.809 & 18.951 & 20.599 & 4.113 & 28.459 & 19.291 & 10.616 \\
\hline 10/4/10 4:00 & 35.779 & 18.969 & 20.564 & 4.192 & 28.449 & 19.263 & 10.597 \\
\hline 10/4/10 8:00 & 35.752 & 18.997 & 20.562 & 4.271 & 28.435 & 19.238 & 10.581 \\
\hline 10/4/10 12:00 & 35.724 & 19.030 & 20.524 & 4.350 & 28.424 & 19.205 & 10.555 \\
\hline 10/4/10 16:00 & 35.683 & 18.997 & 20.441 & 4.427 & 28.412 & 19.170 & 10.530 \\
\hline 10/4/10 20:00 & 35.651 & 18.986 & 20.448 & 4.506 & 28.398 & 19.144 & 10.506 \\
\hline 10/5/10 0:00 & 35.623 & 19.021 & 20.494 & 4.585 & 28.391 & 19.114 & 10.490 \\
\hline
\end{tabular}

TABLE S1.2 Page 10 of 58 


\begin{tabular}{|c|c|c|c|c|c|c|c|}
\hline \multirow[b]{2}{*}{ Date and Time } & \multicolumn{7}{|c|}{ Water Level (ft below reference point) } \\
\hline & SB43S & SB44S & SB45S & SB46S & SB47S & SB48S & SB49S \\
\hline 10/5/10 4:00 & 35.596 & 19.044 & 20.469 & 4.664 & 28.377 & 19.088 & 10.471 \\
\hline 10/5/10 8:00 & 35.568 & 19.077 & 20.471 & 4.743 & 28.370 & 19.065 & 10.455 \\
\hline 10/5/10 12:00 & 35.543 & 19.118 & 20.438 & 4.820 & 28.356 & 19.032 & 10.436 \\
\hline 10/5/10 16:00 & 35.504 & 19.095 & 20.336 & 4.895 & 28.344 & 19.000 & 10.408 \\
\hline 10/5/10 20:00 & 35.470 & 19.086 & 20.329 & 4.969 & 28.332 & 18.972 & 10.385 \\
\hline 10/6/10 0:00 & 35.440 & 19.111 & 20.320 & 5.048 & 28.321 & 18.944 & 10.368 \\
\hline 10/6/10 4:00 & 35.408 & 19.114 & 20.369 & 5.120 & 28.309 & 18.911 & 10.347 \\
\hline 10/6/10 8:00 & 35.378 & 19.121 & 20.343 & 5.197 & 28.300 & 18.883 & 10.331 \\
\hline 10/6/10 12:00 & 35.344 & 19.125 & 20.257 & 5.271 & 28.288 & 18.848 & 10.303 \\
\hline 10/6/10 16:00 & 35.302 & 19.095 & 20.222 & 5.343 & 28.276 & 18.815 & 10.277 \\
\hline 10/6/10 20:00 & 35.270 & 19.097 & 20.241 & 5.415 & 28.262 & 18.789 & 10.259 \\
\hline 10/7/10 0:00 & 35.247 & 19.142 & 20.245 & 5.490 & 28.251 & 18.769 & 10.242 \\
\hline 10/7/10 4:00 & 35.222 & 19.186 & 20.268 & 5.562 & 28.244 & 18.745 & 10.228 \\
\hline 10/7/10 8:00 & 35.199 & 19.235 & 20.259 & 5.634 & 28.232 & 18.722 & 10.217 \\
\hline 10/7/10 12:00 & 35.174 & 19.288 & 20.236 & 5.704 & 28.223 & 18.689 & 10.200 \\
\hline 10/7/10 16:00 & 35.135 & 19.267 & 20.124 & 5.769 & 28.209 & 18.659 & 10.172 \\
\hline 10/7/10 20:00 & 35.103 & 19.258 & 20.115 & 5.836 & 28.197 & 18.628 & 10.154 \\
\hline 10/8/10 0:00 & 35.071 & 19.268 & 20.129 & 5.906 & 28.185 & 18.600 & 10.137 \\
\hline 10/8/10 4:00 & 35.037 & 19.270 & 20.131 & 5.974 & 28.174 & 18.572 & 10.116 \\
\hline 10/8/10 8:00 & 35.009 & 19.281 & 20.094 & 6.041 & 28.162 & 18.547 & 10.095 \\
\hline 10/8/10 12:00 & 34.979 & 19.300 & 20.040 & 6.106 & 28.150 & 18.514 & 10.074 \\
\hline 10/8/10 16:00 & 34.940 & 19.281 & 20.040 & 6.171 & 28.139 & 18.484 & 10.049 \\
\hline 10/8/10 20:00 & 34.911 & 19.286 & 20.043 & 6.236 & 28.127 & 18.458 & 10.037 \\
\hline 10/9/10 0:00 & 34.885 & 19.326 & 20.031 & 6.306 & 28.117 & 18.432 & 10.021 \\
\hline 10/9/10 4:00 & 34.856 & 19.351 & 20.075 & 6.371 & 28.108 & 18.407 & 10.002 \\
\hline 10/9/10 8:00 & 34.828 & 19.370 & 20.061 & 6.436 & 28.096 & 18.381 & 9.983 \\
\hline 10/9/10 12:00 & 34.801 & 19.398 & 20.019 & 6.499 & 28.082 & 18.353 & 9.969 \\
\hline 10/9/10 16:00 & 34.766 & 19.398 & 19.952 & 6.564 & 28.068 & 18.320 & 9.946 \\
\hline 10/9/10 20:00 & 34.739 & 19.407 & 19.968 & 6.629 & 28.059 & 18.302 & 9.935 \\
\hline 10/10/10 0:00 & 34.711 & 19.447 & 19.987 & 6.695 & 28.052 & 18.274 & 9.914 \\
\hline 10/10/10 4:00 & 34.679 & 19.461 & 19.959 & 6.760 & 28.038 & 18.248 & 9.897 \\
\hline 10/10/10 8:00 & 34.656 & 19.489 & 19.975 & 6.822 & 28.029 & 18.225 & 9.883 \\
\hline 10/10/10 12:00 & 34.629 & 19.521 & 19.936 & 6.885 & 28.015 & 18.194 & 9.867 \\
\hline 10/10/10 16:00 & 34.594 & 19.514 & 19.854 & 6.948 & 28.005 & 18.166 & 9.846 \\
\hline 10/10/10 20:00 & 34.564 & 19.524 & 19.840 & 7.011 & 27.994 & 18.145 & 9.830 \\
\hline 10/11/10 0:00 & 34.542 & 19.563 & 19.856 & 7.076 & 27.982 & 18.120 & 9.818 \\
\hline 10/11/10 4:00 & 34.509 & 19.582 & 19.826 & 7.139 & 27.973 & 18.094 & 9.801 \\
\hline 10/11/10 8:00 & 34.484 & 19.608 & 19.819 & 6.818 & 27.961 & 18.073 & 9.785 \\
\hline 10/11/10 12:00 & 34.461 & 19.642 & 19.854 & 5.039 & 27.952 & 18.045 & 9.773 \\
\hline 10/11/10 16:00 & 34.427 & 19.635 & 19.815 & 3.806 & 27.940 & 18.017 & 9.755 \\
\hline 10/11/10 20:00 & 34.402 & 19.656 & 19.815 & 3.462 & 27.928 & 17.996 & 9.738 \\
\hline 10/12/10 0:00 & 34.379 & 19.700 & 19.810 & 2.962 & 27.919 & 17.973 & 9.727 \\
\hline 10/12/10 4:00 & 34.351 & 19.724 & 19.833 & 0.869 & 27.907 & 17.951 & 9.710 \\
\hline 10/12/10 8:00 & 34.333 & 19.768 & 19.840 & -0.270 & 27.900 & 17.935 & 9.701 \\
\hline 10/12/10 12:00 & 34.315 & 19.840 & 19.826 & -0.196 & 27.889 & 17.914 & 9.692 \\
\hline $10 / 12 / 1016: 00$ & 34.289 & 19.868 & 19.801 & 0.130 & 27.877 & 17.891 & 9.682 \\
\hline 10/12/10 20:00 & 34.271 & 19.912 & 19.838 & 0.204 & 27.868 & 17.877 & 9.675 \\
\hline 10/13/10 0:00 & 34.257 & 19.994 & 19.847 & 0.155 & 27.860 & 17.860 & 9.673 \\
\hline 10/13/10 4:00 & 34.237 & 20.052 & 19.810 & 0.157 & 27.849 & 17.842 & 9.659 \\
\hline 10/13/10 8:00 & 34.221 & 20.106 & 19.810 & 0.109 & 27.844 & 17.828 & 9.652 \\
\hline 10/13/10 12:00 & 34.200 & 20.157 & 19.791 & 0.188 & 27.832 & 17.802 & 9.643 \\
\hline 10/13/10 16:00 & 34.168 & 20.148 & 19.698 & 0.506 & 27.821 & 17.774 & 9.626 \\
\hline 10/13/10 20:00 & 34.141 & 20.155 & 19.694 & 0.611 & 27.809 & 17.753 & 9.615 \\
\hline 10/14/10 0:00 & 34.118 & 20.185 & 19.717 & 0.623 & 27.797 & 17.730 & 9.598 \\
\hline
\end{tabular}

TABLE S1.2 Page 11 of 58 


\begin{tabular}{|c|c|c|c|c|c|c|c|}
\hline \multirow[b]{2}{*}{ Date and Time } & \multicolumn{7}{|c|}{ Water Level (ft below reference point) } \\
\hline & SB43S & SB44S & SB45S & SB46S & SB47S & SB48S & SB49S \\
\hline 10/14/10 4:00 & 34.088 & 20.187 & 19.666 & 0.699 & 27.786 & 17.704 & 9.582 \\
\hline 10/14/10 8:00 & 34.062 & 20.185 & 19.677 & 0.755 & 27.774 & 17.678 & 9.568 \\
\hline $10 / 14 / 1012: 00$ & 34.024 & 20.166 & 19.666 & 0.995 & 27.765 & 17.641 & 9.545 \\
\hline 10/14/10 16:00 & 33.976 & 20.080 & 19.619 & 1.536 & 27.751 & 17.611 & 9.517 \\
\hline 10/14/10 20:00 & 33.955 & 20.073 & 19.621 & 1.632 & 27.741 & 17.594 & 9.505 \\
\hline 10/15/10 0:00 & 33.932 & 20.134 & 19.659 & 1.741 & 27.727 & 17.575 & 9.495 \\
\hline 10/15/10 4:00 & 33.914 & 20.185 & 19.682 & 1.846 & 27.718 & 17.557 & 9.486 \\
\hline 10/15/10 8:00 & 33.895 & 20.243 & 19.677 & 1.948 & 27.709 & 17.540 & 9.477 \\
\hline 10/15/10 12:00 & 33.875 & 20.295 & 19.652 & 2.050 & 27.699 & 17.515 & 9.470 \\
\hline 10/15/10 16:00 & 33.840 & 20.278 & 19.545 & 2.153 & 27.685 & 17.487 & 9.449 \\
\hline 10/15/10 20:00 & 33.811 & 20.269 & 19.538 & 2.251 & 27.674 & 17.466 & 9.430 \\
\hline 10/16/10 0:00 & 33.785 & 20.290 & 19.531 & 2.353 & 27.662 & 17.443 & 9.418 \\
\hline 10/16/10 4:00 & 33.758 & 20.292 & 19.517 & 2.451 & 27.653 & 17.419 & 9.400 \\
\hline 10/16/10 8:00 & 33.728 & 20.292 & 19.496 & 2.546 & 27.639 & 17.396 & 9.390 \\
\hline 10/16/10 12:00 & 33.705 & 20.325 & 19.500 & 2.644 & 27.627 & 17.375 & 9.376 \\
\hline 10/16/10 16:00 & 33.680 & 20.334 & 19.498 & 2.746 & 27.618 & 17.354 & 9.362 \\
\hline 10/16/10 20:00 & 33.662 & 20.383 & 19.538 & 2.843 & 27.608 & 17.338 & 9.353 \\
\hline 10/17/10 0:00 & 33.646 & 20.446 & 19.584 & 2.946 & 27.599 & 17.321 & 9.348 \\
\hline 10/17/10 4:00 & 33.627 & 20.486 & 19.596 & 3.043 & 27.587 & 17.305 & 9.339 \\
\hline 10/17/10 8:00 & 33.604 & 20.518 & 19.566 & 3.139 & 27.580 & 17.284 & 9.327 \\
\hline 10/17/10 12:00 & 33.579 & 20.534 & 19.517 & 3.234 & 27.566 & 17.253 & 9.313 \\
\hline 10/17/10 16:00 & 33.542 & 20.490 & 19.440 & 3.322 & 27.554 & 17.225 & 9.292 \\
\hline 10/17/10 20:00 & 33.513 & 20.474 & 19.431 & 3.418 & 27.543 & 17.207 & 9.276 \\
\hline 10/18/10 0:00 & 33.487 & 20.488 & 19.431 & 3.511 & 27.531 & 17.183 & 9.260 \\
\hline 10/18/10 4:00 & 33.462 & 20.500 & 19.440 & 3.604 & 27.519 & 17.162 & 9.250 \\
\hline 10/18/10 8:00 & 33.444 & 20.534 & 19.477 & 3.697 & 27.510 & 17.151 & 9.239 \\
\hline 10/18/10 12:00 & 33.423 & 20.581 & 19.489 & 3.790 & 27.498 & 17.130 & 9.234 \\
\hline 10/18/10 16:00 & 33.398 & 20.593 & 19.428 & 3.883 & 27.489 & 17.109 & 9.218 \\
\hline 10/18/10 20:00 & 33.380 & 20.625 & 19.456 & 3.976 & 27.477 & 17.095 & 9.213 \\
\hline 10/19/10 0:00 & 33.361 & 20.672 & 19.480 & 4.069 & 27.468 & 17.074 & 9.204 \\
\hline 10/19/10 4:00 & 33.336 & 20.688 & 19.454 & 4.160 & 27.456 & 17.053 & 9.192 \\
\hline 10/19/10 8:00 & 33.315 & 20.721 & 19.466 & 4.250 & 27.447 & 17.034 & 9.183 \\
\hline 10/19/10 12:00 & 33.295 & 20.758 & 19.452 & 4.339 & 27.435 & 17.008 & 9.171 \\
\hline 10/19/10 16:00 & 33.261 & 20.725 & 19.349 & 4.425 & 27.421 & 16.985 & 9.152 \\
\hline 10/19/10 20:00 & 33.233 & 20.714 & 19.345 & 4.513 & 27.410 & 16.964 & 9.141 \\
\hline 10/20/10 0:00 & 33.210 & 20.732 & 19.363 & 4.599 & 27.400 & 16.945 & 9.124 \\
\hline 10/20/10 4:00 & 33.187 & 20.751 & 19.345 & 4.690 & 27.389 & 16.922 & 9.113 \\
\hline 10/20/10 8:00 & 33.164 & 20.763 & 19.349 & 4.795 & 27.377 & 16.908 & 9.108 \\
\hline 10/20/10 12:00 & 33.146 & 20.807 & 19.356 & 4.864 & 27.368 & 16.885 & 9.101 \\
\hline 10/20/10 16:00 & 33.116 & 20.798 & 19.324 & 4.948 & 27.354 & 16.861 & 9.082 \\
\hline 10/20/10 20:00 & 33.093 & 20.805 & 19.342 & 5.036 & 27.342 & 16.847 & 9.068 \\
\hline 10/21/10 0:00 & 33.077 & 20.851 & 19.375 & 5.122 & 27.333 & 16.831 & 9.066 \\
\hline 10/21/10 4:00 & 33.061 & 20.893 & 19.380 & 5.213 & 27.321 & 16.819 & 9.059 \\
\hline 10/21/10 8:00 & 33.045 & 20.940 & 19.407 & 5.311 & 27.314 & 16.803 & 9.055 \\
\hline 10/21/10 12:00 & 33.027 & 20.979 & 19.356 & 5.385 & 27.302 & 16.780 & 9.045 \\
\hline 10/21/10 16:00 & 32.997 & 20.960 & 19.272 & 5.464 & 27.288 & 16.756 & 9.033 \\
\hline 10/21/10 20:00 & 32.976 & 20.963 & 19.282 & 5.546 & 27.272 & 16.740 & 9.019 \\
\hline 10/22/10 0:00 & 32.951 & 20.982 & 19.298 & 5.625 & 27.260 & 16.717 & 9.008 \\
\hline 10/22/10 4:00 & 32.926 & 20.984 & 19.298 & 5.701 & 27.246 & 16.698 & 9.001 \\
\hline 10/22/10 8:00 & 32.903 & 20.996 & 19.326 & 5.804 & 27.232 & 16.679 & 8.987 \\
\hline $10 / 22 / 1012: 00$ & 32.878 & 21.000 & 19.272 & 5.860 & 27.216 & 16.658 & 8.973 \\
\hline 10/22/10 16:00 & 32.853 & 20.998 & 19.240 & 5.936 & 27.199 & 16.635 & 8.954 \\
\hline 10/22/10 20:00 & 32.832 & 21.000 & 19.249 & 6.013 & 27.185 & 16.616 & 8.949 \\
\hline 10/23/10 0:00 & 32.804 & 21.012 & 19.235 & 5.518 & 27.171 & 16.595 & 8.940 \\
\hline
\end{tabular}

TABLE S1.2 Page 12 of 58 


\begin{tabular}{|c|c|c|c|c|c|c|c|}
\hline \multirow[b]{2}{*}{ Date and Time } & \multicolumn{7}{|c|}{ Water Level (ft below reference point) } \\
\hline & SB43S & SB44S & SB45S & SB46S & SB47S & SB48S & SB49S \\
\hline 10/23/10 4:00 & 32.779 & 20.995 & 19.252 & 3.050 & 27.155 & 16.574 & 8.924 \\
\hline 10/23/10 8:00 & 32.754 & 20.763 & 19.224 & 1.695 & 27.141 & 16.553 & 8.912 \\
\hline 10/23/10 12:00 & 32.727 & 20.514 & 19.240 & 0.320 & 27.125 & 16.527 & 8.900 \\
\hline 10/23/10 16:00 & 32.697 & 20.364 & 19.205 & 0.683 & 27.113 & 16.504 & 8.879 \\
\hline 10/23/10 20:00 & 32.681 & 20.306 & 19.214 & 0.764 & 27.101 & 16.488 & 8.877 \\
\hline 10/24/10 0:00 & 32.658 & 20.302 & 19.210 & 0.799 & 27.090 & 16.467 & 8.863 \\
\hline 10/24/10 4:00 & 32.632 & 20.269 & 19.245 & 0.892 & 27.076 & 16.448 & 8.849 \\
\hline 10/24/10 8:00 & 32.607 & 20.255 & 19.233 & 0.990 & 27.064 & 16.427 & 8.837 \\
\hline $10 / 24 / 1012: 00$ & 32.587 & 20.264 & 19.226 & 1.081 & 27.054 & 16.406 & 8.828 \\
\hline 10/24/10 16:00 & 32.562 & 20.262 & 19.214 & 1.260 & 27.040 & 16.385 & 8.816 \\
\hline 10/24/10 20:00 & 32.539 & 20.274 & 19.189 & 1.341 & 27.026 & 16.366 & 8.805 \\
\hline 10/25/10 0:00 & 32.511 & 20.283 & 19.205 & 1.197 & 27.012 & 16.345 & 8.791 \\
\hline 10/25/10 4:00 & 32.481 & 20.252 & 19.189 & 1.297 & 26.998 & 16.317 & 8.774 \\
\hline 10/25/10 8:00 & 32.447 & 20.208 & 19.096 & 1.397 & 26.987 & 16.289 & 8.756 \\
\hline 10/25/10 12:00 & 32.412 & 20.173 & 19.070 & 1.497 & 26.973 & 16.254 & 8.735 \\
\hline 10/25/10 16:00 & 32.367 & 20.059 & 19.061 & 1.590 & 26.959 & 16.224 & 8.704 \\
\hline 10/25/10 20:00 & 32.339 & 20.031 & 19.037 & 1.690 & 26.945 & 16.208 & 8.693 \\
\hline 10/26/10 0:00 & 32.323 & 20.083 & 19.086 & 1.792 & 26.935 & 16.196 & 8.686 \\
\hline 10/26/10 4:00 & 32.314 & 20.183 & 19.156 & 0.899 & 26.935 & 16.184 & 8.686 \\
\hline 10/26/10 8:00 & 32.303 & 20.264 & 19.144 & 0.216 & 26.921 & 16.177 & 8.681 \\
\hline 10/26/10 12:00 & 32.298 & 20.346 & 19.161 & 0.199 & 26.919 & 16.166 & 8.683 \\
\hline 10/26/10 16:00 & 32.277 & 20.385 & 19.161 & 0.385 & 26.905 & 16.149 & 8.676 \\
\hline 10/26/10 20:00 & 32.266 & 20.444 & 19.133 & 0.434 & 26.900 & 16.142 & 8.676 \\
\hline 10/27/10 0:00 & 32.257 & 20.520 & 19.135 & 0.413 & 26.889 & 16.128 & 8.672 \\
\hline 10/27/10 4:00 & 32.241 & 20.574 & 19.147 & 0.418 & 26.882 & 16.117 & 8.667 \\
\hline 10/27/10 8:00 & 32.229 & 20.637 & 19.156 & 0.392 & 26.872 & 16.105 & 8.665 \\
\hline 10/27/10 12:00 & 32.222 & 20.721 & 19.172 & 0.420 & 26.861 & 16.091 & 8.665 \\
\hline 10/27/10 16:00 & 32.209 & 20.774 & 19.163 & 0.597 & 26.856 & 16.089 & 8.662 \\
\hline 10/27/10 20:00 & 32.213 & 20.905 & 19.163 & 0.490 & 26.854 & 16.086 & 8.676 \\
\hline 10/28/10 0:00 & 32.206 & 21.019 & 19.189 & 0.416 & 26.847 & 16.077 & 8.674 \\
\hline 10/28/10 4:00 & 32.195 & 21.084 & 19.172 & 0.395 & 26.837 & 16.068 & 8.676 \\
\hline 10/28/10 8:00 & 32.188 & 21.158 & 19.189 & 0.353 & 26.830 & 16.063 & 8.674 \\
\hline 10/28/10 12:00 & 32.179 & 21.228 & 19.177 & 0.411 & 26.821 & 16.047 & 8.676 \\
\hline 10/28/10 16:00 & 32.156 & 21.235 & 19.151 & 0.716 & 26.809 & 16.030 & 8.665 \\
\hline 10/28/10 20:00 & 32.140 & 21.245 & 19.156 & 2.050 & 26.800 & 16.016 & 8.655 \\
\hline 10/29/10 0:00 & 32.122 & 21.261 & 19.161 & 2.167 & 26.791 & 15.995 & 8.648 \\
\hline 10/29/10 4:00 & 32.096 & 21.247 & 19.128 & 2.271 & 26.776 & 15.979 & 8.641 \\
\hline 10/29/10 8:00 & 32.073 & 21.231 & 19.079 & 2.374 & 26.767 & 15.958 & 8.629 \\
\hline 10/29/10 12:00 & 32.044 & 21.193 & 19.047 & 2.474 & 26.753 & 16.422 & 8.613 \\
\hline 10/29/10 16:00 & 31.995 & 21.079 & 19.037 & 2.581 & 26.730 & 15.899 & 8.571 \\
\hline 10/29/10 20:00 & 31.968 & 21.026 & 19.065 & $N A^{b}$ & 26.716 & 15.878 & 8.555 \\
\hline 10/30/10 0:00 & 31.943 & 21.005 & 19.051 & & 26.702 & 15.857 & 8.541 \\
\hline 10/30/10 4:00 & 31.911 & 20.963 & 19.063 & & 26.688 & 15.836 & 8.524 \\
\hline 10/30/10 8:00 & 31.892 & 20.965 & 19.070 & & 26.678 & 15.820 & 8.520 \\
\hline 10/30/10 12:00 & 31.874 & 20.996 & 19.075 & & 26.667 & 15.806 & 8.510 \\
\hline 10/30/10 16:00 & 31.851 & 21.007 & 19.068 & & 26.660 & 15.790 & 8.501 \\
\hline 10/30/10 20:00 & 31.837 & 21.058 & 19.070 & & 26.650 & 15.778 & 8.499 \\
\hline 10/31/10 0:00 & 31.826 & 21.117 & 19.072 & & 26.641 & 15.769 & 8.494 \\
\hline 10/31/10 4:00 & 31.810 & 21.165 & 19.075 & & 26.632 & 15.752 & 8.489 \\
\hline 10/31/10 8:00 & 31.794 & 21.193 & 19.084 & & 26.622 & 15.743 & 8.480 \\
\hline 10/31/10 12:00 & 31.782 & 21.252 & 19.075 & & 26.615 & 15.722 & 8.480 \\
\hline 10/31/10 16:00 & 31.762 & 21.228 & 19.065 & & 26.604 & 15.710 & 8.466 \\
\hline 10/31/10 20:00 & 31.746 & 21.277 & 19.070 & & 26.597 & 15.701 & 8.466 \\
\hline 11/1/10 0:00 & 31.732 & 21.326 & 19.070 & & 26.590 & 15.685 & 8.459 \\
\hline
\end{tabular}

TABLE S1.2 Page 13 of 58 


\begin{tabular}{|c|c|c|c|c|c|c|c|}
\hline \multirow[b]{2}{*}{ Date and Time } & \multicolumn{7}{|c|}{ Water Level (ft below reference point) } \\
\hline & SB43S & SB44S & SB45S & SB46S & SB47S & SB48S & SB49S \\
\hline 11/1/10 4:00 & 31.716 & 21.340 & 19.068 & & 26.578 & 15.673 & 8.450 \\
\hline 11/1/10 8:00 & 31.704 & 21.384 & 19.072 & & 26.569 & 15.664 & 8.447 \\
\hline 11/1/10 12:00 & 31.691 & 21.424 & 19.072 & & 26.559 & 15.647 & 8.445 \\
\hline 11/1/10 16:00 & 31.672 & 21.429 & 19.070 & & 26.552 & 15.631 & 8.438 \\
\hline 11/1/10 20:00 & 31.659 & 21.461 & 19.070 & & 26.543 & 15.624 & 8.438 \\
\hline $11 / 2 / 100: 00$ & 31.642 & 21.503 & 19.072 & & 26.536 & 15.610 & 8.433 \\
\hline 11/2/10 4:00 & 31.627 & 21.510 & 19.068 & & 26.524 & 15.591 & 8.426 \\
\hline 11/2/10 8:00 & 31.608 & 21.515 & 19.065 & & 26.515 & 15.582 & 8.419 \\
\hline 11/2/10 12:00 & 31.590 & 21.541 & 19.065 & & 26.505 & 15.559 & 8.415 \\
\hline 11/2/10 16:00 & 31.562 & 21.494 & 19.051 & & 26.494 & 15.542 & 8.396 \\
\hline $11 / 2 / 1020: 00$ & 31.544 & 21.484 & 19.047 & & 26.484 & 15.524 & 8.387 \\
\hline 11/3/10 0:00 & 31.523 & 21.487 & 19.042 & & 26.475 & 15.505 & 8.382 \\
\hline 11/3/10 4:00 & 31.498 & 21.473 & 19.035 & & 26.463 & 15.489 & 8.368 \\
\hline 11/3/10 8:00 & 31.480 & 21.464 & 19.033 & & 26.452 & 15.477 & 8.364 \\
\hline $11 / 3 / 1012: 00$ & 31.464 & 21.496 & 19.033 & & 26.445 & 15.458 & 8.359 \\
\hline $11 / 3 / 1016: 00$ & 31.441 & 21.487 & 19.028 & & 26.431 & 15.442 & 8.349 \\
\hline $11 / 3 / 1020: 00$ & 31.422 & 21.498 & 19.024 & & 26.421 & 15.428 & 8.333 \\
\hline 11/4/10 0:00 & 31.409 & 21.531 & 19.026 & & 26.414 & 15.416 & 8.335 \\
\hline 11/4/10 4:00 & 31.390 & 21.554 & 19.021 & & 26.403 & 15.405 & 8.326 \\
\hline 11/4/10 8:00 & 31.372 & 21.561 & 19.019 & & 26.393 & 15.391 & 8.319 \\
\hline 11/4/10 12:00 & 31.356 & 21.578 & 19.005 & & 26.384 & 15.367 & 8.310 \\
\hline 11/4/10 16:00 & 31.331 & 21.550 & 18.970 & & 26.375 & 15.356 & 8.300 \\
\hline 11/4/10 20:00 & 31.317 & 21.578 & 19.012 & & 26.363 & 15.344 & 8.298 \\
\hline 11/5/10 0:00 & 31.303 & 21.610 & 19.017 & & 26.354 & 15.335 & 8.291 \\
\hline 11/5/10 4:00 & 31.287 & 21.634 & 19.014 & & 26.344 & 15.323 & 8.286 \\
\hline $11 / 5 / 108: 00$ & 31.276 & 21.664 & 19.014 & & 26.335 & 15.311 & 8.287 \\
\hline $11 / 5 / 1012: 00$ & 31.260 & 21.696 & 19.014 & & 26.328 & 15.293 & 8.284 \\
\hline 11/5/10 16:00 & 31.237 & 21.675 & 19.010 & & 26.314 & 15.276 & 8.272 \\
\hline 11/5/10 20:00 & 31.219 & 21.678 & 19.005 & & 26.309 & 15.265 & 8.263 \\
\hline 11/6/10 0:00 & 31.205 & 21.708 & 19.007 & & 26.298 & 15.253 & 8.258 \\
\hline 11/6/10 4:00 & 31.186 & 21.729 & 19.005 & & 26.286 & 15.239 & 8.254 \\
\hline 11/6/10 8:00 & 31.175 & 21.748 & 19.005 & & 26.279 & 15.229 & 8.251 \\
\hline 11/6/10 12:00 & 31.159 & 21.776 & 18.993 & & 26.270 & 15.208 & 8.247 \\
\hline 11/6/10 16:00 & 31.131 & 21.736 & 18.947 & & 26.258 & 15.192 & 8.233 \\
\hline 11/6/10 20:00 & 31.115 & 21.736 & 18.961 & & 26.246 & 15.178 & 8.230 \\
\hline 11/7/10 0:00 & 31.095 & 21.746 & 18.961 & & 26.237 & 15.164 & 8.219 \\
\hline $11 / 7 / 104: 00$ & 31.076 & 21.750 & 18.991 & & 26.223 & 15.150 & 8.214 \\
\hline 11/7/10 8:00 & 31.060 & 21.748 & 18.989 & & 26.216 & 15.136 & 8.203 \\
\hline 11/7/10 12:00 & 31.042 & 21.764 & 18.986 & & 26.206 & 15.115 & 8.195 \\
\hline 11/7/10 16:00 & 31.015 & 21.720 & 18.972 & & 26.192 & 15.094 & 8.181 \\
\hline 11/7/10 20:00 & 30.992 & 21.704 & 18.970 & & 26.183 & 15.080 & 8.177 \\
\hline 11/8/10 0:00 & 30.971 & 21.704 & 18.963 & & 26.174 & 15.061 & 8.168 \\
\hline $11 / 8 / 104: 00$ & 30.946 & 21.675 & 18.956 & & 26.160 & 15.045 & 8.156 \\
\hline 11/8/10 8:00 & 30.927 & 21.666 & 18.951 & & 26.150 & 15.033 & 8.147 \\
\hline 11/8/10 12:00 & 30.909 & 21.682 & 18.951 & & 26.141 & 15.015 & 8.144 \\
\hline $11 / 8 / 1016: 00$ & 30.886 & 21.673 & 18.947 & & 26.127 & 14.998 & 8.125 \\
\hline $11 / 8 / 1020: 00$ & 30.870 & 21.680 & 18.942 & & 26.122 & 14.987 & 8.125 \\
\hline $11 / 9 / 100: 00$ & 30.856 & 21.706 & 18.951 & & 26.111 & 14.973 & 8.118 \\
\hline 11/9/10 4:00 & 30.836 & 21.722 & 18.935 & & 26.101 & 14.959 & 8.114 \\
\hline 11/9/10 8:00 & 30.822 & 21.734 & 18.947 & & 26.092 & 14.947 & 8.109 \\
\hline $11 / 9 / 10$ 12:00 & 30.804 & 21.759 & 18.933 & & 26.085 & 14.931 & 8.100 \\
\hline 11/9/10 16:00 & 30.781 & 21.743 & 18.933 & & 26.071 & 14.921 & 8.091 \\
\hline $11 / 9 / 1020: 00$ & 30.767 & 21.764 & 18.937 & & 26.064 & 14.907 & 8.086 \\
\hline 11/10/10 0:00 & 30.753 & 21.811 & 18.940 & & 26.057 & 14.898 & 8.088 \\
\hline
\end{tabular}

TABLE S1.2 Page 14 of 58 


\begin{tabular}{|c|c|c|c|c|c|c|c|}
\hline \multirow[b]{2}{*}{ Date and Time } & \multicolumn{7}{|c|}{ Water Level (ft below reference point) } \\
\hline & SB43S & SB44S & SB45S & SB46S & SB47S & SB48S & SB49S \\
\hline 11/10/10 4:00 & 30.740 & 21.831 & 18.940 & & 26.050 & 14.886 & 8.079 \\
\hline 11/10/10 8:00 & 30.726 & 21.845 & 18.940 & & 26.038 & 14.875 & 8.081 \\
\hline 11/10/10 12:00 & 30.710 & 21.871 & 18.942 & & 26.029 & 14.863 & 8.076 \\
\hline 11/10/10 16:00 & 30.703 & 21.906 & 18.944 & & 26.024 & 14.861 & 8.079 \\
\hline 11/10/10 20:00 & 30.703 & 21.995 & 18.961 & & 26.020 & 14.861 & 8.086 \\
\hline $11 / 11 / 100: 00$ & 30.698 & 22.074 & 18.970 & & 26.013 & 14.856 & 8.093 \\
\hline $11 / 11 / 104: 00$ & 30.691 & 22.130 & 18.977 & & 26.006 & 14.849 & 8.090 \\
\hline 11/11/10 8:00 & 30.687 & 22.179 & 18.986 & & 25.999 & 14.844 & 8.095 \\
\hline $11 / 11 / 10$ 12:00 & 30.678 & 22.223 & 18.991 & & 25.992 & 14.833 & 8.100 \\
\hline 11/11/10 16:00 & 30.657 & 22.209 & 18.984 & & 25.985 & 14.819 & 8.090 \\
\hline $11 / 11 / 1020: 00$ & 30.643 & 22.218 & 18.989 & & 25.971 & 14.805 & 8.090 \\
\hline $11 / 12 / 100: 00$ & 30.623 & 22.220 & 18.984 & & 25.961 & 14.784 & 8.076 \\
\hline $11 / 12 / 104: 00$ & 30.600 & 22.176 & 18.977 & & 25.952 & 14.774 & 8.067 \\
\hline 11/12/10 8:00 & 30.588 & 22.204 & 18.977 & & 25.945 & 14.767 & 8.062 \\
\hline $11 / 12 / 1012: 00$ & 30.565 & 22.172 & 18.975 & & 25.933 & 14.744 & 8.055 \\
\hline 11/12/10 16:00 & 30.540 & 22.046 & 18.963 & & 25.921 & 14.725 & 8.044 \\
\hline $11 / 12 / 1020: 00$ & 30.531 & 21.950 & 18.965 & & 25.910 & 14.709 & 8.039 \\
\hline 11/13/10 0:00 & 30.494 & 21.571 & 18.933 & & 25.898 & 14.690 & 8.020 \\
\hline 11/13/10 4:00 & 30.481 & 21.345 & 18.949 & & 25.889 & 14.681 & 8.013 \\
\hline 11/13/10 8:00 & 30.469 & 21.221 & 18.949 & & 25.879 & 14.672 & 8.013 \\
\hline $11 / 13 / 1012: 00$ & 30.458 & 21.156 & 18.956 & & 25.875 & 14.660 & 8.011 \\
\hline 11/13/10 16:00 & 30.437 & 21.107 & 18.949 & & 25.863 & 14.646 & 8.002 \\
\hline 11/13/10 20:00 & 30.426 & 21.107 & 18.949 & & 25.854 & 14.634 & 7.999 \\
\hline 11/14/10 0:00 & 30.410 & 21.100 & 18.940 & & 25.844 & 14.618 & 7.992 \\
\hline 11/14/10 4:00 & 30.389 & 21.084 & 18.903 & & 25.835 & 14.606 & 7.985 \\
\hline $11 / 14 / 108: 00$ & 30.368 & 21.072 & 18.891 & & 25.826 & 14.590 & 7.976 \\
\hline $11 / 14 / 1012: 00$ & 30.352 & 21.065 & 18.889 & & 25.814 & 14.569 & 7.971 \\
\hline $11 / 14 / 1016: 00$ & 30.325 & 21.023 & 18.921 & & 25.802 & 14.555 & 7.953 \\
\hline $11 / 14 / 1020: 00$ & 30.309 & 21.019 & 18.916 & & 25.793 & 14.541 & 7.948 \\
\hline 11/15/10 0:00 & 30.291 & 21.021 & 18.914 & & 25.784 & 14.525 & 7.939 \\
\hline 11/15/10 4:00 & 30.267 & 21.000 & 18.905 & & 25.772 & 14.508 & 7.929 \\
\hline 11/15/10 8:00 & 30.251 & 20.995 & 18.903 & & 25.763 & 14.497 & 7.922 \\
\hline 11/15/10 12:00 & 30.238 & 21.040 & 18.903 & & 25.756 & 14.480 & 7.915 \\
\hline 11/15/10 16:00 & 30.217 & 21.047 & 18.898 & & 25.742 & 14.469 & 7.908 \\
\hline $11 / 15 / 10$ 20:00 & 30.206 & 21.077 & 18.898 & & 25.735 & 14.459 & 7.908 \\
\hline 11/16/10 0:00 & 30.190 & 21.103 & 18.893 & & 25.725 & 14.447 & 7.901 \\
\hline 11/16/10 4:00 & 30.169 & 21.098 & 18.891 & & 25.714 & 14.429 & 7.901 \\
\hline 11/16/10 8:00 & 30.151 & 21.100 & 18.889 & & 25.704 & 14.417 & 7.885 \\
\hline 11/16/10 12:00 & 30.132 & 21.107 & 18.884 & & 25.695 & 14.401 & 7.876 \\
\hline $11 / 16 / 1016: 00$ & 30.112 & 21.093 & 18.877 & & 25.683 & 14.389 & 7.866 \\
\hline 11/16/10 20:00 & 30.100 & 21.133 & 18.879 & & 25.674 & 14.382 & 7.864 \\
\hline 11/17/10 0:00 & 30.093 & 21.210 & 18.889 & & 25.667 & 14.380 & 7.866 \\
\hline 11/17/10 4:00 & 30.089 & 21.291 & 18.896 & & 25.662 & 14.375 & 7.873 \\
\hline 11/17/10 8:00 & 30.086 & 21.389 & 18.907 & & 25.655 & 14.375 & 7.878 \\
\hline 11/17/10 12:00 & 30.089 & 21.515 & 18.921 & & 25.650 & 14.368 & 7.885 \\
\hline $11 / 17 / 1016: 00$ & 30.070 & 21.536 & 18.919 & & 25.641 & 14.364 & 7.880 \\
\hline $11 / 17 / 1020: 00$ & 30.068 & 21.610 & 18.930 & & 25.632 & 14.359 & 7.880 \\
\hline $11 / 18 / 100: 00$ & 30.059 & 21.673 & 18.935 & & 25.629 & 14.350 & 7.880 \\
\hline 11/18/10 4:00 & 30.045 & 21.696 & 18.935 & & 25.618 & 14.340 & 7.873 \\
\hline 11/18/10 8:00 & 30.031 & 21.717 & 18.937 & & 25.611 & 14.329 & 7.871 \\
\hline $11 / 18 / 1012: 00$ & 30.018 & 21.748 & 18.937 & & 25.601 & 14.315 & 7.871 \\
\hline 11/18/10 16:00 & 29.997 & 21.699 & 18.928 & & 25.587 & 14.294 & 7.852 \\
\hline $11 / 18 / 1020: 00$ & 29.970 & 21.641 & 18.919 & & 25.576 & 14.277 & 7.843 \\
\hline 11/19/10 0:00 & 29.949 & 21.606 & 18.912 & & 25.564 & 14.261 & 7.836 \\
\hline
\end{tabular}

TABLE S1.2 Page 15 of 58 


\begin{tabular}{|c|c|c|c|c|c|c|c|}
\hline \multirow[b]{2}{*}{ Date and Time } & \multicolumn{7}{|c|}{ Water Level (ft below reference point) } \\
\hline & SB43S & SB44S & SB45S & SB46S & SB47S & SB48S & SB49S \\
\hline $11 / 19 / 104: 00$ & 29.921 & 21.552 & 18.900 & & 25.550 & 14.242 & 7.813 \\
\hline 11/19/10 8:00 & 29.899 & 21.503 & 18.889 & & 25.541 & 14.233 & 7.806 \\
\hline $11 / 19 / 1012: 00$ & 29.887 & 21.538 & 18.893 & & 25.534 & 14.219 & 7.801 \\
\hline $11 / 19 / 1016: 00$ & 29.871 & 21.543 & 18.889 & & 25.524 & 14.212 & 7.789 \\
\hline $11 / 19 / 1020: 00$ & 29.860 & 21.594 & 18.896 & & 25.515 & 14.205 & 7.799 \\
\hline 11/20/10 0:00 & 29.853 & 21.657 & 18.900 & & 25.510 & 14.196 & 7.796 \\
\hline $11 / 20 / 104: 00$ & 29.834 & 21.682 & 18.900 & & 25.499 & 14.186 & 7.794 \\
\hline 11/20/10 8:00 & 29.823 & 21.694 & 18.900 & & 25.487 & 14.177 & 7.787 \\
\hline $11 / 20 / 1012: 00$ & 29.802 & 21.699 & 18.896 & & 25.478 & 14.153 & 7.778 \\
\hline 11/20/10 16:00 & 29.777 & 21.643 & 18.884 & & 25.466 & 14.142 & 7.766 \\
\hline $11 / 20 / 1020: 00$ & 29.756 & 21.624 & 18.877 & & 25.454 & 14.125 & 7.754 \\
\hline 11/21/10 0:00 & 29.740 & 21.622 & 18.877 & & 25.445 & 14.114 & 7.750 \\
\hline $11 / 21 / 104: 00$ & 29.717 & 21.608 & 18.868 & & 25.433 & 14.097 & 7.736 \\
\hline 11/21/10 8:00 & 29.697 & 21.573 & 18.861 & & 25.424 & 14.086 & 7.726 \\
\hline $11 / 21 / 1012: 00$ & 29.683 & 21.599 & 18.865 & & 25.415 & 14.076 & 7.726 \\
\hline $11 / 21 / 1016: 00$ & 29.669 & 21.620 & 18.863 & & 25.403 & 14.067 & 7.722 \\
\hline $11 / 21 / 1020: 00$ & 29.660 & 21.675 & 18.870 & & 25.396 & 14.060 & 7.717 \\
\hline 11/22/10 0:00 & 29.644 & 21.703 & 18.865 & & 25.384 & 14.046 & 7.712 \\
\hline $11 / 22 / 104: 00$ & 29.619 & 21.673 & 18.858 & & 25.375 & 14.027 & 7.698 \\
\hline 11/22/10 8:00 & 29.598 & 21.631 & 18.851 & & 25.361 & 14.020 & 7.689 \\
\hline $11 / 22 / 1012: 00$ & 29.601 & 21.715 & 18.865 & & 25.358 & 14.018 & 7.698 \\
\hline $11 / 22 / 1016: 00$ & 29.589 & 21.787 & 18.868 & & 25.347 & 14.011 & 7.698 \\
\hline $11 / 22 / 1020: 00$ & 29.589 & 21.869 & 18.884 & & 25.347 & 14.016 & 7.705 \\
\hline $11 / 23 / 100: 00$ & 29.585 & 21.957 & 18.891 & & 25.340 & 14.011 & 7.708 \\
\hline $11 / 23 / 104: 00$ & 29.575 & 22.011 & 18.898 & & 25.330 & 14.009 & 7.708 \\
\hline $11 / 23 / 108: 00$ & 29.573 & 22.076 & 18.909 & & 25.326 & 14.004 & 7.715 \\
\hline $11 / 23 / 1012: 00$ & 29.564 & 22.132 & 18.912 & & 25.319 & 13.992 & 7.715 \\
\hline $11 / 23 / 1016: 00$ & 29.543 & 22.104 & 18.907 & & 25.307 & 13.974 & 7.708 \\
\hline $11 / 23 / 1020: 00$ & 29.523 & 22.060 & 18.900 & & 25.295 & 13.962 & 7.696 \\
\hline 11/24/10 0:00 & 29.500 & 22.020 & 18.891 & & 25.281 & 13.939 & 7.682 \\
\hline 11/24/10 4:00 & 29.472 & 21.943 & 18.877 & & 25.270 & 13.922 & 7.666 \\
\hline $11 / 24 / 108: 00$ & 29.445 & 21.866 & 18.863 & & 25.253 & 13.899 & 7.649 \\
\hline $11 / 24 / 1012: 00$ & 29.413 & 21.804 & 18.849 & & 25.239 & 13.878 & 7.633 \\
\hline $11 / 24 / 1016: 00$ & 29.390 & 21.752 & 18.840 & & 25.228 & 13.871 & 7.619 \\
\hline $11 / 24 / 1020: 00$ & 29.392 & 21.815 & 18.856 & & 25.225 & 13.873 & 7.626 \\
\hline $11 / 25 / 100: 00$ & 29.392 & 21.946 & 18.868 & & 25.223 & 13.878 & 7.637 \\
\hline $11 / 25 / 104: 00$ & 29.390 & 22.046 & 18.879 & & 25.216 & 13.873 & 7.642 \\
\hline 11/25/10 8:00 & 29.378 & 22.097 & 18.884 & & 25.207 & 13.866 & 7.645 \\
\hline $11 / 25 / 1012: 00$ & 29.369 & 22.134 & 18.886 & & 25.197 & 13.855 & 7.642 \\
\hline $11 / 25 / 1016: 00$ & 29.353 & 22.132 & 18.886 & & 25.190 & 13.848 & 7.631 \\
\hline 11/25/10 20:00 & 29.346 & 22.172 & 18.891 & & 25.183 & 13.843 & 7.635 \\
\hline $11 / 26 / 100: 00$ & 29.337 & 22.199 & 18.896 & & 25.174 & 13.834 & 7.633 \\
\hline $11 / 26 / 104: 00$ & 29.323 & 22.209 & 18.896 & & 25.167 & 13.824 & 7.631 \\
\hline 11/26/10 8:00 & 29.307 & 22.197 & 18.893 & & 25.155 & 13.817 & 7.624 \\
\hline $11 / 26 / 1012: 00$ & 29.294 & 22.216 & 18.893 & & 25.146 & 13.801 & 7.619 \\
\hline $11 / 26 / 1016: 00$ & 29.275 & 22.195 & 18.891 & & 25.137 & 13.794 & 7.612 \\
\hline $11 / 26 / 1020: 00$ & 29.268 & 22.232 & 18.896 & & 25.127 & 13.789 & 7.612 \\
\hline $11 / 27 / 100: 00$ & 29.261 & 22.260 & 18.900 & & 25.120 & 13.782 & 7.614 \\
\hline $11 / 27 / 104: 00$ & 29.248 & 22.281 & 18.903 & & 25.113 & 13.775 & 7.610 \\
\hline 11/27/10 8:00 & 29.239 & 22.304 & 18.907 & & 25.104 & 13.768 & 7.610 \\
\hline $11 / 27 / 1012: 00$ & 29.227 & 22.328 & 18.893 & & 25.094 & 13.757 & 7.610 \\
\hline 11/27/10 16:00 & 29.206 & 22.297 & 18.863 & & 25.085 & 13.743 & 7.598 \\
\hline $11 / 27 / 1020: 00$ & 29.190 & 22.274 & 18.868 & & 25.076 & 13.733 & 7.591 \\
\hline 11/28/10 0:00 & 29.174 & 22.267 & 18.898 & & 25.066 & 13.719 & 7.586 \\
\hline
\end{tabular}

TABLE S1.2 Page 16 of 58 


\begin{tabular}{|c|c|c|c|c|c|c|c|}
\hline \multirow[b]{2}{*}{ Date and Time } & \multicolumn{7}{|c|}{ Water Level (ft below reference point) } \\
\hline & SB43S & SB44S & SB45S & SB46S & SB47S & SB48S & SB49S \\
\hline 11/28/10 4:00 & 29.156 & 22.244 & 18.893 & & 25.055 & 13.708 & 7.574 \\
\hline 11/28/10 8:00 & 29.140 & 22.232 & 18.889 & & 25.045 & 13.696 & 7.570 \\
\hline 11/28/10 12:00 & 29.117 & 22.213 & 18.884 & & 25.034 & 13.675 & 7.561 \\
\hline 11/28/10 16:00 & 29.090 & 22.146 & 18.870 & & 25.022 & 13.659 & 7.544 \\
\hline 11/28/10 20:00 & 29.074 & 22.118 & 18.865 & & 25.008 & 13.654 & 7.535 \\
\hline 11/29/10 0:00 & 29.064 & 22.151 & 18.868 & & 24.994 & 13.642 & 7.535 \\
\hline 11/29/10 4:00 & 29.046 & 22.144 & 18.865 & & 24.973 & 13.628 & 7.530 \\
\hline 11/29/10 8:00 & 29.028 & 22.134 & 18.861 & & 24.947 & 13.619 & 7.521 \\
\hline 11/29/10 12:00 & 29.014 & 22.132 & 18.858 & & 24.929 & 13.600 & 7.512 \\
\hline 11/29/10 16:00 & 28.996 & 22.113 & 18.856 & & 24.905 & 13.610 & 7.509 \\
\hline 11/29/10 20:00 & 29.009 & 22.253 & 18.879 & & 24.922 & 13.610 & 7.526 \\
\hline 11/30/10 0:00 & 29.002 & 22.342 & 18.886 & & 24.917 & 13.607 & 7.530 \\
\hline 11/30/10 4:00 & 28.996 & 22.386 & 18.893 & & 24.910 & 13.600 & 7.533 \\
\hline 11/30/10 8:00 & 28.989 & 22.414 & 18.900 & & 24.898 & 13.600 & 7.535 \\
\hline $11 / 30 / 1012: 00$ & 28.984 & 22.470 & 18.907 & & 24.894 & 13.591 & 7.537 \\
\hline 11/30/10 16:00 & 28.975 & 22.488 & 18.909 & & 24.884 & 13.589 & 7.537 \\
\hline 11/30/10 20:00 & 28.966 & 22.519 & 18.914 & & 24.873 & 13.579 & 7.540 \\
\hline 12/1/10 0:00 & 28.954 & 22.530 & 18.916 & & 24.856 & 13.575 & 7.533 \\
\hline 12/1/10 4:00 & 28.947 & 22.546 & 18.923 & & 24.847 & 13.570 & 7.535 \\
\hline 12/1/10 8:00 & 28.938 & 22.572 & 18.928 & & 24.835 & 13.565 & 7.535 \\
\hline 12/1/10 12:00 & 28.931 & 22.605 & 18.935 & & 24.823 & 13.556 & 7.537 \\
\hline 12/1/10 16:00 & 28.915 & 22.591 & 18.928 & & 24.803 & 13.547 & 7.530 \\
\hline 12/1/10 20:00 & 28.904 & 22.586 & 18.916 & & 24.786 & 13.542 & 7.530 \\
\hline 12/2/10 0:00 & 28.890 & 22.591 & 18.954 & & 24.767 & 13.528 & 7.521 \\
\hline $12 / 2 / 104: 00$ & 28.879 & 22.586 & 18.986 & & 24.749 & 13.521 & 7.519 \\
\hline 12/2/10 8:00 & 28.863 & 22.577 & 18.956 & & 24.728 & 13.514 & 7.516 \\
\hline $12 / 2 / 1012: 00$ & 28.854 & 22.605 & 19.014 & & 24.716 & 13.505 & 7.514 \\
\hline 12/2/10 16:00 & 28.840 & 22.600 & 18.935 & & 24.697 & 13.500 & 7.512 \\
\hline $12 / 2 / 1020: 00$ & 28.837 & 22.642 & 18.942 & & 24.693 & 13.500 & 7.514 \\
\hline 12/3/10 0:00 & 28.828 & 22.675 & 18.947 & & 24.681 & 13.488 & 7.516 \\
\hline $12 / 3 / 104: 00$ & 28.815 & 22.670 & 18.947 & & 24.662 & 13.479 & 7.512 \\
\hline 12/3/10 8:00 & 28.799 & 22.651 & 18.944 & & 24.639 & 13.469 & 7.507 \\
\hline $12 / 3 / 1012: 00$ & 28.782 & 22.633 & 18.940 & & 24.616 & 13.451 & 7.498 \\
\hline 12/3/10 16:00 & 28.755 & 22.551 & 18.926 & & 24.578 & 13.432 & 7.484 \\
\hline $12 / 3 / 10$ 20:00 & 28.739 & 22.516 & 18.923 & & 24.555 & 13.430 & 7.477 \\
\hline 12/4/10 0:00 & 28.730 & 22.533 & 18.926 & & 24.541 & 13.425 & 7.477 \\
\hline $12 / 4 / 104: 00$ & 28.732 & 22.598 & 18.937 & & 24.543 & 13.427 & 7.477 \\
\hline 12/4/10 8:00 & 28.730 & 22.684 & 18.951 & & 24.543 & 13.432 & 7.488 \\
\hline $12 / 4 / 1012: 00$ & 28.737 & 22.782 & 18.968 & & 24.553 & 13.430 & 7.502 \\
\hline 12/4/10 16:00 & 28.727 & 22.812 & 18.972 & & 24.541 & 13.430 & 7.500 \\
\hline $12 / 4 / 10$ 20:00 & 28.725 & 22.854 & 18.982 & & 24.539 & 13.425 & 7.507 \\
\hline 12/5/10 0:00 & 28.716 & 22.882 & 18.986 & & 24.524 & 13.421 & 7.505 \\
\hline $12 / 5 / 104: 00$ & 28.707 & 22.896 & 18.989 & & 24.515 & 13.418 & 7.507 \\
\hline 12/5/10 8:00 & 28.700 & 22.912 & 18.996 & & 24.503 & 13.416 & 7.512 \\
\hline 12/5/10 12:00 & 28.695 & 22.940 & 19.000 & & 24.494 & 13.402 & 7.509 \\
\hline 12/5/10 16:00 & 28.679 & 22.912 & 18.998 & & 24.471 & 13.397 & 7.502 \\
\hline 12/5/10 20:00 & 28.668 & 22.907 & 18.998 & & 24.457 & 13.388 & 7.502 \\
\hline 12/6/10 0:00 & 28.656 & 22.912 & 18.998 & & 24.440 & 13.379 & 7.495 \\
\hline $12 / 6 / 104: 00$ & 28.640 & 22.905 & 18.998 & & 24.422 & 13.369 & 7.495 \\
\hline 12/6/10 8:00 & 28.629 & 22.887 & 18.996 & & 24.398 & 13.360 & 7.488 \\
\hline 12/6/10 12:00 & 28.613 & 22.880 & 18.996 & & 24.380 & 13.350 & 7.481 \\
\hline $12 / 6 / 1016: 00$ & 28.595 & 22.833 & 18.986 & & 24.349 & 13.337 & 7.474 \\
\hline $12 / 6 / 10$ 20:00 & 28.579 & 22.800 & 18.984 & & 24.328 & 13.329 & 7.465 \\
\hline 12/7/10 0:00 & 28.565 & 22.796 & 18.984 & & 24.310 & 13.318 & 7.465 \\
\hline
\end{tabular}

TABLE S1.2 Page 17 of 58 


\begin{tabular}{|c|c|c|c|c|c|c|c|}
\hline \multirow[b]{2}{*}{ Date and Time } & \multicolumn{7}{|c|}{ Water Level (ft below reference point) } \\
\hline & SB43S & SB44S & SB45S & SB46S & SB47S & SB48S & SB49S \\
\hline 12/7/10 4:00 & 28.553 & 22.800 & 18.984 & & 24.293 & 13.313 & 7.458 \\
\hline 12/7/10 8:00 & 28.546 & 22.817 & 18.984 & & 24.277 & 13.309 & 7.456 \\
\hline 12/7/10 12:00 & 28.540 & 22.861 & 18.993 & & 24.272 & 13.302 & 7.460 \\
\hline 12/7/10 16:00 & 28.524 & 22.856 & 18.991 & & 24.249 & 13.295 & 7.456 \\
\hline 12/7/10 20:00 & 28.517 & 22.887 & 18.996 & & 24.239 & 13.288 & 7.456 \\
\hline 12/8/10 0:00 & 28.507 & 22.901 & 18.998 & & 24.225 & 13.283 & 7.460 \\
\hline 12/8/10 4:00 & 28.494 & 22.905 & 18.996 & & 24.204 & 13.274 & 7.449 \\
\hline $12 / 8 / 108: 00$ & 28.482 & 22.903 & 18.998 & & 24.188 & 13.267 & 7.446 \\
\hline $12 / 8 / 1012: 00$ & 28.473 & 22.919 & 19.000 & & 24.174 & 13.257 & 7.446 \\
\hline 12/8/10 16:00 & 28.457 & 22.891 & 18.993 & & 24.151 & 13.248 & 7.437 \\
\hline 12/8/10 20:00 & 28.443 & 22.889 & 18.993 & & 24.132 & 13.239 & 7.432 \\
\hline 12/9/10 0:00 & 28.430 & 22.870 & 18.991 & & 24.113 & 13.225 & 7.425 \\
\hline 12/9/10 4:00 & 28.411 & 22.842 & 18.984 & & 24.085 & 13.213 & 7.418 \\
\hline $12 / 9 / 108: 00$ & 28.388 & 22.796 & 18.975 & & 24.055 & 13.201 & 7.407 \\
\hline $12 / 9 / 1012: 00$ & 28.372 & 22.761 & 18.970 & & 24.029 & 13.187 & 7.400 \\
\hline 12/9/10 16:00 & 28.356 & 22.740 & 18.970 & & 24.008 & 13.183 & 7.393 \\
\hline 12/9/10 20:00 & 28.354 & 22.791 & 18.977 & & 24.004 & 13.183 & 7.397 \\
\hline 12/10/10 0:00 & 28.347 & 22.840 & 18.981 & & 23.994 & 13.178 & 7.397 \\
\hline $12 / 10 / 104: 00$ & 28.340 & 22.873 & 18.986 & & 23.982 & 13.171 & 7.402 \\
\hline 12/10/10 8:00 & 28.329 & 22.884 & 18.988 & & 23.968 & 13.166 & 7.397 \\
\hline 12/10/10 12:00 & 28.317 & 22.905 & 18.989 & & 23.954 & 13.155 & 7.395 \\
\hline 12/10/10 16:00 & 28.299 & 22.856 & 18.979 & & 23.922 & 13.141 & 7.386 \\
\hline 12/10/10 20:00 & 28.285 & 22.840 & 18.977 & & 23.903 & 13.134 & 7.383 \\
\hline 12/11/10 0:00 & 28.269 & 22.812 & 18.977 & & 23.877 & 13.122 & 7.374 \\
\hline 12/11/10 4:00 & 28.251 & 22.796 & 18.970 & & 23.854 & 13.108 & 7.365 \\
\hline 12/11/10 8:00 & 28.242 & 22.784 & 18.972 & & 23.840 & 13.113 & 7.365 \\
\hline $12 / 11 / 1012: 00$ & 28.239 & 22.868 & 18.984 & & 23.833 & 13.108 & 7.369 \\
\hline $12 / 11 / 1016: 00$ & 28.232 & 22.898 & 18.984 & & 23.826 & 13.103 & 7.374 \\
\hline 12/11/10 20:00 & 28.223 & 22.931 & 18.993 & & 23.810 & 13.101 & 7.372 \\
\hline 12/12/10 0:00 & 28.226 & 22.973 & 19.000 & & 23.812 & 13.105 & 7.376 \\
\hline $12 / 12 / 104: 00$ & 28.228 & 23.040 & 19.014 & & 23.812 & 13.105 & 7.386 \\
\hline 12/12/10 8:00 & 28.228 & 23.110 & 19.028 & & 23.814 & 13.110 & 7.395 \\
\hline $12 / 12 / 1012: 00$ & 28.228 & 23.161 & 19.037 & & 23.812 & 13.103 & 7.407 \\
\hline 12/12/10 16:00 & 28.221 & 23.175 & 19.040 & & 23.803 & 13.103 & 7.404 \\
\hline $12 / 12 / 1020: 00$ & 28.212 & 23.203 & 19.047 & & 23.793 & 13.098 & 7.407 \\
\hline 12/13/10 0:00 & 28.203 & 23.208 & 19.049 & & 23.779 & 13.089 & 7.407 \\
\hline 12/13/10 4:00 & 28.193 & 23.196 & 19.047 & & 23.763 & 13.082 & 7.404 \\
\hline 12/13/10 8:00 & 28.180 & 23.189 & 19.049 & & 23.744 & 13.080 & 7.400 \\
\hline 12/13/10 12:00 & 28.175 & 23.210 & 19.054 & & 23.737 & 13.070 & 7.402 \\
\hline $12 / 13 / 1016: 00$ & 28.161 & 23.185 & 19.051 & & 23.711 & 13.061 & 7.393 \\
\hline $12 / 13 / 1020: 00$ & 28.148 & 23.173 & 19.049 & & 23.695 & 13.056 & 7.390 \\
\hline 12/14/10 0:00 & 28.141 & 23.182 & 19.054 & & 23.681 & 13.049 & 7.390 \\
\hline $12 / 14 / 104: 00$ & 28.127 & 23.173 & 19.051 & & 23.662 & 13.040 & 7.386 \\
\hline 12/14/10 8:00 & 28.111 & 23.145 & 19.044 & & 23.639 & 13.031 & 7.381 \\
\hline 12/14/10 12:00 & 28.100 & 23.140 & 19.047 & & 23.623 & 13.019 & 7.379 \\
\hline $12 / 14 / 1016: 00$ & 28.074 & 23.087 & 19.035 & & 23.590 & 13.010 & 7.362 \\
\hline $12 / 14 / 1020: 00$ & 28.061 & 23.068 & 19.035 & & 23.571 & 13.000 & 7.358 \\
\hline 12/15/10 0:00 & 28.049 & 23.061 & 19.037 & & 23.553 & 12.991 & 7.355 \\
\hline 12/15/10 4:00 & 28.033 & 23.043 & 19.030 & & 23.532 & 12.979 & 7.351 \\
\hline 12/15/10 8:00 & 28.015 & 23.017 & 19.026 & & 23.506 & 12.972 & 7.341 \\
\hline $12 / 15 / 1012: 00$ & 28.001 & 23.003 & 19.021 & & 23.483 & 12.956 & 7.337 \\
\hline $12 / 15 / 1016: 00$ & 27.983 & 22.973 & 19.019 & & 23.459 & 12.954 & 7.330 \\
\hline 12/15/10 20:00 & 27.978 & 23.008 & 19.021 & & 23.450 & 12.949 & 7.332 \\
\hline 12/16/10 0:00 & 27.973 & 23.043 & 19.028 & & 23.440 & 12.944 & 7.334 \\
\hline
\end{tabular}

TABLE S1.2 Page 18 of 58 


\begin{tabular}{|c|c|c|c|c|c|c|c|}
\hline \multirow[b]{2}{*}{ Date and Time } & \multicolumn{7}{|c|}{ Water Level (ft below reference point) } \\
\hline & SB43S & SB44S & SB45S & SB46S & SB47S & SB48S & SB49S \\
\hline $12 / 16 / 104: 00$ & 27.967 & 23.066 & 19.030 & & 23.429 & 12.942 & 7.334 \\
\hline 12/16/10 8:00 & 27.964 & 23.112 & 19.040 & & 23.424 & 12.944 & 7.339 \\
\hline 12/16/10 12:00 & 27.964 & 23.180 & 19.051 & & 23.424 & 12.942 & 7.346 \\
\hline $12 / 16 / 1016: 00$ & 27.957 & 23.210 & 19.054 & & 23.410 & 12.942 & 7.346 \\
\hline 12/16/10 20:00 & 27.955 & 23.257 & 19.063 & & 23.403 & 12.942 & 7.353 \\
\hline 12/17/10 0:00 & 27.951 & 23.282 & 19.070 & & 23.396 & 12.937 & 7.360 \\
\hline 12/17/10 4:00 & 27.946 & 23.313 & 19.079 & & 23.389 & 12.935 & 7.355 \\
\hline 12/17/10 8:00 & 27.941 & 23.331 & 19.084 & & 23.377 & 12.937 & 7.360 \\
\hline $12 / 17 / 1012: 00$ & 27.941 & 23.369 & 19.093 & & 23.375 & 12.930 & 7.365 \\
\hline $12 / 17 / 1016: 00$ & 27.928 & 23.357 & 19.091 & & 23.359 & 12.926 & 7.367 \\
\hline $12 / 17 / 1020: 00$ & 27.918 & 23.357 & 19.096 & & 23.342 & 12.921 & 7.360 \\
\hline 12/18/10 0:00 & 27.912 & 23.364 & 19.096 & & 23.328 & 12.914 & 7.358 \\
\hline 12/18/10 4:00 & 27.900 & 23.357 & 19.096 & & 23.312 & 12.905 & 7.355 \\
\hline 12/18/10 8:00 & 27.889 & 23.345 & 19.096 & & 23.296 & 12.900 & 7.358 \\
\hline $12 / 18 / 1012: 00$ & 27.880 & 23.348 & 19.098 & & 23.282 & 12.888 & 7.353 \\
\hline 12/18/10 16:00 & 27.861 & 23.310 & 19.091 & & 23.256 & 12.884 & 7.346 \\
\hline 12/18/10 20:00 & 27.852 & 23.301 & 19.091 & & 23.237 & 12.877 & 7.341 \\
\hline 12/19/10 0:00 & 27.841 & 23.308 & 19.091 & & 23.223 & 12.872 & 7.339 \\
\hline 12/19/10 4:00 & 27.834 & 23.310 & 19.093 & & 23.207 & 12.863 & 7.341 \\
\hline 12/19/10 8:00 & 27.822 & 23.308 & 19.093 & & 23.193 & 12.860 & 7.337 \\
\hline 12/19/10 12:00 & 27.813 & 23.327 & 19.093 & & 23.179 & 12.849 & 7.339 \\
\hline 12/19/10 16:00 & 27.797 & 23.299 & 19.091 & & 23.137 & 12.842 & 7.325 \\
\hline 12/19/10 20:00 & 27.790 & 23.299 & 19.091 & & 23.104 & 12.837 & 7.327 \\
\hline 12/20/10 0:00 & 27.779 & 23.308 & 19.091 & & 23.071 & 12.830 & 7.325 \\
\hline 12/20/10 4:00 & 27.765 & 23.303 & 19.089 & & 23.041 & 12.818 & 7.320 \\
\hline $12 / 20 / 108: 00$ & 27.749 & 23.271 & 19.084 & & 23.013 & 12.814 & 7.311 \\
\hline 12/20/10 12:00 & 27.735 & 23.261 & 19.079 & & 22.994 & 12.804 & 7.304 \\
\hline $12 / 20 / 1016: 00$ & 27.719 & 23.227 & 19.072 & & 22.936 & 12.795 & 7.295 \\
\hline $12 / 20 / 10$ 20:00 & 27.719 & 23.273 & 19.084 & & 22.931 & 12.795 & 7.306 \\
\hline 12/21/10 0:00 & 27.715 & 23.324 & 19.093 & & 22.924 & 12.795 & 7.309 \\
\hline 12/21/10 4:00 & 27.715 & 23.366 & 19.100 & & 22.920 & 12.797 & 7.313 \\
\hline 12/21/10 8:00 & 27.712 & 23.413 & 19.110 & & 22.920 & 12.800 & 7.320 \\
\hline $12 / 21 / 1012: 00$ & 27.715 & 23.469 & 19.121 & & 22.917 & 12.795 & 7.327 \\
\hline 12/21/10 16:00 & 27.710 & 23.483 & 19.126 & & 22.910 & 12.800 & 7.332 \\
\hline $12 / 21 / 1020: 00$ & 27.712 & 23.529 & 19.140 & & 22.913 & 12.804 & 7.339 \\
\hline 12/22/10 0:00 & 27.708 & 23.569 & 19.147 & & 22.910 & 12.802 & 7.341 \\
\hline $12 / 22 / 104: 00$ & 27.710 & 23.606 & 19.158 & & 22.908 & 12.802 & 7.353 \\
\hline 12/22/10 8:00 & 27.708 & 23.629 & 19.165 & & 22.901 & 12.805 & 7.358 \\
\hline $12 / 22 / 1012: 00$ & 27.708 & 23.669 & 19.175 & & 22.898 & 12.802 & 7.365 \\
\hline $12 / 22 / 1016: 00$ & 27.698 & 23.662 & 19.175 & & 22.884 & 12.798 & 7.362 \\
\hline $12 / 22 / 1020: 00$ & 27.692 & 23.674 & 19.179 & & 22.875 & 12.797 & 7.365 \\
\hline 12/23/10 0:00 & 27.685 & 23.685 & 19.184 & & 22.866 & 12.790 & 7.365 \\
\hline $12 / 23 / 104: 00$ & 27.676 & 23.674 & 19.184 & & 22.845 & 12.786 & 7.358 \\
\hline 12/23/10 8:00 & 27.666 & 23.662 & 19.184 & & 22.833 & 12.779 & 7.360 \\
\hline $12 / 23 / 10$ 12:00 & 27.655 & 23.650 & 19.184 & & 22.814 & 12.767 & 7.355 \\
\hline $12 / 23 / 1016: 00$ & 27.639 & 23.597 & 19.177 & & 22.789 & 12.760 & 7.344 \\
\hline $12 / 23 / 1020: 00$ & 27.625 & 23.574 & 19.175 & & 22.770 & 12.753 & 7.339 \\
\hline 12/24/10 0:00 & 27.616 & 23.569 & 19.175 & & 22.754 & 12.746 & 7.339 \\
\hline $12 / 24 / 104: 00$ & 27.609 & 23.571 & 19.177 & & 22.740 & 12.742 & 7.337 \\
\hline 12/24/10 8:00 & 27.598 & 23.564 & 19.175 & & 22.723 & 12.739 & 7.334 \\
\hline 12/24/10 12:00 & 27.588 & 23.569 & 19.177 & & 22.712 & 12.732 & 7.334 \\
\hline $12 / 24 / 1016: 00$ & 27.579 & 23.560 & 19.177 & & 22.691 & 12.728 & 7.330 \\
\hline $12 / 24 / 1020: 00$ & 27.575 & 23.595 & 19.184 & & 22.674 & 12.730 & 7.332 \\
\hline 12/25/10 0:00 & 27.575 & 23.643 & 19.191 & & 22.665 & 12.732 & 7.339 \\
\hline
\end{tabular}

TABLE S1.2 Page 19 of 58 


\begin{tabular}{|c|c|c|c|c|c|c|c|}
\hline \multirow[b]{2}{*}{ Date and Time } & \multicolumn{7}{|c|}{ Water Level (ft below reference point) } \\
\hline & SB43S & SB44S & SB45S & SB46S & SB47S & SB48S & SB49S \\
\hline $12 / 25 / 104: 00$ & 27.572 & 23.690 & 19.203 & & 22.660 & 12.732 & 7.344 \\
\hline 12/25/10 8:00 & 27.570 & 23.716 & 19.207 & & 22.651 & 12.732 & 7.346 \\
\hline $12 / 25 / 1012: 00$ & 27.568 & 23.741 & 19.214 & & 22.646 & 12.728 & 7.351 \\
\hline $12 / 25 / 1016: 00$ & 27.556 & 23.725 & 19.212 & & 22.627 & 12.720 & 7.348 \\
\hline $12 / 25 / 1020: 00$ & 27.552 & 23.741 & 19.219 & & 22.618 & 12.723 & 7.348 \\
\hline $12 / 26 / 100: 00$ & 27.545 & 23.748 & 19.221 & & 22.604 & 12.716 & 7.348 \\
\hline $12 / 26 / 104: 00$ & 27.536 & 23.744 & 19.224 & & 22.595 & 12.714 & 7.348 \\
\hline $12 / 26 / 108: 00$ & 27.529 & 23.741 & 19.223 & & 22.579 & 12.711 & 7.346 \\
\hline $12 / 26 / 1012: 00$ & 27.524 & 23.760 & 19.228 & & 22.569 & 12.702 & 7.351 \\
\hline $12 / 26 / 1016: 00$ & 27.511 & 23.732 & 19.223 & & 22.548 & 12.695 & 7.341 \\
\hline $12 / 26 / 1020: 00$ & 27.497 & 23.713 & 19.221 & & 22.529 & 12.690 & 7.334 \\
\hline $12 / 27 / 100: 00$ & 27.488 & 23.699 & 19.221 & & 22.513 & 12.685 & 7.334 \\
\hline $12 / 27 / 104: 00$ & 27.478 & 23.702 & 19.221 & & 22.499 & 12.678 & 7.332 \\
\hline 12/27/10 8:00 & 27.469 & 23.690 & 19.221 & & 22.483 & 12.674 & 7.332 \\
\hline $12 / 27 / 1012: 00$ & 27.462 & 23.699 & 19.224 & & 22.469 & 12.664 & 7.327 \\
\hline $12 / 27 / 1016: 00$ & 27.440 & 23.650 & 19.212 & & 22.441 & 12.657 & 7.313 \\
\hline $12 / 27 / 1020: 00$ & 27.435 & 23.646 & 19.214 & & 22.427 & 12.653 & 7.318 \\
\hline 12/28/10 0:00 & 27.426 & 23.657 & 19.216 & & 22.415 & 12.648 & 7.316 \\
\hline $12 / 28 / 104: 00$ & 27.417 & 23.660 & 19.219 & & 22.401 & 12.643 & 7.313 \\
\hline 12/28/10 8:00 & 27.412 & 23.669 & 19.221 & & 22.389 & 12.641 & 7.313 \\
\hline $12 / 28 / 1012: 00$ & 27.405 & 23.692 & 19.224 & & 22.378 & 12.634 & 7.316 \\
\hline $12 / 28 / 1016: 00$ & 27.387 & 23.662 & 19.219 & & 22.357 & 12.627 & 7.306 \\
\hline $12 / 28 / 1020: 00$ & 27.378 & 23.650 & 19.217 & & 22.331 & 12.620 & 7.304 \\
\hline 12/29/10 0:00 & 27.364 & 23.634 & 19.212 & & 22.310 & 12.611 & 7.295 \\
\hline $12 / 29 / 104: 00$ & 27.348 & 23.604 & 19.210 & & 22.286 & 12.599 & 7.290 \\
\hline $12 / 29 / 108: 00$ & 27.334 & 23.576 & 19.200 & & 22.263 & 12.592 & 7.278 \\
\hline $12 / 29 / 1012: 00$ & 27.318 & 23.557 & 19.196 & & 22.223 & 12.576 & 7.276 \\
\hline $12 / 29 / 1016: 00$ & 27.293 & 23.494 & 19.179 & & 22.179 & 12.566 & 7.257 \\
\hline $12 / 29 / 1020: 00$ & 27.284 & 23.476 & 19.177 & & 22.158 & 12.559 & 7.250 \\
\hline 12/30/10 0:00 & 27.272 & 23.473 & 19.177 & & 22.142 & 12.552 & 7.248 \\
\hline 12/30/10 4:00 & 27.261 & 23.452 & 19.172 & & 22.118 & 12.543 & 7.239 \\
\hline 12/30/10 8:00 & 27.247 & 23.441 & 19.168 & & 22.097 & 12.541 & 7.239 \\
\hline $12 / 30 / 1012: 00$ & 27.240 & 23.464 & 19.175 & & 22.086 & 12.536 & 7.239 \\
\hline $12 / 30 / 1016: 00$ & 27.233 & 23.464 & 19.175 & & 22.072 & 12.541 & 7.239 \\
\hline $12 / 30 / 1020: 00$ & 27.242 & 23.562 & 19.196 & & 22.086 & 12.548 & 7.253 \\
\hline 12/31/10 0:00 & 27.249 & 23.657 & 19.210 & & 22.088 & 12.550 & 7.264 \\
\hline 12/31/10 4:00 & 27.238 & 23.671 & 19.205 & & 22.074 & 12.543 & 7.262 \\
\hline $12 / 31 / 108: 00$ & 27.226 & 23.641 & 19.205 & & 22.055 & 12.534 & 7.260 \\
\hline 12/31/10 12:00 & 27.224 & 23.648 & 19.210 & & 22.046 & 12.538 & 7.257 \\
\hline $12 / 31 / 1016: 00$ & 27.226 & 23.709 & 19.223 & & 22.041 & 12.545 & 7.269 \\
\hline $12 / 31 / 1020: 00$ & 27.233 & 23.783 & 19.240 & & 22.046 & 12.550 & 7.281 \\
\hline $1 / 1 / 110: 00$ & 27.231 & 23.827 & 19.247 & & 22.041 & 12.548 & 7.288 \\
\hline 1/1/11 4:00 & 27.229 & 23.839 & 19.251 & & 22.034 & 12.548 & 7.290 \\
\hline 1/1/11 8:00 & 27.226 & 23.860 & 19.256 & & 22.030 & 12.552 & 7.292 \\
\hline 1/1/11 12:00 & 27.231 & 23.911 & 19.272 & & 22.032 & 12.552 & 7.304 \\
\hline $1 / 1 / 1116: 00$ & 27.226 & 23.930 & 19.277 & & 22.025 & 12.557 & 7.306 \\
\hline $1 / 1 / 1120: 00$ & 27.229 & 23.960 & 19.289 & & 22.027 & 12.555 & 7.318 \\
\hline $1 / 2 / 110: 00$ & 27.224 & 23.976 & 19.293 & & 22.018 & 12.552 & 7.316 \\
\hline $1 / 2 / 114: 00$ & 27.215 & 23.974 & 19.293 & & 22.001 & 12.548 & 7.316 \\
\hline $1 / 2 / 118: 00$ & 27.210 & 23.965 & 19.298 & & 21.990 & 12.545 & 7.313 \\
\hline $1 / 2 / 11$ 12:00 & 27.201 & 23.967 & 19.298 & & 21.976 & 12.536 & 7.311 \\
\hline $1 / 2 / 1116: 00$ & 27.121 & 23.916 & 19.293 & & 21.943 & 12.531 & 7.306 \\
\hline 1/2/11 20:00 & 27.176 & 23.914 & 19.293 & & 21.915 & 12.529 & 7.306 \\
\hline 1/3/11 0:00 & 27.171 & 23.925 & 19.298 & & 21.906 & 12.524 & 7.306 \\
\hline
\end{tabular}

TABLE S1.2 Page 20 of 58 


\begin{tabular}{|c|c|c|c|c|c|c|c|}
\hline \multirow[b]{2}{*}{ Date and Time } & \multicolumn{7}{|c|}{ Water Level (ft below reference point) } \\
\hline & SB43S & SB44S & SB45S & SB46S & SB47S & SB48S & SB49S \\
\hline $1 / 3 / 114: 00$ & 27.162 & 23.928 & 19.298 & & 21.866 & 12.520 & 7.302 \\
\hline 1/3/11 8:00 & 27.153 & 23.916 & 19.296 & & 21.805 & 12.515 & 7.302 \\
\hline $1 / 3 / 11$ 12:00 & 27.146 & 23.923 & 19.300 & & 21.777 & 12.508 & 7.299 \\
\hline 1/3/11 16:00 & 27.132 & 23.890 & 19.293 & & 21.871 & 12.508 & 7.295 \\
\hline $1 / 3 / 1120: 00$ & 27.132 & 23.920 & 19.303 & & 21.866 & 12.510 & 7.297 \\
\hline 1/4/11 0:00 & 27.132 & 23.965 & 19.310 & & 21.861 & 12.510 & 7.304 \\
\hline $1 / 4 / 114: 00$ & 27.128 & 23.988 & 19.314 & & 21.852 & 12.508 & 7.304 \\
\hline 1/4/11 8:00 & 27.126 & 24.002 & 19.319 & & 21.847 & 12.508 & 7.306 \\
\hline $1 / 4 / 11$ 12:00 & 27.119 & 24.014 & 19.324 & & 21.836 & 12.501 & 7.309 \\
\hline $1 / 4 / 11$ 16:00 & 27.105 & 23.967 & 19.317 & & 21.810 & 12.492 & 7.299 \\
\hline $1 / 4 / 1120: 00$ & 27.093 & 23.944 & 19.314 & & 21.796 & 12.489 & 7.297 \\
\hline 1/5/11 0:00 & 27.084 & 23.937 & 19.314 & & 21.782 & 12.482 & 7.295 \\
\hline 1/5/11 4:00 & 27.075 & 23.925 & 19.312 & & 21.766 & 12.475 & 7.290 \\
\hline 1/5/11 8:00 & 27.064 & 23.904 & 19.310 & & 21.744 & 12.471 & 7.285 \\
\hline $1 / 5 / 11$ 12:00 & 27.057 & 23.904 & 19.312 & & 21.735 & 12.466 & 7.288 \\
\hline 1/5/11 16:00 & 27.050 & 23.897 & 19.312 & & 21.716 & 12.466 & 7.281 \\
\hline $1 / 5 / 1120: 00$ & 27.045 & 23.925 & 19.317 & & 21.712 & 12.461 & 7.283 \\
\hline 1/6/11 0:00 & 27.041 & 23.953 & 19.321 & & 21.705 & 12.459 & 7.285 \\
\hline 1/6/11 4:00 & 27.034 & 23.958 & 19.321 & & 21.691 & 12.457 & 7.285 \\
\hline 1/6/11 8:00 & 27.022 & 23.946 & 19.321 & & 21.672 & 12.452 & 7.281 \\
\hline $1 / 6 / 11$ 12:00 & 27.015 & 23.944 & 19.321 & & 21.660 & 12.447 & 7.281 \\
\hline $1 / 6 / 11$ 16:00 & 27.004 & 23.930 & 19.319 & & 21.642 & 12.443 & 7.276 \\
\hline 1/6/11 20:00 & 26.995 & 23.923 & 19.317 & & 21.628 & 12.436 & 7.274 \\
\hline 1/7/11 0:00 & 26.983 & 23.897 & 19.312 & & 21.604 & 12.426 & 7.264 \\
\hline 1/7/11 4:00 & 26.967 & 23.869 & 19.305 & & 21.581 & 12.422 & 7.257 \\
\hline 1/7/11 8:00 & 26.963 & 23.883 & 19.310 & & 21.574 & 12.426 & 7.264 \\
\hline $1 / 7 / 11$ 12:00 & 26.965 & 23.937 & 19.319 & & 21.572 & 12.422 & 7.269 \\
\hline 1/7/11 16:00 & 26.963 & 23.965 & 19.326 & & 21.565 & 12.426 & 7.271 \\
\hline $1 / 7 / 1120: 00$ & 26.961 & 24.002 & 19.333 & & 21.562 & 12.426 & 7.274 \\
\hline 1/8/11 0:00 & 26.958 & 24.028 & 19.337 & & 21.551 & 12.426 & 7.283 \\
\hline 1/8/11 4:00 & 26.958 & 24.049 & 19.347 & & 21.546 & 12.429 & 7.283 \\
\hline 1/8/11 8:00 & 26.961 & 24.077 & 19.354 & & 21.546 & 12.433 & 7.288 \\
\hline $1 / 8 / 11$ 12:00 & 26.963 & 24.123 & 19.368 & & 21.548 & 12.433 & 7.297 \\
\hline $1 / 8 / 11$ 16:00 & 26.954 & 24.121 & 19.365 & & 21.532 & 12.429 & 7.295 \\
\hline 1/8/11 20:00 & 26.954 & 24.140 & 19.375 & & 21.530 & 12.431 & 7.299 \\
\hline 1/9/11 0:00 & 26.951 & 24.153 & 19.379 & & 21.520 & 12.431 & 7.306 \\
\hline $1 / 9 / 114: 00$ & 26.949 & 24.167 & 19.382 & & 21.513 & 12.426 & 7.309 \\
\hline 1/9/11 8:00 & 26.942 & 24.172 & 19.386 & & 21.504 & 12.429 & 7.306 \\
\hline $1 / 9 / 11$ 12:00 & 26.942 & 24.193 & 19.396 & & 21.499 & 12.426 & 7.311 \\
\hline 1/9/11 16:00 & 26.933 & 24.177 & 19.396 & & 21.483 & 12.422 & 7.306 \\
\hline $1 / 9 / 1120: 00$ & 26.928 & 24.188 & 19.398 & & 21.476 & 12.419 & 7.313 \\
\hline 1/10/11 0:00 & 26.922 & 24.186 & 19.400 & & 21.462 & 12.422 & 7.313 \\
\hline 1/10/11 4:00 & 26.922 & 24.212 & 19.405 & & 21.459 & 12.417 & 7.313 \\
\hline 1/10/11 8:00 & 26.912 & 24.207 & 19.407 & & 21.446 & 12.417 & 7.311 \\
\hline 1/10/11 12:00 & 26.908 & 24.212 & 19.410 & & 21.436 & 12.410 & 7.313 \\
\hline 1/10/11 16:00 & 26.899 & 24.191 & 19.410 & & 21.420 & 12.415 & 7.309 \\
\hline 1/10/11 20:00 & 26.896 & 24.212 & 19.412 & & 21.413 & 12.410 & 7.311 \\
\hline 1/11/11 0:00 & 26.894 & 24.223 & 19.419 & & 21.403 & 12.410 & 7.313 \\
\hline $1 / 11 / 114: 00$ & 26.892 & 24.237 & 19.424 & & 21.401 & 12.412 & 7.320 \\
\hline 1/11/11 8:00 & 26.889 & 24.258 & 19.433 & & 21.396 & 12.417 & 7.323 \\
\hline 1/11/11 12:00 & 26.894 & 24.296 & 19.445 & & 21.396 & 12.417 & 7.334 \\
\hline 1/11/11 16:00 & 26.894 & 24.314 & 19.452 & & 21.394 & 12.420 & 7.337 \\
\hline 1/11/11 20:00 & 26.894 & 24.337 & 19.458 & & 21.392 & 12.419 & 7.341 \\
\hline 1/12/11 0:00 & 26.894 & 24.354 & 19.465 & & 21.389 & 12.424 & 7.339 \\
\hline
\end{tabular}

TABLE S1.2 Page 21 of 58 


\begin{tabular}{|c|c|c|c|c|c|c|c|}
\hline \multirow[b]{2}{*}{ Date and Time } & \multicolumn{7}{|c|}{ Water Level (ft below reference point) } \\
\hline & SB43S & SB44S & SB45S & SB46S & SB47S & SB48S & SB49S \\
\hline $1 / 12 / 114: 00$ & 26.896 & 24.375 & 19.475 & & 21.385 & 12.424 & 7.351 \\
\hline 1/12/11 8:00 & 26.894 & 24.393 & 19.482 & & 21.378 & 12.429 & 7.355 \\
\hline $1 / 12 / 11$ 12:00 & 26.896 & 24.417 & 19.491 & & 21.375 & 12.424 & 7.367 \\
\hline 1/12/11 16:00 & 26.887 & 24.396 & 19.491 & & 21.359 & 12.424 & 7.360 \\
\hline 1/12/11 20:00 & 26.883 & 24.398 & 19.493 & & 21.350 & 12.419 & 7.355 \\
\hline 1/13/11 0:00 & 26.871 & 24.389 & 19.489 & & 21.333 & 12.413 & 7.358 \\
\hline $1 / 13 / 114: 00$ & 26.862 & 24.361 & 19.489 & & 21.319 & 12.408 & 7.353 \\
\hline 1/13/11 8:00 & 26.851 & 24.330 & 19.482 & & 21.298 & 12.401 & 7.346 \\
\hline 1/13/11 12:00 & 26.837 & 24.305 & 19.477 & & 21.277 & 12.387 & 7.339 \\
\hline $1 / 13 / 11$ 16:00 & 26.823 & 24.240 & 19.468 & & 21.249 & 12.382 & 7.325 \\
\hline $1 / 13 / 1120: 00$ & 26.814 & 24.235 & 19.466 & & 21.238 & 12.380 & 7.325 \\
\hline 1/14/11 0:00 & 26.807 & 24.233 & 19.468 & & 21.226 & 12.375 & 7.330 \\
\hline $1 / 14 / 114: 00$ & 26.802 & 24.239 & 19.470 & & 21.214 & 12.373 & 7.325 \\
\hline 1/14/11 8:00 & 26.798 & 24.244 & 19.473 & & 21.205 & 12.378 & 7.325 \\
\hline $1 / 14 / 11$ 12:00 & 26.796 & 24.272 & 19.480 & & 21.200 & 12.371 & 7.330 \\
\hline $1 / 14 / 11$ 16:00 & 26.784 & 24.256 & 19.475 & & 21.177 & 12.366 & 7.323 \\
\hline $1 / 14 / 1120: 00$ & 26.777 & 24.244 & 19.475 & & 21.168 & 12.364 & 7.320 \\
\hline 1/15/11 0:00 & 26.770 & 24.244 & 19.475 & & 21.153 & 12.361 & 7.318 \\
\hline 1/15/11 4:00 & 26.768 & 24.251 & 19.482 & & 21.144 & 12.361 & 7.325 \\
\hline 1/15/11 8:00 & 26.768 & 24.284 & 19.486 & & 21.142 & 12.364 & 7.330 \\
\hline $1 / 15 / 11$ 12:00 & 26.766 & 24.319 & 19.496 & & 21.137 & 12.361 & 7.332 \\
\hline $1 / 15 / 11$ 16:00 & 26.759 & 24.324 & 19.494 & & 21.125 & 12.361 & 7.332 \\
\hline 1/15/11 20:00 & 26.757 & 24.347 & 19.500 & & 21.118 & 12.361 & 7.334 \\
\hline 1/16/11 0:00 & 26.754 & 24.354 & 19.503 & & 21.109 & 12.359 & 7.334 \\
\hline $1 / 16 / 114: 00$ & 26.747 & 24.356 & 19.505 & & 21.100 & 12.359 & 7.334 \\
\hline 1/16/11 8:00 & 26.741 & 24.354 & 19.505 & & 21.086 & 12.354 & 7.334 \\
\hline 1/16/11 12:00 & 26.736 & 24.351 & 19.507 & & 21.074 & 12.345 & 7.332 \\
\hline 1/16/11 16:00 & 26.718 & 24.300 & 19.496 & & 21.053 & 12.338 & 7.320 \\
\hline 1/16/11 20:00 & 26.708 & 24.275 & 19.494 & & 21.032 & 12.331 & 7.316 \\
\hline 1/17/11 0:00 & 26.695 & 24.244 & 19.484 & & 21.009 & 12.319 & 7.309 \\
\hline 1/17/11 4:00 & 26.676 & 24.200 & 19.475 & & 20.978 & 12.308 & 7.295 \\
\hline 1/17/11 8:00 & 26.658 & 24.151 & 19.461 & & 20.953 & 12.298 & 7.283 \\
\hline 1/17/11 12:00 & 26.649 & 24.135 & 19.461 & & 20.936 & 12.291 & 7.278 \\
\hline 1/17/11 16:00 & 26.637 & 24.137 & 19.456 & & 20.913 & 12.291 & 7.278 \\
\hline $1 / 17 / 1120: 00$ & 26.640 & 24.160 & 19.468 & & 20.894 & 12.298 & 7.278 \\
\hline 1/18/11 0:00 & 26.647 & 24.167 & 19.479 & & 20.880 & 12.305 & 7.290 \\
\hline 1/18/11 4:00 & 26.651 & 24.181 & 19.493 & & 20.866 & 12.310 & 7.304 \\
\hline 1/18/11 8:00 & 26.658 & 24.284 & 19.503 & & 20.871 & 12.322 & 7.313 \\
\hline 1/18/11 12:00 & 26.660 & 24.335 & 19.514 & & 20.871 & 12.315 & 7.323 \\
\hline 1/18/11 16:00 & 26.653 & 24.326 & 19.514 & & 20.859 & 12.319 & 7.316 \\
\hline 1/18/11 20:00 & 26.656 & 24.354 & 19.526 & & 20.859 & 12.319 & 7.325 \\
\hline 1/19/11 0:00 & 26.651 & 24.365 & 19.531 & & 20.850 & 12.319 & 7.330 \\
\hline 1/19/11 4:00 & 26.647 & 24.370 & 19.533 & & 20.845 & 12.319 & 7.332 \\
\hline 1/19/11 8:00 & 26.647 & 24.379 & 19.538 & & 20.833 & 12.319 & 7.334 \\
\hline 1/19/11 12:00 & 26.644 & 24.403 & 19.542 & & 20.829 & 12.315 & 7.337 \\
\hline 1/19/11 16:00 & 26.630 & 24.375 & 19.538 & & 20.812 & 12.312 & 7.332 \\
\hline 1/19/11 20:00 & 26.635 & 24.391 & 19.547 & & 20.810 & 12.317 & 7.337 \\
\hline 1/20/11 0:00 & 26.635 & 24.423 & 19.552 & & 20.808 & 12.319 & 7.341 \\
\hline $1 / 20 / 114: 00$ & 26.633 & 24.435 & 19.559 & & 20.798 & 12.319 & 7.341 \\
\hline 1/20/11 8:00 & 26.631 & 24.442 & 19.563 & & 20.789 & 12.317 & 7.346 \\
\hline 1/20/11 12:00 & 26.626 & 24.449 & 19.563 & & 20.782 & 12.312 & 7.348 \\
\hline 1/20/11 16:00 & 26.617 & 24.419 & 19.561 & & 20.766 & 12.310 & 7.344 \\
\hline 1/20/11 20:00 & 26.610 & 24.419 & 19.563 & & 20.756 & 12.305 & 7.341 \\
\hline 1/21/11 0:00 & 26.605 & 24.412 & 19.563 & & 20.740 & 12.298 & 7.341 \\
\hline
\end{tabular}

TABLE S1.2 Page 22 of 58 


\begin{tabular}{|c|c|c|c|c|c|c|c|}
\hline \multirow[b]{2}{*}{ Date and Time } & \multicolumn{7}{|c|}{ Water Level (ft below reference point) } \\
\hline & SB43S & SB44S & SB45S & SB46S & SB47S & SB48S & SB49S \\
\hline 1/21/11 4:00 & 26.594 & 24.389 & 19.561 & & 20.726 & 12.296 & 7.332 \\
\hline 1/21/11 8:00 & 26.585 & 24.363 & 19.556 & & 20.707 & 12.291 & 7.334 \\
\hline $1 / 21 / 1112: 00$ & 26.573 & 24.344 & 19.554 & & 20.693 & 12.282 & 7.327 \\
\hline 1/21/11 16:00 & 26.564 & 24.317 & 19.547 & & 20.675 & 12.282 & 7.320 \\
\hline 1/21/11 20:00 & 26.562 & 24.324 & 19.554 & & 20.668 & 12.279 & 7.325 \\
\hline 1/22/11 0:00 & 26.555 & 24.326 & 19.552 & & 20.656 & 12.277 & 7.320 \\
\hline 1/22/11 4:00 & 26.548 & 24.326 & 19.552 & & 20.640 & 12.272 & 7.318 \\
\hline 1/22/11 8:00 & 26.543 & 24.317 & 19.554 & & 20.630 & 12.272 & 7.316 \\
\hline 1/22/11 12:00 & 26.539 & 24.335 & 19.559 & & 20.621 & 12.268 & 7.318 \\
\hline $1 / 22 / 1116: 00$ & 26.530 & 24.324 & 19.556 & & 20.607 & 12.268 & 7.318 \\
\hline $1 / 22 / 1120: 00$ & 26.530 & 24.351 & 19.566 & & 20.605 & 12.270 & 7.323 \\
\hline 1/23/11 0:00 & 26.532 & 24.391 & 19.572 & & 20.602 & 12.275 & 7.327 \\
\hline 1/23/11 4:00 & 26.537 & 24.428 & 19.579 & & 20.602 & 12.279 & 7.337 \\
\hline 1/23/11 8:00 & 26.539 & 24.470 & 19.591 & & 20.605 & 12.282 & 7.341 \\
\hline $1 / 23 / 11$ 12:00 & 26.537 & 24.494 & 19.596 & & 20.600 & 12.279 & 7.346 \\
\hline $1 / 23 / 11$ 16:00 & 26.527 & 24.459 & 19.591 & & 20.579 & 12.275 & 7.341 \\
\hline $1 / 23 / 1120: 00$ & 26.523 & 24.454 & 19.591 & & 20.569 & 12.272 & 7.344 \\
\hline 1/24/11 0:00 & 26.514 & 24.445 & 19.593 & & 20.555 & 12.265 & 7.337 \\
\hline 1/24/11 4:00 & 26.505 & 24.426 & 19.589 & & 20.537 & 12.265 & 7.334 \\
\hline 1/24/11 8:00 & 26.502 & 24.433 & 19.593 & & 20.530 & 12.265 & 7.339 \\
\hline 1/24/11 12:00 & 26.502 & 24.461 & 19.598 & & 20.527 & 12.265 & 7.344 \\
\hline 1/24/11 16:00 & 26.500 & 24.477 & 19.605 & & 20.518 & 12.268 & 7.342 \\
\hline 1/24/11 20:00 & 26.500 & 24.505 & 19.610 & & 20.518 & 12.270 & 7.351 \\
\hline 1/25/11 0:00 & 26.498 & 24.519 & 19.614 & & 20.511 & 12.270 & 7.351 \\
\hline $1 / 25 / 114: 00$ & 26.495 & 24.528 & 19.619 & & 20.506 & 12.268 & 7.353 \\
\hline 1/25/11 8:00 & 26.491 & 24.521 & 19.619 & & 20.490 & 12.268 & 7.351 \\
\hline $1 / 25 / 11$ 12:00 & 26.486 & 24.528 & 19.624 & & 20.485 & 12.261 & 7.355 \\
\hline 1/25/11 16:00 & 26.475 & 24.491 & 19.617 & & 20.464 & 12.249 & 7.346 \\
\hline $1 / 25 / 1120: 00$ & 26.470 & 24.473 & 19.615 & & 20.453 & 12.249 & 7.341 \\
\hline 1/26/11 0:00 & 26.466 & 24.473 & 19.619 & & 20.443 & 12.247 & 7.344 \\
\hline 1/26/11 4:00 & 26.461 & 24.468 & 19.621 & & 20.434 & 12.247 & 7.351 \\
\hline 1/26/11 8:00 & 26.459 & 24.482 & 19.628 & & 20.429 & 12.251 & 7.351 \\
\hline 1/26/11 12:00 & 26.466 & 24.524 & 19.638 & & 20.432 & 12.256 & 7.358 \\
\hline $1 / 26 / 11$ 16:00 & 26.459 & 24.531 & 19.640 & & 20.418 & 12.251 & 7.355 \\
\hline $1 / 26 / 1120: 00$ & 26.456 & 24.533 & 19.642 & & 20.411 & 12.249 & 7.358 \\
\hline 1/27/11 0:00 & 26.447 & 24.514 & 19.640 & & 20.397 & 12.244 & 7.355 \\
\hline 1/27/11 4:00 & 26.433 & 24.480 & 19.633 & & 20.378 & 12.233 & 7.346 \\
\hline 1/27/11 8:00 & 26.422 & 24.433 & 19.621 & & 20.357 & 12.230 & 7.339 \\
\hline $1 / 27 / 11$ 12:00 & 26.417 & 24.431 & 19.626 & & 20.343 & 12.228 & 7.339 \\
\hline 1/27/11 16:00 & 26.413 & 24.433 & 19.626 & & 20.331 & 12.230 & 7.339 \\
\hline 1/27/11 20:00 & 26.413 & 24.459 & 19.633 & & 20.327 & 12.228 & 7.344 \\
\hline 1/28/11 0:00 & 26.411 & 24.468 & 19.638 & & 20.322 & 12.226 & 7.346 \\
\hline 1/28/11 4:00 & 26.406 & 24.459 & 19.638 & & 20.310 & 12.226 & 7.349 \\
\hline 1/28/11 8:00 & 26.401 & 24.442 & 19.640 & & 20.303 & 12.226 & 7.341 \\
\hline 1/28/11 12:00 & 26.395 & 24.430 & 19.638 & & 20.289 & 12.216 & 7.344 \\
\hline 1/28/11 16:00 & 26.383 & 24.400 & 19.633 & & 20.273 & 12.216 & 7.337 \\
\hline 1/28/11 20:00 & 26.381 & 24.447 & 19.638 & & 20.266 & 12.214 & 7.341 \\
\hline 1/29/11 0:00 & 26.383 & 24.416 & 19.645 & & 20.263 & 12.221 & 7.346 \\
\hline 1/29/11 4:00 & 26.381 & 24.384 & 19.652 & & 20.256 & 12.221 & 7.348 \\
\hline 1/29/11 8:00 & 26.378 & 24.354 & 19.652 & & 20.250 & 12.219 & 7.351 \\
\hline 1/29/11 12:00 & 26.378 & 24.382 & 19.661 & & 20.245 & 12.219 & 7.353 \\
\hline 1/29/11 16:00 & 26.374 & 24.379 & 19.661 & & 20.238 & 12.221 & 7.353 \\
\hline 1/29/11 20:00 & 26.376 & 24.398 & 19.666 & & 20.235 & 12.219 & 7.360 \\
\hline 1/30/11 0:00 & 26.378 & 24.414 & 19.677 & & 20.238 & 12.226 & 7.369 \\
\hline
\end{tabular}

TABLE S1.2 Page 23 of 58 


\begin{tabular}{|c|c|c|c|c|c|c|c|}
\hline \multirow[b]{2}{*}{ Date and Time } & \multicolumn{7}{|c|}{ Water Level (ft below reference point) } \\
\hline & SB43S & SB44S & SB45S & SB46S & SB47S & SB48S & SB49S \\
\hline 1/30/11 4:00 & 26.383 & 24.431 & 19.684 & & 20.235 & 12.230 & 7.377 \\
\hline 1/30/11 8:00 & 26.385 & 24.449 & 19.693 & & 20.233 & 12.233 & 7.381 \\
\hline 1/30/11 12:00 & 26.385 & 24.466 & 19.698 & & 20.233 & 12.228 & 7.388 \\
\hline $1 / 30 / 1116: 00$ & 26.376 & 24.438 & 19.693 & & 20.214 & 12.228 & 7.383 \\
\hline 1/30/11 20:00 & 26.372 & 24.424 & 19.696 & & 20.207 & 12.223 & 7.381 \\
\hline 1/31/11 0:00 & 26.367 & 24.424 & 19.696 & & 20.198 & 12.221 & 7.386 \\
\hline 1/31/11 4:00 & 26.358 & 24.412 & 19.696 & & 20.186 & 12.216 & 7.379 \\
\hline 1/31/11 8:00 & 26.349 & 24.379 & 19.687 & & 20.168 & 12.212 & 7.374 \\
\hline 1/31/11 12:00 & 26.344 & 24.386 & 19.691 & & 20.158 & 12.209 & 7.374 \\
\hline 1/31/11 16:00 & 26.335 & 24.365 & 19.684 & & 20.144 & 12.210 & 7.372 \\
\hline 1/31/11 20:00 & 26.335 & 24.391 & 19.694 & & 20.140 & 12.212 & 7.377 \\
\hline $2 / 1 / 110: 00$ & 26.340 & 24.433 & 19.700 & & 20.142 & 12.212 & 7.379 \\
\hline 2/1/11 4:00 & 26.337 & 24.449 & 19.703 & & 20.135 & 12.212 & 7.381 \\
\hline 2/1/11 8:00 & 26.330 & 24.449 & 19.703 & & 20.123 & 12.205 & 7.381 \\
\hline 2/1/11 12:00 & 26.323 & 24.426 & 19.703 & & 20.109 & 12.203 & 7.377 \\
\hline $2 / 1 / 1116: 00$ & 26.317 & 24.403 & 19.700 & & 20.100 & 12.200 & 7.379 \\
\hline 2/1/11 20:00 & 26.314 & 24.421 & 19.705 & & 20.091 & 12.207 & 7.381 \\
\hline 2/2/11 0:00 & 26.323 & 24.475 & 19.717 & & 20.095 & 12.214 & 7.388 \\
\hline 2/2/11 4:00 & 26.330 & 24.526 & 19.729 & & 20.100 & 12.219 & 7.400 \\
\hline 2/2/11 8:00 & 26.337 & 24.570 & 19.738 & & 20.105 & 12.226 & 7.407 \\
\hline 2/2/11 12:00 & 26.342 & 24.603 & 19.749 & & 20.109 & 12.230 & 7.419 \\
\hline 2/2/11 16:00 & 26.339 & 24.622 & 19.756 & & 20.105 & 12.230 & 7.421 \\
\hline 2/2/11 20:00 & 26.340 & 24.640 & 19.759 & & 20.102 & 12.233 & 7.428 \\
\hline 2/3/11 0:00 & 26.340 & 24.640 & 19.761 & & 20.093 & 12.228 & 7.426 \\
\hline 2/3/11 4:00 & 26.330 & 24.617 & 19.759 & & 20.079 & 12.223 & 7.423 \\
\hline 2/3/11 8:00 & 26.323 & 24.593 & 19.756 & & 20.065 & 12.221 & 7.421 \\
\hline $2 / 3 / 1112: 00$ & 26.317 & 24.580 & 19.756 & & 20.056 & 12.212 & 7.421 \\
\hline 2/3/11 16:00 & 26.303 & 24.535 & 19.747 & & 20.030 & 12.207 & 7.405 \\
\hline 2/3/11 20:00 & 26.296 & 24.517 & 19.742 & & 20.018 & 12.205 & 7.402 \\
\hline 2/4/11 0:00 & 26.289 & 24.510 & 19.742 & & 20.007 & 12.198 & 7.402 \\
\hline 2/4/11 4:00 & 26.278 & 24.475 & 19.731 & & 19.985 & 12.196 & 7.393 \\
\hline 2/4/11 8:00 & 26.271 & 24.463 & 19.733 & & 19.974 & 12.189 & 7.388 \\
\hline 2/4/11 12:00 & 26.264 & 24.442 & 19.728 & & 19.960 & 12.179 & 7.388 \\
\hline 2/4/11 16:00 & 26.248 & 24.391 & 19.715 & & 19.934 & 12.172 & 7.374 \\
\hline 2/4/11 20:00 & 26.236 & 24.365 & 19.710 & & 19.918 & 12.165 & 7.367 \\
\hline 2/5/11 0:00 & 26.227 & 24.344 & 19.707 & & 19.899 & 12.156 & 7.360 \\
\hline 2/5/11 4:00 & 26.211 & 24.307 & 19.696 & & 19.878 & 12.151 & 7.351 \\
\hline 2/5/11 8:00 & 26.204 & 24.282 & 19.689 & & 19.862 & 12.146 & 7.349 \\
\hline 2/5/11 12:00 & 26.197 & 24.282 & 19.691 & & 19.850 & 12.144 & 7.346 \\
\hline 2/5/11 16:00 & 26.191 & 24.274 & 19.691 & & 19.841 & 12.144 & 7.342 \\
\hline 2/5/11 20:00 & 26.195 & 24.307 & 19.696 & & 19.836 & 12.144 & 7.349 \\
\hline 2/6/11 0:00 & 26.193 & 24.326 & 19.698 & & 19.831 & 12.149 & 7.351 \\
\hline 2/6/11 4:00 & 26.193 & 24.349 & 19.705 & & 19.827 & 12.151 & 7.356 \\
\hline 2/6/11 8:00 & 26.197 & 24.386 & 19.717 & & 19.831 & 12.158 & 7.367 \\
\hline 2/6/11 12:00 & 26.204 & 24.442 & 19.726 & & 19.838 & 12.161 & 7.377 \\
\hline $2 / 6 / 1116: 00$ & 26.206 & 24.477 & 19.736 & & 19.834 & 12.168 & 7.381 \\
\hline $2 / 6 / 1120: 00$ & 26.209 & 24.519 & 19.745 & & 19.841 & 12.172 & 7.391 \\
\hline 2/7/11 0:00 & 26.216 & 24.549 & 19.754 & & 19.838 & 12.175 & 7.400 \\
\hline 2/7/11 4:00 & 26.211 & 24.561 & 19.756 & & 19.831 & 12.172 & 7.398 \\
\hline 2/7/11 8:00 & 26.211 & 24.561 & 19.759 & & 19.822 & 12.172 & 7.400 \\
\hline 2/7/11 12:00 & 26.209 & 24.573 & 19.763 & & 19.817 & 12.170 & 7.405 \\
\hline 2/7/11 16:00 & 26.202 & 24.554 & 19.759 & & 19.806 & 12.170 & 7.400 \\
\hline 2/7/11 20:00 & 26.202 & 24.575 & 19.768 & & 19.803 & 12.177 & 7.405 \\
\hline 2/8/11 0:00 & 26.207 & 24.607 & 19.775 & & 19.806 & 12.182 & 7.414 \\
\hline
\end{tabular}

TABLE S1.2 Page 24 of 58 


\begin{tabular}{|c|c|c|c|c|c|c|c|}
\hline \multirow[b]{2}{*}{ Date and Time } & \multicolumn{7}{|c|}{ Water Level (ft below reference point) } \\
\hline & SB43S & SB44S & SB45S & SB46S & SB47S & SB48S & SB49S \\
\hline 2/8/11 4:00 & 26.209 & 24.640 & 19.784 & & 19.806 & 12.189 & 7.421 \\
\hline 2/8/11 8:00 & 26.218 & 24.678 & 19.798 & & 19.813 & 12.193 & 7.435 \\
\hline 2/8/11 12:00 & 26.223 & 24.701 & 19.805 & & 19.810 & 12.191 & 7.435 \\
\hline 2/8/11 16:00 & 26.211 & 24.666 & 19.798 & & 19.792 & 12.186 & 7.433 \\
\hline 2/8/11 20:00 & 26.211 & 24.666 & 19.801 & & 19.787 & 12.191 & 7.435 \\
\hline 2/9/11 0:00 & 26.209 & 24.687 & 19.805 & & 19.782 & 12.184 & 7.437 \\
\hline 2/9/11 4:00 & 26.197 & 24.657 & 19.798 & & 19.764 & 12.182 & 7.433 \\
\hline 2/9/11 8:00 & 26.195 & 24.638 & 19.801 & & 19.757 & 12.177 & 7.428 \\
\hline 2/9/11 12:00 & 26.191 & 24.633 & 19.801 & & 19.745 & 12.177 & 7.430 \\
\hline 2/9/11 16:00 & 26.181 & 24.617 & 19.798 & & 19.731 & 12.172 & 7.423 \\
\hline 2/9/11 20:00 & 26.179 & 24.614 & 19.796 & & 19.722 & 12.172 & 7.423 \\
\hline 2/10/11 0:00 & 26.172 & 24.610 & 19.796 & & 19.710 & 12.168 & 7.423 \\
\hline 2/10/11 4:00 & 26.163 & 24.589 & 19.791 & & 19.696 & 12.161 & 7.419 \\
\hline 2/10/11 8:00 & 26.152 & 24.549 & 19.784 & & 19.677 & 12.156 & 7.407 \\
\hline 2/10/11 12:00 & 26.149 & 24.540 & 19.784 & & 19.670 & 12.154 & 7.412 \\
\hline 2/10/11 16:00 & 26.138 & 24.521 & 19.780 & & 19.651 & 12.149 & 7.405 \\
\hline 2/10/11 20:00 & 26.138 & 24.521 & 19.784 & & 19.644 & 12.147 & 7.407 \\
\hline 2/11/11 0:00 & 26.133 & 24.528 & 19.782 & & 19.635 & 12.144 & 7.402 \\
\hline 2/11/11 4:00 & 26.129 & 24.524 & 19.782 & & 19.626 & 12.142 & 7.400 \\
\hline 2/11/11 8:00 & 26.124 & 24.528 & 19.784 & & 19.616 & 12.147 & 7.405 \\
\hline 2/11/11 12:00 & 26.122 & 24.547 & 19.789 & & 19.612 & 12.144 & 7.407 \\
\hline 2/11/11 16:00 & 26.119 & 24.552 & 19.787 & & 19.602 & 12.147 & 7.412 \\
\hline 2/11/11 20:00 & 26.122 & 24.589 & 19.798 & & 19.602 & 12.149 & 7.414 \\
\hline 2/12/11 0:00 & 26.126 & 24.624 & 19.803 & & 19.600 & 12.151 & 7.421 \\
\hline $2 / 12 / 114: 00$ & 26.124 & 24.635 & 19.808 & & 19.593 & 12.149 & 7.416 \\
\hline 2/12/11 8:00 & 26.119 & 24.631 & 19.808 & & 19.586 & 12.151 & 7.421 \\
\hline $2 / 12 / 11$ 12:00 & 26.113 & 24.617 & 19.805 & & 19.574 & 12.142 & 7.419 \\
\hline 2/12/11 16:00 & 26.099 & 24.575 & 19.796 & & 19.556 & 12.142 & 7.412 \\
\hline 2/12/11 20:00 & 26.101 & 24.566 & 19.803 & & 19.551 & 12.140 & 7.416 \\
\hline 2/13/11 0:00 & 26.094 & 24.535 & 19.801 & & 19.542 & 12.137 & 7.416 \\
\hline 2/13/11 4:00 & 26.087 & 24.479 & 19.796 & & 19.525 & 12.128 & 7.407 \\
\hline 2/13/11 8:00 & 26.078 & 24.428 & 19.791 & & 19.509 & 12.126 & 7.402 \\
\hline 2/13/11 12:00 & 26.069 & 24.396 & 19.789 & & 19.497 & 12.119 & 7.398 \\
\hline 2/13/11 16:00 & 26.060 & 24.347 & 19.777 & & 19.479 & 12.121 & 7.388 \\
\hline 2/13/11 20:00 & 26.067 & 24.279 & 19.787 & & 19.472 & 12.128 & 7.400 \\
\hline 2/14/11 0:00 & 26.080 & 24.086 & 19.796 & & 19.472 & 12.121 & 7.416 \\
\hline 2/14/11 4:00 & 26.083 & 23.862 & 19.794 & & 19.460 & 12.121 & 7.423 \\
\hline 2/14/11 8:00 & 26.085 & 23.736 & 19.798 & & 19.460 & 12.126 & 7.426 \\
\hline 2/14/11 12:00 & 26.092 & 23.695 & 19.801 & & 19.458 & 12.126 & 7.430 \\
\hline 2/14/11 16:00 & 26.085 & 23.657 & 19.768 & & 19.439 & 12.116 & 7.430 \\
\hline 2/14/11 20:00 & 26.081 & 23.394 & 19.728 & & 19.411 & 12.107 & 7.426 \\
\hline 2/15/11 0:00 & 26.074 & 23.105 & 19.721 & & 19.394 & 12.093 & 7.423 \\
\hline 2/15/11 4:00 & 26.065 & 22.924 & 19.717 & & 19.371 & 12.072 & 7.414 \\
\hline 2/15/11 8:00 & 26.055 & 22.800 & 19.740 & & 19.352 & 12.053 & 7.402 \\
\hline 2/15/11 12:00 & 26.046 & 22.742 & 19.731 & & 19.336 & 12.046 & 7.400 \\
\hline 2/15/11 16:00 & 26.037 & 22.693 & 19.719 & & 19.301 & 12.035 & 7.393 \\
\hline 2/15/11 20:00 & 26.032 & 22.397 & 19.712 & & 19.275 & 12.023 & 7.391 \\
\hline 2/16/11 0:00 & 26.032 & 22.134 & 19.707 & & 19.266 & 12.011 & 7.398 \\
\hline 2/16/11 4:00 & 26.025 & 22.029 & 19.698 & & 19.245 & 11.997 & 7.388 \\
\hline 2/16/11 8:00 & 26.019 & 21.945 & 19.687 & & 19.222 & 11.979 & 7.381 \\
\hline 2/16/11 12:00 & 26.012 & 21.869 & 19.682 & & 19.203 & 11.965 & 7.381 \\
\hline 2/16/11 16:00 & 25.996 & 21.747 & 19.661 & & 19.175 & 11.941 & 7.365 \\
\hline 2/16/11 20:00 & 25.982 & 21.186 & 19.647 & & 19.159 & 11.918 & 7.356 \\
\hline 2/17/11 0:00 & 25.971 & 20.681 & 19.638 & & 18.871 & 11.897 & 7.351 \\
\hline
\end{tabular}

TABLE S1.2 Page 25 of 58 


\begin{tabular}{|c|c|c|c|c|c|c|c|}
\hline \multirow[b]{2}{*}{ Date and Time } & \multicolumn{7}{|c|}{ Water Level (ft below reference point) } \\
\hline & SB43S & SB44S & SB45S & SB46S & SB47S & SB48S & SB49S \\
\hline 2/17/11 4:00 & 25.961 & 19.947 & 19.619 & & 18.813 & 11.878 & 7.342 \\
\hline 2/17/11 8:00 & 25.952 & 19.262 & 19.610 & & 18.780 & 11.862 & 7.335 \\
\hline 2/17/11 12:00 & 25.948 & 18.888 & 19.596 & & 18.796 & 11.850 & 7.328 \\
\hline 2/17/11 16:00 & 25.938 & 18.689 & 19.593 & & 18.829 & 11.848 & 7.325 \\
\hline 2/17/11 20:00 & 25.950 & 18.867 & 19.603 & & 18.883 & 11.848 & 7.339 \\
\hline 2/18/11 0:00 & 25.959 & 19.142 & 19.610 & & 18.890 & 11.843 & 7.351 \\
\hline 2/18/11 4:00 & 25.961 & 19.351 & 19.615 & & 18.892 & 11.838 & 7.358 \\
\hline 2/18/11 8:00 & 25.961 & 19.509 & 19.612 & & 18.895 & 11.831 & 7.367 \\
\hline 2/18/11 12:00 & 25.961 & 19.649 & 19.615 & & 18.895 & 11.822 & 7.372 \\
\hline 2/18/11 16:00 & 25.952 & 19.689 & 19.603 & & 18.883 & 11.808 & 7.365 \\
\hline 2/18/11 20:00 & 25.945 & 19.698 & 19.596 & & 18.874 & 11.799 & 7.363 \\
\hline 2/19/11 0:00 & 25.936 & 19.733 & 19.589 & & 18.862 & 11.789 & 7.358 \\
\hline 2/19/11 4:00 & 25.927 & 19.754 & 19.584 & & 18.852 & 11.778 & 7.358 \\
\hline 2/19/11 8:00 & 25.918 & 19.751 & 19.575 & & 18.838 & 11.764 & 7.353 \\
\hline 2/19/11 12:00 & 25.909 & 19.761 & 19.566 & & 18.827 & 11.750 & 7.346 \\
\hline 2/19/11 16:00 & 25.893 & 19.705 & 19.549 & & 18.806 & 11.731 & 7.342 \\
\hline 2/19/11 20:00 & 25.879 & 19.658 & 19.535 & & 18.787 & 11.717 & 7.330 \\
\hline 2/20/11 0:00 & 25.861 & 19.605 & 19.519 & & 18.768 & 11.701 & 7.321 \\
\hline 2/20/11 4:00 & 25.838 & 19.530 & 19.500 & & 18.743 & 11.680 & 7.304 \\
\hline 2/20/11 8:00 & 25.817 & 19.412 & 19.480 & & 18.719 & 11.661 & 7.286 \\
\hline 2/20/11 12:00 & 25.796 & 19.332 & 19.459 & & 18.694 & 11.640 & 7.274 \\
\hline 2/20/11 16:00 & 25.776 & 19.258 & 19.442 & & 18.668 & 11.633 & 7.258 \\
\hline 2/20/11 20:00 & 25.780 & 19.339 & 19.449 & & 18.675 & 11.635 & 7.269 \\
\hline 2/21/11 0:00 & 25.778 & 19.472 & 19.449 & & 18.677 & 11.626 & 7.272 \\
\hline $2 / 21 / 114: 00$ & 25.773 & 19.547 & 19.445 & & 18.668 & 11.619 & 7.272 \\
\hline 2/21/11 8:00 & 25.766 & 19.595 & 19.445 & & 18.661 & 11.612 & 7.272 \\
\hline 2/21/11 12:00 & 25.764 & 19.679 & 19.445 & & 18.663 & 11.607 & 7.279 \\
\hline 2/21/11 16:00 & 25.757 & 19.717 & 19.442 & & 18.654 & 11.600 & 7.269 \\
\hline 2/21/11 20:00 & 25.755 & 19.782 & 19.442 & & 18.654 & 11.598 & 7.276 \\
\hline 2/22/11 0:00 & 25.751 & 19.859 & 19.442 & & 18.649 & 11.591 & 7.276 \\
\hline $2 / 22 / 114: 00$ & 25.746 & 19.901 & 19.440 & & 18.642 & 11.586 & 7.276 \\
\hline 2/22/11 8:00 & 25.739 & 19.936 & 19.438 & & 18.638 & 11.582 & 7.276 \\
\hline 2/22/11 12:00 & 25.735 & 19.992 & 19.440 & & 18.628 & 11.570 & 7.276 \\
\hline $2 / 22 / 11$ 16:00 & 25.718 & 19.963 & 19.424 & & 18.614 & 11.554 & 7.267 \\
\hline 2/22/11 20:00 & 25.705 & 19.901 & 19.412 & & 18.596 & 11.549 & 7.258 \\
\hline 2/23/11 0:00 & 25.691 & 19.894 & 19.407 & & 18.586 & 11.535 & 7.251 \\
\hline 2/23/11 4:00 & 25.675 & 19.852 & 19.393 & & 18.565 & 11.521 & 7.241 \\
\hline 2/23/11 8:00 & 25.659 & 19.789 & 19.382 & & 18.546 & 11.509 & 7.234 \\
\hline 2/23/11 12:00 & 25.647 & 19.751 & 19.373 & & 18.532 & 11.498 & 7.227 \\
\hline 2/23/11 16:00 & 25.634 & 19.719 & 19.361 & & 18.514 & 11.488 & 7.216 \\
\hline 2/23/11 20:00 & 25.629 & 19.775 & 19.363 & & 18.511 & 11.491 & 7.218 \\
\hline 2/24/11 0:00 & 25.627 & 19.868 & 19.365 & & 18.509 & 11.484 & 7.220 \\
\hline $2 / 24 / 114: 00$ & 25.618 & 19.912 & 19.363 & & 18.500 & 11.477 & 7.220 \\
\hline 2/24/11 8:00 & 25.608 & 19.910 & 19.351 & & 18.488 & 11.465 & 7.211 \\
\hline 2/24/11 12:00 & 25.602 & 19.929 & 19.349 & & 18.479 & 11.453 & 7.213 \\
\hline 2/24/11 16:00 & 25.576 & 19.847 & 19.331 & & 18.451 & 11.444 & 7.192 \\
\hline 2/24/11 20:00 & 25.572 & 19.845 & 19.331 & & 18.448 & 11.439 & 7.195 \\
\hline 2/25/11 0:00 & 25.570 & 19.922 & 19.335 & & 18.444 & 11.439 & 7.197 \\
\hline $2 / 25 / 114: 00$ & 25.567 & 20.027 & 19.340 & & 18.444 & 11.435 & 7.204 \\
\hline 2/25/11 8:00 & 25.565 & 20.101 & 19.338 & & 18.437 & 11.435 & 7.206 \\
\hline 2/25/11 12:00 & 25.558 & 20.161 & 19.340 & & 18.432 & 11.425 & 7.202 \\
\hline 2/25/11 16:00 & 25.544 & 20.141 & 19.328 & & 18.409 & 11.414 & 7.192 \\
\hline 2/25/11 20:00 & 25.533 & 20.115 & 19.319 & & 18.392 & 11.407 & 7.185 \\
\hline 2/26/11 0:00 & 25.521 & 20.101 & 19.310 & & 18.376 & 11.393 & 7.183 \\
\hline
\end{tabular}

TABLE S1.2 Page 26 of 58 


\begin{tabular}{|c|c|c|c|c|c|c|c|}
\hline \multirow[b]{2}{*}{ Date and Time } & \multicolumn{7}{|c|}{ Water Level (ft below reference point) } \\
\hline & SB43S & SB44S & SB45S & SB46S & SB47S & SB48S & SB49S \\
\hline 2/26/11 4:00 & 25.505 & 20.052 & 19.300 & & 18.355 & 11.381 & 7.169 \\
\hline 2/26/11 8:00 & 25.485 & 19.987 & 19.286 & & 18.334 & 11.374 & 7.157 \\
\hline 2/26/11 12:00 & 25.471 & 19.952 & 19.279 & & 18.315 & 11.360 & 7.151 \\
\hline 2/26/11 16:00 & 25.453 & 19.882 & 19.263 & & 18.292 & 11.348 & 7.139 \\
\hline 2/26/11 20:00 & 25.441 & 19.870 & 19.256 & & 18.275 & 11.341 & 7.132 \\
\hline 2/27/11 0:00 & 25.430 & 19.880 & 19.247 & & 18.259 & 11.330 & 7.127 \\
\hline 2/27/11 4:00 & 25.414 & 19.850 & 19.238 & & 18.243 & 11.318 & 7.116 \\
\hline 2/27/11 8:00 & 25.398 & 19.819 & 19.228 & & 18.224 & 11.311 & 7.108 \\
\hline 2/27/11 12:00 & 25.386 & 19.826 & 19.221 & & 18.212 & 11.297 & 7.104 \\
\hline $2 / 27 / 1116: 00$ & 25.370 & 19.772 & 19.207 & & 18.191 & 11.285 & 7.092 \\
\hline 2/27/11 20:00 & 25.361 & 19.782 & 19.207 & & 18.182 & 11.292 & 7.095 \\
\hline $2 / 28 / 110: 00$ & 25.356 & 19.891 & 19.212 & & 18.182 & 11.295 & 7.094 \\
\hline 2/28/11 4:00 & 25.361 & 19.975 & 19.219 & & 18.184 & 11.292 & 7.108 \\
\hline 2/28/11 8:00 & 25.370 & 20.112 & 19.233 & & 18.198 & 11.292 & 7.120 \\
\hline $2 / 28 / 1112: 00$ & 25.368 & 20.204 & 19.238 & & 18.198 & 11.281 & 7.127 \\
\hline 2/28/11 16:00 & 25.359 & 20.234 & 19.233 & & 18.187 & 11.283 & 7.122 \\
\hline 2/28/11 20:00 & 25.350 & 20.231 & 19.228 & & 18.175 & 11.278 & 7.120 \\
\hline 3/1/11 0:00 & 25.336 & 20.185 & 19.221 & & 18.161 & 11.257 & 7.113 \\
\hline 3/1/11 4:00 & 25.320 & 20.096 & 19.207 & & 18.142 & 11.236 & 7.106 \\
\hline 3/1/11 8:00 & 25.311 & 20.059 & 19.203 & & 18.128 & 11.227 & 7.097 \\
\hline 3/1/11 12:00 & 25.301 & 20.064 & 19.196 & & 18.117 & 11.220 & 7.092 \\
\hline 3/1/11 16:00 & 25.288 & 20.108 & 19.189 & & 18.103 & 11.211 & 7.087 \\
\hline 3/1/11 20:00 & 25.283 & 20.078 & 19.191 & & 18.096 & 11.204 & 7.090 \\
\hline 3/2/11 0:00 & 25.281 & 20.059 & 19.193 & & 18.091 & 11.199 & 7.092 \\
\hline 3/2/11 4:00 & 25.276 & 20.087 & 19.196 & & 18.089 & 11.192 & 7.097 \\
\hline $3 / 2 / 118: 00$ & 25.272 & 20.115 & 19.193 & & 18.082 & 11.185 & 7.097 \\
\hline $3 / 2 / 1112: 00$ & 25.265 & 20.129 & 19.191 & & 18.075 & 11.169 & 7.092 \\
\hline 3/2/11 16:00 & 25.244 & 20.038 & 19.175 & & 18.051 & 11.152 & 7.080 \\
\hline 3/2/11 20:00 & 25.233 & 19.940 & 19.165 & & 18.033 & 11.136 & 7.069 \\
\hline 3/3/11 0:00 & 25.216 & 19.884 & 19.154 & & 18.016 & 11.122 & 7.064 \\
\hline 3/3/11 4:00 & 25.198 & 19.803 & 19.138 & & 17.993 & 11.108 & 7.045 \\
\hline $3 / 3 / 118: 00$ & 25.187 & 19.761 & 19.133 & & 17.979 & 11.096 & 7.043 \\
\hline 3/3/11 12:00 & 25.173 & 19.719 & 19.121 & & 17.960 & 11.077 & 7.031 \\
\hline 3/3/11 16:00 & 25.155 & 19.619 & 19.105 & & 17.937 & 11.063 & 7.015 \\
\hline 3/3/11 20:00 & 25.143 & 19.572 & 19.100 & & 17.923 & 11.054 & 7.013 \\
\hline 3/4/11 0:00 & 25.132 & 19.577 & 19.093 & & 17.911 & 11.040 & 7.006 \\
\hline 3/4/11 4:00 & 25.120 & 19.547 & 19.086 & & 17.897 & 11.031 & 7.001 \\
\hline 3/4/11 8:00 & 25.116 & 19.579 & 19.086 & & 17.892 & 11.026 & 7.001 \\
\hline 3/4/11 12:00 & 25.106 & 19.633 & 19.084 & & 17.883 & 11.019 & 6.999 \\
\hline 3/4/11 16:00 & 25.100 & 19.649 & 19.082 & & 17.876 & 11.012 & 6.994 \\
\hline 3/4/11 20:00 & 25.100 & 19.703 & 19.084 & & 17.871 & 11.012 & 7.001 \\
\hline 3/5/11 0:00 & 25.097 & 19.791 & 19.086 & & 17.869 & 11.007 & 7.003 \\
\hline 3/5/11 4:00 & 25.093 & 19.847 & 19.089 & & 17.864 & 11.001 & 7.006 \\
\hline 3/5/11 8:00 & 25.088 & 19.884 & 19.084 & & 17.860 & 10.996 & 7.004 \\
\hline 3/5/11 12:00 & 25.084 & 19.926 & 19.084 & & 17.850 & 10.984 & 7.006 \\
\hline 3/5/11 16:00 & 25.068 & 19.896 & 19.075 & & 17.834 & 10.975 & 6.992 \\
\hline $3 / 5 / 1120: 00$ & 25.061 & 19.870 & 19.072 & & 17.825 & 10.970 & 6.992 \\
\hline 3/6/11 0:00 & 25.052 & 19.887 & 19.068 & & 17.813 & 10.958 & 6.985 \\
\hline 3/6/11 4:00 & 25.038 & 19.857 & 19.058 & & 17.794 & 10.949 & 6.975 \\
\hline 3/6/11 8:00 & 25.026 & 19.821 & 19.049 & & 17.780 & 10.940 & 6.971 \\
\hline 3/6/11 12:00 & 25.013 & 19.807 & 19.044 & & 17.766 & 10.926 & 6.964 \\
\hline 3/6/11 16:00 & 24.994 & 19.728 & 19.028 & & 17.743 & 10.912 & 6.952 \\
\hline $3 / 6 / 1120: 00$ & 24.983 & 19.665 & 19.021 & & 17.726 & 10.905 & 6.943 \\
\hline 3/7/11 0:00 & 24.974 & 19.670 & 19.017 & & 17.713 & 10.893 & 6.938 \\
\hline
\end{tabular}

TABLE S1.2 Page 27 of 58 


\begin{tabular}{|c|c|c|c|c|c|c|c|}
\hline \multirow[b]{2}{*}{ Date and Time } & \multicolumn{7}{|c|}{ Water Level (ft below reference point) } \\
\hline & SB43S & SB44S & SB45S & SB46S & SB47S & SB48S & SB49S \\
\hline 3/7/11 4:00 & 24.958 & 19.644 & 19.005 & & 17.696 & 10.881 & 6.933 \\
\hline 3/7/11 8:00 & 24.948 & 19.633 & 19.000 & & 17.685 & 10.877 & 6.924 \\
\hline 3/7/11 12:00 & 24.939 & 19.652 & 18.996 & & 17.673 & 10.867 & 6.919 \\
\hline 3/7/11 16:00 & 24.926 & 19.616 & 18.986 & & 17.656 & 10.856 & 6.910 \\
\hline 3/7/11 20:00 & 24.916 & 19.654 & 18.982 & & 17.635 & 10.851 & 6.905 \\
\hline 3/8/11 0:00 & 24.907 & 19.656 & 18.977 & & 17.619 & 10.840 & 6.905 \\
\hline 3/8/11 4:00 & 24.893 & 19.642 & 18.970 & & 17.603 & 10.833 & 6.896 \\
\hline 3/8/11 8:00 & 24.882 & 19.619 & 18.965 & & 17.589 & 10.825 & 6.891 \\
\hline 3/8/11 12:00 & 24.873 & 19.626 & 18.958 & & 17.577 & 10.816 & 6.889 \\
\hline 3/8/11 16:00 & 24.855 & 19.633 & 18.944 & & 17.549 & 10.809 & 6.873 \\
\hline 3/8/11 20:00 & 24.843 & 19.579 & 18.942 & & 17.530 & 10.802 & 6.875 \\
\hline 3/9/11 0:00 & 24.829 & 19.521 & 18.935 & & 17.514 & 10.790 & 6.866 \\
\hline 3/9/11 4:00 & 24.818 & 19.444 & 18.930 & & 17.502 & 10.786 & 6.859 \\
\hline 3/9/11 8:00 & 24.813 & 19.395 & 18.930 & & 17.495 & 10.781 & 6.859 \\
\hline 3/9/11 12:00 & 24.811 & 19.416 & 18.935 & & 17.493 & 10.774 & 6.863 \\
\hline 3/9/11 16:00 & 24.802 & 19.435 & 18.933 & & 17.479 & 10.762 & 6.866 \\
\hline 3/9/11 20:00 & 24.800 & 19.488 & 18.937 & & 17.481 & 10.758 & 6.873 \\
\hline 3/10/11 0:00 & 24.795 & 19.537 & 18.940 & & 17.477 & 10.751 & 6.868 \\
\hline 3/10/11 4:00 & 24.788 & 19.561 & 18.933 & & 17.467 & 10.746 & 6.866 \\
\hline 3/10/11 8:00 & 24.781 & 19.588 & 18.935 & & 17.458 & 10.741 & 6.863 \\
\hline 3/10/11 12:00 & 24.774 & 19.616 & 18.933 & & 17.448 & 10.728 & 6.863 \\
\hline 3/10/11 16:00 & 24.761 & 19.565 & 18.919 & & 17.430 & 10.716 & 6.849 \\
\hline 3/10/11 20:00 & 24.744 & 19.514 & 18.912 & & 17.411 & 10.702 & 6.842 \\
\hline 3/11/11 0:00 & 24.733 & 19.486 & 18.903 & & 17.395 & 10.688 & 6.833 \\
\hline 3/11/11 4:00 & 24.717 & 19.442 & 18.891 & & 17.376 & 10.674 & 6.826 \\
\hline 3/11/11 8:00 & 24.699 & 19.372 & 18.879 & & 17.355 & 10.660 & 6.814 \\
\hline $3 / 11 / 11$ 12:00 & 24.683 & 19.314 & 18.863 & & 17.332 & 10.646 & 6.798 \\
\hline $3 / 11 / 11$ 16:00 & 24.664 & 19.230 & 18.851 & & 17.306 & 10.627 & 6.784 \\
\hline 3/11/11 20:00 & 24.660 & 19.286 & 18.856 & & 17.308 & 10.620 & 6.789 \\
\hline 3/12/11 0:00 & 24.657 & 19.395 & 18.858 & & 17.299 & 10.606 & 6.791 \\
\hline 3/12/11 4:00 & 24.648 & 19.435 & 18.854 & & 17.287 & 10.592 & 6.784 \\
\hline 3/12/11 8:00 & 24.641 & 19.470 & 18.851 & & 17.280 & 10.585 & 6.784 \\
\hline $3 / 12 / 11$ 12:00 & 24.637 & 19.544 & 18.854 & & 17.276 & 10.573 & 6.786 \\
\hline 3/12/11 16:00 & 24.628 & 19.563 & 18.849 & & 17.266 & 10.562 & 6.782 \\
\hline 3/12/11 20:00 & 24.621 & 19.591 & 18.849 & & 17.257 & 10.559 & 6.782 \\
\hline 3/13/11 0:00 & 24.616 & 19.661 & 18.849 & & 17.252 & 10.548 & 6.782 \\
\hline 3/13/11 4:00 & 24.611 & 19.693 & 18.847 & & 17.243 & 10.541 & 6.777 \\
\hline 3/13/11 8:00 & 24.602 & 19.714 & 18.844 & & 17.234 & 10.538 & 6.775 \\
\hline 3/13/11 12:00 & 24.598 & 19.777 & 18.847 & & 17.227 & 10.527 & 6.779 \\
\hline 3/13/11 16:00 & 24.586 & 19.777 & 18.837 & & 17.213 & 10.515 & 6.770 \\
\hline 3/13/11 20:00 & 24.577 & 19.777 & 18.837 & & 17.201 & 10.508 & 6.765 \\
\hline 3/14/11 0:00 & 24.570 & 19.821 & 18.837 & & 17.196 & 10.496 & 6.765 \\
\hline 3/14/11 4:00 & 24.556 & 19.798 & 18.826 & & 17.180 & 10.489 & 6.754 \\
\hline 3/14/11 8:00 & 24.547 & 19.824 & 18.826 & & 17.171 & 10.478 & 6.756 \\
\hline $3 / 14 / 11$ 12:00 & 24.538 & 19.815 & 18.819 & & 17.154 & 10.466 & 6.747 \\
\hline 3/14/11 16:00 & 24.522 & 19.749 & 18.807 & & 17.133 & 10.447 & 6.733 \\
\hline 3/14/11 20:00 & 24.511 & 19.700 & 18.802 & & 17.119 & 10.440 & 6.730 \\
\hline 3/15/11 0:00 & 24.499 & 19.703 & 18.798 & & 17.110 & 10.431 & 6.726 \\
\hline 3/15/11 4:00 & 24.488 & 19.686 & 18.791 & & 17.093 & 10.417 & 6.716 \\
\hline 3/15/11 8:00 & 24.476 & 19.656 & 18.786 & & 17.079 & 10.408 & 6.712 \\
\hline 3/15/11 12:00 & 24.467 & 19.673 & 18.782 & & 17.068 & 10.396 & 6.707 \\
\hline 3/15/11 16:00 & 24.453 & 19.621 & 18.770 & & 17.047 & 10.384 & 6.695 \\
\hline 3/15/11 20:00 & 24.442 & 19.593 & 18.765 & & 17.035 & 10.377 & 6.690 \\
\hline 3/16/11 0:00 & 24.435 & 19.628 & 18.765 & & 17.026 & 10.368 & 6.693 \\
\hline
\end{tabular}

TABLE S1.2 Page 28 of 58 


\begin{tabular}{|c|c|c|c|c|c|c|c|}
\hline \multirow[b]{2}{*}{ Date and Time } & \multicolumn{7}{|c|}{ Water Level (ft below reference point) } \\
\hline & SB43S & SB44S & SB45S & SB46S & SB47S & SB48S & SB49S \\
\hline 3/16/11 4:00 & 24.424 & 19.635 & 18.758 & & 17.012 & 10.359 & 6.684 \\
\hline 3/16/11 8:00 & 24.412 & 19.628 & 18.756 & & 17.000 & 10.349 & 6.679 \\
\hline 3/16/11 12:00 & 24.401 & 19.621 & 18.747 & & 16.984 & 10.333 & 6.670 \\
\hline 3/16/11 16:00 & 24.385 & 19.540 & 18.735 & & 16.963 & 10.316 & 6.658 \\
\hline 3/16/11 20:00 & 24.369 & 19.486 & 18.726 & & 16.946 & 10.307 & 6.649 \\
\hline 3/17/11 0:00 & 24.355 & 19.472 & 18.716 & & 16.930 & 10.293 & 6.644 \\
\hline 3/17/11 4:00 & 24.343 & 19.426 & 18.709 & & 16.909 & 10.279 & 6.635 \\
\hline 3/17/11 8:00 & 24.330 & 19.393 & 18.702 & & 16.897 & 10.272 & 6.630 \\
\hline 3/17/11 12:00 & 24.323 & 19.432 & 18.702 & & 16.888 & 10.268 & 6.628 \\
\hline 3/17/11 16:00 & 24.318 & 19.484 & 18.702 & & 16.883 & 10.265 & 6.630 \\
\hline 3/17/11 20:00 & 24.318 & 19.603 & 18.714 & & 16.888 & 10.270 & 6.637 \\
\hline 3/18/11 0:00 & 24.318 & 19.726 & 18.719 & & 16.888 & 10.267 & 6.639 \\
\hline 3/18/11 4:00 & 24.311 & 19.798 & 18.723 & & 16.883 & 10.265 & 6.644 \\
\hline 3/18/11 8:00 & 24.309 & 19.849 & 18.726 & & 16.879 & 10.265 & 6.649 \\
\hline $3 / 18 / 1112: 00$ & 24.307 & 19.936 & 18.730 & & 16.874 & 10.258 & 6.651 \\
\hline 3/18/11 16:00 & 24.295 & 19.922 & 18.726 & & 16.864 & 10.253 & 6.644 \\
\hline 3/18/11 20:00 & 24.288 & 19.929 & 18.723 & & 16.855 & 10.249 & 6.644 \\
\hline 3/19/11 0:00 & 24.282 & 19.963 & 18.723 & & 16.846 & 10.244 & 6.639 \\
\hline 3/19/11 4:00 & 24.270 & 19.975 & 18.721 & & 16.836 & 10.235 & 6.635 \\
\hline 3/19/11 8:00 & 24.263 & 19.963 & 18.716 & & 16.825 & 10.228 & 6.630 \\
\hline $3 / 19 / 11$ 12:00 & 24.247 & 19.933 & 18.709 & & 16.806 & 10.214 & 6.625 \\
\hline 3/19/11 16:00 & 24.233 & 19.875 & 18.695 & & 16.785 & 10.202 & 6.614 \\
\hline 3/19/11 20:00 & 24.217 & 19.814 & 18.691 & & 16.771 & 10.188 & 6.604 \\
\hline 3/20/11 0:00 & 24.208 & 19.765 & 18.684 & & 16.755 & 10.181 & 6.600 \\
\hline 3/20/11 4:00 & 24.190 & 19.745 & 18.672 & & 16.736 & 10.170 & 6.590 \\
\hline $3 / 20 / 118: 00$ & 24.178 & 19.738 & 18.665 & & 16.720 & 10.155 & 6.581 \\
\hline $3 / 20 / 11$ 12:00 & 24.165 & 19.691 & 18.658 & & 16.703 & 10.144 & 6.576 \\
\hline 3/20/11 16:00 & 24.153 & 19.635 & 18.651 & & 16.687 & 10.139 & 6.567 \\
\hline 3/20/11 20:00 & 24.144 & 19.658 & 18.649 & & 16.680 & 10.137 & 6.565 \\
\hline 3/21/11 0:00 & 24.144 & 19.763 & 18.658 & & 16.678 & 10.137 & 6.574 \\
\hline 3/21/11 4:00 & 24.135 & 19.822 & 18.658 & & 16.671 & 10.132 & 6.574 \\
\hline $3 / 21 / 118: 00$ & 24.128 & 19.831 & 18.656 & & 16.661 & 10.121 & 6.572 \\
\hline 3/21/11 12:00 & 24.116 & 19.833 & 18.651 & & 16.647 & 10.114 & 6.562 \\
\hline 3/21/11 16:00 & 24.098 & 19.765 & 18.637 & & 16.626 & 10.102 & 6.553 \\
\hline 3/21/11 20:00 & 24.085 & 19.714 & 18.633 & & 16.610 & 10.095 & 6.546 \\
\hline 3/22/11 0:00 & 24.075 & 19.719 & 18.626 & & 16.601 & 10.083 & 6.544 \\
\hline 3/22/11 4:00 & 24.057 & 19.672 & 18.619 & & 16.580 & 10.067 & 6.530 \\
\hline 3/22/11 8:00 & 24.041 & 19.595 & 18.605 & & 16.561 & 10.055 & 6.518 \\
\hline $3 / 22 / 11$ 12:00 & 24.020 & 19.542 & 18.593 & & 16.540 & 10.041 & 6.506 \\
\hline 3/22/11 16:00 & 24.009 & 19.484 & 18.586 & & 16.519 & 10.027 & 6.501 \\
\hline 3/22/11 20:00 & 24.000 & 19.456 & 18.581 & & 16.509 & 10.027 & 6.497 \\
\hline 3/23/11 0:00 & 23.997 & 19.563 & 18.588 & & 16.507 & 10.020 & 6.499 \\
\hline 3/23/11 4:00 & 23.988 & 19.616 & 18.588 & & 16.498 & 10.015 & 6.499 \\
\hline 3/23/11 8:00 & 23.988 & 19.675 & 18.595 & & 16.498 & 10.025 & 6.506 \\
\hline 3/23/11 12:00 & 23.986 & 19.791 & 18.600 & & 16.498 & 10.022 & 6.511 \\
\hline 3/23/11 16:00 & 23.986 & 19.877 & 18.607 & & 16.495 & 10.025 & 6.520 \\
\hline $3 / 23 / 1120: 00$ & 23.988 & 19.991 & 18.619 & & 16.502 & 10.027 & 6.522 \\
\hline 3/24/11 0:00 & 23.986 & 20.096 & 18.623 & & 16.502 & 10.030 & 6.532 \\
\hline 3/24/11 4:00 & 23.981 & 20.152 & 18.628 & & 16.498 & 10.027 & 6.534 \\
\hline 3/24/11 8:00 & 23.979 & 20.199 & 18.630 & & 16.493 & 10.029 & 6.541 \\
\hline 3/24/11 12:00 & 23.972 & 20.245 & 18.633 & & 16.488 & 10.020 & 6.539 \\
\hline 3/24/11 16:00 & 23.956 & 20.180 & 18.621 & & 16.463 & 10.009 & 6.527 \\
\hline 3/24/11 20:00 & 23.938 & 20.080 & 18.607 & & 16.442 & 10.009 & 6.513 \\
\hline 3/25/11 0:00 & 23.933 & 20.138 & 18.607 & & 16.435 & 9.999 & 6.513 \\
\hline
\end{tabular}

TABLE S1.2 Page 29 of 58 


\begin{tabular}{|c|c|c|c|c|c|c|c|}
\hline \multirow[b]{2}{*}{ Date and Time } & \multicolumn{7}{|c|}{ Water Level (ft below reference point) } \\
\hline & SB43S & SB44S & SB45S & SB46S & SB47S & SB48S & SB49S \\
\hline 3/25/11 4:00 & 23.922 & 20.101 & 18.602 & & 16.423 & 9.988 & 6.511 \\
\hline 3/25/11 8:00 & 23.915 & 20.073 & 18.602 & & 16.411 & 9.987 & 6.506 \\
\hline 3/25/11 12:00 & 23.906 & 20.029 & 18.602 & & 16.402 & 9.978 & 6.506 \\
\hline 3/25/11 16:00 & 23.897 & 19.968 & 18.598 & & 16.386 & 9.971 & 6.499 \\
\hline 3/25/11 20:00 & 23.892 & 19.940 & 18.598 & & 16.379 & 9.969 & 6.499 \\
\hline 3/26/11 0:00 & 23.883 & 19.956 & 18.598 & & 16.369 & 9.959 & 6.501 \\
\hline 3/26/11 4:00 & 23.871 & 19.903 & 18.591 & & 16.358 & 9.953 & 6.494 \\
\hline 3/26/11 8:00 & 23.860 & 19.873 & 18.586 & & 16.344 & 9.950 & 6.490 \\
\hline 3/26/11 12:00 & 23.855 & 19.903 & 18.591 & & 16.339 & 9.948 & 6.492 \\
\hline 3/26/11 16:00 & 23.851 & 19.933 & 18.591 & & 16.332 & 9.943 & 6.490 \\
\hline 3/26/11 20:00 & 23.844 & 19.961 & 18.591 & & 16.327 & 9.943 & 6.492 \\
\hline 3/27/11 0:00 & 23.841 & 20.010 & 18.595 & & 16.325 & 9.938 & 6.494 \\
\hline 3/27/11 4:00 & 23.832 & 20.022 & 18.591 & & 16.313 & 9.936 & 6.487 \\
\hline 3/27/11 8:00 & 23.830 & 20.043 & 18.595 & & 16.311 & 9.934 & 6.492 \\
\hline 3/27/11 12:00 & 23.823 & 20.078 & 18.593 & & 16.299 & 9.927 & 6.490 \\
\hline 3/27/11 16:00 & 23.810 & 20.031 & 18.584 & & 16.288 & 9.920 & 6.485 \\
\hline 3/27/11 20:00 & 23.800 & 20.024 & 18.584 & & 16.278 & 9.917 & 6.478 \\
\hline 3/28/11 0:00 & 23.796 & 20.061 & 18.586 & & 16.271 & 9.915 & 6.483 \\
\hline 3/28/11 4:00 & 23.789 & 20.080 & 18.586 & & 16.262 & 9.908 & 6.480 \\
\hline 3/28/11 8:00 & 23.784 & 20.092 & 18.584 & & 16.255 & 9.910 & 6.480 \\
\hline 3/28/11 12:00 & 23.775 & 20.124 & 18.581 & & 16.248 & 9.903 & 6.480 \\
\hline 3/28/11 16:00 & 23.764 & 20.080 & 18.577 & & 16.231 & 9.894 & 6.466 \\
\hline 3/28/11 20:00 & 23.757 & 20.061 & 18.574 & & 16.222 & 9.894 & 6.469 \\
\hline 3/29/11 0:00 & 23.750 & 20.110 & 18.577 & & 16.217 & 9.892 & 6.469 \\
\hline 3/29/11 4:00 & 23.743 & 20.133 & 18.577 & & 16.208 & 9.887 & 6.469 \\
\hline 3/29/11 8:00 & 23.741 & 20.180 & 18.581 & & 16.206 & 9.889 & 6.473 \\
\hline $3 / 29 / 1112: 00$ & 23.734 & 20.220 & 18.581 & & 16.201 & 9.885 & 6.471 \\
\hline $3 / 29 / 11$ 16:00 & 23.725 & 20.196 & 18.577 & & 16.187 & 9.880 & 6.464 \\
\hline 3/29/11 20:00 & 23.716 & 20.194 & 18.574 & & 16.182 & 9.878 & 6.464 \\
\hline 3/30/11 0:00 & 23.711 & 20.229 & 18.577 & & 16.171 & 9.873 & 6.462 \\
\hline 3/30/11 4:00 & 23.700 & 20.199 & 18.567 & & 16.159 & 9.866 & 6.457 \\
\hline 3/30/11 8:00 & 23.690 & 20.185 & 18.570 & & 16.152 & 9.866 & 6.457 \\
\hline 3/30/11 12:00 & 23.683 & 20.199 & 18.565 & & 16.140 & 9.859 & 6.452 \\
\hline 3/30/11 16:00 & 23.670 & 20.162 & 18.556 & & 16.124 & 9.850 & 6.445 \\
\hline 3/30/11 20:00 & 23.656 & 20.175 & 18.549 & & 16.108 & 9.843 & 6.436 \\
\hline 3/31/11 0:00 & 23.647 & 20.234 & 18.547 & & 16.096 & 9.836 & 6.438 \\
\hline 3/31/11 4:00 & 23.635 & 20.264 & 18.540 & & 16.084 & 9.829 & 6.427 \\
\hline 3/31/11 8:00 & 23.624 & 20.290 & 18.535 & & 16.068 & 9.822 & 6.424 \\
\hline 3/31/11 12:00 & 23.615 & 20.317 & 18.530 & & 16.059 & 9.815 & 6.420 \\
\hline 3/31/11 16:00 & 23.603 & 20.331 & 18.523 & & 16.042 & 9.810 & 6.410 \\
\hline 3/31/11 20:00 & 23.594 & 20.374 & 18.521 & & 16.033 & 9.808 & 6.406 \\
\hline 4/1/11 0:00 & 23.587 & 20.429 & 18.521 & & 16.024 & 9.801 & 6.406 \\
\hline 4/1/11 4:00 & 23.578 & 20.464 & 18.521 & & 16.017 & 9.798 & 6.406 \\
\hline 4/1/11 8:00 & 23.578 & 20.543 & 18.526 & & 16.014 & 9.801 & 6.408 \\
\hline 4/1/11 12:00 & 23.569 & 20.627 & 18.530 & & 16.007 & 9.801 & 6.410 \\
\hline 4/1/11 16:00 & 23.560 & 20.667 & 18.526 & & 15.998 & 9.798 & 6.414 \\
\hline 4/1/11 20:00 & 23.557 & 20.739 & 18.526 & & 15.998 & 9.796 & 6.414 \\
\hline 4/2/11 0:00 & 23.553 & 20.818 & 18.533 & & 15.991 & 9.796 & 6.414 \\
\hline 4/2/11 4:00 & 23.551 & 20.879 & 18.535 & & 15.986 & 9.794 & 6.416 \\
\hline 4/2/11 8:00 & 23.544 & 20.930 & 18.537 & & 15.981 & 9.794 & 6.418 \\
\hline 4/2/11 12:00 & 23.539 & 20.988 & 18.537 & & 15.974 & 9.787 & 6.421 \\
\hline 4/2/11 16:00 & 23.521 & 20.935 & 18.523 & & 15.953 & 9.777 & 6.405 \\
\hline 4/2/11 20:00 & 23.507 & 20.876 & 18.516 & & 15.960 & 9.773 & 6.395 \\
\hline 4/3/11 0:00 & 23.493 & 20.851 & 18.509 & & 15.953 & 9.759 & 6.384 \\
\hline
\end{tabular}

TABLE S1.2 Page 30 of 58 


\begin{tabular}{|c|c|c|c|c|c|c|c|}
\hline \multirow[b]{2}{*}{ Date and Time } & \multicolumn{7}{|c|}{ Water Level (ft below reference point) } \\
\hline & SB43S & SB44S & SB45S & SB46S & SB47S & SB48S & SB49S \\
\hline 4/3/11 4:00 & 23.473 & 20.762 & 18.493 & & 15.935 & 9.742 & 6.377 \\
\hline 4/3/11 8:00 & 23.459 & 20.683 & 18.484 & & 15.918 & 9.740 & 6.367 \\
\hline $4 / 3 / 1112: 00$ & 23.447 & 20.676 & 18.479 & & 15.909 & 9.728 & 6.360 \\
\hline $4 / 3 / 1116: 00$ & 23.434 & 20.641 & 18.467 & & 15.890 & 9.728 & 6.353 \\
\hline $4 / 3 / 1120: 00$ & 23.434 & 20.723 & 18.479 & & 15.895 & 9.738 & 6.360 \\
\hline 4/4/11 0:00 & 23.441 & 20.925 & 18.495 & & 15.904 & 9.742 & 6.381 \\
\hline 4/4/11 4:00 & 23.447 & 21.065 & 18.507 & & 15.907 & 9.742 & 6.384 \\
\hline 4/4/11 8:00 & 23.447 & 21.184 & 18.516 & & 15.911 & 9.752 & 6.395 \\
\hline 4/4/11 12:00 & 23.445 & 21.286 & 18.521 & & 15.907 & 9.749 & 6.395 \\
\hline $4 / 4 / 1116: 00$ & 23.440 & 21.328 & 18.523 & & 15.900 & 9.752 & 6.402 \\
\hline $4 / 4 / 1120: 00$ & 23.438 & 21.370 & 18.526 & & 15.895 & 9.752 & 6.402 \\
\hline 4/5/11 0:00 & 23.434 & 21.428 & 18.530 & & 15.893 & 9.747 & 6.407 \\
\hline 4/5/11 4:00 & 23.427 & 21.438 & 18.526 & & 15.881 & 9.747 & 6.400 \\
\hline 4/5/11 8:00 & 23.418 & 21.426 & 18.523 & & 15.872 & 9.738 & 6.395 \\
\hline 4/5/11 12:00 & 23.406 & 21.396 & 18.519 & & 15.858 & 9.733 & 6.386 \\
\hline 4/5/11 16:00 & 23.388 & 21.345 & 18.507 & & 15.839 & 9.731 & 6.384 \\
\hline 4/5/11 20:00 & 23.383 & 21.314 & 18.505 & & 15.830 & 9.724 & 6.379 \\
\hline 4/6/11 0:00 & 23.372 & 21.293 & 18.500 & & 15.818 & 9.719 & 6.372 \\
\hline 4/6/11 4:00 & 23.363 & 21.286 & 18.498 & & 15.806 & 9.714 & 6.372 \\
\hline 4/6/11 8:00 & 23.360 & 21.333 & 18.502 & & 15.804 & 9.719 & 6.377 \\
\hline 4/6/11 12:00 & 23.360 & 21.438 & 18.512 & & 15.804 & 9.721 & 6.381 \\
\hline 4/6/11 16:00 & 23.358 & 21.515 & 18.514 & & 15.802 & 9.724 & 6.386 \\
\hline 4/6/11 20:00 & 23.358 & 21.585 & 18.523 & & 15.799 & 9.728 & 6.391 \\
\hline 4/7/11 0:00 & 23.356 & 21.650 & 18.528 & & 15.799 & 9.728 & 6.393 \\
\hline 4/7/11 4:00 & 23.353 & 21.694 & 18.530 & & 15.790 & 9.728 & 6.395 \\
\hline 4/7/11 8:00 & 23.347 & 21.708 & 18.528 & & 15.783 & 9.726 & 6.395 \\
\hline 4/7/11 12:00 & 23.340 & 21.726 & 18.530 & & 15.774 & 9.724 & 6.395 \\
\hline 4/7/11 16:00 & 23.328 & 21.696 & 18.516 & & 15.755 & 9.714 & 6.379 \\
\hline 4/7/11 20:00 & 23.321 & 21.524 & 18.521 & & 15.750 & 9.714 & 6.381 \\
\hline 4/8/11 0:00 & 23.312 & 21.454 & 18.519 & & 15.741 & 9.712 & 6.379 \\
\hline 4/8/11 4:00 & 23.308 & 21.389 & 18.516 & & 15.734 & 9.714 & 6.379 \\
\hline 4/8/11 8:00 & 23.305 & 21.347 & 18.523 & & 15.732 & 9.712 & 6.384 \\
\hline 4/8/11 12:00 & 23.303 & 21.349 & 18.526 & & 15.729 & 9.710 & 6.386 \\
\hline 4/8/11 16:00 & 23.294 & 21.314 & 18.523 & & 15.717 & 9.705 & 6.381 \\
\hline 4/8/11 20:00 & 23.285 & 21.270 & 18.519 & & 15.703 & 9.705 & 6.377 \\
\hline 4/9/11 0:00 & 23.280 & 21.282 & 18.519 & & 15.701 & 9.698 & 6.379 \\
\hline 4/9/11 4:00 & 23.273 & 21.265 & 18.516 & & 15.692 & 9.698 & 6.374 \\
\hline 4/9/11 8:00 & 23.269 & 21.298 & 18.519 & & 15.685 & 9.696 & 6.379 \\
\hline 4/9/11 12:00 & 23.262 & 21.305 & 18.516 & & 15.675 & 9.689 & 6.372 \\
\hline 4/9/11 16:00 & 23.248 & 21.244 & 18.505 & & 15.657 & 9.682 & 6.363 \\
\hline 4/9/11 20:00 & 23.236 & 21.205 & 18.502 & & 15.647 & 9.679 & 6.358 \\
\hline 4/10/11 0:00 & 23.232 & 21.216 & 18.500 & & 15.638 & 9.675 & 6.358 \\
\hline 4/10/11 4:00 & 23.223 & 21.219 & 18.495 & & 15.626 & 9.672 & 6.351 \\
\hline 4/10/11 8:00 & 23.216 & 21.224 & 18.495 & & 15.619 & 9.670 & 6.351 \\
\hline $4 / 10 / 1112: 00$ & 23.209 & 21.237 & 18.495 & & 15.612 & 9.665 & 6.346 \\
\hline 4/10/11 16:00 & 23.202 & 21.272 & 18.498 & & 15.608 & 9.667 & 6.351 \\
\hline 4/10/11 20:00 & 23.207 & 21.363 & 18.505 & & 15.608 & 9.675 & 6.355 \\
\hline $4 / 11 / 110: 00$ & 23.211 & 21.484 & 18.514 & & 15.612 & 9.677 & 6.372 \\
\hline 4/11/11 4:00 & 23.209 & 21.547 & 18.514 & & 15.608 & 9.679 & 6.369 \\
\hline 4/11/11 8:00 & 23.209 & 21.603 & 18.523 & & 15.608 & 9.684 & 6.372 \\
\hline $4 / 11 / 11$ 12:00 & 23.207 & 21.687 & 18.526 & & 15.603 & 9.682 & 6.376 \\
\hline 4/11/11 16:00 & 23.200 & 21.715 & 18.528 & & 15.596 & 9.684 & 6.376 \\
\hline $4 / 11 / 1120: 00$ & 23.200 & 21.759 & 18.530 & & 15.596 & 9.689 & 6.381 \\
\hline 4/12/11 0:00 & 23.202 & 21.827 & 18.537 & & 15.594 & 9.689 & 6.388 \\
\hline
\end{tabular}

TABLE S1.2 Page 31 of 58 


\begin{tabular}{|c|c|c|c|c|c|c|c|}
\hline \multirow[b]{2}{*}{ Date and Time } & \multicolumn{7}{|c|}{ Water Level (ft below reference point) } \\
\hline & SB43S & SB44S & SB45S & SB46S & SB47S & SB48S & SB49S \\
\hline $4 / 12 / 114: 00$ & 23.198 & 21.866 & 18.540 & & 15.591 & 9.691 & 6.391 \\
\hline 4/12/11 8:00 & 23.198 & 21.908 & 18.547 & & 15.589 & 9.693 & 6.390 \\
\hline $4 / 12 / 11$ 12:00 & 23.193 & 21.941 & 18.547 & & 15.580 & 9.691 & 6.393 \\
\hline 4/12/11 16:00 & 23.182 & 21.901 & 18.540 & & 15.566 & 9.684 & 6.386 \\
\hline 4/12/11 20:00 & 23.172 & 21.866 & 18.535 & & 15.559 & 9.684 & 6.381 \\
\hline 4/13/11 0:00 & 23.168 & 21.885 & 18.535 & & 15.551 & 9.686 & 6.381 \\
\hline 4/13/11 4:00 & 23.163 & 21.883 & 18.537 & & 15.544 & 9.684 & 6.376 \\
\hline 4/13/11 8:00 & 23.161 & 21.903 & 18.540 & & 15.537 & 9.684 & 6.381 \\
\hline 4/13/11 12:00 & 23.154 & 21.920 & 18.535 & & 15.530 & 9.677 & 6.383 \\
\hline 4/13/11 16:00 & 23.143 & 21.890 & 18.530 & & 15.516 & 9.677 & 6.374 \\
\hline $4 / 13 / 1120: 00$ & 23.136 & 21.883 & 18.533 & 13.765 & 15.512 & 9.677 & 6.369 \\
\hline 4/14/11 0:00 & 23.133 & 21.917 & 18.533 & 13.802 & 15.505 & 9.677 & 6.372 \\
\hline 4/14/11 4:00 & 23.131 & 21.934 & 18.535 & 13.839 & 15.500 & 9.677 & 6.372 \\
\hline 4/14/11 8:00 & 23.124 & 21.950 & 18.533 & 13.876 & 15.491 & 9.672 & 6.369 \\
\hline 4/14/11 12:00 & 23.113 & 21.901 & 18.526 & 13.907 & 15.479 & 9.665 & 6.367 \\
\hline 4/14/11 16:00 & 23.099 & 21.808 & 18.514 & 13.944 & 15.458 & 9.658 & 6.353 \\
\hline 4/14/11 20:00 & 23.088 & 21.764 & 18.512 & 13.977 & 15.449 & 9.670 & 6.344 \\
\hline 4/15/11 0:00 & 23.081 & 21.806 & 18.507 & 14.009 & 15.437 & 9.646 & 6.351 \\
\hline 4/15/11 4:00 & 23.074 & 21.400 & 18.502 & 14.042 & 15.425 & 9.644 & 6.341 \\
\hline 4/15/11 8:00 & 23.065 & 21.142 & 18.495 & 14.065 & 15.404 & 9.639 & 6.332 \\
\hline 4/15/11 12:00 & 23.056 & 20.960 & 18.491 & 14.091 & 15.395 & 9.639 & 6.327 \\
\hline 4/15/11 16:00 & 23.051 & 20.872 & 18.488 & 14.123 & 15.383 & 9.632 & 6.323 \\
\hline 4/15/11 20:00 & 23.049 & 20.704 & 18.493 & 14.149 & 15.379 & 9.635 & 6.330 \\
\hline 4/16/11 0:00 & 23.049 & 20.595 & 18.498 & 14.191 & 15.376 & 9.637 & 6.339 \\
\hline 4/16/11 4:00 & 23.051 & 20.599 & 18.505 & 14.214 & 15.374 & 9.644 & 6.341 \\
\hline 4/16/11 8:00 & 23.056 & 20.667 & 18.514 & 14.240 & 15.379 & 9.646 & 6.355 \\
\hline 4/16/11 12:00 & 23.058 & 20.732 & 18.521 & 14.275 & 15.381 & 9.644 & 6.358 \\
\hline 4/16/11 16:00 & 23.051 & 20.709 & 18.519 & 14.303 & 15.374 & 9.637 & 6.355 \\
\hline 4/16/11 20:00 & 23.044 & 20.662 & 18.516 & 14.328 & 15.362 & 9.642 & 6.353 \\
\hline 4/17/11 0:00 & 23.044 & 20.690 & 18.516 & 14.354 & 15.362 & 9.639 & 6.358 \\
\hline 4/17/11 4:00 & 23.035 & 20.695 & 18.514 & 14.382 & 15.355 & 9.632 & 6.353 \\
\hline 4/17/11 8:00 & 23.028 & 20.667 & 18.509 & 14.405 & 15.346 & 9.628 & 6.348 \\
\hline 4/17/11 12:00 & 23.019 & 20.630 & 18.500 & 14.431 & 15.334 & 9.616 & 6.341 \\
\hline 4/17/11 16:00 & 23.005 & 20.555 & 18.488 & 14.454 & 15.320 & 9.609 & 6.332 \\
\hline 4/17/11 20:00 & 22.996 & 20.513 & 18.484 & 14.475 & 15.306 & 9.607 & 6.325 \\
\hline 4/18/11 0:00 & 22.991 & 20.532 & 18.481 & 14.503 & 15.304 & 9.607 & 6.320 \\
\hline 4/18/11 4:00 & 22.991 & 20.595 & 18.486 & 14.531 & 15.304 & 9.607 & 6.325 \\
\hline 4/18/11 8:00 & 22.989 & 20.664 & 18.491 & 14.561 & 15.306 & 9.611 & 6.330 \\
\hline 4/18/11 12:00 & 22.989 & 20.734 & 18.495 & 14.589 & 15.302 & 9.604 & 6.337 \\
\hline 4/18/11 16:00 & 22.978 & 20.711 & 18.481 & 14.615 & 15.288 & 9.602 & 6.323 \\
\hline 4/18/11 20:00 & 22.973 & 20.702 & 18.479 & 14.643 & 15.281 & 9.597 & 6.323 \\
\hline 4/19/11 0:00 & 22.966 & 20.713 & 18.477 & 14.671 & 15.273 & 9.593 & 6.313 \\
\hline 4/19/11 4:00 & 22.948 & 20.634 & 18.463 & 14.689 & 15.255 & 9.583 & 6.304 \\
\hline 4/19/11 8:00 & 22.948 & 20.620 & 18.463 & 14.715 & 15.250 & 9.581 & 6.302 \\
\hline 4/19/11 12:00 & 22.939 & 20.655 & 18.463 & 14.741 & 15.245 & 9.581 & 6.304 \\
\hline 4/19/11 16:00 & 22.939 & 20.716 & 18.465 & 14.771 & 15.245 & 9.583 & 6.302 \\
\hline 4/19/11 20:00 & 22.941 & 20.816 & 18.474 & 14.799 & 15.250 & 9.590 & 6.309 \\
\hline 4/20/11 0:00 & 22.948 & 20.953 & 18.484 & 14.834 & 15.257 & 9.595 & 6.320 \\
\hline $4 / 20 / 114: 00$ & 22.948 & 21.039 & 18.491 & 14.864 & 15.257 & 9.600 & 6.327 \\
\hline 4/20/11 8:00 & 22.950 & 21.126 & 18.498 & 14.897 & 15.257 & 9.604 & 6.334 \\
\hline 4/20/11 12:00 & 22.952 & 21.214 & 18.505 & 14.929 & 15.262 & 9.607 & 6.334 \\
\hline 4/20/11 16:00 & 22.946 & 21.228 & 18.502 & 14.955 & 15.255 & 9.602 & 6.332 \\
\hline 4/20/11 20:00 & 22.941 & 21.226 & 18.500 & 14.983 & 15.250 & 9.602 & 6.330 \\
\hline 4/21/11 0:00 & 22.936 & 21.254 & 18.500 & 15.011 & 15.243 & 9.602 & 6.330 \\
\hline
\end{tabular}

TABLE S1.2 Page 32 of 58 


\begin{tabular}{|c|c|c|c|c|c|c|c|}
\hline \multirow[b]{2}{*}{ Date and Time } & \multicolumn{7}{|c|}{ Water Level (ft below reference point) } \\
\hline & SB43S & SB44S & SB45S & SB46S & SB47S & SB48S & SB49S \\
\hline $4 / 21 / 114: 00$ & 22.932 & 21.275 & 18.500 & 15.036 & 15.243 & 9.600 & 6.330 \\
\hline 4/21/11 8:00 & 22.927 & 21.251 & 18.498 & 15.064 & 15.231 & 9.597 & 6.323 \\
\hline $4 / 21 / 11$ 12:00 & 22.911 & 21.207 & 18.486 & 15.088 & 15.217 & 9.581 & 6.313 \\
\hline 4/21/11 16:00 & 22.895 & 21.079 & 18.472 & 15.106 & 15.199 & 9.579 & 6.299 \\
\hline 4/21/11 20:00 & 22.888 & 21.030 & 18.467 & 15.130 & 15.189 & 9.576 & 6.292 \\
\hline 4/22/11 0:00 & 22.879 & 21.004 & 18.463 & 15.150 & 15.178 & 9.569 & 6.288 \\
\hline $4 / 22 / 114: 00$ & 22.868 & 20.937 & 18.451 & 15.174 & 15.164 & 9.553 & 6.276 \\
\hline $4 / 22 / 118: 00$ & 22.858 & 20.881 & 18.449 & 15.195 & 15.154 & 9.560 & 6.269 \\
\hline 4/22/11 12:00 & 22.847 & 20.858 & 18.439 & 15.218 & 15.140 & 9.553 & 6.262 \\
\hline $4 / 22 / 11$ 16:00 & 22.845 & 20.867 & 18.439 & 15.246 & 15.138 & 9.551 & 6.262 \\
\hline $4 / 22 / 1120: 00$ & 22.838 & 20.886 & 18.437 & 15.269 & 15.133 & 9.555 & 6.260 \\
\hline 4/23/11 0:00 & 22.840 & 20.956 & 18.446 & 15.297 & 15.133 & 9.555 & 6.262 \\
\hline $4 / 23 / 114: 00$ & 22.833 & 21.000 & 18.444 & 15.323 & 15.124 & 9.555 & 6.264 \\
\hline 4/23/11 8:00 & 22.836 & 21.060 & 18.453 & 15.351 & 15.131 & 9.560 & 6.269 \\
\hline $4 / 23 / 11$ 12:00 & 22.838 & 21.172 & 18.458 & 15.379 & 15.133 & 9.560 & 6.269 \\
\hline $4 / 23 / 11$ 16:00 & 22.831 & 21.195 & 18.458 & 15.404 & 15.126 & 9.560 & 6.269 \\
\hline $4 / 23 / 1120: 00$ & 22.829 & 21.214 & 18.458 & 15.430 & 15.122 & 9.565 & 6.269 \\
\hline 4/24/11 0:00 & 22.826 & 21.272 & 18.463 & 15.460 & 15.122 & 9.562 & 6.269 \\
\hline 4/24/11 4:00 & 22.822 & 21.289 & 18.463 & 15.481 & 15.115 & 9.560 & 6.271 \\
\hline 4/24/11 8:00 & 22.817 & 21.286 & 18.460 & 15.507 & 15.110 & 9.562 & 6.264 \\
\hline $4 / 24 / 11$ 12:00 & 22.810 & 21.314 & 18.460 & 15.535 & 15.100 & 9.555 & 6.262 \\
\hline $4 / 24 / 11$ 16:00 & 22.799 & 21.263 & 18.449 & 15.556 & 15.089 & 9.551 & 6.253 \\
\hline 4/24/11 20:00 & 22.792 & 21.214 & 18.446 & 15.579 & 15.079 & 9.548 & 6.253 \\
\hline 4/25/11 0:00 & 22.787 & 21.226 & 18.446 & 15.602 & 15.075 & 9.544 & 6.250 \\
\hline $4 / 25 / 114: 00$ & 22.778 & 21.188 & 18.439 & 15.623 & 15.065 & 9.539 & 6.241 \\
\hline 4/25/11 8:00 & 22.767 & 21.151 & 18.432 & 15.642 & 15.052 & 9.539 & 6.234 \\
\hline $4 / 25 / 11$ 12:00 & 22.758 & 21.168 & 18.430 & 15.661 & 15.040 & 9.530 & 6.225 \\
\hline 4/25/11 16:00 & 22.742 & 21.065 & 18.416 & 15.684 & 15.023 & 9.523 & 6.218 \\
\hline $4 / 25 / 1120: 00$ & 22.732 & 20.977 & 18.409 & 15.702 & 15.012 & 9.518 & 6.208 \\
\hline 4/26/11 0:00 & 22.721 & 20.937 & 18.405 & 15.719 & 15.000 & 9.513 & 6.204 \\
\hline 4/26/11 4:00 & 22.714 & 20.951 & 18.398 & 15.737 & 14.988 & 9.511 & 6.197 \\
\hline 4/26/11 8:00 & 22.712 & 20.928 & 18.400 & 15.763 & 14.984 & 9.513 & 6.201 \\
\hline 4/26/11 12:00 & 22.707 & 20.958 & 18.407 & 15.791 & 14.981 & 9.516 & 6.201 \\
\hline 4/26/11 16:00 & 22.707 & 21.000 & 18.407 & 15.812 & 14.981 & 9.513 & 6.204 \\
\hline 4/26/11 20:00 & 22.703 & 21.037 & 18.407 & 15.835 & 14.974 & 9.509 & 6.201 \\
\hline 4/27/11 0:00 & 22.700 & 21.049 & 18.407 & 15.856 & 14.970 & 9.513 & 6.204 \\
\hline 4/27/11 4:00 & 22.693 & 21.060 & 18.405 & 15.877 & 14.963 & 9.513 & 6.201 \\
\hline 4/27/11 8:00 & 22.691 & 21.107 & 18.409 & 15.903 & 14.963 & 9.513 & 6.204 \\
\hline 4/27/11 12:00 & 22.689 & 21.165 & 18.414 & 15.926 & 14.963 & 9.513 & 6.208 \\
\hline 4/27/11 16:00 & 22.684 & 21.195 & 18.414 & 15.952 & 14.958 & 9.513 & 6.206 \\
\hline 4/27/11 20:00 & 22.684 & 21.237 & 18.416 & 15.970 & 14.953 & 9.518 & 6.206 \\
\hline 4/28/11 0:00 & 22.682 & 21.307 & 18.421 & 15.996 & 14.956 & 9.518 & 6.213 \\
\hline 4/28/11 4:00 & 22.677 & 21.333 & 18.421 & 16.022 & 14.946 & 9.520 & 6.213 \\
\hline 4/28/11 8:00 & 22.680 & 21.379 & 18.428 & 16.045 & 14.949 & 9.525 & 6.218 \\
\hline $4 / 28 / 11$ 12:00 & 22.675 & 21.433 & 18.428 & 16.073 & 14.944 & 9.525 & 6.218 \\
\hline 4/28/11 16:00 & 22.668 & 21.447 & 18.428 & 16.098 & 14.937 & 9.525 & 6.213 \\
\hline $4 / 28 / 1120: 00$ & 22.666 & 21.461 & 18.430 & 16.115 & 14.935 & 9.527 & 6.218 \\
\hline 4/29/11 0:00 & 22.664 & 21.487 & 18.430 & 16.140 & 14.928 & 9.525 & 6.218 \\
\hline 4/29/11 4:00 & 22.657 & 21.487 & 18.430 & 16.159 & 14.925 & 9.523 & 6.215 \\
\hline 4/29/11 8:00 & 22.652 & 21.489 & 18.428 & 16.187 & 14.916 & 9.516 & 6.208 \\
\hline 4/29/11 12:00 & 22.643 & 21.456 & 18.421 & 16.203 & 14.904 & 9.511 & 6.204 \\
\hline 4/29/11 16:00 & 22.629 & 21.335 & 18.402 & 16.220 & 14.883 & 9.506 & 6.183 \\
\hline 4/29/11 20:00 & 22.616 & 21.291 & 18.405 & 16.240 & 14.876 & 9.504 & 6.187 \\
\hline 4/30/11 0:00 & 22.611 & 21.279 & 18.400 & 16.247 & 14.867 & 9.502 & 6.178 \\
\hline
\end{tabular}

TABLE S1.2 Page 33 of 58 


\begin{tabular}{|c|c|c|c|c|c|c|c|}
\hline \multirow[b]{2}{*}{ Date and Time } & \multicolumn{7}{|c|}{ Water Level (ft below reference point) } \\
\hline & SB43S & SB44S & SB45S & SB46S & SB47S & SB48S & SB49S \\
\hline $4 / 30 / 114: 00$ & 22.602 & 21.268 & 18.395 & 16.257 & 14.862 & 9.499 & 6.180 \\
\hline 4/30/11 8:00 & 22.602 & 21.279 & 18.400 & 16.273 & 14.858 & 9.504 & 6.176 \\
\hline 4/30/11 12:00 & 22.600 & 21.372 & 18.407 & 16.301 & 14.862 & 9.509 & 6.183 \\
\hline 4/30/11 16:00 & 22.597 & 21.438 & 18.409 & 16.327 & 14.855 & 9.509 & 6.187 \\
\hline 4/30/11 20:00 & 22.600 & 21.494 & 18.416 & 16.352 & 14.860 & 9.513 & 6.190 \\
\hline 5/1/11 0:00 & 22.602 & 21.596 & 18.428 & 16.378 & 14.860 & 9.520 & 6.204 \\
\hline $5 / 1 / 114: 00$ & 22.604 & 21.685 & 18.432 & 16.399 & 14.862 & 9.527 & 6.204 \\
\hline 5/1/11 8:00 & 22.606 & 21.766 & 18.442 & 16.424 & 14.867 & 9.532 & 6.215 \\
\hline $5 / 1 / 1112: 00$ & 22.604 & 21.824 & 18.446 & 16.452 & 14.862 & 9.532 & 6.215 \\
\hline 5/1/11 16:00 & 22.602 & 21.824 & 18.442 & 16.476 & 14.858 & 9.530 & 6.213 \\
\hline $5 / 1 / 1120: 00$ & 22.595 & 21.799 & 18.439 & 16.497 & 14.851 & 9.532 & 6.211 \\
\hline 5/2/11 0:00 & 22.595 & 21.857 & 18.446 & 16.522 & 14.851 & 9.537 & 6.215 \\
\hline $5 / 2 / 114: 00$ & 22.590 & 21.885 & 18.449 & 16.543 & 14.844 & 9.539 & 6.215 \\
\hline $5 / 2 / 118: 00$ & 22.597 & 21.950 & 18.460 & 16.571 & 14.848 & 9.546 & 6.229 \\
\hline $5 / 2 / 1112: 00$ & 22.595 & 22.018 & 18.463 & 16.599 & 14.846 & 9.544 & 6.229 \\
\hline $5 / 2 / 1116: 00$ & 22.586 & 21.990 & 18.458 & 16.618 & 14.837 & 9.544 & 6.222 \\
\hline $5 / 2 / 1120: 00$ & 22.583 & 21.985 & 18.460 & 16.641 & 14.832 & 9.548 & 6.225 \\
\hline 5/3/11 0:00 & 22.581 & 22.013 & 18.463 & 16.664 & 14.827 & 9.546 & 6.225 \\
\hline $5 / 3 / 114: 00$ & 22.577 & 22.022 & 18.465 & 16.685 & 14.822 & 9.548 & 6.227 \\
\hline $5 / 3 / 118: 00$ & 22.577 & 22.055 & 18.467 & 16.713 & 14.822 & 9.551 & 6.229 \\
\hline $5 / 3 / 1112: 00$ & 22.572 & 22.085 & 18.467 & 16.732 & 14.818 & 9.551 & 6.232 \\
\hline $5 / 3 / 1116: 00$ & 22.567 & 22.057 & 18.467 & 16.753 & 14.806 & 9.548 & 6.225 \\
\hline $5 / 3 / 1120: 00$ & 22.561 & 22.036 & 18.465 & 16.774 & 14.801 & 9.553 & 6.227 \\
\hline 5/4/11 0:00 & 22.561 & 22.064 & 18.472 & 16.797 & 14.799 & 9.553 & 6.225 \\
\hline $5 / 4 / 114: 00$ & 22.556 & 22.074 & 18.472 & 16.818 & 14.794 & 9.555 & 6.227 \\
\hline 5/4/11 8:00 & 22.556 & 22.097 & 18.477 & 16.841 & 14.790 & 9.555 & 6.227 \\
\hline $5 / 4 / 1112: 00$ & 22.549 & 22.092 & 18.472 & 16.860 & 14.783 & 9.551 & 6.222 \\
\hline 5/4/11 16:00 & 22.535 & 22.020 & 18.463 & 16.886 & 14.769 & 9.548 & 6.218 \\
\hline 5/4/11 20:00 & 22.528 & 21.973 & 18.463 & 16.897 & 14.759 & 9.546 & 6.211 \\
\hline 5/5/11 0:00 & 22.526 & 21.990 & 18.465 & 16.923 & 14.755 & 9.548 & 6.213 \\
\hline $5 / 5 / 114: 00$ & 22.522 & 22.011 & 18.463 & 16.942 & 14.750 & 9.551 & 6.211 \\
\hline 5/5/11 8:00 & 22.519 & 22.029 & 18.465 & 16.963 & 14.748 & 9.551 & 6.213 \\
\hline $5 / 5 / 1112: 00$ & 22.517 & 22.057 & 18.465 & 16.986 & 14.741 & 9.546 & 6.211 \\
\hline $5 / 5 / 1116: 00$ & 22.506 & 22.013 & 18.458 & 17.002 & 14.729 & 9.546 & 6.206 \\
\hline $5 / 5 / 1120: 00$ & 22.501 & 22.006 & 18.460 & 17.023 & 14.724 & 9.548 & 6.204 \\
\hline 5/6/11 0:00 & 22.496 & 22.025 & 18.463 & 17.046 & 14.720 & 9.546 & 6.210 \\
\hline 5/6/11 4:00 & 22.492 & 22.013 & 18.460 & 17.063 & 14.710 & 9.543 & 6.206 \\
\hline 5/6/11 8:00 & 22.487 & 22.006 & 18.460 & 17.081 & 14.703 & 9.544 & 6.201 \\
\hline $5 / 6 / 11$ 12:00 & 22.476 & 21.988 & 18.453 & 17.102 & 14.692 & 9.536 & 6.199 \\
\hline 5/6/11 16:00 & 22.464 & 21.908 & 18.446 & 17.116 & 14.678 & 9.532 & 6.187 \\
\hline 5/6/11 20:00 & 22.455 & 21.862 & 18.439 & 17.133 & 14.666 & 9.532 & 6.180 \\
\hline 5/7/11 0:00 & 22.455 & 21.887 & 18.446 & 17.156 & 14.666 & 9.532 & 6.187 \\
\hline $5 / 7 / 114: 00$ & 22.446 & 21.885 & 18.440 & 17.170 & 14.656 & 9.532 & 6.180 \\
\hline 5/7/11 8:00 & 22.448 & 21.922 & 18.449 & 17.191 & 14.659 & 9.536 & 6.185 \\
\hline $5 / 7 / 11$ 12:00 & 22.446 & 21.987 & 18.449 & 17.216 & 14.654 & 9.536 & 6.192 \\
\hline 5/7/11 16:00 & 22.441 & 21.999 & 18.451 & 17.235 & 14.647 & 9.536 & 6.192 \\
\hline 5/7/11 20:00 & 22.439 & 22.034 & 18.453 & 17.258 & 14.645 & 9.541 & 6.194 \\
\hline 5/8/11 0:00 & 22.439 & 22.097 & 18.458 & 17.277 & 14.645 & 9.541 & 6.196 \\
\hline $5 / 8 / 114: 00$ & 22.437 & 22.125 & 18.458 & 17.296 & 14.640 & 9.541 & 6.199 \\
\hline 5/8/11 8:00 & 22.432 & 22.160 & 18.463 & 17.319 & 14.635 & 9.541 & 6.201 \\
\hline 5/8/11 12:00 & 22.425 & 22.150 & 18.458 & 17.337 & 14.624 & 9.539 & 6.196 \\
\hline 5/8/11 16:00 & 22.414 & 22.104 & 18.449 & 17.351 & 14.612 & 9.536 & 6.189 \\
\hline $5 / 8 / 1120: 00$ & 22.409 & 22.080 & 18.449 & 17.370 & 14.605 & 9.536 & 6.185 \\
\hline 5/9/11 0:00 & 22.407 & 22.108 & 18.451 & 17.391 & 14.603 & 9.534 & 6.189 \\
\hline
\end{tabular}

TABLE S1.2 Page 34 of 58 


\begin{tabular}{|c|c|c|c|c|c|c|c|}
\hline \multirow[b]{2}{*}{ Date and Time } & \multicolumn{7}{|c|}{ Water Level (ft below reference point) } \\
\hline & SB43S & SB44S & SB45S & SB46S & SB47S & SB48S & SB49S \\
\hline $5 / 9 / 114: 00$ & 22.400 & 22.111 & 18.449 & 17.405 & 14.593 & 9.534 & 6.185 \\
\hline 5/9/11 8:00 & 22.396 & 22.125 & 18.451 & 17.428 & 14.586 & 9.532 & 6.189 \\
\hline $5 / 9 / 1112: 00$ & 22.391 & 22.111 & 18.447 & 17.447 & 14.579 & 9.534 & 6.182 \\
\hline 5/9/11 16:00 & 22.386 & 22.104 & 18.446 & 17.466 & 14.572 & 9.529 & 6.180 \\
\hline 5/9/11 20:00 & 22.384 & 22.106 & 18.446 & 17.482 & 14.568 & 9.536 & 6.178 \\
\hline 5/10/11 0:00 & 22.382 & 22.174 & 18.453 & 17.501 & 14.570 & 9.536 & 6.187 \\
\hline 5/10/11 4:00 & 22.377 & 22.197 & 18.453 & 17.521 & 14.561 & 9.536 & 6.189 \\
\hline 5/10/11 8:00 & 22.373 & 22.223 & 18.456 & 17.540 & 14.561 & 9.541 & 6.199 \\
\hline 5/10/11 12:00 & 22.375 & 22.281 & 18.463 & 17.566 & 14.556 & 9.541 & 6.194 \\
\hline 5/10/11 16:00 & 22.370 & 22.306 & 18.463 & 17.587 & 14.554 & 9.543 & 6.199 \\
\hline 5/10/11 20:00 & 22.370 & 22.346 & 18.470 & 17.603 & 14.556 & 9.548 & 6.199 \\
\hline 5/11/11 0:00 & 22.370 & 22.404 & 18.472 & 17.626 & 14.551 & 9.555 & 6.206 \\
\hline 5/11/11 4:00 & 22.370 & 22.453 & 18.479 & 17.647 & 14.551 & 9.557 & 6.210 \\
\hline 5/11/11 8:00 & 22.373 & 22.509 & 18.486 & 17.668 & 14.551 & 9.562 & 6.217 \\
\hline 5/11/11 12:00 & 22.368 & 22.528 & 18.486 & 17.689 & 14.547 & 9.560 & 6.215 \\
\hline 5/11/11 16:00 & 22.361 & 22.507 & 18.484 & 17.708 & 14.535 & 9.560 & 6.215 \\
\hline 5/11/11 20:00 & 22.359 & 22.521 & 18.486 & 17.729 & 14.533 & 9.562 & 6.217 \\
\hline 5/12/11 0:00 & 22.357 & 22.574 & 18.488 & 17.747 & 14.530 & 9.564 & 6.218 \\
\hline 5/12/11 4:00 & 22.356 & 22.546 & 18.491 & 17.768 & 14.528 & 9.569 & 6.220 \\
\hline 5/12/11 8:00 & 22.357 & 22.544 & 18.498 & 17.787 & 14.526 & 9.571 & 6.227 \\
\hline 5/12/11 12:00 & 22.354 & 22.556 & 18.500 & 17.808 & 14.523 & 9.569 & 6.229 \\
\hline 5/12/11 16:00 & 22.350 & 22.528 & 18.500 & 17.829 & 14.516 & 9.571 & 6.227 \\
\hline 5/12/11 20:00 & 22.345 & 22.514 & 18.498 & 17.843 & 14.509 & 9.571 & 6.224 \\
\hline 5/13/11 0:00 & 22.343 & 22.518 & 18.500 & 17.862 & 14.507 & 9.574 & 6.229 \\
\hline 5/13/11 4:00 & 22.341 & 22.537 & 18.502 & 17.878 & 14.505 & 9.576 & 6.229 \\
\hline 5/13/11 8:00 & 22.338 & 22.584 & 18.509 & 17.899 & 14.502 & 9.578 & 6.236 \\
\hline 5/13/11 12:00 & 22.345 & 22.670 & 18.519 & 17.922 & 14.505 & 9.585 & 6.243 \\
\hline 5/13/11 16:00 & 22.343 & 22.705 & 18.523 & 17.943 & 14.502 & 9.590 & 6.245 \\
\hline $5 / 13 / 1120: 00$ & 22.345 & 22.747 & 18.528 & 17.962 & 14.502 & 9.595 & 6.252 \\
\hline 5/14/11 0:00 & 22.345 & 22.786 & 18.533 & 17.980 & 14.498 & 9.592 & 6.257 \\
\hline 5/14/11 4:00 & 22.341 & 22.789 & 18.533 & 18.001 & 14.495 & 9.597 & 6.257 \\
\hline 5/14/11 8:00 & 22.343 & 22.809 & 18.535 & 18.020 & 14.498 & 9.599 & 6.257 \\
\hline $5 / 14 / 11$ 12:00 & 22.338 & 22.835 & 18.540 & 18.043 & 14.493 & 9.604 & 6.262 \\
\hline 5/14/11 16:00 & 22.336 & 22.835 & 18.537 & 18.060 & 14.484 & 9.599 & 6.262 \\
\hline 5/14/11 20:00 & 22.331 & 22.840 & 18.540 & 18.078 & 14.481 & 9.604 & 6.262 \\
\hline 5/15/11 0:00 & 22.331 & 22.875 & 18.544 & 18.099 & 14.479 & 9.604 & 6.266 \\
\hline 5/15/11 4:00 & 22.327 & 22.861 & 18.544 & 18.118 & 14.472 & 9.604 & 6.264 \\
\hline 5/15/11 8:00 & 22.325 & 22.875 & 18.547 & 18.136 & 14.467 & 9.606 & 6.266 \\
\hline 5/15/11 12:00 & 22.327 & 22.907 & 18.551 & 18.157 & 14.465 & 9.609 & 6.271 \\
\hline 5/15/11 16:00 & 22.325 & 22.931 & 18.556 & 18.174 & 14.465 & 9.611 & 6.273 \\
\hline 5/15/11 20:00 & 22.322 & 22.949 & 18.556 & 18.199 & 14.460 & 9.618 & 6.273 \\
\hline 5/16/11 0:00 & 22.322 & 22.984 & 18.563 & 18.216 & 14.463 & 9.620 & 6.278 \\
\hline 5/16/11 4:00 & 22.322 & 22.993 & 18.565 & 18.234 & 14.456 & 9.623 & 6.280 \\
\hline 5/16/11 8:00 & 22.322 & 23.033 & 18.572 & 18.255 & 14.456 & 9.625 & 6.287 \\
\hline 5/16/11 12:00 & 22.325 & 23.061 & 18.574 & 18.276 & 14.453 & 9.625 & 6.292 \\
\hline 5/16/11 16:00 & 22.315 & 23.038 & 18.570 & 18.292 & 14.444 & 9.623 & 6.290 \\
\hline 5/16/11 20:00 & 22.313 & 23.024 & 18.572 & 18.309 & 14.437 & 9.625 & 6.287 \\
\hline 5/17/11 0:00 & 22.311 & 23.056 & 18.577 & 18.330 & 14.439 & 9.630 & 6.285 \\
\hline 5/17/11 4:00 & 22.311 & 23.061 & 18.574 & 18.344 & 14.430 & 9.627 & 6.290 \\
\hline 5/17/11 8:00 & 22.308 & 23.075 & 18.577 & 18.367 & 14.430 & 9.630 & 6.288 \\
\hline 5/17/11 12:00 & 22.306 & 23.082 & 18.579 & 18.386 & 14.423 & 9.627 & 6.292 \\
\hline 5/17/11 16:00 & 22.297 & 23.042 & 18.572 & 18.400 & 14.414 & 9.625 & 6.285 \\
\hline 5/17/11 20:00 & 22.292 & 23.021 & 18.574 & 18.416 & 14.407 & 9.627 & 6.290 \\
\hline 5/18/11 0:00 & 22.292 & 23.052 & 18.577 & 18.437 & 14.404 & 9.627 & 6.292 \\
\hline
\end{tabular}

TABLE S1.2 Page 35 of 58 


\begin{tabular}{|c|c|c|c|c|c|c|c|}
\hline \multirow[b]{2}{*}{ Date and Time } & \multicolumn{7}{|c|}{ Water Level (ft below reference point) } \\
\hline & SB43S & SB44S & SB45S & SB46S & SB47S & SB48S & SB49S \\
\hline 5/18/11 4:00 & 22.290 & 23.052 & 18.577 & 18.448 & 14.400 & 9.630 & 6.290 \\
\hline 5/18/11 8:00 & 22.288 & 23.077 & 18.579 & 18.469 & 14.397 & 9.632 & 6.290 \\
\hline 5/18/11 12:00 & 22.288 & 23.094 & 18.581 & 18.488 & 14.395 & 9.632 & 6.297 \\
\hline 5/18/11 16:00 & 22.281 & 23.087 & 18.581 & 18.504 & 14.385 & 9.630 & 6.292 \\
\hline 5/18/11 20:00 & 22.279 & 23.087 & 18.584 & 18.521 & 14.383 & 9.637 & 6.295 \\
\hline 5/19/11 0:00 & 22.281 & 23.136 & 18.588 & 18.542 & 14.383 & 9.634 & 6.301 \\
\hline 5/19/11 4:00 & 22.276 & 23.138 & 18.588 & 18.556 & 14.378 & 9.637 & 6.299 \\
\hline 5/19/11 8:00 & 22.276 & 23.156 & 18.593 & 18.574 & 14.376 & 9.644 & 6.304 \\
\hline 5/19/11 12:00 & 22.276 & 23.201 & 18.598 & 18.595 & 14.376 & 9.641 & 6.304 \\
\hline 5/19/11 16:00 & 22.274 & 23.201 & 18.598 & 18.612 & 14.369 & 9.644 & 6.306 \\
\hline 5/19/11 20:00 & 22.274 & 23.217 & 18.600 & 18.630 & 14.367 & 9.646 & 6.306 \\
\hline 5/20/11 0:00 & 22.274 & 23.248 & 18.607 & 18.649 & 14.364 & 9.648 & 6.315 \\
\hline 5/20/11 4:00 & 22.270 & 23.240 & 18.602 & 18.665 & 14.357 & 9.648 & 6.311 \\
\hline 5/20/11 8:00 & 22.270 & 23.257 & 18.607 & 18.681 & 14.355 & 9.651 & 6.315 \\
\hline $5 / 20 / 11$ 12:00 & 22.265 & 23.264 & 18.607 & 18.700 & 14.348 & 9.651 & 6.313 \\
\hline 5/20/11 16:00 & 22.260 & 23.226 & 18.605 & 18.719 & 14.346 & 9.648 & 6.313 \\
\hline 5/20/11 20:00 & 22.258 & 23.159 & 18.605 & 18.730 & 14.336 & 9.653 & 6.315 \\
\hline 5/21/11 0:00 & 22.256 & 23.124 & 18.607 & 18.747 & 14.334 & 9.651 & 6.313 \\
\hline 5/21/11 4:00 & 22.256 & 23.096 & 18.609 & 18.763 & 14.327 & 9.653 & 6.313 \\
\hline 5/21/11 8:00 & 22.253 & 23.110 & 18.614 & 18.779 & 14.332 & 9.658 & 6.318 \\
\hline $5 / 21 / 11$ 12:00 & 22.253 & 23.138 & 18.616 & 18.800 & 14.325 & 9.655 & 6.322 \\
\hline 5/21/11 16:00 & 22.249 & 23.115 & 18.612 & 18.809 & 14.318 & 9.653 & 6.320 \\
\hline 5/21/11 20:00 & 22.247 & 23.101 & 18.614 & 18.826 & 14.315 & 9.658 & 6.318 \\
\hline 5/22/11 0:00 & 22.244 & 23.119 & 18.614 & 18.837 & 14.311 & 9.655 & 6.320 \\
\hline 5/22/11 4:00 & 22.240 & 23.124 & 18.614 & 18.854 & 14.304 & 9.655 & 6.318 \\
\hline 5/22/11 8:00 & 22.240 & 23.133 & 18.616 & 18.868 & 14.301 & 9.658 & 6.322 \\
\hline $5 / 22 / 11$ 12:00 & 22.240 & 23.154 & 18.619 & 18.886 & 14.297 & 9.658 & 6.325 \\
\hline 5/22/11 16:00 & 22.233 & 23.159 & 18.616 & 18.903 & 14.290 & 9.660 & 6.325 \\
\hline $5 / 22 / 1120: 00$ & 22.233 & 23.166 & 18.619 & 18.919 & 14.290 & 9.660 & 6.327 \\
\hline 5/23/11 0:00 & 22.237 & 23.194 & 18.626 & 18.935 & 14.292 & 9.667 & 6.334 \\
\hline 5/23/11 4:00 & 22.237 & 23.250 & 18.633 & 18.954 & 14.292 & 9.672 & 6.339 \\
\hline 5/23/11 8:00 & 22.237 & 23.280 & 18.633 & 18.968 & 14.285 & 9.674 & 6.339 \\
\hline $5 / 23 / 11$ 12:00 & 22.240 & 23.331 & 18.640 & 18.989 & 14.290 & 9.674 & 6.346 \\
\hline 5/23/11 16:00 & 22.237 & 23.347 & 18.642 & 19.005 & 14.285 & 9.676 & 6.348 \\
\hline 5/23/11 20:00 & 22.235 & 23.352 & 18.642 & 19.021 & 14.280 & 9.679 & 6.348 \\
\hline 5/24/11 0:00 & 22.240 & 23.399 & 18.651 & 19.038 & 14.283 & 9.683 & 6.357 \\
\hline 5/24/11 4:00 & 22.237 & 23.420 & 18.654 & 19.054 & 14.278 & 9.686 & 6.360 \\
\hline 5/24/11 8:00 & 22.240 & 23.452 & 18.658 & 19.070 & 14.276 & 9.686 & 6.362 \\
\hline $5 / 24 / 11$ 12:00 & 22.237 & 23.459 & 18.658 & 19.084 & 14.276 & 9.688 & 6.362 \\
\hline 5/24/11 16:00 & 22.233 & 23.359 & 18.656 & 19.098 & 14.266 & 9.681 & 6.355 \\
\hline 5/24/11 20:00 & 22.228 & 23.208 & 18.654 & 19.110 & 14.259 & 9.686 & 6.358 \\
\hline 5/25/11 0:00 & 22.226 & 23.089 & 18.651 & 19.122 & 14.252 & 9.681 & 6.355 \\
\hline $5 / 25 / 114: 00$ & 22.215 & 22.574 & 18.644 & 19.124 & 14.241 & 9.679 & 6.346 \\
\hline 5/25/11 8:00 & 22.215 & 22.230 & 18.644 & 19.133 & 14.238 & 9.679 & 6.348 \\
\hline $5 / 25 / 11$ 12:00 & 22.212 & 22.057 & 18.642 & 19.143 & 14.231 & 9.676 & 6.346 \\
\hline 5/25/11 16:00 & 22.212 & 21.997 & 18.644 & 19.150 & 14.229 & 9.686 & 6.350 \\
\hline 5/25/11 20:00 & 22.219 & 21.668 & 18.654 & 19.161 & 14.231 & 9.693 & 6.357 \\
\hline 5/26/11 0:00 & 22.228 & 21.303 & 18.663 & 19.170 & 14.238 & 9.697 & 6.372 \\
\hline 5/26/11 4:00 & 22.231 & 21.147 & 18.665 & 19.177 & 14.238 & 9.704 & 6.376 \\
\hline 5/26/11 8:00 & 22.237 & 21.109 & 18.668 & 19.184 & 14.243 & 9.707 & 6.383 \\
\hline $5 / 26 / 11$ 12:00 & 22.240 & 21.126 & 18.672 & 19.191 & 14.243 & 9.707 & 6.390 \\
\hline 5/26/11 16:00 & 22.237 & 21.112 & 18.668 & 19.194 & 14.241 & 9.707 & 6.386 \\
\hline 5/26/11 20:00 & 22.233 & 21.079 & 18.665 & 19.198 & 14.234 & 9.707 & 6.385 \\
\hline 5/27/11 0:00 & 22.235 & 21.105 & 18.665 & 19.201 & 14.234 & 9.707 & 6.390 \\
\hline
\end{tabular}

TABLE S1.2 Page 36 of 58 


\begin{tabular}{|c|c|c|c|c|c|c|c|}
\hline \multirow[b]{2}{*}{ Date and Time } & \multicolumn{7}{|c|}{ Water Level (ft below reference point) } \\
\hline & SB43S & SB44S & SB45S & SB46S & SB47S & SB48S & SB49S \\
\hline $5 / 27 / 114: 00$ & 22.230 & 21.112 & 18.661 & 19.205 & 14.224 & 9.704 & 6.385 \\
\hline 5/27/11 8:00 & 22.226 & 21.102 & 18.656 & 19.203 & 14.224 & 9.707 & 6.383 \\
\hline $5 / 27 / 11$ 12:00 & 22.224 & 21.116 & 18.654 & 19.208 & 14.215 & 9.700 & 6.383 \\
\hline 5/27/11 16:00 & 22.217 & 21.070 & 18.647 & 19.210 & 14.208 & 9.700 & 6.374 \\
\hline 5/27/11 20:00 & 22.210 & 21.084 & 18.644 & 19.210 & 14.203 & 9.700 & 6.374 \\
\hline 5/28/11 0:00 & 22.215 & 21.130 & 18.644 & 19.217 & 14.205 & 9.700 & 6.374 \\
\hline $5 / 28 / 114: 00$ & 22.208 & 21.156 & 18.644 & 19.222 & 14.201 & 9.697 & 6.378 \\
\hline $5 / 28 / 118: 00$ & 22.205 & 21.172 & 18.637 & 19.226 & 14.196 & 9.700 & 6.371 \\
\hline $5 / 28 / 11$ 12:00 & 22.203 & 21.219 & 18.637 & 19.231 & 14.194 & 9.700 & 6.371 \\
\hline $5 / 28 / 11$ 16:00 & 22.201 & 21.216 & 18.633 & 19.233 & 14.189 & 9.700 & 6.371 \\
\hline $5 / 28 / 1120: 00$ & 22.198 & 21.254 & 18.633 & 19.238 & 14.189 & 9.702 & 6.371 \\
\hline 5/29/11 0:00 & 22.196 & 21.310 & 18.633 & 19.245 & 14.184 & 9.697 & 6.371 \\
\hline $5 / 29 / 114: 00$ & 22.187 & 21.279 & 18.628 & 19.245 & 14.177 & 9.697 & 6.362 \\
\hline 5/29/11 8:00 & 22.187 & 21.277 & 18.626 & 19.247 & 14.173 & 9.695 & 6.362 \\
\hline $5 / 29 / 11$ 12:00 & 22.182 & 21.310 & 18.623 & 19.254 & 14.170 & 9.697 & 6.364 \\
\hline 5/29/11 16:00 & 22.182 & 21.342 & 18.619 & 19.261 & 14.166 & 9.697 & 6.357 \\
\hline 5/29/11 20:00 & 22.178 & 21.386 & 18.623 & 19.266 & 14.166 & 9.704 & 6.360 \\
\hline 5/30/11 0:00 & 22.185 & 21.475 & 18.626 & 19.278 & 14.168 & 9.702 & 6.362 \\
\hline $5 / 30 / 114: 00$ & 22.180 & 21.515 & 18.626 & 19.285 & 14.166 & 9.707 & 6.364 \\
\hline $5 / 30 / 118: 00$ & 22.180 & 21.570 & 18.628 & 19.287 & 14.168 & 9.711 & 6.364 \\
\hline 5/30/11 12:00 & 22.182 & 21.624 & 18.628 & 19.299 & 14.166 & 9.711 & 6.369 \\
\hline 5/30/11 16:00 & 22.180 & 21.664 & 18.630 & 19.308 & 14.163 & 9.714 & 6.369 \\
\hline 5/30/11 20:00 & 22.180 & 21.701 & 18.628 & 19.310 & 14.161 & 9.716 & 6.369 \\
\hline 5/31/11 0:00 & 22.171 & 21.713 & 18.626 & 19.320 & 14.159 & 9.714 & 6.367 \\
\hline $5 / 31 / 114: 00$ & 22.182 & 21.822 & 18.637 & 19.331 & 14.168 & 9.723 & 6.383 \\
\hline 5/31/11 8:00 & 22.182 & 21.901 & 18.642 & 19.343 & 14.168 & 9.730 & 6.376 \\
\hline $5 / 31 / 11$ 12:00 & 22.192 & 21.980 & 18.651 & 19.357 & 14.175 & 9.737 & 6.392 \\
\hline 5/31/11 16:00 & 22.192 & 22.008 & 18.654 & 19.366 & 14.175 & 9.739 & 6.395 \\
\hline 5/31/11 20:00 & 22.192 & 22.020 & 18.656 & 19.375 & 14.173 & 9.742 & 6.395 \\
\hline 6/1/11 0:00 & 22.194 & 22.062 & 18.658 & 19.382 & 14.175 & 9.744 & 6.399 \\
\hline $6 / 1 / 114: 00$ & 22.189 & 22.069 & 18.658 & 19.387 & 14.173 & 9.744 & 6.399 \\
\hline 6/1/11 8:00 & 22.192 & 22.099 & 18.661 & 19.399 & 14.170 & 9.749 & 6.402 \\
\hline $6 / 1 / 11$ 12:00 & 22.187 & 22.118 & 18.661 & 19.408 & 14.168 & 9.744 & 6.402 \\
\hline $6 / 1 / 11$ 16:00 & 22.180 & 22.080 & 18.654 & 19.413 & 14.159 & 9.737 & 6.395 \\
\hline $6 / 1 / 1120: 00$ & 22.171 & 22.013 & 18.644 & 19.413 & 14.149 & 9.739 & 6.385 \\
\hline 6/2/11 0:00 & 22.169 & 22.029 & 18.642 & 19.420 & 14.145 & 9.739 & 6.385 \\
\hline $6 / 2 / 114: 00$ & 22.171 & 22.087 & 18.647 & 19.429 & 14.145 & 9.742 & 6.388 \\
\hline $6 / 2 / 118: 00$ & 22.162 & 22.004 & 18.640 & 19.429 & 14.131 & 9.737 & 6.381 \\
\hline $6 / 2 / 1112: 00$ & 22.160 & 21.857 & 18.637 & 19.436 & 14.126 & 9.735 & 6.378 \\
\hline $6 / 2 / 11$ 16:00 & 22.153 & 21.727 & 18.633 & 19.438 & 14.117 & 9.732 & 6.374 \\
\hline $6 / 2 / 1120: 00$ & 22.148 & 21.636 & 18.628 & 19.441 & 14.112 & 9.732 & 6.374 \\
\hline 6/3/11 0:00 & 22.146 & 21.603 & 18.628 & 19.445 & 14.107 & 9.735 & 6.371 \\
\hline $6 / 3 / 114: 00$ & 22.143 & 21.601 & 18.626 & 19.450 & 14.105 & 9.735 & 6.371 \\
\hline 6/3/11 8:00 & 22.146 & 21.622 & 18.628 & 19.452 & 14.107 & 9.735 & 6.371 \\
\hline $6 / 3 / 11$ 12:00 & 22.141 & 21.647 & 18.626 & 19.462 & 14.103 & 9.737 & 6.371 \\
\hline 6/3/11 16:00 & 22.137 & 21.675 & 18.623 & 19.464 & 14.100 & 9.737 & 6.371 \\
\hline $6 / 3 / 1120: 00$ & 22.137 & 21.703 & 18.626 & 19.473 & 14.100 & 9.739 & 6.371 \\
\hline 6/4/11 0:00 & 22.139 & 21.773 & 18.630 & 19.478 & 14.105 & 9.744 & 6.376 \\
\hline $6 / 4 / 114: 00$ & 22.141 & 21.841 & 18.633 & 19.485 & 14.103 & 9.749 & 6.378 \\
\hline 6/4/11 8:00 & 22.143 & 21.910 & 18.635 & 19.494 & 14.107 & 9.753 & 6.383 \\
\hline 6/4/11 12:00 & 22.143 & 21.959 & 18.635 & 19.501 & 14.103 & 9.751 & 6.385 \\
\hline $6 / 4 / 11$ 16:00 & 22.139 & 21.983 & 18.635 & 19.511 & 14.100 & 9.753 & 6.383 \\
\hline $6 / 4 / 1120: 00$ & 22.134 & 21.997 & 18.633 & 19.515 & 14.096 & 9.751 & 6.385 \\
\hline 6/5/11 0:00 & 22.134 & 22.025 & 18.633 & 19.518 & 14.098 & 9.753 & 6.385 \\
\hline
\end{tabular}

TABLE S1.2 Page 37 of 58 


\begin{tabular}{|c|c|c|c|c|c|c|c|}
\hline \multirow[b]{2}{*}{ Date and Time } & \multicolumn{7}{|c|}{ Water Level (ft below reference point) } \\
\hline & SB43S & SB44S & SB45S & SB46S & SB47S & SB48S & SB49S \\
\hline $6 / 5 / 114: 00$ & 22.132 & 22.046 & 18.635 & 19.525 & 14.093 & 9.753 & 6.385 \\
\hline 6/5/11 8:00 & 22.130 & 22.064 & 18.633 & 19.531 & 14.091 & 9.756 & 6.385 \\
\hline $6 / 5 / 1112: 00$ & 22.130 & 22.092 & 18.630 & 19.541 & 14.086 & 9.756 & 6.383 \\
\hline 6/5/11 16:00 & 22.123 & 22.087 & 18.626 & 19.548 & 14.084 & 9.753 & 6.381 \\
\hline $6 / 5 / 1120: 00$ & 22.121 & 22.074 & 18.623 & 19.548 & 14.077 & 9.753 & 6.378 \\
\hline 6/6/11 0:00 & 22.116 & 22.090 & 18.623 & 19.555 & 14.075 & 9.751 & 6.376 \\
\hline 6/6/11 4:00 & 22.111 & 22.083 & 18.619 & 19.557 & 14.070 & 9.753 & 6.374 \\
\hline 6/6/11 8:00 & 22.111 & 22.108 & 18.619 & 19.562 & 14.068 & 9.753 & 6.376 \\
\hline $6 / 6 / 11$ 12:00 & 22.107 & 22.129 & 18.619 & 19.573 & 14.063 & 9.753 & 6.374 \\
\hline 6/6/11 16:00 & 22.105 & 22.134 & 18.614 & 19.580 & 14.058 & 9.748 & 6.371 \\
\hline $6 / 6 / 1120: 00$ & 22.100 & 22.134 & 18.612 & 19.583 & 14.053 & 9.753 & 6.362 \\
\hline 6/7/11 0:00 & 22.100 & 22.174 & 18.614 & 19.590 & 14.053 & 9.753 & 6.371 \\
\hline $6 / 7 / 114: 00$ & 22.098 & 22.190 & 18.612 & 19.594 & 14.051 & 9.756 & 6.371 \\
\hline 6/7/11 8:00 & 22.095 & 22.220 & 18.614 & 19.599 & 14.049 & 9.753 & 6.371 \\
\hline $6 / 7 / 11$ 12:00 & 22.091 & 22.230 & 18.609 & 19.608 & 14.044 & 9.751 & 6.369 \\
\hline 6/7/11 16:00 & 22.086 & 22.220 & 18.607 & 19.611 & 14.037 & 9.751 & 6.367 \\
\hline 6/7/11 20:00 & 22.084 & 22.234 & 18.607 & 19.620 & 14.035 & 9.753 & 6.364 \\
\hline 6/8/11 0:00 & 22.084 & 22.278 & 18.609 & 19.627 & 14.030 & 9.753 & 6.369 \\
\hline $6 / 8 / 114: 00$ & 22.084 & 22.302 & 18.609 & 19.632 & 14.028 & 9.755 & 6.367 \\
\hline 6/8/11 8:00 & 22.082 & 22.339 & 18.612 & 19.639 & 14.028 & 9.758 & 6.374 \\
\hline $6 / 8 / 11$ 12:00 & 22.082 & 22.374 & 18.612 & 19.650 & 14.023 & 9.760 & 6.369 \\
\hline 6/8/11 16:00 & 22.077 & 22.397 & 18.612 & 19.662 & 14.023 & 9.760 & 6.371 \\
\hline 6/8/11 20:00 & 22.079 & 22.425 & 18.614 & 19.667 & 14.023 & 9.765 & 6.374 \\
\hline 6/9/11 0:00 & 22.082 & 22.486 & 18.619 & 19.676 & 14.025 & 9.767 & 6.376 \\
\hline $6 / 9 / 114: 00$ & 22.077 & 22.519 & 18.616 & 19.681 & 14.021 & 9.769 & 6.378 \\
\hline 6/9/11 8:00 & 22.079 & 22.567 & 18.621 & 19.692 & 14.023 & 9.772 & 6.378 \\
\hline $6 / 9 / 11$ 12:00 & 22.082 & 22.574 & 18.623 & 19.699 & 14.018 & 9.767 & 6.383 \\
\hline 6/9/11 16:00 & 22.072 & 22.465 & 18.616 & 19.706 & 14.011 & 9.765 & 6.378 \\
\hline 6/9/11 20:00 & 22.068 & 22.444 & 18.616 & 19.709 & 14.007 & 9.769 & 6.381 \\
\hline 6/10/11 0:00 & 22.068 & 22.467 & 18.616 & 19.718 & 14.007 & 9.769 & 6.378 \\
\hline $6 / 10 / 114: 00$ & 22.070 & 22.507 & 18.621 & 19.727 & 14.007 & 9.777 & 6.378 \\
\hline 6/10/11 8:00 & 22.072 & 22.598 & 18.628 & 19.739 & 14.014 & 9.784 & 6.385 \\
\hline $6 / 10 / 11$ 12:00 & 22.072 & 22.646 & 18.630 & 19.748 & 14.009 & 9.781 & 6.390 \\
\hline $6 / 10 / 11$ 16:00 & 22.070 & 22.663 & 18.630 & 19.755 & 14.009 & 9.781 & 6.392 \\
\hline 6/10/11 20:00 & 22.070 & 22.681 & 18.633 & 19.762 & 14.007 & 9.783 & 6.392 \\
\hline 6/11/11 0:00 & 22.072 & 22.728 & 18.635 & 19.776 & 14.007 & 9.786 & 6.397 \\
\hline $6 / 11 / 114: 00$ & 22.072 & 22.777 & 18.637 & 19.785 & 14.007 & 9.790 & 6.399 \\
\hline 6/11/11 8:00 & 22.077 & 22.823 & 18.644 & 19.795 & 14.009 & 9.793 & 6.411 \\
\hline $6 / 11 / 11$ 12:00 & 22.075 & 22.858 & 18.644 & 19.806 & 14.007 & 9.793 & 6.406 \\
\hline $6 / 11 / 11$ 16:00 & 22.072 & 22.863 & 18.644 & 19.816 & 14.002 & 9.790 & 6.406 \\
\hline $6 / 11 / 1120: 00$ & 22.068 & 22.858 & 18.640 & 19.823 & 13.997 & 9.790 & 6.404 \\
\hline 6/12/11 0:00 & 22.068 & 22.896 & 18.647 & 19.832 & 13.997 & 9.793 & 6.409 \\
\hline $6 / 12 / 114: 00$ & 22.070 & 22.910 & 18.649 & 19.841 & 13.997 & 9.797 & 6.411 \\
\hline $6 / 12 / 118: 00$ & 22.072 & 22.961 & 18.656 & 19.853 & 14.000 & 9.797 & 6.418 \\
\hline $6 / 12 / 11$ 12:00 & 22.070 & 22.977 & 18.649 & 19.862 & 13.995 & 9.798 & 6.418 \\
\hline 6/12/11 16:00 & 22.068 & 22.977 & 18.651 & 19.869 & 13.990 & 9.797 & 6.413 \\
\hline $6 / 12 / 1120: 00$ & 22.066 & 22.986 & 18.651 & 19.879 & 13.988 & 9.800 & 6.413 \\
\hline 6/13/11 0:00 & 22.066 & 23.019 & 18.656 & 19.886 & 13.988 & 9.800 & 6.418 \\
\hline $6 / 13 / 114: 00$ & 22.061 & 23.017 & 18.654 & 19.893 & 13.981 & 9.802 & 6.418 \\
\hline 6/13/11 8:00 & 22.061 & 23.024 & 18.654 & 19.902 & 13.981 & 9.800 & 6.416 \\
\hline $6 / 13 / 11$ 12:00 & 22.059 & 23.029 & 18.651 & 19.913 & 13.976 & 9.802 & 6.418 \\
\hline $6 / 13 / 11$ 16:00 & 22.052 & 23.026 & 18.651 & 19.918 & 13.972 & 9.800 & 6.416 \\
\hline $6 / 13 / 1120: 00$ & 22.054 & 23.042 & 18.651 & 19.927 & 13.969 & 9.797 & 6.413 \\
\hline 6/14/11 0:00 & 22.050 & 23.042 & 18.651 & 19.937 & 13.965 & 9.797 & 6.413 \\
\hline
\end{tabular}

TABLE S1.2 Page 38 of 58 


\begin{tabular}{|c|c|c|c|c|c|c|c|}
\hline \multirow[b]{2}{*}{ Date and Time } & \multicolumn{7}{|c|}{ Water Level (ft below reference point) } \\
\hline & SB43S & SB44S & SB45S & SB46S & SB47S & SB48S & SB49S \\
\hline $6 / 14 / 114: 00$ & 22.050 & 23.042 & 18.649 & 19.939 & 13.962 & 9.800 & 6.416 \\
\hline 6/14/11 8:00 & 22.047 & 23.070 & 18.651 & 19.953 & 13.960 & 9.797 & 6.418 \\
\hline $6 / 14 / 11$ 12:00 & 22.045 & 23.056 & 18.649 & 19.955 & 13.953 & 9.797 & 6.409 \\
\hline 6/14/11 16:00 & 22.038 & 23.040 & 18.647 & 19.965 & 13.946 & 9.797 & 6.406 \\
\hline 6/14/11 20:00 & 22.038 & 23.042 & 18.644 & 19.972 & 13.941 & 9.797 & 6.409 \\
\hline 6/15/11 0:00 & 22.045 & 23.105 & 18.658 & 19.986 & 13.953 & 9.802 & 6.418 \\
\hline $6 / 15 / 114: 00$ & 22.045 & 23.143 & 18.656 & 19.993 & 13.946 & 9.807 & 6.418 \\
\hline 6/15/11 8:00 & 22.045 & 23.210 & 18.665 & 20.007 & 13.953 & 9.809 & 6.425 \\
\hline $6 / 15 / 11$ 12:00 & 22.047 & 23.252 & 18.668 & 20.018 & 13.951 & 9.811 & 6.430 \\
\hline $6 / 15 / 11$ 16:00 & 22.045 & 23.275 & 18.665 & 20.028 & 13.946 & 9.811 & 6.432 \\
\hline 6/15/11 20:00 & 22.045 & 23.289 & 18.668 & 20.035 & 13.944 & 9.814 & 6.432 \\
\hline 6/16/11 0:00 & 22.045 & 23.315 & 18.668 & 20.044 & 13.944 & 9.814 & 6.430 \\
\hline $6 / 16 / 114: 00$ & 22.045 & 23.322 & 18.670 & 20.053 & 13.939 & 9.814 & 6.434 \\
\hline 6/16/11 8:00 & 22.045 & 23.343 & 18.675 & 20.065 & 13.937 & 9.821 & 6.437 \\
\hline $6 / 16 / 11$ 12:00 & 22.045 & 23.382 & 18.677 & 20.070 & 13.937 & 9.814 & 6.439 \\
\hline 6/16/11 16:00 & 22.043 & 23.354 & 18.677 & 20.081 & 13.934 & 9.816 & 6.439 \\
\hline 6/16/11 20:00 & 22.038 & 23.350 & 18.675 & 20.090 & 13.927 & 9.816 & 6.437 \\
\hline 6/17/11 0:00 & 22.040 & 23.387 & 18.677 & 20.102 & 13.930 & 9.821 & 6.439 \\
\hline 6/17/11 4:00 & 22.036 & 23.392 & 18.672 & 20.104 & 13.920 & 9.821 & 6.434 \\
\hline 6/17/11 8:00 & 22.036 & 23.364 & 18.679 & 20.116 & 13.920 & 9.821 & 6.444 \\
\hline $6 / 17 / 11$ 12:00 & 22.038 & 23.333 & 18.682 & 20.128 & 13.923 & 9.825 & 6.444 \\
\hline 6/17/11 16:00 & 22.038 & 23.324 & 18.684 & 20.137 & 13.918 & 9.825 & 6.448 \\
\hline 6/17/11 20:00 & 22.038 & 23.338 & 18.686 & 20.146 & 13.920 & 9.828 & 6.448 \\
\hline 6/18/11 0:00 & 22.043 & 23.373 & 18.691 & 20.156 & 13.918 & 9.828 & 6.458 \\
\hline $6 / 18 / 114: 00$ & 22.043 & 23.366 & 18.691 & 20.165 & 13.916 & 9.839 & 6.453 \\
\hline 6/18/11 8:00 & 22.040 & 23.331 & 18.691 & 20.163 & 13.911 & 9.832 & 6.453 \\
\hline $6 / 18 / 11$ 12:00 & 22.043 & 23.143 & 18.698 & 20.181 & 13.916 & 9.832 & 6.455 \\
\hline 6/18/11 16:00 & 22.043 & 22.998 & 18.700 & 20.188 & 13.913 & 9.835 & 6.462 \\
\hline 6/18/11 20:00 & 22.043 & 22.912 & 18.700 & 20.193 & 13.911 & 9.832 & 6.462 \\
\hline 6/19/11 0:00 & 22.038 & 22.861 & 18.700 & 20.195 & 13.906 & 9.835 & 6.460 \\
\hline 6/19/11 4:00 & 22.038 & 22.842 & 18.693 & 20.200 & 13.902 & 9.832 & 6.460 \\
\hline 6/19/11 8:00 & 22.040 & 22.851 & 18.700 & 20.205 & 13.901 & 9.835 & 6.462 \\
\hline 6/19/11 12:00 & 22.038 & 22.868 & 18.698 & 20.214 & 13.897 & 9.832 & 6.460 \\
\hline 6/19/11 16:00 & 22.033 & 22.851 & 18.695 & 20.219 & 13.892 & 9.832 & 6.460 \\
\hline 6/19/11 20:00 & 22.031 & 22.851 & 18.693 & 20.223 & 13.888 & 9.830 & 6.460 \\
\hline 6/20/11 0:00 & 22.029 & 22.849 & 18.691 & 20.226 & 13.883 & 9.828 & 6.458 \\
\hline $6 / 20 / 114: 00$ & 22.022 & 22.835 & 18.686 & 20.223 & 13.876 & 9.832 & 6.455 \\
\hline 6/20/11 8:00 & 22.027 & 22.898 & 18.696 & 20.237 & 13.881 & 9.832 & 6.460 \\
\hline 6/20/11 12:00 & 22.027 & 22.928 & 18.693 & 20.242 & 13.881 & 9.832 & 6.460 \\
\hline 6/20/11 16:00 & 22.029 & 22.952 & 18.691 & 20.251 & 13.876 & 9.832 & 6.460 \\
\hline 6/20/11 20:00 & 22.024 & 22.959 & 18.691 & 20.256 & 13.871 & 9.839 & 6.458 \\
\hline 6/21/11 0:00 & 22.031 & 23.024 & 18.698 & 20.260 & 13.874 & 9.835 & 6.467 \\
\hline $6 / 21 / 114: 00$ & 22.022 & 22.903 & 18.693 & 20.260 & 13.867 & 9.832 & 6.465 \\
\hline $6 / 21 / 118: 00$ & 22.024 & 22.835 & 18.693 & 20.270 & 13.867 & 9.832 & 6.462 \\
\hline $6 / 21 / 11$ 12:00 & 22.029 & 22.842 & 18.700 & 20.274 & 13.869 & 9.837 & 6.471 \\
\hline 6/21/11 16:00 & 22.029 & 22.854 & 18.700 & 20.286 & 13.867 & 9.839 & 6.474 \\
\hline $6 / 21 / 1120: 00$ & 22.031 & 22.886 & 18.707 & 20.293 & 13.869 & 9.846 & 6.481 \\
\hline 6/22/11 0:00 & 22.033 & 22.938 & 18.712 & 20.300 & 13.871 & 9.846 & 6.481 \\
\hline $6 / 22 / 114: 00$ & 22.036 & 22.968 & 18.714 & 20.305 & 13.871 & 9.851 & 6.486 \\
\hline 6/22/11 8:00 & 22.038 & 23.017 & 18.716 & 20.316 & 13.869 & 9.853 & 6.493 \\
\hline 6/22/11 12:00 & 22.040 & 23.061 & 18.721 & 20.326 & 13.874 & 9.856 & 6.493 \\
\hline $6 / 22 / 11$ 16:00 & 22.043 & 23.098 & 18.721 & 20.328 & 13.874 & 9.858 & 6.497 \\
\hline $6 / 22 / 1120: 00$ & 22.043 & 23.136 & 18.723 & 20.335 & 13.874 & 9.863 & 6.500 \\
\hline 6/23/11 0:00 & 22.045 & 23.182 & 18.728 & 20.344 & 13.871 & 9.863 & 6.507 \\
\hline
\end{tabular}

TABLE S1.2 Page 39 of 58 


\begin{tabular}{|c|c|c|c|c|c|c|c|}
\hline \multirow[b]{2}{*}{ Date and Time } & \multicolumn{7}{|c|}{ Water Level (ft below reference point) } \\
\hline & SB43S & SB44S & SB45S & SB46S & SB47S & SB48S & SB49S \\
\hline $6 / 23 / 114: 00$ & 22.047 & 23.205 & 18.728 & 20.349 & 13.871 & 9.865 & 6.507 \\
\hline 6/23/11 8:00 & 22.050 & 23.250 & 18.735 & 20.358 & 13.876 & 9.872 & 6.511 \\
\hline 6/23/11 12:00 & 22.050 & 23.287 & 18.735 & 20.368 & 13.871 & 9.870 & 6.521 \\
\hline 6/23/11 16:00 & 22.050 & 23.328 & 18.728 & 20.377 & 13.871 & 9.853 & 6.514 \\
\hline 6/23/11 20:00 & 22.047 & 23.342 & 18.728 & 20.384 & 13.866 & 9.853 & 6.518 \\
\hline 6/24/11 0:00 & 22.050 & 23.367 & 18.733 & 20.391 & 13.866 & 9.856 & 6.518 \\
\hline $6 / 24 / 114: 00$ & 22.052 & 23.393 & 18.733 & 20.398 & 13.866 & 9.858 & 6.523 \\
\hline 6/24/11 8:00 & 22.052 & 23.426 & 18.735 & 20.405 & 13.864 & 9.860 & 6.523 \\
\hline 6/24/11 12:00 & 22.054 & 23.456 & 18.735 & 20.419 & 13.862 & 9.858 & 6.528 \\
\hline $6 / 24 / 11$ 16:00 & 22.052 & 23.437 & 18.735 & 20.421 & 13.857 & 9.858 & 6.523 \\
\hline 6/24/11 20:00 & 22.050 & 23.442 & 18.733 & 20.428 & 13.852 & 9.858 & 6.523 \\
\hline 6/25/11 0:00 & 22.052 & 23.465 & 18.735 & 20.431 & 13.852 & 9.860 & 6.525 \\
\hline 6/25/11 4:00 & 22.050 & 23.467 & 18.733 & 20.438 & 13.850 & 9.860 & 6.528 \\
\hline 6/25/11 8:00 & 22.061 & 23.523 & 18.744 & 20.451 & 13.857 & 9.860 & 6.539 \\
\hline 6/25/11 12:00 & 22.052 & 23.444 & 18.735 & 20.451 & 13.848 & 9.863 & 6.528 \\
\hline 6/25/11 16:00 & 22.054 & 23.342 & 18.740 & 20.461 & 13.845 & 9.863 & 6.530 \\
\hline 6/25/11 20:00 & 22.054 & 23.265 & 18.742 & 20.468 & 13.845 & 9.867 & 6.532 \\
\hline 6/26/11 0:00 & 22.054 & 23.234 & 18.742 & 20.472 & 13.845 & 9.867 & 6.535 \\
\hline 6/26/11 4:00 & 22.054 & 23.220 & 18.744 & 20.477 & 13.843 & 9.872 & 6.539 \\
\hline 6/26/11 8:00 & 22.056 & 23.248 & 18.744 & 20.484 & 13.846 & 9.870 & 6.539 \\
\hline 6/26/11 12:00 & 22.054 & 23.246 & 18.742 & 20.491 & 13.836 & 9.865 & 6.544 \\
\hline 6/26/11 16:00 & 22.050 & 23.209 & 18.735 & 20.491 & 13.831 & 9.865 & 6.532 \\
\hline 6/26/11 20:00 & 22.050 & 23.213 & 18.737 & 20.496 & 13.829 & 9.865 & 6.535 \\
\hline 6/27/11 0:00 & 22.054 & 23.239 & 18.740 & 20.503 & 13.829 & 9.867 & 6.537 \\
\hline 6/27/11 4:00 & 22.052 & 22.352 & 18.775 & 20.505 & 13.836 & 9.874 & 6.539 \\
\hline 6/27/11 8:00 & 22.059 & 19.685 & 18.740 & 20.496 & 13.829 & 9.870 & 6.546 \\
\hline 6/27/11 12:00 & 22.063 & 18.090 & 18.740 & 20.489 & 13.836 & 9.867 & 6.549 \\
\hline 6/27/11 16:00 & 22.061 & 17.403 & 18.735 & 20.479 & 13.834 & 9.860 & 6.549 \\
\hline 6/27/11 20:00 & 22.063 & 17.258 & 18.728 & 20.465 & 13.836 & 9.858 & 6.549 \\
\hline 6/28/11 0:00 & 22.063 & 17.377 & 18.726 & 20.454 & 13.841 & 9.853 & 6.551 \\
\hline 6/28/11 4:00 & 22.059 & 17.533 & 18.719 & 20.435 & 13.838 & 9.844 & 6.546 \\
\hline 6/28/11 8:00 & 22.056 & 17.680 & 18.714 & 20.426 & 13.843 & 9.839 & 6.544 \\
\hline $6 / 28 / 11$ 12:00 & 22.052 & 17.845 & 18.710 & 20.412 & 13.841 & 9.832 & 6.539 \\
\hline 6/28/11 16:00 & 22.045 & 17.938 & 18.698 & 20.400 & 13.834 & 9.823 & 6.530 \\
\hline 6/28/11 20:00 & 22.033 & 17.997 & 18.686 & 20.382 & 13.829 & 9.816 & 6.518 \\
\hline 6/29/11 0:00 & 22.031 & 18.078 & 18.681 & 20.365 & 13.829 & 9.809 & 6.514 \\
\hline 6/29/11 4:00 & 22.022 & 18.143 & 18.672 & 20.351 & 13.824 & 9.807 & 6.509 \\
\hline 6/29/11 8:00 & 22.017 & 18.218 & 18.663 & 20.340 & 13.822 & 9.800 & 6.504 \\
\hline 6/29/11 12:00 & 22.011 & 18.276 & 18.658 & 20.326 & 13.822 & 9.790 & 6.500 \\
\hline 6/29/11 16:00 & 22.001 & 18.311 & 18.644 & 20.312 & 13.813 & 9.781 & 6.488 \\
\hline 6/29/11 20:00 & 21.990 & 18.320 & 18.635 & 20.293 & 13.810 & 9.779 & 6.479 \\
\hline 6/30/11 0:00 & 21.985 & 18.337 & 18.628 & 20.281 & 13.808 & 9.776 & 6.472 \\
\hline 6/30/11 4:00 & 21.978 & 18.325 & 18.621 & 20.267 & 13.806 & 9.772 & 6.467 \\
\hline 6/30/11 8:00 & 21.976 & 18.327 & 18.616 & 20.258 & 13.808 & 9.767 & 6.462 \\
\hline 6/30/11 12:00 & 21.967 & 18.346 & 18.607 & 20.244 & 13.803 & 9.762 & 6.455 \\
\hline 6/30/11 16:00 & 21.962 & 18.402 & 18.600 & 20.233 & 13.799 & 9.755 & 6.448 \\
\hline 6/30/11 20:00 & 21.953 & 18.465 & 18.591 & 20.216 & 13.796 & 9.753 & 6.444 \\
\hline 7/1/11 0:00 & 21.949 & 18.544 & 18.584 & 20.205 & 13.794 & 9.748 & 6.441 \\
\hline 7/1/11 4:00 & 21.944 & 18.621 & 18.579 & 20.193 & 13.792 & 9.746 & 6.439 \\
\hline 7/1/11 8:00 & 21.940 & 18.702 & 18.579 & 20.181 & 13.792 & 9.743 & 6.434 \\
\hline 7/1/11 12:00 & 21.933 & 18.770 & 18.570 & 20.170 & 13.789 & 9.739 & 6.432 \\
\hline $7 / 1 / 11$ 16:00 & 21.928 & 18.828 & 18.563 & 20.163 & 13.789 & 9.737 & 6.427 \\
\hline 7/1/11 20:00 & 21.919 & 18.872 & 18.558 & 20.151 & 13.787 & 9.734 & 6.418 \\
\hline 7/2/11 0:00 & 21.917 & 18.933 & 18.554 & 20.139 & 13.789 & 9.732 & 6.423 \\
\hline
\end{tabular}

TABLE S1.2 Page 40 of 58 


\begin{tabular}{|c|c|c|c|c|c|c|c|}
\hline \multirow[b]{2}{*}{ Date and Time } & \multicolumn{7}{|c|}{ Water Level (ft below reference point) } \\
\hline & SB43S & SB44S & SB45S & SB46S & SB47S & SB48S & SB49S \\
\hline $7 / 2 / 114: 00$ & 21.914 & 19.024 & 18.551 & 20.130 & 13.789 & 9.732 & 6.418 \\
\hline $7 / 2 / 118: 00$ & 21.910 & 19.089 & 18.551 & 20.123 & 13.794 & 9.732 & 6.423 \\
\hline $7 / 2 / 11$ 12:00 & 21.907 & 19.168 & 18.547 & 20.118 & 13.792 & 9.729 & 6.423 \\
\hline $7 / 2 / 11$ 16:00 & 21.903 & 19.203 & 18.540 & 20.109 & 13.787 & 9.725 & 6.416 \\
\hline $7 / 2 / 1120: 00$ & 21.896 & 19.233 & 18.533 & 20.097 & 13.782 & 9.722 & 6.411 \\
\hline 7/3/11 0:00 & 21.889 & 19.261 & 18.526 & 20.086 & 13.780 & 9.718 & 6.406 \\
\hline $7 / 3 / 114: 00$ & 21.885 & 19.282 & 18.521 & 20.074 & 13.778 & 9.713 & 6.406 \\
\hline $7 / 3 / 118: 00$ & 21.880 & 19.329 & 18.519 & 20.070 & 13.780 & 9.718 & 6.406 \\
\hline $7 / 3 / 1112: 00$ & 21.868 & 19.343 & 18.507 & 20.056 & 13.768 & 9.708 & 6.390 \\
\hline $7 / 3 / 11$ 16:00 & 21.859 & 19.042 & 18.498 & 20.046 & 13.766 & 9.699 & 6.383 \\
\hline $7 / 3 / 1120: 00$ & 21.852 & 18.861 & 18.488 & 20.032 & 13.759 & 9.699 & 6.381 \\
\hline 7/4/11 0:00 & 21.848 & 18.835 & 18.486 & 20.018 & 13.759 & 9.692 & 6.378 \\
\hline $7 / 4 / 114: 00$ & 21.841 & 18.826 & 18.479 & 20.007 & 13.757 & 9.690 & 6.381 \\
\hline $7 / 4 / 118: 00$ & 21.841 & 18.861 & 18.475 & 19.995 & 13.757 & 9.687 & 6.374 \\
\hline $7 / 4 / 11$ 12:00 & 21.834 & 18.893 & 18.470 & 19.986 & 13.754 & 9.685 & 6.369 \\
\hline 7/4/11 16:00 & 21.827 & 18.912 & 18.460 & 19.974 & 13.750 & 9.681 & 6.360 \\
\hline $7 / 4 / 1120: 00$ & 21.820 & 18.921 & 18.454 & 19.962 & 13.747 & 9.678 & 6.357 \\
\hline 7/5/11 0:00 & 21.813 & 18.975 & 18.451 & 19.951 & 13.745 & 9.673 & 6.355 \\
\hline $7 / 5 / 114: 00$ & 21.811 & 19.014 & 18.444 & 19.937 & 13.743 & 9.671 & 6.355 \\
\hline 7/5/11 8:00 & 21.807 & 19.054 & 18.440 & 19.932 & 13.743 & 9.669 & 6.350 \\
\hline 7/5/11 12:00 & 21.802 & 19.100 & 18.437 & 19.923 & 13.740 & 9.664 & 6.348 \\
\hline 7/5/11 16:00 & 21.795 & 19.119 & 18.428 & 19.913 & 13.731 & 9.659 & 6.343 \\
\hline $7 / 5 / 1120: 00$ & 21.788 & 19.149 & 18.423 & 19.904 & 13.733 & 9.657 & 6.339 \\
\hline 7/6/11 0:00 & 21.781 & 19.196 & 18.414 & 19.890 & 13.729 & 9.653 & 6.332 \\
\hline $7 / 6 / 114: 00$ & 21.781 & 19.198 & 18.414 & 19.886 & 13.731 & 9.655 & 6.329 \\
\hline 7/6/11 8:00 & 21.777 & 19.210 & 18.409 & 19.874 & 13.729 & 9.650 & 6.332 \\
\hline $7 / 6 / 11$ 12:00 & 21.770 & 19.215 & 18.402 & 19.869 & 13.724 & 9.645 & 6.329 \\
\hline 7/6/11 16:00 & 21.763 & 19.177 & 18.395 & 19.860 & 13.717 & 9.641 & 6.320 \\
\hline $7 / 6 / 1120: 00$ & 21.754 & 19.138 & 18.388 & 19.848 & 13.715 & 9.638 & 6.318 \\
\hline 7/7/11 0:00 & 21.749 & 19.152 & 18.386 & 19.841 & 13.710 & 9.634 & 6.313 \\
\hline $7 / 7 / 114: 00$ & 21.740 & 19.105 & 18.370 & 19.825 & 13.705 & 9.636 & 6.304 \\
\hline 7/7/11 8:00 & 21.736 & 19.128 & 18.365 & 19.818 & 13.703 & 9.629 & 6.304 \\
\hline $7 / 7 / 11$ 12:00 & 21.731 & 18.935 & 18.358 & 19.806 & 13.696 & 9.620 & 6.297 \\
\hline 7/7/11 16:00 & 21.722 & 18.623 & 18.349 & 19.792 & 13.691 & 9.615 & 6.290 \\
\hline 7/7/11 20:00 & 21.715 & 18.451 & 18.342 & 19.776 & 13.686 & 9.613 & 6.290 \\
\hline 7/8/11 0:00 & 21.713 & 18.469 & 18.337 & 19.764 & 13.686 & 9.610 & 6.287 \\
\hline $7 / 8 / 114: 00$ & 21.708 & 18.535 & 18.333 & 19.750 & 13.684 & 9.608 & 6.280 \\
\hline $7 / 8 / 118: 00$ & 21.703 & 18.595 & 18.328 & 19.743 & 13.682 & 9.601 & 6.280 \\
\hline $7 / 8 / 11$ 12:00 & 21.697 & 18.644 & 18.321 & 19.732 & 13.677 & 9.597 & 6.276 \\
\hline 7/8/11 16:00 & 21.690 & 18.670 & 18.309 & 19.715 & 13.675 & 9.589 & 6.273 \\
\hline 7/8/11 20:00 & 21.683 & 18.686 & 18.302 & 19.702 & 13.668 & 9.587 & 6.266 \\
\hline 7/9/11 0:00 & 21.681 & 18.735 & 18.300 & 19.692 & 13.670 & 9.585 & 6.264 \\
\hline $7 / 9 / 114: 00$ & 21.674 & 18.772 & 18.291 & 19.676 & 13.665 & 9.578 & 6.259 \\
\hline 7/9/11 8:00 & 21.669 & 18.819 & 18.286 & 19.669 & 13.663 & 9.575 & 6.259 \\
\hline 7/9/11 12:00 & 21.662 & 18.840 & 18.277 & 19.660 & 13.656 & 9.568 & 6.250 \\
\hline 7/9/11 16:00 & 21.655 & 18.861 & 18.270 & 19.646 & 13.654 & 9.566 & 6.245 \\
\hline $7 / 9 / 1120: 00$ & 21.648 & 18.893 & 18.263 & 19.634 & 13.651 & 9.561 & 6.238 \\
\hline 7/10/11 0:00 & 21.642 & 18.928 & 18.258 & 19.622 & 13.647 & 9.559 & 6.238 \\
\hline $7 / 10 / 114: 00$ & 21.635 & 18.949 & 18.246 & 19.608 & 13.640 & 9.552 & 6.229 \\
\hline 7/10/11 8:00 & 21.630 & 18.968 & 18.242 & 19.599 & 13.637 & 9.552 & 6.229 \\
\hline $7 / 10 / 11$ 12:00 & 21.628 & 19.031 & 18.237 & 19.592 & 13.640 & 9.547 & 6.226 \\
\hline $7 / 10 / 11$ 16:00 & 21.621 & 19.080 & 18.233 & 19.583 & 13.635 & 9.545 & 6.222 \\
\hline 7/10/11 20:00 & 21.614 & 19.107 & 18.223 & 19.573 & 13.628 & 9.540 & 6.212 \\
\hline 7/11/11 0:00 & 21.610 & 19.126 & 18.218 & 19.559 & 13.626 & 9.533 & 6.215 \\
\hline
\end{tabular}

TABLE S1.2 Page 41 of 58 


\begin{tabular}{|c|c|c|c|c|c|c|c|}
\hline \multirow[b]{2}{*}{ Date and Time } & \multicolumn{7}{|c|}{ Water Level (ft below reference point) } \\
\hline & SB43S & SB44S & SB45S & SB46S & SB47S & SB48S & SB49S \\
\hline $7 / 11 / 114: 00$ & 21.607 & 19.187 & 18.218 & 19.555 & 13.630 & 9.533 & 6.215 \\
\hline 7/11/11 8:00 & 21.610 & 19.294 & 18.216 & 19.550 & 13.633 & 9.536 & 6.222 \\
\hline 7/11/11 12:00 & 21.603 & 19.354 & 18.212 & 19.545 & 13.628 & 9.534 & 6.215 \\
\hline 7/11/11 16:00 & 21.598 & 19.387 & 18.205 & 19.538 & 13.626 & 9.529 & 6.212 \\
\hline 7/11/11 20:00 & 21.591 & 19.415 & 18.197 & 19.529 & 13.619 & 9.529 & 6.208 \\
\hline 7/12/11 0:00 & 21.587 & 19.466 & 18.195 & 19.522 & 13.621 & 9.522 & 6.210 \\
\hline $7 / 12 / 114: 00$ & 21.582 & 19.504 & 18.188 & 19.513 & 13.616 & 9.522 & 6.203 \\
\hline $7 / 12 / 118: 00$ & 21.577 & 19.538 & 18.184 & 19.508 & 13.616 & 9.519 & 6.201 \\
\hline $7 / 12 / 11$ 12:00 & 21.575 & 19.587 & 18.179 & 19.501 & 13.612 & 9.515 & 6.201 \\
\hline $7 / 12 / 11$ 16:00 & 21.566 & 19.613 & 18.170 & 19.497 & 13.609 & 9.512 & 6.198 \\
\hline 7/12/11 20:00 & 21.561 & 19.643 & 18.165 & 19.487 & 13.605 & 9.512 & 6.194 \\
\hline 7/13/11 0:00 & 21.564 & 19.739 & 18.170 & 19.485 & 13.609 & 9.512 & 6.201 \\
\hline $7 / 13 / 114: 00$ & 21.555 & 19.764 & 18.160 & 19.476 & 13.602 & 9.508 & 6.194 \\
\hline $7 / 13 / 118: 00$ & 21.552 & 19.822 & 18.160 & 19.471 & 13.605 & 9.508 & 6.191 \\
\hline 7/13/11 12:00 & 21.550 & 19.860 & 18.156 & 19.469 & 13.600 & 9.505 & 6.191 \\
\hline 7/13/11 16:00 & 21.541 & 19.874 & 18.146 & 19.464 & 13.591 & 9.501 & 6.184 \\
\hline 7/13/11 20:00 & 21.536 & 19.874 & 18.142 & 19.455 & 13.588 & 9.501 & 6.184 \\
\hline 7/14/11 0:00 & 21.534 & 19.925 & 18.137 & 19.452 & 13.591 & 9.494 & 6.182 \\
\hline $7 / 14 / 114: 00$ & 21.527 & 19.939 & 18.130 & 19.445 & 13.581 & 9.491 & 6.184 \\
\hline 7/14/11 8:00 & 21.525 & 19.960 & 18.128 & 19.441 & 13.584 & 9.491 & 6.175 \\
\hline $7 / 14 / 11$ 12:00 & 21.518 & 19.992 & 18.123 & 19.436 & 13.577 & 9.487 & 6.173 \\
\hline 7/14/11 16:00 & 21.513 & 19.997 & 18.114 & 19.431 & 13.572 & 9.482 & 6.168 \\
\hline 7/14/11 20:00 & 21.504 & 19.988 & 18.109 & 19.424 & 13.565 & 9.482 & 6.168 \\
\hline 7/15/11 0:00 & 21.504 & 20.037 & 18.107 & 19.420 & 13.567 & 9.477 & 6.166 \\
\hline $7 / 15 / 114: 00$ & 21.500 & 20.076 & 18.100 & 19.413 & 13.563 & 9.477 & 6.159 \\
\hline 7/15/11 8:00 & 21.497 & 20.116 & 18.100 & 19.415 & 13.563 & 9.475 & 6.166 \\
\hline 7/15/11 12:00 & 21.493 & 20.158 & 18.093 & 19.410 & 13.558 & 9.473 & 6.166 \\
\hline 7/15/11 16:00 & 21.486 & 20.176 & 18.088 & 19.410 & 13.551 & 9.470 & 6.161 \\
\hline $7 / 15 / 11$ 20:00 & 21.479 & 20.181 & 18.083 & 19.401 & 13.551 & 9.468 & 6.152 \\
\hline 7/16/11 0:00 & 21.479 & 20.237 & 18.086 & 19.401 & 13.549 & 9.466 & 6.159 \\
\hline $7 / 16 / 114: 00$ & 21.474 & 20.291 & 18.081 & 19.399 & 13.549 & 9.470 & 6.159 \\
\hline 7/16/11 8:00 & 21.477 & 20.365 & 18.081 & 19.396 & 13.546 & 9.468 & 6.163 \\
\hline 7/16/11 12:00 & 21.472 & 20.402 & 18.079 & 19.396 & 13.546 & 9.468 & 6.159 \\
\hline 7/16/11 16:00 & 21.470 & 20.449 & 18.074 & 19.399 & 13.544 & 9.468 & 6.161 \\
\hline 7/16/11 20:00 & 21.465 & 20.486 & 18.072 & 19.396 & 13.544 & 9.466 & 6.161 \\
\hline 7/17/11 0:00 & 21.465 & 20.533 & 18.072 & 19.392 & 13.539 & 9.466 & 6.163 \\
\hline $7 / 17 / 114: 00$ & 21.461 & 20.572 & 18.070 & 19.392 & 13.539 & 9.470 & 6.161 \\
\hline 7/17/11 8:00 & 21.463 & 20.640 & 18.072 & 19.392 & 13.542 & 9.468 & 6.163 \\
\hline 7/17/11 12:00 & 21.461 & 20.710 & 18.072 & 19.396 & 13.539 & 9.468 & 6.168 \\
\hline 7/17/11 16:00 & 21.458 & 20.740 & 18.067 & 19.399 & 13.539 & 9.461 & 6.166 \\
\hline 7/17/11 20:00 & 21.451 & 20.754 & 18.063 & 19.396 & 13.532 & 9.463 & 6.161 \\
\hline 7/18/11 0:00 & 21.449 & 20.782 & 18.060 & 19.392 & 13.532 & 9.463 & 6.168 \\
\hline $7 / 18 / 114: 00$ & 21.447 & 20.803 & 18.060 & 19.392 & 13.528 & 9.463 & 6.161 \\
\hline 7/18/11 8:00 & 21.445 & 20.847 & 18.058 & 19.394 & 13.528 & 9.463 & 6.166 \\
\hline 7/18/11 12:00 & 21.442 & 20.889 & 18.053 & 19.394 & 13.523 & 9.461 & 6.163 \\
\hline 7/18/11 16:00 & 21.438 & 20.908 & 18.051 & 19.396 & 13.518 & 9.456 & 6.163 \\
\hline 7/18/11 20:00 & 21.433 & 20.912 & 18.044 & 19.394 & 13.516 & 9.456 & 6.161 \\
\hline 7/19/11 0:00 & 21.428 & 20.938 & 18.044 & 19.392 & 13.516 & 9.454 & 6.161 \\
\hline 7/19/11 4:00 & 21.424 & 20.959 & 18.039 & 19.389 & 13.509 & 9.454 & 6.159 \\
\hline 7/19/11 8:00 & 21.422 & 20.964 & 18.037 & 19.394 & 13.504 & 9.454 & 6.159 \\
\hline 7/19/11 12:00 & 21.417 & 20.971 & 18.035 & 19.394 & 13.500 & 9.452 & 6.159 \\
\hline 7/19/11 16:00 & 21.415 & 20.989 & 18.030 & 19.392 & 13.497 & 9.447 & 6.154 \\
\hline 7/19/11 20:00 & 21.408 & 21.003 & 18.023 & 19.389 & 13.493 & 9.445 & 6.152 \\
\hline 7/20/11 0:00 & 21.403 & 21.003 & 18.021 & 19.389 & 13.488 & 9.445 & 6.149 \\
\hline
\end{tabular}

TABLE S1.2 Page 42 of 58 


\begin{tabular}{|c|c|c|c|c|c|c|c|}
\hline \multirow[b]{2}{*}{ Date and Time } & \multicolumn{7}{|c|}{ Water Level (ft below reference point) } \\
\hline & SB43S & SB44S & SB45S & SB46S & SB47S & SB48S & SB49S \\
\hline $7 / 20 / 114: 00$ & 21.401 & 21.048 & 18.018 & 19.389 & 13.486 & 9.447 & 6.152 \\
\hline $7 / 20 / 118: 00$ & 21.401 & 21.082 & 18.018 & 19.392 & 13.483 & 9.445 & 6.152 \\
\hline $7 / 20 / 1112: 00$ & 21.399 & 21.108 & 18.016 & 19.392 & 13.478 & 9.445 & 6.154 \\
\hline $7 / 20 / 1116: 00$ & 21.392 & 21.127 & 18.014 & 19.396 & 13.476 & 9.442 & 6.152 \\
\hline $7 / 20 / 1120: 00$ & 21.387 & 21.099 & 18.007 & 19.394 & 13.469 & 9.442 & 6.149 \\
\hline 7/21/11 0:00 & 21.387 & 21.061 & 18.009 & 19.394 & 13.469 & 9.442 & 6.147 \\
\hline $7 / 21 / 114: 00$ & 21.385 & 21.010 & 18.007 & 19.396 & 13.467 & 9.442 & 6.156 \\
\hline $7 / 21 / 118: 00$ & 21.387 & 20.994 & 18.011 & 19.399 & 13.469 & 9.445 & 6.161 \\
\hline $7 / 21 / 11$ 12:00 & 21.383 & 21.003 & 18.009 & 19.406 & 13.467 & 9.442 & 6.159 \\
\hline $7 / 21 / 11$ 16:00 & 21.378 & 21.006 & 18.007 & 19.403 & 13.460 & 9.440 & 6.156 \\
\hline $7 / 21 / 11$ 20:00 & 21.371 & 21.008 & 18.002 & 19.406 & 13.455 & 9.442 & 6.154 \\
\hline $7 / 22 / 11$ 0:00 & 21.369 & 21.045 & 18.002 & 19.406 & 13.455 & 9.440 & 6.159 \\
\hline $7 / 22 / 114: 00$ & 21.369 & 21.103 & 18.004 & 19.413 & 13.457 & 9.442 & 6.159 \\
\hline $7 / 22 / 118: 00$ & 21.367 & 21.169 & 18.002 & 19.413 & 13.455 & 9.445 & 6.161 \\
\hline $7 / 22 / 11$ 12:00 & 21.364 & 21.218 & 18.004 & 19.417 & 13.453 & 9.442 & 6.161 \\
\hline $7 / 22 / 11$ 16:00 & 21.360 & 21.248 & 18.000 & 19.420 & 13.448 & 9.442 & 6.163 \\
\hline $7 / 22 / 11$ 20:00 & 21.357 & 21.280 & 17.997 & 19.422 & 13.443 & 9.445 & 6.166 \\
\hline 7/23/11 0:00 & 21.355 & 21.320 & 18.002 & 19.424 & 13.443 & 9.447 & 6.163 \\
\hline $7 / 23 / 114: 00$ & 21.355 & 21.367 & 18.000 & 19.429 & 13.441 & 9.447 & 6.170 \\
\hline 7/23/11 8:00 & 21.353 & 21.390 & 18.007 & 19.436 & 13.441 & 9.452 & 6.175 \\
\hline $7 / 23 / 11$ 12:00 & 21.353 & 21.437 & 18.004 & 19.441 & 13.441 & 9.449 & 6.177 \\
\hline 7/23/11 16:00 & 21.351 & 21.488 & 18.002 & 19.445 & 13.436 & 9.452 & 6.177 \\
\hline 7/23/11 20:00 & 21.348 & 21.530 & 18.002 & 19.448 & 13.434 & 9.454 & 6.180 \\
\hline 7/24/11 0:00 & 21.351 & 21.604 & 18.007 & 19.452 & 13.434 & 9.456 & 6.182 \\
\hline $7 / 24 / 114: 00$ & 21.351 & 21.646 & 18.009 & 19.455 & 13.434 & 9.461 & 6.187 \\
\hline 7/24/11 8:00 & 21.348 & 21.718 & 18.007 & 19.462 & 13.434 & 9.459 & 6.189 \\
\hline $7 / 24 / 11$ 12:00 & 21.351 & 21.683 & 18.014 & 19.469 & 13.436 & 9.459 & 6.196 \\
\hline $7 / 24 / 11$ 16:00 & 21.346 & 21.634 & 18.011 & 19.473 & 13.432 & 9.459 & 6.196 \\
\hline $7 / 24 / 1120: 00$ & 21.344 & 21.579 & 18.009 & 19.471 & 13.427 & 9.463 & 6.191 \\
\hline 7/25/11 0:00 & 21.346 & 21.646 & 18.011 & 19.478 & 13.429 & 9.461 & 6.203 \\
\hline $7 / 25 / 114: 00$ & 21.344 & 21.613 & 18.014 & 19.478 & 13.425 & 9.461 & 6.198 \\
\hline $7 / 25 / 118: 00$ & 21.344 & 21.634 & 18.014 & 19.480 & 13.425 & 9.461 & 6.203 \\
\hline $7 / 25 / 11$ 12:00 & 21.344 & 21.674 & 18.011 & 19.485 & 13.418 & 9.461 & 6.203 \\
\hline 7/25/11 16:00 & 21.337 & 21.667 & 18.007 & 19.487 & 13.413 & 9.456 & 6.205 \\
\hline 7/25/11 20:00 & 21.332 & 21.653 & 18.004 & 19.490 & 13.408 & 9.454 & 6.203 \\
\hline 7/26/11 0:00 & 21.332 & 21.681 & 18.004 & 19.490 & 13.408 & 9.456 & 6.203 \\
\hline $7 / 26 / 114: 00$ & 21.330 & 21.702 & 18.002 & 19.490 & 13.401 & 9.456 & 6.201 \\
\hline $7 / 26 / 118: 00$ & 21.328 & 21.723 & 18.002 & 19.494 & 13.401 & 9.454 & 6.201 \\
\hline 7/26/11 12:00 & 21.328 & 21.756 & 18.002 & 19.501 & 13.399 & 9.454 & 6.205 \\
\hline 7/26/11 16:00 & 21.321 & 21.760 & 17.997 & 19.501 & 13.394 & 9.449 & 6.203 \\
\hline $7 / 26 / 11$ 20:00 & 21.318 & 21.760 & 17.995 & 19.501 & 13.385 & 9.452 & 6.203 \\
\hline 7/27/11 0:00 & 21.318 & 21.788 & 17.995 & 19.506 & 13.385 & 9.454 & 6.201 \\
\hline $7 / 27 / 114: 00$ & 21.316 & 21.825 & 17.993 & 19.508 & 13.380 & 9.452 & 6.205 \\
\hline $7 / 27 / 118: 00$ & 21.316 & 21.865 & 17.995 & 19.508 & 13.380 & 9.449 & 6.208 \\
\hline 7/27/11 12:00 & 21.314 & 21.868 & 17.990 & 19.511 & 13.376 & 9.452 & 6.208 \\
\hline 7/27/11 16:00 & 21.309 & 21.898 & 17.993 & 19.520 & 13.371 & 9.452 & 6.203 \\
\hline $7 / 27 / 11$ 20:00 & 21.309 & 21.928 & 17.990 & 19.520 & 13.371 & 9.454 & 6.208 \\
\hline 7/28/11 0:00 & 21.312 & 21.998 & 17.998 & 19.529 & 13.373 & 9.456 & 6.212 \\
\hline $7 / 28 / 114: 00$ & 21.314 & 22.065 & 18.000 & 19.534 & 13.371 & 9.461 & 6.219 \\
\hline 7/28/11 8:00 & 21.316 & 22.130 & 18.004 & 19.541 & 13.373 & 9.466 & 6.224 \\
\hline $7 / 28 / 11$ 12:00 & 21.318 & 22.205 & 18.007 & 19.548 & 13.376 & 9.468 & 6.231 \\
\hline $7 / 28 / 11$ 16:00 & 21.321 & 22.252 & 18.009 & 19.557 & 13.376 & 9.468 & 6.233 \\
\hline 7/28/11 20:00 & 21.321 & 22.280 & 18.014 & 19.562 & 13.373 & 9.470 & 6.238 \\
\hline 7/29/11 0:00 & 21.325 & 22.345 & 18.021 & 19.569 & 13.378 & 9.475 & 6.245 \\
\hline
\end{tabular}

TABLE S1.2 Page 43 of 58 


\begin{tabular}{|c|c|c|c|c|c|c|c|}
\hline \multirow[b]{2}{*}{ Date and Time } & \multicolumn{7}{|c|}{ Water Level (ft below reference point) } \\
\hline & SB43S & SB44S & SB45S & SB46S & SB47S & SB48S & SB49S \\
\hline $7 / 29 / 114: 00$ & 21.325 & 22.389 & 18.023 & 19.573 & 13.376 & 9.477 & 6.250 \\
\hline 7/29/11 8:00 & 21.328 & 22.422 & 18.023 & 19.583 & 13.373 & 9.477 & 6.254 \\
\hline $7 / 29 / 11$ 12:00 & 21.328 & 22.447 & 18.023 & 19.587 & 13.371 & 9.477 & 6.254 \\
\hline 7/29/11 16:00 & 21.325 & 22.461 & 18.023 & 19.592 & 13.366 & 9.477 & 6.257 \\
\hline $7 / 29 / 1120: 00$ & 21.325 & 22.468 & 18.025 & 19.597 & 13.364 & 9.480 & 6.259 \\
\hline 7/30/11 0:00 & 21.325 & 22.482 & 18.028 & 19.606 & 13.362 & 9.480 & 6.262 \\
\hline $7 / 30 / 114: 00$ & 21.328 & 22.438 & 18.030 & 19.611 & 13.362 & 9.491 & 6.266 \\
\hline $7 / 30 / 118: 00$ & 21.328 & 21.625 & 18.028 & 19.608 & 13.341 & 9.477 & 6.262 \\
\hline $7 / 30 / 11$ 12:00 & 21.325 & 20.172 & 18.025 & 19.601 & 13.341 & 9.475 & 6.266 \\
\hline $7 / 30 / 11$ 16:00 & 21.323 & 19.538 & 18.016 & 19.590 & 13.345 & 9.473 & 6.262 \\
\hline $7 / 30 / 1120: 00$ & 21.323 & 19.361 & 18.011 & 19.580 & 13.345 & 9.470 & 6.262 \\
\hline 7/31/11 0:00 & 21.325 & 19.375 & 18.009 & 19.571 & 13.343 & 9.470 & 6.266 \\
\hline $7 / 31 / 114: 00$ & 21.323 & 19.420 & 18.007 & 19.559 & 13.341 & 9.468 & 6.266 \\
\hline 7/31/11 8:00 & 21.325 & 19.490 & 18.004 & 19.552 & 13.343 & 9.466 & 6.266 \\
\hline $7 / 31 / 11$ 12:00 & 21.323 & 19.555 & 18.000 & 19.548 & 13.338 & 9.463 & 6.266 \\
\hline $7 / 31 / 11$ 16:00 & 21.321 & 19.590 & 17.993 & 19.538 & 13.334 & 9.459 & 6.264 \\
\hline $7 / 31 / 1120: 00$ & 21.321 & 19.624 & 17.990 & 19.529 & 13.331 & 9.456 & 6.264 \\
\hline 8/1/11 0:00 & 21.318 & 19.676 & 17.986 & 19.520 & 13.329 & 9.454 & 6.262 \\
\hline 8/1/11 4:00 & 21.316 & 19.727 & 17.979 & 19.511 & 13.327 & 9.449 & 6.262 \\
\hline 8/1/11 8:00 & 21.316 & 19.771 & 17.976 & 19.504 & 13.324 & 9.447 & 6.262 \\
\hline 8/1/11 12:00 & 21.312 & 19.811 & 17.974 & 19.499 & 13.320 & 9.445 & 6.259 \\
\hline 8/1/11 16:00 & 21.307 & 19.832 & 17.965 & 19.492 & 13.310 & 9.442 & 6.252 \\
\hline 8/1/11 20:00 & 21.302 & 19.848 & 17.958 & 19.480 & 13.308 & 9.438 & 6.247 \\
\hline 8/2/11 0:00 & 21.300 & 19.895 & 17.953 & 19.471 & 13.303 & 9.435 & 6.247 \\
\hline 8/2/11 4:00 & 21.296 & 19.911 & 17.946 & 19.462 & 13.299 & 9.435 & 6.243 \\
\hline 8/2/11 8:00 & 21.293 & 19.944 & 17.944 & 19.455 & 13.296 & 9.433 & 6.243 \\
\hline $8 / 2 / 11$ 12:00 & 21.291 & 19.997 & 17.935 & 19.452 & 13.294 & 9.428 & 6.240 \\
\hline 8/2/11 16:00 & 21.289 & 20.046 & 17.932 & 19.445 & 13.287 & 9.428 & 6.243 \\
\hline $8 / 2 / 1120: 00$ & 21.289 & 20.109 & 17.930 & 19.441 & 13.289 & 9.431 & 6.240 \\
\hline 8/3/11 0:00 & 21.291 & 20.195 & 17.930 & 19.436 & 13.292 & 9.431 & 6.243 \\
\hline 8/3/11 4:00 & 21.289 & 20.267 & 17.930 & 19.434 & 13.292 & 9.431 & 6.243 \\
\hline 8/3/11 8:00 & 21.291 & 20.342 & 17.928 & 19.429 & 13.289 & 9.433 & 6.245 \\
\hline $8 / 3 / 11$ 12:00 & 21.291 & 20.423 & 17.928 & 19.429 & 13.289 & 9.431 & 6.252 \\
\hline 8/3/11 16:00 & 21.289 & 20.449 & 17.921 & 19.424 & 13.287 & 9.426 & 6.247 \\
\hline 8/3/11 20:00 & 21.284 & 20.470 & 17.918 & 19.420 & 13.282 & 9.426 & 6.245 \\
\hline 8/4/11 0:00 & 21.284 & 20.533 & 17.916 & 19.413 & 13.282 & 9.428 & 6.247 \\
\hline 8/4/11 4:00 & 21.284 & 20.603 & 17.914 & 19.415 & 13.278 & 9.426 & 6.247 \\
\hline 8/4/11 8:00 & 21.282 & 20.645 & 17.909 & 19.410 & 13.278 & 9.424 & 6.245 \\
\hline $8 / 4 / 11$ 12:00 & 21.280 & 20.714 & 17.907 & 19.408 & 13.275 & 9.426 & 6.247 \\
\hline 8/4/11 16:00 & 21.277 & 20.754 & 17.904 & 19.406 & 13.273 & 9.421 & 6.245 \\
\hline 8/4/11 20:00 & 21.275 & 20.784 & 17.902 & 19.403 & 13.271 & 9.421 & 6.243 \\
\hline 8/5/11 0:00 & 21.275 & 20.840 & 17.900 & 19.401 & 13.268 & 9.421 & 6.245 \\
\hline 8/5/11 4:00 & 21.273 & 20.873 & 17.895 & 19.399 & 13.264 & 9.421 & 6.247 \\
\hline 8/5/11 8:00 & 21.277 & 20.926 & 17.902 & 19.401 & 13.271 & 9.419 & 6.252 \\
\hline 8/5/11 12:00 & 21.270 & 20.887 & 17.895 & 19.396 & 13.261 & 9.421 & 6.245 \\
\hline 8/5/11 16:00 & 21.268 & 20.852 & 17.890 & 19.399 & 13.257 & 9.419 & 6.240 \\
\hline 8/5/11 20:00 & 21.268 & 20.798 & 17.886 & 19.392 & 13.254 & 9.417 & 6.243 \\
\hline 8/6/11 0:00 & 21.266 & 20.791 & 17.886 & 19.392 & 13.252 & 9.414 & 6.245 \\
\hline 8/6/11 4:00 & 21.261 & 20.789 & 17.881 & 19.382 & 13.250 & 9.414 & 6.245 \\
\hline 8/6/11 8:00 & 21.266 & 20.833 & 17.883 & 19.385 & 13.250 & 9.414 & 6.250 \\
\hline 8/6/11 12:00 & 21.263 & 20.877 & 17.879 & 19.382 & 13.245 & 9.412 & 6.247 \\
\hline 8/6/11 16:00 & 21.259 & 20.884 & 17.874 & 19.385 & 13.240 & 9.412 & 6.245 \\
\hline 8/6/11 20:00 & 21.259 & 20.898 & 17.872 & 19.380 & 13.240 & 9.417 & 6.243 \\
\hline 8/7/11 0:00 & 21.261 & 20.964 & 17.876 & 19.380 & 13.243 & 9.412 & 6.250 \\
\hline
\end{tabular}

TABLE S1.2 Page 44 of 58 


\begin{tabular}{|c|c|c|c|c|c|c|c|}
\hline \multirow[b]{2}{*}{ Date and Time } & \multicolumn{7}{|c|}{ Water Level (ft below reference point) } \\
\hline & SB43S & SB44S & SB45S & SB46S & SB47S & SB48S & SB49S \\
\hline 8/7/11 4:00 & 21.257 & 20.985 & 17.869 & 19.375 & 13.236 & 9.407 & 6.250 \\
\hline 8/7/11 8:00 & 21.254 & 21.024 & 17.867 & 19.375 & 13.236 & 9.407 & 6.247 \\
\hline 8/7/11 12:00 & 21.257 & 21.068 & 17.869 & 19.378 & 13.236 & 9.412 & 6.247 \\
\hline 8/7/11 16:00 & 21.250 & 21.082 & 17.860 & 19.373 & 13.226 & 9.403 & 6.243 \\
\hline 8/7/11 20:00 & 21.247 & 21.073 & 17.858 & 19.371 & 13.222 & 9.400 & 6.243 \\
\hline 8/8/11 0:00 & 21.250 & 21.094 & 17.860 & 19.368 & 13.224 & 9.400 & 6.247 \\
\hline 8/8/11 4:00 & 21.247 & 21.157 & 17.853 & 19.371 & 13.217 & 9.400 & 6.240 \\
\hline 8/8/11 8:00 & 21.250 & 21.192 & 17.858 & 19.373 & 13.219 & 9.398 & 6.247 \\
\hline $8 / 8 / 11$ 12:00 & 21.247 & 21.197 & 17.853 & 19.371 & 13.215 & 9.398 & 6.247 \\
\hline 8/8/11 16:00 & 21.243 & 21.225 & 17.851 & 19.373 & 13.210 & 9.398 & 6.247 \\
\hline 8/8/11 20:00 & 21.241 & 21.245 & 17.846 & 19.368 & 13.207 & 9.398 & 6.243 \\
\hline 8/9/11 0:00 & 21.243 & 21.297 & 17.848 & 19.373 & 13.207 & 9.398 & 6.245 \\
\hline 8/9/11 4:00 & 21.243 & 21.339 & 17.846 & 19.371 & 13.203 & 9.398 & 6.245 \\
\hline 8/9/11 8:00 & 21.243 & 21.390 & 17.851 & 19.373 & 13.205 & 9.398 & 6.250 \\
\hline $8 / 9 / 11$ 12:00 & 21.243 & 21.451 & 17.851 & 19.380 & 13.205 & 9.396 & 6.254 \\
\hline 8/9/11 16:00 & 21.243 & 21.465 & 17.846 & 19.380 & 13.200 & 9.398 & 6.250 \\
\hline 8/9/11 20:00 & 21.241 & 21.502 & 17.846 & 19.382 & 13.198 & 9.396 & 6.252 \\
\hline 8/10/11 0:00 & 21.243 & 21.546 & 17.848 & 19.385 & 13.198 & 9.398 & 6.257 \\
\hline 8/10/11 4:00 & 21.241 & 21.574 & 17.844 & 19.385 & 13.198 & 9.398 & 6.259 \\
\hline 8/10/11 8:00 & 21.245 & 21.642 & 17.851 & 19.387 & 13.198 & 9.398 & 6.264 \\
\hline $8 / 10 / 11$ 12:00 & 22.336 & 22.352 & & 41.248 & 13.193 & 9.398 & 6.264 \\
\hline 8/10/11 16:00 & & 21.804 & 18.847 & 41.108 & 33.898 & 11.165 & 7.410 \\
\hline 8/10/11 20:00 & 39.001 & 21.749 & 18.840 & 41.215 & 28.244 & 10.502 & 21.933 \\
\hline 8/11/11 0:00 & 38.955 & 21.795 & 18.837 & 41.196 & 28.220 & 10.505 & 21.856 \\
\hline $8 / 11 / 114: 00$ & 38.912 & 21.825 & 18.830 & 41.209 & 28.206 & 10.507 & 21.796 \\
\hline 8/11/11 8:00 & 38.877 & 21.865 & 18.828 & 41.211 & 28.195 & 10.509 & 21.742 \\
\hline $8 / 11 / 11$ 12:00 & 38.843 & 21.921 & 18.823 & 40.593 & 28.185 & 28.863 & 21.691 \\
\hline 8/11/11 16:00 & 38.799 & 33.361 & 18.812 & 39.925 & 28.174 & 28.795 & 21.625 \\
\hline $8 / 11 / 1120: 00$ & 38.760 & 26.712 & 34.559 & 29.882 & 28.167 & 28.762 & 21.565 \\
\hline 8/12/11 0:00 & 38.731 & 23.500 & 34.462 & 29.882 & 28.160 & 28.741 & 21.515 \\
\hline $8 / 12 / 114: 00$ & 38.708 & 22.403 & 34.387 & 29.852 & 28.155 & 28.725 & 21.466 \\
\hline 8/12/11 8:00 & 38.664 & 22.056 & 34.294 & 29.812 & 28.146 & 28.711 & 21.403 \\
\hline $8 / 12 / 11$ 12:00 & 38.639 & 21.977 & 34.217 & 29.768 & 28.141 & 28.695 & 21.352 \\
\hline $8 / 12 / 11$ 16:00 & 38.598 & 21.814 & 34.131 & 29.724 & 28.134 & 28.681 & 21.289 \\
\hline 8/12/11 20:00 & 38.566 & 21.739 & 34.048 & 29.672 & 28.124 & 28.672 & 21.238 \\
\hline 8/13/11 0:00 & 38.538 & 21.723 & 33.971 & 29.628 & 28.120 & 28.660 & 21.186 \\
\hline $8 / 13 / 114: 00$ & 38.506 & 21.728 & 33.887 & 29.581 & 28.110 & 28.648 & 21.135 \\
\hline 8/13/11 8:00 & 38.476 & 21.749 & 33.806 & 29.533 & 28.108 & 28.636 & 21.081 \\
\hline $8 / 13 / 11$ 12:00 & 38.449 & 21.786 & 33.729 & 29.491 & 28.101 & 28.622 & 21.035 \\
\hline 8/13/11 16:00 & 38.414 & 21.821 & 33.647 & 29.442 & 28.094 & 28.599 & 20.981 \\
\hline $8 / 13 / 1120: 00$ & 38.385 & 21.844 & 33.566 & 29.393 & 28.087 & 28.580 & 20.932 \\
\hline 8/14/11 0:00 & 38.355 & 21.895 & 33.491 & 29.346 & 28.082 & 28.559 & 20.881 \\
\hline $8 / 14 / 114: 00$ & 38.325 & 21.935 & 33.415 & 29.300 & 28.075 & 28.541 & 20.832 \\
\hline 8/14/11 8:00 & 38.295 & 21.979 & 33.338 & 29.258 & 28.071 & 28.520 & 20.783 \\
\hline $8 / 14 / 11$ 12:00 & 38.263 & 22.016 & 33.261 & 29.216 & 28.064 & 28.499 & 20.736 \\
\hline 8/14/11 16:00 & 38.226 & 22.023 & 33.175 & 29.167 & 28.059 & 28.480 & 20.675 \\
\hline $8 / 14 / 1120: 00$ & 38.187 & 22.014 & 33.091 & 29.116 & 28.052 & 28.459 & 20.612 \\
\hline 8/15/11 0:00 & 38.155 & 22.028 & 33.012 & 29.069 & 28.045 & 28.438 & 20.565 \\
\hline $8 / 15 / 114: 00$ & 38.128 & 22.044 & 32.940 & 29.027 & 28.043 & 28.417 & 20.519 \\
\hline 8/15/11 8:00 & 38.091 & 22.137 & 32.859 & 28.983 & 28.036 & 28.398 & 20.470 \\
\hline $8 / 15 / 11$ 12:00 & 38.064 & 22.098 & 32.786 & 28.941 & 28.029 & 28.377 & 20.421 \\
\hline 8/15/11 16:00 & 38.027 & 22.196 & 32.705 & 28.890 & 28.022 & 28.359 & 20.362 \\
\hline 8/15/11 20:00 & 37.993 & 22.151 & 32.628 & 28.846 & 28.017 & 28.338 & 20.309 \\
\hline 8/16/11 0:00 & 37.963 & 22.086 & 32.556 & 28.806 & 28.010 & 28.319 & 20.262 \\
\hline
\end{tabular}

TABLE S1.2 Page 45 of 58 


\begin{tabular}{|c|c|c|c|c|c|c|c|}
\hline \multirow[b]{2}{*}{ Date and Time } & \multicolumn{7}{|c|}{ Water Level (ft below reference point) } \\
\hline & SB43S & SB44S & SB45S & SB46S & SB47S & SB48S & SB49S \\
\hline 8/16/11 4:00 & 37.926 & 22.117 & 32.475 & 28.759 & 28.008 & 28.298 & 20.211 \\
\hline 8/16/11 8:00 & 37.899 & 22.068 & 32.405 & 28.713 & 27.998 & 28.277 & 20.159 \\
\hline $8 / 16 / 11$ 12:00 & 37.874 & 22.063 & 32.335 & 28.673 & 27.991 & 28.261 & 20.117 \\
\hline 8/16/11 16:00 & 37.837 & 21.895 & 32.261 & 28.624 & 27.987 & 28.240 & 20.066 \\
\hline 8/16/11 20:00 & 37.812 & 21.802 & 32.191 & 28.585 & 27.980 & 28.221 & 20.022 \\
\hline 8/17/11 0:00 & 37.789 & 21.790 & 32.128 & 28.543 & 27.975 & 28.200 & 19.980 \\
\hline 8/17/11 4:00 & 37.761 & 21.804 & 32.056 & 28.501 & 27.966 & 28.184 & 19.938 \\
\hline 8/17/11 8:00 & 37.741 & 21.832 & 31.993 & 28.459 & 27.959 & 28.165 & 19.896 \\
\hline $8 / 17 / 11$ 12:00 & 37.713 & 21.884 & 31.926 & 28.422 & 27.952 & 28.142 & 19.854 \\
\hline $8 / 17 / 11$ 16:00 & 37.681 & 21.902 & 31.851 & 28.375 & 27.947 & 28.123 & 19.800 \\
\hline $8 / 17 / 1120: 00$ & 37.644 & 21.893 & 31.774 & 28.331 & 27.938 & 28.102 & 19.746 \\
\hline 8/18/11 0:00 & 37.621 & 21.916 & 31.707 & 28.287 & 27.935 & 28.081 & 19.704 \\
\hline 8/18/11 4:00 & 37.587 & 21.937 & 31.637 & 28.245 & 27.924 & 28.062 & 19.653 \\
\hline 8/18/11 8:00 & 37.562 & 21.961 & 31.570 & 28.203 & 27.917 & 28.041 & 19.606 \\
\hline $8 / 18 / 11$ 12:00 & 37.527 & 21.981 & 31.495 & 28.158 & 27.910 & 28.022 & 19.557 \\
\hline 8/18/11 16:00 & 37.486 & 21.958 & 31.414 & 28.117 & 27.903 & 27.999 & 19.499 \\
\hline 8/18/11 20:00 & 37.454 & 21.923 & 31.342 & 28.070 & 27.891 & 27.978 & 19.450 \\
\hline 8/19/11 0:00 & 37.429 & 21.758 & 31.272 & 28.028 & 27.889 & 27.962 & 19.403 \\
\hline 8/19/11 4:00 & 37.390 & 21.290 & 31.200 & 27.977 & 27.879 & 27.941 & 19.349 \\
\hline 8/19/11 8:00 & 37.369 & 20.922 & 31.141 & 27.935 & 27.872 & 27.922 & 19.310 \\
\hline $8 / 19 / 11$ 12:00 & 37.344 & 21.050 & 31.076 & 27.898 & 27.865 & 27.903 & 19.265 \\
\hline $8 / 19 / 11$ 16:00 & 37.310 & 20.968 & 31.002 & 27.849 & 27.856 & 27.885 & 19.212 \\
\hline 8/19/11 20:00 & 37.280 & 20.945 & 30.934 & 27.804 & 27.853 & 27.861 & 19.167 \\
\hline 8/20/11 0:00 & 37.255 & 20.973 & 30.872 & 27.760 & 27.842 & 27.843 & 19.128 \\
\hline $8 / 20 / 114: 00$ & 37.243 & 21.029 & 30.811 & 27.721 & 27.837 & 27.824 & 19.083 \\
\hline 8/20/11 8:00 & 37.207 & 20.717 & 30.741 & 27.669 & 27.828 & 27.803 & 19.037 \\
\hline $8 / 20 / 11$ 12:00 & 37.181 & 20.544 & 30.678 & 27.627 & 27.823 & 27.782 & 18.995 \\
\hline 8/20/11 16:00 & 37.147 & 20.463 & 30.606 & 27.578 & 27.816 & 27.761 & 18.948 \\
\hline 8/20/11 20:00 & 37.120 & 20.416 & 30.539 & 27.532 & 27.809 & 27.742 & 18.894 \\
\hline 8/21/11 0:00 & 37.094 & 20.426 & 30.474 & 27.488 & 27.800 & 27.721 & 18.854 \\
\hline $8 / 21 / 114: 00$ & 37.067 & 20.440 & 30.408 & 27.441 & 27.793 & 27.700 & 18.808 \\
\hline 8/21/11 8:00 & 37.042 & 20.477 & 30.346 & 27.397 & 27.786 & 27.679 & 18.761 \\
\hline $8 / 21 / 11$ 12:00 & 37.019 & 20.526 & 30.283 & 27.355 & 27.776 & 27.658 & 18.724 \\
\hline $8 / 21 / 11$ 16:00 & 36.980 & 20.537 & 30.208 & 27.308 & 27.772 & 27.637 & 18.668 \\
\hline $8 / 21 / 1120: 00$ & 36.952 & 20.535 & 30.143 & 27.264 & 27.762 & 27.616 & 18.616 \\
\hline 8/22/11 0:00 & 36.927 & 20.575 & 30.080 & 27.220 & 27.755 & 27.598 & 18.577 \\
\hline $8 / 22 / 114: 00$ & 36.897 & 20.591 & 30.015 & 27.173 & 27.751 & 27.574 & 18.530 \\
\hline 8/22/11 8:00 & 36.877 & 20.605 & 29.959 & 27.134 & 27.744 & 27.556 & 18.497 \\
\hline $8 / 22 / 11$ 12:00 & 36.840 & 19.645 & 29.885 & 27.085 & 27.737 & 27.535 & 18.434 \\
\hline $8 / 22 / 11$ 16:00 & 36.803 & 18.854 & 29.811 & 27.033 & 27.727 & 27.514 & 18.376 \\
\hline $8 / 22 / 1120: 00$ & 36.769 & 18.530 & 29.736 & 26.978 & 27.720 & 27.495 & 18.322 \\
\hline 8/23/11 0:00 & 36.737 & 18.458 & 29.669 & 26.926 & 27.713 & 27.472 & 18.271 \\
\hline 8/23/11 4:00 & 36.707 & 18.446 & 29.596 & 26.870 & 27.706 & 27.451 & 18.220 \\
\hline 8/23/11 8:00 & 36.668 & 18.453 & 29.524 & 26.822 & 27.697 & 27.425 & 18.171 \\
\hline $8 / 23 / 11$ 12:00 & 36.634 & 18.472 & 29.455 & 26.773 & 27.690 & 27.399 & 18.117 \\
\hline 8/23/11 16:00 & 36.597 & 18.483 & 29.380 & 26.717 & 27.681 & 27.373 & 18.058 \\
\hline 8/23/11 20:00 & 36.570 & 18.523 & 29.317 & 26.665 & 27.674 & 27.352 & 18.009 \\
\hline 8/24/11 0:00 & 36.537 & 18.572 & 29.247 & 26.619 & 27.667 & 27.327 & 17.956 \\
\hline $8 / 24 / 114: 00$ & 36.508 & 18.576 & 29.180 & 26.568 & 27.660 & 27.303 & 17.907 \\
\hline 8/24/11 8:00 & 36.480 & 18.609 & 29.119 & 26.521 & 27.655 & 27.280 & 17.862 \\
\hline 8/24/11 12:00 & 36.455 & 18.688 & 29.061 & 26.477 & 27.648 & 27.254 & 17.823 \\
\hline $8 / 24 / 11$ 16:00 & 36.430 & 18.765 & 29.005 & 26.435 & 27.641 & 27.231 & 17.781 \\
\hline $8 / 24 / 1120: 00$ & 36.402 & 18.828 & 28.940 & 26.386 & 27.634 & 27.208 & 17.736 \\
\hline 8/25/11 0:00 & 36.382 & 18.896 & 28.884 & 26.342 & 27.625 & 27.182 & 17.697 \\
\hline
\end{tabular}

TABLE S1.2 Page 46 of 58 


\begin{tabular}{|c|c|c|c|c|c|c|c|}
\hline \multirow[b]{2}{*}{ Date and Time } & \multicolumn{7}{|c|}{ Water Level (ft below reference point) } \\
\hline & SB43S & SB44S & SB45S & SB46S & SB47S & SB48S & SB49S \\
\hline $8 / 25 / 114: 00$ & 36.356 & 18.961 & 28.829 & 26.297 & 27.620 & 27.161 & 17.655 \\
\hline 8/25/11 8:00 & 36.331 & 19.014 & 28.768 & 26.256 & 27.613 & 27.138 & 17.613 \\
\hline 8/25/11 12:00 & 36.304 & 19.068 & 28.708 & 26.216 & 27.606 & 27.114 & 17.571 \\
\hline 8/25/11 16:00 & 36.267 & 19.073 & 28.638 & 26.167 & 27.601 & 27.091 & 17.519 \\
\hline 8/25/11 20:00 & 36.230 & 19.061 & 28.573 & 26.118 & 27.592 & 27.065 & 17.468 \\
\hline 8/26/11 0:00 & 36.203 & 19.073 & 28.510 & 26.074 & 27.587 & 27.042 & 17.426 \\
\hline $8 / 26 / 114: 00$ & 36.171 & 19.091 & 28.449 & 26.030 & 27.578 & 27.019 & 17.379 \\
\hline 8/26/11 8:00 & 36.148 & 19.138 & 28.391 & 25.988 & 27.571 & 26.995 & 17.340 \\
\hline 8/26/11 12:00 & 36.111 & 19.149 & 28.328 & 25.941 & 27.564 & 26.974 & 17.291 \\
\hline 8/26/11 16:00 & 36.077 & 19.147 & 28.261 & 25.897 & 27.557 & 26.951 & 17.244 \\
\hline 8/26/11 20:00 & 36.054 & 19.143 & 28.196 & 25.853 & 27.550 & 26.928 & 17.195 \\
\hline 8/27/11 0:00 & 36.029 & 19.180 & 28.142 & 25.811 & 27.547 & 26.907 & 17.157 \\
\hline 8/27/11 4:00 & 36.001 & 19.212 & 28.079 & 25.771 & 27.536 & 26.883 & 17.118 \\
\hline 8/27/11 8:00 & 35.971 & 19.245 & 28.024 & 25.727 & 27.529 & 26.860 & 17.076 \\
\hline 8/27/11 12:00 & 35.942 & 19.275 & 27.965 & 25.687 & 27.519 & 26.839 & 17.031 \\
\hline 8/27/11 16:00 & 35.905 & 19.282 & 27.900 & 25.645 & 27.515 & 26.813 & 16.985 \\
\hline 8/27/11 20:00 & 35.871 & 19.278 & 27.835 & 25.601 & 27.505 & 26.792 & 16.938 \\
\hline 8/28/11 0:00 & 35.843 & 19.301 & 27.779 & 25.559 & 27.498 & 26.771 & 16.896 \\
\hline 8/28/11 4:00 & 35.811 & 19.315 & 27.719 & 25.517 & 27.489 & 26.748 & 16.852 \\
\hline 8/28/11 8:00 & 35.779 & 19.326 & 27.661 & 25.475 & 27.482 & 26.724 & 16.807 \\
\hline 8/28/11 12:00 & 35.749 & 19.347 & 27.602 & 25.436 & 27.477 & 26.703 & 16.765 \\
\hline 8/28/11 16:00 & 35.710 & 19.361 & 27.535 & 25.391 & 27.468 & 26.678 & 16.719 \\
\hline 8/28/11 20:00 & 35.676 & 19.333 & 27.477 & 25.352 & 27.456 & 26.657 & 16.674 \\
\hline 8/29/11 0:00 & 35.655 & 19.387 & 27.428 & 25.315 & 27.452 & 26.636 & 16.639 \\
\hline 8/29/11 4:00 & 35.630 & 19.436 & 27.372 & 25.277 & 27.447 & 26.615 & 16.597 \\
\hline 8/29/11 8:00 & 35.602 & 19.473 & 27.319 & 25.238 & 27.438 & 26.591 & 16.562 \\
\hline 8/29/11 12:00 & 35.575 & 19.529 & 27.263 & 25.203 & 27.433 & 26.568 & 16.523 \\
\hline 8/29/11 16:00 & 35.538 & 19.527 & 27.202 & 25.166 & 27.421 & 26.549 & 16.481 \\
\hline 8/29/11 20:00 & 35.506 & 19.529 & 27.146 & 25.126 & 27.414 & 26.524 & 16.436 \\
\hline 8/30/11 0:00 & 35.479 & 19.550 & 27.090 & 25.086 & 27.407 & 26.503 & 16.401 \\
\hline 8/30/11 4:00 & 35.444 & 19.559 & 27.035 & 25.044 & 27.400 & 26.479 & 16.350 \\
\hline 8/30/11 8:00 & 35.419 & 19.555 & 26.983 & 25.009 & 27.391 & 26.458 & 16.312 \\
\hline 8/30/11 12:00 & 35.385 & 19.543 & 26.923 & 24.972 & 27.382 & 26.437 & 16.271 \\
\hline 8/30/11 16:00 & 35.350 & 19.480 & 26.865 & 24.933 & 27.375 & 26.416 & 16.226 \\
\hline 8/30/11 20:00 & 35.323 & 19.441 & 26.811 & 24.895 & 27.365 & 26.395 & 16.184 \\
\hline 8/31/11 0:00 & 35.295 & 19.466 & 26.762 & 24.858 & 27.358 & 26.372 & 16.154 \\
\hline 8/31/11 4:00 & 35.266 & 19.475 & 26.709 & 24.819 & 27.351 & 26.349 & 16.114 \\
\hline 8/31/11 8:00 & 35.243 & 19.492 & 26.660 & 24.786 & 27.342 & 26.330 & 16.081 \\
\hline 8/31/11 12:00 & 35.213 & 19.517 & 26.609 & 24.751 & 27.335 & 26.307 & 16.039 \\
\hline 8/31/11 16:00 & 35.179 & 19.529 & 26.553 & 24.718 & 27.326 & 26.286 & 16.002 \\
\hline 8/31/11 20:00 & 35.151 & 19.543 & 26.499 & 24.679 & 27.318 & 26.262 & 15.958 \\
\hline 9/1/11 0:00 & 35.124 & 19.573 & 26.451 & 24.644 & 27.309 & 26.241 & 15.923 \\
\hline 9/1/11 4:00 & 35.096 & 19.597 & 26.399 & 24.607 & 27.300 & 26.220 & 15.888 \\
\hline 9/1/11 8:00 & 35.071 & 19.634 & 26.353 & 24.574 & 27.293 & 26.199 & 15.853 \\
\hline 9/1/11 12:00 & 35.041 & 19.666 & 26.302 & 24.539 & 27.283 & 26.176 & 15.813 \\
\hline 9/1/11 16:00 & 35.009 & 19.659 & 26.246 & 24.502 & 27.274 & 26.155 & 15.773 \\
\hline 9/1/11 20:00 & 34.977 & 19.669 & 26.195 & 24.469 & 27.267 & 26.132 & 15.736 \\
\hline 9/2/11 0:00 & 34.954 & 19.715 & 26.150 & 24.437 & 27.258 & 26.111 & 15.703 \\
\hline 9/2/11 4:00 & 34.926 & 19.753 & 26.102 & 24.402 & 27.248 & 26.092 & 15.666 \\
\hline 9/2/11 8:00 & 34.904 & 19.801 & 26.057 & 24.374 & 27.239 & 26.073 & 15.636 \\
\hline 9/2/11 12:00 & 34.878 & 19.862 & 26.009 & 24.343 & 27.232 & 26.052 & 15.601 \\
\hline 9/2/11 16:00 & 34.844 & 19.876 & 25.957 & 24.311 & 27.223 & 26.029 & 15.559 \\
\hline 9/2/11 20:00 & 34.814 & 19.878 & 25.906 & 24.276 & 27.209 & 26.008 & 15.524 \\
\hline 9/3/11 0:00 & 34.787 & 19.911 & 25.860 & 24.243 & 27.202 & 25.984 & 15.488 \\
\hline
\end{tabular}

TABLE S1.2 Page 47 of 58 


\begin{tabular}{|c|c|c|c|c|c|c|c|}
\hline \multirow[b]{2}{*}{ Date and Time } & \multicolumn{7}{|c|}{ Water Level (ft below reference point) } \\
\hline & SB43S & SB44S & SB45S & SB46S & SB47S & SB48S & SB49S \\
\hline 9/3/11 4:00 & 34.759 & 19.944 & 25.813 & 24.213 & 27.192 & 25.963 & 15.456 \\
\hline 9/3/11 8:00 & 34.732 & 19.969 & 25.764 & 24.180 & 27.178 & 25.945 & 15.416 \\
\hline 9/3/11 12:00 & 34.709 & 20.002 & 25.722 & 24.152 & 27.169 & 25.924 & 15.386 \\
\hline 9/3/11 16:00 & 34.679 & 20.048 & 25.673 & 24.120 & 27.155 & 25.900 & 15.351 \\
\hline 9/3/11 20:00 & 34.661 & 20.088 & 25.634 & 24.092 & 27.146 & 25.882 & 15.318 \\
\hline 9/4/11 0:00 & 34.640 & 20.128 & 25.594 & 24.066 & 27.134 & 25.863 & 15.290 \\
\hline 9/4/11 4:00 & 34.617 & 20.132 & 25.552 & 24.041 & 27.125 & 25.842 & 15.264 \\
\hline 9/4/11 8:00 & 34.599 & 20.162 & 25.515 & 24.015 & 27.111 & 25.819 & 15.234 \\
\hline 9/4/11 12:00 & 34.574 & 20.193 & 25.473 & 23.985 & 27.101 & 25.798 & 15.199 \\
\hline 9/4/11 16:00 & 34.541 & 20.181 & 25.420 & 23.954 & 27.090 & 25.779 & 15.164 \\
\hline 9/4/11 20:00 & 34.514 & 20.183 & 25.378 & 23.926 & 27.083 & 25.758 & 15.134 \\
\hline 9/5/11 0:00 & 34.493 & 20.223 & 25.341 & 23.899 & 27.073 & 25.737 & 15.103 \\
\hline 9/5/11 4:00 & 34.466 & 20.246 & 25.294 & 23.868 & 27.064 & 25.711 & 15.068 \\
\hline 9/5/11 8:00 & 34.443 & 20.274 & 25.252 & 23.845 & 27.059 & 25.693 & 15.038 \\
\hline 9/5/11 12:00 & 34.415 & 20.312 & 25.208 & 23.817 & 27.052 & 25.669 & 15.003 \\
\hline 9/5/11 16:00 & 34.379 & 20.298 & 25.157 & 23.789 & 27.045 & 25.646 & 14.963 \\
\hline 9/5/11 20:00 & 34.351 & 20.286 & 25.115 & 23.759 & 27.033 & 25.627 & 14.928 \\
\hline 9/6/11 0:00 & 34.328 & 20.316 & 25.075 & 23.733 & 27.024 & 25.604 & 14.898 \\
\hline 9/6/11 4:00 & 34.303 & 20.356 & 25.034 & 23.703 & 27.017 & 25.581 & 14.868 \\
\hline 9/6/11 8:00 & 34.282 & 20.400 & 24.994 & 23.682 & 27.010 & 25.560 & 14.840 \\
\hline 9/6/11 12:00 & 34.257 & 20.444 & 24.952 & 23.656 & 27.001 & 25.539 & 14.807 \\
\hline 9/6/11 16:00 & 34.223 & 20.447 & 24.906 & 23.631 & 26.991 & 25.515 & 14.772 \\
\hline 9/6/11 20:00 & 34.200 & 20.456 & 24.866 & 23.603 & 26.982 & 25.494 & 14.735 \\
\hline 9/7/11 0:00 & 34.177 & 20.512 & 24.827 & 23.579 & 26.977 & 25.471 & 14.711 \\
\hline 9/7/11 4:00 & 34.154 & 20.549 & 24.787 & 23.556 & 26.968 & 25.455 & 14.679 \\
\hline 9/7/11 8:00 & 34.134 & 20.607 & 24.752 & 23.531 & 26.961 & 25.431 & 14.655 \\
\hline 9/7/11 12:00 & 34.108 & 20.656 & 24.713 & 23.512 & 26.952 & 25.410 & 14.625 \\
\hline 9/7/11 16:00 & 34.079 & 20.663 & 24.668 & 23.484 & 26.942 & 25.389 & 14.590 \\
\hline 9/7/11 20:00 & 34.051 & 20.663 & 24.629 & 23.461 & 26.935 & 25.361 & 14.557 \\
\hline 9/8/11 0:00 & 34.030 & 20.710 & 24.592 & 23.435 & 26.928 & 25.326 & 14.532 \\
\hline 9/8/11 4:00 & 34.003 & 20.735 & 24.552 & 23.412 & 26.921 & 25.291 & 14.494 \\
\hline 9/8/11 8:00 & 33.980 & 20.761 & 24.515 & 23.391 & 26.912 & 25.256 & 14.464 \\
\hline 9/8/11 12:00 & 33.955 & 20.799 & 24.475 & 23.370 & 26.905 & 25.212 & 14.438 \\
\hline 9/8/11 16:00 & 33.920 & 20.768 & 24.429 & 23.344 & 26.893 & 25.170 & 14.399 \\
\hline 9/8/11 20:00 & 33.891 & 20.742 & 24.387 & 23.319 & 26.886 & 25.135 & 14.366 \\
\hline 9/9/11 0:00 & 33.865 & 20.749 & 24.347 & 23.293 & 26.877 & 25.095 & 14.331 \\
\hline 9/9/11 4:00 & 33.838 & 20.749 & 24.305 & 23.267 & 26.868 & 25.055 & 14.301 \\
\hline 9/9/11 8:00 & 33.813 & 20.766 & 24.266 & 23.249 & 26.861 & 25.023 & 14.268 \\
\hline 9/9/11 12:00 & 33.785 & 20.784 & 24.229 & 23.225 & 26.851 & 24.978 & 14.237 \\
\hline 9/9/11 16:00 & 33.749 & 20.754 & 24.182 & 23.200 & 26.840 & 24.939 & 14.200 \\
\hline 9/9/11 20:00 & 33.728 & 20.754 & 24.145 & 23.176 & 26.830 & 24.908 & 14.172 \\
\hline 9/10/11 0:00 & 33.705 & 20.805 & 24.112 & 23.158 & 26.823 & 24.876 & 14.144 \\
\hline 9/10/11 4:00 & 33.682 & 20.838 & 24.077 & 23.132 & 26.818 & 24.843 & 14.116 \\
\hline 9/10/11 8:00 & 33.661 & 20.884 & 24.045 & 23.116 & 26.809 & 24.817 & 14.093 \\
\hline 9/10/11 12:00 & 33.639 & 20.943 & 24.010 & 23.100 & 26.802 & 24.778 & 14.062 \\
\hline 9/10/11 16:00 & 33.611 & 20.957 & 23.973 & 23.076 & 26.795 & 24.743 & 14.032 \\
\hline 9/10/11 20:00 & 33.588 & 20.975 & 23.938 & 23.055 & 26.786 & 24.714 & 14.004 \\
\hline 9/11/11 0:00 & 33.567 & 21.027 & 23.907 & 23.037 & 26.781 & 24.679 & 13.978 \\
\hline 9/11/11 4:00 & 33.545 & 21.054 & 23.873 & 23.018 & 26.769 & 24.651 & 13.955 \\
\hline 9/11/11 8:00 & 33.524 & 21.103 & 23.840 & 22.999 & 26.765 & 24.621 & 13.932 \\
\hline 9/11/11 12:00 & 33.501 & 21.155 & 23.810 & 22.983 & 26.758 & 24.586 & 13.904 \\
\hline 9/11/11 16:00 & 33.471 & 21.157 & 23.770 & 22.965 & 26.748 & 24.544 & 13.873 \\
\hline 9/11/11 20:00 & 33.446 & 21.159 & 23.735 & 22.946 & 26.739 & 24.516 & 13.841 \\
\hline 9/12/11 0:00 & 33.425 & 21.194 & 23.703 & 22.925 & 26.730 & 24.481 & 13.815 \\
\hline
\end{tabular}

TABLE S1.2 Page 48 of 58 


\begin{tabular}{|c|c|c|c|c|c|c|c|}
\hline \multirow[b]{2}{*}{ Date and Time } & \multicolumn{7}{|c|}{ Water Level (ft below reference point) } \\
\hline & SB43S & SB44S & SB45S & SB46S & SB47S & SB48S & SB49S \\
\hline 9/12/11 4:00 & 33.400 & 21.211 & 23.668 & 22.906 & 26.725 & 24.448 & 13.787 \\
\hline 9/12/11 8:00 & 33.377 & 21.232 & 23.635 & 22.888 & 26.718 & 24.413 & 13.761 \\
\hline 9/12/11 12:00 & 33.350 & 21.252 & 23.598 & 22.871 & 26.706 & 24.371 & 13.736 \\
\hline 9/12/11 16:00 & 33.318 & 21.220 & 23.561 & 22.848 & 26.697 & 24.332 & 13.696 \\
\hline 9/12/11 20:00 & 33.295 & 21.211 & 23.524 & 22.829 & 26.690 & 24.304 & 13.668 \\
\hline 9/13/11 0:00 & 33.272 & 21.262 & 23.493 & 22.813 & 26.681 & 24.266 & 13.645 \\
\hline 9/13/11 4:00 & 33.251 & 21.322 & 23.461 & 22.797 & 26.674 & 24.245 & 13.612 \\
\hline 9/13/11 8:00 & 33.235 & 21.427 & 23.440 & 22.781 & 26.667 & 24.220 & 13.598 \\
\hline 9/13/11 12:00 & 33.217 & 21.544 & 23.412 & 22.769 & 26.657 & 24.185 & 13.577 \\
\hline 9/13/11 16:00 & 33.189 & 21.602 & 23.379 & 22.755 & 26.650 & 24.154 & 13.549 \\
\hline 9/13/11 20:00 & 33.166 & 21.651 & 23.349 & 22.739 & 26.643 & 24.124 & 13.523 \\
\hline 9/14/11 0:00 & 33.146 & 21.711 & 23.319 & 22.725 & 26.636 & 24.091 & 13.498 \\
\hline 9/14/11 4:00 & 33.127 & 21.756 & 23.291 & 22.708 & 26.629 & 24.068 & 13.474 \\
\hline 9/14/11 8:00 & 33.109 & 21.804 & 23.270 & 22.701 & 26.622 & 24.047 & 13.455 \\
\hline 9/14/11 12:00 & 33.093 & 21.870 & 23.244 & 22.687 & 26.613 & 24.016 & 13.437 \\
\hline 9/14/11 16:00 & 33.072 & 21.907 & 23.214 & 22.680 & 26.608 & 23.988 & 13.418 \\
\hline 9/14/11 20:00 & 33.054 & 21.937 & 23.191 & 22.664 & 26.599 & 23.967 & 13.395 \\
\hline 9/15/11 0:00 & 33.038 & 22.005 & 23.170 & 22.657 & 26.594 & 23.939 & 13.374 \\
\hline 9/15/11 4:00 & 33.017 & 22.035 & 23.144 & 22.643 & 26.585 & 23.911 & 13.355 \\
\hline 9/15/11 8:00 & 33.001 & 22.070 & 23.119 & 22.636 & 26.578 & 23.888 & 13.334 \\
\hline 9/15/11 12:00 & 32.981 & 22.110 & 23.093 & 22.625 & 26.571 & 23.851 & 13.311 \\
\hline 9/15/11 16:00 & 32.951 & 22.091 & 23.058 & 22.608 & 26.561 & 23.813 & 13.288 \\
\hline 9/15/11 20:00 & 32.924 & 22.058 & 23.026 & 22.594 & 26.552 & 23.781 & 13.252 \\
\hline 9/16/11 0:00 & 32.901 & 22.051 & 22.998 & 22.583 & 26.545 & 23.748 & 13.227 \\
\hline 9/16/11 4:00 & 32.875 & 22.049 & 22.970 & 22.571 & 26.533 & 23.715 & 13.199 \\
\hline 9/16/11 8:00 & 32.852 & 22.063 & 22.944 & 22.555 & 26.527 & 23.687 & 13.175 \\
\hline 9/16/11 12:00 & 32.830 & 22.075 & 22.914 & 22.545 & 26.520 & 23.650 & 13.150 \\
\hline 9/16/11 16:00 & 32.804 & 22.047 & 22.884 & 22.527 & 26.510 & 23.615 & 13.122 \\
\hline 9/16/11 20:00 & 32.779 & 22.019 & 22.853 & 22.515 & 26.503 & 23.587 & 13.098 \\
\hline 9/17/11 0:00 & 32.759 & 22.035 & 22.828 & 22.506 & 26.494 & 23.557 & 13.075 \\
\hline 9/17/11 4:00 & 32.736 & 22.028 & 22.800 & 22.492 & 26.484 & 23.524 & 13.047 \\
\hline 9/17/11 8:00 & 32.710 & 22.030 & 22.774 & 22.478 & 26.477 & 23.491 & 13.021 \\
\hline 9/17/11 12:00 & 32.690 & 22.042 & 22.749 & 22.468 & 26.468 & 23.456 & 13.000 \\
\hline 9/17/11 16:00 & 32.662 & 22.023 & 22.716 & 22.452 & 26.459 & 23.421 & 12.965 \\
\hline 9/17/11 20:00 & 32.639 & 22.009 & 22.688 & 22.436 & 26.449 & 23.391 & 12.942 \\
\hline 9/18/11 0:00 & 32.616 & 22.021 & 22.665 & 22.424 & 26.445 & 23.358 & 12.921 \\
\hline 9/18/11 4:00 & 32.594 & 22.023 & 22.637 & 22.413 & 26.433 & 23.328 & 12.893 \\
\hline 9/18/11 8:00 & 32.573 & 22.040 & 22.614 & 22.403 & 26.426 & 23.300 & 12.877 \\
\hline 9/18/11 12:00 & 32.552 & 22.058 & 22.586 & 22.392 & 26.419 & 23.269 & 12.853 \\
\hline 9/18/11 16:00 & 32.527 & 22.063 & 22.560 & 22.382 & 26.410 & 23.237 & 12.825 \\
\hline 9/18/11 20:00 & 32.509 & 22.075 & 22.539 & 22.368 & 26.403 & 23.216 & 12.804 \\
\hline 9/19/11 0:00 & 32.493 & 22.091 & 22.521 & 22.359 & 26.393 & 23.188 & 12.785 \\
\hline 9/19/11 4:00 & 32.474 & 22.075 & 22.495 & 22.350 & 26.386 & 23.164 & 12.765 \\
\hline 9/19/11 8:00 & 32.458 & 22.077 & 22.476 & 22.340 & 26.382 & 23.139 & 12.751 \\
\hline 9/19/11 12:00 & 32.440 & 22.103 & 22.453 & 22.331 & 26.372 & 23.104 & 12.732 \\
\hline 9/19/11 16:00 & 32.412 & 22.082 & 22.425 & 22.317 & 26.363 & 23.071 & 12.701 \\
\hline 9/19/11 20:00 & 32.387 & 22.063 & 22.400 & 22.303 & 26.354 & 23.043 & 12.678 \\
\hline 9/20/11 0:00 & 32.369 & 22.082 & 22.376 & 22.291 & 26.347 & 23.010 & 12.655 \\
\hline 9/20/11 4:00 & 32.346 & 22.089 & 22.351 & 22.280 & 26.337 & 22.978 & 12.634 \\
\hline 9/20/11 8:00 & 32.325 & 22.098 & 22.328 & 22.268 & 26.330 & 22.954 & 12.606 \\
\hline 9/20/11 12:00 & 32.305 & 22.142 & 22.304 & 22.259 & 26.323 & 22.919 & 12.587 \\
\hline 9/20/11 16:00 & 32.280 & 22.121 & 22.279 & 22.247 & 26.312 & 22.891 & 12.562 \\
\hline 9/20/11 20:00 & 32.264 & 22.151 & 22.260 & 22.240 & 26.305 & 22.868 & 12.543 \\
\hline 9/21/11 0:00 & 32.252 & 22.221 & 22.246 & 22.233 & 26.300 & 22.852 & 12.531 \\
\hline
\end{tabular}

TABLE S1.2 Page 49 of 58 


\begin{tabular}{|c|c|c|c|c|c|c|c|}
\hline \multirow[b]{2}{*}{ Date and Time } & \multicolumn{7}{|c|}{ Water Level (ft below reference point) } \\
\hline & SB43S & SB44S & SB45S & SB46S & SB47S & SB48S & SB49S \\
\hline 9/21/11 4:00 & 32.234 & 22.280 & 22.227 & 22.226 & 26.288 & 22.821 & 12.510 \\
\hline 9/21/11 8:00 & 32.218 & 22.312 & 22.207 & 22.217 & 26.286 & 22.800 & 12.492 \\
\hline 9/21/11 12:00 & 32.199 & 22.363 & 22.188 & 22.210 & 26.276 & 22.770 & 12.473 \\
\hline 9/21/11 16:00 & 32.179 & 22.377 & 22.165 & 22.203 & 26.272 & 22.740 & 12.450 \\
\hline 9/21/11 20:00 & 32.163 & 22.403 & 22.144 & 22.191 & 26.262 & 22.716 & 12.433 \\
\hline 9/22/11 0:00 & 32.144 & 22.450 & 22.127 & 22.184 & 26.255 & 22.690 & 12.417 \\
\hline 9/22/11 4:00 & 32.124 & 22.471 & 22.104 & 22.177 & 26.246 & 22.662 & 12.394 \\
\hline 9/22/11 8:00 & 32.110 & 22.505 & 22.090 & 22.170 & 26.237 & 22.641 & 12.379 \\
\hline 9/22/11 12:00 & 32.092 & 22.540 & 22.069 & 22.163 & 26.230 & 22.604 & 12.356 \\
\hline 9/22/11 16:00 & 32.064 & 22.515 & 22.041 & 22.154 & 26.220 & 22.574 & 12.328 \\
\hline 9/22/11 20:00 & 32.053 & 22.515 & 22.023 & 22.145 & 26.213 & 22.550 & 12.312 \\
\hline 9/23/11 0:00 & 32.037 & 22.561 & 22.004 & 22.138 & 26.204 & 22.522 & 12.293 \\
\hline 9/23/11 4:00 & 32.016 & 22.575 & 21.983 & 22.131 & 26.199 & 22.494 & 12.274 \\
\hline 9/23/11 8:00 & 32.000 & 22.594 & 21.965 & 22.124 & 26.190 & 22.471 & 12.253 \\
\hline 9/23/11 12:00 & 31.970 & 22.617 & 21.946 & 22.119 & 26.181 & & 12.232 \\
\hline 9/23/11 16:00 & 31.943 & 22.594 & 21.918 & Removed $^{c}$ & 26.171 & 22.431 & 12.202 \\
\hline 9/23/11 20:00 & 31.922 & 22.590 & 21.899 & & 26.160 & 22.403 & 12.181 \\
\hline 9/24/11 0:00 & 31.904 & 22.613 & 21.878 & & 26.150 & 22.377 & 12.165 \\
\hline 9/24/11 4:00 & 31.883 & 22.622 & 21.858 & & 26.146 & 22.347 & 12.141 \\
\hline 9/24/11 8:00 & 31.867 & 22.638 & 21.841 & & 26.136 & 22.321 & 12.123 \\
\hline 9/24/11 12:00 & 31.844 & 22.661 & 21.818 & & 26.129 & 22.291 & 12.102 \\
\hline 9/24/11 16:00 & 31.819 & 22.641 & 21.797 & & 26.118 & 22.258 & 12.074 \\
\hline 9/24/11 20:00 & 31.805 & 22.671 & 21.781 & & 26.111 & 22.237 & 12.060 \\
\hline 9/25/11 0:00 & 31.789 & 22.710 & 21.764 & & 26.104 & 22.209 & 12.039 \\
\hline 9/25/11 4:00 & 31.769 & 22.727 & 21.746 & & 26.097 & 22.181 & 12.020 \\
\hline 9/25/11 8:00 & 31.752 & 22.759 & 21.730 & & 26.087 & 22.158 & 12.004 \\
\hline 9/25/11 12:00 & 31.734 & 22.790 & 21.711 & & 26.083 & 22.130 & 11.985 \\
\hline 9/25/11 16:00 & 31.709 & 22.771 & 21.690 & & 26.071 & 22.095 & 11.959 \\
\hline 9/25/11 20:00 & 31.693 & 22.792 & 21.674 & & 26.066 & 22.074 & 11.948 \\
\hline 9/26/11 0:00 & 31.675 & 22.829 & 21.655 & & 26.057 & 22.046 & 11.924 \\
\hline 9/26/11 4:00 & 31.656 & 22.841 & 21.636 & & 26.050 & 22.018 & 11.903 \\
\hline 9/26/11 8:00 & 31.642 & 22.885 & 21.625 & & 26.043 & 21.994 & 11.891 \\
\hline 9/26/11 12:00 & 31.626 & 22.932 & 21.609 & & 26.036 & 21.973 & 11.875 \\
\hline 9/26/11 16:00 & 31.601 & 22.918 & 21.585 & & 26.029 & 21.938 & 11.854 \\
\hline 9/26/11 20:00 & 31.583 & 22.922 & 21.571 & & 26.020 & 21.913 & 11.831 \\
\hline 9/27/11 0:00 & 31.569 & 22.967 & 21.555 & & 26.013 & 21.892 & 11.817 \\
\hline 9/27/11 4:00 & 31.553 & 22.997 & 21.541 & & 26.003 & 21.866 & 11.798 \\
\hline 9/27/11 8:00 & 31.544 & 23.060 & 21.532 & & 25.999 & 21.847 & 11.789 \\
\hline 9/27/11 12:00 & 31.528 & 23.104 & 21.515 & & 25.994 & 21.824 & 11.773 \\
\hline 9/27/11 16:00 & 31.507 & 23.113 & 21.497 & & 25.985 & 21.796 & 11.754 \\
\hline 9/27/11 20:00 & 31.489 & 23.127 & 21.485 & & 25.977 & 21.770 & 11.735 \\
\hline 9/28/11 0:00 & 31.473 & 23.158 & 21.469 & & 25.966 & 21.747 & 11.719 \\
\hline 9/28/11 4:00 & 31.455 & 23.169 & 21.453 & & 25.959 & 21.721 & 11.698 \\
\hline 9/28/11 8:00 & 31.439 & 23.195 & 21.439 & & 25.952 & 21.698 & 11.681 \\
\hline 9/28/11 12:00 & 31.422 & 23.213 & 21.422 & & 25.945 & 21.672 & 11.667 \\
\hline 9/28/11 16:00 & 31.402 & 23.211 & 21.406 & & 25.935 & 21.642 & 11.646 \\
\hline 9/28/11 20:00 & 31.386 & 23.227 & 21.392 & & 25.928 & 21.616 & 11.630 \\
\hline 9/29/11 0:00 & 31.372 & 23.260 & 21.381 & & 25.921 & 21.598 & 11.614 \\
\hline 9/29/11 4:00 & 31.354 & 23.281 & 21.367 & & 25.917 & 21.572 & 11.600 \\
\hline 9/29/11 8:00 & 31.349 & 23.323 & 21.362 & & 25.910 & 21.558 & 11.588 \\
\hline 9/29/11 12:00 & 31.340 & 23.400 & 21.355 & & 25.905 & 21.544 & 11.586 \\
\hline 9/29/11 16:00 & 31.324 & 23.442 & 21.343 & & 25.896 & 21.523 & 11.567 \\
\hline 9/29/11 20:00 & 31.312 & 23.465 & 21.334 & & 25.889 & 21.502 & 11.555 \\
\hline 9/30/11 0:00 & 31.301 & 23.519 & 21.325 & & 25.886 & 21.481 & 11.546 \\
\hline
\end{tabular}

TABLE S1.2 Page 50 of 58 


\begin{tabular}{|c|c|c|c|c|c|c|c|}
\hline \multirow[b]{2}{*}{ Date and Time } & \multicolumn{7}{|c|}{ Water Level (ft below reference point) } \\
\hline & SB43S & SB44S & SB45S & SB46S & SB47S & SB48S & SB49S \\
\hline 9/30/11 4:00 & 31.290 & 23.558 & 21.250 & & 25.879 & 21.462 & 11.535 \\
\hline 9/30/11 8:00 & 31.278 & 23.593 & 21.148 & & 25.872 & 21.444 & 11.523 \\
\hline 9/30/11 12:00 & 31.262 & 23.623 & 21.432 & & 25.863 & 21.420 & 11.504 \\
\hline 9/30/11 16:00 & 31.241 & 23.607 & 21.276 & & 25.856 & 21.392 & 11.488 \\
\hline 9/30/11 20:00 & 31.223 & 23.600 & 21.262 & & 25.847 & 21.366 & 11.469 \\
\hline 10/1/11 0:00 & 31.212 & 23.635 & 21.252 & & 25.844 & 21.345 & 11.455 \\
\hline 10/1/11 4:00 & 31.200 & 23.670 & 21.173 & & 25.833 & 21.324 & 11.439 \\
\hline 10/1/11 8:00 & 31.191 & 23.707 & 21.059 & & 25.828 & 21.306 & 11.432 \\
\hline 10/1/11 12:00 & 31.177 & 23.744 & 21.339 & & 25.823 & 21.287 & 11.420 \\
\hline 10/1/11 16:00 & 31.157 & 23.733 & 21.208 & & 25.814 & 21.259 & 11.399 \\
\hline 10/1/11 20:00 & 31.141 & 23.731 & 21.197 & & 25.805 & 21.231 & 11.383 \\
\hline 10/2/11 0:00 & 31.125 & 23.754 & 21.185 & & 25.798 & 21.212 & 11.366 \\
\hline 10/2/11 4:00 & 31.111 & 23.775 & 21.101 & & 25.791 & 21.189 & 11.350 \\
\hline 10/2/11 8:00 & 31.099 & 23.796 & 21.032 & & 25.784 & 21.170 & 11.338 \\
\hline 10/2/11 12:00 & 31.086 & 23.821 & 21.320 & & 25.777 & 21.145 & 11.324 \\
\hline 10/2/11 16:00 & 31.060 & 23.798 & 21.136 & & 25.767 & 21.114 & 11.303 \\
\hline 10/2/11 20:00 & 31.044 & 23.796 & 21.127 & & 25.760 & 21.091 & 11.285 \\
\hline 10/3/11 0:00 & 31.033 & 23.828 & 21.115 & & 25.753 & 21.072 & 11.271 \\
\hline 10/3/11 4:00 & 31.019 & 23.849 & 21.083 & & 25.746 & 21.051 & 11.259 \\
\hline 10/3/11 8:00 & 31.008 & 23.873 & 21.015 & & 25.737 & 21.030 & 11.247 \\
\hline 10/3/11 12:00 & 30.992 & 23.898 & 21.085 & & 25.732 & 21.007 & 11.240 \\
\hline 10/3/11 16:00 & 30.971 & 23.884 & 21.071 & & 25.723 & 20.979 & 11.212 \\
\hline 10/3/11 20:00 & 30.957 & 23.894 & 21.059 & & 25.716 & 20.958 & 11.200 \\
\hline 10/4/11 0:00 & 30.944 & 23.924 & 21.052 & & 25.709 & 20.937 & 11.184 \\
\hline 10/4/11 4:00 & 30.932 & 23.942 & 21.043 & & 25.704 & 20.916 & 11.173 \\
\hline 10/4/11 8:00 & 30.918 & 23.963 & 21.029 & & 25.695 & 20.895 & 11.161 \\
\hline 10/4/11 12:00 & 30.902 & 23.980 & 21.022 & & 25.688 & 20.874 & 11.147 \\
\hline 10/4/11 16:00 & 30.884 & 23.968 & 21.008 & & 25.681 & 20.846 & 11.126 \\
\hline 10/4/11 20:00 & 30.870 & 23.977 & 20.999 & & 25.671 & 20.823 & 11.114 \\
\hline 10/5/11 0:00 & 30.856 & 24.008 & 20.992 & & 25.664 & 20.802 & 11.102 \\
\hline 10/5/11 4:00 & 30.843 & 24.026 & 20.983 & & 25.657 & 20.783 & 11.086 \\
\hline 10/5/11 8:00 & 30.831 & 24.050 & 20.931 & & 25.650 & 20.764 & 11.072 \\
\hline 10/5/11 12:00 & 30.815 & 24.066 & 20.964 & & 25.643 & 20.741 & 11.061 \\
\hline 10/5/11 16:00 & 30.797 & 24.052 & 20.950 & & 25.636 & 20.713 & 11.040 \\
\hline 10/5/11 20:00 & 30.781 & 24.054 & 20.941 & & 25.627 & 20.689 & 11.026 \\
\hline 10/6/11 0:00 & 30.772 & 24.085 & 20.931 & & 25.620 & 20.673 & 11.011 \\
\hline 10/6/11 4:00 & 30.756 & 24.098 & 20.915 & & 25.613 & 20.650 & 11.000 \\
\hline 10/6/11 8:00 & 30.744 & 24.117 & 20.836 & & 25.606 & 20.631 & 10.990 \\
\hline 10/6/11 12:00 & 30.728 & 24.136 & 21.064 & & 25.599 & 20.605 & 10.977 \\
\hline 10/6/11 16:00 & 30.707 & 24.122 & 20.889 & & 25.587 & 20.580 & 10.953 \\
\hline 10/6/11 20:00 & 30.696 & 24.126 & 20.883 & & 25.580 & 20.556 & 10.939 \\
\hline 10/7/11 0:00 & 30.682 & 24.159 & 20.873 & & 25.576 & 20.538 & 10.927 \\
\hline 10/7/11 4:00 & 30.671 & 24.182 & 20.869 & & 25.569 & 20.519 & 10.921 \\
\hline 10/7/11 8:00 & 30.662 & 24.210 & 20.864 & & 25.564 & 20.505 & 10.909 \\
\hline 10/7/11 12:00 & 30.648 & 24.236 & 20.855 & & 25.555 & 20.484 & 10.895 \\
\hline 10/7/11 16:00 & 30.632 & 24.241 & 20.845 & & 25.545 & 20.461 & 10.879 \\
\hline 10/7/11 20:00 & 30.618 & 24.255 & 20.838 & & 25.538 & 20.440 & 10.869 \\
\hline 10/8/11 0:00 & 30.611 & 24.287 & 20.834 & & 25.536 & 20.426 & 10.860 \\
\hline 10/8/11 4:00 & 30.597 & 24.308 & 20.827 & & 25.527 & 20.405 & 10.851 \\
\hline 10/8/11 8:00 & 30.588 & 24.334 & 20.822 & & 25.520 & 20.391 & 10.841 \\
\hline 10/8/11 12:00 & 30.577 & 24.364 & 20.820 & & 25.513 & 20.374 & 10.829 \\
\hline 10/8/11 16:00 & 30.561 & 24.357 & 20.806 & & 25.503 & 20.346 & 10.813 \\
\hline 10/8/11 20:00 & 30.552 & 24.380 & 20.801 & & 25.499 & 20.330 & 10.804 \\
\hline 10/9/11 0:00 & 30.542 & 24.415 & 20.797 & & 25.492 & 20.314 & 10.795 \\
\hline
\end{tabular}

TABLE S1.2 Page 51 of 58 


\begin{tabular}{|c|c|c|c|c|c|c|c|}
\hline \multirow[b]{2}{*}{ Date and Time } & \multicolumn{7}{|c|}{ Water Level (ft below reference point) } \\
\hline & SB43S & SB44S & SB45S & SB46S & SB47S & SB48S & SB49S \\
\hline 10/9/11 4:00 & 30.529 & 24.436 & 20.792 & & 25.487 & 20.295 & 10.783 \\
\hline 10/9/11 8:00 & 30.522 & 24.459 & 20.787 & & 25.480 & 20.276 & 10.778 \\
\hline 10/9/11 12:00 & 30.506 & 24.476 & 20.778 & & 25.471 & 20.258 & 10.767 \\
\hline 10/9/11 16:00 & 30.487 & 24.457 & 20.764 & & 25.464 & 20.230 & 10.745 \\
\hline 10/9/11 20:00 & 30.476 & 24.473 & 20.759 & & 25.454 & 20.209 & 10.734 \\
\hline 10/10/11 0:00 & 30.465 & 24.499 & 20.752 & & 25.450 & 20.192 & 10.722 \\
\hline 10/10/11 4:00 & 30.451 & 24.506 & 20.745 & & 25.442 & 20.171 & 10.706 \\
\hline 10/10/11 8:00 & 30.435 & 24.520 & 20.741 & & 25.438 & 20.150 & 10.694 \\
\hline 10/10/11 12:00 & 30.428 & 24.539 & 20.731 & & 25.428 & 20.134 & 10.685 \\
\hline 10/10/11 16:00 & 30.407 & 24.534 & 20.722 & & 25.419 & 20.106 & 10.671 \\
\hline 10/10/11 20:00 & 30.396 & 24.543 & 20.715 & & 25.412 & 20.087 & 10.657 \\
\hline 10/11/11 0:00 & 30.384 & 24.562 & 20.710 & & 25.405 & 20.066 & 10.650 \\
\hline 10/11/11 4:00 & 30.371 & 24.569 & 20.701 & & 25.398 & 20.045 & 10.629 \\
\hline 10/11/11 8:00 & 30.357 & 24.581 & 20.696 & & 25.391 & 20.027 & 10.622 \\
\hline 10/11/11 12:00 & 30.343 & 24.592 & 20.687 & & 25.382 & 20.008 & 10.608 \\
\hline 10/11/11 16:00 & 30.322 & 24.578 & 20.675 & & 25.372 & 19.980 & 10.594 \\
\hline 10/11/11 20:00 & 30.311 & 24.581 & 20.666 & & 25.368 & 19.956 & 10.577 \\
\hline 10/12/11 0:00 & 30.297 & 24.599 & 20.661 & & 25.356 & 19.938 & 10.568 \\
\hline 10/12/11 4:00 & 30.284 & 24.602 & 20.652 & & 25.349 & 19.917 & 10.549 \\
\hline 10/12/11 8:00 & 30.267 & 24.604 & 20.645 & & 25.342 & 19.893 & 10.540 \\
\hline 10/12/11 12:00 & 30.256 & 24.620 & 20.638 & & 25.333 & 19.875 & 10.528 \\
\hline 10/12/11 16:00 & 30.240 & 24.616 & 20.629 & & 25.326 & 19.849 & 10.512 \\
\hline 10/12/11 20:00 & 30.233 & 24.646 & 20.631 & & 25.323 & 19.837 & 10.510 \\
\hline 10/13/11 0:00 & 30.226 & 24.676 & 20.629 & & 25.316 & 19.823 & 10.505 \\
\hline 10/13/11 4:00 & 30.217 & 24.692 & 20.624 & & 25.307 & 19.805 & 10.493 \\
\hline 10/13/11 8:00 & 30.208 & 24.716 & 20.622 & & 25.302 & 19.791 & 10.486 \\
\hline 10/13/11 12:00 & 30.201 & 24.741 & 20.620 & & 25.295 & 19.774 & 10.475 \\
\hline 10/13/11 16:00 & 30.183 & 24.737 & 20.608 & & 25.286 & 19.751 & 10.463 \\
\hline 10/13/11 20:00 & 30.174 & 24.753 & 20.606 & & 25.279 & 19.735 & 10.454 \\
\hline 10/14/11 0:00 & 30.164 & 24.779 & 20.603 & & 25.274 & 19.721 & 10.447 \\
\hline 10/14/11 4:00 & 30.155 & 24.800 & 20.603 & & 25.267 & 19.704 & 10.440 \\
\hline 10/14/11 8:00 & 30.151 & 24.827 & 20.601 & & 25.263 & 19.693 & 10.437 \\
\hline 10/14/11 12:00 & 30.146 & 24.862 & 20.606 & & 25.256 & 19.681 & 10.430 \\
\hline 10/14/11 16:00 & 30.132 & 24.874 & 20.599 & & 25.251 & 19.662 & 10.419 \\
\hline 10/14/11 20:00 & 30.125 & 24.893 & 20.599 & & 25.242 & 19.646 & 10.414 \\
\hline 10/15/11 0:00 & 30.119 & 24.918 & 20.594 & & 25.239 & 19.632 & 10.407 \\
\hline 10/15/11 4:00 & 30.109 & 24.937 & 20.594 & & 25.230 & 19.616 & 10.400 \\
\hline 10/15/11 8:00 & 30.100 & 24.958 & 20.592 & & 25.223 & 19.602 & 10.391 \\
\hline $10 / 15 / 11$ 12:00 & 30.089 & 24.970 & 20.587 & & 25.216 & 19.583 & 10.384 \\
\hline 10/15/11 16:00 & 30.066 & 24.944 & 20.568 & & 25.207 & 19.553 & 10.363 \\
\hline 10/15/11 20:00 & 30.050 & 24.925 & 20.562 & & 25.197 & 19.529 & 10.346 \\
\hline 10/16/11 0:00 & 30.036 & 24.935 & 20.554 & & 25.188 & 19.506 & 10.332 \\
\hline 10/16/11 4:00 & 30.018 & 24.937 & 20.548 & & 25.179 & 19.487 & 10.323 \\
\hline 10/16/11 8:00 & 30.018 & 24.981 & 20.554 & & 25.176 & 19.478 & 10.321 \\
\hline $10 / 16 / 11$ 12:00 & 30.020 & 25.035 & 20.562 & & 25.174 & 19.473 & 10.321 \\
\hline 10/16/11 16:00 & 30.009 & 25.051 & 20.557 & & 25.167 & 19.455 & 10.311 \\
\hline 10/16/11 20:00 & 30.002 & 25.070 & 20.557 & & 25.160 & 19.441 & 10.304 \\
\hline 10/17/11 0:00 & 29.990 & 25.081 & 20.552 & & 25.150 & 19.422 & 10.295 \\
\hline 10/17/11 4:00 & 29.981 & 25.091 & 20.547 & & 25.146 & 19.401 & 10.286 \\
\hline 10/17/11 8:00 & 29.967 & 25.100 & 20.543 & & 25.136 & 19.385 & 10.274 \\
\hline 10/17/11 12:00 & 29.958 & 25.114 & 20.538 & & 25.129 & 19.368 & 10.267 \\
\hline 10/17/11 16:00 & 29.947 & 25.112 & 20.531 & & 25.120 & 19.347 & 10.258 \\
\hline 10/17/11 20:00 & 29.940 & 25.142 & 20.534 & & 25.115 & 19.335 & 10.251 \\
\hline 10/18/11 0:00 & 29.928 & 25.154 & 20.531 & & 25.106 & 19.319 & 10.244 \\
\hline
\end{tabular}

TABLE S1.2 Page 52 of 58 


\begin{tabular}{|c|c|c|c|c|c|c|c|}
\hline \multirow[b]{2}{*}{ Date and Time } & \multicolumn{7}{|c|}{ Water Level (ft below reference point) } \\
\hline & SB43S & SB44S & SB45S & SB46S & SB47S & SB48S & SB49S \\
\hline 10/18/11 4:00 & 29.915 & 25.158 & 20.524 & & 25.101 & 19.298 & 10.232 \\
\hline 10/18/11 8:00 & 29.905 & 25.174 & 20.524 & & 25.094 & 19.282 & 10.220 \\
\hline 10/18/11 12:00 & 29.901 & 25.195 & 20.524 & & 25.087 & 19.270 & 10.216 \\
\hline 10/18/11 16:00 & 29.889 & 25.207 & 20.522 & & 25.080 & 19.254 & 10.206 \\
\hline 10/18/11 20:00 & 29.882 & 25.221 & 20.520 & & 25.076 & 19.235 & 10.204 \\
\hline 10/19/11 0:00 & 29.871 & 25.235 & 20.506 & & 25.066 & 19.219 & 10.190 \\
\hline 10/19/11 4:00 & 29.860 & 25.244 & 20.429 & & 25.055 & 19.200 & 10.178 \\
\hline 10/19/11 8:00 & 29.853 & 25.258 & 20.375 & & 25.048 & 19.186 & 10.176 \\
\hline 10/19/11 12:00 & 29.846 & 25.277 & 20.564 & & 25.036 & 19.170 & 10.169 \\
\hline 10/19/11 16:00 & 29.832 & 25.284 & 20.506 & & 25.020 & 19.151 & 10.157 \\
\hline 10/19/11 20:00 & 29.823 & 25.298 & 20.503 & & 25.008 & 19.137 & 10.148 \\
\hline 10/20/11 0:00 & 29.811 & 25.310 & 20.499 & & 24.996 & 19.118 & 10.143 \\
\hline 10/20/11 4:00 & 29.802 & 25.319 & 20.473 & & 24.980 & 19.100 & 10.132 \\
\hline 10/20/11 8:00 & 29.795 & 25.333 & 20.412 & & 24.971 & 19.086 & 10.127 \\
\hline 10/20/11 12:00 & 29.791 & 25.354 & 20.594 & & 24.961 & 19.074 & 10.120 \\
\hline 10/20/11 16:00 & 29.777 & 25.356 & 20.489 & & 24.945 & 19.051 & 10.108 \\
\hline 10/20/11 20:00 & 29.770 & 25.375 & 20.492 & & 24.936 & 19.039 & 10.104 \\
\hline 10/21/11 0:00 & 29.766 & 25.398 & 20.494 & & 24.929 & 19.025 & 10.101 \\
\hline 10/21/11 4:00 & 29.756 & 25.414 & 20.494 & & 24.919 & 19.013 & 10.097 \\
\hline 10/21/11 8:00 & 29.752 & 25.431 & 20.494 & & 24.912 & 18.997 & 10.087 \\
\hline $10 / 21 / 11$ 12:00 & 29.740 & 25.445 & 20.489 & & 24.898 & 18.985 & 10.080 \\
\hline 10/21/11 16:00 & 29.727 & 25.433 & 20.485 & & 24.877 & 18.960 & 10.066 \\
\hline 10/21/11 20:00 & 29.717 & 25.452 & 20.480 & & 24.865 & 18.946 & 10.064 \\
\hline 10/22/11 0:00 & 29.708 & 25.470 & 20.482 & & 24.851 & 18.929 & 10.050 \\
\hline 10/22/11 4:00 & 29.699 & 25.489 & 20.480 & & 24.840 & 18.913 & 10.043 \\
\hline 10/22/11 8:00 & 29.695 & 25.515 & 20.482 & & 24.833 & 18.901 & 10.038 \\
\hline $10 / 22 / 11$ 12:00 & 29.685 & 25.542 & 20.480 & & 24.823 & 18.887 & 10.034 \\
\hline 10/22/11 16:00 & 29.672 & 25.549 & 20.473 & & 24.802 & 18.866 & 10.022 \\
\hline 10/22/11 20:00 & 29.660 & 25.561 & 20.471 & & 24.788 & 18.850 & 10.015 \\
\hline 10/23/11 0:00 & 29.651 & 25.575 & 20.468 & & 24.772 & 18.834 & 10.003 \\
\hline 10/23/11 4:00 & 29.640 & 25.584 & 20.464 & & 24.756 & 18.815 & 9.996 \\
\hline 10/23/11 8:00 & 29.633 & 25.596 & 20.461 & & 24.742 & 18.799 & 9.987 \\
\hline $10 / 23 / 11$ 12:00 & 29.621 & 25.610 & 20.461 & & 24.730 & 18.785 & 9.980 \\
\hline 10/23/11 16:00 & 29.607 & 25.608 & 20.454 & & 24.709 & 18.761 & 9.971 \\
\hline 10/23/11 20:00 & 29.601 & 25.622 & 20.452 & & 24.700 & 18.745 & 9.959 \\
\hline 10/24/11 0:00 & 29.601 & 25.654 & 20.461 & & 24.700 & 18.740 & 9.964 \\
\hline 10/24/11 4:00 & 29.594 & 25.673 & 20.461 & & 24.690 & 18.731 & 9.959 \\
\hline 10/24/11 8:00 & 29.589 & 25.696 & 20.466 & & 24.688 & 18.724 & 9.959 \\
\hline $10 / 24 / 11$ 12:00 & 29.582 & 25.708 & 20.464 & & 24.674 & 18.703 & 9.952 \\
\hline 10/24/11 16:00 & 29.562 & 25.692 & 20.454 & & 24.648 & 18.680 & 9.933 \\
\hline 10/24/11 20:00 & 29.550 & 25.696 & 20.450 & & 24.627 & 18.659 & 9.924 \\
\hline 10/25/11 0:00 & 29.539 & 25.717 & 20.447 & & 24.613 & 18.644 & 9.915 \\
\hline 10/25/11 4:00 & 29.525 & 25.729 & 20.441 & & 24.590 & 18.621 & 9.903 \\
\hline 10/25/11 8:00 & 29.511 & 25.740 & 20.433 & & 24.571 & 18.602 & 9.891 \\
\hline $10 / 25 / 11$ 12:00 & 29.500 & 25.764 & 20.429 & & 24.555 & 18.586 & 9.880 \\
\hline 10/25/11 16:00 & 29.488 & 25.785 & 20.429 & & 24.538 & 18.567 & 9.873 \\
\hline 10/25/11 20:00 & 29.493 & 25.834 & 20.436 & & 24.545 & 18.565 & 9.873 \\
\hline 10/26/11 0:00 & 29.491 & 25.876 & 20.443 & & 24.543 & 18.556 & 9.875 \\
\hline 10/26/11 4:00 & 29.488 & 25.908 & 20.443 & & 24.536 & 18.546 & 9.877 \\
\hline 10/26/11 8:00 & 29.486 & 25.934 & 20.354 & & 24.531 & 18.537 & 9.875 \\
\hline 10/26/11 12:00 & 29.479 & 25.952 & 20.461 & & 24.522 & 18.526 & 9.868 \\
\hline 10/26/11 16:00 & 29.468 & 25.952 & 20.447 & & 24.506 & 18.507 & 9.861 \\
\hline 10/26/11 20:00 & 29.463 & 25.973 & 20.450 & & 24.499 & 18.500 & 9.856 \\
\hline 10/27/11 0:00 & 29.456 & 25.985 & 20.450 & & 24.489 & 18.484 & 9.851 \\
\hline
\end{tabular}

TABLE S1.2 Page 53 of 58 


\begin{tabular}{|c|c|c|c|c|c|c|c|}
\hline \multirow[b]{2}{*}{ Date and Time } & \multicolumn{7}{|c|}{ Water Level (ft below reference point) } \\
\hline & SB43S & SB44S & SB45S & SB46S & SB47S & SB48S & SB49S \\
\hline 10/27/11 4:00 & 29.449 & 25.994 & 20.450 & & 24.478 & 18.469 & 9.847 \\
\hline 10/27/11 8:00 & 29.442 & 26.008 & 20.410 & & 24.471 & 18.460 & 9.842 \\
\hline $10 / 27 / 11$ 12:00 & 29.440 & 26.027 & 20.499 & & 24.466 & 18.451 & 9.842 \\
\hline 10/27/11 16:00 & 29.429 & 26.020 & 20.450 & & 24.447 & 18.432 & 9.831 \\
\hline 10/27/11 20:00 & 29.422 & 26.036 & 20.452 & & 24.438 & 18.421 & 9.826 \\
\hline 10/28/11 0:00 & 29.417 & 26.050 & 20.447 & & 24.429 & 18.409 & 9.826 \\
\hline 10/28/11 4:00 & 29.410 & 26.059 & 20.380 & & 24.422 & 18.395 & 9.817 \\
\hline 10/28/11 8:00 & 29.404 & 26.066 & 20.350 & & 24.410 & 18.383 & 9.812 \\
\hline 10/28/11 12:00 & 29.394 & 26.076 & 20.540 & & 24.398 & 18.367 & 9.805 \\
\hline 10/28/11 16:00 & 29.385 & 26.076 & 20.452 & & 24.380 & 18.351 & 9.798 \\
\hline 10/28/11 20:00 & 29.381 & 26.092 & 20.452 & & 24.375 & 18.339 & 9.796 \\
\hline 10/29/11 0:00 & 29.371 & 26.099 & 20.454 & & 24.361 & 18.325 & 9.789 \\
\hline 10/29/11 4:00 & 29.360 & 26.104 & 20.422 & & 24.347 & 18.308 & 9.782 \\
\hline 10/29/11 8:00 & 29.355 & 26.111 & 20.382 & & 24.335 & 18.294 & 9.775 \\
\hline 10/29/11 12:00 & 29.342 & 26.111 & 20.617 & & 24.312 & 18.276 & 9.763 \\
\hline 10/29/11 16:00 & 29.321 & 26.090 & 20.433 & & 24.284 & 18.250 & 9.746 \\
\hline 10/29/11 20:00 & 29.312 & 26.099 & 20.431 & & 24.272 & 18.236 & 9.742 \\
\hline 10/30/11 0:00 & 29.303 & 26.120 & 20.433 & & 24.260 & 18.217 & 9.732 \\
\hline 10/30/11 4:00 & 29.296 & 26.134 & 20.433 & & 24.246 & 18.203 & 9.730 \\
\hline 10/30/11 8:00 & 29.294 & 26.160 & 20.438 & & 24.242 & 18.199 & 9.728 \\
\hline $10 / 30 / 11$ 12:00 & 29.296 & 26.183 & 20.443 & & 24.237 & 18.192 & 9.730 \\
\hline 10/30/11 16:00 & 29.289 & 26.190 & 20.443 & & 24.228 & 18.178 & 9.721 \\
\hline 10/30/11 20:00 & 29.280 & 26.204 & 20.443 & & 24.216 & 18.166 & 9.716 \\
\hline 10/31/11 0:00 & 29.275 & 26.218 & 20.445 & & 24.209 & 18.154 & 9.709 \\
\hline 10/31/11 4:00 & 29.271 & 26.230 & 20.366 & & 24.200 & 18.143 & 9.711 \\
\hline 10/31/11 8:00 & 29.264 & 26.241 & 20.329 & & 24.190 & 18.131 & 9.704 \\
\hline $10 / 31 / 11$ 12:00 & 29.257 & 26.248 & 20.513 & & 24.176 & 18.117 & 9.702 \\
\hline 10/31/11 16:00 & 29.241 & 26.234 & 20.440 & & 24.153 & 18.094 & 9.688 \\
\hline 10/31/11 20:00 & 29.234 & 26.246 & 20.441 & & 24.139 & 18.082 & 9.681 \\
\hline 11/1/11 0:00 & 29.225 & 26.260 & 20.438 & & 24.127 & 18.068 & 9.669 \\
\hline 11/1/11 4:00 & 29.218 & 26.264 & 20.436 & & 24.113 & 18.052 & 9.667 \\
\hline 11/1/11 8:00 & 29.209 & 26.274 & 20.438 & & 24.099 & 18.038 & 9.662 \\
\hline $11 / 1 / 11$ 12:00 & 29.200 & 26.283 & 20.436 & & 24.087 & 18.024 & 9.660 \\
\hline $11 / 1 / 11$ 16:00 & 29.186 & 26.281 & 20.431 & & 24.071 & 18.005 & 9.646 \\
\hline $11 / 1 / 1120: 00$ & 29.186 & 26.299 & 20.436 & & 24.059 & 17.998 & 9.641 \\
\hline 11/2/11 0:00 & 29.179 & 26.313 & 20.438 & & 24.050 & 17.984 & 9.646 \\
\hline $11 / 2 / 114: 00$ & 29.177 & 26.330 & 20.438 & & 24.043 & 17.977 & 9.639 \\
\hline 11/2/11 8:00 & 29.174 & 26.348 & 20.445 & & 24.038 & 17.968 & 9.639 \\
\hline $11 / 2 / 11$ 12:00 & 29.167 & 26.358 & 20.445 & & 24.031 & 17.958 & 9.632 \\
\hline $11 / 2 / 11$ 16:00 & 29.163 & 26.374 & 20.447 & & 24.022 & 17.949 & 9.634 \\
\hline $11 / 2 / 1120: 00$ & 29.161 & 26.386 & 20.394 & & 24.015 & 17.940 & 9.634 \\
\hline 11/3/11 0:00 & 29.156 & 26.383 & 20.387 & & 24.006 & 17.926 & 9.630 \\
\hline $11 / 3 / 114: 00$ & 29.151 & 26.381 & 20.359 & & 23.999 & 17.914 & 9.625 \\
\hline $11 / 3 / 118: 00$ & 29.151 & 26.400 & 20.308 & & 23.994 & 17.907 & 9.623 \\
\hline $11 / 3 / 11$ 12:00 & 29.149 & 26.416 & 20.401 & & 23.992 & 17.902 & 9.620 \\
\hline 11/3/11 16:00 & 29.142 & 26.416 & 20.543 & & 23.978 & 17.886 & 9.618 \\
\hline $11 / 3 / 1120: 00$ & 29.138 & 26.427 & 20.389 & & 23.971 & 17.877 & 9.616 \\
\hline 11/4/11 0:00 & 29.135 & 26.439 & 20.317 & & 23.964 & 17.865 & 9.613 \\
\hline $11 / 4 / 114: 00$ & 29.126 & 26.437 & 20.331 & & 23.950 & 17.851 & 9.606 \\
\hline 11/4/11 8:00 & 29.119 & 26.448 & 20.331 & & 23.940 & 17.839 & 9.604 \\
\hline 11/4/11 12:00 & 29.112 & 26.453 & 20.517 & & 23.929 & 17.825 & 9.602 \\
\hline $11 / 4 / 11$ 16:00 & 29.096 & 26.434 & 20.559 & & 23.901 & 17.804 & 9.585 \\
\hline $11 / 4 / 1120: 00$ & 29.085 & 26.437 & 20.485 & & 23.887 & 17.788 & 9.576 \\
\hline 11/5/11 0:00 & 29.078 & 26.453 & 20.440 & & 23.870 & 17.774 & 9.569 \\
\hline
\end{tabular}

TABLE S1.2 Page 54 of 58 


\begin{tabular}{|c|c|c|c|c|c|c|c|}
\hline \multirow[b]{2}{*}{ Date and Time } & \multicolumn{7}{|c|}{ Water Level (ft below reference point) } \\
\hline & SB43S & SB44S & SB45S & SB46S & SB47S & SB48S & SB49S \\
\hline $11 / 5 / 114: 00$ & 29.067 & 26.455 & 20.450 & & 23.856 & 17.760 & 9.562 \\
\hline 11/5/11 8:00 & 29.062 & 26.462 & 20.445 & & 23.842 & 17.725 & 9.557 \\
\hline $11 / 5 / 11$ 12:00 & 29.051 & 26.462 & 20.445 & & 23.821 & 17.727 & 9.548 \\
\hline 11/5/11 16:00 & 29.030 & 26.448 & 20.436 & & 23.798 & 17.706 & 9.536 \\
\hline 11/5/11 20:00 & 29.019 & 26.444 & 20.433 & & 23.779 & 17.690 & 9.525 \\
\hline 11/6/11 0:00 & 29.014 & 26.460 & 20.433 & & 23.770 & 17.678 & 9.520 \\
\hline 11/6/11 4:00 & 29.007 & 26.467 & 20.433 & & 23.756 & 17.664 & 9.520 \\
\hline 11/6/11 8:00 & 29.005 & 26.479 & 20.440 & & 23.753 & 17.655 & 9.518 \\
\hline 11/6/11 12:00 & 29.014 & 26.511 & 20.450 & & 23.756 & 17.657 & 9.525 \\
\hline $11 / 6 / 11$ 16:00 & 29.007 & 26.518 & 20.447 & & 23.746 & 17.643 & 9.520 \\
\hline $11 / 6 / 1120: 00$ & 29.009 & 26.535 & 20.457 & & 23.742 & 17.638 & 9.525 \\
\hline 11/7/11 0:00 & 29.007 & 26.551 & 20.459 & & 23.737 & 17.631 & 9.522 \\
\hline $11 / 7 / 114: 00$ & 29.005 & 26.558 & 20.459 & & 23.732 & 17.624 & 9.522 \\
\hline 11/7/11 8:00 & 29.000 & 26.572 & 20.382 & & 23.725 & 17.615 & 9.522 \\
\hline 11/7/11 12:00 & 28.996 & 26.577 & 20.550 & & 23.714 & 17.603 & 9.518 \\
\hline 11/7/11 16:00 & 28.982 & 26.567 & 20.547 & & 23.693 & 17.585 & 9.504 \\
\hline 11/7/11 20:00 & 28.977 & 26.570 & 20.412 & & 23.686 & 17.573 & 9.501 \\
\hline 11/8/11 0:00 & 28.961 & 26.285 & 20.478 & & 23.660 & 17.547 & 9.487 \\
\hline 11/8/11 4:00 & 28.952 & 25.675 & 20.464 & & 23.644 & 17.536 & 9.480 \\
\hline 11/8/11 8:00 & 28.950 & 25.198 & 20.424 & & 23.639 & 17.526 & 9.476 \\
\hline $11 / 8 / 11$ 12:00 & 28.941 & 24.886 & 20.426 & & 23.627 & 17.512 & 9.469 \\
\hline $11 / 8 / 11$ 16:00 & 28.931 & 24.658 & 20.475 & & 23.609 & 17.491 & 9.457 \\
\hline 11/8/11 20:00 & 28.927 & 24.429 & 20.434 & & 23.597 & 17.484 & 9.455 \\
\hline 11/9/11 0:00 & 28.918 & 23.963 & 20.333 & & 23.590 & 17.468 & 9.448 \\
\hline $11 / 9 / 114: 00$ & 28.920 & 23.425 & 20.212 & & 23.585 & 17.461 & 9.441 \\
\hline 11/9/11 8:00 & 28.922 & 23.050 & 20.189 & & 23.585 & 17.452 & 9.448 \\
\hline 11/9/11 12:00 & 28.920 & 22.792 & 20.454 & & 23.585 & 17.445 & 9.448 \\
\hline 11/9/11 16:00 & 28.915 & 22.552 & 20.513 & & 23.574 & 17.428 & 9.445 \\
\hline 11/9/11 20:00 & 28.911 & 22.366 & 20.352 & & 23.567 & 17.417 & 9.438 \\
\hline 11/10/11 0:00 & 28.904 & 22.228 & 20.301 & & 23.552 & 17.400 & 9.434 \\
\hline 11/10/11 4:00 & 28.895 & 22.133 & 20.291 & & 23.543 & 17.386 & 9.424 \\
\hline 11/10/11 8:00 & 28.886 & 22.079 & 20.259 & & 23.536 & 17.370 & 9.420 \\
\hline 11/10/11 12:00 & 28.881 & 22.079 & 20.405 & & 23.529 & 17.358 & 9.417 \\
\hline 11/10/11 16:00 & 28.867 & 22.035 & 20.431 & & 23.508 & 17.335 & 9.406 \\
\hline $11 / 10 / 1120: 00$ & 28.854 & 21.998 & 20.350 & & 23.487 & 17.314 & 9.392 \\
\hline 11/11/11 0:00 & 28.840 & 21.979 & 20.326 & & 23.468 & 17.293 & 9.380 \\
\hline 11/11/11 4:00 & 28.821 & 21.935 & 20.394 & & 23.443 & 17.270 & 9.366 \\
\hline 11/11/11 8:00 & 28.801 & 21.877 & 20.329 & & 23.415 & 17.230 & 9.345 \\
\hline 11/11/11 12:00 & 28.792 & 21.884 & 20.282 & & 23.408 & 17.223 & 9.340 \\
\hline 11/11/11 16:00 & 28.780 & 21.912 & 20.271 & & 23.389 & 17.209 & 9.331 \\
\hline 11/11/11 20:00 & 28.771 & 21.960 & 20.259 & & 23.377 & 17.190 & 9.324 \\
\hline 11/12/11 0:00 & 28.762 & 22.019 & 20.250 & & 23.363 & 17.176 & 9.317 \\
\hline $11 / 12 / 114: 00$ & 28.748 & 22.044 & 20.236 & & 23.347 & 17.158 & 9.305 \\
\hline $11 / 12 / 118: 00$ & 28.734 & 22.054 & 20.224 & & 23.331 & 17.137 & 9.291 \\
\hline $11 / 12 / 11$ 12:00 & 28.721 & 22.068 & 20.210 & & 23.307 & 17.113 & 9.280 \\
\hline 11/12/11 16:00 & 28.702 & 22.047 & 20.196 & & 23.288 & 17.095 & 9.261 \\
\hline 11/12/11 20:00 & 28.691 & 22.065 & 20.184 & & 23.272 & 17.076 & 9.259 \\
\hline 11/13/11 0:00 & 28.679 & 22.117 & 20.173 & & 23.258 & 17.060 & 9.247 \\
\hline $11 / 13 / 114: 00$ & 28.654 & 22.093 & 20.152 & & 23.228 & 17.032 & 9.226 \\
\hline 11/13/11 8:00 & 28.654 & 22.151 & 20.152 & & 23.230 & 17.025 & 9.228 \\
\hline $11 / 13 / 11$ 12:00 & 28.656 & 22.289 & 20.152 & & 23.225 & 17.018 & 9.228 \\
\hline 11/13/11 16:00 & 28.649 & 22.384 & 20.145 & & 23.221 & 17.006 & 9.221 \\
\hline 11/13/11 20:00 & 28.645 & 22.484 & 20.143 & & 23.218 & 16.999 & 9.219 \\
\hline 11/14/11 0:00 & 28.636 & 22.543 & 20.133 & & 23.207 & 16.983 & 9.210 \\
\hline
\end{tabular}

TABLE S1.2 Page 55 of 58 


\begin{tabular}{|c|c|c|c|c|c|c|c|}
\hline \multirow[b]{2}{*}{ Date and Time } & \multicolumn{7}{|c|}{ Water Level (ft below reference point) } \\
\hline & SB43S & SB44S & SB45S & SB46S & SB47S & SB48S & SB49S \\
\hline $11 / 14 / 114: 00$ & 28.620 & 22.554 & 20.122 & & 23.188 & 16.964 & 9.203 \\
\hline 11/14/11 8:00 & 28.613 & 22.594 & 20.117 & & 23.181 & 16.952 & 9.196 \\
\hline $11 / 14 / 1112: 00$ & 28.611 & 22.685 & 20.117 & & 23.181 & 16.945 & 9.191 \\
\hline $11 / 14 / 1116: 00$ & 28.604 & 22.745 & 20.108 & & 23.174 & 16.931 & 9.189 \\
\hline $11 / 14 / 1120: 00$ & 28.599 & 22.815 & 20.103 & & 23.169 & 16.924 & 9.184 \\
\hline 11/15/11 0:00 & 28.590 & 22.859 & 20.098 & & 23.155 & 16.908 & 9.175 \\
\hline 11/15/11 4:00 & 28.576 & 22.866 & 20.089 & & 23.139 & 16.891 & 9.161 \\
\hline 11/15/11 8:00 & 28.567 & 22.897 & 20.082 & & 23.130 & 16.877 & 9.158 \\
\hline $11 / 15 / 1112: 00$ & 28.562 & 22.964 & 20.077 & & 23.127 & 16.868 & 9.154 \\
\hline 11/15/11 16:00 & 28.555 & 23.002 & 20.073 & & 23.118 & 16.856 & 9.147 \\
\hline $11 / 15 / 1120: 00$ & 28.560 & 23.111 & 20.077 & & 23.130 & 16.859 & 9.156 \\
\hline 11/16/11 0:00 & 28.558 & 23.213 & 20.080 & & 23.130 & 16.854 & 9.156 \\
\hline $11 / 16 / 114: 00$ & 28.556 & 23.283 & 20.075 & & 23.125 & 16.842 & 9.149 \\
\hline 11/16/11 8:00 & 28.553 & 23.335 & 20.024 & & 23.123 & 16.835 & 9.149 \\
\hline $11 / 16 / 1112: 00$ & 28.551 & 23.432 & 20.045 & & 23.123 & 16.831 & 9.151 \\
\hline $11 / 16 / 1116: 00$ & 28.540 & 23.463 & 20.143 & & 23.111 & 16.817 & 9.140 \\
\hline 11/16/11 20:00 & 28.535 & 23.505 & 19.973 & & 23.109 & 16.807 & 9.135 \\
\hline 11/17/11 0:00 & 28.528 & 23.540 & 19.894 & & 23.102 & 16.796 & 9.128 \\
\hline 11/17/11 4:00 & 28.521 & 23.574 & 19.887 & & 23.092 & 16.784 & 9.121 \\
\hline 11/17/11 8:00 & 28.512 & 23.588 & 19.889 & & 23.081 & 16.770 & 9.114 \\
\hline $11 / 17 / 1112: 00$ & 28.498 & 23.605 & 20.094 & & 23.069 & 16.756 & 9.105 \\
\hline $11 / 17 / 11$ 16:00 & 28.482 & 23.556 & 20.145 & & 23.043 & 16.733 & 9.093 \\
\hline 11/17/11 20:00 & 28.464 & 23.512 & 20.082 & & 23.025 & 16.714 & 9.074 \\
\hline $11 / 18 / 110: 00$ & 28.452 & 23.488 & 20.056 & & 23.006 & 16.695 & 9.060 \\
\hline $11 / 18 / 114: 00$ & 28.434 & 23.463 & 20.082 & & 22.985 & 16.675 & 9.044 \\
\hline $11 / 18 / 118: 00$ & 28.418 & 23.430 & 20.089 & & 22.966 & 16.656 & 9.032 \\
\hline $11 / 18 / 1112: 00$ & 28.404 & 23.425 & 20.094 & & 22.950 & 16.642 & 9.023 \\
\hline 11/18/11 16:00 & 28.386 & 23.379 & 20.052 & & 22.926 & 16.618 & 9.004 \\
\hline $11 / 18 / 1120: 00$ & 28.379 & 23.393 & 19.952 & & 22.917 & 16.609 & 9.000 \\
\hline 11/19/11 0:00 & 28.370 & 23.428 & 19.922 & & 22.908 & 16.595 & 8.990 \\
\hline 11/19/11 4:00 & 28.354 & 23.416 & 19.989 & & 22.884 & 16.579 & 8.981 \\
\hline $11 / 19 / 118: 00$ & 28.342 & 23.395 & 20.056 & & 22.868 & 16.558 & 8.967 \\
\hline $11 / 19 / 11$ 12:00 & 28.326 & 23.397 & 19.938 & & 22.854 & 16.544 & 8.958 \\
\hline 11/19/11 16:00 & 28.333 & 23.458 & 19.838 & & 22.863 & 16.544 & 8.958 \\
\hline 11/19/11 20:00 & 28.345 & 23.651 & 19.784 & & 22.884 & 16.551 & 8.972 \\
\hline 11/20/11 0:00 & 28.347 & 23.779 & 19.956 & & 22.887 & 16.551 & 8.979 \\
\hline $11 / 20 / 114: 00$ & 28.345 & 23.847 & 19.959 & & 22.887 & 16.546 & 8.974 \\
\hline 11/20/11 8:00 & 28.342 & 23.894 & 19.961 & & 22.880 & 16.537 & 8.972 \\
\hline $11 / 20 / 1112: 00$ & 28.336 & 23.940 & 19.956 & & 22.875 & 16.527 & 8.965 \\
\hline $11 / 20 / 1116: 00$ & 28.322 & 23.933 & 19.952 & & 22.861 & 16.511 & 8.953 \\
\hline $11 / 20 / 1120: 00$ & 28.315 & 23.954 & 19.945 & & 22.849 & 16.499 & 8.948 \\
\hline 11/21/11 0:00 & 28.306 & 23.975 & 19.942 & & 22.835 & 16.485 & 8.939 \\
\hline $11 / 21 / 114: 00$ & 28.297 & 23.984 & 19.938 & & 22.826 & 16.474 & 8.932 \\
\hline 11/21/11 8:00 & 28.287 & 23.998 & 19.933 & & 22.817 & 16.462 & 8.925 \\
\hline $11 / 21 / 1112: 00$ & 28.276 & 24.022 & 19.929 & & 22.803 & 16.450 & 8.918 \\
\hline $11 / 21 / 1116: 00$ & 28.262 & 23.987 & 19.922 & & 22.782 & 16.432 & 8.904 \\
\hline $11 / 21 / 1120: 00$ & 28.251 & 23.982 & 19.915 & & 22.768 & 16.413 & 8.892 \\
\hline $11 / 22 / 110: 00$ & 28.242 & 24.008 & 19.912 & & 22.761 & 16.406 & 8.890 \\
\hline $11 / 22 / 114: 00$ & 28.230 & 23.991 & 19.903 & & 22.744 & 16.387 & 8.876 \\
\hline 11/22/11 8:00 & 28.219 & 23.989 & 19.915 & & 22.733 & 16.376 & 8.866 \\
\hline $11 / 22 / 11$ 12:00 & 28.209 & 24.003 & 19.929 & & 22.716 & 16.364 & 8.859 \\
\hline $11 / 22 / 11$ 16:00 & 28.200 & 24.001 & 19.940 & & 22.709 & 16.350 & 8.848 \\
\hline $11 / 22 / 1120: 00$ & 28.198 & 24.033 & 19.835 & & 22.705 & 16.345 & 8.850 \\
\hline 11/23/11 0:00 & 28.198 & 24.082 & 19.796 & & 22.707 & 16.338 & 8.850 \\
\hline
\end{tabular}

TABLE S1.2 Page 56 of 58 


\begin{tabular}{|c|c|c|c|c|c|c|c|}
\hline \multirow[b]{2}{*}{ Date and Time } & \multicolumn{7}{|c|}{ Water Level (ft below reference point) } \\
\hline & SB43S & SB44S & SB45S & SB46S & SB47S & SB48S & SB49S \\
\hline $11 / 23 / 114: 00$ & 28.189 & 24.103 & 19.819 & & 22.695 & 16.329 & 8.843 \\
\hline 11/23/11 8:00 & 28.182 & 24.124 & 19.801 & & 22.688 & 16.320 & 8.836 \\
\hline $11 / 23 / 1112: 00$ & 28.177 & 24.152 & 19.850 & & 22.679 & 16.308 & 8.829 \\
\hline $11 / 23 / 1116: 00$ & 28.161 & 24.140 & 19.994 & & 22.662 & 16.292 & 8.817 \\
\hline $11 / 23 / 1120: 00$ & 28.154 & 24.147 & 19.877 & & 22.653 & 16.282 & 8.813 \\
\hline 11/24/11 0:00 & 28.145 & 24.164 & 19.847 & & 22.641 & 16.271 & 8.803 \\
\hline $11 / 24 / 114: 00$ & 28.134 & 24.166 & 19.845 & & 22.627 & 16.257 & 8.796 \\
\hline 11/24/11 8:00 & 28.125 & 24.171 & 19.854 & & 22.616 & 16.245 & 8.789 \\
\hline $11 / 24 / 1112: 00$ & 28.113 & 24.180 & 20.001 & & 22.604 & 16.229 & 8.778 \\
\hline $11 / 24 / 11$ 16:00 & 28.100 & 24.143 & 19.970 & & 22.581 & 16.210 & 8.768 \\
\hline $11 / 24 / 1120: 00$ & 28.088 & 24.138 & 19.910 & & 22.569 & 16.198 & 8.757 \\
\hline 11/25/11 0:00 & 28.081 & 24.150 & 19.842 & & 22.555 & 16.187 & 8.752 \\
\hline $11 / 25 / 114: 00$ & 28.067 & 24.140 & 19.868 & & 22.539 & 16.173 & 8.736 \\
\hline 11/25/11 8:00 & 28.058 & 24.136 & 19.861 & & 22.527 & 16.159 & 8.731 \\
\hline $11 / 25 / 1112: 00$ & 28.047 & 24.145 & 19.910 & & 22.515 & 16.145 & 8.722 \\
\hline $11 / 25 / 1116: 00$ & 28.031 & 24.115 & 19.940 & & 22.494 & 16.128 & 8.708 \\
\hline $11 / 25 / 1120: 00$ & 28.022 & 24.115 & 19.919 & & 22.480 & 16.114 & 8.701 \\
\hline 11/26/11 0:00 & 28.015 & 24.140 & 19.880 & & 22.471 & 16.105 & 8.696 \\
\hline 11/26/11 4:00 & 28.008 & 24.171 & 19.856 & & 22.462 & 16.096 & 8.687 \\
\hline 11/26/11 8:00 & 28.003 & 24.196 & 19.733 & & 22.464 & 16.091 & 8.687 \\
\hline $11 / 26 / 11$ 12:00 & 28.006 & 24.250 & 19.684 & & 22.464 & 16.089 & 8.691 \\
\hline $11 / 26 / 11$ 16:00 & 28.001 & 24.243 & 19.693 & & 22.462 & 16.082 & 8.687 \\
\hline $11 / 26 / 1120: 00$ & 28.006 & 24.275 & 19.761 & & 22.464 & 16.079 & 8.689 \\
\hline $11 / 27 / 110: 00$ & 27.996 & 24.292 & 19.714 & & 22.457 & 16.072 & 8.689 \\
\hline $11 / 27 / 114: 00$ & 27.987 & 24.303 & 19.733 & & 22.450 & 16.063 & 8.682 \\
\hline $11 / 27 / 118: 00$ & 27.985 & 24.324 & 19.840 & & 22.443 & 16.056 & 8.680 \\
\hline $11 / 27 / 1112: 00$ & 27.983 & 24.357 & 19.842 & & 22.441 & 16.047 & 8.673 \\
\hline $11 / 27 / 1116: 00$ & 27.969 & 24.355 & 19.835 & & 22.424 & 16.035 & 8.668 \\
\hline $11 / 27 / 1120: 00$ & 27.964 & 24.376 & 19.835 & & 22.417 & 16.026 & 8.663 \\
\hline 11/28/11 0:00 & 27.955 & 24.387 & 19.833 & & 22.403 & 16.012 & 8.654 \\
\hline 11/28/11 4:00 & 27.941 & 24.383 & 19.828 & & 22.389 & 15.998 & 8.647 \\
\hline $11 / 28 / 118: 00$ & 27.937 & 24.394 & 19.828 & & 22.380 & 15.986 & 8.640 \\
\hline $11 / 28 / 11$ 12:00 & 27.923 & 24.401 & 19.824 & & 22.366 & 15.974 & 8.628 \\
\hline $11 / 28 / 1116: 00$ & 27.909 & 24.373 & 19.814 & & 22.347 & 15.958 & 8.621 \\
\hline $11 / 28 / 1120: 00$ & 27.902 & 24.387 & 19.814 & & 22.340 & 15.949 & 8.617 \\
\hline 11/29/11 0:00 & 27.896 & 24.408 & 19.814 & & 22.328 & 15.939 & 8.607 \\
\hline $11 / 29 / 114: 00$ & 27.891 & 24.446 & 19.817 & & 22.328 & 15.932 & 8.610 \\
\hline 11/29/11 8:00 & 27.891 & 24.487 & 19.819 & & 22.326 & 15.928 & 8.605 \\
\hline $11 / 29 / 11$ 12:00 & 27.889 & 24.532 & 19.824 & & 22.321 & 15.923 & 8.607 \\
\hline $11 / 29 / 11$ 16:00 & 27.877 & 24.534 & 19.819 & & 22.307 & 15.909 & 8.596 \\
\hline $11 / 29 / 1120: 00$ & 27.870 & 24.553 & 19.817 & & 22.298 & 15.899 & 8.593 \\
\hline 11/30/11 0:00 & 27.859 & 24.564 & 19.814 & & 22.286 & 15.888 & 8.584 \\
\hline $11 / 30 / 114: 00$ & 27.852 & 24.562 & 19.810 & & 22.272 & 15.876 & 8.579 \\
\hline 11/30/11 8:00 & 27.840 & 24.548 & 19.808 & & 22.258 & 15.864 & 8.568 \\
\hline $11 / 30 / 1112: 00$ & 27.829 & 24.557 & 19.803 & & 22.242 & 15.850 & 8.561 \\
\hline $11 / 30 / 1116: 00$ & 27.811 & 24.515 & 19.794 & & 22.221 & 15.829 & 8.547 \\
\hline $11 / 30 / 1120: 00$ & 27.804 & 24.511 & 19.791 & & 22.209 & 15.818 & 8.542 \\
\hline $12 / 1 / 110: 00$ & 27.802 & 24.550 & 19.796 & & 22.205 & 15.815 & 8.535 \\
\hline $12 / 1 / 114: 00$ & 27.795 & 24.585 & 19.796 & & 22.198 & 15.806 & 8.533 \\
\hline $12 / 1 / 118: 00$ & 27.797 & 24.629 & 19.805 & & 22.198 & 15.801 & 8.535 \\
\hline $12 / 1 / 1112: 00$ & 27.797 & 24.678 & 19.808 & & 22.200 & 15.799 & 8.537 \\
\hline $12 / 1 / 11$ 16:00 & 27.792 & 24.716 & 19.810 & & 22.198 & 15.797 & 8.537 \\
\hline $12 / 1 / 1120: 00$ & 27.797 & 24.774 & 19.819 & & 22.202 & 15.797 & 8.540 \\
\hline 12/2/11 0:00 & 27.795 & 24.816 & 19.824 & & 22.200 & 15.790 & 8.540 \\
\hline
\end{tabular}

TABLE S1.2 Page 57 of 58 


\begin{tabular}{|c|c|c|c|c|c|c|c|}
\hline \multirow[b]{2}{*}{ Date and Time } & \multicolumn{7}{|c|}{ Water Level (ft below reference point) } \\
\hline & SB43S & SB44S & SB45S & SB46S & SB47S & SB48S & SB49S \\
\hline $12 / 2 / 114: 00$ & 27.788 & 24.834 & 19.821 & & 22.186 & 15.780 & 8.528 \\
\hline $12 / 2 / 118: 00$ & 27.779 & 24.837 & 19.819 & & 22.174 & 15.766 & 8.523 \\
\hline $12 / 2 / 1112: 00$ & & 24.844 & 19.814 & & 22.158 & 15.755 & 8.516 \\
\hline
\end{tabular}

${ }^{a}$ Wells SB44S, SB46S, and SB48S were dry when recorders were installed in the other wells in July 2010. Recorders were not installed in these wells until August 20, 2010.

${ }^{\mathrm{b}} \mathrm{NA}$, data from indicated date and time until next reported value are missing because of logger electrical malfunction.

c Recorder in well SB46S was removed on September 23, 2011, to prevent winter damage; see Section 3.5 . 
TABLE S1.3 Automatically recorded groundwater level data for deep monitoring wells SB43D-SB49D, July 2010 to December 2011.

\begin{tabular}{|c|c|c|c|c|c|c|c|}
\hline \multirow[b]{2}{*}{ Date and Time } & \multicolumn{7}{|c|}{ Water Level (ft below reference point) } \\
\hline & SB43D & SB44D & SB45D & SB46D & SB47D & SB48D & SB49D \\
\hline 7/7/10 16:00 & 56.323 & 58.055 & & 55.723 & & & \\
\hline 7/7/10 20:00 & 55.682 & 58.055 & & 55.728 & & & \\
\hline $7 / 8 / 100: 00$ & 55.547 & 58.055 & & 55.732 & & & \\
\hline $7 / 8 / 104: 00$ & 55.490 & 58.041 & & 55.732 & & & \\
\hline $7 / 8 / 108: 00$ & 55.481 & 58.051 & & 55.744 & & & \\
\hline 7/8/10 12:00 & 55.476 & 58.060 & & 55.749 & & & \\
\hline 7/8/10 16:00 & 55.453 & 58.051 & & 55.721 & & & \\
\hline 7/8/10 20:00 & 55.441 & 58.044 & & 55.723 & & & \\
\hline 7/9/10 0:00 & 55.441 & 58.048 & & 55.730 & & & \\
\hline 7/9/10 4:00 & 55.431 & 58.039 & & 55.718 & & & \\
\hline 7/9/10 8:00 & 55.438 & 58.046 & & 55.742 & & & \\
\hline 7/9/10 12:00 & 55.445 & 58.062 & & 55.756 & & & \\
\hline 7/9/10 16:00 & 55.429 & 58.046 & & 55.725 & & & \\
\hline 7/9/10 20:00 & 55.422 & 58.043 & & 55.720 & & & \\
\hline $7 / 10 / 100: 00$ & 55.427 & 58.053 & & 55.732 & & & \\
\hline 7/10/10 4:00 & 55.424 & 58.041 & & 55.723 & & & \\
\hline 7/10/10 8:00 & 55.427 & 58.043 & & 55.735 & & & \\
\hline $7 / 10 / 10$ 12:00 & 55.436 & 58.057 & & 55.753 & & & \\
\hline 7/10/10 16:00 & 55.427 & 58.050 & & 55.728 & & & \\
\hline $7 / 10 / 1020: 00$ & 55.412 & 58.038 & & 55.711 & & & \\
\hline $7 / 11 / 100: 00$ & 55.419 & 58.041 & & 55.725 & & & \\
\hline 7/11/10 4:00 & 55.410 & 58.031 & & 55.706 & & & \\
\hline 7/11/10 8:00 & 55.398 & 58.015 & & 55.772 & & & \\
\hline $7 / 11 / 1012: 00$ & 55.405 & 58.029 & & 55.779 & & & \\
\hline $7 / 11 / 1016: 00$ & 55.403 & 58.022 & 49.311 & 55.704 & & & \\
\hline 7/11/10 20:00 & 55.386 & 57.999 & 49.283 & 55.676 & 36.255 & 36.205 & 40.165 \\
\hline 7/12/10 0:00 & 55.407 & 58.020 & 49.318 & 55.713 & 36.293 & 36.219 & 40.188 \\
\hline 7/12/10 4:00 & 55.398 & 58.013 & 49.302 & 55.697 & 36.293 & 36.214 & 40.179 \\
\hline 7/12/10 8:00 & 55.400 & 58.015 & 49.304 & 55.702 & 36.295 & 36.216 & 40.179 \\
\hline $7 / 12 / 10$ 12:00 & 55.431 & 58.041 & 49.348 & 55.746 & 36.319 & 36.240 & 40.207 \\
\hline 7/12/10 16:00 & 55.497 & 58.038 & 49.321 & 55.720 & 36.314 & 36.233 & 40.202 \\
\hline $7 / 12 / 10$ 20:00 & 55.443 & 58.029 & 49.309 & 55.709 & 36.307 & 36.228 & 40.190 \\
\hline 7/13/10 0:00 & 55.362 & 58.034 & 49.327 & 55.723 & 36.321 & 36.235 & 40.195 \\
\hline 7/13/10 4:00 & 55.362 & 58.038 & 49.321 & 55.723 & 36.319 & 36.237 & 40.197 \\
\hline 7/13/10 8:00 & 55.327 & 58.041 & 49.330 & 55.727 & 36.323 & 36.242 & 40.199 \\
\hline $7 / 13 / 10$ 12:00 & 55.450 & 58.057 & 49.355 & 55.756 & 36.347 & 36.263 & 40.216 \\
\hline $7 / 13 / 10$ 16:00 & 55.441 & 58.066 & 49.358 & 55.758 & 36.361 & 36.270 & 40.225 \\
\hline $7 / 13 / 10$ 20:00 & 55.436 & 58.057 & 49.339 & 55.739 & 36.354 & 36.263 & 40.218 \\
\hline 7/14/10 0:00 & 55.445 & 58.073 & 49.360 & 55.760 & 36.370 & 36.275 & 40.230 \\
\hline 7/14/10 4:00 & 55.457 & 58.075 & 49.367 & 55.770 & 36.382 & 36.284 & 40.234 \\
\hline 7/14/10 8:00 & 55.459 & 58.073 & 49.360 & 55.765 & 36.384 & 36.286 & 40.234 \\
\hline $7 / 14 / 10$ 12:00 & 55.467 & 58.092 & 49.388 & 55.791 & 36.396 & 36.305 & 40.251 \\
\hline $7 / 14 / 10$ 16:00 & 55.485 & 58.106 & 49.399 & 55.805 & 36.417 & 36.319 & 40.262 \\
\hline $7 / 14 / 10$ 20:00 & 55.485 & 58.101 & 49.383 & 55.786 & 36.419 & 36.314 & 40.260 \\
\hline 7/15/10 0:00 & 55.471 & 58.066 & 49.358 & 55.837 & 36.666 & 36.420 & 40.232 \\
\hline $7 / 15 / 104: 00$ & 55.424 & 58.071 & 49.351 & 55.730 & 36.605 & 36.212 & 40.225 \\
\hline 7/15/10 8:00 & 55.436 & 58.059 & 49.344 & 55.744 & 36.518 & 36.216 & 40.220 \\
\hline $7 / 15 / 10$ 12:00 & 55.443 & 58.068 & 49.360 & 55.758 & 36.352 & 36.228 & 40.225 \\
\hline 7/15/10 16:00 & 55.455 & 58.085 & 49.369 & 55.772 & 36.347 & 36.251 & 40.239 \\
\hline $7 / 15 / 10$ 20:00 & 55.448 & 58.078 & 49.351 & 55.753 & 36.330 & 36.240 & 40.234 \\
\hline 7/16/10 0:00 & 55.448 & 58.075 & 49.360 & 55.760 & 36.316 & 36.247 & 40.234 \\
\hline
\end{tabular}

TABLE S1.3 Page 1 of 58 


\begin{tabular}{|c|c|c|c|c|c|c|c|}
\hline \multirow[b]{2}{*}{ Date and Time } & \multicolumn{7}{|c|}{ Water Level (ft below reference point) } \\
\hline & SB43D & SB44D & SB45D & SB46D & SB47D & SB48D & SB49D \\
\hline $7 / 16 / 104: 00$ & 55.450 & 58.080 & 49.372 & 55.772 & 36.318 & 36.249 & 40.241 \\
\hline 7/16/10 8:00 & 55.445 & 58.075 & 49.360 & 55.762 & 36.309 & 36.242 & 40.234 \\
\hline $7 / 16 / 10$ 12:00 & 55.436 & 58.078 & 49.360 & 55.762 & 36.304 & 36.235 & 40.232 \\
\hline 7/16/10 16:00 & 55.405 & 58.054 & 49.321 & 55.758 & 36.408 & 36.261 & 40.206 \\
\hline 7/16/10 20:00 & 55.398 & 58.047 & 49.316 & 55.720 & 36.243 & 36.176 & 40.202 \\
\hline 7/17/10 0:00 & 55.396 & 58.043 & 49.323 & 55.720 & 36.227 & 36.172 & 40.199 \\
\hline $7 / 17 / 104: 00$ & 55.395 & 58.050 & 49.332 & 55.734 & 36.220 & 36.172 & 40.204 \\
\hline 7/17/10 8:00 & 55.386 & 58.045 & 49.323 & 55.727 & 36.206 & 36.162 & 40.202 \\
\hline 7/17/10 12:00 & 55.377 & 58.040 & 49.318 & 55.723 & 36.189 & 36.155 & 40.199 \\
\hline 7/17/10 16:00 & 55.362 & 58.047 & 49.321 & 55.723 & 36.182 & 36.148 & 40.199 \\
\hline 7/17/10 20:00 & 55.355 & 58.040 & 49.311 & 55.716 & 36.168 & 36.144 & 40.195 \\
\hline 7/18/10 0:00 & 55.348 & 58.036 & 49.309 & 55.713 & 36.166 & 36.134 & 40.193 \\
\hline $7 / 18 / 104: 00$ & 55.336 & 58.115 & 49.302 & 55.770 & 36.206 & 36.216 & 40.174 \\
\hline 7/18/10 8:00 & 55.325 & 58.024 & 49.309 & 55.746 & 36.257 & 36.394 & 40.183 \\
\hline 7/18/10 12:00 & 55.313 & 58.019 & 49.302 & 55.720 & 36.314 & 36.219 & 40.176 \\
\hline 7/18/10 16:00 & 55.303 & 58.012 & 49.295 & 55.699 & 36.114 & 36.069 & 40.172 \\
\hline 7/18/10 20:00 & 55.296 & 58.012 & 49.295 & 55.699 & 36.102 & 36.062 & 40.172 \\
\hline 7/19/10 0:00 & 55.294 & 58.015 & 49.295 & 55.699 & 36.097 & 36.064 & 40.172 \\
\hline $7 / 19 / 104: 00$ & 55.275 & 58.010 & 49.288 & 55.694 & 36.083 & 36.064 & 40.165 \\
\hline 7/19/10 8:00 & 55.279 & 58.012 & 49.302 & 55.704 & 36.079 & 36.067 & 40.169 \\
\hline 7/19/10 12:00 & 55.272 & 58.010 & 49.290 & 55.694 & 36.069 & 36.062 & 40.169 \\
\hline 7/19/10 16:00 & 55.253 & 58.001 & 49.274 & 55.680 & 36.058 & 36.053 & 40.158 \\
\hline 7/19/10 20:00 & 55.249 & 58.001 & 49.279 & 55.685 & 36.060 & 36.048 & 40.158 \\
\hline 7/20/10 0:00 & 55.249 & 57.998 & 49.283 & 55.687 & 36.058 & 36.046 & 40.160 \\
\hline $7 / 20 / 104: 00$ & 55.251 & 58.003 & 49.295 & 55.697 & 36.062 & 36.055 & 40.160 \\
\hline 7/20/10 8:00 & 55.230 & 57.994 & 49.286 & 55.692 & 36.055 & 36.181 & 40.153 \\
\hline $7 / 20 / 10$ 12:00 & 55.242 & 58.003 & 49.297 & 55.702 & 36.074 & 36.032 & 40.162 \\
\hline $7 / 20 / 10$ 16:00 & 55.227 & 57.991 & 49.269 & 55.678 & 36.053 & 36.036 & 40.153 \\
\hline $7 / 20 / 10$ 20:00 & 55.232 & 57.994 & 49.286 & 55.690 & 36.043 & 36.030 & 40.158 \\
\hline 7/21/10 0:00 & 55.227 & 57.998 & 49.286 & 55.690 & 36.015 & 36.029 & 40.158 \\
\hline $7 / 21 / 104: 00$ & 55.225 & 57.994 & 49.288 & 55.694 & 36.034 & 36.025 & 40.158 \\
\hline 7/21/10 8:00 & 55.237 & 58.010 & 49.314 & 55.716 & 36.046 & 36.041 & 40.172 \\
\hline $7 / 21 / 10$ 12:00 & 55.239 & 58.012 & 49.311 & 55.716 & 36.053 & 36.048 & 40.176 \\
\hline $7 / 21 / 10$ 16:00 & 55.218 & 57.996 & 49.279 & 55.685 & 36.036 & 36.032 & 40.162 \\
\hline $7 / 21 / 10$ 20:00 & 55.206 & 57.994 & 49.274 & 55.678 & 36.029 & 36.023 & 40.153 \\
\hline 7/22/10 0:00 & 55.206 & 57.993 & 49.274 & 55.680 & 36.027 & 36.027 & 40.155 \\
\hline $7 / 22 / 104: 00$ & 55.194 & 57.982 & 49.262 & 55.669 & 36.025 & 36.015 & 40.146 \\
\hline 7/22/10 8:00 & 55.204 & 57.991 & 49.286 & 55.692 & 36.027 & 36.027 & 40.153 \\
\hline $7 / 22 / 10$ 12:00 & 55.204 & 57.991 & 49.283 & 55.692 & 36.034 & 36.039 & 40.158 \\
\hline $7 / 22 / 10$ 16:00 & 55.194 & 57.989 & 49.262 & 55.671 & 36.029 & 36.030 & 40.146 \\
\hline $7 / 22 / 10$ 20:00 & 55.192 & 57.986 & 49.265 & 55.673 & 36.032 & 36.018 & 40.148 \\
\hline 7/23/10 0:00 & 55.201 & 57.991 & 49.279 & 55.687 & 36.043 & 36.025 & 40.155 \\
\hline $7 / 23 / 104: 00$ & 55.199 & 57.989 & 49.272 & 55.678 & 36.046 & 36.022 & 40.153 \\
\hline 7/23/10 8:00 & 55.213 & 58.000 & 49.300 & 55.706 & 36.062 & 36.039 & 40.165 \\
\hline $7 / 23 / 10$ 12:00 & 55.225 & 58.019 & 49.316 & 55.725 & 36.079 & 36.064 & 40.183 \\
\hline 7/23/10 16:00 & 55.220 & 58.010 & 49.297 & 55.706 & 36.081 & 36.058 & 40.176 \\
\hline $7 / 23 / 10$ 20:00 & 55.220 & 58.012 & 49.293 & 55.702 & 36.088 & 36.053 & 40.174 \\
\hline $7 / 24 / 100: 00$ & 55.235 & 58.019 & 49.316 & 55.723 & 36.102 & 36.069 & 40.186 \\
\hline $7 / 24 / 104: 00$ & 55.230 & 58.010 & 49.300 & 55.706 & 36.105 & 36.069 & 40.174 \\
\hline 7/24/10 8:00 & 55.242 & 58.024 & 49.325 & 55.730 & 36.121 & 36.086 & 40.187 \\
\hline $7 / 24 / 10$ 12:00 & 55.265 & 58.047 & 49.353 & 55.755 & 36.130 & 36.107 & 40.206 \\
\hline $7 / 24 / 10$ 16:00 & 55.275 & 58.038 & 49.344 & 55.732 & 36.100 & 36.104 & 40.206 \\
\hline $7 / 24 / 10$ 20:00 & 55.235 & 58.019 & 49.311 & 55.713 & 36.086 & 36.074 & 40.181 \\
\hline 7/25/10 0:00 & 55.263 & 58.037 & 49.348 & 55.753 & 36.090 & 36.107 & 40.202 \\
\hline
\end{tabular}

TABLE S1.3 Page 2 of 58 


\begin{tabular}{|c|c|c|c|c|c|c|c|}
\hline \multirow[b]{2}{*}{ Date and Time } & \multicolumn{7}{|c|}{ Water Level (ft below reference point) } \\
\hline & SB43D & SB44D & SB45D & SB46D & SB47D & SB48D & SB49D \\
\hline $7 / 25 / 104: 00$ & 55.256 & 58.028 & 49.323 & 55.725 & 36.149 & 36.104 & 40.193 \\
\hline 7/25/10 8:00 & 55.268 & 58.037 & 49.344 & 55.746 & 36.163 & 36.114 & 40.199 \\
\hline $7 / 25 / 1012: 00$ & 55.287 & 58.059 & 49.372 & 55.776 & 36.191 & 36.137 & 40.223 \\
\hline $7 / 25 / 10$ 16:00 & 55.287 & 58.058 & 49.353 & 55.758 & 36.196 & 36.139 & 40.223 \\
\hline $7 / 25 / 1020: 00$ & 55.282 & 58.051 & 49.341 & 55.744 & 36.194 & 36.132 & 40.216 \\
\hline 7/26/10 0:00 & 55.298 & 58.066 & 49.365 & 55.769 & 36.210 & 36.151 & 40.227 \\
\hline $7 / 26 / 104: 00$ & 55.296 & 58.058 & 49.351 & 55.755 & 36.210 & 36.149 & 40.220 \\
\hline $7 / 26 / 108: 00$ & 55.303 & 58.061 & 49.360 & 55.765 & 36.222 & 36.156 & 40.223 \\
\hline $7 / 26 / 1012: 00$ & 55.315 & 58.075 & 49.381 & 55.786 & 36.236 & 36.174 & 40.236 \\
\hline $7 / 26 / 10$ 16:00 & 55.313 & 58.073 & 49.362 & 55.769 & 36.238 & 36.174 & 40.234 \\
\hline $7 / 26 / 10$ 20:00 & 55.312 & 58.068 & 49.355 & 55.760 & 36.238 & 36.174 & 40.230 \\
\hline 7/27/10 0:00 & 55.329 & 58.084 & 49.381 & 55.783 & 36.257 & 36.191 & 40.241 \\
\hline $7 / 27 / 104: 00$ & 55.334 & 58.082 & 49.374 & 55.779 & 36.264 & 36.191 & 40.243 \\
\hline 7/27/10 8:00 & 55.336 & 58.079 & 49.374 & 55.781 & 36.267 & 36.196 & 40.241 \\
\hline $7 / 27 / 10$ 12:00 & 55.348 & 58.093 & 49.404 & 55.805 & 36.288 & 36.217 & 40.255 \\
\hline $7 / 27 / 10$ 16:00 & 55.358 & 58.103 & 49.399 & 55.805 & 36.300 & 36.224 & 40.265 \\
\hline $7 / 27 / 10$ 20:00 & 55.355 & 58.098 & 49.383 & 55.786 & 36.292 & 36.219 & 40.258 \\
\hline 7/28/10 0:00 & 55.374 & 58.114 & 49.413 & 55.816 & 36.316 & 36.233 & 40.274 \\
\hline $7 / 28 / 104: 00$ & 55.381 & 58.114 & 49.416 & 55.819 & 36.325 & 36.242 & 40.276 \\
\hline 7/28/10 8:00 & 55.391 & 58.117 & 49.418 & 55.826 & 36.332 & 36.249 & 40.281 \\
\hline $7 / 28 / 1012: 00$ & 55.400 & 58.138 & 49.446 & 55.849 & 36.351 & 36.268 & 40.297 \\
\hline 7/28/10 16:00 & 55.414 & 58.152 & 49.448 & 55.854 & 36.365 & 36.280 & 40.308 \\
\hline $7 / 28 / 10$ 20:00 & 55.419 & 58.147 & 49.437 & 55.842 & 36.375 & 36.280 & 40.308 \\
\hline 7/29/10 0:00 & 55.435 & 58.163 & 49.469 & 55.870 & 36.396 & 36.298 & 40.323 \\
\hline $7 / 29 / 104: 00$ & 55.447 & 58.173 & 49.476 & 55.877 & 36.410 & 36.310 & 40.332 \\
\hline 7/29/10 8:00 & 55.452 & 58.168 & 49.471 & 55.872 & 36.415 & 36.315 & 40.332 \\
\hline $7 / 29 / 10$ 12:00 & 55.459 & 58.184 & 49.488 & 55.891 & 36.429 & 36.329 & 40.346 \\
\hline $7 / 29 / 10$ 16:00 & 55.468 & 58.194 & 49.490 & 55.896 & 36.443 & 36.338 & 40.352 \\
\hline $7 / 29 / 10$ 20:00 & 55.466 & 58.184 & 49.474 & 55.877 & 36.440 & 36.333 & 40.348 \\
\hline 7/30/10 0:00 & 55.476 & 58.191 & 49.490 & 55.891 & 36.455 & 36.348 & 40.352 \\
\hline $7 / 30 / 104: 00$ & 55.483 & 58.194 & 49.492 & 55.896 & 36.462 & 36.352 & 40.358 \\
\hline 7/30/10 8:00 & 55.483 & 58.187 & 49.481 & 55.882 & 36.462 & 36.350 & 40.348 \\
\hline $7 / 30 / 10$ 12:00 & 55.485 & 58.193 & 49.490 & 55.896 & 36.464 & 36.352 & 40.355 \\
\hline $7 / 30 / 10$ 16:00 & 55.485 & 58.194 & 49.488 & 55.891 & 36.471 & 36.354 & 40.353 \\
\hline 7/30/10 20:00 & 55.487 & 58.191 & 49.478 & 55.884 & 36.469 & 36.354 & 40.348 \\
\hline 7/31/10 0:00 & 55.499 & 58.201 & 49.499 & 55.901 & 36.480 & 36.366 & 40.362 \\
\hline $7 / 31 / 104: 00$ & 55.513 & 58.212 & 49.511 & 55.915 & 36.495 & 36.383 & 40.374 \\
\hline 7/31/10 8:00 & 55.528 & 58.217 & 49.518 & 55.922 & 36.506 & 36.387 & 40.381 \\
\hline $7 / 31 / 10$ 12:00 & 55.532 & 58.226 & 49.529 & 55.936 & 36.520 & 36.399 & 40.390 \\
\hline $7 / 31 / 10$ 16:00 & 55.544 & 58.245 & 49.543 & 55.950 & 36.537 & 36.413 & 40.404 \\
\hline 7/31/10 20:00 & 55.551 & 58.245 & 49.536 & 55.945 & 36.542 & 36.415 & 40.404 \\
\hline 8/1/10 0:00 & 55.565 & 58.256 & 49.557 & 55.961 & 36.553 & 36.432 & 40.418 \\
\hline 8/1/10 4:00 & 55.577 & 58.266 & 49.569 & 55.973 & 36.572 & 36.443 & 40.427 \\
\hline 8/1/10 8:00 & 55.589 & 58.270 & 49.573 & 55.978 & 36.581 & 36.453 & 40.434 \\
\hline 8/1/10 12:00 & 55.589 & 58.277 & 49.576 & 55.980 & 36.591 & 36.460 & 40.441 \\
\hline 8/1/10 16:00 & 55.599 & 58.289 & 49.585 & 55.992 & 36.603 & 36.474 & 40.450 \\
\hline 8/1/10 20:00 & 55.608 & 58.291 & 49.585 & 55.990 & 36.614 & 36.476 & 40.455 \\
\hline 8/2/10 0:00 & 55.617 & 58.301 & 49.594 & 55.999 & 36.617 & 36.488 & 40.457 \\
\hline 8/2/10 4:00 & 55.627 & 58.310 & 49.606 & 56.013 & 36.633 & 36.497 & 40.467 \\
\hline 8/2/10 8:00 & 55.627 & 58.298 & 49.594 & 56.013 & 36.631 & 36.490 & 40.455 \\
\hline 8/2/10 12:00 & 55.627 & 58.301 & 49.599 & 56.001 & 36.638 & 36.497 & 40.459 \\
\hline 8/2/10 16:00 & 55.629 & 58.310 & 49.604 & 56.011 & 36.650 & 36.509 & 40.466 \\
\hline 8/2/10 20:00 & 55.643 & 58.317 & 49.613 & 56.015 & 36.661 & 36.516 & 40.476 \\
\hline 8/3/10 0:00 & 55.655 & 58.324 & 49.620 & 56.025 & 36.673 & 36.528 & 40.485 \\
\hline
\end{tabular}

TABLE S1.3 Page 3 of 58 


\begin{tabular}{|c|c|c|c|c|c|c|c|}
\hline \multirow[b]{2}{*}{ Date and Time } & \multicolumn{7}{|c|}{ Water Level (ft below reference point) } \\
\hline & SB43D & SB44D & SB45D & SB46D & SB47D & SB48D & SB49D \\
\hline 8/3/10 4:00 & 55.667 & 58.335 & 49.636 & 56.043 & 36.687 & 36.539 & 40.495 \\
\hline 8/3/10 8:00 & 55.688 & 58.349 & 49.657 & 56.065 & 36.706 & 36.556 & 40.511 \\
\hline 8/3/10 12:00 & 55.695 & 58.359 & 49.659 & 56.067 & 36.713 & 36.570 & 40.520 \\
\hline 8/3/10 16:00 & 55.698 & 58.366 & 49.659 & 56.067 & 36.727 & 36.575 & 40.527 \\
\hline 8/3/10 20:00 & 55.710 & 58.377 & 49.669 & 56.074 & 36.737 & 36.584 & 40.534 \\
\hline 8/4/10 0:00 & 55.722 & 58.382 & 49.680 & 56.083 & 36.751 & 36.600 & 40.541 \\
\hline 8/4/10 4:00 & 55.736 & 58.394 & 49.694 & 56.100 & 36.765 & 36.614 & 40.553 \\
\hline 8/4/10 8:00 & 55.755 & 58.405 & 49.715 & 56.118 & 36.786 & 36.631 & 40.571 \\
\hline 8/4/10 12:00 & 55.766 & 58.415 & 49.722 & 56.128 & 36.793 & 36.642 & 40.578 \\
\hline 8/4/10 16:00 & 55.769 & 58.422 & 49.717 & 56.121 & 36.798 & 36.645 & 40.583 \\
\hline 8/4/10 20:00 & 55.774 & 58.424 & 49.722 & 56.130 & 36.809 & 36.654 & 40.585 \\
\hline 8/5/10 0:00 & 55.790 & 58.433 & 49.736 & 56.139 & 36.823 & 36.668 & 40.594 \\
\hline 8/5/10 4:00 & 55.795 & 58.440 & 49.743 & 56.147 & 36.835 & 36.677 & 40.599 \\
\hline 8/5/10 8:00 & 55.818 & 58.457 & 49.778 & 56.177 & 36.852 & 36.703 & 40.625 \\
\hline 8/5/10 12:00 & 55.835 & 58.471 & 49.778 & 56.182 & 36.870 & 36.715 & 40.632 \\
\hline 8/5/10 16:00 & 55.821 & 58.471 & 49.757 & 56.165 & 36.866 & 36.710 & 40.627 \\
\hline 8/5/10 20:00 & 55.833 & 58.482 & 49.773 & 56.177 & 36.880 & 36.717 & 40.639 \\
\hline 8/6/10 0:00 & 55.854 & 58.492 & 49.789 & 56.196 & 36.894 & 36.738 & 40.651 \\
\hline 8/6/10 4:00 & 55.859 & 58.494 & 49.794 & 56.196 & 36.906 & 36.740 & 40.658 \\
\hline 8/6/10 8:00 & 55.882 & 58.515 & 49.829 & 56.229 & 36.927 & 36.766 & 40.676 \\
\hline 8/6/10 12:00 & 55.913 & 58.529 & 49.838 & 56.243 & 36.948 & 36.783 & 40.692 \\
\hline 8/6/10 16:00 & 56.022 & 58.524 & 49.813 & 56.217 & 36.941 & 36.780 & 40.683 \\
\hline 8/6/10 20:00 & 55.925 & 58.529 & 49.820 & 56.226 & 36.946 & 36.780 & 40.687 \\
\hline 8/7/10 0:00 & 55.844 & 58.540 & 49.838 & 56.245 & 36.967 & 36.799 & 40.702 \\
\hline 8/7/10 4:00 & 55.854 & 58.538 & 49.831 & 56.236 & 36.969 & 36.797 & 40.699 \\
\hline 8/7/10 8:00 & 55.854 & 58.554 & 49.861 & 56.264 & 36.986 & 36.815 & 40.716 \\
\hline 8/7/10 12:00 & 55.963 & 58.573 & 49.875 & 56.282 & 37.002 & 36.834 & 40.732 \\
\hline 8/7/10 16:00 & 55.944 & 58.568 & 49.852 & 56.259 & 37.004 & 36.829 & 40.727 \\
\hline 8/7/10 20:00 & 55.939 & 58.568 & 49.854 & 56.259 & 37.002 & 36.829 & 40.727 \\
\hline 8/8/10 0:00 & 55.963 & 58.582 & 49.882 & 56.287 & 37.028 & 36.848 & 40.745 \\
\hline 8/8/10 4:00 & 55.965 & 58.582 & 49.873 & 56.280 & 37.025 & 36.848 & 40.746 \\
\hline 8/8/10 8:00 & 55.977 & 58.596 & 49.898 & 56.301 & 37.037 & 36.862 & 40.755 \\
\hline 8/8/10 12:00 & 55.998 & 58.620 & 49.926 & 56.332 & 37.058 & 36.885 & 40.776 \\
\hline 8/8/10 16:00 & 55.996 & 58.615 & 49.903 & 56.311 & 37.061 & 36.881 & 40.776 \\
\hline 8/8/10 20:00 & 55.998 & 58.620 & 49.910 & 56.315 & 37.063 & 36.883 & 40.778 \\
\hline 8/9/10 0:00 & 56.022 & 58.636 & 49.936 & 56.346 & 37.089 & 36.904 & 40.799 \\
\hline 8/9/10 4:00 & 56.029 & 58.641 & 49.938 & 56.341 & 37.094 & 36.913 & 40.799 \\
\hline 8/9/10 8:00 & 56.048 & 58.657 & 49.968 & 56.371 & 37.110 & 36.932 & 40.820 \\
\hline 8/9/10 12:00 & 56.064 & 58.683 & 49.996 & 56.400 & 37.138 & 36.953 & 40.844 \\
\hline 8/9/10 16:00 & 56.072 & 58.685 & 49.977 & 56.385 & 37.143 & 36.958 & 40.845 \\
\hline 8/9/10 20:00 & 56.074 & 58.687 & 49.977 & 56.383 & 37.150 & 36.958 & 40.848 \\
\hline 8/10/10 0:00 & 56.098 & 58.711 & 50.015 & 56.421 & 37.176 & 36.986 & 40.869 \\
\hline 8/10/10 4:00 & 56.105 & 58.711 & 50.012 & 56.416 & 37.183 & 36.991 & 40.873 \\
\hline 8/10/10 8:00 & 56.112 & 58.715 & 50.019 & 56.425 & 37.188 & 37.000 & 40.878 \\
\hline 8/10/10 12:00 & 56.131 & 58.743 & 50.054 & 56.460 & 37.218 & 37.023 & 40.904 \\
\hline 8/10/10 16:00 & 56.140 & 58.750 & 50.042 & 56.453 & 37.220 & 37.028 & 40.911 \\
\hline 8/10/10 20:00 & 56.140 & 58.752 & 50.040 & 56.444 & 37.220 & 37.030 & 40.908 \\
\hline 8/11/10 0:00 & 56.157 & 58.762 & 50.066 & 56.468 & 37.239 & 37.042 & 40.920 \\
\hline 8/11/10 4:00 & 56.168 & 58.769 & 50.070 & 56.475 & 37.251 & 37.051 & 40.931 \\
\hline 8/11/10 8:00 & 56.173 & 58.771 & 50.068 & 56.477 & 37.256 & 37.058 & 40.931 \\
\hline 8/11/10 12:00 & 56.190 & 58.797 & 50.103 & 56.510 & 37.282 & 37.082 & 40.955 \\
\hline 8/11/10 16:00 & 56.199 & 58.808 & 50.103 & 56.510 & 37.291 & 37.096 & 40.966 \\
\hline 8/11/10 20:00 & 56.204 & 58.806 & 50.096 & 56.500 & 37.291 & 37.093 & 40.964 \\
\hline 8/12/10 0:00 & 56.221 & 58.822 & 50.126 & 56.533 & 37.312 & 37.112 & 40.985 \\
\hline
\end{tabular}

TABLE S1.3 Page 4 of 58 


\begin{tabular}{|c|c|c|c|c|c|c|c|}
\hline \multirow[b]{2}{*}{ Date and Time } & \multicolumn{7}{|c|}{ Water Level (ft below reference point) } \\
\hline & SB43D & SB44D & SB45D & SB46D & SB47D & SB48D & SB49D \\
\hline $8 / 12 / 104: 00$ & 56.235 & 58.831 & 50.131 & 56.538 & 37.324 & 37.122 & 40.992 \\
\hline 8/12/10 8:00 & 56.237 & 58.831 & 50.126 & 56.531 & 37.329 & 37.124 & 40.992 \\
\hline $8 / 12 / 1012: 00$ & 56.244 & 58.848 & 50.149 & 56.554 & 37.340 & 37.140 & 41.008 \\
\hline $8 / 12 / 1016: 00$ & 56.256 & 58.859 & 50.149 & 56.559 & 37.357 & 37.150 & 41.017 \\
\hline $8 / 12 / 1020: 00$ & 56.258 & 58.857 & 50.140 & 56.547 & 37.352 & 37.150 & 41.018 \\
\hline 8/13/10 0:00 & 56.275 & 58.873 & 50.168 & 56.575 & 37.376 & 37.166 & 41.034 \\
\hline $8 / 13 / 104: 00$ & 56.291 & 58.885 & 50.184 & 56.589 & 37.385 & 37.185 & 41.045 \\
\hline 8/13/10 8:00 & 56.291 & 58.883 & 50.175 & 56.580 & 37.385 & 37.182 & 41.043 \\
\hline $8 / 13 / 1012: 00$ & 56.296 & 58.894 & 50.186 & 56.596 & 37.394 & 37.194 & 41.055 \\
\hline 8/13/10 16:00 & 56.310 & 58.908 & 50.203 & 56.606 & 37.408 & 37.203 & 41.069 \\
\hline $8 / 13 / 10$ 20:00 & 56.197 & 58.838 & 50.091 & 56.557 & 37.277 & 37.236 & 40.985 \\
\hline 8/14/10 0:00 & 56.223 & 58.852 & 50.131 & 56.428 & 37.307 & 37.103 & 41.009 \\
\hline $8 / 14 / 104: 00$ & 56.247 & 58.862 & 50.151 & 56.393 & 37.324 & 37.126 & 41.017 \\
\hline 8/14/10 8:00 & 56.256 & 58.859 & 50.149 & 56.528 & 37.321 & 37.136 & 41.020 \\
\hline $8 / 14 / 10$ 12:00 & 56.261 & 58.869 & 50.158 & 56.566 & 37.336 & 37.143 & 41.029 \\
\hline 8/14/10 16:00 & 56.277 & 58.887 & 50.184 & 56.587 & 37.347 & 37.159 & 41.050 \\
\hline 8/14/10 20:00 & 56.291 & 58.899 & 50.189 & 56.594 & 37.359 & 37.168 & 41.057 \\
\hline 8/15/10 0:00 & 56.306 & 58.911 & 50.212 & 56.610 & 37.366 & 37.182 & 41.071 \\
\hline 8/15/10 4:00 & 56.327 & 58.932 & 50.240 & 56.643 & 37.392 & 37.201 & 41.095 \\
\hline 8/15/10 8:00 & 56.336 & 58.936 & 50.242 & 56.646 & 37.392 & 37.213 & 41.104 \\
\hline 8/15/10 12:00 & 56.336 & 58.943 & 50.247 & 56.648 & 37.404 & 37.217 & 41.106 \\
\hline 8/15/10 16:00 & 56.344 & 58.957 & 50.256 & 56.662 & 37.406 & 37.229 & 41.117 \\
\hline 8/15/10 20:00 & 56.348 & 58.962 & 50.254 & 56.655 & 37.410 & 37.229 & 41.120 \\
\hline 8/16/10 0:00 & 56.362 & 58.971 & 50.270 & 56.676 & 37.420 & 37.238 & 41.134 \\
\hline 8/16/10 4:00 & 56.377 & 58.988 & 50.293 & 56.699 & 37.437 & 37.255 & 41.148 \\
\hline 8/16/10 8:00 & 56.388 & 58.992 & 50.298 & 56.702 & 37.446 & 37.266 & 41.159 \\
\hline 8/16/10 12:00 & 56.384 & 58.999 & 50.293 & 56.699 & 37.448 & 37.269 & 41.159 \\
\hline 8/16/10 16:00 & 56.384 & 59.004 & 50.293 & 56.697 & 37.453 & 37.271 & 41.164 \\
\hline 8/16/10 20:00 & 56.388 & 59.004 & 50.291 & 56.695 & 37.451 & 37.271 & 41.166 \\
\hline 8/17/10 0:00 & 56.405 & 59.015 & 50.312 & 56.718 & 37.470 & 37.285 & 41.176 \\
\hline 8/17/10 4:00 & 56.412 & 59.025 & 50.323 & 56.728 & 37.477 & 37.294 & 41.183 \\
\hline 8/17/10 8:00 & 56.424 & 59.032 & 50.340 & 56.742 & 37.486 & 37.304 & 41.197 \\
\hline $8 / 17 / 10$ 12:00 & 56.429 & 59.039 & 50.335 & 56.737 & 37.488 & 37.306 & 41.199 \\
\hline 8/17/10 16:00 & 56.424 & 59.036 & 50.328 & 56.735 & 37.491 & 37.309 & 41.201 \\
\hline 8/17/10 20:00 & 56.426 & 59.041 & 50.333 & 56.737 & 37.488 & 37.309 & 41.203 \\
\hline 8/18/10 0:00 & 56.440 & 59.050 & 50.346 & 56.751 & 37.502 & 37.318 & 41.213 \\
\hline 8/18/10 4:00 & 56.445 & 59.060 & 50.356 & 56.760 & 37.505 & 37.325 & 41.220 \\
\hline 8/18/10 8:00 & 56.457 & 59.069 & 50.372 & 56.777 & 37.512 & 37.337 & 41.231 \\
\hline 8/18/10 12:00 & 56.462 & 59.076 & 50.367 & 56.772 & 37.514 & 37.341 & 41.236 \\
\hline 8/18/10 16:00 & 56.452 & 59.071 & 50.351 & 56.760 & 37.509 & 37.334 & 41.229 \\
\hline 8/18/10 20:00 & 56.455 & 59.076 & 50.356 & 56.765 & 37.516 & 37.339 & 41.234 \\
\hline 8/19/10 0:00 & 56.471 & 59.083 & 50.372 & 56.777 & 37.531 & 37.349 & 41.245 \\
\hline 8/19/10 4:00 & 56.471 & 59.085 & 50.374 & 56.779 & 37.531 & 37.346 & 41.245 \\
\hline 8/19/10 8:00 & 56.483 & 59.095 & 50.395 & 56.798 & 37.538 & 37.362 & 41.255 \\
\hline 8/19/10 12:00 & 56.490 & 59.106 & 50.400 & 56.807 & 37.554 & 37.372 & 41.269 \\
\hline 8/19/10 16:00 & 56.483 & 59.104 & 50.379 & 56.788 & 37.545 & 37.365 & 41.262 \\
\hline 8/19/10 20:00 & 56.488 & 59.109 & 50.391 & 56.796 & 37.552 & 37.372 & 41.267 \\
\hline 8/20/10 0:00 & 56.502 & 59.116 & 50.405 & 56.810 & 37.559 & 37.379 & 41.278 \\
\hline $8 / 20 / 104: 00$ & 56.499 & 59.113 & 50.398 & 56.805 & 37.563 & 37.381 & 41.276 \\
\hline 8/20/10 8:00 & 56.511 & 59.125 & 50.423 & 56.828 & 37.573 & 37.393 & 41.290 \\
\hline $8 / 20 / 1012: 00$ & 56.516 & 59.134 & 50.435 & 56.842 & 37.587 & 37.402 & 41.296 \\
\hline 8/20/10 16:00 & 56.459 & 59.095 & 50.374 & 56.723 & 37.516 & 37.318 & 41.250 \\
\hline 8/20/10 20:00 & 56.391 & 59.064 & 50.335 & 56.728 & 37.500 & 37.281 & 41.217 \\
\hline 8/21/10 0:00 & 56.422 & 59.071 & 50.356 & 56.756 & 37.477 & 37.301 & 41.231 \\
\hline
\end{tabular}

TABLE S1.3 Page 5 of 58 


\begin{tabular}{|c|c|c|c|c|c|c|c|}
\hline \multirow[b]{2}{*}{ Date and Time } & \multicolumn{7}{|c|}{ Water Level (ft below reference point) } \\
\hline & SB43D & SB44D & SB45D & SB46D & SB47D & SB48D & SB49D \\
\hline $8 / 21 / 104: 00$ & 56.436 & 59.078 & 50.360 & 56.760 & 37.477 & 37.313 & 41.231 \\
\hline 8/21/10 8:00 & 56.455 & 59.085 & 50.393 & 56.791 & 37.491 & 37.330 & 41.250 \\
\hline $8 / 21 / 10$ 12:00 & 56.478 & 59.111 & 50.418 & 56.819 & 37.509 & 37.353 & 41.273 \\
\hline $8 / 21 / 10$ 16:00 & 56.476 & 59.111 & 50.405 & 56.805 & 37.512 & 37.353 & 41.276 \\
\hline $8 / 21 / 1020: 00$ & 56.481 & 59.118 & 50.414 & 56.814 & 37.512 & 37.353 & 41.278 \\
\hline $8 / 22 / 100: 00$ & 56.500 & 59.130 & 50.439 & 56.838 & 37.528 & 37.372 & 41.296 \\
\hline $8 / 22 / 104: 00$ & 56.497 & 59.127 & 50.428 & 56.826 & 37.528 & 37.369 & 41.294 \\
\hline 8/22/10 8:00 & 56.509 & 59.141 & 50.453 & 56.852 & 37.540 & 37.384 & 41.308 \\
\hline $8 / 22 / 1012: 00$ & 56.526 & 59.160 & 50.472 & 56.873 & 37.556 & 37.400 & 41.324 \\
\hline $8 / 22 / 1016: 00$ & 56.518 & 59.160 & 50.453 & 56.854 & 37.552 & 37.395 & 41.322 \\
\hline $8 / 22 / 1020: 00$ & 56.516 & 59.158 & 50.451 & 56.849 & 37.549 & 37.395 & 41.320 \\
\hline 8/23/10 0:00 & 56.530 & 59.169 & 50.472 & 56.870 & 37.566 & 37.405 & 41.334 \\
\hline $8 / 23 / 104: 00$ & 56.533 & 59.162 & 50.463 & 56.861 & 37.563 & 37.404 & 41.329 \\
\hline 8/23/10 8:00 & 56.535 & 59.172 & 50.479 & 56.877 & 37.568 & 37.411 & 41.336 \\
\hline $8 / 23 / 10$ 12:00 & 56.547 & 59.188 & 50.497 & 56.896 & 37.582 & 37.428 & 41.352 \\
\hline 8/23/10 16:00 & 56.547 & 59.193 & 50.479 & 56.882 & 37.578 & 37.421 & 41.352 \\
\hline 8/23/10 20:00 & 56.544 & 59.186 & 50.476 & 56.880 & 37.580 & 37.421 & 41.350 \\
\hline 8/24/10 0:00 & 56.566 & 59.202 & 50.509 & 56.908 & 37.599 & 37.440 & 41.366 \\
\hline $8 / 24 / 104: 00$ & 56.580 & 59.209 & 50.514 & 56.913 & 37.610 & 37.447 & 41.376 \\
\hline 8/24/10 8:00 & 56.582 & 59.213 & 50.525 & 56.922 & 37.613 & 37.454 & 41.383 \\
\hline $8 / 24 / 1012: 00$ & 56.753 & 59.241 & 50.669 & & 37.641 & 37.509 & 41.417 \\
\hline $8 / 24 / 10$ 16:00 & & 59.290 & 50.562 & 56.976 & 37.636 & 37.614 & 41.502 \\
\hline $8 / 24 / 1020: 00$ & 57.034 & 59.269 & 50.558 & 56.976 & 37.704 & 37.556 & 41.448 \\
\hline 8/25/10 0:00 & 56.772 & 59.271 & 50.586 & 56.999 & 37.669 & 37.561 & 41.452 \\
\hline $8 / 25 / 104: 00$ & 56.703 & 59.272 & 50.574 & 56.990 & 37.657 & 37.556 & 41.448 \\
\hline 8/25/10 8:00 & 56.684 & 59.272 & 50.581 & 56.997 & 37.662 & 37.556 & 41.445 \\
\hline $8 / 25 / 1012: 00$ & 56.689 & 59.288 & 50.604 & 57.020 & 37.679 & 37.568 & 41.464 \\
\hline 8/25/10 16:00 & 56.684 & 59.285 & 50.590 & 57.006 & 37.683 & 37.568 & 41.462 \\
\hline $8 / 25 / 1020: 00$ & 56.681 & 59.285 & 50.586 & 57.002 & 37.679 & 37.564 & 41.459 \\
\hline 8/26/10 0:00 & 56.696 & 59.297 & 50.613 & 57.025 & 37.700 & 37.582 & 41.473 \\
\hline $8 / 26 / 104: 00$ & 56.703 & 59.300 & 50.606 & 57.023 & 37.702 & 37.585 & 41.476 \\
\hline 8/26/10 8:00 & 56.703 & 59.299 & 50.611 & 57.025 & 37.704 & 37.589 & 41.473 \\
\hline $8 / 26 / 10$ 12:00 & 56.710 & 59.311 & 50.634 & 57.051 & 37.716 & 37.603 & 41.485 \\
\hline 8/26/10 16:00 & 56.710 & 59.316 & 50.623 & 57.039 & 37.719 & 37.606 & 41.489 \\
\hline $8 / 26 / 1020: 00$ & 56.708 & 59.309 & 50.609 & 57.027 & 37.719 & 37.596 & 41.483 \\
\hline 8/27/10 0:00 & 56.722 & 59.327 & 50.639 & 57.053 & 37.733 & 37.610 & 41.496 \\
\hline $8 / 27 / 104: 00$ & 56.729 & 59.323 & 50.637 & 57.053 & 37.740 & 37.617 & 41.501 \\
\hline 8/27/10 8:00 & 56.731 & 59.323 & 50.632 & 57.048 & 37.740 & 37.617 & 41.499 \\
\hline $8 / 27 / 10$ 12:00 & 56.736 & 59.334 & 50.651 & 57.067 & 37.754 & 37.629 & 41.508 \\
\hline $8 / 27 / 10$ 16:00 & 56.738 & 59.337 & 50.644 & 57.060 & 37.751 & 37.631 & 41.510 \\
\hline $8 / 27 / 1020: 00$ & 56.741 & 59.335 & 50.637 & 57.053 & 37.751 & 37.631 & 41.511 \\
\hline 8/28/10 0:00 & 56.757 & 59.351 & 50.662 & 57.081 & 37.768 & 37.643 & 41.524 \\
\hline $8 / 28 / 104: 00$ & 56.764 & 59.356 & 50.667 & 57.084 & 37.782 & 37.652 & 41.531 \\
\hline 8/28/10 8:00 & 56.767 & 59.353 & 50.660 & 57.077 & 37.775 & 37.647 & 41.529 \\
\hline $8 / 28 / 10$ 12:00 & 56.771 & 59.365 & 50.678 & 57.093 & 37.791 & 37.659 & 41.538 \\
\hline 8/28/10 16:00 & 56.776 & 59.372 & 50.678 & 57.098 & 37.796 & 37.668 & 41.543 \\
\hline $8 / 28 / 1020: 00$ & 56.781 & 59.372 & 50.674 & 57.091 & 37.798 & 37.668 & 41.545 \\
\hline 8/29/10 0:00 & 56.800 & 59.388 & 50.704 & 57.119 & 37.817 & 37.685 & 41.562 \\
\hline $8 / 29 / 104: 00$ & 56.812 & 59.398 & 50.713 & 57.130 & 37.827 & 37.694 & 41.573 \\
\hline 8/29/10 8:00 & 56.819 & 59.397 & 50.711 & 57.126 & 37.831 & 37.697 & 41.576 \\
\hline 8/29/10 12:00 & 56.821 & 59.411 & 50.723 & 57.140 & 37.843 & 37.706 & 41.582 \\
\hline 8/29/10 16:00 & 56.826 & 59.418 & 50.725 & 57.142 & 37.852 & 37.713 & 41.592 \\
\hline 8/29/10 20:00 & 56.835 & 59.423 & 50.727 & 57.142 & 37.857 & 37.718 & 41.594 \\
\hline 8/30/10 0:00 & 56.854 & 59.437 & 50.755 & 57.168 & 37.881 & 37.736 & 41.613 \\
\hline
\end{tabular}

TABLE S1.3 Page 6 of 58 


\begin{tabular}{|c|c|c|c|c|c|c|c|}
\hline \multirow[b]{2}{*}{ Date and Time } & \multicolumn{7}{|c|}{ Water Level (ft below reference point) } \\
\hline & SB43D & SB44D & SB45D & SB46D & SB47D & SB48D & SB49D \\
\hline 8/30/10 4:00 & 56.871 & 59.453 & 50.771 & 57.189 & 37.890 & 37.750 & 41.627 \\
\hline 8/30/10 8:00 & 56.880 & 59.456 & 50.774 & 57.187 & 37.899 & $N A^{a}$ & 41.634 \\
\hline 8/30/10 12:00 & 56.878 & 59.460 & 50.771 & 57.191 & 37.902 & & 41.636 \\
\hline 8/30/10 16:00 & 56.878 & 59.463 & 50.774 & 57.187 & 37.914 & & 41.638 \\
\hline 8/30/10 20:00 & 56.883 & 59.465 & 50.769 & 57.184 & 37.908 & & 41.638 \\
\hline 8/31/10 0:00 & 56.894 & 59.472 & 50.788 & 57.201 & 37.930 & & 41.648 \\
\hline 8/31/10 4:00 & 56.909 & 59.486 & 50.806 & 57.222 & 37.939 & & 41.662 \\
\hline 8/31/10 8:00 & 56.916 & 59.486 & 50.799 & 57.215 & 37.944 & & 41.662 \\
\hline 8/31/10 12:00 & 56.913 & 59.486 & 50.795 & 57.210 & 37.944 & & 41.664 \\
\hline 8/31/10 16:00 & 56.916 & 59.493 & 50.799 & 57.217 & 37.951 & & 41.669 \\
\hline 8/31/10 20:00 & 56.930 & 59.500 & 50.813 & 57.229 & 37.956 & & 41.675 \\
\hline 9/1/10 0:00 & 56.883 & 59.474 & 50.781 & 57.205 & 37.909 & & 41.638 \\
\hline 9/1/10 4:00 & 56.864 & 59.465 & 50.790 & 57.238 & 37.890 & & 41.636 \\
\hline 9/1/10 8:00 & 56.833 & 59.442 & 50.739 & 57.262 & 37.871 & & 41.613 \\
\hline 9/1/10 12:00 & 56.856 & 59.444 & 50.746 & 57.163 & 37.871 & & 41.617 \\
\hline 9/1/10 16:00 & 56.845 & 59.435 & 50.720 & 57.140 & 37.852 & & 41.601 \\
\hline 9/1/10 20:00 & 56.861 & 59.439 & 50.736 & 57.154 & 37.864 & & 41.613 \\
\hline 9/2/10 0:00 & 56.868 & 59.439 & 50.743 & 57.161 & 37.862 & & 41.615 \\
\hline 9/2/10 4:00 & 56.845 & 59.421 & 50.725 & 57.259 & 37.845 & & 41.592 \\
\hline 9/2/10 8:00 & 56.852 & 59.432 & 50.746 & 57.161 & 37.845 & & 41.608 \\
\hline 9/2/10 12:00 & 56.864 & 59.437 & 50.743 & 57.159 & 37.852 & & 41.613 \\
\hline 9/2/10 16:00 & 56.873 & 59.444 & 50.757 & 57.170 & 37.855 & & 41.622 \\
\hline 9/2/10 20:00 & 56.875 & 59.446 & 50.762 & 57.175 & 37.859 & & 41.627 \\
\hline 9/3/10 0:00 & 56.894 & 59.463 & 50.783 & 57.198 & 37.869 & & 41.643 \\
\hline 9/3/10 4:00 & 56.897 & 59.470 & 50.792 & 57.208 & 37.874 & & 41.652 \\
\hline 9/3/10 8:00 & 56.906 & 59.481 & 50.808 & 57.222 & 37.883 & & 41.664 \\
\hline 9/3/10 12:00 & 56.909 & 59.490 & 50.801 & 57.215 & 37.890 & & 41.669 \\
\hline 9/3/10 16:00 & 56.894 & 59.479 & 50.785 & 57.198 & 37.883 & & 41.659 \\
\hline 9/3/10 20:00 & 56.904 & 59.486 & 50.792 & 57.210 & 37.888 & & 41.666 \\
\hline 9/4/10 0:00 & 56.911 & 59.490 & 50.804 & 57.217 & 37.895 & & 41.671 \\
\hline 9/4/10 4:00 & 56.913 & 59.495 & 50.804 & 57.217 & 37.892 & & 41.673 \\
\hline 9/4/10 8:00 & 56.925 & 59.509 & 50.827 & 57.243 & 37.909 & & 41.685 \\
\hline 9/4/10 12:00 & 56.927 & 59.509 & 50.818 & 57.236 & 37.909 & & 41.689 \\
\hline 9/4/10 16:00 & 56.904 & 59.490 & 50.785 & 57.203 & 37.899 & & 41.669 \\
\hline 9/4/10 20:00 & 56.904 & 59.493 & 50.790 & 57.203 & 37.890 & & 41.669 \\
\hline 9/5/10 0:00 & 56.913 & 59.497 & 50.799 & 57.212 & 37.902 & & 41.673 \\
\hline 9/5/10 4:00 & 56.906 & 59.486 & 50.788 & 57.203 & 37.892 & & 41.666 \\
\hline 9/5/10 8:00 & 56.911 & 59.495 & 50.804 & 57.220 & 37.899 & & 41.673 \\
\hline 9/5/10 12:00 & 56.918 & 59.502 & 50.808 & 57.229 & 37.906 & & 41.682 \\
\hline 9/5/10 16:00 & 56.901 & 59.486 & 50.771 & 57.191 & 37.888 & & 41.662 \\
\hline 9/5/10 20:00 & 56.901 & 59.488 & 50.783 & 57.201 & 37.888 & & 41.666 \\
\hline 9/6/10 0:00 & 56.918 & 59.504 & 50.804 & 57.222 & 37.904 & & 41.680 \\
\hline 9/6/10 4:00 & 56.909 & 59.488 & 50.783 & 57.203 & 37.897 & & 41.671 \\
\hline 9/6/10 8:00 & 56.913 & 59.497 & 50.799 & 57.220 & 37.902 & & 41.675 \\
\hline 9/6/10 12:00 & 56.932 & 59.516 & 50.825 & 57.245 & 37.925 & & 41.694 \\
\hline 9/6/10 16:00 & 56.932 & 59.516 & 50.813 & 57.229 & 37.916 & & 41.692 \\
\hline 9/6/10 20:00 & 56.968 & 59.542 & 50.864 & 57.278 & 37.944 & & 41.722 \\
\hline 9/7/10 0:00 & 56.989 & 59.567 & 50.897 & 57.311 & 37.972 & & 41.750 \\
\hline 9/7/10 4:00 & 56.991 & 59.567 & 50.880 & 57.299 & 37.982 & & 41.750 \\
\hline 9/7/10 8:00 & 56.998 & 59.579 & 50.904 & 57.316 & 37.991 & & 41.762 \\
\hline 9/7/10 12:00 & 57.020 & 59.607 & 50.936 & 57.351 & 38.024 & & 41.787 \\
\hline 9/7/10 16:00 & 57.017 & 59.602 & 50.906 & 57.327 & 38.017 & & 41.780 \\
\hline 9/7/10 20:00 & 57.017 & 59.602 & 50.911 & 57.327 & 38.024 & & 41.780 \\
\hline 9/8/10 0:00 & 57.036 & 59.616 & 50.936 & 57.353 & 38.045 & & 41.799 \\
\hline
\end{tabular}

TABLE S1.3 Page 7 of 58 


\begin{tabular}{|c|c|c|c|c|c|c|c|}
\hline \multirow[b]{2}{*}{ Date and Time } & \multicolumn{7}{|c|}{ Water Level (ft below reference point) } \\
\hline & SB43D & SB44D & SB45D & SB46D & SB47D & SB48D & SB49D \\
\hline 9/8/10 4:00 & 57.039 & 59.612 & 50.918 & 57.334 & 38.043 & & 41.794 \\
\hline 9/8/10 8:00 & 57.041 & 59.614 & 50.929 & 57.344 & 38.047 & & 41.794 \\
\hline 9/8/10 12:00 & 57.053 & 59.635 & 50.952 & 57.365 & 38.066 & & 41.808 \\
\hline 9/8/10 16:00 & 57.051 & 59.623 & 50.927 & 57.344 & 38.064 & & 41.803 \\
\hline 9/8/10 20:00 & 57.043 & 59.619 & 50.918 & 57.332 & 38.059 & & 41.796 \\
\hline 9/9/10 0:00 & 57.060 & 59.635 & 50.950 & 57.367 & 38.083 & & 41.815 \\
\hline 9/9/10 4:00 & 57.058 & 59.628 & 50.929 & 57.344 & 38.071 & & 41.806 \\
\hline 9/9/10 8:00 & 57.053 & 59.619 & 50.918 & 57.334 & 38.069 & & 41.801 \\
\hline 9/9/10 12:00 & 57.062 & 59.633 & 50.943 & 57.360 & 38.080 & & 41.810 \\
\hline 9/9/10 16:00 & 57.060 & 59.630 & 50.925 & 57.339 & 38.083 & & 41.810 \\
\hline 9/9/10 20:00 & 57.062 & 59.628 & 50.925 & 57.339 & 38.076 & & 41.803 \\
\hline 9/10/10 0:00 & 57.079 & 59.644 & 50.957 & 57.372 & 38.097 & & 41.822 \\
\hline 9/10/10 4:00 & 57.084 & 59.644 & 50.950 & 57.365 & 38.099 & & 41.822 \\
\hline 9/10/10 8:00 & 57.076 & 59.633 & 50.931 & 57.346 & 38.092 & & 41.813 \\
\hline 9/10/10 12:00 & 57.081 & 59.642 & 50.950 & 57.367 & 38.101 & & 41.822 \\
\hline 9/10/10 16:00 & 57.076 & 59.635 & 50.934 & 57.442 & 38.087 & & 41.810 \\
\hline 9/10/10 20:00 & 57.074 & 59.630 & 50.929 & 57.344 & 38.087 & & 41.810 \\
\hline 9/11/10 0:00 & 57.100 & 59.661 & 50.978 & 57.391 & 38.111 & & 41.838 \\
\hline 9/11/10 4:00 & 57.126 & 59.677 & 50.996 & 57.412 & 38.132 & & 41.859 \\
\hline 9/11/10 8:00 & 57.131 & 59.679 & 50.992 & 57.407 & 38.137 & & 41.861 \\
\hline 9/11/10 12:00 & 57.136 & 59.691 & 51.008 & 57.421 & 38.153 & & 41.873 \\
\hline 9/11/10 16:00 & 57.140 & 59.702 & 51.010 & 57.423 & 38.156 & & 41.882 \\
\hline 9/11/10 20:00 & 57.147 & 59.705 & 51.010 & 57.428 & 38.167 & & 41.885 \\
\hline 9/12/10 0:00 & 57.162 & 59.719 & 51.036 & 57.449 & 38.179 & & 41.899 \\
\hline 9/12/10 4:00 & 57.176 & 59.728 & 51.045 & 57.461 & 38.203 & & 41.910 \\
\hline 9/12/10 8:00 & 57.181 & 59.728 & 51.036 & 57.456 & 38.200 & & 41.910 \\
\hline 9/12/10 12:00 & 57.181 & 59.737 & 51.043 & 57.459 & 38.207 & & 41.915 \\
\hline 9/12/10 16:00 & 57.183 & 59.737 & 51.038 & 57.456 & 38.214 & & 41.917 \\
\hline 9/12/10 20:00 & 57.190 & 59.740 & 51.038 & 57.456 & 38.221 & & 41.920 \\
\hline 9/13/10 0:00 & 57.202 & 59.749 & 51.057 & 57.473 & 38.238 & & 41.929 \\
\hline 9/13/10 4:00 & 57.216 & 59.761 & 51.075 & 57.491 & 38.249 & & 41.943 \\
\hline 9/13/10 8:00 & 57.228 & 59.765 & 51.075 & 57.487 & 38.254 & & 41.943 \\
\hline 9/13/10 12:00 & 57.223 & 59.768 & 51.068 & 57.484 & 38.259 & & 41.943 \\
\hline 9/13/10 16:00 & 57.218 & 59.763 & 51.059 & 57.475 & 38.264 & & 41.941 \\
\hline 9/13/10 20:00 & 57.233 & 59.765 & 51.071 & 57.484 & 38.264 & & 41.948 \\
\hline 9/14/10 0:00 & 57.216 & 59.760 & 51.061 & 57.519 & 38.247 & & 41.938 \\
\hline 9/14/10 4:00 & 57.211 & 59.754 & 51.055 & 57.566 & 38.240 & & 41.924 \\
\hline 9/14/10 8:00 & 57.221 & 59.763 & 51.068 & 57.482 & 38.249 & & 41.941 \\
\hline 9/14/10 12:00 & 57.228 & 59.765 & 51.068 & 57.482 & 38.259 & & 41.943 \\
\hline 9/14/10 16:00 & 57.223 & 59.765 & 51.057 & 57.475 & 38.256 & & 41.943 \\
\hline 9/14/10 20:00 & 57.237 & 59.772 & 51.066 & 57.482 & 38.264 & & 41.950 \\
\hline 9/15/10 0:00 & 57.244 & 59.777 & 51.075 & 57.491 & 38.273 & & 41.955 \\
\hline 9/15/10 4:00 & 57.254 & 59.782 & 51.085 & 57.503 & 38.282 & & 41.961 \\
\hline 9/15/10 8:00 & 57.259 & 59.786 & 51.087 & 57.501 & 38.289 & & 41.964 \\
\hline 9/15/10 12:00 & 57.230 & 59.768 & 51.055 & 57.459 & 38.268 & & 41.941 \\
\hline 9/15/10 16:00 & 57.249 & 59.775 & 51.064 & 57.482 & 38.278 & & 41.952 \\
\hline 9/15/10 20:00 & 57.256 & 59.779 & 51.073 & 57.491 & 38.278 & & 41.957 \\
\hline 9/16/10 0:00 & 57.268 & 59.791 & 51.085 & 57.503 & 38.292 & & 41.966 \\
\hline 9/16/10 4:00 & 57.282 & 59.798 & 51.106 & 57.519 & 38.306 & & 41.980 \\
\hline 9/16/10 8:00 & 57.299 & 59.812 & 51.129 & 57.545 & 38.318 & & 41.996 \\
\hline 9/16/10 12:00 & 57.315 & 59.828 & 51.138 & 57.552 & 38.332 & & 42.013 \\
\hline 9/16/10 16:00 & 57.306 & 59.821 & 51.122 & 57.538 & 38.322 & & 42.003 \\
\hline 9/16/10 20:00 & 57.303 & 59.828 & 51.122 & 57.538 & 38.331 & & 42.006 \\
\hline 9/17/10 0:00 & 57.315 & 59.831 & 51.131 & 57.545 & 38.339 & & 42.013 \\
\hline
\end{tabular}

TABLE S1.3 Page 8 of 58 


\begin{tabular}{|c|c|c|c|c|c|c|c|}
\hline \multirow[b]{2}{*}{ Date and Time } & \multicolumn{7}{|c|}{ Water Level (ft below reference point) } \\
\hline & SB43D & SB44D & SB45D & SB46D & SB47D & SB48D & SB49D \\
\hline 9/17/10 4:00 & 57.318 & 59.835 & 51.131 & 57.548 & 38.341 & & 42.015 \\
\hline 9/17/10 8:00 & 57.329 & 59.847 & 51.152 & 57.566 & 38.353 & & 42.027 \\
\hline 9/17/10 12:00 & 57.337 & 59.849 & 51.150 & 57.566 & 38.358 & & 42.031 \\
\hline 9/17/10 16:00 & 57.325 & 59.845 & 51.131 & 57.548 & 38.355 & & 42.020 \\
\hline 9/17/10 20:00 & 57.337 & 59.852 & 51.147 & 57.562 & 38.367 & & 42.029 \\
\hline 9/18/10 0:00 & 57.353 & 59.861 & 51.161 & 57.576 & 38.374 & & 42.041 \\
\hline 9/18/10 4:00 & 57.356 & 59.861 & 51.159 & 57.576 & 38.381 & & 42.043 \\
\hline 9/18/10 8:00 & 57.374 & 59.879 & 51.185 & 57.604 & 38.397 & & 42.059 \\
\hline 9/18/10 12:00 & 57.386 & 59.891 & 51.194 & 57.611 & 38.412 & & 42.068 \\
\hline 9/18/10 16:00 & 57.382 & 59.887 & 51.182 & 57.597 & 38.416 & & 42.068 \\
\hline 9/18/10 20:00 & 57.389 & 59.894 & 51.194 & 57.606 & 38.416 & & 42.073 \\
\hline 9/19/10 0:00 & 57.403 & 59.903 & 51.203 & 57.620 & 38.430 & & 42.082 \\
\hline 9/19/10 4:00 & 57.398 & 59.898 & 51.194 & 57.606 & 38.428 & & 42.080 \\
\hline 9/19/10 8:00 & 57.415 & 59.912 & 51.219 & 57.634 & 38.444 & & 42.094 \\
\hline 9/19/10 12:00 & 57.426 & 59.921 & 51.226 & 57.644 & 38.459 & & 42.101 \\
\hline 9/19/10 16:00 & 57.417 & 59.914 & 51.201 & 57.620 & 38.446 & & 42.092 \\
\hline 9/19/10 20:00 & 57.422 & 59.919 & 51.210 & 57.630 & 38.454 & & 42.099 \\
\hline 9/20/10 0:00 & 57.436 & 59.926 & 51.222 & 57.639 & 38.463 & & 42.108 \\
\hline 9/20/10 4:00 & 57.426 & 59.914 & 51.203 & 57.618 & 38.459 & & 42.094 \\
\hline 9/20/10 8:00 & 57.436 & 59.924 & 51.222 & 57.639 & 38.468 & & 42.103 \\
\hline 9/20/10 12:00 & 57.445 & 59.935 & 51.231 & 57.651 & 38.475 & & 42.115 \\
\hline 9/20/10 16:00 & 57.436 & 59.926 & 51.201 & 57.620 & 38.470 & & 42.103 \\
\hline 9/20/10 20:00 & 57.441 & 59.928 & 51.208 & 57.630 & 38.473 & & 42.103 \\
\hline 9/21/10 0:00 & 57.460 & 59.942 & 51.236 & 57.651 & 38.491 & & 42.120 \\
\hline 9/21/10 4:00 & 57.460 & 59.938 & 51.219 & 57.639 & 38.494 & & 42.117 \\
\hline 9/21/10 8:00 & 57.467 & 59.945 & 51.240 & 57.658 & 38.499 & & 42.122 \\
\hline 9/21/10 12:00 & 57.476 & 59.956 & 51.254 & 57.669 & 38.510 & & 42.138 \\
\hline 9/21/10 16:00 & 57.483 & 59.959 & 51.245 & 57.665 & 38.513 & & 42.136 \\
\hline 9/21/10 20:00 & 57.365 & 59.903 & 51.157 & 57.646 & 38.414 & & 42.057 \\
\hline 9/22/10 0:00 & 57.379 & 59.831 & 51.185 & 57.601 & 38.409 & & 42.080 \\
\hline 9/22/10 4:00 & 57.405 & 59.800 & 51.171 & 57.592 & 38.402 & & 42.073 \\
\hline 9/22/10 8:00 & 57.417 & 59.788 & 51.180 & 57.601 & 38.402 & & 42.075 \\
\hline 9/22/10 12:00 & 57.431 & 59.866 & 51.201 & 57.618 & 38.409 & & 42.087 \\
\hline 9/22/10 16:00 & 57.419 & 59.901 & 51.168 & 57.585 & 38.393 & & 42.078 \\
\hline 9/22/10 20:00 & 57.412 & 59.896 & 51.168 & 57.585 & 38.381 & & 42.068 \\
\hline 9/23/10 0:00 & 57.424 & 59.905 & 51.189 & 57.608 & 38.385 & & 42.082 \\
\hline 9/23/10 4:00 & 57.417 & 59.896 & 51.168 & 57.587 & 38.376 & & 42.075 \\
\hline 9/23/10 8:00 & 57.408 & 59.891 & 51.164 & 57.585 & 38.362 & & 42.068 \\
\hline 9/23/10 12:00 & 57.408 & 59.898 & 51.180 & 57.601 & 38.369 & & 42.073 \\
\hline 9/23/10 16:00 & 57.396 & 59.889 & 51.154 & 57.578 & 38.353 & & 42.061 \\
\hline 9/23/10 20:00 & 57.339 & 59.863 & 51.126 & 57.615 & 38.296 & & 42.031 \\
\hline 9/24/10 0:00 & 57.320 & 59.875 & 51.152 & 57.590 & 38.287 & & 42.048 \\
\hline 9/24/10 4:00 & 57.353 & 59.879 & 51.157 & 57.576 & 38.292 & & 42.059 \\
\hline 9/24/10 8:00 & 57.367 & 59.882 & 51.164 & 57.580 & 38.287 & & 42.064 \\
\hline 9/24/10 12:00 & 57.379 & 59.894 & 51.185 & 57.604 & 38.294 & & 42.075 \\
\hline 9/24/10 16:00 & 57.374 & 59.891 & 51.166 & 57.585 & 38.278 & & 42.071 \\
\hline 9/24/10 20:00 & 57.370 & 59.889 & 51.159 & 57.583 & 38.266 & & 42.066 \\
\hline 9/25/10 0:00 & 57.374 & 59.898 & 51.185 & 57.601 & 38.268 & & 42.078 \\
\hline 9/25/10 4:00 & 57.365 & 59.889 & 51.164 & 57.580 & 38.247 & & 42.068 \\
\hline 9/25/10 8:00 & 57.334 & 59.870 & 51.131 & 57.548 & 38.226 & & 42.048 \\
\hline 9/25/10 12:00 & 57.329 & 59.870 & 51.147 & 57.562 & 38.217 & & 42.048 \\
\hline 9/25/10 16:00 & 57.334 & 59.872 & 51.145 & 57.559 & 38.214 & & 42.052 \\
\hline 9/25/10 20:00 & 57.325 & 59.865 & 51.140 & 57.559 & 38.202 & & 42.045 \\
\hline 9/26/10 0:00 & 57.329 & 59.877 & 51.166 & 57.585 & 38.210 & & 42.059 \\
\hline
\end{tabular}

TABLE S1.3 Page 9 of 58 


\begin{tabular}{|c|c|c|c|c|c|c|c|}
\hline \multirow[b]{2}{*}{ Date and Time } & \multicolumn{7}{|c|}{ Water Level (ft below reference point) } \\
\hline & SB43D & SB44D & SB45D & SB46D & SB47D & SB48D & SB49D \\
\hline 9/26/10 4:00 & 57.327 & 59.875 & 51.157 & 57.576 & 38.202 & & 42.054 \\
\hline 9/26/10 8:00 & 57.313 & 59.863 & 51.143 & 57.559 & 38.186 & & 42.045 \\
\hline 9/26/10 12:00 & 57.303 & 59.863 & 51.150 & 57.566 & 38.181 & & 42.045 \\
\hline 9/26/10 16:00 & 57.289 & 59.856 & 51.133 & 57.552 & 38.169 & & 42.038 \\
\hline 9/26/10 20:00 & 57.282 & 59.849 & 51.122 & 57.541 & 38.160 & & 42.031 \\
\hline 9/27/10 0:00 & 57.280 & 59.851 & 51.136 & 57.555 & 38.160 & & 42.034 \\
\hline 9/27/10 4:00 & 57.277 & 59.849 & 51.129 & 57.550 & 38.156 & & 42.031 \\
\hline 9/27/10 8:00 & 57.263 & 59.838 & 51.106 & 57.529 & 38.139 & & 42.015 \\
\hline 9/27/10 12:00 & 57.251 & 59.833 & 51.110 & 57.531 & 38.134 & & 42.015 \\
\hline 9/27/10 16:00 & 57.242 & 59.828 & 51.096 & 57.517 & 38.123 & & 42.003 \\
\hline 9/27/10 20:00 & 57.230 & 59.814 & 51.082 & 57.503 & 38.113 & & 41.994 \\
\hline 9/28/10 0:00 & 57.225 & 59.814 & 51.092 & 57.512 & 38.106 & & 41.992 \\
\hline 9/28/10 4:00 & 57.235 & 59.819 & 51.101 & 57.519 & 38.115 & & 41.999 \\
\hline 9/28/10 8:00 & 57.235 & 59.814 & 51.099 & 57.515 & 38.108 & & 41.994 \\
\hline 9/28/10 12:00 & 57.221 & 59.812 & 51.099 & 57.515 & 38.113 & & 41.999 \\
\hline 9/28/10 16:00 & 57.214 & 59.812 & 51.089 & 57.508 & 38.108 & & 41.994 \\
\hline 9/28/10 20:00 & 57.216 & 59.809 & 51.089 & 57.510 & 38.106 & & 41.989 \\
\hline 9/29/10 0:00 & 57.216 & 59.814 & 51.099 & 57.519 & 38.106 & & 41.994 \\
\hline 9/29/10 4:00 & 57.221 & 59.819 & 51.110 & 57.529 & 38.111 & & 41.999 \\
\hline 9/29/10 8:00 & 57.218 & 59.814 & 51.101 & 57.519 & 38.111 & & 41.996 \\
\hline 9/29/10 12:00 & 57.207 & 59.807 & 51.089 & 57.510 & 38.104 & & 41.989 \\
\hline 9/29/10 16:00 & 57.202 & 59.807 & 51.089 & 57.510 & 38.103 & & 41.987 \\
\hline 9/29/10 20:00 & 57.209 & 59.809 & 51.099 & 57.517 & 38.108 & & 41.991 \\
\hline 9/30/10 0:00 & 57.225 & 59.826 & 51.124 & 57.543 & 38.125 & & 42.013 \\
\hline 9/30/10 4:00 & 57.235 & 59.840 & 51.143 & 57.562 & 38.139 & & 42.024 \\
\hline 9/30/10 8:00 & 57.244 & 59.847 & 51.150 & 57.566 & 38.151 & & 42.029 \\
\hline 9/30/10 12:00 & 57.242 & 59.847 & 51.145 & 57.564 & 38.151 & & 42.029 \\
\hline 9/30/10 16:00 & 57.233 & 59.847 & 51.138 & 57.555 & 38.156 & & 42.027 \\
\hline 9/30/10 20:00 & 57.242 & 59.852 & 51.147 & 57.564 & 38.160 & & 42.034 \\
\hline 10/1/10 0:00 & 57.249 & 59.856 & 51.154 & 57.571 & 38.172 & & 42.038 \\
\hline 10/1/10 4:00 & 57.256 & 59.863 & 51.164 & 57.583 & 38.181 & & 42.045 \\
\hline 10/1/10 8:00 & 57.266 & 59.870 & 51.175 & 57.592 & 38.193 & & 42.052 \\
\hline 10/1/10 12:00 & 57.259 & 59.863 & 51.157 & 57.576 & 38.188 & & 42.045 \\
\hline 10/1/10 16:00 & 57.244 & 59.856 & 51.143 & 57.562 & 38.181 & & 42.036 \\
\hline 10/1/10 20:00 & 57.254 & 59.861 & 51.150 & 57.573 & 38.188 & & 42.041 \\
\hline 10/2/10 0:00 & 57.251 & 59.856 & 51.147 & 57.569 & 38.188 & & 42.038 \\
\hline 10/2/10 4:00 & 57.270 & 59.870 & 51.175 & 57.592 & 38.205 & & 42.054 \\
\hline 10/2/10 8:00 & 57.294 & 59.891 & 51.212 & 57.630 & 38.231 & & 42.080 \\
\hline 10/2/10 12:00 & 57.301 & 59.898 & 51.208 & 57.627 & 38.238 & & 42.085 \\
\hline 10/2/10 16:00 & 57.289 & 59.893 & 51.189 & 57.608 & 38.233 & & 42.078 \\
\hline 10/2/10 20:00 & 57.299 & 59.903 & 51.208 & 57.627 & 38.247 & & 42.085 \\
\hline 10/3/10 0:00 & 57.308 & 59.908 & 51.212 & 57.634 & 38.257 & & 42.092 \\
\hline 10/3/10 4:00 & 57.311 & 59.910 & 51.215 & 57.632 & 38.259 & & 42.092 \\
\hline 10/3/10 8:00 & 57.327 & 59.924 & 51.243 & 57.660 & 38.275 & & 42.108 \\
\hline 10/3/10 12:00 & 57.332 & 59.926 & 51.236 & 57.655 & 38.285 & & 42.113 \\
\hline 10/3/10 16:00 & 57.315 & 59.912 & 51.205 & 57.625 & 38.273 & & 42.094 \\
\hline 10/3/10 20:00 & 57.322 & 59.919 & 51.219 & 57.641 & 38.280 & & 42.099 \\
\hline 10/4/10 0:00 & 57.332 & 59.924 & 51.224 & 57.644 & 38.287 & & 42.106 \\
\hline 10/4/10 4:00 & 57.329 & 59.919 & 51.219 & 57.637 & 38.284 & & 42.099 \\
\hline 10/4/10 8:00 & 57.341 & 59.931 & 51.243 & 57.660 & 38.301 & & 42.113 \\
\hline 10/4/10 12:00 & 57.353 & 59.938 & 51.247 & 57.667 & 38.310 & & 42.122 \\
\hline 10/4/10 16:00 & 57.334 & 59.926 & 51.215 & 57.634 & 38.296 & & 42.103 \\
\hline 10/4/10 20:00 & 57.341 & 59.931 & 51.236 & 57.653 & 38.304 & & 42.113 \\
\hline 10/5/10 0:00 & 57.356 & 59.942 & 51.247 & 57.667 & 38.318 & & 42.124 \\
\hline
\end{tabular}

TABLE S1.3 Page 10 of 58 


\begin{tabular}{|c|c|c|c|c|c|c|c|}
\hline \multirow[b]{2}{*}{ Date and Time } & \multicolumn{7}{|c|}{ Water Level (ft below reference point) } \\
\hline & SB43D & SB44D & SB45D & SB46D & SB47D & SB48D & SB49D \\
\hline 10/5/10 4:00 & 57.353 & 59.933 & 51.231 & 57.651 & 38.313 & & 42.115 \\
\hline 10/5/10 8:00 & 57.358 & 59.945 & 51.254 & 57.674 & 38.327 & & 42.124 \\
\hline 10/5/10 12:00 & 57.374 & 59.959 & 51.268 & 57.688 & 38.341 & & 42.141 \\
\hline 10/5/10 16:00 & 57.360 & 59.945 & 51.236 & 57.660 & 38.329 & & 42.122 \\
\hline 10/5/10 20:00 & 57.365 & 59.952 & 51.256 & 57.672 & 38.334 & & 42.131 \\
\hline 10/6/10 0:00 & 57.382 & 59.963 & 51.270 & 57.693 & 38.353 & & 42.145 \\
\hline 10/6/10 4:00 & 57.372 & 59.949 & 51.240 & 57.662 & 38.341 & & 42.129 \\
\hline 10/6/10 8:00 & 57.370 & 59.949 & 51.250 & 57.674 & 38.346 & & 42.129 \\
\hline 10/6/10 12:00 & 57.379 & 59.961 & 51.263 & 57.688 & 38.355 & & 42.143 \\
\hline 10/6/10 16:00 & 57.372 & 59.949 & 51.238 & 57.660 & 38.345 & & 42.129 \\
\hline 10/6/10 20:00 & 57.379 & 59.956 & 51.254 & 57.674 & 38.355 & & 42.136 \\
\hline 10/7/10 0:00 & 57.400 & 59.975 & 51.284 & 57.704 & 38.374 & & 42.157 \\
\hline 10/7/10 4:00 & 57.403 & 59.970 & 51.266 & 57.688 & 38.374 & & 42.152 \\
\hline 10/7/10 8:00 & 57.400 & 59.973 & 51.275 & 57.695 & 38.371 & & 42.152 \\
\hline 10/7/10 12:00 & 57.415 & 59.989 & 51.301 & 57.723 & 38.397 & & 42.171 \\
\hline 10/7/10 16:00 & 57.408 & 59.982 & 51.275 & 57.700 & 38.390 & & 42.161 \\
\hline 10/7/10 20:00 & 57.410 & 59.982 & 51.280 & 57.702 & 38.393 & & 42.161 \\
\hline 10/8/10 0:00 & 57.424 & 59.998 & 51.303 & 57.726 & 38.412 & & 42.178 \\
\hline 10/8/10 4:00 & 57.424 & 59.989 & 51.280 & 57.704 & 38.402 & & 42.168 \\
\hline 10/8/10 8:00 & 57.415 & 59.979 & 51.273 & 57.693 & 38.395 & & 42.161 \\
\hline 10/8/10 12:00 & 57.422 & 59.993 & 51.294 & 57.719 & 38.414 & & 42.173 \\
\hline 10/8/10 16:00 & 57.422 & 59.991 & 51.277 & 57.702 & 38.411 & & 42.168 \\
\hline 10/8/10 20:00 & 57.426 & 59.991 & 51.284 & 57.707 & 38.409 & & 42.168 \\
\hline 10/9/10 0:00 & 57.445 & 60.010 & 51.317 & 57.740 & 38.430 & & 42.189 \\
\hline 10/9/10 4:00 & 57.450 & 60.010 & 51.305 & 57.733 & 38.433 & & 42.189 \\
\hline 10/9/10 8:00 & 57.443 & 60.003 & 51.291 & 57.714 & 38.430 & & 42.180 \\
\hline 10/9/10 12:00 & 57.448 & 60.010 & 51.310 & 57.733 & 38.437 & & 42.189 \\
\hline 10/9/10 16:00 & 57.450 & 60.014 & 51.303 & 57.728 & 38.444 & & 42.189 \\
\hline 10/9/10 20:00 & 57.455 & 60.014 & 51.310 & 57.730 & 38.444 & & 42.192 \\
\hline 10/10/10 0:00 & 57.471 & 60.033 & 51.338 & 57.761 & 38.468 & & 42.210 \\
\hline 10/10/10 4:00 & 57.478 & 60.033 & 51.331 & 57.756 & 38.468 & & 42.213 \\
\hline 10/10/10 8:00 & 57.476 & 60.026 & 51.317 & 57.742 & 38.465 & & 42.206 \\
\hline 10/10/10 12:00 & 57.476 & 60.033 & 51.328 & 57.754 & 38.472 & & 42.210 \\
\hline 10/10/10 16:00 & 57.481 & 60.038 & 51.328 & 57.754 & 38.475 & & 42.215 \\
\hline 10/10/10 20:00 & 57.481 & 60.035 & 51.326 & 57.751 & 38.475 & & 42.215 \\
\hline 10/11/10 0:00 & 57.500 & 60.049 & 51.356 & 57.779 & 38.498 & & 42.231 \\
\hline 10/11/10 4:00 & 57.509 & 60.059 & 51.359 & 57.782 & 38.503 & & 42.234 \\
\hline 10/11/10 8:00 & 57.505 & 60.049 & 51.340 & 57.768 & 38.494 & & 42.229 \\
\hline $10 / 11 / 1012: 00$ & 57.507 & 60.052 & 51.352 & 57.772 & 38.501 & & 42.231 \\
\hline 10/11/10 16:00 & 57.505 & 60.049 & 51.347 & 57.770 & 38.503 & & 42.229 \\
\hline 10/11/10 20:00 & 57.514 & 60.056 & 51.352 & 57.777 & 38.510 & & 42.236 \\
\hline 10/12/10 0:00 & 57.521 & 60.061 & 51.366 & 57.829 & 38.513 & & 42.240 \\
\hline 10/12/10 4:00 & 57.528 & 60.068 & 51.377 & 57.805 & 38.520 & & 42.250 \\
\hline 10/12/10 8:00 & 57.533 & 60.073 & 51.373 & 57.772 & 38.522 & & 42.250 \\
\hline $10 / 12 / 1012: 00$ & 57.538 & 60.077 & 51.379 & 57.803 & 38.534 & & 42.257 \\
\hline 10/12/10 16:00 & 57.545 & 60.089 & 51.389 & 57.810 & 38.543 & & 42.264 \\
\hline 10/12/10 20:00 & 57.557 & 60.096 & 51.398 & 57.824 & 38.552 & & 42.275 \\
\hline 10/13/10 0:00 & 57.575 & 60.112 & 51.421 & 57.847 & 38.574 & & 42.294 \\
\hline 10/13/10 4:00 & 57.587 & 60.126 & 51.440 & 57.864 & 38.588 & & 42.308 \\
\hline 10/13/10 8:00 & 57.604 & 60.136 & 51.444 & 57.868 & 38.597 & & 42.315 \\
\hline 10/13/10 12:00 & 57.599 & 60.138 & 51.442 & 57.864 & 38.604 & & 42.320 \\
\hline 10/13/10 16:00 & 57.597 & 60.138 & 51.438 & 57.859 & 38.609 & & 42.317 \\
\hline 10/13/10 20:00 & 57.609 & 60.145 & 51.442 & 57.868 & 38.616 & & 42.324 \\
\hline 10/14/10 0:00 & 57.618 & 60.150 & 51.451 & 57.875 & 38.627 & & 42.329 \\
\hline
\end{tabular}

TABLE S1.3 Page 11 of 58 


\begin{tabular}{|c|c|c|c|c|c|c|c|}
\hline \multirow[b]{2}{*}{ Date and Time } & \multicolumn{7}{|c|}{ Water Level (ft below reference point) } \\
\hline & SB43D & SB44D & SB45D & SB46D & SB47D & SB48D & SB49D \\
\hline 10/14/10 4:00 & 57.623 & 60.152 & 51.454 & 57.878 & 38.632 & & 42.331 \\
\hline 10/14/10 8:00 & 57.627 & 60.157 & 51.451 & 57.878 & 38.642 & & 42.333 \\
\hline $10 / 14 / 1012: 00$ & 57.618 & 60.145 & 51.428 & 57.854 & 38.632 & & 42.322 \\
\hline 10/14/10 16:00 & 57.606 & 60.133 & 51.414 & 57.838 & 38.621 & & 42.308 \\
\hline 10/14/10 20:00 & 57.620 & 60.143 & 51.433 & 57.857 & 38.630 & & 42.317 \\
\hline 10/15/10 0:00 & 57.635 & 60.152 & 51.449 & 57.871 & 38.646 & & 42.329 \\
\hline 10/15/10 4:00 & 57.646 & 60.166 & 51.465 & 57.887 & 38.656 & & 42.338 \\
\hline 10/15/10 8:00 & 57.663 & 60.175 & 51.482 & 57.904 & 38.670 & & 42.352 \\
\hline 10/15/10 12:00 & 57.668 & 60.178 & 51.472 & 57.899 & 38.677 & & 42.354 \\
\hline 10/15/10 16:00 & 57.658 & 60.175 & 51.458 & 57.887 & 38.670 & & 42.350 \\
\hline 10/15/10 20:00 & 57.665 & 60.180 & 51.468 & 57.892 & 38.681 & & 42.354 \\
\hline 10/16/10 0:00 & 57.672 & 60.182 & 51.470 & 57.894 & 38.682 & & 42.357 \\
\hline 10/16/10 4:00 & 57.672 & 60.182 & 51.468 & 57.897 & 38.686 & & 42.357 \\
\hline 10/16/10 8:00 & 57.680 & 60.187 & 51.479 & 57.904 & 38.694 & & 42.364 \\
\hline 10/16/10 12:00 & 57.689 & 60.191 & 51.484 & 57.906 & 38.698 & & 42.366 \\
\hline 10/16/10 16:00 & 57.689 & 60.191 & 51.477 & 57.904 & 38.701 & & 42.368 \\
\hline 10/16/10 20:00 & 57.708 & 60.208 & 51.505 & 57.932 & 38.724 & & 42.384 \\
\hline 10/17/10 0:00 & 57.722 & 60.217 & 51.514 & 57.939 & 38.731 & & 42.396 \\
\hline 10/17/10 4:00 & 57.724 & 60.219 & 51.516 & 57.941 & 38.738 & & 42.399 \\
\hline 10/17/10 8:00 & 57.736 & 60.231 & 51.533 & 57.960 & 38.750 & & 42.410 \\
\hline 10/17/10 12:00 & 57.739 & 60.233 & 51.526 & 57.960 & 38.757 & & 42.410 \\
\hline 10/17/10 16:00 & 57.724 & 60.224 & 51.500 & 57.936 & 38.745 & & 42.394 \\
\hline 10/17/10 20:00 & 57.734 & 60.229 & 51.516 & 57.948 & 38.752 & & 42.401 \\
\hline 10/18/10 0:00 & 57.743 & 60.231 & 51.516 & 57.950 & 38.761 & & 42.406 \\
\hline 10/18/10 4:00 & 57.743 & 60.229 & 51.509 & 57.946 & 38.759 & & 42.401 \\
\hline 10/18/10 8:00 & 57.758 & 60.240 & 51.537 & 57.967 & 38.775 & & 42.417 \\
\hline 10/18/10 12:00 & 57.772 & 60.252 & 51.544 & 57.976 & 38.783 & & 42.429 \\
\hline 10/18/10 16:00 & 57.767 & 60.250 & 51.528 & 57.960 & 38.785 & & 42.424 \\
\hline 10/18/10 20:00 & 57.781 & 60.261 & 51.554 & 57.986 & 38.794 & & 42.438 \\
\hline 10/19/10 0:00 & 57.798 & 60.271 & 51.563 & 57.997 & 38.811 & & 42.450 \\
\hline 10/19/10 4:00 & 57.793 & 60.264 & 51.549 & 57.983 & 38.806 & & 42.440 \\
\hline 10/19/10 8:00 & 57.798 & 60.273 & 51.565 & 58.000 & 38.820 & & 42.450 \\
\hline 10/19/10 12:00 & 57.812 & 60.285 & 51.574 & 58.011 & 38.829 & & 42.459 \\
\hline 10/19/10 16:00 & 57.800 & 60.275 & 51.551 & 57.986 & 38.820 & & 42.445 \\
\hline 10/19/10 20:00 & 57.807 & 60.282 & 51.565 & 58.000 & 38.829 & & 42.452 \\
\hline 10/20/10 0:00 & 57.819 & 60.289 & 51.574 & 58.007 & 38.842 & & 42.459 \\
\hline 10/20/10 4:00 & 57.817 & 60.282 & 51.556 & 57.990 & 38.837 & & 42.454 \\
\hline 10/20/10 8:00 & 57.824 & 60.287 & 51.574 & 58.007 & 38.842 & & 42.461 \\
\hline 10/20/10 12:00 & 57.838 & 60.303 & 51.593 & 58.026 & 38.860 & & 42.473 \\
\hline 10/20/10 16:00 & 57.831 & 60.296 & 51.572 & 58.004 & 38.851 & & 42.468 \\
\hline 10/20/10 20:00 & 57.843 & 60.306 & 51.593 & 58.026 & 38.867 & & 42.480 \\
\hline 10/21/10 0:00 & 57.864 & 60.324 & 51.614 & 58.049 & 38.879 & & 42.494 \\
\hline 10/21/10 4:00 & 57.871 & 60.322 & 51.607 & 58.040 & 38.881 & & 42.496 \\
\hline 10/21/10 8:00 & 57.876 & 60.329 & 51.619 & 58.047 & 38.889 & & 42.501 \\
\hline 10/21/10 12:00 & 57.888 & 60.343 & 51.637 & 58.068 & 38.909 & & 42.517 \\
\hline $10 / 21 / 1016: 00$ & 57.883 & 60.338 & 51.612 & 58.047 & 38.905 & & 42.508 \\
\hline 10/21/10 20:00 & 57.890 & 60.345 & 51.626 & 58.061 & 38.914 & & 42.515 \\
\hline 10/22/10 0:00 & 57.904 & 60.357 & 51.642 & 58.075 & 38.926 & & 42.529 \\
\hline 10/22/10 4:00 & 57.902 & 60.350 & 51.623 & 58.058 & 38.923 & & 42.520 \\
\hline 10/22/10 8:00 & 57.899 & 60.345 & 51.619 & 58.056 & 38.924 & & 42.517 \\
\hline $10 / 22 / 1012: 00$ & 57.909 & 60.357 & 51.635 & 58.072 & 38.938 & & 42.526 \\
\hline 10/22/10 16:00 & 57.907 & 60.355 & 51.621 & 58.056 & 38.933 & & 42.522 \\
\hline 10/22/10 20:00 & 57.914 & 60.357 & 51.635 & 58.070 & 38.938 & & 42.526 \\
\hline 10/23/10 0:00 & 57.921 & 60.359 & 51.642 & 58.143 & 38.938 & & 42.529 \\
\hline
\end{tabular}

TABLE S1.3 Page 12 of 58 


\begin{tabular}{|c|c|c|c|c|c|c|c|}
\hline \multirow[b]{2}{*}{ Date and Time } & \multicolumn{7}{|c|}{ Water Level (ft below reference point) } \\
\hline & SB43D & SB44D & SB45D & SB46D & SB47D & SB48D & SB49D \\
\hline 10/23/10 4:00 & 57.895 & 60.320 & 51.593 & 58.103 & 38.895 & & 42.480 \\
\hline 10/23/10 8:00 & 57.829 & 60.315 & 51.558 & 57.997 & 38.858 & & 42.475 \\
\hline 10/23/10 12:00 & 57.838 & 60.310 & 51.572 & 58.011 & 38.860 & & 42.475 \\
\hline 10/23/10 16:00 & 57.845 & 60.306 & 51.558 & 57.997 & 38.860 & & 42.471 \\
\hline 10/23/10 20:00 & 57.857 & 60.308 & 51.577 & 58.011 & 38.867 & & 42.473 \\
\hline 10/24/10 0:00 & 57.876 & 60.322 & 51.600 & 58.037 & 38.883 & & 42.492 \\
\hline 10/24/10 4:00 & 57.873 & 60.313 & 51.579 & 58.016 & 38.876 & & 42.485 \\
\hline 10/24/10 8:00 & 57.862 & 60.303 & 51.565 & 58.000 & 38.865 & & 42.471 \\
\hline $10 / 24 / 1012: 00$ & 57.873 & 60.315 & 51.584 & 58.023 & 38.879 & & 42.485 \\
\hline 10/24/10 16:00 & 57.876 & 60.317 & 51.581 & 58.018 & 38.881 & & 42.485 \\
\hline 10/24/10 20:00 & 57.878 & 60.315 & 51.584 & 58.021 & 38.881 & & 42.482 \\
\hline 10/25/10 0:00 & 57.883 & 60.327 & 51.602 & 58.035 & 38.896 & & 42.492 \\
\hline 10/25/10 4:00 & 57.881 & 60.322 & 51.579 & 58.018 & 38.883 & & 42.487 \\
\hline 10/25/10 8:00 & 57.862 & 60.296 & 51.549 & 57.986 & 38.863 & & 42.464 \\
\hline 10/25/10 12:00 & 57.852 & 60.292 & 51.542 & 57.981 & 38.862 & & 42.459 \\
\hline 10/25/10 16:00 & 57.836 & 60.273 & 51.523 & 57.955 & 38.839 & & 42.438 \\
\hline 10/25/10 20:00 & 57.836 & 60.268 & 51.519 & 57.960 & 38.844 & & 42.436 \\
\hline 10/26/10 0:00 & 57.859 & 60.292 & 51.565 & 57.997 & 38.858 & & 42.459 \\
\hline 10/26/10 4:00 & 57.871 & 60.308 & 51.574 & 58.018 & 38.862 & & 42.471 \\
\hline 10/26/10 8:00 & 57.873 & 60.306 & 51.574 & NA & 38.867 & & 42.473 \\
\hline 10/26/10 12:00 & 57.888 & 60.322 & 51.600 & & 38.888 & & 42.492 \\
\hline 10/26/10 16:00 & 57.897 & 60.329 & 51.605 & & 38.898 & & 42.503 \\
\hline 10/26/10 20:00 & 57.911 & 60.343 & 51.619 & & 38.905 & & 42.513 \\
\hline 10/27/10 0:00 & 57.928 & 60.364 & 51.649 & & 38.931 & & 42.531 \\
\hline 10/27/10 4:00 & 57.944 & 60.376 & 51.660 & & 38.947 & & 42.543 \\
\hline 10/27/10 8:00 & 57.951 & 60.376 & 51.656 & & 38.954 & & 42.547 \\
\hline 10/27/10 12:00 & 57.959 & 60.390 & 51.672 & & 38.968 & & 42.557 \\
\hline 10/27/10 16:00 & 57.975 & 60.403 & 51.693 & & 38.987 & & 42.573 \\
\hline 10/27/10 20:00 & 58.008 & 60.429 & 51.725 & & 39.015 & & 42.603 \\
\hline 10/28/10 0:00 & 58.027 & 60.448 & 51.749 & & 39.041 & & 42.624 \\
\hline 10/28/10 4:00 & 58.046 & 60.464 & 51.765 & & 39.057 & & 42.638 \\
\hline 10/28/10 8:00 & 58.060 & 60.473 & 51.765 & & 39.076 & & 42.645 \\
\hline 10/28/10 12:00 & 58.065 & 60.480 & 51.769 & & 39.083 & & 42.650 \\
\hline 10/28/10 16:00 & 58.067 & 60.487 & 51.769 & & 39.095 & & 42.654 \\
\hline 10/28/10 20:00 & 58.077 & 60.490 & 51.772 & & 39.104 & & 42.659 \\
\hline 10/29/10 0:00 & 58.084 & 60.497 & 51.779 & & 39.116 & & 42.661 \\
\hline 10/29/10 4:00 & 58.091 & 60.499 & 51.783 & & 39.125 & & 42.668 \\
\hline 10/29/10 8:00 & 58.093 & 60.497 & 51.769 & & 39.125 & & 42.664 \\
\hline 10/29/10 12:00 & 58.079 & 60.485 & 51.744 & & 39.116 & & 42.647 \\
\hline 10/29/10 16:00 & 58.065 & 60.469 & 51.728 & & 39.109 & 38.901 & 42.631 \\
\hline 10/29/10 20:00 & 58.074 & 60.469 & 51.730 & & 39.116 & 38.903 & 42.631 \\
\hline 10/30/10 0:00 & 58.074 & 60.469 & 51.723 & & 39.109 & 38.903 & 42.626 \\
\hline 10/30/10 4:00 & 58.072 & 60.462 & 51.723 & & 39.109 & 38.901 & 42.626 \\
\hline 10/30/10 8:00 & 58.086 & 60.469 & 51.735 & & 39.119 & 38.910 & 42.636 \\
\hline 10/30/10 12:00 & 58.089 & 60.471 & 51.732 & & 39.121 & 38.912 & 42.633 \\
\hline 10/30/10 16:00 & 58.091 & 60.476 & 51.737 & & 39.125 & 38.912 & 42.638 \\
\hline 10/30/10 20:00 & 58.110 & 60.492 & 51.760 & & 39.144 & 38.924 & 42.652 \\
\hline $10 / 31 / 100: 00$ & 58.122 & 60.499 & 51.767 & & 39.149 & 38.936 & 42.664 \\
\hline 10/31/10 4:00 & 58.127 & 60.506 & 51.774 & & 39.161 & 38.945 & 42.668 \\
\hline 10/31/10 8:00 & 58.141 & 60.518 & 51.795 & & 39.172 & 38.959 & 42.680 \\
\hline 10/31/10 12:00 & 58.148 & 60.520 & 51.790 & & 39.180 & 38.971 & 42.687 \\
\hline 10/31/10 16:00 & 58.143 & 60.518 & 51.781 & & 39.177 & 38.964 & 42.680 \\
\hline 10/31/10 20:00 & 58.162 & 60.534 & 51.811 & & 39.196 & 38.980 & 42.696 \\
\hline 11/1/10 0:00 & 58.171 & 60.543 & 51.809 & & 39.205 & 38.992 & 42.703 \\
\hline
\end{tabular}

TABLE S1.3 Page 13 of 58 


\begin{tabular}{|c|c|c|c|c|c|c|c|}
\hline \multirow[b]{2}{*}{ Date and Time } & \multicolumn{7}{|c|}{ Water Level (ft below reference point) } \\
\hline & SB43D & SB44D & SB45D & SB46D & SB47D & SB48D & SB49D \\
\hline 11/1/10 4:00 & 58.174 & 60.539 & 51.811 & & 39.205 & 38.992 & 42.705 \\
\hline $11 / 1 / 108: 00$ & 58.190 & 60.557 & 51.834 & & 39.224 & 39.011 & 42.722 \\
\hline $11 / 1 / 1012: 00$ & 58.200 & 60.562 & 51.832 & & 39.236 & 39.025 & 42.726 \\
\hline $11 / 1 / 1016: 00$ & 58.195 & 60.562 & 51.827 & & 39.236 & 39.020 & 42.726 \\
\hline $11 / 1 / 1020: 00$ & 58.216 & 60.578 & 51.860 & & 39.257 & 39.037 & 42.743 \\
\hline 11/2/10 0:00 & 58.230 & 60.588 & 51.858 & & 39.266 & 39.053 & 42.750 \\
\hline $11 / 2 / 104: 00$ & 58.223 & 60.581 & 51.839 & & 39.262 & 39.048 & 42.743 \\
\hline $11 / 2 / 108: 00$ & 58.231 & 60.588 & 51.860 & & 39.273 & 39.060 & 42.750 \\
\hline $11 / 2 / 1012: 00$ & 58.242 & 60.595 & 51.862 & & 39.283 & 39.072 & 42.757 \\
\hline 11/2/10 16:00 & 58.226 & 60.581 & 51.837 & & 39.271 & 39.060 & 42.743 \\
\hline $11 / 2 / 1020: 00$ & 58.238 & 60.595 & 51.860 & & 39.288 & 39.072 & 42.754 \\
\hline 11/3/10 0:00 & 58.249 & 60.595 & 51.858 & & 39.288 & 39.079 & 42.757 \\
\hline $11 / 3 / 104: 00$ & 58.238 & 60.583 & 51.830 & & 39.273 & 39.069 & 42.743 \\
\hline $11 / 3 / 108: 00$ & 58.242 & 60.583 & 51.848 & & 39.290 & 39.076 & 42.747 \\
\hline $11 / 3 / 1012: 00$ & 58.257 & 60.599 & 51.865 & & 39.306 & 39.091 & 42.761 \\
\hline $11 / 3 / 1016: 00$ & 58.247 & 60.588 & 51.844 & & 39.295 & 39.084 & 42.754 \\
\hline 11/3/10 20:00 & 58.264 & 60.602 & 51.867 & & 39.306 & 39.100 & 42.764 \\
\hline 11/4/10 0:00 & 58.283 & 60.615 & 51.883 & & 39.328 & 39.114 & 42.782 \\
\hline $11 / 4 / 104: 00$ & 58.278 & 60.606 & 51.862 & & 39.313 & 39.109 & 42.768 \\
\hline $11 / 4 / 108: 00$ & 58.273 & 60.604 & 51.867 & & 39.320 & 39.112 & 42.768 \\
\hline $11 / 4 / 1012: 00$ & 58.285 & 60.615 & 51.881 & & 39.328 & 39.126 & 42.780 \\
\hline $11 / 4 / 1016: 00$ & 58.278 & 60.609 & 51.855 & & 39.325 & 39.121 & 42.773 \\
\hline $11 / 4 / 10$ 20:00 & 58.294 & 60.625 & 51.888 & & 39.342 & 39.133 & 42.787 \\
\hline $11 / 5 / 100: 00$ & 58.316 & 60.641 & 51.909 & & 39.356 & 39.152 & 42.803 \\
\hline $11 / 5 / 104: 00$ & 58.313 & 60.629 & 51.890 & & 39.349 & 39.152 & 42.796 \\
\hline $11 / 5 / 108: 00$ & 58.311 & 60.634 & 51.893 & & 39.351 & 39.154 & 42.794 \\
\hline $11 / 5 / 1012: 00$ & 58.325 & 60.646 & 51.913 & & 39.370 & 39.168 & 42.810 \\
\hline 11/5/10 16:00 & 58.318 & 60.641 & 51.892 & & 39.365 & 39.163 & 42.803 \\
\hline 11/5/10 20:00 & 58.325 & 60.648 & 51.909 & & 39.382 & 39.170 & 42.808 \\
\hline 11/6/10 0:00 & 58.349 & 60.669 & 51.939 & & 39.394 & 39.194 & 42.829 \\
\hline 11/6/10 4:00 & 58.351 & 60.662 & 51.916 & & 39.387 & 39.189 & 42.824 \\
\hline $11 / 6 / 108: 00$ & 58.346 & 60.657 & 51.916 & & 39.384 & 39.189 & 42.819 \\
\hline $11 / 6 / 1012: 00$ & 58.354 & 60.669 & 51.930 & & 39.400 & 39.199 & 42.829 \\
\hline $11 / 6 / 1016: 00$ & 58.346 & 60.660 & 51.909 & & 39.391 & 39.192 & 42.817 \\
\hline $11 / 6 / 1020: 00$ & 58.354 & 60.664 & 51.923 & & 39.403 & 39.199 & 42.824 \\
\hline $11 / 7 / 100: 00$ & 58.368 & 60.681 & 51.946 & & 39.419 & 39.213 & 42.840 \\
\hline $11 / 7 / 104: 00$ & 58.372 & 60.678 & 51.927 & & 39.415 & 39.213 & 42.838 \\
\hline 11/7/10 8:00 & 58.363 & 60.669 & 51.909 & & 39.410 & 39.203 & 42.826 \\
\hline 11/7/10 12:00 & 58.368 & 60.674 & 51.927 & & 39.414 & 39.210 & 42.831 \\
\hline $11 / 7 / 1016: 00$ & 58.361 & 60.660 & 51.906 & & 39.407 & 39.203 & 42.824 \\
\hline $11 / 7 / 1020: 00$ & 58.363 & 60.667 & 51.913 & & 39.405 & 39.206 & 42.826 \\
\hline $11 / 8 / 100: 00$ & 58.370 & 60.674 & 51.930 & & 39.422 & 39.215 & 42.836 \\
\hline 11/8/10 4:00 & 58.372 & 60.671 & 51.916 & & 39.419 & 39.215 & 42.831 \\
\hline 11/8/10 8:00 & 58.363 & 60.660 & 51.897 & & 39.408 & 39.203 & 42.815 \\
\hline $11 / 8 / 1012: 00$ & 58.368 & 60.667 & 51.916 & & 39.414 & 39.210 & 42.824 \\
\hline $11 / 8 / 1016: 00$ & 58.368 & 60.662 & 51.906 & & 39.417 & 39.210 & 42.819 \\
\hline $11 / 8 / 1020: 00$ & 58.375 & 60.667 & 51.913 & & 39.414 & 39.213 & 42.824 \\
\hline $11 / 9 / 100: 00$ & 58.389 & 60.683 & 51.941 & & 39.433 & 39.227 & 42.840 \\
\hline 11/9/10 4:00 & 58.398 & 60.688 & 51.939 & & 39.436 & 39.234 & 42.845 \\
\hline 11/9/10 8:00 & 58.396 & 60.678 & 51.925 & & 39.436 & 39.229 & 42.838 \\
\hline $11 / 9 / 1012: 00$ & 58.391 & 60.681 & 51.930 & & 39.438 & 39.231 & 42.840 \\
\hline 11/9/10 16:00 & 58.394 & 60.683 & 51.927 & & 39.440 & 39.234 & 42.840 \\
\hline $11 / 9 / 1020: 00$ & 58.403 & 60.690 & 51.937 & & 39.447 & 39.236 & 42.845 \\
\hline 11/10/10 0:00 & 58.420 & 60.706 & 51.960 & & 39.459 & 39.253 & 42.864 \\
\hline
\end{tabular}

TABLE S1.3 Page 14 of 58 


\begin{tabular}{|c|c|c|c|c|c|c|c|}
\hline \multirow[b]{2}{*}{ Date and Time } & \multicolumn{7}{|c|}{ Water Level (ft below reference point) } \\
\hline & SB43D & SB44D & SB45D & SB46D & SB47D & SB48D & SB49D \\
\hline 11/10/10 4:00 & 58.429 & 60.713 & 51.969 & & 39.468 & 39.264 & 42.873 \\
\hline 11/10/10 8:00 & 58.429 & 60.711 & 51.955 & & 39.464 & 39.262 & 42.868 \\
\hline 11/10/10 12:00 & 58.429 & 60.709 & 51.958 & & 39.471 & 39.267 & 42.871 \\
\hline 11/10/10 16:00 & 58.446 & 60.725 & 51.983 & & 39.487 & 39.278 & 42.885 \\
\hline 11/10/10 20:00 & 58.469 & 60.741 & 52.004 & & 39.501 & 39.297 & 42.901 \\
\hline 11/11/10 0:00 & 58.486 & 60.762 & 52.032 & & 39.527 & 39.278 & 42.924 \\
\hline 11/11/10 4:00 & 58.505 & 60.776 & 52.046 & & 39.546 & 39.335 & 42.938 \\
\hline 11/11/10 8:00 & 58.517 & 60.786 & 52.053 & & 39.555 & 39.337 & 42.947 \\
\hline 11/11/10 12:00 & 58.517 & 60.788 & 52.048 & & 39.560 & 39.349 & 42.947 \\
\hline 11/11/10 16:00 & 58.512 & 60.788 & 52.048 & & 39.565 & 39.344 & 42.949 \\
\hline $11 / 11 / 1020: 00$ & 58.521 & 60.792 & 52.050 & & 39.574 & 39.318 & 42.954 \\
\hline 11/12/10 0:00 & 58.509 & 60.776 & 52.029 & & 39.556 & 39.304 & 42.933 \\
\hline 11/12/10 4:00 & 58.507 & 60.781 & 52.032 & & 39.562 & 39.307 & 42.938 \\
\hline 11/12/10 8:00 & 58.514 & 60.772 & 52.025 & & 39.565 & 39.330 & 42.931 \\
\hline $11 / 12 / 1012: 00$ & 58.472 & 60.760 & 51.995 & & 39.520 & 39.276 & 42.915 \\
\hline 11/12/10 16:00 & 58.460 & 60.744 & 51.978 & & 39.516 & 39.285 & 42.901 \\
\hline 11/12/10 20:00 & 58.441 & 60.718 & 51.948 & & 39.546 & 39.257 & 42.868 \\
\hline 11/13/10 0:00 & 58.396 & 60.709 & 51.927 & & 39.452 & 39.227 & 42.857 \\
\hline 11/13/10 4:00 & 58.415 & 60.711 & 51.946 & & 39.457 & 39.243 & 42.866 \\
\hline 11/13/10 8:00 & 58.434 & 60.716 & 51.955 & & 39.464 & 39.262 & 42.873 \\
\hline 11/13/10 12:00 & 58.441 & 60.718 & 51.960 & & 39.468 & 39.269 & 42.873 \\
\hline $11 / 13 / 1016: 00$ & 58.439 & 60.713 & 51.957 & & 39.468 & 39.269 & 42.870 \\
\hline 11/13/10 20:00 & 58.451 & 60.722 & 51.969 & & 39.471 & 39.278 & 42.882 \\
\hline 11/14/10 0:00 & 58.451 & 60.718 & 51.962 & & 39.468 & 39.278 & 42.878 \\
\hline 11/14/10 4:00 & 58.446 & 60.718 & 51.960 & & 39.464 & 39.278 & 42.875 \\
\hline 11/14/10 8:00 & 58.448 & 60.716 & 51.962 & & 39.466 & 39.281 & 42.875 \\
\hline $11 / 14 / 1012: 00$ & 58.443 & 60.711 & 51.948 & & 39.459 & 39.276 & 42.871 \\
\hline $11 / 14 / 1016: 00$ & 58.432 & 60.704 & 51.939 & & 39.457 & 39.269 & 42.859 \\
\hline 11/14/10 20:00 & 58.441 & 60.706 & 51.950 & & 39.461 & 39.274 & 42.866 \\
\hline 11/15/10 0:00 & 58.441 & 60.706 & 51.946 & & 39.457 & 39.269 & 42.864 \\
\hline 11/15/10 4:00 & 58.432 & 60.701 & 51.934 & & 39.450 & 39.262 & 42.856 \\
\hline 11/15/10 8:00 & 58.439 & 60.704 & 51.944 & & 39.459 & 39.267 & 42.859 \\
\hline $11 / 15 / 1012: 00$ & 58.443 & 60.706 & 51.948 & & 39.457 & 39.271 & 42.864 \\
\hline 11/15/10 16:00 & 58.439 & 60.706 & 51.944 & & 39.457 & 39.269 & 42.861 \\
\hline 11/15/10 20:00 & 58.453 & 60.718 & 51.964 & & 39.464 & 39.283 & 42.877 \\
\hline 11/16/10 0:00 & 58.453 & 60.716 & 51.957 & & 39.466 & 39.283 & 42.873 \\
\hline 11/16/10 4:00 & 58.443 & 60.706 & 51.944 & & 39.457 & 39.278 & 42.866 \\
\hline 11/16/10 8:00 & 58.448 & 60.711 & 51.953 & & 39.466 & 39.288 & 42.868 \\
\hline 11/16/10 12:00 & 58.448 & 60.709 & 51.948 & & 39.464 & 39.278 & 42.868 \\
\hline 11/16/10 16:00 & 58.439 & 60.704 & 51.939 & & 39.454 & 39.264 & 42.861 \\
\hline 11/16/10 20:00 & 58.458 & 60.720 & 51.969 & & 39.474 & 39.262 & 42.877 \\
\hline 11/17/10 0:00 & 58.477 & 60.732 & 51.985 & & 39.490 & 39.297 & 42.892 \\
\hline 11/17/10 4:00 & 58.481 & 60.737 & 51.988 & & 39.497 & 39.302 & 42.899 \\
\hline 11/17/10 8:00 & 58.495 & 60.755 & 52.018 & & 39.518 & 39.314 & 42.915 \\
\hline 11/17/10 12:00 & 58.512 & 60.772 & 52.036 & & 39.537 & 39.321 & 42.936 \\
\hline 11/17/10 16:00 & 58.505 & 60.769 & 52.022 & & 39.534 & 39.316 & 42.931 \\
\hline 11/17/10 20:00 & 58.524 & 60.792 & 52.060 & & 39.563 & 39.354 & 42.952 \\
\hline 11/18/10 0:00 & 58.538 & 60.802 & 52.067 & & 39.572 & 39.377 & 42.964 \\
\hline 11/18/10 4:00 & 58.531 & 60.797 & 52.043 & & 39.569 & 39.368 & 42.954 \\
\hline 11/18/10 8:00 & 58.531 & 60.800 & 52.055 & & 39.572 & 39.356 & 42.959 \\
\hline 11/18/10 12:00 & 58.536 & 60.806 & 52.055 & & 39.581 & 39.358 & 42.963 \\
\hline 11/18/10 16:00 & 58.524 & 60.790 & 52.032 & & 39.577 & 39.346 & 42.947 \\
\hline 11/18/10 20:00 & 58.526 & 60.790 & 52.039 & & 39.574 & 39.351 & 42.950 \\
\hline 11/19/10 0:00 & 58.533 & 60.793 & 52.036 & & 39.577 & 39.358 & 42.949 \\
\hline
\end{tabular}

TABLE S1.3 Page 15 of 58 


\begin{tabular}{|c|c|c|c|c|c|c|c|}
\hline \multirow[b]{2}{*}{ Date and Time } & \multicolumn{7}{|c|}{ Water Level (ft below reference point) } \\
\hline & SB43D & SB44D & SB45D & SB46D & SB47D & SB48D & SB49D \\
\hline $11 / 19 / 104: 00$ & 58.514 & 60.767 & 51.990 & & 39.555 & 39.328 & 42.924 \\
\hline 11/19/10 8:00 & 58.507 & 60.760 & 51.997 & & 39.551 & 39.323 & 42.919 \\
\hline 11/19/10 12:00 & 58.524 & 60.774 & 52.018 & & 39.565 & 39.344 & 42.931 \\
\hline 11/19/10 16:00 & 58.521 & 60.769 & 52.009 & & 39.567 & 39.335 & 42.926 \\
\hline 11/19/10 20:00 & 58.538 & 60.785 & 52.041 & & 39.581 & 39.356 & 42.945 \\
\hline 11/20/10 0:00 & 58.557 & 60.802 & 52.062 & & 39.598 & 39.370 & 42.964 \\
\hline $11 / 20 / 104: 00$ & 58.545 & 60.797 & 52.036 & & 39.591 & 39.363 & 42.952 \\
\hline 11/20/10 8:00 & 58.540 & 60.795 & 52.039 & & 39.593 & 39.363 & 42.950 \\
\hline 11/20/10 12:00 & 58.543 & 60.797 & 52.039 & & 39.584 & 39.368 & 42.954 \\
\hline 11/20/10 16:00 & 58.529 & 60.783 & 52.011 & & 39.575 & 39.365 & 42.938 \\
\hline 11/20/10 20:00 & 58.533 & 60.783 & 52.022 & & 39.579 & 39.361 & 42.940 \\
\hline $11 / 21 / 100: 00$ & 58.547 & 60.797 & 52.046 & & 39.595 & 39.375 & 42.952 \\
\hline $11 / 21 / 104: 00$ & 58.536 & 60.779 & 52.009 & & 39.582 & 39.363 & 42.936 \\
\hline 11/21/10 8:00 & 58.521 & 60.767 & 51.995 & & 39.567 & 39.361 & 42.924 \\
\hline $11 / 21 / 1012: 00$ & 58.535 & 60.781 & 52.022 & & 39.579 & 39.358 & 42.938 \\
\hline 11/21/10 16:00 & 58.538 & 60.778 & 52.015 & & 39.579 & 39.368 & 42.935 \\
\hline $11 / 21 / 1020: 00$ & 58.545 & 60.790 & 52.036 & & 39.586 & 39.384 & 42.947 \\
\hline 11/22/10 0:00 & 58.557 & 60.802 & 52.050 & & 39.602 & 39.382 & 42.959 \\
\hline $11 / 22 / 104: 00$ & 58.545 & 60.790 & 52.018 & & 39.588 & 39.379 & 42.945 \\
\hline 11/22/10 8:00 & 58.526 & 60.769 & 51.992 & & 39.572 & 39.358 & 42.926 \\
\hline 11/22/10 12:00 & 58.557 & 60.797 & 52.048 & & 39.602 & 39.379 & 42.957 \\
\hline $11 / 22 / 1016: 00$ & 58.559 & 60.802 & 52.048 & & 39.609 & 39.382 & 42.961 \\
\hline 11/22/10 20:00 & 58.578 & 60.823 & 52.085 & & 39.631 & 39.403 & 42.982 \\
\hline 11/23/10 0:00 & 58.599 & 60.851 & 52.115 & & 39.650 & 39.424 & 43.008 \\
\hline $11 / 23 / 104: 00$ & 58.607 & 60.858 & 52.111 & & 39.659 & 39.438 & 43.014 \\
\hline $11 / 23 / 108: 00$ & 58.604 & 60.851 & 52.104 & & 39.659 & 39.433 & 43.012 \\
\hline $11 / 23 / 1012: 00$ & 58.609 & 60.865 & 52.120 & & 39.673 & 39.440 & 43.019 \\
\hline 11/23/10 16:00 & 58.602 & 60.853 & 52.094 & & 39.671 & 39.440 & 43.012 \\
\hline 11/23/10 20:00 & 58.595 & 60.846 & 52.087 & & 39.666 & 39.433 & 43.005 \\
\hline 11/24/10 0:00 & 58.599 & 60.851 & 52.094 & & 39.668 & 39.436 & 43.010 \\
\hline 11/24/10 4:00 & 58.592 & 60.841 & 52.069 & & 39.656 & 39.424 & 42.996 \\
\hline 11/24/10 8:00 & 58.564 & 60.809 & 52.029 & & 39.633 & 39.398 & 42.963 \\
\hline $11 / 24 / 1012: 00$ & 58.555 & 60.800 & 52.020 & & 39.624 & 39.389 & 42.949 \\
\hline $11 / 24 / 1016: 00$ & 58.552 & 60.793 & 52.015 & & 39.621 & 39.379 & 42.947 \\
\hline $11 / 24 / 1020: 00$ & 58.576 & 60.811 & 52.060 & & 39.635 & 39.400 & 42.968 \\
\hline 11/25/10 0:00 & 58.604 & 60.837 & 52.101 & & 39.666 & 39.431 & 42.996 \\
\hline 11/25/10 4:00 & 58.630 & 60.865 & 52.125 & & 39.685 & 39.454 & 43.024 \\
\hline 11/25/10 8:00 & 58.618 & 60.858 & 52.104 & & 39.680 & 39.447 & 43.017 \\
\hline $11 / 25 / 1012: 00$ & 58.616 & 60.858 & 52.099 & & 39.687 & 39.452 & 43.017 \\
\hline $11 / 25 / 10$ 16:00 & 58.621 & 60.865 & 52.108 & & 39.692 & 39.462 & 43.024 \\
\hline $11 / 25 / 1020: 00$ & 58.630 & 60.872 & 52.113 & & 39.699 & 39.466 & 43.031 \\
\hline $11 / 26 / 100: 00$ & 58.637 & 60.883 & 52.134 & & 39.710 & 39.483 & 43.040 \\
\hline 11/26/10 4:00 & 58.647 & 60.888 & 52.139 & & 39.720 & 39.492 & 43.049 \\
\hline 11/26/10 8:00 & 58.642 & 60.881 & 52.118 & & 39.715 & 39.480 & 43.038 \\
\hline $11 / 26 / 1012: 00$ & 58.637 & 60.874 & 52.111 & & 39.710 & 39.485 & 43.033 \\
\hline $11 / 26 / 1016: 00$ & 58.642 & 60.879 & 52.118 & & 39.718 & 39.471 & 43.033 \\
\hline $11 / 26 / 1020: 00$ & 58.652 & 60.886 & 52.127 & & 39.725 & 39.478 & 43.045 \\
\hline $11 / 27 / 100: 00$ & 58.661 & 60.897 & 52.143 & & 39.736 & 39.487 & 43.054 \\
\hline 11/27/10 4:00 & 58.673 & 60.909 & 52.157 & & 39.751 & 39.497 & 43.064 \\
\hline 11/27/10 8:00 & 58.677 & 60.906 & 52.150 & & 39.750 & 39.499 & 43.065 \\
\hline 11/27/10 12:00 & 58.673 & 60.902 & 52.141 & & 39.748 & 39.499 & 43.061 \\
\hline $11 / 27 / 1016: 00$ & 58.668 & 60.900 & 52.136 & & 39.743 & 39.497 & 43.056 \\
\hline 11/27/10 20:00 & 58.673 & 60.902 & 52.136 & & 39.748 & 39.504 & 43.057 \\
\hline 11/28/10 0:00 & 58.670 & 60.897 & 52.129 & & 39.750 & 39.501 & 43.052 \\
\hline
\end{tabular}

TABLE S1.3 Page 16 of 58 


\begin{tabular}{|c|c|c|c|c|c|c|c|}
\hline \multirow[b]{2}{*}{ Date and Time } & \multicolumn{7}{|c|}{ Water Level (ft below reference point) } \\
\hline & SB43D & SB44D & SB45D & SB46D & SB47D & SB48D & SB49D \\
\hline $11 / 28 / 104: 00$ & 58.670 & 60.899 & 52.134 & & 39.750 & 39.506 & 43.052 \\
\hline 11/28/10 8:00 & 58.673 & 60.895 & 52.127 & & 39.746 & 39.504 & 43.052 \\
\hline $11 / 28 / 1012: 00$ & 58.656 & 60.883 & 52.104 & & 39.734 & 39.492 & 43.040 \\
\hline $11 / 28 / 1016: 00$ & 58.642 & 60.869 & 52.092 & & 39.725 & 39.480 & 43.022 \\
\hline $11 / 28 / 1020: 00$ & 58.656 & 60.872 & 52.099 & & 39.734 & 39.487 & 43.026 \\
\hline 11/29/10 0:00 & 58.661 & 60.876 & 52.101 & & 39.732 & 39.490 & 43.031 \\
\hline 11/29/10 4:00 & 58.659 & 60.879 & 52.101 & & 39.734 & 39.490 & 43.031 \\
\hline 11/29/10 8:00 & 58.661 & 60.874 & 52.106 & & 39.734 & 39.487 & 43.031 \\
\hline $11 / 29 / 1012: 00$ & 58.656 & 60.872 & 52.094 & & 39.732 & 39.483 & 43.026 \\
\hline 11/29/10 16:00 & 58.654 & 60.867 & 52.101 & & 39.727 & 39.480 & 43.024 \\
\hline $11 / 29 / 1020: 00$ & 58.694 & 60.900 & 52.155 & & 39.762 & 39.518 & 43.061 \\
\hline 11/30/10 0:00 & 58.704 & 60.911 & 52.155 & & 39.774 & 39.525 & 43.071 \\
\hline $11 / 30 / 104: 00$ & 58.703 & 60.913 & 52.159 & & 39.776 & 39.532 & 43.073 \\
\hline 11/30/10 8:00 & 58.715 & 60.930 & 52.173 & & 39.788 & 39.541 & 43.084 \\
\hline $11 / 30 / 1012: 00$ & 58.725 & 60.937 & 52.178 & & 39.802 & 39.551 & 43.094 \\
\hline $11 / 30 / 1016: 00$ & 58.727 & 60.939 & 52.183 & & 39.807 & 39.558 & 43.096 \\
\hline $11 / 30 / 1020: 00$ & 58.741 & 60.953 & 52.204 & & 39.821 & 39.570 & 43.112 \\
\hline $12 / 1 / 100: 00$ & 58.746 & 60.958 & 52.199 & & 39.828 & 39.577 & 43.115 \\
\hline $12 / 1 / 104: 00$ & 58.746 & 60.953 & 52.190 & & 39.826 & 39.577 & 43.112 \\
\hline $12 / 1 / 108: 00$ & 58.753 & 60.963 & 52.206 & & 39.840 & 39.586 & 43.119 \\
\hline $12 / 1 / 1012: 00$ & 58.765 & 60.970 & 52.213 & & 39.849 & 39.595 & 43.129 \\
\hline $12 / 1 / 1016: 00$ & 58.758 & 60.965 & 52.201 & & 39.844 & 39.588 & 43.119 \\
\hline 12/1/10 20:00 & 58.772 & 60.974 & 52.222 & & 39.858 & 39.602 & 43.133 \\
\hline $12 / 2 / 100: 00$ & 58.779 & 60.979 & 52.220 & & 39.863 & 39.609 & 43.135 \\
\hline $12 / 2 / 104: 00$ & 58.770 & 60.967 & 52.197 & & 39.854 & 39.598 & 43.121 \\
\hline $12 / 2 / 108: 00$ & 58.770 & 60.967 & 52.206 & & 39.861 & 39.602 & 43.124 \\
\hline $12 / 2 / 1012: 00$ & 58.782 & 60.979 & 52.220 & & 39.870 & 39.614 & 43.136 \\
\hline $12 / 2 / 1016: 00$ & 58.777 & 60.974 & 52.210 & & 39.863 & 39.609 & 43.133 \\
\hline $12 / 2 / 1020: 00$ & 58.803 & 61.000 & 52.250 & & 39.887 & 39.633 & 43.156 \\
\hline 12/3/10 0:00 & 58.817 & 61.011 & 52.257 & & 39.901 & 39.642 & 43.168 \\
\hline 12/3/10 4:00 & 58.803 & 60.998 & 52.227 & & 39.889 & 39.633 & 43.152 \\
\hline $12 / 3 / 108: 00$ & 58.796 & 60.991 & 52.222 & & 39.884 & 39.628 & 43.147 \\
\hline $12 / 3 / 1012: 00$ & 58.796 & 60.993 & 52.220 & & 39.887 & 39.628 & 43.145 \\
\hline $12 / 3 / 1016: 00$ & 58.774 & 60.972 & 52.187 & & 39.868 & 39.612 & 43.124 \\
\hline $12 / 3 / 1020: 00$ & 58.786 & 60.981 & 52.215 & & 39.878 & 39.619 & 43.135 \\
\hline $12 / 4 / 100: 00$ & 58.805 & 60.993 & 52.236 & & 39.891 & 39.635 & 43.154 \\
\hline $12 / 4 / 104: 00$ & 58.815 & 61.002 & 52.238 & & 39.896 & 39.640 & 43.161 \\
\hline $12 / 4 / 108: 00$ & 58.824 & 61.009 & 52.257 & & 39.905 & 39.654 & 43.170 \\
\hline $12 / 4 / 1012: 00$ & 58.850 & 61.037 & 52.292 & & 39.936 & 39.678 & 43.194 \\
\hline $12 / 4 / 1016: 00$ & 58.852 & 61.039 & 52.287 & & 39.938 & 39.682 & 43.201 \\
\hline $12 / 4 / 1020: 00$ & 58.867 & 61.060 & 52.310 & & 39.957 & 39.696 & 43.214 \\
\hline $12 / 5 / 100: 00$ & 58.885 & 61.072 & 52.329 & & 39.974 & 39.715 & 43.233 \\
\hline 12/5/10 4:00 & 58.883 & 61.070 & 52.310 & & 39.969 & 39.713 & 43.228 \\
\hline $12 / 5 / 108: 00$ & 58.881 & 61.067 & 52.308 & & 39.971 & 39.713 & 43.226 \\
\hline $12 / 5 / 1012: 00$ & 58.890 & 61.079 & 52.324 & & 39.985 & 39.724 & 43.236 \\
\hline $12 / 5 / 1016: 00$ & 58.885 & 61.072 & 52.310 & & 39.981 & 39.717 & 43.228 \\
\hline $12 / 5 / 1020: 00$ & 58.890 & 61.074 & 52.320 & & 39.990 & 39.722 & 43.233 \\
\hline $12 / 6 / 100: 00$ & 58.907 & 61.093 & 52.343 & & 40.004 & 39.741 & 43.249 \\
\hline $12 / 6 / 104: 00$ & 58.907 & 61.086 & 52.324 & & 40.002 & 39.739 & 43.245 \\
\hline $12 / 6 / 108: 00$ & 58.893 & 61.074 & 52.303 & & 39.992 & 39.724 & 43.231 \\
\hline $12 / 6 / 1012: 00$ & 58.895 & 61.074 & 52.313 & & 39.995 & 39.732 & 43.233 \\
\hline 12/6/10 16:00 & 58.890 & 61.067 & 52.294 & & 39.985 & 39.724 & 43.222 \\
\hline $12 / 6 / 1020: 00$ & 58.885 & 61.067 & 52.294 & & 39.981 & 39.720 & 43.219 \\
\hline 12/7/10 0:00 & 58.902 & 61.079 & 52.322 & & 39.997 & 39.736 & 43.238 \\
\hline
\end{tabular}

TABLE S1.3 Page 17 of 58 


\begin{tabular}{|c|c|c|c|c|c|c|c|}
\hline \multirow[b]{2}{*}{ Date and Time } & \multicolumn{7}{|c|}{ Water Level (ft below reference point) } \\
\hline & SB43D & SB44D & SB45D & SB46D & SB47D & SB48D & SB49D \\
\hline 12/7/10 4:00 & 58.909 & 61.081 & 52.317 & & 39.997 & 39.738 & 43.236 \\
\hline 12/7/10 8:00 & 58.900 & 61.077 & 52.301 & & 39.995 & 39.732 & 43.228 \\
\hline 12/7/10 12:00 & 58.907 & 61.081 & 52.322 & & 40.004 & 39.743 & 43.238 \\
\hline 12/7/10 16:00 & 58.909 & 61.079 & 52.315 & & 40.000 & 39.776 & 43.238 \\
\hline 12/7/10 20:00 & 58.916 & 61.088 & 52.324 & & 40.009 & 39.786 & 43.245 \\
\hline 12/8/10 0:00 & 58.931 & 61.105 & 52.350 & & 40.026 & 39.804 & 43.259 \\
\hline 12/8/10 4:00 & 58.935 & 61.107 & 52.340 & & 40.025 & 39.807 & 43.261 \\
\hline $12 / 8 / 108: 00$ & 58.926 & 61.093 & 52.324 & & 40.018 & 39.799 & 43.250 \\
\hline $12 / 8 / 1012: 00$ & 58.924 & 61.100 & 52.334 & & 40.021 & 39.804 & 43.252 \\
\hline 12/8/10 16:00 & 58.926 & 61.093 & 52.322 & & 40.021 & 39.807 & 43.247 \\
\hline 12/8/10 20:00 & 58.926 & 61.091 & 52.317 & & 40.021 & 39.800 & 43.247 \\
\hline 12/9/10 0:00 & 58.931 & 61.098 & 52.331 & & 40.025 & 39.811 & 43.254 \\
\hline 12/9/10 4:00 & 58.930 & 61.100 & 52.327 & & 40.025 & 39.814 & 43.252 \\
\hline $12 / 9 / 108: 00$ & 58.912 & 61.077 & 52.289 & & 40.002 & 39.828 & 43.233 \\
\hline $12 / 9 / 1012: 00$ & 58.900 & 61.074 & 52.292 & & 39.995 & 39.814 & 43.226 \\
\hline 12/9/10 16:00 & 58.909 & 61.077 & 52.296 & & 39.997 & 39.816 & 43.226 \\
\hline 12/9/10 20:00 & 58.923 & 61.088 & 52.313 & & 40.014 & 39.821 & 43.240 \\
\hline 12/10/10 0:00 & 58.935 & 61.098 & 52.336 & & 40.025 & 39.840 & 43.254 \\
\hline $12 / 10 / 104: 00$ & 58.947 & 61.109 & 52.345 & & 40.032 & 39.861 & 43.264 \\
\hline 12/10/10 8:00 & 58.942 & 61.105 & 52.329 & & 40.028 & 39.847 & 43.258 \\
\hline 12/10/10 12:00 & 58.940 & 61.105 & 52.331 & & 40.032 & 39.837 & 43.259 \\
\hline 12/10/10 16:00 & 58.930 & 61.100 & 52.317 & & 40.023 & 39.832 & 43.252 \\
\hline 12/10/10 20:00 & 58.933 & 61.095 & 52.317 & & 40.023 & 39.840 & 43.250 \\
\hline 12/11/10 0:00 & 58.928 & 61.095 & 52.315 & & 40.018 & 39.825 & 43.245 \\
\hline 12/11/10 4:00 & 58.928 & 61.091 & 52.313 & & 40.018 & 39.830 & 43.245 \\
\hline 12/11/10 8:00 & 58.928 & 61.088 & 52.310 & & 40.019 & 39.823 & 43.243 \\
\hline 12/11/10 12:00 & 58.940 & 61.095 & 52.329 & & 40.032 & 39.837 & 43.256 \\
\hline $12 / 11 / 1016: 00$ & 58.945 & 61.105 & 52.340 & & 40.035 & 39.849 & 43.264 \\
\hline 12/11/10 20:00 & 58.952 & 61.109 & 52.338 & & 40.037 & 39.863 & 43.266 \\
\hline 12/12/10 0:00 & 58.957 & 61.116 & 52.352 & & 40.044 & 39.886 & 43.278 \\
\hline $12 / 12 / 104: 00$ & 58.980 & 61.139 & 52.385 & & 40.068 & 39.922 & 43.298 \\
\hline 12/12/10 8:00 & 58.994 & 61.151 & 52.392 & & 40.086 & 39.929 & 43.310 \\
\hline $12 / 12 / 1012: 00$ & 59.004 & 61.156 & 52.403 & & 40.093 & 39.940 & 43.317 \\
\hline 12/12/10 16:00 & 59.008 & 61.165 & 52.410 & & 40.100 & 39.957 & 43.326 \\
\hline $12 / 12 / 1020: 00$ & 59.023 & 61.177 & 52.415 & & 40.112 & 39.959 & 43.336 \\
\hline 12/13/10 0:00 & 59.020 & 61.177 & 52.410 & & 40.119 & 39.959 & 43.333 \\
\hline 12/13/10 4:00 & 59.023 & 61.182 & 52.415 & & 40.122 & 39.966 & 43.338 \\
\hline 12/13/10 8:00 & 59.023 & 61.174 & 52.410 & & 40.122 & 39.966 & 43.333 \\
\hline 12/13/10 12:00 & 59.025 & 61.181 & 52.410 & & 40.126 & 39.971 & 43.333 \\
\hline 12/13/10 16:00 & 59.025 & 61.174 & 52.405 & & 40.126 & 39.973 & 43.331 \\
\hline $12 / 13 / 1020: 00$ & 59.037 & 61.184 & 52.410 & & 40.136 & 39.987 & 43.335 \\
\hline 12/14/10 0:00 & 59.039 & 61.179 & 52.412 & & 40.140 & 39.992 & 43.338 \\
\hline 12/14/10 4:00 & 59.035 & 61.179 & 52.406 & & 40.140 & 39.990 & 43.333 \\
\hline 12/14/10 8:00 & 59.032 & 61.177 & 52.399 & & 40.133 & 39.980 & 43.329 \\
\hline 12/14/10 12:00 & 59.032 & 61.174 & 52.394 & & 40.133 & 39.983 & 43.321 \\
\hline $12 / 14 / 1016: 00$ & 59.020 & 61.165 & 52.380 & & 40.122 & 39.976 & 43.312 \\
\hline $12 / 14 / 1020: 00$ & 59.032 & 61.170 & 52.392 & & 40.136 & 39.980 & 43.321 \\
\hline 12/15/10 0:00 & 59.032 & 61.165 & 52.385 & & 40.133 & 39.973 & 43.322 \\
\hline 12/15/10 4:00 & 59.023 & 61.158 & 52.373 & & 40.122 & 39.971 & 43.312 \\
\hline 12/15/10 8:00 & 59.018 & 61.151 & 52.364 & & 40.119 & 39.980 & 43.303 \\
\hline 12/15/10 12:00 & 59.013 & 61.146 & 52.357 & & 40.117 & 39.962 & 43.298 \\
\hline $12 / 15 / 1016: 00$ & 59.011 & 61.144 & 52.364 & & 40.113 & 40.060 & 43.296 \\
\hline 12/15/10 20:00 & 59.032 & 61.156 & 52.389 & & 40.126 & 40.088 & 43.312 \\
\hline 12/16/10 0:00 & 59.044 & 61.170 & 52.394 & & 40.133 & 40.095 & 43.321 \\
\hline
\end{tabular}

TABLE S1.3 Page 18 of 58 


\begin{tabular}{|c|c|c|c|c|c|c|c|}
\hline \multirow[b]{2}{*}{ Date and Time } & \multicolumn{7}{|c|}{ Water Level (ft below reference point) } \\
\hline & SB43D & SB44D & SB45D & SB46D & SB47D & SB48D & SB49D \\
\hline $12 / 16 / 104: 00$ & 59.039 & 61.165 & 52.389 & & 40.131 & 40.098 & 43.319 \\
\hline $12 / 16 / 108: 00$ & 59.051 & 61.181 & 52.410 & & 40.147 & 40.114 & 43.333 \\
\hline $12 / 16 / 1012: 00$ & 59.068 & 61.191 & 52.429 & & 40.164 & 40.131 & 43.347 \\
\hline $12 / 16 / 1016: 00$ & 59.070 & 61.198 & 52.431 & & 40.167 & 40.133 & 43.351 \\
\hline $12 / 16 / 1020: 00$ & 59.094 & 61.221 & 52.461 & & 40.183 & 40.159 & 43.373 \\
\hline 12/17/10 0:00 & 59.103 & 61.230 & 52.468 & & 40.197 & 40.171 & 43.382 \\
\hline 12/17/10 4:00 & 59.105 & 61.233 & 52.457 & & 40.197 & 40.175 & 43.382 \\
\hline 12/17/10 8:00 & 59.110 & 61.237 & 52.468 & & 40.209 & 40.187 & 43.389 \\
\hline $12 / 17 / 1012: 00$ & 59.122 & 61.244 & 52.480 & & 40.220 & 40.206 & 43.398 \\
\hline $12 / 17 / 1016: 00$ & 59.115 & 61.237 & 52.470 & & 40.216 & 40.192 & 43.391 \\
\hline $12 / 17 / 1020: 00$ & 59.127 & 61.254 & 52.491 & & 40.227 & 40.217 & 43.405 \\
\hline 12/18/10 0:00 & 59.141 & 61.261 & 52.491 & & 40.241 & 40.206 & 43.415 \\
\hline $12 / 18 / 104: 00$ & 59.129 & 61.249 & 52.470 & & 40.230 & 40.206 & 43.403 \\
\hline $12 / 18 / 108: 00$ & 59.127 & 61.244 & 52.468 & & 40.230 & 40.213 & 43.398 \\
\hline $12 / 18 / 1012: 00$ & 59.131 & 61.251 & 52.480 & & 40.234 & 40.213 & 43.403 \\
\hline $12 / 18 / 1016: 00$ & 59.120 & 61.237 & 52.457 & & 40.227 & 40.208 & 43.389 \\
\hline 12/18/10 20:00 & 59.129 & 61.249 & 52.475 & & 40.232 & 40.208 & 43.396 \\
\hline 12/19/10 0:00 & 59.141 & 61.254 & 52.487 & & 40.241 & 40.229 & 43.410 \\
\hline $12 / 19 / 104: 00$ & 59.134 & 61.247 & 52.466 & & 40.234 & 40.217 & 43.398 \\
\hline $12 / 19 / 108: 00$ & 59.129 & 61.244 & 52.464 & & 40.225 & 40.213 & 43.393 \\
\hline $12 / 19 / 1012: 00$ & 59.136 & 61.251 & 52.475 & & 40.239 & 40.227 & 43.403 \\
\hline $12 / 19 / 1016: 00$ & 59.125 & 61.240 & 52.452 & & 40.227 & 40.206 & 43.389 \\
\hline $12 / 19 / 1020: 00$ & 59.134 & 61.249 & 52.480 & & 40.239 & 40.220 & 43.401 \\
\hline $12 / 20 / 100: 00$ & 59.153 & 61.261 & 52.496 & & 40.248 & 40.225 & 43.415 \\
\hline $12 / 20 / 104: 00$ & 59.143 & 61.249 & 52.464 & & 40.239 & 40.220 & 43.403 \\
\hline $12 / 20 / 108: 00$ & 59.122 & 61.233 & 52.440 & & 40.223 & 40.203 & 43.384 \\
\hline $12 / 20 / 1012: 00$ & 59.127 & 61.237 & 52.450 & & 40.230 & 40.234 & 43.387 \\
\hline $12 / 20 / 1016: 00$ & 59.120 & 61.230 & 52.440 & & 40.218 & 40.133 & 43.379 \\
\hline $12 / 20 / 1020: 00$ & 59.141 & 61.247 & 52.475 & & 40.239 & 40.159 & 43.400 \\
\hline 12/21/10 0:00 & 59.165 & 61.272 & 52.512 & & 40.258 & 40.234 & 43.426 \\
\hline $12 / 21 / 104: 00$ & 59.174 & 61.277 & 52.503 & & 40.265 & 40.236 & 43.431 \\
\hline $12 / 21 / 108: 00$ & 59.169 & 61.277 & 52.503 & & 40.267 & 40.182 & 43.431 \\
\hline $12 / 21 / 1012: 00$ & 59.188 & 61.293 & 52.529 & & 40.286 & 40.269 & 43.449 \\
\hline $12 / 21 / 1016: 00$ & 59.198 & 61.303 & 52.531 & & 40.293 & 40.264 & 43.459 \\
\hline $12 / 21 / 1020: 00$ & 59.212 & 61.321 & 52.554 & & 40.305 & 40.283 & 43.475 \\
\hline $12 / 22 / 100: 00$ & 59.233 & 61.344 & 52.594 & & 40.331 & 40.307 & 43.500 \\
\hline $12 / 22 / 104: 00$ & 59.250 & 61.356 & 52.594 & & 40.340 & 40.321 & 43.510 \\
\hline 12/22/10 8:00 & 59.243 & 61.347 & 52.577 & & 40.340 & 40.309 & 43.505 \\
\hline $12 / 22 / 1012: 00$ & 59.254 & 61.361 & 52.603 & & 40.352 & 40.330 & 43.514 \\
\hline $12 / 22 / 1016: 00$ & 59.254 & 61.361 & 52.594 & & 40.354 & 40.330 & 43.515 \\
\hline $12 / 22 / 1020: 00$ & 59.259 & 61.363 & 52.596 & & 40.359 & 40.330 & 43.519 \\
\hline $12 / 23 / 100: 00$ & 59.276 & 61.382 & 52.624 & & 40.380 & 40.349 & 43.535 \\
\hline $12 / 23 / 104: 00$ & 59.283 & 61.382 & 52.612 & & 40.380 & 40.354 & 43.536 \\
\hline $12 / 23 / 108: 00$ & 59.264 & 61.368 & 52.584 & & 40.366 & 40.344 & 43.521 \\
\hline $12 / 23 / 1012: 00$ & 59.264 & 61.368 & 52.594 & & 40.368 & 40.349 & 43.519 \\
\hline $12 / 23 / 1016: 00$ & 59.257 & 61.361 & 52.575 & & 40.361 & 40.344 & 43.510 \\
\hline $12 / 23 / 1020: 00$ & 59.255 & 61.351 & 52.570 & & 40.359 & 40.340 & 43.505 \\
\hline $12 / 24 / 100: 00$ & 59.264 & 61.363 & 52.591 & & 40.368 & 40.351 & 43.517 \\
\hline $12 / 24 / 104: 00$ & 59.278 & 61.370 & 52.598 & & 40.378 & 40.363 & 43.524 \\
\hline $12 / 24 / 108: 00$ & 59.262 & 61.356 & 52.568 & & 40.357 & 40.344 & 43.505 \\
\hline $12 / 24 / 1012: 00$ & 59.257 & 61.351 & 52.575 & & 40.359 & 40.347 & 43.503 \\
\hline $12 / 24 / 1016: 00$ & 59.262 & 61.358 & 52.577 & & 40.359 & 40.354 & 43.510 \\
\hline $12 / 24 / 1020: 00$ & 59.266 & 61.358 & 52.584 & & 40.368 & 40.358 & 43.512 \\
\hline 12/25/10 0:00 & 59.281 & 61.377 & 52.612 & & 40.380 & 40.377 & 43.531 \\
\hline
\end{tabular}

TABLE S1.3 Page 19 of 58 


\begin{tabular}{|c|c|c|c|c|c|c|c|}
\hline \multirow[b]{2}{*}{ Date and Time } & \multicolumn{7}{|c|}{ Water Level (ft below reference point) } \\
\hline & SB43D & SB44D & SB45D & SB46D & SB47D & SB48D & SB49D \\
\hline $12 / 25 / 104: 00$ & 59.302 & 61.391 & 52.633 & & 40.399 & 40.394 & 43.552 \\
\hline $12 / 25 / 108: 00$ & 59.304 & 61.393 & 52.621 & & 40.399 & 40.393 & 43.550 \\
\hline $12 / 25 / 1012: 00$ & 59.304 & 61.398 & 52.628 & & 40.401 & 40.398 & 43.554 \\
\hline $12 / 25 / 1016: 00$ & 59.309 & 61.403 & 52.633 & & 40.408 & 40.426 & 43.556 \\
\hline $12 / 25 / 1020: 00$ & 59.316 & 61.405 & 52.635 & & 40.411 & 40.426 & 43.558 \\
\hline 12/26/10 0:00 & 59.316 & 61.410 & 52.638 & & 40.415 & 40.426 & 43.563 \\
\hline $12 / 26 / 104: 00$ & 59.326 & 61.414 & 52.645 & & 40.425 & 40.429 & 43.570 \\
\hline 12/26/10 8:00 & 59.325 & 61.412 & 52.640 & & 40.425 & 40.426 & 43.568 \\
\hline $12 / 26 / 1012: 00$ & 59.323 & 61.412 & 52.638 & & 40.425 & 40.431 & 43.566 \\
\hline $12 / 26 / 1016: 00$ & 59.325 & 61.412 & 52.638 & & 40.427 & 40.431 & 43.565 \\
\hline $12 / 26 / 1020: 00$ & 59.325 & 61.414 & 52.628 & & 40.422 & 40.424 & 43.563 \\
\hline 12/27/10 0:00 & 59.321 & 61.407 & 52.626 & & 40.420 & 40.424 & 43.561 \\
\hline $12 / 27 / 104: 00$ & 59.328 & 61.414 & 52.638 & & 40.425 & 40.438 & 43.566 \\
\hline $12 / 27 / 108: 00$ & 59.325 & 61.410 & 52.628 & & 40.425 & 40.438 & 43.561 \\
\hline $12 / 27 / 1012: 00$ & 59.321 & 61.407 & 52.621 & & 40.420 & 40.431 & 43.558 \\
\hline $12 / 27 / 1016: 00$ & 59.314 & 61.396 & 52.617 & & 40.415 & 40.426 & 43.550 \\
\hline $12 / 27 / 1020: 00$ & 59.328 & 61.410 & 52.628 & & 40.422 & 40.436 & 43.561 \\
\hline 12/28/10 0:00 & 59.325 & 61.405 & 52.621 & & 40.420 & 40.431 & 43.556 \\
\hline $12 / 28 / 104: 00$ & 59.325 & 61.410 & 52.626 & & 40.422 & 40.436 & 43.561 \\
\hline $12 / 28 / 108: 00$ & 59.332 & 61.412 & 52.631 & & 40.425 & 40.441 & 43.563 \\
\hline $12 / 28 / 1012: 00$ & 59.332 & 61.414 & 52.628 & & 40.425 & 40.438 & 43.566 \\
\hline $12 / 28 / 1016: 00$ & 59.325 & 61.407 & 52.624 & & 40.425 & 40.436 & 43.558 \\
\hline $12 / 28 / 1020: 00$ & 59.335 & 61.412 & 52.635 & & 40.429 & 40.433 & 43.566 \\
\hline $12 / 29 / 100: 00$ & 59.323 & 61.403 & 52.607 & & 40.418 & 40.422 & 43.554 \\
\hline $12 / 29 / 104: 00$ & 59.314 & 61.391 & 52.596 & & 40.411 & 40.417 & 43.542 \\
\hline $12 / 29 / 108: 00$ & 59.306 & 61.389 & 52.594 & & 40.401 & 40.410 & 43.537 \\
\hline $12 / 29 / 1012: 00$ & 59.300 & 61.379 & 52.580 & & 40.396 & 40.398 & 43.528 \\
\hline $12 / 29 / 1016: 00$ & 59.281 & 61.363 & 52.559 & & 40.375 & 40.335 & 43.510 \\
\hline $12 / 29 / 1020: 00$ & 59.292 & 61.370 & 52.582 & & 40.382 & 40.349 & 43.522 \\
\hline 12/30/10 0:00 & 59.292 & 61.370 & 52.573 & & 40.380 & 40.349 & 43.519 \\
\hline $12 / 30 / 104: 00$ & 59.278 & 61.354 & 52.549 & & 40.368 & 40.337 & 43.503 \\
\hline $12 / 30 / 108: 00$ & 59.278 & 61.354 & 52.556 & & 40.368 & 40.337 & 43.505 \\
\hline $12 / 30 / 1012: 00$ & 59.285 & 61.358 & 52.563 & & 40.371 & 40.347 & 43.512 \\
\hline $12 / 30 / 1016: 00$ & 59.290 & 61.370 & 52.575 & & 40.373 & 40.358 & 43.515 \\
\hline $12 / 30 / 1020: 00$ & 59.332 & 61.410 & 52.638 & & 40.408 & 40.396 & 43.558 \\
\hline $12 / 31 / 100: 00$ & 59.349 & 61.426 & 52.649 & & 40.427 & 40.410 & 43.577 \\
\hline $12 / 31 / 104: 00$ & 59.335 & 61.410 & 52.619 & & 40.420 & 40.401 & 43.565 \\
\hline 12/31/10 8:00 & 59.307 & 61.391 & 52.605 & & 40.396 & 40.384 & 43.544 \\
\hline $12 / 31 / 1012: 00$ & 59.321 & 61.403 & 52.621 & & 40.408 & 40.391 & 43.554 \\
\hline $12 / 31 / 1016: 00$ & 59.340 & 61.414 & 52.640 & & 40.425 & 40.410 & 43.570 \\
\hline $12 / 31 / 1020: 00$ & 59.368 & 61.447 & 52.684 & & 40.451 & 40.436 & 43.603 \\
\hline $1 / 1 / 110: 00$ & 59.382 & 61.466 & 52.693 & & 40.469 & 40.450 & 43.619 \\
\hline 1/1/11 4:00 & 59.375 & 61.461 & 52.677 & & 40.458 & 40.445 & 43.614 \\
\hline 1/1/11 8:00 & 59.380 & 61.463 & 52.689 & & 40.469 & 40.450 & 43.619 \\
\hline $1 / 1 / 1112: 00$ & 59.403 & 61.482 & 52.717 & & 40.490 & 40.462 & 43.637 \\
\hline $1 / 1 / 1116: 00$ & 59.408 & 61.484 & 52.717 & & 40.500 & 40.497 & 43.643 \\
\hline $1 / 1 / 1120: 00$ & 59.430 & 61.510 & 52.744 & & 40.521 & 40.516 & 43.663 \\
\hline $1 / 2 / 110: 00$ & 59.444 & 61.526 & 52.756 & & 40.535 & 40.541 & 43.679 \\
\hline $1 / 2 / 114: 00$ & 59.437 & 61.512 & 52.726 & & 40.528 & 40.532 & 43.666 \\
\hline $1 / 2 / 118: 00$ & 59.425 & 61.508 & 52.721 & & 40.523 & 40.544 & 43.659 \\
\hline $1 / 2 / 1112: 00$ & 59.427 & 61.508 & 52.726 & & 40.533 & 40.534 & 43.659 \\
\hline 1/2/11 16:00 & 59.422 & 61.498 & 52.707 & & 40.521 & 40.513 & 43.650 \\
\hline $1 / 2 / 1120: 00$ & 59.434 & 61.505 & 52.733 & & 40.533 & 40.520 & 43.661 \\
\hline 1/3/11 0:00 & 59.453 & 61.524 & 52.754 & & 40.549 & 40.565 & 43.677 \\
\hline
\end{tabular}

TABLE S1.3 Page 20 of 58 


\begin{tabular}{|c|c|c|c|c|c|c|c|}
\hline \multirow[b]{2}{*}{ Date and Time } & \multicolumn{7}{|c|}{ Water Level (ft below reference point) } \\
\hline & SB43D & SB44D & SB45D & SB46D & SB47D & SB48D & SB49D \\
\hline $1 / 3 / 114: 00$ & 59.448 & 61.519 & 52.733 & & 40.547 & 40.541 & 43.672 \\
\hline 1/3/11 8:00 & 59.434 & 61.510 & 52.714 & & 40.533 & 40.530 & 43.658 \\
\hline $1 / 3 / 1112: 00$ & 59.441 & 61.515 & 52.733 & & 40.547 & 40.541 & 43.665 \\
\hline 1/3/11 16:00 & 59.434 & 61.510 & 52.717 & & 40.530 & 40.532 & 43.656 \\
\hline $1 / 3 / 1120: 00$ & 59.456 & 61.519 & 52.747 & & 40.547 & 40.549 & 43.675 \\
\hline 1/4/11 0:00 & 59.479 & 61.547 & 52.779 & & 40.570 & 40.567 & 43.698 \\
\hline $1 / 4 / 114: 00$ & 59.484 & 61.545 & 52.765 & & 40.573 & 40.570 & 43.703 \\
\hline 1/4/11 8:00 & 59.474 & 61.540 & 52.751 & & 40.568 & 40.563 & 43.696 \\
\hline $1 / 4 / 1112: 00$ & 59.479 & 61.547 & 52.768 & & 40.577 & 40.572 & 43.698 \\
\hline $1 / 4 / 1116: 00$ & 59.467 & 61.536 & 52.742 & & 40.563 & 40.560 & 43.684 \\
\hline $1 / 4 / 1120: 00$ & 59.467 & 61.536 & 52.747 & & 40.566 & 40.565 & 43.686 \\
\hline 1/5/11 0:00 & 59.481 & 61.552 & 52.772 & & 40.580 & 40.586 & 43.700 \\
\hline $1 / 5 / 114: 00$ & 59.484 & 61.547 & 52.754 & & 40.577 & 40.572 & 43.698 \\
\hline 1/5/11 8:00 & 59.467 & 61.529 & 52.730 & & 40.563 & 40.558 & 43.682 \\
\hline $1 / 5 / 1112: 00$ & 59.467 & 61.531 & 52.747 & & 40.570 & 40.558 & 43.684 \\
\hline 1/5/11 16:00 & 59.472 & 61.531 & 52.744 & & 40.573 & 40.581 & 43.684 \\
\hline 1/5/11 20:00 & 59.479 & 61.540 & 52.754 & & 40.575 & 40.581 & 43.691 \\
\hline 1/6/11 0:00 & 59.498 & 61.559 & 52.786 & & 40.594 & 40.595 & 43.710 \\
\hline $1 / 6 / 114: 00$ & 59.503 & 61.563 & 52.777 & & 40.591 & 40.598 & 43.714 \\
\hline 1/6/11 8:00 & 59.484 & 61.545 & 52.749 & & 40.577 & 40.577 & 43.698 \\
\hline 1/6/11 12:00 & 59.486 & 61.549 & 52.758 & & 40.587 & 40.579 & 43.698 \\
\hline 1/6/11 16:00 & 59.486 & 61.545 & 52.749 & & 40.587 & 40.595 & 43.696 \\
\hline 1/6/11 20:00 & 59.484 & 61.540 & 52.744 & & 40.580 & 40.586 & 43.691 \\
\hline 1/7/11 0:00 & 59.484 & 61.545 & 52.754 & & 40.587 & 40.588 & 43.696 \\
\hline $1 / 7 / 114: 00$ & 59.486 & 61.542 & 52.742 & & 40.577 & 40.584 & 43.693 \\
\hline 1/7/11 8:00 & 59.484 & 61.540 & 52.742 & & 40.577 & 40.581 & 43.689 \\
\hline $1 / 7 / 1112: 00$ & 59.496 & 61.554 & 52.770 & & 40.594 & 40.595 & 43.705 \\
\hline 1/7/11 16:00 & 59.512 & 61.566 & 52.782 & & 40.606 & 40.607 & 43.721 \\
\hline $1 / 7 / 1120: 00$ & 59.512 & 61.566 & 52.782 & & 40.603 & 40.635 & 43.719 \\
\hline 1/8/11 0:00 & 59.526 & 61.582 & 52.805 & & 40.620 & 40.621 & 43.737 \\
\hline 1/8/11 4:00 & 59.543 & 61.596 & 52.816 & & 40.634 & 40.633 & 43.751 \\
\hline 1/8/11 8:00 & 59.545 & 61.598 & 52.814 & & 40.636 & 40.638 & 43.754 \\
\hline $1 / 8 / 1112: 00$ & 59.560 & 61.615 & 52.840 & & 40.657 & 40.654 & 43.768 \\
\hline 1/8/11 16:00 & 59.559 & 61.622 & 52.837 & & 40.653 & 40.661 & 43.768 \\
\hline $1 / 8 / 1120: 00$ & 59.569 & 61.619 & 52.837 & & 40.660 & 40.666 & 43.772 \\
\hline 1/9/11 0:00 & 59.576 & 61.631 & 52.851 & & 40.671 & 40.675 & 43.782 \\
\hline $1 / 9 / 114: 00$ & 59.588 & 61.638 & 52.858 & & 40.683 & 40.689 & 43.794 \\
\hline 1/9/11 8:00 & 59.586 & 61.636 & 52.849 & & 40.681 & 40.685 & 43.791 \\
\hline 1/9/11 12:00 & 59.593 & 61.645 & 52.863 & & 40.693 & 40.696 & 43.793 \\
\hline 1/9/11 16:00 & 59.593 & 61.645 & 52.865 & & 40.690 & 40.696 & 43.793 \\
\hline 1/9/11 20:00 & 59.597 & 61.650 & 52.858 & & 40.693 & 40.703 & 43.798 \\
\hline 1/10/11 0:00 & 59.600 & 61.652 & 52.867 & & 40.697 & 40.708 & 43.803 \\
\hline $1 / 10 / 114: 00$ & 59.609 & 61.657 & 52.874 & & 40.707 & 40.717 & 43.807 \\
\hline $1 / 10 / 118: 00$ & 59.597 & 61.640 & 52.856 & & 40.700 & 40.708 & 43.793 \\
\hline $1 / 10 / 11$ 12:00 & 59.590 & 61.633 & 52.851 & & 40.685 & 40.701 & 43.787 \\
\hline 1/10/11 16:00 & 59.583 & 61.629 & 52.849 & & 40.685 & 40.699 & 43.779 \\
\hline 1/10/11 20:00 & 59.588 & 61.629 & 52.844 & & 40.688 & 40.701 & 43.782 \\
\hline 1/11/11 0:00 & 59.588 & 61.631 & 52.849 & & 40.690 & 40.701 & 43.784 \\
\hline $1 / 11 / 114: 00$ & 59.597 & 61.643 & 52.867 & & 40.695 & 40.713 & 43.796 \\
\hline 1/11/11 8:00 & 59.604 & 61.645 & 52.865 & & 40.702 & 40.722 & 43.800 \\
\hline $1 / 11 / 11$ 12:00 & 59.616 & 61.661 & 52.884 & & 40.714 & 40.734 & 43.812 \\
\hline $1 / 11 / 11$ 16:00 & 59.631 & 61.671 & 52.905 & & 40.730 & 40.753 & 43.826 \\
\hline $1 / 11 / 1120: 00$ & 59.645 & 61.682 & 52.909 & & 40.742 & 40.767 & 43.838 \\
\hline 1/12/11 0:00 & 59.647 & 61.689 & 52.914 & & 40.751 & 40.774 & 43.840 \\
\hline
\end{tabular}

TABLE S1.3 Page 21 of 58 


\begin{tabular}{|c|c|c|c|c|c|c|c|}
\hline \multirow[b]{2}{*}{ Date and Time } & \multicolumn{7}{|c|}{ Water Level (ft below reference point) } \\
\hline & SB43D & SB44D & SB45D & SB46D & SB47D & SB48D & SB49D \\
\hline $1 / 12 / 114: 00$ & 59.659 & 61.699 & 52.928 & & 40.758 & 40.786 & 43.854 \\
\hline 1/12/11 8:00 & 59.666 & 61.708 & 52.932 & & 40.765 & 40.793 & 43.859 \\
\hline 1/12/11 12:00 & 59.673 & 61.713 & 52.937 & & 40.777 & 40.800 & 43.868 \\
\hline 1/12/11 16:00 & 59.671 & 61.715 & 52.935 & & 40.775 & 40.802 & 43.868 \\
\hline 1/12/11 20:00 & 59.680 & 61.720 & 52.944 & & 40.786 & 40.811 & 43.875 \\
\hline 1/13/11 0:00 & 59.675 & 61.710 & 52.923 & & 40.772 & 40.807 & 43.865 \\
\hline 1/13/11 4:00 & 59.668 & 61.708 & 52.919 & & 40.775 & 40.807 & 43.858 \\
\hline 1/13/11 8:00 & 59.661 & 61.699 & 52.905 & & 40.768 & 40.802 & 43.851 \\
\hline 1/13/11 12:00 & 59.652 & 61.689 & 52.891 & & 40.763 & 40.788 & 43.837 \\
\hline 1/13/11 16:00 & 59.640 & 61.685 & 52.893 & & 40.747 & 40.781 & 43.833 \\
\hline 1/13/11 20:00 & 59.652 & 61.689 & 52.905 & & 40.758 & 40.793 & 43.842 \\
\hline 1/14/11 0:00 & 59.654 & 61.689 & 52.895 & & 40.754 & 40.795 & 43.840 \\
\hline 1/14/11 4:00 & 59.654 & 61.687 & 52.893 & & 40.754 & 40.788 & 43.840 \\
\hline 1/14/11 8:00 & 59.659 & 61.689 & 52.902 & & 40.758 & 40.793 & 43.843 \\
\hline 1/14/11 12:00 & 59.664 & 61.701 & 52.914 & & 40.768 & 40.797 & 43.847 \\
\hline 1/14/11 16:00 & 59.659 & 61.694 & 52.902 & & 40.758 & 40.795 & 43.844 \\
\hline 1/14/11 20:00 & 59.668 & 61.708 & 52.923 & & 40.768 & 40.807 & 43.856 \\
\hline 1/15/11 0:00 & 59.673 & 61.710 & 52.916 & & 40.768 & 40.807 & 43.858 \\
\hline 1/15/11 4:00 & 59.671 & 61.703 & 52.914 & & 40.765 & 40.807 & 43.859 \\
\hline 1/15/11 8:00 & 59.678 & 61.715 & 52.925 & & 40.777 & 40.649 & 43.863 \\
\hline 1/15/11 12:00 & 59.690 & 61.720 & 52.937 & & 40.782 & 40.654 & 43.875 \\
\hline 1/15/11 16:00 & 59.685 & 61.720 & 52.932 & & 40.784 & 40.729 & 43.872 \\
\hline 1/15/11 20:00 & 59.704 & 61.741 & 52.965 & & 40.801 & 40.786 & 43.893 \\
\hline 1/16/11 0:00 & 59.713 & 61.750 & 52.965 & & 40.810 & 40.788 & 43.900 \\
\hline 1/16/11 4:00 & 59.704 & 61.743 & 52.946 & & 40.798 & 40.781 & 43.891 \\
\hline 1/16/11 8:00 & 59.697 & 61.731 & 52.942 & & 40.796 & 40.840 & 43.884 \\
\hline 1/16/11 12:00 & 59.702 & 61.734 & 52.946 & & 40.803 & 40.847 & 43.889 \\
\hline 1/16/11 16:00 & 59.682 & 61.717 & 52.919 & & 40.784 & 40.835 & 43.868 \\
\hline 1/16/11 20:00 & 59.690 & 61.727 & 52.935 & & 40.794 & 40.844 & 43.877 \\
\hline 1/17/11 0:00 & 59.692 & 61.724 & 52.923 & & 40.789 & 40.844 & 43.872 \\
\hline 1/17/11 4:00 & 59.666 & 61.699 & 52.884 & & 40.763 & 40.821 & 43.849 \\
\hline 1/17/11 8:00 & 59.640 & 61.680 & 52.863 & & 40.742 & 40.797 & 43.826 \\
\hline 1/17/11 12:00 & 59.647 & 61.682 & 52.877 & & 40.749 & 40.800 & 43.830 \\
\hline 1/17/11 16:00 & 59.633 & 61.666 & 52.860 & & 40.728 & 40.783 & 43.815 \\
\hline 1/17/11 20:00 & 59.654 & 61.694 & 52.902 & & 40.751 & 40.804 & 43.840 \\
\hline 1/18/11 0:00 & 59.690 & 61.720 & 52.944 & & 40.772 & 40.833 & 43.875 \\
\hline 1/18/11 4:00 & 59.699 & 61.729 & 52.939 & & 40.782 & 40.840 & 43.882 \\
\hline 1/18/11 8:00 & 59.704 & 61.736 & 52.953 & & 40.789 & 40.849 & 43.891 \\
\hline 1/18/11 12:00 & 59.720 & 61.757 & 52.974 & & 40.808 & 40.865 & 43.910 \\
\hline 1/18/11 16:00 & 59.716 & 61.752 & 52.963 & & 40.805 & 40.863 & 43.903 \\
\hline 1/18/11 20:00 & 59.730 & 61.766 & 52.986 & & 40.822 & 40.882 & 43.919 \\
\hline 1/19/11 0:00 & 59.749 & 61.785 & 53.009 & & 40.838 & 40.898 & 43.940 \\
\hline 1/19/11 4:00 & 59.749 & 61.782 & 52.988 & & 40.831 & 40.901 & 43.933 \\
\hline 1/19/11 8:00 & 59.737 & 61.773 & 52.974 & & 40.826 & 40.891 & 43.926 \\
\hline 1/19/11 12:00 & 59.749 & 61.785 & 53.000 & & 40.841 & 40.912 & 43.937 \\
\hline 1/19/11 16:00 & 59.739 & 61.775 & 52.974 & & 40.831 & 40.905 & 43.923 \\
\hline 1/19/11 20:00 & 59.739 & 61.768 & 52.988 & & 40.831 & 40.908 & 43.923 \\
\hline 1/20/11 0:00 & 59.751 & 61.785 & 53.016 & & 40.846 & 40.922 & 43.938 \\
\hline 1/20/11 4:00 & 59.751 & 61.782 & 52.997 & & 40.841 & 40.912 & 43.935 \\
\hline 1/20/11 8:00 & 59.742 & 61.773 & 52.977 & & 40.838 & 40.903 & 43.926 \\
\hline 1/20/11 12:00 & 59.749 & 61.778 & 52.995 & & 40.845 & 40.912 & 43.930 \\
\hline 1/20/11 16:00 & 59.744 & 61.775 & 52.983 & & 40.841 & 40.910 & 43.924 \\
\hline 1/20/11 20:00 & 59.739 & 61.773 & 52.974 & & 40.833 & 40.929 & 43.923 \\
\hline 1/21/11 0:00 & 59.756 & 61.785 & 53.004 & & 40.850 & 40.940 & 43.937 \\
\hline
\end{tabular}

TABLE S1.3 Page 22 of 58 


\begin{tabular}{|c|c|c|c|c|c|c|c|}
\hline \multirow[b]{2}{*}{ Date and Time } & \multicolumn{7}{|c|}{ Water Level (ft below reference point) } \\
\hline & SB43D & SB44D & SB45D & SB46D & SB47D & SB48D & SB49D \\
\hline $1 / 21 / 114: 00$ & 59.756 & 61.780 & 52.986 & & 40.848 & 40.931 & 43.931 \\
\hline 1/21/11 8:00 & 59.732 & 61.759 & 52.956 & & 40.831 & 40.912 & 43.912 \\
\hline $1 / 21 / 1112: 00$ & 59.730 & 61.759 & 52.965 & & 40.831 & 40.917 & 43.910 \\
\hline 1/21/11 16:00 & 59.730 & 61.757 & 52.956 & & 40.826 & 40.912 & 43.905 \\
\hline $1 / 21 / 1120: 00$ & 59.732 & 61.759 & 52.958 & & 40.829 & 40.915 & 43.907 \\
\hline 1/22/11 0:00 & 59.739 & 61.771 & 52.979 & & 40.833 & 40.922 & 43.916 \\
\hline $1 / 22 / 114: 00$ & 59.749 & 61.773 & 52.977 & & 40.841 & 40.929 & 43.923 \\
\hline $1 / 22 / 118: 00$ & 59.737 & 61.761 & 52.960 & & 40.829 & 40.915 & 43.914 \\
\hline $1 / 22 / 1112: 00$ & 59.742 & 61.771 & 52.979 & & 40.836 & 40.922 & 43.919 \\
\hline $1 / 22 / 11$ 16:00 & 59.746 & 61.773 & 52.979 & & 40.836 & 40.929 & 43.923 \\
\hline $1 / 22 / 1120: 00$ & 59.751 & 61.780 & 52.979 & & 40.843 & 40.924 & 43.929 \\
\hline 1/23/11 0:00 & 59.756 & 61.780 & 53.000 & & 40.845 & 40.933 & 43.933 \\
\hline $1 / 23 / 114: 00$ & 59.761 & 61.787 & 53.009 & & 40.850 & 40.936 & 43.937 \\
\hline 1/23/11 8:00 & 59.761 & 61.785 & 52.993 & & 40.845 & 40.931 & 43.935 \\
\hline $1 / 23 / 11$ 12:00 & 59.761 & 61.789 & 53.004 & & 40.850 & 40.936 & 43.942 \\
\hline $1 / 23 / 11$ 16:00 & 59.758 & 61.789 & 53.002 & & 40.857 & 40.940 & 43.942 \\
\hline $1 / 23 / 1120: 00$ & 59.756 & 61.782 & 52.986 & & 40.848 & 40.933 & 43.938 \\
\hline 1/24/11 0:00 & 59.753 & 61.782 & 52.988 & & 40.852 & 40.934 & 43.930 \\
\hline $1 / 24 / 114: 00$ & 59.756 & 61.782 & 52.988 & & 40.852 & 40.938 & 43.936 \\
\hline $1 / 24 / 118: 00$ & 59.758 & 61.782 & 52.986 & & 40.855 & 40.936 & 43.933 \\
\hline $1 / 24 / 11$ 12:00 & 59.763 & 61.794 & 53.002 & & 40.859 & 40.948 & 43.942 \\
\hline $1 / 24 / 11$ 16:00 & 59.777 & 61.806 & 53.023 & & 40.876 & 40.964 & 43.956 \\
\hline $1 / 24 / 1120: 00$ & 59.789 & 61.810 & 53.025 & & 40.883 & 40.971 & 43.966 \\
\hline 1/25/11 0:00 & 59.789 & 61.817 & 53.025 & & 40.883 & 40.973 & 43.968 \\
\hline $1 / 25 / 114: 00$ & 59.798 & 61.824 & 53.039 & & 40.892 & 40.983 & 43.977 \\
\hline 1/25/11 8:00 & 59.796 & 61.820 & 53.023 & & 40.892 & 40.980 & 43.972 \\
\hline $1 / 25 / 11$ 12:00 & 59.796 & 61.829 & 53.030 & & 40.892 & 40.990 & 43.975 \\
\hline 1/25/11 16:00 & 59.794 & 61.824 & 53.030 & & 40.895 & 40.980 & 43.968 \\
\hline $1 / 25 / 1120: 00$ & 59.798 & 61.827 & 53.030 & & 40.892 & 40.985 & 43.977 \\
\hline 1/26/11 0:00 & 59.796 & 61.822 & 53.025 & & 40.890 & 40.980 & 43.972 \\
\hline $1 / 26 / 114: 00$ & 59.801 & 61.827 & 53.037 & & 40.895 & 40.990 & 43.979 \\
\hline 1/26/11 8:00 & 59.810 & 61.834 & 53.044 & & 40.899 & 40.997 & 43.989 \\
\hline $1 / 26 / 11$ 12:00 & 59.822 & 61.845 & 53.062 & & 40.916 & 41.006 & 44.000 \\
\hline $1 / 26 / 11$ 16:00 & 59.822 & 61.850 & 53.067 & & 40.918 & 41.018 & 44.003 \\
\hline $1 / 26 / 1120: 00$ & 59.836 & 61.864 & 53.069 & & 40.927 & 41.020 & 44.014 \\
\hline 1/27/11 0:00 & 59.824 & 61.859 & 53.053 & & 40.918 & 41.011 & 44.007 \\
\hline $1 / 27 / 114: 00$ & 59.810 & 61.843 & 53.035 & & 40.909 & 40.997 & 43.991 \\
\hline 1/27/11 8:00 & 59.798 & 61.829 & 53.023 & & 40.895 & 40.987 & 43.982 \\
\hline $1 / 27 / 11$ 12:00 & 59.803 & 61.834 & 53.032 & & 40.899 & 40.994 & 43.982 \\
\hline $1 / 27 / 11$ 16:00 & 59.808 & 61.836 & 53.044 & & 40.904 & 40.997 & 43.986 \\
\hline $1 / 27 / 1120: 00$ & 59.824 & 61.859 & 53.069 & & 40.923 & 41.011 & 44.008 \\
\hline 1/28/11 0:00 & 59.831 & 61.866 & 53.065 & & 40.920 & 41.016 & 44.012 \\
\hline $1 / 28 / 114: 00$ & 59.824 & 61.857 & 53.053 & & 40.911 & 41.018 & 44.010 \\
\hline $1 / 28 / 118: 00$ & 59.827 & 61.859 & 53.058 & & 40.920 & 41.018 & 44.010 \\
\hline $1 / 28 / 11$ 12:00 & 59.824 & 61.855 & 53.053 & & 40.918 & 41.016 & 44.005 \\
\hline 1/28/11 16:00 & 59.815 & 61.852 & 53.051 & & 40.911 & 41.006 & 44.000 \\
\hline $1 / 28 / 1120: 00$ & 59.831 & 61.873 & 53.076 & & 40.920 & 41.018 & 44.019 \\
\hline 1/29/11 0:00 & 59.846 & 61.883 & 53.090 & & 40.932 & 41.032 & 44.033 \\
\hline $1 / 29 / 114: 00$ & 59.843 & 61.880 & 53.079 & & 40.925 & 41.034 & 44.028 \\
\hline 1/29/11 8:00 & 59.846 & 61.883 & 53.081 & & 40.932 & 41.051 & 44.033 \\
\hline $1 / 29 / 1112: 00$ & 59.853 & 61.897 & 53.095 & & 40.942 & 41.048 & 44.040 \\
\hline $1 / 29 / 11$ 16:00 & 59.850 & 61.894 & 53.097 & & 40.939 & 41.048 & 44.042 \\
\hline $1 / 29 / 1120: 00$ & 59.869 & 61.913 & 53.132 & & 40.958 & 41.070 & 44.063 \\
\hline 1/30/11 0:00 & 59.888 & 61.929 & 53.148 & & 40.970 & 41.081 & 44.082 \\
\hline
\end{tabular}

TABLE S1.3 Page 23 of 58 


\begin{tabular}{|c|c|c|c|c|c|c|c|}
\hline \multirow[b]{2}{*}{ Date and Time } & \multicolumn{7}{|c|}{ Water Level (ft below reference point) } \\
\hline & SB43D & SB44D & SB45D & SB46D & SB47D & SB48D & SB49D \\
\hline $1 / 30 / 114: 00$ & 59.886 & 61.925 & 53.134 & & 40.970 & 41.084 & 44.079 \\
\hline 1/30/11 8:00 & 59.893 & 61.934 & 53.148 & & 40.979 & 41.088 & 44.086 \\
\hline $1 / 30 / 11$ 12:00 & 59.907 & 61.946 & 53.153 & & 40.986 & 41.098 & 44.091 \\
\hline $1 / 30 / 11$ 16:00 & 59.893 & 61.932 & 53.134 & & 40.974 & 41.093 & 44.082 \\
\hline 1/30/11 20:00 & 59.898 & 61.941 & 53.153 & & 40.989 & 41.098 & 44.089 \\
\hline 1/31/11 0:00 & 59.912 & 61.948 & 53.165 & & 40.996 & 41.110 & 44.098 \\
\hline $1 / 31 / 114: 00$ & 59.900 & 61.932 & 53.132 & & 40.984 & 41.100 & 44.084 \\
\hline 1/31/11 8:00 & 59.879 & 61.915 & 53.118 & & 40.963 & 41.079 & 44.065 \\
\hline $1 / 31 / 11$ 12:00 & 59.888 & 61.920 & 53.130 & & 40.977 & 41.091 & 44.072 \\
\hline $1 / 31 / 11$ 16:00 & 59.879 & 61.913 & 53.109 & & 40.967 & 41.081 & 44.063 \\
\hline $1 / 31 / 1120: 00$ & 59.893 & 61.929 & 53.139 & & 40.979 & 41.114 & 44.079 \\
\hline 2/1/11 0:00 & 59.917 & 61.957 & 53.174 & & 41.000 & 41.126 & 44.103 \\
\hline 2/1/11 4:00 & 59.910 & 61.946 & 53.148 & & 40.993 & 41.107 & 44.098 \\
\hline 2/1/11 8:00 & 59.902 & 61.934 & 53.134 & & 40.984 & 41.096 & 44.089 \\
\hline 2/1/11 12:00 & 59.895 & 61.925 & 53.139 & & 40.977 & 41.095 & 44.082 \\
\hline 2/1/11 16:00 & 59.876 & 61.908 & 53.120 & & 40.958 & 41.074 & 44.065 \\
\hline 2/1/11 20:00 & 59.860 & 61.892 & 53.109 & & 40.946 & 41.084 & 44.056 \\
\hline 2/2/11 0:00 & 59.886 & 61.922 & 53.148 & & 40.967 & 41.095 & 44.077 \\
\hline 2/2/11 4:00 & 59.893 & 61.927 & 53.141 & & 40.970 & 41.091 & 44.082 \\
\hline 2/2/11 8:00 & 59.898 & 61.932 & 53.144 & & 40.981 & 41.098 & 44.089 \\
\hline $2 / 2 / 11$ 12:00 & 59.921 & 61.950 & 53.181 & & 41.000 & 41.121 & 44.107 \\
\hline $2 / 2 / 11$ 16:00 & 59.919 & 61.955 & 53.174 & & 41.003 & 41.126 & 44.107 \\
\hline 2/2/11 20:00 & 59.924 & 61.960 & 53.176 & & 41.007 & 41.133 & 44.119 \\
\hline 2/3/11 0:00 & 59.938 & 61.971 & 53.192 & & 41.026 & 41.143 & 44.128 \\
\hline $2 / 3 / 114: 00$ & 59.931 & 61.962 & 53.167 & & 41.019 & 41.138 & 44.114 \\
\hline 2/3/11 8:00 & 59.917 & 61.950 & 53.153 & & 41.010 & 41.128 & 44.105 \\
\hline 2/3/11 12:00 & 59.921 & 61.950 & 53.162 & & 41.014 & 41.128 & 44.103 \\
\hline 2/3/11 16:00 & 59.902 & 61.936 & 53.137 & & 40.998 & 41.121 & 44.086 \\
\hline $2 / 3 / 1120: 00$ & 59.898 & 61.934 & 53.134 & & 40.993 & 41.114 & 44.084 \\
\hline 2/4/11 0:00 & 59.912 & 61.943 & 53.155 & & 41.005 & 41.126 & 44.098 \\
\hline 2/4/11 4:00 & 59.903 & 61.932 & 53.123 & & 40.993 & 41.114 & 44.082 \\
\hline 2/4/11 8:00 & 59.891 & 61.920 & 53.113 & & 40.981 & 41.105 & 44.075 \\
\hline 2/4/11 12:00 & 59.893 & 61.922 & 53.120 & & 40.981 & 41.110 & 44.068 \\
\hline $2 / 4 / 11$ 16:00 & 59.872 & 61.906 & 53.093 & & 40.965 & 41.102 & 44.051 \\
\hline 2/4/11 20:00 & 59.862 & 61.897 & 53.083 & & 40.958 & 41.093 & 44.044 \\
\hline 2/5/11 0:00 & 59.869 & 61.906 & 53.097 & & 40.963 & 41.098 & 44.051 \\
\hline 2/5/11 4:00 & 59.865 & 61.892 & 53.076 & & 40.956 & 41.088 & 44.044 \\
\hline 2/5/11 8:00 & 59.850 & 61.876 & 53.060 & & 40.937 & 41.077 & 44.028 \\
\hline 2/5/11 12:00 & 59.855 & 61.878 & 53.083 & & 40.946 & 41.088 & 44.033 \\
\hline 2/5/11 16:00 & 59.860 & 61.887 & 53.083 & & 40.949 & 41.098 & 44.035 \\
\hline 2/5/11 20:00 & 59.865 & 61.892 & 53.090 & & 40.951 & 41.100 & 44.042 \\
\hline 2/6/11 0:00 & 59.876 & 61.904 & 53.111 & & 40.963 & 41.105 & 44.051 \\
\hline 2/6/11 4:00 & 59.891 & 61.915 & 53.123 & & 40.970 & 41.114 & 44.068 \\
\hline 2/6/11 8:00 & 59.898 & 61.925 & 53.130 & & 40.977 & 41.121 & 44.075 \\
\hline 2/6/11 12:00 & 59.917 & 61.943 & 53.165 & & 40.998 & 41.135 & 44.100 \\
\hline 2/6/11 16:00 & 59.931 & 61.960 & 53.172 & & 41.012 & 41.147 & 44.112 \\
\hline 2/6/11 20:00 & 59.938 & 61.966 & 53.178 & & 41.019 & 41.154 & 44.123 \\
\hline 2/7/11 0:00 & 59.947 & 61.978 & 53.197 & & 41.031 & NA & 44.135 \\
\hline 2/7/11 4:00 & 59.957 & 61.990 & 53.197 & & 41.038 & & 44.140 \\
\hline 2/7/11 8:00 & 59.952 & 61.985 & 53.185 & & 41.043 & & 44.137 \\
\hline 2/7/11 12:00 & 59.962 & 61.994 & 53.204 & & 41.050 & & 44.144 \\
\hline 2/7/11 16:00 & 59.962 & 61.992 & 53.202 & & 41.059 & & 44.144 \\
\hline 2/7/11 20:00 & 59.964 & 61.994 & 53.199 & & 41.052 & & 44.147 \\
\hline 2/8/11 0:00 & 59.980 & 62.008 & 53.225 & & 41.071 & & 44.165 \\
\hline
\end{tabular}

TABLE S1.3 Page 24 of 58 


\begin{tabular}{|c|c|c|c|c|c|c|c|}
\hline \multirow[b]{2}{*}{ Date and Time } & \multicolumn{7}{|c|}{ Water Level (ft below reference point) } \\
\hline & SB43D & SB44D & SB45D & SB46D & SB47D & SB48D & SB49D \\
\hline 2/8/11 4:00 & 59.997 & 62.027 & 53.239 & & 41.085 & & 44.182 \\
\hline 2/8/11 8:00 & 60.011 & 62.039 & 53.253 & & 41.097 & & 44.189 \\
\hline 2/8/11 12:00 & 60.018 & 62.041 & 53.262 & & 41.108 & & 44.198 \\
\hline 2/8/11 16:00 & 60.009 & 62.036 & 53.246 & & 41.106 & & 44.189 \\
\hline 2/8/11 20:00 & 60.018 & 62.043 & 53.246 & & 41.108 & & 44.198 \\
\hline 2/9/11 0:00 & 60.014 & 62.034 & 53.241 & & 41.104 & & 44.191 \\
\hline 2/9/11 4:00 & 59.999 & 62.029 & 53.225 & & 41.101 & & 44.179 \\
\hline 2/9/11 8:00 & 59.999 & 62.025 & 53.230 & & 41.097 & & 44.177 \\
\hline 2/9/11 12:00 & 60.004 & 62.022 & 53.237 & & 41.097 & & 44.177 \\
\hline 2/9/11 16:00 & 60.006 & 62.032 & 53.237 & & 41.101 & & 44.182 \\
\hline 2/9/11 20:00 & 60.009 & 62.032 & 53.232 & & 41.101 & & 44.184 \\
\hline 2/10/11 0:00 & 60.006 & 62.029 & 53.225 & & 41.099 & & 44.179 \\
\hline 2/10/11 4:00 & 60.002 & 62.018 & 53.213 & & 41.104 & & 44.172 \\
\hline 2/10/11 8:00 & 59.990 & 62.004 & 53.195 & & 41.080 & & 44.161 \\
\hline 2/10/11 12:00 & 59.990 & 62.004 & 53.206 & & 41.085 & & 44.154 \\
\hline 2/10/11 16:00 & 59.985 & 62.006 & 53.209 & & 41.085 & & 44.156 \\
\hline 2/10/11 20:00 & 59.997 & 62.018 & 53.218 & & 41.090 & & 44.168 \\
\hline 2/11/11 0:00 & 59.992 & 62.008 & 53.206 & & 41.082 & & 44.163 \\
\hline 2/11/11 4:00 & 59.990 & 62.011 & 53.206 & & 41.085 & & 44.161 \\
\hline 2/11/11 8:00 & 59.999 & 62.015 & 53.211 & & 41.087 & & 44.165 \\
\hline 2/11/11 12:00 & 60.002 & 62.013 & 53.216 & & 41.092 & & 44.163 \\
\hline 2/11/11 16:00 & 60.004 & 62.025 & 53.232 & & 41.097 & & 44.172 \\
\hline 2/11/11 20:00 & 60.025 & 62.043 & 53.255 & & 41.113 & & 44.196 \\
\hline 2/12/11 0:00 & 60.032 & 62.048 & 53.255 & & 41.115 & & 44.202 \\
\hline 2/12/11 4:00 & 60.030 & 62.050 & 53.250 & & 41.115 & & 44.200 \\
\hline 2/12/11 8:00 & 60.028 & 62.048 & 53.243 & & 41.118 & & 44.198 \\
\hline 2/12/11 12:00 & 60.025 & 62.046 & 53.239 & & 41.120 & & 44.191 \\
\hline 2/12/11 16:00 & 60.016 & 62.046 & 53.234 & & 41.118 & & 44.191 \\
\hline 2/12/11 20:00 & 60.030 & 62.067 & 53.262 & & 41.120 & & 44.216 \\
\hline 2/13/11 0:00 & 60.030 & 62.062 & 53.253 & & 41.120 & & 44.212 \\
\hline 2/13/11 4:00 & 60.016 & 62.043 & 53.227 & & 41.108 & & 44.193 \\
\hline 2/13/11 8:00 & 60.009 & 62.036 & 53.223 & & 41.104 & & 44.184 \\
\hline 2/13/11 12:00 & 60.011 & 62.029 & 53.216 & & 41.099 & & 44.182 \\
\hline 2/13/11 16:00 & 59.997 & 62.043 & 53.209 & & 41.075 & & 44.186 \\
\hline 2/13/11 20:00 & 60.018 & 62.081 & 53.281 & & 41.115 & & 44.228 \\
\hline 2/14/11 0:00 & 60.054 & 62.090 & 53.292 & & 41.132 & & 44.242 \\
\hline 2/14/11 4:00 & 60.056 & 62.088 & 53.274 & & 41.122 & & 44.235 \\
\hline 2/14/11 8:00 & 60.063 & 62.085 & 53.288 & & 41.115 & & 44.237 \\
\hline 2/14/11 12:00 & 60.073 & 62.092 & 53.292 & & 41.115 & & 44.244 \\
\hline 2/14/11 16:00 & 60.056 & 62.111 & 53.276 & & 41.101 & & 44.247 \\
\hline 2/14/11 20:00 & 60.051 & 62.104 & 53.281 & & 41.101 & & 44.247 \\
\hline 2/15/11 0:00 & 60.051 & 62.092 & 53.269 & & 41.080 & & 44.233 \\
\hline 2/15/11 4:00 & 60.021 & 62.055 & 53.211 & & 41.040 & & 44.198 \\
\hline 2/15/11 8:00 & 60.002 & 62.034 & 53.199 & & 41.012 & & 44.179 \\
\hline 2/15/11 12:00 & 59.997 & 62.025 & 53.192 & & 40.998 & & 44.168 \\
\hline 2/15/11 16:00 & 59.962 & 62.022 & 53.165 & & 41.108 & & 44.154 \\
\hline 2/15/11 20:00 & 59.959 & 62.022 & 53.188 & & 41.045 & & 44.158 \\
\hline 2/16/11 0:00 & 59.971 & 62.013 & 53.195 & & 40.862 & & 44.158 \\
\hline 2/16/11 4:00 & 59.950 & 61.987 & 53.151 & & 40.815 & & 44.128 \\
\hline 2/16/11 8:00 & 59.924 & 61.964 & 53.132 & & 40.794 & & 44.105 \\
\hline 2/16/11 12:00 & 59.917 & 61.960 & 53.130 & & 40.808 & & 44.098 \\
\hline 2/16/11 16:00 & 59.869 & 61.932 & 53.076 & & 40.949 & & 44.068 \\
\hline 2/16/11 20:00 & 59.857 & 61.925 & 53.076 & & 40.951 & & 44.058 \\
\hline 2/17/11 0:00 & 59.862 & 61.918 & 53.081 & & 40.932 & & 44.054 \\
\hline
\end{tabular}

TABLE S1.3 Page 25 of 58 


\begin{tabular}{|c|c|c|c|c|c|c|c|}
\hline \multirow[b]{2}{*}{ Date and Time } & \multicolumn{7}{|c|}{ Water Level (ft below reference point) } \\
\hline & SB43D & SB44D & SB45D & SB46D & SB47D & SB48D & SB49D \\
\hline 2/17/11 4:00 & 59.836 & 61.897 & 53.028 & & 40.890 & & 44.026 \\
\hline 2/17/11 8:00 & 59.815 & 61.873 & 53.004 & & 40.857 & & 44.003 \\
\hline 2/17/11 12:00 & 59.810 & 61.871 & 53.018 & & 40.833 & & 44.005 \\
\hline 2/17/11 16:00 & 59.803 & 61.855 & 53.002 & & 40.688 & & 43.989 \\
\hline 2/17/11 20:00 & 59.813 & 61.862 & 53.039 & & 40.690 & & 44.000 \\
\hline 2/18/11 0:00 & 59.824 & 61.880 & 53.076 & & 40.707 & & 44.021 \\
\hline 2/18/11 4:00 & 59.815 & 61.866 & 53.051 & & 40.688 & & 44.014 \\
\hline 2/18/11 8:00 & 59.794 & 61.855 & 53.037 & & 40.674 & & 44.000 \\
\hline 2/18/11 12:00 & 59.789 & 61.855 & 53.053 & & 40.676 & & 44.005 \\
\hline 2/18/11 16:00 & 59.763 & 61.841 & 53.011 & & 40.650 & & 43.982 \\
\hline 2/18/11 20:00 & 59.737 & 61.815 & 52.983 & & 40.629 & & 43.958 \\
\hline 2/19/11 0:00 & 59.730 & 61.815 & 52.995 & & 40.629 & & 43.958 \\
\hline 2/19/11 4:00 & 59.716 & 61.794 & 52.967 & & 40.608 & & 43.940 \\
\hline 2/19/11 8:00 & 59.682 & 61.764 & 52.935 & & 40.580 & & 43.910 \\
\hline 2/19/11 12:00 & 59.673 & 61.757 & 52.944 & & 40.577 & & 43.903 \\
\hline 2/19/11 16:00 & 59.647 & 61.736 & 52.900 & & 40.542 & & 43.877 \\
\hline 2/19/11 20:00 & 59.612 & 61.703 & 52.863 & & 40.514 & & 43.842 \\
\hline 2/20/11 0:00 & 59.590 & 61.689 & 52.856 & & 40.490 & & 43.828 \\
\hline 2/20/11 4:00 & 59.567 & 61.659 & 52.814 & & 40.472 & & 43.800 \\
\hline 2/20/11 8:00 & 59.526 & 61.619 & 52.768 & & 40.429 & & 43.758 \\
\hline 2/20/11 12:00 & 59.503 & 61.605 & 52.761 & & 40.408 & & 43.740 \\
\hline 2/20/11 16:00 & 59.481 & 61.580 & 52.742 & & 40.385 & & 43.721 \\
\hline 2/20/11 20:00 & 59.484 & 61.582 & 52.761 & & 40.382 & & 43.726 \\
\hline 2/21/11 0:00 & 59.479 & 61.582 & 52.777 & & 40.387 & & 43.730 \\
\hline 2/21/11 4:00 & 59.472 & 61.580 & 52.763 & & 40.375 & & 43.726 \\
\hline 2/21/11 8:00 & 59.460 & 61.568 & 52.751 & & 40.364 & & 43.712 \\
\hline 2/21/11 12:00 & 59.453 & 61.573 & 52.768 & & 40.366 & & 43.721 \\
\hline 2/21/11 16:00 & 59.453 & 61.573 & 52.768 & & 40.368 & & 43.719 \\
\hline 2/21/11 20:00 & 59.446 & 61.566 & 52.756 & & 40.357 & & 43.712 \\
\hline 2/22/11 0:00 & 59.434 & 61.556 & 52.758 & & 40.359 & & 43.707 \\
\hline $2 / 22 / 114: 00$ & 59.432 & 61.559 & 52.758 & & 40.352 & & 43.707 \\
\hline 2/22/11 8:00 & 59.418 & 61.545 & 52.740 & & 40.342 & & 43.691 \\
\hline 2/22/11 12:00 & 59.415 & 61.547 & 52.751 & & 40.345 & & 43.691 \\
\hline 2/22/11 16:00 & 59.401 & 61.536 & 52.730 & & 40.338 & & 43.679 \\
\hline 2/22/11 20:00 & 59.387 & 61.517 & 52.703 & & 40.319 & & 43.661 \\
\hline 2/23/11 0:00 & 59.366 & 61.505 & 52.686 & & 40.298 & & 43.644 \\
\hline 2/23/11 4:00 & 59.351 & 61.482 & 52.670 & & 40.286 & & 43.626 \\
\hline 2/23/11 8:00 & 59.332 & 61.463 & 52.642 & & 40.265 & & 43.607 \\
\hline 2/23/11 12:00 & 59.318 & 61.449 & 52.645 & & 40.260 & & 43.596 \\
\hline 2/23/11 16:00 & 59.314 & 61.452 & 52.645 & & 40.251 & & 43.593 \\
\hline 2/23/11 20:00 & 59.323 & 61.452 & 52.654 & & 40.256 & & 43.600 \\
\hline 2/24/11 0:00 & 59.314 & 61.449 & 52.647 & & 40.248 & & 43.596 \\
\hline 2/24/11 4:00 & 59.302 & 61.440 & 52.638 & & 40.246 & & 43.586 \\
\hline 2/24/11 8:00 & 59.285 & 61.426 & 52.624 & & 40.230 & & 43.575 \\
\hline 2/24/11 12:00 & 59.280 & 61.419 & 52.628 & & 40.230 & & 43.568 \\
\hline 2/24/11 16:00 & 59.247 & 61.389 & 52.591 & & 40.187 & & 43.533 \\
\hline 2/24/11 20:00 & 59.240 & 61.377 & 52.584 & & 40.178 & & 43.524 \\
\hline 2/25/11 0:00 & 59.214 & 61.358 & 52.573 & & 40.162 & & 43.510 \\
\hline 2/25/11 4:00 & 59.214 & 61.361 & 52.573 & & 40.166 & & 43.512 \\
\hline 2/25/11 8:00 & 59.214 & 61.363 & 52.575 & & 40.169 & & 43.512 \\
\hline 2/25/11 12:00 & 59.212 & 61.365 & 52.580 & & 40.164 & & 43.512 \\
\hline 2/25/11 16:00 & 59.200 & 61.351 & 52.566 & & 40.159 & & 43.500 \\
\hline 2/25/11 20:00 & 59.195 & 61.349 & 52.563 & & 40.154 & & 43.498 \\
\hline 2/26/11 0:00 & 59.183 & 61.335 & 52.542 & & 40.147 & & 43.482 \\
\hline
\end{tabular}

TABLE S1.3 Page 26 of 58 


\begin{tabular}{|c|c|c|c|c|c|c|c|}
\hline \multirow[b]{2}{*}{ Date and Time } & \multicolumn{7}{|c|}{ Water Level (ft below reference point) } \\
\hline & SB43D & SB44D & SB45D & SB46D & SB47D & SB48D & SB49D \\
\hline 2/26/11 4:00 & 59.162 & 61.314 & 52.515 & & 40.124 & & 43.461 \\
\hline 2/26/11 8:00 & 59.146 & 61.300 & 52.498 & & 40.112 & & 43.444 \\
\hline 2/26/11 12:00 & 59.136 & 61.291 & 52.491 & & 40.100 & & 43.435 \\
\hline 2/26/11 16:00 & 59.120 & 61.275 & 52.470 & & 40.084 & & 43.421 \\
\hline 2/26/11 20:00 & 59.122 & 61.277 & 52.484 & & 40.089 & & 43.421 \\
\hline 2/27/11 0:00 & 59.115 & 61.265 & 52.470 & & 40.079 & & 43.417 \\
\hline 2/27/11 4:00 & 59.094 & 61.251 & 52.450 & & 40.056 & & 43.396 \\
\hline 2/27/11 8:00 & 59.079 & 61.240 & 52.438 & & 40.046 & & 43.384 \\
\hline 2/27/11 12:00 & 59.084 & 61.235 & 52.440 & & 40.044 & & 43.382 \\
\hline 2/27/11 16:00 & 59.058 & 61.223 & 52.417 & & 40.028 & & 43.368 \\
\hline 2/27/11 20:00 & 59.060 & 61.228 & 52.445 & & 40.021 & & 43.370 \\
\hline 2/28/11 0:00 & 59.058 & 61.244 & 52.450 & & 40.025 & & 43.386 \\
\hline 2/28/11 4:00 & 59.063 & 61.244 & 52.461 & & 40.030 & & 43.393 \\
\hline 2/28/11 8:00 & 59.079 & 61.265 & 52.498 & & 40.046 & & 43.414 \\
\hline 2/28/11 12:00 & 59.091 & 61.272 & 52.508 & & 40.058 & & 43.424 \\
\hline 2/28/11 16:00 & 59.077 & 61.268 & 52.487 & & 40.037 & & 43.414 \\
\hline 2/28/11 20:00 & 59.075 & 61.272 & 52.491 & & 40.037 & & 43.419 \\
\hline 3/1/11 0:00 & 59.075 & 61.270 & 52.489 & & 40.030 & & 43.419 \\
\hline $3 / 1 / 114: 00$ & 59.046 & 61.242 & 52.443 & & 39.995 & & 43.389 \\
\hline 3/1/11 8:00 & 59.037 & 61.235 & 52.450 & & 39.983 & & 43.382 \\
\hline $3 / 1 / 11$ 12:00 & 59.034 & 61.235 & 52.454 & & 39.981 & & 43.386 \\
\hline 3/1/11 16:00 & 59.016 & 61.230 & 52.438 & & 39.959 & & 43.372 \\
\hline $3 / 1 / 1120: 00$ & 59.018 & 61.240 & 52.461 & & 39.959 & & 43.386 \\
\hline 3/2/11 0:00 & 59.030 & 61.249 & 52.480 & & 39.957 & & 43.400 \\
\hline $3 / 2 / 114: 00$ & 59.025 & 61.247 & 52.468 & & 39.943 & & 43.398 \\
\hline 3/2/11 8:00 & 59.011 & 61.244 & 52.466 & & 39.927 & & 43.396 \\
\hline $3 / 2 / 11$ 12:00 & 59.011 & 61.244 & 52.468 & & 39.915 & & 43.398 \\
\hline $3 / 2 / 11$ 16:00 & 58.978 & 61.223 & 52.424 & & 39.880 & & 43.375 \\
\hline 3/2/11 20:00 & 58.961 & 61.216 & 52.422 & & 39.861 & & 43.363 \\
\hline 3/3/11 0:00 & 58.954 & 61.212 & 52.429 & & 39.851 & & 43.363 \\
\hline $3 / 3 / 114: 00$ & 58.926 & 61.188 & 52.382 & & 39.814 & & 43.335 \\
\hline 3/3/11 8:00 & 58.902 & 61.172 & 52.375 & & 39.790 & & 43.324 \\
\hline 3/3/11 12:00 & 58.890 & 61.167 & 52.371 & & 39.776 & & 43.314 \\
\hline 3/3/11 16:00 & 58.862 & 61.142 & 52.334 & & 39.741 & & 43.289 \\
\hline $3 / 3 / 1120: 00$ & 58.845 & 61.135 & 52.340 & & 39.727 & & 43.284 \\
\hline 3/4/11 0:00 & 58.845 & 61.139 & 52.350 & & 39.720 & & 43.291 \\
\hline 3/4/11 4:00 & 58.831 & 61.125 & 52.329 & & 39.701 & & 43.277 \\
\hline 3/4/11 8:00 & 58.817 & 61.125 & 52.336 & & 39.692 & & 43.277 \\
\hline $3 / 4 / 11$ 12:00 & 58.815 & 61.130 & 52.350 & & 39.689 & & 43.284 \\
\hline 3/4/11 16:00 & 58.810 & 61.121 & 52.334 & & 39.678 & & 43.277 \\
\hline 3/4/11 20:00 & 58.798 & 61.121 & 52.343 & & 39.673 & & 43.279 \\
\hline 3/5/11 0:00 & 58.807 & 61.135 & 52.368 & & 39.680 & & 43.296 \\
\hline 3/5/11 4:00 & 58.800 & 61.132 & 52.357 & & 39.675 & & 43.291 \\
\hline 3/5/11 8:00 & 58.786 & 61.128 & 52.347 & & 39.666 & & 43.284 \\
\hline 3/5/11 12:00 & 58.789 & 61.132 & 52.368 & & 39.673 & & 43.291 \\
\hline 3/5/11 16:00 & 58.770 & 61.118 & 52.334 & & 39.654 & & 43.272 \\
\hline $3 / 5 / 1120: 00$ & 58.753 & 61.107 & 52.324 & & 39.640 & & 43.263 \\
\hline 3/6/11 0:00 & 58.755 & 61.114 & 52.338 & & 39.645 & & 43.265 \\
\hline 3/6/11 4:00 & 58.741 & 61.095 & 52.308 & & 39.626 & & 43.252 \\
\hline 3/6/11 8:00 & 58.722 & 61.079 & 52.292 & & 39.612 & & 43.235 \\
\hline $3 / 6 / 11$ 12:00 & 58.718 & 61.079 & 52.299 & & 39.612 & & 43.235 \\
\hline 3/6/11 16:00 & 58.699 & 61.063 & 52.266 & & 39.588 & & 43.217 \\
\hline $3 / 6 / 1120: 00$ & 58.682 & 61.046 & 52.252 & & 39.569 & & 43.198 \\
\hline 3/7/11 0:00 & 58.677 & 61.049 & 52.262 & & 39.567 & & 43.198 \\
\hline
\end{tabular}

TABLE S1.3 Page 27 of 58 


\begin{tabular}{|c|c|c|c|c|c|c|c|}
\hline \multirow[b]{2}{*}{ Date and Time } & \multicolumn{7}{|c|}{ Water Level (ft below reference point) } \\
\hline & SB43D & SB44D & SB45D & SB46D & SB47D & SB48D & SB49D \\
\hline 3/7/11 4:00 & 58.666 & 61.035 & 52.241 & & 39.558 & & 43.186 \\
\hline 3/7/11 8:00 & 58.651 & 61.021 & 52.234 & & 39.544 & & 43.175 \\
\hline 3/7/11 12:00 & 58.651 & 61.028 & 52.248 & & 39.544 & & 43.177 \\
\hline 3/7/11 16:00 & 58.642 & 61.014 & 52.229 & & 39.530 & & 43.165 \\
\hline 3/7/11 20:00 & 58.616 & 60.992 & 52.204 & & 39.511 & & 43.142 \\
\hline 3/8/11 0:00 & 58.611 & 60.995 & 52.215 & & 39.508 & & 43.147 \\
\hline 3/8/11 4:00 & 58.604 & 60.986 & 52.201 & & 39.501 & & 43.142 \\
\hline 3/8/11 8:00 & 58.587 & 60.969 & 52.183 & & 39.485 & & 43.124 \\
\hline $3 / 8 / 11$ 12:00 & 58.583 & 60.969 & 52.190 & & 39.483 & & 43.121 \\
\hline 3/8/11 16:00 & 58.554 & 60.944 & 52.155 & & 39.497 & & 43.096 \\
\hline 3/8/11 20:00 & 58.526 & 60.930 & 52.134 & & 39.511 & & 43.082 \\
\hline 3/9/11 0:00 & 58.514 & 60.925 & 52.136 & & 39.506 & & 43.077 \\
\hline 3/9/11 4:00 & 58.512 & 60.918 & 52.134 & & 39.464 & & 43.075 \\
\hline 3/9/11 8:00 & 58.509 & 60.916 & 52.134 & & 39.384 & & 43.070 \\
\hline 3/9/11 12:00 & 58.514 & 60.932 & 52.166 & & 39.398 & & 43.086 \\
\hline 3/9/11 16:00 & 58.519 & 60.937 & 52.169 & & 39.391 & & 43.091 \\
\hline 3/9/11 20:00 & 58.512 & 60.932 & 52.162 & & 39.384 & & 43.091 \\
\hline 3/10/11 0:00 & 58.505 & 60.934 & 52.166 & & 39.377 & & 43.093 \\
\hline 3/10/11 4:00 & 58.498 & 60.934 & 52.166 & & 39.365 & & 43.091 \\
\hline 3/10/11 8:00 & 58.491 & 60.930 & 52.157 & & 39.356 & & 43.086 \\
\hline 3/10/11 12:00 & 58.481 & 60.930 & 52.162 & & 39.349 & & 43.089 \\
\hline 3/10/11 16:00 & 58.469 & 60.923 & 52.148 & & 39.335 & & 43.079 \\
\hline 3/10/11 20:00 & 58.453 & 60.909 & 52.129 & & 39.313 & & 43.065 \\
\hline 3/11/11 0:00 & 58.431 & 60.895 & 52.108 & & 39.290 & & 43.047 \\
\hline $3 / 11 / 114: 00$ & 58.412 & 60.879 & 52.094 & & 39.271 & & 43.035 \\
\hline 3/11/11 8:00 & 58.389 & 60.860 & 52.069 & & 39.248 & & 43.017 \\
\hline 3/11/11 12:00 & 58.368 & 60.844 & 52.050 & & 39.226 & & 43.003 \\
\hline 3/11/11 16:00 & 58.353 & 60.837 & 52.050 & & 39.210 & & 42.993 \\
\hline 3/11/11 20:00 & 58.365 & 60.848 & 52.080 & & 39.219 & & 43.010 \\
\hline 3/12/11 0:00 & 58.356 & 60.851 & 52.074 & & 39.208 & & 43.005 \\
\hline $3 / 12 / 114: 00$ & 58.344 & 60.846 & 52.064 & & 39.201 & & 43.000 \\
\hline 3/12/11 8:00 & 58.339 & 60.839 & 52.067 & & 39.194 & & 43.000 \\
\hline 3/12/11 12:00 & 58.337 & 60.844 & 52.074 & & 39.196 & & 43.003 \\
\hline 3/12/11 16:00 & 58.330 & 60.846 & 52.078 & & 39.191 & & 43.003 \\
\hline 3/12/11 20:00 & 58.335 & 60.848 & 52.085 & & 39.196 & & 43.010 \\
\hline 3/13/11 0:00 & 58.327 & 60.846 & 52.078 & & 39.189 & & 43.007 \\
\hline 3/13/11 4:00 & 58.313 & 60.837 & 52.067 & & 39.182 & & 42.998 \\
\hline 3/13/11 8:00 & 58.308 & 60.834 & 52.071 & & 39.180 & & 42.996 \\
\hline 3/13/11 12:00 & 58.311 & 60.839 & 52.076 & & 39.184 & & 42.998 \\
\hline 3/13/11 16:00 & 58.299 & 60.830 & 52.062 & & 39.175 & & 42.991 \\
\hline 3/13/11 20:00 & 58.304 & 60.834 & 52.078 & & 39.184 & & 42.996 \\
\hline 3/14/11 0:00 & 58.299 & 60.827 & 52.062 & & 39.177 & & 42.991 \\
\hline $3 / 14 / 114: 00$ & 58.273 & 60.804 & 52.036 & & 39.151 & & 42.968 \\
\hline 3/14/11 8:00 & 58.268 & 60.804 & 52.041 & & 39.158 & & 42.966 \\
\hline 3/14/11 12:00 & 58.263 & 60.799 & 52.032 & & 39.151 & & 42.956 \\
\hline 3/14/11 16:00 & 58.245 & 60.788 & 52.013 & & 39.133 & & 42.945 \\
\hline 3/14/11 20:00 & 58.249 & 60.790 & 52.027 & & 39.133 & & 42.952 \\
\hline 3/15/11 0:00 & 58.247 & 60.788 & 52.015 & & 39.130 & & 42.947 \\
\hline $3 / 15 / 114: 00$ & 58.226 & 60.764 & 51.988 & & 39.109 & & 42.924 \\
\hline 3/15/11 8:00 & 58.221 & 60.762 & 51.997 & & 39.107 & & 42.924 \\
\hline 3/15/11 12:00 & 58.219 & 60.762 & 51.992 & & 39.104 & & 42.921 \\
\hline 3/15/11 16:00 & 58.197 & 60.746 & 51.969 & & 39.086 & & 42.905 \\
\hline 3/15/11 20:00 & 58.202 & 60.755 & 51.992 & & 39.088 & & 42.910 \\
\hline 3/16/11 0:00 & 58.211 & 60.760 & 51.997 & & 39.095 & & 42.919 \\
\hline
\end{tabular}

TABLE S1.3 Page 28 of 58 


\begin{tabular}{|c|c|c|c|c|c|c|c|}
\hline \multirow[b]{2}{*}{ Date and Time } & \multicolumn{7}{|c|}{ Water Level (ft below reference point) } \\
\hline & SB43D & SB44D & SB45D & SB46D & SB47D & SB48D & SB49D \\
\hline 3/16/11 4:00 & 58.190 & 60.741 & 51.967 & & 39.078 & & 42.900 \\
\hline 3/16/11 8:00 & 58.181 & 60.736 & 51.971 & & 39.074 & & 42.896 \\
\hline 3/16/11 12:00 & 58.176 & 60.732 & 51.962 & & 39.067 & & 42.891 \\
\hline 3/16/11 16:00 & 58.155 & 60.711 & 51.930 & & 39.048 & & 42.870 \\
\hline 3/16/11 20:00 & 58.150 & 60.711 & 51.941 & & 39.041 & & 42.868 \\
\hline 3/17/11 0:00 & 58.152 & 60.715 & 51.941 & & 39.043 & & 42.870 \\
\hline $3 / 17 / 114: 00$ & 58.124 & 60.687 & 51.902 & & 39.022 & & 42.845 \\
\hline 3/17/11 8:00 & 58.114 & 60.681 & 51.906 & & 39.013 & & 42.838 \\
\hline $3 / 17 / 11$ 12:00 & 58.126 & 60.694 & 51.932 & & 39.020 & & 42.849 \\
\hline 3/17/11 16:00 & 58.122 & 60.687 & 51.925 & & 39.017 & & 42.849 \\
\hline 3/17/11 20:00 & 58.138 & 60.711 & 51.964 & & 39.036 & & 42.875 \\
\hline 3/18/11 0:00 & 58.157 & 60.729 & 51.988 & & 39.055 & & 42.893 \\
\hline 3/18/11 4:00 & 58.152 & 60.720 & 51.962 & & 39.053 & & 42.884 \\
\hline 3/18/11 8:00 & 58.150 & 60.727 & 51.983 & & 39.057 & & 42.889 \\
\hline 3/18/11 12:00 & 58.167 & 60.746 & 52.004 & & 39.081 & & 42.907 \\
\hline 3/18/11 16:00 & 58.152 & 60.729 & 51.969 & & 39.067 & & 42.891 \\
\hline 3/18/11 20:00 & 58.148 & 60.729 & 51.974 & & 39.067 & & 42.886 \\
\hline 3/19/11 0:00 & 58.162 & 60.739 & 51.999 & & 39.081 & & 42.903 \\
\hline 3/19/11 4:00 & 58.157 & 60.727 & 51.971 & & 39.074 & & 42.889 \\
\hline 3/19/11 8:00 & 58.140 & 60.718 & 51.967 & & 39.069 & & 42.879 \\
\hline 3/19/11 12:00 & 58.148 & 60.725 & 51.976 & & 39.071 & & 42.882 \\
\hline 3/19/11 16:00 & 58.138 & 60.697 & 51.916 & & 39.057 & & 42.856 \\
\hline 3/19/11 20:00 & 58.105 & 60.680 & 51.911 & & 39.034 & & 42.838 \\
\hline 3/20/11 0:00 & 58.119 & 60.687 & 51.934 & & 39.046 & & 42.849 \\
\hline $3 / 20 / 114: 00$ & 58.103 & 60.671 & 51.902 & & 39.027 & & 42.826 \\
\hline 3/20/11 8:00 & 58.081 & 60.655 & 51.885 & & 39.008 & & 42.812 \\
\hline 3/20/11 12:00 & 58.091 & 60.664 & 51.911 & & 39.017 & & 42.821 \\
\hline 3/20/11 16:00 & 58.086 & 60.655 & 51.888 & & 39.010 & & 42.817 \\
\hline 3/20/11 20:00 & 58.079 & 60.655 & 51.890 & & 39.001 & & 42.812 \\
\hline 3/21/11 0:00 & 58.098 & 60.676 & 51.925 & & 39.027 & & 42.833 \\
\hline $3 / 21 / 114: 00$ & 58.098 & 60.676 & 51.918 & & 39.024 & & 42.835 \\
\hline 3/21/11 8:00 & 58.086 & 60.667 & 51.906 & & 39.015 & & 42.824 \\
\hline $3 / 21 / 11$ 12:00 & 58.091 & 60.671 & 51.927 & & 39.024 & & 42.833 \\
\hline 3/21/11 16:00 & 58.081 & 60.662 & 51.895 & & 39.015 & & 42.819 \\
\hline 3/21/11 20:00 & 58.062 & 60.646 & 51.869 & & 38.999 & & 42.803 \\
\hline 3/22/11 0:00 & 58.065 & 60.648 & 51.890 & & 39.001 & & 42.807 \\
\hline $3 / 22 / 114: 00$ & 58.055 & 60.634 & 51.867 & & 38.992 & & 42.793 \\
\hline 3/22/11 8:00 & 58.034 & 60.613 & 51.837 & & 38.966 & & 42.775 \\
\hline $3 / 22 / 11$ 12:00 & 58.032 & 60.611 & 51.841 & & 38.968 & & 42.768 \\
\hline $3 / 22 / 11$ 16:00 & 58.027 & 60.611 & 51.837 & & 38.963 & & 42.768 \\
\hline 3/22/11 20:00 & 58.018 & 60.599 & 51.830 & & 38.949 & & 42.759 \\
\hline 3/23/11 0:00 & 58.027 & 60.611 & 51.848 & & 38.961 & & 42.770 \\
\hline $3 / 23 / 114: 00$ & 58.029 & 60.613 & 51.855 & & 38.963 & & 42.773 \\
\hline 3/23/11 8:00 & 58.039 & 60.625 & 51.874 & & 38.973 & & 42.784 \\
\hline 3/23/11 12:00 & 58.051 & 60.641 & 51.902 & & 38.994 & & 42.803 \\
\hline 3/23/11 16:00 & 58.072 & 60.662 & 51.927 & & 39.015 & & 42.826 \\
\hline 3/23/11 20:00 & 58.079 & 60.671 & 51.932 & & 39.024 & & 42.838 \\
\hline 3/24/11 0:00 & 58.079 & 60.671 & 51.937 & & 39.027 & & 42.840 \\
\hline $3 / 24 / 114: 00$ & 58.086 & 60.678 & 51.944 & & 39.043 & & 42.845 \\
\hline 3/24/11 8:00 & 58.091 & 60.681 & 51.948 & & 39.050 & & 42.847 \\
\hline $3 / 24 / 11$ 12:00 & 58.096 & 60.692 & 51.955 & & 39.057 & & 42.854 \\
\hline 3/24/11 16:00 & 58.091 & 60.683 & 51.934 & & 39.055 & & 42.842 \\
\hline 3/24/11 20:00 & 58.072 & 60.664 & 51.902 & & 39.036 & & 42.821 \\
\hline 3/25/11 0:00 & 58.058 & 60.646 & 51.885 & & 39.024 & & 42.805 \\
\hline
\end{tabular}

TABLE S1.3 Page 29 of 58 


\begin{tabular}{|c|c|c|c|c|c|c|c|}
\hline \multirow[b]{2}{*}{ Date and Time } & \multicolumn{7}{|c|}{ Water Level (ft below reference point) } \\
\hline & SB43D & SB44D & SB45D & SB46D & SB47D & SB48D & SB49D \\
\hline 3/25/11 4:00 & 58.034 & 60.629 & 51.867 & & 38.999 & & 42.784 \\
\hline 3/25/11 8:00 & 58.036 & 60.636 & 51.883 & & 39.003 & & 42.796 \\
\hline $3 / 25 / 1112: 00$ & 58.036 & 60.634 & 51.883 & & 39.006 & & 42.796 \\
\hline $3 / 25 / 1116: 00$ & 58.041 & 60.636 & 51.890 & & 39.008 & & 42.796 \\
\hline 3/25/11 20:00 & 58.048 & 60.641 & 51.897 & & 39.013 & & 42.800 \\
\hline 3/26/11 0:00 & 58.044 & 60.639 & 51.883 & & 39.008 & & 42.798 \\
\hline 3/26/11 4:00 & 58.036 & 60.629 & 51.872 & & 39.001 & & 42.786 \\
\hline 3/26/11 8:00 & 58.036 & 60.627 & 51.872 & & 38.999 & & 42.789 \\
\hline 3/26/11 12:00 & 58.044 & 60.629 & 51.888 & & 39.003 & & 42.798 \\
\hline 3/26/11 16:00 & 58.048 & 60.641 & 51.895 & & 39.010 & & 42.800 \\
\hline 3/26/11 20:00 & 58.058 & 60.648 & 51.909 & & 39.013 & & 42.812 \\
\hline $3 / 27 / 110: 00$ & 58.058 & 60.653 & 51.904 & & 39.010 & & 42.812 \\
\hline 3/27/11 4:00 & 58.048 & 60.641 & 51.890 & & 39.003 & & 42.807 \\
\hline 3/27/11 8:00 & 58.055 & 60.653 & 51.913 & & 39.008 & & 42.817 \\
\hline 3/27/11 12:00 & 58.055 & 60.650 & 51.904 & & 39.008 & & 42.814 \\
\hline 3/27/11 16:00 & 58.044 & 60.650 & 51.892 & & 38.999 & & 42.807 \\
\hline 3/27/11 20:00 & 58.053 & 60.657 & 51.909 & & 39.006 & & 42.819 \\
\hline 3/28/11 0:00 & 58.055 & 60.657 & 51.909 & & 39.006 & & 42.821 \\
\hline 3/28/11 4:00 & 58.046 & 60.648 & 51.895 & & 38.994 & & 42.812 \\
\hline 3/28/11 8:00 & 58.048 & 60.655 & 51.909 & & 38.999 & & 42.819 \\
\hline $3 / 28 / 11$ 12:00 & 58.048 & 60.655 & 51.904 & & 38.999 & & 42.817 \\
\hline 3/28/11 16:00 & 58.036 & 60.648 & 51.885 & & 38.989 & & 42.807 \\
\hline $3 / 28 / 1120: 00$ & 58.041 & 60.655 & 51.904 & & 38.996 & & 42.814 \\
\hline $3 / 29 / 110: 00$ & 58.053 & 60.655 & 51.906 & & 39.001 & & 42.821 \\
\hline 3/29/11 4:00 & 58.041 & 60.653 & 51.895 & & 38.996 & & 42.814 \\
\hline 3/29/11 8:00 & 58.048 & 60.660 & 51.916 & & 39.006 & & 42.824 \\
\hline 3/29/11 12:00 & 58.051 & 60.667 & 51.916 & & 39.003 & & 42.826 \\
\hline 3/29/11 16:00 & 58.039 & 60.653 & 51.892 & & 38.996 & & 42.814 \\
\hline 3/29/11 20:00 & 58.044 & 60.660 & 51.909 & & 38.999 & & 42.821 \\
\hline 3/30/11 0:00 & 58.051 & 60.664 & 51.913 & & 39.008 & & 42.826 \\
\hline 3/30/11 4:00 & 58.034 & 60.646 & 51.883 & & 38.989 & & 42.807 \\
\hline 3/30/11 8:00 & 58.034 & 60.650 & 51.899 & & 38.992 & & 42.814 \\
\hline $3 / 30 / 11$ 12:00 & 58.036 & 60.650 & 51.895 & & 38.992 & & 42.812 \\
\hline 3/30/11 16:00 & 58.020 & 60.629 & 51.865 & & 38.970 & & 42.791 \\
\hline $3 / 30 / 11$ 20:00 & 58.015 & 60.625 & 51.865 & & 38.970 & & 42.786 \\
\hline 3/31/11 0:00 & 58.020 & 60.627 & 51.869 & & 38.973 & & 42.789 \\
\hline 3/31/11 4:00 & 58.008 & 60.613 & 51.846 & & 38.961 & & 42.777 \\
\hline 3/31/11 8:00 & 57.999 & 60.606 & 51.851 & & 38.956 & & 42.768 \\
\hline 3/31/11 12:00 & 58.001 & 60.608 & 51.853 & & 38.956 & & 42.770 \\
\hline 3/31/11 16:00 & 57.987 & 60.594 & 51.830 & & 38.940 & & 42.754 \\
\hline $3 / 31 / 1120: 00$ & 57.982 & 60.594 & 51.839 & & 38.945 & & 42.759 \\
\hline 4/1/11 0:00 & 57.996 & 60.606 & 51.853 & & 38.949 & & 42.768 \\
\hline 4/1/11 4:00 & 57.987 & 60.594 & 51.837 & & 38.940 & & 42.761 \\
\hline 4/1/11 8:00 & 57.994 & 60.606 & 51.862 & & 38.949 & & 42.770 \\
\hline 4/1/11 12:00 & 58.011 & 60.618 & 51.874 & & 38.963 & & 42.782 \\
\hline 4/1/11 16:00 & 58.003 & 60.611 & 51.855 & & 38.947 & & 42.773 \\
\hline 4/1/11 20:00 & 58.006 & 60.618 & 51.867 & & 38.949 & & 42.779 \\
\hline 4/2/11 0:00 & 58.022 & 60.632 & 51.892 & & 38.970 & & 42.793 \\
\hline 4/2/11 4:00 & 58.020 & 60.627 & 51.874 & & 38.968 & & 42.789 \\
\hline 4/2/11 8:00 & 58.018 & 60.632 & 51.888 & & 38.973 & & 42.791 \\
\hline 4/2/11 12:00 & 58.029 & 60.643 & 51.899 & & 38.985 & & 42.803 \\
\hline 4/2/11 16:00 & 58.008 & 60.620 & 51.851 & & 38.963 & & 42.782 \\
\hline 4/2/11 20:00 & 57.996 & 60.606 & 51.841 & & 38.952 & & 42.768 \\
\hline 4/3/11 0:00 & 57.994 & 60.601 & 51.837 & & 38.952 & & 42.763 \\
\hline
\end{tabular}

TABLE S1.3 Page 30 of 58 


\begin{tabular}{|c|c|c|c|c|c|c|c|}
\hline \multirow[b]{2}{*}{ Date and Time } & \multicolumn{7}{|c|}{ Water Level (ft below reference point) } \\
\hline & SB43D & SB44D & SB45D & SB46D & SB47D & SB48D & SB49D \\
\hline 4/3/11 4:00 & 57.977 & 60.580 & 51.804 & & 38.930 & & 42.740 \\
\hline 4/3/11 8:00 & 57.958 & 60.566 & 51.793 & & 38.921 & & 42.724 \\
\hline 4/3/11 12:00 & 57.968 & 60.573 & 51.811 & & 38.923 & & 42.731 \\
\hline 4/3/11 16:00 & 57.958 & 60.562 & 51.786 & & 38.912 & & 42.719 \\
\hline $4 / 3 / 1120: 00$ & 57.963 & 60.573 & 51.827 & & 38.921 & & 42.740 \\
\hline 4/4/11 0:00 & 58.003 & 60.601 & 51.865 & & 38.954 & & 42.768 \\
\hline 4/4/11 4:00 & 58.013 & 60.617 & 51.876 & & 38.963 & & 42.782 \\
\hline 4/4/11 8:00 & 58.015 & 60.627 & 51.890 & & 38.975 & & 42.789 \\
\hline 4/4/11 12:00 & 58.029 & 60.646 & 51.918 & & 38.996 & & 42.814 \\
\hline 4/4/11 16:00 & 58.036 & 60.648 & 51.904 & & 39.003 & & 42.812 \\
\hline 4/4/11 20:00 & 58.029 & 60.643 & 51.895 & & 38.999 & & 42.807 \\
\hline 4/5/11 0:00 & 58.041 & 60.657 & 51.913 & & 39.017 & & 42.819 \\
\hline 4/5/11 4:00 & 58.044 & 60.650 & 51.902 & & 39.010 & & 42.814 \\
\hline 4/5/11 8:00 & 58.034 & 60.641 & 51.885 & & 39.008 & & 42.803 \\
\hline 4/5/11 12:00 & 58.036 & 60.643 & 51.899 & & 39.017 & & 42.805 \\
\hline 4/5/11 16:00 & 58.034 & 60.636 & 51.872 & & 39.008 & & 42.796 \\
\hline 4/5/11 20:00 & 58.022 & 60.625 & 51.855 & & 38.996 & & 42.782 \\
\hline 4/6/11 0:00 & 58.020 & 60.622 & 51.860 & & 38.994 & & 42.779 \\
\hline 4/6/11 4:00 & 58.022 & 60.618 & 51.853 & & 38.992 & & 42.779 \\
\hline 4/6/11 8:00 & 58.029 & 60.625 & 51.876 & & 39.003 & & 42.786 \\
\hline 4/6/11 12:00 & 58.048 & 60.648 & 51.911 & & 39.029 & & 42.807 \\
\hline 4/6/11 16:00 & 58.062 & 60.657 & 51.916 & & 39.041 & & 42.819 \\
\hline 4/6/11 20:00 & 58.065 & 60.662 & 51.911 & & 39.039 & & 42.824 \\
\hline 4/7/11 0:00 & 58.070 & 60.664 & 51.920 & & 39.048 & & 42.826 \\
\hline 4/7/11 4:00 & 58.077 & 60.669 & 51.925 & & 39.057 & & 42.831 \\
\hline 4/7/11 8:00 & 58.074 & 60.667 & 51.913 & & 39.055 & & 42.826 \\
\hline 4/7/11 12:00 & 58.079 & 60.664 & 51.920 & & 39.062 & & 42.826 \\
\hline 4/7/11 16:00 & 58.029 & 60.629 & 51.858 & & 39.010 & & 42.775 \\
\hline 4/7/11 20:00 & 58.032 & 60.634 & 51.874 & & 39.008 & & 42.791 \\
\hline 4/8/11 0:00 & 58.015 & 60.620 & 51.860 & & 39.001 & & 42.782 \\
\hline 4/8/11 4:00 & 58.010 & 60.620 & 51.855 & & 38.996 & & 42.779 \\
\hline 4/8/11 8:00 & 58.022 & 60.625 & 51.869 & & 39.001 & & 42.784 \\
\hline 4/8/11 12:00 & 58.034 & 60.638 & 51.890 & & 39.008 & & 42.796 \\
\hline 4/8/11 16:00 & 58.036 & 60.634 & 51.881 & & 39.008 & & 42.796 \\
\hline 4/8/11 20:00 & 58.029 & 60.627 & 51.860 & & 38.999 & & 42.784 \\
\hline 4/9/11 0:00 & 58.029 & 60.622 & 51.858 & & 38.999 & & 42.782 \\
\hline 4/9/11 4:00 & 58.022 & 60.618 & 51.865 & & 38.992 & & 42.777 \\
\hline 4/9/11 8:00 & 58.025 & 60.620 & 51.862 & & 38.987 & & 42.777 \\
\hline 4/9/11 12:00 & 58.022 & 60.620 & 51.862 & & 38.989 & & 42.779 \\
\hline 4/9/11 16:00 & 58.018 & 60.618 & 51.855 & & 38.980 & & 42.773 \\
\hline 4/9/11 20:00 & 58.022 & 60.615 & 51.846 & & 38.975 & & 42.773 \\
\hline 4/10/11 0:00 & 58.010 & 60.611 & 51.841 & & 38.970 & & 42.766 \\
\hline 4/10/11 4:00 & 58.003 & 60.604 & 51.839 & & 38.961 & & 42.763 \\
\hline 4/10/11 8:00 & 58.001 & 60.604 & 51.839 & & 38.959 & & 42.766 \\
\hline 4/10/11 12:00 & 57.999 & 60.601 & 51.844 & & 38.956 & & 42.766 \\
\hline 4/10/11 16:00 & 58.015 & 60.622 & 51.865 & & 38.973 & & 42.773 \\
\hline 4/10/11 20:00 & 58.034 & 60.639 & 51.892 & & 38.985 & & 42.800 \\
\hline 4/11/11 0:00 & 58.041 & 60.646 & 51.899 & & 38.992 & & 42.810 \\
\hline 4/11/11 4:00 & 58.036 & 60.643 & 51.892 & & 38.989 & & 42.807 \\
\hline 4/11/11 8:00 & 58.046 & 60.655 & 51.913 & & 39.003 & & 42.819 \\
\hline 4/11/11 12:00 & 58.051 & 60.664 & 51.916 & & 39.013 & & 42.826 \\
\hline 4/11/11 16:00 & 58.055 & 60.669 & 51.925 & & 39.020 & & 42.833 \\
\hline 4/11/11 20:00 & 58.072 & 60.683 & 51.937 & & 39.031 & & 42.845 \\
\hline 4/12/11 0:00 & 58.077 & 60.681 & 51.937 & & 39.036 & & 42.847 \\
\hline
\end{tabular}

TABLE S1.3 Page 31 of 58 


\begin{tabular}{|c|c|c|c|c|c|c|c|}
\hline \multirow[b]{2}{*}{ Date and Time } & \multicolumn{7}{|c|}{ Water Level (ft below reference point) } \\
\hline & SB43D & SB44D & SB45D & SB46D & SB47D & SB48D & SB49D \\
\hline 4/12/11 4:00 & 58.072 & 60.681 & 51.932 & & 39.036 & & 42.845 \\
\hline 4/12/11 8:00 & 58.081 & 60.690 & 51.950 & & 39.048 & & 42.854 \\
\hline 4/12/11 12:00 & 58.079 & 60.690 & 51.941 & & 39.053 & & 42.852 \\
\hline 4/12/11 16:00 & 58.072 & 60.683 & 51.925 & & 39.046 & & 42.842 \\
\hline 4/12/11 20:00 & 58.084 & 60.692 & 51.939 & & 39.053 & & 42.849 \\
\hline 4/13/11 0:00 & 58.084 & 60.685 & 51.930 & & 39.048 & & 42.847 \\
\hline 4/13/11 4:00 & 58.074 & 60.678 & 51.920 & & 39.046 & & 42.840 \\
\hline 4/13/11 8:00 & 58.081 & 60.683 & 51.937 & & 39.053 & & 42.847 \\
\hline 4/13/11 12:00 & 58.084 & 60.685 & 51.934 & & 39.055 & & 42.847 \\
\hline 4/13/11 16:00 & 58.074 & 60.678 & 51.913 & & 39.048 & & 42.838 \\
\hline 4/13/11 20:00 & 58.086 & 60.669 & 51.939 & 58.393 & 39.060 & & 42.849 \\
\hline 4/14/11 0:00 & 58.093 & 60.669 & 51.937 & 58.393 & 39.062 & & 42.854 \\
\hline 4/14/11 4:00 & 58.081 & 60.660 & 51.920 & 58.372 & 39.050 & & 42.840 \\
\hline 4/14/11 8:00 & 58.070 & 60.653 & 51.920 & 58.372 & 39.043 & & 42.831 \\
\hline 4/14/11 12:00 & 58.072 & 60.648 & 51.906 & 58.365 & 39.041 & & 42.828 \\
\hline 4/14/11 16:00 & 58.048 & 60.627 & 51.869 & 58.323 & 39.017 & & 42.807 \\
\hline 4/14/11 20:00 & 58.053 & 60.636 & 51.897 & 58.339 & 39.027 & & 42.812 \\
\hline 4/15/11 0:00 & 57.987 & 60.613 & 51.832 & 58.299 & 38.968 & & 42.773 \\
\hline 4/15/11 4:00 & 57.973 & 60.589 & 51.814 & 58.266 & 38.940 & & 42.766 \\
\hline 4/15/11 8:00 & 57.963 & 60.578 & 51.816 & 58.264 & 38.928 & & 42.749 \\
\hline 4/15/11 12:00 & 57.965 & 60.578 & 51.814 & 58.269 & 38.916 & & 42.752 \\
\hline 4/15/11 16:00 & 57.932 & 60.555 & 51.781 & 58.234 & 38.938 & & 42.726 \\
\hline 4/15/11 20:00 & 57.932 & 60.559 & 51.807 & 58.252 & 38.876 & & 42.735 \\
\hline 4/16/11 0:00 & 57.954 & 60.569 & 51.827 & 58.266 & 38.881 & & 42.752 \\
\hline 4/16/11 4:00 & 57.951 & 60.566 & 51.818 & 58.269 & 38.865 & & 42.747 \\
\hline 4/16/11 8:00 & 57.958 & 60.583 & 51.853 & 58.301 & 38.869 & & 42.773 \\
\hline 4/16/11 12:00 & 57.975 & 60.603 & 51.874 & 58.323 & 38.874 & & 42.786 \\
\hline 4/16/11 16:00 & 57.956 & 60.592 & 51.834 & 58.290 & 38.844 & & 42.770 \\
\hline 4/16/11 20:00 & 57.937 & 60.585 & 51.825 & 58.278 & 38.822 & & 42.763 \\
\hline 4/17/11 0:00 & 57.944 & 60.594 & 51.851 & 58.299 & 38.827 & & 42.777 \\
\hline 4/17/11 4:00 & 57.928 & 60.575 & 51.820 & 58.273 & 38.799 & & 42.763 \\
\hline 4/17/11 8:00 & 57.909 & 60.571 & 51.818 & 58.269 & 38.785 & & 42.756 \\
\hline 4/17/11 12:00 & 57.904 & 60.576 & 51.825 & 58.276 & 38.775 & & 42.756 \\
\hline 4/17/11 16:00 & 57.876 & 60.550 & 51.774 & 58.231 & 38.740 & & 42.728 \\
\hline 4/17/11 20:00 & 57.852 & 60.534 & 51.762 & 58.215 & 38.721 & & 42.714 \\
\hline 4/18/11 0:00 & 57.850 & 60.536 & 51.781 & 58.234 & 38.714 & & 42.721 \\
\hline 4/18/11 4:00 & 57.850 & 60.541 & 51.788 & 58.234 & 38.710 & & 42.724 \\
\hline 4/18/11 8:00 & 57.840 & 60.541 & 51.800 & 58.241 & 38.707 & & 42.724 \\
\hline 4/18/11 12:00 & 57.854 & 60.557 & 51.820 & 58.271 & 38.719 & & 42.742 \\
\hline 4/18/11 16:00 & 57.828 & 60.536 & 51.776 & 58.229 & 38.696 & & 42.721 \\
\hline 4/18/11 20:00 & 57.807 & 60.522 & 51.765 & 58.210 & 38.674 & & 42.703 \\
\hline 4/19/11 0:00 & 57.800 & 60.520 & 51.765 & 58.213 & 38.670 & & 42.705 \\
\hline 4/19/11 4:00 & 57.772 & 60.496 & 51.723 & 58.171 & 38.641 & & 42.675 \\
\hline 4/19/11 8:00 & 57.767 & 60.480 & 51.725 & 58.177 & 38.630 & & 42.670 \\
\hline 4/19/11 12:00 & 57.764 & 60.492 & 51.760 & 58.203 & 38.634 & & 42.677 \\
\hline 4/19/11 16:00 & 57.767 & 60.489 & 51.753 & 58.201 & 38.634 & & 42.684 \\
\hline 4/19/11 20:00 & 57.757 & 60.489 & 51.744 & 58.192 & 38.632 & & 42.680 \\
\hline 4/20/11 0:00 & 57.767 & 60.510 & 51.783 & 58.224 & 38.644 & & 42.698 \\
\hline 4/20/11 4:00 & 57.774 & 60.520 & 51.783 & 58.236 & 38.651 & & 42.705 \\
\hline 4/20/11 8:00 & 57.772 & 60.520 & 51.788 & 58.234 & 38.651 & & 42.705 \\
\hline 4/20/11 12:00 & 57.786 & 60.538 & 51.818 & 58.269 & 38.672 & & 42.724 \\
\hline 4/20/11 16:00 & 57.786 & 60.538 & 51.804 & 58.255 & 38.670 & & 42.724 \\
\hline 4/20/11 20:00 & 57.769 & 60.522 & 51.776 & 58.227 & 38.656 & & 42.707 \\
\hline 4/21/11 0:00 & 57.767 & 60.520 & 51.783 & 58.229 & 38.658 & & 42.705 \\
\hline
\end{tabular}

TABLE S1.3 Page 32 of 58 


\begin{tabular}{|c|c|c|c|c|c|c|c|}
\hline \multirow[b]{2}{*}{ Date and Time } & \multicolumn{7}{|c|}{ Water Level (ft below reference point) } \\
\hline & SB43D & SB44D & SB45D & SB46D & SB47D & SB48D & SB49D \\
\hline $4 / 21 / 114: 00$ & 57.764 & 60.517 & 51.781 & 58.227 & 38.660 & & 42.703 \\
\hline 4/21/11 8:00 & 57.753 & 60.503 & 51.762 & 58.210 & 38.651 & & 42.686 \\
\hline 4/21/11 12:00 & 57.743 & 60.492 & 51.749 & 58.203 & 38.641 & & 42.677 \\
\hline 4/21/11 16:00 & 57.734 & 60.487 & 51.735 & 58.185 & 38.630 & & 42.666 \\
\hline 4/21/11 20:00 & 57.717 & 60.468 & 51.709 & 58.161 & 38.609 & & 42.649 \\
\hline 4/22/11 0:00 & 57.701 & 60.450 & 51.695 & 58.140 & 38.597 & & 42.633 \\
\hline $4 / 22 / 114: 00$ & 57.689 & 60.434 & 51.684 & 58.131 & 38.580 & & 42.619 \\
\hline 4/22/11 8:00 & 57.670 & 60.415 & 51.672 & 58.114 & 38.569 & & 42.600 \\
\hline 4/22/11 12:00 & 57.658 & 60.413 & 51.665 & 58.112 & 38.552 & & 42.591 \\
\hline 4/22/11 16:00 & 57.667 & 60.424 & 51.681 & 58.131 & 38.566 & & 42.603 \\
\hline 4/22/11 20:00 & 57.660 & 60.415 & 51.667 & 58.117 & 38.552 & & 42.593 \\
\hline 4/23/11 0:00 & 57.660 & 60.420 & 51.677 & 58.126 & 38.555 & & 42.600 \\
\hline 4/23/11 4:00 & 57.656 & 60.417 & 51.677 & 58.121 & 38.550 & & 42.598 \\
\hline 4/23/11 8:00 & 57.667 & 60.429 & 51.704 & 58.147 & 38.564 & & 42.614 \\
\hline 4/23/11 12:00 & 57.672 & 60.438 & 51.711 & 58.156 & 38.573 & & 42.624 \\
\hline 4/23/11 16:00 & 57.675 & 60.443 & 51.714 & 58.159 & 38.578 & & 42.626 \\
\hline 4/23/11 20:00 & 57.675 & 60.441 & 51.709 & 58.156 & 38.578 & & 42.626 \\
\hline 4/24/11 0:00 & 57.672 & 60.436 & 51.702 & 58.149 & 38.578 & & 42.624 \\
\hline 4/24/11 4:00 & 57.665 & 60.436 & 51.704 & 58.147 & 38.578 & & 42.619 \\
\hline 4/24/11 8:00 & 57.663 & 60.434 & 51.702 & 58.140 & 38.571 & & 42.619 \\
\hline 4/24/11 12:00 & 57.660 & 60.427 & 51.693 & 58.142 & 38.573 & & 42.612 \\
\hline 4/24/11 16:00 & 57.656 & 60.422 & 51.686 & 58.133 & 38.569 & & 42.605 \\
\hline 4/24/11 20:00 & 57.653 & 60.420 & 51.679 & 58.126 & 38.571 & & 42.600 \\
\hline 4/25/11 0:00 & 57.653 & 60.415 & 51.672 & 58.124 & 38.564 & & 42.598 \\
\hline 4/25/11 4:00 & 57.637 & 60.406 & 51.663 & 58.107 & 38.557 & & 42.587 \\
\hline 4/25/11 8:00 & 57.630 & 60.392 & 51.651 & 58.096 & 38.545 & & 42.573 \\
\hline 4/25/11 12:00 & 57.606 & 60.373 & 51.635 & 58.070 & 38.526 & & 42.556 \\
\hline 4/25/11 16:00 & 57.594 & 60.361 & 51.612 & 58.058 & 38.510 & & 42.540 \\
\hline 4/25/11 20:00 & 57.589 & 60.352 & 51.600 & 58.056 & 38.503 & & 42.533 \\
\hline 4/26/11 0:00 & 57.575 & 60.338 & 51.588 & 58.035 & 38.486 & & 42.519 \\
\hline 4/26/11 4:00 & 57.556 & 60.324 & 51.577 & 58.019 & 38.472 & & 42.505 \\
\hline 4/26/11 8:00 & 57.563 & 60.333 & 51.595 & 58.044 & 38.479 & & 42.517 \\
\hline 4/26/11 12:00 & 57.571 & 60.338 & 51.607 & 58.056 & 38.486 & & 42.524 \\
\hline 4/26/11 16:00 & 57.568 & 60.343 & 51.607 & 58.054 & 38.486 & & 42.526 \\
\hline 4/26/11 20:00 & 57.573 & 60.345 & 51.609 & 58.063 & 38.486 & & 42.531 \\
\hline 4/27/11 0:00 & 57.575 & 60.347 & 51.616 & 58.063 & 38.489 & & 42.531 \\
\hline 4/27/11 4:00 & 57.563 & 60.333 & 51.600 & 58.044 & 38.475 & & 42.521 \\
\hline 4/27/11 8:00 & 57.571 & 60.345 & 51.618 & 58.063 & 38.491 & & 42.531 \\
\hline 4/27/11 12:00 & 57.578 & 60.354 & 51.628 & 58.072 & 38.498 & & 42.542 \\
\hline 4/27/11 16:00 & 57.571 & 60.354 & 51.618 & 58.063 & 38.501 & & 42.535 \\
\hline 4/27/11 20:00 & 57.580 & 60.361 & 51.637 & 58.082 & 38.505 & & 42.545 \\
\hline 4/28/11 0:00 & 57.589 & 60.371 & 51.649 & 58.093 & 38.512 & & 42.552 \\
\hline 4/28/11 4:00 & 57.580 & 60.361 & 51.632 & 58.077 & 38.508 & & 42.545 \\
\hline 4/28/11 8:00 & 57.592 & 60.373 & 51.658 & 58.100 & 38.526 & & 42.559 \\
\hline 4/28/11 12:00 & 57.599 & 60.380 & 51.660 & 58.107 & 38.533 & & 42.568 \\
\hline 4/28/11 16:00 & 57.592 & 60.375 & 51.646 & 58.089 & 38.531 & & 42.559 \\
\hline $4 / 28 / 1120: 00$ & 57.601 & 60.385 & 51.663 & 58.105 & 38.540 & & 42.566 \\
\hline 4/29/11 0:00 & 57.606 & 60.385 & 51.663 & 58.107 & 38.545 & & 42.570 \\
\hline 4/29/11 4:00 & 57.599 & 60.375 & 51.649 & 58.091 & 38.540 & & 42.559 \\
\hline 4/29/11 8:00 & 57.601 & 60.377 & 51.656 & 58.098 & 38.540 & & 42.561 \\
\hline 4/29/11 12:00 & 57.597 & 60.373 & 51.642 & 58.091 & 38.538 & & 42.554 \\
\hline 4/29/11 16:00 & 57.573 & 60.343 & 51.586 & 58.037 & 38.510 & & 42.519 \\
\hline 4/29/11 20:00 & 57.575 & 60.349 & 51.614 & 58.056 & 38.524 & & 42.528 \\
\hline 4/30/11 0:00 & 57.580 & 60.347 & 51.612 & 58.061 & 38.519 & & 42.528 \\
\hline
\end{tabular}

TABLE S1.3 Page 33 of 58 


\begin{tabular}{|c|c|c|c|c|c|c|c|}
\hline \multirow[b]{2}{*}{ Date and Time } & \multicolumn{7}{|c|}{ Water Level (ft below reference point) } \\
\hline & SB43D & SB44D & SB45D & SB46D & SB47D & SB48D & SB49D \\
\hline $4 / 30 / 114: 00$ & 57.571 & 60.340 & 51.598 & 58.044 & 38.519 & & 42.519 \\
\hline 4/30/11 8:00 & 57.578 & 60.347 & 51.628 & 58.063 & 38.524 & & 42.531 \\
\hline 4/30/11 12:00 & 57.597 & 60.366 & 51.651 & 58.093 & 38.545 & & 42.549 \\
\hline 4/30/11 16:00 & 57.597 & 60.366 & 51.637 & 58.082 & 38.543 & & 42.547 \\
\hline 4/30/11 20:00 & 57.606 & 60.378 & 51.660 & 58.100 & 38.559 & & 42.561 \\
\hline 5/1/11 0:00 & 57.625 & 60.396 & 51.688 & 58.128 & 38.578 & & 42.582 \\
\hline $5 / 1 / 114: 00$ & 57.627 & 60.394 & 51.679 & 58.119 & 38.583 & & 42.582 \\
\hline 5/1/11 8:00 & 57.641 & 60.415 & 51.721 & 58.149 & 38.604 & & 42.598 \\
\hline $5 / 1 / 1112: 00$ & 57.658 & 60.422 & 51.721 & 58.161 & 38.620 & & 42.609 \\
\hline 5/1/11 16:00 & 57.646 & 60.415 & 51.688 & 58.131 & 38.616 & & 42.598 \\
\hline $5 / 1 / 1120: 00$ & 57.644 & 60.410 & 51.690 & 58.128 & 38.609 & & 42.591 \\
\hline 5/2/11 0:00 & 57.663 & 60.424 & 51.718 & 58.154 & 38.630 & & 42.610 \\
\hline $5 / 2 / 114: 00$ & 57.660 & 60.413 & 51.702 & 58.138 & 38.625 & & 42.600 \\
\hline $5 / 2 / 118: 00$ & 57.679 & 60.434 & 51.732 & 58.178 & 38.656 & & 42.621 \\
\hline $5 / 2 / 1112: 00$ & 57.694 & 60.450 & 51.751 & 58.192 & 38.670 & & 42.635 \\
\hline $5 / 2 / 1116: 00$ & 57.689 & 60.443 & 51.718 & 58.161 & 38.670 & & 42.624 \\
\hline $5 / 2 / 1120: 00$ & 57.684 & 60.436 & 51.716 & 58.159 & 38.665 & & 42.619 \\
\hline 5/3/11 0:00 & 57.696 & 60.443 & 51.735 & 58.173 & 38.679 & & 42.628 \\
\hline $5 / 3 / 114: 00$ & 57.698 & 60.443 & 51.728 & 58.168 & 38.679 & & 42.626 \\
\hline $5 / 3 / 118: 00$ & 57.703 & 60.448 & 51.742 & 58.178 & 38.688 & & 42.631 \\
\hline $5 / 3 / 1112: 00$ & 57.717 & 60.459 & 51.760 & 58.199 & 38.705 & & 42.642 \\
\hline $5 / 3 / 1116: 00$ & 57.715 & 60.452 & 51.732 & 58.180 & 38.700 & & 42.635 \\
\hline $5 / 3 / 1120: 00$ & 57.710 & 60.445 & 51.725 & 58.166 & 38.696 & & 42.628 \\
\hline 5/4/11 0:00 & 57.722 & 60.457 & 51.749 & 58.187 & 38.712 & & 42.638 \\
\hline $5 / 4 / 114: 00$ & 57.727 & 60.457 & 51.737 & 58.180 & 38.714 & & 42.638 \\
\hline 5/4/11 8:00 & 57.727 & 60.457 & 51.746 & 58.182 & 38.712 & & 42.640 \\
\hline $5 / 4 / 1112: 00$ & 57.734 & 60.464 & 51.753 & 58.194 & 38.724 & & 42.642 \\
\hline 5/4/11 16:00 & 57.729 & 60.448 & 51.725 & 58.171 & 38.714 & & 42.633 \\
\hline 5/4/11 20:00 & 57.715 & 60.438 & 51.707 & 58.147 & 38.707 & & 42.614 \\
\hline 5/5/11 0:00 & 57.727 & 60.445 & 51.730 & 58.168 & 38.717 & & 42.626 \\
\hline $5 / 5 / 114: 00$ & 57.731 & 60.450 & 51.728 & 58.171 & 38.724 & & 42.628 \\
\hline 5/5/11 8:00 & 57.729 & 60.445 & 51.728 & 58.168 & 38.719 & & 42.626 \\
\hline $5 / 5 / 1112: 00$ & 57.741 & 60.455 & 51.749 & 58.187 & 38.738 & & 42.638 \\
\hline $5 / 5 / 1116: 00$ & 57.736 & 60.448 & 51.723 & 58.163 & 38.726 & & 42.626 \\
\hline $5 / 5 / 1120: 00$ & 57.727 & 60.441 & 51.707 & 58.149 & 38.721 & & 42.617 \\
\hline 5/6/11 0:00 & 57.729 & 60.441 & 51.716 & 58.156 & 38.721 & & 42.619 \\
\hline 5/6/11 4:00 & 57.731 & 60.436 & 51.711 & 58.154 & 38.724 & & 42.619 \\
\hline 5/6/11 8:00 & 57.724 & 60.431 & 51.707 & 58.145 & 38.719 & & 42.610 \\
\hline $5 / 6 / 11$ 12:00 & 57.727 & 60.436 & 51.711 & 58.154 & 38.724 & & 42.612 \\
\hline 5/6/11 16:00 & 57.724 & 60.434 & 51.697 & 58.138 & 38.721 & & 42.605 \\
\hline 5/6/11 20:00 & 57.717 & 60.415 & 51.674 & 58.119 & 38.705 & & 42.593 \\
\hline 5/7/11 0:00 & 57.722 & 60.419 & 51.695 & 58.135 & 38.712 & & 42.600 \\
\hline $5 / 7 / 114: 00$ & 57.717 & 60.410 & 51.683 & 58.124 & 38.707 & & 42.593 \\
\hline 5/7/11 8:00 & 57.727 & 60.424 & 51.700 & 58.140 & 38.726 & & 42.605 \\
\hline $5 / 7 / 11$ 12:00 & 57.738 & 60.436 & 51.721 & 58.163 & 38.738 & & 42.617 \\
\hline 5/7/11 16:00 & 57.750 & 60.450 & 51.725 & 58.171 & 38.747 & & 42.624 \\
\hline 5/7/11 20:00 & 57.755 & 60.445 & 51.723 & 58.163 & 38.747 & & 42.626 \\
\hline 5/8/11 0:00 & 57.755 & 60.450 & 51.728 & 58.168 & 38.757 & & 42.628 \\
\hline $5 / 8 / 114: 00$ & 57.757 & 60.452 & 51.728 & 58.168 & 38.757 & & 42.633 \\
\hline 5/8/11 8:00 & 57.760 & 60.452 & 51.732 & 58.171 & 38.766 & & 42.631 \\
\hline 5/8/11 12:00 & 57.757 & 60.450 & 51.725 & 58.163 & 38.764 & & 42.631 \\
\hline 5/8/11 16:00 & 57.760 & 60.448 & 51.718 & 58.166 & 38.764 & & 42.628 \\
\hline 5/8/11 20:00 & 57.764 & 60.448 & 51.716 & 58.156 & 38.768 & & 42.624 \\
\hline 5/9/11 0:00 & 57.762 & 60.443 & 51.711 & 58.154 & 38.768 & & 42.624 \\
\hline
\end{tabular}

TABLE S1.3 Page 34 of 58 


\begin{tabular}{|c|c|c|c|c|c|c|c|}
\hline \multirow[b]{2}{*}{ Date and Time } & \multicolumn{7}{|c|}{ Water Level (ft below reference point) } \\
\hline & SB43D & SB44D & SB45D & SB46D & SB47D & SB48D & SB49D \\
\hline 5/9/11 4:00 & 57.762 & 60.445 & 51.711 & 58.154 & 38.771 & & 42.621 \\
\hline 5/9/11 8:00 & 57.767 & 60.443 & 51.714 & 58.159 & 38.768 & & 42.624 \\
\hline 5/9/11 12:00 & 57.762 & 60.440 & 51.709 & 58.152 & 38.766 & & 42.614 \\
\hline 5/9/11 16:00 & 57.772 & 60.445 & 51.723 & 58.168 & 38.780 & & 42.628 \\
\hline 5/9/11 20:00 & 57.783 & 60.457 & 51.725 & 58.171 & 38.792 & & 42.633 \\
\hline 5/10/11 0:00 & 57.788 & 60.457 & 51.730 & 58.173 & 38.797 & & 42.638 \\
\hline 5/10/11 4:00 & 57.786 & 60.454 & 51.728 & 58.168 & 38.799 & & 42.633 \\
\hline 5/10/11 8:00 & 57.795 & 60.466 & 51.751 & 58.185 & 38.811 & & 42.645 \\
\hline 5/10/11 12:00 & 57.802 & 60.473 & 51.751 & 58.192 & 38.820 & & 42.649 \\
\hline 5/10/11 16:00 & 57.814 & 60.485 & 51.760 & 58.203 & 38.832 & & 42.661 \\
\hline 5/10/11 20:00 & 57.833 & 60.499 & 51.779 & 58.220 & 38.851 & & 42.675 \\
\hline 5/11/11 0:00 & 57.835 & 60.494 & 51.767 & 58.208 & 38.851 & & 42.670 \\
\hline 5/11/11 4:00 & 57.840 & 60.499 & 51.781 & 58.220 & 38.862 & & 42.680 \\
\hline 5/11/11 8:00 & 57.857 & 60.513 & 51.797 & 58.241 & 38.879 & & 42.693 \\
\hline $5 / 11 / 11$ 12:00 & 57.859 & 60.513 & 51.786 & 58.231 & 38.886 & & 42.691 \\
\hline 5/11/11 16:00 & 57.854 & 60.510 & 51.776 & 58.222 & 38.888 & & 42.686 \\
\hline 5/11/11 20:00 & 57.873 & 60.522 & 51.804 & 58.238 & 38.900 & & 42.700 \\
\hline 5/12/11 0:00 & 57.850 & 60.499 & 51.765 & 58.213 & 38.876 & & 42.673 \\
\hline 5/12/11 4:00 & 57.850 & 60.499 & 51.779 & 58.215 & 38.883 & & 42.680 \\
\hline 5/12/11 8:00 & 57.866 & 60.513 & 51.800 & 58.238 & 38.900 & & 42.693 \\
\hline $5 / 12 / 11$ 12:00 & 57.876 & 60.515 & 51.793 & 58.236 & 38.902 & & 42.691 \\
\hline $5 / 12 / 11$ 16:00 & 57.869 & 60.510 & 51.783 & 58.224 & 38.900 & & 42.689 \\
\hline 5/12/11 20:00 & 57.880 & 60.515 & 51.795 & 58.231 & 38.912 & & 42.693 \\
\hline 5/13/11 0:00 & 57.887 & 60.517 & 51.795 & 58.236 & 38.919 & & 42.696 \\
\hline 5/13/11 4:00 & 57.885 & 60.517 & 51.795 & 58.224 & 38.921 & & 42.696 \\
\hline 5/13/11 8:00 & 57.902 & 60.531 & 51.823 & 58.255 & 38.938 & & 42.712 \\
\hline $5 / 13 / 11$ 12:00 & 57.921 & 60.545 & 51.834 & 58.271 & 38.954 & & 42.726 \\
\hline 5/13/11 16:00 & 57.916 & 60.536 & 51.816 & 58.250 & 38.949 & & 42.717 \\
\hline $5 / 13 / 1120: 00$ & 57.928 & 60.548 & 51.837 & 58.271 & 38.966 & & 42.728 \\
\hline 5/14/11 0:00 & 57.937 & 60.552 & 51.837 & 58.271 & 38.977 & & 42.735 \\
\hline 5/14/11 4:00 & 57.932 & 60.547 & 51.827 & 58.264 & 38.975 & & 42.728 \\
\hline 5/14/11 8:00 & 57.944 & 60.561 & 51.853 & 58.285 & 38.987 & & 42.742 \\
\hline $5 / 14 / 11$ 12:00 & 57.958 & 60.569 & 51.858 & 58.294 & 38.999 & & 42.749 \\
\hline 5/14/11 16:00 & 57.949 & 60.555 & 51.827 & 58.266 & 38.994 & & 42.735 \\
\hline 5/14/11 20:00 & 57.951 & 60.564 & 51.844 & 58.278 & 38.996 & & 42.742 \\
\hline 5/15/11 0:00 & 57.968 & 60.571 & 51.853 & 58.290 & 39.008 & & 42.749 \\
\hline 5/15/11 4:00 & 57.961 & 60.559 & 51.832 & 58.271 & 38.996 & & 42.740 \\
\hline 5/15/11 8:00 & 57.973 & 60.571 & 51.860 & 58.290 & 39.015 & & 42.749 \\
\hline $5 / 15 / 11$ 12:00 & 57.994 & 60.585 & 51.881 & 58.311 & 39.034 & & 42.768 \\
\hline 5/15/11 16:00 & 57.984 & 60.580 & 51.853 & 58.290 & 39.027 & & 42.759 \\
\hline 5/15/11 20:00 & 57.982 & 60.580 & 51.858 & 58.292 & 39.027 & & 42.756 \\
\hline 5/16/11 0:00 & 58.001 & 60.594 & 51.876 & 58.313 & 39.046 & & 42.770 \\
\hline 5/16/11 4:00 & 58.001 & 60.587 & 51.867 & 58.301 & 39.039 & & 42.766 \\
\hline 5/16/11 8:00 & 58.013 & 60.599 & 51.892 & 58.325 & 39.060 & & 42.779 \\
\hline 5/16/11 12:00 & 58.034 & 60.620 & 51.909 & 58.348 & 39.078 & & 42.796 \\
\hline 5/16/11 16:00 & 58.025 & 60.608 & 51.874 & 58.315 & 39.074 & & 42.784 \\
\hline 5/16/11 20:00 & 58.020 & 60.603 & 51.869 & 58.304 & 39.064 & & 42.777 \\
\hline 5/17/11 0:00 & 58.034 & 60.611 & 51.890 & 58.327 & 39.078 & & 42.789 \\
\hline 5/17/11 4:00 & 58.036 & 60.604 & 51.881 & 58.318 & 39.086 & & 42.784 \\
\hline 5/17/11 8:00 & 58.039 & 60.613 & 51.897 & 58.329 & 39.088 & & 42.789 \\
\hline 5/17/11 12:00 & 58.058 & 60.629 & 51.916 & 58.351 & 39.107 & & 42.805 \\
\hline 5/17/11 16:00 & 58.053 & 60.618 & 51.881 & 58.325 & 39.093 & & 42.793 \\
\hline 5/17/11 20:00 & 58.046 & 60.606 & 51.869 & 58.308 & 39.090 & & 42.784 \\
\hline 5/18/11 0:00 & 58.055 & 60.615 & 51.892 & 58.327 & 39.104 & & 42.793 \\
\hline
\end{tabular}

TABLE S1.3 Page 35 of 58 


\begin{tabular}{|c|c|c|c|c|c|c|c|}
\hline \multirow[b]{2}{*}{ Date and Time } & \multicolumn{7}{|c|}{ Water Level (ft below reference point) } \\
\hline & SB43D & SB44D & SB45D & SB46D & SB47D & SB48D & SB49D \\
\hline 5/18/11 4:00 & 58.060 & 60.617 & 51.883 & 58.320 & 39.107 & & 42.791 \\
\hline 5/18/11 8:00 & 58.062 & 60.618 & 51.892 & 58.330 & 39.109 & & 42.793 \\
\hline 5/18/11 12:00 & 58.079 & 60.636 & 51.918 & 58.353 & 39.125 & & 42.810 \\
\hline 5/18/11 16:00 & 58.086 & 60.634 & 51.904 & 58.344 & 39.123 & & 42.807 \\
\hline 5/18/11 20:00 & 58.074 & 60.625 & 51.890 & 58.325 & 39.121 & & 42.798 \\
\hline 5/19/11 0:00 & 58.086 & 60.634 & 51.909 & 58.348 & 39.135 & & 42.810 \\
\hline 5/19/11 4:00 & 58.088 & 60.636 & 51.906 & 58.337 & 39.140 & & 42.810 \\
\hline 5/19/11 8:00 & 58.093 & 60.636 & 51.913 & 58.346 & 39.140 & & 42.812 \\
\hline 5/19/11 12:00 & 58.112 & 60.655 & 51.941 & 58.376 & 39.161 & & 42.831 \\
\hline 5/19/11 16:00 & 58.119 & 60.655 & 51.932 & 58.374 & 39.168 & & 42.833 \\
\hline 5/19/11 20:00 & 58.112 & 60.650 & 51.916 & 58.353 & 39.158 & & 42.821 \\
\hline 5/20/11 0:00 & 58.114 & 60.655 & 51.925 & 58.360 & 39.168 & & 42.828 \\
\hline 5/20/11 4:00 & 58.117 & 60.648 & 51.918 & 58.355 & 39.158 & & 42.824 \\
\hline 5/20/11 8:00 & 58.119 & 60.653 & 51.930 & 58.362 & 39.168 & & 42.828 \\
\hline 5/20/11 12:00 & 58.124 & 60.641 & 51.920 & 58.362 & 39.165 & & 42.814 \\
\hline 5/20/11 16:00 & 58.105 & 60.653 & 51.918 & 58.350 & 39.156 & & 42.814 \\
\hline 5/20/11 20:00 & 58.098 & 60.634 & 51.892 & 58.332 & 39.144 & & 42.807 \\
\hline 5/21/11 0:00 & 58.100 & 60.634 & 51.897 & 58.332 & 39.144 & & 42.805 \\
\hline 5/21/11 4:00 & 58.105 & 60.634 & 51.904 & 58.339 & 39.149 & & 42.810 \\
\hline 5/21/11 8:00 & 58.110 & 60.636 & 51.909 & 58.344 & 39.158 & & 42.812 \\
\hline 5/21/11 12:00 & 58.122 & 60.646 & 51.920 & 58.358 & 39.168 & & 42.817 \\
\hline 5/21/11 16:00 & 58.129 & 60.650 & 51.916 & 58.362 & 39.168 & & 42.824 \\
\hline 5/21/11 20:00 & 58.129 & 60.645 & 51.911 & 58.355 & 39.168 & & 42.819 \\
\hline 5/22/11 0:00 & 58.124 & 60.641 & 51.904 & 58.344 & 39.168 & & 42.817 \\
\hline 5/22/11 4:00 & 58.126 & 60.643 & 51.909 & 58.346 & 39.177 & & 42.814 \\
\hline 5/22/11 8:00 & 58.129 & 60.643 & 51.911 & 58.348 & 39.177 & & 42.814 \\
\hline 5/22/11 12:00 & 58.133 & 60.652 & 51.918 & 58.360 & 39.184 & & 42.824 \\
\hline 5/22/11 16:00 & 58.148 & 60.660 & 51.930 & 58.374 & 39.194 & & 42.833 \\
\hline $5 / 22 / 1120: 00$ & 58.159 & 60.667 & 51.932 & 58.372 & 39.203 & & 42.838 \\
\hline 5/23/11 0:00 & 58.162 & 60.669 & 51.936 & 58.376 & 39.210 & & 42.845 \\
\hline 5/23/11 4:00 & 58.174 & 60.683 & 51.953 & 58.390 & 39.222 & & 42.854 \\
\hline 5/23/11 8:00 & 58.176 & 60.678 & 51.946 & 58.386 & 39.224 & & 42.854 \\
\hline $5 / 23 / 11$ 12:00 & 58.185 & 60.692 & 51.964 & 58.404 & 39.236 & & 42.866 \\
\hline 5/23/11 16:00 & 58.197 & 60.701 & 51.976 & 58.414 & 39.250 & & 42.875 \\
\hline 5/23/11 20:00 & 58.209 & 60.704 & 51.976 & 58.414 & 39.257 & & 42.877 \\
\hline 5/24/11 0:00 & 58.214 & 60.711 & 51.981 & 58.416 & 39.266 & & 42.882 \\
\hline 5/24/11 4:00 & 58.216 & 60.708 & 51.978 & 58.418 & 39.269 & & 42.882 \\
\hline 5/24/11 8:00 & 58.226 & 60.720 & 51.992 & 58.428 & 39.278 & & 42.891 \\
\hline 5/24/11 12:00 & 58.228 & 60.722 & 52.004 & 58.423 & 39.283 & & 42.893 \\
\hline 5/24/11 16:00 & 58.171 & 60.699 & 51.946 & 58.386 & 39.231 & & 42.863 \\
\hline 5/24/11 20:00 & 58.174 & 60.692 & 51.946 & 58.381 & 39.229 & & 42.859 \\
\hline 5/25/11 0:00 & 58.112 & 60.660 & 51.876 & 58.320 & 39.163 & & 42.807 \\
\hline 5/25/11 4:00 & 58.096 & 60.638 & 51.865 & 58.306 & 39.147 & & 42.803 \\
\hline 5/25/11 8:00 & 58.112 & 60.641 & 51.885 & 58.325 & 39.156 & & 42.810 \\
\hline 5/25/11 12:00 & 58.117 & 60.638 & 51.876 & 58.320 & 39.154 & & 42.803 \\
\hline 5/25/11 16:00 & 58.103 & 60.622 & 51.869 & 58.306 & 39.133 & & 42.786 \\
\hline 5/25/11 20:00 & 58.055 & 60.624 & 51.867 & 58.301 & 39.095 & & 42.789 \\
\hline 5/26/11 0:00 & 58.088 & 60.627 & 51.881 & 58.315 & 39.107 & & 42.798 \\
\hline 5/26/11 4:00 & 58.100 & 60.625 & 51.883 & 58.318 & 39.102 & & 42.800 \\
\hline 5/26/11 8:00 & 58.119 & 60.639 & 51.909 & 58.341 & 39.111 & & 42.812 \\
\hline 5/26/11 12:00 & 58.124 & 60.641 & 51.902 & 58.341 & 39.109 & & 42.814 \\
\hline 5/26/11 16:00 & 58.112 & 60.634 & 51.879 & 58.320 & 39.090 & & 42.805 \\
\hline 5/26/11 20:00 & 58.112 & 60.634 & 51.881 & 58.323 & 39.081 & & 42.803 \\
\hline 5/27/11 0:00 & 58.110 & 60.629 & 51.881 & 58.320 & 39.078 & & 42.800 \\
\hline
\end{tabular}

TABLE S1.3 Page 36 of 58 


\begin{tabular}{|c|c|c|c|c|c|c|c|}
\hline \multirow[b]{2}{*}{ Date and Time } & \multicolumn{7}{|c|}{ Water Level (ft below reference point) } \\
\hline & SB43D & SB44D & SB45D & SB46D & SB47D & SB48D & SB49D \\
\hline 5/27/11 4:00 & 58.096 & 60.613 & 51.865 & 58.301 & 39.064 & & 42.786 \\
\hline 5/27/11 8:00 & 58.088 & 60.615 & 51.869 & 58.299 & 39.055 & & 42.782 \\
\hline $5 / 27 / 11$ 12:00 & 58.084 & 60.606 & 51.848 & 58.297 & 39.046 & & 42.777 \\
\hline 5/27/11 16:00 & 58.060 & 60.587 & 51.832 & 58.266 & 39.022 & & 42.759 \\
\hline 5/27/11 20:00 & 58.058 & 60.582 & 51.830 & 58.269 & 39.015 & & 42.754 \\
\hline 5/28/11 0:00 & 58.060 & 60.587 & 51.834 & 58.283 & 39.017 & & 42.761 \\
\hline 5/28/11 4:00 & 58.046 & 60.576 & 51.830 & 58.271 & 39.006 & & 42.747 \\
\hline 5/28/11 8:00 & 58.044 & 60.580 & 51.837 & 58.273 & 39.006 & & 42.752 \\
\hline 5/28/11 12:00 & 58.046 & 60.578 & 51.832 & 58.278 & 39.003 & & 42.749 \\
\hline $5 / 28 / 11$ 16:00 & 58.029 & 60.566 & 51.811 & 58.252 & 38.985 & & 42.738 \\
\hline $5 / 28 / 1120: 00$ & 58.027 & 60.566 & 51.825 & 58.259 & 38.987 & & 42.740 \\
\hline 5/29/11 0:00 & 58.029 & 60.566 & 51.820 & 58.264 & 38.989 & & 42.740 \\
\hline 5/29/11 4:00 & 58.010 & 60.552 & 51.802 & 58.236 & 38.966 & & 42.724 \\
\hline 5/29/11 8:00 & 58.008 & 60.557 & 51.816 & 58.250 & 38.968 & & 42.726 \\
\hline $5 / 29 / 11$ 12:00 & 58.013 & 60.559 & 51.816 & 58.255 & 38.975 & & 42.731 \\
\hline 5/29/11 16:00 & 58.003 & 60.548 & 51.795 & 58.238 & 38.961 & & 42.719 \\
\hline 5/29/11 20:00 & 58.003 & 60.550 & 51.806 & 58.248 & 38.970 & & 42.721 \\
\hline 5/30/11 0:00 & 58.018 & 60.562 & 51.823 & 58.264 & 38.980 & & 42.735 \\
\hline 5/30/11 4:00 & 58.008 & 60.554 & 51.811 & 58.252 & 38.973 & & 42.728 \\
\hline 5/30/11 8:00 & 58.015 & 60.562 & 51.832 & 58.266 & 38.985 & & 42.735 \\
\hline $5 / 30 / 11$ 12:00 & 58.027 & 60.573 & 51.841 & 58.283 & 38.996 & & 42.742 \\
\hline 5/30/11 16:00 & 58.022 & 60.566 & 51.820 & 58.269 & 38.994 & & 42.740 \\
\hline 5/30/11 20:00 & 58.018 & 60.569 & 51.823 & 58.264 & 38.999 & & 42.735 \\
\hline 5/31/11 0:00 & 58.022 & 60.561 & 51.825 & 58.266 & 38.999 & & 42.738 \\
\hline 5/31/11 4:00 & 58.034 & 60.566 & 51.846 & 58.283 & 39.008 & & 42.740 \\
\hline 5/31/11 8:00 & 58.015 & 60.571 & 51.848 & 58.278 & 38.999 & & 42.742 \\
\hline 5/31/11 12:00 & 58.051 & 60.601 & 51.890 & 58.329 & 39.034 & & 42.775 \\
\hline 5/31/11 16:00 & 58.053 & 60.599 & 51.867 & 58.311 & 39.039 & & 42.773 \\
\hline 5/31/11 20:00 & 58.051 & 60.597 & 51.865 & 58.304 & 39.046 & & 42.770 \\
\hline 6/1/11 0:00 & 58.065 & 60.604 & 51.883 & 58.320 & 39.055 & & 42.779 \\
\hline 6/1/11 4:00 & 58.062 & 60.597 & 51.869 & 58.306 & 39.053 & & 42.770 \\
\hline 6/1/11 8:00 & 58.067 & 60.601 & 51.878 & 58.315 & 39.062 & & 42.775 \\
\hline $6 / 1 / 11$ 12:00 & 58.079 & 60.618 & 51.895 & 58.334 & 39.076 & & 42.786 \\
\hline $6 / 1 / 11$ 16:00 & 58.074 & 60.601 & 51.865 & 58.304 & 39.069 & & 42.773 \\
\hline $6 / 1 / 11$ 20:00 & 58.051 & 60.576 & 51.830 & 58.266 & 39.048 & & 42.747 \\
\hline 6/2/11 0:00 & 58.058 & 60.580 & 51.841 & 58.280 & 39.057 & & 42.747 \\
\hline $6 / 2 / 114: 00$ & 58.055 & 60.564 & 51.832 & 58.283 & 39.050 & & 42.735 \\
\hline 6/2/11 8:00 & 58.013 & 60.566 & 51.816 & 58.245 & 39.017 & & 42.724 \\
\hline $6 / 2 / 11$ 12:00 & 58.032 & 60.573 & 51.834 & 58.278 & 39.029 & & 42.738 \\
\hline $6 / 2 / 11$ 16:00 & 58.029 & 60.561 & 51.816 & 58.257 & 39.022 & & 42.728 \\
\hline $6 / 2 / 1120: 00$ & 58.018 & 60.543 & 51.797 & 58.234 & 39.010 & & 42.712 \\
\hline 6/3/11 0:00 & 58.025 & 60.543 & 51.809 & 58.245 & 39.017 & & 42.717 \\
\hline $6 / 3 / 114: 00$ & 58.025 & 60.540 & 51.802 & 58.243 & 39.020 & & 42.710 \\
\hline 6/3/11 8:00 & 58.027 & 60.543 & 51.809 & 58.245 & 39.017 & & 42.714 \\
\hline $6 / 3 / 11$ 12:00 & 58.041 & 60.557 & 51.834 & 58.273 & 39.034 & & 42.726 \\
\hline $6 / 3 / 11$ 16:00 & 58.048 & 60.559 & 51.825 & 58.271 & 39.043 & & 42.735 \\
\hline 6/3/11 20:00 & 58.046 & 60.559 & 51.816 & 58.252 & 39.034 & & 42.728 \\
\hline 6/4/11 0:00 & 58.055 & 60.569 & 51.841 & 58.276 & 39.048 & & 42.740 \\
\hline $6 / 4 / 114: 00$ & 58.062 & 60.571 & 51.844 & 58.278 & 39.057 & & 42.742 \\
\hline 6/4/11 8:00 & 58.070 & 60.576 & 51.853 & 58.287 & 39.067 & & 42.749 \\
\hline $6 / 4 / 11$ 12:00 & 58.079 & 60.590 & 51.867 & 58.306 & 39.081 & & 42.759 \\
\hline 6/4/11 16:00 & 58.091 & 60.597 & 51.867 & 58.311 & 39.090 & & 42.768 \\
\hline $6 / 4 / 1120: 00$ & 58.081 & 60.589 & 51.846 & 58.283 & 39.083 & & 42.756 \\
\hline 6/5/11 0:00 & 58.084 & 60.585 & 51.853 & 58.290 & 39.086 & & 42.754 \\
\hline
\end{tabular}

TABLE S1.3 Page 37 of 58 


\begin{tabular}{|c|c|c|c|c|c|c|c|}
\hline \multirow[b]{2}{*}{ Date and Time } & \multicolumn{7}{|c|}{ Water Level (ft below reference point) } \\
\hline & SB43D & SB44D & SB45D & SB46D & SB47D & SB48D & SB49D \\
\hline 6/5/11 4:00 & 58.088 & 60.585 & 51.855 & 58.292 & 39.090 & & 42.756 \\
\hline 6/5/11 8:00 & 58.086 & 60.580 & 51.848 & 58.285 & 39.090 & & 42.752 \\
\hline 6/5/11 12:00 & 58.093 & 60.589 & 51.867 & 58.304 & 39.104 & & 42.761 \\
\hline 6/5/11 16:00 & 58.103 & 60.599 & 51.865 & 58.306 & 39.114 & & 42.768 \\
\hline 6/5/11 20:00 & 58.100 & 60.587 & 51.841 & 58.285 & 39.107 & & 42.759 \\
\hline 6/6/11 0:00 & 58.098 & 60.585 & 51.846 & 58.283 & 39.107 & & 42.756 \\
\hline 6/6/11 4:00 & 58.100 & 60.585 & 51.846 & 58.285 & 39.114 & & 42.754 \\
\hline 6/6/11 8:00 & 58.100 & 60.587 & 51.848 & 58.283 & 39.109 & & 42.756 \\
\hline 6/6/11 12:00 & 58.103 & 60.589 & 51.858 & 58.294 & 39.118 & & 42.759 \\
\hline 6/6/11 16:00 & 58.117 & 60.601 & 51.867 & 58.306 & 39.130 & & 42.770 \\
\hline 6/6/11 20:00 & 58.122 & 60.596 & 51.851 & 58.292 & 39.135 & & 42.767 \\
\hline 6/7/11 0:00 & 58.122 & 60.599 & 51.860 & 58.294 & 39.135 & & 42.766 \\
\hline 6/7/11 4:00 & 58.126 & 60.601 & 51.862 & 58.301 & 39.144 & & 42.770 \\
\hline 6/7/11 8:00 & 58.131 & 60.601 & 51.865 & 58.301 & 39.149 & & 42.770 \\
\hline 6/7/11 12:00 & 58.129 & 60.599 & 51.860 & 58.297 & 39.149 & & 42.768 \\
\hline 6/7/11 16:00 & 58.138 & 60.608 & 51.872 & 58.308 & 39.161 & & 42.777 \\
\hline 6/7/11 20:00 & 58.150 & 60.613 & 51.869 & 58.311 & 39.165 & & 42.782 \\
\hline 6/8/11 0:00 & 58.150 & 60.610 & 51.872 & 58.308 & 39.170 & & 42.779 \\
\hline 6/8/11 4:00 & 58.155 & 60.617 & 51.881 & 58.318 & 39.180 & & 42.786 \\
\hline 6/8/11 8:00 & 58.167 & 60.615 & 51.890 & 58.325 & 39.191 & & 42.793 \\
\hline 6/8/11 12:00 & 58.169 & 60.624 & 51.888 & 58.329 & 39.194 & & 42.793 \\
\hline 6/8/11 16:00 & 58.178 & 60.634 & 51.902 & 58.339 & 39.208 & & 42.805 \\
\hline 6/8/11 20:00 & 58.195 & 60.648 & 51.911 & 58.348 & 39.217 & & 42.814 \\
\hline 6/9/11 0:00 & 58.200 & 60.650 & 51.913 & 58.350 & 39.226 & & 42.819 \\
\hline 6/9/11 4:00 & 58.202 & 60.650 & 51.920 & 58.350 & 39.234 & & 42.819 \\
\hline 6/9/11 8:00 & 58.223 & 60.662 & 51.937 & 58.372 & 39.255 & & 42.833 \\
\hline 6/9/11 12:00 & 58.219 & 60.662 & 51.925 & 58.362 & 39.252 & & 42.831 \\
\hline 6/9/11 16:00 & 58.216 & 60.657 & 51.913 & 58.353 & 39.250 & & 42.826 \\
\hline $6 / 9 / 1120: 00$ & 58.230 & 60.662 & 51.923 & 58.358 & 39.259 & & 42.831 \\
\hline 6/10/11 0:00 & 58.228 & 60.657 & 51.913 & 58.353 & 39.257 & & 42.824 \\
\hline 6/10/11 4:00 & 58.235 & 60.666 & 51.936 & 58.369 & 39.271 & & 42.838 \\
\hline 6/10/11 8:00 & 58.261 & 60.685 & 51.967 & 58.402 & 39.295 & & 42.859 \\
\hline 6/10/11 12:00 & 58.256 & 60.678 & 51.939 & 58.381 & 39.288 & & 42.847 \\
\hline 6/10/11 16:00 & 58.254 & 60.680 & 51.946 & 58.379 & 39.292 & & 42.852 \\
\hline 6/10/11 20:00 & 58.261 & 60.683 & 51.953 & 58.388 & 39.299 & & 42.856 \\
\hline 6/11/11 0:00 & 58.271 & 60.692 & 51.957 & 58.393 & 39.304 & & 42.863 \\
\hline 6/11/11 4:00 & 58.273 & 60.697 & 51.962 & 58.395 & 39.313 & & 42.868 \\
\hline 6/11/11 8:00 & 58.294 & 60.718 & 51.997 & 58.430 & 39.330 & & 42.889 \\
\hline $6 / 11 / 11$ 12:00 & 58.299 & 60.718 & 51.985 & 58.423 & 39.337 & & 42.886 \\
\hline $6 / 11 / 11$ 16:00 & 58.294 & 60.715 & 51.971 & 58.409 & 39.332 & & 42.882 \\
\hline 6/11/11 20:00 & 58.299 & 60.713 & 51.974 & 58.414 & 39.342 & & 42.882 \\
\hline 6/12/11 0:00 & 58.313 & 60.722 & 51.988 & 58.428 & 39.356 & & 42.893 \\
\hline $6 / 12 / 114: 00$ & 58.316 & 60.725 & 51.990 & 58.425 & 39.360 & & 42.896 \\
\hline 6/12/11 8:00 & 58.334 & 60.746 & 52.027 & 58.458 & 39.382 & & 42.917 \\
\hline $6 / 12 / 11$ 12:00 & 58.339 & 60.739 & 52.006 & 58.444 & 39.379 & & 42.907 \\
\hline 6/12/11 16:00 & 58.330 & 60.734 & 51.988 & 58.423 & 39.374 & & 42.903 \\
\hline $6 / 12 / 1120: 00$ & 58.332 & 60.734 & 51.997 & 58.428 & 39.379 & & 42.903 \\
\hline 6/13/11 0:00 & 58.344 & 60.741 & 52.004 & 58.435 & 39.391 & & 42.910 \\
\hline $6 / 13 / 114: 00$ & 58.342 & 60.736 & 51.995 & 58.425 & 39.393 & & 42.903 \\
\hline 6/13/11 8:00 & 58.349 & 60.739 & 52.011 & 58.442 & 39.403 & & 42.912 \\
\hline 6/13/11 12:00 & 58.368 & 60.750 & 52.020 & 58.453 & 39.412 & & 42.921 \\
\hline 6/13/11 16:00 & 58.356 & 60.736 & 51.990 & 58.421 & 39.405 & & 42.907 \\
\hline 6/13/11 20:00 & 58.353 & 60.739 & 51.997 & 58.425 & 39.405 & & 42.907 \\
\hline 6/14/11 0:00 & 58.360 & 60.739 & 51.999 & 58.430 & 39.410 & & 42.907 \\
\hline
\end{tabular}

TABLE S1.3 Page 38 of 58 


\begin{tabular}{|c|c|c|c|c|c|c|c|}
\hline \multirow[b]{2}{*}{ Date and Time } & \multicolumn{7}{|c|}{ Water Level (ft below reference point) } \\
\hline & SB43D & SB44D & SB45D & SB46D & SB47D & SB48D & SB49D \\
\hline $6 / 14 / 114: 00$ & 58.358 & 60.736 & 51.990 & 58.423 & 39.407 & & 42.903 \\
\hline 6/14/11 8:00 & 58.370 & 60.739 & 52.011 & 58.442 & 39.424 & & 42.912 \\
\hline $6 / 14 / 11$ 12:00 & 58.379 & 60.752 & 52.020 & 58.451 & 39.431 & & 42.921 \\
\hline 6/14/11 16:00 & 58.370 & 60.743 & 51.992 & 58.428 & 39.424 & & 42.910 \\
\hline 6/14/11 20:00 & 58.368 & 60.739 & 51.983 & 58.423 & 39.421 & & 42.905 \\
\hline 6/15/11 0:00 & 58.394 & 60.757 & 52.034 & 58.460 & 39.445 & & 42.928 \\
\hline $6 / 15 / 114: 00$ & 58.394 & 60.755 & 52.018 & 58.451 & 39.447 & & 42.926 \\
\hline 6/15/11 8:00 & 58.408 & 60.769 & 52.039 & 58.470 & 39.461 & & 42.938 \\
\hline $6 / 15 / 11$ 12:00 & 58.427 & 60.792 & 52.066 & 58.500 & 39.485 & & 42.959 \\
\hline $6 / 15 / 11$ 16:00 & 58.431 & 60.792 & 52.050 & 58.488 & 39.490 & & 42.961 \\
\hline 6/15/11 20:00 & 58.427 & 60.785 & 52.036 & 58.472 & 39.487 & & 42.952 \\
\hline 6/16/11 0:00 & 58.438 & 60.794 & 52.050 & 58.486 & 39.497 & & 42.961 \\
\hline $6 / 16 / 114: 00$ & 58.441 & 60.794 & 52.046 & 58.479 & 39.504 & & 42.959 \\
\hline 6/16/11 8:00 & 58.446 & 60.797 & 52.057 & 58.486 & 39.506 & & 42.963 \\
\hline $6 / 16 / 11$ 12:00 & 58.457 & 60.804 & 52.069 & 58.496 & 39.520 & & 42.970 \\
\hline 6/16/11 16:00 & 58.457 & 60.801 & 52.060 & 58.493 & 39.515 & & 42.968 \\
\hline 6/16/11 20:00 & 58.443 & 60.783 & 52.029 & 58.463 & 39.508 & & 42.952 \\
\hline 6/17/11 0:00 & 58.460 & 60.799 & 52.064 & 58.493 & 39.520 & & 42.968 \\
\hline $6 / 17 / 114: 00$ & 58.434 & 60.776 & 52.011 & 58.458 & 39.487 & & 42.938 \\
\hline $6 / 17 / 118: 00$ & 58.434 & 60.783 & 52.029 & 58.463 & 39.497 & & 42.945 \\
\hline $6 / 17 / 11$ 12:00 & 58.455 & 60.801 & 52.066 & 58.500 & 39.520 & & 42.968 \\
\hline 6/17/11 16:00 & 58.467 & 60.811 & 52.067 & 58.505 & 39.539 & & 42.977 \\
\hline 6/17/11 20:00 & 58.467 & 60.811 & 52.055 & 58.491 & 39.530 & & 42.975 \\
\hline 6/18/11 0:00 & 58.481 & 60.813 & 52.067 & 58.507 & 39.539 & & 42.979 \\
\hline $6 / 18 / 114: 00$ & 58.479 & 60.818 & 52.071 & 58.500 & 39.539 & & 42.982 \\
\hline 6/18/11 8:00 & 58.410 & 60.785 & 52.002 & 58.430 & 39.483 & & 42.945 \\
\hline 6/18/11 12:00 & 58.436 & 60.809 & 52.057 & 58.488 & 39.499 & & 42.970 \\
\hline 6/18/11 16:00 & 58.453 & 60.813 & 52.060 & 58.495 & 39.511 & & 42.977 \\
\hline $6 / 18 / 1120: 00$ & 58.453 & 60.804 & 52.039 & 58.479 & 39.508 & & 42.968 \\
\hline 6/19/11 0:00 & 58.450 & 60.799 & 52.046 & 58.474 & 39.508 & & 42.963 \\
\hline $6 / 19 / 114: 00$ & 58.455 & 60.792 & 52.029 & 58.470 & 39.513 & & 42.959 \\
\hline 6/19/11 8:00 & 58.462 & 60.795 & 52.048 & 58.479 & 39.511 & & 42.961 \\
\hline $6 / 19 / 11$ 12:00 & 58.464 & 60.801 & 52.055 & 58.489 & 39.518 & & 42.966 \\
\hline 6/19/11 16:00 & 58.474 & 60.811 & 52.060 & 58.495 & 39.520 & & 42.975 \\
\hline 6/19/11 20:00 & 58.472 & 60.804 & 52.043 & 58.477 & 39.520 & & 42.968 \\
\hline 6/20/11 0:00 & 58.467 & 60.799 & 52.043 & 58.474 & 39.518 & & 42.963 \\
\hline $6 / 20 / 114: 00$ & 58.464 & 60.792 & 52.036 & 58.467 & 39.513 & & 42.961 \\
\hline 6/20/11 8:00 & 58.479 & 60.806 & 52.060 & 58.481 & 39.532 & & 42.968 \\
\hline $6 / 20 / 11$ 12:00 & 58.481 & 60.806 & 52.060 & 58.493 & 39.530 & & 42.972 \\
\hline 6/20/11 16:00 & 58.498 & 60.825 & 52.076 & 58.514 & 39.544 & & 42.991 \\
\hline 6/20/11 20:00 & 58.495 & 60.820 & 52.062 & 58.498 & 39.544 & & 42.984 \\
\hline 6/21/11 0:00 & 58.472 & 60.815 & 52.048 & 58.486 & 39.523 & & 42.972 \\
\hline $6 / 21 / 114: 00$ & 58.462 & 60.811 & 52.046 & 58.484 & 39.510 & & 42.970 \\
\hline 6/21/11 8:00 & 58.472 & 60.808 & 52.053 & 58.486 & 39.525 & & 42.975 \\
\hline $6 / 21 / 11$ 12:00 & 58.483 & 60.818 & 52.071 & 58.505 & 39.534 & & 42.984 \\
\hline 6/21/11 16:00 & 58.498 & 60.829 & 52.085 & 58.519 & 39.546 & & 42.998 \\
\hline $6 / 21 / 1120: 00$ & 58.509 & 60.832 & 52.090 & 58.526 & 39.553 & & 43.000 \\
\hline $6 / 22 / 110: 00$ & 58.509 & 60.836 & 52.090 & 58.521 & 39.555 & & 43.003 \\
\hline $6 / 22 / 114: 00$ & 58.519 & 60.843 & 52.104 & 58.535 & 39.562 & & 43.007 \\
\hline $6 / 22 / 118: 00$ & 58.528 & 60.848 & 52.108 & 58.540 & 39.574 & & 43.017 \\
\hline $6 / 22 / 11$ 12:00 & 58.538 & 60.855 & 52.118 & 58.549 & 39.584 & & 43.024 \\
\hline $6 / 22 / 11$ 16:00 & 58.545 & 60.864 & 52.122 & 58.556 & 39.591 & & 43.031 \\
\hline $6 / 22 / 1120: 00$ & 58.550 & 60.871 & 52.129 & 58.563 & 39.598 & & 43.038 \\
\hline 6/23/11 0:00 & 58.554 & 60.872 & 52.129 & 58.563 & 39.607 & & 43.042 \\
\hline
\end{tabular}

TABLE S1.3 Page 39 of 58 


\begin{tabular}{|c|c|c|c|c|c|c|c|}
\hline \multirow[b]{2}{*}{ Date and Time } & \multicolumn{7}{|c|}{ Water Level (ft below reference point) } \\
\hline & SB43D & SB44D & SB45D & SB46D & SB47D & SB48D & SB49D \\
\hline $6 / 23 / 114: 00$ & 58.561 & 60.874 & 52.141 & 58.570 & 39.614 & & 43.045 \\
\hline 6/23/11 8:00 & 58.573 & 60.888 & 52.155 & 58.582 & 39.626 & & 43.056 \\
\hline $6 / 23 / 11$ 12:00 & 58.578 & 60.895 & 52.152 & 58.589 & 39.631 & & 43.061 \\
\hline 6/23/11 16:00 & 58.580 & 60.897 & 52.157 & 58.617 & 39.892 & 39.445 & 43.068 \\
\hline 6/23/11 20:00 & 58.587 & 60.904 & 52.155 & 58.617 & 39.896 & 39.445 & 43.068 \\
\hline 6/24/11 0:00 & 58.590 & 60.899 & 52.152 & 58.617 & 39.896 & 39.445 & 43.070 \\
\hline $6 / 24 / 114: 00$ & 58.597 & 60.906 & 52.164 & 58.627 & 39.906 & 39.449 & 43.075 \\
\hline 6/24/11 8:00 & 58.611 & 60.916 & 52.180 & 58.643 & 39.922 & 39.459 & 43.086 \\
\hline 6/24/11 12:00 & 58.616 & 60.920 & 52.171 & 58.641 & 39.932 & 39.463 & 43.086 \\
\hline $6 / 24 / 11$ 16:00 & 58.611 & 60.916 & 52.164 & 58.629 & 39.927 & 39.459 & 43.084 \\
\hline 6/24/11 20:00 & 58.618 & 60.925 & 52.171 & 58.634 & 39.932 & 39.463 & 43.089 \\
\hline 6/25/11 0:00 & 58.623 & 60.925 & 52.176 & 58.636 & 39.936 & 39.468 & 43.093 \\
\hline $6 / 25 / 114: 00$ & 58.623 & 60.923 & 52.178 & 58.634 & 39.939 & 39.471 & 43.089 \\
\hline 6/25/11 8:00 & 58.651 & 60.927 & 52.194 & 58.673 & 39.965 & 39.494 & 43.103 \\
\hline $6 / 25 / 11$ 12:00 & 58.597 & 60.920 & 52.157 & 58.622 & 39.915 & 39.421 & 43.079 \\
\hline 6/25/11 16:00 & 58.602 & 60.923 & 52.164 & 58.627 & 39.918 & 39.440 & 43.084 \\
\hline 6/25/11 20:00 & 58.613 & 60.925 & 52.173 & 58.636 & 39.925 & 39.456 & 43.089 \\
\hline 6/26/11 0:00 & 58.618 & 60.923 & 52.171 & 58.634 & 39.929 & 39.466 & 43.089 \\
\hline 6/26/11 4:00 & 58.623 & 60.923 & 52.178 & 58.636 & 39.934 & 39.471 & 43.091 \\
\hline 6/26/11 8:00 & 58.637 & 60.934 & 52.192 & 58.652 & 39.946 & 39.487 & 43.100 \\
\hline 6/26/11 12:00 & 58.640 & 60.927 & 52.176 & 58.648 & 39.948 & 39.485 & 43.098 \\
\hline 6/26/11 16:00 & 58.625 & 60.918 & 52.155 & 58.622 & 39.932 & 39.473 & 43.082 \\
\hline 6/26/11 20:00 & 58.632 & 60.923 & 52.169 & 58.634 & 39.941 & 39.480 & 43.089 \\
\hline 6/27/11 0:00 & 58.632 & 60.902 & 52.150 & 58.622 & 39.946 & 39.473 & 43.063 \\
\hline $6 / 27 / 114: 00$ & 58.481 & 60.851 & 52.085 & 58.519 & 39.763 & 39.318 & 43.017 \\
\hline 6/27/11 8:00 & 58.621 & 60.850 & 52.025 & 58.498 & 39.711 & 39.252 & 42.991 \\
\hline 6/27/11 12:00 & 58.590 & 60.848 & 52.046 & 58.517 & 39.716 & 39.287 & 43.000 \\
\hline 6/27/11 16:00 & 58.547 & 60.822 & 52.018 & 58.486 & 39.680 & 39.271 & 42.979 \\
\hline 6/27/11 20:00 & 58.521 & 60.813 & 52.020 & 58.484 & 39.650 & 39.264 & 42.972 \\
\hline 6/28/11 0:00 & 58.502 & 60.806 & 52.022 & 58.484 & 39.626 & 39.255 & 42.966 \\
\hline $6 / 28 / 114: 00$ & 58.467 & 60.788 & 51.997 & 58.461 & 39.586 & 39.231 & 42.947 \\
\hline 6/28/11 8:00 & 58.446 & 60.783 & 52.008 & 58.465 & 39.561 & 39.219 & 42.942 \\
\hline $6 / 28 / 11$ 12:00 & 58.434 & 60.781 & 52.004 & 58.468 & 39.544 & 39.210 & 42.940 \\
\hline 6/28/11 16:00 & 58.394 & 60.750 & 51.953 & 58.421 & 39.504 & 39.174 & 42.912 \\
\hline 6/28/11 20:00 & 58.365 & 60.739 & 51.941 & 58.402 & 39.474 & 39.149 & 42.893 \\
\hline 6/29/11 0:00 & 58.351 & 60.727 & 51.941 & 58.402 & 39.459 & 39.134 & 42.886 \\
\hline 6/29/11 4:00 & 58.320 & 60.704 & 51.916 & 58.374 & 39.434 & 39.109 & 42.866 \\
\hline 6/29/11 8:00 & 58.304 & 60.697 & 51.916 & 58.379 & 39.413 & 39.097 & 42.859 \\
\hline 6/29/11 12:00 & 58.297 & 60.699 & 51.923 & 58.388 & 39.408 & 39.092 & 42.856 \\
\hline 6/29/11 16:00 & 58.266 & 60.676 & 51.878 & 58.348 & 39.382 & 39.067 & 42.835 \\
\hline 6/29/11 20:00 & 58.240 & 60.657 & 51.865 & 58.330 & 39.356 & 39.040 & 42.817 \\
\hline 6/30/11 0:00 & 58.230 & 60.650 & 51.865 & 58.330 & 39.342 & 39.033 & 42.812 \\
\hline $6 / 30 / 114: 00$ & 58.211 & 60.634 & 51.851 & 58.313 & 39.328 & 39.015 & 42.796 \\
\hline 6/30/11 8:00 & 58.200 & 60.632 & 51.860 & 58.320 & 39.321 & 39.008 & 42.793 \\
\hline 6/30/11 12:00 & 58.197 & 60.634 & 51.867 & 58.330 & 39.326 & 39.008 & 42.793 \\
\hline 6/30/11 16:00 & 58.185 & 60.620 & 51.837 & 58.306 & 39.311 & 38.991 & 42.782 \\
\hline 6/30/11 20:00 & 58.164 & 60.606 & 51.820 & 58.283 & 39.293 & 38.972 & 42.768 \\
\hline 7/1/11 0:00 & 58.159 & 60.606 & 51.832 & 58.295 & 39.295 & 38.972 & 42.768 \\
\hline 7/1/11 4:00 & 58.152 & 60.594 & 51.820 & 58.285 & 39.288 & 38.965 & 42.756 \\
\hline 7/1/11 8:00 & 58.143 & 60.594 & 51.830 & 58.290 & 39.288 & 38.958 & 42.756 \\
\hline $7 / 1 / 11$ 12:00 & 58.150 & 60.599 & 51.846 & 58.309 & 39.297 & 38.968 & 42.766 \\
\hline 7/1/11 16:00 & 58.143 & 60.596 & 51.830 & 58.297 & 39.297 & 38.961 & 42.761 \\
\hline $7 / 1 / 1120: 00$ & 58.129 & 60.580 & 51.806 & 58.271 & 39.281 & 38.946 & 42.747 \\
\hline $7 / 2 / 11$ 0:00 & 58.131 & 60.587 & 51.830 & 58.288 & 39.290 & 38.954 & 42.752 \\
\hline
\end{tabular}

TABLE S1.3 Page 40 of 58 


\begin{tabular}{|c|c|c|c|c|c|c|c|}
\hline \multirow[b]{2}{*}{ Date and Time } & \multicolumn{7}{|c|}{ Water Level (ft below reference point) } \\
\hline & SB43D & SB44D & SB45D & SB46D & SB47D & SB48D & SB49D \\
\hline $7 / 2 / 114: 00$ & 58.129 & 60.582 & 51.820 & 58.285 & 39.293 & 38.951 & 42.747 \\
\hline $7 / 2 / 118: 00$ & 58.124 & 60.580 & 51.830 & 58.285 & 39.293 & 38.951 & 42.747 \\
\hline $7 / 2 / 1112: 00$ & 58.133 & 60.596 & 51.855 & 58.313 & 39.307 & 38.963 & 42.763 \\
\hline 7/2/11 16:00 & 58.136 & 60.594 & 51.841 & 58.306 & 39.311 & 38.966 & 42.761 \\
\hline $7 / 2 / 1120: 00$ & 58.119 & 60.575 & 51.809 & 58.276 & 39.300 & 38.949 & 42.742 \\
\hline 7/3/11 0:00 & 58.117 & 60.573 & 51.820 & 58.278 & 39.304 & 38.949 & 42.738 \\
\hline $7 / 3 / 114: 00$ & 58.117 & 60.571 & 51.813 & 58.281 & 39.302 & 38.949 & 42.738 \\
\hline $7 / 3 / 118: 00$ & 58.117 & 60.566 & 51.823 & 58.276 & 39.304 & 38.946 & 42.733 \\
\hline $7 / 3 / 11$ 12:00 & 58.020 & 60.517 & 51.753 & 58.210 & 39.220 & 38.846 & 42.670 \\
\hline $7 / 3 / 11$ 16:00 & 58.029 & 60.515 & 51.748 & 58.217 & 39.229 & 38.860 & 42.675 \\
\hline $7 / 3 / 1120: 00$ & 58.022 & 60.501 & 51.728 & 58.187 & 39.210 & 38.850 & 42.660 \\
\hline 7/4/11 0:00 & 58.022 & 60.494 & 51.737 & 58.199 & 39.215 & 38.857 & 42.659 \\
\hline $7 / 4 / 114: 00$ & 58.025 & 60.489 & 51.735 & 58.196 & 39.215 & 38.857 & 42.654 \\
\hline 7/4/11 8:00 & 58.018 & 60.480 & 51.730 & 58.189 & 39.206 & 38.855 & 42.649 \\
\hline $7 / 4 / 11$ 12:00 & 58.020 & 60.492 & 51.746 & 58.206 & 39.213 & 38.857 & 42.656 \\
\hline $7 / 4 / 11$ 16:00 & 58.027 & 60.496 & 51.744 & 58.210 & 39.215 & 38.862 & 42.661 \\
\hline $7 / 4 / 1120: 00$ & 58.015 & 60.487 & 51.728 & 58.187 & 39.206 & 38.848 & 42.649 \\
\hline 7/5/11 0:00 & 58.013 & 60.485 & 51.732 & 58.196 & 39.206 & 38.850 & 42.649 \\
\hline $7 / 5 / 114: 00$ & 58.015 & 60.487 & 51.739 & 58.201 & 39.210 & 38.852 & 42.652 \\
\hline $7 / 5 / 118: 00$ & 58.008 & 60.478 & 51.730 & 58.189 & 39.203 & 38.845 & 42.647 \\
\hline $7 / 5 / 11$ 12:00 & 58.006 & 60.480 & 51.735 & 58.196 & 39.208 & 38.850 & 42.649 \\
\hline $7 / 5 / 11$ 16:00 & 58.015 & 60.489 & 51.744 & 58.208 & 39.215 & 38.855 & 42.654 \\
\hline 7/5/11 20:00 & 58.020 & 60.489 & 51.739 & 58.206 & 39.218 & 38.852 & 42.656 \\
\hline 7/6/11 0:00 & 57.999 & 60.473 & 51.716 & 58.180 & 39.203 & 38.838 & 42.638 \\
\hline $7 / 6 / 114: 00$ & 58.015 & 60.487 & 51.753 & 58.213 & 39.222 & 38.857 & 42.656 \\
\hline $7 / 6 / 118: 00$ & 58.013 & 60.485 & 51.746 & 58.203 & 39.218 & 38.855 & 42.654 \\
\hline $7 / 6 / 11$ 12:00 & 58.006 & 60.480 & 51.737 & 58.199 & 39.215 & 38.850 & 42.647 \\
\hline 7/6/11 16:00 & 58.006 & 60.485 & 51.739 & 58.206 & 39.225 & 38.852 & 42.649 \\
\hline $7 / 6 / 1120: 00$ & 58.006 & 60.478 & 51.728 & 58.194 & 39.215 & 38.848 & 42.644 \\
\hline 7/7/11 0:00 & 57.999 & 60.478 & 51.728 & 58.189 & 39.215 & 38.843 & 42.640 \\
\hline $7 / 7 / 114: 00$ & 57.996 & 60.475 & 51.735 & 58.187 & 39.210 & 38.843 & 42.640 \\
\hline 7/7/11 8:00 & 57.956 & 60.445 & 51.688 & 58.159 & 39.173 & 38.782 & 42.600 \\
\hline $7 / 7 / 11$ 12:00 & 57.904 & 60.429 & 51.663 & 58.131 & 39.131 & 38.745 & 42.582 \\
\hline $7 / 7 / 11$ 16:00 & 57.909 & 60.429 & 51.667 & 58.133 & 39.131 & 38.756 & 42.584 \\
\hline 7/7/11 20:00 & 57.921 & 60.424 & 51.667 & 58.131 & 39.131 & 38.763 & 42.589 \\
\hline $7 / 8 / 11$ 0:00 & 57.921 & 60.417 & 51.663 & 58.129 & 39.126 & 38.766 & 42.584 \\
\hline $7 / 8 / 114: 00$ & 57.928 & 60.424 & 51.681 & 58.140 & 39.126 & 38.773 & 42.589 \\
\hline 7/8/11 8:00 & 57.930 & 60.424 & 51.681 & 58.147 & 39.128 & 38.775 & 42.593 \\
\hline $7 / 8 / 11$ 12:00 & 57.923 & 60.422 & 51.672 & 58.140 & 39.124 & 38.770 & 42.589 \\
\hline 7/8/11 16:00 & 57.918 & 60.419 & 51.667 & 58.136 & 39.116 & 38.763 & 42.587 \\
\hline $7 / 8 / 1120: 00$ & 57.921 & 60.424 & 51.672 & 58.136 & 39.112 & 38.761 & 42.589 \\
\hline 7/9/11 0:00 & 57.918 & 60.422 & 51.676 & 58.138 & 39.109 & 38.761 & 42.591 \\
\hline $7 / 9 / 114: 00$ & 57.913 & 60.424 & 51.676 & 58.140 & 39.107 & 38.758 & 42.589 \\
\hline $7 / 9 / 118: 00$ & 57.923 & 60.429 & 51.697 & 58.159 & 39.121 & 38.766 & 42.603 \\
\hline $7 / 9 / 11$ 12:00 & 57.913 & 60.424 & 51.676 & 58.143 & 39.105 & 38.758 & 42.593 \\
\hline 7/9/11 16:00 & 57.904 & 60.422 & 51.670 & 58.136 & 39.102 & 38.751 & 42.589 \\
\hline $7 / 9 / 1120: 00$ & 57.906 & 60.424 & 51.677 & 58.143 & 39.105 & 38.751 & 42.593 \\
\hline 7/10/11 0:00 & 57.906 & 60.427 & 51.674 & 58.140 & 39.105 & 38.749 & 42.591 \\
\hline $7 / 10 / 114: 00$ & 57.899 & 60.422 & 51.667 & 58.133 & 39.100 & 38.747 & 42.587 \\
\hline 7/10/11 8:00 & 57.906 & 60.426 & 51.690 & 58.154 & 39.105 & 38.754 & 42.596 \\
\hline 7/10/11 12:00 & 57.913 & 60.433 & 51.695 & 58.164 & 39.114 & 38.758 & 42.605 \\
\hline 7/10/11 16:00 & 57.904 & 60.427 & 51.679 & 58.150 & 39.105 & 38.751 & 42.598 \\
\hline $7 / 10 / 11$ 20:00 & 57.899 & 60.429 & 51.676 & 58.145 & 39.107 & 38.747 & 42.596 \\
\hline 7/11/11 0:00 & 57.902 & 60.429 & 51.681 & 58.145 & 39.107 & 38.749 & 42.596 \\
\hline
\end{tabular}

TABLE S1.3 Page 41 of 58 


\begin{tabular}{|c|c|c|c|c|c|c|c|}
\hline \multirow[b]{2}{*}{ Date and Time } & \multicolumn{7}{|c|}{ Water Level (ft below reference point) } \\
\hline & SB43D & SB44D & SB45D & SB46D & SB47D & SB48D & SB49D \\
\hline $7 / 11 / 114: 00$ & 57.909 & 60.433 & 51.702 & 58.161 & 39.116 & 38.756 & 42.605 \\
\hline $7 / 11 / 118: 00$ & 57.928 & 60.454 & 51.728 & 58.196 & 39.133 & 38.775 & 42.624 \\
\hline $7 / 11 / 11$ 12:00 & 57.930 & 60.457 & 51.725 & 58.194 & 39.138 & 38.775 & 42.628 \\
\hline 7/11/11 16:00 & 57.921 & 60.452 & 51.707 & 58.175 & 39.133 & 38.770 & 42.621 \\
\hline 7/11/11 20:00 & 57.921 & 60.452 & 51.711 & 58.175 & 39.142 & 38.770 & 42.621 \\
\hline 7/12/11 0:00 & 57.932 & 60.461 & 51.725 & 58.187 & 39.149 & 38.780 & 42.630 \\
\hline $7 / 12 / 114: 00$ & 57.928 & 60.454 & 51.714 & 58.180 & 39.147 & 38.777 & 42.624 \\
\hline $7 / 12 / 118: 00$ & 57.935 & 60.466 & 51.737 & 58.196 & 39.152 & 38.787 & 42.633 \\
\hline $7 / 12 / 11$ 12:00 & 57.949 & 60.480 & 51.744 & 58.215 & 39.175 & 38.796 & 42.645 \\
\hline $7 / 12 / 11$ 16:00 & 57.939 & 60.471 & 51.721 & 58.192 & 39.168 & 38.791 & 42.638 \\
\hline $7 / 12 / 1120: 00$ & 57.942 & 60.468 & 51.728 & 58.187 & 39.166 & 38.789 & 42.635 \\
\hline 7/13/11 0:00 & 57.956 & 60.475 & 51.741 & 58.213 & 39.182 & 38.805 & 42.652 \\
\hline $7 / 13 / 114: 00$ & 57.949 & 60.471 & 51.732 & 58.196 & 39.180 & 38.801 & 42.642 \\
\hline 7/13/11 8:00 & 57.961 & 60.482 & 51.758 & 58.220 & 39.196 & 38.813 & 42.652 \\
\hline $7 / 13 / 11$ 12:00 & 57.973 & 60.499 & 51.772 & 58.234 & 39.208 & 38.824 & 42.668 \\
\hline $7 / 13 / 11$ 16:00 & 57.965 & 60.485 & 51.737 & 58.208 & 39.203 & 38.815 & 42.656 \\
\hline $7 / 13 / 11$ 20:00 & 57.961 & 60.485 & 51.739 & 58.203 & 39.199 & 38.813 & 42.654 \\
\hline 7/14/11 0:00 & 57.973 & 60.492 & 51.753 & 58.220 & 39.210 & 38.824 & 42.663 \\
\hline $7 / 14 / 114: 00$ & 57.970 & 60.485 & 51.739 & 58.203 & 39.208 & 38.820 & 42.654 \\
\hline 7/14/11 8:00 & 57.973 & 60.487 & 51.758 & 58.217 & 39.220 & 38.827 & 42.659 \\
\hline $7 / 14 / 11$ 12:00 & 57.987 & 60.506 & 51.774 & 58.238 & 39.229 & 38.838 & 42.675 \\
\hline $7 / 14 / 11$ 16:00 & 57.984 & 60.499 & 51.751 & 58.222 & 39.227 & 38.834 & 42.668 \\
\hline 7/14/11 20:00 & 57.975 & 60.492 & 51.739 & 58.206 & 39.213 & 38.824 & 42.659 \\
\hline 7/15/11 0:00 & 57.991 & 60.503 & 51.765 & 58.231 & 39.236 & 38.838 & 42.675 \\
\hline $7 / 15 / 114: 00$ & 57.991 & 60.501 & 51.755 & 58.224 & 39.239 & 38.838 & 42.668 \\
\hline 7/15/11 8:00 & 57.994 & 60.501 & 51.769 & 58.231 & 39.246 & 38.845 & 42.673 \\
\hline $7 / 15 / 11$ 12:00 & 58.010 & 60.522 & 51.793 & 58.262 & 39.257 & 38.862 & 42.691 \\
\hline $7 / 15 / 11$ 16:00 & 58.015 & 60.522 & 51.779 & 58.248 & 39.262 & 38.864 & 42.689 \\
\hline $7 / 15 / 1120: 00$ & 58.010 & 60.515 & 51.767 & 58.234 & 39.264 & 38.855 & 42.684 \\
\hline 7/16/11 0:00 & 58.027 & 60.534 & 51.802 & 58.267 & 39.281 & 38.874 & 42.700 \\
\hline $7 / 16 / 114: 00$ & 58.034 & 60.531 & 51.800 & 58.264 & 39.290 & 38.878 & 42.703 \\
\hline 7/16/11 8:00 & 58.036 & 60.536 & 51.800 & 58.267 & 39.293 & 38.883 & 42.705 \\
\hline $7 / 16 / 11$ 12:00 & 58.051 & 60.552 & 51.830 & 58.295 & 39.314 & 38.899 & 42.724 \\
\hline $7 / 16 / 11$ 16:00 & 58.065 & 60.566 & 51.827 & 58.297 & 39.326 & 38.911 & 42.731 \\
\hline 7/16/11 20:00 & 58.060 & 60.554 & 51.814 & 58.281 & 39.330 & 38.907 & 42.728 \\
\hline $7 / 17 / 110: 00$ & 58.074 & 60.571 & 51.837 & 58.302 & 39.340 & 38.921 & 42.735 \\
\hline $7 / 17 / 114: 00$ & 58.081 & 60.571 & 51.837 & 58.302 & 39.349 & 38.928 & 42.740 \\
\hline 7/17/11 8:00 & 58.086 & 60.578 & 51.844 & 58.306 & 39.351 & 38.935 & 42.745 \\
\hline $7 / 17 / 11$ 12:00 & 58.105 & 60.596 & 51.871 & 58.337 & 39.380 & 38.954 & 42.766 \\
\hline $7 / 17 / 11$ 16:00 & 58.119 & 60.606 & 51.871 & 58.344 & 39.391 & 38.963 & 42.773 \\
\hline $7 / 17 / 11$ 20:00 & 58.117 & 60.599 & 51.853 & 58.323 & 39.396 & 38.958 & 42.768 \\
\hline 7/18/11 0:00 & 58.124 & 60.604 & 51.871 & 58.337 & 39.401 & 38.970 & 42.777 \\
\hline $7 / 18 / 114: 00$ & 58.136 & 60.608 & 51.874 & 58.341 & 39.413 & 38.979 & 42.777 \\
\hline 7/18/11 8:00 & 58.138 & 60.606 & 51.869 & 58.337 & 39.420 & 38.982 & 42.777 \\
\hline $7 / 18 / 11$ 12:00 & 58.148 & 60.620 & 51.892 & 58.358 & 39.431 & 38.989 & 42.789 \\
\hline 7/18/11 16:00 & 58.159 & 60.624 & 51.890 & 58.362 & 39.445 & 39.003 & 42.796 \\
\hline 7/18/11 20:00 & 58.159 & 60.622 & 51.874 & 58.344 & 39.438 & 38.998 & 42.791 \\
\hline 7/19/11 0:00 & 58.164 & 60.624 & 51.890 & 58.355 & 39.450 & 39.008 & 42.796 \\
\hline $7 / 19 / 114: 00$ & 58.174 & 60.634 & 51.890 & 58.360 & 39.462 & 39.017 & 42.798 \\
\hline 7/19/11 8:00 & 58.176 & 60.624 & 51.890 & 58.358 & 39.467 & 39.019 & 42.798 \\
\hline 7/19/11 12:00 & 58.183 & 60.638 & 51.899 & 58.369 & 39.474 & 39.026 & 42.807 \\
\hline 7/19/11 16:00 & 58.193 & 60.648 & 51.909 & 58.376 & 39.485 & 39.038 & 42.814 \\
\hline 7/19/11 20:00 & 58.195 & 60.641 & 51.888 & 58.358 & 39.481 & 39.036 & 42.807 \\
\hline 7/20/11 0:00 & 58.195 & 60.645 & 51.899 & 58.363 & 39.488 & 39.036 & 42.810 \\
\hline
\end{tabular}

TABLE S1.3 Page 42 of 58 


\begin{tabular}{|c|c|c|c|c|c|c|c|}
\hline \multirow[b]{2}{*}{ Date and Time } & \multicolumn{7}{|c|}{ Water Level (ft below reference point) } \\
\hline & SB43D & SB44D & SB45D & SB46D & SB47D & SB48D & SB49D \\
\hline $7 / 20 / 114: 00$ & 58.214 & 60.652 & 51.916 & 58.383 & 39.506 & 39.052 & 42.821 \\
\hline 7/20/11 8:00 & 58.214 & 60.648 & 51.906 & 58.372 & 39.511 & 39.052 & 42.819 \\
\hline $7 / 20 / 11$ 12:00 & 58.216 & 60.652 & 51.916 & 58.383 & 39.516 & 39.059 & 42.824 \\
\hline 7/20/11 16:00 & 58.245 & 60.666 & 51.927 & 58.398 & 39.525 & 39.069 & 42.835 \\
\hline $7 / 20 / 1120: 00$ & 58.275 & 60.664 & 51.918 & 58.386 & 39.535 & 39.071 & 42.831 \\
\hline 7/21/11 0:00 & 58.292 & 60.673 & 51.934 & 58.398 & 39.544 & 39.083 & 42.840 \\
\hline $7 / 21 / 114: 00$ & 58.287 & 60.683 & 51.946 & 58.414 & 39.563 & 39.099 & 42.852 \\
\hline $7 / 21 / 118: 00$ & 58.287 & 60.692 & 51.955 & 58.421 & 39.568 & 39.109 & 42.863 \\
\hline $7 / 21 / 11$ 12:00 & 58.278 & 60.697 & 51.957 & 58.426 & 39.575 & 39.111 & 42.866 \\
\hline $7 / 21 / 11$ 16:00 & 58.280 & 60.706 & 51.957 & 58.428 & 39.584 & 39.118 & 42.870 \\
\hline $7 / 21 / 1120: 00$ & 58.285 & 60.704 & 51.953 & 58.423 & 39.593 & 39.120 & 42.873 \\
\hline $7 / 22 / 110: 00$ & 58.292 & 60.711 & 51.967 & 58.433 & 39.600 & 39.130 & 42.877 \\
\hline $7 / 22 / 114: 00$ & 58.311 & 60.725 & 51.992 & 58.456 & 39.622 & 39.149 & 42.893 \\
\hline $7 / 22 / 118: 00$ & 58.313 & 60.725 & 51.985 & 58.451 & 39.624 & 39.153 & 42.893 \\
\hline $7 / 22 / 11$ 12:00 & 58.316 & 60.732 & 51.990 & 58.456 & 39.631 & 39.158 & 42.898 \\
\hline $7 / 22 / 11$ 16:00 & 58.323 & 60.736 & 51.988 & 58.458 & 39.643 & 39.165 & 42.903 \\
\hline $7 / 22 / 1120: 00$ & 58.337 & 60.743 & 51.999 & 58.465 & 39.657 & 39.174 & 42.912 \\
\hline 7/23/11 0:00 & 58.344 & 60.748 & 52.006 & 58.468 & 39.662 & 39.181 & 42.917 \\
\hline $7 / 23 / 114: 00$ & 58.356 & 60.757 & 52.020 & 58.484 & 39.676 & 39.196 & 42.926 \\
\hline $7 / 23 / 118: 00$ & 58.372 & 60.769 & 52.036 & 58.500 & 39.692 & 39.210 & 42.938 \\
\hline $7 / 23 / 11$ 12:00 & 58.377 & 60.773 & 52.032 & 58.503 & 39.699 & 39.214 & 42.942 \\
\hline $7 / 23 / 11$ 16:00 & 58.382 & 60.780 & 52.036 & 58.505 & 39.706 & 39.221 & 42.947 \\
\hline $7 / 23 / 1120: 00$ & 58.396 & 60.790 & 52.043 & 58.512 & 39.716 & 39.231 & 42.956 \\
\hline $7 / 24 / 11$ 0:00 & 58.408 & 60.794 & 52.053 & 58.522 & 39.732 & 39.245 & 42.963 \\
\hline $7 / 24 / 114: 00$ & 58.420 & 60.811 & 52.076 & 58.531 & 39.746 & 39.257 & 42.975 \\
\hline $7 / 24 / 118: 00$ & 58.389 & 60.783 & 52.036 & 58.510 & 39.720 & 39.210 & 42.942 \\
\hline $7 / 24 / 11$ 12:00 & 58.401 & 60.797 & 52.053 & 58.524 & 39.730 & 39.228 & 42.963 \\
\hline $7 / 24 / 11$ 16:00 & 58.403 & 60.799 & 52.048 & 58.517 & 39.732 & 39.236 & 42.966 \\
\hline $7 / 24 / 1120: 00$ & 58.415 & 60.801 & 52.050 & 58.521 & 39.734 & 39.247 & 42.968 \\
\hline 7/25/11 0:00 & 58.434 & 60.806 & 52.043 & 58.526 & 39.763 & 39.264 & 42.968 \\
\hline $7 / 25 / 114: 00$ & 58.431 & 60.809 & 52.064 & 58.528 & 39.760 & 39.268 & 42.975 \\
\hline 7/25/11 8:00 & 58.450 & 60.820 & 52.087 & 58.552 & 39.779 & 39.285 & 42.989 \\
\hline $7 / 25 / 11$ 12:00 & 58.455 & 60.820 & 52.080 & 58.550 & 39.779 & 39.290 & 42.991 \\
\hline $7 / 25 / 11$ 16:00 & 58.450 & 60.815 & 52.062 & 58.533 & 39.772 & 39.285 & 42.984 \\
\hline $7 / 25 / 1120: 00$ & 58.457 & 60.820 & 52.069 & 58.535 & 39.781 & 39.287 & 42.986 \\
\hline $7 / 26 / 110: 00$ & 58.467 & 60.827 & 52.078 & 58.545 & 39.793 & 39.297 & 42.993 \\
\hline $7 / 26 / 114: 00$ & 58.467 & 60.820 & 52.073 & 58.540 & 39.795 & 39.299 & 42.989 \\
\hline 7/26/11 8:00 & 58.481 & 60.839 & 52.097 & 58.564 & 39.800 & 39.311 & 43.005 \\
\hline $7 / 26 / 11$ 12:00 & 58.488 & 60.843 & 52.099 & 58.568 & 39.812 & 39.318 & 43.012 \\
\hline $7 / 26 / 11$ 16:00 & 58.483 & 60.843 & 52.078 & 58.550 & 39.807 & 39.313 & 43.005 \\
\hline $7 / 26 / 1120: 00$ & 58.488 & 60.836 & 52.085 & 58.552 & 39.814 & 39.315 & 43.005 \\
\hline 7/27/11 0:00 & 58.498 & 60.846 & 52.097 & 58.561 & 39.826 & 39.327 & 43.012 \\
\hline $7 / 27 / 114: 00$ & 58.502 & 60.844 & 52.094 & 58.561 & 39.826 & 39.332 & 43.012 \\
\hline $7 / 27 / 118: 00$ & 58.517 & 60.857 & 52.120 & 58.585 & 39.842 & 39.346 & 43.026 \\
\hline $7 / 27 / 11$ 12:00 & 58.524 & 60.867 & 52.115 & 58.585 & 39.847 & 39.351 & 43.031 \\
\hline 7/27/11 16:00 & 58.524 & 60.864 & 52.106 & 58.578 & 39.852 & 39.351 & 43.031 \\
\hline $7 / 27 / 1120: 00$ & 58.531 & 60.874 & 52.120 & 58.585 & 39.866 & 39.358 & 43.038 \\
\hline $7 / 28 / 110: 00$ & 58.550 & 60.883 & 52.141 & 58.608 & 39.885 & 39.377 & 43.054 \\
\hline $7 / 28 / 114: 00$ & 58.559 & 60.890 & 52.143 & 58.610 & 39.892 & 39.386 & 43.059 \\
\hline 7/28/11 8:00 & 58.578 & 60.904 & 52.173 & 58.638 & 39.918 & 39.405 & 43.075 \\
\hline $7 / 28 / 11$ 12:00 & 58.599 & 60.930 & 52.194 & 58.662 & 39.936 & 39.426 & 43.093 \\
\hline $7 / 28 / 11$ 16:00 & 58.599 & 60.927 & 52.176 & 58.648 & 39.936 & 39.426 & 43.093 \\
\hline $7 / 28 / 1120: 00$ & 58.602 & 60.932 & 52.185 & 58.645 & 39.932 & 39.431 & 43.096 \\
\hline 7/29/11 0:00 & 58.625 & 60.948 & 52.213 & 58.676 & 39.960 & 39.452 & 43.114 \\
\hline
\end{tabular}

TABLE S1.3 Page 43 of 58 


\begin{tabular}{|c|c|c|c|c|c|c|c|}
\hline \multirow[b]{2}{*}{ Date and Time } & \multicolumn{7}{|c|}{ Water Level (ft below reference point) } \\
\hline & SB43D & SB44D & SB45D & SB46D & SB47D & SB48D & SB49D \\
\hline $7 / 29 / 114: 00$ & 58.609 & 60.932 & 52.185 & 58.650 & 39.948 & 39.435 & 43.096 \\
\hline 7/29/11 8:00 & 58.618 & 60.934 & 52.203 & 58.664 & 39.960 & 39.447 & 43.105 \\
\hline 7/29/11 12:00 & 58.637 & 60.955 & 52.222 & 58.688 & 39.974 & 39.463 & 43.121 \\
\hline 7/29/11 16:00 & 58.635 & 60.948 & 52.194 & 58.664 & 39.967 & 39.459 & 43.114 \\
\hline $7 / 29 / 11$ 20:00 & 58.625 & 60.941 & 52.187 & 58.655 & 39.958 & 39.454 & 43.107 \\
\hline 7/30/11 0:00 & 58.616 & 60.946 & 52.187 & 58.657 & 39.955 & 39.433 & 43.105 \\
\hline 7/30/11 4:00 & 58.599 & 60.906 & 52.155 & 58.631 & 39.929 & 39.402 & 43.072 \\
\hline 7/30/11 8:00 & 58.408 & 60.871 & 52.053 & 58.528 & 39.758 & 39.250 & 43.007 \\
\hline $7 / 30 / 11$ 12:00 & 58.476 & 60.872 & 52.080 & 58.554 & 39.786 & 39.283 & 43.024 \\
\hline $7 / 30 / 11$ 16:00 & 58.486 & 60.855 & 52.048 & 58.524 & 39.774 & 39.285 & 43.010 \\
\hline 7/30/11 20:00 & 58.479 & 60.834 & 52.039 & 58.507 & 39.767 & 39.280 & 42.993 \\
\hline 7/31/11 0:00 & 58.493 & 60.839 & 52.057 & 58.528 & 39.770 & 39.292 & 42.996 \\
\hline 7/31/11 4:00 & 58.493 & 60.825 & 52.043 & 58.517 & 39.763 & 39.290 & 42.989 \\
\hline 7/31/11 8:00 & 58.486 & 60.818 & 52.043 & 58.510 & 39.748 & 39.287 & 42.982 \\
\hline 7/31/11 12:00 & 58.493 & 60.830 & 52.066 & 58.533 & 39.760 & 39.297 & 42.991 \\
\hline 7/31/11 16:00 & 58.491 & 60.827 & 52.046 & 58.521 & 39.756 & 39.287 & 42.986 \\
\hline 7/31/11 20:00 & 58.479 & 60.808 & 52.027 & 58.498 & 39.739 & 39.275 & 42.972 \\
\hline 8/1/11 0:00 & 58.481 & 60.815 & 52.048 & 58.514 & 39.741 & 39.278 & 42.977 \\
\hline 8/1/11 4:00 & 58.479 & 60.804 & 52.034 & 58.505 & 39.734 & 39.273 & 42.970 \\
\hline 8/1/11 8:00 & 58.464 & 60.792 & 52.020 & 58.489 & 39.718 & 39.264 & 42.954 \\
\hline 8/1/11 12:00 & 58.464 & 60.804 & 52.041 & 58.512 & 39.730 & 39.271 & 42.968 \\
\hline 8/1/11 16:00 & 58.469 & 60.804 & 52.029 & 58.505 & 39.727 & 39.266 & 42.966 \\
\hline 8/1/11 20:00 & 58.455 & 60.792 & 52.008 & 58.477 & 39.716 & 39.252 & 42.952 \\
\hline 8/2/11 0:00 & 58.457 & 60.792 & 52.025 & 58.493 & 39.713 & 39.257 & 42.961 \\
\hline $8 / 2 / 114: 00$ & 58.455 & 60.787 & 52.015 & 58.486 & 39.709 & 39.252 & 42.954 \\
\hline 8/2/11 8:00 & 58.446 & 60.776 & 52.006 & 58.470 & 39.706 & 39.240 & 42.942 \\
\hline $8 / 2 / 11$ 12:00 & 58.446 & 60.785 & 52.020 & 58.489 & 39.706 & 39.247 & 42.949 \\
\hline 8/2/11 16:00 & 58.457 & 60.797 & 52.029 & 58.503 & 39.718 & 39.254 & 42.961 \\
\hline $8 / 2 / 1120: 00$ & 58.457 & 60.795 & 52.020 & 58.491 & 39.706 & 39.252 & 42.959 \\
\hline 8/3/11 0:00 & 58.462 & 60.801 & 52.041 & 58.507 & 39.720 & 39.259 & 42.968 \\
\hline 8/3/11 4:00 & 58.474 & 60.811 & 52.053 & 58.521 & 39.730 & 39.268 & 42.975 \\
\hline 8/3/11 8:00 & 58.469 & 60.799 & 52.041 & 58.510 & 39.730 & 39.266 & 42.970 \\
\hline 8/3/11 12:00 & 58.469 & 60.806 & 52.055 & 58.521 & 39.734 & 39.271 & 42.977 \\
\hline 8/3/11 16:00 & 58.476 & 60.815 & 52.057 & 58.526 & 39.737 & 39.268 & 42.982 \\
\hline 8/3/11 20:00 & 58.476 & 60.804 & 52.036 & 58.510 & 39.732 & 39.259 & 42.972 \\
\hline 8/4/11 0:00 & 58.481 & 60.811 & 52.053 & 58.521 & 39.739 & 39.268 & 42.975 \\
\hline 8/4/11 4:00 & 58.491 & 60.818 & 52.066 & 58.533 & 39.753 & 39.280 & 42.986 \\
\hline 8/4/11 8:00 & 58.483 & 60.815 & 52.055 & 58.521 & 39.741 & 39.278 & 42.979 \\
\hline 8/4/11 12:00 & 58.481 & 60.813 & 52.053 & 58.521 & 39.744 & 39.278 & 42.979 \\
\hline 8/4/11 16:00 & 58.483 & 60.820 & 52.060 & 58.531 & 39.753 & 39.283 & 42.986 \\
\hline 8/4/11 20:00 & 58.486 & 60.820 & 52.053 & 58.521 & 39.756 & 39.280 & 42.984 \\
\hline 8/5/11 0:00 & 58.488 & 60.822 & 52.064 & 58.528 & 39.756 & 39.283 & 42.989 \\
\hline 8/5/11 4:00 & 58.495 & 60.827 & 52.073 & 58.535 & 39.760 & 39.290 & 42.993 \\
\hline 8/5/11 8:00 & 58.502 & 60.836 & 52.087 & 58.547 & 39.767 & 39.299 & 43.005 \\
\hline 8/5/11 12:00 & 58.450 & 60.806 & 52.039 & 58.503 & 39.720 & 39.233 & 42.966 \\
\hline 8/5/11 16:00 & 58.455 & 60.811 & 52.041 & 58.514 & 39.727 & 39.245 & 42.972 \\
\hline 8/5/11 20:00 & 58.457 & 60.801 & 52.034 & 58.503 & 39.725 & 39.247 & 42.966 \\
\hline 8/6/11 0:00 & 58.460 & 60.801 & 52.039 & 58.507 & 39.727 & 39.257 & 42.968 \\
\hline 8/6/11 4:00 & 58.462 & 60.804 & 52.048 & 58.510 & 39.730 & 39.259 & 42.968 \\
\hline 8/6/11 8:00 & 58.481 & 60.815 & 52.064 & 58.533 & 39.746 & 39.275 & 42.984 \\
\hline 8/6/11 12:00 & 58.472 & 60.811 & 52.039 & 58.512 & 39.741 & 39.268 & 42.977 \\
\hline 8/6/11 16:00 & 58.469 & 60.808 & 52.046 & 58.517 & 39.741 & 39.268 & 42.979 \\
\hline 8/6/11 20:00 & 58.476 & 60.815 & 52.050 & 58.519 & 39.744 & 39.268 & 42.979 \\
\hline 8/7/11 0:00 & 58.486 & 60.825 & 52.073 & 58.531 & 39.756 & 39.280 & 42.996 \\
\hline
\end{tabular}

TABLE S1.3 Page 44 of 58 


\begin{tabular}{|c|c|c|c|c|c|c|c|}
\hline \multirow[b]{2}{*}{ Date and Time } & \multicolumn{7}{|c|}{ Water Level (ft below reference point) } \\
\hline & SB43D & SB44D & SB45D & SB46D & SB47D & SB48D & SB49D \\
\hline 8/7/11 4:00 & 58.483 & 60.820 & 52.067 & 58.526 & 39.756 & 39.280 & 42.989 \\
\hline 8/7/11 8:00 & 58.498 & 60.829 & 52.073 & 58.540 & 39.772 & 39.292 & 42.996 \\
\hline $8 / 7 / 1112: 00$ & 58.498 & 60.834 & 52.076 & 58.542 & 39.774 & 39.294 & 43.000 \\
\hline 8/7/11 16:00 & 58.476 & 60.818 & 52.043 & 58.496 & 39.753 & 39.273 & 42.984 \\
\hline 8/7/11 20:00 & 58.491 & 60.825 & 52.055 & 58.524 & 39.765 & 39.285 & 42.989 \\
\hline 8/8/11 0:00 & 58.495 & 60.834 & 52.071 & 58.528 & 39.767 & 39.290 & 42.998 \\
\hline 8/8/11 4:00 & 58.500 & 60.825 & 52.055 & 58.535 & 39.777 & 39.294 & 42.989 \\
\hline 8/8/11 8:00 & 58.517 & 60.832 & 52.092 & 58.564 & 39.786 & 39.306 & 43.003 \\
\hline 8/8/11 12:00 & 58.509 & 60.832 & 52.078 & 58.550 & 39.786 & 39.301 & 43.003 \\
\hline 8/8/11 16:00 & 58.500 & 60.832 & 52.064 & 58.533 & 39.781 & 39.297 & 42.996 \\
\hline 8/8/11 20:00 & 58.505 & 60.836 & 52.073 & 58.538 & 39.784 & 39.299 & 43.003 \\
\hline 8/9/11 0:00 & 58.517 & 60.846 & 52.087 & 58.552 & 39.795 & 39.311 & 43.012 \\
\hline 8/9/11 4:00 & 58.517 & 60.841 & 52.083 & 58.550 & 39.800 & 39.311 & 43.010 \\
\hline 8/9/11 8:00 & 58.533 & 60.862 & 52.115 & 58.578 & 39.812 & 39.330 & 43.028 \\
\hline 8/9/11 12:00 & 58.545 & 60.867 & 52.115 & 58.587 & 39.824 & 39.339 & 43.040 \\
\hline 8/9/11 16:00 & 58.540 & 60.864 & 52.099 & 58.571 & 39.826 & 39.334 & 43.035 \\
\hline 8/9/11 20:00 & 58.547 & 60.871 & 52.108 & 58.578 & 39.833 & 39.339 & 43.042 \\
\hline 8/10/11 0:00 & 58.559 & 60.881 & 52.125 & 58.592 & 39.847 & 39.351 & 43.047 \\
\hline 8/10/11 4:00 & 58.554 & 60.874 & 52.115 & 58.580 & 39.845 & 39.351 & 43.045 \\
\hline 8/10/11 8:00 & 58.576 & 60.895 & 52.152 & 58.615 & 39.866 & 39.372 & 43.061 \\
\hline 8/10/11 12:00 & 58.961 & 60.899 & & & 39.875 & 39.379 & 43.077 \\
\hline 8/10/11 16:00 & 58.613 & 60.899 & 52.157 & & 39.951 & 39.412 & 43.079 \\
\hline 8/10/11 20:00 & 59.032 & 60.941 & 52.162 & & 39.923 & 39.113 & 43.124 \\
\hline 8/11/11 0:00 & 58.706 & 60.930 & 52.180 & & 39.906 & 39.440 & 43.096 \\
\hline 8/11/11 4:00 & 58.663 & 60.920 & 52.171 & & 39.904 & 39.428 & 43.084 \\
\hline 8/11/11 8:00 & 58.661 & 60.923 & 52.194 & & 39.918 & 39.440 & 43.091 \\
\hline 8/11/11 12:00 & 58.677 & & 52.213 & & 39.936 & 39.456 & 43.184 \\
\hline 8/11/11 16:00 & 58.668 & 60.962 & 52.185 & 58.641 & 39.925 & 39.449 & 43.121 \\
\hline 8/11/11 20:00 & 58.661 & 60.955 & 52.234 & 58.645 & 39.920 & 39.445 & 43.105 \\
\hline 8/12/11 0:00 & 58.675 & 60.958 & 52.183 & 58.650 & 39.939 & 39.461 & 43.112 \\
\hline 8/12/11 4:00 & 58.687 & 60.946 & 52.176 & 58.659 & 39.948 & 39.471 & 43.105 \\
\hline 8/12/11 8:00 & 58.640 & 60.934 & 52.162 & 58.622 & 39.911 & 39.419 & 43.091 \\
\hline 8/12/11 12:00 & 58.642 & 60.941 & 52.162 & 58.634 & 39.915 & 39.412 & 43.089 \\
\hline 8/12/11 16:00 & 58.644 & 60.934 & 52.148 & 58.624 & 39.908 & 39.419 & 43.086 \\
\hline 8/12/11 20:00 & 58.640 & 60.925 & 52.141 & 58.613 & 39.906 & 39.421 & 43.079 \\
\hline 8/13/11 0:00 & 58.656 & 60.939 & 52.164 & 58.634 & 39.922 & 39.438 & 43.091 \\
\hline 8/13/11 4:00 & 58.658 & 60.927 & 52.150 & 58.622 & 39.922 & 39.440 & 43.084 \\
\hline 8/13/11 8:00 & 58.661 & 60.934 & 52.162 & 58.629 & 39.922 & 39.445 & 43.089 \\
\hline 8/13/11 12:00 & 58.682 & 60.953 & 52.190 & 58.659 & 39.941 & 39.466 & 43.112 \\
\hline 8/13/11 16:00 & 58.687 & 60.953 & 52.178 & 58.650 & 39.948 & 39.466 & 43.114 \\
\hline 8/13/11 20:00 & 58.684 & 60.953 & 52.178 & 58.643 & 39.948 & 39.463 & 43.112 \\
\hline 8/14/11 0:00 & 58.703 & 60.964 & 52.201 & 58.669 & 39.962 & 39.480 & 43.124 \\
\hline 8/14/11 4:00 & 58.706 & 60.962 & 52.194 & 58.659 & 39.967 & 39.482 & 43.121 \\
\hline 8/14/11 8:00 & 58.703 & 60.967 & 52.196 & 58.659 & 39.965 & 39.485 & 43.121 \\
\hline 8/14/11 12:00 & 58.718 & 60.983 & 52.217 & 58.687 & 39.986 & 39.499 & 43.135 \\
\hline 8/14/11 16:00 & 58.722 & 60.986 & 52.201 & 58.676 & 39.983 & 39.496 & 43.138 \\
\hline 8/14/11 20:00 & 58.715 & 60.974 & 52.190 & 58.657 & 39.979 & 39.489 & 43.126 \\
\hline 8/15/11 0:00 & 58.725 & 60.983 & 52.208 & 58.676 & 39.990 & 39.501 & 43.138 \\
\hline 8/15/11 4:00 & 58.734 & 60.988 & 52.210 & 58.681 & 40.000 & 39.508 & 43.142 \\
\hline 8/15/11 8:00 & 58.720 & 60.964 & 52.183 & 58.652 & 39.986 & 39.496 & 43.121 \\
\hline 8/15/11 12:00 & 58.736 & 60.979 & 52.210 & 58.690 & 40.005 & 39.510 & 43.135 \\
\hline 8/15/11 16:00 & 58.725 & 60.976 & 52.201 & 58.669 & 39.995 & 39.501 & 43.131 \\
\hline 8/15/11 20:00 & 58.720 & 60.971 & 52.187 & 58.655 & 39.981 & 39.494 & 43.126 \\
\hline 8/16/11 0:00 & 58.729 & 60.981 & 52.208 & 58.674 & 39.993 & 39.506 & 43.135 \\
\hline
\end{tabular}

TABLE S1.3 Page 45 of 58 


\begin{tabular}{|c|c|c|c|c|c|c|c|}
\hline \multirow[b]{2}{*}{ Date and Time } & \multicolumn{7}{|c|}{ Water Level (ft below reference point) } \\
\hline & SB43D & SB44D & SB45D & SB46D & SB47D & SB48D & SB49D \\
\hline 8/16/11 4:00 & 58.732 & 60.981 & 52.192 & 58.666 & 39.993 & 39.506 & 43.131 \\
\hline 8/16/11 8:00 & 58.713 & 60.960 & 52.185 & 58.641 & 39.969 & 39.471 & 43.105 \\
\hline $8 / 16 / 11$ 12:00 & 58.694 & 60.976 & 52.192 & 58.662 & 39.962 & 39.452 & 43.126 \\
\hline 8/16/11 16:00 & 58.703 & 60.976 & 52.194 & 58.662 & 39.965 & 39.468 & 43.128 \\
\hline 8/16/11 20:00 & 58.706 & 60.974 & 52.187 & 58.657 & 39.962 & 39.473 & 43.126 \\
\hline 8/17/11 0:00 & 58.720 & 60.985 & 52.213 & 58.678 & 39.981 & 39.492 & 43.138 \\
\hline 8/17/11 4:00 & 58.734 & 60.988 & 52.215 & 58.685 & 39.990 & 39.503 & 43.142 \\
\hline 8/17/11 8:00 & 58.736 & 60.988 & 52.217 & 58.683 & 39.993 & 39.506 & 43.145 \\
\hline $8 / 17 / 11$ 12:00 & 58.744 & 60.999 & 52.231 & 58.699 & 40.000 & 39.515 & 43.156 \\
\hline $8 / 17 / 11$ 16:00 & 58.751 & 61.006 & 52.229 & 58.702 & 40.009 & 39.520 & 43.158 \\
\hline $8 / 17 / 1120: 00$ & 58.744 & 60.992 & 52.210 & 58.678 & 40.000 & 39.513 & 43.149 \\
\hline 8/18/11 0:00 & 58.755 & 61.007 & 52.236 & 58.699 & 40.016 & 39.525 & 43.161 \\
\hline 8/18/11 4:00 & 58.758 & 61.002 & 52.234 & 58.697 & 40.021 & 39.529 & 43.161 \\
\hline 8/18/11 8:00 & 58.760 & 61.002 & 52.229 & 58.692 & 40.014 & 39.530 & 43.158 \\
\hline $8 / 18 / 11$ 12:00 & 58.755 & 61.004 & 52.224 & 58.694 & 40.014 & 39.529 & 43.158 \\
\hline 8/18/11 16:00 & 58.758 & 61.007 & 52.213 & 58.687 & 40.021 & 39.527 & 43.156 \\
\hline 8/18/11 20:00 & 58.760 & 60.999 & 52.213 & 58.690 & 40.021 & 39.527 & 43.156 \\
\hline 8/19/11 0:00 & 58.663 & 60.964 & 52.145 & 58.629 & 39.927 & 39.384 & 43.109 \\
\hline 8/19/11 4:00 & 58.670 & 60.960 & 52.162 & 58.631 & 39.932 & 39.433 & 43.107 \\
\hline 8/19/11 8:00 & 58.692 & 60.958 & 52.169 & 58.636 & 39.885 & 39.362 & 43.110 \\
\hline $8 / 19 / 11$ 12:00 & 58.696 & 60.962 & 52.171 & 58.638 & 39.960 & 39.445 & 43.114 \\
\hline $8 / 19 / 11$ 16:00 & 58.701 & 60.962 & 52.164 & 58.638 & 39.941 & 39.579 & 43.114 \\
\hline 8/19/11 20:00 & 58.706 & 60.960 & 52.162 & 58.634 & 39.943 & 39.489 & 43.112 \\
\hline 8/20/11 0:00 & 58.694 & 60.955 & 52.171 & 58.620 & 39.929 & 39.454 & 43.105 \\
\hline $8 / 20 / 114: 00$ & 58.658 & 60.948 & 52.152 & 58.617 & 39.899 & 39.311 & 43.089 \\
\hline 8/20/11 8:00 & 58.649 & 60.937 & 52.143 & 58.610 & 39.887 & 39.339 & 43.089 \\
\hline $8 / 20 / 11$ 12:00 & 58.658 & 60.934 & 52.143 & 58.613 & 39.889 & 39.365 & 43.086 \\
\hline $8 / 20 / 11$ 16:00 & 58.658 & 60.927 & 52.138 & 58.608 & 39.882 & 39.442 & 43.084 \\
\hline 8/20/11 20:00 & 58.661 & 60.927 & 52.129 & 58.599 & 39.868 & 39.431 & 43.079 \\
\hline 8/21/11 0:00 & 58.661 & 60.927 & 52.136 & 58.603 & 39.868 & 39.362 & 43.082 \\
\hline $8 / 21 / 114: 00$ & 58.663 & 60.930 & 52.143 & 58.613 & 39.868 & 39.358 & 43.084 \\
\hline 8/21/11 8:00 & 58.666 & 60.927 & 52.143 & 58.613 & 39.866 & 39.341 & 43.086 \\
\hline $8 / 21 / 11$ 12:00 & 58.661 & 60.925 & 52.143 & 58.613 & 39.861 & 39.391 & 43.084 \\
\hline $8 / 21 / 11$ 16:00 & 58.649 & 60.918 & 52.127 & 58.599 & 39.849 & 39.527 & 43.077 \\
\hline $8 / 21 / 1120: 00$ & 58.647 & 60.920 & 52.127 & 58.596 & 39.842 & 39.449 & 43.072 \\
\hline 8/22/11 0:00 & 58.649 & 60.920 & 52.131 & 58.601 & 39.847 & 39.353 & 43.077 \\
\hline $8 / 22 / 114: 00$ & 58.644 & 60.920 & 52.143 & 58.603 & 39.840 & 39.360 & 43.079 \\
\hline 8/22/11 8:00 & 58.573 & 60.916 & 52.057 & 58.535 & 39.727 & 39.193 & 43.028 \\
\hline $8 / 22 / 11$ 12:00 & 58.557 & 60.864 & 52.032 & 58.507 & 39.716 & 39.334 & 43.012 \\
\hline $8 / 22 / 11$ 16:00 & 58.554 & 60.853 & 52.013 & 58.489 & 39.699 & 39.440 & 42.996 \\
\hline $8 / 22 / 1120: 00$ & 58.545 & 60.832 & 52.001 & 58.472 & 39.671 & 39.386 & 42.982 \\
\hline 8/23/11 0:00 & 58.535 & 60.815 & 51.988 & 58.461 & 39.645 & 39.280 & 42.966 \\
\hline 8/23/11 4:00 & 58.514 & 60.794 & 51.976 & 58.444 & 39.617 & 39.252 & 42.952 \\
\hline 8/23/11 8:00 & 58.495 & 60.783 & 51.960 & 58.430 & 39.584 & 39.259 & 42.933 \\
\hline $8 / 23 / 11$ 12:00 & 58.474 & 60.767 & 51.934 & 58.416 & 39.558 & 39.292 & 42.919 \\
\hline 8/23/11 16:00 & 58.446 & 60.750 & 51.913 & 58.386 & 39.528 & 39.170 & 42.900 \\
\hline 8/23/11 20:00 & 58.434 & 60.743 & 51.916 & 58.388 & 39.518 & 39.158 & 42.896 \\
\hline 8/24/11 0:00 & 58.417 & 60.727 & 51.897 & 58.367 & 39.492 & 39.134 & 42.877 \\
\hline $8 / 24 / 114: 00$ & 58.398 & 60.711 & 51.888 & 58.360 & 39.471 & 39.118 & 42.866 \\
\hline 8/24/11 8:00 & 58.394 & 60.715 & 51.909 & 58.376 & 39.467 & 39.113 & 42.868 \\
\hline 8/24/11 12:00 & 58.384 & 60.713 & 51.902 & 58.372 & 39.462 & 39.106 & 42.866 \\
\hline $8 / 24 / 11$ 16:00 & 58.368 & 60.701 & 51.888 & 58.358 & 39.450 & 39.092 & 42.859 \\
\hline $8 / 24 / 1120: 00$ & 58.363 & 60.701 & 51.892 & 58.360 & 39.450 & 39.085 & 42.856 \\
\hline 8/25/11 0:00 & 58.360 & 60.699 & 51.892 & 58.372 & 39.448 & 39.085 & 42.856 \\
\hline
\end{tabular}

TABLE S1.3 Page 46 of 58 


\begin{tabular}{|c|c|c|c|c|c|c|c|}
\hline \multirow[b]{2}{*}{ Date and Time } & \multicolumn{7}{|c|}{ Water Level (ft below reference point) } \\
\hline & SB43D & SB44D & SB45D & SB46D & SB47D & SB48D & SB49D \\
\hline $8 / 25 / 114: 00$ & 58.346 & 60.692 & 51.885 & 58.360 & 39.441 & 39.073 & 42.847 \\
\hline 8/25/11 8:00 & 58.346 & 60.690 & 51.904 & 58.372 & 39.445 & 39.076 & 42.849 \\
\hline $8 / 25 / 11$ 12:00 & 58.346 & 60.692 & 51.897 & 58.374 & 39.448 & 39.076 & 42.849 \\
\hline $8 / 25 / 11$ 16:00 & 58.323 & 60.669 & 51.858 & 58.341 & 39.431 & 39.055 & 42.828 \\
\hline $8 / 25 / 1120: 00$ & 58.311 & 60.664 & 51.858 & 58.332 & 39.420 & 39.045 & 42.817 \\
\hline 8/26/11 0:00 & 58.308 & 60.657 & 51.855 & 58.330 & 39.422 & 39.043 & 42.814 \\
\hline $8 / 26 / 114: 00$ & 58.297 & 60.643 & 51.844 & 58.313 & 39.413 & 39.031 & 42.800 \\
\hline 8/26/11 8:00 & 58.297 & 60.648 & 51.858 & 58.334 & 39.424 & 39.036 & 42.803 \\
\hline $8 / 26 / 11$ 12:00 & 58.294 & 60.645 & 51.855 & 58.332 & 39.420 & 39.036 & 42.805 \\
\hline $8 / 26 / 11$ 16:00 & 58.278 & 60.629 & 51.823 & 58.302 & 39.408 & 39.019 & 42.784 \\
\hline 8/26/11 20:00 & 58.261 & 60.620 & 51.818 & 58.285 & 39.396 & 39.005 & 42.773 \\
\hline 8/27/11 0:00 & 58.275 & 60.627 & 51.834 & 58.309 & 39.408 & 39.015 & 42.784 \\
\hline $8 / 27 / 114: 00$ & 58.263 & 60.610 & 51.813 & 58.290 & 39.403 & 39.005 & 42.770 \\
\hline 8/27/11 8:00 & 58.261 & 60.615 & 51.827 & 58.302 & 39.405 & 39.008 & 42.770 \\
\hline $8 / 27 / 11$ 12:00 & 58.268 & 60.622 & 51.841 & 58.318 & 39.415 & 39.015 & 42.779 \\
\hline 8/27/11 16:00 & 58.252 & 60.610 & 51.804 & 58.285 & 39.405 & 39.001 & 42.761 \\
\hline 8/27/11 20:00 & 58.242 & 60.599 & 51.802 & 58.274 & 39.398 & 38.991 & 42.754 \\
\hline 8/28/11 0:00 & 58.249 & 60.601 & 51.818 & 58.292 & 39.408 & 38.998 & 42.761 \\
\hline 8/28/11 4:00 & 58.240 & 60.582 & 51.793 & 58.271 & 39.398 & 38.989 & 42.745 \\
\hline 8/28/11 8:00 & 58.233 & 60.582 & 51.797 & 58.269 & 39.394 & 38.984 & 42.740 \\
\hline $8 / 28 / 11$ 12:00 & 58.242 & 60.596 & 51.820 & 58.295 & 39.410 & 38.996 & 42.754 \\
\hline 8/28/11 16:00 & 58.228 & 60.575 & 51.776 & 58.260 & 39.394 & 38.982 & 42.731 \\
\hline 8/28/11 20:00 & 58.214 & 60.568 & 51.774 & 58.248 & 39.384 & 38.968 & 42.724 \\
\hline 8/29/11 0:00 & 58.233 & 60.582 & 51.811 & 58.283 & 39.403 & 38.984 & 42.742 \\
\hline $8 / 29 / 114: 00$ & 58.230 & 60.575 & 51.790 & 58.269 & 39.405 & 38.982 & 42.735 \\
\hline 8/29/11 8:00 & 58.223 & 60.571 & 51.800 & 58.267 & 39.401 & 38.977 & 42.731 \\
\hline 8/29/11 12:00 & 58.233 & 60.592 & 51.820 & 58.295 & 39.417 & 38.993 & 42.747 \\
\hline 8/29/11 16:00 & 58.226 & 60.583 & 51.795 & 58.271 & 39.413 & 38.984 & 42.738 \\
\hline 8/29/11 20:00 & 58.214 & 60.571 & 51.786 & 58.262 & 39.405 & 38.975 & 42.728 \\
\hline 8/30/11 0:00 & 58.223 & 60.582 & 51.809 & 58.283 & 39.417 & 38.984 & 42.740 \\
\hline $8 / 30 / 114: 00$ & 58.219 & 60.575 & 51.793 & 58.267 & 39.415 & 38.979 & 42.733 \\
\hline 8/30/11 8:00 & 58.204 & 60.559 & 51.797 & 58.250 & 39.403 & 38.968 & 42.724 \\
\hline $8 / 30 / 11$ 12:00 & 58.174 & 60.547 & 51.772 & 58.246 & 39.375 & 38.923 & 42.700 \\
\hline 8/30/11 16:00 & 58.176 & 60.538 & 51.758 & 58.238 & 39.375 & 38.930 & 42.698 \\
\hline 8/30/11 20:00 & 58.169 & 60.538 & 51.755 & 58.227 & 39.366 & 38.928 & 42.693 \\
\hline 8/31/11 0:00 & 58.183 & 60.550 & 51.786 & 58.255 & 39.387 & 38.944 & 42.710 \\
\hline 8/31/11 4:00 & 58.188 & 60.550 & 51.779 & 58.253 & 39.387 & 38.946 & 42.707 \\
\hline 8/31/11 8:00 & 58.178 & 60.538 & 51.765 & 58.236 & 39.377 & 38.939 & 42.698 \\
\hline 8/31/11 12:00 & 58.181 & 60.550 & 51.786 & 58.260 & 39.387 & 38.949 & 42.707 \\
\hline 8/31/11 16:00 & 58.188 & 60.554 & 51.786 & 58.260 & 39.396 & 38.954 & 42.714 \\
\hline 8/31/11 20:00 & 58.183 & 60.552 & 51.774 & 58.248 & 39.391 & 38.946 & 42.710 \\
\hline 9/1/11 0:00 & 58.193 & 60.564 & 51.800 & 58.271 & 39.403 & 38.958 & 42.724 \\
\hline 9/1/11 4:00 & 58.202 & 60.568 & 51.804 & 58.283 & 39.410 & 38.968 & 42.728 \\
\hline 9/1/11 8:00 & 58.195 & 60.561 & 51.788 & 58.264 & 39.408 & 38.963 & 42.721 \\
\hline 9/1/11 12:00 & 58.193 & 60.566 & 51.797 & 58.274 & 39.413 & 38.965 & 42.724 \\
\hline 9/1/11 16:00 & 58.197 & 60.573 & 51.800 & 58.276 & 39.420 & 38.968 & 42.726 \\
\hline 9/1/11 20:00 & 58.197 & 60.568 & 51.795 & 58.271 & 39.422 & 38.968 & 42.728 \\
\hline 9/2/11 0:00 & 58.209 & 60.580 & 51.825 & 58.295 & 39.438 & 38.982 & 42.742 \\
\hline 9/2/11 4:00 & 58.223 & 60.589 & 51.839 & 58.313 & 39.452 & 38.993 & 42.754 \\
\hline 9/2/11 8:00 & 58.228 & 60.594 & 51.832 & 58.309 & 39.457 & 38.996 & 42.754 \\
\hline 9/2/11 12:00 & 58.223 & 60.599 & 51.837 & 58.313 & 39.459 & 38.998 & 42.756 \\
\hline 9/2/11 16:00 & 58.228 & 60.603 & 51.841 & 58.318 & 39.467 & 39.005 & 42.766 \\
\hline 9/2/11 20:00 & 58.230 & 60.606 & 51.837 & 58.311 & 39.469 & 39.005 & 42.761 \\
\hline 9/3/11 0:00 & 58.237 & 60.613 & 51.855 & 58.327 & 39.481 & 39.015 & 42.775 \\
\hline
\end{tabular}

TABLE S1.3 Page 47 of 58 


\begin{tabular}{|c|c|c|c|c|c|c|c|}
\hline \multirow[b]{2}{*}{ Date and Time } & \multicolumn{7}{|c|}{ Water Level (ft below reference point) } \\
\hline & SB43D & SB44D & SB45D & SB46D & SB47D & SB48D & SB49D \\
\hline $9 / 3 / 114: 00$ & 58.252 & 60.622 & 51.869 & 58.344 & 39.499 & 39.031 & 42.786 \\
\hline 9/3/11 8:00 & 58.254 & 60.624 & 51.862 & 58.339 & 39.499 & 39.033 & 42.784 \\
\hline 9/3/11 12:00 & 58.256 & 60.631 & 51.869 & 58.348 & 39.506 & 39.038 & 42.791 \\
\hline 9/3/11 16:00 & 58.256 & 60.631 & 51.874 & 58.346 & 39.504 & 39.038 & 42.793 \\
\hline 9/3/11 20:00 & 58.256 & 60.610 & 51.862 & 58.337 & 39.495 & 39.019 & 42.770 \\
\hline 9/4/11 0:00 & 58.245 & 60.634 & 51.881 & 58.353 & 39.499 & 39.019 & 42.796 \\
\hline $9 / 4 / 114: 00$ & 58.261 & 60.650 & 51.902 & 58.374 & 39.516 & 39.043 & 42.812 \\
\hline 9/4/11 8:00 & 58.280 & 60.657 & 51.918 & 58.393 & 39.535 & 39.059 & 42.824 \\
\hline 9/4/11 12:00 & 58.278 & 60.657 & 51.906 & 58.381 & 39.535 & 39.062 & 42.824 \\
\hline 9/4/11 16:00 & 58.275 & 60.655 & 51.909 & 58.379 & 39.539 & 39.064 & 42.828 \\
\hline 9/4/11 20:00 & 58.289 & 60.673 & 51.916 & 58.393 & 39.546 & 39.073 & 42.835 \\
\hline 9/5/11 0:00 & 58.297 & 60.680 & 51.930 & 58.400 & 39.558 & 39.083 & 42.845 \\
\hline 9/5/11 4:00 & 58.306 & 60.685 & 51.941 & 58.412 & 39.565 & 39.095 & 42.852 \\
\hline 9/5/11 8:00 & 58.320 & 60.697 & 51.957 & 58.428 & 39.565 & 39.106 & 42.866 \\
\hline 9/5/11 12:00 & 58.320 & 60.699 & 51.946 & 58.423 & 39.636 & 39.109 & 42.863 \\
\hline 9/5/11 16:00 & 58.311 & 60.697 & 51.932 & 58.407 & 39.579 & 39.102 & 42.859 \\
\hline 9/5/11 20:00 & 58.320 & 60.701 & 51.941 & 58.414 & 39.584 & 39.106 & 42.863 \\
\hline 9/6/11 0:00 & 58.327 & 60.708 & 51.950 & 58.426 & 39.598 & 39.116 & 42.873 \\
\hline 9/6/11 4:00 & 58.337 & 60.713 & 51.962 & 58.437 & 39.600 & 39.127 & 42.879 \\
\hline 9/6/11 8:00 & 58.353 & 60.729 & 51.985 & 58.458 & 39.598 & 39.142 & 42.893 \\
\hline 9/6/11 12:00 & 58.358 & 60.736 & 51.981 & 58.461 & 39.687 & 39.149 & 42.900 \\
\hline 9/6/11 16:00 & 58.351 & 60.732 & 51.962 & 58.444 & 39.619 & 39.139 & 42.893 \\
\hline 9/6/11 20:00 & 58.358 & 60.736 & 51.978 & 58.454 & 39.633 & 39.146 & 42.903 \\
\hline 9/7/11 0:00 & 58.370 & 60.743 & 51.990 & 58.463 & 39.645 & 39.158 & 42.912 \\
\hline 9/7/11 4:00 & 58.375 & 60.743 & 51.995 & 58.470 & 39.645 & 39.165 & 42.914 \\
\hline 9/7/11 8:00 & 58.394 & 60.769 & 52.025 & 58.498 & 39.577 & 39.184 & 42.935 \\
\hline 9/7/11 12:00 & 58.403 & 60.773 & 52.022 & 58.503 & 39.687 & 39.193 & 42.940 \\
\hline 9/7/11 16:00 & 58.394 & 60.769 & 52.004 & 58.479 & 39.671 & 39.186 & 42.933 \\
\hline 9/7/11 20:00 & 58.398 & 60.776 & 52.015 & 58.489 & 39.683 & 39.193 & 42.940 \\
\hline 9/8/11 0:00 & 58.415 & 60.783 & 52.027 & 58.505 & 39.694 & 39.205 & 42.949 \\
\hline 9/8/11 4:00 & 58.415 & 60.780 & 52.022 & 58.498 & 39.699 & 39.207 & 42.947 \\
\hline 9/8/11 8:00 & 58.427 & 60.794 & 52.046 & 58.519 & 39.709 & 39.219 & 42.961 \\
\hline 9/8/11 12:00 & 58.441 & 60.806 & 52.053 & 58.528 & 39.727 & 39.231 & 42.970 \\
\hline 9/8/11 16:00 & 58.427 & 60.794 & 52.020 & 58.498 & 39.709 & 39.219 & 42.956 \\
\hline 9/8/11 20:00 & 58.429 & 60.794 & 52.025 & 58.503 & 39.713 & 39.228 & 42.956 \\
\hline 9/9/11 0:00 & 58.441 & 60.801 & 52.034 & 58.512 & 39.727 & 39.238 & 42.963 \\
\hline 9/9/11 4:00 & 58.436 & 60.794 & 52.022 & 58.503 & 39.720 & 39.236 & 42.959 \\
\hline 9/9/11 8:00 & 58.446 & 60.801 & 52.046 & 58.517 & 39.737 & 39.245 & 42.966 \\
\hline 9/9/11 12:00 & 58.460 & 60.811 & 52.053 & 58.533 & 39.744 & 39.257 & 42.979 \\
\hline 9/9/11 16:00 & 58.446 & 60.797 & 52.022 & 58.503 & 39.734 & 39.245 & 42.966 \\
\hline 9/9/11 20:00 & 58.453 & 60.806 & 52.039 & 58.517 & 39.741 & 39.250 & 42.972 \\
\hline 9/10/11 0:00 & 58.469 & 60.820 & 52.060 & 58.535 & 39.760 & 39.268 & 42.986 \\
\hline 9/10/11 4:00 & 58.469 & 60.815 & 52.046 & 58.524 & 39.758 & 39.266 & 42.977 \\
\hline 9/10/11 8:00 & 58.479 & 60.827 & 52.073 & 58.545 & 39.774 & 39.278 & 42.993 \\
\hline 9/10/11 12:00 & 58.500 & 60.846 & 52.092 & 58.568 & 39.788 & 39.297 & 43.012 \\
\hline 9/10/11 16:00 & 58.495 & 60.844 & 52.069 & 58.550 & 39.786 & 39.292 & 43.005 \\
\hline 9/10/11 20:00 & 58.495 & 60.846 & 52.078 & 58.557 & 39.791 & 39.294 & 43.010 \\
\hline 9/11/11 0:00 & 58.512 & 60.860 & 52.097 & 58.575 & 39.814 & 39.311 & 43.024 \\
\hline 9/11/11 4:00 & 58.514 & 60.853 & 52.090 & 58.564 & 39.807 & 39.313 & 43.019 \\
\hline 9/11/11 8:00 & 58.521 & 60.862 & 52.104 & 58.578 & 39.819 & 39.320 & 43.028 \\
\hline 9/11/11 12:00 & 58.540 & 60.883 & 52.127 & 58.606 & 39.840 & 39.339 & 43.047 \\
\hline 9/11/11 16:00 & 58.540 & 60.878 & 52.101 & 58.587 & 39.835 & 39.337 & 43.040 \\
\hline 9/11/11 20:00 & 58.538 & 60.878 & 52.113 & 58.585 & 39.845 & 39.337 & 43.042 \\
\hline 9/12/11 0:00 & 58.554 & 60.890 & 52.131 & 58.606 & 39.857 & 39.353 & 43.056 \\
\hline
\end{tabular}

TABLE S1.3 Page 48 of 58 


\begin{tabular}{|c|c|c|c|c|c|c|c|}
\hline \multirow[b]{2}{*}{ Date and Time } & \multicolumn{7}{|c|}{ Water Level (ft below reference point) } \\
\hline & SB43D & SB44D & SB45D & SB46D & SB47D & SB48D & SB49D \\
\hline $9 / 12 / 114: 00$ & 58.557 & 60.878 & 52.113 & 58.594 & 39.861 & 39.353 & 43.049 \\
\hline 9/12/11 8:00 & 58.557 & 60.885 & 52.120 & 58.594 & 39.859 & 39.355 & 43.052 \\
\hline 9/12/11 12:00 & 58.569 & 60.899 & 52.134 & 58.615 & 39.875 & 39.367 & 43.059 \\
\hline 9/12/11 16:00 & 58.566 & 60.888 & 52.111 & 58.596 & 39.871 & 39.358 & 43.054 \\
\hline 9/12/11 20:00 & 58.569 & 60.885 & 52.118 & 58.594 & 39.873 & 39.355 & 43.052 \\
\hline 9/13/11 0:00 & 58.595 & 60.899 & 52.143 & 58.622 & 39.892 & 39.374 & 43.065 \\
\hline 9/13/11 4:00 & 58.604 & 60.897 & 52.138 & 58.617 & 39.901 & 39.379 & 43.065 \\
\hline 9/13/11 8:00 & 58.613 & 60.902 & 52.155 & 58.624 & 39.908 & 39.393 & 43.075 \\
\hline 9/13/11 12:00 & 58.632 & 60.927 & 52.180 & 58.655 & 39.932 & 39.419 & 43.096 \\
\hline 9/13/11 16:00 & 58.635 & 60.930 & 52.169 & 58.645 & 39.936 & 39.424 & 43.100 \\
\hline 9/13/11 20:00 & 58.632 & 60.937 & 52.171 & 58.643 & 39.936 & 39.426 & 43.103 \\
\hline 9/14/11 0:00 & 58.647 & 60.953 & 52.194 & 58.669 & 39.960 & 39.445 & 43.117 \\
\hline 9/14/11 4:00 & 58.661 & 60.958 & 52.192 & 58.676 & 39.969 & 39.456 & 43.126 \\
\hline 9/14/11 8:00 & 58.654 & 60.953 & 52.196 & 58.666 & 39.969 & 39.452 & 43.121 \\
\hline 9/14/11 12:00 & 58.670 & 60.971 & 52.224 & 58.692 & 39.986 & 39.473 & 43.142 \\
\hline 9/14/11 16:00 & 58.680 & 60.981 & 52.220 & 58.699 & 40.000 & 39.482 & 43.149 \\
\hline 9/14/11 20:00 & 58.687 & 60.990 & 52.229 & 58.699 & 40.002 & 39.487 & 43.156 \\
\hline 9/15/11 0:00 & 58.710 & 61.011 & 52.261 & 58.737 & 40.030 & 39.513 & 43.179 \\
\hline 9/15/11 4:00 & 58.718 & 61.016 & 52.259 & 58.732 & 40.037 & 39.520 & 43.184 \\
\hline 9/15/11 8:00 & 58.720 & 61.016 & 52.259 & 58.730 & 40.042 & 39.522 & 43.186 \\
\hline 9/15/11 12:00 & 58.729 & 61.027 & 52.275 & 58.746 & 40.056 & 39.536 & 43.198 \\
\hline 9/15/11 16:00 & 58.732 & 61.025 & 52.257 & 58.737 & 40.059 & 39.536 & 43.193 \\
\hline 9/15/11 20:00 & 58.727 & 61.020 & 52.245 & 58.720 & 40.049 & 39.529 & 43.186 \\
\hline 9/16/11 0:00 & 58.736 & 61.032 & 52.271 & 58.739 & 40.061 & 39.543 & 43.196 \\
\hline 9/16/11 4:00 & 58.748 & 61.034 & 52.268 & 58.744 & 40.075 & 39.548 & 43.200 \\
\hline 9/16/11 8:00 & 58.744 & 61.020 & 52.252 & 58.727 & 40.066 & 39.543 & 43.189 \\
\hline 9/16/11 12:00 & 58.734 & 61.020 & 52.250 & 58.727 & 40.061 & 39.536 & 43.182 \\
\hline 9/16/11 16:00 & 58.736 & 61.020 & 52.245 & 58.725 & 40.061 & 39.536 & 43.184 \\
\hline 9/16/11 20:00 & 58.736 & 61.018 & 52.245 & 58.716 & 40.063 & 39.536 & 43.182 \\
\hline 9/17/11 0:00 & 58.746 & 61.027 & 52.264 & 58.737 & 40.068 & 39.548 & 43.191 \\
\hline 9/17/11 4:00 & 58.755 & 61.030 & 52.264 & 58.741 & 40.077 & 39.555 & 43.198 \\
\hline 9/17/11 8:00 & 58.753 & 61.027 & 52.250 & 58.730 & 40.070 & 39.553 & 43.193 \\
\hline 9/17/11 12:00 & 58.755 & 61.032 & 52.257 & 58.734 & 40.073 & 39.553 & 43.196 \\
\hline 9/17/11 16:00 & 58.751 & 61.027 & 52.252 & 58.727 & 40.070 & 39.550 & 43.193 \\
\hline 9/17/11 20:00 & 58.748 & 61.023 & 52.243 & 58.718 & 40.066 & 39.548 & 43.189 \\
\hline 9/18/11 0:00 & 58.755 & 61.035 & 52.261 & 58.737 & 40.073 & 39.555 & 43.198 \\
\hline 9/18/11 4:00 & 58.767 & 61.039 & 52.273 & 58.744 & 40.082 & 39.562 & 43.203 \\
\hline 9/18/11 8:00 & 58.767 & 61.034 & 52.259 & 58.741 & 40.077 & 39.562 & 43.200 \\
\hline 9/18/11 12:00 & 58.760 & 61.034 & 52.259 & 58.737 & 40.070 & 39.557 & 43.198 \\
\hline 9/18/11 16:00 & 58.762 & 61.037 & 52.261 & 58.739 & 40.077 & 39.560 & 43.200 \\
\hline 9/18/11 20:00 & 58.748 & 61.032 & 52.255 & 58.732 & 40.063 & 39.536 & 43.191 \\
\hline 9/19/11 0:00 & 58.755 & 61.044 & 52.271 & 58.744 & 40.073 & 39.548 & 43.205 \\
\hline 9/19/11 4:00 & 58.770 & 61.051 & 52.285 & 58.760 & 40.082 & 39.562 & 43.214 \\
\hline 9/19/11 8:00 & 58.779 & 61.051 & 52.287 & 58.762 & 40.091 & 39.572 & 43.219 \\
\hline 9/19/11 12:00 & 58.779 & 61.053 & 52.285 & 58.762 & 40.091 & 39.574 & 43.219 \\
\hline 9/19/11 16:00 & 58.777 & 61.053 & 52.275 & 58.755 & 40.091 & 39.572 & 43.219 \\
\hline 9/19/11 20:00 & 58.779 & 61.055 & 52.278 & 58.755 & 40.094 & 39.574 & 43.221 \\
\hline 9/20/11 0:00 & 58.784 & 61.058 & 52.282 & 58.760 & 40.101 & 39.579 & 43.224 \\
\hline 9/20/11 4:00 & 58.791 & 61.062 & 52.294 & 58.772 & 40.113 & 39.588 & 43.228 \\
\hline 9/20/11 8:00 & 58.800 & 61.067 & 52.296 & 58.776 & 40.117 & 39.595 & 43.233 \\
\hline 9/20/11 12:00 & 58.800 & 61.067 & 52.289 & 58.774 & 40.120 & 39.597 & 43.231 \\
\hline 9/20/11 16:00 & 58.798 & 61.072 & 52.289 & 58.769 & 40.120 & 39.597 & 43.233 \\
\hline 9/20/11 20:00 & 58.815 & 61.079 & 52.313 & 58.788 & 40.134 & 39.609 & 43.249 \\
\hline 9/21/11 0:00 & 58.829 & 61.093 & 52.329 & 58.802 & 40.148 & 39.621 & 43.258 \\
\hline
\end{tabular}

TABLE S1.3 Page 49 of 58 


\begin{tabular}{|c|c|c|c|c|c|c|c|}
\hline \multirow[b]{2}{*}{ Date and Time } & \multicolumn{7}{|c|}{ Water Level (ft below reference point) } \\
\hline & SB43D & SB44D & SB45D & SB46D & SB47D & SB48D & SB49D \\
\hline 9/21/11 4:00 & 58.841 & 61.100 & 52.343 & 58.816 & 40.164 & 39.635 & 43.268 \\
\hline 9/21/11 8:00 & 58.855 & 61.114 & 52.359 & 58.830 & 40.176 & 39.647 & 43.282 \\
\hline 9/21/11 12:00 & 58.859 & 61.116 & 52.350 & 58.825 & 40.185 & 39.654 & 43.286 \\
\hline 9/21/11 16:00 & 58.857 & 61.118 & 52.345 & 58.825 & 40.183 & 39.654 & 43.284 \\
\hline 9/21/11 20:00 & 58.871 & 61.130 & 52.364 & 58.837 & 40.200 & 39.666 & 43.296 \\
\hline 9/22/11 0:00 & 58.881 & 61.132 & 52.368 & 58.844 & 40.207 & 39.675 & 43.303 \\
\hline 9/22/11 4:00 & 58.883 & 61.132 & 52.368 & 58.842 & 40.214 & 39.677 & 43.305 \\
\hline 9/22/11 8:00 & 58.902 & 61.153 & 52.398 & 58.872 & 40.230 & 39.698 & 43.321 \\
\hline 9/22/11 12:00 & 58.907 & 61.156 & 52.387 & 58.865 & 40.232 & 39.701 & 43.324 \\
\hline 9/22/11 16:00 & 58.895 & 61.144 & 52.366 & 58.844 & 40.228 & 39.689 & 43.310 \\
\hline 9/22/11 20:00 & 58.904 & 61.158 & 52.385 & 58.861 & 40.239 & 39.701 & 43.321 \\
\hline 9/23/11 0:00 & 58.919 & 61.163 & 52.394 & 58.870 & 40.249 & 39.713 & 43.328 \\
\hline 9/23/11 4:00 & 58.916 & 61.163 & 52.389 & 58.861 & 40.249 & 39.713 & 43.326 \\
\hline 9/23/11 8:00 & 58.933 & 61.172 & 52.415 & 58.886 & 40.263 & 39.727 & 43.340 \\
\hline 9/23/11 12:00 & 58.935 & & 52.410 & 58.891 & 40.270 & 39.734 & 43.347 \\
\hline 9/23/11 16:00 & 58.923 & 61.231 & 52.378 & 58.856 & 40.051 & 39.717 & 43.333 \\
\hline 9/23/11 20:00 & 58.930 & 61.240 & 52.394 & Removed $^{b}$ & 40.058 & 39.724 & 43.342 \\
\hline 9/24/11 0:00 & 58.942 & 61.243 & 52.398 & & 40.075 & 39.736 & 43.349 \\
\hline 9/24/11 4:00 & 58.937 & 61.231 & 52.389 & & 40.070 & 39.729 & 43.342 \\
\hline 9/24/11 8:00 & 58.949 & 61.247 & 52.419 & & 40.082 & 39.745 & 43.354 \\
\hline 9/24/11 12:00 & 58.961 & 61.254 & 52.417 & & 40.094 & 39.755 & 43.363 \\
\hline 9/24/11 16:00 & 58.949 & 61.245 & 52.391 & & 40.079 & 39.741 & 43.351 \\
\hline 9/24/11 20:00 & 58.961 & 61.257 & 52.419 & & 40.096 & 39.750 & 43.365 \\
\hline 9/25/11 0:00 & 58.978 & 61.270 & 52.429 & & 40.108 & 39.767 & 43.375 \\
\hline 9/25/11 4:00 & 58.971 & 61.261 & 52.410 & & 40.103 & 39.762 & 43.365 \\
\hline 9/25/11 8:00 & 58.980 & 61.268 & 52.433 & & 40.115 & 39.774 & 43.375 \\
\hline 9/25/11 12:00 & 58.994 & 61.280 & 52.443 & & 40.131 & 39.788 & 43.386 \\
\hline 9/25/11 16:00 & 58.985 & 61.273 & 52.417 & & 40.117 & 39.774 & 43.377 \\
\hline 9/25/11 20:00 & 58.990 & 61.275 & 52.440 & & 40.124 & 39.781 & 43.386 \\
\hline 9/26/11 0:00 & 59.006 & 61.289 & 52.452 & & 40.141 & 39.799 & 43.396 \\
\hline 9/26/11 4:00 & 59.001 & 61.280 & 52.436 & & 40.136 & 39.792 & 43.389 \\
\hline 9/26/11 8:00 & 59.006 & 61.291 & 52.452 & & 40.143 & 39.799 & 43.396 \\
\hline 9/26/11 12:00 & 59.027 & 61.310 & 52.475 & & 40.166 & 39.821 & 43.414 \\
\hline 9/26/11 16:00 & 59.018 & 61.298 & 52.443 & & 40.157 & 39.809 & 43.403 \\
\hline 9/26/11 20:00 & 59.020 & 61.301 & 52.463 & & 40.159 & 39.809 & 43.410 \\
\hline 9/27/11 0:00 & 59.044 & 61.319 & 52.489 & & 40.180 & 39.832 & 43.428 \\
\hline 9/27/11 4:00 & 59.042 & 61.315 & 52.470 & & 40.176 & 39.832 & 43.421 \\
\hline 9/27/11 8:00 & 59.046 & 61.319 & 52.484 & & 40.188 & 39.839 & 43.428 \\
\hline 9/27/11 12:00 & 59.063 & 61.340 & 52.510 & & 40.202 & 39.856 & 43.449 \\
\hline 9/27/11 16:00 & 59.065 & 61.338 & 52.491 & & 40.202 & 39.856 & 43.442 \\
\hline 9/27/11 20:00 & 59.065 & 61.341 & 52.496 & & 40.206 & 39.856 & 43.447 \\
\hline 9/28/11 0:00 & 59.084 & 61.357 & 52.524 & & 40.227 & 39.875 & 43.461 \\
\hline 9/28/11 4:00 & 59.089 & 61.352 & 52.505 & & 40.227 & 39.875 & 43.456 \\
\hline 9/28/11 8:00 & 59.082 & 61.345 & 52.505 & & 40.223 & 39.870 & 43.451 \\
\hline 9/28/11 12:00 & 59.091 & 61.357 & 52.524 & & 40.239 & 39.882 & 43.465 \\
\hline 9/28/11 16:00 & 59.098 & 61.364 & 52.515 & & 40.242 & 39.886 & 43.468 \\
\hline 9/28/11 20:00 & 59.098 & 61.366 & 52.519 & & 40.239 & 39.886 & 43.468 \\
\hline 9/29/11 0:00 & 59.122 & 61.385 & 52.556 & & 40.265 & 39.910 & 43.489 \\
\hline 9/29/11 4:00 & 59.127 & 61.385 & 52.542 & & 40.270 & 39.915 & 43.489 \\
\hline 9/29/11 8:00 & 59.131 & 61.382 & 52.547 & & 40.272 & 39.917 & 43.493 \\
\hline 9/29/11 12:00 & 59.150 & 61.408 & 52.584 & & 40.300 & 39.943 & 43.517 \\
\hline 9/29/11 16:00 & 59.165 & 61.420 & 52.584 & & 40.305 & 39.950 & 43.528 \\
\hline 9/29/11 20:00 & 59.167 & 61.422 & 52.591 & & 40.310 & 39.955 & 43.531 \\
\hline 9/30/11 0:00 & 59.193 & 61.448 & 52.626 & & 40.338 & 39.978 & 43.554 \\
\hline
\end{tabular}

TABLE S1.3 Page 50 of 58 


\begin{tabular}{|c|c|c|c|c|c|c|c|}
\hline \multirow[b]{2}{*}{ Date and Time } & \multicolumn{7}{|c|}{ Water Level (ft below reference point) } \\
\hline & SB43D & SB44D & SB45D & SB46D & SB47D & SB48D & SB49D \\
\hline 9/30/11 4:00 & 59.209 & 61.459 & 52.633 & & 40.352 & 39.995 & 43.565 \\
\hline 9/30/11 8:00 & 59.207 & 61.457 & 52.621 & & 40.354 & 39.995 & 43.563 \\
\hline 9/30/11 12:00 & 59.207 & 61.459 & 52.626 & & 40.359 & 39.999 & 43.568 \\
\hline 9/30/11 16:00 & 59.209 & 61.459 & 52.617 & & 40.361 & 39.999 & 43.565 \\
\hline 9/30/11 20:00 & 59.209 & 61.459 & 52.614 & & 40.357 & 39.999 & 43.561 \\
\hline 10/1/11 0:00 & 59.228 & 61.473 & 52.647 & & 40.378 & 40.018 & 43.582 \\
\hline 10/1/11 4:00 & 59.250 & 61.489 & 52.661 & & 40.397 & 40.034 & 43.593 \\
\hline 10/1/11 8:00 & 59.252 & 61.494 & 52.654 & & 40.404 & 40.039 & 43.596 \\
\hline 10/1/11 12:00 & 59.254 & 61.494 & 52.658 & & 40.406 & 40.042 & 43.600 \\
\hline 10/1/11 16:00 & 59.254 & 61.494 & 52.654 & & 40.406 & 40.042 & 43.600 \\
\hline 10/1/11 20:00 & 59.257 & 61.494 & 52.649 & & 40.408 & 40.042 & 43.600 \\
\hline 10/2/11 0:00 & 59.269 & 61.506 & 52.672 & & 40.422 & 40.056 & 43.607 \\
\hline $10 / 2 / 114: 00$ & 59.283 & 61.517 & 52.684 & & 40.434 & 40.070 & 43.624 \\
\hline 10/2/11 8:00 & 59.290 & 61.517 & 52.684 & & 40.444 & 40.074 & 43.624 \\
\hline 10/2/11 12:00 & 59.288 & 61.517 & 52.677 & & 40.441 & 40.072 & 43.621 \\
\hline 10/2/11 16:00 & 59.283 & 61.517 & 52.668 & & 40.437 & 40.070 & 43.619 \\
\hline 10/2/11 20:00 & 59.290 & 61.522 & 52.677 & & 40.444 & 40.074 & 43.624 \\
\hline 10/3/11 0:00 & 59.299 & 61.529 & 52.691 & & 40.453 & 40.084 & 43.631 \\
\hline 10/3/11 4:00 & 59.314 & 61.536 & 52.707 & & 40.467 & 40.098 & 43.642 \\
\hline 10/3/11 8:00 & 59.323 & 61.545 & 52.707 & & 40.474 & 40.105 & 43.647 \\
\hline 10/3/11 12:00 & 59.323 & 61.545 & 52.703 & & 40.474 & 40.105 & 43.649 \\
\hline 10/3/11 16:00 & 59.318 & 61.545 & 52.698 & & 40.472 & 40.103 & 43.644 \\
\hline 10/3/11 20:00 & 59.328 & 61.548 & 52.710 & & 40.484 & 40.110 & 43.651 \\
\hline 10/4/11 0:00 & 59.337 & 61.562 & 52.721 & & 40.493 & 40.119 & 43.661 \\
\hline 10/4/11 4:00 & 59.347 & 61.564 & 52.730 & & 40.500 & 40.128 & 43.668 \\
\hline 10/4/11 8:00 & 59.358 & 61.571 & 52.742 & & 40.512 & 40.140 & 43.682 \\
\hline 10/4/11 12:00 & 59.358 & 61.576 & 52.728 & & 40.512 & 40.138 & 43.675 \\
\hline 10/4/11 16:00 & 59.349 & 61.573 & 52.723 & & 40.509 & 40.136 & 43.672 \\
\hline 10/4/11 20:00 & 59.363 & 61.581 & 52.740 & & 40.516 & 40.145 & 43.682 \\
\hline 10/5/11 0:00 & 59.373 & 61.583 & 52.742 & & 40.528 & 40.154 & 43.686 \\
\hline 10/5/11 4:00 & 59.377 & 61.587 & 52.754 & & 40.535 & 40.161 & 43.693 \\
\hline 10/5/11 8:00 & 59.392 & 61.604 & 52.768 & & 40.549 & 40.173 & 43.705 \\
\hline 10/5/11 12:00 & 59.394 & 61.604 & 52.758 & & 40.547 & 40.173 & 43.705 \\
\hline 10/5/11 16:00 & 59.387 & 61.592 & 52.744 & & 40.540 & 40.166 & 43.698 \\
\hline 10/5/11 20:00 & 59.394 & 61.604 & 52.763 & & 40.549 & 40.175 & 43.705 \\
\hline 10/6/11 0:00 & 59.403 & 61.611 & 52.768 & & 40.559 & 40.185 & 43.712 \\
\hline 10/6/11 4:00 & 59.403 & 61.613 & 52.768 & & 40.563 & 40.187 & 43.712 \\
\hline 10/6/11 8:00 & 59.418 & 61.618 & 52.786 & & 40.573 & 40.199 & 43.724 \\
\hline 10/6/11 12:00 & 59.425 & 61.627 & 52.777 & & 40.577 & 40.201 & 43.726 \\
\hline 10/6/11 16:00 & 59.413 & 61.615 & 52.763 & & 40.566 & 40.192 & 43.714 \\
\hline 10/6/11 20:00 & 59.422 & 61.625 & 52.786 & & 40.575 & 40.201 & 43.726 \\
\hline 10/7/11 0:00 & 59.437 & 61.636 & 52.786 & & 40.587 & 40.213 & 43.733 \\
\hline 10/7/11 4:00 & 59.437 & 61.634 & 52.793 & & 40.592 & 40.213 & 43.737 \\
\hline 10/7/11 8:00 & 59.448 & 61.648 & 52.819 & & 40.599 & 40.232 & 43.751 \\
\hline 10/7/11 12:00 & 59.463 & 61.653 & 52.816 & & 40.613 & 40.237 & 43.758 \\
\hline 10/7/11 16:00 & 59.453 & 61.648 & 52.800 & & 40.603 & 40.227 & 43.754 \\
\hline 10/7/11 20:00 & 59.460 & 61.664 & 52.826 & & 40.613 & 40.239 & 43.763 \\
\hline 10/8/11 0:00 & 59.477 & 61.676 & 52.835 & & 40.627 & 40.253 & 43.777 \\
\hline 10/8/11 4:00 & 59.477 & 61.674 & 52.826 & & 40.622 & 40.251 & 43.772 \\
\hline 10/8/11 8:00 & 59.486 & 61.683 & 52.853 & & 40.629 & 40.262 & 43.786 \\
\hline 10/8/11 12:00 & 59.500 & 61.697 & 52.863 & & 40.646 & 40.274 & 43.798 \\
\hline 10/8/11 16:00 & 59.489 & 61.690 & 52.837 & & 40.632 & 40.262 & 43.784 \\
\hline 10/8/11 20:00 & 59.500 & 61.697 & 52.867 & & 40.643 & 40.274 & 43.803 \\
\hline 10/9/11 0:00 & 59.517 & 61.715 & 52.879 & & 40.657 & 40.288 & 43.814 \\
\hline
\end{tabular}

TABLE S1.3 Page 51 of 58 


\begin{tabular}{|c|c|c|c|c|c|c|c|}
\hline \multirow[b]{2}{*}{ Date and Time } & \multicolumn{7}{|c|}{ Water Level (ft below reference point) } \\
\hline & SB43D & SB44D & SB45D & SB46D & SB47D & SB48D & SB49D \\
\hline 10/9/11 4:00 & 59.512 & 61.709 & 52.863 & & 40.653 & 40.286 & 43.810 \\
\hline 10/9/11 8:00 & 59.519 & 61.718 & 52.884 & & 40.664 & 40.293 & 43.819 \\
\hline $10 / 9 / 1112: 00$ & 59.531 & 61.727 & 52.886 & & 40.667 & 40.300 & 43.828 \\
\hline $10 / 9 / 1116: 00$ & 59.519 & 61.713 & 52.860 & & 40.653 & 40.284 & 43.814 \\
\hline $10 / 9 / 1120: 00$ & 59.519 & 61.725 & 52.884 & & 40.657 & 40.293 & 43.821 \\
\hline 10/10/11 0:00 & 59.538 & 61.734 & 52.898 & & 40.671 & 40.307 & 43.835 \\
\hline 10/10/11 4:00 & 59.531 & 61.727 & 52.874 & & 40.662 & 40.300 & 43.823 \\
\hline 10/10/11 8:00 & 59.526 & 61.720 & 52.886 & & 40.660 & 40.298 & 43.826 \\
\hline 10/10/11 12:00 & 59.541 & 61.739 & 52.900 & & 40.671 & 40.309 & 43.840 \\
\hline 10/10/11 16:00 & 59.531 & 61.730 & 52.874 & & 40.660 & 40.300 & 43.826 \\
\hline $10 / 10 / 1120: 00$ & 59.531 & 61.732 & 52.891 & & 40.662 & 40.302 & 43.833 \\
\hline 10/11/11 0:00 & 59.545 & 61.746 & 52.905 & & 40.671 & 40.314 & 43.844 \\
\hline $10 / 11 / 114: 00$ & 59.541 & 61.736 & 52.888 & & 40.664 & 40.307 & 43.835 \\
\hline $10 / 11 / 118: 00$ & 59.533 & 61.729 & 52.888 & & 40.657 & 40.302 & 43.833 \\
\hline $10 / 11 / 1112: 00$ & 59.541 & 61.741 & 52.902 & & 40.667 & 40.309 & 43.844 \\
\hline $10 / 11 / 1116: 00$ & 59.533 & 61.734 & 52.874 & & 40.655 & 40.298 & 43.833 \\
\hline 10/11/11 20:00 & 59.531 & 61.734 & 52.888 & & 40.657 & 40.298 & 43.835 \\
\hline 10/12/11 0:00 & 59.543 & 61.748 & 52.905 & & 40.662 & 40.309 & 43.847 \\
\hline $10 / 12 / 114: 00$ & 59.538 & 61.739 & 52.881 & & 40.655 & 40.302 & 43.837 \\
\hline $10 / 12 / 118: 00$ & 59.522 & 61.732 & 52.872 & & 40.639 & 40.291 & 43.828 \\
\hline $10 / 12 / 1112: 00$ & 59.538 & 61.741 & 52.900 & & 40.655 & 40.302 & 43.842 \\
\hline $10 / 12 / 1116: 00$ & 59.533 & 61.736 & 52.884 & & 40.650 & 40.298 & 43.835 \\
\hline 10/12/11 20:00 & 59.538 & 61.736 & 52.898 & & 40.650 & 40.298 & 43.842 \\
\hline 10/13/11 0:00 & 59.552 & 61.758 & 52.923 & & 40.667 & 40.314 & 43.858 \\
\hline $10 / 13 / 114: 00$ & 59.557 & 61.760 & 52.911 & & 40.664 & 40.316 & 43.858 \\
\hline $10 / 13 / 118: 00$ & 59.552 & 61.757 & 52.911 & & 40.662 & 40.314 & 43.858 \\
\hline $10 / 13 / 11$ 12:00 & 59.562 & 61.771 & 52.935 & & 40.676 & 40.326 & 43.872 \\
\hline 10/13/11 16:00 & 59.559 & 61.769 & 52.921 & & 40.676 & 40.324 & 43.870 \\
\hline $10 / 13 / 1120: 00$ & 59.562 & 61.774 & 52.930 & & 40.676 & 40.326 & 43.872 \\
\hline 10/14/11 0:00 & 59.583 & 61.790 & 52.958 & & 40.697 & 40.345 & 43.896 \\
\hline 10/14/11 4:00 & 59.590 & 61.797 & 52.951 & & 40.704 & 40.349 & 43.896 \\
\hline $10 / 14 / 118: 00$ & 59.590 & 61.795 & 52.956 & & 40.707 & 40.352 & 43.898 \\
\hline 10/14/11 12:00 & 59.602 & 61.811 & 52.981 & & 40.718 & 40.366 & 43.916 \\
\hline 10/14/11 16:00 & 59.609 & 61.816 & 52.974 & & 40.723 & 40.371 & 43.919 \\
\hline 10/14/11 20:00 & 59.612 & 61.825 & 52.981 & & 40.723 & 40.373 & 43.921 \\
\hline $10 / 15 / 110: 00$ & 59.630 & 61.839 & 53.009 & & 40.751 & 40.394 & 43.942 \\
\hline $10 / 15 / 114: 00$ & 59.640 & 61.846 & 53.009 & & 40.758 & 40.401 & 43.944 \\
\hline 10/15/11 8:00 & 59.635 & 61.837 & 52.995 & & 40.751 & 40.396 & 43.940 \\
\hline 10/15/11 12:00 & 59.635 & 61.844 & 53.004 & & 40.758 & 40.399 & 43.942 \\
\hline 10/15/11 16:00 & 59.628 & 61.834 & 52.976 & & 40.751 & 40.392 & 43.930 \\
\hline 10/15/11 20:00 & 59.621 & 61.830 & 52.970 & & 40.747 & 40.387 & 43.923 \\
\hline 10/16/11 0:00 & 59.628 & 61.834 & 52.988 & & 40.753 & 40.394 & 43.930 \\
\hline 10/16/11 4:00 & 59.635 & 61.837 & 52.988 & & 40.763 & 40.401 & 43.930 \\
\hline 10/16/11 8:00 & 59.642 & 61.846 & 53.016 & & 40.768 & 40.408 & 43.942 \\
\hline $10 / 16 / 1112: 00$ & 59.666 & 61.862 & 53.037 & & 40.791 & 40.429 & 43.968 \\
\hline $10 / 16 / 11$ 16:00 & 59.673 & 61.872 & 53.032 & & 40.798 & 40.434 & 43.970 \\
\hline $10 / 16 / 1120: 00$ & 59.680 & 61.876 & 53.035 & & 40.810 & 40.439 & 43.975 \\
\hline 10/17/11 0:00 & 59.685 & 61.883 & 53.048 & & 40.810 & 40.450 & 43.982 \\
\hline 10/17/11 4:00 & 59.697 & 61.890 & 53.053 & & 40.822 & 40.457 & 43.991 \\
\hline 10/17/11 8:00 & 59.692 & 61.886 & 53.037 & & 40.815 & 40.455 & 43.982 \\
\hline 10/17/11 12:00 & 59.692 & 61.883 & 53.039 & & 40.827 & 40.455 & 43.984 \\
\hline 10/17/11 16:00 & 59.694 & 61.886 & 53.044 & & 40.827 & 40.457 & 43.986 \\
\hline $10 / 17 / 1120: 00$ & 59.706 & 61.893 & 53.053 & & 40.836 & 40.469 & 43.993 \\
\hline 10/18/11 0:00 & 59.711 & 61.900 & 53.058 & & 40.841 & 40.476 & 43.998 \\
\hline
\end{tabular}

TABLE S1.3 Page 52 of 58 


\begin{tabular}{|c|c|c|c|c|c|c|c|}
\hline \multirow[b]{2}{*}{ Date and Time } & \multicolumn{7}{|c|}{ Water Level (ft below reference point) } \\
\hline & SB43D & SB44D & SB45D & SB46D & SB47D & SB48D & SB49D \\
\hline 10/18/11 4:00 & 59.716 & 61.906 & 53.067 & & 40.848 & 40.481 & 44.005 \\
\hline 10/18/11 8:00 & 59.720 & 61.906 & 53.062 & & 40.850 & 40.481 & 44.005 \\
\hline 10/18/11 12:00 & 59.725 & 61.911 & 53.067 & & 40.855 & 40.488 & 44.010 \\
\hline 10/18/11 16:00 & 59.730 & 61.916 & 53.074 & & 40.864 & 40.495 & 44.016 \\
\hline 10/18/11 20:00 & 59.737 & 61.918 & 53.079 & & 40.864 & 40.497 & 44.021 \\
\hline 10/19/11 0:00 & 59.739 & 61.918 & 53.079 & & 40.871 & 40.504 & 44.023 \\
\hline 10/19/11 4:00 & 59.746 & 61.927 & 53.093 & & 40.881 & 40.509 & 44.030 \\
\hline 10/19/11 8:00 & 59.756 & 61.927 & 53.097 & & 40.885 & 40.519 & 44.035 \\
\hline 10/19/11 12:00 & 59.756 & 61.932 & 53.090 & & 40.890 & 40.519 & 44.033 \\
\hline 10/19/11 16:00 & 59.753 & 61.930 & 53.093 & & 40.888 & 40.519 & 44.035 \\
\hline 10/19/11 20:00 & 59.768 & 61.944 & 53.102 & & 40.892 & 40.528 & 44.042 \\
\hline 10/20/11 0:00 & 59.768 & 61.944 & 53.100 & & 40.904 & 40.530 & 44.042 \\
\hline 10/20/11 4:00 & 59.772 & 61.946 & 53.111 & & 40.909 & 40.535 & 44.049 \\
\hline 10/20/11 8:00 & 59.784 & 61.953 & 53.118 & & 40.921 & 40.547 & 44.054 \\
\hline 10/20/11 12:00 & 59.789 & 61.958 & 53.113 & & 40.921 & 40.549 & 44.056 \\
\hline 10/20/11 16:00 & 59.784 & 61.955 & 53.111 & & 40.918 & 40.544 & 44.056 \\
\hline 10/20/11 20:00 & 59.798 & 61.969 & 53.130 & & 40.928 & 40.559 & 44.065 \\
\hline 10/21/11 0:00 & 59.810 & 61.976 & 53.134 & & 40.939 & 40.568 & 44.075 \\
\hline 10/21/11 4:00 & 59.815 & 61.981 & 53.144 & & 40.949 & 40.573 & 44.082 \\
\hline 10/21/11 8:00 & 59.829 & 61.991 & 53.165 & & 40.960 & 40.589 & 44.096 \\
\hline $10 / 21 / 11$ 12:00 & 59.829 & 61.990 & 53.146 & & 40.963 & 40.587 & 44.089 \\
\hline 10/21/11 16:00 & 59.820 & 61.981 & 53.134 & & 40.953 & 40.573 & 44.079 \\
\hline 10/21/11 20:00 & 59.831 & 61.995 & 53.155 & & 40.965 & 40.584 & 44.091 \\
\hline 10/22/11 0:00 & 59.839 & 61.997 & 53.153 & & 40.972 & 40.591 & 44.093 \\
\hline 10/22/11 4:00 & 59.836 & 61.995 & 53.153 & & 40.972 & 40.594 & 44.093 \\
\hline 10/22/11 8:00 & 59.853 & 62.014 & 53.178 & & 40.991 & 40.610 & 44.109 \\
\hline $10 / 22 / 11$ 12:00 & 59.862 & 62.018 & 53.171 & & 41.003 & 40.617 & 44.114 \\
\hline 10/22/11 16:00 & 59.850 & 62.007 & 53.158 & & 40.989 & 40.608 & 44.100 \\
\hline 10/22/11 20:00 & 59.857 & 62.009 & 53.174 & & 41.000 & 40.617 & 44.112 \\
\hline 10/23/11 0:00 & 59.865 & 62.016 & 53.171 & & 41.003 & 40.622 & 44.114 \\
\hline 10/23/11 4:00 & 59.857 & 62.009 & 53.155 & & 40.998 & 40.615 & 44.105 \\
\hline 10/23/11 8:00 & 59.867 & 62.018 & 53.174 & & 41.003 & 40.627 & 44.114 \\
\hline 10/23/11 12:00 & 59.876 & 62.023 & 53.178 & & 41.014 & 40.634 & 44.119 \\
\hline 10/23/11 16:00 & 59.862 & 62.011 & 53.155 & & 41.007 & 40.622 & 44.107 \\
\hline 10/23/11 20:00 & 59.879 & 62.025 & 53.190 & & 41.014 & 40.636 & 44.126 \\
\hline 10/24/11 0:00 & 59.907 & 62.049 & 53.216 & & 41.043 & 40.660 & 44.149 \\
\hline 10/24/11 4:00 & 59.902 & 62.044 & 53.199 & & 41.038 & 40.657 & 44.142 \\
\hline 10/24/11 8:00 & 59.914 & 62.058 & 53.225 & & 41.052 & 40.671 & 44.154 \\
\hline $10 / 24 / 11$ 12:00 & 59.926 & 62.065 & 53.225 & & 41.066 & 40.676 & 44.165 \\
\hline 10/24/11 16:00 & 59.907 & 62.044 & 53.190 & & 41.047 & 40.655 & 44.142 \\
\hline 10/24/11 20:00 & 59.905 & 62.053 & 53.204 & & 41.047 & 40.662 & 44.144 \\
\hline 10/25/11 0:00 & 59.926 & 62.058 & 53.213 & & 41.069 & 40.674 & 44.154 \\
\hline 10/25/11 4:00 & 59.910 & 62.037 & 53.178 & & 41.054 & 40.660 & 44.133 \\
\hline 10/25/11 8:00 & 59.902 & 62.025 & 53.181 & & 41.050 & 40.657 & 44.128 \\
\hline $10 / 25 / 11$ 12:00 & 59.917 & 62.042 & 53.197 & & 41.057 & 40.669 & 44.140 \\
\hline 10/25/11 16:00 & 59.917 & 62.037 & 53.188 & & 41.057 & 40.662 & 44.135 \\
\hline 10/25/11 20:00 & 59.940 & 62.070 & 53.239 & & 41.085 & 40.690 & 44.165 \\
\hline 10/26/11 0:00 & 59.969 & 62.093 & 53.269 & & 41.106 & 40.718 & 44.193 \\
\hline 10/26/11 4:00 & 59.971 & 62.093 & 53.250 & & 41.111 & 40.723 & 44.193 \\
\hline 10/26/11 8:00 & 59.976 & 62.098 & 53.257 & & 41.108 & 40.728 & 44.196 \\
\hline 10/26/11 12:00 & 59.983 & 62.109 & 53.274 & & 41.130 & 40.739 & 44.209 \\
\hline 10/26/11 16:00 & 59.980 & 62.105 & 53.260 & & 41.125 & 40.735 & 44.205 \\
\hline 10/26/11 20:00 & 59.990 & 62.121 & 53.281 & & 41.137 & 40.747 & 44.216 \\
\hline 10/27/11 0:00 & 60.009 & 62.135 & 53.306 & & 41.155 & 40.765 & 44.235 \\
\hline
\end{tabular}

TABLE S1.3 Page 53 of 58 


\begin{tabular}{|c|c|c|c|c|c|c|c|}
\hline \multirow[b]{2}{*}{ Date and Time } & \multicolumn{7}{|c|}{ Water Level (ft below reference point) } \\
\hline & SB43D & SB44D & SB45D & SB46D & SB47D & SB48D & SB49D \\
\hline 10/27/11 4:00 & 60.014 & 62.135 & 53.281 & & 41.153 & 40.765 & 44.233 \\
\hline 10/27/11 8:00 & 60.006 & 62.132 & 53.283 & & 41.148 & 40.761 & 44.226 \\
\hline 10/27/11 12:00 & 60.018 & 62.142 & 53.304 & & 41.170 & 40.777 & 44.242 \\
\hline 10/27/11 16:00 & 60.021 & 62.139 & 53.290 & & 41.167 & 40.772 & 44.237 \\
\hline 10/27/11 20:00 & 60.025 & 62.142 & 53.306 & & 41.174 & 40.779 & 44.242 \\
\hline 10/28/11 0:00 & 60.047 & 62.165 & 53.339 & & 41.193 & 40.801 & 44.265 \\
\hline 10/28/11 4:00 & 60.054 & 62.170 & 53.322 & & 41.200 & 40.805 & 44.263 \\
\hline 10/28/11 8:00 & 60.042 & 62.156 & 53.306 & & 41.191 & 40.796 & 44.254 \\
\hline 10/28/11 12:00 & 60.047 & 62.163 & 53.322 & & 41.193 & 40.803 & 44.261 \\
\hline 10/28/11 16:00 & 60.049 & 62.165 & 53.313 & & 41.200 & 40.803 & 44.263 \\
\hline 10/28/11 20:00 & 60.059 & 62.170 & 53.329 & & 41.200 & 40.810 & 44.268 \\
\hline 10/29/11 0:00 & 60.070 & 62.184 & 53.348 & & 41.219 & 40.824 & 44.282 \\
\hline 10/29/11 4:00 & 60.075 & 62.186 & 53.341 & & 41.221 & 40.829 & 44.282 \\
\hline 10/29/11 8:00 & 60.066 & 62.174 & 53.322 & & 41.214 & 40.819 & 44.270 \\
\hline 10/29/11 12:00 & 60.056 & 62.172 & 53.315 & & 41.207 & 40.812 & 44.265 \\
\hline 10/29/11 16:00 & 60.051 & 62.158 & 53.299 & & 41.198 & 40.803 & 44.254 \\
\hline 10/29/11 20:00 & 60.051 & 62.160 & 53.306 & & 41.198 & 40.803 & 44.254 \\
\hline 10/30/11 0:00 & 60.063 & 62.170 & 53.327 & & 41.214 & 40.817 & 44.265 \\
\hline 10/30/11 4:00 & 60.077 & 62.181 & 53.339 & & 41.226 & 40.829 & 44.277 \\
\hline 10/30/11 8:00 & 60.085 & 62.181 & 53.339 & & 41.228 & 40.831 & 44.279 \\
\hline 10/30/11 12:00 & 60.092 & 62.191 & 53.350 & & 41.240 & 40.838 & 44.291 \\
\hline 10/30/11 16:00 & 60.094 & 62.195 & 53.357 & & 41.245 & 40.848 & 44.295 \\
\hline 10/30/11 20:00 & 60.103 & 62.205 & 53.357 & & 41.254 & 40.852 & 44.300 \\
\hline 10/31/11 0:00 & 60.113 & 62.214 & 53.378 & & 41.259 & 40.864 & 44.312 \\
\hline 10/31/11 4:00 & 60.129 & 62.226 & 53.387 & & 41.275 & 40.878 & 44.326 \\
\hline 10/31/11 8:00 & 60.129 & 62.223 & 53.378 & & 41.268 & 40.876 & 44.321 \\
\hline 10/31/11 12:00 & 60.125 & 62.223 & 53.373 & & 41.273 & 40.873 & 44.319 \\
\hline 10/31/11 16:00 & 60.118 & 62.217 & 53.364 & & 41.268 & 40.869 & 44.312 \\
\hline 10/31/11 20:00 & 60.125 & 62.223 & 53.371 & & 41.273 & 40.871 & 44.314 \\
\hline 11/1/11 0:00 & 60.127 & 62.226 & 53.373 & & 41.275 & 40.878 & 44.319 \\
\hline 11/1/11 4:00 & 60.134 & 62.230 & 53.383 & & 41.282 & 40.885 & 44.323 \\
\hline 11/1/11 8:00 & 60.139 & 62.233 & 53.383 & & 41.282 & 40.887 & 44.326 \\
\hline 11/1/11 12:00 & 60.137 & 62.226 & 53.373 & & 41.285 & 40.885 & 44.321 \\
\hline 11/1/11 16:00 & 60.129 & 62.226 & 53.376 & & 41.282 & 40.880 & 44.319 \\
\hline 11/1/11 20:00 & 60.146 & 62.235 & 53.390 & & 41.292 & 40.890 & 44.333 \\
\hline 11/2/11 0:00 & 60.153 & 62.242 & 53.399 & & 41.301 & 40.899 & 44.337 \\
\hline 11/2/11 4:00 & 60.167 & 62.254 & 53.420 & & 41.315 & 40.913 & 44.351 \\
\hline 11/2/11 8:00 & 60.179 & 62.265 & 53.427 & & 41.329 & 40.925 & 44.365 \\
\hline 11/2/11 12:00 & 60.179 & 62.263 & 53.418 & & 41.322 & 40.923 & 44.363 \\
\hline 11/2/11 16:00 & 60.184 & 62.265 & 53.422 & & 41.332 & 40.932 & 44.363 \\
\hline 11/2/11 20:00 & 60.174 & 62.263 & 53.418 & & 41.325 & 40.920 & 44.363 \\
\hline 11/3/11 0:00 & 60.179 & 62.265 & 53.418 & & 41.327 & 40.925 & 44.363 \\
\hline 11/3/11 4:00 & 60.184 & 62.268 & 53.431 & & 41.332 & 40.932 & 44.368 \\
\hline 11/3/11 8:00 & 60.200 & 62.277 & 53.450 & & 41.343 & 40.946 & 44.382 \\
\hline 11/3/11 12:00 & 60.210 & 62.286 & 53.445 & & 41.350 & 40.953 & 44.389 \\
\hline 11/3/11 16:00 & 60.205 & 62.289 & 53.445 & & 41.348 & 40.951 & 44.389 \\
\hline 11/3/11 20:00 & 60.219 & 62.307 & 53.464 & & 41.362 & 40.963 & 44.400 \\
\hline 11/4/11 0:00 & 60.229 & 62.307 & 53.466 & & 41.369 & 40.970 & 44.405 \\
\hline 11/4/11 4:00 & 60.224 & 62.307 & 53.464 & & 41.369 & 40.970 & 44.402 \\
\hline 11/4/11 8:00 & 60.234 & 62.312 & 53.476 & & 41.379 & 40.977 & 44.409 \\
\hline 11/4/11 12:00 & 60.234 & 62.312 & 53.464 & & 41.379 & 40.977 & 44.407 \\
\hline 11/4/11 16:00 & 60.217 & 62.298 & 53.443 & & 41.367 & 40.963 & 44.391 \\
\hline 11/4/11 20:00 & 60.224 & 62.303 & 53.457 & & 41.374 & 40.970 & 44.395 \\
\hline 11/5/11 0:00 & 60.226 & 62.305 & 53.450 & & 41.374 & 40.972 & 44.398 \\
\hline
\end{tabular}

TABLE S1.3 Page 54 of 58 


\begin{tabular}{|c|c|c|c|c|c|c|c|}
\hline \multirow[b]{2}{*}{ Date and Time } & \multicolumn{7}{|c|}{ Water Level (ft below reference point) } \\
\hline & SB43D & SB44D & SB45D & SB46D & SB47D & SB48D & SB49D \\
\hline $11 / 5 / 114: 00$ & 60.219 & 62.296 & 53.438 & & 41.367 & 40.967 & 44.389 \\
\hline 11/5/11 8:00 & 60.224 & 62.300 & 53.452 & & 41.369 & 40.970 & 44.395 \\
\hline $11 / 5 / 11$ 12:00 & 60.222 & 62.298 & 53.436 & & 41.367 & 40.970 & 44.391 \\
\hline 11/5/11 16:00 & 60.205 & 62.286 & 53.420 & & 41.350 & 40.953 & 44.377 \\
\hline 11/5/11 20:00 & 60.212 & 62.284 & 53.434 & & 41.357 & 40.958 & 44.379 \\
\hline 11/6/11 0:00 & 60.222 & 62.293 & 53.427 & & 41.365 & 40.965 & 44.391 \\
\hline $11 / 6 / 114: 00$ & 60.212 & 62.296 & 53.413 & & 41.357 & 40.958 & 44.393 \\
\hline 11/6/11 8:00 & 60.224 & 62.317 & 53.448 & & 41.369 & 40.972 & 44.412 \\
\hline 11/6/11 12:00 & 60.255 & 62.344 & 53.473 & & 41.397 & 40.998 & 44.444 \\
\hline $11 / 6 / 11$ 16:00 & 60.250 & 62.347 & 53.466 & & 41.395 & 40.993 & 44.444 \\
\hline $11 / 6 / 1120: 00$ & 60.269 & 62.365 & 53.494 & & 41.412 & 41.012 & 44.468 \\
\hline 11/7/11 0:00 & 60.288 & 62.382 & 53.503 & & 41.428 & 41.028 & 44.482 \\
\hline $11 / 7 / 114: 00$ & 60.283 & 62.382 & 53.492 & & 41.421 & 41.026 & 44.479 \\
\hline 11/7/11 8:00 & 60.290 & 62.389 & 53.510 & & 41.435 & 41.033 & 44.491 \\
\hline 11/7/11 12:00 & 60.300 & 62.398 & 53.513 & & 41.442 & 41.043 & 44.498 \\
\hline 11/7/11 16:00 & 60.288 & 62.384 & 53.485 & & 41.435 & 41.031 & 44.482 \\
\hline 11/7/11 20:00 & 60.262 & 62.363 & 53.473 & & 41.402 & 40.986 & 44.451 \\
\hline 11/8/11 0:00 & 60.118 & 62.342 & 53.380 & & 41.266 & 40.812 & 44.423 \\
\hline 11/8/11 4:00 & 60.132 & 62.319 & 53.362 & & 41.266 & 40.845 & 44.405 \\
\hline 11/8/11 8:00 & 60.139 & 62.300 & 53.376 & & 41.268 & 40.862 & 44.395 \\
\hline $11 / 8 / 11$ 12:00 & 60.153 & 62.298 & 53.380 & & 41.271 & 40.873 & 44.391 \\
\hline $11 / 8 / 11$ 16:00 & 60.125 & 62.272 & 53.343 & & 41.240 & 40.843 & 44.365 \\
\hline 11/8/11 20:00 & 60.087 & 62.272 & 53.334 & & 41.200 & 40.803 & 44.347 \\
\hline 11/9/11 0:00 & 60.051 & 62.258 & 53.318 & & 41.160 & 40.772 & 44.340 \\
\hline $11 / 9 / 114: 00$ & 60.035 & 62.219 & 53.285 & & 41.127 & 40.756 & 44.309 \\
\hline 11/9/11 8:00 & 60.032 & 62.228 & 53.304 & & 41.123 & 40.754 & 44.319 \\
\hline 11/9/11 12:00 & 60.051 & 62.240 & 53.322 & & 41.137 & 40.772 & 44.333 \\
\hline 11/9/11 16:00 & 60.044 & 62.242 & 53.313 & & 41.123 & 40.765 & 44.330 \\
\hline 11/9/11 20:00 & 60.054 & 62.240 & 53.327 & & 41.127 & 40.779 & 44.333 \\
\hline 11/10/11 0:00 & 60.068 & 62.244 & 53.334 & & 41.132 & 40.791 & 44.337 \\
\hline 11/10/11 4:00 & 60.061 & 62.228 & 53.304 & & 41.118 & 40.784 & 44.321 \\
\hline 11/10/11 8:00 & 60.049 & 62.216 & 53.301 & & 41.101 & 40.772 & 44.312 \\
\hline 11/10/11 12:00 & 60.056 & 62.219 & 53.313 & & 41.108 & 40.779 & 44.316 \\
\hline 11/10/11 16:00 & 60.040 & 62.205 & 53.283 & & 41.090 & 40.761 & 44.298 \\
\hline $11 / 10 / 1120: 00$ & 60.028 & 62.191 & 53.278 & & 41.078 & 40.749 & 44.289 \\
\hline 11/11/11 0:00 & 60.030 & 62.195 & 53.285 & & 41.073 & 40.751 & 44.291 \\
\hline 11/11/11 4:00 & 60.009 & 62.174 & 53.241 & & 41.047 & 40.728 & 44.265 \\
\hline 11/11/11 8:00 & 59.973 & 62.146 & 53.209 & & 41.017 & 40.697 & 44.237 \\
\hline 11/11/11 12:00 & 59.976 & 62.147 & 53.232 & & 41.019 & 40.700 & 44.242 \\
\hline 11/11/11 16:00 & 59.973 & 62.137 & 53.218 & & 41.010 & 40.690 & 44.235 \\
\hline 11/11/11 20:00 & 59.969 & 62.137 & 53.223 & & 41.010 & 40.685 & 44.233 \\
\hline 11/12/11 0:00 & 59.978 & 62.144 & 53.241 & & 41.014 & 40.695 & 44.242 \\
\hline $11 / 12 / 114: 00$ & 59.966 & 62.135 & 53.213 & & 40.996 & 40.683 & 44.228 \\
\hline $11 / 12 / 118: 00$ & 59.943 & 62.112 & 53.188 & & 40.975 & 40.662 & 44.207 \\
\hline $11 / 12 / 11$ 12:00 & 59.933 & 62.105 & 53.188 & & 40.967 & 40.653 & 44.205 \\
\hline 11/12/11 16:00 & 59.924 & 62.095 & 53.169 & & 40.953 & 40.638 & 44.191 \\
\hline 11/12/11 20:00 & 59.914 & 62.093 & 53.171 & & 40.949 & 40.631 & 44.186 \\
\hline 11/13/11 0:00 & 59.924 & 62.095 & 53.188 & & 40.953 & 40.638 & 44.193 \\
\hline $11 / 13 / 114: 00$ & 59.905 & 62.079 & 53.151 & & 40.932 & 40.620 & 44.172 \\
\hline 11/13/11 8:00 & 59.905 & 62.074 & 53.165 & & 40.935 & 40.617 & 44.177 \\
\hline $11 / 13 / 11$ 12:00 & 59.914 & 62.088 & 53.188 & & 40.944 & 40.629 & 44.189 \\
\hline 11/13/11 16:00 & 59.921 & 62.100 & 53.195 & & 40.951 & 40.634 & 44.196 \\
\hline 11/13/11 20:00 & 59.926 & 62.102 & 53.202 & & 40.951 & 40.638 & 44.202 \\
\hline 11/14/11 0:00 & 59.928 & 62.109 & 53.206 & & 40.956 & 40.643 & 44.205 \\
\hline
\end{tabular}

TABLE S1.3 Page 55 of 58 


\begin{tabular}{|c|c|c|c|c|c|c|c|}
\hline \multirow[b]{2}{*}{ Date and Time } & \multicolumn{7}{|c|}{ Water Level (ft below reference point) } \\
\hline & SB43D & SB44D & SB45D & SB46D & SB47D & SB48D & SB49D \\
\hline $11 / 14 / 114: 00$ & 59.926 & 62.105 & 53.195 & & 40.953 & 40.638 & 44.200 \\
\hline 11/14/11 8:00 & 59.914 & 62.093 & 53.183 & & 40.949 & 40.629 & 44.193 \\
\hline $11 / 14 / 1112: 00$ & 59.924 & 62.105 & 53.206 & & 40.958 & 40.638 & 44.205 \\
\hline $11 / 14 / 1116: 00$ & 59.926 & 62.109 & 53.206 & & 40.960 & 40.641 & 44.205 \\
\hline $11 / 14 / 1120: 00$ & 59.931 & 62.109 & 53.213 & & 40.963 & 40.643 & 44.212 \\
\hline 11/15/11 0:00 & 59.933 & 62.118 & 53.216 & & 40.972 & 40.648 & 44.212 \\
\hline 11/15/11 4:00 & 59.936 & 62.114 & 53.211 & & 40.972 & 40.648 & 44.212 \\
\hline 11/15/11 8:00 & 59.928 & 62.107 & 53.197 & & 40.965 & 40.641 & 44.202 \\
\hline $11 / 15 / 1112: 00$ & 59.928 & 62.107 & 53.209 & & 40.970 & 40.645 & 44.209 \\
\hline 11/15/11 16:00 & 59.933 & 62.116 & 53.220 & & 40.977 & 40.648 & 44.214 \\
\hline $11 / 15 / 1120: 00$ & 59.957 & 62.128 & 53.243 & & 40.998 & 40.669 & 44.235 \\
\hline 11/16/11 0:00 & 59.969 & 62.144 & 53.262 & & 41.014 & 40.683 & 44.249 \\
\hline $11 / 16 / 114: 00$ & 59.980 & 62.158 & 53.274 & & 41.026 & 40.695 & 44.258 \\
\hline $11 / 16 / 118: 00$ & 59.983 & 62.158 & 53.269 & & 41.022 & 40.695 & 44.261 \\
\hline $11 / 16 / 1112: 00$ & 59.990 & 62.167 & 53.276 & & 41.033 & 40.704 & 44.265 \\
\hline $11 / 16 / 1116: 00$ & 59.990 & 62.165 & 53.278 & & 41.038 & 40.704 & 44.270 \\
\hline 11/16/11 20:00 & 59.997 & 62.172 & 53.276 & & 41.052 & 40.711 & 44.270 \\
\hline 11/17/11 0:00 & 59.999 & 62.174 & 53.288 & & 41.054 & 40.714 & 44.275 \\
\hline $11 / 17 / 114: 00$ & 60.006 & 62.184 & 53.292 & & 41.059 & 40.723 & 44.282 \\
\hline $11 / 17 / 118: 00$ & 60.006 & 62.174 & 53.281 & & 41.059 & 40.721 & 44.277 \\
\hline $11 / 17 / 1112: 00$ & 59.997 & 62.170 & 53.267 & & 41.059 & 40.714 & 44.265 \\
\hline $11 / 17 / 11$ 16:00 & 59.983 & 62.158 & 53.250 & & 41.045 & 40.702 & 44.254 \\
\hline 11/17/11 20:00 & 59.978 & 62.151 & 53.239 & & 41.040 & 40.692 & 44.249 \\
\hline $11 / 18 / 110: 00$ & 59.973 & 62.142 & 53.232 & & 41.031 & 40.690 & 44.240 \\
\hline $11 / 18 / 114: 00$ & 59.969 & 62.135 & 53.227 & & 41.026 & 40.683 & 44.233 \\
\hline $11 / 18 / 118: 00$ & 59.962 & 62.123 & 53.220 & & 41.024 & 40.678 & 44.223 \\
\hline $11 / 18 / 1112: 00$ & 59.952 & 62.118 & 53.204 & & 41.014 & 40.671 & 44.212 \\
\hline 11/18/11 16:00 & 59.938 & 62.109 & 53.197 & & 41.005 & 40.657 & 44.205 \\
\hline $11 / 18 / 1120: 00$ & 59.952 & 62.118 & 53.213 & & 41.007 & 40.664 & 44.216 \\
\hline 11/19/11 0:00 & 59.954 & 62.118 & 53.209 & & 41.010 & 40.667 & 44.214 \\
\hline 11/19/11 4:00 & 59.945 & 62.112 & 53.202 & & 41.003 & 40.660 & 44.207 \\
\hline $11 / 19 / 118: 00$ & 59.945 & 62.112 & 53.197 & & 41.005 & 40.657 & 44.205 \\
\hline $11 / 19 / 1112: 00$ & 59.943 & 62.102 & 53.185 & & 40.996 & 40.653 & 44.200 \\
\hline $11 / 19 / 1116: 00$ & 59.962 & 62.125 & 53.241 & & 41.012 & 40.671 & 44.230 \\
\hline 11/19/11 20:00 & 60.002 & 62.163 & 53.290 & & 41.054 & 40.711 & 44.268 \\
\hline $11 / 20 / 110: 00$ & 60.018 & 62.177 & 53.295 & & 41.073 & 40.725 & 44.279 \\
\hline $11 / 20 / 114: 00$ & 60.018 & 62.184 & 53.299 & & 41.076 & 40.732 & 44.286 \\
\hline 11/20/11 8:00 & 60.025 & 62.195 & 53.311 & & 41.090 & 40.739 & 44.298 \\
\hline $11 / 20 / 1112: 00$ & 60.035 & 62.198 & 53.295 & & 41.094 & 40.747 & 44.298 \\
\hline $11 / 20 / 1116: 00$ & 60.018 & 62.188 & 53.292 & & 41.087 & 40.735 & 44.289 \\
\hline $11 / 20 / 1120: 00$ & 60.032 & 62.195 & 53.313 & & 41.101 & 40.749 & 44.298 \\
\hline $11 / 21 / 110: 00$ & 60.037 & 62.198 & 53.295 & & 41.104 & 40.751 & 44.295 \\
\hline $11 / 21 / 114: 00$ & 60.025 & 62.191 & 53.285 & & 41.097 & 40.742 & 44.286 \\
\hline 11/21/11 8:00 & 60.030 & 62.191 & 53.299 & & 41.099 & 40.749 & 44.291 \\
\hline $11 / 21 / 1112: 00$ & 60.035 & 62.195 & 53.285 & & 41.104 & 40.749 & 44.289 \\
\hline $11 / 21 / 1116: 00$ & 60.018 & 62.179 & 53.274 & & 41.090 & 40.735 & 44.277 \\
\hline $11 / 21 / 1120: 00$ & 60.028 & 62.186 & 53.288 & & 41.101 & 40.742 & 44.284 \\
\hline $11 / 22 / 110: 00$ & 60.042 & 62.193 & 53.290 & & 41.108 & 40.754 & 44.293 \\
\hline $11 / 22 / 114: 00$ & 60.023 & 62.172 & 53.260 & & 41.087 & 40.735 & 44.270 \\
\hline 11/22/11 8:00 & 60.004 & 62.156 & 53.257 & & 41.073 & 40.716 & 44.254 \\
\hline $11 / 22 / 1112: 00$ & 60.014 & 62.167 & 53.262 & & 41.083 & 40.721 & 44.263 \\
\hline $11 / 22 / 11$ 16:00 & 60.009 & 62.165 & 53.260 & & 41.076 & 40.721 & 44.261 \\
\hline $11 / 22 / 1120: 00$ & 60.025 & 62.184 & 53.299 & & 41.094 & 40.739 & 44.284 \\
\hline 11/23/11 0:00 & 60.049 & 62.205 & 53.313 & & 41.111 & 40.758 & 44.302 \\
\hline
\end{tabular}

TABLE S1.3 Page 56 of 58 


\begin{tabular}{|c|c|c|c|c|c|c|c|}
\hline \multirow[b]{2}{*}{ Date and Time } & \multicolumn{7}{|c|}{ Water Level (ft below reference point) } \\
\hline & SB43D & SB44D & SB45D & SB46D & SB47D & SB48D & SB49D \\
\hline $11 / 23 / 114: 00$ & 60.037 & 62.191 & 53.283 & & 41.104 & 40.749 & 44.289 \\
\hline $11 / 23 / 118: 00$ & 60.035 & 62.186 & 53.292 & & 41.103 & 40.749 & 44.289 \\
\hline $11 / 23 / 11$ 12:00 & 60.047 & 62.198 & 53.301 & & 41.118 & 40.758 & 44.298 \\
\hline 11/23/11 16:00 & 60.035 & 62.191 & 53.278 & & 41.106 & 40.747 & 44.286 \\
\hline 11/23/11 20:00 & 60.042 & 62.198 & 53.304 & & 41.108 & 40.756 & 44.298 \\
\hline 11/24/11 0:00 & 60.059 & 62.212 & 53.315 & & 41.125 & 40.768 & 44.309 \\
\hline $11 / 24 / 114: 00$ & 60.047 & 62.195 & 53.283 & & 41.113 & 40.758 & 44.293 \\
\hline $11 / 24 / 118: 00$ & 60.032 & 62.186 & 53.278 & & 41.106 & 40.747 & 44.284 \\
\hline $11 / 24 / 11$ 12:00 & 60.040 & 62.191 & 53.285 & & 41.111 & 40.754 & 44.289 \\
\hline 11/24/11 16:00 & 60.028 & 62.179 & 53.267 & & 41.099 & 40.739 & 44.277 \\
\hline 11/24/11 20:00 & 60.030 & 62.186 & 53.281 & & 41.101 & 40.744 & 44.282 \\
\hline 11/25/11 0:00 & 60.049 & 62.200 & 53.301 & & 41.118 & 40.761 & 44.295 \\
\hline 11/25/11 4:00 & 60.042 & 62.182 & 53.262 & & 41.104 & 40.749 & 44.279 \\
\hline 11/25/11 8:00 & 60.023 & 62.174 & 53.257 & & 41.092 & 40.737 & 44.270 \\
\hline $11 / 25 / 11$ 12:00 & 60.030 & 62.177 & 53.269 & & 41.099 & 40.742 & 44.275 \\
\hline 11/25/11 16:00 & 60.018 & 62.167 & 53.250 & & 41.085 & 40.730 & 44.261 \\
\hline 11/25/11 20:00 & 60.018 & 62.165 & 53.262 & & 41.090 & 40.732 & 44.265 \\
\hline 11/26/11 0:00 & 60.040 & 62.188 & 53.295 & & 41.111 & 40.751 & 44.286 \\
\hline 11/26/11 4:00 & 60.032 & 62.181 & 53.267 & & 41.094 & 40.735 & 44.277 \\
\hline $11 / 26 / 118: 00$ & 60.023 & 62.177 & 53.271 & & 41.085 & 40.725 & 44.272 \\
\hline $11 / 26 / 11$ 12:00 & 60.037 & 62.200 & 53.295 & & 41.101 & 40.739 & 44.295 \\
\hline 11/26/11 16:00 & 60.042 & 62.198 & 53.301 & & 41.104 & 40.747 & 44.300 \\
\hline 11/26/11 20:00 & 60.059 & 62.212 & 53.318 & & 41.125 & 40.765 & 44.314 \\
\hline 11/27/11 0:00 & 60.075 & 62.226 & 53.346 & & 41.134 & 40.784 & 44.333 \\
\hline 11/27/11 4:00 & 60.082 & 62.230 & 53.334 & & 41.141 & 40.791 & 44.335 \\
\hline 11/27/11 8:00 & 60.075 & 62.226 & 53.322 & & 41.136 & 40.784 & 44.328 \\
\hline 11/27/11 12:00 & 60.077 & 62.233 & 53.334 & & 41.144 & 40.791 & 44.330 \\
\hline 11/27/11 16:00 & 60.077 & 62.230 & 53.325 & & 41.144 & 40.786 & 44.328 \\
\hline 11/27/11 20:00 & 60.080 & 62.233 & 53.329 & & 41.148 & 40.791 & 44.330 \\
\hline 11/28/11 0:00 & 60.087 & 62.242 & 53.343 & & 41.155 & 40.801 & 44.337 \\
\hline $11 / 28 / 114: 00$ & 60.092 & 62.240 & 53.334 & & 41.160 & 40.801 & 44.340 \\
\hline $11 / 28 / 118: 00$ & 60.085 & 62.228 & 53.320 & & 41.151 & 40.794 & 44.328 \\
\hline 11/28/11 12:00 & 60.077 & 62.228 & 53.318 & & 41.146 & 40.789 & 44.326 \\
\hline 11/28/11 16:00 & 60.075 & 62.223 & 53.315 & & 41.144 & 40.786 & 44.319 \\
\hline $11 / 28 / 1120: 00$ & 60.075 & 62.221 & 53.315 & & 41.139 & 40.786 & 44.321 \\
\hline 11/29/11 0:00 & 60.085 & 62.233 & 53.336 & & 41.151 & 40.794 & 44.330 \\
\hline 11/29/11 4:00 & 60.103 & 62.247 & 53.355 & & 41.167 & 40.810 & 44.349 \\
\hline 11/29/11 8:00 & 60.106 & 62.249 & 53.343 & & 41.170 & 40.812 & 44.349 \\
\hline 11/29/11 12:00 & 60.106 & 62.249 & 53.350 & & 41.170 & 40.817 & 44.354 \\
\hline 11/29/11 16:00 & 60.108 & 62.254 & 53.353 & & 41.177 & 40.817 & 44.356 \\
\hline 11/29/11 20:00 & 60.111 & 62.258 & 53.353 & & 41.177 & 40.819 & 44.356 \\
\hline 11/30/11 0:00 & 60.111 & 62.261 & 53.355 & & 41.177 & 40.819 & 44.356 \\
\hline 11/30/11 4:00 & 60.118 & 62.263 & 53.355 & & 41.184 & 40.824 & 44.358 \\
\hline 11/30/11 8:00 & 60.108 & 62.251 & 53.334 & & 41.172 & 40.815 & 44.349 \\
\hline 11/30/11 12:00 & 60.101 & 62.242 & 53.327 & & 41.167 & 40.810 & 44.340 \\
\hline 11/30/11 16:00 & 60.089 & 62.235 & 53.320 & & 41.162 & 40.801 & 44.333 \\
\hline 11/30/11 20:00 & 60.096 & 62.235 & 53.322 & & 41.165 & 40.803 & 44.335 \\
\hline 12/1/11 0:00 & 60.106 & 62.251 & 53.343 & & 41.174 & 40.812 & 44.347 \\
\hline $12 / 1 / 114: 00$ & 60.118 & 62.256 & 53.355 & & 41.186 & 40.822 & 44.356 \\
\hline 12/1/11 8:00 & 60.129 & 62.265 & 53.369 & & 41.191 & 40.833 & 44.365 \\
\hline 12/1/11 12:00 & 60.137 & 62.275 & 53.371 & & 41.202 & 40.840 & 44.372 \\
\hline $12 / 1 / 11$ 16:00 & 60.144 & 62.286 & 53.392 & & 41.212 & 40.852 & 44.389 \\
\hline $12 / 1 / 1120: 00$ & 60.163 & 62.305 & 53.411 & & 41.235 & 40.869 & 44.407 \\
\hline 12/2/11 0:00 & 60.172 & 62.307 & 53.413 & & 41.235 & 40.876 & 44.412 \\
\hline
\end{tabular}

TABLE S1.3 Page 57 of 58 


\begin{tabular}{lllllll}
\hline & \multicolumn{5}{c}{ Water Level (ft below reference point) } \\
\cline { 2 - 7 } Date and Time & SB43D & SB44D & SB45D & SB46D & SB47D & SB48D \\
\hline 12/2/11 4:00 & 60.172 & 62.310 & 53.411 & 41.242 & 40.878 & 44.409 \\
$12 / 2 / 118: 00$ & 60.170 & 62.310 & 53.401 & 41.240 & 40.876 & 44.407 \\
$12 / 2 / 11$ 12:00 & 60.165 & 62.303 & 53.387 & 41.235 & 40.873 & 44.398 \\
\hline
\end{tabular}

a NA, data from indicated date and time until next reported value are missing because logger electrical malfunction.

b Recorder in well SB46D was removed on September 23, 2011, to prevent winter damage; see Section 3.5. 
TABLE S1.4 Summary of daily rainfall events at St. Joseph, Missouri, 5/1/09-12/3/11.

\begin{tabular}{|c|c|c|c|c|c|}
\hline Date & Rainfall (in.) & Date & Rainfall (in.) & Date & Rainfall (in.) \\
\hline $5 / 1 / 2009$ & 0.00 & 9/9/2009 & 0.09 & 3/9/2010 & 0.47 \\
\hline $5 / 8 / 2009$ & 0.17 & $9 / 21 / 2009$ & 1.09 & $3 / 10 / 2010$ & 0.44 \\
\hline $5 / 12 / 2009$ & 0.09 & $9 / 25 / 2009$ & 0.24 & $3 / 11 / 2010$ & 0.08 \\
\hline $5 / 15 / 2009$ & 2.10 & $9 / 26 / 2009$ & 0.20 & $3 / 12 / 2010$ & 0.02 \\
\hline $5 / 25 / 2009$ & 0.02 & $10 / 1 / 2009$ & 0.44 & $3 / 14 / 2010$ & 0.01 \\
\hline $5 / 26 / 2009$ & 1.32 & $10 / 6 / 2009$ & 0.24 & $3 / 19 / 2010$ & 0.09 \\
\hline $5 / 27 / 2009$ & 0.04 & $10 / 8 / 2009$ & 0.46 & $3 / 20 / 2010$ & 0.02 \\
\hline $6 / 2 / 2009$ & 0.12 & $10 / 13 / 2009$ & 0.22 & $3 / 21 / 2010$ & 0.08 \\
\hline $6 / 6 / 2009$ & 0.03 & $10 / 14 / 2009$ & 0.11 & $3 / 24 / 2010$ & 0.81 \\
\hline $6 / 7 / 2009$ & 0.03 & $10 / 15 / 2009$ & 0.01 & $3 / 27 / 2010$ & 0.66 \\
\hline $6 / 8 / 2009$ & 0.17 & $10 / 17 / 2009$ & 0.03 & $4 / 2 / 2010$ & 0.50 \\
\hline $6 / 9 / 2009$ & 0.55 & $10 / 21 / 2009$ & 0.65 & $4 / 15 / 2010$ & 0.15 \\
\hline $6 / 10 / 2009$ & 0.16 & $10 / 22 / 2009$ & 1.24 & $4 / 16 / 2010$ & 0.52 \\
\hline $6 / 11 / 2009$ & 0.85 & $10 / 23 / 2009$ & 0.03 & $4 / 21 / 2010$ & 0.03 \\
\hline $6 / 12 / 2009$ & 0.05 & $10 / 25 / 2009$ & 0.22 & $4 / 22 / 2010$ & 0.38 \\
\hline $6 / 15 / 2009$ & 1.04 & $10 / 29 / 2009$ & 0.57 & $4 / 23 / 2010$ & 0.61 \\
\hline $6 / 16 / 2009$ & 0.03 & $11 / 9 / 2009$ & 0.06 & 4/24/2010 & 0.82 \\
\hline $6 / 17 / 2009$ & 0.77 & $11 / 15 / 2009$ & 0.11 & $4 / 25 / 2010$ & 0.13 \\
\hline 6/20/2009 & 0.16 & $11 / 16 / 2009$ & 1.30 & 4/26/2010 & 0.05 \\
\hline $6 / 21 / 2009$ & 0.08 & $11 / 17 / 2009$ & 0.45 & 4/30/2010 & 0.41 \\
\hline $6 / 24 / 2009$ & 0.07 & $11 / 18 / 2009$ & 0.05 & 5/7/2010 & 0.56 \\
\hline $6 / 25 / 2009$ & 0.03 & $11 / 21 / 2009$ & 0.01 & $5 / 10 / 2010$ & 2.29 \\
\hline 7/3/2009 & 0.73 & $11 / 23 / 2009$ & 0.29 & $5 / 12 / 2010$ & 1.66 \\
\hline $7 / 4 / 2009$ & 0.04 & $11 / 24 / 2009$ & 0.29 & $5 / 13 / 2010$ & 0.54 \\
\hline $7 / 5 / 2009$ & 0.01 & $11 / 25 / 2009$ & 0.03 & $5 / 15 / 2010$ & 0.22 \\
\hline $7 / 8 / 2009$ & 0.04 & $12 / 11 / 2009$ & 0.01 & $5 / 16 / 2010$ & 0.12 \\
\hline $7 / 10 / 2009$ & 1.06 & $12 / 12 / 2009$ & 0.22 & $5 / 17 / 2010$ & 0.44 \\
\hline $7 / 12 / 2009$ & 0.55 & $12 / 23 / 2009$ & 0.20 & $5 / 19 / 2010$ & 0.27 \\
\hline $7 / 14 / 2009$ & 1.07 & $12 / 24 / 2009$ & 0.12 & $5 / 20 / 2010$ & 0.40 \\
\hline $7 / 16 / 2009$ & 0.37 & $12 / 28 / 2009$ & 0.04 & $5 / 30 / 2010$ & 0.05 \\
\hline $7 / 20 / 2009$ & 0.54 & $12 / 30 / 2009$ & 0.20 & 6/1/2010 & 1.87 \\
\hline $7 / 21 / 2009$ & 0.07 & $1 / 11 / 2010$ & 0.01 & $6 / 2 / 2010$ & 1.49 \\
\hline $7 / 28 / 2009$ & 0.38 & $1 / 13 / 2010$ & 0.08 & 6/4/2010 & 0.37 \\
\hline 8/1/2009 & 0.60 & $1 / 19 / 2010$ & 0.03 & 6/8/2010 & 0.66 \\
\hline $8 / 3 / 2009$ & 0.05 & $1 / 20 / 2010$ & 0.11 & $6 / 12 / 2010$ & 1.26 \\
\hline $8 / 4 / 2009$ & 0.51 & $1 / 23 / 2010$ & 0.01 & 6/13/2010 & 0.95 \\
\hline 8/9/2009 & 1.06 & $2 / 5 / 2010$ & 0.12 & 6/14/2010 & 0.70 \\
\hline $8 / 10 / 2009$ & 0.32 & $2 / 6 / 2010$ & 0.12 & 6/15/2010 & 0.36 \\
\hline $8 / 15 / 2009$ & 0.48 & 2/7/2010 & 0.07 & 6/16/2010 & 0.02 \\
\hline $8 / 16 / 2009$ & 2.41 & 2/10/2010 & 0.01 & 6/18/2010 & 0.22 \\
\hline $8 / 17 / 2009$ & 1.89 & 2/19/2010 & 0.22 & 6/19/2010 & 0.74 \\
\hline $8 / 19 / 2009$ & 0.33 & $2 / 20 / 2010$ & 0.02 & 6/20/2010 & 0.70 \\
\hline $8 / 20 / 2009$ & 0.01 & $2 / 24 / 2010$ & 0.01 & 6/21/2010 & 0.01 \\
\hline $8 / 26 / 2009$ & 0.01 & $2 / 25 / 2010$ & 0.10 & 6/27/2010 & 0.03 \\
\hline $8 / 27 / 2009$ & 0.02 & 2/26/2010 & 0.16 & $7 / 3 / 2010$ & 0.05 \\
\hline 9/2/2009 & 0.02 & 3/6/2010 & 0.02 & $7 / 4 / 2010$ & 1.22 \\
\hline 9/8/2009 & 0.19 & $3 / 8 / 2010$ & 0.12 & 7/5/2010 & 0.10 \\
\hline
\end{tabular}

TABLE S1.4 Page 1 of 2 


\begin{tabular}{|c|c|c|c|c|c|}
\hline Date & Rainfall (in.) & Date & Rainfall (in.) & Date & Rainfall (in.) \\
\hline $7 / 7 / 2010$ & 0.07 & $1 / 24 / 2011$ & 0.02 & $6 / 21 / 2011$ & 0.06 \\
\hline $7 / 8 / 2010$ & 0.03 & $1 / 25 / 2011$ & 0.14 & $6 / 25 / 2011$ & 0.72 \\
\hline $7 / 11 / 2010$ & 0.81 & 2/4/2011 & 0.08 & $6 / 26 / 2011$ & 0.61 \\
\hline $7 / 14 / 2010$ & 1.09 & $2 / 5 / 2011$ & 0.01 & $6 / 27 / 2011$ & 1.61 \\
\hline $7 / 15 / 2010$ & 0.01 & $2 / 11 / 2011$ & 0.01 & $7 / 3 / 2011$ & 1.23 \\
\hline $7 / 16 / 2010$ & 0.33 & $2 / 25 / 2011$ & 0.12 & $7 / 5 / 2011$ & 0.01 \\
\hline $7 / 18 / 2010$ & 0.02 & $2 / 27 / 2011$ & 0.33 & 7/7/2011 & 1.22 \\
\hline $7 / 20 / 2010$ & 0.08 & $3 / 4 / 2011$ & 0.08 & $7 / 12 / 2011$ & 0.10 \\
\hline $7 / 21 / 2010$ & 0.21 & $3 / 7 / 2011$ & 0.07 & $7 / 13 / 2011$ & 0.01 \\
\hline $7 / 24 / 2010$ & 0.04 & $3 / 8 / 2011$ & 0.47 & $7 / 24 / 2011$ & 0.19 \\
\hline $7 / 28 / 2010$ & 0.02 & $3 / 14 / 2011$ & 0.19 & $7 / 28 / 2011$ & 0.12 \\
\hline $7 / 30 / 2010$ & 0.31 & 3/19/2011 & 0.19 & $7 / 29 / 2011$ & 0.28 \\
\hline $8 / 2 / 2010$ & 0.34 & $3 / 24 / 2011$ & 0.01 & $7 / 30 / 2011$ & 1.50 \\
\hline $8 / 13 / 2010$ & 0.99 & $3 / 25 / 2011$ & 0.47 & $8 / 5 / 2011$ & 0.95 \\
\hline $8 / 17 / 2010$ & 0.02 & $4 / 3 / 2011$ & 0.01 & $8 / 7 / 2011$ & 0.54 \\
\hline $8 / 20 / 2010$ & 2.18 & 4/7/2011 & 0.18 & 8/8/2011 & 0.01 \\
\hline $8 / 31 / 2010$ & 1.53 & 4/8/2011 & 0.27 & $8 / 12 / 2011$ & 0.47 \\
\hline $9 / 1 / 2010$ & 0.94 & 4/9/2011 & 0.01 & $8 / 15 / 2011$ & 0.40 \\
\hline $9 / 2 / 2010$ & 0.22 & $4 / 14 / 2011$ & 0.66 & $8 / 16 / 2011$ & 0.71 \\
\hline 9/6/2010 & 0.01 & $4 / 15 / 2011$ & 0.36 & 8/18/2011 & 1.11 \\
\hline 9/9/2010 & 0.04 & $4 / 16 / 2011$ & 0.05 & $8 / 19 / 2011$ & 0.21 \\
\hline 9/10/2010 & 0.22 & $4 / 19 / 2011$ & 0.06 & $8 / 20 / 2011$ & 0.59 \\
\hline 9/13/2010 & 0.92 & $4 / 22 / 2011$ & 0.83 & $8 / 22 / 2011$ & 0.86 \\
\hline 9/14/2010 & 0.24 & $4 / 25 / 2011$ & 0.30 & $8 / 30 / 2011$ & 0.75 \\
\hline 9/18/2010 & 0.14 & $4 / 26 / 2011$ & 0.10 & $9 / 3 / 2011$ & 0.16 \\
\hline 9/19/2010 & 0.01 & $5 / 5 / 2011$ & 0.29 & $9 / 9 / 2011$ & 0.13 \\
\hline 9/21/2010 & 0.17 & $5 / 11 / 2011$ & 0.07 & 9/10/2011 & 0.01 \\
\hline 9/22/2010 & 0.07 & $5 / 12 / 2011$ & 0.01 & 9/14/2011 & 0.15 \\
\hline 9/23/2010 & 1.17 & $5 / 13 / 2011$ & 0.04 & 9/15/2011 & 0.01 \\
\hline 9/25/2010 & 0.14 & $5 / 19 / 2011$ & 0.09 & 9/16/2011 & 0.61 \\
\hline 10/11/2010 & 0.16 & $5 / 20 / 2011$ & 0.43 & 9/18/2011 & 0.27 \\
\hline 10/22/2010 & 0.15 & $5 / 21 / 2011$ & 0.15 & 9/19/2011 & 0.01 \\
\hline 10/23/2010 & 0.68 & $5 / 24 / 2011$ & 1.26 & $10 / 12 / 2011$ & 0.02 \\
\hline 10/25/2010 & 0.05 & $5 / 25 / 2011$ & 0.75 & $10 / 17 / 2011$ & 0.07 \\
\hline 10/26/2010 & 0.09 & $5 / 27 / 2011$ & 0.01 & $11 / 2 / 2011$ & 0.33 \\
\hline 11/11/2010 & 0.14 & $5 / 31 / 2011$ & 0.50 & $11 / 3 / 2011$ & 0.03 \\
\hline $11 / 12 / 2010$ & 1.53 & 6/1/2011 & 0.03 & $11 / 7 / 2011$ & 1.97 \\
\hline $11 / 13 / 2010$ & 0.01 & $6 / 2 / 2011$ & 0.24 & $11 / 8 / 2011$ & 0.87 \\
\hline 11/17/2010 & 0.01 & $6 / 9 / 2011$ & 0.10 & $11 / 9 / 2011$ & 0.19 \\
\hline $12 / 11 / 2010$ & 0.04 & $6 / 10 / 2011$ & 0.72 & $11 / 22 / 2011$ & 0.13 \\
\hline $12 / 19 / 2010$ & 0.08 & $6 / 14 / 2011$ & 0.01 & $11 / 25 / 2011$ & 0.01 \\
\hline $12 / 24 / 2010$ & 0.01 & $6 / 16 / 2011$ & 0.06 & $11 / 26 / 2011$ & 0.38 \\
\hline $12 / 28 / 2010$ & 0.06 & $6 / 17 / 2011$ & 0.15 & $12 / 2 / 2011$ & 0.07 \\
\hline $1 / 2 / 2011$ & 0.07 & $6 / 18 / 2011$ & 0.28 & $12 / 3 / 2011$ & 1.16 \\
\hline $1 / 17 / 2011$ & 0.04 & $6 / 19 / 2011$ & 0.02 & & \\
\hline $1 / 22 / 2011$ & 0.07 & $6 / 20 / 2011$ & 0.32 & & \\
\hline
\end{tabular}

Source of data: MUE (2011).

TABLE S1.4 Page 2 of 2 
Supplement 2:

Quality Control for Sample Collection, Handling, and Analysis 


\section{Supplement 2:}

\section{Quality Control for Sample Collection, Handling, and Analysis}

Soil and groundwater sampling was conducted during the 2010-2011 Phase II investigation to complete the scope of work presented in the report of Phase I work at Savannah (Argonne 2010). The QA/QC procedures followed for sample collection, handling, and analysis are described in detail in the Master Work Plan (Argonne 2002).

The sections below discuss the quality of the analytical data generated during the investigation. Evaluation of the organic analytical data was consistent with regulatory guidelines (EPA 1994).

\section{S2.1 Sampling to Monitor Sampling Collection, Handling, and Analysis Procedures}

Sample collection and handling activities were monitored by the documentation of samples as they were collected and the use of chain-of-custody forms and custody seals to ensure sample integrity during handling and shipment. Field blanks, equipment rinsates, and trip blanks were collected to monitor sample collection and handling activities. Field replicate samples were collected, and samples were selected for duplicate analyses as a measure of analytical precision. The QA/QC samples are listed in Table S2.1. Analytical results for carbon tetrachloride and chloroform in QA/QC samples collected to monitor sample collection and handling activities are in Table S2.2.

\section{S2.1.1 Field Blanks}

To verify that contamination detected did not derive from water used for equipment decontamination, 4 field blanks were collected of waters used during the sampling activities. Carbon tetrachloride was not detected in the field blanks. Chloroform was detected at low concentrations in water obtained from the public water supply during the June-July 2010 sampling event. 


\section{S2.1.2 Equipment Rinsates}

To verify that adequate equipment decontamination procedures were followed during the field efforts, 8 equipment rinsates were collected. Neither carbon tetrachloride nor chloroform was detected in the rinsate samples.

\section{S2.1.3 Trip Blanks}

As an indicator of potential cross-contamination of samples during shipment, 20 trip blanks were prepared and shipped with soil or water samples sent to laboratories for organic analysis. Included in this total were 9 water trip blanks and 6 soil trip blanks sent to the AGEM Laboratory and 4 water trip blanks and 1 soil trip blank sent to TestAmerica. The analytical results, shown in Table S2.2, indicate that sample handling procedures were followed during the investigation and that cross-contamination did not occur during shipment.

\section{S2.1.4 Replicate Samples and Duplicate Analyses}

As an indicator of the consistency of the sampling methodology followed and to provide a measure of analytical precision, replicate soil and groundwater samples were collected, and samples were selected by the AGEM Laboratory for duplicate organic analysis. In addition, selected soil and groundwater samples were submitted for verification organic analysis at a secondary laboratory. Replicate samples, samples selected for duplicate analysis, and samples selected for verification organic analysis are listed in Table S2.1.

\section{S2.2 Quality Control for Organic Analysis of Soil and Water Samples at the AGEM Laboratory}

To obtain additional information on the possible occurrence of a soil source of carbon tetrachloride to groundwater in the vicinity of the former CCC/USDA facility, four locations (SB43-SB46) were investigated in June 2010. Borings SB43 and SB44 were positioned along the northern margin of the property formerly occupied by the CCC/USDA grain storage facility. Boring SB46 was located on the MoDOT property immediately west of the contaminated MoDOT well. Boring SB45 was positioned downgradient of the MoDOT well. At each location, soil samples were collected for VOCs analysis at 4 - $\mathrm{ft}$ intervals from $4 \mathrm{ft}$ BGL to the top of 
bedrock. Soil samples were quick-frozen on dry ice as they were collected and shipped to the AGEM Laboratory for VOCs analysis by two analytical methods: (1) the headspace method to provide a rapid-turnaround qualitative measure of contaminant distribution for evaluation in the field and (2) the purge-and-trap method to provide a quantitative measure of contaminant distribution.

The soil samples were first analyzed at the AGEM Laboratory (on a rapid-turnaround basis) by using a modification of the protocol in EPA Method 5021 (headspace analysis using a GC with electron capture detection; EPA 1998). An 11-point calibration of the GC system was established on the basis of the mass of known quantities of carbon tetrachloride and chloroform, from $0.125 \mathrm{ng}$ to $4 \mathrm{ng}$. Typical detection limits achieved were $0.10 \mu \mathrm{g} / \mathrm{kg}$ for carbon tetrachloride and $0.75 \mu \mathrm{g} / \mathrm{kg}$ for chloroform. The analytical data obtained with this method are acceptable for qualitative determination of contaminant distribution.

Aliquots of the samples were subsequently analyzed for carbon tetrachloride, chloroform, and methylene chloride (contaminants of concern in the investigation) by using a modification of EPA Method 8260B (a purge-and-trap method; EPA 1998) to achieve a quantitation limit of $10 \mu \mathrm{g} / \mathrm{kg}$. At the laboratory, the VOCs in each soil sample were extracted with methanol from the sample matrix. For the purge-and-trap soil analyses, an aliquot of the methanol extract was purged, and the volatile species were transferred to a sorbent tube. After purging, the sorbent tube was heated and backflushed with an inert gas to desorb the components into the GC-MS system.

In total, 119 soil samples were analyzed, including 4 replicate soil samples and 6 trip blanks collected for QC purposes.

Groundwater sampling was conducted during the Phase II investigation in three sampling events. In June-July 2010, the monitoring well network initially installed during Phase I was expanded and sampled, and the two contaminated private wells (the Morgan well and the MoDOT well) were resampled. In August 2010 and again in August 2011, the entire monitoring well network was sampled to provide a quantitative measure of contaminant distribution. Water samples (and associated QC samples) were sent to the AGEM Laboratory for VOCs analysis by modified EPA Method 524.2 (EPA 1995) to achieve a quantitation limit of $1.0 \mu \mathrm{g} / \mathrm{L}$. For these purge-and-trap analyses with a GC-MS system, VOCs in the groundwater samples were extracted (purged) from the sample matrix by bubbling an inert gas through each sample. The 
purged components were trapped in a sorbent tube. After the purging, the sorbent tube was heated and backflushed with an inert gas to desorb the components into the GC-MS system.

For both the soil and water analyses, the compounds eluting from the GC column were identified by retention time and by comparison with reference library spectra. The concentration of each component was calculated by comparison of the MS response for the quantitation ion to corresponding calibration curves and/or the response for internal standards. The internal standard recovery limits were $80-120 \%$. Calibration checks with each sample delivery group (SDG) were required to be within $\pm 20 \%$ of the standard.

Samples submitted to the AGEM Laboratory for organic analysis by the purge-and-trap method were analyzed in 19 SDGs, as shown in Table S2.3. The QA/QC procedures followed included analysis of instrument calibration check standards, analysis of laboratory blanks, monitoring of surrogate spike recovery, and duplicate laboratory analyses. Significant results include the following:

- Samples shipped to the AGEM Laboratory were received with custody seals intact and at the appropriate temperature. Samples were analyzed within required holding times.

- Carbon tetrachloride and chloroform were not detected in laboratory method blanks analyzed with the samples.

- For each SDG, analytical instrument calibration was monitored by the analysis of calibration check standards. Table S2.3 shows the relative percent difference (RPD) between the known and calculated concentrations of the standards. The concentrations of calibration check standards measured in all SDGs were within the acceptable range of $\pm 20 \%$.

- Surrogate standard determinations were performed on samples and blanks by using surrogate spike compounds fluorobenzene, dichlorobenzene- $\mathrm{d}_{4}$, and bromofluorobenzene. Table S2.3 shows the percent recovery of these systemmonitoring compounds for each of the analyses. The surrogate recoveries were within the specified range of $80-120 \%$ for all samples, in either the initial analysis or a successful reanalysis. 
- Secondary QC analyses of soil and groundwater samples at the AGEM Laboratory by the purge-and-trap method were conducted throughout the investigation as a measure of the consistency in the sampling and analytical methodologies. This was accomplished through the analysis of replicate samples submitted to the laboratory or duplicate analysis of samples selected by the laboratory. Table S2.4 summarizes the analytical results for carbon tetrachloride and chloroform in the primary samples and the associated replicate or duplicate analyses. Consistency in both the sampling and analytical methodologies is indicated by the average RPD values of $7 \%$ for carbon tetrachloride and $6 \%$ for chloroform in the dual analyses with the contaminants present above the method quantitation limit.

The analytical data from the AGEM Laboratory are acceptable for quantitative determination of contaminant distribution.

\section{S2.3 Quality Control for Verification Organic Analysis of Soil and Groundwater Samples by TestAmerica}

In accordance with the QA/QC procedures defined in the Master Work Plan (Argonne 2002), selected soil and groundwater samples analyzed at the AGEM Laboratory were subjected to verification analysis at a second laboratory with CLP methodology. Of the 109 vertical-profile soil samples analyzed at the AGEM Laboratory, 11 samples ( $10 \%$ of the soil samples) were also analyzed by TestAmerica. In addition, 18 of the 88 groundwater samples analyzed at the AGEM Laboratory (20\% of the groundwater samples) were also analyzed by TestAmerica. The results, reported by TestAmerica in 5 SDGs (137933, 137941, 138033, 200-1286, and 200-6522), are included as Supplement 3 (on CD).

The QA/QC procedures followed included initial and continuing calibration of instruments, analysis of laboratory blanks, monitoring of surrogate spike recovery, and analyses of laboratory QC samples. Significant results include the following:

- Soil and groundwater samples shipped to TestAmerica were received with custody seals intact and at the appropriate preservation conditions. All samples were analyzed within the required holding times. 
- Analytical instruments were properly tuned; initial and continuing calibration checks remained within the allowable limits. Surrogate standard determinations were also within allowable limits.

- Carbon tetrachloride and chloroform, the primary contaminants of concern in the investigation, were not detected in the associated blanks analyzed with the samples.

The verification organic results for the soil and groundwater samples analyzed at the AGEM Laboratory and TestAmerica are summarized in Table S2.5. Agreement is good over the range of contaminant concentrations detected. The concentrations detected in soil and groundwater during analyses at the AGEM Laboratory are supported by the TestAmerica verification analyses. 
TABLE S2.1 Quality control samples collected during the Phase II investigation.

\begin{tabular}{|c|c|c|c|c|c|c|c|c|}
\hline Location & $\begin{array}{l}\text { Sample } \\
\text { Date }\end{array}$ & $\begin{array}{l}\text { Sample } \\
\text { Time }\end{array}$ & Sample & $\begin{array}{l}\text { Sample } \\
\text { Medium }\end{array}$ & $\begin{array}{c}\text { Depth } \\
\text { (ft BGL) }\end{array}$ & $\begin{array}{l}\text { Chain of } \\
\text { Custody }\end{array}$ & $\begin{array}{l}\text { Shipping } \\
\text { Date }\end{array}$ & Sample Description \\
\hline
\end{tabular}

Field blanks

$\begin{array}{llll}\text { QC } & 8 / 25 / 10 & 15: 46 & \text { SVDIH2O-W-25846 } \\ & & & \\ \text { QC } & 6 / 21 / 10 & 17: 35 & \text { SVTRUCK-W-25667 } \\ \text { QC } & 6 / 21 / 10 & 17: 45 & \text { SVTAP1-W-25668 } \\ \text { QC } & 8 / 11 / 11 & 19: 24 & \text { SVDIH2O-W-25890 }\end{array}$

$\begin{array}{lll}\text { Water } & - & 2740 \\ & & \\ \text { Water } & - & 2875 \\ \text { Water } & - & 2875 \\ \text { Water } & - & 2990\end{array}$

8/25/10 Blank of water used during August 2010 monitoring event.

6/21/10 Blank of water in field support truck

$6 / 21 / 10$ Blank of water from city tap for field support truck.

8/11/11 Blank of water used during August 2011 monitoring event.

\section{Equipment rinsates}

$\begin{array}{llrl}\text { QC } & 8 / 24 / 10 & 13: 26 & \text { SVQCIR-W-25847 } \\ \text { QC } & 8 / 24 / 10 & 16: 34 & \text { SVQCIR-W-25848 } \\ \text { QC } & 8 / 25 / 10 & 9: 56 & \text { SVQCIR-W-25849 } \\ \text { QC } & 8 / 25 / 10 & 10: 33 & \text { SVQCIR-W-25850 } \\ \text { QC } & 8 / 10 / 11 & 12: 40 & \text { SVQCIR-W-25891 } \\ \text { QC } & 8 / 10 / 11 & 19: 50 & \text { SVQCIR-W-25892 } \\ \text { QC } & 8 / 11 / 11 & 13: 42 & \text { SVQCIR-W-25894 } \\ \text { QC } & 8 / 11 / 11 & 15: 23 & \text { SVQCIR-W-25893 }\end{array}$

$\begin{array}{lll}\text { Water } & - & 2739 \\ \text { Water } & - & 2739 \\ \text { Water } & - & 2740 \\ \text { Water } & - & 2740 \\ \text { Water } & - & 2989 \\ \text { Water } & - & 2989 \\ \text { Water } & - & 2990 \\ \text { Water } & - & 2990\end{array}$

8/24/10 Rinsate of decontaminated sampling line after collection of sample SVSB44D-W-25833.

8/24/10 Rinsate of decontaminated sampling line after collection of sample SVSB48D-W-25841.

8/25/10 Rinsate of decontaminated sampling line after collection of sample SVSB45S-W-25834.

8/25/10 Rinsate of decontaminated sampling line after collection of sample SVSB47S-W-25838.

8/10/11 Rinsate of decontaminated sampling bailer after collection of sample SVSB38GRAB-W-25857.

8/10/11 Rinsate of decontaminated sampling line after collection of sample SVSB49S-W-25886

8/11/11 Rinsate of decontaminated sampling line after collection of sample SVSB44S-W-25876.

8/11/11 Rinsate of decontaminated sampling line after collection of sample SVSB46D-W-25881 and replicate SVSB46DDUP-W-25889.

Trip blanks

\begin{tabular}{|c|c|c|c|}
\hline QC & $6 / 21 / 10$ & $16: 10$ & SVQCTB-S-25671 \\
\hline QC & $6 / 21 / 10$ & $17: 55$ & SVSB43-W-25669 \\
\hline
\end{tabular}

Soil

Water
2874

2875
6/21/10 Trip blank sent to the AGEM Laboratory for organic analysis with soil samples listed on chain-ofcustody forms (COCs) 2873 and 2874.

6/21/10 Trip blank sent to the AGEM Laboratory for organic analysis with water samples listed on COC 2875 


\begin{tabular}{|c|c|c|c|c|c|c|c|c|}
\hline Location & $\begin{array}{l}\text { Sample } \\
\text { Date }\end{array}$ & $\begin{array}{l}\text { Sample } \\
\text { Time }\end{array}$ & Sample & $\begin{array}{l}\text { Sample } \\
\text { Medium }\end{array}$ & $\begin{array}{l}\text { Depth } \\
\text { (ft BGL) }\end{array}$ & $\begin{array}{l}\text { Chain of } \\
\text { Custody }\end{array}$ & $\begin{array}{l}\text { Shipping } \\
\text { Date }\end{array}$ & Sample Description \\
\hline \multicolumn{9}{|c|}{ Trip blanks (cont.) } \\
\hline QC & $6 / 22 / 10$ & 17:09 & SVSB43-S-25675 & Soil & - & 2876 & $6 / 22 / 10$ & $\begin{array}{l}\text { Trip blank sent to the AGEM Laboratory for organic } \\
\text { analysis with soil samples listed on COC } 2876 \text {. }\end{array}$ \\
\hline QC & $6 / 23 / 10$ & $10: 40$ & SVSB44-S-25707 & Soil & - & 2877 & $6 / 23 / 10$ & $\begin{array}{l}\text { Trip blank sent to the AGEM Laboratory for organic } \\
\text { analysis with soil samples listed on COCs } 2878, \\
2877 \text {, and } 2879 \text {. }\end{array}$ \\
\hline QC & $6 / 24 / 10$ & 12:01 & SVSB45-S-25746 & Soil & - & 2880 & $6 / 24 / 10$ & $\begin{array}{l}\text { Trip blank sent to the AGEM Laboratory for organic } \\
\text { analysis with soil samples listed on COCs } 2881 \\
\text { and } 2880 .\end{array}$ \\
\hline QC & $6 / 25 / 10$ & $14: 00$ & SVSB46-S-25765 & Soil & - & 2882 & $6 / 25 / 10$ & $\begin{array}{l}\text { Trip blank sent to the AGEM Laboratory for organic } \\
\text { analysis with soil samples listed on COCs } 2882 \\
\text { and } 2883 \text {. }\end{array}$ \\
\hline QC & $6 / 25 / 10$ & $18: 30$ & SVQCTB-W-25777 & Water & - & 2665 & $6 / 25 / 10$ & $\begin{array}{l}\text { Trip blank sent to the AGEM Laboratory for organic } \\
\text { analysis with water samples listed on COC } 2665 \text {. }\end{array}$ \\
\hline QC & $6 / 25 / 10$ & $18: 37$ & SVQCTB-W-25852 & Water & - & 2740 & $6 / 25 / 10$ & $\begin{array}{l}\text { Trip blank sent to the AGEM Laboratory for organic } \\
\text { analysis with water samples listed on COC } 2740 \text {. }\end{array}$ \\
\hline QC & $6 / 25 / 10$ & 18:37 & SVQCTB-W-25777 & Water & - & 2666 & $6 / 25 / 10$ & $\begin{array}{l}\text { Trip blank sent to TestAmerica for verification } \\
\text { organic analysis with water samples listed on } \\
\text { COC } 2666 \text {. }\end{array}$ \\
\hline QC & $6 / 26 / 10$ & 19:20 & SVQCTB-S-25715 & Soil & - & 2670 & $6 / 28 / 10$ & $\begin{array}{l}\text { Trip blank sent to the AGEM Laboratory for organic } \\
\text { analysis with soil samples listed on COC } 2670 \text {. }\end{array}$ \\
\hline QC & $6 / 28 / 10$ & 19:14 & SVQCTB-W-25780 & Water & - & 2663 & $6 / 28 / 10$ & $\begin{array}{l}\text { Trip blank sent to the AGEM Laboratory for organic } \\
\text { analysis with water samples listed on COC } 2663 \text {. }\end{array}$ \\
\hline QC & $6 / 28 / 10$ & 19:47 & SVQCTB-W-25780 & Water & - & 2663 & $6 / 28 / 10$ & $\begin{array}{l}\text { Trip blank sent to TestAmerica for verification } \\
\text { organic analysis with water samples listed on } \\
\text { COC } 2671 \text {. }\end{array}$ \\
\hline QC & $7 / 1 / 10$ & 9:15 & SV-S-MEOH BLANK 1JUL10 & Soil & - & 4047 & $7 / 1 / 10$ & $\begin{array}{l}\text { Trip blank sent to TestAmerica for verification } \\
\text { organic analysis with soil samples listed on COC } \\
4047 \text {. }\end{array}$ \\
\hline QC & $7 / 6 / 10$ & $12: 00$ & SVQCTB-W-25814 & Water & - & 2865 & $7 / 6 / 10$ & $\begin{array}{l}\text { Trip blank sent to the AGEM Laboratory for organic } \\
\text { analysis with water samples listed on COC } 2865 \text {. }\end{array}$ \\
\hline QC & $8 / 24 / 10$ & $18: 30$ & SVQCTB-W-25829 & Water & - & 2678 & $8 / 24 / 10$ & $\begin{array}{l}\text { Trip blank sent to the AGEM Laboratory for organic } \\
\text { analysis with water samples listed on COC } 2678 \text {. }\end{array}$ \\
\hline QC & $8 / 25 / 10$ & $15: 30$ & SVQCTB-W-25852 & Water & - & 2740 & $8 / 25 / 10$ & $\begin{array}{l}\text { Trip blank sent to the AGEM Laboratory for organic } \\
\text { analysis with water samples listed on COC } 2740 \text {. }\end{array}$ \\
\hline $\mathrm{QC}$ & $8 / 10 / 11$ & $15: 25$ & SVQCTB-W-25895 & Water & - & 2988 & 8/10/11 & $\begin{array}{l}\text { Trip blank sent to the AGEM Laboratory for organic } \\
\text { analysis with water samples listed on COC } 2988 .\end{array}$ \\
\hline
\end{tabular}


TABLE S2.1 (Cont.)

\begin{tabular}{|c|c|c|c|c|c|c|c|c|}
\hline Location & $\begin{array}{c}\text { Sample } \\
\text { Date }\end{array}$ & $\begin{array}{c}\text { Sample } \\
\text { Time }\end{array}$ & Sample & $\begin{array}{l}\text { Sample } \\
\text { Medium }\end{array}$ & $\begin{array}{l}\text { Depth } \\
\text { (ft BGL) }\end{array}$ & $\begin{array}{l}\text { Chain of } \\
\text { Custody }\end{array}$ & $\begin{array}{l}\text { Shipping } \\
\text { Date }\end{array}$ & Sample Description \\
\hline
\end{tabular}

Trip blanks (cont.)

\begin{tabular}{|c|c|c|c|c|c|c|}
\hline QC & $8 / 10 / 11$ & 19:00 & SVQCTB-W-25896 & Water & - & 2989 \\
\hline QC & $8 / 11 / 11$ & 19:00 & SVQCTB-W-25897 & Water & - & 2991 \\
\hline QC & $8 / 11 / 11$ & $18: 30$ & SVQCTB-W-25897 & Water & - & 2990 \\
\hline \multicolumn{7}{|c|}{ Replicate samples } \\
\hline SB43 & $6 / 21 / 10$ & $14: 18$ & SVSB43-S-25650 & Soil & 48 & 2873 \\
\hline SB43 & $6 / 22 / 10$ & $7: 58$ & SVSB43-S-25673 & Soil & 104 & 2876 \\
\hline SB44 & $6 / 23 / 10$ & $10: 07$ & SVSB44-S-25705 & Soil & 80 & 2879 \\
\hline SB45 & $6 / 24 / 10$ & $15: 00$ & SVSB45-S-25747 & Soil & 100 & 2880 \\
\hline SB46 & $6 / 25 / 10$ & $10: 54$ & SVSB46-S-25766 & Soil & 36 & 2882 \\
\hline SB49D & $8 / 24 / 10$ & $11: 42$ & SVSB49DDUP-W-25844 & Water & $77-97$ & 2739 \\
\hline SB48S & $8 / 25 / 10$ & $11: 13$ & SVSB48SDUP-W-25845 & Water & $25-35$ & 2740 \\
\hline SB43D & $8 / 10 / 11$ & $17: 15$ & SVSB43DDUP-W-25888 & Water & $95-105$ & 2989 \\
\hline SB46D & $8 / 11 / 11$ & $14: 03$ & SVSB46DDUP-W-25889 & Water & $90-110$ & 2990 \\
\hline
\end{tabular}

8/10/11 Trip blank sent to the AGEM Laboratory for organic analysis with water samples listed on COC 2989.

8/11/11 Trip blank sent to TestAmerica for verification

organic analysis with water samples listed on COC 2991.

8/11/11 Trip blank sent to the AGEM Laboratory for organic analysis with water samples listed on COC 2990.

Samples selected for duplicate organic analysis by the AGEM Laboratory

$\begin{array}{lrrlcrr}\text { SB43 } & 6 / 21 / 10 & 14: 18 & \text { SVSB43-S-25648 } & \text { Soil } & 48 & 2873 \\ \text { SB43 } & 6 / 21 / 10 & 16: 10 & \text { SVSB43-S-25658 } & \text { Soil } & 80 & 2874 \\ \text { SB43 } & 6 / 21 / 10 & 18: 32 & \text { SVSB43-W-25666 } & \text { Water } & 95-100 & 2875 \\ & & & & & & \\ \text { SB44 } & 6 / 23 / 10 & 8: 53 & \text { SVSB44-S-25688 } & \text { Soil } & 52 & 2878 \\ \text { SB44 } & 6 / 23 / 10 & 9: 35 & \text { SVSB44-S-25693 } & \text { Soil } & 72 & 2877 \\ \text { SB45 } & 6 / 24 / 10 & 11: 36 & \text { SVSB45-S-25731 } & \text { Soil } & 44 & 2881 \\ \text { SB46 } & 6 / 25 / 10 & 14: 48 & \text { SVSB46-S-25761 } & \text { Soil } & 88 & 2882 \\ \text { MoDOT } & 6 / 25 / 10 & 16: 26 & \text { SVMODOTLFB-W-25775 } & \text { Water } & 44 & 2665 \\ \text { MoDOT } & 6 / 27 / 10 & 16: 44 & \text { SVMODOT3X-W-25778 } & \text { Water } & 44 & 2663 \\ \text { Morgan } & 6 / 28 / 10 & 14: 56 & \text { SVMORGAN3X-W-25779 } & \text { Water } & 60.58 & 2663 \\ \text { SB44S } & 8 / 24 / 10 & 17: 24 & \text { SVSB44S-W-25832 } & \text { Water } & 46.5-56.5 & 2739 \\ \text { SB47D } & 8 / 24 / 10 & 18: 42 & \text { SVSB47D-W-25839 } & \text { Water } & 77-97 & 2739 \\ \text { SB48S } & 8 / 25 / 10 & 11: 13 & \text { SVSB48S-W-25840 } & \text { Water } & 25-35 & 2740\end{array}$

6/21/10 Replicate of sample SVSB43-S-25648.

6/22/10 Replicate of SVSB44-S-25672.

6/23/10 Replicate of SVSB44-S-25695.

6/24/10 Replicate of sample SVSB45-S-25745

6/25/10 Replicate of sample SVSB46-S-25718.

8/24/10 Replicate of sample SVSB49D-W-25843.

8/25/10 Replicate of sample SVSB48S-W-25840.

8/10/11 Replicate of sample SVSB43D-W-25875

8/11/11 Replicate of sample SVSB46D-W-25881.

6/21/10 CPT subsurface soil sample.

6/21/10 CPT subsurface soil sample.

$6 / 21 / 10$ Collected from open borehole via bailer during recovery of core.

6/23/10 CPT subsurface soil sample.

$6 / 23 / 10$ CPT subsurface soil sample.

6/24/10 CPT subsurface soil sample.

6/25/10 CPT subsurface soil sample.

6/25/10 Low-flow sample.

$6 / 28 / 10$ Three-well-volume purge sample.

$6 / 28 / 10$ Three-well-volume purge sample.

$8 / 24 / 10$

$8 / 24 / 10$

$8 / 25 / 10$ 
TABLE S2.1 (Cont.)

\begin{tabular}{|c|c|c|c|c|c|c|c|c|}
\hline Location & $\begin{array}{l}\text { Sample } \\
\text { Date }\end{array}$ & $\begin{array}{l}\text { Sample } \\
\text { Time }\end{array}$ & Sample & $\begin{array}{l}\text { Sample } \\
\text { Medium }\end{array}$ & $\begin{array}{c}\text { Depth } \\
\text { (ft BGL) }\end{array}$ & $\begin{array}{l}\text { Chain of } \\
\text { Custody }\end{array}$ & $\begin{array}{l}\text { Shipping } \\
\text { Date }\end{array}$ & Sample Description \\
\hline
\end{tabular}

Samples selected for duplicate organic analysis by the AGEM Laboratory (cont.)

\begin{tabular}{|c|c|c|c|c|c|c|c|c|}
\hline SB44S & $8 / 10 / 11$ & $11: 16$ & SVSB44SGRAB-W-25860 & Water & $46.5-56.5$ & 2988 & $8 / 10 / 11$ & $\begin{array}{l}\text { Grab sample collected without purging. Bailer } \\
\text { lowered to bottom of well and withdrawn. }\end{array}$ \\
\hline SB49D & $8 / 10 / 11$ & $12: 25$ & SVSB49DGRAB-W-25871 & Water & $77-97$ & 2989 & $8 / 10 / 11$ & $\begin{array}{l}\text { Grab sample collected without purging. Bailer } \\
\text { lowered to bottom of well and withdrawn. }\end{array}$ \\
\hline SB48S & $8 / 10 / 11$ & $14: 43$ & SVSB48SGRAB-W-25868 & Water & $25-35$ & 2988 & $8 / 10 / 11$ & Grab sample collected without purging. \\
\hline \multicolumn{9}{|c|}{ Samples selected for verification organic analysis at TestAmerica } \\
\hline SB43 & $6 / 21 / 10$ & $12: 30$ & SVSB43-S-25643 & Soil & 18 & 2873 & $6 / 21 / 10$ & CPT subsurface soil sample. \\
\hline SB43 & $6 / 21 / 10$ & 14:18 & SVSB43-S-25650 & Soil & 48 & 2873 & $6 / 21 / 10$ & Replicate of sample SVSB43-S-25648. \\
\hline SB43 & $6 / 21 / 10$ & 15:08 & SVSB43-S-25654 & Soil & 64 & 2874 & $6 / 21 / 10$ & CPT subsurface soil sample. \\
\hline SB44 & $6 / 23 / 10$ & $7: 32$ & SVSB44-S-25677 & Soil & 8 & 2878 & $6 / 23 / 10$ & CPT subsurface soil sample. \\
\hline SB44 & $6 / 23 / 10$ & $8: 53$ & SVSB44-S-25688 & Soil & 52 & 2878 & $6 / 23 / 10$ & CPT subsurface soil sample. \\
\hline SB44 & $6 / 23 / 10$ & $11: 22$ & SVSB44-S-25698 & Soil & 89 & 2877 & $6 / 23 / 10$ & CPT subsurface soil sample. \\
\hline Morgan & $6 / 23 / 10$ & $14: 14$ & SVMORGANLFB-W-25770 & Water & 60.58 & 2665 & $6 / 25 / 10$ & Low-flow sample. \\
\hline Morgan & $6 / 23 / 10$ & $15: 27$ & SVMORGANLEM-W-25771 & Water & 60.58 & 2665 & $6 / 25 / 10$ & Low-flow sample. \\
\hline SB38 & $6 / 23 / 10$ & $17: 55$ & SVSB38LFB-W-25772 & Water & $50-90$ & 2665 & $6 / 25 / 10$ & Low-flow sample. \\
\hline SB45 & $6 / 24 / 10$ & $10: 57$ & SVSB45-S-25723 & Soil & 16 & 2881 & $6 / 24 / 10$ & CPT subsurface soil sample. \\
\hline SB38 & $6 / 24 / 10$ & $12: 22$ & SVSB38LFM-W-25773 & Water & $50-90$ & 2665 & $6 / 25 / 10$ & Low-flow sample. \\
\hline SB45 & $6 / 24 / 10$ & $13: 00$ & SVSB45-S-25736 & Soil & 64 & 2880 & $6 / 24 / 10$ & CPT subsurface soil sample. \\
\hline SB45 & $6 / 24 / 10$ & $15: 00$ & SVSB45-S-25745 & Soil & 100 & 2880 & $6 / 24 / 10$ & CPT subsurface soil sample. \\
\hline SB46 & $6 / 25 / 10$ & $10: 58$ & SVSB46-S-25719 & Soil & 40 & 2883 & $6 / 25 / 10$ & CPT subsurface soil sample. \\
\hline SB46 & $6 / 25 / 10$ & $12: 34$ & SVSB46-S-25756 & Soil & 68 & 2883 & $6 / 25 / 10$ & CPT subsurface soil sample. \\
\hline SB24 & $6 / 25 / 10$ & $14: 54$ & SVSB24LFB-W-25774 & Water & $50-60$ & 2665 & $6 / 25 / 10$ & Low-flow sample. \\
\hline MoDOT & $6 / 25 / 10$ & $16: 26$ & SVMODOTLFB-W-25775 & Water & 44 & 2665 & $6 / 25 / 10$ & Low-flow sample. \\
\hline MoDOT & $6 / 25 / 10$ & $17: 58$ & SVMODOTLFM-W-25776 & Water & 44 & 2665 & $6 / 25 / 10$ & Low-flow sample. \\
\hline MoDOT & $6 / 27 / 10$ & $16: 44$ & SVMODOT3X-W-25778 & Water & 44 & 2663 & $6 / 28 / 10$ & Three-well-volume purge sample. \\
\hline Morgan & $6 / 28 / 10$ & $14: 56$ & SVMORGAN3X-W-25779 & Water & 60.58 & 2663 & $6 / 28 / 10$ & Three-well-volume purge sample. \\
\hline SB49D & $8 / 24 / 10$ & $15: 00$ & SVSB49D-W-25843 & Water & $77-97$ & 2739 & $8 / 24 / 10$ & \\
\hline SB48D & $8 / 24 / 10$ & $15: 28$ & SVSB48D-W-25841 & Water & $77-97$ & 2739 & $8 / 24 / 10$ & \\
\hline SB43D & $8 / 24 / 10$ & $16: 18$ & SVSB43D-W-25831 & Water & $95-105$ & 2739 & $8 / 24 / 10$ & \\
\hline SB47S & $8 / 25 / 10$ & $10: 10$ & SVSB47S-W-25838 & Water & $25-35$ & 2740 & $8 / 25 / 10$ & \\
\hline SB46S & $8 / 25 / 10$ & $12: 22$ & SVSB46S-W-25836 & Water & $42-52$ & 2740 & $8 / 25 / 10$ & \\
\hline
\end{tabular}




\begin{tabular}{|c|c|c|c|c|c|c|c|c|}
\hline Location & $\begin{array}{l}\text { Sample } \\
\text { Date }\end{array}$ & $\begin{array}{l}\text { Sample } \\
\text { Time }\end{array}$ & Sample & $\begin{array}{l}\text { Sample } \\
\text { Medium }\end{array}$ & $\begin{array}{l}\text { Depth } \\
\text { (ft BGL) }\end{array}$ & $\begin{array}{l}\text { Chain of } \\
\text { Custody }\end{array}$ & $\begin{array}{l}\text { Shipping } \\
\text { Date }\end{array}$ & Sample Description \\
\hline \multicolumn{9}{|c|}{ Samples selected for verification organic analysis at TestAmerica (cont.) } \\
\hline SB43S & $8 / 10 / 11$ & 16:08 & SVSB43S-W-25874 & Water & $45-55$ & 2989 & $8 / 10 / 11$ & $\begin{array}{l}\text { Depth to water }=24.30 \mathrm{ft} \text {. Depth of } 2 \text {-in. well }= \\
55.03 \mathrm{ft} \text {. Sample collected by using low-flow } \\
\text { bladder pump after purging of } 11 \mathrm{~L} \text {. Pump intake } \\
\text { positioned at } 53 \mathrm{ft} \text {. }\end{array}$ \\
\hline SB47D & $8 / 10 / 11$ & 18:14 & SVSB47D-W-25883 & Water & $77-97$ & 2989 & $8 / 10 / 11$ & $\begin{array}{l}\text { Depth to water }=40.53 \mathrm{ft} \text {. Depth of } 2 \text {-in. well }= \\
97.65 \mathrm{ft} \text {. Sample collected by using low-flow } \\
\text { bladder pump after purging of } 9 \text { L. Pump intake } \\
\text { positioned at } 95 \mathrm{ft} \text {. }\end{array}$ \\
\hline SB44D & $8 / 11 / 11$ & 12:10 & SVSB44D-W-25877 & Water & $92-102$ & 2990 & $8 / 11 / 11$ & $\begin{array}{l}\text { Depth to water }=61.70 \mathrm{ft} \text {. Depth of } 2 \text {-in. well }= \\
102.01 \mathrm{ft} \text {. Sample collected by using low-flow } \\
\text { bladder pump after purging of } 9 \mathrm{~L} \text {. Pump intake } \\
\text { positioned at } 100 \mathrm{ft} \text {. }\end{array}$ \\
\hline SB46S & $8 / 11 / 11$ & 16:13 & SVSB46S-W-25880 & Water & $42-52$ & 2990 & $8 / 11 / 11$ & $\begin{array}{l}\text { Depth to water }=21.72 \mathrm{ft} \text {. Depth of 2-in. well = } \\
51.8 \mathrm{ft} \text {. Sample collected by using low-flow } \\
\text { bladder pump after purging of } 6 \text { L. Pump intake } \\
\text { positioned at } 50 \mathrm{ft} \text {. }\end{array}$ \\
\hline
\end{tabular}
$5.03 \mathrm{ft}$. Sample collected by using low-flow bladder pump after purging of $11 \mathrm{~L}$. Pump intake tioned at $53 \mathrm{ft}$ bladder pump after purging of $9 \mathrm{~L}$. Pump intake

bladder pump after purging of $6 \mathrm{~L}$. Pump intake 
TABLE S2.2 Results from the AGEM Laboratory for samples collected to monitoring sample collection and handling activities during the Phase II investigation.

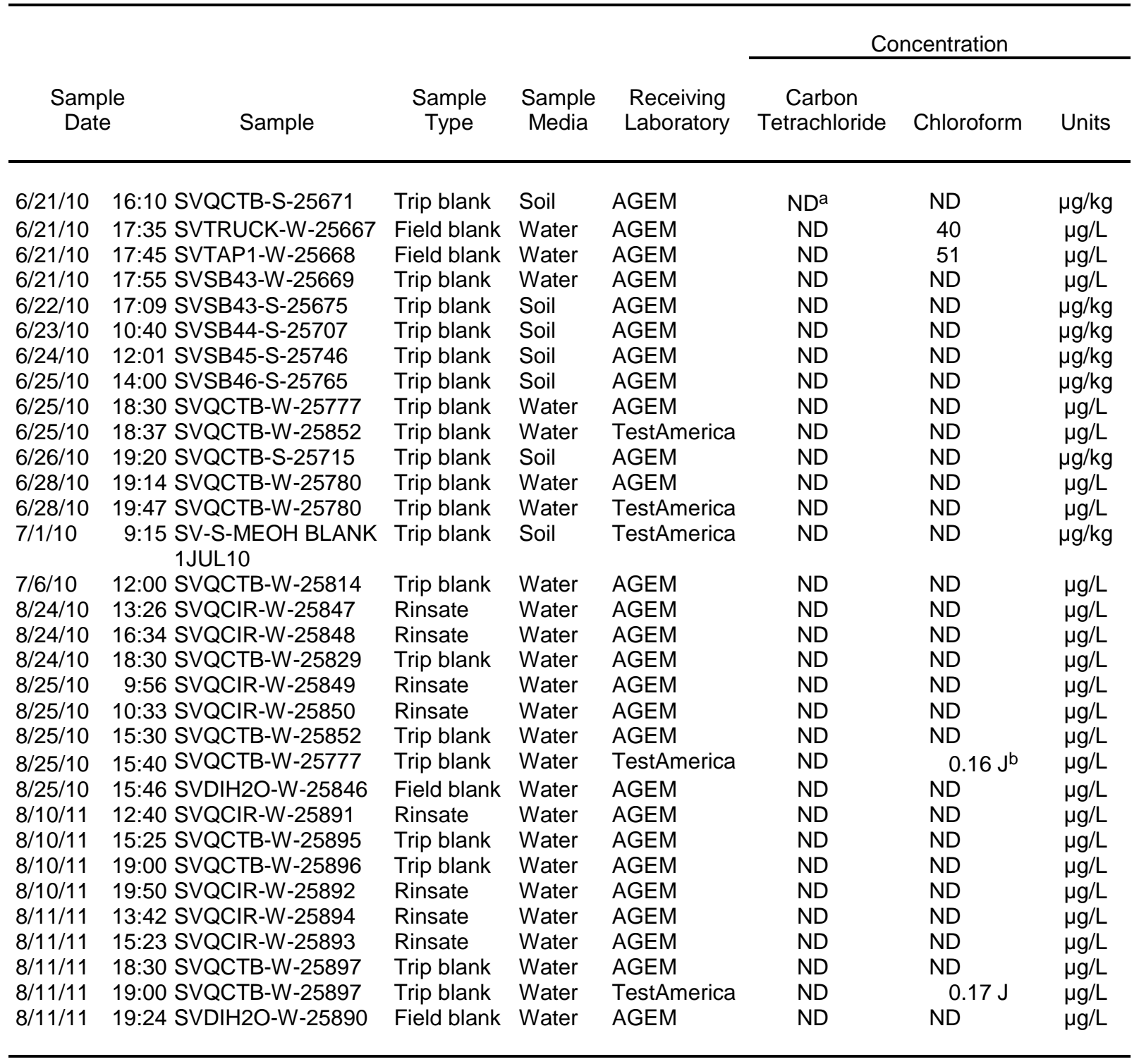

a ND, not detected at an instrument detection limit of $0.1 \mu \mathrm{g} / \mathrm{L}$ for water or $1.0 \mu \mathrm{g} / \mathrm{kg}$ for soil.

b J, estimated concentration below the method quantitation limit. 
TABLE S2.3 Calibration and surrogate recovery during organic analyses of soil and water samples at the AGEM Laboratory.

\begin{tabular}{|c|c|c|c|c|c|c|c|c|}
\hline \multirow[b]{3}{*}{ Sample } & \multicolumn{3}{|c|}{ Recovery of Surrogate Compounds ${ }^{\mathrm{a}}(\%)$} & \multicolumn{5}{|c|}{$\begin{array}{l}\text { Measured Concentration and RPD Value for } \\
\text { Calibration Check Standard }\end{array}$} \\
\hline & & Bromo- & Dichloro- & $\begin{array}{l}\text { Carbon } \\
\text { Tetrachloride }\end{array}$ & & oform & $\begin{array}{r}\text { Met } \\
\text { Ch }\end{array}$ & $\begin{array}{l}\text { lene } \\
\text { ride }\end{array}$ \\
\hline & Fluorobenzene & fluorobenzene & benzene- $d_{4}$ & $\mathrm{ppb}^{\mathrm{b}} \quad \mathrm{RPD}^{\mathrm{c}}$ & $\mathrm{ppb}^{\mathrm{b}}$ & $\mathrm{RPD}^{\mathrm{c}}$ & $\mathrm{ppb}^{\mathrm{b}}$ & $\mathrm{RPD}^{\mathrm{c}}$ \\
\hline
\end{tabular}

SDG 10-6-23, analysis date June 23, 2010

20-ppb standard

Laboratory blank

SVSB43-W-25666

SVSB43-W-25666DUP

SVSB43-W-25669

SVTRUCK-W-25667

SVTAP1-W-25668

$\begin{array}{rr}104 & 115 \\ 113 & 114 \\ 95 & 103 \\ 110 & 115 \\ 107 & 116 \\ 89 & 94 \\ 82 & 89\end{array}$

SDG 10-6-24a, analysis date June 24, 2010

20-ppb standard
Methanol blank
SVSB43-S-25664
SVSB43-S-25663
SVSB43-S-25670
SVSB43-S-25643
SVSB43-S-25645
SVSB43-S-25658
SVSB43-S-25658DUP
Methanol blank 2
SVSB43-S-25659
SVSB43-S-25661
SVSB43-S-25656
SVSB43-S-25665
SVSB43-S-25640
SVSB43-S-25646
SVSB43-S-25641
SVSB43-S-25650
SVSB43-S-25644
SVSB43-S-25652
SVSB43-S-25653

$\begin{array}{rr}100 & 100 \\ 95 & 96 \\ 110 & 114 \\ 114 & 114 \\ 100 & 104 \\ 94 & 94 \\ 98 & 114 \\ 97 & 99 \\ 86 & 83 \\ 94 & 90 \\ 100 & 105 \\ 91 & 92 \\ 89 & 82 \\ 87 & 87 \\ 76^{\mathrm{d}} & 76^{\mathrm{d}} \\ 61^{\mathrm{d}} & 56^{\mathrm{d}} \\ 80 & 82 \\ 90 & 89 \\ 89 & 95 \\ 90 & 86 \\ 90 & 86\end{array}$

SVSB43-S-25653
Reanalyzed in SDG 07-10-31b with recovery limit met.

$17.3 \quad 14.5$

19.14

4.4

20

0.0

$$
\begin{array}{r}
103 \\
114 \\
109 \\
96 \\
90
\end{array}
$$$$
111
$$

100
93
117
115
108
98
113
100
86
90
105
91
85
92
80
62
83
90
95
90
88

117

115

113

100

905

91

85

92

80

83

95

90

88
18.74
6.5
19.47
2.7
19.87
0.7

Reanalyzed in SDG 10-6-28 with recovery limit met.

Reanalyzed in SDG 10-6-28 with recovery limit met. 


\begin{tabular}{|c|c|c|c|c|c|c|c|c|c|}
\hline \multirow[b]{3}{*}{ Sample } & \multicolumn{3}{|c|}{ Recovery of Surrogate Compounds ${ }^{a}(\%)$} & \multicolumn{6}{|c|}{$\begin{array}{l}\text { Measured Concentration and RPD Value for } \\
\text { Calibration Check Standard }\end{array}$} \\
\hline & \multirow[b]{2}{*}{ Fluorobenzene } & \multirow{2}{*}{$\begin{array}{l}\text { Bromo- } \\
\text { fluorobenzene }\end{array}$} & \multirow{2}{*}{$\begin{array}{c}\text { Dichloro- } \\
\text { benzene-d }\end{array}$} & \multicolumn{2}{|c|}{$\begin{array}{c}\text { Carbon } \\
\text { Tetrachloride }\end{array}$} & \multicolumn{2}{|c|}{ Chloroform } & \multicolumn{2}{|c|}{$\begin{array}{l}\text { Methylene } \\
\text { Chloride }\end{array}$} \\
\hline & & & & $\mathrm{ppb}^{\mathrm{b}}$ & $\mathrm{RPD}^{\mathrm{c}}$ & $\mathrm{ppb}^{\mathrm{b}}$ & $\mathrm{RPD}^{\mathrm{C}}$ & $\mathrm{ppb}^{\mathrm{b}}$ & $\mathrm{RPD}^{\mathrm{c}}$ \\
\hline \multicolumn{10}{|c|}{ SDG 10-6-24b, analysis date June 24, 2010} \\
\hline 20-ppb standard & 100 & 100 & 100 & 16.57 & 18.8 & 17.07 & 15.8 & 23.95 & 18.0 \\
\hline Methanol blank & 82 & 82 & 86 & & & & & & \\
\hline SVSB43-S-25655 & 97 & 97 & 101 & & & & & & \\
\hline SVSB43-S-25673 & 96 & 90 & 96 & & & & & & \\
\hline SVSB43-S-25642 & 90 & 92 & 100 & & & & & & \\
\hline SVSB43-S-25672 & 95 & 93 & 96 & & & & & & \\
\hline SVSB43-S-25647 & 104 & 98 & 103 & & & & & & \\
\hline SVSB43-S-25648 & 109 & 104 & 107 & & & & & & \\
\hline SVSB43-S-25648DUP & 90 & 96 & 101 & & & & & & \\
\hline Methanol blank 2 & 100 & 100 & 100 & & & & & & \\
\hline SVSB43-S-25660 & 112 & $123^{d}$ & $124^{d}$ & Reanals & zed in 5 & $10-6-2$ & ith reco & nit met. & \\
\hline SVSB43-S-25657 & 108 & 116 & 120 & & & & & & \\
\hline SVSB43-S-25651 & 102 & 110 & 116 & & & & & & \\
\hline SVSB43-S-25662 & 91 & 98 & 102 & & & & & & \\
\hline SVSB43-S-25649 & 83 & 89 & 99 & & & & & & \\
\hline SVSB43-S-25654 & 81 & 91 & 100 & & & & & & \\
\hline \multicolumn{10}{|c|}{ SDG 10-6-25, analysis date June 25, 2010} \\
\hline 20-ppb standard & 100 & 100 & 100 & 18.59 & 7.3 & 19.13 & 4.4 & 18.8 & 6.2 \\
\hline Methanol blank & 111 & 110 & 108 & & & & & & \\
\hline SVQCTB1-S-25671 & 98 & 103 & 104 & & & & & & \\
\hline SVSB43-S-25675 & 101 & 106 & 111 & & & & & & \\
\hline SVSB44-S-25700 & 96 & 105 & 110 & & & & & & \\
\hline SVSB44-S-25692 & 97 & 97 & 102 & & & & & & \\
\hline SVSB44-S-25702 & 88 & 92 & 99 & & & & & & \\
\hline SVSB44-S-25708 & 88 & 94 & 97 & & & & & & \\
\hline SVSB44-S-25679 & 90 & 96 & 99 & & & & & & \\
\hline SVSB44-S-25685 & 85 & 92 & 93 & & & & & & \\
\hline SVSB44-S-25681 & 84 & 82 & 90 & & & & & & \\
\hline SVSB44-S-25690 & 83 & 81 & 90 & & & & & & \\
\hline
\end{tabular}

SDG 10-6-24b, analysis date June 24, 2010

SVSB43-S-25655

SDG 10-6-25, analysis date June 25, 2010 
SDG 10-6-25, analysis date June 25, 2010 (cont.)

SVSB44-S-25694
SVSB44-S-25688
SVSB44-S-25688DUP
Methanol blank 2
SVSB44-S-25697
SVSB44-S-25689
SVSB44-S-25699
SVSB44-S-25704
SVSB44-S-25683
SVSB44-S-25684
SVSB44-S-25676
SVSB44-S-25698
SVSB44-S-25705

$\begin{array}{rrr}81 & 79^{d} & 85 \\ 92 & 101 & 103 \\ 88 & 90 & 98 \\ 89 & 90 & 92 \\ 101 & 103 & 101 \\ 105 & 107 & 110 \\ 94 & 97 & 100 \\ 96 & 97 & 102 \\ 99 & 97 & 99 \\ 91 & 91 & 99 \\ 95 & 96 & 100 \\ 90 & 86 & 92 \\ 89 & 91 & 95\end{array}$

SDG 10-6-28, analysis date June 28, 2010

SVMORGANLFB-W-25770

SVMORGANLEM-W-25771

SVSB38LFB-W-25772

SVSB38LFM-W-25773

SVSB24LFB-W-25774

SVMODOTLFB-W-25775

SVMDOTLFB-W-25775DUP

SVMODOTLFM-W-25776

SVQCTB-W-25777

Methanol blank

SVSB43-S-25640

SVSB43-S-25646

SVSB43-S-25660

SVSB44-S-25701

108
107
101
106
101
101
99
95
92
94
90
100
102
98
99
101

112
109
103
109
105
104
107
100
94
91
89
100
112
102
100
109

Measured Concentration and RPD Value for Calibration Check Standard

Bromo- Dichloro-

Carbon

Tetrachloride

$\mathrm{ppb}^{\mathrm{b}} \mathrm{RPD}^{\mathrm{c}}$

ppbb

$\mathrm{RPD}^{\mathrm{C}}$

Methylene

Chloride

$\mathrm{ppb}^{\mathrm{b}} \quad \mathrm{RPD}^{\mathrm{c}}$
Accepted. 
SDG 10-6-28, analysis date June 28, 2010 (cont.)

SVSB44-S-25687

SVSB44-S-25682

SVSB44-S-25696

SVSB44-S-25680

SVSB44-S-25693

SVSB44-S-25693DUP

Methanol blank 2

SVSB44-S-25691

$\begin{array}{rr}104 & 101 \\ 107 & 116 \\ 101 & 102 \\ 98 & 97 \\ 97 & 98 \\ 93 & 88 \\ 106 & 103 \\ 104 & 106\end{array}$

SDG 10-6-29, analysis date June 29, 2010

\section{0-ppb standard}

Laboratory blank

SVMODOT3X-W-25778

103
100

86

SVMORGAN3X-W-25779

SVMRGAN3X-W-25779DUP

SVQCTB-W-25780

Methanol blank

SVSB45-S-25721

SVSB45-S-25736

SVSB45-S-25725

SVSB45-S-25734

SVSB45-S-25730

SVSB44-S-25686

SVSB45-S-25735

SVSB45-S-25728

SVSB45-S-25732

SVSB45-S-25742

Methanol blank 2

SVSB45-S-25747

SVSB45-S-25745
Measured Concentration and RPD Value for Calibration Check Standard

Bromo- Dichloro-

Carbon

Tetrachloride Chloroform

Methylene

ppb $^{b} \quad R^{\circ} D^{c}$

$\mathrm{RPD}^{\mathrm{C}}$

$\mathrm{ppb}^{\mathrm{b}} \quad \mathrm{RPD}^{\mathrm{c}}$ 


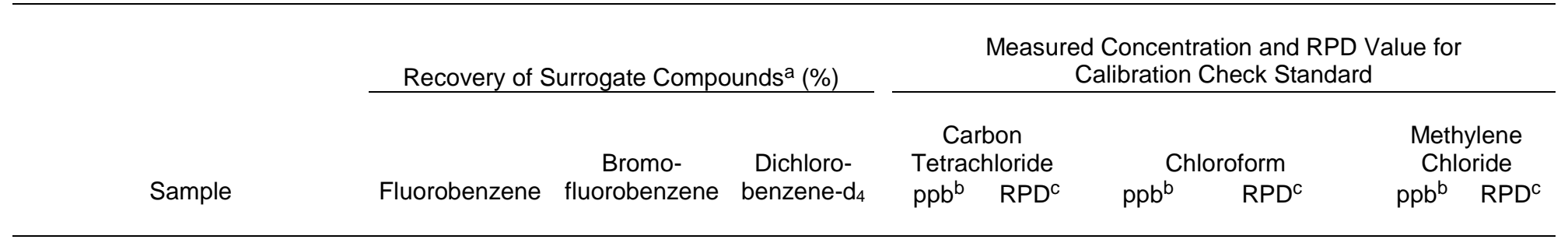

SDG 10-6-29, analysis date June 29, 2010 (cont.)

$\begin{array}{lrrl}\text { SVSB45-S-25743 } & 113 & 114 & 121^{\mathrm{d}} \\ \text { SVSB45-S-25741 } & 108 & 109 & 112 \\ \text { SVSB45-S-25737 } & 108 & 106 & 109 \\ \text { SVSB45-S-25720 } & 106 & 107 & 113 \\ \text { SVSB45-S-25727 } & 106 & 109 & 115 \\ \text { SVSB44-S-25695 } & 107 & 99 & 106\end{array}$

SDG 10-6-30, analysis date June 30, 2010

$\begin{array}{lrrr}\text { 20-ppb standard } & 99 & 101 & 103 \\ \text { Methanol blank } & 100 & 100 & 100 \\ \text { SVSB44-S-25709 } & 106 & 114 & 116 \\ \text { SVSB44-S-25677 } & 104 & 108 & 115 \\ \text { SVSB44-S-25678 } & 99 & 105 & 112 \\ \text { SVSB44-S-25707 } & 95 & 97 & 101 \\ \text { SVSB45-S-25722 } & 104 & 108 & 112 \\ \text { SVSB45-S-25724 } & 103 & 83 & 85 \\ \text { SVSB45-S-25744 } & 103 & 108 & 113 \\ \text { SVSB45-S-25726 } & 101 & 108 & 113 \\ \text { SVSB45-S-25729 } & 98 & 97 & 102 \\ \text { SVSB45-S-25738 } & 95 & 100 & 105 \\ \text { SVSB45-S-25733 } & 99 & 99 & 108 \\ \text { SVSB45-S-25740 } & 93 & 94 & 100 \\ \text { SVSB45-S-25731 } & 98 & 97 & 105 \\ \text { SVSB45-S-25731DUP } & 98 & 94 & 100 \\ \text { Methanol blank 2 } & 92 & 90 & 97 \\ \text { Laboratory blank } & 100 & 100 & 100 \\ \text { SVMODOT3X-W-25778 } & 94 & 98 & 102 \\ \text { SVMODOT3X-W-25778DUP } & 95 & 97 & 103\end{array}$

Reanalyzed in SDG 10-7-1 with recovery limit met.

113

106

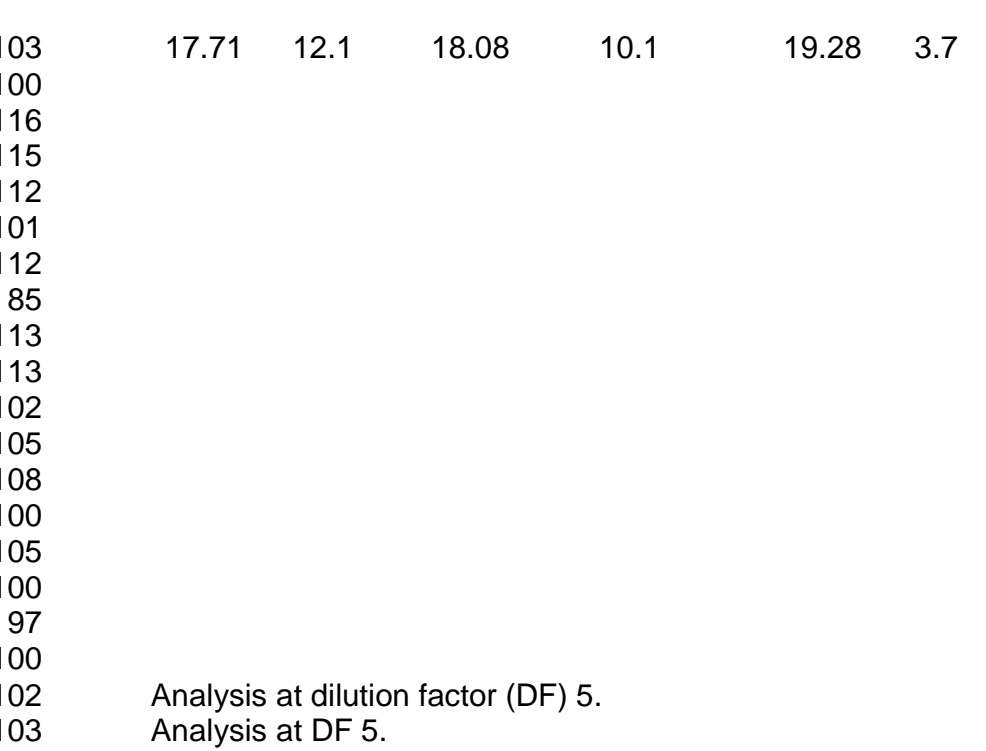


SDG 10-7-1, analysis date July 1, 2010

$\begin{array}{lr}\text { 20-ppb standard } & 100 \\ \text { Methanol blank } & 96 \\ \text { SVSB45-S-25743 } & 116 \\ \text { SVSB45-S-25739 } & 103 \\ \text { SVSB45-S-25746 } & 104 \\ \text { SVSB45-S-25746DUP } & 98 \\ \text { SVSB45-S-25723 } & 105 \\ \text { SVSB46-S-25718 } & 106 \\ \text { SVSB46-S-25760 } & 98 \\ \text { SVSB46-S-25755 } & 96 \\ \text { SVSB46-S-25751 } & 95 \\ \text { SVSB46-S-25766 } & 94 \\ \text { SVSB46-S-25767 } & 97 \\ \text { SVSB46-S-25757 } & 96 \\ \text { SVSB46-S-25758 } & 90 \\ \text { SVSB46-S-25712 } & \end{array}$

100
96
116
103
104
98
105
106
98
96
95
94
97
101
96
90

Measured Concentration and RPD Value for

Recovery of Surrogate Compounds ${ }^{\mathrm{a}}(\%)$ Calibration Check Standard

Bromo- Dichloro-

$\begin{array}{cc}\text { Carbon } & \\ \text { Tetrachloride } & \text { Methylene }\end{array}$

$\mathrm{ppb}^{\mathrm{b}} \quad \mathrm{RPD}^{\mathrm{c}}$

$\mathrm{ppb}^{\mathrm{b}} \quad \mathrm{RPD}^{\mathrm{c}}$

SVSB46-S-25712

$\begin{array}{rr}100 & 100 \\ 90 & 93 \\ 118 & 115 \\ 99 & 97 \\ 110 & 107 \\ 94 & 98 \\ 100 & 102 \\ 97 & 101 \\ 103 & 108 \\ 89 & 90 \\ 95 & 96 \\ 94 & 98 \\ 93 & 91 \\ 97 & 103 \\ 93 & 96 \\ 91 & 91\end{array}$

SDG 10-7-2, analysis date July 2, 2010

$\begin{array}{lr}\text { 20-ppb standard } & 100 \\ \text { Methanol blank } & 98 \\ \text { SVSB46-S-25719 } & 104 \\ \text { SVSB46-S-25710 } & 119 \\ \text { SVSB46-S-25716 } & 101 \\ \text { SVSB46-S-25754 } & 100 \\ \text { SVSB46-S-25768 } & 95 \\ \text { SVSB46-S-25762 } & 108 \\ \text { SVSB46-S-25763 } & 96 \\ \text { SVSB46-S-25711 } & 81 \\ \text { SVSB46-S-25753 } & 94 \\ \text { SVSB46-S-25764 } & 88 \\ \text { SVSB46-S-25756 } & 88\end{array}$

$\begin{array}{rr}100 & 100 \\ 90 & 88 \\ 100 & 103 \\ 113 & 113 \\ 104 & 110 \\ 101 & 105 \\ 98 & 107 \\ 106 & 112 \\ 96 & 102 \\ 84 & 90 \\ 94 & 94 \\ 91 & 97 \\ 88 & 96\end{array}$

$\begin{array}{llllll}16.51 & 19.1 & 17.16 & 15.3 & 17.91 & 11.0\end{array}$




\begin{tabular}{|c|c|c|c|c|c|c|c|}
\hline \multirow[b]{3}{*}{ Sample } & \multicolumn{2}{|c|}{ Recovery of Surrogate Compounds ${ }^{\mathrm{a}}(\%)$} & \multicolumn{5}{|c|}{$\begin{array}{l}\text { Measured Concentration and RPD Value for } \\
\text { Calibration Check Standard }\end{array}$} \\
\hline & Bromo- & Dichloro- & $\begin{array}{c}\text { Carbon } \\
\text { Tetrachloride }\end{array}$ & & oform & $\begin{array}{r}\text { Met } \\
\text { Ch }\end{array}$ & $\begin{array}{l}\text { lene } \\
\text { ride }\end{array}$ \\
\hline & Fluorobenzene fluorobenzene & benzene- $d_{4}$ & $\mathrm{ppb}^{\mathrm{b}} \quad \mathrm{RPD}^{\mathrm{c}}$ & $\mathrm{ppb}^{\mathrm{b}}$ & $\mathrm{RPD}^{\mathrm{C}}$ & $\mathrm{ppb}^{\mathrm{b}}$ & $\mathrm{RPD}^{\mathrm{C}}$ \\
\hline
\end{tabular}

SDG 10-7-2, analysis date July 2, 2010 (cont.)

SVSB46-S-25750

SVSB46-S-25761

SVSB46-S-25761DUP

Methanol blank 2

SVSB46-S-25752

SVSB46-S-25759

SVSB46-S-25765

SVQCTB-S-25715

SVSB46-S-25713

SVSB46-S-25714

SDG 10-7-7, analysis date July 7, 2010

20-ppb standard

Laboratory blank

SVSB46S-W-25801

SVSB46S-W-25801

SVSB45D-W-25803

SVSB49D-W-25804

SVSB49S-W-25805

SVSB48D-W-25806

SVSB48S-W-25807

SVSB47D-W-25808

SVSB47S-W-25809

SVSB44D-W-25810

SVSB44S-W-25811

SVSB43S-W-25812

SVSB43D-W-25813

SVQCTB-W-25814

$\begin{array}{rrr}86 & 81 & 91 \\ 89 & 88 & 94 \\ 88 & 83 & 90 \\ 88 & 86 & 90 \\ 90 & 91 & 94 \\ 97 & 98 & 98 \\ 91 & 91 & 93 \\ 94 & 104 & 101 \\ 90 & 90 & 94 \\ 90 & 93 & 97\end{array}$

$\begin{array}{rrr}98 & 98 & 100 \\ 100 & 100 & 100 \\ 94 & 99 & 100 \\ 96 & 92 & 103 \\ 93 & 94 & 102 \\ 100 & 100 & 100 \\ 94 & 96 & 99 \\ 82 & 80 & 94 \\ 80 & 88^{d} & 81 \\ 79^{d} & 79^{d} & 84 \\ 80 & 74^{d} & 80 \\ 77^{d} & 75^{d} & 85 \\ 75^{d} & 69^{d} & 73^{d} \\ 70^{d} & 66^{d} & 69^{d} \\ 72^{d} & 67^{d} & 72^{d} \\ 67^{d} & 61^{d} & 65^{d} \\ 136^{d} & 132^{d} & 134^{d}\end{array}$
19.57
2.2
19.55
2.3
16.97
16.4

Reanalyzed in SDG 10-7-8 with recovery limit met. Reanalyzed in SDG 10-7-8 with recovery limit met. Reanalyzed in SDG 10-7-8 with recovery limit met. Reanalyzed in SDG 10-7-8 with recovery limit met. Reanalyzed in SDG 10-7-8 with recovery limit met. Accepted. Insufficient sample to reanalyze.

Reanalyzed in SDG 10-7-8 with recovery limit met. Reanalyzed in SDG 10-7-8 with recovery limit met.

Reanalyzed in SDG 10-7-8 with recovery limit met. 


\begin{tabular}{|c|c|c|c|c|c|c|c|c|c|}
\hline \multirow[b]{3}{*}{ Sample } & \multicolumn{3}{|c|}{ Recovery of Surrogate Compounds ${ }^{\mathrm{a}}(\%)$} & \multicolumn{6}{|c|}{$\begin{array}{l}\text { Measured Concentration and RPD Value for } \\
\text { Calibration Check Standard }\end{array}$} \\
\hline & \multirow[b]{2}{*}{ Fluorobenzene } & \multirow{2}{*}{$\begin{array}{l}\text { Bromo- } \\
\text { fluorobenzene }\end{array}$} & \multirow{2}{*}{$\begin{array}{l}\text { Dichloro- } \\
\text { benzene-d }\end{array}$} & \multicolumn{2}{|c|}{$\begin{array}{l}\text { Carbon } \\
\text { Tetrachloride }\end{array}$} & \multicolumn{2}{|c|}{ Chloroform } & \multicolumn{2}{|c|}{$\begin{array}{l}\text { Methylene } \\
\text { Chloride }\end{array}$} \\
\hline & & & & $\mathrm{ppb}^{\mathrm{b}}$ & $\mathrm{RPD}^{\mathrm{C}}$ & $\mathrm{ppb}^{\mathrm{b}}$ & $\mathrm{RPD}^{\mathrm{C}}$ & $\mathrm{ppb}^{\mathrm{b}}$ & $\mathrm{RPD}^{\mathrm{C}}$ \\
\hline \multicolumn{10}{|c|}{ SDG 10-7-8, analysis date July 8, 2010} \\
\hline 20-ppb standard & 90 & 96 & 87 & 21.93 & 9.2 & 22.21 & 10.5 & 21.67 & 8.0 \\
\hline Laboratory blank & 100 & 100 & 100 & & & & & & \\
\hline SVSB48D-W-25806 & 91 & 94 & 99 & & & & & & \\
\hline SVSB48S-W-25807 & 93 & 102 & 102 & & & & & & \\
\hline SVSB47D-W-25808 & 96 & 99 & 104 & & & & & & \\
\hline SVSB47S-W-25809 & 97 & 108 & 114 & & & & & & \\
\hline SVSB44D-W-25810 & 93 & 94 & 97 & & & & & & \\
\hline SVSB43S-W-25812 & 92 & 93 & 100 & & & & & & \\
\hline SVSB43D-W-25813 & 92 & 96 & 96 & & & & & & \\
\hline SVQCTB-W-25814 & 90 & 92 & 88 & & & & & & \\
\hline \multicolumn{10}{|c|}{ SDG 10-8-25a, analysis date August 25, 2010} \\
\hline 20-ppb standard & 106 & 106 & 103 & 22.71 & 12.7 & 23.91 & 17.8 & 22.15 & 10.2 \\
\hline Laboratory blank & 114 & 118 & 119 & & & & & & \\
\hline SVSB43S-W-25815 & 100 & 98 & 103 & & & & & & \\
\hline SVSB43D-W-25816 & 104 & 102 & 103 & & & & & & \\
\hline SVSB44S-W-25817 & 100 & 97 & 100 & & & & & & \\
\hline SVSB44D-W-25818 & 96 & 89 & 96 & & & & & & \\
\hline SVSB45S-W-25819 & 95 & 90 & 93 & & & & & & \\
\hline SVSB45D-W-25820 & 95 & 87 & 89 & & & & & & \\
\hline SVSB46S-W-25821 & 89 & 83 & 83 & & & & & & \\
\hline SVSB46D-W-25822 & 93 & 83 & 86 & & & & & & \\
\hline SVSB47S-W-25823 & 88 & 82 & 83 & & & & & & \\
\hline SVSB47D-W-25824 & 88 & 82 & 81 & & & & & & \\
\hline SVSB48S-W-25825 & 88 & $78^{d}$ & $78^{d}$ & \multirow{4}{*}{\multicolumn{6}{|c|}{$\begin{array}{l}\text { Reanalyzed in SDG } 10-8-26 \text { a with recovery limit met. } \\
\text { Reanalyzed in SDG 10-8-26a with recovery limit met. } \\
\text { Reanalyzed in SDG 10-8-26a with recovery limit met. } \\
\text { Reanalyzed in SDG } 10-8-26 \text { a with recovery limit met. }\end{array}$}} \\
\hline SVSB48D-W-25826 & 86 & $77^{d}$ & $76^{d}$ & & & & & & \\
\hline SVSB49S-W-25827 & 83 & $74^{d}$ & $72^{d}$ & & & & & & \\
\hline SVSB49D-W-25828 & 83 & $73^{d}$ & $73^{d}$ & & & & & & \\
\hline
\end{tabular}

SDG 10-7-8, analysis date July 8,2010

-ppb standard

Reanalyzed in SDG 10-8-26a with recovery limit met. 


\begin{tabular}{|c|c|c|c|c|c|c|c|c|c|}
\hline \multirow[b]{3}{*}{ Sample } & \multicolumn{3}{|c|}{ Recovery of Surrogate Compounds ${ }^{a}(\%)$} & \multicolumn{6}{|c|}{$\begin{array}{l}\text { Measured Concentration and RPD Value for } \\
\text { Calibration Check Standard }\end{array}$} \\
\hline & \multirow[b]{2}{*}{ Fluorobenzene } & \multirow{2}{*}{$\begin{array}{l}\text { Bromo- } \\
\text { fluorobenzene }\end{array}$} & \multirow{2}{*}{$\begin{array}{c}\text { Dichloro- } \\
\text { benzene-d }\end{array}$} & \multicolumn{2}{|c|}{$\begin{array}{l}\text { Carbon } \\
\text { Tetrachloride }\end{array}$} & \multicolumn{2}{|c|}{ Chloroform } & \multicolumn{2}{|c|}{$\begin{array}{l}\text { Methylene } \\
\text { Chloride }\end{array}$} \\
\hline & & & & $\mathrm{ppb}^{\mathrm{b}}$ & $\mathrm{RPD}^{\mathrm{C}}$ & $\mathrm{ppb}^{\mathrm{b}}$ & $\mathrm{RPD}^{\mathrm{c}}$ & $\mathrm{ppb}^{\mathrm{b}}$ & $\mathrm{RPD}^{\mathrm{C}}$ \\
\hline \multicolumn{10}{|c|}{ SDG 10-8-25b, analysis date August 25, 2010} \\
\hline 20-ppb standard & 100 & 100 & 100 & 22.42 & 11.4 & 23.38 & 15.6 & 20 & 0.0 \\
\hline Laboratory blank & 116 & 117 & 113 & & & & & & \\
\hline SVQCTB-W-25829 & 115 & 120 & 119 & & & & & & \\
\hline SVSB43S-W-25830 & 106 & 111 & 112 & & & & & & \\
\hline SVSB43D-W-25831 & 95 & 105 & 100 & & & & & & \\
\hline SVSB44S-W-25832 & 100 & 99 & 105 & \multicolumn{6}{|c|}{$\begin{array}{l}\text { Outside calibration range for carbon tetrachloride at zero } \\
\text { dilution. Analyzed at dilution in SDG } 10-8-26 a \text {. }\end{array}$} \\
\hline SVSB47D-W-25839 & 92 & 95 & 103 & & & & & & \\
\hline SVSB47D-W-25839DUP & 90 & 88 & 91 & & & & & & \\
\hline SVSB44D-W-25833 & 89 & 94 & 120 & & & & & & \\
\hline SVSB45D-W-25835 & 88 & 96 & 98 & & & & & & \\
\hline SVSB46D-W-25837 & 90 & 97 & 100 & & & & & & \\
\hline Laboratory blank 2 & 84 & 83 & 87 & & & & & & \\
\hline SVSB48D-W-25841 & 84 & 81 & 87 & & & & & & \\
\hline SVSB49D-W-25843 & 82 & 85 & $136^{d}$ & Reanaly & zed in $\varsigma$ & $10-8-2$ & with rec & limit met. & \\
\hline SVSB49DDUP-W-25844 & 83 & 92 & 98 & & & & & & \\
\hline SVQCIR-W-25847 & 81 & 107 & 90 & & & & & & \\
\hline SVQCIR-W-25848 & $33^{d}$ & $36^{d}$ & $41^{d}$ & Reanaly & zed in $S$ & 10-8-2 & with rec & limit met. & \\
\hline \multicolumn{10}{|c|}{ SDG 10-8-26a, analysis date August 26, 2010} \\
\hline 20-ppb standard & 85 & 91 & 90 & 19.38 & 3.1 & 20.94 & 4.6 & 19.26 & 3.8 \\
\hline Laboratory blank & 100 & 100 & 100 & & & & & & \\
\hline SVSB48D-W-25826 & 100 & 105 & 110 & & & & & & \\
\hline SVSB49S-W-25827 & 94 & 100 & 110 & & & & & & \\
\hline SVSB49D-W-25828 & 93 & 93 & 101 & & & & & & \\
\hline SVSB44S-W-25832 & 86 & 91 & 96 & & & & & & \\
\hline SVSB44S-W-25832DUP & 85 & 87 & 90 & & & & & & \\
\hline SVSB49D-W-25843 & 81 & 88 & 102 & & & & & & \\
\hline SVQCIR-W-25848 & 87 & 86 & 89 & & & & & & \\
\hline
\end{tabular}

SDG 10-8-25b, analysis date August 25, 2010 


\begin{tabular}{|c|c|c|c|c|c|c|c|}
\hline \multirow[b]{3}{*}{ Sample } & \multicolumn{3}{|c|}{ Recovery of Surrogate Compounds ${ }^{a}(\%)$} & \multicolumn{4}{|c|}{$\begin{array}{l}\text { Measured Concentration and RPD Value for } \\
\text { Calibration Check Standard }\end{array}$} \\
\hline & & Bromo- & Dichloro- & $\begin{array}{l}\text { Carbon } \\
\text { Tetrachloride }\end{array}$ & & fform & $\begin{array}{l}\text { Methylene } \\
\text { Chloride }\end{array}$ \\
\hline & Fluorobenzene & fluorobenzene & benzene- $d_{4}$ & $\mathrm{ppb}^{\mathrm{b}} \quad \mathrm{RPD}^{\mathrm{c}}$ & $\mathrm{ppb}^{\mathrm{b}}$ & $\mathrm{RPD}^{\mathrm{c}}$ & $\mathrm{ppb}^{\mathrm{b}} \quad \mathrm{RPD}^{\mathrm{c}}$ \\
\hline
\end{tabular}

SDG 10-8-26a, analysis date August 26, 2010 (cont.)

SVSB48S-W-25825

SVSB46S-W-25836

86
91

SDG 10-8-26b, analysis date August 26, 2010

$\begin{array}{lc}\text { 20-ppb standard } & 100 \\ \text { Laboratory blank } & 115 \\ \text { SVSB45S-W-25834 } & 102 \\ \text { SVSB47S-W-25838 } & 98 \\ \text { SVSB48S-W-25840 } & 86 \\ \text { SVSB48S-W-25840DUP } & 96 \\ \text { SVSB49S-W-25842 } & 97 \\ \text { SVSB48SDUP-W-25845 } & 82 \\ \text { SVDIH2O-W-25846 } & 87 \\ \text { SVQCIR-W-25849 } & 92 \\ \text { SVQCIR-W-25850 } & 91 \\ \text { SVQCTB-W-25852 } & 85 \\ \text { SVSB24-W-25854 } & 74^{\mathrm{d}} \\ \text { SVSB38-W-25855 } & 108\end{array}$

SVSB38-W-25855

SDG 10-8-27, analysis date August 27, 2010

$\begin{array}{lrr}\text { 20-ppb standard } & 86 & \\ \text { Laboratory blank } & 100 & 100 \\ \text { SVSB24-W-25854 } & 95 & 90 \\ \text { SVSB24-W-25854DUP } & 97\end{array}$

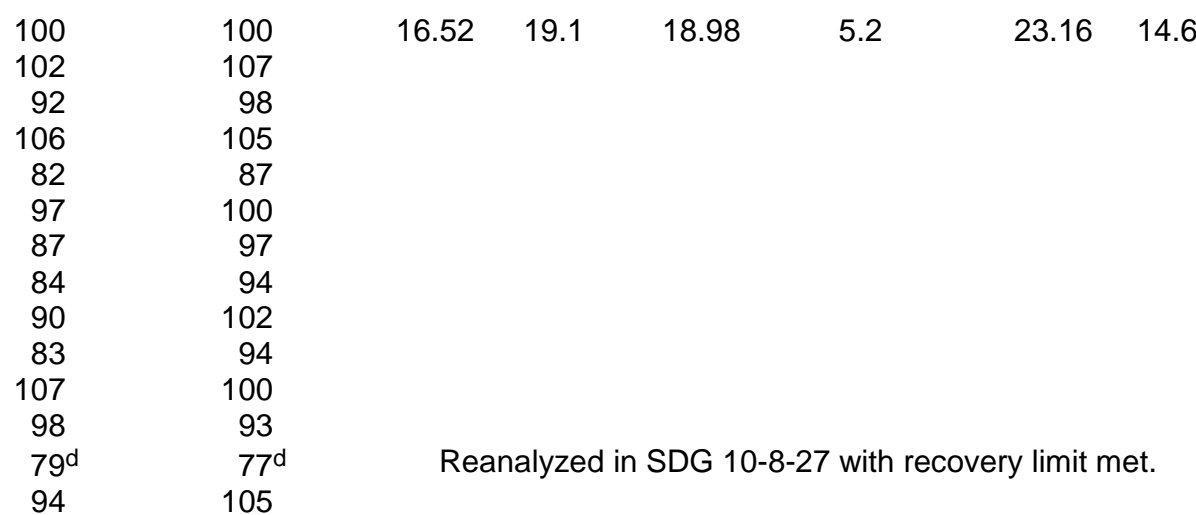

SDG 11-8-11, analysis date August 11, 2011

20-ppb standard

Laboratory blank

SVSB24GRAB-W-25856

93
100

101 
SDG 11-8-11, analysis date August 11, 2011 (cont.)

SVSB38GRAB-W-25857

SVSB43SGRAB-W-25858

SVSB43DGRAB-W-25859

SVSB44SGRAB-W-25860

92
96
94 $\quad 89$

SVSB44DGRAB-W-25861

SVSB45SGRAB-W-25862

SVSB45DGRAB-W-25863

SVSB46SGRAB-W-25864

SVSB46DGRAB-W-25865

SVSB47SGRAB-W-25866

SVSB47DGRAB-W-25867

SVSB48SGRAB-W-25868

SVSB48SGRAB-W-25868DUP

Laboratory blank 2

SVSB48DGRAB-W-25869

SVQCTB-W-25895

SVSB49SGRAB-W-25870

SVSB49DGRAB-W-25871

SVSB49DGRAB-W-25871DUP

SVSB43S-W-25874

SVSB43D-W-25875

SVSB47S-W-25882

91

97

95

89

92

90

91

88

90
92

99

91

92

90

92

92

92

SDG 11-8-12, analysis date August 12, 2011

\section{0-ppb standard}

Laboratory blank

SVSB47D-W-25883

SVSB48D-W-25885

SVSB49S-W-25886

SVSB49D-W-25887

$\begin{array}{rrr}82 & 106 & 89 \\ 100 & 100 & 100 \\ 102 & 107 & 105 \\ 91 & 95 & 98 \\ 88 & 92 & 107 \\ 90 & 96 & 99\end{array}$

Measured Concentration and RPD Value for

Bromo- Dichlorofluorobenzene benzene- $\mathrm{d}_{4}$

\section{Carbon}

Tetrachloride

$\mathrm{ppb}^{\mathrm{b}} \quad \mathrm{RPD}^{\mathrm{c}}$
Calibration Check Standard

Methylene

Chloride

$\mathrm{ppb}^{\mathrm{b}} \quad \mathrm{RPD}^{\mathrm{c}}$
Outside calibration range for carbon tetrachloride at zero dilution. Analyzed at dilution in SDG 11-8-15.

$\begin{array}{rrrrrrr}89 & 18.39 & 8.4 & 18.12 & 9.9 & 18.6 & 7.3 \\ 100 & & & & & & \\ 105 & & & & & & \\ 98 & & & & & & \\ 107 & & & & & & \\ 99 & & & & \end{array}$




\begin{tabular}{|c|c|c|c|c|c|c|c|c|c|}
\hline \multirow[b]{3}{*}{ Sample } & \multicolumn{3}{|c|}{ Recovery of Surrogate Compounds ${ }^{a}(\%)$} & \multicolumn{6}{|c|}{$\begin{array}{l}\text { Measured Concentration and RPD Value for } \\
\text { Calibration Check Standard }\end{array}$} \\
\hline & \multirow[b]{2}{*}{ Fluorobenzene } & \multirow{2}{*}{$\begin{array}{l}\text { Bromo- } \\
\text { fluorobenzene }\end{array}$} & \multirow{2}{*}{$\begin{array}{l}\text { Dichloro- } \\
\text { benzene-d }\end{array}$} & \multicolumn{2}{|c|}{$\begin{array}{l}\text { Carbon } \\
\text { Tetrachloride }\end{array}$} & \multicolumn{2}{|c|}{ Chloroform } & \multicolumn{2}{|c|}{$\begin{array}{l}\text { Methylene } \\
\text { Chloride }\end{array}$} \\
\hline & & & & $\mathrm{ppb}^{\mathrm{b}}$ & $\mathrm{RPD}^{\mathrm{C}}$ & $\mathrm{ppb}^{\mathrm{b}}$ & $\mathrm{RPD}^{\mathrm{c}}$ & $\mathrm{ppb}^{\mathrm{b}}$ & $\mathrm{RPD}^{\mathrm{c}}$ \\
\hline \multicolumn{10}{|c|}{ SDG 11-8-12, analysis date August 12, 2011 (cont.) } \\
\hline SVSB43DDUP-W-25888 & 91 & 95 & 95 & & & & & & \\
\hline SVQCIR-W-25891 & 89 & 109 & 104 & & & & & & \\
\hline SVQCIR-W-25892 & 91 & 116 & 110 & & & & & & \\
\hline SVQCTB-W-25896 & 89 & 106 & 103 & & & & & & \\
\hline SVSB24-W-25872 & 96 & 103 & 97 & & & & & & \\
\hline SVSB38-W-25873 & 91 & 104 & 95 & & & & & & \\
\hline SVSB44S-W-25876 & 94 & 94 & 95 & \multicolumn{6}{|c|}{$\begin{array}{l}\text { Outside calibration range for carbon tetrachloride at zero } \\
\text { dilution. Analyzed at dilution in SDG } 11-8-15 \text {. }\end{array}$} \\
\hline SVSB44D-W-25877 & 87 & 93 & 95 & & & & & & \\
\hline SVSB45S-W-25878 & 84 & 83 & 85 & & & & & & \\
\hline \multicolumn{10}{|c|}{ SDG 11-8-15, analysis date August 15, 2011} \\
\hline 20-ppb standard & 96 & 117 & 100 & 19.21 & 4.0 & 16.59 & 18.6 & 18.55 & 7.5 \\
\hline Laboratory blank & 100 & 100 & 100 & & & & & & \\
\hline SVSB44SGRAB-W-25860 & 99 & 96 & 99 & \multicolumn{6}{|c|}{ Analysis at DF 5.} \\
\hline SVSB44S-W-25876 & 101 & 98 & 104 & \multicolumn{6}{|c|}{ Analysis at DF 5.} \\
\hline SVSB45D-W-25879 & 100 & 96 & 100 & 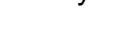 & & & & & \\
\hline SVSB46S-W-25880 & 97 & 94 & 99 & & & & & & \\
\hline SVSB46D-W-25881 & 97 & 97 & 98 & & & & & & \\
\hline SVSB48S-W-25884 & 92 & 88 & 94 & & & & & & \\
\hline SVSB46DDUP-W-25889 & 93 & 94 & 94 & & & & & & \\
\hline SVQCIR-W-25893 & 93 & 117 & 101 & & & & & & \\
\hline SVQCIR-W-25894 & 96 & 110 & 104 & & & & & & \\
\hline SVDIH2O-W-25890 & 96 & 97 & 93 & & & & & & \\
\hline SVQCTB-W-25897 & 92 & 84 & 83 & & & & & & \\
\hline SVSB44S-W-25876DUP & 104 & 92 & 92 & \multicolumn{6}{|c|}{ Duplicate analysis at DF 5.} \\
\hline
\end{tabular}

SDG 11-8-12, analysis date August 12, 2011 (cont.)

Analysis at DF 5.

Analysis at DF 5 


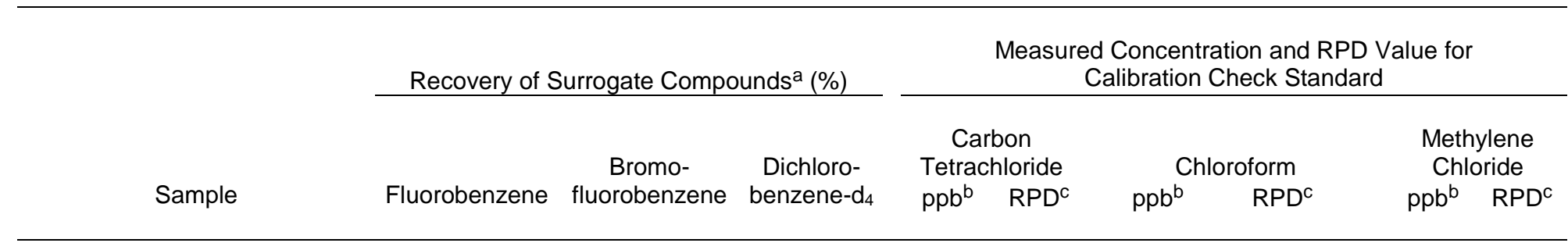

a Quality control range for recovery $=80-120 \%$.

b Concentration in parts per billion ( $\mu \mathrm{g} / \mathrm{L}$ in water or $\mu \mathrm{g} / \mathrm{kg}$ in soil).

c Quality control range for RPD $= \pm 20 \%$.

d Surrogate recovery outside QC limit. 
TABLE S2.4 Results from the AGEM Laboratory for dual analyses of samples.

\begin{tabular}{|c|c|c|c|c|c|c|c|c|c|c|}
\hline \multirow[b]{2}{*}{ Location } & \multirow[b]{2}{*}{ Sample } & \multirow[b]{2}{*}{$\begin{array}{l}\text { Sample } \\
\text { Date }\end{array}$} & \multirow[b]{2}{*}{$\begin{array}{l}\text { Analysis } \\
\text { Type }\end{array}$} & \multirow[b]{2}{*}{$\begin{array}{c}\text { Groundwater } \\
\text { Collection } \\
\text { Method }^{\mathrm{a}}\end{array}$} & \multirow[b]{2}{*}{$\begin{array}{l}\text { Installation } \\
\text { Type }^{b}\end{array}$} & \multirow[b]{2}{*}{$\begin{array}{c}\text { Depth } \\
\text { (ft BGL) }\end{array}$} & \multirow[b]{2}{*}{$\begin{array}{l}\text { Sample } \\
\text { Medium }\end{array}$} & \multicolumn{3}{|c|}{ Concentration } \\
\hline & & & & & & & & $\begin{array}{c}\text { Carbon } \\
\text { Tetrachloride }\end{array}$ & Chloroform & Units \\
\hline \multicolumn{11}{|c|}{ June-July 2010 sampling } \\
\hline SB43 & SVSB43-S-25648 & $6 / 21 / 10$ & Primary & - & CPT & 48 & Soil & ND & ND & $\mu \mathrm{g} / \mathrm{kg}$ \\
\hline SB43 & SVSB43-S-25648DUP & $6 / 21 / 10$ & Duplicate & - & CPT & 48 & Soil & ND & ND & $\mu \mathrm{g} / \mathrm{kg}$ \\
\hline SB43 & SVSB43-S-25650 & $6 / 21 / 10$ & Replicate & - & СРT & 48 & Soil & ND & ND & $\mu \mathrm{g} / \mathrm{kg}$ \\
\hline SB43 & SVSB43-S-25658 & $6 / 21 / 10$ & Primary & - & CPT & 80 & Soil & ND & ND & $\mu \mathrm{g} / \mathrm{kg}$ \\
\hline SB43 & SVSB43-S-25658DUP & $6 / 21 / 10$ & Duplicate & - & CPT & 80 & Soil & ND & ND & $\mu \mathrm{g} / \mathrm{kg}$ \\
\hline SB43 & SVSB43-S-25664 & $6 / 21 / 10$ & Primary & - & CPT & 100 & Soil & ND & ND & $\mu \mathrm{g} / \mathrm{kg}$ \\
\hline SB43 & SVSB43-S-25665 & $6 / 21 / 10$ & Replicate & - & СРT & 100 & Soil & ND & ND & $\mu \mathrm{g} / \mathrm{kg}$ \\
\hline SB43 & SVSB43-S-25672 & $6 / 22 / 10$ & Primary & - & CPT & 104 & Soil & ND & ND & $\mu \mathrm{g} / \mathrm{kg}$ \\
\hline SB43 & SVSB43-S-25673 & $6 / 22 / 10$ & Replicate & - & СРT & 104 & Soil & ND & ND & $\mu \mathrm{g} / \mathrm{kg}$ \\
\hline SB44 & SVSB44-S-25688 & $6 / 23 / 10$ & Primary & - & CPT & 52 & Soil & 139 & ND & $\mu \mathrm{g} / \mathrm{kg}$ \\
\hline SB44 & SVSB44-S-25688DUP & $6 / 23 / 10$ & Duplicate & - & CPT & 52 & Soil & 151 & ND & $\mu \mathrm{g} / \mathrm{kg}$ \\
\hline SB44 & SVSB44-S-25693 & $6 / 23 / 10$ & Primary & - & CPT & 72 & Soil & ND & ND & $\mu \mathrm{g} / \mathrm{kg}$ \\
\hline SB44 & SVSB44-S-25693DUP & $6 / 23 / 10$ & Duplicate & - & CPT & 72 & Soil & ND & ND & $\mu \mathrm{g} / \mathrm{kg}$ \\
\hline SB44 & SVSB44-S-25695 & $6 / 23 / 10$ & Primary & - & CPT & 80 & Soil & ND & ND & $\mu \mathrm{g} / \mathrm{kg}$ \\
\hline SB44 & SVSB44-S-25705 & $6 / 23 / 10$ & Replicate & - & CPT & 80 & Soil & ND & ND & $\mu \mathrm{g} / \mathrm{kg}$ \\
\hline SB45 & SVSB45-S-25731 & $6 / 24 / 10$ & Primary & - & CPT & 44 & Soil & 63 & $1.7 \mathrm{~J}$ & $\mu \mathrm{g} / \mathrm{kg}$ \\
\hline SB45 & SVSB45-S-25731DUP & $6 / 24 / 10$ & Duplicate & - & CPT & 44 & Soil & 65 & $1.9 \mathrm{~J}$ & $\mu \mathrm{g} / \mathrm{kg}$ \\
\hline SB45 & SVSB45-S-25745 & $6 / 24 / 10$ & Primary & - & CPT & 100 & Soil & ND & ND & $\mu \mathrm{g} / \mathrm{kg}$ \\
\hline SB45 & SVSB45-S-25747 & $6 / 24 / 10$ & Replicate & - & CPT & 100 & Soil & ND & ND & $\mu \mathrm{g} / \mathrm{kg}$ \\
\hline SB46 & SVSB46-S-25718 & $6 / 25 / 10$ & Primary & - & CPT & 36 & Soil & $8.7 \mathrm{~J}$ & ND & $\mu \mathrm{g} / \mathrm{kg}$ \\
\hline SB46 & SVSB46-S-25766 & $6 / 25 / 10$ & Replicate & - & СРT & 36 & Soil & $7.2 \mathrm{~J}$ & ND & $\mu \mathrm{g} / \mathrm{kg}$ \\
\hline SB46 & SVSB46-S-25761 & $6 / 25 / 10$ & Primary & - & CPT & 88 & Soil & ND & ND & $\mu \mathrm{g} / \mathrm{kg}$ \\
\hline SB46 & SVSB46-S-25761DUP & $6 / 25 / 10$ & Duplicate & - & CPT & 88 & Soil & ND & ND & $\mu \mathrm{g} / \mathrm{kg}$ \\
\hline
\end{tabular}


June-July 2010 sampling (cont.)

$\begin{array}{ll}\text { SB43 } & \text { SVSB43-W-25666 } \\ \text { SB43 } & \text { SVSB43-W-25666DUP } \\ & \\ \text { MODOT } & \text { SVMODOTLFB-W-25775 } \\ \text { MODOT } & \text { SVMDOTLFB-W-25775DUP } \\ \text { MODOT } & \text { SVMODOT3X-W-25778 } \\ \text { MODOT } & \text { SVMODOT3X-W-25778DUP } \\ & \\ \text { MORGAN } & \text { SVMORGAN3X-W-25779 } \\ \text { MORGAN } & \text { SVMRGAN3X-W-25779DUP }\end{array}$

August 2010 sampling

$\begin{array}{ll}\text { SB44S } & \text { SVSB44S-W-25832 } \\ \text { SB44S } & \text { SVSB44S-W-25832DUP } \\ & \\ \text { SB47D } & \text { SVSB47D-W-25839 } \\ \text { SB47D } & \text { SVSB47D-W-25839DUP } \\ & \\ \text { SB48S } & \text { SVSB48S-W-25840 } \\ \text { SB48S } & \text { SVSB48S-W-25840DUP } \\ \text { SB48S } & \text { SVSB48SDUP-W-25845 } \\ & \\ \text { SB49D } & \text { SVSB49D-W-25843 } \\ \text { SB49D } & \text { SVSB49DDUP-W-25844 }\end{array}$

August 2011 sampling

$\begin{array}{ll}\text { SB43D } & \text { SVSB43D-W-25875 } \\ \text { SB43D } & \text { SVSB43DDUP-W-25888 } \\ & \\ \text { SB44S } & \text { SVSB44SGRAB-W-25860 } \\ \text { SB44S } & \text { SVSB44SGRAB-W-25860DUP }\end{array}$

$\begin{array}{lll}\text { 6/21/10 } & \text { Primary } & \text { Grab } \\ \text { 6/21/10 } & \text { Duplicate } & \text { Grab } \\ \text { 6/25/10 } & \text { Primary } & \\ \text { 6/25/10 } & \text { Duplicate } & \text { LF } \\ \text { 6/27/10 } & \text { Primary } & 3 W \\ 6 / 27 / 10 & \text { Duplicate } & 3 \\ & \\ \text { 6/28/10 } & \text { Primary } & 3 W \\ 6 / 28 / 10 & \text { Duplicate } & 3 W V\end{array}$

Grab

LF

LF

$3 W V$

$3 W V$

$6 / 28 / 10$ Duplicate

\begin{abstract}
8/24/10 Primary 8/24/10 Duplicate

8/24/10 Primary

8/24/10 Duplicate

8/25/10 Primary

8/25/10 Duplicate

8/25/10 Replicate

8/24/10 Primary

8/24/10 Replicate
\end{abstract}

8/10/11 Primary

8/10/11 Replicate

8/10/11 Primary

8/10/11 Duplicate
Concentration

Carbon

Tetrachloride Chloroform Units 
August 2011 sampling (cont.)

\begin{tabular}{|c|c|c|c|c|c|c|c|c|c|c|}
\hline SB46D & SVSB46D-W-25881 & $8 / 11 / 11$ & Primary & LF & MW & $90-110$ & Water & ND & ND & $\mu g / L$ \\
\hline SB46D & SVSB46DDUP-W-25889 & $8 / 11 / 11$ & Replicate & LF & MW & $90-110$ & Water & ND & ND & $\mu g / L$ \\
\hline SB48S & SVSB48SGRAB-W-25868 & $8 / 10 / 11$ & Primary & Grab & MW & $25-35$ & Water & ND & ND & $\mu g / L$ \\
\hline SB48S & SVSB48SGRAB-W-25868DUP & $8 / 10 / 11$ & Duplicate & Grab & MW & $25-35$ & Water & ND & ND & $\mu g / L$ \\
\hline SB49D & SVSB49DGRAB-W-25871 & $8 / 10 / 11$ & Primary & Grab & MW & $77-97$ & Water & ND & 1.3 & $\mu g / L$ \\
\hline SB49D & SVSB49DGRAB-W-25871DUP & $8 / 10 / 11$ & Duplicate & Grab & MW & $77-97$ & Water & ND & 1.3 & $\mu \mathrm{g} / \mathrm{L}$ \\
\hline
\end{tabular}

a Collection method abbreviations: $3 \mathrm{WV}$, three-well-volume purge; LF, low flow.

b Installation types: CPT, cone penetrometer; DW, domestic well; MW, monitoring well. 
TABLE S2.5 Analytical results for samples submitted for verification organic analyses during the Phase II investigation.

\begin{tabular}{|c|c|c|c|c|c|c|c|c|c|}
\hline \multirow[b]{3}{*}{ Location } & \multirow[b]{3}{*}{ Sample } & \multirow[b]{3}{*}{$\begin{array}{l}\text { Sample } \\
\text { Medium }\end{array}$} & \multirow[b]{3}{*}{$\begin{array}{l}\text { Sample } \\
\text { Date }\end{array}$} & \multirow[b]{3}{*}{$\begin{array}{l}\text { Depth } \\
\text { (ft BGL) }\end{array}$} & \multicolumn{5}{|c|}{ Concentration $(\mu \mathrm{g} / \mathrm{L})$} \\
\hline & & & & & \multicolumn{2}{|c|}{ AGEM Laboratory } & \multicolumn{3}{|c|}{ TestAmerica } \\
\hline & & & & & $\begin{array}{c}\text { Carbon } \\
\text { Tetrachloride }\end{array}$ & Chloroform & $\begin{array}{c}\text { Carbon } \\
\text { Tetrachloride }\end{array}$ & Chloroform & $\begin{array}{c}\text { Sample } \\
\text { Delivery } \\
\text { Group }\end{array}$ \\
\hline \multicolumn{10}{|c|}{ June-July 2010 sampling } \\
\hline SB43 & SVSB43-S-25643 & Soil & 6/21/10 & 18 & $N D^{a}$ & ND & ND & ND & 138033 \\
\hline SB43 & SVSB43-S-25650 & Soil & $6 / 21 / 10$ & 48 & ND & ND & ND & ND & 138033 \\
\hline SB43 & SVSB43-S-25654 & Soil & $6 / 21 / 10$ & 64 & ND & ND & ND & ND & 138033 \\
\hline SB44 & SVSB44-S-25677 & Soil & $6 / 23 / 10$ & 8 & ND & ND & ND & ND & 138033 \\
\hline SB44 & SVSB44-S-25688 & Soil & $6 / 23 / 10$ & 52 & 139 & ND & 12 & ND & 138033 \\
\hline SB44 & SVSB44-S-25698 & Soil & $6 / 23 / 10$ & 89 & ND & ND & ND & ND & 138033 \\
\hline SB45 & SVSB45-S-25723 & Soil & $6 / 24 / 10$ & 16 & ND & ND & ND & ND & 138033 \\
\hline SB45 & SVSB45-S-25736 & Soil & $6 / 24 / 10$ & 64 & ND & ND & ND & ND & 138033 \\
\hline SB45 & SVSB45-S-25745 & Soil & $6 / 24 / 10$ & 100 & ND & ND & ND & ND & 138033 \\
\hline SB46 & SVSB46-S-25719 & Soil & $6 / 25 / 10$ & 40 & 81 & ND & 7.5 & $0.22 \mathrm{~J}^{\mathrm{b}}$ & 138033 \\
\hline SB46 & SVSB46-S-25756 & Soil & $6 / 25 / 10$ & 68 & ND & ND & ND & ND & 138033 \\
\hline QC & SV-S-MEOH BLANK 1JUL10 & Soil & $7 / 1 / 10$ & - & - & - & ND & ND & 138033 \\
\hline SB24 & SVSB24LFB-W-25774 & Water & $6 / 25 / 10$ & $50-60$ & 4.5 & 6.0 & 4.4 & 5.9 & 137933 \\
\hline SB38 & SVSB38LFM-W-25773 & Water & $6 / 24 / 10$ & $50-90$ & 3.7 & 3.2 & 3.7 & 3.2 & 137933 \\
\hline SB38 & SVSB38LFB-W-25772 & Water & $6 / 23 / 10$ & $50-90$ & 1.9 & 2.1 & 1.8 & 2.5 & 137933 \\
\hline MODOT & SVMODOTLFM-W-25776 & Water & $6 / 25 / 10$ & 44 & 210 & 6.8 & 180 & 6.3 & 137933 \\
\hline MODOT & SVMODOTLFB-W-25775 & Water & $6 / 25 / 10$ & 44 & 179 & 6.2 & 170 & 5.8 & 137933 \\
\hline MODOT & SVMODOT3X-W-25778 & Water & $6 / 27 / 10$ & 44 & 196 & 6.8 & 190 & 6.0 & 137941 \\
\hline MORGAN & SVMORGANLEM-W-25771 & Water & $6 / 23 / 10$ & 60.58 & 13 & 5.0 & 13 & 5.7 & 137933 \\
\hline MORGAN & SVMORGANLFB-W-25770 & Water & $6 / 23 / 10$ & 60.58 & 13 & 5.0 & 13 & 5.4 & 137933 \\
\hline MORGAN & SVMORGAN3X-W-25779 & Water & $6 / 28 / 10$ & 60.58 & 13 & 3.4 & 13 & 5.7 & 137941 \\
\hline
\end{tabular}




\begin{tabular}{|c|c|c|c|c|c|c|c|c|c|}
\hline \multirow[b]{3}{*}{ Location } & \multirow[b]{3}{*}{ Sample } & \multirow[b]{3}{*}{$\begin{array}{l}\text { Sample } \\
\text { Medium }\end{array}$} & \multirow[b]{3}{*}{$\begin{array}{l}\text { Sample } \\
\text { Date }\end{array}$} & \multirow[b]{3}{*}{$\begin{array}{c}\text { Depth } \\
\text { (ft BGL) }\end{array}$} & \multicolumn{5}{|c|}{ Concentration $(\mu \mathrm{g} / \mathrm{L})$} \\
\hline & & & & & \multicolumn{2}{|c|}{ AGEM Laboratory } & \multicolumn{3}{|c|}{ TestAmerica } \\
\hline & & & & & $\begin{array}{c}\text { Carbon } \\
\text { Tetrachloride }\end{array}$ & Chloroform & $\begin{array}{c}\text { Carbon } \\
\text { Tetrachloride }\end{array}$ & Chloroform & $\begin{array}{c}\text { Sample } \\
\text { Delivery } \\
\text { Group }\end{array}$ \\
\hline \multicolumn{10}{|c|}{ June-July 2010 sampling (cont.) } \\
\hline QC & SVQCTB-W-25777 & Water & $6 / 25 / 10$ & - & ND & ND & ND & ND & 137933 \\
\hline QC & SVQCTB-W-25780 & Water & $6 / 28 / 10$ & - & ND & ND & ND & ND & 137941 \\
\hline \multicolumn{10}{|c|}{ August 2010 sampling } \\
\hline SB43D & SVSB43D-W-25831 & Water & $8 / 24 / 10$ & $95-105$ & ND & 1.3 & ND & 0.97 & $200-1286$ \\
\hline SB46S & SVSB46S-W-25836 & Water & $8 / 25 / 10$ & $42-52$ & 15 & 1.8 & 12 & 2.0 & $200-1286$ \\
\hline SB47S & SVSB47S-W-25838 & Water & $8 / 25 / 10$ & $25-35$ & ND & 5.6 & ND & 4.4 & $200-1286$ \\
\hline SB48D & SVSB48D-W-25841 & Water & $8 / 24 / 10$ & $77-97$ & ND & ND & ND & $0.09 \mathrm{~J}$ & $200-1286$ \\
\hline SB49D & SVSB49D-W-25843 & Water & $8 / 24 / 10$ & $77-97$ & ND & 3.0 & ND & 2.9 & $200-1286$ \\
\hline $\mathrm{QC}$ & SVQCTB-W-25852 & Water & $8 / 25 / 10$ & - & ND & ND & ND & $0.16 \mathrm{~J}$ & $200-1286$ \\
\hline \multicolumn{10}{|c|}{ August 2011 sampling } \\
\hline SB43S & SVSB43S-W-25874 & Water & $8 / 10 / 11$ & $45-55$ & ND & 3.2 & $0.042 \mathrm{~J}$ & 2.8 & $200-6522$ \\
\hline SB44D & SVSB44D-W-25877 & Water & $8 / 11 / 11$ & $92-102$ & ND & ND & $0.039 \mathrm{~J}$ & $0.41 \mathrm{~J}$ & $200-6522$ \\
\hline SB46S & SVSB46S-W-25880 & Water & $8 / 11 / 11$ & $42-52$ & 17 & 7.8 & 13 & 5.7 & $200-6522$ \\
\hline SB47D & SVSB47D-W-25883 & Water & $8 / 10 / 11$ & $77-97$ & ND & ND & ND & $0.09 \mathrm{~J}$ & $200-6522$ \\
\hline $\mathrm{QC}$ & SVQCTB-W-25897 & Water & $8 / 11 / 11$ & - & ND & ND & ND & $0.17 \mathrm{~J}$ & $200-6522$ \\
\hline
\end{tabular}

Footnotes on next page. 
a ND, not detected at the following instrument detection limits:

\begin{tabular}{lcc} 
& \multicolumn{2}{c}{ Concentration } \\
\cline { 2 - 3 } Laboratory & $\begin{array}{c}\text { Soil } \\
(\mu \mathrm{g} / \mathrm{kg})\end{array}$ & $\begin{array}{c}\text { Water } \\
(\mu \mathrm{g} / \mathrm{L})\end{array}$ \\
\hline AGEM & 1.0 & 0.1 \\
TestAmerica & 0.1 & 0.01
\end{tabular}

b J, estimated concentration below the following method quantitation limits:

\begin{tabular}{lrr} 
& \multicolumn{2}{c}{ Concentration } \\
\cline { 2 - 3 } Laboratory & $\begin{array}{c}\text { Soil } \\
(\mu \mathrm{g} / \mathrm{kg})\end{array}$ & $\begin{array}{c}\text { Water } \\
(\mu \mathrm{g} / \mathrm{L})\end{array}$ \\
\hline AGEM & 10 & 1.0 \\
TestAmerica & 5 & 0.5
\end{tabular}


Supplement 3:

Verification Laboratory Data 


\section{Supplement 3 Contents}

Report for TA SDG 137933 (Water) .............................................................. 3 of 419

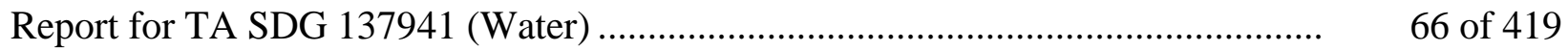

Report for TA SDG 138033 (Soil).................................................................. 108 of 419

Report for TA SDG 200-1286 (Water) ........................................................... 177 of 419

Report for TA SDG 200-6522 (Water) ............................................................ 224 of 419 


\section{TestAmerica}

IHE LEAOER IN ENVIRONMENTAL TESTING

TestAmerica Laboratories, Inc.

July 9, 2010

Mr. Clyde Dennis

Argonne National Laboratory

9700 S. Cass Avenue

Bldg. 203, Office B149

Argonne, IL 60439

Re: Laboratory Project No. 21005

Case: SAVANNAH; SDG: 137933

Dear Mr. Dennis:

Enclosed are analytical results for samples that were received by TestAmerica Burlington on June $26^{\text {th }}, 2010$. Laboratory identification numbers were assigned, and designated as follows:

$\begin{array}{llll}\text { Lab ID } & \begin{array}{l}\text { Client } \\ \text { Sample ID }\end{array} & \begin{array}{l}\text { Sample } \\ \text { Date }\end{array} & \begin{array}{l}\text { Sample } \\ \text { Matrix }\end{array} \\ & \text { Received: 06/26/10 ETR No: } 137933 & \\ 834001 & \text { SVMORGANLFB-W-25770 } & 06 / 23 / 10 & \text { WATER } \\ 834002 & \text { SVMORGANLFM-W-25771 } & 06 / 23 / 10 & \text { WATER } \\ 834003 & \text { SVSB38LFB-W-25772 } & 06 / 23 / 10 & \text { WATER } \\ 834004 & \text { SVSB38LFM-W-25773 } & 06 / 24 / 10 & \text { WATER } \\ 834005 & \text { SVSB24LFB-W-25774 } & 06 / 25 / 10 & \text { WATER } \\ 834006 & \text { SVMODOTLFB-W-25775 } & 06 / 25 / 10 & \text { WATER } \\ 834007 & \text { SVMODOTLFM-W-25776 } & 06 / 25 / 10 & \text { WATER } \\ 834008 & \text { SVQCTB-W-25777 } & 06 / 25 / 10 & \text { WATER } \\ 834009 & \text { VHBLK01 } & 06 / 28 / 10 & \text { WATER }\end{array}$

Documentation of the condition of the samples at the time of their receipt and any exception to the laboratory's Sample Acceptance Policy is documented in the Sample Handling section of this submittal. The samples, as received, were not acid preserved. On that basis, the laboratory did provide for the analytical work to be performed within seven days of sample collection.

In order to accommodate field length limitations in processing the data summary forms, the laboratory did, in certain instances, abbreviate the sample identifier. The electronically formatted data provides for the full sample identifier.

\section{SOM01.2 Volatile Organics (Trace Level Water)}

A storage blank was prepared for volatile organics analysis, and stored in association with the storage of the samples. That storage blank, identified as VHBLK01, was carried through the 


\section{TestAmerica}

THE LEADER IN ENVIRONMENTAL TESTING

holding period with the samples, and analyzed.

Samples SVMODOTLFB-W-25775 and SVMODOTLFM-W-25776 were analyzed at a dilution in order to provide quantification within the range of calibrated instrument response. An additional, more concentrated analysis was performed on each sample in order to provide for a lower reporting limit for those compounds that were not identified in the primary analysis. Both sets of results for the analysis of samples SVMODOTLFB-W-25775 and SVMODOTLFM-W-25776 are included in this submittal.

Each of the analyses associated with the sample set exhibited an acceptable internal standard performance. There was an acceptable recovery of each deuterated monitoring compound (DMC) in the analysis of each method blank and instrument blank associated with the analytical work, and in the analysis of the storage blank associated with the sample set. The analysis of the samples in this sample set did meet the technical acceptance criteria specific to DMC recoveries, although not all DMC recoveries were within the control range in each analysis. The technical acceptance criteria does provide for the recovery of up to three DMCs to fall outside of the control range in the analysis of field samples. Matrix spike and matrix spike duplicate analyses were not performed on samples in this sample set. Trace concentrations of acetone and carbon disulfide were identified in the analysis of the method blank associated with the analytical work. The concentration of each compound in that analysis was below the established reporting limit, and the analysis did meet the technical acceptance criteria for a compliant method blank analysis. Trace concentrations of acetone, carbon disulfide, methyl acetate, and carbon tetrachloride were identified in the analysis of one of the instrument blanks associated with the analytical work, and trace concentrations of acetone and carbon tetrachloride were identified in the analysis of the second instrument blank. The concentration of each compound in each analysis was below the established reporting limit, and each analysis did meet the technical acceptance criteria for a compliant instrument blank analysis. Trace concentrations of acetone and methyl acetate were identified in the analysis of the storage blank associated with the sample set. The concentration of each compound in that analysis was below the established reporting limit, and the analysis did meet the technical acceptance criteria for a compliant storage blank analysis. Present in the method blank, instrument blank, and storage blank analyses was a non-target constituent that represented a compound that is related to the DMC formulation. The fact that the presence of this compound is not within the laboratory's control is at issue. The derived results for that compound have been qualified with an "X" qualifier to reflect the source of the contamination.

The responses for each of the target analytes met the relative standard deviation criterion in the initial calibration. The response for each target analyte met the percent difference criterion in the continuing calibration check acquisition. The response for each target analyte met the 50.0 percent difference criterion in the closing calibration check acquisition.

The primary quantitation mass for methylcyclohexane that is specified in the Statement of Work is mass 83 . The laboratory did identify a contribution to mass 83 from 1,2-dichloropropane- $d_{61}$ one of the deuterated monitoring compounds (DMCs). The laboratory did change the primary quantitation mass assignment to mass 55 for the quantification of methylcyclohexane.

Manual integration was employed in deriving certain of the analytical results. The values that have been derived from manual integration are qualified on the quantitation reports. Extracted ion current profiles for each manual integration are included in the data package, and further 


\section{TestAmerica}

THE LEADER IN ENVIRONMENTAL TESTING

documented in the Sample Preparation section of this submittal.

Any reference within this report to Severn Trent Laboratories, Inc. or STL, should be understood to refer to TestAmerica Laboratories, Inc. (formerly known as Severn Trent Laboratories, Inc.) The analytical results associated with the samples presented in this test report were generated under a quality system that adheres to requirements specified in the NELAC standard. Release of the data in this test report and any associated electronic deliverables is authorized by the Laboratory Director's designee as verified by the following signature.

If there are any questions regarding this submittal, please contact me at 802 660-1990.

Sincerely,

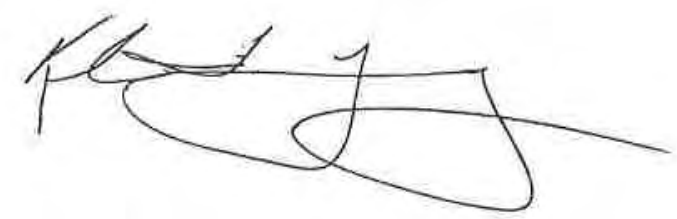

Kirk F. Young

Project Manager

KFY/hsf

Enclosure 


\section{TestAmerica Burlington Data Qualifier Definitions}

\section{$\underline{\text { Organic }}$}

$\mathrm{U}$ : Compound analyzed but not detected at a concentration above the reporting limit.

J: $\quad$ Estimated value.

$\mathrm{N}$ : Indicates presumptive evidence of a compound. This flag is used only for tentatively identified compounds (TICs) where the identification of a compound is based on a mass spectral library search.

P: $\quad$ SW-846: The relative percent difference for detected concentrations between two GC columns is greater than $40 \%$. Unless otherwise specified the higher of the two values is reported on the Form I.

CLP SOW: Greater than $25 \%$ difference for detected concentrations between two $\mathrm{GC}$ columns. Unless otherwise specified the lower of the two values is reported on the Form I.

C: $\quad$ Pesticide result whose identification has been confirmed by GC/MS .

B: $\quad$ Analyte is found in the sample and the associated method blank. The flag is used for tentatively identified compounds as well as positively identified compounds.

E: Compounds whose concentrations exceed the upper limit of the calibration range of the instrument for that specific analysis.

D: Concentrations identified from analysis of the sample at a secondary dilution.

A: Tentatively identified compound is a suspected aldol condensation product.

$X, Y, Z$ : Laboratory defined flags that may be used alone or combined, as needed. If used, the description of the flag is defined in the project narrative.

\section{Inorganic/Metals}

E: $\quad$ Reported value is estimated due to the presence of interference.

$\mathrm{N}: \quad$ Matrix spike sample recovery is not within control limits.

* Duplicate sample analysis is not within control limits.

B: The result reported is less than the reporting limit but greater than the instrument detection limit.

$\mathrm{U}: \quad$ Analyte was analyzed for but not detected above the reporting limit.

\section{Method Codes:}

P ICP-AES

MS ICP-MS

CV Cold Vapor AA

AS Semi-Automated Spectrophotometric 


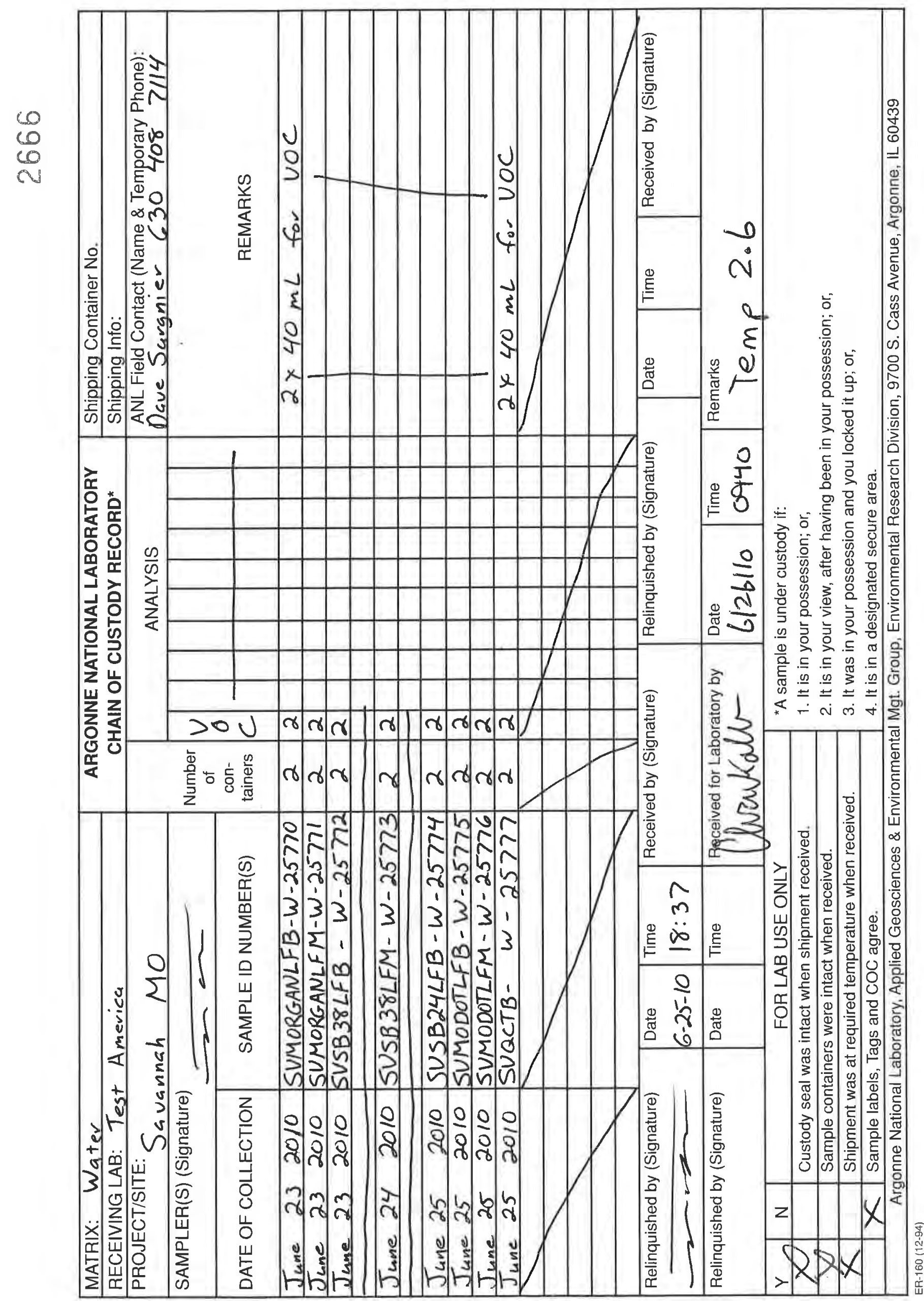




\section{TestAmerica}

THE LEADER IN ENVIRONMENTAL TESTING

\section{Sample Data Summary - SOM01.2 Volatiles - Trace}


$1 A$ - FORM I VOA-I

VOLATILE ORGANICS ANALYSIS DATA SHEET
EPA SAMPLE NO.

MODOTLFB - 5
Lab Name: TESTAMERICA BURLINGTON

Lab Code: STLV Case No.: SAVANNAH Mod. Ref No.:
Contract : 21005

\section{Matrix: (SOIL/SED/WATER) Water}

Sample wt/vol: 25.0

$(\mathrm{g} / \mathrm{mL}) \mathrm{mL}$

Level: (TRACE/LOW/MED) TRACE

\% Moisture: not dec.

GC Column: DB-624

Soil Extract Volume:

ID $: 0.20$

Purge volume: 25.0
Lab Sample ID: 834006

Lab File ID: $834006 \mathrm{D} 2$

Date Received: 06/26/2010

Date Analyzed: 06/30/2010

Dilution Factor: 1.1

Soil Aliquot volume:

(UL)

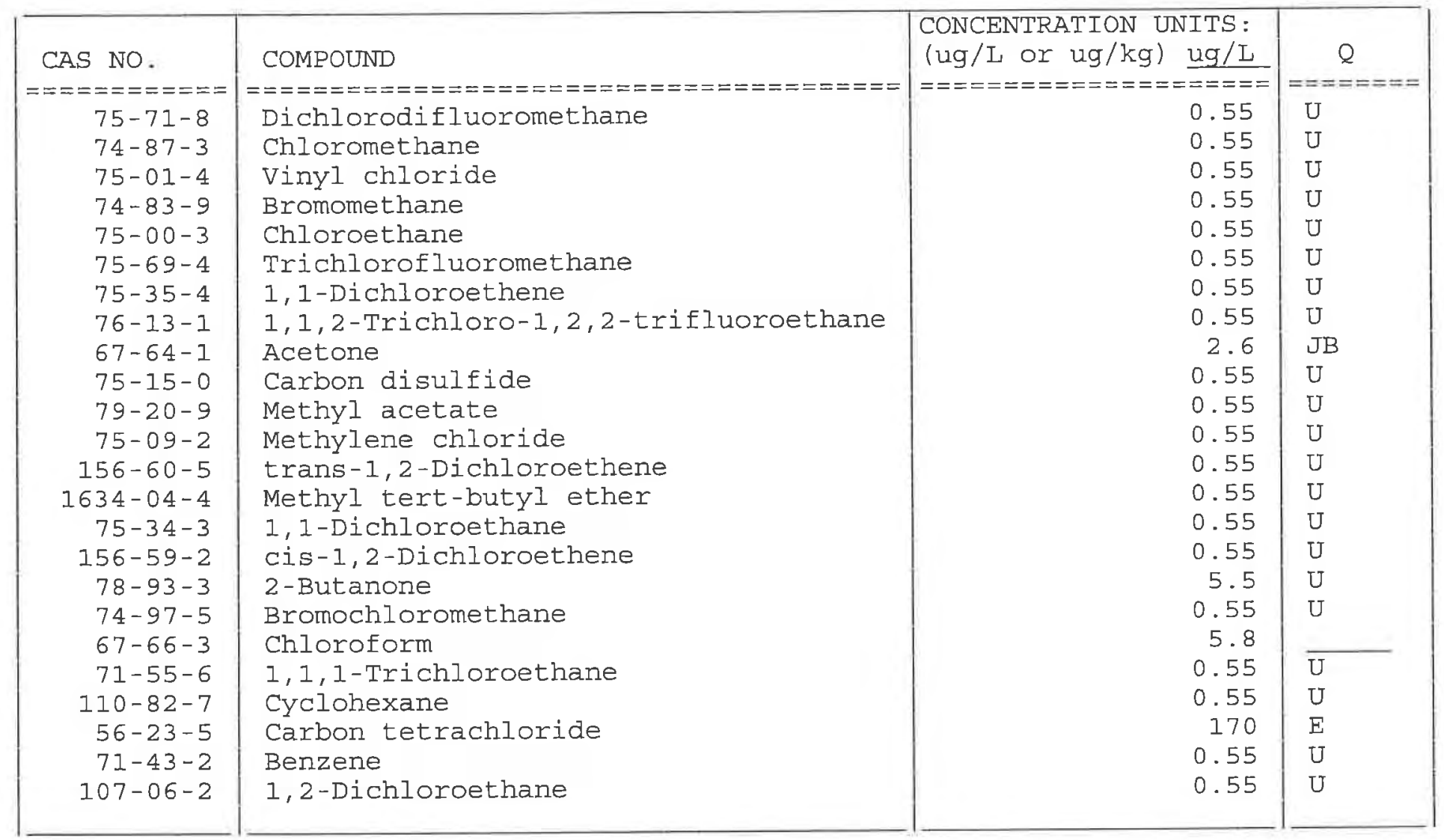

Report 1,4-Dioxane for Low-Medium VOA analysis only 
$1 B$ - FORM I VOA-2

VOLATILE ORGANICS ANALYSIS DATA SHEET
EPA SAMPLE NO.

MODOTLFB - 5
Lab Name: TESTAMERICA BURLINGTON

Lab code: STLV Case No.: SAVANNAH Mod. Ref No.:
Contract: 21005
Matrix: (SOIL/SED/WATER) Water

Sample wt/vol: 25.0

$(\mathrm{g} / \mathrm{mL}) \mathrm{mI}$

Level: (TRACE/LOW/MED) TRACE

\% Moisture: not dec.

GC Column: DB-624

ID : 0.20

Soil Extract Volume:

Purge Volume: 25.0
Lab Sample ID: 834006

Lab File ID: $834006 D 2$

Date Received: 06/26/2010

Date Analyzed: 06/30/2010

Dilution Factor: 1.1

Soil Aliquot Volume:

(uL)

\begin{tabular}{|c|c|c|c|}
\hline CAS NO. & COMPOUND & $\begin{array}{l}\text { CONCENTRATION UNITS: } \\
(\mathrm{ug} / \mathrm{L} \text { or } u g / \mathrm{kg}) \mathrm{ug} / \mathrm{L}\end{array}$ & Q \\
\hline 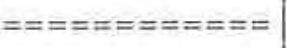 & 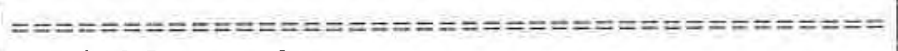 & 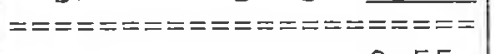 & $===== \pm==$ \\
\hline $79-01-6$ & Trichloroethene & 0.55 & $\mathrm{U}$ \\
\hline $108-87-2$ & Methylcyclohexane & 0.55 & $\mathrm{U}$ \\
\hline $78-87-5$ & 1,2-Dichloropropane & 0.55 & $\mathrm{U}$ \\
\hline $75-27-4$ & Bromodichloromethane & 0.55 & $\mathrm{U}$ \\
\hline $10061-01-5$ & cis-1,3-Dichloropropene & 0.55 & $U$ \\
\hline $108-10-1$ & 4-Methyl-2-pentanone & 5.5 & U \\
\hline $108-88-3$ & Toluene & 0.11 & J \\
\hline $10061-02-6$ & trans-1,3-Dichloropropene & 0.55 & $\mathrm{U}$ \\
\hline $79-00-5$ & $1,1,2-$ Trichloroethane & 0.55 & U \\
\hline $127-18-4$ & Tetrachloroethene & 0.55 & $\mathrm{U}$ \\
\hline $591-78-6$ & 2-Hexanone & 5.5 & $\mathrm{U}$ \\
\hline $124-48-1$ & Dibromochloromethane & 0.55 & $\mathrm{U}$ \\
\hline $106-93-4$ & 1,2-Dibromoethane & 0.55 & $\mathrm{U}$ \\
\hline $108-90-7$ & Chlorobenzene & 0.55 & $\mathrm{U}$ \\
\hline $100-41-4$ & Ethylbenzene & 0.55 & $\mathrm{U}$ \\
\hline $95-47-6$ & o-Xylene & 0.55 & $\mathrm{U}$ \\
\hline $179601-23-1$ & $\mathrm{~m}, \mathrm{p}-\mathrm{Xy}$ lene & 0.090 & J \\
\hline $100-42-5$ & styrene & 0.55 & $\mathrm{U}$ \\
\hline $75-25-2$ & Bromoform & 0.55 & $\mathrm{U}$ \\
\hline $98-82-8$ & Isopropylbenzene & 0.55 & $\mathrm{U}$ \\
\hline $79-34-5$ & $1,1,2,2$-Tetrachloroethane & 0.55 & $\mathrm{U}$ \\
\hline $541-73-1$ & 1,3 -Dichlorobenzene & 0.55 & $\mathrm{U}$ \\
\hline $106-46-7$ & 1,4-Dichlorobenzene & 0.55 & $\mathrm{U}$ \\
\hline $95-50-1$ & 1,2 -Dichlorobenzene & 0.55 & $\mathrm{U}$ \\
\hline $96-12-8$ & 1,2 -Dibromo-3-chloropropane & 0.55 & $\mathrm{U}$ \\
\hline $120-82-1$ & $1,2,4-T r i c h l o r o b e n z e n e$ & 0.55 & $\mathrm{U}$ \\
\hline $87-61-6$ & $1,2,3$-Trichlorobenzene & 0.55 & $\mathrm{U}$ \\
\hline
\end{tabular}


$1 J$ - FORM I VOA-TIC

VOLATILE ORGANICS ANALYSIS DATA SHEET

TENTATIVELY IDENTIFIED COMPOUNDS
EPA SAMPLE NO.

MODOTLFB- 5
Lab Name: TESTAMERICA BURLINGTON

Lab Code: STLV Case No.: SAVANNAH

Matrix: (SOIL/SED/WATER) Water

Sample wt/vol: $25.0(\mathrm{~g} / \mathrm{mL}) \mathrm{mL}$

Level: (TRACE or LOW/MED) TRACE

\% Moisture: not dec.

GC Column: DB-624

ID $: 0.20$

Soil Extract volume:

CONCENTRATION UNITS: (ug/L or $\mathrm{ug} / \mathrm{kg}) \mathrm{ug} / \mathrm{L}$
Contract: 21005

Mod. Ref No.:

SDG No.: 137933
Lab Sample ID: 834006

Lab File ID: 834006D2

Date Received: 06/26/2010

Date Analyzed: 06/30/2010

(mm) Dilution Factor: 1.1

(uL) Soil Aliquot volume:

(uL)

Purge Volume: 25.0

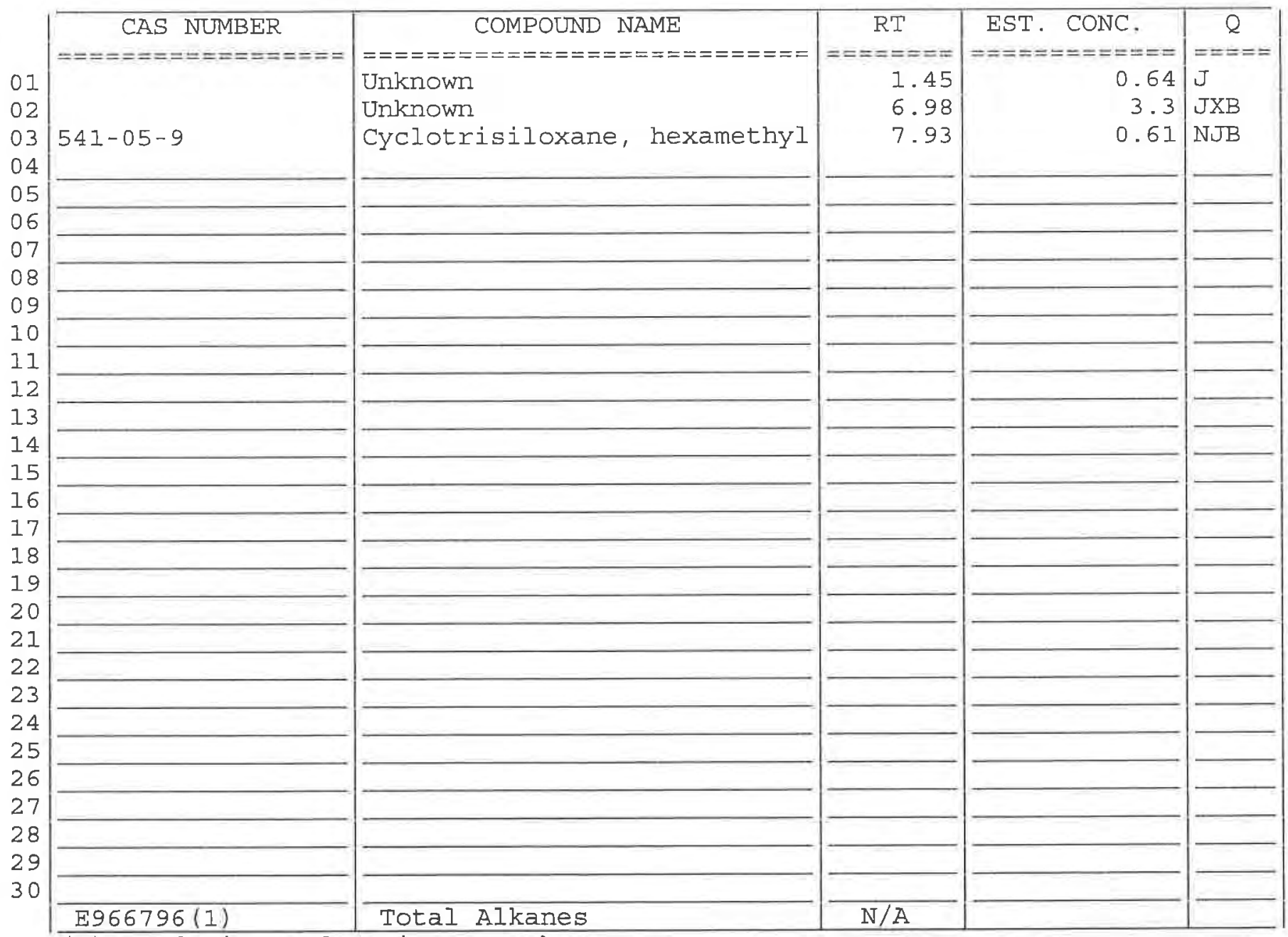

(1) EPA-designated Registry Number. 
$1 A$ - FORM I VOA-I

VOLATILE ORGANICS ANALYSIS DATA SHEET

EPA SAMPLE NO.

Lab Name: TESTAMERICA BURIINGTON

Contract: 21005

Lab Code: STLV Case No.: SAVANNAH Mod. Ref No.:

SDG NO.: 137933

Matrix: (SOIL/SED/WATER) water

Sample wt/vol: $25.0 \quad(\mathrm{~g} / \mathrm{mL}) \mathrm{mL}$

Level: (TRACE/LOW/MED) TRACE

\% Moisture: not dec.

GC Column: DB-624 ID: 0.20 (mm) Dilution Factor: 14.2

Soil Extract Volume:

Purge Volume: 25.0
Lab Sample ID: $834006 \mathrm{DI}$

Lab File ID: $834006 D$

Date Received: 06/26/2010

Date Analyzed: 06/30/2010

(uI) Soil Aliquot Volume:

(uL)

$(m I)$

\begin{tabular}{|c|c|c|c|}
\hline CAS NO. & COMPOUND & $\begin{array}{l}\text { CONCENTRATION UNITS: } \\
(u g / \mathrm{L} \text { or } u g / \mathrm{kg}) \mathrm{ug} / \mathrm{L}\end{array}$ & $Q$ \\
\hline 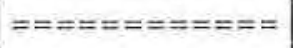 & 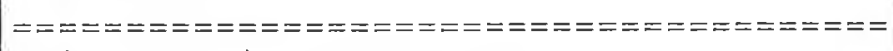 & 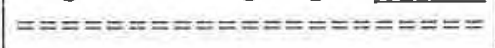 & 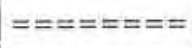 \\
\hline $75-71-8$ & Dichlorodifluoromethane & 7.1 & $\mathrm{U}$ \\
\hline $74-87-3$ & Chloromethane & 7.1 & $\mathrm{U}$ \\
\hline $75-01-4$ & vinyl chloride & 7.1 & $\mathrm{U}$ \\
\hline $74-83-9$ & Bromomethane & 7.1 & $\mathrm{U}$ \\
\hline $75-00-3$ & Chloroethane & 7.1 & $\mathrm{U}$ \\
\hline $75-69-4$ & Trichlorofluoromethane & 7.1 & U \\
\hline $75-35-4$ & 1,1-Dichloroethene & 7.1 & $\mathrm{U}$ \\
\hline $76-13-1$ & 1,1,2-Trichloro-1,2,2-trifluoroethane & 7.1 & $\mathrm{U}$ \\
\hline $67-64-1$ & Acetone & 46 & DJB \\
\hline $75-15-0$ & Carbon disulfide & 7.1 & $\mathrm{U}$ \\
\hline $79-20-9$ & Methyl acetate & 7.1 & $\mathrm{U}$ \\
\hline $75-09-2$ & Methylene chloride & 7.1 & $\mathrm{U}$ \\
\hline $156-60-5$ & trans-1,2-Dichloroethene & 7.1 & $\mathrm{U}$ \\
\hline $1634-04-4$ & Methyl tert-butyl ether & 7.1 & $\mathrm{U}$ \\
\hline $75-34-3$ & 1,1-Dichloroethane & 7.1 & $\mathrm{U}$ \\
\hline $156-59-2$ & cis-1,2-Dichloroethene & 7.1 & $\mathrm{U}$ \\
\hline $78-93-3$ & 2-Butanone & 71 & U \\
\hline $74-97-5$ & Bromochloromethane & 7.1 & $\mathrm{U}$ \\
\hline $67-66-3$ & Chloroform & 6.6 & DJ \\
\hline $71-55-6$ & 1,1,1-Trichloroethane & 7.1 & U \\
\hline $110-82-7$ & Cyclohexane & 7.1 & U \\
\hline $56-23-5$ & Carbon tetrachloride & 170 & $\mathrm{D}$ \\
\hline $71-43-2$ & Benzene & 7.1 & $\mathrm{U}$ \\
\hline $107-06-2$ & 1,2-Dichloroethane & 7.1 & $\mathrm{U}$ \\
\hline
\end{tabular}

Report 1,4-Dioxane for Low-Medium VOA analysis only 


\author{
IB - FORM I VOA-2 \\ VOLATILE ORGANICS ANALYSIS DATA SHEET
}

EPA SAMPLE NO.
Lab Name: TESTAMERICA BURLINGTON

Lab Code: STLV Case No.: SAVANNAH Mod. Ref No.:
Contract: 21005
Matrix: (SOIL/SED/WATER) Water

Sample wt/vol: 25.0

$(\mathrm{g} / \mathrm{mL}) \mathrm{mL}$

Level: (TRACE/LOW/MED) TRACE

\% Moisture: not dec.

GC Column: DB-624

ID $: 0.20$

Soil Extract Volume:

Purge Volume: 25.0
Lab Sample ID: $834006 \mathrm{DI}$

Lab File ID: $834006 \mathrm{D}$

Date Received: 06/26/2010

Date Analyzed: 06/30/2010

Dilution Factor: 14.2

Soil Aliquot Volume:

(uL)

\begin{tabular}{|c|c|c|c|}
\hline CAS NO. & COMPOUND & $\begin{array}{l}\text { CONCENTRATION UNITS: } \\
(\mathrm{ug} / \mathrm{L} \text { or } \mathrm{ug} / \mathrm{kg} \text { ) ug/I }\end{array}$ & Q \\
\hline 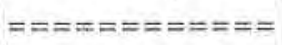 & 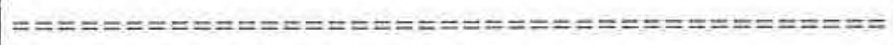 & 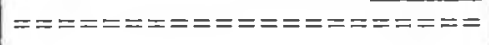 & $==x===0$ \\
\hline $79-01-6$ & Trichloroethene & 7.1 & $\mathrm{U}$ \\
\hline $108-87-2$ & Methylcyclohexane & 7.1 & $\mathrm{U}$ \\
\hline $78-87-5$ & 1,2-Dichloropropane & 7.1 & $\mathrm{U}$ \\
\hline $75-27-4$ & Bromodichloromethane & 7.1 & $\mathrm{U}$ \\
\hline $10061-01-5$ & Cis-1,3-Dichloropropene & 7.1 & $\mathrm{U}$ \\
\hline $108-10-1$ & 4-Methyl-2-pentanone & 71 & $\mathrm{U}$ \\
\hline $108-88-3$ & Toluene & 7.1 & $\mathrm{U}$ \\
\hline $10061-02-6$ & trans-1,3-Dichloropropene & 7.1 & U \\
\hline $79-00-5$ & 1,1,2-Trichloroethane & 7.1 & $\mathrm{U}$ \\
\hline $127-18-4$ & Tetrachloroethene & 7.1 & $\mathrm{U}$ \\
\hline $591-78-6$ & 2-Hexanone & 71 & $\mathrm{U}$ \\
\hline $124-48-1$ & Dibromochloromethane & 7.1 & $\mathrm{U}$ \\
\hline $106-93-4$ & 1,2-Dibromoethane & 7.1 & $\mathrm{U}$ \\
\hline $108-90-7$ & Chlorobenzene & 7.1 & $\mathrm{U}$ \\
\hline $100-41-4$ & Ethylbenzene & 7.1 & $\mathrm{U}$ \\
\hline $95-47-6$ & o-Xylene & 7.1 & $\mathrm{U}$ \\
\hline $179601-23-1$ & $\mathrm{~m}, \mathrm{p}-\mathrm{xy}$ lene & 7.1 & $\mathrm{U}$ \\
\hline $100-42-5$ & styrene & 7.1 & $\mathrm{U}$ \\
\hline $75-25-2$ & Bromoform & 7.1 & $\mathrm{U}$ \\
\hline $98-82-8$ & Isopropylbenzene & 7.1 & $\mathrm{U}$ \\
\hline $79-34-5$ & $1,1,2,2$-Tetrachloroethane & 7.1 & $\mathrm{U}$ \\
\hline $541-73-1$ & 1,3-Dichlorobenzene & 7.1 & $\mathrm{U}$ \\
\hline $106-46-7$ & 1,4-Dichlorobenzene & 7.1 & $\mathrm{U}$ \\
\hline $95-50-1$ & 1,2-Dichlorobenzene & 7.1 & $\mathrm{U}$ \\
\hline $96-12-8$ & 1,2-Dibromo-3-chloropropane & 7.1 & U \\
\hline $120-82-1$ & $1,2,4$-Trichlorobenzene & 7.1 & $\mathrm{U}$ \\
\hline $87-61-6$ & $1,2,3$-Trichlorobenzene & 7.1 & $\mathrm{U}$ \\
\hline
\end{tabular}


IJ - FORM I VOA-TIC

VOLATILE ORGANICS ANALYSIS DATA SHEET

TENTATIVELY IDENTIFIED COMPOUNDS
EPA SAMPLE NO. MODOTLFB-5DL
Lab Name: TESTAMERICA BURLINGTON

Lab Code: STLV Case No.: SAVANNAH

Matrix: (SOIL/SED/WATER) Water

Sample wt/vol: $25.0 \quad(\mathrm{~g} / \mathrm{mL}) \mathrm{mL}$

Level: (TRACE Or LOW/MED) TRACE

$\div$ Moisture: not dec.

GC Column: DB-624

Soil Extract Volume:

ID : 0.20

CONCENTRATION UNITS: (ug/L Or $\mathrm{ug} / \mathrm{kg}$ ) ug/L
Contract : 21005

Mod. Ref No.:

SDG No.: 137933

Lab Sample ID: 834006D1

Lab File ID: 834006D

Date Received: 06/26/2010

Date Analyzed: 06/30/2010

(mm) Dilution Factor: 14.2

(uL) Soil Aliquot Volume:

(uL)

Purge Volume: 25.0

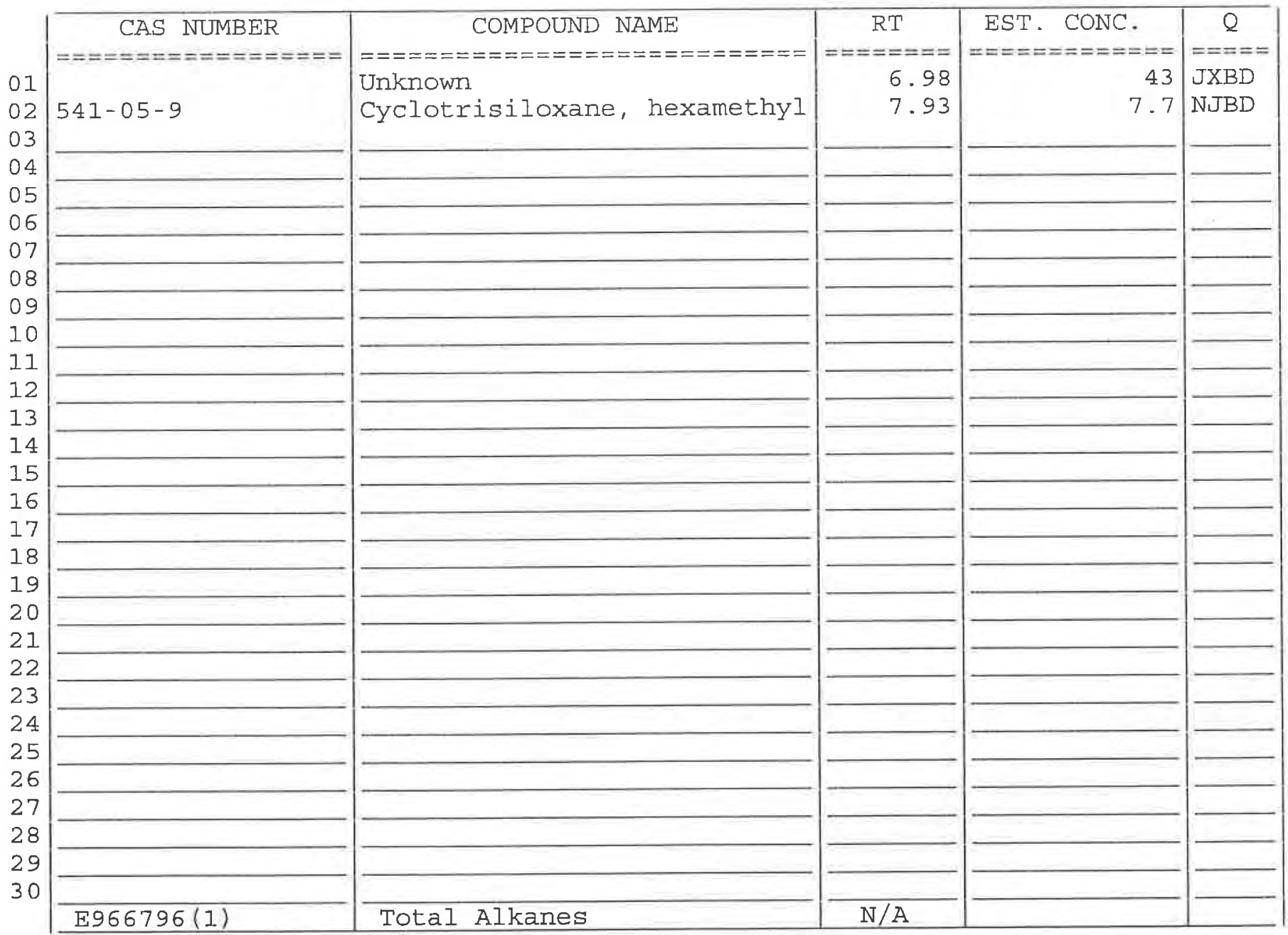

(1) EPA-designated Registry Number. 
Lab Name: TESTAMERICA BURLINGTON

Lab Code: STLV Case No.: SAVANNAH Mod. Ref No.:
Contract: 21005
Matrix: (SOIL/SED/WATER) Water

Sample wt/vol: 25.0

(g/mL) $\mathrm{mL}$

Level: (TRACE/LOW/MED) TRACE

\% Moisture: not dec.

GC Column: DB-624

ID $: 0.20$

Soil Extract Volume:

Purge Volume: 25.0
Lab sample ID: 834007

Lab File ID: $834007 D 2$

Date Received: 06/26/2010

Date Analyzed: 06/30/2010

Dilution Factor: 1.1

Soil Aliquot Volume:

(uL)

$(\mathrm{mL})$

\begin{tabular}{|c|c|c|c|}
\hline $\begin{array}{c}\text { CAS NO. } \\
========== \\
75-71-8 \\
74-87-3 \\
75-01-4 \\
74-83-9 \\
75-00-3 \\
75-69-4 \\
75-35-4 \\
76-13-1 \\
67-64-1 \\
75-15-0 \\
79-20-9 \\
75-09-2 \\
156-60-5 \\
1634-04-4 \\
75-34-3 \\
156-59-2 \\
78-93-3 \\
74-97-5 \\
67-66-3 \\
71-55-6 \\
110-82-7 \\
56-23-5 \\
71-43-2 \\
107-06-2\end{array}$ & 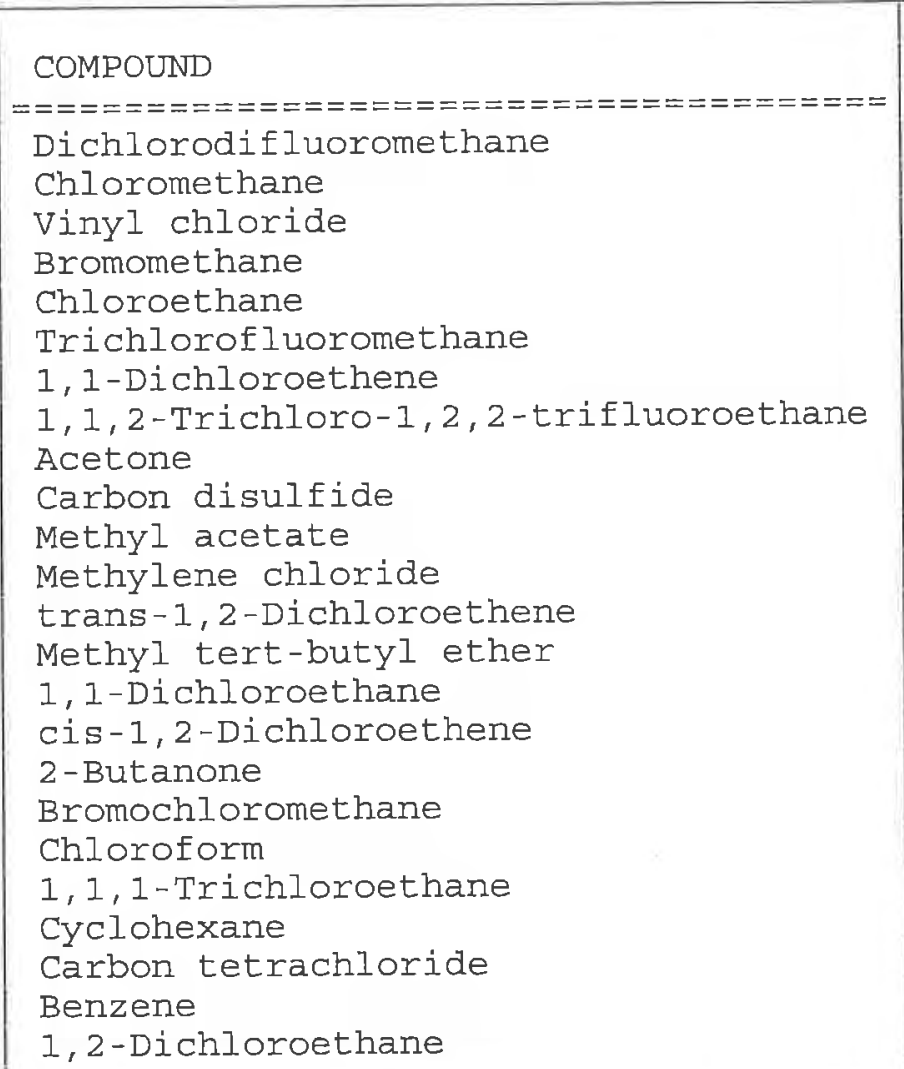 & \begin{tabular}{|cc} 
CONCENTRATION UNITS: \\
$(\mathrm{ug} / \mathrm{L}$ or $\mathrm{ug} / \mathrm{kg})$ & $\mathrm{ug} / \mathrm{L}$ \\
$=================$ \\
0.55 \\
0.55 \\
0.55 \\
0.55 \\
0.55 \\
0.55 \\
0.55 \\
0.55 \\
2.6 \\
0.55 \\
0.55 \\
0.55 \\
0.55 \\
0.55 \\
0.55 \\
0.55 \\
5.5 \\
0.55 \\
6.3 \\
0.55 \\
0.55 \\
190 \\
0.55 \\
0.55
\end{tabular} & $\begin{array}{l}======= \\
U \\
U \\
U \\
U \\
U \\
U \\
U \\
U \\
J B \\
U \\
U \\
U \\
U \\
U \\
U \\
U \\
U \\
U\end{array}$ \\
\hline
\end{tabular}

Report 1,4-Dioxane for Low-Medium VOA analysis only 
Lab Name: TESTAMERICA BURLINGTON

Lab Code: STLV Case No.: SAVANNAH Mod. Ref No.:
Contract: 21005
Matrix: (SOIL/SED/WATER) water

Sample wt/vol: 25.0

$(\mathrm{g} / \mathrm{mL}) \mathrm{mL}$

Level: (TRACE/LOW/MED) TRACE

\% Moisture: not dec.

GC Column: $\mathrm{DB}-624$

ID $: 0.20$

Soil Extract Volume:

Purge Volume: 25.0
Lab Sample ID: 834007

Lab File ID: $834007 \mathrm{D} 2$

Date Received: 06/26/2010

Date Analyzed: 06/30/2010

Dilution Factor: 1.1

Soil Aliquot Volume:

(uL)

(mL)

\begin{tabular}{|c|c|c|c|}
\hline & & CONCENTRATION UNITS: & 0 \\
\hline CAS NO. & COMPOUND & (ug/L or ug/kg) ug/L & $\stackrel{Q}{Q}=====$ \\
\hline $\begin{aligned}== & ======= \\
& 79-01-6\end{aligned}$ & 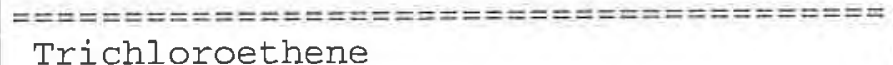 & 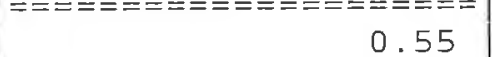 & $\begin{array}{l}= \pm===== \\
\mathrm{U}\end{array}$ \\
\hline $\begin{array}{r}79-01-6 \\
108-87-2\end{array}$ & Methylcyclohexane & 0.55 & $\mathrm{U}$ \\
\hline $78-87-5$ & 1,2-Dichloropropane & 0.55 & $\mathrm{U}$ \\
\hline $75-27-4$ & Bromodichloromethane & 0.55 & $\mathrm{U}$ \\
\hline $10061-01-5$ & cis-1,3-Dichloropropene & 0.55 & $\mathrm{U}$ \\
\hline $108-10-1$ & 4-Methyl-2-pentanone & 5.5 & $\mathrm{U}$ \\
\hline $108-88-3$ & Toluene & 0.10 & $\mathrm{~J}$ \\
\hline $10061-02-6$ & trans-1,3-Dichloropropene & 0.55 & $\mathrm{U}$ \\
\hline $79-00-5$ & $1,1,2$-Trichloroethane & 0.55 & $\mathrm{U}$ \\
\hline $127-18-4$ & Tetrachloroethene & 0.55 & $\mathrm{U}$ \\
\hline $591-78-6$ & 2 -Hexanone & 5.5 & $\mathrm{U}$ \\
\hline $124-48-1$ & Dibromochloromethane & 0.55 & $\mathrm{U}$ \\
\hline $106-93-4$ & 1,2 -Dibromoethane & 0.55 & $\mathrm{U}$ \\
\hline $108-90-7$ & Chlorobenzene & 0.55 & U \\
\hline $100-41-4$ & Ethylbenzene & 0.55 & U \\
\hline $95-47-6$ & o-xylene & 0.55 & $\mathrm{U}$ \\
\hline $179601-23-1$ & $\mathrm{~m}, \mathrm{p}$-xylene & 0.087 & $\mathrm{~J}$ \\
\hline $100-42-5$ & styrene & 0.55 & $\mathrm{U}$ \\
\hline $75-25-2$ & Bromoform & 0.55 & $\mathrm{U}$ \\
\hline $98-82-8$ & Isopropylbenzene & 0.55 & $\mathrm{U}$ \\
\hline $79-34-5$ & $1,1,2,2$-Tetrachloroethane & 0.55 & $\mathrm{U}$ \\
\hline $541-73-1$ & 1,3-Dichlorobenzene & 0.55 & $\mathrm{U}$ \\
\hline $106-46-7$ & 1,4-Dichlorobenzene & 0.55 & $\mathrm{U}$ \\
\hline $95-50-1$ & 1,2-Dichlorobenzene & 0.55 & $\mathrm{U}$ \\
\hline $96-12-8$ & 1,2 -Dibromo-3-chloropropane & 0.55 & U \\
\hline $120-82-1$ & $1,2,4$-Trichlorobenzene & 0.55 & $\mathrm{U}$ \\
\hline $87-61-6$ & $1,2,3$-Trichlorobenzene & 0.55 & $\mathrm{U}$ \\
\hline
\end{tabular}


IJ - FORM I VOA-TIC

VOLATILE ORGANICS ANALYSIS DATA SHEET TENTATIVELY IDENTIFIED COMPOUNDS
EPA SAMPLE NO. MODOTLFM- 6
Lab Name: TESTAMERICA BURLINGTON

Lab Code: STLV Case No.: SAVANNAH

Matrix: (SOIL/SED/WATER) water

Sample wt/vol: $25.0 \quad(\mathrm{~g} / \mathrm{mL}) \mathrm{mL}$

Level: (TRACE or LOW/MED) TRACE

\% Moisture: not dec.

GC Column: $\mathrm{DB}-624$

Soil Extract Volume:
Contract: 21005

Mod. Ref No.:
Lab Sample ID: 834007

Lab File ID: $834007 D 2$

Date Received: 06/26/2010

Date Analyzed: 06/30/2010

(mm) Dilution Factor: 1.1

(uL) Soil Aliquot Volume:

(uL)

Purge Volume: 25.0

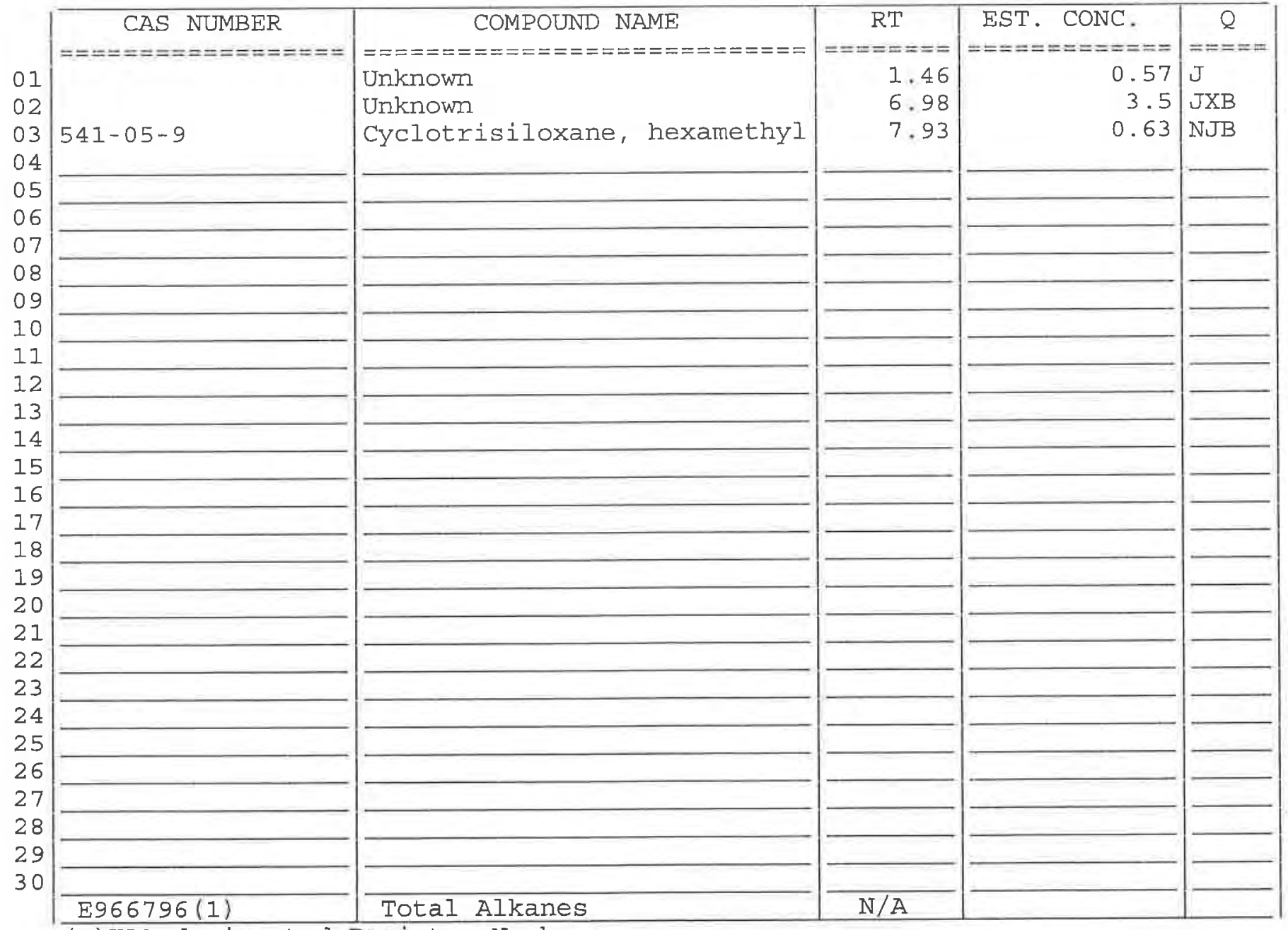

(1) EPA-designated Registry Number. 
$1 A$ - FORM I VOA-1

VOLATILE ORGANICS ANALYSIS DATA SHEET
EPA SAMPLE NO.

MODOTLFM-6DL
Lab Name: TESTAMERICA BURLINGTON

Lab Code: STIV Case No.: SAVANNAH Mod. Ref No.:
Contract : 21005
Matrix: (SOIL/SED/WATER) Water

Sample wt/vol: 25.0

$(\mathrm{g} / \mathrm{mL}) \mathrm{mL}$

Level: (TRACE/LOW/MED) TRACE

\% Moisture: not dec.

GC Column: DB-624

ID : 0.20

Soil Extract Volume:

Purge Volume: 25.0
Lab Sample ID: 834007D1

Lab File ID: $834007 D$

Date Received: 06/26/2010

Date Analyzed: 06/30/2010

Dilution Factor: 15.7

Soil Aliquot Volume:

(uL)

\begin{tabular}{|c|c|c|c|}
\hline CAS NO. & COMPOUND & $\begin{array}{l}\text { CONCENTRATION UNITS: } \\
(u g / I \text { or } u g / k g) u g / L\end{array}$ & $Q$ \\
\hline 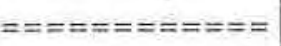 & 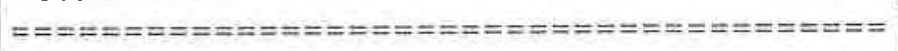 & 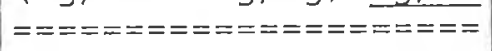 & $===== \pm==$ \\
\hline $75-71-8$ & Dichlorodifluoromethane & 7.9 & $\mathrm{U}$ \\
\hline $74-87-3$ & Chloromethane & 7.9 & $\mathrm{U}$ \\
\hline $75-01-4$ & vinyl chloride & 7.9 & U \\
\hline $74-83-9$ & Bromomethane & 7.9 & $\mathrm{U}$ \\
\hline $75-00-3$ & Chloroethane & 7.9 & $\mathrm{U}$ \\
\hline $75-69-4$ & Trichlorofluoromethane & 7.9 & $\mathrm{U}$ \\
\hline $75-35-4$ & 1,1 -Dichloroethene & 7.9 & $\mathrm{U}$ \\
\hline $76-13-1$ & $1,1,2$-Trichloro-1,2,2-trifluoroethane & 7.9 & $\mathrm{U}$ \\
\hline $67-64-1$ & Acetone & 38 & $\mathrm{DJB}$ \\
\hline $75-15-0$ & Carbon disulfide & 7.9 & U \\
\hline $79-20-9$ & Methyl acetate & 7.9 & $\mathrm{U}$ \\
\hline $75-09-2$ & Methylene chloride & 7.9 & $\mathrm{U}$ \\
\hline $156-60-5$ & trans-1,2-Dichloroethene & 7.9 & U \\
\hline $1634-04-4$ & Methyl tert-butyl ether & 7.9 & $\mathrm{U}$ \\
\hline $75-34-3$ & 1,1-Dichloroethane & 7.9 & $\mathrm{U}$ \\
\hline $156-59-2$ & cis-1,2-Dichloroethene & 7.9 & $\mathrm{U}$ \\
\hline $78-93-3$ & 2-Butanone & 79 & $U$ \\
\hline $74-97-5$ & Bromochloromethane & 7.9 & U \\
\hline $67-66-3$ & Chloroform & 6.6 & DJ \\
\hline $71-55-6$ & 1,1,1-Trichloroethane & 7.9 & $\mathrm{U}$ \\
\hline $110-82-7$ & Cyclohexane & 7.9 & $\mathrm{U}$ \\
\hline $56-23-5$ & Carbon tetrachloride & 180 & $\mathrm{D}$ \\
\hline $71-43-2$ & Benzene & 7.9 & $\mathrm{U}$ \\
\hline $107-06-2$ & 1,2-Dichloroethane & 7.9 & $\mathrm{U}$ \\
\hline
\end{tabular}

Report 1,4-Dioxane for Low-Medium VOA analysis only 
Lab Name: TESTAMERICA BURLINGTON

Contract: 21005

Lab Code: STLV Case No.: SAVANNAH Mod. Ref No.:

SDG No.: 137933

Matrix: (SOIL/SED/WATER) water

Sample wt/vol: $25.0 \quad(\mathrm{~g} / \mathrm{mL}) \mathrm{mL}$

Leve1: (TRACE/LOW/MED) TRACE

: Moisture: not dec.

GC Column: DB-624

ID : 0.20

$(\mathrm{mm})$

(uL)

Soil Aliquot Volume:

(uI)

(mI)

\begin{tabular}{|c|c|c|c|}
\hline CAS NO. & COMPOUND & $\begin{array}{l}\text { CONCENTRATION UNITS: } \\
(\mathrm{ug} / \mathrm{L} \text { or } \mathrm{ug} / \mathrm{kg}) \mathrm{ug} / \mathrm{L}\end{array}$ & $Q$ \\
\hline$=======+==+=$ & 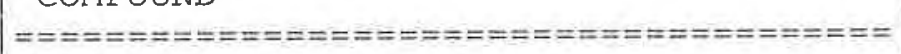 & $== \pm==== \pm==0====\equiv====$ & $========$ \\
\hline $79-01-6$ & Trichloroethene & 7.9 & $\mathrm{U}$ \\
\hline $108-87-2$ & Methylcyclohexane & 7.9 & $\mathrm{U}$ \\
\hline $78-87-5$ & 1,2-Dichloropropane & 7.9 & U \\
\hline $75-27-4$ & Bromodichloromethane & 7.9 & $\mathrm{U}$ \\
\hline $10061-01-5$ & cis-1,3-Dichloropropene & 7.9 & $\mathrm{U}$ \\
\hline $108-10-1$ & 4 -Methyl-2-pentanone & 79 & $\mathrm{U}$ \\
\hline $108-88-3$ & Toluene & 7.9 & $\mathrm{U}$ \\
\hline $10061-02-6$ & trans-1,3-Dichloropropene & 7.9 & $\mathrm{U}$ \\
\hline $79-00-5$ & $1,1,2$-Trichloroethane & 7.9 & $\mathrm{U}$ \\
\hline $127-18-4$ & Tetrachloroethene & 7.9 & $\mathrm{U}$ \\
\hline $591-78-6$ & 2 -Hexanone & 79 & U \\
\hline $124-48-1$ & Dibromochloromethane & 7.9 & $\mathrm{U}$ \\
\hline $106-93-4$ & 1,2 -Dibromoethane & 7.9 & $\mathrm{U}$ \\
\hline $108-90-7$ & Chlorobenzene & 7.9 & $\mathrm{U}$ \\
\hline $100-41-4$ & Ethylbenzene & 7.9 & $\mathrm{U}$ \\
\hline $95-47-6$ & o-xylene & 7.9 & $\mathrm{U}$ \\
\hline $179601-23-1$ & $\mathrm{~m}, \mathrm{p}$-Xylene & 7.9 & $\mathrm{U}$ \\
\hline $100-42-5$ & Styrene & 7.9 & $\mathrm{U}$ \\
\hline $75-25-2$ & Bromoform & 7.9 & $\mathrm{U}$ \\
\hline $98-82-8$ & Isopropylbenzene & 7.9 & $\mathrm{U}$ \\
\hline $79-34-5$ & $1,1,2,2$-Tetrachloroethane & 7.9 & $\mathrm{U}$ \\
\hline $541-73-1$ & 1,3-Dichlorobenzene & 7.9 & U \\
\hline $106-46-7$ & 1,4-Dichlorobenzene & 7.9 & $\mathrm{U}$ \\
\hline $95-50-1$ & 1,2 -Dichlorobenzene & 7.9 & U \\
\hline $96-12-8$ & 1,2 -Dibromo-3-chloropropane & 7.9 & $\mathrm{U}$ \\
\hline $120-82-1$ & $1,2,4$-Trichlorobenzene & 7.9 & $\mathrm{U}$ \\
\hline $87-61-6$ & $1,2,3$-Trichlorobenzene & 7.9 & $\mathrm{U}$ \\
\hline
\end{tabular}


IJ - FORM I VOA-TIC

VOLATILE ORGANICS ANALYSIS DATA SHEET

TENTATIVELY IDENTIFIED COMPOUNDS
EPA SAMPLE NO.

MODOTLFM- 6DL
Lab Name: TESTAMERICA BURLINGTON

Lab Code: STLV Case No.: SAVANNAH

Matrix: (SOIL/SED/WATER) Water

Sample wt/vol: $25.0 \quad(\mathrm{~g} / \mathrm{mL}) \mathrm{mL}$

Level: (TRACE or LOW/MED) TRACE

\% Moisture: not dec.

GC Column: DB-624

Soil Extract Volume:

ID $: 0.20$

CONCENTRATION UNITS: $(\mathrm{ug} / \mathrm{L}$ or $u g / \mathrm{kg}) \mathrm{ug} / \mathrm{L}$
Contract: 21005

Mod. Ref No.:

SDG No.: 137933

Lab File ID: 834007D

Date Received: 06/26/2010

Date Analyzed: 06/30/2010

(mm) Dilution Factor: 15.7

(uL) Soil Aliquot Volume:

Purge Volume: 25.0

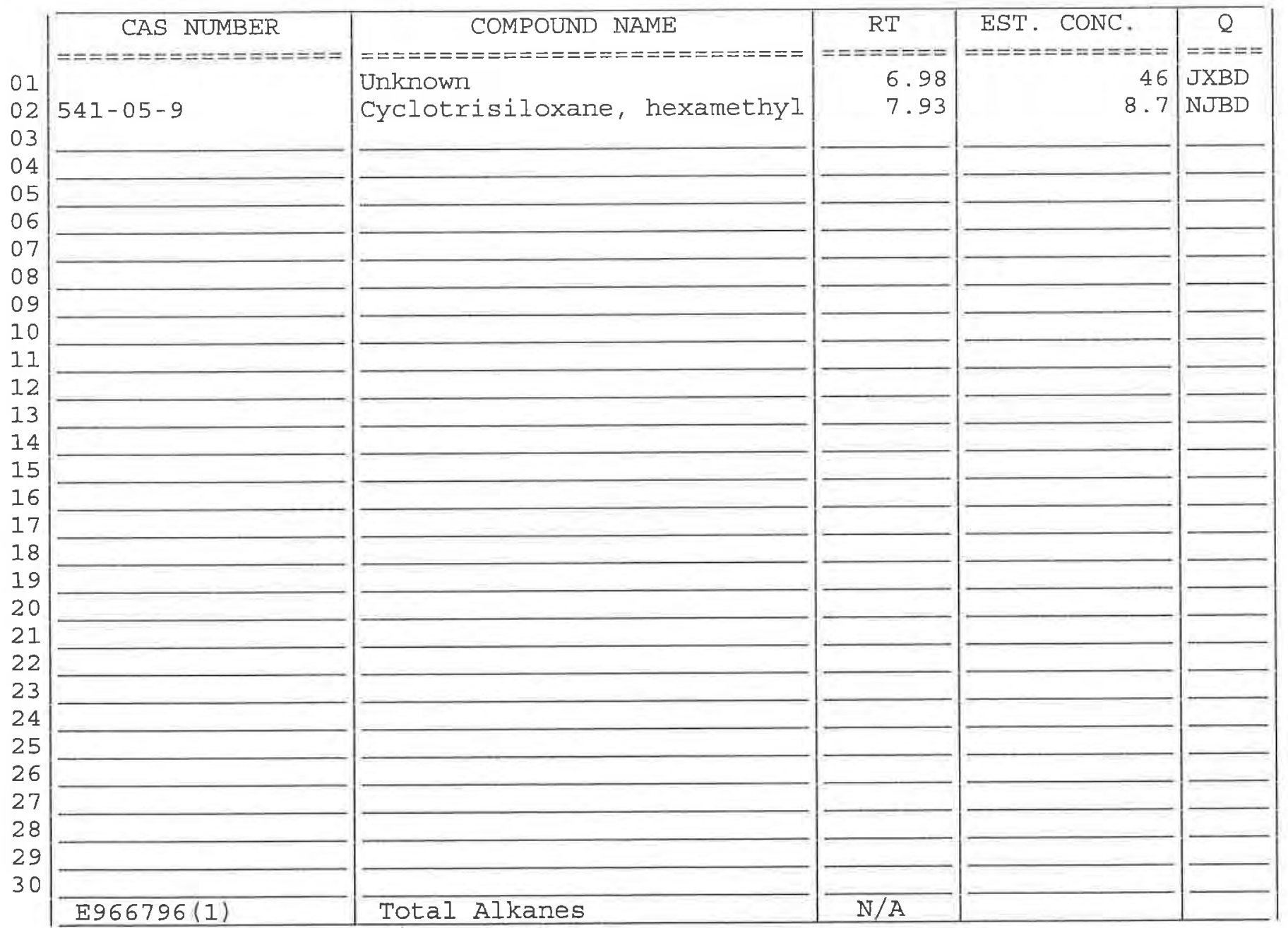

(1) EPA-designated Registry Number. 
$1 A$ - FORM I VOA-1

VOLATILE ORGANICS ANALYSIS DATA SHEET
EPA SAMPLE NO.

MORGANLFB-0
Lab Name: TESTAMERICA BURLINGTON

Lab Code: STLV Case No.: SAVANNAH Mod. Ref No.:
Contract: 21005
Matrix: (SOIL/SED/WATER) Water

Sample wt/vol: $25.0 \quad(\mathrm{~g} / \mathrm{mL}) \mathrm{mL}$

Level: (TRACE/LOW/MED) TRACE

\% Moisture: not dec.

GC Column: DB-624

Soil Extract Volume:

ID $: 0.20$

$(m m)$

(uL)

(mI)

Purge Volume: 25.0
Lab Sample ID: 834001

Lab File ID: 834001

Date Received: 06/26/2010

Date Analyzed: 06/30/2010

Dilution Factor: 1.0

Soil Aliquot Volume:

(uL)
SDG No. : 137933

\begin{tabular}{|c|c|c|c|}
\hline CAS NO. & COMPOUND & $\begin{array}{l}\text { CONCENTRATION UNITS: } \\
(\mathrm{ug} / \mathrm{L} \text { or } \mathrm{ug} / \mathrm{kg}) \mathrm{ug} / \mathrm{L}\end{array}$ & $Q$ \\
\hline 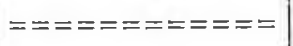 & 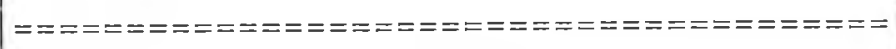 & 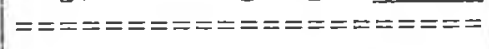 & $======-=$ \\
\hline $75-71-8$ & Dichlorodifluoromethane & 0.50 & $\mathrm{U}$ \\
\hline $74-87-3$ & Chloromethane & 0.50 & $\mathrm{U}$ \\
\hline $75-01-4$ & Vinyl chloride & 0.50 & $\mathrm{U}$ \\
\hline $74-83-9$ & Bromomethane & 0.50 & $\mathrm{U}$ \\
\hline $75-00-3$ & Chloroethane & 0.50 & $\mathrm{U}$ \\
\hline $75-69-4$ & Trichlorofluoromethane & 0.50 & $\mathrm{U}$ \\
\hline $75-35-4$ & 1,1-Dichloroethene & 0.50 & $\mathrm{U}$ \\
\hline $76-13-1$ & $1,1,2$-Trichloro-1,2,2-trifluoroethane & 0.50 & $\mathrm{U}$ \\
\hline $67-64-1$ & Acetone & 2.5 & JB \\
\hline $75-15-0$ & Carbon disulfide & 0.52 & $\mathrm{~B}$ \\
\hline $79-20-9$ & Methyl acetate & 0.50 & $\mathrm{U}$ \\
\hline $75-09-2$ & Methylene chloride & 0.50 & $\mathrm{U}$ \\
\hline $156-60-5$ & trans-1,2-Dichloroethene & 0.50 & $\mathrm{U}$ \\
\hline $1634-04-4$ & Methyl tert-butyl ether & 0.50 & $\mathrm{U}$ \\
\hline $75-34-3$ & 1,1-Dichloroethane & 0.50 & U \\
\hline $156-59-2$ & cis-1,2-Dichloroethene & 0.50 & $\mathrm{U}$ \\
\hline $78-93-3$ & 2-Butanone & 5.0 & $\mathrm{U}$ \\
\hline $74-97-5$ & Bromochloromethane & 0.50 & $\mathrm{U}$ \\
\hline $67-66-3$ & Chloroform & 5.4 & \\
\hline $71-55-6$ & $1,1,1$-Trichloroethane & 0.50 & $\mathrm{U}$ \\
\hline $110-82-7$ & Cyclohexane & 0.50 & $\mathrm{U}$ \\
\hline $56-23-5$ & Carbon tetrachloride & 13 & \\
\hline $71-43-2$ & Benzene & 0.50 & $\mathrm{U}$ \\
\hline $107-06-2$ & 1,2-Dichloroethane & 0.50 & $\mathrm{U}$ \\
\hline
\end{tabular}

Report 1,4-Dioxane for Low-Medium VOA analysis only 
IB - FORM I VOA-2

VOLATILE ORGANICS ANALYSIS DATA SHEET
EPA SAMPLE NO. MORGANLFB - 0

Lab Name: TESTAMERICA BURLINGTON

Contract: 21005

Lab Code: STLV Case No.: SAVANNAH Mod. Ref No.:

SDG No.: 137933

Matrix: (SOIL/SED/WATER) Water

Sample wt/vol: 25.0

$(\mathrm{g} / \mathrm{mL}) \mathrm{mL}$

Level: (TRACE/LOW/MED) TRACE

\% Moisture: not dec.

GC Column: DB-624

ID $: 0.20$

Soil Extract Volume:

Purge Volume: 25.0
Lab Sample ID: 834001

Lab File ID: 834001

Date Received: 06/26/2010

Date Analyzed: 06/30/2010

Dilution Factor: 1.0

Soil Aliquot Volume:

(uL)

\begin{tabular}{|c|c|c|c|}
\hline CAS NO. & COMPOUND & $\begin{array}{l}\text { CONCENTRATION UNITS: } \\
(\mathrm{ug} / \mathrm{L} \text { or } \mathrm{ug} / \mathrm{kg}) \mathrm{ug} / \mathrm{L}\end{array}$ & Q \\
\hline 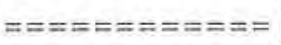 & 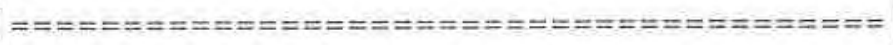 & 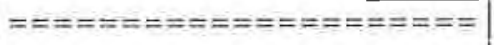 & $=====-=$ \\
\hline $79-01-6$ & Trichloroethene & 0.50 & U \\
\hline $108-87-2$ & Methylcyclohexane & 0.50 & $\mathrm{U}$ \\
\hline $78-87-5$ & 1,2-Dichloropropane & 0.50 & $\mathrm{U}$ \\
\hline $75-27-4$ & Bromodichloromethane & 2.5 & \\
\hline $10061-01-5$ & cis-1,3-Dichloropropene & 0.50 & $\overline{\mathrm{U}}$ \\
\hline $108-10-1$ & 4-Methyl-2-pentanone & 5.0 & $\mathrm{U}$ \\
\hline $108-88-3$ & Toluene & 0.12 & 丁 \\
\hline $10061-02-6$ & trans-1,3-Dichloropropene & 0.50 & $\mathrm{U}$ \\
\hline $79-00-5$ & $1,1,2$-Trichloroethane & 0.50 & $\mathrm{U}$ \\
\hline $127-18-4$ & Tetrachloroethene & 0.11 & J \\
\hline $591-78-6$ & 2 -Hexanone & 5.0 & $\mathrm{U}$ \\
\hline $124-48-1$ & Dibromochloromethane & 1.3 & \\
\hline $106-93-4$ & 1,2-Dibromoethane & 0.50 & $\overline{\mathrm{U}}$ \\
\hline $108-90-7$ & Chlorobenzene & 0.50 & $\mathrm{U}$ \\
\hline $100-41-4$ & Ethylbenzene & 0.50 & $\mathrm{U}$ \\
\hline $95-47-6$ & o-Xylene & 0.50 & $\mathrm{U}$ \\
\hline $179601-23-1$ & $\mathrm{~m}, \mathrm{p}$-xylene & 0.11 & $\mathrm{~J}$ \\
\hline $100-42-5$ & Styrene & 0.50 & $\mathrm{U}$ \\
\hline $75-25-2$ & Bromoform & 0.50 & $\mathrm{U}$ \\
\hline $98-82-8$ & Isopropylbenzene & 0.50 & $\mathrm{U}$ \\
\hline $79-34-5$ & $1,1,2,2$-Tetrachloroethane & 0.50 & $\mathrm{U}$ \\
\hline $541-73-1$ & 1,3-Dichlorobenzene & 0.50 & $\mathrm{U}$ \\
\hline $106-46-7$ & 1,4-Dichlorobenzene & 0.50 & U \\
\hline $95-50-1$ & 1,2-Dichlorobenzene & 0.50 & $\mathrm{U}$ \\
\hline $96-12-8$ & 1,2 -Dibromo-3-chloropropane & 0.50 & $\mathrm{U}$ \\
\hline $120-82-1$ & $1,2,4$-Trichlorobenzene & 0.50 & $\mathrm{U}$ \\
\hline $87-61-6$ & $1,2,3$-Trichlorobenzene & 0.50 & $\mathrm{U}$ \\
\hline
\end{tabular}


IJ - FORM I VOA-TIC

VOLATILE ORGANICS ANALYSIS DATA SHEET

TENTATIVELY IDENTIFIED COMPOUNDS
EPA SAMPLE NO. MORGANLFB-O
Lab Name: TESTAMERICA BURIINGTON

Lab Code: STLV Case No.: SAVANNAH

Matrix: (SOIL/SED/WATER) Water

Sample wt/vol: $25.0 \quad(\mathrm{~g} / \mathrm{mL}) \mathrm{mL}$

Level: (TRACE or LOW/MED) TRACE

\% Moisture: not dec.

GC Column: DB-624

ID $: 0.20$

Soil Extract Volume:

CONCENTRATION UNITS: (ug/L or ug/kg) ug/L
Contract: 21005

Mod. Ref No.:

SDG NO. : 137933
Lab Sample ID: 834001

Lab File ID: 834001

Date Received: 06/26/2010

Date Analyzed: 06/30/2010

(mm) Dilution Factor: 1.0

(uL) Soil Aliquot Volume:

(uL)

Purge Volume: 25.0

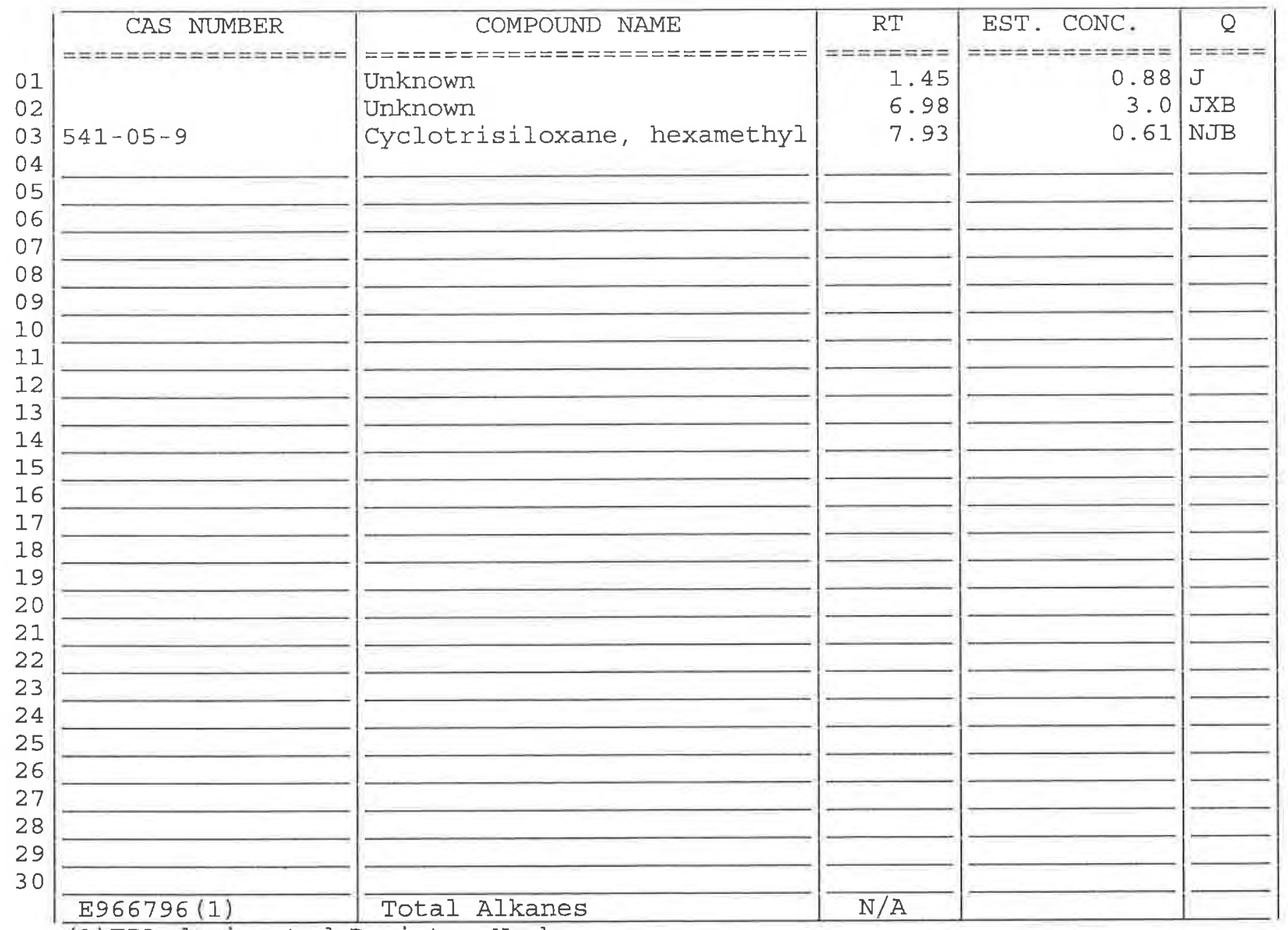

(1) EPA-designated Registry Number. 
Lab Name: TESTAMERICA BURLINGTON

Contract: 21005

Lab Code: STLV Case No.: SAVANNAH Mod. Ref No.:

SDG NO.: 137933

Matrix: (SOIL/SED/WATER) Water

Sample wt/vol: 25.0

$(\mathrm{g} / \mathrm{mL}) \mathrm{mL}$

Level: (TRACE/LOW/MED) TRACE

\% Moisture: not dec.

GC Column: DB-624

ID : 0.20

Soil Extract Volume:

Purge Volume: 25.0
Lab Sample ID: 834002

Lab File ID: 834002

Date Received: 06/26/2010

Date Analyzed: 06/30/2010

Dilution Factor: 1.0

(mL)

\begin{tabular}{|c|c|c|c|}
\hline CAS NO. & COMPOUND & $\begin{array}{l}\text { CONCENTRATION UNITS: } \\
(\mathrm{ug} / \mathrm{L} \text { or } \mathrm{ug} / \mathrm{kg}) \mathrm{ug} / \mathrm{L}\end{array}$ & $Q$ \\
\hline 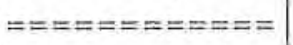 & 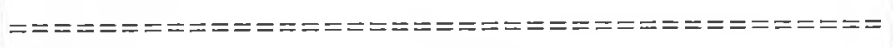 & 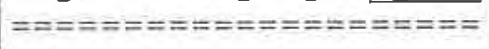 & $=======$ \\
\hline $75-71-8$ & Dichlorodifluoromethane & 0.50 & $\mathrm{U}$ \\
\hline $74-87-3$ & Chloromethane & 0.50 & $\mathrm{U}$ \\
\hline $75-01-4$ & vinyl chloride & 0.50 & $\mathrm{U}$ \\
\hline $74-83-9$ & Bromomethane & 0.50 & $\mathrm{U}$ \\
\hline $75-00-3$ & Chloroethane & 0.50 & $\mathrm{U}$ \\
\hline $75-69-4$ & Trichlorofluoromethane & 0.50 & $\mathrm{U}$ \\
\hline $75-35-4$ & 1,1 -Dichloroethene & 0.50 & $\mathrm{U}$ \\
\hline $76-13-1$ & $1,1,2$-Trichloro-1,2,2-trifluoroethane & 0.50 & $\mathrm{U}$ \\
\hline $67-64-1$ & Acetone & 2.8 & JB \\
\hline $75-15-0$ & Carbon disulfide & 0.21 & JB \\
\hline $79-20-9$ & Methyl acetate & 0.50 & $\mathrm{U}$ \\
\hline $75-09-2$ & Methylene chloride & 0.50 & $U$ \\
\hline $156-60-5$ & trans-1,2-Dichloroethene & 0.50 & $\mathrm{U}$ \\
\hline $1634-04-4$ & Methyl tert-butyl ether & 0.50 & $\mathrm{U}$ \\
\hline $75-34-3$ & 1,1-Dichloroethane & 0.50 & $\mathrm{U}$ \\
\hline $156-59-2$ & cis-1,2-Dichloroethene & 0.50 & U \\
\hline $78-93-3$ & 2-Butanone & 5.0 & $\mathrm{U}$ \\
\hline $74-97-5$ & Bromochloromethane & 0.50 & $\mathrm{U}$ \\
\hline $67-66-3$ & Chloroform & 5.7 & \\
\hline $71-55-6$ & $1,1,1$-Trichloroethane & 0.50 & $\bar{U}$ \\
\hline $110-82-7$ & Cyclohexane & 0.50 & $U$ \\
\hline $56-23-5$ & Carbon tetrachloride & 13 & \\
\hline $71-43-2$ & Benzene & 0.50 & $\overline{\mathrm{U}}$ \\
\hline $107-06-2$ & 1,2-Di chloroethane & 0.50 & U \\
\hline
\end{tabular}

Report 1,4-Dioxane for Low-Medium VOA analysis only 
1B - FORM I VOA-2
VOLATILE ORGANICS ANALYSIS DATA SHEET
EPA SAMPLE NO.

MORGANLFM- 1
Lab Name: TESTAMERICA BURLINGTON

Lab Code: STIV

Case No.: SAVANNAH
Contract: 21005
Matrix: (SOIL/SED/WATER) Water

Sample wt/vol: 25.0

$(\mathrm{g} / \mathrm{mL}) \mathrm{mL}$

Level: (TRACE/LOW/MED) TRACE

\% Moisture: not dec.

GC Column: DB-624

ID : 0.20

Soil Extract Volume:

Purge Volume: 25.0
Lab Sample ID: 834002

Lab File ID: 834002

Date Received: 06/26/2010

Date Analyzed: 06/30/2010

(mm) Dilution Factor: 1.0

(uL) Soil Aliquot Volume:

(uL)

(mL)

\begin{tabular}{|c|c|c|c|}
\hline CAS NO. & COMPOUND & $\begin{array}{l}\text { CONCENTRATION UNITS: } \\
(\mathrm{ug} / \mathrm{L} \text { or } \mathrm{ug} / \mathrm{kg}) \mathrm{ug} / \mathrm{I}\end{array}$ & $Q$ \\
\hline$===:=====$ & 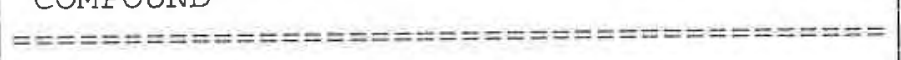 & $========= \pm=========$ & $=======$ \\
\hline $79-0 I-6$ & Trichloroethene & 0.50 & $\mathrm{U}$ \\
\hline $108-87-2$ & Methylcyclohexane & 0.50 & $\mathrm{U}$ \\
\hline $78-87-5$ & 1,2-Dichloropropane & 0.50 & $\mathrm{U}$ \\
\hline $75-27-4$ & Bromodichloromethane & 2.6 & \\
\hline $10061-01-5$ & cis-1,3-Dichloropropene & 0.50 & $\mathrm{U}$ \\
\hline $108-10-1$ & 4-Methyl-2-pentanone & 5.0 & $\mathrm{U}$ \\
\hline $108-88-3$ & Toluene & 0.10 & $\mathrm{~J}$ \\
\hline $10061-02-6$ & trans-1,3-Dichloropropene & 0.50 & $\mathrm{U}$ \\
\hline $79-00-5$ & $1,1,2$-Trichloroethane & 0.50 & $\mathrm{U}$ \\
\hline $127-18-4$ & Tetrachloroethene & 0.11 & $\mathrm{~J}$ \\
\hline $591-78-6$ & 2-Hexanone & 5.0 & $\mathrm{U}$ \\
\hline $124-48-1$ & Dibromochloromethane & 1.4 & \\
\hline $106-93-4$ & 1,2-Dibromoethane & 0.50 & $\mathrm{U}$ \\
\hline $108-90-7$ & Chlorobenzene & 0.50 & $\mathrm{U}$ \\
\hline $100-41-4$ & Ethylbenzene & 0.50 & $\mathrm{U}$ \\
\hline $95-47-6$ & o-Xylene & 0.50 & $\mathrm{U}$ \\
\hline $179601-23-1$ & $\mathrm{~m}, \mathrm{p}-\mathrm{XYl}$ lene & 0.10 & $\mathrm{~J}$ \\
\hline $100-42-5$ & Styrene & 0.50 & $\mathrm{U}$ \\
\hline $75-25-2$ & Bromoform & 0.50 & $\mathrm{U}$ \\
\hline $98-82-8$ & Isopropylbenzene & 0.50 & U \\
\hline $79-34-5$ & $1,1,2,2$-Tetrachloroethane & 0.50 & U \\
\hline $541-73-1$ & 1,3-Dichlorobenzene & 0.50 & $\mathrm{U}$ \\
\hline $106-46-7$ & 1,4-Dichlorobenzene & 0.50 & $\mathrm{U}$ \\
\hline $95-50-1$ & 1,2 -Dichlorobenzene & 0.50 & $\mathrm{U}$ \\
\hline $96-12-8$ & 1,2-Dibromo-3-chloropropane & 0.50 & $\mathrm{U}$ \\
\hline $120-82-1$ & $1,2,4$-Trichlorobenzene & 0.50 & $\mathrm{U}$ \\
\hline $87-61-6$ & $1,2,3$-Trichlorobenzene & 0.50 & $\mathrm{U}$ \\
\hline
\end{tabular}


IJ - FORM I VOA-TIC

VOLATILE ORGANICS ANALYSIS DATA SHEET

TENTATIVELY IDENTIFIED COMPOUNDS
EPA SAMPLE NO.

MORGANLFM - I

Contract: 21005

Mod. Ref No.:

SDG No.: 137933

Lab Sample ID: 834002

Lab File ID: 834002

Date Received: 06/26/2010

Date Analyzed: 06/30/2010

\% Moisture: not dec.

(mm) Dilution Factor: 1.0

GC Column: DB-624

ID: 0.20

(uL) Soil Aliquot Volume:

(uL)

Soil Extract Volume:

Purge Volume: 25.0

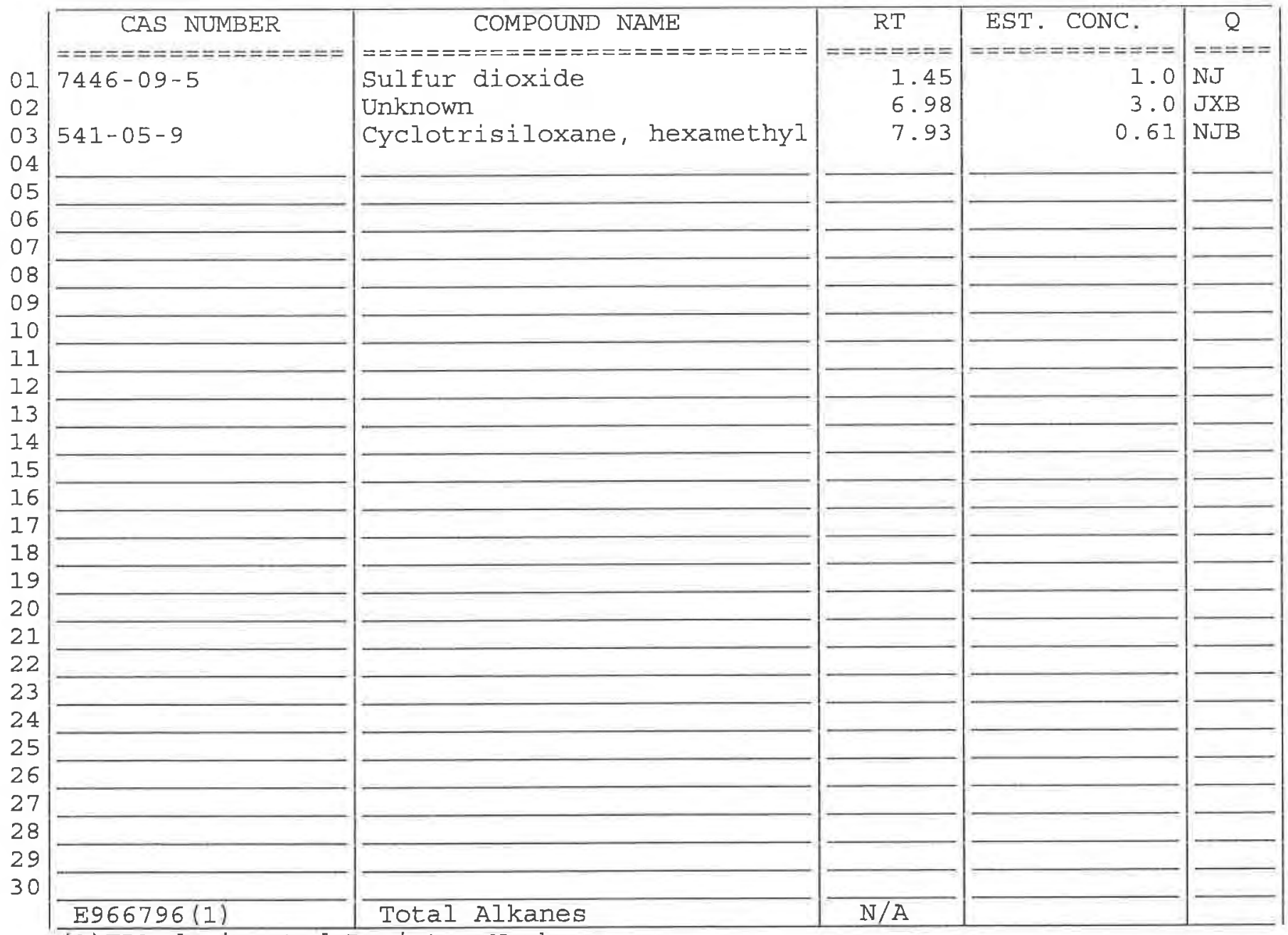

(1) EPA-designated Registry Number. 
$1 A$ - FORM I VOA-1

VOLATILE ORGANICS ANALYSIS DATA SHEET
EPA SAMPLE NO.

QCTB - 7
Lab Name: TESTAMERICA BURLINGTON

Lab Code: STLV

Case No. : SAVANNA
Contract : 21005
Matrix: (SOIL/SED/WATER) Water

Sample wt/vol: 25.0

$(g / m L) \quad m L$

Level: (TRACE/LOW/MED) TRACE

$\%$ Moisture: not dec.

GC Column: DB-624

ID $: 0.20$

Soil Extract Volume:

Purge Volume: 25.0
Mod. Ref No.:
Lab Sample ID: 834008

Lab File ID: 834008

Date Received: 06/26/2010

Date Analyzed: 06/30/2010

Dilution Factor: 1.0

Soil Aliquot Volume:

$(\mathrm{mL})$

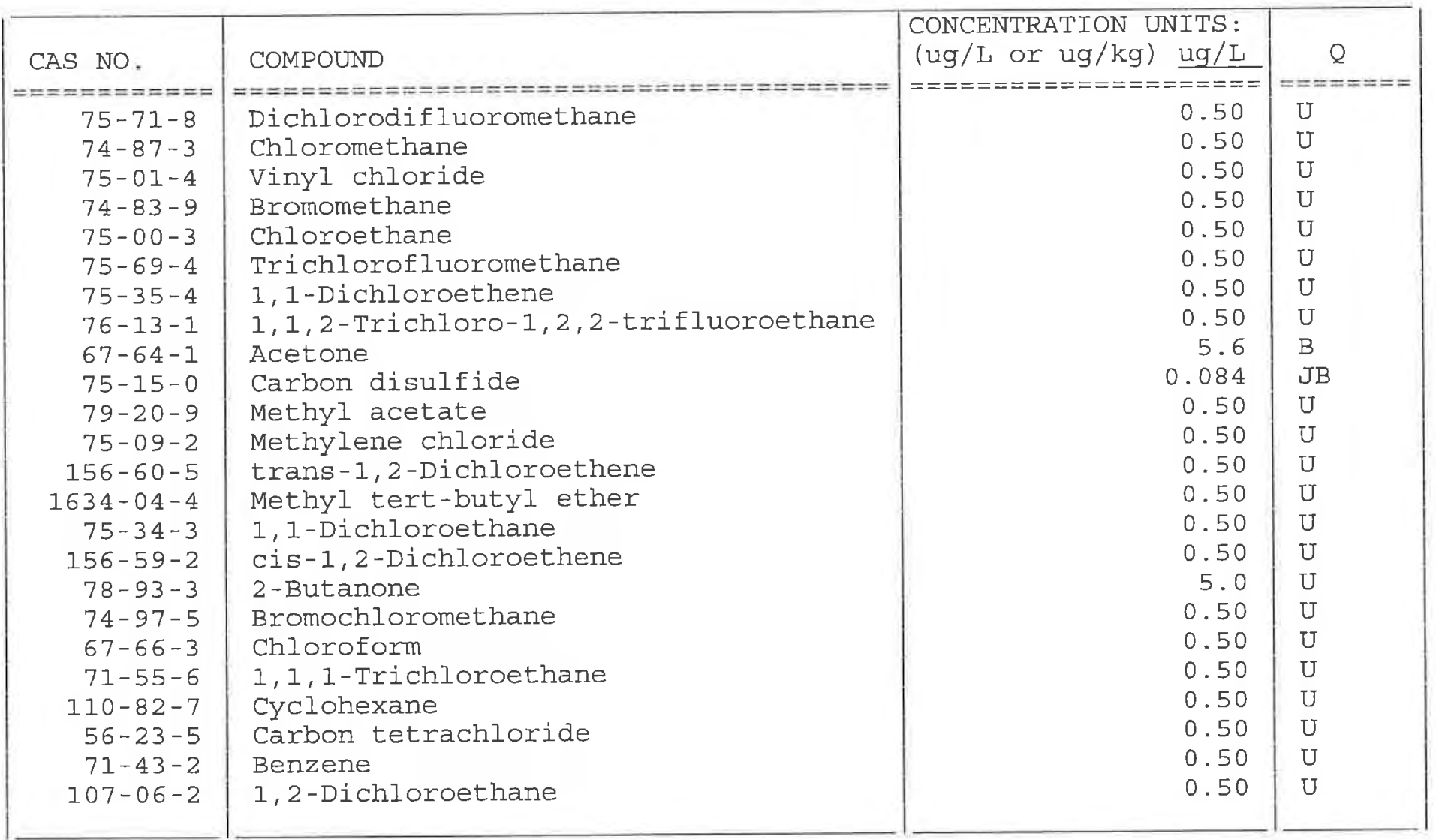

Report 1,4-Dioxane for Low-Medium VOA analysis only 
1B - FORM I VOA-2
VOLATILE ORGANICS ANALYSIS DATA SHEET
EPA SAMPLE NO.

QCTB -7

Lab Name: TESTAMERICA BURLINGTON

Contract: 21005

Lab Code: STLV Case No.: SAVANNAH Mod. Ref No.:

SDG NO.: 137933

Matrix: (SOIL/SED/WATER) Water

Sample wt/vol: 25.0

$(\mathrm{g} / \mathrm{mL}) \mathrm{mL}$

Level: (TRACE/LOW/MED) TRACE

\% Moisture: not dec.

GC Column: DB-624

Soil Extract Volume:

ID : 0.20

(mm)

(uI)

(mL)

Purge Volume: 25.0
Lab Sample ID: 834008

Lab File ID: 834008

Date Received: 06/26/2010

Date Analyzed: 06/30/2010

Dilution Factor: 1.0

Soil Aliquot Volume:

(uL)

\begin{tabular}{|c|c|c|c|}
\hline CAS NO. & COMPOUND & $\begin{array}{l}\text { CONCENTRATION UNITS: } \\
(\mathrm{ug} / \mathrm{L} \text { or } \mathrm{ug} / \mathrm{kg}) \mathrm{ug} / \mathrm{L}\end{array}$ & $Q$ \\
\hline 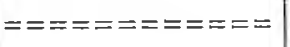 & 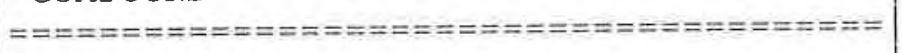 & 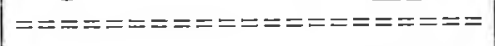 & $===-==\pi=$ \\
\hline $79-01-6$ & Trichloroethene & 0.50 & $\mathrm{U}$ \\
\hline $108-87-2$ & Methylcyclohexane & 0.50 & $\mathrm{U}$ \\
\hline $78-87-5$ & 1,2-Dichloropropane & 0.50 & U \\
\hline $75-27-4$ & Bromodichloromethane & 0.50 & U \\
\hline $10061-01-5$ & cis-1,3-Dichloropropene & 0.50 & $\mathrm{U}$ \\
\hline $108-10-1$ & 4 -Methyl-2-pentanone & 5.0 & $\mathrm{U}$ \\
\hline $108-88-3$ & Toluene & 0.076 & J \\
\hline $10061-02-6$ & trans-1,3-Dichloropropene & 0.50 & U \\
\hline $79-00-5$ & 1,1,2-Trichloroethane & 0.50 & $\mathrm{U}$ \\
\hline $127-18-4$ & Tetrachloroethene & 0.50 & $\mathrm{U}$ \\
\hline $591-78-6$ & 2-Hexanone & 5.0 & $\mathrm{U}$ \\
\hline $124-48-1$ & Dibromochloromethane & 0.50 & $\mathrm{U}$ \\
\hline $106-93-4$ & 1,2-Dibromoethane & 0.50 & $\mathrm{U}$ \\
\hline $108-90-7$ & Chlorobenzene & 0.50 & $\mathrm{U}$ \\
\hline $100-41-4$ & Ethylbenzene & 0.50 & $\mathrm{U}$ \\
\hline $95-47-6$ & o-XYlene & 0.50 & U \\
\hline $179601-23-1$ & $\mathrm{~m}, \mathrm{p}$-Xylene & 0.50 & $\mathrm{U}$ \\
\hline $100-42-5$ & Styrene & 0.50 & $\mathrm{U}$ \\
\hline $75-25-2$ & Bromoform & 0.50 & $\mathrm{U}$ \\
\hline $98-82-8$ & Isopropylbenzene & 0.50 & U \\
\hline $79-34-5$ & $1,1,2,2$-Tetrachloroethane & 0.50 & $\mathrm{U}$ \\
\hline $541-73-1$ & 1,3-Dichlorobenzene & 0.50 & $\mathrm{U}$ \\
\hline $106-46-7$ & 1,4-Dichlorobenzene & 0.50 & $\mathrm{U}$ \\
\hline $95-50-1$ & 1,2-Dichlorobenzene & 0.50 & $\mathrm{U}$ \\
\hline $96-12-8$ & 1,2-Dibromo-3-chloropropane & 0.50 & $\mathrm{U}$ \\
\hline $120-82-1$ & $1,2,4$-Trichlorobenzene & 0.50 & $\mathrm{U}$ \\
\hline $87-61-6$ & $1,2,3$-Trichlorobenzene & 0.50 & $\mathrm{U}$ \\
\hline
\end{tabular}


IJ - FORM I VOA-TIC

VOLATILE ORGANICS ANALYSIS DATA SHEET

TENTATIVELY IDENTIFIED COMPOUNDS
EPA SAMPLE NO.

$\mathrm{QCTB}-7$
Lab Name: TESTAMERICA BURLINGTON

Lab Code: STLV Case No.: SAVANNAH

Matrix: (SOIL/SED/WATER) Water

Sample wt/vol: $25.0(\mathrm{~g} / \mathrm{mL}) \mathrm{mL}$

Level: (TRACE or LOW/MED) TRACE

\% Moisture: not dec.

GC Column: $\mathrm{DB}-624$

ID $: 0.20$

Soil Extract Volume:

CONCENTRATION UNITS: (ug/L or ug/ $\mathrm{kg}$ ) ug/L
Contract: 21005

Mod. Ref No.:

SDG No.: 137933
Lab Sample ID: 834008

Lab File ID: 834008

Date Received: $06 / 26 / 2010$

Date Analyzed: 06/30/2010

(mm) Dilution Factor: 1.0

(uL) Soil Aliquot Volume:

(uL)

Purge Volume: 25.0

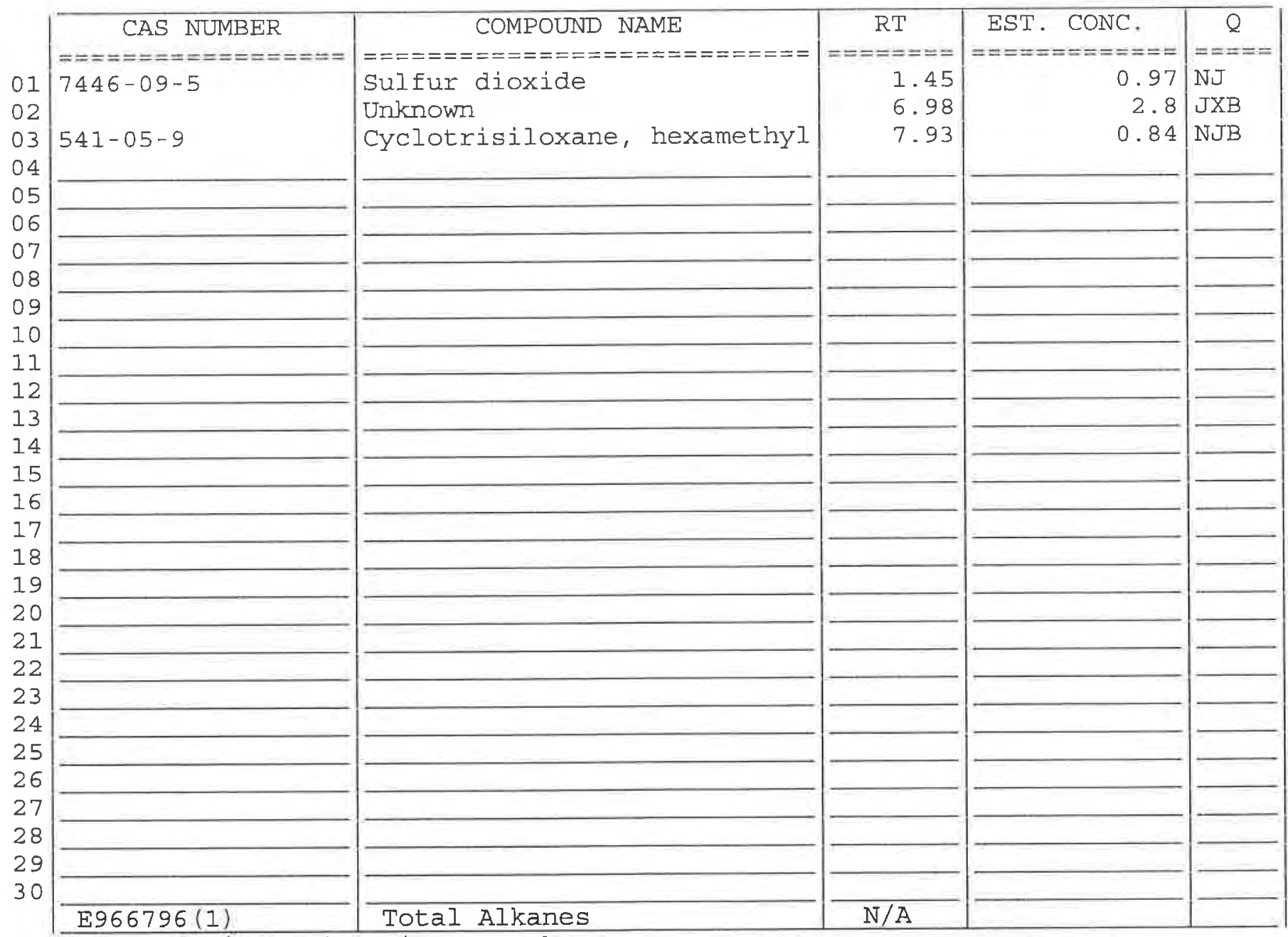

(1) EPA-designated Registry Number. 
$1 A$ - FORM I VOA-I

VOLATILE ORGANICS ANALYSIS DATA SHEET
EPA SAMPLE NO.

SB2 4LFB - 4
Lab Name: TESTAMERICA BURIINGTON

Lab Code: STLV Case No.: SAVANNAH Mod. Ref No.:
Contract: 21005
Matrix: (SOIL/SED/WATER) Water

Sample wt/vol: 25.0

$(\mathrm{g} / \mathrm{mL}) \mathrm{mL}$

Level: (TRACE/LOW/MED) TRACE

\% Moisture: not dec.

GC Column: DB-624

ID $: 0.20$

Soil Extract Volume:

Purge Volume: 25.0
Lab Sample ID: 834005

Lab File ID: 834005

Date Received: 06/26/2010

Date Analyzed: 06/30/2010

Dilution Factor: 1.0

Soil Aliquot Volume:

\begin{tabular}{|c|c|c|c|}
\hline CAS NO. & COMPOUND & $\begin{array}{l}\text { CONCENTRATION UNITS: } \\
(\mathrm{ug} / \mathrm{L} \text { or } \mathrm{ug} / \mathrm{kg} \text { ) ug/I }\end{array}$ & Q \\
\hline 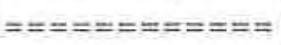 & 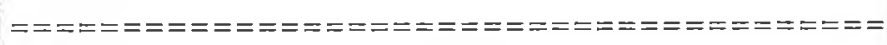 & 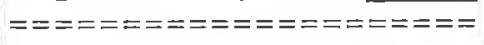 & 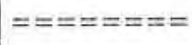 \\
\hline $75-71-8$ & Dichlorodifluoromethane & 0.50 & $\mathrm{U}$ \\
\hline $74-87-3$ & Chloromethane & 0.11 & $\mathrm{~J}$ \\
\hline $75-01-4$ & Vinyl chloride & 0.50 & $\mathrm{U}$ \\
\hline $74-83-9$ & Bromomethane & 0.50 & U \\
\hline $75-00-3$ & Chloroethane & 0.50 & $\mathrm{U}$ \\
\hline $75-69-4$ & Trichlorofluoromethane & 0.50 & $\mathrm{U}$ \\
\hline $75-35-4$ & 1,1 -Dichloroethene & 0.50 & $\mathrm{U}$ \\
\hline $76-13-1$ & $1,1,2$-Trichloro-1,2,2-trifluoroethane & 0.50 & $\mathrm{U}$ \\
\hline $67-64-1$ & Acetone & 3.3 & JB \\
\hline $75-15-0$ & Carbon disulfide & 0.083 & JB \\
\hline $79-20-9$ & Methyl acetate & 0.50 & $\mathrm{U}$ \\
\hline $75-09-2$ & Methylene chloride & 0.068 & J \\
\hline $156-60-5$ & trans-1,2-Dichloroethene & 0.50 & $\mathrm{U}$ \\
\hline $1634-04-4$ & Methyl tert-butyl ether & 0.50 & $\mathrm{U}$ \\
\hline $75-34-3$ & 1,1-Dichloroethane & 0.50 & $\mathrm{U}$ \\
\hline $156-59-2$ & cis-1,2-Dichloroethene & 0.50 & $\mathrm{U}$ \\
\hline $78-93-3$ & 2 -Butanone & 5.0 & $\mathrm{U}$ \\
\hline $74-97-5$ & Bromochloromethane & 0.50 & $\mathrm{U}$ \\
\hline $67-66-3$ & Chloroform & 5.9 & \\
\hline $71-55-6$ & $1,1,1$-Trichloroethane & 0.50 & $\mathrm{U}$ \\
\hline $110-82-7$ & Cyclohexane & 0.50 & $\mathrm{U}$ \\
\hline $56-23-5$ & Carbon tetrachloride & 4.4 & \\
\hline $71-43-2$ & Benzene & 0.050 & $\mathrm{~J}$ \\
\hline $107-06-2$ & 1,2-Dichloroethane & 0.50 & $\mathrm{U}$ \\
\hline
\end{tabular}

Report 1,4-Dioxane for Low-Medium VOA analysis only 
Lab Name: TESTAMERICA BURLINGTON

Lab Code: STLV Case No.: SAVANNAH Mod. Ref No.:
Contract : 21005
SDG No. : 137933
Matrix: (SOIL/SED/WATER) Water

Sample wt/vol: 25.0

$(\mathrm{g} / \mathrm{mL}) \mathrm{mI}$

Level: (TRACE/LOW/MED) TRACE

\% Moisture: not dec.

GC Column: DB-624

ID $: 0.20$

(mm)

(uL)

Soil Aliquot Volume:

(mL)

Lab Sample ID: 834005

Lab File ID: 834005

Date Received: 06/26/2010

Date Analyzed: 06/30/2010

Dilution Factor: 1.0

Soil Extract Volume:

Purge Volume: 25.0

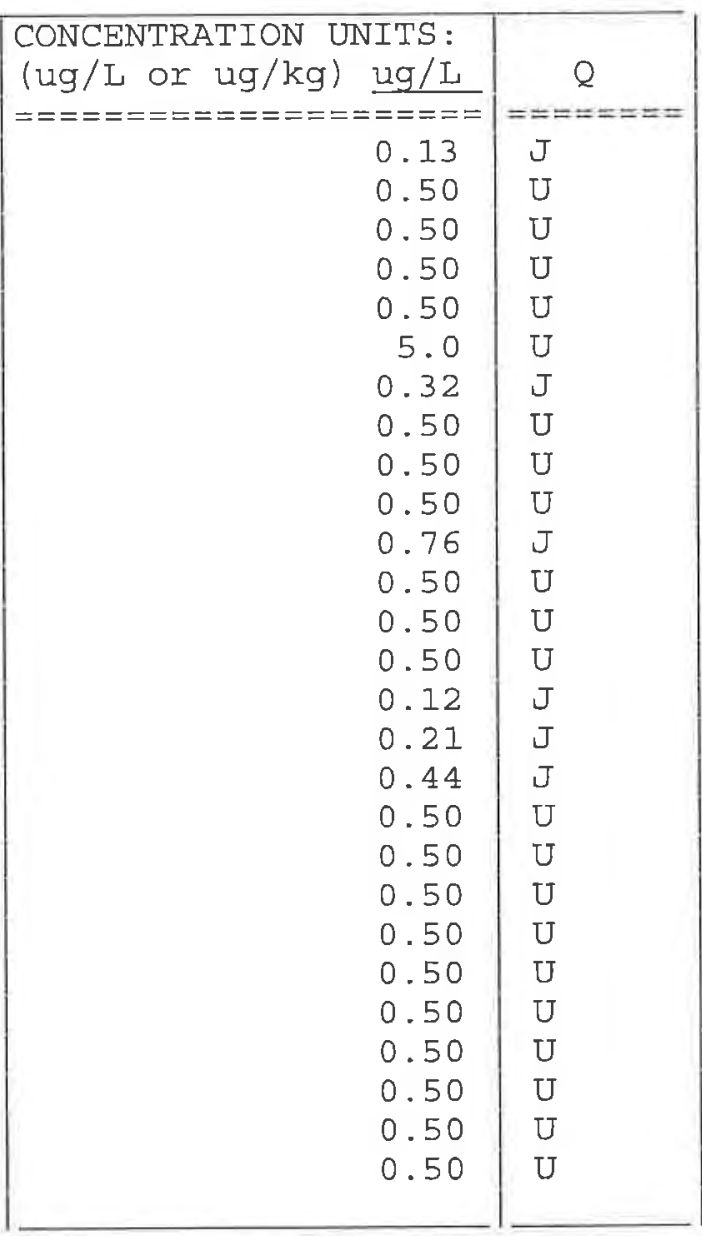


IJ - FORM I VOA-TIC

VOIATILE ORGANICS ANALYSIS DATA SHEET

TENTATIVELY IDENTIFIED COMPOUNDS
EPA SAMPLE NO.

SB2 4LFB-4

Contract: 21005

Lab Name: TESTAMERICA BURLINGTON

Mod. Ref No.:

SDG No. : 137933

Lab Sample ID: 834005

Lab File ID: 834005

Date Received: 06/26/2010

Date Analyzed: 06/30/2010

$\div$ Moisture: not dec.

GC Column: DB-624

ID : 0.20

$(m i n)$

Dilution Factor: 1.0

Soil Extract Volume:

(uL) Soil Aliquot Volume:

(uL)

Purge Volume: 25.0

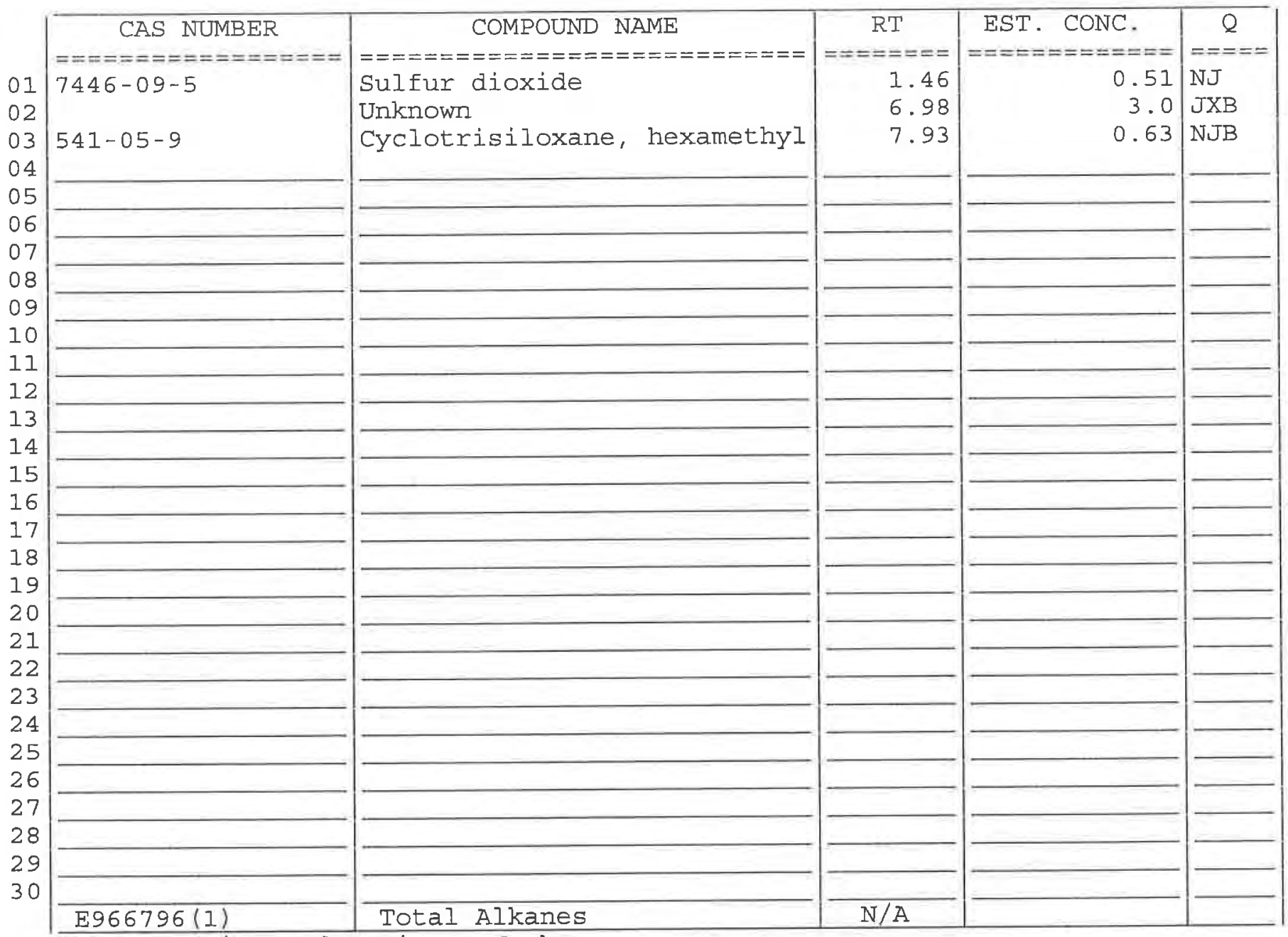

(1) EPA-designated Registry Number. 
IA - FORM I VOA-I

VOLATILE ORGANICS ANALYSIS DATA SHEET
EPA SAMPLE NO.

SB 38 LFB -2
Lab Name: TESTAMERICA BURLINGTON

Lab Code: STLV Case No.: SAVANNAH Mod. Ref No.:
Contract : 21005
Matrix: (SOIL/SED/WATER) water

Sample wt/vol: 25.0

$(g / \mathrm{mL}) \mathrm{mL}$

Level: (TRACE/LOW/MED) TRACE

\% Moisture: not dec.

GC Column: DB-624

ID : 0.20

Soil Extract Volume:

Purge Volume: 25.0
Lab Sample ID: 834003

Lab File ID: 834003

Date Received: 06/26/2010

Date Analyzed: 06/30/2010

Dilution Factor: 1.0

Soil Aliquot Volume:

(mL)

\begin{tabular}{|c|c|c|c|}
\hline $\begin{array}{c}\text { CAS NO. } \\
========== \\
75-71-8 \\
74-87-3 \\
75-01-4 \\
74-83-9 \\
75-00-3 \\
75-69-4 \\
75-35-4 \\
76-13-1 \\
67-64-1 \\
75-15-0 \\
79-20-9 \\
75-09-2 \\
156-60-5 \\
1634-04-4 \\
75-34-3 \\
156-59-2 \\
78-93-3 \\
74-97-5 \\
67-66-3 \\
71-55-6 \\
110-82-7 \\
56-23-5 \\
71-43-2 \\
107-06-2\end{array}$ & 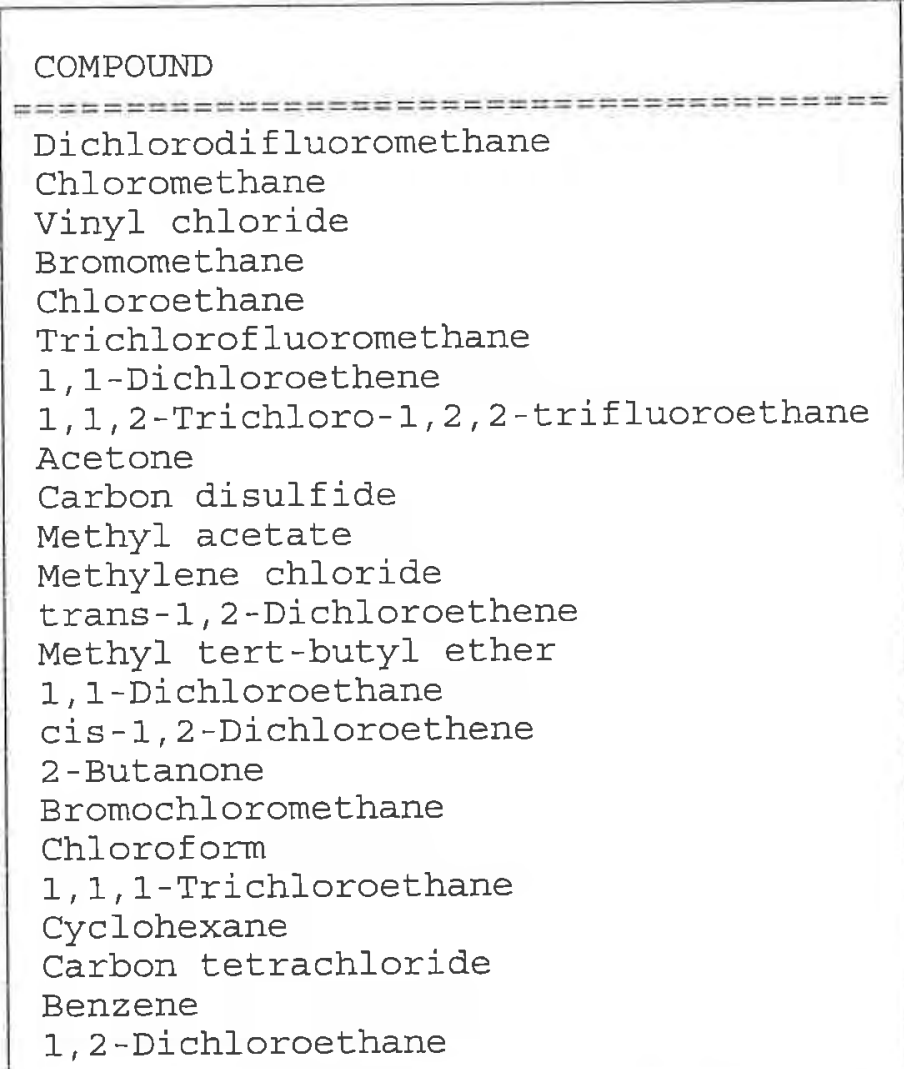 & 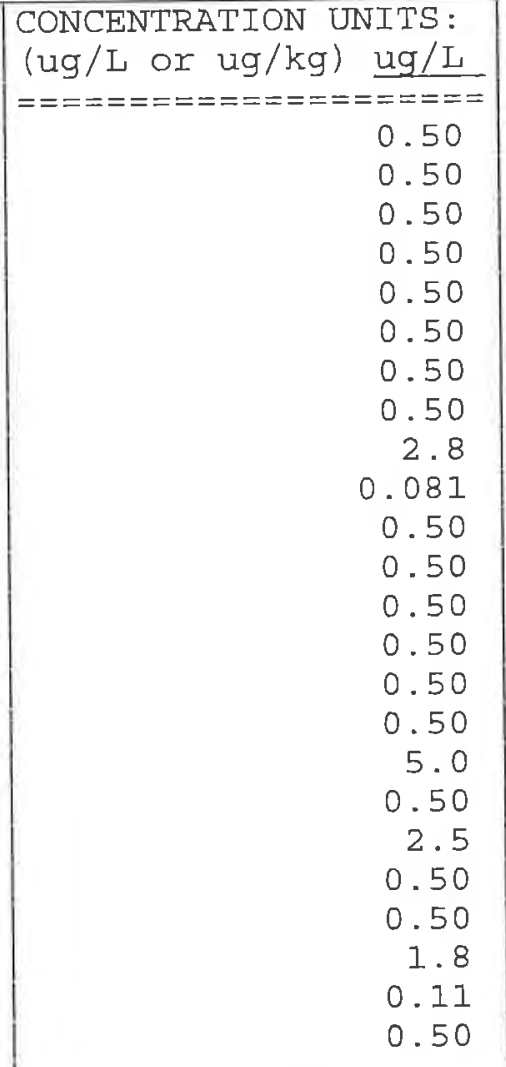 & $\begin{array}{l}======= \\
\mathrm{U} \\
\mathrm{U} \\
\mathrm{U} \\
\mathrm{U} \\
\mathrm{U} \\
\mathrm{U} \\
\mathrm{U} \\
\mathrm{U} \\
\mathrm{JB} \\
\mathrm{JB} \\
\mathrm{U} \\
\mathrm{U} \\
\mathrm{U} \\
\mathrm{U} \\
\mathrm{U} \\
\mathrm{U} \\
\mathrm{U} \\
\mathrm{U}\end{array}$ \\
\hline
\end{tabular}

Report 1,4-Dioxane for Low-Medium VOA analysis only 
Lab Name: TESTAMERICA BURLINGTON

Lab Code: STLV Case No.: SAVANNAH Mod. Ref No.:

Contract: 21005
Matrix: (SOIL/SED/WATER) Water

Sample wt/vol: 25.0

$(g / \mathrm{mL}) \mathrm{mL}$

Level: (TRACE/LOW/MED) TRACE

\% Moisture: not dec.

GC Column: DB-624

Soil Extract Volume:

ID : 0.20

(mm)

(uL)

(m工)

Purge Volume: 25.0
Lab Sample ID: 834003

Lab File ID: 834003

Date Received: 06/26/2010

Date Analyzed: 06/30/2010

Dilution Factor: 1.0

Soil Aliquot Volume:

(uL)

(m)

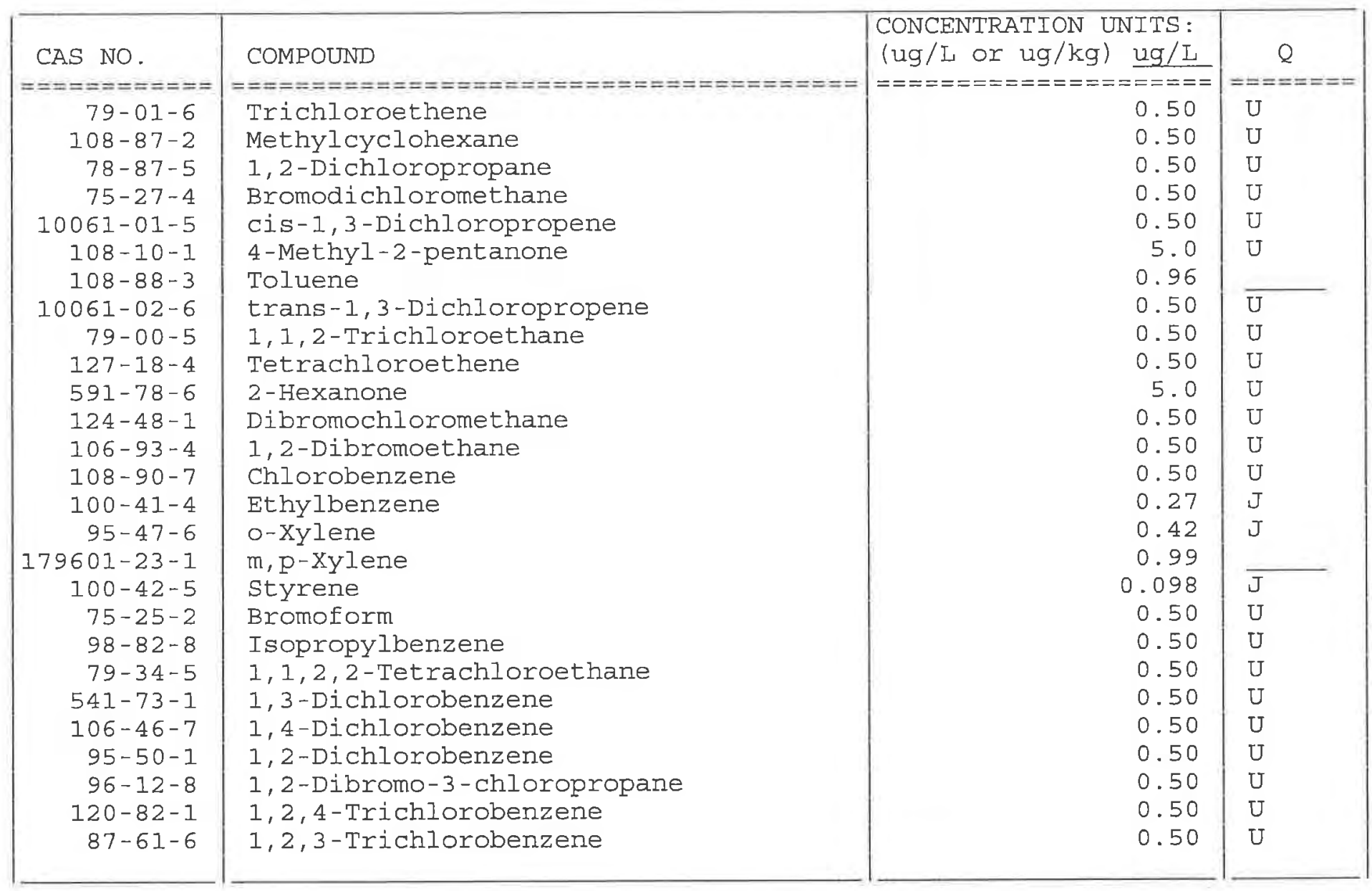


IJ - FORM I VOA-TIC

VOLATILE ORGANICS ANALYSIS DATA SHEET

TENTATIVELY IDENTIFIED COMPOUNDS
EPA SAMPLE NO.

SB3 8LFB-2
Lab Name: TESTAMERICA BURLINGTON

Lab Code: STLV Case No.: SAVANNAH

Matrix: (SOIL/SED/WATER) Water

Sample wt/vol: $25.0 \quad(\mathrm{~g} / \mathrm{mL}) \mathrm{mL}$

Level: (TRACE or LOW/MED) TRACE

\% Moisture: not dec.

GC Column: DB-624 ID: 0.20

Soil Extract Volume:

CONCENTRATION UNITS: (ug/L or ug/kg) ug/L
Contract: 21005

Mod. Ref No.:

SDG No.: 137933
Lab Sample ID: 834003

Lab File ID: 834003

Date Received: 06/26/2010

Date Analyzed: 06/30/2010

(mm) Dilution Factor: 1.0

(uL) Soil Aliquot Volume:

(uI)

Purge Volume: 25.0

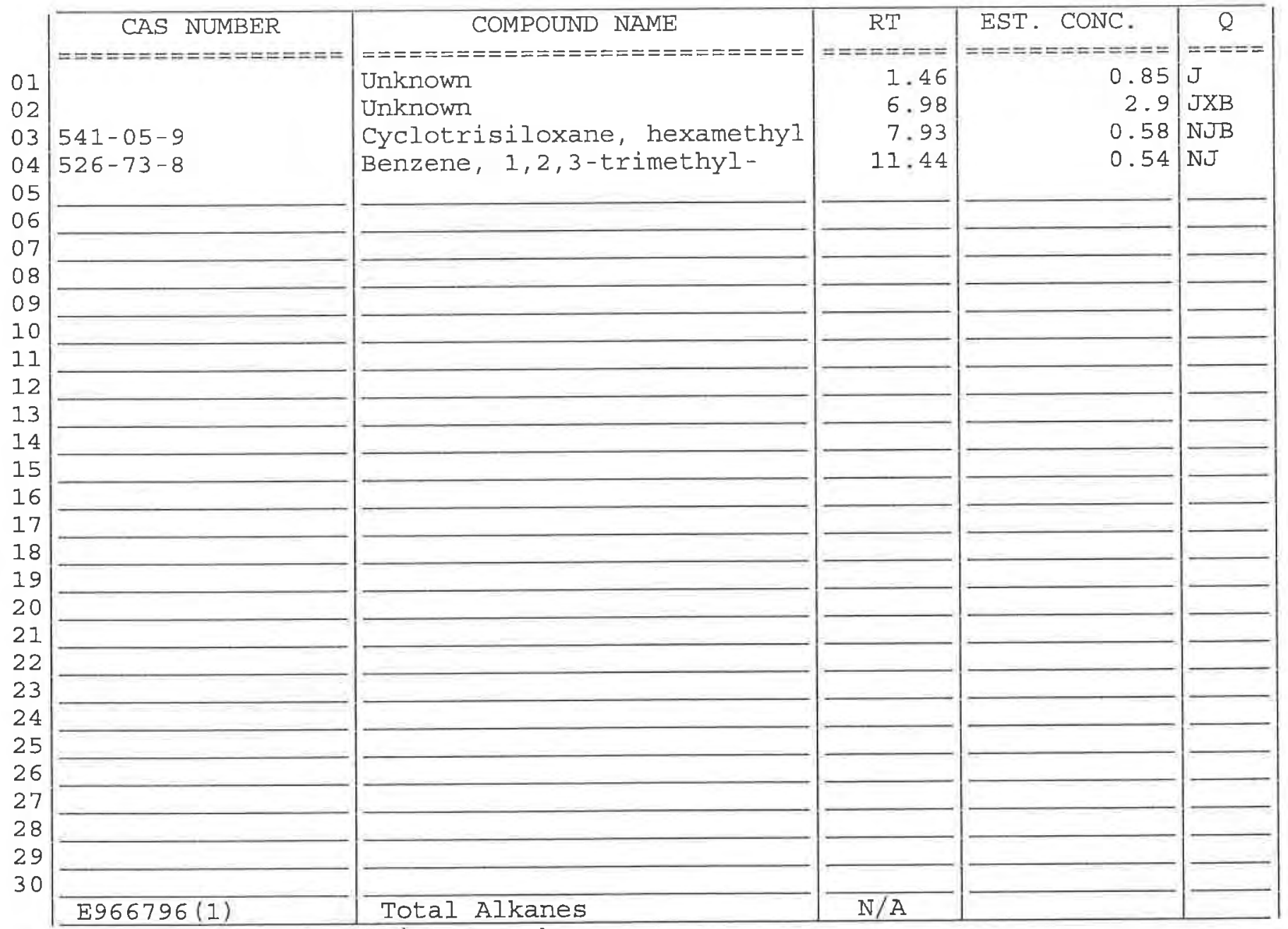

(1) EPA-designated Registry Number. 
$1 A$ - FORM I VOA-1

VOLATILE ORGANICS ANALYSIS DATA SHEET
EPA SAMPLE NO.

SB3 8LFM- 3
Lab Name: TESTAMERICA BURLINGTON

Lab Code: STLV
Matrix: (SOIL/SED/WATER) Water

Sample wt/vol: 25.0

$(\mathrm{g} / \mathrm{mL}) \mathrm{mL}$

Level: (TRACE/LOW/MED) TRACE

$\%$ Moisture: not dec.

GC Column: DB-624

ID $: 0.20$

Soil Extract Volume:

Purge Volume: 25.0
Lab Sample ID: 834004

Lab File ID: 834004

Date Received: 06/26/2010

Date Analyzed: 06/30/2010

Dilution Factor: 1.0

Soil Aliquot Volume:

$(\mathrm{mL})$

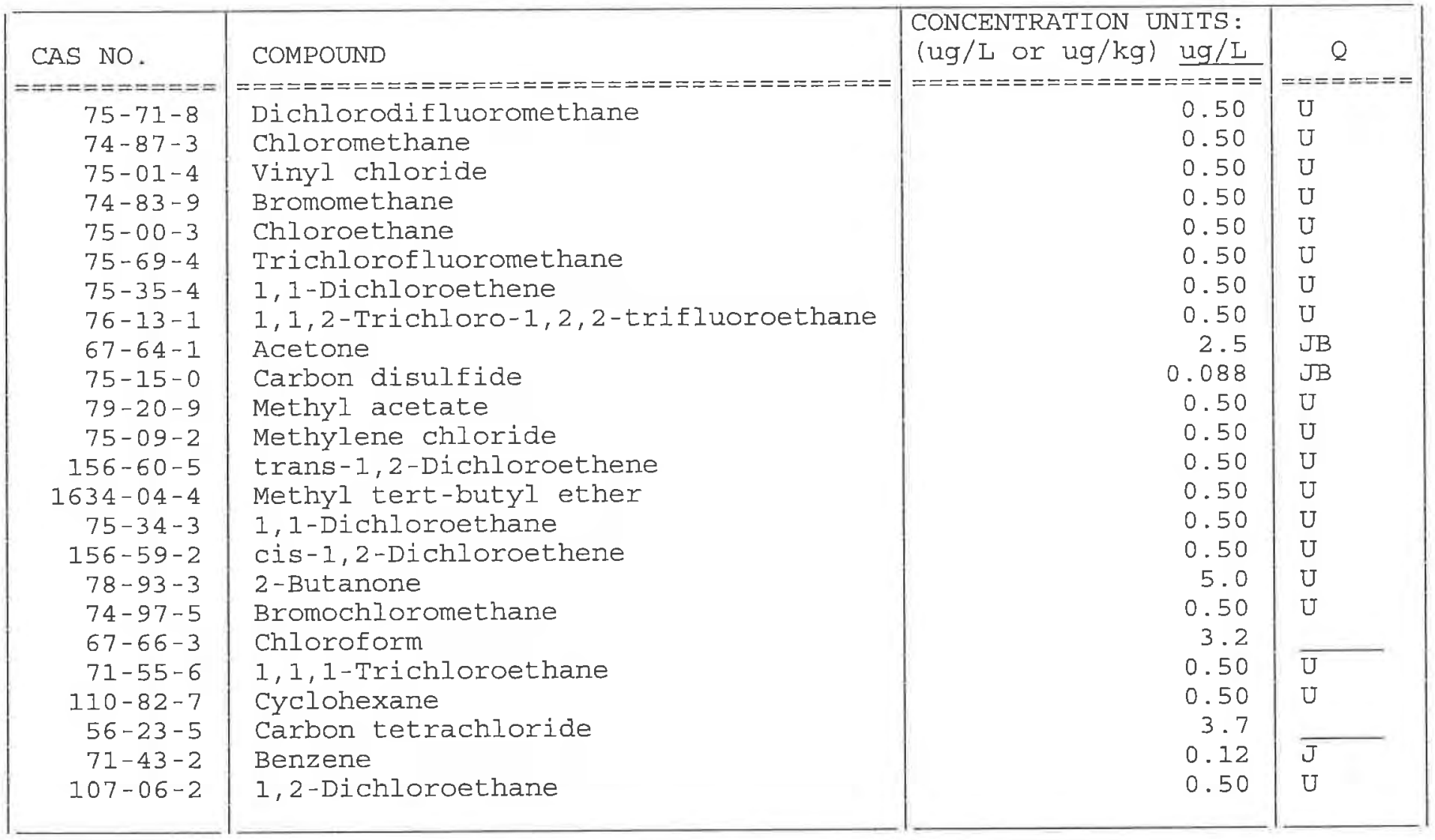

Report 1,4-Dioxane for Low-Medium VOA analysis only 
Lab Name: TESTAMERICA BURLINGTON

Lab Code: STLV Case No.: SAVANNAH Mod. Ref No.:
Contract: 21005
Matrix: (SOIL/SED/WATER) Water

Sample wt/vol: 25.0

(g/mL) $\mathrm{mI}$

Level: (TRACE/LOW/MED) TRACE

\% Moisture: not dec.

GC Column: DB-624

Soil Extract Volume:

ID: 0.20

(mm)

(uL)

$(m L)$

Purge Volume: 25.0
Lab Sample ID: 834004

Lab File ID: 834004

Date Received: 06/26/2010

Date Analyzed: 06/30/2010

Dilution Factor: 1.0

Soil Aliquot Volume:

(uL)

SDG NO.: 137933

\begin{tabular}{|c|c|c|c|}
\hline CAS NO. & COMPOUND & $\begin{array}{l}\text { CONCENTRATION UNITS: } \\
(\mathrm{ug} / \mathrm{L} \text { or } u g / \mathrm{kg}) \mathrm{ug} / \mathrm{L}\end{array}$ & Q \\
\hline$=======+===$ & 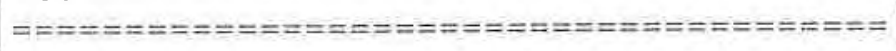 & 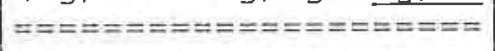 & $======-=$ \\
\hline $79-01-6$ & Trichloroethene & 0.50 & $\mathrm{U}$ \\
\hline $108-87-2$ & Methylcyclohexane & 0.50 & $\mathrm{U}$ \\
\hline $78-87-5$ & 1,2 -Dichloropropane & 0.50 & $\mathrm{U}$ \\
\hline $75-27-4$ & Bromodichloromethane & 0.50 & U \\
\hline $10061-01-5$ & cis-1,3-Dichloropropene & 0.50 & $\mathrm{U}$ \\
\hline $108-10-1$ & 4-Methyl-2-pentanone & 5.0 & $\mathrm{U}$ \\
\hline $108-88-3$ & Toluene & 0.96 & \\
\hline $10061-02-6$ & trans-1,3-Dichloropropene & 0.50 & $\overline{\mathrm{U}}$ \\
\hline $79-00-5$ & $1,1,2$-Trichloroethane & 0.50 & $\mathrm{U}$ \\
\hline $127-18-4$ & Tetrachloroethene & 0.50 & $\mathrm{U}$ \\
\hline $591-78-6$ & 2 -Hexanone & 5.0 & U \\
\hline $124-48-1$ & Dibromochloromethane & 0.50 & U \\
\hline $106-93-4$ & 1,2 -Dibromoethane & 0.50 & U \\
\hline $108-90-7$ & Chlorobenzene & 0.50 & $\mathrm{U}$ \\
\hline $100-41-4$ & Ethylbenzene & 0.24 & $\mathrm{~J}$ \\
\hline $95-47-6$ & o-xylene & 0.46 & $\mathrm{~J}$ \\
\hline $179601-23-1$ & $\mathrm{~m}, \mathrm{p}$-Xylene & 0.93 & \\
\hline $100-42-5$ & styrene & 0.098 & $\overline{\mathrm{J}}$ \\
\hline $75-25-2$ & Bromoform & 0.50 & $\mathrm{U}$ \\
\hline $98-82-8$ & Isopropylbenzene & 0.50 & $\mathrm{U}$ \\
\hline $79-34-5$ & $1,1,2,2$-Tetrachloroethane & 0.50 & $\mathrm{U}$ \\
\hline $541-73-1$ & 1,3-Dichlorobenzene & 0.50 & $\mathrm{U}$ \\
\hline $106-46-7$ & 1,4-Dichlorobenzene & 0.50 & $\mathrm{U}$ \\
\hline $95-50-1$ & 1,2-Dichlorobenzene & 0.50 & $\mathrm{U}$ \\
\hline $96-12-8$ & 1,2 -Dibromo-3-chloropropane & 0.50 & $U$ \\
\hline $120-82-1$ & $1,2,4$-Trichlorobenzene & 0.50 & $\mathrm{U}$ \\
\hline $87-61-6$ & $1,2,3$-Trichlorobenzene & 0.50 & $\mathrm{U}$ \\
\hline
\end{tabular}


IJ - FORM I. VOA-TIC

VOLATILE ORGANICS ANALYSIS DATA SHEET

TENTATIVELY IDENTIFIED COMPOUNDS
EPA SAMPLE NO.

SB3 8LFM-3

Contract: 21005

Mod. Ref No.:

SDG NO.: 137933

Lab Sample ID: 834004

Lab File ID: 834004

Date Received: 06/26/2010

Date Analyzed: 06/30/2010

\% Moisture: not dec.

GC Column: DB-624 ID: 0.20 (mm) Dilution Factor: 1.0

Soil Extract Volume:

(uL) Soil Aliquot Volume:

(uL)

Purge Volume: 25.0

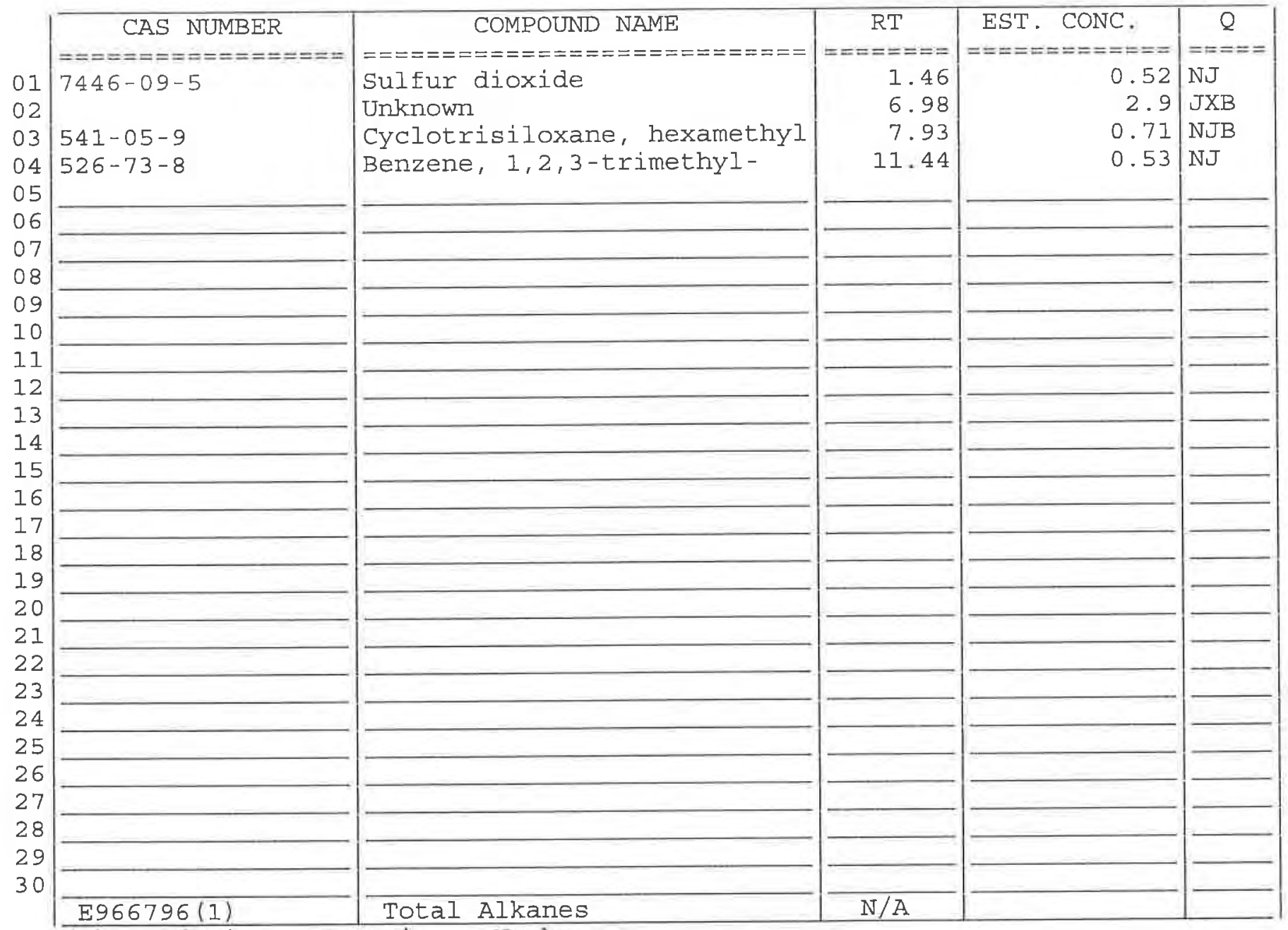

(1) EPA-designated Registry Number. 
1A - FORM I VOA-1

VOLATILE ORGANICS ANALYSIS DATA SHEET
EPA SAMPLE NO.

VBLKJH

Contract: 21005
Lab Name: TESTAMERICA BURLINGTON

Lab Code: STLV Case No.: SAVANNAH Mod. Ref No.:
SDG No.: 137933
Lab Sample ID: VBLKJH

Lab File ID: JBEB03C

Date Received:

Date Analyzed: 06/30/2010

\% Moisture: not dec.

(mm) Dilution Factor: 1.0

(uI) Soil Aliquot volume:

(uL)

(mL)

\begin{tabular}{|c|c|c|c|}
\hline CAS NO. & COMPOUND & $\begin{array}{l}\text { CONCENTRATION UNITS: } \\
(\mathrm{ug} / \mathrm{L} \text { or } \mathrm{ug} / \mathrm{kg}) \mathrm{ug} / \mathrm{L}\end{array}$ & $Q$ \\
\hline 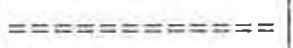 & 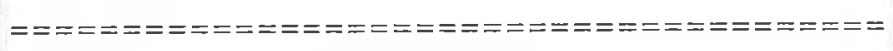 & 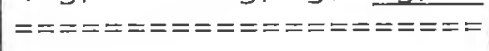 & $====\pi===$ \\
\hline $75-71-8$ & Dichlorodifluoromethane & 0.50 & $\mathrm{U}$ \\
\hline $74-87-3$ & Chloromethane & 0.50 & $\mathrm{U}$ \\
\hline $75-01-4$ & Vinyl chloride & 0.50 & $U$ \\
\hline $74-83-9$ & Bromomethane & 0.50 & U \\
\hline $75-00-3$ & Chloroethane & 0.50 & $\mathrm{U}$ \\
\hline $75-69-4$ & Trichlorofluoromethane & 0.50 & $\mathrm{U}$ \\
\hline $75-35-4$ & 1,1-Dichloroethene & 0.50 & $\mathrm{U}$ \\
\hline $76-13-1$ & 1, 1,2-Trichloro-1,2,2-trifluoroethane & 0.50 & U \\
\hline $67-64-1$ & Acetone & 1.6 & $\mathrm{~J}$ \\
\hline $75-15-0$ & Carbon disulfide & 0.070 & J \\
\hline $79-20-9$ & Methyl acetate & 0.50 & $\mathrm{U}$ \\
\hline $75-09-2$ & Methylene chloride & 0.50 & $\mathrm{U}$ \\
\hline $156-60-5$ & trans-1,2-Dichloroethene & 0.50 & $\mathrm{U}$ \\
\hline $1634-04-4$ & Methyl tert-butyl ether & 0.50 & $\mathrm{U}$ \\
\hline $75-34-3$ & 1,1 -Dichloroethane & 0.50 & $\mathrm{U}$ \\
\hline $156-59-2$ & cis-1,2-Dichloroethene & 0.50 & $\mathrm{U}$ \\
\hline $78-93-3$ & 2-Butanone & 5.0 & $\mathrm{U}$ \\
\hline $74-97-5$ & Bromochloromethane & 0.50 & $\mathrm{U}$ \\
\hline $67-66-3$ & Chloroform & 0.50 & $\mathrm{U}$ \\
\hline $71-55-6$ & 1,1,1-Trichloroethane & 0.50 & U \\
\hline $110-82-7$ & Cyclohexane & 0.50 & $\mathrm{U}$ \\
\hline $56-23-5$ & Carbon tetrachloride & 0.50 & $\mathrm{U}$ \\
\hline $71-43-2$ & Benzene & 0.50 & $\mathrm{U}$ \\
\hline $107-06-2$ & 1,2-Dichloroethane & 0.50 & $\mathrm{U}$ \\
\hline
\end{tabular}

Report 1,4-Dioxane for Low-Medium VOA analysis only 
IB - FORM I VOA-2
VOLATILE ORGANICS ANALYSIS DATA SHEET

Lab Name: TESTAMERICA BURLINGTON

Contract: 21005

Lab Code: STLV Case No.: SAVANNAH Mod. Ref No.:

SDG No.: 137933
Matrix: (SOIL/SED/WATER) water

Sample wt/vol: $25.0 \quad(\mathrm{~g} / \mathrm{mL}) \mathrm{mL}$

Level: (TRACE/LOW/MED) TRACE

\% Moisture: not dec.

GC Column: DB-624

ID $: 0.20$

Soil Extract Volume:

Purge Volume: 25.0
Lab Sample ID: VBLKJH

Lab File ID: JBEB03C

Date Received:

Date Analyzed: 06/30/2010

Dilution Factor: 1.0

Soil Aliquot Volume:

(uL)

\begin{tabular}{|c|c|c|c|}
\hline CAS NO. & COMPOUND & $\begin{array}{l}\text { CONCENTRATION UNITS: } \\
(\mathrm{ug} / \mathrm{L} \text { or } u g / \mathrm{kg}) \mathrm{ug} / \mathrm{L}\end{array}$ & $Q$ \\
\hline 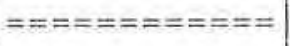 & 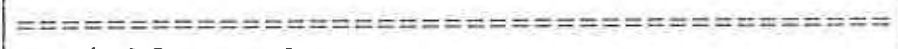 & 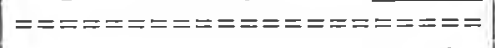 & $== \pm===x$ \\
\hline $79-01-6$ & Trichloroethene & 0.50 & $\mathrm{U}$ \\
\hline $108-87-2$ & Methylcyclohexane & 0.50 & $\mathrm{U}$ \\
\hline $78-87-5$ & 1,2 -Dichloropropane & 0.50 & $\mathrm{U}$ \\
\hline $75-27-4$ & Bromodichloromethane & 0.50 & $\mathrm{U}$ \\
\hline $10061-01-5$ & cis-1,3-Dichloropropene & 0.50 & $\mathrm{U}$ \\
\hline $108-10-1$ & 4-Methyl-2-pentanone & 5.0 & $\mathrm{U}$ \\
\hline $108-88-3$ & Toluene & 0.50 & $\mathrm{U}$ \\
\hline $10061-02-6$ & trans-1,3-Dichloropropene & 0.50 & $\mathrm{U}$ \\
\hline $79-00-5$ & $1,1,2$-Trichloroethane & 0.50 & $\mathrm{U}$ \\
\hline $127-18-4$ & Tetrachloroethene & 0.50 & $\mathrm{U}$ \\
\hline $591-78-6$ & 2-Hexanone & 5.0 & $\mathrm{U}$ \\
\hline $124-48-1$ & Dibromochloromethane & 0.50 & $\mathrm{U}$ \\
\hline $106-93-4$ & 1,2-Dibromoethane & 0.50 & $\mathrm{U}$ \\
\hline $108-90-7$ & Chlorobenzene & 0.50 & $\mathrm{U}$ \\
\hline $100-41-4$ & Ethylbenzene & 0.50 & $\mathrm{U}$ \\
\hline $95-47-6$ & o-Xylene & 0.50 & $\mathrm{U}$ \\
\hline $179601-23-1$ & $\mathrm{~m}, \mathrm{p}$-Xylene & 0.50 & $\mathrm{U}$ \\
\hline $100-42-5$ & styrene & 0.50 & $\mathrm{U}$ \\
\hline $75-25-2$ & Bromoform & 0.50 & $\mathrm{U}$ \\
\hline $98-82-8$ & Isopropylbenzene & 0.50 & $\mathrm{U}$ \\
\hline $79-34-5$ & $1,1,2,2$-Tetrachloroethane & 0.50 & $\mathrm{U}$ \\
\hline $541-73-1$ & 1,3-Dichlorobenzene & 0.50 & $\mathrm{U}$ \\
\hline $106-46-7$ & 1,4-Dichlorobenzene & 0.50 & $\mathrm{U}$ \\
\hline $95-50-1$ & 1,2-Dichlorobenzene & 0.50 & $\mathrm{U}$ \\
\hline $96-12-8$ & 1,2-Dibromo-3-chloropropane & 0.50 & $\mathrm{U}$ \\
\hline $120-82-1$ & 1,2,4-Trichlorobenzene & 0.50 & $\mathrm{U}$ \\
\hline $87-61-6$ & $1,2,3$-Trichlorobenzene & 0.50 & $\mathrm{U}$ \\
\hline
\end{tabular}


$1 \mathrm{~J}$ - FORM I VOA-TIC

VOLATILE ORGANICS ANALYSIS DATA SHEET TENTATIVELY IDENTIFIED COMPOUNDS

EPA SAMPIE NO.
VBIKJH

Lab Name: TESTAMERICA BURLINGTON

Contract: 21005

Lab Code: STLV Case No.: SAVANNAH Mod. Ref No.:

SDG No.: 137933

Lab sample ID: VBLKJH

Matrix: (SOIL/SED/WATER) Water

Sample wt/vol: $25.0 \quad(\mathrm{~g} / \mathrm{mL}) \mathrm{mL}$

Lab File ID: JBEBO3C

Level: (TRACE or LOW/MED) TRACE

Date Received:

\% Moisture: not dec.

Date Analyzed: 06/30/2010

GC Column: DB-624

ID $: 0.20$

$(\mathrm{mm})$

Dilution Factor: 1.0

Soil Extract Volume:

(uL) Soil Aliquot Volume:

(uL)

CONCENTRATION UNITS: (ug/L or ug/kg) ug/L Purge Volume: 25.0

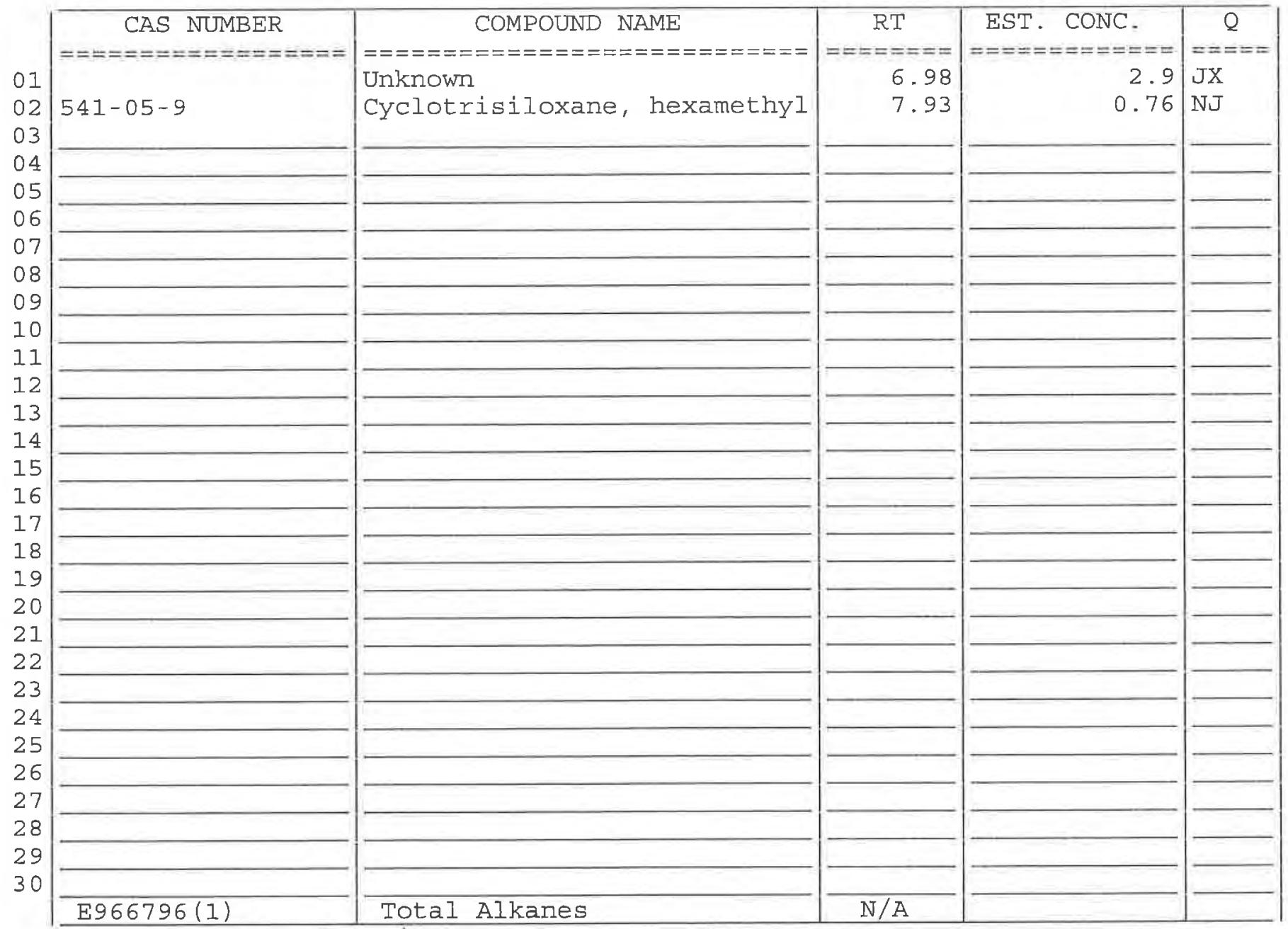

(1) EPA-designated Registry Number. 
IA - FORM I VOA-1

VOLATILE ORGANICS ANALYSIS DATA SHEET
EPA SAMPLE NO.

VHBLKO 1

Contract: 21005
Lab Name: TESTAMERICA BURIINGTON

Lab Code: STLV Case No.: SAVANNAH Mod. Ref No.:
SDG No. : 137933
Matrix: (SOIL/SED/WATER) Water

Sample wt/vol: 25.0

$(\mathrm{g} / \mathrm{mL}) \mathrm{mL}$

Level: (TRACE/LOW/MED) TRACE

\% Moisture: not dec.

GC Column: DB-624

Soil Extract Volume:

ID : 0.20

Purge Volume: 25.0
Lab Sample ID: 834009

Lab File ID: 834009

Date Received:

Date Analyzed: 06/30/2010

Dilution Factor: 1.0

Soil Aliquot Volume:

(uL)

$(\mathrm{mL})$

\begin{tabular}{|c|c|c|c|}
\hline CAS NO. & COMPOUND & $\begin{array}{l}\text { CONCENTRATION UNITS: } \\
(\mathrm{ug} / \mathrm{L} \text { or } u g / \mathrm{kg}) \text { ug } / \mathrm{L}\end{array}$ & $Q$ \\
\hline 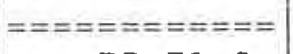 & 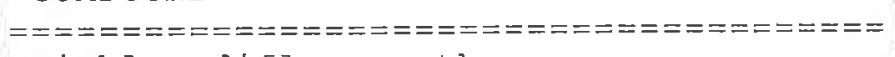 & 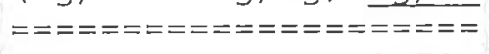 & $=======$ \\
\hline $75-71-8$ & Dichlorodifluoromethane & 0.50 & $\mathrm{U}$ \\
\hline $74-87-3$ & Chloromethane & 0.50 & U \\
\hline $75-01-4$ & Vinyl chloride & 0.50 & $\mathrm{U}$ \\
\hline $74-83-9$ & Bromomethane & 0.50 & $\mathrm{U}$ \\
\hline $75-00-3$ & Chloroethane & 0.50 & $\mathrm{U}$ \\
\hline $75-69-4$ & Trichlorofluoromethane & 0.50 & U \\
\hline $75-35-4$ & 1,1-Dichloroethene & 0.50 & $\mathrm{U}$ \\
\hline $76-13-1$ & $1,1,2$-Trichloro-1,2,2-trifluoroethane & 0.50 & $\mathrm{U}$ \\
\hline $67-64-1$ & Acetone & 1.8 & JB \\
\hline $75-15-0$ & Carbon disulfide & 0.50 & U \\
\hline $79-20-9$ & Methyl acetate & 0.16 & $\mathrm{~J}$ \\
\hline $75-09-2$ & Methylene chloride & 0.50 & $\mathrm{U}$ \\
\hline $156-60-5$ & trans-1,2-Dichloroethene & 0.50 & $\mathrm{U}$ \\
\hline $1634-04-4$ & Methyl tert-butyl ether & 0.50 & $\mathrm{U}$ \\
\hline $75-34-3$ & 1,1 -Dichloroethane & 0.50 & $\mathrm{U}$ \\
\hline $156-59-2$ & cis-1,2-Dichloroethene & 0.50 & U \\
\hline $78-93-3$ & 2 -Butanone & 5.0 & $\mathrm{U}$ \\
\hline $74-97-5$ & Bromochloromethane & 0.50 & $\mathrm{U}$ \\
\hline $67-66-3$ & Chloroform & 0.50 & $\mathrm{U}$ \\
\hline $71-55-6$ & 1,1,1-Trichloroethane & 0.50 & $\mathrm{U}$ \\
\hline $110-82-7$ & Cyclohexane & 0.50 & $\mathrm{U}$ \\
\hline $56-23-5$ & Carbon tetrachloride & 0.50 & $\mathrm{U}$ \\
\hline $71-43-2$ & Benzene & 0.50 & $\mathrm{U}$ \\
\hline $107-06-2$ & 1,2-Dichloroethane & 0.50 & U \\
\hline
\end{tabular}

Report 1,4-Dioxane for Low-Medium VOA analysis only 
IB - FORM I VOA-2
VOLATILE ORGANICS ANALYSIS DATA SHEET

Contract : 21005

Lab Code: STLV Case No.: SAVANNAH Mod. Ref No.:

SDG No.: 137933

Matrix: (SOIL/SED/WATER) Water

Sample wt/vol: 25.0

$(\mathrm{g} / \mathrm{mL}) \mathrm{mL}$

Level: (TRACE/LOW/MED) TRACE

$\because$ Moisture: not dec.

GC Column: DB-624

ID : 0.20

SoiI Extract Volume:

Purge Volume: 25.0
Lab Sample ID: 834009

Lab File ID: 834009

Date Received:

Date Analyzed: 06/30/2010

Dilution Factor: 1.0

Soil Aliquot Volume:

(uL)

\begin{tabular}{|c|c|c|c|}
\hline CAS NO. & COMPOUND & $\begin{array}{l}\text { CONCENTRATION UNITS: } \\
(\mathrm{ug} / \mathrm{L} \text { or } \mathrm{ug} / \mathrm{kg}) \mathrm{ug} / \mathrm{L}\end{array}$ & Q \\
\hline$== \pm========$ & 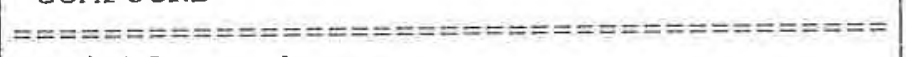 & 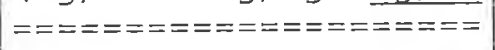 & $========$ \\
\hline $79-01-6$ & Trichloroethene & 0.50 & $\mathrm{U}$ \\
\hline $108-87-2$ & Methylcyclohexane & 0.50 & $\mathrm{U}$ \\
\hline $78-87-5$ & 1,2-Dichloropropane & 0.50 & $\mathrm{U}$ \\
\hline $75-27-4$ & Bromodichloromethane & 0.50 & U \\
\hline $10061-01-5$ & cis-1,3-Dichloropropene & 0.50 & U \\
\hline $108-10-1$ & 4-Methyl-2-pentanone & 5.0 & U \\
\hline $108-88-3$ & Toluene & 0.50 & U \\
\hline $10061-02-6$ & trans-1,3-Dichloropropene & 0.50 & U \\
\hline $79-00-5$ & $1,1,2$-Trichloroethane & 0.50 & U \\
\hline $127-18-4$ & Tetrachloroethene & 0.50 & $\mathrm{U}$ \\
\hline $591-78-6$ & 2 -Hexanone & 5.0 & $\mathrm{U}$ \\
\hline $124-48-1$ & Dibromochloromethane & 0.50 & $\mathrm{U}$ \\
\hline $106-93-4$ & 1,2-Dibromoethane & 0.50 & $\mathrm{U}$ \\
\hline $108-90-7$ & Chlorobenzene & 0.50 & $\mathrm{U}$ \\
\hline $100-41-4$ & Ethylbenzene & 0.50 & $\mathrm{U}$ \\
\hline $95-47-6$ & o-Xylene & 0.50 & $\mathrm{U}$ \\
\hline $179601-23-1$ & m,p-Xylene & 0.50 & $\mathrm{U}$ \\
\hline $100-42-5$ & styrene & 0.50 & $\mathrm{U}$ \\
\hline $75-25-2$ & Bromoform & 0.50 & $\mathrm{U}$ \\
\hline $98-82-8$ & Isopropylbenzene & 0.50 & U \\
\hline $79-34-5$ & $1,1,2,2$-Tetrachloroethane & 0.50 & U \\
\hline $541-73-1$ & 1,3-Dichlorobenzene & 0.50 & $U$ \\
\hline $106-46-7$ & 1,4-Dichlorobenzene & 0.50 & U \\
\hline $95-50-1$ & 1,2-Dichlorobenzene & 0.50 & U \\
\hline $96-12-8$ & 1,2-Dibromo-3-chloropropane & 0.50 & $\mathrm{U}$ \\
\hline $120-82-1$ & $1,2,4$-Trichlorobenzene & 0.50 & U \\
\hline $87-61-6$ & $1,2,3$-Trichlorobenzene & 0.50 & $\mathrm{U}$ \\
\hline
\end{tabular}


IJ - FORM I VOA-TIC

VOLATILE ORGANICS ANALYSIS DATA SHEET

TENTATIVELY IDENTIFIED COMPOUNDS
EPA SAMPLE NO.

VHBLK01
Lab Name: TESTAMERICA BURLINGTON

Lab Code: STLV Case No.: SAVANNAH

Matrix: (SOIL/SED/WATER) Water

Sample wt/vol: $25.0 \quad(\mathrm{~g} / \mathrm{mL}) \mathrm{mL}$

Level: (TRACE or LOW/MED) TRACE

\% Moisture: not dec.

GC Column: DB-624

ID : 0.20

Soil Extract Volume:

CONCENTRATION UNITS: (ug/L or ug/kg) ug/L
Contract: 21005

Mod. Ref No.:

SDG No.: 137933
Lab Sample ID: 834009

Lab File ID: 834009

Date Received:

Date Analyzed: 06/30/2010

(mm) Dilution Factor: 1.0

(uL) Soil Aliquot Volume:

(uL)

Purge Volume: 25.0

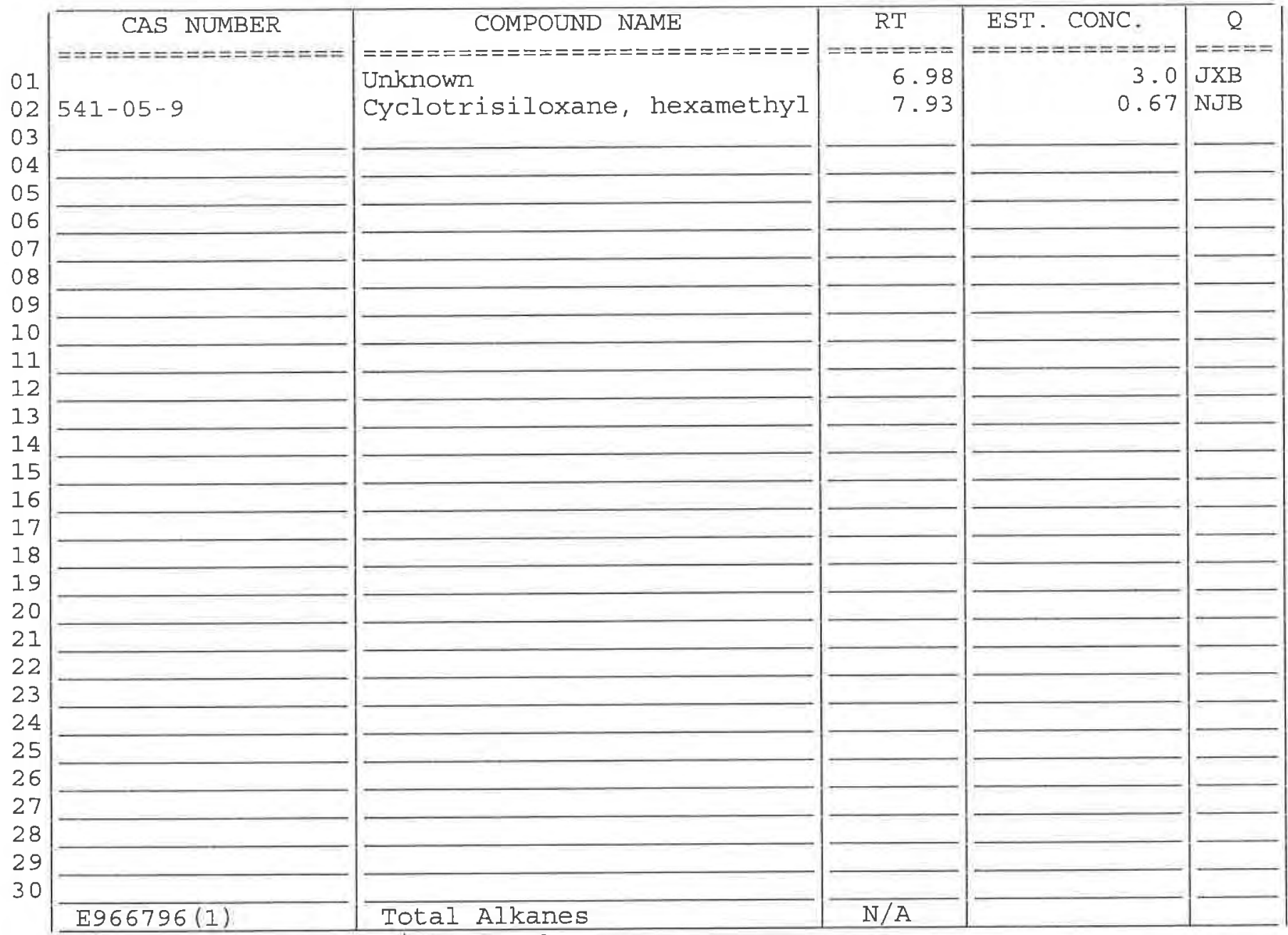

(1) EPA-designated Registry Number. 
IA - FORM I VOA-I

VOLATILE ORGANICS ANALYSIS DATA SHEET
EPA SAMPLE NO.

VIBLKJK

Contract: 21005
Lab Name: TESTAMERICA BURIINGTON

Lab Code: STLV

Case No.: SAVANNAH

SDG NO.: 137933

Matrix: (SOIL/SED/WATER) Water

Sample wt/vol: 25.0

$(g / m L) \mathrm{mL}$

Level: (TRACE/LOW/MED) TRACE

\% Moisture: not dec.

GC Column: $\mathrm{DB}-624$

ID : 0.20

Soil Extract Volume:

Purge Volume: 25.0
Lab sample ID: VIBLKJK

Lab File ID: JBEBO4C

Date Received:

Date Analyzed: 06/30/2010

(mm) Dilution Factor: 1.0

(uI) Soil Aliquot Volume:

(uL)

(mL)

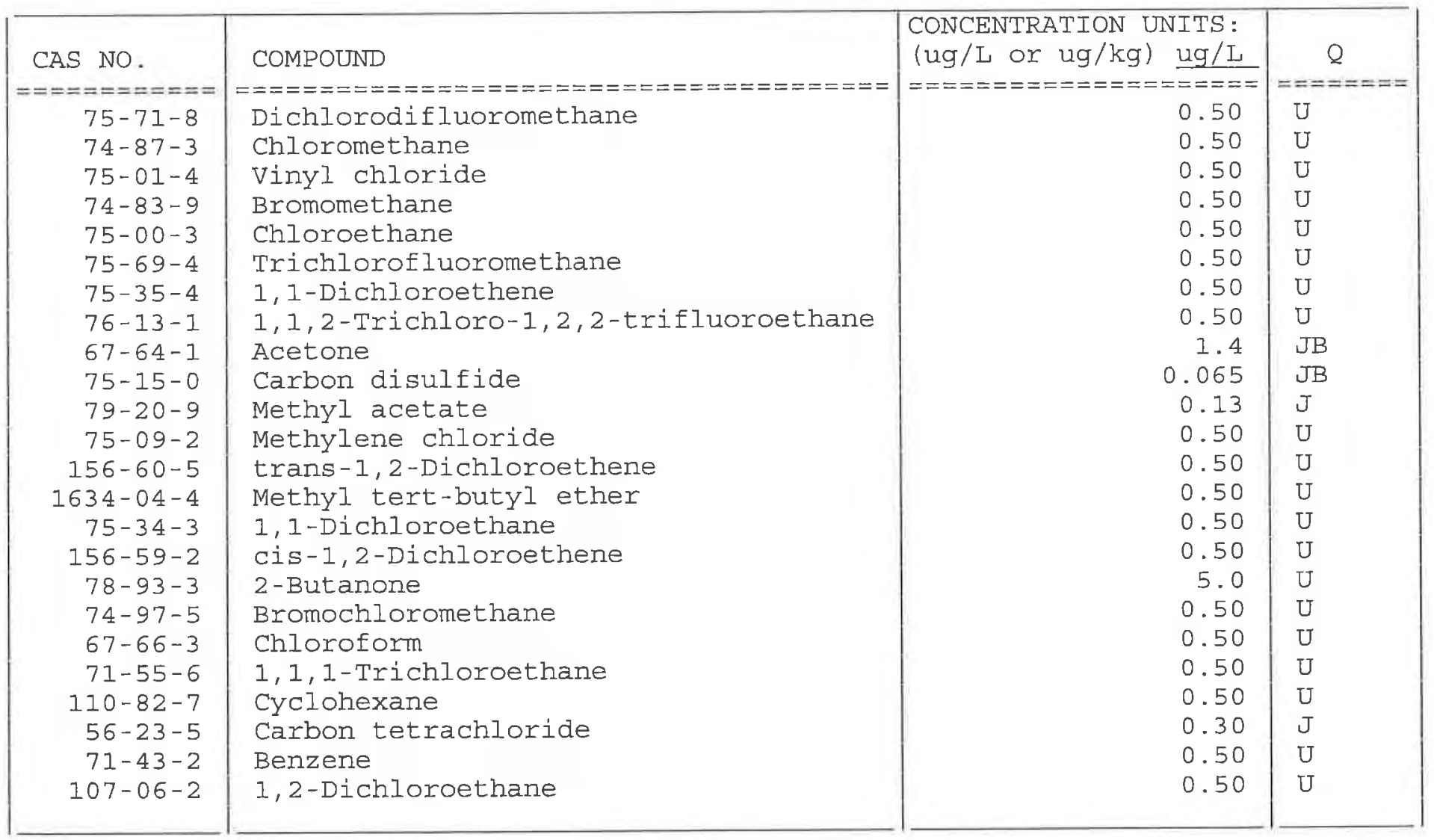

Report 1,4-Dioxane for Low-Medium VOA analysis only 
IB - FORM I VOA-2

VOLATILE ORGANICS ANALYSIS DATA SHEET
EPA SAMPLE NO.

VIBLKJK

Contract: 21005
Lab Name: TESTAMERICA BURIINGTON

Lab Code: STLV Case No.: SAVANNAH Mod. Ref No.:
Lab Sample ID: VIBLKJK

Lab File ID: JBEB04C

Date Received:

Date Analyzed: 06/30/2010

\% Moisture: not dec.

GC Column: DB-624 ID: 0.20 (mm) Dilution Factor: 1.0

Soil Extract Volume:

(uL) Soil Aliquot Volume:

(uL)

(mL)

\begin{tabular}{|c|c|c|c|}
\hline CAS NO. & COMPOUND & $\begin{array}{l}\text { CONCENTRATION UNITS: } \\
(\mathrm{ug} / \mathrm{L} \text { or } \mathrm{ug} / \mathrm{kg}) \mathrm{ug} / \mathrm{L}\end{array}$ & $Q$ \\
\hline$==ニ=ニ=====-$ & 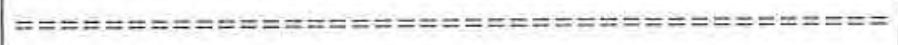 & $=============-====$ & $== \pm=== \pm=$ \\
\hline $79-01-6$ & Trichloroethene & 0.50 & $\mathrm{U}$ \\
\hline $108-87-2$ & Methylcyclohexane & 0.50 & $\mathrm{U}$ \\
\hline $78-87-5$ & 1,2-Dichloropropane & 0.50 & $\mathrm{U}$ \\
\hline $75-27-4$ & Bromodichloromethane & 0.50 & $\mathrm{U}$ \\
\hline $10061-01-5$ & cis-1,3-Dichloropropene & 0.50 & $\mathrm{U}$ \\
\hline $108-10-1$ & 4-Methyl-2-pentanone & 5.0 & $\mathrm{U}$ \\
\hline $108-88-3$ & Toluene & 0.50 & $\mathrm{U}$ \\
\hline $10061-02-6$ & trans-1,3-Dichloropropene & 0.50 & $\mathrm{U}$ \\
\hline $79-00-5$ & $1,1,2-T r i c h l o r o e t h a n e$ & 0.50 & $\mathrm{U}$ \\
\hline $127-18-4$ & Tetrachloroethene & 0.50 & $\mathrm{U}$ \\
\hline $591-78-6$ & 2-Hexanone & 5.0 & $\mathrm{U}$ \\
\hline $124-48-1$ & Dibromochloromethane & 0.50 & $\mathrm{U}$ \\
\hline $106-93-4$ & 1,2-Dibromoethane & 0.50 & $\mathrm{U}$ \\
\hline $108-90-7$ & Chlorobenzene & 0.50 & $\mathrm{U}$ \\
\hline $100-41-4$ & Ethylbenzene & 0.50 & $\mathrm{U}$ \\
\hline $95-47-6$ & o-Xylene & 0.50 & $\mathrm{U}$ \\
\hline $179601-23-1$ & $\mathrm{~m}, \mathrm{p}-\mathrm{xy}$ lene & 0.50 & $\mathrm{U}$ \\
\hline $100-42-5$ & Styrene & 0.50 & $\mathrm{U}$ \\
\hline $75-25-2$ & Bromoform & 0.50 & $\mathrm{U}$ \\
\hline $98-82-8$ & Isopropylbenzene & 0.50 & $\mathrm{U}$ \\
\hline $79-34-5$ & $1,1,2,2$-Tetrachloroethane & 0.50 & $\mathrm{U}$ \\
\hline $541-73-1$ & 1,3-Dichlorobenzene & 0.50 & $\mathrm{U}$ \\
\hline $106-46-7$ & 1,4-Dichlorobenzene & 0.50 & $\mathrm{U}$ \\
\hline $95-50-1$ & 1,2 -Dichlorobenzene & 0.50 & $\mathrm{U}$ \\
\hline $96-12-8$ & 1,2-Dibromo-3-chloropropane & 0.50 & $\mathrm{U}$ \\
\hline $120-82-1$ & $1,2,4-T r i c h l o r o b e n z e n e$ & 0.50 & $\mathrm{U}$ \\
\hline $87-61-6$ & $1,2,3-T r i c h l o r o b e n z e n e$ & 0.50 & $\mathrm{U}$ \\
\hline
\end{tabular}


$1 J$ - FORM I VOA-TIC

VOLATILE ORGANICS ANALYSIS DATA SHEET

TENTATIVELY IDENTIFIED COMPOUNDS
EPA SAMPLE NO.

VIBLKJK
Lab Name: TESTAMERICA BURIINGTON

Lab Code: STLV Case No.: SAVANNAH

Matrix: (SOIL/SED/WATER) Water

Sample wt/vol: $25.0(\mathrm{~g} / \mathrm{mL}) \mathrm{mL}$

Level: (TRACE or LOW/MED) TRACE

\% Moisture: not dec.

GC Column: DB-624

ID: 0.20

Soil Extract Volume:

CONCENTRATION UNITS: (ug/L or ug/kg) ug/L
Contract: 21005

Mod. Ref No.:

SDG No.: 137933

Lab Sample ID: VIBLKJK

Lab File ID: JBEBO4C

Date Received:

Date Analyzed: 06/30/2010

(mm) Dilution Factor: 1.0

(uJ) Soil Aliquot Volume:

(uL)

Purge Volume: 25.0

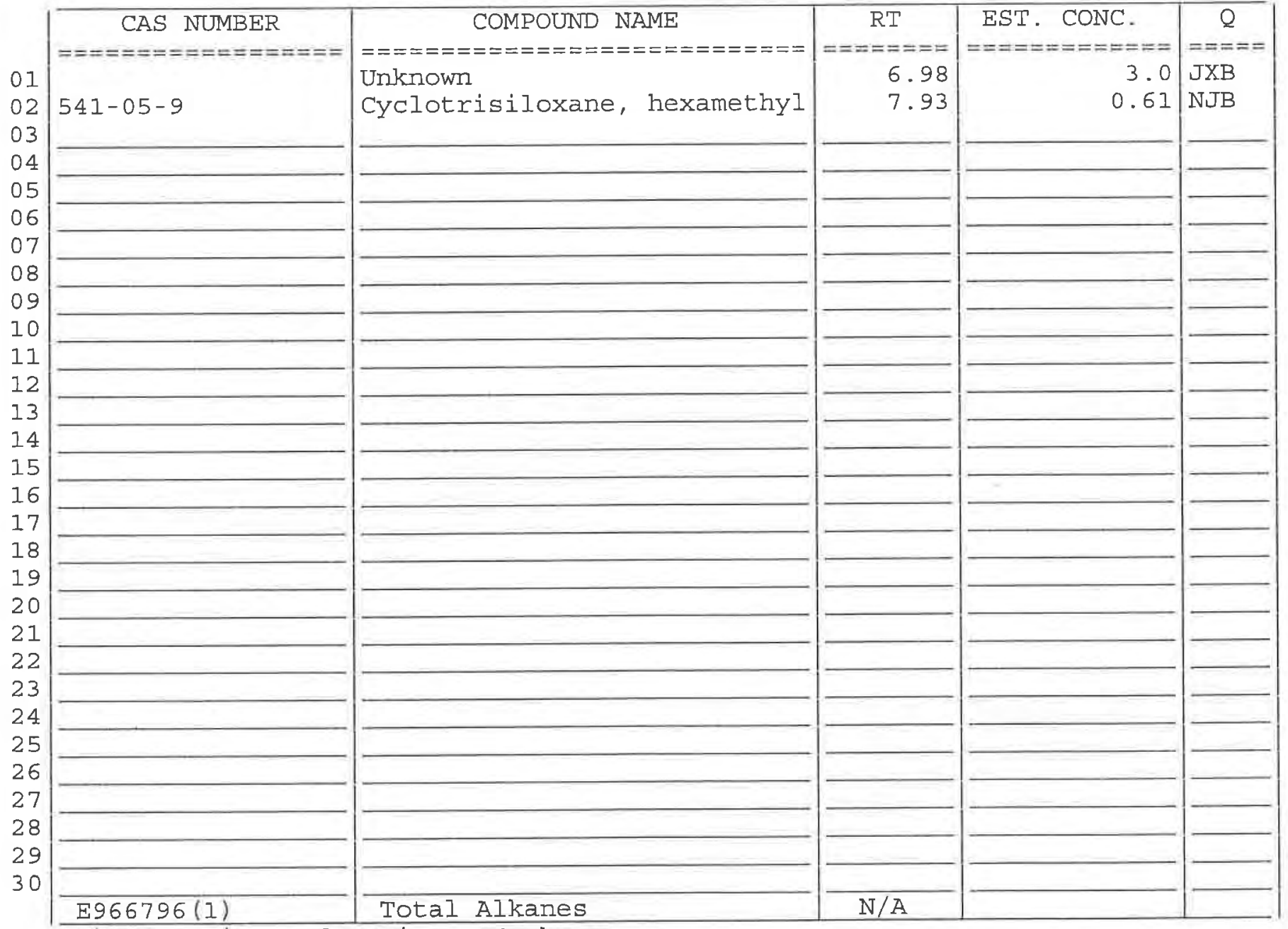

(1) EPA-designated Registry Number. 
$1 A$ - FORM I VOA-1

VOLATILE ORGANICS ANALYSIS DATA SHEET
EPA SAMPLE NO.

VIBLKJI

Contract: 21005
Lab Name: TESTAMERICA BURLINGTON

Lab Code: STLV Case No.: SAVANNAH Mod. Ref No.:
SDG NO. : 137933
Matrix: (SOIL/SED/WATER) Water

Sample wt/vol: $25.0 \quad(\mathrm{~g} / \mathrm{mL}) \mathrm{mL}$

Level: (TRACE/LOW/MED) TRACE

\% Moisture: not dec.

GC Column: DB-624

Soil Extract Volume:

ID : 0.20

(mm)

(uI)

Soil Aliquot Volume:

(mI)
Lab sample ID: VIBLKJL

Lab File ID: JBEB05C

Date Received:

Date Analyzed: 06/30/2010

Dilution Factor: 1.0

(uL)

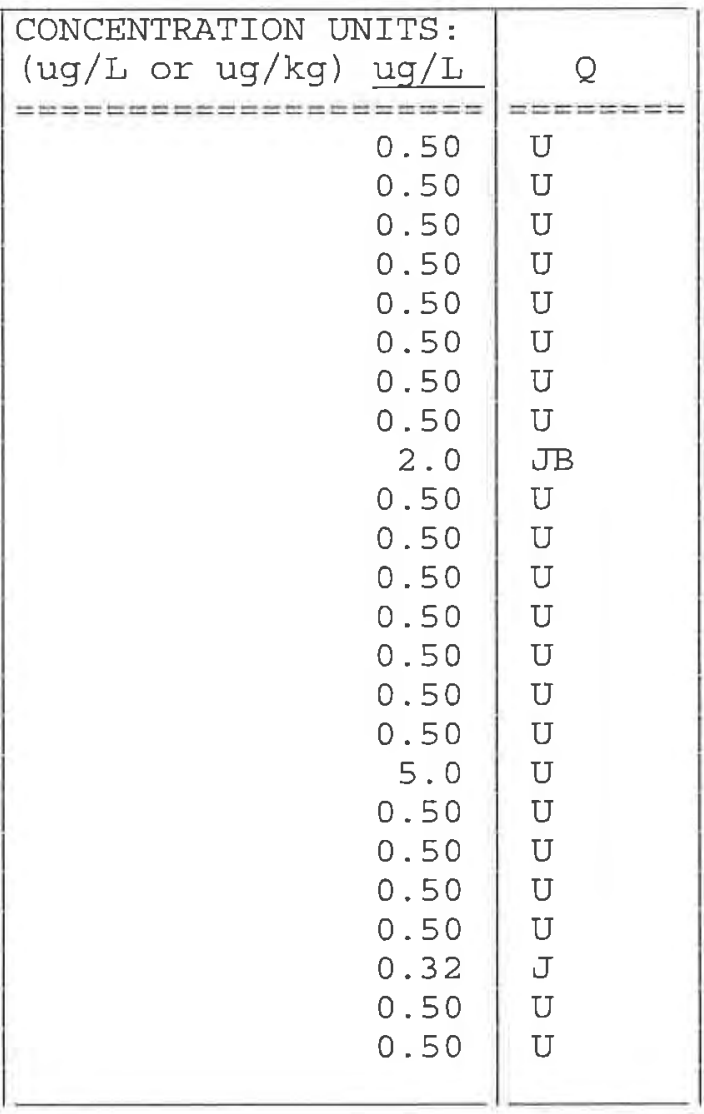

Report 1,4-Dioxane for Low-Medium VOA analysis only 
IB - FORM I VOA-2

VOLATILE ORGANICS ANALYSIS DATA SHEET
EPA SAMPLE NO.

VIBLKJL

Contract: 21005
Lab Name: TESTAMERICA BURLINGTON

Lab Code: STLV Case No.: SAVANNAH Mod. Ref No.:
SDG No.: 137933
Lab sample ID: VIBLKJL

Matrix: (SOIL/SED/WATER) Water

Sample wt/vol: 25.0

$(\mathrm{g} / \mathrm{mL}) \mathrm{mL}$

Level: (TRACE/LOW/MED) TRACE

\% Moisture: not dec.

GC Column: $\mathrm{DB}-624$

ID $: 0.20$

$(\mathrm{mm})$

(UI)

Soil Aliquot Volume:

(mL)

Lab File ID: JBEB05C

Date Received:

Date Analyzed: 06/30/2010

Dilution Factor: 1.0

Soil Extract Volume:

Purge volume: 25.0

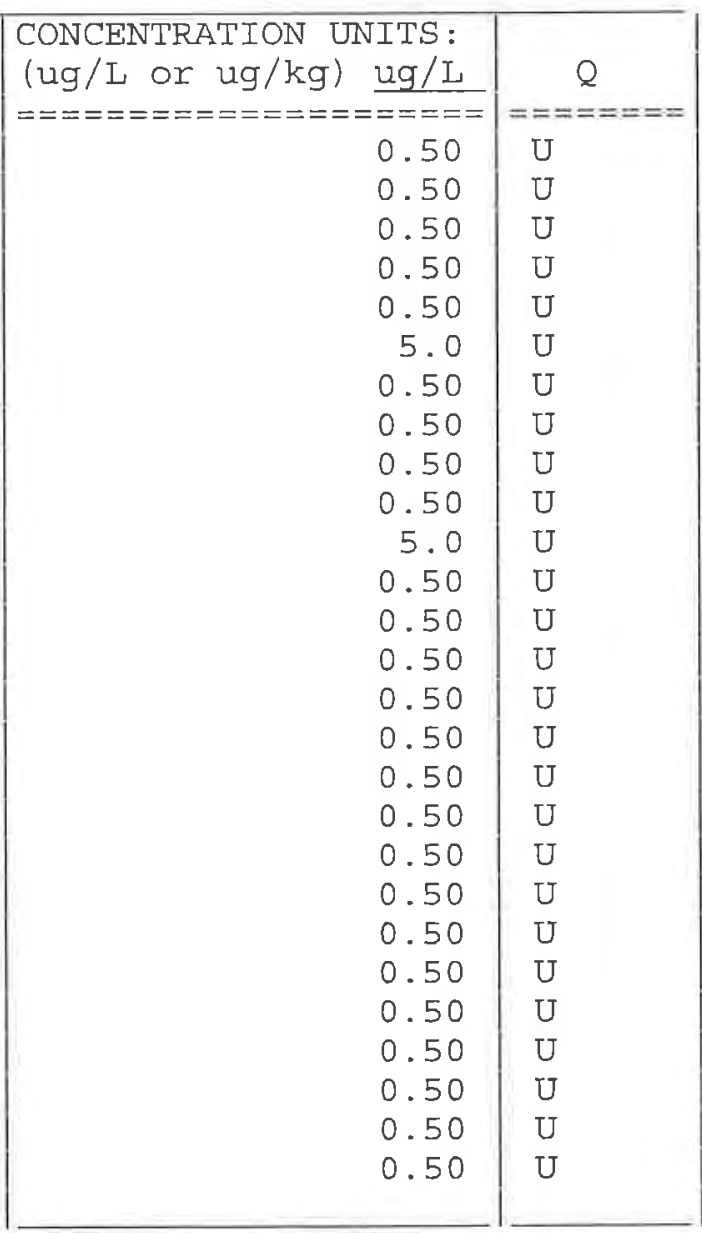


IJ - FORM I VOA-TIC

VOLATILE ORGANICS ANALYSIS DATA SHEET

TENTATIVELY IDENTIFIED COMPOUNDS
EPA SAMPLE NO.

VIBLKJI
Lab Name: TESTAMERICA BURLINGTON

Lab Code: STLV Case No.: SAVANNAH

Matrix: (SOIL/SED/WATER) Water

Sample wt/vol: $25.0 \quad(\mathrm{~g} / \mathrm{mL}) \mathrm{mL}$

Level: (TRACE or LOW/MED) TRACE

\% Moisture: not dec.

GC Column: DB-624

ID : 0.20

Soil Extract Volume:

CONCENTRATION UNITS: (ug/L or ug/kg) ug/L
Contract: 21005

Mod. Ref No.:

SDG NO.: 137933
Lab sample ID: VIBLKJL

Lab File ID: JBEBO5C

Date Received:

Date Analyzed: 06/30/2010

(mm) Dilution Factor: 1.0

(uL) Soil Aliquot Volume:

(uL)

Purge Volume: 25.0

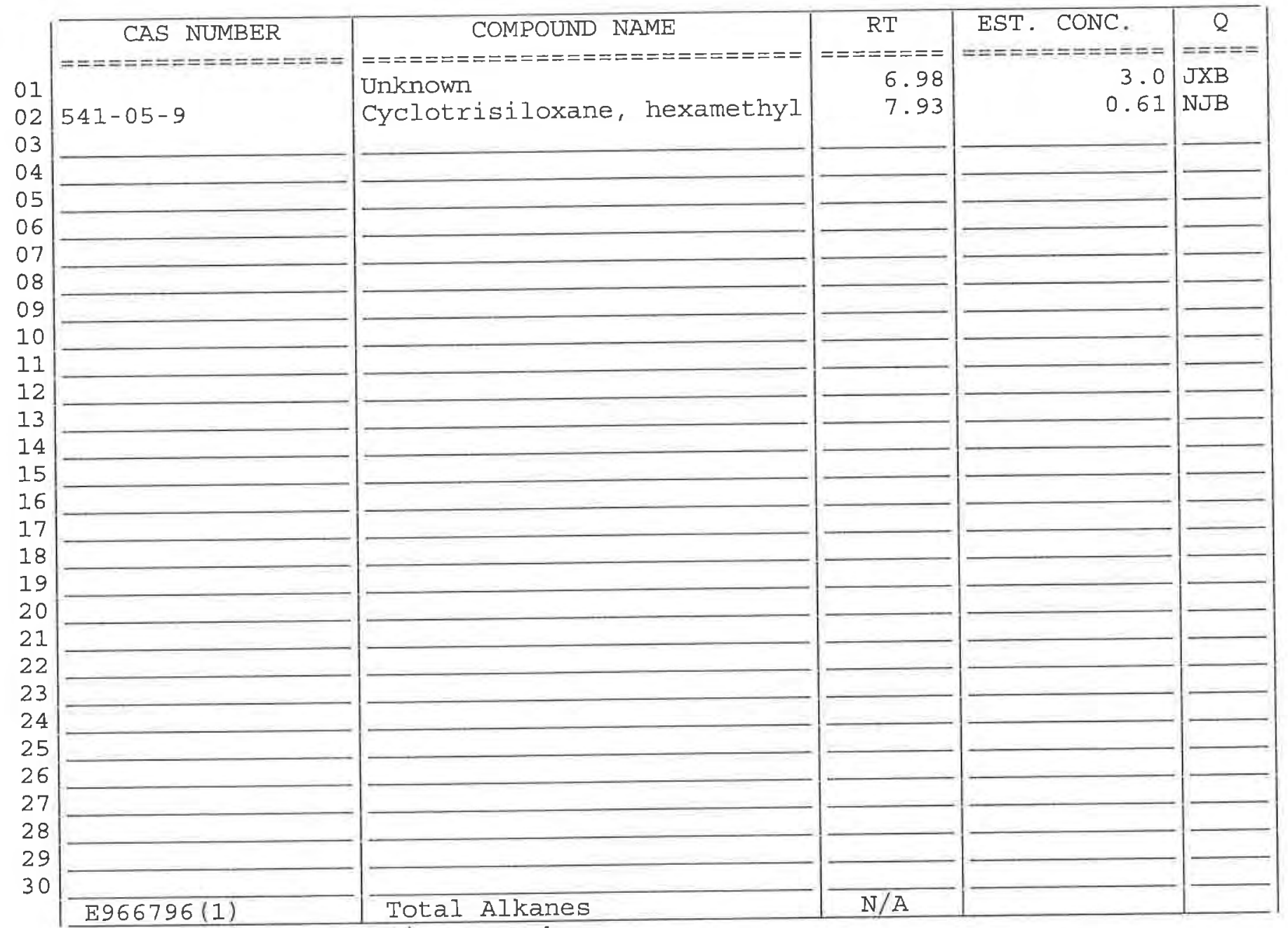

(1) EPA-designated Registry Number. 
$2 A$ - FORM II VOA-1

WATER VOLATILE DEUTERATED MONITORING COMPOUND RECOVERY

Lab Name: TESTAMERICA BURLINGTON

Contract: 21005

Lab Code: STLV Case No.: SAVANNAH Mod. Ref No.:

SDG No.: 137933

Level: (TRACE or LOW) TRACE

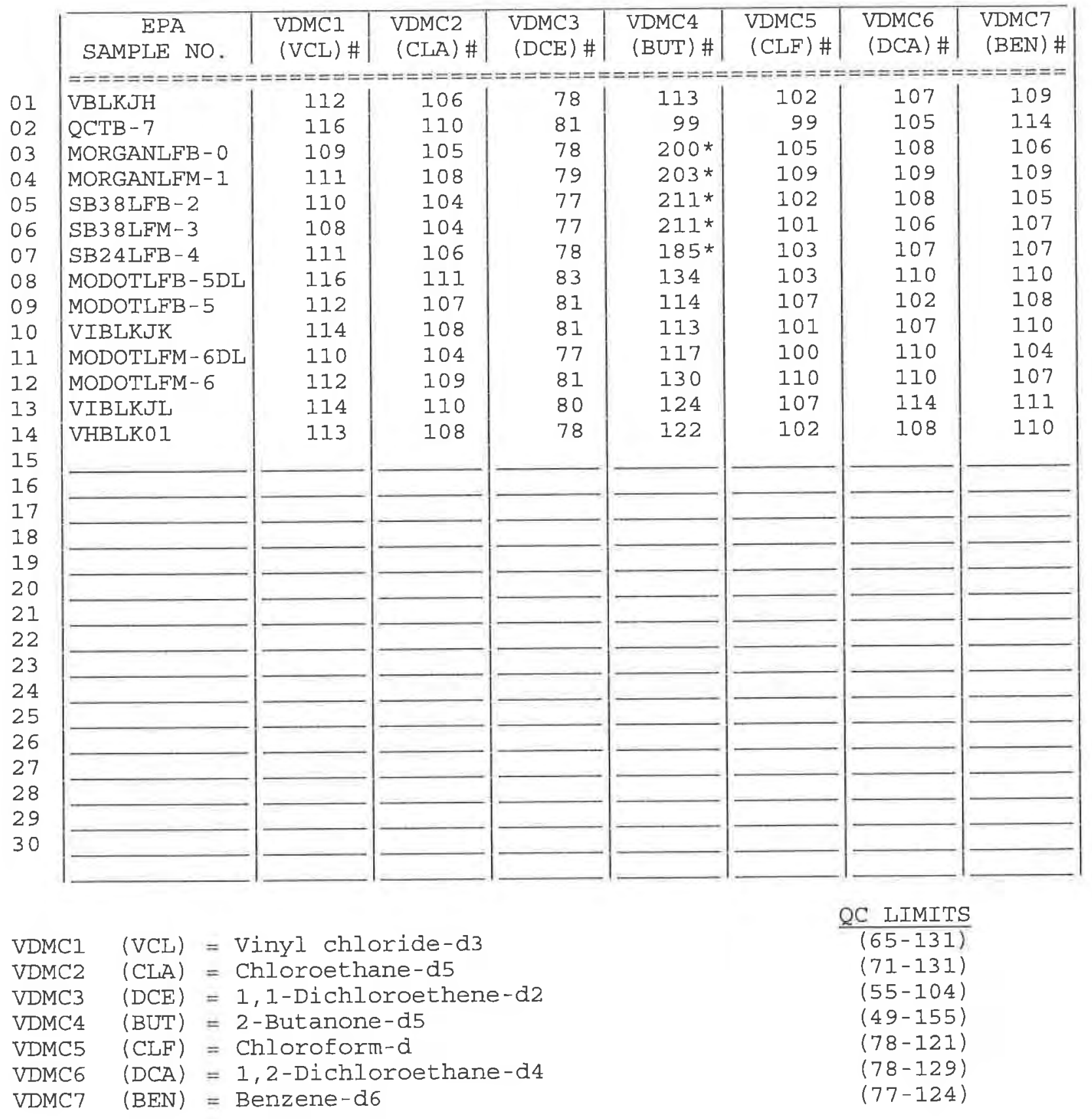

\# Column to be used to flag recovery values

* Values outside of contract required QC limits Page 1 of 2 
$2 B$ - FORM II $V O A-2$

WATER VOLATILE DEUTERATED MONITORING COMPOUND RECOVERY

Lab Name: TESTAMERICA BURLINGTON

Contract: 21005

Lab Code: STLV Case No.: SAVANNAH Mod, Ref No.:

SDG No.: 137933

Level: (TRACE or LOW) TRACE

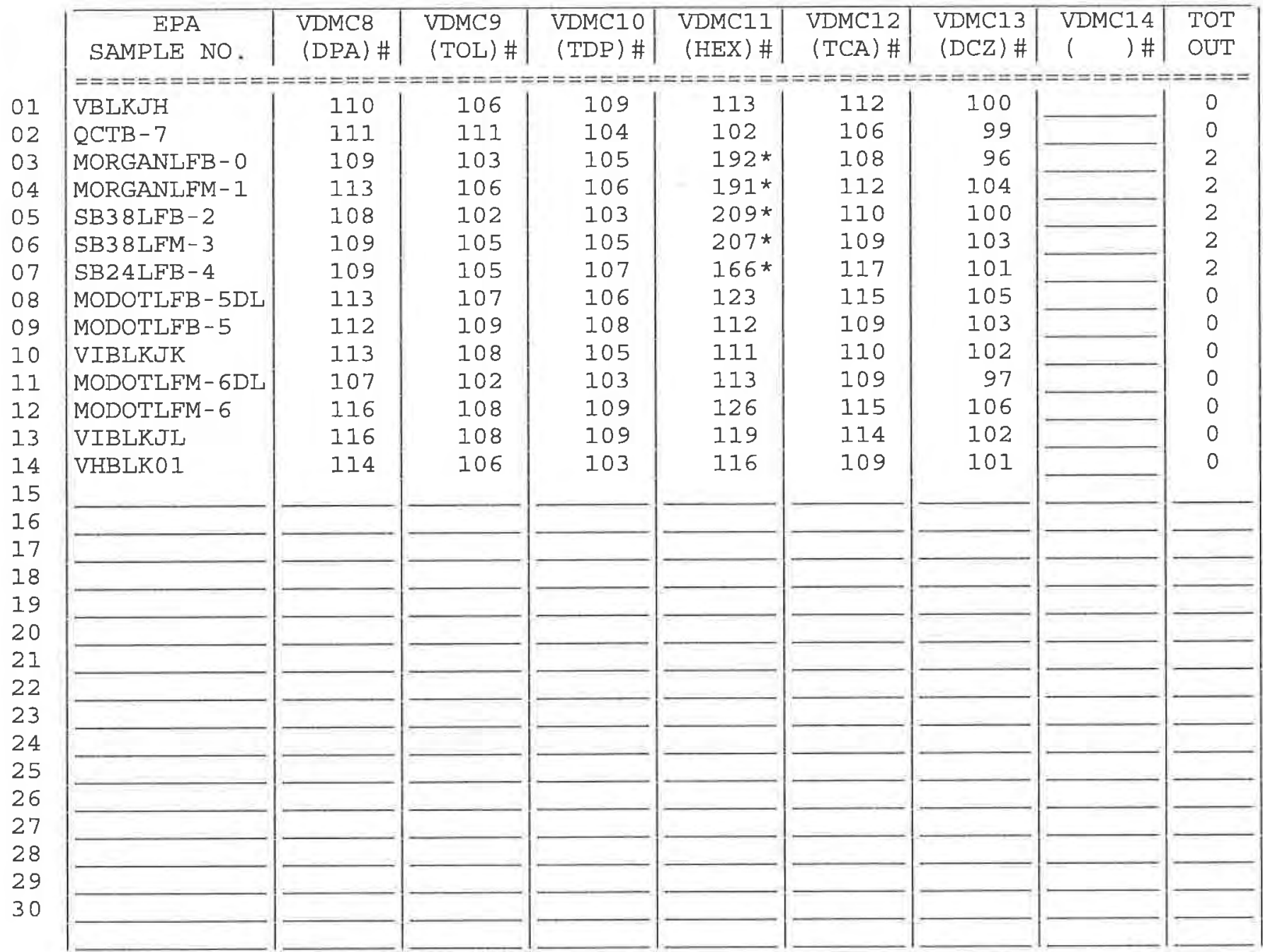

VDMC8 $(D P A)=1,2-$ Dichloropropane-d 6

VDMC9 $($ TOL $)=$ Toluene- $d 8$

VDMC10 $($ TDP $)=$ trans $-1,3-\mathrm{Dichloropropene}-\mathrm{d} 4$

VDMC11 (HEX) $=2$-Hexanone-d5

VDMC12 $(T C A)=1,1,2,2-$ Tetrachloroethane-d2

VDMC13 $(D C Z)=1,2-$ Dichlorobenzene- $\mathrm{d} 4$
QC LIMITS

$(79-124)$

$(77-121)$

$(73-121)$

$(28-135)$

$(73-125)$

$(80-131)$

\# Column to be used to flag recovery values

* Values outside of contract required QC limits

Report 1,4-Dioxane-d8 for Low-Medium VOA analysis only Page 2 of 2 
$4 A$ - FORM IV VOA

VOLATILE METHOD BLANK SUMMARY
EPA SAMPLE NO.

VBLKJH

Contract: 21005
Lab Name: TESTAMERICA BURIINGTON

Case No.: SAVANNAH Mod. Ref No.:
SDG No.: 137933
Lab File ID: JBEBO3C

Instrument ID: J.i

Matrix: (SOIL/SED/WATER) Water

Level: (TRACE or LOW/MED) TRACE

GC Column: DB-624
ID: 0.20
Lab Sample ID: VBLKJH

Date Analyzed: 06/30/2010

Time Analyzed: 0721

(mm) Heated Purge: (Y/N) N

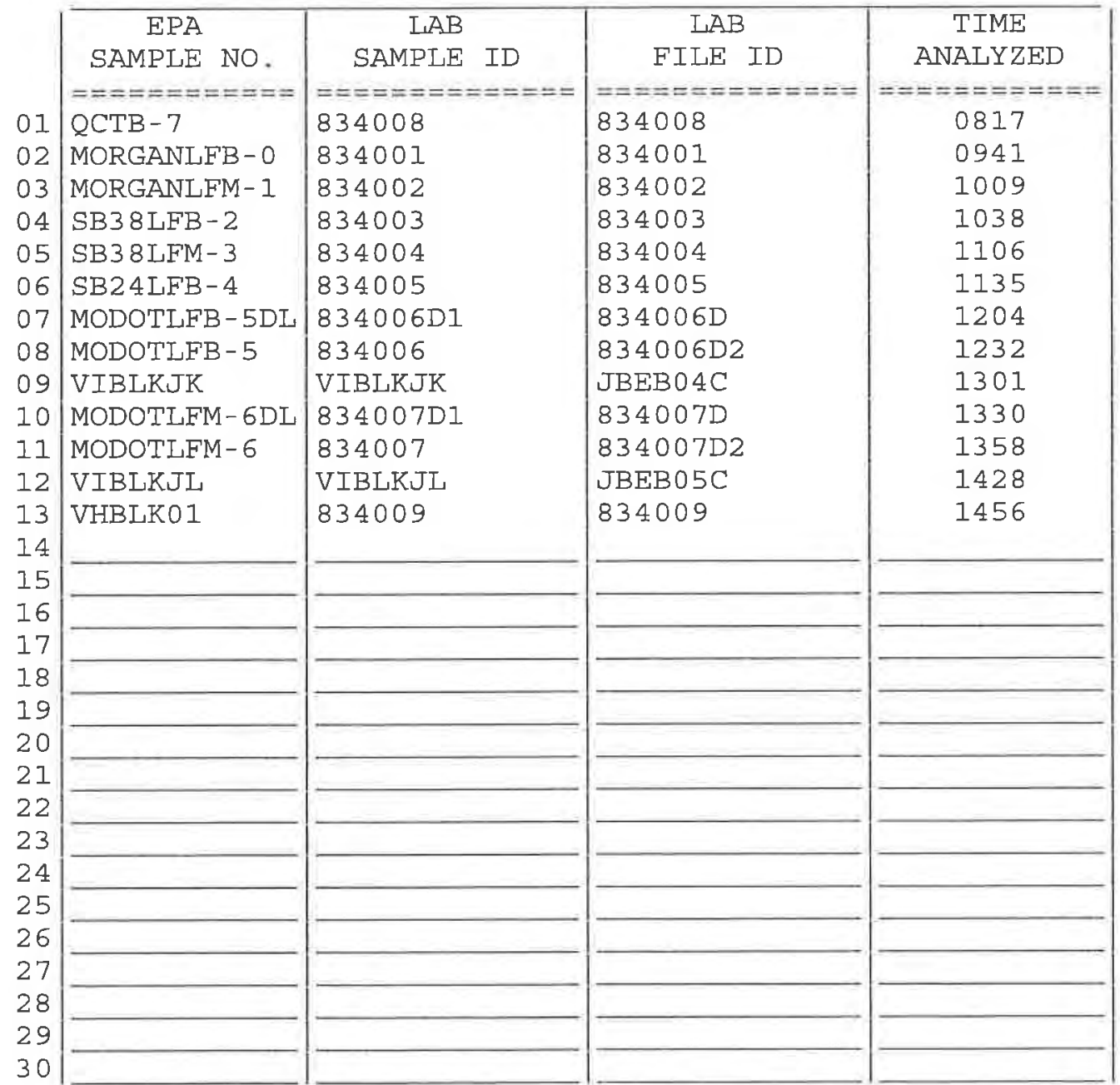

COMMENTS : 
$5 A$ - FORM V VOA

VOLATILE ORGANIC INSTRUMENT

PERFORMANCE CHECK

BROMOFLUOROBENZENE (BFB)
EPA SAMPLE NO.

BFBJE

Contract: 21005

Lab Name: TESTAMERICA BURLINGTON

Case No.: SAVANNAH Mod. Ref No.:

SDG No.: 137933

BFB Injection Date: 06/25/2010

Lab File ID: JBE02PV

BFB Injection Time: 1015

Instrument ID: J.i

ID $: 0.20 \quad(\mathrm{~mm})$

GC Column: DB-624

\begin{tabular}{|c|c|c|}
\hline $\begin{array}{c}\mathrm{m} / \mathrm{e} \\
===== \\
50\end{array}$ & 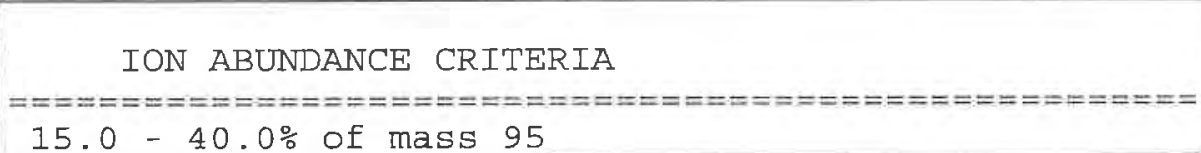 & 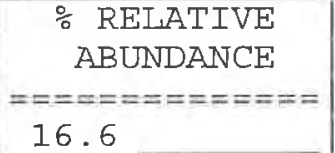 \\
\hline 75 & $30.0-80.0 \%$ of mass 95 & 48.1 \\
\hline 95 & Base Peak, $100 \%$ relative abundance & 100.0 \\
\hline 96 & $5.0-9.0 \%$ of mass 95 & 6.0 \\
\hline 173 & Less than $2.0 \%$ of mass 174 & 0.01 \\
\hline 174 & $50.0-120.0 \%$ of mass 95 & 67.2 \\
\hline 175 & $5.0-9.0 \%$ of mass 174 & 5.81 \\
\hline 176 & $95.0-101.0 \%$ of mass 174 & $66.4(98.8) 1$ \\
\hline 177 & $5.0-9.0 \%$ of mass 176 & 4.01 \\
\hline
\end{tabular}

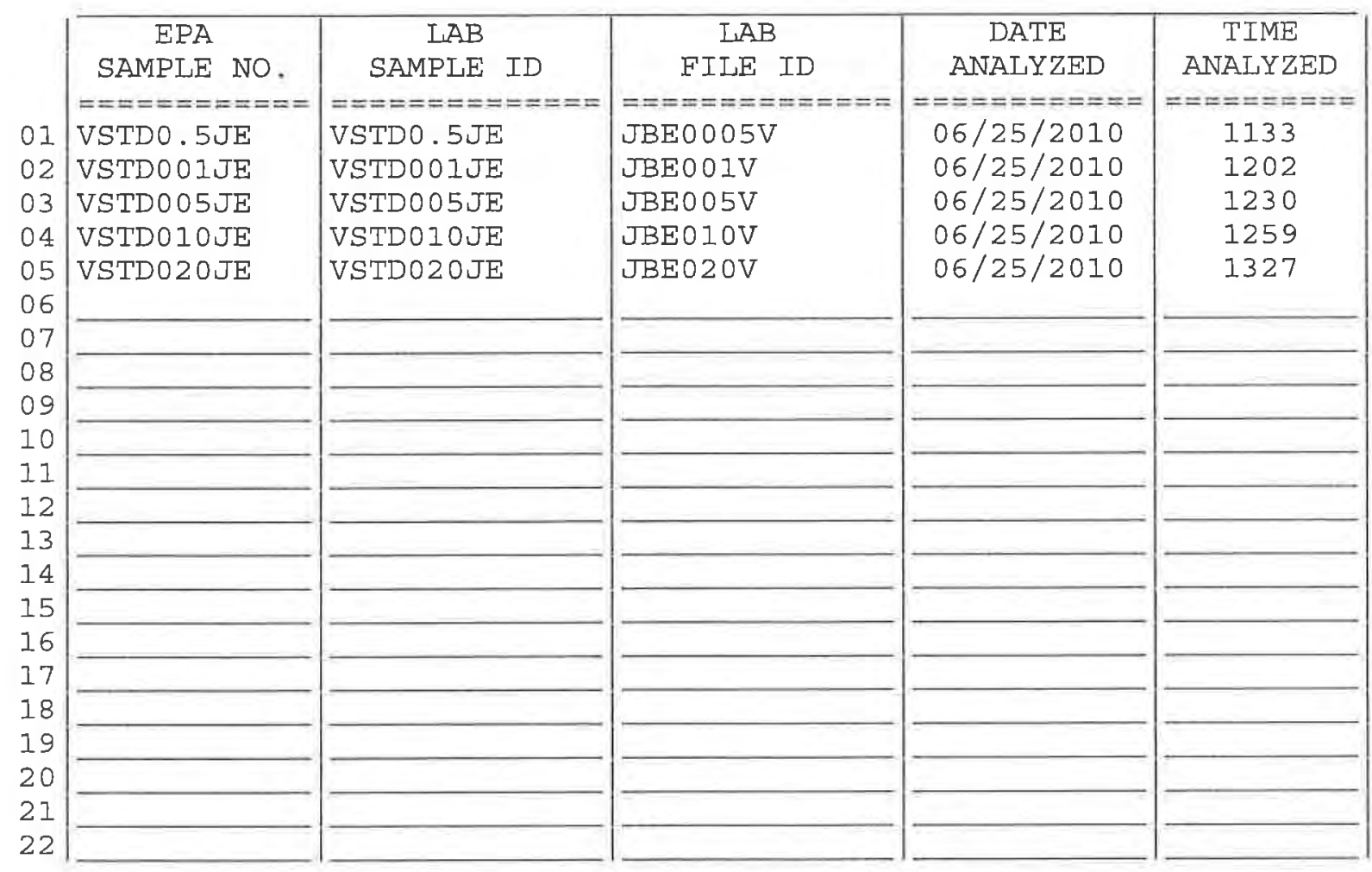


$5 A$ - FORM V VOA

VOLATILE ORGANIC INSTRUMENT

PERFORMANCE CHECK

BROMOFLUOROBENZENE (BFB)
EPA SAMPLE NO.

Contract: 21005
Lab Name: TESTAMERICA BURLINGTON

Lab Code: STLV Case No.: SAVANNAH Mod. Ref No.:
BFBJH

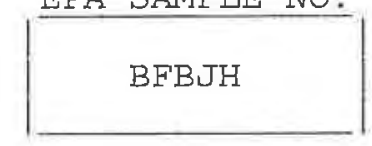

SDG NO.: 137933

BFB Injection Date: 06/30/2010

Lab File ID: JBE08PV

BFB Injection Time: 0535

Instrument ID: J.i

ID $: 0.20 \quad(\mathrm{~mm})$

GC Column: DB-624

ID: .20.

\begin{tabular}{|c|c|c|}
\hline $\begin{array}{c}\mathrm{m} / \mathrm{e} \\
===== \\
50\end{array}$ & 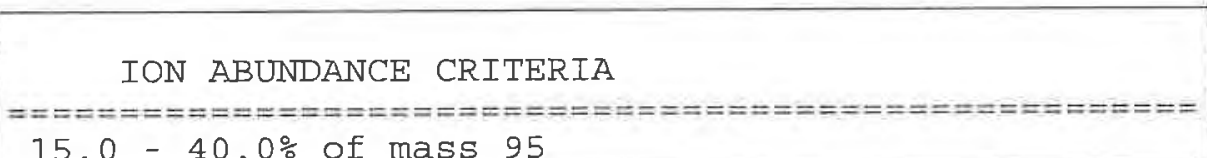 & $\begin{array}{c}\text { \% RELATIVE } \\
\text { ABUNDANCE } \\
============= \\
17.5\end{array}$ \\
\hline 75 & $30.0-80.0 \%$ of mass 95 & 48.1 \\
\hline 95 & Base Peak, $100 \%$ relative abundance & 100.0 \\
\hline 96 & $5.0-9.0 \%$ of mass 95 & 6.4 \\
\hline 173 & Less than $2.0 \%$ of mass 174 & $0.3 \bar{l}$ \\
\hline 174 & $50.0-120.0 \%$ of mass 95 & 61.4 \\
\hline 175 & $5.0-9.0 \%$ of mass 174 & $5 . 1 \longdiv { ( 8 . 3 ) 1 }$ \\
\hline 176 & $95.0-101.0 \%$ of mass 174 & $60.1(97.9) 1$ \\
\hline 177 & $5.0-9.0 \%$ of mass 176 & $3.8(6.4) 2$ \\
\hline
\end{tabular}

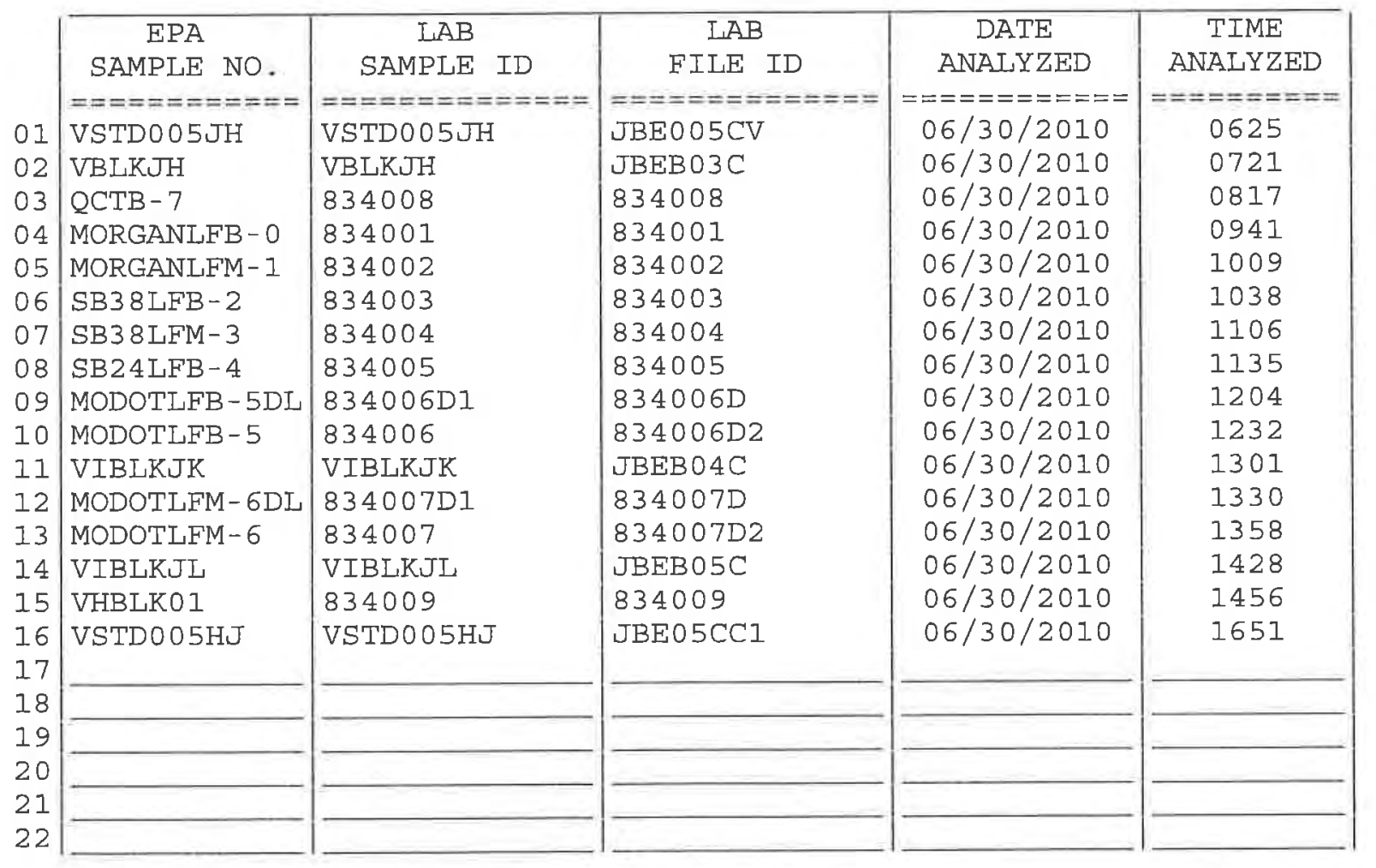


$6 \mathrm{~A}$ - FORM VI VOA-1

VOLATILE ORGANICS INITIAL CALIBRATION DATA

Lab Name: TESTAMERICA BURIINGTON

Lab Code: STLV Case No.: SAVANNAH Mod. Ref No.:
Heated Purge: $(Y / N) N$

Purge Volume: 25.0

GC Column: DB-624
Calibration Date(s): 06/25/2010 06/25/2010

Calibration Time(s): $1133 \quad 1327$
Contract: 21005

SDG No.: 137933

(mL)

ID $: 0.20$ (mm) Length: $25 \quad$ (m)

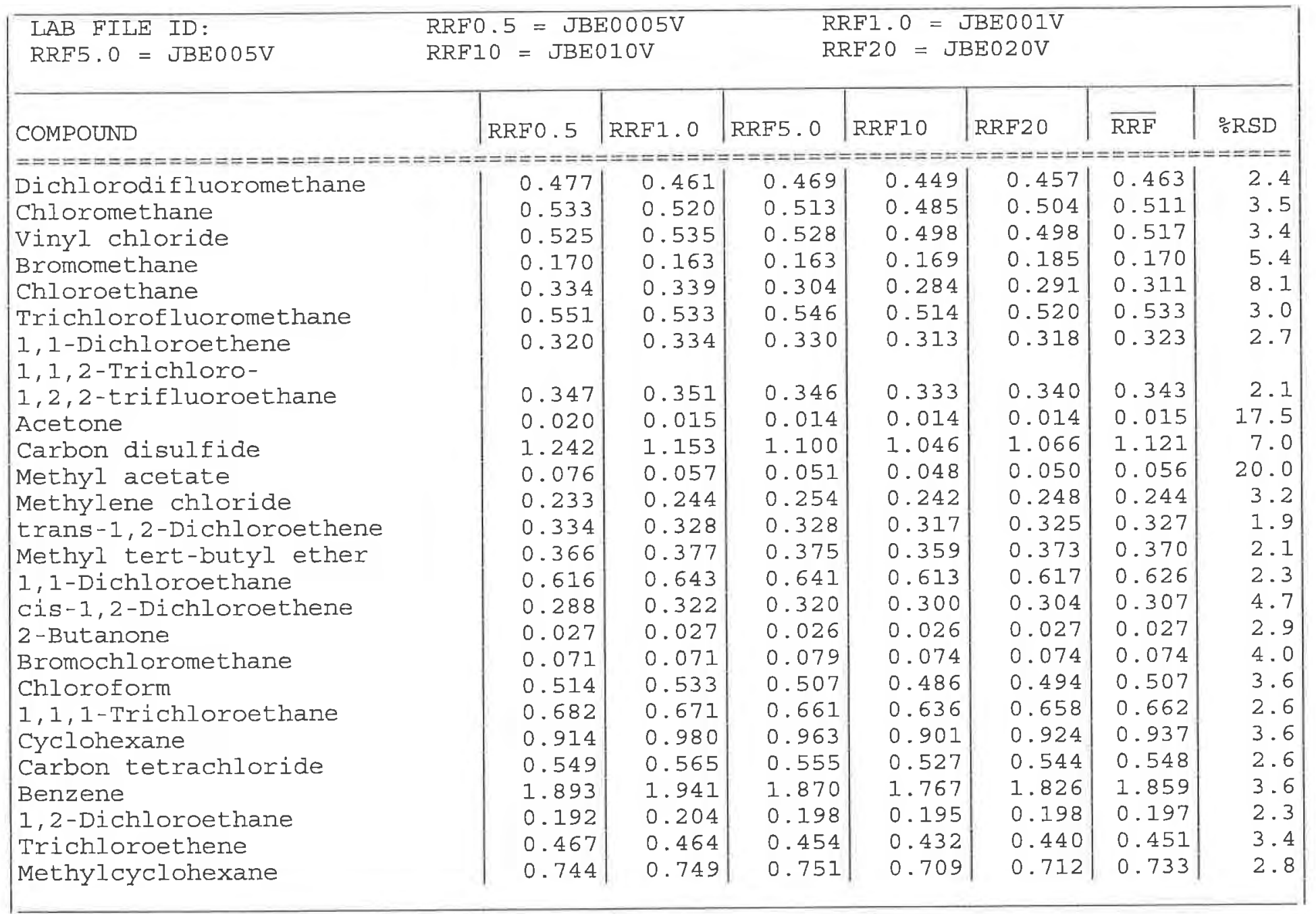

Report 1,4-Dioxane for Low-Medium VOA analysis only 
$6 \mathrm{~B}$ - FORM VI VOA-2

VOLATILE ORGANICS INITIAL CALIBRATION DATA

Lab Name: TESTAMERICA BURLINGTON

Lab Code: STLV Case No.: SAVANNAH Mod. Ref No.:
SDG No. : 137933

Calibration Date(s): 06/25/2010 06/25/2010

Calibration Time(s): $1133 \quad 1327$

(mL)

Purge Volume: 25.0

GC Column: DB-624 ID: 0.20 (mm) Length: 25 (m)

\begin{tabular}{|c|c|c|c|c|c|c|c|}
\hline $\begin{array}{l}\text { LAAB FILE ID: } \\
\text { RRF5.0 = JBE005V }\end{array}$ & $\begin{array}{l}0.5=\mathrm{JBE} \\
10=\mathrm{JBEC}\end{array}$ & $\begin{array}{l}80005 \mathrm{~V} \\
10 \mathrm{~V}\end{array}$ & & $\begin{array}{l}\mathrm{RF1.0}=\mathrm{L} \\
\mathrm{RF} 20=\mathrm{JE}\end{array}$ & $\begin{array}{l}\mathrm{JBEO01V} \\
\mathrm{BE0} 20 \mathrm{~V}\end{array}$ & & \\
\hline COMPOUND & RRF0. 5 & RRF1.0 & RRF5. 0 & RRF10 & RRF20 & $\overline{R R F}$ & $\because \mathrm{RSD}$ \\
\hline 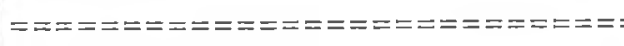 & $======$ & $=====$ & $====-=$ & $=======$ & $======$ & $==== \pm=$ & $====$ \\
\hline 1,2-Dichloropropane & 0.381 & 0.401 & 0.384 & 0.366 & 0.376 & 0.382 & 3.4 \\
\hline Bromodichloromethane & 0.368 & 0.396 & 0.391 & 0.372 & 0.389 & 0.383 & 3.2 \\
\hline cis-1,3-Dichloropropene & 0.481 & 0.516 & 0.511 & 0.491 & 0.504 & 0.501 & 2.9 \\
\hline 4-Methyl-2-pentanone & 0.084 & 0.085 & 0.088 & 0.087 & 0.091 & 0.087 & 3.5 \\
\hline Toluene & 1.916 & 1.972 & 1.949 & 1.828 & 1.908 & 1.915 & 2.9 \\
\hline trans-1,3-Dichloropropene & 0.345 & 0.354 & 0.350 & 0.331 & 0.350 & 0.346 & 2.6 \\
\hline $1,1,2-$ Trichloroethane & 0.167 & 0.144 & 0.161 & 0.144 & 0.147 & 0.153 & 7.0 \\
\hline Tetrachloroethene & 0.343 & 0.333 & 0.329 & 0.310 & 0.321 & 0.327 & 3.8 \\
\hline 2 -Hexanone & 0.056 & 0.055 & 0.057 & 0.056 & 0.059 & 0.057 & 3.1 \\
\hline Dibromochloromethane & 0.182 & 0.171 & 0.170 & 0.166 & 0.173 & 0.172 & 3.5 \\
\hline 1,2-Dibromoethane & 0.123 & 0.128 & 0.127 & 0.123 & 0.130 & 0.126 & 2.5 \\
\hline Chlorobenzene & 0.991 & 0.983 & 0.982 & 0.947 & 0.985 & 0.978 & 1.8 \\
\hline Ethylbenzene & 2.119 & 2.211 & 2.186 & 2.096 & 2.180 & 2.159 & 2.3 \\
\hline o-xylene & 0.705 & 0.707 & 0.722 & 0.684 & 0.724 & 0.709 & 2.3 \\
\hline $\mathrm{m}, \mathrm{p}-\mathrm{xy}$ lene & 0.769 & 0.810 & 0.790 & 0.767 & 0.805 & 0.788 & 2.5 \\
\hline styrene & 0.963 & 1.035 & 1.045 & 1.022 & 1.066 & 1.026 & 3.8 \\
\hline Bromoform & 0.167 & 0.176 & 0.177 & 0.175 & 0.174 & 0.174 & 2.4 \\
\hline Isopropylbenzene & 2.048 & 2.039 & 2.105 & 2.027 & 2.123 & 2.068 & 2.1 \\
\hline $1,1,2,2$-Tetrachloroethane & 0.133 & 0.145 & 0.147 & 0.135 & 0.144 & 0.141 & 4.6 \\
\hline 1,3-Dichlorobenzene & 1.613 & 1.586 & 1.588 & 1.548 & 1.604 & 1.588 & 1.6 \\
\hline 1,4-Dichlorobenzene & I. 527 & 1.528 & 1.521 & 1.467 & 1.504 & 1.509 & 1.7 \\
\hline 1,2-Dichlorobenzene & 1.114 & 1.162 & 1.162 & 1.118 & 1.155 & 1.142 & 2.1 \\
\hline 1,2-Dibromo-3-chloropropane & 0.042 & 0.044 & 0.048 & 0.046 & 0.048 & 0.046 & 5.8 \\
\hline $1,2,4$-Trichlorobenzene & 0.651 & 0.713 & 0.676 & 0.685 & 0.698 & 0.685 & 3.4 \\
\hline 1,2,3-Trichlorobenzene & 0.427 & 0.481 & 0.491 & 0.473 & 0.503 & 0.475 & 6.2 \\
\hline
\end{tabular}


$6 C$ - FORM VI VOA-3

VOLATILE ORGANICS INITIAL CALIBRATION DATA

Lab Name: TESTAMERICA BURLINGTON

Lab Code: STLV Case No.: SAVANNAH Mod. Ref No.:

Instrument ID: J.i

Heated Purge: $(\mathrm{Y} / \mathrm{N}) \mathrm{N}$

Purge Volume: 25.0

GC Column: DB-624

(mL)
Contract: 21005

Calibration Date(s): 06/25/2010 06/25/2010

Calibration Time(s): $1133 \quad 1327$

ID $: 0.20 \quad(\mathrm{~mm})$ Length: $25 \quad(\mathrm{~m})$

\begin{tabular}{|c|c|c|c|c|c|c|c|}
\hline $\begin{array}{l}\text { IAB FILE ID: } \\
\text { RRF5.0 = JBE005V }\end{array}$ & $\begin{array}{l}.5=\mathrm{JB} \\
0=\mathrm{JBE}\end{array}$ & $\begin{array}{l}\text { E0005V } \\
010 \mathrm{~V}\end{array}$ & & $\begin{array}{l}\mathrm{RF} 1.0= \\
\mathrm{RF} 20=\mathrm{J}\end{array}$ & $\begin{array}{l}\text { JBE001V } \\
3 E 020 \mathrm{~V}\end{array}$ & & \\
\hline COMPOUND & RRF0. 5 & RRF1. 0 & RRF5. 0 & RRF10 & RRF 20 & $\overline{\mathrm{RRF}}$ & $\%$ RSD \\
\hline 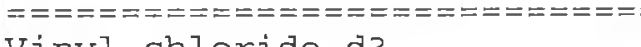 & & -1 & $====$ & $=====$ & $======$ & $====$ & \\
\hline $\begin{array}{l}\text { Vinyl chloride-c } \\
\text { Chloroethane-d5 }\end{array}$ & $\begin{array}{l}0.420 \\
0.332\end{array}$ & $\begin{array}{l}0.434 \\
0.361\end{array}$ & $\begin{array}{l}0.441 \\
0.350\end{array}$ & $\begin{array}{l}0.419 \\
0.332\end{array}$ & $\begin{array}{l}0.425 \\
0.331\end{array}$ & $\begin{array}{l}0.428 \\
0.341\end{array}$ & $\begin{array}{l}2.2 \\
4.0\end{array}$ \\
\hline 1,1 -Dichloroethene-d2 & 0.705 & 0.737 & 0.733 & 0.692 & 0.703 & 0.714 & 2.8 \\
\hline 2-Butanone-d5 & 0.022 & 0.023 & 0.025 & 0.025 & 0.027 & 0.024 & 7.2 \\
\hline Chloroform-d & 0.523 & 0.528 & 0.533 & 0.510 & 0.517 & 0.522 & 1.7 \\
\hline 1,2-Dichloroethane-d4 & 0.153 & 0.164 & 0.159 & 0.152 & 0.158 & 0.158 & 3.0 \\
\hline Benzene-d6 & 1.700 & 1.750 & 1.729 & 1.643 & 1.685 & 1.701 & 2.4 \\
\hline 1,2-Dichloropropane-d6 & 0.413 & 0.455 & 0.444 & 0.413 & 0.425 & 0.430 & 4.4 \\
\hline Toluene-d8 & 1.538 & 1.566 & 1.563 & 1.487 & 1.530 & 1.537 & 2.1 \\
\hline trans-1,3-Dichloropropene-d4 & 0.303 & 0.304 & 0.301 & 0.292 & 0.306 & 0.301 & 1.9 \\
\hline 2 -Hexanone-d5 & 0.027 & 0.025 & 0.030 & 0.029 & 0.031 & 0.028 & 8.1 \\
\hline $1,1,2,2$-Tetrachloroethane-d2 & 0.131 & 0.142 & 0.138 & 0.133 & 0.141 & 0.137 & 3.7 \\
\hline 1,2-Dichlorobenzene-c & 0.709 & 0.743 & 0.739 & 0.684 & 0.711 & 0.717 & 3.4 \\
\hline
\end{tabular}

Report 1,4-Dioxane-d8 for Low-Medium VOA analysis only 
$7 A$ - FORM VII VOA-1

VOLATILE CONTINUING CALIBRATION DATA

Lab Name: TESTAMERICA BURLINGTON

Lab Code: STLV Case No.: SAVANNAH Mod. Ref No.:
Contract: 21005

Calibration Date: 06/30/2010 Time: 0625

Init. Calib. Date(s): 06/25/2010 06/25/2010

EPA Sample No. (VSTD\#\#\#\#\#): VSTD005JH Init. Calib. Time(s): 1133

Heated Purge: (Y/N)N GC Column: DB-624 ID: 0.20 (mm) Length: 25

$(\mathrm{m})$

Purge Volume: 25.0

$(\mathrm{mL})$

\begin{tabular}{|c|c|c|c|c|c|}
\hline COMPOUND & $\widehat{\mathrm{RRF}}$ & RRF5. 0 & $\begin{array}{l}\text { MIN } \\
\text { RRF }\end{array}$ & $\div \mathrm{D}$ & MAX $\div D$ \\
\hline \multicolumn{6}{|c|}{ 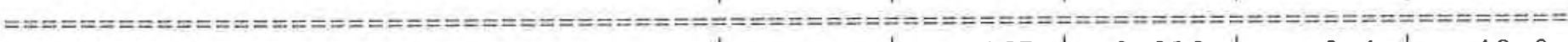 } \\
\hline Dichlorodifluoromethane & 0.463 & 0.465 & 0.010 & 0.4 & 40.0 \\
\hline Chloromethane & 0.511 & 0.535 & 0.010 & 4.7 & 40.0 \\
\hline Vinyl chloride & 0.517 & 0.544 & 0.100 & 5.2 & 30.0 \\
\hline Bromomethane & 0.170 & 0.189 & 0.100 & 11.1 & 30.0 \\
\hline Chloroethane & 0.311 & 0.314 & 0.010 & 1.1 & 40.0 \\
\hline Trichlorofluoromethane & 0.533 & 0.511 & 0.010 & $-4 \cdot 1$ & 40.0 \\
\hline 1,1-Dichloroethene & 0.323 & 0.305 & 0.100 & -5.4 & 30.0 \\
\hline 1,1,2-Trichloro-1,2,2-trifluoroethane & 0.343 & 0.339 & 0.010 & -1.3 & 40.0 \\
\hline Acetone & 0.015 & 0.015 & 0.010 & -5.5 & 40.0 \\
\hline Carbon disulfide & 1.121 & 1.061 & 0.010 & -5.4 & 40.0 \\
\hline Methyl acetate & 0.056 & 0.052 & 0.010 & -7.3 & 40.0 \\
\hline Methylene chloride & 0.244 & 0.245 & 0.010 & 0.4 & 40.0 \\
\hline trans-1,2-Dichloroethene & 0.327 & 0.328 & 0.010 & 0.5 & 40.0 \\
\hline Methyl tert-butyl ether & 0.370 & 0.373 & 0.010 & 0.7 & 40.0 \\
\hline 1,1-Dichloroethane & 0.626 & 0.652 & 0.200 & 4.1 & 30.0 \\
\hline cis-1,2-Dichloroethene & 0.307 & 0.310 & 0.010 & 1.1 & 40.0 \\
\hline 2-Butanone & 0.027 & 0.030 & 0.010 & 10.9 & 40.0 \\
\hline Bromochloromethane & 0.074 & 0.075 & 0.050 & 1. 2 & 30.0 \\
\hline Chloroform & 0.507 & 0.508 & 0.200 & 0.1 & 30.0 \\
\hline $1,1,1$-Trichloroethane & 0.662 & 0.667 & 0.100 & 0.8 & 30.0 \\
\hline Cyclohexane & 0.937 & 0.995 & 0.010 & 6.2 & 40.0 \\
\hline Carbon tetrachloride & 0.548 & 0.544 & 0.100 & -0.6 & 30.0 \\
\hline Benzene & 1.859 & 1.926 & 0.400 & 3.6 & 30.0 \\
\hline 1,2-Dichloroethane & 0.197 & 0.208 & 0.100 & 5.4 & 30.0 \\
\hline Trichloroethene & 0.451 & 0.457 & 0.300 & 1.1 & 30.0 \\
\hline Methylcyclohexane & 0.733 & 0.780 & 0.010 & 6.4 & 40.0 \\
\hline
\end{tabular}

Report 1,4-Dioxane for Low-Medium VOA analysis only 
$7 B$ - FORM VII VOA-2

VOLATILE CONTINUING CAIIBRATION DATA

Lab Name: TESTAMERICA BURLINGTON

Contract: 21005

Lab Code: STLV Case No.: SAVANNAH Mod. Ref No.:

SDG No. : 137933

Instrument ID: J.i

Calibration Date: 06/30/2010 Time: 0625

Lab File ID: JBE005CV

Init. Calib. Date(s): 06/25/2010 06/25/2010

EPA Sample No. (VSTD\#\#\#\#) : VSTD005JH Init. Calib. Time(s): 11331327

Heated Purge: ( $Y / N) N$ GC Column: DB-624 ID: 0.20 (mm) Length: 25 (m)

Purge Volume: 25.0

(mL)

\begin{tabular}{|c|c|c|c|c|c|}
\hline COMPOUND & $\overline{R R F}$ & RRF5.0 & $\begin{array}{l}\text { MIN } \\
\text { RRF }\end{array}$ & $\% \mathrm{D}$ & MAX $\div D$ \\
\hline \multicolumn{6}{|c|}{ 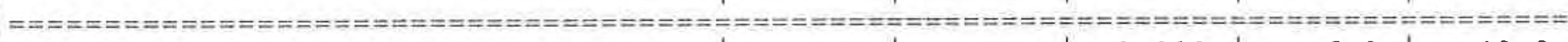 } \\
\hline 1,2-Dichloropropane & 0.382 & 0.404 & 0.010 & 6.0 & 40.0 \\
\hline Bromodichloromethane & 0.383 & 0.386 & 0.200 & 0.7 & 30.0 \\
\hline cis-1,3-Dichloropropene & 0.501 & 0.520 & 0.200 & 3.9 & 30.0 \\
\hline 4-Methyl-2-pentanone & 0.087 & 0.098 & 0.010 & 12.3 & 40.0 \\
\hline Toluene & 1.915 & 1.950 & 0.400 & 1.8 & 30.0 \\
\hline trans-1,3-Dichloropropene & 0.346 & 0.345 & 0.100 & -0.2 & 30.0 \\
\hline 1,1,2-Trichloroethane & 0.153 & 0.156 & 0.100 & 2.2 & 30.0 \\
\hline Tetrachloroethene & 0.327 & 0.308 & 0.100 & -6.0 & 30.0 \\
\hline 2-Hexanone & 0.057 & 0.062 & 0.010 & 8.9 & 40.0 \\
\hline Dibromochloromethane & 0.172 & 0.164 & 0.100 & -4.8 & 30.0 \\
\hline 1,2-Dibromoethane & 0.126 & 0.127 & 0.010 & 0.6 & 30.0 \\
\hline Chlorobenzene & 0.978 & 0.979 & 0.500 & 0.1 & 30.0 \\
\hline Ethylbenzene & 2.159 & 2.213 & 0.100 & 2.5 & 30.0 \\
\hline o-Xylene & 0.709 & 0.718 & 0.300 & 1.3 & 30.0 \\
\hline $\mathrm{m}, \mathrm{p}$-xylene & 0.788 & 0.807 & 0.300 & 2.4 & 30.0 \\
\hline styrene & 1.026 & 1.023 & 0.300 & -0.3 & 30.0 \\
\hline Bromoform & 0.174 & 0.169 & 0.050 & -2.8 & 30.0 \\
\hline Isopropylbenzene & 2.068 & 2.106 & 0.010 & 1.8 & 40.0 \\
\hline $1,1,2,2$-Tetrachloroethane & 0.141 & 0.146 & 0.100 & 3.4 & 30.0 \\
\hline 1,3-Dichlorobenzene & 1.588 & 1.612 & 0.400 & 1.5 & 30.0 \\
\hline 1,4-Dichlorobenzene & 1.509 & 1.536 & 0.400 & 1.7 & 30.0 \\
\hline 1,2-Dichlorobenzene & 1.142 & 1.155 & 0.400 & 1.1 & 30.0 \\
\hline 1,2-Dibromo-3-chloropropane & 0.046 & 0.051 & 0.010 & 11.3 & 40.0 \\
\hline $1,2,4$-Trichlorobenzene & 0.685 & 0.663 & 0.200 & -3.2 & 30.0 \\
\hline $1,2,3$-Trichlorobenzene & 0.475 & 0.477 & 0.200 & 0.5 & 30.0 \\
\hline
\end{tabular}


$7 C$ - FORM VII VOA-3

VOLATILE CONTINUING CALIBRATION DATA

Lab Name: TESTAMERICA BURLINGTON

Contract: 21005

Lab Code: STLV Case No.: SAVANNAH Mod. Ref No.:

SDG No.: 137933

Instrument ID: J.i

Calibration Date: 06/30/2010 Time: 0625

Lab File ID: JBE005CV

Init. Calib. Date (s): 06/25/2010 06/25/2010

EPA Sample No. (VSTD\#\#\#\#\#): VSTD005JH

Init. Calib. Time (s): $1133 \quad 1327$

Heated Purge: (Y/N)N GC Column: DB-624 ID: 0.20 (mm) Length: 25

$(\mathrm{m})$

Purge Volume: 25.0

$(\mathrm{mL})$

\begin{tabular}{|c|c|c|c|c|c|}
\hline COMPOUND & RRF & RRF5. 0 & $\begin{array}{l}\text { MIN } \\
\text { RRF }\end{array}$ & $\frac{\circ D}{\partial}$ & $M A X \div D$ \\
\hline \multicolumn{6}{|c|}{ 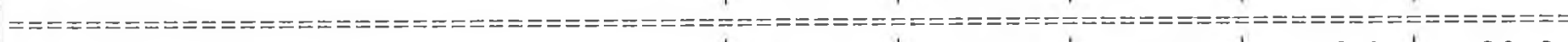 } \\
\hline Vinyl chloride-d3 & 0.428 & 0.455 & 0.010 & 6.3 & 30.0 \\
\hline Chloroethane- d5 & 0.341 & 0.342 & 0.010 & 0.3 & 40.0 \\
\hline I,I-Dichloroethene-d2 & 0.714 & 0.715 & 0.010 & 0.1 & 30.0 \\
\hline 2-Butanone-d5 & 0.024 & 0.027 & 0.010 & 12.2 & 40.0 \\
\hline Chloroform-d & 0.522 & 0.531 & 0.010 & 1.6 & 30.0 \\
\hline 1,2-Dichloroethane-d4 & 0.158 & 0.162 & 0.010 & 2.6 & 30.0 \\
\hline Benzene-d 6 & 1.701 & 1.739 & 0.010 & 2.2 & 30.0 \\
\hline 1,2 -Dichloropropane-d6 & 0.430 & 0.457 & 0.010 & 6.3 & 40.0 \\
\hline Toluene-d8 & 1.537 & 1.566 & 0.010 & 1.9 & 30.0 \\
\hline trans-1,3-Dichloropropene-d4 & 0.301 & 0.308 & 0.010 & $2 \cdot 3$ & 30.0 \\
\hline 2-Hexanone-d5 & 0.028 & 0.032 & 0.010 & 11.1 & 40.0 \\
\hline $1,1,2,2$-Tetrachloroethane-d2 & 0.137 & 0.152 & 0.010 & 10.8 & 30.0 \\
\hline 1,2 -Dichlorobenzene-d4 & 0.717 & 0.729 & 0.010 & 1.6 & 30.0 \\
\hline
\end{tabular}

Report 1,4-Dioxane-d8 for Low-Medium VOA analysis only 
$7 A$ - FORM VII VOA-1

VOLATILE CONTINUING CALIBRATION DATA

Lab Name: TESTAMERICA BURIINGTON

Contract: 21005

Lab Code: STLV Case No.: SAVANNAH Mod. Ref No.:

SDG No.: 137933

Instrument ID: J.i

Calibration Date: 06/30/2010 Time: 1651

Lab File ID: JBE05CC1

Init. Calib. Date(s): 06/25/2010 06/25/2010

EPA Sample No. (VSTD\#\#\#\#) : VSTD005HJ Init. Calib. Time(s): 11331327

Heated Purge: (Y/N)N GC Column: DB-624 ID: 0.20 (mm) Length: 25

$(\mathrm{m})$

Purge volume: 25.0

$(\mathrm{mL})$

\begin{tabular}{|c|c|c|c|c|c|}
\hline COMPOUND & $\overline{\mathrm{RRF}}$ & RRF5. 0 & $\begin{array}{l}\text { MIN } \\
\text { RRF }\end{array}$ & $\% \mathrm{D}$ & $\operatorname{MAX} \div \mathrm{D}$ \\
\hline \multicolumn{6}{|c|}{ 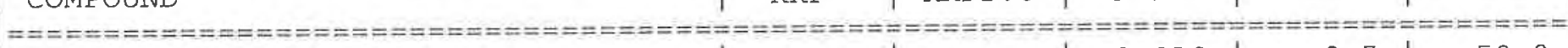 } \\
\hline Dichlorodifluoromethane & 0.463 & 0.461 & 0.010 & -0.5 & 50.0 \\
\hline Chloromethane & 0.511 & 0.556 & 0.010 & 8.8 & 50.0 \\
\hline Vinyl chloride & 0.517 & 0.539 & 0.010 & 4.4 & 50.0 \\
\hline Bromomethane & 0.170 & 0.194 & 0.010 & 13.9 & 50.0 \\
\hline Chloroethane & 0.311 & 0.304 & 0.010 & -2.2 & 50.0 \\
\hline Trichlorofluoromethane & 0.533 & 0.503 & 0.010 & -5.6 & 50.0 \\
\hline $1,1-\mathrm{Dich}$ loroethene & 0.323 & 0.310 & 0.010 & -3.8 & 50.0 \\
\hline $1,1,2$-Trichloro-1,2,2-trifluoroethane & 0.343 & 0.332 & 0.010 & -3.2 & 50.0 \\
\hline Acetone & 0.015 & 0.015 & 0.010 & -3.2 & 50.0 \\
\hline Carbon disulfide & 1.121 & 1.052 & 0.010 & -6.2 & 50.0 \\
\hline Methyl acetate & 0.056 & 0.052 & 0.010 & -7.9 & 50.0 \\
\hline Methylene chloride & 0.244 & 0.253 & 0.010 & 3.9 & 50.0 \\
\hline trans-1,2-Dichloroethene & 0.327 & 0.339 & 0.010 & 3.7 & 50.0 \\
\hline Methyl tert-butyl ether & 0.370 & 0.395 & 0.010 & 6.7 & 50.0 \\
\hline 1, 1-Dichloroethane & 0.626 & 0.680 & 0.010 & 8.6 & 50.0 \\
\hline cis-1,2-Dichloroethene & 0.307 & 0.324 & 0.010 & 5.7 & 50.0 \\
\hline 2-Butanone & 0.027 & 0.032 & 0.010 & 20.1 & 50.0 \\
\hline Bromochloromethane & 0.074 & 0.078 & 0.010 & 6.2 & 50.0 \\
\hline Chloroform & 0.507 & 0.523 & 0.010 & 3.1 & 50.0 \\
\hline $1,1,1-T r i c h l o r o e t h a n e$ & 0.662 & 0.679 & 0.010 & 2.6 & 50.0 \\
\hline Cyclohexane & 0.937 & 1.045 & 0.010 & 11.6 & 50.0 \\
\hline Carbon tetrachloride & 0.548 & 0.539 & 0.010 & -1.6 & 50.0 \\
\hline Benzene & 1.859 & 2.006 & 0.010 & 7.9 & 50.0 \\
\hline 1,2-Dichloroethane & 0.197 & 0.216 & 0.010 & 9.3 & 50.0 \\
\hline Trichloroethene & 0.451 & 0.457 & 0.010 & 1.2 & 50.0 \\
\hline Methylcyclohexane & 0.733 & 0.795 & 0.010 & 8.4 & 50.0 \\
\hline
\end{tabular}

Report 1,4-Dioxane for Low-Medium VOA analysis only 
$7 \mathrm{~B}$ - FORM VII VOA-2

VOLATILE CONTINUING CALIBRATION DATA

Lab Name: TESTAMERICA BURLINGTON

Contract: 21005

Lab Code: STLV Case No.: SAVAnNaH Mod. Ref No.:

SDG No.: 137933

Instrument ID: J.i

Calibration Date: 06/30/2010 Time: 1651

Lab File ID: JBE05CC1

Init. Calib. Date(s): 06/25/2010 06/25/2010

EPA Sample No. (VSTD\#\#\#\#\#): VSTD005HJ Init. Calib. Time(s): $1133 \quad 1327$

Heated Purge: (Y/N)N GC Column: DB-624 ID: 0.20 (mm) Length: 25

(m)

Purge Volume: 25.0

$(\mathrm{mL})$

\begin{tabular}{|c|c|c|c|c|c|}
\hline COMPOUND & $\overline{R R F}$ & RRF5. 0 & $\begin{array}{l}\text { MIN } \\
\text { RRF }\end{array}$ & $\because \mathrm{D}$ & $\mathrm{MAX} \div \mathrm{D}$ \\
\hline \multicolumn{6}{|c|}{ 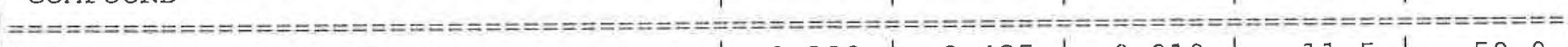 } \\
\hline 1,2-Dichloropropane & 0.382 & 0.425 & 0.010 & 11.5 & 50.0 \\
\hline Bromodichloromethane & 0.383 & 0.416 & 0.010 & 8.7 & 50.0 \\
\hline cis-1,3-Dichloropropene & 0.501 & 0.552 & 0.010 & 10.4 & 50.0 \\
\hline 4-Methyl-2-pentanone & 0.087 & 0.106 & 0.010 & 21.9 & 50.0 \\
\hline Toluene & 1.915 & 2.039 & 0.010 & 6.5 & 50.0 \\
\hline trans-1,3-Dichloropropene & 0.346 & 0.381 & 0.010 & 10.1 & 50.0 \\
\hline 1,1,2-Trichloroethane & 0.153 & 0.161 & 0.010 & 5.6 & 50.0 \\
\hline Tetrachloroethene & 0.327 & 0.310 & 0.010 & -5.3 & 50.0 \\
\hline 2-Hexanone & 0.057 & 0.068 & 0.010 & 19.9 & 50.0 \\
\hline Dibromochloromethane & 0.172 & 0.174 & 0.010 & 0.7 & 50.0 \\
\hline 1,2-Dibromoethane & 0.126 & 0.135 & 0.010 & 6.6 & 50.0 \\
\hline Chlorobenzene & 0.978 & 1.035 & 0.010 & 5.8 & 50.0 \\
\hline Ethylbenzene & 2.159 & 2.281 & 0.010 & 5.7 & 50.0 \\
\hline o-xylene & 0.709 & 0.746 & 0.010 & 5.2 & 50.0 \\
\hline $\mathrm{m}, \mathrm{p}-\mathrm{xy}$ lene & 0.788 & 0.827 & 0.010 & 4.9 & 50.0 \\
\hline styrene & 1.026 & 1.101 & 0.010 & 7.3 & 50.0 \\
\hline Bromoform & 0.174 & 0.170 & 0.010 & -2.0 & 50.0 \\
\hline Isopropylbenzene & 2.068 & 2.168 & 0.010 & 4.8 & 50.0 \\
\hline $1,1,2,2$-Tetrachloroethane & 0.141 & 0.161 & 0.010 & 14.2 & 50.0 \\
\hline 1,3-Dichlorobenzene & 1.588 & 1.584 & 0.010 & -0.2 & 50.0 \\
\hline 1,4-Dichlorobenzene & 1.509 & 1.489 & 0.010 & -1.3 & 50.0 \\
\hline 1,2-Dichlorobenzene & 1.142 & 1.181 & 0.010 & 3.4 & 50.0 \\
\hline 1,2-Dibromo-3-chloropropane & 0.046 & 0.050 & 0.010 & 10.4 & 50.0 \\
\hline 1,2,4-Trichlorobenzene & 0.685 & 0.670 & 0.010 & -2.2 & 50.0 \\
\hline $1,2,3$-Trichlorobenzene & 0.475 & 0.473 & 0.010 & -0.3 & 50.0 \\
\hline
\end{tabular}




$$
7 C \text { - FORM VII VOA-3 }
$$

VOLATILE CONTINUING CALIBRATION DATA

Lab Name: TESTAMERICA BURLINGTON

Contract: 21005

Lab Code: STlv Case No.: SAVANNAH Mod. Ref No.:

SDG No.: 137933

Instrument ID: J.i

Calibration Date: 06/30/2010 Time: 1651

Lab File ID: JBE05CC1

Init. Calib. Date(s): 06/25/2010 06/25/2010

EPA Sample No. (VSTD\#\#\#\#\#): VSTD005HJ

Init. Calib. Time(s): 1133

1327

Heated Purge: (Y/N)N GC Column: DB-624

ID $: 0.20$

(mm) Length: 25

(m)

Purge Volume: 25.0

(mL)

\begin{tabular}{|c|c|c|c|c|c|}
\hline COMPOUND & $\overline{\mathrm{RRF}}$ & RRF5. 0 & $\begin{array}{l}\text { MIN } \\
\text { RRF }\end{array}$ & $\div \mathrm{D}$ & $\mathrm{MAX} \div \mathrm{D}$ \\
\hline \multicolumn{6}{|c|}{ 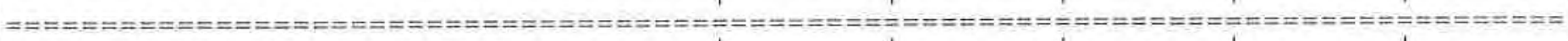 } \\
\hline Vinyl chloride-d3 & 0.428 & 0.454 & 0.010 & 6.1 & 50.0 \\
\hline Chloroethane-d5 & 0.341 & 0.353 & 0.010 & 3.6 & 50.0 \\
\hline 1,1-Dichloroethene- $\mathrm{d} 2$ & 0.714 & 0.716 & 0.010 & 0.3 & 50.0 \\
\hline 2 -Butanone- 25 & 0.024 & 0.030 & 0.010 & 24.4 & 50.0 \\
\hline Chloroform-d & 0.522 & 0.546 & 0.010 & 4.5 & 50.0 \\
\hline $1,2-D i c h l o r o e t h a n e-d 4$ & 0.158 & 0.174 & 0.010 & 10.7 & 50.0 \\
\hline Benzene-d6 & 1.701 & 1.825 & 0.010 & 7.3 & 50.0 \\
\hline 1,2-Dichloropropane- 26 & 0.430 & 0.480 & 0.010 & 11.8 & 50.0 \\
\hline Toluene-d8 & 1.537 & 1.612 & 0.010 & 4.9 & 50.0 \\
\hline trans-1,3-Dichloropropene-d4 & 0.301 & 0.320 & 0.010 & 6.4 & 50.0 \\
\hline 2 -Hexanone-d5 & 0.028 & 0.036 & 0.010 & 26.5 & 50.0 \\
\hline $1,1,2,2$-Tetrachloroethane-d2 & 0.137 & 0.160 & 0.010 & 17.2 & 50.0 \\
\hline 1,2 -Dichlorobenzene-d4 & 0.717 & 0.703 & 0.010 & -2.0 & 50.0 \\
\hline
\end{tabular}

Report 1,4-Dioxane-d8 for Low-Medium VOA analysis only 
$8 A$ - FORM VIII VOA

VOLATILE INTERNAL STANDARD AREA AND RETENTION TIME SUMMARY

Lab Name: TESTAMERICA BURLINGTON

Contract: 21005

Lab Code: STLV Case No.: SAVANNAH Mod. Ref No.:

SDG No.: 137933

GC Column: DB-624 ID: 0.20 (mm)

Init. Calib. Date(s): 06/25/2010 06/25/2010

EPA Sample No. (VSTD\#\#\#\#\#): VSTD005JH

Date Analyzed: 06/30/2010

Lab File ID (Standard): JBE005CV

Time Analyzed: 0625

Instrument ID: J.i

Heated Purge: (Y/N) N

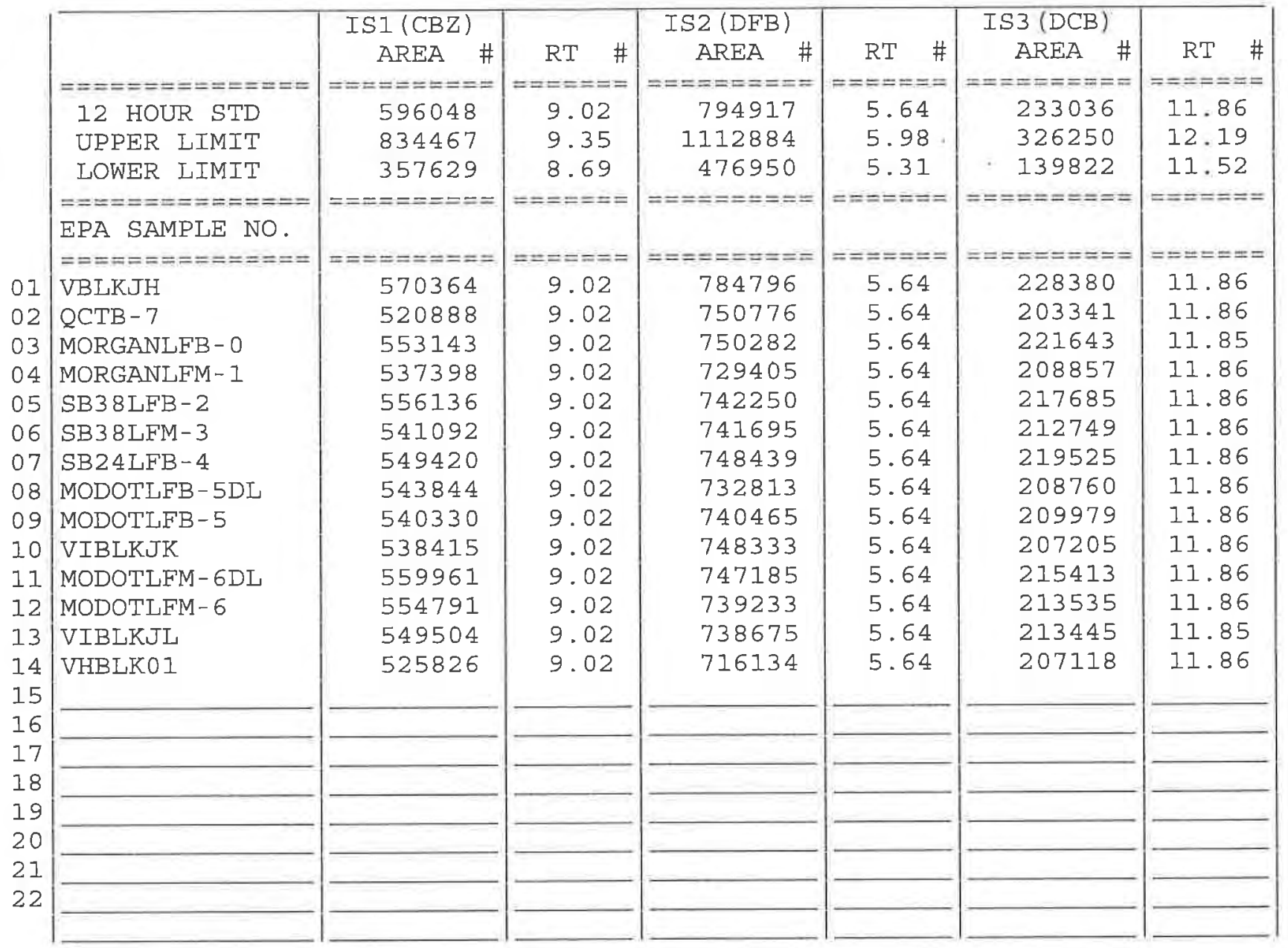

IS1 (CBZ) = Chlorobenzene $-\mathrm{d} 5$

IS2 (DFB) = 1,4-Difluorobenzene

IS3 $(\mathrm{DCB})=1,4-\mathrm{Dich}$ orobenzene $-\mathrm{d} 4$

AREA UPPER LIMIT = 200\% (Low-Medium Volatiles) and 140\% (Trace Volatiles) of internal standard area

AREA LOWER LIMIT $=50 \%$ (Low-Medium Volatiles) and $60 \%$ (Trace Volatiles) of internal standard area

RT UPPER LIMIT $=+0.50$ (Low-Medium Volatiles) and +0.33 (Trace Volatiles) minutes of internal standard RT

RT LOWER IIMIT $=-0.50$ (Low-Medium Volatiles) and - 0.33 (Trace Volatiles) minutes of internal standard RT

\# Column used to flag values outside QC limits with an asterisk

Page 1 of 1

SOMO1.2 


\section{TestAmerica}

THE LEADER IN ENVIRONMENTAL TESTING

TestAmerica Laboratories, Inc.

July 13,2010

Mr. Clyde Dennis

Argonne National Laboratory

9700 S. Cass Avenue

Bldg. 203, Office B149

Argonne, IL 60439

Re: Laboratory Project No. 21005

Case: SAVANNAH; SDG: 137941

Dear Mr. Dennis:

Enclosed are analytical results for samples that were received by TestAmerica Burlington on June $29^{\text {th }}, 2010$. Laboratory identification numbers were assigned, and designated as follows:

$\begin{array}{llll}\text { Lab ID } & \text { Client } & \text { Sample } & \text { Sample } \\ & \text { Sample ID } & \underline{\text { Date }} & \underline{\text { Matrix }}\end{array}$

Received: 06/29/10 ETR No: 137941

$\begin{array}{llll}834123 & \text { SVMODO3X-W-25778 } & 06 / 27 / 10 & \text { WATER } \\ 834124 & \text { SVMORGAN3X-W-25779 } & 06 / 28 / 10 & \text { WATER } \\ 834125 & \text { SVQCTB-W-25780 } & 06 / 28 / 10 & \text { WATER } \\ 834126 & \text { VHBLK01 } & 06 / 29 / 10 & \text { WATER }\end{array}$

Documentation of the condition of the samples at the time of their receipt and any exception to the laboratory's Sample Acceptance Policy is documented in the Sample Handling section of this submittal. The samples, as received, were not acid preserved. On that basis, the laboratory attempted to provide for the analytical work to be performed within seven days of sample collection. The analysis of sample SVMODO3X-W-25778 did occur on the tenth day from the date that the sample was collected, and the analysis of samples SVMORGAN3X-W-25779 and SVQCTB-W-25780 did occur on the ninth day from their collection date.

In order to accommodate field length limitations in processing the data summary forms, the laboratory did, in certain instances, abbreviate the sample identifier. The electronically formatted data provides for the full sample identifier.

\section{SOM01.2 Volatile Organics (Trace Level Water)}

A storage blank was prepared for volatile organics analysis, and stored in association with the storage of the samples. That storage blank, identified as VHBLK01, was carried through the holding period with the samples, and analyzed. 


\section{TestAmerica}

THE LEADER IN ENVIRONMENTAL TESTING

Sample SVMODO3X-W-25778 was analyzed at a dilution in order to provide quantification within the range of calibrated instrument response. An additional, more concentrated analysis was performed on the sample in order to provide for a lower reporting limit for those compounds that were not identified in the primary analysis. Both sets of results for the analysis of sample SVMODO3X-W-25778 are included in this submittal.

Each of the analyses associated with the sample set exhibited an acceptable internal standard performance. There was an acceptable recovery of each deuterated monitoring compound (DMC) in the analysis of each method blank and instrument blank associated with the analytical work, and in the analysis of the storage blank associated with the sample set. The analysis of the samples in this sample set did meet the technical acceptance criteria specific to DMC recoveries, although not all DMC recoveries were within the control range in each analysis. The technical acceptance criteria does provide for the recovery of up to three DMCs to fall outside of the control range in the analysis of field samples. Matrix spike and matrix spike duplicate analyses were not performed on samples in this sample set. A trace concentration of acetone was identified in the analysis of the method blank associated with the analytical work. The concentration of acetone in that analysis was below the established reporting limit, and the analysis did meet the technical acceptance criteria for a compliant method blank analysis. Trace concentrations of acetone, carbon disulfide, and carbon tetrachloride were identified in the analysis of the instrument blank associated with the analytical work. The concentration of each compound in that analysis was below the established reporting limit, and the analysis did meet the technical acceptance criteria for a compliant instrument blank analysis. A trace concentration of acetone was identified in the analysis of the storage blank associated with the sample set. The concentration of acetone in that analysis was below the established reporting limit, and the analysis did meet the technical acceptance criteria for a compliant storage blank analysis. Present in the method blank, instrument blank, and storage blank analyses was a nontarget constituent that represented a compound that is related to the DMC formulation. The fact that the presence of this compound is not within the laboratory's control is at issue. The derived results for that compound have been qualified with an " $\mathrm{X}$ " qualifier to reflect the source of the contamination.

The responses for each of the target analytes met the relative standard deviation criterion in the initial calibration. The response for each target analyte met the percent difference criterion in the continuing calibration check acquisition. The response for each target analyte met the 50.0 percent difference criterion in the closing calibration check acquisition.

The primary quantitation mass for methylcyclohexane that is specified in the Statement of Work is mass 83 . The laboratory did identify a contribution to mass 83 from 1,2-dichloropropane- $d_{6}$, one of the deuterated monitoring compounds (DMCs). The laboratory did change the primary quantitation mass assignment to mass 55 for the quantification of methylcyclohexane.

Manual integration was employed in deriving certain of the analytical results. The values that have been derived from manual integration are qualified on the quantitation reports. Extracted ion current profiles for each manual integration are included in the data package, and further documented in the Sample Preparation section of this submittal.

Any reference within this report to Severn Trent Laboratories, Inc. or STL, should be understood to refer to TestAmerica Laboratories, Inc. (formerly known as Severn Trent Laboratories, Inc.) The analytical results associated with the samples presented in this test report were generated 


\section{TestAmerica}

THE LEADER IN ENVIRONMENTAL TESTING

under a quality system that adheres to requirements specified in the NELAC standard. Release of the data in this test report and any associated electronic deliverables is authorized by the Laboratory Director's designee as verified by the following signature.

If there are any questions regarding this submittal, please contact me at 802 660-1990.

Sincerely,

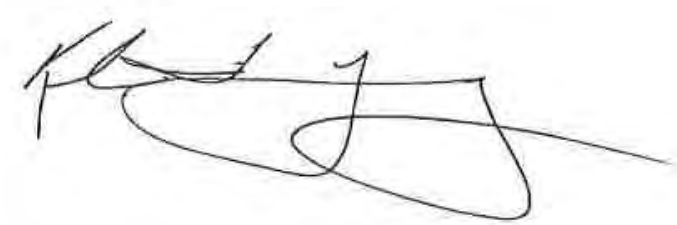

Kirk F. Young

Project Manager

$\mathrm{KFY} / \mathrm{hsf}$

Enclosure 


\section{Organic}

$\mathrm{U}$ : Compound analyzed but not detected at a concentration above the reporting limit.

J: Estimated value.

$\mathrm{N}$ : Indicates presumptive evidence of a compound. This flag is used only for tentatively identified compounds (TICs) where the identification of a compound is based on a mass spectral library search.

P: $\quad$ SW-846: The relative percent difference for detected concentrations between two GC columns is greater than $40 \%$. Unless otherwise specified the higher of the two values is reported on the Form I.

CLP SOW: Greater than $25 \%$ difference for detected concentrations between two GC columns. Unless otherwise specified the lower of the two values is reported on the Form I.

C: Pesticide result whose identification has been confirmed by GC/MS.

B: $\quad$ Analyte is found in the sample and the associated method blank. The flag is used for tentatively identified compounds as well as positively identified compounds.

E: Compounds whose concentrations exceed the upper limit of the calibration range of the instrument for that specific analysis.

D: Concentrations identified from analysis of the sample at a secondary dilution.

A: Tentatively identified compound is a suspected aldol condensation product.

$X, Y, Z$ : Laboratory defined flags that may be used alone or combined, as needed. If used, the description of the flag is defined in the project narrative.

\section{Inorganic/Metals}

E: $\quad$ Reported value is estimated due to the presence of interference.

N: $\quad$ Matrix spike sample recovery is not within control limits.

* Duplicate sample analysis is not within control limits.

B: The result reported is less than the reporting limit but greater than the instrument detection limit.

$\mathrm{U}: \quad$ Analyte was analyzed for but not detected above the reporting limit.

\section{Method Codes:}

P ICP-AES

MS ICP-MS

CV Cold Vapor AA

AS Semi-Automated Spectrophotometric 


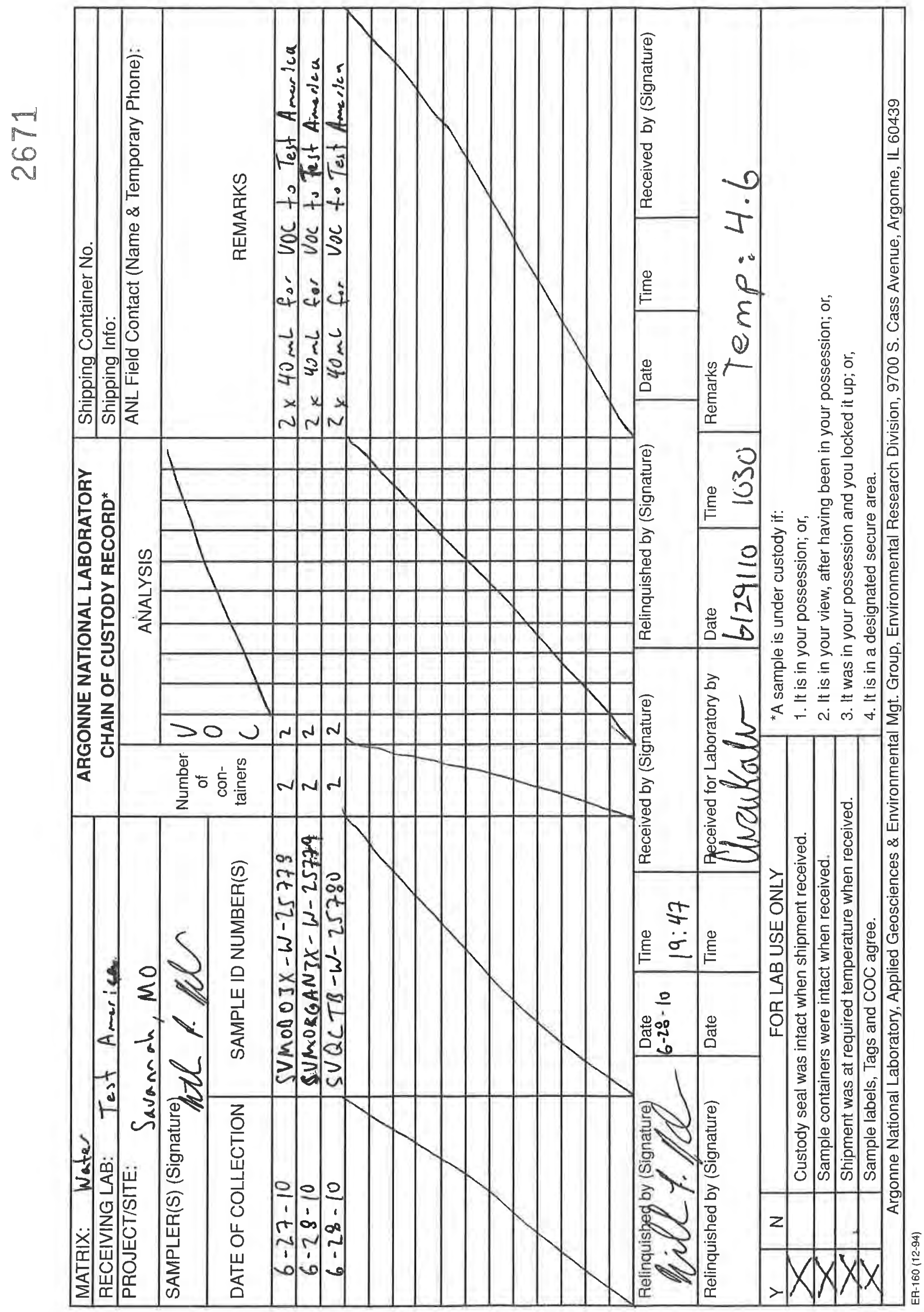




\section{TestAmerica \\ THE LEADER IN ENVIRONMENTAL TESTING}

\section{Sample Data Summary - SOM01.2 Volatiles - Trace}


$1 A$ - FORM I VOA-1

VOLATILE ORGANICS ANALYSIS DATA SHEET
EPA SAMPLE NO.

MODO $3 \times 778$
Lab Name: TESTAMERICA BURLINGTON

Lab Code: STLV Case No.: SAVANNAH Mod. Ref No.:
Contract: 21005

SDG NO.: 137941

Matrix: (SOIL/SED/WATER) water

Sample wt/vol: 25.0

$(g / m L) m L$

Level: (TRACE/LOW/MED) TRACE

\% Moisture: not dec.

GC Column: DB-624

Soil Extract Volume:

ID : 0.20

$(\mathrm{mm})$

(uL)

Lab Sample ID: 834123

Lab File ID: $834123 D 2$

Date Received: 06/29/2010

Date Analyzed: 07/07/2010

Purge Volume: 25.0
$(\mathrm{mL})$

\begin{tabular}{|c|c|c|c|}
\hline CAS NO. & COMPOUND & $\begin{array}{l}\text { CONCENTRATION UNITS: } \\
(\mathrm{ug} / \mathrm{L} \text { or } \mathrm{ug} / \mathrm{kg}) \mathrm{ug} / \mathrm{L}\end{array}$ & Q \\
\hline$==\mathbf{=}=\mathbf{=}=\mathbf{=}=\mathbf{=}=$ & 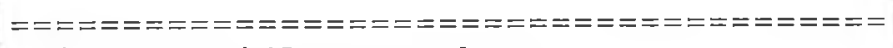 & 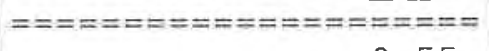 & 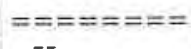 \\
\hline $75-71-8$ & Dichlorodifluoromethane & 0.55 & $\mathrm{U}$ \\
\hline $74-87-3$ & Chloromethane & 0.55 & $\mathrm{U}$ \\
\hline $75-01-4$ & Vinyl chloride & 0.55 & $\mathrm{U}$ \\
\hline $74-83-9$ & Bromomethane & 0.55 & $\mathrm{U}$ \\
\hline $75-00-3$ & Chloroethane & 0.55 & $\mathrm{U}$ \\
\hline $75-69-4$ & Trichlorofluoromethane & 0.55 & $\mathrm{U}$ \\
\hline $75-35-4$ & 1,1-Dichloroethene & 0.55 & $\mathrm{U}$ \\
\hline $76-13-1$ & $1,1,2$-Trichloro-1,2,2-trifluoroethane & 0.55 & $\mathrm{U}$ \\
\hline $67-64-1$ & Acetone & 2.4 & JB \\
\hline $75-15-0$ & Carbon disulfide & 0.14 & $\mathrm{~J}$ \\
\hline $79-20-9$ & Methyl acetate & 0.55 & $\mathrm{U}$ \\
\hline $75-09-2$ & Methylene chloride & 0.55 & $\mathrm{U}$ \\
\hline $156-60-5$ & trans-1,2-Dichloroethene & 0.55 & U \\
\hline $1634-04-4$ & Methyl tert-butyl ether & 0.55 & $\mathrm{U}$ \\
\hline $75-34-3$ & 1,1-Dichloroethane & 0.55 & $\mathrm{U}$ \\
\hline $156-59-2$ & cis-1,2-Dichloroethene & 0.55 & $\mathrm{U}$ \\
\hline $78-93-3$ & 2 -Butanone & 5.5 & $\mathrm{U}$ \\
\hline $74-97-5$ & Bromochloromethane & 0.55 & U \\
\hline $67-66-3$ & Chloroform & 6.0 & \\
\hline $71-55-6$ & 1,1,1-Trichloroethane & 0.55 & $\overline{\mathrm{U}}$ \\
\hline $110-82-7$ & Cyclohexane & 0.55 & $\mathrm{U}$ \\
\hline $56-23-5$ & Carbon tetrachloride & 170 & $\mathrm{E}$ \\
\hline $71-43-2$ & Benzene & 0.55 & $\mathrm{U}$ \\
\hline $107-06-2$ & 1,2 -Dichloroethane & 0.55 & U \\
\hline
\end{tabular}

Report 1,4-Dioxane for Low-Medium VOA analysis only 
IB - FORM I VOA-2

VOLATILE ORGANICS ANALYSIS DATA SHEET
EPA SAMPLE NO.

$\operatorname{MODO} 3 \times 778$
Lab Name: TESTAMERICA BURLINGTON

Lab Code: STLV Case No.: SAVANNAH Mod. Ref No.:
Contract: 21005
Matrix: (SOIL/SED/WATER) Water

Sample wt/vol: 25.0

$(\mathrm{g} / \mathrm{mL}) \mathrm{mL}$

Level: (TRACE/LOW/MED) TRACE

\% Moisture: not dec.

GC Column: DB-624

Soil Extract Volume:

ID $: 0.20$

(mm)

(uL)

(mL)

Purge Volume: 25.0
Lab Sample ID: 834123

Lab File ID: $834123 \mathrm{D} 2$

Date Received: 06/29/2010

Date Analyzed: 07/07/2010

Dilution Factor: 1.1

Soil Aliquot Volume:

(uL)

\section{(mi)}

\begin{tabular}{|c|c|c|c|}
\hline CAS NO. & COMPOUND & $\begin{array}{l}\text { CONCENTRATION UNITS: } \\
(\mathrm{ug} / \mathrm{L} \text { or } \mathrm{ug} / \mathrm{kg}) \mathrm{ug} / \mathrm{L}\end{array}$ & Q \\
\hline 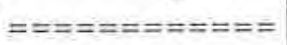 & 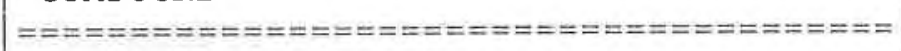 & $=============\overline{====}$ & $======-=$ \\
\hline $79-01-6$ & Trichloroethene & 0.55 & $\mathrm{U}$ \\
\hline $108-87-2$ & Methylcyclohexane & 0.55 & $\mathrm{U}$ \\
\hline $78-87-5$ & 1,2-Dichloropropane & 0.55 & $\mathrm{U}$ \\
\hline $75-27-4$ & Bromodichloromethane & 0.55 & $\mathrm{U}$ \\
\hline $10061-01-5$ & cis-1,3-Dichloropropene & 0.55 & $\mathrm{U}$ \\
\hline $108-10-1$ & 4-Methyl-2-pentanone & 5.5 & $\mathrm{U}$ \\
\hline $108-88-3$ & Toluene & 0.55 & $\mathrm{U}$ \\
\hline $10061-02-6$ & trans-1,3-Dichloropropene & 0.55 & $\mathrm{U}$ \\
\hline $79-00-5$ & $1,1,2-$ Trichloroethane & 0.55 & $\mathrm{U}$ \\
\hline $127-18-4$ & Tetrachloroethene & 0.067 & 丁 \\
\hline $591-78-6$ & 2-Hexanone & 5.5 & $\mathrm{U}$ \\
\hline $124-48-1$ & Dibromochloromethane & 0.55 & $\mathrm{U}$ \\
\hline $106-93-4$ & 1,2 -Dibromoethane & 0.55 & $\mathrm{U}$ \\
\hline $108-90-7$ & Chlorobenzene & 0.55 & $\mathrm{U}$ \\
\hline $100-41-4$ & Ethylbenzene & 0.55 & $\mathrm{U}$ \\
\hline $95-47-6$ & o-xylene & 0.55 & $\mathrm{U}$ \\
\hline $179601-23-1$ & $\mathrm{~m}, \mathrm{p}-\mathrm{Xy}$ lene & 0.55 & $\mathrm{U}$ \\
\hline $100-42-5$ & styrene & 0.55 & $\mathrm{U}$ \\
\hline $75-25-2$ & Bromoform & 0.55 & U \\
\hline $98-82-8$ & Isopropylbenzene & 0.55 & $\mathrm{U}$ \\
\hline $79-34-5$ & $1,1,2,2$-Tetrachloroethane & 0.55 & $\mathrm{U}$ \\
\hline $541-73-1$ & 1,3 -Dichlorobenzene & 0.55 & $\mathrm{U}$ \\
\hline $106-46-7$ & 1,4-Dichlorobenzene & 0.55 & $\mathrm{U}$ \\
\hline $95-50-1$ & 1,2 -Dichlorobenzene & 0.55 & $\mathrm{U}$ \\
\hline $96-12-8$ & 1,2 -Dibromo-3-chloropropane & 0.55 & $\mathrm{U}$ \\
\hline $120-82-1$ & $1,2,4$-Trichlorobenzene & 0.55 & $\mathrm{U}$ \\
\hline $87-61-6$ & $1,2,3$-Trichlorobenzene & 0.55 & $\mathrm{U}$ \\
\hline
\end{tabular}


$1 \mathrm{~J}$ - FORM I VOA-TIC

VOLATILE ORGANICS ANALYSIS DATA SHEET

TENTATIVELY IDENTIFIED COMPOUNDS
EPA SAMPLE NO.

MODO3X778
Lab Name: TESTAMERICA BURLINGTON

Lab Code: STLV Case No.: SAVANNAH

Matrix: (SOIL/SED/WATER) Water

Sample wt/vol: $25.0 \quad(\mathrm{~g} / \mathrm{mL}) \mathrm{mL}$

Level: (TRACE or LOW/MED) TRACE

\% Moisture: not dec.

GC Column: DB-624

Soil Extract Volume:

ID $: 0.20$

CONCENTRATION UNITS: (ug/L or $\mathrm{ug} / \mathrm{kg}$ ) $\mathrm{ug} / \mathrm{L}$
Contract: 21005

Mod. Ref No.:

SDG No.: 137941
Lab File ID: 834123D2

Date Received: 06/29/2010

Date Analyzed: 07/07/2010

(mm) Dilution Factor: 1.1

(uL) Soil Aliquot Volume:

(UL)

Purge Volume: 25.0

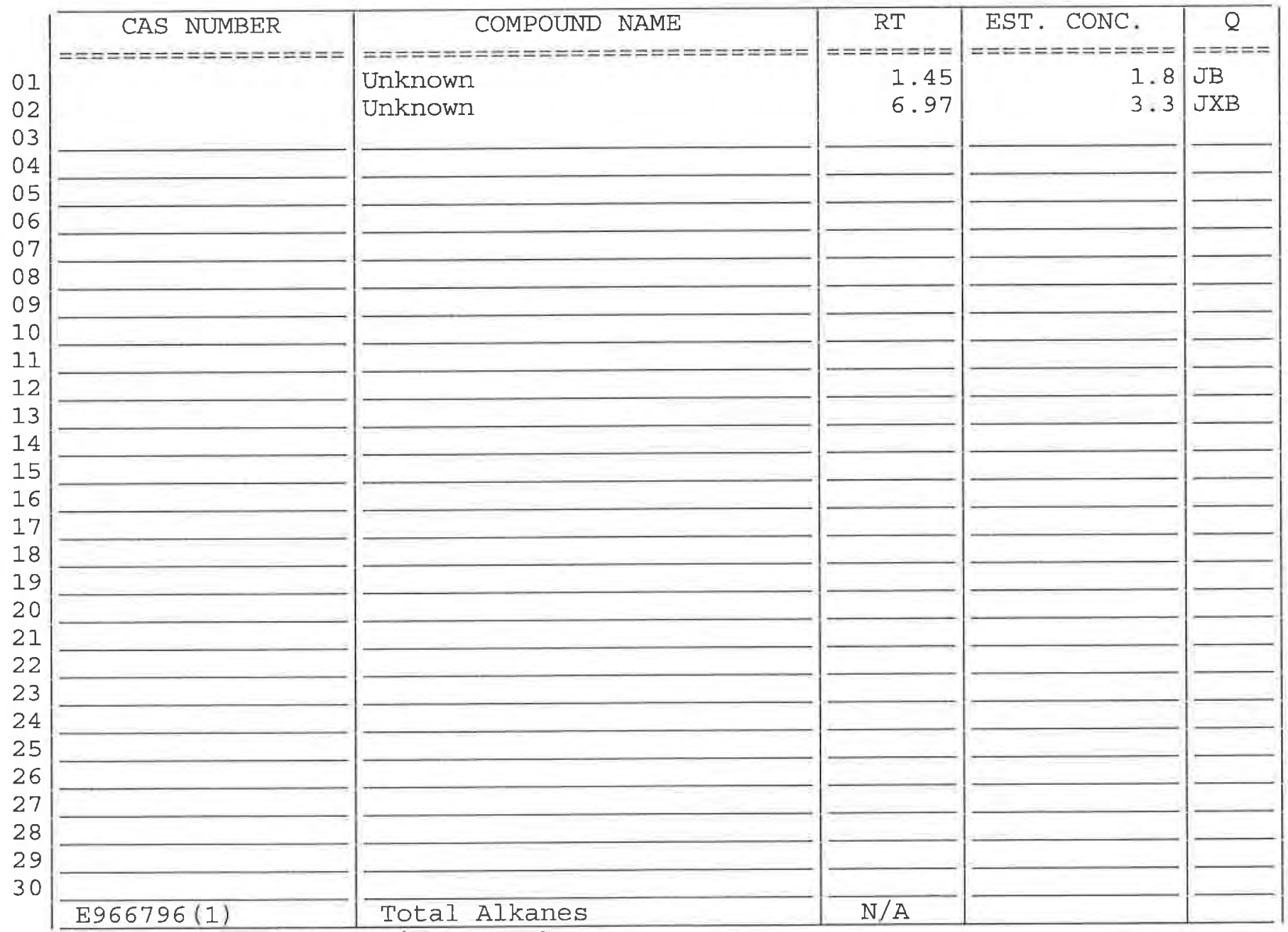

(1) EPA-designated Registry Number. 
$1 A$ - FORM I VOA-1

VOLATILE ORGANICS ANALYSIS DATA SHEET
EPA SAMPLE NO.

MODO $3 \times 778 D L$
Lab Name: TESTAMERICA BURLINGTON

Lab Code: STLV

Case No.: SAVANNAH

Matrix: (SOIL/SED/WATER) water

Sample wt/vol: 25.0

$(\mathrm{g} / \mathrm{mL}) \mathrm{mL}$

Level: (TRACE/LOW/MED) TRACE

\% Moisture: not dec.

GC Column: DB-624

ID : 0.20

(mm)

(uL)

(mL)
Soil Extract Volume:

Purge Volume: 25.0
Contract: 21005

SDG No.: 137941
Soil Aliquot Volume:

Lab Sample ID: $834123 \mathrm{Dl}$

Lab File ID: $834123 \mathrm{D}$

Date Received: 06/29/2010

Date Analyzed: 07/07/2010

Dilution Factor: 14.7

(uL)

\begin{tabular}{|c|c|c|c|}
\hline CAS NO. & COMPOUND & $\begin{array}{l}\text { CONCENTRATION UNITS: } \\
(\mathrm{ug} / \mathrm{L} \text { or } \mathrm{ug} / \mathrm{kg}) \mathrm{ug} / \mathrm{L}\end{array}$ & $Q$ \\
\hline$==========$ & 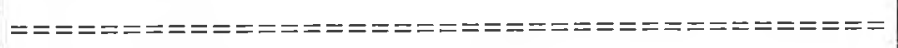 & 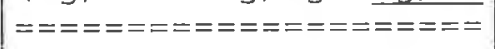 & $==== \pm==-$ \\
\hline $75-71-8$ & Dichlorodifluoromethane & 7.4 & $\mathrm{U}$ \\
\hline $74-87-3$ & Chloromethane & 7.4 & $\mathrm{U}$ \\
\hline $75-01-4$ & Vinyl chloride & 7.4 & $\mathrm{U}$ \\
\hline $74-83-9$ & Bromomethane & 7.4 & $\mathrm{U}$ \\
\hline $75-00-3$ & Chloroethane & 7.4 & $\mathrm{U}$ \\
\hline $75-69-4$ & Trichlorofluoromethane & 7.4 & $\mathrm{U}$ \\
\hline $75-35-4$ & 1,1 -Dichloroethene & 7.4 & $\mathrm{U}$ \\
\hline $76-13-1$ & 1,1,2-Trichloro-1,2,2-trifluoroethane & 7.4 & $\mathrm{U}$ \\
\hline $67-64-1$ & Acetone & 34 & DJB \\
\hline $75-15-0$ & Carbon disulfide & 1.3 & DJ \\
\hline $79-20-9$ & Methyl acetate & 7.4 & $\mathrm{U}$ \\
\hline $75-09-2$ & Methylene chloride & 7.4 & $\mathrm{U}$ \\
\hline $156-60-5$ & trans-1,2-Dichloroethene & 7.4 & $\mathrm{U}$ \\
\hline $1634-04-4$ & Methyl tert-butyl ether & 7.4 & U \\
\hline $75-34-3$ & 1,1 -Dichloroethane & 7.4 & $\mathrm{U}$ \\
\hline $156-59-2$ & cis-1,2-Dichloroethene & 7.4 & $\mathrm{U}$ \\
\hline $78-93-3$ & 2 -Butanone & 74 & $\mathrm{U}$ \\
\hline $74-97-5$ & Bromochloromethane & 7.4 & $\mathrm{U}$ \\
\hline $67-66-3$ & Chloroform & 7.3 & DJ \\
\hline $71-55-6$ & $1,1,1$-Trichloroethane & 7.4 & $\mathrm{U}$ \\
\hline $110-82-7$ & Cyclohexane & 7.4 & $\mathrm{U}$ \\
\hline $56-23-5$ & Carbon tetrachloride & 190 & $\mathrm{D}$ \\
\hline $71-43-2$ & Benzene & 7.4 & $\mathrm{U}$ \\
\hline $107-06-2$ & 1,2-Dichloroethane & 7.4 & $\mathrm{U}$ \\
\hline
\end{tabular}

Report 1,4-Dioxane for Low-Medium VOA analysis only 
IB - FORM I VOA-2

VOIATILE ORGANICS ANALYSIS DATA SHEET
EPA SAMPLE NO.

MODO3X778DL
Lab Name: TESTAMERICA BURLINGTON

Lab Code: STLV Case No.: SAVANNAH Mod. Ref No.:
Contract: 21005
Matrix: (SOIL/SED/WATER) Water

Sample wt/vol: 25.0

$(g / m L) \mathrm{mL}$

Level: (TRACE/LOW/MED) TRACE

\% Moisture: not dec.

GC Column: DB-624

Soil Extract Volume:

ID : 0.20

(mm)

(uL)

Lab Sample ID: $834123 D 1$

Lab File ID: $834123 \mathrm{D}$

Date Received: 06/29/2010

Date Analyzed: 07/07/2010

Purge Volume: 25.0

(mL)

\begin{tabular}{|c|c|c|c|}
\hline & & $\begin{array}{l}\text { CONCENTRATION UNITS: } \\
\text { (ug/L or ug/kg) ug/L }\end{array}$ & 0 \\
\hline CAS NO. & COMPUUND & $=================$ & $===\simeq====$ \\
\hline $\begin{aligned}=== & ====== \\
& 79-01-6\end{aligned}$ & 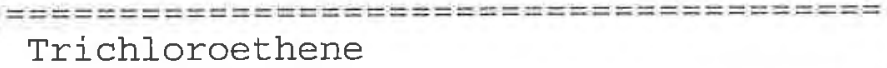 & 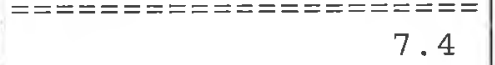 & $\begin{array}{l}==-==+= \\
\mathrm{U}\end{array}$ \\
\hline $108-87-2$ & Methylcyclohexane & 7.4 & $\mathrm{U}$ \\
\hline $78-87-5$ & 1,2-Dichloropropane & 7.4 & $\mathrm{U}$ \\
\hline $75-27-4$ & Bromodichloromethane & 7.4 & $\mathrm{U}$ \\
\hline $10061-01-5$ & cis-1,3-Dichloropropene & 7.4 & $\mathrm{U}$ \\
\hline $108-10-1$ & 4-Methyl-2-pentanone & 74 & $\mathrm{U}$ \\
\hline $108-88-3$ & Toluene & 7.4 & $\mathrm{U}$ \\
\hline $10061-02-6$ & trans-1,3-Dichloropropene & 7.4 & U \\
\hline $79-00-5$ & 1,1,2-Trichloroethane & 7.4 & $\mathrm{U}$ \\
\hline $127-18-4$ & Tetrachloroethene & 7.4 & $\mathrm{U}$ \\
\hline $591-78-6$ & 2-Hexanone & 74 & $\mathrm{U}$ \\
\hline $124-48-1$ & Dibromochloromethane & 7.4 & $\mathrm{U}$ \\
\hline $106-93-4$ & 1,2-Dibromoethane & 7.4 & $\mathrm{U}$ \\
\hline $108-90-7$ & Chlorobenzene & 7.4 & $\mathrm{U}$ \\
\hline $100-41-4$ & Ethylbenzene & 7.4 & $\mathrm{U}$ \\
\hline $95-47-6$ & o-Xylene & 7.4 & $\mathrm{U}$ \\
\hline $179601-23-1$ & $\mathrm{~m}, \mathrm{p}-\mathrm{Xy}$ lene & 7.4 & $\mathrm{U}$ \\
\hline $100-42-5$ & styrene & 7.4 & $\mathrm{U}$ \\
\hline $75-25-2$ & Bromoform & 7.4 & $\mathrm{U}$ \\
\hline $98-82-8$ & Isopropylbenzene & 7.4 & $\mathrm{U}$ \\
\hline $79-34-5$ & $1,1,2,2$-Tetrachloroethane & 7.4 & U \\
\hline $541-73-1$ & 1,3-Dichlorobenzene & 7.4 & U \\
\hline $106-46-7$ & 1,4-Dichlorobenzene & 7.4 & U \\
\hline $95-50-1$ & 1,2-Dichlorobenzene & 7.4 & U \\
\hline $96-12-8$ & 1,2-Dibromo-3-chloropropane & 7.4 & U \\
\hline $120-82-1$ & 1,2,4-Trichlorobenzene & 7.4 & $\mathrm{U}$ \\
\hline $87-61-6$ & 1,2,3-Trichlorobenzene & 7.4 & $\mathrm{U}$ \\
\hline
\end{tabular}


IJ - FORM I VOA-TIC

VOLATILE ORGANICS ANALYSIS DATA SHEET

TENTATIVELY IDENTIFIED COMPOUNDS
EPA SAMPLE NO. MODO3X778DL
Lab Name: TESTAMERICA BURLINGTON

Lab Code: STLV Case No.: SAVANNAH

Matrix: (SOIL/SED/WATER) Water

Sample wt/vol: $25.0 \quad(\mathrm{~g} / \mathrm{mL}) \mathrm{mL}$

Level: (TRACE or LOW/MED) TRACE

\% Moisture: not dec.

GC Column: DB-624

ID : 0.20

Soil Extract Volume:

CONCENTRATION UNITS: (ug/L or $\mathrm{ug} / \mathrm{kg}$ ) ug/L
Contract: 21005

Mod. Ref No.:

SDG No.: 137941

Lab Sample ID: 834123D1

Lab File ID: 834123D

Date Received: 06/29/2010

Date Analyzed: 07/07/2010

(mm) Dilution Factor: 14.7

(uL) Soil Aliquot Volume:

(UL)

Purge Volume: 25.0

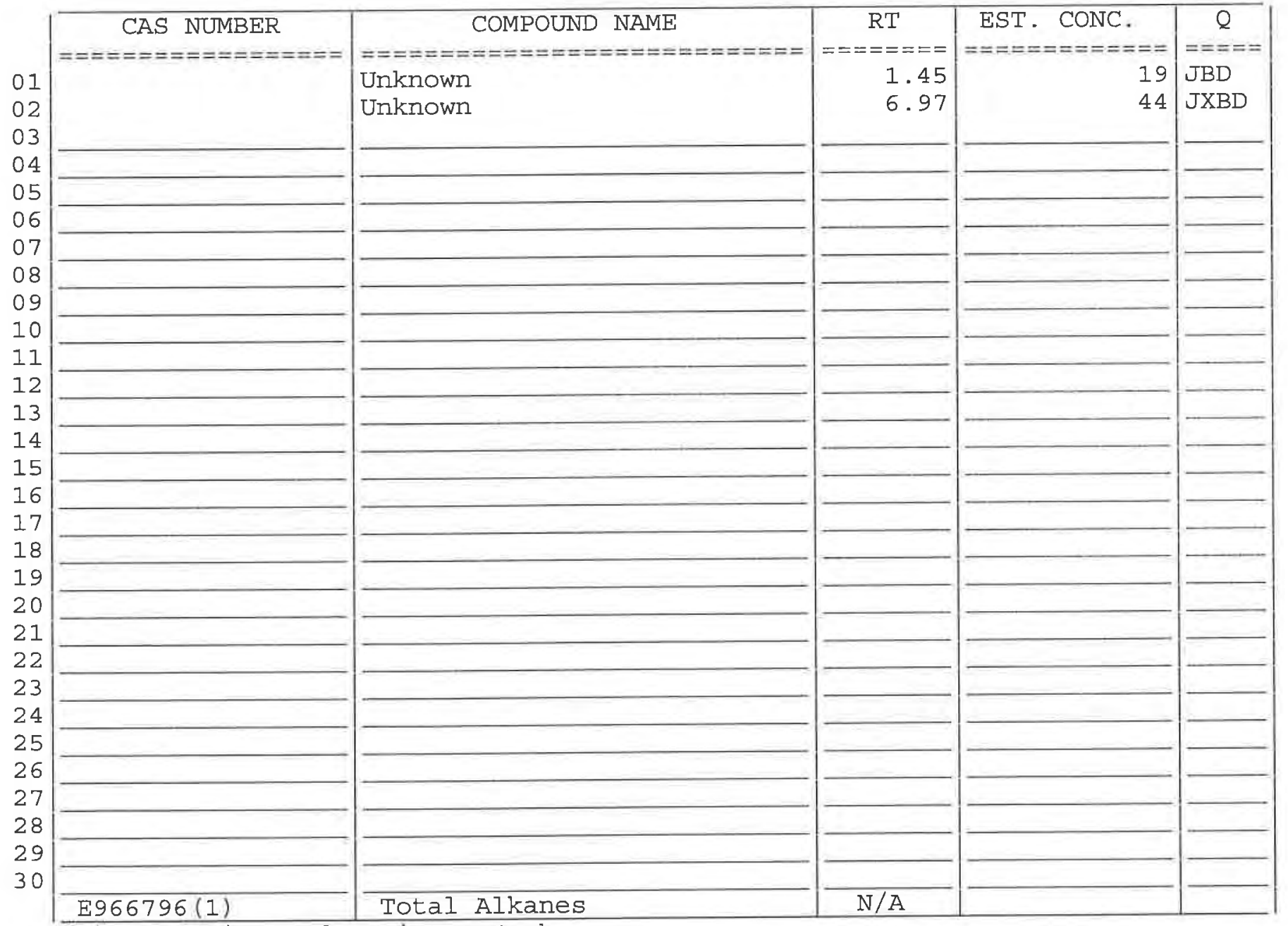

(1) EPA-designated Registry Number. 
$1 \mathrm{~A}$ - FORM I VOA-1

VOLATILE ORGANICS ANALYSIS DATA SHEET
EPA SAMPLE NO.

MORGAN3X779
Lab Name: TESTAMERICA BURLINGTON

Lab Code: STLV Case No.: SAVANNAH Mod. Ref No.:
Contract: 21005
Matrix: (SOIL/SED/WATER) water

Sample wt/vol: 25.0

$(\mathrm{g} / \mathrm{mL}) \mathrm{mL}$

Level: (TRACE/LOW/MED) TRACE

\% Moisture: not dec.

GC Column: DB-624

Soil Extract Volume:

ID : 0.20

(mm)

(uL)

(mL)

Purge Volume: 25.0
Soil Aliquot Volume:

Lab Sample ID: 834124

Lab File ID: 834124

Date Received: 06/29/2010

Date Analyzed: 07/07/2010

Dilution Factor: 1.0

(uL)

SDG No.: 137941

\begin{tabular}{|c|c|c|c|}
\hline CAS NO. & COMPOUND & $\begin{array}{l}\text { CONCENTRATION UNITS: } \\
(\mathrm{ug} / \mathrm{L} \text { or } \mathrm{ug} / \mathrm{kg}) \mathrm{ug} / \mathrm{L}\end{array}$ & $Q$ \\
\hline 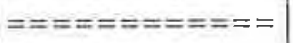 & 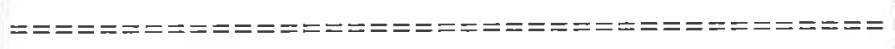 & 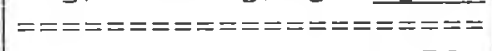 & $======-$ \\
\hline $75-71-8$ & Dichlorodifluoromethane & 0.50 & $\mathrm{U}$ \\
\hline $74-87-3$ & Chloromethane & 0.50 & $\mathrm{U}$ \\
\hline $75-01-4$ & Vinyl chloride & 0.50 & $\mathrm{U}$ \\
\hline $74-83-9$ & Bromomethane & 0.50 & $\mathrm{U}$ \\
\hline $75-00-3$ & Chloroethane & 0.50 & $\mathrm{U}$ \\
\hline $75-69-4$ & Trichlorofluoromethane & 0.50 & $\mathrm{U}$ \\
\hline $75-35-4$ & 1,1-Dichloroethene & 0.50 & $\mathrm{U}$ \\
\hline $76-13-1$ & $1,1,2$-Trichloro-1,2,2-trifluoroethane & 0.50 & $\mathrm{U}$ \\
\hline $67-64-1$ & Acetone & 2.7 & JB \\
\hline $75-15-0$ & Carbon disulfide & 0.23 & $\mathrm{~J}$ \\
\hline $79-20-9$ & Methyl acetate & 0.50 & $\mathrm{U}$ \\
\hline $75-09-2$ & Methylene chloride & 0.50 & $\mathrm{U}$ \\
\hline $156-60-5$ & trans-1,2-Dichloroethene & 0.50 & $\mathrm{U}$ \\
\hline $1634-04-4$ & Methyl tert-butyl ether & 0.50 & $U$ \\
\hline $75-34-3$ & 1,1-Dichloroethane & 0.50 & $\mathrm{U}$ \\
\hline $156-59-2$ & cis-1,2-Dichloroethene & 0.50 & $\mathrm{U}$ \\
\hline $78-93-3$ & 2-Butanone & 5.0 & $\mathrm{U}$ \\
\hline $74-97-5$ & Bromochloromethane & 0.50 & $\mathrm{U}$ \\
\hline $67-66-3$ & Chloroform & 5.7 & \\
\hline $71-55-6$ & $1,1,1$-Trichloroethane & 0.50 & $\mathrm{U}$ \\
\hline $110-82-7$ & Cyclohexane & 0.50 & $\mathrm{U}$ \\
\hline $56-23-5$ & Carbon tetrachloride & 13 & \\
\hline $71-43-2$ & Benzene & 0.50 & $\mathrm{U}$ \\
\hline $107-06-2$ & 1,2-Dichloroethane & 0.50 & $\mathrm{U}$ \\
\hline
\end{tabular}

Report 1,4-Dioxane for Low-Medium VOA analysis only 
IB - FORM I VOA-2

VOLATILE ORGANICS ANALYSIS DATA SHEET
EPA SAMPLE NO.

MORGAN3X779

Lab Name: TESTAMERICA BURLINGTON

Contract: 21005

Lab Code: STlV Case No.: SAVANNAH Mod, Ref No.:

SDG No.: 137941

Matrix: (SOIL/SED/WATER) Water

Lab Sample ID: 834124

Sample wt/vol: 25.0

$(\mathrm{g} / \mathrm{mL}) \mathrm{mL}$

Lab File ID: 834124

LeVel: (TRACE/LOW/MED) TRACE

Date Received: 06/29/2010

\% Moisture: not dec.

Date Analyzed: 07/07/2010

GC Column: DB-624

ID : 0.20

(mm)

Dilution Factor: 1.0

Soil Extract Volume:

(uL) Soil Aliquot volume:

(uL)

Purge Volume: 25.0

$(\mathrm{mL})$

\begin{tabular}{|c|c|c|c|}
\hline CAS NO. & COMPOUND & $\begin{array}{l}\text { CONCENTRATION UNITS: } \\
(\mathrm{ug} / \mathrm{L} \text { or } \mathrm{ug} / \mathrm{kg}) \mathrm{ug} / \mathrm{L}\end{array}$ & Q \\
\hline 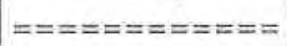 & 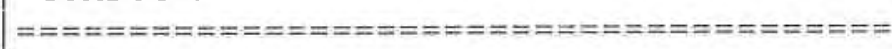 & l= =e==" & 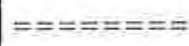 \\
\hline $79-01-6$ & Trichloroethene & 0.50 & $\mathrm{U}$ \\
\hline $108-87-2$ & Methylcyclohexane & 0.50 & $\mathrm{U}$ \\
\hline $78-87-5$ & 1,2-Dichloropropane & 0.50 & U \\
\hline $75-27-4$ & Bromodichloromethane & 2.8 & \\
\hline $10061-01-5$ & cis-1,3-Dichloropropene & 0.50 & $\overline{\mathrm{U}}$ \\
\hline $108-10-1$ & 4-Methyl-2-pentanone & 5.0 & U \\
\hline $108-88-3$ & Toluene & 0.50 & $\mathrm{U}$ \\
\hline $10061-02-6$ & trans-1,3-Dichloropropene & 0.50 & $\mathrm{U}$ \\
\hline $79-00-5$ & $1,1,2$-Trichloroethane & 0.50 & $\mathrm{U}$ \\
\hline $127-18-4$ & Tetrachloroethene & 0.11 & 丁 \\
\hline $591-78-6$ & 2 -Hexanone & 5.0 & U \\
\hline $124-48-1$ & Dibromochloromethane & 1.5 & \\
\hline $106-93-4$ & 1,2-Dibromoethane & 0.50 & $\mathrm{U}$ \\
\hline $108-90-7$ & Chlorobenzene & 0.50 & $\mathrm{U}$ \\
\hline $100-41-4$ & Ethylbenzene & 0.50 & $\mathrm{U}$ \\
\hline $95-47-6$ & o-Xylene & 0.50 & $\mathrm{U}$ \\
\hline $179601-23-1$ & $\mathrm{~m}, \mathrm{p}$-Xylene & 0.50 & $\mathrm{U}$ \\
\hline $100-42-5$ & Styrene & 0.50 & U \\
\hline $75-25-2$ & Bromoform & 0.50 & U \\
\hline $98-82-8$ & Isopropylbenzene & 0.50 & $\mathrm{U}$ \\
\hline $79-34-5$ & $1,1,2,2$-Tetrachloroethane & 0.50 & U \\
\hline $541-73-1$ & 1,3-Dichlorobenzene & 0.50 & $\mathrm{U}$ \\
\hline $106-46-7$ & 1,4-Dichlorobenzene & 0.50 & $\mathrm{U}$ \\
\hline $95-50-1$ & 1,2-Dichlorobenzene & 0.50 & $\mathrm{U}$ \\
\hline $96-12-8$ & 1,2-Dibromo-3-chloropropane & 0.50 & $\mathrm{U}$ \\
\hline $120-82-1$ & $1,2,4$-Trichlorobenzene & 0.50 & $\mathrm{U}$ \\
\hline $87-61-6$ & 1,2,3-Trichlorobenzene & 0.50 & U \\
\hline
\end{tabular}


1J - FORM I VOA-TIC

VOLATILE ORGANICS ANALYSIS DATA SHEET

TENTATIVELY IDENTIFIED COMPOUNDS
EPA SAMPLE NO.

MORGAN3X779
Lab Name: TESTAMERICA BURLINGTON

Lab Code: STLV Case No.: SAVANNAH

Matrix: (SOIL/SED/WATER) Water

Sample wt/vol: $25.0 \quad(\mathrm{~g} / \mathrm{mL}) \mathrm{mL}$

Level: (TRACE or LOW/MED) TRACE

응 Moisture: not dec.

GC Column: DB-624

ID $: 0.20$

Soil Extract Volume:

CONCENTRATION UNITS: (ug/L or ug/kg) ug/L
Contract: 21005

Mod. Ref No.:

SDG No.: 137941

Lab Sample ID: 834124

Lab File ID: 834124

Date Received: 06/29/2010

Date Analyzed: 07/07/2010

(mm) Dilution Factor: 1.0

(uL) Soil Aliquot Volume:

(uI)

Purge Volume: 25.0

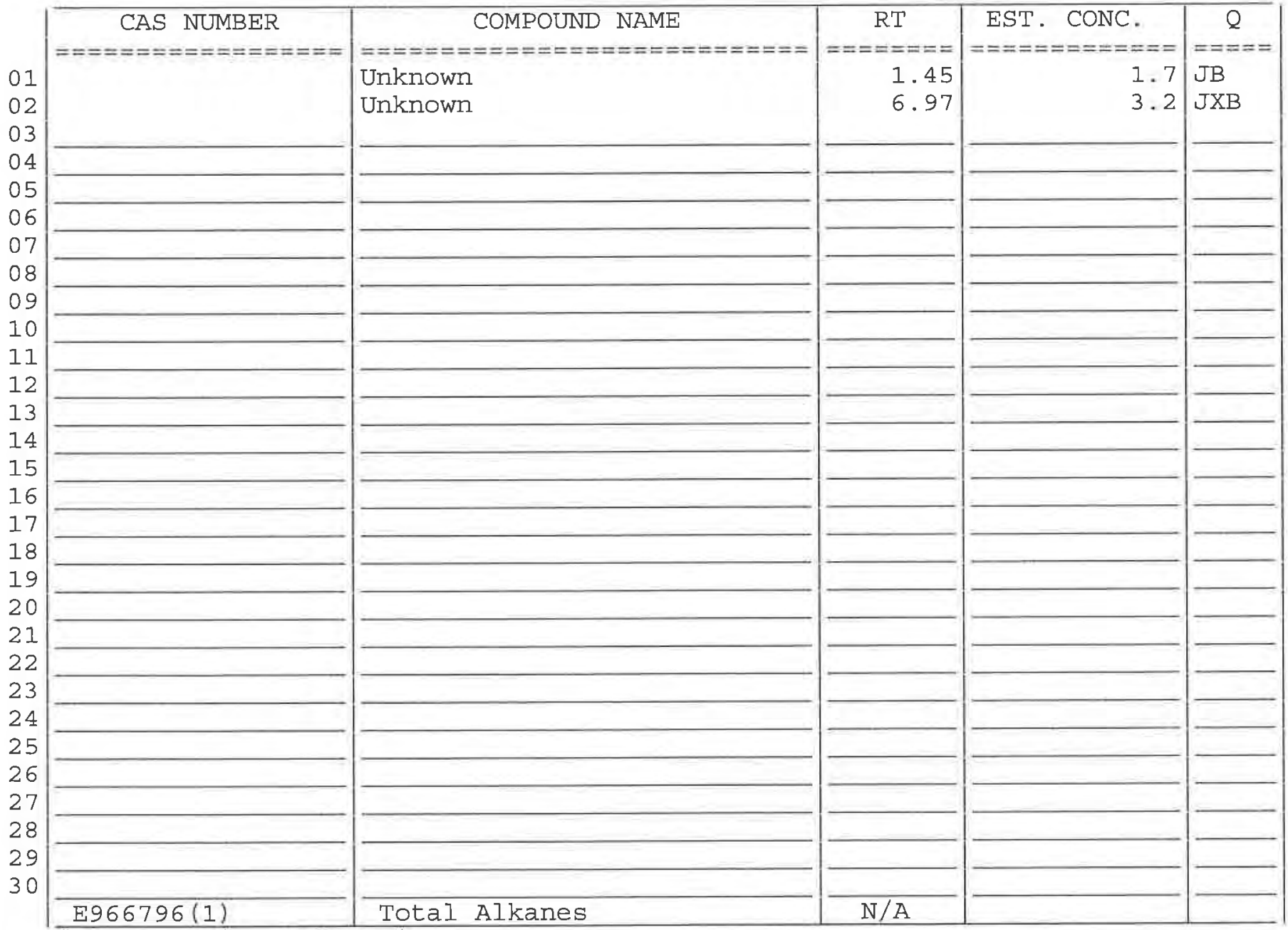

(1) EPA-designated Registry Number. 
$1 A$ - FORM I VOA-1

VOLATILE ORGANICS ANALYSIS DATA SHEET
EPA SAMPLE NO.

QCTB 780
Lab Name: TESTAMERICA BURLINGTON

Case No.: SAVANNAH

Matrix: (SOIL/SED/WATER) Water

Sample wt/vol: 25.0

(g/mL) $\mathrm{mL}$

Level: (TRACE/LOW/MED) TRACE

\% Moisture: not dec.

GC Column: DB-624

Soil Extract Volume:

ID : 0.20

$(\mathrm{mm})$

Contract: 21005
(uL)

Lab Sample ID: 834125

Lab File ID: 834125

Date Received: 06/29/2010

Date Analyzed: 07/07/2010

Dilution Factor: 1.0

Soil Aliquot Volume:

(uL)

(mL)

\begin{tabular}{|c|c|c|c|}
\hline CAS NO. & COMPOUND & $\begin{array}{l}\text { CONCENTRATION UNITS: } \\
(u g / L \text { or } u g / k g) ~ u g / L\end{array}$ & \\
\hline 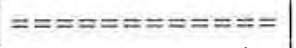 & 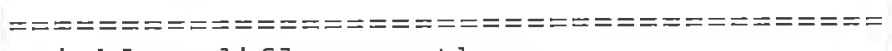 & 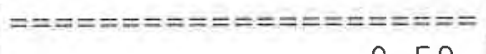 & $=======$ \\
\hline $75-71-8$ & Dichlorodifluoromethane & 0.50 & $\mathrm{U}$ \\
\hline $74-87-3$ & Chloromethane & 0.59 & \\
\hline $75-01-4$ & Vinyl chloride & 0.50 & U \\
\hline $74-83-9$ & Bromomethane & 0.50 & $\mathrm{U}$ \\
\hline $75-00-3$ & Chloroethane & 0.50 & $\mathrm{U}$ \\
\hline $75-69-4$ & Trichlorofluoromethane & 0.50 & $\mathrm{U}$ \\
\hline $75-35-4$ & 1,1-Dichloroethene & 0.50 & $\mathrm{U}$ \\
\hline $76-13-1$ & 1,1,2-Trichloro-1,2,2-trifluoroethane & 0.50 & $\mathrm{U}$ \\
\hline $67-64-1$ & Acetone & 14 & B \\
\hline $75-15-0$ & Carbon disulfide & 0.094 & J \\
\hline $79-20-9$ & Methyl acetate & 0.50 & $\mathrm{U}$ \\
\hline $75-09-2$ & Methylene chloride & 0.50 & $\mathrm{U}$ \\
\hline $156-60-5$ & trans-1,2-Dichloroethene & 0.50 & $\mathrm{U}$ \\
\hline $1634-04-4$ & Methyl tert-butyl ether & 0.50 & $\mathrm{U}$ \\
\hline $75-34-3$ & 1,1-Dichloroethane & 0.50 & $\mathrm{U}$ \\
\hline $156-59-2$ & cis-1,2-Dichloroethene & 0.50 & U \\
\hline $78-93-3$ & 2-Butanone & 0.75 & $\mathrm{~J}$ \\
\hline $74-97-5$ & Bromochloromethane & 0.50 & $\mathrm{U}$ \\
\hline $67-66-3$ & Chloroform & 0.50 & $\mathrm{U}$ \\
\hline $71-55-6$ & 1,1,1-Trichloroethane & 0.50 & $\mathrm{U}$ \\
\hline $110-82-7$ & Cyclohexane & 0.50 & $\mathrm{U}$ \\
\hline $56-23-5$ & Carbon tetrachloride & 0.50 & $\mathrm{U}$ \\
\hline $71-43-2$ & Benzene & 0.056 & $\mathrm{~J}$ \\
\hline $107-06-2$ & 1,2-Dichloroethane & 0.50 & U \\
\hline
\end{tabular}

Report 1,4-Dioxane for Low-Medium VOA analysis only 
$1 B$ - FORM I VOA-2

VOLATILE ORGANICS ANALYSIS DATA SHEET
Lab Name: TESTAMERICA BURLINGTON

Lab Code: STLV Case No.: SAVANNAH Mod, Ref No.:
Contract: 21005
EPA SAMPLE NO.

QCTB780

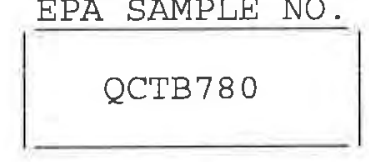

SDG No. : 137941
Matrix: (SOIL/SED/WATER) Water

Sample wt/vol: 25.0

$(\mathrm{g} / \mathrm{mL}) \mathrm{mL}$

Level: (TRACE/LOW/MED) TRACE

$\%$ Moisture: not dec.

GC Column: DB-624

Soil Extract Volume:

ID : 0.20

(mm)

(uL)

Soil Aliquot Volume:

$(\mathrm{mL})$

Lab Sample ID: 834125

Lab File ID: 834125

Date Received: 06/29/2010

Date Analyzed: 07/07/2010

Dilution Factor: 1.0

Purge Volume: 25.0

\begin{tabular}{|c|c|c|c|}
\hline & & $\begin{array}{l}\text { CONCENTRATION UNITS: } \\
\text { (ug/I or ug/kg) ug/L }\end{array}$ & 0 \\
\hline CAS NO. & 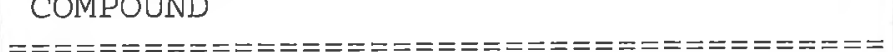 & $===================$ & $========$ \\
\hline $\begin{aligned}==x= & ==x== \\
& 79-01-6\end{aligned}$ & 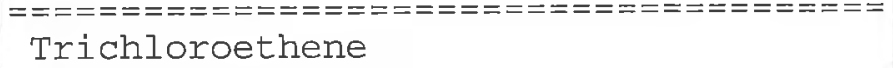 & $\begin{array}{rl}0.50 & 0 .=-1\end{array}$ & $\mathrm{U}$ \\
\hline $108-87-2$ & Methylcyclohexane & 0.50 & $\mathrm{U}$ \\
\hline $78-87-5$ & 1,2-Dichloropropane & 0.50 & U \\
\hline $75-27-4$ & Bromodichloromethane & 0.50 & $\mathrm{U}$ \\
\hline $10061-01-5$ & cis-1,3-Dichloropropene & 0.50 & U \\
\hline $108-10-1$ & 4-Methyl-2-pentanone & 5.0 & $\mathrm{U}$ \\
\hline $108-88-3$ & Toluene & 0.039 & J \\
\hline $10061-02-6$ & trans-1,3-Dichloropropene & 0.50 & $\mathrm{U}$ \\
\hline $79-00-5$ & 1,1,2-Trichloroethane & 0.50 & $\mathrm{U}$ \\
\hline $127-18-4$ & Tetrachloroethene & 0.50 & $\mathrm{U}$ \\
\hline $591-78-6$ & 2-Hexanone & 5.0 & $\mathrm{U}$ \\
\hline $124-48-1$ & Dibromochloromethane & 0.50 & $\mathrm{U}$ \\
\hline $106-93-4$ & 1,2-Dibromoethane & 0.50 & U \\
\hline $108-90-7$ & Chlorobenzene & 0.50 & $\mathrm{U}$ \\
\hline $100-41-4$ & Ethylbenzene & 0.50 & $\mathrm{U}$ \\
\hline $95-47-6$ & o-Xylene & 0.044 & $\mathrm{~J}$ \\
\hline $179601-23-1$ & $\mathrm{~m}, \mathrm{p}$-Xylene & 0.076 & J \\
\hline $100-42-5$ & styrene & 0.50 & $\mathrm{U}$ \\
\hline $75-25-2$ & Bromoform & 0.50 & $\mathrm{U}$ \\
\hline $98-82-8$ & Isopropylbenzene & 0.50 & $\mathrm{U}$ \\
\hline $79-34-5$ & $1,1,2,2$-Tetrachloroethane & 0.50 & $\mathrm{U}$ \\
\hline $541-73-1$ & 1,3-Dichlorobenzene & 0.50 & $U$ \\
\hline $106-46-7$ & 1,4-Dichlorobenzene & 0.50 & $\mathrm{U}$ \\
\hline $95-50-1$ & 1,2-Dichlorobenzene & 0.50 & $\mathrm{U}$ \\
\hline $96-12-8$ & 1,2-Dibromo-3-chloropropane & 0.50 & $\mathrm{U}$ \\
\hline $120-82-1$ & $1,2,4$-Trichlorobenzene & 0.50 & $\mathrm{U}$ \\
\hline $87-61-6$ & 1,2,3-Trichlorobenzene & 0.50 & $\mathrm{U}$ \\
\hline
\end{tabular}


$1 \mathrm{~J}$ - FORM I VOA-TIC

VOLATILE ORGANICS ANALYSIS DATA SHEET

TENTATIVELY IDENTIFIED COMPOUNDS
EPA SAMPLE NO.

QCTB 780
Lab Name: TESTAMERICA BURIINGTON

Lab Code: STLV Case No.: SAVANNAH

Matrix: (SOIL/SED/WATER) Water

Sample wt/vol: $25.0 \quad(\mathrm{~g} / \mathrm{mL}) \mathrm{mL}$

Level: (TRACE or LOW/MED) TRACE

\% Moisture: not dec.

GC Column: DB-624

ID : 0.20

Soil Extract Volume:

CONCENTRATION UNITS: (ug/L or $\mathrm{ug} / \mathrm{kg}$ ) ug/L
Contract: 21005

Mod. Ref No.:

SDG No.: 137941
Lab Sample ID: 834125

Lab File ID: 834125

Date Received: $06 / 29 / 2010$

Date Analyzed: 07/07/2010

(mm) Dilution Factor: 1.0

(uL) Soil Aliquot Volume:

(uL)

Purge Volume: 25.0

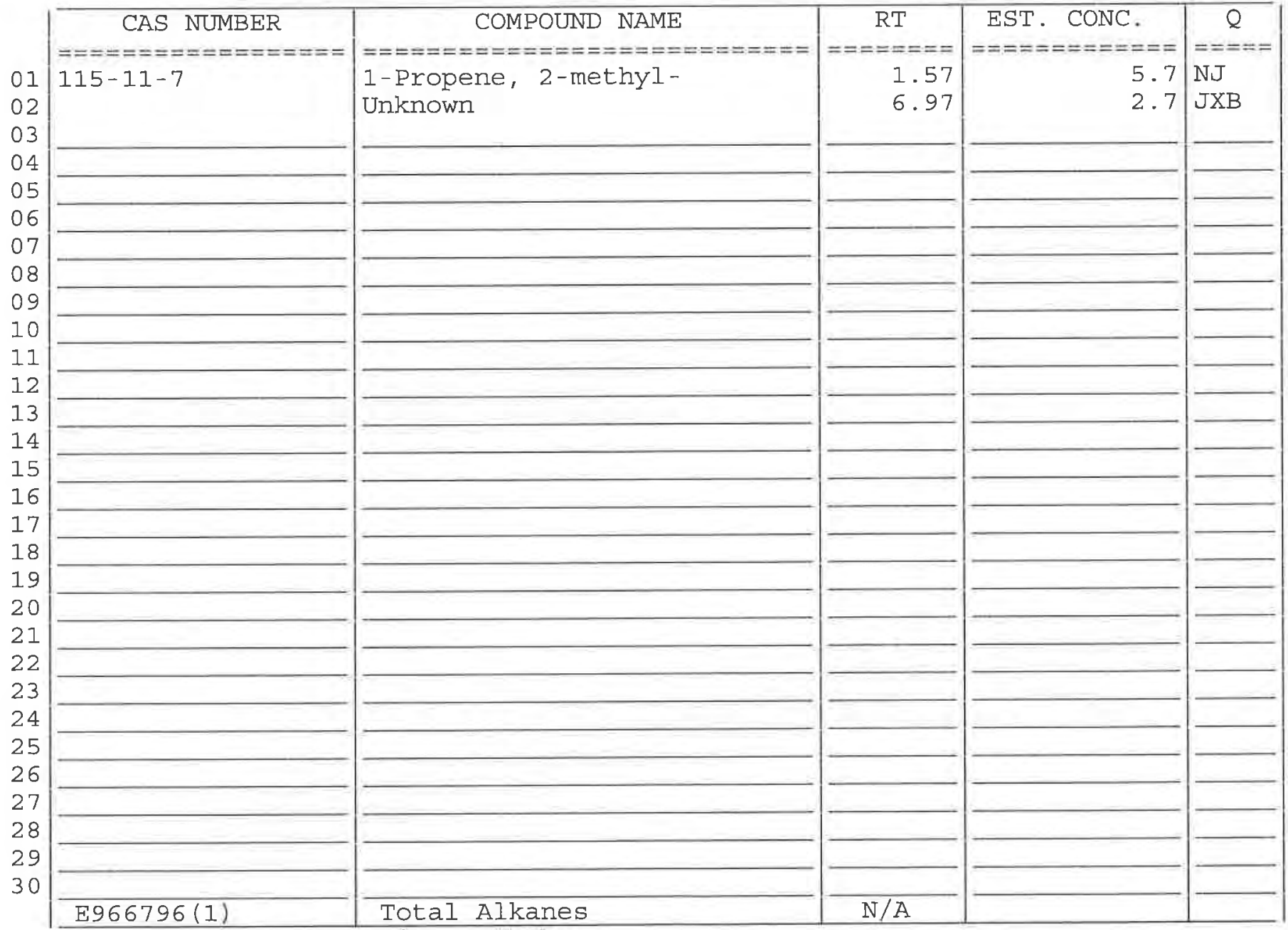

(1) EPA-designated Registry Number. 
$1 A$ - FORM I VOA-1

VOLATILE ORGANICS ANALYSIS DATA SHEET
EPA SAMPLE NO.

VBLKJQ
Lab Name: TESTAMERICA BURLINGTON

Lab Code: STLV Case No.: SAVANNAH Mod. Ref No.:
Contract: 21005
Matrix: (SOIL/SED/WATER) Water

Sample wt/vol: 25.0

$(g / \mathrm{mL}) \mathrm{mL}$

Level: (TRACE/LOW/MED) TRACE

\% Moisture: not dec.

GC Column: DB-624

Soil Extract Volume:

ID $: 0.20$

$(\mathrm{mm})$

(uL)

Soil Aliquot Volume:

(mL)

Lab Sample ID: VBLKJQ

Lab File ID: JBEBO $2 \mathrm{H}$

Date Received:

Date Analyzed: 07/07/2010

Dilution Factor: 1.0

Purge Volume: 25.0
SDG No.: 137941

\begin{tabular}{|c|c|c|c|}
\hline CAS NO. & COMPOUND & $\begin{array}{l}\text { CONCENTRATION UNITS: } \\
(\mathrm{ug} / \mathrm{L} \text { or } \mathrm{ug} / \mathrm{kg}) \mathrm{ug} / \mathrm{L}\end{array}$ & $Q$ \\
\hline 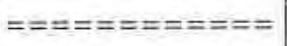 & 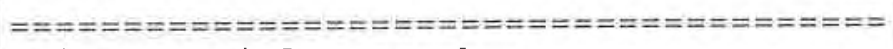 & 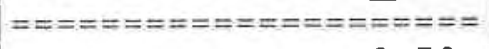 & $==\mathbf{=}==\boldsymbol{=}=$ \\
\hline $75-71-8$ & Dichlorodifluoromethane & 0.50 & U \\
\hline $74-87-3$ & Chloromethane & 0.50 & $\mathrm{U}$ \\
\hline $75-01-4$ & vinyl chloride & 0.50 & $\mathrm{U}$ \\
\hline $74-83-9$ & Bromomethane & 0.50 & $\mathrm{U}$ \\
\hline $75-00-3$ & Chloroethane & 0.50 & $\mathrm{U}$ \\
\hline $75-69-4$ & Trichlorofluoromethane & 0.50 & $\mathrm{U}$ \\
\hline $75-35-4$ & 1,1-Dichloroethene & 0.50 & $\mathrm{U}$ \\
\hline $76-13-1$ & $1,1,2$-Trichloro-1,2,2-trifluoroethane & 0.50 & $\mathrm{U}$ \\
\hline $67-64-1$ & Acetone & 1.7 & $\mathrm{~J}$ \\
\hline $75-15-0$ & Carbon disulfide & 0.50 & $\mathrm{U}$ \\
\hline $79-20-9$ & Methyl acetate & 0.50 & $\mathrm{U}$ \\
\hline $75-09-2$ & Methylene chloride & 0.50 & $\mathrm{U}$ \\
\hline $156-60-5$ & trans-1,2-Dichloroethene & 0.50 & $\mathrm{U}$ \\
\hline $1634-04-4$ & Methyl tert-butyl ether & 0.50 & $\mathrm{U}$ \\
\hline $75-34-3$ & 1,1-Dichloroethane & 0.50 & $\mathrm{U}$ \\
\hline $156-59-2$ & Cis-1,2-Dichloroethene & 0.50 & $\mathrm{U}$ \\
\hline $78-93-3$ & 2-Butanone & 5.0 & $\mathrm{U}$ \\
\hline $74-97-5$ & Bromochloromethane & 0.50 & $\mathrm{U}$ \\
\hline $67-66-3$ & Chloroform & 0.50 & $\mathrm{U}$ \\
\hline $71-55-6$ & $1,1,1$-Trichloroethane & 0.50 & $\mathrm{U}$ \\
\hline $110-82-7$ & Cyclohexane & 0.50 & $\mathrm{U}$ \\
\hline $56-23-5$ & Carbon tetrachloride & 0.50 & $\mathrm{U}$ \\
\hline $71-43-2$ & Benzene & 0.50 & $\mathrm{U}$ \\
\hline $107-06-2$ & 1,2-Dichloroethane & 0.50 & $\mathrm{U}$ \\
\hline
\end{tabular}

Report 1,4-Dioxane for Low-Medium VOA analysis only 
IB - FORM I VOA-2

VOLATILE ORGANICS ANALYSIS DATA SHEET
EPA SAMPLE NO.

VBLKJQ

Contract: 21005
Lab Name: TESTAMERICA BURLINGTON

Lab Code: STLV Case No.: SAVANNAH Mod. Ref No.:
Lab Sample ID: VBLKJQ

Lab File ID: JBEBO $2 \mathrm{H}$

Date Received:

Date Analyzed: 07/07/2010

Dilution Factor: 1.0

Soil Aliquot Volume:

(uL)

(mL)

\begin{tabular}{|c|c|c|c|}
\hline CAS NO. & COMPOUND & $\begin{array}{l}\text { CONCENTRATION UNITS: } \\
(\mathrm{ug} / \mathrm{L} \text { or } \mathrm{ug} / \mathrm{kg}) \mathrm{ug} / \mathrm{L}\end{array}$ & $Q$ \\
\hline$===-=======$ & 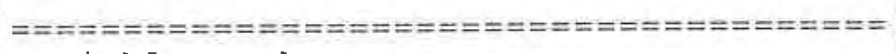 & 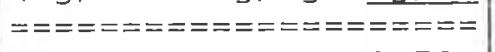 & $=======-$ \\
\hline $79-01-6$ & Trichloroethene & 0.50 & $\mathrm{U}$ \\
\hline $108-87-2$ & Methylcyclohexane & 0.50 & $\mathrm{U}$ \\
\hline $78-87-5$ & 1,2-Dichloropropane & 0.50 & U \\
\hline $75-27-4$ & Bromodichloromethane & 0.50 & $\mathrm{U}$ \\
\hline $10061-01-5$ & cis-1,3-Dichloropropene & 0.50 & $\mathrm{U}$ \\
\hline $108-10-1$ & 4-Methyl-2-pentanone & 5.0 & $\mathrm{U}$ \\
\hline $108-88-3$ & Toluene & 0.50 & $\mathrm{U}$ \\
\hline $10061-02-6$ & trans-1,3-Dichloropropene & 0.50 & $\mathrm{U}$ \\
\hline $79-00-5$ & $1,1,2$-Trichloroethane & 0.50 & $\mathrm{U}$ \\
\hline $127-18-4$ & Tetrachloroethene & 0.50 & $\mathrm{U}$ \\
\hline $591-78-6$ & 2 -Hexanone & 5.0 & $\mathrm{U}$ \\
\hline $124-48-1$ & Dibromochloromethane & 0.50 & $\mathrm{U}$ \\
\hline $106-93-4$ & 1,2 -Dibromoethane & 0.50 & $\mathrm{U}$ \\
\hline $108-90-7$ & Chlorobenzene & 0.50 & $\mathrm{U}$ \\
\hline $100-41-4$ & Ethylbenzene & 0.50 & $\mathrm{U}$ \\
\hline $95-47-6$ & o-xylene & 0.50 & $\mathrm{U}$ \\
\hline $179601-23-1$ & $\mathrm{~m}, \mathrm{p}-\mathrm{Xy}$ lene & 0.50 & U \\
\hline $100-42-5$ & styrene & 0.50 & $\mathrm{U}$ \\
\hline $75-25-2$ & Bromoform & 0.50 & $\mathrm{U}$ \\
\hline $98-82-8$ & Isopropylbenzene & 0.50 & $\mathrm{U}$ \\
\hline $79-34-5$ & $1,1,2,2$-Tetrachloroethane & 0.50 & $\mathrm{U}$ \\
\hline $541-73-1$ & 1,3-Dichlorobenzene & 0.50 & $\mathrm{U}$ \\
\hline $106-46-7$ & 1,4-Dichlorobenzene & 0.50 & $\mathrm{U}$ \\
\hline $95-50-1$ & 1,2-Dichlorobenzene & 0.50 & $\mathrm{U}$ \\
\hline $96-12-8$ & 1,2-Dibromo-3-chloropropane & 0.50 & $\mathrm{U}$ \\
\hline $120-82-1$ & $1,2,4$-Trichlorobenzene & 0.50 & $\mathrm{U}$ \\
\hline $87-61-6$ & $1,2,3$-Trichlorobenzene & 0.50 & $\mathrm{U}$ \\
\hline
\end{tabular}


IJ - FORM I VOA-TIC

VOLATILE ORGANICS ANALYSIS DATA SHEET TENTATIVELY IDENTIFIED COMPOUNDS
EPA SAMPLE NO.

VBLKJQ
Lab Name: TESTAMERICA BURLINGTON

Lab Code: STLV Case No.: SAVANNAH

Matrix: (SOIL/SED/WATER) Water

Sample wt/vol: 25.0 (g/mL) $\mathrm{mL}$

Level: (TRACE or LOW/MED) TRACE

\% Moisture: not dec.

GC Column: DB-624

ID : 0.20

Soil Extract Volume:

CONCENTRATION UNITS: (ug/L or ug/kg) ug/L
Contract: 21005

Mod. Ref No.:

SDG No.: 137941
Lab sample ID: VBLKJQ

Lab File ID: JBEBO2H

Date Received:

Date Analyzed: 07/07/2010

(mm) Dilution Factor: 1.0

(uL) Soil Aliquot Volume:

(uL)

Purge Volume: 25.0

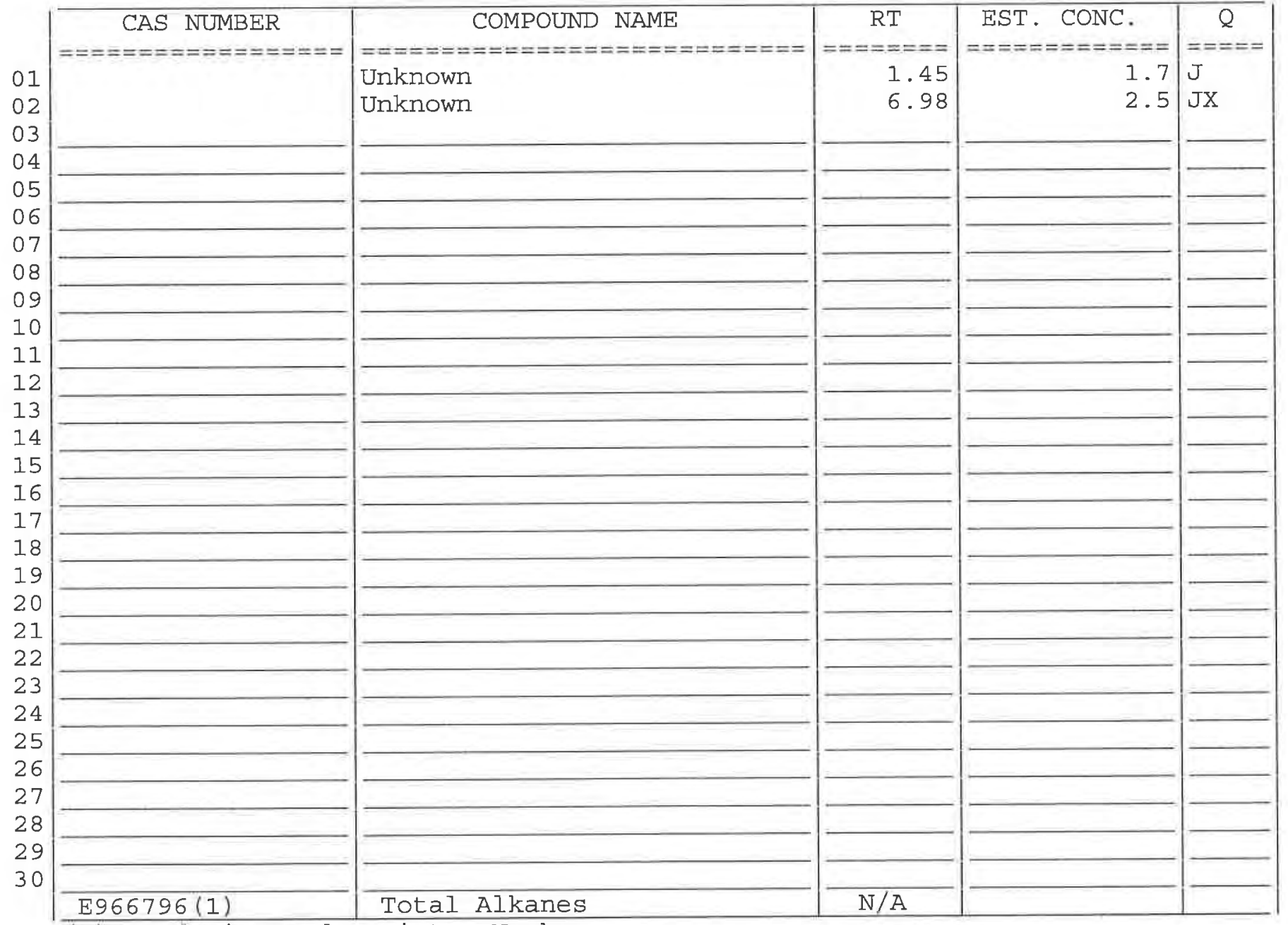

(1) EPA-designated Registry Number. 
$1 A$ - FORM I VOA-1

VOLATILE ORGANICS ANALYSIS DATA SHEET
EPA SAMPLE NO.

VHBLKOI
Lab Name: TESTAMERICA BURLINGTON

Contract : 21005

Lab Code: STLV Case No.: SAVANNAH Mod. Ref No.:

SDG No.: 137941

Matrix: (SOIL/SED/WATER) Water

Sample wt/vol: 25.0

$(\mathrm{g} / \mathrm{mL}) \mathrm{mL}$

Level: (TRACE/LOW/MED) TRACE

\% Moisture: not dec.

GC Column: DB-624

Soil Extract Volume:

Purge Volume: 25.0
Lab Sample ID: 834126

Lab File ID: 834126

Date Received:

Date Analyzed: 07/07/2010

Dilution Factor: 1.0

Soil Aliquot Volume:

(uL)

(mL)

\begin{tabular}{|c|c|c|c|}
\hline CAS NO. & COMPOUND & $\begin{array}{l}\text { CONCENTRATION UNITS: } \\
(\mathrm{ug} / \mathrm{L} \text { or } \mathrm{ug} / \mathrm{kg}) \mathrm{ug} / \mathrm{L}\end{array}$ & Q \\
\hline$==x===x=====$ & 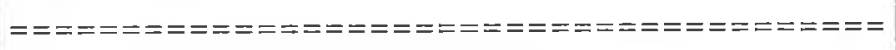 & 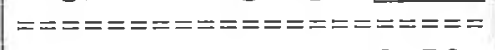 & $==E=====$ \\
\hline $75-71-8$ & Dichlorodifluoromethane & 0.50 & $\mathrm{U}$ \\
\hline $74-87-3$ & Chloromethane & 0.50 & $\mathrm{U}$ \\
\hline $75-01-4$ & Vinyl chloride & 0.50 & $\mathrm{U}$ \\
\hline $74-83-9$ & Bromomethane & 0.50 & $\mathrm{U}$ \\
\hline $75-00-3$ & Chloroethane & 0.50 & $\mathrm{U}$ \\
\hline $75-69-4$ & Trichlorofluoromethane & 0.50 & $\mathrm{U}$ \\
\hline $75-35-4$ & 1,1-Dichloroethene & 0.50 & $\mathrm{U}$ \\
\hline $76-13-1$ & 1,1,2-Trichloro-1,2,2-trifluoroethane & 0.50 & U \\
\hline $67-64-1$ & Acetone & 1.3 & JB \\
\hline $75-15-0$ & Carbon disulfide & 0.50 & $\mathrm{U}$ \\
\hline $79-20-9$ & Methyl acetate & 0.50 & $\mathrm{U}$ \\
\hline $75-09-2$ & Methylene chloride & 0.50 & $\mathrm{U}$ \\
\hline $156-60-5$ & trans-1,2-Dichloroethene & 0.50 & $\mathrm{U}$ \\
\hline $1634-04-4$ & Methyl tert-butyl ether & 0.50 & $\mathrm{U}$ \\
\hline $75-34-3$ & 1,1-Dichloroethane & 0.50 & $\mathrm{U}$ \\
\hline $156-59-2$ & cis-1,2-Dichloroethene & 0.50 & U \\
\hline $78-93-3$ & 2-Butanone & 5.0 & U \\
\hline $74-97-5$ & Bromochloromethane & 0.50 & $\mathrm{U}$ \\
\hline $67-66-3$ & Chloroform & 0.50 & $\mathrm{U}$ \\
\hline $71-55-6$ & 1,1,1-Trichloroethane & 0.50 & $\mathrm{U}$ \\
\hline $110-82-7$ & Cyclohexane & 0.50 & $\mathrm{U}$ \\
\hline $56-23-5$ & Carbon tetrachloride & 0.50 & $\mathrm{U}$ \\
\hline $71-43-2$ & Benzene & 0.50 & $\mathrm{U}$ \\
\hline $107-06-2$ & 1,2-Dichloroethane & 0.50 & $\mathrm{U}$ \\
\hline
\end{tabular}

Report 1,4-Dioxane for Low-Medium VOA analysis only 
IB - FORM I VOA-2

VOLATILE ORGANICS ANALYSIS DATA SHEET
EPA SAMPLE NO.

VHBLKOI
Lab Name: TESTAMERICA BURLINGTON

Case No.: SAVANNAH

Matrix: (SOIL/SED/WATER) Water

Sample wt/vol: 25.0

$(\mathrm{g} / \mathrm{mL}) \mathrm{mL}$

Level: (TRACE/LOW/MED) TRACE

\% Moisture: not dec.

GC Column: DB-624

Soil Extract Volume:

ID : 0.20

(mm)

Contract: 21005
(uL)

Lab Sample ID: 834126

Lab File ID: 834126

Date Received:

Date Analyzed: 07/07/2010

Dilution Factor: 1.0

Soil Aliquot Volume:

(uL)

(mL)

\begin{tabular}{|c|c|c|c|}
\hline CAS NO. & COMPOUND & $\begin{array}{l}\text { CONCENTRATION UNITS: } \\
(\mathrm{ug} / \mathrm{L} \text { or } \mathrm{ug} / \mathrm{kg}) \mathrm{ug} / \mathrm{L}\end{array}$ & $Q$ \\
\hline 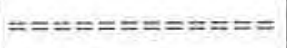 & 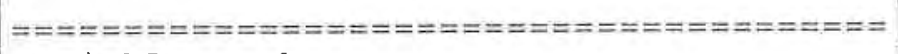 & 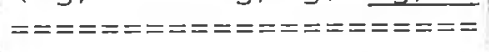 & $========$ \\
\hline $79-01-6$ & Trichloroethene & 0.50 & $\mathrm{U}$ \\
\hline $108-87-2$ & Methylcyclohexane & 0.50 & $\mathrm{U}$ \\
\hline $78-87-5$ & 1,2-Dichloropropane & 0.50 & $\mathrm{U}$ \\
\hline $75-27-4$ & Bromodichloromethane & 0.50 & $\mathrm{U}$ \\
\hline $10061-01-5$ & cis-1,3-Dichloropropene & 0.50 & $\mathrm{U}$ \\
\hline $108-10-1$ & 4-Methyl-2-pentanone & 5.0 & $\mathrm{U}$ \\
\hline $108-88-3$ & Toluene & 0.50 & $\mathrm{U}$ \\
\hline $10061-02-6$ & trans-1,3-Dichloropropene & 0.50 & $\mathrm{U}$ \\
\hline $79-00-5$ & $1,1,2$-Trichloroethane & 0.50 & U \\
\hline $127-18-4$ & Tetrachloroethene & 0.50 & $\mathrm{U}$ \\
\hline $591-78-6$ & 2-Hexanone & 5.0 & $\mathrm{U}$ \\
\hline $124-48-1$ & Dibromochloromethane & 0.50 & $\mathrm{U}$ \\
\hline $106-93-4$ & 1,2 -Dibromoethane & 0.50 & $\mathrm{U}$ \\
\hline $108-90-7$ & Chlorobenzene & 0.50 & $\mathrm{U}$ \\
\hline $100-41-4$ & Ethylbenzene & 0.50 & $\mathrm{U}$ \\
\hline $95-47-6$ & o-Xylene & 0.50 & $\mathrm{U}$ \\
\hline $179601-23-1$ & $\mathrm{~m}, \mathrm{p}-\mathrm{xy}$ lene & 0.50 & $\mathrm{U}$ \\
\hline $100-42-5$ & Styrene & 0.50 & $\mathrm{U}$ \\
\hline $75-25-2$ & Bromoform & 0.50 & $\mathrm{U}$ \\
\hline $98-82-8$ & Isopropylbenzene & 0.50 & $\mathrm{U}$ \\
\hline $79-34-5$ & $1,1,2,2$-Tetrachloroethane & 0.50 & U \\
\hline $541-73-1$ & 1,3 -Dichlorobenzene & 0.50 & $\mathrm{U}$ \\
\hline $106-46-7$ & 1,4-Dichlorobenzene & 0.50 & U \\
\hline $95-50-1$ & 1,2 -Dichlorobenzene & 0.50 & $\mathrm{U}$ \\
\hline $96-12-8$ & 1,2-Dibromo-3-chloropropane & 0.50 & $\mathrm{U}$ \\
\hline $120-82-1$ & $1,2,4$-Trichlorobenzene & 0.50 & $\mathrm{U}$ \\
\hline $87-61-6$ & 1,2,3-Trichlorobenzene & 0.50 & $\mathrm{U}$ \\
\hline
\end{tabular}


1J - FORM I VOA-TIC

VOLATILE ORGANICS ANALYSIS DATA SHEET

TENTATIVELY IDENTIFIED COMPOUNDS
EPA SAMPLE NO.

VHBLKO 1
Lab Name: TESTAMERICA BURLINGTON

Lab Code: STLV Case No.: SAVANNAH

Matrix: (SOIL/SED/WATER) water

Sample wt/vol: $25.0 \quad$ (g/mL) $\mathrm{mL}$

Level: (TRACE or LOW/MED) TRACE

\% Moisture: not dec.

GC Column: DB-624

ID $: 0.20$

Soil Extract Volume:

CONCENTRATION UNITS: (ug/L or ug/kg) ug/L
Contract: 21005

Mod. Ref No.:

SDG No.: 137941
Lab Sample ID: 834126

Lab File ID: 834126

Date Received:

Date Analyzed: 07/07/2010

(mm) Dilution Factor: 1.0

(uL) Soil Aliquot Volume:

(uL)

Purge Volume: 25.0

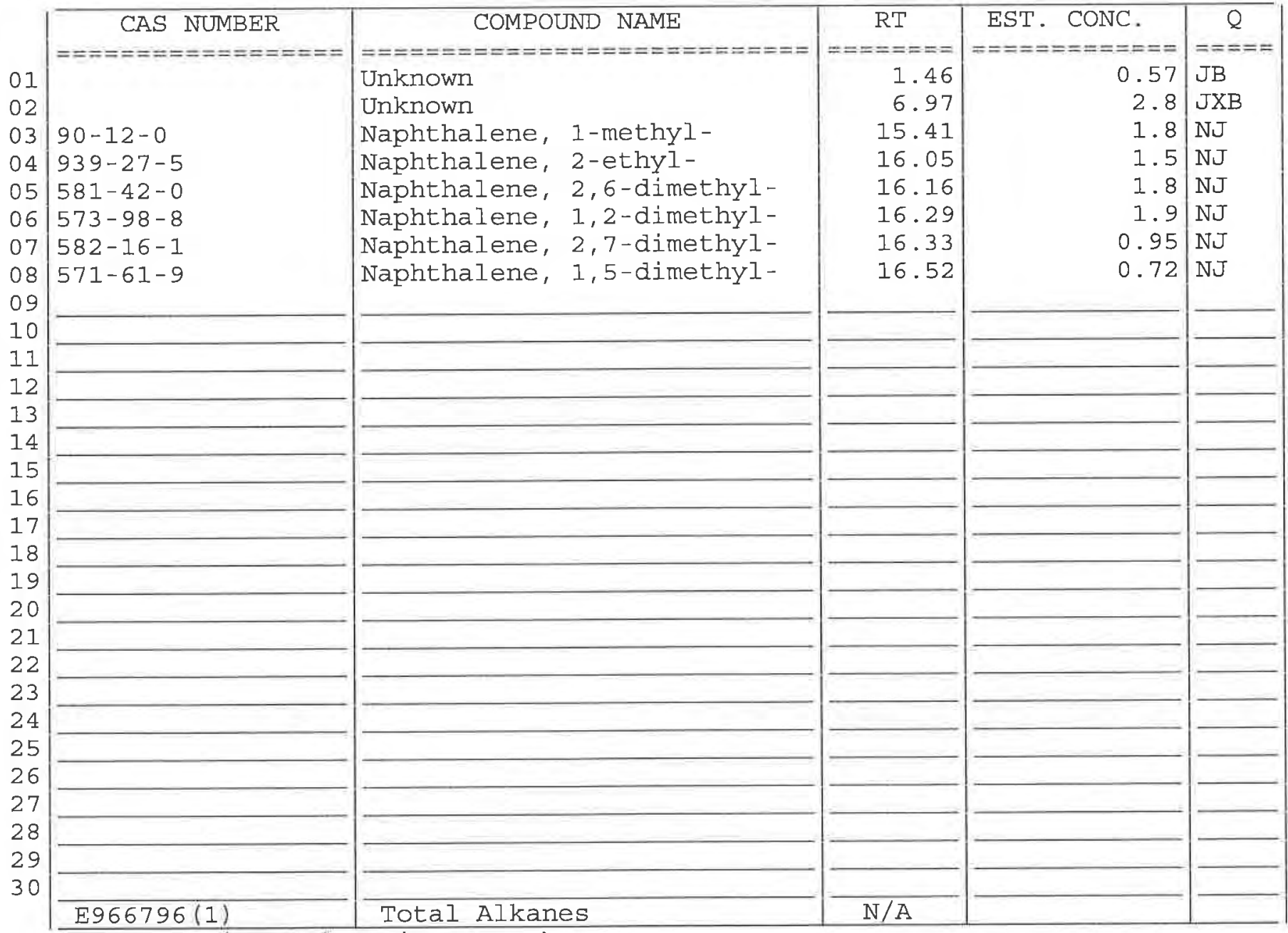

(1) EPA-designated Registry Number. 
IA - FORM I VOA-1

VOLATILE ORGANICS ANALYSIS DATA SHEET
EPA SAMPLE NO.

VIBLKJA
Lab Name: TESTAMERICA BURLINGTON

Lab Code: STLV Case No.: SAVANNAH Mod. Ref No.:
Contract: 21005

SDG No.: 137941

Matrix: (SOIL/SED/WATER) water

Sample wt/vol: 25.0

$(g / m L) m L$

Level: (TRACE/LOW/MED) TRACE

\% Moisture: not dec.

GC Column: DB-624

ID : 0.20

Soil Extract Volume:

Purge volume: 25.0
Lab sample ID: VIBLKJA

Lab File ID: JBEBO3H

Date Received:

Date Analyzed: 07/07/2010

Dilution Factor: 1.0

(uL) Soil Aliquot Volume:

(u工)

(mL)

\begin{tabular}{|c|c|c|c|}
\hline CAS NO. & COMPOUND & $\begin{array}{l}\text { CONCENTRATION UNITS: } \\
(\mathrm{ug} / \mathrm{L} \text { or } \mathrm{ug} / \mathrm{kg}) \mathrm{ug} / \mathrm{L}\end{array}$ & Q \\
\hline$==========1$ & 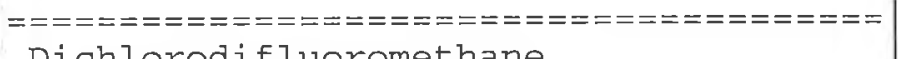 & 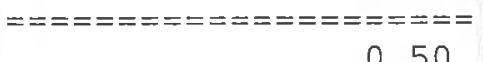 & $=======$ \\
\hline $\begin{array}{l}75-71-8 \\
74-87-3\end{array}$ & $\begin{array}{l}\text { Dichloroditluoromethane } \\
\text { Chloromethane }\end{array}$ & 0.50 & $\mathrm{U}$ \\
\hline $75-01-4$ & Vinyl chloride & 0.50 & $\mathrm{U}$ \\
\hline $74-83-9$ & Bromomethane & 0.50 & $\mathrm{U}$ \\
\hline $75-00-3$ & Chloroethane & 0.50 & $\mathrm{U}$ \\
\hline $75-69-4$ & Trichlorofluoromethane & 0.50 & $\mathrm{U}$ \\
\hline $75-35-4$ & 1,1-Dichloroethene & 0.50 & $\mathrm{U}$ \\
\hline $76-13-1$ & $1,1,2$-Trichloro-1,2,2-trifluoroethane & 0.50 & $\mathrm{U}$ \\
\hline $67-64-1$ & Acetone & 1.6 & JB \\
\hline $75-15-0$ & Carbon disulfide & 0.090 & J \\
\hline $79-20-9$ & Methyl acetate & 0.50 & $\mathrm{U}$ \\
\hline $75-09-2$ & Methylene chloride & 0.50 & $\mathrm{U}$ \\
\hline $156-60-5$ & trans-1,2-Dichloroethene & 0.50 & $\mathrm{U}$ \\
\hline $1634-04-4$ & Methyl tert-butyl ether & 0.50 & $\mathrm{U}$ \\
\hline $75-34-3$ & 1,1-Dichloroethane & 0.50 & $\mathrm{U}$ \\
\hline $156-59-2$ & cis-1,2-Dichloroethene & 0.50 & $\mathrm{U}$ \\
\hline $78-93-3$ & 2-Butanone & 5.0 & $\mathrm{U}$ \\
\hline $74-97-5$ & Bromochloromethane & 0.50 & $\mathrm{U}$ \\
\hline $67-66-3$ & Chloroform & 0.50 & $\mathrm{U}$ \\
\hline $71-55-6$ & $1,1,1$-Trichloroethane & 0.50 & $\mathrm{U}$ \\
\hline $110-82-7$ & Cyclohexane & 0.50 & $\mathrm{U}$ \\
\hline $56-23-5$ & Carbon tetrachloride & 0.31 & J \\
\hline $71-43-2$ & Benzene & 0.50 & $\mathrm{U}$ \\
\hline $107-06-2$ & 1,2-Dichloroethane & 0.50 & $\mathrm{U}$ \\
\hline
\end{tabular}

Report 1,4-Dioxane for Low-Medium VOA analysis only 
$1 B$ - FORM I VOA-2

VOLATILE ORGANICS ANALYSIS DATA SHEET
EPA SAMPLE NO.

VIBLKJA
Lab Name: TESTAMERICA BURLINGTON

Contract: 21005

Lab Code: STLV Case No.: SAVANNAH Mod. Ref No.:

SDG No. : 137941

Matrix: (SOIL/SED/WATER) Water

Sample wt/vol: 25.0

$(\mathrm{g} / \mathrm{mL}) \mathrm{mL}$

Level: (TRACE/LOW/MED) TRACE

\% Moisture: not dec.

GC Column: DB-624

ID : 0.20

$(\mathrm{mm})$

(uL)

Soil Aliquot Volume:

(mL)

Lab Sample ID: VIBLKJA

Lab File ID: JBEBO3H

Date Received:

Date Analyzed: 07/07/2010

Dilution Factor: 1.0

Soil Extract Volume:

Purge Volume: 25.0

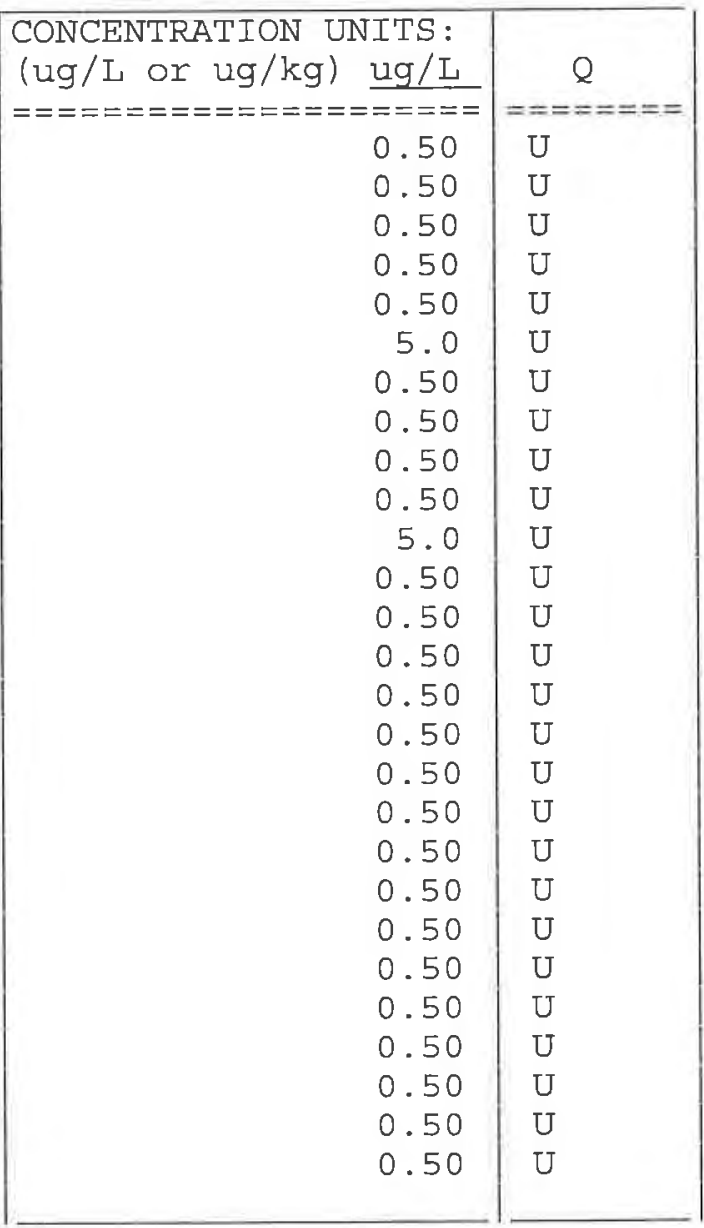


IJ - FORM I VOA-TIC

VOLATILE ORGANICS ANALYSIS DATA SHEET

TENTATIVELY IDENTIFIED COMPOUNDS
EPA SAMPLE NO.

VIBLKJA
Lab Name: TESTAMERICA BURLINGTON

Lab Code: STLV Case No.: SAVANNAH

Matrix: (SOIL/SED/WATER) water

Sample wt/vol: $25.0 \quad(\mathrm{~g} / \mathrm{mL}) \mathrm{mL}$

Level: (TRACE or LOW/MED) TRACE

\% Moisture: not dec.

GC Column: DB-624

ID $: 0.20$

Soil Extract Volume:

CONCENTRATION UNITS: (ug/L or ug/kg) ug/L
Contract: 21005

Mod. Ref No.:

SDG No.: 137941
Lab File ID: JBEBO3H

Date Received:

Date Analyzed: 07/07/2010

(mm) Dilution Factor: 1.0

(uL) Soil Aliquot Volume:

(uL)

Purge Volume: 25.0

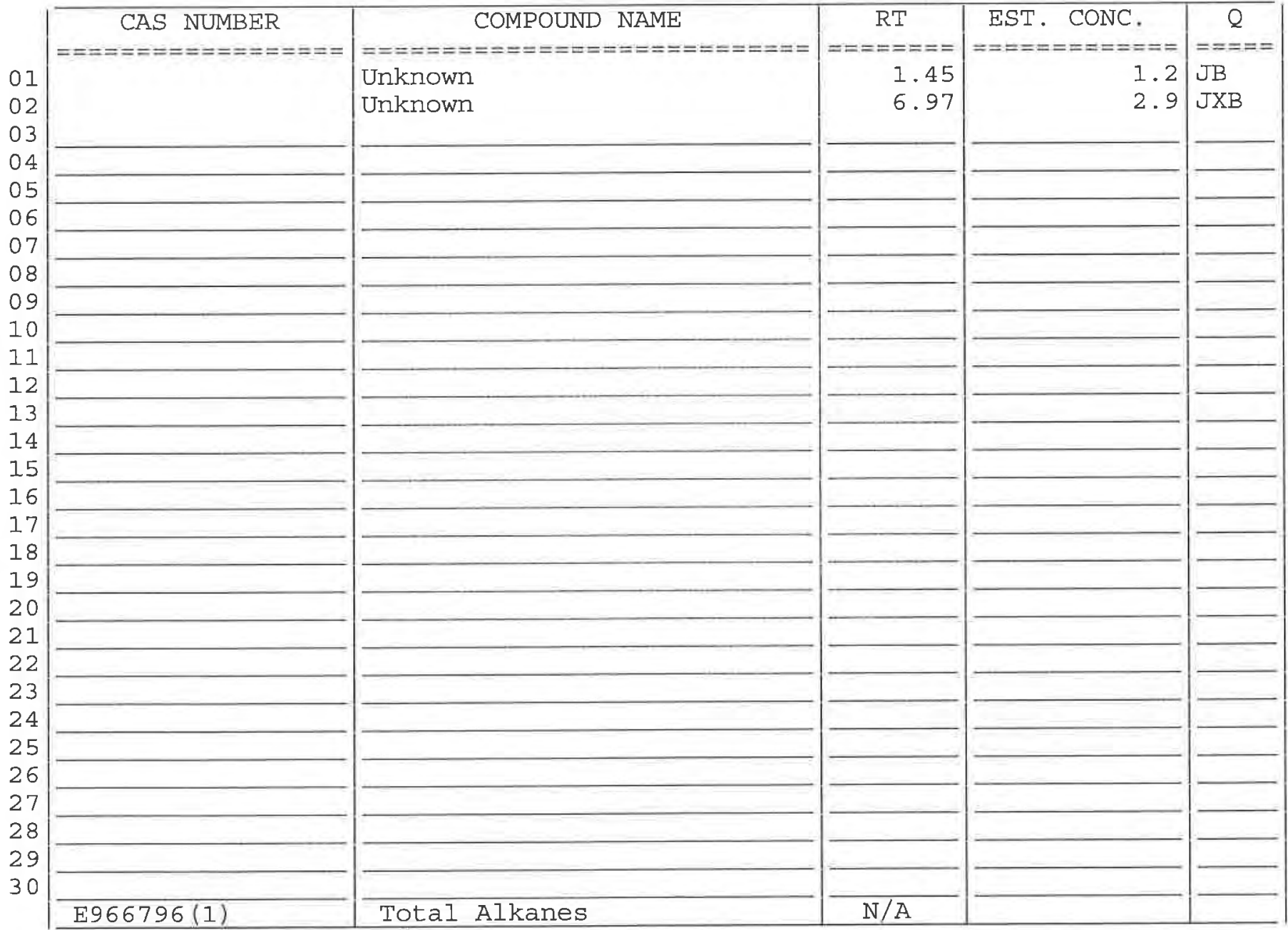

(1) EPA-designated Registry Number. 
$2 A$ - FORM II VOA-1

WATER VOLATILE DEUTERATED MONITORING COMPOUND RECOVERY

Lab Name: TESTAMERICA BURLINGTON

Contract: 21005

Lab Code: STLV Case No.: SAVANNAH Mod. Ref No.:

SDG No.: 137941

Level: (TRACE or LOW) TRACE

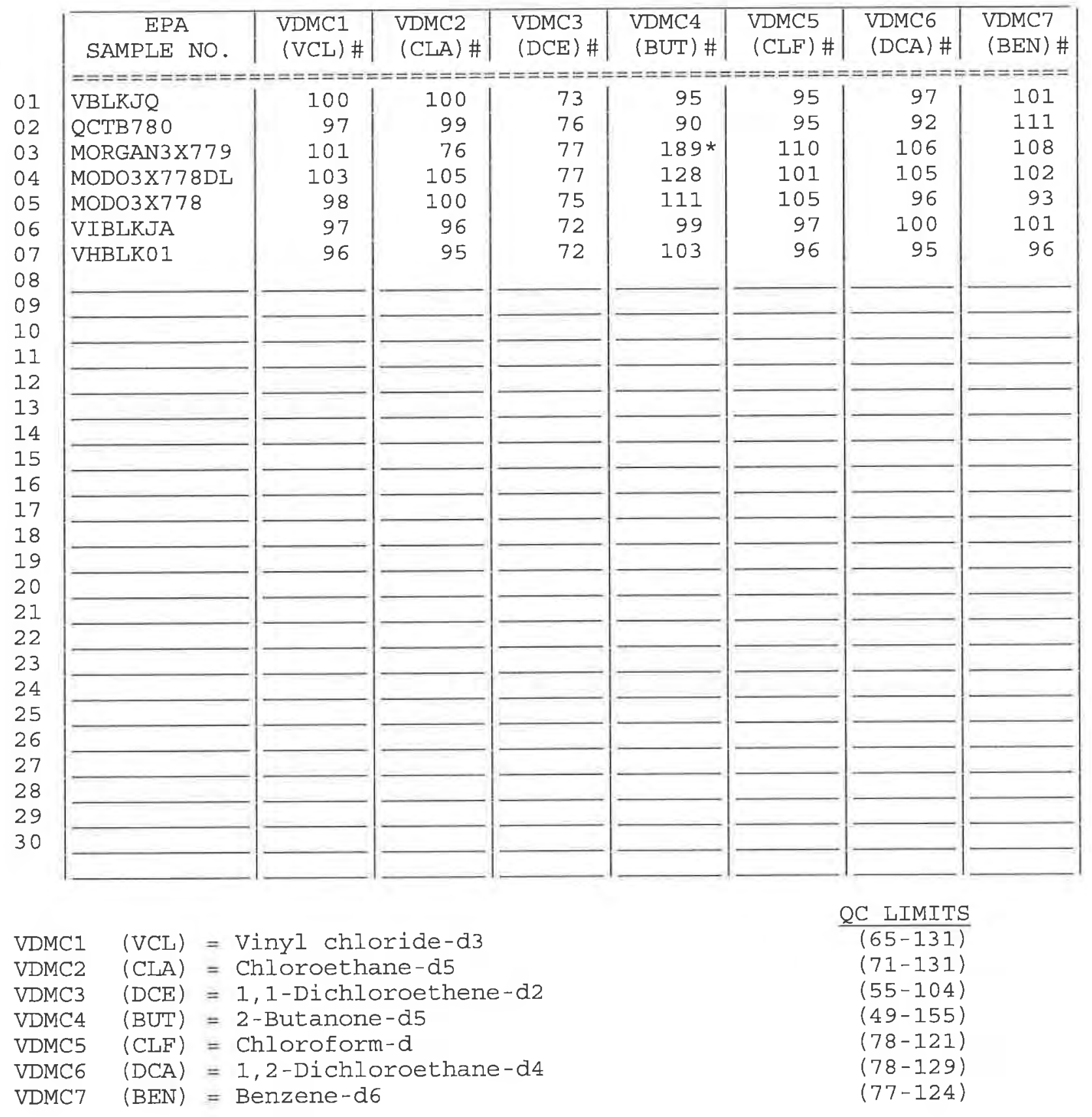

\# Column to be used to flag recovery values

* Values outside of contract required QC limits

Page 1 of 2

SOMO1.2 
2B - FORM II VOA-2

WATER VOLATILE DEUTERATED MONITORING COMPOUND RECOVERY

Lab Name: TESTAMERICA BURLINGTON

Contract: 21005

Lab Code: STLV Case No.: SAVANNAH Mod. Ref No.:

SDG No.: 137941

Level: (TRACE or LOW) TRACE

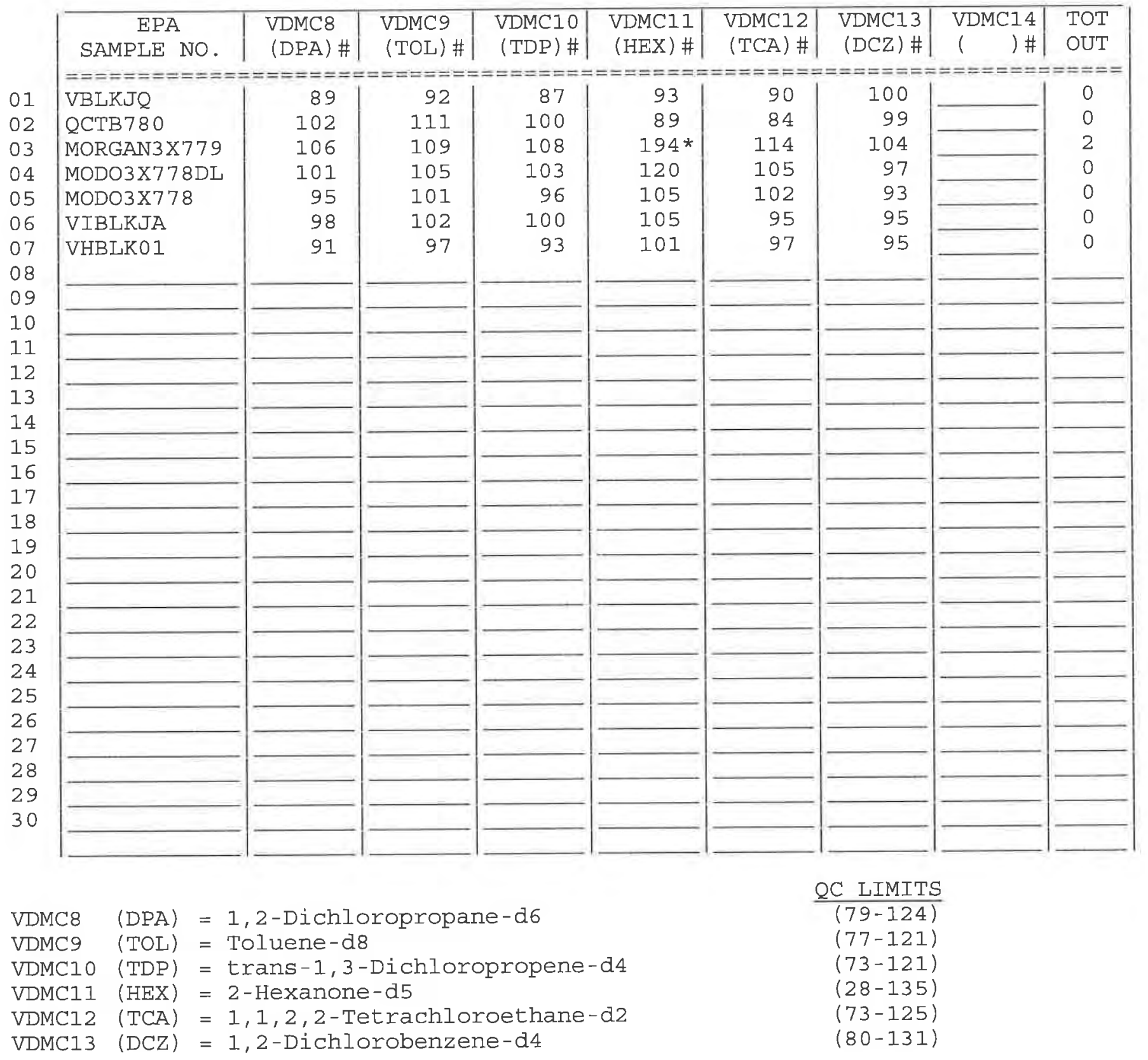

\# Column to be used to flag recovery values

* Values outside of contract required QC limits

Report 1,4-Dioxane-d8 for Low-Medium VOA analysis only Page 2 of 2 
$4 A$ - FORM IV VOA

VOLATILE METHOD BLANK SUMMARY
EPA SAMPLE NO.

VBLKJQ

Lab Name: TESTAMERICA BURLINGTON

Contract: 21005

Lab Code: STLV Case No.: SAVANNAH Mod. Ref No.:

Lab File ID: JBEBO2H

Instrument ID: J.i

Matrix: (SOIL/SED/WATER) Water

Level: (TRACE or LOW/MED) TRACE

GC Column: $\mathrm{DB}-624$
ID : 0.20

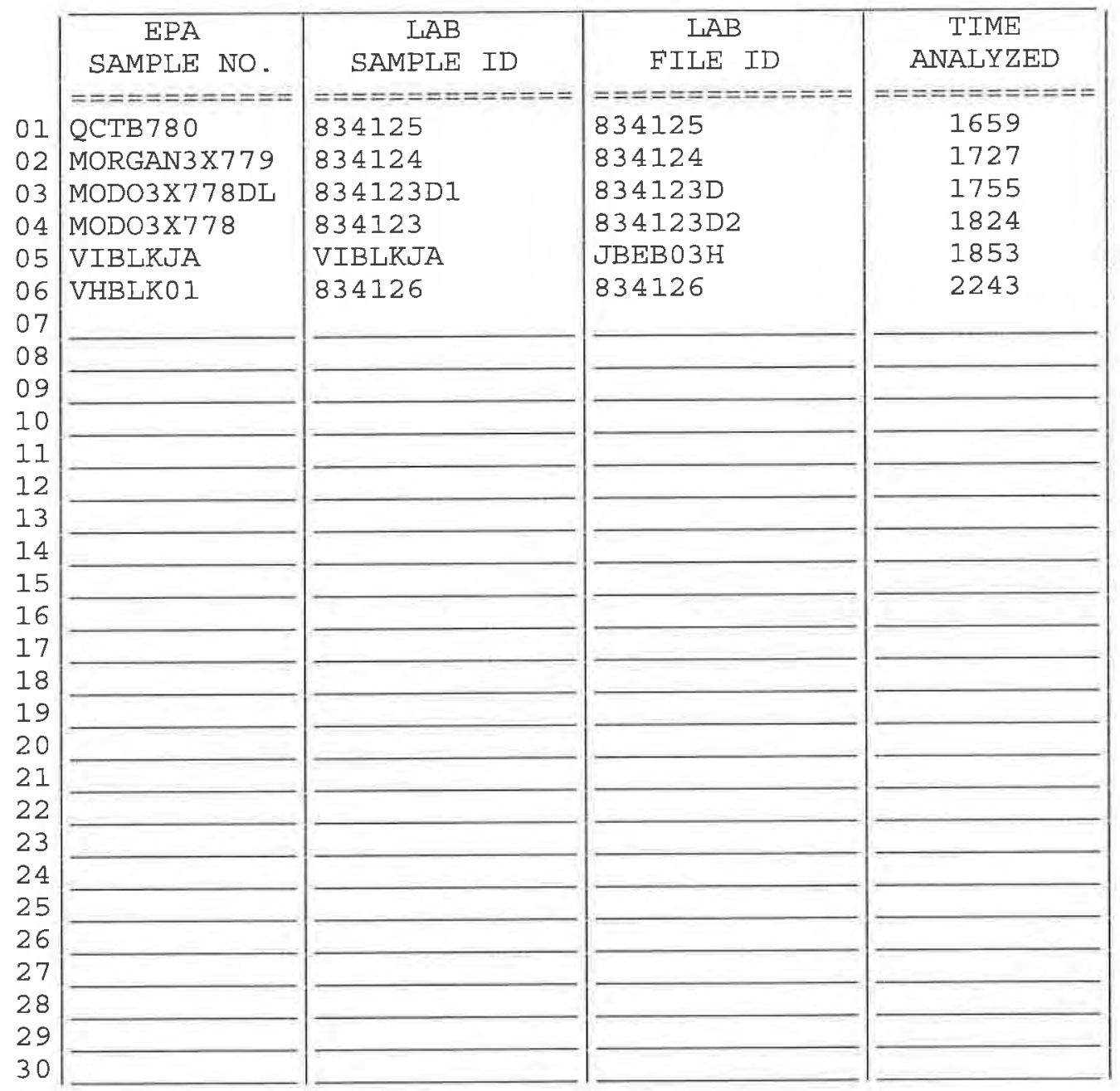

COMMENTS :

Date Analyzed: 07/07/2010

Time Analyzed: 1611

(mm) Heated Purge: (Y/N) N 
$5 A$ - FORM V VOA

VOLATILE ORGANIC INSTRUMENT

PERFORMANCE CHECK

BROMOFLUOROBENZENE (BFB)
EPA SAMPLE NO.

BFBJE

Lab Name: TESTAMERICA BURLINGTON

Contract: 21005

Lab Code: STLV Case No.: SAVANNAH Mod. Ref No.:

SDG No.: 137941

Lab File ID: JBE02PV

BFB Injection Date: 06/25/2010

Instrument ID: J.i

BFB Injection Time: 1015

GC Column: DB-624

ID: $0.20 \quad(\mathrm{~mm})$

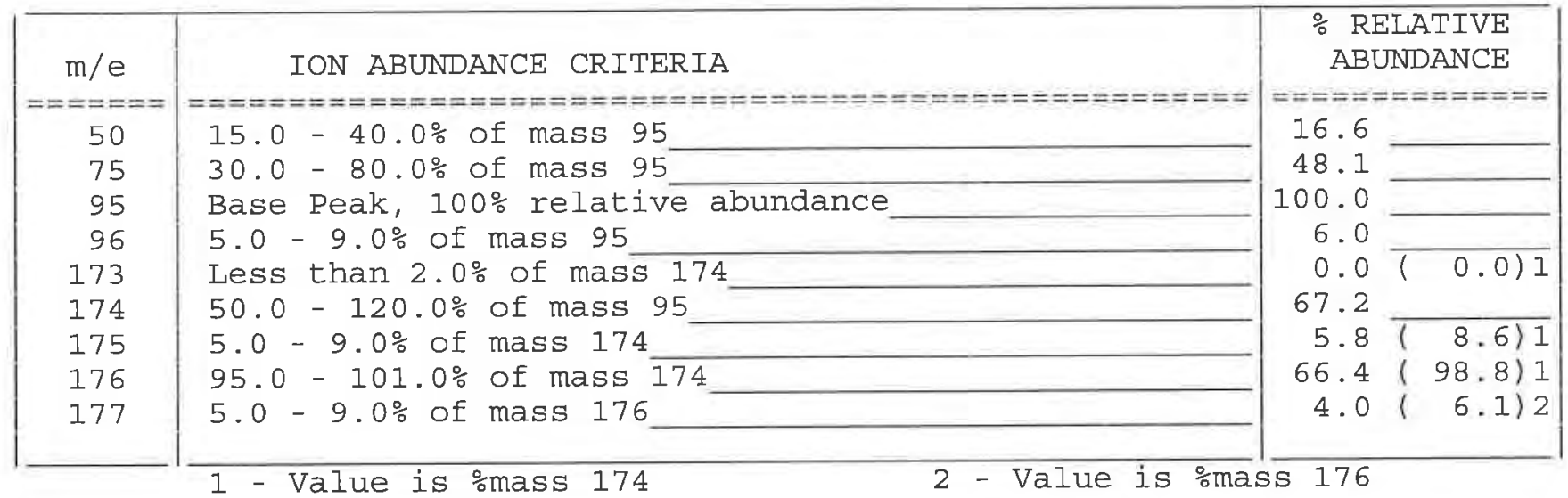

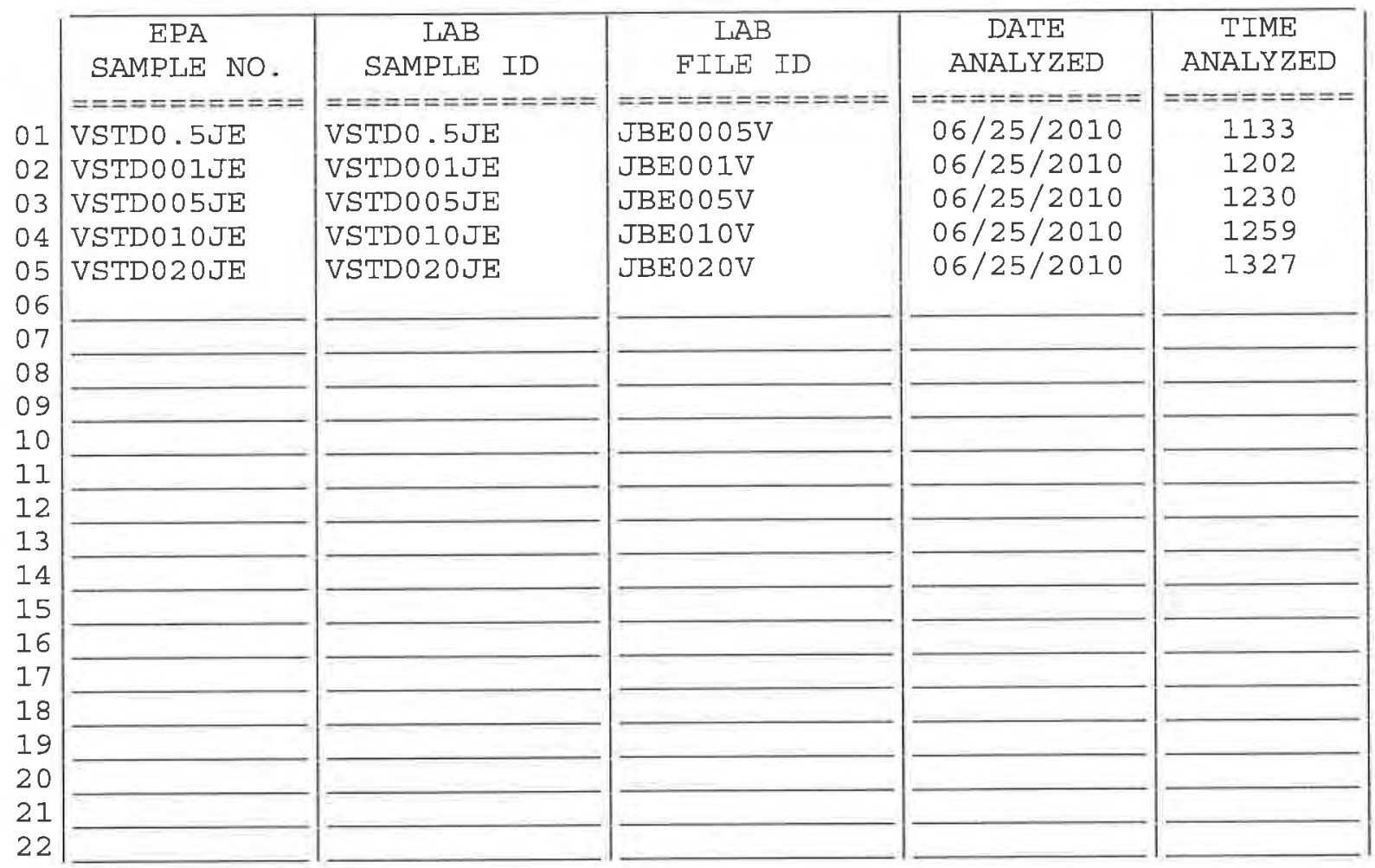


$5 A$ - FORM V VOA

VOLATILE ORGANIC INSTRUMENT

PERFORMANCE CHECK

BROMOFLUOROBENZENE (BFB)
EPA SAMPLE NO.

BFBJQ

SDG No.: 137941

Lab Code: STLV Case No.: SAVANNAH Mod. Ref No.:

BFB Injection Date: 07/07/2010

Lab File ID: JBE22PV

BFB Injection Time: 1455

Instrument ID: J.j

ID $: 0.20 \quad(\mathrm{~mm})$

GC Column: DB-624

(1):

\begin{tabular}{|c|c|c|}
\hline$==\begin{array}{c}\mathrm{m} / \mathrm{e} \\
50\end{array}$ & 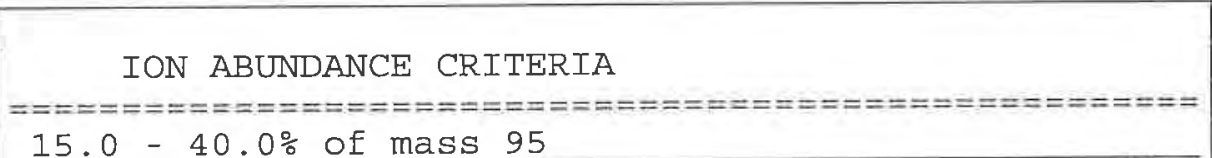 & 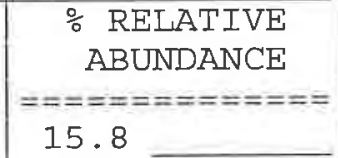 \\
\hline 75 & $30.0-80.0 \%$ of mass 95 & 45.1 \\
\hline 95 & Base Peak, $100 \%$ relative abundance & 100.0 \\
\hline 96 & $5.0-9.0 \%$ of mass 95 & 6.4 \\
\hline 173 & Less than $2.0 \%$ of mass 174 & 0.61 \\
\hline 174 & $50.0-120.0 \%$ of mass 95 & 65.2 \\
\hline 175 & $5.0-9.0 \%$ of mass 174 & 5.71 \\
\hline 176 & $95.0-101.0 \%$ of mass 174 & $64.3(98.6) 1$ \\
\hline 177 & $5.0-9.0 \%$ of mass 176 & 4.21 \\
\hline
\end{tabular}

\begin{tabular}{|c|c|c|c|c|c|}
\hline & EPA & LAB & $\stackrel{L A B}{A}$ & $\begin{array}{c}\text { DATE } \\
\text { ANATYZED }\end{array}$ & $\begin{array}{c}\text { TIME } \\
\text { ANALYZED }\end{array}$ \\
\hline & $\begin{array}{l}\text { SAMPLE NO. } \\
===========\end{array}$ & $\begin{array}{c}\text { SAMPLE ID } \\
============\end{array}$ & 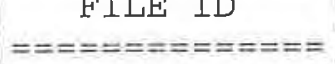 & $\begin{array}{c}\text { ANA } \perp Y Z E D \\
============\end{array}$ & $\begin{array}{l}\text { ANA } \perp Y Z E D \\
=========\end{array}$ \\
\hline & VSTD005JQ & VSTD005JQ & JBE0 05HV & $07 / 07 / 2010$ & 1516 \\
\hline & VBLKJQ & VBLKJQ & JBEBO2H & $07 / 07 / 2010$ & 1611 \\
\hline & QCTB780 & 834125 & 834125 & $07 / 07 / 2010$ & 1659 \\
\hline & MORGAN3X'779 & 834124 & 834124 & $07 / 07 / 2010$ & 1727 \\
\hline & MODO3X778DL & $834123 D 1$ & $834123 D$ & $07 / 07 / 2010$ & 1755 \\
\hline & MODO3X778 & 834123 & $834123 D 2$ & $07 / 07 / 2010$ & 1824 \\
\hline & VIBLKJA & VIBLKJA & JBEB03H & $07 / 07 / 2010$ & 1853 \\
\hline & VHBLKO 1 & 834126 & 834126 & $07 / 07 / 2010$ & 2243 \\
\hline & VSTD005QJ & VSTD005QJ & JBE05HC1 & $07 / 07 / 2010$ & 2311 \\
\hline & & & & & \\
\hline & & & & & \\
\hline & & & & & \\
\hline & & & & & \\
\hline & & & & & \\
\hline & & & & & \\
\hline & & & & & \\
\hline & & & & & \\
\hline & & & & & \\
\hline & & & & & \\
\hline & & & & & \\
\hline & & & & & \\
\hline
\end{tabular}


$6 A$ - FORM VI VOA-1

VOLATILE ORGANICS INITIAL CALIBRATION DATA

Lab Name: TESTAMERICA BURLINGTON

Lab Code: STLV Case No.: SAVANNAH Mod. Ref No.: contract: 21005

Calibration Date(s): 06/25/2010 06/25/2010

Calibration Time (s): $1133 \quad 1327$

(mL)

ID : 0.20 (mm) Length: $25 \quad$ (m)

\begin{tabular}{|c|c|c|c|c|c|c|c|c|}
\hline $\begin{array}{l}\text { LAB FILE ID: } \\
\text { RRF5.0 = JBE005V }\end{array}$ & \multicolumn{3}{|c|}{$\begin{array}{l}\text { RRF0.5 = JBE0005V } \\
\text { RRF10 = JBE010V }\end{array}$} & \multicolumn{3}{|c|}{$\begin{array}{l}\text { RRF1.0 = JBE001V } \\
\text { RRF20 = JBE020V }\end{array}$} & \multirow{2}{*}{\multicolumn{2}{|c|}{$\% R S D$}} \\
\hline COMPOUND & & RRF 0.5 & RRF1. 0 & RRF5. 0 & RRF10 & RRF20 & & \\
\hline \multicolumn{9}{|c|}{ 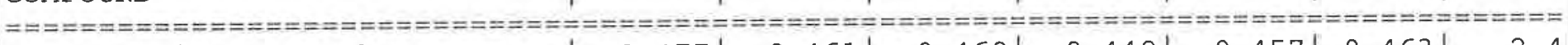 } \\
\hline Dichlorodifluoromethane & & 0.477 & 0.461 & 0.469 & 0.449 & 0.457 & 0.463 & 2.4 \\
\hline Chloromethane & & 0.533 & 0.520 & 0.513 & 0.485 & 0.504 & 0.511 & 3.5 \\
\hline Vinyl chloride & & 0.525 & 0.535 & 0.528 & 0.498 & 0.498 & 0.517 & 3.4 \\
\hline Bromomethane & & 0.170 & 0.163 & 0.163 & 0.169 & 0.185 & 0.170 & 5.4 \\
\hline Chloroethane & & 0.334 & 0.339 & 0.304 & 0.284 & 0.291 & 0.311 & 8.1 \\
\hline Trichlorofluoromethane & & 0.551 & 0.533 & 0.546 & 0.514 & 0.520 & 0.533 & 3.0 \\
\hline 1,1-Dichloroethene & & 0.320 & 0.334 & 0.330 & 0.313 & 0.318 & 0.323 & 2.7 \\
\hline $1,1,2$-Trichloro- & & & & & & & & \\
\hline $1,2,2$-trifluoroethane & & 0.347 & 0.351 & 0.346 & 0.333 & 0.340 & 0.343 & 2.1 \\
\hline Acetone & & 0.020 & 0.015 & 0.014 & 0.014 & 0.014 & 0.015 & 17.5 \\
\hline Carbon disulfide & & 1.242 & 1.153 & 1.100 & 1.046 & 1.066 & 1.121 & 7.0 \\
\hline Methyl acetate & & 0.076 & 0.057 & 0.051 & 0.048 & 0.050 & 0.056 & 20.0 \\
\hline Methylene chloride & & 0.233 & 0.244 & 0.254 & 0.242 & 0.248 & 0.244 & 3.2 \\
\hline $\operatorname{trans}-1,2$-Dichloroethene & & 0.334 & 0.328 & 0.328 & 0.317 & 0.325 & 0.327 & 1.9 \\
\hline Methyl tert-butyl ether & & 0.366 & 0.377 & 0.375 & 0.359 & 0.373 & 0.370 & 2.1 \\
\hline 1,1-Dichloroethane & & 0.616 & 0.643 & 0.641 & 0.613 & 0.617 & 0.626 & 2.3 \\
\hline cis-1,2-Dichloroethene & & 0.288 & 0.322 & 0.320 & 0.300 & 0.304 & 0.307 & 4.7 \\
\hline 2-Butanone & & 0.027 & 0.027 & 0.026 & 0.026 & 0.027 & 0.027 & 2.9 \\
\hline Bromochloromethane & * & 0.071 & 0.071 & 0.079 & 0.074 & 0.074 & 0.074 & 4.0 \\
\hline Chloroform & & 0.514 & 0.533 & 0.507 & 0.486 & 0.494 & 0.507 & 3.6 \\
\hline $1,1,1$-Trichloroethane & & 0.582 & 0.671 & 0.661 & 0.636 & 0.658 & 0.662 & 2.6 \\
\hline Cyclohexane & & 0.914 & 0.980 & 0.963 & 0.901 & 0.924 & 0.937 & 3.6 \\
\hline Carbon tetrachloride & & 0.549 & 0.565 & 0.555 & 0.527 & 0.544 & 0.548 & 2.6 \\
\hline Benzene & & 1.893 & 1.941 & 1.870 & 1.767 & 1.826 & 1.859 & 3.6 \\
\hline 1,2-Dichloroethane & & 0.192 & 0.204 & 0.198 & 0.195 & 0.198 & 0.197 & 2.3 \\
\hline Trichloroethene & & 0.467 & 0.464 & 0.454 & 0.432 & 0.440 & 0.451 & 3.4 \\
\hline Methylcyclohexane & & 0.744 & 0.749 & 0.751 & 0.709 & 0.712 & 0.733 & 2.8 \\
\hline
\end{tabular}

Report 1,4-Dioxane for Low-Medium VOA analysis only 
$6 \mathrm{~B}$ - FORM VI VOA-2

VOLATILE ORGANICS INITIAL CALIBRATION DATA

Lab Name: TESTAMERICA BURLINGTON

Lab Code: STLV Case No.: SAVANNAH Mod. Ref No.:
Contract: 21005

Calibration Date (s): 06/25/2010 06/25/2010

Calibration Time (s): $1133 \quad 1327$

(m工)

Purge Volume: 25.0

GC Column: DB-624

ID : $0.20 \quad(\mathrm{~mm})$

Length: 25

(m)

\begin{tabular}{|c|c|c|c|c|c|c|c|}
\hline $\begin{array}{l}\text { LAB FILE ID: } \\
\text { RRF5.0 = JBE005V }\end{array}$ & \multicolumn{2}{|c|}{$\begin{array}{l}\text { RRF0.5 = JBE0005V } \\
\text { RRF10 = JBE010V }\end{array}$} & \multicolumn{3}{|c|}{$\begin{array}{l}\text { RRF1.0 = JBE001V } \\
\text { RRF20 = JBE020V }\end{array}$} & \multirow{2}{*}{\multicolumn{2}{|c|}{$\because \mathrm{RSD}$}} \\
\hline COMPOUND & RRF0. 5 & RRF1. 0 & RRF5.0 & RRF10 & RRF20 & & \\
\hline \multicolumn{8}{|c|}{ 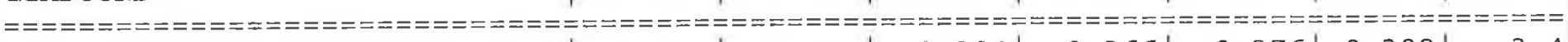 } \\
\hline 1,2-Dichloropropane & 0.381 & 0.401 & 0.384 & 0.366 & 0.376 & 0.382 & 3.4 \\
\hline Bromodichloromethane & 0.368 & 0.396 & 0.391 & 0.372 & 0.389 & 0.383 & 3.2 \\
\hline Cis-1,3-Dichloropropene & 0.481 & 0.516 & 0.511 & 0.491 & 0.504 & 0.501 & 2.9 \\
\hline 4-Methyl-2-pentanone & 0.084 & 0.085 & 0.088 & 0.087 & 0.091 & 0.087 & 3.5 \\
\hline Toluene & 1.916 & 1.972 & 1.949 & 1.828 & 1.908 & 1.915 & 2.9 \\
\hline trans-1,3-Dichloropropene & 0.345 & 0.354 & 0.350 & 0.331 & 0.350 & 0.346 & 2.6 \\
\hline 1,1,2-Trichloroethane & 0.167 & 0.144 & 0.161 & 0.144 & 0.147 & 0.153 & 7.0 \\
\hline Tetrachloroethene & 0.343 & 0.333 & 0.329 & 0.310 & 0.321 & 0.327 & 3.8 \\
\hline 2-Hexanone & 0.056 & 0.055 & 0.057 & 0.056 & 0.059 & 0.057 & 3.1 \\
\hline Dibromochloromethane & 0.182 & 0.171 & 0.170 & 0.166 & 0.173 & 0.172 & 3.5 \\
\hline 1,2-Dibromoethane & 0.123 & 0.128 & 0.127 & 0.123 & 0.130 & 0.126 & 2.5 \\
\hline Chlorobenzene & 0.991 & 0.983 & 0.982 & 0.947 & 0.985 & 0.978 & 1.8 \\
\hline Ethylbenzene & 2.119 & 2.211 & 2.186 & 2.096 & 2.180 & 2.159 & 2.3 \\
\hline o-Xylene & 0.705 & 0.707 & 0.722 & 0.684 & 0.724 & 0.709 & 2.3 \\
\hline $\mathrm{m}, \mathrm{p}$-xylene & 0.769 & 0.810 & 0.790 & 0.767 & 0.805 & 0.788 & 2.5 \\
\hline styrene & 0.963 & 1.035 & 1.045 & 1.022 & 1.066 & 1.026 & 3.8 \\
\hline Bromoform & 0.167 & 0.176 & 0.177 & 0.175 & 0.174 & 0.174 & 2.4 \\
\hline Isopropylbenzene & 2.048 & 2.039 & 2.105 & 2.027 & 2.123 & 2.068 & 2.1 \\
\hline $1,1,2,2$-Tetrachloroethane & 0.133 & 0.145 & 0.147 & 0.135 & 0.144 & 0.141 & 4.6 \\
\hline 1,3-Dichlorobenzene & 1.613 & 1.586 & 1.588 & 1.548 & 1.604 & 1.588 & 1.6 \\
\hline 1,4-Dichlorobenzene & 1.527 & 1.528 & 1.521 & 1.467 & 1.504 & 1.509 & 1.7 \\
\hline 1,2-Dichlorobenzene & 1.114 & 1.162 & 1.162 & 1.118 & 1.155 & 1.142 & 2.1 \\
\hline 1,2-Dibromo-3-chloropropane & 0.042 & 0.044 & 0.048 & 0.046 & 0.048 & 0.046 & 5.8 \\
\hline $1,2,4$-Trichlorobenzene & 0.651 & 0.713 & 0.676 & 0.685 & 0.698 & 0.685 & 3.4 \\
\hline 1,2,3-Trichlorobenzene & 0.427 & 0.481 & 0.491 & 0.473 & 0.503 & 0.475 & 6.2 \\
\hline
\end{tabular}




$$
\text { 6C - FORM VI VOA-3 }
$$

VOLATILE ORGANICS INITIAL CALIBRATION DATA

Lab Name: TESTAMERICA BURIINGTON

Lab Code: STLV Case No.: SAVANNAH

Instrument ID: J.i

Heated Purge: $(Y / N) N$

Purge volume: 25.0

GC Column: DB-624

$(\mathrm{mL})$
Contract : 21005

Mod. Ref No.:

SDG No. : 137941

Calibration Date(s): 06/25/2010 06/25/2010

Calibration Time(s): $1133 \quad 1327$

ID: $0.20 \quad(\mathrm{~mm}) \quad$ Length: $25 \quad$ (m)

\begin{tabular}{|c|c|c|c|c|c|c|c|}
\hline $\begin{array}{l}\text { LAB FILE ID: } \\
\text { RRF5.0 = JBE005V }\end{array}$ & $\begin{array}{l}0.5=\mathrm{JBE} \\
10=\mathrm{JBEC}\end{array}$ & $\begin{array}{l}80005 \mathrm{~V} \\
010 \mathrm{~V}\end{array}$ & & $\begin{array}{l}\operatorname{RF1} 10= \\
\operatorname{RF} 20=\mathrm{J}\end{array}$ & $\begin{array}{l}\text { JBE001V } \\
\text { BE020V }\end{array}$ & & \\
\hline COMPOUND & RRF0. 5 & RRF1.0 & RRF5. 0 & RRF10 & RRF20 & $\begin{array}{c}\overrightarrow{R R F} \\
==== \pm\end{array}$ & $\because \mathrm{RSD}$ \\
\hline & & & 0 & $==== \pm$ & 0.425 & 0.428 & $===$ \\
\hline $\begin{array}{l}\text { Vinyı chlorlde-ds } \\
\text { Chloroethane-d5 }\end{array}$ & 0.420 & 0.434 & $\begin{array}{l}0.441 \\
0.350\end{array}$ & $\begin{array}{l}0.419 \\
0.332\end{array}$ & 0.331 & 0.341 & $\begin{array}{l}2.2 \\
4.0\end{array}$ \\
\hline $\begin{array}{l}\text { Chloroethane- } d b \\
1,1-\text { Dichloroethene- } d 2\end{array}$ & 0.332 & $\begin{array}{l}0.361 \\
0.737\end{array}$ & 0.733 & 0.692 & 0.703 & 0.714 & $\begin{array}{l}4.0 \\
2.8\end{array}$ \\
\hline & $\begin{array}{l}0.705 \\
0.022\end{array}$ & 0.023 & 0.025 & 0.025 & 0.027 & 0.024 & 7.2 \\
\hline Chloroform-d & 0.523 & 0.528 & 0.533 & 0.510 & 0.517 & 0.522 & 1.7 \\
\hline 1,2 -Dichloroethane-d4 & 0.153 & 0.164 & 0.159 & 0.152 & 0.158 & 0.158 & 3.0 \\
\hline Benzene-d6 & 1.700 & 1.750 & 1.729 & 1.643 & 1.685 & 1.701 & 2.4 \\
\hline 1,2-Dichloropropane-d6 & 0.413 & 0.455 & 0.444 & 0.413 & 0.425 & 0.430 & 4.4 \\
\hline Toluene-d8 & 1.538 & 1.566 & 1.563 & 1.487 & 1.530 & 1.537 & 2.1 \\
\hline trans-1,3-Dichloropropene-d4 & 0.303 & 0.304 & 0.301 & 0.292 & 0.306 & 0.301 & 1.9 \\
\hline 2 -Hexanone-d5 & 0.027 & 0.025 & 0.030 & 0.029 & 0.031 & 0.028 & 8.1 \\
\hline $1,1,2,2$-Tetrachloroethane-d2 & 0.131 & 0.142 & 0.138 & 0.133 & 0.141 & 0.137 & 3.7 \\
\hline 1,2-Dichlorobenzene-d4 & 0.709 & 0.743 & 0.739 & 0.684 & 0.711 & 0.717 & 3.4 \\
\hline
\end{tabular}

Report 1,4-Dioxane-d8 for Low-Medium VOA analysis only 
$7 A$ - FORM VII VOA-1

VOLATILE CONTINUING CALIBRATION DATA

Lab Name: TESTAMERICA BURLINGTON

Lab Code: STLV Case No.: SAVANNAH Mod. Ref No.:
Contract : 21005

Calibration Date: 07/07/2010 Time: 1516

Init. Calib. Date(s): 06/25/2010 06/25/2010

EPA Sample No. (VSTD\#\#\#\#): VSTD005JQ Init. Calib. Time(s): 113311327

Heated Purge: ( $\mathrm{Y} / \mathrm{N}$ ) N GC Column: DB-624 ID: 0.20 (mm) Length: 25 (m)

Purge Volume: 25.0

$(\mathrm{mL})$

\begin{tabular}{|c|c|c|c|c|c|}
\hline COMPOUND & $\overline{\mathrm{RRF}}$ & RRF5. 0 & $\begin{array}{l}\text { MIN } \\
\text { RRF }\end{array}$ & $\div \mathrm{D}$ & $\mathrm{MAX} \div \mathrm{D}$ \\
\hline \multicolumn{6}{|c|}{ 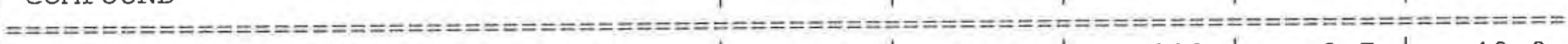 } \\
\hline Dichlorodifluoromethane & 0.463 & 0.418 & 0.010 & -9.7 & 40.0 \\
\hline Chloromethane & 0.511 & 0.423 & 0.010 & -17.3 & 40.0 \\
\hline Vinyl chloride & 0.517 & 0.462 & 0.100 & -10.5 & 30.0 \\
\hline Bromomethane & 0.170 & 0.194 & 0.100 & 13.9 & 30.0 \\
\hline Chloroethane & 0.311 & 0.376 & 0.010 & 21.0 & 40.0 \\
\hline Trichlorofluoromethane & 0.533 & 0.533 & 0.010 & -0.0 & 40.0 \\
\hline 1,1-Dichloroethene & 0.323 & 0.320 & 0.100 & -0.9 & 30.0 \\
\hline $1,1,2$-Trichloro-1,2,2-trifluoroethane & 0.343 & 0.349 & 0.010 & 1.8 & 40.0 \\
\hline Acetone & 0.015 & 0.013 & 0.010 & -16.6 & 40.0 \\
\hline Carbon disulfide & 1.121 & 1.012 & 0.010 & -9.8 & 40.0 \\
\hline Methyl acetate & 0.056 & 0.043 & 0.010 & -24.5 & 40.0 \\
\hline Methylene chloride & 0.244 & 0.235 & 0.010 & -3.8 & 40.0 \\
\hline trans-1,2-Dichloroethene & 0.327 & 0.337 & 0.010 & 3.2 & 40.0 \\
\hline Methyl tert-butyl ether & 0.370 & 0.337 & 0.010 & -8.8 & 40.0 \\
\hline 1,1-Dichloroethane & 0.626 & 0.567 & 0.200 & -9.5 & 30.0 \\
\hline cis-1,2-Dichloroethene & 0.307 & 0.308 & 0.010 & 0.5 & 40.0 \\
\hline 2-Butanone & 0.027 & 0.022 & 0.010 & -18.6 & 40.0 \\
\hline Bromochloromethane & 0.074 & 0.081 & 0.050 & 9.6 & 30.0 \\
\hline Chloroform & 0.507 & 0.484 & 0.200 & -4.5 & 30.0 \\
\hline $1,1,1-T r i c h l o r o e t h a n e$ & 0.662 & 0.652 & 0.100 & -1.4 & 30.0 \\
\hline Cyclohexane & 0.937 & 0.831 & 0.010 & -11.3 & 40.0 \\
\hline Carbon tetrachloride & 0.548 & 0.559 & 0.100 & 2.1 & 30.0 \\
\hline Benzene & 1.859 & 1.704 & 0.400 & -8.3 & 30.0 \\
\hline 1,2-Dichloroethane & 0.197 & 0.177 & 0.100 & -10.5 & 30.0 \\
\hline Trichloroethene & 0.451 & 0.429 & 0.300 & -4.9 & 30.0 \\
\hline Methylcyclohexane & 0.733 & 0.651 & 0.010 & -11.2 & 40.0 \\
\hline
\end{tabular}

Report 1,4-Dioxane for Low-Medium VOA analysis only 
$7 \mathrm{~B}$ - FORM VII VOA-2

VOLATILE CONTINUING CALIBRATION DATA

Lab Name: TESTAMERICA BURLINGTON

Lab Code: STLV Case No.: SAVANNAH Mod. Ref No.:
Contract : 21005

Calibration Date: 07/07/2010 Time: 1516

Init. Calib. Date(s): 06/25/2010 06/25/2010

EPA Sample No. (VSTD\#\#\#\#\#): VSTD005JQ Init. Calib. Time(s): 11331327

Heated Purge: (Y/N)N GC Column: DB-624 ID: 0.20 (mm) Length: 25 (m)

Purge Volume: 25.0

$(\mathrm{mL})$

\begin{tabular}{|c|c|c|c|c|c|}
\hline COMPOUND & RRF & RRF5. 0 & $\begin{array}{l}\text { MIN } \\
\text { RRF }\end{array}$ & $\because \mathrm{D}$ & MAX $\div \mathrm{D}$ \\
\hline \multicolumn{6}{|c|}{ 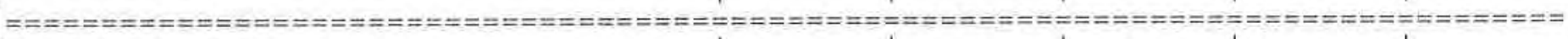 } \\
\hline 1,2-Dichloropropane & 0.382 & 0.334 & 0.010 & -12.5 & 40.0 \\
\hline Bromodichloromethane & 0.383 & 0.348 & 0.200 & -9.3 & 30.0 \\
\hline cis-1,3-Dichloropropene & 0.501 & 0.457 & 0.200 & -8.8 & 30.0 \\
\hline 4-Methyl-2-pentanone & 0.087 & 0.074 & 0.010 & -14.7 & 40.0 \\
\hline Toluene & 1.915 & 1.845 & 0.400 & $-3 \cdot 6$ & 30.0 \\
\hline trans-1,3-Dichloropropene & 0.346 & 0.301 & 0.100 & -13.0 & 30.0 \\
\hline 1,1,2-Trichloroethane & 0.153 & 0.141 & 0.100 & -7.8 & 30.0 \\
\hline Tetrachloroethene & 0.327 & 0.339 & 0.100 & 3.5 & 30.0 \\
\hline 2-Hexanone & 0.057 & 0.044 & 0.010 & -21.6 & 40.0 \\
\hline Dibromochloromethane & 0.172 & 0.173 & 0.100 & 0.2 & 30.0 \\
\hline 1,2-Dibromoethane & 0.126 & 0.122 & 0.010 & -3.1 & 30.0 \\
\hline Chlorobenzene & 0.978 & 0.969 & 0.500 & -0.8 & 30.0 \\
\hline Ethylbenzene & 2.159 & 2.079 & 0.100 & -3.7 & 30.0 \\
\hline o-xylene & 0.709 & 0.701 & 0.300 & -1.0 & 30.0 \\
\hline $\mathrm{m}, \mathrm{p}-\mathrm{xy}$ lene & 0.788 & 0.784 & 0.300 & -0.6 & 30.0 \\
\hline styrene & 1.026 & 1.010 & 0.300 & -1.6 & 30.0 \\
\hline Bromoform & 0.174 & 0.158 & 0.050 & -9.3 & 30.0 \\
\hline Isopropylbenzene & 2.068 & 2.072 & 0.010 & 0.2 & 40.0 \\
\hline $1,1,2,2$-Tetrachloroethane & 0.141 & 0.130 & 0.100 & -7.6 & 30.0 \\
\hline 1,3-Dichlorobenzene & 1.588 & 1.494 & 0.400 & -5.9 & 30.0 \\
\hline 1,4-Dichlorobenzene & 1.509 & 1.396 & 0.400 & -7.5 & 30.0 \\
\hline 1,2-Dichlorobenzene & 1.142 & 1.108 & 0.400 & -3.0 & 30.0 \\
\hline 1,2-Dibromo-3-chloropropane & 0.046 & 0.040 & 0.010 & $-13 \cdot 5$ & 40.0 \\
\hline $1,2,4$-Trichlorobenzene & 0.685 & 0.586 & 0.200 & -14.4 & 30.0 \\
\hline 1,2,3-Trichlorobenzene & 0.475 & 0.412 & 0.200 & $-13 \cdot 2$ & 30.0 \\
\hline
\end{tabular}


7C - FORM VII VOA-3

VOLATILE CONTINUING CALIBRATION DATA

Lab Name: TESTAMERICA BURLINGTON

Contract: 21005

Lab Code: STLV Case No.: SAVANNAH Mod. Ref No.:

SDG No.: 137941

Instrument ID: J.i

Calibration Date: 07/07/2010 Time: 1516

Lab File ID: JBE005HV

Init. Calib. Date(s): 06/25/2010 06/25/2010

EPA Sample No. (VSTD\#\#\#\#): VSTD005JQ Init. Calib. Time(s): $1133 \quad 1327$

Heated Purge: (Y/N)N GC Column: DB-624 ID: $0.20 \quad$ (mm) Length: 25 (m)

Purge Volume: 25.0

(mL)

\begin{tabular}{|c|c|c|c|c|c|}
\hline COMPOUND & $\mathrm{RRF}$ & RRF5.0 & $\begin{array}{l}\text { MIN } \\
\text { RRF }\end{array}$ & $\div D$ & $\operatorname{MAX} \div \mathrm{D}$ \\
\hline \multicolumn{6}{|c|}{ 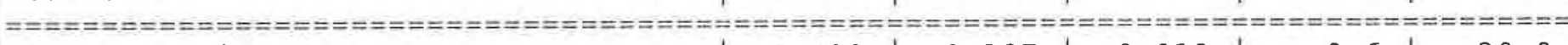 } \\
\hline Vinyl chloride-d3 & 0.428 & 0.387 & 0.010 & -9.6 & 30.0 \\
\hline Chloroethane-d5 & 0.341 & 0.308 & 0.010 & -9.6 & 40.0 \\
\hline 1,1-Dichloroethene-d2 & 0.714 & 0.659 & 0.010 & -7.6 & 30.0 \\
\hline 2-Butanone-d5 & 0.024 & 0.021 & 0.010 & -15.4 & 40.0 \\
\hline Chloroform- $d$ & 0.522 & 0.503 & 0.010 & -3.7 & 30.0 \\
\hline 1,2-Dichloroethane-d4 & 0.158 & 0.138 & 0.010 & -12.3 & 30.0 \\
\hline Benzene-d6 & 1.701 & 1.580 & 0.010 & -7.1 & 30.0 \\
\hline 1,2-Dichloropropane-d6 & 0.430 & 0.386 & 0.010 & -10.3 & 40.0 \\
\hline Toluene-d8 & 1.537 & 1.503 & 0.010 & -2.2 & 30.0 \\
\hline trans-1,3-Dichloropropene-d4 & 0.301 & 0.276 & 0.010 & -8.3 & 30.0 \\
\hline 2-Hexanone-d5 & 0.028 & 0.026 & 0.010 & -9.5 & 40.0 \\
\hline $1,1,2,2$-Tetrachloroethane-d2 & 0.137 & 0.128 & 0.010 & -6.8 & 30.0 \\
\hline 1,2-Dichlorobenzene-d4 & 0.717 & 0.661 & 0.010 & -7.8 & 30.0 \\
\hline
\end{tabular}

Report 1,4-Dioxane-d8 for Low-Medium VOA analysis only 
$7 A$ - FORM VII VOA-1

VOLATILE CONTINUING CALIBRATION DATA

Lab Name: TESTAMERICA BURLINGTON

Lab Code: STLV Case No.: SAVANNAH Mod. Ref No.:
Contract: 21005

Calibration Date: 07/07/2010 Time: 2311

Init. Calib. Date(s): 06/25/2010 06/25/2010

EPA Sample No. (VSTD\#\#\#\#\#): VSTD005QJ Init. Calib. Time(s): 113311327

Heated Purge: (Y/N)N GC Column: DB-624 ID: 0.20 (mm) Length: 25 (m)

Purge Volume: 25.0

$(\mathrm{mL})$

\begin{tabular}{|c|c|c|c|c|c|}
\hline COMPOUND & RRF & RRF5.0 & $\begin{array}{l}\text { MIN } \\
\text { RRF }\end{array}$ & $\because \mathrm{D}$ & $\mathrm{MAX} \div \mathrm{D}$ \\
\hline \multicolumn{6}{|c|}{ 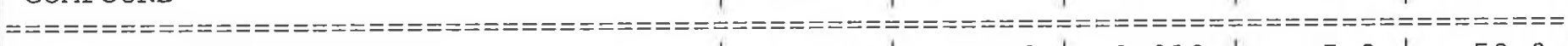 } \\
\hline Dichlorodifluoromethane & 0.463 & 0.436 & 0.010 & -5.8 & 50.0 \\
\hline Chloromethane & 0.511 & 0.463 & 0.010 & -9.4 & 50.0 \\
\hline Vinyl chloride & 0.517 & 0.493 & 0.010 & -4.7 & 50.0 \\
\hline Bromomethane & 0.170 & 0.200 & 0.010 & 17.6 & 50.0 \\
\hline Chloroethane & 0.311 & 0.288 & 0.010 & -7.1 & 50.0 \\
\hline Trichlorofluoromethane & 0.533 & 0.538 & 0.010 & 1.0 & 50.0 \\
\hline 1,1-Dichloroethene & 0.323 & 0.329 & 0.010 & 1.8 & 50.0 \\
\hline $1,1,2$-Trichloro-1,2,2-trifluoroethane & 0.343 & 0.352 & 0.010 & 2.7 & 50.0 \\
\hline Acetone & 0.015 & 0.013 & 0.010 & -13.5 & 50.0 \\
\hline Carbon disulfide & 1.121 & 1.047 & 0.010 & -6.6 & 50.0 \\
\hline Methyl acetate & 0.056 & 0.042 & 0.010 & $-25 \cdot 3$ & 50.0 \\
\hline Methylene chloride & 0.244 & 0.250 & 0.010 & 2.6 & 50.0 \\
\hline trans-1,2-Dichloroethene & 0.327 & 0.348 & 0.010 & 6.4 & 50.0 \\
\hline Methyl tert-butyl ether & 0.370 & 0.377 & 0.010 & 1.8 & 50.0 \\
\hline 1,1-Dichloroethane & 0.626 & 0.628 & 0.010 & 0.3 & 50.0 \\
\hline cis-1,2-Dichloroethene & 0.307 & 0.321 & 0.010 & 4.6 & 50.0 \\
\hline 2-Butanone & 0.027 & 0.026 & 0.010 & -2.6 & 50.0 \\
\hline Bromochloromethane & 0.074 & 0.080 & 0.010 & 7.8 & 50.0 \\
\hline Chloroform & 0.507 & 0.512 & 0.010 & 0.9 & 50.0 \\
\hline 1,1,1-Trichloroethane & 0.662 & 0.669 & 0.010 & 1.1 & 50.0 \\
\hline Cyclohexane & 0.937 & 0.898 & 0.010 & -4.1 & 50.0 \\
\hline Carbon tetrachloride & 0.548 & 0.572 & 0.010 & 4.3 & 50.0 \\
\hline Benzene & 1.859 & 1.815 & 0.010 & -2.4 & 50.0 \\
\hline 1,2-Dichloroethane & 0.197 & 0.199 & 0.010 & 1.0 & 50.0 \\
\hline Trichloroethene & 0.451 & 0.453 & 0.010 & 0.4 & 50.0 \\
\hline Methylcyclohexane & 0.733 & 0.675 & 0.010 & -7.9 & 50.0 \\
\hline
\end{tabular}

Report 1,4-Dioxane for Low-Medium VOA analysis only 
$7 B$ - FORM VII VOA-2

VOLATILE CONTINUING CALIBRATION DATA

Lab Name: TESTAMERICA BURLINGTON

Lab Code: STLV Case No.: SAVANNAH Mod. Ref No.:
Contract: 21005

SDG No.: 137941
Instrument ID: J.i

Lab File ID: JBE05HCl

EPA Sample No. (VSTD\#\#\#\#\#) : VSTD0050T

Purge Volume: $25.0 \quad$ (mL)

Heated Purge: ( $\mathrm{Y} / \mathrm{N}$ ) N GC Column: DB-624 ID: 0.20 (mm) Length: 25 (m)

Calibration Date: 07/07/2010 Time: 2311

Init. Calib. Date(s): 06/25/2010 06/25/2010

Init. Calib. Time(s): $1133 \quad 1327$

\begin{tabular}{|c|c|c|c|c|c|}
\hline COMPOUND & RRF & RRF5.0 & $\begin{array}{l}\text { MIN } \\
\text { RRF }\end{array}$ & $\div \mathrm{D}$ & $\mathrm{MAX} \div \mathrm{D}$ \\
\hline \multicolumn{6}{|c|}{ 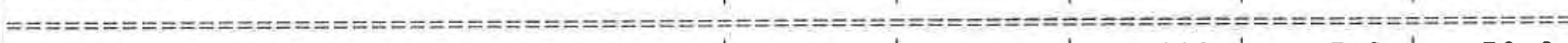 } \\
\hline 1,2-Dichloropropane & 0.382 & 0.362 & 0.010 & -5.2 & 50.0 \\
\hline Bromodichloromethane & 0.383 & 0.382 & 0.010 & -0.3 & 50.0 \\
\hline cis-1,3-Dichloropropene & 0.501 & 0.488 & 0.010 & -2.5 & 50.0 \\
\hline 4-Methyl-2-pentanone & 0.087 & 0.085 & 0.010 & -1.9 & 50.0 \\
\hline Toluene & 1.915 & 1.937 & 0.010 & 1.1 & 50.0 \\
\hline trans-1,3-Dichloropropene & 0.346 & 0.338 & 0.010 & -2.3 & 50.0 \\
\hline 1,1,2-Trichloroethane & 0.153 & 0.151 & 0.010 & -1.4 & 50.0 \\
\hline Tetrachloroethene & 0.327 & 0.325 & 0.010 & -0.8 & 50.0 \\
\hline 2-Hexanone & 0.057 & 0.053 & 0.010 & -5.9 & 50.0 \\
\hline Dibromochloromethane & 0.172 & 0.175 & 0.010 & 1.7 & 50.0 \\
\hline 1,2-Dibromoethane & 0.126 & 0.130 & 0.010 & 3.2 & 50.0 \\
\hline Chlorobenzene & 0.978 & 1.006 & 0.010 & 2.9 & 50.0 \\
\hline Ethylbenzene & 2.159 & 2.198 & 0.010 & 1.8 & 50.0 \\
\hline o-xylene & 0.709 & 0.741 & 0.010 & 4.6 & 50.0 \\
\hline $\mathrm{m}, \mathrm{p}-\mathrm{xy}$ lene & 0.788 & 0.809 & 0.010 & 2.7 & 50.0 \\
\hline styrene & 1.026 & 1.068 & 0.010 & 4.0 & 50.0 \\
\hline Bromoform & 0.174 & 0.165 & 0.010 & -5.3 & 50.0 \\
\hline Isopropylbenzene & 2.068 & 2.116 & 0.010 & 2.3 & 50.0 \\
\hline $1,1,2,2$-Tetrachloroethane & 0.141 & 0.143 & 0.010 & 1.8 & 50.0 \\
\hline 1,3-Dichlorobenzene & 1.588 & 1.530 & 0.010 & -3.6 & 50.0 \\
\hline 1,4-Dichlorobenzene & 1.509 & 1.454 & 0.010 & -3.6 & 50.0 \\
\hline 1,2-Dichlorobenzene & 1.142 & 1.131 & 0.010 & -1.0 & 50.0 \\
\hline 1,2-Dibromo-3-chloropropane & 0.046 & 0.044 & 0.010 & -4.2 & 50.0 \\
\hline $1,2,4$-Trichlorobenzene & 0.685 & 0.625 & 0.010 & -8.7 & 50.0 \\
\hline $1,2,3$-Trichlorobenzene & 0.475 & 0.422 & 0.010 & -11.1 & 50.0 \\
\hline
\end{tabular}


$7 \mathrm{C}$ - FORM VII VOA-3

VOLATILE CONTINUING CALIBRATION DATA

Lab Name: TESTAMERICA BURLINGTON

Contract: 21005

Lab Code: STLV Case No.: SAVANNAH Mod. Ref No.:

SDG No. : 137941

Instrument ID: J.i

Calibration Date: 07/07/2010 Time: 2311

Lab File ID: JBE05HC1

Init. Calib. Date(s): 06/25/2010 06/25/2010

EPA Sample No. (VSTD\#\#\#\#) : VSTD005QJ Init. Calib. Time(s): 113311327

Heated Purge: (Y/N)N GC Column: DB-624 ID: 0.20 (mm) Length: 25

$(m)$

Purge Volume: 25.0

(mL)

\begin{tabular}{|c|c|c|c|c|c|}
\hline COMPOUND & RRF & RRF5. 0 & $\begin{array}{l}\text { MIN } \\
\text { RRF }\end{array}$ & $\div \mathrm{D}$ & MAX $\div D$ \\
\hline \multicolumn{6}{|c|}{ 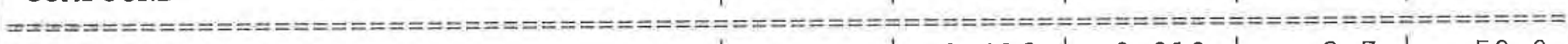 } \\
\hline Vinyl chloride-d3 & 0.428 & 0.416 & 0.010 & -2.7 & 50.0 \\
\hline Chloroethane-d5 & 0.341 & 0.330 & 0.010 & -3.1 & 50.0 \\
\hline 1,1-Dichloroethene-d2 & 0.714 & 0.690 & 0.010 & $-3 \cdot 3$ & 50.0 \\
\hline 2-Butanone-d5 & 0.024 & 0.026 & 0.010 & 6.2 & 50.0 \\
\hline Chloroform-d & 0.522 & 0.543 & 0.010 & 3.9 & 50.0 \\
\hline 1,2-Dichloroethane-d4 & 0.158 & 0.155 & 0.010 & -1.8 & 50.0 \\
\hline Benzene-d6 & 1.701 & 1.694 & 0.010 & -0.4 & 50.0 \\
\hline 1,2-Dichloropropane-d6 & 0.430 & 0.426 & 0.010 & -0.9 & 50.0 \\
\hline Toluene-d8 & 1.537 & 1.560 & 0.010 & 1.5 & 50.0 \\
\hline trans-1,3-Dichloropropene-d4 & 0.301 & 0.294 & 0.010 & -2.4 & 50.0 \\
\hline 2 -Hexanone-d5 & 0.028 & 0.031 & 0.010 & 7.6 & 50.0 \\
\hline $1,1,2,2$-Tetrachloroethane-d2 & 0.137 & 0.145 & 0.010 & 6.0 & 50.0 \\
\hline $1,2-\mathrm{Dich}$ lorobenzene- $\mathrm{d} 4$ & 0.717 & 0.690 & 0.010 & $-3 \cdot 9$ & 50.0 \\
\hline
\end{tabular}

Report 1,4-Dioxane-d8 for Low-Medium VOA analysis only 
$8 A$ - FORM VIII VOA

VOLATILE INTERNAL STANDARD AREA AND RETENTION TIME SUMMARY

Lab Name: TESTAMERICA BURLINGTON

Contract: 21005

Lab Code: STLV Case No.: SAVANNAH Mod. Ref No.:

SDG No.: 137941

GC Column: DB-624 ID: 0.20 (mm)

Init. Calib. Date(s): 06/25/2010 06/25/2010

EPA Sample No. (VSTD\#\#\#\#\#): VSTD005JQ

Date Analyzed: 07/07/2010

Lab File ID (Standard): JBE005HV

Time Analyzed: 1516

Instrument ID: J.i

Heated Purge: (Y/N) N

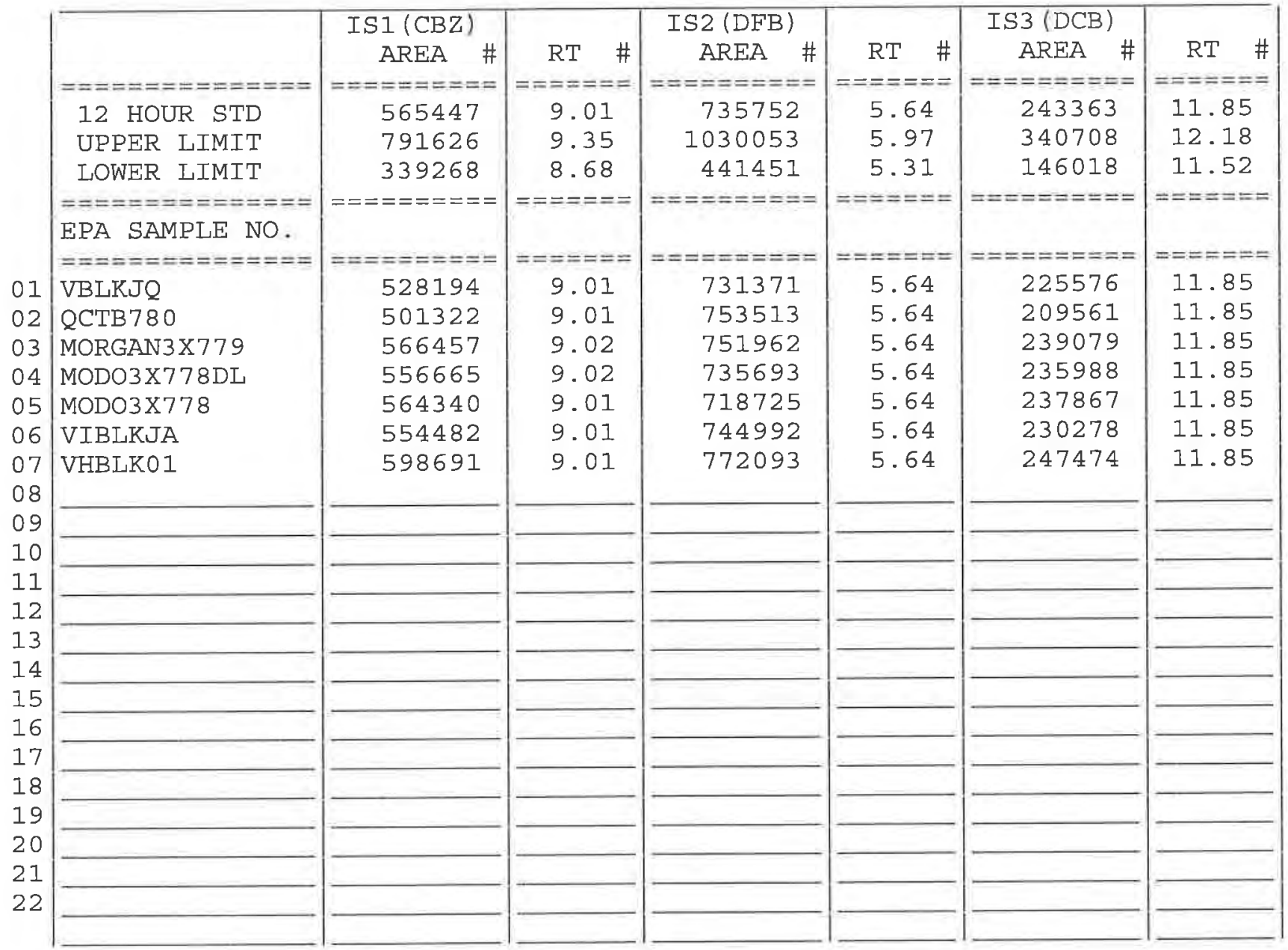

IS1 $(\mathrm{CBZ})=$ Chlorobenzene $-\mathrm{d} 5$

IS2 $(\mathrm{DFB})=1,4$-Difluorobenzene

IS3 $(\mathrm{DCB})=1,4$-Dichlorobenzene-d4

AREA UPPER LIMIT $=200 \%$ (Low-Medium Volatiles) and $140 \%$ (Trace Volatiles) of internal standard area

AREA LOWER LIMIT $=50 \%$ (LOW-Medium Volatiles) and $60 \%$ (Trace Volatiles) of internal standard area

RT UPPER LIMIT $=+0.50$ (Low-Medium Volatiles) and +0.33 (Trace Volatiles) minutes of internal standard RT

RT LOWER LIMIT $=-0.50$ (Low-Medium Volatiles) and -0.33 (Trace Volatiles) minutes of internal standard RT

\# Column used to flag values outside QC limits with an asterisk

Page 1 of 1

SOMO1. 2 


\section{TesłAmerica}

THE LEADER IN ENVIRONMENTAL. TESTING

TestAmerica Laboratories, Inc.

July 26, 2010

Mr. Clyde Dennis

Argonne National Laboratory

9700 S. Cass Avenue

Building 203, Office B149

Argonne, IL 60439

Re: Laboratory Project No. 21005

Case: SAVANNAH; SDG: 138033

Dear Mr. Dennis:

Enclosed are analytical results for samples that were received by TestAmerica Burlington on July $8^{\text {th }}, 2010$. Laboratory identification numbers were assigned, and designated as follows:

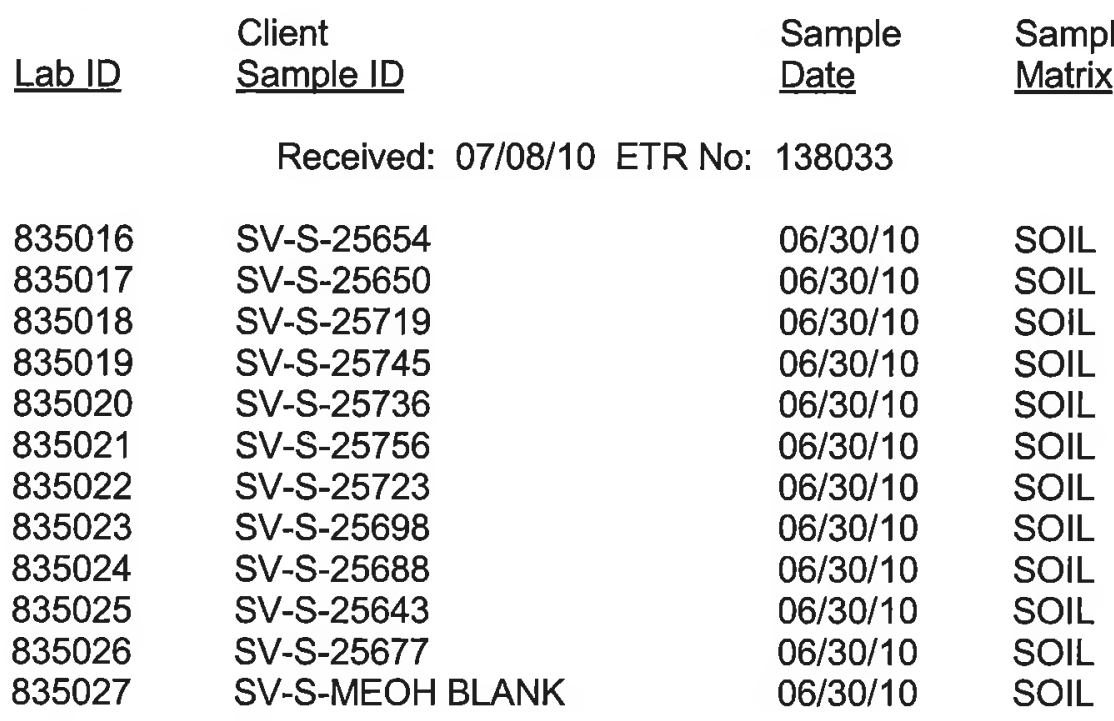

Documentation of the condition of the samples at the time of their receipt and any exception to the laboratory's Sample Acceptance Policy is documented in the Sample Handling section of this submittal.

The samples were analyzed by Method $8260 \mathrm{~B}$, using a low-level calibration. In performing the analytical work, 500 microliters of the methanol extract were added to the 5 milliliter purge volume. Each analysis associated with the samples in this sample set did exhibit an acceptable internal standard performance. The derived recovery of one or more of the surrogate controls was high in the analysis of samples SV-S-25698 and SV-S-25688. Two types of laboratory control sample analyses were performed in the analytical sequence. One was performed to evaluate 


\section{TestAmerica}

THE LEADER IN ENYIRONMENTAL TESTING

method performance, and one was performed with 500 microliters of methanol added to the purge volume in order to characterize the affect on the analytical process. There was an acceptable recovery of each target analyte in the laboratory control sample analysis that defined method performance. In the laboratory control sample analysis with methanol, several of the earlier eluting compounds did exhibit a lower recovery performance. Most significantly affected was the recovery performance of chloroethane, trichlorofluoromethane, acrolein, acetone, and methyl iodide, for which the recovery was below 50 percent. Additionally, the recovery of several of the target analytes was high in the laboratory control sample analysis with methanol, this included carbon tetrachloride (120 percent). With the exception of the high recovery of carbon tetrachloride in the laboratory control sample analysis with methanol, there was an acceptable recovery of both chloroform and carbon tetrachloride in each of the laboratory control sample analyses. Matrix spike and matrix spike duplicate analyses were not performed on samples in this sample set. Trace concentrations of chloromethane, bromomethane, methyl iodide, trans-1,3-dichloropropene, 1,4-dichlorobenzene, 1,2,4-trichlorobenzene, naphthalene, and 1,2,3-trichlorobenzene were identified in the analysis sample SV-S-MEOH BLANK. The laboratory did associate the analysis of SV-S-MEOH BLANK with the analysis of each of the field samples in order to reference the blank association, and accordingly qualify the reported results. Trace concentrations of chloromethane, bromomethane, methyl iodide, 1,4-dioxane, and naphthalene were identified in the analysis of the instrument blank associated with the analytical work.

Any reference within this report to Severn Trent Laboratories, Inc. or STL, should be understood to refer to TestAmerica Laboratories, Inc. (formerly known as Severn Trent Laboratories, Inc.) The analytical results associated with the samples presented in this test report were generated under a quality system that adheres to requirements specified in the NELAC standard. Release of the data in this test report and any associated electronic deliverables is authorized by the Laboratory Director's designee as verified by the following signature.

If there are any questions regarding this submittal, please contact me at 802 660-1990.

Sincerely,

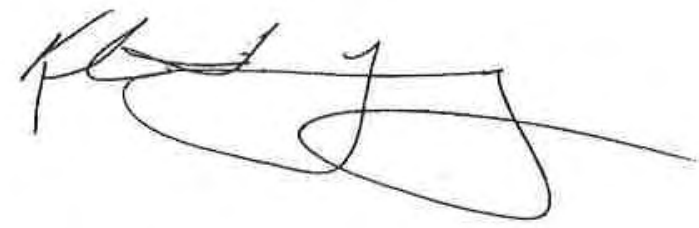

Kirk F. Young

Project Manager

KFY/hsf

Enclosure 


\section{TestAmerica Burlington Data Qualifier Definitions}

\section{Organic}

U: Compound analyzed but not detected at a concentration above the reporting limit.

$\mathrm{J}: \quad$ Estimated value.

$\mathrm{N}$ : Indicates presumptive evidence of a compound. This flag is used only for tentatively identified compounds (TICs) where the identification of a compound is based on a mass spectral library search.

P: $\quad$ SW-846: The relative percent difference for detected concentrations between two GC columns is greater than $40 \%$. Unless otherwise specified the higher of the two values is reported on the Form I.

CLP SOW: Greater than 25\% difference for detected concentrations between two GC columns. Unless otherwise specified the lower of the two values is reported on the Form I.

C: Pesticide result whose identification has been confirmed by GC/MS.

B: $\quad$ Analyte is found in the sample and the associated method blank. The flag is used for tentatively identified compounds as well as positively identified compounds.

E: Compounds whose concentrations exceed the upper limit of the calibration range of the instrument for that specific analysis.

D: Concentrations identified from analysis of the sample at a secondary dilution.

A: Tentatively identified compound is a suspected aldol condensation product.

$X, Y, Z$ : Laboratory defined flags that may be used alone or combined, as needed. If used, the description of the flag is defined in the project narrative.

\section{Inorganic/Metals}

$\mathrm{E}$ : $\quad$ Reported value is estimated due to the presence of interference.

$\mathrm{N}$ : Matrix spike sample recovery is not within control limits.

* Duplicate sample analysis is not within control limits.

B: The result reported is less than the reporting limit but greater than the instrument detection limit.

U: $\quad$ Analyte was analyzed for but not detected above the reporting limit.

Method Codes:

P ICP-AES

MS ICP-MS

CV Cold Vapor AA

AS Semi-Automated Spectrophotometric 


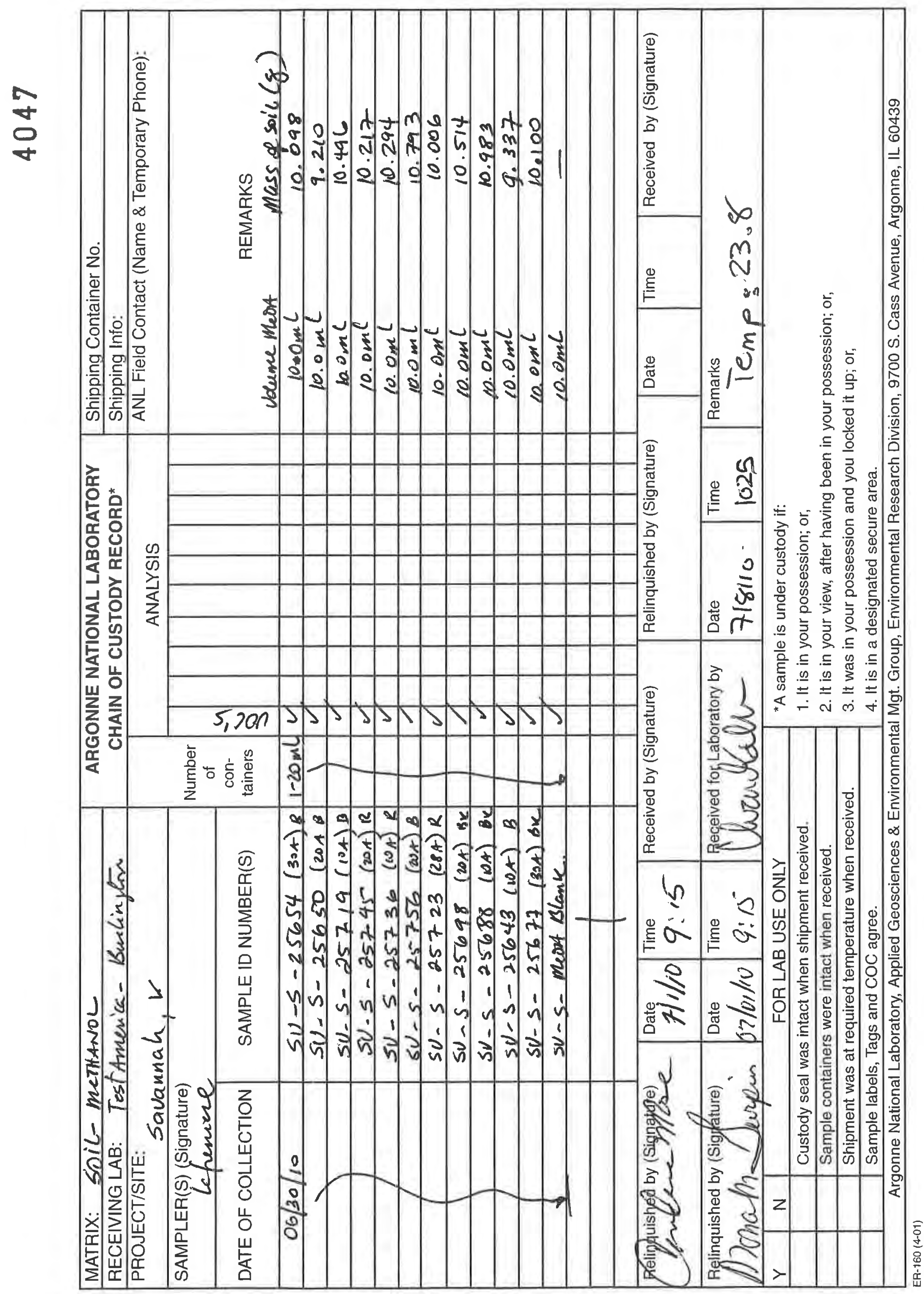




\section{TestAmerica

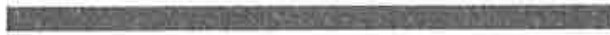 \\ THE LEADER IN ENVIRONMENTAL TESTING}

\section{Sample Data Summary - 8260B Low Waters}


FORM 1

VOLATILE ORGANICS ANALYSIS DATA SHEET
ARGLAB SAMPLE NO.

$S V-S-25643$
Lab Name: TESTAMERICA BURLINGTON

Contract: $8 E-00302$

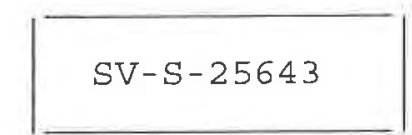

SDG NO.: 138033

Iub code: STIV Case No.: SAVANNAH SAS No.:

Lab Sample ID: 835025

Matrix: (soil/water) soIL

Lab File ID: 835025

Sample wt/vol:

$5.0(\mathrm{~g} / \mathrm{mL}) \mathrm{G}$

Date Received: 07/08/10

Level: (low/med) LOW

Date Analyzed: 07/14/10

\% Moisture: not dec.

GC Column: DB-624 ID: 0.53 (mm)

Dilution Factor: 1.0

Soil Extract Volume:

(uL)

Soil Aliquot Volume:

(UL)

CAS NO.

COMPOUND

CONCENTRATION UNITS:

(ug/L or ug/Kg) UG/KG

$Q$

$5.0 \mathrm{U}$

$0.46 \mathrm{JB}$

$5.0 \mathrm{U}$

2.1 JB

5. $0 \mathrm{U}$

5. $0 \mathrm{U}$

$5.0 \mathrm{U}$

5. $0 \mathrm{U}$

$5.0 \mathrm{U}$

$5.0 \mathrm{U}$

5. 0 U

$5.0 \mathrm{U}$

$5.0 \mathrm{U}$

$5.0 \mathrm{U}$

5. $0 \mathrm{U}$

5. $0 \mathrm{U}$

5. $0 \mathrm{U}$

$5.0 \mathrm{U}$

$5.0 \mathrm{U}$

$5.0 \mathrm{U}$

$5.0 \mathrm{U}$

5. $0 \mathrm{U}$

$5.0 \mathrm{U}$

$5.0 \mathrm{U}$

$20 \mathrm{U}$

$5.0 \mathrm{U}$

5. $0 \mathrm{U}$

$50 \mathrm{U}$

$5.0 \mathrm{U}$

$0.24 \mathrm{~J}$

$5.0 \mathrm{U}$

$5.0 \mathrm{U}$

$250 \mathrm{U}$ 
FORM 1
VOLATILE ORGANICS ANALYSIS DATA SHEET

ARGLAB SAMPLE NO.

$S V-S-25643$

Lab Name: TESTAMERICA BURLINGTON

Contract : $8 \mathrm{E}-00302$

Lab Code: STIV Case No.: SAVANNAH SAS No.:

SDG No.: 138033

Matrix: (soil/water) soIL

Lab Sample ID: 835025

Sample wt/vol:

$5.0(\mathrm{~g} / \mathrm{mL}) \mathrm{G}$

Lab File ID: $\quad 835025$

Level : (low/med) LOW

Date Received: 07/08/10

\% Moisture: not dec.

GC Column: DB-624 ID: 0.53 (mm)

Soil Extract Volume:

(uL)
Date Analyzed: 07/14/10

Dilution Factor: 1.0

Soil Aliquot Volume: (uI)

CAS NO. COMPOUND (ug/L or $\mathrm{ug} / \mathrm{Kg}) \mathrm{UG} / \mathrm{KG}$

$71-43-2--------B e n z e n e$

107-06-2------1,2-Dichloroethane

79-01-6-.....-. Trichloroethene

$78-87-5 \ldots \ldots-\ldots 1,2-\mathrm{Di}$ chloropropane

74-95-3-..---Dibromomethane

80-62-6-...-- Methyl Methacrylate

123-91-1-...-1,4-Dioxane

75-27-4-...---Bromodichloromethane

110-75-8---.--2-Chloroethyl Vinyl Ether

10061-01-5----cis-1,3-Dichloropropene

108-10-1------4-Methyl-2-pentanone

108-88-3---.-- Toluene

10061-02-6-...-trans-1,3-Dichloropropene

97-63-2-...-.-Ethyl Methacrylate

79-00-5-...--1, 1, 2-Trichloroethane

127-18-4--..-- Tetrachloroethene

142-28-9-..---1,3-Dichloropropane

591-78-6-...--2-Hexanone

124-48-1-...-.-Dibromochloromethane

106-93-4-..--1, 2-Dibromoethane

108-90-7-...-- Chlorobenzene

630-20-6-...-.1,1,1,2-Tetrachloroethane

100-41-4_....-. Ethylbenzene

$1330-20-7-\ldots .-x_{y}$ lene $(\mathrm{m}, \mathrm{p})$

95-47-6--.---.xylene (o)

1330-20-7-...-. Xylene (total)

100-42-5--.----styrene

75-25-2--.---Bromoform

98-82-8-- - - - - Isopropylbenzene

108-86-1 -...---Bromobenzene

79-34-5-.....-1, 1, 2, 2-Tetrachloroethane

96-18-4-.----1, 2, 3-Trichloropropane

110-57-6--.--trans-1,4-Dichloro-2-butene
$5.0 \mathrm{U}$

$5.0 \mathrm{U}$

$5.0 \mathrm{U}$

$5.0 \mathrm{U}$

$5.0 \mathrm{U}$

$5.0 \mathrm{U}$

$50 \mathrm{U}$

$5.0 \mathrm{U}$

$5.0 \mathrm{U}$

$5.0 \mathrm{U}$

$5.0 \mathrm{U}$

$5.0 \mathrm{U}$

5. $0 \mathrm{U}$

$5.0 \mathrm{U}$

$5.0 \mathrm{U}$

$5.0 \mathrm{U}$

$5.0 \mathrm{U}$

$5.0 \mathrm{U}$

$5.0 \mathrm{U}$

$5.0 \mathrm{U}$

$5.0 \mathrm{U}$

$5.0 \mathrm{U}$

$5.0 \mathrm{U}$

$5.0 \mathrm{U}$

5. $0 \mathrm{U}$

$5.0 \mathrm{U}$

5. $0 \mathrm{U}$

$5.0 \mathrm{U}$

$5.0 \mathrm{U}$

$5.0 \mathrm{U}$

5. $01 \mathrm{U}$

$5.0 \mathrm{U}$

5. $0 \mathrm{U}$ 
FORM 1 VOLATILE ORGANICS ANALYSIS DATA SHEET
ARGLAB SAMPLE NO.

$S V-S-25643$

Lab Name: TESTAMERICA BURLINGTON Contract: 8E-00302

SDG NO.: 138033 Lab Code: STLV Case No.: SAVANNAH SAS No.: Lab Sample ID: 835025

Matrix: (soil/water) SoIL Lab File ID: 835025 Sample wt/vol:

Date Received: 07/08/10

Level: (low/med) LOW

Date Analyzed: 07/14/10

\% Moisture: not dec.

GC Column: DB-624 ID: 0.53 (mm)

Dilution Factor: 1.0

Soil Extract Volume: (uL)

Soil Aliquot volume: (uL) CONCENTRATION UNITS :

CAS NO.

COMPOUND

( $\mathrm{ug} / \mathrm{L}$ or $\mathrm{ug} / \mathrm{Kg}$ ) UG/KG

Q

103-65-1-...-n-Propylbenzene 95-49-8-....--2-Chlorotoluene 106-43-4-...-.4-Chlorotoluene $108-67-8-\ldots-\cdots-1,3,5-$ Trimethyl benzene

98-06-6-...-.-tert-Butylbenzene

95-63-6-.....-1,2,4-Trimethylbenzene

$135-98-8$-...-.-sec-Butylbenzene

541-73-1_...--1,3-Dichlorobenzene

99-87-6-..---4-Isopropyltoluene

$106-46-7-\ldots .-1,4-\mathrm{Dich}$-..-robenzene

95-50-1-...---1,2-Dichlorobenzene

104-51-8-....-n-Butylbenzene

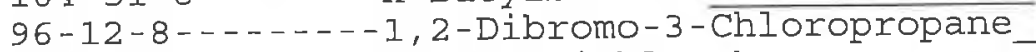

120-82-1-...--1,2,4-Trichlorobenzene

87-68-3-.....-Hexachlorobutadiene

91-20-3-..--- Naphthalene

87-61-6.....-.1,2,3-Trichlorobenzene
5. $0 \mathrm{U}$

5. $0 \mathrm{U}$

5. $0 \mathrm{U}$

$5.0 \mathrm{U}$

$5.0 \mathrm{U}$

$5.0 \mathrm{U}$

$5.0 \mathrm{U}$

$5.0 \mathrm{U}$

$5.0 \mathrm{U}$

$5.0 \mathrm{U}$

$5.0 \mathrm{U}$

5. $0 \mathrm{U}$

$5.0 \mathrm{U}$

$5.0 \mathrm{U}$

$5.0 \mathrm{U}$

$5.0 \mathrm{U}$

$5.0 \mathrm{U}$ 
FORM 1 VOLATILE ORGANICS ANALYSIS DATA SHEET

Lab Name: TESTAMERICA BURLINGTON

Contract: $8 E-00302$

Lab Code: STLV Case No.: SAVANNAH SAS No.:

Lab Sample ID: 835017

Matrix: (soil/water) SOIL

Sample wt/vol:

$5.0(\mathrm{~g} / \mathrm{mL}) \mathrm{G}$

Level: $\quad($ low $/$ med) LOW

\% Moisture: not dec.

GC Column: DB-624 ID: 0.53 (mm)

Soil Extract Volume:

(UL)

Lab File ID: 835017

Date Received: $07 / 08 / 10$

Date Analyzed: 07/14/10

Dilution Factor: 1.0

Soil Aliquot Volume:
ARGIAB SAMPLE NO.

$S V-S-25650$

SDG NO.: 138033 (uL) CONCENTRATION UNITS :

CAS NO.

COMPOUND

( $\mathrm{ug} / \mathrm{L}$ or $\mathrm{ug} / \mathrm{Kg}$ ) UG/KG

Q

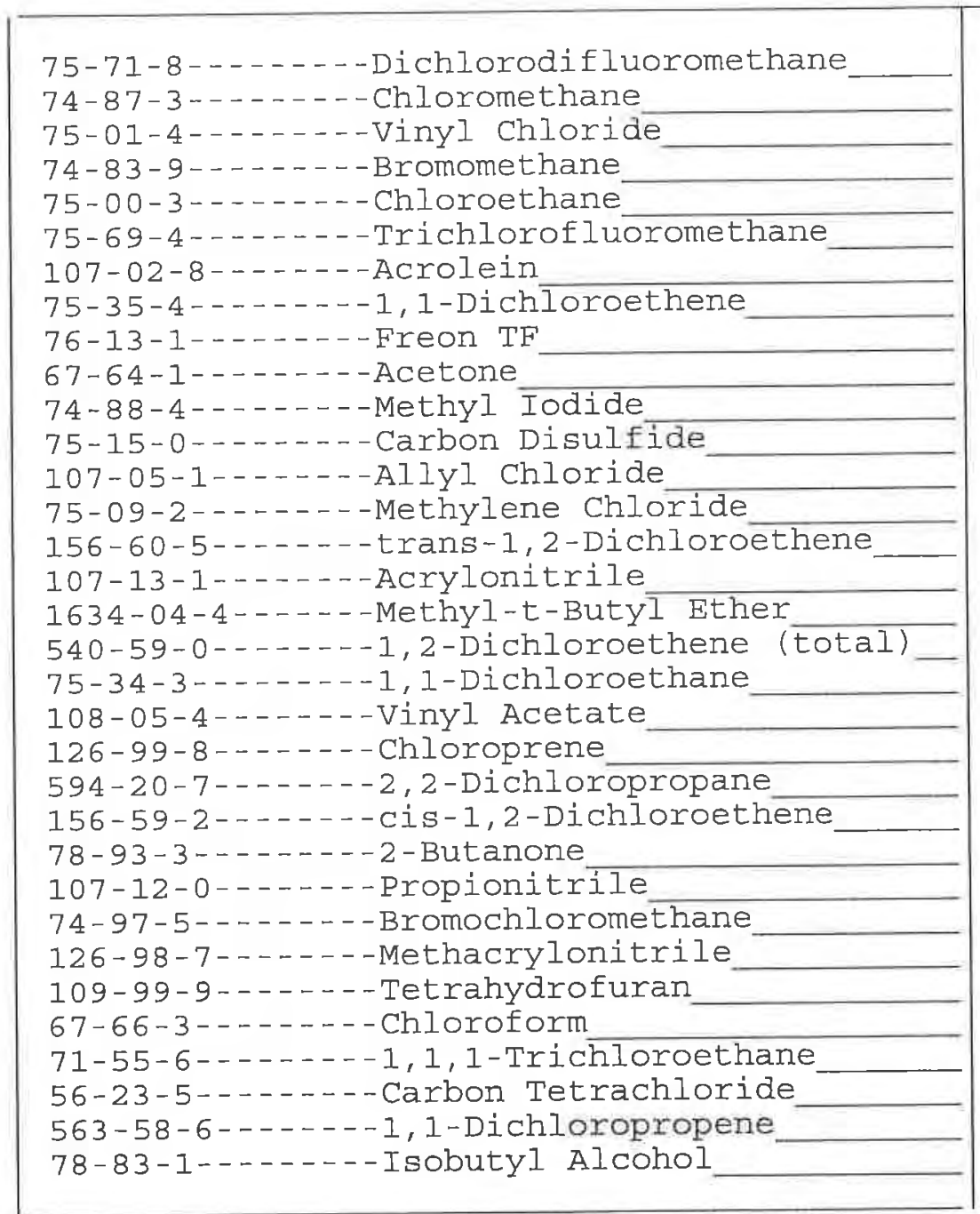

$5.0 \mathrm{U}$

$0.57 \mathrm{JB}$

5. 0 U

$2.3 \mathrm{JB}$

$5.0 \mathrm{U}$

$5.0 \mathrm{U}$

$5.0 \mathrm{U}$

$5.0 \mathrm{U}$

$5.0 \mathrm{U}$

$5.0 \mathrm{U}$

$0.98 \mathrm{JB}$

$5.0 \mathrm{U}$

$5.0 \mathrm{U}$

5. $0 \mathrm{U}$

$5.0 \mathrm{U}$

$5.0 \mathrm{U}$

5.0 U

$5.0 \mathrm{U}$

$5.0 \mathrm{U}$

$5.0 \mathrm{U}$

$5.0 \mathrm{U}$

$5.0 \mathrm{U}$

$5.0 \mathrm{U}$

$5.0 \mathrm{U}$

$20 \mathrm{U}$

5. $0 \mathrm{U}$

$5.0 \mathrm{U}$

$50 \mathrm{U}$

$5.0 \mathrm{U}$

$0.20 \mathrm{~J}$

$5.0 \mathrm{U}$

$5.0 \mathrm{U}$

250 U 
FORM 1

VOLATILE ORGANICS ANALYSIS DATA SHEET
ARGLAB SAMPLE NO.

$S V-S-25650$

Lab Name: TESTAMERICA BURIINGTON Contract: 8E-00302

Lab Code: STLV Case No.: SAVANNAH SAS No.:

SDG NO. : 138033

Matrix: (soil/water) SOIL

Sample wt/vol:

$5.0(\mathrm{~g} / \mathrm{mL}) \mathrm{G}$

Level: (low/med) LOW

\% Moisture: not dec.

GC Column: DB-624 ID: 0.53 (mm)

Soil Extract Volume:

(uL)
Lab Sample ID: 835017

Lab File ID: 835017

Date Received: 07/08/10

Date Analyzed: 07/14/10

Dilution Factor: 1.0

Soil Aliquot Volume: (uL)

CONCENIRATION UNITS:

$71-43-2-\ldots-\ldots-$ Benzene

107-06-2-...-1, 2-Dichloroethane

79-01-6-...-.-Trichloroethene

78-87-5-...... 1, 2-Dichloropropane

$74-95-3 \ldots-\ldots-$ Dibromomethane

80-62-6......-. Methyl Methacrylate

$123-91-1 \ldots-\ldots-1,4-$ Dioxane

75-27-4-...--Bromodichloromethane

110-75-8--.---2-Chloroethyl vinyl Ether

10061-01-5-..-cis-1,3-Dichloropropene

108-10-1------4-Methyl-2-pentanone

$108-88-3 \ldots-\ldots-$ Toluene

10061-02-6--.--trans-1,3-Dichloropropene

97-63-2-...-.-Ethyl Methacrylate

79-00-5-...--1, 1,2-Trichloroethane

127-18-4-...-- Tetrachloroethene

$142-28-9 \ldots-\ldots-1,3-$ Dichloropropane

591-78-6-..-.-2-Hexanone

124-48-1-...- Dibromochloromethane

106-93-4-...-. 1, 2-Dibromoethane

108-90-7--.--- Chlorobenzene

630-20-6-...--1,1,1,2-Tetrachloroethane

100-41-4-...-.-Ethylbenzene

$1330-20-7-\ldots .-x_{y}$ lene $(m, p)$

95-47-6.......-Xylene (o)

1330-20-7-..--xylene (total)

100-42-5-..---styrene

$75-25-2-\ldots-\cdots-$ Bromoform

98-82-8-.....-. Isopropylbenzene

108-86-1-- - - - Bromobenzene

79-34-5-...-.-1, 1,2,2-Tetrachloroethane

96-18-4-...-.-1, 2, 3-Trichloropropane

110-57-6--.---trans-1,4-Dichloro-2-butene
$5.0 \mathrm{U}$

5. $0 \mathrm{U}$

$5.0 \mathrm{U}$

5. $0 \mathrm{U}$

$5.0 \mathrm{U}$

$5.0 \mathrm{U}$

$50 \mathrm{U}$

$5.0 \mathrm{U}$

$5.0 \mathrm{U}$

$5.0 \mathrm{U}$

$5.0 \mathrm{U}$

$5.0 \mathrm{U}$

$5.0 \mathrm{U}$

$5.0 \mathrm{U}$

$5.0 \mathrm{U}$

5. $0 \mathrm{U}$

$5.0 \mathrm{U}$

$5.0 \mathrm{U}$

$5.0 \mathrm{U}$

$5.0 \mathrm{U}$

$5.0 \mathrm{U}$

$5.0 \mathrm{U}$

$5.0 \mathrm{U}$

5. $0 \mathrm{U}$

$5.0 \mathrm{U}$

$5.0 \mathrm{U}$

$5.0 \mathrm{U}$

5. $0 \mathrm{U}$

$5.0 \mathrm{U}$

$5.0 \mathrm{U}$

$5.0 \mathrm{U}$

5. $0 \mathrm{U}$

$5.0 \mathrm{U}$ 
FORM 1

VOLATILE ORGANICS ANALYSIS DATA SHEET

Lab Name: TESTAMERICA BURIINGTON Contract: 8E-00302

ARGLAB SAMPLE NO.

$S V-S-25650$

Lab Code: STLV Case No.: SAVANNAH SAS No.:

SDG No. : 138033

Matrix: (soil/water) soIL

Lab Sample ID: 835017

sample wt/vol:

$5.0(\mathrm{~g} / \mathrm{mL}) \mathrm{G}$

Lab File ID: 835017

Level: (low/med) LOW

Date Received: 07/08/10

\% Moisture: not dec.

GC Column: DB-624 ID: 0.53 (mm)

Date Analyzed: 07/14/10

Soil Extract Volume: (uL)

Dilution Factor: 1.0

Soil Aliquot Volume: (uL) CONCENTRATION UNITS:

CAS NO.

COMPOUND

(ug/L or ug/Kg) UG/KG

$Q$

103-65-1------n-Propylbenzene

95-49-8-...-.-2-Chlorotoluene

106-43-4-- - - -4-Chlorotoluene

$108-67-8-\ldots-\ldots-1,3,5-T r i m e t h y l$ benzene

98-06-6-..-.--tert-Butylbenzene

95-63-6--...--1,2,4-Trimethylbenzene

135-98-8-...--sec-Butylbenzene

541-73-1-..--1, 3-Dichlorobenzene

99-87-6-...---4-Isopropyl toluene

106-46-7-..--1,4-Dichlorobenzene

95-50-1-....-1,2-Dichlorobenzene

104-51-8-...-n-Butylbenzene

96-12-8-...-- - 1, 2-Dibromo-3-Chloropropane

120-82-1-.-.-.1,2,4-Trichlorobenzene.

87-68-3-...-.-Hexachlorobutadiene

$91-20-3 \ldots-\ldots-N a p h t h a l e n e$

87-61-6-....--1,2,3-Trichlorobenzene

$5.0 \mathrm{U}$

$5.0 \mathrm{U}$

5. $0 \mathrm{U}$

5. $0 \mathrm{U}$

$5.0 \mathrm{U}$

$5.0 \mathrm{U}$

$5.0 \mathrm{U}$

$5.0 \mathrm{U}$

$5.0 \mathrm{U}$

$5.0 \mathrm{U}$

$5.0 \mathrm{U}$

$5.0 \mathrm{U}$

$5.0 \mathrm{U}$

$5.0 \mathrm{U}$

$5.0 \mathrm{U}$

$5.0 \mathrm{U}$

5. $0 \mathrm{U}$ 
FORM 1

VOLATILE ORGANICS ANALYSIS DATA SHEET
ARGLAB SAMPLE NO.

$S V-S-25654$

Lab Name: TESTAMERICA BURLINGTON Contract: 8E-00302

SDG No.: 138033

Lab Code: STLV Case No.: SAVANNAH SAS No.:

Lab Sample ID: 835016

Matrix: (soil/water) SoIL

Lab File ID: 835016

Sample wt/vol: $\quad 5.0(\mathrm{~g} / \mathrm{mL}) \mathrm{G}$

Date Received: 07/08/10

Level: (low/med) Low

Date Analyzed: 07/14/10

\% Moisture: not dec.

GC Column: DB-624 ID: 0.53 (mm)

Dilution Factor: 1.0

Soil Extract Volume:

(UL)

Soil Aliquot Volume: $(u L)$

CONCENTRATION UNITS:

CAS NO. COMPOUND $(\mathrm{ug} / \mathrm{L}$ or $\mathrm{ug} / \mathrm{Kg}) \mathrm{UG} / \mathrm{KG}$

Q

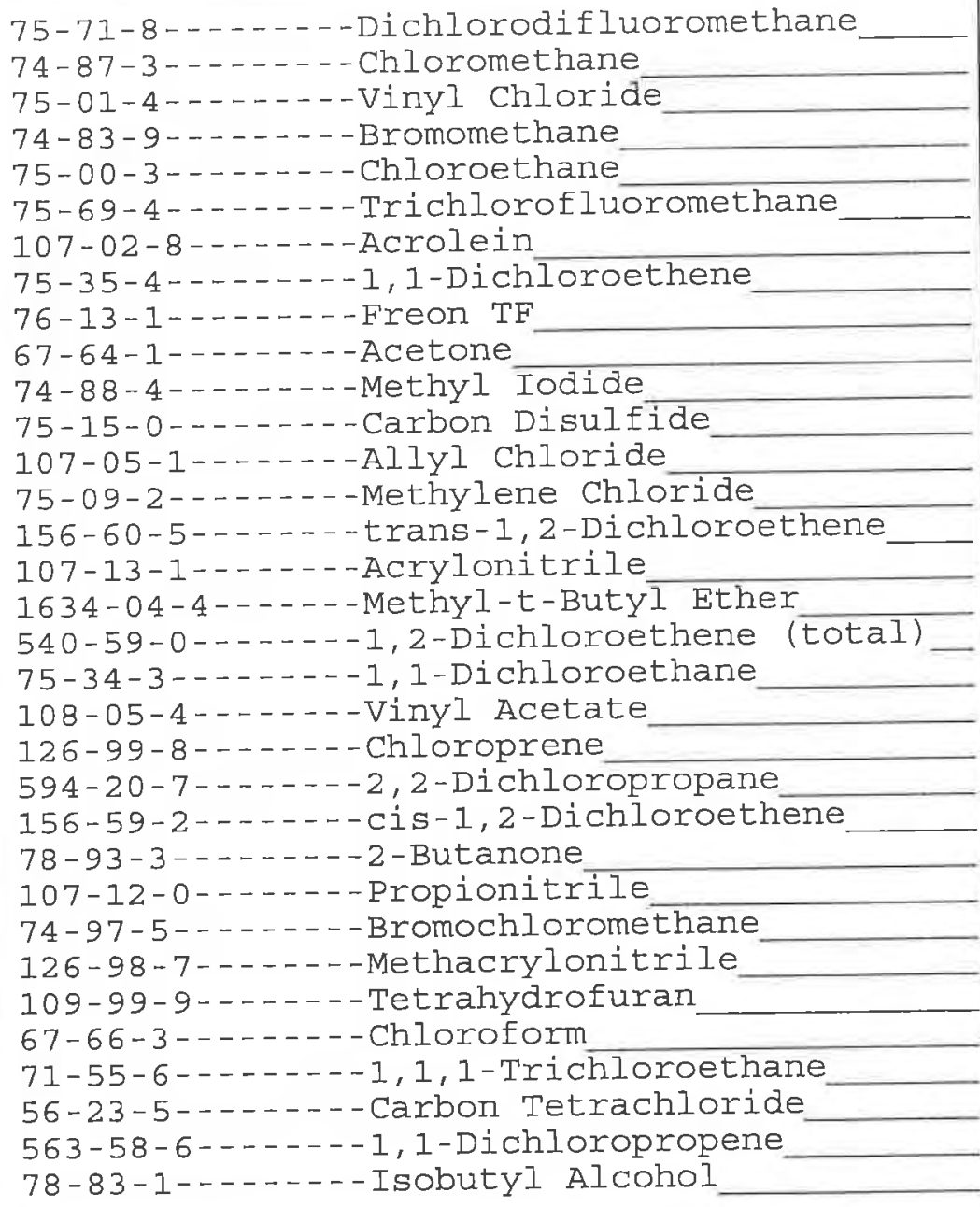

$5.0 \mathrm{U}$

$0.78 \mathrm{JB}$

$5.0 \mathrm{U}$

3.2 JB

$5.0 \mathrm{U}$

$5.0 \mathrm{U}$

$5.0 \mathrm{U}$

$5.0 \mathrm{U}$

$5.0 \mathrm{U}$

$5.0 \mathrm{U}$

$0.90 \mathrm{JB}$

$5.0 \mathrm{U}$

$5.0 \mathrm{U}$

$5.0 \mathrm{U}$

$5.0 \mathrm{U}$

$5.0 \mathrm{U}$

$5.0 \mathrm{U}$

$5.0 \mathrm{U}$

$5.0 \mathrm{U}$

$5.0 \mathrm{U}$

$5.0 \mathrm{U}$

$5.0 \mathrm{U}$

$5.0 \mathrm{U}$

$5.0 \mathrm{U}$

$20 \mathrm{U}$

$5.0 \mathrm{U}$

$5.0 \mathrm{U}$

$50 \mathrm{U}$

$5.0 \mathrm{U}$

$0.32 \mathrm{~J}$

$5.0 \mathrm{U}$

$5.0 \mathrm{U}$

$250 \mathrm{U}$ 
FORM 1

VOLATILE ORGANICS ANALYSIS DATA SHEET
ARGLAB SAMPLE NO.

SV-S-25654

Lab Name: TESTAMERICA BURLINGTON Contract: 8E-00302

SDG NO. : 138033

Lab code: STLV Case No.: SAVANNAH SAS No.:

Lab Sample ID: 835016

Matrix: (soil/water) soIL

Lab File ID: $\quad 835016$

Sample wt/vol:

$5.0(\mathrm{~g} / \mathrm{mL}) \mathrm{G}$

Date Received: 07/08/10

Level : (low/med) LOW

Date Analyzed: 07/14/10

\% Moisture: not dec.

GC Column: DB-624 ID: 0.53 (mm)

Dilution Factor: 1.0

Soil Extract Volume:

(uL)

Soil Aliquot Volume:

(uL)

CONCENTRATION UNITS:

CAS NO. COMPOUND (ug/L or ug/ $\mathrm{Kg}) \mathrm{UG} / \mathrm{KG}$

$Q$

5. $0 \mathrm{U}$

$5.0 \mathrm{U}$

$5.0 \mathrm{U}$

$5.0 \mathrm{U}$

$5.0 \mathrm{U}$

$5.0 \mathrm{U}$

$50 \mathrm{U}$

$5.0 \mathrm{U}$

$5.0 \mathrm{U}$

5. $0 \mathrm{U}$

$5.0 \mathrm{U}$

$5.0 \mathrm{U}$

$5.0 \mathrm{U}$

$5.0 \mathrm{U}$

$5.0 \mathrm{U}$

5. $0 \mathrm{U}$

5. $0 \mathrm{U}$

5. $0 \mathrm{U}$

$5.0 \mathrm{U}$

$5.0 \mathrm{U}$

$5.0 \mathrm{U}$

$5.0 \mathrm{U}$

5. $0 \mathrm{U}$

$5.0 \mathrm{U}$

$5.0 \mathrm{U}$

5. $0 \mathrm{U}$

5. $0 \mathrm{U}$

5. $0 \mathrm{U}$

$5.0 \mathrm{U}$

5. $0 \mathrm{U}$

5.0 U

$5.0 \mathrm{U}$

$5.0 \mathrm{U}$ 
FORM 1

VOLATILE ORGANICS ANALYSIS DATA SHEET
ARGLAB SAMPLE NO.

SV-S- 25654

Lab Name: TESTAMERICA BURLINGTON Contract: 8E-00302

SDG No. : 138033

Lab Code: STLV Case No.: SAVANNAH SAS No.:

Lab Sample ID: 835016

Matrix: (soil/water) SOIL

Lab File ID: 835016

Sample wt/vol:

$5.0(\mathrm{~g} / \mathrm{mL}) \mathrm{G}$

Date Received: 07/08/10

Level : (low/med) LOW

Date Analyzed: 07/14/10

\% Moisture: not dec.

GC Column: DB-624 ID: 0.53 (mm)

Dilution Factor: 1.0

Soil Extract Volume:

(UI)

Soil Aliquot Volume:

(uL)

CONCENTRATION UNITS:

CAS NO. COMPOUND $(\mathrm{ug} / \mathrm{L}$ or ug/Kg) UG/KG

Q

$5.0 \mathrm{U}$

5. $0 \mathrm{U}$

$5.0 \mathrm{U}$

$5.0 \mathrm{U}$

$5.0 \mathrm{U}$

$5.0 \mathrm{U}$

$5.0 \mathrm{U}$

$5.0 \mathrm{U}$

$5.0 \mathrm{U}$

$5.0 \mathrm{U}$

$5.0 \mathrm{U}$

5. $0 \mathrm{U}$

5. $0 \mathrm{U}$

5. $0 \mathrm{U}$

5. $0 \mathrm{U}$

5. $0 \mathrm{U}$

5. $0 \mathrm{U}$ 
FORM 1 VOLATILE ORGANICS ANALYSIS DATA SHEET
ARGLAB SAMPLE NO.

SV-S -25677
Lab Name: TESTAMERICA BURLINGTON Contract: 8E-00302

Lab Code: STLV Case No.: SAVANNAH SAS No.:

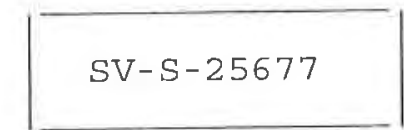

SDG No.: 138033
Matrix: (soil/water) soII

Sample wt/vol:

$5.0(\mathrm{~g} / \mathrm{mL}) \mathrm{G}$

Level: (low/med) LOW

\% Moisture: not dec.

GC Column: DB-624 ID: 0.53 (mm)

Soil Extract Volume:

(uL)
Lab Sample ID: 835026

Lab File ID: 835026

Date Received: 07/08/10

Date Analyzed: 07/14/10

Dilution Factor: 1.0

Soil Aliquot Volume: (uL)

CAS NO.

COMPOUND

CONCENTRATION UNITS:

(ug/L or $\mathrm{ug} / \mathrm{Kg}$ ) UG/KG

Q

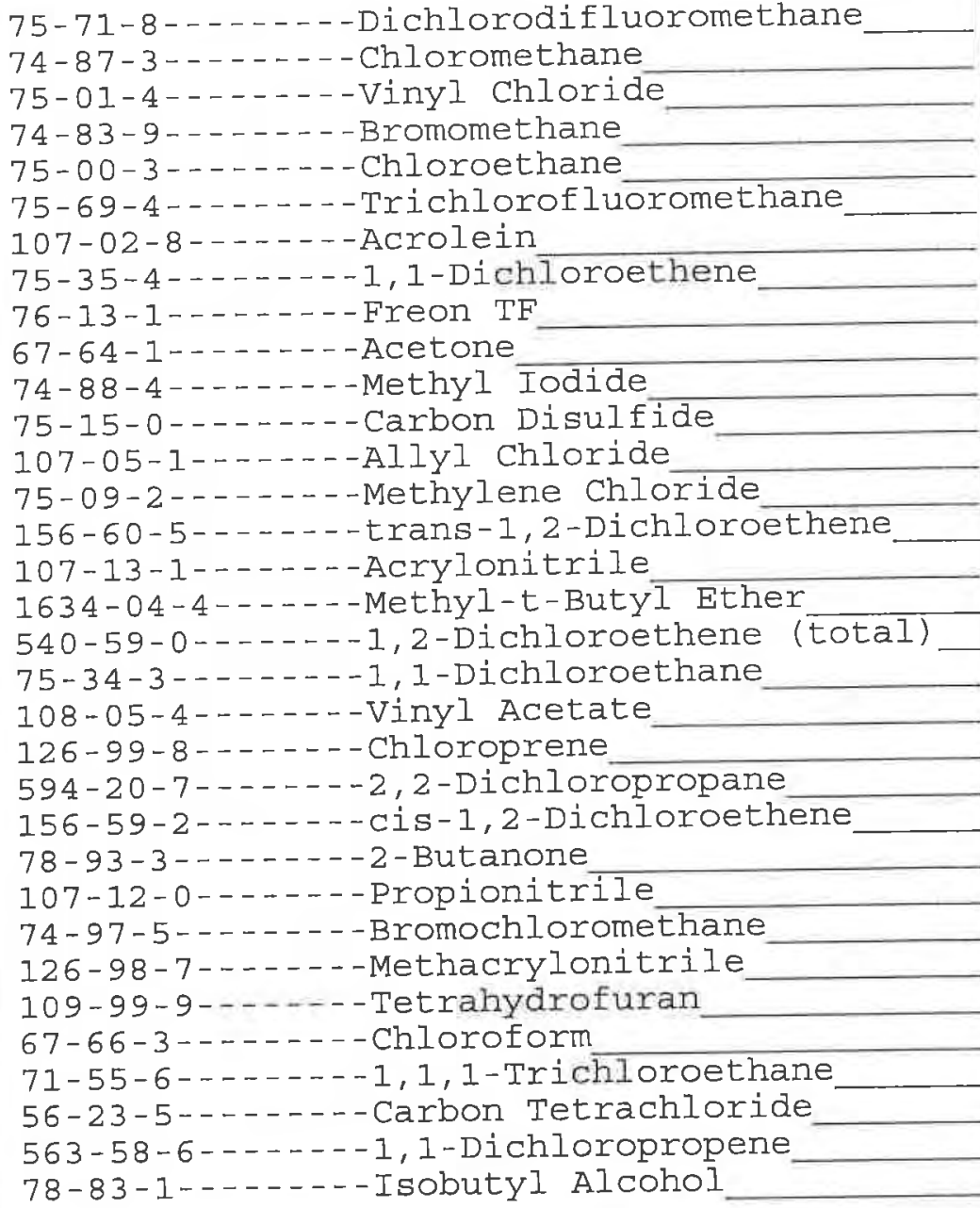

$5.0 \mathrm{U}$

$0.43 \mathrm{JB}$

$5.0 \mathrm{U}$

1. $5 \mathrm{JB}$

5. 0 U

$5.0 \mathrm{U}$

$5.0 \mathrm{U}$

$5.0 \mathrm{U}$

$5.0 \mathrm{U}$

$5.0 \mathrm{U}$

$0.40 \mathrm{JB}$

$5.0 \mathrm{U}$

5. $0 \mathrm{U}$

$5.0 \mathrm{U}$

5. $0 \mathrm{U}$

$5.0 \mathrm{U}$

$5.0 \mathrm{U}$

$5.0 \mathrm{U}$

$5.0 \mathrm{U}$

$5.0 \mathrm{U}$

$5.0 \mathrm{U}$

$5.0 \mathrm{U}$

$5.0 \mathrm{U}$

$5.0 \mathrm{U}$

$20 \mathrm{U}$

$5.0 \mathrm{U}$

$5.0 \mathrm{U}$

$50 \mathrm{U}$

$5.0 \mathrm{U}$

$5.0 \mathrm{U}$

$5.0 \mathrm{U}$

$5.0 \mathrm{U}$

$250 \mathrm{U}$

78-83-1-...--Isobutyl Alcohol 
FORM 1

VOLATILE ORGANICS ANALYSIS DATA SHEET
ARGLAB SAMPLE NO.

$S V-S-25677$

Lab Name: TESTAMERICA BURLINGTON Contract: 8E-00302

SDG No.: 138033

Lab Code: STLV Case No.: SAVANNAH SAS No.:

Lab Sample ID: 835026

Matrix: (soil/water) SoIL

Lab File ID: $\quad 835026$

Sample wt/vol:

$5.0(\mathrm{~g} / \mathrm{mL}) \mathrm{G}$

Level: (low/med) Low

Date Received: 07/08/10

\% Moisture: not dec.

GC Column: DB-624 ID: 0.53 (mm)

Date Analyzed: 07/14/10

Soil Extract Volume: (uL)

Dilution Factor: 1.0

Soil Aliquot Volume: (uL) CONCENTRATION UNITS:

CAS NO. COMPOUND (ug/L or ug/Kg) UG/KG

$71-43-2-------B e n z e n e$

107-06-2-...-1, 2-Dichloroethane

79-01-6-...-.-Trichloroethene

78-87-5--.---1, 2-Dichloropropane

74-95-3-...-.-Dibromomethane

80-62-6-...--.-Methyl Methacrylate

123-91-1-....-1,4-Dioxane

75-27-4-...-.-Bromodichloromethane

110-75-8-...--2-Chloroethyl Vinyl Ether

10061-01-5--.--cis-1,3-Dichloropropene

108-10-1...-.-4-Methyl-2-pentanone

$108-88-3------$ Toluene

10061-02-6--.--trans-1,3-Dichloropropene

97-63-2-...-...thyl Methacrylate

79-00-5-.....-1,1,2-Trichloroethane

127-18-4--...--Tetrachloroethene

142-28-9--..--1,3-Dichloropropane

591-78-6-- - - - 2-Hexanone

124-48-1.....-Dibromochloromethane

106-93-4------1,2-Dibromoethane

108-90-7-...-- Chlorobenzene

630-20-6-...-1, 1, 1, 2-Tetrachloroethane

100-41-4-...--Ethylbenzene

$1330-20-7-\ldots .-x_{\text {Xlene }}(\mathrm{m}, \mathrm{p})$

95-47-6-...-.-.xylene (o)

1330-20-7-..-.-Xylene (total)

100-42-5-..----styrene

$75-25-2-\ldots-\cdots-$ Bromoform

98-82-8-..--- - Isopropylbenzene

108-86-1.....--Bromobenzene

$79-34-5-\ldots-\ldots-1,1,2,2-$ Tetrachloroethane

96-18-4-...-.-1,2,3-Trichloropropane

110-57-6--.-.-trans-1,4-Dichloro-2-bütene
$5.0 \mathrm{U}$

5. $0 \mathrm{U}$

5. $0 \mathrm{U}$

5. $0 \mathrm{U}$

$5.0 \mathrm{U}$

$5.0 \mathrm{U}$

$50 \mathrm{U}$

$5.0 \mathrm{U}$

$5.0 \mathrm{U}$

$5.0 \mathrm{U}$

$5.0 \mathrm{U}$

$5.0 \mathrm{U}$

$5.0 \mathrm{U}$

$5.0 \mathrm{U}$

$5.0 \mathrm{U}$

$5.0 \mathrm{U}$

5. $0 \mathrm{U}$

$5.0 \mathrm{U}$

5. 0 U

5. $0 \mathrm{U}$

$5.0 \mathrm{U}$

$5.0 \mathrm{U}$

$5.0 \mathrm{U}$

$5.0 \mathrm{U}$

$5.0 \mathrm{U}$

$5.0 \mathrm{U}$

$5.0 \mathrm{U}$

$5.0 \mathrm{U}$

$5.0 \mathrm{U}$

5.0 U

$5.0 \mathrm{U}$

5. $0 \mathrm{U}$

5. $0 \mathrm{U}$ 
FORM 1

VOLATILE ORGANICS ANALYSIS DATA SHEET

Lab Name: TESTAMERICA BURLINGTON

Contract : $8 \mathrm{E}-00302$

Lab code: STLV Case No.: SAVANNAH SAS No.:

Lab Sample ID: 835026

Matrix: (soil/water) soIL

Sample wt/vol:

$5.0(\mathrm{~g} / \mathrm{mI}) \mathrm{G}$

Level: (low/med) LOW

\% Moisture: not dec.

GC Column: DB-624 ID: 0.53 (mm)

Soil Extract Volume: (uL) Lab File ID: $\quad 835026$

Date Received: 07/08/10

Date Analyzed: 07/14/10

Dilution Factor: 1.0

Soil Aliquot Volume:
ARGLAB SAMPLE NO.

$S V-S-25677$

SDG No.: 138033 (uL) CONCENTRATION UNITS :

CAS NO. COMPOUND (ug/L or ug/ $\mathrm{Kg}) \mathrm{UG} / \mathrm{KG}$

Q

103-65-1-...--n-Propylbenzene

95-49-8-...-.-2-Chlorotoluene

$106-43-4 \ldots-\ldots-4-$ Chlorotoluene

$108-67-8-\ldots---1,3,5-$ Trimethyl benzene

98-06-6-...-.-.tert-Butylbenzene

95-63-6-...-.-1, 2,4-Trimethylbenzene

135-98-8-....- sec-Butylbenzene

541-73-1-.....-1, 3-Dichlorobenzene

99-87-6-....-4-Isopropyltoluene

$106-46-7 \ldots \ldots-1,4-D i c h l o r o b e n z e n e$

95-50-1-.....-1, 2-Dichlorobenzene

104-51-8-...-n-Butylbenzene

96-12-8-...- - 1, 2-Dibromo-3-Chloropropane

120-82-1-..---1,2,4-Trichlorobenzene

87-68-3-....-.-Hexachlorobutadiene

91-20-3-...--Naphthalene

87-61-6-..... 1, 2, 3-Trichlorobenzene

\begin{tabular}{|l|l|l|}
5.0 & $\mathrm{U}$ \\
5.0 & $\mathrm{U}$ \\
5.0 & $\mathrm{U}$ \\
5.0 & $\mathrm{U}$ \\
5.0 & $\mathrm{U}$ \\
5.0 & $\mathrm{U}$ \\
5.0 & $\mathrm{U}$ \\
5.0 & $\mathrm{U}$ \\
5.0 & $\mathrm{U}$ \\
5.0 & $\mathrm{U}$ \\
5.0 & $\mathrm{U}$ \\
5.0 & $\mathrm{U}$ \\
5.0 & $\mathrm{U}$ \\
5.0 & $\mathrm{U}$ \\
5.0 & $\mathrm{U}$ \\
5.0 & $\mathrm{U}$ \\
5.0 & $\mathrm{U}$ \\
\hline
\end{tabular}


FORM 1

VOLATILE ORGANICS ANALYSIS DATA SHEET
ARGLAB SAMPLE NO.

$S V-S-25688$

Lab Name: TESTAMERICA BURLINGTON Contract: 8E-00302

Lab Code: STLV Case No.: SAVANNAH SAS No.:

Lab Sample ID: 835024

Matrix: (soil/water) SoIL

Sample wt/vol:

$5.0(\mathrm{~g} / \mathrm{mL}) \quad \mathrm{G}$

Lab File ID: $\quad 835024$ I2

Level: (low/med) LOW

․․ Moisture: not dec.

GC Column: $\mathrm{DB}-624$ ID: 0.53 (mm)

Soil Extract Volume:

(uL)
Date Received: 07/08/10

Date Analyzed: 07/14/10

Dilution Factor: 1.0

Soil Aliquot Volume:

(uL)

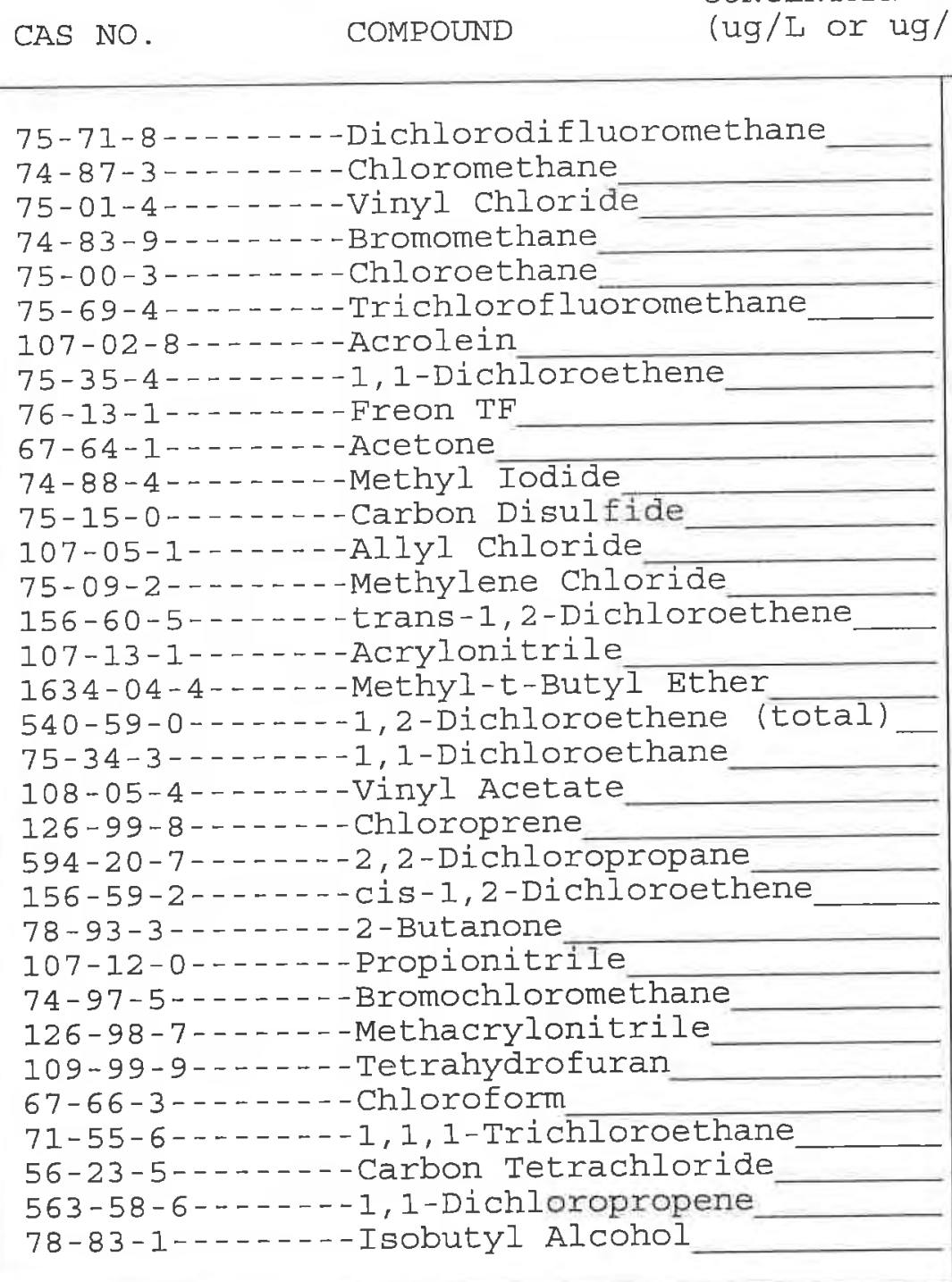

$5.0 \mathrm{U}$

$0.60 \mathrm{JB}$

$5.0 \mathrm{U}$

$2.3 \mathrm{JB}$

$5.0 \mathrm{U}$

$5.0 \mathrm{U}$

$5.0 \mathrm{U}$

$5.0 \mathrm{U}$

$5.0 \mathrm{U}$

$5.0 \mathrm{U}$

$5.0 \mathrm{U}$

$5.0 \mathrm{U}$

$5.0 \mathrm{U}$

$5.0 \mathrm{U}$

$5.0 \mathrm{U}$

5. $0 \mathrm{U}$

5. $0 \mathrm{U}$

$5.0 \mathrm{U}$

$5.0 \mathrm{U}$

$5.0 \mathrm{U}$

$5.0 \mathrm{U}$

$5.0 \mathrm{U}$

$5.0 \mathrm{U}$

$5.0 \mathrm{U}$

$20 \mathrm{U}$

$5.0 \mathrm{U}$

$5.0 \mathrm{U}$

$50 \mathrm{U}$

$5.0 \mathrm{U}$

$5.0 \mathrm{U}$

12

$5.0 \mathrm{U}$

$250 \mathrm{U}$ 
FORM 1.

VOLATILE ORGANICS ANALYSIS DATA SHEET
ARGLAB SAMPLE NO.

$S V-S-25688$

Lab Name: TESTAMERICA BURLINGTON Contract: 8E-00302

SDG NO. : 138033

Lab Code: STLV Case No.: SAVANNAH SAS No.:

Lab Sample ID: 835024

Matrix: (soil/water) soIL

Lab File ID: 835024 I2

Sample wt/vol:

$5.0(\mathrm{~g} / \mathrm{mL}) \mathrm{G}$

Date Received: 07/08/10

Level: (low/med) LOW

Date Analyzed: 07/14/10

$\div$ Moisture: not dec.

GC Column: $\mathrm{DB}-624$ ID: 0.53 (mm)

Dilution Factor: 1.0

Soil Extract Volume:

(uL)

Soil Aliquot Volume: (uL)

CONCENTRATION UNITS:

CAS NO. COMPOUND (ug/L or ug/Kg) UG/KG

Q

$71-43-2---\cdots-$ - Benzene

107-06-2--..-1,2-Dichloroethane

79-01-6-...-- Trichloroethene

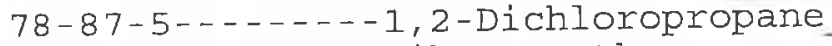

74-95-3-...--Dibromomethane.

80-62-6-....-. Methyl Methacrylate

123-91-1-...--1,4-Dioxane

75-27-4-.....-Bromodichloromethane

110-75-8--..-2-Chloroethyl vinyl Ether

10061-01-5-----cis-1,3-Dichloropropene

108-10-1-....-4-Methyl-2-pentanone

108-88-3-...--Toluene

10061-02-6-----trans-1,3-Dichloropropene

97-63-2-..-.-. Ethyl Methacrylate

79-00-5-...--1, 1,2-Trichloroethane

127-18-4-....-. Tetrachloroethene

142-28-9--.---1,3-Dichloropropane

$591-78-6 \ldots-\ldots-2-$ Hexanone

124-48-1-....-Dibromochloromethane

106-93-4-...--1,2-Dibromoethane

108-90-7-..-- - Chlorobenzene

630-20-6-...-1,1,1,2-Tetrachloroethane

100-41-4---.---Ethylbenzene

1330-20-7-----xylene (m, p)

95-47-6-....-.xylene (o)

$1330-20-7 \ldots-.-x y l e n e$ (total)

$100-42-5-\ldots-\ldots-$ Styrene

75-25-2-....--Bromoform

98-82-8-..----Isopropylbenzene

108-86-1-..-.--Bromobenzene

79-34-5-..-.--1, 1,2,2-Tetrachloroethane

96-18-4-...-- 1,2,3-Trichloropropane

110-57-6-....-trans-1,4-Dichloro-2-butene
$5.0 \mathrm{U}$

5. $0 \mathrm{U}$

5. $0 \mathrm{U}$

$5.0 \mathrm{U}$

5. $0 \mathrm{U}$

$5.0 \mathrm{U}$

$50 \mathrm{U}$

$5.0 \mathrm{U}$

$5.0 \mathrm{U}$

$5.0 \mathrm{U}$

$5.0 \mathrm{U}$

5. $0 \mathrm{U}$

5.0 U

$5.0 \mathrm{U}$

$5.0 \mathrm{U}$

$5.0 \mathrm{U}$

5. $0 \mathrm{U}$

$5.0 \mathrm{U}$

$5.0 \mathrm{U}$

$5.0 \mathrm{U}$

5.0 U

$5.0 \mathrm{U}$

5. $0 \mathrm{U}$

5. $0 \mathrm{U}$

$5.0 \mathrm{U}$

5. $0 \mathrm{U}$

5. $0 \mathrm{U}$

5. 0 U

5. $0 \mathrm{U}$

$5.0 \mathrm{U}$

$5.0 \mathrm{U}$

5. 0 U

5. $0 \mathrm{U}$ 
FORM 1

VOLATILE ORGANICS ANALYSIS DATA SHEET

Lab Name: TESTAMERICA BURLINGTON

Contract: $8 \mathrm{E}-00302$

Lab Code: STLV Case No.: SAVANNAH SAS No.:

ARGLAB SAMPLE NO.

SV-S- 25688

Lab Sample ID: 835024

Matrix: (soil/water) solL

Lab File ID: 835024 I2

Sample wt/vol:

$5.0(\mathrm{~g} / \mathrm{mL}) \mathrm{G}$

Level: (low/med) LOW

Date Received: 07/08/10

\% Moisture: not dec.

GC Column: DB-624 ID: 0.53 (mm)

Date Analyzed: 07/14/10

Soil Extract Volume: (uL)

Dilution Factor: 1.0

Soil Aliquot Volume: (uL)

CONCENTRATION UNITS:

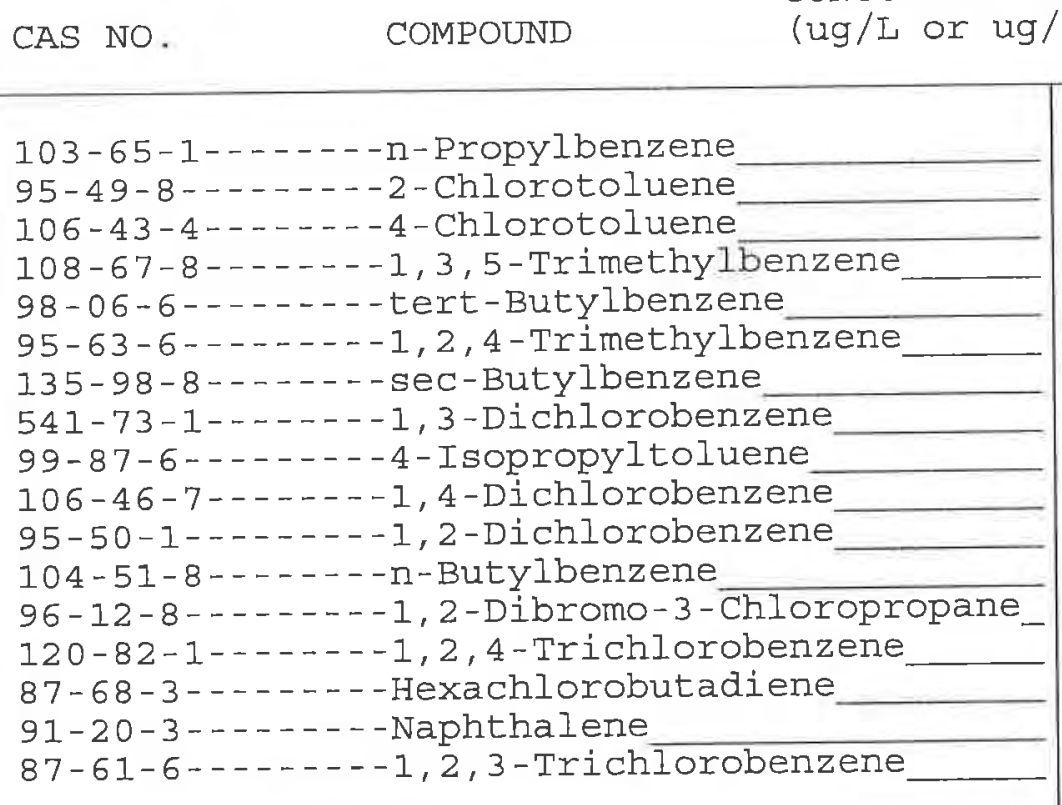

$5.0 \mathrm{U}$

$5.0 \mathrm{U}$

$5.0 \mathrm{U}$

$5.0 \mathrm{U}$

$5.0 \mathrm{U}$

$5.0 \mathrm{U}$

$5.0 \mathrm{U}$

$5.0 \mathrm{U}$

$5.0 \mathrm{U}$

$5.0 \mathrm{U}$

$5.0 \mathrm{U}$

$5.0 \mathrm{U}$

$5.0 \mathrm{U}$

$5.0 \mathrm{U}$

$5.0 \mathrm{U}$

$5.0 \mathrm{U}$

$5.0 \mathrm{U}$

Q 
FORM 1 VOLATILE ORGANICS ANALYSIS DATA SHEET
ARGIAB SAMPLE NO.

SV $-S-25698$

Lab Name: TESTAMERICA BURLINGTON Contract: 8E-00302

SDG NO. : 138033

Lab Code: STLV Case No.: SAVANNAH SAS No.:

Lab Sample ID: 835023

Matrix: (soil/water) SOIL

Lab File ID: $\quad 835023 I 2$

Sample wt/vol:

$5.0(\mathrm{~g} / \mathrm{mL}) \mathrm{G}$

Date Received: 07/08/10

Level: (low/med) LOW

Date Analyzed: 07/14/10

\% Moisture: not dec.

GC Column: DB-624 ID: 0.53 (mm)

Dilution Factor: 1.0

Soil Extract Volume: (uL)

Soil Aliquot Volume: (uL)

CONCENTRATION UNITS:

CAS NO.

COMPOUND

( $\mathrm{ug} / \mathrm{L}$ or $\mathrm{ug} / \mathrm{Kg}$ ) UG/KG

$Q$

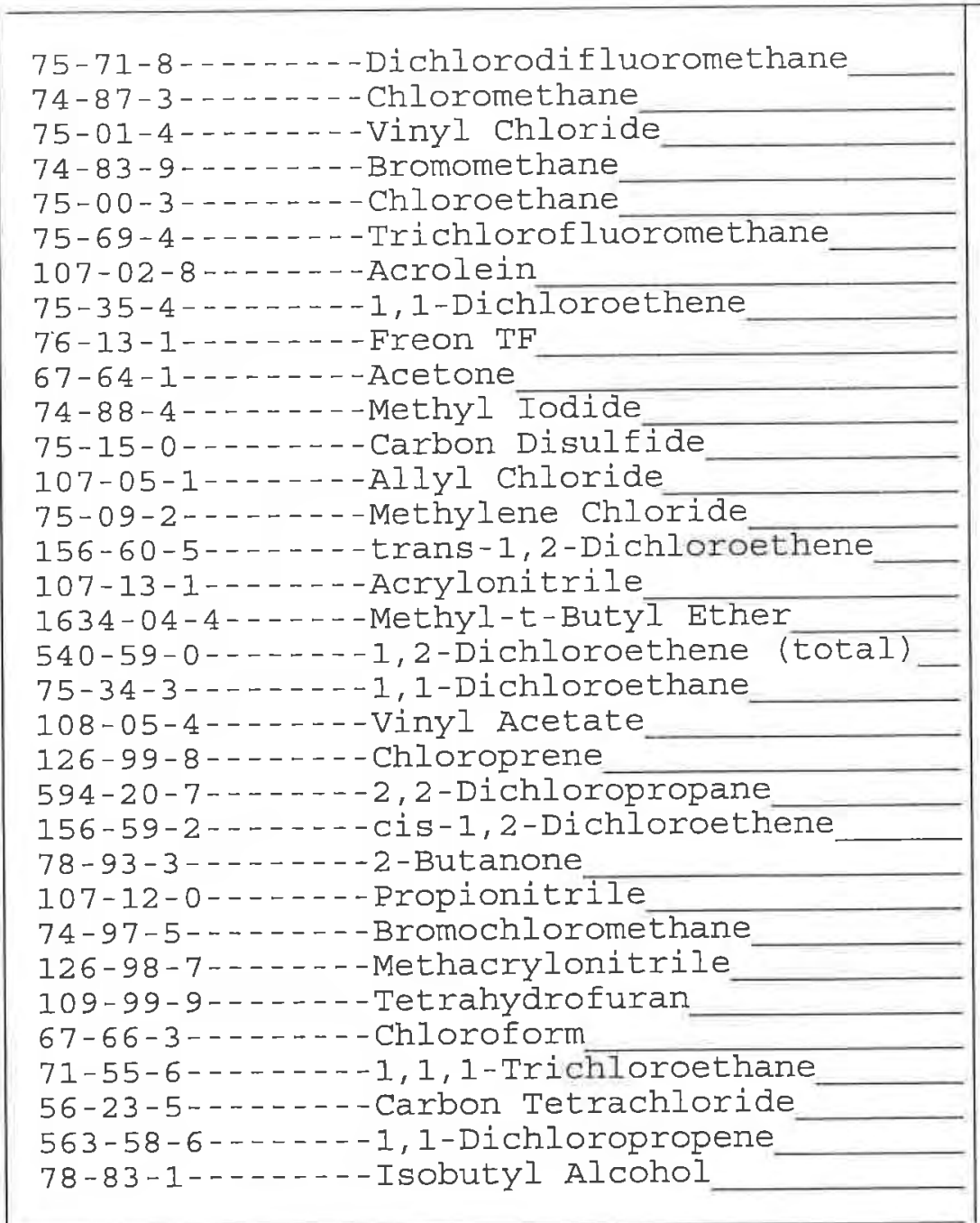

$5.0 \mathrm{U}$

$0.45 \mathrm{JB}$

$5.0 \mathrm{U}$

$2.1 \mathrm{JB}$

$5.0 \mathrm{U}$

$5.0 \mathrm{U}$

$5.0 \mathrm{U}$

$5.0 \mathrm{U}$

$5.0 \mathrm{U}$

$5.0 \mathrm{U}$

$0.54 \mathrm{JB}$

$5.0 \mathrm{U}$

$5.0 \mathrm{U}$

$5.0 \mathrm{U}$

$5.0 \mathrm{U}$

$5.0 \mathrm{U}$

5. $0 \mathrm{U}$

$5.0 \mathrm{U}$

$5.0 \mathrm{U}$

$5.0 \mathrm{U}$

$5.0 \mathrm{U}$

$5.0 \mathrm{U}$

5. $0 \mathrm{U}$

$5.0 \mathrm{U}$

$20 \mathrm{U}$

$5.0 \mathrm{U}$

$5.0 \mathrm{U}$

$50 \mathrm{U}$

$5.0 \mathrm{U}$

$5.0 \mathrm{U}$

$5.0 \mathrm{U}$

$5.0 \mathrm{U}$

$250 \mathrm{U}$ 
FORM 1

VOLATILE ORGANICS ANALYSIS DATA SHEET
ARGLAB SAMPLE NO.

$S V-S-25698$

Lab Name: TESTAMERICA BURIINGTON Contract: 8E-00302

SDG No. : 138033

Lab Code: STLV Case No.: SAVANNAH SAS No.:

Lab Sample ID: 835023

Matrix: (soil/water) SOIL

Lab File ID: $\quad 835023 I 2$

Sample wt/vol: $\quad 5.0(\mathrm{~g} / \mathrm{mL}) \mathrm{G}$

Date Received: 07/08/10

Level: (low/med) LOW

Date Analyzed: 07/14/10

\% Moisture: not dec.

GC Column: DB-624 ID: 0.53 (mm)

Soil Extract Volume:

(uL)

Dilution Factor: 1.0

Soil Aliquot volume: (uL) CONCENTRATION UNITS:

CAS NO. COMPOUND (ug/L or ug/ $\mathrm{Kg}) \mathrm{UG} / \mathrm{KG}$

$Q$

$5.0 \mathrm{U}$

5. 0 U

$5.0 \mathrm{U}$

$5.0 \mathrm{U}$

5. $0 \mathrm{U}$

5. $0 \mathrm{U}$

$50 \mathrm{U}$

$5.0 \mathrm{U}$

5. $0 \mathrm{U}$

$5.0 \mathrm{U}$

5. $0 \mathrm{U}$

5. $0 \mathrm{U}$

$5.0 \mathrm{U}$

$5.0 \mathrm{U}$

$5.0 \mathrm{U}$

$5.0 \mathrm{U}$

$5.0 \mathrm{U}$

$5.0 \mathrm{U}$

$5.0 \mathrm{U}$

$5.0 \mathrm{U}$

$5.0 \mathrm{U}$

$5.0 \mathrm{U}$

$5.0 \mathrm{U}$

$5.0 \mathrm{U}$

5. $0 \mathrm{U}$

5. $0 \mathrm{U}$

5. $0 \mathrm{U}$

5. $0 \mathrm{U}$

5. 0 U

$5.0 \mathrm{U}$

5. $0 \mathrm{U}$

$5.0 \mathrm{U}$

$5.0 \mathrm{U}$ 
FORM 1

VOLATILE ORGANICS ANALYSIS DATA SHEET
ARGLAB SAMPLE NO.

SV $-S-25698$

Lab Name: TESTAMERICA BURLINGTON Contract: 8E-00302

SDG No. : 138033

Lab Code: STLV Case No.: SAVANNAH SAS No.:

Lab Sample ID: 835023

Matrix: (soil/water) SOIL

Lab File ID: 835023 I2

Sample wt/vol:

$5.0(\mathrm{~g} / \mathrm{mL}) \quad \mathrm{G}$

Date Received: 07/08/10

Level: (low/med) LOW

Date Analyzed: 07/14/10

$\because$ Moisture: not dec.

GC Column: DB-624 ID: 0.53 (mm)

Dilution Factor: 1.0

Soil Extract Volume:

(uL)

Soil Aliquot Volume:

(UI)

CONCENTRATION UNITS:

CAS NO.

COMPOUND

$(u g / I$ or $u g / K g) ~ U G / K G$

Q

103-65-1-...--n-Propylbenzene

95-49-8--.---2-Chlorotoluene

106-43-4--..-4-Chlorotoluene

108-67-8-...-1,3,5-Trimethylbenzene

98-06-6--..--tert-Butylbenzene

95-63-6-...-1,2,4-Trimethylbenzene

135-98-8-...-sec-Butylbenzene

541-73-1-..--1,3-Dichlorobenzene

99-87-6--.-.-4-Isopropyltoluene

106-46-7-...-1,4-Dichlorobenzene

95-50-1-...-1, 2-Dichlorobenzene

104-51-8--.---n-Butylbenzene

96-12-8-- - - - 1, 2-Dibromo-3-Chloropropane

120-82-1-...-1,2,4-Trichlorobenzene

87-68-3-...... Hexachlorobutadiene

91-20-3-...--Naphthalene

87-61-6......-1,2,3-Trichlorobenzene
$5.0 \mathrm{U}$

$5.0 \mathrm{U}$

$5.0 \mathrm{U}$

$5.0 \mathrm{U}$

$5.0 \mathrm{U}$

$5.0 \mathrm{U}$

$5.0 \mathrm{U}$

$5.0 \mathrm{U}$

$5.0 \mathrm{U}$

$5.0 \mathrm{U}$

$5.0 \mathrm{U}$

$5.0 \mathrm{U}$

$5.0 \mathrm{U}$

$5.0 \mathrm{U}$

$5.0 \mathrm{U}$

$5.0 \mathrm{U}$

$5.0 \mathrm{U}$ 
FORM 1

VOLATILE ORGANICS ANALYSIS DATA SHEET
ARGLAB SAMPLE NO.

$S V-S-25719$

SDG No.: 138033

Lab Code: STLV Case No.: SAVANNAH SAS No.:

Lab Sample ID: 835018

Matrix: (soil/water) soIL

Lab File ID: $\quad 835018$

Sample wt/vol: $\quad 5.0(\mathrm{~g} / \mathrm{mL}) \mathrm{G}$

Date Received: 07/08/10

Level: (low/med) LOW

Date Analyzed: 07/14/10

\% Moisture: not dec.

GC Column: $\mathrm{DB}-624$ ID: 0.53 (mm)

Dilution Factor: 1.0

Soil Extract Volume:

(uL)

Soil Aliquot Volume:

(UI)

CONCENTRATION UNITS:

CAS NO.

COMPOUND

(ug/L or ug/Kg) UG/KG

$Q$

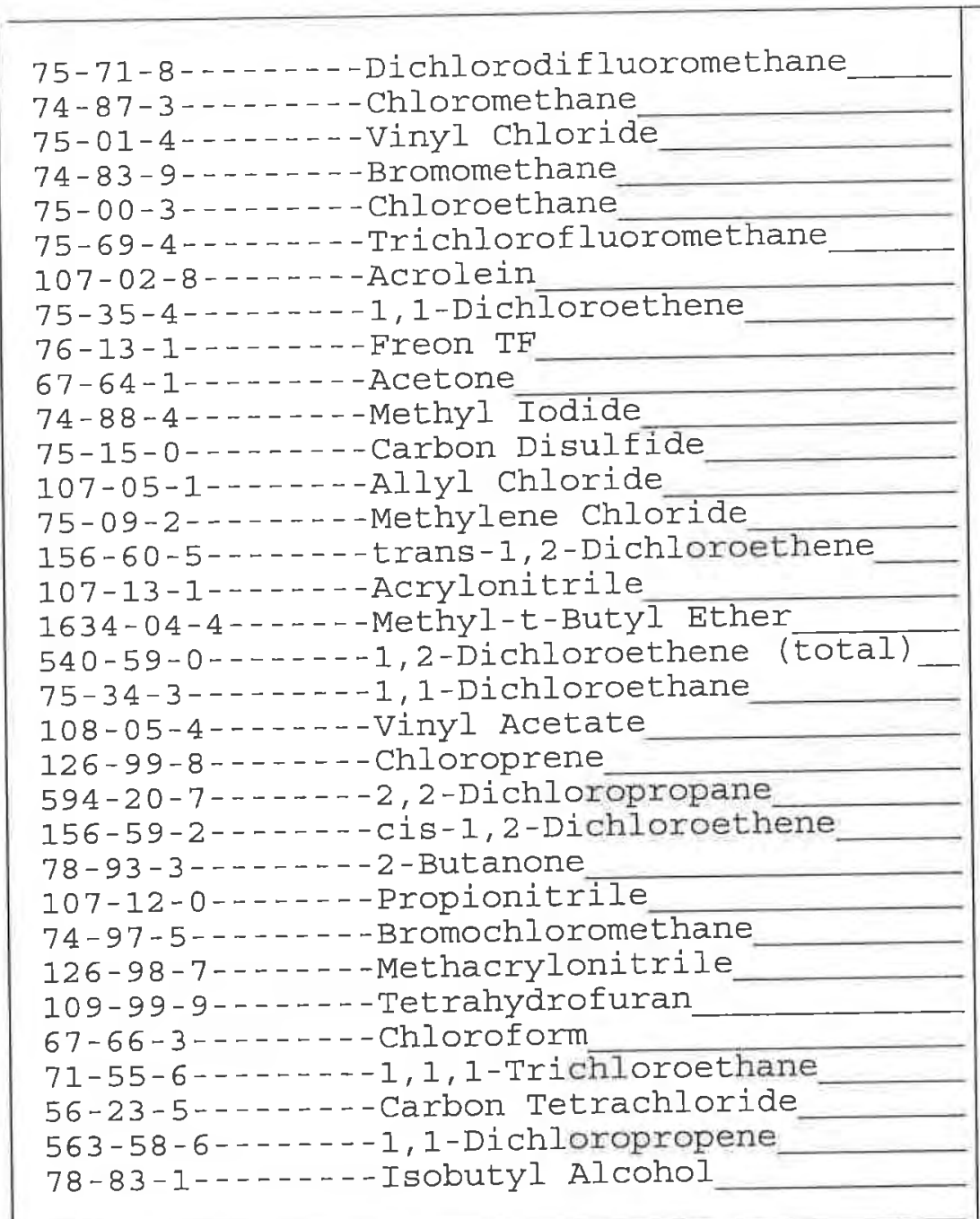

$5.0 \mathrm{U}$

$0.71 \mathrm{JB}$

$5.0 \mathrm{U}$

2.4 JB

$5.0 \mathrm{U}$

$5.0 \mathrm{U}$

$5.0 \mathrm{U}$

$5.0 \mathrm{U}$

$5.0 \mathrm{U}$

$5.0 \mathrm{U}$

$0.86 \mathrm{JB}$

$5.0 \mathrm{U}$

$5.0 \mathrm{U}$

$5.0 \mathrm{U}$

$5.0 \mathrm{U}$

$5.0 \mathrm{U}$

$5.0 \mathrm{U}$

$5.0 \mathrm{U}$

$5.0 \mathrm{U}$

$5.0 \mathrm{U}$

$5.0 \mathrm{U}$

$5.0 \mathrm{U}$

$5.0 \mathrm{U}$

$5.0 \mathrm{U}$

$20 \mathrm{U}$

$5.0 \mathrm{U}$

$5.0 \mathrm{U}$

$50 \mathrm{U}$

$0.22 \mathrm{~J}$

$5.0 \mathrm{U}$

7.5

$5.0 \mathrm{U}$

$250 \mathrm{U}$ 
FORM 1

VOLATILE ORGANICS ANALYSIS DATA SHEET

Lab Name: TESTAMERICA BURLINGTON Contract: 8E-00302

Lab Code: STLV Case No.: SAVANNAH SAS No.:

ARGLAB SAMPLE NO.

SV-S-25719

SDG NO.: 138033
Matrix: (soil/water) SOII

Sample wt/vol:

$5.0(\mathrm{~g} / \mathrm{mL}) \mathrm{G}$

Level: (low/med) LOW

$\div$ Moisture: not dec.

GC Column: DB-624 ID: 0.53 (mm)

Soil Extract Volume:
(uL)
Lab Sample ID: 835018

Lab File ID: 835018

Date Received: 07/08/10

Date Analyzed: 07/14/10

Dilution Factor: 1.0

Soil Aliquot Volume: (uL)

CONCENTRATION UNITS:

CAS NO.

COMPOUND

( $\mathrm{ug} / \mathrm{L}$ or $\mathrm{ug} / \mathrm{Kg}$ ) UG/KG

Q

$5.0 \mathrm{U}$

$5.0 \mathrm{U}$

$5.0 \mathrm{U}$

$5.0 \mathrm{U}$

$5.0 \mathrm{U}$

$5.0 \mathrm{U}$

$50 \mathrm{U}$

$5.0 \mathrm{U}$

$5.0 \mathrm{U}$

$5.0 \mathrm{U}$

$5.0 \mathrm{U}$

$5.0 \mathrm{U}$

$5.0 \mathrm{U}$

$5.0 \mathrm{U}$

$5.0 \mathrm{U}$

$5.0 \mathrm{U}$

$5.0 \mathrm{U}$

$5.0 \mathrm{U}$

$5.0 \mathrm{U}$

$5.0 \mathrm{U}$

$5.0 \mathrm{U}$

$5.0 \mathrm{U}$

$5.0 \mathrm{U}$

$5.0 \mathrm{U}$

$5.0 \mathrm{U}$

$5.0 \mathrm{U}$

$5.0 \mathrm{U}$

$5.0 \mathrm{U}$

$5.0 \mathrm{U}$

$5.0 \mathrm{U}$

$5.0 \mathrm{U}$

$5.0 \mathrm{U}$

$5.0 \mathrm{U}$ 
FORM 1

VOLATILE ORGANICS ANALYSIS DATA SHEET
ARGLAB SAMPLE NO.

SV-S-25719

Lab Name: TESTAMERICA BURLINGTON Contract: 8E-00302

SDG NO.: 138033

Lab Code: STLV Case No.: SAVANNAH SAS No.:

Lab Sample ID: 835018

Matrix: (soil/water) SOIL

Lab File ID: 835018

Sample wt/vol:

$5.0(\mathrm{~g} / \mathrm{mL}) \quad \mathrm{G}$

Level: (low/med) LOW

Date Received: 07/08/10

$\div$ Moisture: not dec.

Date Analyzed: 07/14/10

GC Column: DB-624 ID: 0.53 (mm)

Dilution Factor: 1.0

Soil Extract Volume:

(uL)

Soil Aliquot Volume:

(uL)

CONCENTRATION UNITS :

CAS NO. COMPOUND (ug/L or ug/Kg) UG/KG

Q

103-65-1-...-n-Propylbenzene

95-49-8--..-2-Chlorotoluene

106-43-4-..--4-Chlorotoluene

108-67-8-..--1,3,5-Trimethylbenzene

98-06-6-...--tert-Butylbenzene

95-63-6-...--1,2,4-Trimethylbenzene

135-98-8------sec-Butylbenzene

541-73-1-...-1,3-Dichlorobenzene

99-87-6-..---4-I sopropyltoluene

106-46-7--.--1,4-Dichlorobenzene

95-50-1-....-1,2-Dichlorobenzene

104-51-8-....-n-Butylbenzene

96-12-8--..--1,2-Dibromo-3-Chloropropane_

120-82-1-..--1,2,4-Trichlorobenzene

87-68-3-...-.-Hexachlorobutadiene

91-20-3-- - - -Naphthalene

87-61-6-...--1,2,3-Trichlorobenzene

\begin{tabular}{|l|l|l|}
5.0 & $\mathrm{U}$ \\
5.0 & $\mathrm{U}$ \\
5.0 & $\mathrm{U}$ \\
5.0 & $\mathrm{U}$ \\
5.0 & $\mathrm{U}$ \\
5.0 & $\mathrm{U}$ \\
5.0 & $\mathrm{U}$ \\
5.0 & $\mathrm{U}$ \\
5.0 & $\mathrm{U}$ \\
5.0 & $\mathrm{U}$ \\
5.0 & $\mathrm{U}$ \\
5.0 & $\mathrm{U}$ \\
5.0 & $\mathrm{U}$ \\
5.0 & $\mathrm{U}$ \\
5.0 & $\mathrm{U}$ \\
5.0 & $\mathrm{U}$ \\
5.0 & $\mathrm{U}$ \\
\hline
\end{tabular}

FORM I VOA 
FORM 1

VOLATILE ORGANICS ANALYSIS DATA SHEET
ARGLAB SAMPLE NO.

$S V-S-25723$
Lab Name: TESTAMERICA BURLINGTON Contract: 8E-00302

Lab Code: STLV Case No.: SAVANNAH SAS No.:
SDG No.: 138033
Matrix: (soil/water) SoIL

Sample wt/vol:

$5.0(\mathrm{~g} / \mathrm{mL}) \mathrm{G}$

Level: (low/med) LOW

\% Moisture: not dec.

GC Column: DB-624 ID: 0.53 (mm)

Soil Extract Volume:

(UI)
Lab Sample ID: 835022

Lab File ID: $\quad 835022$

Date Received: 07/08/10

Date Analyzed: 07/14/10

Dilution Factor: 1.0

Soil Aliquot Volume: (uL)

(ug/L or $\mathrm{ug} / \mathrm{Kg}$ ) UG/KG

Q

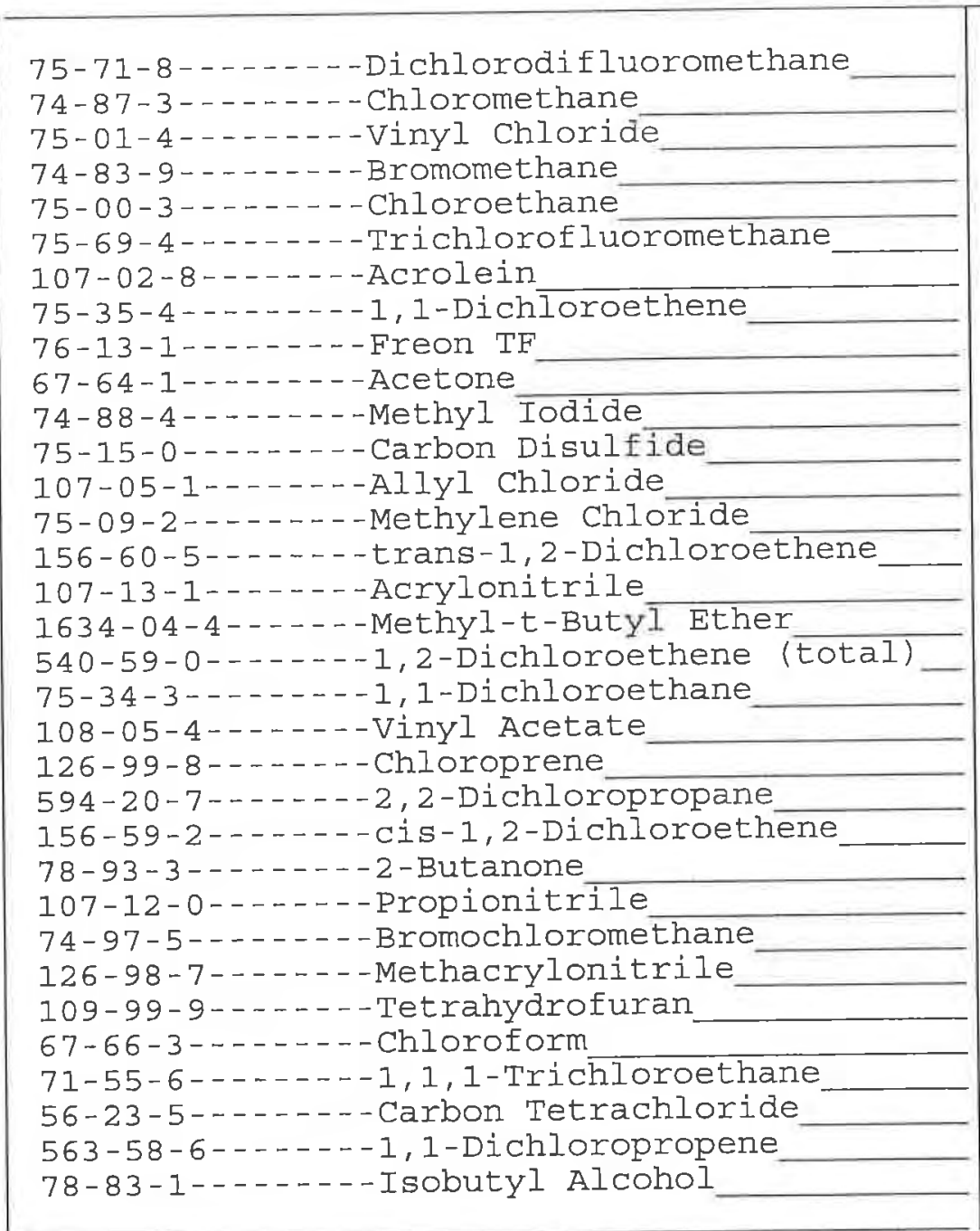

$5.0 \mathrm{U}$

$0.58 \mathrm{JB}$

$5.0 \mathrm{U}$

1.9 JB

$5.0 \mathrm{U}$

$5.0 \mathrm{U}$

$5.0 \mathrm{U}$

$5.0 \mathrm{U}$

$5.0 \mathrm{U}$

$5.0 \mathrm{U}$

$0.53 \mathrm{JB}$

$5.0 \mathrm{U}$

$5.0 \mathrm{U}$

$5.0 \mathrm{U}$

$5.0 \mathrm{U}$

5. $0 \mathrm{U}$

$5.0 \mathrm{U}$

$5.0 \mathrm{U}$

$5.0 \mathrm{U}$

$5.0 \mathrm{U}$

$5.0 \mathrm{U}$

$5.0 \mathrm{U}$

5. $0 \mathrm{U}$

5. $0 \mathrm{U}$

$20 \mathrm{U}$

$5.0 \mathrm{U}$

$5.0 \mathrm{U}$

$50 \mathrm{U}$

$5.0 \mathrm{U}$

$5.0 \mathrm{U}$

$5.0 \mathrm{U}$

$5.0 \mathrm{U}$

$250 \mathrm{U}$ 
FORM 1

VOLATILE ORGANICS ANALYSIS DATA SHEET
ARGLAB SAMPLE NO.

SV $-S-25723$

Lab Name: TESTAMERICA BURLINGTON Contract: 8E-00302

Lab code: STLV Case No.: SAVANNAH SAS No.:

SDG No.: 138033

Matrix: (soil/water) SOIL

Lab Sample ID: 835022

Sample wt/vol:

$5.0(\mathrm{~g} / \mathrm{mL}) \mathrm{G}$

Lab File ID: $\quad 835022$

Level: (low/med) LOW

Date Received: $07 / 08 / 10$

\% Moisture: not dec.

Date Analyzed: 07/14/10

GC Column: DB-624 ID: 0.53 (mm)

Dilution Factor: 1.0

Soil Extract Volume:

(uI)

Soil Aliquot Volume:

(uL)

CONCENTRATION UNITS:

CAS NO. COMPOUND $(u g / L$ or $u g / \mathrm{Kg}) \mathrm{UG} / \mathrm{KG}$

$Q$

$71-43-2 \ldots-\ldots---B e n z e n e$

107-06-2------1,2-Dichloroethane

79-01-6-...-.-Trichloroethene

$78-87-5 \ldots-\ldots-1,2-\mathrm{Dichloropropane}$

74-95-3--.----Dibromomethane

80-62-6-............ Methyl Methacrylate

$123-91-1 \ldots-\cdots-1,4-$ Dioxane

75-27-4-....-Bromodichloromethane

110-75-8-....-2-Chloroethyl vinyl Ether

10061-01-5----cis-1,3-Dichloropropene

108-10-1-...--4-Methyl-2-pentanone

108-88-3--.--- Toluene

10061-02-6-..--trans-1,3-Dichloropropene

97-63-2--..---Ethyl Methacrylate

79-00-5-.....-1, 1, 2-Trichloroethane

127-18-4-...-- Tetrachloroethene

142-28-9-...--1,3-Dichloropropane

591-78-6-....-2-Hexanone

124-48-1-...--Dibromochloromethane

106-93-4-...-1, 2-Dibromoethane

108-90-7--..-- Chlorobenzene

630-20-6--.---1,1,1,2-Tetrachloroethane

$100-41-4 \ldots-\ldots-$ Ethylbenzene

1330-20-7-....xylene (m, p)

95-47-6.........xylene (o)

$1330-20-7-\cdots--x y l e n e$ (total)

100-42-5-...---styrene

75-25-2-....-. Bromoform

98-82-8-..--.--Isopropyl benzene

108-86-1--.---Bromobenzene

79-34-5-..--- 1, 1,2,2-Tetrachloroethane

96-18-4-...--1,2,3-Trichloropropane

110-57-6-...--trans-1,4-Dichloro-2-butene
$5.0 \mathrm{U}$

$5.0 \mathrm{U}$

$5.0 \mathrm{U}$

$5.0 \mathrm{U}$

$5.0 \mathrm{U}$

$5.0 \mathrm{U}$

$50 \mathrm{U}$

$5.0 \mathrm{U}$

$5.0 \mathrm{U}$

$5.0 \mathrm{U}$

$5.0 \mathrm{U}$

5. $0 \mathrm{U}$

$5.0 \mathrm{U}$

$5.0 \mathrm{U}$

$5.0 \mathrm{U}$

$5.0 \mathrm{U}$

$5.0 \mathrm{U}$

$5.0 \mathrm{U}$

$5.0 \mathrm{U}$

$5.0 \mathrm{U}$

$5.0 \mathrm{U}$

$5.0 \mathrm{U}$

5. $0 \mathrm{U}$

$5.0 \mathrm{U}$

$5.0 \mathrm{U}$

5. $0 \mathrm{U}$

5. $0 \mathrm{U}$

5. $0 \mathrm{U}$

5. $0 \mathrm{U}$

5. $0 \mathrm{U}$

$5.0 \mathrm{U}$

$5.0 \mathrm{U}$

5. $0 \mathrm{U}$ 
FORM 1

VOLATILE ORGANICS ANALYSIS DATA SHEET

Lab Name: TESTAMERICA BURLINGTON Contract: 8E-00302

Lab Code: STLV Case No.: SAVANNAH SAS No.:

Lab Sample ID: 835022

Matrix: (soil/water) SOIL

$5.0(\mathrm{~g} / \mathrm{mL}) \mathrm{G}$

Sample wt/vol:

Level: (low/med) LOW

$\div$ Moisture: not dec.

GC Column: DB-624 ID: 0.53 (mm)

soil Extract volume: (UL)

Lab File ID: 835022

Date Received: 07/08/10

Date Analyzed: 07/14/10

Dilution Factor: 1.0

Soil Aliquot Volume:
ARGLAB SAMPLE NO.

$S V-S-25723$

SDG NO.: 138033 (uL) CONCENTRATION UNITS:

CAS NO. COMPOUNDD (ug/L or ug/ $\mathrm{Kg}$ ) UG/KG

Q

103-65-1-...--n-Propylbenzene

95-49-8-...--2-Chlorotoluene

106-43-4-.-.-4-Chlorotoluene

108-67-8-...-1,3,5-Trimethylbenzene

98-06-6-...--tert-Butylbenzene

95-63-6-...-1, 2,4-Trimethylbenzene

135-98-8-....-sec-Butylbenzene

541-73-1-..--1,3-Dichlorobenzene

99-87-6------4-Isopropyltoluene

106-46-7-..--1, 4-Dichlorobenzene

95-50-1--.--1, 2-Dichlorobenzene

104-51-8-..--n-Butylbenzene

96-12-8-...-1, 2-Dibromo-3-Chloropropane

120-82-1-...-1,2,4-Trichlorobenzene

87-68-3-..---Hexachlorobutadiene

91-20-3-..--Naphthalene

87-61-6-...-1, 2, 3-Trichlorobenzene

$5.0 \mathrm{U}$

$5.0 \mathrm{U}$

5. $0 \mathrm{U}$

5. $0 \mathrm{U}$

$5.0 \mathrm{U}$

$5.0 \mathrm{U}$

$5.0 \mathrm{U}$

$5.0 \mathrm{U}$

5. $0 \mathrm{U}$

5. $0 \mathrm{U}$

$5.0 \mathrm{U}$

$5.0 \mathrm{U}$

5. $0 \mathrm{U}$

5. $0 \mathrm{U}$

$5.0 \mathrm{U}$

$5.0 \mathrm{U}$

$5.0 \mathrm{U}$

FORM I VOA 
FORM 1 VOLATILE ORGANICS ANALYSIS DATA SHEET
ARGLAB SAMPLE NO.

$S V-S-25736$

Lab Name: TESTAMERICA BURLINGTON Contract: 8E-00302

Lab Code: STLV Case No.: SAVANNAH SAS No.:

SDG NO. : 138033

Lab Sample ID: 835020

Matrix: (soil/water) solL

Lab File ID: 835020

Sample wt/vol: $\quad 5.0(\mathrm{~g} / \mathrm{mL}) \mathrm{G}$

Date Received: 07/08/10

Level: (low/med) LOW

Date Analyzed: 07/14/10

\% Moisture: not dec.

GC Column: DB-624 ID: 0.53 (mm)

Soil Extract Volume:

(uL)
Dilution Factor: 1.0

Soil Aliquot volume:

(uL)

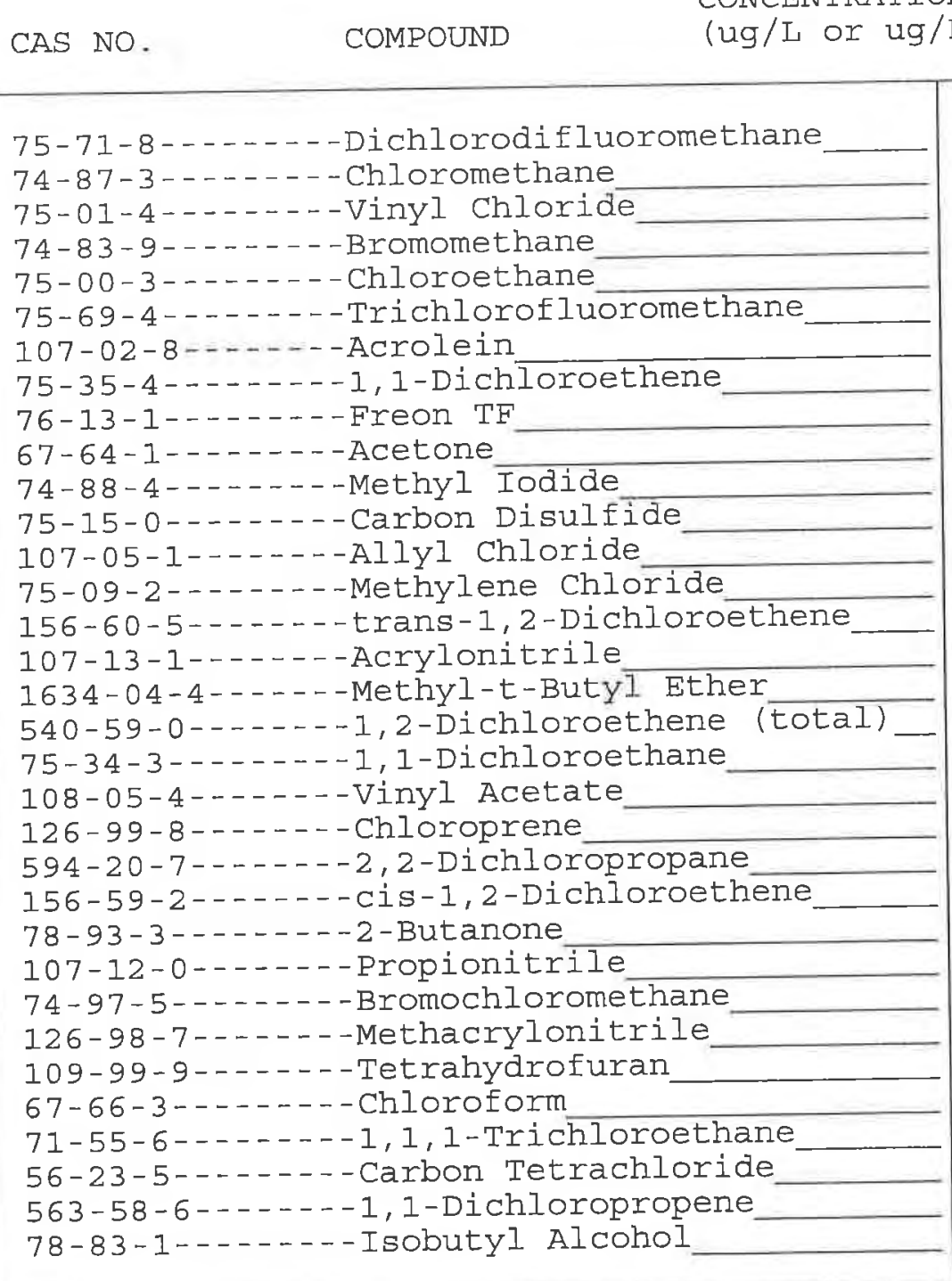

$5.0 \mathrm{U}$

$0.44 \mathrm{JB}$

$5.0 \mathrm{U}$

$1.9 \mathrm{JB}$

$5.0 \mathrm{U}$

$5.0 \mathrm{U}$

$5.0 \mathrm{U}$

$5.0 \mathrm{U}$

$5.0 \mathrm{U}$

$5.0 \mathrm{U}$

$0.55 \mathrm{JB}$

$5.0 \mathrm{U}$

$5.0 \mathrm{U}$

$5.0 \mathrm{U}$

$5.0 \mathrm{U}$

$5.0 \mathrm{U}$

$5.0 \mathrm{U}$

$5.0 \mathrm{U}$

$5.0 \mathrm{U}$

$5.0 \mathrm{U}$

$5.0 \mathrm{U}$

$5.0 \mathrm{U}$

5.0 U

$5.0 \mathrm{U}$

$20 \mathrm{U}$

$5.0 \mathrm{U}$

$5.0 \mathrm{U}$

$50 \mathrm{U}$

$5.0 \mathrm{U}$

$5.0 \mathrm{U}$

$5.0 \mathrm{U}$

$5.0 \mathrm{U}$

$250 \mathrm{U}$ 
FORM 1

VOLATILE ORGANICS ANAIYSIS DATA SHEET
ARGLAB SAMPLE NO.

SV $-S-25736$

Lab Name: TESTAMERICA BURLINGTON Contract: 8E-00302

SDG NO.: 138033

Lab Code: STLV Case No.: SAVANNAH SAS No.:

Lab Sample ID: 835020

Matrix: (soil/water) SOIL

Lab File ID: 835020

Sample wt/vol: $\quad 5.0(\mathrm{~g} / \mathrm{mL}) \mathrm{G}$

Date Received: 07/08/10

Level: (low/med) LOW

Date Analyzed: 07/14/10

\% Moisture: not dec.

GC Column: DB-624 ID: 0.53 (mm)

Dilution Factor: 1.0

Soil Extract Volume:

(uL)

Soil Aliquot Volume:

(uL)

CONCENTRATION UNITS:

CAS NO.

COMPOUND

(ug/L or $\mathrm{ug} / \mathrm{Kg}$ ) UG/KG

$Q$

$5.0 \mathrm{U}$

$5.0 \mathrm{U}$

$5.0 \mathrm{U}$

$5.0 \mathrm{U}$

$5.0 \mathrm{U}$

$5.0 \mathrm{U}$

$50 \mathrm{U}$

$5.0 \mathrm{U}$

$5.0 \mathrm{U}$

$5.0 \mathrm{U}$

$5.0 \mathrm{U}$

$5.0 \mathrm{U}$

$5.0 \mathrm{U}$

$5.0 \mathrm{U}$

$5.0 \mathrm{U}$

$5.0 \mathrm{U}$

$5.0 \mathrm{U}$

$5.0 \mathrm{U}$

$5.0 \mathrm{U}$

$5.0 \mathrm{U}$

$5.0 \mathrm{U}$

$5.0 \mathrm{U}$

$5.0 \mathrm{U}$

$5.0 \mathrm{U}$

$5.0 \mathrm{U}$

$5.0 \mathrm{U}$

$5.0 \mathrm{U}$

$5.0 \mathrm{U}$

$5.0 \mathrm{U}$

$5.0 \mathrm{U}$

$5.0 \mathrm{U}$

$5.0 \mathrm{U}$

$5.0 \mathrm{U}$ 
FORM 1

VOLATILE ORGANICS ANALYSIS DATA SHEET
ARGLAB SAMPLE NO.

SV $-S-25736$

Lab Name: TESTAMERICA BURLINGTON Contract: 8E-00302

SDG NO.: 138033

Lab Code: STLV Case No.: SAVANNAH SAS No.:

Lab Sample ID: 835020

Matrix: (soil/water) SOII

Lab File ID: $\quad 835020$

Sample wt/vol:

$5.0(\mathrm{~g} / \mathrm{mL}) \mathrm{G}$

Date Received: 07/08/10

Level: (low/med) LOW

Date Analyzed: 07/14/10

․․․ Moisture: not dec.

GC Column: DB-624 ID: 0.53 (mm)

Dilution Factor: 1.0

Soil Extract Volume: (uL)

Soil Aliquot Volume: (UL)

CONCENTRATION UNITS:

CAS NO. COMPOUND (ug/L or ug/Kg) UG/KG

Q

103-65-1-...-n--nropylbenzene

95-49-8-...-2-Chlorotoluene

106-43-4-...-4-Chlorotoluene

108-67-8-...-1,3,5-Trimethylbenzene

98-06-6-...-.-tert-Butylbenzene

95-63-6-.....1,2,4-Trimethylbenzene

135-98-8-...--sec-Butylbenzene

541-73-1-...-1, 3-Dichlorobenzene

99-87-6-...--4-Isopropyltoluene

106-46-7--.--1,4-Dichlorobenzene

95-50-1-....-1,2-Dichlorobenzene

104-51-8-..--n-Butylbenzene

96-12-8-.....-1,2-Dibromo-3-Chloropropane

120-82-1-...-1,2,4-Trichlorobenzene

87-68-3-...-.-Hexachlorobutadiene

91-20-3-.....-Naphthalene

87-61-6-....-1,2,3-Trichlorobenzene

$5.0 \mathrm{U}$

$5.0 \mathrm{U}$

$5.0 \mathrm{U}$

5. $0 \mathrm{U}$

$5.0 \mathrm{U}$

$5.0 \mathrm{U}$

$5.0 \mathrm{U}$

5. $0 \mathrm{U}$

$5.0 \mathrm{U}$

$5.0 \mathrm{U}$

$5.0 \mathrm{U}$

$5.0 \mathrm{U}$

$5.0 \mathrm{U}$

$5.0 \mathrm{U}$

$5.0 \mathrm{U}$

$5.0 \mathrm{U}$

5. $0 \mathrm{U}$ 
FORM 1

VOLATILE ORGANICS ANALYSIS DATA SHEET
ARGLAB SAMPLE NO.

$S V-S-25745$

Lab Name: TESTAMERICA BURLINGTON Contract: 8E-00302

SDG NO. : 138033

Lab Code: STLV Case No.: SAVANNAH SAS No.:

Lab Sample ID: 835019

Matrix: (soil/water) SOIL

Lab File ID: 835019

Sample wt/vol:

$5.0(\mathrm{~g} / \mathrm{mL}) \mathrm{G}$

Date Received: 07/08/10

Level : (low/med) LOW

\% Moisture: not dec.

GC Column: DB-624 ID: 0.53 (mm)

Date Analyzed: $07 / 14 / 10$

Soil Extract Volume: (uL)

Dilution Factor: 1.0

Soil Aliquot Volume: (uL)

CONCENTRATION UNITS:

CAS NO. COMPOUND $(\mathrm{ug} / \mathrm{L}$ or $\mathrm{ug} / \mathrm{Kg}) \mathrm{UG} / \mathrm{KG}$

Q

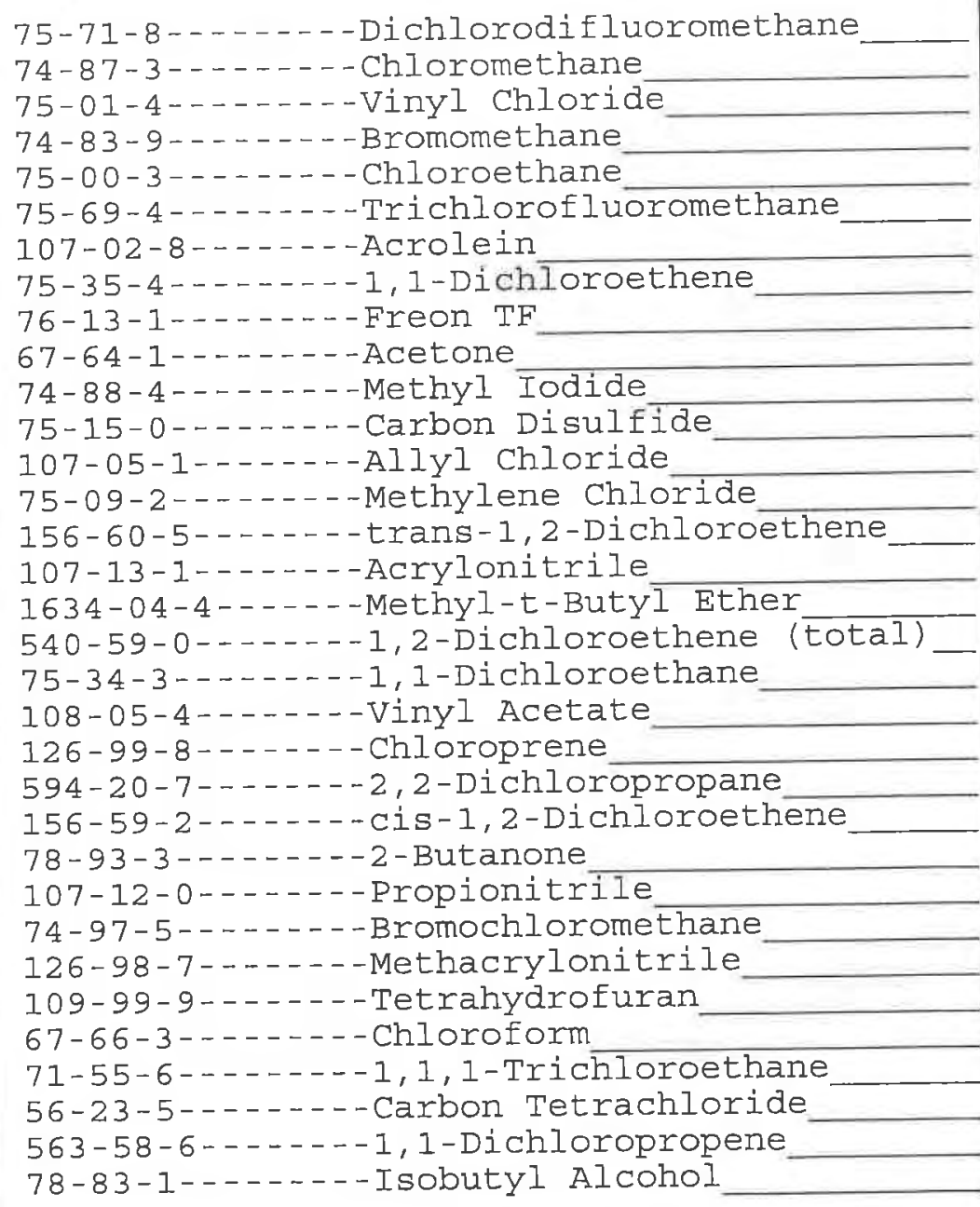

$5.0 \mathrm{U}$

$0.58 \mathrm{JB}$

$5.0 \mathrm{U}$

$2.6 \mathrm{JB}$

$5.0 \mathrm{U}$

$5.0 \mathrm{U}$

$5.0 \mathrm{U}$

$5.0 \mathrm{U}$

5. $0 \mathrm{U}$

$5.0 \mathrm{U}$

$0.73 \mathrm{JB}$

5. $0 \mathrm{U}$

$5.0 \mathrm{U}$

$5.0 \mathrm{U}$

5. $0 \mathrm{U}$

$5.0 \mathrm{U}$

$5.0 \mathrm{U}$

5. $0 \mathrm{U}$

$5.0 \mathrm{U}$

$5.0 \mathrm{U}$

$5.0 \mathrm{U}$

5. $0 \mathrm{U}$

$5.0 \mathrm{U}$

$5.0 \mathrm{U}$

20 U

$5.0 \mathrm{U}$

$5.0 \mathrm{U}$

$50 \mathrm{U}$

$5.0 \mathrm{U}$

$5.0 \mathrm{U}$

$5.0 \mathrm{U}$

$5.0 \mathrm{U}$

$250 \mathrm{U}$ 
FORM 1

VOLATILE ORGANICS ANALYSIS DATA SHEET
ARGLAB SAMPLE NO.

SV-S -25745

Lab Name: TESTAMERICA BURLINGTON Contract: 8E-00302

SDG NO.: 138033

Lab code: STLV Case No.: SAVANNAH SAS No.:

Lab Sample ID: 835019

Matrix: (soil/water) SoIL

Lab File ID: 835019

Sample wt/vol:

$5.0(\mathrm{~g} / \mathrm{mL}) \quad \mathrm{G}$

Date Received: 07/08/10

Level: (low/med) LOW

Date Analyzed: 07/14/10

\% Moisture: not dec.

GC Column: DB-624 ID: 0.53 (mm)

Dilution Factor: 1.0

Soil Extract Volume:

(uI)

Soil Aliquot Volume:

$(u I)$

CONCENTRATION UNITS:

CAS NO. COMPOUND (ug/L or ug/Kg) UG/KG

$Q$

$71-43-2 \ldots-\ldots-$ - Benzene

107-06-2 -...-1, 2-Dichloroethane

79-01-6-....-. Trichloroethene.

78-87-5------ 1, 2-Dichloropropane

74-95-3-...--Dibromomethane

80-62-6-...--Methyl Methacrylate

$123-91-1 \ldots-\ldots-1,4-D i o x a n e$

75-27-4-....--Bromodichloromethane

110-75-8-..---2-Chloroethyl vinyl Ether

10061-01-5----cis-1,3-Dichloropropene

108-10-1-...--4-Methyl-2-pentanone

108-88-3-..--- Toluene

10061-02-6-...-trans-1,3-Dichloropropene

97-63-2-....-. Ethyl Methacrylate

79-00-5-....-1, 1, 2-Trichloroethane

127-18-4-...-.-Tetrachloroethene

142-28-9-...-1, 3-Dichloropropane

591-78-6-...--2-Hexanone

124-48-1-...--Dibromochloromethane

106-93-4-....-1, 2-Dibromoethane

108-90-7-..--- Chlorobenzene

$630-20-6 \ldots-\ldots-1,1,1,2-$ Tetrachloroethane

100-41-4-...--. Ethylbenzene

1330-20-7-......xylene (m, p)

95-47-6-...-.-Xylene (o)

1330-20-7....-xylene (total)

100-42-5--.---styrene

75-25-2-....-Bromoform

98-82-8-...-.-. Isopropylbenzene

108-86-1-...-.-Bromobenzene

$79-34-5 \ldots \ldots-\ldots, 1,2,2-$ Tetrachloroethane

96-18-4_....- - 1, 2, 3-Trichloropropane

110-57-6--..--trans-1,4-Dichloro-2-butene
$5.0 \mathrm{U}$

$5.0 \mathrm{U}$

5. $0 \mathrm{U}$

5. $0 \mathrm{U}$

$5.0 \mathrm{U}$

$5.0 \mathrm{U}$

$50 \mathrm{U}$

$5.0 \mathrm{U}$

$5.0 \mathrm{U}$

5. $0 \mathrm{U}$

$5.0 \mathrm{U}$

5. 0 U

$5.0 \mathrm{U}$

$5.0 \mathrm{U}$

$5.0 \mathrm{U}$

$5.0 \mathrm{U}$

5. 0 U

$5.0 \mathrm{U}$

$5.0 \mathrm{U}$

$5.0 \mathrm{U}$

$5.0 \mathrm{U}$

$5.0 \mathrm{U}$

5. 0 U

$5.0 \mathrm{U}$

5. $0 \mathrm{U}$

$5.0 \mathrm{U}$

5. $0 \mathrm{U}$

$5.0 \mathrm{U}$

5. $0 \mathrm{U}$

5. $0 \mathrm{U}$

5. 0 U

5. $0 \mathrm{U}$

5. $0 \mathrm{U}$ 
FORM 1

VOLATILE ORGANICS ANALYSIS DATA SHEET
ARGLAB SAMPLE NO.

SV-S-25745

Lab Name: TESTAMERICA BURLINGTON Contract: 8E-00302

SDG No.: 138033

Lab Code: STLV Case No.: SAVANNAH SAS No.:

Lab Sample ID: 835019

Matrix: (soil/water) soIL

Lab File ID: 835019

Sample wt/vol:

$5.0(\mathrm{~g} / \mathrm{mL}) \mathrm{G}$

Date Received: 07/08/10

Level: (low/med) LOW

Date Analyzed: 07/14/10

$\%$ Moisture: not dec.

GC Column: DB-624 ID: 0.53 (mm)

Dilution Factor: 1.0

Soil Extract Volume: (UL)

Soil Aliquot Volume: (UL)

CONCENTRATION UNITS:

CAS NO.

COMPOUND

(ug/L or $\mathrm{ug} / \mathrm{Kg}$ ) UG/KG

Q

103-65-1--.---n-Propylbenzene

95-49-8-...--2-Chlorotoluene

106-43-4-...-4-Chlorotoluene

108-67-8-n-.-1, 3,5-Trimethylbenzene

98-06-6------tert-Butylbenzene

95-63-6-..... 1,2,4-Trimethylbenzene

135-98-8-...--sec-Butylbenzene

541-73-1-..-1, 3-Dichlorobenzene

99-87-6--..--4-Isopropyltoluene

106-46-7-..--1,4-Dichlorobenzene

95-50-1-....-1,2-Dichlorobenzene

104-51-8-...--n-Butylbenzene

96-12-8-...-1, 2-Dibromo-3-Chloropropane 120-82-1-.....1,2,4-Trichlorobenzene.

87-68-3-..---Hexachlorobutadiene

91-20-3-....-Naphthalene

87-61-6-....-1, 2,3-Trichlorobenzene

$5.0 \mathrm{U}$

5. $0 \mathrm{U}$

$5.0 \mathrm{U}$

$5.0 \mathrm{U}$

$5.0 \mathrm{U}$

$5.0 \mathrm{U}$

$5.0 \mathrm{U}$

$5.0 \mathrm{U}$

$5.0 \mathrm{U}$

$5.0 \mathrm{U}$

$5.0 \mathrm{U}$

$5.0 \mathrm{U}$

$5.0 \mathrm{U}$

$5.0 \mathrm{U}$

5. $0 \mathrm{U}$

$5.0 \mathrm{U}$

$5.0 \mathrm{U}$ 
FORM 1

VOLATILE ORGANICS ANALYSIS DATA SHEET
ARGLAB SAMPLE NO.

SV - S -25756
Lab Name: TESTAMERICA BURLINGTON Contract: 8E-00302

Lab Code: STLV Case No.: SAVANNAH SAS No.:
Lab sample ID: 835021

Lab File ID: 835021

Date Received: 07/08/10

Date Analyzed: 07/14/10

Dilution Factor: 1.0

Soil Aliquot Volume: (uL)
Soil Extract Volume: (uL)
CONCENTRATION UNITS:

(ug/L or $\mathrm{ug} / \mathrm{Kg}$ ) UG/KG
CAS NO.

\begin{tabular}{rl|l|}
5.0 & $\mathrm{U}$ \\
0.53 & $\mathrm{UB}$ \\
5.0 & $\mathrm{U}$ \\
1.9 & $\mathrm{JB}$ \\
5.0 & $\mathrm{U}$ \\
5.0 & $\mathrm{U}$ \\
5.0 & $\mathrm{U}$ \\
5.0 & $\mathrm{U}$ \\
5.0 & $\mathrm{U}$ \\
5.0 & $\mathrm{U}$ \\
0.46 & $\mathrm{JB}$ \\
5.0 & $\mathrm{U}$ \\
5.0 & $\mathrm{U}$ \\
5.0 & $\mathrm{U}$ \\
5.0 & $\mathrm{U}$ \\
5.0 & $\mathrm{U}$ \\
5.0 & $\mathrm{U}$ \\
5.0 & $\mathrm{U}$ \\
5.0 & $\mathrm{U}$ \\
5.0 & $\mathrm{U}$ \\
5.0 & $\mathrm{U}$ \\
5.0 & $\mathrm{U}$ \\
5.0 & $\mathrm{U}$ \\
5.0 & $\mathrm{U}$ \\
20 & $\mathrm{U}$ \\
5.0 & $\mathrm{U}$ \\
5.0 & $\mathrm{U}$ \\
50 & $\mathrm{U}$ \\
5.0 & $\mathrm{U}$ \\
5.0 & $\mathrm{U}$ \\
5.0 & $\mathrm{U}$ \\
5.0 & $\mathrm{U}$ \\
250 & $\mathrm{U}$ \\
& \\
\hline 5 & \\
5.5
\end{tabular}


FORM 1

VOLATILE ORGANICS ANALYSIS DATA SHEET
ARGLAB SAMPLE NO.

SV-S-25756

Lab Name: TESTAMERICA BURLINGTON Contract: 8E-00302

SDG No.: 138033

Lab Code: STLV Case No.: SAVANNAH SAS No.:

Lab Sample ID: 835021

Matrix: (soil/water) SOIL

Lab File ID: 835021

Sample wt/vol:

$5.0(\mathrm{~g} / \mathrm{mL}) \mathrm{G}$

Level: (low/med) LOW

Date Received: 07/08/10

\% Moisture: not dec.

GC Column: DB-624 ID: 0.53 (mm)

Date Analyzed: 07/14/10

Soil Extract Volume:

(UL)

Dilution Factor: 1.0

Soil Aliquot volume: (uL)

CONCENTRATION UNITS:

CAS NO.

COMPOUND

( ug/I or ug/Kg) UG/KG

Q

$5.0 \mathrm{U}$

$5.0 \mathrm{U}$

$5.0 \mathrm{U}$

$5.0 \mathrm{U}$

$5.0 \mathrm{U}$

$5.0 \mathrm{U}$

$50 \mathrm{U}$

$5.0 \mathrm{U}$

$5.0 \mathrm{U}$

$5.0 \mathrm{U}$

$5.0 \mathrm{U}$

$5.0 \mathrm{U}$

$5.0 \mathrm{U}$

$5.0 \mathrm{U}$

$5.0 \mathrm{U}$

$5.0 \mathrm{U}$

$5.0 \mathrm{U}$

$5.0 \mathrm{U}$

$5.0 \mathrm{U}$

$5.0 \mathrm{U}$

$5.0 \mathrm{U}$

$5.0 \mathrm{U}$

$5.0 \mathrm{U}$

$5.0 \mathrm{U}$

$5.0 \mathrm{U}$

$5.0 \mathrm{U}$

$5.0 \mathrm{U}$

$5.0 \mathrm{U}$

$5.0 \mathrm{U}$

$5.0 \mathrm{U}$

$5.0 \mathrm{U}$

$5.0 \mathrm{U}$

$5.0 \mathrm{U}$ 
FORM 1

VOLATILE ORGANICS ANALYSIS DATA SHEET
ARGLAB SAMPLE NO.

$S V-S-25756$

Lab Name: TESTAMERICA BURLINGTON Contract: 8E-00302

SDG NO.: 138033

Lab Code: STLV Case No.: SAVANNAH SAS No.:

Lab Sample ID: 835021

Matrix: (soil/water) SOIL

Lab File ID: 835021

Sample wt/vol:

$5.0(\mathrm{~g} / \mathrm{mL}) \mathrm{G}$

Date Received: 07/08/10

Level: (low/med) LOW

Date Analyzed: 07/14/10

$\%$ Moisture: not dec.

GC Column: DB-624 ID: 0.53 (mm)

Dilution Factor: 1.0

Soil Extract Volume: (uL)

Soil Aliquot Volume: (UL)

CONCENTRATION UNITS:

CAS NO.

COMPOUND

(ug/L or $\mathrm{ug} / \mathrm{Kg}$ ) UG/KG

Q

103-65-1-...-n-Propylbenzene

95-49-8-n---2-Chlorotoluene

106-43-4-...-4-Chlorotoluene

108-67-8-...-1,3,5-Trimethylbenzene

98-06-6--..--tert-Butylbenzene

95-63-6-...--1, 2,4-Trimethylbenzene

135-98-8-..---sec-Butylbenzene

541-73-1-..-1, 3-Dichlorobenzene

99-87-6-...--4-Isopropyltoluene

106-46-7-..--1,4-Dichlorobenzene

95-50-1--.--1,2-Dichlorobenzene

104-51-8-...-n-Butylbenzene

96-12-8------1, 2-Dibromo-3-Chloropropane

120-82-1-...-1,2,4-Trichlorobenzene

87-68-3-....-Hexachlorobutadiene

91-20-3-....-Naphthalene

87-61-6------1,2,3-Trichlorobenzene

$5.0 \mathrm{U}$

$5.0 \mathrm{U}$

$5.0 \mathrm{U}$

$5.0 \mathrm{U}$

$5.0 \mathrm{U}$

$5.0 \mathrm{U}$

$5.0 \mathrm{U}$

$5.0 \mathrm{U}$

$5.0 \mathrm{U}$

$5.0 \mathrm{U}$

$5.0 \mathrm{U}$

$5.0 \mathrm{U}$

$5.0 \mathrm{U}$

$5.0 \mathrm{U}$

$5.0 \mathrm{U}$

$5.0 \mathrm{U}$

$5.0 \mathrm{U}$ 
FORM 1

VOLATILE ORGANICS ANALYSIS DATA SHEET

Lab Name: TESTAMERICA BURLINGTON Contract: 8E-00302

Lab Code: STLV Case No.: SAVANNAH SAS No.:

Lab Sample ID: 835027

Matrix: (soil/water) soIL

Sample wt/vol: $\quad 5.0(\mathrm{~g} / \mathrm{mL}) \mathrm{G}$

Level: $\quad$ (low/med) LOW

\% Moisture: not dec.

GC Column: DB-624 ID: 0.53 (mm)

Soil Extract Volume:

(uL)

Lab File ID: $\quad 835027$

Date Received: 07/08/10

Date Analyzed: 07/14/10

Dilution Factor: 1.0

Soil Aliquot Volume:
ARGLAB SAMPLE NO. SVSMEOHBLANK

SDG NO.: 138033 (uI)

CONCENTRATION UNITS:

CAS NO. COMPOUND (ug/L or ug/ $\mathrm{Kg}) \mathrm{UG} / \mathrm{KG}$

Q

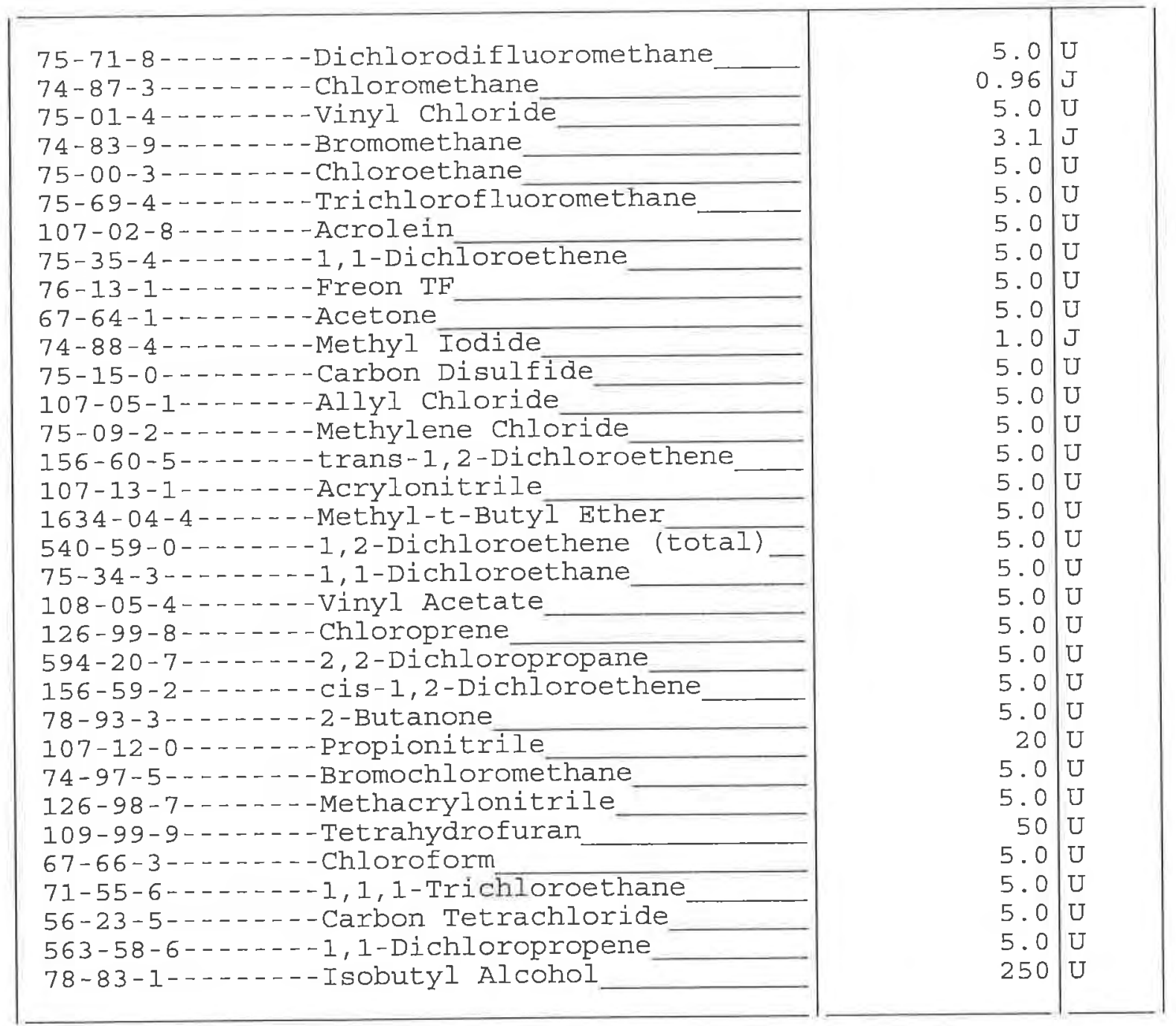


FORM 1

VOLATILE ORGANICS ANALYSIS DATA SHEET
ARGLAB SAMPLE NO.

SVSMEOHBLANK

Lab Name: TESTAMERICA BURLINGTON Contract: 8E-00302

SDG NO.: 138033

Lab Code: STLV Case No.: SAVANNAH SAS No.:

Lab Sample ID: 835027

Matrix: (soil/water) soIL

Lab File ID: 835027

Sample wt/vol: $\quad 5.0(\mathrm{~g} / \mathrm{mL}) \mathrm{G}$

Date Received: 07/08/10

Level: (low/med) LOW

Date Analyzed: 07/14/10

\% Moisture: not dec.

GC column: DB-624 ID: 0.53 (mm)

Dilution Factor: 1.0

Soil Extract Volume:

(UL)

Soil Aliquot Volume:

(uL)

CONCENTRATION UNITS:

CAS NO. COMPOUND (ug/L or ug/Kg) UG/KG

71-43-2--..---Benzene

107-06-2-..---1, 2-Dichloroethane

79-01-6-.....-Trichloroethene

78-87-5--- - - 1,2-Dichloropropane

74-95-3-..---Dibromomethane.

80-62-6-..---.Methyl Methacrylate

$123-91-1 \ldots-\ldots-1,4-D i o x a n e$

75-27-4-.....-Bromodichloromethane

110-75-8-..---2-Chloroethyl Vinyl Ether

10061-01-5-----cis-1,3-Dichloropropene

108-10-1--..--4-Methyl-2-pentanone

$108-88-3-\ldots-\ldots-$ Toluene

10061-02-6----trans-1,3-Dichloropropene

97-63-2--.----Ethyl Methacrylate

79-00-5-....-. 1, 1, 2-Trichloroethane

127-18-4--..-- Tetrachloroethene

142-28-9-...-1,3-Dichloropropane

59l-78-6-...---2-Hexanone

124-48-1-.....-Dibromochloromethane

106-93-4-...--1, 2-Dibromoethane

108-90-7-....-.-Chlorobenzene

630-20-6-..---1, 1, 1, 2-Tetrachloroethane

100-41-4-..--- Ethylbenzene

$1330-20-7 \ldots . .-x_{y}$ ene $(m, p)$

95-47-6-....-.xylene (o)

$1330-20-7 \ldots . .-$ Xylene (total)

100-42-5-...-.-styrene

$75-25-2-\cdots-\cdots-$ Bromoform

98-82-8-..---.-Isopropylbenzene

108-86-1-...-- Bromobenzene

79-34-5-....-1, 1,2,2-Tetrachloroethane

96-18-4--.----1,2,3-Trichloropropane

110-57-6--..--trans-1,4-Dichloro-2-butene
$5.0 \mathrm{U}$

$5.0 \mathrm{U}$

$5.0 \mathrm{U}$

$5.0 \mathrm{U}$

$5.0 \mathrm{U}$

$5.0 \mathrm{U}$

$50 \mathrm{U}$

$5.0 \mathrm{U}$

$5.0 \mathrm{U}$

5. $0 \mathrm{U}$

$5.0 \mathrm{U}$

$5.0 \mathrm{U}$

$0.28 \mathrm{~J}$

$5.0 \mathrm{U}$

$5.0 \mathrm{U}$

$5.0 \mathrm{U}$

$5.0 \mathrm{U}$

5. $0 \mathrm{U}$

$5.0 \mathrm{U}$

$5.0 \mathrm{U}$

$5.0 \mathrm{U}$

$5.0 \mathrm{U}$

$5.0 \mathrm{U}$

5. $0 \mathrm{U}$

$5.0 \mathrm{U}$

$5.0 \mathrm{U}$

5. $0 \mathrm{U}$

5. $0 \mathrm{U}$

$5.0 \mathrm{U}$

$5.0 \mathrm{U}$

5. 0 U

$5.0 \mathrm{U}$

$5.0 \mathrm{U}$ 
FORM 1

VOLATILE ORGANICS ANALYSIS DATA SHEET
ARGLAB SAMPLE NO. SVSMEOHBLANK

Lab Name: TESTAMERICA BURLINGTON Contract: 8E-00302

SDG NO. : 138033

Lab Code: STLV Case No.: SAVANNAH SAS No.:

Lab Sample ID: 835027

Matrix: (soil/water) SOIL

Lab File ID: $\quad 835027$

Sample wt/vol:

$5.0(\mathrm{~g} / \mathrm{mL}) \mathrm{G}$

Date Received: 07/08/10

Level: (low/med) LOW

Date Analyzed: 07/14/10

음 Moisture: not dec.

GC Column: DB-624 ID: 0.53 (mm)

Dilution Factor: 1.0

Soil Extract Volume: (uL)

Soil Aliquot Volume: (uL) CONCENTRATION UNITS: CAS NO. COMPOUND (ug/L or ug/Kg) UG/KG $Q$ 103-65-1-...-.n-Propylbenzene 95-49-8--..---2-Chlorotoluene $106-43-4-\ldots---4-$ Chlorotoluene 108-67-8--..--1,3,5-Trimethylbenzene 98-06-6-...-.-tert-Butylbenzene 95-63-6-....-1, 2,4-Trimethylbenzene 135-98-8-...-.-sec-Butylbenzene 541-73-1--.---1,3-Dichlorobenzene 99-87-6--...--4-Isopropyltoluene 106-46-7-...--1,4-Dichlorobenzene. 95-50-1-....-1, 2-Dichlorobenzene 104-51-8-...-.n-Butylbenzene 96-12-8--.----1, 2-Dibromo-3-Chloropropane $120-82-1 \ldots \ldots-\ldots, 2,4-$ Trichlorobenzene 87-68-3-..--.-Hexachlorobutadiene 91-20-3-...-.-Naphthalene 87-61-6-...-.-1,2,3-Trichlorobenzene

\begin{tabular}{|r|r|l|}
5.0 & $\mathrm{U}$ \\
5.0 & $\mathrm{U}$ \\
5.0 & $\mathrm{U}$ \\
5.0 & $\mathrm{U}$ \\
5.0 & $\mathrm{U}$ \\
5.0 & $\mathrm{U}$ \\
5.0 & $\mathrm{U}$ \\
5.0 & $\mathrm{U}$ \\
5.0 & $\mathrm{U}$ \\
0.24 & $\mathrm{~J}$ \\
5.0 & $\mathrm{U}$ \\
5.0 & $\mathrm{U}$ \\
5.0 & $\mathrm{U}$ \\
0.33 & $\mathrm{~J}$ \\
5.0 & $\mathrm{U}$ \\
0.32 & $\mathrm{~J}$ \\
0.27 & $\mathrm{~J}$ \\
\hline
\end{tabular}


FORM 1

VOLATILE ORGANICS ANALYSIS DATA SHEET
CLIENT SAMPLE NO.

MBLK071410LA

Lab Name: TESTAMERICA BURLINGTON Contract: 8E-00302

SDG NO.: 138033

Lab Code: STLV Case No.: SAVANNAH SAS No.:

Lab Sample ID: MBLK071410LA

Matrix: (soil/water) WATER

Lab File ID: LEKB03D

Sample wt/vol:

$5.000(\mathrm{~g} / \mathrm{mL}) \mathrm{ML}$

Date Received:

Level: (low/med) LOW

Date Analyzed: 07/14/10

: Moisture: not dec.

GC Column: $\mathrm{DB}-624$ ID: 0.53 (mm)

Dilution Factor: 1.0

Soil Extract Volume:

(UL)

Soil Aliquot Volume:

(uL)

CONCENTRATION UNITS:

CAS NO.

COMPOUND

(ug/L or ug/Kg) UG/L

Q

$1.0 \mathrm{U}$

$0.25 \mathrm{~J}$

1.0 U

$0.97 \mathrm{~J}$

$1.0 \mathrm{U}$

$1.0 \mathrm{U}$

$5.0 \mathrm{U}$

$1.0 \mathrm{U}$

$1.0 \mathrm{U}$

$5.0 \mathrm{U}$

$0.48 \mathrm{~J}$

$1.0 \mathrm{U}$

$1.0 \mathrm{U}$

$1.0 \mathrm{U}$

$1.0 \mathrm{U}$

$1.0 \mathrm{U}$

$1.0 \mathrm{U}$

$1.0 \mathrm{U}$

$1.0 \mathrm{U}$

$1.0 \mathrm{U}$

$1.0 \mathrm{U}$

$1.0 \mathrm{U}$

$1.0 \mathrm{U}$

$5.0 \mathrm{U}$

$4.0 \mathrm{U}$

$1.0 \mathrm{U}$

$1.0 \mathrm{U}$

$14 \mathrm{U}$

$1.0 \mathrm{U}$

$1.0 \mathrm{U}$

$1.0 \mathrm{U}$

$1.0 \mathrm{U}$

$50 \mathrm{U}$ 
FORM 1

VOLATILE ORGANICS ANALYSIS DATA SHEET
CLIENT SAMPLE NO.

MBLK071410LA

SDG NO.: 138033
Lab Code: STLV Case No.: SAVANNAH SAS No.:

Matrix: (soil/water) WATER

Sample wt/vol:

$5.000(\mathrm{~g} / \mathrm{mL}) \mathrm{ML}$

Level: (low/med) LOW

: Moisture: not dec.

GC Column: DB-624 ID: 0.53 (mm)

Soil Extract Volume:
Contract: $8 E-00302$

Lab Sample ID: MBLK071410LA

Lab File ID: LEKB03D

Date Received:

Date Analyzed: 07/14/10

Dilution Factor: 1.0

Soil Aliquot Volume:

(uL)

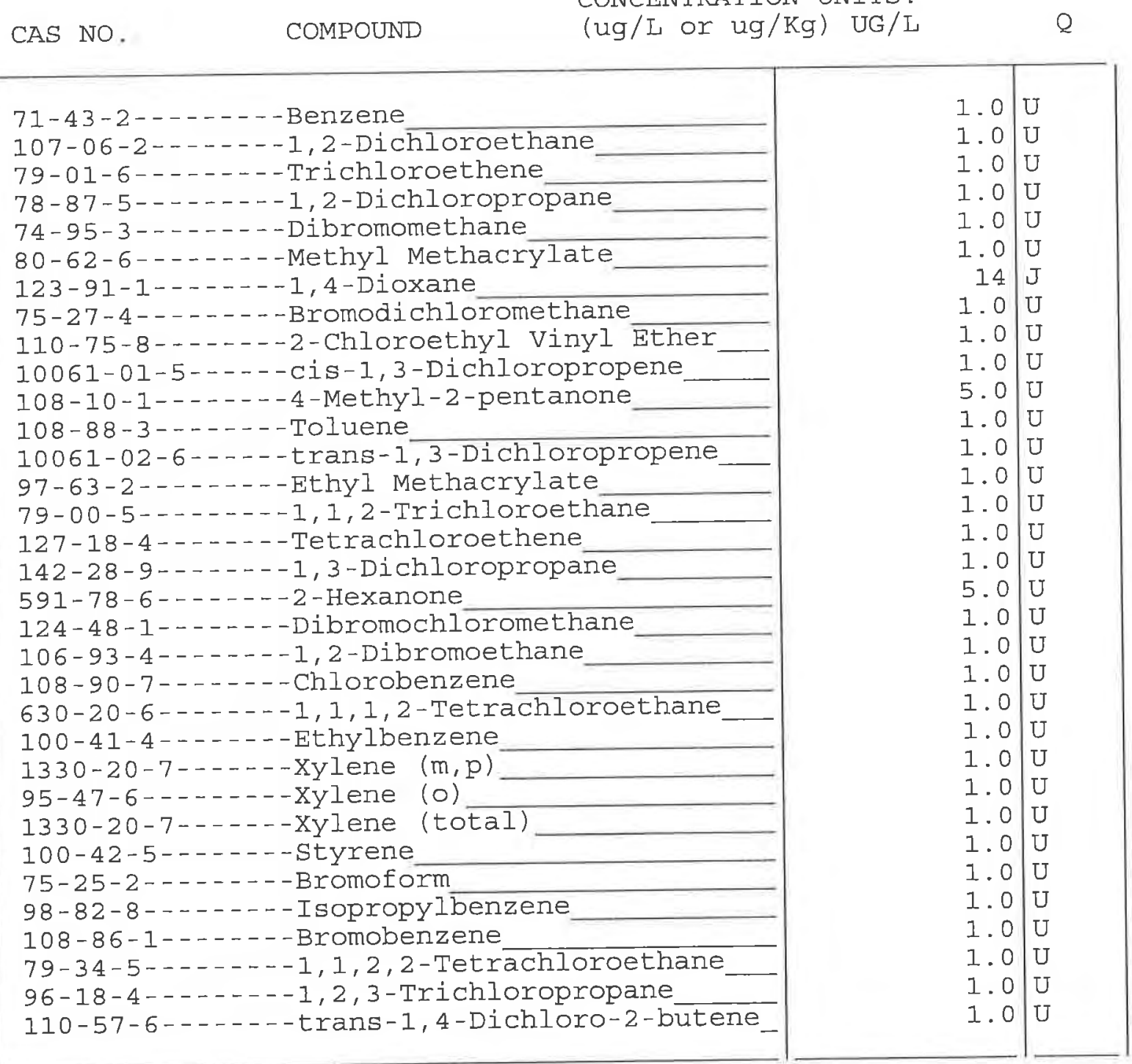


FORM 1

VOLATILE ORGANICS ANALYSIS DATA SHEET
CLIENT SAMPLE NO.

MBLKO 71410LA

Lab Name: TESTAMERICA BURLINGTON Contract: 8E-00302

SDG No.: 138033

Lab Code: STLV Case No.: SAVANNAH SAS No.:

Lab Sample ID: MBLK071410LA

Matrix: (soil/water) WATER

Lab File ID: LEKB03D

Sample wt/vol:

$5.000(\mathrm{~g} / \mathrm{mL}) \mathrm{ML}$

Level: (low/med) LOW

Date Received:

\% Moisture: not dec.

GC Column: DB-624 ID: 0.53 (mm)

Date Analyzed: 07/14/10

Soil Extract Volume: (uL)

Dilution Factor: 1.0

Soil Aliquot Volume: (uL) CONCENTRATION UNITS:

CAS NO. COMPOUND $(\mathrm{ug} / \mathrm{L}$ or $\mathrm{ug} / \mathrm{Kg}) \mathrm{UG} / \mathrm{L}$

$Q$

103-65-1-..-.-n-Propylbenzene

95-49-8-..-.-. 2-Chlorotoluene

106-43-4-..--4-Chlorotoluene

108-67-8-..--1, 3,5-Trimethyl benzene

98-06-6-...--.-tert-Butylbenzene

95-63-6--.----1,2,4-Trimethylbenzene

135-98-8-...---sec-Butylbenzene

541-73-1-...-1, 3-Dichlorobenzene

99-87-6-------4-Isopropyltoluene

$106-46-7-\ldots-\ldots-1,4-\mathrm{Dich}$ lorobenzene

95-50-1-.....-1,2-Dichlorobenzene

104-51-8-..--n-Butylbenzene

96-12-8-..--- 1, 2-Dibromo-3-Chloropropane

120-82-1-....-1,2,4-Trichlorobenzene

87-68-3-....-.-Hexachlorobutadiene

91-20-3-...--Naphthalene

87-61-6-..----1,2,3-Trichlorobenzene
$1.0 \mathrm{U}$

$1.0 \mathrm{U}$

1. $0 \mathrm{U}$

1. $0 \mathrm{U}$

1. $0 \mathrm{U}$

1. $0 \mathrm{U}$

1. $0 \mathrm{U}$

$1.0 \mathrm{U}$

$1.0 \mathrm{U}$

1. $0 \mathrm{U}$

$1.0 \mathrm{U}$

$1.0 \mathrm{U}$

I. $0 \mathrm{U}$

1.0 U

1. $0 \mathrm{U}$

$0.33 \mathrm{~J}$

1.0 U 
FORM 1

VOLATILE ORGANICS ANALYSIS DATA SHEET
CLIENT SAMPLE NO.

LAO 71410LCS
Lab Name: TESTAMERICA BURLINGTON

Lab code: STLV Case No.: SAVANNAH SAS No.:
Contract: $8 \mathrm{E}-00302$
Matrix: (soil/water) WATER

Sample wt/vol:

$5.000(\mathrm{~g} / \mathrm{mL}) \mathrm{ML}$

Level: (low/med) LOW

\% Moisture: not dec.

GC Column: DB-624 ID: 0.53 (mm)

Soil Extract Volume:

(uL)
Lab Sample ID: LA071410LCS

Lab File ID: LEKOIODQ

Date Received:

Date Analyzed: 07/14/10

Dilution Factor: 1.0

Soil Aliquot Volume: (uL)

CAS NO.

COMPOUND

CONCENTRATION UNITS :

$(\mathrm{ug} / \mathrm{L}$ or $\mathrm{ug} / \mathrm{Kg}$ ) UG/L

Q

75-71-8-..---Dichlorodifluoromethane

74-87-3-..--- Chloromethane

75-01-4......-Vinyl Chloride

74-83-9-...-.-Bromomethane

75-00-3-...---Chloroethane

75-69-4-...-.- Trichlorofluoromethane

107-02-8-...--Acrolein

75-35-4-....-1, 1-Dich Joroethene

76-13-1-.....-Freon TF

67-64-1-....-Acetone

74-88-4-..-.--Methyl Iodide

75-15-0-..-.-.-Carbon Disulfide

107-05-1-.....Allyl Chloride

75-09-2.......-Methylene Chloride

156-60-5-...--trans-1,2-Dichloroethene

107-13-1--..-Acrylonitrile

1634-04-4-..--Methyl-t-Butyl Ether

540-59-0.....-1,2-Dichloroethene (total)

75-34-3-...-.-1, 1-Dichloroethane

108-05-4-..---Vinyl Acetate.

126-99-8--..-- Chloroprene

594-20-7-..---2,2-Dichloropropane

156-59-2-...-- Cis-1,2-Dichloroethene

78-93-3-...--2-Butanone

107-12-0-...--Propionitrile

74-97-5-....--Bromochloromethane

126-98-7--..--Methacrylonitrile

109-99-9-...-- Tetrahydrofuran

67-66-3-...-- Chloroform

71-55-6-...--1, 1, 1-Trichloroethane

56-23-5-...-.--Carbon Tetrachloride

563-58-6-----1, 1-Dichloropropene

78-83-1-....-.-. Isobutyl Alcohol

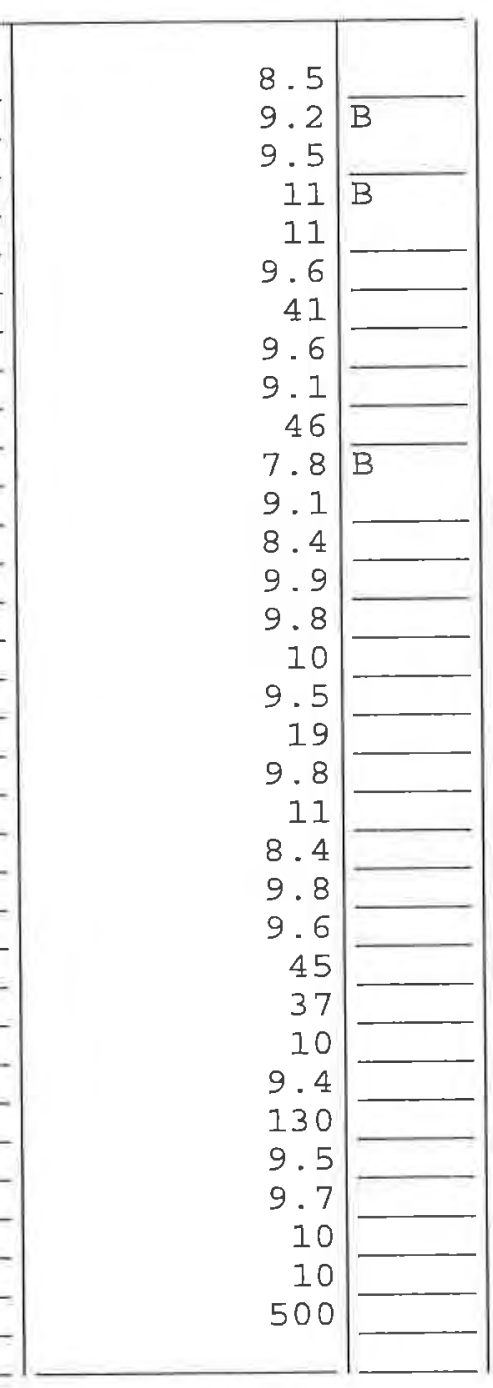


FORM 1

VOLATILE ORGANICS ANALYSIS DATA SHEET
CLIENT SAMPLE NO.

LA071410LCS

Lab Name: TESTAMERICA BURLINGTON Contract: 8E-00302

Lab code: STLV Case No.: SAVANNAH SAS No.:

Lab Sample ID: LA071410LCS

Matrix: (soil/water) WATER

Sample wt/vol: $\quad 5.000(\mathrm{~g} / \mathrm{mL}) \mathrm{ML}$

Level : (low/med) IJOW

Lab File ID: LEKO10DQ

Date Received:

\% Moisture: not dec.

GC Column: DB-624 ID: 0.53 (mm)

Soil Extract Volume:

(uL)

Date Analyzed: 07/14/10

Dilution Factor: 1.0

Soil Aliquot Volume:

(uL)

CONCENTRATION UNITS:

( $\mathrm{ug} / \mathrm{L}$ or $\mathrm{ug} / \mathrm{Kg}$ ) UG/L Q

CAS NO.

COMPOUND

\begin{tabular}{r|l}
9.9 & \\
9.7 & $\square .8$ \\
9.8 & $\square$ \\
10 & $\square .1$ \\
560 & $\bar{B}$ \\
10 & \\
11 \\
10 \\
46 \\
10 \\
9.7 \\
9.5 \\
9.9 \\
10 \\
9.7 \\
46 \\
10 \\
9.9 \\
9.9 \\
10 \\
9.7 \\
19 \\
9.8 \\
30 \\
9.7 \\
9.9 \\
9.4 \\
9.6 \\
9.6 \\
8.6 \\
9.4 \\
\end{tabular}


FORM 1

VOLATILE ORGANICS ANALYSIS DATA SHEET

Lab Name: TESTAMERICA BURLINGTON Contract: 8E-00302
CLIENT SAMPIE NO.

I_AO 71410LCS

Lab Code: STLV Case No.: SAVANNAH SAS No.:

SDG NO.: 138033

Matrix: (soil/water) WATER

Sample wt/vol:

$5.000(\mathrm{~g} / \mathrm{mL}) \mathrm{ML}$

Level: (low/med) LOW

\% Moisture: not dec.

GC Column: DB-624 ID: 0.53 (mm)

Soil Extract Volume: (uL)
Lab Sample ID: LA071410LCS

Lab File ID: LEKO1ODQ

Date Received:

Date Analyzed: 07/14/10

Dilution Factor: 1.0

Soil Aliquot Volume: (uL)

CAS NO.

COMPOUND

CONCENTRATION UNITS :

(ug/L or ug/Kg) UG/L

Q

103-65-1......n-Propylbenzene

95-49-8-....--2-Chlorotoluene

106-43-4------4-Chlorotoluene

$108-67-8-\ldots-\cdots-1,3,5-$ Trimethyl benzene

98-06-6-.....-tert-Butylbenzene

95-63-6-...-.-1,2,4-Trimethylbenzene

135-98-8-.----sec-Butylbenzene

541-73-1-...-1, 3-Dichlorobenzene

99-87-6---.--4- Isopropyltoluene

106-46-7-...--1,4-Dichlorobenzene

95-50-1-....-1, 2-Dichlorobenzene

104-51-8--.--n-Butylbenzene

96-12-8--..---1,2-Dibromo-3-Chloropropane

120-82-1--..--1,2,4-Trichlorobenzene

87-68-3-.....-Hexachlorobutadiene

91-20-3-...-- Naphthalene

87-61-6-....-.1,2,3-Trichlorobenzene

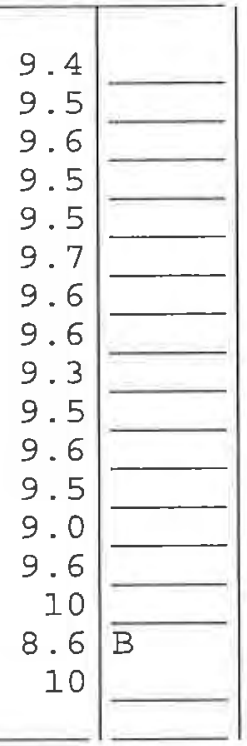


FORM 1
VOLATILE ORGANICS ANALYSIS DATA SHEET

CLIENT SAMPLE NO.

MEOHLCS

Lab Name: TESTAMERICA BURLINGTON Contract: 8E-00302

Lab Code: STLV Case No.: SAVANNAH SAS No.:

SDG No.: 138033

Lab Sample ID: MEOHLCS

Matrix: (soil/water) SOIL

Lab File ID: LEK10DQ2

Sample wt/vol:

$5.0(\mathrm{~g} / \mathrm{mL}) \mathrm{G}$

Date Received:

Level: (low/med) LOW

Date Analyzed: 07/14/10

\% Moisture: not dec.

GC Column: DB-624 ID: 0.53 (mm)

Dilution Factor: 1.0

Soil Extract Volume:

(uL)

Soil Aliquot Volume:

(uL)

CONCENTRATION UNITS :

CAS NO. COMPOUND (ug/L or ug/ $\mathrm{Kg}) \mathrm{UG} / \mathrm{KG}$

Q

75-71-8-.....-Dichlorodifluoromethane

74-87-3-..--- Chloromethane

75-01-4-...-.-.vinyl Chloride

74-83-9-....--Bromomethane

75-00-3--...--Chloroethane

75-69-4_.....- Trichlorofluoromethane

107-02-8--.---Acrolein

$75-35-4 \ldots-\cdots-1,1-D i c h l o r o e t h e n e$

76-13-1.....-. Freon TF

67-64-1-....-Acetone

74-88-4-...---Methyl Iodide

75-15-0-...--.-Carbon Disulfide

107-05-1-....-Allyl Chloride

75-09-2-....--Methylene Chloride

156-60-5-.....trans-1,2-Dichloroethene

107-13-1-...-Acrylonitrile

1634-04-4--.---Methyl-t-Butyl Ether

540-59-0-.-.--1,2-Dichloroethene (total)

75-34-3-...-.-1, 1-Dichloroethane

108-05-4--.---Vinyl Acetate

126-99-8-...-- Chloroprene

594-20-7-...--2, 2-Dichloropropane

156-59-2-...-. cis-1,2-Dichloroethene

78-93-3-..----2-Butanone

107-12-0-..---Propionitrile

74-97-5-.....-Bromochloromethane

126-98-7-...--. Methacrylonitrile

109-99-9-...-.- Tetrahydrofuran

67-66-3-..-.-- Chloroform

$71-55-6 \ldots-\ldots-1,1,1-\operatorname{Tr} i c h l o r o e t h a n e$

56-23-5-..---. Carbon Tetrachloride

563-58-6------1, 1-Dichloropropene

78-83-1-...-.-. Isobutyl Alcohol

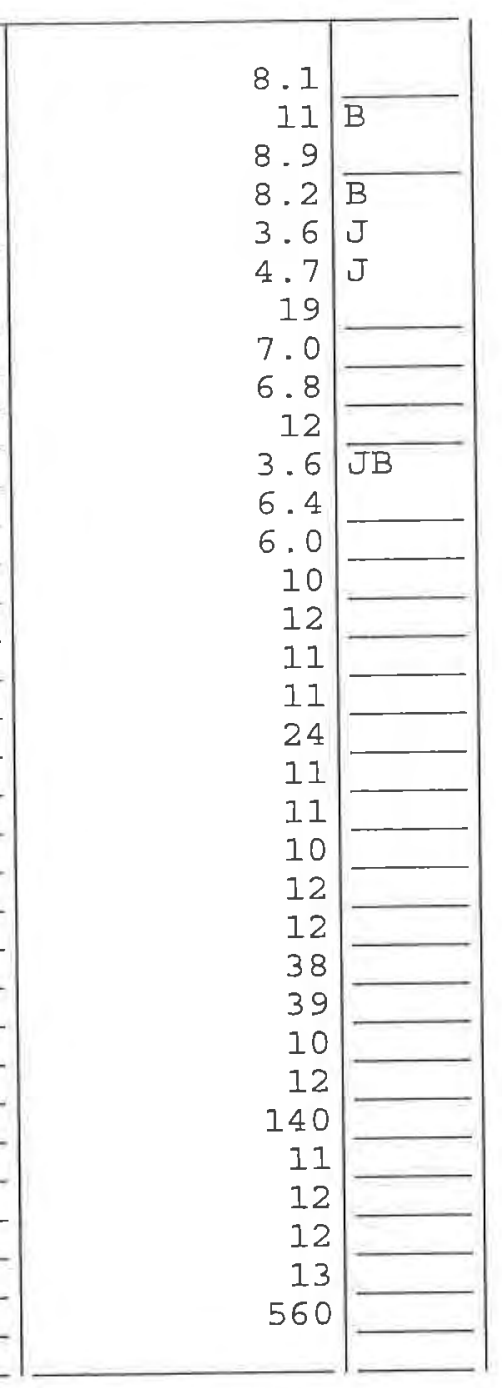


FORM 1 VOLATILE ORGANICS ANALYSIS DATA SHEET
CLIENT SAMPLE NO.

MEOHLCS

Lab Name: TESTAMERICA BURIINGTON Contract: 8E-00302

Lab Code: STLV Case No.: SAVANNAH SAS No.:

Lab Sample ID: MEOHLCS

Matrix: (soil/water) SoIL

Sample wt/vol: $\quad 5.0(\mathrm{~g} / \mathrm{mL}) \mathrm{G}$

Lab File ID: LEKIODQ2

Level: (low/med) LOW

Date Received:

\% Moisture: not dec.

GC Column: DB-624 ID: 0.53 (mm)

Soil Extract Volume:

(uL)

Date Analyzed: 07/14/10

Dilution Factor: 1.0

Soil Aliquot Volume: (uL)

CAS NO. COMPOUND (ug/L or $u g / \mathrm{Kg}) \mathrm{UG} / \mathrm{KG}$

Q

$71-43-2-\ldots-\ldots-$ Benzene

107-06-2 - - - 1, 2-Dichloroethane

79-01-6-....-Trichloroethene

$78-87-5 \ldots \ldots-\ldots-1,2-$ Dichloropropane

74-95-3--.-.--Dibromomethane

80-62-6-..--- Methyl Methacrylate

123-91-1--.---1,4-Dioxane

75-27-4-...-.-. Bromodichloromethane

110-75-8--.---2-Chloroethyl Vinyl Ether

10061-01-5-.---cis-1,3-Dichloropropene

108-10-1-.-.--4-Methyl-2-pentanone

108-88-3-...-. Toluene

10061-02-6--.--trans-1,3-Dichloropropene

97-63-2-...-.-Ethyl Methacrylate

79-00-5-..---1, 1, 2-Trichloroethane

127-18-4-...- Tetrachloroethene

142-28-9-...--1,3-Dichloropropane

591-78-6-...-.-2-Hexanone

124-48-1-....-Dibromochloromethane

106-93-4--.---1, 2-Dibromoethane

108-90-7-...-- Chlorobenzene

630-20-6-...-. 1, 1, 1, 2-Tetrachloroethane

100-41-4---.---Ethylbenzene

$1330-20-7 \ldots-\ldots$ - Xylene $(\mathrm{m}, \mathrm{p})$

95-47-6......-Xylene (o)

1330-20-7-...-xylene (total)

$100-42-5-\ldots-.--$ Styrene

75-25-2-....-Bromoform

98-82-8-- - - - - Isopropylbenzene

108-86-1-...--Bromobenzene

79-34-5-...--1, 1,2, 2-Tetrachloroethane

96-18-4-...--1,2,3-Trichloropropane

110-57-6-...--trans-1,4-Dichloro-2-butene

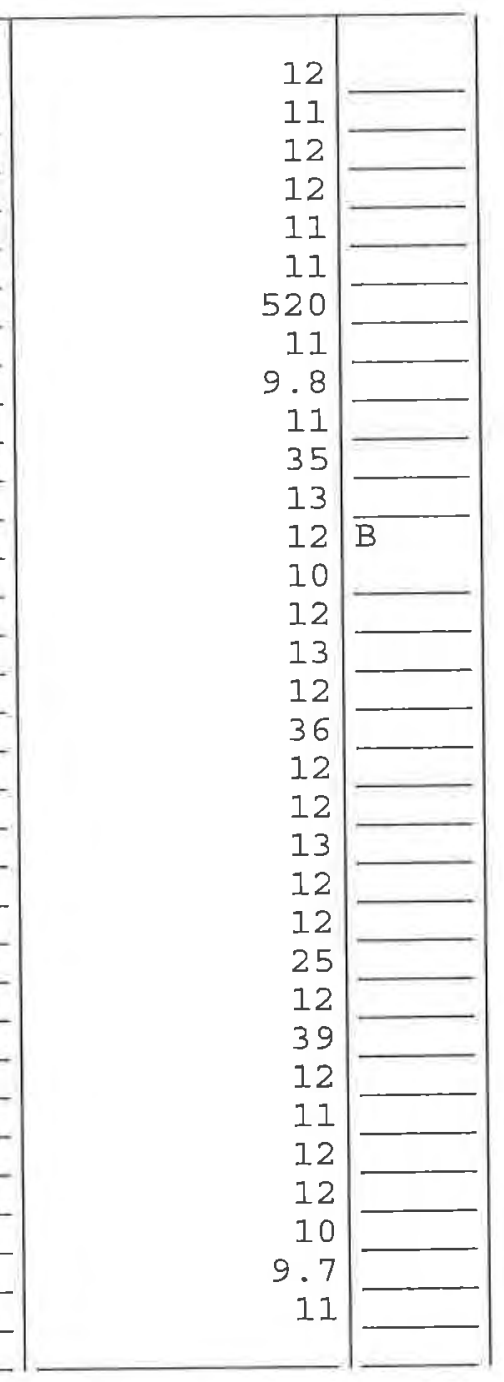


FORM 1

VOIATILE ORGANICS ANALYSIS DATA SHEET
CLIENT SAMPLE NO.

MEOHICS

Lab Name: TESTAMERICA BURLINGTON Contract: 8E-00302

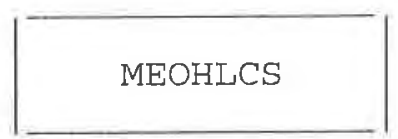

Lab Code: STLV Case No.: SAVANNAH SAS No.:

SDG NO.: 138033

Matrix: (soil/water) SoIL

Lab Sample ID: MEOHLCS

Sample wt/vol:

$5.0(\mathrm{~g} / \mathrm{mL}) \quad \mathrm{G}$

Lab File ID: LEK10DQ2

Level: (low/med) LOW

Date Received:

\% Moisture: not dec.

Date Analyzed: 07/14/10

GC Column: DB-624 ID: 0.53 (mm)

Dilution Factor: 1.0

Soil Extract Volume:

(UI)

Soil Aliquot Volume: (uL) CONCENTRATION UNITS:

CAS NO. COMPOUND (ug/L or ug/ $\mathrm{Kg}) \mathrm{UG} / \mathrm{KG}$

$Q$

103-65-1...--n-Propylbenzene

95-49-8--.--- 2-Chlorotoluene

106-43-4-...--4-Chlorotoluene

$108-67-8 \ldots-\ldots-1,3,5-$ Trimethylbenzene

98-06-6--..-.-tert-Butylbenzene

95-63-6-- - - - - 1, 2, 4-Trimethylbenzene

135-98-8-- - - - sec-Butylbenzene

541-73-1-...-1, 3-Dichlorobenzene

99-87-6-..--- 4-Isopropyltoluene

106-46-7-..--1,4-Dichlorobenzene

95-50-1-..--- 1, 2-Dichlorobenzene

104-51-8--.-.-n-Butylbenzene

96-12-8-....--1,2-Dibromo-3-Chloropropane

120-82-1-.....-1,2,4-Trichlorobenzene

87-68-3-....-.-Hexachlorobutadiene

91-20-3-....-. Naphthalene

87-61-6......-.1,2,3-Trichlorobenzene

$-\mid$\begin{tabular}{rll} 
& \\
12 & \\
12 & \\
12 & \\
12 & \\
12 & \\
13 & - \\
13 & - \\
12 & \\
13 & \\
12 & $\mathrm{~B}$ \\
12 & \\
13 & \\
9.2 & \\
13 & $\mathrm{~B}$ \\
14 & \\
9.3 & $\mathrm{~B}$ \\
12 & $\mathrm{~B}$ \\
\hline
\end{tabular}


FORM 2

SOIL VOLATILE SYSTEM MONITORING COMPOUND RECOVERY

Lab Name: TESTAMERICA BURLINGTON Contract: 8E-00302

Lab Code: STLV Case No.: SAVANNAH SAS No.: $\quad$ SDG No.: 138033

Level: (low/med) LOW

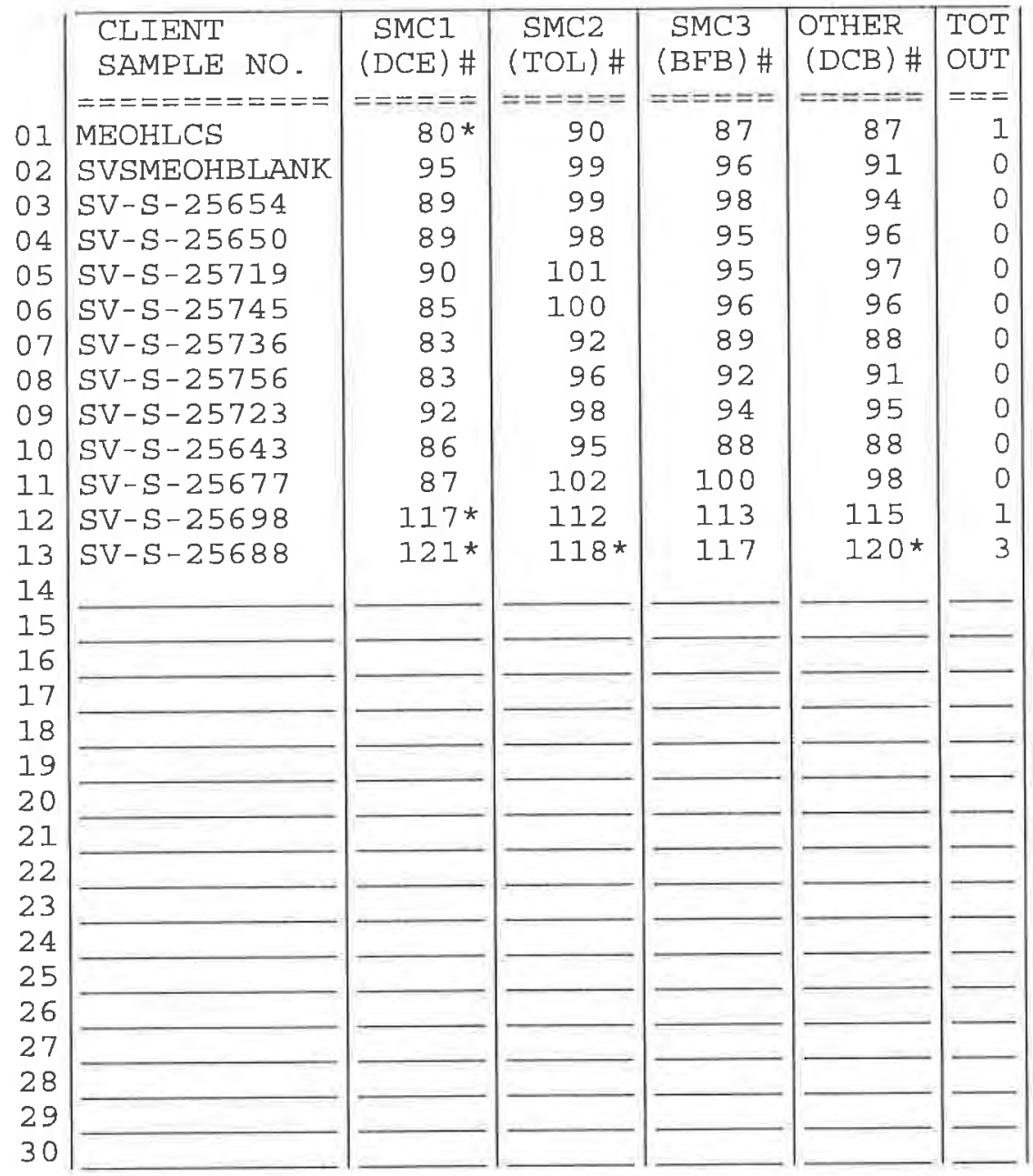

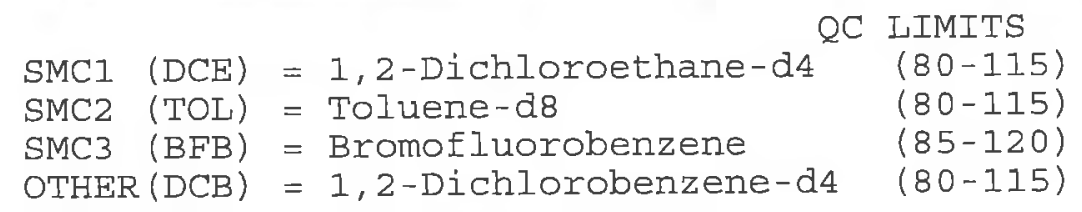

\# Column to be used to flag recovery values

* Values outside of contract required QC limits

D System Monitoring Compound diluted out 
FORM 2

WATER VOLATILE SYSTEM MONITORING COMPOUND RECOVERY

Lab Name: TESTAMERICA BURLINGTON Contract: 8E-00302

Lab Code: STLV Case No.: SAVANNAH SAS No.: $\quad$ SDG No.: 138033

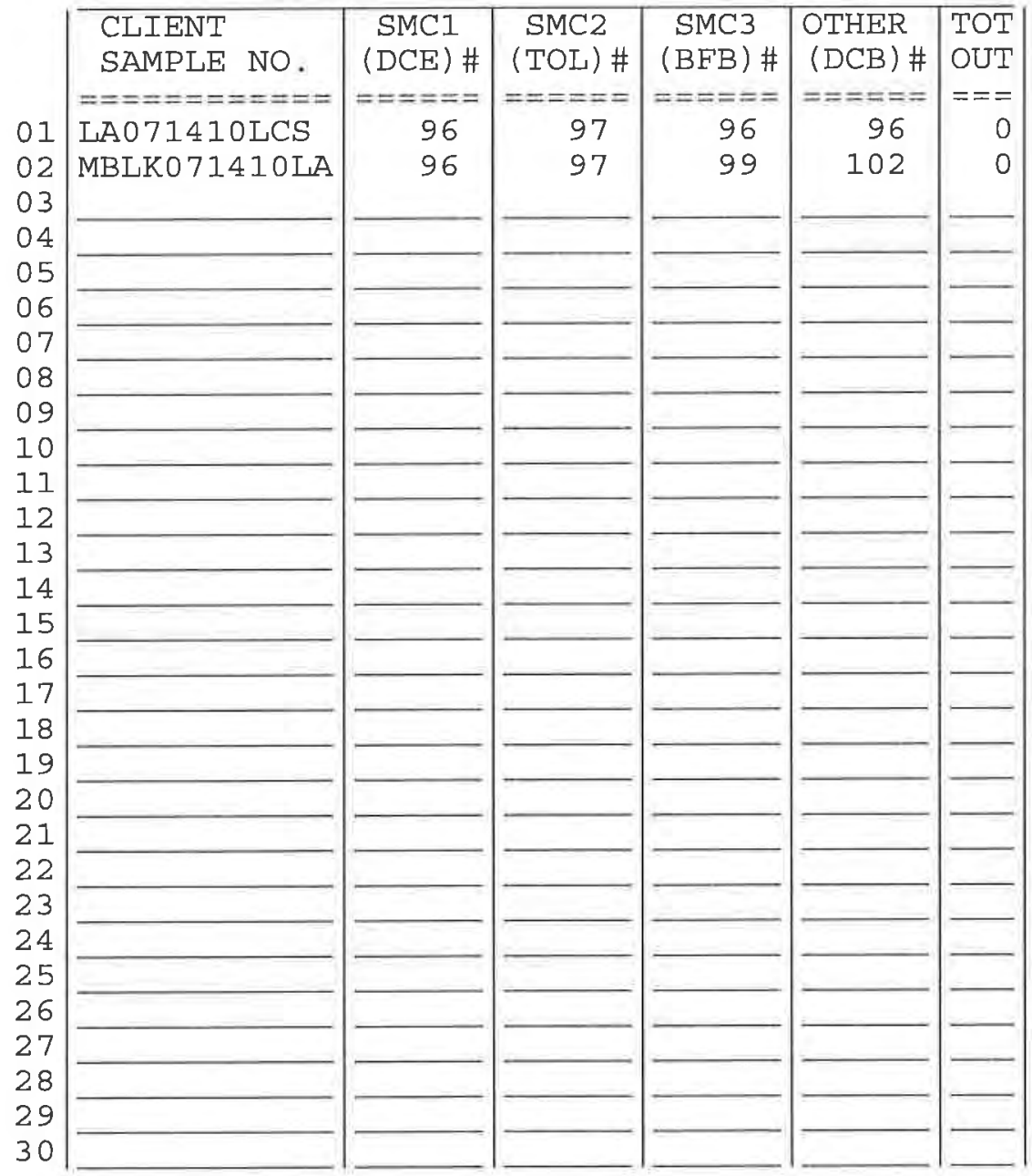

$$
\begin{aligned}
& \text { SMC1 }(\mathrm{DCE})=1,2-\mathrm{Dich} \text { loroethane-d4 }(80-115) \\
& \text { SMC2 }(\text { TOL) = Toluene-d8 (80-115) } \\
& \text { SMC3 }(\mathrm{BFB})=\text { Bromofluorobenzene }(85-120) \\
& \operatorname{OTHER}(\mathrm{DCB})=1,2-\mathrm{Dich} \text { lorobenzene-d4 (80-115) }
\end{aligned}
$$

\# Column to be used to flag recovery values

* Values outside of contract required QC limits

D System Monitoring Compound diluted out 
FORM 3

WATER VOLATILE LAB CONTROL SAMPLE

Lab Name: TESTAMERICA BURLINGTON Contract: 8E-00302

Lab code: STLV Case No.: SAVANNAH SAS No.: $\quad$ SDG No.: 138033

Matrix Spike - Sample No.: LA071410LCS

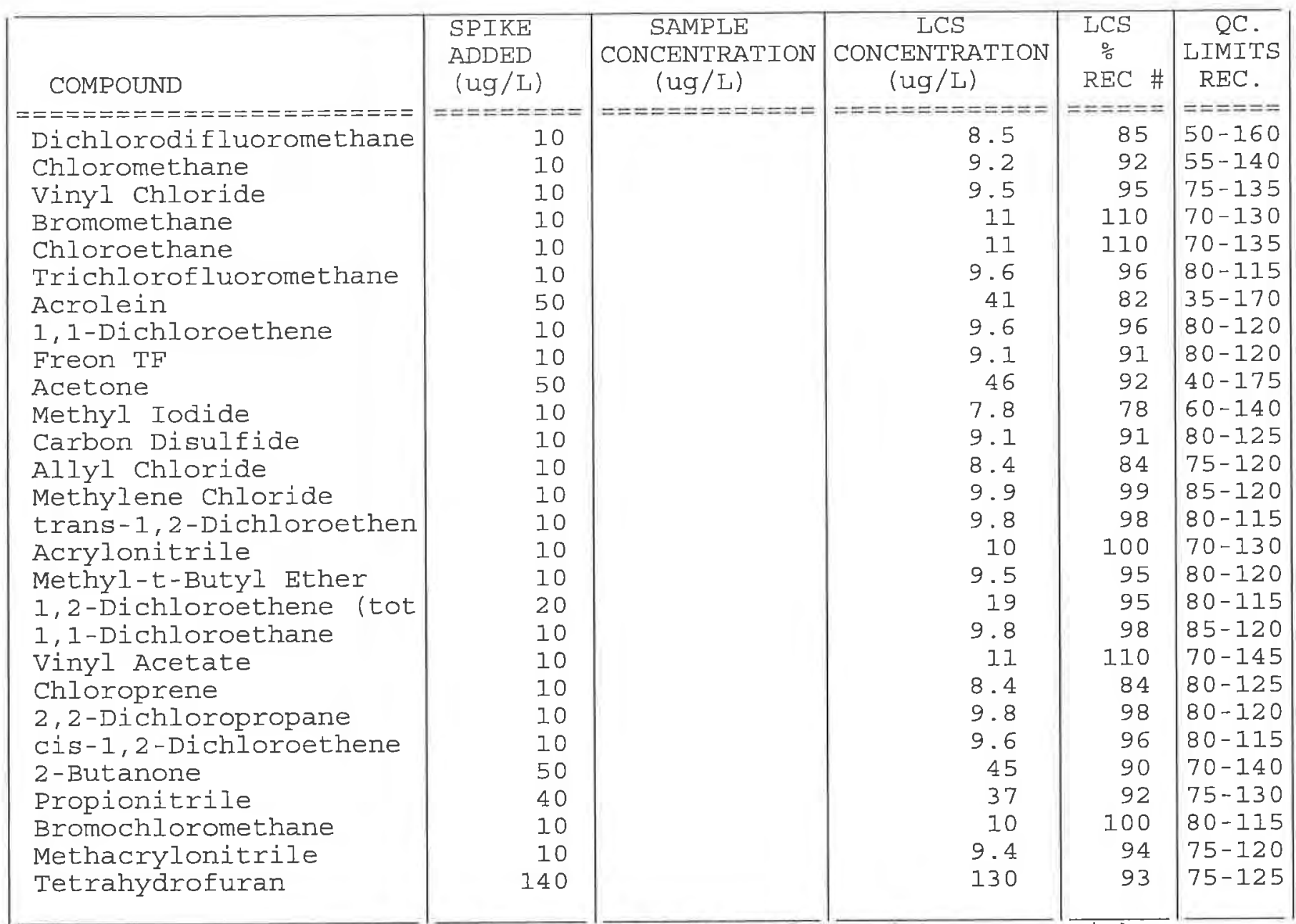

\# Column to be used to flag recovery and RPD values with an asterisk

* Values outside of QC limits

COMMENTS : 
FORM 3

WATER VOLATILE LAB CONTROL SAMPLE

Lab Name: TESTAMERICA BURLINGTON Contract: 8E-00302

Lab Code: STLV Case No.: SAVANNAH SAS No.: SDG No.: 138033

Matrix Spike - Sample NO.: LAO71410LCS

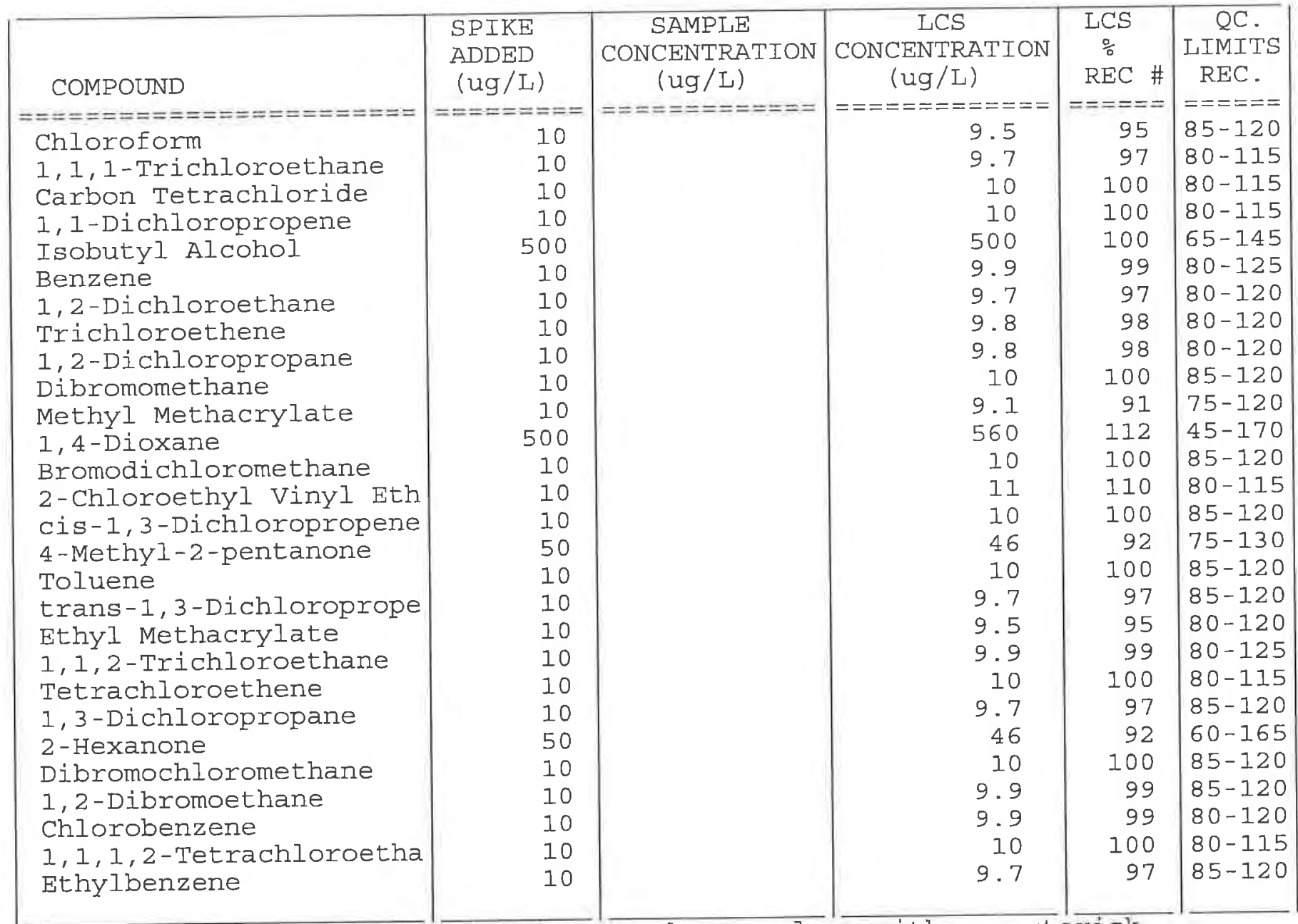

\# Column to be used to flag recovery and RPD values with an asterisk

* Values outside of QC limits

COMMENTS : 
FORM 3

WATER VOLATILE LAB CONTROL SAMPLE

Lab Name: TESTAMERICA BURIINGTON Contract: 8E-00302

Lab Code: STLV Case No.: SAVANNAH SAS No.: $\quad$ SDG No.: 138033

Matrix Spike - Sample No.: LA071410LCS

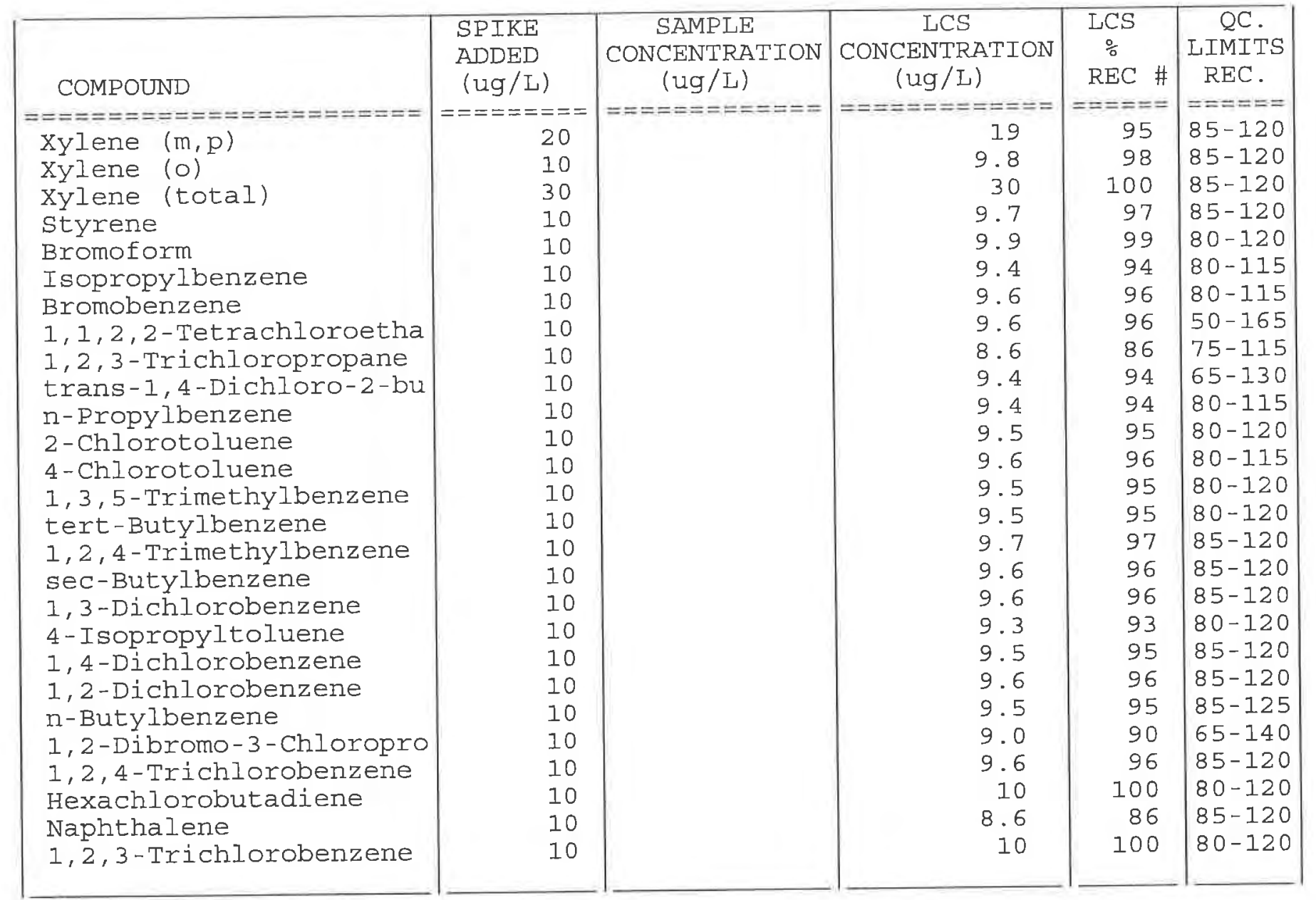

\# Column to be used to flag recovery and $R P D$ values with an asterisk

* Values outside of QC limits

RPD: 0 out of 0 outside limits

Spike Recovery: 0 out of 83 outside limits

COMMENTS : 
FORM 3

SOIL VOLATILE LAB CONTROL SAMPLE

Lab Name: TESTAMERICA BURLINGTON Contract: 8E-00302

Lab Code: STLV Case No.: SAVANNAH SAS No.: SDG No.: 138033

Matrix Spike - Sample No.: MEOHLCS Level:(low/med) LOW

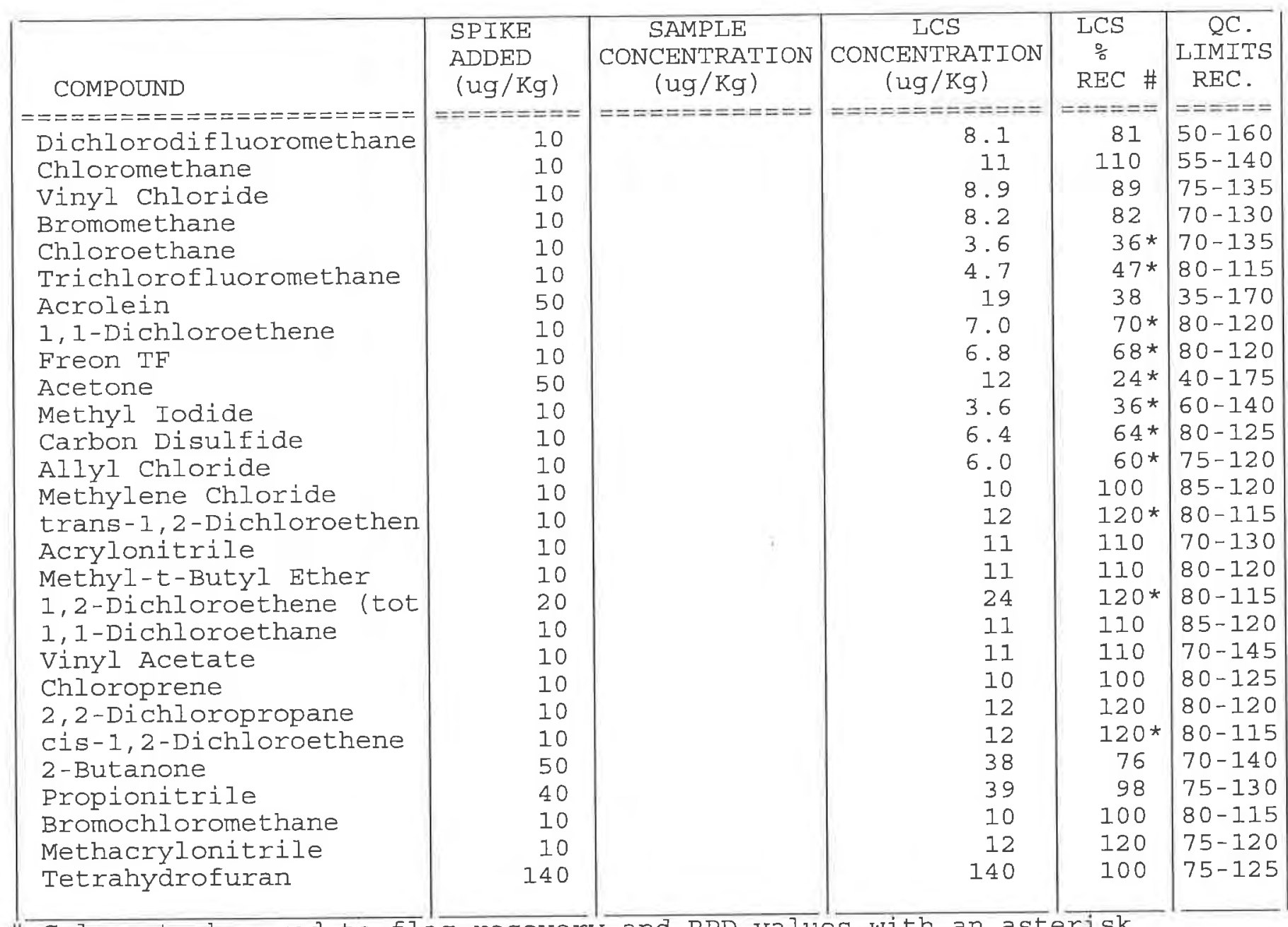

\# Column to be used to flag recovery and RPD values with an asterisk

* Values outside of QC limits

COMMENTS : 
FORM 3

SOII VOLATILE LAB CONTROL SAMPLE

Lab Name: TESTAMERICA BURLINGTON Contract: 8E-00302

Lab Code: STLV Case No.: SAVANNAH SAS No.: $\quad$ SDG No.: 138033

Matrix Spike - Sample No.: MEOHLCS Level:(low/med) LOW

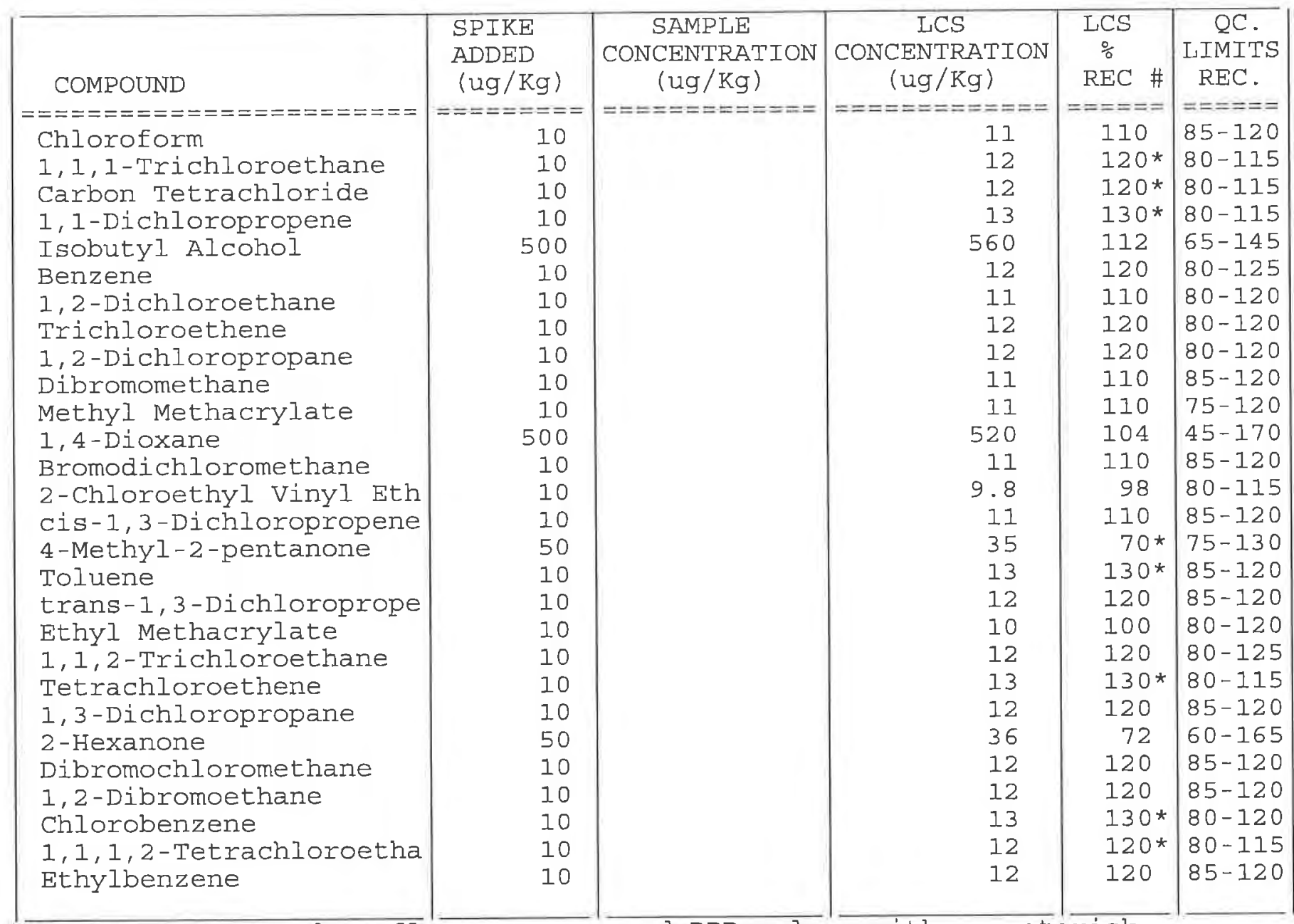

Column to be used to flag recovery and RPD values with an asterisk

* Values outside of QC limits

COMMENTS : 
FORM 3

SOIL VOLATILE LAB CONTROL SAMPLE

Lab Name: TESTAMERICA BURLINGTON Contract: 8E-00302

Lab Code: STLV Case No.: SAVANNAH SAS No.: SDG No.: 138033

Matrix Spike - Sample No.: MEOHLCS Level:(low/med) LOW

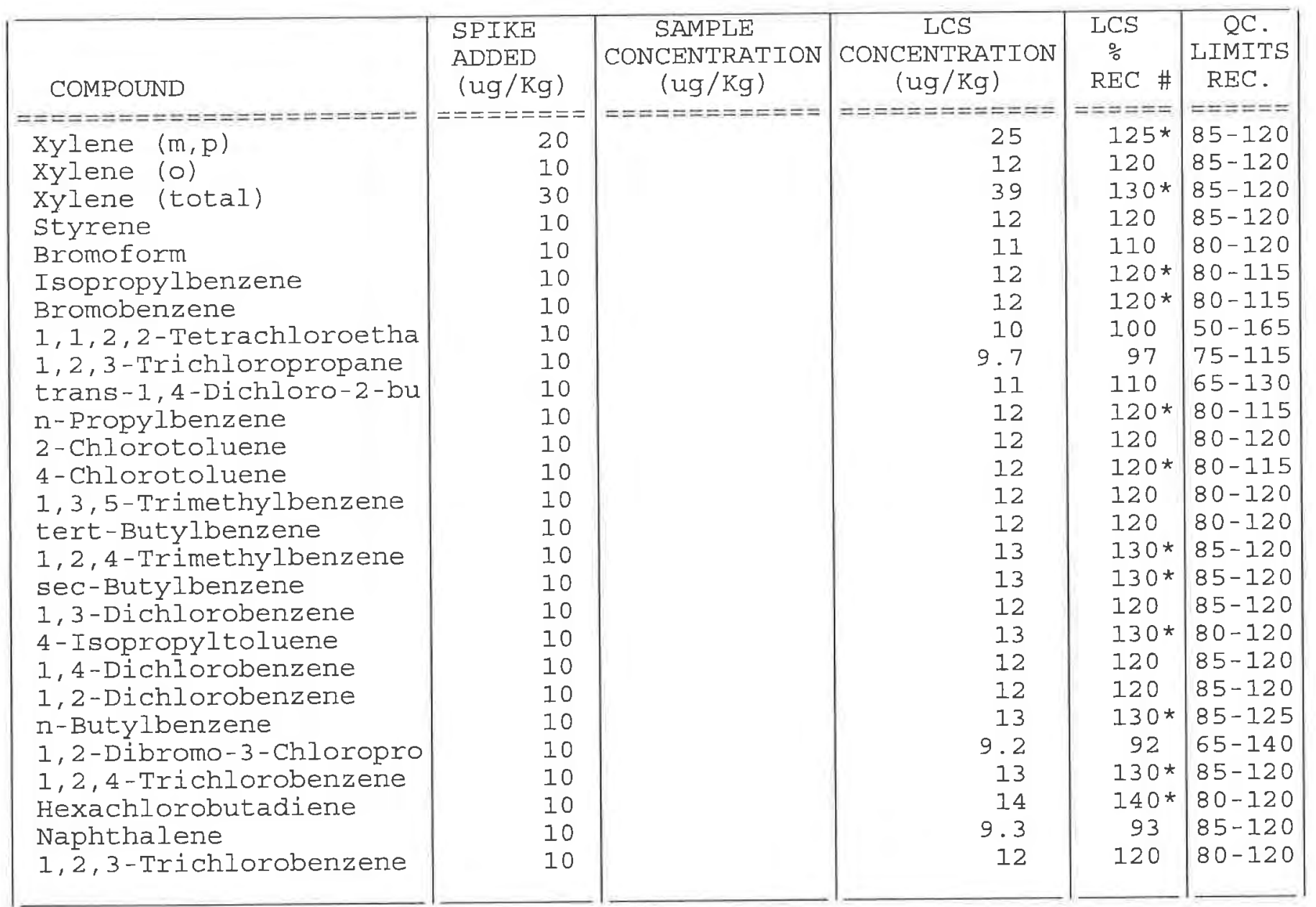

\# Column to be used to flag recovery and RPD values with an asterisk

* Values outside of QC limits

RPD: 0 out of 0 outside limits

Spike Recovery: 31 out of 83 outside limits

COMMENTS : 
FORM 4

VOLATILE METHOD BLANK SUMMARY
CLIENT SAMPLE NO.

MBLKO 71410LA

Lab Name: TESTAMERICA BURLINGTON Contract: 8E-00302 Lab Code: STLV Case No.: SAVANNAH SAS No.: Lab Sample ID: MBLK071410LA Lab File ID: LEKBO3D Time Analyzed: 0944 Date Analyzed: 07/14/10 Heated Purge: ( $\mathrm{Y} / \mathrm{N}$ ) N

GC Column: DB-624 ID: 0.53 (mm)

Instrument ID: I

THIS METHOD BLANK APPLIES TO THE FOLLOWING SAMPLES, MS and MSD:

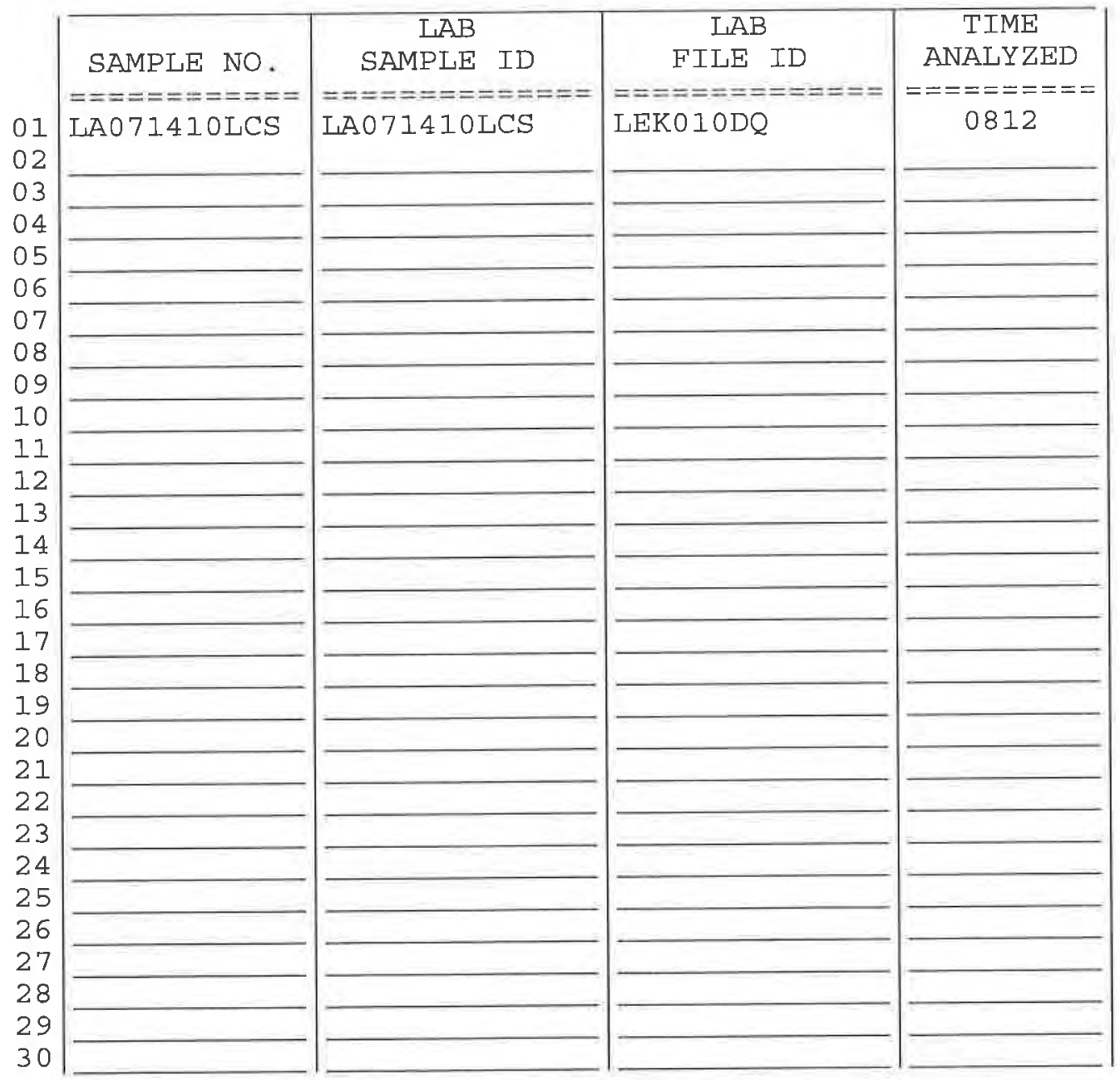

\section{COMMENTS :}

page 1 of 1 
FORM 4

VOLATILE METHOD BLANK SUMMARY

Lab Name: TESTAMERICA BURLINGTON Contract: 8E-00302

Lab Code: STLV Case No.: SAVANNAH SAS No.:

Lab Sample ID: 835027

Lab File ID: 835027

Date Analyzed: $07 / 14 / 10$

GC Column: DB-624 ID: 0.53 (mm)

Instrument ID: L

Time Analyzed: 1050

Heated Purge: (Y/N) N
ARGIAAB SAMPLE NO.

SVSMEOHBLANK

SDG No.: 138033

THIS METHOD BLANK APPLIES TO THE FOLLOWING SAMPLES, MS and MSD:

\begin{tabular}{|c|c|c|c|c|}
\hline & $\begin{array}{l}\text { ARGLAB } \\
\text { SAMPLE NO. }\end{array}$ & $\begin{aligned} \text { LAAB } \\
\text { SAMPLE ID }\end{aligned}$ & $\begin{array}{l}\text { IAB } \\
\text { FILE ID }\end{array}$ & $\begin{array}{c}\text { TIME } \\
\text { ANALYZED }\end{array}$ \\
\hline & $===-===-====$ & $=== \pm===:=== \pm==$ & $=======x======$ & $==== \pm==x==$ \\
\hline & MEOHLCS & MEOHLCS & LEK10DQ2 & 0843 \\
\hline & $S V-S-25654$ & 835016 & 835016 & 1121 \\
\hline & $S V-S-25650$ & 835017 & 835017 & 1151 \\
\hline & SV-S-25719 & 835018 & 835018 & 1222 \\
\hline & $S V-S-25745$ & 835019 & 835019 & 1252 \\
\hline & SV-S-25736 & 835020 & 835020 & 1322 \\
\hline & SV-S-25756 & 835021 & 835021 & 1353 \\
\hline & SV-S-25723 & 835022 & 835022 & 1423 \\
\hline & SV-S-25643 & 835025 & 835025 & 1651 \\
\hline & SV-S-25677 & 835026 & 835026 & 1722 \\
\hline & SV-S-25698 & 835023 & $835023 I 2$ & 1752 \\
\hline & $S V-S-25688$ & 835024 & $835024 I 2$ & 1822 \\
\hline & & & & \\
\hline & & & & \\
\hline & & & & \\
\hline & & & & \\
\hline & & & & \\
\hline & & & & \\
\hline & & & & \\
\hline & & & & \\
\hline & & & & \\
\hline & & & & \\
\hline & & & & \\
\hline & & & & \\
\hline & & & & \\
\hline & & & & \\
\hline & & & & \\
\hline & & & & \\
\hline
\end{tabular}

COMMENTS :

page 1 of 1 
FORM 5

VOLATILE ORGANIC INSTRUMENT PERFORMANCE CHECK BROMOFLUOROBENZENE (BFB)

Lab Name: TESTAMERICA BURLINGTON Contract: 8E-00302

Lab Code: STLV Case No.: SAVANNAH SAS No.: $\quad$ SDG No.: 138033

Lab File ID: LEK001

Instrument ID: L

GC Column: DB-624
ID : $0.53(\mathrm{~mm})$
BFB Injection Date: 07/09/10

BFB Injection Time: 1958

Heated Purge: ( $\mathrm{Y} / \mathrm{N}) \mathrm{N}$

\begin{tabular}{|c|c|c|}
\hline $\begin{array}{l}\mathrm{m} / \mathrm{e} \\
=====\end{array}$ & 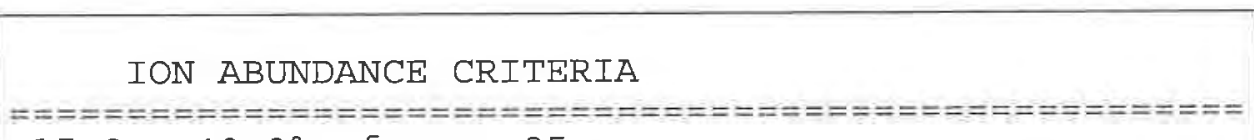 & $\begin{array}{c}\% \text { RELATIVE } \\
\text { ABUNDANCE } \\
=============\end{array}$ \\
\hline 50 & $15.0-40.0 \%$ of mass 95 & 20.1 \\
\hline 75 & $30.0-60.0 \%$ of mass $95^{-}$ & 44.9 \\
\hline 95 & Base Peak, $100 \%$ relative abundance & 100.0 \\
\hline 96 & $5.0-9.0 \%$ of mass 95 & 6.5 \\
\hline 173 & Less than $2.0 \%$ of mass 174 & $0.3(0.4) 1$ \\
\hline 174 & $50.0-120.0 \%$ of mass 95 & 70.3 \\
\hline 175 & $5.0-9.0 \%$ of mass 174 & $4.9(6.9) 1$ \\
\hline 176 & $95.0-101.0 \%$ of mass 174 & $67.1(95.4) 1$ \\
\hline 177 & $5.0-9.0 \%$ of mass 176 & $4.5(6.8) 2$ \\
\hline
\end{tabular}

THIS CHECK APPLIES TO THE FOLLOWING SAMPLES, MS, MSD, BLANKS, AND STANDARDS:

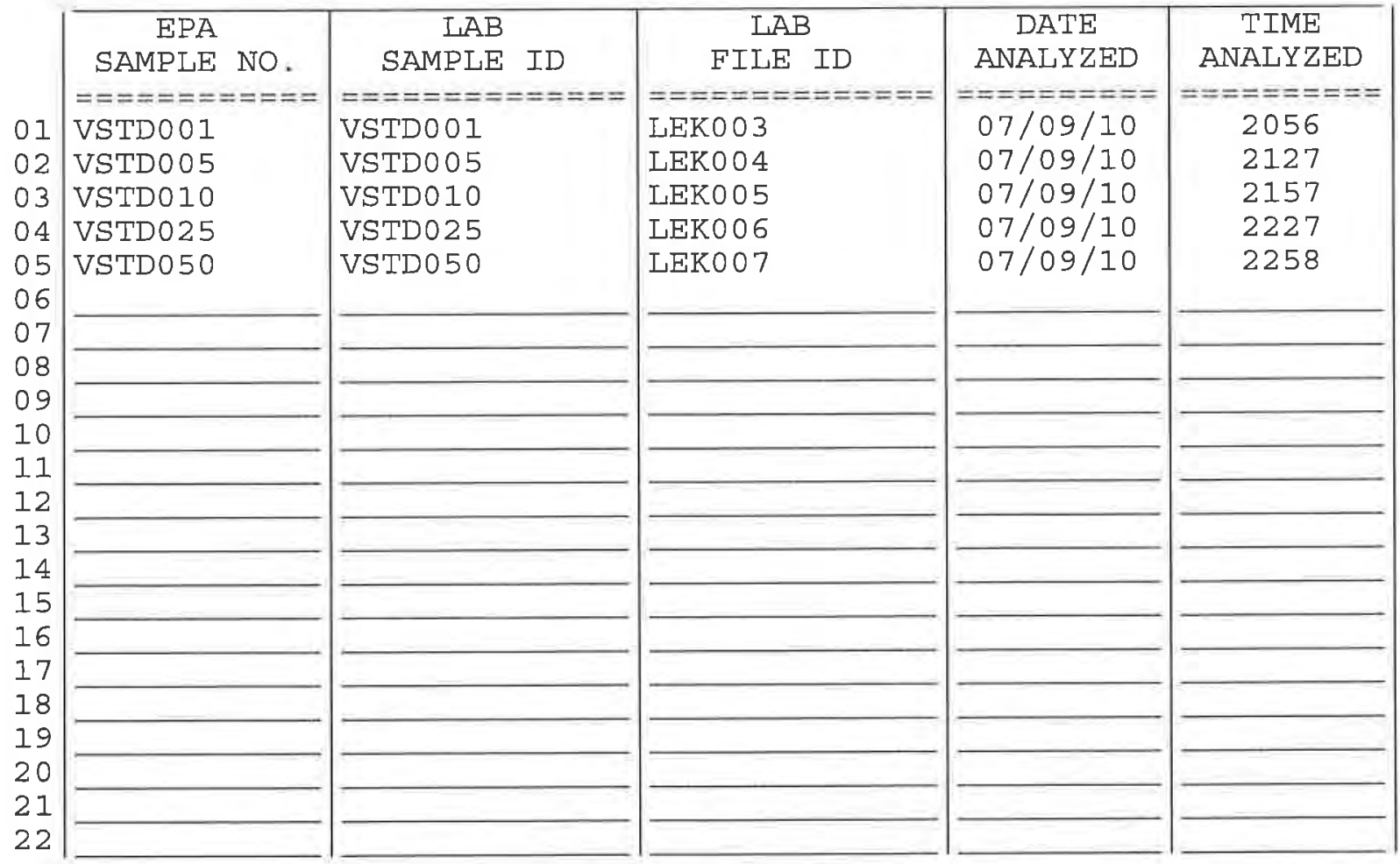

page 1 of 1 
FORM 6

VOLATILE ORGANICS INITIAL CALIBRATION DATA

Lab Name: TESTAMERICA BURLINGTON Contract: 8E-00302

Lab Code: STLV Case No.: SAVANNAH SAS No.:

SDG NO.: 138033

Instrument ID: L

Calibration Date(s): 07/09/10 07/09/10

Heated Purge: (Y/N) N Calibration Time(s): $2056 \quad 2258$

GC Column: DB-624 ID: 0.53 (mm)

\begin{tabular}{|c|c|c|c|c|c|c|c|}
\hline $\begin{array}{l}\text { LAB FILE ID : } \\
\text { RRF10 =LEK005 }\end{array}$ & $\begin{array}{l}=\text { LEKO } 03 \\
=\text { LEKO0 } 6\end{array}$ & & $\begin{array}{l}\text { RRF5 } \\
\text { RRF5 }\end{array}$ & $\begin{aligned} & =\text { LEKO } \\
0 & =\text { LEKO }\end{aligned}$ & & & \\
\hline COMPOUND & RRF1 & RRF5 & RRFIO & RRF2 5 & RRF50 & $\overline{\mathrm{RRF}}$ & RSD \\
\hline 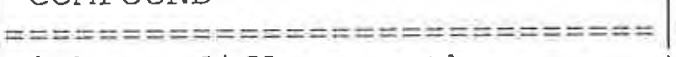 & $== \pm===$ & $=== \pm=$ & 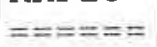 & 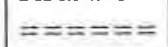 & $=====$ & $== \pm===$ & $=====$ \\
\hline Dichlorodifluoromethane & 0.585 & 0.620 & 0.613 & 0.608 & 0.607 & 0.607 & $2.2 *$ \\
\hline Chloromethane & $\times \quad 0.278$ & 0.263 & 0.259 & 0.267 & 0.266 & 0.267 & $2.6 *$ \\
\hline Vinyl Chloride & $* 0.303$ & 0.325 & 0.313 & 0.320 & 0.324 & 0.317 & $2.8 *$ \\
\hline Bromomethane & $\times 0.247$ & 0.228 & 0.212 & 0.215 & 0.222 & 0.225 & $6.2^{\star}$ \\
\hline Chloroethane & $\star 0.172$ & 0.180 & 0.183 & 0.178 & 0.175 & 0.178 & $2.3 *$ \\
\hline Trichlorofluoromethane & $* 0.590$ & 0.641 & 0.622 & 0.630 & 0.624 & 0.621 & $3.1 *$ \\
\hline Acrolein & $\star 0.025$ & 0.024 & 0.024 & 0.025 & 0.026 & 0.025 & $3.1 *$ \\
\hline 1,1-Dichloroethene & $\star 0.282$ & 0.287 & 0.275 & 0.284 & 0.284 & 0.282 & $1.6 *$ \\
\hline Freon TF & $\star 0.570$ & 0.616 & 0.606 & 0.614 & 0.617 & 0.605 & 3.2 * \\
\hline Acetone & $\star 0.052$ & 0.044 & 0.045 & 0.048 & 0.048 & 0.047 & 7.0 * \\
\hline Methyl Iodide & $\star 0.328$ & 0.333 & 0.377 & 0.442 & 0.502 & 0.396 & $18.9 *$ \\
\hline Carbon Disulfide & $\star 0.836$ & 0.816 & 0.795 & 0.802 & 0.811 & 0.812 & $2.0 *$ \\
\hline Allyl Chloride. & $* 0.514$ & 0.525 & 0.516 & 0.523 & 0.527 & 0.521 & $1.1 *$ \\
\hline Methylene Chloride & 0.283 & 0.277 & 0.274 & 0.278 & 0.281 & 0.279 & $1.2 *$ \\
\hline trans-1,2-Dichloroethene & 0.303 & 0.318 & 0.304 & 0.308 & 0.310 & 0.309 & $1.9 *$ \\
\hline Acrylonitrile & 0.050 & 0.043 & 0.055 & 0.058 & 0.058 & 0.053 & $12.5 *$ \\
\hline Methyl-t-Butyl Ether & 0.651 & 0.679 & 0.662 & 0.685 & 0.683 & 0.672 & $2.2 *$ \\
\hline 1,2-Dichloroethene (total) & 0.314 & 0.323 & 0.315 & 0.318 & 0.321 & 0.318 & 1.2 * \\
\hline 1,1-Dichloroethane & 0.612 & 0.618 & 0.609 & 0.615 & 0.614 & 0.614 & $0.6 *$ \\
\hline Vinyl Acetate & 0.546 & 0.577 & 0.558 & 0.568 & 0.564 & 0.563 & $2.0 *$ \\
\hline Chloroprene & * 0.487 & 0.508 & 0.506 & 0.516 & 0.513 & 0.506 & $2.2 *$ \\
\hline 2,2-Dichloropropane & * 0.554 & 0.541 & 0.529 & 0.521 & 0.511 & 0.531 & $3.2 *$ \\
\hline cis-1,2-Dichloroethene & 0.326 & 0.328 & 0.326 & 0.327 & 0.331 & 0.328 & $0.7 *$ \\
\hline 2 -Butanone & 0.020 & 0.021 & 0.021 & 0.022 & 0.022 & 0.021 & $3.1 *$ \\
\hline Propionitrile & $\star 0.020$ & 0.020 & 0.020 & 0.021 & 0.021 & 0.020 & 3.3 * \\
\hline Bromochloromethane & * 0.173 & 0.182 & 0.184 & 0.190 & 0.190 & 0.184 & $3.8 *$ \\
\hline Methacrylonitrile & $\star 0.068$ & 0.071 & 0.068 & 0.073 & 0.074 & 0.071 & $4.1 *$ \\
\hline Tetrahydrofuran & 0.068 & 0.068 & 0.067 & 0.070 & 0.071 & 0.069 & $2.6 *$ \\
\hline Chloroform & $\star 0.635$ & 0.658 & 0.650 & 0.661 & 0.659 & 0.653 & $1.6 *$ \\
\hline 1, 1, 1-Trichloroethane & $\star 0.541$ & 0.575 & 0.570 & 0.572 & 0.564 & 0.564 & $2.4 *$ \\
\hline Carbon Tetrachloride & * 0.472 & 0.520 & 0.524 & 0.529 & 0.523 & 0.514 & $4.6 *$ \\
\hline 1,1-Dichloropropene & $\star 0.483$ & 0.504 & 0.500 & 0.509 & 0.513 & 0.502 & $2.3 *$ \\
\hline Isobutyl Alcohol & $\star 0.008$ & 0.009 & 0.009 & 0.009 & 0.009 & 0.009 & $7.1 *$ \\
\hline Benzene & * 0.866 & 0.932 & 0.932 & 0.959 & 0.967 & 0.931 & 4. $3 *$ \\
\hline 1,2-Dichloroethane & $\star 0.328$ & 0.355 & 0.350 & 0.357 & 0.352 & 0.348 & $3.4 *$ \\
\hline Trichloroethene & $\star 0.385$ & 0.387 & 0.384 & 0.396 & 0.403 & 0.391 & $2.1 *$ \\
\hline 1,2-Dichloropropane & $\star 0.355$ & 0.370 & 0.363 & 0.374 & 0.373 & 0.367 & $2.1 *$ \\
\hline
\end{tabular}

Compounds with required minimum RRF and maximim $\% \overline{R S D}$ values.

All other compounds must meet a minimim RRF of 0.010 .

page 1 of 3 
FORM 6

VOLATILE ORGANICS INITIAL CALIBRATION DATA

Lab Name: TESTAMERICA BURLINGTON Contract: 8E-00302

Lab Code: STLV Case No.: SAVANNAH SAS No.: SDG No.: 138033

Instrument ID: L Calibration Date(s): 07/09/10 07/09/10

Heated Purge: (Y/N) N Calibration Time(s): $2056 \quad 2258$

GC Column: DB-624 ID: $0.53 \quad(\mathrm{~mm})$

\begin{tabular}{|c|c|c|c|c|c|c|c|}
\hline $\begin{array}{l}\text { LAB FILE ID: } \\
\text { RRF10 =LEK005 }\end{array}$ & $\begin{array}{l}=\text { LEKO } 03 \\
=L E K 006\end{array}$ & & $\begin{array}{l}\text { RRF5 } \\
\text { RRF 5 }\end{array}$ & $\begin{array}{l}=\text { LEKO } \\
=\text { LEKO }\end{array}$ & & & \\
\hline COMPOUND & $R F$ & & PRE7 10 & - & D. & $\overrightarrow{\mathrm{RRF}}$ & RS \\
\hline $\begin{array}{l}========================= \\
\text { Dibromomethane }\end{array}$ & $======$ & $==$ & $==$ & & & & - \\
\hline $\begin{array}{l}\text { Dibromomethar } \\
\text { Methyl Methar }\end{array}$ & $\begin{array}{l}0.288 \\
0.235\end{array}$ & $\begin{array}{l}0.285 \\
0.250\end{array}$ & $\begin{array}{l}0.282 \\
0.241\end{array}$ & $\begin{array}{l}0.291 \\
0.248\end{array}$ & $\begin{array}{l}0.294 \\
0.251\end{array}$ & $\begin{array}{l}0.288 \\
0.245\end{array}$ & $1.1^{\pi}$ \\
\hline 4-Dioxane & $\star 0.002$ & 0.002 & 0.002 & 0.002 & 0.002 & 0.002 & $7.7 *$ \\
\hline Bromodichlorc & 0.570 & & 0.600 & 0.622 & & & $3.5 *$ \\
\hline 2-Chloroethyl & 0.176 & & 0.182 & 0.189 & & .182 & 4. $0 *$ \\
\hline is-1,3-Dichlor & $\star 0.482$ & 0.494 & 0.512 & 0.532 & & 0.510 & $4 \cdot 3 *$ \\
\hline hyl-2-pel & $\star 0$. & & 0.304 & 0.311 & & 0.304 & $3.2 *$ \\
\hline le & 0. & 0. & 0.763 & 0.776 & & & 1.8 * \\
\hline$-1,3-$ Dichlo & 0. & & 0.542 & 0.560 & & 0.555 & $1.4 *$ \\
\hline Methac: & 0 . & 0. & 0.410 & 0.427 & & 2 & $4.7^{*}$ \\
\hline -Trichlc & $\star 0$. & 0.346 & 0.333 & 0.335 & & 5 & $2.6 *$ \\
\hline chlor & 0. & & 0.589 & 0.600 & & & $2.6^{\star}$ \\
\hline chloro & $\star 0$. & 0 . & 0.619 & 0.641 & & & $3.2 *$ \\
\hline none & & & 0.257 & 0.263 & & 4 & $4.9 *$ \\
\hline lochloro & * 0. & & 0.600 & 0.630 & & .6 & $5.2^{\star}$ \\
\hline bromoe & * 0 . & & 0.530 & 0.554 & & 0 & $3.4 *$ \\
\hline benze & $\star 0.918$ & & 0. & 61 & & & $2.0 *$ \\
\hline 2-Tetrachlor & $\star 0.468$ & & 0. & 0.512 & & 6 & 3.4 * \\
\hline thylbenzene & $\star 1$. & & 1.6 & 1.684 & & 1 & $2.7 *$ \\
\hline lene $(\mathrm{m}, \mathrm{p})$ & $\star 0.580$ & 0. & 0.603 & 0.612 & 30 & 07 & $2.9 *$ \\
\hline lene (o) & 0.539 & 0. & 0. & 0.586 & & 74 & $3.6 *$ \\
\hline ylene (to & * 0. & & 0.571 & 86 & & 74 & $3.6 *$ \\
\hline & * 0.887 & & 0.943 & 0.990 & & 56 & 5.0 \\
\hline Eorm & * 0 . & & 0 . & 0.477 & & 55 & 6.7 \\
\hline ylber & * 3. & & 3. & 3. & & & 1.7 \\
\hline 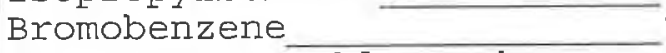 & $\star 0.880$ & 0 . & 0.901 & 0.898 & & 99 & 1.5 \\
\hline $1,1,2,2$-Tetrachloroethar & $\star \quad 1.206$ & & 1.107 & 1.084 & & 4 & 4.7 \\
\hline 1,2,3-Trichloropropar & $\star 0.290$ & & 0.282 & 0.278 & & 0.283 & 2.4 \\
\hline Loro-2-buten & $\star 0$. & 0 . & 0.224 & 0.223 & & 0.220 & 4.4 \\
\hline 1 & $\star 0$. & & 0.746 & 0.728 & & & 2.1 \\
\hline rotolue & $* 0.667$ & & 0.701 & 0.677 & & & 2.2 \\
\hline 4-Chlorotoluene & $\star 0.640$ & & 0.686 & 0.693 & & 0 . & 3.4 \\
\hline $1,3,5$-Trimethyl & $\star 2.181$ & & 2.308 & 2.268 & & 2.261 & 2.1 \\
\hline & * 2.3 & & 2.384 & 2.332 & 2.333 & 2.3 & 1.3 \\
\hline - Trime & $\star 1.903$ & 2.095 & 2.159 & 2.171 & 2.205 & & 5.7 \\
\hline sec-Butylben: & $\star 3.315$ & 3.431 & 3.466 & 3.420 & & 3.2 & 1.8 \\
\hline 1,3 -Dichlorobenzer & $\star 1.436$ & 1.446 & 1.476 & 1.482 & 1.465 & 1.461 & 1.3 \\
\hline
\end{tabular}

* Compounds with required minimum RRF and maximim \%RSD values.

All other compounds must meet a minimim RRF of 0.010 .

page 2 of 3 


\section{FORM 6 \\ VOLATILE ORGANICS INITIAL CALIBRATION DATA}

Lab Name: TESTAMERICA BURLINGTON Contract: 8E-00302

Lab Code: STLV Case No.: SAVANNAH SAS No.:

SDG NO.: 138033

Instrument ID: L

Calibration Date(s) : 07/09/10 07/09/10

Heated Purge: ( $\mathrm{Y} / \mathrm{N}) \mathrm{N}$

Calibration Time(s): 2056

2258

GC Column: DB-624 ID: 0.53 (mm)

\begin{tabular}{|c|c|c|c|c|c|c|c|}
\hline $\begin{array}{ll}\text { LAB FILE ID : } & \text { RRF1 } \\
\text { RRF } 10=L E K 005 & \text { RRF25 }\end{array}$ & $\begin{array}{l}=\text { LEKO03 } \\
=\text { LEKO066 }\end{array}$ & & $\begin{array}{l}\text { RRF5 } \\
\text { RRF50 }\end{array}$ & $\begin{array}{l}=\text { LEKOC } \\
=\text { LEKOC }\end{array}$ & & & \\
\hline COMPOUND & RRF 1 & RRF5 & RRF10 & RRF 25 & RRF50 & $\overline{\mathrm{RRF}}$ & $\begin{array}{c}\frac{0}{0} \\
\text { RSD }\end{array}$ \\
\hline 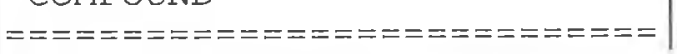 & $=====$ & $=====$ & $=====$ & $======$ & $======$ & $=====\pi$ & $=====$ \\
\hline 4-Isopropyltoluene & 2.393 & 2.504 & 2.580 & 2.582 & 2.626 & 2.537 & $3.6 *$ \\
\hline I,4-Dichlorobenzene & $\times 1.518$ & 1.590 & 1.559 & 1.549 & 1.597 & 1.563 & $2.0 *$ \\
\hline 1,2-Dichlorobenzene & +1.324 & 1.356 & 1.342 & 1.351 & 1.369 & 1.348 & $1.3 *$ \\
\hline n-Butylbenzene & +2.033 & 2.234 & 2.309 & 2.423 & 2.514 & 2.303 & $8.0 *$ \\
\hline 1,2-Dibromo-3-Chloropropane_* & 0.265 & 0.206 & 0.203 & 0.206 & 0.203 & 0.217 & $12.5 *$ \\
\hline $1,2,4$-Trichlorobenzene & 0.848 & 0.858 & 0.922 & 0.952 & 0.994 & 0.915 & $6.8 *$ \\
\hline Hexachlorobutadiene & 0.647 & 0.688 & 0.686 & 0.678 & 0.670 & 0.674 & 2.4 * \\
\hline Naphthalene & 2.264 & 1.571 & 1.544 & 1.513 & 1.554 & 1.689 & $19.1 *$ \\
\hline $1,2,3$-Trichlorobenzene & +0.733 & 0.765 & 0.805 & 0.841 & 0.864 & 0.802 & $6.7 *$ \\
\hline 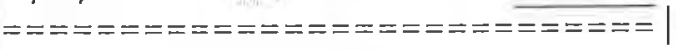 & $======$ & $======$ & $======$ & $==== \pm=$ & $======$ & $======$ & $=====\mid$ \\
\hline 1,2 -Dichloroethane- $\mathrm{d}^{4}$ & 0.287 & 0.276 & 0.278 & 0.275 & 0.271 & 0.277 & $2.1 *$ \\
\hline Toluene-d8 & $\times 1.133$ & 1.188 & 1.148 & 1.174 & 1.185 & 1.166 & $2.0 *$ \\
\hline Bromofluorobenzene & * 1.258 & 1.273 & 1.232 & 1.221 & 1.206 & 1.238 & $2.2 *$ \\
\hline 1,2-Dichlorobenzene-d4 & $\times 0.832$ & 0.853 & 0.872 & 0.871 & 0.864 & 0.858 & $2.0 *$ \\
\hline & & & & & & & \\
\hline & & & & & & & \\
\hline & & & & & & & \\
\hline & & & & & & & \\
\hline & & & & & & & \\
\hline & & & & & & & \\
\hline & & & & & & & \\
\hline & & & & & & & \\
\hline & & & & & & & \\
\hline & & & & & & & \\
\hline & & & & & & & \\
\hline & & & & & & & \\
\hline & & & & & & & \\
\hline & & & & & & & \\
\hline & & & - & 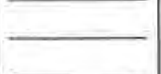 & & & 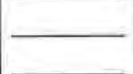 \\
\hline & & & & & & & \\
\hline
\end{tabular}

Compounds with required minimum RRF and maximim $\div$ RSD values.

All other compounds must meet a minimim RRF of 0.010 . 
FORM 7

VOLATILE CONTINUING CALIBRATION CHECK

Lab Name: TESTAMERICA BURLINGTON Contract: 8E-00302

Lab Code: STLV Case No.: SAVANNAH SAS No.:

SDG NO.: 138033

Instrument ID: L

Calibration Date: 07/14/10 Time: 0742

Lab File ID: LEKOIODV

Init. Calib. Date(s): 07/09/10 07/09/10

Heated Purge: $(\mathrm{Y} / \mathrm{N}) \mathrm{N}$

Init. Calib. Times: $2056 \quad 2258$

GC Column: DB-624 ID: 0.53 (mm)

\begin{tabular}{|c|c|c|c|c|c|}
\hline COMPOUND & $\overline{\mathrm{RRF}}$ & RRF10 & $\begin{array}{l}\text { MIN } \\
\text { RRF }\end{array}$ & $\because \mathrm{D}$ & $\begin{array}{l}\text { MAX } \\
\circ D\end{array}$ \\
\hline 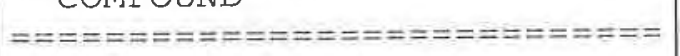 & $====-===0$ & $========$ & $===== \pm==$ & $======$ & $====$ \\
\hline Dichlorodifluoromethane & 0.607 & 0.511 & & 15.8 & 20.0 \\
\hline Chloromethane & 0.267 & 0.230 & 0.1 & 13.8 & 20.0 \\
\hline Vinyl Chloride & 0.317 & 0.275 & & 13.2 & 20.0 \\
\hline Bromomethane & 0.225 & 0.222 & & 1.3 & 20.0 \\
\hline Chloroethane & 0.178 & 0.162 & & 9.0 & 20.0 \\
\hline Trichlorof Iuoromethane & 0.621 & 0.558 & & 10.1 & 20.0 \\
\hline Acrolein & 0.025 & 0.024 & & 4.0 & 20.0 \\
\hline 1.1-Dichloroethene & 0.282 & 0.266 & & 5.7 & 20.0 \\
\hline Freon $\mathrm{TF}$ & 0.605 & 0.596 & & 1.5 & 20.0 \\
\hline Acetone & 0.047 & 0.040 & & 14.9 & 20.0 \\
\hline Methyl Iodide & 0.396 & 0.315 & & 20.4 & 20.0 \\
\hline Carbon Disulfide & 0.812 & 0.800 & & 1.5 & 20.0 \\
\hline Allyl Chloride & 0.521 & 0.518 & & 0.6 & 20.0 \\
\hline Methylene Chloride & 0.279 & 0.276 & & 1.1 & 20.0 \\
\hline trans-1,2-Dichloroethene & 0.309 & 0.305 & & 1.3 & 20.0 \\
\hline Acrylonitrile & 0.053 & 0.049 & & 7.5 & 20.0 \\
\hline Methyl-t-Buty ether & 0.672 & 0.645 & & 4.0 & 20.0 \\
\hline 1,2 -Dichloroethene (total) & 0.318 & 0.309 & & 2.8 & 20.0 \\
\hline 1,1-Dichloroethane & 0.614 & 0.599 & 0.1 & 2.4 & 20.0 \\
\hline Vinyl Acetate & 0.563 & 0.628 & & 11.5 & 20.0 \\
\hline Chloroprene & 0.506 & 0.499 & & 1.4 & 20.0 \\
\hline 2,2-Dichloropropane & 0.531 & 0.533 & & 0.4 & 20.0 \\
\hline cis-1,2-Dichloroethene & 0.328 & 0.314 & & 4.3 & 20.0 \\
\hline 2-Butanone & 0.021 & 0.020 & & 4.8 & 20.0 \\
\hline Propionitrile & 0.020 & 0.019 & & 5.0 & 20.0 \\
\hline Bromochloromethane & 0.184 & 0.179 & & 2.7 & 20.0 \\
\hline Methacrylonitrile & 0.071 & 0.063 & & 11.3 & 20.0 \\
\hline Tetrahydrofuran & 0.069 & 0.066 & & 4.3 & 20.0 \\
\hline Chloroform & 0.653 & 0.642 & & 1.7 & 20.0 \\
\hline 1,1,1-Trichloroethane & 0.564 & 0.555 & & 1.6 & 20.0 \\
\hline Carbon Tetrachloride & 0.514 & 0.510 & & 0.8 & 20.0 \\
\hline 1,1-Dichloropropene & 0.502 & 0.497 & & 1.0 & 20.0 \\
\hline Isobutyl Alcohol & 0.009 & 0.009 & & 0.0 & 20.0 \\
\hline Benzene & 0.931 & 0.912 & & 2.0 & 20.0 \\
\hline 1,2-Dichloroethane & 0.348 & 0.345 & & 0.9 & 20.0 \\
\hline Trichloroethene & 0.391 & 0.377 & & 3.6 & 20.0 \\
\hline 1,2-Di chloropropane & 0.367 & 0.355 & & 3.3 & 20.0 \\
\hline
\end{tabular}

page 1 of 3 
FORM 7

VOLATILE CONTINUING CALIBRATION CHECK

Lab Name: TESTAMERICA BURIINGTON Contract: 8E-00302

Lab Code: STLV Case No.: SAVANNAH SAS No.:

SDG NO.: 138033

Instrument ID: L

Calibration Date: 07/14/10 Time: 0742

Lab File ID: LEKOIODV

Init. Calib. Date(s): 07/09/10 07/09/10

Heated Purge: $(Y / N)$ N

Init. Calib. Times: 2056

2258

GC Column: DB-624 ID: 0.53 (mm)

\begin{tabular}{|c|c|c|c|c|c|}
\hline & & & $\begin{array}{l}\text { MIN } \\
\text { RRF }\end{array}$ & $\because D$ & $\begin{array}{l}\mathrm{MAX} \\
\div \mathrm{D}\end{array}$ \\
\hline COMPOUND & & $\begin{array}{c}\text { RRF } 10 \\
========\end{array}$ & $\begin{array}{c}\operatorname{RRF} \\
=======\end{array}$ & $======$ & $====$ \\
\hline $\begin{array}{l}=========================x= \\
\text { Dibromomethane }\end{array}$ & $\begin{array}{r}======= \\
0.288\end{array}$ & $\begin{aligned}=== & ==== \\
& 0.282\end{aligned}$ & $==-== \pm=$ & $\begin{array}{r}=-1.1 \\
2.1\end{array}$ & 20.0 \\
\hline Methyl Methacrylate & 0.245 & 0.239 & & 2.4 & 20.0 \\
\hline 1,4 -Dioxane & 0.002 & 0.002 & & 0.0 & 20.0 \\
\hline Bromodichloromethane & 0.603 & 0.594 & & 1.5 & 20.0 \\
\hline 2-Chloroethyl Vinyl Ether & 0.182 & 0.182 & & 0.0 & 20.0 \\
\hline cis-1,3-Dichloropropene & 0.510 & 0.508 & & 0.4 & 20.0 \\
\hline 4-Methyl-2-pentanone & 0.304 & 0.306 & & 0.6 & 20.0 \\
\hline Toluene & 0.773 & 0.756 & & 2.2 & 20.0 \\
\hline trans-1,3-Dichloropropene & 0.555 & 0.541 & & 2.5 & 20.0 \\
\hline Ethyl Methacrylate & 0.412 & 0.404 & & 1.9 & 20.0 \\
\hline $1,1,2$-Trichloroethane & 0.335 & 0.327 & & 2.4 & 20.0 \\
\hline Tetrachloroethene & 0.592 & 0.577 & & 2.5 & 20.0 \\
\hline 1,3-Dichloropropane & 0.624 & 0.607 & & 2.7 & 20.0 \\
\hline 2-Hexanone & 0.254 & 0.254 & & 0.0 & 20.0 \\
\hline Dibromochloromethane & 0.605 & 0.607 & & 0.3 & 20.0 \\
\hline 1,2-Dibromoethane & 0.540 & 0.526 & & 2.6 & 20.0 \\
\hline Chlorobenzene & 0.948 & 0.940 & 0.3 & 0.8 & 20.0 \\
\hline $1,1,1,2$-Tetrachloroethane & 0.496 & 0.490 & & 1.2 & 20.0 \\
\hline Ethylbenzene & 1.661 & 1.624 & & 2.2 & 20.0 \\
\hline Xylene $(m, p)$ & 0.607 & 0.592 & & 2.5 & 20.0 \\
\hline xylene (o) & 0.574 & 0.563 & & 1.9 & 20.0 \\
\hline xylene (total) & 0.574 & 0.563 & & 1.9 & 20.0 \\
\hline styrene & 0.956 & 0.934 & & 2.3 & 20.0 \\
\hline Bromoform & 0.455 & 0.454 & 0.1 & 0.2 & 20.0 \\
\hline Isopropylbenzene & 3.086 & 2.932 & & 5.0 & 20.0 \\
\hline Bromobenzene & 0.899 & 0.864 & & 3.9 & 20.0 \\
\hline $1,1,2,2$-Tetrachloroethane & 1.124 & 1.071 & 0.3 & 4.7 & 20.0 \\
\hline 1,2,3-Trichloropropane & 0.283 & 0.274 & & 3.2 & 20.0 \\
\hline trans-1,4-Dichloro-2-butene & 0.220 & 0.222 & & 0.9 & 20.0 \\
\hline n-Propylbenzene & 0.732 & 0.697 & & 4.8 & 20.0 \\
\hline 2-Chlorotoluene & 0.685 & 0.656 & & 4.2 & 20.0 \\
\hline 4-Chlorotoluene & 0.680 & 0.664 & & 2.4 & 20.0 \\
\hline $1,3,5$-Trimethylbenzene & 2.261 & 2.158 & & 4.6 & 20.0 \\
\hline tert-Butylbenzene & 2.366 & 2.264 & & $4 \cdot 3$ & 20.0 \\
\hline $1,2,4$-Trimethylbenzene & 2.107 & 2.022 & & 4.0 & 20.0 \\
\hline sec-Butylbenzene & 3.418 & 3.305 & & 3.3 & 20.0 \\
\hline 1,3-Dichlorobenzen & 1.461 & 1.395 & & 4.5 & 20.0 \\
\hline
\end{tabular}


FORM 7

VOLATILE CONTINUING CALIBRATION CHECK

Lab Name: TESTAMERICA BURLINGTON Contract: 8E-00302

Lab Code: STLV Case No.: SAVANNAH SAS No.:

SDG NO.: 138033

Instrument ID: L

Calibration Date: 07/14/10 Time: 0742

Lab File ID: LEKOIODV

Init. Calib. Date(s): 07/09/10 07/09/10

Heated Purge: $(\mathrm{Y} / \mathrm{N}) \mathrm{N}$

Init. Calib. Times: $2056 \quad 2258$

GC Column: DB-624 ID: 0.53 (mm)

\begin{tabular}{|c|c|c|c|c|c|}
\hline UND & $\overline{\mathrm{RRF}}$ & RRFI0 & $\begin{array}{l}\text { MIN } \\
\text { RRF }\end{array}$ & $\div \mathrm{D}$ & $\begin{array}{l}\text { MAX } \\
\because D\end{array}$ \\
\hline 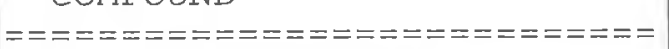 & $=== \pm=====$ & $========$ & $=== \pm===\approx$ & $==-===$ & $====$ \\
\hline 4-Isopropyltoluene & 2.537 & 2.462 & & 3.0 & 20.0 \\
\hline 1,4-Dichlorobenzene & 1.563 & 1.488 & & 4.8 & 20.0 \\
\hline 1,2-Dichlorobenzene & 1.348 & 1.285 & & 4.7 & 20.0 \\
\hline $\mathrm{n}-\mathrm{Bu}$ & 2.303 & 2.234 & & 3.0 & 20.0 \\
\hline Chloropropane & 0.217 & 0.203 & & 6.4 & 20.0 \\
\hline $1,2,4$ & 0.915 & 0.897 & & 2.0 & 20.0 \\
\hline Hexa & 0.674 & 0.660 & & 2.1 & 20.0 \\
\hline Naph & 1.689 & 1.364 & & 19.2 & 20.0 \\
\hline $1,2,3$-Trichlorobenzene & 0.802 & 0.792 & & 1.2 & 20.0 \\
\hline 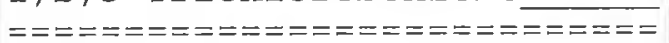 & $=========$ & $====$ = = $=$ = & $===\approx==\approx=$ & $==x= \pm=$ & $====$ \\
\hline Domothona & 0.277 & 0.259 & & 6.5 & 20.0 \\
\hline Tolu & 1.166 & 1.136 & & 2.6 & 20.0 \\
\hline Bro & 1.238 & 1.203 & & 2.8 & 20.0 \\
\hline 1,2-Dichlorobenzene-d4 & 0.858 & 0.838 & & 2.3 & 20.0 \\
\hline
\end{tabular}

page 3 of 3 
FORM 8

VOLATILE INTERNAL STANDARD AREA AND RT SUMMARY

Lab Name: TESTAMERICA BURLINGTON Contract: 8E-00302

Lab Code: STLV Case No.: SAVANNAH SAS No.: $\quad$ SDG No.: 138033

Lab File ID (Standard): LEKoloDV Date Analyzed: 07/14/10

Instrument ID: L

Time Analyzed: 0742

GC Column: DB-624 ID: 0.53 (mm) Heated Purge: (Y/N) N

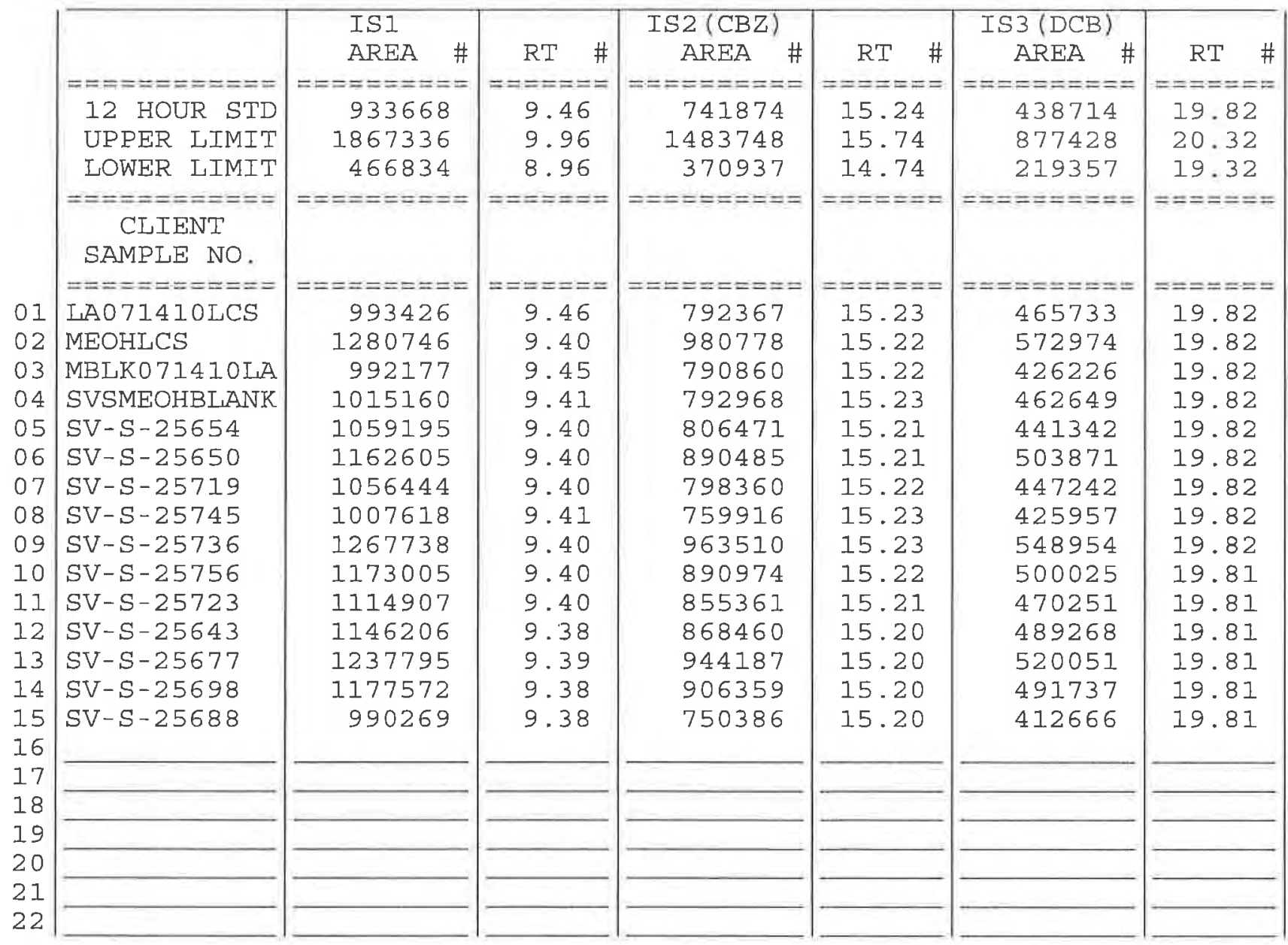

IS1 $=$ Fluorobenzene

IS2 $(\mathrm{CBZ})=$ Chlorobenzene $-\mathrm{d} 5$

IS3 $(\mathrm{DCB})=1,4-\mathrm{Dichlorobenzene-d4}$

AREA UPPER LIMIT $=+100 \%$ of internal standard area

AREA LOWER LIMIT $=-50 \%$ of internal standard area

RT UPPER LIMIT $=+0.50$ minutes of internal standard RT

RT LOWER LIMIT $=-0.50$ minutes of internal standard $\mathrm{RT}$

\# Column used to flag values outside QC limits with an asterisk.

* Values outside of QC limits.

page 1 of 1 


\title{
TestAmerica
}

THE LEA.DER IN ENVIRONMENTAL. TESTING

TestAmerica Laboratories, Inc.

\section{CASE NARRATIVE}

\author{
Client: Argonne National Laboratory \\ Project: SAVANNAH (200-1286)
}

Report Number: 200-1286-1

With the exceptions noted as flags or footnotes, standard analytical protocols were followed in the analysis of the samples and no problems were encountered or anomalies observed. In addition all laboratory quality control samples were within established control limits. Each sample in the sample set was analyzed without a dilution.

Calculations are performed before rounding to avoid round-off errors in calculated results.

All holding times were met and proper preservation noted for the methods performed on these samples, unless otherwise detailed in the individual sections below.

\section{$\underline{\text { Receipt }}$}

The samples were received on $08 / 26 / 2010$. Documentation of the condition of the samples at the time of their receipt and any exception to the laboratory's Sample Acceptance Policy is documented in the Sample Handling section of this submittal. The samples, as received, were not acid preserved. On that basis, the laboratory did provide for the analytical work to be performed within seven days of sample collection.

\section{SOM01.2 Volatile Organics (Trace Level Water)}

A storage blank was prepared for volatile organics analysis, and stored in association with the storage of the samples. That storage blank, identified as VHBLK01, was carried through the holding period with the samples, and analyzed.

Each sample was analyzed without a dilution. Each of the analyses associated with the sample set exhibited an acceptable internal standard performance. There was an acceptable recovery of each deuterated monitoring compound (DMC) in the analysis of the method blank associated with the analytical work, and in the analysis of the storage blank associated with the sample set. The analysis of the samples in this sample set did meet the technical acceptance criteria specific to $\mathrm{DMC}$ recoveries, although not all DMC recoveries were within the control range in each analysis. The technical acceptance criteria does provide for the recovery of up to three DMCs to fall outside of the control range in the analysis of field samples. Matrix spike and matrix spike duplicate analyses were not performed on samples in this sample set. Trace concentrations of acetone and 1,2,3-trichlorobenzene were identified in the analysis of the method blank associated with the analytical work. The concentration of each compound in that analysis was 


\section{Test America \\ THE LEADER IN ENVIRONMENTAL TESTING}

below the established reporting limit, and the analysis did meet the technical acceptance criteria for a compliant method blank analysis. Trace concentrations of acetone, carbon disulfide, and benzene were identified in the analysis of the storage blank associated with the sample set. The concentration of each compound in that analysis was below the established reporting limit, and the analysis did meet the technical acceptance criteria for a compliant storage blank analysis. Present in the method blank and storage blank analyses was a non-target constituent that represented a compound that is related to the DMC formulation. The fact that the presence of this compound is not within the laboratory's control is at issue. The derived results for that compound have been qualified with an " $X$ " qualifier to reflect the source of the contamination.

The responses for each of the target analytes met the relative standard deviation criterion in the initial calibration. The response for each target analyte met the percent difference criterion in the continuing calibration check acquisition. The response for each target analyte met the 50.0 percent difference criterion in the closing calibration check acquisition.

The primary quantitation mass for methylcyclohexane that is specified in the Statement of Work is mass 83 . The laboratory did identify a contribution to mass 83 from 1,2-dichloropropane- $\mathrm{d}_{6}$, one of the deuterated monitoring compounds (DMCs). The laboratory did change the primary quantitation mass assignment to mass 55 for the quantification of methylcyclohexane.

Manual integration was employed in deriving certain of the analytical results. The values that have been derived from manual integration are qualified on the quantitation reports. Extracted ion current profiles for each manual integration are included in the data package, and further documented in the Sample Preparation section of this submittal.

The analytical results associated with the samples presented in this test report were generated under a quality system that adheres to requirements specified in the NELAC standard. Release of the data in this test report and any associated electronic deliverables is authorized by the Laboratory Director's designee as verified by the following signature.

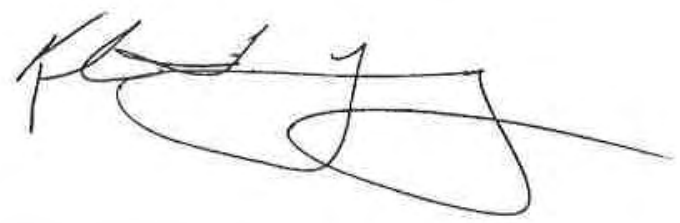

Kirk F. Young

Project Manager 


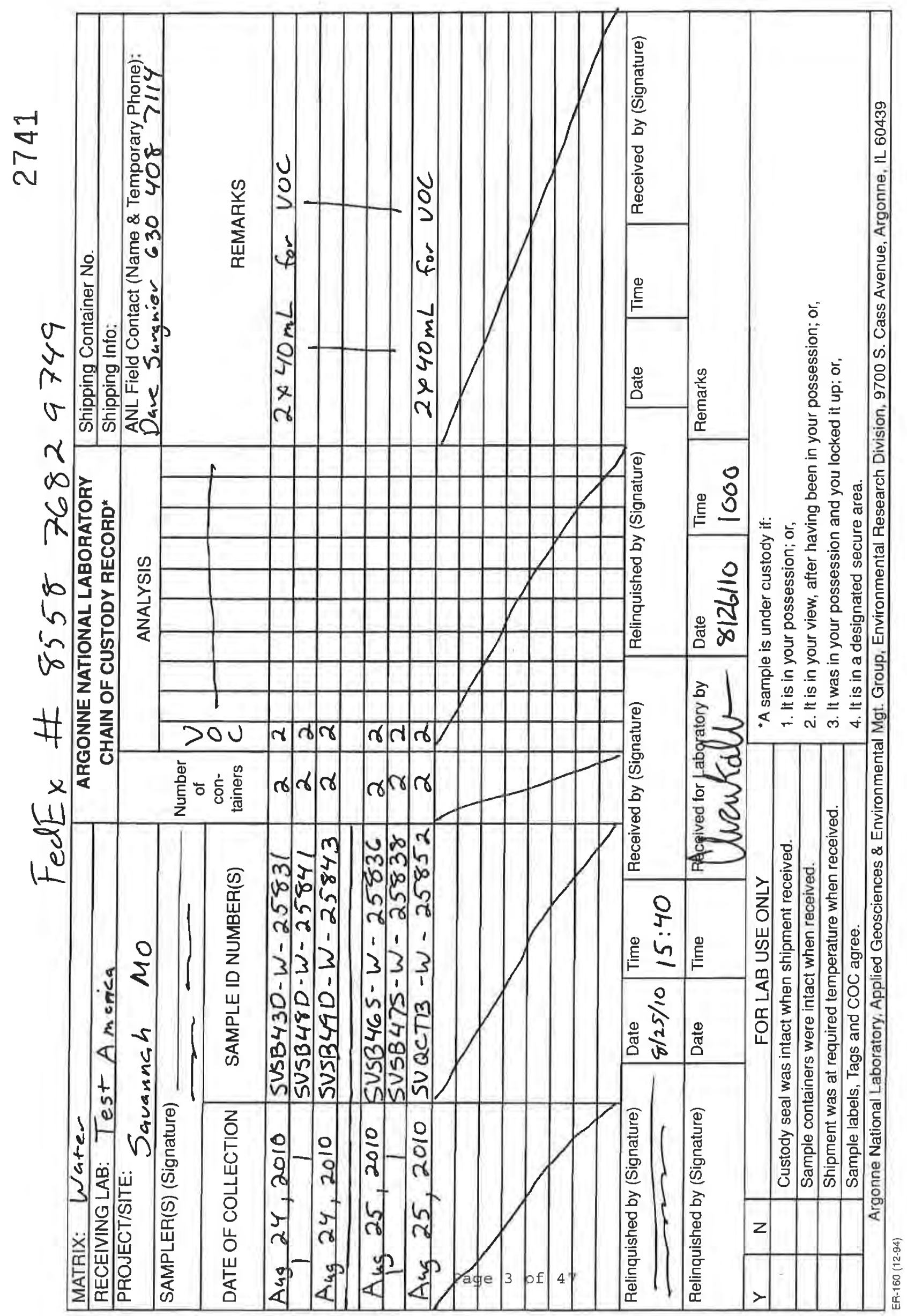




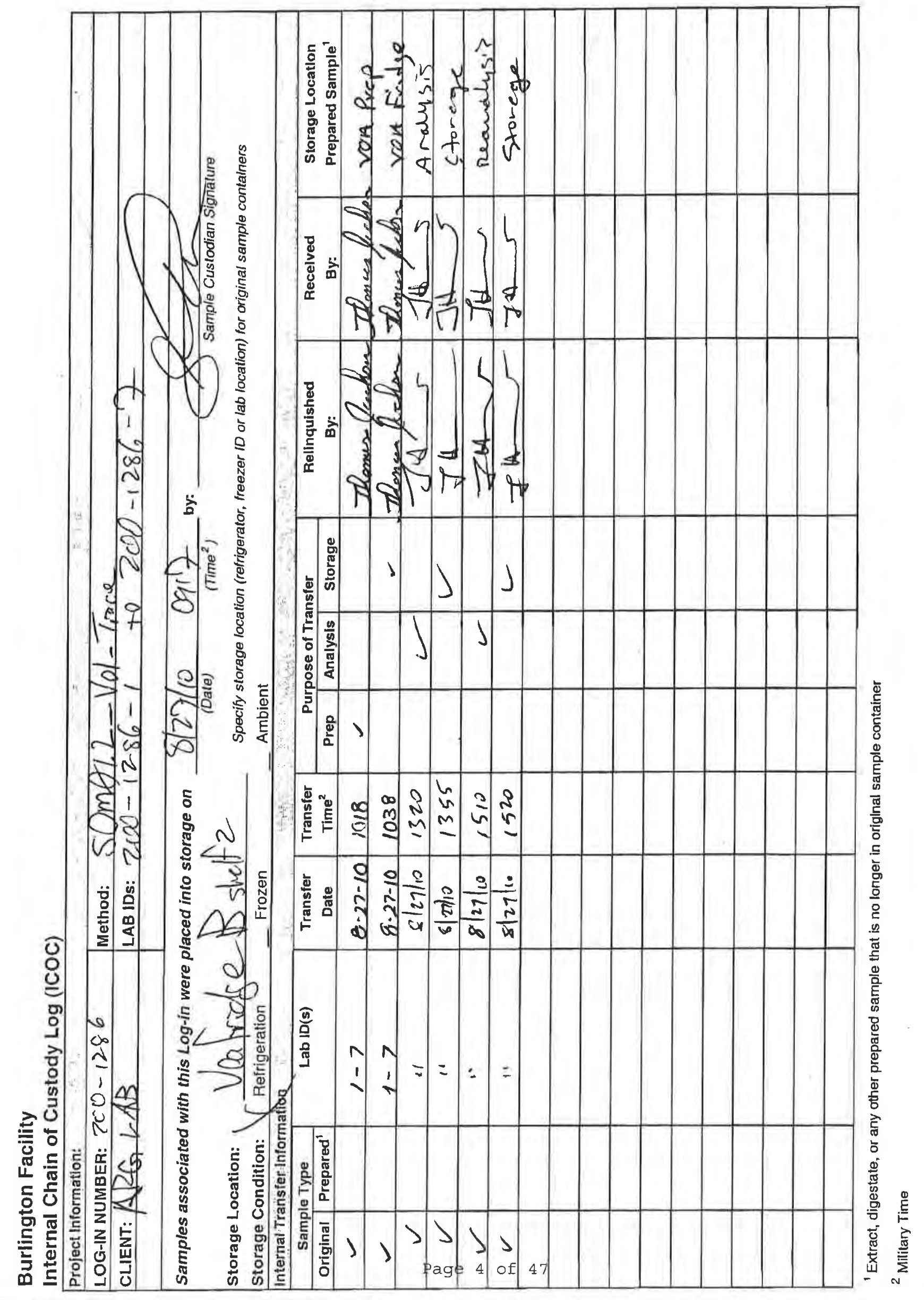




\section{SAMPLE SUMMARY}

Client: Argonne National Laboratory

Job Number: $\quad 200-1286-1$

Sdg Number: SAVANNAH (200-1286)

\begin{tabular}{|c|c|c|c|c|c|c|}
\hline \multirow{2}{*}{$\begin{array}{l}\text { Lab Sample ID } \\
200-1286-1\end{array}$} & \multirow{2}{*}{$\begin{array}{l}\text { Client Sample ID } \\
\text { SVSB43D-W-25831 }\end{array}$} & \multirow{2}{*}{$\begin{array}{l}\text { Client Matrix } \\
\text { Water }\end{array}$} & \multicolumn{2}{|c|}{$\begin{array}{l}\text { Date/Time } \\
\text { Sampled }\end{array}$} & \multicolumn{2}{|c|}{$\begin{array}{l}\text { Date/Time } \\
\text { Received }\end{array}$} \\
\hline & & & $08 / 24 / 2010$ & 0000 & $08 / 26 / 2010$ & 1000 \\
\hline $200-1286-2$ & SVSB48D-W-25841 & Water & $08 / 24 / 2010$ & 0000 & $08 / 26 / 2010$ & 1000 \\
\hline $200-1286-3$ & SVSB49D-W-25843 & Water & $08 / 24 / 2010$ & 0000 & $08 / 26 / 2010$ & 1000 \\
\hline $200-1286-4$ & SVSB46S-W-25836 & Water & $08 / 25 / 2010$ & 0000 & $08 / 26 / 2010$ & 1000 \\
\hline $200-1286-5$ & SVSB47S-W-25838 & Water & $08 / 25 / 2010$ & 0000 & $08 / 26 / 2010$ & 1000 \\
\hline $200-1286-6$ & SVQCTB-W-25852 & Water & $08 / 25 / 2010$ & 0000 & $08 / 26 / 2010$ & 1000 \\
\hline $200-1286-7$ & VHBLK01 & Water & $08 / 27 / 2010$ & 0915 & $08 / 26 / 2010$ & 1000 \\
\hline
\end{tabular}

TestAmerica Burlington 


\section{METHOD SUMMARY}

Client: Argonne National Laboratory

Description

Matrix: Water

Trace Water

Volatile sample preservation, Field Preserved Water

Volatile sample preservation, Unpreserved Water

Lab References:

TAL BUR = TestAmerica Burlington

Method References:

SOM01.2 = U.S. Environmental Protection Agency
Job Number: 200-1286-1

Sdg Number: SAVANNAH (200-1286)

Lab Location Method Preparation Method

TAL BUR

TAL BUR

SOM01.2 SOM01.2NOA_Tr

TAL BUR

SOM01.2 SOM01.2NOA_PR

SOM01.2 SOM01.2NOA_PR

\section{TestAmerica Burlington}




\section{METHOD / ANALYST SUMMARY}

Client: Argonne National Laboratory

Job Number: $\quad$ 200-1286-1

Sdg Number: SAVANNAH (200-1286)

Method

Analyst

Analyst ID

SOM01.2 SOM01.2NOA_Tr

Heald, John

JRH

TestAmerica Burlington 


\section{DATA REPORTING QUALIFIERS}

Client: Argonne National Laboratory

Job Number: 200-1286-1

Sodg Number: SAVANNAH (200-1286)

GC/MS VOA

U

$J$

J

*

$\mathrm{X}$

B

N
Analyzed for but not detected.

Indicates an Estimated Value for TICs

Indicates an estimated value.

Recovery or RPD exceeds control limits

See case narrative notes for explanation of the ' $X$ ' flag

The analyte was found in an associated blank, as well as in the sample.

This flag indicates the presumptive evidence of a compound. 
2A - FORM II VOA-1

WATER VOLATILE DEUTERATED MONITORING COMPOUND RECOVERY

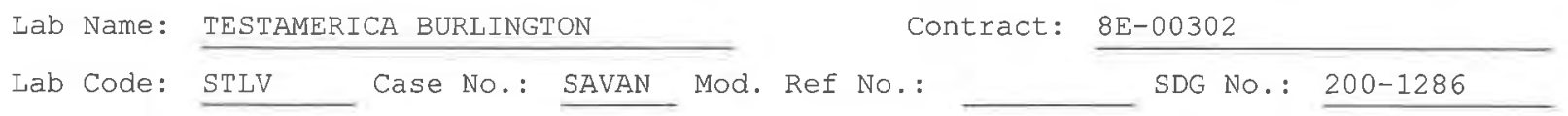

Level: (TRACE or LOW) TRACE

\begin{tabular}{|c|c|c|c|c|c|c|c|}
\hline $\begin{array}{c}\text { EPA } \\
\text { SAMPLE NO. }\end{array}$ & $\begin{array}{l}\text { VDMC1 } \\
\text { (VCL) \# }\end{array}$ & $\begin{array}{c}\text { VDMC2 } \\
\text { (CLA) \# }\end{array}$ & $\begin{array}{l}\text { VDMC3 } \\
\text { (DCE) \# }\end{array}$ & $\begin{array}{l}\text { VDMC4 } \\
\text { (BUT) \# }\end{array}$ & $\begin{array}{c}\text { VDMC5 } \\
(\mathrm{CLF}) \#\end{array}$ & $\begin{array}{l}\text { VDMC6 } \\
\text { (DCA) \# }\end{array}$ & $\begin{array}{l}\text { VDMC7 } \\
\text { (BEN) \# }\end{array}$ \\
\hline VBLKJD & 97 & 95 & 72 & 106 & $\overline{994}$ & 101 & 100 \\
\hline $\begin{array}{l}\text { SVSB43D-W-2583 } \\
1\end{array}$ & 99 & 96 & 72 & 181 * & 95 & 102 & 105 \\
\hline $\begin{array}{l}\text { SVSB48D-W-2584 } \\
1\end{array}$ & 103 & 104 & 74 & $206 *$ & 97 & 105 & 106 \\
\hline $\begin{array}{l}\text { SVSB4 9D-W-2584 } \\
3\end{array}$ & 103 & 103 & 74 & $224 *$ & 99 & 107 & 103 \\
\hline $\begin{array}{l}\text { SVSB } 47 S-W-2583 \\
8\end{array}$ & 98 & 98 & 74 & 146 & 99 & 104 & 104 \\
\hline SVQCTB-W-25852 & 99 & 97 & 73 & $232 \star$ & 96 & 104 & 103 \\
\hline $\begin{array}{l}\text { SVSB4 6S-W-2583 } \\
6\end{array}$ & 105 & 103 & 75 & 231 * & 90 & 105 & 102 \\
\hline VHBLK01 & 98 & 98 & 72 & 111 & 97 & 105 & 104 \\
\hline
\end{tabular}

$\begin{array}{ll}\text { VDMC1 } & (\mathrm{VCL})=\text { Vinyl Chloride-d3 } \\ \text { VDMC2 } & \text { (CLA) }=\text { Chloroethane-d5 } \\ \text { VDMC3 } & (\mathrm{DCE})=1,1-\text { Dichloroethene-d2 } \\ \text { VDMC4 } & (\mathrm{BUT})=2-\text { Butanone-d5 } \\ \text { VDMC5 } & (\mathrm{CLF})=\text { Chloroform-d } \\ \text { VDMC6 } & (\mathrm{DCA})=1,2-\text { Dichloroethane-d4 } \\ \text { VDMC7 } & (\mathrm{BEN})=\text { Benzene-d6 }\end{array}$

\# Column to be used to flag recovery values

* Values outside of contract required QC limits
QC LIMITS

$(65-131)$

(71-131)

(55-104)

(49-155)

(78-121)

(78-129)

$(77-124)$ 
2B - FORM II VOA-2

WATER VOLATILE DEUTERATED MONITORING COMPOUND RECOVERY

Lab Name: TESTAMERICA BURLINGTON

Contract: $8 \mathrm{E}-00302$

Lab Code: STLV Case No.: SAVAN Mod. Ref No.:

SDG No.: $200-1286$

Level: (TRACE or LOW) TRACE

\begin{tabular}{|c|c|c|c|c|c|c|c|c|}
\hline $\begin{array}{c}\text { EPA } \\
\text { SAMPLE NO. }\end{array}$ & $\begin{array}{l}\text { VDMC8 } \\
(\mathrm{DPA}) \quad \#\end{array}$ & $\begin{array}{l}\text { VDMC9 } \\
\text { (TOL) \# }\end{array}$ & $\begin{array}{l}\text { VDMC10 } \\
\text { (TDP) \# }\end{array}$ & $\begin{array}{l}\text { VDMC11 } \\
\text { (HEX) \# }\end{array}$ & $\begin{array}{l}\text { VDMC12 } \\
\text { (TCA) \# }\end{array}$ & $\begin{array}{l}\text { VDMC13 } \\
\text { (DCZ) \# }\end{array}$ & OTHER & $\begin{array}{l}\text { TOT } \\
\text { OUT }\end{array}$ \\
\hline VBLKJD & 102 & 97 & 95 & 110 & 93 & 97 & & 0 \\
\hline $\begin{array}{l}\text { SVSB43D-W-2583 } \\
1\end{array}$ & 102 & 100 & 98 & 206 * & 93 & 96 & & 2 \\
\hline $\begin{array}{l}\text { SVSB48D-W-2584 } \\
1\end{array}$ & 105 & 102 & 99 & $229 \star$ & 102 & 103 & & 2 \\
\hline $\begin{array}{l}\text { SVSB } 49 D-W-2584 \\
3\end{array}$ & 102 & 100 & 99 & 228 * & 99 & 106 & & 2 \\
\hline $\begin{array}{l}\text { SVSB } 47 S-W-2583 \\
8\end{array}$ & 104 & 98 & 96 & 129 & 94 & 101 & & 0 \\
\hline SVQCTB-W-25852 & 103 & 99 & 97 & 239 * & 98 & 100 & & 2 \\
\hline $\begin{array}{l}\text { SVSB } 46 S-W-2583 \\
6\end{array}$ & 102 & 99 & 101 & $244 \star$ & 86 & 104 & & 2 \\
\hline VHBLKO 1 & 104 & 99 & 99 & 112 & 95 & 102 & & 0 \\
\hline
\end{tabular}

\footnotetext{
VDMC8 $(D P A)=1,2-$ Dichloropropane-d6

VDMC9 $($ TOL $)=$ Toluene-d8

VDMC10 $(T D P)=$ trans-1,3-Dichloropropene-d4

VDMC11 $(\mathrm{HEX})=2-$ Hexanone-d5

VDMC12 $(T C A)=1,1,2,2-$ Tetrachloroethane- $d 2$

VDMC13 $(D C Z)=1,2-$ Dichlorobenzene-d4
}

QC LIMITS

$(79-124)$

$(77-121)$

$(73-121)$

$(28-135)$

$(73-125)$

$(80-131)$

\# Column to be used to flag recovery values

* Values outside of contract required QC limits

Report 1,4-Dioxane-d8 for Low-Medium VOA analysis only

Page 1 of 1

Page 10 of 47

SOM01.2 (4/2007) 
$4 A$ - FORM IV VOA

VOLATILE METHOD BLANK SUMMARY
EPA SAMPLE NO.

VBLKJD

Lab Name: TESTAMERICA BURLINGTON

Contract: $8 \mathrm{E}-00302$

Lab Code: STLV Case No.: SAVAN Mod. Ref No.: SDG No.: 200-1286

Lab File ID: JBIR05.D

Lab Sample ID: MB 200-5901/5

Instrument ID: J.i

Matrix: (SOIL/SED/WATER)

Water

Date Analyzed: 08/27/2010

Level: (TRACE or LOW/MED) TRACE

Time Analyzed: 0945

GC Column: DB-624

ID: 0.20 $(\mathrm{mm})$

Heated Purge: $(\mathrm{Y} / \mathrm{N}) \mathrm{N}$

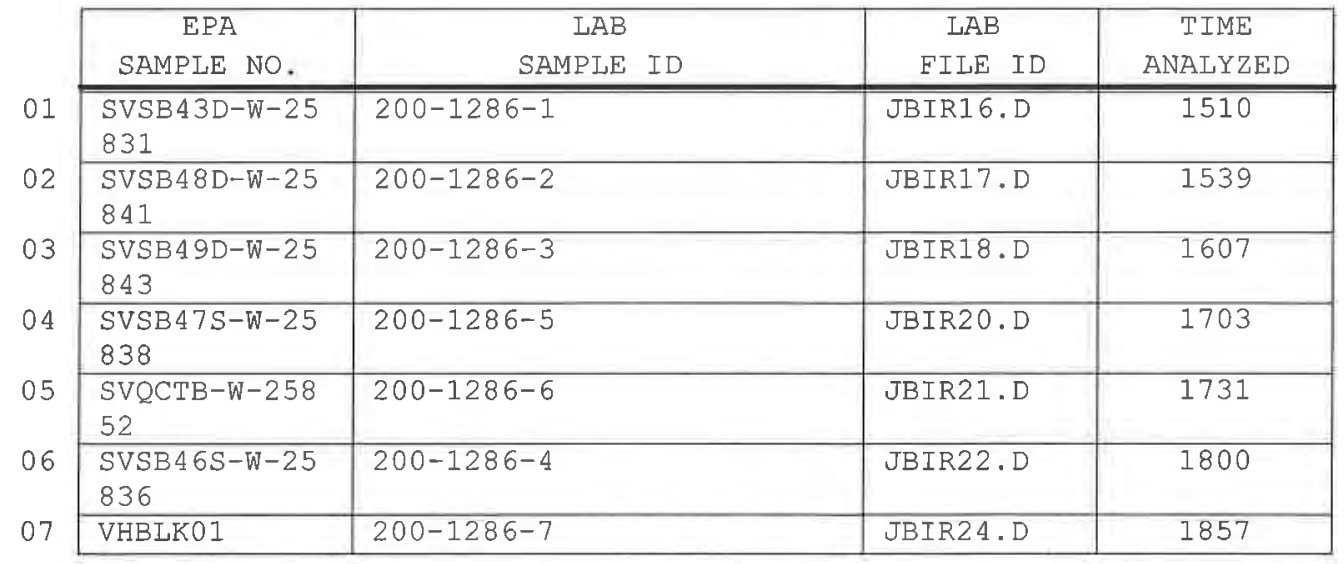

COMMENTS :

Page 1 of 1 
$5 A-F O R M V V O A$

VOLATILE ORGANICS INSTRUMENT

PERFORMANCE CHECK

BROMOFLUOROBENZENE

(BFB)
EPA SAMPLE NO.

BFBJI
Lab Name: TESTAMERICA BURLINGTON

Lab Code: STLV Case No.: SAVAN Mod. Ref No.:

Lab File Id: JBI03PV.D

Instrument Id: J.i

GC Column: DB-624

ID: 0.20 $(\mathrm{mm})$
Contract: $8 \mathrm{E}-00302$

SDG No.: 200-1286

BFB Injection Time: 1610

\begin{tabular}{|c|c|c|}
\hline $\mathrm{m} / \mathrm{e}$ & ION ABUNDANCE CRITERIA & $\begin{array}{l}\% \text { RELATIVE } \\
\text { ABUNDANCE }\end{array}$ \\
\hline 50 & $15.0-40.0 \%$ of mass 95 & 17.2 \\
\hline 75 & $30.0-80.0 \%$ of mass 95 & 50.3 \\
\hline 95 & Base peak, $100 \%$ relative abundance & 100 \\
\hline 96 & $5.0-9.0 \%$ of mass 95 & 6.9 \\
\hline 173 & Less than $2.0 \%$ of mass 174 & 0.41 \\
\hline 174 & $50.0-120 \%$ of mass 95 & 84.3 \\
\hline 175 & $5.0-9.0 \%$ of mass 174 & 5.91 \\
\hline 176 & $95.0-101 \%$ of mass 174 & $81.7(96.9) 1$ \\
\hline 177 & $5.0-9.0 \%$ of mass 176 & 5.51 \\
\hline
\end{tabular}

1 - Value is \%mass 1742 - Value is \%mass 176

\begin{tabular}{|c|c|c|c|c|}
\hline $\begin{array}{c}\text { EPA } \\
\text { SAMPLE NO. }\end{array}$ & $\begin{array}{c}\text { LAB } \\
\text { SAMPLE ID }\end{array}$ & $\begin{array}{c}\text { LAB } \\
\text { EILE ID }\end{array}$ & $\begin{array}{c}\text { DATE } \\
\text { ANALYZED }\end{array}$ & $\begin{array}{c}\text { TIME } \\
\text { ANALYZED }\end{array}$ \\
\hline VSTD0.5JI & IC $200-5303 / 5$ & JBI0005V.D & $07 / 27 / 2010$ & 1657 \\
\hline VSTD001JI & IC $200-5303 / 6$ & JBI001V.D & $07 / 27 / 2010$ & 1725 \\
\hline VSTD005JI & ICIS $200-5303 / 8$ & JBI005V.D & $07 / 27 / 2010$ & 1753 \\
\hline VSTD010JI & IC $200-5303 / 9$ & JBI010V.D & $07 / 27 / 2010$ & 1822 \\
\hline VSTD020JI & IC $200-5303 / 10$ & JBI020V.D & $07 / 27 / 2010$ & 1850 \\
\hline
\end{tabular}


$5 A$ - FORM V VOA

VOLATILE ORGANICS INSTRUMENT

PERFORMANCE CHECK

BROMOFLUOROBENZENE

Lab Name: TESTAMERICA BURLINGTON

Lab Code: STLV

Case No.: SAVAN

Lab File Id: JBIROI.D

Instrument Id: J.i

GC Column: DB-624

ID: 0.20
EPA SAMPLE NO.

BFBJD

\begin{tabular}{|c|c|c|}
\hline $\mathrm{m} / \mathrm{e}$ & ION ABUNDANCE CRITERIA & $\begin{array}{l}\text { \% RELATIVE } \\
\text { ABUNDANCE }\end{array}$ \\
\hline 50 & $15.0-40.0 \%$ of mass 95 & 17.5 \\
\hline 75 & $30.0-80.0 \div$ of mass 95 & 49.0 \\
\hline 95 & Base peak, $100 \%$ relative abundance & 100 \\
\hline 96 & $5.0-9.0 \%$ of mass 95 & 6.7 \\
\hline 173 & Less than $2.0 \%$ of mass 174 & $0.5(0.7) 1$ \\
\hline 174 & $50.0-120 \%$ of mass 95 & 76.6 \\
\hline 175 & $5.0-9.0 \%$ of mass 174 & $5.4(7.0) 1$ \\
\hline 176 & $95.0-101 \%$ of mass 174 & $74.6(97.3) 1$ \\
\hline 177 & $5.0-9.0 \%$ of mass 176 & $5.4(7.2) 2$ \\
\hline
\end{tabular}

1 - Value is omass 1742 - Value is omass 176

\begin{tabular}{|c|c|c|c|c|}
\hline $\begin{array}{c}\text { EPA } \\
\text { SAMPLE NO. }\end{array}$ & $\begin{array}{r}\text { LAB } \\
\text { SAMPLE ID } \\
\end{array}$ & $\begin{array}{c}\text { LAB } \\
\text { FILE ID }\end{array}$ & $\begin{array}{c}\text { DATE } \\
\text { ANALYZED } \\
\end{array}$ & $\begin{array}{c}\text { TIME } \\
\text { ANALYZED } \\
\end{array}$ \\
\hline VSTD005JD & CCVIS $200-5901 / 3$ & JBIR03.D & $08 / 27 / 2010$ & 0849 \\
\hline VBLKJD & MB 200-5901/5 & JBIR05.D & $08 / 27 / 2010$ & 0945 \\
\hline $\begin{array}{l}\text { SVSBA3D-W- } \\
25831\end{array}$ & $200-1286-1$ & JBIR16.D & $08 / 27 / 2010$ & 1510 \\
\hline $\begin{array}{l}\text { SVSB } 48 D-W- \\
25841\end{array}$ & $200-1286-2$ & JBIR17.D & $08 / 27 / 2010$ & 1539 \\
\hline $\begin{array}{l}\text { SVSB 49D-W- } \\
25843\end{array}$ & $200-1286-3$ & JBIR18.D & $08 / 27 / 2010$ & 1607 \\
\hline $\begin{array}{l}\text { SVSB4 7S-W- } \\
25838\end{array}$ & $200-1286-5$ & JBIR20.D & $08 / 27 / 2010$ & 1703 \\
\hline $\begin{array}{l}\text { SVQCTB-W-2 } \\
5852\end{array}$ & $200-1286-6$ & JBIR21.D & $08 / 27 / 2010$ & 1731 \\
\hline $\begin{array}{l}\text { SVSB } 46 S-W- \\
25836\end{array}$ & $200-1286-4$ & JBIR22 . D & $08 / 27 / 2010$ & 1800 \\
\hline VHBLK01 & $200-1286-7$ & JBIR24.D & $08 / 27 / 2010$ & 1857 \\
\hline VSTD005DJ & CCVC $200-5901 / 25$ & JBIR25.D & $08 / 27 / 2010$ & 1926 \\
\hline
\end{tabular}

Page 1 of 1 
$8 \mathrm{~A}$ - FORM VIII VOA

VOLATILE INTERNAL STANDARD AREA AND RETENTION TIME SUMMARY

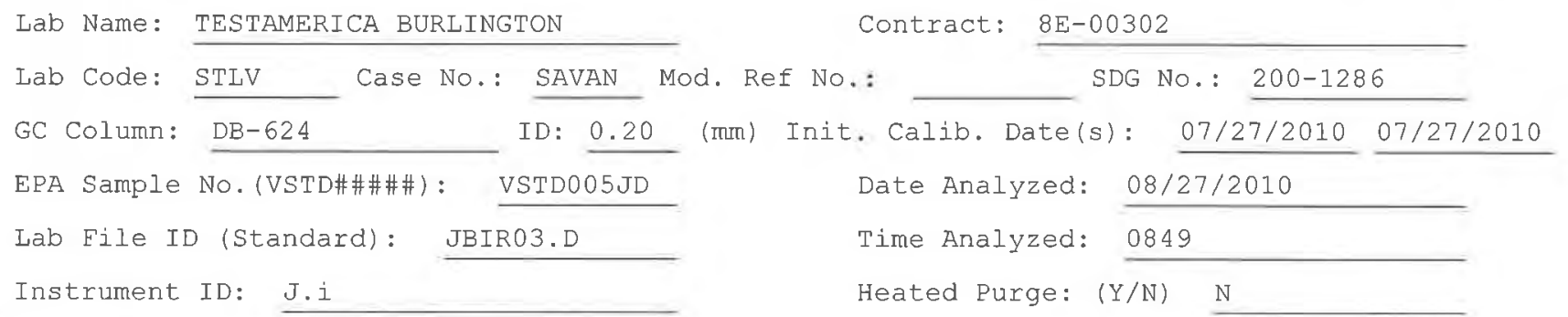

\begin{tabular}{|c|c|c|c|c|c|c|}
\hline & $\begin{array}{c}\text { IS1 (CBZ) } \\
\text { AREA }\end{array}$ & RT \# & $\begin{array}{c}\text { IS2 (DFB) } \\
\text { AREA }\end{array}$ & RT \# & $\begin{array}{c}\text { IS3 (DCB) } \\
\text { AREA }\end{array}$ & RT \# \\
\hline 12 HOUR STD & 639873 & 8.98 & 841683 & 5.60 & 282364 & 11.81 \\
\hline UPPER LIMIT & 895822 & 9.31 & 1178356 & 5.93 & 395310 & 12.14 \\
\hline LOWER LIMIT & 383924 & 8.65 & 505010 & 5.27 & 169418 & 11.48 \\
\hline EPA SAMPLE NO. & & & & & & \\
\hline$\overline{\text { VBLKJD }}$ & $\overline{597871}$ & 8.98 & 805517 & 5.60 & 256582 & 11.81 \\
\hline $\begin{array}{l}\text { SVSB4 3D-W-2583 } \\
1\end{array}$ & 572625 & 8.98 & 797509 & 5.60 & 246745 & 11.81 \\
\hline $\begin{array}{l}\text { SVSB48D-W-2584 } \\
1\end{array}$ & 527175 & 8.98 & 718642 & 5.60 & 228643 & 11.81 \\
\hline $\begin{array}{l}\text { SVSB49D-W-2584 } \\
3\end{array}$ & 579154 & 8.98 & 774685 & 5.60 & 250882 & 11.81 \\
\hline $\begin{array}{l}\text { SVSB47S-W-2583 } \\
8\end{array}$ & 590193 & 8.98 & 802306 & 5.60 & 249013 & 11.81 \\
\hline SVQCTB-W-25852 & 574622 & 8.98 & 776786 & 5.60 & 244138 & 11.81 \\
\hline $\begin{array}{l}\text { SVSB 46S-W-2583 } \\
6\end{array}$ & 549349 & 8.98 & 735566 & 5.60 & 243152 & 11.81 \\
\hline VHBLK01 & 553336 & 8.98 & 748398 & 5.60 & 233345 & 11.81 \\
\hline
\end{tabular}

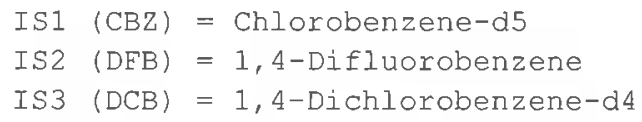


$1 A$ - FORM I VOA-1

VOLATILE ORGANICS ANALYSIS DATA SHEET
EPA SAMPLE NO.

SVQCTB-W-25852
Lab Name: TESTAMERICA BURLINGTON

Lab Code: STLV

Matrix: (SOIL/SED/WATER)

Sample wt/vol: 25.0

Level: (TRACE/LOW/MED) Case No.: SAVA

Water

$(\mathrm{g} / \mathrm{mL}) \quad \mathrm{mL}$

\% Moisture: not dec.

GC Column: DB-624 ID: $0.20 \quad(\mathrm{~mm})$

Soil Extract Volume: TRACE

Purge volume: 25.0 (uL) (mL)
Contract: $8 \mathrm{E}-00302$

SDG NO.: 200-1286

\begin{tabular}{|c|c|c|c|}
\hline CAS NO. & COMPOUND & $\begin{array}{l}\text { CONCENTRATION UNITS: } \\
(\mathrm{ug} / \mathrm{L} \text { or } \mathrm{ug} / \mathrm{kg}) \mathrm{ug} / \mathrm{L}\end{array}$ & $Q$ \\
\hline $75-71-8$ & Dichlorodifluoromethane & 0.50 & $\mathrm{U}$ \\
\hline $74-87-3$ & Chloromethane & 0.50 & $\mathrm{U}$ \\
\hline $75-01-4$ & Vinyl chloride & 0.50 & $\mathrm{U}$ \\
\hline $74-83-9$ & Bromomethane & 0.50 & $\mathrm{U}$ \\
\hline $75-00-3$ & Chloroethane & 0.50 & $\mathrm{U}$ \\
\hline $75-69-4$ & Trichlorofluoromethane & 0.50 & $\mathrm{U}$ \\
\hline $75-35-4$ & 1,1-Dichloroethene & 0.50 & $\mathrm{U}$ \\
\hline $76-13-1$ & 1,1,2-Trichloro-1,2,2-trifluoroethane & 0.50 & $\mathrm{U}$ \\
\hline $67-64-1$ & Acetone & 4.6 & $\mathrm{~J} B$ \\
\hline $75-15-0$ & Carbon disulfide & 0.064 & $\mathrm{~J}$ \\
\hline $79-20-9$ & Methyl acetate & 0.50 & $\mathrm{U}$ \\
\hline $75-09-2$ & Methylene Chloride & 0.50 & $\mathrm{U}$ \\
\hline $156-60-5$ & trans-1,2-Dichloroethene & 0.50 & $\mathrm{U}$ \\
\hline $1634-04-4$ & Methyl tert-butyl ether & 0.50 & $\mathrm{U}$ \\
\hline $75-34-3$ & 1,1-Dichloroethane & 0.50 & $\mathrm{U}$ \\
\hline $156-59-2$ & cis-1,2-Dichloroethene & 0.50 & $\mathrm{U}$ \\
\hline $78-93-3$ & 2-Butanone & 5.0 & $\mathrm{U}$ \\
\hline $74-97-5$ & Bromochloromethane & 0.50 & $\mathrm{U}$ \\
\hline $67-66-3$ & Chloroform & 0.16 & $\mathrm{~J}$ \\
\hline $71-55-6$ & 1,1,1-Trichloroethane & 0.50 & $\mathrm{U}$ \\
\hline $110-82-7$ & Cyclohexane & 0.50 & $\mathrm{U}$ \\
\hline $56-23-5$ & Carbon tetrachloride & 0.50 & $\mathrm{U}$ \\
\hline $71-43-2$ & Benzene & 0.50 & $\mathrm{U}$ \\
\hline $107-06-2$ & 1,2-Dichloroethane & 0.50 & $\mathrm{U}$ \\
\hline
\end{tabular}

Report 1,4-Dioxane for Low-Medium VOA analysis only

Lab Sample ID: 200-1286-6

Lab File ID: JBIR21.D

Date Received: $08 / 26 / 2010$

Date Analyzed: 08/27/2010

Dilution Factor: 1.0

Soil Aliquot Volume:

(uL) 
$1 B$ - FORM I VOA-2

VOLATILE ORGANICS ANALYSIS DATA SHEET
EPA SAMPLE NO.

SVQCTB-W-25852
Contract: $8 \mathrm{E}-00302$

SDG No.: 200-1286
Lab Code: STLV Case No.: SAVAN Mod. Ref No.:

Matrix: (SOIL/SED/WATER) Watex

Sample wt/vol: 25.0

$(\mathrm{g} / \mathrm{mL}) \quad \mathrm{mL}$

Level: (TRACE/LOW/MED) TRACE

\% Moisture: not dec.

GC Column: DB-624

ID: 0.20

(mm)

Soil Extract Volume:

(uI)

( $\mathrm{nL})$

Purge Volume: 25.0
Lab Sample ID: 200-1286-6

Lab File ID: JBIR21.D

Date Received: 08/26/2010

Date Analyzed: 08/27/2010

Dilution Factor: 1.0

Soil Aliquot Volume:

(uL)

\begin{tabular}{|c|c|c|c|}
\hline CAS NO. & COMPOUND & $\begin{array}{l}\text { CONCENTRATION UNITS: } \\
(\mathrm{ug} / \mathrm{L} \text { or } \mathrm{ug} / \mathrm{kg}) \mathrm{ug} / \mathrm{L}\end{array}$ & $Q$ \\
\hline $79-01-6$ & Trichloroethene & 0.50 & $\bar{U}$ \\
\hline $108-87-2$ & Methylcyclohexane & 0.50 & $\mathrm{U}$ \\
\hline $78-87-5$ & 1,2-Dichloropropane & 0.50 & $\mathrm{U}$ \\
\hline $75-27-4$ & Bromodichloromethane & 0.50 & $\mathrm{U}$ \\
\hline $10061-01-5$ & cis-1,3-Dichloropropene & 0.50 & $\mathrm{U}$ \\
\hline $108-10-1$ & 4-Methyl-2-pentanone & 5.0 & $\mathrm{U}$ \\
\hline $108-88-3$ & Toluene & 0.48 & $\mathrm{~J}$ \\
\hline $10061-02-6$ & trans-1,3-Dichloropropene & 0.50 & $\mathrm{U}$ \\
\hline $79-00-5$ & 1,1,2-Trichloroethane & 0.50 & $\mathrm{U}$ \\
\hline $127-18-4$ & Tetrachloroethene & 0.50 & $\mathrm{U}$ \\
\hline $591-78-6$ & 2-Hexanone & 5.0 & U \\
\hline $124-48-1$ & Dibromochloromethane & 0.50 & $\mathrm{U}$ \\
\hline $106-93-4$ & 1,2-Dibromoethane & 0.50 & $\mathrm{U}$ \\
\hline $108-90-7$ & Chlorobenzene & 0.50 & $\mathrm{U}$ \\
\hline $100-41-4$ & Ethylbenzene & 0.50 & $\mathrm{U}$ \\
\hline $95-47-6$ & o-Xylene & 0.50 & $\mathrm{U}$ \\
\hline $179601-23-1$ & $\mathrm{~m}, \mathrm{p}$-Xylene & 0.061 & $\mathrm{~J}$ \\
\hline $100-42-5$ & Styrene & 0.50 & $\mathrm{U}$ \\
\hline $75-25-2$ & Bromoform & 0.50 & $\mathrm{U}$ \\
\hline $98-82-8$ & Isopropylbenzene & 0.50 & $\mathrm{U}$ \\
\hline $79-34-5$ & $1,1,2,2$-Tetrachloroethane & 0.50 & $\mathrm{U}$ \\
\hline $541-73-1$ & 1,3-Dichlorobenzene & 0.50 & $\mathrm{U}$ \\
\hline $106-46-7$ & 1,4-Dichlorobenzene & 0.50 & $\mathrm{U}$ \\
\hline $95-50-1$ & 1,2-Dichlorobenzene & 0.50 & $\mathrm{U}$ \\
\hline $96-12-8$ & 1,2-Dibromo-3-Chloropropane & 0.50 & $\mathrm{U}$ \\
\hline $120-82-1$ & 1,2,4-Trichlorobenzene & 0.50 & $\mathrm{U}$ \\
\hline $87-61-6$ & 1,2,3-Trichlorobenzene & 0.50 & $\mathrm{U}$ \\
\hline
\end{tabular}


$1 \mathrm{~J}$ - FORM I VOA-TIC

VOLATILE ORGANICS ANALYSIS DATA SHEET

TENTATIVELY IDENTIFIED COMPOUNDS
EPA SAMPLE NO.

SVQCTB-W-25852

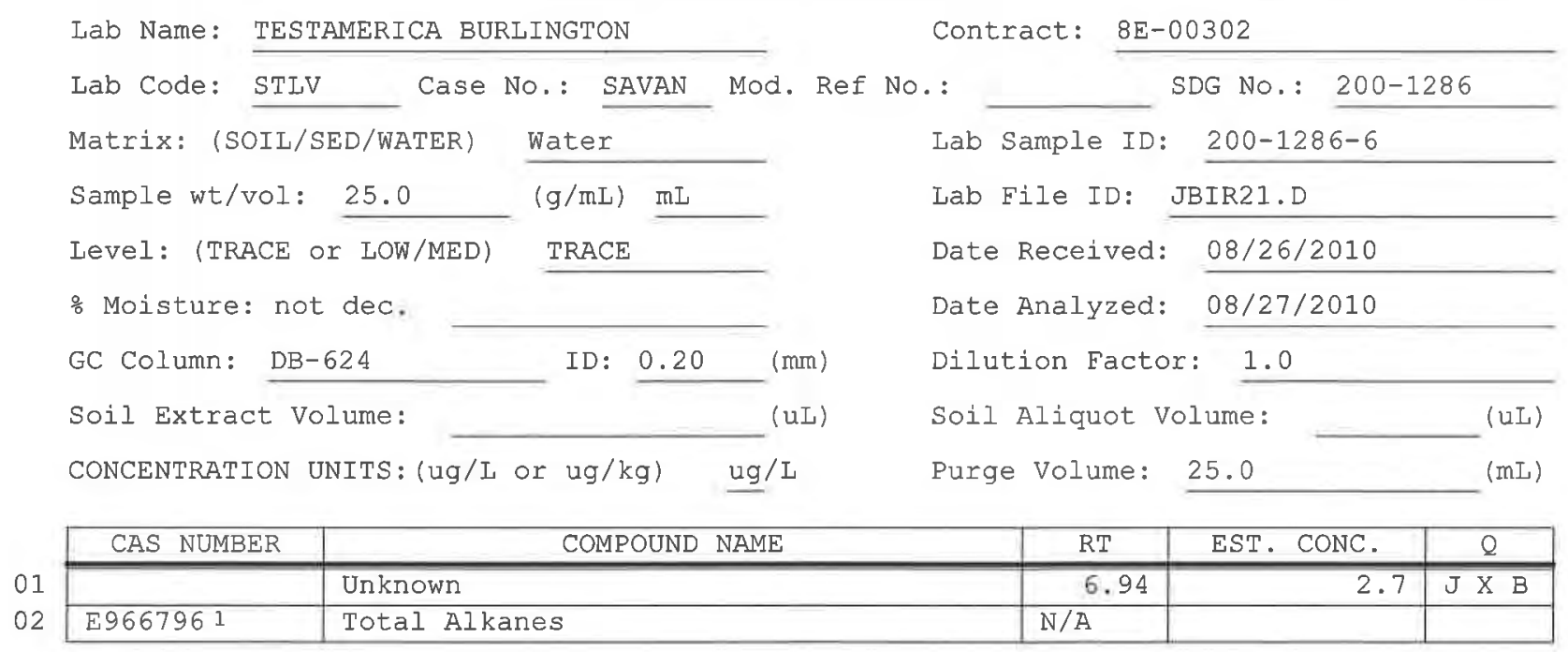

1 EPA-designated Registry Number. 
$1 A$ - FORM I VOA-1

VOLATILE ORGANICS ANALYSIS DATA SHEET
EPA SAMPLE NO.

SVSB43D-W-25831
Lab Name: TESTAMERICA BURLINGTON

Lab Code: STLV Case No.: SAVAN Mod. Ref No.: Matrix: (SOIL/SED/WATER) Water

Sample wt/vol: 25.0 $(g / m L) \quad m L$

Level: (TRACE/LOW/MED) TRACE

응 Moisture: not dec.

GC Column: $\mathrm{DB}-624$ ID: $0.20 \quad(\mathrm{~mm})$

Soil Extract Volume: (uL)

Purge volume: 25.0 (mL)
Contract: $8 \mathrm{E}-00302$ SDG No.: 200-1286
Lab Sample ID: 200-1286-1

Lab File ID: JBIR16.D

Date Received: $08 / 26 / 2010$

Date Analyzed: 08/27/2010

Dilution Factor: 1.0

Soil Aliquot Volume:

\begin{tabular}{|c|c|c|c|}
\hline CAS NO. & COMPOUND & $\begin{array}{l}\text { CONCENTRATION UNITS: } \\
(\mathrm{ug} / \mathrm{L} \text { or } \mathrm{ug} / \mathrm{kg}) \mathrm{ug} / \mathrm{L}\end{array}$ & Q \\
\hline $75-71-8$ & Dichlorodifluoromethane & 0.50 & $\overline{\mathrm{U}}$ \\
\hline $74-87-3$ & Chloromethane & 0.50 & $\mathrm{U}$ \\
\hline $75-01-4$ & Vinyl chloride & 0.50 & $\mathrm{U}$ \\
\hline $74-83-9$ & Bromomethane & 0.50 & $\mathrm{U}$ \\
\hline $75-00-3$ & Chloroethane & 0.50 & $\mathrm{U}$ \\
\hline $75-69-4$ & Trichlorofluoromethane & 0.50 & $\mathrm{U}$ \\
\hline $75-35-4$ & 1,1-Dichloroethene & 0.50 & $\mathrm{U}$ \\
\hline $76-13-1$ & 1,1,2-Trichloro-1,2,2-trifluoroethane & 0.50 & $\mathrm{U}$ \\
\hline $67-64-1$ & Acetone & 1.7 & $\mathrm{~J} B$ \\
\hline $75-15-0$ & Carbon disulfide & 0.075 & $\mathrm{~J}$ \\
\hline $79-20-9$ & Methyl acetate & 0.50 & $\mathrm{U}$ \\
\hline $75-09-2$ & Methylene Chloride & 0.50 & $\mathrm{U}$ \\
\hline $156-60-5$ & trans-1,2-Dichloroethene & 0.50 & $\mathrm{U}$ \\
\hline $1634-04-4$ & Methyl tert-butyl ether & 0.50 & $\mathrm{U}$ \\
\hline $75-34-3$ & 1,1-Dichloroethane & 0.50 & $\mathrm{U}$ \\
\hline $156-59-2$ & cis-1,2-Dichloroethene & 0.50 & $\mathrm{U}$ \\
\hline $78-93-3$ & 2-Butanone & 5.0 & $\mathrm{U}$ \\
\hline $74-97-5$ & Bromochloromethane & 0.50 & $\mathrm{U}$ \\
\hline $67-66-3$ & Chloroform & 0.97 & \\
\hline $71-55-6$ & 1,1,1-Trichloroethane & 0.50 & $\mathrm{U}$ \\
\hline $110-82-7$ & Cyclohexane & 0.50 & $\mathrm{U}$ \\
\hline $56-23-5$ & Carbon tetrachloride & 0.50 & $\mathrm{U}$ \\
\hline $71-43-2$ & Benzene & 0.50 & $\bar{U}$ \\
\hline $107-06-2$ & 1,2-Dichloroethane & 0.50 & $\mathrm{U}$ \\
\hline
\end{tabular}

Report 1,4-Dioxane for Low-Medium VOA analysis only 
$1 \mathrm{~B}-$ FORM I VOA-2

VOLATILE ORGANICS ANALYSIS DATA SHEET
EPA SAMPLE NO.

SVSB $43 D-W-25831$
Lab Name: TESTAMERICA BURLINGTON

Lab Code: STLV

Matrix: (SOIL/SED/WATER)

Sample wt/vol: 25.0

Sample wt/vol:

$(\mathrm{g} / \mathrm{mL}) \mathrm{mL}$

Level: (TRACE/LOW/MED) TRACE

$\because$ Moisture: not dec.

GC Column: DB-624

Soil Extract Volume:

ID: $0.20 \quad(\mathrm{~mm})$

Purge Volume: 25.0

Contract: 8E-00302

SDG No.: 200-1286

Lab Sample ID: 200-1286-1

Lab File ID: JBIR16.D

Date Received: 08/26/2010

Date Analyzed: 08/27/2010

Dilution Factor: 1.0

Soil Aliquot Volume:

(uL)

\begin{tabular}{|c|c|c|c|}
\hline CAS NO. & COMPOUND & $\begin{array}{l}\text { CONCENTRATION UNITS: } \\
(\mathrm{ug} / \mathrm{L} \text { or } \mathrm{ug} / \mathrm{kg}) \mathrm{ug} / \mathrm{L}\end{array}$ & $Q$ \\
\hline $79-01-6$ & Trichloroethene & 0.50 & $\overline{\mathrm{U}}$ \\
\hline $108-87-2$ & Methylcyclohexane & 0.50 & $\mathrm{U}$ \\
\hline $78-87-5$ & 1,2-Dichloropropane & 0.50 & $\mathrm{U}$ \\
\hline $75-27-4$ & Bromodichloromethane & 0.20 & $\mathrm{~J}$ \\
\hline $10061-01-5$ & cis-1,3-Dichloropropene & 0.50 & $\mathrm{U}$ \\
\hline $108-10-1$ & 4-Methyl-2-pentanone & 5.0 & $\mathrm{U}$ \\
\hline $108-88-3$ & Toluene & 0.073 & $\mathrm{~J}$ \\
\hline $10061-02-6$ & trans-1,3-Dichloropropene & 0.50 & $\mathrm{U}$ \\
\hline $79-00-5$ & 1,1,2-Trichloroethane & 0.50 & $\mathrm{U}$ \\
\hline $127-18-4$ & Tetrachloroethene & 0.073 & $\mathrm{~J}$ \\
\hline $591-78-6$ & 2-Hexanone & 5.0 & $\mathrm{U}$ \\
\hline $124-48-1$ & Dibromochloromethane & 0.084 & $\mathrm{~J}$ \\
\hline $106-93-4$ & 1,2-Dibromoethane & 0.50 & $\mathrm{U}$ \\
\hline $108-90-7$ & Chlorobenzene & 0.50 & $\mathrm{U}$ \\
\hline $100-41-4$ & Ethylbenzene & 0.50 & $\mathrm{U}$ \\
\hline $95-47-6$ & o-Xylene & 0.50 & $\mathrm{U}$ \\
\hline $179601-23-1$ & $\mathrm{~m}, \mathrm{p}$-Xylene & 0.50 & $\mathrm{U}$ \\
\hline $100-42-5$ & styrene & 0.43 & $\mathrm{~J}$ \\
\hline $75-25-2$ & Bromoform & 0.50 & $\mathrm{U}$ \\
\hline $98-82-8$ & Isopropylbenzene & 0.50 & $\mathrm{U}$ \\
\hline $79-34-5$ & $1,1,2,2$-Tetrachloroethane & 0.50 & $\mathrm{U}$ \\
\hline $541-73-1$ & 1,3-Dichlorobenzene & 0.50 & $\mathrm{U}$ \\
\hline $106-46-7$ & 1,4-Dichlorobenzene & 0.50 & $\mathrm{U}$ \\
\hline $95-50-1$ & 1,2-Dichlorobenzene & 0.50 & $\mathrm{U}$ \\
\hline $96-12-8$ & 1,2-Dibromo-3-Chloropropane & 0.50 & $\mathrm{U}$ \\
\hline $120-82-1$ & 1,2,4-Trichlorobenzene & 0.50 & $\mathrm{U}$ \\
\hline $87-61-6$ & 1,2,3-Trichlorobenzene & 0.50 & $\mathrm{U}$ \\
\hline
\end{tabular}


$1 \mathrm{~J}$ - FORM I VOA-TIC

VOLATILE ORGANICS ANALYSIS DATA SHEET

TENTATIVELY IDENTIFIED COMPOUNDS
EPA SAMPLE NO.

SVSB $43 D-W-25831$

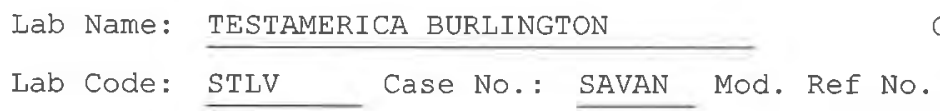

Matrix: (SOIL/SED/WATER) Water

Sample wt/vol: 25.0

Level: (TRACE or LOW/MED)

$(\mathrm{g} / \mathrm{mL}) \mathrm{mL}$

o Moisture: not dec.

GC Column: DB-624

Soil Extract Volume:

TRACE

CONCENTRATION UNITS: (ug/L or ug/ $\mathrm{kg}$ )

ID: 0.20 (mm)

(uI)

$\underline{\mathrm{ug} / \mathrm{L}}$
Contract: $8 \mathrm{E}-00302$

SDG NO.: 200-1286

Lab Sample ID: 200-1286-1

Lab File ID: JBIR16.D

Date Received: 08/26/2010

Date Analyzed: 08/27/2010

Dilution Factor: 1.0

Soil Aliquot volume: (uL)

Purge Volume: 25.0 (mL)

01

02

03

04

\begin{tabular}{|c|c|c|c|c|}
\hline CAS NUMBER & COMPOUND NAME & $\mathrm{RT}$ & EST. CONC. & $Q$ \\
\hline & Unknown & 6.94 & 2.6 & $\overline{J X B}$ \\
\hline $541-05-9$ & Cyclotrisiloxane, hexamethyl- & 7.90 & 0.56 & \\
\hline & Unknown & 10.74 & 0.61 & $\mathrm{~J}$ \\
\hline E9667961 & Total Alkanes & $\mathrm{N} / \mathrm{A}$ & & \\
\hline
\end{tabular}

1 EPA-designated Registry Number. 
$1 A$ - FORM I VOA-1

VOLATILE ORGANICS ANALYSIS DATA SHEET
EPA SAMPLE NO.

SVSB $46 S-W-25836$
Lab Name: TESTAMERICA BURLINGTON

Lab Code: STLV

Matrix: (SOIL/SED/WATER)

Sample wt/vol: 25.0

Water

$(\mathrm{g} / \mathrm{mL}) \quad \mathrm{mL}$

Level: (TRACE/LOW/MED) TRACE

응 Moisture: not dec.

GC Column: DB-624

Soil Extract Volume:

Purge Volume: 25.0 ID: $0.20 \quad(\mathrm{~mm})$ (uL) (mL)

Contract: $8 \mathrm{E}-00302$

\begin{tabular}{|c|c|c|c|}
\hline CAS NO. & COMPOUND & $\begin{array}{l}\text { CONCENTRATION UNITS: } \\
(\mathrm{ug} / \mathrm{L} \text { or } \mathrm{ug} / \mathrm{kg}) \mathrm{ug} / \mathrm{L}\end{array}$ & $Q$ \\
\hline $75-71-8$ & Dichlorodifluoromethane & 0.50 & $\mathrm{U}$ \\
\hline $74-87-3$ & Chloromethane & 0.50 & $\mathrm{U}$ \\
\hline $75-01-4$ & Vinyl chloride & 0.50 & $\mathrm{U}$ \\
\hline $74-83-9$ & Bromomethane & 0.50 & $\mathrm{U}$ \\
\hline $75-00-3$ & Chloroethane & 0.50 & $\mathrm{U}$ \\
\hline $75-69-4$ & Trichlorofluoromethane & 0.50 & $\mathrm{U}$ \\
\hline $75-35-4$ & 1,1-Dichloroethene & 0.50 & $\mathrm{U}$ \\
\hline $76-13-1$ & 1,1,2-Trichloro-1,2,2-trifluoroethane & 0.50 & $\mathrm{U}$ \\
\hline $67-64-1$ & Acetone & 36 & $\mathrm{~B}$ \\
\hline $75-15-0$ & Carbon disulfide & 0.070 & $\mathrm{~J}$ \\
\hline $79-20-9$ & Methyl acetate & 0.50 & $\mathrm{U}$ \\
\hline $75-09-2$ & Methylene Chloride & 0.50 & $\mathrm{U}$ \\
\hline $156-60-5$ & trans-1,2-Dichloroethene & 0.50 & $\mathrm{U}$ \\
\hline $1634-04-4$ & Methyl tert-butyl ether & 0.50 & $\mathrm{U}$ \\
\hline $75-34-3$ & 1,1-Dichloroethane & 0.50 & $\mathrm{U}$ \\
\hline $156-59-2$ & cis-1,2-Dichloroethene & 0.50 & $\mathrm{U}$ \\
\hline $78-93-3$ & 2-Butanone & 6.6 & \\
\hline $74-97-5$ & Bromochloromethane & 0.50 & $\mathrm{U}$ \\
\hline $67-66-3$ & Chloroform & 2.0 & \\
\hline $71-55-6$ & 1,1,1-Trichloroethane & 0.50 & $\mathrm{U}$ \\
\hline $110-82-7$ & Cyclohexane & 0.50 & $\mathrm{U}$ \\
\hline $56-23-5$ & Carbon tetrachloride & 12 & \\
\hline $71-43-2$ & Benzene & 0.047 & $\mathrm{~J}$ \\
\hline $107-06-2$ & 1,2-Dichloroethane & 0.50 & $\mathrm{U}$ \\
\hline
\end{tabular}

Report 1,4-Dioxane for Low-Medium VOA analysis only

Lab Sample ID: 200-1286-4

Lab File ID: JBIR22.D

Date Received: 08/26/2010

Date Analyzed: 08/27/2010

Dilution Factor: 1.0

Soil Aliquot Volume:

(UL) 
$1 B$ - FORM I VOA-2

VOLATILE ORGANICS ANALYSIS DATA SHEET
EPA SAMPLE NO.

SVSB 46 S-W -25836
Lab Name: TESTAMERICA BURLINGTON

Lab Code: STLV Matrix: (SOIL/SED/WATER)

Sample wt/vol: 25.0

Level: (TRACE/LOW/MED) Case No.: SAVAN

Water

$(\mathrm{g} / \mathrm{mL}) \quad \mathrm{mL}$

\% Moisture: not dec.

GC Column: DB-624 ID: $0.20 \quad$ (mm)

Soil Extract Volume: (uL)

Purge volume: 25.0 (mL)
Contract: $8 \mathrm{E}-00302$

SDG NO.: 200-1286

Lab Sample ID: 200-1286-4

Lab File ID: JBIR22.D

Date Received: 08/26/2010

Date Analyzed: 08/27/2010

Dilution Factor: 1.0

Soil Aliquot volume:

(uL)

\begin{tabular}{|c|c|c|c|}
\hline CAS NO. & COMPOUND & $\begin{array}{l}\text { CONCENTRATION UNITS: } \\
(\mathrm{ug} / \mathrm{L} \text { or } \mathrm{ug} / \mathrm{kg}) \mathrm{ug} / \mathrm{L}\end{array}$ & Q \\
\hline $79-01-6$ & Trichloroethene & 0.50 & $\mathrm{U}$ \\
\hline $108-87-2$ & Methylcyclohexane & 0.50 & $\mathrm{U}$ \\
\hline $78-87-5$ & 1,2-Dichloropropane & 0.50 & $\mathrm{U}$ \\
\hline $75-27-4$ & Bromodichloromethane & 0.50 & $\mathrm{U}$ \\
\hline $10061-01-5$ & cis-1,3-Dichloropropene & 0.50 & $\mathrm{U}$ \\
\hline $108-10-1$ & 4-Methyl-2-pentanone & 5.0 & $\mathrm{U}$ \\
\hline $108-88-3$ & Toluene & 0.33 & $\mathrm{~J}$ \\
\hline $10061-02-6$ & trans-1,3-Dichloropropene & 0.50 & $\mathrm{U}$ \\
\hline $79-00-5$ & 1,1,2-Trichloroethane & 0.50 & $\mathrm{U}$ \\
\hline $127-18-4$ & Tetrachloroethene & 0.11 & $\mathrm{~J}$ \\
\hline $591-78-6$ & 2-Hexanone & 5.0 & $\mathrm{U}$ \\
\hline $124-48-1$ & Dibromochloromethane & 0.50 & $\mathrm{U}$ \\
\hline $106-93-4$ & 1,2-Dibromoethane & 0.50 & $\mathrm{U}$ \\
\hline $108-90-7$ & Chlorobenzene & 0.50 & $\mathrm{U}$ \\
\hline $100-41-4$ & Ethylbenzene & 0.50 & $\mathrm{U}$ \\
\hline $95-47-6$ & o-Xylene & 0.055 & $\mathrm{~J}$ \\
\hline $179601-23-1$ & m,p-Xylene & 0.10 & $\mathrm{~J}$ \\
\hline $100-42-5$ & Styrene & 0.29 & $\mathrm{~J}$ \\
\hline $75-25-2$ & Bromoform & 0.50 & $\mathrm{U}$ \\
\hline $98-82-8$ & Isopropylbenzene & 0.50 & $\mathrm{U}$ \\
\hline $79-34-5$ & $1,1,2,2$-Tetrachloroethane & 0.50 & $\mathrm{U}$ \\
\hline $541-73-1$ & 1,3-Dichlorobenzene & 0.50 & $\mathrm{U}$ \\
\hline $106-46-7$ & 1,4-Dichlorobenzene & 0.50 & $\mathrm{U}$ \\
\hline $95-50-1$ & 1,2-Dichlorobenzene & 0.50 & $\mathrm{U}$ \\
\hline $96-12-8$ & 1,2-Dibromo-3-Chloropropane & 0.50 & $\mathrm{U}$ \\
\hline $120-82-1$ & 1,2,4-Trichlorobenzene & 0.50 & $\mathrm{U}$ \\
\hline $87-61-6$ & 1,2,3-Trichlorobenzene & 0.50 & $\mathrm{U}$ \\
\hline
\end{tabular}


$1 \mathrm{~J}-$ FORM I VOA-TIC

VOLATILE ORGANICS ANALYSIS DATA SHEET

TENTATIVELY IDENTIEIED COMPOUNDS
EPA SAMPLE NO.

SVSB $46 S-W-25836$

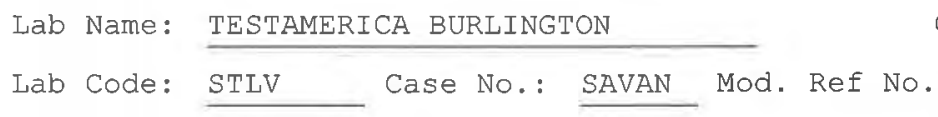

Contract: $8 E-00302$

Matrix: (SOIL/SED/WATER) water

Sample wt/vol: 25.0 $(\mathrm{g} / \mathrm{mL}) \mathrm{mL}$

Level: (TRACE or LOW/MED)

TRACE

\% Moisture: not dec.

GC Column: DB-624

ID: 0.20 $(\mathrm{mm})$

Soil Extract Volume: (uL)

CONCENTRATION UNITS: (ug/L or ug/kg) ug/L
SDG No.: 200-1286

Lab Sample ID: 200-1286-4

Lab File ID: JBIR22.D

Date Received: $08 / 26 / 2010$

Date Analyzed: $08 / 27 / 2010$

Dilution Factor: 1.0

Soil Aliquot Volume: $(\mathrm{uL})$

Purge Volume: 25.0 (mL)

\begin{tabular}{|c|c|c|c|c|}
\hline CAS NUMBER & COMPOUND NAME & $\mathrm{RT}$ & EST. CONC. & $Q$ \\
\hline & Unknown & 5.88 & 0.63 & $\bar{J}$ \\
\hline & Unknown & 6.94 & 2.8 & $\mathrm{JXB}$ \\
\hline & Unknown & 12.14 & 0.64 & $\mathrm{~J}$ \\
\hline E9667961 & Total Alkanes & $N / A$ & & \\
\hline
\end{tabular}

1 EPA-designated Registry Number. 
$1 A$ - FORM I VOA-1

VOLATILE ORGANICS ANALYSIS DATA SHEET
EPA SAMPLE NO.

SVSB $475-W-25838$
Lab Name: TESTAMERICA BURLINGTON

Lab Code: STLV

Matrix: (SOIL/SED/WATER)

Sample wt/vol: 25.0

Level: (TRACE/LOW/MED

Case No.: SAVAN

Mod. Ref No.:

\section{Water}

$(\mathrm{g} / \mathrm{mL}) \mathrm{mL}$
TRACE
Contract: 8E-00302

SDG No.: 200-1286
\% Moisture: not dec.

GC Column: DB-624

Soil Extract Volume:

Purge Volume: 25.0

ID: $0.20 \quad(\mathrm{~mm})$

(uL)

(mL)

Lab Sample ID: 200-1286-5

Lab File ID: JBIR20.D

Date Received: 08/26/2010

Date Analyzed: 08/27/2010

Dilution Factor: 1.0

Soil Aliquot Volume:

(uL)

\begin{tabular}{|c|c|c|c|}
\hline CAS NO. & COMPOUND & $\begin{array}{l}\text { CONCENTRATION UNITS: } \\
(\mathrm{ug} / \mathrm{L} \text { or } \mathrm{ug} / \mathrm{kg}) \mathrm{ug} / \mathrm{L}\end{array}$ & Q \\
\hline $75-71-8$ & Dichlorodifluoromethane & 0.50 & $\mathrm{U}$ \\
\hline $74-87-3$ & Chloromethane & 0.50 & $\mathrm{U}$ \\
\hline $75-01-4$ & Vinyl chloride & 0.50 & $\mathrm{U}$ \\
\hline $74-83-9$ & Bromomethane & 0.50 & $\mathrm{U}$ \\
\hline $75-00-3$ & Chloroethane & 0.50 & $\mathrm{U}$ \\
\hline $75-69-4$ & Trichlorofluoromethane & 0.50 & $\mathrm{U}$ \\
\hline $75-35-4$ & 1,1-Dichloroethene & 0.50 & $\mathrm{U}$ \\
\hline $76-13-1$ & 1,1,2-Trichloro-1,2,2-trifluoroethane & 0.50 & $\mathrm{U}$ \\
\hline $67-64-1$ & Acetone & 4.6 & $\mathrm{~J} B$ \\
\hline $75-15-0$ & Carbon disulfide & 0.091 & $\mathrm{~J}$ \\
\hline $79-20-9$ & Methyl acetate & 0.50 & $\mathrm{U}$ \\
\hline $75-09-2$ & Methylene Chloride & 0.50 & $\mathrm{U}$ \\
\hline $156-60-5$ & trans-1,2-Dichloroethene & 0.50 & $\mathrm{U}$ \\
\hline $1634-04-4$ & Methyl tert-butyl ether & 0.50 & $\mathrm{U}$ \\
\hline $75-34-3$ & 1,1-Dichloroethane & 0.50 & $\mathrm{U}$ \\
\hline $156-59-2$ & cis-1,2-Dichloroethene & 0.50 & $\mathrm{U}$ \\
\hline $78-93-3$ & 2-Butanone & 5.0 & $\mathrm{U}$ \\
\hline $74-97-5$ & Bromochloromethane & 0.50 & $\mathrm{U}$ \\
\hline $67-66-3$ & Chloroform & 4.4 & \\
\hline $71-55-6$ & 1,1,1-Trichloroethane & 0.50 & $\mathrm{U}$ \\
\hline $110-82-7$ & Cyclohexane & 0.50 & $\mathrm{U}$ \\
\hline $56-23-5$ & Carbon tetrachloride & 0.50 & $\mathrm{U}$ \\
\hline $71-43-2$ & Benzene & 0.50 & $\mathrm{U}$ \\
\hline $107-06-2$ & 1,2-Dichloroethane & 0.50 & $\mathrm{U}$ \\
\hline
\end{tabular}

Report 1,4-Dioxane for Low-Medium VOA analysis only 
Lab Name: TESTAMERICA BURLINGTON

Lab Code: STLV Case No.: SAVAN Mod. Ref No.:

Contract: 8E-00302 ( $\mathrm{mm})$ (uI) Matrix: (SOIL/SED/WATER)

Sample wt/vol: 25.0

Level: (TRACE/LOW/MED) TRACE

o Moisture: not dec.

GC Column: DB-624

ID: 0.20

Soil Extract Volume:

Water

$(\mathrm{g} / \mathrm{mL}) \quad \mathrm{mI}$

Purge volume: 25.0 (mL)

Lab Sample ID: 200-1286-5

Lab File ID: JBIR20.D

Date Received: 08/26/2010

Date Analyzed: 08/27/2010

Dilution Factor: 1.0

Soil Aliquot Volume:

(uI)

\begin{tabular}{|c|c|c|c|}
\hline CAS NO. & COMPOUND & $\begin{array}{l}\text { CONCENTRATION UNITS: } \\
(\mathrm{ug} / \mathrm{L} \text { or } \mathrm{ug} / \mathrm{kg}) \mathrm{ug} / \mathrm{L}\end{array}$ & Q \\
\hline $79-01-6$ & Trichloroethene & 0.50 & $\mathrm{U}$ \\
\hline $108-87-2$ & Methylcyclohexane & 0.50 & $\mathrm{U}$ \\
\hline $78-87-5$ & 1,2-Dichloropropane & 0.50 & $\mathrm{U}$ \\
\hline $75-27-4$ & Bromodichloromethane & 1.7 & \\
\hline $10061-01-5$ & cis-1,3-Dichloropropene & 0.50 & $\mathrm{U}$ \\
\hline $108-10-1$ & 4-Methyl-2-pentanone & 5.0 & $\mathrm{U}$ \\
\hline $108-88-3$ & Toluene & 0.11 & $\mathrm{~J}$ \\
\hline $10061-02-6$ & trans-1,3-Dichloropropene & 0.50 & $\mathrm{U}$ \\
\hline $79-00-5$ & 1,1,2-Trichloroethane & 0.50 & $\mathrm{U}$ \\
\hline $127-18-4$ & Tetrachloroethene & 0.10 & $\mathrm{~J}$ \\
\hline $591-78-6$ & 2-Hexanone & 5.0 & $\mathrm{U}$ \\
\hline $124-48-1$ & Dibromochloromethane & 0.72 & \\
\hline $106-93-4$ & 1,2-Dibromoethane & 0.50 & $\mathrm{U}$ \\
\hline $108-90-7$ & Chlorobenzene & 0.50 & $\mathrm{U}$ \\
\hline $100-41-4$ & Ethylbenzene & 0.50 & U \\
\hline $95-47-6$ & o-xylene & 0.50 & $\mathrm{U}$ \\
\hline $179601-23-1$ & m,p-Xylene & 0.50 & $\mathrm{U}$ \\
\hline $100-42-5$ & Styrene & 0.30 & $\mathrm{~J}$ \\
\hline $75-25-2$ & Bromoform & 0.50 & $\mathrm{U}$ \\
\hline $98-82-8$ & Isopropylbenzene & 0.50 & U \\
\hline $79-34-5$ & $1,1,2,2$-Tetrachloroethane & 0.50 & $\mathrm{U}$ \\
\hline $541-73-1$ & 1,3-Dichlorobenzene & 0.50 & $\mathrm{U}$ \\
\hline $106-46-7$ & 1,4-Dichlorobenzene & 0.50 & $\mathrm{U}$ \\
\hline $95-50-1$ & 1,2-Dichlorobenzene & 0.50 & $\mathrm{U}$ \\
\hline $96-12-8$ & 1,2-Dibromo-3-Chloropropane & 0.50 & $\mathrm{U}$ \\
\hline $120-82-1$ & 1,2,4-Trichlorobenzene & 0.50 & $\mathrm{U}$ \\
\hline $87-61-6$ & 1,2,3-Trichlorobenzene & 0.50 & $\mathrm{U}$ \\
\hline
\end{tabular}


$1 \mathrm{~J}$ - FORM I VOA-TIC

VOLATILE ORGANICS ANALYSIS DATA SHEET

TENTATIVELY IDENTIEIED COMPOUNDS
EPA SAMPLE NO.

SVSB $47 S-W-25838$

Lab Name: TESTAMERICA BURLINGTON

Contract: $\quad 8 \mathrm{E}-00302$

Lab Code: STLV Case No.: SAVAN

Mod. Ref No.: SDG No.: $200-1286$

Matrix: (SOIL/SED/WATER) Water

Sample wt/vol: 25.0

$(\mathrm{g} / \mathrm{mL}) \mathrm{mL}$

Level: (TRACE or LOW/MED)

TRACE

\% Moisture: not dec.

GC Column: DB-624

ID: $0.20 \quad(\mathrm{~mm})$

Soil Extract Volume: (UL)

CONCENTRATION UNITS: (ug/L or $\mathrm{ug} / \mathrm{kg}) \mathrm{ug} / \mathrm{L}$

Lab Sample ID: 200-1286-5

Lab File ID: JBIR20.D

Date Received: 08/26/2010

Date Analyzed: 08/27/2010

Dilution Factor: 1.0

Soil Aliquot Volume:

(uL)

Purge Volume: 25.0 $(\mathrm{mL})$

\begin{tabular}{|l|l|l|r|r|r|}
\hline \multicolumn{1}{|c|}{ CAS NUMBER } & \multicolumn{1}{|c|}{ COMPOUND NAME } & RT & EST. CONC. & Q \\
\cline { 2 - 6 } 01 & & Unknown & 6.94 & $\mathrm{~J} \times \mathrm{B}$ \\
\cline { 2 - 6 } 02 & E9667961 & Total Alkanes & N/A & & \\
\cline { 2 - 6 }
\end{tabular}

1 EPA-designated Registry Number. 
$1 A$ - FORM I VOA-1

VOLATILE ORGANICS ANALYSIS DATA SHEET
EPA SAMPLE NO.

SVSB $48 \mathrm{D}-\mathrm{W}-25841$
Lab Name: TESTAMERICA BURLINGTON

Lab Code: STLV

Matrix: (SOIL/SED/WATER)

Sample wt/vol: 25.0

Level: (TRACE/LOW/MED)

응 Moisture: not dec.

GC Column: DB-624

Soil Extract Volume: ID: $0.20 \quad(\mathrm{~mm})$

Purge Volume: 25.0

Contract: $8 \mathrm{E}-00302$

Case No.: SAVAN Mod. Ref No.: SDG No.: 200-1286

Lab Sample ID: 200-1286-2

Lab File ID: JBIR17.D

Date Received: $\quad 08 / 26 / 2010$

Date Analyzed: 08/27/2010

Dilution Factor: 1.0

Soil Aliquot Volume:

(UI)

\begin{tabular}{|c|c|c|c|}
\hline CAS NO. & COMPOUND & $\begin{array}{l}\text { CONCENTRATION UNITS: } \\
\text { (ug/L or ug/kg) ug/L }\end{array}$ & c \\
\hline $75-71-8$ & Dichlorodifluoromethane & 0.50 & $\overline{\mathrm{U}}$ \\
\hline $74-87-3$ & Chloromethane & 0.50 & $\mathrm{U}$ \\
\hline $75-01-4$ & Vinyl chloride & 0.50 & $\mathrm{U}$ \\
\hline $74-83-9$ & Bromomethane & 0.50 & $\mathrm{U}$ \\
\hline $75-00-3$ & Chloroethane & 0.50 & $\mathrm{U}$ \\
\hline $75-69-4$ & Trichlorofluoromethane & 0.50 & $\mathrm{U}$ \\
\hline $75-35-4$ & 1,1-Dichloroethene & 0.50 & $\mathrm{U}$ \\
\hline $76-13-1$ & 1,1,2-Trichloro-1,2,2-trifluoroethane & 0.50 & $\mathrm{U}$ \\
\hline $67-64-1$ & Acetone & 3.7 & $\mathrm{~J} B$ \\
\hline $75-15-0$ & Carbon disulfide & 0.50 & $\mathrm{U}$ \\
\hline $79-20-9$ & Methyl acetate & 0.50 & $\mathrm{U}$ \\
\hline $75-09-2$ & Methylene Chloride & 0.50 & $\mathrm{U}$ \\
\hline $156-60-5$ & trans-1,2-Dichloroethene & 0.50 & $\mathrm{U}$ \\
\hline $1634-04-4$ & Methyl tert-butyl ether & 0.50 & $\mathrm{U}$ \\
\hline $75-34-3$ & 1,1-Dichloroethane & 0.50 & $\mathrm{U}$ \\
\hline $156-59-2$ & cis-1,2-Dichloroethene & 0.50 & $\mathrm{U}$ \\
\hline $78-93-3$ & 2-Butanone & 1.1 & $\mathrm{~J}$ \\
\hline $74-97-5$ & Bromochloromethane & 0.50 & $\mathrm{U}$ \\
\hline $67-66-3$ & Chloroform & 0.091 & $\mathrm{~J}$ \\
\hline $71-55-6$ & 1,1,1-Trichloroethane & 0.50 & $\mathrm{U}$ \\
\hline $110-82-7$ & Cyclohexane & 0.50 & $\mathrm{U}$ \\
\hline $56-23-5$ & Carbon tetrachloride & 0.50 & $\mathrm{U}$ \\
\hline $71-43-2$ & Benzene & 0.50 & $\mathrm{U}$ \\
\hline $107-06-2$ & 1,2-Dichloroethane & 0.50 & $\mathrm{U}$ \\
\hline
\end{tabular}

Report 1,4-Dioxane for Low-Medium VOA analysis only 
$1 B$ - FORM I VOA-2

VOLATILE ORGANICS ANALYSIS DATA SHEET
EPA SAMPLE NO.

SVSB $48 D-W-25841$
Lab Name: TESTAMERICA BURLINGTON

Lab Code: STLV

Matrix: (SOIL/SED/WATER)

Sample wt/vol: 25.0

Level: (TRACE/LOW/MED) Case No.: SAVAN

Water

$(\mathrm{g} / \mathrm{mL}) \mathrm{mL}$

\% Moisture: not dec.

GC Column: DB-624

ID: 0.20 (mm)

Soil Extract Volume: (uI)

Purge Volume: 25.0 (mL)
Contract: $8 \mathrm{E}-00302$

SDG No.: 200-1286

Lab Sample ID: 200-1286-2

Lab File ID: JBIR17.D

Date Received: 08/26/2010

Date Analyzed: 08/27/2010

Dilution Factor: 1.0

Soil Aliquot Volume:

(uL)

\begin{tabular}{|c|c|c|c|}
\hline CAS NO. & COMPOUND & $\begin{array}{l}\text { CONCENTRATION UNITS: } \\
(\mathrm{ug} / \mathrm{L} \text { or } \mathrm{ug} / \mathrm{kg}) \mathrm{ug} / \mathrm{L}\end{array}$ & $Q$ \\
\hline $79-01-6$ & Trichloroethene & 0.50 & $\mathrm{U}$ \\
\hline $108-87-2$ & Methylcyclohexane & 0.50 & $\mathrm{U}$ \\
\hline $78-87-5$ & 1,2-Dichloropropane & 0.50 & $\mathrm{U}$ \\
\hline $75-27-4$ & Bromodichloromethane & 0.50 & $\mathrm{U}$ \\
\hline $10061-01-5$ & cis-1,3-Dichloropropene & 0.50 & $\mathrm{U}$ \\
\hline $108-10-1$ & 4-Methyl-2-pentanone & 5.0 & $\mathrm{U}$ \\
\hline $108-88-3$ & Toluene & 0.056 & $\mathrm{~J}$ \\
\hline $10061-02-6$ & trans-1,3-Dichloropropene & 0.50 & $\mathrm{U}$ \\
\hline $79-00-5$ & 1,1,2-Trichloroethane & 0.50 & $\mathrm{U}$ \\
\hline $127-18-4$ & Tetrachloroethene & 0.50 & $\mathrm{U}$ \\
\hline $591-78-6$ & 2-Hexanone & 5.0 & $\mathrm{U}$ \\
\hline $124-48-1$ & Dibromochloromethane & 0.50 & $\mathrm{U}$ \\
\hline $106-93-4$ & 1,2-Dibromoethane & 0.50 & $\mathrm{U}$ \\
\hline $108-90-7$ & Chlorobenzene & 0.50 & $\mathrm{U}$ \\
\hline $100-41-4$ & Ethylbenzene & 0.50 & $\mathrm{U}$ \\
\hline $95-47-6$ & o-Xylene & 0.50 & $\mathrm{U}$ \\
\hline $179601-23-1$ & $\mathrm{~m}, \mathrm{p}$-Xylene & 0.50 & $\mathrm{U}$ \\
\hline $100-42-5$ & Styrene & 0.50 & $\mathrm{U}$ \\
\hline $75-25-2$ & Bromoform & 0.50 & $\mathrm{U}$ \\
\hline $98-82-8$ & Isopropylbenzene & 0.50 & $\mathrm{U}$ \\
\hline $79-34-5$ & $1,1,2,2$-Tetrachloroethane & 0.50 & $\mathrm{U}$ \\
\hline $541-73-1$ & 1,3-Dichlorobenzene & 0.50 & $\mathrm{U}$ \\
\hline $106-46-7$ & 1,4-Dichlorobenzene & 0.50 & $\mathrm{U}$ \\
\hline $95-50-1$ & 1,2-Dichlorobenzene & 0.50 & $\mathrm{U}$ \\
\hline $96-12-8$ & 1,2-Dibromo-3-Chloropropane & 0.50 & $\mathrm{U}$ \\
\hline $120-82-1$ & 1,2,4-Trichlorobenzene & 0.50 & $\mathrm{U}$ \\
\hline $87-61-6$ & 1,2,3-Trichlorobenzene & 0.50 & $\mathrm{U}$ \\
\hline
\end{tabular}


IJ - FORM I VOA-TIC

VOLATILE ORGANICS ANALYSIS DATA SHEET

TENTATIVELY IDENTIFIED COMPOUNDS
EPA SAMPLE NO.

SVSB 48D-W-25841
Lab Name: TESTAMERICA BURIINGTON Case No.: SAVAN Mod. Ref No.:

Lab Code: STLV

Matrix: (SOIL/SED/WATER)

Water

Sample wt/vol: 25.0 $(\mathrm{g} / \mathrm{mL}) \quad \mathrm{mL}$

Level: (TRACE or LOW/MED)

TRACE

哈 Moisture: not dec.

GC Column: DB-624 ID: 0.20 (mm)

Soil Extract Volume: (uL)

CONCENTRATION UNITS: (ug/L or $\mathrm{ug} / \mathrm{kg}$ )

$\mathrm{ug} / \mathrm{L}$
Contract: $8 \mathrm{E}-00302$ SDG No.: 200-1286

Lab Sample ID: 200-1286-2

Lab File ID: JBIR17.D

Date Received: 08/26/2010

Date Analyzed: 08/27/2010

Dilution Factor: 1.0

Soil Aliquot Volume: (UL)

Purge Volume: 25.0 (mL)

01

\begin{tabular}{|l|l|c|c|c|}
\hline CAS NUMBER & \multicolumn{1}{|c|}{ COMPOUND NAME } & RT & EST. CONC. & Q \\
\hline & Unknown & 6.94 & & 2.7 \\
\hline E9667961 & Total Alkanes & N/A & & \\
\hline
\end{tabular}

1 EPA-designated Registry Number. 
$1 A$ - FORM I VOA-1

VOLATILE ORGANICS ANALYSIS DATA SHEET
EPA SAMPLE NO.

SVSB49D-W-25843
Lab Name: TESTAMERICA BURLINGTON

Lab Code: STLV

Matrix: (SOIL/SED/WATER)

Sample wt/vol: 25.0

Case No.: SAVAN

\section{R)}

Water

$(\mathrm{g} / \mathrm{mL}) \quad \mathrm{mL}$

Level: (TRACE/LOW/MED) TRACE

\% Moisture: not dec.

GC Column:

$$
\mathrm{DB}-624
$$
ID: $0.20 \quad(\mathrm{~mm})$

Soil Extract Volume:

Purge Volume: 25.0
Contract: $8 \mathrm{E}-00302$ SDG No,: 200-1286

\begin{tabular}{|c|c|c|c|}
\hline CAS NO. & COMPOUND & $\begin{array}{l}\text { CONCENTRATION UNITS: } \\
(\mathrm{ug} / \mathrm{L} \text { or } \mathrm{ug} / \mathrm{kg}) \mathrm{ug} / \mathrm{L}\end{array}$ & $Q$ \\
\hline $75-71-8$ & Dichlorodifluoromethane & 0.50 & $\mathrm{U}$ \\
\hline $74-87-3$ & Chloromethane & 0.50 & $\mathrm{U}$ \\
\hline $75-01-4$ & Vinyl chloride & 0.50 & $\mathrm{U}$ \\
\hline $74-83-9$ & Bromomethane & 0.50 & $\mathrm{U}$ \\
\hline $75-00-3$ & Chloroethane & 0.50 & $\mathrm{U}$ \\
\hline $75-69-4$ & Trichlorofluoromethane & 0.50 & $\mathrm{U}$ \\
\hline $75-35-4$ & 1,1-Dichloroethene & 0.50 & $\mathrm{U}$ \\
\hline $76-13-1$ & 1,1,2-Trichloro-1,2,2-trifluoroethane & 0.50 & $\mathrm{U}$ \\
\hline $67-64-1$ & Acetone & 7.0 & $\mathrm{~B}$ \\
\hline $75-15-0$ & Carbon disulfide & 0.21 & $\mathrm{~J}$ \\
\hline $79-20-9$ & Methyl acetate & 0.50 & $\mathrm{U}$ \\
\hline $75-09-2$ & Methylene Chloride & 0.13 & $\mathrm{~J}$ \\
\hline $156-60-5$ & trans-1,2-Dichloroethene & 0.50 & $\mathrm{U}$ \\
\hline $1634-04-4$ & Methyl tert-butyl ether & 0.50 & $\mathrm{U}$ \\
\hline $75-34-3$ & 1,1-Dichloroethane & 0.50 & $\mathrm{U}$ \\
\hline $156-59-2$ & cis-1,2-Dichloroethene & 0.50 & $\mathrm{U}$ \\
\hline $78-93-3$ & 2-Butanone & 1.1 & $\mathrm{~J}$ \\
\hline $74-97-5$ & Bromochloromethane & 0.50 & $\mathrm{U}$ \\
\hline $67-66-3$ & Chloroform & 2.9 & \\
\hline $71-55-6$ & 1,1,1-Trichloroethane & 0.50 & $\mathrm{U}$ \\
\hline $110-82-7$ & Cyclohexane & 0.50 & $\mathrm{U}$ \\
\hline $56-23-5$ & Carbon tetrachloride & 0.50 & $\mathrm{U}$ \\
\hline $71-43-2$ & Benzene & 0.059 & $\mathrm{~J}$ \\
\hline $107-06-2$ & 1,2-Dichloroethane & 0.50 & $\mathrm{U}$ \\
\hline
\end{tabular}

Report 1,4-Dioxane for Low-Medium VOA analysis only 
$1 B$ - FORM I VOA-2

VOLATILE ORGANICS ANALYSIS DATA SHEET
EPA SAMPLE NO.

SVSB49D-W-25843

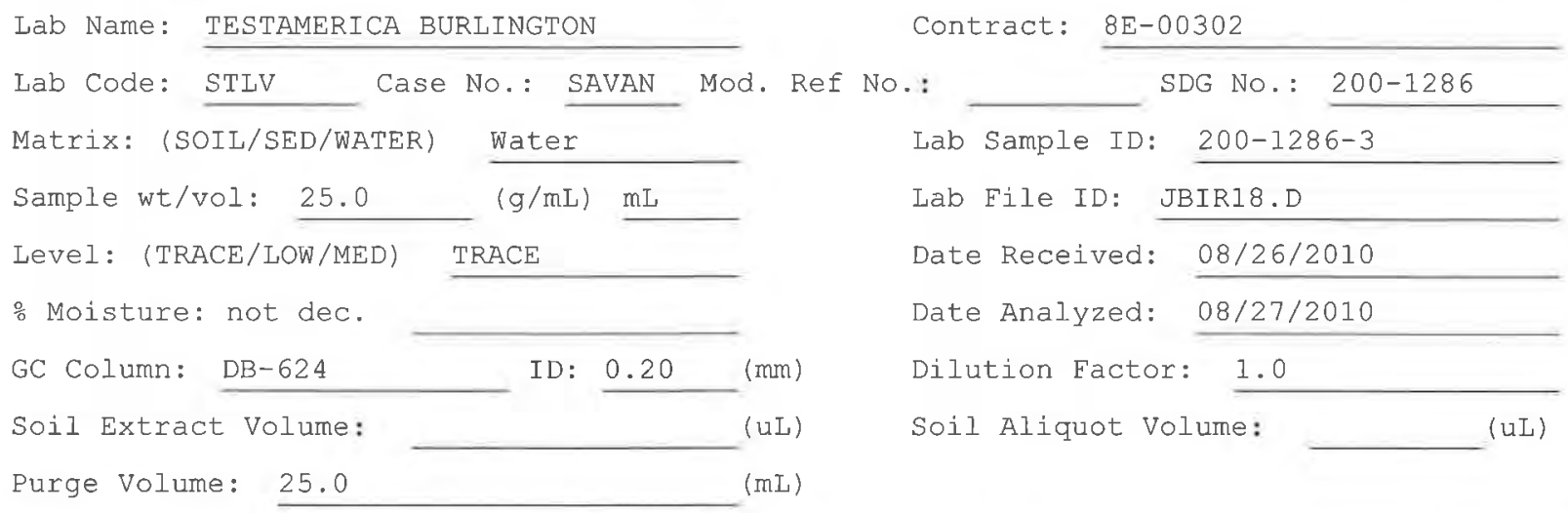

\begin{tabular}{|c|c|c|c|}
\hline CAS NO. & COMPOUND & $\begin{array}{l}\text { CONCENTRATION UNITS: } \\
(\mathrm{ug} / \mathrm{L} \text { or } \mathrm{ug} / \mathrm{kg}) \mathrm{ug} / \mathrm{L}\end{array}$ & 8 \\
\hline $79-01-6$ & Trichloroethene & 0.50 & $\mathrm{U}$ \\
\hline $108-87-2$ & Methylcyclohexane & 0.50 & $\mathrm{U}$ \\
\hline $78-87-5$ & 1,2-Dichloropropane & 0.50 & $\mathrm{U}$ \\
\hline $75-27-4$ & Bromodichloromethane & 0.50 & $\mathrm{U}$ \\
\hline $10061-01-5$ & cis-1,3-Dichloropropene & 0.50 & $\mathrm{U}$ \\
\hline $108-10-1$ & 4-Methyl-2-pentanone & 5.0 & $U$ \\
\hline $108-88-3$ & Toluene & 0.33 & $\mathrm{~J}$ \\
\hline $10061-02-6$ & trans-1,3-Dichloropropene & 0.50 & $\mathrm{U}$ \\
\hline $79-00-5$ & 1,1,2-Trichloroethane & 0.50 & $\mathrm{U}$ \\
\hline $127-18-4$ & Tetrachloroethene & 0.50 & $\mathrm{U}$ \\
\hline $591-78-6$ & 2-Hexanone & 5.0 & $\mathrm{U}$ \\
\hline $124-48-1$ & Dibromochloromethane & 0.50 & $\mathrm{U}$ \\
\hline $106-93-4$ & 1,2-Dibromoethane & 0.50 & $\mathrm{U}$ \\
\hline $108-90-7$ & Chlorobenzene & 0.50 & $\mathrm{U}$ \\
\hline $100-41-4$ & Ethylbenzene & 0.50 & $\mathrm{U}$ \\
\hline $95-47-6$ & o-Xylene & 0.50 & $\mathrm{U}$ \\
\hline $179601-23-1$ & $\mathrm{~m}, \mathrm{p}-\mathrm{Xy}$ lene & 0.084 & $\mathrm{~J}$ \\
\hline $100-42-5$ & Styrene & 0.50 & $\mathrm{U}$ \\
\hline $75-25-2$ & Bromoform & 0.50 & $\mathrm{U}$ \\
\hline $98-82-8$ & Isopropylbenzene & 0.50 & $\mathrm{U}$ \\
\hline $79-34-5$ & $1,1,2,2$-Tetrachloroethane & 0.50 & $\mathrm{U}$ \\
\hline $541-73-1$ & 1,3-Dichlorobenzene & 0.50 & $\mathrm{U}$ \\
\hline $106-46-7$ & 1,4-Dichlorobenzene & 0.50 & $\mathrm{U}$ \\
\hline $95-50-1$ & 1,2-Dichlorobenzene & 0.50 & $\mathrm{U}$ \\
\hline $96-12-8$ & 1,2-Dibromo-3-Chloropropane & 0.50 & $\mathrm{U}$ \\
\hline $120-82-1$ & 1,2,4-Trichlorobenzene & 0.50 & $\mathrm{U}$ \\
\hline $87-61-6$ & 1,2,3-Trichlorobenzene & 0.50 & $\mathrm{U}$ \\
\hline
\end{tabular}


IJ - FORM I VOA-TIC

EPA SAMPLE NO.

VOLATILE ORGANICS ANALYSIS DATA SHEET

TENTATIVELY IDENTIEIED COMPOUNDS

SVSB4 9D-W-25843

Lab Name: TESTAMERICA BURLINGTON

Contract: $8 E-00302$

Lab Code: STLV Case No.: SAVAN Mod. Ref No.:

SDG No.: 200-1286

Matrix: (SOIL/SED/WATER) Water

Sample wt/vol: 25.0

$(\mathrm{g} / \mathrm{mL}) \quad \mathrm{mL}$

Lab Sample ID: 200-1286-3

Level: (TRACE or LOW/MED)

TRACE

Lab File ID: JBIR18.D

응 Moisture: not dec.

GC Column: DB-624

ID: 0.20 (mm)

Soil Extract Volume:

(uL)

Date Received: 08/26/2010

Date Analyzed: 08/27/2010

CONCENTRATION UNITS: (ug/L or $\mathrm{ug} / \mathrm{kg}) \mathrm{ug} / \mathrm{L}$

Dilution Factor: 1.0

Soil Aliquot Volume:

(uL)

Purge Volume: 25.0

(mL)

\begin{tabular}{|c|c|c|c|c|}
\hline CAS NUMBER & COMPOUND NAME & $\mathrm{RT}$ & EST. CONC. & Q \\
\hline $420-12-2$ & Thiirane & 3.58 & 0.62 & $\mathrm{JN}$ \\
\hline 02 & Unknown & 6.94 & 2.7 & $J X B$ \\
\hline $123-05-7$ & Hexanal, 2-ethyl- & 10.91 & 2.9 & $\mathrm{~J} \mathrm{~N}$ \\
\hline $104-76-7$ & 1-Hexanol, 2-ethyl- & 12.14 & 15 & \\
\hline E9667961 & Total Alkanes & $\mathrm{N} / \mathrm{A}$ & 16 & $\mathrm{~J}$ \\
\hline
\end{tabular}

1 EPA-designated Registry Number. 
$1 A$ - FORM I VOA-1

VOLATILE ORGANICS ANALYSIS DATA SHEET
EPA SAMPLE NO.

VHBLKO1
Lab Name: TESTAMERICA BURLINGTON

Lab Code: STLV

Matrix: (SOIL/SED/WATER)

Sample wt/vol: 25.0

Level: (TRACE/LOW/MED)

\% Moisture: not dec.

GC Column: DB-624

Soil Extract Volume: ID $: 0.20 \quad(\mathrm{~mm})$

Purge Volume: 25.0 ( $\mathrm{mL})$

Contract: $8 \mathrm{E}-00302$ Case No.: SAVAN Mod. Ref No.: SDG NO.: 200-1286

Lab Sample ID: 200-1286-7

Lab File ID: JBIR24.D

Date Received: $08 / 26 / 2010$

Date Analyzed: 08/27/2010

Dilution Factor: 1.0

Soil Aliquot Volume: (uL)

\begin{tabular}{|c|c|c|c|}
\hline CAS NO. & COMPOUND & $\begin{array}{l}\text { CONCENTRATION UNITS: } \\
(\mathrm{ug} / \mathrm{L} \text { or } \mathrm{ug} / \mathrm{kg}) \mathrm{ug} / \mathrm{L}\end{array}$ & $Q$ \\
\hline $75-71-8$ & Dichlorodifluoromethane & 0.50 & $\mathrm{U}$ \\
\hline $74-87-3$ & Chloromethane & 0.50 & $\mathrm{U}$ \\
\hline $75-01-4$ & Vinyl chloride & 0.50 & $\mathrm{U}$ \\
\hline $74-83-9$ & Bromomethane & 0.50 & $\mathrm{U}$ \\
\hline $75-00-3$ & Chloroethane & 0.50 & $\mathrm{U}$ \\
\hline $75-69-4$ & Trichlorofluoromethane & 0.50 & $\mathrm{U}$ \\
\hline $75-35-4$ & 1,1-Dichloroethene & 0.50 & $\mathrm{U}$ \\
\hline $76-13-1$ & 1,1,2-Trichloro-1,2,2-trifluoroethane & 0.50 & $\mathrm{U}$ \\
\hline $67-64-1$ & Acetone & 2.2 & $\mathrm{~J} \mathrm{~B}$ \\
\hline $75-15-0$ & Carbon disulfide & 0.063 & $\mathrm{~J}$ \\
\hline $79-20-9$ & Methyl acetate & 0.50 & $\mathrm{U}$ \\
\hline $75-09-2$ & Methylene Chloride & 0.50 & $\mathrm{U}$ \\
\hline $156-60-5$ & trans-1,2-Dichloroethene & 0.50 & $\mathrm{U}$ \\
\hline $1634-04-4$ & Methyl tert-butyl ether & 0.50 & $\mathrm{U}$ \\
\hline $75-34-3$ & 1,1-Dichloroethane & 0.50 & $\mathrm{U}$ \\
\hline $156-59-2$ & cis-1,2-Dichloroethene & 0.50 & $\mathrm{U}$ \\
\hline $78-93-3$ & 2-Butanone & 5.0 & $\mathrm{U}$ \\
\hline $74-97-5$ & Bromochloromethane & 0.50 & $\mathrm{U}$ \\
\hline $67-66-3$ & Chloroform & 0.50 & $\mathrm{U}$ \\
\hline $71-55-6$ & 1,1,1-Trichloroethane & 0.50 & $\mathrm{U}$ \\
\hline $110-82-7$ & Cyclohexane & 0.50 & $\mathrm{U}$ \\
\hline $56-23-5$ & Carbon tetrachloride & 0.50 & $\mathrm{U}$ \\
\hline $71-43-2$ & Benzene & 0.040 & $\mathrm{~J}$ \\
\hline $107-06-2$ & 1,2-Dichloroethane & 0.50 & $\mathrm{U}$ \\
\hline
\end{tabular}

Report 1,4-Dioxane for Low-Medium VOA analysis only 
1B - FORM I VOA-2

VOLATILE ORGANICS ANALYSIS DATA SHEET
EPA SAMPLE NO.

VHBLK01
Lab Name: TESTAMERICA BURLINGTON

Lab Code: STLV

Matrix: (SOIL/SED/WATER)

Sample wt/vol: 25.0

Level: (TRACE/LOW/MED)

\% Moisture: not dec.

GC Column: DB-624

Soil Extract volume: Case No.: SAVAN Mod. Ref No.:

Contract: $8 \mathrm{E}-00302$

Purge volume: 25.0
Water

$(\mathrm{g} / \mathrm{mL}) \quad \mathrm{mL}$

TRACE

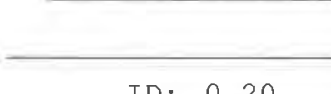
(mm) (uL) $(m L)$
SDG No.: 200-1286

Lab Sample ID: 200-1286-7

Lab File ID: JBIR24.D

Date Received: 08/26/2010

Date Analyzed: 08/27/2010

Dilution Factor: 1.0

Soil Aliquot Volume: (uL)

\begin{tabular}{|c|c|c|c|}
\hline CAS NO. & COMPOUND & $\begin{array}{l}\text { CONCENTRATION UNITS: } \\
(\mathrm{ug} / \mathrm{L} \text { or } \mathrm{ug} / \mathrm{kg}) \mathrm{ug} / \mathrm{L}\end{array}$ & 8 \\
\hline $79-01-6$ & Trichloroethene & 0.50 & $\mathrm{U}$ \\
\hline $108-87-2$ & Methylcyclohexane & 0.50 & $\mathrm{U}$ \\
\hline $78-87-5$ & 1,2-Dichloropropane & 0.50 & $\mathrm{U}$ \\
\hline $75-27-4$ & Bromodichloromethane & 0.50 & $\mathrm{U}$ \\
\hline $10061-01-5$ & cis-1,3-Dichloropropene & 0.50 & $\mathrm{U}$ \\
\hline $108-10-1$ & 4-Methyl-2-pentanone & 5.0 & $\mathrm{U}$ \\
\hline $108-88-3$ & Toluene & 0.50 & $\mathrm{U}$ \\
\hline $10061-02-6$ & trans-1,3-Dichloropropene & 0.50 & $\mathrm{U}$ \\
\hline $79-00-5$ & 1, 1,2-Trichloroethane & 0.50 & $\mathrm{U}$ \\
\hline $127-18-4$ & Tetrachloroethene & 0.50 & $\mathrm{U}$ \\
\hline $591-78-6$ & 2-Hexanone & 5.0 & $\mathrm{U}$ \\
\hline $124-48-1$ & Dibromochloromethane & 0.50 & $\mathrm{U}$ \\
\hline $106-93-4$ & 1,2-Dibromoethane & 0.50 & $\mathrm{U}$ \\
\hline $108-90-7$ & Chlorobenzene & 0.50 & $\mathrm{U}$ \\
\hline $100-41-4$ & Ethylbenzene & 0.50 & $\mathrm{U}$ \\
\hline $95-47-6$ & o-Xylene & 0.50 & $\mathrm{U}$ \\
\hline $179601-23-1$ & $\mathrm{~m}, \mathrm{p}$-xylene & 0.50 & $\mathrm{U}$ \\
\hline $100-42-5$ & Styrene & 0.50 & $\mathrm{U}$ \\
\hline $75-25-2$ & Bromoform & 0.50 & $\mathrm{U}$ \\
\hline $98-82-8$ & Isopropylbenzene & 0.50 & $\mathrm{U}$ \\
\hline $79-34-5$ & $1,1,2,2$-Tetrachloroethane & 0.50 & $\mathrm{U}$ \\
\hline $541-73-1$ & 1,3-Dichlorobenzene & 0.50 & $\mathrm{U}$ \\
\hline $106-46-7$ & 1,4-Dichlorobenzene & 0.50 & $\mathrm{U}$ \\
\hline $95-50-1$ & 1,2-Dichlorobenzene & 0.50 & $\mathrm{U}$ \\
\hline $96-12-8$ & 1,2-Dibromo-3-Chloropropane & 0.50 & $\mathrm{U}$ \\
\hline $120-82-1$ & 1,2,4-Trichlorobenzene & 0.50 & $\mathrm{U}$ \\
\hline $87-61-6$ & 1,2,3-Trichlorobenzene & 0.50 & $\mathrm{U}$ \\
\hline
\end{tabular}


IJ - FORM I VOA-TIC

VOLATILE ORGANICS ANALYSIS DATA SHEET

TENTATIVELY IDENTIEIED COMPOUNDS
EPA SAMPLE NO.

VHBLKOI
Lab Name: TESTAMERICA BURLINGTON Case No.: SAVAN

Lab Code: STLV Matrix: (SOIL/SED/WATER)

Sample wt/vol: 25.0

Water

25.00.

$(\mathrm{g} / \mathrm{mL}) \quad \mathrm{mL}$

Level: (TRACE or LOW/MED)

TRACE

응 Moisture: not dec.

GC Column: DB-624

ID: $0.20 \quad(\mathrm{~mm})$

Soil Extract Volume:

CONCENTRATION UNITS: (ug/L or $u g / \mathrm{kg}$ )
Contract: $8 E-00302$ SDG No.: 200-1286

Lab Sample ID: 200-1286-7

Lab File ID: JBIR24.D

Date Received: 08/26/2010

Date Analyzed: 08/27/2010

Dilution Factor: 1.0

Soil Aliquot Volume: (uL)

Purge Volume: 25.0 (mL)

01

02

\begin{tabular}{|c|c|c|c|c|}
\hline CAS NUMBER & COMPOUND NAME & $\mathrm{RT}$ & EST. CONC. & Q \\
\hline & Unknown & 6.94 & 2.7 & $\mathrm{JXB}$ \\
\hline E9667961 & Total Alkanes & $\mathrm{N} / \mathrm{A}$ & & \\
\hline
\end{tabular}

1 EPA-designated Registry Number. 
$6 A$ - FORM VI VOA-1

VOLATILE ORGANICS INITIAL CALIBRATION DATA

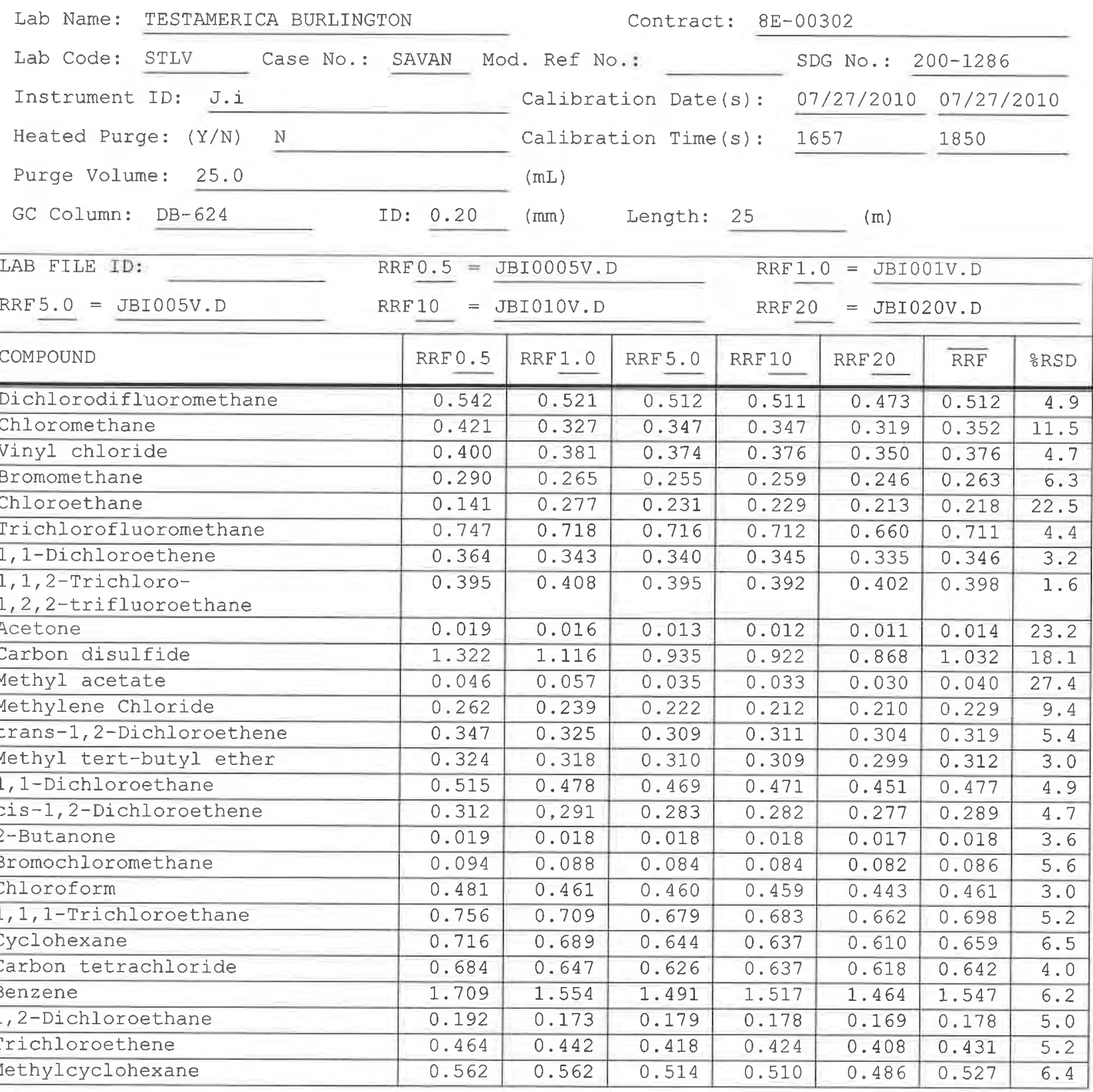

Report 1,4-Dioxane for Low-Medium VoA analysis only 
$6 \mathrm{~B}$ - FORM VI VOA-2

VOLATILE ORGANICS INITIAL CALIBRATION DATA

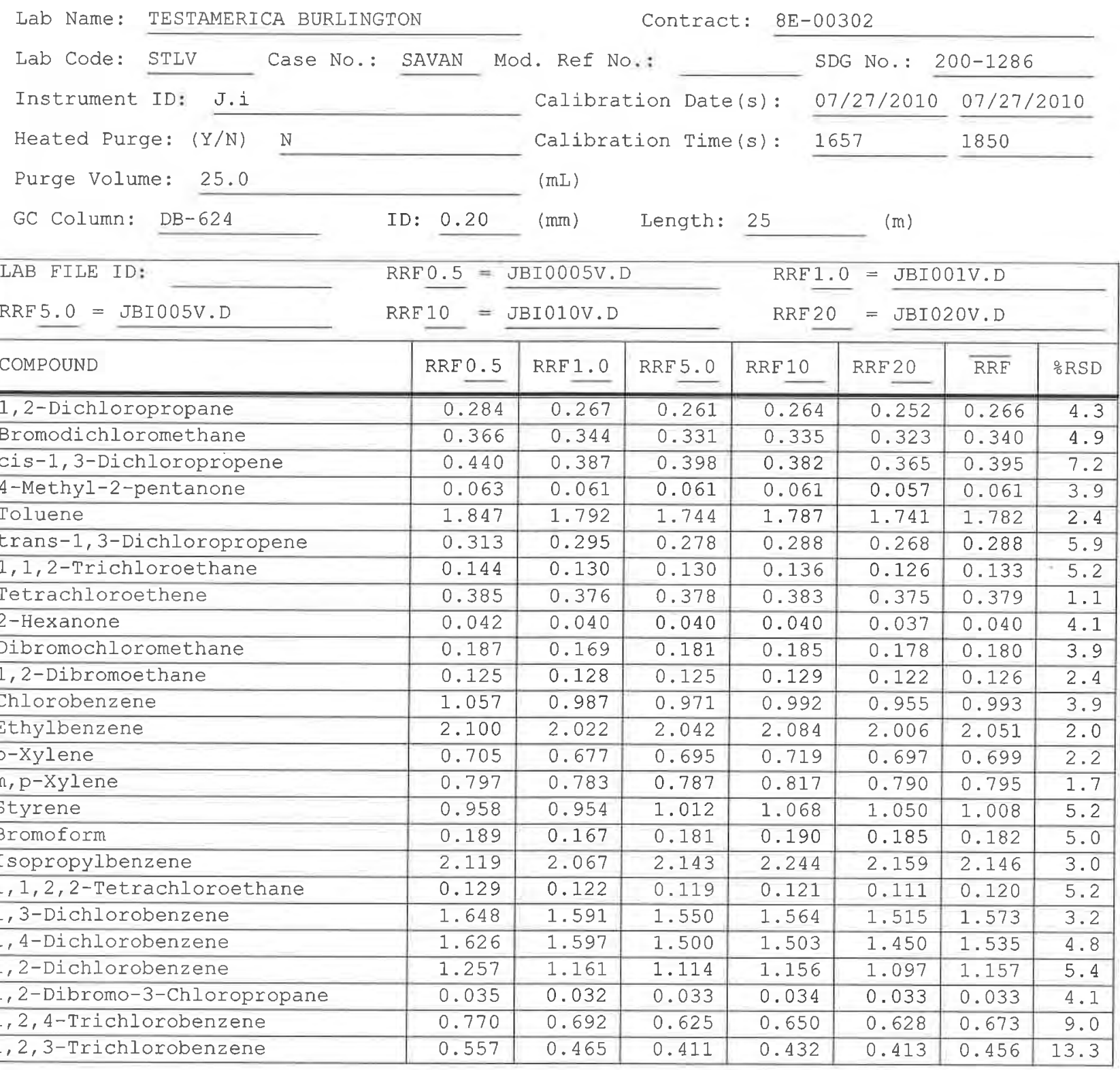


$6 \mathrm{C}$ - FORM VI VOA-3

VOLATILE ORGANICS INITIAL CALIBRATION DATA

Lab Name: TESTAMERICA BURLINGTON

Contract: $8 \mathrm{E}-00302$

Lab Code: STLV Case No.: SAVAN Mod. Ref No.:

SDG No.: 200-1286

Instrument ID: J.i

Calibration Date(s): 07/27/2010 07/27/2010

Heated Purge: ( $\mathrm{Y} / \mathrm{N}) \quad \mathrm{N}$

Calibration Time (s):

1657

1850

Purge Volume: 25.0

$(\mathrm{mL})$

GC Column: DB-624

ID: 0.20

(mm) Length: 25

(m)

\begin{tabular}{|c|c|c|c|c|c|c|c|}
\hline \multirow{2}{*}{$\begin{array}{l}\text { LAB FILE ID: } \\
\text { RRF } 5.0=\text { JBI005V.D }\end{array}$} & \multicolumn{3}{|c|}{ RRF $0.5=J B I 0005 \mathrm{~V} . \mathrm{D}$} & \multicolumn{4}{|c|}{$\mathrm{RRF} 1.0=$ JBI001V.D } \\
\hline & RRE 10 & $31010 \mathrm{~V} . \mathrm{D}$ & & RRF 20 & $=\mathrm{JBIO}$ & OV.D & \\
\hline COMPOUND & $\mathrm{RRE} 0.5$ & RRF 1.0 & RRF 5.0 & RRF 10 & RRF 20 & $\overline{\mathrm{RRF}}$ & $\because \mathrm{RSD}$ \\
\hline Vinyl Chloride-d3 & 0.368 & 0.351 & 0.340 & 0.338 & 0.315 & 0.342 & 5.7 \\
\hline Chloroethane-d5 & 0.331 & 0.322 & 0.291 & 0.296 & 0.274 & 0.303 & 7.7 \\
\hline 1,1-Dichloroethene-d2 & 0.708 & 0.677 & 0.667 & 0.668 & 0.644 & 0.673 & 3.4 \\
\hline 2-Butanone-d5 & 0.018 & 0.018 & 0.018 & 0.017 & 0.017 & 0.018 & 2.3 \\
\hline Chloroform-d & 0.528 & 0.498 & 0.494 & 0.496 & 0.484 & 0.500 & 3.3 \\
\hline 1,2-Dichloroethane-d4 & 0.162 & 0.155 & 0.148 & 0.146 & 0.140 & 0.150 & 5.6 \\
\hline Benzene-d 6 & 1.562 & 1.470 & 1.418 & 1.443 & 1.408 & 1.460 & 4.2 \\
\hline 1,2-Dichloropropane-d6 & 0.385 & 0.322 & 0.314 & 0.316 & 0.298 & 0.327 & 10.3 \\
\hline Toluene-d8 & 1.505 & 1.463 & 1.453 & 1.501 & 1.461 & 1.477 & 1.7 \\
\hline trans-1,3-Dichloropropene-d4 & 0.281 & 0.262 & 0.264 & 0.266 & 0.253 & 0.265 & 3.9 \\
\hline 2-Hexanone-d5 & 0.021 & 0.020 & 0.022 & 0.023 & 0.021 & 0.021 & 4.7 \\
\hline $1,1,2,2$-Tetrachloroethane-d2 & 0.118 & 0.123 & 0.122 & 0.121 & 0.114 & 0.120 & 3.1 \\
\hline 1,2-Dichlorobenzene-d4 & 0.765 & 0.742 & 0.703 & 0.725 & 0.695 & 0.726 & 3.9 \\
\hline
\end{tabular}

Report 1,4-Dioxane-d8 for Low-Medium VOA analysis only 
$7 A$ - FORM VII VOA-1

VOLATILE CONTINUING CALIBRATION DATA

Lab Name: $\frac{\text { TESTAMERICA BURLINGTON }}{\text { Lab Code: } \frac{\text { STLV Case No.: SAVAN Mod }}{\text { Instrument ID: J.i }}}$
Lab File Id: JBIR03.D

EPA Sample No. (VSTD\#\#\#\#): VSTD005JD

Heated Purge: (Y/N)

Purge Volume: 25.0
Contract: $8 \mathrm{E}-00302$

SDG No.: 200-1286

Calibration Date: 08/27/2010 Time: 0849 Init. Calib. Date(s): 07/27/2010 07/27/2010

Init. Calib. Time(s): $1657 \quad 1850$ GC Column: DB-624 ID: 0.20 (mm) Length: 25 (m)

$(\mathrm{mL})$

\begin{tabular}{|c|c|c|c|c|c|}
\hline COMPOUND & $\overline{R R F}$ & RRE5.0 & $\begin{array}{l}\text { MIN } \\
\text { RRE }\end{array}$ & $\div \mathrm{D}$ & $\operatorname{MAX} \div \mathrm{D}$ \\
\hline Dichlorodifluoromethane & 0.512 & 0.455 & 0.010 & -11.0 & 40.0 \\
\hline Chloromethane & 0.352 & 0.358 & 0.010 & 1.7 & 40.0 \\
\hline Vinyl chloride & 0.376 & 0.359 & 0.010 & -4.6 & 30.0 \\
\hline Bromomethane & 0.263 & 0.215 & 0.100 & -18.1 & 30.0 \\
\hline Chloroethane & 0.218 & 0.233 & 0.010 & 6.8 & 40.0 \\
\hline Trichlorofluoromethane & 0.711 & 0.619 & 0.010 & -12.9 & 40.0 \\
\hline 1,1-Dichloroethene & 0.346 & 0.291 & 0.100 & -15.8 & 30.0 \\
\hline 1,1,2-Trichloro-1,2,2-trifluoroethane & 0.398 & 0.330 & 0.010 & -17.2 & 40.0 \\
\hline Acetone & 0.014 & 0.013 & 0.010 & $-11 \cdot 3$ & 40.0 \\
\hline Carbon disulfide & 1.032 & 0.824 & 0.010 & -20.2 & 40.0 \\
\hline Methyl acetate & 0.040 & 0.035 & 0.010 & $-12 \cdot 6$ & 40.0 \\
\hline Methylene Chloride & 0.229 & 0.209 & 0.010 & -8.7 & 40.0 \\
\hline trans-1,2-Dichloroethene & 0.319 & 0.289 & 0.010 & -9.4 & 40.0 \\
\hline Methyl tert-butyl ether & 0.312 & 0.303 & 0.010 & -3.0 & 40.0 \\
\hline 1,1-Dichloroethane & 0.477 & 0.477 & 0.200 & 0.0 & 30.0 \\
\hline cis-1,2-Dichloroethene & 0.289 & 0.276 & 0.010 & -4.5 & 40.0 \\
\hline 2-Butanone & 0.018 & 0.019 & 0.010 & 1.9 & 40.0 \\
\hline Bromochloromethane & 0.086 & 0.077 & 0.050 & -11.4 & 30.0 \\
\hline Chloroform & 0.461 & 0.453 & 0.200 & -1.6 & 30.0 \\
\hline 1,1,1-Trichloroethane & 0.698 & 0.663 & 0.100 & -5.0 & 30.0 \\
\hline Cyclohexane & 0.659 & 0.649 & 0.010 & -1.5 & 40.0 \\
\hline Carbon tetrachloride & 0.642 & 0.609 & 0.100 & -5.2 & 30.0 \\
\hline Benzene & 1.547 & 1.475 & 0.400 & -4.7 & 30.0 \\
\hline 1,2-Dichloroethane & 0.178 & 0.187 & 0.100 & 5.0 & 30.0 \\
\hline Trichloroethene & 0.431 & 0.404 & 0.300 & -6.2 & 30.0 \\
\hline Methylcyclohexane & 0.527 & 0.535 & 0.010 & 1.6 & 40.0 \\
\hline
\end{tabular}

Report 1,4-Dioxane for Low/Medium VOA analysis only 
$7 B$ - FORM VII VOA-2

VOLATILE CONTINUING CALIBRATION DATA

Lab Name: TESTAMERICA BURLINGTON

Contract: $8 \mathrm{E}-00302$

Lab Code: STLV Case No.: SAVAN Mod. Ref No. SDG No.: 200-1286

Instrument ID: J.i

Lab File Id: JBIR03.D

Calibration Date: 08/27/2010 Time: 0849

I
Init. Calib. Date(s): 07/27/2010 07/27/2010

EPA Sample No.(VSTD\#\#\#\#): VSTD005JD

Init. Calib. Time(s): $1657 \quad 1850$

Heated Purge: ( $Y / N) \quad N$

GC Column: DB-624

ID: $0.20(\mathrm{~mm})$ Length: 25 (m)

Purge Volume: 25.0

(mL)

\begin{tabular}{|c|c|c|c|c|c|}
\hline COMPOUND & $\overline{\mathrm{RRF}}$ & RRF5. 0 & $\begin{array}{l}\text { MIN } \\
\text { RRF }\end{array}$ & $\because \mathrm{D}$ & MAX $\because D$ \\
\hline 1,2-Dichloropropane & 0.266 & 0.279 & 0.010 & 4.9 & 40.0 \\
\hline Bromodichloromethane & 0.340 & 0.333 & 0.200 & -2.1 & 30.0 \\
\hline cis-1,3-Dichloropropene & 0.395 & 0.393 & 0.200 & -0.3 & 30.0 \\
\hline 4-Methyl-2-pentanone & 0.061 & 0.065 & 0.010 & 6.1 & 40.0 \\
\hline Toluene & 1.782 & 1.614 & 0.400 & -9.4 & 30.0 \\
\hline trans-1,3-Dichloropropene & 0.288 & 0.282 & 0.100 & -2.3 & 30.0 \\
\hline 1,1,2-Trichloroethane & 0.133 & 0.127 & 0.100 & -4.9 & 30.0 \\
\hline Tetrachloroethene & 0.379 & 0.369 & 0.100 & -2.8 & 30.0 \\
\hline 2-Hexanone & 0.040 & 0.041 & 0.010 & 3.6 & 40.0 \\
\hline Dibromochloromethane & 0.180 & 0.172 & 0.100 & -4.5 & 30.0 \\
\hline 1,2-Dibromoethane & 0.126 & 0.118 & 0.010 & -5.8 & 40.0 \\
\hline Chlorobenzene & 0.993 & 0.936 & 0.500 & -5.7 & 30.0 \\
\hline Ethylbenzene & 2.051 & 1.924 & 0.100 & -6.2 & 30.0 \\
\hline o-Xylene & 0.699 & 0.648 & 0.300 & -7.3 & 30.0 \\
\hline $\mathrm{m}, \mathrm{p}$-Xylene & 0.795 & 0.749 & 0.300 & -5.8 & 30.0 \\
\hline Styrene & 1.008 & 0.934 & 0.300 & -7.4 & 30.0 \\
\hline Bromoform & 0.182 & 0.165 & 0.050 & -9.3 & 30.0 \\
\hline Isopropylbenzene & 2.146 & 1.976 & 0.010 & -7.9 & 40.0 \\
\hline $1,1,2,2$-Tetrachloroethane & 0.120 & 0.117 & 0.100 & -2.5 & 30.0 \\
\hline 1,3-Dichlorobenzene & 1.573 & 1.485 & 0.400 & -5.6 & 30.0 \\
\hline 1,4-Dichlorobenzene & 1.535 & 1.417 & 0.400 & -7.7 & 30.0 \\
\hline 1,2-Dichlorobenzene & 1.157 & 1.081 & 0.400 & -6.6 & 30.0 \\
\hline 1,2-Dibromo-3-Chloropropane & 0.033 & 0.033 & 0.010 & -2.5 & 40.0 \\
\hline 1,2,4-Trichlorobenzene & 0.673 & 0.651 & 0.200 & -3.3 & 30.0 \\
\hline 1,2,3-Trichlorobenzene & 0.456 & 0.426 & 0.200 & -6.4 & 30.0 \\
\hline
\end{tabular}


$7 C$ - FORM VII VOA-3

VOLATILE CONTINUING CALIBRATION DATA

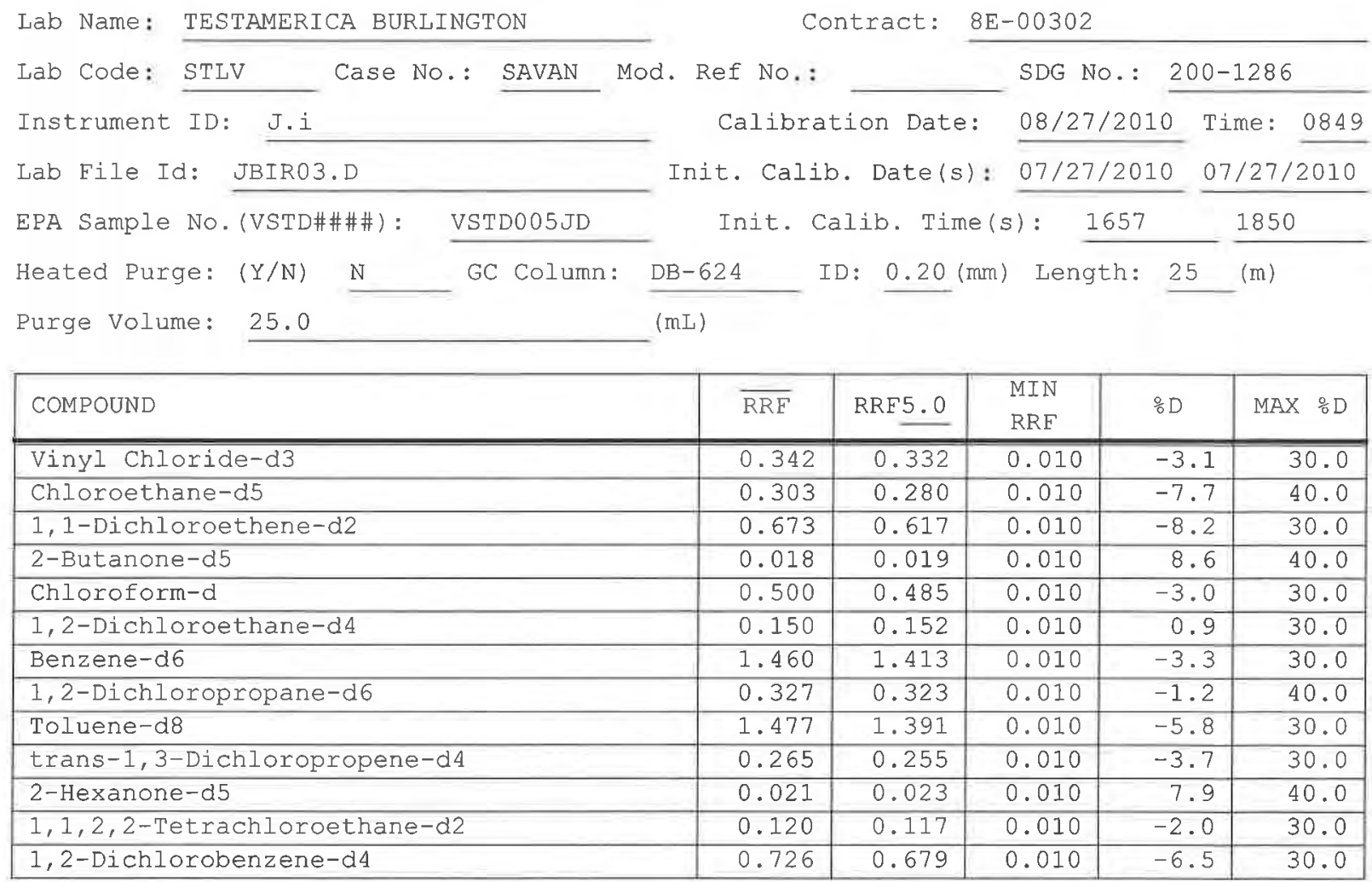

Report 1,4-Dioxane-d8 for Low/Medium VoA analysis only 
$7 A$ - FORM VII VOA-1

VOLATILE CONTINUING CALIBRATION DATA

Lab Name: TESTAMERICA BURLINGTON

Contract: $8 \mathrm{E}-00302$

Lab Code: STLV Case No.: SAVAN Mod. Ref No.: SDG No.: 200-1286

Instrument ID: J.i

Calibration Date: 08/27/2010 Time: 1926

Lab File Id: JBIR25.D Init. Calib. Date(s): 07/27/2010 07/27/2010

EPA Sample No. (VSTD\#\#\#\#)

VSTD005DJ

Init. Calib. Time(s): $1657 \quad 1850$

Heated Puxge: ( $Y / N) \quad N$

GC Column: DB-624

ID: $0.20(\mathrm{~mm})$ Length: 25 (m)

Purge Volume: 25.0

(mL)

\begin{tabular}{|c|c|c|c|c|c|}
\hline COMPOUND & $\overline{\mathrm{RRE}}$ & RRF5. 0 & $\begin{array}{l}\text { MIN } \\
\text { RRF }\end{array}$ & $\because D$ & $M A X \div D$ \\
\hline Dichlorodifluoromethane & 0.512 & 0.457 & 0.010 & -10.7 & 50.0 \\
\hline Chloromethane & 0.352 & 0.374 & 0.010 & 6.2 & 50.0 \\
\hline Vinyl chloride & 0.376 & 0.371 & 0.010 & -1.3 & 50.0 \\
\hline Bromomethane & 0.263 & 0.217 & 0.010 & -17.5 & 50.0 \\
\hline Chloroethane & 0.218 & 0.240 & 0.010 & 9.9 & 50.0 \\
\hline Trichlorofluoromethane & 0.711 & 0.639 & 0.010 & -10.1 & 50.0 \\
\hline 1,1-Dichloroethene & 0.346 & 0.307 & 0.010 & -11.1 & 50.0 \\
\hline 1,1,2-Trichloro-1,2,2-trifluoroethane & 0.398 & 0.346 & 0.010 & -13.2 & 50.0 \\
\hline Acetone & 0.014 & 0.012 & 0.010 & -15.5 & 50.0 \\
\hline Carbon disulfide & 1.032 & 0.849 & 0.010 & -17.8 & 50.0 \\
\hline Methyl acetate & 0.040 & 0.033 & 0.010 & -16.7 & 50.0 \\
\hline Methylene Chloride & 0.229 & 0.208 & 0.010 & -9.1 & 50.0 \\
\hline trans-1,2-Dichloroethene & 0.319 & 0.305 & 0.010 & -4.5 & 50.0 \\
\hline Methyl tert-butyl ether & 0.312 & 0.307 & 0.010 & -1.6 & 50.0 \\
\hline 1,1-Dichloroethane & 0.477 & 0.499 & 0.010 & 4.7 & 50.0 \\
\hline cis-1,2-Dichloroethene & 0.289 & 0.281 & 0.010 & -2.8 & 50.0 \\
\hline 2-Butanone & 0.018 & 0.018 & 0.010 & 1.1 & 50.0 \\
\hline Bromochloromethane & 0.086 & 0.077 & 0.010 & -10.5 & 50.0 \\
\hline Chloroform & 0.461 & 0.469 & 0.010 & 1.8 & 50.0 \\
\hline 1,1,1-Trichloroethane & 0.698 & 0.691 & 0.010 & -0.9 & 50.0 \\
\hline Cyclohexane & 0.659 & 0.679 & 0.010 & 3.0 & 50.0 \\
\hline Carbon tetrachloride & 0.642 & 0.636 & 0.010 & -1.0 & 50.0 \\
\hline Benzene & 1.547 & 1.558 & 0.010 & 0.7 & 50.0 \\
\hline 1,2-Dichloroethane & 0.178 & 0.193 & 0.010 & 8.5 & 50.0 \\
\hline Trichloroethene & 0.431 & 0.424 & 0.010 & -1.6 & 50.0 \\
\hline Methylcyclohexane & 0.527 & 0.558 & 0.010 & 6.0 & 50.0 \\
\hline
\end{tabular}

Report 1,4-Dioxane for Low/Medium VOA analysis only 
$7 B$ - FORM VII VOA-2

VOLATILE CONTINUING CALIBRATION DATA

Lab Name: TESTAMERICA BURLINGTON

Contract: $8 \mathrm{E}-00302$

Lab Code: STLV Case No.: SAVAN Mod. Ref No.

SDG NO.: 200-1286

Instrument ID: J.i

Lab File Id: JBIR25.D

Calibration Date: 08/27/2010 Time: 1926

EPA Sample No. (VSTD\#\#\#\#)

VSTD005DJ

Init. Calib. Date(s): 07/27/2010 07/27/2010

Heated Purge: ( $Y / N)$

GC Column: DB-624

Init. Calib. Time(s): 1657 1850

Purge Volume: 25.0

$(\mathrm{mL})$

\begin{tabular}{|c|c|c|c|c|c|}
\hline COMPOUND & $\overline{\mathrm{RRF}}$ & $\mathrm{RRF} 5.0$ & $\begin{array}{l}\text { MIN } \\
\text { RRF }\end{array}$ & $\because \mathrm{D}$ & MAX $\div D$ \\
\hline 1,2-Dichloropropane & 0.266 & 0.283 & 0.010 & 6.6 & 50.0 \\
\hline Bromodichloromethane & 0.340 & 0.337 & 0.010 & -1.0 & 50.0 \\
\hline cis-1,3-Dichloropropene & 0.395 & 0.379 & 0.010 & -4.1 & 50.0 \\
\hline 4-Methyl-2-pentanone & 0.061 & 0.065 & 0.010 & 7.2 & 50.0 \\
\hline Toluene & 1.782 & 1.711 & 0.010 & -4.0 & 50.0 \\
\hline trans-1,3-Dichloropropene & 0.288 & 0.285 & 0.010 & -1.3 & 50.0 \\
\hline 1,1,2-Trichloroethane & 0.133 & 0.132 & 0.010 & -1.2 & 50.0 \\
\hline Tetrachloroethene & 0.379 & 0.383 & 0.010 & 0.9 & 50.0 \\
\hline 2-Hexanone & 0.040 & 0.042 & 0.010 & 5.4 & 50.0 \\
\hline Dibromochloromethane & 0.180 & 0.171 & 0.010 & -4.9 & 50.0 \\
\hline 1,2-Dibromoethane & 0.126 & 0.116 & 0.010 & -7.9 & 50.0 \\
\hline Chlorobenzene & 0.993 & 0.970 & 0.010 & -2.3 & 50.0 \\
\hline Ethylbenzene & 2.051 & 1.995 & 0.010 & -2.7 & 50.0 \\
\hline o-Xylene & 0.699 & 0.677 & 0.010 & -3.1 & 50.0 \\
\hline $\mathrm{m}, \mathrm{p}$-Xylene & 0.795 & 0.772 & 0.010 & -2.8 & 50.0 \\
\hline Styrene & 1.008 & 0.932 & 0.010 & -7.5 & 50.0 \\
\hline Bromoform & 0.182 & 0.173 & 0.010 & -5.0 & 50.0 \\
\hline Isopropylbenzene & 2.146 & 2.056 & 0.010 & -4.2 & 50.0 \\
\hline $1,1,2,2$-Tetrachloroethane & 0.120 & 0.113 & 0.010 & -5.8 & 50.0 \\
\hline 1,3-Dichlorobenzene & 1.573 & 1.532 & 0.010 & -2.6 & 50.0 \\
\hline 1,4-Dichlorobenzene & 1.535 & 1.457 & 0.010 & -5.1 & 50.0 \\
\hline 1,2-Dichlorobenzene & 1.157 & 1.115 & 0.010 & -3.7 & 50.0 \\
\hline 1,2-Dibromo-3-Chloropropane & 0.033 & 0.032 & 0.010 & -4.1 & 50.0 \\
\hline 1,2,4-Trichlorobenzene & 0.673 & 0.672 & 0.010 & -0.1 & 50.0 \\
\hline 1,2,3-Trichlorobenzene & 0.456 & 0.428 & 0.010 & -5.9 & 50.0 \\
\hline
\end{tabular}


$7 C$ - FORM VII VOA-3

VOLATILE CONTINUING CALIBRATION DATA

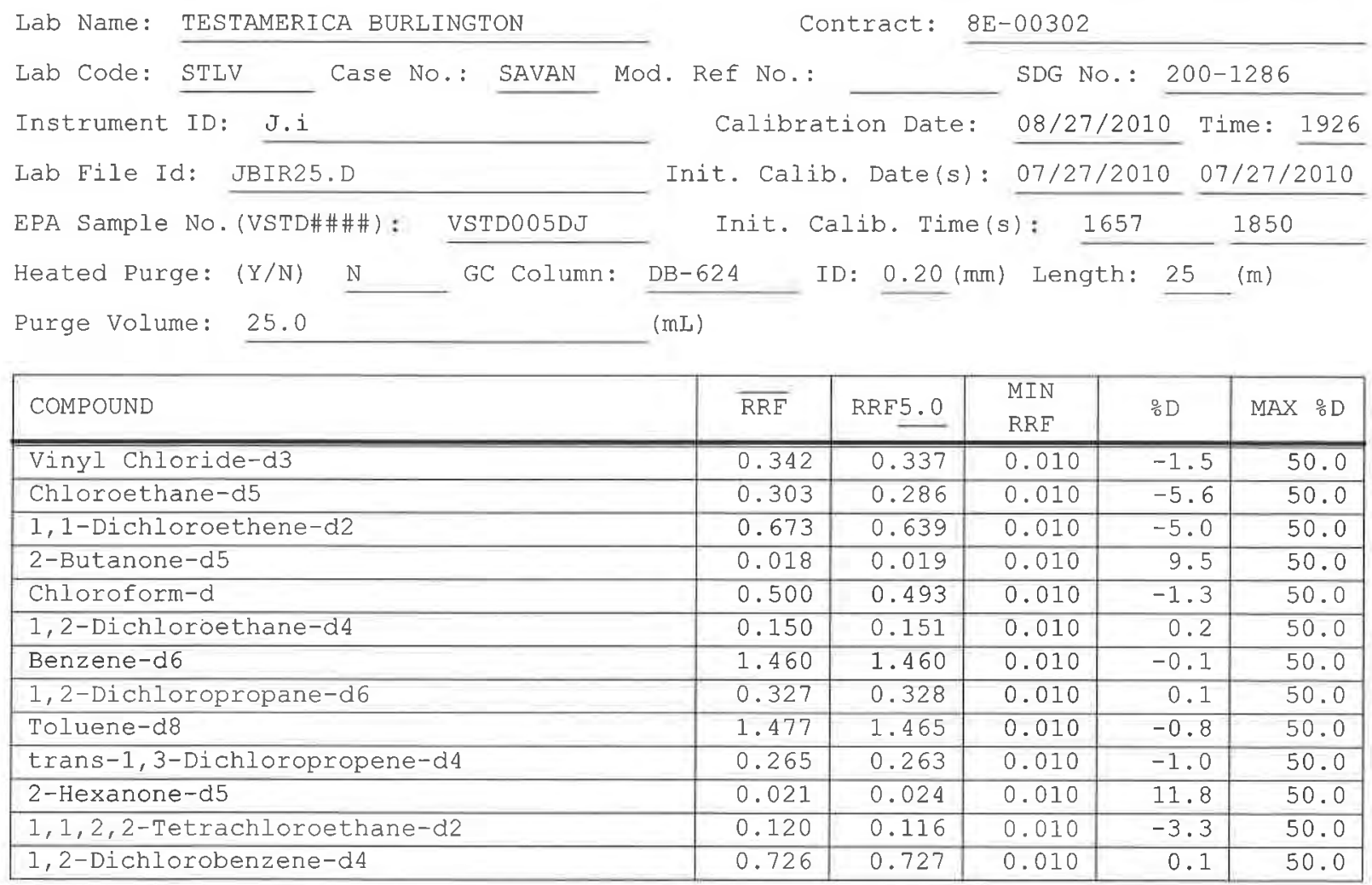


IA - FORM I VOA-1

VOLATILE ORGANICS ANALYSIS DATA SHEET
EPA SAMPLE NO.

VBLKJD
Lab Name: TESTAMERICA BURLINGTON

Lab Code: STLV

Matrix: (SOIL/SED/WATER)

Sample wt/vol: 25.0

Level: (TRACE/LOW/MED)

TRACE

응 Moisture: not dec.

GC Column: DB-624

ID: 0.20 (mm)

Soil Extract Volume:

(uL)

Purge Volume: 25.0 (mL)
Contract: $8 \mathrm{E}-00302$

SDG No.: 200-1286

Lab Sample ID: MB 200-5901/5

Lab File ID: JBIR05.D

Date Received:

Date Analyzed: 08/27/2010

Dilution Factor: 1.0

Soil Aliquot Volume:

(uL)

\begin{tabular}{|c|c|c|c|}
\hline CAS NO. & COMPOUND & $\begin{array}{l}\text { CONCENTRATION UNITS: } \\
(\mathrm{ug} / \mathrm{L} \text { or } \mathrm{ug} / \mathrm{kg}) \mathrm{ug} / \mathrm{L}\end{array}$ & Q \\
\hline $75-71-8$ & Dichlorodifluoromethane & 0.50 & $\mathrm{U}$ \\
\hline $74-87-3$ & Chloromethane & 0.50 & $\mathrm{U}$ \\
\hline $75-01-4$ & Vinyl chloride & 0.50 & $\mathrm{U}$ \\
\hline $74-83-9$ & Bromomethane & 0.50 & $\mathrm{U}$ \\
\hline $75-00-3$ & Chloroethane & 0.50 & $\mathrm{U}$ \\
\hline $75-69-4$ & Trichlorofluoromethane & 0.50 & $\mathrm{U}$ \\
\hline $75-35-4$ & 1,1-Dichloroethene & 0.50 & $\mathrm{U}$ \\
\hline $76-13-1$ & 1,1,2-Trichloro-1,2,2-trifluoroethane & 0.50 & $\mathrm{U}$ \\
\hline $67-64-1$ & Acetone & 1.6 & $\mathrm{~J}$ \\
\hline $75-15-0$ & Carbon disulfide & 0.50 & $\mathrm{U}$ \\
\hline $79-20-9$ & Methyl acetate & 0.50 & $\mathrm{U}$ \\
\hline $75-09-2$ & Methylene Chloride & 0.50 & $\mathrm{U}$ \\
\hline $156-60-5$ & trans-1,2-Dichloroethene & 0.50 & $\mathrm{U}$ \\
\hline $1634-04-4$ & Methyl tert-butyl ether & 0.50 & $\mathrm{U}$ \\
\hline $75-34-3$ & 1,1-Dichloroethane & 0.50 & $\mathrm{U}$ \\
\hline $156-59-2$ & cis-1,2-Dichloroethene & 0.50 & $\mathrm{U}$ \\
\hline $78-93-3$ & 2-Butanone & 5.0 & $\mathrm{U}$ \\
\hline $74-97-5$ & Bromochloromethane & 0.50 & $\mathrm{U}$ \\
\hline $67-66-3$ & Chloroform & 0.50 & $\mathrm{U}$ \\
\hline $71-55-6$ & 1,1,1-Trichloroethane & 0.50 & $\mathrm{U}$ \\
\hline $110-82-7$ & Cyclohexane & 0.50 & $\mathrm{U}$ \\
\hline $56-23-5$ & Carbon tetrachloride & 0.50 & $\mathrm{U}$ \\
\hline $71-43-2$ & Benzene & 0.50 & $\mathrm{U}$ \\
\hline $107-06-2$ & 1,2-Dichloroethane & 0.50 & $\mathrm{U}$ \\
\hline
\end{tabular}

Report 1,4-Dioxane for Low-Medium VOA analysis only 


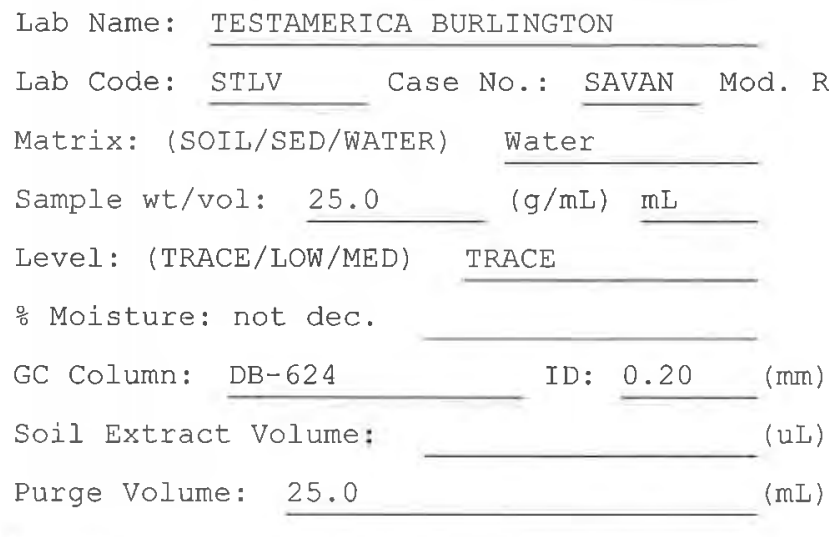

Contract: $\quad 8 \mathrm{E}-00302$

\begin{tabular}{|c|c|c|c|}
\hline CAS NO. & COMPOUND & $\begin{array}{l}\text { CONCENTRATION UNITS: } \\
(\mathrm{ug} / \mathrm{L} \text { or } \mathrm{ug} / \mathrm{kg}) \mathrm{ug} / \mathrm{L}\end{array}$ & $Q$ \\
\hline $79-01-6$ & Trichloroethene & 0.50 & $\mathrm{U}$ \\
\hline $108-87-2$ & Methylcyclohexane & 0.50 & $\mathrm{U}$ \\
\hline $78-87-5$ & 1,2-Dichloropropane & 0.50 & $\mathrm{U}$ \\
\hline $75-27-4$ & Bromodichloromethane & 0.50 & $\mathrm{U}$ \\
\hline $10061-01-5$ & cis-1,3-Dichloropropene & 0.50 & $\mathrm{U}$ \\
\hline $108-10-1$ & 4-Methyl-2-pentanone & 5.0 & $\mathrm{U}$ \\
\hline $108-88-3$ & Toluene & 0.50 & $\mathrm{U}$ \\
\hline $10061-02-6$ & trans-1, 3-Dichloropropene & 0.50 & $\mathrm{U}$ \\
\hline $79-00-5$ & 1,1,2-Trichloroethane & 0.50 & $\mathrm{U}$ \\
\hline $127-18-4$ & Tetrachloroethene & 0.50 & $\mathrm{U}$ \\
\hline $591-78-6$ & 2-Hexanone & 5.0 & $\mathrm{U}$ \\
\hline $124-48-1$ & Dibromochloromethane & 0.50 & $\mathrm{U}$ \\
\hline $106-93-4$ & 1,2-Dibromoethane & 0.50 & U \\
\hline $108-90-7$ & Chlorobenzene & 0.50 & $\mathrm{U}$ \\
\hline $100-41-4$ & Ethylbenzene & 0.50 & $\mathrm{U}$ \\
\hline $95-47-6$ & o-Xylene & 0.50 & $\mathrm{U}$ \\
\hline $179601-23-1$ & m,p-Xylene & 0.50 & $\mathrm{U}$ \\
\hline $100-42-5$ & Styrene & 0.50 & $\mathrm{U}$ \\
\hline $75-25-2$ & Bromoform & 0.50 & $\mathrm{U}$ \\
\hline $98-82-8$ & Isopropylbenzene & 0.50 & $\mathrm{U}$ \\
\hline $79-34-5$ & $1,1,2,2$-Tetrachloroethane & 0.50 & $\mathrm{U}$ \\
\hline $541-73-1$ & 1,3-Dichlorobenzene & 0.50 & $\mathrm{U}$ \\
\hline $106-46-7$ & 1,4-Dichlorobenzene & 0.50 & $\mathrm{U}$ \\
\hline $95-50-1$ & 1,2-Dichlorobenzene & 0.50 & $\mathrm{U}$ \\
\hline $96-12-8$ & 1,2-Dibromo-3-Chloropropane & 0.50 & $\mathrm{U}$ \\
\hline $120-82-1$ & 1,2,4-Trichlorobenzene & 0.50 & $\mathrm{U}$ \\
\hline $87-61-6$ & 1,2,3-Trichlorobenzene & 0.054 & $\mathrm{~J}$ \\
\hline
\end{tabular}


IJ - FORM I VOA-TIC

VOLATILE ORGANICS ANALYSIS DATA SHEET TENTATIVELY IDENTIFIED COMPOUNDS
EPA SAMPLE NO.

VBLKJD

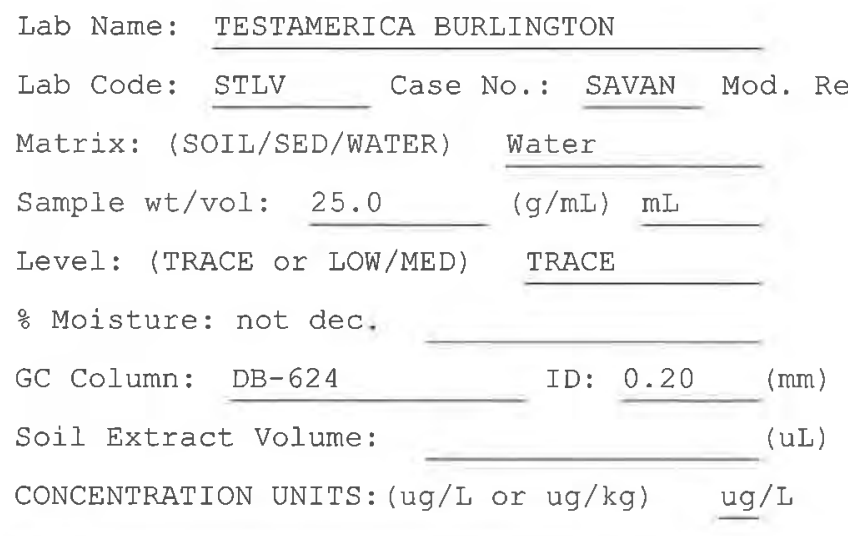

Contract: 8E-00302 SDG No.: 200-1286
Lab Sample ID: MB 200-5901/5

Lab File ID: JBIR05.D

Date Received:

Date Analyzed: 08/27/2010

Dilution Factor: 1.0

Soil Aliquot Volume:

(uL)

Purge Volume: 25.0 (mL)

\begin{tabular}{|c|c|c|c|c|c|}
\hline & CAS NUMBER & COMPOUND NAME & $\mathrm{RT}$ & EST. CONC. & 0 \\
\hline 01 & & Unknown & 6.94 & 2.7 & $\mathrm{XJ}$ \\
\hline 02 & & Unknown & 10.74 & 0.51 & $\mathrm{~J}$ \\
\hline 03 & E9667961 & Total Alkanes & $\mathrm{N} / \mathrm{A}$ & & \\
\hline
\end{tabular}

$1 E P A$-designated Registry Number. 


\section{TestAmerica}

THE LEADER IN ENYIRONMENTAL TESTING

\section{ANALYTICAL REPORT}

Job Number: 200-6522-1

SDG Number: 200-6522

Job Description: Savannah (200-6522)

Contract Number: EP-W-09-044

For:

Argonne National Laboratory

9700 South Cass Avenue

Building 203

Office B-149

Argonne, IL 60439

Attention: Mr. Clyde Dennis

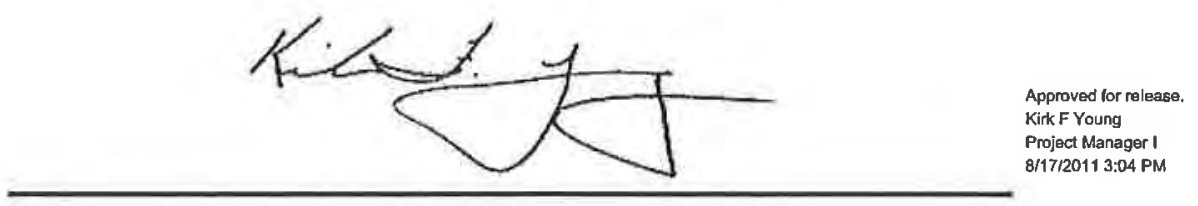

Kirk F Young

Project Manager I

kirk.young@testamericainc.com

$08 / 17 / 2011$

The test results in this report relate only to sample(s) as received by the laboratory. These test results were derived under a quality system that adheres to the requirements of NELAC. Pursuant to NELAC, this report may not be produced in full without written approval from the laboratory 


\section{Table of Contents}

Cover Title Page . . . . . . . . . . . . . . . . . 1

Report Narrative . . . . . . . . . . . . . . . . . . 4

Case Narrative .............................. 4

Qualifier Definition .............................. 6

External Chain of Custody . . . . . . . . . . . . . . . 7

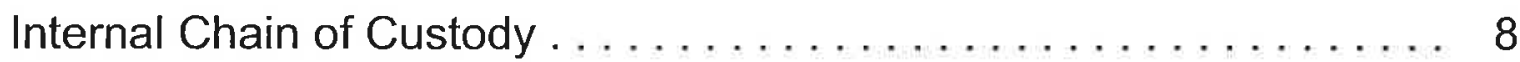

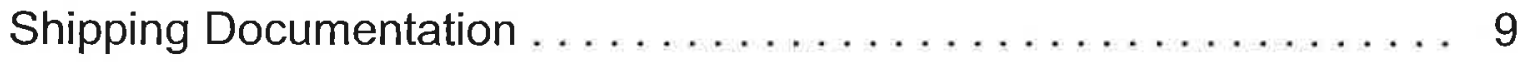

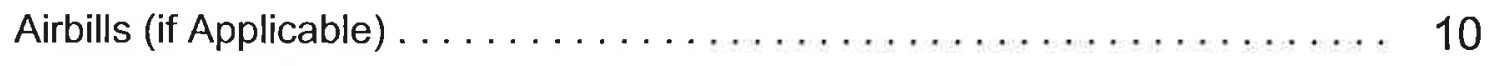

Sample Receipt and Log In Check List .................... 11

Methodology Review . . . . . . . . . . . . . . . . . . . . . 13

QC Summary - SOM01.2 Volatiles-Trace . . . . . . . . . . . . . . . 14

QC Summary - SOM01.2 Volatiles-Trace . . . . . . . . . . . . . . . 14

Deuterated Monitoring Compound Summary $\ldots \ldots \ldots \ldots \ldots \ldots \ldots \ldots \ldots \ldots \ldots \ldots$

Method Blank $\ldots \ldots \ldots \ldots \ldots, \ldots, \ldots, \ldots \ldots \ldots \ldots \ldots, 16$

GC/MS Instrument Performance Check $\ldots \ldots \ldots \ldots \ldots \ldots \ldots \ldots \ldots \ldots \ldots \ldots \ldots \ldots \ldots \ldots$

Internal Standard Area and RT Summary $\ldots \ldots \ldots \ldots \ldots \ldots \ldots \ldots \ldots \ldots \ldots \ldots \ldots \ldots \ldots \ldots$

Sample Data - SOM01.2 Volatiles-Trace . . . . . . . . . . . . 20

Sample Data - SOM01.2 Volatiles-Trace . . . . . . . . . . . . . . 20

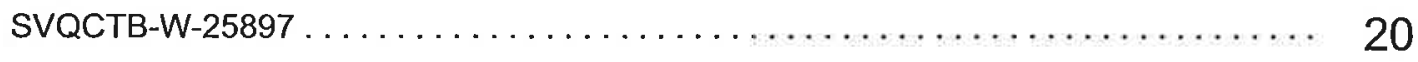

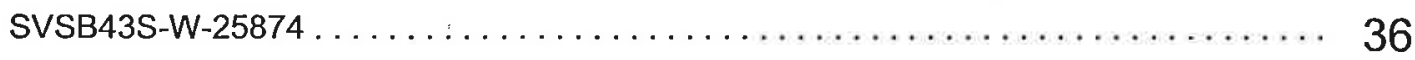

SVSB44D-W-25877 ...................................... 53

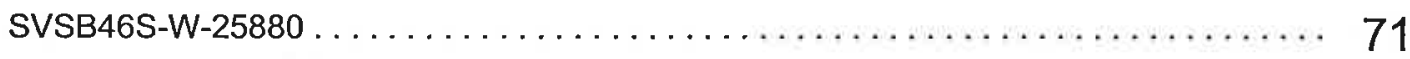

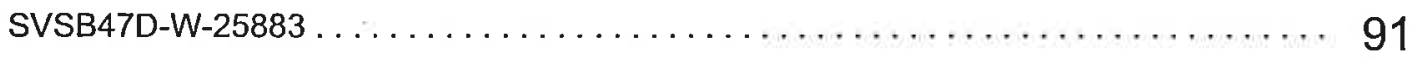

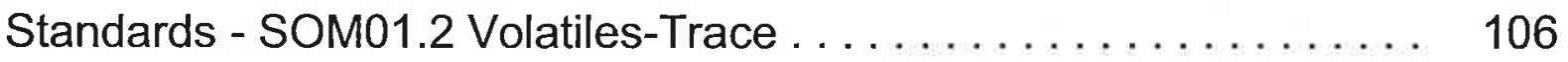

Standards - SOM01.2 Volatiles-Trace ..................... 106

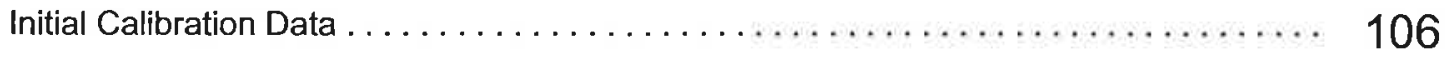

$\begin{array}{ll}\text { Page } 2 \text { of } 196 & 08 / 17 / 2011\end{array}$ 


\section{Table of Contents}

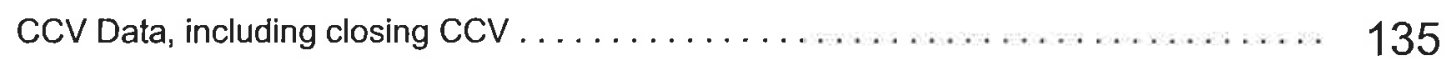

Raw Qc Data - SOM01.2 Volatiles-Trace . . . . . . . . . . . . . . . . 149

Raw Qc Data - SOM01.2 Volatiles-Trace . . . . . . . . . . . . . . . . . . 149

Raw Qc Data - SOM01.2 Volatiles-Trace ...................... 149

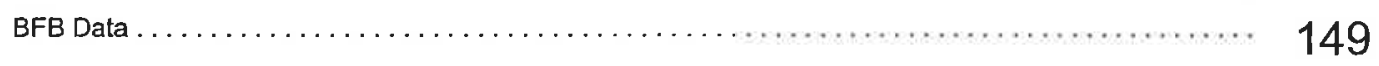

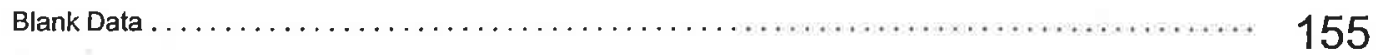

Log Book Documentation . . . . . . . . . . . . . . . . . . . 178

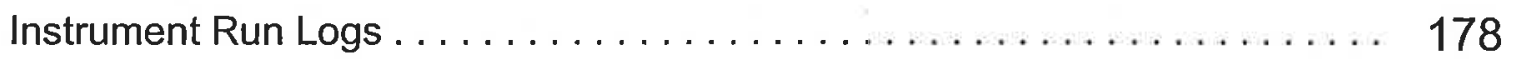

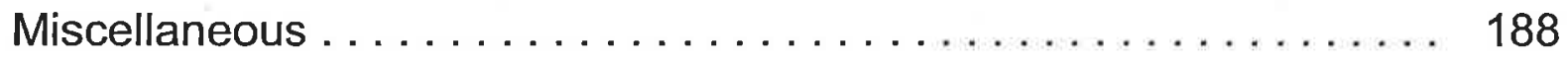

Sample Preparation . . . . . . . . . . . . . . . . . . . . . . 188

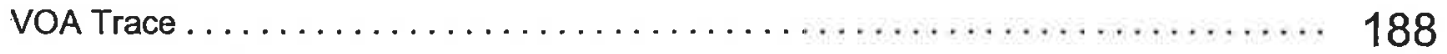

Manual Integrations $\ldots \ldots \ldots \ldots \ldots \ldots \ldots \ldots \ldots \ldots \ldots \ldots \ldots \ldots \ldots \ldots \ldots \ldots \ldots, 188$

Standards Traceability . . . . . . . . . . . . . . . . 189 


\title{
CASE NARRATIVE
}

\author{
Client: Argonne National Laboratory \\ Project: Savannah (200-6522)
}

Report Number: 200-6522-1

\begin{abstract}
Enclosed is the data set for the referenced project work. With the exceptions noted as flags or footnotes, standard analytical protocols were followed in performing the analytical work and the applied control limits were met.
\end{abstract}

Calculations were performed before rounding to avoid round-off errors in calculated results.

All holding times were met and proper preservation noted for the methods performed on these samples, unless otherwise detailed in the individual sections below.

\section{Receipt}

The samples were received on 08/12/2011. Documentation of the condition of the samples at the time of their receipt and any exception to the laboratory's Sample Acceptance Policy is documented in the Shipping and Receiving section of this submittal. The samples, as received, were not acid preserved. On that basis, the laboratory did provide for the analytical work to be performed within seven days of sample collection.

\section{SOM01.2 Volatile Organics (Trace Level Water)}

A storage blank was prepared for volatile organics analysis, and stored in association with the storage of the samples. That storage blank, identified as VHBLK01, was carried through the holding period with the samples, and analyzed.

Each sample in the sample set was analyzed without a dilution. Each of the analyses associated with the sample set exhibited an acceptable internal standard performance. There was an acceptable recovery of each deuterated monitoring compound (DMC) in the analysis of the method blank associated with the analytical work, and in the analysis of the storage blank associated with the sample set. The analysis of the samples in this sample set did meet the technical acceptance criteria specific to DMC recoveries, although not all DMC recoveries were within the control range in each analysis. The technical acceptance criteria does provide for the recovery of up to three DMCs to fall outside of the control range in the analysis of field samples. Matrix spike and matrix spike duplicate analyses were not performed on samples in this sample set. Trace concentration of carbon disulfide, methylene chloride, benzene, and toluene were identified in the analysis of the method blank associated with the analytical work. The concentration of each analyte in that analysis was below the established reporting limit, and the analysis did meet the technical acceptance criteria for a compliant method blank analysis. The analysis of the storage blank associated with the sample set was free of analyte contamination. Present in the method blank and storage blank analyses was a non-target constituent that represents a compound that is related to the DMC formulation. The fact that the presence of this compound is not within the laboratory's control is at issue. The derived results for that compound have been qualified with an "X" qualifier to reflect the source of the contamination.

The responses for each of the target analytes met the relative standard deviation criterion in the initial calibration. The response for each target analyte met the percent difference criterion in 
each opening/continuing calibration check acquisition. The response for each target analyte met the 50.0 percent difference criterion in each closing calibration check acquisition.

The primary quantitation mass for methylcyclohexane that is specified in the Statement of Work is mass 83 . The laboratory did identify a contribution to mass 83 from 1,2-dichloropropane- $d_{6}$, one of the deuterated monitoring compounds (DMCs). The laboratory did change the primary quantitation mass assignment to mass 55 for the quantification of methylcyclohexane.

Manual integration was employed in deriving certain of the analytical results. The values that have been derived from manual integration are qualified on the quantitation reports. Extracted ion current profiles for each manual integration are included in the data package, and further documented at the end of this submittal. 


\section{DATA REPORTING QUALIFIERS}

Client: Argonne National Laboratory

Job Number: 200-6522-1

Sdg Number: 200-6522

GC/MS VOA

U

J

J

$x$

B
Analyzed for but not detected.

Indicates an Estimated Value for TICs

Indicates an estimated value.

See case narrative notes for explanation of the ' $X$ ' flag

Surrogate exceeds the control limit

The analyte was found in an associated blank, as well as in the sample. 


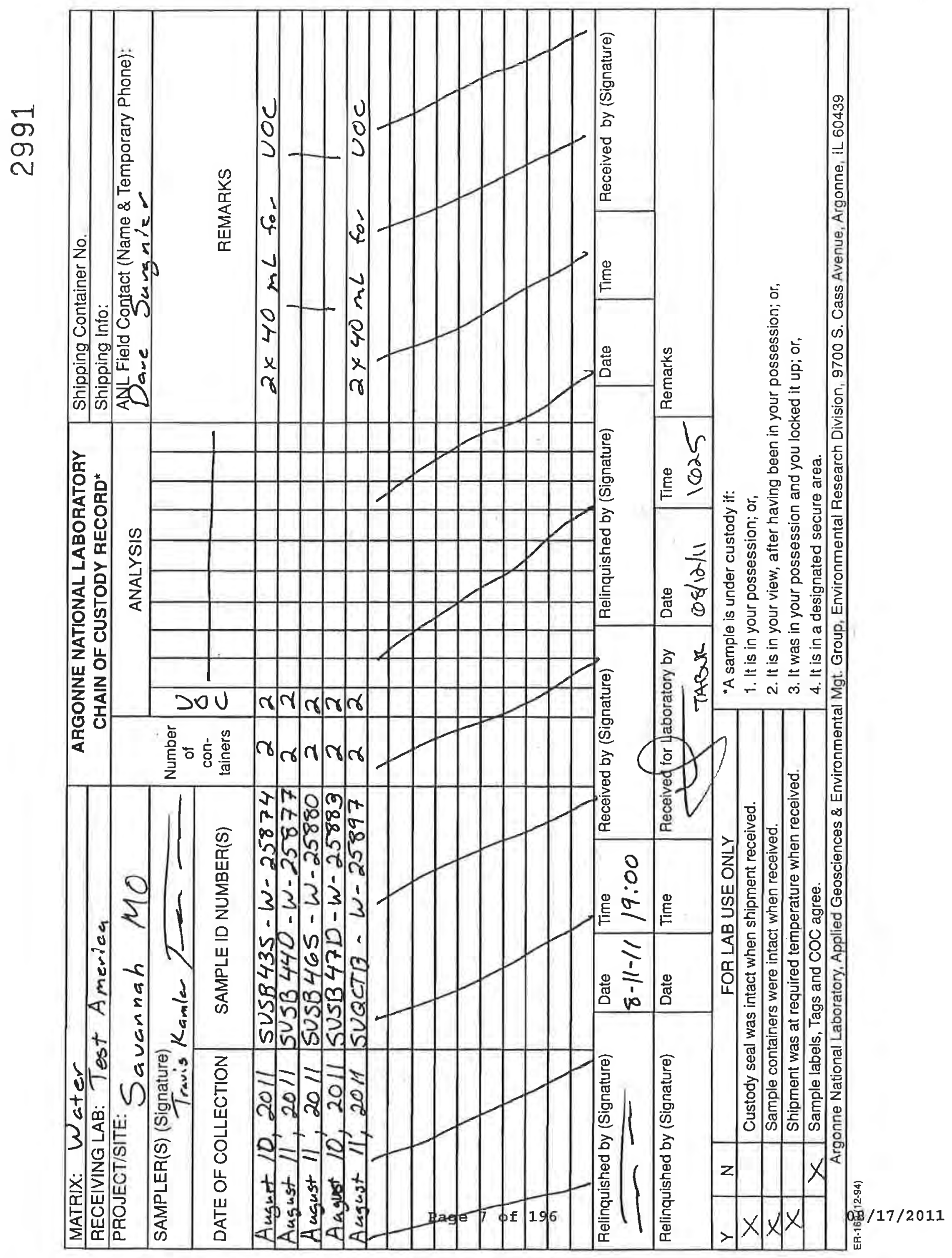




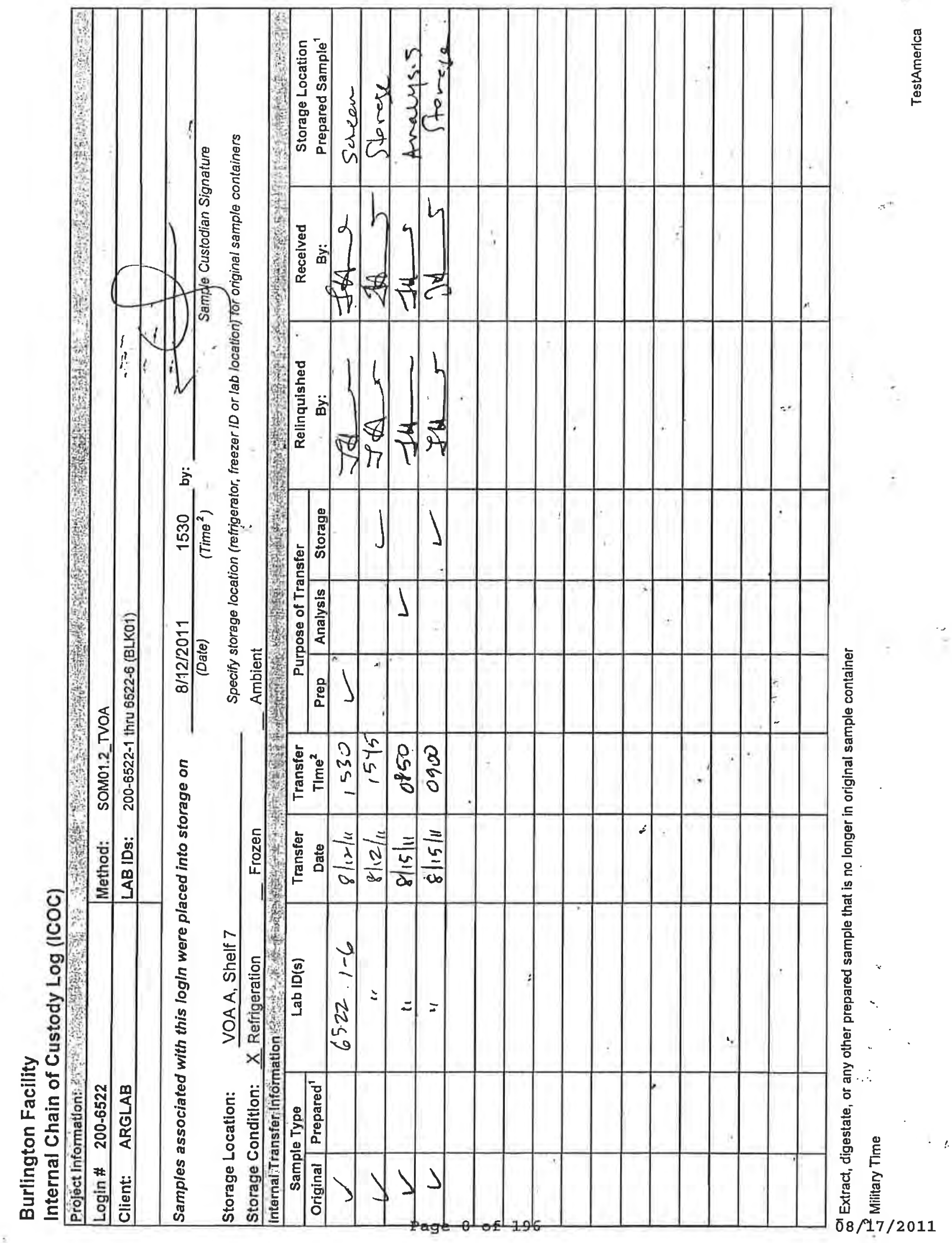




\section{Shipping and Receiving Documents}




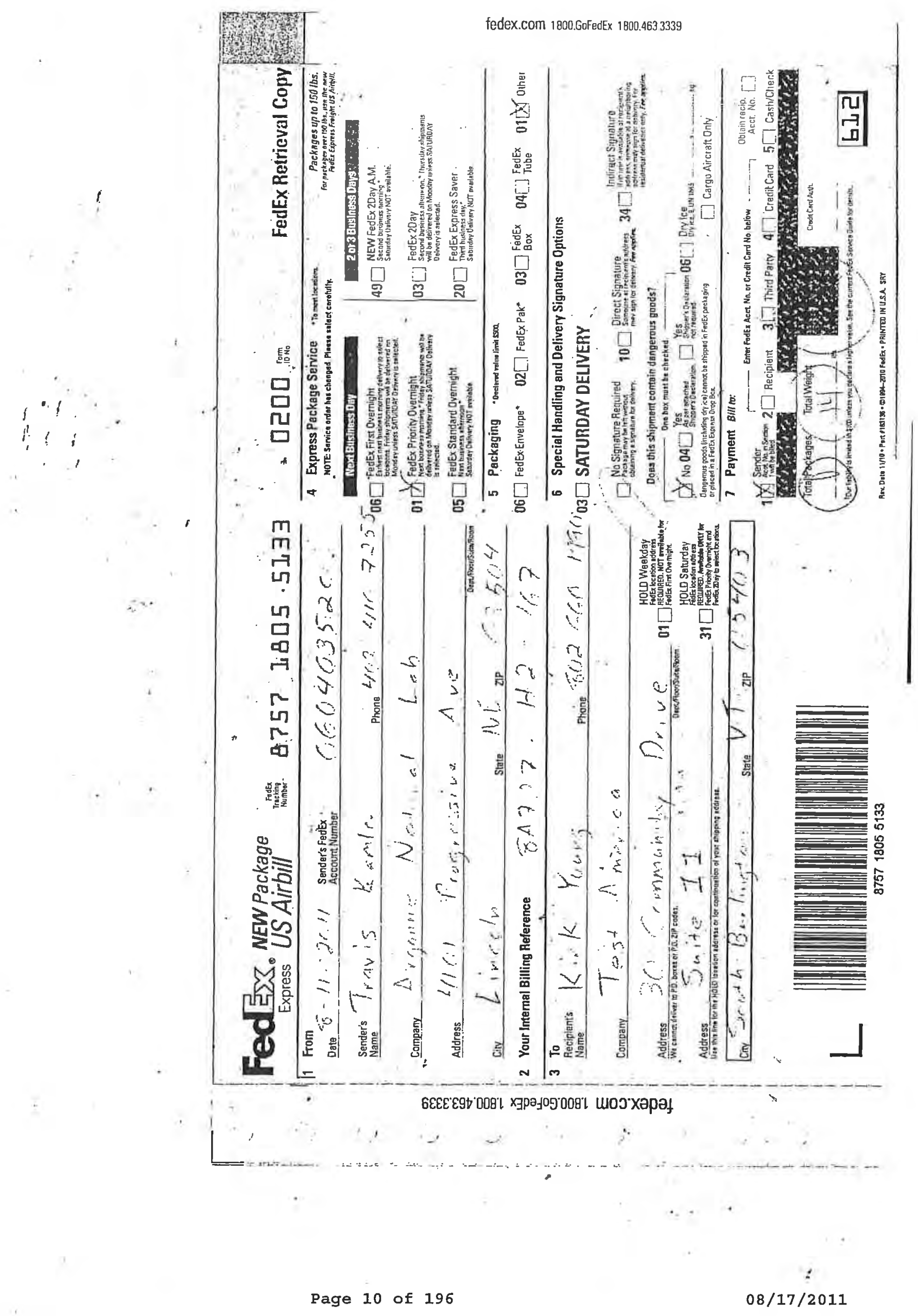




\section{Login Sample Receipt Checklist}

Client: Argonne National Laboratory

Job Number: 200-6522-1

SDG Number: 200-6522

Login Number: 6522

List Source: TestAmerica Burlington

List Number: 2

Creator: Matot, Wade $\mathrm{M}$

Question

Answe

Radioactivity either was not measured or, if measured, is at or below background

The cooler's custody seal, if present, is intact.

The cooler or samples do not appear to have been compromised or tampered with.

Samples were received on ice.

N/A

Comment

Cooler Temperature is acceptable.

Cooler Temperature is recorded.

$\mathrm{COC}$ is present.

COC is filled out in ink and legible.

$\mathrm{COC}$ is filled out with all pertinent information.

Is the Field Sampler's name present on COC?

There are no discrepancies between the sample IDs on the containers and the COC.

Samples are received within Holding Time.

True

Lab does not accept radioactive samples.

Sample containers have legible labels.

True

No numbers

True

Containers are not broken or leaking.

True

True

$2.6^{\circ} \mathrm{C}$, IR gun ID $96, \mathrm{CF}=0$

True

True

True

True

False

Moinor Discrepacies

Sample collection date/times are provided.

True

Appropriate sample containers are used.

True

True

True

Sample bottles are completely filled.

Sample Preservation Verified.

True

True

There is sufficient vol. for all requested analyses, incl. any requested

N/A

Sample volumes were received unpreserved.

MS/MSDs

VOA sample vials do not have headspace or bubble is $<6 \mathrm{~mm}\left(1 / 4^{\prime \prime}\right)$ in

True

diameter.

Multiphasic samples are not present.

True

Samples do not require splitting or compositing.

True

Residual Chlorine Checked.

True

N/A

Check done at department level as required. 


\section{Sample Login Acknowledgement}

Job 200-6522-1

Client Job Description:

Purchase Order \#:

Work Order \#:

Project Manager:

Job Due Date:

Job TAT:

Max Deliverable Leve:

Earliest Deliverable Due:
Savannah (200-6522)

$8 \mathrm{E}-00302$

$8 \mathrm{E}-00302$

Kirk $F$ Young

$8 / 26 / 2011$

14 Days

IV

$8 / 26 / 2011$

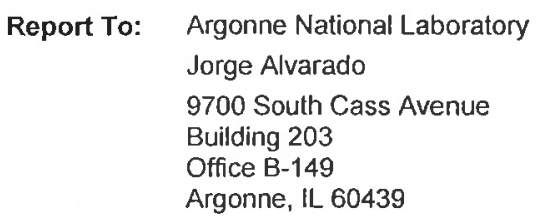

Report To: Argonne National Laboratory

Jorge Alvarado

9700 South Cass Avenue

Building 203

Office B-149

Argonne, IL 60439

Bill To: $\quad$ Argonne National Laboratory

Accounts Payable

Chief Financial Offices

9700 S. Cass Ave.

Building 201

Argonne, IL 60439

Login 200-6522

\begin{tabular}{|c|c|}
\hline Sample Receipt: & $8 / 12 / 2011 \quad 10: 25: 00 \mathrm{AM}$ \\
\hline lethod of Delivery: & FedEx Priority Overnight \\
\hline $\begin{array}{l}\text { Lab Sample \# } \\
\text { Method }\end{array}$ & $\begin{array}{l}\text { Client Sample ID } \\
\text { Method Description / Work Location }\end{array}$ \\
\hline \multirow{2}{*}{$\begin{array}{l}200-6522-1 \\
\text { SOM01.2_Vol_Tr }\end{array}$} & SVSB43S-W-25874 \\
\hline & SOM01.2 Trace Volatile Organics / In-Lab \\
\hline \multirow{2}{*}{$\begin{array}{l}200-6522-2 \\
\text { SOM01.2_Vol_Tr }\end{array}$} & SVSB44D-W-25877 \\
\hline & SOM01.2 Trace Volatile Organics / In-Lab \\
\hline \multirow{2}{*}{$\begin{array}{l}\text { 200-6522-3 S } \\
\text { SOM01.2_Vol_Tr }\end{array}$} & SVSB46S-W-25880 \\
\hline & SOM01.2 Trace Volatile Organics / In-Lab \\
\hline \multirow{2}{*}{$\begin{array}{l}200-6522-4 \\
\text { SOM01.2_Vol_Tr }\end{array}$} & SVSB47D-W-25883 \\
\hline & SOM01.2 Trace Volatile Organics / In-Lab \\
\hline \multirow{2}{*}{$\begin{array}{l}200-6522-5 \quad S \\
\text { SOM01.2_Vol_Tr }\end{array}$} & SVQCTB-W-25897 \\
\hline & SOM01.2 Trace Volatile Organics / In-Lab \\
\hline \multirow{2}{*}{$\begin{array}{l}\text { 200-6522-6 } \\
\text { SOM01.2_Vol_Tr }\end{array}$} & VHBLK01 \\
\hline & SOM01.2 Tr \\
\hline
\end{tabular}

$\begin{array}{ll}\text { Number of Coolers: } & 1 \\ \text { Cooler Temperature }(s)\left(C^{\circ}\right): & 2.6\end{array}$

Date Sampled

Matrix

8/10/2011 12:00:00 AM Water

Rpt Basis Dry / Wet *

Total Wet

8/11/2011 12:00:00 AM Water Total Wet

8/11/2011 12:00:00 AM Water

8/10/2011 12:00:00 AM Water

Total Wet

8/11/2011 12:00:00 AM Water Total Wet

8/12/2011 3:10:00 PM Water 


\section{METHODOLOGY SUMMARY}

\begin{tabular}{|c|c|c|c|}
\hline Laboratory: & TestAmerica Laboratories & Project Nc & \\
\hline Location: & South Burlington, Vermont & SDG No: & $200-6522$ \\
\hline VOA & & & \\
\hline
\end{tabular}


$2 \mathrm{~A}$ - FORM II $\mathrm{VOA}-1$

WATER VOLATILE DEUTERATED MONITORING COMPOUND RECOVERY

Lab Name: TESTAMERICA BURLINGTON Contract: 8E-00302

Lab Code: STLV Case No.: SAVANN Mod. Ref No.: 1 SDG No.: 200-6522

Level: (TRACE or LOW) TRACE

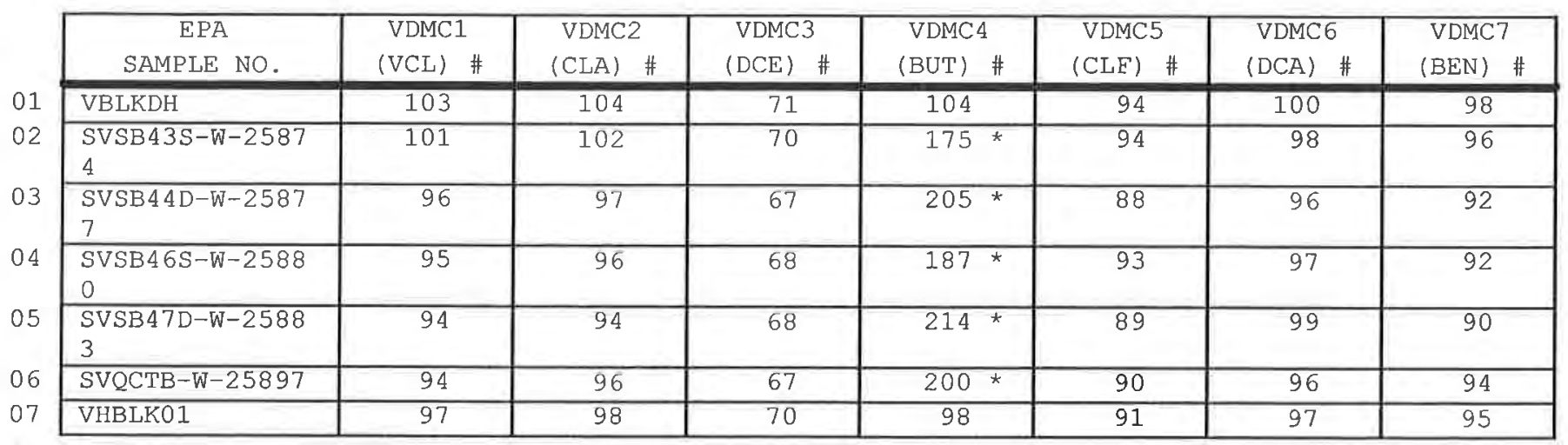

\footnotetext{
VDMC1 (VCL) = Vinyl Chloride-d3

VDMC2 $\quad(C L A)=$ Chloroethane-d5

VDMC3 $(D C E)=1,1-D i c h l o r o e t h e n e-d 2$

VDMC4 $($ BUT $)=2-$ Butanone-d5

VDMC5 $(\mathrm{CLF})=$ Chloroform-d

VDMC6 $(\mathrm{DCA})=1,2-$ Dichloroethane-d4

VDMC7 $(\mathrm{BEN})=$ Benzene-d6
}

QC LIMITS

(65-131)

$(71-131)$

(55-104)

$(49-155)$

$(78-121)$

(78-129)

$(77-124)$

\# Column to be used to flag recovery values

* Values outside of contract required QC limits 
2B - FORM II VOA-2

WATER VOLATILE DEUTERATED MONITORING COMPOUND RECOVERY

Lab Name: TESTAMERICA BURLINGTON

Contract: $8 \mathrm{E}-00302$

Lab Code: STLV Case No.: SAVANN Mod. Ref No.:

SDG No.: 200-6522

Level: (TRACE or LOW) TRACE

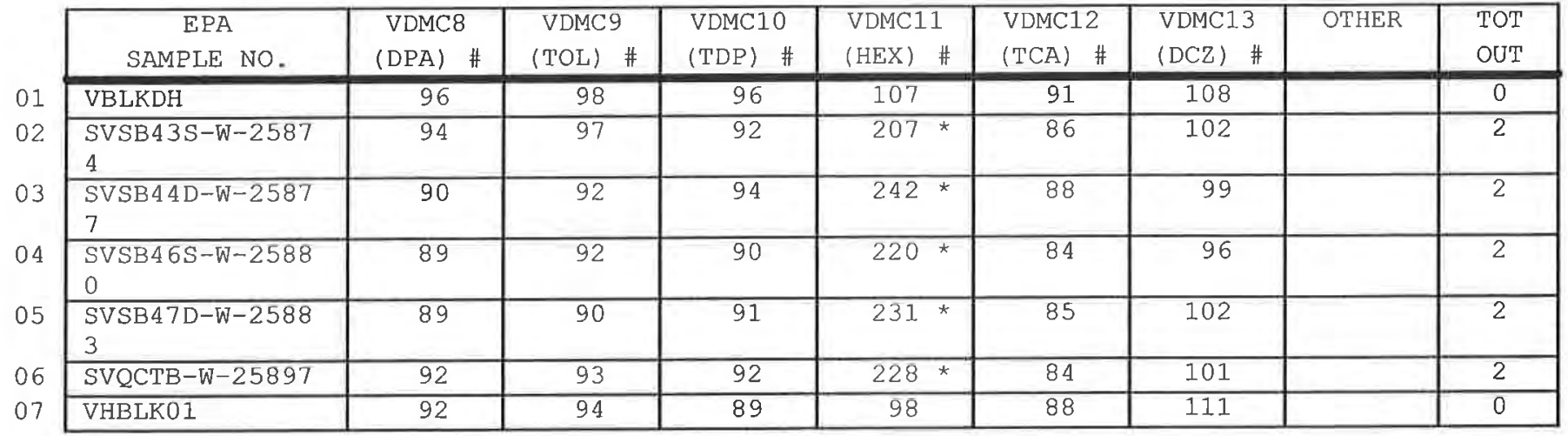

$\begin{array}{ll}\text { VDMC8 } & (\text { DPA }=1,2-\text { Dichloropropane-d6 } \\ \text { VDMC9 } & \text { (TOL) }=\text { Toluene-d8 } \\ \text { VDMC10 } & (\text { TDP }=\text { trans-1,3-Dichloropropene-d4 } \\ \text { VDMC11 } & (\mathrm{HEX})=2-\text { Hexanone-d5 } \\ \text { VDMC12 } & (\mathrm{TCA})=1,1,2,2-\text { Tetrachloroethane-d2 } \\ \text { VDMC13 } & (\mathrm{DCZ})=1,2-\text { Dichlorobenzene-d4 }\end{array}$

\# Column to be used to flag recovery values

* Values outside of contract required QC limits

Report 1,4-Dioxane-d8 for Low-Medium VOA analysis only

Page 1 of 1
QC LIMITS

$(79-124)$

$(77-121)$

$(73-121)$

$(28-135)$

$(73-125)$

(80-131) 
$4 A$ - FORM IV VOA

VOLATILE METHOD BLANK SUMMARY
EPA SAMPLE NO.

VBLKDH
Lab Name: TESTAMERICA BURLINGTON Case No.: SAVANN Mod. Ref No.:

Lab Code: STLV

Lab File ID: DHPK05.D

Instrument ID: D.i

Matrix: (SOIL/SED/WATER)

Water

Level: (TRACE or LOW/MED)

GC Column: DB-624
TRACE

ID : 0.20
Contract: $8 \mathrm{E}-00302$

SDG No.: 200-6522

\begin{tabular}{c|l|l|l|c|}
\hline \multicolumn{1}{|c|}{$\begin{array}{c}\text { EPA } \\
\text { SAMPLE NO. }\end{array}$} & \multicolumn{1}{|c|}{$\begin{array}{c}\text { LAB } \\
\text { SAMPLE ID }\end{array}$} & $\begin{array}{c}\text { LAB } \\
\text { FILE ID }\end{array}$ & $\begin{array}{c}\text { TIME } \\
\text { ANALYZED }\end{array}$ \\
\cline { 2 - 5 } 01 & $\begin{array}{l}\text { SVSB43S-W-25 } \\
874\end{array}$ & $200-6522-1$ & DHPK06.D & 0927 \\
02 & $\begin{array}{l}\text { SVSB44D-W-25 } \\
877\end{array}$ & $200-6522-2$ & DHPK07.D & 0951 \\
\cline { 2 - 5 } 03 & $\begin{array}{l}\text { SVSB46S-W-25 } \\
880\end{array}$ & $200-6522-3$ & DHPK08.D & 1016 \\
\cline { 2 - 5 } 04 & $\begin{array}{l}\text { SVSB47D-W-25 } \\
883\end{array}$ & $200-6522-4$ & DHPK09.D & 1040 \\
\cline { 2 - 5 } 05 & $\begin{array}{l}\text { SVQCTB-W-258 } \\
97\end{array}$ & $200-6522-5$ & DHPK10.D & 1105 \\
\cline { 2 - 5 } 06 & VHBLK01 & $200-6522-6$ & DHPK21.D & 1536 \\
\cline { 2 - 5 } & & & \\
\hline
\end{tabular}

COMMENTS :

Page 1 of 1 
$5 A$ - FORM V VOA

VOLATILE ORGANICS INSTRUMENT

PERFORMANCE CHECK

BROMOFLUOROBENZENE (BFB)
EPA SAMPLE NO.

BFBDV

Lab Name: TESTAMERICA BURLINGTON

Contract: $\quad 8 \mathrm{E}-00302$

Lab Code: STLV Case No.: SAVANN Mod. Ref No.: SDG No.: 200-6522

Lab File Id: DHP01.D

BFB Injection Date: 07/07/2011

Instrument Id: D.i

BFB Injection Time: 1005

GC Column: DB-624

ID $: 0.20$ (mm)

\begin{tabular}{|c|c|c|c|}
\hline $\mathrm{m} / \mathrm{e}$ & ION ABUNDANCE CRITERIA & $\begin{array}{r}\text { RELATIVE } \\
\text { ABUNDANCE }\end{array}$ & \\
\hline 50 & $15.0-40.0 \%$ of mass 95 & 26.1 & \\
\hline 75 & $30.0-80.0 \%$ of mass 95 & 46.4 & \\
\hline 95 & Base peak, $100 \%$ relative abundance & 100 & \\
\hline 96 & $5.0-9.0 \%$ of mass 95 & 6.3 & \\
\hline 173 & Less than $2.0 \%$ of mass 174 & $0 \quad 1$ & 0) 1 \\
\hline 174 & $50.0-120 \%$ of mass 95 & 77.7 & \\
\hline 175 & $5.0-9.0$ 응 of mass 174 & 6.01 & 7.8) 1 \\
\hline 176 & $95.0-101$ 음 of mass 174 & 78.21 & 101) 1 \\
\hline 177 & $5.0-9.0 \%$ of mass 176 & 5.21 & $6 . 6 \longdiv { 2 }$ \\
\hline
\end{tabular}

1 - Value is omass 174

2 - Value is omass 176

\begin{tabular}{|c|c|l|l|c|c|}
\cline { 2 - 6 } & \multicolumn{1}{|c|}{$\begin{array}{c}\text { EPA } \\
\text { SAMPLE NO. }\end{array}$} & \multicolumn{1}{c|}{ LAB } & DATE & TIME \\
SAMPLE ID & FILE ID & ANALYZED & ANALYZED \\
\cline { 2 - 7 } 01 & VSTD0.5DV & IC 200-21023/4 & DHP04.D & $07 / 07 / 2011$ & 1117 \\
\cline { 2 - 7 } 02 & VSTD001DV & IC 200-21023/5 & DHP05.D & $07 / 07 / 2011$ & 1141 \\
\cline { 2 - 7 } 04 & VSTD005DV & ICIS 200-21023/6 & DHP06.D & $07 / 07 / 2011$ & 1206 \\
\cline { 2 - 7 } 05 & VSTD010DV & IC 200-21023/7 & DHP07.D & $07 / 07 / 2011$ & 1231 \\
\cline { 2 - 7 } & VSTD020DV & IC 200-21023/8 & DHP08.D & $07 / 07 / 2011$ & 1256 \\
\hline
\end{tabular}


$5 A$ - FORM V VOA

VOLATILE ORGANICS INSTRUMENT

PERFORMANCE CHECK

BROMOFLUOROBENZENE (BEB)
EPA SAMPLE NO.

BEBDH
Lab Name: TESTAMERICA BURLINGTON

Lab Code: STLV

Lab File Id: DHPK02.D

Instrument Id: D.i

GC Colunn: DB-624

ID $: 0.20$
Contract: $\quad 8 \mathrm{E}-00302$ SDG No.: 200-6522

BEB Injection Date: 08/15/2011

BFB Injection Time: 0736 $(\mathrm{mm})$

\begin{tabular}{|r|l|r|}
\hline $\mathrm{m} / \mathrm{e}$ & ION ABUNDANCE CRITERIA & \multicolumn{2}{|c|}{$\begin{array}{c}\text { RELATIVE } \\
\text { ABUNDANCE }\end{array}$} \\
\hline 50 & $15.0-40.0 \%$ of mass 95 & 26.7 \\
\hline 75 & $30.0-80.0 \%$ of mass 95 & 47.3 \\
\hline 95 & Base peak, 100\% relative abundance & 700 \\
\hline 96 & $5.0-9.0 \%$ of mass 95 & 7.2 \\
\hline 173 & Less than 2.0\% of mass 174 & 0 \\
\hline 174 & $50.0-120 \%$ of mass 95 & 5.9 \\
\hline 175 & $5.0-9.0 \%$ of mass 174 & $(1.5) 1$ \\
\hline 176 & $95.0-101 \%$ of mass 174 & $75.2(95.9) 1$ \\
\hline 177 & $5.0-9.0 \%$ of mass 176 & 4.1( \\
\hline
\end{tabular}

1 - Value is ogmass $174 \quad 2$ - Value is onass 176

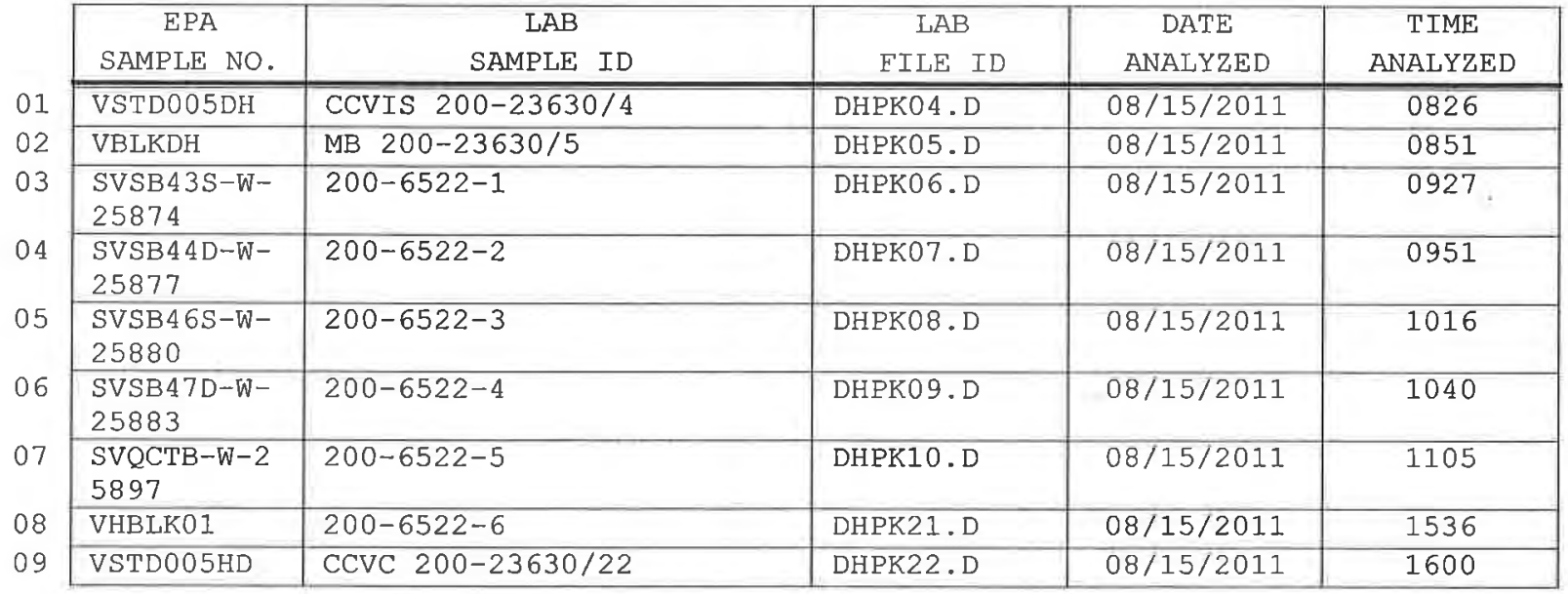


$8 A$ - FORM VIII VOA

VOLATILE INTERNAL STANDARD AREA AND RETENTION TIME SUMMARY

Lab Name: TESTAMERICA BURLINGTON

Contract: $8 \mathrm{E}-00302$

Lab Code: STLV Case No.: SAVANN Mod. Ref No.: SDG No.: $200-6522$

GC Column: DB-624 ID: 0.20 (mm) Init.

Calib. Date(s): 07/07/2011 07/07/2011

EPA Sample No. (VSTD\#\#\#\#\#): VSTD005DH

Date Analyzed: 08/15/2011

Lab File ID (Standard): DHPK04.D

Time Analyzed: 0826

Instrument ID: D.i

Heated Purge: ( $\mathrm{Y} / \mathrm{N}) \mathrm{N}$

\begin{tabular}{|c|c|c|c|c|c|c|}
\hline & $\begin{array}{c}\text { IS1 (CBZ) } \\
\text { AREA }\end{array}$ & RT \# & $\begin{array}{c}\text { IS2 (DFB) } \\
\text { AREA }\end{array}$ & RT \# & $\begin{array}{c}\text { IS3 (DCB) } \\
\text { AREA }\end{array}$ & $\mathrm{RT} \quad \#$ \\
\hline 12 HOUR STD & 154132 & 8.83 & 178846 & 5.47 & 75451 & 11.66 \\
\hline UPPER LIMIT & 215785 & 9.16 & 250384 & 5.80 & 105631 & 11.99 \\
\hline LOWER LIMIT & 92479 & 8.50 & 107308 & 5.14 & 45271 & 11.33 \\
\hline EPA SAMPLE NO. & & & & & & \\
\hline VBLKDH & 152278 & 8.83 & 179847 & 5.47 & 61195 & 11.66 \\
\hline $\begin{array}{l}\text { SVSB43S-W-2587 } \\
4\end{array}$ & 149249 & 8.83 & 177084 & 5.47 & 61580 & 11.66 \\
\hline $\begin{array}{l}\text { SVSB44D-W-2587 } \\
7\end{array}$ & 155219 & 8.83 & 180995 & 5.47 & 64486 & 11.66 \\
\hline $\begin{array}{l}\text { SVSB } 46 S-W-2588 \\
0\end{array}$ & 149001 & 8.83 & 174229 & 5.47 & 62589 & 11.66 \\
\hline $\begin{array}{l}\text { SVSB 47D-W-2588 } \\
3\end{array}$ & 151139 & 8.83 & 172350 & 5.47 & 59220 & 11.66 \\
\hline SVQCTB-W-25897 & 148796 & 8.83 & 175136 & 5.47 & 60493 & 11.66 \\
\hline VHBLK01 & 136100 & 8.84 & 159578 & 5.47 & 51738 & 11.66 \\
\hline
\end{tabular}

IS1 (CBZ) = Chlorobenzene-d5

IS2 $(\mathrm{DFB})=1,4$-Difluorobenzene

IS3 $(\mathrm{DCB})=1,4$-Dichlorobenzene-d4

AREA UPPER LIMIT $=140 \%$ (Trace Volatiles) of internal standard area AREA LOWER LIMIT $=60 \%$ (Trace Volatiles) of internal standard area RT UPPER LIMIT $=+0.33$ (Trace Volatiles) minutes of internal standard RT RT LOWER LIMIT $=-0.33$ (Trace Volatiles) minutes of internal standard RT

\# Column used to flag values outside contract required QC limits with an asterisk. 
$1 A$ - FORM I VOA-1

VOLATILE ORGANICS ANALYSIS DATA SHEET
EPA SAMPLE NO.

SVQCTB-W-25897
Lab Name: TESTAMERICA BURLINGTON

Lab Code: STLV

Matrix: (SOIL/SED/WATER)

Sample wt/vol: 25.0

Water

$(\mathrm{g} / \mathrm{mL}) \quad \mathrm{mI}$

Level: (TRACE/LOW/MED) TRACE

o Moisture: not dec.

GC Column: DB-624

ID $: 0.20$

Soil Extract Volume:

Purge Volume: 25.0

(mI)
Contract: $8 \mathrm{E}-00302$

SDG No.: 200-6522

Lab Sample ID: 200-6522-5

Lab File ID: DHPK10.D

Date Received: 08/12/2011

Date Analyzed: 08/15/2011

Dilution Factor: 1.0

Soil Aliquot Volume:

(uL)

\begin{tabular}{|c|c|c|c|}
\hline CAS NO. & COMPOUND & $\begin{array}{l}\text { CONCENTRATION UNITS: } \\
(\mathrm{ug} / \mathrm{L} \text { or } \mathrm{ug} / \mathrm{kg}) \mathrm{ug} / \mathrm{L}\end{array}$ & $Q$ \\
\hline $75-71-8$ & Dichlorodifluoromethane & 0.50 & $\mathrm{U}$ \\
\hline $74-87-3$ & Chloromethane & 0.50 & $\mathrm{U}$ \\
\hline $75-01-4$ & Vinyl chloride & 0.50 & $\mathrm{U}$ \\
\hline $74-83-9$ & Bromomethane & 0.50 & $\mathrm{U}$ \\
\hline $75-00-3$ & Chloroethane & 0.50 & $\mathrm{U}$ \\
\hline $75-69-4$ & Trichlorofluoromethane & 0.50 & $\mathrm{U}$ \\
\hline $75-35-4$ & 1,1-Dichloroethene & 0.50 & $\mathrm{U}$ \\
\hline $76-13-1$ & 1,1,2-Trichloro-1,2,2-trifluoroethane & 0.50 & $\mathrm{U}$ \\
\hline $67-64-1$ & Acetone & 2.7 & $\mathrm{~J}$ \\
\hline $75-15-0$ & Carbon disulfide & 0.50 & $\mathrm{U}$ \\
\hline $79-20-9$ & Methyl acetate & 0.50 & $\mathrm{U}$ \\
\hline $75-09-2$ & Methylene Chloride & 0.50 & $\mathrm{U}$ \\
\hline $156-60-5$ & trans-1,2-Dichloroethene & 0.50 & $\mathrm{U}$ \\
\hline $1634-04-4$ & Methyl tert-butyl ether & 0.50 & $\mathrm{U}$ \\
\hline $75-34-3$ & 1,1-Dichloroethane & 0.50 & $\mathrm{U}$ \\
\hline $156-59-2$ & cis-1,2-Dichloroethene & 0.50 & $\mathrm{U}$ \\
\hline $78-93-3$ & 2-Butanone & 5.0 & $\mathrm{U}$ \\
\hline $74-97-5$ & Bromochloromethane & 0.50 & $\mathrm{U}$ \\
\hline $67-66-3$ & Chloroform & 0.17 & $\mathrm{~J}$ \\
\hline $71-55-6$ & 1,1,1-Trichloroethane & 0.50 & $\mathrm{U}$ \\
\hline $110-82-7$ & Cyclohexane & 0.50 & $\mathrm{U}$ \\
\hline $56-23-5$ & Carbon tetrachloride & 0.50 & $\mathrm{U}$ \\
\hline $71-43-2$ & Benzene & 0.0045 & $\mathrm{~J} \mathrm{~B}$ \\
\hline $107-06-2$ & 1,2-Dichloroethane & 0.50 & $\mathrm{U}$ \\
\hline
\end{tabular}

Report 1,4-Dioxane for Low-Medium VOA analysis only 
Lab Name: TESTAMERICA BURLINGTON

Lab Code: STLV Case No.: SAVANN Mod. Ref No.:
Contract: $8 \mathrm{E}-00302$

SDG No.: 200-6522
Matrix: (SOIL/SED/WATER) Water

Sample wt/vol: 25.0 $(\mathrm{g} / \mathrm{mL}) \quad \mathrm{mL}$

Level: (TRACE/LOW/MED) TRACE

웅 Moisture: not dec.

GC Column: DB-624 ID: $0.20 \quad(\mathrm{~mm})$

Soil Extract Volume: (uL)

Purge Volume: 25.0
Lab Sample ID: 200-6522-5

Lab File ID: DHPK10.D

Date Received: 08/12/2011

Date Analyzed: 08/15/2011

Dilution Factor: 1.0

Soil Aliquot Volume: $\left(u I^{\prime}\right)$

\begin{tabular}{|c|c|c|c|}
\hline CAS NO. & COMPOUND & $\begin{array}{l}\text { CONCENTRATION UNITS: } \\
(\mathrm{ug} / \mathrm{I} \text { or } \mathrm{ug} / \mathrm{kg}) \mathrm{ug} / \mathrm{I}\end{array}$ & $Q$ \\
\hline $79-01-6$ & Trichloroethene & 0.50 & $\overline{\mathrm{U}}$ \\
\hline $108-87-2$ & Methylcyclohexane & 0.50 & $\mathrm{U}$ \\
\hline $78-87-5$ & 1,2-Dichloropropane & 0.50 & $\mathrm{U}$ \\
\hline $75-27-4$ & Bromodichloromethane & 0.50 & $\mathrm{U}$ \\
\hline $10061-01-5$ & cis-1,3-Dichloropropene & 0.50 & $\mathrm{U}$ \\
\hline $108-10-1$ & 4-Methyl-2-pentanone & 5.0 & $\mathrm{U}$ \\
\hline $108-88-3$ & Toluene & 0.18 & $\mathrm{~J} \mathrm{~B}$ \\
\hline $10061-02-6$ & trans-1,3-Dichloropropene & 0.50 & $\mathrm{U}$ \\
\hline $79-00-5$ & 1,1,2-Trichloroethane & 0.50 & $\mathrm{U}$ \\
\hline $127-18-4$ & Tetrachloroethene & 0.50 & $\mathrm{U}$ \\
\hline $591-78-6$ & 2-Hexanone & 5.0 & $\mathrm{U}$ \\
\hline $124-48-1$ & Dibromochloromethane & 0.50 & $\mathrm{U}$ \\
\hline $106-93-4$ & 1,2-Dibromoethane & 0.50 & $\mathrm{U}$ \\
\hline $108-90-7$ & Chlorobenzene & 0.50 & $\mathrm{U}$ \\
\hline $100-41-4$ & Ethylbenzene & 0.50 & $\mathrm{U}$ \\
\hline $95-47-6$ & o-xylene & 0.023 & $\mathrm{~J}$ \\
\hline $179601-23-1$ & $\mathrm{~m}, \mathrm{p}$-Xylene & 0.018 & $\mathrm{~J}$ \\
\hline $100-42-5$ & Styrene & 0.049 & $\mathrm{~J}$ \\
\hline $75-25-2$ & Bromoform & 0.50 & $\mathrm{U}$ \\
\hline $98-82-8$ & Isopropylbenzene & 0.50 & $\mathrm{U}$ \\
\hline $79-34-5$ & $1,1,2,2$-Tetrachloroethane & 0.50 & $\mathrm{U}$ \\
\hline $541-73-1$ & 1,3-Dichlorobenzene & 0.50 & U \\
\hline $106-46-7$ & 1,4-Dichlorobenzene & 0.50 & $\mathrm{U}$ \\
\hline $95-50-1$ & 1,2-Dichlorobenzene & 0.50 & $\mathrm{U}$ \\
\hline $96-12-8$ & 1,2-Dibromo-3-Chloropropane & 0.50 & $\mathrm{U}$ \\
\hline $120-82-1$ & 1,2,4-Trichlorobenzene & 0.50 & $\mathrm{U}$ \\
\hline $87-61-6$ & 1,2,3-Trichlorobenzene & 0.50 & $\mathrm{U}$ \\
\hline
\end{tabular}


IJ - FORM I VOA-TIC

VOLATILE ORGANICS ANALYSIS DATA SHEET TENTATIVELY IDENTIFIED COMPOUNDS
EPA SAMPLE NO.

SVQCTB-W-25897

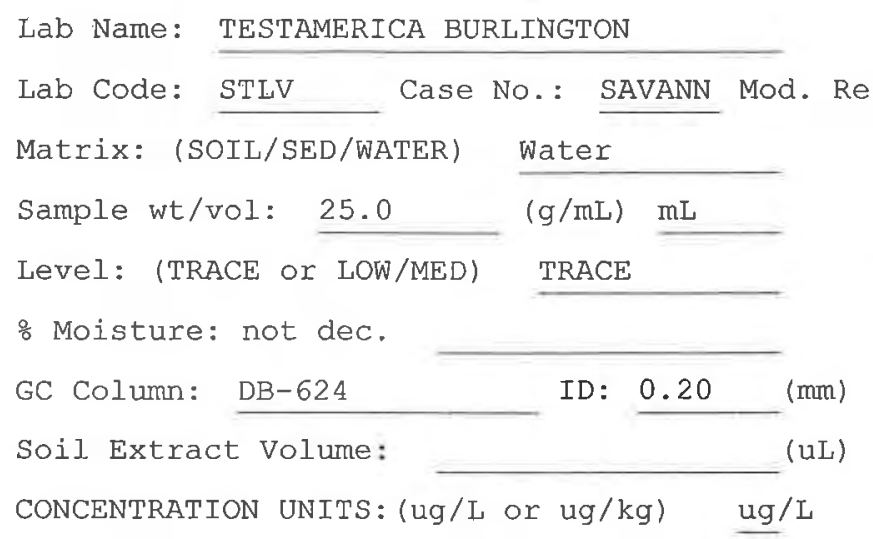

Contract: $8 \mathrm{E}-00302$ SDG No.: 200-6522

Lab Sample ID: 200-6522-5

Lab File ID: DHPK10.D

Date Received: 08/12/2011

Date Analyzed: 08/15/2011

Dilution Factor: 1.0

Soil Aliquot Volume: (uL)

Purge Volume: 25.0 (mL)

\begin{tabular}{l|l|l|l|r|r|}
\hline \multicolumn{1}{|c|}{ CAS NUMBER } & \multicolumn{1}{|c|}{ COMPOUND NAME } & RT & EST. CONC. & Q \\
\cline { 2 - 6 } 01 & & Unknown & 6.79 & B X J \\
\cline { 2 - 7 } 02 & Total Alkanes & N/A & & \\
\cline { 2 - 6 }
\end{tabular}

1 EPA-designated Registry Number. 


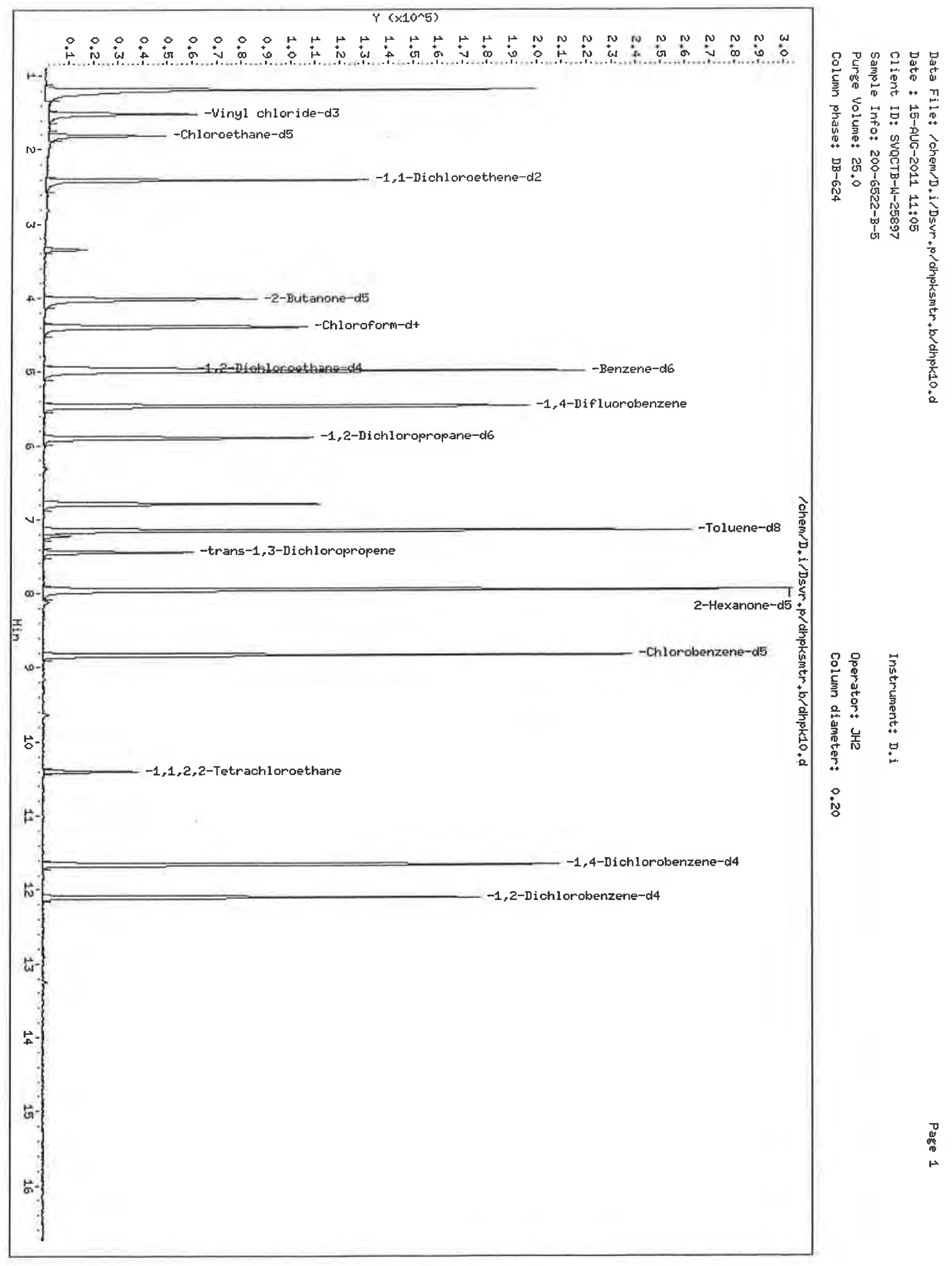

Page 23 of 196 
Lab Sample Id: 200-6522-5

Client Smp ID: SVQCTB-W-25897

Inj Date : 15-AUG-2011 11:05

Operator : JH2

Smp Info : 200-6522-B-5

Inst ID: D.i

Misc Info : 1,5

Comment :

Method : /chem/D.i/Dsvr.p/dhpksmtr.b/somtr5t.m

Meth Date : 15-Aug-2011 16:21 jh2 Quant Type: ISTD

Cal Date : 07-JUL-2011 12:56 Cal File: dhp08.d

Als bottle: 8

Dil Factor: 1.00000

Integrator: HP RTE

Target Version: 3.50

Processing Host: chemsvr6

Concentration Formula: Amt * DF * Uf/Vo * CpndVariable

\begin{tabular}{clc} 
Name & Value & Description \\
\hline DE & 1.00000 & Dilution Factor \\
Uf & 1.00000 & ng unit correction factor \\
Vo & 25.00000 & Sample volume purged (mL)
\end{tabular}

Cpnd Variable

Local Compound Variable

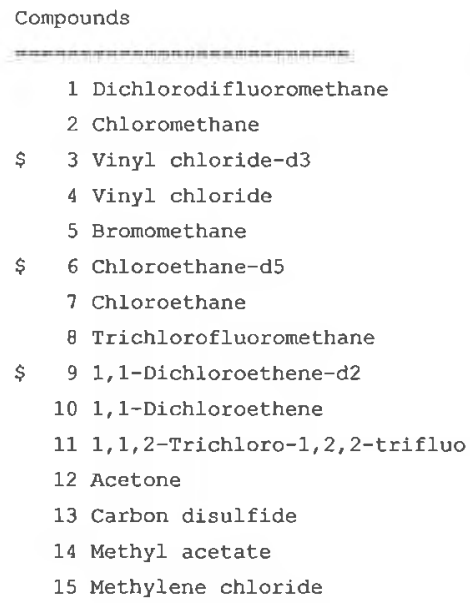

CONCENTRATIONS

QUANT SIG
MASS
\#\#rr
85
50
65
62
94
69
64
101
63
96
101
43
76
43
84

\section{RT EXP RT REL RT RESPONSE}

Compound Not Detected.

Compound Not Detected.

$1.5151 .521(0.277)$

Compound Not Detected. Compound Not Detected.

$\begin{array}{llll}1.807 & 1.807 & (0.331)\end{array}$

Compound Not Detected. Compound Not Detected.

$2.405 \quad 2.411 \quad(0.440)$

Compound Not Detected.

Compound Not Detected.

$2.447 \quad 2.448 \quad(0.448)$

Compound Not Detected.

Compound Not Detected.

Compound Not Detected. nd Sublist: all.sub 


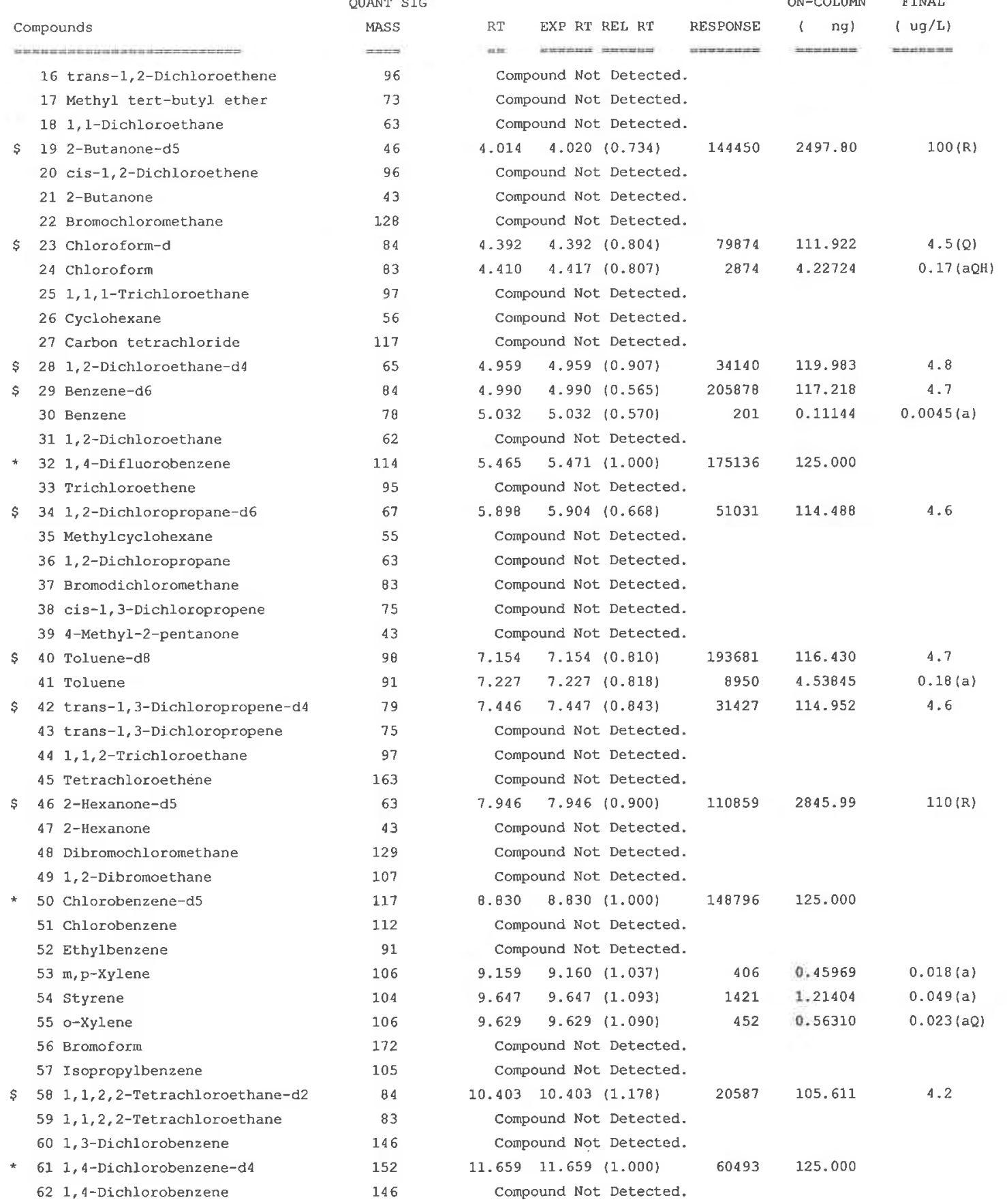




\begin{tabular}{|c|c|c|c|c|c|c|}
\hline \multirow{3}{*}{ Compounds } & \multirow[b]{2}{*}{ QUANT SIG } & & & & \multicolumn{2}{|c|}{ CONCENTRATIONS } \\
\hline & & & & & ON-COLUMN & FINAL \\
\hline & MASS & $\mathrm{RT}$ & EXP RT REL RT & RESPONSE & ( $\mathrm{ng})$ & $(\mathrm{ug} / \mathrm{L})$ \\
\hline 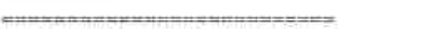 & 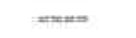 & $=$ & 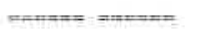 & $=\mathrm{re=merm}$ & $== \pm===$ & $=======$ \\
\hline$\$ 631,2-$ Dichlorobenzene-d4 & 152 & 12.104 & $12.104(1.038)$ & 50485 & 125.742 & 5.0 \\
\hline 64 1,2-Dichlorobenzene & 146 & Comp & ound Not Detected & & & \\
\hline 65 1,2-Dibromo-3-chloropropane & 75 & Comp & ound Not Detected & & & \\
\hline $661,2,4$-Trichlorobenzene & 180 & Comp & ound Not Detected & & & \\
\hline 67 1,2,3-Trichlorobenzene & 180 & Comp & ound Not Detected & & & \\
\hline
\end{tabular}

QC Flag Legend

a - Target compound detected but, quantitated amount Below Limit of Quantitation (BLOQ).

Q - Qualifier signal failed the ratio test.

$R$ - Spike/Surrogate failed recovery limits.

H - Operator selected an alternate compound hit. 


\section{TestAmerica Burlington}

Lab Sample Id: 200-6522-5

Client Smp ID: SVQCTB-W-25897

Inj Date : 15-AUG-2011 11:05

Operator : $\mathrm{JH} 2$

Inst ID: D.i

Smp Info : 200-6522-B-5

Misc Info: 1,5

Comment :

Method : /chem/D.i/Dsvr.p/dhpksmtr.b/somtr5t.m

Meth Date : 15-Aug-2011 16:21 jh2 Quant Type: ISTD

Cal Date : 07-JUL-2011 12:56 Cal File: dhp08.d

Als bottle: 8

Dil Factor: 1.00000

Integrator: HP RTE

Target Version: 3.50

Processing Host: chemsvr6

Concentration Formula: Ant * DE * Uf/Vo * CpndVariable

$\begin{array}{clc}\text { Name } & \text { Value } & \text { Description } \\ \text { DE } & 1.00000 & \text { Dilution Factor } \\ \text { Uf } & 1.00000 & \text { ng unit correction factor } \\ \text { Vo } & 25.00000 & \text { Sample Volume purged (mL) }\end{array}$

Cpnd Variable

ISTD

$=====:=$

* $321,4-$ Difluorobenzene

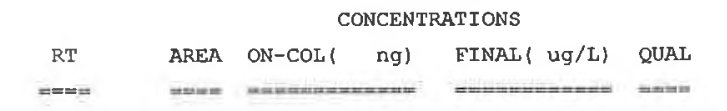

Unknown

6.794

$219834 \quad 69.7894972$
Local Compound Variable

$\begin{array}{lll}\text { RT } & \begin{array}{l}\text { AREA } \\ ======\end{array} & \begin{array}{l}\text { AMOUNT } \\ ======\end{array} \\ 5.465 & 393744 & 125.000\end{array}$

\begin{tabular}{ccc}
\multicolumn{2}{c}{ QUANT } \\
LIBRARY & LIB ENTRY & CPND \# \\
$=====$ & $========$
\end{tabular}

CAS H:
$0 \quad 32$ 
Data File: dhpk10.d

Lab Sample ID: 200-6522-5

Date: 15-AUG-2011 11:05

Client ID: SVQCTB-W-25897

Instrument: D.i

Sample Info: 200-6522-B-5

Operator: JH2

12 Acetone
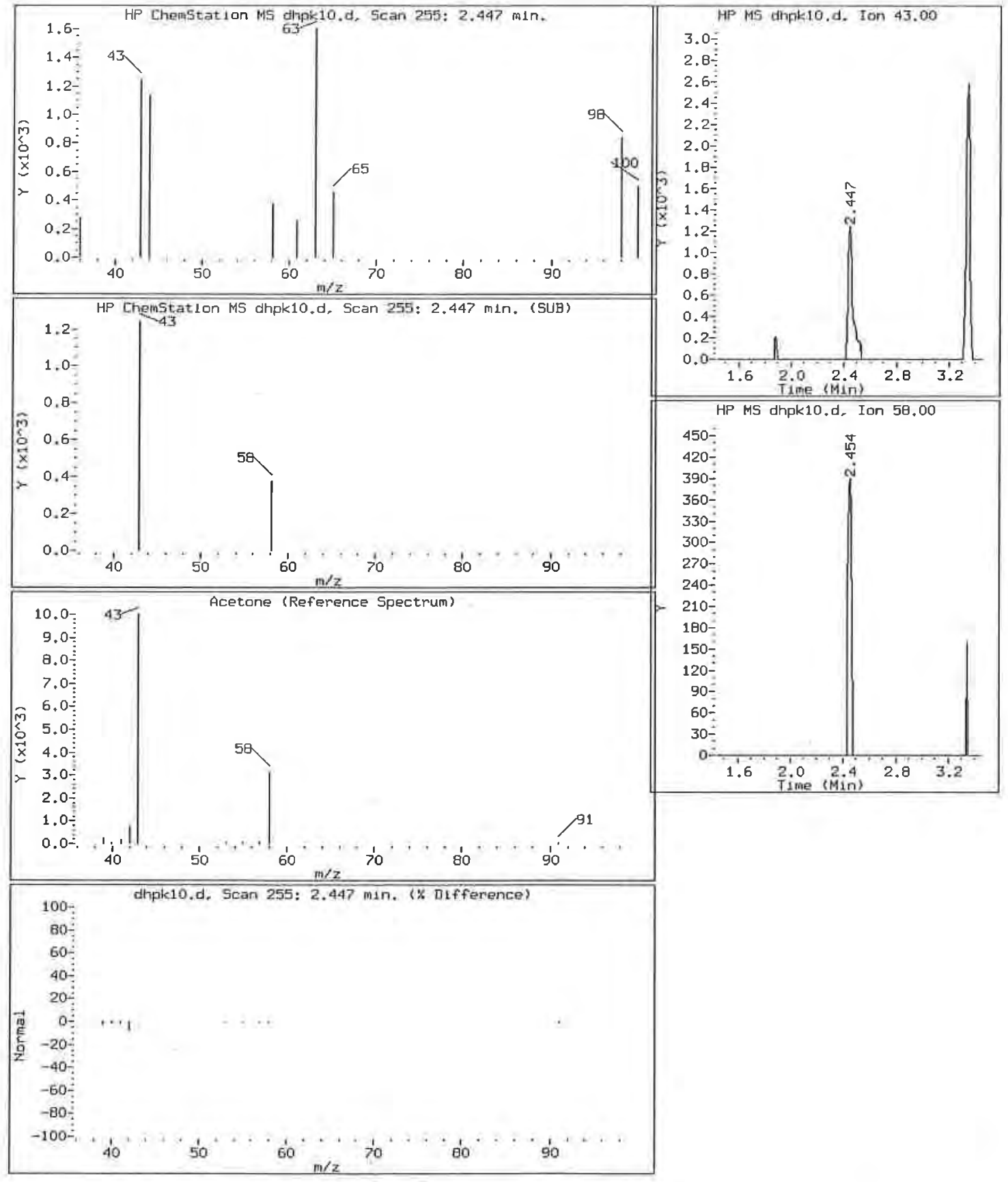
Data File: dhpk10.d

Lab Sample ID: 200-6522-5

Date: 15-AUG-2011 11:05

Client ID: SVQCTB-W-25897

Instrument: D.i

Sample Info: 200-6522-B-5

Operator: JH2

\section{Chloroform}
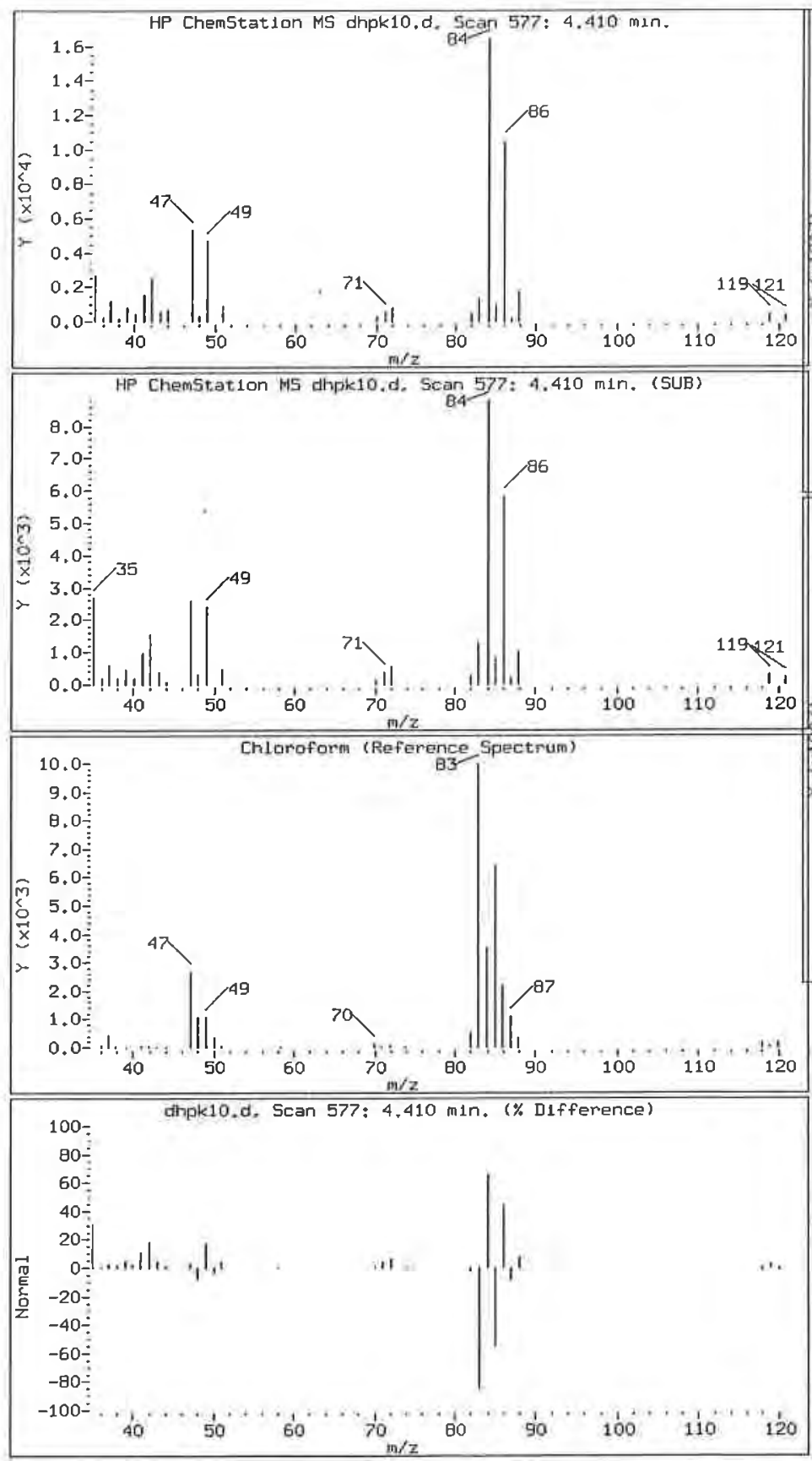
Data File: dhpk10.d

Lab Sample ID: 200-6522-5

Client ID: SVQCTB-W-25897

Sample Info: 200-6522-B-5
Date: 15-AUG-2011 11:05

Instrument: D.i

Operator: JH2

30 Benzene

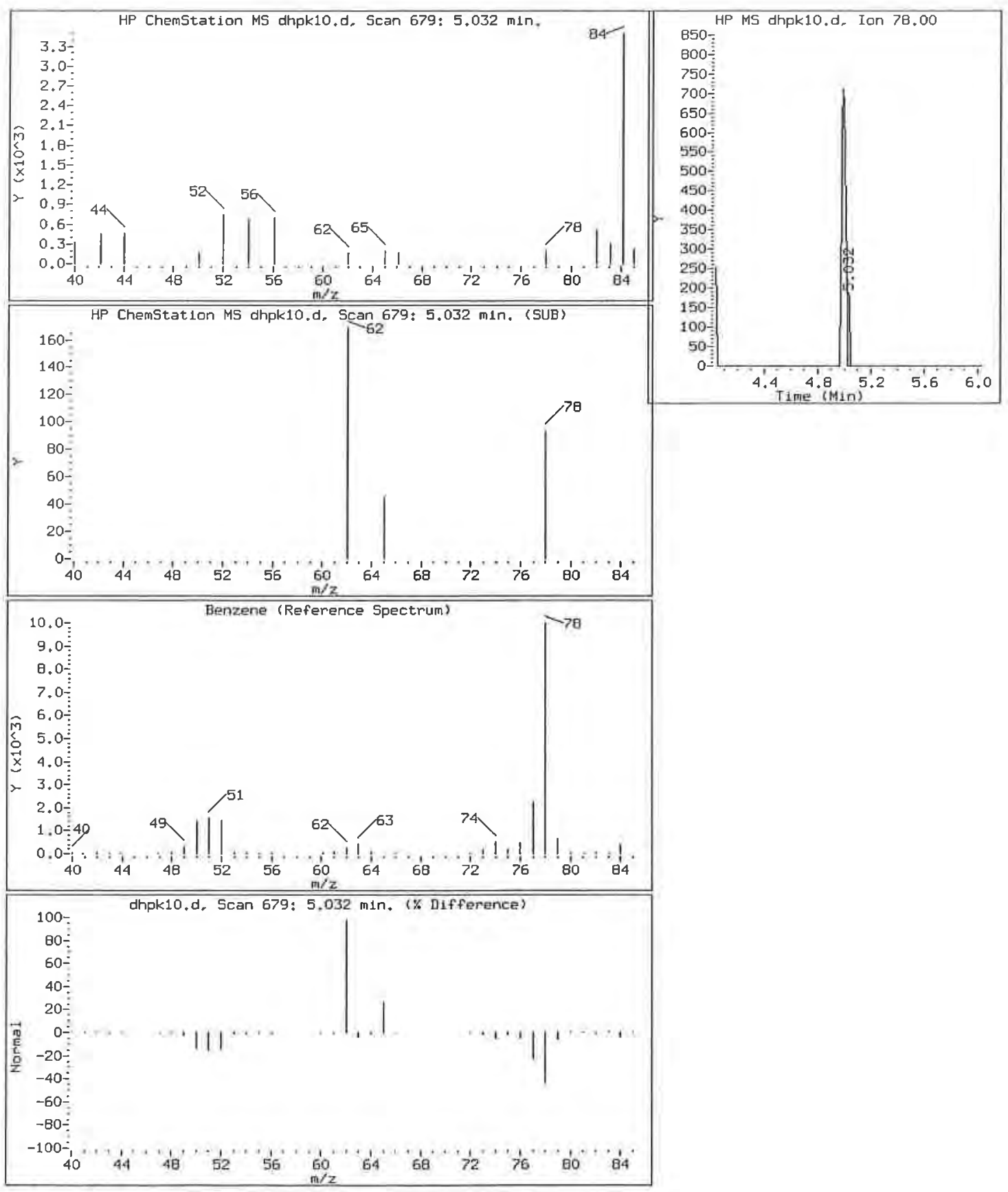


Data Eile: dhpk10.d

Lab Sample ID: 200-6522-5

Date: 15-AUG-2011 11:05

Client ID: SVQCTB-W-25897

Instrument: D.i

Sample Info: 200-6522-B-5

Operator: JH2

41 Toluene
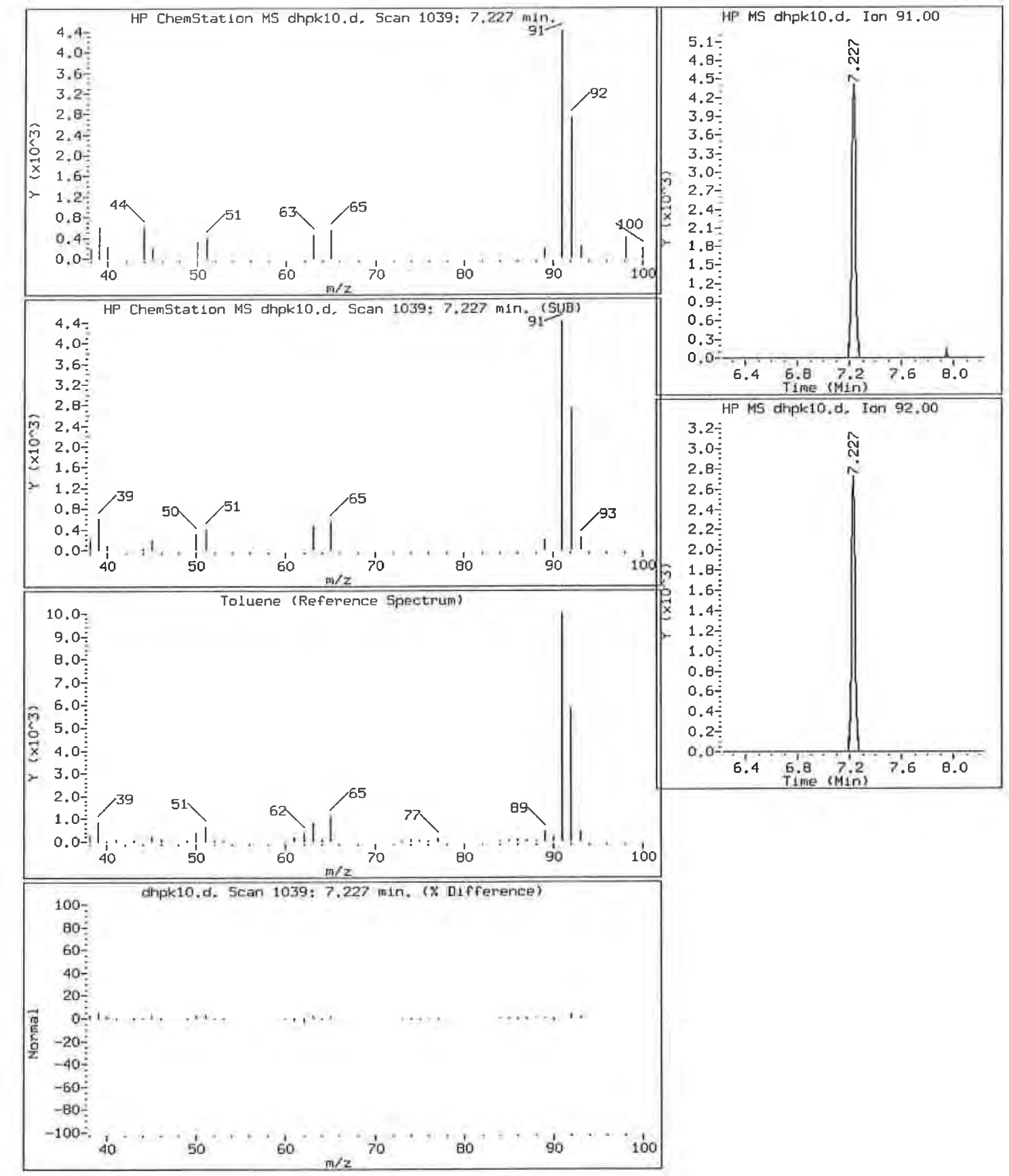
0 
Data File: dhpk10.d

Lab Sample ID: 200-6522-5

Date: 15-AUG-2011 11:05

Client ID: SVQCTB-W-25897

Instrument: D.i

Sample Info: 200-6522-B-5

Operator: JH2

$53 \mathrm{~m}, \mathrm{p}-\mathrm{xy}$ lene
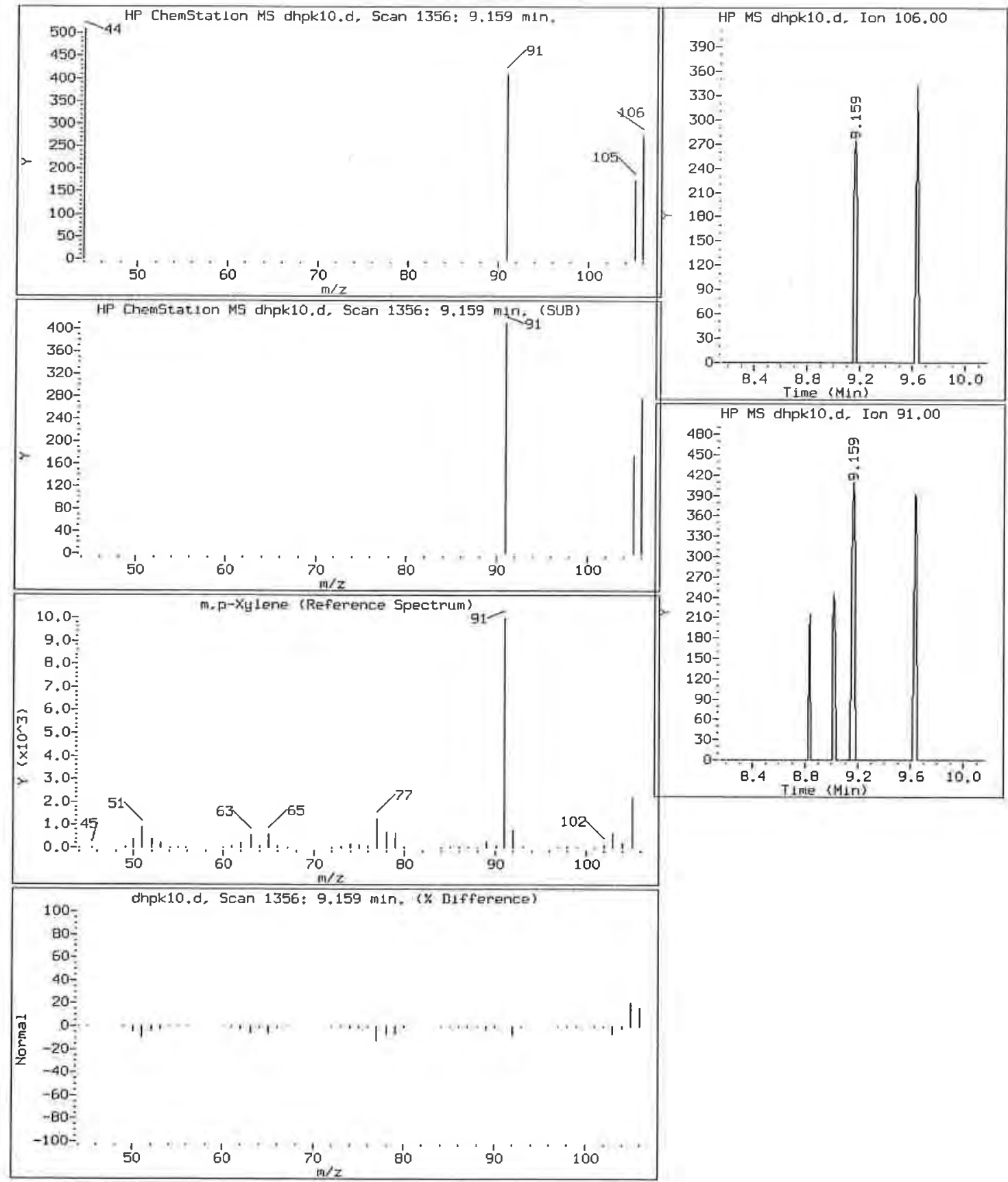
Data File: dhpk10.d
Lab Sample ID: 200-6522-5
Date: 15-AUG-2011 11:05
Client ID: SVQCTB-W-25897
Instrument: D.i
Sample Info: 200-6522-B-5
Operator: JH2

55 o-xylene
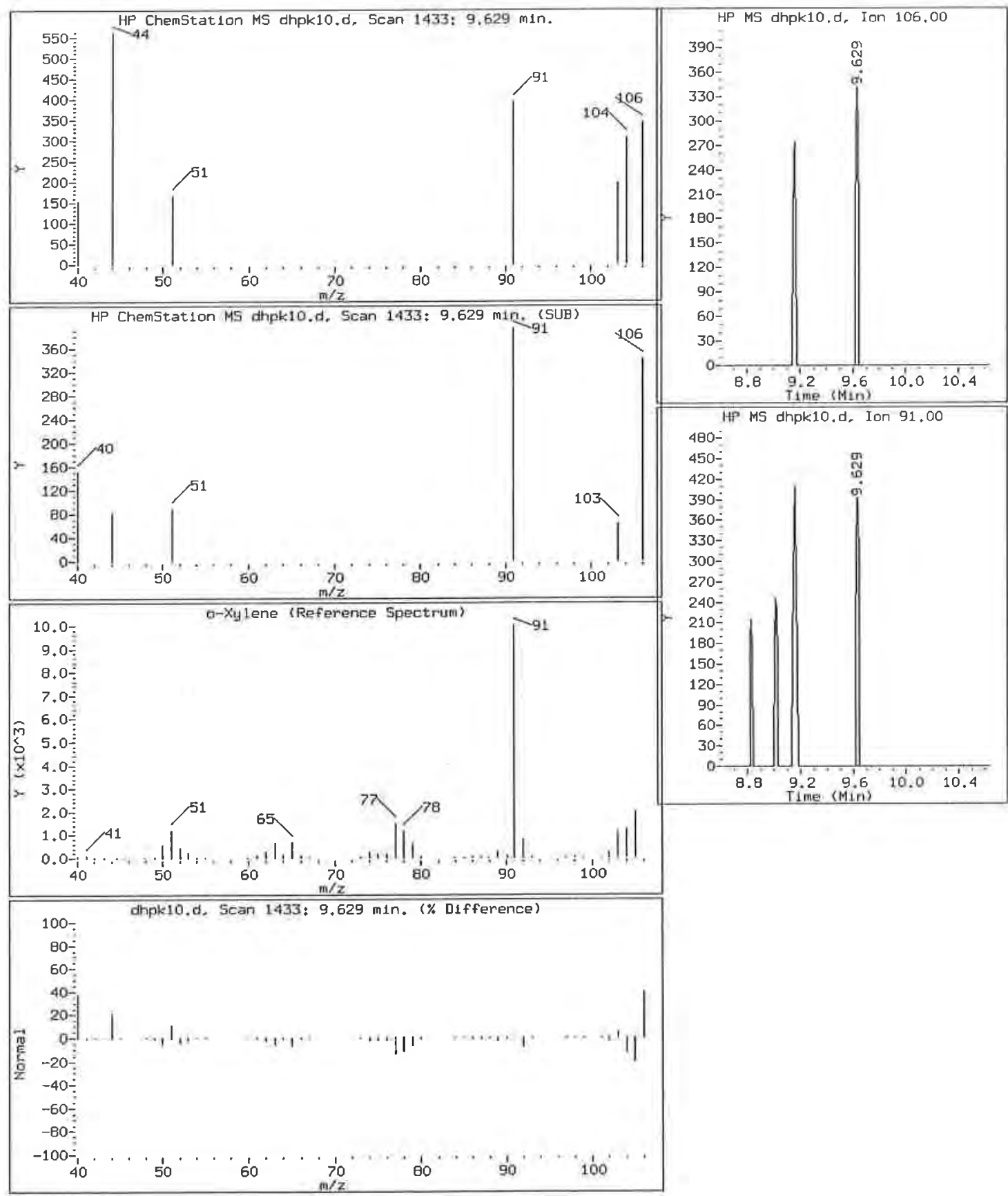
Data File: dhpk10.d

Lab Sample ID: 200-6522-5

Date: 15-AUG-2011 11:05

Client ID: SVQCTB-W-25897

Instrument: D.i

Sample Info: 200-6522-B-5

Operator: JH2

\section{4 styrene}
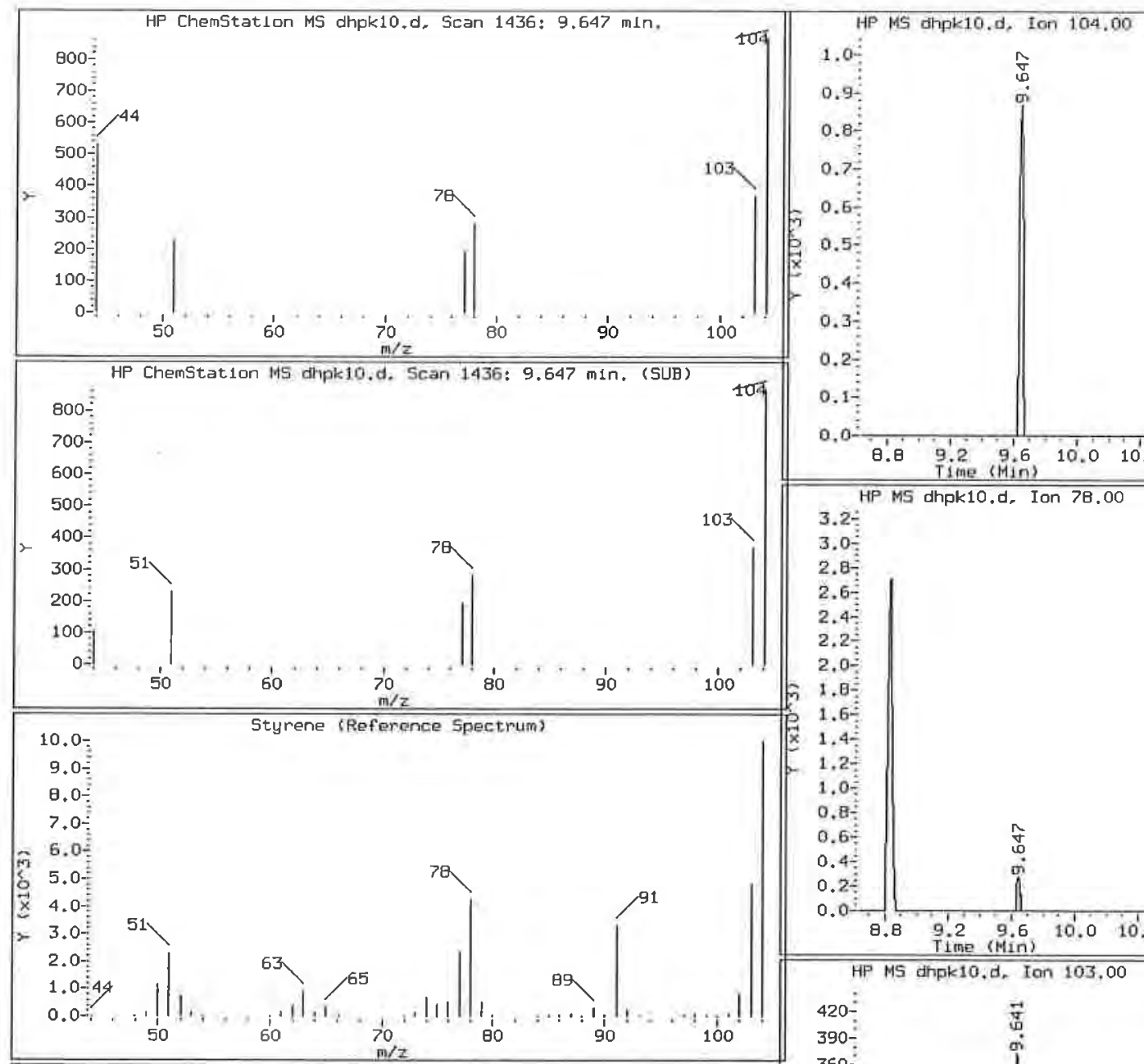

0.2

$0.1 \frac{1}{-}$
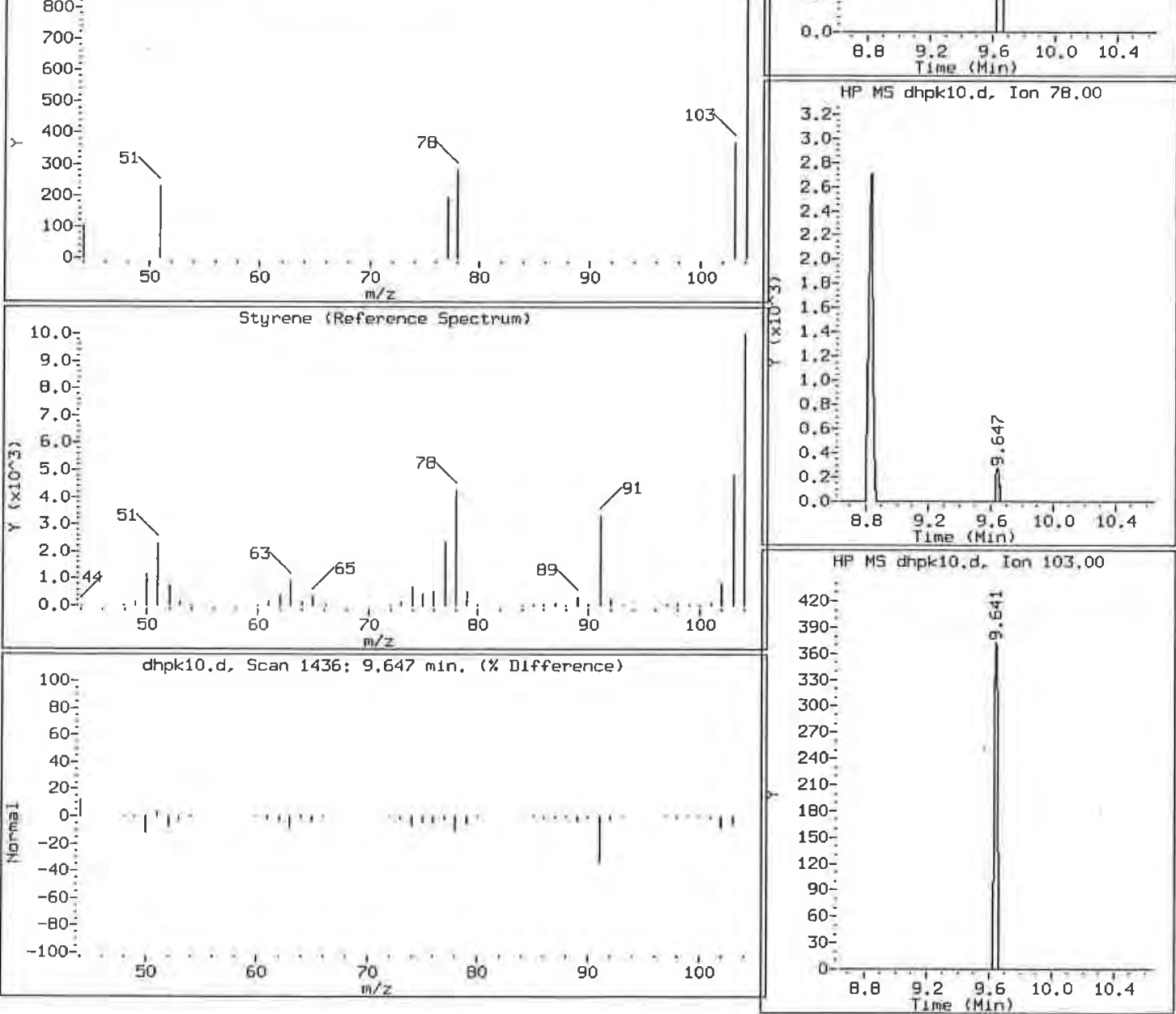
Data File: dhpk10.d

Lab Sample ID: 200-6522-5

Client ID: SVQCTB-W-25897

Sample Info: 200-6522-B-5

Retention Time: $\quad 6.79$

Library Search Compound Match

Unknown

Carbonothioic dichloride

2-Propanol, 1,3-dichloro-

Methane, oxybis[chloro-
Date: 15-AUG-2011 11:05

Instrument: D.i

Operator: JH2

$\begin{array}{llllll}\text { CAS Number } & \text { Library } & \text { Entry } & \text { Quality } & \text { Formula } & \text { Weight } \\ & & & & & \\ \text { 463-71-8 } & \text { NIST05.1 } & 7475 & 47 & \text { CC12S } & 114 \\ 96-23-1 & \text { NIST05.1 } & 11615 & 25 & \text { C3H6Cl2O } & 128 \\ 542-88-1 & \text { NIST05.1 } & 6855 & 23 & \text { C2H4Cl2O } & 114\end{array}$
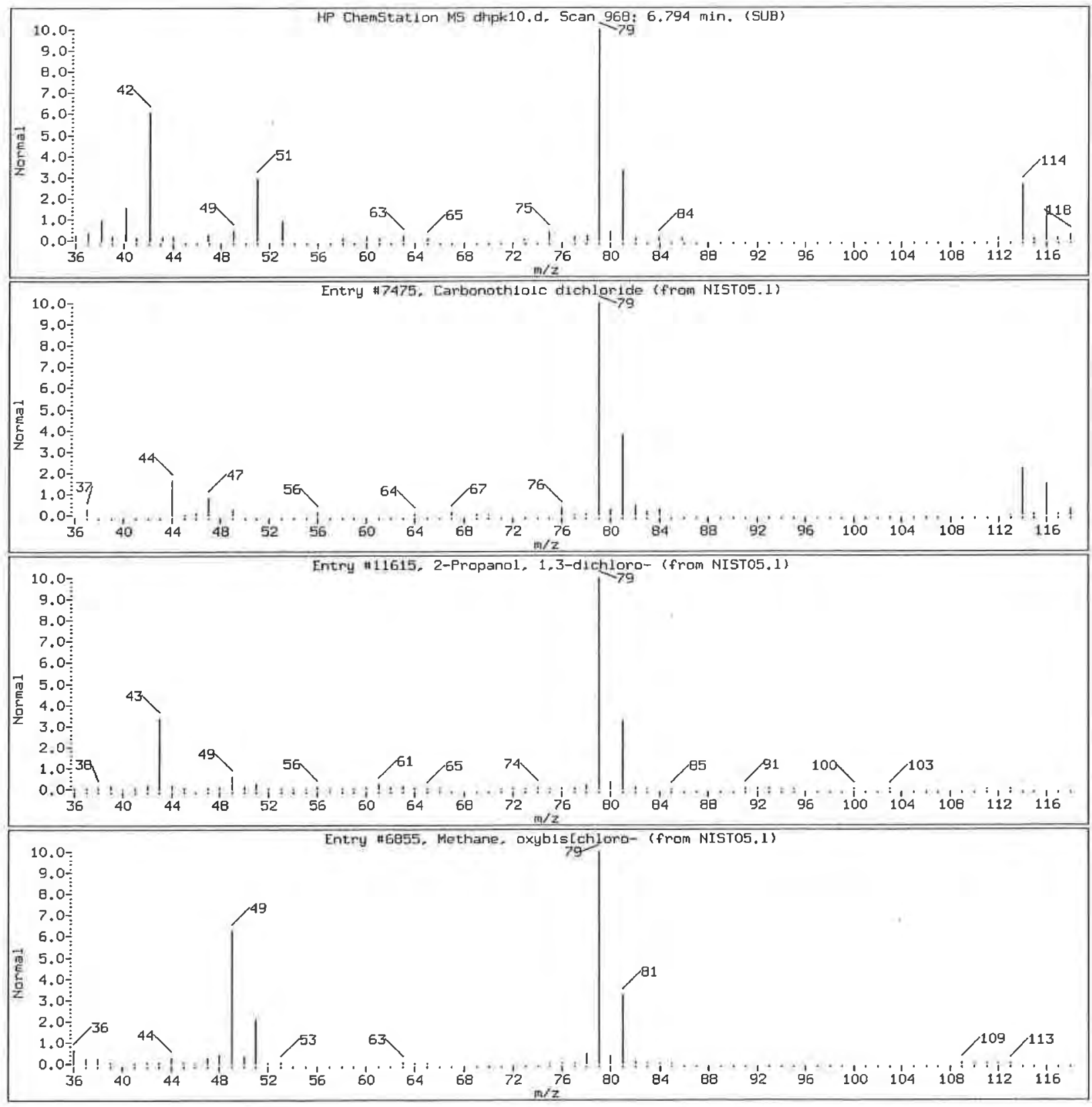
$1 A$ - EORM I VOA-1

VOLATILE ORGANICS ANALYSIS DATA SHEET
EPA SAMPLE NO.

SVSB $43 S-W-25874$
Lab Name: TESTAMERICA BURLINGTON

Lab Code: STLV

Matrix: (SOIL/SED/WATER)

Sample wt/vol: 25.0

Level: (TRACE/LOW/MED) Case No.: SAVANN Mod. Ref No.:

Contract: $\quad 8 \mathrm{E}-00302$

o Moisture: not dec.

GC Column: DB-624

ID $: 0.20$

Water

$(\mathrm{g} / \mathrm{mL}) \quad \mathrm{mL}$

RACE

Soil Extract Volume:

Purge Volume: 25.0

$(m i)$
SDG No.: 200-6522

Lab Sample ID: 200-6522-1

Lab File ID: DHPK06.D

Date Received: 08/12/2011

Date Analyzed: 08/15/2011

Dilution Factor: 1.0

Soil Aliquot Volume: (uL)

\begin{tabular}{|c|c|c|c|}
\hline CAS NO. & COMPOUND & $\begin{array}{l}\text { CONCENTRATION UNITS: } \\
(\mathrm{ug} / \mathrm{L} \text { or } \mathrm{ug} / \mathrm{kg} \text { ) } \mathrm{ug} / \mathrm{L}\end{array}$ & $\mathrm{Q}$ \\
\hline $75-71-8$ & Dichlorodifluoromethane & 0.50 & $\overline{\mathrm{U}}$ \\
\hline $74-87-3$ & Chloromethane & 0.10 & $\mathrm{~J}$ \\
\hline $75-01-4$ & Vinyl chloride & 0.50 & $\mathrm{U}$ \\
\hline $74-83-9$ & Bromomethane & 0.50 & $\mathrm{U}$ \\
\hline $75-00-3$ & Chloroethane & 0.50 & $\mathrm{U}$ \\
\hline $75-69-4$ & Trichlorofluoromethane & 0.50 & $\mathrm{U}$ \\
\hline $75-35-4$ & 1,1-Dichloroethene & 0.50 & $\mathrm{U}$ \\
\hline $76-13-1$ & 1,1,2-Trichloro-1,2,2-trifluoroethane & 0.50 & $\mathrm{U}$ \\
\hline $67-64-1$ & Acetone & 0.73 & $\mathrm{~J}$ \\
\hline $75-15-0$ & Carbon disulfide & 0.50 & $\mathrm{U}$ \\
\hline $79-20-9$ & Methyl acetate & 0.50 & $\mathrm{U}$ \\
\hline $75-09-2$ & Methylene Chloride & 0.27 & $\mathrm{~J} B$ \\
\hline $156-60-5$ & trans-1,2-Dichloroethene & 0.50 & $\mathrm{U}$ \\
\hline $1634-04-4$ & Methyl tert-butyl ether & 0.50 & $\mathrm{U}$ \\
\hline $75-34-3$ & 1,1-Dichloroethane & 0.50 & $\mathrm{U}$ \\
\hline $156-59-2$ & cis-1,2-Dichloroethene & 0.50 & $\mathrm{U}$ \\
\hline $78-93-3$ & 2-Butanone & 5.0 & $\mathrm{U}$ \\
\hline $74-97-5$ & Bromochloromethane & 0.50 & $\mathrm{U}$ \\
\hline $67-66-3$ & Chloroform & 2.8 & \\
\hline $71-55-6$ & 1,1,1-Trichloroethane & 0.50 & $\mathrm{U}$ \\
\hline $110-82-7$ & Cyclohexane & 0.50 & $\mathrm{U}$ \\
\hline $56-23-5$ & Carbon tetrachloride & 0.042 & $\mathrm{~J}$ \\
\hline $71-43-2$ & Benzene & 0.0040 & $\mathrm{~J} \mathrm{~B}$ \\
\hline $107-06-2$ & 1,2-Dichloroethane & 0.50 & $\mathrm{U}$ \\
\hline
\end{tabular}

Report 1,4-Dioxane for Low-Medium VOA analysis only 
$1 B$ - FORM I VOA-2

VOLATILE ORGANICS ANALYSIS DATA SHEET
EPA SAMPLE NO.

SVSB43S-W-25874
Lab Name: TESTAMERICA BURLINGTON

Lab Code: STLV Case No.: SAVANN Mod. Ref No.:
Contract: $8 \mathrm{E}-00302$

SDG No.: 200-6522
Matrix: (SOIL/SED/WATER)

Sample wt/vol: 25.0

Water

$(\mathrm{g} / \mathrm{mL}) \quad \mathrm{mL}$

Level: (TRACE/LOW/MED) TRACE

응 Moisture: not dec.

GC Column: DB-624

Soil Extract Volume:

Purge Volume: 25.0
ID: $0.20 \quad$ (mm)

(uL)

(mL)
Lab Sample ID: 200-6522-1

Lab File ID: DHPK06.D

Date Received: 08/12/2011

Date Analyzed: 08/15/2011

Dilution Factor: 1.0

Soil Aliquot Volume: (uL)

\begin{tabular}{|c|c|c|c|}
\hline CAS NO. & COMPOUND & $\begin{array}{l}\text { CONCENTRATION UNITS: } \\
(u g / L \text { or } u g / k g) u g / L\end{array}$ & $\mathrm{Q}$ \\
\hline $79-01-6$ & Trichloroethene & 0.50 & $\mathrm{U}$ \\
\hline $108-87-2$ & Methylcyclohexane & 0.50 & $\mathrm{U}$ \\
\hline $78-87-5$ & 1,2-Dichloropropane & 0.50 & $\mathrm{U}$ \\
\hline $75-27-4$ & Bromodichloromethane & 0.50 & $\mathrm{U}$ \\
\hline $10061-01-5$ & cis-1,3-Dichloropropene & 0.50 & $\mathrm{U}$ \\
\hline $108-10-1$ & 4-Methyl-2-pentanone & 5.0 & $\mathrm{U}$ \\
\hline $108-88-3$ & Toluene & 0.032 & $\mathrm{~J} \mathrm{~B}$ \\
\hline $10061-02-6$ & trans-1,3-Dichloropropene & 0.50 & $\mathrm{U}$ \\
\hline $79-00-5$ & 1,1,2-Trichloroethane & 0.50 & $\mathrm{U}$ \\
\hline $127-18-4$ & Tetrachloroethene & 0.073 & $\mathrm{~J}$ \\
\hline $591-78-6$ & 2-Hexanone & 5.0 & $\mathrm{U}$ \\
\hline $124-48-1$ & Dibromochloromethane & 0.50 & $\mathrm{U}$ \\
\hline $106-93-4$ & 1,2-Dibromoethane & 0.50 & $\mathrm{U}$ \\
\hline $108-90-7$ & Chlorobenzene & 0.50 & $\mathrm{U}$ \\
\hline $100-41-4$ & Ethylbenzene & 0.50 & $\mathrm{U}$ \\
\hline $95-47-6$ & o-Xylene & 0.50 & $\mathrm{U}$ \\
\hline $179601-23-1$ & $\mathrm{~m}, \mathrm{p}$-Xylene & 0.50 & $\mathrm{U}$ \\
\hline $100-42-5$ & Styrene & 0.50 & $\mathrm{U}$ \\
\hline $75-25-2$ & Bromoform & 0.50 & $\mathrm{U}$ \\
\hline $98-82-8$ & Isopropylbenzene & 0.50 & $\mathrm{U}$ \\
\hline $79-34-5$ & $1,1,2,2$-Tetrachloroethane & 0.50 & U \\
\hline $541-73-1$ & 1,3-Dichlorobenzene & 0.50 & $\mathrm{U}$ \\
\hline $106-46-7$ & 1,4-Dichlorobenzene & 0.50 & $\mathrm{U}$ \\
\hline $95-50-1$ & 1,2-Dichlorobenzene & 0.50 & $\mathrm{U}$ \\
\hline $96-12-8$ & 1,2-Dibromo-3-Chloropropane & 0.50 & $\mathrm{U}$ \\
\hline $120-82-1$ & $1,2,4$-Trichlorobenzene & 0.50 & $\mathrm{U}$ \\
\hline $87-61-6$ & $1,2,3$-Trichlorobenzene & 0.50 & $\mathrm{U}$ \\
\hline
\end{tabular}


IJ - FORM I VOA-TIC

VOLATILE ORGANICS ANALYSIS DATA SHEET

TENTATIVELY IDENTIEIED COMPOUNDS
EPA SAMPLE NO.

SVSB43S-W-25874

Lab Name: TESTAMERICA BURLINGTON

Contract: $8 \mathrm{E}-00302$

Lab Code: STLV Case No.: SAVANN Mod. Ref No.:

Matrix: (SOIL/SED/WATER)

Sample wt/vol: 25.0

Water

Level: (TRACE or LOW/MED) $(\mathrm{g} / \mathrm{mL}) \mathrm{mL}$

o Moisture: not dec.

GC Column: DB-624 ID : 0.20 (mrn)

Soil Extract Volume: (uL)

CONCENTRATION UNITS: (ug/L or $\mathrm{ug} / \mathrm{kg}$ ) ug/L SDG No.: 200-6522

$$
\text { : }
$$

Lab Sample ID: 200-6522-1

Lab File ID: DHPK06.D

Date Received: 08/12/2011

Date Analyzed: 08/15/2011

Dilution Factor: 1.0

Soil Aliquot Volume: (uL)

Purge Volume: 25.0 (mL)

\begin{tabular}{|l|l|l|r|r|r|}
\hline \multicolumn{1}{|c|}{ CAS NUMBER } & \multicolumn{1}{|c|}{ COMPOUND NAME } & RT & EST. CONC. & Q \\
\cline { 2 - 6 } 01 & & Unknown & 6.79 & 2.9 & B X J \\
\cline { 2 - 6 } & E9667961 & Total Alkanes & N/A & & \\
\hline
\end{tabular}

1 EPA-designated Registry Number. 


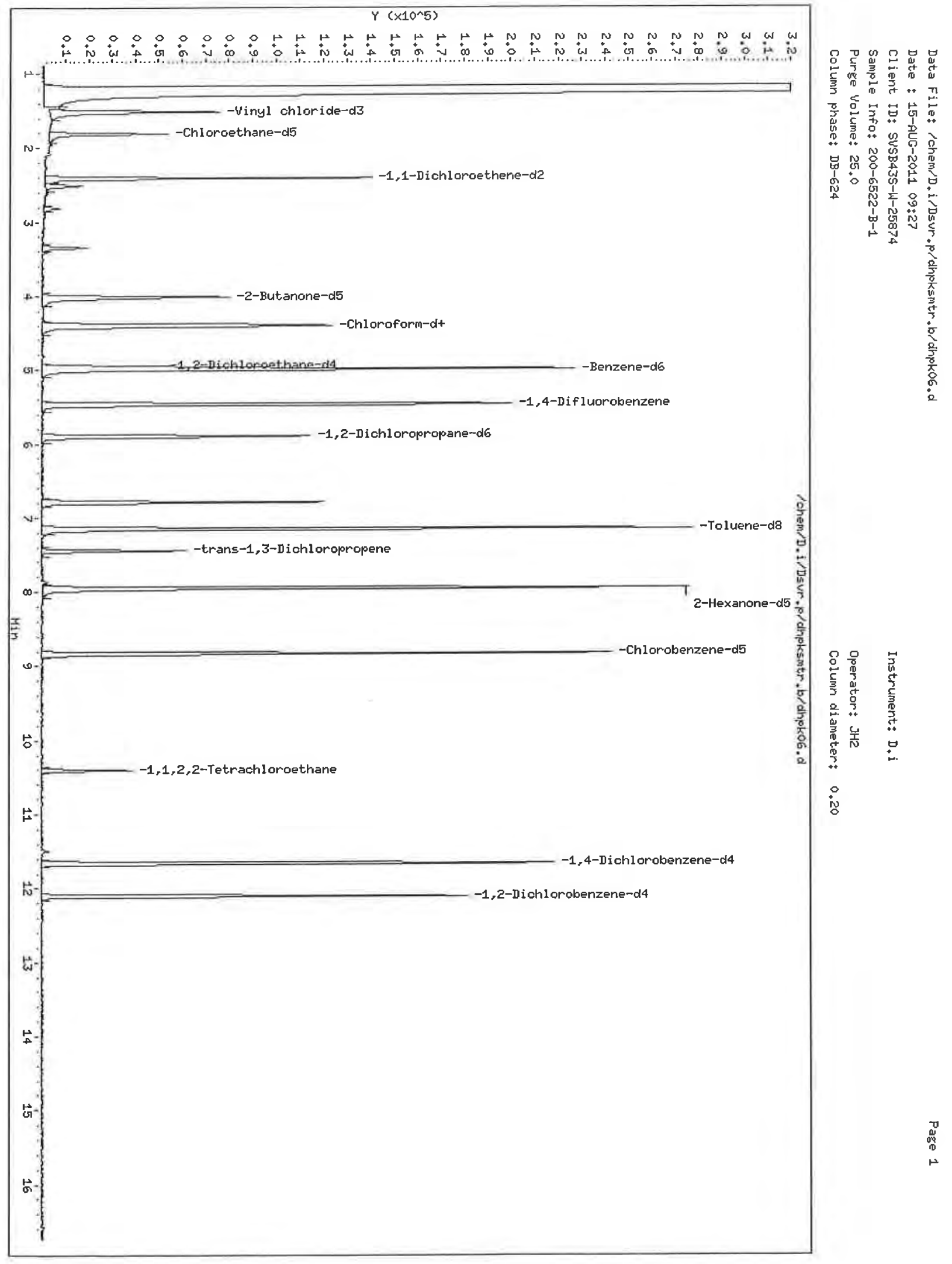


TestAmerica Burlington

Lab Sample Id: 200-6522-1

LOW CONCENTRATION VOLATILE QUANTITATION REPORT

Client Smp ID: SVSB43S-W-25874

Inj Date : 15-AUG-2011 09:27

Operator : JH2

Smp Info : 200-6522-B-1

Inst ID: D.i

Misc Info: 1,5

Comment :

Method : /chem/D.i/Dsvr.p/dhpksmtr.b/somtr5t.m

Meth Date : 15-Aug-2011 16:21 jh2 Quant Type: ISTD

Cal Date : 07-JUL-2011 12:56 Cal File: dhp08.d

Als bottle: 4

Dil Factor: 1.00000

Integrator: HP RTE Compound Sublist: all.sub

Target Version: 3.50

Processing Host: chemsvr6

Concentration Formula: Amt * DF * Uf/Vo * CpndVariable

\begin{tabular}{|c|c|c|}
\hline Name & Value & Description \\
\hline $\mathrm{DF}$ & 1.00000 & Dilution Factor \\
\hline Uf & 1.00000 & ng unit correction factor \\
\hline Vo & 25.00000 & Sample Volume purged (mL) \\
\hline
\end{tabular}

\section{Compounds}

1 Dichlorodifluoromethane

2 chloromethane

$\$ 3$ Vinyl chloride-d3

4 vinyl chloride

5 Bromomethane

\$ 6 Chloroethane-d5

7 Chloroethane

8 Trichlorofluoromethane

\$ 9 1,1-Dichloroethene-d2

10 1,1-Dichloroethene

11 1,1,2-Trichloro-1,2,2-trifluo

12 Acetone

13 Carbon disulfide

14 Methyl acetate

15 Methylene chloride

16 trans-1,2-Dichloroethene

\section{QUANT SIG}

MASS

85

50

65

62

94

69

64

101

63

96

101

43

76

43

84

96
RT EXP RT REL RT
$=\quad$ REms
Compound Not Detected.

$1.454 \quad 1.454 \quad(0.266)$

$1.515 \quad 1.521(0.277)$

Compound Not Detected.

Compound Not Detected.

$1.808 \quad 1.807 \quad(0.331)$

Compound Not Detected.

Compound Not Detected.

$2.405 \quad 2.411 \quad(0.440)$.

Compound Not Detected.

Compound Not Detected.

$2.448 \quad 2.448 \quad(0.448)$

Compound Not Detected.

Compound Not Detected.

$2.813 \quad 2.813 \quad(0.515)$

Compound Not Detected.
CONCENTRAT IONS

ON-COLUMN FINAT

$\begin{array}{rcc}\text { RESPONSE } & \begin{array}{c}\text { ON-COLUMN } \\ (\mathrm{ng})\end{array} & \begin{array}{c}\text { FINAT } \\ (\mathrm{ug} / \mathrm{L}) \\ =======\end{array} \\ 2427 & \begin{array}{c}2.49389 \\ 77675\end{array} & \begin{array}{c}0.100(\mathrm{a}) \\ 125.861\end{array} \\ & & 5.0 \\ 51.165 & 127.779 & 5.1 \\ & & \\ 99213 & 88.0817 & 3.5 \\ & & 0.73(\mathrm{a}) \\ 922 & 18.2397 & \\ & & 0.27(\mathrm{a})\end{array}$




\begin{tabular}{|c|c|c|}
\hline \multicolumn{2}{|c|}{ Compounds } & \multirow{2}{*}{$\begin{array}{l}\text { MASS } \\
=\mathrm{m}=\end{array}$} \\
\hline ment & 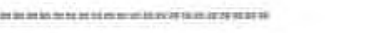 & \\
\hline 17 & Methyl tert-butyl ether & 73 \\
\hline 18 & 1,1-Dichloroethane & 63 \\
\hline 19 & 2-Butanone-d5 & 46 \\
\hline 20 & cis-1,2-Dichloroethene & 96 \\
\hline 21 & 2-Butanone & 43 \\
\hline 22 & Bromochloromethane & 128 \\
\hline 23 & Chloroform-d & 84 \\
\hline 24 & Chloroform & 83 \\
\hline 25 & 1,1,1-Trichloroethane & 97 \\
\hline 26 & Cyclohexane & 56 \\
\hline 27 & Carbon tetrachloride & 117 \\
\hline 28 & 1,2-Dichloroethane-d4 & 65 \\
\hline 29 & Benzene-d6 & 84 \\
\hline 30 & Benzene & 78 \\
\hline 31 & 1,2-Dichloroethane & 62 \\
\hline 32 & 1,4-Difluorobenzene & 114 \\
\hline 33 & Trichloroethene & 95 \\
\hline 34 & 1,2-Dichloropropane-d6 & 67 \\
\hline 35 & Methylcyclohexane & 55 \\
\hline 36 & 1,2-Dichloropropane & 63 \\
\hline 37 & Bromodichloromethane & 83 \\
\hline 38 & cis-1,3-Dichloropropene & 75 \\
\hline 39 & 4-Methyl-2-pentanone & 43 \\
\hline 40 & Toluene-d8 & 98 \\
\hline 41 & Toluene & 91 \\
\hline 42 & trans-1,3-Dichloropropene-d4 & 79 \\
\hline 43 & trans-1,3-Dichloropropene & 75 \\
\hline 44 & 1,1,2-Trichloroethane & 97 \\
\hline 45 & Tetrachloroethene & 164 \\
\hline 46 & 2-Hexanone-d5 & 63 \\
\hline 47 & 2-Hexanone & 43 \\
\hline 48 & Dibromochloromethane & 129 \\
\hline 49 & 1,2-Dibromoethane & 107 \\
\hline 50 & Chlorobenzene-d5 & 117 \\
\hline 51 & Chlorobenzene & 112 \\
\hline 52 & Ethylbenzene & 91 \\
\hline 53 & m,p-Xylene & 106 \\
\hline 54 & Styrene & 104 \\
\hline 55 & o-Xylene & 106 \\
\hline 56 & Bromoform & 172 \\
\hline 57 & Isopropylbenzene & 105 \\
\hline 58 & $1,1,2,2$-Tetrachloroethane-d2 & 84 \\
\hline 59 & $1,1,2,2$-Tetrachloroethane & 83 \\
\hline 60 & 1,3-Dichlorobenzene & 146 \\
\hline 61 & 1,4-Dichlorobenzene-d4 & 152 \\
\hline 62 & 1,4-Dichlorobenzene & 146 \\
\hline 63 & 1,2-Dichlorobenzene-d4 & 152 \\
\hline 64 & 1,2-Dichlorobenzene & 146 \\
\hline
\end{tabular}

\begin{tabular}{|c|c|c|c|c|c|}
\hline \multirow[b]{2}{*}{ QUANT SIG } & & & & \multicolumn{2}{|c|}{ CONCENTRATIONS } \\
\hline & & & & ON-COLUMN & FINAL \\
\hline MASS & RT & EXP RT REL RT & RESPONSE & ( $\mathrm{ng})$ & $(\mathrm{ug} / \mathrm{L})$ \\
\hline$= \pm=$ & $=$ & 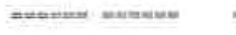 & 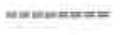 & 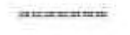 & $=\sin =0=$ \\
\hline 73 & \multicolumn{3}{|c|}{ Compound Not Detected. } & & \\
\hline 63 & \multicolumn{3}{|c|}{ Compound Not Detected. } & & \\
\hline 46 & 4.014 & $4.020(0.735)$ & 127954 & 2188.21 & $88(R)$ \\
\hline 96 & \multicolumn{3}{|c|}{ Compound Not Detected. } & & \\
\hline 43 & \multicolumn{3}{|c|}{ Compound Not Detected. } & & \\
\hline 128 & \multicolumn{3}{|c|}{ Compound Not Detected. } & & \\
\hline 84 & 4.392 & $4.392(0.804)$ & 84414 & 116.983 & 4.7 \\
\hline 83 & 4.411 & $4.417(0.807)$ & 48903 & 71.1381 & 2.8 \\
\hline 97 & \multicolumn{3}{|c|}{ Compound Not Detected. } & & \\
\hline 56 & \multicolumn{3}{|c|}{ Compound Not Detected. } & & \\
\hline 117 & 4.819 & $4.819(0.546)$ & 599 & 1.06224 & $0.042(\mathrm{aQ})$ \\
\hline 65 & 4.953 & $4.959(0.906)$ & 35272 & 122.598 & 4.9 \\
\hline 84 & 4.990 & $4.990(0.565)$ & 211642 & 120.134 & 4.8 \\
\hline 78 & 5.039 & $5.032(0.571)$ & 183 & 0.10115 & $0.0040(a)$ \\
\hline 62 & \multicolumn{3}{|c|}{ Compound Not Detected. } & & \\
\hline 114 & 5.465 & $5.471(1.000)$ & 177084 & 125.000 & \\
\hline 95 & \multicolumn{3}{|c|}{ Compound Not Detected. } & & \\
\hline 67 & 5.898 & $5.904 \quad(0.668)$ & 52746 & 117.976 & 4.7 \\
\hline 55 & \multicolumn{3}{|c|}{ Compound Not Detected. } & & \\
\hline 63 & \multicolumn{3}{|c|}{ Compound Not Detected. } & & \\
\hline 83 & \multicolumn{3}{|c|}{ Compound Not Detected. } & & . \\
\hline 75 & \multicolumn{3}{|c|}{ Compound Not Detected. } & & \\
\hline 43 & \multicolumn{3}{|c|}{ Compound Not Detected. } & & \\
\hline 98 & 7.148 & $7.154 \quad(0.809)$ & 201805 & 120.945 & 4.8 \\
\hline 91 & 7.227 & $7.227(0.818)$ & 1579 & 0.79826 & 0.032 (a) \\
\hline 79 & 7.447 & $7.447(0.843)$ & 31627 & 115.333 & 4.6 \\
\hline 75 & \multicolumn{3}{|c|}{ Compound Not Detected. } & & \\
\hline 97 & \multicolumn{3}{|c|}{ Compound Not Detected. } & & \\
\hline 164 & 7.873 & $7.867 \quad(0.892)$ & 666 & 1.82392 & $0.073(a)$ \\
\hline 63 & 7.947 & $7.946(0.900)$ & 101172 & 2589.42 & $100(\mathrm{R})$ \\
\hline 43 & \multicolumn{2}{|c|}{ Compound Not Detected. } & & & \\
\hline 129 & \multicolumn{2}{|c|}{ Compound Not Detected. } & & & \\
\hline 107 & Comp & ound Not Detected. & & & \\
\hline 117 & 8.831 & $8.830(1.000)$ & 149249 & 125.000 & \\
\hline 112 & Comp & ound Not Detected. & & & \\
\hline 91 & Comp & ound Not Detected. & & & \\
\hline 106 & Comp & ound Not Detected. & & & \\
\hline 104 & Comp & ound Not Detected. & & & \\
\hline 106 & Comp & ound Not Detected. & & & \\
\hline 172 & Comp & ound Not Detected. & & & \\
\hline 105 & Comp & ound Not Detected. & & & \\
\hline 84 & 10.403 & $10.403(1.178)$ & 21005 & 107.428 & 4.3 \\
\hline 83 & Comp & ound Not Detected. & & & \\
\hline 146 & Comp & ound Not Detected. & & & \\
\hline 152 & 11.659 & $11.659(1.000)$ & 61580 & 125.000 & (Q) \\
\hline 146 & Comp & ound Not Detected. & & & \\
\hline 152 & 12.104 & $12.104(1.038)$ & 52239 & 127.814 & 5.1 \\
\hline 146 & Comp & ound Not Detected. & & & \\
\hline
\end{tabular}


Data File:/chem/D.i/Dsvr.p/dhpksmtr.b/dhpk06.d Report Date: 15-Aug-2011 20:11

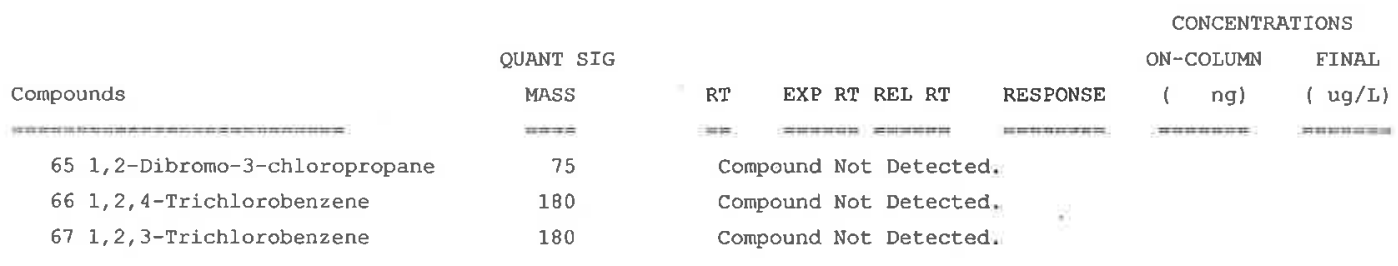

QC Flag Legend

a - Target compound detected but, quantitated amount Below Limit of Quantitation(BLOQ).

Q - Qualifier signal failed the ratio test.

R - Spike/Surrogate failed recovery limits. 


\section{TestAmerica Burlington}

LOW CONCENTRATION VOLATILE QUANTITATION REPORT

Lab Sample Id: 200-6522-1

Client Smp ID: SVSB43S-W-25874

Inj Date : 15-AUG-2011 09:27

operator : JH2

Inst ID: D.i

Smp Info: $200-6522-B-1$

Misc Info: 1,5

Comment :

Method : /chem/D.i/Dsvr.p/dhpksmtr.b/somtr5t.m

Meth Date : 15-Aug-2011 16:21 jh2 Quant Type: ISTD

Cal Date : 07-JUL-2011 12:56 Cal File: dhp08.d

Als bottle: 4

Dil Factor: 1.00000

Integrator: HP RTE

Target Version: 3.50

Processing Host: chemsvr6

Concentration Formula: Amt * DF * Uf/Vo * CpndVariable

\begin{tabular}{clc} 
Name & Value & Description \\
\hline DF & 1.00000 & Dilution Factor \\
Uf & 1.00000 & ng unit correction factor \\
Vo & 25.00000 & Sample volume purged (mL)
\end{tabular}

Cpnd Variable

$\stackrel{\text { ISTD }}{======}$

* $321,4-D i f l u o r o b e n z e n e$
Local Compound Variable

$\begin{array}{lll}\text { RT } & \begin{array}{l}\text { AREA } \\ ======\end{array} & \begin{array}{l}\text { AMOUNT } \\ =====\end{array} \\ 5.465 & 397201 & 125.000\end{array}$

CONCENTRATIONS

RT AREA ON-COL( ng) FINAL ( ug/L) QUAL

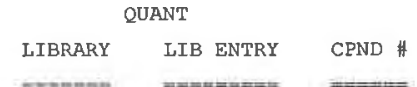

CAS \#:

Unknown

6.788

$231958 \quad 72.9975327$
$=$

0
$0 \quad 32$ 
Data File: dhpk06.d

Lab Sample ID: 200-6522-1

Date: 15-AUG-2011 09:27

Client ID: SVSB43S-W-25874

Instrument: D.i

Sample Info: 200-6522-B-1

Operator: JH2

2 Chloromethane

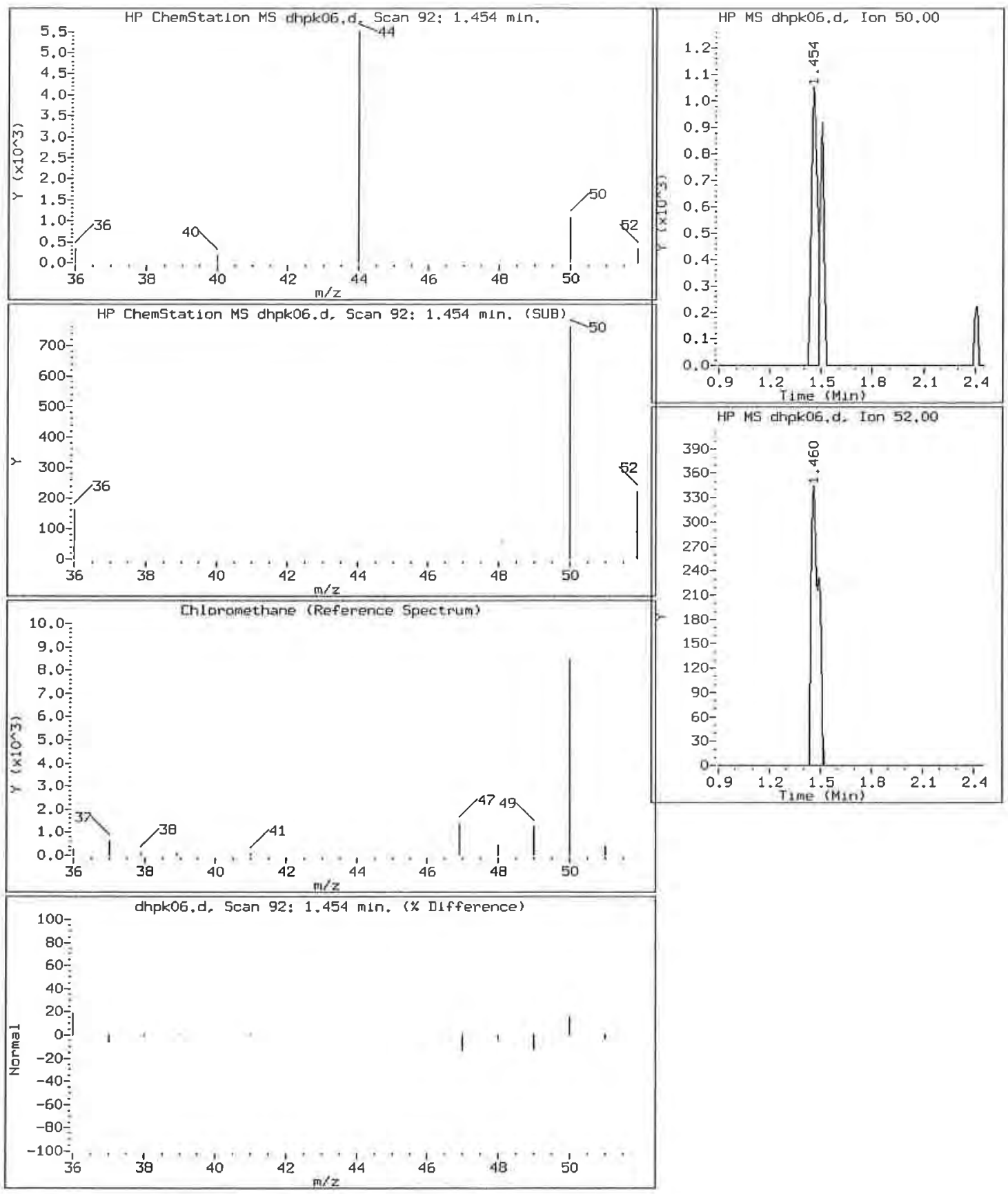


Data File: dhpk06.d

Lab Sample ID: 200-6522-1

Date: 15-AUG-2011 09:27

Client ID: SVSB43S-W-25874

Instrument: D.j

Sample Info: 200-6522-B-1

Operator: JH2

12 Acetone
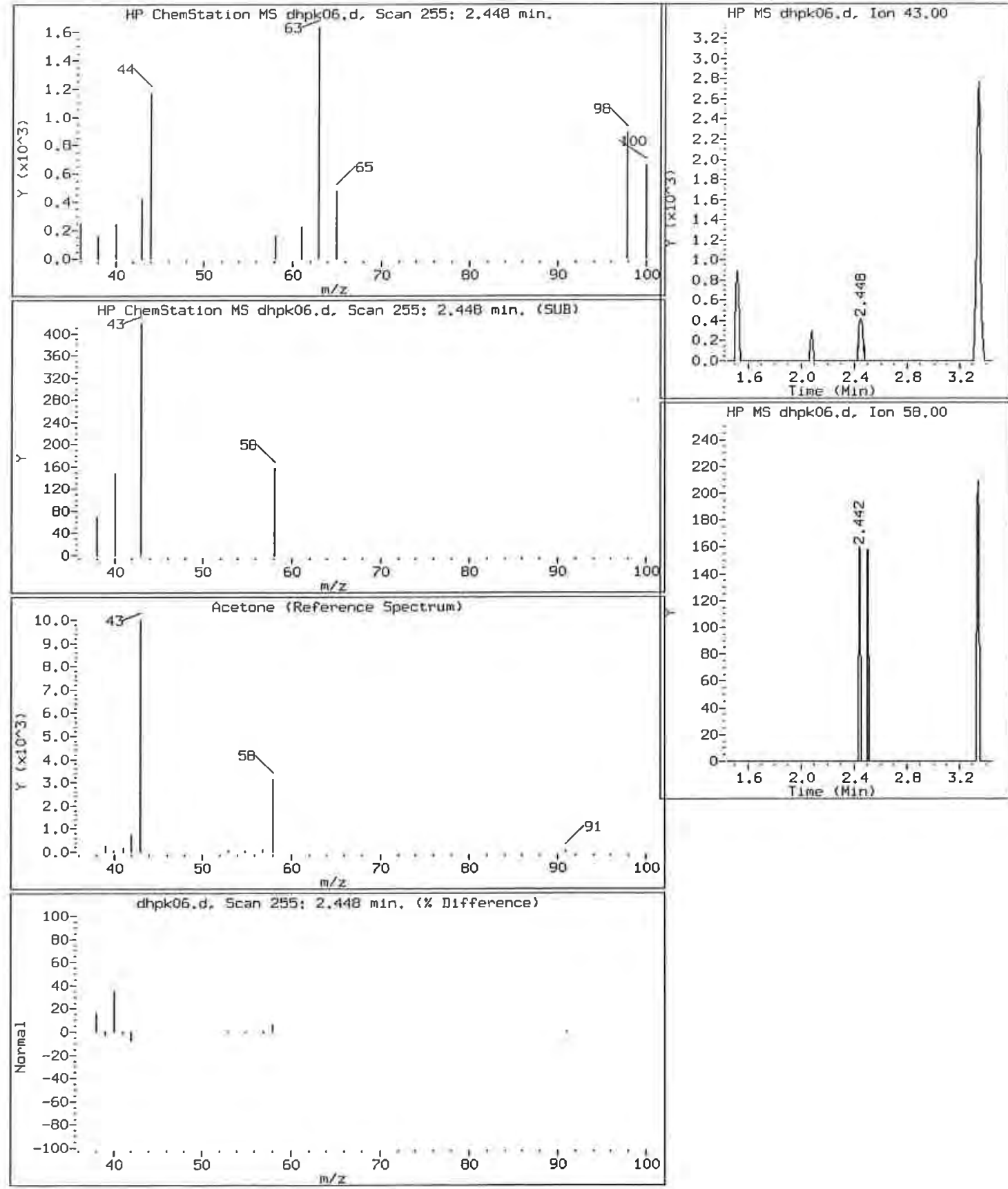
Data File: dhpk06.d

Lab Sample ID: 200-6522-1

Date: 15-AUG-2011 09:27

Client ID: SVSB43S-W-25874

Instrument: D.i

Sample Info: 200-6522-B-1

Operator: JH2

15 Methylene chloride
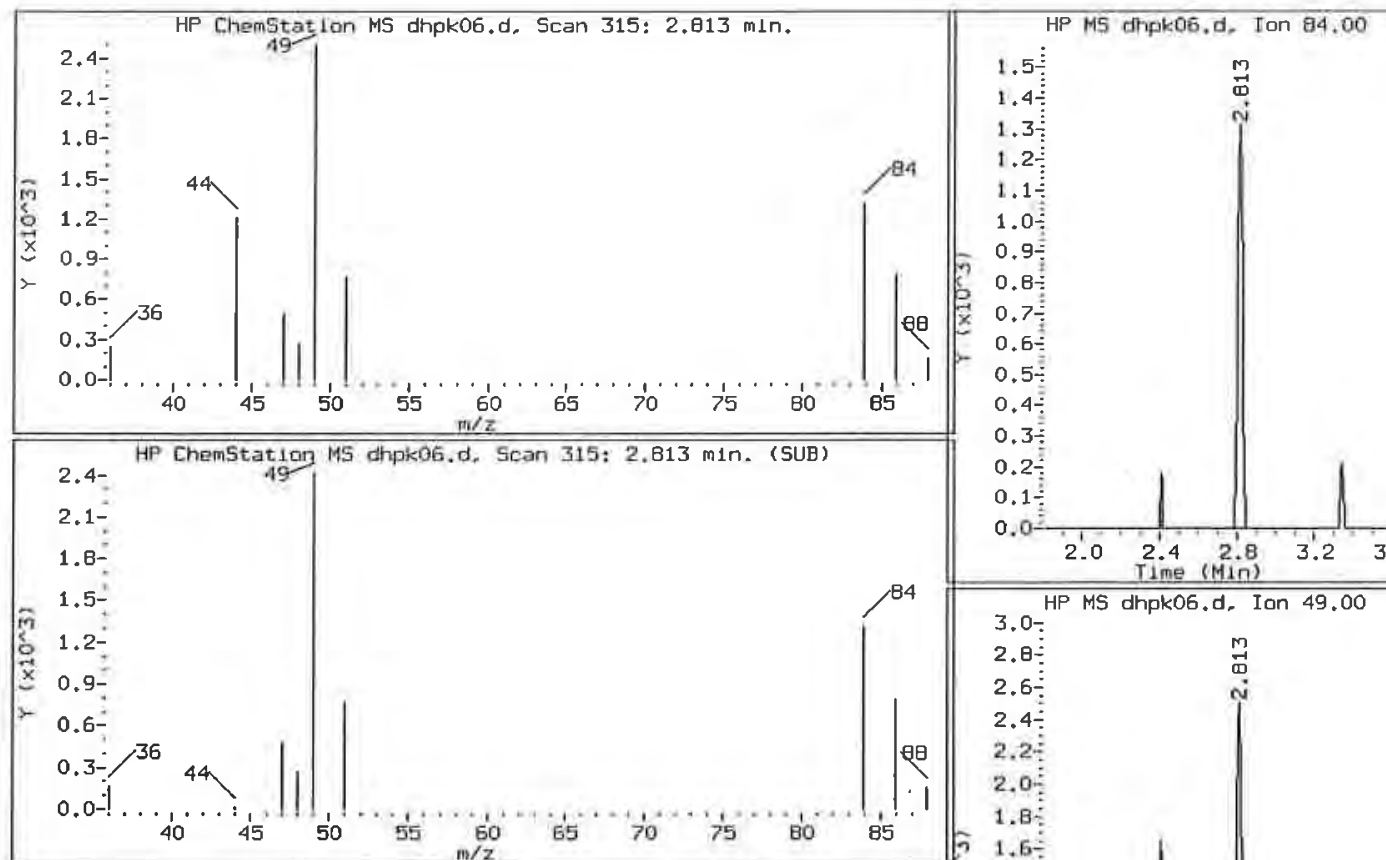

1.5

1.4

1.3

1.0

$0.9=$

$0.8 \stackrel{3}{=}$

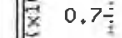

$0.6 \div$

$0.4=$

$0.3=$

$0.2 \div$

$0.1 \frac{1}{2}$
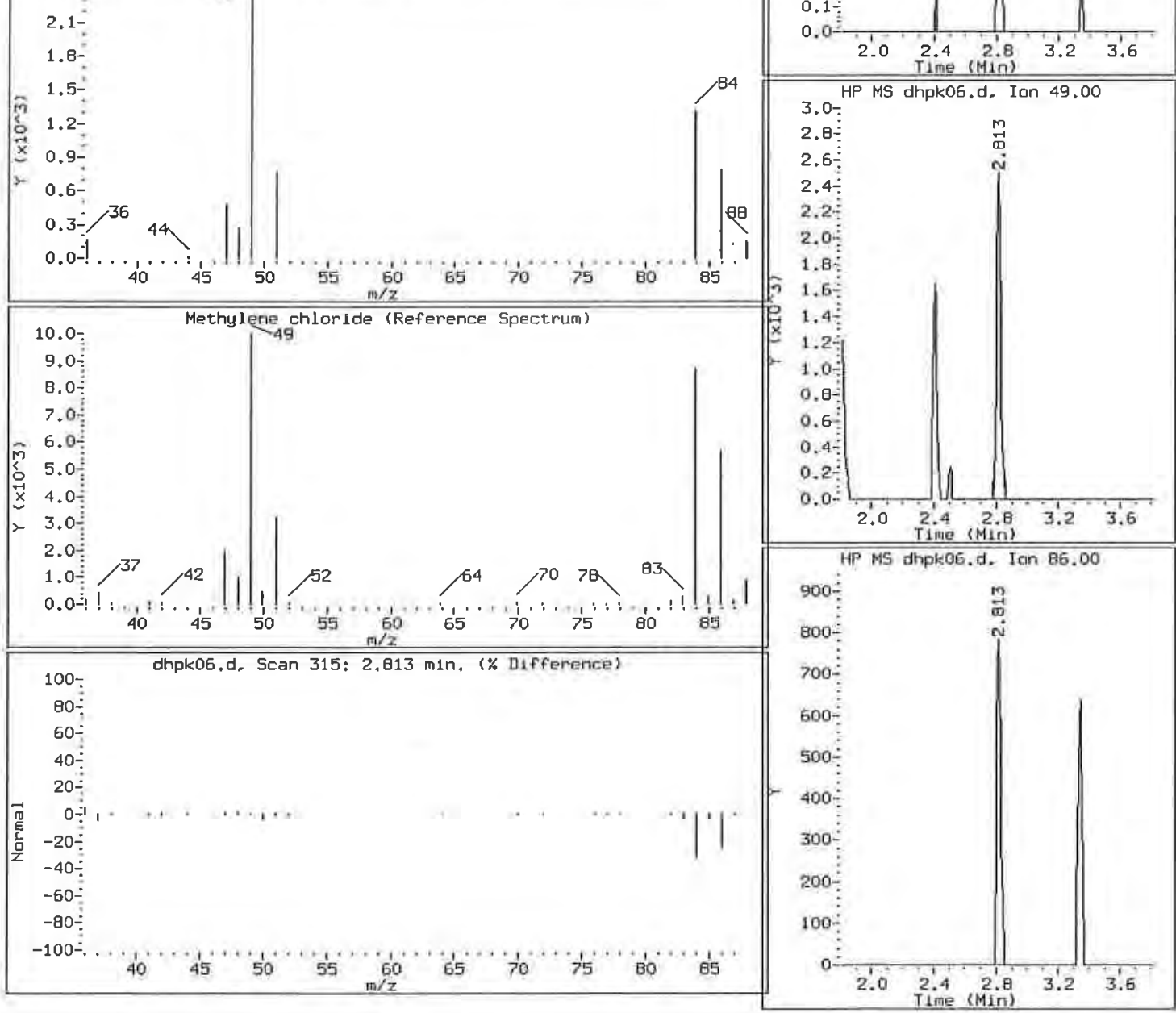
Data File: dhpk06.d

Lab Sample ID: 200-6522-1

Date: 15-AUG-2011 09:27

Client ID: SVSB43S-W-25874

Instrument: D.i

Sample Info: 200-6522-B-1

Operator: JH2

24 Chloroform
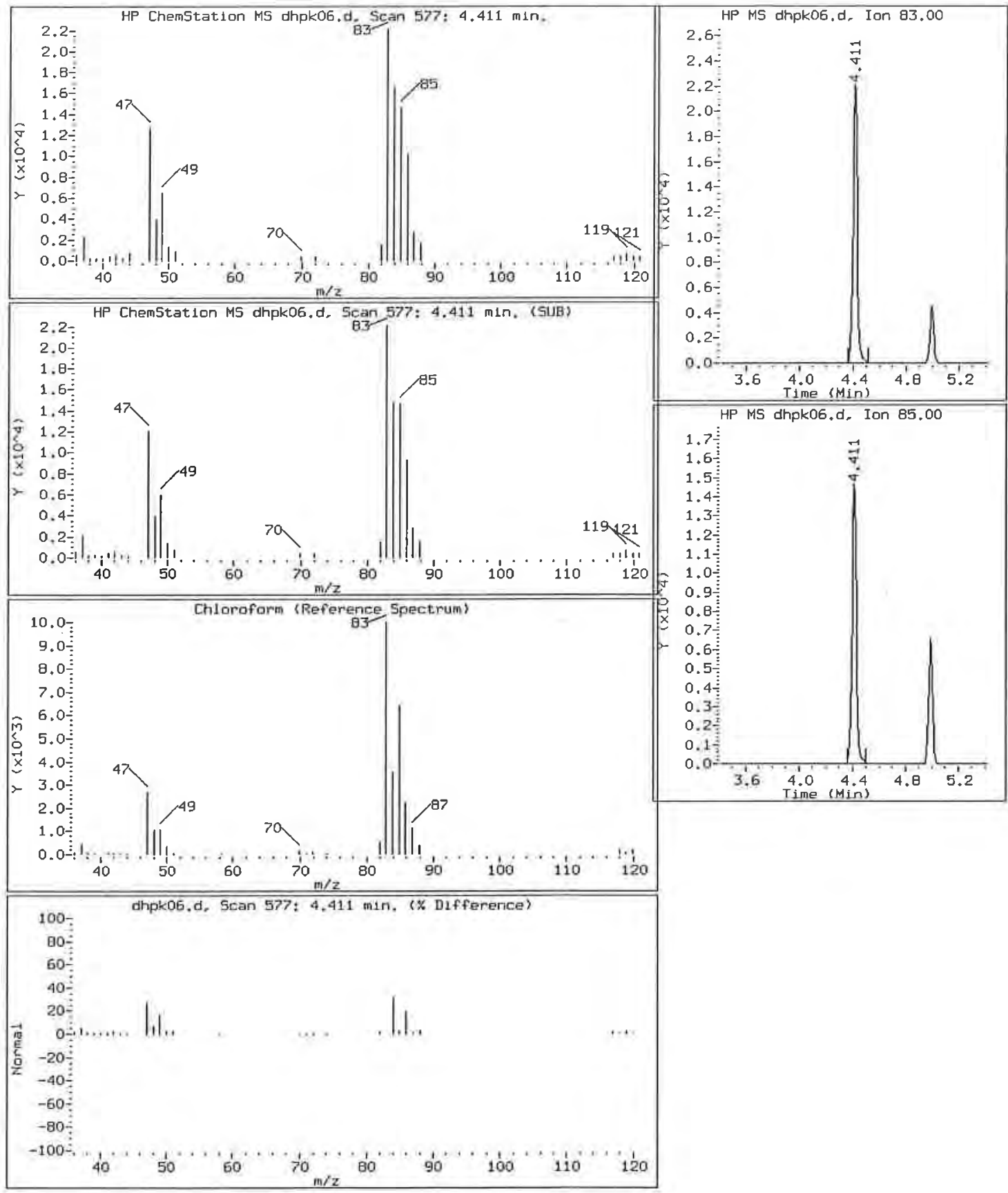
Data File: dhpk06.d

Lab Sample ID: 200-6522-1

Date: 15-AUG-2011 09:27

Client ID: SVSB43S-W-25874

Instrument: D.i

Sample Info: 200-6522-B-1

Operator: JH2

27 Carbon tetrachloride
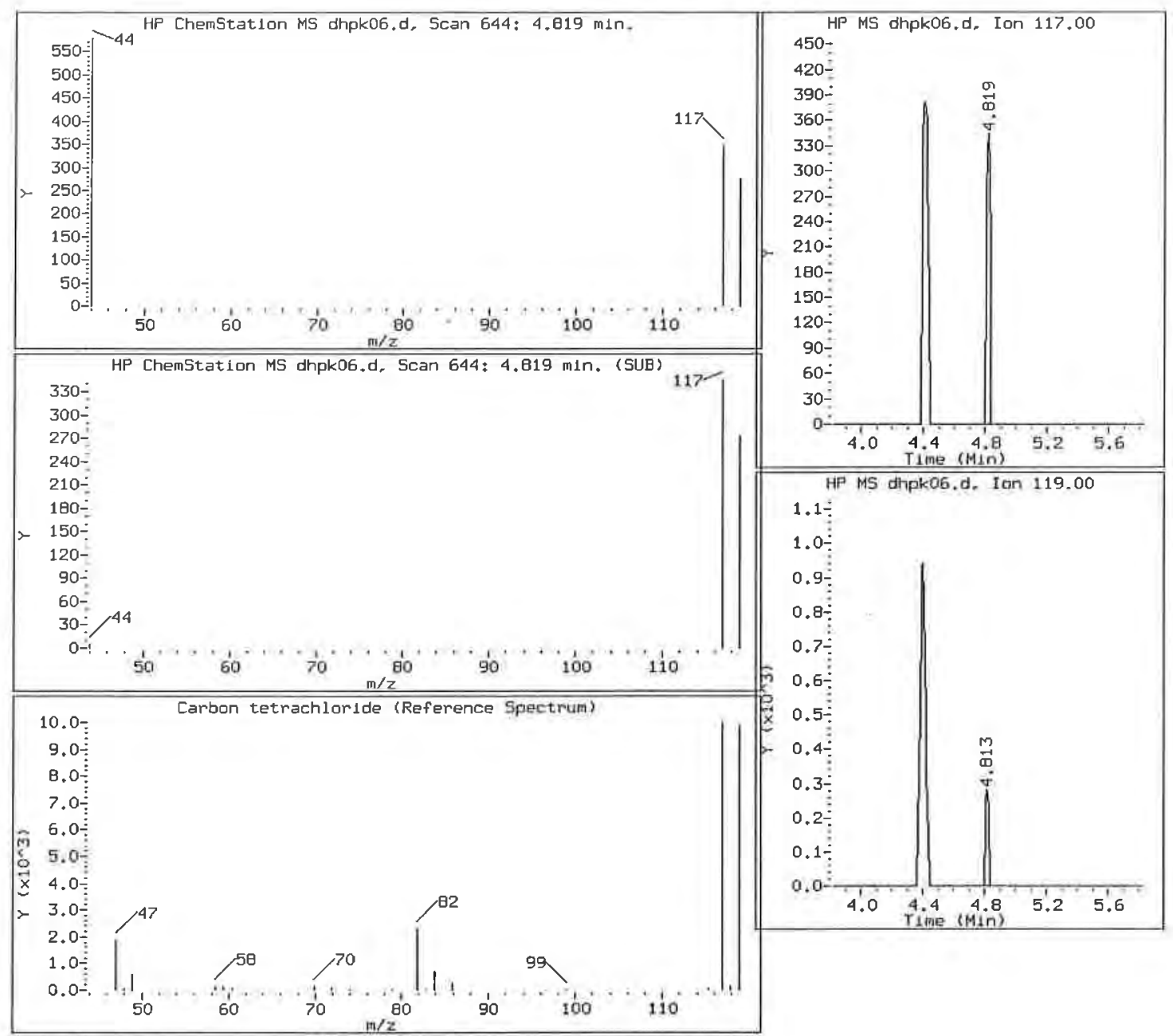

HP MS dhpk06.d. Ion 119.00

$1.0 \div$

$0.9 \div$

$8 \div$

$0.6 \div$

$0.5 \div$
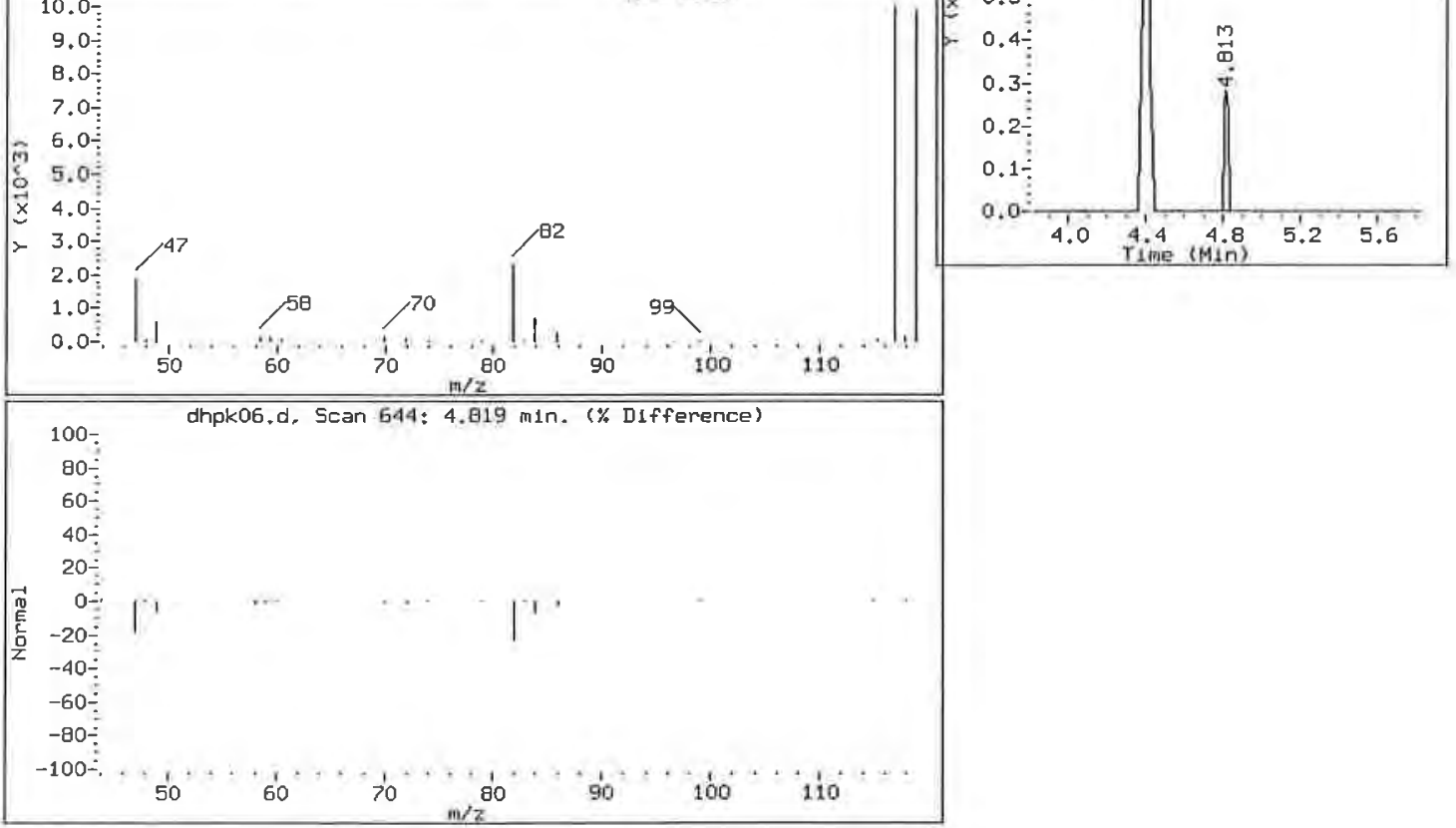
Data File: dhpk06.d

Lab Sample ID: 200-6522-1

Date: 15-AUG-2011 09:27

Client ID: SVSB43S-W-25874

Instrument: D.i

Sample Info: 200-6522-B-1

Operator: JH2

30 Benzene
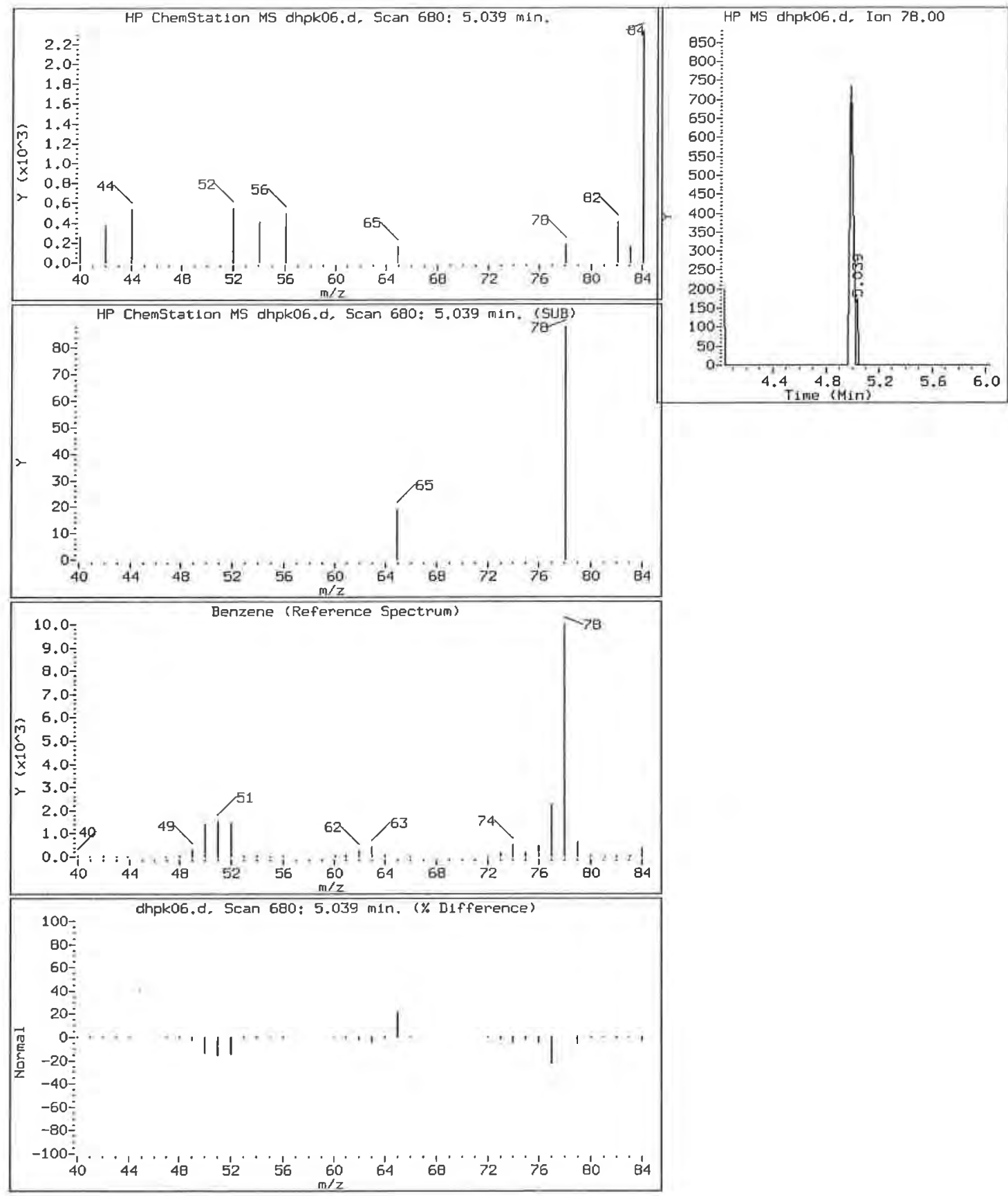
Data File: dhpk06.d

Lab Sample ID: 200-6522-1

Date: 15-AUG-2011 09:27

Client ID: SVSB43S-W-25874

Instrument: D.i

Sample Info: 200-6522-B-1

operator: JH2

41 Toluene
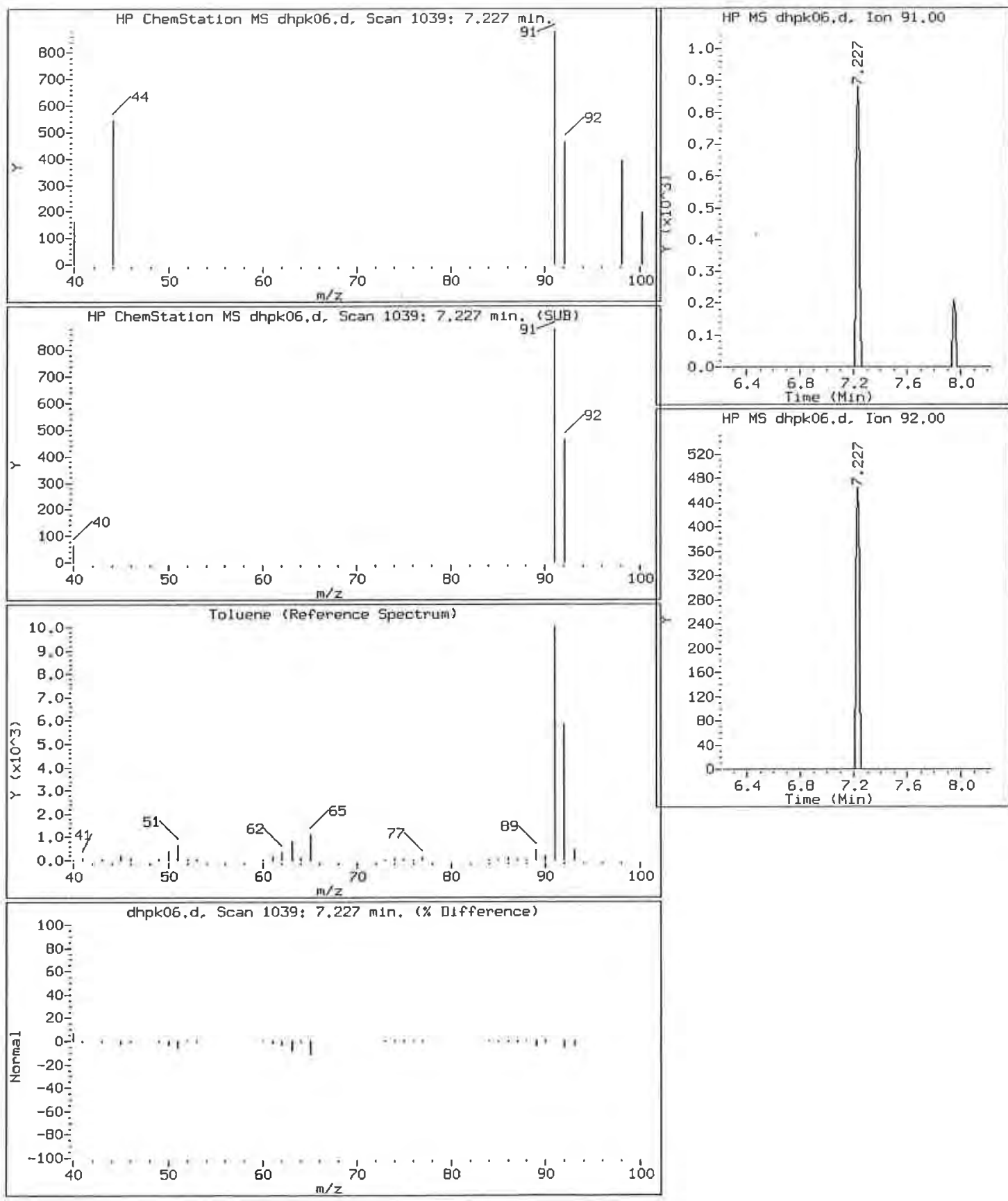
Data File: dhpk06.d

Lab Sample ID: 200-6522-1

Date: 15-AUG-2011 09:27

Client ID: SVSB43S-W-25874

Instrument: D.i

Sample Info: 200-6522-B-1

Operator: JH2

45 Tetrachloroethene
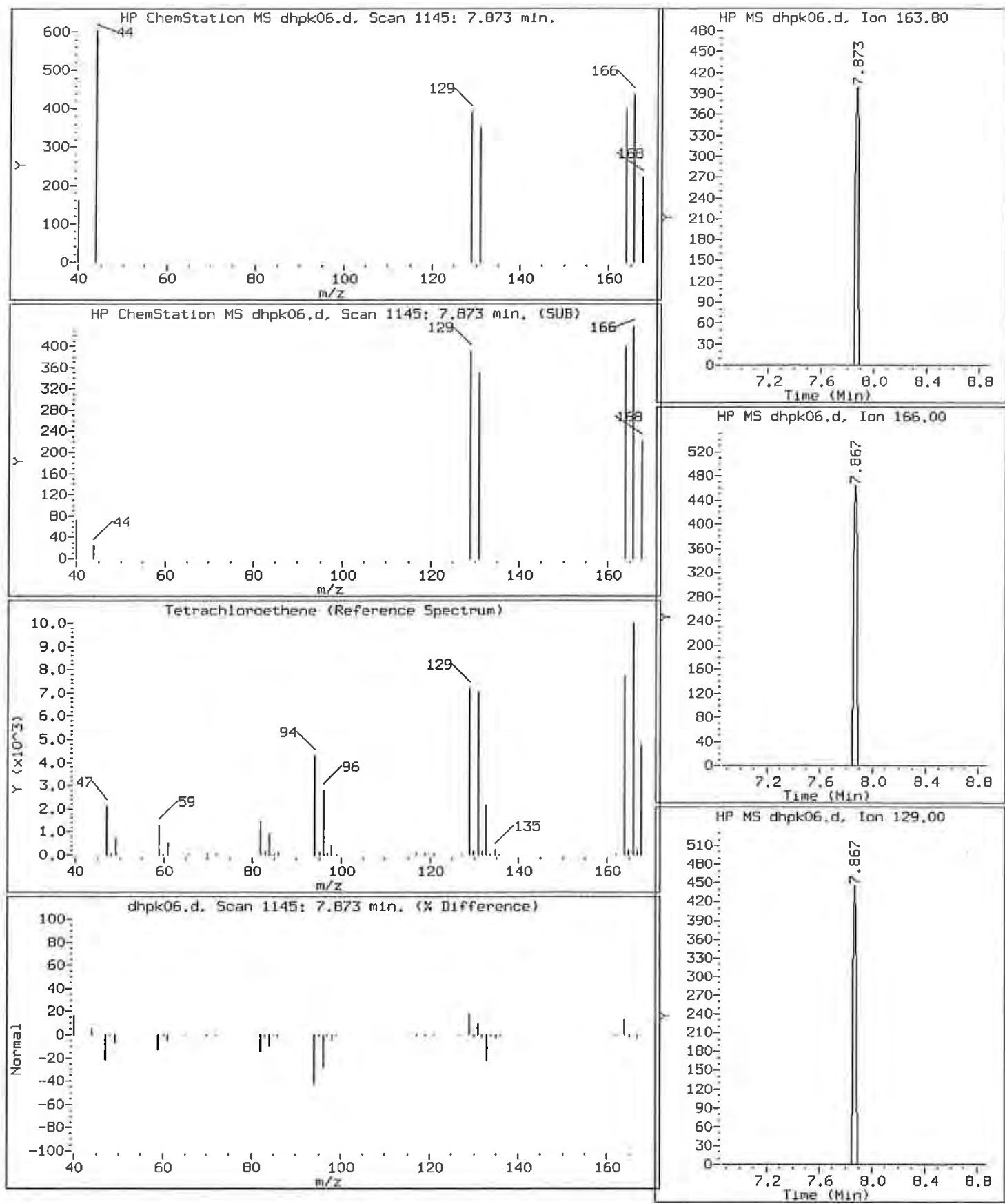
Data File: dhpk06.d

Lab Sample ID: 200-6522-1

Client ID: SVSB43S-W-25874

Sample Info: 200-6522-B-1

Retention Time: $\quad 6.79$
Date: 15-AUG-2011 09:27

Instrument: D. i

Operator: JH2
Library Search Compound Match Unknown

Carbonothioic dichloride Methane, oxybis[chloro-

2-Propanol, 1,3-dichloro-
CAS Number Library

$463-71-8$

$542-88-1$

96-23-1
NIST05.1

NIST05. 1

NIST05.1
Entry

7475

6855

11615

$\begin{array}{cll}\text { Quality } & \text { Formula } & \text { Weight } \\ 37 & \mathrm{CC} 12 \mathrm{~S} & 114 \\ 32 & \mathrm{C} 2 \mathrm{H} 4 \mathrm{Cl} 2 \mathrm{O} & 114 \\ 25 & \mathrm{C} 3 \mathrm{H} 6 \mathrm{Cl} 2 \mathrm{O} & 128\end{array}$

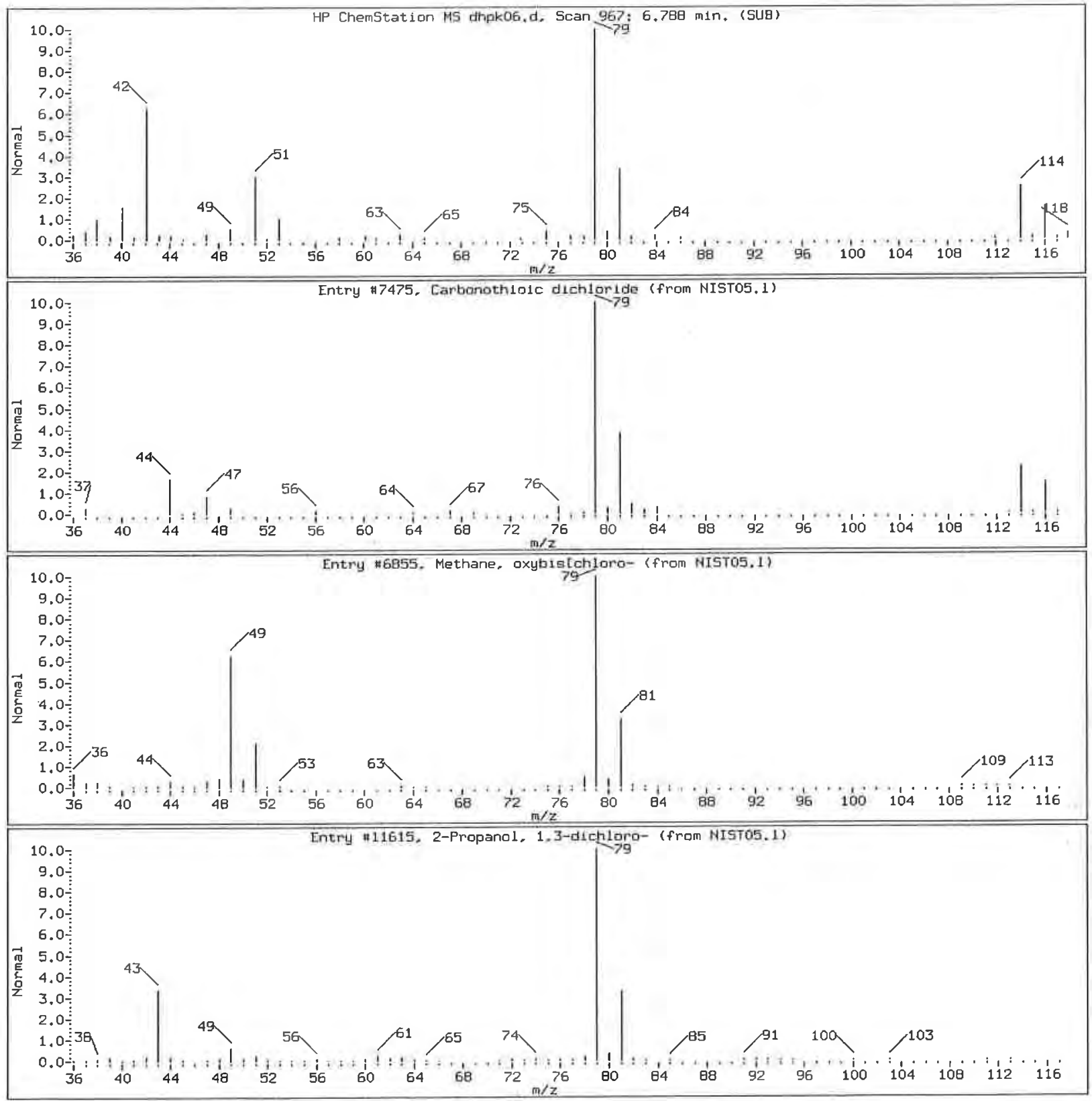

Page 52 of 196

$08 / 17 / 2011$ 
Lab Name: TESTAMERICA BURLINGTON

Contract: $8 \mathrm{E}-00302$

Lab Code: STLV Case No.: SAVANN Mod. Ref No.: SDG No.: 200-6522

Matrix: (SOIL/SED/WATER)

Sample wt/vol: 25.0

Level: (TRACE/LOW./MED)

Water

$(\mathrm{g} / \mathrm{mL}) \quad \mathrm{mL}$

TRACE

․ Moisture: not dec.

GC Column: DB-624 ID : $0.20 \quad(\mathrm{~mm})$

Soil Extract Volume: (uL)

Purge Volume: 25.0 (mL)
Lab Sample ID: 200-6522-2

Lab Eile ID: DHPK07.D

Date Received: 08/12/2011

Date Analyzed: 08/15/2011

Dilution Factor: 1.0

Soil Aliquot Volume: (uL)

\begin{tabular}{|c|c|c|c|}
\hline CAS NO. & COMPOUND & $\begin{array}{l}\text { CONCENTRATION UNITS: } \\
(\mathrm{ug} / \mathrm{L} \text { or } \mathrm{ug} / \mathrm{kg}) \mathrm{ug} / \mathrm{L}\end{array}$ & $Q$ \\
\hline $75-71-8$ & Dichlorodifluoromethane & 0.50 & $\bar{U}$ \\
\hline $74-87-3$ & Chloromethane & 0.094 & $\mathrm{~J}$ \\
\hline $75-01-4$ & Vinyl chloride & 0.50 & $\mathrm{U}$ \\
\hline $74-83-9$ & Bromomethane & 0.50 & $\mathrm{U}$ \\
\hline $75-00-3$ & Chloroethane & 0.50 & $\mathrm{U}$ \\
\hline $75-69-4$ & Trichlorofluoromethane & 0.50 & $\mathrm{U}$ \\
\hline $75-35-4$ & 1,1-Dichloroethene & 0.50 & $\mathrm{U}$ \\
\hline $76-13-1$ & 1,1,2-Trichloro-1,2,2-trifluoroethane & 0.50 & $\mathrm{U}$ \\
\hline $67-64-1$ & Acetone & 5.0 & $\mathrm{U}$ \\
\hline $75-15-0$ & Carbon disulfide & 0.11 & $\mathrm{~J} \mathrm{~B}$ \\
\hline $79-20-9$ & Methyl acetate & 0.50 & $\mathrm{U}$ \\
\hline $75-09-2$ & Methylene Chloride & 0.052 & $\mathrm{~J} \mathrm{~B}$ \\
\hline $156-60-5$ & trans-1,2-Dichloroethene & 0.50 & $\mathrm{U}$ \\
\hline $1634-04-4$ & Methyl tert-butyl ether & 0.50 & $\mathrm{U}$ \\
\hline $75-34-3$ & 1,1-Dichloroethane & 0.50 & $\mathrm{U}$ \\
\hline $156-59-2$ & cis-1,2-Dichloroethene & 0.50 & $\mathrm{U}$ \\
\hline $78-93-3$ & 2-Butanone & 5.0 & $\mathrm{U}$ \\
\hline $74-97-5$ & Bromochloromethane & 0.50 & $\mathrm{U}$ \\
\hline $67-66-3$ & Chloroform & 0.41 & $\mathrm{~J}$ \\
\hline $71-55-6$ & 1,1,1-Trichloroethane & 0.50 & $\mathrm{U}$ \\
\hline $110-82-7$ & Cyclohexane & 0.50 & $\mathrm{U}$ \\
\hline $56-23-5$ & Carbon tetrachloride & 0.039 & $\mathrm{~J}$ \\
\hline $71-43-2$ & Benzene & 0.50 & $\mathrm{U}$ \\
\hline $107-06-2$ & 1,2-Dichloroethane & 0.50 & $\mathrm{U}$ \\
\hline
\end{tabular}

Report 1,4-Dioxane for Low-Medium VOA analysis only 
1B - FORM I VOA-2

VOLATILE ORGANICS ANALYSIS DATA SHEET
EPA SAMPLE NO.

SVSB 4 4D-W-25877
Lab Name: TESTAMERICA BURLINGTON

Lab Code: STLV

Matrix: (SOIL/SED/WATER)

Water

Sample wt/vol: 25.0 $(\mathrm{g} / \mathrm{mL}) \quad \mathrm{mL}$

Level: (TRACE/LOW/MED) TRACE

용 Moisture: not dec.

GC Column: DB-624 ID: 0.20 $(\mathrm{mm})$

Soil Extract Volume: (uL)

Purge Volume: 25.0 (mL)
Contract: $8 \mathrm{E}-00302$

SDG No.: 200-6522
Lab Sample ID: 200-6522-2

Lab File ID: DHPK07.D

Date Received: 08/12/2011

Date Analyzed: 08/15/2011

Dilution Factor: 1.0

Soil Aliquot Volume:

(uL)

\begin{tabular}{|c|c|c|c|}
\hline CAS NO. & COMPOUND & $\begin{array}{l}\text { CONCENTRATION UNITS: } \\
(\mathrm{ug} / \mathrm{L} \text { or } \mathrm{ug} / \mathrm{kg}) \mathrm{ug} / \mathrm{L}\end{array}$ & $Q$ \\
\hline $79-01-6$ & Trichloroethene & 0.50 & $\overline{\mathrm{U}}$ \\
\hline $108-87-2$ & Methylcyclohexane & 0.50 & $\mathrm{U}$ \\
\hline $78-87-5$ & 1,2-Dichloropropane & 0.50 & $\mathrm{U}$ \\
\hline $75-27-4$ & Bromodichloromethane & 0.50 & U \\
\hline $10061-01-5$ & cis-1,3-Dichloropropene & 0.50 & $\mathrm{U}$ \\
\hline $108-10-1$ & 4-Methyl-2-pentanone & 5.0 & $\mathrm{U}$ \\
\hline $108-88-3$ & Toluene & 0.049 & J B \\
\hline $10061-02-6$ & trans-1,3-Dichloropropene & 0.50 & $\mathrm{U}$ \\
\hline $79-00-5$ & 1,1,2-Trichloroethane & 0.50 & $\mathrm{U}$ \\
\hline $127-18-4$ & Tetrachloroethene & 0.034 & $\mathrm{~J}$ \\
\hline $591-78-6$ & 2-Hexanone & 5.0 & $\mathrm{U}$ \\
\hline $124-48-1$ & Dibromochloromethane & 0.50 & $\mathrm{U}$ \\
\hline $106-93-4$ & 1,2-Dibromoethane & 0.50 & $\mathrm{U}$ \\
\hline $108-90-7$ & Chlorobenzene & 0.50 & $\mathrm{U}$ \\
\hline $100-41-4$ & Ethylbenzene & 0.50 & $\mathrm{U}$ \\
\hline $95-47-6$ & o-Xylene & 0.50 & $\mathrm{U}$ \\
\hline $179601-23-1$ & $m, p-x y$ lene & 0.020 & $\mathrm{~J}$ \\
\hline $100-42-5$ & Styrene & 0.054 & $\mathrm{~J}$ \\
\hline $75-25-2$ & Bromoform & 0.50 & $\mathrm{U}$ \\
\hline $98-82-8$ & Isopropylbenzene & 0.50 & $\mathrm{U}$ \\
\hline $79-34-5$ & $1,1,2,2$-Tetrachloroethane & 0.50 & $\mathrm{U}$ \\
\hline $541-73-1$ & 1,3-Dichlorobenzene & 0.50 & $\mathrm{U}$ \\
\hline $106-46-7$ & 1,4-Dichlorobenzene & 0.50 & $\mathrm{U}$ \\
\hline $95-50-1$ & 1,2-Dichlorobenzene & 0.50 & $\mathrm{U}$ \\
\hline $96-12-8$ & 1,2-Dibromo-3-Chloropropane & 0.50 & $\mathrm{U}$ \\
\hline $120-82-1$ & $1,2,4$-Trichlorobenzene & 0.50 & $\mathrm{U}$ \\
\hline $87-61-6$ & 1,2,3-Trichlorobenzene & 0.50 & $\mathrm{U}$ \\
\hline
\end{tabular}




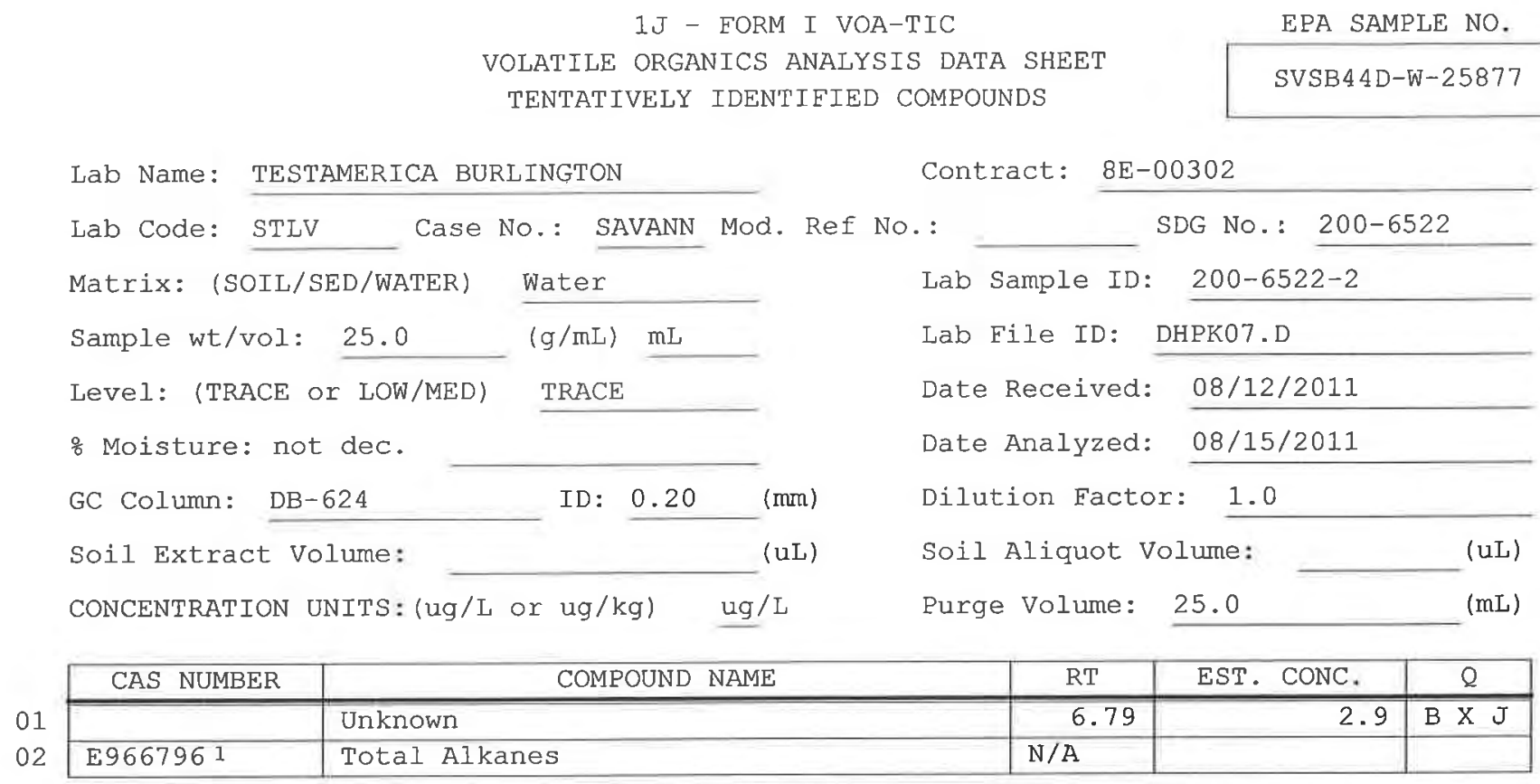

1 EPA-designated Registry Number. 


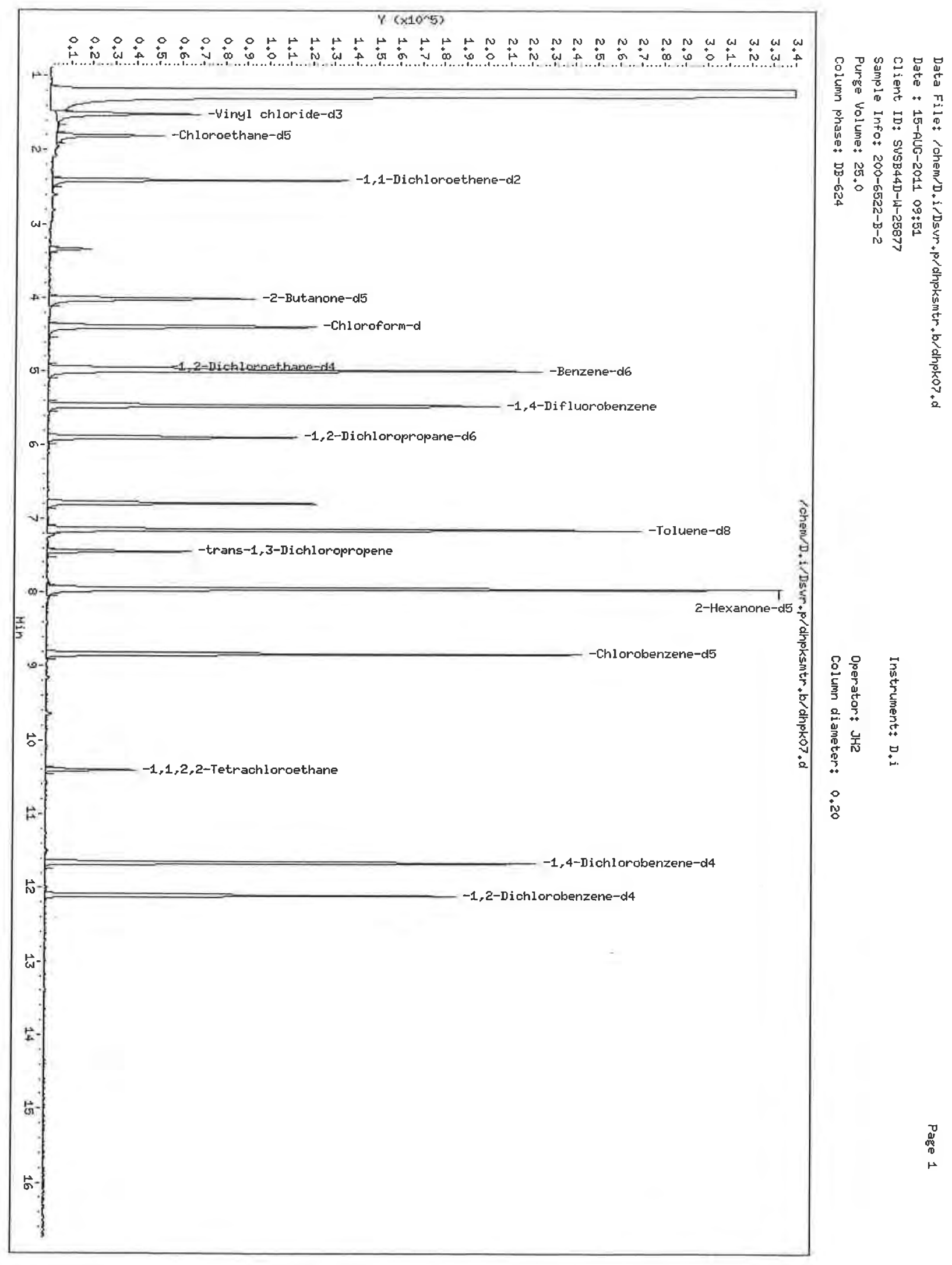


Lab Sample Id: 200-6522-2

Client Smp ID: SVSB44D-W-25877

Inj Date : 15-AUG-2011 09:51

Operator : JH2

Smp Info : 200-6522-B-2

Inst ID: D.i

Misc Info : 1,5

Comment :

Method : /chem/D.i/Dsvr.p/dhpksmtr.b/somtr5t.m

Meth Date : 15-Aug-2011 16:21 jh2 Quant Type: ISTD

Cal Date : 07-JUL-2011 12:56 Cal File: dhp08.d

Als bottle: 5

Dil Factor: 1.00000

Integrator: HP RTE

Target Version: 3.50

Processing Host: chemsvr6

Concentration Eormula: Ant * DF * Uf/Vo * CpndVariable

\begin{tabular}{ccc} 
Name & Value & Description \\
\hline DF & 1.00000 & Dilution Factor \\
Uf & 1.00000 & ng unit correction factor \\
Vo & 25.00000 & Sample volume purged (mL)
\end{tabular}

Cpnd Variable

Local Compound Variable

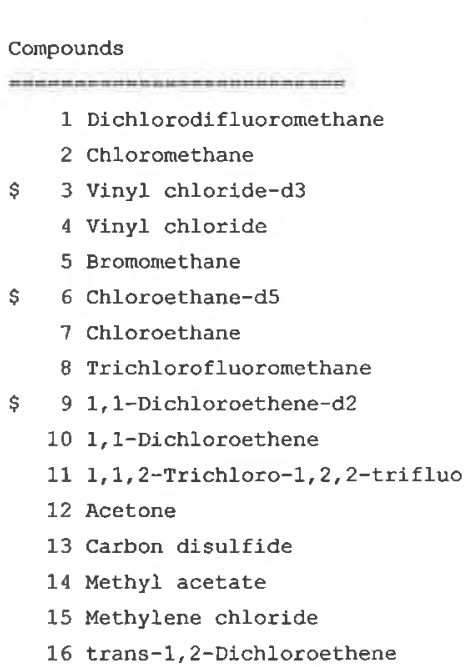

\begin{tabular}{c} 
QUANT SIG \\
MASS \\
\hline $9=7$ \\
85 \\
50 \\
65 \\
62 \\
94 \\
69 \\
64 \\
101 \\
63 \\
96 \\
101 \\
43 \\
76 \\
43 \\
84 \\
96
\end{tabular}

\begin{tabular}{|c|c|c|c|c|}
\hline \multirow[b]{2}{*}{ RT } & \multirow[b]{2}{*}{ EXP RT REL RT } & \multirow[b]{2}{*}{ RESPONSE } & ON-COLUMN & \multirow{2}{*}{$\begin{array}{l}\text { FINAL } \\
(\mathrm{ug} / \mathrm{L})\end{array}$} \\
\hline & & & ( $\mathrm{ng})$ & \\
\hline$=$ & 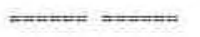 & 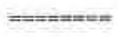 & 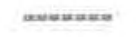 & prentate= \\
\hline \multicolumn{5}{|c|}{ Compound Not Detected. } \\
\hline 1.454 & $1.454(0.266)$ & 2335 & 2.34751 & $0.094(a)$ \\
\hline 1.515 & $1.521(0.277)$ & 75449 & 119.613 & 4.8 \\
\hline \multicolumn{5}{|c|}{$\begin{array}{l}\text { Compound Not Detected. } \\
\text { Compound Not Detected. }\end{array}$} \\
\hline 1.807 & $1.807(0.331)$ & 49421 & 120.756 & 4.8 \\
\hline \multicolumn{5}{|c|}{$\begin{array}{l}\text { Compound Not Detected. } \\
\text { Compound Not Detected. }\end{array}$} \\
\hline 2.405 & $2.411(0.440)$ & 96389 & 83.7254 & 3.3 \\
\hline \multicolumn{5}{|c|}{ Compound Not Detected. } \\
\hline \multicolumn{5}{|c|}{ Compound Not Detected. } \\
\hline \multicolumn{5}{|c|}{ Compound Not Detected. } \\
\hline 2.606 & $2.612(0.477)$ & 3005 & 2.79728 & 0.11 (a) \\
\hline \multicolumn{5}{|c|}{ Compound Not Detected. } \\
\hline 2.813 & $2.813(0.515)$ & 473 & 1.30571 & $0.052(\mathrm{aQ})$ \\
\hline
\end{tabular}




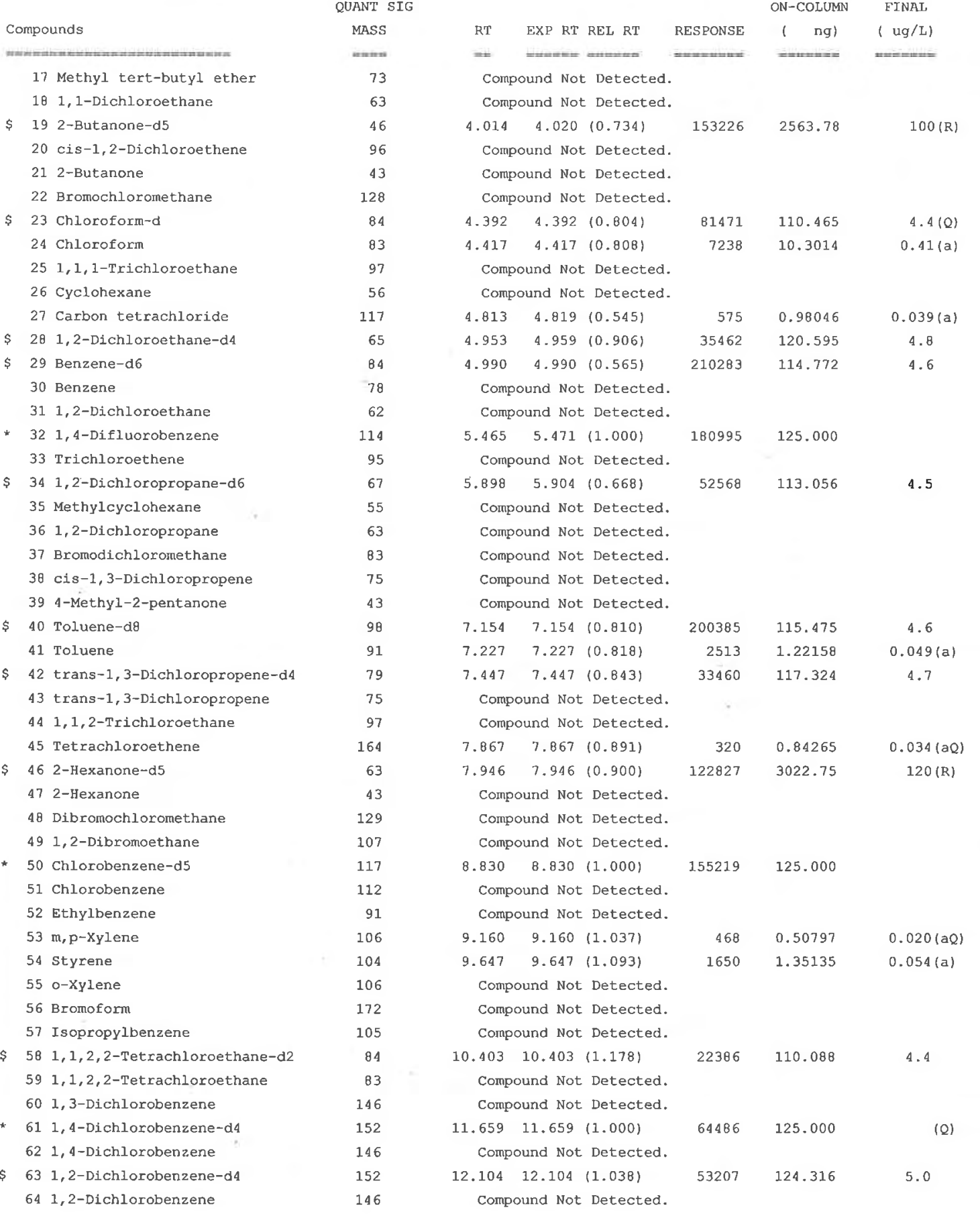


Data File: /chem/D.i/Dsvr.p/dhpksmtr.b/dhpk07.d

Page 3 Report Date: 15-Aug-2011 20:11

\begin{tabular}{|c|c|c|c|c|c|c|}
\hline \multirow[b]{3}{*}{ Compounds } & \multirow[b]{2}{*}{ QUANT SIG } & & & & \multicolumn{2}{|c|}{ CONCENTRATIONS } \\
\hline & & & & & ON-COLUMN & FINAL \\
\hline & MASS & RT & EXP RT REL RT & RESPONSE & ( ng) & $(\mathrm{ug} / \mathrm{L})$ \\
\hline 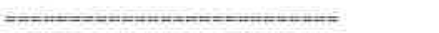 & $=\pi n=$ & $=0$ & 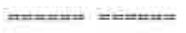 & 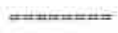 & enturatus= & 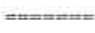 \\
\hline 65 1,2-Dibromo-3-chloropropane & 75 & & ound Not Detect & & & \\
\hline $661,2,4$-Trichlorobenzene & 180 & & ound Not Detect & & & \\
\hline $671,2,3$-Trichlorobenzene & 180 & Co & ound Not Detect & & & \\
\hline
\end{tabular}

QC Flag Legend

a - Target compound detected but, quantitated amount Below Limit of Quantitation (BLOQ):

Q - Qualifier signal failed the ratio test.

$\mathrm{R}$ - Spike/Surrogate failed recovery limits. 
Data File: /chem/D.i/Dsvr.p/dhpksmtr.b/dhpk07.d

Page 1

Report Date: 15-Aug-2011 20:11

TestAmerica Burlington

Lab Sample Id: 200-6522-2

Client Smp ID: SVSB44D-W-25877

Inj Date : 15-AUG-2011 09:51

Operator : JH2

Smp Info : 200-6522-B-2

Inst ID: D.i

Misc Info : 1,5

Comment :

Method : /chem/D.i/Dsvr.p/dhpksmtr.b/somtr5t.m

Meth Date : 15-Aug-2011 16:21 jh2 Quant Type: ISTD

Cal Date : 07-JUL-2011 12:56 Cal File: dhp08.d

Als bottle: 5

Dil Eactor: 1.00000

Integrator: HP RTE

Target Version: 3.50

Processing Host: chemsvr6

Concentration Formula: Amt * DF * Uf/Vo * CpndVariable

\begin{tabular}{cll} 
Name & \multicolumn{1}{c}{ Value } & Description \\
\hline DE & 1.00000 & Dilution Factor \\
Uf & 1.00000 & ng unit correction factor \\
Vo & 25.00000 & Sample Volume purged (mL)
\end{tabular}

Cpnd Variable

Local Compound Variable

ISTD

$== \pm= \pm= \pm=$

* 32 1,4-Difluorobenzene

CONCENTRATIONS

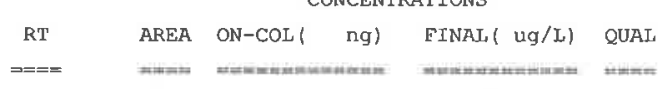

Unknown

6.794

$236186 \quad 71.9351289$

$\begin{array}{lll}\text { RT } & \begin{array}{l}\text { AREA } \\ ==== \pm==\end{array} & \begin{array}{l}\text { AMOUNT } \\ ======\end{array} \\ 5.465 & 410414 & 125.000\end{array}$

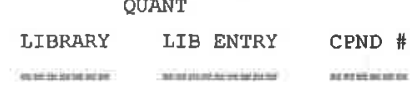

CAS \#:

0
$0 \quad 32$ 
Data File: dhpk07.d

Lab Sample ID: 200-6522-2

Date: 15-AUG-2011 09:51

Client ID: SVSB44D-W-25877

Instrument: D.i

Sample Info: 200-6522-B-2

Operator: JH2

2 Chloromethane
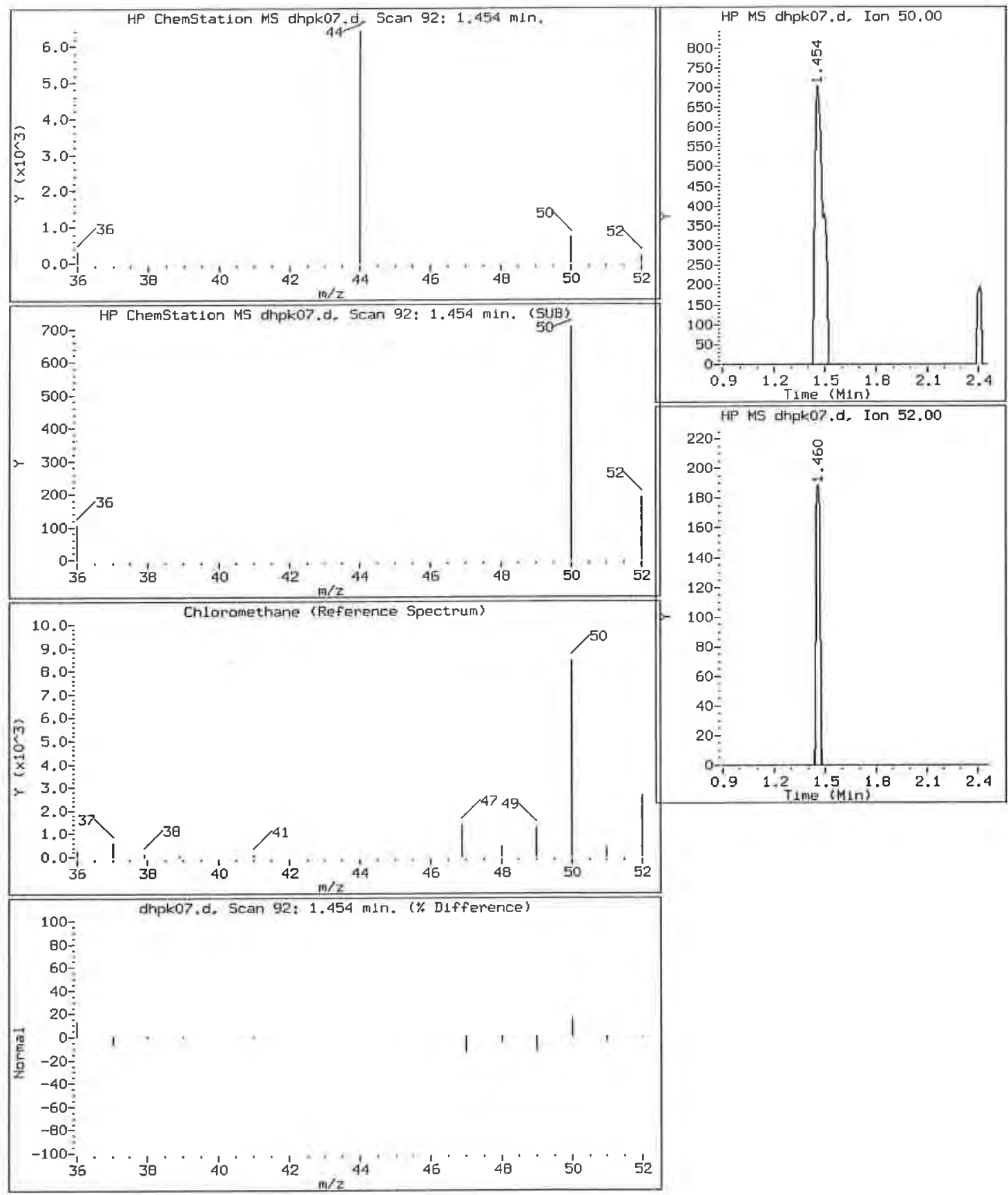
Data File: dhpk07.d

Lab Sample ID: 200-6522-2

Date: 15-AUG-2011 09:51

Client ID: SVSB44D-W-25877

Instrument: D.i

Sample Info: 200-6522-B-2

Operator: JH2

\section{Carbon disulfide}
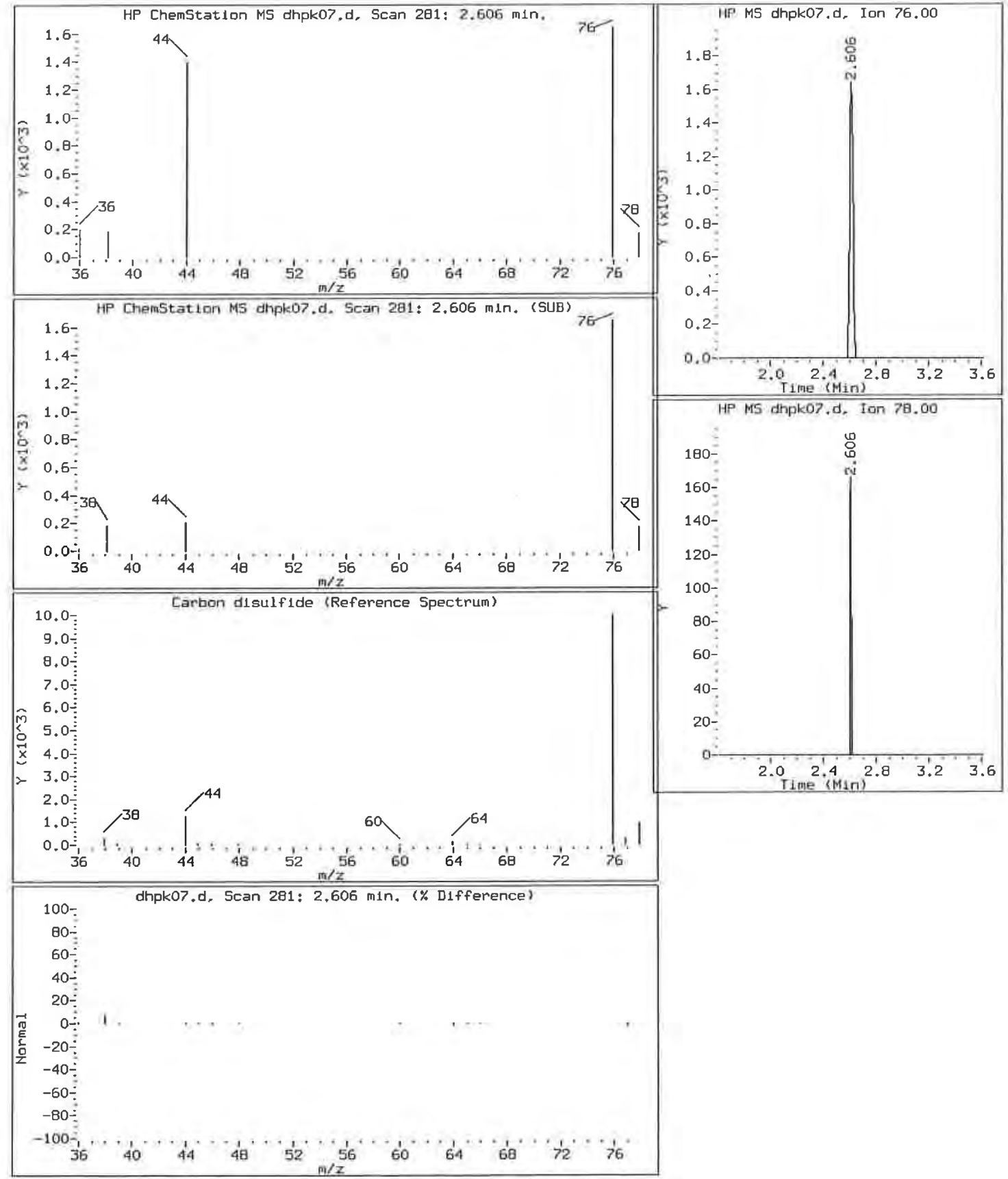
Data File: dhpk07.d

Iab Sample ID: 200-6522-2

Date: 15-AUG-2011 09:51

Client ID: SVSB44D-W-25877

Instrument: D.i

Sample Info: 200-6522-B-2

Operator: JH2

15 Methylene chloride
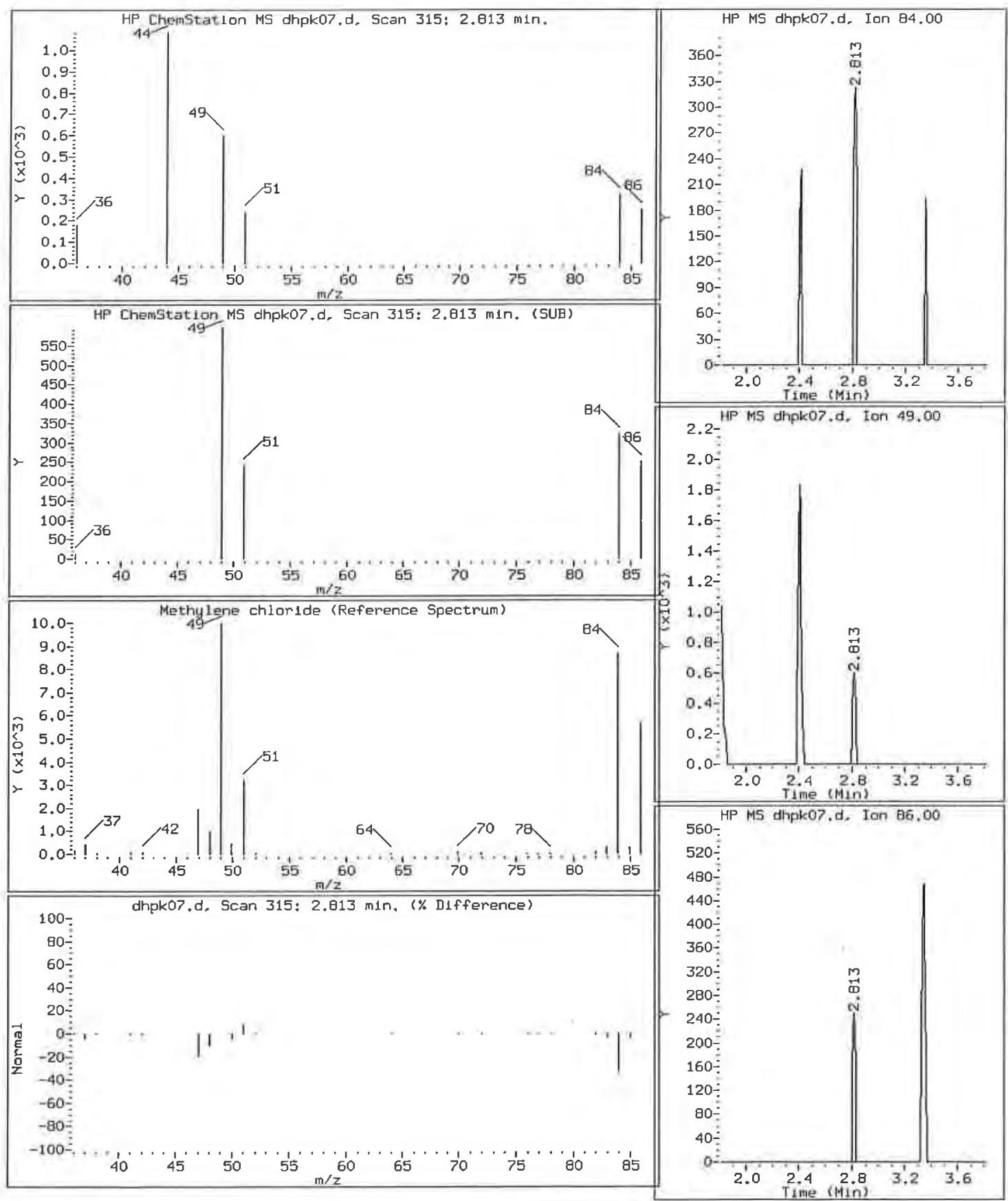
Data File: dhpk07.d

Lab Sample ID: 200-6522-2

Client ID: SVSB44D-W-25877

Sample Info: 200-6522-B-2
Date: 15-AUG-2011 09:51

Instrument: D.i

Operator: JH2

\section{Chloroform}
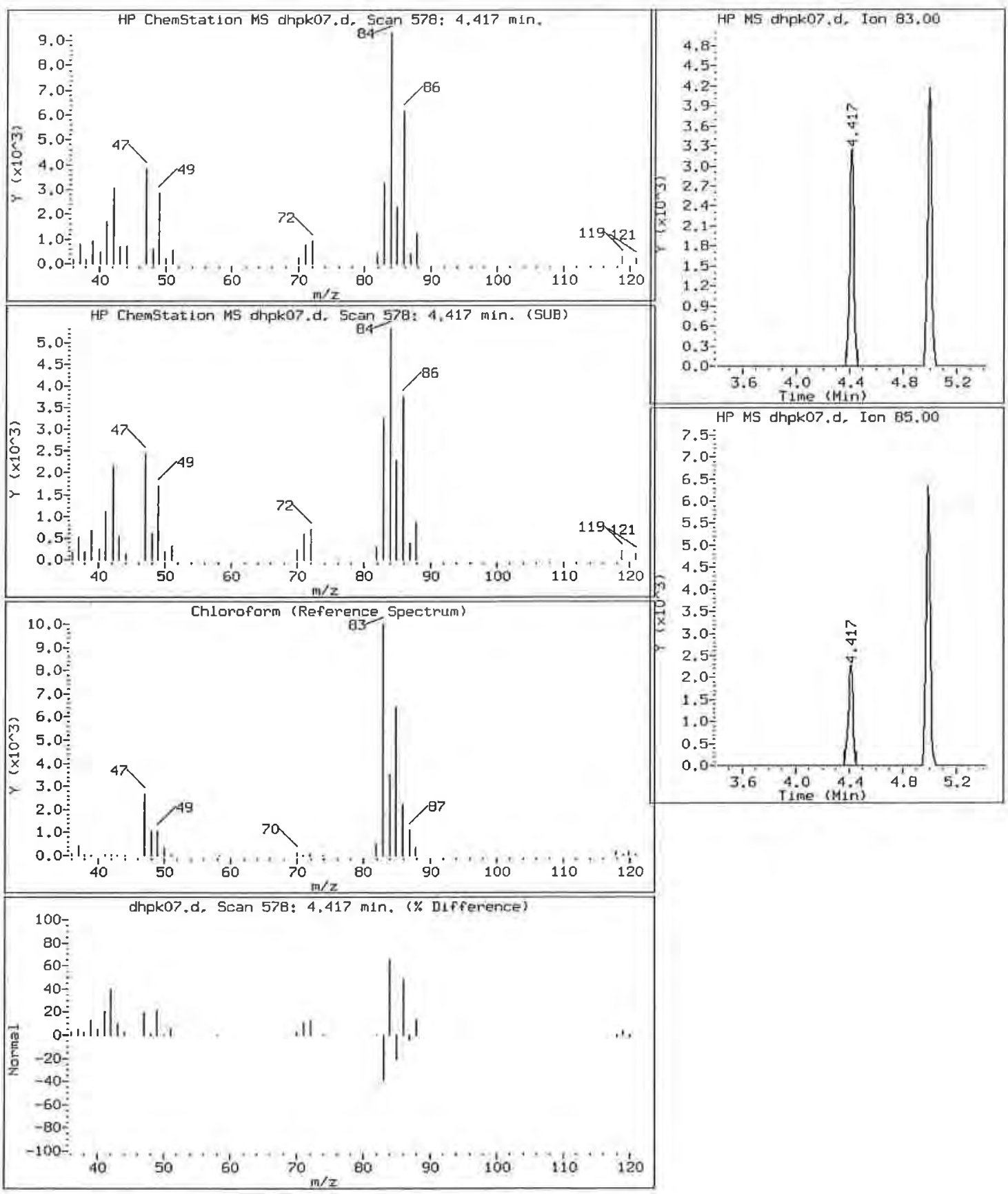
Data File: dhpk07.d

Lab Sample ID: 200-6522-2

Date: 15-AUG-2011 09:51

Client ID: SVSB44D-W-25877

Instrument: D.i

Sample Info: 200-6522-B-2

Operator: JH2

27 Carbon tetrachloride
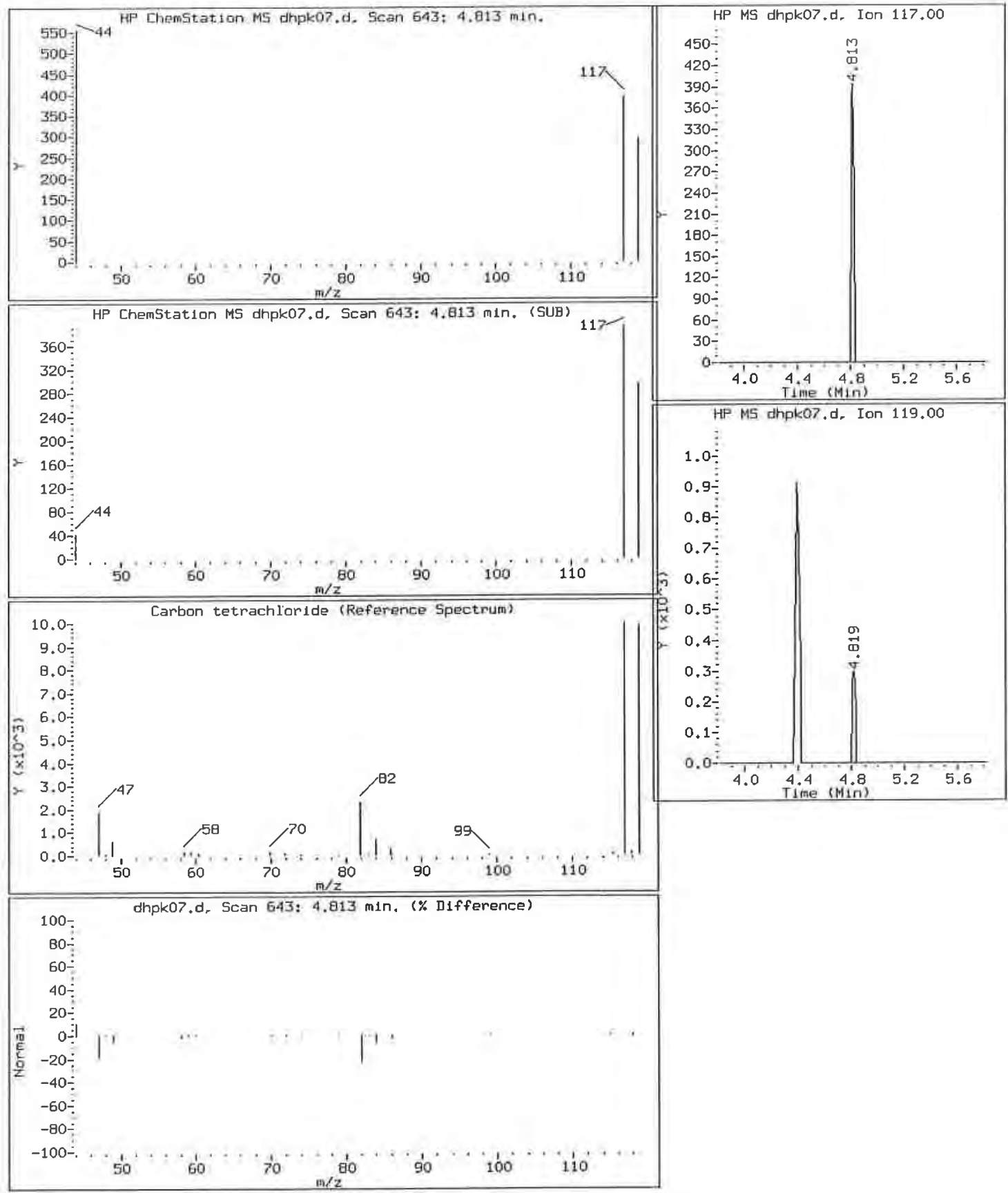
Data File: dhpk07.d

Lab Sample ID: 200-6522-2

Date: 15-AUG-2011 09:51

Client ID: SVSB44D-W-25877

Instrument: D.i

Sample Info: 200-6522-B-2

Operator: JH2

41 Toluene
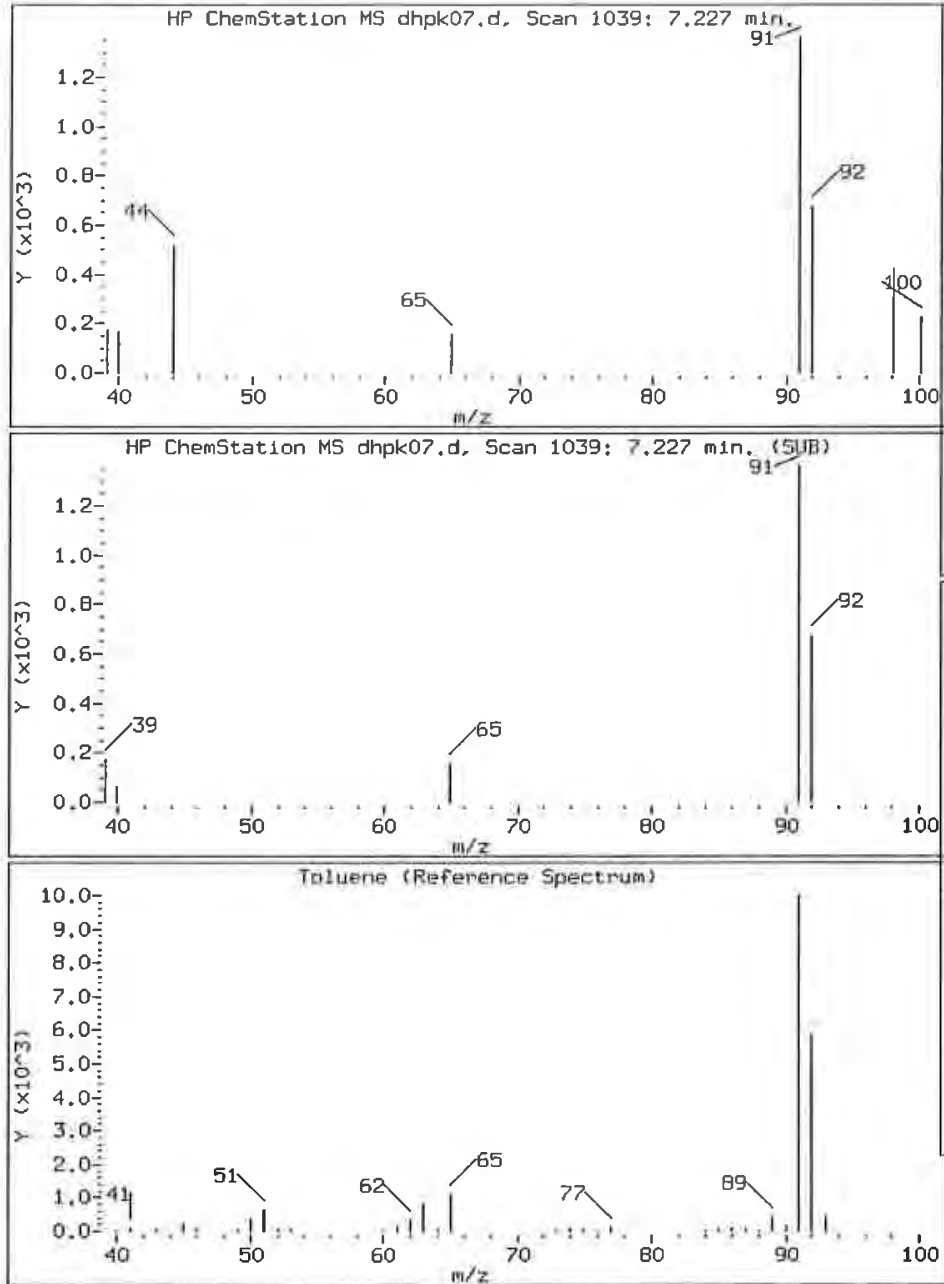

HP MS dhpk07,d, Ion 91,00

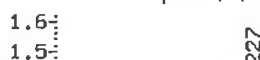

1.5

1.4

1.3

1.2

1.0

$0.9 \div$

0.0

$0.7 \vdots$

0.6

0.4

$0.3+$

0.2

$0,1+2$
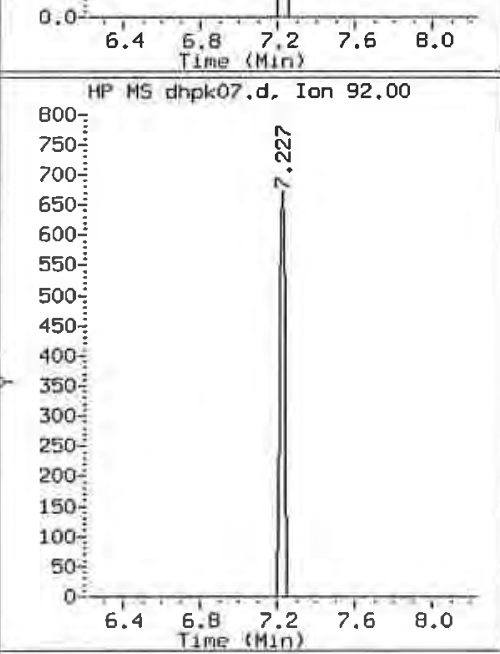

$60-70$ 90 100

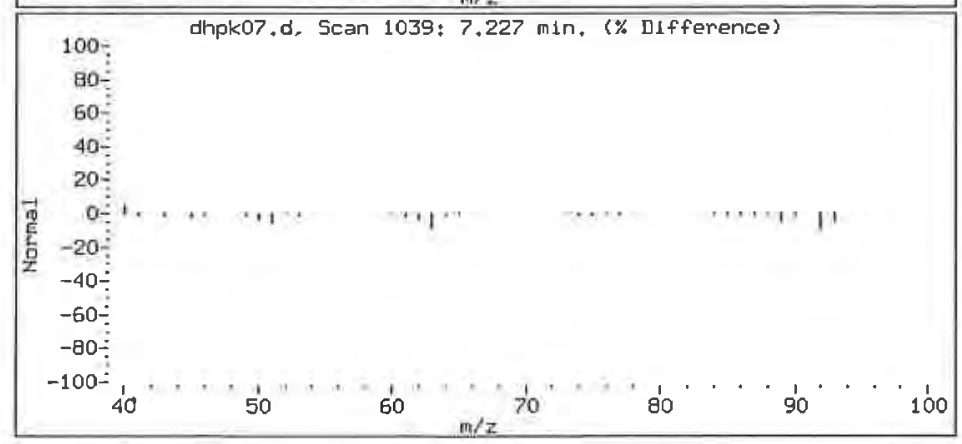


Data File: dhpk07.d

Lab Sample ID: 200-6522-2

Date: 15-AUG-2011 09:51

Client ID: SVSB44D-W-25877

Instrument: D.i

Sample Info: 200-6522-B-2

Operator: JH2

45 Tetrachloroethene
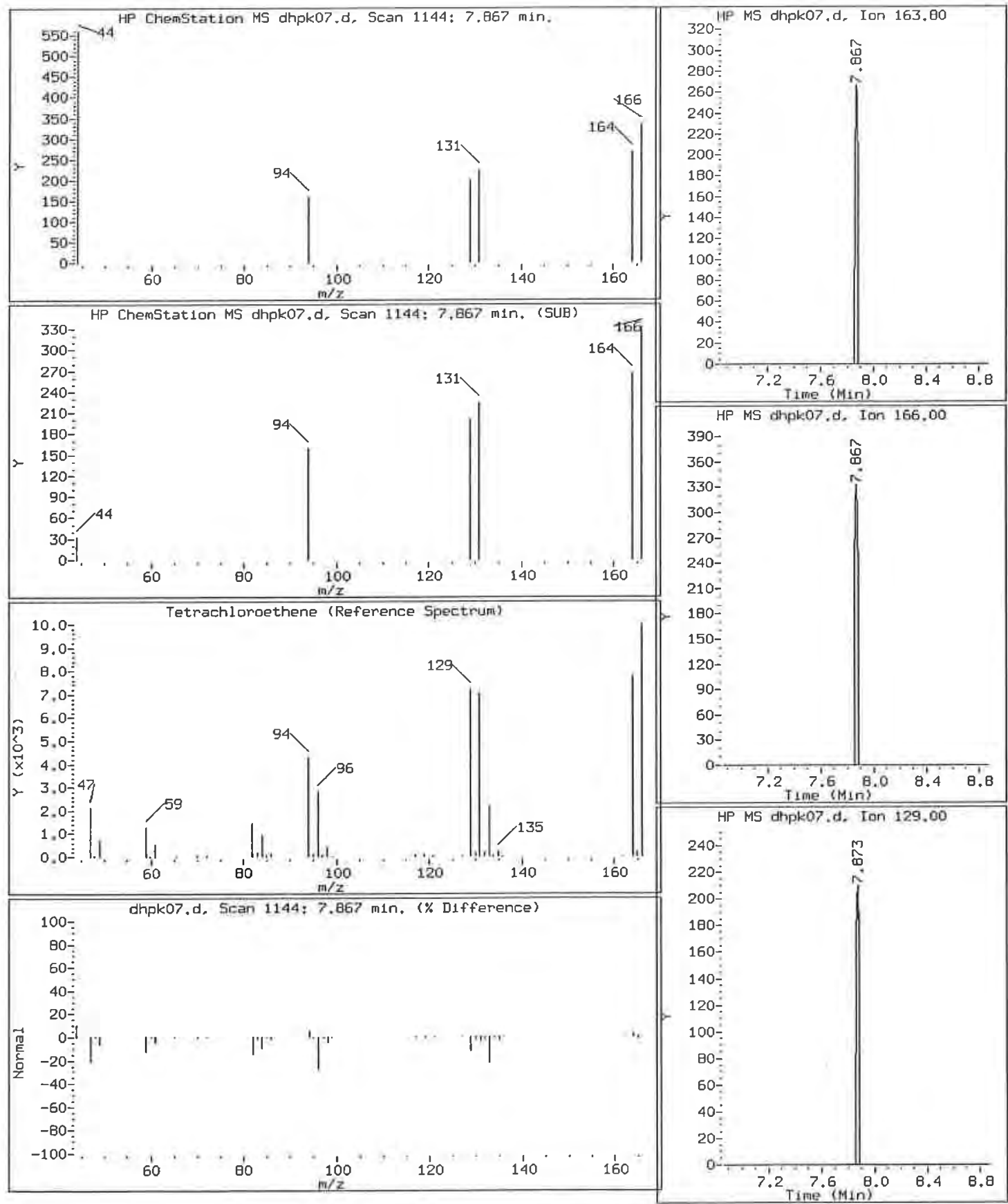
Data File: dhpk07.d

Lab Sample ID: 200-6522-2

Date: 15-AUG-2011 09:51

Client ID: SVSB44D-W-25877

Instrument: D.i

Sample Info: 200-6522-B-2

Operator: JH2

$53 \mathrm{~m}, \mathrm{p}$-xylene
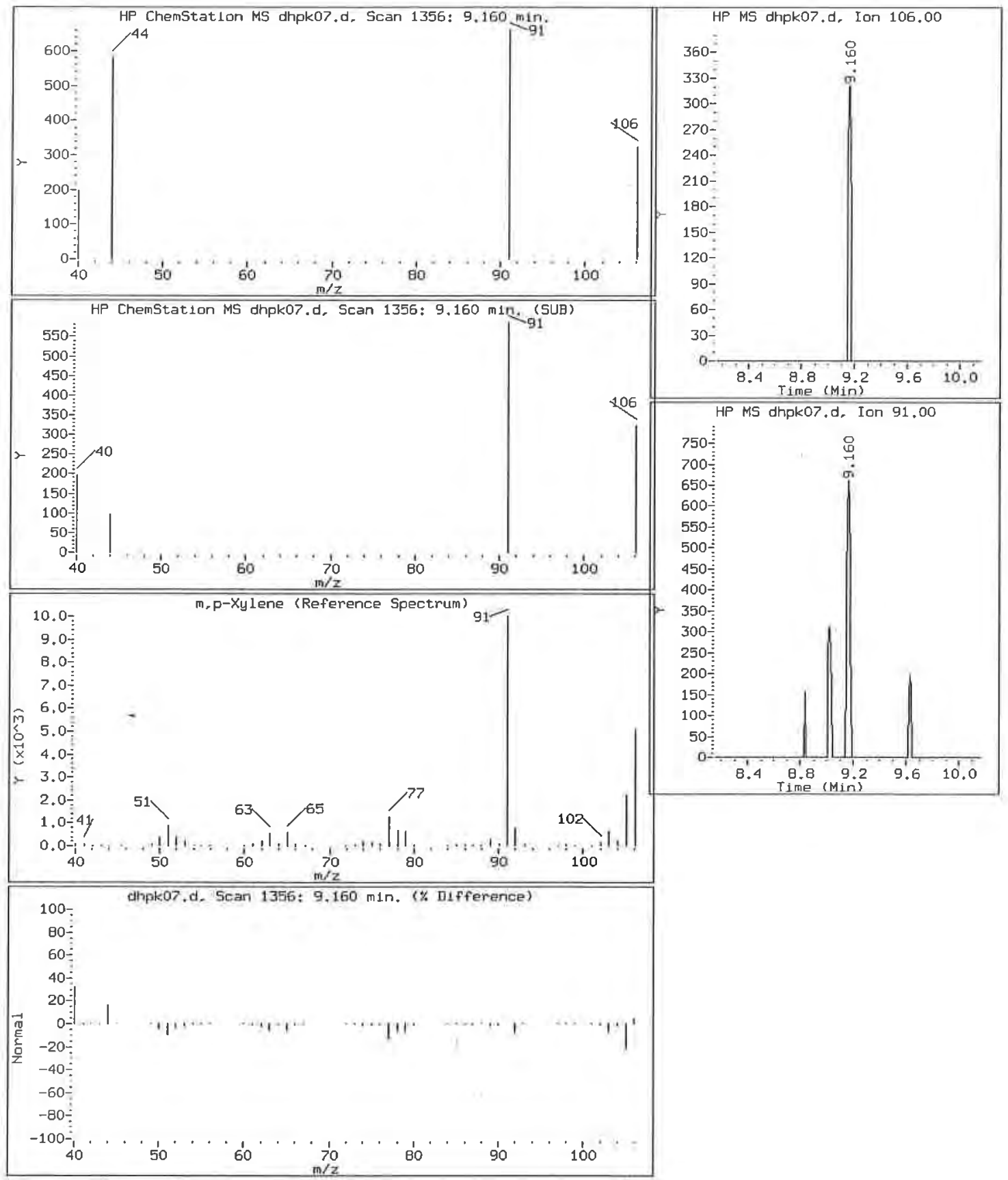
Data File: dhpk07.d

Lab Sample ID: 200-6522-2

Date: 15-AUG-2011 09:51

Client ID: SVSB44D-W-25877

Instrument: D.i

Sample Info: 200-6522-B-2

operator: JH2

54 styrene
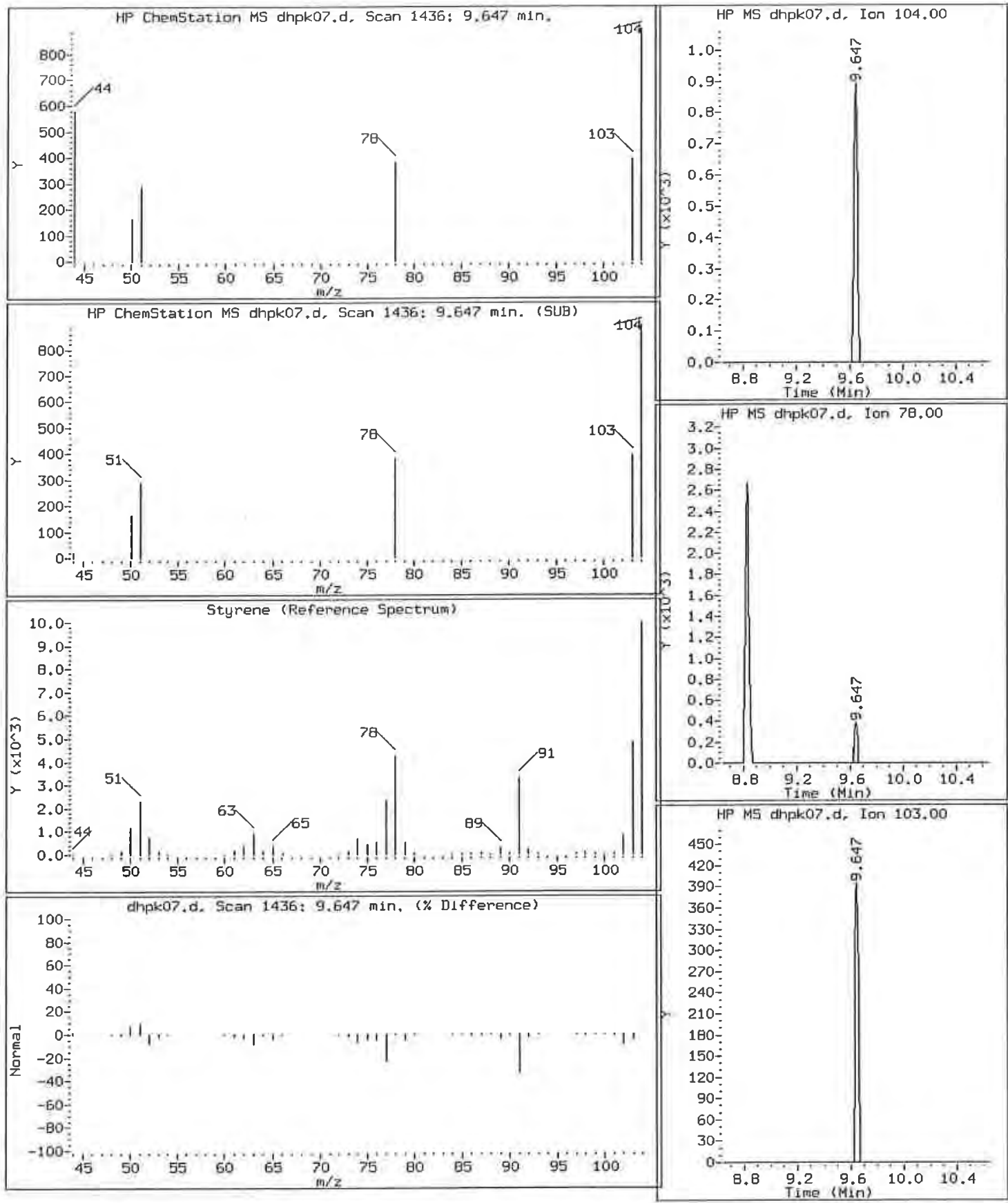
Data File: dhpk07.d

Lab Sample ID: 200 6522-2

Client ID: SVSB44D-W-25877

Sample Info: $200-6522-B-2$

Retention Time: 6.79

Library Search Compound Match

Unknown

Carbonothioic dichloride

Methane, oxybis [chloro-

Methane, oxybis [chloro-
Date: 15-AUG-2011 09:51

Instrument: D.i

Operator: $\mathrm{JH} 2$
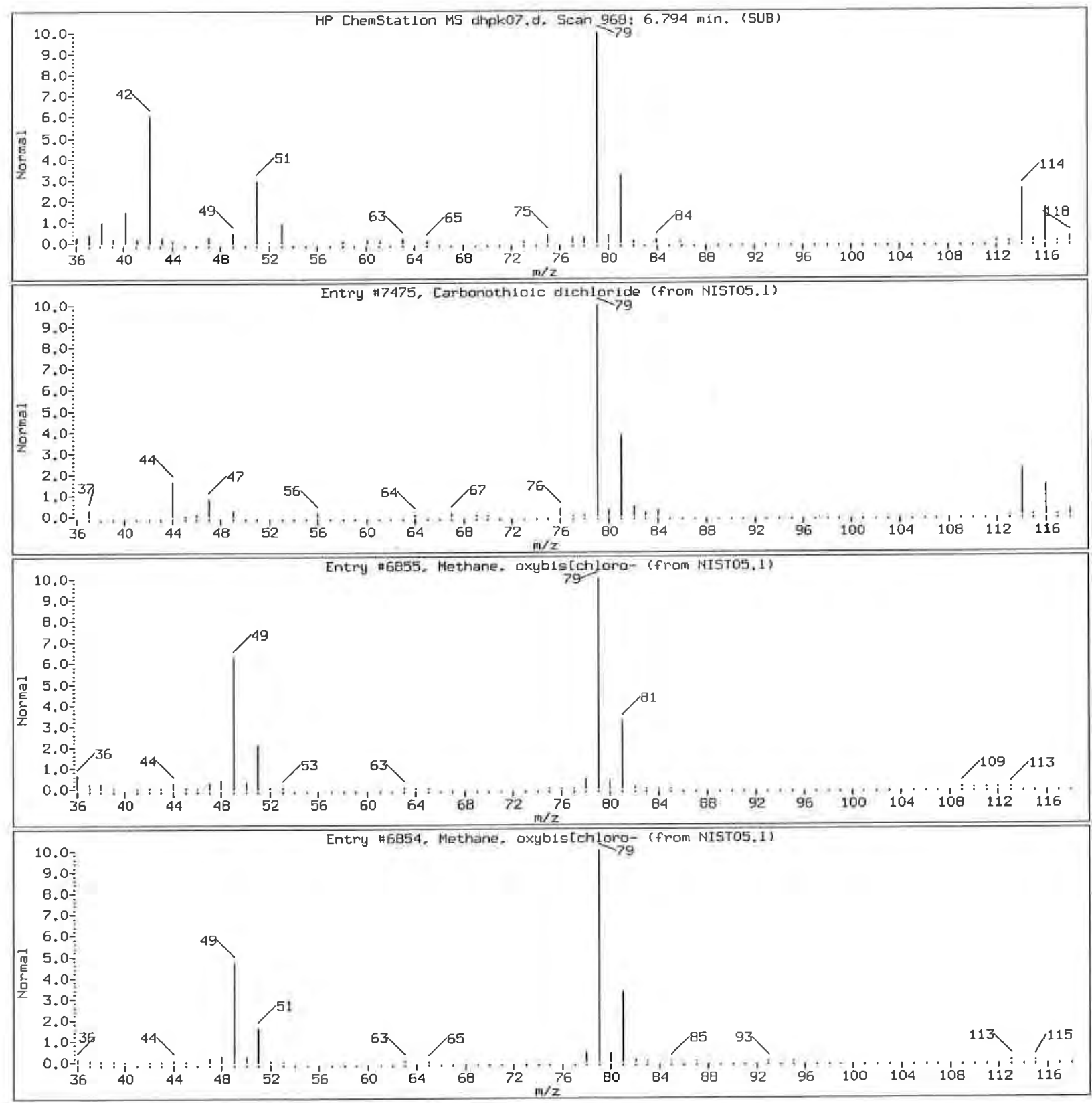
$1 A$ - FORM I VOA-1

VOLATILE ORGANICS ANALYSIS DATA SHEET
EPA SAMPLE NO.

SVSB $46 S-W-25880$
Lab Name: TESTAMERICA BURLINGTON

Lab code: STLV $\mathrm{Ca}$

Matrix: (SOIL/SED/WATER)

Sample wt/vol: 25.0 $(\mathrm{g} / \mathrm{mL}) \mathrm{mL}$

Level: (TRACE/LOW/MED) TRACE

\% Moisture: not dec.

GC Column: DB-624 ID: $0.20 \quad(\mathrm{~mm})$

Soil Extract Volume: (UL) (mL)

Purge Volume: 25.0
Contract: $8 \mathrm{E}-00302$

SDG No.: 200-6522 o.: SAVANN Mod. Ref No.:

Lab Sample ID: 200-6522-3

Lab File ID: DHPK08.D

Date Received: 08/12/2011

Date Analyzed: 08/15/2011

Dilution Factor: 1.0

Soil Aliquot Volume:

(uL)

\begin{tabular}{|c|c|c|c|}
\hline CAS NO. & COMPOUND & $\begin{array}{l}\text { CONCENTRATION UNITS: } \\
(\mathrm{ug} / \mathrm{L} \text { or } \mathrm{ug} / \mathrm{kg}) \mathrm{ug} / \mathrm{L}\end{array}$ & Q \\
\hline $75-71-8$ & Dichlorodifluoromethane & 0.50 & $\mathrm{U}$ \\
\hline $74-87-3$ & Chloromethane & 0.13 & $\mathrm{~J}$ \\
\hline $75-01-4$ & Vinyl chloride & 1.1 & \\
\hline $74-83-9$ & Bromomethane & 0.50 & $\mathrm{U}$ \\
\hline $75-00-3$ & Chloroethane & 0.091 & $\mathrm{~J}$ \\
\hline $75-69-4$ & Trichlorofluoromethane & 0.50 & $\mathrm{U}$ \\
\hline $75-35-4$ & 1,1-Dichloroethene & 0.50 & $\mathrm{U}$ \\
\hline $76-13-1$ & 1,1,2-Trichloro-1,2,2-trifluoroethane & 0.50 & $\mathrm{U}$ \\
\hline $67-64-1$ & Acetone & 2.1 & $\mathrm{~J}$ \\
\hline $75-15-0$ & Carbon disulfide & 0.50 & $\mathrm{U}$ \\
\hline $79-20-9$ & Methyl acetate & 0.50 & $\mathrm{U}$ \\
\hline $75-09-2$ & Methylene Chloride & 0.098 & $\mathrm{~J} B$ \\
\hline $156-60-5$ & trans-1,2-Dichloroethene & 0.50 & $\mathrm{U}$ \\
\hline $1634-04-4$ & Methyl tert-butyl ether & 0.50 & $\mathrm{U}$ \\
\hline $75-34-3$ & 1,1-Dichloroethane & 0.50 & $\mathrm{U}$ \\
\hline $156-59-2$ & cis-1,2-Dichloroethene & 0.50 & $\mathrm{U}$ \\
\hline $78-93-3$ & 2-Butanone & 5.0 & $\mathrm{U}$ \\
\hline $74-97-5$ & Bromochloromethane & 0.50 & $\mathrm{U}$ \\
\hline $67-66-3$ & Chloroform & 5.7 & \\
\hline $71-55-6$ & 1,1,1-Trichloroethane & 0.50 & $\mathrm{U}$ \\
\hline $110-82-7$ & Cyclohexane & 0.50 & $\mathrm{U}$ \\
\hline $56-23-5$ & Carbon tetrachloride & 13 & \\
\hline $71-43-2$ & Benzene & 0.0029 & $\mathrm{~J} \mathrm{~B}$ \\
\hline $107-06-2$ & 1,2-Dichloroethane & 0.50 & $\mathrm{U}$ \\
\hline
\end{tabular}

Report 1,4-Dioxane for Low-Medium VOA analysis only 
$1 B$ - FORM I VOA-2

VOLATILE ORGANICS ANALYSIS DATA SHEET
EPA SAMPLE NO.

SVSB $46 S-W-25880$

Lab Name: TESTAMERICA BURLINGTON

Lab Code: STLV Case No.: SAVANN Mod. Ref No,:

Contract: $\quad 8 \mathrm{E}-00302$

Matrix: (SOIL/SED/WATER) Water

Sample wt/vol: 25.0 $(g / m L) \quad m L$

Level: (TRACE/LOW/MED) TRACE

o Moisture: not dec.

GC Column: DB-624 ID: $0.20 \quad(\mathrm{~mm})$

Soil Extract Volume: (uL) (mL)

Purge Volume: 25.0
Lab Sample ID: 200-6522-3

Lab File ID: DHPK08.D

Date Received: 08/12/2011

Date Analyzed: 08/15/2011

Dilution Factor: 1.0

Soil Aliquot Volume:

(uL)

\begin{tabular}{|c|c|c|c|}
\hline CAS NO. & COMPOUND & $\begin{array}{l}\text { CONCENTRATION UNITS: } \\
(\mathrm{ug} / \mathrm{L} \text { or } \mathrm{ug} / \mathrm{kg}) \mathrm{ug} / \mathrm{L}\end{array}$ & $\mathrm{Q}$ \\
\hline $79-01-6$ & Trichloroethene & 0.50 & $\mathrm{U}$ \\
\hline $108-87-2$ & Methylcyclohexane & 0.50 & $\mathrm{U}$ \\
\hline $78-87-5$ & 1,2-Dichloropropane & 0.50 & $\mathrm{U}$ \\
\hline $75-27-4$ & Bromodichloromethane & 0.50 & $\mathrm{U}$ \\
\hline $10061-01-5$ & cis-1,3-Dichloropropene & 0.50 & $\mathrm{U}$ \\
\hline $108-10-1$ & 4-Methyl-2-pentanone & 5.0 & $\mathrm{U}$ \\
\hline $108-88-3$ & Toluene & 0.12 & $\mathrm{~J} \mathrm{~B}$ \\
\hline $10061-02-6$ & trans-1,3-Dichloropropene & 0.50 & $\mathrm{U}$ \\
\hline $79-00-5$ & 1,1,2-Trichloroethane & 0.50 & $\mathrm{U}$ \\
\hline $127-18-4$ & Tetrachloroethene & 0.057 & $\mathrm{~J}$ \\
\hline $591-78-6$ & 2-Hexanone & 5.0 & $\mathrm{U}$ \\
\hline $124-48-1$ & Dibromochloromethane & 0.50 & $\mathrm{U}$ \\
\hline $106-93-4$ & 1,2-Dibromoethane & 0.50 & $\mathrm{U}$ \\
\hline $108-90-7$ & Chlorobenzene & 0.50 & $\mathrm{U}$ \\
\hline $100-41-4$ & Ethylbenzene & 0.50 & $\mathrm{U}$ \\
\hline $95-47-6$ & o-xylene & 0.50 & $\mathrm{U}$ \\
\hline 179601-23-1 & $\mathrm{m}, \mathrm{p}-\mathrm{xy}$ lene & 0.0055 & $\mathrm{~J}$ \\
\hline $100-42-5$ & Styrene & 0.50 & $\mathrm{U}$ \\
\hline $75-25-2$ & Bromoform & 0.50 & $\mathrm{U}$ \\
\hline $98-82-8$ & Isopropylbenzene & 0.50 & $\mathrm{U}$ \\
\hline $79-34-5$ & $1,1,2,2$-Tetrachloroethane & 0.50 & $\mathrm{U}$ \\
\hline $541-73-1$ & 1,3-Dichlorobenzene & 0.50 & $\mathrm{U}$ \\
\hline $106-46-7$ & 1,4-Dichlorobenzene & 0.50 & $\mathrm{U}$ \\
\hline $95-50-1$ & 1,2-Dichlorobenzene & 0.50 & $\mathrm{U}$ \\
\hline $96-12-8$ & 1,2-Dibromo-3-Chloropropane & 0.50 & $\mathrm{U}$ \\
\hline $120-82-1$ & 1,2,4-Trichlorobenzene & 0.50 & $\mathrm{U}$ \\
\hline $87-61-6$ & 1,2,3-Trichlorobenzene & 0.50 & $\mathrm{U}$ \\
\hline
\end{tabular}




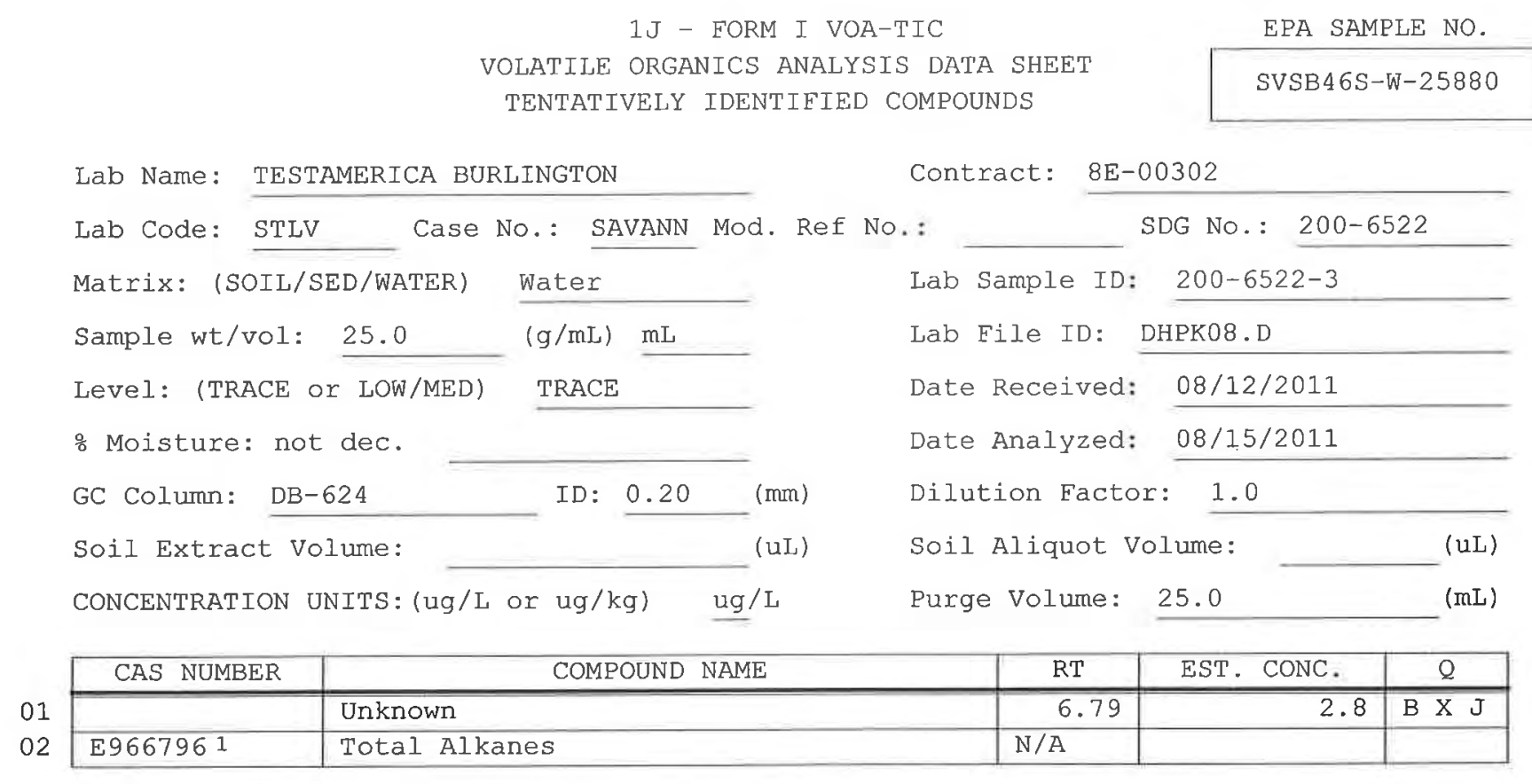

1 EPA-designated Registry Number. 


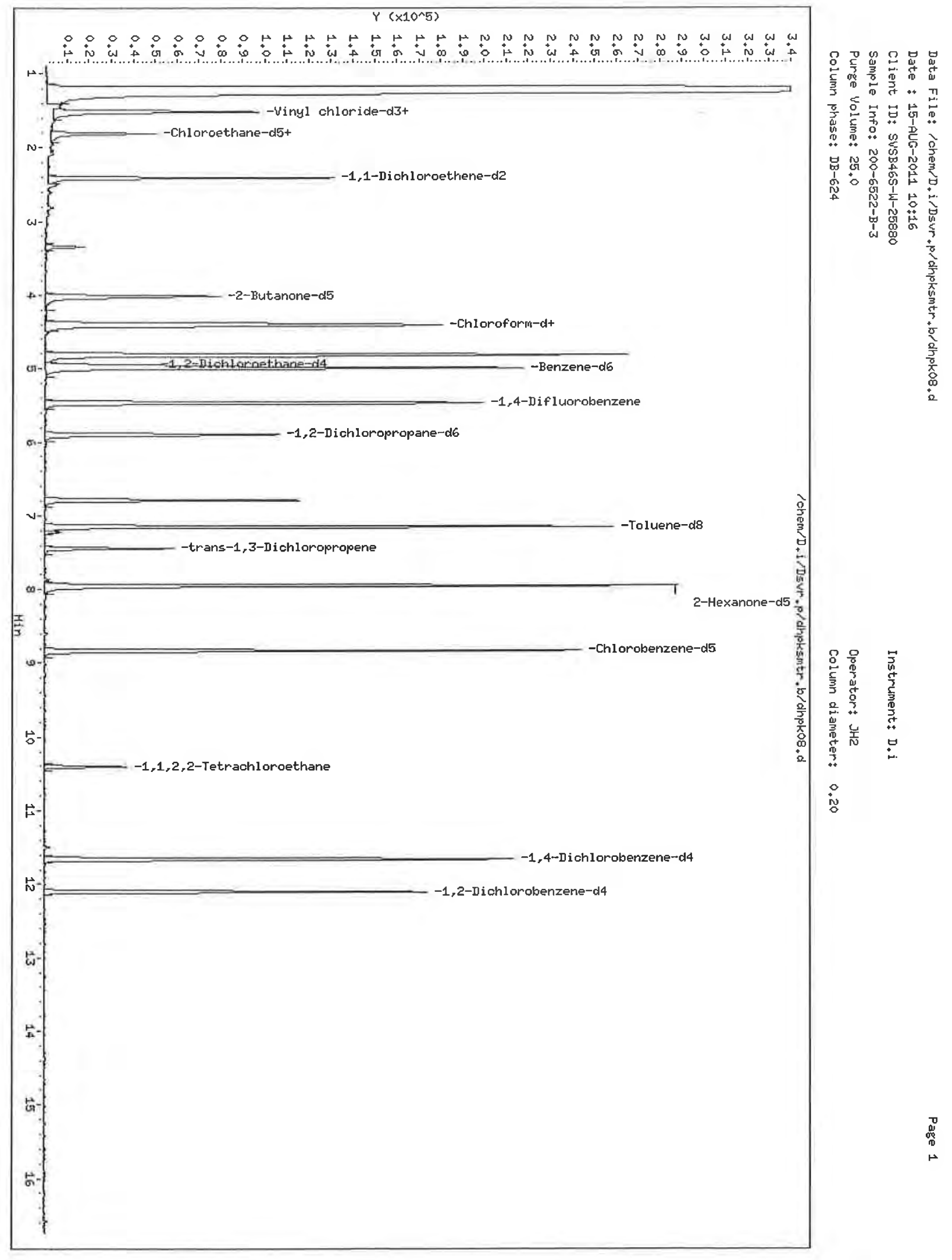




\section{TestAmerica Burlington}

Lab Sample Id: 200-6522-3

Client Smp ID: SVSB46S-W-25880

Inj Date : 15-AUG-2011 10:16

Operator : JH2

Smp Info : 200-6522-B-3

Inst ID: D. i

Misc Info : 1,5

Comment :

Method : /chem/D.i/Dsvr.p/dhpksmtr.b/somtr5t.m

Meth Date : 15-Aug-2011 16:21 jh2 Quant Type: ISTD

Cal Date : 07-JUL-2011 12:56 Cal File: dhp08.d

Als bottle: 6

Dil Factor: 1.00000

Integrator: HP RTE

Target Version: 3.50

Processing Host: chemsvr6

Concentration Formula: Amt * DF * Uf/Vo * CpndVariable

\begin{tabular}{clc} 
Name & Value & Description \\
\hline DE & 1.00000 & Dilution Factor \\
Uf & 1.00000 & ng unit correction factor \\
Vo & 25.00000 & Sample volume purged (mL)
\end{tabular}

Cpnd Variable

Local Compound Variable

Compounds
1 1 Dichlorodifluoromethane
2 Chloromethane
\$ 3 Vinyl chloride-d3
4 Vinyl chloride
5 Bromomethane
6 Chloroethane-d5
7 Chloroethane
9 Trlchlorofluoromethane
9 1,1-Dichloroethene-d2
10 1,1-Dichloroethene
11 1, 1,2-Trichloro-1,2,2-trifluo
12 Acetone
13 Carbon disulfide
14 Methyl acetate
15 Methylene chloride
16 trans-1,2-Dichloroethene

QUANT SIG
MASS
85
85
50
65
62
94
69
64
101
63
96
101
43
76
43
84
96

\begin{tabular}{|c|c|c|c|c|}
\hline \multirow[b]{2}{*}{$\mathrm{RT}$} & \multirow[b]{2}{*}{ EXP RT REL RT } & \multirow[b]{2}{*}{ RESPONSE } & ON-COLUMN & \multirow{2}{*}{$\begin{array}{l}\text { FINAL } \\
(\mathrm{ug} / \mathrm{L})\end{array}$} \\
\hline & & & ( ng) & \\
\hline$=$ & 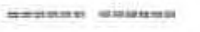 & 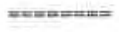 & menteres & 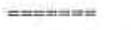 \\
\hline \multicolumn{5}{|c|}{ Compound Not Detected. } \\
\hline 1.454 & $1.454(0.266)$ & 3039 & 3.17393 & $0.13(a)$ \\
\hline 1.515 & $1.521(0.277)$ & 71970 & 118.528 & 4.7 \\
\hline 1.521 & $1.521(0.27 \theta)$ & 19494 & 28.1838 & 1.1 \\
\hline \multicolumn{5}{|c|}{ Compound Not Detected. } \\
\hline 1.807 & $1.807(0.331)$ & 47307 & 120.080 & 4.8 \\
\hline 1.826 & $1.832(0.334)$ & 859 & 2.26538 & 0.091 (a) \\
\hline \multicolumn{5}{|c|}{ Compound Not Detected. } \\
\hline 2.405 & $2.411(0.440)$ & 93860 & 84.6947 & 3.4 \\
\hline \multicolumn{5}{|c|}{ Compound Not Detected. } \\
\hline \multicolumn{5}{|c|}{ Compound Not Detected. } \\
\hline 2.448 & $2.448 \quad(0.448)$ & 2598 & 52.2379 & 2.1 (a) \\
\hline \multicolumn{5}{|c|}{ Compound Not Detected. } \\
\hline \multicolumn{5}{|c|}{ Compound Not Detected. } \\
\hline 2.813 & $2.813(0.515)$ & 852 & 2.44326 & $0.098(a)$ \\
\hline
\end{tabular}




\begin{tabular}{|c|c|c|c|}
\hline \multicolumn{3}{|c|}{ Compounds } & \multirow{2}{*}{$\begin{array}{l}\text { MASS } \\
=\end{array}$} \\
\hline & $10=$ & 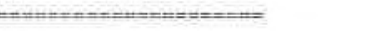 & \\
\hline & 17 & Methyl tert-butyl ether & 73 \\
\hline & 18 & 1,1-Dichloroethane & 63 \\
\hline \multirow[t]{4}{*}{$\$$} & 19 & 2-Butanone-d5 & 46 \\
\hline & 20 & cis-1,2-Dichloroethene & 96 \\
\hline & 21 & 2-Butanone & 43 \\
\hline & 22 & Bromochloromethane & 128 \\
\hline \multirow[t]{5}{*}{$\$$} & 23 & Chloroform-d & 84 \\
\hline & 24 & Chloroform & 83 \\
\hline & 25 & $1,1,1$-Trichloroethane & 97 \\
\hline & 26 & Cyclohexane & 56 \\
\hline & 27 & Carbon tetrachloride & 117 \\
\hline$\$$ & 28 & 1,2-Dichloroethane-d4 & 65 \\
\hline \multirow[t]{3}{*}{$\$$} & 29 & Benzene- $d 6$ & 84 \\
\hline & 30 & Benzene & 78 \\
\hline & 31 & 1,2-Dichloroethane & 62 \\
\hline \multirow[t]{2}{*}{ * } & 32 & 1,4-Difluorobenzene & 114 \\
\hline & 33 & Trichloroethene & 95 \\
\hline \multirow[t]{6}{*}{$\$$} & 34 & 1,2-Dichloropropane-d6 & 67 \\
\hline & 35 & Methylcyclohexane & 55 \\
\hline & 36 & 1,2-Dichloropropane & 63 \\
\hline & 37 & Bromodichloromethane & 83 \\
\hline & 38 & cis-1,3-Dichloropropene & 75 \\
\hline & 39 & 4-Methyl-2-pentanone & 43 \\
\hline \multirow[t]{2}{*}{$\$$} & 40 & Toluene-d8 & 98 \\
\hline & 41 & Toluene & 91 \\
\hline \multirow[t]{4}{*}{$\$$} & 42 & trans-1,3-Dichloropropene-d4 & 79 \\
\hline & 43 & trans-1, 3-Dichloropropene & 75 \\
\hline & 44 & 1,1,2-Trichloroethane & 97 \\
\hline & 45 & Tetrachloroethene & 164 \\
\hline \multirow[t]{4}{*}{$\$$} & 46 & 2-Hexanone-d5 & 63 \\
\hline & 47 & 2-Hexanone & 43 \\
\hline & 48 & Dibromochloromethane & 129 \\
\hline & 49 & 1,2-Dibromoethane & 107 \\
\hline \multirow[t]{8}{*}{ * } & 50 & Chlorobenzene-d5 & 117 \\
\hline & 51 & Chlorobenzene & 112 \\
\hline & 52 & Ethylbenzene & 91 \\
\hline & 53 & m,p-Xylene & 106 \\
\hline & 54 & Styrene & 104 \\
\hline & 55 & o-xylene & 106 \\
\hline & 56 & Bromoform & 172 \\
\hline & 57 & Isopropylbenzene & 105 \\
\hline \multirow[t]{3}{*}{$\$$} & 58 & $1,1,2,2$-Tetrachloroethane-d2 & 84 \\
\hline & 59 & $1,1,2,2$-Tetrachloroethane & 83 \\
\hline & 60 & 1,3-Dichlorobenzene & 146 \\
\hline \multirow[t]{2}{*}{ * } & 61 & 1,4-Dichlorobenzene-d4 & 152 \\
\hline & 62 & 1,4-Dichlorobenzene & 146 \\
\hline \multirow[t]{2}{*}{ \$ } & 63 & 1,2-Dichlorobenzene-d4 & 152 \\
\hline & 64 & 1,2-Dichlorobenzene & 146 \\
\hline
\end{tabular}

\begin{tabular}{|c|c|c|c|c|c|}
\hline \multirow[b]{2}{*}{ UANT SIG } & \multirow[b]{3}{*}{ RT } & \multirow[b]{3}{*}{ EXP RT REL RT } & \multirow[b]{3}{*}{ RESPONSE } & \multicolumn{2}{|c|}{ CONCENTRATIONS } \\
\hline & & & & ON-COLUMN & FINAL \\
\hline MASS & & & & ( ng) & $(\mathrm{ug} / \mathrm{L})$ \\
\hline$=$ & $=$ & 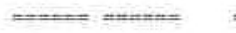 & strenture & +mentre & enement= \\
\hline 73 & \multicolumn{2}{|c|}{ Compound Not Detected. } & & & \\
\hline 63 & \multicolumn{2}{|c|}{ Compound Not Detected. } & & & \\
\hline 46 & 4.014 & $4.020 \quad(0.735)$ & 134648 & 2340.42 & $94(R)$ \\
\hline 96 & \multicolumn{2}{|c|}{ Compound Not Detected. } & & & \\
\hline 43 & \multicolumn{2}{|c|}{ Compound Not Detected. } & & & \\
\hline 128 & \multicolumn{2}{|c|}{ Compound Not Detected. } & & & \\
\hline 84 & 4.392 & $4.392(0.804)$ & 82846 & 116.691 & 4.7 \\
\hline 83 & 4.417 & $4.417(0.808)$ & 96423 & 142.563 & 5.7 \\
\hline 97 & \multicolumn{2}{|c|}{ Compound Not Detected. } & & & \\
\hline 56 & \multicolumn{2}{|c|}{ Compound Not Detected. } & & & \\
\hline 117 & 4.813 & $4.819(0.545)$ & 182910 & 324.904 & 13 \\
\hline 65 & 4.953 & $4.959(0.906)$ & 34330 & 121.279 & 4.9 \\
\hline 84 & 4.990 & $4.990 \quad(0.565)$ & 202272 & 115.007 & 4.6 \\
\hline 78 & 5.039 & $5.032(0.571)$ & 130 & 0.07197 & $0.0029(a)$ \\
\hline 62 & \multicolumn{2}{|c|}{ Compound Not Detected. } & & & \\
\hline 114 & 5.465 & $5.471(1.000)$ & 174229 & 125.000 & \\
\hline 95 & \multicolumn{2}{|c|}{ Compound Not Detected. } & & & \\
\hline 67 & 5.898 & $5.904 \quad(0.668)$ & 49850 & 111.685 & 4.5 \\
\hline 55 & \multicolumn{2}{|c|}{ Compound Not Detected. } & & & \\
\hline 63 & \multicolumn{2}{|c|}{ Compound Not Detected. } & & & \\
\hline 83 & \multicolumn{2}{|c|}{ Compound Not Detected. } & & & \\
\hline 75 & \multicolumn{2}{|c|}{ Compound Not Detected. } & & & \\
\hline 43 & \multicolumn{2}{|c|}{ Compound Not Detected. } & & & \\
\hline 98 & 7.154 & $7.154(0.810)$ & 190898 & 114.599 & 4.6 \\
\hline 91 & 7.227 & $7.227 \quad(0.818)$ & 5707 & 2.88998 & $0.12(a)$ \\
\hline 79 & 7.447 & $7.447 \quad(0.843)$ & 30663 & 112.004 & 4.5 \\
\hline 75 & \multicolumn{3}{|c|}{ Compound Not Detected. } & & \\
\hline 97 & Comr & ound Not Detected. & & & \\
\hline 164 & 7.867 & $7.867(0.891)$ & 522 & 1.43194 & $0.057(a)$ \\
\hline 63 & 7.947 & $7.946(0.900)$ & 107142 & 2746.78 & $110(\mathrm{R})$ \\
\hline 43 & Comp & ound Not Detected. & & & \\
\hline 129 & Comr & ound Not Detected. & & & \\
\hline 107 & Comp & ound Not Detected. & & & \\
\hline 117 & 8.831 & $8.830 \quad(1.000)$ & 149001 & 125.000 & \\
\hline 112 & Comy & ound Not Detected. & & & \\
\hline 91 & Comp & ound Not Detected. & & & \\
\hline 106 & 9.166 & $9.160 \quad(1.038)$ & 121 & 0.13681 & $0.0055(a Q)$ \\
\hline 104 & Coms & ound Not Detected. & & & \\
\hline 106 & Comr & ound Not Detected. & & & \\
\hline 172 & Comr & ound Not Detected. & & & \\
\hline 105 & Comr & ound Not Detected. & & & \\
\hline 84 & 10.403 & $10.403 \quad(1.1 .78)$ & 20537 & 105.210 & 4.2 \\
\hline 83 & Conn & ound Not Detected. & & & \\
\hline 146 & Coms & ound Not Detected. & & & \\
\hline 152 & 11.659 & $11.659(1.000)$ & 62589 & 125.000 & (Q) \\
\hline 146 & Coms & ound Not Detected. & & & \\
\hline 152 & 12.104 & $12.104(1.038)$ & 49865 & 120.038 & 4.8 \\
\hline 146 & Comp & ound Not Detected. & & & \\
\hline
\end{tabular}


Data File: /chem/D.i/Dsvr.p/dhpksmtr.b/dhpk08.d

Page 3 Report Date: 15-Aug-2011 20:11

\begin{tabular}{|c|c|c|c|c|c|c|}
\hline \multirow[b]{3}{*}{ Compounds } & \multirow[b]{2}{*}{ QUANTT SIG } & & & & \multicolumn{2}{|c|}{ CONCENTRATIONS } \\
\hline & & & & & ON-COLUMN & FINAL \\
\hline & MASS & RT & EXP RT REL RT & RESPONSE & ng) & $(\mathrm{ug} / \mathrm{L})$ \\
\hline 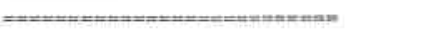 & mसн= & $=$ & 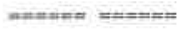 & $=-=+$ nenter & $======$ & $==== \pm==$ \\
\hline 65 1,2-Dibromo-3-chloropropane & 75 & \multicolumn{3}{|c|}{ Compound Not Detected. } & & \\
\hline $661,2,4$-Trichlorobenzene & 180 & \multicolumn{3}{|c|}{ Compound Not Detected. } & & \\
\hline 67 1,2,3-Trichlorobenzene & 180 & \multicolumn{3}{|c|}{ Compound Not Detected. } & & \\
\hline
\end{tabular}

QC Flag Legend

a - Target compound detected but, quantitated amount Below Limit of Quantitation (BLOQ).

Q - Qualifier signal failed the ratio test.

$\mathrm{R}$ - Spike/Surrogate failed recovery limits. 
Lab Sample Id: 200-6522-3

Client Smp ID: SVSB46S-W-25880

Inj Date : 15-AUG-2011 10:16

Operator : JH2

Inst ID: D.i

Smp Info : 200-6522-B-3

Misc Info : 1,5

Comment :

Method : /chem/D.i/Dsvr.p/dhpksmtr.b/somtr5t.m

Meth Date : 15-Aug-2011 16:21 jh2 Quant Type: ISTD

Cal Date : 07-JUL-2011 12:56 Cal File: dhp08.d

Als bottle: 6

Dil Factor: 1.00000

Integrator: HP RTE

Target Version: 3.50

Processing Host: chemsvr6

Concentration Eormula: Ant * DE * Uf/Vo * CpndVariable

\begin{tabular}{clc} 
Name & \multicolumn{1}{c}{ Value } & Description \\
\hline DF & 1.00000 & Dilution Factor \\
Uf & 1.00000 & ng unit correction factor \\
Vo & 25.00000 & Sample volume purged (mL)
\end{tabular}

Cpnd Variable

ISTD

$===== \pm=$

* 321,4 -Difluorobenzene

CONCENTRATIONS

RT AREA ON-COL ( ng) FINAL ( ug/L) QUAL

Unknown

6.794

22302470.5494180
Local Compound Variable

$\begin{array}{lll}\begin{array}{l}\mathrm{RT} \\ ===\end{array} & \begin{array}{c}\text { AREA } \\ == \pm===\end{array} & \begin{array}{l}\text { AMOUNT } \\ =====\end{array} \\ 5.465 & 395154 & 125.000\end{array}$

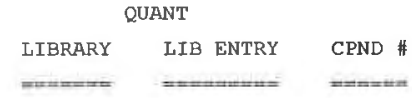

CAS H:
$0 \quad 32$ 
Data File: dhpk08.d

Lab Sample ID: 200-6522-3

Date: 15-AUG-2011 10:16

Client ID: SVSB46S-W-25880

Instrument: D.i

Sample Info: 200-6522-B-3

Operator: JH2

2 Chloromethane
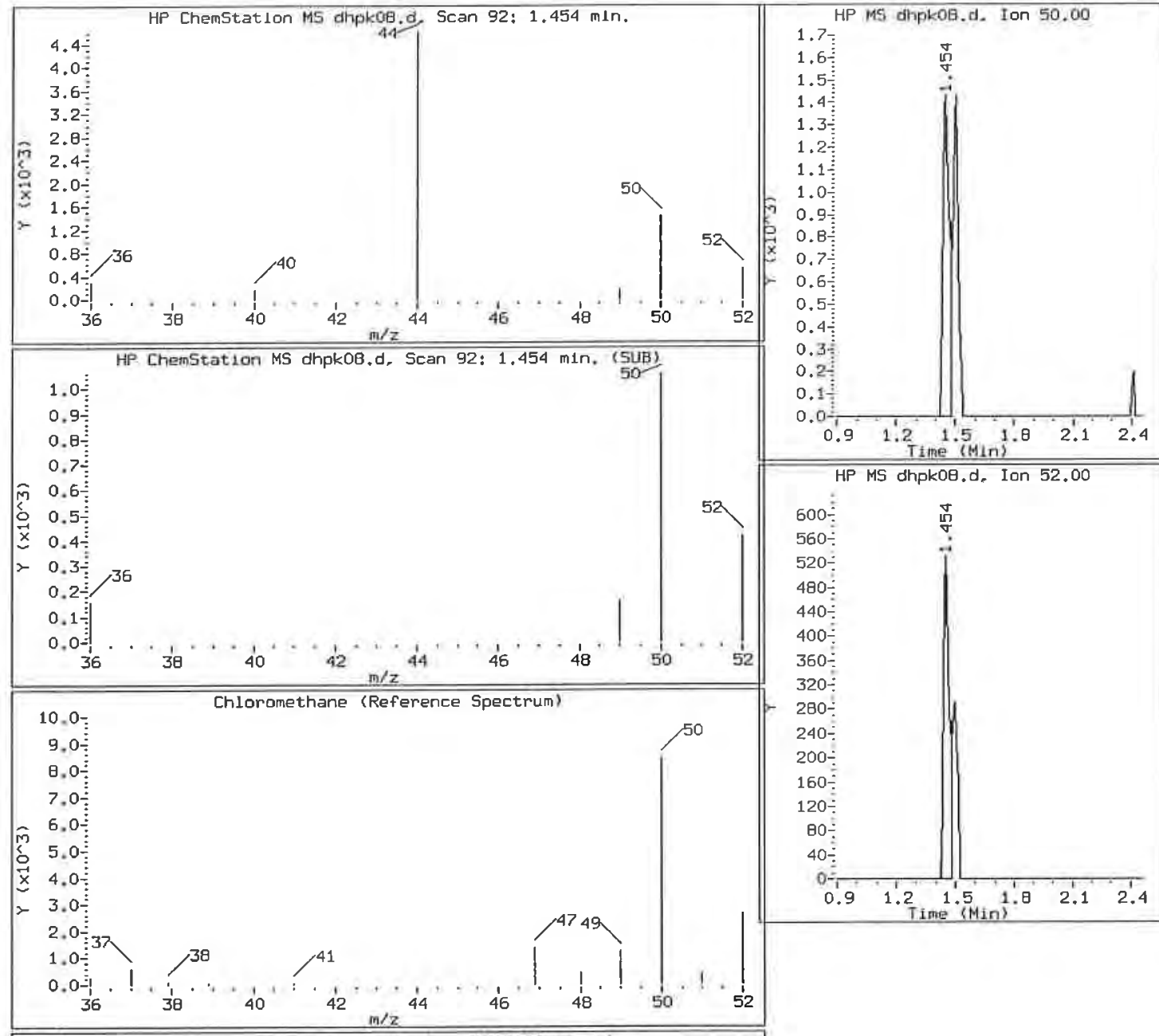

लิ 0.6

0.5

$\therefore \quad 0.4$

$0.3 \div$

0,1

$0.0=$

$10.0-$

9,0

$7.0-$

6,0

5.0

4,0

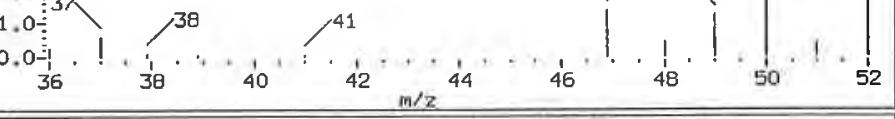

HP MS dhpk00.d. Ion 52.00
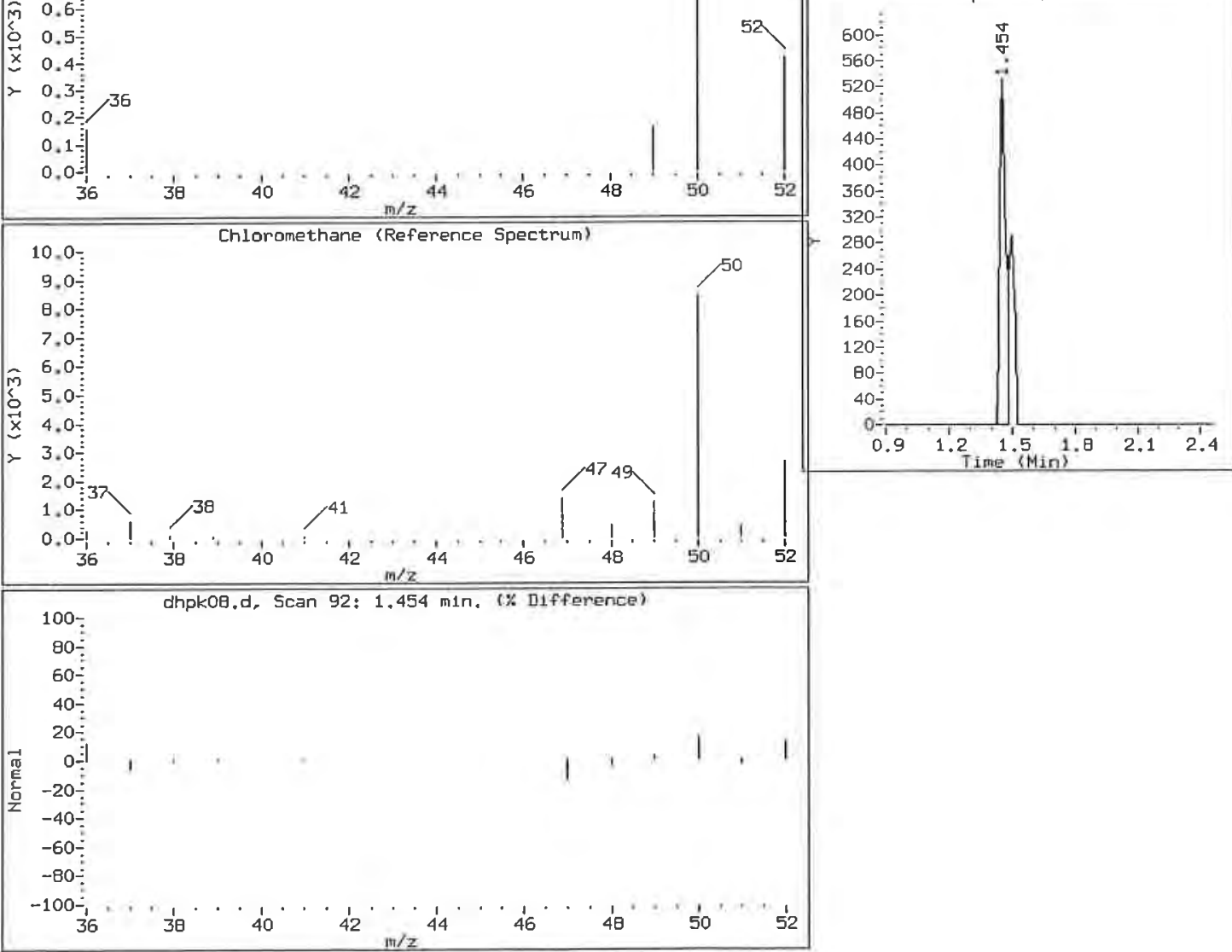
Data File: dhpk08.d
Lab Sample ID: 200-6522-3
Date: 15-AUG-2011 10:16
Client ID: SVSB $46 S-W-25880$
Instrument: D.i
Sample Info: 200-6522-B-3
Operator: JH2

4 Vinyl chloride

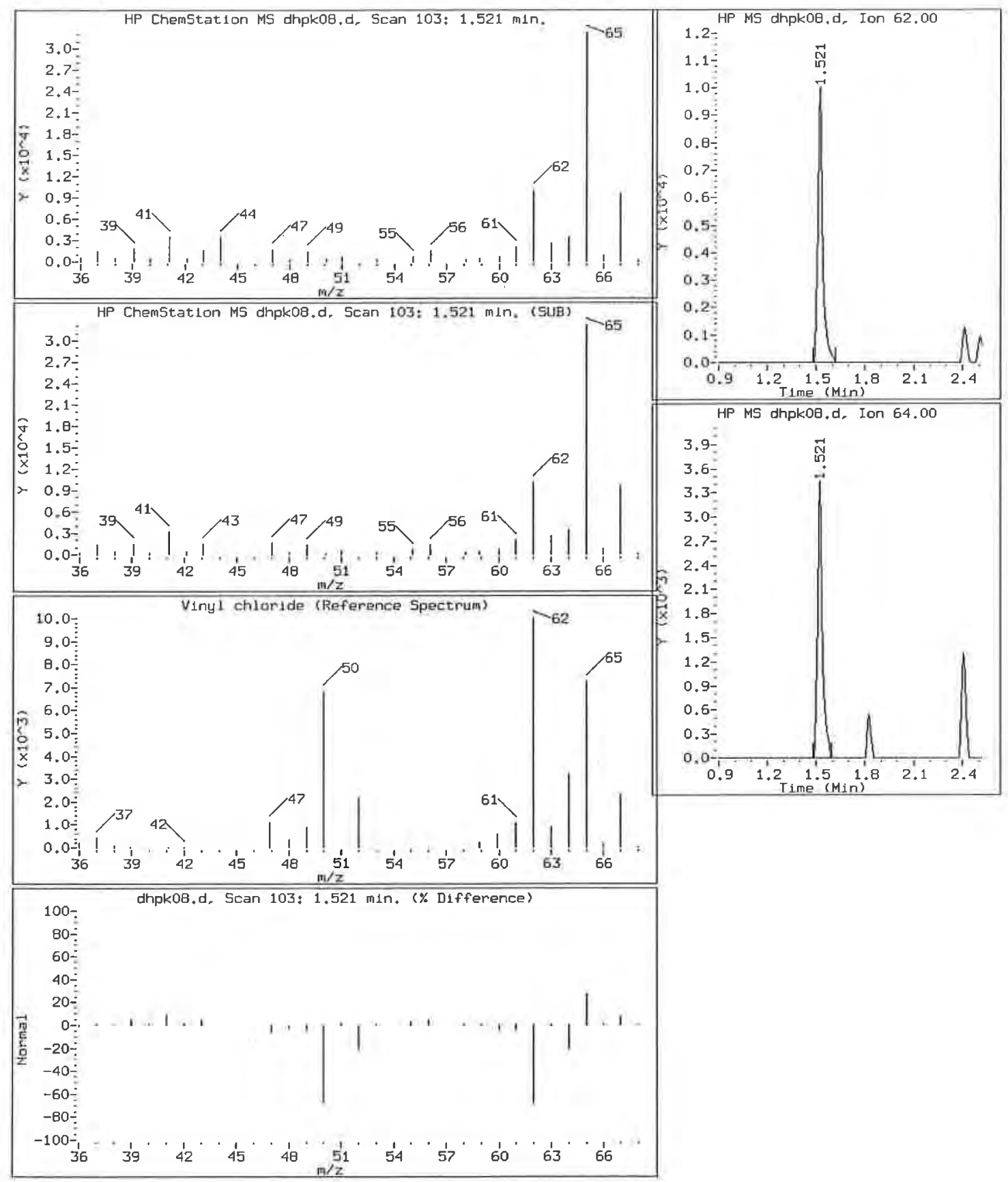


Data File: dhpk08.d

Lab Sample ID: 200-6522-3

Date: 15-AUG-2011 10:16

Client ID: SVSB46S-W-25880

Instrument: D.i

Sample Info: 200-6522-B-3

Operator: JH2

7 Chloroethane
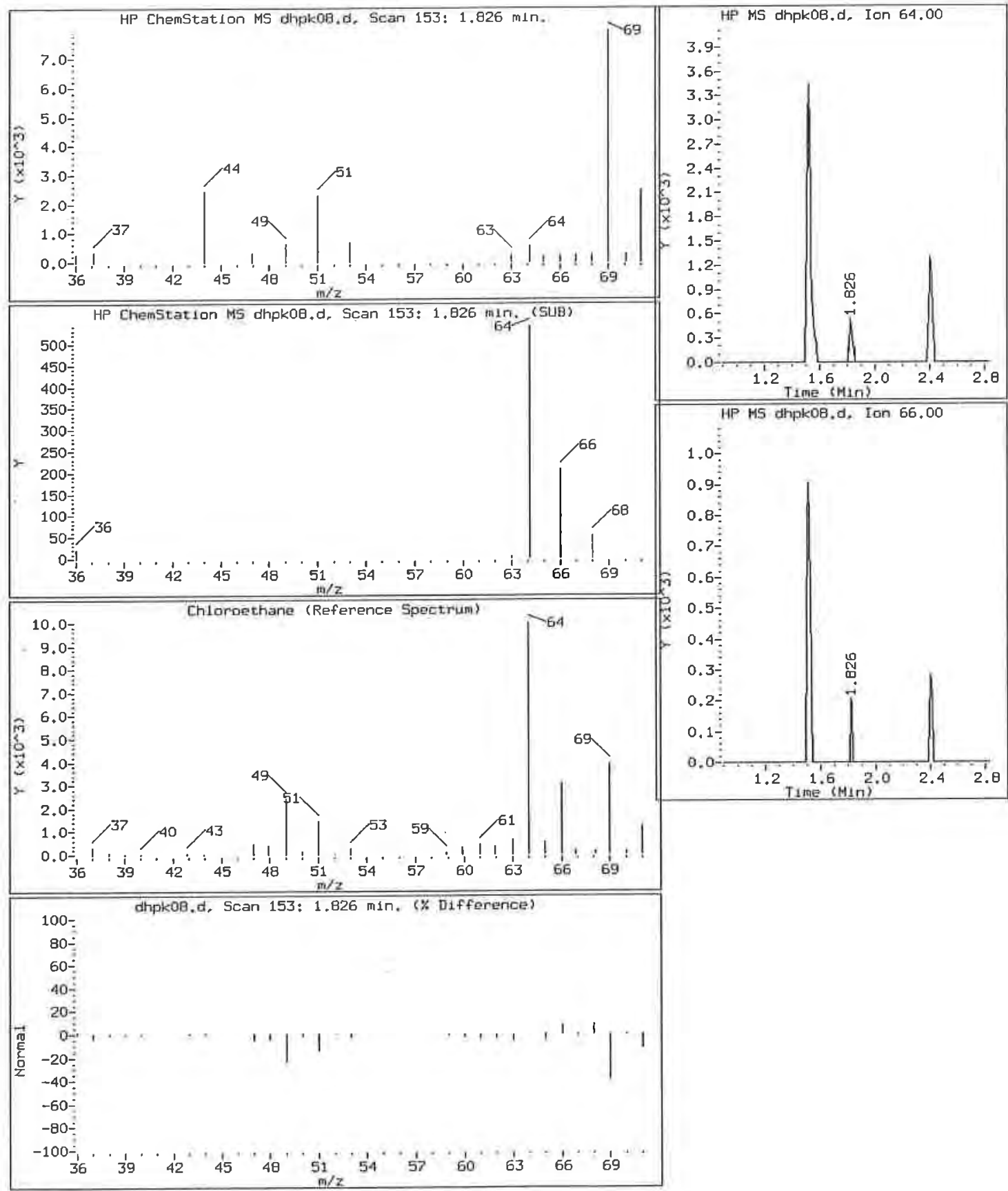
Data File: dhpk08.d

Lab Sample ID: 200-6522-3

Date: 15-AUG-2011 10:16

Client ID: SVSB46S-W-25880

Instrument: D.i

Sample Info: 200-6522-B-3

Operator: JH2

12 Acetone
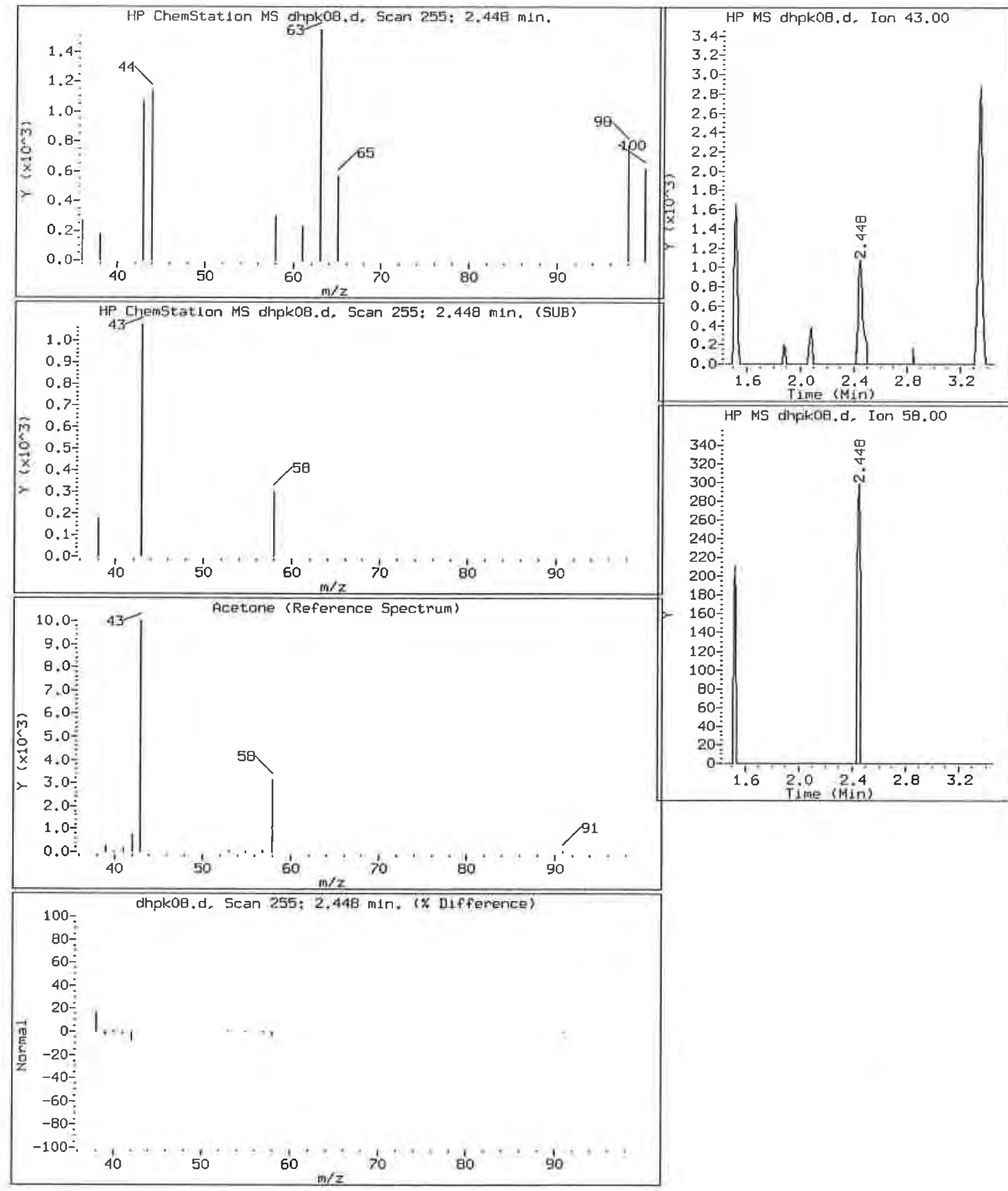
Data File: dhpk08.d

Lab Sample ID: 200-6522-3

Date: 15-AUG-2011 10:16

Client ID: SVSB46S-W-25880

Instrument: D.i

Sample Info: 200-6522-B-3

Operator: JH2

15 Methylene chloride

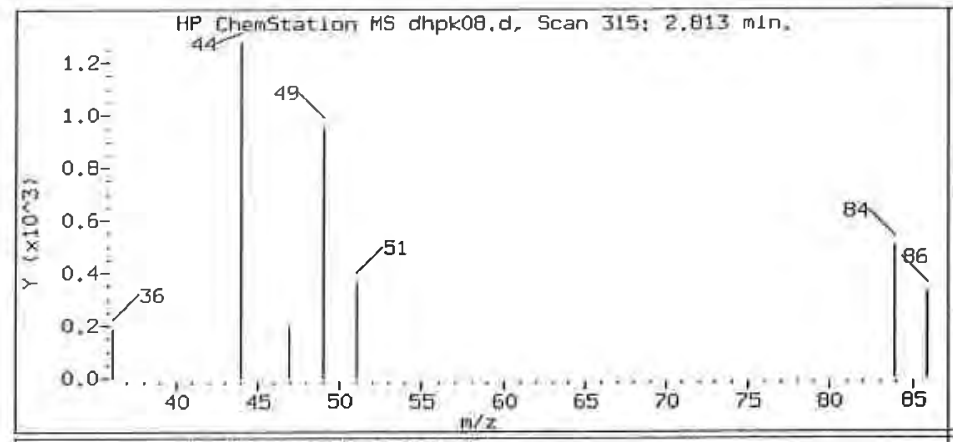

6
5
5
4
4
4
3
3
2
2

HF MS dhpk08.d, Ion 84.00
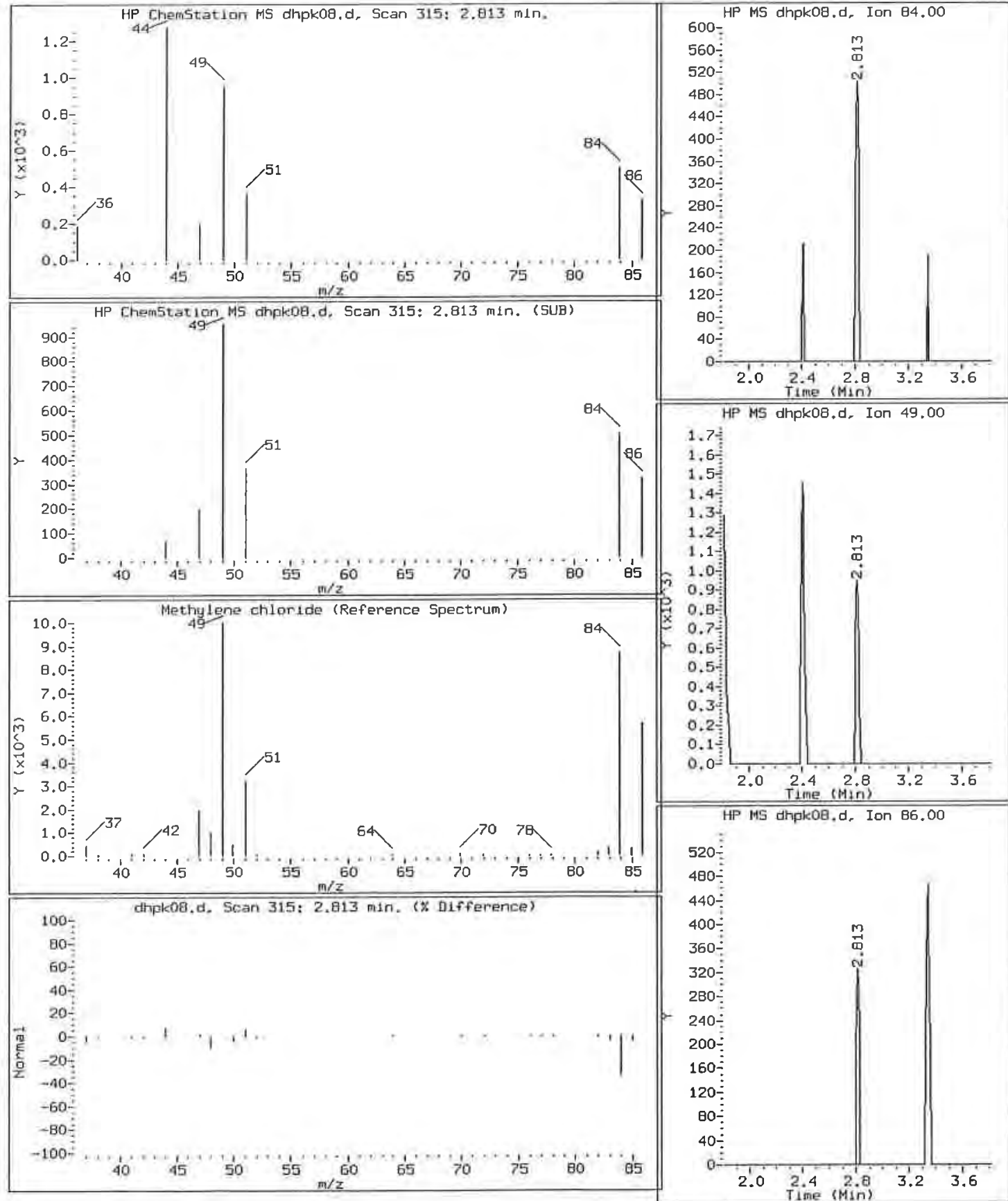
Data File: dhpk08.d

Lab Sample ID: 200-6522-3

Client ID: SVSB46S-W-25880

Sample Info: 200-6522-B-3
Date: 15-AUG-2011 10:16

Instrument: D.i

Operator: JH2

24 Chloroform
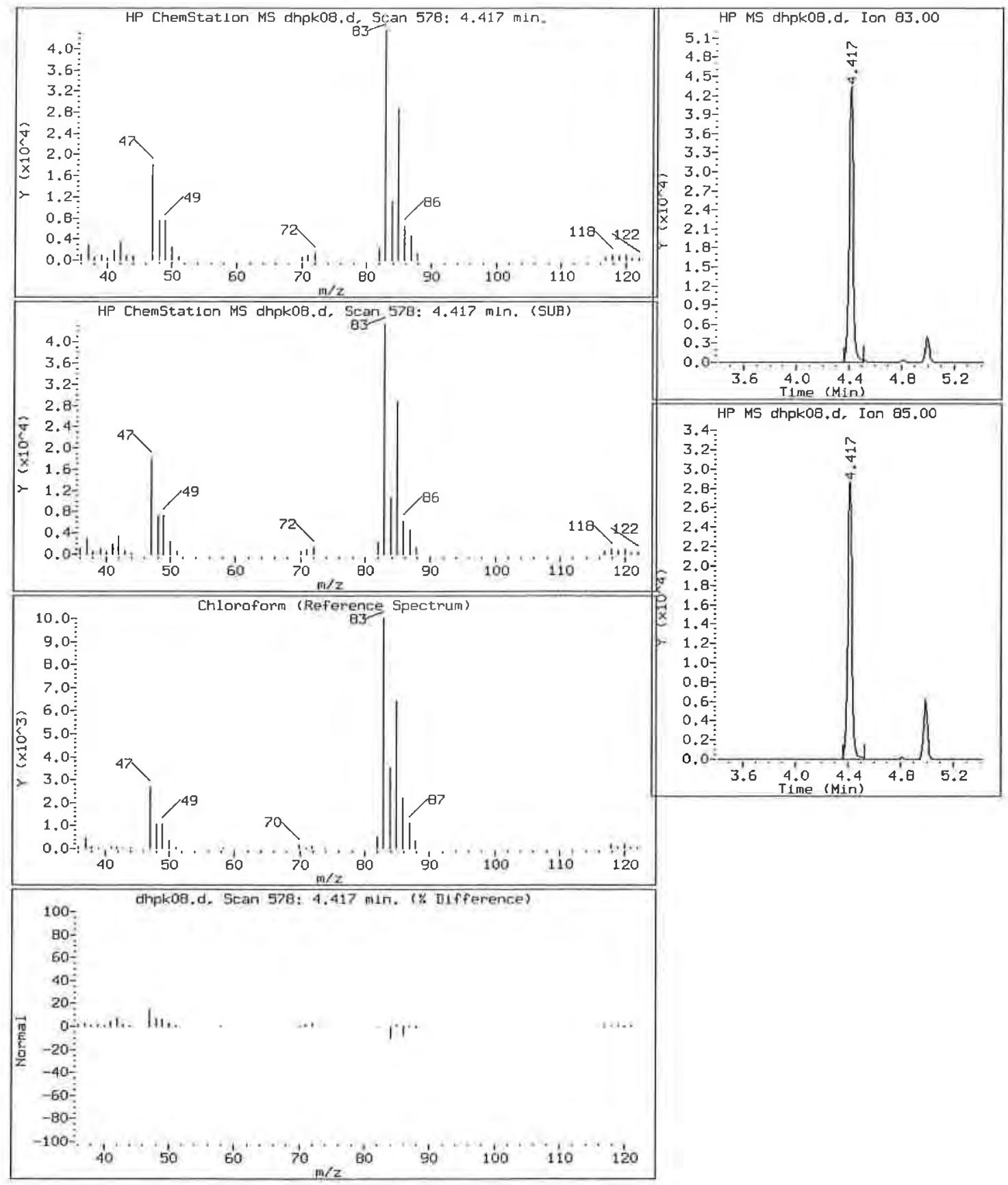
Data File: dhpk08.d

Lab Sample ID: 200-6522-3

Date: 15-AUG-2011 10:16

Client ID: SVSB46S-W-25880

Instrument: D.i

Sample Info: 200-6522-B-3

Operator: JH2

27 Carbon tetrachloride

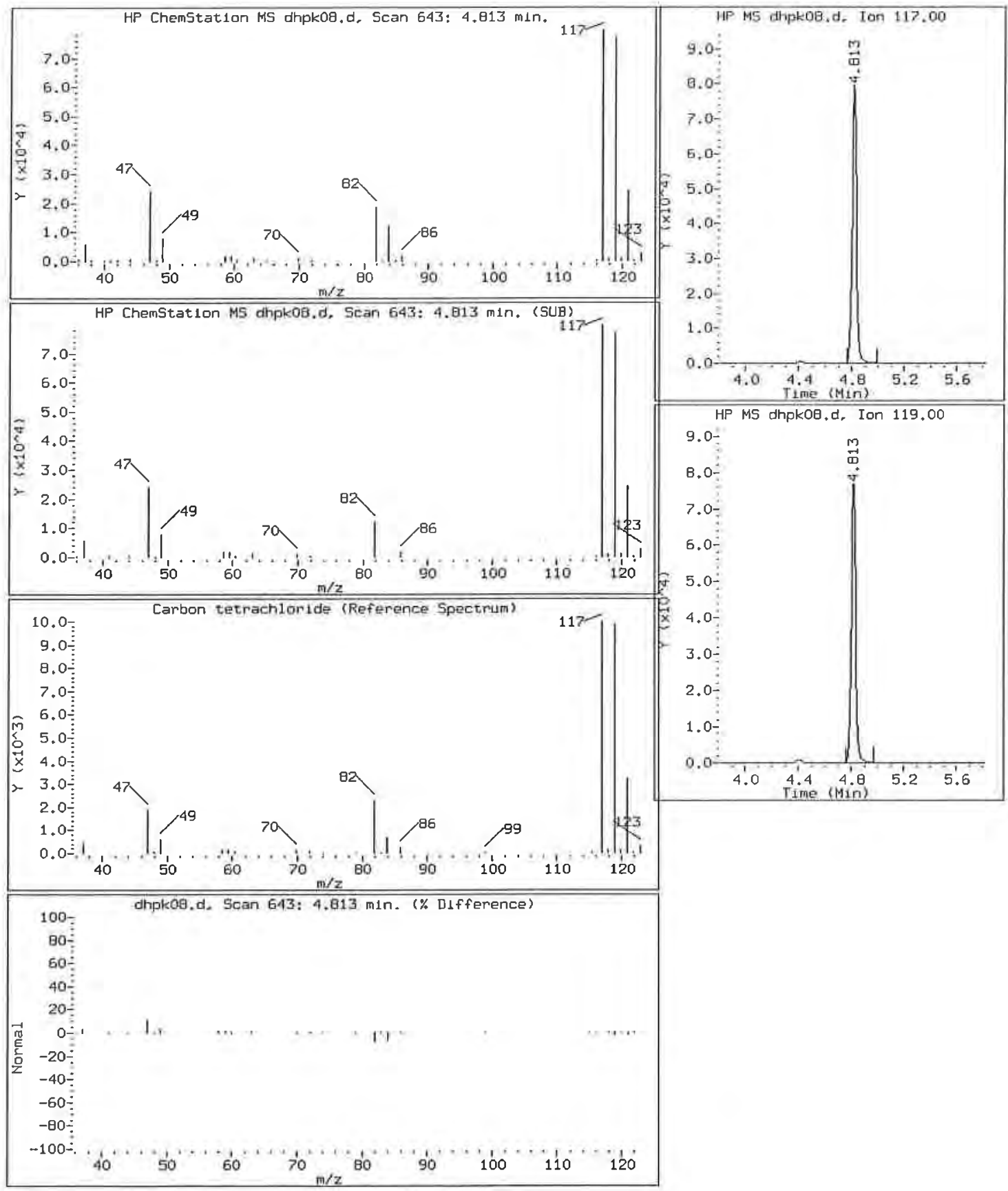


Data File: dhpk08.d

Lab Sample ID: 200-6522-3

Date: 15-AUG-2011 10:16

Client ID: SVSB $46 \mathrm{~S}-\mathrm{W}-25880$

Instrument: D.i

Sample Info: 200-6522-B-3

Operator: JH2

30 Benzene
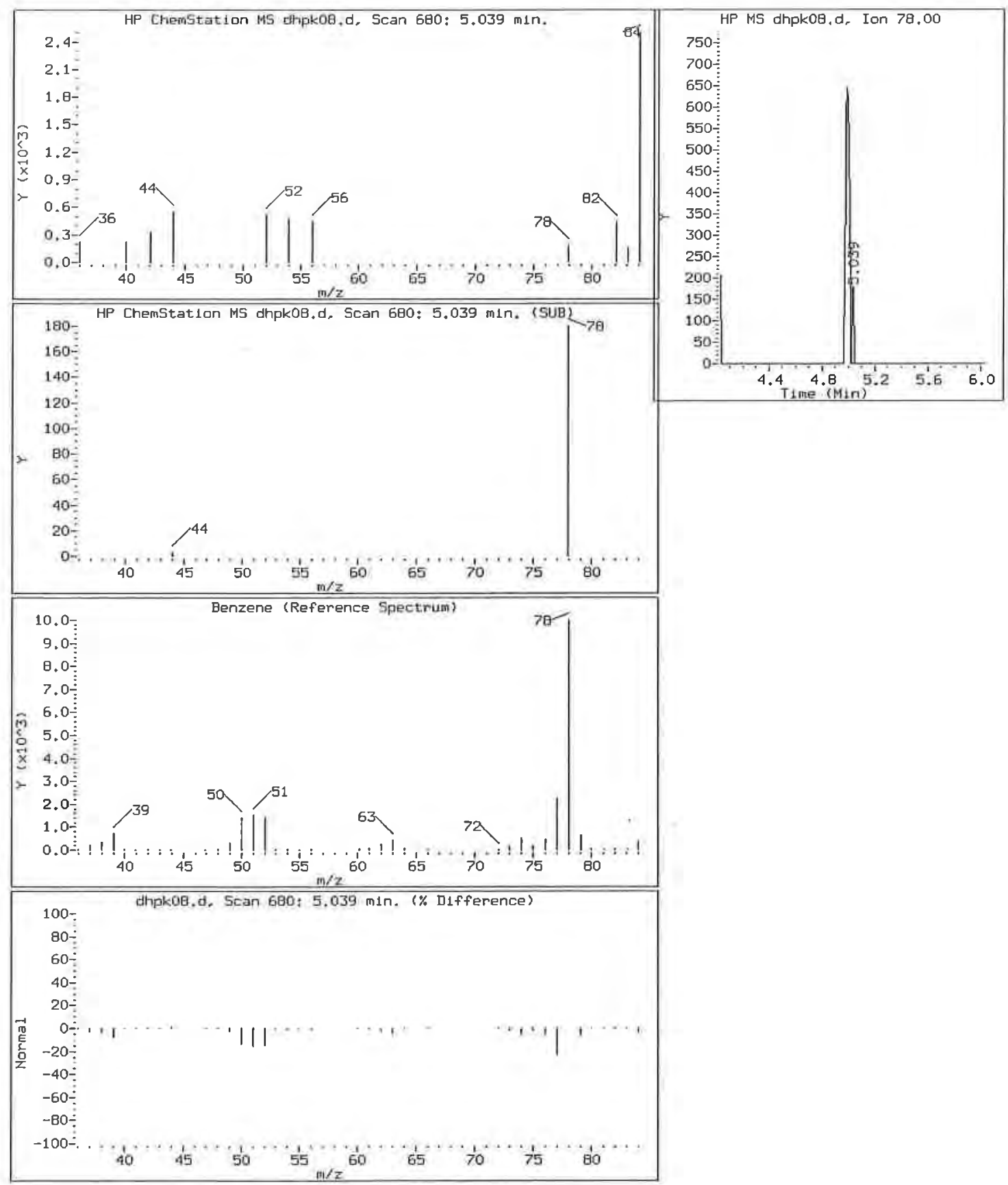
Data File: dhpk08.d

Lab Sample ID: 200-6522-3

Date: 15-AUG-2011 10:16

Client ID: SVSB46S-W-25880

Instrument: D.j

Sample Info: 200-6522-B-3

Operator: JH2

41 Toluene
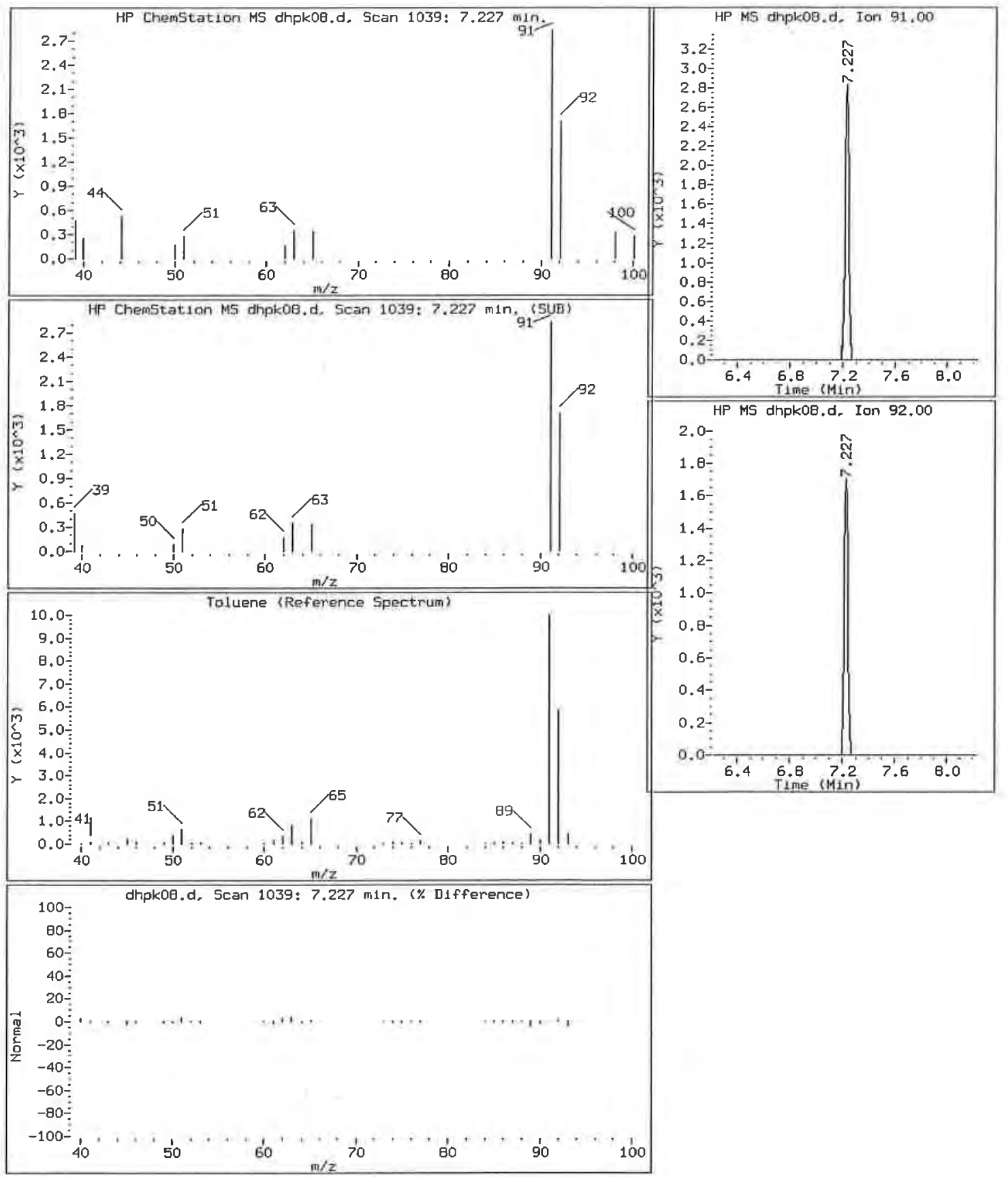
Data File: dhpk08.d

Lab Sample ID: 200-6522-3

Date: 15-AUG-2011 10:16

Client ID: SVSB46S-W-25880

Instrument: D.i

Sample Info: 200-6522-B-3

Operator: JH2

45 Tetrachloroethene
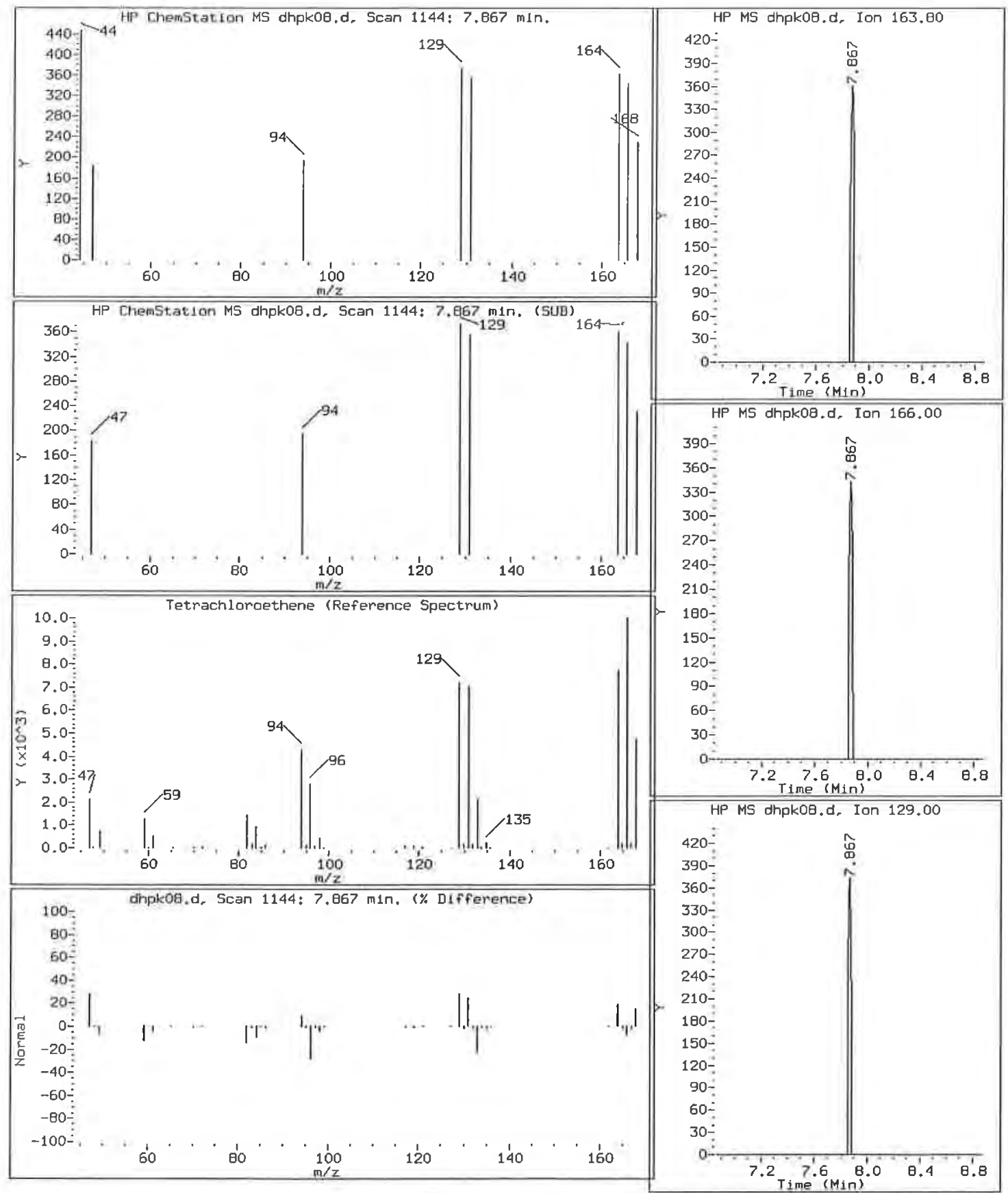
Data File: dhpk08.d

Lab Sample ID: 200-6522-3

Date: 15-AUG-2011 10:16

Client ID: SVSB46S-W-25880

Instrument: D.i

Sample Info: 200-6522-B-3

Operator: JH2

$53 \mathrm{~m}, \mathrm{p}$-Xylene
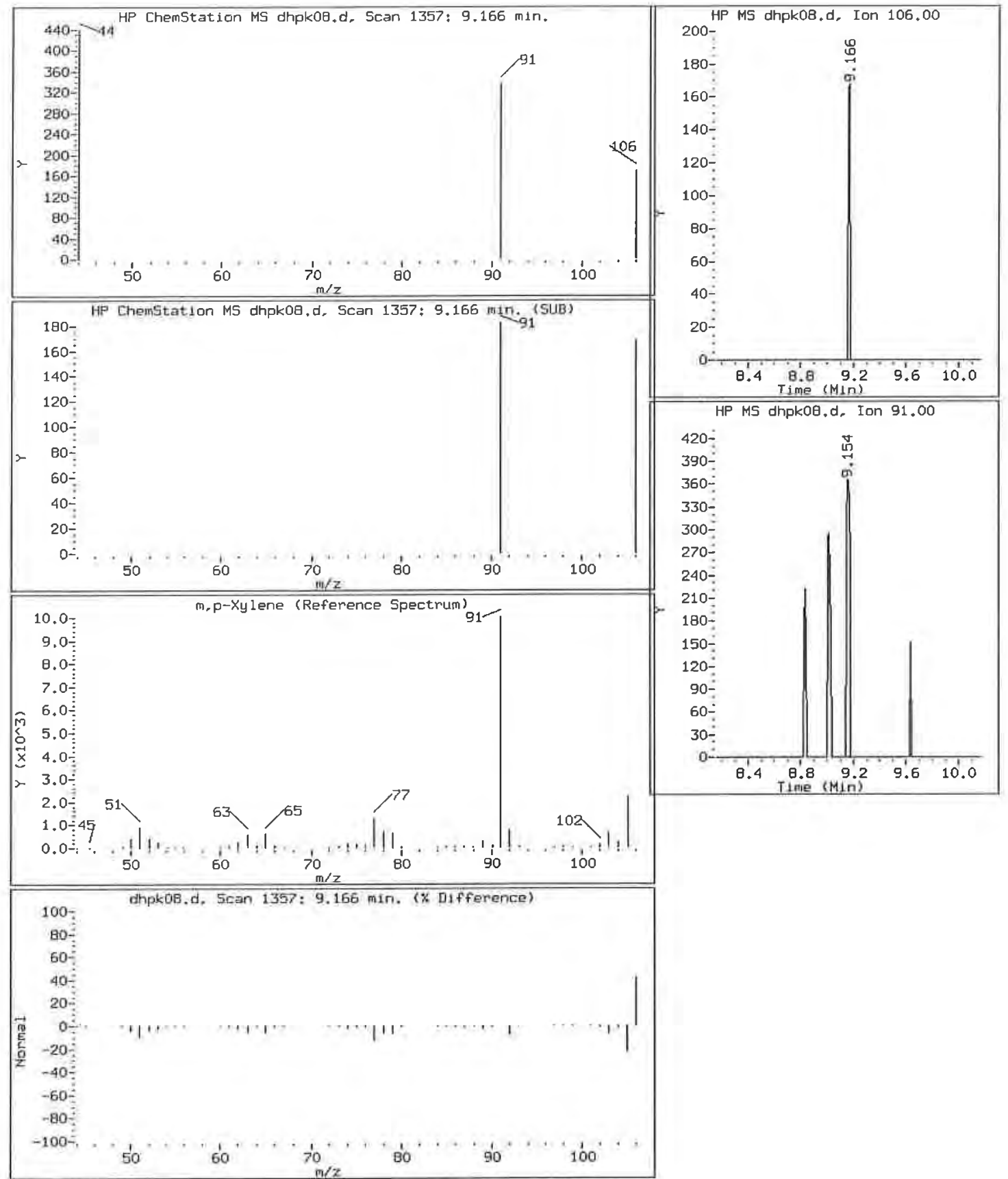
Data File: dhpk08.d

Lab Sample ID: 200-6522-3

Client ID: SVSB $465-\omega-25880$

Sample Info: 200-6522-B-3

Retention Time:

6.79

Library Search Compound Match Unknown

Carbonothioic dichloride

Methane, oxybis[chloro-

2-Propanol, 1,3-dichloro-
Date: 15-AUG-2011 10:16

Instrument: D. 1

Operator: JH2
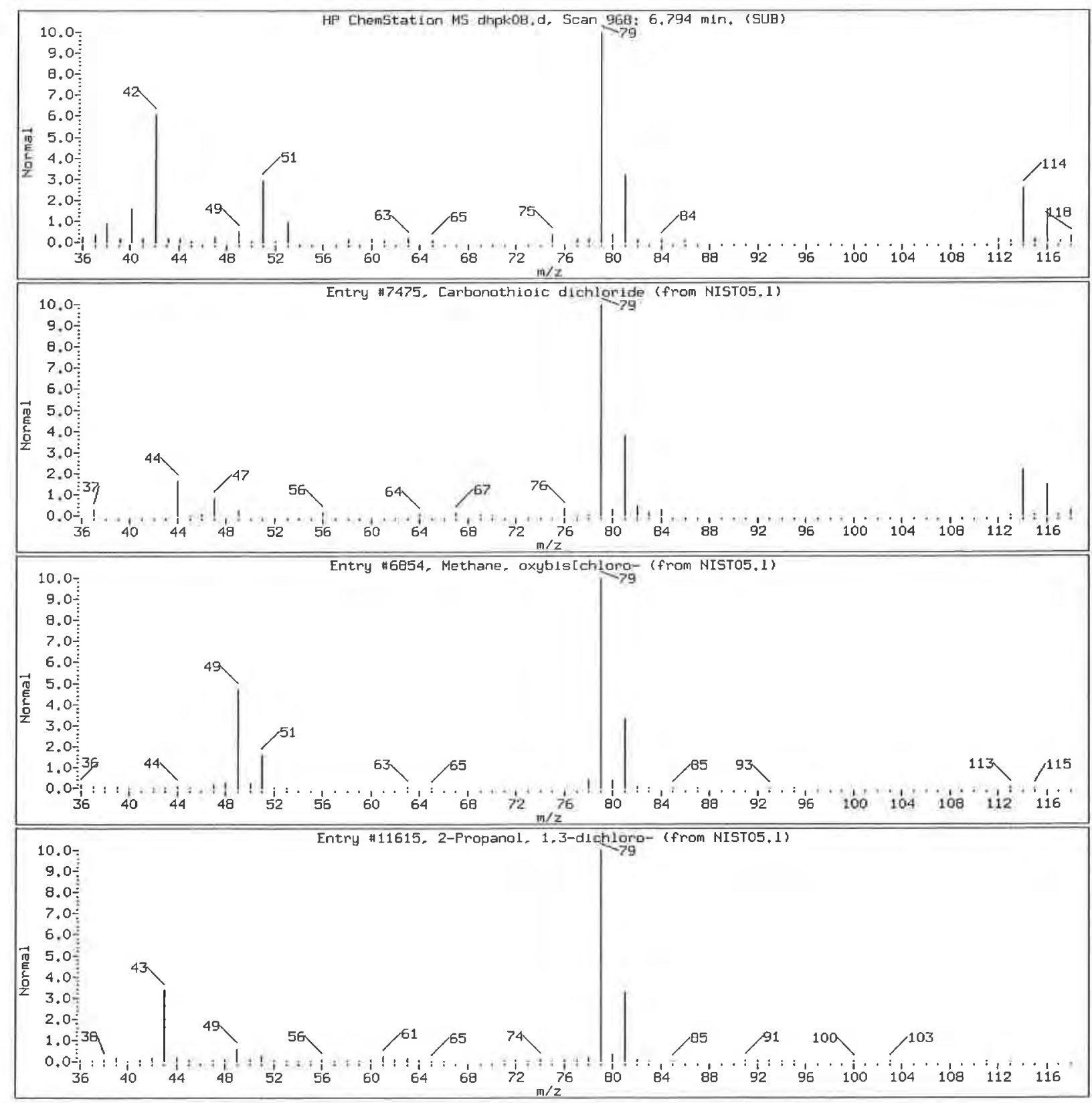
$1 A$ - FORM I VOA-1

VOLATILE ORGANICS ANALYSIS DATA SHEET
EPA SAMPLE NO.

SVSB 47D-W-25883
Lab Name: TESTAMERICA BURLINGTON

Lab Code: STLV Ca

Matrix: (SOIL/SED/WATER)

water

Sample wt/vol: 25.0

$(\mathrm{g} / \mathrm{mL}) \mathrm{mL}$

Level: (TRACE/LOW/MED) TRACE

음 Moisture: not dec.

GC Column: DB-624 ID: $0.20 \quad$ (mm)

Soil Extract Volume:

Purge Volume: 25.0

Contract: $8 \mathrm{E}-00302$

SDG No.: 200-6522

Lab Sample ID: 200-6522-4

Lab File ID: DHPK09.D

Date Received: 08/12/2011

Date Analyzed: 08/15/2011

Dilution Factor: 1.0

Soil Aliquot Volume:

(uL)

\begin{tabular}{|c|c|c|c|}
\hline CAS NO. & COMPOUND & $\begin{array}{l}\text { CONCENTRATION UNITS: } \\
(\mathrm{ug} / \mathrm{L} \text { or } \mathrm{ug} / \mathrm{kg}) \mathrm{ug} / \mathrm{L}\end{array}$ & $Q$ \\
\hline $75-71-8$ & Dichlorodifluoromethane & 0.50 & $\bar{U}$ \\
\hline $74-87-3$ & Chloromethane & 0.049 & $\mathrm{~J}$ \\
\hline $75-01-4$ & Vinyl chloride & 0.50 & $\mathrm{U}$ \\
\hline $74-83-9$ & Bromomethane & 0.50 & $\mathrm{U}$ \\
\hline $75-00-3$ & Chloroethane & 0.50 & $\mathrm{U}$ \\
\hline $75-69-4$ & Trichlorofluoromethane & 0.50 & $\mathrm{U}$ \\
\hline $75-35-4$ & 1,1-Dichloroethene & 0.50 & $\mathrm{U}$ \\
\hline $76-13-1$ & 1,1,2-Trichloro-1,2,2-trifluoroethane & 0.50 & $\mathrm{U}$ \\
\hline $67-64-1$ & Acetone & 5.0 & $\mathrm{U}$ \\
\hline $75-15-0$ & Carbon disulfide & 0.50 & $\mathrm{U}$ \\
\hline $79-20-9$ & Methyl acetate & 0.50 & $\mathrm{U}$ \\
\hline $75-09-2$ & Methylene Chloride & 0.039 & $\mathrm{~J} \mathrm{~B}$ \\
\hline $156-60-5$ & trans-1,2-Dichloroethene & 0.50 & $\mathrm{U}$ \\
\hline $1634-04-4$ & Methyl tert-butyl ether & 0.50 & $\mathrm{U}$ \\
\hline $75-34-3$ & 1,1-Dichloroethane & 0.50 & $\mathrm{U}$ \\
\hline $156-59-2$ & cis-1,2-Dichloroethene & 0.50 & $\mathrm{U}$ \\
\hline $78-93-3$ & 2-Butanone & 5.0 & $\mathrm{U}$ \\
\hline $74-97-5$ & Bromochloromethane & 0.50 & $\mathrm{U}$ \\
\hline $67-66-3$ & Chloroform & 0.089 & $\mathrm{~J}$ \\
\hline $71-55-6$ & 1,1,1-Trichloroethane & 0.50 & $\mathrm{U}$ \\
\hline $110-82-7$ & Cyclohexane & 0.50 & $\mathrm{U}$ \\
\hline $56-23-5$ & Carbon tetrachloride & 0.50 & $\mathrm{U}$ \\
\hline $71-43-2$ & Benzene & 0.50 & $\mathrm{U}$ \\
\hline $107-06-2$ & 1,2-Dichloroethane & 0.50 & $\mathrm{U}$ \\
\hline
\end{tabular}

Report 1,4-Dioxane for Low-Medium VOA analysis only 
$1 B$ - FORM I VOA-2

VOLATILE ORGANICS ANALYSIS DATA SHEET
EPA SAMPLE NO. SVSB 47D-W-25883
Lab Name: TESTAMERICA BURLINGTON

Lab Code: STLV Case No.: SAVANN Mod. Ref No.:
Contract: $8 \mathrm{E}-00302$

SDG No.: 200-6522
Matrix: (SOIL/SED/WATER)

Sample wt/vol: 25.0

Level: (TRACE/LOW/MED) TRACE

\% Moisture: not dec.

GC Column: DB-624

Soil Extract Volume:

Water

$(\mathrm{g} / \mathrm{mL}) \quad \mathrm{mL}$

Purge Volume: 25.0
ID $: 0.20 \quad(\mathrm{~mm})$

(uL)

(mL)
Lab Sample ID: 200-6522-4

Lab File ID: DHPK09.D

Date Received: 08/12/2011

Date Analyzed: 08/15/2011

Dilution Factor: 1.0

Soil Aliquot Volume:

(uL)

\begin{tabular}{|c|c|c|c|}
\hline CAS NO. & COMPOUND & $\begin{array}{l}\text { CONCENTRATION UNITS: } \\
(\mathrm{ug} / \mathrm{L} \text { or } \mathrm{ug} / \mathrm{kg}) \mathrm{ug} / \mathrm{L}\end{array}$ & $Q$ \\
\hline $79-01-6$ & Trichloroethene & 0.50 & $\bar{U}$ \\
\hline $108-87-2$ & Methylcyclohexane & 0.50 & $\mathrm{U}$ \\
\hline $78-87-5$ & 1,2-Dichloropropane & 0.50 & $\mathrm{U}$ \\
\hline $75-27-4$ & Bromodichloromethane & 0.50 & $\mathrm{U}$ \\
\hline $10061-01-5$ & cis-1,3-Dichloropropene & 0.50 & $\mathrm{U}$ \\
\hline $108-10-1$ & 4-Methyl-2-pentanone & 5.0 & $\mathrm{U}$ \\
\hline $108-88-3$ & Toluene & 0.025 & $\mathrm{~J} \mathrm{~B}$ \\
\hline $10061-02-6$ & trans-1,3-Dichloropropene & 0.50 & $\mathrm{U}$ \\
\hline $79-00-5$ & 1,1,2-Trichloroethane & 0.50 & $\mathrm{U}$ \\
\hline $127-18-4$ & Tetrachloroethene & 0.041 & $\mathrm{~J}$ \\
\hline $591-78-6$ & 2-Hexanone & 5.0 & $\mathrm{U}$ \\
\hline $124-48-1$ & Dibromochloromethane & 0.50 & $\mathrm{U}$ \\
\hline $106-93-4$ & 1,2-Dibromoethane & 0.50 & $\mathrm{U}$ \\
\hline $108-90-7$ & Chlorobenzene & 0.50 & $\mathrm{U}$ \\
\hline $100-41-4$ & Ethylbenzene & 0.50 & $\mathrm{U}$ \\
\hline $95-47-6$ & o-Xylene & 0.50 & $\mathrm{U}$ \\
\hline $179601-23-1$ & $\mathrm{~m}, \mathrm{p}-\mathrm{XY}_{\mathrm{l}} \mathrm{lene}$ & 0.0051 & $\mathrm{~J}$ \\
\hline $100-42-5$ & Styrene & 0.50 & $\mathrm{U}$ \\
\hline $75-25-2$ & Bromoform & 0.50 & $\mathrm{U}$ \\
\hline $98-82-8$ & Isopropylbenzene & 0.50 & $\mathrm{U}$ \\
\hline $79-34-5$ & $1,1,2,2$-Tetrachloroethane & 0.50 & $\mathrm{U}$ \\
\hline $541-73-1$ & 1,3-Dichlorobenzene & 0.50 & $\mathrm{U}$ \\
\hline $106-46-7$ & 1,4-Dichlorobenzene & 0.50 & $\mathrm{U}$ \\
\hline $95-50-1$ & 1,2-Dichlorobenzene & 0.50 & $\mathrm{U}$ \\
\hline $96-12-8$ & 1,2-Dibromo-3-Chloropropane & 0.50 & $\mathrm{U}$ \\
\hline $120-82-1$ & $1,2,4$-Trichlorobenzene & 0.50 & $\mathrm{U}$ \\
\hline $87-61-6$ & $1,2,3$-Trichlorobenzene & 0.50 & $\mathrm{U}$ \\
\hline
\end{tabular}




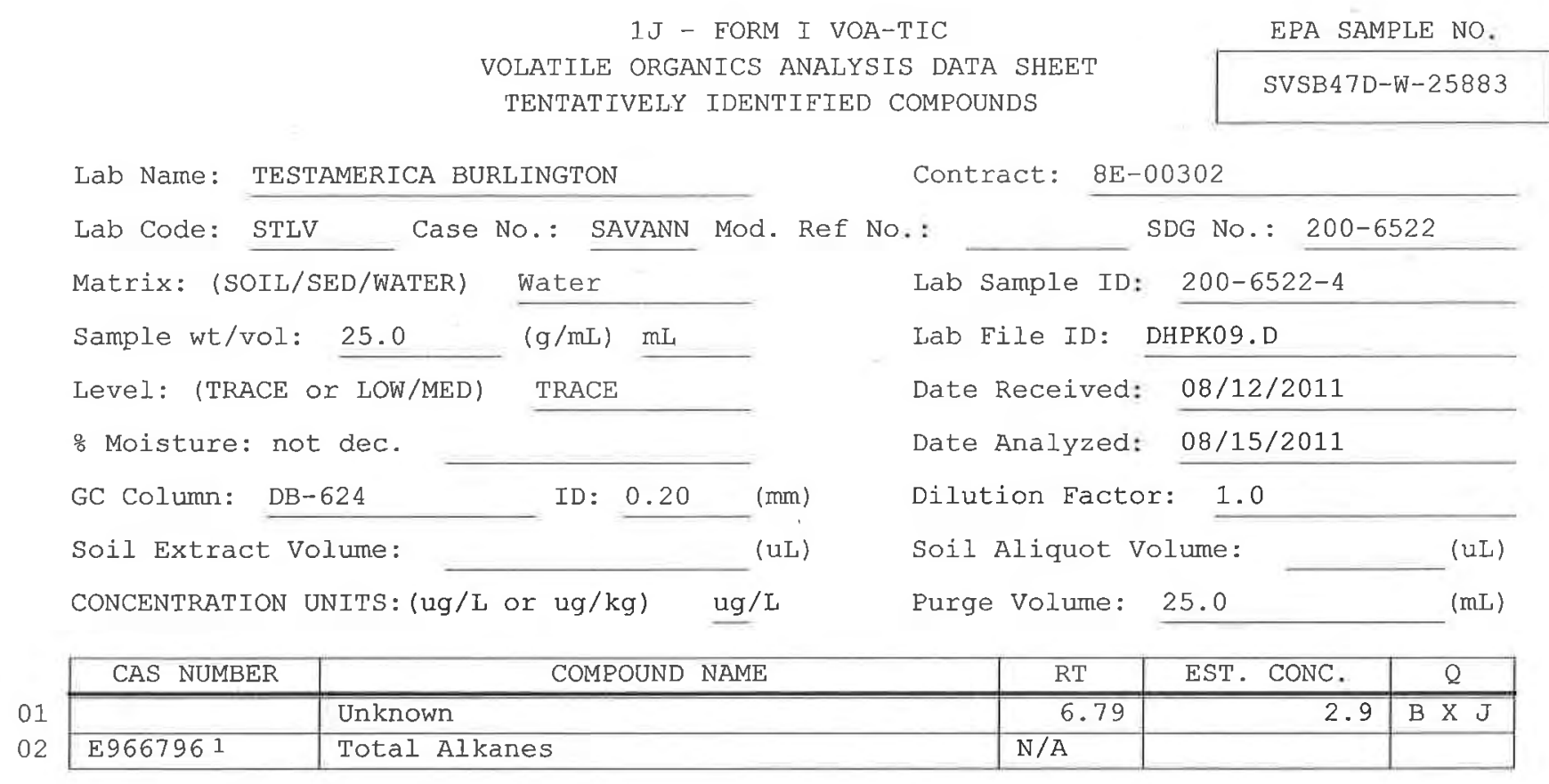

1 EPA-designated Registry Number. 


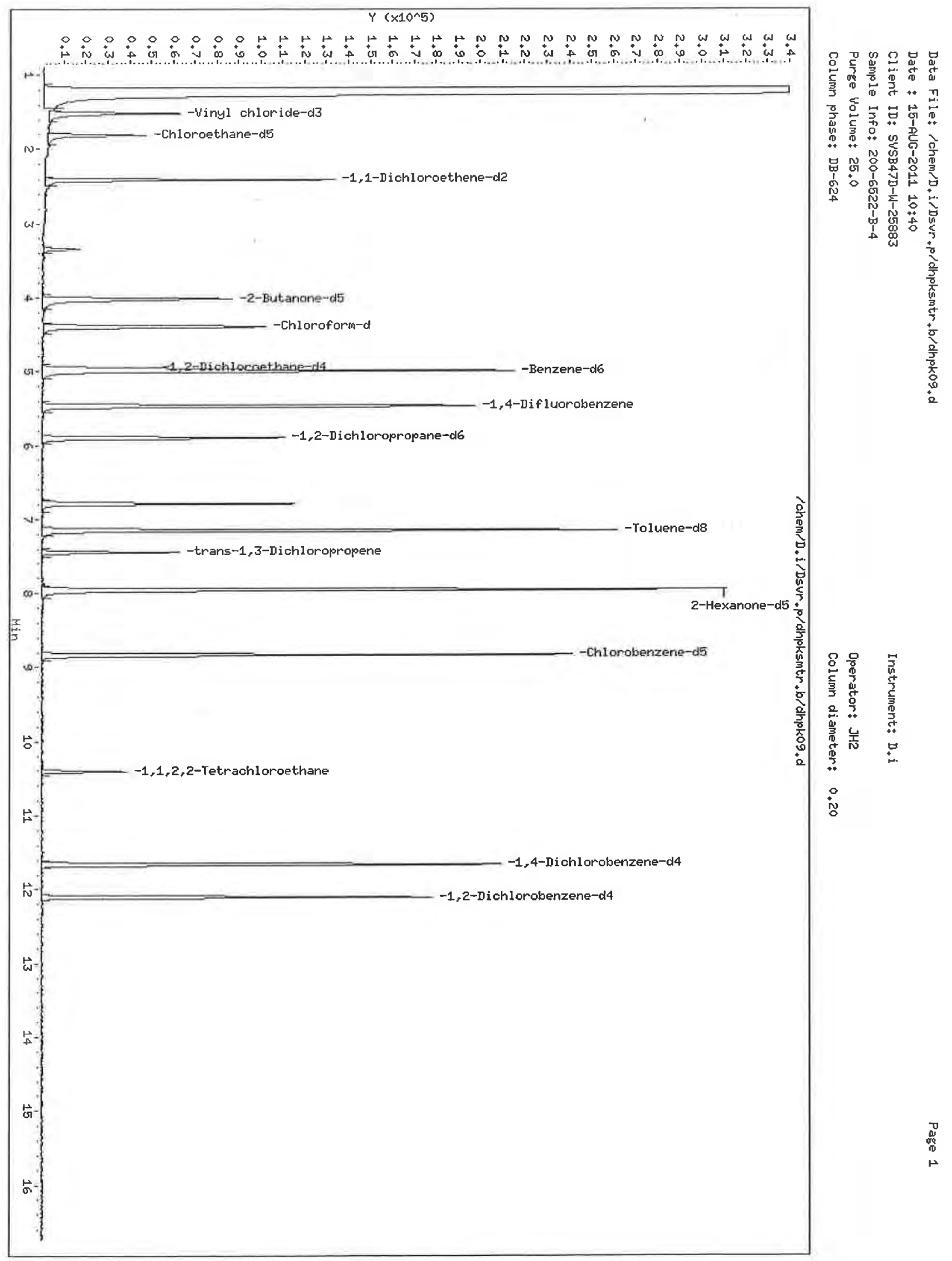


Lab Sample Id: 200-6522-4

Client Smp ID: SVSB47D-W-25883

Inj Date : 15-AUG-2011 10:40

Operator : $\mathrm{JH} 2$

Inst ID: D.i

Smp Info : 200-6522-B-4

Misc Info: 1,5

Comment :

Method : /chem/D.i/Dsvr.p/dhpksmtr.b/somtr5t.m

Meth Date : 15-Aug-2011 16:21 jh2 Quant Type: ISTD

Cal Date : 07-JUL-2011 12:56 Cal File: dhp08.d

Als bottle: 7

Dil Factor: 1.00000

Integrator: HP RTE

Target Version: 3.50

Processing Host: chemsvr6

Concentration Formula: Amt * DF * Uf/Vo * CpndVariable

\begin{tabular}{clc} 
Name & Value & Description \\
\hline DE & 1.00000 & Dilution Factor \\
Uf & 1.00000 & ng unit correction factor \\
Vo & 25.00000 & Sample Volume purged (mL)
\end{tabular}

Cpnd Variable

Local Compound Variable

Compounds
1 Dichlorodifluoromethane
2 Chloromethane
3 Vinyl chloride-d3
4 Vinyl chloride
5 Bromomethane
6 Chloroethane-d5
7 Chloroethane
8 Trichlorofluoromethane
$91,1-D i c h l o r o e t h e n e-d 2$
$101,1-D i c h l o r o e t h e n e$
11 1, $1,2-T r i c h l o r o-1,2,2-t r i f l u o$
12 Acetone
13 Carbon disulfide
14 Methyl acetate
15 Methylene chloride
16 trang-1,2-Dichloroethene

QUANT SIG
MASS
rस=
85
50
65
62
94
69
64
101
63
96
101
43
76
43
84
96

\begin{tabular}{|c|c|c|c|c|}
\hline & & & CONCENTR & CIONS \\
\hline & & & ON-COLUMN & FINAL \\
\hline RT & EXP RT REL RT & RESPONSE & $(n g)$ & $(\mathrm{ug} / \mathrm{L})$ \\
\hline$=$ & 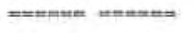 & 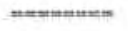 & 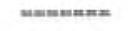 & mumuna \\
\hline Comp & ound Not Detecte & & & \\
\hline 1.448 & $1.454 \quad(0.265)$ & 1152 & 1.21626 & 0.049 (a) \\
\hline 1.515 & $1.521(0.277)$ & 70760 & 117.806 & 4.7 \\
\hline Comp & ound Not Detect & & & \\
\hline Comp & ound Not Detect & & & \\
\hline 1.601 & $1.807 \quad(0.330)$ & 45889 & 117.750 & 4.7 \\
\hline Comp & ound Not Detecte & & & \\
\hline Comp & ound Not Detecte & & & \\
\hline 2.405 & $2.411(0.440)$ & 93097 & 84.9221 & 3.4 \\
\hline Comp & ound Not Detecte & & & \\
\hline Comp & ound Not Detect & & & \\
\hline Comp & ound Not Detect & & & \\
\hline Comp & ound Not Detecte & & & \\
\hline Comp & ound Not Detecte & & & \\
\hline 2.813 & $2.813 \quad(0.515)$ & 337 & 0.97694 & $0.039(\mathrm{aQ})$ \\
\hline
\end{tabular}




\section{Compounds}

17 Methyl tert-butyl ether

18 1,1-Dichloroethane

$\$ 19$ 2-Butanone-d5

20 cis-1,2-Dichloroethene

21 2-Butanone

22 Bromochloromethane

\$ 23 Chloroform-d

24 Chloroform

25 1,1,1-Trichloroethane

26 Cyclohexane

27 Carbon tetrachloride

$\$ 28$ 1,2-Dichloroethane-d4

$\$ 29$ Benzene-d6

30 Benzene

31 1,2-Dichloroethane

* 32 1,4-Difluorobenzene

33 Trichloroethene

\$ 34 1,2-Dichloropropane-d6 35 Methylcyclohexane

36 1,2-Dichloropropane

37 Bromodichloromethane

38 cis-1, 3-D1chloropropene

39 4-Methyl-2-pentanone

$\$ 40$ Toluene-d8

41 Toluene

\$ 42 trans-1,3-Dichloropropene-d4

43 trans-1, 3-Dichloropropene

44 1,1,2-Trichloroethane

45 Tetrachloroethene

$\$ 46$ 2-Hexanone-d5

47 2-Hexanone

48 Dibromochloromethane

49 1,2-Dibromoethane

* 50 Chlorobenzene-d5

51 Chlorobenzene

52 Ethylbenzene

$53 \mathrm{~m}, \mathrm{p}$-xylene

54 Styrene

55 o-xylene

56 Bromoform

57 Isopropylbenzene

\$ 58 1,1,2,2-Tetrachloroethane-d2 59 1, 1,2,2-Tetrachloroethane 601,3 -Dichlorobenzene

* 61 1,4-Dichlorobenzene-d4

62 1,4-Dichlorobenzene

\$ 63 1,2-Dichlorobenzene-d4

64 1,2-Dichlorobenzene
QUANT SIG

MASS

watem

73

63

46

96

43

128

83

56

117

84

78

62

114

95

67

55

63

83

98

91

79

75

97

164

63

43

129

107

117

112

91

106

104

106

172

105

84

83

146

152

146

152

146
CONCENTRATIONS

ON-COLUMN FINAL

EXP RT REL RT

RESPONSE

( ng) ( ug/L)

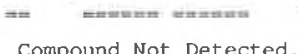

Compound Not Detected.

$4.014 \quad 4.020 \quad(0.734)$

Compound Not Detected.

Compound Not Detected.

Compound Not Detected.

$4.392 \quad 4.392(0.804)$

$4.417 \quad 4.417 \quad(0.808)$

Compound Not Detected.

Compound Not Detected.

Compound Not Detected.

$4.953 \quad 4.959(0.906)$

$4.990 \quad 4.990 \quad(0.565)$

Compound Not Detected.

Compound Not Detected.

$5.465 \quad 5.471(1.000)$

Compound Not Detected.

$5.898 \quad 5.904 \quad(0.668)$

Compound Not Detected.

Compound Not Detected.

Compound Not Detected.

Compound Not Detected.

Compound Not Detected.

$\begin{array}{llll}7.148 & 7.154 & (0.809)\end{array}$

$7.227 \quad 7.227 \quad(0.818)$

$190204 \quad 112.567$

0.63003

$7.447 \quad 7.447 \quad(0.843)$

113.466

Compound Not Detected.

Compound Not Detected.

$\begin{array}{llll}7.867 & 7.867 & (0.891)\end{array}$

$7.946 \quad 7.946 \quad(0.900)$

$379 \quad 1.02496$

2892.85

$0.041(\mathrm{aQ})$

Compound Not Detected.

Compound Not Detected.

Compound Not Detected.

$8.830 \quad 8.830 \quad(1.000)$

Compound Not Detected. Compound Not Detected.

$9.160 \quad 9.160 \quad(1.037)$

$151139 \quad 125.000$

Compound Not Detected.

Compound Not Detected.

Compound Not Detected.

Compound Not Detected.

$10.403 \quad 10.403 \quad(1.178)$

$20993 \quad 106.024 \quad 4.2$

Compound Not Detected. Compound Not Detected.

11.65911 .659 (1.000)

Compound Not Detected.

$12.104 \quad 12.104$ (1.038)

$59220 \quad 125.000$

(Q)

Compound Not Detected.

$\begin{array}{lll}49925 & 127.020 & 5.1\end{array}$ 
Data File: /chem/D.i/Dsvr.p/dhpksmtr.b/dhpk09.d

Report Date: 15-Aug-2011 20:11

\begin{tabular}{|c|c|c|c|c|c|c|}
\hline \multirow[b]{3}{*}{ Compounds } & \multirow[b]{2}{*}{ QUANT SIG } & & & & \multicolumn{2}{|c|}{ CONCENTRATIONS } \\
\hline & & & & & ON-COLUMN & FINAL \\
\hline & MASS & RT & EXP RT REL RT & RESPONSE & ( $n g)$ & $(\mathrm{ug} / \mathrm{L})$ \\
\hline 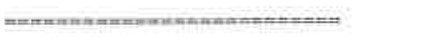 & $==-$ & $=$ & 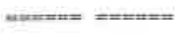 & entrenture & 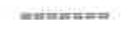 & 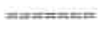 \\
\hline 65 1,2-Dibromo-3-chloropropane & 75 & \multicolumn{5}{|c|}{ Compound Not Detected. } \\
\hline 66 1,2,4-Trichlorobenzene & 180 & \multicolumn{5}{|c|}{ Compound Not Detected. } \\
\hline 67 1,2,3-Trichlorobenzene & 180 & \multicolumn{5}{|c|}{ Compound Not Detected. } \\
\hline
\end{tabular}

QC Flag Legend

a - Target compound detected but, quantitated amount Below Limit of Quantitation (BLOQ).

Q - Qualifier signal failed the ratio test.

$\mathrm{R}$ - Spike/Surrogate failed recovery limits. 


\section{TestAmerica Burlington}

Lab Sample Id: 200-6522-4

Client Smp ID: SVSB47D-W-25883

Inj Date : 15-AUG-2011 10:40

Operator : JH2

Smp Info : 200-6522-B-4

Inst ID: D.i

Misc Info : 1,5

Comment :

Method : /chem/D.i/Dsvr.p/dhpksmtr.b/somtr5t.m

Meth Date : 15-Aug-2011 16:21 jh2 Quant Type: ISTD

Cal Date : 07-JUL-2011 12:56 Cal File: dhp08.d

Als bottle: 7

Dil Factor: 1.00000

Integrator: HP RTE

Target Version: 3.50

Processing Host: chemsvr6

Concentration Formula: Amt * DF * Uf/Vo * CpndVariable

$\begin{array}{clc}\text { Name } & \text { Value } & \text { Description } \\ \text { DF } & 1.00000 & \text { Dilution Factor } \\ \text { Uf } & 1.00000 & \text { ng unit correction factor } \\ \text { Vo } & 25.00000 & \text { Sample volume purged (mL) }\end{array}$

Cpnd Variable

ISTD
$======$

* $321,4-$ Difluorobenzene

CONCENTRATIONS

RT AREA ON-COL( ng) FINAL( ug/L) QUAL

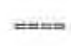

$=$

Unknown

6.788

$227150 \quad 73.0223051$
Local Compound Variable

$\begin{array}{lll}\text { RT } & \begin{array}{l}\text { AREA } \\ =======\end{array} & \begin{array}{l}\text { AMOUNT } \\ =====\end{array} \\ 5.465 & 388836 & 125.000\end{array}$

QUANT

LIBRARY LIB ENTRY CPND \#

CAS \#:

$\begin{array}{llll}2.9 & 0 & 0 & 32\end{array}$ 
Data File: dhpk09.d

Lab Sample ID: 200-6522-4

Date: 15-AUG-2011 10:40

Client ID: SVSB47D-W-25883

Instrument: D.i

Sample Info: 200-6522-B-4

Operator: JH2

2 Chloromethane
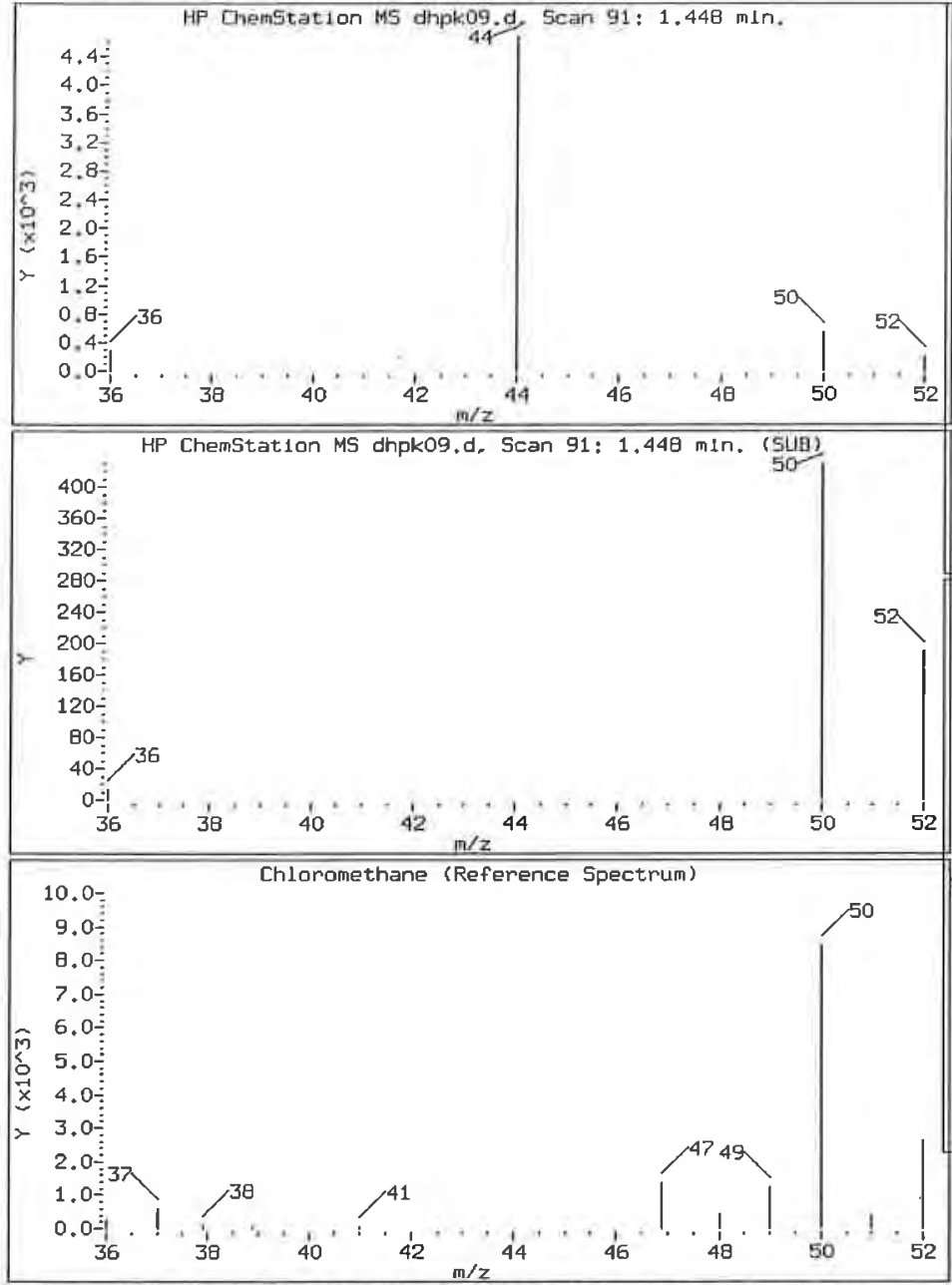

540
600
56
520
480
440
400
36
32
28
240
200
160

HP MS dhpk09,d, Ion $\mathbf{5 0 , 0 0}$ 640-: $600 \div$

$520 \div$

$440 \div$

$400 \div$

$320 \div$

$280 \div$

$240 \div$

200-

120

80

0

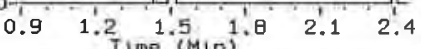

HP MS dipkk09.d. Ion 52.00

220

$200-$

180-:

$160-$

$140-$

$120 \div$

$100-$

$80-$

$60-$

$40 \div$

$20 \div$

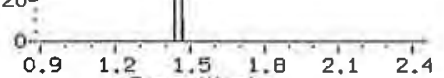

$\begin{array}{llllll}0.9 & 1.2 & 1.5 & 1.8 & 2.1 & 2.4\end{array}$

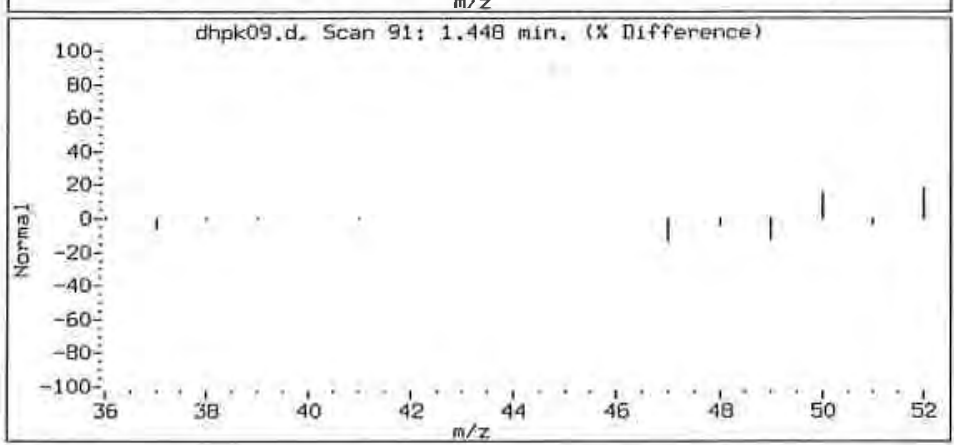


Data File: dhpk09.d

Lab Sample ID: 200-6522-4

Date: 15-AUG-2011 10:40

Client ID: SVSB47D-W-25883

Instrument: D.i

Sample Info: 200-6522-B-4

Operator: JH2

15 Methylene chloride
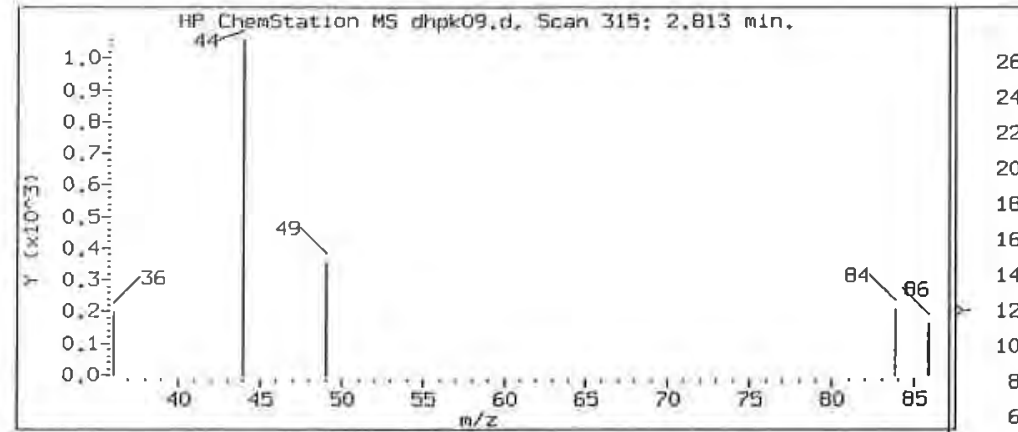

HP MS dhpk09,d, Ion 84,00
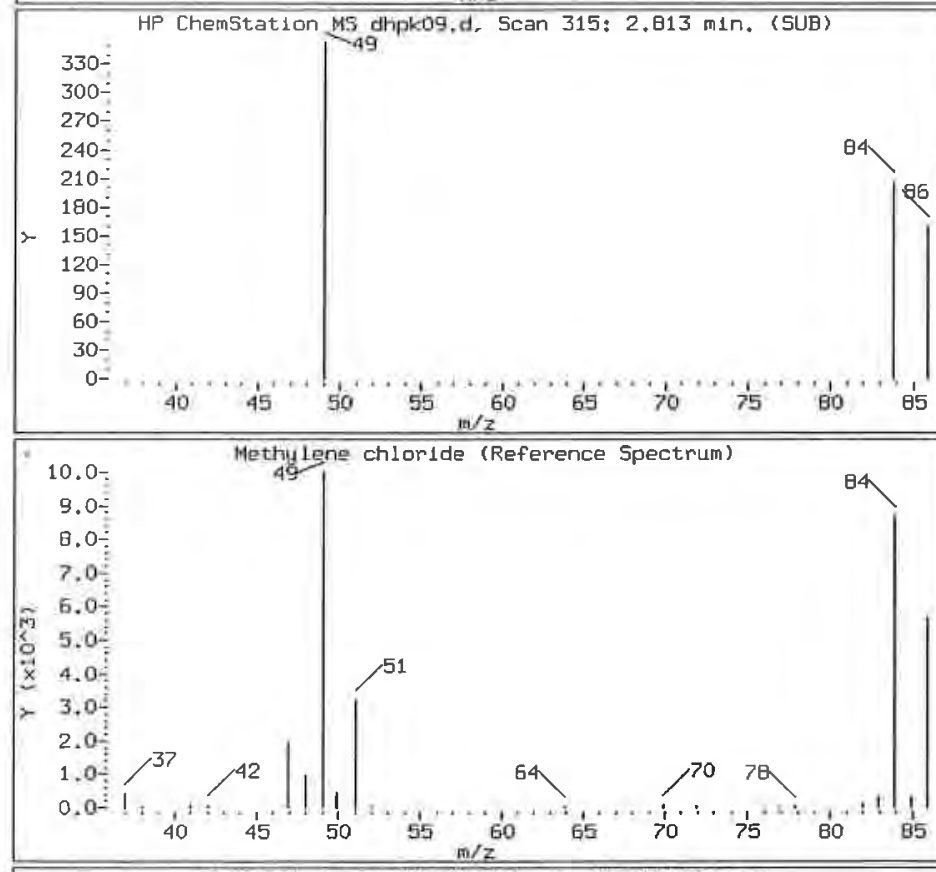

(5) $1.0=$
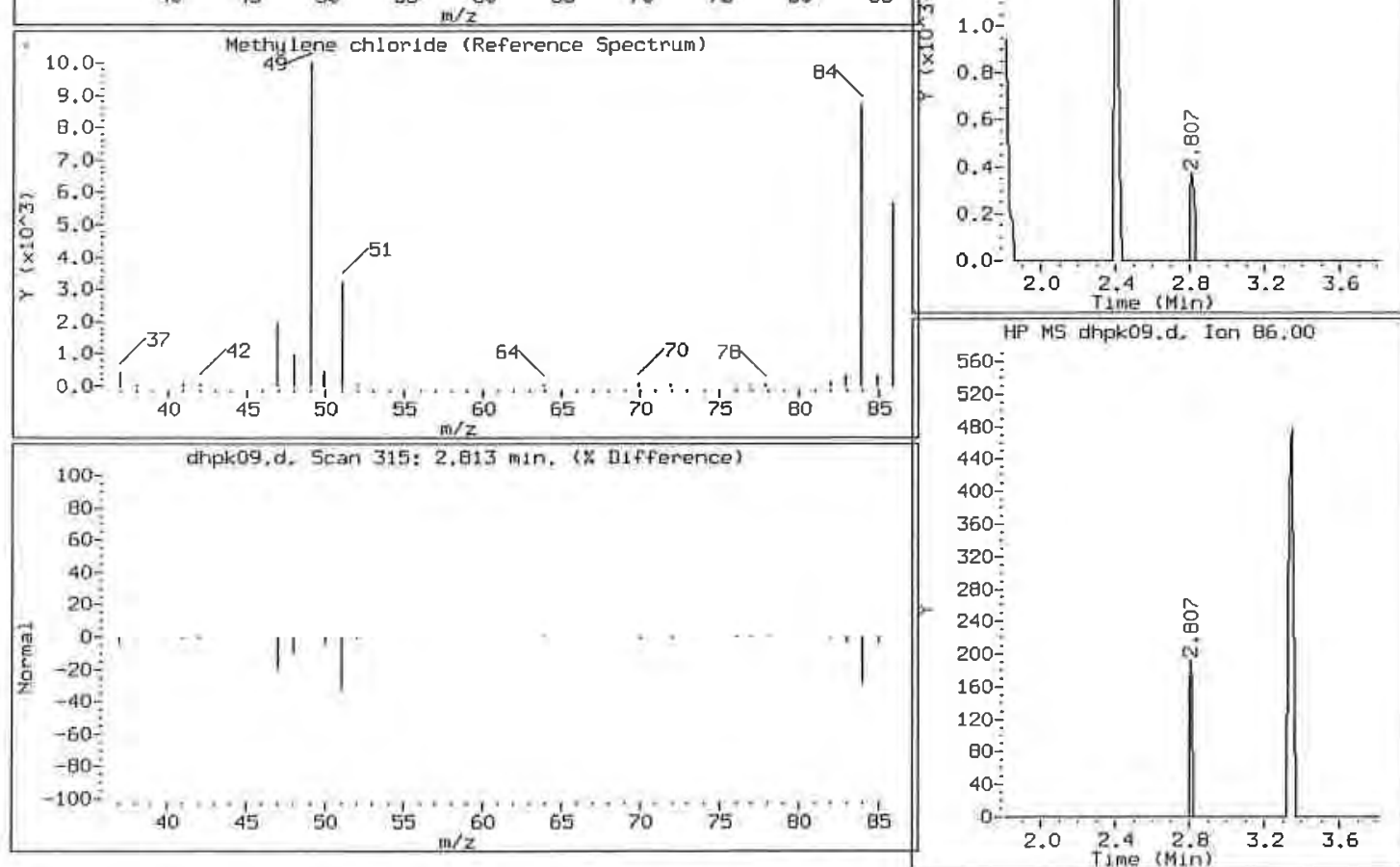
Data File: dhpk09.d

Lab Sample ID: 200-6522-4

Date: 15-AUG-2011 10:40

Client ID: SVSB47D-W-25883

Instrument: D.i

Sample Info: 200-6522-B-4

Operator: JH2

24 Chloroform
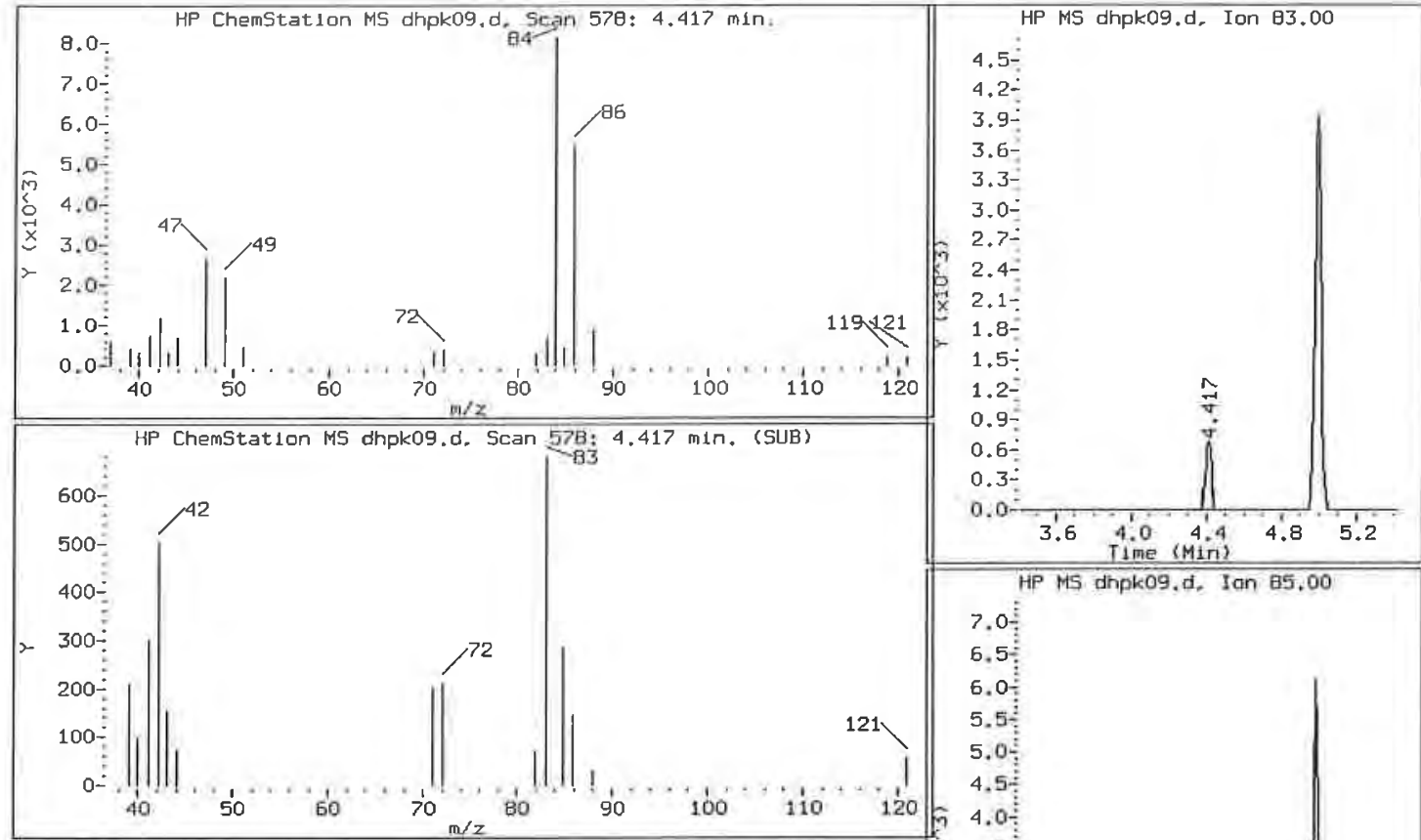

HP MS dhpk09, d. Ian 85.00
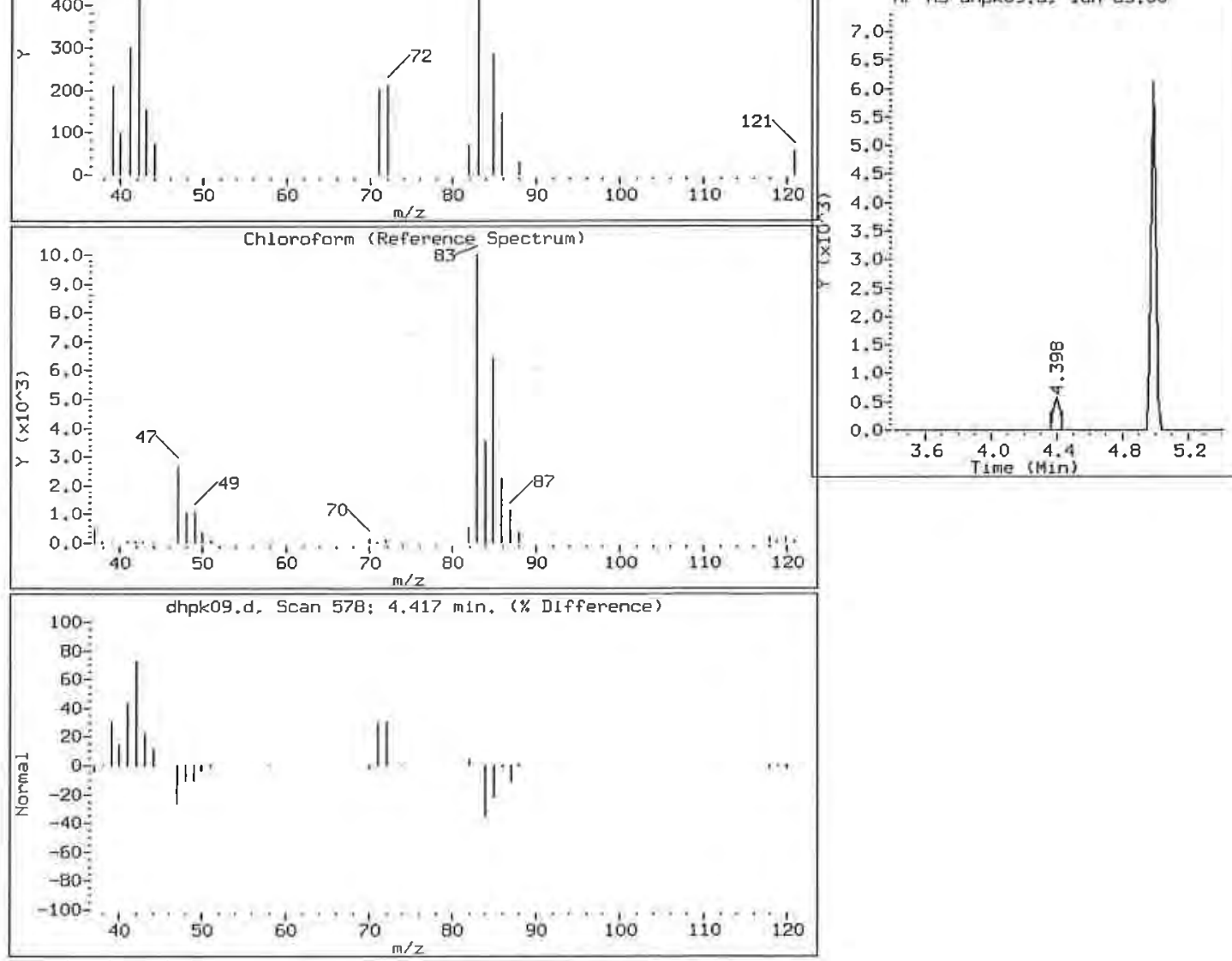
Data File: dhpk09.d

Lab Sample ID: 200-6522-4

Date: 15-AUG-2011 10:40

Client ID: SVSB47D-W-25883

Instrument: D.i

Sample Info: 200-6522-B-4

Operator: JH2

41 Toluene
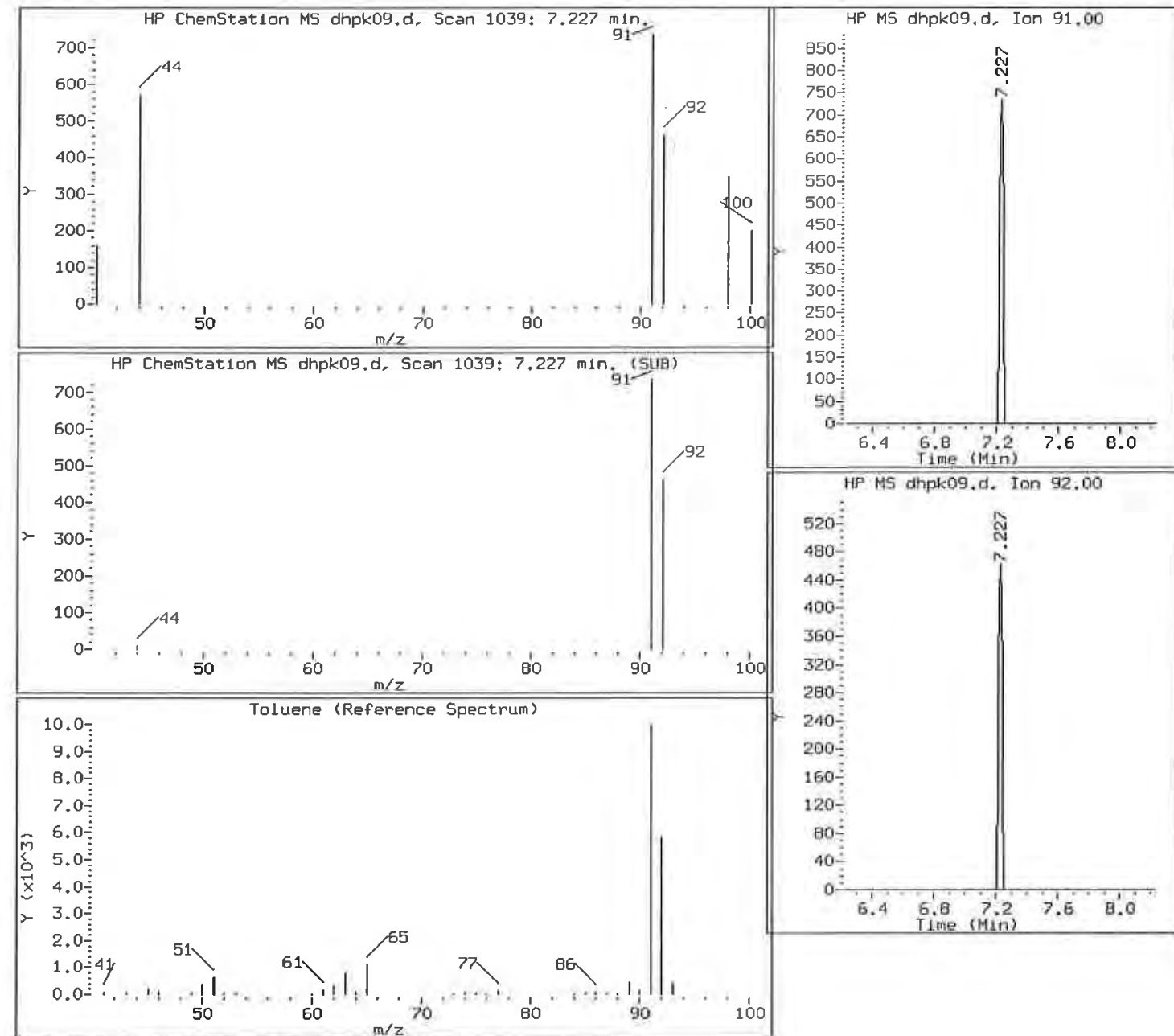

HP MS dhpk09.d. Ion 92.00
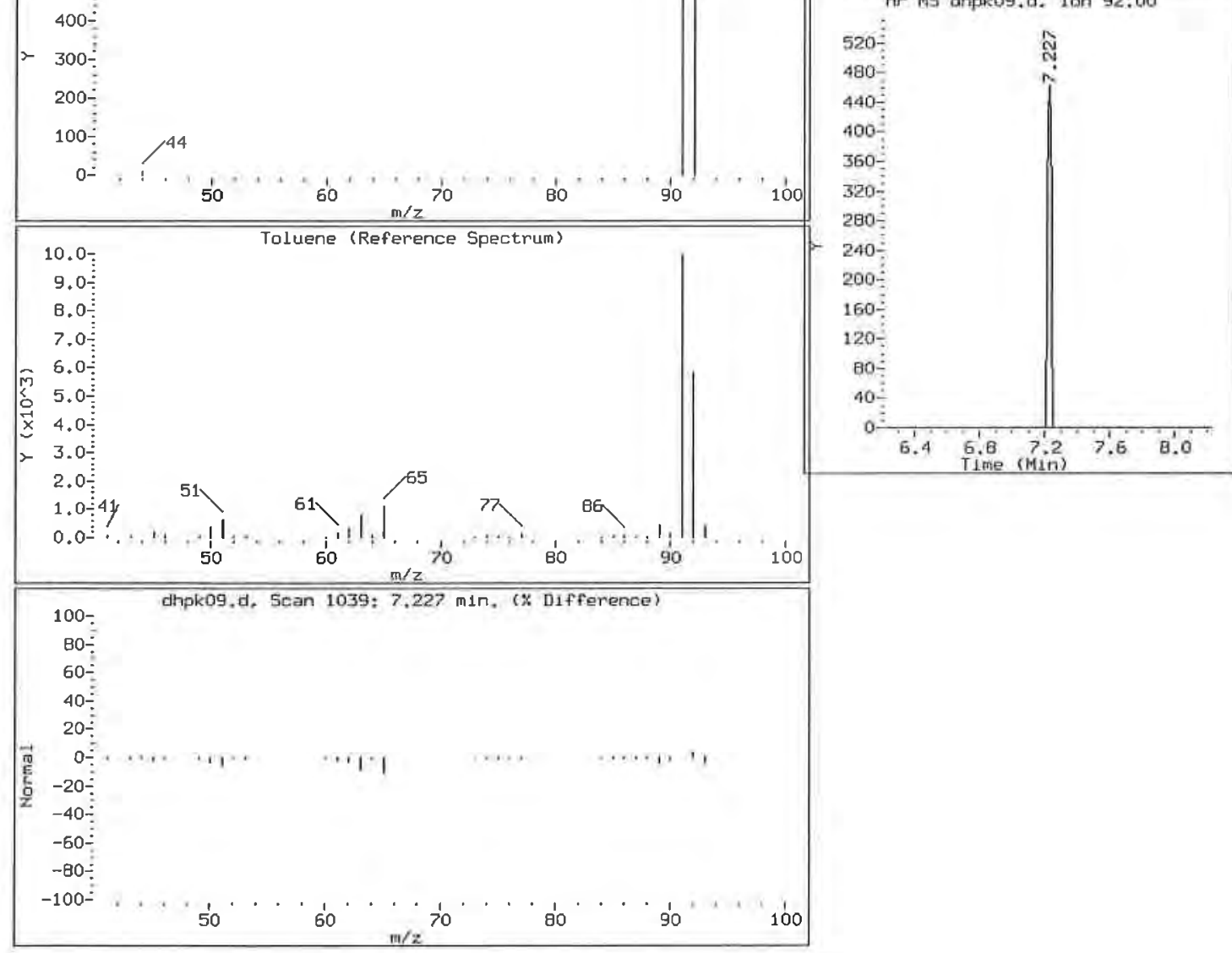
Data File: dhpk09.d

Lab Sample ID: 200-6522-4

Date: 15-AUG-2011 10:40

Client ID: SVSB47D-W-25883

Instrument: D.i

Sample Info: 200-6522-B-4

Operator: JH2

45 Tetrachloroethene
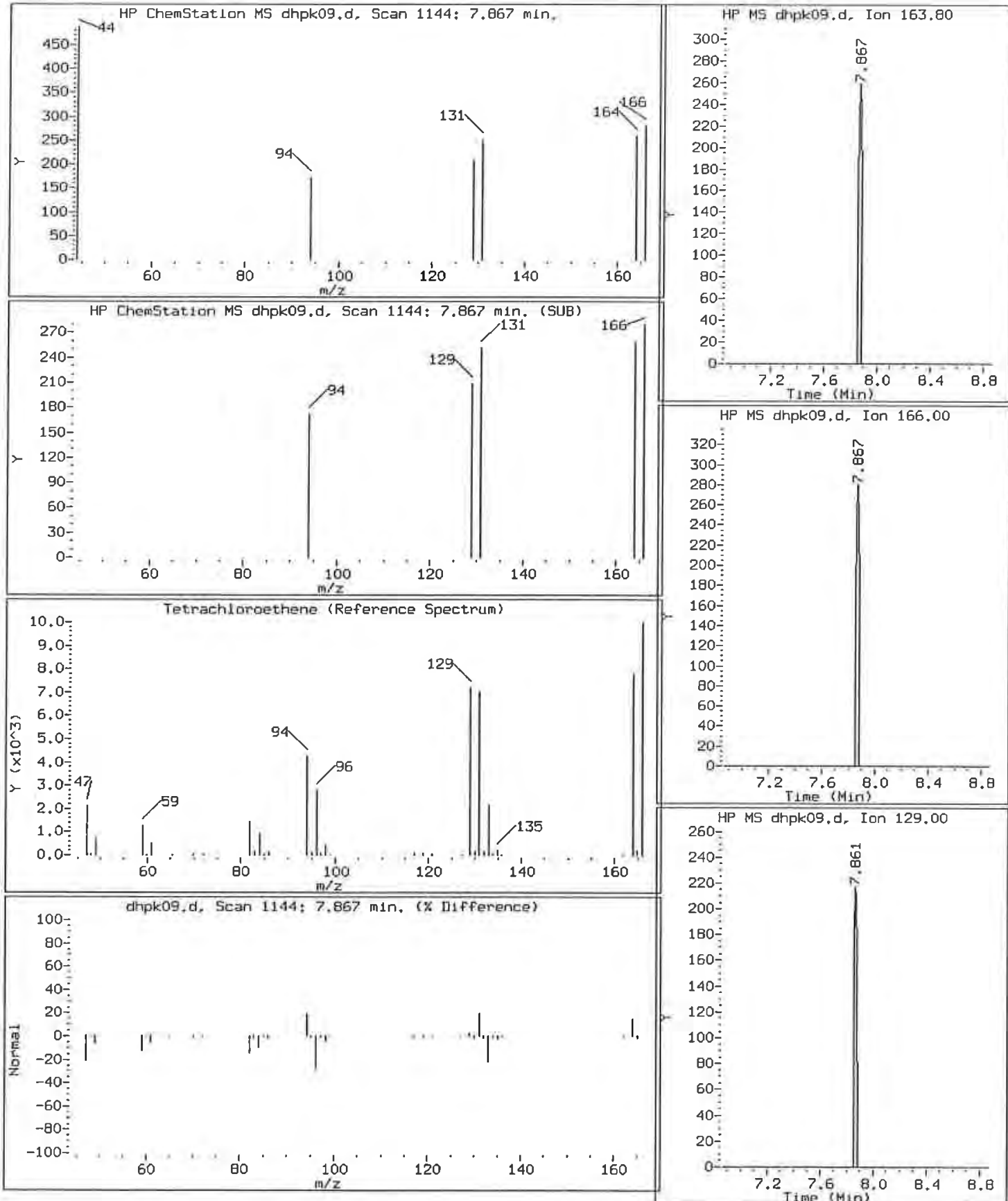
Data File: dhpk09.d

Lab Sample ID: 200-6522-4

Date: 15-AUG-2011 10:40

Client ID: SVSB47D-W-25883

Instrument: D.i

Sample Info: 200-6522-B-4

Operator: JH2

$53 \mathrm{~m}, \mathrm{p}$-xylene
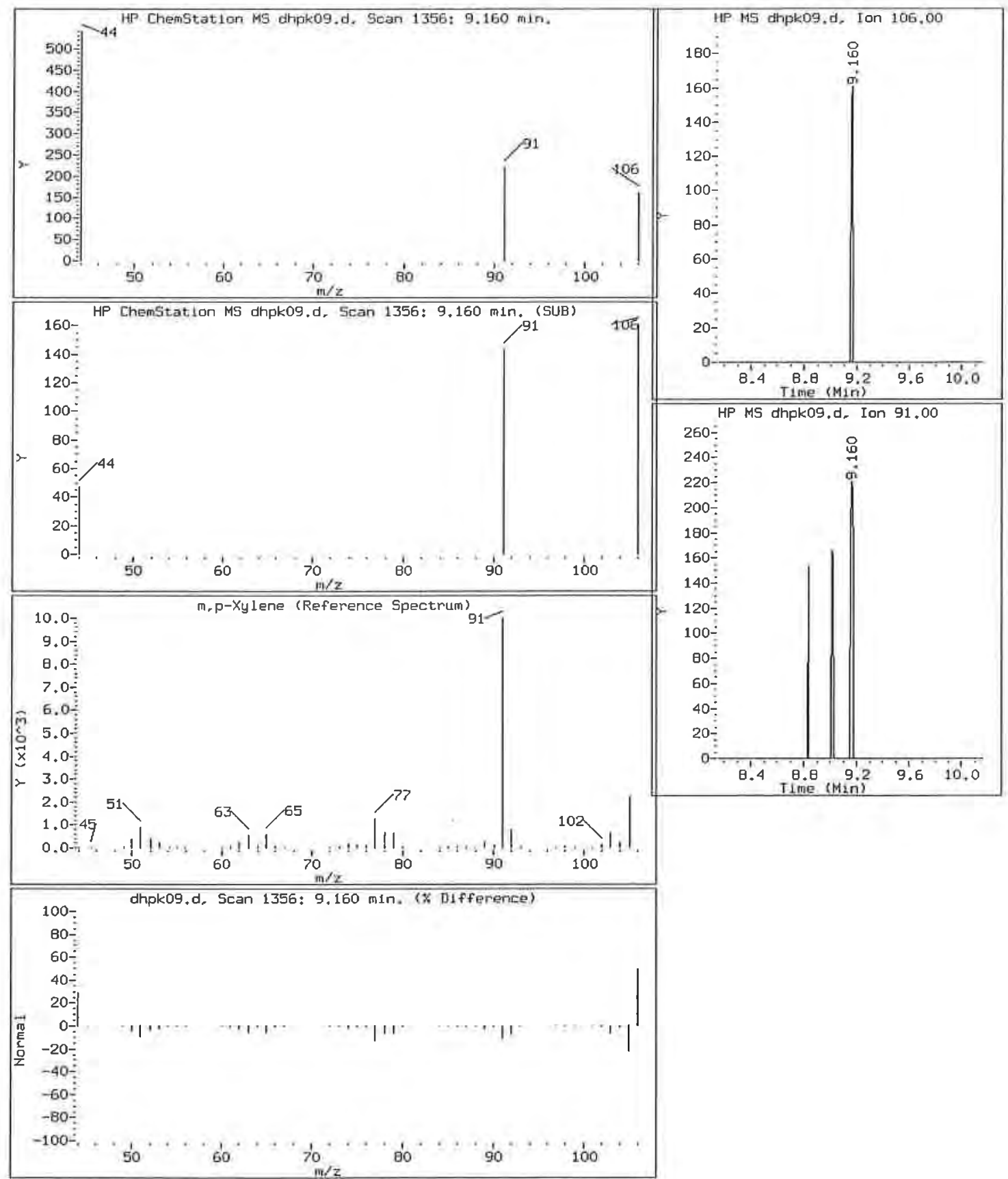
Data File: dhpk09.d

Lab Sample ID: 200-6522-4

Client ID: SVSB47D-W-25883

Sample Info: 200-6522-B-4

Retention Time: $\quad 6.79$
Date: 15-AUG-2011 10:40

Instrument: D.i

operator: JH2

$\begin{array}{lllllll}\text { Library Search Compound Match } & \text { CAS Number } & \text { Library } & \text { Entry } & \text { Quality } & \text { Eormula } & \text { Weight } \\ \text { Unknown } & & & & & \text { CCl2S } & 114 \\ \text { Carbonothioic dichloride } & 463-71-8 & \text { NIST05.1 } & 7475 & 49 & \text { C2H4Cl2O } & 114 \\ \text { Methane, oxybis [chloro- } & 542-88-1 & \text { NIST05.1 } & 6855 & 32 & \text { C3H6Cl2O } & 128\end{array}$
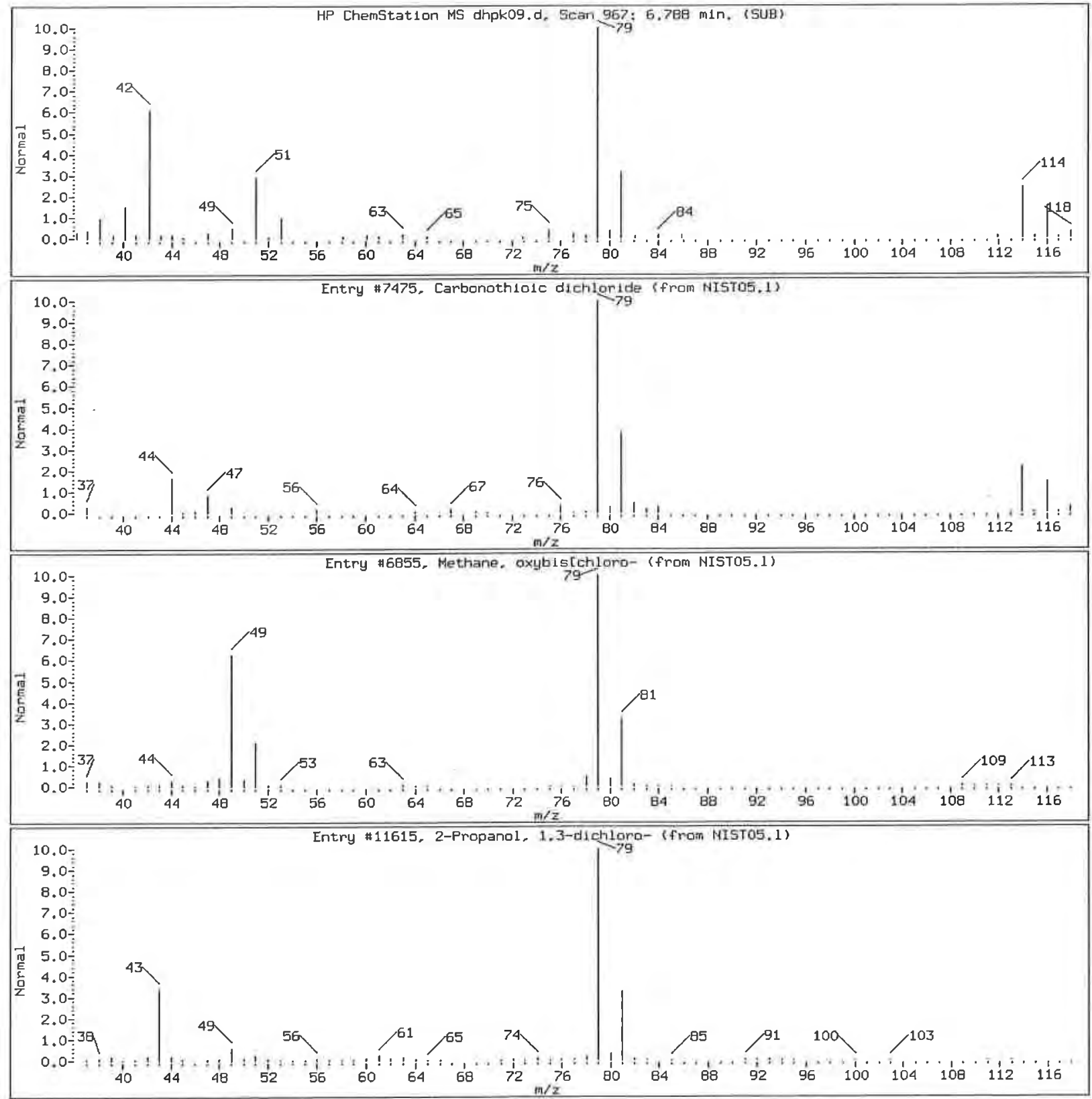
$6 \mathrm{~A}$ - FORM VI VOA-1

VOLATILE ORGANICS INITIAL CALIBRATION DATA

Lab Name: TESTAMERICA BURLINGTON

Lab Code: STlv Case No.: SAVANN Mod. Ref No.:

Instrument ID: D.i

Heated Purge: $(\mathrm{Y} / \mathrm{N}) \mathrm{N}$

Purge Volume: 25.0

GC Column: DB-624
ID: 0.20
Contract: $8 \mathrm{E}-00302$

SDG No.: $200-6522$

Calibration Date(s): 07/07/2011 07/07/2011
Calibration Time(s): $1117 \quad 1256$ (mL)

(Inm) Length: 25

(m)

\begin{tabular}{|c|c|c|c|c|c|c|c|}
\hline \multirow{2}{*}{$\begin{array}{l}\text { LAB FILE ID: } \\
\text { RRE } 5.0=\text { DHP06.D }\end{array}$} & \multicolumn{3}{|c|}{ RRE $0.5=$ DHP0 $4 . D$} & \multicolumn{4}{|c|}{ RRE $1.0=$ DHP05.D } \\
\hline & RRF 10 & HP07.D & & RRE 20 & $=\mathrm{DHPO}$ & . $\mathrm{D}$ & \\
\hline COMPOUND & RRE 0.5 & RRF 1.0 & RRF 5.0 & RRE 10 & RRF 20 & $\overline{\mathrm{RRF}}$ & $\circ \mathrm{RSD}$ \\
\hline Dichlorodifluoromethane & 0.418 & 0.398 & 0.390 & 0.409 & 0.398 & 0.403 & 2.7 \\
\hline Chloromethane & 0.769 & 0.713 & 0.655 & 0.659 & 0.639 & 0.687 & 7.8 \\
\hline Vinyl chloride & 0.524 & 0.491 & 0.475 & 0.499 & 0.491 & 0.496 & 3.6 \\
\hline Bromomethane & 0.180 & 0.203 & 0.205 & 0.219 & 0.239 & 0.209 & 10.3 \\
\hline Chloroethane & 0.292 & 0.280 & 0.259 & 0.269 & 0.259 & 0.272 & 5.2 \\
\hline Trichlorofluoromethane & 0.612 & 0.609 & 0.587 & 0.608 & 0.611 & 0.606 & 1.7 \\
\hline 1,1-Dichloroethene & 0.318 & 0.307 & 0.298 & 0.303 & 0.297 & 0.305 & 2.8 \\
\hline $\begin{array}{l}1,1,2 \text {-Trichloro- } \\
1,2,2-\text { trifluoroethane }\end{array}$ & 0.377 & 0.358 & 0.336 & 0.358 & 0.347 & 0.355 & 4.3 \\
\hline Acetone & 0.047 & 0.038 & 0.032 & 0.032 & 0.030 & 0.036 & 19.3 \\
\hline Carbon disulfide & 0.758 & 0.707 & 0.719 & 0.760 & 0.766 & 0.742 & 3.6 \\
\hline Methyl acetate & 0.108 & 0.094 & 0.076 & 0.079 & 0.074 & 0.086 & 16.7 \\
\hline Methylene Chloride & 0.264 & 0.257 & 0.242 & 0.248 & 0.241 & 0.250 & 3.9 \\
\hline trans-1,2-Dichloroethene & 0.323 & 0.298 & 0.301 & 0.312 & 0.306 & 0.308 & 3.3 \\
\hline Methyl tert-butyl ether & 0.304 & 0.278 & 0.292 & 0.319 & 0.318 & 0.302 & 5.7 \\
\hline 1,1-Dichloroethane & 0.642 & 0.586 & 0.594 & 0.617 & 0.607 & 0.609 & 3.6 \\
\hline cis-1,2-Dichloroethene & 0.302 & 0.289 & 0.300 & 0.312 & 0.306 & 0.302 & 2.7 \\
\hline 2-Butanone & 0.045 & 0.046 & 0.047 & 0.049 & 0.048 & 0.047 & 3.4 \\
\hline Bromochloromethane & 0.104 & 0.103 & 0.100 & 0.099 & 0.101 & 0.101 & 2.0 \\
\hline Chloroform & 0.502 & 0.482 & 0.473 & 0.493 & 0.477 & 0.485 & 2.5 \\
\hline 1,1,1-Trichloroethane & 0.514 & 0.490 & 0.494 & 0.490 & 0.485 & 0.494 & 2.3 \\
\hline Cyclohexane & 0.761 & 0.795 & 0.901 & 0.909 & 0.883 & 0.850 & 7.9 \\
\hline Carbon tetrachloride & 0.487 & 0.459 & 0.477 & 0.474 & 0.464 & 0.472 & 2.4 \\
\hline Benzene & 1.617 & 1.501 & 1.518 & 1.495 & 1.447 & 1.515 & 4.1 \\
\hline 1,2-Dichloroethane & 0.291 & 0.271 & 0.277 & 0.284 & 0.285 & 0.282 & 2.7 \\
\hline Trichloroethene & 0.397 & 0.380 & 0.357 & 0.355 & 0.351 & 0.368 & 5.4 \\
\hline Methylcyclohexane & 0.703 & 0.708 & 0.769 & 0.757 & 0.751 & 0.737 & 4.1 \\
\hline
\end{tabular}

Report 1,4-Dioxane for Low-Medium VOA analysis only 
$6 B$ - FORM VI VOA-2

VOLATILE ORGANICS INITIAL CALIBRATION DATA

Lab Name: TESTAMERICA BURLINGTON

Contract: $8 \mathrm{E}-00302$

Lab Code: STLV Case No.: SAVANN Mod. Ref No.: SDG No.: 200-6522

Instrument ID: D.i Calibration Date(s): 07/07/2011 07/07/2011

Heated Purge: ( $\mathrm{Y} / \mathrm{N}) \mathrm{N}$ Calibration Time(s) : $1117 \quad 1256$

Purge Volume: 25.0 (mL)

GC Colunn: DB-624

ID: $0.20 \quad(\mathrm{~mm}) \quad$ Length: 25

(m)

\begin{tabular}{|c|c|c|c|c|c|c|c|}
\hline \multirow{2}{*}{$\begin{array}{l}\text { LAB FILE ID: } \\
\text { RRF } 5.0=\text { DHPO6.D }\end{array}$} & \multicolumn{3}{|c|}{ RRF0.5 = DHP0 4.D } & \multicolumn{4}{|c|}{ RRE1.0 $=$ DHP05.D } \\
\hline & RRE 10 & HP07.D & & RRF 20 & $=\mathrm{DHPC}$ & . $D$ & \\
\hline COMPOUND & RRF 0.5 & RRE 1.0 & RRF 5.0 & RRE 10 & RRE 20 & $\overline{\mathrm{RRF}}$ & $\% \mathrm{RSD}$ \\
\hline 1,2-Dichloropropane & 0.363 & 0.373 & 0.367 & 0.371 & 0.355 & 0.366 & 2.0 \\
\hline Bromodichloromethane & 0.310 & 0.296 & 0.307 & 0.305 & 0.306 & 0.305 & 1.6 \\
\hline cis-1,3-Dichloropropene & 0.310 & 0.338 & 0.406 & 0.414 & 0.418 & 0.377 & 13.2 \\
\hline 4-Methyl-2-pentanone & 0.104 & 0.114 & 0.137 & 0.140 & 0.137 & 0.126 & 12.7 \\
\hline Toluene & 1.608 & 1.623 & 1.695 & 1.690 & 1.667 & 1.657 & 2.4 \\
\hline trans-1,3-Dichloropropene & 0.216 & 0.225 & 0.285 & 0.302 & 0.307 & 0.267 & 16.2 \\
\hline 1,1,2-Trichloroethane & 0.155 & 0.162 & 0.160 & 0.156 & 0.157 & 0.158 & 1.9 \\
\hline Tetrachloroethene & 0.311 & 0.304 & 0.306 & 0.308 & 0.299 & 0.306 & 1.5 \\
\hline 2-Hexanone & 0.076 & 0.085 & 0.096 & 0.099 & 0.096 & 0.090 & 10.6 \\
\hline Dibromochloromethane & 0.172 & 0.176 & 0.180 & 0.178 & 0.192 & 0.180 & 4.1 \\
\hline 1,2-Dibromoethane & 0.132 & 0.135 & 0.138 & 0.145 & 0.145 & 0.139 & 4.2 \\
\hline Chlorobenzene & 1.079 & 1.036 & 1.034 & 1.041 & 1.021 & 1.042 & 2.1 \\
\hline Ethylbenzene & 1.594 & 1.651 & 1.933 & 1.968 & 1.978 & 1.825 & 10.2 \\
\hline o-Xylene & 0.539 & 0.600 & 0.727 & 0.749 & 0.755 & 0.674 & 14.6 \\
\hline m,p-Xylene & 0.644 & 0.689 & 0.779 & 0.797 & 0.802 & 0.742 & 9.6 \\
\hline Styrene & 0.763 & 0.886 & 1.048 & 1.096 & 1.123 & 0.983 & 15.6 \\
\hline Bromoform & 0.107 & 0.116 & 0.126 & 0.119 & 0.137 & 0.121 & 9.2 \\
\hline Isopropylbenzene & 1.400 & 1.553 & 2.026 & 2.103 & 2.121 & 1.841 & 18.4 \\
\hline $1,1,2,2$-Tetrachloroethane & 0.168 & 0.154 & 0.168 & 0.164 & 0.164 & 0.164 & 3.6 \\
\hline 1,3-Dichlorobenzene & 1.494 & 1.468 & 1.569 & 1.629 & 1.647 & 1.562 & 5.1 \\
\hline 1,4-Dichlorobenzene & 1.827 & 1.736 & 1.615 & 1.641 & 1.610 & 1.686 & 5.5 \\
\hline 1,2-Dichlorobenzene & 1.377 & 1.388 & 1.407 & 1.392 & 1.355 & 1.384 & 1.4 \\
\hline 1,2-Dibromo-3-Chloropropane & 0.021 & 0.027 & 0.039 & 0.035 & 0.040 & 0.032 & 24.3 \\
\hline 1,2,4-Trichlorobenzene & 0.603 & 0.574 & 0.686 & 0.751 & 0.756 & 0.674 & 12.4 \\
\hline 1,2,3-Trichlorobenzene & 0.433 & 0.469 & 0.522 & 0.573 & 0.575 & 0.514 & 12.3 \\
\hline
\end{tabular}


$6 \mathrm{C}$ - FORM VI VOA-3

VOLATILE ORGANICS INITIAL CALIBRATION DATA

\begin{tabular}{|c|c|c|c|c|c|c|c|c|}
\hline \multicolumn{2}{|c|}{ TESTAMERICA BURLINGTON } & \multicolumn{3}{|c|}{ Contract: } & \multicolumn{4}{|c|}{$8 E-00302$} \\
\hline \multicolumn{2}{|l|}{ STLV } & \multicolumn{3}{|c|}{ Mod. Ref No.: } & \multicolumn{2}{|c|}{ SDG No.: } & \multicolumn{2}{|c|}{$200-6522$} \\
\hline Instrument ID: D.i & & \multirow{2}{*}{\multicolumn{3}{|c|}{$\begin{array}{l}\text { Calibration Date(s): } \\
\text { Calibration Time (s): }\end{array}$}} & \multicolumn{2}{|c|}{$07 / 07 / 2011$} & \multicolumn{2}{|c|}{$07 / 07 / 2011$} \\
\hline Heated Purge: $(Y / N)$ & $\mathrm{N}$ & & & & \multicolumn{2}{|c|}{1117} & \multicolumn{2}{|l|}{1256} \\
\hline Purge Volume: 25.0 & & \multirow{2}{*}{\multicolumn{7}{|c|}{$\begin{array}{l}(\mathrm{mL}) \\
(\mathrm{mm}) \quad \text { Length: } 25\end{array}$}} \\
\hline GC Column: $\quad$ DB-624 & ID $: 0.20$ & & & & & & & \\
\hline LAB FILE ID: & \multicolumn{3}{|c|}{$\mathrm{RRF} 0.5=\mathrm{DHPO} 4 . \mathrm{D}$} & \multicolumn{5}{|c|}{ RRF 1.0 $=$ DHP05.D } \\
\hline RRF5.0 $=$ DHP06.D & \multicolumn{3}{|c|}{$R R E 10=D H P 07 . D$} & \multicolumn{5}{|c|}{ RRE 20 $=$ DHP08.D } \\
\hline COMPOUND & RRF 0.5 & RRE 1.0 & RRF 5.0 & RRF 1 & & RRF 20 & $\overline{\mathrm{RRE}}$ & \& RSD \\
\hline Vinyl Chloride-d3 & 0.479 & 0.428 & 0.415 & 0.4 & & 0.426 & 0.436 & 5.7 \\
\hline Chloroethane-d5 & 0.304 & 0.290 & 0.269 & 0.2 & & 0.274 & 0.283 & 5.0 \\
\hline 1,1-Dichloroethene-d2 & 0.849 & 0.795 & 0.757 & 0.7 & & 0.787 & 0.795 & 4.2 \\
\hline 2-Butanone-d5 & 0.041 & 0.038 & 0.040 & 0.0 & & 0.043 & 0.041 & 5.5 \\
\hline Chloroform-d & 0.512 & 0.515 & 0.502 & 0.5 & & 0.503 & 0.509 & 1.3 \\
\hline 1,2-Dichloroethane-d4 & 0.218 & 0.203 & 0.199 & 0.2 & & 0.194 & 0.203 & 4.3 \\
\hline Benzene-d6 & 1.573 & 1.471 & 1.478 & 1.4 & & 1.403 & 1.475 & 4.2 \\
\hline 1,2-Dichloropropane-d6 & 0.410 & 0.374 & 0.367 & 0.3 & & 0.356 & 0.374 & 5.7 \\
\hline Toluene-d8 & 1.315 & 1.370 & 1.458 & 1.4 & & 1.404 & 1.397 & 4.1 \\
\hline trans-1,3-Dichloropropene-d4 & 0.197 & 0.207 & 0.232 & 0.2 & & 0.264 & 0.230 & 12.2 \\
\hline 2-Hexanone-d5 & 0.023 & 0.026 & 0.037 & 0.0 & & 0.039 & 0.033 & 23.1 \\
\hline $1,1,2,2$-Tetrachloroethane-d2 & 0.151 & 0.161 & 0.167 & 0.1 & & 0.169 & 0.164 & 4.8 \\
\hline 1,2-Dichlorobenzene-d4 & 0.885 & 0.843 & 0.815 & 0.8 & & 0.799 & 0.830 & 4.2 \\
\hline
\end{tabular}

Report 1,4-Dioxane-d8 for Low-Medium VOA analysis only 


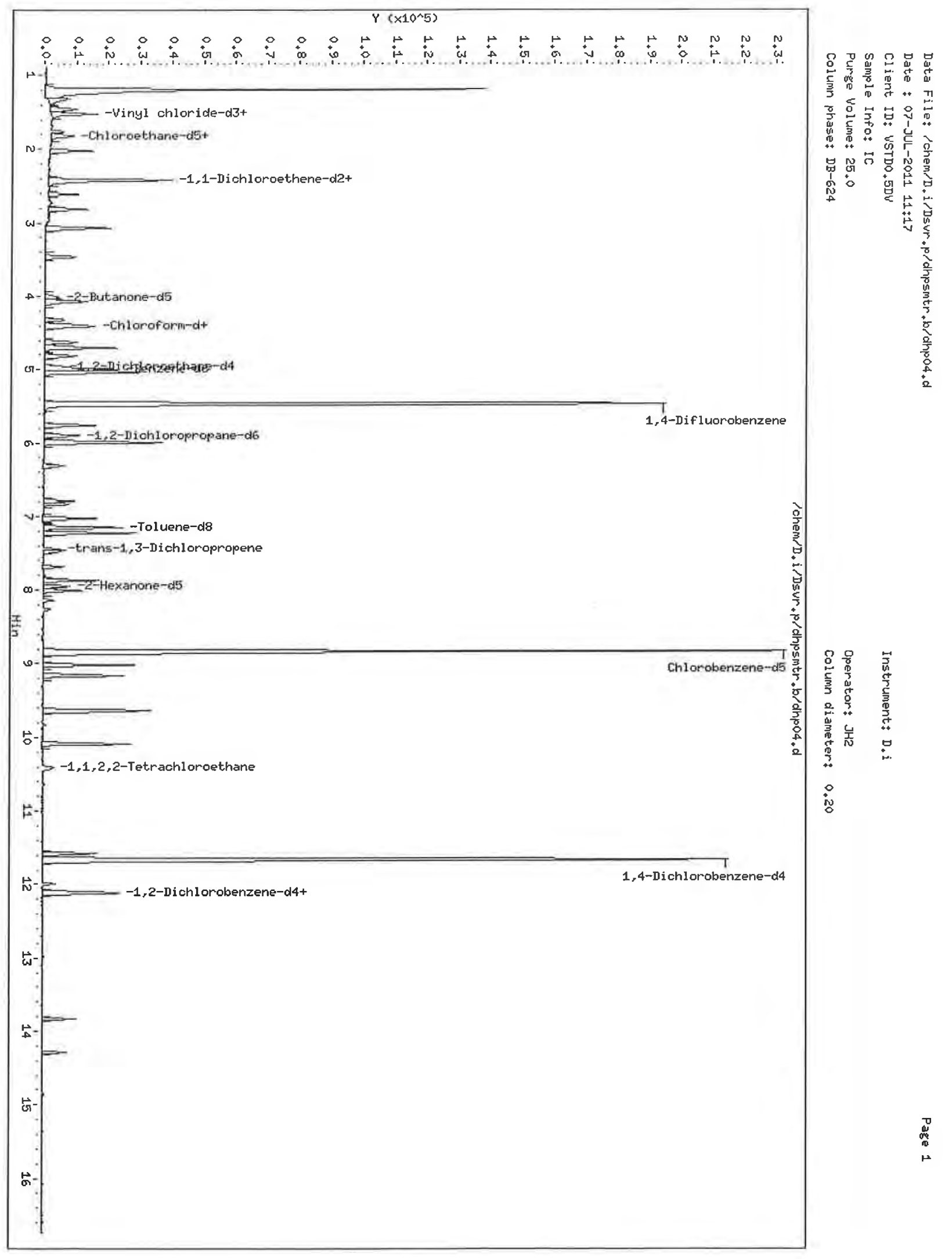




\section{TestAmerica Burlington}

LOW CONCENTRATION VOLATILE QUANTITATION REPORT

Data file : /chem/D.i/Dsvr.p/dhpsmtr.b/dhp04.d

Lab Smp Id: IC

Inj Date : 07-JUL-2011 11:17

Operator : JH2

Smp Info : IC

Misc Info : 1,5

Comment :

Method : /chem/D.i/Dsvr.p/dhpsmtr.b/somtr5t.m

Meth Date : 07-Jul-2011 19:24 jh2 Quant Type: ISTD

Cal Date : 07-JUL-2011 11:17

Als bottle: 80

Dil Factor: 1.00000

Integrator: HP RTE

Target Version: 3.50

Client Smp ID: VSTDO.5DV

Processing Host: chemsvr6

Concentration Formula: Amt * DF * Uf/Vo * CpndVariable

\begin{tabular}{ccc} 
Name & Value & Description \\
\hline DE & 1.00000 & Dilution Factor \\
Uf & 1.00000 & ng unit correction factor \\
Vo & 25.00000 & Sample volume purged (mL)
\end{tabular}

Cpnd Variable

Local Compound Variable

Compounds
1 Dichlorodifluoromethane
2 Chloromethane
3 Vinyl chloride-d3
4 Vinyl chloride
5 Bromomethane
6 Chloroethane-d5
7 Chloroethane
8 Trichlorofluoromethane
91,1 -Dichloroethene-d2
10 1, 1-Dichloroethene
11 1, 1,2-Trichloro-1,2,2-trifluo
12 Acetone
13 Carbon disulfide
14 Methyl acetate
15 Methylene chloride

\begin{tabular}{r} 
QUANT SIG \\
MASS \\
\hline rate= \\
85 \\
50 \\
65 \\
62 \\
94 \\
69 \\
64 \\
101 \\
63 \\
96 \\
101 \\
43 \\
76 \\
43 \\
84
\end{tabular}




\section{Compounds}

\begin{tabular}{|c|c|c|}
\hline & 16 & trans-1,2-Dichloroethene \\
\hline & 17 & Methyl tert-butyl ether \\
\hline & 18 & 1,1-Dichloroethane \\
\hline$\$$ & 19 & 2-Butanone-d5 \\
\hline & 20 & cis-1,2-Dichloroethene \\
\hline & 21 & 2-Butanone \\
\hline & 22 & Bromochloromethane \\
\hline \$ & 23 & Chloroform-d \\
\hline & 24 & Chloroform \\
\hline & 25 & 1,1,1-Trichloroethane \\
\hline & 26 & Cyclohexane \\
\hline & 27 & Carbon tetrachloride \\
\hline$\$$ & 28 & 1,2-Dichloroethane-d4 \\
\hline$\$$ & 29 & Benzene-d6 \\
\hline & 30 & Benzene \\
\hline & 31 & 1,2-Dichloroethane \\
\hline * & 32 & 1,4-Difluorobenzene \\
\hline & 33 & Trichloroethene \\
\hline$\$$ & 34 & 1,2-Dichloropropane-d6 \\
\hline & 35 & Methylcyclohexane \\
\hline & 36 & 1,2-Dichloropropane \\
\hline & 37 & Bromodichloromethane \\
\hline & 38 & cis-1,3-Dichloropropene \\
\hline & 39 & 4-Methyl-2-pentanone \\
\hline$\$$ & 40 & Toluene-d8 \\
\hline & 41 & Toluene \\
\hline$\$$ & 42 & trans-1,3-Dichloropropene-d4 \\
\hline & 43 & trans-1,3-Dichloropropene \\
\hline & 44 & 1,1,2-Trichloroethane \\
\hline & 45 & Tetrachloroethene \\
\hline$\$$ & 46 & 2-Hexanone-d5 \\
\hline & 47 & 2-Hexanone \\
\hline & 48 & Dibromochloromethane \\
\hline & 49 & 1,2-Dibromoethane \\
\hline * & 50 & Chlorobenzene-d5 \\
\hline & 51 & Chlorobenzene \\
\hline & 52 & Ethylbenzene \\
\hline & 53 & $\mathrm{~m}, \mathrm{p}$-Xylene \\
\hline & 54 & Styrene \\
\hline & 55 & o-Xylene \\
\hline & 56 & Bromoform \\
\hline & 57 & Isopropylbenzene \\
\hline$\$$ & 58 & $1,1,2,2$-Tetrachloroethane-d2 \\
\hline & 59 & $1,1,2,2$-Tetrachloroethane \\
\hline & 60 & 1,3-Dichlorobenzene \\
\hline * & 61 & 1,4-Dichlorobenzene-d4 \\
\hline & 62 & 1,4-Dichlorobenzene \\
\hline
\end{tabular}

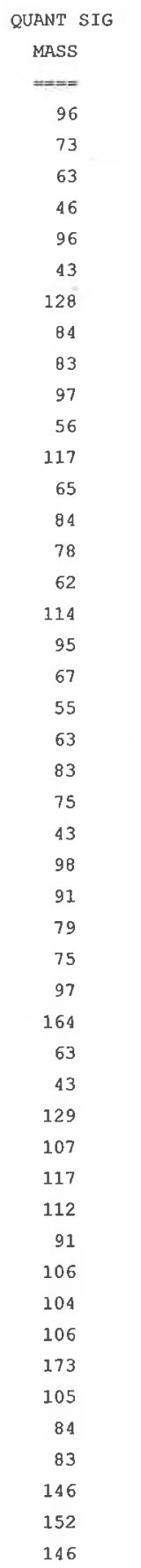

\begin{tabular}{|c|c|c|}
\hline RT & EXP RT & REL RT \\
\hline$m$ & 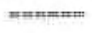 & $=m=$ \\
\hline 3.069 & 3.069 & $(0.562)$ \\
\hline 3.075 & 3.082 & $(0.563)$ \\
\hline 3.466 & 3.466 & $(0.634)$ \\
\hline 4.020 & 4.020 & $(0.736)$ \\
\hline 4.069 & 4.069 & $(0.745)$ \\
\hline 4.087 & 4.061 & $(0.748)$ \\
\hline 4.325 & 4.325 & $(0.791)$ \\
\hline 4.392 & 4.392 & $(0.804)$ \\
\hline 4.417 & 4.417 & $(0.808)$ \\
\hline 4.630 & 4.630 & $(0.524)$ \\
\hline 4.703 & 4.703 & $(0.533)$ \\
\hline 4.813 & 4.819 & $(0.545)$ \\
\hline 4. 959 & 4.959 & $(0.907)$ \\
\hline 4.990 & 4.990 & $(0.565)$ \\
\hline 5.032 & 5.032 & $(0.570)$ \\
\hline 5.038 & 5.039 & $(0.922)$ \\
\hline 5.465 & 5.471 & $(1.000)$ \\
\hline 5.758 & 5.758 & $(0.652)$ \\
\hline 5.898 & 5.904 & $(0.668)$ \\
\hline 5.989 & 5.990 & $(0.678)$ \\
\hline 6.002 & 6.002 & $(0.680)$ \\
\hline 6.312 & 6.313 & $(0.715)$ \\
\hline 6.837 & 6.837 & $(0.774)$ \\
\hline 7.026 & 7.026 & $(0.796)$ \\
\hline 7.154 & 7.154 & $(0.810)$ \\
\hline 7.227 & 7.227 & $(0.818)$ \\
\hline 7.446 & 7.447 & $(0.843)$ \\
\hline 7.477 & 7.477 & $(0.847)$ \\
\hline 7.684 & 7.684 & $(0.870)$ \\
\hline 7.867 & 7.867 & $(0.891)$ \\
\hline 7.946 & 7.946 & $(0.900)$ \\
\hline 8.007 & 8.007 & $(0.907)$ \\
\hline 8.141 & 8.142 & $(0.922)$ \\
\hline 8.263 & 8.264 & $(0.936)$ \\
\hline 8.830 & 8.830 & $(1.000)$ \\
\hline 8. 867 & 8.867 & $(1.004)$ \\
\hline 9.013 & 9.019 & $(1.021)$ \\
\hline 9.159 & 9.160 & $(1.037)$ \\
\hline 9.647 & 9.647 & $(1.093)$ \\
\hline 9.635 & 9.629 & $(1.091)$ \\
\hline 9.836 & 9.836 & $(0.844)$ \\
\hline 10.086 & 10.086 & $(1.142)$ \\
\hline 10.403 & 10.403 & (1.178) \\
\hline 10.434 & 10.434 & $(1.182)$ \\
\hline 11.580 & 11.580 & $(0.993)$ \\
\hline 11.659 & 11.659 & $(1.000)$ \\
\hline 11.690 & 11.690 & $(1.003)$ \\
\hline
\end{tabular}

\begin{tabular}{|c|c|c|}
\hline \multirow[b]{3}{*}{ RESPONSE } & \multicolumn{2}{|c|}{ AMOUNTS } \\
\hline & CAL-AMT & $\mathrm{ON}-\mathrm{COL}$ \\
\hline & $(\mathrm{ng})$ & $\mathrm{ng})$ \\
\hline 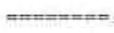 & nemertive & nowementes \\
\hline 5577 & 12.5000 & 13 \\
\hline 5251 & 12.5000 & 13 \\
\hline 11078 & 12.5000 & 13 \\
\hline 7093 & 125.000 & $120(a)$ \\
\hline 5203 & 12.5000 & $12(a)$ \\
\hline 7753 & 125.000 & $120(a)$ \\
\hline 1788 & 12.5000 & $13(0)$ \\
\hline 8843 & 12.5000 & 13 \\
\hline 8662 & 12.5000 & 13 \\
\hline 7331 & 12.5000 & 13 \\
\hline 10859 & 12.5000 & 11 (a) \\
\hline 6957 & 12.5000 & 13 \\
\hline 3757 & 12.5000 & 13 \\
\hline 22443 & 12.5000 & 13. \\
\hline 23075 & 12.5000 & 13 \\
\hline 5019 & 12.5000 & 13 \\
\hline 172557 & 125.000 & \\
\hline 5668 & 12.5000 & 13 \\
\hline 5857 & 12.5000 & $14(\mathrm{H})$ \\
\hline 10030 & 12.5000 & 12 (a) \\
\hline 5180 & 12.5000 & $12(\mathrm{aQ})$ \\
\hline 4418 & 12.5000 & 13 \\
\hline 4425 & 12.5000 & $10(a)$ \\
\hline 14887 & 125.000 & $100(a)$ \\
\hline 18761 & 12.5000 & $12(a)$ \\
\hline 22955 & 12.5000 & $12(a)$ \\
\hline 2810 & 12.5000 & $11(a)$ \\
\hline 3083 & 12.5000 & $10(a)$ \\
\hline 2207 & 12.5000 & $12(a)$ \\
\hline 4439 & 12.5000 & 13 \\
\hline 3288 & 125.000 & $8 \theta(a)$ \\
\hline 10908 & 125.000 & $110(a)$ \\
\hline 2459 & 12.5000 & $12(a)$ \\
\hline 1888 & 12.5000 & 12 (aM) \\
\hline 142712 & 125.000 & \\
\hline 15402 & 12.5000 & 13 \\
\hline 22743 & 12.5000 & $11(a)$ \\
\hline 9186 & 12.5000 & 11 (a) \\
\hline 10895 & 12.5000 & $9.7(a)$ \\
\hline 7694 & 12.5000 & $10(a)$ \\
\hline 662 & 12.5000 & $11(a M)$ \\
\hline 19982 & 12.5000 & $9.5(a)$ \\
\hline 2155 & 12.5000 & $12(a)$ \\
\hline 2402 & 12.5000 & $13(\mathrm{M})$ \\
\hline 9259 & 12.5000 & $12(a)$ \\
\hline 61965 & 125.000 & \\
\hline 11318 & 12.5000 & 14 \\
\hline
\end{tabular}


Data File: /chem/D.i/Dsvr.p/dhpsmtr.b/dhp04.d

Page 3

Report Date: 07-Jul-2011 19:24

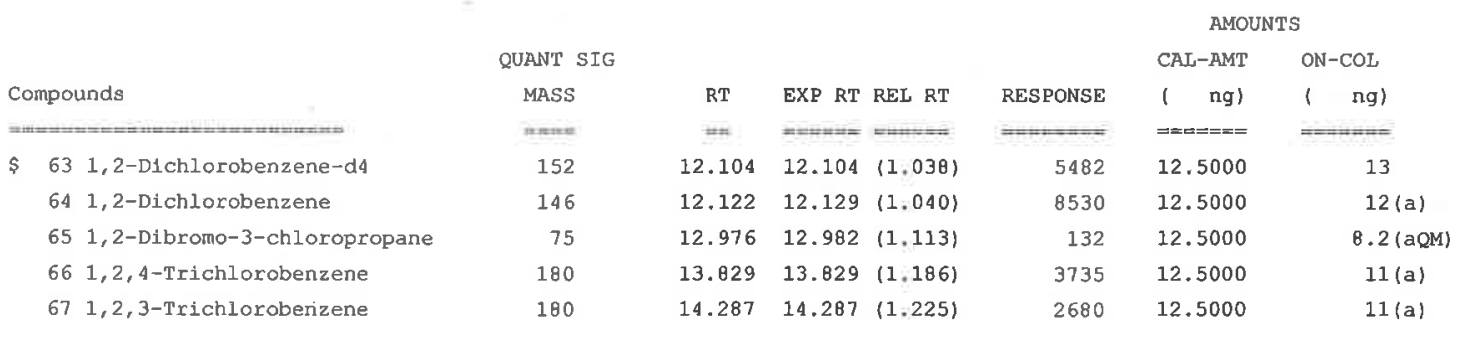

QC Flag Legend

a - Target compound detected but, quantitated amount Below Limit of Quantitation (BLOQ).

Q - Qualifier signal failed the ratio test.

M - Compound response manually integrated.

$\mathrm{H}$ - Operator selected an alternate compound hit. 
Data File: dhp04.d

Lab Sample ID: IC

Inj. Date and Time: 07-JUL-2011 11:17

Instrument ID: D.i

Client ID: VSTD0.5DV

Compound: $491,2-$ Dibromoethane

CAS \# : 106-93-4

Report Date: 07/07/2011

Not Detected

Expected RT: 8.26

Processing Integration Results

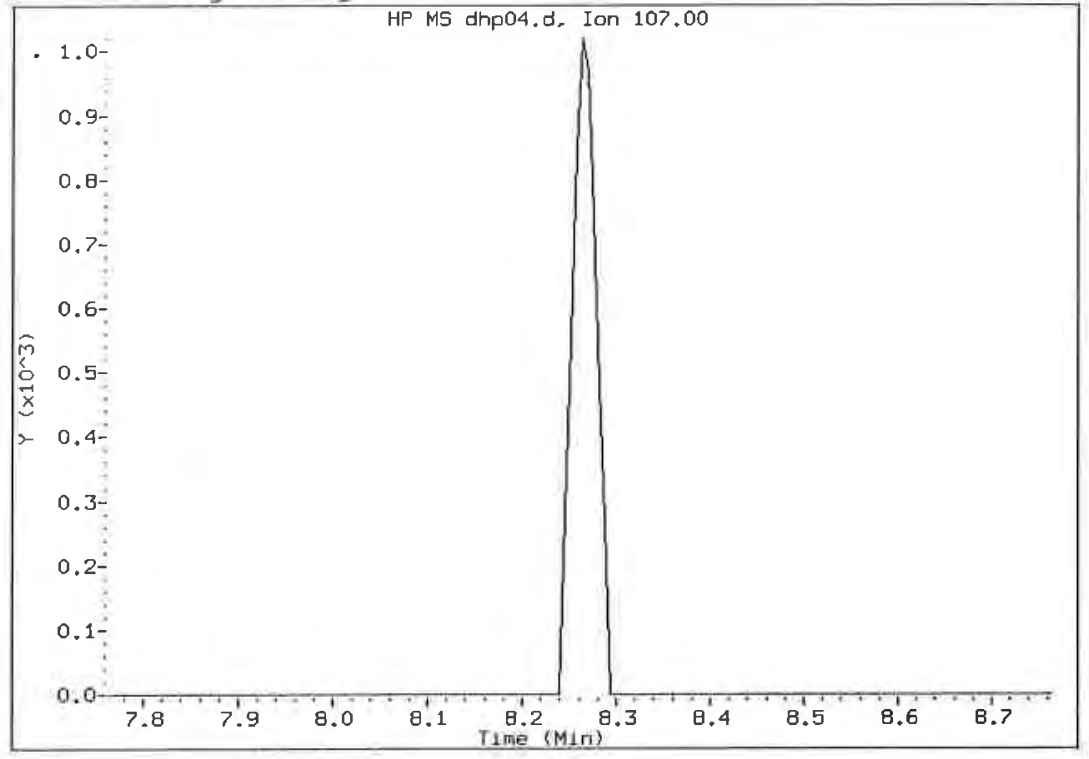

Manual Integration Results

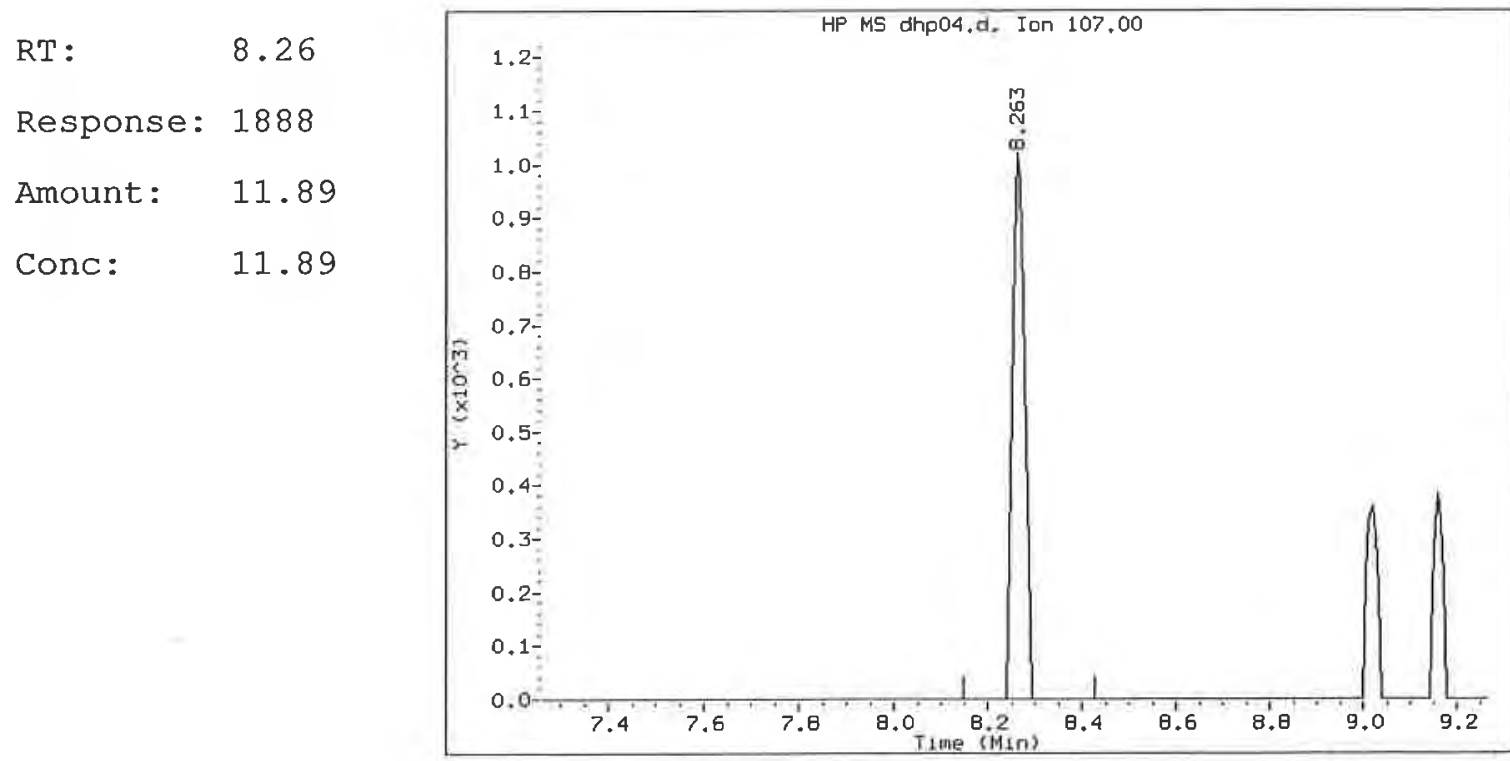

File Uploaded By: jh2

Manual Integration Reason: Analyte not identified by the data system 
Data Eile: dhp04.d

Lab Sample ID: IC

Inj. Date and Time: 07-JUL-2011 11:17

Instrument ID: D.i

Client ID: VSTD0.5DV

Compound: 56 Bromoform

CAS \# : 75-25-2

Report Date: 07/07/2011

Not Detected

Expected RT: 9.84

Processing Integration Results

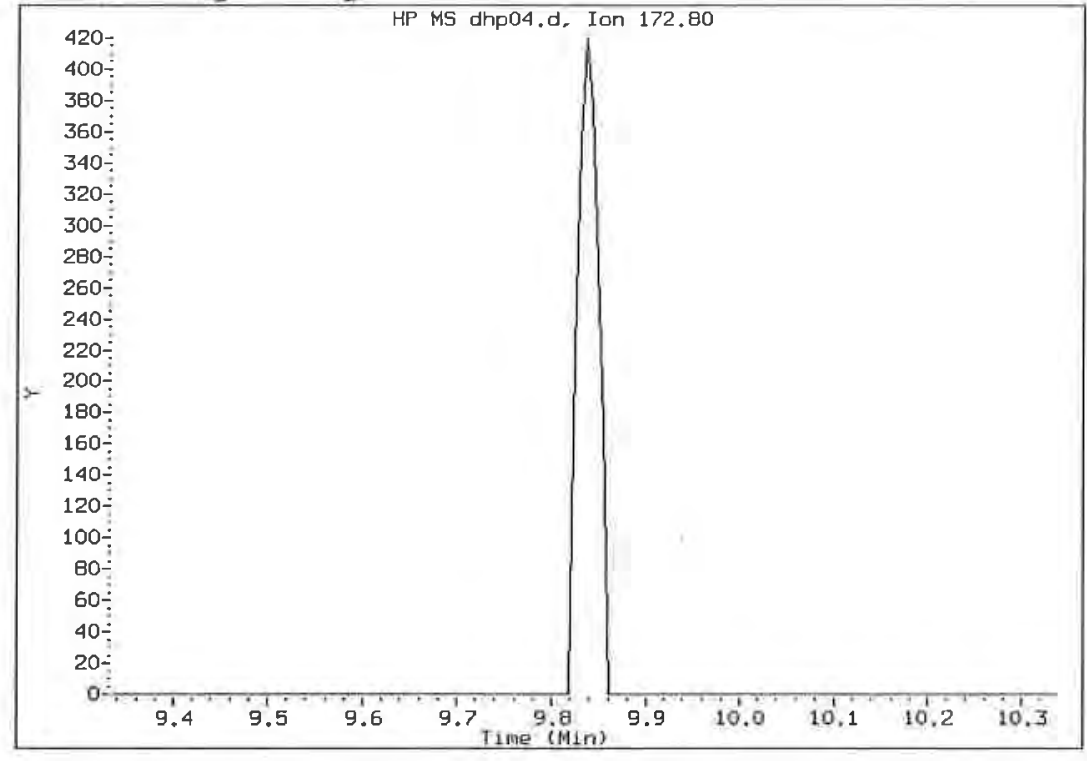

Manual Integration Results

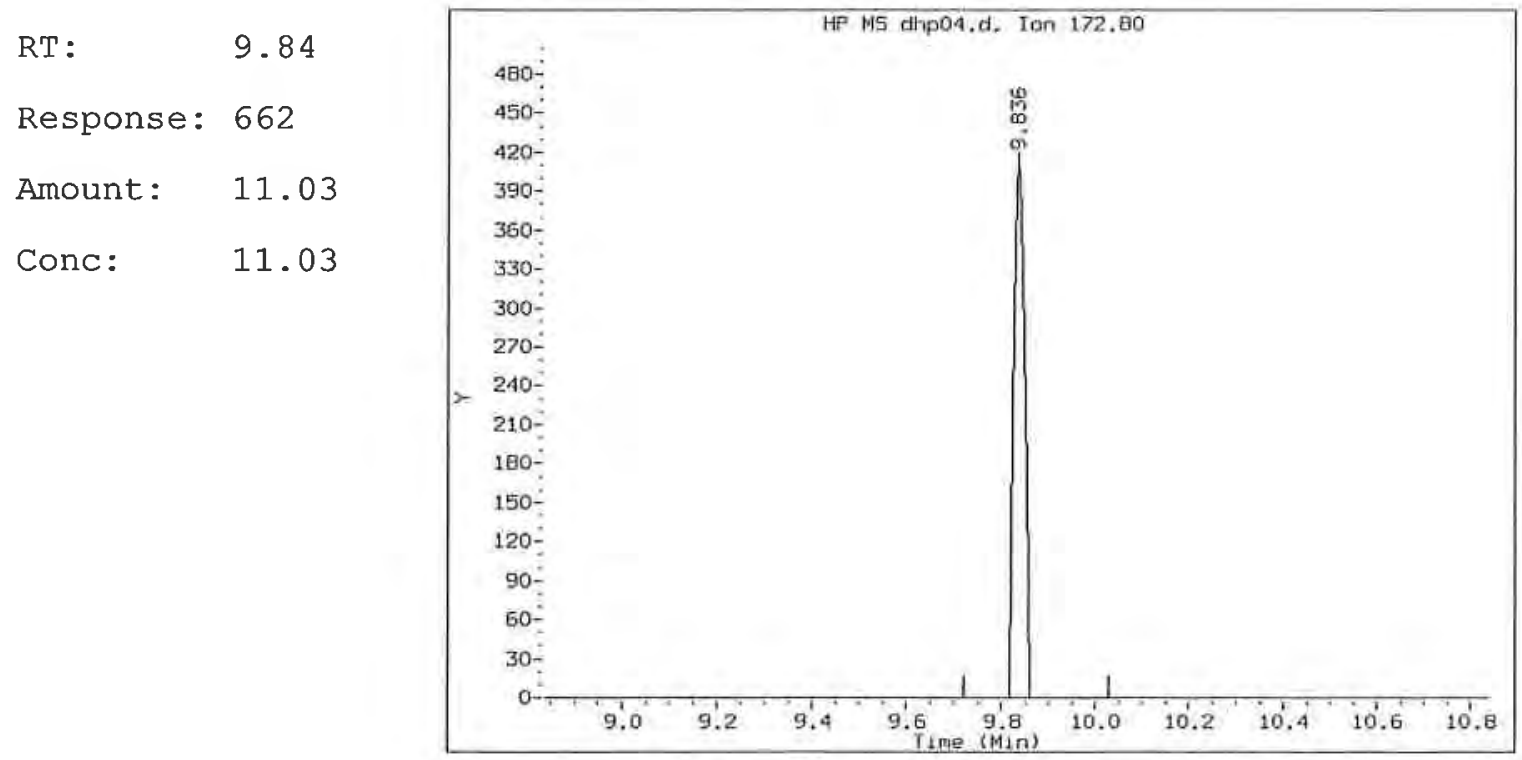

File Uploaded By: jh2

Manual Integration Reason: 
Data Eile: dhp04.d

Lab Sample ID: IC

Inj. Date and Time: 07-JUL-2011 11:17

Instrument ID: D.i

Client ID: VSTD0.5DV

Compound: $591,1,2,2$-Tetrachloroethane

CAS \#: 79-34-5

Report Date: 07/07/2011

Processing Integration Results

Not Detected

Expected RT: 10.43

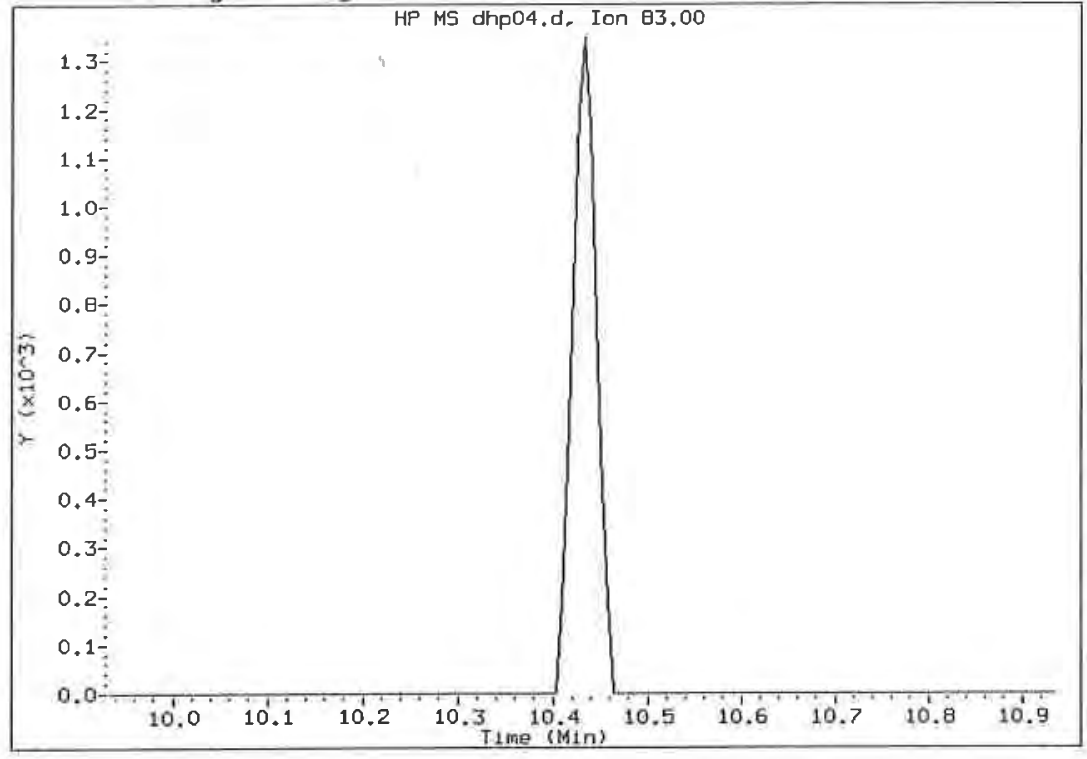

\section{Manual. Integration Results}

$\begin{array}{ll}\text { RT: } & 10.43 \\ \text { Response: } & 2402 \\ \text { Amount: } & 12.84 \\ \text { Conc: } & 12.84\end{array}$

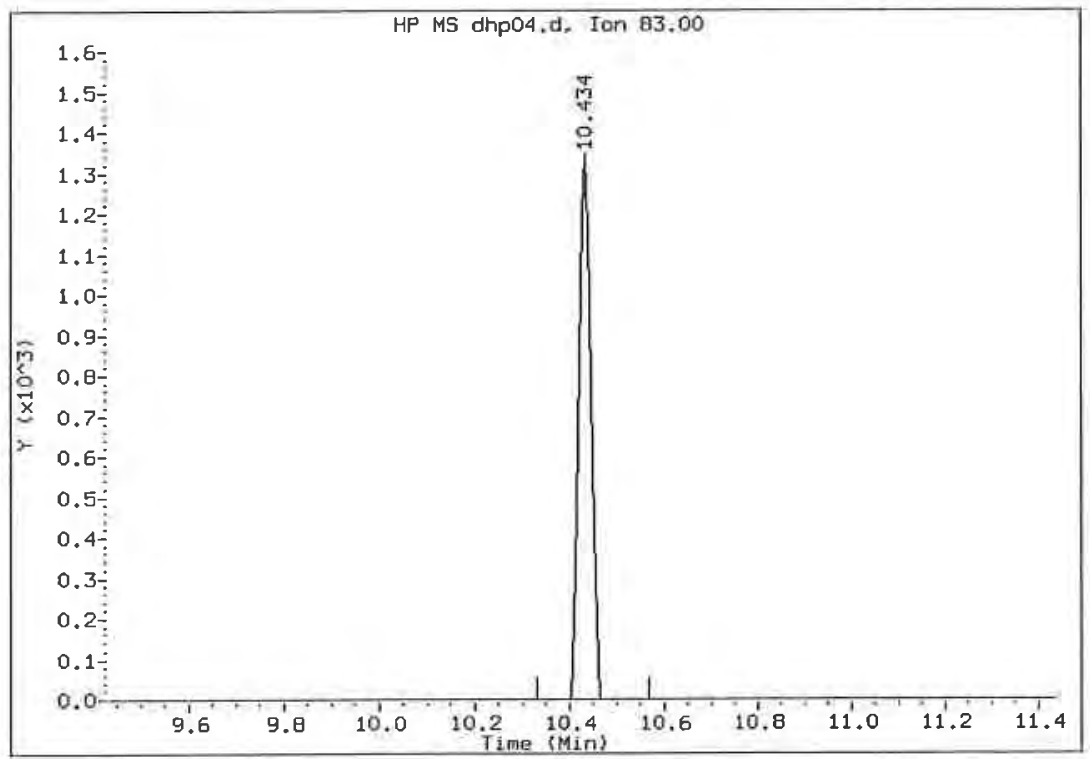

File Uploaded By: jh2

Manual Integration Reason: 
Manual Integration Report

Data File: dhp04.d

Lab Sample ID: IC

Inj. Date and Time: 07-JUL-2011 11:17

Instrument ID: D.i

Client ID: VSTDO.5DV

Compound: 65 1,2-Dibromo-3-chloropropane

CAS \# : 96-12-8

Report Date: 07/07/2011

Processing Integration Results

Not Detected

Expected RT: 12.98

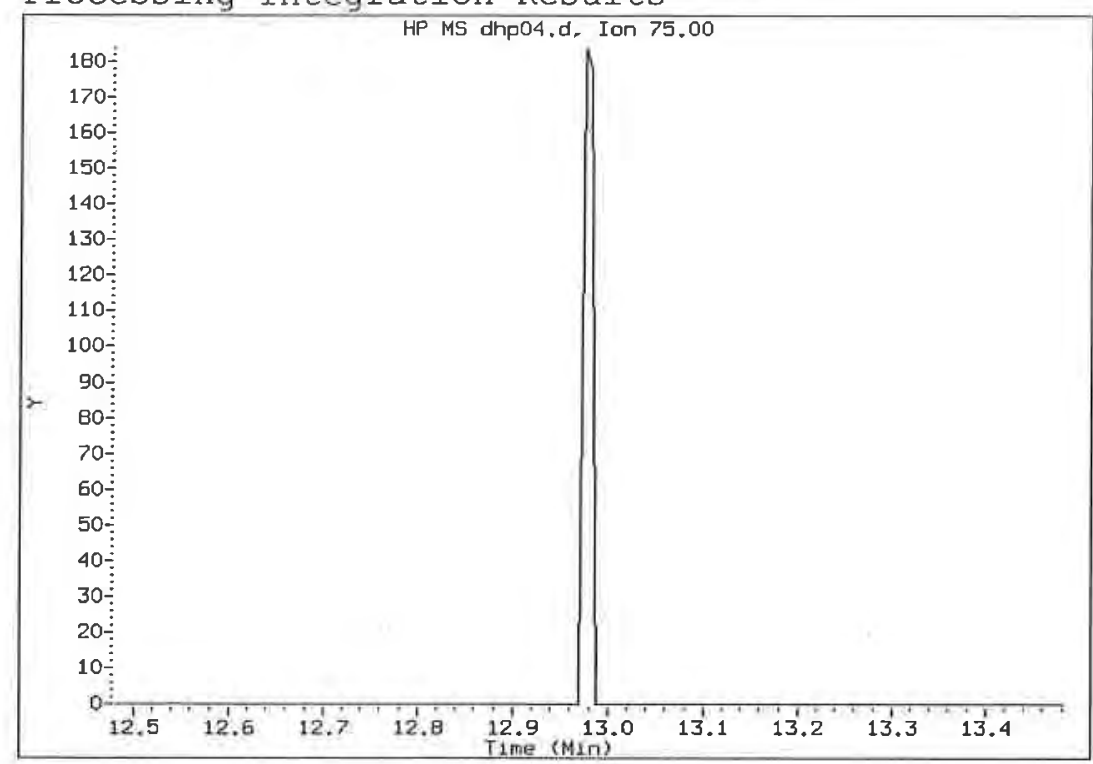

Manual Integration Results

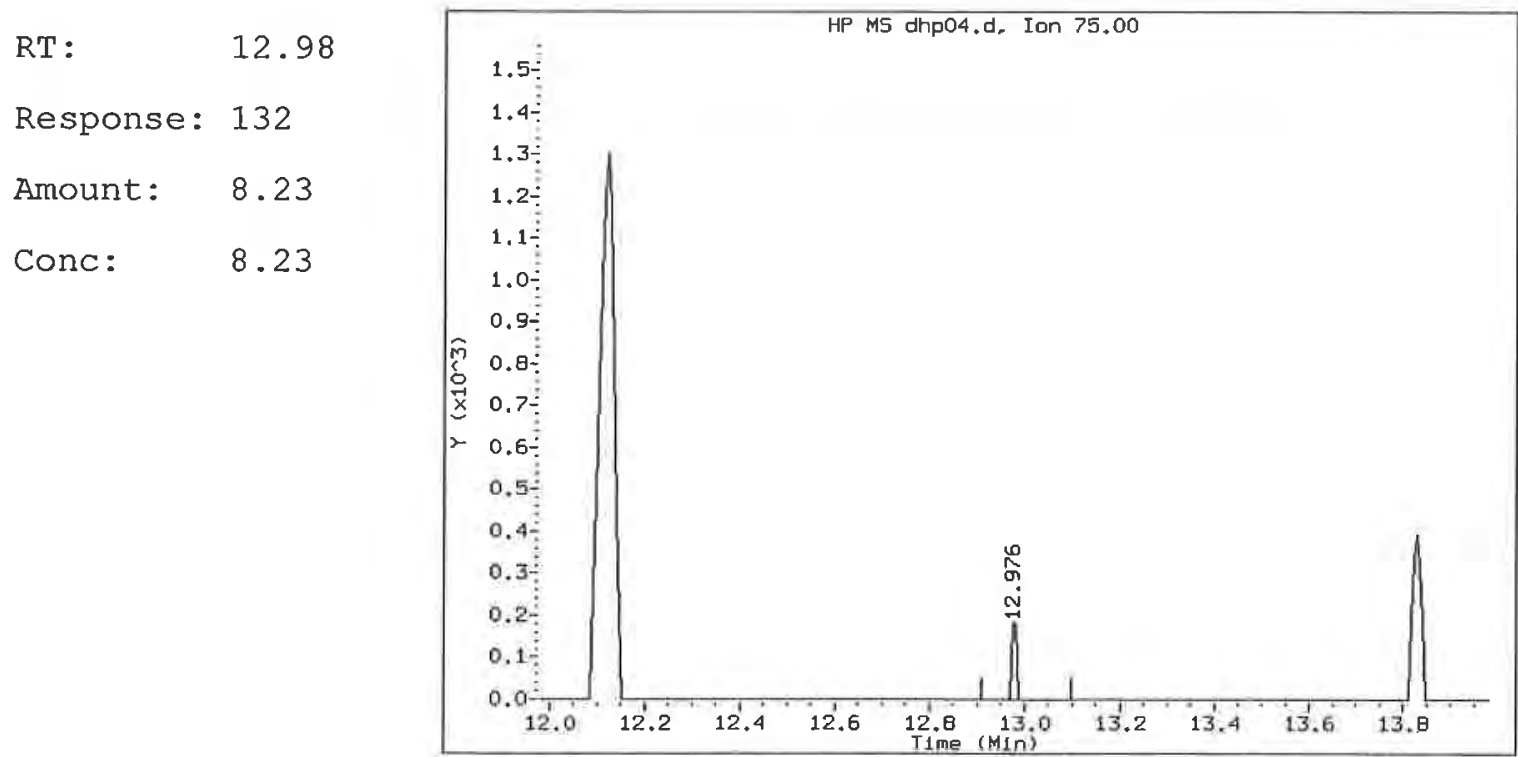

File Uploaded By: jh2

Manual Integration Reason: Analyte not identified by the data system 


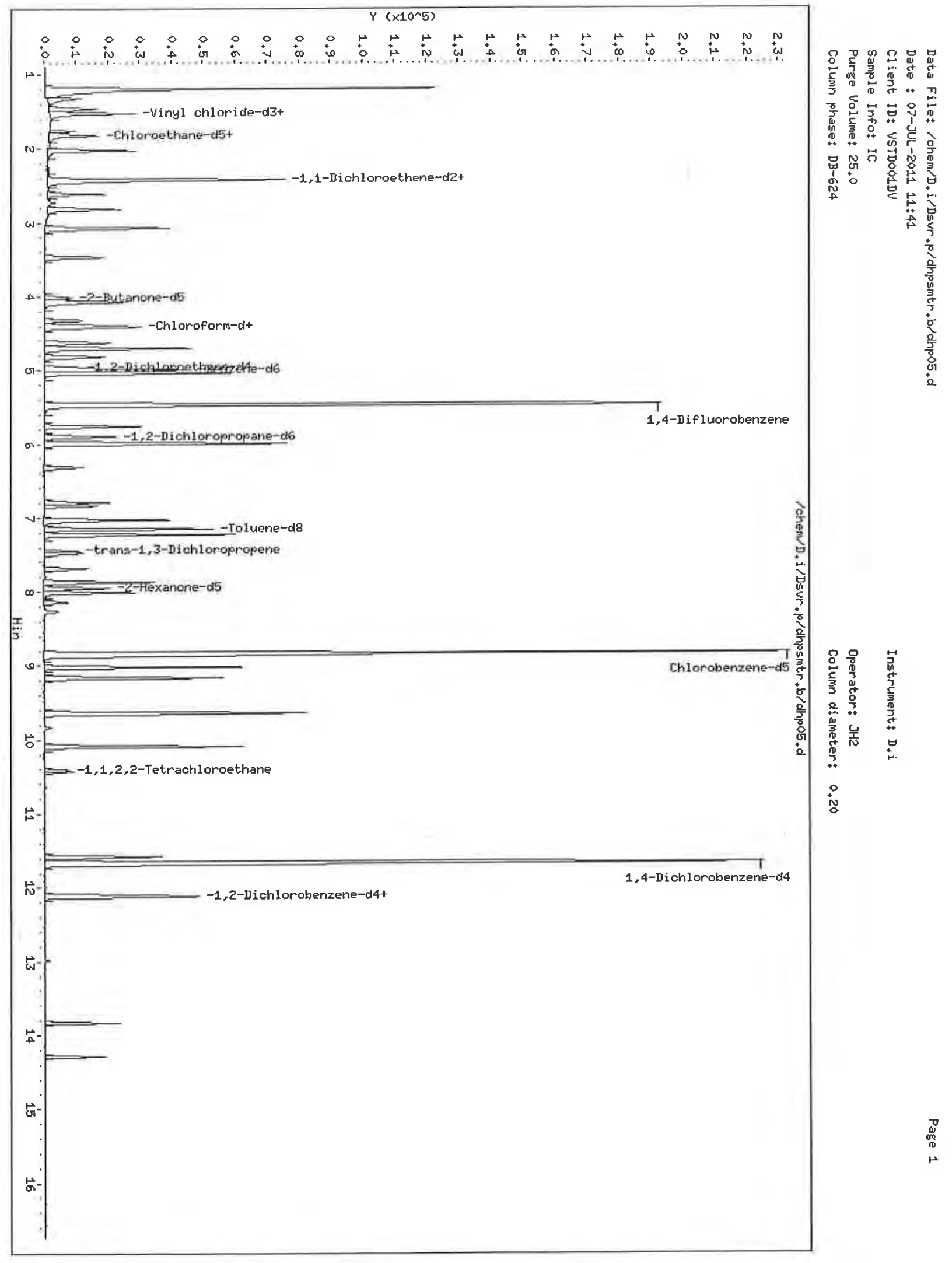


LOW CONCENTRATION VOLATILE QUANTITATION REPORT

Data file : /chem/D.i/Dsvr.p/dhpsmtr.b/dhp05.d

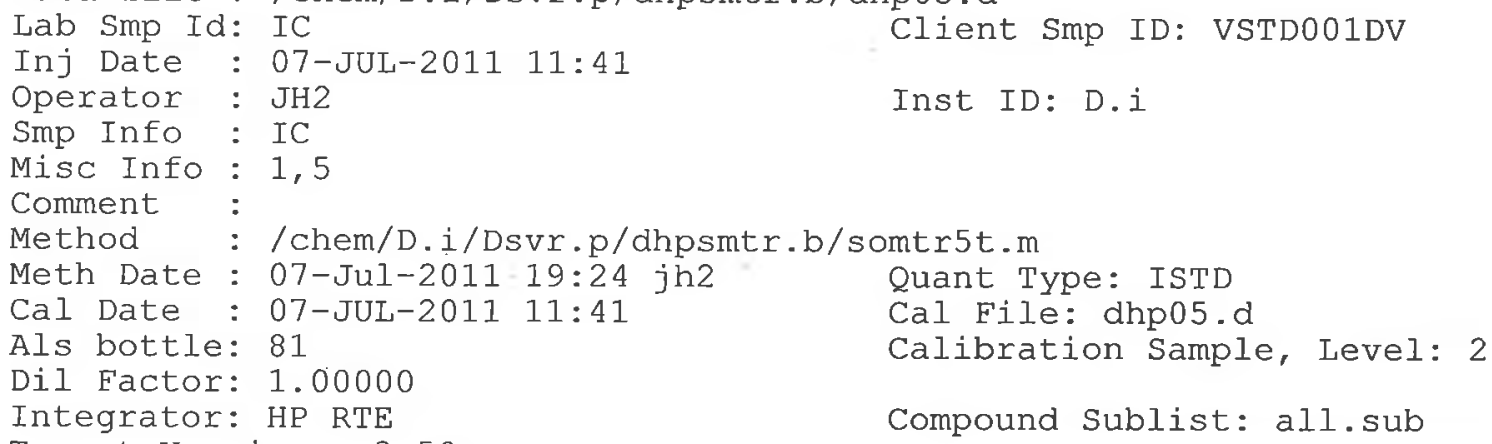

Calibration Sample, Level: 2

Compound Sublist: all.sub

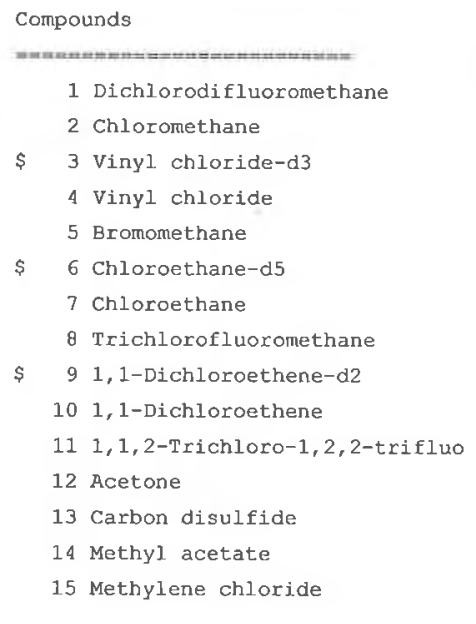

15 Methylene chloride 


\section{Compounds}

16 trans-1,2-Dichloroethene

17 Methyl tert-butyl ether

18 1,1-Dichloroethane

\$ 19 2-Butanone-d5

20 cis-1,2-Dichloroethene

21 2-Butanone

22 Bromochloromethane

\$ 23 Chloroform-d

24 chloroform

25 1,1,1-Trichloroethane

26 Cyclohexane

27 Carbon tetrachloride

\$ 28 1,2-Dichloroethane-d4

$\$ 29$ Benzene-d6

30 Benzene

31 1,2-Dichloroethane

* 32 1,4-Difluorobenzene

33 Trichloroethene

\$34 1,2-Dichloropropane-d6

35 Methylcyclohexane

36 1,2-Dichloropropane

37 Bromodichloromethane

38 cis-1,3-Dichloropropene

39 4-Methyl-2-pentanone

$\$ 40$ Toluene-d8

41 Toluene

\$ 42 trans-1,3-Dichloropropene-d4

43 trans-1,3-Dichloropropene

44 1,1,2-Trichloroethane

45 Tetrachloroethene

$\$ 46$ 2-Hexanone-d5

47 2-Hexanone

48 Dibromochloromethane

49 1,2-Dibromoethane

* 50 Chlorobenzene-d5

51 Chlorobenzene

52 Ethylbenzene

$53 \mathrm{~m}, \mathrm{p}$-xylene

54 styrene

55 o-xylene

56 Bromoform

57 Isopropylbenzene

\$ $501,1,2,2$-Tetrachloroethane-d2

59 1, 2,2,2-Tetrachloroethane

60 1,3-Dichlorobenzene

* 61 1,4-Dichlorobenzene-d4

62 1,4-Dichlorobenzene
QUANT SIG
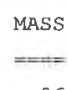

96

73

63

46

96

43

128

84

83

97

56

117

65

84

78

62

114

95

67

55

63

83

75

43

98

91

79

75

97

164

63

43

129

107

117

112

91

106

104

106

173

105

84

83

146

152

146
RT EXP RT REL RT

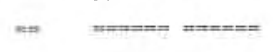

$3.069 \quad 3.069 \quad(0.561)$

$\begin{array}{llll}3.082 & 3.082 & (0.563)\end{array}$

$3.466 \quad 3.466 \quad(0.633)$

$4.020 \quad 4.020 \quad(0.735)$

$4.069 \quad 4.069(0.744)$

$4.088 \quad 4.081 \quad(0.747)$

$4.325 \quad 4.325 \quad(0.791)$

$4.392 \quad 4.392 \quad(0.803)$

$4.417 \quad 4.417 \quad(0.807)$

$4.630 \quad 4.630 \quad(0.524)$

$4.703 \quad 4.703 \quad(0.533)$

$\begin{array}{llll}4.819 & 4.819 & (0.546)\end{array}$

$4.959 \quad 4.959 \quad(0.906)$

$4.990 \quad 4.990 \quad(0.565)$

$5.039 \quad 5.032 \quad(0.571)$

$5.039 \quad 5.039 \quad(0.921)$

$5.4715 .471(1.000)$

$5.764 \quad 5.758 \quad(0.653)$

$5.904 \quad 5.904 \quad(0.669)$

$5.990 \quad 5.990(0.678)$

$6.002 \quad 6.002(0.680)$

$6.313 \quad 6.313(0.715)$

$6.837 \quad 6.837 \quad(0.774)$

$\begin{array}{llll}7.032 & 7.026 & (0.796)\end{array}$

$7.154 \quad 7.154 \quad(0.810)$

$7.227 \quad 7.227 \quad(0.818)$

$\begin{array}{llll}7.447 & 7.447 \quad(0.843)\end{array}$

$7.477 \quad 7.477 \quad(0.847)$

$\begin{array}{llll}7.684 & 7.684 & (0.870)\end{array}$

$\begin{array}{llll}7.867 & 7.867 & (0.891)\end{array}$

$7.953 \quad 7.946 \quad(0.901)$

$\begin{array}{llll}8.008 & 8.007 \quad(0.907)\end{array}$

$8.142 \quad 8.142 \quad(0.922)$

$8.264 \quad 8.264 \quad(0.936)$

$8.831 \quad 8.830 \quad(1.000)$

$8.867 \quad 0.867 \quad(1.004)$

$9.020 \quad 9.019 \quad(1.021)$

$9.160 \quad 9.160 \quad(1.037)$

$9.647 \quad 9.647$ (1.093)

$9.629 \quad 9.629 \quad(1.090)$

$9.836 \quad 9.836 \quad(0.844)$

$10.086 \quad 10.086(1.142)$

$10.403 \quad 10.403(1.178)$

$10.434 \quad 10.434 \quad(1.182)$

$11.580 \quad 11.580 \quad(0.993)$

$11.659 \quad 11.659 \quad(1.000)$

$11.690 \quad 11.690 \quad(1.003)$
AMOUNTS

CAL-AMT ON-COL

RESPONSE

( ng) ( ng)

misturiase =

$\begin{array}{ll}25.0000 & 24 \\ 25.0000 & 23\end{array}$

$25.0000 \quad 24$

$250.000 \quad 230$

$25.0000 \quad 24$

$250.000 \quad 240$

$25.0000 \quad 25$

$25.0000 \quad 25$

$25.0000 \quad 25$

$25.0000 \quad 25$

$25.0000 \quad 23$

$25.0000 \quad 24$

$25.0000 \quad 25$

$25.0000 \quad 25$

$25.0000 \quad 25$

$9396 \quad 25.0000 \quad 24$

$173166 \quad 125.000$

$11057 \quad 25.0000$

$10885 \quad 25.0000$

2059225.0000

$10843 \quad 25.0000$

$8622 \quad 25.0000$

$9842 \quad 25.0000$

$33182 \quad 250.000$

$39850 \quad 25.0000$

$47194 \quad 25.0000$

601125.0000

$6541 \quad 25.0000$

$4712 \quad 25.0000$

883525.0000

$7614 \quad 250.000$

$24613 \quad 250.000$

$5119 \quad 25.0000$

$3930 \quad 25.0000$

$145424 \quad 125.000$

$30136 \quad 25.0000$

$48025 \quad 25.0000$

$20029 \quad 25.0000$

$25758 \quad 25.0000$

$17457 \quad 25.0000$

$1501 \quad 25.0000$

$45177 \quad 25.0000$

$4694 \quad 25.0000$

$4480 \quad 25.0000$

$18960 \quad 25.0000$

$64569 \quad 125.000$

$22415 \quad 25.0000$ 
Data File: /chem/D.i/Dsvr.p/dhpsmtr.b/dhp05.d Report Date: 07-Jul-2011 19:24

\begin{tabular}{|c|c|c|c|c|c|c|}
\hline \multirow[b]{3}{*}{ Compounds } & \multirow{2}{*}{\multicolumn{2}{|c|}{ QUANT SIG }} & \multirow[b]{3}{*}{ REL RT } & \multirow[b]{3}{*}{ RESPONSE } & \multicolumn{2}{|c|}{ AMOUNTS } \\
\hline & & & & & CAL-AMT & ON-COL \\
\hline & MASS & $\mathrm{RT}$ & & & ng) & ng) \\
\hline 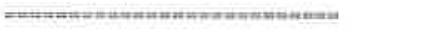 & manten & सक & 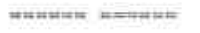 & 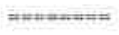 & =-nuture & entantur \\
\hline$\$ 631,2-D i c h l o r o b e n z e n e-d 4$ & 152 & 12.104 & $12.104(1.038)$ & 10890 & 25.0000 & 25 \\
\hline 64 1,2-Dichlorobenzene & 146 & 12.129 & $12.129(1.040)$ & 17922 & 25.0000 & 25 \\
\hline 65 1,2-Dibromo-3-chloropropane & 75 & 12.982 & $12.982(1.113)$ & 350 & 25.0000 & 21 \\
\hline 66 1,2,4-Trichlorobenzene & 180 & 13.830 & $13.829(1.186)$ & 7408 & 25.0000 & 21 \\
\hline 67 1,2,3-Trichlorobenzene & 180 & 14.287 & $14.287 \quad(1.225)$ & 6062 & 25.0000 & 23 \\
\hline
\end{tabular}

QC Flag Legend

Q - Qualifier signal failed the ratio test.

M - Compound response manually integrated. 
Data File: dhp05.d

Lab Sample ID: IC

Inj. Date and Time: 07-JUL-2011 11:41

Instrument ID: D.i

Client ID: VSTD001DV

Compound: 49 1,2-Dibromoethane

CAS \# : 106-93-4

Report Date: 07/07/2011

Processing Integration Results

Not Detected

Expected RT: 8.26

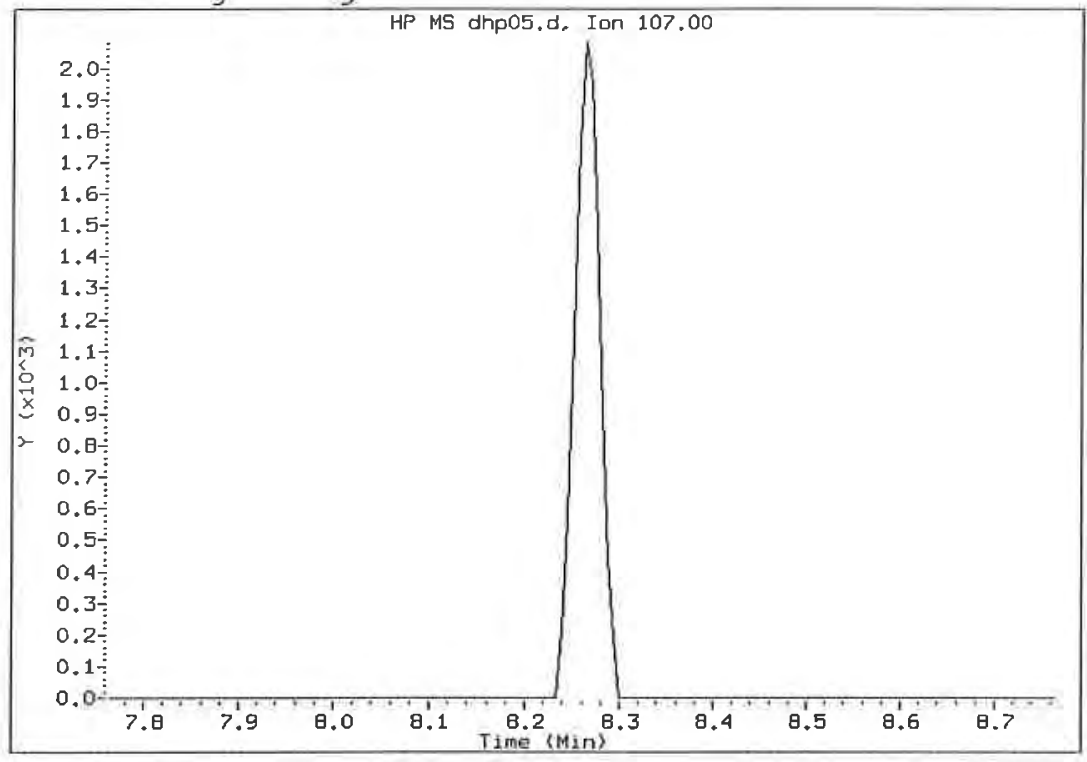

Manual Integration Results

$\mathrm{RT}: \quad 8.26$

Response: 3930

Amount: 24.28

Conc: $\quad 24.28$

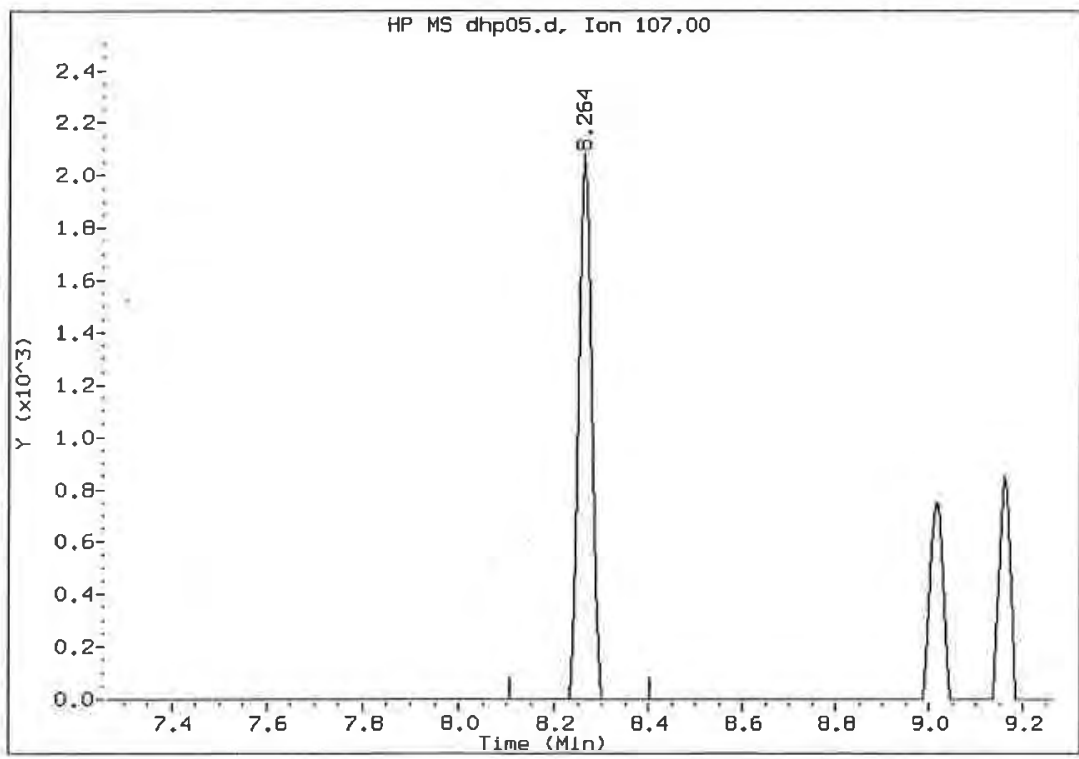

File Uploaded By: jh2

Manual Integration Reason: Analyte not identified by the data system 
Data File: dhp05.d

Lab Sample ID: IC

Inj. Date and Time: 07-JUL-2011 11:41

Instrument ID: D.i

Client ID: VSTD001DV

Compound: 56 Bromoform

CAS \# : 75-25-2

Report Date: 07/07/2011

Processing Integration Results

Not Detected

Expected RT: 9.84

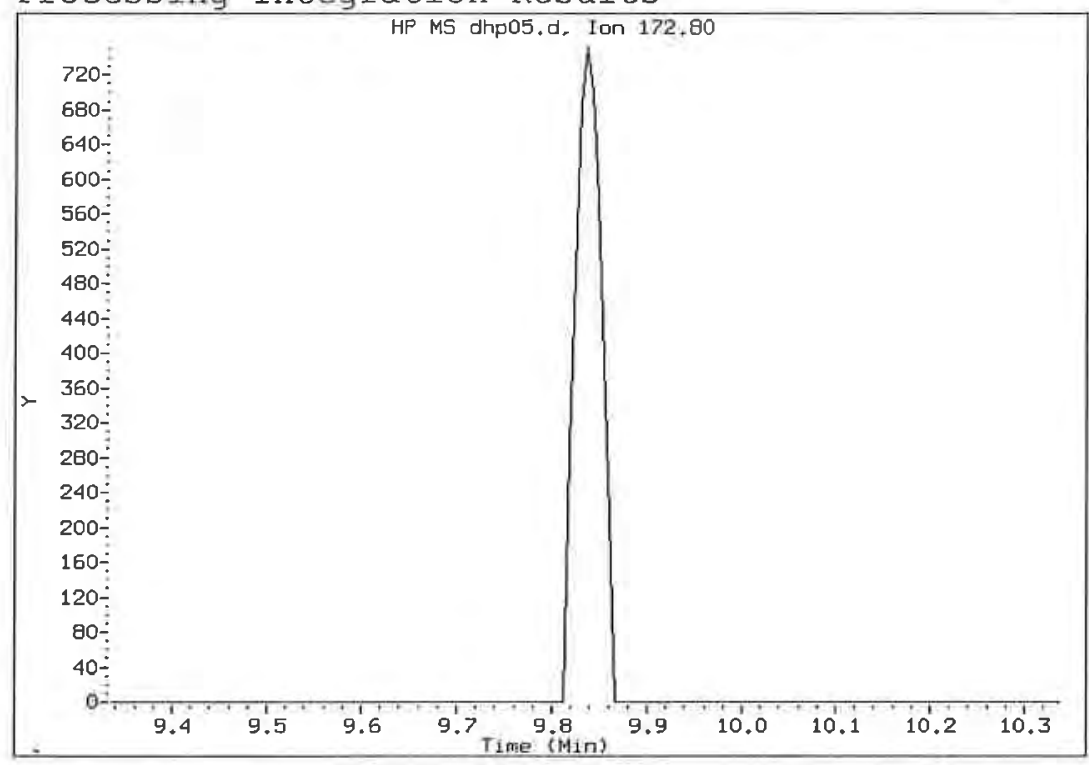

Manual Integration Results

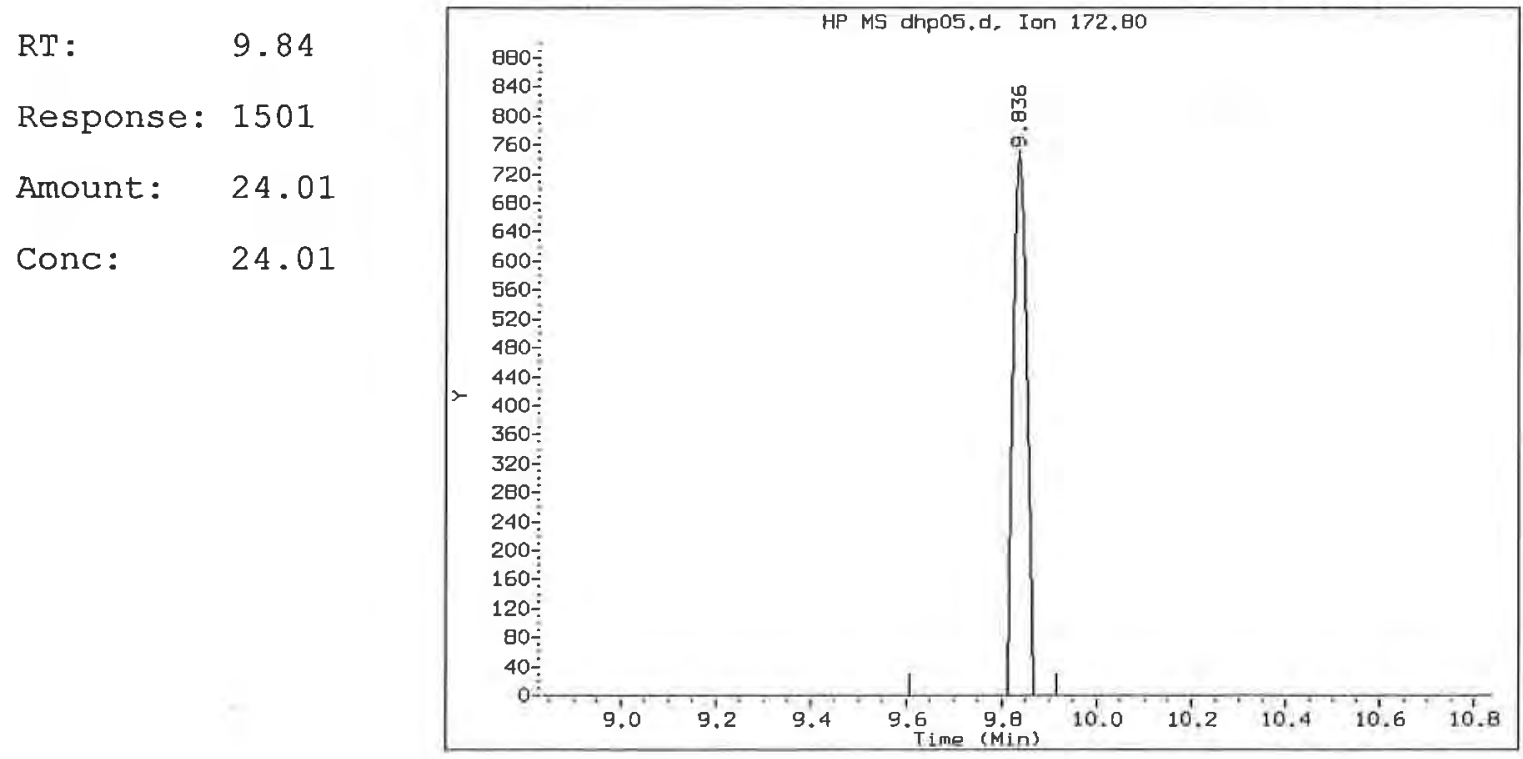

File Uploaded By: jh2

Manual Integration Reason: Analyte not identified by the data system 


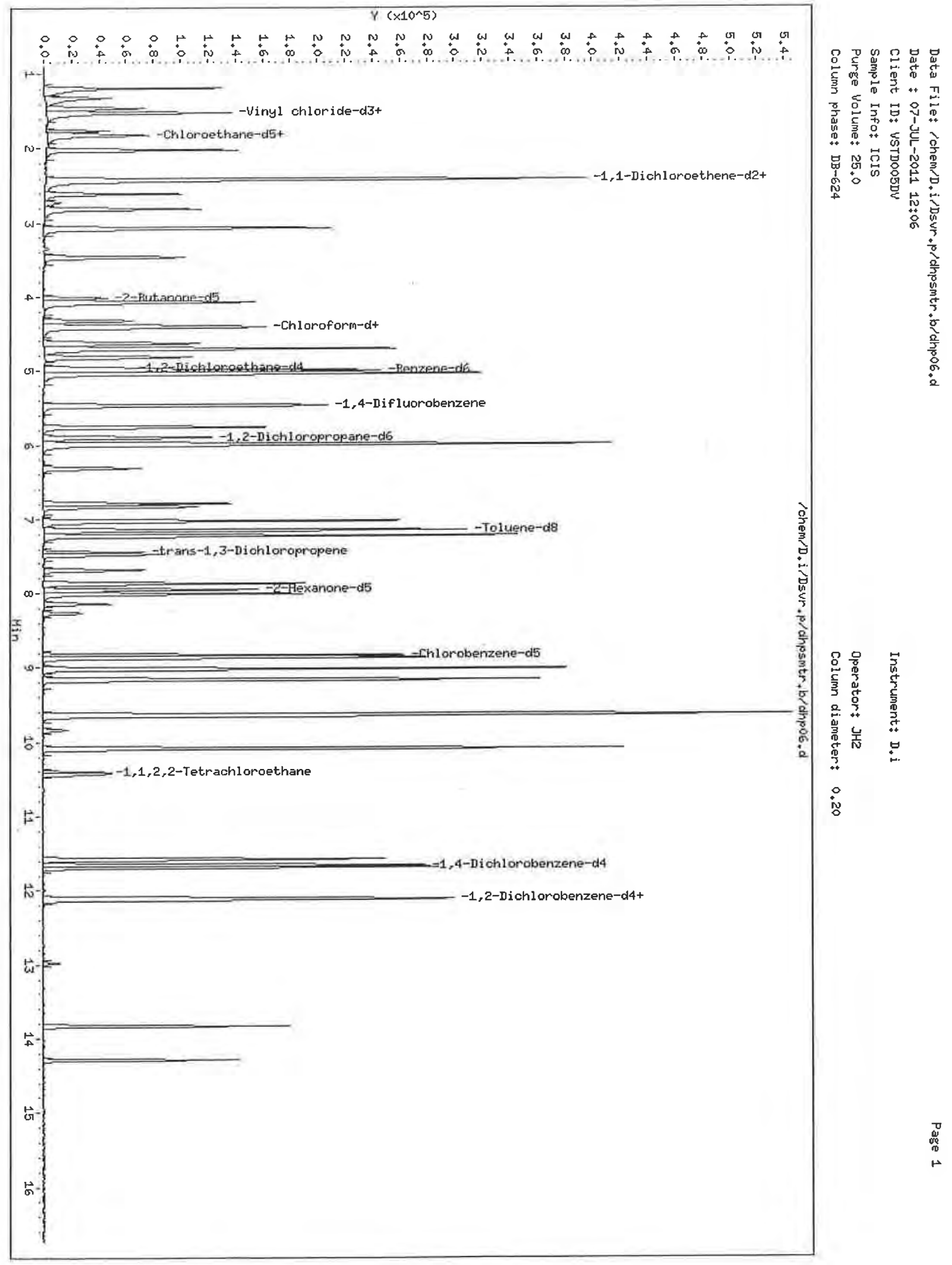




\section{TestAmerica Burlington}

LOW CONCENTRATION VOLATILE QUANTITATION, REPORT

Data file : /chem/D.i/Dsvr.p/dhpsmtr.b/dhp06.d

Lab Smp Id: ICIS

Inj Date : 07-JUL-2011 12:06

Operator : JH2

Client smp ID: VSTD005DV

Smp Info : ICIS

Misc Info : 1,5

Comment :

Method : /chem/D.i/Dsvr.p/dhpsmtr.b/somtr5t.m

Meth Date : 07-Jul-2011 19:24 jh2 Quant Type: ISTD

Cal Date : 07-JUL-2011 12:06 Cal File: dhp06.d

Als bottle: 82

Dil Eactor: 1.00000

Integrator: HP RTE

Target Version: 3.50

Processing Host: chemsvr6

Concentration Formula: Amt * DF * Uf/Vo * CpndVariable

\begin{tabular}{clc} 
Name & Value & Description \\
\hline DF & 1.00000 & Dilution Factor \\
Uf & 1.00000 & ng unit correction factor \\
Vo & 25.00000 & Sample volume purged (mL)
\end{tabular}

Cpnd Variable

Local Compound Variable

Compounds
1 Dichlorodifluoronethane
2 Chloromethane
3 Vinyl chloride-d3
4 Vinyl chloride
5 Bromomethane
6 Chloroethane-d5
7 Chloroethane
8 Trichlorofluoromethane
9 1,1-Dichloroethene-d2
10 1,1-Dichloroethene
11 1,1,2-Trichloro-1,2,2-trifluo
12 Acetone
13 Carbon disulfide
14 Methyl acetate
15 Methylene chloride

\begin{tabular}{c} 
QUANT SIG \\
MASS \\
\hline$=15$ \\
85 \\
50 \\
65 \\
62 \\
94 \\
69 \\
64 \\
101 \\
63 \\
96 \\
101 \\
43 \\
76 \\
43 \\
84
\end{tabular}

\begin{tabular}{|c|c|c|}
\hline $\mathrm{RT}$ & EXP RT & REL RT \\
\hline$=\pi$ & 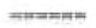 & 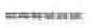 \\
\hline 1.320 & 1.320 & $(0.241)$ \\
\hline 1.454 & 1.454 & $(0.266)$ \\
\hline 1.521 & 1.521 & $(0.278)$ \\
\hline 1.521 & 1.521 & $(0.278)$ \\
\hline 1.759 & 1.759 & $(0.321)$ \\
\hline 1.807 & 1.807 & $(0.330\rangle$ \\
\hline 1.832 & 1.832 & $(0.335)$ \\
\hline 2.021 & 2.021 & $(0.369)$ \\
\hline 2.411 & 2.411 & $(0.441)$ \\
\hline 2.423 & 2.423 & $(0.443)$ \\
\hline 2.423 & 2.423 & $(0.443)$ \\
\hline 2.448 & 2.448 & $(0.447)$ \\
\hline 2.612 & 2.612 & $(0.477)$ \\
\hline 2.728 & 2.728 & $(0.499)$ \\
\hline 2.813 & 2.813 & $(0.514)$ \\
\hline
\end{tabular}

\begin{tabular}{|c|c|}
\hline \multicolumn{2}{|c|}{ AMOUNTS } \\
\hline CAL-AMT & $\mathrm{ON}-\mathrm{COL}$ \\
\hline$(\mathrm{ng})$ & ( $n g)$ \\
\hline 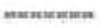 & 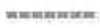 \\
\hline 125.000 & 120 \\
\hline 125.000 & 120 \\
\hline 125.000 & 120 \\
\hline 125.000 & 120 \\
\hline 125.000 & 120 \\
\hline 125.000 & 120 \\
\hline 125.000 & 120 \\
\hline 125.000 & 120 \\
\hline 125.000 & 120 \\
\hline 125.000 & 120 \\
\hline 125.000 & 120 \\
\hline 1250.00 & 1100 \\
\hline 125.000 & 120 \\
\hline 125.000 & 110 \\
\hline 125.000 & 120 \\
\hline
\end{tabular}




\section{Compounds}

16 trans-1,2-Dichloroethene

17 Methyl tert-butyl ether

18 1,1-Dichloroethane

$\$ 19$ 2-Butanone-d5

20 cis-1,2-Dichloroethene

21 2-Butanone

22 Bromochloromethane

$\$ 23$ Chloroform-d

24 Chloroform

25 1,1,1-Trichloroethane

26 Cyclohexane

27 Carbon tetrachloride

\$ 28 1,2-Dichloroethane-d4

$\$ 29$ Benzene-d6

30 Benzene

31 1,2-Dichloroethane

* 32 1,4-Difluorobenzene 33 Trichloroethene

\$ 34 1,2-Dichloropropane-d6 35 Methylcyclohexane

36 1,2-Dichloropropane

37 Bromodichloromethane

38 cis-1, 3-Dichloropropene

39 4-Methyl-2-pentanone

$\$ 40$ Toluene- $d B$

41 Toluene

\$ 42 trans-1,3-Dichloropropene-d4

43 trans-1,3-Dichloropropene

44 1, 1,2-Trichloroethane

45 Tetrachloroethene

$\$ 46$ 2-Hexanone-d5

47 2-Hexanone

48 Dibromochloromethane

49 1,2-Dibromoethane

* 50 Chlorobenzene-d5

51 Chlorobenzene

52 Ethylbenzene

$53 \mathrm{~m}, \mathrm{p}$-xylene

54 styrene

55 o-xylene

56 Bromoform

57 Isopropylbenzene

$\$ 581,1,2,2$-Tetrachloroethane-d2

59 1, 1,2, 2-Tetrachloroethane

60 1,3-Dichlorobenzene

* 61 1,4-Dichlorobenzene-d4.

62 1,4-Dichlorobenzene

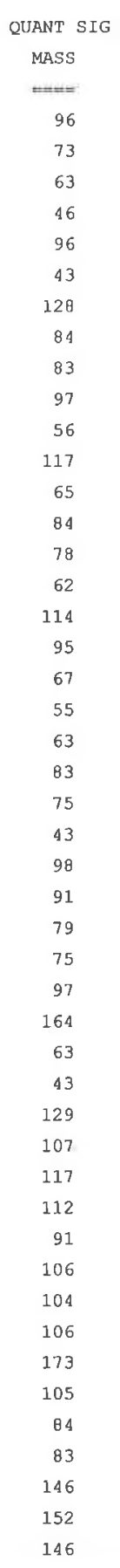

AMOUNTS

CAL-AMT ON-COL,

$\begin{array}{ll}1 \mathrm{ng}) & (\mathrm{ng}) \\ 125.000 & =0\end{array}$

RESPONSE

56580

55036

111871

75473

56499

89175

18877

94559

$8897 \mathrm{~B}$

78842

143812

76170

37533

235875

242195

52079

188233

56914

58598

122648

58533

49061

64773

218529

232618

270555

37000

45468

25569

48902

58453

153296

28804

22012

159591

165081

308475

124379

167227

116094

9703

323391

26651

26678

120760

76959

124310
$125.000 \quad 120$

$125.000 \quad 120$

$125.000 \quad 120$

$1250.00 \quad 1200$

$125.000 \quad 120$

$1250.00 \quad 1300$

$125.000 \quad 120$

$125.000 \quad 120$

$125.000 \quad 120$

$125.000 \quad 120$

$125.000 \quad 130$

$125.000 \quad 130$

$125.000 \quad 120$

$125.000 \quad 130$

$125.000 \quad 130$

$125.000 \quad 120$

125.000

$125.000 \quad 120$

$125.000 \quad 120$

$125.000 \quad 130$

$125.000 \quad 130$

$125.000 \quad 130$

$125.000 \quad 130$

$1250.00 \quad 1400$

$125.000 \quad 130$

$125.000 \quad 130$

$125.000 \quad 130$

$125.000 \quad 130$

$.125 .000 \quad 130$

$125.000 \quad 130$

$1250.00 \quad 1400$

$1250.00 \quad 1300$

$125.000 \quad 130$

$125.000 \quad 120$

125.000

125.000

125.000

125.000

125.000

125.000

125.000

125.000

125.000

125.000

125.000

125.000

125.000
120

130

130

130

130

130

140

130

130

130

120 
Data File: /chem/D.i/Dsvr.p/dhpsmtr.b/dhp06.d

Page 3

Report Date: 07-Jul-2011 19:24

\begin{tabular}{|c|c|c|c|c|c|c|}
\hline \multirow{4}{*}{ Compounds } & \multirow{2}{*}{\multicolumn{2}{|c|}{ QUANT SIG }} & \multirow[b]{3}{*}{ REL RT } & \multirow[b]{3}{*}{ RESPONSE } & \multicolumn{2}{|c|}{ AMOUNTS } \\
\hline & & & & & CAL-AMT & $\mathrm{ON}-\mathrm{COL}$ \\
\hline & MASS & $\mathrm{RT}$ & & & ng) & ng) \\
\hline & $=$ & $\Rightarrow$ & 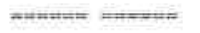 & 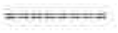 & perentres & entrert=s \\
\hline$\$ 63$ 1,2-Dichlorobenzene-d 4 & 152 & 12.104 & $12.104(1.038)$ & 62691 & 125.000 & 120 \\
\hline 64 1,2-Dichlorobenzene & 146 & 12.129 & $12.129(1.040)$ & 108304 & 125.000 & 130 \\
\hline 65 1,2-Dibromo-3-chloropropane & 75 & 12.982 & $12.982(1.113)$ & 2964 & 125.000 & 150 \\
\hline $661,2,4$-Trichlorobenzene & 180 & 13.829 & $13.829 \quad(1.186)$ & 52828 & 125.000 & 130 \\
\hline $671,2,3$-Trichlorobenzene & 180 & 14.287 & $14.287 \quad(1.225)$ & 40161 & 125.000 & 130 \\
\hline
\end{tabular}




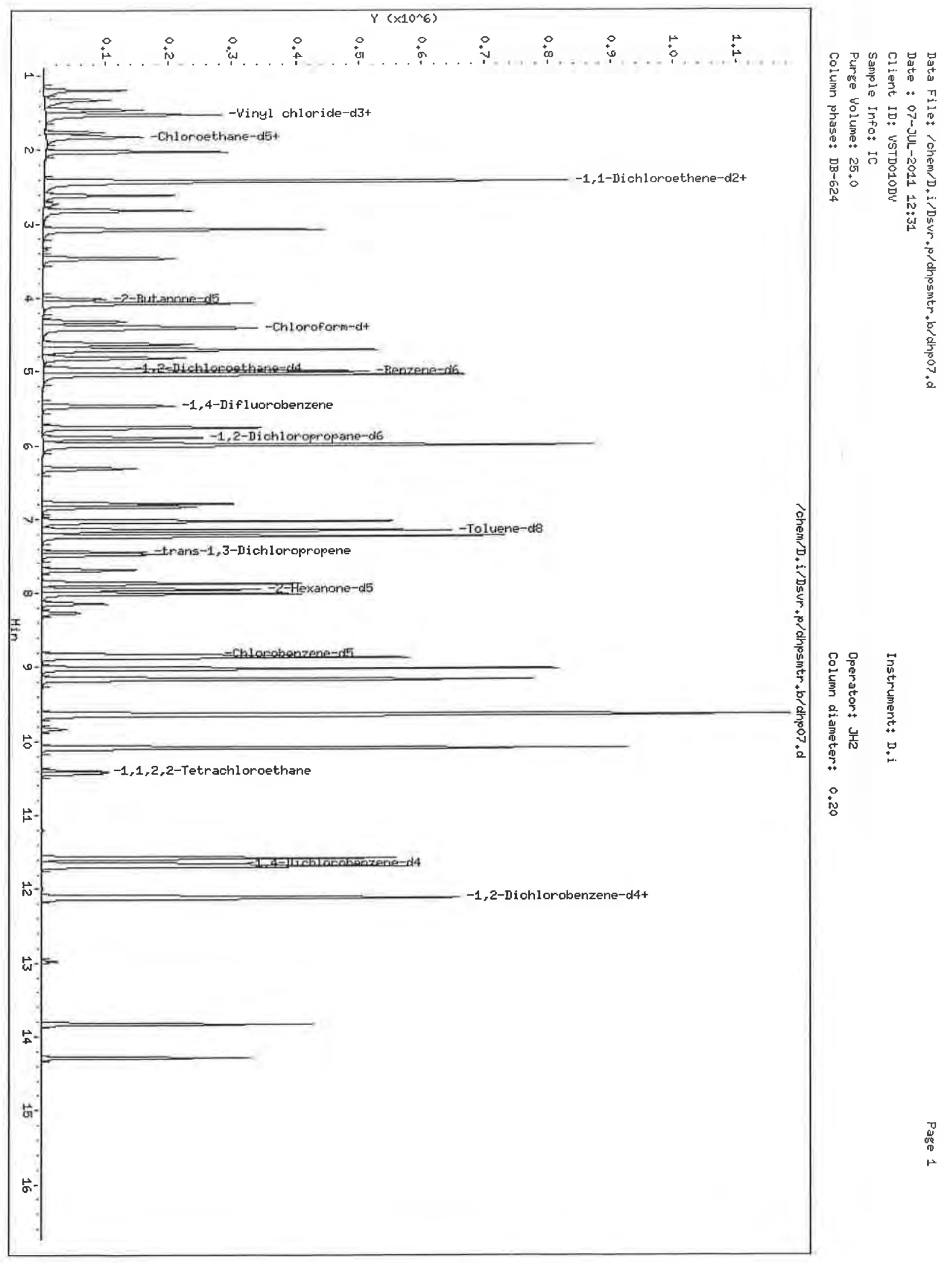




\section{TestAmerica Burlington}

LOW CONCENTRATION VOLATILE QUANTITATION REPORT

Data file: /chem/D.i/Dsvr.p/dhpsmtr.b/dhp07.d

Lab Smp Id: IC

Inj Date : 07-JUL-2011 12:31

Operator : JH2

Smp Info : IC

Misc Info : 1,5

Comment :

Method : /chem/D.i/Dsvr.p/dhpsmtr.b/somtr5t.m

Meth Date : 07-Jul-2011 19:24 jh2 Quant Type: ISTD

Cal Date : 07-JUL-2011 12:31

Als bottle: 83

Dil Factor: 1.00000

Integrator: HP RTE

Target Version: 3.50

Client Smp ID: VSTD010DV

Processing Host: chemsvr6

Concentration Formula: Amt * DF * Uf/Vo * CpndVariable

$\begin{array}{clc}\text { Name } & \text { Value } & \text { Description } \\ \text { DF } & 1.00000 & \text { Dilution Factor } \\ \text { Uf } & 1.00000 & \text { ng unit correction factor } \\ \text { Vo } & 25.00000 & \text { Sample volume purged (mL) }\end{array}$

Local Compound Variable

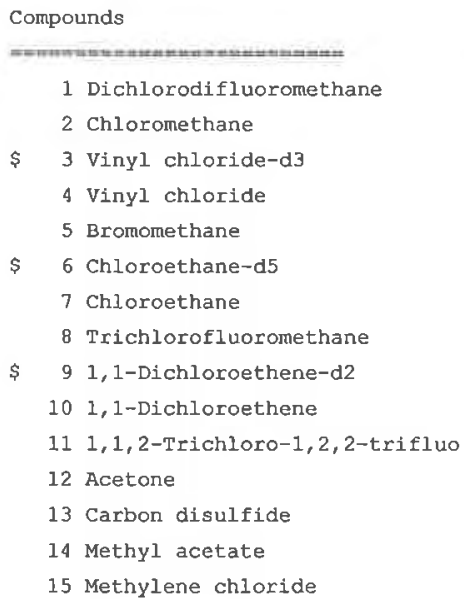




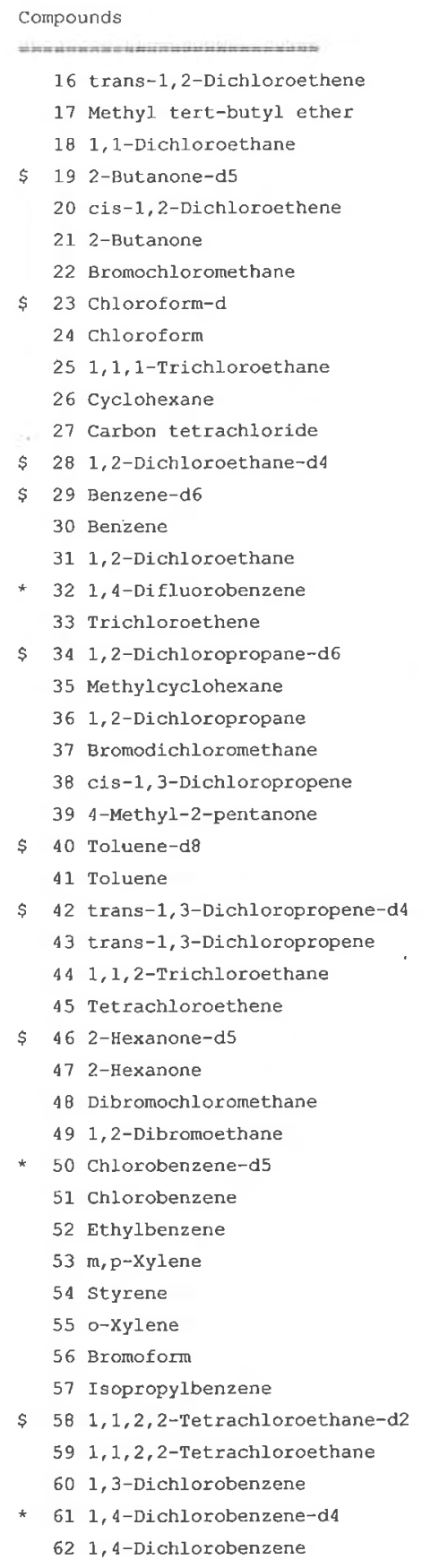

\begin{tabular}{|c|c|c|c|c|c|c|}
\hline \multirow[b]{2}{*}{ QUANT SIG } & \multirow[b]{3}{*}{ RT } & \multirow[b]{3}{*}{ EXP RT } & \multirow[b]{3}{*}{ REL RT } & \multirow[b]{3}{*}{ RESPONSE } & \multicolumn{2}{|c|}{ AMOUNTS } \\
\hline & & & & & CAL-AMT & $\mathrm{ON}-\mathrm{COL}$ \\
\hline MASS & & & & & ( $\mathrm{ng})$ & ng) \\
\hline$m=$ & $=$ & денसне & p=enu= & 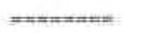 & 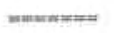 & prowere \\
\hline 96 & 3.069 & 3.069 & $(0.562)$ & 119063 & 250.000 & 250 \\
\hline 73 & 3.075 & 3.082 & $(0.563)$ & 121562 & 250.000 & 260 \\
\hline 63 & 3.466 & 3.466 & $(0.634)$ & 235201 & 250.000 & 250 \\
\hline 46 & 4.014 & 4.020 & $(0.734)$ & 167398 & 2500.00 & 2700 \\
\hline 96 & 4.069 & 4.069 & $(0.745)$ & 118853 & 250.000 & 260 \\
\hline 43 & 4.081 & 4.081 & $(0.747)$ & 186280 & 2500.00 & 2600 \\
\hline 128 & 4.325 & 4.325 & $(0.791)$ & 37569 & 250.000 & 240 \\
\hline 84 & 4.392 & 4.392 & $(0.804)$ & 196014 & 250.000 & 250 \\
\hline 83 & 4.417 & 4.417 & $(0.808)$ & 187732 & 250.000 & 250 \\
\hline 97 & 4.630 & 4.630 & $(0.524)$ & 166670 & 250.000 & 250 \\
\hline 56 & 4.703 & 4.703 & $(0.533)$ & 309331 & 250.000 & 270 \\
\hline 117 & 4.813 & 4.819 & $(0.545)$ & 161186 & 250.000 & 250 \\
\hline 65 & 4.959 & 4.959 & $(0.907)$ & 76448 & 250.000 & 250 \\
\hline 84 & 4.990 & 4.990 & $(0.565)$ & 494102 & 250.000 & 250 \\
\hline 78 & 5.032 & 5.032 & $(0.570)$ & 508412 & 250.000 & 250 \\
\hline 62 & 5.038 & 5.039 & $(0.922)$ & 108082 & 250.000 & 250 \\
\hline 114 & 5.465 & 5.471 & $(1.000)$ & 190530 & 125.000 & \\
\hline 95 & 5.758 & 5.758 & $(0.652)$ & 120753 & 250.000 & 240 \\
\hline 67 & 5.904 & 5.904 & $(0.669)$ & 124061 & 250.000 & 240 \\
\hline 55 & 5.989 & 5.990 & $(0.678)$ & 257323 & 250.000 & 260 \\
\hline 63 & 6.002 & 6.002 & $(0.680)$ & 126084 & 250.000 & 250 \\
\hline 83 & 6.313 & 6.313 & $(0.715)$ & 103839 & 250.000 & 250 \\
\hline 75 & 6.837 & 6.837 & $(0.774)$ & 140970 & 250.000 & 270 \\
\hline 43 & 7.026 & 7.026 & $(0.796)$ & 474597 & 2500.00 & 2800 \\
\hline 98 & 7.154 & 7.154 & $(0.810)$ & 490073 & 250.000 & 260 \\
\hline 91 & 7.227 & 7.227 & $(0.818)$ & 574773 & 250.000 & 260 \\
\hline 79 & 7.446 & 7.447 & $(0.843)$ & 84810 & 250.000 & 270 \\
\hline 75 & 7.477 & 7.477 & $(0.847)$ & 102777 & 250.000 & 280 \\
\hline 97 & 7.684 & 7.684 & $(0.870)$ & 53061 & 250.000 & 250 \\
\hline 164 & 7.867 & 7.867 & $(0.891)$ & 104927 & 250.000 & 250 \\
\hline 63 & 7.952 & 7.946 & $(0.901)$ & 130743 & 2500.00 & 2900 \\
\hline 43 & 8.007 & 0.007 & $(0.907)$ & 335715 & 2500.00 & 2700 \\
\hline 129 & 8.141 & 8.142 & $(0.922)$ & 60404 & 250.000 & 250 \\
\hline 107 & 8.263 & 8.264 & $(0.936)$ & 49404 & 250.000 & 260 \\
\hline 117 & 8.830 & 0.830 & $(1.000)$ & 170070 & 125.000 & \\
\hline 112 & 8.867 & 8.867 & $(1.004)$ & 354056 & 250.000 & 250 \\
\hline 91 & 9.019 & 9.019 & $(1.021)$ & 669343 & 250.000 & 270 \\
\hline 106 & 9.160 & 9.160 & $(1.037)$ & 270935 & 250.000 & 270 \\
\hline 104 & 9.647 & 9.647 & $(1.093)$ & 372915 & 250.000 & 280 \\
\hline 106 & 9.629 & 9.629 & $(1.090)$ & 254902 & 250.000 & 280 \\
\hline 173 & 9.836 & 9.836 & $(0.844)$ & 19994 & 250.000 & 250 \\
\hline 105 & 10.086 & 10.086 & $(1.142)$ & 715478 & 250.000 & 290 \\
\hline 84 & 10.403 & 10.403 & $(1.178)$ & 58030 & 250.000 & 260 \\
\hline 83 & 10.434 & 10.434 & $(1.182)$ & 55885 & 250.000 & 250 \\
\hline 146 & 11.580 & 11.580 & $(0.993)$ & 272872 & 250.000 & 260 \\
\hline 152 & 11.659 & 11.659 & $(1.000)$ & 83731 & 125.000 & \\
\hline 146 & 11.690 & 11.690 & $(1.003)$ & 274868 & 250.000 & 240 \\
\hline
\end{tabular}


Data File: /chem/D.i/Dsvr.p/dhpsmtr.b/dhp07.d

Page 3 Report Date: 07-Jul-2011 19:24

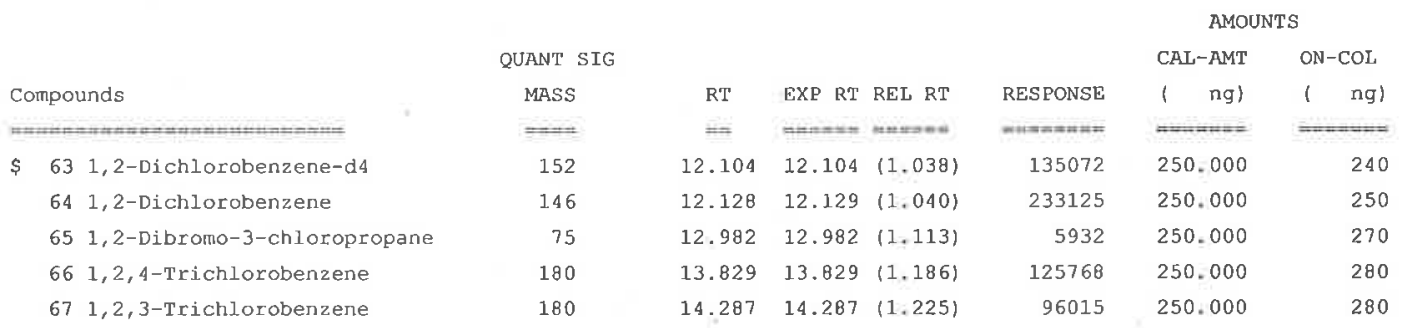




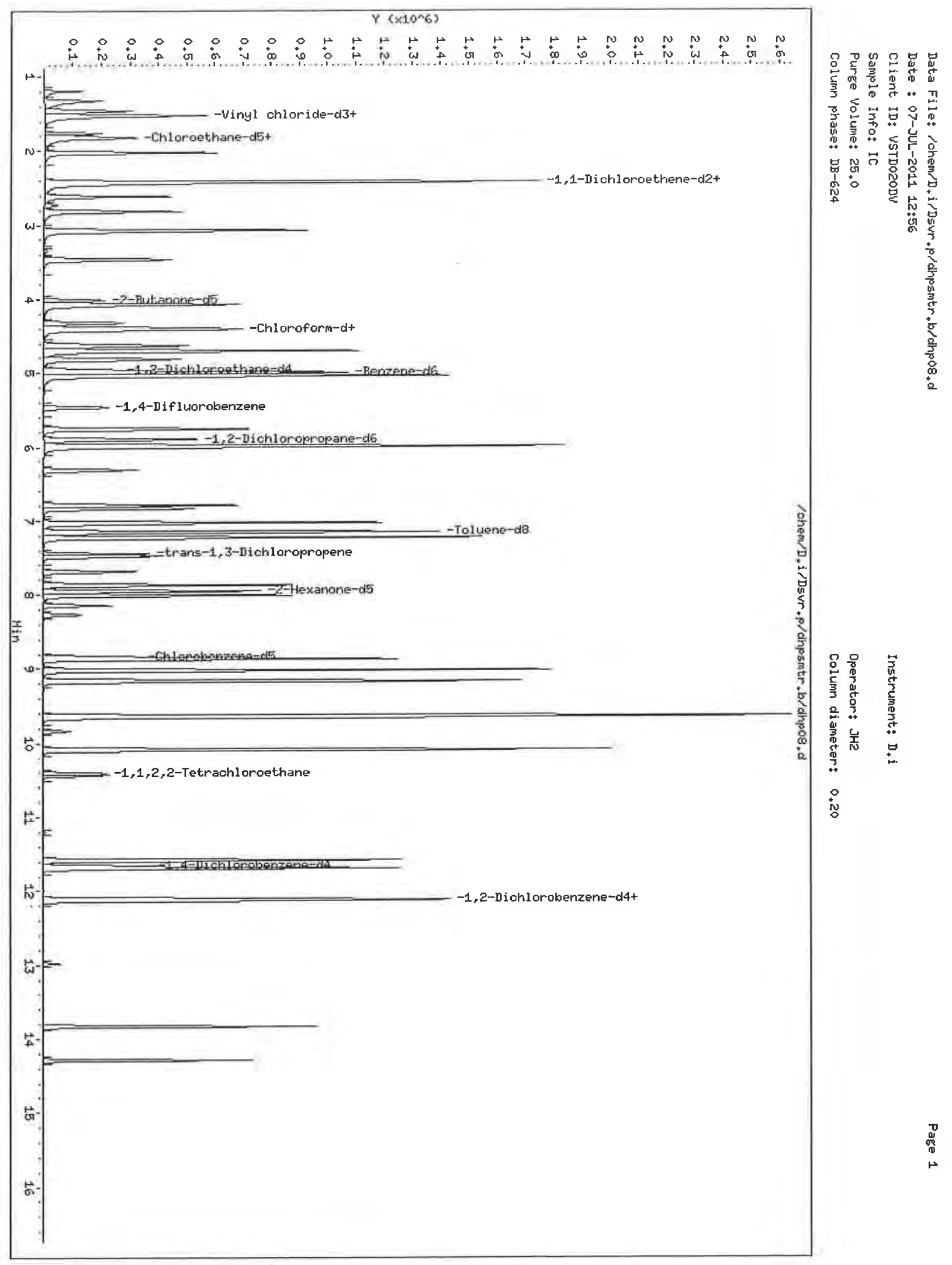




\section{TestAmerica Burlington}

LOW CONCENTRATION VOLATILE QUANTITATION REPORT

Data file : /chem/D.i/Dsvr.p/dhpsmtr.b/dhp08.d

Lab Smp Id: IC

Inj Date : 07-JUL-2011 12:56

Operator : JH2

Smp Info : IC

Misc Info : 1,5

Comment :

Method : /chem/D.i/Dsvr.p/dhpsmtr.b/somtr5t.m

Meth Date : 07-Jul-2011 19:24 jh2 Quant Type: ISTD

Cal Date : 07-JUL-2011 12:56

Als bottle: 84

Dil Factor: 1.00000

Integrator: HP RTE

Target Version: 3.50

Client Smp ID: VSTD020DV

Processing Host: chemsvr6

Concentration Formula: Amt * DF * Uf/Vo * CpndVariable

$\begin{array}{clc}\text { Name } & \text { Value } & \text { Description } \\ \text { DF } & 1.00000 & \text { Dilution Factor } \\ \text { Uf } & 1.00000 & \text { ng unit correction factor } \\ \text { Vo } & 25.00000 & \text { Sample volume purged (mL) }\end{array}$

Cpnd Variable

Local Compound Variable

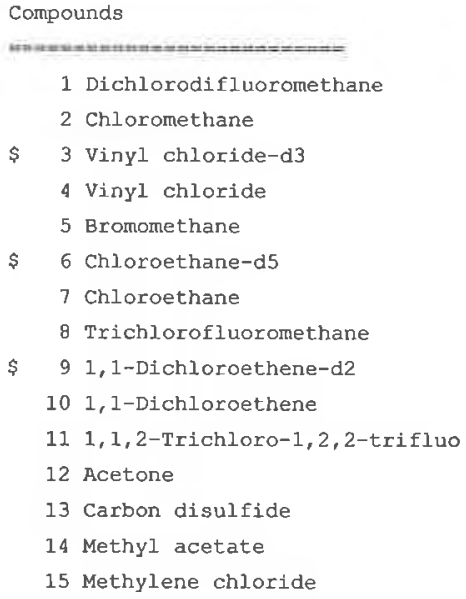

Cal File: dhp08.d

Calibration Sample, Level: 5

Compound Sublist: all.sub 
Data File: /chem/D.i/Dsvr.p/dhpsmtr.b/dhp08.d

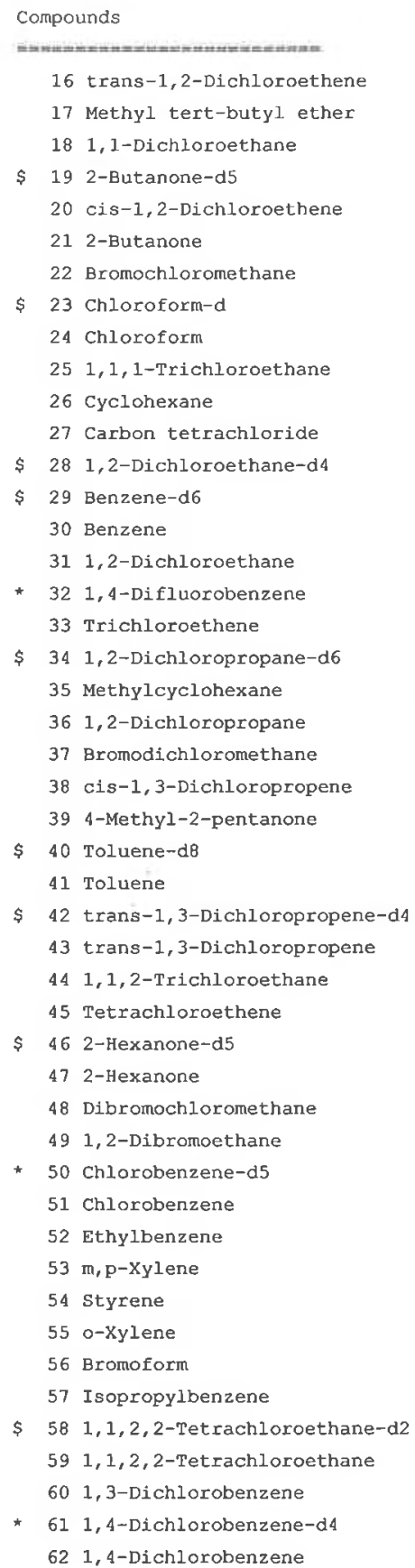

\begin{tabular}{|c|c|c|c|c|c|c|}
\hline \multicolumn{5}{|c|}{ QUANT SIG } & CAL-AMT & $\mathrm{ON}-\mathrm{COL}$ \\
\hline MASS & RT & EXP RT & REL RT & RESPONSE & ng) & ng) \\
\hline$== \pm$ & $=$ & 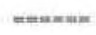 & mankes & 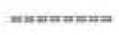 & 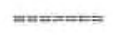 & 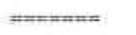 \\
\hline 96 & 3.069 & 3.069 & $(0.561)$ & 248803 & 500.000 & 500 \\
\hline 73 & 3.082 & 3.082 & $(0.563)$ & 258880 & 500.000 & $530(\mathrm{~A})$ \\
\hline 63 & 3.466 & 3.466 & $(0.633)$ & 494377 & 500.000 & 500 \\
\hline 46 & 4.020 & 4.020 & $(0.735)$ & 350284 & 5000.00 & $5200(A)$ \\
\hline 96 & 4.069 & 4.069 & $(0.744)$ & 248802 & 500.000 & 510 (A) \\
\hline 43 & 4.081 & 4.081 & $(0.746)$ & 392312 & 5000.00 & $5100(\mathrm{~A})$ \\
\hline 128 & 4.325 & 4.325 & $(0.791)$ & 82089 & 500.000 & 500 \\
\hline 84 & 4.392 & 4. 392 & $(0.803)$ & 409229 & 500.000 & 490 \\
\hline 83 & 4.417 & 4.417 & $(0.807)$ & 388397 & 500.000 & 490 \\
\hline 97 & 4.630 & 4.630 & $(0.524)$ & 355388 & 500.000 & 490 \\
\hline 56 & 4.703 & 4.703 & $(0.532)$ & 647503 & 500.000 & $520(A)$ \\
\hline 117 & 4.819 & 4.819 & $(0.545)$ & 340520 & 500.000 & 490 \\
\hline 65 & 4.959 & 4.959 & $(0.906)$ & 158193 & 500.000 & 480 \\
\hline 84 & 4.996 & 4.990 & $(0.565)$ & 1029105 & 500.000 & 480 \\
\hline 78 & 5.038 & 5.032 & $(0.570)$ & 1061044 & 500.000 & 480 \\
\hline 62 & 5.045 & 5.039 & $(0.922)$ & 232139 & 500.000 & 510 (A) \\
\hline 114 & 5.471 & 5.471 & $(1.000)$ & 203576 & 125.000 & \\
\hline 95 & 5.758 & 5.758 & $(0.652)$ & 257754 & 500.000 & 480 \\
\hline 67 & 5.904 & 5.904 & $(0.668)$ & 260882 & 500.000 & 470 \\
\hline 55 & 5.990 & 5.990 & $(0.678)$ & 550877 & 500.000 & $510(A)$ \\
\hline 63 & 6.002 & 6.002 & $(0.679)$ & 260033 & 500.000 & 480 \\
\hline 83 & 6.313 & 6.313 & $(0.714)$ & 224219 & 500.000 & $500(A)$ \\
\hline 75 & 6.837 & 6.837 & $(0.774)$ & 306681 & 500.000 & $550(A)$ \\
\hline 43 & 7.032 & 7.026 & $(0.796)$ & 1005397 & 5000.00 & $5400(A)$ \\
\hline 98 & 7.154 & 7.154 & $(0.810)$ & 1029967 & 500.000 & $500(A)$ \\
\hline 91 & 7.227 & 7.227 & $(0.818)$ & 1222776 & 500.000 & $500(A)$ \\
\hline 79 & 7.447 & 7.447 & $(0.843)$ & 193342 & 500.000 & $570(\mathrm{~A})$ \\
\hline 75 & 7.483 & 7.477 & $(0.847)$ & 225130 & 500.000 & $570(A)$ \\
\hline 97 & 7.684 & 7.684 & $(0.870)$ & 115486 & 500.000 & 500 \\
\hline 164 & 7.867 & 7.867 & $(0.890)$ & 219595 & 500.000 & 490 \\
\hline 63 & 7.953 & 7.946 & $(0.900)$ & 288505 & 5000.00 & $6000(A)$ \\
\hline 43 & 8.007 & 8.007 & $(0.906)$ & 707446 & 5000.00 & $5300(A)$ \\
\hline 129 & B. 142 & 8.142 & $(0.921)$ & 140620 & 500.000 & 530 (A) \\
\hline 107 & 8.270 & 8.264 & $(0.936)$ & 106378 & 500.000 & $520(\mathrm{~A})$ \\
\hline 117 & 8.837 & 8.830 & $(1.000)$ & 183370 & 125.000 & \\
\hline 112 & 8.867 & 8.867 & $(1.003)$ & 748659 & 500.000 & 490 \\
\hline 91 & 9.019 & 9.019 & $(1.021)$ & 1451019 & 500.000 & $540(A)$ \\
\hline 106 & 9.160 & 9.160 & $(1.037)$ & 587917 & 500.000 & $540(\mathrm{~B})$ \\
\hline 104 & 9.647 & 9.647 & $(1.092)$ & 823832 & 500.000 & 570 (A) \\
\hline 106 & 9.635 & 9.629 & $(1.090)$ & 554094 & 500.000 & $560(A)$ \\
\hline 173 & 9.836 & 9.836 & $(0.844)$ & 50048 & 500.000 & $560(\mathrm{~A})$ \\
\hline 105 & 10.086 & 10.086 & $(1.141)$ & 1556065 & 500.000 & $580(A)$ \\
\hline 84 & 10.403 & 10.403 & $(1.177)$ & 123807 & 500.000 & $520(A)$ \\
\hline 83 & 10.434 & 10.434 & $(1.181)$ & 120239 & 500.000 & $500(A)$ \\
\hline 146 & 11.580 & 11.580 & $(0.993)$ & 603266 & 500.000 & $530(\mathrm{~A})$ \\
\hline 152 & 11.659 & 11.659 & $(1.000)$ & 91561 & 125.000 & (Q) \\
\hline 146 & 11.690 & 11.690 & $(1.003)$ & 589778 & 500.000 & 480 \\
\hline
\end{tabular}


Data File: /chem/D.i/Dsvr.p/dhpsmtr.b/dhp08.d

Page 3

Report Date: 07-Jul-2011 19:24

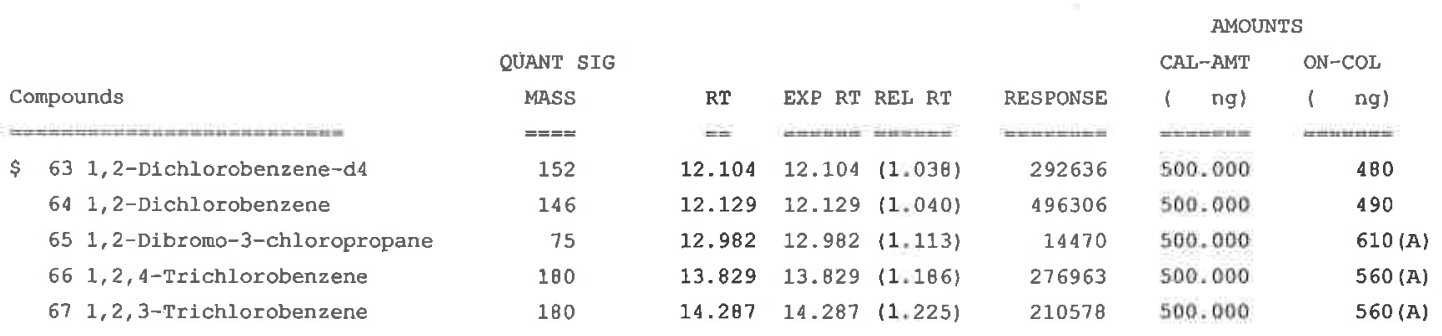

\section{QC Flag Legend}

A - Target compound detected but, quantitated amount exceeded maximum amount.

Q - Qualifier signal failed the ratio test. 
$7 A$ - FORM VII VOA-1

VOLATILE CONTINUING CALIBRATION DATA

Lab Name: TESTAMERICA BURLINGTON

Contract: $8 \mathrm{E}-00302$

Lab Code: STLV Case No.: SAVANN Mod. Ref No.:

SDG No.: 200-6522

Instrument ID: D.i

Calibration Date: 08/15/2011 Time: 0826

Lab File Id: DHPK04.D

Init. Calib. Date(s): 07/07/2011 07/07/2011

EPA Sample No. (VSTD\#\#\#\#): VSTD005DH

Init. Calib. Time(s): $1117 \quad 1256$

Heated Purge: $(\mathrm{Y} / \mathrm{N})$

GC Column:

ID: $0.20(\mathrm{~mm})$ Length: 25 (m)

Purge Volume: 25.0 $(\mathrm{mL})$

\begin{tabular}{|c|c|c|c|c|c|}
\hline COMPOUND & $\overline{\mathrm{RRF}}$ & RRF5.0 & $\begin{array}{l}\text { MIN } \\
\text { RRF }\end{array}$ & $\circ \mathrm{D}$ & MAX $\because D$ \\
\hline Dichlorodifluoromethane & 0.403 & 0.532 & 0.010 & 32.1 & 40.0 \\
\hline Chloromethane & 0.687 & 0.737 & 0.010 & 7.4 & 40.0 \\
\hline Vinyl chloride & 0.496 & 0.544 & 0.010 & 9.6 & 30.0 \\
\hline Bromomethane & 0.209 & 0.194 & 0.010 & -7.4 & 30.0 \\
\hline Chloroethane & 0.272 & 0.281 & 0.010 & 3.4 & 40.0 \\
\hline Trichlorofluoromethane & 0.606 & 0.647 & 0.010 & 6.9 & 40.0 \\
\hline 1,1-Dichloroethene & 0.305 & 0.319 & 0.010 & 4.9 & 30.0 \\
\hline 1,1,2-Trichloro-1,2,2-trifluoroethane & 0.355 & 0.377 & 0.010 & 6.2 & 40.0 \\
\hline Acetone & 0.036 & 0.033 & 0.010 & -7.9 & 40.0 \\
\hline Carbon disulfide & 0.742 & 0.805 & 0.010 & 8.5 & 40.0 \\
\hline Methyl acetate & 0.086 & 0.088 & 0.010 & 1.8 & 40.0 \\
\hline Methylene Chloride & 0.250 & 0.263 & 0.010 & 4.9 & 40.0 \\
\hline trans-1,2-Dichloroethene & 0.308 & 0.307 & 0.010 & -0.4 & 40.0 \\
\hline Methyl tert-butyl ether & 0.302 & 0.303 & 0.010 & 0.1 & 40.0 \\
\hline 1,1-Dichloroethane & 0.609 & 0.626 & 0.010 & 2.8 & 30.0 \\
\hline cis-1,2-Dichloroethene & 0.302 & 0.313 & 0.010 & 3.6 & 40.0 \\
\hline 2-Butanone & 0.047 & 0.047 & 0.010 & -0.7 & 40.0 \\
\hline Bromochloromethane & 0.101 & 0.105 & 0.010 & 3.4 & 30.0 \\
\hline Chloroform & 0.485 & 0.497 & 0.010 & 2.3 & 30.0 \\
\hline 1,1,1-Trichloroethane & 0.494 & 0.506 & 0.010 & 2.2 & 30.0 \\
\hline Cyclohexane & 0.850 & 0.942 & 0.010 & 10.8 & 40.0 \\
\hline Carbon tetrachloride & 0.472 & 0.485 & 0.010 & 2.6 & 30.0 \\
\hline Benzene & 1.515 & 1.552 & 0.010 & 2.4 & 30.0 \\
\hline 1,2-Dichloroethane & 0.282 & 0.297 & 0.010 & 5.6 & 30.0 \\
\hline Trichloroethene & 0.368 & 0.369 & 0.010 & 0.2 & 30.0 \\
\hline Methylcyclohexane & 0.737 & 0.803 & 0.010 & 8.9 & 40.0 \\
\hline
\end{tabular}

Report 1,4-Dioxane for Low/Medium VOA analysis only 
$7 \mathrm{~B}$ - FORM VII VOA-2

VOLATILE CONTINUING CALIBRATION DATA

Lab Name: TESTAMERICA BURLINGTON

Lab Code: STLV Case No.: SAVANN Mod. Ref No.:

Contract: $8 \mathrm{E}-00302$
Instrument ID: D.i
Lab File Id: DHPKO4.D

EPA Sample No. (VSTD\#\#\#\#): VSTD005DH

Heated Purge: ( $\mathrm{Y} / \mathrm{N}) \quad \mathrm{N}$

N GC Column: $\mathrm{DB}-624$
Calibration Date: 08/15/2011 Time: 0826 Init. Calib. Date(s): 07/07/2011 07/07/2011

Init. Calib. Time(s): $1117 \quad 1256$

ID: $0.20(\mathrm{~mm})$ Length: 25 (m)

Purge Volume: 25.0

$(\mathrm{mL})$

\begin{tabular}{|c|c|c|c|c|c|}
\hline COMPOUND & $\overline{\mathrm{RRF}}$ & RRE5.0 & $\begin{array}{l}\text { MIN } \\
\text { RRF }\end{array}$ & $\because \mathrm{D}$ & $\operatorname{MAX} \because \mathrm{D}$ \\
\hline 1,2-Dichloropropane & 0.366 & 0.394 & 0.010 & 7.7 & 40.0 \\
\hline Bromodichloromethane & 0.305 & 0.317 & 0.010 & 4.1 & 30.0 \\
\hline cis-1,3-Dichloropropene & 0.377 & 0.414 & 0.010 & 9.7 & 30.0 \\
\hline 4-Methyl-2-pentanone & 0.126 & 0.134 & 0.010 & 5.8 & 40.0 \\
\hline Toluene & 1.657 & 1.741 & 0.010 & 5.1 & 30.0 \\
\hline trans-1,3-Dichloropropene & 0.267 & 0.299 & 0.010 & 12.0 & 30.0 \\
\hline 1,1,2-Trichloroethane & 0.158 & 0.172 & 0.010 & 8.8 & 30.0 \\
\hline Tetrachloroethene & 0.306 & 0.321 & 0.010 & 4.9 & 30.0 \\
\hline 2-Hexanone & 0.090 & 0.094 & 0.010 & 4.2 & 40.0 \\
\hline Dibromochloromethane & 0.180 & 0.189 & 0.010 & 5.5 & 30.0 \\
\hline 1,2-Dibromoethane & 0.139 & 0.146 & 0.010 & 4.9 & 40.0 \\
\hline Chlorobenzene & 1.042 & 1.110 & 0.010 & 6.5 & 30.0 \\
\hline Ethylbenzene & 1.825 & 1.962 & 0.010 & 7.5 & 30.0 \\
\hline o-Xylene & 0.674 & 0.758 & 0.010 & 12.4 & 30.0 \\
\hline$m, p-x y l e n e$ & 0.742 & 0.807 & 0.010 & 8.8 & 30.0 \\
\hline Styrene & 0.983 & 1.096 & 0.010 & 11.4 & 30.0 \\
\hline Bromoform & 0.121 & 0.138 & 0.010 & 14.0 & 30.0 \\
\hline Isopropylbenzene & 1.841 & 2.114 & 0.010 & 14.8 & 40.0 \\
\hline $1,1,2,2$-Tetrachloroethane & 0.164 & 0.167 & 0.010 & 2.0 & 30.0 \\
\hline 1,3-Dichlorobenzene & 1.562 & 1.639 & 0.010 & 5.0 & 30.0 \\
\hline 1,4-Dichlorobenzene & 1.686 & 1.745 & 0.010 & 3.5 & 30.0 \\
\hline 1,2-Dichlorobenzene & 1.384 & 1.481 & 0.010 & 7.0 & 30.0 \\
\hline 1,2-Dibromo-3-Chloropropane & 0.032 & 0.038 & 0.010 & 18.7 & 40.0 \\
\hline 1,2,4-Trichlorobenzene & 0.674 & 0.730 & 0.010 & 8.3 & 30.0 \\
\hline 1,2,3-Trichlorobenzene & 0.514 & 0.587 & 0.010 & 14.2 & 30.0 \\
\hline
\end{tabular}


Lab Name: TESTAMERICA BURLINGTON

Contract: $\quad 8 \mathrm{E}-00302$

Lab Code: STLV Case No.: SAVANN Mod. Ref No.:

SDG No.: 200-6522

Instrument ID: D.i

Calibration Date: 08/15/2011 Time: 0826

Lab File Id: DHPKO4.D Init. Calib. Date(s): 07/07/2011 07/07/2011

EPA Sample No. (VSTD\#\#\#\#): VSTD005DH

Init. Calib. Time(s): $1117 \quad 1256$

Heated Purge: $(\mathrm{Y} / \mathrm{N}) \mathrm{N}$

GC Column: DB-624 ID: $0.20(\mathrm{~mm})$ Length: 25

(m)

Purge Volume: 25.0 (mL)

\begin{tabular}{|c|c|c|c|c|c|}
\hline COMPOUND & $\overline{\mathrm{RRF}}$ & RRE5.0 & $\begin{array}{l}\text { MIN } \\
\text { RRF }\end{array}$ & 읗 D & MAX $\div D$ \\
\hline Vinyl Chloride-d3 & 0.436 & 0.396 & 0.010 & -9.1 & 30.0 \\
\hline Chloroethane-d5 & 0.283 & 0.270 & 0.010 & -4.6 & 40.0 \\
\hline 1,1-Dichloroethene-d2 & 0.795 & 0.790 & 0.010 & -0.7 & 30.0 \\
\hline 2-Butanone-d5 & 0.041 & 0.040 & 0.010 & -2.5 & 40.0 \\
\hline Chloroform-d & 0.509 & 0.506 & 0.010 & -0.6 & 30.0 \\
\hline 1,2-Dichloroethane-d4 & 0.203 & 0.204 & 0.010 & 0.3 & 30.0 \\
\hline Benzene-d6 & 1.475 & 1.478 & 0.010 & 0.2 & 30.0 \\
\hline 1,2-Dichloropropane-d6 & 0.374 & 0.367 & 0.010 & -1.9 & 40.0 \\
\hline Toluene-d8 & 1.397 & 1.465 & 0.010 & 4.8 & 30.0 \\
\hline trans-1,3-Dichloropropene-d4 & 0.230 & 0.234 & 0.010 & 1.9 & 30.0 \\
\hline 2-Hexanone-d5 & 0.033 & 0.035 & 0.010 & 8.0 & 40.0 \\
\hline $1,1,2,2$-Tetrachloroethane-d2 & 0.164 & 0.171 & 0.010 & 4.4 & 30.0 \\
\hline 1,2-Dichlorobenzene-d4 & 0.830 & 0.830 & 0.010 & 0.0 & 30.0 \\
\hline
\end{tabular}

Report 1,4-Dioxane-d8 for Low/Medium VOA analysis only 


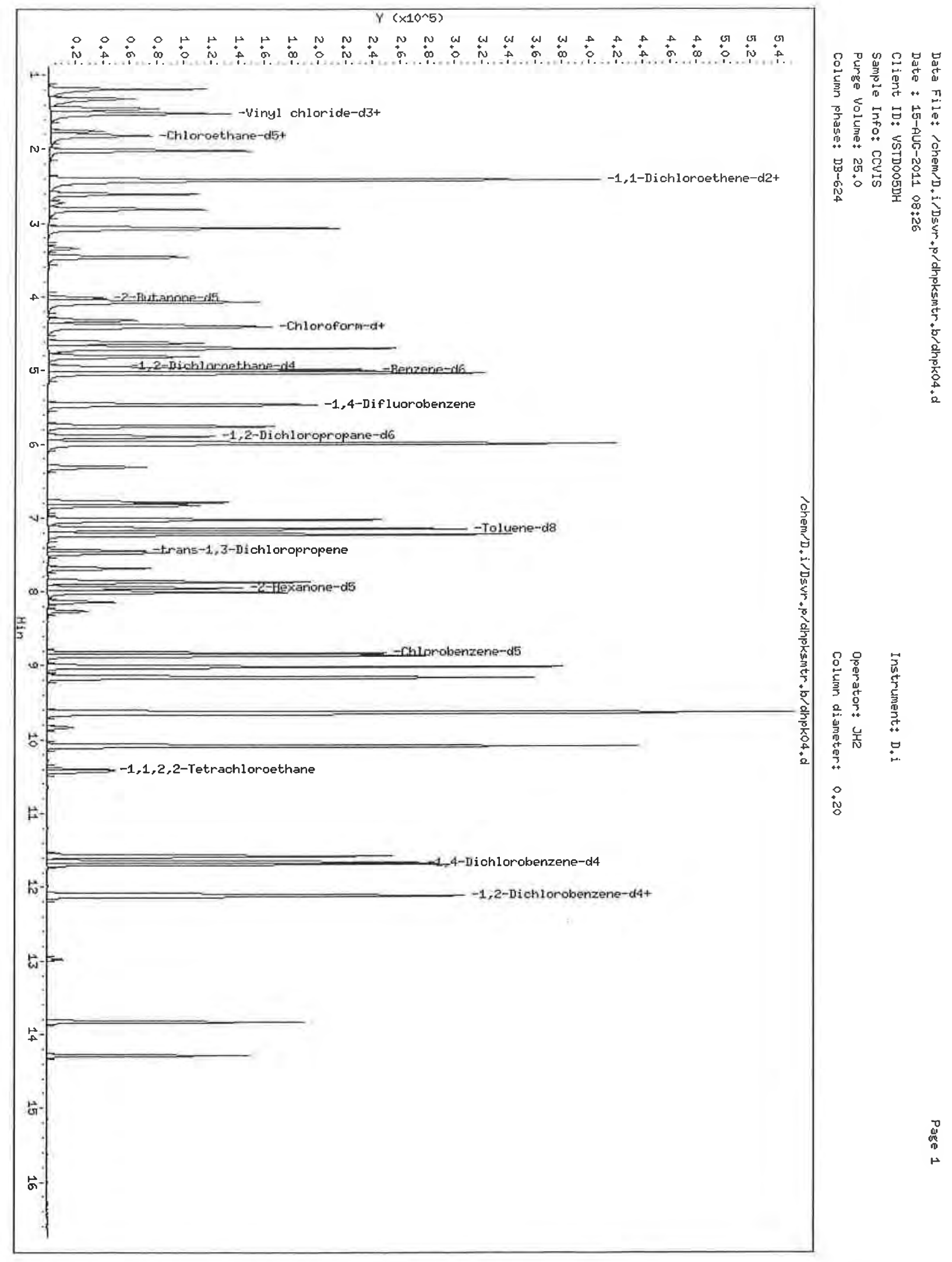


Data File:/chem/D.i/Dsvr.p/dhpksmtr.b/dhpk04.d

Page 1

Report Date: 15-Aug-2011 16:21

TestAmerica Burlington

LOW CONCENTRATION VOLATILE QUANTITATION REPORT

Data file : /chem/D.i/Dsvr.p/dhpksmtr.b/dhpk04.d

Lab Smp Id: CCVIS

Client Smp ID: VSTD005DH

Inj Date : 15-AUG-2011 08:26

Operator : JH2

Inst ID: D.i

Smp Info : CCVIS

Misc Info : 1,5

Comment :

Method : /chem/D.i/Dsvr.p/dhpksmtr.b/somtr5t.m

Meth Date : 15-Aug-2011 16:21 jh2 Quant Type: ISTD

Cal Date : 07-JUL-2011 12:56 Cal File: dhp08.d

Als bottle: 2

Dil Factor: 1.00000

Integrator: HP RTE

Target Version: 3.50

Processing Host: chemsvr6

Concentration Formula: Ant * DF * Uf/Vo * CpndVariable

\begin{tabular}{ccc} 
Name & Value & Description \\
\hline DF & 1.00000 & Dilution Factor \\
Uf & 1.00000 & ng unit correction factor \\
Vo & 25.00000 & Sample volume purged (mL)
\end{tabular}

Cpnd Variable

Local Compound Variable

Compounds
1 Dichlorodifluoromethane
2 Chloronethane
3 Vinyl chloride-d3
4 Vinyl chloride
5 Bromomethane
6 Chloroethane-d5
7 Chloroethane
8 Trichlorofluoromethane
9 1,1-Dichloroethene-d2
10 1, 1-Dichloroethene
11 1, 1,2-Trichloro-1,2,2-trifluo
12 Acetone
13 Carbon disulfide
14 Methyl acetate
15 Methylene chloride

QUANT SIG

MASS
85
50
65
62
94
69
64
101
63
96
101
43
76
43
84

AMOUNTS

CAL-AMT ON-COL

$\begin{array}{lll}\text { RT } & \text { EXP RT } & \text { REL RT } \\ =1.320 & 1.320 & (0.241) \\ 1.454 & 1.454 & (0.266) \\ 1.515 & 1.521 & (0.277) \\ 1.521 & 1.521 & (0.278) \\ 1.758 & 1.759 & (0.322) \\ 1.807 & 1.807 & (0.331) \\ 1.826 & 1.832 & (0.334) \\ 2.021 & 2.021 & (0.370) \\ 2.411 & 2.411 & (0.441) \\ 2.417 & 2.423 & (0.442) \\ 2.423 & 2.423 & (0.443) \\ 2.441 & 2.448 & (0.447) \\ 2.606 & 2.612 & (0.477) \\ 2.722 & 2.728 & (0.498) \\ 2.813 & 2.813 & (0.515)\end{array}$

( ng)

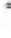

95136

$131897 \quad 125.000 \quad 130$

$\begin{array}{lll}70858 & 125.000 & 110\end{array}$

$97294 \quad 125.000 \quad 140$

$\begin{array}{lll}34625 & 125.000 & 120\end{array}$

$\begin{array}{lll}48242 & 125.000 & 120\end{array}$

$\begin{array}{lll}50318 & 125.000 \quad 130\end{array}$

$\begin{array}{lll}115776 \quad 125.000 & 130\end{array}$

$141216 \quad 125.000 \quad 120$

$57124 \quad 125.000 \quad 130$

$\begin{array}{lll}67458 & 125.000 \quad 130\end{array}$

$\begin{array}{lll}58789 & 1250.00 \quad 1200\end{array}$

$144012 \quad 125.000 \quad 140$

$\begin{array}{lll}15658 & 125.000 \quad 130\end{array}$

$\begin{array}{lll}46958 & 125.000 & 130\end{array}$ 


\section{Compounds}

16 trans-1,2-Dichloroethene

17 Methyl tert-butyl ether

18 1,1-Dichloroethane

\$ 19 2-Butanone-d5

20 cis-1,2-Dichloroethene

21 2-Butanone

22 Bromochlorome thane

\$ 23 Chloroform-d

24 Chloroform

25 1, 1,1-Trichloroethane

26 Cyclohexane

27 Carbon tetrachloride

$\$ 28$ 1,2-Dichloroethane-d4

\$ 29 Benzene-d6

30 Benzene

31 1,2-Dichloroethane

* 32 1,4-Difluorobenzene 33 Trichloroethene

\$ 34 1,2-Dichloropropane-d6 35 Methylcyclohexane

36 1,2-Dichloropropane

37 Bromodichloromethane

38 cis-1,3-Dichloropropene

39 4-Methyl-2-pentanone

$\$ 40$ Toluene-d8

41 Toluene

$\$ 42$ trans-1,3-Dichloropropene-d4

43 trans-1,3-Dichloropropene

44 1, 1,2-Trichloroethane

45 Tetrachloroethene

\$ 46 2-Hexanone-d5

47 2-Hexanone

48 Dibromochloromethane

49 1,2-Dibromoethane

- 50 Chlorobenzene-d5

51 Chlorobenzene

52 Ethylbenzene

$53 \mathrm{~m}, \mathrm{p}$-xylene

54 styrene

55 o-Xylene

56 Bromoform

57 Isopropylbenzene

\$ 58 1,1,2,2-Tetrachloroethane-d2 59 1, 1,2,2-Tetrachloroethane 60 1,3-Dichlorobenzene

* 61 1,4-Dichlorobenzene-d4

62 1,4-Dichlorobenzene
QUANT SIG

MASS

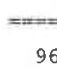

96

63

46

96

43

128

84

83

97

56

117

65

84

78

62

114

95

67

55

63

83

75

43

98

91

79

75

97

164

63

43

129

107

117

112

91

106

104

106

173

105

84

83

146

152

146
AMOUN'S

CAL-AMT ON-COL,

RT EXP RT REL RT RESPONSE ( ng) ( ng)

$\begin{array}{lllll}3.069 & 3.069 & (0.562) & 54845 & 125.000\end{array}$

$\begin{array}{llllll}3.075 & 3.082 & (0.563) & 54117 & 125.000 & 130\end{array}$

$\begin{array}{llllll}3.459 & 3.466 & (0.633) & 112039 & 125.000 & 130\end{array}$

$\begin{array}{llllll}4.014 & 4.020 & (0.734) & 71997 & 1250.00 & 1200\end{array}$

$\begin{array}{llllll}4.063 & 4.069 & (0.743) & 55917 & 125.000 & 130\end{array}$

$\begin{array}{llllll}4.081 & 4.081 & (0.747) & 83598 & 1250.00 & 1200\end{array}$

$\begin{array}{llllll}4.319 & 4.325 & (0.790) & 18716 & 125.000 & 130\end{array}$

$\begin{array}{lllll}4.392 & 4.392(0.804) & 90522 & 125.000 & 120\end{array}$

$\begin{array}{llllll}4.410 & 4.417 & (0.807) & 88818 & 125.000 & 130\end{array}$

$\begin{array}{llllll}4.624 & 4.630 & (0.524) & 77916 & 125.000 & 130\end{array}$

$\begin{array}{llllll}4.697 & 4.703 & (0.532) & 145171 & 125.000 & 140\end{array}$

$\begin{array}{llllll}4.813 & 4.819 & (0.545) & 74688 & 125.000 & 130\end{array}$

$\begin{array}{llllll}4.953 & 4.959 & (0.906) & 36442 & 125.000 & \cdots\end{array}$

$\begin{array}{llllll}4.990 & 4.990 & (0.565) & 227817 & 125.000 & 130\end{array}$

$\begin{array}{llllll}5.032 & 5.032 & (0.570) & 239225 & 125.000 & 130\end{array}$

$\begin{array}{llllll}5.038 & 5.039 & (0.922) & 53180 & 125.000 & 130\end{array}$

$5.465 \quad 5.471(1.000) \quad 178846 \quad 125.000$

$\begin{array}{llllll}5.758 & 5.758 & (0.652) & 56846 & 125.000 & 130\end{array}$

$\begin{array}{llllll}5.898 & 5.904 & (0.668) & 56639 & 125.000 & 120\end{array}$

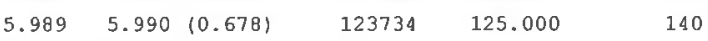

$\begin{array}{llllll}6.002 & 6.002 & (0.680) & 60678 & 125.000 & 130\end{array}$

$\begin{array}{llllll}6.312 & 6.313 & (0.715) & 48929 & 125.000 & 130\end{array}$

$\begin{array}{llllll}6.831 & 6.837 & (0.774) & 63826 & 125.000 & 140\end{array}$

$\begin{array}{llllll}7.026 & 7.026 & (0.796) & 206196 & 1250.00 & 1300\end{array}$

$\begin{array}{llllll}7.148 & 7.154 & (0.809) & 225768 & 125.000 & 130\end{array}$

$\begin{array}{llllll}7.227 & 7.227 & (0.818) & 268281 & 125.000 & 130\end{array}$

$\begin{array}{llllll}7.446 & 7.447 & (0.843) & 36079 & 125.000 & 130\end{array}$

$\begin{array}{llllll}7.477 & 7.477 & (0.847) & 46079 & 125.000 & 140\end{array}$

$\begin{array}{llllll}7.684 & 7.684 & (0.870) & 26498 & 125.000 & 140\end{array}$

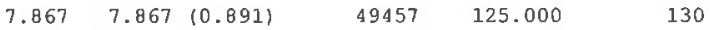

$\begin{array}{llllll}7.946 & 7.946 & (0.900) & 54450 & 1250.00 & 1300\end{array}$

$\begin{array}{llllll}8.007 & 8.007 & (0.907) & 145303 & 1250.00 & 1300\end{array}$

$\begin{array}{llllll}8.141 & 8.142 & (0.922) & 29197 & 125.000 & 130\end{array}$

$\begin{array}{llllll}8.263 & 8.264 & (0.936) & 22493 & 125.000 & 130\end{array}$

$8.830 \quad 8.830(1.000)$

$8.867 \quad 0.867(1.004)$

$9.013 \quad 9.019(1.021)$

$9.1599 .160(1.037)$

$9.647 \quad 9.647$ (1.093)

$9.629 \quad 9.629(1.090)$

$9.836 \quad 9.836 \quad(0.844)$

$10.08610 .086(1.142)$

$10.403 \quad 10.403$ (1.178)

$10.434 \quad 10.434 \quad(1.182)$

$11.580 \quad 11.580 \quad(0.993)$

$11.65911 .659(1.000)$

$11.68911 .690(1.003)$

$154132 \quad 125.000$

$\begin{array}{lll}171152 \quad 125.000 & 130\end{array}$

$302353 \quad 125.000 \quad 130$

$124414 \quad 125.000 \quad 140$

$168884 \quad 125.000 \quad 140$

$116787 \quad 125.000 \quad 140$

$10415 \quad 125.000 \quad 140$

$325846 \quad 125.000 \quad 140$

$26342 \quad 125.000 \quad 130$

$25743 \quad 125.000 \quad 130$

$123685 \quad 125.000 \quad 130$

$75451 \quad 125.000$

$\begin{array}{lll}131630 & 125.000 & 130\end{array}$ 


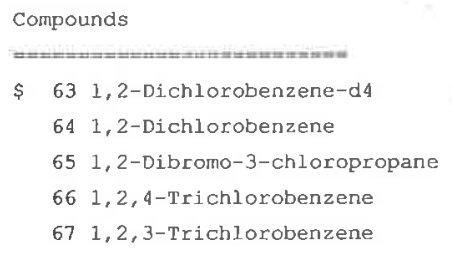

QUANT SIG
MASS
===-
152
146
75
180
180

\begin{tabular}{|c|c|c|c|c|c|}
\hline \multirow[b]{3}{*}{ RT } & \multirow[b]{3}{*}{ EXP RT } & \multirow[b]{3}{*}{ REL RT } & \multirow[b]{3}{*}{ RESPONSE } & \multicolumn{2}{|c|}{ AMOUNTS } \\
\hline & & & & CAL-AMT & $\mathrm{ON}-\mathrm{COL}$ \\
\hline & & & & ( $\mathrm{ng})$ & ng) \\
\hline$=$ & mostatsus & 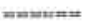 & 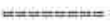 & 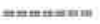 & 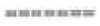 \\
\hline 12.104 & 12.104 & $(1.038)$ & 62619 & 125.000 & 130 \\
\hline 12.122 & 12.129 & $(1.040)$ & 111729 & 125.000 & 130 \\
\hline 12.982 & 12.982 & $(1.113)$ & 2899 & 125.000 & 150 \\
\hline 13.829 & 13.829 & $(1.186)$ & 55061 & 125.000 & 140 \\
\hline 14.287 & 14.287 & $(1.225)$ & 44319 & 125.000 & 140 \\
\hline
\end{tabular}


$7 A$ - EORM VII VOA-1

VOLATILE CONTINUING CALIBRATION DATA

Lab Name: TESTAMERICA BURLINGTON

Contract: $8 \mathrm{E}-00302$

Lab Code: STLV Case No.: SAVANN Mod. Ref No.:

SDG No.: 200-6522

Instrument ID: D.i

Calibration Date: 08/15/2011 Time: 1600

Lab File Id: DHPK22.D

Init. Calib. Date(s): 07/07/2011 07/07/2011

EPA Sample No. (VSTD\#\#\#\#): VSTD005HD

Init. Calib. Time(s): $1117 \quad 1256$

Heated Purge: ( $\mathrm{Y} / \mathrm{N})$

GC Column: DB-624

ID: $0.20(\mathrm{~mm})$ Length: 25

(m)

Purge Volume: 25.0

(mL)

\begin{tabular}{|c|c|c|c|c|c|}
\hline COMPOUND & $\overline{\mathrm{RRF}}$ & RRF5.0 & $\begin{array}{l}\text { MIN } \\
\text { RRE }\end{array}$ & 웅 D & MAX $\% \mathrm{D}$ \\
\hline Dichlorodifluoromethane & 0.403 & 0.495 & 0.010 & 22.8 & 50.0 \\
\hline Chloromethane & 0.687 & 0.712 & 0.010 & 3.7 & 50.0 \\
\hline Vinyl chloride & 0.496 & 0.516 & 0.100 & 3.9 & 50.0 \\
\hline Bromomethane & 0.209 & 0.180 & 0.100 & -14.0 & 50.0 \\
\hline Chloroethane & 0.272 & 0.266 & 0.010 & -2.3 & 50.0 \\
\hline Trichlorofluoromethane & 0.606 & 0.622 & 0.010 & 2.8 & 50.0 \\
\hline 1,1-Dichloroethene & 0.305 & 0.294 & 0.100 & -3.6 & 50.0 \\
\hline 1,1,2-Trichloro-1,2,2-trifluoroethane & 0.355 & 0.363 & 0.010 & 2.2 & 50.0 \\
\hline Acetone & 0.036 & 0.030 & 0.010 & -15.3 & 50.0 \\
\hline Carbon disulfide & 0.742 & 0.725 & 0.010 & -2.3 & 50.0 \\
\hline Methyl acetate & 0.086 & 0.083 & 0.010 & -4.0 & 50.0 \\
\hline Methylene Chloride & 0.250 & 0.244 & 0.010 & -2.3 & 50.0 \\
\hline trans-1,2-Dichloroethene & 0.308 & 0.298 & 0.010 & -3.1 & 50.0 \\
\hline Methyl tert-butyl ether & 0.302 & 0.286 & 0.010 & -5.5 & 50.0 \\
\hline 1,1-Dichloroethane & 0.609 & 0.598 & 0.200 & -1.9 & 50.0 \\
\hline cis-1,2-Dichloroethene & 0.302 & 0.293 & 0.010 & -2.8 & 50.0 \\
\hline 2-Butanone & 0.047 & 0.046 & 0.010 & -2.0 & 50.0 \\
\hline Bromochloromethane & 0.101 & 0.102 & 0.050 & 0.4 & 50.0 \\
\hline Chloroform & 0.485 & 0.477 & 0.200 & -1.7 & 50.0 \\
\hline 1,1,1-Trichloroethane & 0.494 & 0.464 & 0.100 & -6.2 & 50.0 \\
\hline Cyclohexane & 0.850 & 0.879 & 0.010 & 3.5 & 50.0 \\
\hline Carbon tetrachloride & 0.472 & 0.462 & 0.100 & -2.2 & 50.0 \\
\hline Benzene & 1.515 & 1.446 & 0.400 & -4.5 & 50.0 \\
\hline 1,2-Dichloroethane & 0.282 & 0.282 & 0.100 & 0.2 & 50.0 \\
\hline Trichloroethene & 0.368 & 0.336 & 0.300 & -8.8 & 50.0 \\
\hline Methylcyclohexane & 0.737 & 0.755 & 0.010 & 2.4 & 50.0 \\
\hline
\end{tabular}

Report 1,4-Dioxane for Low/Mediurn VOA analysis only 
$7 B$ - FORM VII VOA-2

VOLATILE CONTINUING CALIBRATION DATA

Lab Name: TESTAMERICA BURLINGTON

Contract: $8 \mathrm{E}-00302$

Lab Code: STLV Case No.: SAVANN Mod. Ref No.:

SDG No.: 200-6522

Instrument ID: D.i

Calibration Date: 08/15/2011 Time: 1600

Lab File Id: DHPK22.D

Init. Calib. Date(s): 07/07/2011 07/07/2011

EPA Sample No. (VSTD\#\#\#\#): VSTD005HD

Init. Calib. Time(s): $1117 \quad 1256$

Heated Purge: $(Y / N) \quad N$

GC Column:

ID: $0.20(\mathrm{~mm})$ Length: 25

(m)

Purge Volume: 25.0

(mL)

\begin{tabular}{|c|c|c|c|c|c|}
\hline COMPOUND & $\overline{\mathrm{RRF}}$ & RRF5.0 & $\begin{array}{l}\text { MIN } \\
\text { RRF }\end{array}$ & $\frac{\circ}{\partial} \mathrm{D}$ & MAX $\because D$ \\
\hline 1,2-Dichloropropane & 0.366 & 0.354 & 0.010 & -3.2 & 50.0 \\
\hline Bromodichloromethane & 0.305 & 0.299 & 0.200 & -2.0 & 50.0 \\
\hline cis-1,3-Dichloropropene & 0.377 & 0.381 & 0.200 & 1.0 & 50.0 \\
\hline 4-Methyl-2-pentanone & 0.126 & 0.130 & 0.010 & 2.6 & 50.0 \\
\hline Toluene & 1.657 & 1.633 & 0.400 & -1.4 & 50.0 \\
\hline trans-1,3-Dichloropropene & 0.267 & 0.269 & 0.100 & 0.7 & 50.0 \\
\hline 1,1,2-Trichloroethane & 0.158 & 0.157 & 0.100 & -0.7 & 50.0 \\
\hline Tetrachloroethene & 0.306 & 0.293 & 0.100 & -4.1 & 50.0 \\
\hline 2-Hexanone & 0.090 & 0.091 & 0.010 & 1.0 & 50.0 \\
\hline Dibromochloromethane & 0.180 & 0.172 & 0.100 & -4.0 & 50.0 \\
\hline 1,2-Dibromoethane & 0.139 & 0.137 & 0.010 & -1.3 & 50.0 \\
\hline Chlorobenzene & 1.042 & 1.029 & 0.500 & -1.3 & 50.0 \\
\hline Ethylbenzene & 1.825 & 1.851 & 0.100 & 1.4 & 50.0 \\
\hline o-Xylene & 0.674 & 0.712 & 0.300 & 5.6 & 50.0 \\
\hline $\mathrm{m}, \mathrm{p}$-Xylene & 0.742 & 0.771 & 0.300 & 3.9 & 50.0 \\
\hline Styrene & 0.983 & 1.072 & 0.300 & 9.0 & 50.0 \\
\hline Bromoform & 0.121 & 0.115 & 0.050 & -4.8 & 50.0 \\
\hline Isopropylbenzene & 1.841 & 1.961 & 0.010 & 6.5 & 50.0 \\
\hline $1,1,2,2$-Tetrachloroethane & 0.164 & 0.160 & 0.100 & -2.4 & 50.0 \\
\hline 1,3-Dichlorobenzene & 1.562 & 1.543 & 0.400 & -1.2 & 50.0 \\
\hline 1,4-Dichlorobenzene & 1.686 & 1.641 & 0.400 & -2.6 & 50.0 \\
\hline 1,2-Dichlorobenzene & 1.384 & 1.387 & 0.400 & 0.2 & 50.0 \\
\hline 1,2-Dibromo-3-Chloropropane & 0.032 & 0.030 & 0.010 & -7.7 & 50.0 \\
\hline 1,2,4-Trichlorobenzene & 0.674 & 0.661 & 0.200 & -2.0 & 50.0 \\
\hline 1,2,3-Trichlorobenzene & 0.514 & 0.536 & 0.200 & 4.2 & 50.0 \\
\hline
\end{tabular}


$7 C$ - EORM VII VOA-3

VOLATILE CONTINUING CALIBRATION DATA

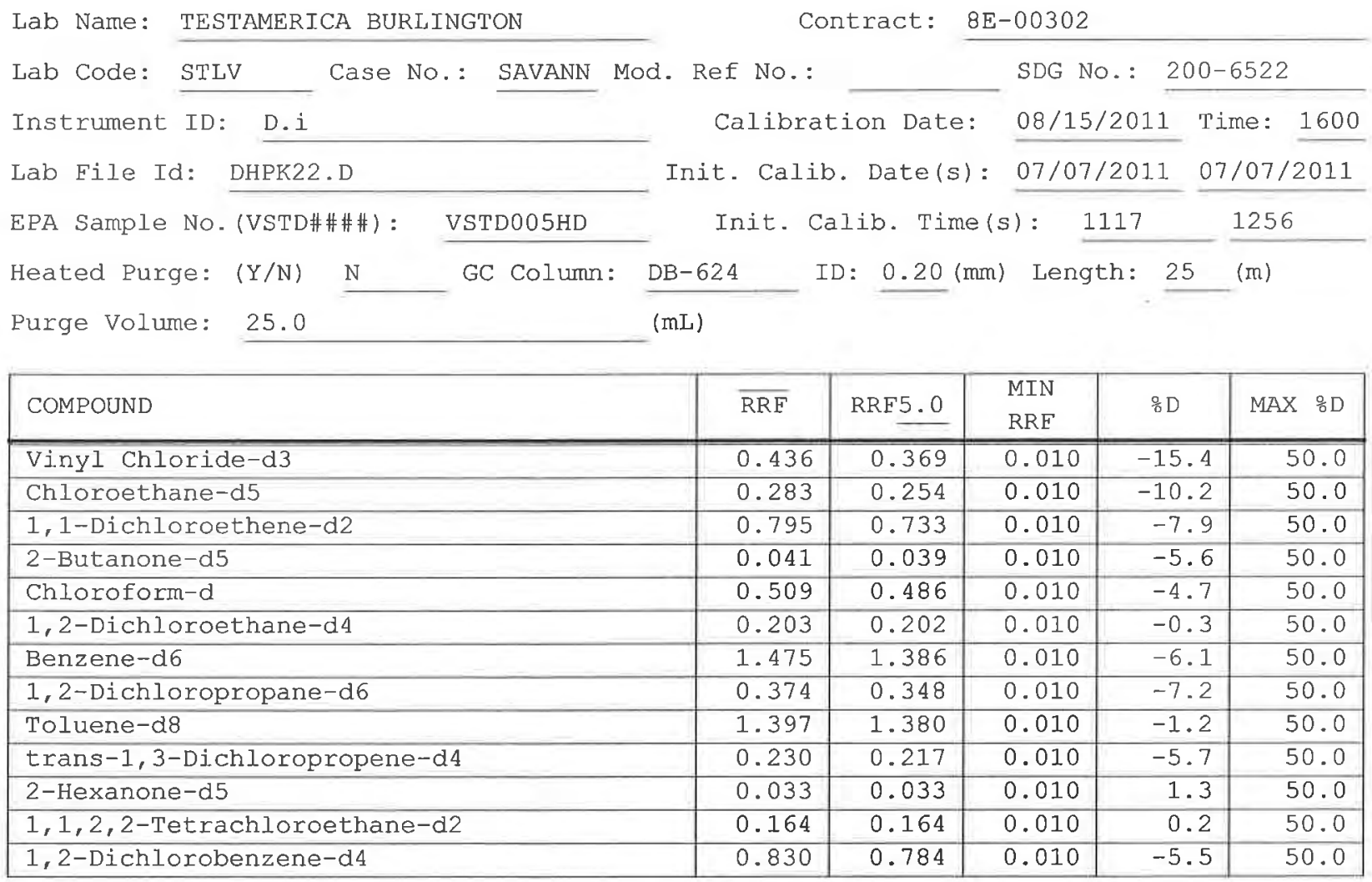




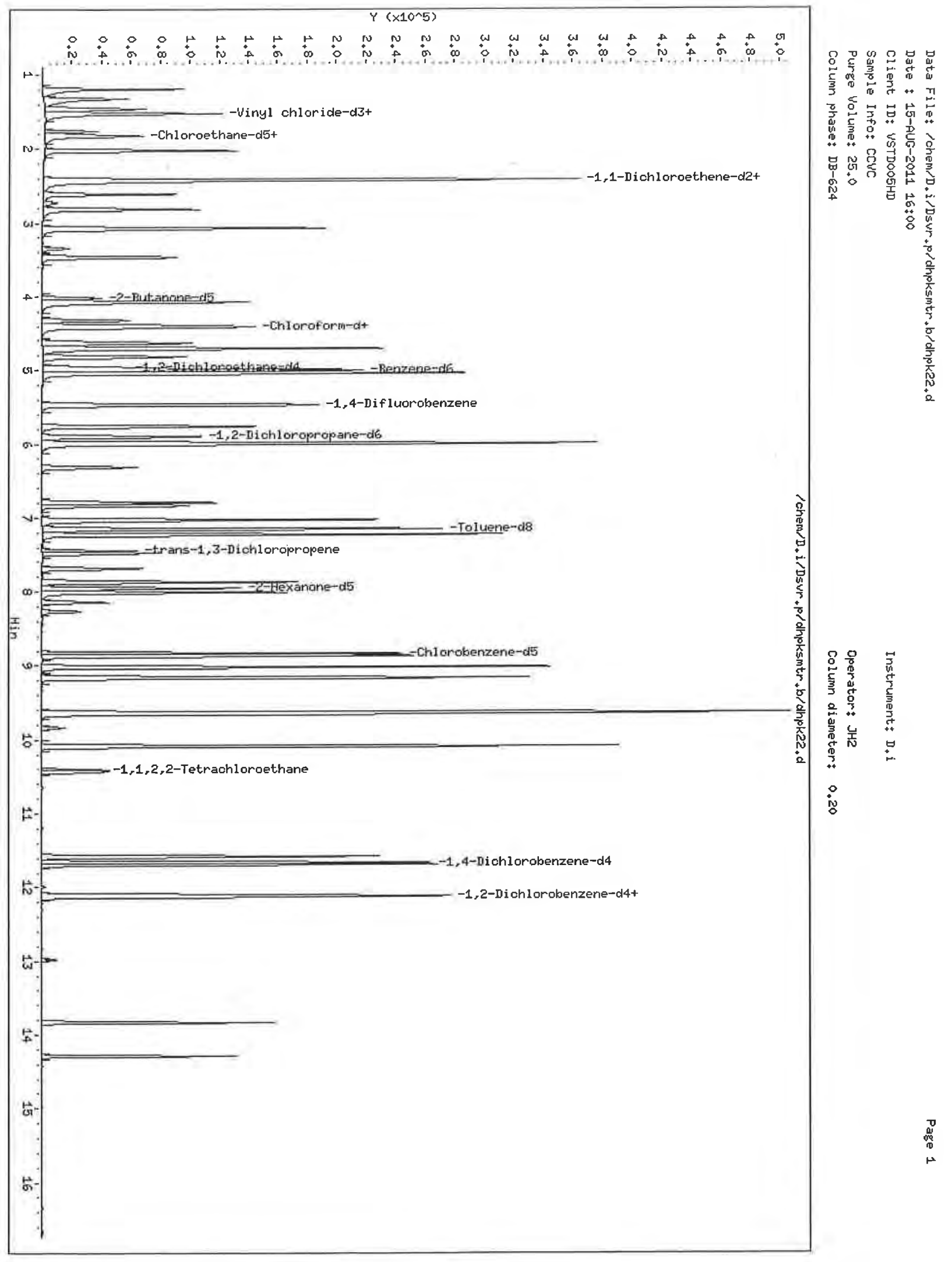




\section{TestAmerica Burlington}

LOW CONCENTRATION VOLATILE QUANTITATION REPORT

Data file : /chem/D.i/Dsvr.p/dhpksmtr.b/dhpk22.d

Lab Smp Id: CCVC

Client Smp ID: VSTD005HD

Inj Date : 15-AUG-2011 16:00

Operator : JH2

Smp Info: CCVC

Misc Info : 1,5

Comment :

Method : /chem/D.i/Dsvr.p/dhpksmtr.b/somtr5t.m

Meth Date : 15-Aug-2011 16:19 jh2 Quant Type: ISTD

Cal Date : 07-JUL-2011 12:56 Cal File: dhp08.d

Als bottle: 20

Dil Factor: 1.00000

Integrator: HP RTE

Target Version: 3.50

Inst ID: D.i

Processing Host: chemsvr6

Concentration Formula: Amt * DF * Uf/Vo * CpndVariable

$\begin{array}{ccc}\text { Name } & \text { Value } & \text { Description } \\ \text { DF } & 1.00000 & \text { Dilution Factor } \\ \text { Uf } & 1.00000 & \text { ng unit correction factor } \\ \text { Vo } & 25.00000 & \text { Sample Volume purged (mL) } \\ \text { Cpnd Variable } & \text { Local Compound Variable }\end{array}$

Compounds

1 Dichlorodifluoromethane

2 Chloromethane

\$ 3 Vinyl chloride-d3

4 Vinyl chloride

5 Bromomethane

$\$ 6$ Chloroethane-d5

7 Chloroethane

8 Trichlorofluoromethane

\$ 9 1,1-Dichloroethene-d2

10 1,1-Dichloroethene

11 1,1,2-Trichloro-1,2,2-trifluo

12 Acetone

13 Carbon disulfide

14 Methyl acetate

15 Methylene chloride
Continuing Calibration Sample

Compound Sublist: all.sub

\begin{tabular}{|c|c|c|c|c|c|c|}
\hline \multirow[b]{2}{*}{ QUANT SIG } & \multirow[b]{3}{*}{ RT } & \multirow[b]{3}{*}{ EXP RT } & \multirow[b]{3}{*}{ REL RT } & \multirow[b]{3}{*}{ RESPONSE } & \multicolumn{2}{|c|}{ AMOUNTS } \\
\hline & & & & & CAL-AMT & $\mathrm{ON}-\mathrm{COL}$ \\
\hline MASS & & & & & ng) & ng) \\
\hline$= \pm=0$ & $=$ & 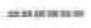 & онимина & 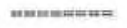 & 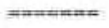 & 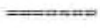 \\
\hline 85 & 1.320 & 1.320 & $(0.241)$ & 82852 & 125.000 & 150 \\
\hline 50 & 1.454 & 1.454 & $(0.266)$ & 119342 & 125.000 & 130 \\
\hline 65 & 1.515 & 1.521 & $(0.277)$ & 61773 & 125.000 & 110 \\
\hline 62 & 1.521 & 1.521 & $(0.278)$ & 86422 & 125.000 & 130 \\
\hline 94 & 1.759 & 1.759 & $(0.321)$ & 30139 & 125.000 & 110 \\
\hline 69 & 1.807 & 1.807 & $(0.330)$ & 42506 & 125.000 & 110 \\
\hline 64 & 1.826 & 1.832 & $(0.334)$ & 44523 & 125.000 & 120 \\
\hline 101 & 2.021 & 2.021 & $(0.369)$ & 104281 & 125.000 & 130 \\
\hline 63 & 2.411 & 2.411 & $(0.441)$ & 122734 & 125.000 & 120 \\
\hline 96 & 2.417 & 2.423 & $(0.442)$ & 49176 & 125.000 & 120 \\
\hline 101 & 2.423 & 2.423 & $(0.443)$ & 60794 & 125.000 & 130 \\
\hline 43 & 2.448 & 2.448 & $(0.447)$ & 50622 & 1250.00 & 1100 \\
\hline 76 & 2.606 & 2.612 & $(0.476)$ & 121424 & 125.000 & 120 \\
\hline 43 & 2.728 & 2.728 & $(0.499)$ & 13833 & 125.000 & 120 \\
\hline 84 & 2.813 & 2.813 & $(0.514)$ & 40958 & 125.000 & 120 \\
\hline
\end{tabular}




\section{Compounds}

16 trans-1,2-Dichloroethene

17 Methyl tert-butyl ether

18 1,1-Dichloroethane

$\$ 192$-Butanone-d5

20 cis-1,2-Dichloroethene

21 2-Butanone

22 Bromochloromethane

$\$ 23$ Chloroform-d

24 chloroform

25 1, 1, 1-Trichloroethane

26 Cyclohexane

27 Carbon tetrachloride

\$ 28 1,2-Dichloroethane-d4

$\$ 29$ Benzene-d6

30 Benzene

31 1,2-Dichloroethane

* 32 1,4-Difluorobenzene 33 Trichloroethene

\$ 34 1,2-Dichloropropane-d6

35 Methylcyclohexane

36 1,2-Dichloropropane

37 Bromodichloromethane

38 cis-1,3-Dichloropropene

39 4-Methyl-2-pentanone

$\$ 40$ Toluene-d8

41 Toluene

\$ 42 trans-1,3-Dichloropropene-d4

43 trans-1,3-Dichloropropene

44 1, 1, 2-Trichloroethane

45 Tetrachloroethene

$\$ 46$ 2-Hexanone-d5

47 2-hexanone

48 Dibromochloromethane

49 1,2-Dibromoethane

* 50 Chlorobenzene-d5

51 Chlorobenzene

52 Ethylbenzene

$53 \mathrm{~m}, \mathrm{p}$-Xylene

54 styrene

55 o-xylene

56 Bromoform

57 Isopropylbenzene

\$ $581,1,2,2$-Tetrachloroethane-d2

$591,1,2,2$-Tetrachloroethane

601,3 -Dichlorobenzene

* 61 1,4-Dichlorobenzene-d4

62 1,4-Dichlorobenzene

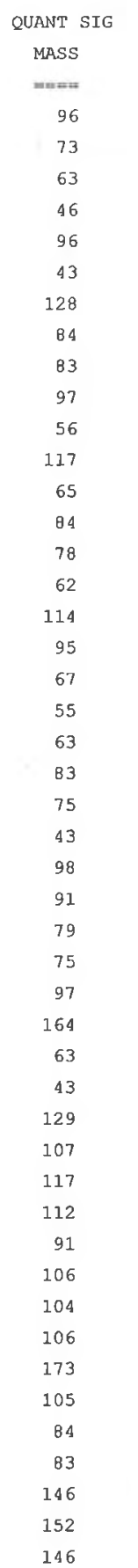

AMOUNTS

CAL-AMT ON-COL

RT EXP RT REL RT

RESPONSE

( ng) ( ng)

$125.000 \quad 120$

$125.000 \quad 120$

$125.000 \quad 120$

$1250.00 \quad 1200$

$125.000 \quad 120$

$1250.00 \quad 1200$

$125.000 \quad 130$

$125.000 \quad 120$

$125.000 \quad 120$

$125.000 \quad 120$

$125.000 \quad 130$

$125.000 \quad 120$

$125.000 \quad 120$

$125.000 \quad 120$

$125.000 \quad 120$

$125.000 \quad 130$

125.000

$125.000 \quad 110$

$125.000 \quad 120$

$125.000 \quad 130$

$125.000 \quad 120$

$125.000 \quad 120$

$125.000 \quad 130$

$1250.00 \quad 1300$

$125.000 \quad 120$

$125.000 \quad 120$

$125.000 \quad 120$

$125.000 \quad 130$

$125.000 \quad 120$

$125.000 \quad 120$

$1250.00 \quad 1300$

$1250.00 \quad 1300$

$125.000 \quad 120$

$125.000 \quad 120$

125.000

$125.000 \quad 120$

$125.000 \quad 130$

$125.000 \quad 130$

$125.000 \quad 140$

$125.000 \quad 130$

$125.000 \quad 120$

$125.000 \quad 130$

$125.000 \quad 130$

$125.000 \quad 120$

$125.000 \quad 120$

125.000

$125.000 \quad 120$ 
Data File: /chem/D.i/Dsvr.p/dhpksmtr.b/dhpk22.d

Report Date: 15-Aug-2011 16:19

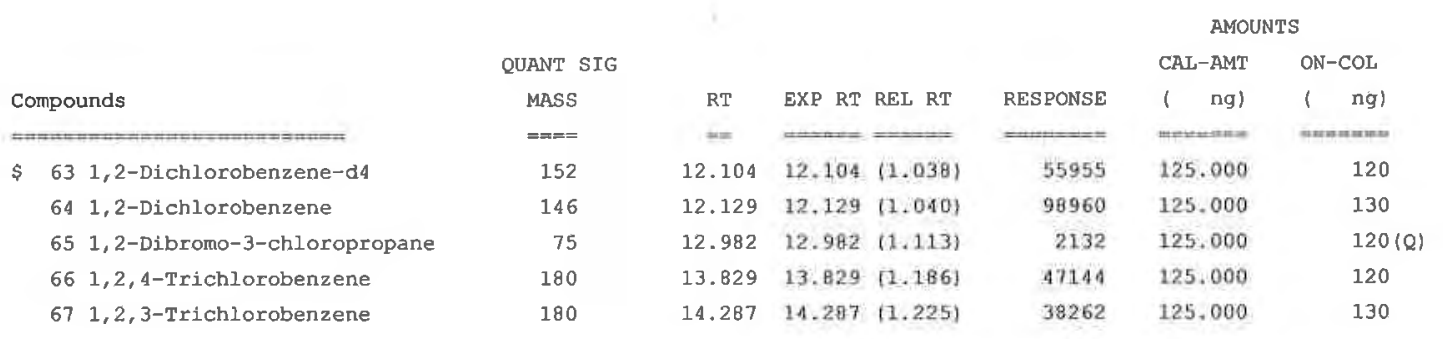

QC Flag Legend

Q - Qualifier signal failed the ratio test. 

Data File:
dhp01.d
Date:
07-JUL-2011 10:05
Client ID:
BFBDV
Instrument: D.i
$\begin{array}{ll}\text { Operator: } & \text { JH2 } \\ \text { Column Type: } & \text { Capillary }\end{array}$
Inj Vol:
Diameter:
1.0 (ul)
Stationary Phase: DB-624
Sample Info: 50NG BEB
Lab Sample ID: BFB
$1 \mathrm{bfb}$

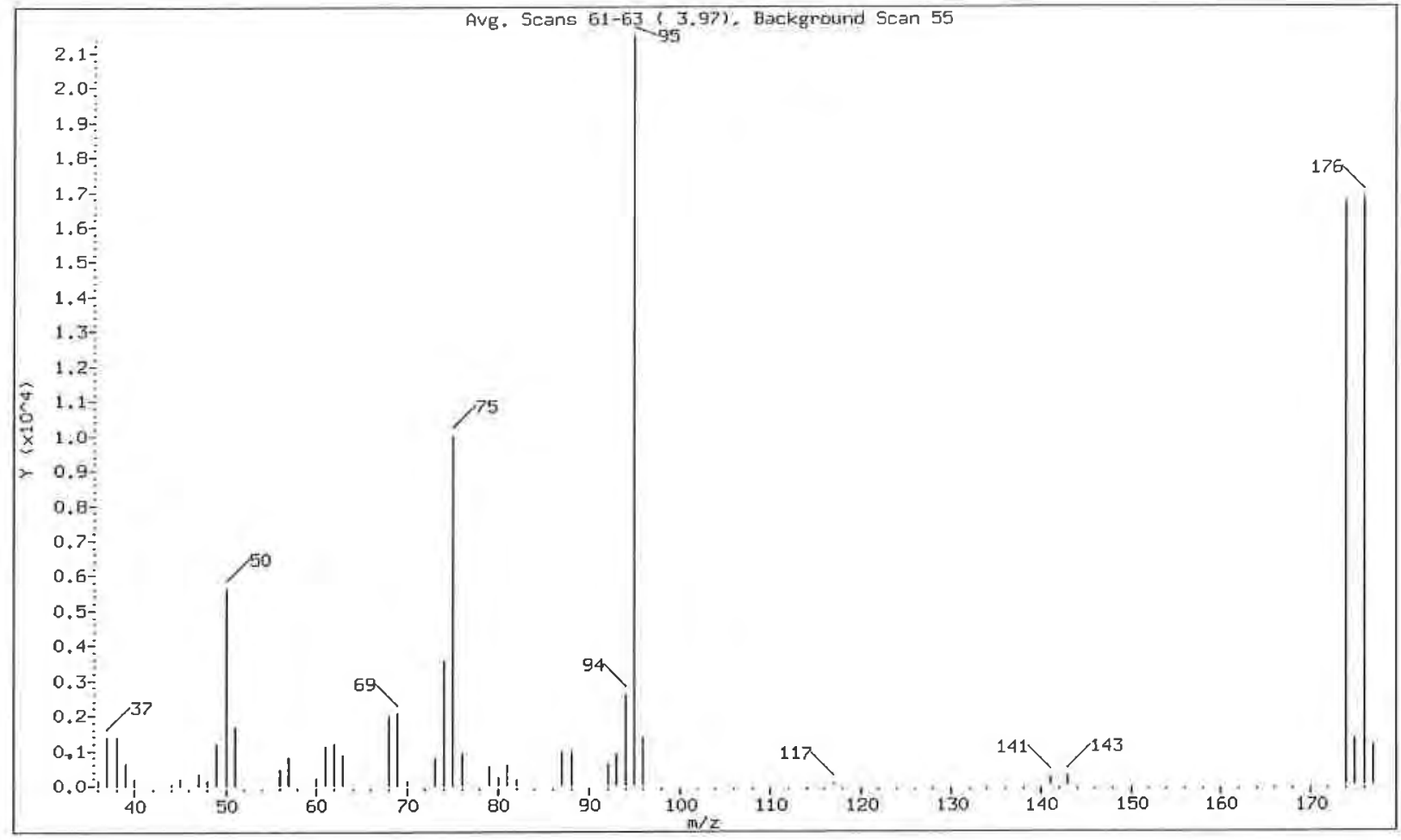

\begin{tabular}{|c|c|c|c|c|c|}
\hline $\mathrm{m} / \mathrm{e}$ & ION ABUNDANCE CRITERIA & \multicolumn{4}{|c|}{$\begin{array}{l}\text { RELATIVE } \\
\text { ABUNDANCE }\end{array}$} \\
\hline | & 1 & $i$ & & & \\
\hline 95 & I Base Peak, 100\% relative abundance & i & 100.00 & & \\
\hline 50 & $15.00-40.00 \%$ of mass 95 & i & 26.08 & & \\
\hline 75 & $30.00-80.00 \%$ of mass 95 & i & 46.40 & & \\
\hline 96 & $5.00-9.00 \%$ of mass 95 & $i$ & 6.26 & & \\
\hline 173 & Less than $2.00 \%$ of mass 174 & i & 0.00 & $0.00)$ & \\
\hline 174 & $50.00-120.00 \%$ of mass 95 & i & 77.67 & & \\
\hline 175 & $5.00-9.00 \%$ of mass 174 & i & 6.05 & $7.79)$ & \\
\hline 176 & $95.00-101.00 \%$ of mass 174 & $i$ & 78.23 & $(100.72)$ & \\
\hline 177 & i $5.00-9.00 \%$ of mass 176 & 1 & 5.20 & $6.65)$ & \\
\hline
\end{tabular}




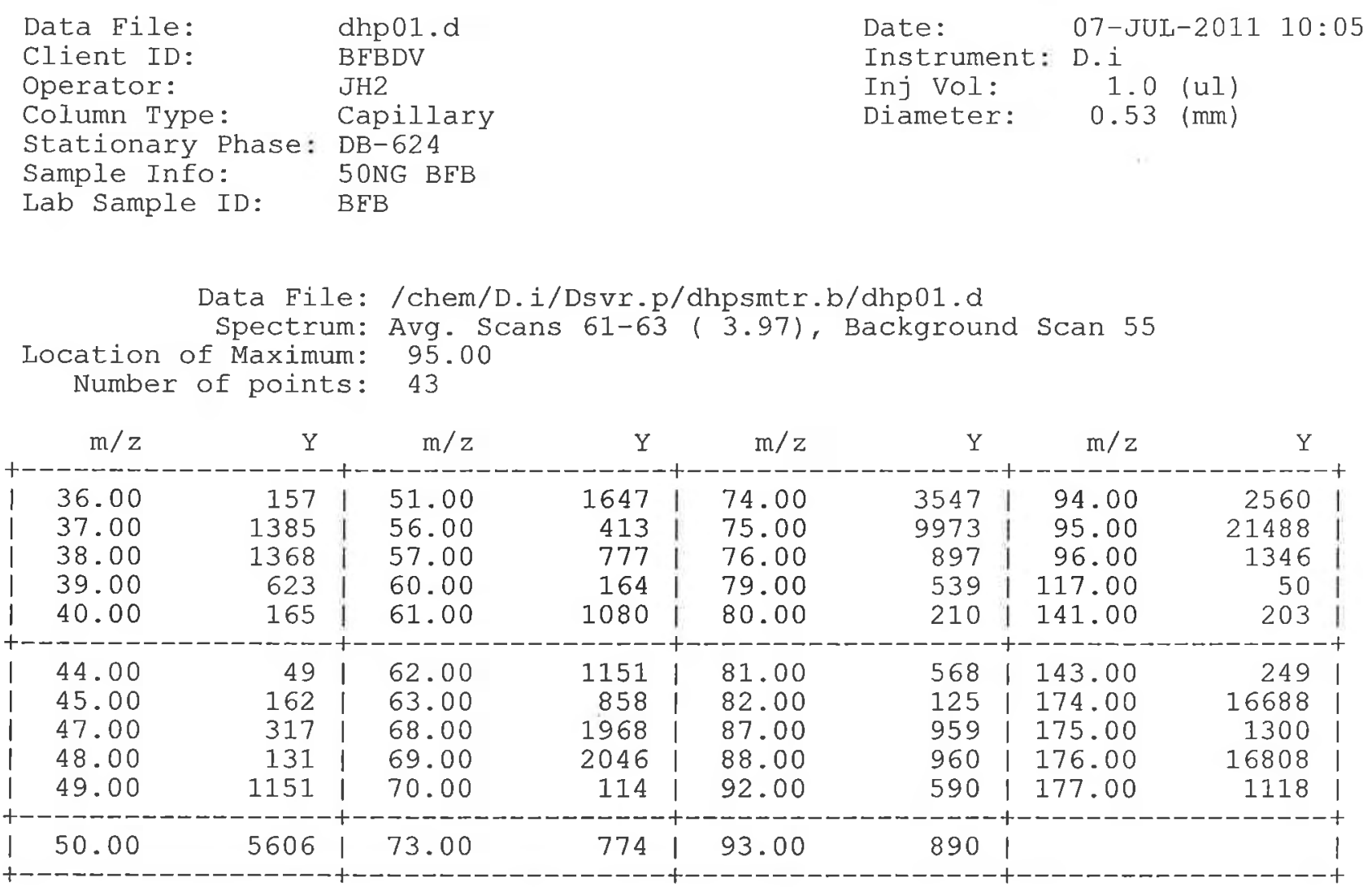



Data File:
dhp01.d
Date:
07-JUL-2011 10:05
Client ID:
BEBDV
Operator:
JH2
Column Type: Capillary
Instrument: D.i
Stationary phase: DB-624
Sample Info: 50NG BFB
Lab Sample ID: BFB

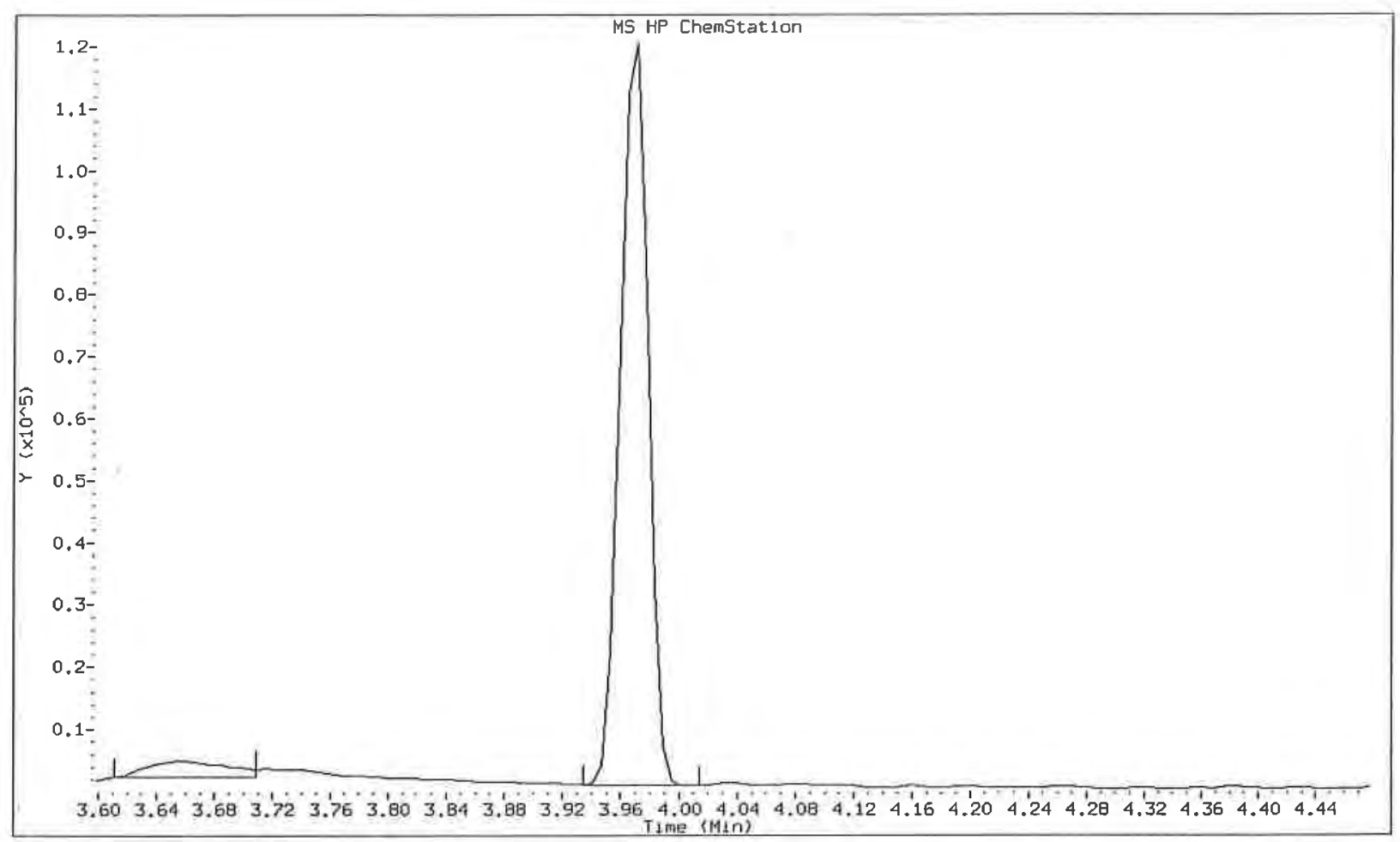



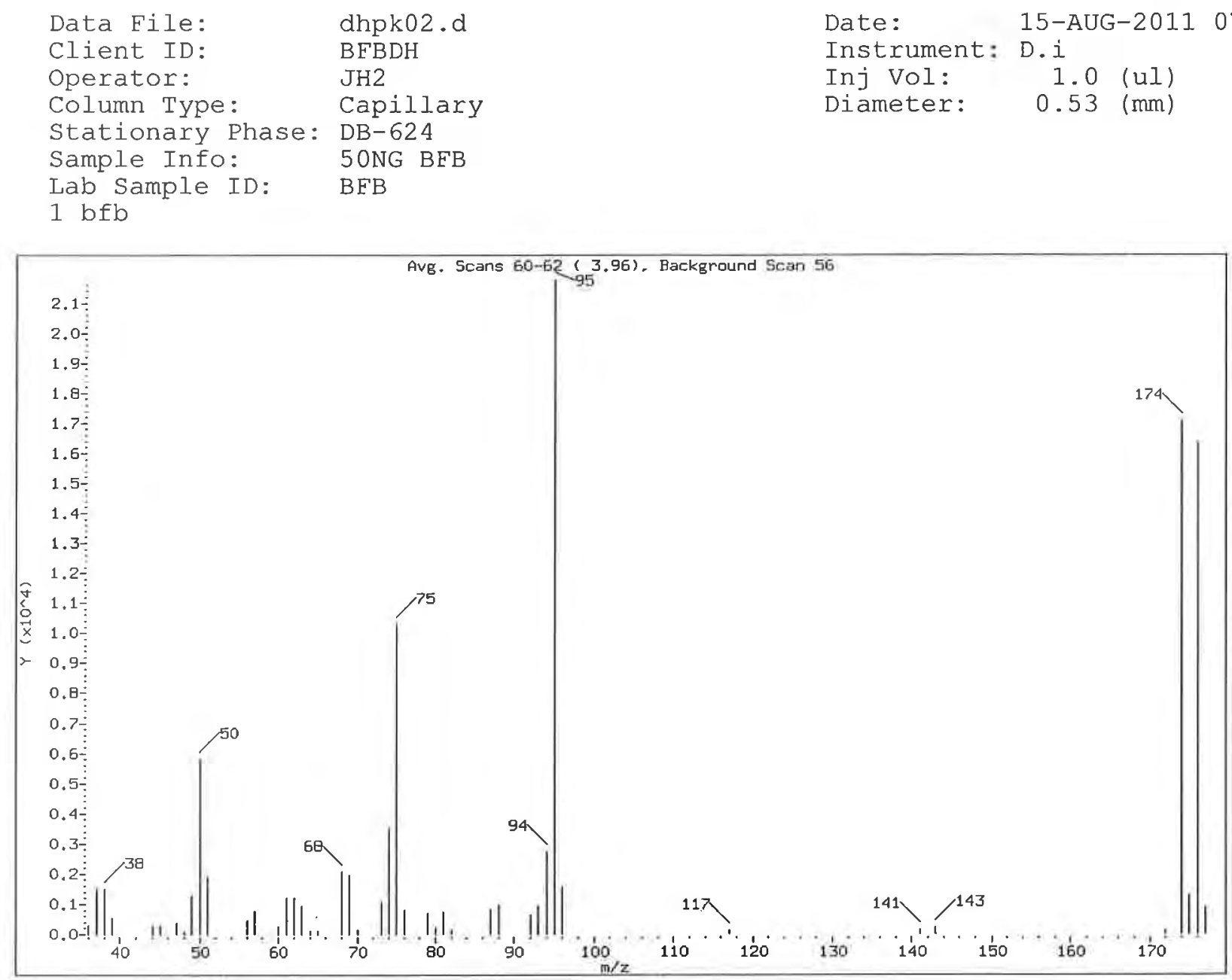

\begin{tabular}{|c|c|c|c|c|c|c|}
\hline $\mathrm{m} / \mathrm{e}$ & ION ABUNDANCE CRITERIA & \multicolumn{4}{|c|}{$\begin{array}{l}\circ \text { RELATIVE } \\
\text { ABUNDANCE }\end{array}$} & \\
\hline & & 1 & & & & \\
\hline 95 & Base Peak, $100 \%$ relative abundance & I & 100.00 & & & \\
\hline 50 & $15.00-40.00 \%$ of mass 95 & 1 & 26.73 & & & \\
\hline 75 & $30.00-80.00 \%$ of mass 95 & i & 47.25 & & & \\
\hline 96 & $5.00-9.00 \%$ of mass 95 & I & 7.24 & & & \\
\hline 173 & Less than $2.00 \%$ of mass 174 & 1 & 0.00 & ( & $0.00)$ & \\
\hline 174 & $50.00-120.00 \%$ of mass 95 & i & 78.35 & & & \\
\hline 175 & $5.00-9.00 \%$ of mass 174 & $i$ & 5.86 & ( & $7.47)$ & \\
\hline 176 & $95.00-101.00 \%$ of mass 174 & 1 & 75.16 & ( & $95.93)$ & \\
\hline 177 & $5.00-9.00 \%$ of mass 176 & i & 4.12 & i & $5.48)$ & \\
\hline
\end{tabular}




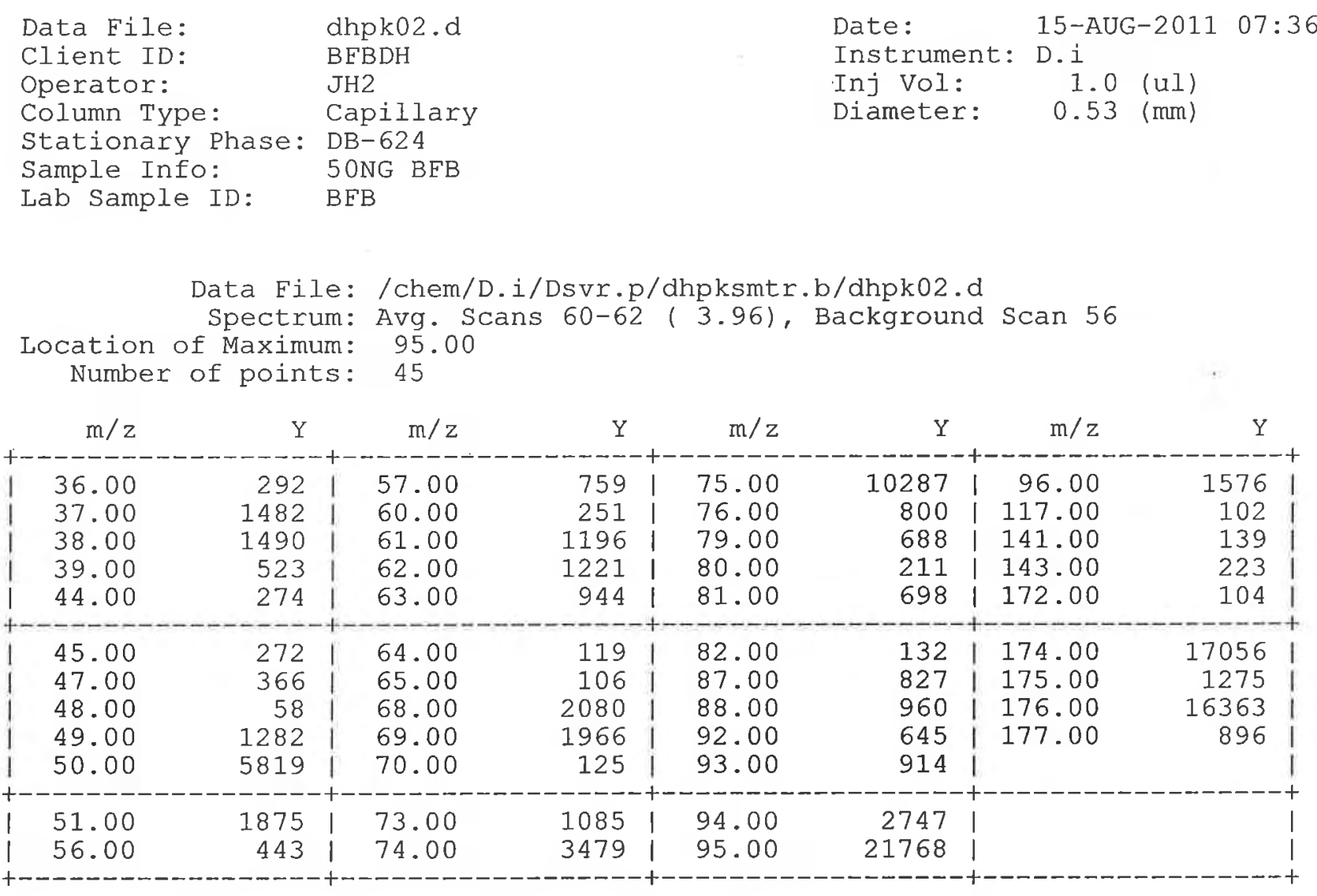




$\begin{array}{llll}\text { Data File: } & \text { dhpk02.d } & \text { Date: } & 15-\text { AUG-2011 } 07: 36 \\ \text { Client ID: } & \text { BFBDH } & \text { Instrument: D.i } \\ \text { Operator: } & \text { JH2 } & \text { Inj Vol: } & \text { 1.0 (ul) } \\ \text { Column Type: } & \text { Capillary } & \text { Diameter: } & 0.53 \text { (mm) } \\ \text { Stationary Phase: } & \text { DB-624 } & \\ \text { Sample Info: } & \text { 50NG BEB } & \\ \text { Lab Sample ID: } & \text { BFB } & \end{array}$

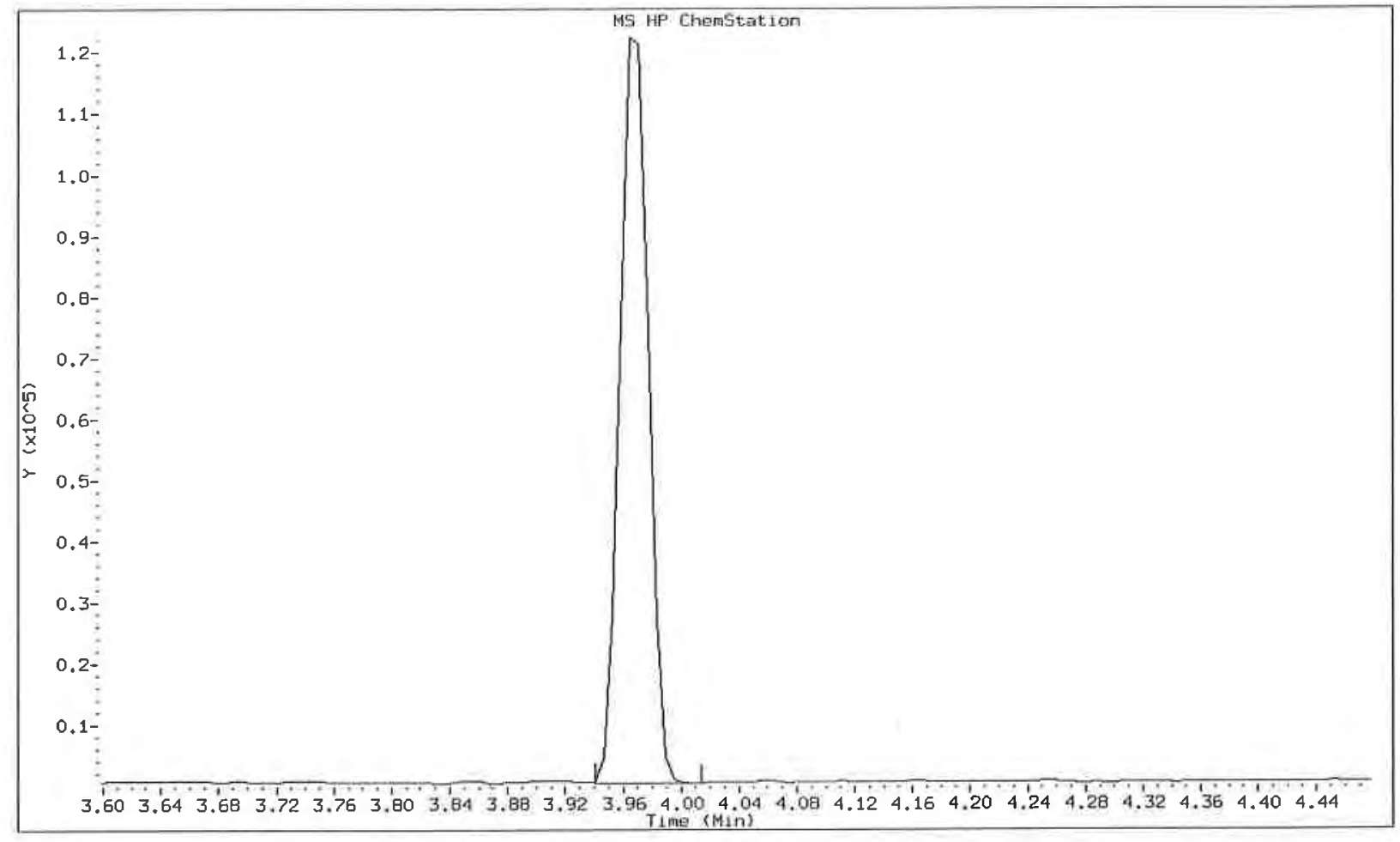


$1 A$ - FORM I VOA-1

VOLATILE ORGANICS ANALYSIS DATA SHEET
EPA SAMPLE NO. VBLKDH
Lab Name: TESTAMERICA BURLINGTON

Lab Code: STLV Case No.: SAVANN Mod. Ref No.:
Contract: $8 \mathrm{E}-00302$

SDG No.: 200-6522
Matrix: (SOIL/SED/WATER)

Sample wt/vol: 25.0

Water

$(\mathrm{g} / \mathrm{mL}) \quad \mathrm{mL}$

Level: (TRACE/LOW/MED) TRACE

응 Moisture: not dec.

GC Column: DB-624

ID $: 0.20$ (mm)

Soil Extract Volume:

(uL)

(mL)

Purge Volume: 25.0
Lab Sample ID: MB 200-23630/5

Lab File ID: DHPK05.D

Date Received:

Date Analyzed: 08/15/2011

Dilution Factor: 1.0

Soil Aliquot Volume:

(uL)

CONCENTRATION UNITS :

\begin{tabular}{|c|c|c|c|}
\hline CAS NO. & COMPOUND & $\begin{array}{l}\text { CONCENTRATION UNITS: } \\
(\mathrm{ug} / \mathrm{J} \text { or } \mathrm{ug} / \mathrm{kg}) \mathrm{ug} / \mathrm{I}\end{array}$ & $Q$ \\
\hline $75-71-8$ & Dichlorodifluoromethane & 0.50 & $\overline{\mathrm{U}}$ \\
\hline $74-87-3$ & Chloromethane & 0.50 & $\mathrm{U}$ \\
\hline $75-01-4$ & Vinyl chloride & 0.50 & $\mathrm{U}$ \\
\hline $74-83-9$ & Bromomethane & 0.50 & $\mathrm{U}$ \\
\hline $75-00-3$ & Chloroethane & 0.50 & $\mathrm{U}$ \\
\hline $75-69-4$ & Trichlorofluoromethane & 0.50 & $\mathrm{U}$ \\
\hline $75-35-4$ & 1,1-Dichloroethene & 0.50 & $\mathrm{U}$ \\
\hline $76-13-1$ & 1,1,2-Trichloro-1,2,2-trifluoroethane & 0.50 & U \\
\hline $67-64-1$ & Acetone & 5.0 & $\mathrm{U}$ \\
\hline $75-15-0$ & Carbon disulfide & 0.067 & $\mathrm{~J}$ \\
\hline $79-20-9$ & Methyl acetate & 0.50 & $U$ \\
\hline $75-09-2$ & Methylene Chloride & 0.048 & $\mathrm{~J}$ \\
\hline $156-60-5$ & trans $-1,2-$ Dichloroethene & 0.50 & $\mathrm{U}$ \\
\hline $1634-04-4$ & Methyl tert-butyl ether & 0.50 & $\mathrm{U}$ \\
\hline $75-34-3$ & 1,1-Dichloroethane & 0.50 & $\mathrm{U}$ \\
\hline $156-59-2$ & cis-1,2-Dichloroethene & 0.50 & $\mathrm{U}$ \\
\hline $78-93-3$ & 2-Butanone & 5.0 & $\mathrm{U}$ \\
\hline $74-97-5$ & Bromochloromethane & 0.50 & $\mathrm{U}$ \\
\hline $67-66-3$ & Chloroform & 0.50 & $\mathrm{U}$ \\
\hline $71-55-6$ & 1,1,1-Trichloroethane & 0.50 & $\mathrm{U}$ \\
\hline $110-82-7$ & Cyclohexane & 0.50 & $\mathrm{U}$ \\
\hline $56-23-5$ & Carbon tetrachloride & 0.50 & $\mathrm{U}$ \\
\hline $71-43-2$ & Benzene & 0.0027 & $\mathrm{~J}$ \\
\hline $107-06-2$ & 1,2-Dichloroethane & 0.50 & $\mathrm{U}$ \\
\hline
\end{tabular}

Report 1,4-Dioxane for Low-Medium VOA analysis only 
$1 B$ - FORM I VOA-2

VOLATILE ORGANICS ANALYSIS DATA SHEET
EPA SAMPLE NO.

VBLKDH
Lab Name: TESTAMERICA BURLINGTON

Lab Code: STLV Cas

Matrix: (SOIL/SED/WATER)

Sample wt/vol: 25.0

Water

$(\mathrm{g} / \mathrm{mL}) \quad \mathrm{mL}$

Level: (TRACE/LOW/MED)

TRACE

\% Moisture: not dec.

GC Column: DB-624 ID: $0.20 \quad(\mathrm{~mm})$

Soil Extract Volume:

(uL)

(mI)

Purge Volume: 25.0
Contract: $\quad 8 \mathrm{E}-00302$

SDG No.: 200-6522

\begin{tabular}{|c|c|c|c|}
\hline CAS NO. & COMPOUND & $\begin{array}{l}\text { CONCENTRATION UNITS: } \\
(\mathrm{ug} / \mathrm{L} \text { or } \mathrm{ug} / \mathrm{kg}) \mathrm{ug} / \mathrm{L}\end{array}$ & $Q$ \\
\hline $79-01-6$ & Trichloroethene & 0.50 & $\mathrm{U}$ \\
\hline $108-87-2$ & Methylcyclohexane & 0.50 & $\mathrm{U}$ \\
\hline $78-87-5$ & 1.2-Dichloropropane & 0.50 & $\mathrm{U}$ \\
\hline $75-27-4$ & Bromodichloromethane & 0.50 & $\mathrm{U}$ \\
\hline $10061-01-5$ & cis-1,3-Dichloropropene & 0.50 & $\mathrm{U}$ \\
\hline $108-10-1$ & 4-Methyl-2-pentanone & 5.0 & $\mathrm{U}$ \\
\hline $108-88-3$ & Toluene & 0.0097 & $\mathrm{~J}$ \\
\hline $10061-02-6$ & trans-1,3-Dichloropropene & 0.50 & $\mathrm{U}$ \\
\hline $79-00-5$ & 1,1,2-Trichloroethane & 0.50 & $\mathrm{U}$ \\
\hline $127-18-4$ & Tetrachloroethene & 0.50 & $\mathrm{U}$ \\
\hline $591-78-6$ & 2-Hexanone & 5.0 & $\mathrm{U}$ \\
\hline $124-48-1$ & Dibromochloromethane & 0.50 & $\mathrm{U}$ \\
\hline $106-93-4$ & 1,2-Dibromoethane & 0.50 & $\mathrm{U}$ \\
\hline $108-90-7$ & Chlorobenzene & 0.50 & $\mathrm{U}$ \\
\hline $100-41-4$ & Ethylbenzene & 0.50 & $\mathrm{U}$ \\
\hline $95-47-6$ & o-Xylene & 0.50 & $\mathrm{U}$ \\
\hline $179601-23-1$ & $\mathrm{~m}, \mathrm{p}$-Xylene & 0.50 & $\mathrm{U}$ \\
\hline $100-42-5$ & Styrene & 0.50 & $\mathrm{U}$ \\
\hline $75-25-2$ & Bromoform & 0.50 & $\mathrm{U}$ \\
\hline $98-82-8$ & Isopropylbenzene & 0.50 & $\mathrm{U}$ \\
\hline $79-34-5$ & $1,1,2,2$-Tetrachloroethane & 0.50 & $\mathrm{U}$ \\
\hline $541-73-1$ & 1,3-Dichlorobenzene & 0.50 & $\mathrm{U}$ \\
\hline $106-46-7$ & 1,4-Dichlorobenzene & 0.50 & $\mathrm{U}$ \\
\hline $95-50-1$ & 1,2-Dichlorobenzene & 0.50 & $\mathrm{U}$ \\
\hline $96-12-8$ & 1,2-Dibromo-3-Chloropropane & 0.50 & $\mathrm{U}$ \\
\hline $120-82-1$ & 1,2,4-Trichlorobenzene & 0.50 & $\mathrm{U}$ \\
\hline $87-61-6$ & 1,2,3-Trichlorobenzene & 0.50 & $\mathrm{U}$ \\
\hline
\end{tabular}


$1 \mathrm{~J}$ - FORM I VOA-TIC

VOLATILE ORGANICS ANALYSIS DATA SHEET TENTATIVELY IDENTIFIED COMPOUNDS
EPA SAMPLE NO.

VBLKDH
Lab Name: TESTAMERICA BURLINGTON

Lab Code: STLV Case No.

Matrix: (SOIL/SED/WATER)

Sample wt/vol: 25.0

Level: (TRACE or LOW/MED)

Water

$(\mathrm{g} / \mathrm{mL}) \mathrm{mL}$

\% Moisture: not dec.

GC Column: DB-624

Soil Extract Volume: TRACE

CONCENTRATION UNITS: (ug/I or $\mathrm{ug} / \mathrm{kg}$ )
Contract: 8E-00302 SDG No.: 200-6522
Lab Sample ID: MB 200-23630/5

Lab File ID: DHPK05.D

Date Received:

Date Analyzed: 08/15/2011

Dilution Factor: 1.0

Soil Aliquot Volume: (uL) (uL) $\operatorname{ug} / \mathrm{L}$
Purge Volume: 25.0 (mL)

\begin{tabular}{|l|l|l|r|r|r|}
\hline \multicolumn{1}{|c|}{ CAS NUMBER } & \multicolumn{1}{|c|}{ COMPOUND NAME } & RT & EST. CONC. & Q \\
\cline { 2 - 6 } 01 & & 6.79 & $\mathrm{~J}$ \\
\hline
\end{tabular}

\footnotetext{
1 EPA-designated Registry Number.
} 


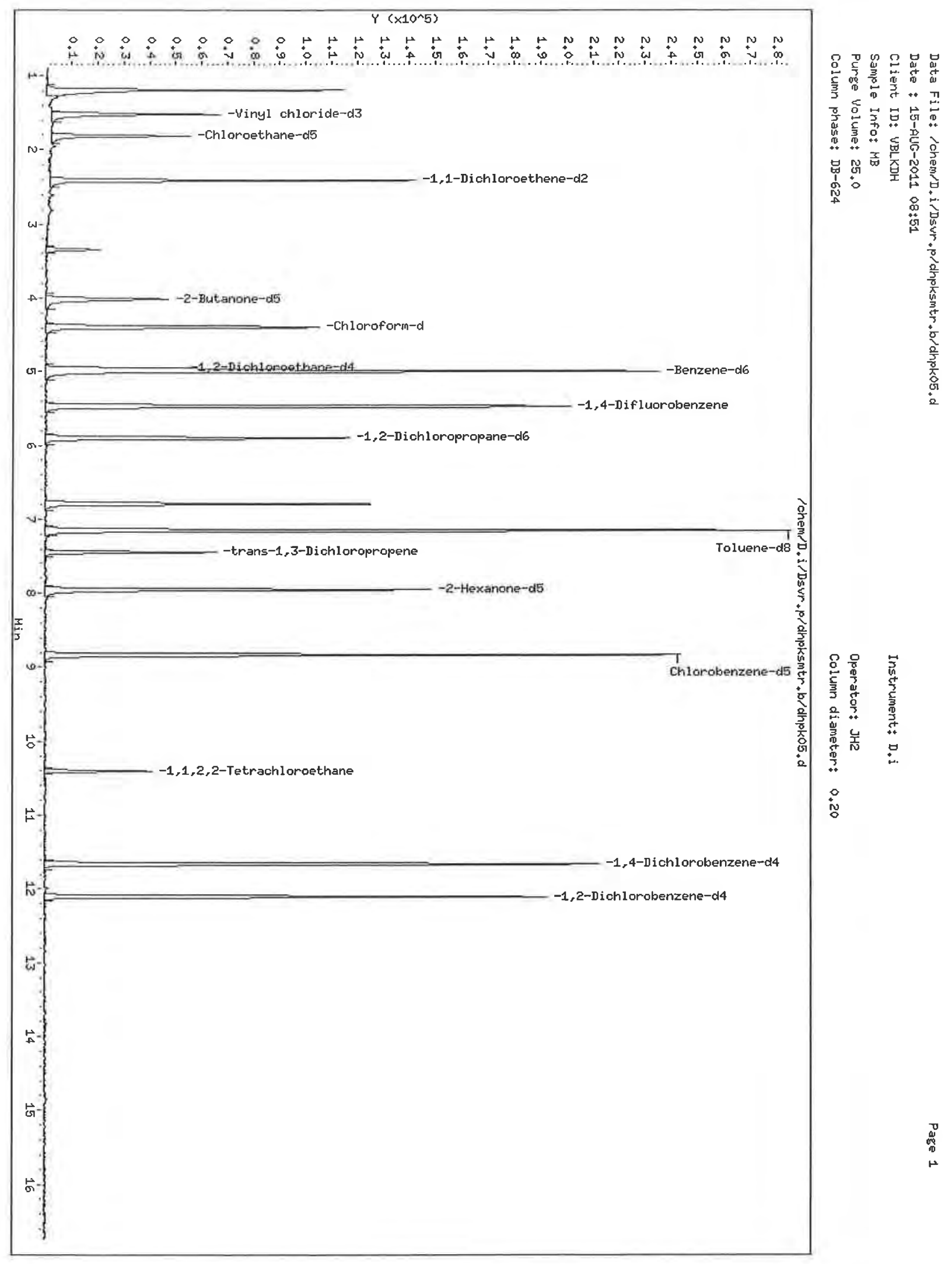




\section{TestAmerica Burlington}

LOW CONCENTRATION VOLATILE QUANTITATION REPORT

Data file : /chem/D.i/Dsvr.p/dhpksmtr.b/dhpk05.d

Lab Smp Id: MB Client Smp ID: VBLKDH

Inj Date : 15-AUG-2011 08:51

Operator : JH2

Inst ID: D.i

Smp Info : $\mathrm{MB}$

Misc Info: 1,5

Comment :

Method : /chem/D.i/Dsvr.p/dhpksmtr.b/somtr5t.m

Meth Date : 15-Aug-2011 16:21 jh2 Quant Type: ISTD

Cal Date : 07-JUL-2011 12:56 Cal File: dhp08.d

Als bottle: 3

Dil Factor: 1.00000

Integrator: HP RTE

Target Version: 3.50

Processing Host: chemsvr6

Concentration Eormula: Amt * DF * Uf/Vo * CpndVariable

\begin{tabular}{clc} 
Name & Value & Description \\
\hline DF & 1.00000 & Dilution Factor \\
Uf & 1.00000 & ng unit correction factor \\
Vo & 25.00000 & Sample volume purged (mL)
\end{tabular}

Cpnd Variable

Local Compound Variable

Compounds

1 Dichlorodifluoromethane

2 Chloromethane

\$ 3 Vinyl chloride-d3

4 Vinyl chloride

5 Bromomethane

\$ 6 Chloroethane-d5

7 Chloroethane

8 Trichlorofluoromethane

\$ 91,1-Dichloroethene-d2

10 1,1-Dichloroethene

11 1, 1,2-Trichloro-1, 2, 2-trifluo

12 Acetone

13 Carbon disulfide

14 Methyl acetate

15 Methylene chloride

16 trans-1,2-Dichloroethene

QUANT SIG
MASS
ma=m
85
50
65
62
94
69
64
101
63
96
101
43
76
43
84
96

CONCENTRATIONS

\begin{tabular}{|c|c|c|c|c|}
\hline & & & ON-COLUMN & FINAL \\
\hline RT & EXP RT REL RT & RESPONSE & ( $n g)$ & $(\mathrm{ug} / \mathrm{L})$ \\
\hline$=$ & 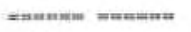 & 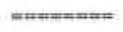 & 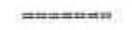 & 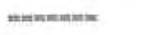 \\
\hline Comp & ound Not Detect & & & \\
\hline Comp & ound Not Detect & & & \\
\hline 1.515 & $1.521 \quad(0.277)$ & 80437 & 128.334 & 5.1 \\
\hline Comp & ound Not Detect & & & \\
\hline Comp & ound Not Detect & & & \\
\hline 1.808 & $1.807 \quad(0.331)$ & 52707 & 129.607 & 5.2 \\
\hline Corng & ound Not Detect & & & \\
\hline Conp & ound Not Detect & $t$ & & \\
\hline 2.405 & $2.411(0.440)$ & 101417 & 88.6551 & 3.5 \\
\hline Comp & ound Not Detect & & & \\
\hline Comp & ound Not Detect & & & \\
\hline Comp & ound Not Detect & & & \\
\hline 2.606 & $2.612(0.477)$ & 1791 & 1.67784 & $0.067(a)$ \\
\hline Comp & ound Not Detect & & & \\
\hline 2.813 & $2.813(0.515)$ & 428 & 1.18903 & $0.048(\mathrm{aQ})$ \\
\hline
\end{tabular}




\begin{tabular}{|c|c|c|c|}
\hline \multicolumn{3}{|c|}{ Compounds } & \multirow{2}{*}{ MASS } \\
\hline & & & \\
\hline & 17 & Methyl tert-butyl ether & 73 \\
\hline & 18 & 1,1-Dichloroethane & 63 \\
\hline \multirow[t]{4}{*}{$\$$} & 19 & 2-Butanone-d5 & 46 \\
\hline & 20 & cis-1,2-Dichloroethene & 96 \\
\hline & 21 & 2-Butanone & 43 \\
\hline & 22 & Bromochloromethane & 128 \\
\hline \multirow[t]{5}{*}{$\$$} & 23 & Chloroform-d & 84 \\
\hline & 24 & Chloroform & 83 \\
\hline & 25 & $1,1,1$-Trichloroethane & 97 \\
\hline & 26 & Cyclohexane & 56 \\
\hline & 27 & Carbon tetrachloride & 117 \\
\hline$\$$ & 28 & 1,2-Dichloroethane-d4 & 65 \\
\hline \multirow[t]{3}{*}{$\$$} & 29 & Benzene-d6 & 84 \\
\hline & 30 & Benzene & 78 \\
\hline & 31 & 1,2-Dichloroethane & 62 \\
\hline \multirow[t]{2}{*}{ * } & 32 & 1,4-Difluorobenzene & 114 \\
\hline & 33 & Trichloroethene & 95 \\
\hline \multirow[t]{6}{*}{$\$$} & 34 & 1,2-Dichloropropane-d6 & 67 \\
\hline & 35 & Methylcyclohexane & 55 \\
\hline & 36 & 1,2-Dichloropropane & 63 \\
\hline & 37 & Bromodichloromethane & 83 \\
\hline & 38 & cis-1,3-Dichloropropene & 75 \\
\hline & 39 & 4-Methyl-2-pentanone & 43 \\
\hline \multirow[t]{2}{*}{$\$$} & 40 & Toluene-d8 & 98 \\
\hline & 41 & Toluene & 91 \\
\hline \multirow[t]{4}{*}{$\$$} & 42 & trans-1,3-Dichloropropene-dd & 79 \\
\hline & 43 & trans-1,3-Dichloropropene & 75 \\
\hline & 44 & 1,1,2-Trichloroethane & 97 \\
\hline & 45 & Tetrachloroethene & 163 \\
\hline \multirow[t]{4}{*}{$\$$} & 46 & 2-Hexanone-d5 & 63 \\
\hline & 47 & 2-Hexanone & 43 \\
\hline & 48 & Dibromochloromethane & 129 \\
\hline & 49 & 1,2-Dibromoethane & 107 \\
\hline \multirow[t]{8}{*}{ * } & 50 & Chlorobenzene-d5 & 117 \\
\hline & 51. & Chlorobenzene & 112 \\
\hline & 52 & Ethylbenzene & 91 \\
\hline & 53 & m,p-Xylene & 106 \\
\hline & 54 & Styrene & 104 \\
\hline & 55 & o-Xylene & 106 \\
\hline & 56 & Bromoform & 172 \\
\hline & 57 & Isopropylbenzene & 105 \\
\hline \multirow[t]{3}{*}{ \$ } & 58 & $1,1,2,2$-Tetrachloroethane-d2 & 84 \\
\hline & 59 & $1,1,2,2$-Tetrachloroethane & 83 \\
\hline & 60 & 1,3-Dichlorobenzene & 146 \\
\hline \multirow[t]{2}{*}{ t } & 61 & 1,4-Dichlorobenzene-d4 & 152 \\
\hline & 62 & 1,4-Dichlorobenzene & 146 \\
\hline \multirow[t]{2}{*}{$\$$} & 63 & 1,2-Dichlorobenzene-d4 & 152 \\
\hline & 64 & 1,2-Dichlorobenzene & 146 \\
\hline
\end{tabular}

\begin{tabular}{|c|c|c|c|c|c|}
\hline \multirow[b]{2}{*}{ QUANT SIG } & & & & \multicolumn{2}{|c|}{ CONCENTRATIONS } \\
\hline & & & & ON-COLUMN & FINAL \\
\hline MASS & $\mathrm{RT}$ & EXP RT REL RT & RESPONSE & $(\mathrm{ng})$ & $(\mathrm{ug} / \mathrm{L})$ \\
\hline =men= & $=$ & 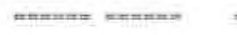 & netmonate & m=ertse & = =mentur \\
\hline 73 & \multicolumn{3}{|c|}{ Compound Not Detected. } & & \\
\hline 63 & \multicolumn{3}{|c|}{ Compound Not Detected. } & & \\
\hline 46 & 4.014 & $4.020(0.735)$ & 77034 & 1297.16 & 52 \\
\hline 96 & \multicolumn{3}{|c|}{ Compound Not Detected. } & & \\
\hline 43 & \multicolumn{2}{|c|}{ Compound Not Detected. } & & & \\
\hline 128 & \multicolumn{2}{|c|}{ Compound Not Detected. } & . & & \\
\hline 84 & 4.392 & $4.392(0.804)$ & 86416 & 117.918 & $4.7(0)$ \\
\hline 83 & \multicolumn{2}{|c|}{ Compound Not Detected. } & & & \\
\hline 97 & \multicolumn{2}{|c|}{ Compound Not Detected. } & & & \\
\hline 56 & \multicolumn{2}{|c|}{ Compound Not Detected. } & & & \\
\hline 117 & \multicolumn{3}{|c|}{ Compound Not Detected. } & & \\
\hline 65 & 4.953 & $4.959(0.906)$ & 36387 & 124.530 & 5.0 \\
\hline 84 & 4.990 & $4.990(0.565)$ & 220517 & 122.682 & 4.9 \\
\hline 78 & 5.032 & $5.032(0.570)$ & 124 & 0.06717 & $0.0027(a)$ \\
\hline 62 & \multicolumn{3}{|c|}{ Compound Not Detected. } & & \\
\hline 114 & 5.465 & $5.471(1.000)$ & 179847 & 125.000 & \\
\hline 95 & \multicolumn{3}{|c|}{ Compound Not Detected. } & & \\
\hline 67 & 5.898 & $5.904 \quad(0.66 \theta)$ & 54563 & 119.613 & 4.8 \\
\hline 55 & \multicolumn{3}{|c|}{ Compound Not Detected. } & & \\
\hline 63 & \multicolumn{3}{|c|}{ Compound Not Detected. } & & \\
\hline 83 & \multicolumn{3}{|c|}{ Compound Not Detected. } & & \\
\hline 75 & \multicolumn{3}{|c|}{ Compound Not Detected. } & & \\
\hline 43 & \multicolumn{3}{|c|}{ Compound Not Detected. } & & \\
\hline 98 & 7.148 & $7.154 \quad(0.809)$ & 209065 & 122.804 & 4.9 \\
\hline 91 & 7.227 & $7.227(0.818)$ & 489 & 0.24230 & $0.0097(a Q)$ \\
\hline 79 & 7.447 & $7.447 \quad(0.843)$ & 33699 & 120.444 & 4.8 \\
\hline 75 & \multicolumn{3}{|c|}{ Compound Not Detected. } & & \\
\hline 97 & \multicolumn{3}{|c|}{ Compound Not Detected. } & & \\
\hline 163 & Comp & ound Not Detected. & & & \\
\hline 63 & 7.947 & $7.946(0.900)$ & 53169 & 1333.75 & 53 \\
\hline 43 & Comp & ound Not Detected. & & & \\
\hline 129 & Comp & ound Not Detected. & & & \\
\hline 107 & Comp & ound Not Detected. & & & \\
\hline 117 & 8.831 & $8.830 \quad(1.000)$ & 152278 & 125.000 & \\
\hline 112 & Comp & ound Not Detected. & & & \\
\hline 91 & Comp & ound Not Detected. & & & \\
\hline 106 & Comp & ound Not Detected. & & & \\
\hline 104 & Comp & ound Not Detected. & & & \\
\hline 106 & Comp & ound Not Detected. & & & \\
\hline 172 & Comp & ound Not Detected. & & & \\
\hline 105 & Comp & ound Not Detected. & & & \\
\hline 84 & 10.403 & $10.403(1.178)$ & 22769 & 114.134 & 4.6 \\
\hline 83 & Cong & ound Not Detected. & & & \\
\hline 146 & Comp & ound Not Detected. & & & \\
\hline 152 & 11.659 & $11.659(1.000)$ & 61195 & 125.000 & (Q) \\
\hline 146 & Comp & ound Not Detected. & & & \\
\hline 152 & 12.104 & $12.104 \quad(1.038)$ & 54591 & 134.409 & 5.4 \\
\hline 146 & Comp & ound Not Detected. & & & \\
\hline
\end{tabular}


Data File: /chem/D.i/Dsvr.p/dhpksmtr.b/dhpk05.d

Report Date: 15-Aug-2011 16:21

\begin{tabular}{|c|c|c|c|c|c|c|}
\hline \multirow[b]{3}{*}{ Compounds } & \multirow[b]{2}{*}{ QUANT SIG } & & & & \multicolumn{2}{|c|}{ CONCENTRATIONS } \\
\hline & & & & & ON-COLUMN & EINAL \\
\hline & MASS & RT & EXP RT REL RT & RESPONSE & ( $\mathrm{ng})$ & $(\mathrm{ug} / \mathrm{L})$ \\
\hline 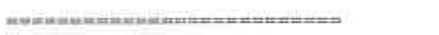 & $==\mathrm{c}=$ & $=$ & 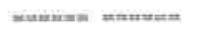 & + & 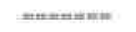 & m=metes \\
\hline 65 1,2-Dibromo-3-chloropropane & 75 & & ound Not Detect & & & \\
\hline $661,2,4$-Trichlorobenzene & 180 & & ound Not Detect & & & \\
\hline 67 1,2,3-Trichlorobenzene & 180 & & ound Not Detect & & & \\
\hline
\end{tabular}

QC Flag Legend

a - Target compound detected but, quantitated amount Below Limit of Quantitation (BLOQ).

Q - Qualifier signal failed the ratio test. 


\section{TestAmerica Burlington}

LOW CONCENTRATION VOLATILE QUANTITATION REPORT

Data file:/chem/D.i/Dsvr.p/dhpksmtr.b/dhpk05.d

Lab Smp Id: MB

Client Smp ID: VBLKDH

Inj Date : 15-AUG-2011 08:51

Operator : JH2

Inst ID: D. i

Smp Info : $\mathrm{MB}$

Misc Info : 1,5

Comment :

Method : /chem/D.i/Dsvr.p/dhpksmtr.b/somtr5t.m

Meth Date : 15-Aug-2011 16:21 jh2 Quant Type: ISTD

Cal Date : 07-JUL-2011 12:56 Cal File: dhp08.d

Als bottle: 3

Dil Factor: 1.00000

Integrator: HP RTE

Target Version: 3.50

Processing Host: chemsvr6

Concentration Eormula: Amt * DF * Uf/Vo * CpndVariable

\begin{tabular}{clc} 
Name & Value & Description \\
\hline DE & 1.00000 & Dilution Factor \\
Uf & 1.00000 & ng unit correction factor \\
Vo & 25.00000 & Sample volume purged (mL)
\end{tabular}

Cpnd Variable

\section{ISTD}

$=== \pm==$

* 32 1,4-Difluorobenzene

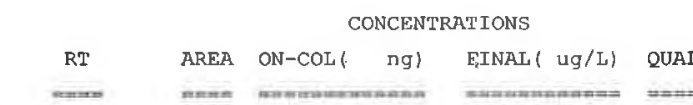

Unknown alkane

$3.344 \quad 41112 \quad 12.7686381$

Unknown

6.794

$244191 \quad 75.8414614$
Local Compound Variable

$\begin{array}{llr}\begin{array}{c}\mathrm{RT} \\ ====\end{array} & \begin{array}{c}\text { AREA } \\ =====\end{array} & \begin{array}{l}\text { AMOUNT } \\ =====\end{array} \\ 5.465 & 402469 & 125.000\end{array}$

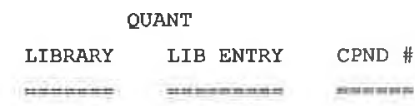

CAS \#:

0

$0 \quad 32$

CAS \#:

$\begin{array}{lll}0 & 0 & 32\end{array}$ 
Data File: dhpk05.d

Lab Sample ID: MB

Date: 15-AUG-2011 08:51

Client ID: VBLKDH

Instrument: D.i

Sample Info: MB

Operator: JH2

13 Carbon disulfide

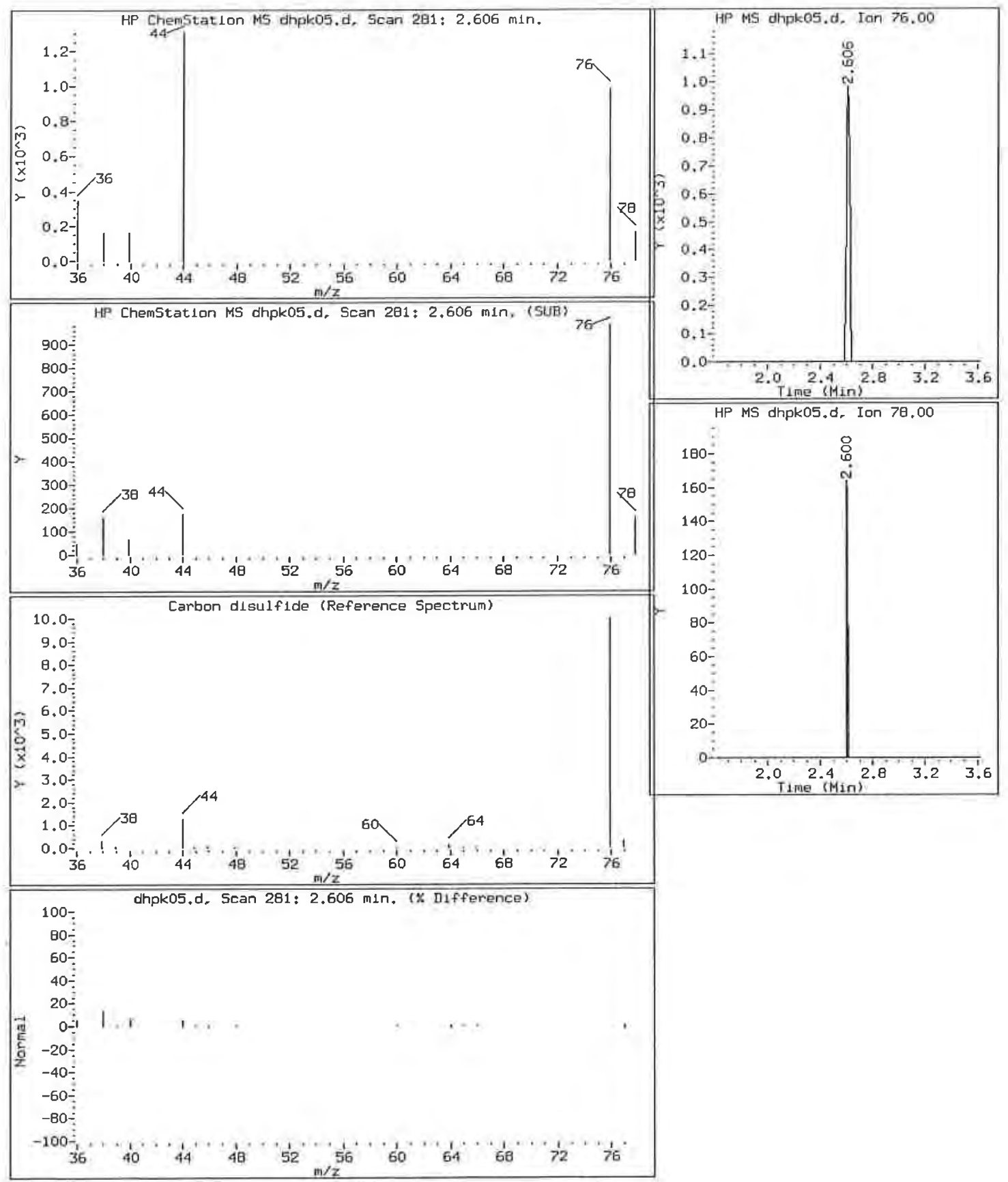


Data Eile: dhpk05.d

Lab Sample ID: MB

Date: 15-AUG-2011 08:51

Client ID: VBLKDH

Instrument: D.i

Sample Info: MB

Operator: JH2

15 Methylene chloride
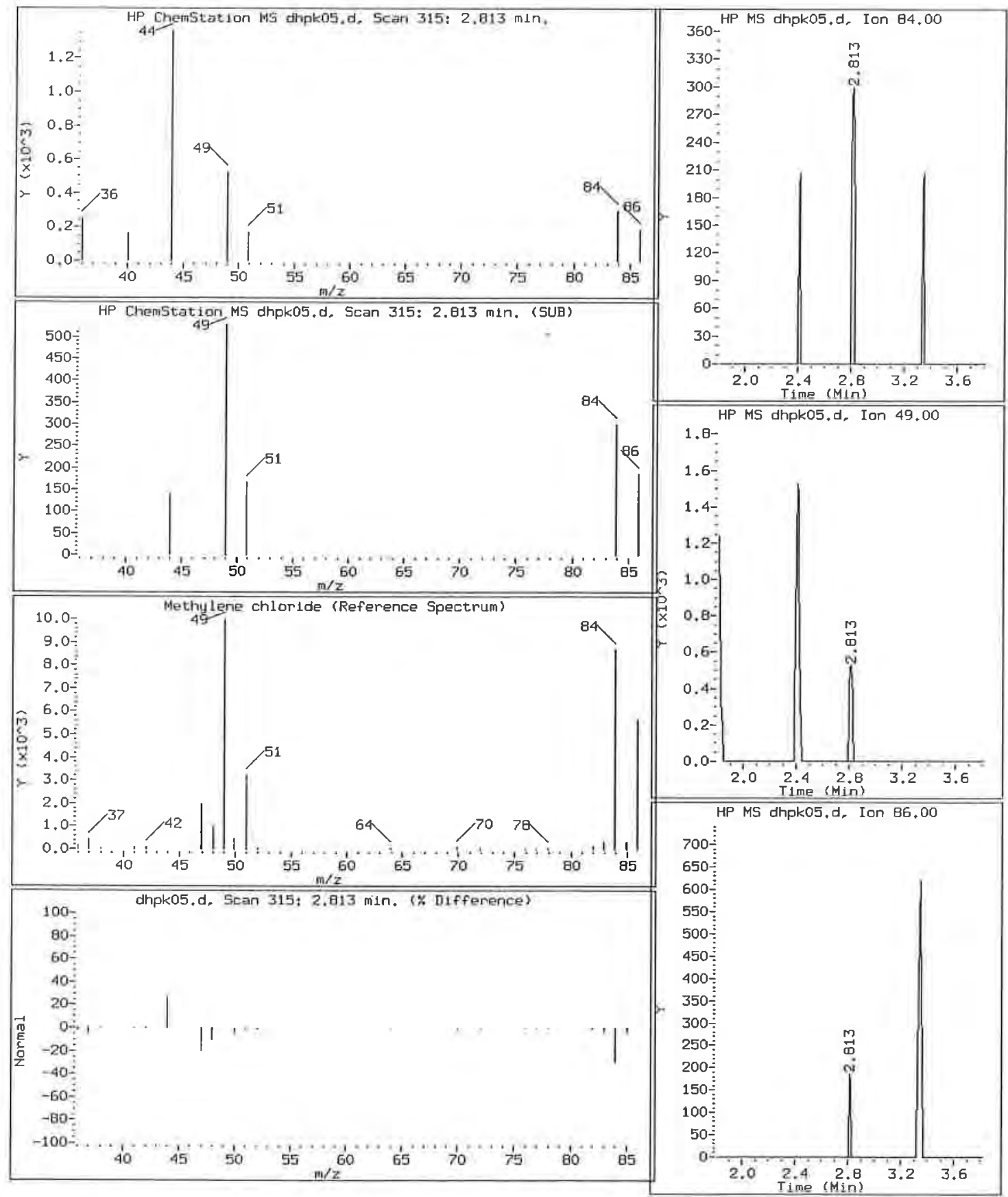
Data File: dhpk05.d

Lab Sample ID: MB

Date: 15-AUG-2011 08:51

Client ID: VBLKDH

Instrument: D.i

Sample Info: MB

Operator: JH2

30 Benzene
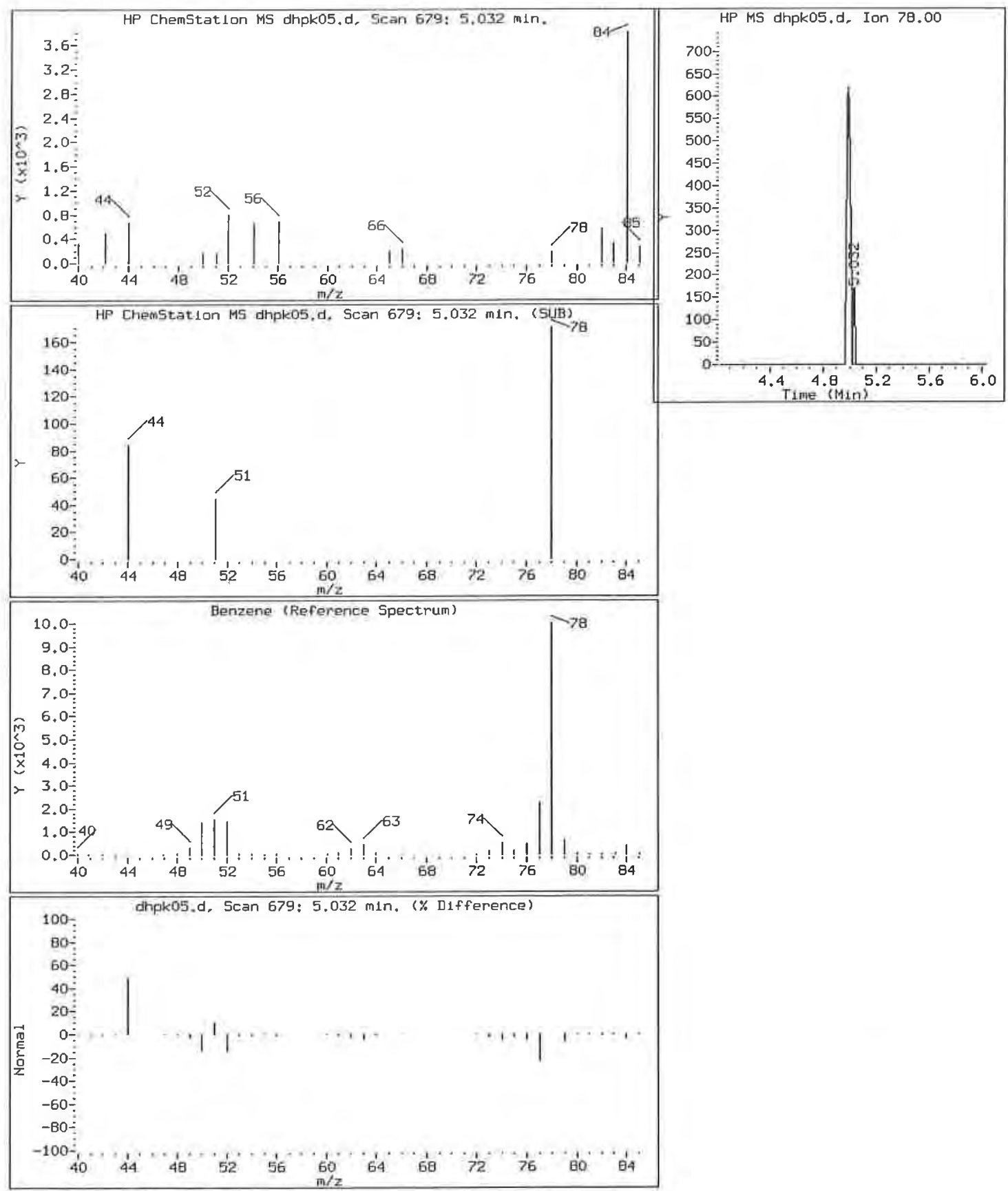
Data File: dhpk05.d

Lab Sample ID: MB

Date: 15-AUG-2011 08:51

Client ID: VBLKDH

Instrument: D.i

Sample Info: MB

Operator: JH2

41 Toluene
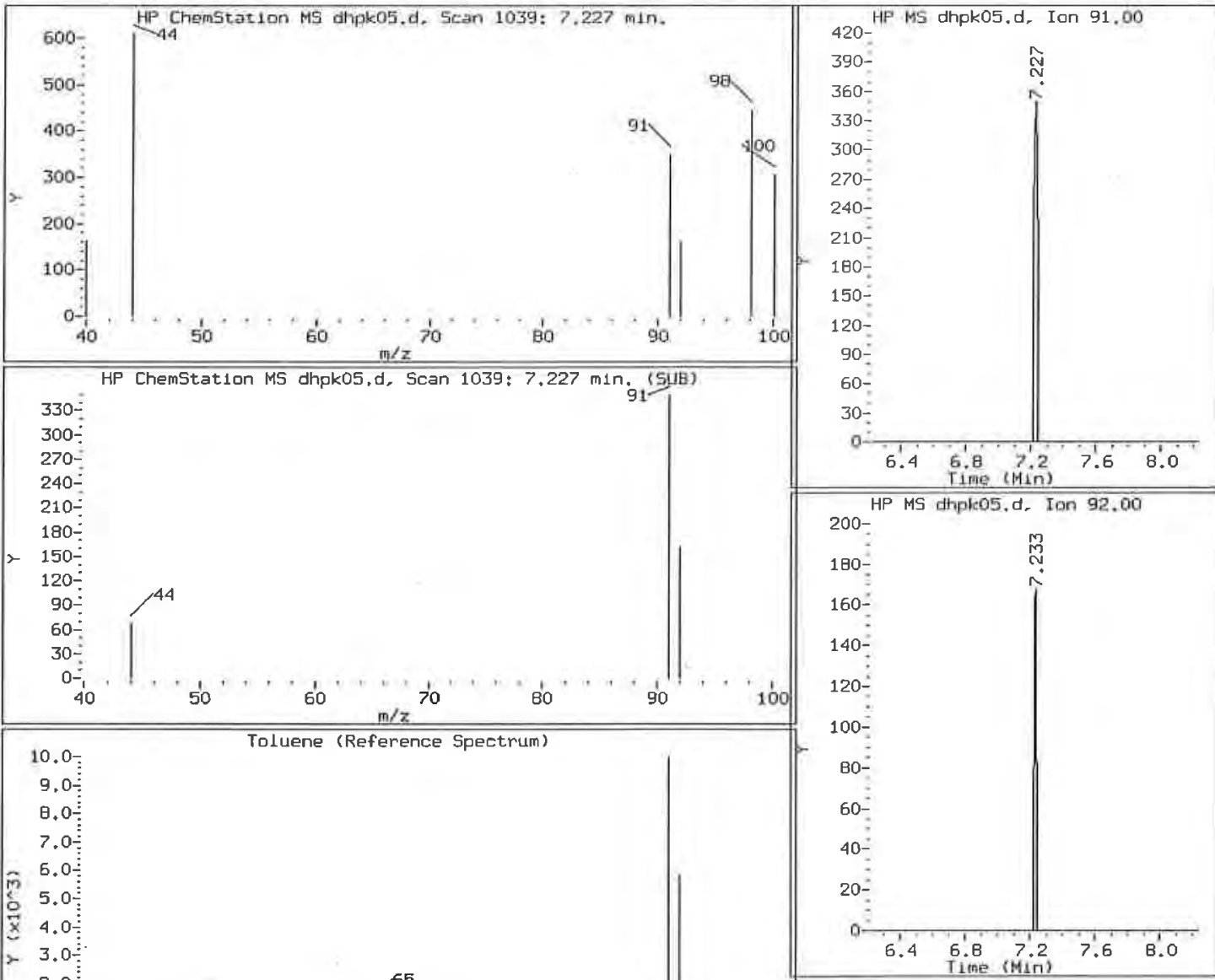

3.0

2.0

0.0
0.0

Toluene (Reference Spectrum)

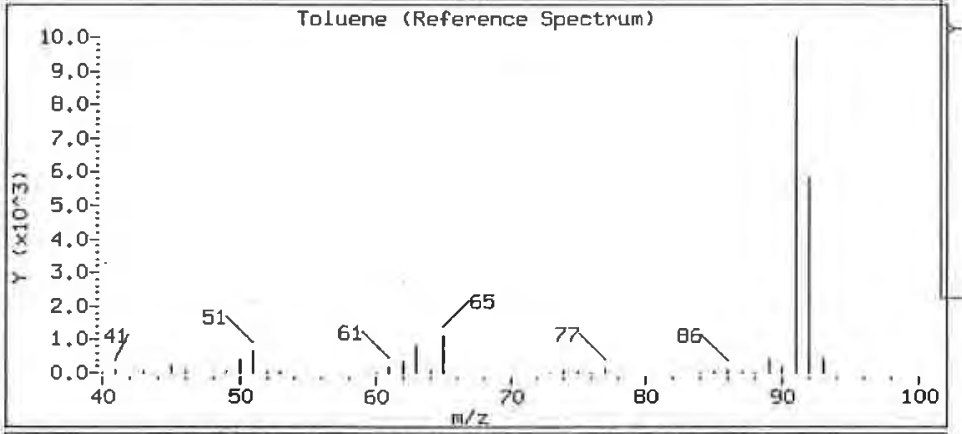

$100-$

$80-$

$60 \div$

$40-$

20

dhpik05,d, Scan 1039: 7.227 $\mathrm{m} 1 \mathrm{n}$. (\% D1fference)

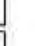

$\overrightarrow{7} \quad 0$

兴 $-20-$

$-40-$

$-60-$

$-80 \div$

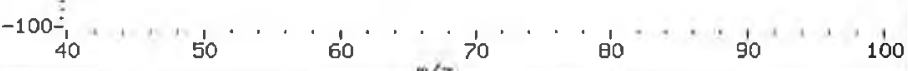


Data File: dhpk05.d

Lab Sample ID: MB

client ID: VBLKDH

Sample Info: $M B$

Retention Time: 3.34
Date: 15-AUG-2011 08:51

Instrument: D. 1

Operator: JH2

$\begin{array}{lcccccc}\text { Library Search Compound Match } & \text { CAS Number } & \text { Library } & \text { Entry } & \text { Quality } & \text { Formula } & \text { Weight } \\ \text { Unknown alkane } & & & & & & \\ \text { Hexane } & 110-54-3 & \text { NIS'05.1 } & 1792 & 86 & \text { C6H14 } & 86 \\ \text { Hexane } & 110-54-3 & \text { NIST05.1 } & 1790 & 80 & \text { C6H14 } & 86 \\ \text { Hexane } & 110-54-3 & \text { NIST05.1 } & 1791 & 59 & \text { C6H14 } & 86\end{array}$
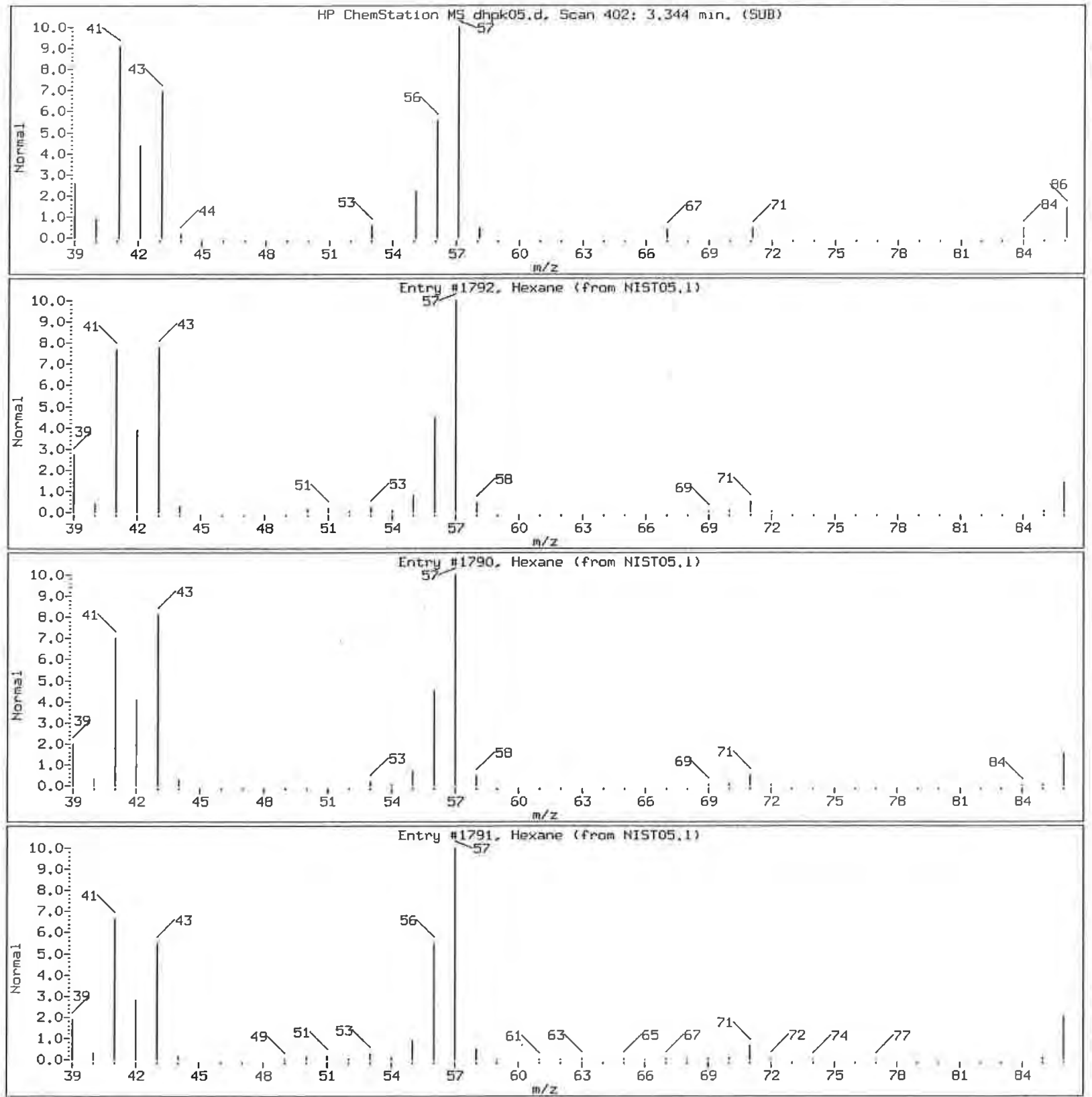
Data File: dhpk05.d

Lab Sample ID: MB

Client ID: VBLKDH

Sample Info: MB

Retention Time:

6.79

Library Search Compound Match Unknown

Carbonothioic dichloride

Methane, oxybis[chloro-

2-Propanol, 1,3-dichloro-
Date: 15-AUG-2011 08:51

Instrument: D.i

Operator: JH2
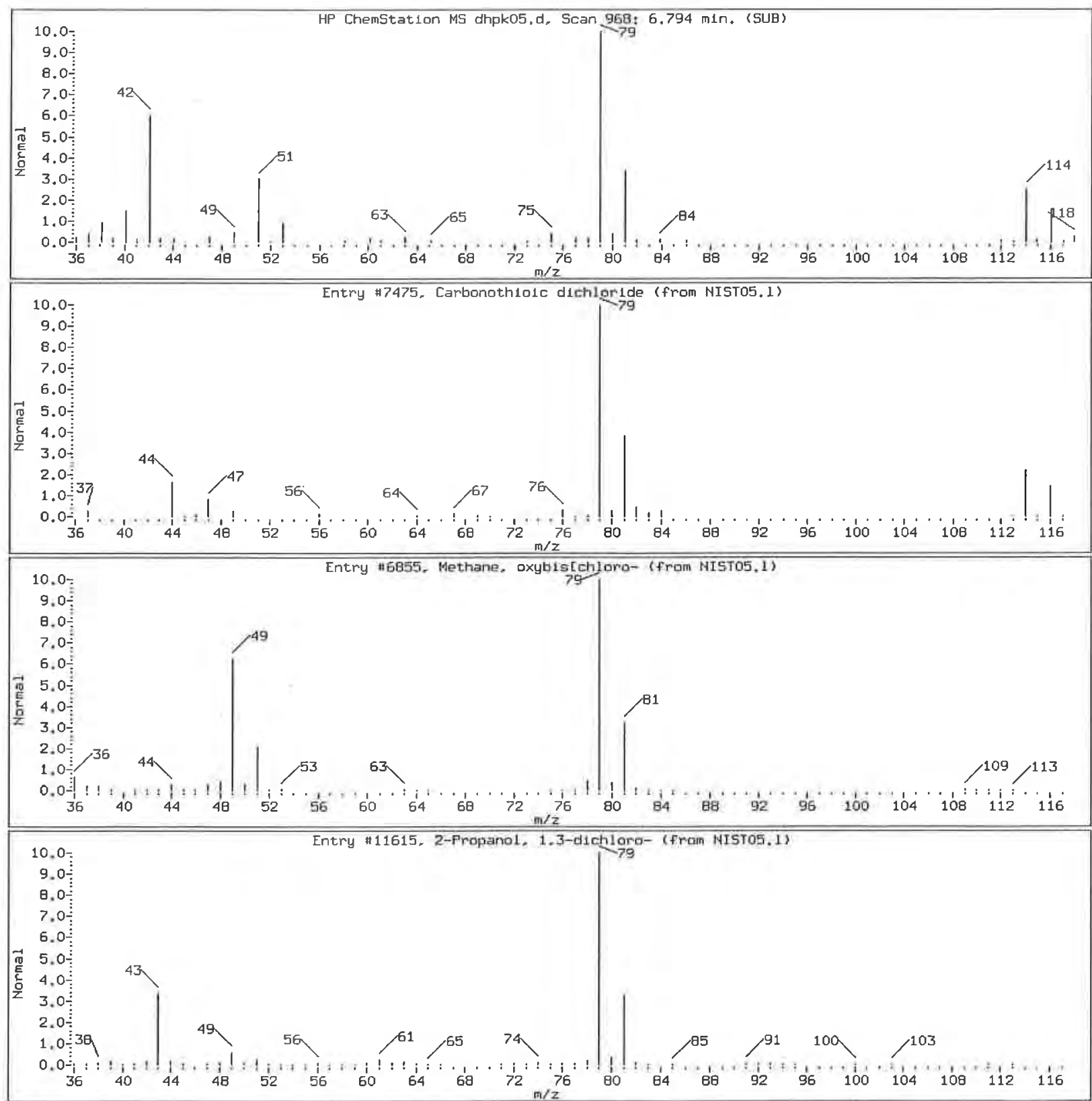
Lab Name: TESTAMERICA BURLINGTON

Contract: $8 \mathrm{E}-00302$

Lab Code: STLV Case No.: SAVANN Mod. Ref No.: SDG No.: 200-6522

Matrix: (SOIL/SED/WATER)

Sample wt/vol: 25.0

Water

$(g / m L) \quad m L$

Level: (TRACE/LOW/MED) TRACE

o Moisture: not dec.

GC Column: DB-624

Soil Extract Volume:

ID: $0.20 \quad$ (mm)

(uL)

(mL)

Purge Volume: 25.0
Lab Sample ID: 200-6522-6

Lab File ID: DHPK21.D

Date Received:

Date Analyzed: 08/15/2011

Dilution Factor: 1.0

Soil Aliquot Volume:

(uL)

\begin{tabular}{|c|c|c|c|}
\hline CAS NO. & COMPOUND & $\begin{array}{l}\text { CONCENTRATION UNITS: } \\
(\mathrm{ug} / \mathrm{L} \text { or } \mathrm{ug} / \mathrm{kg}) \mathrm{ug} / \mathrm{L}\end{array}$ & Q \\
\hline $75-71-8$ & Dichlorodifluoromethane & 0.50 & $\mathrm{U}$ \\
\hline $74-87-3$ & Chloromethane & 0.50 & $\mathrm{U}$ \\
\hline $75-01-4$ & Vinyl chloride & 0.50 & $\mathrm{U}$ \\
\hline $74-83-9$ & Bromomethane & 0.50 & $\mathrm{U}$ \\
\hline $75-00-3$ & Chloroethane & 0.50 & $\mathrm{U}$ \\
\hline $75-69-4$ & Trichlorofluoromethane & 0.50 & $\mathrm{U}$ \\
\hline $75-35-4$ & 1,1-Dichloroethene & 0.50 & $\mathrm{U}$ \\
\hline $76-13-1$ & 1,1,2-Trichloro-1,2,2-trifluoroethane & 0.50 & $\mathrm{U}$ \\
\hline $67-64-1$ & Acetone & 5.0 & $\mathrm{U}$ \\
\hline $75-15-0$ & Carbon disulfide & 0.50 & $\mathrm{U}$ \\
\hline $79-20-9$ & Methyl acetate & 0.50 & $\mathrm{U}$ \\
\hline $75-09-2$ & Methylene Chloride & 0.50 & $\mathrm{U}$ \\
\hline $156-60-5$ & trans-1,2-Dichloroethene & 0.50 & $\mathrm{U}$ \\
\hline $1634-04-4$ & Methyl tert-butyl ether & 0.50 & $\mathrm{U}$ \\
\hline $75-34-3$ & 1,1-Dichloroethane & 0.50 & $\mathrm{U}$ \\
\hline $156-59-2$ & cis-1,2-Dichloroethene & 0.50 & $\mathrm{U}$ \\
\hline $78-93-3$ & 2-Butanone & 5.0 & $\mathrm{U}$ \\
\hline $74-97-5$ & Bromochloromethane & 0.50 & $\mathrm{U}$ \\
\hline $67-66-3$ & Chloroform & 0.50 & $\mathrm{U}$ \\
\hline $71-55-6$ & 1,1,1-Trichloroethane & 0.50 & $\mathrm{U}$ \\
\hline $110-82-7$ & Cyclohexane & 0.50 & $\mathrm{U}$ \\
\hline $56-23-5$ & Carbon tetrachloride & 0.50 & $\mathrm{U}$ \\
\hline $71-43-2$ & Benzene & 0.50 & $\mathrm{U}$ \\
\hline $107-06-2$ & 1,2-Dichloroethane & 0.50 & $\mathrm{U}$ \\
\hline
\end{tabular}

Report 1,4-Dioxane for Low-Medium VOA analysis only 
EPA SAMPLE NO.

Lab Name: TESTAMERICA BURLINGTON

Lab Code: STLV

Contract: 8E-00302

Matrix: (SOIL/SED/WATER)

Sample wt/vol: 25.0

Water

$(\mathrm{g} / \mathrm{mL}) \quad \mathrm{mL}$

Level: (TRACE/LOW/MED) TRACE

? Moisture: not dec.

GC Column: DB-624 ID: $0.20 \quad$ (mm)

Soil Extract Volume:

Purge Volume: 25.0 (mL)
SDG No.: 200-6522

Lab Sample ID: 200-6522-6

Lab File ID: DHPK21.D

Date Received:

Date Analyzed: 08/15/2011

Dilution Factor: 1.0

Soil Aliquot Volume:

(uL)

\begin{tabular}{|c|c|c|c|}
\hline CAS NO. & COMPOUND & $\begin{array}{l}\text { CONCENTRATION UNITS: } \\
(\mathrm{ug} / \mathrm{L} \text { or } u g / k g) \mathrm{ug} / \mathrm{L}\end{array}$ & $Q$ \\
\hline $79-01-6$ & Trichloroethene & 0.50 & $\overline{\mathrm{U}}$ \\
\hline $108-87-2$ & Methylcyclohexane & 0.50 & $\mathrm{U}$ \\
\hline $78-87-5$ & 1,2-Dichloropropane & 0.50 & $\mathrm{U}$ \\
\hline $75-27-4$ & Bromodichloromethane & 0.50 & $\mathrm{U}$ \\
\hline $10061-01-5$ & cis-1,3-Dichloropropene & 0.50 & $\mathrm{U}$ \\
\hline $108-10-1$ & 4-Methyl-2-pentanone & 5.0 & $\mathrm{U}$ \\
\hline $108-88-3$ & Toluene & 0.50 & $\mathrm{U}$ \\
\hline $10061-02-6$ & trans-1,3-Dich1oropropene & 0.50 & $\mathrm{U}$ \\
\hline $79-00-5$ & 1,1,2-Trichloroethane & 0.50 & $\mathrm{U}$ \\
\hline $127-18-4$ & Tetrachloroethene & 0.50 & $\mathrm{U}$ \\
\hline $591-78-6$ & 2-Hexanone & 5.0 & U \\
\hline $124-48-1$ & Dibromochloromethane & 0.50 & $\mathrm{U}$ \\
\hline $106-93-4$ & 1,2-Dibromoethane & 0.50 & $\mathrm{U}$ \\
\hline $108-90-7$ & Chlorobenzene & 0.50 & $\mathrm{U}$ \\
\hline $100-41-4$ & Ethylbenzene & 0.50 & $\mathrm{U}$ \\
\hline $95-47-6$ & o-Xylene & 0.50 & $\mathrm{U}$ \\
\hline $179601-23-1$ & $\mathrm{~m}, \mathrm{p}$-Xylene & 0.50 & $\mathrm{U}$ \\
\hline $100-42-5$ & Styrene & 0.50 & $\mathrm{U}$ \\
\hline $75-25-2$ & Bromoform & 0.50 & U \\
\hline $98-82-8$ & Isopropylbenzene & 0.50 & $\mathrm{U}$ \\
\hline $79-34-5$ & $1,1,2,2$-Tetrachloroethane & 0.50 & $\mathrm{U}$ \\
\hline $541-73-1$ & 1,3-Dichlorobenzene & 0.50 & $\mathrm{U}$ \\
\hline $106-46-7$ & 1,4-Dichlorobenzene & 0.50 & $\mathrm{U}$ \\
\hline $95-50-1$ & 1,2-Dichlorobenzene & 0.50 & $\mathrm{U}$ \\
\hline $96-12-8$ & 1,2-Dibromo-3-Chloropropane & 0.50 & $\mathrm{U}$ \\
\hline $120-82-1$ & 1,2,4-Trichlorobenzene & 0.50 & $\mathrm{U}$ \\
\hline $87-61-6$ & 1,2,3-Trichlorobenzene & 0.50 & $\mathrm{U}$ \\
\hline
\end{tabular}


IJ - FORM I VOA-TIC

VOLATILE ORGANICS ANALYSIS DATA SHEET

TENTATIVELY IDENTIFIED COMPOUNDS
EPA SAMPLE NO.

VHBLK01

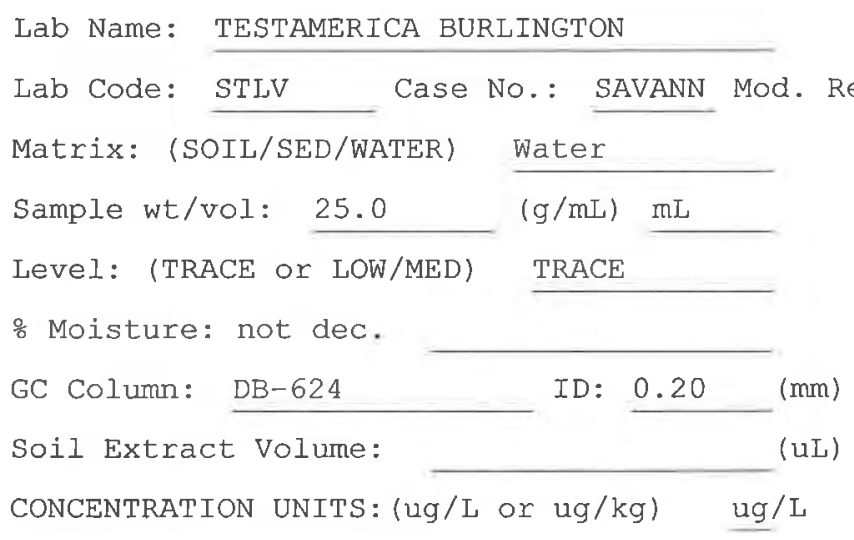

Lab Name: TESTAMERICA BURLINGTON

Contract: 8E-00302 SDG No.: 200-6522
Lab Sample ID: 200-6522-6

Lab File ID: DHPK21.D

Date Received:

Date Analyzed: 08/15/2011

Dilution Factor: 1.0

Soil Aliquot volume: (uL)

Purge Volume: 25.0 (mL)

01

02

\begin{tabular}{|c|l|c|c|c|}
\hline CAS NUMBER & \multicolumn{1}{|c|}{ COMPOUND NAME } & RT & EST. CONC. & Q \\
\hline \hline & Unknown & 6.79 & 2.8 & B X J \\
\hline E9667961 & Total Alkanes & N/A & & \\
\hline
\end{tabular}

1 EPA-designated Registry Number. 


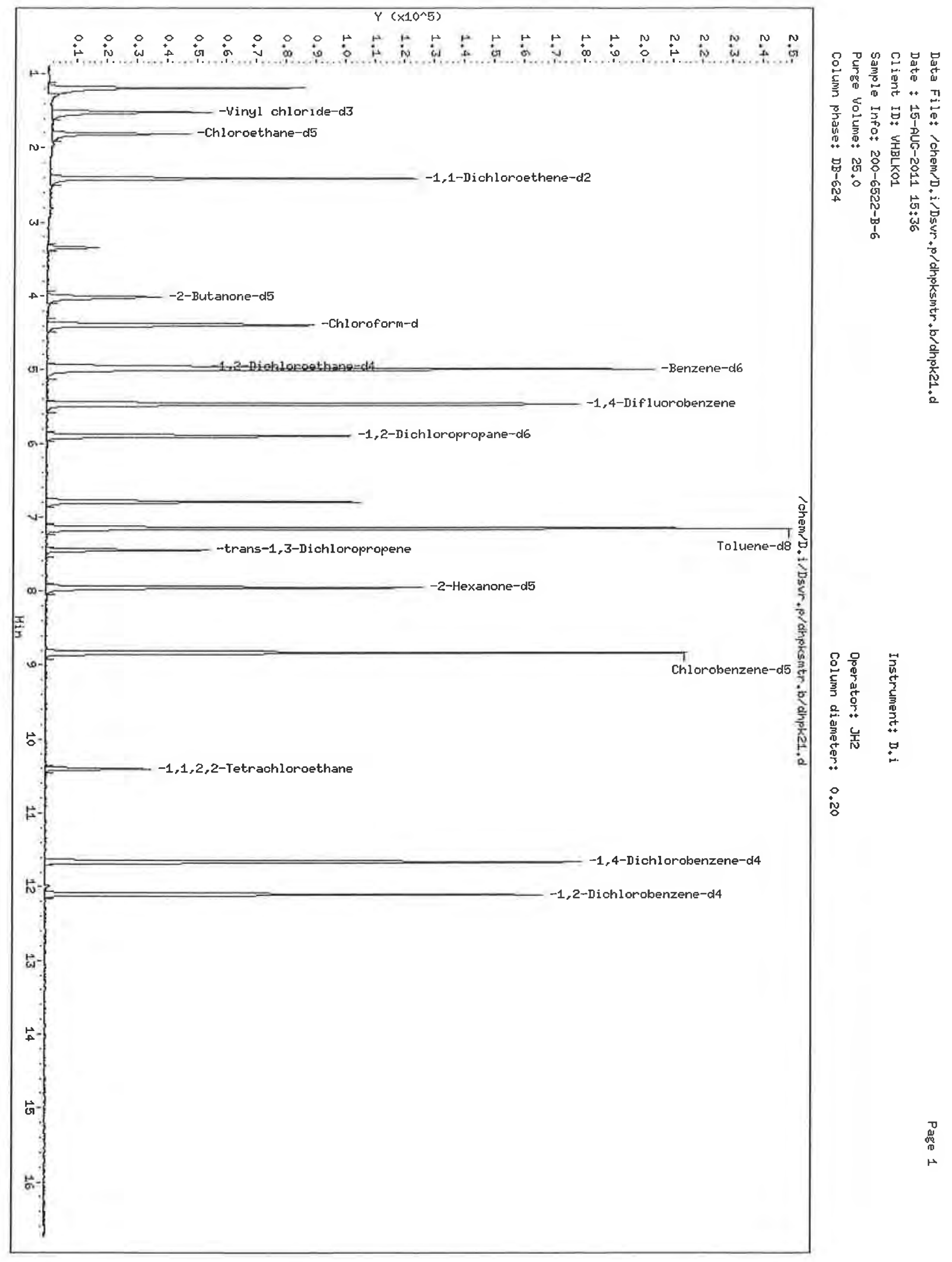




\section{TestAmerica Burlington}

Lab Sample Id: 200-6522-6

Client Smp ID: VHBLKO1

Inj Date : 15-AUG-2011 15:36

Operator : JH2

Inst ID: D.i

Smp Info: 200-6522-B-6

Misc Info: 1,5

Comment :

Method : /chem/D.i/Dsvr.p/dhpksmtr.b/somtr5t.m

Meth Date : 15-Aug-2011 16:21 jh2 Quant Type: ISTD

Cal Date : 07-JUL-2011 12:56 Cal File: dhp08.d

Als bottle: 19

Dil Factor: 1.00000

Integrator: HP RTE

Target Version: 3.50

Compound Sublist: all.sub

Processing Host: chemsvr6

\section{Concentration Formula: Amt * DF * Uf/Vo * CpndVariable}

$\begin{array}{clc}\text { Name } & \text { Value } & \text { Description } \\ \text { DE } & 1.00000 & \text { Dilution Factor } \\ \text { Uf } & 1.00000 & \text { ng unit correction factor } \\ \text { Vo } & 25.00000 & \text { Sample Volume purged (mL) }\end{array}$

Cpnd Variable

Local Compound Variable

Compounds

1 Dichlorodifluoromethane

2 chloromethane

\$ 3 Vinyl chloride-d3

4 Vinyl chloride

5 Bromomethane

$\$ 6$ Chloroethane-d5

7 Chloroethane

8 Trichlorofluoromethane

\$ 9 1,1-Dichloroethene-d2

10 1, 1-Dichloroethene

11 1,1,2-Trichloro-1,2,2-trifluo

12 Acetone

13 Carbon disulfide

14 Methyl acetate

15 Methylene chloride

QUANT SIG
MASS
85
50
65
62
94
69
64
101
63
96
101
43
76
43
84

CONCENTRATIONS

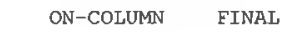

RT EXP RT REL RT RESPONSE ( ng) ( $\mathrm{ug} / \mathrm{L}$ )

Compound Not Detected.

Compound Not Detected.

$1.515 \quad 1.521 \quad(0.277)$

$67261 \quad 120.943 \quad 4.8$

Compound Not Detected.

Compound Not Detected.

$1.807 \quad 1.807 \quad(0.330)$

$44327 \quad 122.846 \quad 4.9$

Compound Not Detected.

Compound Not Detected.

$2.405 \quad 2.411 \quad(0.440)$

$\begin{array}{lll}89194 & 87.8737 & 3.5\end{array}$

Compound Not Detected.

Compound Not Detected.

Compound Not Detected.

Compound Not Detected.

Compound Not Detected.

Compound Not Detected. 


\section{Compounds}

16 trans-1,2-Dichloroethene 17 Methyl tert-butyl ether

18 1,1-Dichloroethane

\$ 19 2-Butanone-d5

20 cis-1,2-Dichloroethene

21 2-Butanone

22 Bromochloromethane

\$ 23 Chloroform-d

24 Chloroform

25 1, 1, 1-Trichloroethane

26 Cyclohexane

27 Carbon tetrachloride

\$ 28 1,2-Dichloroethane-d4

\$ 29 Benzene-d6

30 Benzene

31 1,2-Dichloroethane

- 32 1,4-Difluorobenzene 33 Trichloroethene

\$ 34 1,2-Dichloropropane-d6 35 Methylcyclohexane

36 1,2-Dichloropropane

37 Bromodichloromethane

38 cis-1,3-Dichloropropene

39 4-Methyl-2-pentanone

\$ 40 Toluene-d8

41 Toluene

\$ 42 trans-1,3-Dichloropropene-d4

43 trans-1, 3-Dichloropropene

44 1, 1,2-Trichloroethane

45 Tetrachloroethene

\$. 46 2-Hexanone-d5

47 2-Hexanone

$4 \theta$ Dibromochloromethane

49 1,2-Dibromoethane

- 50 Chlorobenzene-d5

51 Chlorobenzene

52 Ethylbenzene

$53 \mathrm{~m}, \mathrm{p}$-Xylene

54 styrene

55 o-Xylene

56 Bromoform

57 Isopropylbenzene

\$ $581,1,2,2$-Tetrachloroethane-d2

59 1, 1, 2, 2-Tetrachloroethane

60 1,3-Dichlorobenzene

- 61 1,4-Dichlorobenzene-d4

62 1,4-Dichlorobenzene

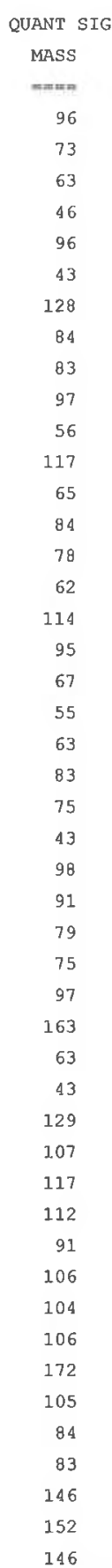

CONCENTRATIONS

ON-COLUMN FINAI

RT EXP RT REL RT RESPONSE ( ng) ( ug/L)

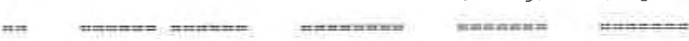

Compound Not Detected.

Compound Not Detected.

Compound Not Detected.

$4.020 \quad 4.020 \quad(0.735)$

Compound Not Detected.

Compound Not Detected.

Compound Not Detected.

$4.3924 .392(0.803)$

Compound Not Detected.

Compound Not Detected.

Compound Not Detected.

Compound Not Detected.

\begin{tabular}{llllll}
4.959 & 4.959 & $(0.906)$ & 31511 & 121.540 & 4.9 \\
4.990 & $4.990(0.565)$ & 191613 & 119.273 & 4.8 \\
\multicolumn{3}{c}{ Compound Not Detected. }
\end{tabular}

Compound Not Detected.

$5.471 \quad 5.471(1.000)$

Compound Not Detected.

$5.898 \quad 5.904 \quad(0.667)$

Compound Not Detected.

Compound Not Detected.

Compound Not Detected.

Compound Not Detected.

Compound Not Detected.

$7.154 \quad 7.154 \quad(0.810)$

$\begin{array}{lll}179591 & 118.031 & 4.7\end{array}$

Compound Not Detected.

$\begin{array}{llll}7.446 & 7.447 & (0.843)\end{array}$

Compound Not Detected.

Compound Not Detected.

Compound Not Detected.

$\begin{array}{lll}7.952 & 7.946 & (0.900)\end{array}$

Compound Not Detected.

Compound Not Detected.

Compound Not Detected.

$8.836 \quad 8.830(1.000)$

Compound Not Detected.

Compound Not Detected.

Compound Not Detected.

Compound Not Detected.

Compound Not Detected.

Compound Not Detected.

Compound Not Detected.

$10.403 \quad 10.403 \quad(1.177)$

$\begin{array}{lll}19589 & 109.866 & 4.4\end{array}$

Compound Not Detected.

Compound Not Detected.

$11.659 \quad 11.659 \quad(1.000)$

$51738 \quad 125.000$

(Q) 
Data File: /chem/D.i/Dsvr.p/dhpksmtr.b/dhpk21.d

Page 3

Report Date: 15-Aug-2011 16:21

\begin{tabular}{|c|c|c|c|c|c|c|}
\hline \multirow[b]{3}{*}{ Compounds } & \multirow[b]{2}{*}{ QUANT SIG } & & & & \multicolumn{2}{|c|}{ CONCENTRATIONS } \\
\hline & & & & & ON-COLUMN & FINAL \\
\hline & MASS & RT & EXP RT REL RT & RESPONSE & ( ng) & $(\mathrm{ug} / \mathrm{L})$ \\
\hline 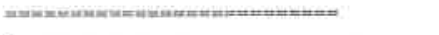 & $=$ & $=$ & 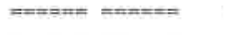 & 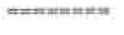 & 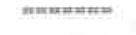 & $== \pm=n=$ \\
\hline$\$ 631,2$-Dichlorobenzene-d4 & 152 & 12.104 & $12.104 \quad(1.038\}$ & 47830 & 139.288 & $5.6(2)$ \\
\hline 641,2 -Dichlorobenzene & 146 & Comp & ound Not Detected. & & & \\
\hline 65 1,2-Dibromo-3-chloropropane & 75 & Comp & ound Not Detected. & & & \\
\hline $661,2,4$-Tr1chlorobenzene & 180 & Comp & ound Not Detected. & & & \\
\hline 67 1,2,3-Trichlorobenzene & 180 & Comp & ound Not Detected. & & & \\
\hline
\end{tabular}

QC Elag Legend

Q - Qualifier signal failed the ratio test. 
Lab Sample Id: 200-6522-6

Client Smp ID: VHBLK01

Inj Date : 15-AUG-2011 15:36

Operator : JH2

Inst ID: D.i

Smp Info : 200-6522-B-6

Misc Info : 1,5

Comment :

Method : /chem/D.i/Dsvr.p/dhpksmtr.b/somtr5t.m

Meth Date : 15-Aug-2011 16:21 jh2 Quant Type: ISTD

Cal Date : 07-JUL-2011 12:56 Cal File: dhp08.d

Als bottle: 19

Dil Factor: 1.00000

Integrator: HP RTE

Target Version: 3.50

Processing Host: chemsvr6

Concentration Formula: Amt * DF * Uf/Vo * CpndVariable

\begin{tabular}{cl} 
Name & Value \\
\hline DF & 1.00000 \\
Uf & 1.00000 \\
Vo & 25.00000
\end{tabular}

Description

Dilution Eactor

ng unit correction factor Sample Volume purged (mL)

Cpnd Variable

Local Compound Variable

ISTD

$= \pm== \pm===$

* 32 1,4-Difluorobenzene

CONCENTRATIONS

RT AREA ON-COL ( ng) FINAL ( ug/L) QUAL

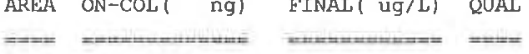

Unknown

6.794

$200304 \quad 69.5388401$

$\begin{array}{lll}\text { RT } & \begin{array}{c}\text { AREA } \\ =====\end{array} & \begin{array}{l}\text { AMOUNT } \\ =====\end{array} \\ 5.471 & 360057 & 125.000\end{array}$

LIBRARY LUANT

CAS \#:
$0 \quad 32$ 
Data File: dhpk21.d

Lab Sample ID: 200-6522-6

Client ID: VHBLK01

Date: 15-AUG-2011 15:36

Sample Info: 200-6522-B-6

Instrument: D.i

Operator: JH2

Retention Time: $\quad 6.79$

Library Search Compound Match Unknown

Carbonothioic dichloride

Methane, oxybis [chloro-

2-Propanol, 1,3-dichloro-

$\begin{array}{llllll}\text { CAS Number } & \text { Library } & \text { Entry } & \text { Quality } & \text { Eormula } & \text { Weight } \\ & & & & & \\ 463-71-8 & \text { NIST05.1 } & 7475 & 47 & \text { CCl2S } & 114 \\ 542-88-1 & \text { NIST05.1 } & 6855 & 32 & \text { C2H4Cl2O } & 114 \\ 96-23-1 & \text { NIST05.I } & 11615 & 25 & \text { C3H6Cl2O } & 128\end{array}$
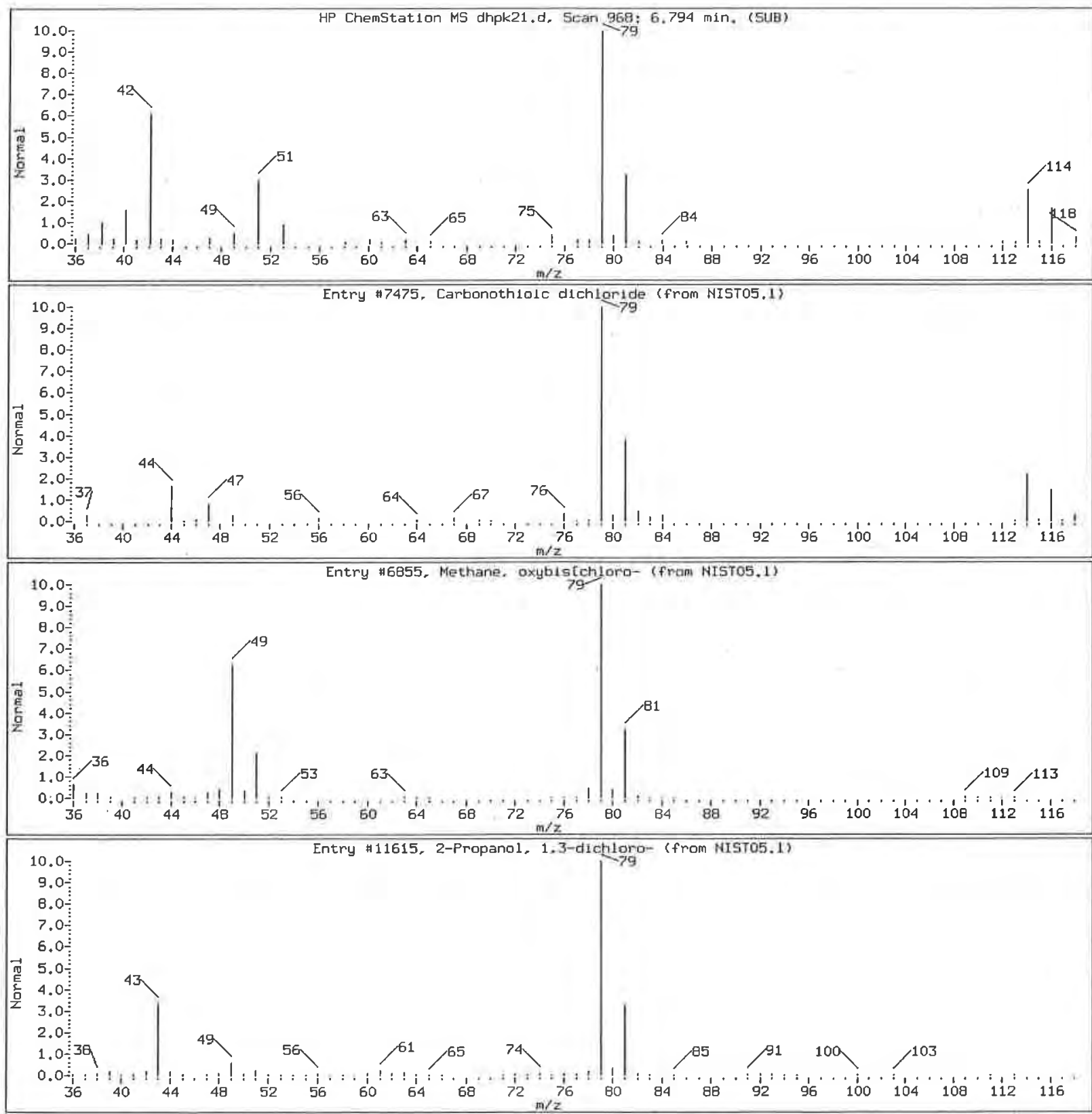

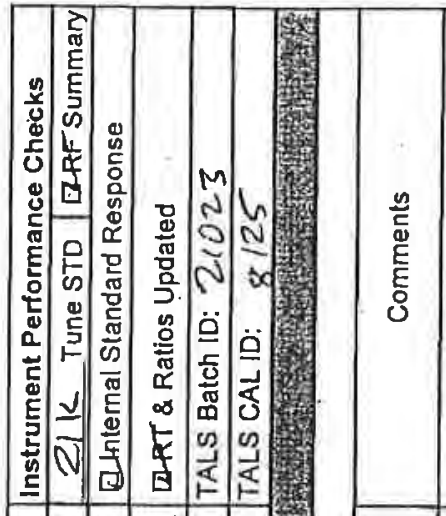

\begin{tabular}{l}
1 \\
3 \\
7 \\
7 \\
\hline
\end{tabular}

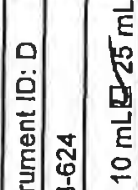

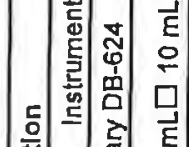

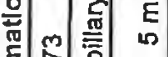

흥영

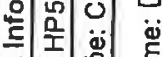

ง)

爷萑

- y unu

c

(

哀这吉

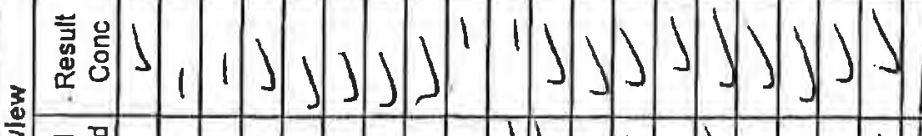

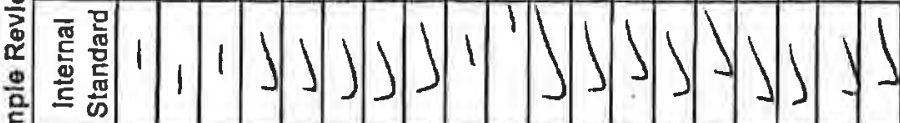

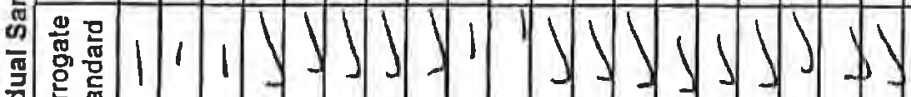

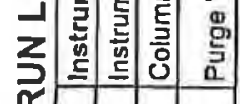

!

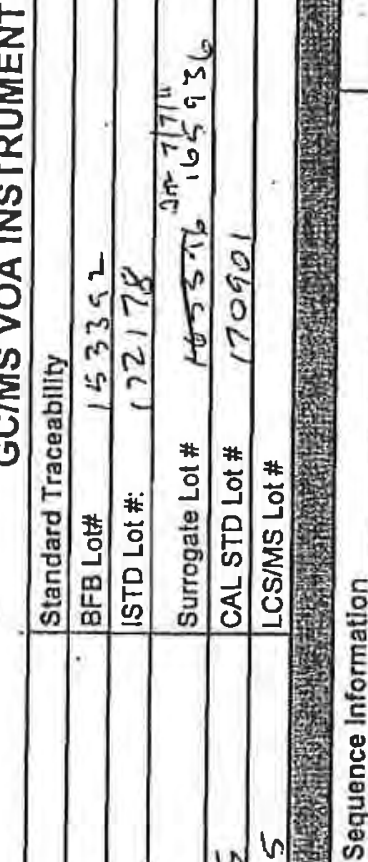
흘 空

$\frac{8}{\stackrel{0}{0}}$

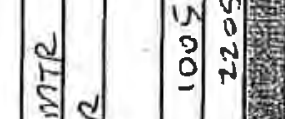

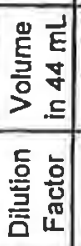

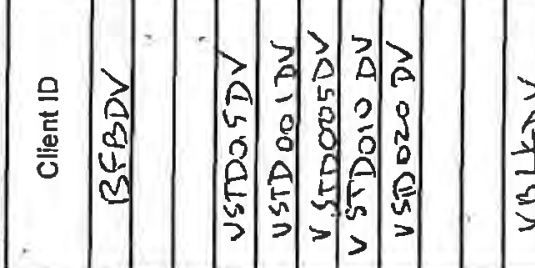

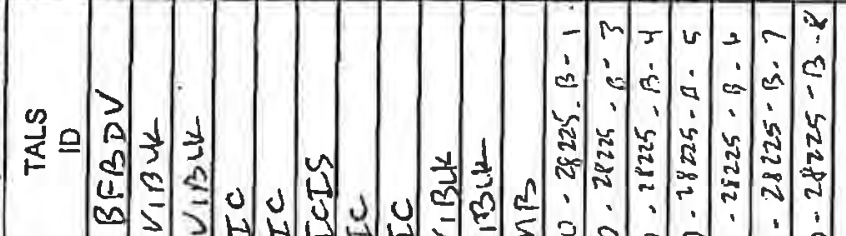

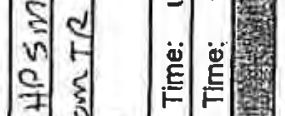

A $n=$

. $r=1+n$

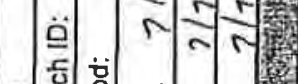

号密密言

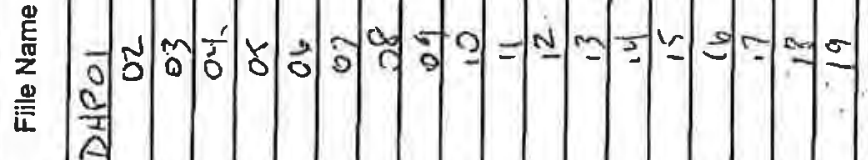

高

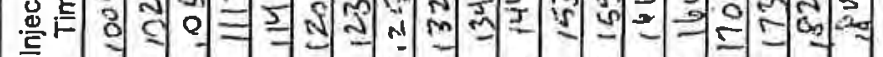

今 

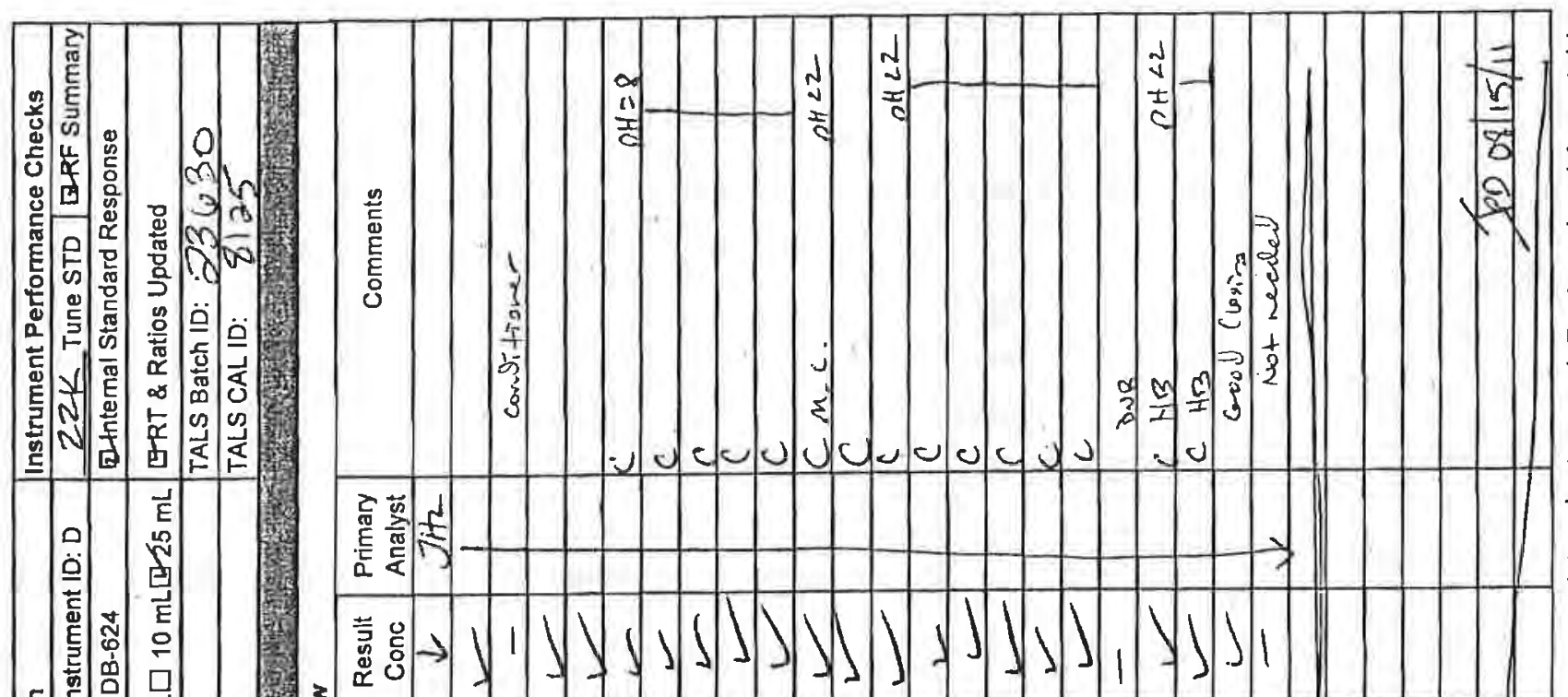

들

藏

을

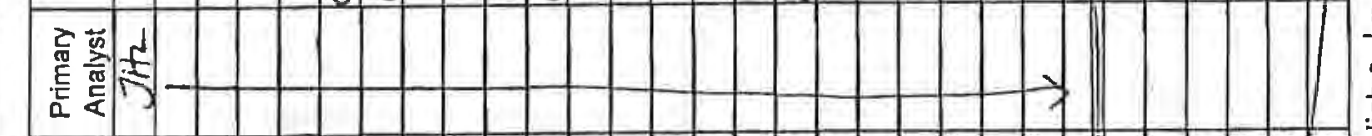

0 突至

i.

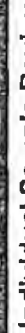

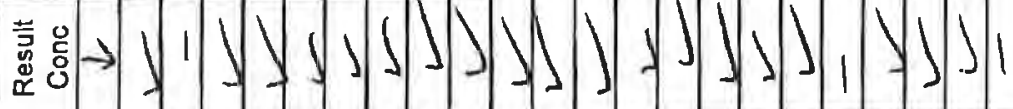

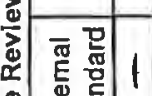

C 3

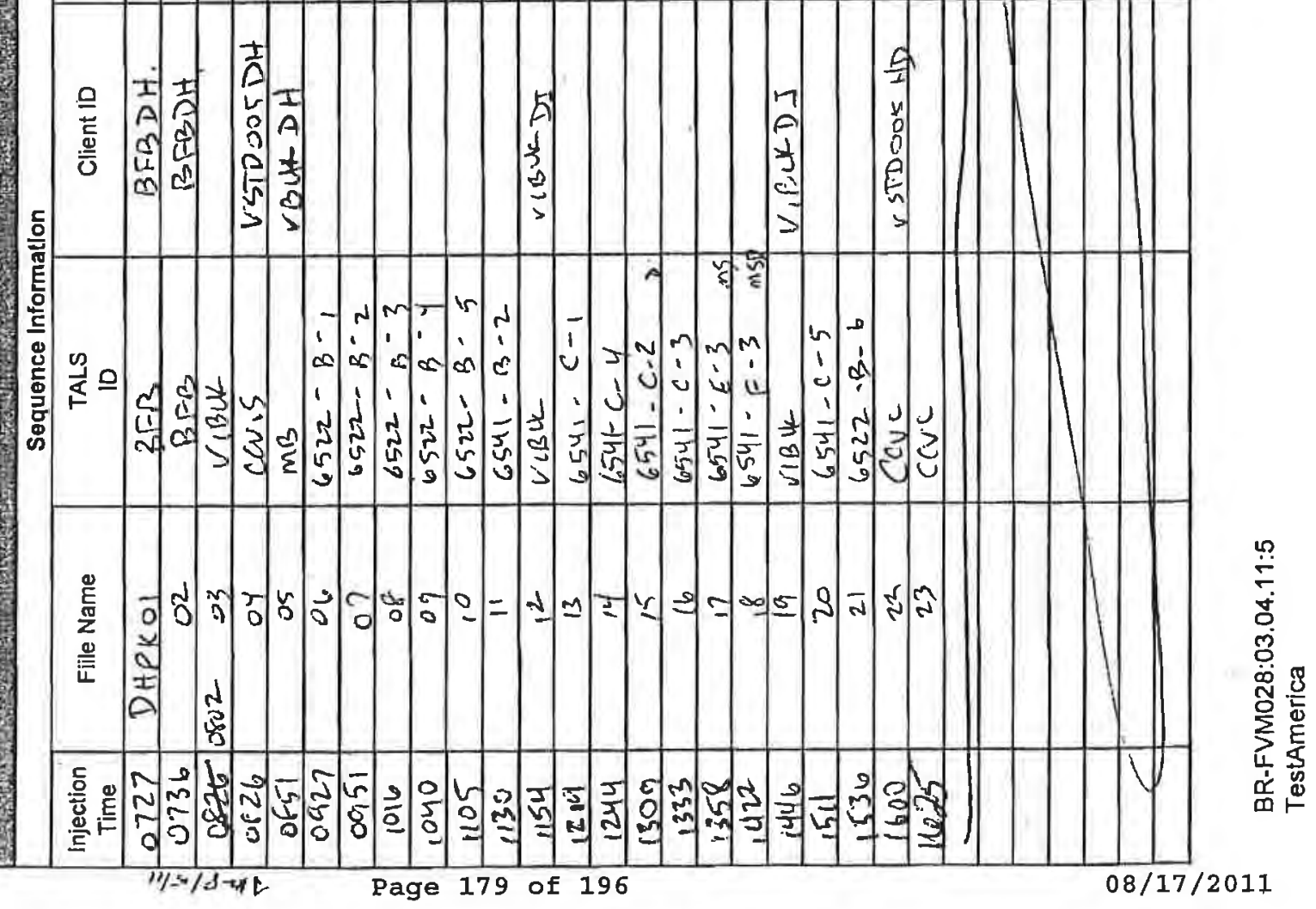


Sample Name: 200-6522-A-1

Data File: C:ICPSpirit5IData2IVoaE072611.0292.RAW

Acquired from Instrument 1 on 8/12/11 5:03:09 PM by

Sample 200-6522-A-1 was diluted 1:5.

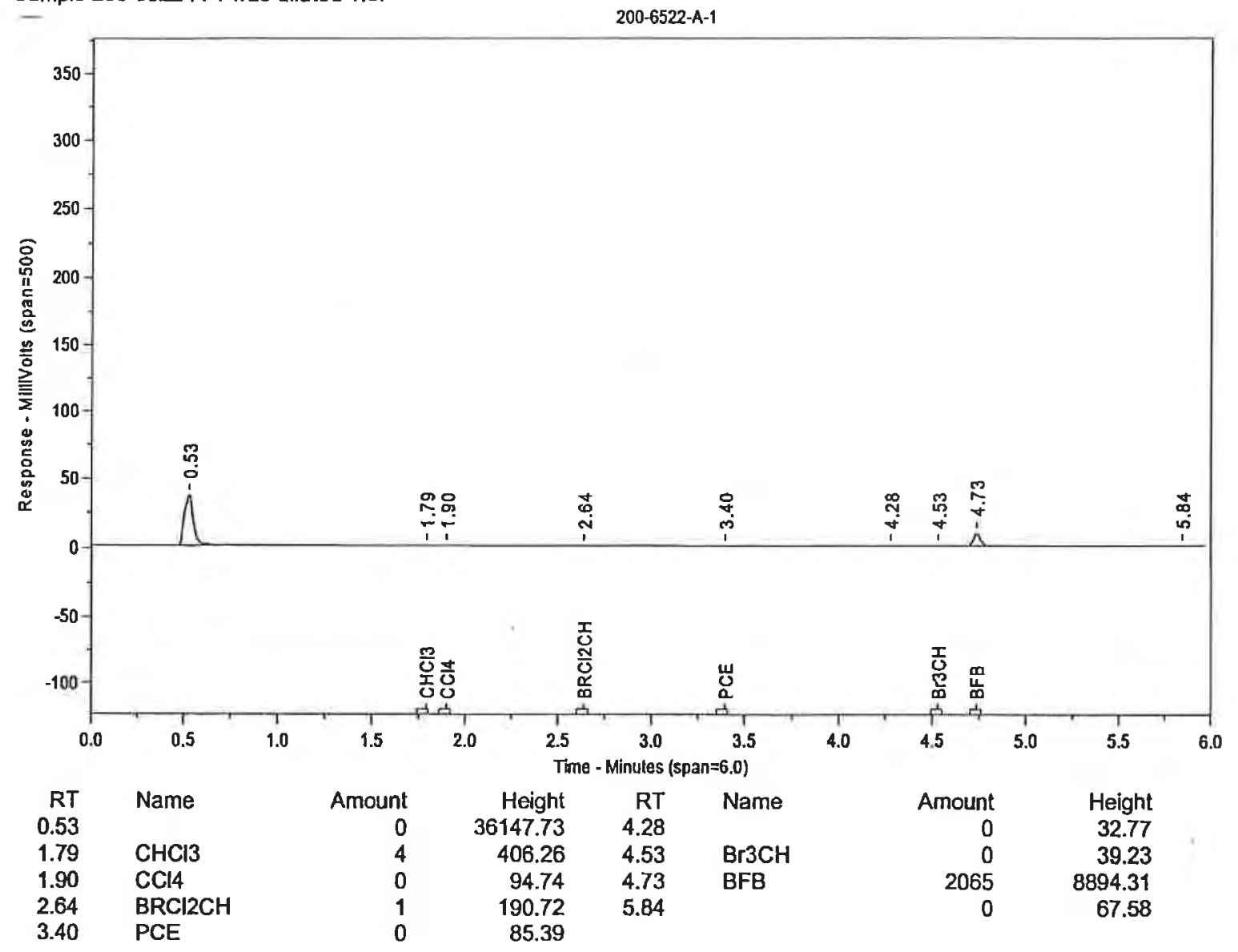

Surrogate BFB recovery is $103.2 \%$ 
Sample Name: 200-6522-A-1

Data File: C:ICPSpirit5IData2IVoaF072611.0292.RAW

Acquired from Instrument 1 on 8/12/11 5:03:09 PM by

Sample 200-6522-A-1 was diluted 1:5.

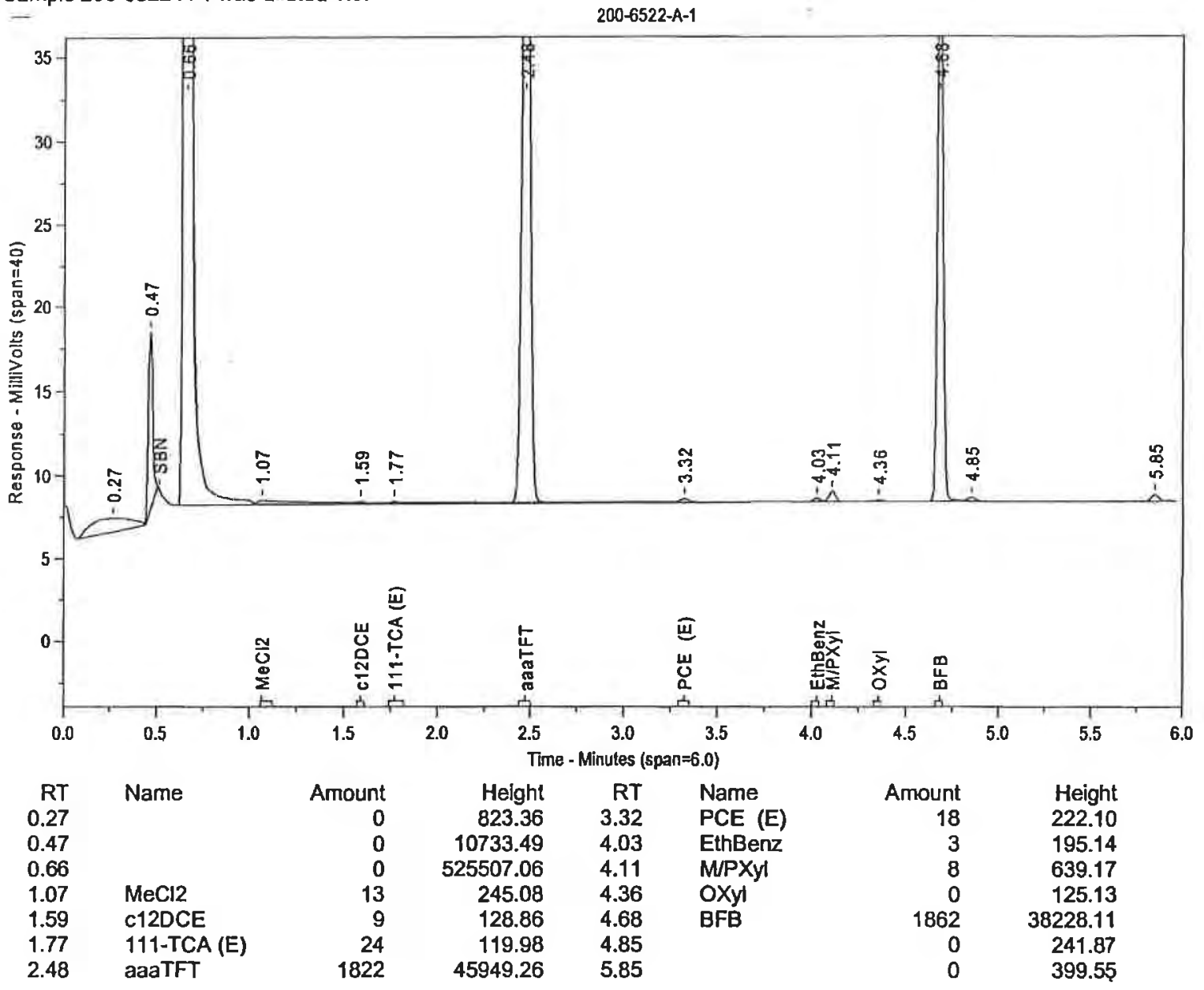

Surrogate aaaTFT recovery is $91.1 \%$

Surrogate BFB recovery is $93.1 \%$ 
Sample Name: 200-6522-A-2

Data File: C:ICPSpirit51Data2IVoaE072611.0293.RAW

Acquired from Instrument 1 on 8/12/11 5:14:50 PM by

Sample 200-6522-A-2 was diluted 1:5.

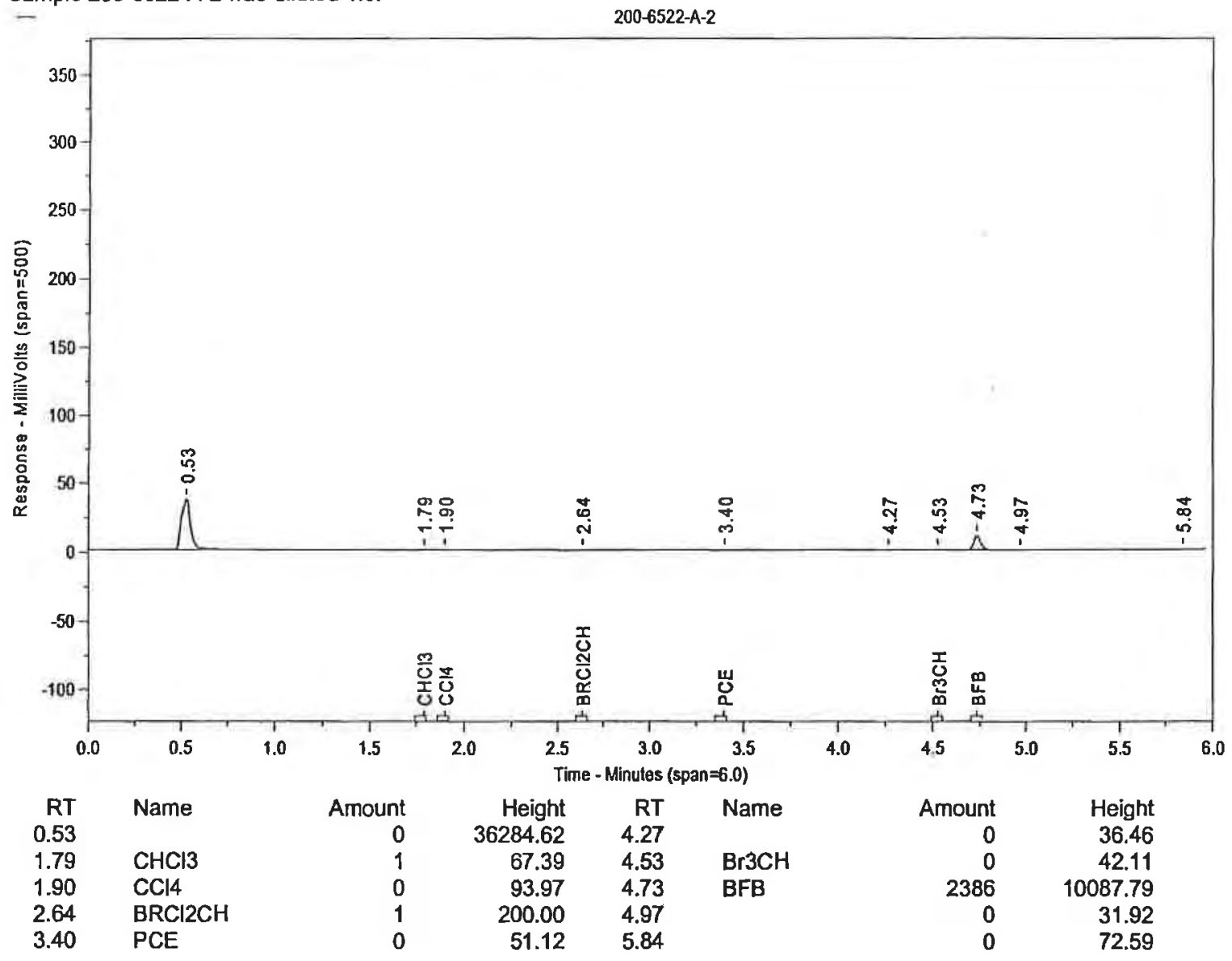

Surrogate BFB recovery is $119.3 \%$ 
Sample Name: 200-6522-A-2

Data File: C:ICPSpirit5IData2IVoaF072611.0293.RAW Acquired from Instrument 1 on 8/12/11 5:14:50 PM by Sample 200-6522-A-2 was diluted 1:5.

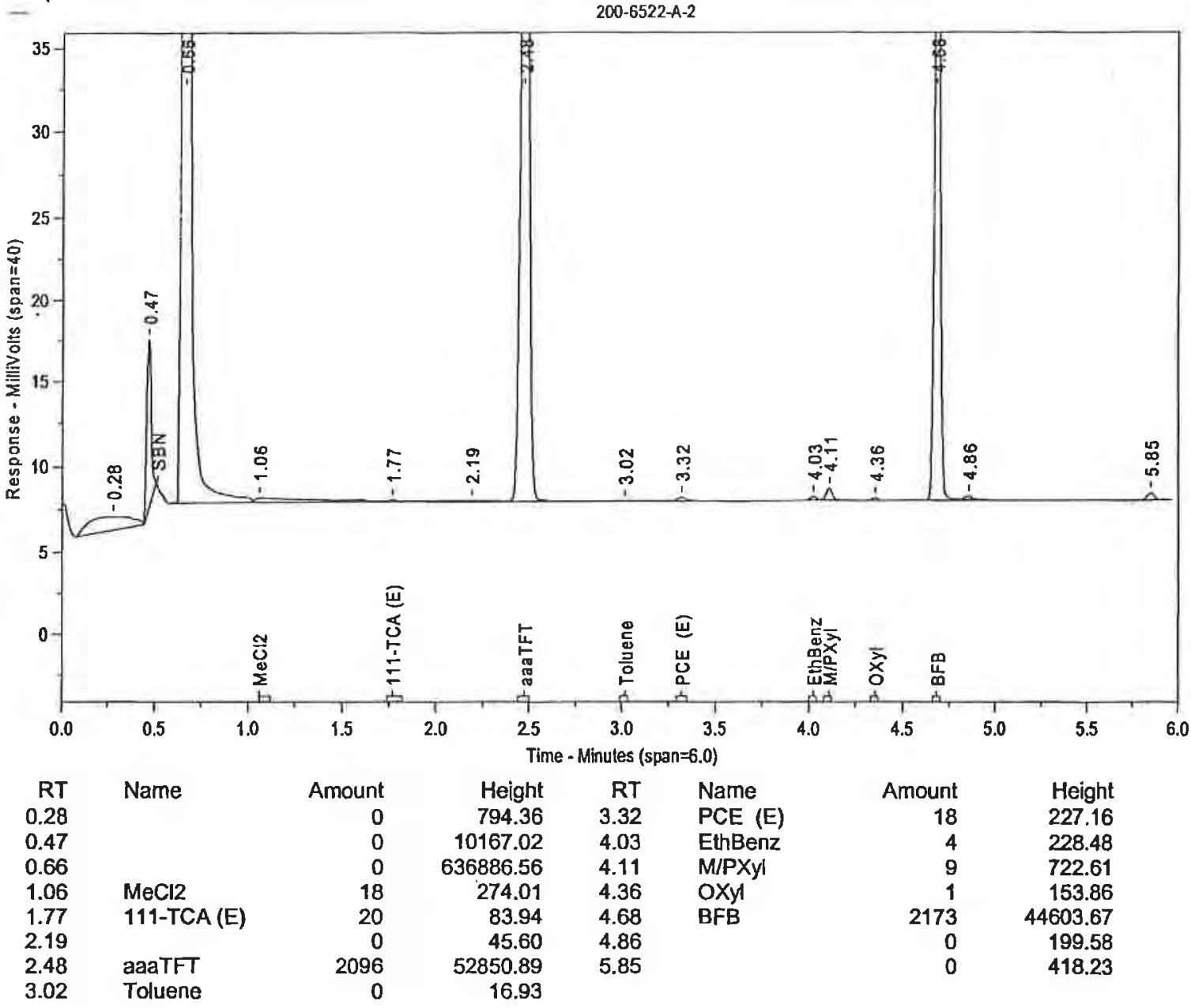

Surrogate aaaTFT recovery is $104.8 \%$ Surrogate BFB recovery is $108.6 \%$ 
Sample Name: 200-6522-A-3

Data File: C:ICPSpirit5IData2IVoaE072611.0294.RAW Acquired from Instrument 1 on 8/12/11 5:26:28 PM by Sample 200-6522-A-3 was diluted 1:5.

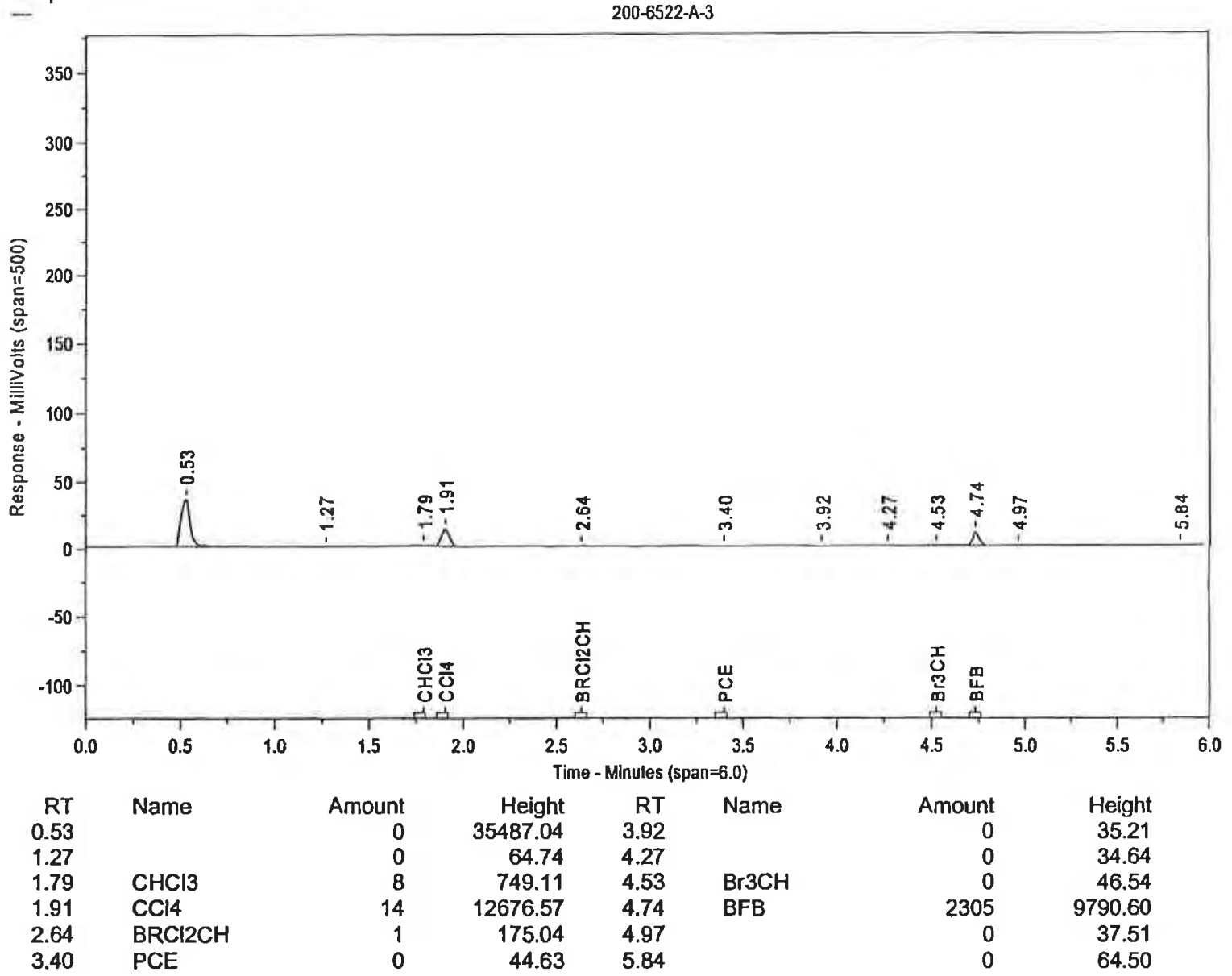

Surrogate BFB recovery is $115.3 \%$ 
Sample Name: 200-6522-A-3

Data File: C:ICPSpirit5lData2IVoaF072611.0294.RAW Acquired from Instrument 1 on 8/12/11 5:26:29 PM by Sample 200-6522-A-3 was diluted 1:5.

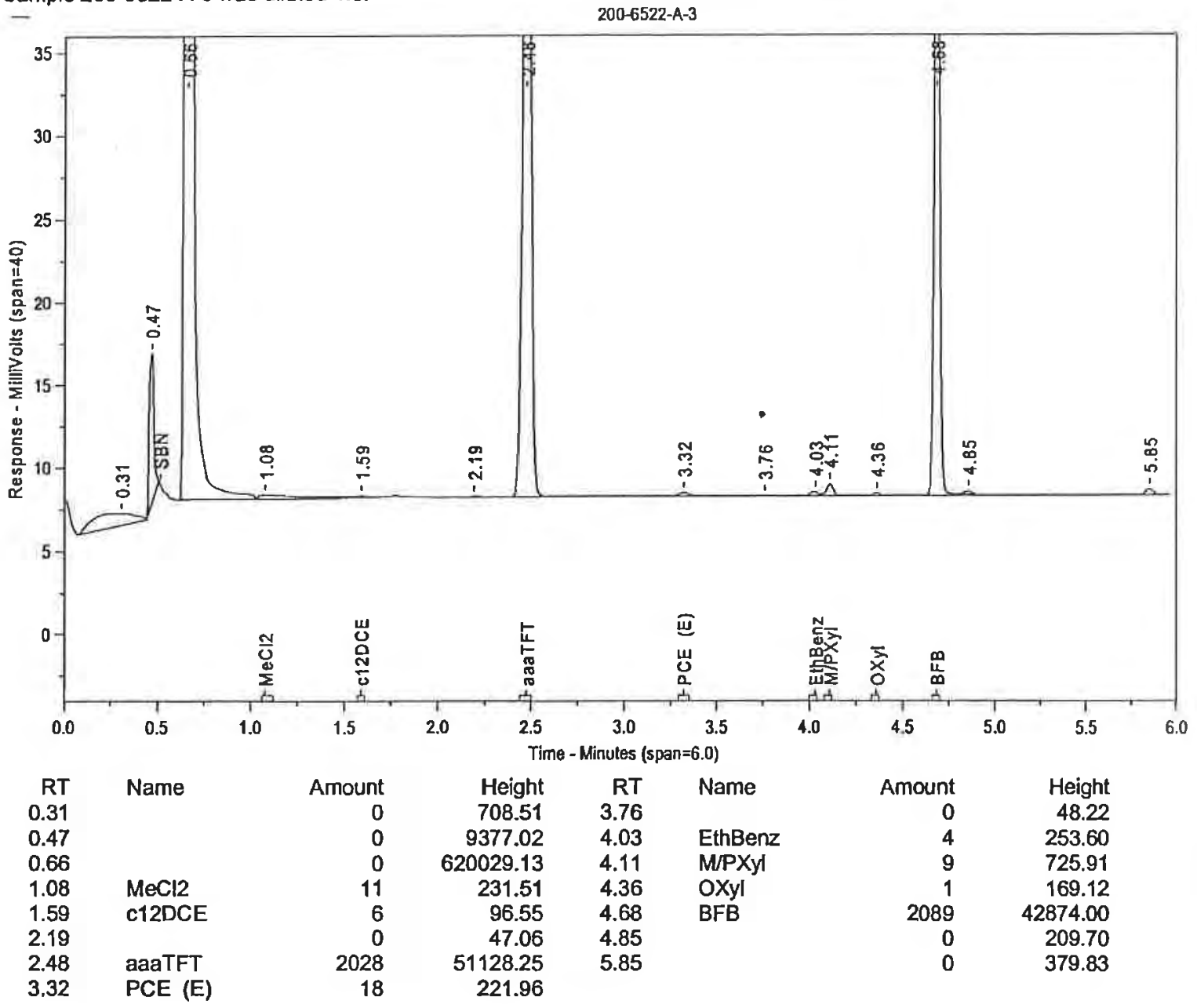

Surrogate aaaTFT recovery is $101.4 \%$ Surrogate BFB recovery is $104.4 \%$ 
Sample Name: 200-6522-A-4

Data File: C:ICPSpirit5IData2IVoaE072611.0295.RAW

Acquired from instrument 1 on 8/12/11 5:38:06 PM by

Sample 200-6522-A-4 was diluted 1:5.

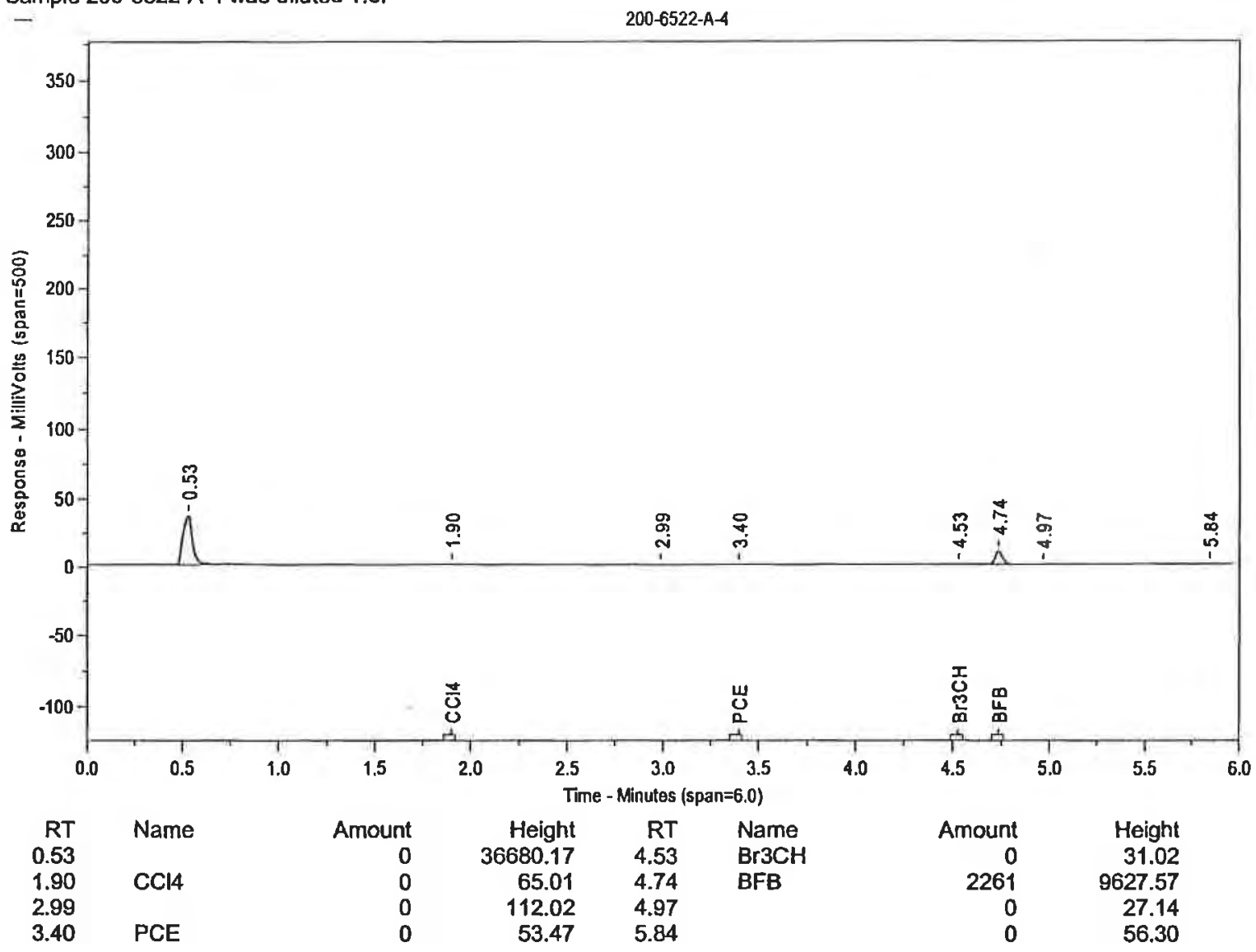

Surrogate BFB recovery is $113.1 \%$ 
Sample Name: 200-6522-A-4

Data File: C:ICPSpirit5IData2IVoaF072611.0295.RAW Acquired from Instrument 1 on 8/12/11 5:38:06 PM by Sample 200-6522-A-4 was diluted 1:5.

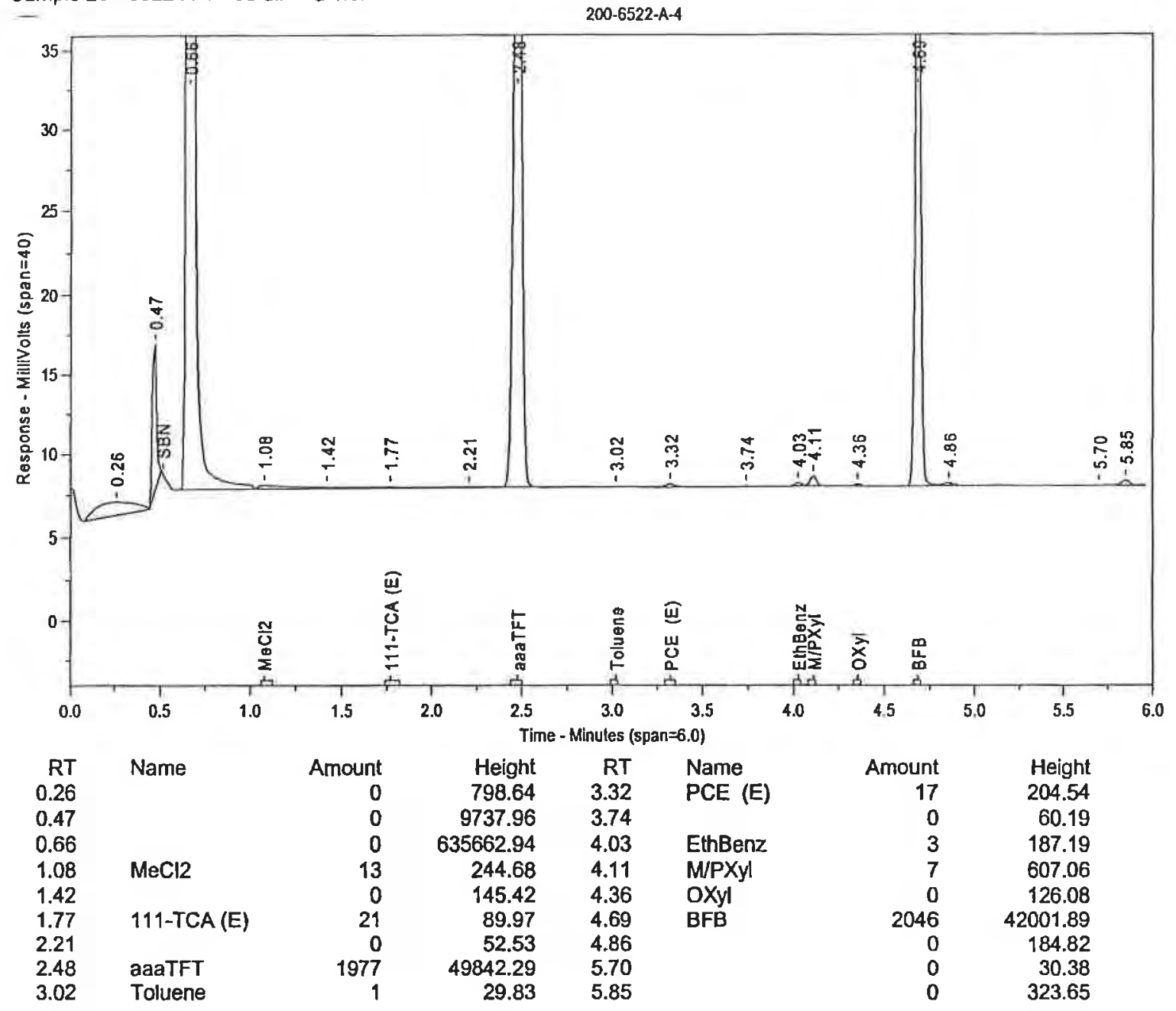

Surrogate aaaTFT recovery is $98.8 \%$

Surrogate BFB recovery is $102.3 \%$ 


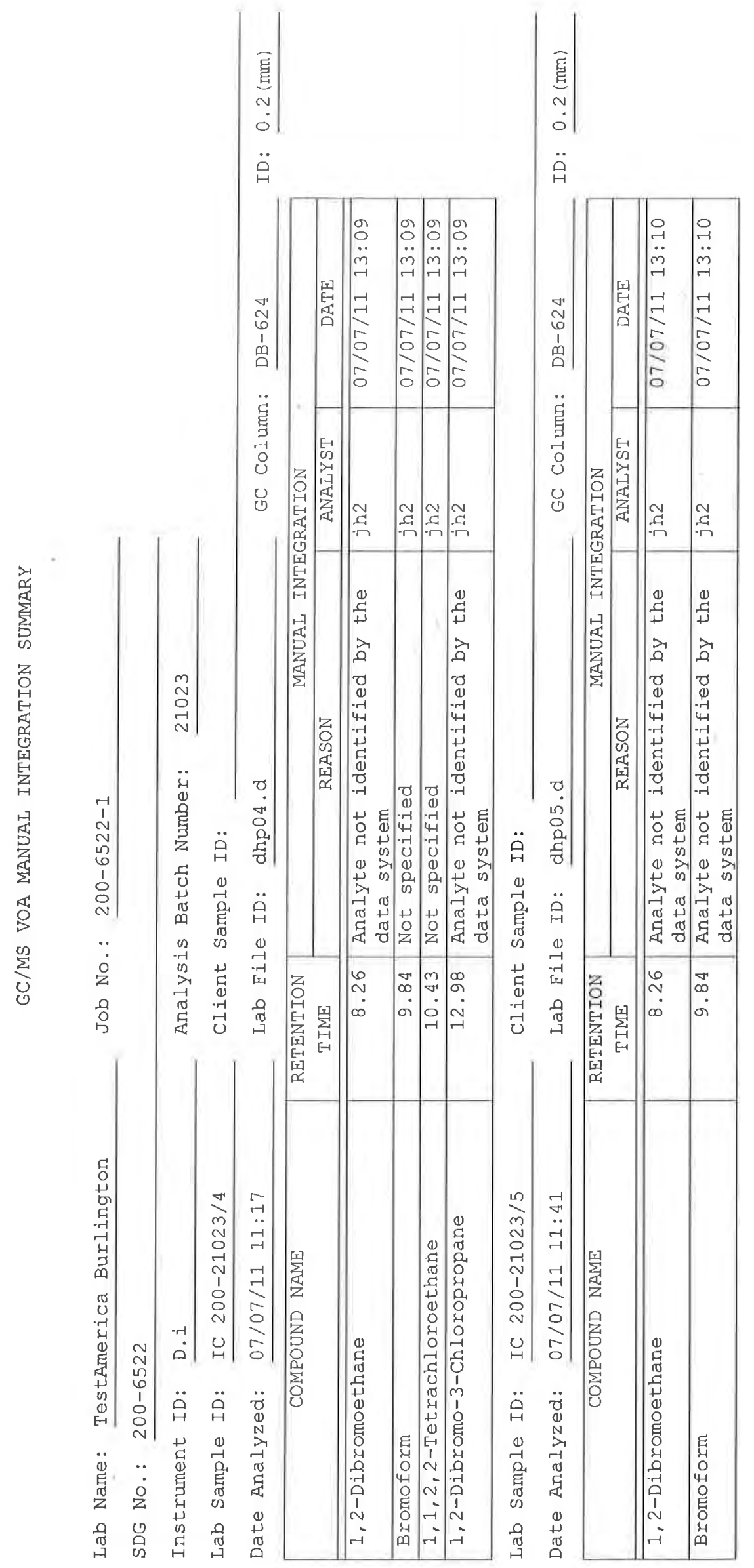


Lab Name: TestAmerica Burlington

SDG No.: 200-6522
Job No.: 200-6522-1

\begin{tabular}{|c|l|l|c|c|}
\hline $\begin{array}{c}\text { Reagent } \\
\text { Container }\end{array}$ & \multicolumn{1}{|c|}{$\begin{array}{c}\text { Reagent } \\
\text { ID }\end{array}$} & \multicolumn{1}{|c|}{$\begin{array}{c}\text { Reagent } \\
\text { Description }\end{array}$} & $\begin{array}{c}\text { Preparation } \\
\text { Date }\end{array}$ & $\begin{array}{c}\text { Expiration } \\
\text { Date }\end{array}$ \\
\hline 153392 & VMBEBw_00006 & BFB TUNE 25 PPM & $06 / 03 / 2011$ & $12 / 03 / 2011$ \\
\hline 170901 & VMSOMTRCALw_00032 & SOM TR CAL 20 PPM & $07 / 05 / 2011$ & $07 / 22 / 2011$ \\
\hline 172178 & VMSOMTRISW_00038 & SOM TR ISTD 20 PPM & $07 / 07 / 2011$ & $08 / 07 / 2011$ \\
\hline 186707 & VMSOMTRCALw_00033 & SOM TR CAL 20 PPM & $07 / 28 / 2011$ & $08 / 20 / 2011$ \\
\hline 193771 & VMSOMTRISw_00039 & SOM TR ISTD 20 PPM & $08 / 08 / 2011$ & $09 / 08 / 2011$ \\
\hline 193793 & VMSOMTRSUw_00034 & SOM TR DMC 20 PPM & $08 / 08 / 2011$ & $09 / 08 / 2011$ \\
\hline
\end{tabular}




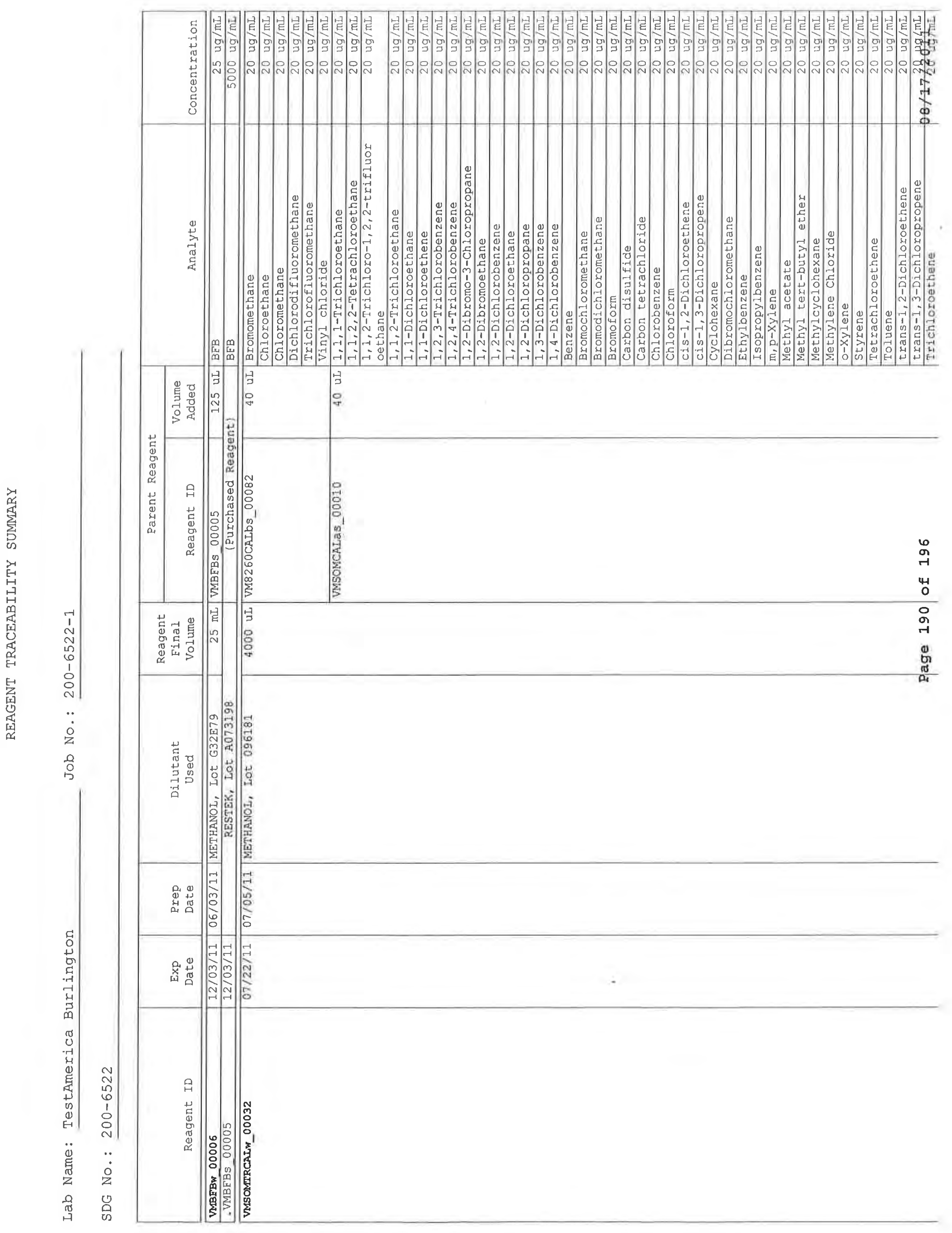




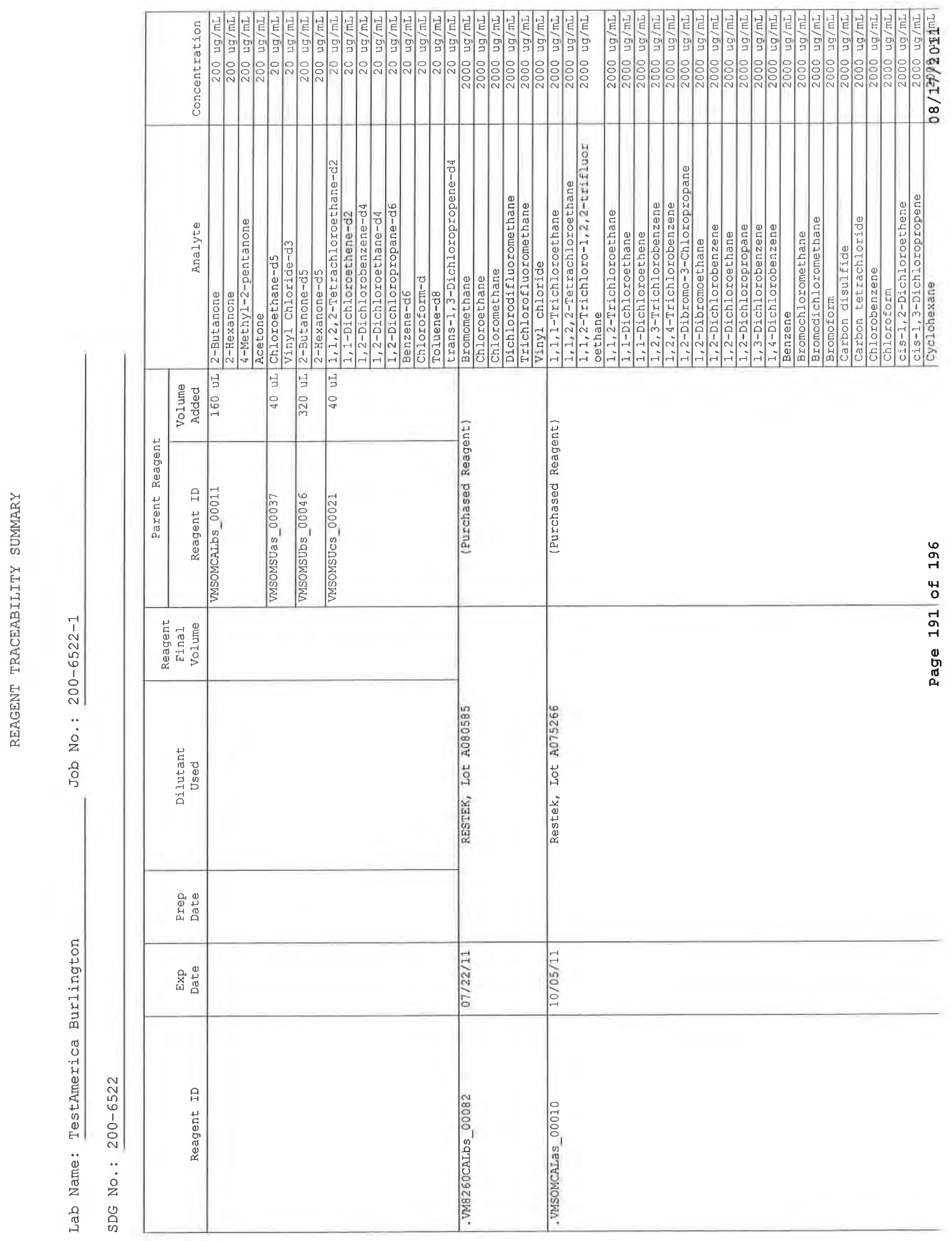




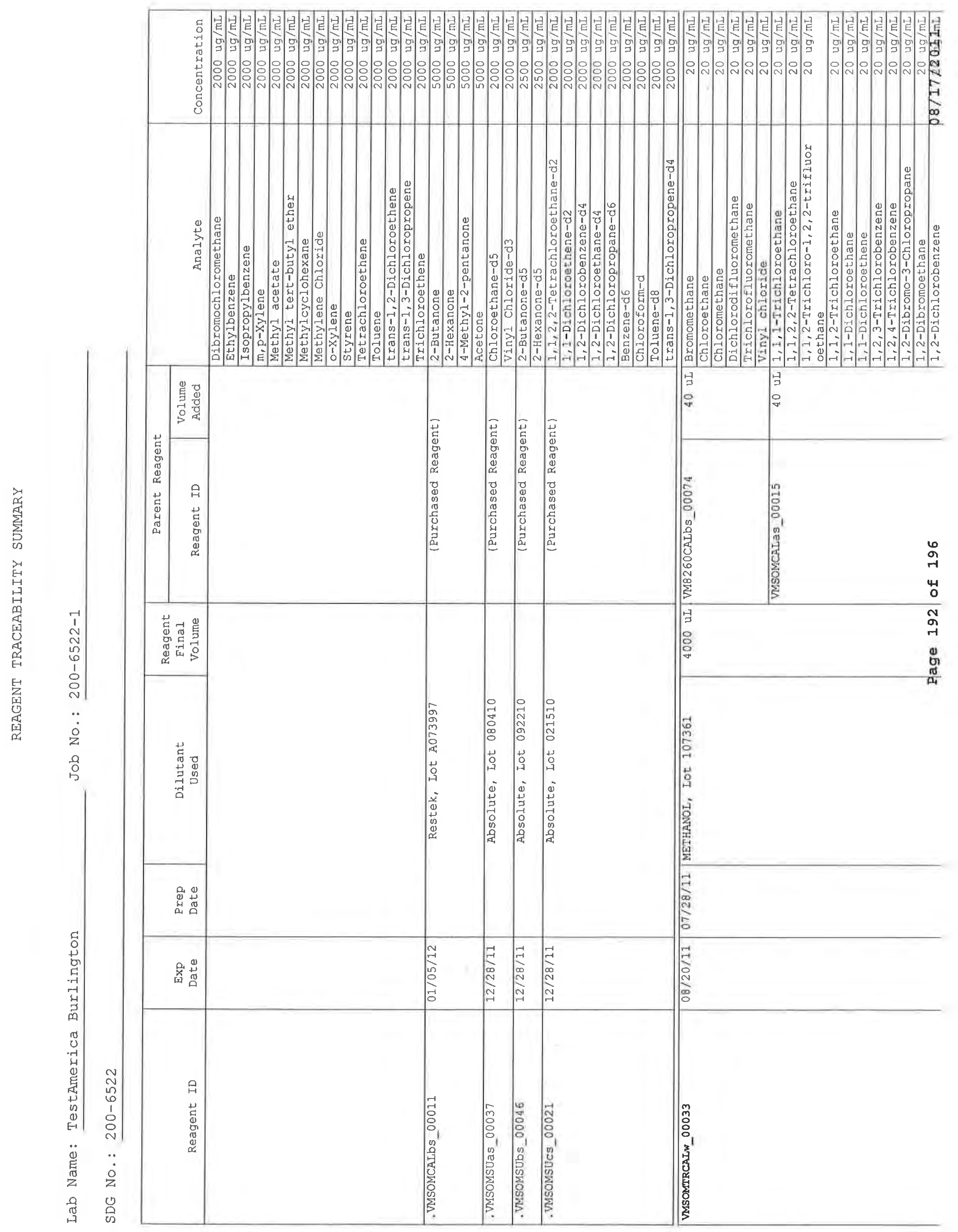




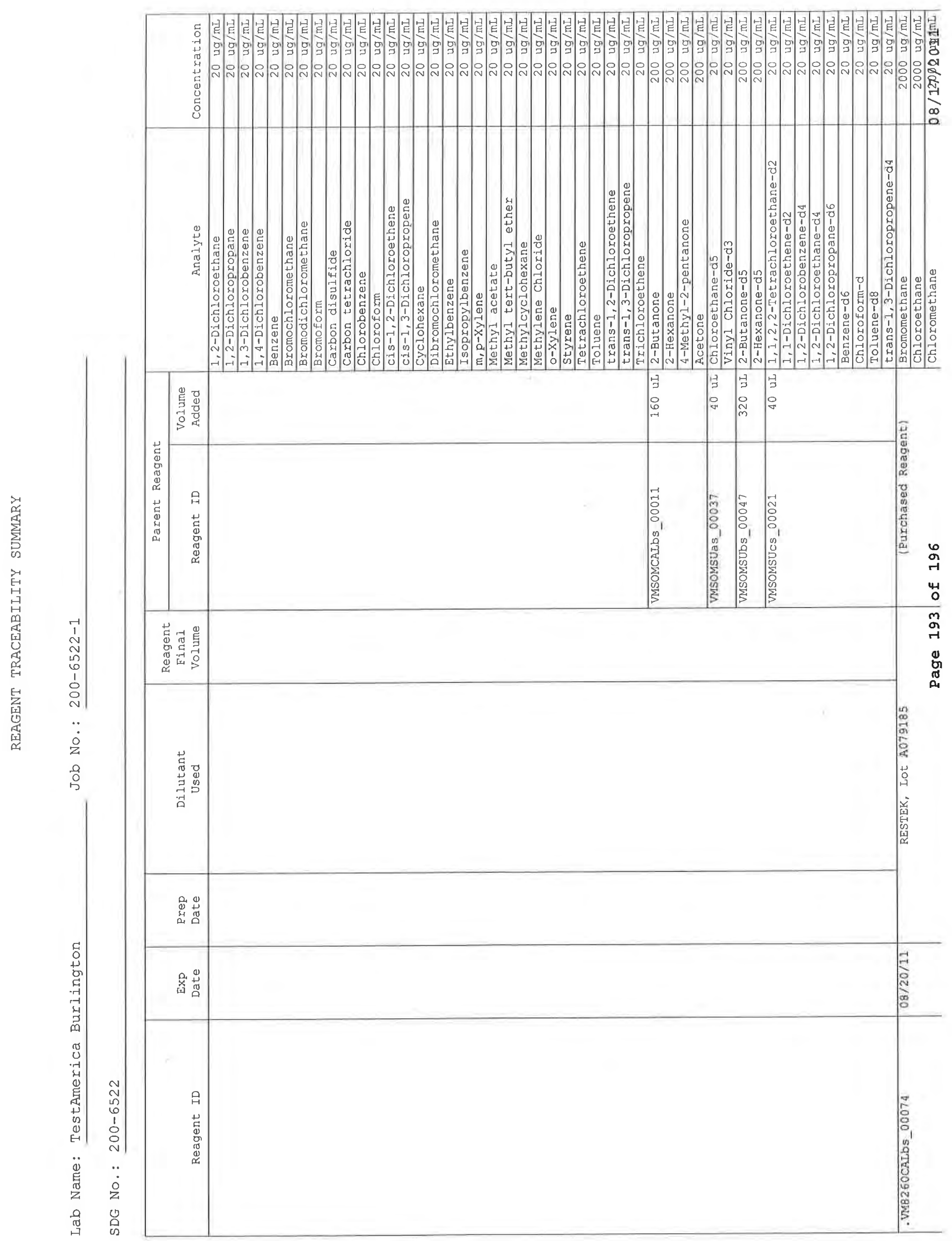




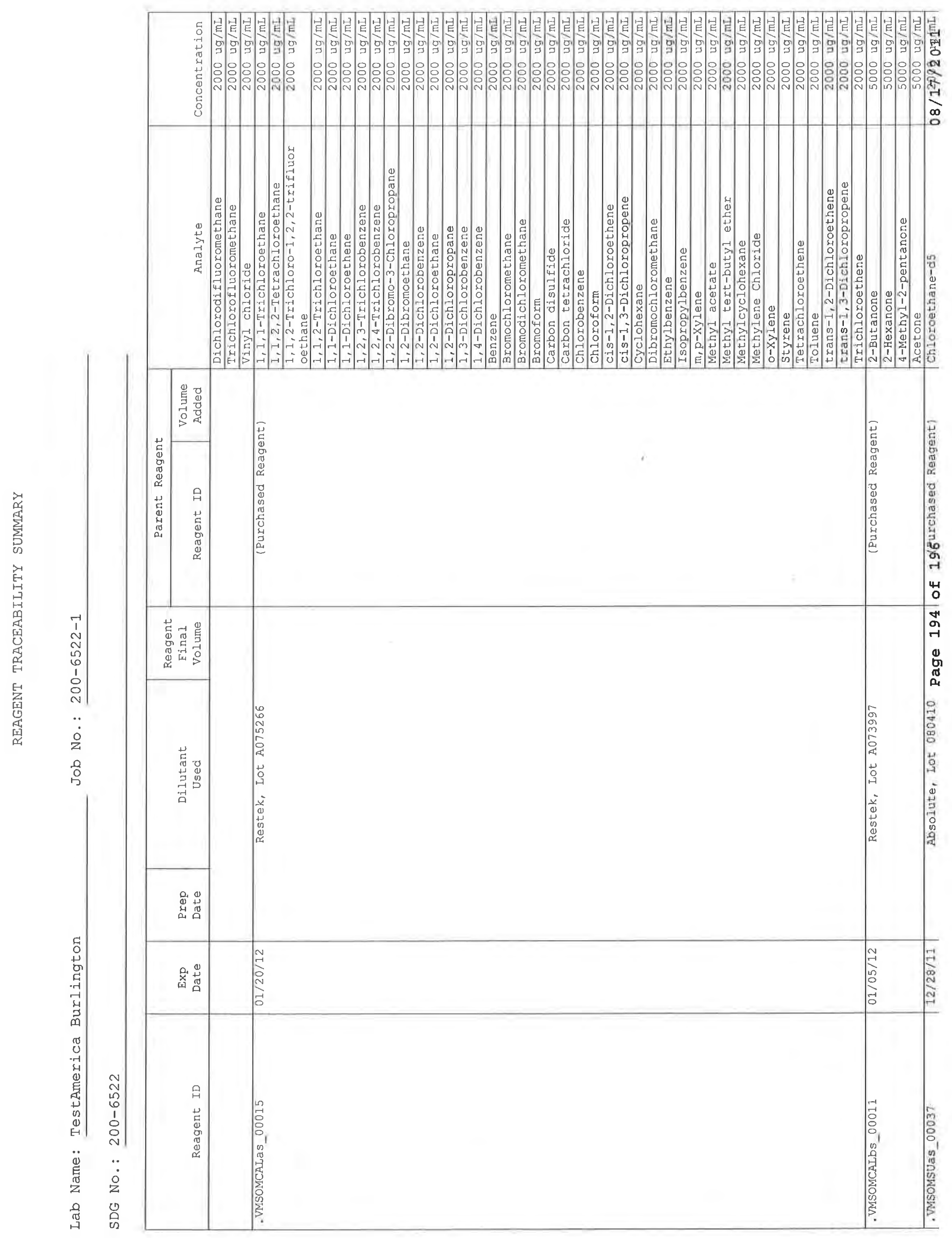




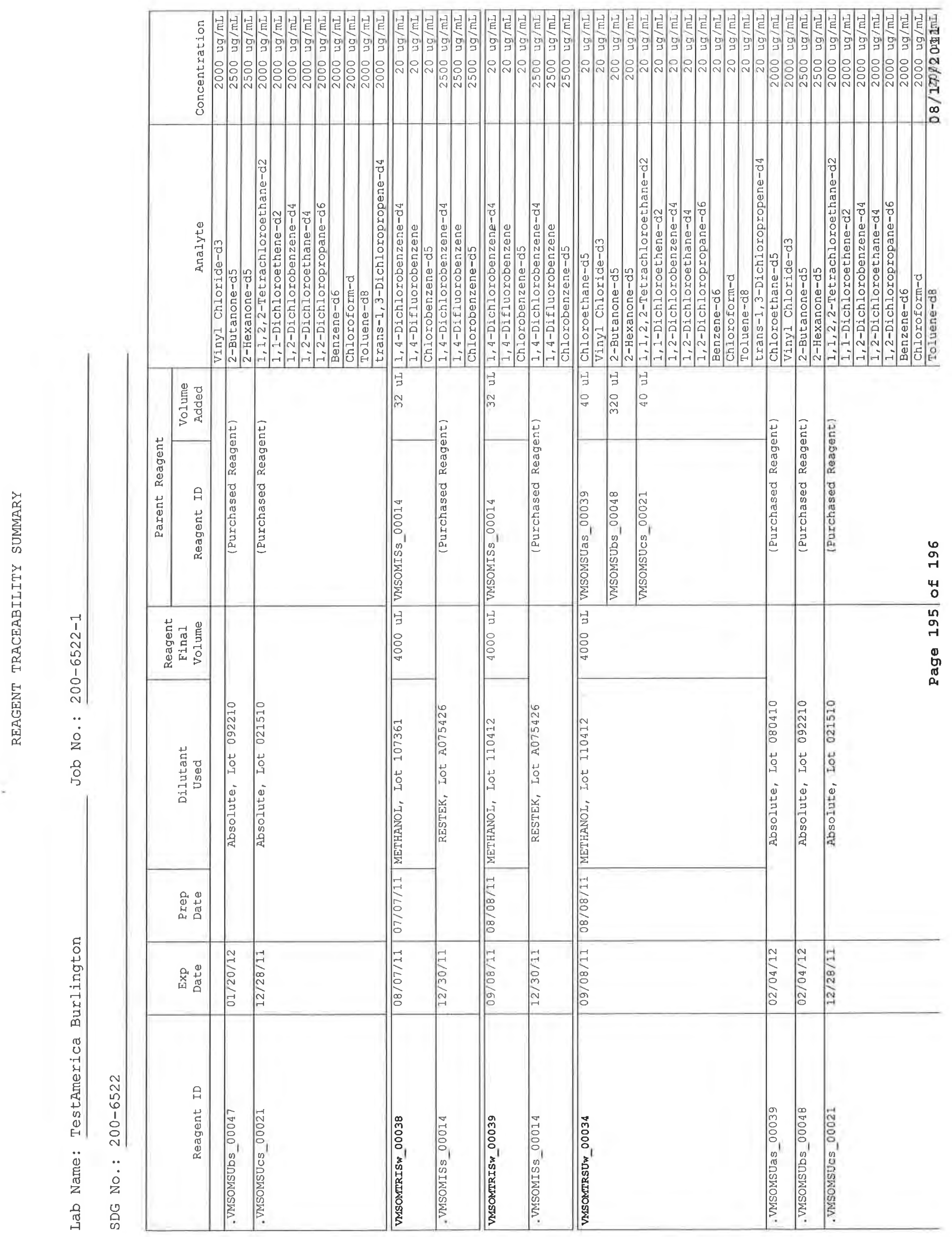




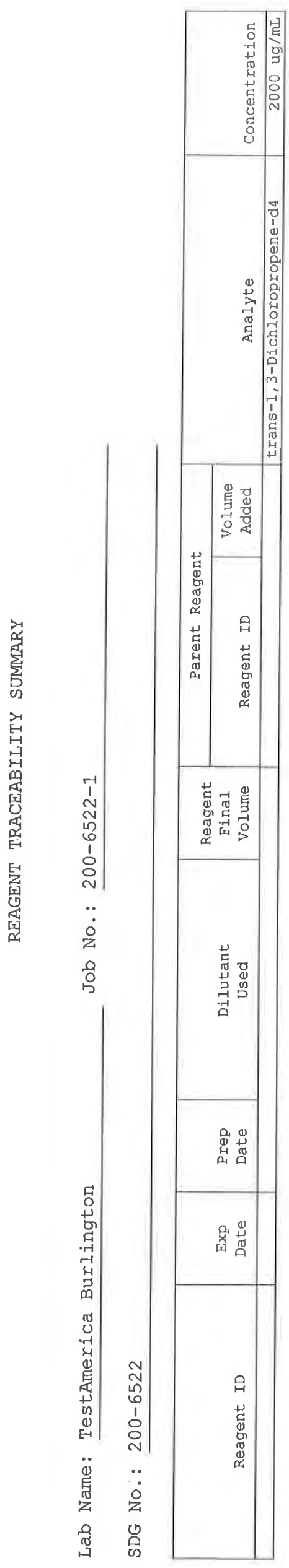


Supplement 4:

Waste Characterization Data 
November 11, 2011

Mr. Travis Kamler

TCW Construction Inc

$141 \mathrm{M}$ Street

Lincoln, NE 68508

RE: Project: KS/MO Waste Water

Pace Project No.: 60109211

Dear Mr. Kamler:

Enclosed are the analytical results for sample(s) received by the laboratory on November 01, 2011.

The results relate only to the samples included in this report. Results reported herein conform to the most current TNI standards and the laboratory's Quality Assurance Manual, where applicable, unless otherwise noted in the body of the report.

If you have any questions concerning this report, please feel free to contact me.

\section{Sincerely,}

$$
\text { suady sigaon }
$$

Trudy Gipson

trudy.gipson@pacelabs.com

Project Manager

Enclosures

cc: Mr. David Surgnier

\section{REPORT OF LABORATORY ANALYSIS}

This report shall not be reproduced, except in full,

without the written consent of Pace Analytical Services, Inc 


\section{CERTIFICATIONS}

Project:

KS/MO Waste Water

Pace Project No.:

60109211

Kansas Certification IDs

9608 Loiret Boulevard, Lenexa, KS 66219

A2LA Certification \#: 2456.01

Arkansas Certification \#: 05-008-0

Illinois Certification \#: 001191

Louisiana Certification \#: 03055

Nevada Certification \#: KS000212008A

Oklahoma Certification \#: 9205/9935

lowa Certification \#: 118

Texas Certification \#: T104704407-08-TX

Kansas/NELAP Certification \#: E-10116 


\title{
SAMPLE SUMMARY
}

\author{
Project: $\quad$ KS/MO Waste Water \\ Pace Project No.: $\quad 60109211$
}

\begin{tabular}{|c|c|c|c|c|}
\hline Lab ID & Sample ID & Matrix & Date Collected & Date Received \\
\hline 60109211001 & AGPURGE-W-10111 & Water & 10/31/11 09:00 & $11 / 01 / 1109: 20$ \\
\hline 60109211002 & BAPURGE-W-10112 & Water & $10 / 31 / 1112: 55$ & $11 / 01 / 1109: 20$ \\
\hline 60109211003 & CNPURGE-W-10113 & Water & 10/31/11 14:02 & 11/01/11 09:20 \\
\hline 60109211004 & EUPURGE-W-10114 & Water & 10/31/11 15:52 & 11/01/11 09:20 \\
\hline 60109211005 & HAPURGE-W-10115 & Water & $10 / 31 / 1112: 27$ & $11 / 01 / 1109: 20$ \\
\hline 60109211006 & MRPURGE-W-10116 & Water & 10/31/11 14:42 & $11 / 01 / 1109: 20$ \\
\hline 60109211007 & SVPURGE-W-10117 & Water & 10/31/11 18:30 & $11 / 01 / 1109: 20$ \\
\hline
\end{tabular}


SAMPLE ANALYTE COUNT

Project: $\quad$ KS/MO Waste Water

Pace Project No.: 60109211

\begin{tabular}{|c|c|c|c|c|}
\hline Lab ID & Sample ID & Method & Analysts & $\begin{array}{l}\text { Analytes } \\
\text { Reported }\end{array}$ \\
\hline \multirow[t]{3}{*}{60109211001} & AGPURGE-W-10111 & EPA 504.1 & NAW & 1 \\
\hline & & EPA $5030 B / 8260$ & HMW & 70 \\
\hline & & EPA 353.2 & AJM & 1 \\
\hline \multirow[t]{3}{*}{60109211002} & BAPURGE-W-10112 & EPA 504.1 & NAW & 1 \\
\hline & & EPA $5030 B / 8260$ & HMW & 70 \\
\hline & & EPA 353.2 & AJM & 1 \\
\hline \multirow[t]{3}{*}{60109211003} & CNPURGE-W-10113 & EPA 504.1 & NAW & 1 \\
\hline & & EPA $5030 B / 8260$ & HMW & 70 \\
\hline & & EPA 353.2 & AJM & 1 \\
\hline \multirow[t]{3}{*}{60109211004} & EUPURGE-W-10114 & EPA 504.1 & NAW & 1 \\
\hline & & EPA $5030 B / 8260$ & HMW & 70 \\
\hline & & EPA 353.2 & AJM & 1 \\
\hline \multirow[t]{3}{*}{60109211005} & HAPURGE-W-10115 & EPA 504.1 & NAW & 1 \\
\hline & & EPA $5030 B / 8260$ & HMW & 70 \\
\hline & & EPA 353.2 & AJM & 1 \\
\hline \multirow[t]{3}{*}{60109211006} & MRPURGE-W-10116 & EPA 504.1 & NAW & 1 \\
\hline & & EPA $5030 B / 8260$ & HMW & 70 \\
\hline & & EPA 353.2 & AJM & 1 \\
\hline \multirow[t]{3}{*}{60109211007} & SVPURGE-W-10117 & EPA 504.1 & NAW & 1 \\
\hline & & EPA 5030B/8260 & HMW & 70 \\
\hline & & EPA 353.2 & AJM & 1 \\
\hline
\end{tabular}




\section{ANALYTICAL RESULTS}

Project: $\quad$ KS/MO Waste Water

Pace Project No.: 60109211

\begin{tabular}{|c|c|c|c|c|c|c|c|c|}
\hline \multirow{2}{*}{$\begin{array}{c}\text { Sample: AGPURGE-W-10111 } \\
\text { Parameters }\end{array}$} & \multirow{2}{*}{\multicolumn{2}{|c|}{$\begin{array}{lr}\text { Lab ID: } & 60109211001 \\
\text { Results } & \text { Units }\end{array}$}} & \multicolumn{2}{|c|}{ Collected: $10 / 31 / 11109: 00$} & \multicolumn{2}{|c|}{ Received: $11 / 01 / 1109: 20$} & \multirow{2}{*}{$\begin{array}{l}\text { Matrix: Water } \\
\text { CAS No. }\end{array}$} & \multirow[b]{2}{*}{ Qual } \\
\hline & & & Report Limit & DF & Prepared & Analyzed & & \\
\hline 504 GCS EDB and DBCP & \multicolumn{7}{|c|}{ Analytical Method: EPA 504.1 Preparation Method: EPA 504.1 } & \\
\hline 1,2-Dibromoethane (EDB) & & VD ug/L & 0.028 & 1 & $11 / 07 / 1100: 00$ & $11 / 08 / 1100: 52$ & $106-93-4$ & \\
\hline 8260 MSV & \multicolumn{7}{|c|}{ Analytical Method: EPA 5030B/8260 } & \\
\hline Acetone & & ND ug/L & 10.0 & 1 & & $11 / 04 / 1120: 18$ & 67-64-1 & \\
\hline Benzene & & VD ug/L & 1.0 & 1 & & $11 / 04 / 1120: 18$ & $71-43-2$ & \\
\hline Bromobenzene & & VD ug/L & 1.0 & 1 & & $11 / 04 / 1120: 18$ & $108-86-1$ & \\
\hline Bromochloromethane & & ND ug/L & 1.0 & 1 & & $11 / 04 / 1120: 18$ & $74-97-5$ & \\
\hline Bromodichloromethane & & ND ug/L & 1.0 & 1 & & $11 / 04 / 1120: 18$ & $75-27-4$ & \\
\hline Bromoform & & ND ug/L & 1.0 & 1 & & $11 / 04 / 1120: 18$ & $75-25-2$ & \\
\hline Bromomethane & & ND ug/L & 1.0 & 1 & & $11 / 04 / 1120: 18$ & $74-83-9$ & \\
\hline 2-Butanone (MEK) & & ND ug/L & 10.0 & 1 & & $11 / 04 / 1120: 18$ & $78-93-3$ & \\
\hline n-Butylbenzene & & ND ug/L & 1.0 & 1 & & $11 / 04 / 1120: 18$ & $104-51-8$ & \\
\hline sec-Butylbenzene & & VD ug/L & 1.0 & 1 & & $11 / 04 / 1120: 18$ & $135-98-8$ & \\
\hline tert-Butylbenzene & & VD ug/L & 1.0 & 1 & & $11 / 04 / 1120: 18$ & $98-06-6$ & \\
\hline Carbon disulfide & & ND ug/L & 5.0 & 1 & & $11 / 04 / 1120: 18$ & $75-15-0$ & \\
\hline Carbon tetrachloride & & $1.8 \mathrm{ug} / \mathrm{L}$ & 1.0 & 1 & & $11 / 04 / 1120: 18$ & $56-23-5$ & \\
\hline Chlorobenzene & & ND ug/L & 1.0 & 1 & & $11 / 04 / 1120: 18$ & $108-90-7$ & \\
\hline Chloroethane & & ND ug/L & 1.0 & 1 & & $11 / 04 / 1120: 18$ & $75-00-3$ & \\
\hline Chloroform & & $1.6 \mathrm{ug} / \mathrm{L}$ & 1.0 & 1 & & $11 / 04 / 1120: 18$ & $67-66-3$ & \\
\hline Chloromethane & & ND ug/L & 1.0 & 1 & & $11 / 04 / 1120: 18$ & $74-87-3$ & \\
\hline 2-Chlorotoluene & & $\mathrm{ND} u g / \mathrm{L}$ & 1.0 & 1 & & $11 / 04 / 1120: 18$ & $95-49-8$ & \\
\hline 4-Chlorotoluene & & ND ug/L & 1.0 & 1 & & $11 / 04 / 1120: 18$ & $106-43-4$ & \\
\hline 1,2-Dibromo-3-chloropropane & & ND ug/L & 2.5 & 1 & & $11 / 04 / 1120: 18$ & $96-12-8$ & \\
\hline Dibromochloromethane & & ND ug/L & 1.0 & 1 & & $11 / 04 / 1120: 18$ & $124-48-1$ & \\
\hline 1,2-Dibromoethane (EDB) & & ND ug/L & 1.0 & 1 & & $11 / 04 / 1120: 18$ & $106-93-4$ & \\
\hline Dibromomethane & & $\mathrm{ND} \mathrm{ug} / \mathrm{L}$ & 1.0 & 1 & & $11 / 04 / 1120: 18$ & $74-95-3$ & \\
\hline 1,2-Dichlorobenzene & & ND ug/L & 1.0 & 1 & & $11 / 04 / 1120: 18$ & $95-50-1$ & \\
\hline 1,3-Dichlorobenzene & & ND ug/L & 1.0 & 1 & & $11 / 04 / 1120: 18$ & $541-73-1$ & \\
\hline 1,4-Dichlorobenzene & & $\mathrm{ND}$ ug/L & 1.0 & 1 & & $11 / 04 / 1120: 18$ & $106-46-7$ & \\
\hline Dichlorodifluoromethane & & ND ug/L & 1.0 & 1 & & $11 / 04 / 1120: 18$ & $75-71-8$ & \\
\hline 1,1-Dichloroethane & & ND ug/L & 1.0 & 1 & & $11 / 04 / 1120: 18$ & $75-34-3$ & \\
\hline 1,2-Dichloroethane & & $\mathrm{ND} u g / \mathrm{L}$ & 1.0 & 1 & & $11 / 04 / 1120: 18$ & $107-06-2$ & \\
\hline 1,2-Dichloroethene (Total) & & ND ug/L & 1.0 & 1 & & 11/04/11 20:18 & $540-59-0$ & \\
\hline 1,1-Dichloroethene & & ND ug/L & 1.0 & 1 & & $11 / 04 / 1120: 18$ & $75-35-4$ & \\
\hline cis-1,2-Dichloroethene & & $\mathrm{ND}$ ug/L & 1.0 & 1 & & $11 / 04 / 1120: 18$ & $156-59-2$ & \\
\hline trans-1,2-Dichloroethene & & ND ug/L & 1.0 & 1 & & $11 / 04 / 1120: 18$ & $156-60-5$ & \\
\hline 1,2-Dichloropropane & & ND ug/L & 1.0 & 1 & & $11 / 04 / 1120: 18$ & $78-87-5$ & \\
\hline 1,3-Dichloropropane & & $\mathrm{ND} \mathrm{ug} / \mathrm{L}$ & 1.0 & 1 & & $11 / 04 / 1120: 18$ & $142-28-9$ & \\
\hline 2,2-Dichloropropane & & ND ug/L & 1.0 & 1 & & $11 / 04 / 1120: 18$ & $594-20-7$ & \\
\hline 1,1-Dichloropropene & & ND ug/L & 1.0 & 1 & & $11 / 04 / 1120: 18$ & $563-58-6$ & \\
\hline cis-1,3-Dichloropropene & & ND ug/L & 1.0 & 1 & & 11/04/11 20:18 & $10061-01-5$ & \\
\hline trans-1,3-Dichloropropene & & ND ug/L & 1.0 & 1 & & $11 / 04 / 1120: 18$ & $10061-02-6$ & \\
\hline Ethylbenzene & & $\mathrm{ND}$ ug/L & 1.0 & 1 & & $11 / 04 / 1120: 18$ & $100-41-4$ & \\
\hline Hexachloro-1,3-butadiene & & ND ug/L & 1.0 & 1 & & $11 / 04 / 1120: 18$ & $87-68-3$ & \\
\hline 2-Hexanone & & JD ug/L & 10.0 & 1 & & $11 / 04 / 1120: 18$ & $591-78-6$ & \\
\hline Isopropylbenzene (Cumene) & & $\mathrm{JD}$ ug/L & 1.0 & 1 & & $11 / 04 / 1120: 18$ & $98-82-8$ & \\
\hline p-Isopropyltoluene & & JD ug/L & 1.0 & 1 & & $11 / 04 / 1120: 18$ & $99-87-6$ & \\
\hline
\end{tabular}

Date: 11/11/2011 11:15 AM 
Pace Analytical Services, Inc. 9608 Loiret Blvd. Lenexa, KS 66219

(913)599-5665

\section{ANALYTICAL RESULTS}

Project: KS/MO Waste Water

Pace Project No.: 60109211

Sample: AGPURGE-W-10111 Lab ID: 60109211001 Collected: 10/31/11 09:00 Received: 11/01/11 09:20 Matrix: Water

\begin{tabular}{|c|c|c|c|c|c|c|}
\hline Parameters & Results & Units & Report Limit & $\mathrm{DF}$ & Prepared & Analyzed \\
\hline
\end{tabular}

8260 MSV

Methylene chloride

4-Methyl-2-pentanone (MIBK)

Methyl-tert-butyl ether

Naphthalene

n-Propylbenzene

Styrene

1,1,1,2-Tetrachloroethane

1,1,2,2-Tetrachloroethane

Tetrachloroethene

Toluene

1,2,3-Trichlorobenzene

1,2,4-Trichlorobenzene

1,1,1-Trichloroethane

1,1,2-Trichloroethane

Trichloroethene

Trichlorofluoromethane

1,2,3-Trichloropropane

1,2,4-Trimethylbenzene

1,3,5-Trimethylbenzene

Vinyl chloride

Xylene (Total)

4-Bromofluorobenzene (S)

Dibromofluoromethane $(S)$

1,2-Dichloroethane-d4 (S)

Toluene-d8 (S)

Preservation $\mathrm{pH}$

353.2 Nitrogen, NO2/NO3 unpres

Nitrogen, Nitrate

Analytical Method: EPA 5030B/8260

\begin{abstract}
ND ug/L
ND ug/L

ND ug/L

ND ug/L

ND ug/L

ND ug/L

ND ug/L

ND ug/L

ND ug/L.

ND ug/L

ND ug/L

ND ug/L

ND ug/L

ND ug/L

ND ug/L

ND ug/L

ND ug/L

ND ug/L

ND ug/L

ND ug/L

ND ug/L

$104 \%$

$103 \%$

$109 \%$

$103 \%$

7.0
\end{abstract}

Analytical Method: EPA 353.2

$14.6 \mathrm{mg} / \mathrm{L}$

$\begin{array}{rr}1.0 & 1 \\ 10.0 & 1 \\ 1.0 & 1 \\ 10.0 & 1 \\ 1.0 & 1 \\ 1.0 & 1 \\ 1.0 & 1 \\ 1.0 & 1 \\ 1.0 & 1 \\ 1.0 & 1 \\ 1.0 & 1 \\ 1.0 & 1 \\ 1.0 & 1 \\ 1.0 & 1 \\ 1.0 & 1 \\ 1.0 & 1 \\ 2.5 & 1 \\ 1.0 & 1 \\ 1.0 & 1 \\ 1.0 & 1 \\ 3.0 & 1 \\ 87-113 & 1 \\ 86-112 & 1 \\ 82-119 & 1 \\ 90-110 & 1 \\ 0.10 & 1\end{array}$

$0.50 \quad 1$
11/04/11 20:18 75-09-2

11/04/11 20:18 108-10-1

11/04/11 20:18 1634-04-4

11/04/11 20:18 91-20-3

11/04/11 20:18 103-65-1

$11 / 04 / 11$ 20:18 100-42-5

11/04/11 20:18 630-20-6

11/04/11 20:18 79-34-5

$11 / 04 / 1120: 18 \quad 127-18-4$

$11 / 04 / 1120: 18$ 108-88-3

11/04/11 20:18 87-61-6

11/04/11 20:18 120-82-1

11/04/11 20:18 71-55-6

11/04/11 20:18 79-00-5

$11 / 04 / 1120: 18 \quad 79-01-6$

11/04/11 20:18 75-69-4

11/04/11 20:18 96-18-4

$11 / 04 / 1120: 18 \quad 95-63-6$

11/04/11 20:18 108-67-8

$11 / 04 / 1120: 18 \quad 75-01-4$

$11 / 04 / 1120: 18 \quad 1330-20-7$

11/04/11 20:18 460-00-4

11/04/11 20:18 1868-53-7

$11 / 04 / 1120: 18 \quad 17060-07-0$

11/04/11 20:18 2037-26-5

11/04/11 20:18

$11 / 02 / 1108: 48$ 


\section{ANALYTICAL RESULTS}

Project: KS/MO Waste Water

Pace Project No.: 60109211

\begin{tabular}{|c|c|c|c|c|c|c|c|c|}
\hline \multirow{2}{*}{$\begin{array}{c}\text { Sample: BAPURGE-W-10112 } \\
\text { Parameters }\end{array}$} & \multirow{2}{*}{\multicolumn{2}{|c|}{$\begin{array}{lr}\text { Lab ID: } & 60109211002 \\
\text { Results } & \text { Units }\end{array}$}} & \multirow{2}{*}{$\begin{array}{c}\text { Collected: } 10 / 31 / 1 \\
\text { Report Limit }\end{array}$} & $12: 55$ & \multirow{2}{*}{\multicolumn{2}{|c|}{$\begin{array}{l}\text { Received: } 11 / 01 / 1109: 20 \\
\text { Prepared } \quad \text { Analyzed }\end{array}$}} & \multirow{2}{*}{$\begin{array}{r}\text { Matrix: Water } \\
\text { CAS No. }\end{array}$} & \multirow[b]{2}{*}{ Qual } \\
\hline & & & & DF & & & & \\
\hline 504 GCS EDB and DBCP & \multicolumn{7}{|c|}{ Analytical Method: EPA 504.1 Preparation Method: EPA 504.1 } & \\
\hline 1,2-Dibromoethane (EDB) & & ND ug/L & 0.029 & 1 & $11 / 07 / 1100: 00$ & $11 / 08 / 1101: 04$ & $106-93-4$ & \\
\hline $8260 \mathrm{MSV}$ & \multicolumn{7}{|c|}{ Analytical Method: EPA 5030B/8260 } & \\
\hline Acetone & & VD ug/L & 10.0 & 1 & & $11 / 04 / 1120: 35$ & $67-64-1$ & \\
\hline Benzene & & ND ug/L & 1.0 & 1 & & $11 / 04 / 1120: 35$ & $71-43-2$ & \\
\hline Bromobenzene & & ND ug/L & 1.0 & 1 & & 11/04/11 20:35 & $108-86-1$ & \\
\hline Bromochloromethane & & ND ug/L & 1.0 & 1 & & $11 / 04 / 1120: 35$ & $74-97-5$ & \\
\hline Bromodichloromethane & & ND ug/L & 1.0 & 1 & & $11 / 04 / 1120: 35$ & $75-27-4$ & \\
\hline Bromoform & & VD ug/L & 1.0 & 1 & & $11 / 04 / 1120: 35$ & $75-25-2$ & \\
\hline Bromomethane & & ND ug/L & 1.0 & 1 & & $11 / 04 / 1120: 35$ & $74-83-9$ & \\
\hline 2-Butanone (MEK) & & ND ug/L & 10.0 & 1 & & $11 / 04 / 1120: 35$ & $78-93-3$ & \\
\hline n-Butylbenzene & & VD ug/L & 1.0 & 1 & & $11 / 04 / 1120: 35$ & $104-51-8$ & \\
\hline sec-Butylbenzene & & ND ug/L & 1.0 & 1 & & $11 / 04 / 1120: 35$ & $135-98-8$ & \\
\hline tert-Butylbenzene & & ND ug/L & 1.0 & 1 & & $11 / 04 / 1120: 35$ & $98-06-6$ & \\
\hline Carbon disulfide & & ND ug/L & 5.0 & 1 & & $11 / 04 / 1120: 35$ & $75-15-0$ & \\
\hline Carbon tetrachloride & & $1.1 \mathrm{ug} / \mathrm{L}$ & 1.0 & 1 & & $11 / 04 / 1120: 35$ & $56-23-5$ & \\
\hline Chlorobenzene & & ND ug/L & 1.0 & 1 & & $11 / 04 / 1120: 35$ & $108-90-7$ & \\
\hline Chloroethane & & ND ug/L. & 1.0 & 1 & & $11 / 04 / 1120: 35$ & $75-00-3$ & \\
\hline Chloroform & & ND ug/L & 1.0 & 1 & & $11 / 04 / 1120: 35$ & $67-66-3$ & \\
\hline Chloromethane & & ND ug/L & 1.0 & 1 & & $11 / 04 / 1120: 35$ & $74-87-3$ & \\
\hline 2-Chlorotoluene & & ND ug/L & 1.0 & 1 & & $11 / 04 / 1120: 35$ & $95-49-8$ & \\
\hline 4-Chlorotoluene & & VD ug/L & 1.0 & 1 & & $11 / 04 / 1120: 35$ & $106-43-4$ & \\
\hline 1,2-Dibromo-3-chloropropane & & ND ug/L & 2.5 & 1 & & $11 / 04 / 1120: 35$ & $96-12-8$ & \\
\hline Dibromochloromethane & & VD ug/L & 1.0 & 1 & & $11 / 04 / 1120: 35$ & $124-48-1$ & \\
\hline 1,2-Dibromoethane (EDB) & & VD ug/L & 1.0 & 1 & & $11 / 04 / 1120: 35$ & $106-93-4$ & \\
\hline Dibromomethane & & VD ug/L & 1.0 & 1 & & $11 / 04 / 1120: 35$ & $74-95-3$ & \\
\hline 1,2-Dichlorobenzene & & VD ug/L & 1.0 & 1 & & $11 / 04 / 1120: 35$ & $95-50-1$ & \\
\hline 1,3-Dichlorobenzene & & ND ug/L & 1.0 & 1 & & $11 / 04 / 1120: 35$ & $541-73-1$ & \\
\hline 1,4-Dichlorobenzene & & ND ug/L & 1.0 & 1 & & $11 / 04 / 1120: 35$ & $106-46-7$ & \\
\hline Dichlorodifluoromethane & & ND ug/L & 1.0 & 1 & & $11 / 04 / 1120: 35$ & $75-71-8$ & \\
\hline 1,1-Dichloroethane & & ND ug/L & 1.0 & 1 & & $11 / 04 / 1120: 35$ & $75-34-3$ & \\
\hline 1,2-Dichloroethane & & ND ug/L & 1.0 & 1 & & $11 / 04 / 1120: 35$ & $107-06-2$ & \\
\hline 1,2-Dichloroethene (Total) & & ND ug/L & 1.0 & 1 & & $11 / 04 / 1120: 35$ & $540-59-0$ & \\
\hline 1,1-Dichloroethene & & ND ug/L & 1.0 & 1 & & $11 / 04 / 1120: 35$ & $75-35-4$ & \\
\hline cis-1,2-Dichloroethene & & ND ug/L & 1.0 & 1 & & $11 / 04 / 1120: 35$ & $156-59-2$ & \\
\hline trans-1,2-Dichloroethene & & ND ug/L & 1.0 & 1 & & $11 / 04 / 1120: 35$ & $156-60-5$ & \\
\hline 1,2-Dichloropropane & & ND ug/L & 1.0 & 1 & & $11 / 04 / 1120: 35$ & $78-87-5$ & \\
\hline 1,3-Dichioropropane & & ND ug/L & 1.0 & 1 & & $11 / 04 / 1120: 35$ & $142-28-9$ & \\
\hline 2,2-Dichloropropane & & ND ug/L & 1.0 & 1 & & $11 / 04 / 1120: 35$ & $594-20-7$ & \\
\hline 1,1-Dichloropropene & & ND ug/L & 1.0 & 1 & & $11 / 04 / 1120: 35$ & $563-58-6$ & \\
\hline cis-1,3-Dichloropropene & & ND ug/L & 1.0 & 1 & & $11 / 04 / 1120: 35$ & $10061-01-5$ & \\
\hline trans-1,3-Dichloropropene & & VD ug/L & 1.0 & 1 & & $11 / 04 / 1120: 35$ & $10061-02-6$ & \\
\hline Ethylbenzene & & ND ug/L & 1.0 & 1 & & $11 / 04 / 1120: 35$ & $100-41-4$ & \\
\hline Hexachloro-1,3-butadiene & & ND ug/L & 1.0 & 1 & & $11 / 04 / 1120: 35$ & $87-68-3$ & \\
\hline 2-Hexanone & & ND ug/L & 10.0 & 1 & & $11 / 04 / 1120: 35$ & $591-78-6$ & \\
\hline Isopropylbenzene (Cumene) & & $\mathrm{ND} \mathrm{ug} / \mathrm{L}$ & 1.0 & 1 & & $11 / 04 / 1120: 35$ & $98-82-8$ & \\
\hline p-Isopropyltoluene & & ND ug/L & 1.0 & 1 & & $11 / 04 / 1120: 35$ & $99-87-6$ & \\
\hline
\end{tabular}

Date: 11/11/2011 11:15 AM 


\section{ANALYTICAL RESULTS}

Project: $\quad$ KS/MO Waste Water

Pace Project No.: 60109211

Sample: BAPURGE-W-10112 Lab ID: 60109211002 Collected: 10/31/11 12:55 Received: 11/01/11 09:20 Matrix: Water

Parameters $\quad$ Results $\_$Units $\longrightarrow$ Report Limit $\longrightarrow$ DF Prepared

\section{MSV}

Methylene chloride 4-Methyl-2-pentanone (MIBK) Methyl-tert-butyl ether Naphthalene n-Propylbenzene Styrene

1,1,1,2-Tetrachloroethane 1,1,2,2-Tetrachloroethane Tetrachloroethene Toluene

1,2,3-Trichlorobenzene

1,2,4-Trichlorobenzene

1,1,1-Trichloroethane

1,1,2-Trichloroethane

Trichloroethene

Trichlorofluoromethane

1,2,3-Trichloropropane

1,2,4-Trimethylbenzene

1,3,5-Trimethylbenzene

Vinyl chloride

Xylene (Total)

4-Bromofluorobenzene (S)

Dibromofluoromethane (S)

1,2-Dichloroethane-d4 (S)

Toluene-d8 (S)

Preservation $\mathrm{pH}$

353.2 Nitrogen, NO2/NO3 unpres

Nitrogen, Nitrate

Analytical Method: EPA 5030B/8260

$$
\begin{aligned}
& \text { ND ug/L } \\
& \text { ND ug/L } \\
& \text { ND ug/L } \\
& \text { ND ug/L } \\
& \text { ND ug/L } \\
& \text { ND ug/L } \\
& \text { ND ug/L } \\
& \text { ND ug/L } \\
& \text { ND ug/L } \\
& \text { ND ug/L } \\
& \text { ND ug/L } \\
& \text { ND ug/L } \\
& \text { ND ug/L } \\
& \text { ND ug/L } \\
& \text { ND ug/L } \\
& \text { ND ug/L } \\
& \text { ND ug/L } \\
& \text { ND ug/L } \\
& \text { ND ug/L } \\
& \text { ND ug/L } \\
& \text { ND ug/L } \\
& 102 \% \\
& 98 \% \\
& 101 \% \\
& 95 \% \\
& 7.0
\end{aligned}
$$

\begin{tabular}{|c|c|}
\hline $1 / 04 / 1120: 35$ & $09-2$ \\
\hline $11 / 04 / 1120: 35$ & $108-10-1$ \\
\hline $1 / 04 / 1120: 35$ & $1634-04-4$ \\
\hline $11 / 04 / 1120: 35$ & $91-20-3$ \\
\hline $11 / 04 / 1120: 35$ & $103-65-1$ \\
\hline $11 / 04 / 1120: 35$ & $100-42-5$ \\
\hline $1 / 04 / 1120: 35$ & $630-20-6$ \\
\hline $11 / 04 / 1120: 35$ & $79-34-5$ \\
\hline $1 / 04 / 1120: 35$ & $127-18-4$ \\
\hline $11 / 04 / 1120: 35$ & $108-88-3$ \\
\hline $11 / 04 / 1120: 35$ & $87-61-6$ \\
\hline $11 / 04 / 1120: 35$ & $120-82-1$ \\
\hline $11 / 04 / 1120: 35$ & $71-55-6$ \\
\hline $11 / 04 / 1120: 35$ & $79-00-5$ \\
\hline $11 / 04 / 1120: 35$ & $79-01-6$ \\
\hline $11 / 04 / 1120: 35$ & $75-69-4$ \\
\hline $11 / 04 / 1120: 35$ & $96-18-4$ \\
\hline 11/04/11 20:35 & $95-63-6$ \\
\hline $11 / 04 / 1120: 35$ & $108-67-8$ \\
\hline $11 / 04 / 1120: 35$ & 75-01-4 \\
\hline $11 / 04 / 1120: 35$ & $1330-20-7$ \\
\hline $11 / 04 / 1120: 35$ & $460-00-4$ \\
\hline $11 / 04 / 1120: 35$ & 1868-53-7 \\
\hline $11 / 04 / 1120: 35$ & $17060-07-0$ \\
\hline $1 / 04 / 1120: 35$ & $2037-26-5$ \\
\hline
\end{tabular}

Analytical Method: EPA 353.2

$$
6.1 \mathrm{mg} / \mathrm{L}
$$

$\begin{array}{rr}1.0 & 1 \\ 10.0 & 1 \\ 1.0 & 1 \\ 10.0 & 1 \\ 1.0 & 1 \\ 1.0 & 1 \\ 1.0 & 1 \\ 1.0 & 1 \\ 1.0 & 1 \\ 1.0 & 1 \\ 1.0 & 1 \\ 1.0 & 1 \\ 1.0 & 1 \\ 1.0 & 1 \\ 1.0 & 1 \\ 1.0 & 1 \\ 2.5 & 1 \\ 1.0 & 1 \\ 1.0 & 1 \\ 1.0 & 1 \\ 3.0 & 1 \\ 87-113 & 1 \\ 86-112 & 1 \\ 82-119 & 1 \\ 90-110 & 1 \\ 0.10 & 1\end{array}$

0.201
$11 / 04 / 1120: 35$

11/02/11 09:17 


\section{ANALYTICAL RESULTS}

Project: KS/MO Waste Water

Pace Project No.: 60109211

\begin{tabular}{|c|c|c|c|c|c|c|c|}
\hline \multirow{2}{*}{$\begin{array}{c}\text { Sample: CNPURGE-W-10113 } \\
\text { Parameters }\end{array}$} & Lab ID: 60109211003 & \multicolumn{2}{|c|}{ Collected: $10 / 31 / 1114: 02$} & \multicolumn{2}{|c|}{ Received: $11 / 01 / 1109: 20$} & \multicolumn{2}{|l|}{ Matrix: Water } \\
\hline & Results & Report Limit & DF & Prepared & Analyzed & CAS No. & Qual \\
\hline 504 GCS EDB and DBCP & \multicolumn{6}{|c|}{ Analytical Method: EPA 504.1 Preparation Method: EPA 504.1 } & \\
\hline 1,2-Dibromoethane (EDB) & ND ug/L & 0.028 & 1 & $11 / 07 / 1100: 00$ & $11 / 08 / 1101: 17$ & $106-93-4$ & \\
\hline $8260 \mathrm{MSV}$ & \multicolumn{6}{|c|}{ Analytical Method: EPA 5030B/8260 } & \\
\hline Acetone & ND ug/L & 10.0 & 1 & & $11 / 04 / 1120: 51$ & $67-64-1$ & \\
\hline Benzene & ND ug/L & 1.0 & 1 & & $11 / 04 / 1120: 51$ & $71-43-2$ & \\
\hline Bromobenzene & ND ug/L & 1.0 & 1 & & $11 / 04 / 1120: 51$ & $108-86-1$ & \\
\hline Bromochloromethane & ND ug/L & 1.0 & 1 & & $11 / 04 / 1120: 51$ & $74-97-5$ & \\
\hline Bromodichloromethane & ND ug/L & 1.0 & 1 & & $11 / 04 / 1120: 51$ & $75-27-4$ & \\
\hline Bromoform & ND ug/L & 1.0 & 1 & & $11 / 04 / 1120: 51$ & $75-25-2$ & \\
\hline Bromomethane & ND ug/L & 1.0 & 1 & & $11 / 04 / 1120: 51$ & $74-83-9$ & \\
\hline 2-Butanone (MEK) & ND ug/L & 10.0 & 1 & & $11 / 04 / 1120: 51$ & $78-93-3$ & \\
\hline n-Butylbenzene & ND ug/L & 1.0 & 1 & & $11 / 04 / 1120: 51$ & $104-51-8$ & \\
\hline sec-Bubylbenzene & ND ug/L & 1.0 & 1 & & $11 / 04 / 1120: 51$ & $135-98-8$ & \\
\hline tert-Butylbenzene & ND ug/L & 1.0 & 1 & & $11 / 04 / 1120: 51$ & $98-06-6$ & \\
\hline Carbon disulfide & ND ug/L & 5.0 & 1 & & $11 / 04 / 1120: 51$ & $75-15-0$ & \\
\hline Carbon tetrachloride & $1.3 \mathrm{ug} / \mathrm{L}$ & 1.0 & 1 & & $11 / 04 / 1120: 51$ & $56-23-5$ & \\
\hline Chlorobenzene & ND ug/L & 1.0 & 1 & & $11 / 04 / 1120: 51$ & $108-90-7$ & \\
\hline Chloroethane & ND ug/L & 1.0 & 1 & & $11 / 04 / 1120: 51$ & $75-00-3$ & \\
\hline Chloroform & ND ug/L & 1.0 & 1 & & $11 / 04 / 1120: 51$ & $67-66-3$ & \\
\hline Chloromethane & ND ug/L & 1.0 & 1 & & $11 / 04 / 1120: 51$ & $74-87-3$ & \\
\hline 2-Chlorotoluene & ND ug/L & 1.0 & 1 & & $\uparrow 1 / 04 / 1120: 51$ & $95-49-8$ & \\
\hline 4-Chlorotoluene & ND ug/L & 1.0 & 1 & & $11 / 04 / 1120: 51$ & $106-43-4$ & \\
\hline 1,2-Dibromo-3-chloropropane & ND ug/L & 2.5 & 1 & & $11 / 04 / 1120: 51$ & $96-12-8$ & \\
\hline Dibromochloromethane & ND ug/L & 1.0 & 1 & & $11 / 04 / 1120: 51$ & $124-48-1$ & \\
\hline 1,2-Dibromoethane (EDB) & ND ug/L & 1.0 & 1 & & $11 / 04 / 1120: 51$ & $106-93-4$ & \\
\hline Dibromomethane & ND ug/L & 1.0 & 1 & & $11 / 04 / 1120: 51$ & $74-95-3$ & \\
\hline 1,2-Dichlorobenzene & ND ug/L & 1.0 & 1 & & $11 / 04 / 1120: 51$ & $95-50-1$ & \\
\hline 1,3-Dichlorobenzene & ND ug/L & 1.0 & 1 & & $11 / 04 / 1120: 51$ & $541-73-1$ & \\
\hline 1,4-Dichlorobenzene & ND ug/L & 1.0 & 1 & & $11 / 04 / 1120: 51$ & $106-46-7$ & \\
\hline Dichlorodifluoromethane & ND ug/L & 1.0 & 1 & & $11 / 04 / 1120: 51$ & $75-71-8$ & \\
\hline 1,1-Dichloroethane & ND ug/L & 1.0 & 1 & & $11 / 04 / 1120: 51$ & $75-34-3$ & \\
\hline 1,2-Dichloroethane & ND ug/L & 1.0 & 1 & & $11 / 04 / 1120: 51$ & $107-06-2$ & \\
\hline 1,2-Dichloroethene (Total) & ND ug/L & 1.0 & 1 & & $11 / 04 / 1120: 51$ & $540-59-0$ & \\
\hline 1,1-Dichloroethene & ND ug/L & 1.0 & 1 & & $11 / 04 / 1120: 51$ & $75-35-4$ & \\
\hline cis-1,2-Dichloroethene & ND ug/L & 1.0 & 1 & & $11 / 04 / 1120: 51$ & $156-59-2$ & \\
\hline trans-1,2-Dichloroethene & ND ug/L & 1.0 & 1 & & $11 / 04 / 1120: 51$ & $156-60-5$ & \\
\hline 1,2-Dichloropropane & ND ug/L & 1.0 & 1 & & $11 / 04 / 1120: 51$ & $78-87-5$ & \\
\hline 1,3-Dichloropropane & ND ug/L & 1.0 & 1 & & $11 / 04 / 1120: 51$ & $142-28-9$ & \\
\hline 2,2-Dichloropropane & ND ug/L & 1.0 & 1 & & $11 / 04 / 1120: 51$ & $594-20-7$ & \\
\hline 1,1-Dichloropropene & ND ug/L & 1.0 & 1 & & $11 / 04 / 1120: 51$ & $563-58-6$ & \\
\hline cis-1,3-Dichloropropene & ND ug/L & 1.0 & 1 & & $11 / 04 / 1120: 51$ & $10061-01-5$ & \\
\hline trans-1,3-Dichloropropene & ND ug/L & 1.0 & 1 & & $11 / 04 / 1120: 51$ & $10061-02-6$ & \\
\hline Ethylbenzene & ND ug/L & 1.0 & 1 & & $11 / 04 / 1120: 51$ & $100-41-4$ & \\
\hline Hexachloro-1,3-butadiene & ND ug/L & 1.0 & 1 & & $11 / 04 / 1120: 51$ & $87-68-3$ & \\
\hline 2-Hexanone & ND ug/L & 10.0 & 1 & & $11 / 04 / 1120: 51$ & $591-78-6$ & \\
\hline Isopropylbenzene (Cumene) & ND ug/L & 1.0 & 1 & & $11 / 04 / 1120: 51$ & $98-82-8$ & \\
\hline p-Isopropyltoluene & ND ug/L & 1.0 & 1 & & $11 / 04 / 1120: 51$ & $99-87-6$ & \\
\hline
\end{tabular}

Date: 11/11/2011 11:15 AM 


\section{ANALYTICAL RESULTS}

Project: KS/MO Waste Water

Pace Project No.: 60109211

Sample: CNPURGE-W-10113

Lab ID: 60109211003 Collected: 10/31/11 14:02 Received: 11/01/11 09:20 Matrix: Water

Parameters $\quad$ Results $\longrightarrow$ Units $\longrightarrow$ Report Limit DF Prepared Analyzed CAS No.

\section{MSV}

Methylene chloride 4-Methyl-2-penlanone (MIBK)

Methyl-tert-butyl ether

Naphthalene

n-Propylbenzene

Styrene

1,1,1,2-Tetrachloroethane

1,1,2,2-Tetrachloroethane

Tetrachloroethene

Toluene

1,2,3-Trichlorobenzene

1,2,4-Trichlorobenzene

1,1,1-Trichloroethane

1,1,2-Trichloroethane

Trichloroethene

Trichlorofluoromethane

1,2,3-Trichloropropane

1,2,4-Trimethylbenzene

1,3,5-Trimethylbenzene

Vinyl chloride

Xylene (Total)

4-Bromofluorobenzene (S)

Dibromofluoromethane (S)

1,2-Dichloroethane-d4 (S)

Toluene-d8 (S)

Preservation $\mathrm{pH}$

\subsection{Nitrogen, NO2/NO3 unpres}

Nitrogen, Nitrate

Analytical Method: EPA 5030B/8260

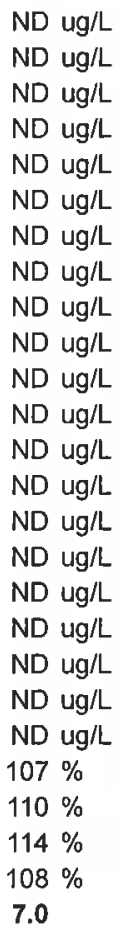

ND ug/L

ND ug/L

ND ug/L

ND ug/L

ND ug/L

ND ug/L

ND ug/L

ND ug/L

ND ug/L

ND ug/L

ND ug/L

ND ug/L

ND ug/L

ND ug/L

ND ug/L

ND ug/L

ND ug/L

$07 \%$

7.0

Analytical Method: EPA 353.2

$4.4 \mathrm{mg} / \mathrm{L}$

$\begin{array}{rr}1.0 & 1 \\ 10.0 & 1 \\ 1.0 & 1 \\ 10.0 & 1 \\ 1.0 & 1 \\ 1.0 & 1 \\ 1.0 & 1 \\ 1.0 & 1 \\ 1.0 & 1 \\ 1.0 & 1 \\ 1.0 & 1 \\ 1.0 & 1 \\ 1.0 & 1 \\ 1.0 & 1 \\ 1.0 & 1 \\ 1.0 & 1 \\ 2.5 & 1 \\ 1.0 & 1 \\ 1.0 & 1 \\ 1.0 & 1 \\ 3.0 & 1 \\ 87-113 & 1 \\ 86-112 & 1 \\ 82-119 & 1 \\ 90-110 & 1 \\ 0.10 & 1\end{array}$

0.101
11/04/11 20:51 75-09-2

11/04/11 20:51 108-10-1

11/04/11 20:51 1634-04-4

11/04/11 20:51 91-20-3

11/04/11 20:51 103-65-1

$11 / 04 / 1120: 51 \quad 100-42-5$

$11 / 04 / 1120: 51 \quad 630-20-6$

11/04/11 20:51 79-34-5

11/04/11 20:51 127-18-4

$11 / 04 / 1120: 51 \quad 108-88-3$

11/04/11 20:51 87-61-6

$11 / 04 / 11$ 20:51 120-82-1

11/04/11 20:51 71-55-6

11/04/11 20:51 79-00-5

11/04/11 20:51 79-01-6

11/04/11 20:51 75-69-4

11/04/11 20:51 96-18-4

11/04/11 20:51 95-63-6

$11 / 04 / 1120: 51 \quad 108-67-8$

11/04/11 20:51 75-01-4

11/04/11 20:51 1330-20-7

$11 / 04 / 1120: 51 \quad 460-00-4$

11/04/11 20:51 1868-53-7

11/04/11 20:51 17060-07-0

11/04/11 20:51 2037-26-5

11/04/11 20:51

11/02/11 09:06 


\title{
ANALYTICAL RESULTS
}

\begin{abstract}
Project: $\quad$ KS/MO Waste Water
Pace Project No.: 60109211
\end{abstract}

Sample: EUPURGE-W-10114 Lab ID: 60109211004 Collected: 10/31/11 $15: 52$ Received: 11/01/11 09:20 Matrix: Water

Parameters $\longrightarrow$ Results $\longrightarrow$ Units $\longrightarrow$ Report Limit DF Prepared $\longrightarrow$ Analyzed CAS No.

504 GCS EDB and DBCP

1,2-Dibromoethane (EDB)

\section{MSV}

\section{Acetone}

Benzene

Bromobenzene

Bromochloromethane

Bromodichloromethane

Bromoform

Bromomethane

2-Butanone (MEK)

n-Butylbenzene

sec-Butylbenzene

tert-Butylbenzene

Carbon disulfide

Carbon tetrachloride

Chlorobenzene

Chloroethane

Chloroform

Chloromethane

2-Chlorotoluene

4-Chlorotoluene

1,2-Dibromo-3-chloropropane

Dibromochloromethane

1,2-Dibromoethane (EDB)

Dibromomethane

1,2-Dichlorobenzene

1,3-Dichlorobenzene

1,4-Dichlorobenzene

Dichlorodifluoromethane

1,1-Dichloroethane

1,2-Dichloroethane

1,2-Dichloroethene (Total)

1,1-Dichloroethene

cis-1,2-Dichloroethene

trans-1,2-Dichloroethene

1,2-Dichloropropane

1,3-Dichloropropane

2,2-Dichloropropane

1,1-Dichloropropene

cis-1,3-Dichloropropene

trans-1,3-Dichloropropene

Ethylbenzene

Hexachloro-1,3-butadiene

2-Hexanone

Isopropylbenzene (Cumene)

p-Isopropyltoluene

Date: 11/11/2011 11:15 AM
Analytical Method: EPA 504.1 Preparation Method: EPA 504.1

$$
\text { ND ug/L }
$$

$0.029 \quad 1$

11/07/11 00:00

$11 / 08 / 11 \quad 01: 30 \quad 106-93-4$

Analytical Method: EPA 5030B/8260

\begin{tabular}{|c|c|c|c|c|}
\hline ND ug/L & 10.0 & 1 & $11 / 04 / 1121: 07$ & $67-64-1$ \\
\hline ND ug/L & 1.0 & 1 & $11 / 04 / 1121: 07$ & $71-43-2$ \\
\hline ND ug/L & 1.0 & 1 & $11 / 04 / 1121: 07$ & $108-86-1$ \\
\hline ND ug/L & 1.0 & 1 & $11 / 04 / 1121: 07$ & $74-97-5$ \\
\hline ND ug/L & 1.0 & 1 & $11 / 04 / 1121: 07$ & $75-27-4$ \\
\hline ND ug/L. & 1.0 & 1 & $11 / 04 / 1121: 07$ & $75-25-2$ \\
\hline ND ug/L & 1.0 & 1 & $11 / 04 / 1121: 07$ & $74-83-9$ \\
\hline ND ug/L & 10.0 & 1 & $11 / 04 / 1121: 07$ & $78-93-3$ \\
\hline ND ug/L & 1.0 & 1 & $11 / 04 / 1121: 07$ & $104-51-8$ \\
\hline ND ug/L & 1.0 & 1 & $11 / 04 / 1121: 07$ & $135-98-8$ \\
\hline ND ug/L & 1.0 & 1 & $11 / 04 / 1121: 07$ & $98-06-6$ \\
\hline ND ug/L & 5.0 & 1 & 11/04/11 21:07 & $75-15-0$ \\
\hline ND ug/L & 1.0 & 1 & $11 / 04 / 1121: 07$ & $56-23-5$ \\
\hline ND ug/L & 1.0 & 1 & $11 / 04 / 1121: 07$ & $108-90-7$ \\
\hline ND ug/L & 1.0 & 1 & $11 / 04 / 1121: 07$ & $75-00-3$ \\
\hline ND ug/L & 1.0 & 1 & $11 / 04 / 1121: 07$ & $67-66-3$ \\
\hline ND ug/L. & 1.0 & 1 & $11 / 04 / 1121: 07$ & $74-87-3$ \\
\hline ND ug/L. & 1.0 & 1 & $11 / 04 / 1121: 07$ & $95-49-8$ \\
\hline ND ug/L. & 1.0 & 1 & $11 / 04 / 1121: 07$ & $106-43-4$ \\
\hline ND ug/L & 2.5 & 1 & $11 / 04 / 1121: 07$ & $96-12-8$ \\
\hline ND ug/L & 1.0 & 1 & $11 / 04 / 1121: 07$ & $124-48-1$ \\
\hline ND ug/L & 1.0 & 1 & $11 / 04 / 1121: 07$ & $106-93-4$ \\
\hline ND ug/L & 1.0 & 1 & $11 / 04 / 1121: 07$ & $74-95-3$ \\
\hline ND ug/L & 1.0 & 1 & $11 / 04 / 1121: 07$ & $95-50-1$ \\
\hline ND ug/L & 1.0 & 1 & $11 / 04 / 1121: 07$ & $541-73-1$ \\
\hline ND ug/L & 1.0 & 1 & $11 / 04 / 1121: 07$ & $106-46-7$ \\
\hline ND ug/L & 1.0 & 1 & $11 / 04 / 1121: 07$ & $75-71-8$ \\
\hline ND ug/L & 1.0 & 1 & $11 / 04 / 1121: 07$ & $75-34-3$ \\
\hline ND ug/L & 1.0 & 1 & $11 / 04 / 1121: 07$ & $107-06-2$ \\
\hline ND ug/L & 1.0 & 1 & $11 / 04 / 1121: 07$ & $540-59-0$ \\
\hline ND ug/L & 1.0 & 1 & $11 / 04 / 1121: 07$ & $75-35-4$ \\
\hline ND ug/L & 1.0 & 1 & $11 / 04 / 1121: 07$ & $156-59-2$ \\
\hline ND ug/L & 1.0 & 1 & $11 / 04 / 1121: 07$ & $156-60-5$ \\
\hline ND ug/L & 1.0 & 1 & $11 / 04 / 1121: 07$ & $78-87-5$ \\
\hline ND ug/L & 1.0 & 1 & $11 / 04 / 1121: 07$ & $142-28-9$ \\
\hline ND ug/L & 1.0 & 1 & $11 / 04 / 1121: 07$ & $594-20-7$ \\
\hline ND ug/L. & 1.0 & 1 & $11 / 04 / 1121: 07$ & $563-58-6$ \\
\hline ND ug/L & 1.0 & 1 & $11 / 04 / 1121: 07$ & $10061-01-5$ \\
\hline ND ug/L. & 1.0 & 1 & $11 / 04 / 1121: 07$ & $10061-02-6$ \\
\hline ND ug/L & 1.0 & 1 & $11 / 04 / 1121: 07$ & $100-41-4$ \\
\hline ND ug/L & 1.0 & 1 & $11 / 04 / 1121: 07$ & $87-68-3$ \\
\hline ND ug/L & 10.0 & 1 & $11 / 04 / 1121: 07$ & $591-78-6$ \\
\hline ND ug/L & 1.0 & 1 & $11 / 04 / 1121: 07$ & $98-82-8$ \\
\hline ND ug/L & 1.0 & 1 & $11 / 04 / 1121: 07$ & $99-87-6$ \\
\hline
\end{tabular}

\section{REPORT OF LABORATORY ANALYSIS}

Page 11 of 26

This report shall not be reproduced, except in full, 


\section{ANALYTICAL RESULTS}

Project: KS/MO Waste Water

Pace Project No.: 60109211

Sample: EUPURGE-W-10114

Lab ID: 60109211004 Collected: 10/31/11 15:52 Received: 11/01/11 09:20 Matrix: Water

Parameters

\section{MSV}

Methylene chloride 4-Methyl-2-pentanone (MIBK)

Methyl-tert-butyl ether

Naphthalene

n-Propylbenzene

Styrene

1,1,1,2-Tetrachloroethane

1,1,2,2-Tetrachloroethane

Tetrachloroethene

Toluene

1,2,3-Trichlorobenzene

1,2,4-Trichlorobenzene

1,1,1-Trichloroethane

1,1,2-Trichloroethane

Trichloroethene

Trichlorofluoromethane

1,2,3-Trichloropropane

1,2,4-Trimethylbenzene

1,3,5-Trimethylbenzene

Vinyl chloride

Xylene (Total)

4-Bromofluorobenzene (S)

Dibromofluoromethane (S)

1,2-Dichloroethane-d4 (S)

Toluene-d8 (S)

Preservation $\mathrm{pH}$

353.2 Nitrogen, NO2/NO3 unpres

Nitrogen, Nitrate

Results

Units Report Limit

DF

Prepare

Analytical Method: EPA 5030B/8260

\begin{abstract}
ND ug/L
ND $u g / L$

ND ug/L

ND ug/L

ND ug/L

ND ug/L

ND ug/L

ND ug/L

ND ug/L

ND ug/L

ND ug/L

ND ug/L

ND ug/L

ND ug/L

ND ug/L

ND ug/L

ND ug/L

ND ug/L

ND ug/L

ND ug/L

ND ug/L

$106 \%$

$108 \%$

$113 \%$

$105 \%$

7.0
\end{abstract}

Analytical Method: EPA 353.2

$10.6 \mathrm{mg} / \mathrm{L}$

$\begin{array}{rr}1.0 & 1 \\ 10.0 & 1 \\ 1.0 & 1 \\ 10.0 & 1 \\ 1.0 & 1 \\ 1.0 & 1 \\ 1.0 & 1 \\ 1.0 & 1 \\ 1.0 & 1 \\ 1.0 & 1 \\ 1.0 & 1 \\ 1.0 & 1 \\ 1.0 & 1 \\ 1.0 & 1 \\ 1.0 & 1 \\ 1.0 & 1 \\ 2.5 & 1 \\ 1.0 & 1 \\ 1.0 & 1 \\ 1.0 & 1 \\ 3.0 & 1 \\ 87-113 & 1 \\ 86-112 & 1 \\ 82-119 & 1 \\ 90-110 & 1 \\ 0.10 & 1\end{array}$

0.50
$11 / 04 / 1121: 07 \quad 75-09-2$

11/04/11 21:07 108-10-1

$11 / 04 / 1121: 07 \quad 1634-04-4$

11/04/11 21:07 91-20-3

$11 / 04 / 1121: 07$ 103-65-1

$11 / 04 / 1121: 07 \quad 100-42-5$

$11 / 04 / 1121: 07$ 630-20-6

11/04/11 21:07 79-34-5

$11 / 04 / 1121: 07 \quad 127-18-4$

$11 / 04 / 1121: 07$ 108-88-3

11/04/11 21:07 87-61-6

$11 / 04 / 1121: 07$ 120-82-1

11/04/11 21:07 71-55-6

11/04/11 21:07 79-00-5

$11 / 04 / 1121: 07 \quad 79-01-6$

11/04/11 21:07 75-69-4

11/04/11 21:07 96-18-4

11/04/11 21:07 95-63-6

$11 / 04 / 1121: 07 \quad 108-67-8$

11/04/11 21:07 75-01-4

$11 / 04 / 1121: 07 \quad 1330-20-7$

11/04/11 21:07 460-00-4

11/04/11 21:07 1868-53-7

$11 / 04 / 1121: 07$ 17060-07-0

11/04/11 21:07 2037-26-5

11/04/11 21:07

$11 / 02 / 1109: 21$ 


\section{ANALYTICAL RESULTS}

Project: $\quad$ KS/MO Waste Water

Pace Project No.: 60109211

Sample: HAPURGE-W-10115 Lab ID: 60109211005 Collected: 10/31/11 12:27 Received: 11/01/11 09:20 Matrix: Water

Parameters U Results - Units - Report Limit DF Prepared Analyzed CAS No.

504 GCS EDB and DBCP

1,2-Dibromoethane (EDB)

8260 MSV

\section{Acetone}

Benzene

Bromobenzene

Bromochloromethane

Bromodichloromethane

Bromoform

Bromomethane

2-Butanone (MEK)

n-Butylbenzene

sec-Butylbenzene

tert-Butylbenzene

Carbon disulfide

Carbon tetrachloride

Chlorobenzene

Chloroethane

Chloroform

Chloromethane

2-Chlorotoluene

4-Chlorotoluene

1,2-Dibromo-3-chloropropane

Dibromochloromethane

1,2-Dibromoethane (EDB)

Dibromomethane

1,2-Dichlorobenzene

1,3-Dichlorobenzene

1,4-Dichlorobenzene

Dichlorodifluoromethane

1,1-Dichloroethane

1,2-Dichloroethane

1,2-Dichloroethene (Total)

1,1-Dichloroethene

cis-1,2-Dichloroethene

trans-1,2-Dichloroethene

1,2-Dichloropropane

1,3-Dichloropropane

2,2-Dichloropropane

1,1-Dichloropropene

cis-1,3-Dichloropropene

trans-1,3-Dichloropropene

Ethylbenzene

Hexachloro-1,3-butadiene

2-Hexanone

Isopropylbenzene (Cumene)

p-Isopropyltoluene

Date: 11/11/2011 11:15 AM
Analytical Method: EPA 504.1 Preparation Method: EPA 504.1

$$
\begin{array}{llllllll}
\text { ND ug/L } & 0.028 & 1 & 11 / 07 / 11 & 00: 00 & 11 / 08 / 11 & 01: 43 & 106-93-4
\end{array}
$$

Analytical Method: EPA 5030B/8260

\begin{tabular}{|c|c|c|c|c|}
\hline ND ug/L & 10.0 & 1 & $11 / 04 / 1121: 24$ & $67-64-1$ \\
\hline ND ug/L & 1.0 & 1 & $11 / 04 / 1121: 24$ & $71-43-2$ \\
\hline ND ug/L & 1.0 & 1 & $11 / 04 / 1121: 24$ & $108-86-1$ \\
\hline ND ug/L & 1.0 & 1 & $11 / 04 / 1121: 24$ & $74-97-5$ \\
\hline ND ug/L & 1.0 & 1 & $11 / 04 / 1121: 24$ & $75-27-4$ \\
\hline ND ug/L & 1.0 & 1 & $11 / 04 / 1121: 24$ & $75-25-2$ \\
\hline ND ug/L & 1.0 & 1 & $11 / 04 / 1121: 24$ & $74-83-9$ \\
\hline ND ug/L & 10.0 & 1 & $11 / 04 / 1121: 24$ & $78-93-3$ \\
\hline ND ug/L & 1.0 & 1 & $11 / 04 / 1121: 24$ & $104-51-8$ \\
\hline ND ug/L & 1.0 & 1 & $11 / 04 / 1121: 24$ & $135-98-8$ \\
\hline ND ug/L & 1.0 & 1 & $11 / 04 / 1121: 24$ & $98-06-6$ \\
\hline ND ug/L & 5.0 & 1 & $11 / 04 / 1121: 24$ & $75-15-0$ \\
\hline $6.1 \mathrm{ug} / \mathrm{L}$ & 1.0 & 1 & $11 / 04 / 1121: 24$ & $56-23-5$ \\
\hline ND ug/L & 1.0 & 1 & $11 / 04 / 1121: 24$ & $108-90-7$ \\
\hline ND ug/L & 1.0 & 1 & $11 / 04 / 1121: 24$ & $75-00-3$ \\
\hline ND ug/L & 1.0 & 1 & $11 / 04 / 1121: 24$ & $67-66-3$ \\
\hline ND ug/L & 1.0 & 1 & $11 / 04 / 1121: 24$ & $74-87-3$ \\
\hline ND ug/L & 1.0 & 1 & $11 / 04 / 1121: 24$ & $95-49-8$ \\
\hline ND ug/L & 1.0 & 1 & $11 / 04 / 1121: 24$ & $106-43-4$ \\
\hline ND ug/L & 2.5 & 1 & $11 / 04 / 1121: 24$ & $96-12-8$ \\
\hline ND ug/L & 1.0 & 1 & $11 / 04 / 1121: 24$ & $124-48-1$ \\
\hline ND ug/L & 1.0 & 1 & $11 / 04 / 1121: 24$ & $106-93-4$ \\
\hline ND ug/L & 1.0 & 1 & $11 / 04 / 1121: 24$ & $74-95-3$ \\
\hline ND ug/L & 1.0 & 1 & $11 / 04 / 1121: 24$ & $95-50-1$ \\
\hline ND ug/L & 1.0 & 1 & $11 / 04 / 1121: 24$ & $541-73-1$ \\
\hline ND ug/L & 1.0 & 1 & $11 / 04 / 1121: 24$ & $106-46-7$ \\
\hline ND ug/L & 1.0 & 1 & $11 / 04 / 1121: 24$ & $75-71-8$ \\
\hline ND ug/L & 1.0 & 1 & $11 / 04 / 1121: 24$ & $75-34-3$ \\
\hline ND ug/L & 1.0 & 1 & $11 / 04 / 1121: 24$ & $107-06-2$ \\
\hline ND ug/L & 1.0 & 1 & $11 / 04 / 1121: 24$ & $540-59-0$ \\
\hline ND ug/L & 1.0 & 1 & $11 / 04 / 1121: 24$ & $75-35-4$ \\
\hline ND ug/L & 1.0 & 1 & $11 / 04 / 1121: 24$ & $156-59-2$ \\
\hline ND ug/L & 1.0 & 1 & $11 / 04 / 1121: 24$ & $156-60-5$ \\
\hline ND ug/L & 1.0 & 1 & $11 / 04 / 1121: 24$ & $78-87-5$ \\
\hline ND ug/L & 1.0 & 1 & $11 / 04 / 1121: 24$ & $142-28-9$ \\
\hline ND ug/L & 1.0 & 1 & $11 / 04 / 1121: 24$ & $594-20-7$ \\
\hline ND ug/L & 1.0 & 1 & $11 / 04 / 1121: 24$ & $563-58-6$ \\
\hline ND ug/L & 1.0 & 1 & $11 / 04 / 1121: 24$ & $10061-01-5$ \\
\hline ND ug/L & 1.0 & 1 & $11 / 04 / 1121: 24$ & $10061-02-6$ \\
\hline ND ug/L & 1.0 & 1 & $11 / 04 / 1121: 24$ & $100-41-4$ \\
\hline ND ug/L & 1.0 & 1 & $11 / 04 / 1121: 24$ & $87-68-3$ \\
\hline ND ug/L & 10.0 & 1 & $11 / 04 / 1121: 24$ & $591-78-6$ \\
\hline ND ug/L & 1.0 & 1 & $11 / 04 / 1121: 24$ & $98-82-8$ \\
\hline ND ug/L & 1.0 & 1 & $11 / 04 / 1121: 24$ & $99-87-6$ \\
\hline
\end{tabular}

\section{REPORT OF LABORATORY ANALYSIS}

This report shall not be reproduced, except in full, without the written consent of Pace Analytical Services, Inc.. 


\section{ANALYTICAL RESULTS}

Project: KS/MO Waste Water

Pace Project No.: 60109211

Sample: HAPURGE-W-10115

Lab ID: 60109211005 Collected: 10/31/11 12:27 Received: 11/01/11 09:20 Matrix: Water

Parameters $\quad$ Results $\longrightarrow$ Units $~ R e p o r t ~ L i m i t ~ D F \quad$ Prepared Analyzed CAS No.

\section{MSV}

Methylene chloride 4-Methyl-2-pentanone (MIBK) Methyl-tert-butyl ether Naphthalene n-Propylbenzene Styrene

1,1,1,2-Tetrachloroethane 1,1,2,2-Tetrachloroethane Tetrachloroethene

Toluene

1,2,3-Trichlorobenzene

1,2,4-Trichlorobenzene

1,1,1-Trichloroethane

1,1,2-Trichloroethane

Trichloroethene

Trichlorofluoromethane

1,2,3-Trichloropropane

1,2,4-Trimethylbenzene

1,3,5-Trimethylbenzene

Vinyl chloride

Xylene (Total)

4-Bromofluorobenzene (S)

Dibromofluoromethane (S)

1,2-Dichloroethane-d4 (S)

Toluene-d8 (S)

Preservation $\mathrm{pH}$

\subsection{Nitrogen, NO2/NO3 unpres}

Nitrogen, Nitrate

Analytical Method: EPA 5030B/8260

$$
\begin{aligned}
& \text { ND ug/L } \\
& \text { ND ug/L } \\
& \text { ND ug/L } \\
& \text { ND ug/L } \\
& \text { ND ug/L } \\
& \text { ND ug/L } \\
& \text { ND ug/L } \\
& \text { ND ug/L } \\
& \text { ND ug/L } \\
& \text { ND ug/L } \\
& \text { ND ug/L } \\
& \text { ND ug/L } \\
& \text { ND ug/L } \\
& \text { ND ug/L } \\
& \text { ND ug/L } \\
& \text { ND ug/L } \\
& \text { ND ug/L } \\
& \text { ND ug/L } \\
& \text { ND ug/L } \\
& \text { ND ug/L } \\
& \text { ND ug/L } \\
& 106 \% \\
& 103 \% \\
& 106 \% \\
& 100 \% \\
& 7.0
\end{aligned}
$$

Analytical Method: EPA 353.2

$5.7 \mathrm{mg} / \mathrm{L}$

$\begin{array}{rr}1.0 & 1 \\ 10.0 & 1 \\ 1.0 & 1 \\ 10.0 & 1 \\ 1.0 & 1 \\ 1.0 & 1 \\ 1.0 & 1 \\ 1.0 & 1 \\ 1.0 & 1 \\ 1.0 & 1 \\ 1.0 & 1 \\ 1.0 & 1 \\ 1.0 & 1 \\ 1.0 & 1 \\ 1.0 & 1 \\ 1.0 & 1 \\ 2.5 & 1 \\ 1.0 & 1 \\ 1.0 & 1 \\ 1.0 & 1 \\ 3.0 & 1 \\ 87-113 & 1 \\ 86-112 & 1 \\ 82-119 & 1 \\ 90-110 & 1 \\ 0.10 & 1\end{array}$

$0.20 \quad 1$
11/04/11 21:24 75-09-2

11/04/11 21:24 108-10-1

11/04/11 21:24 1634-04-4

$11 / 04 / 1121: 24 \quad 91-20-3$

11/04/11 21:24 103-65-1

$11 / 04 / 1121: 24 \quad 100-42-5$

$11 / 04 / 1121: 24$ 630-20-6

11/04/11 21:24 79-34-5

$11 / 04 / 1121: 24$ 127-18-4

$11 / 04 / 1121: 24$ 108-88-3

$11 / 04 / 1121: 24 \quad 87-61-6$

11/04/11 21:24 120-82-1

$11 / 04 / 1121: 24 \quad 71-55-6$

$11 / 04 / 1121: 24 \quad 79-00-5$

11/04/11 21:24 79-01-6

$11 / 04 / 1121: 24 \quad 75-69-4$

11/04/11 21:24 96-18-4

$11 / 04 / 1121: 24$ 95-63-6

11/04/11 21:24 108-67-8

$11 / 04 / 1121: 24 \quad 75-01-4$

11/04/11 21:24 1330-20-7

11/04/11 21:24 460-00-4

11/04/11 21:24 1868-53-7

11/04/11 21:24 17060-07-0

11/04/11 21:24 2037-26-5

$11 / 04 / 1121: 24$

11/02/11 09:16 


\section{ANALYTICAL RESULTS}

Project: KS/MO Waste Water

Pace Project No: 60109211

\begin{tabular}{|c|c|c|c|c|c|c|c|c|}
\hline Sample: MRPURGE-W-10116 & Lab ID: & 60109211006 & Collected: $10 / 31 / 1$ & $14: 42$ & Received: 1 & $11 / 01 / 1109: 20$ & Matrix: Water & \\
\hline Parameters & Results & Units & Report Limit & DF & Prepared & Analyzed & CAS No. & Qual \\
\hline
\end{tabular}

504 GCS EDB and DBCP

1,2-Dibromoethane (EDB)

\section{MSV}

\section{Acetone}

Benzene

Bromobenzene

Bromochloromethane

Bromodichloromethane

Bromoform

Bromomethane

2-Butanone (MEK)

n-Butylbenzene

sec-Butylbenzene

tert-Butylbenzene

Carbon disulfide

Carbon tetrachloride

Chlorobenzene

Chloroethane

Chloroform

Chloromethane

2-Chlorotoluene

4-Chlorotoluene

1,2-Dibromo-3-chloropropane

Dibromochloromethane

1,2-Dibromoethane (EDB)

Dibromomethane

1,2-Dichlorobenzene

1,3-Dichlorobenzene

1,4-Dichlorobenzene

Dichlorodifluoromethane

1,1-Dichloroethane

1,2-Dichloroethane

1,2-Dichloroethene (Total)

1,1-Dichloroethene

cis-1,2-Dichloroethene

trans-1,2-Dichloroethene

1,2-Dichloropropane

1,3-Dichloropropane

2,2-Dichloropropane

1,1-Dichloropropene

cis-1,3-Dichloropropene

trans-1,3-Dichloropropene

Ethylbenzene

Hexachloro-1,3-butadiene

2-Hexanone

Isopropylbenzene (Cumene)

p-Isopropyltoluene

Date: 11/11/2011 11:15 AM
Analytical Method: EPA 504.1 Preparation Method: EPA 504.1

$$
\text { ND ug/L }
$$

$0.029 \quad 1$

$11 / 07 / 1100: 00 \quad 11 / 0$

$11 / 08 / 1101: 55 \quad 106-93-4$

Analytical Method: EPA 5030B/8260

\begin{tabular}{|c|c|c|c|c|}
\hline ND ug/L & 10.0 & 1 & $11 / 04 / 1121: 40$ & $67-64-1$ \\
\hline ND ug/L & 1.0 & 1 & $11 / 04 / 1121: 40$ & $71-43-2$ \\
\hline ND ug/L & 1.0 & 1 & $11 / 04 / 1+21: 40$ & $108-86-1$ \\
\hline ND ug/L & 1.0 & 1 & $11 / 04 / 1121: 40$ & $74-97-5$ \\
\hline ND ug/L & 1.0 & 1 & $11 / 04 / 1121: 40$ & $75-27-4$ \\
\hline ND ug/L & 1.0 & 1 & $11 / 04 / 1121: 40$ & $75-25-2$ \\
\hline ND ug/L & 1.0 & 1 & $11 / 04 / 1121: 40$ & $74-83-9$ \\
\hline ND ug/L & 10.0 & 1 & $11 / 04 / 1121: 40$ & $78-93-3$ \\
\hline ND ug/L & 1.0 & 1 & $11 / 04 / 1121: 40$ & $104-51-8$ \\
\hline ND ug/L & 1.0 & 1 & $11 / 04 / 1121: 40$ & $135-98-8$ \\
\hline ND ug/L & 1.0 & 1 & $11 / 04 / 1121: 40$ & $98-06-6$ \\
\hline ND ug/L & 5.0 & 1 & $11 / 04 / 1121: 40$ & $75-15-0$ \\
\hline $3.4 \mathrm{ug} / \mathrm{L}$ & 1.0 & 1 & $11 / 04 / 1121: 40$ & $56-23-5$ \\
\hline ND ug/L & 1.0 & 1 & $11 / 04 / 1121: 40$ & $108-90-7$ \\
\hline ND ug/L & 1.0 & 1 & $11 / 04 / 1121: 40$ & $75-00-3$ \\
\hline ND ug/L & 1.0 & 1 & $11 / 04 / 1121: 40$ & $67-66-3$ \\
\hline ND ug/L & 1.0 & 1 & $11 / 04 / 1121: 40$ & $74-87-3$ \\
\hline ND ug/L & 1.0 & 1 & $11 / 04 / 1121: 40$ & $95-49-8$ \\
\hline ND ug/L & 1.0 & 1 & $11 / 04 / 1121: 40$ & $106-43-4$ \\
\hline ND ug/L & 2.5 & 1 & $11 / 04 / 1121: 40$ & $96-12-8$ \\
\hline ND ug/L & 1.0 & 1 & $11 / 04 / 1121: 40$ & $124-48-1$ \\
\hline ND ug/L & 1.0 & 1 & $11 / 04 / 1121: 40$ & $106-93-4$ \\
\hline ND ug/L & 1.0 & 1 & $11 / 04 / 1121: 40$ & $74-95-3$ \\
\hline ND ug/L & 1.0 & 1 & $11 / 04 / 1121: 40$ & $95-50-1$ \\
\hline ND ug/L & 1.0 & 1 & $11 / 04 / 1121: 40$ & $541-73-1$ \\
\hline ND ug/L & 1.0 & 1 & $11 / 04 / 1121: 40$ & $106-46-7$ \\
\hline ND ug/L & 1.0 & 1 & $11 / 04 / 1121: 40$ & $75-71-8$ \\
\hline ND ug/L & 1.0 & 1 & $11 / 04 / 1121: 40$ & $75-34-3$ \\
\hline ND ug/L & 1.0 & 1 & $11 / 04 / 1121: 40$ & $107-06-2$ \\
\hline ND ug/L & 1.0 & 1 & $11 / 04 / 1121: 40$ & $540-59-0$ \\
\hline ND ug/L & 1.0 & 1 & $11 / 04 / 1121: 40$ & $75-35-4$ \\
\hline ND ug/L & 1.0 & 1 & $11 / 04 / 1121: 40$ & $156-59-2$ \\
\hline ND ug/L & 1.0 & 1 & $11 / 04 / 1121: 40$ & $156-60-5$ \\
\hline ND ug/L & 1.0 & 1 & $11 / 04 / 1121: 40$ & $78-87-5$ \\
\hline ND ug/L & 1.0 & 1 & $11 / 04 / 1121: 40$ & $142-28-9$ \\
\hline ND ug/L & 1.0 & 1 & $11 / 04 / 1121: 40$ & $594-20-7$ \\
\hline ND ug/L & 1.0 & 1 & $11 / 04 / 1121: 40$ & $563-58-6$ \\
\hline ND ug/L & 1.0 & 1 & $11 / 04 / 1121: 40$ & $10061-01-5$ \\
\hline ND ug/L & 1.0 & 1 & $11 / 04 / 1121: 40$ & $10061-02-6$ \\
\hline ND ug/L & 1.0 & 1 & $11 / 04 / 1121: 40$ & $100-41-4$ \\
\hline ND ug/L & 1.0 & 1 & $11 / 04 / 1121: 40$ & $87-68-3$ \\
\hline ND ug/L & 10.0 & 1 & $11 / 04 / 1121: 40$ & $591-78-6$ \\
\hline ND ug/L & 1.0 & 1 & $11 / 04 / 1121: 40$ & $98-82-8$ \\
\hline ND ug/L & 1.0 & 1 & $11 / 04 / 1121: 40$ & $99-87-6$ \\
\hline
\end{tabular}

REPORT OF LABORATORY ANALYSIS

Page 15 of 26
This report shall not be reproduced, except in full, without the written consent of Pace Analytical Services, Inc. 


\section{ANALYTICAL RESULTS}

Project:

KS/MO Waste Water

Pace Project No.: 60109211

Sample: MRPURGE-W-10116 Lab ID: 60109211006 $\quad$ Collected: 10/31/11 14:42 $\quad$ Received: 11/01/11 09:20 Matrix: Water

Parameters $\quad$ Results $\longrightarrow$ Units Report Limit DF Prepared Analyzed CAS No.

8260 MSV

Methylene chloride 4-Methyl-2-pentanone (MIBK)

Methyl-ter-butyl ether

Naphthalene

n-Propylbenzene

Styrene

1,1,1,2-Tetrachloroethane

1,1,2,2-Tetrachloroethane

Tetrachloroethene

Toluene

1,2,3-Trichlorobenzene

1,2,4-Trichlorobenzene

1,1,1-Trichloroethane

1,1,2-Trichloroethane

Trichloroethene

Trichlorofluoromethane

1,2,3-Trichloropropane

1,2,4-Trimethylbenzene

1,3,5-Trimethylbenzene

Vinyl chloride

Xylene (Total)

4-Bromofluorobenzene (S)

Dibromofluoromethane (S)

1,2-Dichloroethane-d4 (S)

Toluene-d8 (S)

Preservation $\mathrm{pH}$

353.2 Nitrogen, NO2/NO3 unpres

Nitrogen, Nitrate
Analytical Method: EPA 5030B/8260

$$
\begin{aligned}
& \text { ND ug/L } \\
& \text { ND ug/L } \\
& \text { ND ug/L } \\
& \text { ND ug/L } \\
& \text { ND ug/L } \\
& \text { ND ug/L } \\
& \text { ND ug/L } \\
& \text { ND ug/L } \\
& \text { ND ug/L } \\
& \text { ND ug/L } \\
& \text { ND ug/L } \\
& \text { ND ug/L } \\
& \text { ND ug/L } \\
& \text { ND ug/L } \\
& \text { ND ug/L } \\
& \text { ND ug/L } \\
& \text { ND ug/L } \\
& \text { ND ug/L } \\
& \text { ND ug/L } \\
& \text { ND ug/L } \\
& \text { ND ug/L } \\
& 105 \% \\
& 108 \% \\
& 114 \% \\
& 109 \% \\
& 7.0
\end{aligned}
$$

Analytical Method: EPA 353.2

$13.8 \mathrm{mg} / \mathrm{L}$

$\begin{array}{rr}1.0 & 1 \\ 10.0 & 1 \\ 1.0 & 1 \\ 10.0 & 1 \\ 1.0 & 1 \\ 1.0 & 1 \\ 1.0 & 1 \\ 1.0 & 1 \\ 1.0 & 1 \\ 1.0 & 1 \\ 1.0 & 1 \\ 1.0 & 1 \\ 1.0 & 1 \\ 1.0 & 1 \\ 1.0 & 1 \\ 1.0 & 1 \\ 2.5 & 1 \\ 1.0 & 1 \\ 1.0 & 1 \\ 1.0 & 1 \\ 3.0 & 1 \\ 87-113 & 1 \\ 86-112 & 1 \\ 82-119 & 1 \\ 90-110 & 1 \\ 0.10 & 1\end{array}$

$0.50 \quad 1$
11/04/11 21:40 75-09-2

$11 / 04 / 1121: 40 \quad 108-10-1$

$11 / 04 / 1121: 40 \quad 1634-04-4$

$11 / 04 / 1121: 40 \quad 91-20-3$

$11 / 04 / 1121: 40 \quad 103-65-1$

$11 / 04 / 1121: 40 \quad 100-42-5$

$11 / 04 / 1121: 40 \quad 630-20-6$

$11 / 04 / 1121: 40 \quad 79-34-5$

$11 / 04 / 1121: 40 \quad 127-18-4$

$11 / 04 / 1121: 40 \quad 108-88-3$

$11 / 04 / 1121: 40 \quad 87-61-6$

$11 / 04 / 1121: 40 \quad 120-82-1$

$11 / 04 / 1121: 40 \quad 71-55-6$

$11 / 04 / 1121: 40 \quad 79-00-5$

$11 / 04 / 1121: 40 \quad 79-01-6$

$11 / 04 / 1121: 40 \quad 75-69-4$

$11 / 04 / 1121: 40 \quad 96-18-4$

$11 / 04 / 1121: 40 \quad 95-63-6$

$11 / 04 / 1121: 40 \quad 108-67-8$

$11 / 04 / 1121: 40 \quad 75-01-4$

$11 / 04 / 1121: 40 \quad 1330-20-7$

$11 / 04 / 1121: 40 \quad 460-00-4$

11/04/11 21:40 1868-53-7

11/04/11 21:40 17060-07-0

$11 / 04 / 1121: 40 \quad 2037-26-5$

$11 / 04 / 1121: 40$
$11 / 02 / 1109: 20$ 
Pace Analytical Services, Inc. 9608 Loiret Blvd Lenexa, KS 66219

(913)599-5665

\section{ANALYTICAL RESULTS}

Project: $\quad$ KS/MO Waste Water

Pace Project No.: 60109211

Sample: SVPURGE-W-10117 Lab ID: 60109211007 Collected: 10/31/11 18:30 Received: 11/01/11 09:20 Matrix: Water

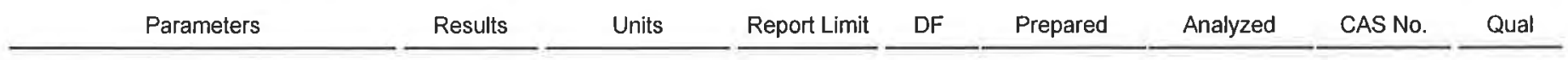

504 GCS EDB and DBCP

1,2-Dibromoethane (EDB)

8260 MSV

\section{Acetone}

Benzene

Bromobenzene

Bromochloromethane

Bromodichloromethane

Bromoform

Bromomethane

2-Butanone (MEK)

n-Butylbenzene

sec-Butylbenzene

tert-Butylbenzene

Carbon disulfide

Carbon tetrachloride

Chlorobenzene

Chloroethane

Chloroform

Chloromethane

2-Chlorotoluene

4-Chlorotoluene

1,2-Dibromo-3-chloropropane

Dibromochloromethane

1,2-Dibromoethane (EDB)

Dibromomethane

1,2-Dichlorobenzene

1,3-Dichlorobenzene

1,4-Dichlorobenzene

Dichlorodifluoromethane

1,1-Dichloroethane

1,2-Dichloroethane

1,2-Dichloroethene (Total)

1,1-Dichloroethene

cis-1,2-Dichloroethene

trans-1,2-Dichloroethene

1,2-Dichloropropane

1,3-Dichloropropane

2,2-Dichloropropane

1,1-Dichloropropene

cis-1,3-Dichloropropene

trans-1,3-Dichloropropene

Ethylbenzene

Hexachloro-1,3-butadiene

2-Hexanone

Isopropylbenzene (Cumene)

p-Isopropyltoluene

Date: 11/11/2011 11:15 AM

Analytical Method: EPA 504.1 Preparation Method: EPA 504.1

ND ug/L

Analytical Method: EPA 5030B/8260

\begin{tabular}{|c|c|}
\hline ND ug/L & 10.0 \\
\hline ND ug/L & 1.0 \\
\hline ND ug/L & 1.0 \\
\hline ND ug/L & 1.0 \\
\hline ND ug/L & 1.0 \\
\hline ND ug/L & 1.0 \\
\hline ND ug/L & 1.0 \\
\hline ND ug/L & 10.0 \\
\hline ND ug/L & 1.0 \\
\hline ND ug/L & 1.0 \\
\hline ND ug/L & 1.0 \\
\hline ND ug/L & 5.0 \\
\hline $6.9 \mathrm{ug} / \mathrm{L}$ & 1.0 \\
\hline ND ug/L & 1.0 \\
\hline ND ug/L & 1.0 \\
\hline $3.3 \mathrm{ug} / \mathrm{L}$ & 1.0 \\
\hline ND ug/L & 1.0 \\
\hline ND ug/L & 1.0 \\
\hline ND ug/L & 1.0 \\
\hline ND ug/L & 2.5 \\
\hline ND ug/L & 1.0 \\
\hline ND ug/L & 1.0 \\
\hline ND $u g / L$ & 1.0 \\
\hline ND ug/L & 1.0 \\
\hline ND ug/L & 1.0 \\
\hline ND ug/L & 1.0 \\
\hline ND ug/L & 1.0 \\
\hline ND ug/L & 1.0 \\
\hline ND ug/L & 1.0 \\
\hline ND ug/L & 1.0 \\
\hline ND ug/L & 1.0 \\
\hline ND ug/L & 1.0 \\
\hline ND ug/L & 1.0 \\
\hline ND ug/L & 1.0 \\
\hline ND ug/L & 1.0 \\
\hline ND ug/L & 1.0 \\
\hline ND ug/L & 1.0 \\
\hline ND ug/L & 1.0 \\
\hline ND ug/L & 1.0 \\
\hline ND ug/L & 1.0 \\
\hline ND ug/L & 1.0 \\
\hline ND ug/L & 10.0 \\
\hline ND ug/L & 1.0 \\
\hline ND ug/L & 1.0 \\
\hline
\end{tabular}

11/04/11 21:56 67-64-1

$11 / 04 / 1121: 56 \quad 71-43-2$

$11 / 04 / 1121: 56 \quad 108-86-1$

11/04/11 21:56 74-97-5

11/04/11 21:56 75-27-4

11/04/11 21:56 75-25-2

11/04/11 21:56 74-83-9

$11 / 04 / 1121: 56 \quad 78-93-3$

$11 / 04 / 1121: 56 \quad 104-51-8$

$11 / 04 / 1121: 56 \quad 135-98-8$

11/04/11 21:56 98-06-6

11/04/11 21:56 75-15-0

11/04/11 21:56 56-23-5

$11 / 04 / 1121: 56 \quad 108-90-7$

11/04/11 21:56 75-00-3

$11 / 04 / 1121: 56 \quad 67-66-3$

11/04/11 21:56 74-87-3

$11 / 04 / 1121: 56 \quad 95-49-8$

$11 / 04 / 1121: 56 \quad 106-43-4$

11/04/11 21:56 96-12-8

11/04/11 21:56 124-48-1

$11 / 04 / 1121: 56 \quad 106-93-4$

11/04/11 21:56 74-95-3

11/04/11 21:56 95-50-1

11/04/11 21:56 541-73-1

11/04/11 21:56 106-46-7

11/04/11 21:56 75-71-8

11/04/11 21:56 75-34-3

$11 / 04 / 1121: 56 \quad 107-06-2$

$11 / 04 / 1121: 56 \quad 540-59-0$

11/04/11 21:56 75-35-4

$11 / 04 / 1121: 56 \quad 156-59-2$

$11 / 04 / 1121: 56 \quad 156-60-5$

11/04/11 21:56 78-87-5

$11 / 04 / 1121: 56 \quad 142-28-9$

11/04/11 21:56 594-20-7

$11 / 04 / 1121: 56 \quad 563-58-6$

$11 / 04 / 1121: 56 \quad 10061-01-5$

$11 / 04 / 1121: 56 \quad 10061-02-6$

$11 / 04 / 1121: 56 \quad 100-41-4$

$11 / 04 / 1121: 56 \quad 87-68-3$

$11 / 04 / 1121: 56 \quad 591-78-6$

$11 / 04 / 1121: 56 \quad 98-82-8$

$11 / 04 / 1121: 56 \quad 99-87-6$ 


\section{ANALYTICAL RESULTS}

Project: KS/MO Waste Water

Pace Project No: $\quad 60109211$

Sample: SVPURGE-W-10117

Lab ID: 60109211007 Collected: 10/31/11 18:30 Received: 11/01/11 09:20 Matrix: Water

Parameters

\section{MSV}

Methylene chloride 4-Methyl-2-pentanone (MIBK)

Methyl-tert-butyl ether

Naphthalene

n-Propylbenzene

Styrene

1,1,1,2-Tetrachloroethane

1,1,2,2-Tetrachloroethane

Tetrachloroethene

Toluene

1,2,3-Trichlorobenzene

1,2,4-Trichlorobenzene

1,1,1-Trichloroethane

1,1,2-Trichloroethane

Trichloroethene

Trichlorofluoromethane

1,2,3-Trichloropropane

1,2,4-Trimethylbenzene

1,3,5-Trimethylbenzene

Vinyl chloride

Xylene (Total)

4-Bromofluorobenzene (S)

Dibromofluoromethane (S)

1,2-Dichloroethane-d4 (S)

Toluene-d8 (S)

Preservation $\mathrm{pH}$

353.2 Nitrogen, NO2/NO3 unpres

Nitrogen, Nitrate

Results

Units Report Limit

DF

Prepare

Analytical Method: EPA 5030B/8260

\begin{abstract}
ND ug/L
ND ug/L

ND ug/L

ND ug/L

ND ug/L

ND ug/L

ND ug/L

ND ug/L

ND ug/L

ND ug/L

ND ug/L

ND ug/L

ND ug/L

ND ug/L

ND ug/L

ND ug/L

ND ug/L

ND ug/L

ND ug/L

ND ug/L

ND ug/L

$90 \%$

$106 \%$

$111 \%$

$110 \%$

7.0
\end{abstract}

Analytical Method: EPA 353.2

$0.41 \mathrm{mg} / \mathrm{L}$

$\begin{array}{rr}1.0 & 1 \\ 10.0 & 1 \\ 1.0 & 1 \\ 10.0 & 1 \\ 1.0 & 1 \\ 1.0 & 1 \\ 1.0 & 1 \\ 1.0 & 1 \\ 1.0 & 1 \\ 1.0 & 1 \\ 1.0 & 1 \\ 1.0 & 1 \\ 1.0 & 1 \\ 1.0 & 1 \\ 1.0 & 1 \\ 1.0 & 1 \\ 2.5 & 1 \\ 1.0 & 1 \\ 1.0 & 1 \\ 1.0 & 1 \\ 3.0 & 1 \\ 87-113 & 1 \\ 86-112 & 1 \\ 82-119 & 1 \\ 90-110 & 1 \\ 0.10 & 1\end{array}$

0.101
11/04/11 21:56 75-09-2

11/04/11 21:56 108-10-1

11/04/11 21:56 1634-04-4

11/04/11 21:56 91-20-3

11/04/11 21:56 103-65-1

$11 / 04 / 1121: 56 \quad 100-42-5$

11/04/11 21:56 630-20-6

11/04/11 21:56 79-34-5

$11 / 04 / 1121: 56 \quad 127-18-4$

$11 / 04 / 1121: 56 \quad 108-88-3$

11/04/11 21:56 87-61-6

$11 / 04 / 1121: 56 \quad 120-82-1$

11/04/11 21:56 71-55-6

11/04/11 21:56 79-00-5

11/04/11 21:56 79-01-6

11/04/11 21:56 75-69-4

$11 / 04 / 1121: 56 \quad 96-18-4$

11/04/11 21:56 95-63-6

$11 / 04 / 1121: 56 \quad 108-67-8$

$11 / 04 / 1121: 56 \quad 75-01-4$

$11 / 04 / 1121: 56 \quad 1330-20-7$

$11 / 04 / 1121: 56 \quad 460-00-4$

$11 / 04 / 1121: 56 \quad 1868-53-7$

$11 / 04 / 1121: 56 \quad 17060-07-0$

11/04/11 21:56 2037-26-5

$11 / 04 / 1121: 56$

11/02/11 09:11 


\section{QUALITY CONTROL DATA}

Project: KS/MO Waste Water

Pace Project No.: 60109211

\begin{tabular}{llll}
\hline QC Batch: & OEXT/31027 & Analysis Method: & EPA 504.1 \\
QC Batch Method: & EPA 504.1 & Analysis Description: & GCS 504 EDB DBCP
\end{tabular}

Associated Lab Samples: $\quad 60109211001,60109211002,60109211003,60109211004,60109211005,60109211006,60109211007$

METHOD BLANK: $906554 \quad$ Matrix: Water

Associated Lab Samples: $\quad 60109211001,60109211002,60109211003,60109211004,60109211005,60109211006,60109211007$

$\begin{gathered}\text { Parameter } \\ \text { 1,2-Dibromoethane (EDB) }\end{gathered} \frac{\text { Units }}{u g / L} \frac{\begin{array}{c}\text { Rlank } \\ \text { Result }\end{array}}{N D} \frac{\begin{array}{c}\text { Reporting } \\ \text { Limit }\end{array}}{0.030} \frac{\text { Analyzed }}{11 / 07 / 1120: 35}-\frac{\text { Qualifiers }}{1}$

\begin{tabular}{|c|c|c|c|c|c|c|c|c|c|c|}
\hline \multirow[t]{2}{*}{ LABORATORY CONTROL SAMPLE \& LCSD: } & 906555 & \multicolumn{5}{|c|}{906556} & \multirow[b]{2}{*}{$\begin{array}{l}\% \operatorname{Rec} \\
\text { Limits }\end{array}$} & \multirow[b]{2}{*}{ RPD } & \multirow[b]{2}{*}{$\begin{array}{l}\text { Max } \\
\text { RPD }\end{array}$} & \multirow[b]{2}{*}{ Qualifiers } \\
\hline & Units & $\begin{array}{l}\text { Spike } \\
\text { Conc. }\end{array}$ & $\begin{array}{l}\text { LCS } \\
\text { Result }\end{array}$ & $\begin{array}{l}\text { LCSD } \\
\text { Result }\end{array}$ & $\begin{array}{l}\text { LCS } \\
\% \operatorname{Rec}\end{array}$ & $\begin{array}{l}\text { LCSD } \\
\% \operatorname{ReC}\end{array}$ & & & & \\
\hline 1,2-Dibromoethane (EDB) & $\mathrm{ug} / \mathrm{L}$ & .25 & 0.23 & 0.21 & 94 & 85 & $70-130$ & 10 & 20 & \\
\hline
\end{tabular}


Pace Analytical Services, Inc. 9608 Loiret Blvd

Lenexa, KS 66219

(913)599-5665

\section{QUALITY CONTROL DATA}

Project: $\quad$ KS/MO Waste Water

Pace Project No.: 60109211

\begin{tabular}{llll}
\hline QC Batch: & MSV/41422 & Analysis Method: & EPA $5030 \mathrm{~B} / 8260$ \\
QC Batch Method: & EPA $5030 \mathrm{~B} / 8260$ & Analysis Description: & 8260 MSV Water 7 day
\end{tabular}

Associated Lab Samples: $\quad 60109211001,60109211002,60109211003,60109211004,60109211005,60109211006,60109211007$

METHOD BLANK: $905182 \quad$ Matrix: Water
Associated Lab Samples: $\quad 60109211001,60109211002,60109211003,60109211004,60109211005,60109211006,60109211007$

Associated Lab Samples: $\quad 60109211001,60109211002,60109211003,60109211004,60109211005,60109211006,60109211007$

Blank Reporting

$$
\text { Parameter }
$$

1,1,1,2-Tetrachloroethane

$1,1,1$-Trichloroethane

1,1,2,2-Tetrachloroethane

1,1,2-Trichloroethane

1,1-Dichloroethane

1,1-Dichloroethene

1,1-Dichloropropene

1,2,3-Trichlorobenzene

1,2,3-Trichloropropane

1,2,4-Trichlorobenzene

1,2,4-Trimethylbenzene

1,2-Dibromo-3-chloropropane

1,2-Dibromoethane (EDB)

1,2-Dichlorobenzene

1,2-Dichloroethane

1,2-Dichloroethene (Total)

1,2-Dichloropropane

1,3,5-Trimethylbenzene

1,3-Dichlorobenzene

1,3-Dichloropropane

1,4-Dichlorobenzene

2,2-Dichloropropane

2-Butanone (MEK)

2-Chlorotoluene

2-Hexanone

4-Chlorotoluene

4-Methyl-2-pentanone (MIBK)

Acetone

Benzene

Bromobenzene

Bromochloromethane

Bromodichloromethane

Bromoform

Bromomethane

Carbon disulfide

Carbon tetrachloride

Chlorobenzene

Chloroethane

Chloroform

Chloromethane

cis-1,2-Dichloroethene

cis-1,3-Dichloropropene

Dibromochloromethane

ug/L
ug/L
$u g / L$
$u g / L$
$u g / L$
$u g / L$
$u g / L$
$u g / L$
$u g / L$
$u g / L$
$u g / L$
$u g / L$
$u g / L$
$u g / L$
$u g / L$
$u g / L$
$u g / L$
$u g / L$
$u g / L$
$u g / L$
$u g / L$

Date: 11/11/2011 11:15 AM

Units Result Limit

\begin{tabular}{|c|c|c|}
\hline ND & 1.0 & $11 / 04 / 1117: 52$ \\
\hline ND & 1.0 & $11 / 04 / 11$ 17:52 \\
\hline ND & 1.0 & $11 / 04 / 1117: 52$ \\
\hline
\end{tabular}

ND $\quad 1.0 \quad 11 / 04 / 1117: 52$

ND $\quad 1.0 \quad 11 / 04 / 1117: 52$

ND $\quad 1.0 \quad 11 / 04 / 11 \quad 17: 52$

ND $\quad 1.0 \quad 11 / 04 / 1117: 52$

ND $\quad 1.0 \quad 11 / 04 / 1117: 52$

ND $\quad 2.5 \quad 11 / 04 / 1117: 52$

ND $\quad 1.0 \quad 11 / 04 / 1117: 52$

ND $\quad 1.0 \quad 11 / 04 / 1117: 52$

ND $\quad 2.5 \quad 11 / 04 / 1117: 52$

ND $\quad 1.0 \quad 11 / 04 / 1117: 52$

ND $\quad 1.0 \quad 11 / 04 / 1117: 52$

ND $\quad 1.0 \quad 11 / 04 / 1117: 52$

ND $\quad 1.0 \quad 11 / 04 / 1117: 52$

ND $\quad 1.0 \quad 11 / 04 / 1117: 52$

ND $\quad 1.0 \quad 11 / 04 / 1117: 52$

ND $\quad 1.0 \quad 11 / 04 / 1117: 52$

ND $\quad 1.0 \quad 11 / 04 / 11 \quad 17: 52$

ND $\quad 1.0 \quad 11 / 04 / 11 \quad 17: 52$

ND $\quad 1.0 \quad 11 / 04 / 1117: 52$

ND $\quad 10.0 \quad 11 / 04 / 1117: 52$

ND $\quad 1.0 \quad 11 / 04 / 1117: 52$

ND $\quad 10.0 \quad 11 / 04 / 1117: 52$

ND $\quad 1.0 \quad 11 / 04 / 1117: 52$

ND $\quad 10.0 \quad 11 / 04 / 1117: 52$

ND $\quad 10.0 \quad 11 / 04 / 1117: 52$

ND $\quad 1.0 \quad 11 / 04 / 1117: 52$

ND $\quad 1.0 \quad 11 / 04 / 1117: 52$

ND $\quad 1.0 \quad 11 / 04 / 1117: 52$

ND $\quad 1.0 \quad 11 / 04 / 1117: 52$

ND $\quad 1.0 \quad 11 / 04 / 1117: 52$

ND $\quad 1.0 \quad 11 / 04 / 1117: 52$

ND $\quad 5.0 \quad 11 / 04 / 1117: 52$

ND $\quad 1.0 \quad 11 / 04 / 1117: 52$

ND $\quad 1.0 \quad 11 / 04 / 11 \quad 17: 52$

ND $\quad 1.0 \quad 11 / 04 / 11 \quad 17: 52$

ND $\quad 1.0 \quad 11 / 04 / 1117: 52$

ND $\quad 1.0 \quad 11 / 04 / 1117: 52$

ND $\quad 1.0 \quad 11 / 04 / 11 \quad 17: 52$

ND $\quad 1.0 \quad 11 / 04 / 1117: 52$

ND $\quad 1.0 \quad 11 / 04 / 1117: 52$ 


\section{QUALITY CONTROL DATA}

Project:

KS/MO Waste Water

Pace Project No: $\quad 60109211$

METHOD BLANK: 905182

Matrix: Water

Associated Lab Samples: $\quad 60109211001,60109211002,60109211003,60109211004,60109211005,60109211006,60109211007$

\begin{tabular}{|c|c|c|c|c|c|}
\hline Parameter & Units & $\begin{array}{l}\text { Blank } \\
\text { Result }\end{array}$ & $\begin{array}{c}\text { Reporting } \\
\text { Limit }\end{array}$ & Analyzed & Qualifiers \\
\hline Dibromomethane & $\mathrm{ug} / \mathrm{L}$ & $\overline{N D}$ & 1.0 & $11 / 04 / 1117: 52$ & \\
\hline Dichlorodifluoromethane & ug/L & ND & 1.0 & $11 / 04 / 1117: 52$ & \\
\hline Ethylbenzene & $u g / L$ & ND & 1.0 & $11 / 04 / 1117: 52$ & \\
\hline Hexachloro-1,3-butadiene & ug/L & ND & 1.0 & $11 / 04 / 1117: 52$ & \\
\hline Isopropylbenzene (Cumene) & $u g / L$ & ND & 1.0 & $11 / 04 / 1117: 52$ & \\
\hline Methyl-tert-butyl ether & $\mathrm{ug} / \mathrm{L}$ & ND & 1.0 & $11 / 04 / 1117: 52$ & \\
\hline Methylene chloride & $\mathrm{ug} / \mathrm{L}$ & ND & 1.0 & $11 / 04 / 1117: 52$ & \\
\hline n-Butylbenzene & $\mathrm{ug} / \mathrm{L}$ & ND & 1.0 & $11 / 04 / 1117: 52$ & \\
\hline n-Propylbenzene & $\mathrm{ug} / \mathrm{L}$ & ND & 1.0 & $11 / 04 / 1117: 52$ & \\
\hline Naphthalene & $\mathrm{ug} / \mathrm{L}$ & ND & 10.0 & $11 / 04 / 1117: 52$ & \\
\hline p-Isopropyltoluene & ug/L & ND & 1.0 & $11 / 04 / 1117: 52$ & \\
\hline sec-Butylbenzene & $u g / L$ & ND & 1.0 & $11 / 04 / 1117: 52$ & \\
\hline Styrene & $\mathrm{ug} / \mathrm{L}$ & ND & 1.0 & $11 / 04 / 1117: 52$ & \\
\hline tert-Butylbenzene & $\mathrm{ug} / \mathrm{L}$ & ND & 1.0 & $11 / 04 / 1117: 52$ & \\
\hline Tetrachloroethene & $\mathrm{ug} / \mathrm{L}$ & ND & 1.0 & $11 / 04 / 1117: 52$ & \\
\hline Toluene & $\mathrm{ug} / \mathrm{L}$ & ND & 1.0 & $11 / 04 / 11 \quad 17: 52$ & \\
\hline trans-1,2-Dichloroethene & ug/L & ND & 1.0 & $11 / 04 / 11 \quad 17: 52$ & \\
\hline trans-1,3-Dichloropropene & $\mathrm{ug} / \mathrm{L}$ & ND & 1.0 & $11 / 04 / 11 \quad 17: 52$ & \\
\hline Trichloroethene & $\mathrm{ug} / \mathrm{L}$ & ND & 1.0 & $11 / 04 / 1117: 52$ & \\
\hline Trichlorofluoromethane & $\mathrm{ug} / \mathrm{L}$ & ND & 1.0 & $11 / 04 / 1117: 52$ & \\
\hline Vinyl chloride & $\mathrm{ug} / \mathrm{L}$ & ND & 1.0 & $11 / 04 / 11 \quad 17: 52$ & \\
\hline Xylene (Total) & $\mathrm{ug} / \mathrm{L}$ & ND & 3.0 & $11 / 04 / 1117: 52$ & \\
\hline 1,2-Dichloroethane-d4 (S) & $\%$ & 107 & $82-119$ & $11 / 04 / 1117: 52$ & \\
\hline 4-Bromofluorobenzene (S) & $\%$ & 97 & $87-113$ & $11 / 04 / 11 \quad 17: 52$ & \\
\hline Dibromofluoromethane (S) & $\%$ & 105 & $86-112$ & $11 / 04 / 11 \quad 17: 52$ & \\
\hline Toluene-d8 (S) & $\%$ & 103 & $90-110$ & $11 / 04 / 1117: 52$ & \\
\hline
\end{tabular}

\begin{tabular}{|c|c|c|c|c|c|c|}
\hline \multirow{2}{*}{$\begin{array}{c}\text { LABORATORY CONTROL SAMPLE: } \\
\text { Parameter }\end{array}$} & \multicolumn{6}{|l|}{905183} \\
\hline & Units & $\begin{array}{l}\text { Spike } \\
\text { Conc. }\end{array}$ & $\begin{array}{l}\text { LCS } \\
\text { Result }\end{array}$ & $\begin{array}{l}\text { LCS } \\
\% \operatorname{ReC}\end{array}$ & $\begin{array}{l}\% \text { Rec } \\
\text { Limits }\end{array}$ & Qualifiers \\
\hline 1,1,1,2-Tetrachloroethane & $\mathrm{ug} / \mathrm{L}$ & 20 & 17.3 & 86 & $81-121$ & \\
\hline 1,1,1-Trichloroethane & $\mathrm{ug} / \mathrm{L}$ & 20 & 17.6 & 88 & $82-119$ & \\
\hline 1,1,2,2-Tetrachloroethane & ug/L & 20 & 18.1 & 91 & $78-124$ & \\
\hline 1,1,2-Trichloroethane & $\mathrm{ug} / \mathrm{L}$ & 20 & 20.3 & 102 & $79-121$ & \\
\hline 1,1-Dichloroethane & ug/L & 20 & 18.3 & 91 & $73-119$ & \\
\hline 1,1-Dichloroethene & $\mathrm{ug} / \mathrm{L}$ & 20 & 17.7 & 89 & $75-120$ & \\
\hline 1.1-Dichloropropene & ug/L & 20 & 18.5 & 93 & $79-123$ & \\
\hline 1,2,3-Trichlorobenzene & $u g / L$ & 20 & 17.8 & 89 & $73-122$ & \\
\hline 1,2,3-Trichloropropane & $\mathrm{ug} / \mathrm{L}$ & 20 & 18.1 & 91 & $77-124$ & \\
\hline 1,2,4-Trichlorobenzene & $\mathrm{ug} / \mathrm{L}$ & 20 & 17.4 & 87 & $75-120$ & \\
\hline $1,2,4$-Trimethylbenzene & $\mathrm{ug} / \mathrm{L}$ & 20 & 18.7 & 94 & $77-120$ & \\
\hline 1,2-Dibromo-3-chloropropane & $\mathrm{ug} / \mathrm{L}$ & 20 & 16.7 & 84 & $69-125$ & \\
\hline 1,2-Dibromoethane (EDB) & $u g / L$ & 20 & 18.8 & 94 & $85-121$ & \\
\hline 1,2-Dichlorobenzene & $u g / L$ & 20 & 19.2 & 96 & $82-115$ & \\
\hline 1,2-Dichloroethane & ug/L & 20 & 19.3 & 96 & $77-125$ & \\
\hline
\end{tabular}

Date: 11/11/2011 11:15 AM 


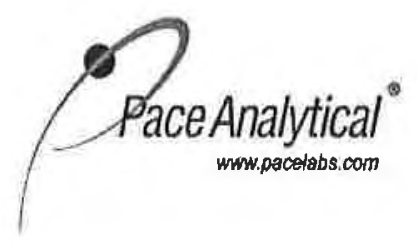

Pace Analytical Services, Inc. 9608 Loiret Blvd. Lenexa, KS 66219

(913)599-5665

\section{QUALITY CONTROL DATA}

Project:

KS/MO Waste Water

Pace Project No.: 60109211

\begin{tabular}{|c|c|c|c|c|c|c|}
\hline \multirow{2}{*}{$\begin{array}{c}\text { LABORATORY CONTROL SAMPLE: } \\
\text { Parameter }\end{array}$} & \multicolumn{6}{|l|}{905183} \\
\hline & Units & $\begin{array}{l}\text { Spike } \\
\text { Conc. }\end{array}$ & $\begin{array}{l}\text { LCS } \\
\text { Result }\end{array}$ & $\begin{array}{c}\text { LCS } \\
\% \operatorname{Rec}\end{array}$ & $\begin{array}{l}\% \operatorname{Rec} \\
\text { Limits }\end{array}$ & Qualifiers \\
\hline 1,2-Dichloroethene (Total) & $u g / L$ & 40 & 40.0 & 100 & $79-120$ & \\
\hline 1,2-Dichloropropane & $\mathrm{ug} / \mathrm{L}$ & 20 & 18.8 & 94 & $83-119$ & \\
\hline 1,3,5-Trimethylbenzene & $u g / L$ & 20 & 18.1 & 91 & $79-121$ & \\
\hline 1,3-Dichlorobenzene & $\mathrm{ug} / \mathrm{L}$ & 20 & 17.7 & 88 & $79-117$ & \\
\hline 1,3-Dichloropropane & ug/L & 20 & 19.2 & 96 & $78-116$ & \\
\hline 1,4-Dichlorobenzene & $\mathrm{ug} / \mathrm{L}$ & 20 & 18.7 & 94 & $83-115$ & \\
\hline 2,2-Dichloropropane & ug/L & 20 & 16.3 & 82 & $66-123$ & \\
\hline 2-Butanone (MEK) & $\mathrm{ug} / \mathrm{L}$ & 100 & 101 & 101 & $43-165$ & \\
\hline 2-Chlorotoluene & ug/L & 20 & 18.6 & 93 & $81-117$ & \\
\hline 2-Hexanone & $u g / L$ & 100 & 98.3 & 98 & $47-159$ & \\
\hline 4-Chlorotoluene & ug/L & 20 & 18.8 & 94 & $84-116$ & \\
\hline 4-Methyl-2-pentanone (MIBK) & $\mathrm{ug} / \mathrm{L}$ & 100 & 90.7 & 91 & $71-129$ & \\
\hline Acelone & ug/L & 100 & 111 & 111 & $18-192$ & \\
\hline Benzene & $\mathrm{ug} / \mathrm{L}$ & 20 & 19.5 & 97 & $82-117$ & \\
\hline Bromobenzene & $\mathrm{ug} / \mathrm{L}$ & 20 & 18.5 & 92 & $83-116$ & \\
\hline Bromochloromethane & $\mathrm{ug} / \mathrm{L}$ & 20 & 18.9 & 94 & $79-121$ & \\
\hline Bromodichloromethane & $\mathrm{ug} / \mathrm{L}$ & 20 & 18.5 & 92 & $79-114$ & \\
\hline Bromoform & $\mathrm{ug} / \mathrm{L}$ & 20 & 17.8 & 89 & $78-121$ & \\
\hline Bromomethane & $\mathrm{ug} / \mathrm{L}$ & 20 & 20.6 & 103 & $36-146$ & \\
\hline Carbon disulfide & ug/L & 20 & 20.6 & 103 & $75-138$ & \\
\hline Carbon tetrachloride & $\mathrm{ug} / \mathrm{L}$ & 20 & 19.3 & 96 & $80-123$ & \\
\hline Chlorobenzene & $\mathrm{ug} / \mathrm{L}$ & 20 & 18.2 & 91 & $83-121$ & \\
\hline Chloroethane & $\mathrm{ug} / \mathrm{L}$ & 20 & 19.6 & 98 & $42-166$ & \\
\hline Chloroform & $\mathrm{ug} / \mathrm{L}$ & 20 & 20.1 & 100 & $82-116$ & \\
\hline Chloromethane & $\mathrm{ug} / \mathrm{L}$ & 20 & 17.7 & 88 & $32-127$ & \\
\hline cis-1,2-Dichloroethene & $\mathrm{ug} / \mathrm{L}$ & 20 & 18.2 & 91 & $80-119$ & \\
\hline cis-1,3-Dichloropropene & $\mathrm{ug} / \mathrm{L}$ & 20 & 17.0 & 85 & $76-119$ & \\
\hline Dibromochioromethane & $\mathrm{ug} / \mathrm{L}$ & 20 & 17.9 & 89 & $81-123$ & \\
\hline Dibromomethane & $\mathrm{ug} / \mathrm{L}$ & 20 & 19.2 & 96 & $79-123$ & \\
\hline Dichlorodifluoromethane & $\mathrm{ug} / \mathrm{L}$ & 20 & 15.2 & 76 & $10-163$ & \\
\hline Ethylbenzene & $\mathrm{ug} / \mathrm{L}$ & 20 & 17.7 & 88 & $79-121$ & \\
\hline Hexachloro-1,3-butadiene & $u g / L$ & 20 & 18.5 & 92 & $78-125$ & \\
\hline Isopropylbenzene (Cumene) & $\mathrm{ug} / \mathrm{L}$ & 20 & 18.7 & 93 & $80-120$ & \\
\hline Methyl-tert-butyl ether & $\mathrm{ug} / \mathrm{L}$ & 20 & 18.1 & 91 & $78-119$ & \\
\hline Methylene chloride & $\mathrm{ug} / \mathrm{L}$ & 20 & 19.6 & 98 & $75-118$ & \\
\hline n-Butylbenzene & $\mathrm{ug} / \mathrm{L}$ & 20 & 18.2 & 91 & $80-126$ & \\
\hline n-Propylbenzene & $u g / L$ & 20 & 18.3 & 91 & $83-116$ & \\
\hline Naphthalene & $\mathrm{ug} / \mathrm{L}$ & 20 & 16.8 & 84 & $66-133$ & \\
\hline p-Isopropyltoluene & $\mathrm{ug} / \mathrm{L}$ & 20 & 17.9 & 89 & $77-120$ & \\
\hline sec-Butylbenzene & ug/L & 20 & 17.8 & 89 & 81.120 & \\
\hline Styrene & $\mathrm{ug} / \mathrm{L}$ & 20 & 18.8 & 94 & $84-115$ & \\
\hline tert-Butylbenzene & $\mathrm{ug} / \mathrm{L}$ & 20 & 18.0 & 90 & $80-117$ & \\
\hline Tetrachloroethene & $u g / L$ & 20 & 19.8 & 99 & $80-124$ & \\
\hline Toluene & $\mathrm{ug} / \mathrm{L}$ & 20 & 19.1 & 95 & $80-120$ & \\
\hline trans-1,2-Dichloroethene & $\mathrm{ug} / \mathrm{L}$ & 20 & 21.8 & 109 & $79-120$ & \\
\hline trans-1,3-Dichloropropene & ug/L & 20 & 18.5 & 92 & $76-118$ & \\
\hline Trichloroethene & $\mathrm{ug} / \mathrm{L}$ & 20 & 17.5 & 88 & $76-122$ & \\
\hline Trichlorofluoromethane & $\mathrm{ug} / \mathrm{L}$ & 20 & 19.0 & 95 & $72-120$ & \\
\hline
\end{tabular}

Date: 11/11/2011 11:15 AM 


\section{QUALITY CONTROL DATA}

Project:

KS/MO Waste Water

Pace Project No.:

60109211

\begin{tabular}{|c|c|c|c|c|c|c|}
\hline LABORATORY CONTROL SAMPLE: & 905183 & & & & & \\
\hline Parameter & Units & $\begin{array}{l}\text { Spike } \\
\text { Conc. }\end{array}$ & $\begin{array}{l}\text { LCS } \\
\text { Result }\end{array}$ & $\begin{array}{l}\text { LCS } \\
\% \operatorname{Rec}\end{array}$ & $\begin{array}{l}\% \text { Rec } \\
\text { Limits }\end{array}$ & Qualifiers \\
\hline Vinyl chloride & $u g / L$ & 20 & 17.5 & 88 & $57-163$ & \\
\hline Xylene (Total) & $u g / L$ & 60 & 53.9 & 90 & $75-120$ & \\
\hline 1,2-Dichloroethane-d4 (S) & $\%$ & & & 103 & $82-119$ & \\
\hline 4-Bromofluorobenzene (S) & $\%$ & & & 104 & $87-113$ & \\
\hline Dibromofluoromethane (S) & $\%$ & & & 101 & $86-112$ & \\
\hline Toluene-d8 (S) & $\%$ & & & 99 & $90-110$ & \\
\hline
\end{tabular}




\section{QUALITY CONTROL DATA}

Project:

KS/MO Waste Water

Pace Project No.: 60109211

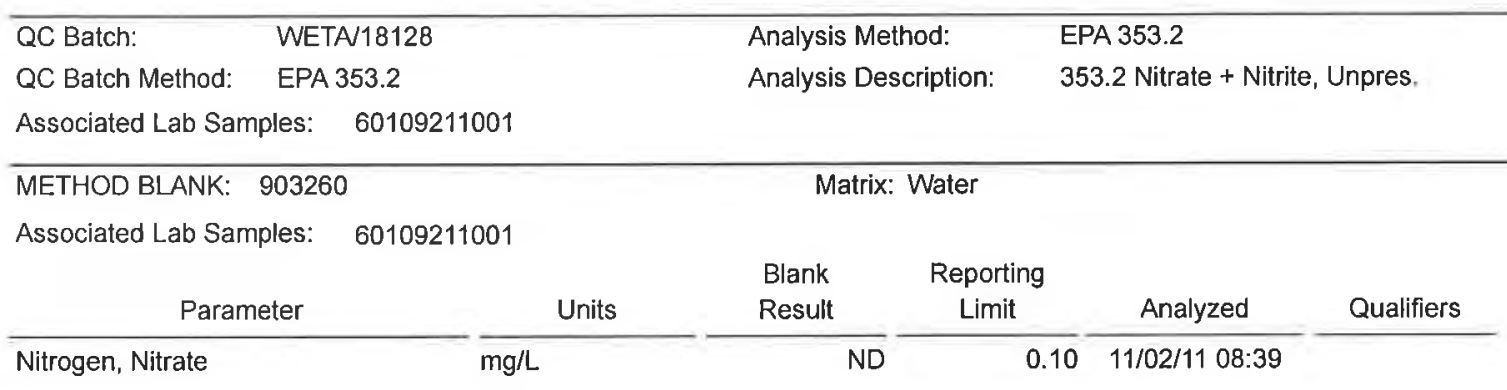

\begin{tabular}{|c|c|c|c|c|c|c|}
\hline LABORATORY CONTROL SAMPLE: & 903261 & & & & & \\
\hline Parameter & Units & $\begin{array}{l}\text { Spike } \\
\text { Conc. }\end{array}$ & $\begin{array}{l}\text { LCS } \\
\text { Result }\end{array}$ & $\begin{array}{l}\text { LCS } \\
\% \operatorname{ReC}\end{array}$ & $\begin{array}{l}\% \text { Rec } \\
\text { Limits }\end{array}$ & Qualifiers \\
\hline Nitrogen, Nitrate & $\mathrm{mg} / \mathrm{L}$ & 1.6 & 1.6 & 97 & $90-110$ & \\
\hline
\end{tabular}

\begin{tabular}{|c|c|c|c|c|c|c|c|}
\hline MATRIX SPIKE SAMPLE: & 903262 & & & & & & \\
\hline Parameter & Units & $\begin{array}{c}60109214001 \\
\text { Result }\end{array}$ & $\begin{array}{l}\text { Spike } \\
\text { Conc. }\end{array}$ & $\begin{array}{c}\text { MS } \\
\text { Result }\end{array}$ & $\begin{array}{c}\text { MS } \\
\% \operatorname{Rec}\end{array}$ & $\begin{array}{l}\% \text { Rec } \\
\text { Limits }\end{array}$ & Qualifiers \\
\hline Nitrogen, Nitrate & $\mathrm{mg} / \mathrm{L}$ & ND & 1.6 & 1.5 & 93 & $90-110$ & \\
\hline
\end{tabular}

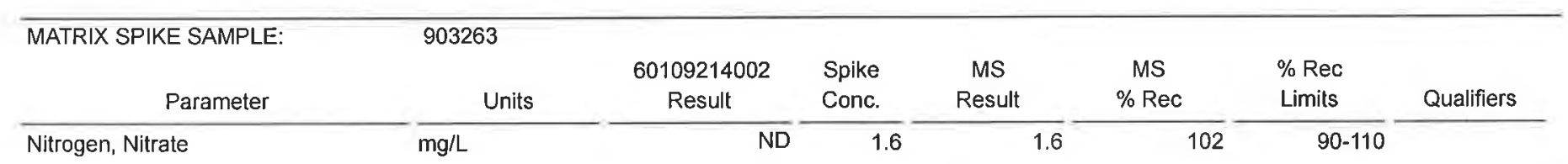

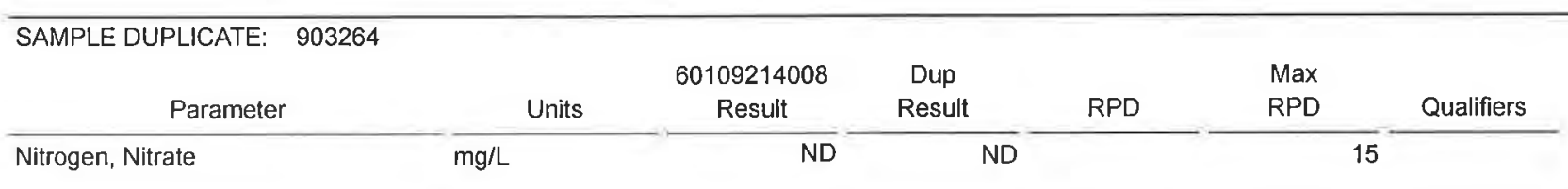




\section{QUALITY CONTROL DATA}

Project:

KS/MO Waste Water

Pace Project No.:

60109211

\begin{tabular}{|c|c|c|c|}
\hline QC Batch: & WETAV18129 & Analysis Method: & EPA 353.2 \\
\hline QC Batch Method: & EPA 353.2 & Analysis Description: & 353.2 Nitrate + Nitrite, Unpres. \\
\hline
\end{tabular}

Associated Lab Samples: $\quad 60109211002,60109211003,60109211004,60109211005,60109211006,60109211007$

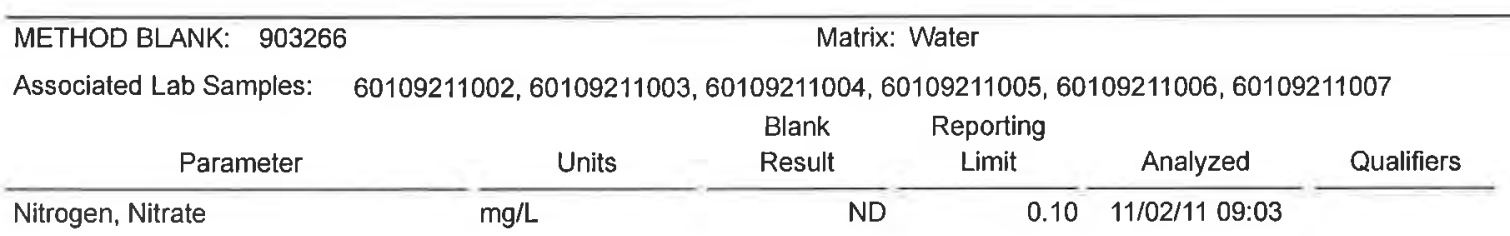

\begin{tabular}{|c|c|c|c|c|c|c|}
\hline LABORATORY CONTROL SAMPLE: & 903267 & & & & & \\
\hline Parameter & Units & $\begin{array}{l}\text { Spike } \\
\text { Conc. }\end{array}$ & $\begin{array}{l}\text { LCS } \\
\text { Result }\end{array}$ & $\begin{array}{l}\text { LCS } \\
\% \operatorname{Rec}\end{array}$ & $\begin{array}{l}\% \text { Rec } \\
\text { Limits }\end{array}$ & Qualifiers \\
\hline Nitrogen, Nitrate & $\mathrm{mg} / \mathrm{L}$ & 1.6 & 1.6 & 98 & $90-110$ & \\
\hline
\end{tabular}

\begin{tabular}{|c|c|c|c|c|c|c|c|}
\hline MATRIX SPIKE SAMPLE: & 903268 & & & & & & \\
\hline Parameter & Units & $\begin{array}{c}60109211007 \\
\text { Result }\end{array}$ & $\begin{array}{l}\text { Spike } \\
\text { Conc. }\end{array}$ & $\begin{array}{c}\text { MS } \\
\text { Result }\end{array}$ & $\begin{array}{c}\text { MS } \\
\% \operatorname{Rec}\end{array}$ & $\begin{array}{l}\% \operatorname{Rec} \\
\text { Limits }\end{array}$ & Qualifiers \\
\hline Nitrogen, Nitrate & $\mathrm{mg} / \mathrm{L}$ & 0.41 & $\overline{1.6}$ & 1.9 & 95 & $90-110$ & \\
\hline
\end{tabular}

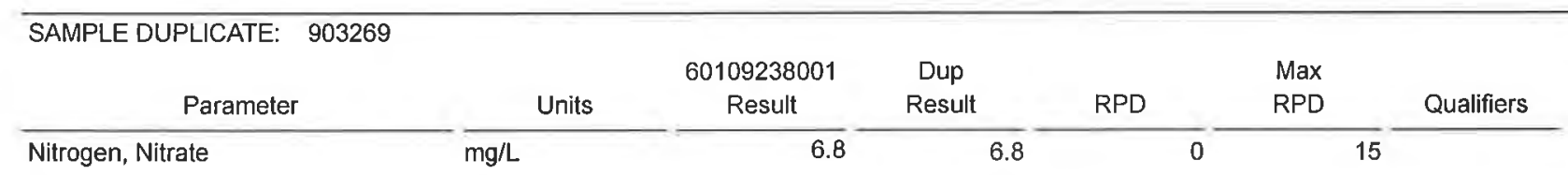




\section{QUALIFIERS}

Project: $\quad$ KS/MO Waste Water

Pace Project No.: 60109211

\section{DEFINITIONS}

DF - Dilution Factor, if reported, represents the factor applied to the reported data due to changes in sample preparation, dilution of the sample aliquot, or moisture content.

ND - Not Detected at or above adjusted reporting limit.

$\mathrm{J}$ - Estimated concentration above the adjusted method detection limit and below the adjusted reporting limit.

MDL - Adjusted Method Detection Limit.

$S$ - Surrogate

1,2-Diphenylhydrazine (8270 listed analyte) decomposes to Azobenzene.

Consistent with EPA guidelines, unrounded data are displayed and have been used to calculate \% recovery and RPD values.

LCS(D) - Laboratory Control Sample (Duplicate)

MS(D) - Matrix Spike (Duplicate)

DUP - Sample Duplicate

RPD - Relative Percent Difference

NC - Not Calculable.

SG - Silica Gel - Clean-Up

$U$ - Indicates the compound was analyzed for, but not detected.

N-Nitrosodiphenylamine decomposes and cannot be separated from Diphenylamine using Method 8270 . The result reported for each analyte is a combined concentration.

Pace Analytical is TNI accredited. Contact your Pace PM for the current list of accredited analytes.

\section{BATCH QUALIFIERS}

Batch: MSV/41422

[M5] A matrix spike/matrix spike duplicate was not performed for this batch due to insufficient sample volume. 


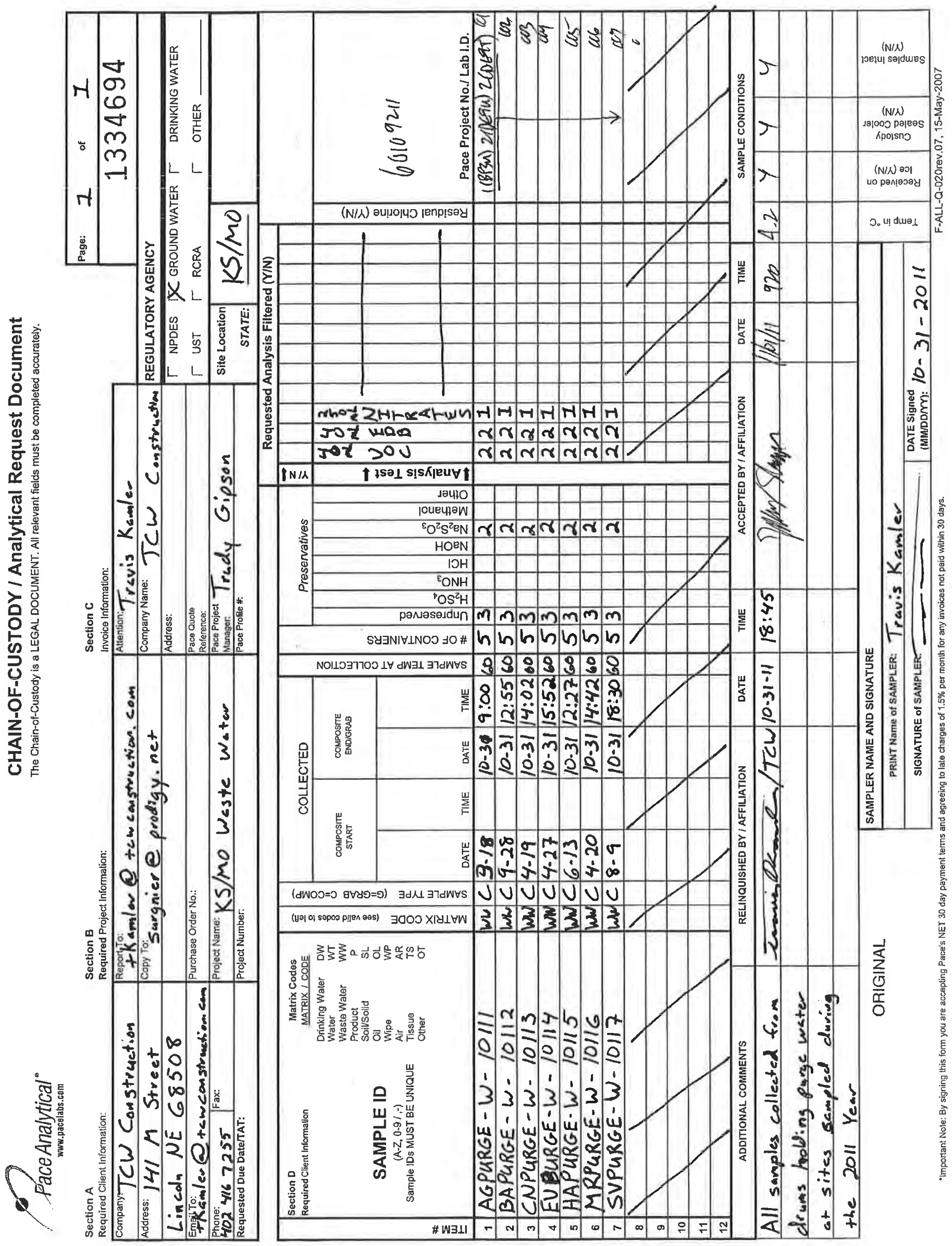




\section{Sample Condition Upon Receipt}

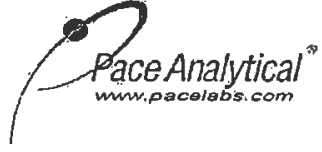

Client Name: TCW Const.

Project \# 60109211

Courier: 凹Fed Ex $\square$ UPS $\square$ USPS $\square$ client $\square$ commercial $\square$ Pace $\square$ other Tracking \#: 875827463563

Custody Seal on Cooler/Box Present:

Thermometer Usedic T-T9P / T-194

Cooler Temperature: $\quad 4,2$

Temperature should be above freezing to $6^{\circ} \mathrm{C}$

Chain of Custody present:

Chain of Custody filled out:

Chain of Custody relinquished:

Sampler name \& signature on COC:

Samples arrived within holding time:

Short Hold Time analyses ( $<72 \mathrm{hr})$ :

Rush Turn Around Time requested:

Sufficient volume:

Correct containers used:

-Pace containers used:

Containers intact:

Unpreserved 5035A soils frozen w/in 48hrs?

Filtered volume received for dissolved tests

Sample labels match $\mathrm{COC}$

-Includes date/time/ID/analyses Matrix

All contalners needing preservation have been checked.

All containers needing preservation are found to be in compliance with EPA recommendation.

Excepllons: VOA, collform, TOC, O\&G, WI-DRO (water), Phenolics

Trip Blank present: Pace Shipping Label Used?

XYes

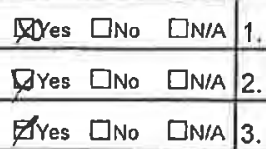

DYes $\square$ No $\square$ N/A 4.

DYes DNo DN/A 5.

GYes 口No DN/A 6. $\mathrm{N}_{3}$

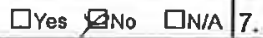

QYes $\square$ No $\square \mathrm{N} / \mathrm{A}$
8.

ZYes $\square$ No $\square$ N/A 9 .

$\square$ Yes $\square$ No $\square$ N/A

$\varangle$ Yres $\square$ No $\square$ N/A 10.

$\square$ Yes $\square$ No $\Xi_{N / A} 11$.

DYes $\square$ No 12 N/A 12.

tres $\square$ No $\square$ N/A 13.

water

$\square$ res $\square$ No $\square$ N/A 14.

$\square$ Yes $\square$ No $\quad$ WN/A

$\square$ Yes $\mathbb{d}_{\text {No }} \square \mathrm{N} / \mathrm{A} 15$

completed $\square$ Yes $区$ No

$\varnothing$ Yes $\square$ No

\begin{tabular}{l} 
Optional \\
\hline $\begin{array}{l}\text { Proj. Due Date: } \\
\text { Proj. Name: }\end{array}$ / $/ 4$
\end{tabular}

$\square$ Bubble Bags $\square$ Foam $\square$ None $\square$ Dther

Type of Ice: Whe None $\square$ Samples on ice, cooling process has begun

Date and Initials of person pxamining contents: IND $11 / 1 / 4$ 1025

\begin{tabular}{l|l|l|}
\hline Ifres $\square$ No & Initial when & Lot \# of added
\end{tabular}

Pace Trip Blank lot \# (if purchased):

Headspace in VOA vials $(>6 \mathrm{~mm})$ :

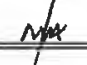

preservative

\begin{tabular}{|c|c|c|c|c|}
\hline Headspace in VOA vials ( $>6 \mathrm{~mm})$ : & $\square$ Yes $\not$ No & $\square$ NA & 16. & \\
\hline Project sampled in USDA Regulated Area: & $\square$ Yes $\square$ No & $\square N / A$ & 17. List State: & $q$ \\
\hline
\end{tabular}

Client Notification/ Resolution:

Copy COC to Client?

$\mathrm{Y} / \mathrm{N}$

Field Data Required?

$Y, N$

Person Contacted:

เ

Date/Time:

Comments/ Resolution:

Comments/Resolution:

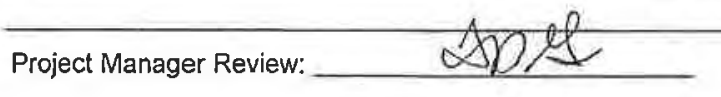

Date: ||$-|-| \mid$

Note: Whenever there is a discrepancy affecting North Carolina compliance samples, a copy of this form will be sent to the North Carolina DEHNR Certification Ofice ( i.e out of hold, incorrect preservative, out of temp, incorrect containers) 
PaceAnalytical"

www.pacelabs.com
INVOICE

Pace Analytical Services, Inc. 9608 Loiret Blvd. Lenexa, KS 66219 Phone: (913)599-5665

\section{Sold To:}

Mr. Travis Kamler TCW Construction Inc $141 \mathrm{M}$ Street Lincoln, NE 68508 402-475-5030
Invoice Number: 116103270

Date: $11 / 11 / 2011$

Total Amount Due: $\$ 1,036.00$

New Remittance Address

Please ensure that payments are now mailed to:

Pace Analytical Services, Inc.

P.O. Box 684056

Chicago, IL 60695-4056

\begin{tabular}{|c|c|c|c|c|}
\hline Client Number/Client ID & Purchase Order No & Pace Project Mgr & Terms & Page \\
\hline 60-508440 / TCW Const & Credit Card & Trudy Gipson & Net 30 Days ${ }^{\star \star}$ & 1 \\
\hline
\end{tabular}

Client Project: KS/MO Waste Water Pace Project No: 60109211 Report Sent To: Mr. David Surgnier, Comments:
Client Name: TCW Construction Inc Sample Received: 11/1/2011

\begin{tabular}{|c|c|c|c|c|c|}
\hline \multicolumn{6}{|c|}{ ANALYTICAL CHARGES } \\
\hline Quantity Unit & Description & Method & Matrix & Price & Total \\
\hline $7 \mathrm{Ea}$ & 353.2 Nitrogen, $\mathrm{NO} 2 / \mathrm{NO} 3$ & EPA 353.2 & Water & $\$ 18.00$ & $\$ 126.00$ \\
\hline $7 \mathrm{Ea}$ & 504 GCS EDB DBCP & EPA 504.1 & Water & $\$ 60.00$ & $\$ 420.00$ \\
\hline \multirow[t]{2}{*}{$7 \mathrm{Ea}$} & 8260 VOC by GC/MS-Full Scan & EPA $5030 B / 8260$ & Water & $\$ 70.00$ & $\$ 490.00$ \\
\hline & & & & Analytical Subtotal & $\$ 1,036.00$ \\
\hline & & er of Charges 21 & & Total Invoice Amount & $\$ 1,036.00$ \\
\hline
\end{tabular}

Samples Received for analysis:

\begin{tabular}{lll} 
Lab ID & Client Sample ID & Received \\
\hline 60109211001 & AGPURGE-W-10111 & $11 / 1 / 20119: 20: 00$ \\
60109211002 & BAPURGE-W-10112 & $11 / 1 / 20119: 20: 00$ \\
60109211003 & CNPURGE-W-10113 & $11 / 1 / 20119: 20: 00$ \\
60109211004 & EUPURGE-W-10114 & $11 / 1 / 20119: 20: 00$ \\
60109211005 & HAPURGE-W-10115 & $11 / 1 / 20119: 20: 00$ \\
60109211006 & MRPURGE-W-10116 & $11 / 1 / 20119: 20: 00$ \\
60109211007 & SVPURGE-W-10117 & $11 / 1 / 20119: 20: 00$
\end{tabular}

If you have any questions or to pay by credit card, please contact Trudy Gipson at Pace. Phone: 1(913)563-1405 Email: trudy.gipson@pacelabs.com

\section{${ }^{*} 1.5 \%$ MONTHLY FINANCE CHARGE ASSESSED AFTER 30 DAYS OR TERMS OF CONTRACT. PLEASE REFERENCE THE INVOICE NUMBER ON ALL REMITTANCE ADVICE.}

Please complete and return copy of invoice with your payment.

INVOICE TOTAL

$\$ 1,036.00$

Amount Paid:

$\$$

Check No: 
Argonne

\section{Environmental Science Division}

Argonne National Laboratory

9700 South Cass Avenue, Bldg. 203

Argonne, IL 60439-4843

www.anl.gov 